ANL-7120

Reactor Technology (TID-4500)

AEC Research and

Development Report

\author{
Argonne National Laboratory \\ 9700 South Cass Avenue \\ Argonne, Illinois 60439
}

\title{
PROCEEDINGS OF THE CONFERENCE ON SAFETY, FUELS, AND CORE DESIGN IN LARGE FAST POWER REACTORS \\ October 11-14, 1965
}

\author{
David Okrent \\ Program Chairman \\ Administrative Arrangements \\ Dorothy Carlson \\ Operated by The University of Chicago \\ under \\ Contract W-31-109-eng-38 \\ with the \\ U. S. Atomic Energy Commission
}




\section{DISCLAIMER}

This report was prepared as an account of work sponsored by an agency of the United States Government. Neither the United States Government nor any agency Thereof, nor any of their employees, makes any warranty, express or implied, or assumes any legal liability or responsibility for the accuracy, completeness, or usefulness of any information, apparatus, product, or process disclosed, or represents that its use would not infringe privately owned rights. Reference herein to any specific commercial product, process, or service by trade name, trademark, manufacturer, or otherwise does not necessarily constitute or imply its endorsement, recommendation, or favoring by the United States Government or any agency thereof. The views and opinions of authors expressed herein do not necessarily state or reflect those of the United States Government or any agency thereof. 


\section{DISCLAIMER}

Portions of this document may be illegible in electronic image products. Images are produced from the best available original document. 


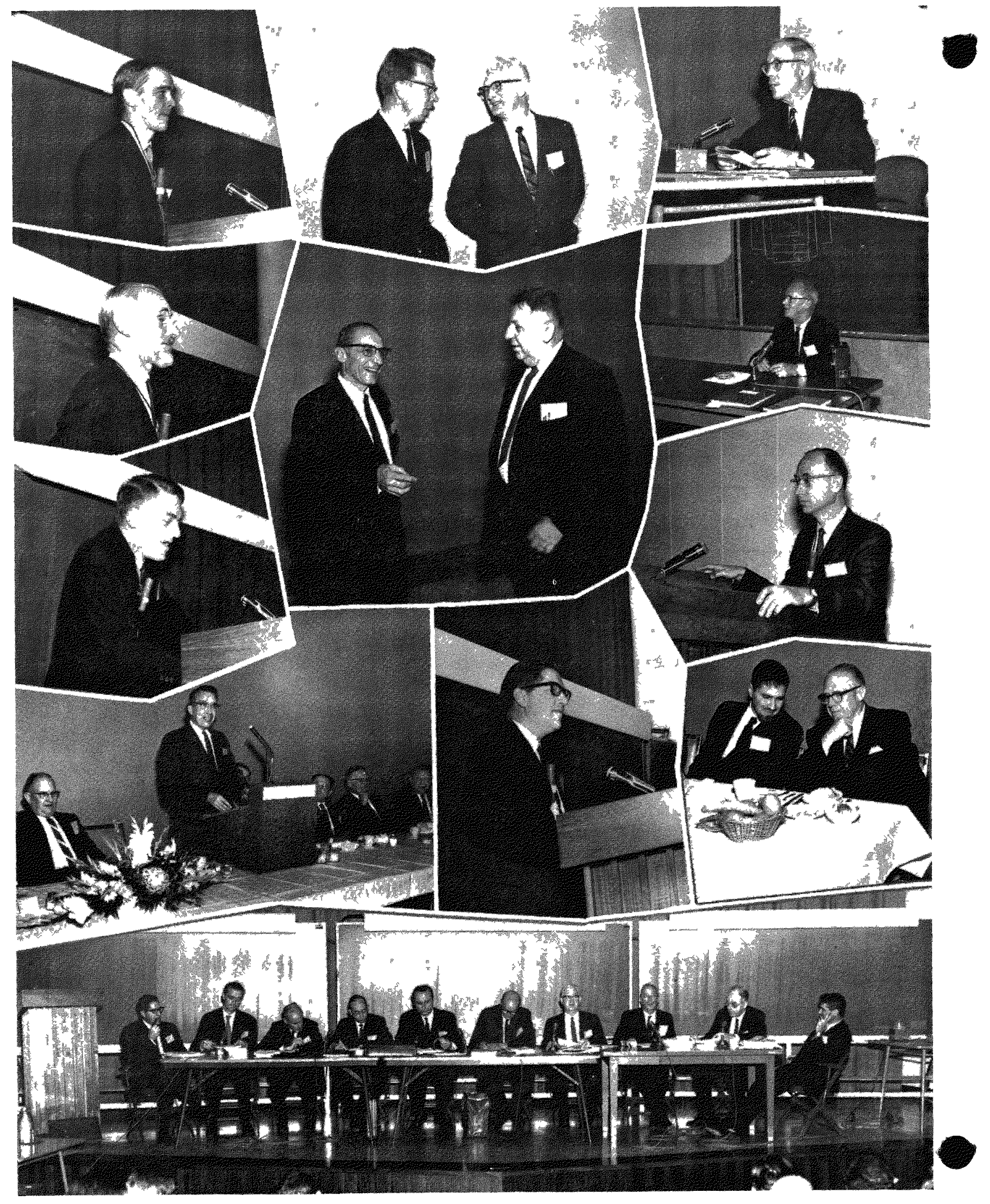




\section{Preface}

This volume contains the proceedings of the second in a series of annual topical conferences sponsored by Argonne National Laboratory and the U. S. Atomic Encrgy Commission on various specific aspects of fast reactor science and technology. The first conference, which was held in Ortober 1963, was entitled "Breeding, Economies and Safety in Large Fast Power Reactors." The proceedings of that conference were issued as ANL-6792. No conference was held in 1964 because the Third International Geneva Conference on the Peaceful Uses of Atomic Energy had been scheduled for that year. In October 1959, a related conference entitled "The Physics of Breeding" was held at Argonne. The proceedings of that conference were issued as ANL-6122.

We at Argonne appreciate very much the assistance of all who participated in this conference. In particular, the open discussion and the willingness to examine controversial questions contribute much to the value of these meetings. We appreciate, too, the cooperation of the $\mathrm{AEC}$ in cosponsoring this program.

These proceedings have been edited by A. Glassner, Joyce Kopta, and D. Okrent. In the interest of hastening publication, the speakers themselves have not been given an opportunity to edit the discussions. The editors hope that a reasonable portrayal of the actual discussion has been accomplished.

David Okrent

Program Chairman 
a

0 


\title{
Table of Contents
}

\author{
SESSION I \\ Octoher 11, 1965 \\ PHYSICS UNCERTAINTIES AND SAFETY STUDIES
}

Chairman: M. Shaw, U.S.A.E.C.

Summary of Intercomparison Calculations Performed in Conjunction with Conference on Safety, Fuels, and Core Design in Large Fast Power Reactors...................... D. Okrent, Argonne

Calculated Physics Parameters and Their Uncertainties in a 1000-MW(e) Fast Ceramic Re-

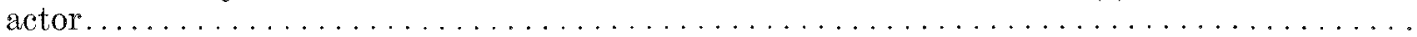

P. Greebler, C. L. Cowan, C. L. Fies, G. L. Gyorey, and J. R. Sueoka, General Electric Safety and Cost Analysis of a 1000-MWe Sodium-cooled Fast Power Reactor..............

D. Smidt, W. Frisch, P. Giondano, G. Heusener, G. Kessler, K. H. Krewer, W. Merk, T. Malmberg, and E. Schönfeld, Karlsruhe

An Analysis of Fast Reactor Transient Response and Safety in Selected Accidents. . . . . . . . .

D. B. Sherer, General Electric

Program Proposed for Safety Studies of the Fast Neutron Project of the EURATOM-CEA

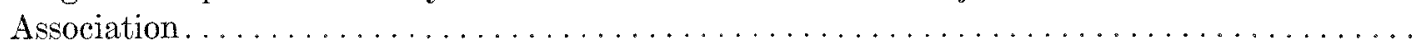

G. Denielou, M. Falgayrettes, J. Fournier, and M. G. Robin, EURATOM-CEA (Cadarache)

Loss-of-Coolant Accidents in a Large Sodium-Cooled Fast Reactor. . . . . . . . . . . . . . .

A. M. JUDD, UKAEA-Winfrith

3

24

33

46

54

67

\section{SESSION II}

October 11, 1965

\section{FAST REACTOR CONCEPTS}

\section{Chairman: W. H. Zinn, Combustion Engineering}

A Large Metal-fueled Fast Reactor Study.

Part I. Neutronies Calculations for a 1,000-MWe Plutonium-Uranium, Metal-Fueled,

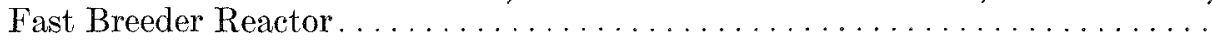

D. K. Butler, W. B. Loewenstein, D. Meneghetti, and K. E. Phillips, Argonne

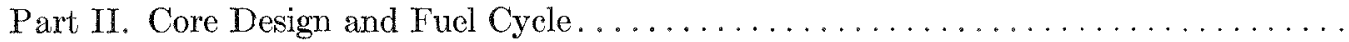

L. E. Link, J. E. Ayer, K. A. Hub, W. J. Mecham, V. G. Trice, Jro, and J. T. WEILLS, Argonne

Selected Optimization Studies in the Use of Carbide Fuel in Large Fast Breeder Reactors. . . . . .

G. A. Sofer, A. H. Kazi, B. R. Teer, and B. H. Cherry, United Nuclear Corp.

A Re-examination of Design Factors for a 1000-MWe Fast Breeder Reactor...............

W.S. Farmer and D. G. STRawson, Allis-Chalmers

Application of the Vented Fuel Concept to a Sodium-cooled Power Breeder with $1000 \mathrm{MWe}$.

D. SMidt and W. Sommer, Karlsruhe

Steam Cooling for Fast Reactors.

R. A. WeBb and D. C. Schluderberg, Babcock \& Wilcox

Safety and Nuclear Considerations in the Design of the FTR . . . . . . . . . . . . . . . .

E. R. Astuey, D. R. de Halas, L. M. Finch, P. L. Hofman, and W. W. Littile, JR., Battelle-Northwest

General Discussion. 


\title{
SESSION III
}

October 12, 1965

\section{PANEL ON SAFETY OF LARGE FAST POWER REACTORS}

\author{
Chairman: D. Okrent, ANL
}

Contribution to Safety Problems Related to a 1000-MWe Fast Power Reactor Project........

G. Denielou, R. Abdon, J. Fournier, J. Ladet, I. Puig, M. Robin, and C. P. Zaleski, EURATOM-CEA (Cadarache)

The Karlsruhe Reference Design of a 1000-MWe Sodium-cooled Fast Breeder Reactor.......

W. HÄFELE, D. SMIDT, and K. WIRTZ, Karlsruhe

Safety and Economic Characteristics of a 1000-MWe Fast Sodium-cooled Reactor Design.... .

K. P. Conen and G. L. O'NenLL, General Electric

Conceptual Design and Preliminary Accident Analysis of a Sodium-cooled, Carbide-fueled, Large Modular Fast Reactor . . . . . . . . . . . . . . . . . . . . . . . . . . . . .

J. H. Wright, W. E. Gunson, F. M. Heck, and R. S. Daleas, Westinghouse

Safety Characteristics of Large Gas-cooled Fast Power Reactors. . . . . . . . . . . . . . . . . . .

Peter Fortescue, R. Shanstrom, J. Broido, J. M. Stein, A. Baxter, and H. Fenech, General Atomic

SESSION IV

Octoher 12, 1965

DISCUSSION OF PAPERS AMONG PANEL

Chairman: D. Okrent, ANL

Panel Discussion

261

SESSION V-A

October 13, 1965

FUELS AND FUEL BEHAVIOR

Chairman: M. Benedict, MIT

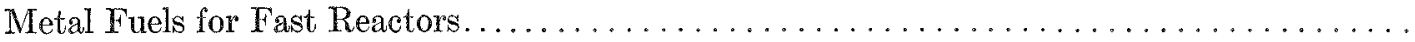

R. E. Macherey, L. R. Kelman, and J. H. Kitter, Argonne

Compatibility of Modified Carbide Fuels with Stainless Steel.

K. R. Jordan, R. J. Herbst, and R. G. Palm, Westinghouse

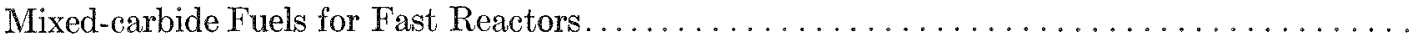

L. A. Neimark, J. E. Ayer, O. L. Kruger, T. W. Latimer, and C. M. Walter, Argonne

An Account of Some Work on the Safety Aspects of Carbide Fast Reactor Fuels. . . . . . . . . 328

B. T. Bradbury, L. E. Russell, and M. B. Waldron, UKAEA-Harwell

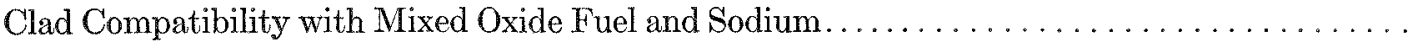

F. A. Compreuti, F. J. Hetzler, and T. A. Lauritzen, General Electric

Effect of Stoichiometry on the Properties of Mixed Oxide U-Pu Fuel . . . . . . . . . . . . . . . .

E. L. Zebroski, W. L. Lyon, and W. E. Batly, General Electric

Comment.

R. E. SKavdaHu, Battelle-Northwest

Plutonium and Fission Product Redistribution in Mixed Oxide Fuels. . . . . . . . . . . . .

P. E. Novak, T. A. Launrtizen, R. Protsik, J. H. Davies, and E. L. Zebroski, General Electric

Microanalytical Studies of Micro- and Macro-segregation in Oxide Fuels...............

A. A. Bauer, H. Beisswenger, G. Giacchetti, E. Patrassi, G. Schumacher, and R. Theisen, Karlsruhe 


\title{
SESSION V-B
}

October 13, 1965

\section{MOSTLY REACTIVITY COEFFICIENTS}

\author{
Chairman: R. Avery, ANL
}

Recent Investigations of Fast Reartor Reactivity Coefficients. . . . . . . . . . . . . . . .

413

H. H. Huminer, R. N. Hwang, and K. Phildips, Argonne

A Comment on the Comparison of Some Sodium Void Coefficients with Experimental Data... . 421

A. Travelli, Argonne

The Influence of Some Important Group Constants on Integral Fast Reactor Quantitics. . . . . .

H. Küsters and M. METzenroth, Karlsruhe

Some Calculations of Fast Reactor Doppler and Sodium Loss Coefficients with Particular Reference to the Effects of Core Lattice St ructure and Radial Fuel Temperature Distribution. .

J. Cond, C. Durston, and Miss W. V. Wright, UKAEA-Winfrith

Results of Doppler Coefficient Calculations for Fast Reactors and Comparison of Different Nethods

Part I: R. Froelich and K. OTt, Karlsruhe

Part II: G. Shaviv and S. IinTAh, Israel AEC

Effect of the Fluctuations in Collision Density on Fast-reactor Doppler Effect Calculations....

R. N. Hwang, Argonne

Comparison of Monte Carlo and Resonance Integral Methods in the Determination of Doppler

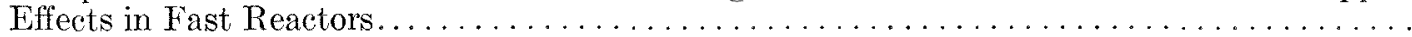

J. E. OlHoeft, H. E. Krug, Westinghouse; and R. N. Hwang, Argonne

Transient Temperature Distributions in Fast Reactor Fuels with Widely Varying Thermal

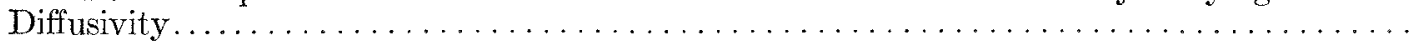

C. K. Sanathanan, J. C. Carter, and R. O. Brittan, Argonne

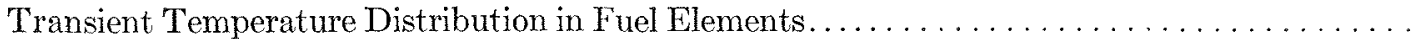

J. E. Godts and E. F. Resteldi, JR., Westinghouse

423

434

440

449

SESSION VI-A

October 13, 1965

\section{FUELS AND FUEL BEHAYIOR}

\section{Chairman: S. Lawroski, ANL}

Fast Reactor Safety Considerations Related to Fuel Macrostructure.

R. E. Peterson and S. Goldsmith, Battelle-Northwest

Molten Plutonium Alloys as Fast Reactor Fuels . . . . . . . . . . . . . . . . . . . . . .

L. D. Krakbride, Los Alamos

The Application of Experimental Data from TREAT Meltdown Studies to Reactor Accident

Analysis.

S. J. Stachura and M. Stlberberg, Atomies International

Mixed Oxide Fuel Movement at Various Power Levels and in Transients . . . . . . . . . . . . . . . . .

S. A. Rabin, W. E. Barly, J. E. Hanson, and F. W. KNIGHT, General Electric

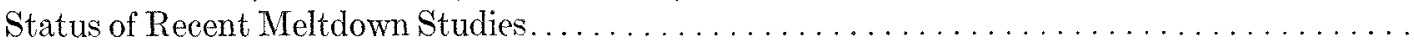

C. E. Dickerman, L. E. Robinson, F. L. Willis, W. Stephany, and C. August, Argonne

Safety and Economic Aspects of Vented Fuel in Sodium-cooled Reactors. . . . . . . . . . . . . 540

G. L. O'Nemi, F. W. Knight, F. J. Leitz, and D. B. Sherer, General Electrie

General Discussion. . . . . . . . . . . . . . . . . . . . . . . . . . . . . . . 


\section{SESSION VI-B \\ October 13, 1965 \\ MOSTLY REACTIVITY COEFFICIENTS \\ Chairman: F. W. Thalgott, ANL}

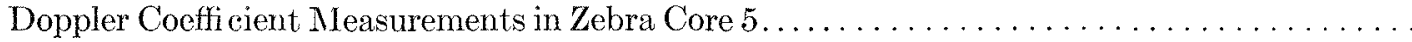

A. R. Baker and R. C. Wheeler, UKAEA.Winfrith

The Interpretation of Doppler and Sodium Reactivity Worth Measurements in Zero Power

Reactors, with Particular Reference to the Zebra Doppler Loop Measurements..............

J. L. Rowlands and D. Wardleworth, UKAEA-Winfrith

Accuracy of Power Reactor Void Coefficients Extrapolated from Measurements in Critical

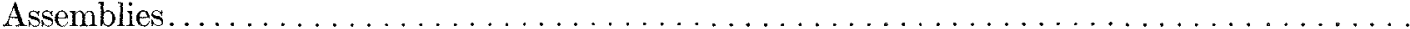

T. C. Fngelder, N. L. Symoow, and T. S. Katyan, Babcock \& Wilcox

Sodium-void Coefficients

W. Y. Kato, G. K. Rusch, and F. H. Helm, Argonne

Measurement and Analysis of Doppler Effect in Plutonium-fueled Fast Reactor Assemblies. . .

G. J. Fischer, D. A. Meneley, R. N. Hwang, E. F. Groh, and C. E. Till, Argonne

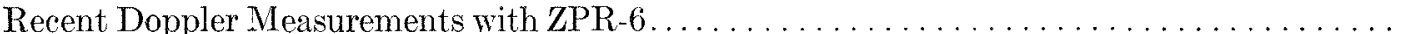

C. E. TILL, R. A. Lewis, and E. F. GroH, Argonne

Recent Results of Doppler Measurements in Fast-neutron Spectra................... .

S. G. Carpenter, L. A. Mountrord, T. H. Springer, and R. J. Tuttle, Atomics International

Doppler Effect in $U^{238}$ Capture and $U^{235}$ Fission in a Fast-neutron Spectrum. . . . . . . . . . .

G. R. Pflasterer, Jr., R. Sher, W. A. Mayer, and F. G. Warzek, General Electric

Transient Flux Distributions by Discontinuous Model Synthesis....................

J. E. Godts, Westinghouse, and A. S. Weinstein, Carnegie Institute

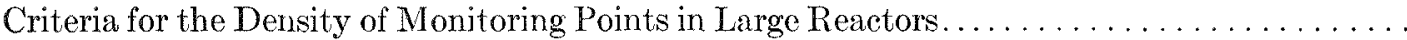

Paul F. Gast, Argonne

General Discussion.

566

585

603

610

614

616

\section{SESSION VII-A}

October 14, 1965

MAXIMUM ACCIDENT AND CONTAINMENT

Chairman: W. J. MeCarthy, Jr., Power Reactor Development Corp.

A Critical Review of the Properties of Materials at the High Temperatures and Pressures

Significant for Fast Reactor Safety . . . . . . . . . . . . . . . . . . . . . . . . . . . .

David Miluer, Argonne

Theoretical Studies on the Fast Reactor Maximum Accident. .

E. P. Hicks and D. C. Menzies, UKAEA-Dounreay

A Parameter Study of Large Fast Reactor Meltdown Aceidents...

R. A. Meyer, B. Wolfe, and N. F. Friedman, General Electric

A Modified Equation of State for Hydrodynamic Calculations in AX1 Numerical Program.... .

V. Z. Jankus, Argonne

A Model Investigation of Explosion Containment in Single Tank Fast Reactors . . . . . . . . . .

N. J. M. REEs, UKAEA-Foulness

Comparison of Pressure Loading Produced by Contained Explosions in Water and Sodium.... .

G. A. V. Drevon, Euratom-CEA (Saclay), M. F. G. Falga yretres, Euratom-CEA (Cadarache), and F. J. WALFORD, UKAEA-Foulness

Design Factors Influencing the Containment of Internal Explosion in Fast Reactors . . . . . . . . .

D. E. J. SAMUELS, UKAEA-Foulness

Response of a Helium-cooled Fast Reactor to Changes in Coolant Flow and Reactivity. . . . . . . .

654

671

686

692

R. S. Carlsmith and J. G. Delene, Oak Ridge 
SESSION VII-B

October 14, 1965

OTHER SYSTEMS AND CONCEPTS

Chairman: K. P. Cohen, General Electric

Mixed Fuel-cycle Fast Breeder Reactors. Nuclear, Safety, and Materials Considerations...... . 751

W. B. Loewerstein and B. Blumenthal, Argonne

Nuclear Design of MPBE.

W. H. Hannum, Los Alamos

Faret Core-I Reference Design

P. J. Persiani, T. R. Bumip, and W. J. Kann, Argonne

Transient Response of Coupled Fast Reactor Cores.

W. F. Gunson, F. M. Heck, and R. S. Daleas, Westinghouse

Enhanced Safety Characteristics of a Sodium-cooled Coupled Reactor.

D. Eggen, A. Gibsox, R. Sevy, and M. Silberberg, Atomies International

Some Safety Considerations Based on Design Experiences in the BNL Settled-bed Fast Re-

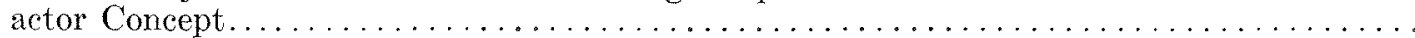

Gerald S. Lellovche, Melvin M. Levine, and Jack Chernick, Brookhaven

Comparison of $\mathrm{He}, \mathrm{CO}_{2}$, and Steam as Coolants of a 1000-MWe Fast Reactor. . . . . . . . . . . . .

M. DaLle- Donne, Karlsruhe

Survey Parameter Study of Large Steam-cooled Fast Power Reactors. . . . . . . . . . . . . . . .

E. KIEFHABER and K. OTt, Karlsruhe

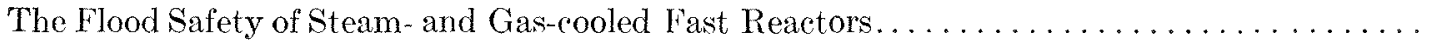

J. W. Hallam, R. K. Haling, P. Krlian, and G. T. Peterson, General Electric

\section{SESSION VIII-A}

October 14, 1965

FISSION PRODUCTS, REACTIONS AND INTERACTIONS

Chairman: J. A. Lieberman, U.S.A.E.C.

Experimental Studies of the High-temperature Interaction of Fuel and Cladding Materials with Liquid Sodium

David L. Swift and Louis Baker, Jre, Argonne

Chemical Considerations in Fast Reactor Safety

A. W. Castleman, Jr. and I. N. Tang, Brookhaven

Fission Product Retention in Sodium and Applications to Vented Fuel Element Design.......

W. Kunkel and S. Berger, Atomics International

Fuel-failure Detection in Sodium-cooled Reactors

K. G. Porges, Argonne

Preliminary Evaluation of a Technique to Study Expulsion of Sodium into Air............

I. Charak and F. A. Smith, Argonne

\section{SESSION VIII-B \\ October 14, 1965 \\ MOSTLY SODIUM BOILING}

Chairman: P. A. Lottes, ANL

Effect of Fission Gas Bubbles on the Response of Molten Fuel Reactors to Fast Transients.....

B. M. Carmichael, Los Alamos

The Development and Growth of In-core Voids Due 10 Boiling during Fast Reactor

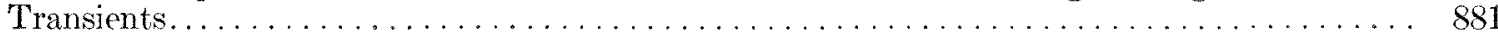

R. C. Noyes, H. Lurie, and A. A. JArretr, Atomics International

Coolant-ejection Studies with Analogy Experiments........................ 890

G. FRIz, Euratom-CCR

Shock Front Conditions in Two-phase Flow Including the Case of Desuperheat . . . . . . . . 895

M. Fischer and W. HäFELE, Karlsruhe

Non-ANL Attendees. . . . . . . . . . . . . . . . . . . . . . . . . . . . . . . 


\section{Session I \\ October 11, 1965}

\section{Physics Uncertainties and Safety Studies}

Chairman: M. SHAW

U. S. Atomic Energy Commission

Secretary: C. E. Dickerman

Argonne National Laboratory 
0

0 


\title{
Summary of Intercomparison Calculations Performed in Conjunction with Conference on Safety, Fuels, and Core Design in Large Fast Power Reactors
}

\author{
D. OKRENT \\ Argonne National Laboratory \\ Argonne, Illinois
}

It was decided to conduct an intercomparison of calculations in connection with this Conference for the following reasons:

1. At the Conference on Breeding, Economics and Safety in I arge Fast Power Reactors held at Argonne National Laboratory in October, 1963, a considerable difference of opinion arose concerning the magnitude of reactivity coefficients to be expected as a function of reactor design. Furthermore, as the group at the 1963 conference discussed large reactor designs, it was difficult to evaluate the relative merits of these concepts, since it was not clear which groups were calculating relatively optimistic numbers, and which groups were obtaining pessimistic numbers for each of the various parameters. Hence, for this conference an intercomparison of calculations on relatively simple systems was planned, to identify areas of difference and to provide more perspective for concept evaluations.

2. In a crude way the intercomparison of calculations was expected to provide some measure of the current uncertainties in predictions of nuclear parameters for large power reactors.

A group of representative, single-region, "bare" cores was defined. In addition, several of these cores were provided with blankets in spherical geometry. Finally, one reactor of a flat, cylindrical shape was specified. For all cases except the pancaked core, it was asked that core radius be varied to achieve criticality. Several parameters of potential interest were suggested in connection with each type of problem. Participation was completely voluntary, and the rarious institutions computed those problems which they found to be of particular interest.

Not all of the resulting numbers could be correlated in the short time available between receipt of the calculated results and the beginning of the Conference. Nor was there sufficient time to identify in detail reasons for the various differences which have appeared. The following tables and graphs, however, provide a rather good indication of the kind of comparison which has resulted.
In some cases, participating institutions did not have cross sections available at the specified fuel temperatures. Where possible, a rough correction to specified fuel temperatures was made at Argonne, but in some cases numbers have been reported for the original calculated temperature.

Most of the participating groups turned in a rather detailed description of the computing methods used, more than could be included in the proceedings. Only an abstract including some of the most vital information has been provided herein for each participant.

A detailed discussion of the resulting differences will not be provided in the proceedings. This is left for the reader to determine in whatever detail he desires. The reader will quickly ascertain that very large discrepancies have appeared in buckling or critical mass, and in the sodium void effect. Excepting the low values obtained by Brookhaven National Laboratory (BNL), differences in calculations of the Doppler effect were significant, but not of such great magnitude. The low values obtained by BNL can be traced rather directly to a reduced fraction of neutrons at lower energies. The variation between computed values of the breeding ratio and of the various cross-section ratios was considerably smaller. There appears to be some correlation between low values of the

$$
\frac{\sigma_{c} \mathrm{C}^{238}}{\sigma_{j} \mathrm{Pu}^{239}}
$$

and high values of buckling or overall reactivity.

In general, there seems to have been a consistent pattern to the discrepancies. Participating institutions tended to obtain either more reactive or less reactive results for all reactors. Similarly, void coefficients tended to be either more positive or more negative for all reactors. This is illustrated quite clearly for buckling on Figures 1 and 2.

Although there is some general trend of agreement for the adjoint flux, considerable differences do occur among the results from some institutions at the high and the low energies. Some have computed adjoints having a much flatter character at low energies, at 
high energies, or both. Although some differcnces were introduced by fission products and $\mathrm{Pu}^{240}$, the general trends of discrepancies observed for clean reactors were not modified markedly when reactors containing fission products and $\mathrm{Pu}^{240}$ were computed.

Further analysis into the causes for some of the discrepancies is being undertaken by H. H. Hummel of Argonne National Laboratory.

In conclusion, we wish to thank all the individuals and installations whose participation in this intercomparison has made possible this interesting group of results.

\section{SPECIFICATIONS OF CALCULATIONS FOR IN'TERCOMPARISON TO BE MADE AT CONFER- ENCE ON SAFETY, FUELS AND CORE DE- SIGN IN LARGE FAST POWER REAC. TORS TO BE HELD AT ARGONNE NATIONAL LABORATORY, OCTOBER, 1965}

Information Desired-Bare Cones

1. Buckling to go critical.

2. Doppler effect, $T(d K / d T)$. Assume:

Fuel OD $=0.25$ in.; clad OD $=0.28$ in.;

center to center distance $=0.38 \mathrm{in}$. (triangular pitch).

2a. (Optional). Compare consequences of specific theoretical assumptions on Doppler effect.

3. Coolant Void Effect ( $50 \%$ Voiding):

a) contribution from elastic, inelastic, and capture terms;

b) adjoint flux as a function of energy.

4. Reactor Spectrum

a) as a function of energy;

b) as characterized by fissions below $25 \mathrm{keV}$ and below $2.1 \mathrm{keV}$;

(c) as characterized by spectral indices:

$$
\frac{\sigma_{f}^{\mathrm{U}-238}}{\sigma_{f}^{\mathrm{Pu}-239}} ; \frac{\sigma_{c}^{\mathrm{U}-238}}{\sigma_{f}^{\mathrm{Pu}_{\mathrm{u}-239}}} ; \frac{\sigma_{c}^{\mathrm{Pu}-239}}{\sigma_{f}^{\mathrm{Pu}_{-239}}} ; \frac{\sigma_{f}^{\mathrm{Pu}-240}}{\sigma_{f}^{\mathrm{Pu}-239}} \cdot
$$

5. Error estimates.

6. Define basis of calculation, particularly method of handling cross sections.

Information Desired-Blanketed Spheres

1. Core radius for criticality, holding compositions fixed, as specified.

2. Critical mass of fissile isotopes. Breeding ratio.

3. Doppler effect, assuming space-independent $\Delta T$ in core, no $\Delta T$ in blanket.

4. Coolant coefficient of reactivity:

a) uniform voiding of core $(50 \%)$;

b) leakage component of coolant coefficient at $100 \%$ coolant density by perturbation theory; c) spatial distribution of coolant worth per gram of coolant.

Information Desired-Blanketed Pancake Core

1. Take pancake core $2 \mathrm{ft}$ high and $10 \mathrm{ft}$ in diameter. Use composition typified by bare-core cases 11-14 (see Table 1). Vary ratio of $\mathrm{Pu}^{239}$ to $\mathrm{U}^{238}$ in core to go critical, holding total heavy atoms and ratio of $\mathrm{Pu}^{240}$ to $\mathrm{Pu}^{239}$ constant. Use 18-in.-thick blanket, radial and axial. Blanket composition, atoms/cc $\left(\times 10^{-24}\right)$, but see Table 2 also.

\begin{tabular}{lcclcc} 
& & & \multicolumn{3}{c}{ Stainless } \\
& $\mathrm{Na}$ & $\mathrm{Pu}^{239}$ & \multicolumn{1}{c}{$\mathrm{U}^{238}$} & Steel & $\mathrm{O}$ \\
Axial & 0.0123 & 0.00007 & 0.0072 & 0.011 & 0.0144 \\
Radial & 0.0069 & 0.00012 & 0.012 & 0.011 & 0.024
\end{tabular}

2. Critical mass and breeding ratio.

3. What is sodium void effect for $50 \%$ loss of sodium from core?

What are leakage and spectral components?

\section{BRIEF DESCRIPTIONS OF METHODS USED}

\author{
E. R. Specht, D. W. Latham, and O. R. Hillig \\ Atomics International
}

The calculations were done with 15-group diffusion and perturbation theory. Homogeneous mixtures of the core materials were assumed in each case. Groupdependent boundary conditions characteristic of a vacuum interface were imposed at the outer radii of the cores.

Multigroup cross-section sets were prepared by averaging energy-dependent data from the AI-evaluated nuclear data tape over ELMOE spectra. Directflux weighting was used for all processes except neutron transport, for which ELMOE current spectra were employed.

Separate spectra, characteristic of each core composition, were used to average the elastic removal and transport cross sections for light and intermediate weight materials ( $\mathrm{Na}, \mathrm{O}, \mathrm{C}$, and $\mathrm{SS}$ ). The spectra of cores 9 and 13 (see Table 1 ) were used to average the nonresonance portion of the heavy element cross sections for the oxide and carbide cores, respectively. This simplification is made possible by the insensitivity of these "smooth" cross sections to the details of the flux fine structure.

Resonance shielding and Doppler broadening of the fuel isotopes were computed by the narrow resonance approximation. Breit-Wigner single-level line shapes were assumed. Statistical sampling from distributions of neutron and fission widths $(\gamma=3)$ for unresolved 
TABLE 1. BARE CORES

Concentration in Atoms/ce $\left(10^{-24}\right)$

\begin{tabular}{|c|c|c|c|c|c|c|c|c|c|c|c|}
\hline Case & $\begin{array}{l}\text { Fuel Den- } \\
\text { sity, }{ }^{* *} \mathrm{~g} / \mathrm{cc}\end{array}$ & $\mathrm{Na}$ & $\mathrm{Pu}^{239}$ & $U^{238}$ & $\mathrm{Pu}^{240}$ & $\mathrm{C}$ & $\begin{array}{c}\text { Stainless } \\
\text { Steel }^{*}\end{array}$ & $\mathrm{O}$ & $\begin{array}{l}\text { Fission } \\
\text { Products }\end{array}$ & $\begin{array}{l}\mathrm{H}_{2} \mathrm{O} \text { (mole } \\
\text { cules) cc }\end{array}$ & $\begin{array}{l}\text { Avg Fuel } \\
\text { Temp, }{ }^{\circ} \mathrm{K}\end{array}$ \\
\hline 1 & 10.77 & 0.0123 & 0.0006 & 0.0066 & - & - & 0.011 & 0.0144 & 一 & 一 & 1500 \\
\hline 2 & 10.77 & 0.0123 & 0.00072 & 0.00648 & - & - & 0.011 & 0.0144 & - & - & 1500 \\
\hline 3 & 10.77 & 0.0123 & 0.0009 & 0.0063 & - & - & 0.011 & 0.0144 & -- & - & 1500 \\
\hline 4 & 10.77 & 0.0123 & 0.0012 & 0.0060 & -- & - & 0.011 & 0.0144 & - & - & 1500 \\
\hline 5 & 10.77 & 0.0123 & 0.0006 & 0.0066 & - & - & 0.011 & 0.0144 & 0.00072 & - & 1500 \\
\hline 6 & 10.77 & 0.0123 & 0.0012 & 0.0060 & & - & 0.011 & 0.0144 & 0.00072 & - & 1500 \\
\hline 7 & 10.77 & 0.0123 & 0.0006 & 0.0066 & 0.0003 & -. & 0.011 & 0.0144 & 0.00072 & - & 1500 \\
\hline 8 & 10.77 & 0.0123 & 0.00072 & 0.00648 & 0.00030 & - & 0.011 & 0.0144 & 0.00072 & - & 1500 \\
\hline 9 & 10.77 & 0.0123 & 0.0009 & 0.0063 & 0.00045 & - & 0.011 & 0.0144 & 0.00072 & - & 1500 \\
\hline 10 & 10.77 & 0.0123 & 00012 & 0.0060 & 0.0006 & - & 0.011 & 0.0144 & 0.00072 & - & 1500 \\
\hline 11 & 12.8 & 0.0123 & 0.00075 & 0.00825 & 0.0003 & 0.009 & 0.011 & 一 & 0.0009 & - & 1100 \\
\hline 12 & 12.8 & 0.0123 & 0.0009 & 0.0081 & 0.00036 & 0.009 & 0.011 & & 0.0009 & - & 1100 \\
\hline 13 & 12.8 & 0.0123 & 0.001125 & 0.007875 & 0.00045 & 0.009 & 0.011 & - & 0.0009 & 一 & 1100 \\
\hline 14 & 12.8 & 0.0123 & 0.0015 & 0.0075 & 0.0006 & 0.009 & 0.011 & & 0.0009 & - & 1100 \\
\hline 15 & 17.6 & 0.0123 & 0.0012 & 0.0108 & 0.0004 & - & 0.011 & & 0.0012 & - & 900 \\
\hline 16 & 10.77 & 一 & 0.0006 & 0.0066 & 0.0003 & - & 0.011 & 0.0144 & 0.00072 & 0.0016 & 1500 \\
\hline 17 & 10.77 & - & 0.00072 & 0.006448 & 0.00036 & - & 0.011 & 0.0144 & 0.00072 & 0.0016 & 1500 \\
\hline 18 & 10.77 & - & 0.0009 & 0.0063 & 0.00045 & - & 0.011 & 0.0144 & 0.00072 & 0.0016 & 1500 \\
\hline 19 & 10.77 & - & 0.0012 & 0.0060 & 0.0006 & - & 0.011 & 0.0144 & 0.00072 & 0.0016 & 1500 \\
\hline
\end{tabular}

* Assume stainless steel composition is like SAE $304(\sim 18 \% \mathrm{Cr}, 8 \% \mathrm{Ni})$. Do not include manganese.

** Density of $\mathrm{Pu}^{239}, \mathrm{U}^{238}$, and appropriate light elements, assuming $30 \mathrm{v} / \mathrm{o}$ fuel.

resonances was used. Resonance overlap was taken into account approximately by neglecting the temperature dependence of the integrated flux in the denominator of the effective cross sections for $\mathrm{Pu}^{239}$. Most of the resonance parameters were taken from Garg et al., ${ }^{(1)}\left(\mathrm{C}^{238}\right)$, Schmitt $\left(\mathrm{Pu}^{239}\right)$, and Barrington $\left(\mathrm{Pu}^{240}\right)$. The resonance weighting functions include only local flux fine structure and neglect more gradual depletion of the flux due to accumulative absorption.

The methods of cross-section generation and eigenvalue analysis used in the present study are the same as those employed in the modular-core work with certain unavoidable exceptions. [The early modular surveys used an old cross-section set derived from Hansen-Roach data and AETR experience. Our present cross-section methods came to completion during the coupled core work and were used in the final analysis of reference modular design.] Our modular analyses required a group structure extending into the thermal energies to describe the effects of strong local moderation. We have retained this structure in order that there be some basis of evaluation of the work to be described at the conference.

(1) See NAA-SR-Memo-11517.
TABLE 2. Blanketed Spherical Reactors (18-IN.-THICK BLANKETS)

Cases 8 and 10

Blanket Composition, Atoms/ec ( $\left.\times 10^{-24}\right)$

$\begin{array}{ccccc}\mathrm{Na} & \mathrm{Pu}^{239} & \mathrm{C}^{238} & \text { Stainless } & \text { Steel } \\ 0.0069 & 0.00012 & 0.012 & 0.011 & 0.024\end{array}$

Case 12

Blanket Composition, Atoms/ce $\left(\times 10^{-24}\right)$

\begin{tabular}{|c|c|c|c|c|}
\hline $\mathrm{Na}$ & $\mathrm{Pu}^{239}$ & $\mathrm{U}^{238}$ & $\begin{array}{l}\text { Stainless } \\
\text { Steel }\end{array}$ & $\mathrm{C}$ \\
\hline 0.0069 & 0.00015 & 0.015 & 0.011 & 0.015 \\
\hline
\end{tabular}

Case 15

Blanket Composition, Atoms/ce ( $\left.\times 10^{-24}\right)$

$\begin{array}{cccc}\mathrm{Na} & \mathrm{Pu}^{23^{\circ}} & \mathrm{U}^{238} & \begin{array}{c}\text { Stainless } \\ \text { Steel }\end{array} \\ 0.0069 & 0.0002 & 0.02 & 0.011\end{array}$

Cases 17 and 19

Blanket Composition, Atoms/cc $\left(\times 10^{-24}\right)$

\begin{tabular}{crccc}
$\mathrm{Pu}^{239}$ & \multicolumn{3}{c}{ Stainless } & $\mathrm{H}_{2} \mathrm{O}$ \\
0.00012 & 0.012 & Steel & $\mathrm{O}$ & $\mathrm{mol} / \mathrm{cc}$ \\
& 0.011 & 0.024 & 0.0016
\end{tabular}




\section{Preparations of Cross Sections}

\author{
H. H. HummeL \\ Argonne National Laboratory
}

Fission and capture cross sections were generated by use of the current version of the Argonne cross-section code $\mathrm{MC}^{2}$. This code calculates cross sections for 0.25 lethargy groups, which are then collapsed to the 22 groups used in the calculations. Fission and capture cross sections above $25 \mathrm{keV}$ were assumed independent of temperature and composition. Below $25 \mathrm{keV}$, resonance integral calculations were performed. At present, $\mathrm{MC}^{2}$ uses Goldstein-Cohen interpolation between narrow and wide resonance calculations. Heterogeneity was taken into account by the use of equivalence theory.

A one-level calculation was used in all cases. The Columbia resonance parameters, extending up to 4 $\mathrm{keV}$, were used for $\mathrm{L}^{238}$. Flux correction factors were used to provide an approximate fertile-fissile overlap correction.

Fundamental mode calculations for Cases 7-15 (see Table 1) were performed directly by ELMOE, using the double lethargy spacing. Apparent transport and elastic removal cross sections for voiding sodium were obtained by subtracting the appropriate macroscopic cross sections for the voided case from those for the unvoided case and dividing by the atoms/ce of sodium roided. For Cases 1-6 of Table 1, the appropriate ELMOE homogenized macroscopic core cross sections were altered by removing $\mathrm{Pu}^{240}$ and fission product contributions. Light-element cross sections were assumed to be unaffected by this process.

Blanket-homogenized macroscopic cross sections were obtained by running ELMOE with $B^{2}=0$ and inputting a fission spectrum source.

\section{Heavy-element Cross Sections}

\section{Thomas Pitterle axd Bey Segal} Atomic Power Development Associates, Inc.

Infinite-dilution cross sections for $\mathrm{U}^{238}, \mathrm{Pu}^{239}$, and $\mathrm{Pu}^{240}$ above $1 \mathrm{keV}$ were taken from the English Data File. (1) Below $1 \mathrm{keV}, \mathrm{Pu}^{239}$ cross sections were those of Schmidt. ${ }^{(2)} \mathrm{U}^{238}$ cross sections up to $3.9 \mathrm{keV}$ were calculated from the resonance parameters. ${ }^{(3)}$

Self-shielding was calculated both at core sodium densities of $100 \%$ and $50 \%$ by means of the APDA Doppler program, IDIOT, which is described in more detail in the section on Doppler calculations. Twenty. four groups were used for the calculations. Groups 15 to 20 were each subdivided into two groups for the

(1) K. Parker, AWRE 0-79/63; A. C. Douglas and J. F. Barry, AWRE 0-79/64; A. C. Douglas, AWRE 0-91/64.

(2) J. J. Schmidt, KFK 120 (EAN1)C-E-35 U).

(3) J. B. Garg et al., Phys. Rev., 134, 5B (1964). cross-section calculation and then flux weighted by the calculated ELAIOE spectrum for the 24-group calculations. Heterogeneity was taken into account using a modified nonresonant cross section given by

$$
\begin{aligned}
\Sigma_{N} & =\Sigma_{p}+\Sigma_{m}+\frac{\Sigma_{s} V_{s} / V_{f}}{1+(1-\tau) \frac{\Sigma_{s} V_{s} l}{V_{f}}} \\
\tau & =\sqrt{\frac{\pi}{2 \sqrt{3}}} \frac{\sqrt{1+\left(V_{s} / V_{f}\right)}-1}{V_{s} / V_{f}}-0.12 .
\end{aligned}
$$

This formula is obtained from the Dancoff corrections given by Sauer ${ }^{(4)}$ for the limiting case where the Dancoff factor $C$ approaches unity. In this formula, $\Sigma_{p}$ is the heavy-element nonresonant scattering in the rod, $\Sigma_{m}$ is the oxygen scattering in the rod, $\Sigma_{s}$ is the homogenized scattering of steel and sodium, $V_{s} / V_{f}$ is the volume ratio of steel and sodium to that of fuel, $\bar{l}$ is the mean chord length for the fuel rod, and $\tau$ is the gcometrical index given by Sauer for a hexagonal lattice. The choice of $V_{s} / V_{f}$ lies between the ratio for the lattice and for the homogenized core. For the calculations presented here, the lattice ratio was chosen. This yields a sodium/fuel ratio of 1.3 and a cladding/ fuel ratio of 0.25 , whereas the homogeneous ratios are estimated to be about 1.86 and 0.43 . This section of lattice ratios tends to give the largest difference between the heterogeneous and homogeneous nonresonance cross sections. Although the effect of the heterogeneity correction is small for criticality calculations, it is more significant for Doppler calculations.

\section{Doppler Calculations}

Self-shielded effective cross sections and temperature derivatives for the Doppler calculations were obtained from the IDIOT Doppler program. This program, written by D. G. Green at APDA, is currently under development. The following approximations are available in this program: resolved resonance calculations with optional use of the intermediate resonance approximation of Goldstein, (s) unresolved resonance calculations in the isolated resonance approximation, overlap corrections to a single resonance sequence using the series expansion of Hwang, ${ }^{(6)}$ and the highenergy Method A approximation. (7) Overlap corrections between different isotopes or different resonance sequences are not calculated as they have been shown to be negligible when the temperature-dependent flux normalization is included in the calculation. The

(4) A. Sauer, Nucl. Sei. Eng., 16, 329 (1963).

(5) R. Goldstein and E. R. Cohen, Nucl. Sei. Eng., 13, 132 (1962).

(6) R. N. Hwang, Nucl. Sci. Eng., 21, 523 (1965).

(7) R. B. Nicholson, APDA-139 (1960). 
Doppler calculations were made by means of the above methods over their appropriate energy ranges. Through an input error in the program, the temperature derivatives were evaluated at a temperature of $1800^{\circ} \mathrm{K}$. A $1 / T$ temperature dependence was applied to obtain the values for the given reactivity coefficients at $1500^{\circ} \mathrm{K}$. It should be noted that the temperature derivatives were evaluated directly rather than using the difference between cross sections over a temperature difference. Heterogeneity was included by use of the nonresonant cross section of Equation 1.

For $\mathrm{U}^{238}$ the resolved resonance data of Garg et al., ${ }^{\langle 3\rangle}$ was used to $3.9 \mathrm{keV}$. Above this energy an arerage spacing of $19.0 \mathrm{eV}$ and strength functions of $0.96 \times 10^{-4}$ and $1.25 \times 10^{-4}$ for $\mathrm{s}$ and $\mathrm{p}$ waves respectively were assumed. The calculated rross sections were then normalized so that the infinite-dilution cross section agreed with the Fnglish Data File. The U ${ }^{238}$ Doppler coefficient was also calculated.

Resonance parameters for $\mathrm{Pu}^{239}$ were obtained by requiring that the parameters yield the fission cross section and alpha values of the English Data File. When only $\mathrm{s}$ waves are present, both the fission and neutron widths are obtained by this fitting procedure. When $p$ waves are present, an s-wave strength function of $1.0 \times 10^{-4}$ was assumed to obtain the s-wave neutron widths, and the fission and p-wave neutron widths were obtained by the fitting procedure. A chi-squared distribution parameter of 2.0 was assumed for the fission widths.

For $\mathrm{Pu}^{240}$, the resonance parameters of Frochlich and $O t^{(8)}$ were used. The s-wave neutron width was obtained from a strength function of $1.0 \times 10^{-4}$ and the $p$-wave widths by requiring that the cross section for compound nuclear formation by $p$ waves be of the same as that for $\mathrm{U}^{235}$.

\section{Calculation of Various Fast Reactor Cores}

\author{
T. S. Katuax \\ Babcock and Wilcox
}

\section{Basis of Calculation}

The calculations were performed utilizing the Babcock and Wilcox LIFE $\mathrm{X}$ code in combination with the current Babcock and Wilcox Standard Fast Cross Section 34 Group Library. The LIFE X nuclear design package consists of a series of individual nuclear design codes arranged in such a manner as to provide a complete program for one-dimensional criticality and core depletion studies. The core depletion portion was not used in this study. Three fast and one thermal macro-

(8) R. Froelich and K. Ott, Nucl. Sci. Eng., 22, 490 (1965). group coefficients were generated by means of RIP, IIUFT 5, and KATE 1 subroutines. RIP calculates the resonance integral; self-shielding and Doppler broadening of neutron resonances are calculated by the Adler-Nordhein method with the Dancoff factor calculated by Sauer's method. IUFT 5 uses a Fourier transform to calculate group constants for the three fast groups. The B1 approximation was used. The capture and fission cross sections used in MUFT 5 consist of a snooth and a resonance portion. The resonance term is only that part due to the neutron resonances. The smooth and resonance portions are combined together for each isotope by use of the relationship

$$
\sigma_{m}=\left(\sigma_{m}^{\text {smooth }}+\frac{R_{m}}{\Delta U_{m}}\right)
$$

where $\sigma_{m}^{\text {smooth }}$ is the smooth cross section of micro-group $m, R_{m}$ is the shielded resonance integral calculated by RIP for micro-group $m$, and $\Delta U_{m}$ is the lethargy width of the micro-group $m$. In the present set of calculations, only $\mathrm{U}^{238}, \mathrm{Pu}^{239}$, and $\mathrm{Pu}^{240}$ have nonzero values of $R_{m}$. The 34-group fast micro-group cross sections are condensed into 3 fast macro-groups by RIP and MUFT 5 . KATE 1 calculates the spectrum of thermal neutrons according to the Wigner-Wilkins theory and averages those quantities important to reactor design over this spectrum. The fast and thermal macro-group few-group constants were then used in the WANDA segment of LIFE $\mathrm{X}$ to calculate $k_{\text {eff }}$ and the few-group fluxes.

A pancake core, $2 \mathrm{ft}$ high and $10 \mathrm{ft}$ in diameter, with a composition typified by bare cores 11-14 (see Table I) was also studied. Axial and radial blankets, 18 in. thick, were placed around the core. The blanket compositions were as follows:

\begin{tabular}{|c|c|c|c|c|c|}
\hline & No & 0239 & $J 238$ & Stainless & 0 \\
\hline Axial & 0.0123 & 0.00007 & 00072 & 0.011 & 0.0144 \\
\hline Radial & 0.0069 & 0.00012 & 0.012 & 0.011 & 0.024 \\
\hline
\end{tabular}

The $\mathrm{Pu} / \mathrm{U}$ ratio was varied in the core portion until the reactor was just critical. The results of these calculations are listed in Table 3.

In order to obtain the perpendicular buckling (and hence the perpendicular leakage), calculations were made for the core in the perpendicular direction with the blanket removed. The core dimension in that direc-

TABLE 3. Pancake Core Results

$\begin{array}{ll}k_{\text {eff }} & =1.00008 \\ k_{\text {eff }}(50 \% \text { void }) & =1.00539 \\ \text { Mass of } \mathrm{U}^{238} \text { in core } & =13,829 \mathrm{~kg} \\ \text { Mass of } \mathrm{Pu}^{239} \text { in core } & =1,884 \mathrm{~kg} \\ \text { Mass of } \mathrm{Pu}^{240} \text { in core } & =757 \mathrm{~kg} \\ \text { Breeding Ratio } & =1.42\end{array}$


tion was increased until $k_{\text {eff }}$ was identical to the blanketed core. This bare-core buckling was then assumed to give the proper perpendicular leakage when the core was calculated in the normal direction.

\section{Melvin M. Levine}

Brookhaven National Laboratory

The AIM-6 and the 20-GRAND multigroup codes with Yiftah, Okrent, and Moldauer cross sections were used for all this work. The cross-section changes due to Doppler effect were obtained with a modification of the GA.T code which allows resonances to have fission as well as capture. The statistical distributions of the neutron and fission widths were also taken into account.

The pancake core was calculated in a two-dimensional representation with the use of six-group cross sections. These were obtained by collapsing the sixteengroup cross sections over spectra obtained from suitable one-dimensional calculations. The overall reactivity change due to sodium voiding was obtained by direct calculation; the breakup into separate components due to leakage, spectral, and capture effects was done by perturbation theory.

J. M. Chaumoxt, G. Neviere, axi J. Ravier Commissariat à l'Energie Atomique, Service d'Etudes de Piles Rapides, Cadarache, France

\section{J. Martix}

Electricite de France, Direction des Etudes et Recherches, Departement Etudes Nucleaires, Paris

\section{Characteristics of the Cross-section Set}

The main part of this intercomparison has been made by using the Russian 26-group set(1) with minor modifications. For each reactor the resonance self-shielding correction factors $f\left(\sigma_{0}, T\right)$ have been applied to the cross sections of fissile and fertile isotopes and to the capture cross sections of higher nuclei.

Transport cross sections and elastic removal cross sections of carbon, oxygen, sodium, iron, chromium, and nickel have been calculated by using ELMIOE and its attached library of $B_{L}$ (Legendre polynomial expansion).(2)

\section{Bare Reactors}

For the six bare reactors the ELAIOE code has been used with 670 energy groups and the $P_{1}$ approximation. Flux and buckling of each reactor have been calculated. Transport and elastic removal cross sections collapsed in 24 groups from the ELMOE calculation have been used by the adjoint "HOBO" code.

(1) L. P. Abagyan et al., Atomizdat (1964).

(2) A. L. Rago and H. H. Hummel, ANL 6805 .
A special code "SEPR 508" starts from direct and adjoint fluxes and computes the Doppler effect.

\section{Blanketed Reactors}

The three blanketed reactors have been calculated by using a one-dimensional diffusion code "MIUDE." Elastic removal cross sections in the core had been given by ELAIOE; removal cross sections in the blanket have been given by the method described below.

\section{Doppler Coefficient}

In the energy range where resonances strongly overlap, the temperature dependence of the effective cross section has been calculated by the classical method A of Nicholson.

In the energy range in which resonances are well isolated, experimental resonance parameters have been used with the one-level Breit-Wigner formula to calculate the effective cross section as a function of the dilution and the temperature.

In the energy range in which resonances weakly overlap, the same formalism as above has been followed; it was assumed that the resonance parameters can be extrapolated from the measured ones at lower energy, using an integration step of $0.1 \mathrm{eV}$.

All the Doppler coefficients have been calculated at $900^{\circ} \mathrm{K}$, assuming a homogeneous medium.

The influence of heterogeneity has been evaluated on blanketed reactor No. 8 (see Table 2). The Bell theory has been applied to the heterogeneous case of two zones (the first one with $\mathrm{U}^{238}-\mathrm{Pu}^{239}$-O-fission products; the second one with $\mathrm{Na}-\mathrm{Fe}-\mathrm{Cr}-\mathrm{Ni}$ ) to calculate the dilutions. Flux and adjoint flux of the homogeneous case have been used.

The various components of the Doppler effect have been increased by $23 \%$ for $\mathrm{Pu}^{239}$, by $42 \%$ for $\mathrm{U}^{238}$, and by $17 \%$ for $\mathrm{Pu}^{240}$; this leads to an overall increase of the Doppler coefficient of $1.8 \%$ only due to compensation between different isotopes.

\section{Comments}

Core data with $100 \% \mathrm{Na}$ and $50 \% \mathrm{Na}$ have been obtained through two successive ELIIOE calculations. Removal cross sections for the blanket have been calculated using the methods described in the section below.

\section{Comparison of Various Cross-section Sets-Influence of the Elastic Slowing Down}

Blanketed reactor No. 8 was chosen for the comparison of the following cross-section sets:

The Russian 26-group set, ${ }^{(1)}$ of which the elastic removal cross sections have been treated by three different methods.

The elastic removal cross sections have been recalcu- 
Table 4. Comparison of the Main Parameters for the Blanketed Reactor No. 8

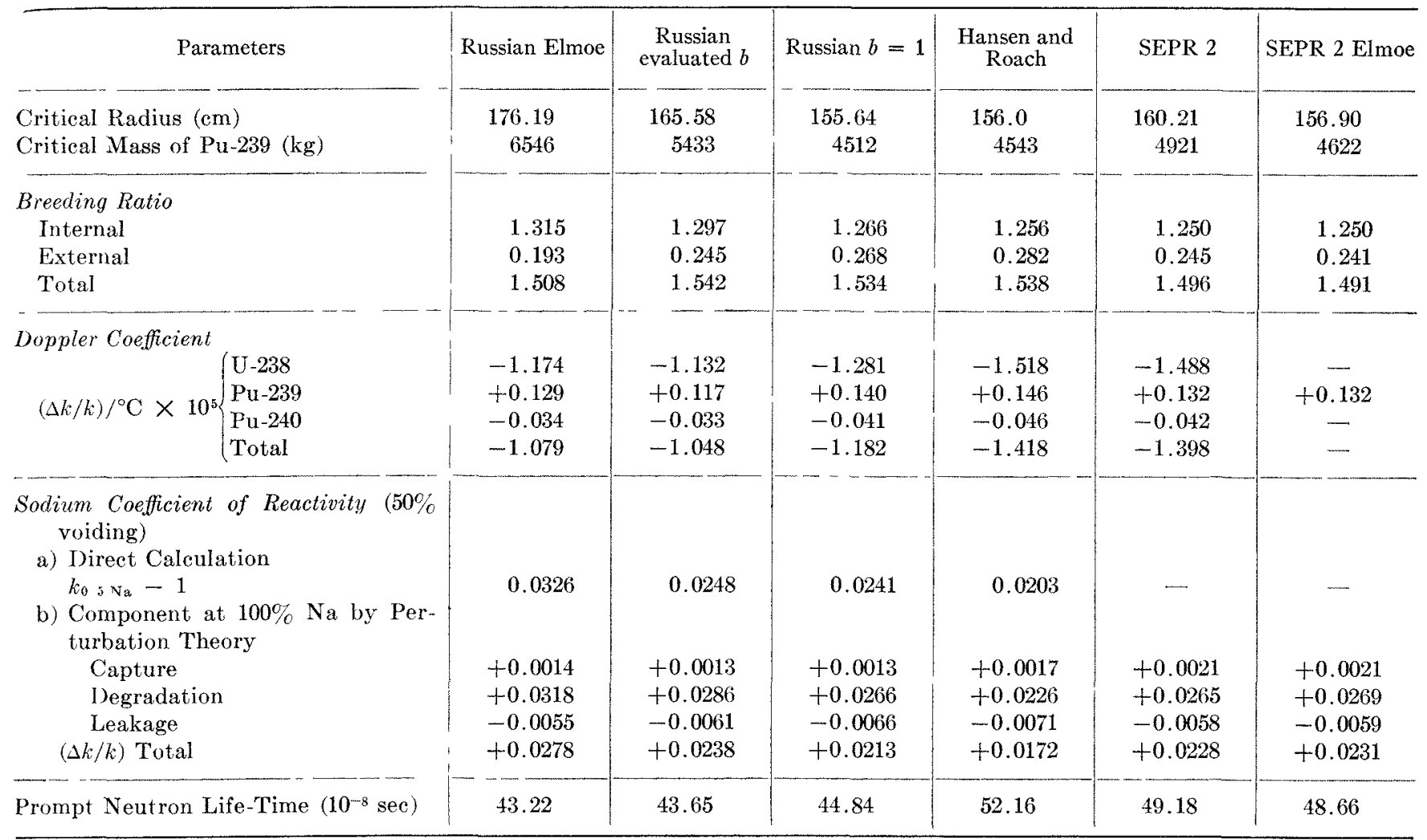

lated through the ELMOE code ("Russian ELMOE" set).

The elastic removal cross sections of Ref. 1 have been corrected by the $b$ factor (ratio of the flux at the lower boundary to the average flux of the group). $b$ was evaluated by a one-iteration procedure ("Russian procedure ("Russian evaluated $b$ " set).

The elastic removal cross sections of Ref. 1 were used without any correction ("Russian $b=1$ " set).

16-group Hansen and Roach set. ${ }^{(3)}$

A special 25-group set designed at Cadarache from the following hypotheses:

Group width is 0.5 between $3.7 \mathrm{MeV}$ and $275 \mathrm{eV}$; the energy range from $14 \mathrm{MeV}$ to $0.4 \mathrm{eV}$ is covered by 24 groups; the 25 th group is thermal.

Fundamental data result from various evaluations: the British ${ }^{(4)}$ for U-238 and Pu-239 at high energy, Na, $\mathrm{O}$, and $\mathrm{Fe}$; the Germans ${ }^{(5)}$ for Pu-239 below $10 \mathrm{keV}$; the French ${ }^{(6)}$ for U-238 at low energy, $\mathrm{Ni}, \mathrm{Cr}$, fission products, $\mathrm{Pu}-240$, and $\nu$ of $\mathrm{Pu}-239$.

Multigroup constants have been calculated by the GALAXY-3 code. ${ }^{(7)}$ The weighting spectrum was

(3) (x. E. Hansen and W. H. Roach, LAMS-2543.

(4) E. Barrington et al., AEEW-R-351 (July 1963).

(5) J. J. Schmidt, KFK-120.

(6) J. Ravier, PNR/SEPR/CA 65.010.

(7) T. Bell et al., AREW-R-379.
$1 / \Sigma_{t} E^{n}$, where $\Sigma_{t}$ is the total macroscopic cross section of the medium and $n$ varies from -2 to +2 according to the energy range.

Below $50 \mathrm{keV}$, resonance self-shielding corrections have been evaluated by two codes:

RDT: a resonance integral code based on the narrow resonance approximation;

SEPR 549 S: a code computing the effective cross section directly from fundamental microscopic cross sections generated by a Doppler-broadened one-level resonance formula. The resonance parameters had been extrapolated from the lower energy region by means of statistical laws.

In the "SEPR 2 ELMIOE" set, removal and transport cross sections have been evaluated through ELAIOE.

Results of this comparison are summarized in Table 4 .

\section{Comments}

In order to explain the differences between the calculated critical radii, the differences between the various calculated core $k_{\infty}$ have been split into the contributions given in Table 5. It can be seen that the Hansen and Roach set is the most reactive. Up to now studies carried out at Cadarache on reactors without $\mathrm{Pu}-240$ and fission products had shown the contrary. ${ }^{(8)}$ This

(8) J. M. Chaumont et al., PNR/SEPR 65.017. 
TABLE 5. CONTRIBUTIONS TO $k_{\infty}$

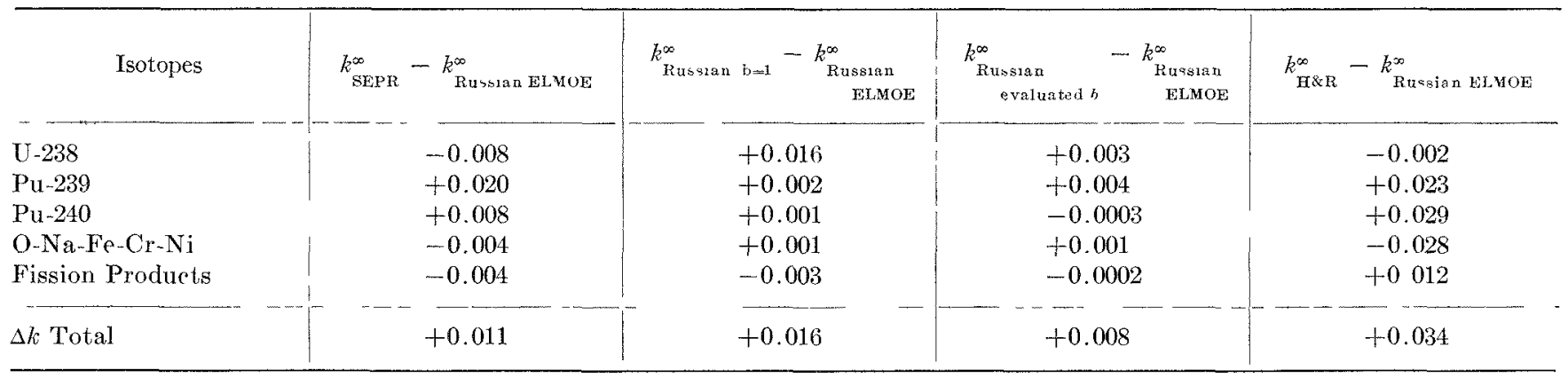

can be explained by the too-weak capture cross sections in both the fission products and Pu-240 of the Hansen and Roach set (without fission products and Pu-240, $\left.k_{\mathrm{H} \& \mathrm{R}}^{\infty}-k_{\mathrm{Ru}}^{\infty} \mathrm{ussan}_{\mathrm{ELMOE}}=-0.007\right)$. On the other hand, capture in chromium and nickel seems to be overevaluated.

The Russian set with three different ways of dealing with elastic scattering gives different results. However, Russian ELMOE and Russian with evaluated $b$ give very similar data.

\section{Geza L. Gyorey General Electric}

The bare cores were calculated by use of 60 energy groups. The basic 60 -group fast reactor data file used at General Electric carries essentially the same information as the ANL set No. 224 (ANL-6858). This 60group file has been adjusted to agree, for all practical purposes, with ANL No. 224, with the following exceptions:

The $\mathrm{Fe} \sigma_{c}$ above $1 \mathrm{MeV}$ is higher in our set. $\mathrm{U}^{238} \sigma_{c}$ above $67 \mathrm{keV}$ is higher (as in ANL-5800), close to the U.K. and Russian sets.

$\mathrm{Pu}^{239} \sigma_{f}$ and $\sigma_{c}$ from 0.5 to $3.3 \mathrm{keV}$ are lower and agree with the data of J. J. Schmidt (KFK 120, EANDC-E-35U, December, 1962).

For the sodium resonance $\Gamma_{\gamma}=0.35$ was used.

For each of the blanketed cores an appropriate zerodimensional calculation was performed with the 60 groups; then, based on the resulting energy distribution of the flux, the cross-section information was condensed to a 24-group set.

\section{E. Kiefhaber axd K. E. Schroeter Kernforschungszentrum, Karlsruhe}

For these calculations the Karlsruhe 26-group set (called KFK 26-10) and partially the Russian 26-group set $^{(1)}$ (called ABN) was used. Both sets are shielded-

(1) L. D. Abagjan et al., Group Constants of Fast and Intermediate Neutrons for Calculation of Reactors, Physical-Energetical Institute of the State Committee for the Use of Atomic Energy in USSR. group-constant sets with self-shielding factors depending on the special composition. The energy boundaries, the lethargy intervals, and the mechanism of shielding is the same.

In the beginning of our calculations the KFK 26-10 SET was available for a $900^{\circ} \mathrm{K}$ fuel temperature only. Therefore all results obtained by the KFK 26-10 SET are valid for $900^{\circ} \mathrm{K}$. For comparison and interpolation we used in some cases (Blanketed Spheres, Cases 8 and 12 of Table 2) the KFK 26-10 SET (at $900^{\circ} \mathrm{K}$ ) and the 26-ABN SET (for 300,900 , and $2100^{\circ} \mathrm{K}$ ), respertively.

For the Doppler-effect calculations all cores were treated as homogeneous.

M. E. Battat, R. J. Labauve, and G. L. Ragan Los Alamos Scientific Laboratory

\section{Calculational Procedure}

A two-dimensional model was used for the calculations. The cross sections used were those of the HansenRoach 16-group library.(1) For fission products, a capture cross section based on Moldauer's data ${ }^{(2)}$ was used, but no scattering cross section. Resonances in the fuel and fertile materials were treated according to the scheme suggested by Hansen and Roach, ${ }^{(3)}$ and cross sections so modified are designated as "shielded" in the results.

Two-dimensional criticality calculations by means of CRAM ${ }^{(4)}$ were done, followed by two-dimensional $S_{n}$ calculations. ${ }^{(5)}$ Also, one-dimensional $S_{n}$ calculations were done in which the reactor was taken to be a "circular" slab, i.e., input data for the 1-D slab was derived. The side leakage was approximated with a buckling equivalent to $\left(j_{0} / R\right)^{2}$.

(1) L. D. Connolly, LAMS-2941 (July 1963).

(2) Proceedings of the Conference on the Physics of Breeding, ANL-6122 (Oct 1959), p. 73 .

(3) G. L. Hansen and W. H. Roach, LAMS-2543 (Dec 1961).

(4) A Hassitt, UKALA TRG Report No. 229(R) (1962).

(5) B. G. Carlson and K. D. Lathrop, I,A-3251-MS (April $1965)$. 


\section{R. W. Suith ANid R. W. Ross UKAEA, Winfrith}

The calculations, corresponding to a fuel temperature of $300^{\circ} \mathrm{K}$, were made with the use of the multi-group diffusion theory program CRAMI, (1) with the 33-group cross-section set called F.D.2. This cross-section set has been constructed for calculations on large dilute fast reactors. Most of the group cross sections have been obtained from U.K.A.E.A. Nuclear Data Library using the data-processing program GALAXY ${ }^{\top(2)}$ with a weighting spectrum approximately representing the spectrum of a large fast reactor core voided of coolant.

The code ELAIOE ${ }^{(3)}$ has been used for selerted group elastic moderation cross sections for oxygen, sodium (the sodium cross sections are the differences between the macroscopic group cross sections for the core with and without sodium per atom of sodium), iron, chromium, and nickel. Use has also been made of the recent compilation of Yiftah and Sicger ${ }^{(4)}$ for nickel and chromium and of Schmidt ${ }^{(5)}$ for iron.

The group structure and the resonance shielding factors were the same as those in the set F.D.1, ${ }^{(6)}$ and ten down-seatter groups are given where appropriate.

In separating the infinite-dilution cross sections given by GALAXY into resonant and nonresonant components, the ratio of resonant to nonresonant was kept approximately equal to that in F.D.1 whilst the sum was kept equal to the GALAXY infinite-dilution cross section.

The only allowance made for the heterogeneity of the composition has been to modify the resonance shielding factors for uranium and plutonium isotopes by using adjusted values of $\sigma_{p}^{g}$, the nonresonant cross section per absorber atom for group $\mathrm{g}$. The contributions to the $\sigma_{p}^{g}$ 's from material outside the fucl (sodium and steel) were reduced by a Bell-type approxination, and these reduced values were input to the programme.

(1) A. Hassitt, TRG Report No. 229(R).

(2) T.J. Bell et al., AEHW-R.379.

(3) A. L. Rago and H. H. Hummel, ANL-6805.

(4) S. Yiftah and M. Sieger, IA.980.

(5) J. J. Schmidt, KFK 120 (EANDC-F-35 U).

(6) J. L. Rowlands and I). Wardleworth, AEEW-11.472.

\section{G. M. S. Computations in Support of the 1965 A. N. L. Intercomparison of Fast Reactor Calculations}

\section{P. Gratton and P. E. Suith UKAEA, Winfrith}

\section{Method of Calculation Used-Cases 16-19 of Table 1}

The method of calculation used was that contained within the computer code GAIS ${ }^{(1)}$ written in FORTRAN for the KDF 9 and the IBII 7030 computers.

(1) C. P. Gratton and P. E. Smith, AEEW-M.458.
GMS is a generalised multigroup system of calculations which has been used for the study of both thermal and fast reactors. The code is coupled with a nuclear data library system and, depending on the reactor, either a data set with emphasis in the thermal-energy region is selected or one appropriate for fast reactor studies. In the present work, the fast reactor data set known as FD $2^{(2)}$ has becn used.

G.IS is written to use 40 energy groups of neutrons, the lowest seven of which cover the region of thermal energy. From Group 1 to (Group 14 the structure follows that of the YONI set, ${ }^{(3)}$ and from Group 15 to Group 33 that of the extension by Rowlands and Wardleworth. ${ }^{(4)}$ The lowest seven groups are included primarily to deal with hydrogen-containing systems, but their inclusion also allows calculations of blanket conditions where thermal-neutron events are of importance.

The Winfrith DSN code ${ }^{(5)}$ is included as a subroutine within GAIS, allowing transport calculations of system heterogeneity and overall reactors to be performed by DSN methods. These calculations are all in one dimension, and include options for slab, cylindrical, and spherical geometry. For many calculations, fewer groups provide a sufficiently accurate solution and because of this, a feature has been included to permit a condensation of the data to fewer groups. To carry out this condensation, a 40-group neutron spectrum characteristic of the system is required. This is obtained in one of two ways:

(i) For a core region a fundamental-mode calculation is carried out using the full 40-group representation.

(ii) For a blanket region a flux spectrum for an infinite medium is evaluated, using as a source a fission spectrum.

Having obtained these spectra for a particular system, calculations with an increasing number of groups can be performed to establish the number required to achieve a sufficiently accurate solution to the problem.

(2) R. W. Smith, to be published.

(3) S. Yiftah, D. Okrent, and P. Moldauer, Fast Reactor Cross Sections, Pergamon Press, New York (1960).

(4) J. L. Rowlands and D. Wardleworth AEEW-M.472.

(5) S. Francescon, AEEW-R.273.

\section{B. H. Cherry \\ United Nuclear Corporation}

The calculations were performed using $\mathrm{DS}_{4}$ approximation to the transport-theory equation for cores with radii less than $110 \mathrm{~cm}$, and ${D S_{2}}_{2}$ for cores with radii greater than $110 \mathrm{~cm}$. The cross sections used are those given by the 16-group infinite-dilution Hansen-Roach ${ }^{(1)}$ set modified as follows:

1. Self-shielding and Doppler broadening were ac-

(1) G. E. Hansen and W. H. Roach, LAMS-2543. 
counted for using the procedures ${ }^{(2)}$ programmed into the United Nuclear BIGSUM code.

2. Sodium cross sections in the region of the 2.85$\mathrm{keV}$ resonance were corrected in terms of the data of Hibdon. ${ }^{(3)}$

(2) C. C. Graves, UNC-5064 (May 1964).

(3) C. T. Hibdon, Phys. Rev., 118, 515 (1960).

\section{J. H. WRIGHT Westinghouse}

Calculations of criticality, spectral indices, and reactivity changes were performed by means of multigroup, one-dimensional diffusion theory. Cross sections were based on the $\mathrm{YOM}^{(1)}$ set, but with the following modifications: (1) energy structure below $10 \mathrm{keV}$ modified

(1) S. Yiftah, D. Okrent, and P. A. Moldauer, Fast Reactor Cross Sections, Pergamon Press, New York (1960). and extended; (2) plutonium and uranium cross sections updated and calculated for self-shielding effects of each case $;^{(2,3,4)}(3)$ scattering cross sections of light-element resonances determined from the ELMOE fine-energystructure code. ${ }^{(3,5)}$

The Doppler coefficients of reactivity were determined from temperature-dependent cross sections of the heavy isotopes. The Doppler-broadened cross sections of $\mathrm{U}^{238}, \mathrm{U}^{239}$, and $\mathrm{Pu}^{240}$ were determined from the ARES-I code, ${ }^{(3)}$ based on resonance integral techniques. The NR (narrow resonance) and NRIM (narrow resonance infinite mass) approximations were assumed. No provision was made for interference effects between resonances.

(2) F. M. Heck, WCAP-3251-1 (1964).

(3) M. G. Blide and H. H. Hummel, ANL-6601 (1962).

(4) F. L. Fillmore, AMTD-130 (1962).

(5) A. L. Rago and H. H. Hummel, ANL-6805 (1964).

\section{SUMMARY AND COMPARISON OF RESULTS}

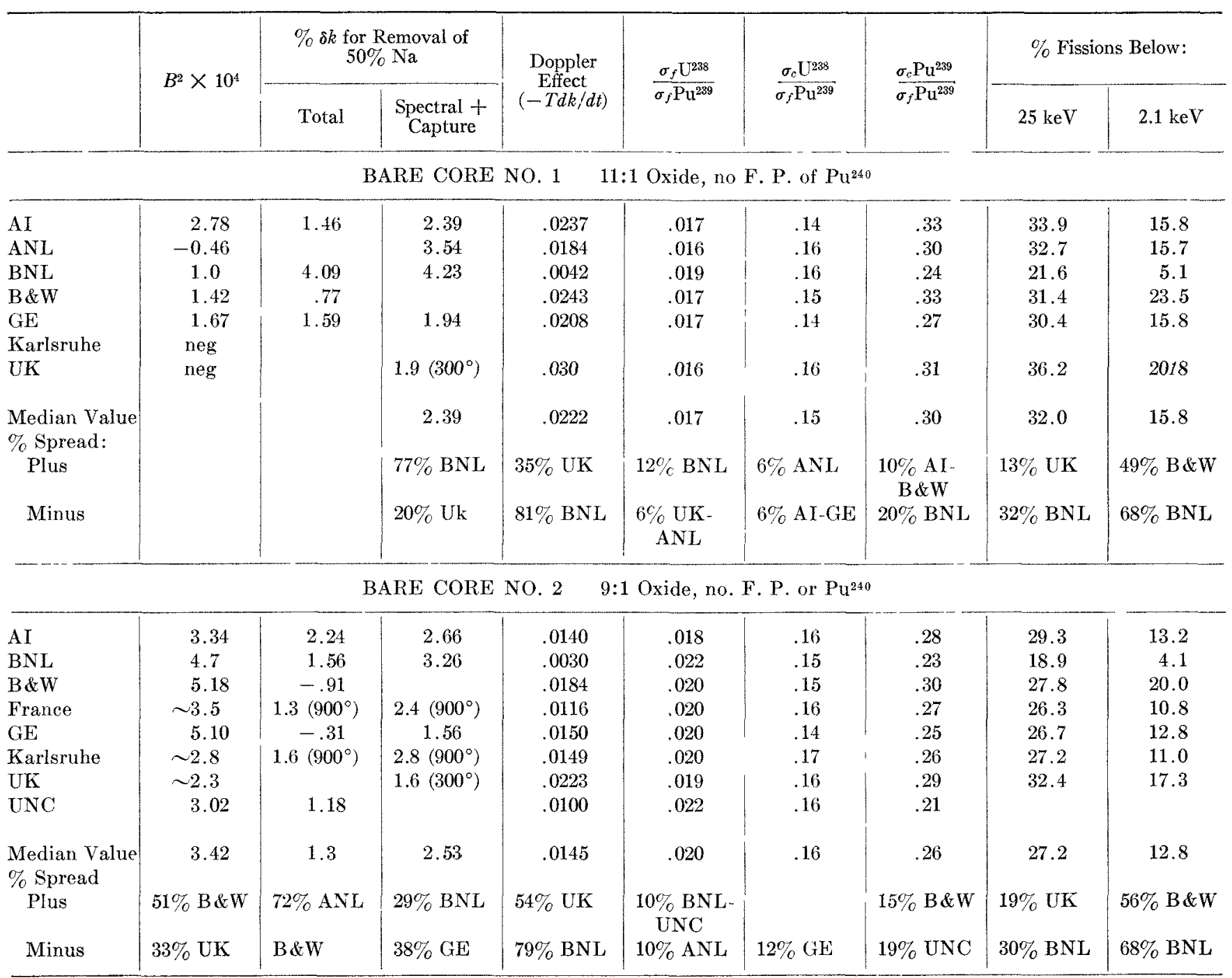




\begin{tabular}{|c|c|c|c|c|c|c|c|c|}
\hline \multirow{2}{*}{$B^{2} \times 10^{4}$} & \multicolumn{2}{|c|}{$\begin{array}{c}\% \delta k \text { for Removal of } \\
50 \% \mathrm{Na}\end{array}$} & \multirow{2}{*}{$\begin{array}{c}\text { Doppler } \\
\text { Effect } \\
(-T d k / d t)\end{array}$} & \multirow{2}{*}{$\frac{\sigma_{f} U^{238}}{\sigma_{f} \mathbf{l}^{2} u^{239}}$} & \multirow{2}{*}{$\frac{\sigma_{c} \mathrm{U}^{238}}{\sigma_{f}} \mathrm{Pu}^{239}$} & \multirow{2}{*}{$\frac{\sigma_{c} \mathrm{Pu}}{\sigma_{f} \mathrm{Pu}^{239}}$} & \multicolumn{2}{|c|}{$\%$ Fissions Below: } \\
\hline & Total & $\begin{array}{l}\text { Spectral }+ \\
\text { Capture }\end{array}$ & & & & & $25 \mathrm{keV}$ & $2.1 \mathrm{keV}$ \\
\hline
\end{tabular}

BARE CORE NO. $3 \quad 7: 1$ Oxide, no F. P. or $\mathrm{Pu}^{240}$

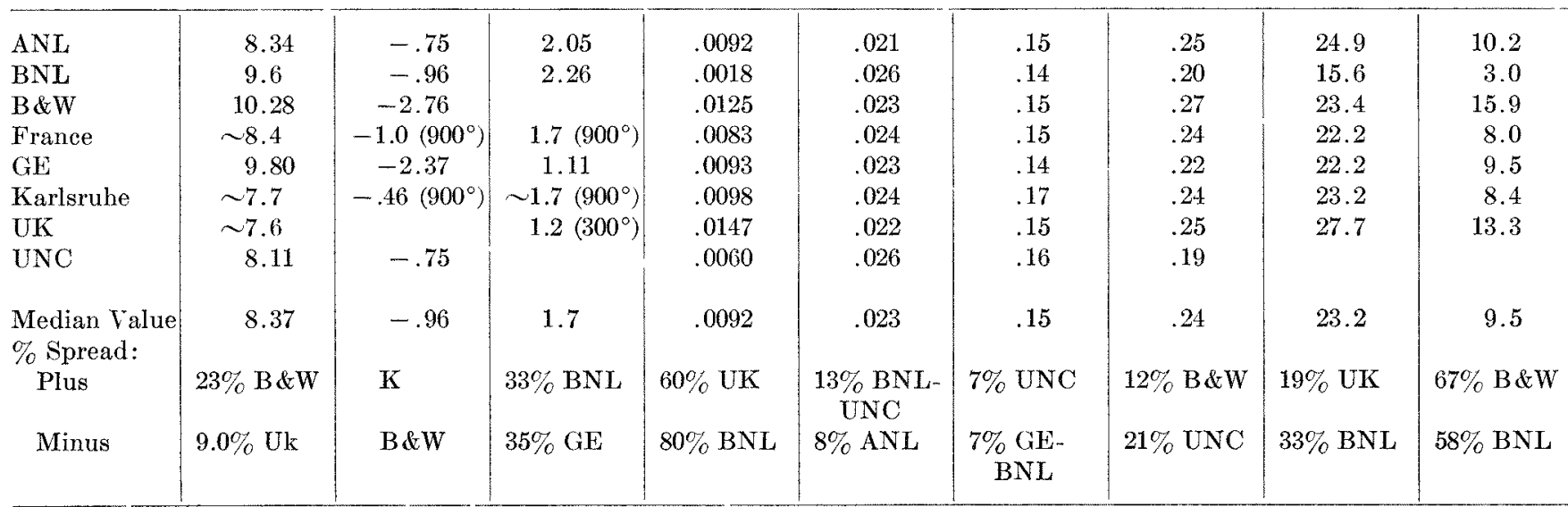

BARE CORE NO. $4 \quad 5: 1$ Oxide, no F. P. or $\mathrm{Pu}^{240}$

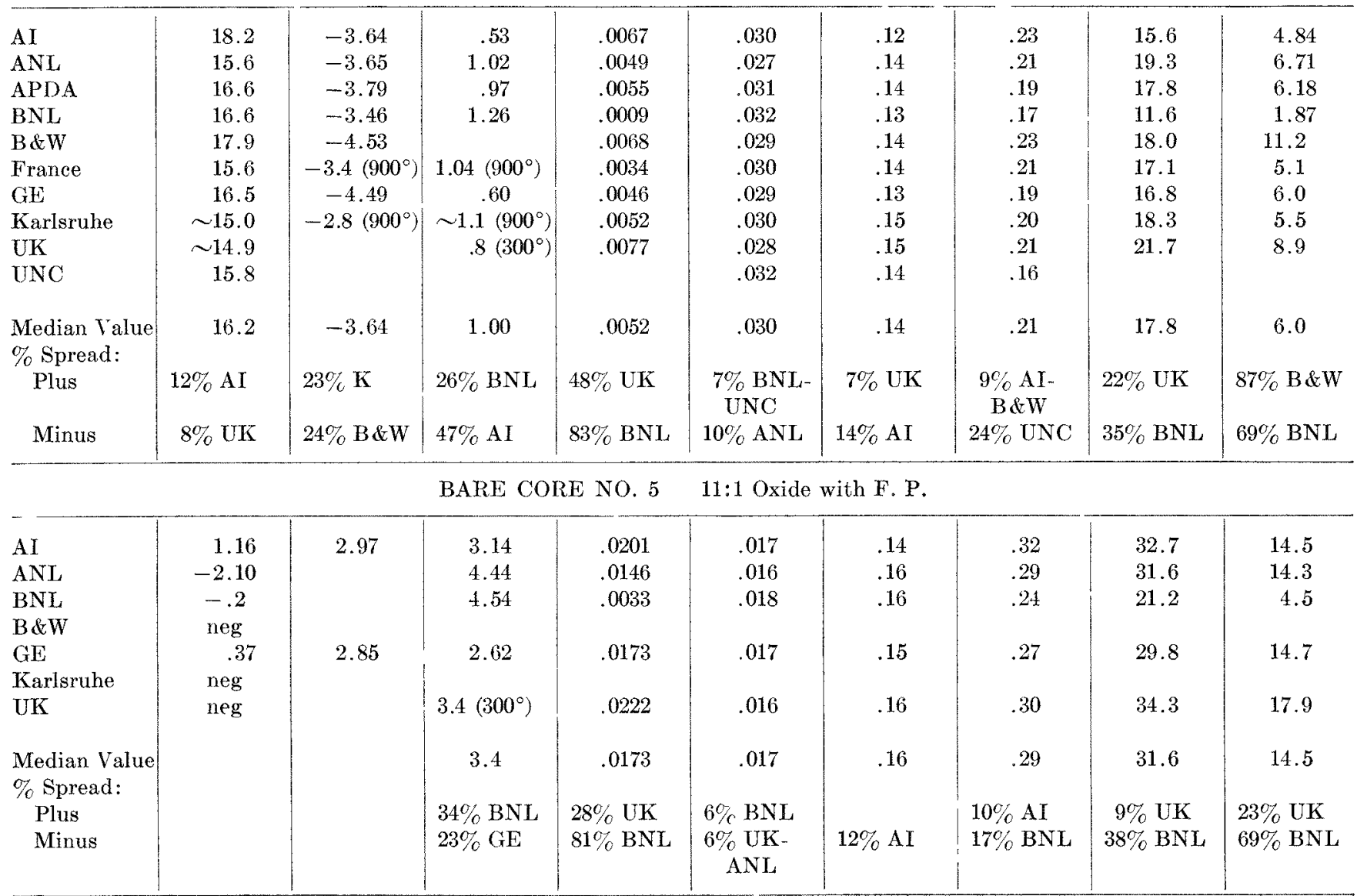




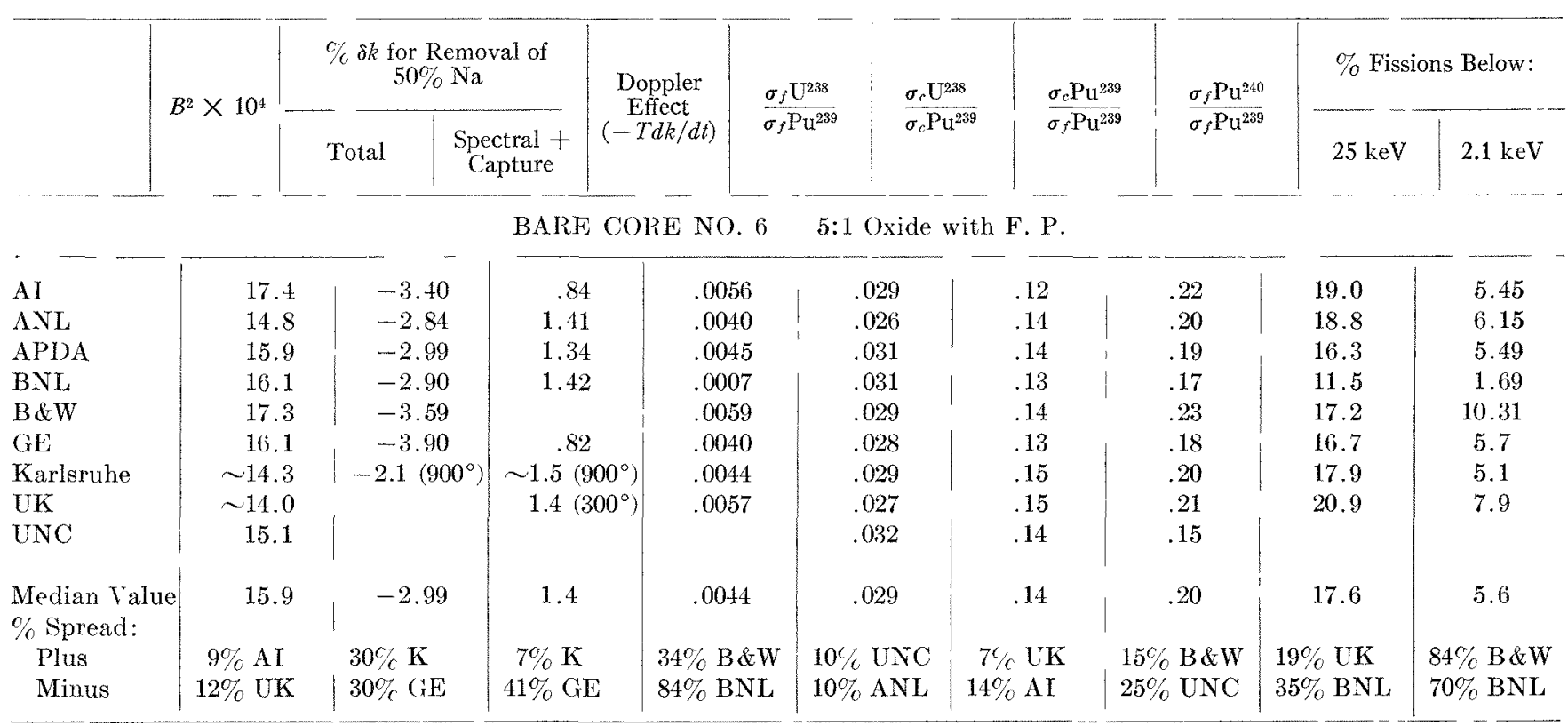

BARE CORE NO. $7 \quad 11: 1$ Oxide with F. P. and $P u^{240}$

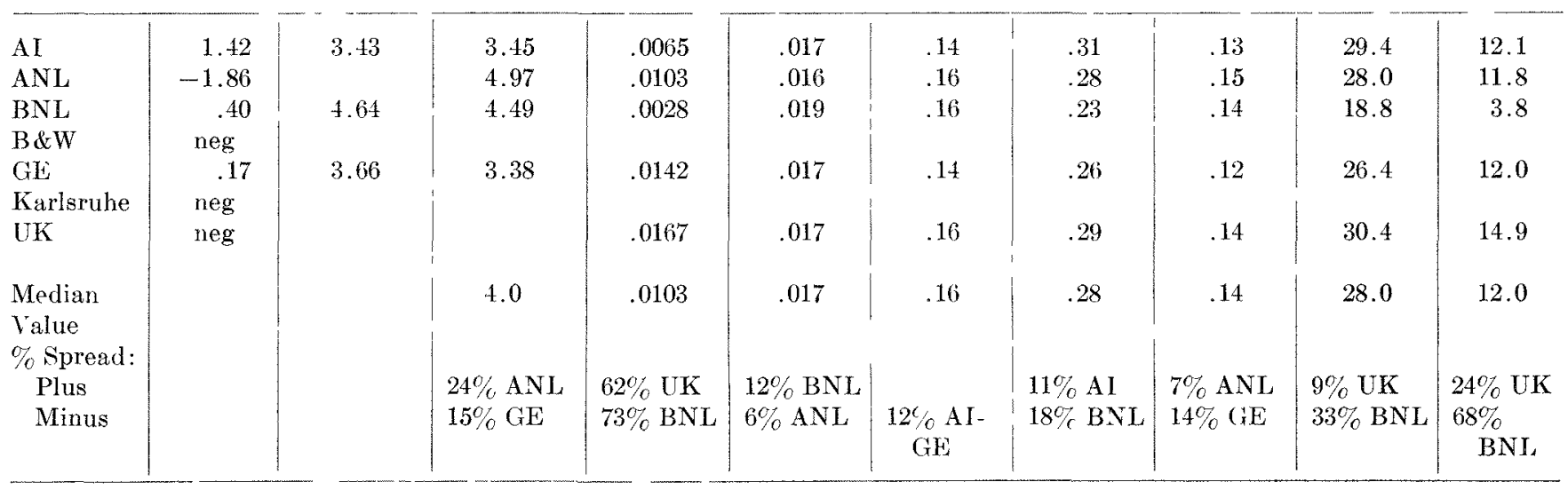

BAIRE CORE NO. $8 \quad 9: 1$ Oxide with F. P. and Pu ${ }^{240}$

\begin{tabular}{|c|c|c|c|c|c|c|c|c|c|c|}
\hline $\mathrm{BNL}$ & 4.50 & 2.31 & 3.58 & .0020 & .022 & .15 & .22 & .16 & 16.2 & 3.0 \\
\hline$B \& W$ & 3.54 & 1.53 & & .0110 & .021 & .15 & .28 & .15 & 22.6 & 15.0 \\
\hline GE & 4.15 & 1.54 & 2.82 & .0100 & .020 & .14 & .23 & .14 & 22.9 & 9.5 \\
\hline Karlsruhe & 1.3 & $3.3\left(900^{\circ}\right)$ & $\sim 3.8\left(900^{\circ}\right)$ & .0096 & .020 & .17 & .25 & .15 & 23.0 & 7.8 \\
\hline UK & $\sim 1.0$ & & $\sim 4.0$ & .0120 & .019 & .16 & .26 & .16 & 26.8 & 12.1 \\
\hline Median & 2.73 & 2.4 & 3.58 & .0096 & .0205 & .15 & .26 & .16 & 23.3 & 9.5 \\
\hline $\begin{array}{l}\text { Value } \\
\text { \% Spread: }\end{array}$ & & & & & & & & & & \\
\hline Plus & $115 \% \mathrm{AI}$ & $45 \%$ ANL & $12 \%$ ANL & & $\begin{array}{c}7 \% \text { BNL. } \\
\text { UNC }\end{array}$ & $\begin{array}{c}7 \% \text { UK- } \\
\text { [NC }\end{array}$ & $\begin{array}{r}8 \% \mathrm{AI}- \\
\mathrm{B} \& \mathrm{~W}\end{array}$ & $6 \% \mathrm{ANL}$ & $15 \%$ UK & $\begin{array}{l}58 \% \\
\quad \text { B\&W }\end{array}$ \\
\hline Minus & $63 \%$ UK & $59 \%$ AI & $26 \% \mathrm{AI}$ & $79 \%$ ANL & $\begin{array}{l}7 \% \text { ANL- } \\
\text { UK }\end{array}$ & $13 \%$ AI & $23 \% \mathrm{UNC}$ & $12 \%(\mathrm{i} E$ & $30 \% \mathrm{BNL}$ & $\begin{array}{l}68 \% \\
\text { BNL }\end{array}$ \\
\hline
\end{tabular}




\begin{tabular}{|c|c|c|c|c|c|c|c|c|c|}
\hline \multirow{2}{*}{$B^{2} \times 10^{4}$} & \multicolumn{2}{|c|}{$\begin{array}{c}\% \delta k \text { for Removal of } \\
50 \% \mathrm{Na}\end{array}$} & \multirow{2}{*}{$\begin{array}{c}\text { Dopplex } \\
\text { Effect } \\
(-T d k / d t)\end{array}$} & \multirow{2}{*}{$\frac{\sigma_{f} \mathrm{U}^{238}}{\sigma_{f} \mathrm{Pu}^{239}}$} & \multirow{2}{*}{$\frac{\sigma_{c} \mathrm{U}^{238}}{\sigma_{c} \mathrm{Pu}^{239}}$} & \multirow{2}{*}{$\frac{\sigma_{\mathcal{c}} \mathrm{Pu}^{239}}{\sigma_{f} \mathrm{Pu}^{239}}$} & \multirow{2}{*}{$\frac{\sigma_{c} \mathrm{Pu}^{239}}{\sigma_{f} \mathrm{Pu}^{239}}$} & \multicolumn{2}{|c|}{$\%$ Fissions Below: } \\
\hline & Total & $\underset{\text { Capture }}{\text { Spectral }+}$ & & & & & & $25 \mathrm{keV}$ & $2.1 \mathrm{keV}$ \\
\hline
\end{tabular}

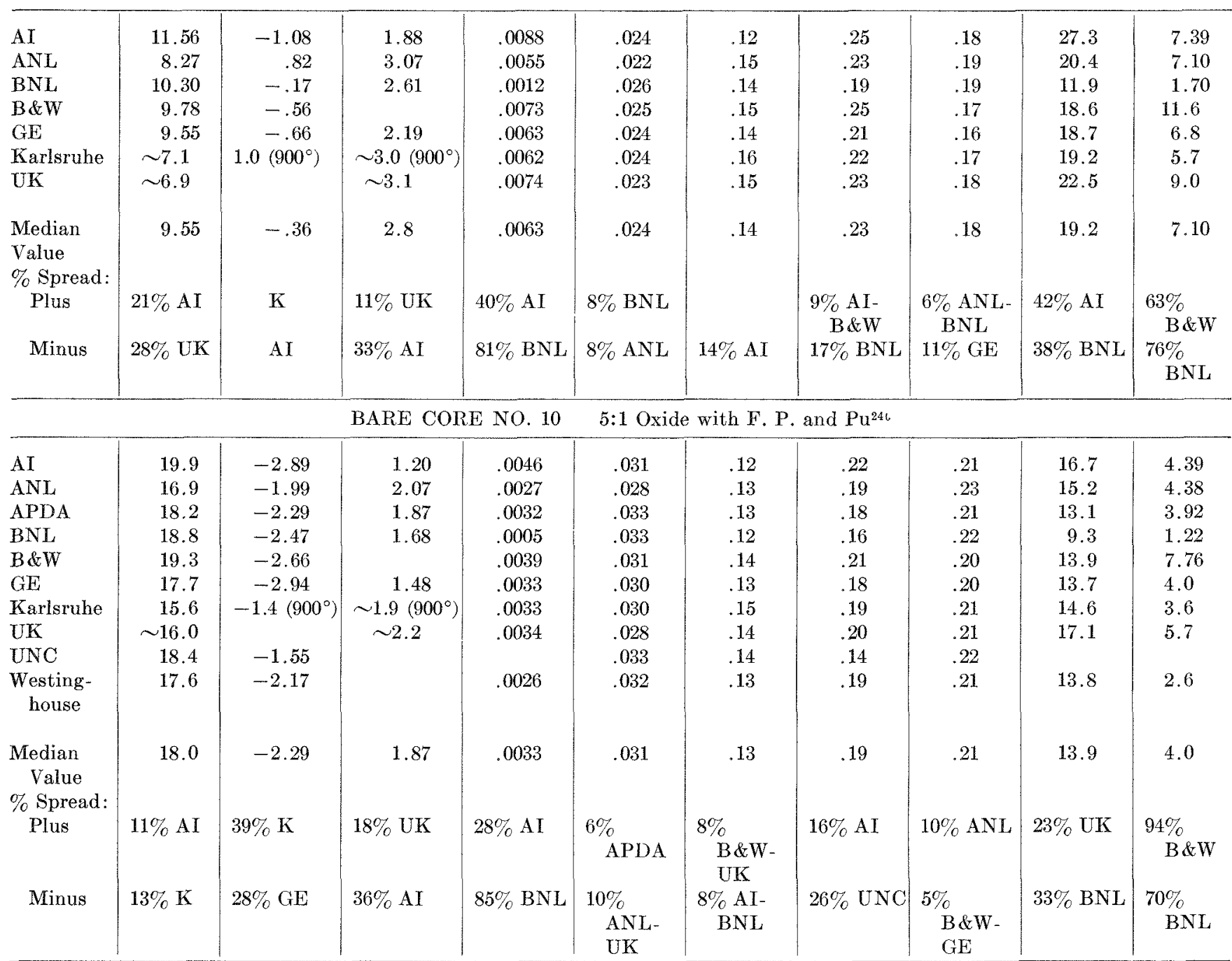

BARE CORE NO. 11 11:1 Carbide with F. P. and $\mathrm{Pu}^{240}$

\begin{tabular}{|c|c|c|c|c|c|c|c|c|c|c|}
\hline ANL & 1.58 & $\sim 4.0$ & 3.96 & .0082 & .019 & .15 & .24 & .18 & 21.7 & 8.25 \\
\hline $\mathrm{BNL}$ & 3.5 & 2.73 & 3.73 & .0019 & .022 & .14 & .20 & .17 & 14.2 & 2.3 \\
\hline$B \& W$ & 1.73 & 2.01 & & .0115 & .020 & .15 & .28 & .15 & 21.8 & 14.4 \\
\hline Karlsruhe & 0.6 & $4.0\left(900^{\circ}\right)$ & $\sim 4.2\left(900^{\circ}\right)$ & .0091 & .020 & .16 & .23 & .17 & 19.8 & 6.3 \\
\hline UK & $\sim 0$ & & $3.7\left(300^{\circ}\right)$ & .0122 & .019 & .15 & .24 & .16 & 22.0 & 9.5 \\
\hline $\mathrm{UNC}$ & 1.19 & 3.50 & & & .021 & .15 & .19 & .16 & & \\
\hline \% Spread: & $\mathrm{AT}$ & $46 \% \mathrm{~K}$ & $13 \% \mathrm{~K}$ & $180 \% \mathrm{AI}$ & $10 \% \mathrm{BNI}$ & & $17^{\circ} \mathrm{B} \& \mathrm{~W}$ & $12 \% \mathrm{ANL}$ & $10 \% \mathrm{AI}$ & $60 \%$ \\
\hline & AI & $\mathbf{4 0 \%} \mathrm{\Omega}$ & & & & & & 1270 ANL & $1070 \mathrm{AI}$ & $\mathrm{B} \& W$ \\
\hline Minus & K-UK & $48 \% \mathrm{AI}$ & $29 \%$ AI & $82 \% \mathrm{BNL}$ & $\begin{array}{c}5 \% \text { UK- } \\
\text { ANL }\end{array}$ & $20 \% \mathrm{AI}$ & $21 \% \mathrm{UNC}$ & $\begin{array}{c}6 \% \mathrm{~B} \& W \\
\mathrm{GE}\end{array}$ & $35 \% \mathrm{BNL}$ & $\begin{array}{l}74 \% \\
\text { BNL }\end{array}$ \\
\hline
\end{tabular}




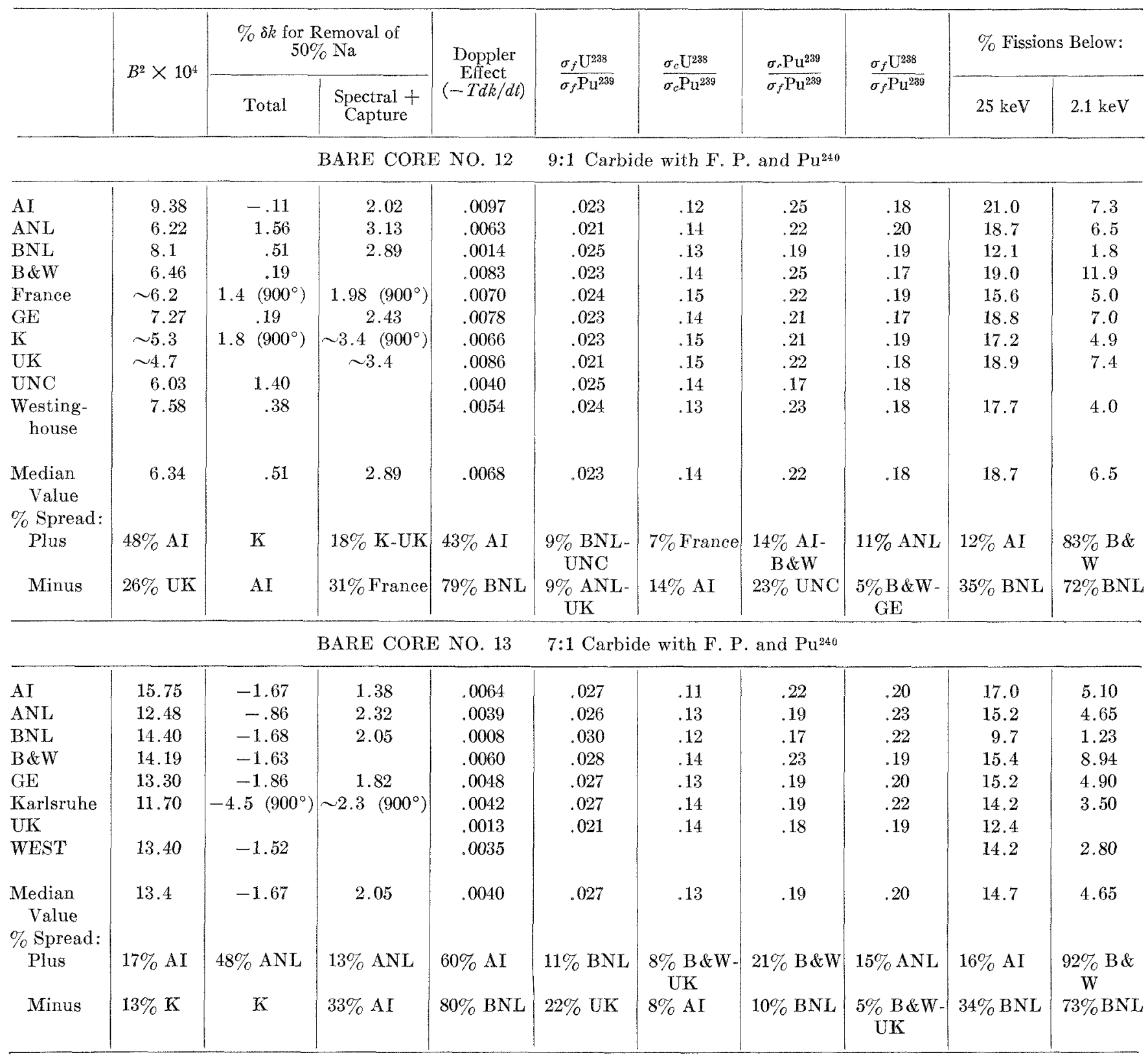

BARE CORE NO. $14 \quad 5: 1$ Carbide with F. P. and $P u^{240}$

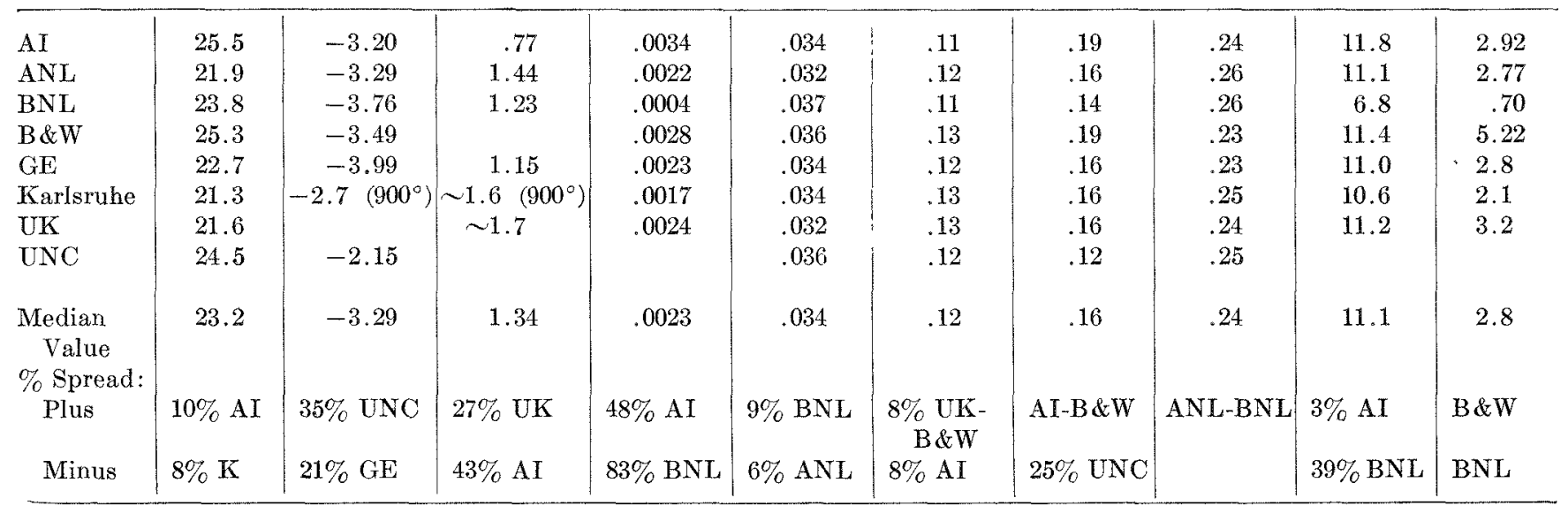




\begin{tabular}{|c|c|c|c|c|c|c|c|c|c|}
\hline \multirow{2}{*}{$B^{2} \times 10^{4}$} & \multicolumn{2}{|c|}{$\begin{array}{c}\% \delta k \text { for Removal of } \\
50 \% \mathrm{Na}\end{array}$} & \multirow{2}{*}{$\begin{array}{c}\text { Dopper } \\
\text { Effect } \\
(-T d k / d t)\end{array}$} & \multirow{2}{*}{$\frac{\sigma_{f} \mathrm{Pu}^{233}}{\sigma_{f} \mathrm{Pu}^{239}}$} & \multirow{2}{*}{$\frac{\sigma_{e} \mathrm{U}^{238}}{\sigma_{e} \mathrm{Pu}^{239}}$} & \multirow{2}{*}{$\frac{\sigma_{f} \mathrm{Pu}^{240}}{\sigma_{f} \mathrm{Pu}^{239}}$} & \multirow{2}{*}{$\frac{\sigma_{f} \mathrm{Pu}^{239}}{\sigma_{f} \mathrm{Pu}^{239}}$} & \multicolumn{2}{|c|}{$\%$ Fissions Below: } \\
\hline & 'Total & $\begin{array}{c}\text { Spectral }+ \\
\text { Capture }\end{array}$ & & & & & & $25 \mathrm{keV}$ & $2.1 \mathrm{keV}$ \\
\hline
\end{tabular}

BARE CORE NO. $15 \quad 9: 1$ Metal with F. P. and $\mathrm{Pu}^{240}$

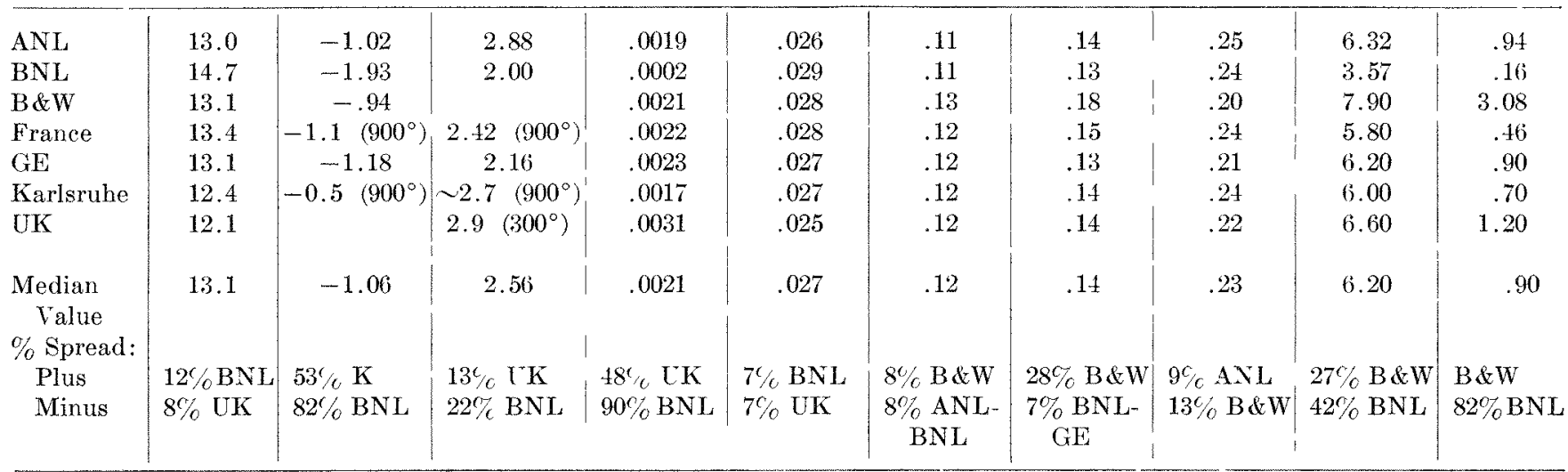

BARE CORE NO. $16 \quad$ Steam-Cooled, 11:1 Oxide

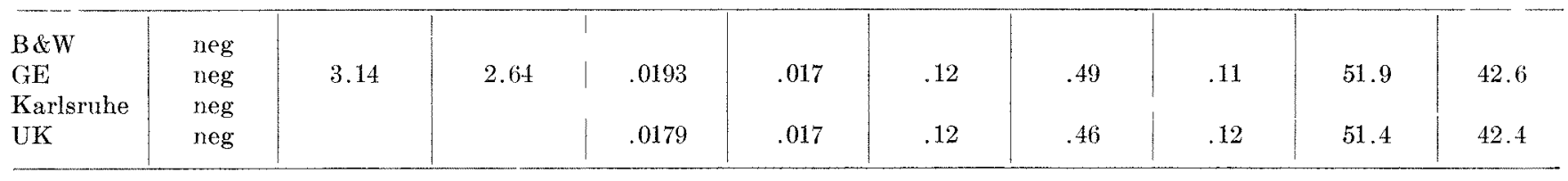

BARE CORE NO. 17 Steam-Cooled, 9:1 Oixde

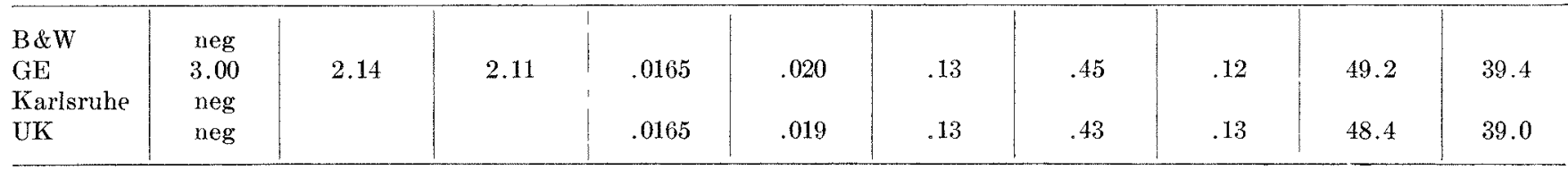

BARE CORE NO. 18 Steam-Cooled 7:1 Oxide

\begin{tabular}{|c|c|c|c|c|c|c|c|c|c|c|}
\hline $\mathrm{GE}$ & 8.05 & .57 & 1.05 & .0131 & .024 & .13 & .41 & .15 & 45.3 & 35.0 \\
\hline Karlsruhe & 4.05 & $3.36\left(900^{\circ}\right)$ & $\sim 3.8\left(900^{\circ}\right)$ & .0191 & .025 & .15 & .42 & .15 & 44.5 & 34.6 \\
\hline UK & $\sim 4.5$ & $\sim 2.5\left(300^{\circ}\right)$ & & .0140 & .023 & .13 & .40 & .16 & 44.5 & 34.6 \\
\hline $\begin{array}{l}\text { Median } \\
\text { Value }\end{array}$ & 5.2 & 2.93 & & .0143 & .024 & & .405 & & 44.5 & 34.8 \\
\hline $\begin{array}{l}\% \text { Spread: } \\
\text { Plus } \\
\text { Minus }\end{array}$ & $\begin{array}{l}55 \% \mathrm{GE} \\
19 \% \mathrm{~K}\end{array}$ & $\begin{array}{l}32 \% \text { B\&W } \\
84 \% \text { GE }\end{array}$ & & $\begin{array}{l}34 \% \mathrm{~K} \\
8 \% \mathrm{GE}\end{array}$ & $\begin{array}{l}4 \% \text { B \&W } \\
4 \% \text { UK }\end{array}$ & & $\begin{array}{l}4 \% \mathrm{~K} \\
4 \% \mathrm{~B} \& \mathrm{~W}\end{array}$ & & $\begin{array}{l}2 \% \mathrm{GE} \\
5 \% \mathrm{~B} \& \mathrm{~W}\end{array}$ & $\begin{array}{c}5 \% \mathrm{~B} \& W \\
0 \% \mathrm{~K}- \\
\text { UK }\end{array}$ \\
\hline
\end{tabular}

BARE CORE NO. 19 Steam-Cooled 5:1 Oxide

\begin{tabular}{|c|c|c|c|c|c|c|c|c|c|c|}
\hline GE & 15.4 & -1.05 & & .0090 & .029 & .13 & .37 & .18 & 39.9 & 29.2 \\
\hline Karlsruhe & 11.6 & $1.2\left(900^{\circ}\right)$ & $\sim 2.2\left(900^{\circ}\right)$ & .0145 & .030 & .15 & .37 & .18 & 39.3 & 29.1 \\
\hline UK & 12.2 & $\sim 1.0\left(300^{\circ}\right)$ & & .0102 & .028 & .14 & .36 & .19 & 38.9 & 28.7 \\
\hline $\begin{array}{l}\text { Median } \\
\text { Value }\end{array}$ & 13.3 & 1.2 & & .0105 & .030 & .14 & .36 & .185 & 39.1 & 29.2 \\
\hline
\end{tabular}




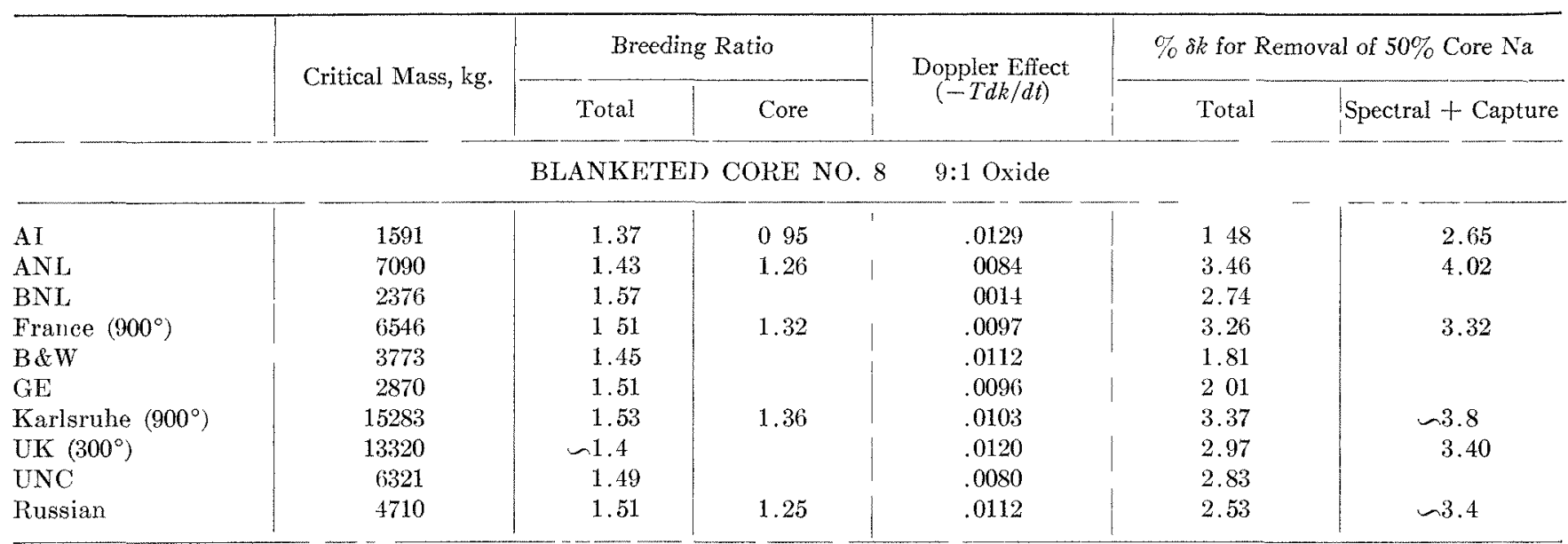

\begin{tabular}{l|r}
\hline API A & 32 \\
ANL & 39 \\
BNL & 304 \\
B\&W & 318 \\
GE & 34 \\
Karlsruhe $\left(900^{\circ}\right)$ & 449 \\
UK $\left(300^{\circ}\right)$ & 429 \\
Westinghouse & 34
\end{tabular}

BLANKETED CORE NO. 10 5:1 Oxide

\begin{tabular}{l|r|r|r|r|r}
\hline 323 & 1.64 & 0.64 & .0036 & 0.10 & \multicolumn{2}{c}{$\begin{array}{l}2.0 \\
391\end{array}$} \\
304 & 152 & 0.70 & .0029 & 0.40 & 2.13 \\
318 & 1.62 & & .0001 & 0.07 & \\
347 & 1.55 & & .0040 & -0.36 & \\
449 & 1.57 & & .0031 & -0.28 & \\
429 & 1.60 & 0.72 & .0036 & 0.41 & 1.95 \\
343 & -1.45 & & .0035 & 0.14 & \\
\hline
\end{tabular}

BLANKFTED CORE NO. 12 9:1 Carbide

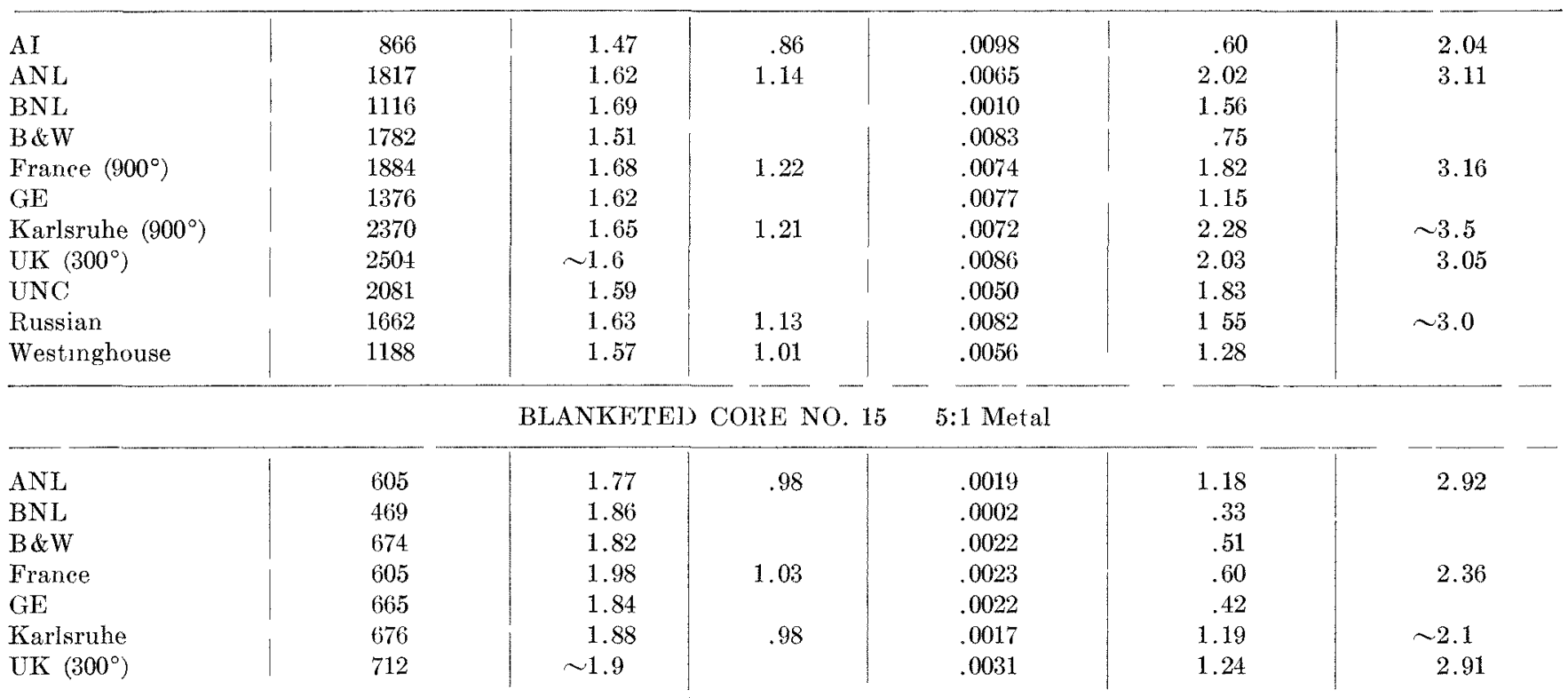

BLANKETED CORE NO. 19 STEAM COOLED, 5:1 Oxide

\begin{tabular}{|c|c|c|c|c|c|}
\hline $\begin{array}{l}\text { B\&W } \\
\text { GE } \\
\text { Karlsruhe }\left(900^{\circ}\right) \\
\text { UK }\left(300^{\circ}\right)\end{array}$ & $\begin{array}{l}463 \\
406 \\
654 \\
558\end{array}$ & $\begin{array}{l}1.23 \\
1.21 \\
1.23 \\
1.18\end{array}$ & $\begin{array}{r}.66 \\
.58\end{array}$ & $\begin{array}{l}.0109 \\
.0086 \\
.0141 \\
.0082\end{array}$ & $\begin{array}{r}1.89 \\
-.31 \\
1.59\end{array}$ \\
\hline
\end{tabular}


TWO-DIMENSIONAL CARBIIEE CYLINDER

Core Diameter: $10 \mathrm{ft}$

Core Height: $2 \mathrm{ft}$

Approx. Ratio $\mathrm{U}^{238}: \mathrm{Pu}^{239} \approx 7$

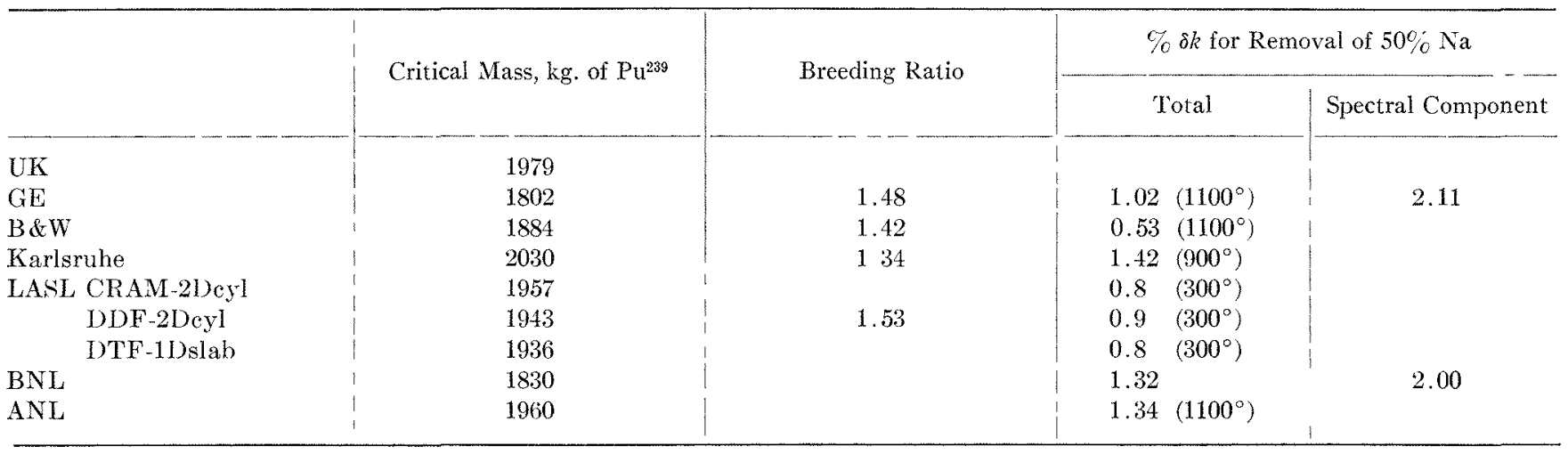

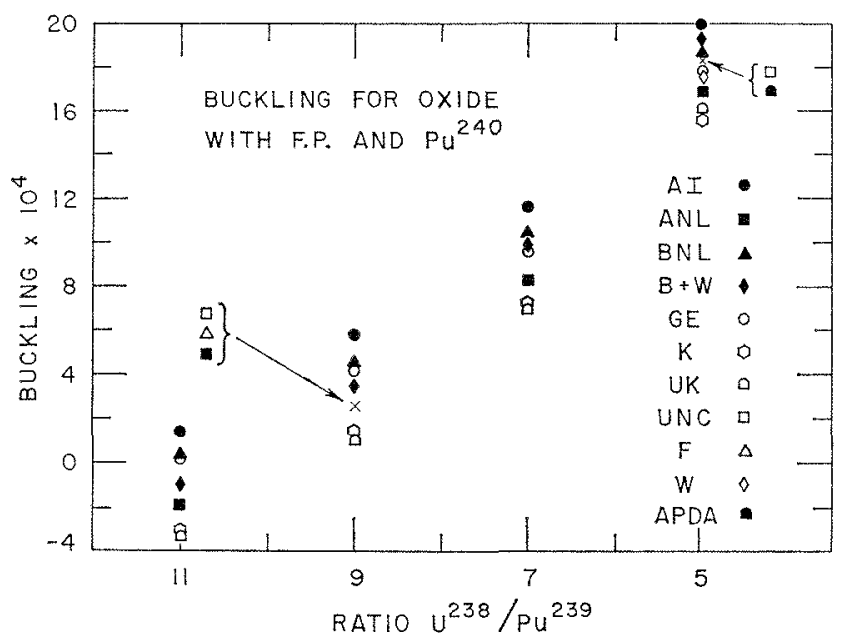

Frg. 1. Buckling for Oxide Reactors

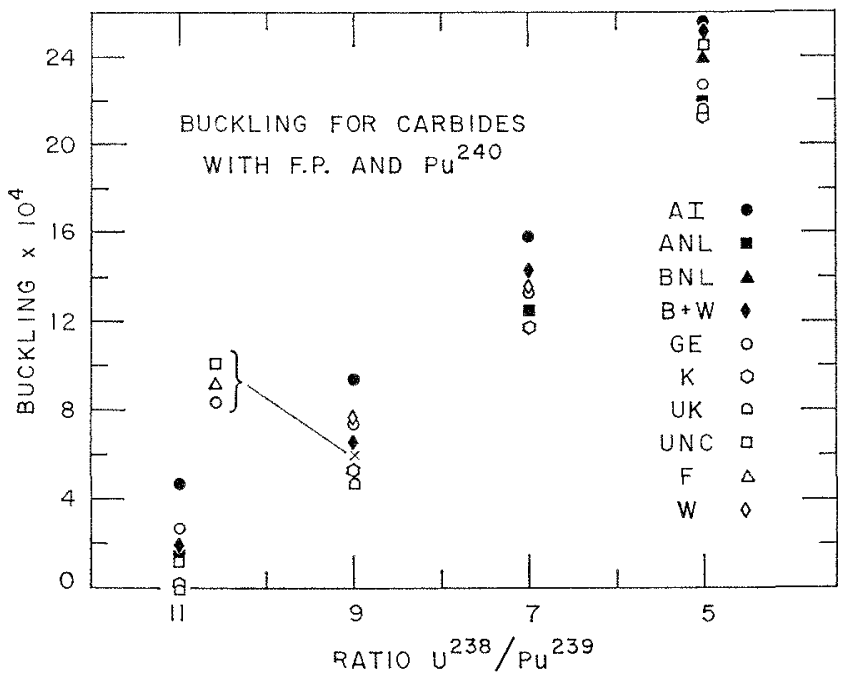

Fıg. 2. Buckling for Carbide Reactors 


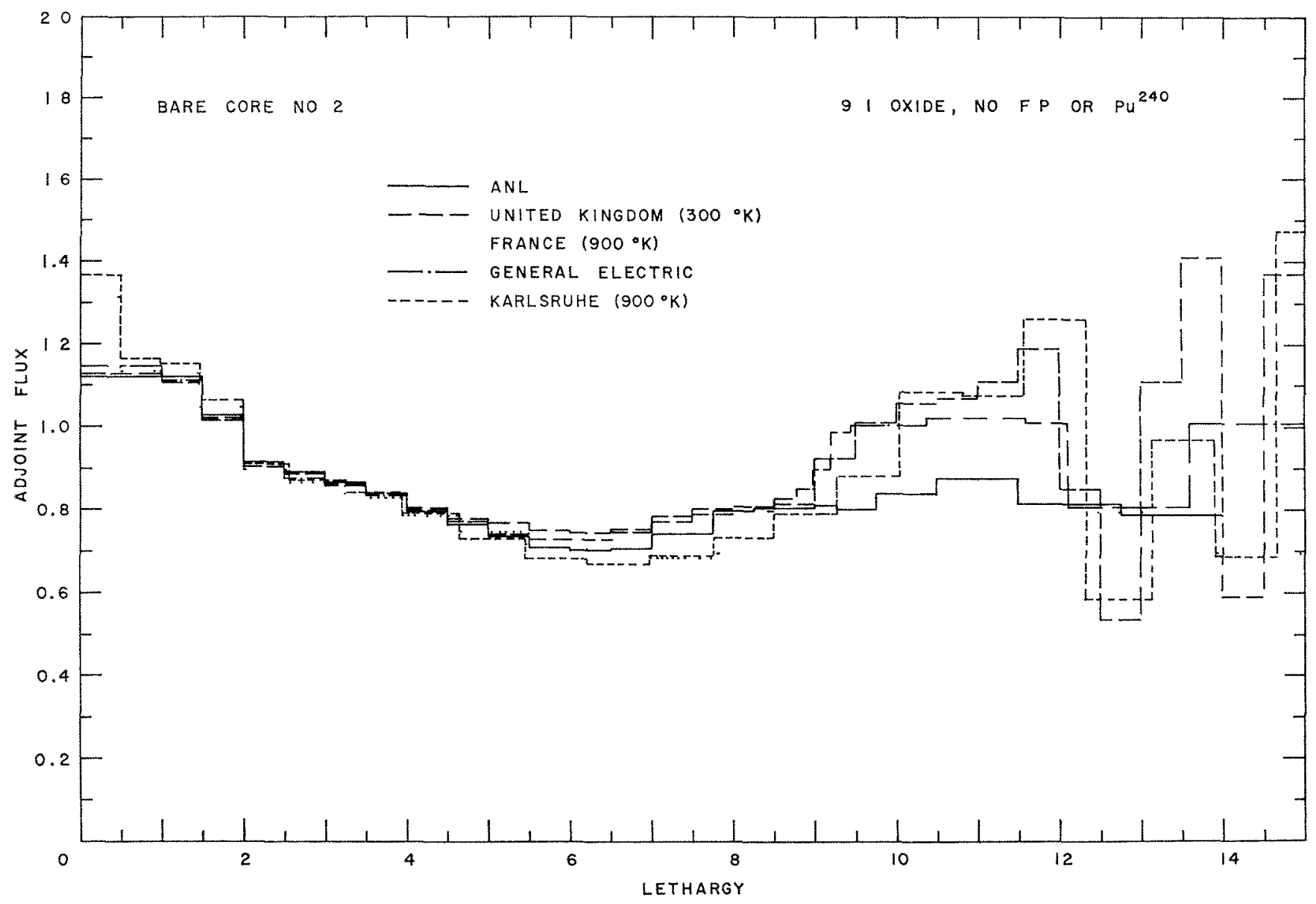

FIG 3 Adjoint Flux for 9:1 Oxide without Fission Produets or Pu ${ }^{240}$ 


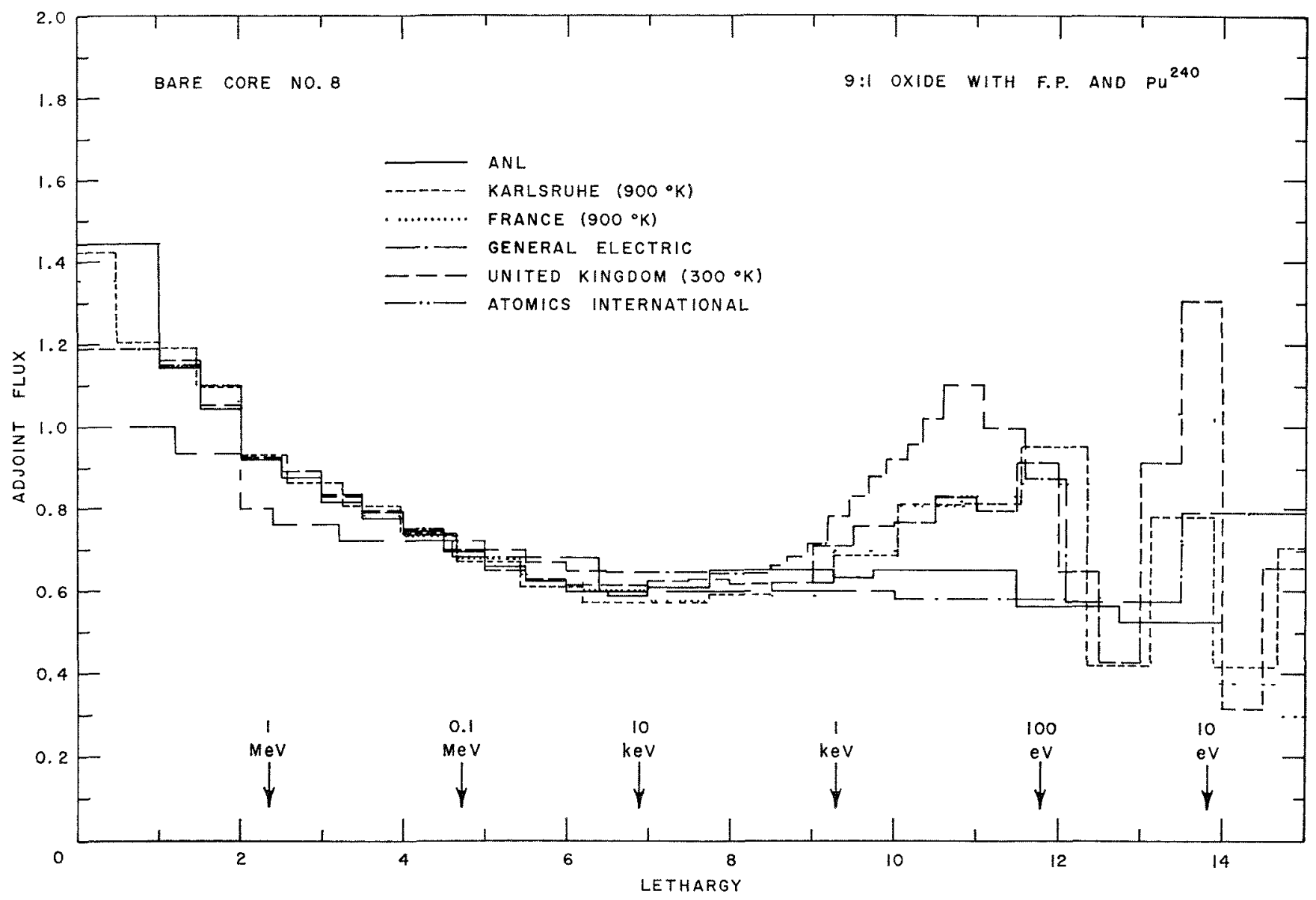

Fir. 4 Adjont Flux for 9:1 Oxide with Fission Product and $\mathrm{Pu}^{2: 0}$ 


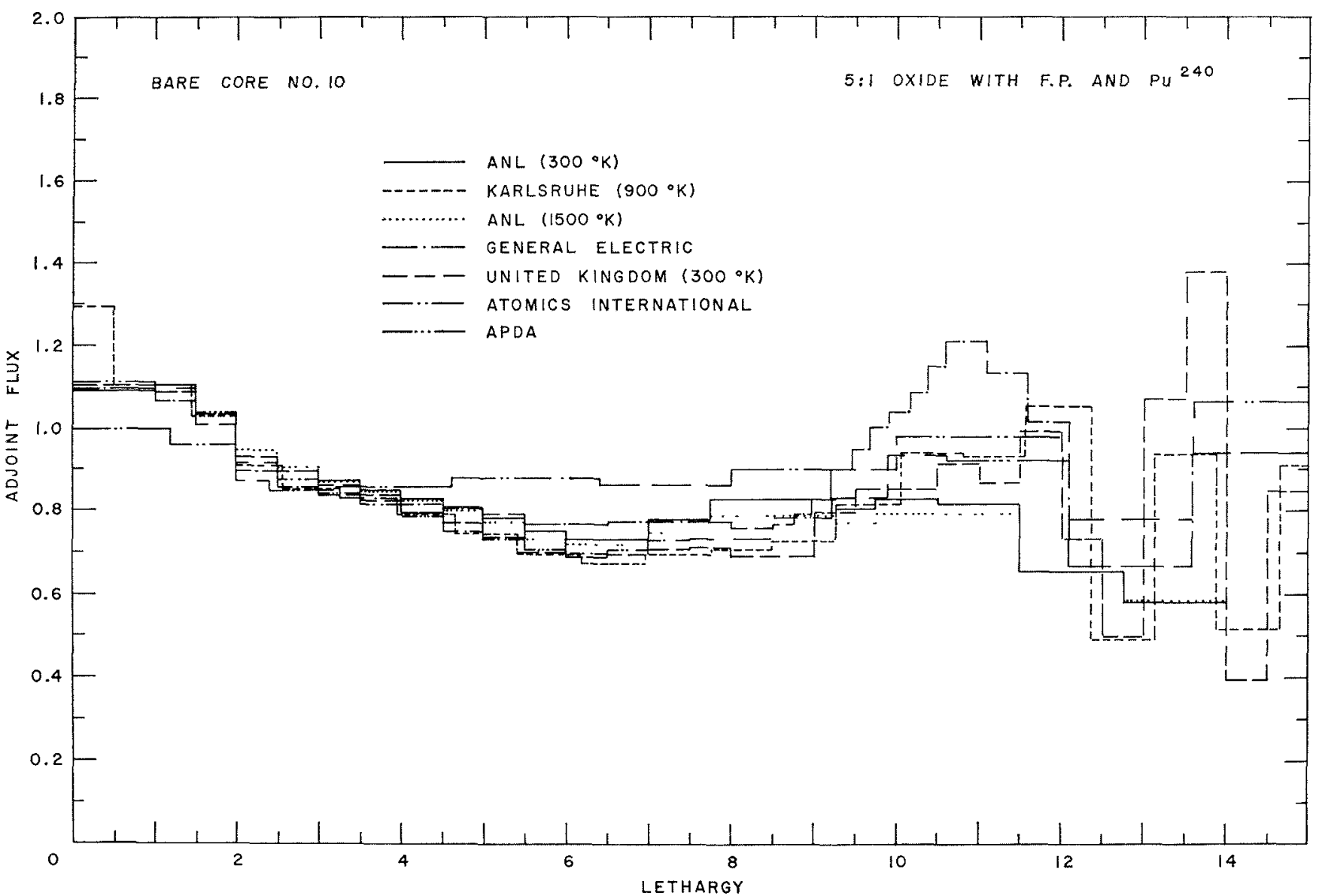

Fig. 5. Adjoint Flux for 5:1 Oxide with Fission Produets and $\mathrm{Pu}^{240}$

\section{Discussion}

Mr. Chernick (BNL): I thought the slides Dr. Okrent showed were very instructive. I've just one brief bit of information to add. It is very clear that people are generally calculating these things accurately except for one or two exceptions which are noted, that is, the machines are behaving themselves these days. The other thing $I$ would like to mention is the fact that it was clear that the Doppler-effect calculations which were made at Brookhaven were quite low, and I'd like to explain this very briefly. We've been interested primarily in very hard spectra at Brookhaven. Therefore we've stuck to the original YOM cross sections. As is well-known, these give a poor picture of the lowenergy spectra from which most of the Doppler effect comes. What we saw on the slides bears this out.

Mr. Greebler (GE): I'd like to comment on a couple of inconsistencies that I noted. In the case of the sodium roid coefficients, the GE value was consistently less positive than that of, for example, the Karlsruhe result. Similarly, the GE result for the steam-cooled void effect was less positive than that of $B$ and $W$. Yet, if we recall the recent Detroit technology meeting, the Karlsruhe people reported a near-zero sodium roid effect with a reactor that was 3 feet high and with a composition similar to that which we had; we had to go to something like 2 feet or less to get a near-zero sodium roid effect. We find a similar comparison with regard to the $B$ and $W$ steam-cooled reactors. I wondered what is the basis of the inconsistency? Are the same people that are calculating these results the ones who are doing the detailed design calculations?

Mr. Okrent: I cannot comment to your question. I have given the names of people signed to the submitted results. Whether they are separate from the design people, one doesn't know.

Mr. Küsters (Karlsruhe): I would like to hear you comment on the high critical masses of the Karlsruhe group. We are using the low plutonium cross sections measured by White (I suppose the UK yeople do this too), and therefore we got an appreciably high critical mass. The plutonium values of White are about $20 \%$ lower in the range from $10 \mathrm{kV}$ to $100 \mathrm{kV}$, and therefore we got so high critical masses. 
Mr. Okerent: This was one of the things that $I$ had in mind when I said, were Argonne to make a change in its cross sections we would become less reactive. I think that the other change that we would hare in mind would involve raising sone of our high-energy $\mathrm{U}$ 238 capture cross sections; again this would make us less reactive, and we would move in your direction for ralues of the eritical mass.

Mr. Perry (ORNL): Not all of the differences noted, of course, are equally important. The large rariations in buckling in the neighborhood of $k_{\infty}=1$, for example, are not necessarily very important. I'd like to ask Dr. Okrent which of the variations he noted seem in his opinion to be most decisive for design of large reactors.

Mr. Okrent: I would think that the large differences in the sodium roid effects require the most attention. Inevitably we can find out what the cricital mass is by building critical experiments. One should not, however, minimize the buckling differenees that were just mentioned. Since interest in large part will eventually be on reactors of large size and many people have been trying to design such reactors to have internal breeding ratios in the vicinity of one (in other words, zero longterm reactivity effects), it's going to be important to know just what it takes to design such a reactor. Assume that you want a reactor having a given volume so that it puts out a certain power. If it turns out that this reactor needs a 7 to 1 enrichment to go critical instead of 9 to 1 , this will leare quite a bit of long-term reactivity loss to be handled. So I don't think one can be too carefree about this buckling difference.

Mr. Küsters: I would like to give the answer to the question of Dr. Greebler. The results presented at the Detroit meeting and Chicago were calculated with the Russian cross-section set. The results presented here wero obtained with the new Karlsruhe set. The people are the same.

$M r$. Okrent: I might note that in the comparison calculations from Karlsruhe they did give results on some reactors with the Russian set and with their new set. The Russian set is certainly much more reactive.

$M r$. Wolfe (GE) : Isn't a large part of the differences shown, especially in the low-enriched reactors, due to the way in which the comparisons were made; that is, when the bucklings came out wrong or the reactivities came out different, the various groups calculated different sized reactors so that any spectral differences, in particular the sodium void effects, would be accentuated by this? I think that if you take the case where people calculate the same size reactors, there'd be an enrichment change, but the various parameters might be much closer togethrer.

Mr. Okrent: I may be wrong, but I would expect the spectral component of the sodiun void effect to be relatively insensitive to this; that is why it was included, as well as the total corlium roid effect, which is influenced by neutron leakage.

Mr. (ireebler: Somewhere midway in the course of the calculations you suggested to us a heterogeneity calculation which would have somewhat overestimated the degrce of heterogeneity. Did all of the participants

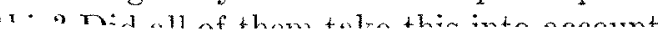

tion of the fact that there is more flux at the very very low energy, say $10 \mathrm{keV}$ and below.

Mr. Okrent: In defonse of my colleagues, I should note that the YOM set ended in the low-energy region where the Doppler effect pretty much begins. The set was intended to analyze the existing criticals in 1960 , none of which had neutrons at such low energies.

Mr. Fenech (MIT): The rrors that we have seen on the slides reflect overall errors which are due to sereral causes: some are due to cross sections and some are due to the matters used to establish these numbers, such as the number of groups and transport diffusion theory or $S_{8}, S_{4}$, etc. Does Dr. Okrent have an idea as to the order of magnitude due to the input into the methods or due to the methods themselves, and whether emphasis should be on the establishment of improving the cross section or on the methods which are used to determine these numbers?

Mr. Okrent: I would assume that the primary difference lies in the cross sections used. Certainly differences in methods of calculations have an effect, but I think similar methods were used for the most part. It appears that the cross-section input is the primary culprit. 



\title{
Calculated Physics Parameters and Their Uncertainties in a 1000-MW(e) Fast Ceramic Reactor
}

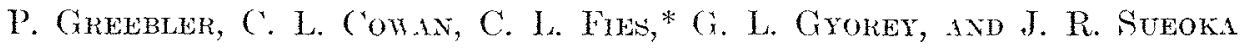 \\ General Electric Company \\ Advancer Products Operation \\ San Jose, rahfoinia \\ (PRLENTEN) BY P. (ARERIBLER)
}

\subsection{Introduction}

I dexign study of a $1000-\mathrm{AIT}(0)$ fast oxide reactor was ronducted to take into account new information in fast reactor technology developed since the last 1000-MW(e) study in late 1963.(1) In the physies area there have been several new cross-section measurements and new sets of group constants, ${ }^{(2-5)}$ dynamies calculations that more clearly defined the role of the 1)oppler and sodium void reactivity effects in reactor safety and stability, ${ }^{(6)}$ and results of seoping studies ${ }^{(7)}$ and other fast reactor design studies ${ }^{(8,9)}$ that offered altermative approaches toward achieving a safe and economical fast power reactor. Lowering of fuel density and of the allowable peak fuel temperature as a result of irradiation and transient fuel test experience ${ }^{(10)}$ and reduction of clad thickness with vented fuel are new material technology factors that affect the physics properties; and they contributed to the need for updating the earlier 1000-MW(e) design.

\subsection{Differences in New 1000-MW(e) Design}

The 2-ft-thick core containing $\mathrm{BeO}$ additive of the earlier 1000-MIW(e) pancake reactor had a high neutron leakage and a degraded spectrum in order to satisfy

* Work performed while on assignment with GE-APO from AEC Chicago Operations Office. stringent safety requirements involving the codium roid athd Doppler effects. Scoping and design studies on alternative eore configurations showed that other core shapes, such an annulat ${ }^{(7,}{ }^{11}$ or modular, ${ }^{(12)}$ could arhieve as good or slightly better sodium roid effect than pancakes for the same core composition and corr neutron leakage. These alternative configurations, hon ever, showed important disadvantages compared with the pancake in the thermal-hydraulies areat ${ }^{(13)}$ and $m$ other aspects of reactor safety such as control requirements. Hence, the pancake-shaped cylindrical core is still the favored design for meeting stringent safory requirements with respect to sodium voiding. More recent considerations on design safety eriteria are discuswed in reference 11.

One of the designs studied in detail is even thimer than the earliex dewign, the core thickness baving been reduced from 24 to $16 \mathrm{in}$. In this design, the soduum volume fraction was reduced from 0.46 to 0.36 and the fuel volume fraction increased from an average of 0.25 to 0.38 . Because of the lower sodium content the core had to be made thimner to make the neutron laakage high enough to produce a zero (slightly negative) reactivity for total sodium loss. This change in core composition and thickness slightly improved the neutron economy without penalizing the safety reartivity cocflicients. $\dagger \mathrm{BeO}$ is used, as in the earlior dorign, to provide an adequate negative Doppler cocfficient. Different $\mathrm{BeO}$ contents in two core zones and a $\mathrm{BeO}$ radial reflector are used in the new design to achieve a suitably flat power distribution. Tantalum is used for control rod material instead of boron carbide as in the earlier design.

Statics physics parameters of this thinner 1000-

$\dagger$ The improvement in fuel cost is not manifest in a direct comparison of the calculated breeding ratio and fissile inventory of the design (Table 1) with those reported for the earlier design. (1) This is because changes in cross sections used, in $\mathrm{Pu}$ isotopic content for the selected mode of recycle, and in fuel density and maximum operating temperature since the earlier design study have all been in the direction of reducing the neutron economy. 
MW(e) design are discussed in Section 2. Discussion of safety criteria upon which the physies design is based and a more complete engineering design description of the reactor will be found in reference 14 .

\subsection{Evaluation of Uncertainties}

Current physics predictions are highly unreliable because of the unsatisfactory status of fast reactor cross-section information and calculation methods. This is evident in the large differences in reactor physics results calculated by different organizations. For example, Karlsruhe and Cadarache report 1000-. $I I W(\mathrm{e})$ designs that have 3 - to 5 - $\mathrm{ft}$ core thicknesses with internal breeding ratios in excess of 0.9 , nearly zero reactivity for total loss of sodium, and Doppler coefficients near $-0.01(T d k / d T)$ without adding any moderating material. ${ }^{(9)}$ General Filectric, on the other hand, using cross-section data very nearly that in ANL Set $224,{ }^{(2)}$ calculates that such Doppler and sodium void effects requires a core thickness below $2 \mathrm{ft}$ with an internal breeding ratio less than 0.7 and $\mathrm{BeO}$ additive. $\left({ }^{1}\right)$ Examination of measured basic cross-section data shows large deviation for many of the material cross sections important in large fast ceramic reactors. Two examples are (1) the U-238 energy point-wise capture cross scetion has a $25 \%$ standard deviation over most of the energy region between 4 and 100 $\mathrm{keV}(15)$ and (2) recent measurements of the Pu-239 fission cross section in the $Y . K$. indicate that it is about $30 \%$ lower than that given by BNL-325 in the energy interval from 24 to $100 \mathrm{keV}$. ${ }^{(16)}$

Calculations were made to evaluate the effects of several cross-section and computation model uncer- tainties on the physics parameters. The reactor model used for this evaluation was an infinite slab approximation of the 1000-MW(e) pancake design. Perturbations in calculated Doppler and sodium void reactivity effects, breeding ratios, fissile "enrichment," and neutron-spectrum indices were obtained for individual changes in important material cross sections and composites of such changes. These cross-section changes represented the estimated range of uncertainty as indicated by differences in basic point-wise cross-section data and in standard sets of group constants. ${ }^{(2-5)}$ This evaluation of uncertainties in the calculated physics parameters is described in Section 3.

\subsection{Physics Parameters of the 1000-MW(e) Design}

Figure 1 shows the core and blanket configuration of the $1000 \mathrm{MW}(\mathrm{e})$ design, and the volumetric composition of each region. $\Lambda$ very thin (16-in.) two-zone core is used with different amounts of $\mathrm{BeO}$ and fuel in the two regions and a thin $\mathrm{BeO}$ reflector around the outer core region. There is no radial blanket, resulting in a small loss of breeding ratio $(\sim 0.03)$ but an improvement in fuel cost. The fissile plutonium "enrichment" of the fresh fuel is the same for the two core zones. Each axial blanket volumetric composition is the same as that of the adjacent core zone.

\subsection{Power Distribution}

The core blanket-reflector configuration of Figure 1 gives a very flat power distribution. The radial peak-toaverage fuel specific power ( $\mathrm{kW} / \mathrm{kg}$ fuel) shown by the white curve in Figure 2, is 1.22 and occurs at the center of the core. The axial peak-to-average is only

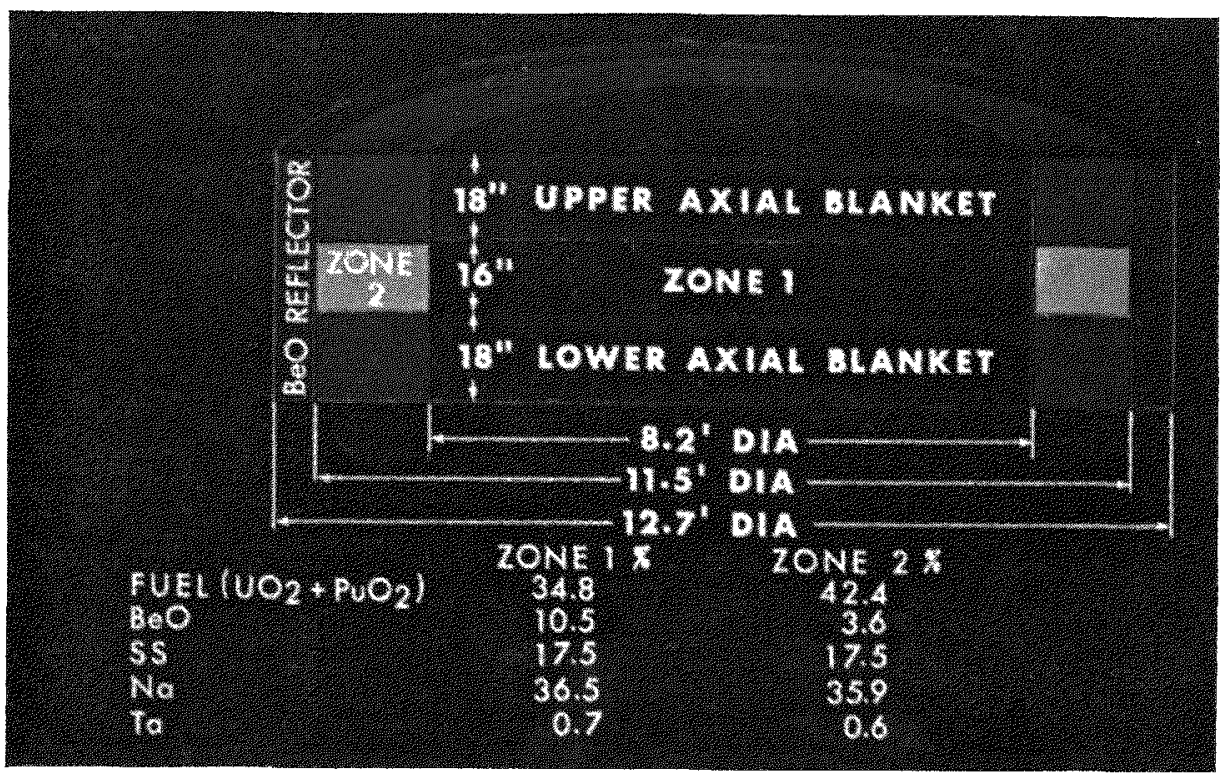

FIG. 1. Core and Blanket Arrangement and Composition 


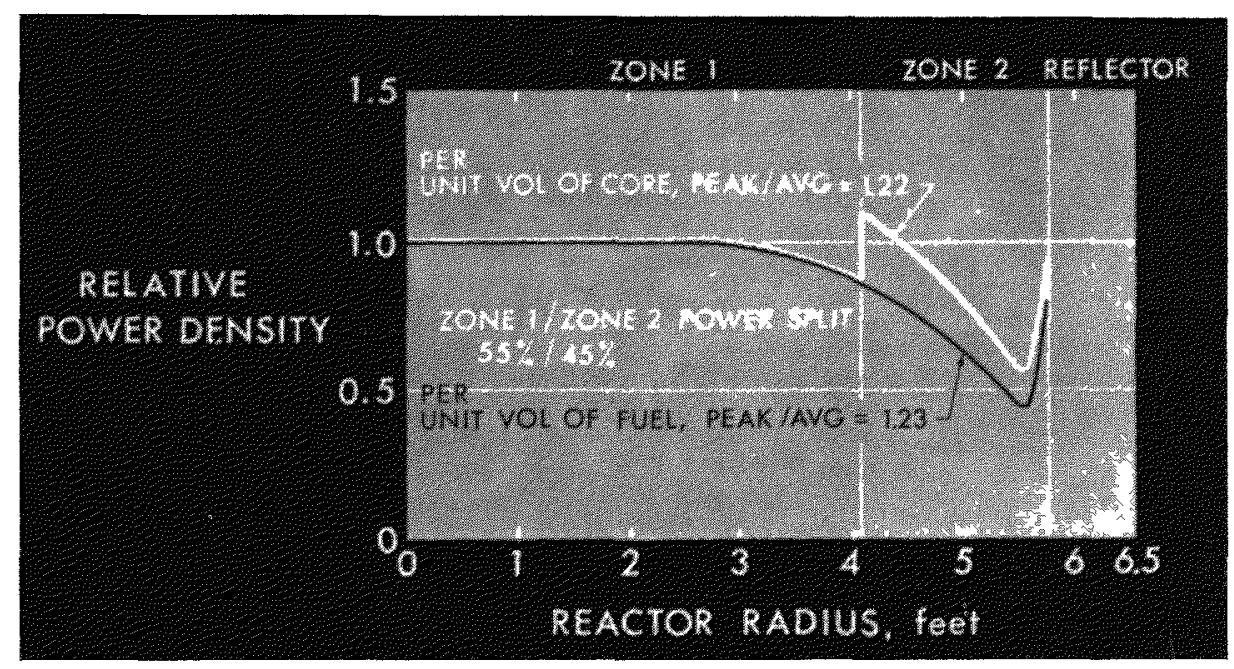

Frg. 2. Core Radial Power I)istribution

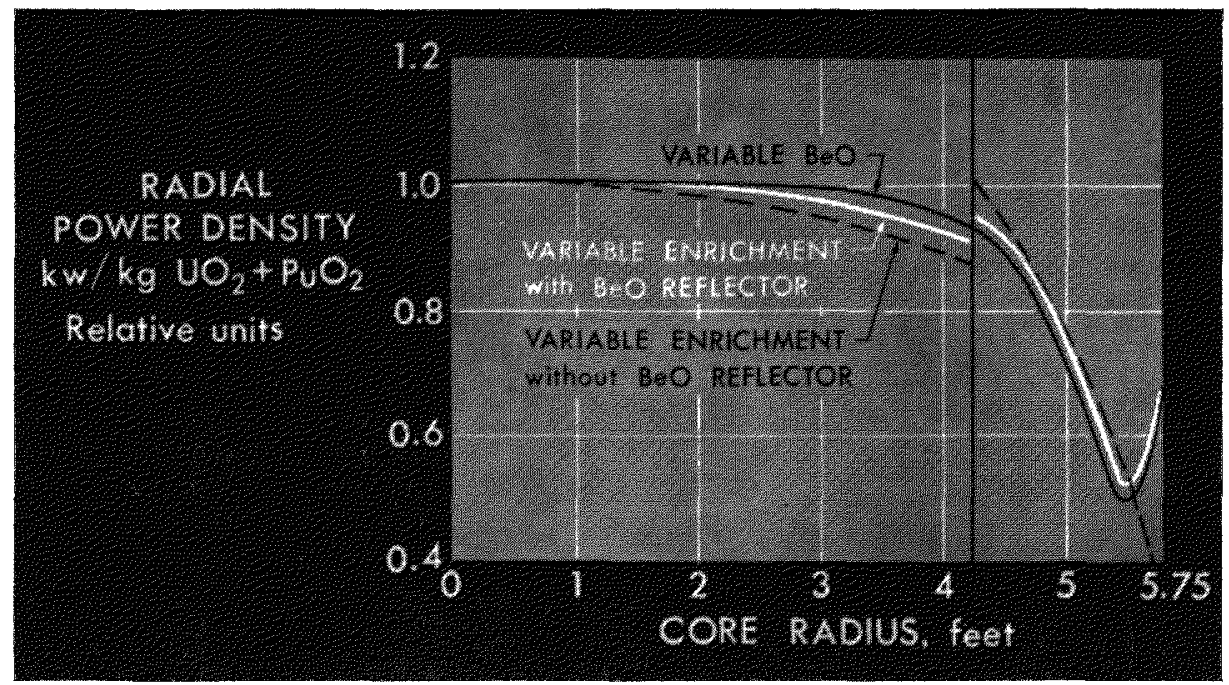

FrG. 3. Comparisons of Power-flattening Methods

1.11 because of the thin core. The radial peak core power density ( $\mathrm{kW} /$ liter) occurs in zone 2 at the interface of the two zones (see black curve in zone 2 region of Figure 2). This is because zone 2 has a higher fuel volume fraction than zone 1 . A power "spike" occurs near the $\mathrm{BeO}$ reflector; however, the power density there is well below the peak value at the center of the core or that at the interface between zones 1 and 2 . It was found that the flat radial power distribution of Figure 2 could be maintained throughout the operating cycle between refuelings by using the shim control rod pattern to contribute to the power flattening at the beginning of the rycle.

Other methods of power flattening were studied. It was found that use of three core zones did not give enough improvement over the power distribution obtainable with two zones to warrant the additional design and refueling eomplexity. In Figure 3 radial power distributions for several of the two-zone approaches that were considered in preliminary scoping studies are compared. With variable fissile "enrichment" in two zones and a $\mathrm{BCO}$ reflector, a power distribution can be obtained that is about as satisfactory as that with variable content of $\mathrm{BeO}$ and fuel in the two zones and a $\mathrm{BeO}$ reflector. Lack of a moderating reflector gives a very sharp power gradient at the outer edge of the core that is about as unsatisfactory as the power "spike" with the $\mathrm{BeO}$ reflector, and the overall power distribution profile is less satisfactory, as seen in Figure 3.

\subsection{Reactivity Coefficients}

Table 1 lists important phycies parameters of the 1000-MW (e) design. The calculated Doppler coefficient 
of $-0.0072 T d k / d T^{\top}$ ( -0.006 with sodium completely removed) and the negative reactivity for total loss of sodium satisfy safety criteria that are discussed in reference 14. Zone 1 has more $\mathrm{BeO}$ and, hence, a softer spectrum than zone 2 (21\% of fissions below $10 \mathrm{keV}$ in zone $1 ; 13 \%$ in zone 2). Accordingly zone 1 had a higher local Doppler power coefficient than zone 2, over and above that due to differences in the power and statistical weight splits between the two zones. The portion of the axial reflector near the core was calculated to make a small $(\sim 5 \%)$ contribution to the Doppler coefficient, the fuel there having both a lower temperature rise and a lower statistical weighting than the average core fuel.

With the 2-zone variable $\mathrm{BeO}$ content, the Doppler coefficient and the neutron economy are sensitive to the power split between the two zones. Table 2 shows the Doppler coefficient and the breeding ratio for the design power split and for other power splits corresponding to abnormal conditions at power or transient overpower (such as having all control rods withdrawn in one zone compensated by an excess of inserted control rods in the other zone). It is seen from Table 2 that an ample Doppler coefficient is present even for postulated transients in which an unusually high fraction of the power occurs in zone 2 .

\subsection{Neutron Economy}

The in-pile fissile inventory of $2470 \mathrm{~kg}$ and reactor power of $2500 \mathrm{MW}(\mathrm{t})$ give a fuel specific power of $1010 \mathrm{kWt} / \mathrm{kg}$ fissile material. This specific power combined with the total breeding ratio of 1.18 , and a load factor of 0.8 and an out-of-pile inventory that is $30 \%$ of the in-pile fissile inventory, results in a doubling

TABLE 1. Physics Parameters of 1000-MW(e) Dusign

\begin{tabular}{|c|c|}
\hline $\begin{array}{l}\text { Fissile Inventory at Mideycle* in Core and } \\
\text { Blankets (kg) }\end{array}$ & 2470 \\
\hline Core Fissile "Enrichment" (a/o) & 15.3 \\
\hline Total Breeding Ratio & 1.18 \\
\hline Core Conversion Ratio & 0.63 \\
\hline Doubling Time $(\mathrm{yr})^{*}$ & 13.4 \\
\hline I oppler Coefficient $\left(T^{\prime} d k / d^{\prime} T^{\prime}\right)$ Sodium In & -0.0072 \\
\hline Sodium Out & -0.006 \\
\hline Sodium Void $s k-$ Total & -0.0007 \\
\hline Core & +0.0037 \\
\hline Worst & +0.0056 \\
\hline$\beta_{\mathrm{eff}}$ & 0.0035 \\
\hline Power Split (MWt)-Core Zone 1 & 1150 \\
\hline Core Zone 2 & 950 \\
\hline BIankets & 400 \\
\hline
\end{tabular}

* Equilibrium fuel cycle with 6 -month operation between refuelings for partial reloading. Input plutonium is recycled plutonium discharged from core and blankets and mixed. Load factor is 0.8 and out of-pile fissile inventory is $30 \%$ of the in-pile inventory.
TABlie 2. Influence of Power Distribution on Dopplei Conffictent and Breeding Ratio

\begin{tabular}{c|c|c|c|c|c|c}
\hline & Power Split (\%) & \multicolumn{2}{|c|}{$\begin{array}{c}\text { Doppler Coefficient } \\
{[T(d k / d T)]}\end{array}$} & $\begin{array}{c}\text { Breeding } \\
\text { Ratio }\end{array}$ \\
& Zone 1 & Zone 2 & Total & Zone 1 & Zone 2 & \\
\hline A & 55 & 45 & -0.0072 & -0.0051 & -0.0021 & 1.18 \\
B & 64.5 & 35.5 & -0.0083 & -0.0072 & -0.0011 & 1.19 \\
C & 51.3 & 48.7 & -0.0069 & -0.0043 & $-\mathbf{0 . 0 0 2 6}$ & 1.17 \\
D & 57.0 & 43.0 & -0.0063 & -0.0043 & $-\mathbf{0 . 0 0 2 0}$ & - \\
\hline
\end{tabular}

Case A: Design power split, approximately uniform shim rod density.

Case B: All zone 2 shim rods inserted; zone 1 shim rods withdrawn to maintain criticality.

Case C: All zone 2 shim rods withdrawn; zone 1 shim rods inserted to maintain criticality.

Case D: Sodium completely voided from zone 1 core and blanket.

Table 3. Evaluation of Physics Parameter UNCERTAINTIES(a)

Cross-section Deviations Contributing to Improved Doppler and Sodium Void Effects

\begin{tabular}{|c|c|c|c|c|c|}
\hline Description $^{(b)}$ & $\begin{array}{c}\text { Doppler } \\
T_{\widetilde{d t}}^{d T}\end{array}$ & $\begin{array}{c}\text { Total } \\
\text { Sodium } \\
\text { Void } \Delta k\end{array}$ & \begin{tabular}{|c|} 
Breeding \\
Ratio
\end{tabular} & $\begin{array}{l}\text { Fissile } \\
\text { Core } \\
\text { a/o }\end{array}$ & $\begin{array}{l}\% \\
\text { Fissions } \\
\text { below } \\
9.1 \mathrm{keV}\end{array}$ \\
\hline Base Case & -0.0074 & +0.0010 & 1.20 & 14.3 & 21.7 \\
\hline High Fuel Inelastic & -0.0076 & -0.0002 & 1.19 & 14.5 & 22.2 \\
\hline $\begin{array}{l}\text { High U-238 Cap- } \\
\text { ture }\end{array}$ & -0.0075 & +0.0003 & 1.24 & 14.8 & 20.4 \\
\hline $\begin{array}{c}\text { Low Pu-239 Fission } \\
(24 \text { to } 170 \mathrm{keV})\end{array}$ & -0.0075 & +0.0006 & 1.20 & 15.1 & 22.6 \\
\hline Low Steel Capture & -0.0078 & +0.0007 & 1.22 & 14.0 & 22.4 \\
\hline $\begin{array}{l}\text { Low Pu-240 Cap- } \\
\text { ture (below } 10 \\
\text { keV) }\end{array}$ & -0.0075 & +0.0001 & 1.20 & 14.2 & 22.1 \\
\hline $\begin{array}{l}\text { Zero Pu-239 1)op- } \\
\text { pler }\end{array}$ & -0.0108 & & & & \\
\hline $\begin{array}{l}\text { Good Coefficient, } \\
\text { Composite }\end{array}$ & -0.0120 & -0.0015 & 1.25 & 15.4 & 22.8 \\
\hline
\end{tabular}

(a) Infinite-slab reactor; 16 -in.-thick core; $37.0 \mathrm{v} / \mathrm{O} \mathrm{PuO}_{2}-$ $\mathrm{UO}_{2}, 20.5 \mathrm{v} / 0$ steel, $33.0 \mathrm{v} / 0$ sodium, $9.1 \mathrm{v} / \mathrm{O} \mathrm{BeO}$, and 0.4 $\mathrm{v} / \mathrm{O} \mathrm{Ta}$. This is a slightly higher steel and lower sodium $\mathrm{v} / \mathrm{o}$ than the two-zone core (see Figure 1). Blankets are $18 \mathrm{in.}$ thick. Mid-burnup condition of refueling cycle with $10^{5}$ MW1)/ $t$ burnup of discharged eore fuel.

(1) See Sections 3.1.1 through 3.1.6.

time of $13.4 \mathrm{yr}$. Reference 14 gives the breakdown of fuel cost.

\subsection{Evaluation of Uncertainties}

An infinite-slab (one-dimensional) representation of the 1000-MW(e) design was used in calculating the sensitivity of physics parameters to uncertainties in cross-section data and computation methods. Footnote (a) of Table 3 gives the reactor geometry and composition. 
Deviations in cross-section data were obtained from the various standard sets of group constants and also from results of recent cross-section measurements that have not yet been incorporated in these standard sets. Group-constant sets considered were the ELMOEcorrected ANL 26-group set, ${ }^{(2)}$ the new U.K. Winfrith 33-group set, the C.S.S.R. 26-group set, ${ }^{(3)}$ the new YOM 16-group set, ${ }^{(5)}$ and the GE 60-group set. Recent cross-section experimental information included (1) U.K. results on the fission cross section of Pu-239 between 24 and $170 \mathrm{keV}$, ${ }^{(16)}$ based on the measured ratio of $\sigma_{f}$ of $\mathrm{Pu}-239$ to that of $\mathrm{U}-235$, which leads to $\mathrm{Pu}-239$ $\sigma_{f}$ values as much as $30 \%$ lower than those in BNL-325. (2) U.K. measurements of the Pu-239 total cross section by Uttley and fission cross section by James below $20 \mathrm{keV}$ that, combined with theoretical evaluation of the scattering cross sections, give high alpha values for $\mathrm{Pu}-239$ below $10 \mathrm{keV}$, and (3) measurement of the absolute $\nu$ value of U-235 at low energy by Colvin and Sowerby ${ }^{(17)}$ that indicates about $2 \%$ lower $\nu$ values for all fissionable materials.

\subsection{Basis for Cross-section Changes in Sensitivity Studies}

\subsubsection{Base Case}

The G.E. 60-group set uses basic cross-section data similar to that in the ELMOE-corrected ANL 26group set. ${ }^{(2)}$ The main differences are slightly higher U-238 capture cross sections between 0.1 and $1 \mathrm{MeV}$ in the G.F. set and independent methods (believed to be similar) used to calculate temperature-dependent

TABLE 4. Evaluation of Physics Parameter UNCERTAINTIES*

Cross-section Deviations Contributing to Worse Doppler and Sodium Void Effects

\begin{tabular}{|c|c|c|c|c|c|}
\hline Description* & $\begin{array}{c}\text { Doppler } \\
T \frac{d k}{d T}\end{array}$ & $\begin{array}{c}\text { Total } \\
\text { Sodium } \\
\text { Void } \Delta k\end{array}$ & $\begin{array}{c}\text { Breeding } \\
\text { Ratio }\end{array}$ & $\begin{array}{l}\text { Fissile } \\
\text { Core } \\
\text { a/o }\end{array}$ & $\begin{array}{l}\text { \%o } \\
\text { Fissions } \\
\text { below } \\
9.1 \mathrm{keV}\end{array}$ \\
\hline BASE CASE & -0.0074 & +0.0010 & 1.20 & 14.3 & 21.7 \\
\hline Low Mastic & -0.0068 & +0.0036 & 1.23 & 13.9 & 19.4 \\
\hline Low U-238 Capture & -0.0078 & +0.0027 & 1.17 & 14.0 & 22.6 \\
\hline $\begin{array}{l}\text { High Pu-239 Alpha } \\
\text { (below } 10 \mathrm{keV} \text { ) }\end{array}$ & -0.0062 & +0.0065 & 1.12 & 15.5 & 19.8 \\
\hline $\begin{array}{l}\text { High Steel Cap- } \\
\text { ture }\end{array}$ & -0.0073 & +0.0007 & 1.19 & 14.4 & 21.4 \\
\hline $\begin{array}{cl}\text { High } \mathrm{I}_{\gamma} & \text { Sodium } \\
2.8 \text {-keV } & \text { Reso- } \\
\text { nance } & \end{array}$ & -0.0073 & +0.0030 & 1.19 & 14.4 & 21.1 \\
\hline $\begin{array}{l}\text { Low } \nu \text { Values }(2.82 \\
\text { for } \mathrm{Pu} 239 \text { at } \\
\text { thermal) }\end{array}$ & -0.0072 & +0.0012 & 1.16 & 15.0 & 21.4 \\
\hline $\begin{array}{l}\text { Poor Coefficient, } \\
\text { Composite }\end{array}$ & -0.0052 & +0.0124 & 1.06 & 15.7 & 17.7 \\
\hline
\end{tabular}

* See Footnotes a and b of Table 3 . self-shielded fuel cross sections. The physics parameters of the Base Case in Tables 3 and 4 were obtained by means of the data of the G.E. 60-group set which was condensed to 26 energy groups for the multigroup one-dimensional calculations. All physies parameters and their variations (see below) were computed by use of 26-group, one-dimensional diffusion theory.

\subsubsection{Inelastic and Elastic Scaltering}

The new U.K. Winfrith 33-group set gave the highest inelastic scattering transfer cross sections for U-238 and plutonium isotopes, both in terms of total inelastic removal and energy loss by the inelastically scattered neutrons. The ANL 26-group and the new U.K. 33group sets have the highest elastic scattering energy transfer cross sections for oxygen and other light clements in the high-encrgy region. The cross-section variation in Table 3 labeled "High Fuel Inelastic" was taken from the new U.K. 33-group set. The cross section variation in Table 4 labeled "Low Elastic" appears in those sets of group constants that probably did not take into account the new values using the ELMOE code; ${ }^{(18)}$ hence, this variation may be more an uncertainty in calculation methods than one due to eross-section discrepancies.

\subsubsection{U-238 Capture}

"Low U-238 Capture" in Table 4 and "High U-238 Capture" in Table 3 are the envelopes of lowest and of highest U-238 $\sigma_{c}$ for all energies obtained from the standard sets of group constants mentioned in Section 3.0. The variations representing ]owest U-238 capture cross sections were obtained from the ANL 26-group set between $67 \mathrm{keV}$ and $825 \mathrm{keV}$, and from the U.K. Winfrith 33 -group set between $12.4 \mathrm{eV}$ and $5.5 \mathrm{keV}$ The variations for highest $U-238$ capture cross sections were obtained from the U.S.S.R. 26-group set between $1 \mathrm{keV}$ and $100 \mathrm{keV}$.*

\subsubsection{Plutonium Fission and Capture}

Measurements of the Pu-239 total cross section by Uttley and of the fission cross section by James below $20 \mathrm{keV}$, combined with theoretical evaluation of the Pu-239 scattering cross section in this energy region, give alpha values between 1.0 and 1.2 over most of the energy region between $100 \mathrm{eV}$ to $10 \mathrm{keV}$. These alpha values are roughly $50 \%$ higher than those deduced from earlier activation measurements. ${ }^{(19)}$ The case in Table 4 labeled "High Pu-239 Alpha" used these high-alpha values in conjunction with James' fission cross sections below $20 \mathrm{keV}$.

"Low Pu-239 Fission" in Table 3 refers to measure-

* Cross sections for other than the indicated variations are the same as the base G.E. 60 -group set. 
ments by White et al. at Aldermaston, ${ }^{(16)}$ which indicate significantly lower Pu-239 $\sigma_{f}$ from $24 \mathrm{keV}$ to $170 \mathrm{keV}$.

"Low Pu-240 Capture" in Table 3 is based on data from the new U.K. Winfrith 33-group set between $91 \mathrm{eV}$ and $5.5 \mathrm{kel}$.

\subsubsection{Steel and Sodium Capture}

"High $\Gamma_{\gamma}$ Sodium 2.8-keV Resonance" in Table 4 is for a $\Gamma_{\gamma}$ of $1.0 \mathrm{eV}$ assumed on the basis of systematics of nuclides. (The new Yiftah 16-group set ${ }^{(5)}$ actually uses a $g \Gamma_{\gamma}=1.0$, which gives even higher capture.) The value $\Gamma_{\gamma}=0.35$ is used for the "Base Case" on the assumption that the $2.8-\mathrm{keV}$ resonance accounts entirely for the thermal capture cross section.

The lowest steel capture cross sections (mainly due to iron and nickel) were found in the new U.K. Winfrith 33-group set for most of the energy region above $2 \mathrm{keV}$. "Low Steel Capture" in Table 3 is for the envelope of lowest iron, nickel, and chromium $\sigma_{c}$ at all energies below $10 \mathrm{MeV}$. For iron this was obtained from the C.K. Winfrith 33 -group set above $3.3 \mathrm{keV}$, and for nickel from the U.K. Winfrith 33-group set between $2.0 \mathrm{keV}$ and $150 \mathrm{keV}$. Low chromium values were taken from the G.E. 60-group set.

"High Steel Capture" in Table 4 is worse than the envelope of highest iron, nickel, and ehromium $\sigma_{c}$ at energies below $1 \mathrm{MeV}$ obtained from the various standard cross-section sets. This is because the calculated resonance integrals of these materials using energy point-wise cross-section duta are 50 to 100 per cent lower than reported resonance integral measurements. ${ }^{(20)}$ There is concern that some capture resonances may be missing in the energy point-wise cross-section data. To take this possibility into account, $25 \%$ was added to the envelope of highest iron, nickel, and chromium $\sigma_{c}$ values below $1 \mathrm{MeV}$. The envelope of highest $\sigma_{c}$ for iron, nickel, and chromium was obtained primarily from the G.E. 60-group set with several specific energy groups from the U.K. Winfrith 33-group set included.

\subsubsection{Values and Pu-239 Doppler}

Measurement of an absolute $\nu$ value of $2.385 \pm$ 0.015 for U-235 by Covin and Sowerby ${ }^{(17)}$ indicates the possibility of about $2 \%$ lower $\nu$ values for all fissionable materials. Using 1.182 as the ratio of $\nu$ for $\mathrm{Pu}-239$ to that for $\mathrm{U}-235^{(17)}$ gives $\nu=2.82$ for $\mathrm{Pu}-239$ at low energies. This was used for the case in Table 4 labeled "Low $\nu$ Values" along with proportionately lower $\nu$ values for $\mathrm{Pu}-239$ at higher energies.

It appears that the calculated positive Doppler coefficient for Pu-239 may be highly overestimated, using a Breit-Wigner single-level resonance model and a chi-squared fission width distribution with three de- grees of freedom. This observation is based on limited experimental information ${ }^{(21,22)}$ in which the errors in some cases may be greater than the desired measured quantity. A one-degree-of-freedom distribution, which is supported by available resonance data, gives a lower Doppler effect, but not enough lower to agree with the near zero (or even negative) values indicated by the experiments. The case in Table 3 labeled "Zero Pu-239 Doppler" discounts the positive Pu-239 Doppler contribution currently computed.

\subsection{Results of Sensitivity Studies}

\subsubsection{Cross-section Deviations Favorable to Reactivity Coefficients}

Table 3 shows calculated Doppler cocfficients, total sodium void reactivity effects, total breeding ratios, core fissile "enrichments," and spectra in terms of fissions below about $10 \mathrm{keV}$ for the Base Case and for those eross-section changes that improved overall Doppler and sodium void effects. The bottom row of Table 3 gives the composite effect of combining all of these cross-section changes.

It is noted that the cross-section deviations contributing to improved coefficients generally softened the neutron spectrum, the only exception being the High U-238 Capture case. The High Fuel Inelastic case, corresponding to the U.K. Winfrith new 33-group inelastic scattering constants for U-238 and plutonium, gave a surprisingly small improvement of the coefficients and degradation of the spectrum $(\sim 5 \%)$. The Low Pu-239 Fission case, corresponding to lower measured $\sigma_{f}$ values between 24 and $170 \mathrm{keV},{ }^{(16)}$ produced very little improvement in the coefficients and significantly increased the required fissile content. (Including this case in the Good Coefficient Composite perhaps imposes an unfair penalty on the combination of cross-section changes that improve the coefficients.) The most significant improvement in the Doppler effect results from neglecting the positive $\mathrm{Pu}-239$ contribution. As discussed in Section 3.1.6, there is experimental evidence that supports a near-zero $\mathrm{Pu}-239$ Doppler effect in a degraded spectrum similar to the calculated Base Case.

\subsubsection{Cross-section Deviations Unfavorable to Reactivity Coefficients}

Table 4 shows the calculated physics parameters, similar to Table 3 but for cross-section changes that worsen overall Doppler and sodium void effects. With a single exception (Low U-238 Capture) these changes tend toward a harder neutron spectrum. The most significant of these variations is that of High $\mathrm{Pu}-239$ Alpha below $10 \mathrm{keV}$ (which also includes slightly lower 
$\sigma_{f}$ values in this energy region) deduced from the Uttley $\sigma_{t}$ and James $\sigma_{f} \mathrm{Pu}-239$ measurements. This potential deviation in cross-section data is harmful not only to the coefficients but also to neutron economy. Therefore, it would not be attractive to overcome it with a variation in core composition or geometry that traded improved coefficients for higher fuel cost, unless other variations in the cross-section data resulted in significant improvement of the economics.

The possibility of $2 \%$ lower $\nu$ values ${ }^{(17)}$ produces mainly a decrease in neutron economy and imposes only a small penalty on the coefficients. The Low Elastic case, representing oxygen and other light-element elastic transfer cross sections that were not ELMOEcorrected, results in a significantly harder spectrum with poorer coefficients and better neutron economy. The deviations in energy-transfer group constants are probably least significant insofar as their long-term effect on fast reactor design is concerned because, when the values of these cross sections become well-established, the deviations from current values can generally be easily compensated by changes in reactor composition. Such adjustment is particularly easy when $\mathrm{BeO}$ or some other moderating additive has been included in the design for the purpose of degrading the spectrum and thereby improving the coefficients.

\subsubsection{Cross-section Deviations that Produce Softer Spectra}

Table 5 compared calculated physics parameters for Base Case, Good Coefficient, Composite taken from
Table 3, and Poor Coefficient, Composite taken from Table 4. A similar comparison is made for a reactor in which the $\mathrm{BeO}$ has been removed and replaced by a proportional amount of fuel and sodium. One other composite of deviations in cross-section data is also included in Table 5 for the $\mathrm{BeO}$ and non-BeO cores: the Soft Spectrum, Composite which is the same as same as that of Table 3, except that Low U-238 Capture replaced High U-238 Capture in calculating the composite effects; hence, Soft Spectrum, Composite represents the combination of cross-section variations that yield the most degraded neutron spectrum.

There is ample experimental evidence that the lowenergy portion of the neutron spertrum is substantially underpredicted with current cross sertions and calculation methods. This includes integral critical measurements, and their comparison with calculations, of neutron lifetime, boron-10 worth, and Doppler effect made in many facilities (see, for example, references 21,22 , 23 , and 24). For the reactor with $\mathrm{BeO}$, the Soft Spectrum, Composite has about $30 \%$ more fissions below $10 \mathrm{keV}$ than the Base Case adjusted for uncorrected elastic removal (Low Elastic case of Table 4). For the reactor without $\mathrm{BeO}$, the difference is slightly larger. The Low Elastic case has roughly the status of crosssection data that was used in some of the earlier calculations of integral experiments and which underpredicted lifetime and boron-10 worth by about $30 \%$ for highly dilute fast critical assemblies.

Table 5. Evaluation of Physics Parameter Uacertainties

Comparisons of Composite Cross-section Deviations for Cores with and without BeO

\begin{tabular}{|c|c|c|c|c|c|c|c|c|c|}
\hline \multirow{2}{*}{ Description } & \multirow{2}{*}{$\mathrm{BeO}, \mathrm{v} / \mathrm{o}$} & \multicolumn{2}{|c|}{$\begin{array}{c}\text { Doppler } \\
{[-T(d k / d T)]}\end{array}$} & \multirow{2}{*}{$\begin{array}{c}\text { Total Sodium } \\
\text { Void } \Delta k\end{array}$} & \multicolumn{2}{|c|}{ Breeding Ratio } & \multirow{2}{*}{$\begin{array}{c}\text { Fissile Core, } \\
a / o\end{array}$} & \multirow{2}{*}{$\begin{array}{l}\text { Fissions } \\
\text { below } \\
9 \mathrm{keV}(\%)\end{array}$} & \multirow{2}{*}{$\begin{array}{l}\text { Doubling } \\
\text { Time, yr }{ }^{(\mathrm{g})}\end{array}$} \\
\hline & & $\mathrm{Na}$ In & Na Out & & Total & Core & & & \\
\hline Base Case ${ }^{(1)}$ & 9 & 0.0074 & 0.0061 & +0.0010 & 1.20 & 0.70 & 14.3 & 21.7 & 13.4 \\
\hline Poor Coefficient, Composite ${ }^{(b)}$ & 9 & 0.0056 & 0.0037 & +0.0124 & 1.06 & 0.61 & 15.8 & 17.7 & 49.1 \\
\hline Good Coeffieient, Composite ${ }^{(a)}$ & 9 & 0.0119 & 0.0098 & -0.0015 & 1.25 & 0.71 & 15.5 & 22.8 & 11.6 \\
\hline Base Case $(d)$ & 0 & 0.0041 & 0.0029 & +0.0086 & 1.39 & 0.76 & 12.7 & 10.6 & 6.1 \\
\hline Poor Coefficient, Composite(d) & 0 & 0.0029 & 0.0018 & +0.0191 & 1.25 & 0.67 & 13.5 & 8.5 & 10.1 \\
\hline Good Coefficient, Composite ${ }^{(d)}$ & 0 & 0.0059 & 0.0042 & +0.0061 & 1.44 & 0.79 & 13,8 & 11.6 & 5.9 \\
\hline Soft Spectrum, Composite (d) & 0 & 0.0061 & 0.0044 & +0.0068 & 1.38 & 0.75 & 13.4 & 12.7 & 6.6 \\
\hline Zero Pu-239 Doppler(e) & 3 & 0.0063 & 0.0049 & 0.0009 & 1.26 & 0.64 & 15.1 & 15.5 & 10.9 \\
\hline
\end{tabular}

(a) Same as in Table 3 .

(b) Same as in Table 4.

(c) Same as Good Coefficient, Composite, except Low U-238 Capture replaces High U-238 Capture (see Section 3.2.3).

(d) Same geometry as BeO case. Volume fractions of fuel, sodium, steel and Ta are $0.429,0.362,0.205$, and 0.004 , respectively.

(e) Volume fractions of fuel, sodium, steel, and Ta are $0.355,0.41,0.20$, and 0.004 , respectively. This case assumes a zero Pu-239 1)oppler effect.

(f) Same as (e) and, in addition, low $\nu$, low $\sigma_{f}$, and high $\alpha$ for $\mathrm{Pu}-239$.

(g) Normalized to agree with two-zone core (see Table 1) for the $9 \mathrm{v} / \mathrm{O}$ BeO case. 
The negative boron-10 reartivity worth was calculated for the core of the Soft Spectrum, Composite case without $\mathrm{BeO}$. This calculation was also made for the same core composition and plutonium content in the fucl but with the cross-section values (hanged to correspond to a "Low Flastic" non-BeO case. The boron-10 worth obtained with the Soft Spectrum, Composite cross sections was found to be $20 \%$ higher than that obtained from the "Low Elastic" cross-section values. Neutron lifetime should show a similar dependence on the cross-section variations as boron-10 worth. This order of improvement in the agreement between predicted and measured parameters that are strongly spectrum dependent tends to make the Soft Spectrum, Composite appear as a reasonable direction for at least part of the cross-section variations that it encompasses. A definite conclusion along these lines, however, can be very hazardous because of the possibilities for compensating errors in cross-section or methods factors that are not being taken into account here (and even for systematic errors in the integral critical measurements!!). In Table 5, the Soft Spectrum, Composite includes the Zero Pu-239 Doppler case since the latter also is justified mainly on the basis of integral critical experiments.

\subsubsection{Heterogeneity Effects}

All of the calculations reported here included a heterogeneity correction which consisted of using an equivalent potential seattering cross section. The simplified approach of a rational approximation leading to Bell's formula ${ }^{(25)}$ was used. Neglecting this heterogeneity correction for the Base Case with $\mathrm{BeO}$ resulted in the following small changes: $5.0 \%$ higher Doppler, 0.0006 higher sodium void $\Delta k, 1.5 \%$ increase in total breeding ratio, and $0.3 \%$ higher fissile content and $0.6 \%$ less fissions below $10 \mathrm{keV}$. These differences are so small that incorrect treatment of heterogeneity is not expected to introduce significant errors in the calculated results.

\subsubsection{Influence of Uncertainties on Design Considerations}

The physics parameters listed in Table 5 for the $\mathrm{BeO}$ and non-BeO cases include Doppler coefficients with sodium removed, core conversion ratios, and fissile doubling times in addition to the parameters listed in Tables 3 and 4 . The doubling times are normalized to make the Base Case value agree with that of the 1000 MW (e) design described in Section 2.

If it be assumed that a minimum Doppler coefficient of $-0.004 T d k / d T$ with sodium removed and a nearzero reactivity for total sodium loss is required to satisfy safety criteria, the variations in data representing Good Coefficient, Composite or Soft Spectrum,
Composite offer considerable scope for adjusting the reactor composition and geometry to achieve these criteria with satisfactory neutron economy. For these favorable composite deviations in the cross-section data, breeding ratios around 1.3 and doubling times below $10 \mathrm{yr}$ appear reasonable, using a significantly smaller amount of $\mathrm{BeO}$ than that in the $\mathrm{BeO}$ Base Case and a higher leakage than that of the Base Case without $\mathrm{BeO}$. If the criterion of non-positive reactivity for total sodium void can be relaxed, then breeding ratios around 1.4 and doubling times around $6 \mathrm{yr}$ appear attainable for the Good Coefficient, and Soft Spectrum, Composite deviations of the cross-section data, corresponding to the non- $\mathrm{BeO}$ reactor.

The Poor Coefficient, Composite deviations, on the other hand, offer a rather dismal possibility. For these cases, in order to achieve a $-0.004 T d k / d T$ Doppler coefficient with sodium removed, it appears necessary to have a tolerance for a significant positive total sodium void reactivity to maintain even a small breeding ratio in excess of unity.

The discussion in Section 3.2.3 of experimental evidence indicating softer neutron spectra than those calculated may provide some assurance that the Poor Coefficient, Composite situation will not arise. In addition, the Doppler measurements with the ZPR-III and ZEBRA assemblies strengthen the argument on a much smaller positive Pu-239 Doppler effect than is indicated by present calculations.

The bottom row of data in Table 5 shows results for a $3 \mathrm{v} / \mathrm{O}$ BeO case based on the Base Case cross sections except for $\mathrm{Pu}-239$. The $\mathrm{Pu}-239$ variations used were (1) low $\nu$ values, (2) high alpha values below 10 $\mathrm{keV}$ (both as in Table 4), (3) the low $\sigma_{f}$ values between 24 and $170 \mathrm{keV}$, which gives slightly better coefficients but significantly increases the fissile inventory (Table 3 ), and (4) the zero Pu-239 Doppler effect as in Table 3. The case with $3 \mathrm{v} / \mathrm{o} \mathrm{BeO}$ gives a Doppler coefficient with sodium removed that meets the -0.004 requirement. This case must be regarded as a realistic (and pessimistic) possibility until it is resolved by convincing experimental results. It is seen in Table 5 that such $\mathrm{Pu}-239$ cross sections would give fuel economics significantly poorer than that of the $9 \mathrm{v} / \mathrm{o}$ BeO Base Case with tolerance for a significant positive reactivity for total sodium voidage. Without such tolerance the neutron economy resulting from this pessimistic $\mathrm{Pu}$ 239 cross section possibility would be much poorer than that calculated for the reference design.

The second-from-the-bottom row of data in Table 5 shows a $3 \mathrm{v} / \mathrm{O} \mathrm{BeO}$ case in which the only variation considered is a zero Pu-239 Doppler coefficient. This case satisfies the Doppler and sodium void design 
safety eriteria with significantly better neutron economy than the $9 \mathrm{v} / 0$ BeO Base Case.

It is evident that the present status of fast reactor cross-section information is very confusing. Until this is resolved by a suitable program of energy point-wise and integral critical measurements, physies predictions of fast power reactor performance will remain highly unreliable.

\section{REFERENCES}

1. Liquid Metal Fast Breeder Reactor Design Study, GEAP. 4418 ( $\operatorname{Tan} 1964)$

2. O'Shea, D., et al, 26-group Cross Sections, ANL-6858 (to be published).

3. Abajan, L. P., et al., Fast and Intermediate Veutron Group Constants for the Calculation of Nuclear Reactors, PhysicoEnergetic Institute of the USSR, Obninsk, USSR (1963).

4. Rowlands, J. L., and Wardleworth, D., A Nineteen-group Extension to the Yiftah, Okrent, and Moldauer C'rosssection Set, ALE Winfrith (1965).

5. Yiftah, S., and Sieger, M., Nuclear Cross Sections for Fast Reactors, IA-980 (July 1964).

6. Sherer, I). B., An Analysis of Fast Reactor Transient Response and Safety in Selected Accidents, GEAP-4787 (to be published).

7. Cowan, C. L., Investigations of Alternate Reactor Concepts, GEAP-4822 (to be published).

8. An Evaluation of Four Design Studies of a 1000-MWe Ceramic-fueled Fast Breeder Reactor, COO-279 (Dec 1964).

9. Zaleski, C. P., and Smidt, I)., Fast Reactor Technology, ANS-100, p. 275 (April 1965).

10. Hanson, J. E., et al., Experimental Studies of Transient Effects in Fast Reactor Fuels, Series II, Mixed Oxide $\left(\mathrm{PuO}_{2}-\mathrm{UO}_{2}\right)$ Irradiations, GEAP-480t (June 1965).

11. Large Fast Reactor Design Study, ACNP-64503 (Jan 1964).

12. Liquid Metal Fast Breeder Reactor Design Study, WCAP3251-1 (Jan 1964).

13. Memorandum, J. M. Borg to I), B. Sherer, Coolant Outlet Temperatures for 1000-MWe Pancake and Annular Core Configurations, April 6, 1965.

14. Cohen, K. P., et al., Safety and Economics Characteristics of a $1000 \mathrm{MW}(e)$ Fast Sodium-cooled Reactor Design, See Paper in Session III of this Conference.

15. Weale, J. W., Review of the Vera Reactor Programme and Related Work at Aldermaston, AWIRE (May 1965).

16. White, P. H., Hodgkinson, J. G., and Wall, G. J., Measurements of $F$ ission Cross Sections for Neutrons of Energies in the Range 40-500 keV, IAEA Conference on the Physies and Chemistry of Fission, Salzburg, Austria, March, 1965.

17. Colvin, 1). W., and Sowerby, M. G., Boron Pile $\bar{\nu}$ Meas urements, SM-60/44, ALRE, Harwell (Feb 1965).

18. Rago, A. L., and Hummel, H. H., ELMOE- An IBM-704 Program Treating Elastic Scattering Resonances in Fast Reactors, ANL-6085 (Jan 1964).
19. Kanne, W. R., Stewart, H. B., and White, F. A., Captureto-Fission Ratio of Pu-239 and U-235 for Intermediate Energy Neutrons, Paper No. 595, Proceedings of the First International Conference on the Peaceful Uses of Atomic Energy, Geneva, Switzerland, 1955.

20. Klimentov, T. B., and Griazev, V. M., Some Neutron Resonance Absorption Integrals, J. Nuclear Energy, 9, 20-27 (1959).

21. Hischer, (Ұ. J., et al., Doppler Effect Measurements in Plutonium-fueled Fast Power Breeder Reactor Spectra, Paper to be published. See also Fischer, G. J., et al., Measurement and Analysis of Doppler Effect in Plutoniumfueled Fast heactor Assemblies, See Paper in Session VI $\mathrm{B}$ of this Conference.

22. Baker, A. R., and Wheeler, R. C., Doppler Coefficient Measurements in Zebra Core 5, See Paper in Session VI B of this Conference.

23. Davey, W. G., A Compartson of Experimental and Calculated Prompt Veutron Lafetimes and Central Reactivity Coefficients in ZPR-III Assemblies and Their Relationship to Other Reactor Parameters, ANL 6682 (June 1963).

24. Sher, R., and Pflasterer, (צ. R., Doppler Effect in U-238 Capture and U-235 Fission in a Fast Neutron Spectrum, Trans. ANS 7, No. 2, Nov 1964.

25. Bell, G. I., A Single Treatment for Effective Resonance Absorption Cross Sections in Dense Lattices, Nuclear Science and Engineering, 5, No. 2, pp. 138-139 (1959).

\section{Discussion}

Mr. Chernick (BNL): Now that the nominal breeding ratios of these big fast reactors are going down towards 1.2, I'd like to ask Dr. Greebler the questions that always bothered Wigner at these last Reartor meetings, namely, are not people being too lackadaisical about losses in processing a fuel? Night this not be just as important? With your goal of maybe $10 \%$ burnup, you have to reprocess only 10 times, but that is still a large number. What is the feeling of General Electric about this problem?

Mr. Greebler: The question of reprocessing losses is an important economic consideration not just from breeding considerations. I'd like to delay answering until tomorrow's panel session at which questions of safety and economies philosophy will be discussed.

Mr. Cohen (GE): I would like to make the following comment on this and the preceding paper: so far we have made intercomparisons without a real point of reference. I think it might be very useful, since we are about to have in ZPR-3 a fairly representative assembly, to invite the people who have taken part in the previous intercomparisons to calculate that SEFOR critical. Let's see where we are . . . before it goes critical. 


\title{
Safety and Cost Analysis of a 1000-MWe Sodium-cooled Fast Power Reactor*
}

\author{
D. Smidt, W. Frisch, P. Giordano, $†$ G. Heusener, G. Kessler, K. H. Krewer, W. Merk, T. Malmberg, and \\ E. SCHÖNFELD \\ Institute for Reactor Development \\ Kernforschungszentrum, Karlsruhe, Germany \\ (PRESENTED BY D. SMIIT)
}

At the end of 196t the Karlsruhe group completed a design study of a 1000-MWe sodium-cooled fast breeder reactor. ${ }^{(1)}$ A detailed safety and cost analysis has now been made. This takes into account good judgement as regards the safcty of large fast reactors in general and for defining the criteria of a second design of a large plant and of the smaller prototype reactor as well. A number of interesting conclusions can be drawn.

\section{Main Features of the Present 1000-MWe Design}

Table 1 shows some of the important design parameters of the core. The main features are a cylindrical shape, oxide fuel of $87 \%$ theoretical density, no moderator like $\mathrm{BeO}$, and a moderate flattening with a heightto-diameter ratio of $1 / 3$. This results in a relatively large negative Doppler coefficient and an internal breeding ratio $\left(B R_{1 n}\right)$ close to unity. The void coefficient will then be somewhat larger than in several other designs. Its effect on overall safety will be one of the topics of this paper.

The coolant volume fraction of $50 \%$ is relatively large, resulting in a small pressure drop and pump size. Special spacers with a small amount of structural materials can be used. The decision not to use $\mathrm{BeO}$ or similar moderators is purely economical. The loss in total breeding gain is evident, whereas the small internal breeding ratio contributes some additional operational difficulties.

The core has 2 zones of equal volume and different enrichment. The 229 subassemblies are of hexagonal shape; the inner radial blanket is of oxide, the outer one of metal. The axial blanket is $40 \mathrm{~cm}$ on either side. A fission gas plenum of $80 \mathrm{~cm}$ is below the core. In another paper during this Conference ${ }^{(5)}$ we give an evaluation of the vented fuel concept.

The characteristics of the plant design will be discussed in detail in our panel paper (see Session IV), so

* This paper has been prepared within the framework of the association ECRATOM-Gesellschaft für Kernforschung $\mathrm{mbH}$. in the field of fast breeder development.

$\dagger$ EURATOM, Brussels, delegated to the Karlsruhe Fast Reactor Project. that we mention only a few important items: The primary circuit is of the loop type with some features of the pool type (mainly rigid connection ducts between reactor vessel and heat exchangers; pumps and heat exchangers are sliding). There are two intermediate heat exchangers and four pumps, as well as four secondary loops. A new type of refueling system has been proposed.

The capital costs, based on studies from industry, have been ealculated to be $\$ 115 / \mathrm{kW}$.

\section{Influence of Group Constants}

The effect of the cross-section set used for calculation of breeding ratio, Doppler effect, and void coefficient defines the reliability of any safety and cost analysis. The results of a world-wide comparison and their interpretation have been presented by Okrent (see first paper of this Session). We have made calculations for our specific reactor with three sets of cross sections:

a) The Russian 26-group set $\Lambda \mathrm{BN}$;

b) $\Lambda$ 60-group set;

c) The new Karlsruhe 26-group set (of J. J. Schmidt and coworkers).

Table 2 shows the results.

\section{Dynamies and Safety of the Reference Reactor}

\subsection{Dynamic Programs}

The dynamir behaviour of our proposed reactor has been studied with the use of 3 programs:

a) An analog program of the core. It included for the simulation:

1) Space-independent neutron kinetics;

2) Fuel heat generation and transfer through fuel and can, and heat removal by the coolant;

3) Reactivity feedback caused by the Doppler effect, structural material, and coolant.

The fuel element was divided into 30 segments. For each segment the heat balance equation was set up; density, specific heat, and thermal conductivity were considered to be constant.

Fuel melting, the tempcrature dependence of the 
Doppler coefficient, and a temperature-dependent heat transfer coefficient between fuel and can were included in the study.

b) An analog program of the total primary circuit, which included:

1) Similarly to the first program, neutron kineties, heat generation, transfer, and removal, and the reactivity feedback (15 segments of the fuel element);

2) The heat exchange in the intermediate heat exchanger (16 segments);

3) The flow coast-down in the main coolant, pumps;

4) The time delay of the pipes between reactor and heat exchanger;

5) The mixing process in the reactor inlet and outlet plenum.

c) $\Lambda$ revised version of the digital code FORL, developed by GE. The Karlsruhe version included, in addition, the temperature dependence of the heat transfer coefficient between fuel and can.

\subsection{General Safety Criteria}

It is impossible to define one general safety criterion for a fast reactor. We have already pointed out ${ }^{(2)}$ that one has to study each possible actual accident or incident more or less separately and evaluate the consequences. Since there is always some uncertainty whether one knows each possible actual cause of an accident, it is nevertheless helpful to study the general behaviour of a system under extraordinary conditions, such as fast reactivity ramps of infinite height, even if the results are not directly applicable to a real case.

TABle 1. Some Design Parameters of A 1000-MWe SODIUM-COOLED FAST REACTOR

Core Height $\mathbf{H}$ :

Core Diameter D: $\mathrm{H} / \mathrm{D}: 1 / 3$

Number of Core Zones:

Max Rod Power $\chi$ :

Rod Diameter $d$ :

Coolant Fraction $\alpha$ :

Structural Fraction $\beta$ :

Pressure Drop (Core and Axial Blanket) $\Delta p: 3.2 \mathrm{~kg} / \mathrm{cm}^{2}$

Internal breeding ratio $B R=\varphi_{\mathrm{rad}} \varphi_{\mathrm{ax}}: \quad 0.80$

Total breeding ratio $B R_{\text {tot }}$ :

Mean specific power $q_{\mathrm{spe}}$ :

Mean power density $q_{m}$ :

$\Delta k_{\mathrm{Void}}$ maximum:

$\Delta k_{\text {void }}$ totalcore

Coolant inlet temperature $\vartheta_{1}$ :

Coolant exit temperature $\vartheta_{2}$ :

Maximum fuel center temperature $\vartheta_{\max }$ :

80-cm fission gas plenum at the bottom; 229 hexagonal subassemblies; $45-\mathrm{cm}$ radial blanket; $40-\mathrm{cm}$ axial blanket.
TABIE 2, Calculations With 3 Cross-section Sets for A 1000-MWe BREEDER*

\begin{tabular}{l|c|c|c}
\hline & 26 Groups & 26 Groups & 60 Groups \\
& $\mathrm{KFK}$ & $\mathrm{ABN}$ & \\
\hline Critical Mass, $\mathrm{kg}$ & 2168 & 2048 & 2010 \\
Internal Breeding Ratio & 0.95 & 0.94 & 0.91 \\
Doppler Coefficient $\left(\times 10^{6}\right)$ & -5.97 & -8.32 & -6.58 \\
$\Delta k_{\text {raax }}$ for Sodium Loss & 0.024 & 0.013 & 0.008 \\
\hline
\end{tabular}

* Slightly revised version which has been used as a reference point for the systems analysis.

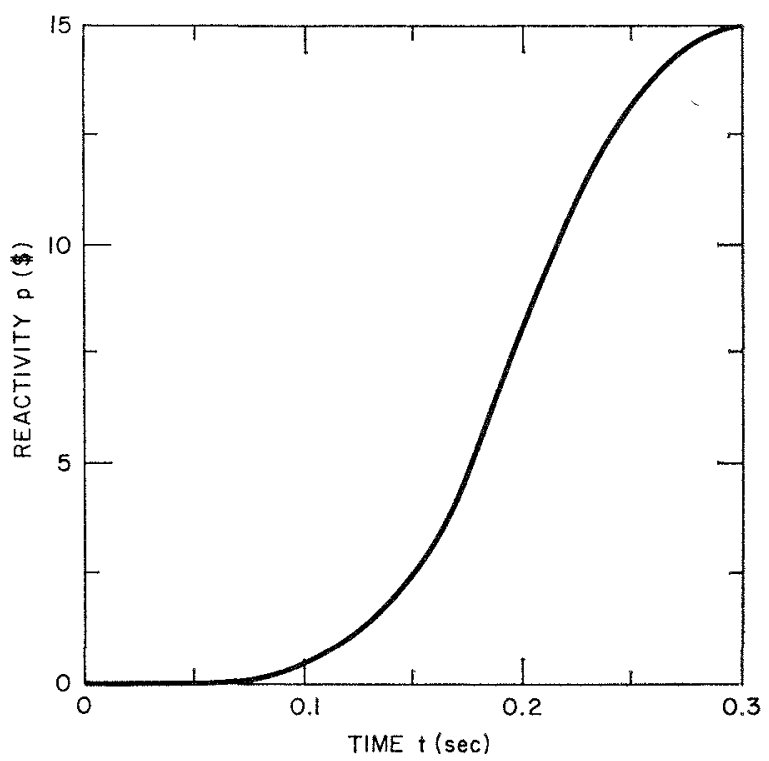

Fra. 1. Reactivity versus time for safety system

The first general safety criterion is the time $t_{m}$ between the beginning of an infinite reactivity ramp input and the starting of melting of the hottest fuel. This gives a reference scale for the possibility of any counteractions by the scram system.

Since any reactivity input above $\$ 0.25$ will lead to fuel melting, and under certain conditions after sufficient time to destruction of the reactor, the reaction of the safety system should be included into the analysis. So our second safety criterion gives the maximum rate of the reactivity ramp, which can be counteracted by the safety system without starting fuel melting. We choose a conventional, spring-driven system with 10 safety rods.

Figure 1 shows the space-time relationship of the safety system, including the specific effects of inertia and friction. Naturally, this criterion is less general, since it depends on a specific safety system, but it is also more practical.

Also the delay time $\tau$ between the onset of the excursion and the beginning of rod movement has to be taken into account. 
A third criterion should be the maximum hypothetical accident (MHA) and its consequences. This depends not only on the core design, but also on the containment and shiclding properties and is even less general. The MHA will be discussed in Sect. 6 .

\subsection{Results with Safety Criterion 1, the Time $t_{m}$ to Reach Start of Fuel Melting}

In Table 3 the input data for the dynamic calculation of the reference reactor are given. In Fig. $2 t_{m}$ is plotted as a function of the Doppler and the structural expansion coefficients. The influence of the Doppler effect is distinct, but not too important. The structural coefficient acts only in the case of slow ramp rates.

In this connection it is interesting to compare $t_{m}$ with $t_{b c}$, the time to reach coolant boiling (at channel exit), and $t_{b f}$, the time to reach fuel boiling. Here $t_{b f}$ may be taken as an equivalent for core decomposition and termination of the excursion.

In Fig. 3 the three time intervals are plotted as a function of the ramp rate $r_{s}$. For large ramp rates always $t_{b c}>t_{b f}>t_{m}$. This is equivalent to the fact that it is impossible in a highly rated oxide core to get coolant boiling before reactor decomposition. Okrent, Cohen, and Loewenstein ${ }^{(3)}$ indicate the same results. Only for small $r_{s}$ is $t_{b c}<t_{b f}$. Then coolant boiling may happen. In connection with safety criterion 2 we shall prove that these slow ramp disturbances can easily be governed by the safety system.

Figure 4 shows the effect of finite ramps. Here $t_{m}$ is plotted as a function of the ramp height for different ramp rates. For $t_{m} \rightarrow \infty$ all curves converge to the same value: $r_{h}=\$ 0.25$, which is defined by static conditions. For this reactivity input the reactor does not need any safety system.

TABLli 3. Basic Constavts for I) yvamic (alleulations of Reverence Reactor

\begin{tabular}{|c|c|}
\hline Thermal Conductivity (W/ & \\
\hline Fuel $\left(1450^{\circ} \mathrm{C}\right)$ & 0.03 \\
\hline $\operatorname{Can}\left(600^{\circ} \mathrm{C}\right)$ & 0.21 \\
\hline Specific Heat (W-sec/g- ${ }^{\circ} \mathrm{C}$ ) & \\
\hline Fuel $\left(1450^{\circ} \mathrm{C}\right)$ & 0343 \\
\hline $\operatorname{Can}\left(600^{\circ} \mathrm{C}\right)$ & 0.503 \\
\hline Coolant $\left(500^{\circ} \mathrm{C}\right)$ & 1.264 \\
\hline Density $\left(\mathrm{g} / \mathrm{cm}^{3}\right)$ & \\
\hline Fuel $\left(1450^{\circ} \mathrm{C}\right)$ & 99 \\
\hline $\operatorname{Can}\left(600^{\circ} \mathrm{C}\right)$ & 8.0 \\
\hline Melting Temperature $\left({ }^{\circ} \mathrm{C}\right)$ & \\
\hline Fuel & 2800 \\
\hline Heat Transfer Coeffeient ( & \\
\hline Fuel $\rightarrow \operatorname{Can}\left(1450^{\circ} \mathrm{C}\right)$ & 0.75 \\
\hline Can $\rightarrow$ Coolant $\left(500^{\circ} \mathrm{C}\right)$ & 14.5 \\
\hline
\end{tabular}

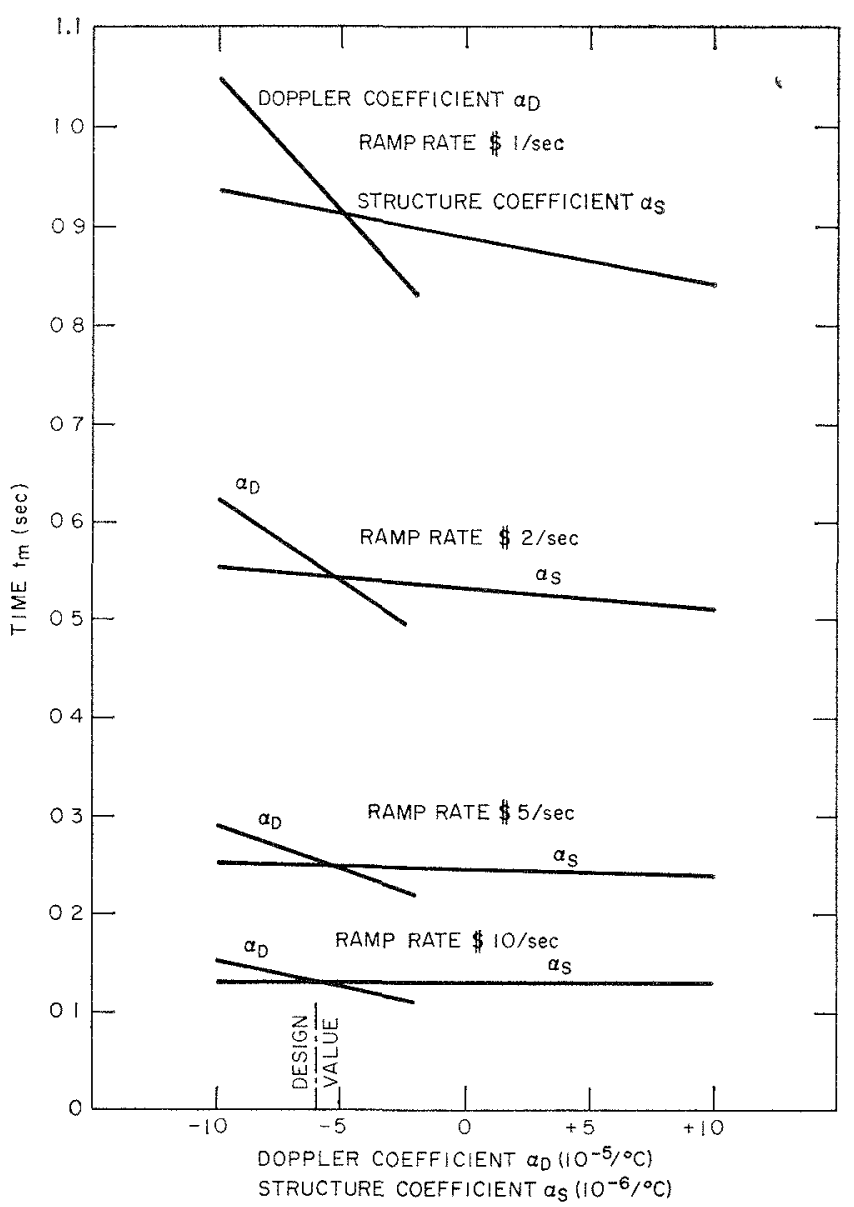

Fic. 2. Time $t_{n b}$ to reach fuel melting versus Doppler coefficient $\alpha_{D}$ and structure coefficient $\alpha_{S}$ for reference reactor.

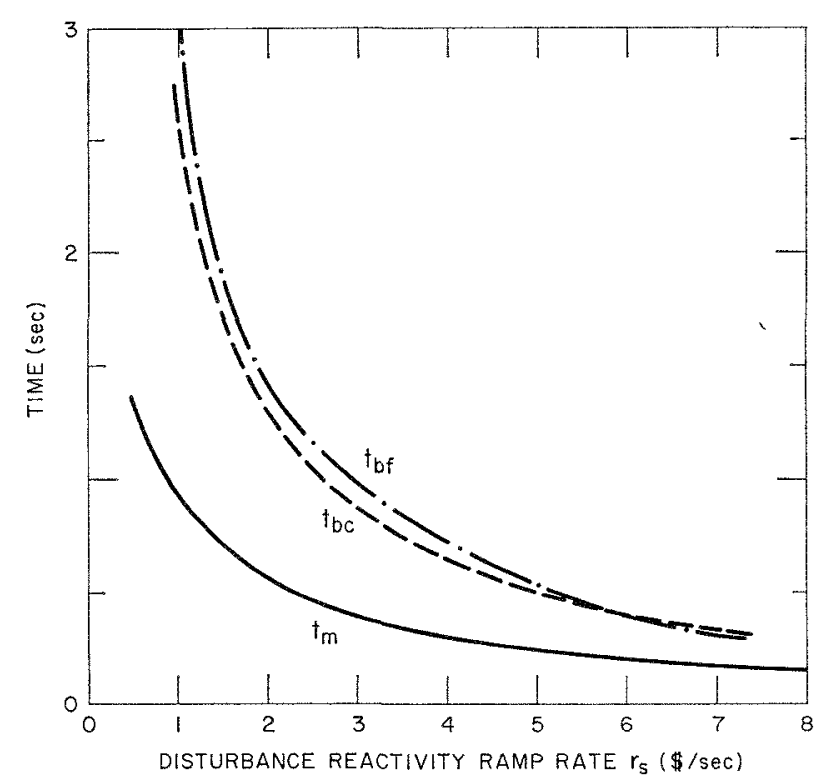

FIa. 3. Time $t_{m}$ to reach fuel melting, time $t_{b c}$ to reach coolant boiling (core exit), and time $t_{b f}$ to reach fuel boiling versus disturbance reactivity ramp rate. 


\subsection{Safety Criterion \&, Maximum Ramp Rate}

Figure 5 shows, for different Doppler coefficients, the maximum ramp rate which can be counteracted without fuel melting by the safety system as a function of the delay time $\tau$ before beginning of rod movement. The safety system consisted of 10 scram rods, earh with a $250-\mathrm{kp} / \mathrm{cm}$ spring and a weight of $100 \mathrm{~kg}$. The total reactivity value of the rods was $\$ 15$. (The spacetime dependence of this system has been given in Fig. 1.)

In. Fig. 6 is plotted the maximum ramp rate which

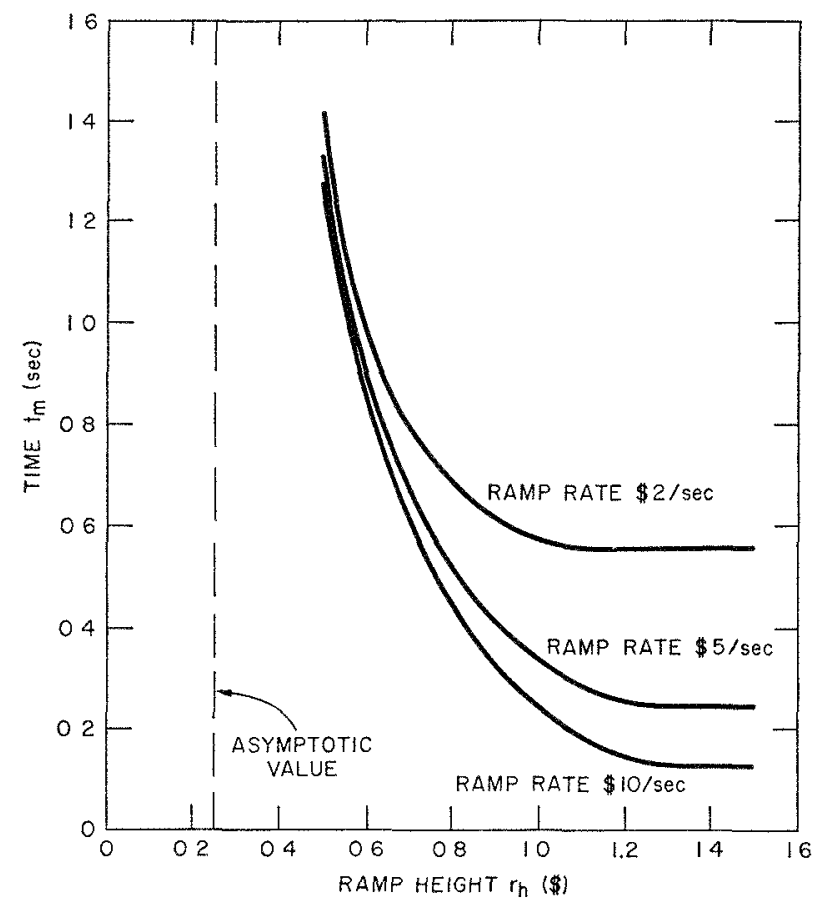

FIG. 4. Time $t_{m}$ to reach fuel melting versus ramp height for different disturbance reactivity ramp rates for the reference reactor.

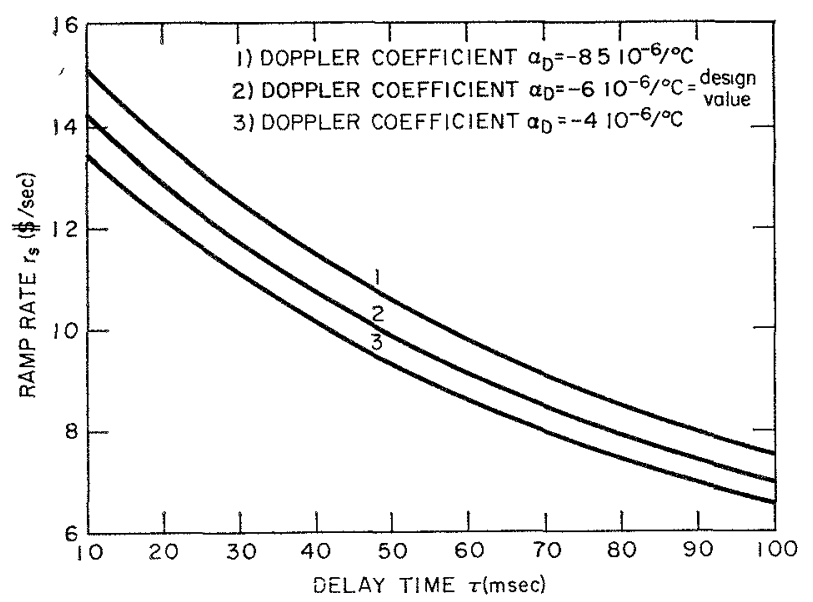

Fra. 5. Maximum allowable disturbance ramp rate $r_{s}$ for different Doppler coefficients versus delay time for the reference reactor.

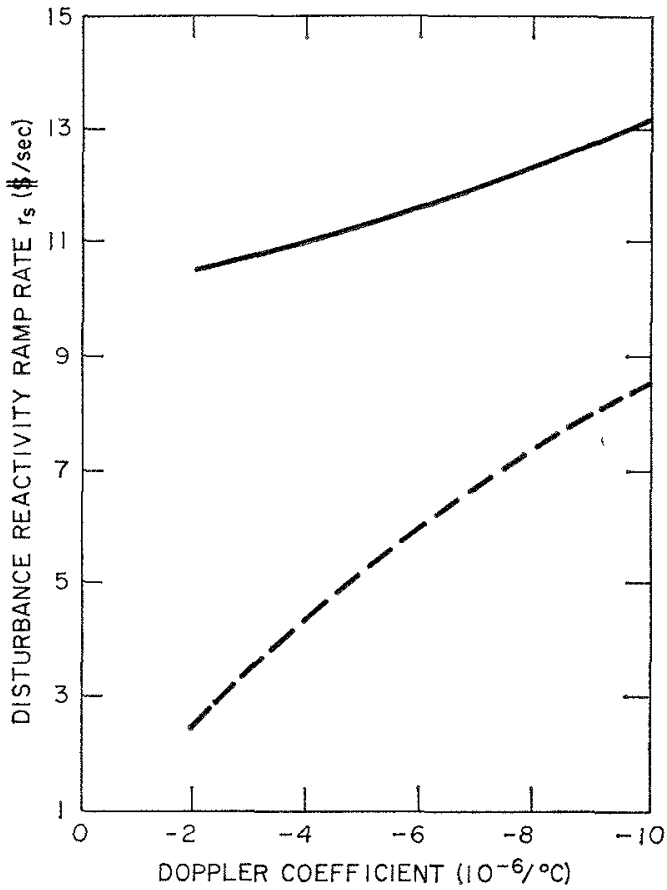

Fya. 6. Maximum disturbance reactivity ramp rate $r_{s}$ for $\tau=30$ msec versus Doppler coefficient. (Dotted line: $r_{s}$ for $\left.t_{b c}=t_{b f}\right)$.

(an be controlled by the above safety system without fuel melting, for a delay time of $30 \mathrm{msec}$, as a function of the Doppler coefficient. By consideration of some of the information of Fig. 3, we get the dotted line; it resembles the ramp rate, for which $t_{b c}=t_{b f}$. For ramp rates above this dotted curve the reactor disassembles before the coolant can boil. The ramp rates for coolant boiling are below the ramp rates, which can be governed by the safety system.

With respect to coolant boiling and void effect, therefore, it is an important fact that it is impossible to generate sodium boiling by any ramp reactivity input as long as the normal conventional safety system is working. It is much easier to destroy the reactor by fuel boiling.

Moreover, in principle the safety action can be accelerated by an additional electromagnetic force according to a proposal of Dosch. ${ }^{(4)}$

Necessarily the values of fuel conductivity, melting temperature, and gap conductivity after some irradiation are not too well known. The influence of these unrertainties is shown in Fig. 7, which shows calculated allowable ramp rates as a function of $\tau$ for different values of the fuel melting temperature.

\subsection{Actual Accidents}

So far we have studied the effect of hypothetical ramp excursions. The actual ramps, i.e., control rod 
runaways, will definitely be kept to the order of $\$ 0.01 /$ sec and below.

Another actual possibility is a loading accident. Suppose the central fuel element, with $\$ 0.7$, is dropped into the cold critical reactor in $500 \mathrm{msec}$. The effect of the resulting perturbation is shown in Fig. 8. All temperatures can be kept below dangerous values.

In Fig. 9 can be seen the result of a simultaneous failure of all 4 primary pumps. The can temperature will reach the boiling point of sodium (about $960^{\circ} \mathrm{C}$ ) after 90 sec.

As is shown in Fig. 10, the structural expansion coefficient will reduce the reactor power to about $75 \%$ during this time. The effect of the uncertainty in this coefficient can also be seen from this figure.

\subsection{Stability}

It can be expected that a reactor system with a fast negative and a slow positive coefficient will be stable in most cases. This has been proved by a detailed analysis. According to the Nyquist diagram, instability may oc-

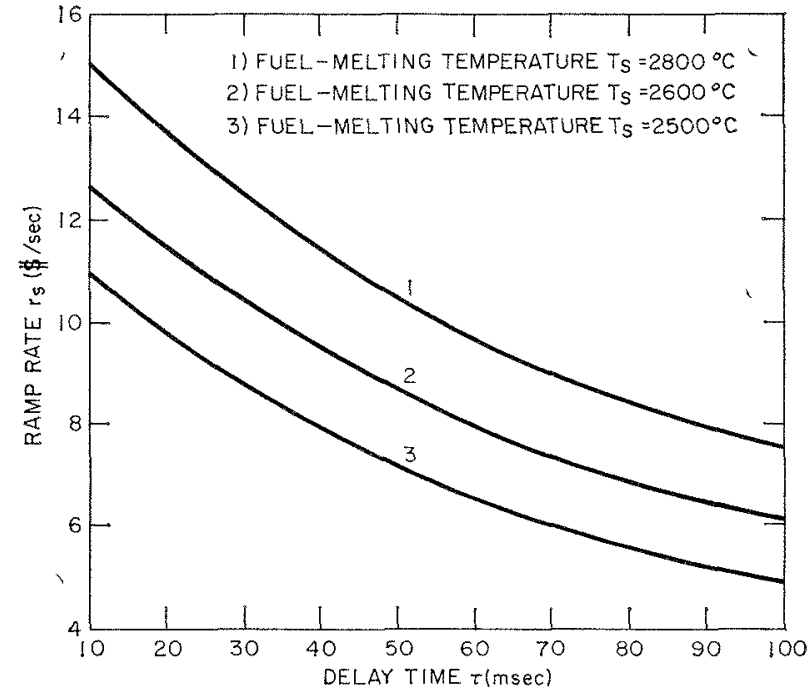

FIc. 7. Maximum allowable disturbance ramp rate $r_{s}$ for different fuel-melting temperature versus delay time for the reference reactor (Doppler coefficient $=6 \times 10^{-6} /{ }^{\circ} \mathrm{C}$ ).

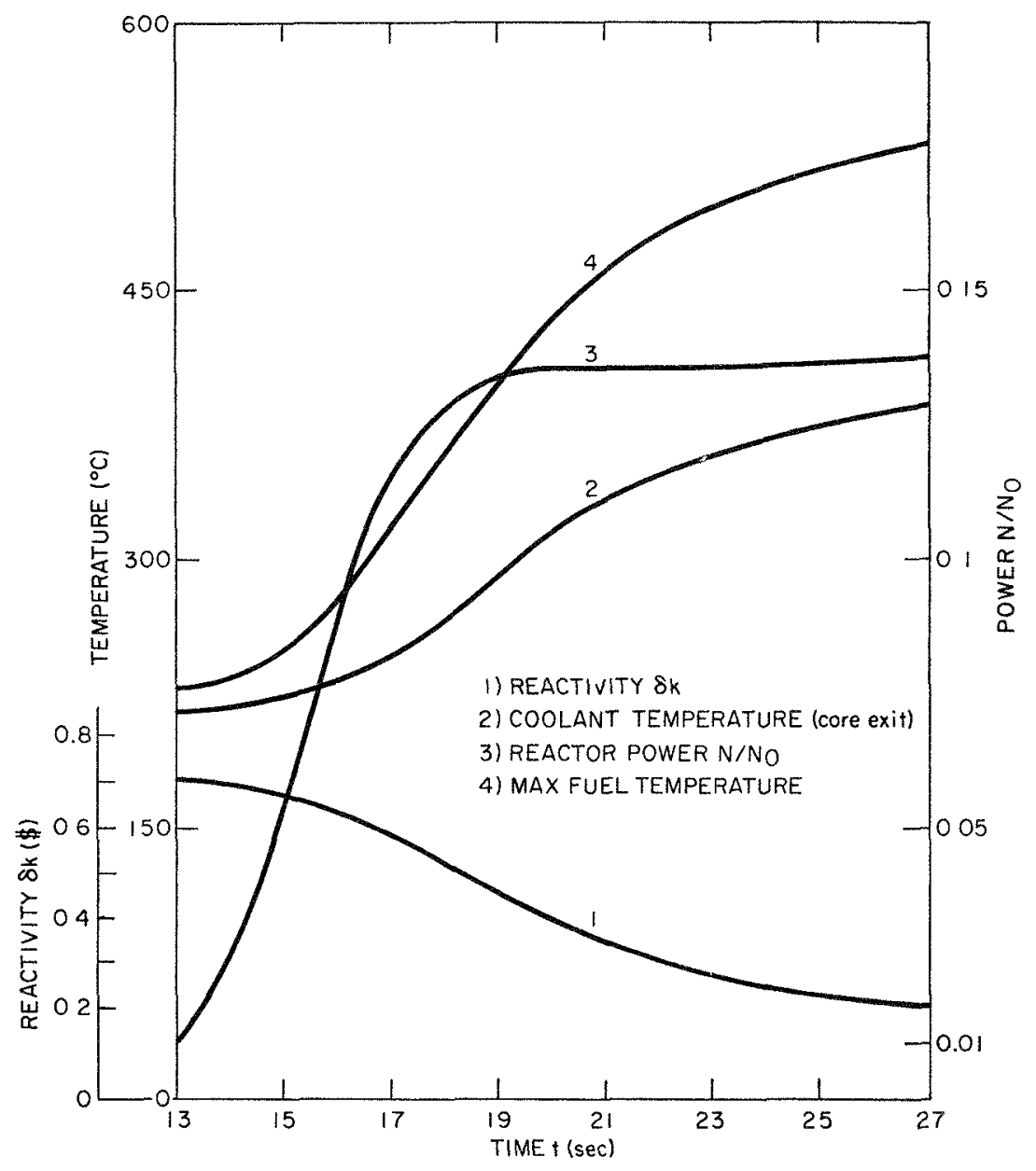

Fig. 8. Loading Accident: reactivity, power, max fuel center temperature, and coolant temperature (core exit) versus time. 


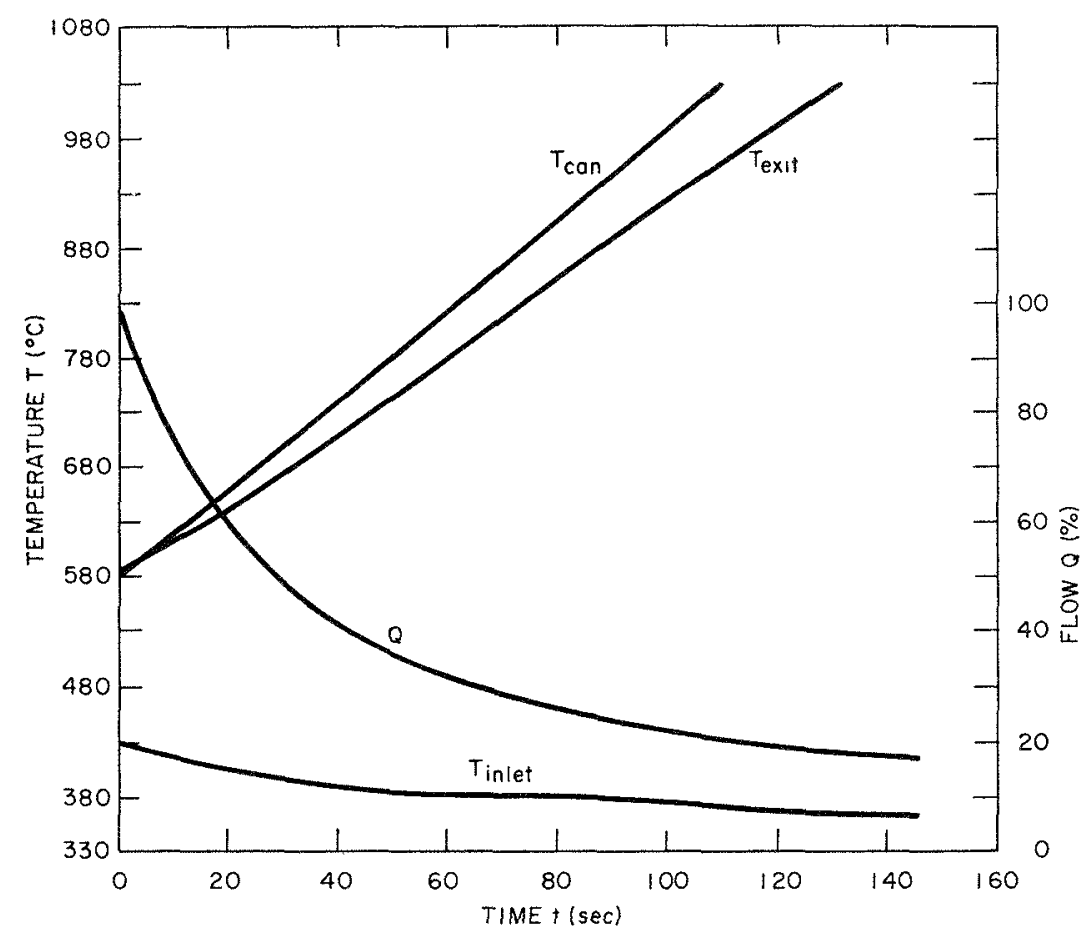

Ficx. 9. Loss of pumping power: coolant fow $Q$, core inlet temperature $T_{\text {inlet }}$, core exit temperature $T_{\text {exit }}$, and can temperature $T_{\text {san }}$ versus time $t$.

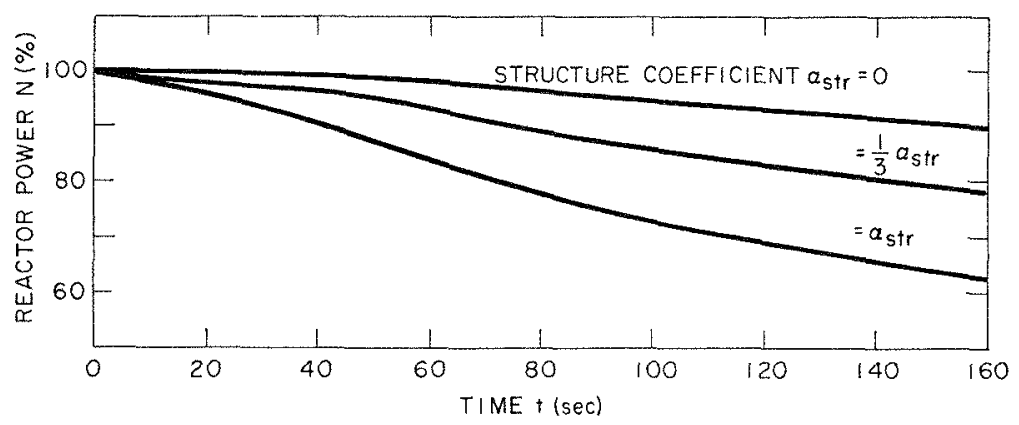

FyG. 10. Loss of pumping power: reactor power $\Lambda^{*}$ versus time for different structure coefficients $\alpha_{s t r}$.

cur if the Doppler coefficient is smaller than $1 / 50$ of its actual value or if the coolant coefficient is 50 times its actual value.

It has been shown that instability may arise also from higher-order delay terms. But there is no reason to assume those.

\section{Parametric Studies; Influence on Cost and Safety}

\subsection{Survey of Variations}

It has been the central point of our analysis to define the influence of the important design parameters on cost and safety and by this to learn where our concept has to be changed. To do this, we systematically varied many values and calculated the effect on safety and costs. Table 4 summarizes the parametric variations, as well as their expected advantages and disadvantages.

In comparison with other parametric surveys, ours refers to a real system. Therefore many detailed effects of an extraordinary importance are included. For example, if the coolant fraction $\alpha$ is lowered (see case 1 in Table 4), the pressure drop rises and therefore more structural material is needed. Moreover, other types of spacers may be needed for geometrical reasons.

Also, for a real system with given heat exchanger, temperatures, pressures, etc., the effect of parametric variations on fuel and capital costs will be more realistic.

For our investigations the following programs were used:

1) One-dimensional multigroup-diffusion program "MIGP"; (12) 
2) Two-dimensional diffusion program "Twenty Grand";(13)

3) Nuclear program system Karlsruhe "NCSYS" [not published];

4) Two-dimensional perturbation Code "2 DPert." (14)

The enrichment of the first and second core zones was determined by one-dimensional diffusions calculations ("MGP") with the 26 KFK-group set. ${ }^{(15)}$ From these results were generated the macroseopic 6-group cross sections by "NUSYS." Then the breeding ratios, critical masses, reactivity coefficients, and power distributions were calculated by the "Twenty Grand" and the twodimensional perturbation code " 2 D-Pert." For the calculation of the Doppler coefficient the one-dimensional perturbation code contained in "NUSYS" were used.

The determination of the core region in which removal of the sodium caused the maximum $\Delta k_{\text {sod loss }}$ effect, was performed by two-dimensional perturbation calculations. The $\Delta k_{\text {sod loss }}$ effect itself was determinated by one- and two-dimensional diffusion calculations.

\subsection{Discussion of Nuclear Results}

In Table 5 the main results are given. In Fig. 11a the most important results are represented graphically.

Case 1: The reduction of the coolant fraction $\alpha$ from 50 to $40 \%$ should result in a larger internal breeding ratio. However, the coolant pressure drop is raised to $13.7 \mathrm{~atm}$, and the larger requirement in structural material cancels the gain in the internal and total breeding ratios.

Case 1a: If $\Delta \theta$ is raised to $200^{\circ} \mathrm{C}$ and $\alpha$ changed in such a way that the pressure drop is the same as for the reference reactor, the internal and total breeding ratios are somewhat higher. The Doppler coefficient stays practically unchanged during these variations, whereas the void effect is somewhat reduced for the smaller values of $\alpha$.

The larger value of $\Delta \vartheta$ also allows for a smaller effective temperature difference between the primary and secondary sodium circuit, and reduces the danger of thermal shocks in the case of a break in the intermediate heat exchanger (IHE). On the other hand, the IHE must be larger or the thermal plant efficiency will be reduced. This will be discussed in our cost considerations.

Case Q: A smaller ratio $H / D$ must be paid for in the internal breeding ratio and Doppler coefficient. The reduction of the void effect is favorable compared with the reference reactor, but does not pay in comparison with Cases 1 and $1 \mathrm{a}$.

Case 3: By addition of $5 \%$ BeO the Doppler constant is nearly doubled, whereas the void effect is reduced by about $\$ 1$. The penalty is purely economical. The advan-
Table 4. List of Parametric Tariations

\begin{tabular}{|c|c|c|c|}
\hline 0) & Original Reactor & Expected Advantages & $\begin{array}{c}\text { Expected } \\
\text { Disadvantages }\end{array}$ \\
\hline 1) & $\begin{array}{l}\text { Smaller } \\
\text { coolant frac- } \\
\text { tion } \alpha\end{array}$ & $\begin{array}{l}\text { Internal } B R \text {; } \\
\text { Total } B R\end{array}$ & $\begin{array}{l}\text { More } \Delta p ; \\
\text { More structural } \\
\text { material }\end{array}$ \\
\hline 1a) & $\begin{array}{c}\text { Smaller } \alpha \text { and } \\
\text { larger } \Delta \vartheta\end{array}$ & $\begin{array}{l}\text { Less structural ma } \\
\text { terial than 1a) }\end{array}$ & $\begin{array}{l}\text { Larger heat ex- } \\
\text { change }\end{array}$ \\
\hline 2) & Smaller $\mathrm{H} / \mathrm{D}$ & Smaller void effect & $\begin{array}{l}\text { Smaller Doppler; } \\
\text { Smaller } B R_{1 \mathrm{n}} \\
\text { Capital costs? }\end{array}$ \\
\hline 3) & $5 \%$ BeO added & Larger Doppler & Smaller $B R$ \\
\hline 4) & $10 \% \mathrm{Mo}$ & $\begin{array}{l}\text { Low fuel tempera- } \\
\text { ture }\end{array}$ & $\begin{array}{l}\text { Smaller Doppler; } \\
\text { Smaller } B R\end{array}$ \\
\hline 5a) & $\begin{array}{l}\mathrm{UC} \text {, same ge- } \\
\text { ometry as } \\
\text { oxide }\end{array}$ & $\begin{array}{l}\text { Low fuel tempera- } \\
\text { ture; } \\
\text { Large internal and } \\
\text { total } B R\end{array}$ & Larger void effect \\
\hline $5 b)$ & $\begin{array}{l}\text { UC compact } \\
\text { core }\end{array}$ & $\begin{array}{l}\text { Compared to } 5 a): \\
\text { capital costs? }\end{array}$ & $\begin{array}{l}\text { Smaller } B R_{1 \mathrm{n}} \text {; } \\
\text { Larger } \Delta p\end{array}$ \\
\hline 6) & Vented fuel & $\begin{array}{l}\text { Larger } B R ; \\
\text { Less pumping } \\
\text { power }\end{array}$ & $\begin{array}{l}\text { Gas-purification } \\
\text { plant }\end{array}$ \\
\hline
\end{tabular}

tages are not too important for operational stability and safety, but are in terms of the maximum hypothetical accident.

Case 4: The addition of molybdenum for a better fuel conductivity is plainly disadvantageous. The breeding ratio is low, the Doppler constant is low, and the void effect is high.

Case 5a: In this carbide core the internal and total breeding ratios are very large, the Doppler coefficient nearly as large as for the oxide cores, but there is a tremendous void effect. The advantage of the low fuel temperature will be discussed in connection with the dynamic behaviour.

Case $5 b$ : Here the breeding is strongly reduced, especially internally. The Doppler effect is smaller, but this is true also for the void effect.

Case 6: The main advantage of the vented fuel concept is the low amount of canning material with a subsequent gain in breeding. It might still be possible that strong gas pressures develop during certain transients and that the can has to withstand them. This might change the evaluation of the vented fuel.

\subsection{Discussion of Economical Results}

In Table 6 and Fig. $11 \mathrm{~b}$ the results of the comparative cost calculation are given. All calculations are based on the same assumptions as for the reference reactor. ${ }^{(1)}$ 
Table 5. Characteristic Data of Different Reactors

\begin{tabular}{|c|c|c|c|c|c|c|c|c|c|}
\hline & \multicolumn{9}{|c|}{ Reactor Number } \\
\hline & 0 & 1 & $1 \mathrm{a}$ & 2 & 3 & 4 & $5 \mathrm{a}$ & $5 b$ & 6 \\
\hline & \multicolumn{9}{|c|}{ Characteristic Data } \\
\hline & $\begin{array}{l}\text { Reference } \\
\text { Reactor }\end{array}$ & $\begin{array}{l}\text { Smaller } \\
\text { Coolant } \\
\text { Fraction } \alpha\end{array}$ & $\begin{array}{c}\text { Smaller } \\
\text { Coolant } \\
\text { Fraction } \alpha \\
\text { and Larger } \Delta \vartheta\end{array}$ & Smaller $H / D$ & $5 \% \mathrm{BeO}$ added & $\begin{array}{l}10 \% \text { Mo in } \\
\text { Fuel }\end{array}$ & $\begin{array}{c}\text { UC Fuel; Same } \\
\text { Geometry as } \\
\text { Oxide }\end{array}$ & $\begin{array}{c}\text { UC Fuel; } \\
\text { Compact Core }\end{array}$ & Vented Fuel \\
\hline Core Volume $\left(\mathrm{m}^{3}\right)$ & 5.9 & 5.3 & 5.2 & 6.0 & 6.6 & 5.9 & 5.9 & 3.2 & 5.9 \\
\hline Core Height (m) & 94 & 91 & 90.5 & 78 & 90 & 94 & 94 & 77 & 94 \\
\hline Height/Diameter: H/D & $1 / 3$ & $1 / 3$ & $1 / 3$ & $1 / 4$ & $1 / 3$ & $1 / 3$ & $1 / 3$ & $1 / 3$ & $1 / 3$ \\
\hline Pressure Drop (atm.) & 4.6 & 13.7 & 4.6 & 2.8 & 5.1 & 4.6 & 4.6 & 12.3 & 3.3 \\
\hline Sodium $(\mathrm{v} / \mathrm{o})$ & 50 & 40 & 44 & 50 & 50 & 50 & 50 & 50 & 50 \\
\hline Fuel $(\mathrm{v} / \mathrm{o})$ & 32.2 & 34.4 & 35.0 & 33.0 & 27.2 & 29.3 & 32.2 & 28.8 & 36.8 \\
\hline Steel $(\mathrm{V} / \mathrm{o})$ & 17.8 & 25.6 & 21.0 & 17.0 & 17.8 & 17.8 & 17.8 & 21.2 & 13.2 \\
\hline Coolant Temp Rise $\Delta \vartheta\left({ }^{\circ} \mathrm{C}\right)$ & 150 & 150 & 200 & 150 & 150 & 150 & 150 & 150 & 150 \\
\hline \multicolumn{10}{|l|}{$\begin{array}{l}\text { Tolume Ratio of } \mathrm{UO}_{2} / \mathrm{PuO}_{2} \\
(\mathrm{UC} / \mathrm{PuC}):\end{array}$} \\
\hline 1 Core Zone & 8.2 & 8.1 & 8.4 & 7.9 & 7.3 & 7.3 & 9.7 & 8.2 & 9.3 \\
\hline 2 Core Zone & 6.0 & 6.0 & 6.1 & 5.8 & 5.3 & 5.2 & 7.3 & 5.6 & 6.7 \\
\hline Critical Mass (kg) & 2168 & 2093 & 2046 & 2320 & 2295 & 2220 & 2370 & 1416 & 2245 \\
\hline \multicolumn{10}{|l|}{ Breeding Ratios: } \\
\hline Core (int.) & 0.95 & 0.95 & 0.97 & 0.91 & 0.89 & 0.84 & 1.12 & 0.91 & 1.04 \\
\hline Blanket & 0.47 & 0.44 & 0.48 & 0.52 & 0.39 & 0.47 & 0.50 & 0.62 & 0.55 \\
\hline Total & 1.42 & 1.39 & 1.45 & 1.43 & 1.28 & 1.31 & 1.62 & 1.53 & 1.59 \\
\hline Doppler Coefficient & $-5.97 \cdot 10^{-6}$ & $-5.99 \cdot 10^{-6}$ & $-5.99 \cdot 10^{-6}$ & $-5.30 \cdot 10^{-6}$ & $-1.05 \cdot 10^{-5}$ & $-4.61 \cdot 10^{-6}$ & $-5.76 \cdot 10^{-6}$ & $-4.72 \cdot 10^{-6}$ & $-6.08 \cdot 10^{-6}$ \\
\hline Max Sodium-loss Effect & & & & & & & & & \\
\hline$\Delta k_{\text {Sod-loss }}$ & 0.0244 & 0.0224 & 0.0221 & 0.0223 & 0.0201 & 0.0271 & 0.0291 & 0.0201 & 0.0263 \\
\hline Total Additional Cost $\left(10^{6} \$\right)$ & 0 & +3.00 & -2.11 & +1.39 & +5.36 & +3.98 & -4.24 & -6.05 & -2.98 \\
\hline
\end{tabular}




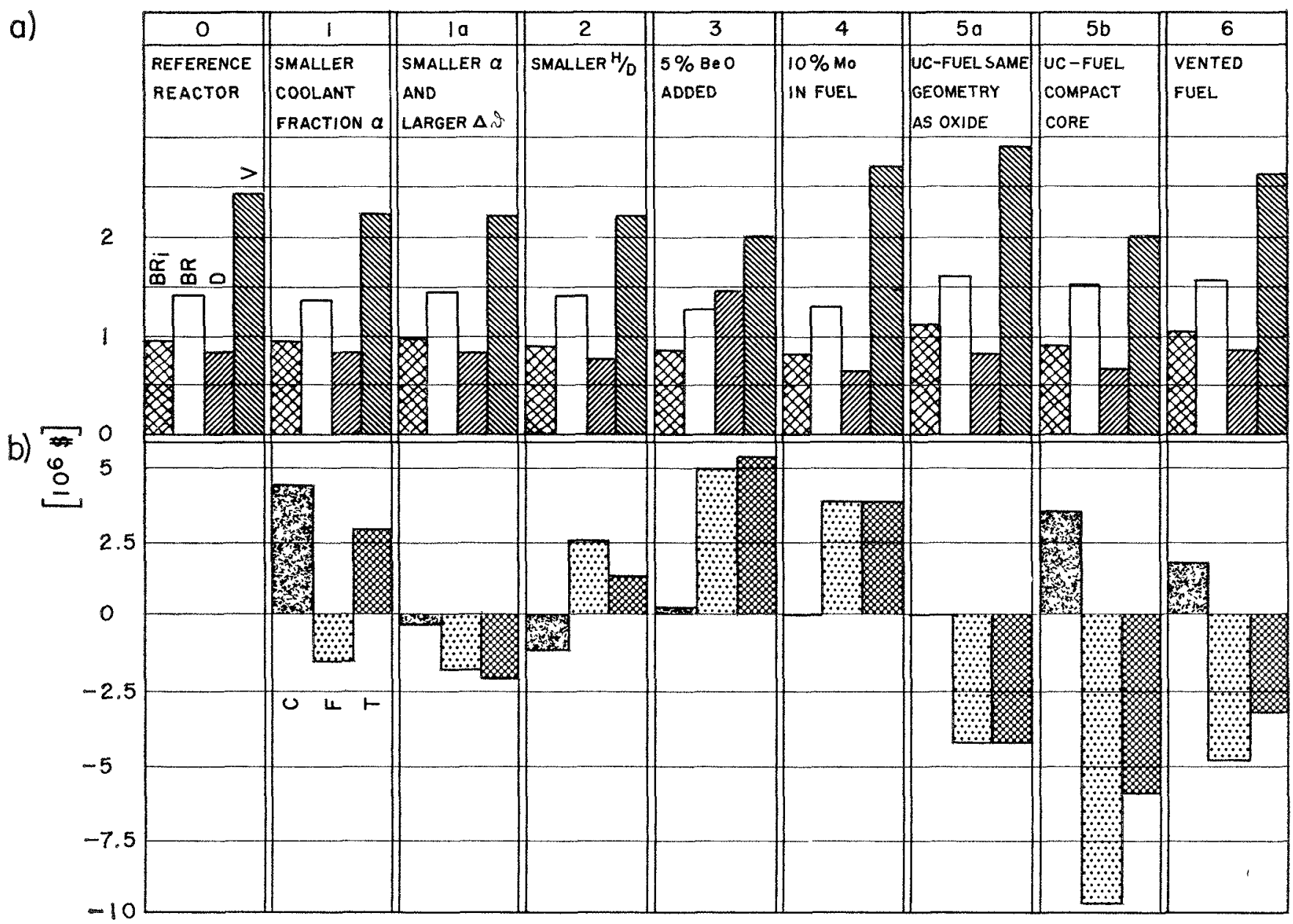

Fig. 11. a) Internal breeding ratio $B \ell_{\imath}$, total breeding ratio $B R$, Doppler constant $D$, and reactivity for complete core sodium loss $V$, for different reactors. b) Total additional costs $T$, and differences in capital costs $C$ and fuel costs $F$.

The main changes of capital costs result from the calculated pressure drop and the corresponding pump size. A relatively moderate power law with an exponent 0.6 has been assumed for the cost dependence on pump. ing power. Other main cost variations come from the fuel and the breeding ratio. These values are capitalized over 15 years for a load factor of 0.8 .

Case 1: Additional costs mainly from pumping power.

Case 1a: Cost savings mainly because of better breeding ratio.

Case 2: Savings in pump size are more than compensated by fuel costs (large number of fuel rods).

Case 3: Somewhat larger capital costs (pressure drop) and much larger fuel costs (breeding). Additional costs of nearly $5 \%$ of the total plant costs.

Case 4: Additional costs mainly by bad breeding.

Case 5a: Considerable cost savings by favourable breeding ratio.

Case 5b: Even with some additional capital costs for pumping power and less breeding ratio than for Case 5a the total net savings are largest ( $5 \%$ of total plant).
This results mainly from lower fuel costs because much less fuel is needed.

Case 6: Savings in pumping power and breeding ratio are partially compensated by gas-purification plant (see Ref. 5). Savings of the order of $2 \%$ of total costs.

\subsection{Discussion of Dynamical Results}

Considerable differences in the dynamical behaviour compared to the reference reactor are found only if other fuels are used. As can be expected, moderate changes in coolant temperature and geometry are of minor importance.

In Fig. $12 t_{m}$ is shown for Cases $0,4,5 \mathrm{a}$, and $5 \mathrm{~b}$ as a function of ramp rate. The two low-temperature cores with molybdenum and carbide have values of $t_{m}$ somewhat above those for the reference core and for the highly rated carbide.

Figure 13 shows the effect of ramp height. The lowtemperature cores naturally can withstand considerable reactivity inputs without any action of the safety system. 
TaBle 6. Capital and Fuet Costs

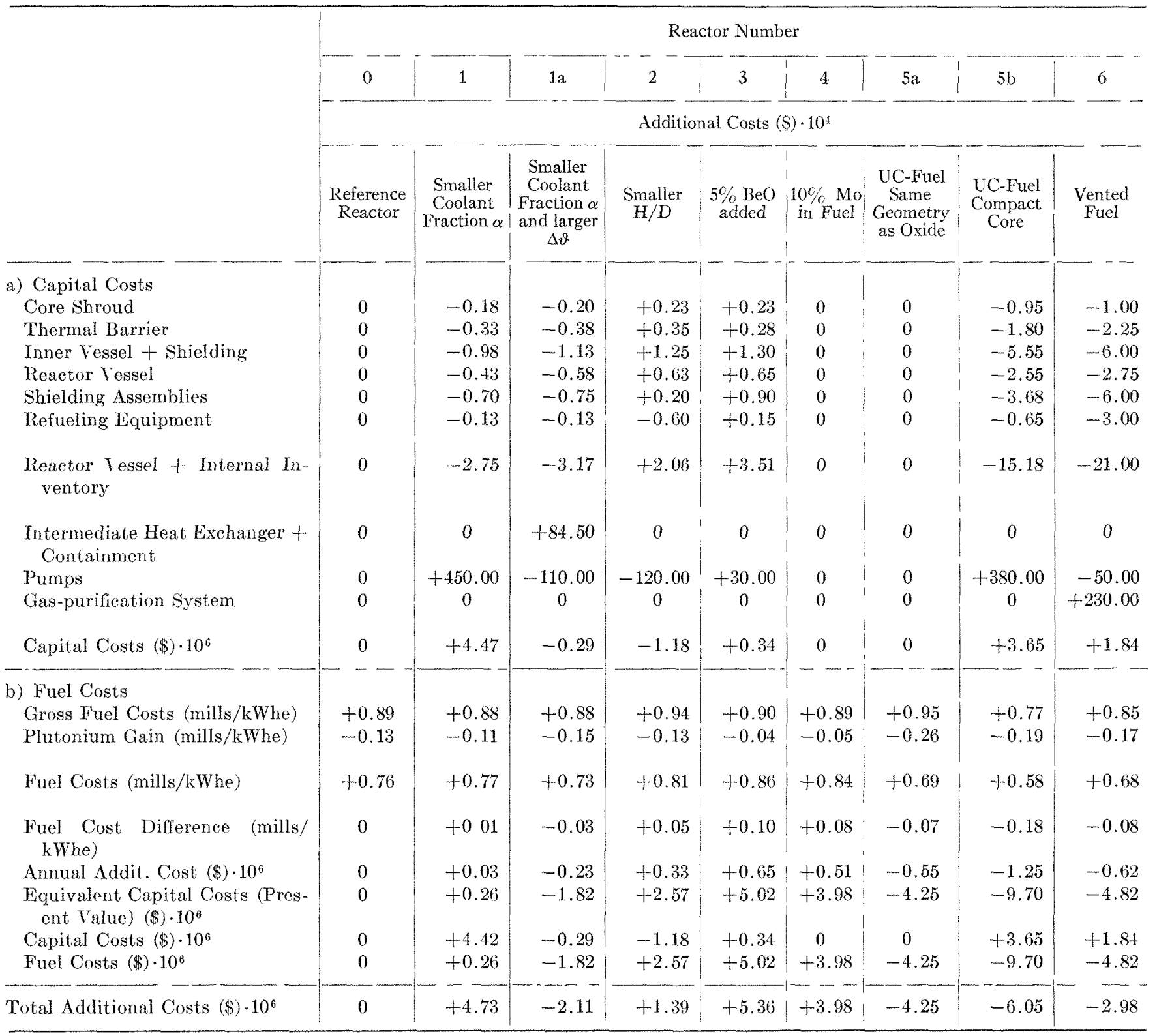

In Fig. 14 is plotted the maximum allowable ramp rate as a function of the delay time of the safety system. The low-temperature cores allow for ramps faster by about 1.5 times.

\section{The Importance of Coolant Boiling}

Partial or complete voiding of the total core may have two causes:

a) loss of coolant flow;

b) a slow excursion.

Both occurrences are improbable to a very high degree. As has been shown, a) requires the simultaneous failure of all primary pumps, of all emergency drives despite a time reserve of about $90 \mathrm{sec}$, and of the safety system (a break of the double-wall primary circuit is excluded). b) requires the simultaneous runaway of several control rods and the failure of the safety system or the runaway of a control rod and the simultaneous failure of all independent safety rods.

It should really be discussed whether a maximum accident beyond this is credible. But if we assume credibility, then one of these accidents would certainly destroy the core. Ramps of rates up to $\$ 100 /$ sec may be generated by coolant evaporation, depending on the initial conditions. The total energy of the excursion is strongly dependent on the Doppler coefficient.

It is only for this type of hypothetical accident, that the void coefficient is of real importance. The dynamic studies 
have shown, that under other conditions boiling in larger parts of the core $(\Delta k>\$ 1)$ cannot occur. (Boiling in a single subassembly will be discussed later.) However, in the light of these considerations two remarks have to be made:

First Remark. The boiling starts at the upper end of the core channels. In this region the void coefficient is negative. Therefore it has been said that an overall positive void coefficient might not be too dangerous under these conditions. This opinion is too optimistic. Because of the large specific volume of sodium vapor, a boiling channel will be blocked very easily by the large pressure drop of the two-phase mixture. For our reactor, boiling begins at $28 \%$ of normal coolant flow, whereas the channel is already blocked at $22 \%$.

If boiling starts at all, chances are very large that a complet flow blockage with subsequent complete voiding occurs.

Second Remark. Some designs aim at a $\Delta k \leqslant 0$ for total voiding, but a positive maximum $\Delta k$. If voiding occurs according to the above-mentioned mechanism of channel blockage it will gradually spread from the core center and at some time the maximum $\Delta k$ will be effective. Therefore, $\Delta l \leqslant \leqslant 0$ for total voiding should not be mistaken for a criterion of a qualitative nature. It might

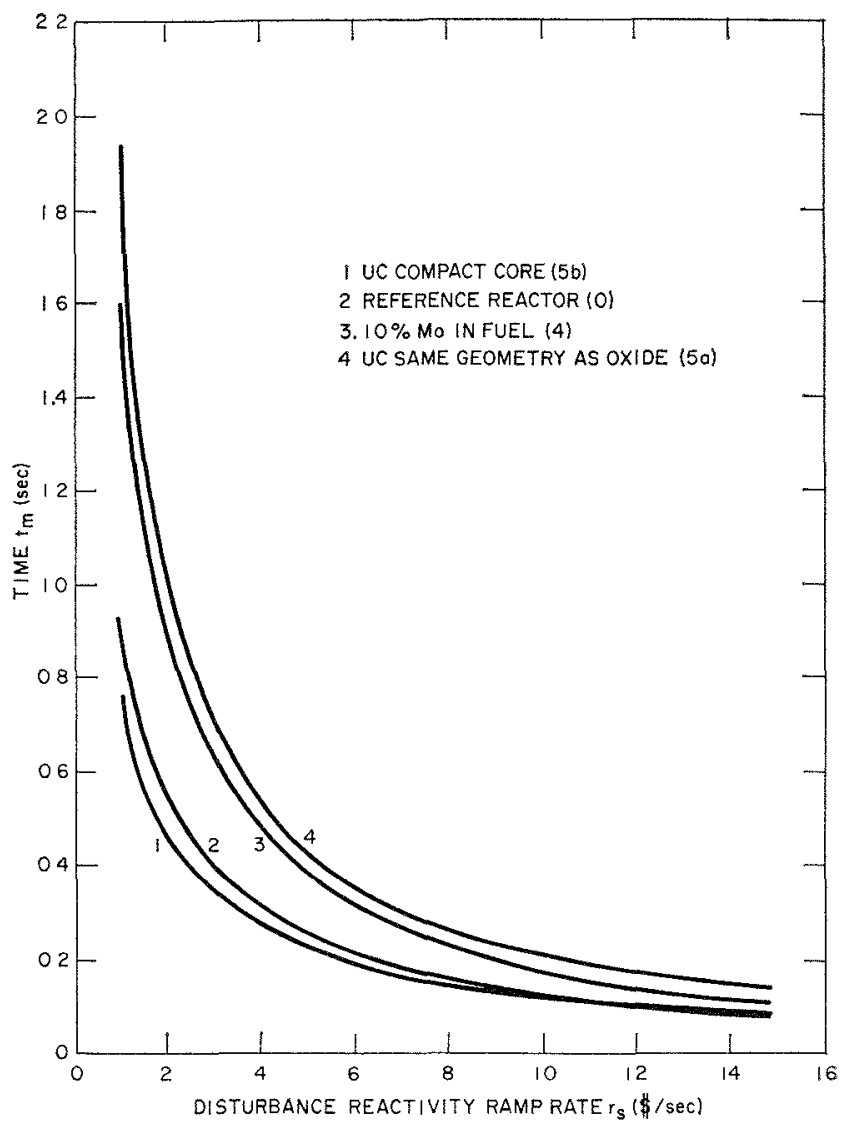

Fig. 12. Time $t_{m}$ to reach fuel melting versus disturbance reactivity ramp rate for different fuel materials.

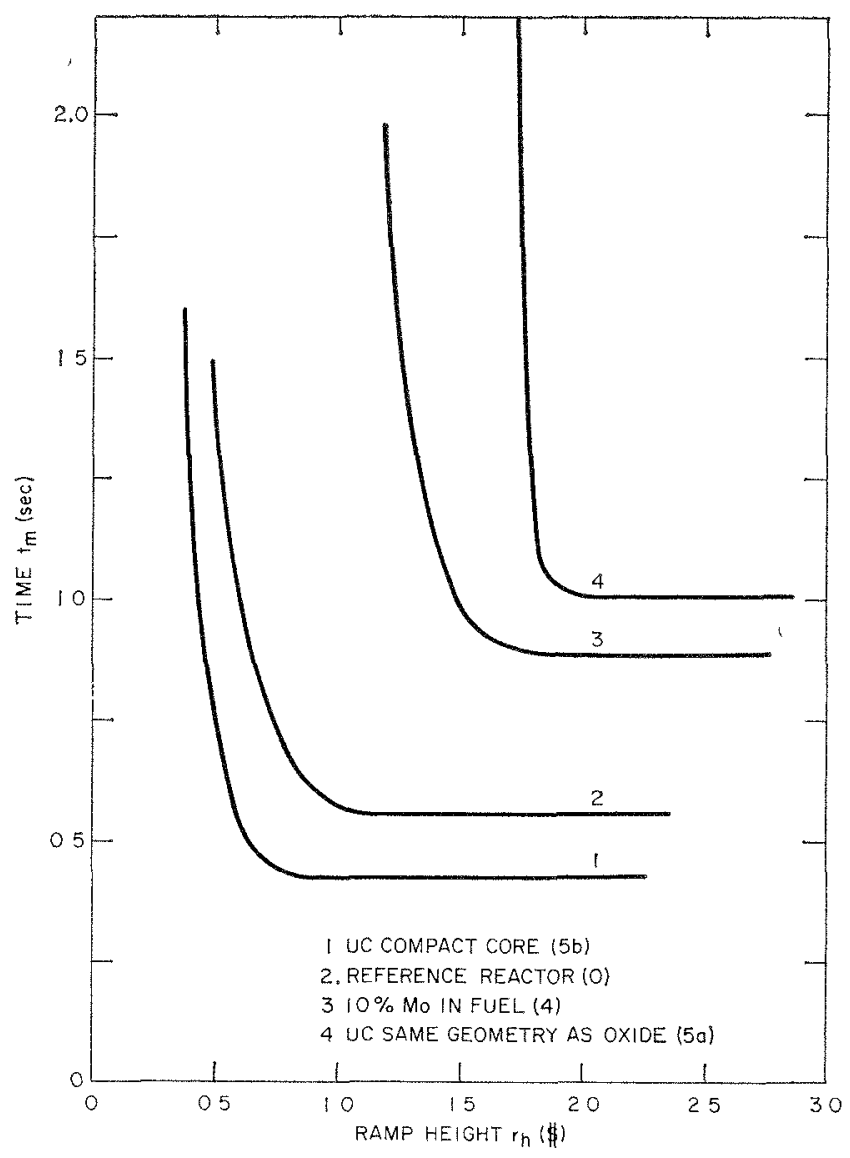

Flg. 13. Time $t_{m}$ to rearh fuel melting versus ramp height for a ramp rate of $2 \$ / \mathrm{sec}$ for different fuels.

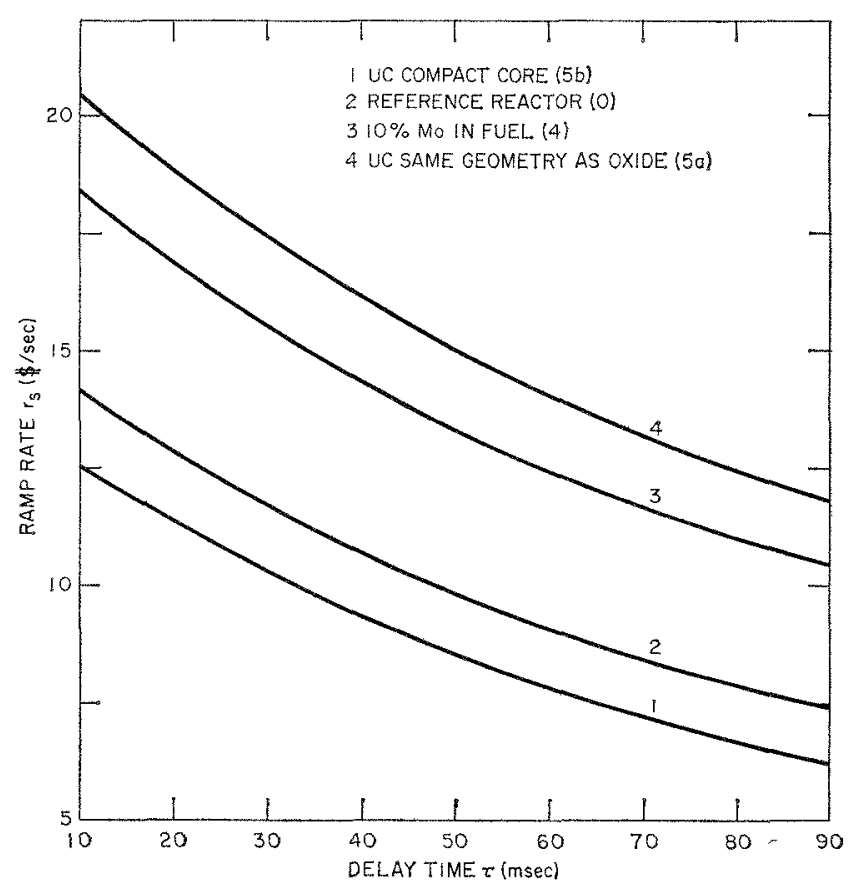

Fig. 14. Maximum allowable disturbance ramp rate $r_{s}$ versus delay time for different fuels. 
be helpful in a quantitative way only insofar as the maximum $\Delta k$ will also be smaller compared to the case in which $\Delta k$ for total voiding is positive.

Although we consider these types of boiling events to be very improbable, the blockage of a single fuel subassembly, for example by something in the sodium flow, is much more probable. The reactivity effect of this can be governed easily by the temperature coefficient (for the central subassembly it amounts to $+\$ 0.08$ ). But it might be disastrous if the sodium superheats to a larger degree.

Experimental results on sodium superheat have been reported. (7-9) The bulk superheat certainly depends on the heat flux and on the surface conditions. Superheating of the order of $200^{\circ} \mathrm{C}$ and even more might be possible. Taking as an example a maximum superheat of $200^{\circ} \mathrm{C}$ and taking into account the temperature distribution in the coolant under reduced flow conditions (21\% of normal flow), a superheating energy of about $70 \mathrm{kcal}$ will be stored in the upper quarter of the subassembly.

If, finally, the superheated liquid is flashed, everything depends on the mechanism as to how the energy is relased in space and time. We have calculated the effect of the worst case, i.c., an instantaneous release of the whole encrgy and its impact on the subassembly wall, by using a one-dimensional model of Symonds and Mentel. ${ }^{(10)}$ Then apparently the subassembly box will be badly deformed and the neighbouring subassemblies will be affected. If the combination of flow reduction and superheat now occurs in the neighbour assemblies, the damage may spread over the core like a chain reaction.

Accordingly, we concentrate an important part of our effort on three subjects:

a) Theoretical and experimental studies of the dynamics of two-phase flow and of the release of superheat in space and time. A first step in this direction is reported by Fischer and Häfele.(11)

b) Development of methods to keep the superheat in a fast power reactor small.

c) Design of the subassembly boxes so that the superheating energy can be dispersed at a maximum rate.

\section{Some Remarks on the Maximum Hypothetical Accident}

The MHA is a fast excursion with the effect of a positive void coefficient. There are two possibilities for the MHA :

a) The core is disassembled by the fastest possible reactivity input rate (first excursion).

b) After the first excursion, the molten core material gathers somewhere in the reactor containment (second excursion).

The maximum reactivity input rate for the first excursion is caused by voiding of the core center. This again can be caused by a slow primary excursion or by a loss of coolant flow. The other possibility for a fast reactivity increase is core meltdown or, at least, a certain stage of fuel slumping.

The consequences of the second excursion may be influenced by the shape of the containment.

Both the first and second excursions are strongly influenced by the Doppler coefficient. In our panel paper (see Session IV) we shall discuss this point in more detail. The released energy has to be contained in the concrete structure surrounding the reactor vessel.

\section{Conclusions}

7.1. According to criteria 1 and 2, our proposed 1000 -MWe reactor is very safe.

7.2. Infinite ramps of the order of $\$ 10 / \mathrm{sec}$ can be counteracted by a spring-driven safety system, much more than will actually occur.

7.3. Coolant boiling in larger regions of the core cannot orcur as long as the safety system is operating.

7.4. The positive void effect is of importance for the maximum hypothetical arcident only. It occurs only if several independent improbable conditions are fulfilled simultaneously.

7.5. Coolant boiling in a single subassembly may be dangerous in combination with sodium superheat.

7.6. Parametric studies show advantages in cost and safety in the direction of lower coolant fractions, larger $\Delta \vartheta$, and a not-too-compact carbide core. The addition of molybdenum to the fuel oxide proves to be very unfavourable.

7.7. Further studies on the MHA and on sodium superheat and sodium two-phase flow are required.

\section{LITERATURF}

1. I). Smidt et al., Referenzstudie für den $1000 \mathrm{MWe}$ natriumgekühlten schnellen Brutreaktor ( $\mathrm{Na}$ 1), KFK 299 (1964).

2. C. P. Zaleski et al., Studies of Large Reactors in France and West Germany, National Topical Meeting, Detroit, April 1965, ANS-100.

3. D. Okrent, K. P. Cohen, and W. B. Loewenstein: Some Nuclear and Safety Considerations in the Design of Large Fast Power Reactors, P/267 of the Third International Conference on the Peaceful Ises of Atomic Energy, (ieneva, Switzerland, 1964

4. P. Dosch, Design and Experimental Evaluation of an Electrumagnetic Acceleration System for Fast Reactor Rods. Physics and Material Problems of Reactor Control Rods, IAEA, Vienna (1964).

5. D. Smidt and W. Sommer, Application of the Vented Fuel Concept to a Sodium Cooled Power Breeder with 1000 ITWe, See Paper in Session II of this Conference.

6. 1). Smidt, Der Siedevorgang und seine Auswirkungen in 
grossen natrium-gekühlten schnellen Reaktoren, Internal Report IRE 18/65, Nuclear Research Center Karlsruhe (1965).

7. A. I. Krakoviak, Superheat Requirements with Boiling Liquid Metals, ORNL-3605 (1963), p. $310 \mathrm{ff}$.

8. H. W. Hoffman and A. T. Krakoviak, Convective Boiling with Liquid Potassum, Proc. of the 1964 Heat Transfer and Fluid Mechanies Institute, Stanford University Press, p. 19 ff.

9. H. Lurie and R. C. Noyes, Boiling Studies for Sodium Reactor Safety. Part II, NAA-SR-9477.

10. P. S. Symonds and T. J. Mentel, Impulsive Loading of Plastic Beams with Axial Constraints, J. Mech. and Phys. of Solids, 6, 186 (1958).

11. M. Fischer and W. Häfele, Shock Front Conditions in Twophase Flow Including the Case of Desuperheat, See Paper in Session VIII-B of this Conference.

12. W. Munzner, MGP. Ein eindimensionales MuligruppenProgramm, GFK Internal Report INR-103/64, Nuelear Research Center, Karlsruhe.

13. M. L. Tobias and T. B. Fowler, The Twenty Grand Program for the Numerical Solution of Few-group Neutron Diffusion Equations in Two Dimensions, ORNI_-3200.

14. J. M. Chaumont and J. A. Koemer, 2 D-Pert, A Twodimensional Perturbation Code, ANL-6555.

15. H. Küsters and M. Metzenroth, The Influence of Some Important Group Constants on Integral Fast Reactor Quantities, See Paper in Session V-B of this Conference.

\section{Discussion}

Mr. Gibson (AI): I noted that your vented fuel elements showed the greatest economic advantage. We performed some studies which showed that the advantage of a vented fuel element depended upon a lack of swelling in the fuel itself to the extent that the fuel swelling did not impose upon the cladding an excessive strain. If one presupposes this condition then, indeed, the vented concept offers the advantage of a reduction in the cladding stress of the order of a third. If, however, the fuel is allowed to expand or one finds that it does until it imposes a cladding strain upon it, then the possible advantage of reduction in cladding thickness is pretty marginal. Did you consider these effects?

Mr. Smidt: First, the vented fuel is not the most economic on this display. Savings as compared to the reference reactor amount to only about $2 \%$ of the total costs, whereas, for instance, the compact carbide core saves about $5 \%$. In comparison with a more optimized reactor, the savings are even less. As you remark, any swelling would make the use of a vented fuel less effective.
Mr. Leitz (GE) : Did your reference fuel show a fission gas plenum at the bottom end of the fuel pin?

Mr. Smidt: Yes; the reference design has the fission gas volume plenum at the bottom to have less stress in the fuel rods.

Mr. Leitz: Did you consider the hazard associated with possible blanketing of adjoining fuel pins in the event of failure of the clad and release of gas from that region? If the fucl pin should fail down in this plenum region and release the fission gas, which is large in inventory and large in pressure, does this not threaten to blanket the adjoining fuel pins and wipe out the subassembly?

Mr. Smidt: This is a danger we are now discussing. The overall reartivity effect when the total subassembly is full of gas, which would be about the worst type of accident, is about $7 / 100$ of a dollar.

Mr. Hall (LASL): You spoke of the danger of fuelelement failure propagating from one subassembly to another and said you were going to take care of this by design consideration. Are you planning any work to substantiate this worry?

Mr. Smidt: Yes. We are working together with ELRATOMI-ISPRA. We are doing some work ourselves on the effect of two-phase flow and the effect of shock waves. The last paper of this conference, by Fischer and Häfele, will give a review of the theoretical approach.

Mr. Häfele (Karlsruhe): If I may additionally comment on that. Investigation of the full mechanism of two-phase flow is parallel to the investigation of the nuclear aspects insofar as safety analysis is concerned. We are paying particular attention to the superheat and plugging accident, as well as to the mechanism of desuperheat with its energy release. More experimental work on desuperheat is needed.

Mr. Bump (ANL): In your vented fuel, does the sodium come in contact with the fuel itself?

Mr. Smidt: Our most optimistic vented fuel is one with a porous plug, the simplest kind of closure. This produces a gain of about $2 \%$ in cost. So we didn't make any detailed experimental design studies.

Mr. Bump: With the porous plug could you keep the sodium away from the fuel then? Is that your assumption?

Mr. Smidt: Yes. 


\title{
An Analysis of Fast Reactor Transient Response and Safety in Selected Accidents
}

\author{
D. B. SHERER \\ General Electric Company \\ Advanced Products Operation \\ San Jose, California
}

\section{INTRODUCTION}

In the development of large fast reactors, attainment of the optimum balance between safety and economics considerations is one of the most important problems to be resolved. Conceptual designs which have been developed ${ }^{(1-4)}$ do reflect little consensus with regard to necessary values for parameters important to safety, such as the Doppler and sodium reactivity coefficients.

A study was therefore made of large fast reactor behavior under accident conditions to provide information which would be helpful in assessing the importance of various parameters to safety, and perhaps also in determining desirable value for these parameters. The FORE computer program ${ }^{(5)}$ was used to calculate the course of accidents in typical large fast reactor cores under a variety of assumptions. Accidents analyzed were nonterminating ramp reactivity insertions at $\$ 0.20 / \mathrm{sec}$ and $\$ 2 / \mathrm{sec}$, and loss of pumping power with resultant flow coastdown.

The inherent safety of the reactor is independent of the system of reactor control, although it has considerable effect on the control-system design. Therefore, although most serious accidents will require a scram to terminate them before core damage occurs, no scram was assumed in this analysis. Instead, the length of time available before a given type of damage or failure occurred was considered to be a measure of the inherent safety of the reactor. A decrease in this time interval requires greater reliance and places greater requirements on the speed and reliability of the reactor-control system. For accidents caused by continuous, constant-rate reactivity insertions, such as were considered in these calculations, the length of time elapsed before core damage is also an indication of the capability of a reactor system to absorb reactivity insertions.

Consideration was given to three principal types of inherent reactivity coefficients which could be present: a prompt coefficient (normally negative) due to changes in fuel temperature, a delayed negative coefficient due to expansion of the reactor structure, and a delayed coefficient due to coolant density change, which may be either negative or positive, depending on core design. The fuel temperature coefficient was assumed to be due essentially to the Doppler effect. In the case of metal fuel, a fuel expansion effect was also assumed, although it may not be dependable if significant burnups are to be achieved. The significance of the coolant reactivity effect is, of course, greatly increased if actual coolant voiding occurs; the reactivity feedback is then a function of both the size and location of the voids, ${ }^{(1,6)}$ and the value of the coefficient for small density changes is not applicable.

The values chosen for the Doppler coefficient range from a small value, $T(d k / d t)=-0.0015$, which might be expected to occur without effort on the part of the reactor designer, to a large value, $T(d k / d t)=-0.01$, which probably requires deliberate spectrum softening to achicre.

The radial structure expansion coefficient represents the combined effects of increasing core size and decreasing atom densities (except for coolant) which result from thermal expansion of the core structure. The value given, $-3.7 \times 10^{-6} /{ }^{\circ} \mathrm{F}$, is the same as that given for two quite different $1000-\mathrm{MWe}$ designs, a pancake and an annular core. ${ }^{(1,2)}$ In the axial direction, the height of the core is defined by the height of the fuel column. Feedback from this source was assumed only for those cases with metal fuel. The sodium coefficient and the axial expansion coefficient are the same as those in Ref. 1, namely, $+0.15 \times 10^{-6} /{ }^{\circ} \mathrm{F}$ and $-1.3 \times$ $10^{-6} /{ }^{\circ} \mathrm{F}$, respectively.

\section{ASSUMED CORE DESIGN}

Core designs investigated were intended to be representative of typical cylindrical, 1000-MWe, oxide-, mental-, and carbide-fueled cores, the metal atoms being nixed uranium and plutonium. On the assumption that carbide has somewhat greater flexibility with respect to its specific power, because of its high conductivity and a sodium-filled fuel-clad gap, both a low $(150 \mathrm{~kW} / \mathrm{kg}$ of carbide) and a high $(300 \mathrm{~kW} / \mathrm{kg})$ 
TABLE 1. Core Designs

Core Power: 2200 MWt; Axial Blankets: 300 MWt

Coolant Inlet: $800^{\circ} \mathrm{F}$

Coolant $\Delta \mathrm{T}: 250^{\circ} \mathrm{F}$

Coolant Velocity: $20 \mathrm{ft} / \mathrm{sec}$

Radial Peaking Factor: 1.7

Axial Reflector Savings: $20 \mathrm{~cm}$

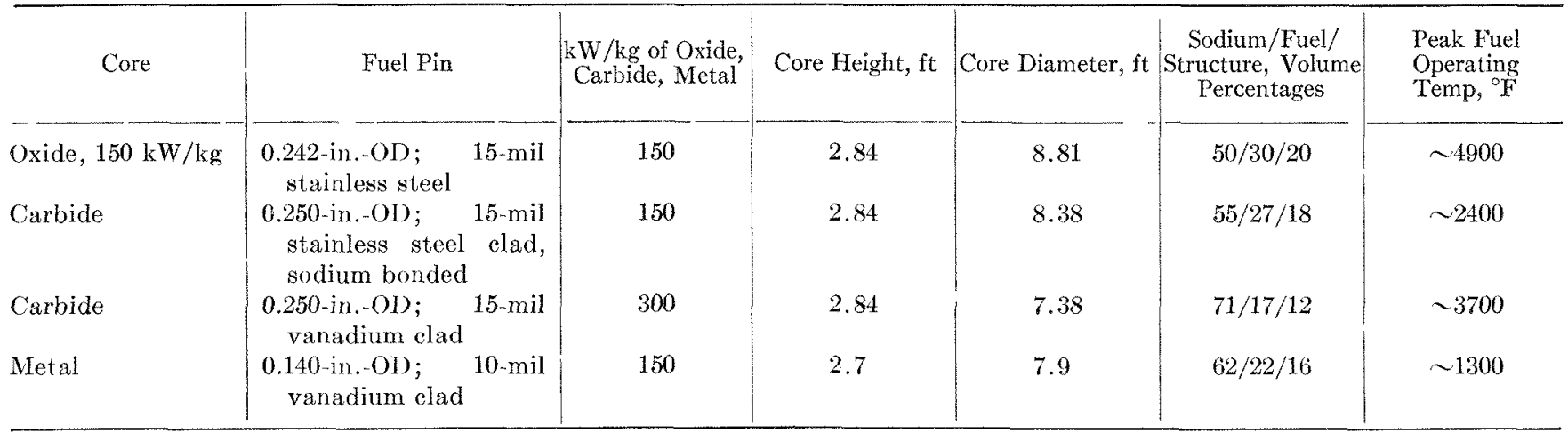

specific power were used for the carbide. For the oxide and metal cases specific power was not a variable.

Table 1 summarizes the core designs.

The fact that these cores are somewhat idealized should be kept in mind when interpreting the results. For example, it should be noted that the radial peaking factor of 1.7 assumed is equivalent to assuming little or no purposeful power flattening in the core, and that local peaking factors were not taken into account. An actual core design might tend to have a lower value of the radial peaking factor due to power flattening, but local peaking factors would offset this. The location of the hottest channel or fuel pin would then not necessarily be in the core center, as it was in this analysis.

Core compositions were picked in terms of volume percentages of sodium, fuel, and structure. The idealization was made that all sodium in the core was effective as a heat transport agent, and space for control was neglected. Thus, specification of core composition combined with a choice of specific power (in $\mathrm{kW} / \mathrm{kg}$ of fuel meat) was sufficient to specify core volume and height. In all cases the volume ratio of fuel to total structure (including clad) was held close to $3 / 2$, a value which should allow enough structure for a good mechanical design. Fuel-pin designs were chosen to be typical of the fuel material (oxide, carbide, or metal) under consideration and consistent with the specific power. Clad was 15 mils thick, and fuel pins were approximately $0.25 \mathrm{in}$. in diameter, except for the metal fuel, which had a 0.14-in. diameter with 10 -mil clad. All fuel except the oxide was sodium bonded. The oxide and lower-specific-power carbide had stainless stecl clad, other cores vanadium clad.
Any of the cores used in the analysis could have been designed with a smaller height-to-diameter ratio if desired, by assuming a lower coolant temperature rise and/or a lower coolant velocity. On the other hand, increasing the height-to-diameter ratio would require higher velocity or larger temperature rises than those chosen, and revisions in this direction are therefore limited.

\section{FAILURE MECHANISMS}

The analysis of the results consists in large part of identifying the failure mechanisms in each transient, the time elapsed before failure, and its location in the core. Failure in this case is intended to mean essentially voiding of coolant, or occurrences which lead very shortly to voiding of coolant, since such voiding can theoretically result in large reactivity insertions, and will in any case cause clad melting and possible core compaction.

Three mechanisms were considered for failure:

1. Fuel clad fails due to pressure from within, possibly causing complete or partial flow blockage and subsequent coolant boiling.

2. Coolant boils due to overheating.

3. Burnout or rapor blanketing of the clad occurs, resulting shortly in clad failure and possible blockage of the coolant channel.

To identify when these might occur is difficult except for the mechanism of coolant boiling because the failure of the clad due to internal pressures is a function of fuel design (smeared density, vented to coolant or not, etc.), and the condition of the clad (neutron irradiation), as well as fuel and clad temperatures. Also, 
the burnout condition for sodium under conditions of interest is not well-known. Consequently, a simplified approach was employed. Clad failure due to internal pressure was considered possible over a range of conditions, and the lower and upper bounds of these conditions were identified respectively with

1. a fuel center temperature above melting, and

2. a fuel center temperature equal to the fuel boiling point at atmospheric pressure.

The first postulated indication, a fuel temperature above the melting point, could cause failure due to fuel volume changes that could not be accommodated within the clad. For example, radial expansion of oxide fuel in going from room temperature to the melting point would be approximately $4 \%,(7,8)$ whereas stainless steel clad operating at an average volumetric temperature of $1200^{\circ} \mathrm{F}$ would expand only about $1.2 \%$. In addition, there is reported to be a volume change in oxide fuel due to melting of $9.6 \%$. $^{(9)}$ Inspection of the radial temperature profiles from computer problems run for this study indicate that $25 \%$ of the fuel is molten or partially molten when the peak fuel temperature begins to rise above the melting point, giving another $1-2 \%$ rolume increase in relation to the overall fuel volume. If the clad should have lost its ductility due to irradiation, these volume changes in the fuel could conceivably cause failure in a fuel pin containing high-density fuel. Similar behavior could presumably occur in the carbide and metal fuel, although the actual percentage rolume inereases would be different. If, on the other hand, ample space exists within the clad for fuel volume changes and clad temperatures are not excessive, an upper bound may be placed on the fuel element transient capability by the vapor pressure of the fuel itself.

Although a high temperature is required for the vapor pressure to become significant, relatively small further increases in temperature will produce very high vapor pressures which would burst the clad. For $\mathrm{UO}_{2}$, an extrapolation of Ackermann's data(10) indicates a vapor pressure of $10 \mathrm{~atm}$ at $6500^{\circ} \mathrm{F}$ and 100 atm at $7250^{\circ} \mathrm{F}$. Since this is subject to great uncertainty at such temperatures, a fuel center temperature of $6500^{\circ} \mathrm{F}$ was assumed to be a failure point due to fuel vapor pressure in the mixed oxide fuel. Experimental confirmation exists that oxide fuel pins will withstand temperatures of this magnitude. ${ }^{(11)}$

The boiling point of uranium carbide at atmospheric pressure has been reported to be $4100-4370^{\circ} \mathrm{C} .{ }^{(12)}$ The lower temperature, $7400^{\circ} \mathrm{F}$, was assumed to represent failure due to excessive vapor pressure for the carbide fuels. Tests have been made in which sodium-bonded carbide pins withstood transient fuel temperatures of about $4200^{\circ} \mathrm{F}{ }^{(13)}$
For the metal fuel a melting point of $2070^{\circ} \mathrm{F}$ was taken. (14) An extrapolation of available correlations for plutonium would indicate a boiling point of $5850^{\circ} \mathrm{F}$. ${ }^{(14)}$ The steady-state temperatures with metal fuel are less than $1500^{\circ} \mathrm{F}$, and the analyses were not carried far enough to observe a temperature of $5850^{\circ} \mathrm{F}$ in the metal fuel. In the accidents analyzed, failure due to high vapor pressure in the metal-fueled cascs would have occurred after all other modes of failure and was therefore considered to be of minor significance.

Bulk boiling of the coolant was assumed to take place if a temperature of $1620^{\circ} \mathrm{F}$, the atmospheric pressure boiling point, was reached. Normally it would be expected that the pumps would pressurize the core slightly and the boiling temperature might be $100^{\circ} \mathrm{F}$ or more higher than was assumed.

The third failure mechanism is burnout. Experimental data on critical heat fluxes at the pressures corresponding to full flow ( $3 \mathrm{~atm}$ or more) are limited. A simple model was used whercin the critical heat flux was the sum of the pool-boiling heat flux and the forced-convection heat flux due to subcooling, and the pool-boiling critical heat flux was assumed to be $1,000,000 \mathrm{Btu} / \mathrm{hr} / \mathrm{ft}^{2}{ }^{(15)}$ This assumption may be quite conservative, depending on the pressure dependence of the critical heat flux. However, increasing the assumed heat flux required for burnout would have little effect on the conclusions to be drawn from the analysis. Since the FORE code has no provision for two-phase flow or boiling heat transfer, the burnout condition was identified by a clad surface temperature of $1720^{\circ} \mathrm{F}$, with $1620^{\circ} \mathrm{F}$ being the saturation temperature at atmosphere pressure and $100^{\circ} \mathrm{F}$ the film drop equivalent to a heat flux of $10^{6} \mathrm{Btu} / \mathrm{hr} / \mathrm{ft}^{2}$.

\section{LOSS OF FLOW ACCIDENTS}

Figure 1 shows the coolant temperature as a function of time for a loss of flow in oxide fuel cores with different Doppler coefficients. The temperature levels off and even starts to fall after the flow reaches its assumed minimum of $13 \%$ of full flow at 10 sec. All of these cases had the normal radial structure expansion, and it can be seen that the Doppler coefficient has very little effect on the accident. What effect it does have is a slight disadvantage because fuel temperatures drop in these accidents, giving a positive Doppler feedback. The large rise in coolant temperature causes a large radial expansion of the core, and the resultant negative feedback is the dominant factor in the accident, causing a drop in power and in fuel temperature.

Similar calculations for the carbide and metal cores give very similar results, with maximum coolant temperature somewhat lower, particularly for the cases of 
metal and low-specific-power carbide. However, the coolant boiling point is exceeded in all cases.

In Figure 2 the effect of radial expansion of the core in the loss-of-flow accident is demonstrated. These cases are all for a Doppler $T(d k / d t)$ of -0.004 . The maximum coolant temperature reached is severely affected if no effective radial expansion fecdback exists. If the radial expansion coefficient were large enough, coolant boiling might be prevented.

Figure 3 shows the average fuel temperatures for the same three cases. In the situation with no radial expansion feedback, core power is not reduced significantly, and fuel temperatures rise.

In all loss-of-flow accidents, coolant boiling was the first failure mechanism. Heat fluxes generally were not high enough to cause burnout, except for carbide fuel operating at $300 \mathrm{~kW} / \mathrm{kg}$.

Two other points should be noted. First, the coolant

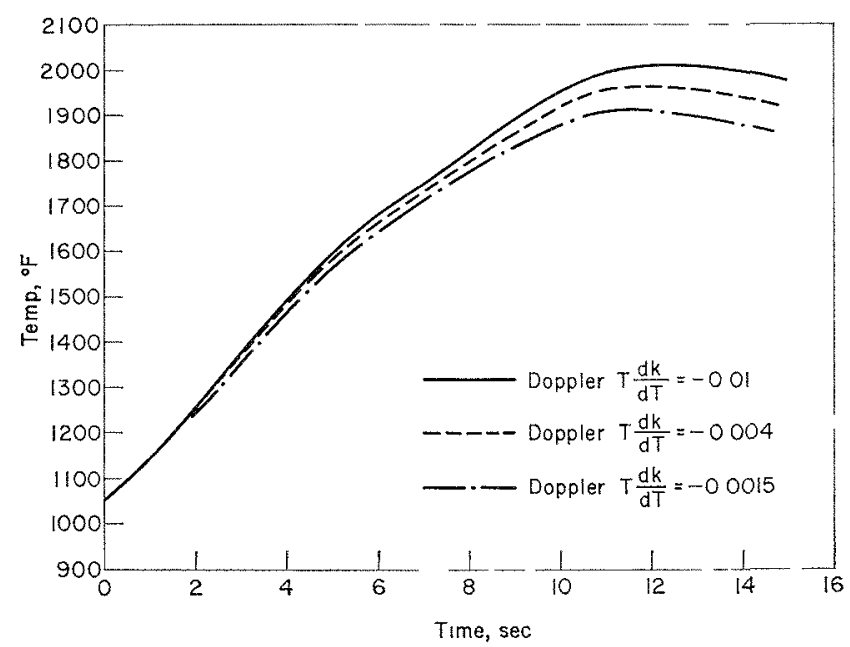

Fig. 1. Coolant Temperature for Loss of Flow in the Case of Oxide Fuel.

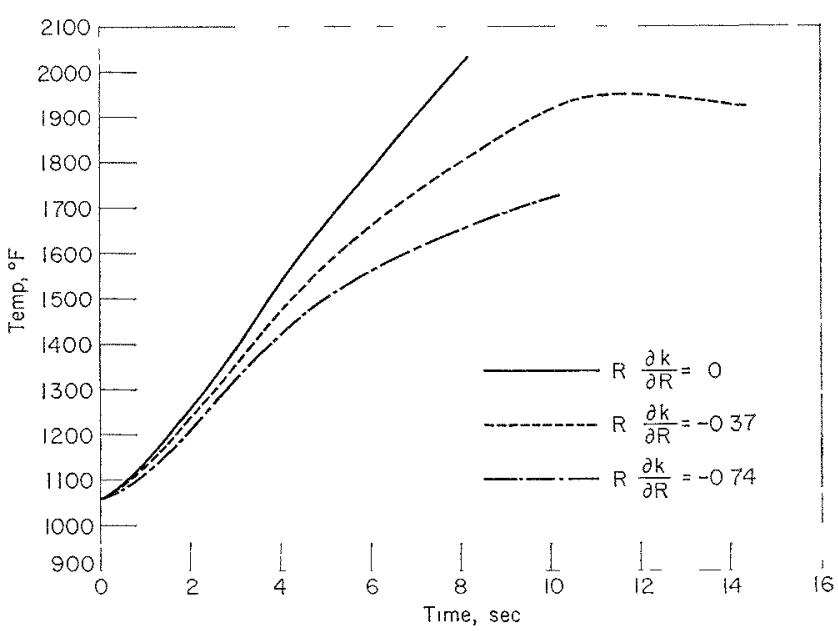

Fit. 2. Effect of Structural Expansion Feedback on Coolant Temperature in Case of Loss of Flow.

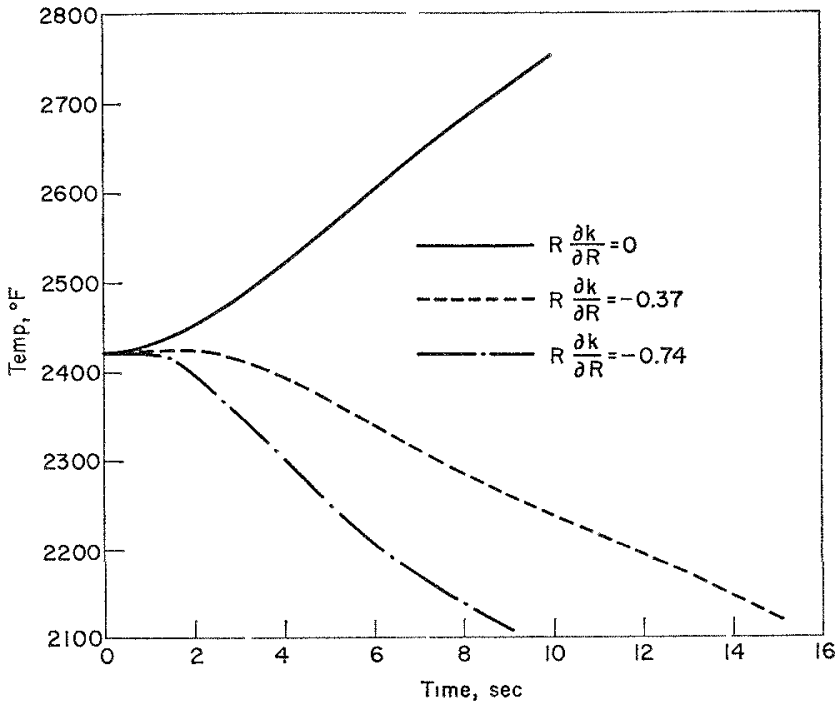

FIG. 3. leffect of Structural Expansion Feedback on Avorage Fuel Temperature in Case of Loss of Flow.

voiding patterns that would result from coolant voiding are relatively favorable from the viewpoint of reactivity effect, since the boiling always begins near the outlet of the core. More basic is the fact that the times required to cause any failure for these accidents are long, of the order of 5 sec or more in every case. Many seram signals due to temperature and flow should be received, and it is difficult to imagine that a control system properly designed to operate in fractions of a second will fail to scram the reactor. This conclusion is, of course, dependent on the length of time required for flow coastdown, but the designer can provide assurance of a sufficient time by the use of several primary loops and by adding inertia to the system if necessary.

\section{REACTIVITY INSERTION ACCIDENTS}

I will not discuss the $\$ 0.20$ /sec insertion, which was calculated for the same cores, because the results appear very similar to the $\$ 2 /$ sec case, but with the time scale expanded by a factor of ten. As for the loss of coolant, the $\$ 0.20$ / sec accidents allow several seconds for detection and corrective action.

Figure 4 shows clad and coolant temperatures in both the peak and average fuel channels for a reactivity insertion of $\$ 2 /$ sec in oxide cores. The effect of the large Doppler coefficient in increasing the time before boiling or burnout occurs is important in these cases.

Figure 5 shows the peak fuel temperatures for the same cases. The time required to reach even the upper limit assumed to indicate failure is shorter than the times required to produce boiling or burnout. Cases 


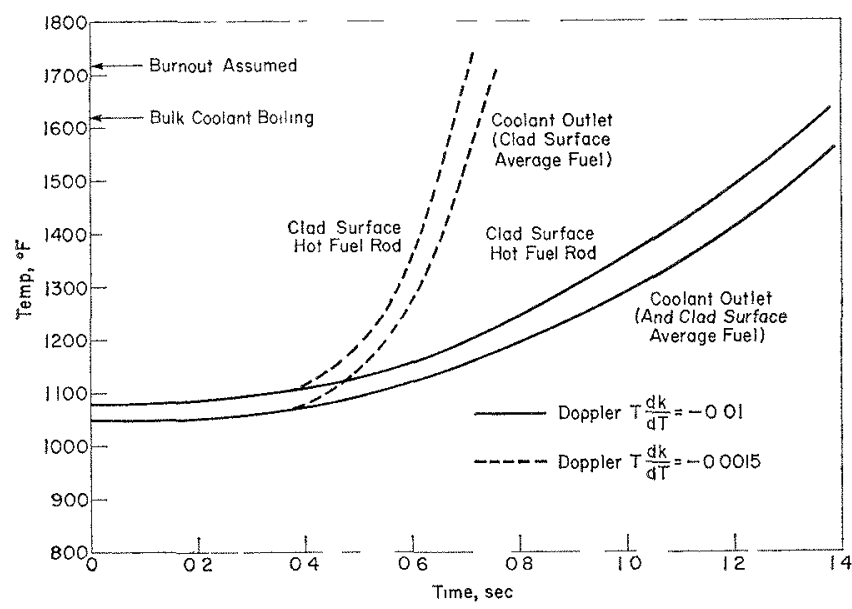

Fig. 4. Clad and Coolant Temperatures of Oxide Fuel for Reactivity Insertions at $\$ 2 / \mathrm{sec}$.

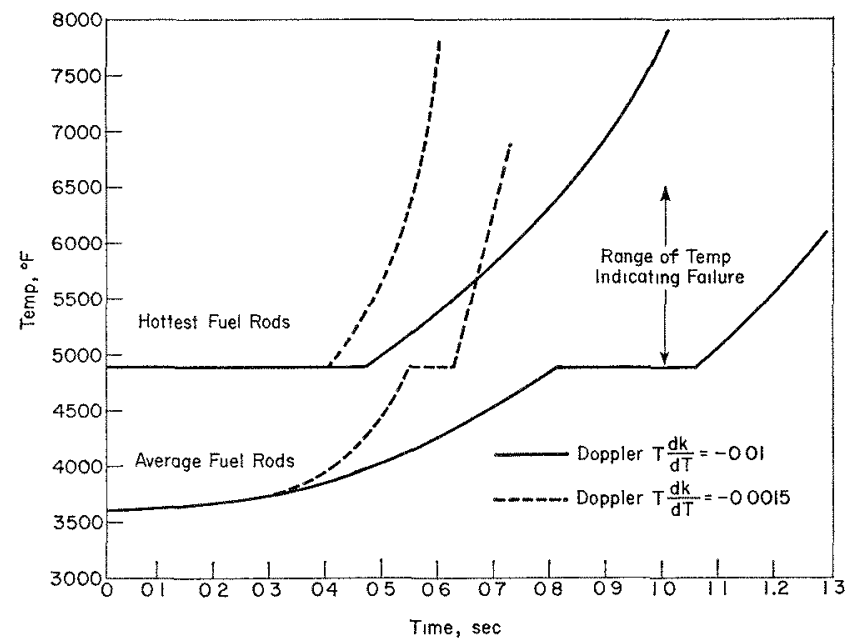

Fik. 5. Peak Fuel Temperatures in Oxide Fuel for Reactivity Insertions at $\$ 2 / \mathrm{sec}$.

were run to examine the effect of reducing the radial core expansion to zero for both rates of reactivity insertion, and it was found to be small.

Inspection of the magnitudes of the various feedback reactivities experienced in these accidents showed that in all the reactivity-insertion accidents, the positive sodium coefficient assumed for the analysis had negligible effect.

Table 2 summarizes most of the results for reactivity insertions at $\$ 2 /$ sec. The beneficial effect of the Doppler coefficient is apparent in most cases. The gain in available time due to the Doppler effect appears to be small in some instances, as between the first two oxide cases in the hot fuel pins. However, the advantage is greater than it might appear, for the following reasons:

1. As the time available for corrective action de- creases, small differences in this time become more important.

2. The relative difference between the first two cases is increased if one subtracts the constant delay time required to produce a scram signal. For example, if the scram signal was due to overpower at $120 \%$ of full power, about 0.1 -sec delay would result in either of the two oxide cases.

3 . Since the hot fuel pin actually represents an unusual combination of hot spots and peaking factors, there may be few or none of them in the core. The average fuel behavior, which shows a greater effect of Doppler, may therefore be as significant as the hottest fuel behavior in evaluating the incentives for a large Doppler coefficient.

It can be scen that failure times for the highspecific-power carbide cases are very similar to those for oxide. In both the oxide and the $300-\mathrm{kW} / \mathrm{kg}$ carbide the first failure indication is to be found in the fuel temperature. The $150-\mathrm{kW} / \mathrm{kg}$ carbide has considerably increased times before failure, due to its low operating temperature. Furthermore, the failure mechanism for the $150-\mathrm{kW} / \mathrm{kg}$ carbide cases may be coolant boiling or clad burnout, at least for the average fuel pin. For the metal fuel, within the accuracy of this analysis, all the failure mechanisms appear to be virtually simultaneous. In interpreting these results, it should be noted that in these cases flow was maintained, causing some pressurization of the core, which would delay both coolant boiling and clad burnout by perhaps a tenth of a second beyond the times shown in the table. Also, a change in the critical heat flux correlation might well result in boiling always occurring before burnout, but this would have little effect on the time of first failure, since boiling generally occurs before or shortly after burnout under the current assumption.

Although it is not shown in Table 2, an oxide case considered was one with a Doppler $T(d k / d t)$ of -0.004 , and a plot of failure time (for any given type of failure) as a function of $T(d k / d t)$ resulted in a straight linc.

If failure is caused either wholly or partially by fuel temperature, it may be expected to occur in the core axial center, due to the axial power peaking factor, whereas coolant boiling and clad burnout occur near or at the outlet. If the assumption is made that resultant sodium void patterns will tend to progress from the point of initial failure, the reactivity effect of roids caused by coolant boiling or burnout might be expected to be less than if caused by failures related to high fuel temperature. The metal and lower- 
TABLE 2. Two Dollar/sec Acciden's

\begin{tabular}{l|c|c|c|c|c|c}
\hline \multirow{2}{*}{ Core } & \multirow{2}{*}[T(dk/dt)]{$_{\text {Dop }}$} & \multicolumn{2}{|c|}{ Hot Fuel Rods } & \multicolumn{2}{c|}{ Average Fuel Rods } & \multirow{2}{*}{ Coolant Boiling, sec } \\
\cline { 3 - 6 } & $\begin{array}{c}\text { Fuel Temp } \\
\text { Limits, sec }\end{array}$ & Burnout, sec & $\begin{array}{c}\text { Fuel Temp } \\
\text { Limits, sec }\end{array}$ & Burnout, sec & \\
\hline Oxide, $150 \mathrm{~kW} / \mathrm{kg}$ & -0.01 & $0.47-0.83$ & 1.45 & $1.06-1.37$ & 1.53 & 1.45 \\
& -0.0015 & $0.40-0.55$ & 0.71 & $0.63-0.71$ & 0.76 & 0.73 \\
\hline Carbide, $150 \mathrm{~kW} / \mathrm{kg}$ & -0.01 & $1.24-1.59$ & 1.23 & $1.67-2.2$ & 1.39 & 1.36 \\
& -0.0015 & $0.61-0.76$ & 0.61 & $0.76-0.94$ & 0.67 & 0.67 \\
\hline Carbide, $300 \mathrm{~kW} / \mathrm{kg}$ & -0.004 & $0.54-0.74$ & 0.63 & $0.75-1.06$ & 0.78 & 0.93 \\
& -0.0015 & $0.39-0.58$ & 0.51 & $0.59-0.74$ & 0.61 & 0.68 \\
\hline Metal, $150 \mathrm{~kW} / \mathrm{kg}$ & -0.004 & $0.85-$ & 0.77 & $0.91-$ & 0.85 & 0.80 \\
& -0.0015 & $0.69-$ & 0.64 & $0.74-$ & 0.71 & 0.67 \\
\hline
\end{tabular}

specific-power carbide cores may thus have some advantages in this respect. The safety advantages of maintaining a low fuel operation temperature in this particular type of accident might provide additional incentive for development of sodium-bonded oxide fuel; all other fuels examined in this analysis were sodium-bonded.

The magnitude and significance of the difference in void patterns are difficult or impossible to estimate at this time. The patterns of sodium voiding will depend not only on the initial failure location but also on the core radial power shape and on the rate of expulsion. The rate of expulsion is not known with confidence. With regard to the rate of radial progression, a powerflattened core with substantial local peaking factors will tend to produce a somewhat incoherent void pattern regardless of failure mechanisms. For any power shape, the lower-power channels may fail by different mechanisms (for example, coolant boiling in the oxide cores) than do the high-power channels, which further complicates the void pattern.

In all of these $\$ 2 / \mathrm{sec}$ reactivity ramps, the times before failure are of the same order of magnitude as those required from the control system to operate. Although in every case it might be expected that a high power level of period scram would prevent any damage, there is little margin for failure of sensor, or other unforeseen delays. However, the credibility of a $\$ 2 / \mathrm{sec}$ ramp at full-power condition should be questioned if proper design measures are taken to avoid mechanisms that might produce such a rate.

If it could be established that credible ramp rates are much less than $\$ 2 /$ sec, the incentive for a large Doppler to increase the time available for scram would be reduced. Other incentives, such as the role of the Doppler in the meltdown accident, might remain. If, on the other hand, ramp rates much greater than $\$ 2 /$ sec are credible, the design of the control system may become very difficult, and the incentives for a large Doppler are, if anything, increased.

\section{REFERENCES}

1. GEAP-4418, Liquid Metal Fast Breeder Reactor Design Study (Jan 1964).

2. ACNP-64503, Large Fast Reactor Design Study (Jan 1964).

3. CEND-200, Liquid Metal Fast Breeder Reactor Design Study (Jan 1964).

4. WCAP-3251-1, Liquid Metal Fast Breeder Reactor Design Study (Jan 1964).

5. P. Greebler, D. B. Sherer, and N. H. Walton, FORE $A$. Computational Program for the Analysis of Fast Reactor Excursions, GEAP-4090 (Oct 1962).

6. K. P. Cohen et al., Reactor Safety and Economics Considerations for Fast Reactors, GEAP-4414 (Nov 11, 1963).

7. T. D. Chikalla, Studies on the Oxides of Plutonium, Memoire 35 , presented at the 2nd International Conference on Plutonium at Grenoble, France, April 1960.

8. J. B. Conway, R. M. Fincel, and R. A. Hein, The Thermal Expansion and Heat Capacity of $\mathrm{UO}_{2}$ to $2200^{\circ} \mathrm{C}$, G.E. NMPO, paper presented at ANS Meeting, Salt Lake City, June 1963.

9. J. A. Christensen, Specific Volume of Molten $\mathrm{CO}_{2}$, paper presented at American Ceramic Society, 15th Pauific Coast Northwest Regional Meeting, Seattle, Washington, Oet 1962.

10. R. J. Ackermann, The High Temperature, High Vacuum Vaporization and Thermodynamics Properties of Uranium Dioxide, ANL-5482.

11. J. Hanson, J. Field, and S. Rabin, Experimental Studies of Transient Effects in Fast Reactor Fuels Series IIMixed Oxide $\left(\mathrm{PuO}_{2}-\mathrm{UO}_{2}\right)$ Irradiations, GEAP-4804 (June 1965).

12. Bolomey et al., Carbide Fuel Development, NDA-2140-2 (Oct 15, 1959).

13. Stachura, Silberberg, and Cordy, Uranium Carbide Transient Heating Experiments-Phase I, NAA-SR-9508 (Aug 1964).

14. The Reactor Handbook, Vol. 3, Sect. 1, First Edition. 
15. H. Lurie, Sodium Boiling Heat Transfer and Hydrodynam$i c s$, in Proceedings of the Conference on Application of High Temperature Instrumentation to Liquid-metal Experiments, September 28-29, 1965, ANL-7100.

\section{Discussion}

Mr. Smidt (Karlsruhe): I think in general our results agreed quite well. I only found a difference in the times to arrive at coolant boiling in the loss-of-flow accident. What assumptions did you make as regards the inertia of the whole system? Are the pumps still running with all probability, or what did you assume?

Mr. Sherer: The assumptions were not specific with regard to the inertia of the system, but we assumed it takes approximately 10 seconds for the flow to decrease to its steady state, that is, to a new steadystate value.

Mr. Lellouche (BNL): What sort of a dynamics model was this? Was it space-dependent, and fully nonlinear or linear, or what?

Mr. Sherer: It was not space-dependent; it was a point kinetics model and nonlinear.

$M r$. Lellouche: You discussed failure of your elements in terms of the melting point, but in the oxides and carbides there will be mobility of fission gases, and a pressure buildup will occur as the temperature increases, which may cause pressure failure at much lower temperatures than the $4900^{\circ} \mathrm{F}$ or $4300^{\circ} \mathrm{F}$ that you indicate for melting. Did you consider this a possible means of failure?

Mr. Sherer: In the first place, these cores were operating for our specific project with the oxide or carbide close to the melting point, so that the amount of fission gas still in the fuel would not be too large. We also have some experimental evidence which indicates that for fuel which is initially operating close to the melting point the sudden release of fission gases should not cause failure as you go through the melting point.

Mr. Häfele (Karlsruhe): I have a more general remark. In connection with evaluation of the importance of the size of power coefficients, I think one finally should arrive at a point where the two consequences are envisaged; that means the power coefficient should be evaluated in a certain light. The first would be the reactivity value which could be withstood without the action of a safety system. And then one has to ask: to what extent is that amount of reactivity influenced by that and that coefficient? This is of a greater importance as regards design characteristics of a safety system, because finally the safety system has to act. Therefore, the meaning of such a power coefficient can be only that insofar as it influences the design charac- teristics of the safety system. If one does that, one finally arrives at the point where at the reactivity rate of $\$ 2 / \mathrm{sec}$, for example, the impact of the Doppler coefficient of the design characteristics of the safety system is not too large. I think you also mentioned that, but I think it should be more explicitly the target of such an investigation. Do you tend to agree with that or do you disagree?

Mr. Sherer: I think I tend to agree with you.

Mr. Gibson (AI): As regards your high powerdensity carbide core, I presume you had a sodium bond. If this is true, did you have any switching mechanism to account for the possible boiling of the bond as you went through the transient with the changing in the transfer coefficients?

Mr. Sherer: No; we did not expect the temperatures of the bond at the sodium-fuel interface to exceed $3000^{\circ} \mathrm{F}$. We more or less made the assumption that there might be some sodium vaporization, but the fuel element would pressurize itself to prevent boiling. I don't exactly know what sodium vapor pressure would be required to cause failure.

Mr. Campise (AI) : Did you consider pressurization of your sodium system? It might be implied from your curve that if you were to pressurize the system upwards of $100 \mathrm{psi}$, you would be able to lengthen the time to boiling considerably.

Mr. Sherer: I think that's right. In fact, by use of 100 psi you can prevent boiling altogether in almost every case, but I think to pressurize to $100 \mathrm{psi}$ is not an easy thing in this system. Also, as regards coolantflow accidents, there will be some pressurization on the sodium due to the pumping, that due to the pressure drop to the core, which was not taken into account in the ramp reactivity insertion during which flow was maintained. This would increase the time required to get to the boiling point slightly.

$M r$. Gast (ANL): Following the lines of $\mathrm{Mr}$. Häfele's comments, do you believe that possibly this kind of a calculation could be inverted, so that if one started from some criteria for a safety system, it would be possible, in some way, to arrive at a criterion as to what the acceptable magnitude of the sodium void coefficient might be in a loss-of-coolant accident?

$M r$. Sherer: I think that one could arrive at a magnitude for the Doppler coefficient to avoid the occurrence of sodium voiding. I think that the acceptable magnitude for the sodium void coefficient is very difficult to fix, because to void sodium on a scale big enough to get a significant reactivity insertion, you are probably going to melt the core anyway. So it's questionable what kind of significance you can attach to that.

Mr. Sofer (UNC) : Do you happen to know the kilo- 
watts per foot rating of the two carbide cases that you considered?

Mr. Sherer: I think that they would correspond to approximately 20 and $40 \mathrm{~kW} / \mathrm{ft}$.

Mr. Persiani (ANL): Did you consider the possible feedback resulting for collapse of core following melting, or did you stop at melting?

Mr. Sherer: Oh, certainly. The analysis was taken only to the point at which core failure began. No consideration was given to what the reactivity rate might be after that point. The value of $\$ 2$ /sec is to be considered as related to things such as control rod runout or shootout or things of that sort.

Mr. Häfele: I would like to come back to the question asked by Mr. Gast and partly to my first remark. If one accepts the situation in which the safety system works, there will be no limit on the sodium void coefficient, and you can accept virtually all void coefficients. Therefore, from that kind of an analysis, virtually a stability analysis or an incident analysis, accepting or assuming the situation in which the safety system works, we at Karlsruhe believe that there will be no lower or upper limit for the sodium void coefficient. That will only come up in the case that the safety system fails. In our panel discussion tomorrow we will come to that point more extensively.

$M r$. Fenech (MTT): I wonder if you have studied the situation in which you lose coolant flow and the sodium superheat was at boiling; after a certain amount of superheat it would flash into vapor and introduce a step reactivity in your core.

$M r$. Sherer: That would occur after either the coolant boiling or the burnout that is analyzed here. Again, therefore, the conclusion would be the same, that is, if the safety system doesn't work....

Mr. Fenech: We have done some experiments on sodium boiling which show that the sodium does not readily boil but rather flashes, and this is a rather violent effect. The work is part of the doctoral thesis of Mr. Marto, who hasn't published it yet. This is work on pool boiling, not forced circulation, but I think it would apply to forced circulation, too.

Mr. Dalle-Donne (Karlsruhe): Referring to this sodium flashing, Oak Ridge has conducted experiments, not with sodium, but with potassium. This is for forced convection, and the flashing was very violent. Not only that, my calculations indicate that the situation with sodium is even worse. 


\title{
Program Proposed for Safety Studies of the Fast Neutron Project of the EURATOM-CEA Association
}

\author{
G. Denielou, M. Falgayrettes, J. Fournier, avi M. G. Robin \\ ET'RATOM-CEA (Cadarache) \\ Bouches-du-Rhône, France
}

(PIRESENTED BY G. DENIELOU)

\section{General: Object, Limits, and Philosophy of the Program}

Safety itself is no goal for research. It is sought after and achieved by a combination of well-known means such as simplicity of design, reliability of elementary components, choice of a suitable staff with good instruction, and adequate administrative procedures, whatever may be the difficulties to obtain them at the same time; and more generally by a reasonable awe with respect to accidents, common to everybody, at all stages of design, construction, or operation.

Actually, safety is nothing else but the lack of accidents. These are the real objects of the investigations. But these studies in turn would have only little interest without the positive care for safety which we dealt with and which is its true basis.

An accident may be defined as any nonqualitative or widely quantitative and nonreversible modification of some of the values which are typical of the normal operation of the reactor.

The widening of the ranges of the values involved is highly interesting, and project teams are permanently on the job. The limits of technology are ever increasingly extended. Power densities or outlet temperatures are increased. But one remains in a qualitatively unchanged field.

It is the part of the accident studies to pass over these ridges separating the normal from the inadmissible. The basic knowledge necessary for these studies is not the same as that used by project teams. In the former case the numerical results can be less accurate; conclusions must not and cannot depend that much on construction details. The accidents studied must deal with the group of reactors and not only with one of them. If the analysis would be too "sharp pointed," it would cease to be convineing. It is, by the way, very good that the safety studies may legitimately be applied to a conceptual design rather than to a Title II. Actually, the acquisition of the basic knowledge necessary to the study of accidents requires resources and a continuity in time which is difficult to conciliate with the financial requirements and the planning of one isolated reactor.

The safety study program of the "Fast Neutron" project nowadays being examined in France illustrates well the preceding arguments.

Most of the accidents we can think of involve actually sodium boiling and/or the vaporization of the fuel. These phenomena themselves are of no interest whatsoever to calculations for the project. Their laws are complex and still badly known. On the other hand, the reasonable, although qualitative, description of the chains of accidental events is still in a very embryonic state. Everybody is harping on BetheTait, that's all.

Thus a long-term double effort has to be pursued:

(i) first of all in imagination, a realistic imagination cleared up by the experience of accidents which have happened elsewhere;

(ii) the numerical knowledge of laws ruling accidental situations.

The program proposed hereafter concerns large fast-neutron reactors with sodium cooling and mixed $\mathrm{UO}_{2}-\mathrm{PuO}_{2}$ oxide fuel. Whatever may be the parameter changes which can be expected following the details of the design and which are important to safety, it seems to us that these reactors have enough points in common to belong to one group:

(i) considerable fuel inventories at high meltdown and vaporization temperature;

(ii) coolant whose nuclear effects are variable from one point to the other and of small amplitude;

(iii) important ratio between specific volumes of the liquid and vapor phases of the coolant;

(iv) existence of a prompt Doppler negative temperature-dependent reactivity effect;

(v) high specific power per $\mathrm{cm}^{3}$ coolant.

The experience gathered with the safety studies of water or gas-graphite reactors is not without interest for these reactors. But it has to be kept in mind that the imaginable sequence of events is certainly qualitatively different here. 


\section{Investigation of Accidents}

\section{II.1. Bethe-Tait Model}

A model of an accident, symbolized by the names of Bethe and Tait, ${ }^{(1)}$ has been widely used in connection with the reactors of the first generation. It is an accident characterized by a fast and unique insertion of reactivity which ends with core disassembly under the pressures which are developed inside. It must be emphasized that the sequence of events considered in the model, in order to lead to the fuel collapse which creates the reactivity insertion, becomes more and more unrealistic when large-dimensioned reactors with flattened core are studied.(2) Because of the essentially improbable nature of the associated events, the model has to be presented as a limiting case for all possible accidents.

But an inconceivable accident cannot be the most important.

It is, on the other hand, not at all certain that the Bethe and Tait model is not optimistic in several points: the core disassembly is a phenomenon which stops the accidental sequence in a very convenient way. Is it even sure that the most serious accident which one can imagine is that which involves the highest power?

The most valuable point of the Bethe-Tait analysis is basically the fact that no serious accident can be conceived which does not involve a partial or total core meltdown.

More detailed pictures of what happens are necessary. Three examples are given below. The events anticipated are related to the different phases: origin, evolution, and effects of the accident, but we do not claim to give a complete description of the latter in placing them side by side.

\section{II.2. First Example: Propagation of a Fuel-pin Ex- plosion}

The fission gases kept under pressure inside the fuel pin are a potential source of energy. It may be asked under which form this energy would be liberated in the course of an ill-timed rupture of the cladding.

It has been suggested that there might exist a mechanism following which the rupture of a pin propagates to the other pins of the subassembly and even to the pins of the neighbouring subassemblies. This would have troublesome consequences on the circulation of the fuel-cooling sodium.

The study of the consequences of a cladding rupture is being tackled experimentally. The rupture could have different kinds of origins:

(a) localized flaws in the cladding, such as an already existing metal defect which might have es- caped manufacturing control or a defect resulting from cladding corrosion in the reaction;

(b) brittleness produced by irradiation of the cladding steel;

(c) defective pin cooling, giving rise to an excessive overheating of the metal.

The tests carried out so far have dealt with RAPSODIE-type pins. The rupture due to a localized defect a) has been simulated by weakening the cladding mechanically and by starting the rupture with the help of an electrical are (see Fig. 1). This kind of flaw never led to an explosion at normal pressure and temperature conditions ( 80 bars and $550^{\circ} \mathrm{C}$ ) (see Fig. $2)$. Bursting has been recorded only at clearly higher pressures (130 bars and $550^{\circ} \mathrm{C}$ ) (see Fig. 3).

These first results lead to the belief that the available energy would be liberated progressively rather than brutally by a bursting. The study is still under way.

\section{II.3. Second Example: Meltdown Propagation in a Fuel Subassembly}

(a) One cannot guarantee that no fuel element will ever melt. In fact, it is pretty sure that some fuel subassembly will melt some day. Flow blockage between fuel pins or plugging of the fuel subassembly inlet are possible events despite any preventive measure.

(b) Such an accident is likely to remain undetected for some time. Noise-analysis detection methods are not yet of practical use. Core outlet thermocouples are not reliable enough to be sure that their readings won't be overlooked. Reactivity effects of sodium voidage and fissile material motion give reliable reactivity indications. Unfortunately they can be of too small amplitude to be detected in time.

(c) One can imagine a mechanism of propagation as follows: the melting fuel gathers over the lower axial blanket of the defective subassembly or even on the support grid of the fuel pins. The molten fuel, serving as an important source of heat, penetrates through the walls which separate it from one of the neighbouring subassemblies. Lumps, solidified by the contact with sodium circulating in the peripheral channels of the neighbouring subassembly, lead to a cooling failure of the adjacent pins, the same process leading step by step to the meltdown at the adjacent element.

This propagation mechanism may not be considered alone. One can imagine that the subassembly meltdown leads also to a plugging of its upper part and that the hexagonal cladding becomes the center of high pressures deforming it. Besides, the pressures origi- 


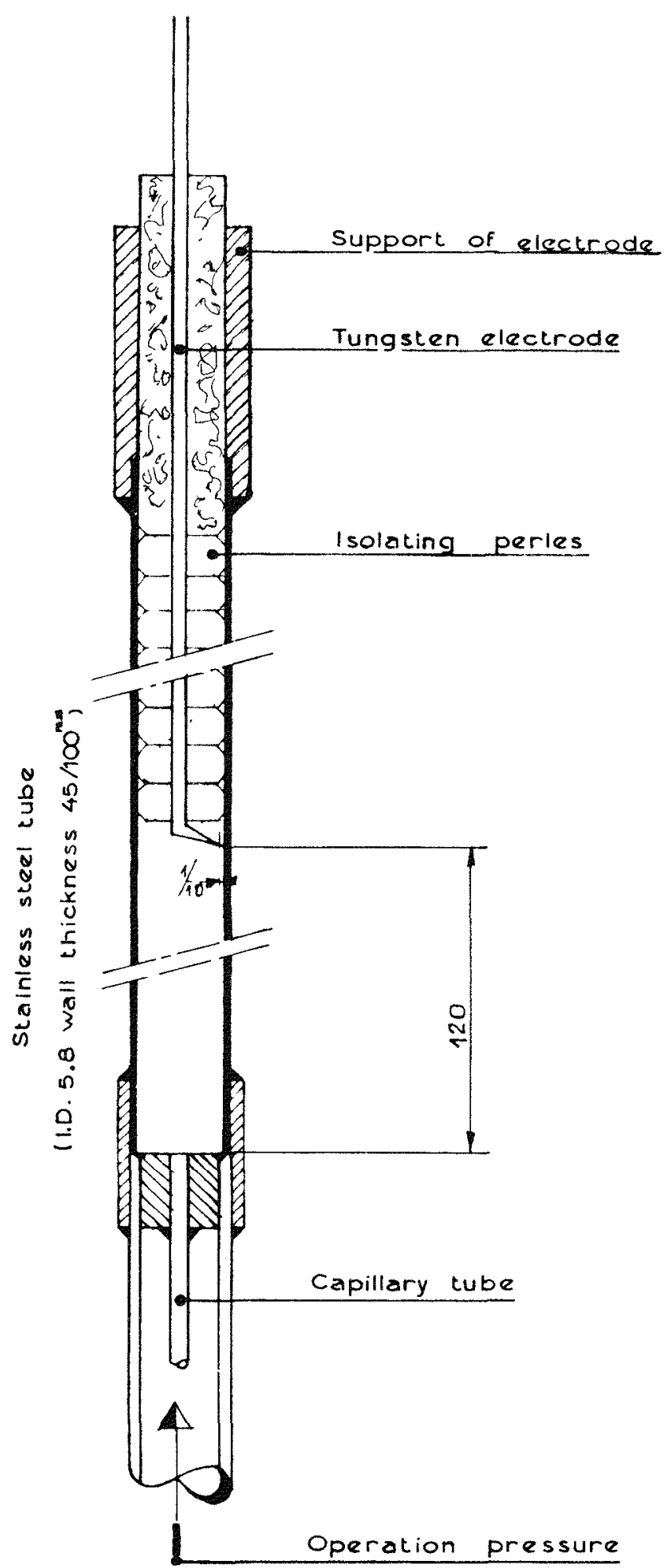

Fra. 1 Experimental Tube for Cladding Rupture Test

nating in the molten fuel itself can take part in the propagation mechanism. It is not proved that this type of incident is no real danger even with scrammed rods.

\section{II.4. Third Example: After-heat Cooling of a Molten} Core

After-heat cooling of a subcritical molten core is in itself a big problem because of the high fission product power of large reactors. In the case of a total loss of heat sink it could lead to sodium boiling in the primary tank and to subsequent loss of tightness of the primary circuit.

Suppose that following a nuclear excursion during a meltdown the primary structure has been able to contain it within its bounds; is it clear that there won't be any fuel reassembly leading to mild power bursts? Is it clear in turn that a mild power burst cannot give rise to a slow disassembly and then to another power burst? In short, can no mechanism be conceived by which or through which energy is exchanged between fissile material and sodium, not in a stcady state but in more or less repetitive transients?

\section{Sodium Boiling}

Future reactors will certainly operate under conditions far removed from those at which sodium boiling occurs, and some doubts may be expressed as to the necessity to assign such important funds to researches on boiling. It must, however, be admitted that a safety review of these reactors will lead to a survey of incidents apt to cause such a temperature rise that the boiling risk does exist. Although sodium boiling is not itself a primary event, it is difficult to imagine a serious accident in which this phenomenon does not take place. When it does, its consequences will always be troublesome.

Once we are convinced that safety requires sodium boiling research, it is not advisable to lay down too strictly utilitarian limitations. We better let the physicists do their work as they are used to doing it. What we lack is a basic quantitative understanding of the phenomenon. For instance, neither exclusively nor mainly do we need the laws governing sodium boiling in a coolant channel between fuel pins. Subassembly meltdown propagation requires knowledge of burnout heat flux in slab geometry or in pool boiling.

We are not so much confident in digital computer codes already written to describe two-phase flow in various geometries because their input data do not seem that reliable.

It is suggested that investigations be carried out on the following points:

(a) Incipient local boiling in free or forced convection. The laws governing $\Delta t_{\text {sat }}$ values have to be studied for various specds, pressures, surface states, etc.

(b) Two-phase pressure drops. S-shaped curve char- 


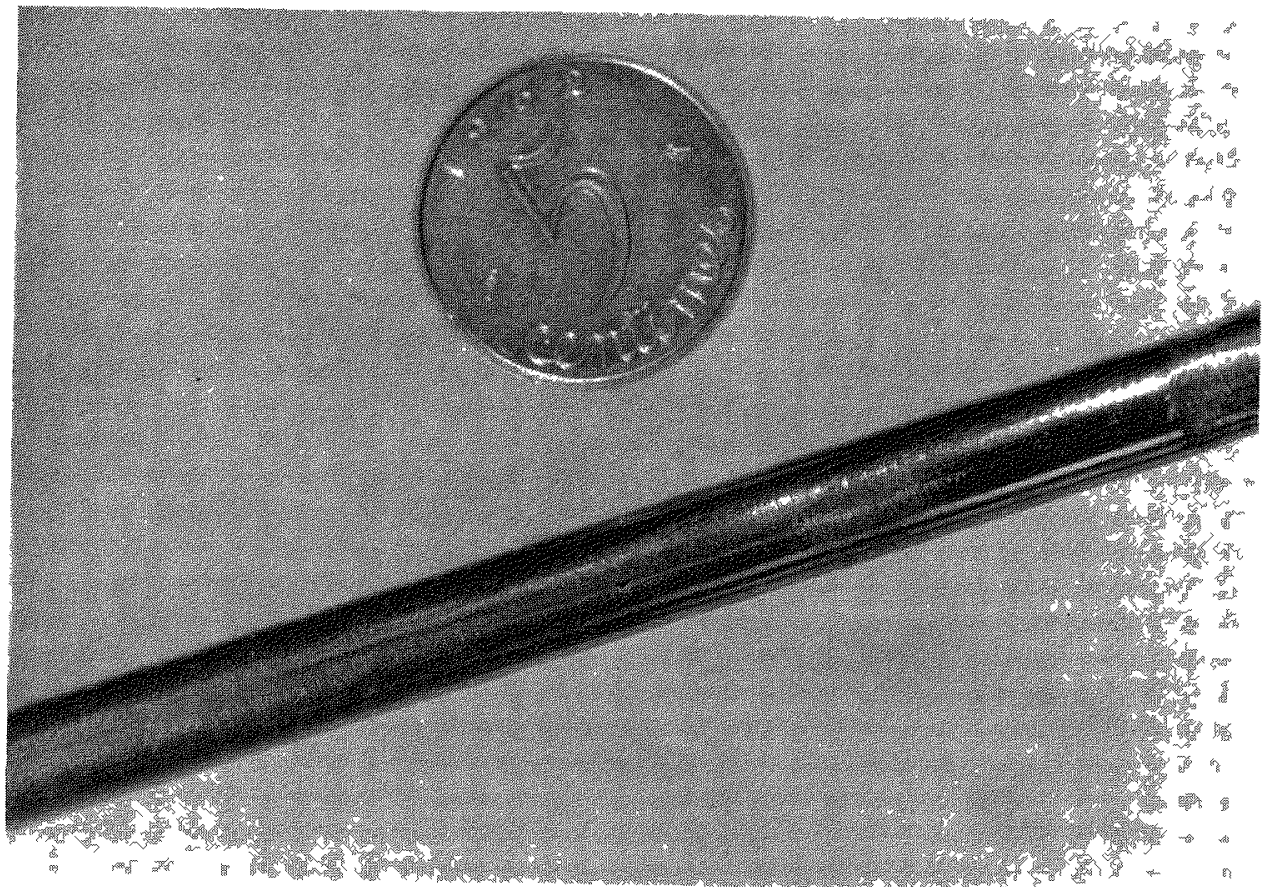

Fig 2 Cladding Rupture Tests Weakenng Brought about with an Electric al Arc The Swelling of the Pin Went Down Slowly Test Conditions $P=80 \mathrm{~kg} / \mathrm{cm}^{2} \quad 7=550^{\circ} \mathrm{C}$ )

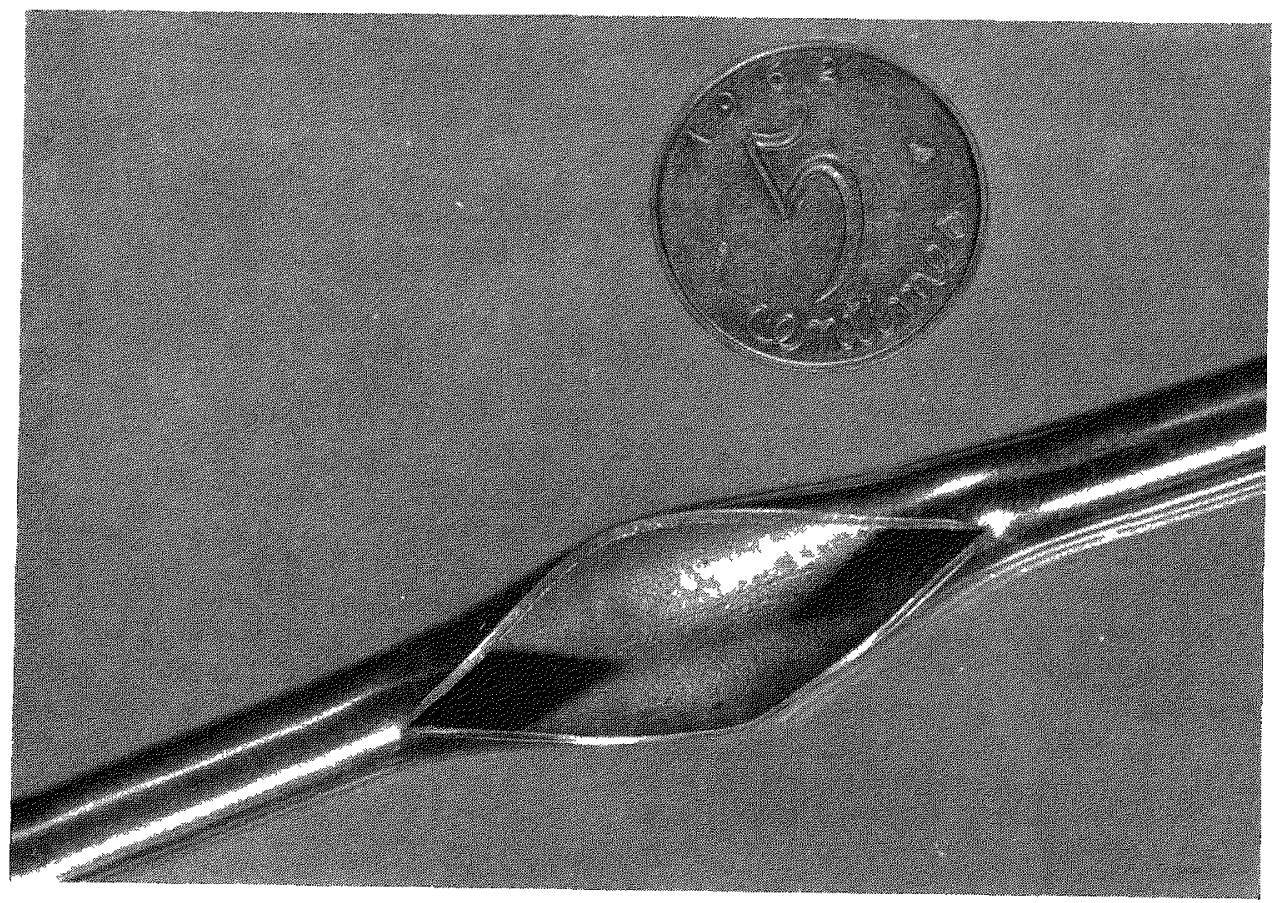

Frg 3 Cladding Rupture Tests Bursted Pin View (Test Conditions $P=130 \mathrm{~kg} / \mathrm{cm}^{2}, T=544^{\circ} \mathrm{C}$ )

acteristics of the flow excursion phenomenon in different geometries have to be determined

(c) Burnout Study of heat exchange through the walls in contact with vaporizing sodium A better understanding of the flow patterns following a flow excursion and of heat transfer before burnout is required

(d) Detection of boiling This investigation will be continued despite the fears that one may have no practicable methods Water-reactor experience is not so 
Flux density $\sim 700 \mathrm{~W} / \mathrm{cm}^{2}$
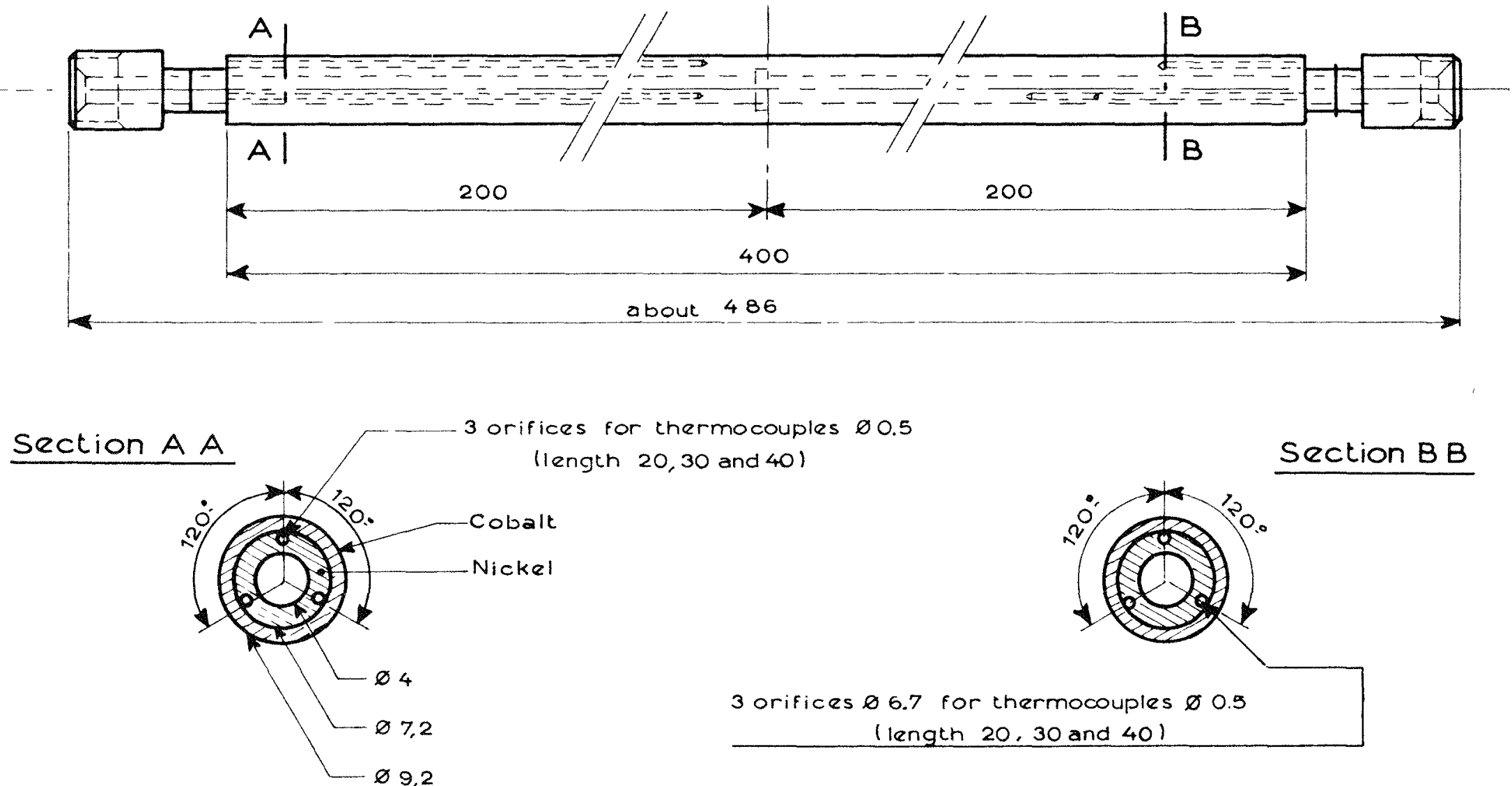

FIa. 4. Induction Heated Tube $(500 \mathrm{kHz})$ 

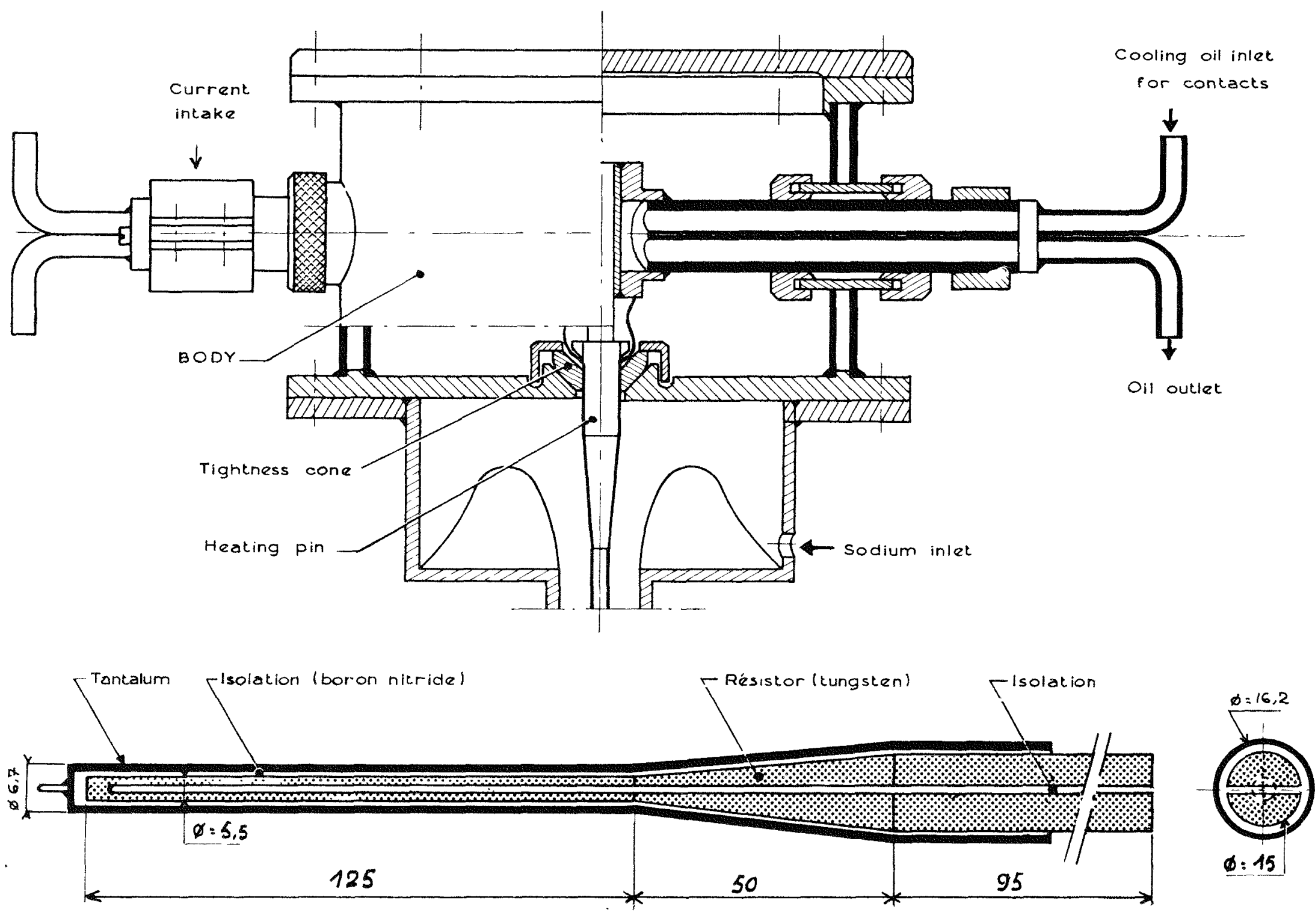

Fra. 5. High-flux, High-temperaure Heating Tube 


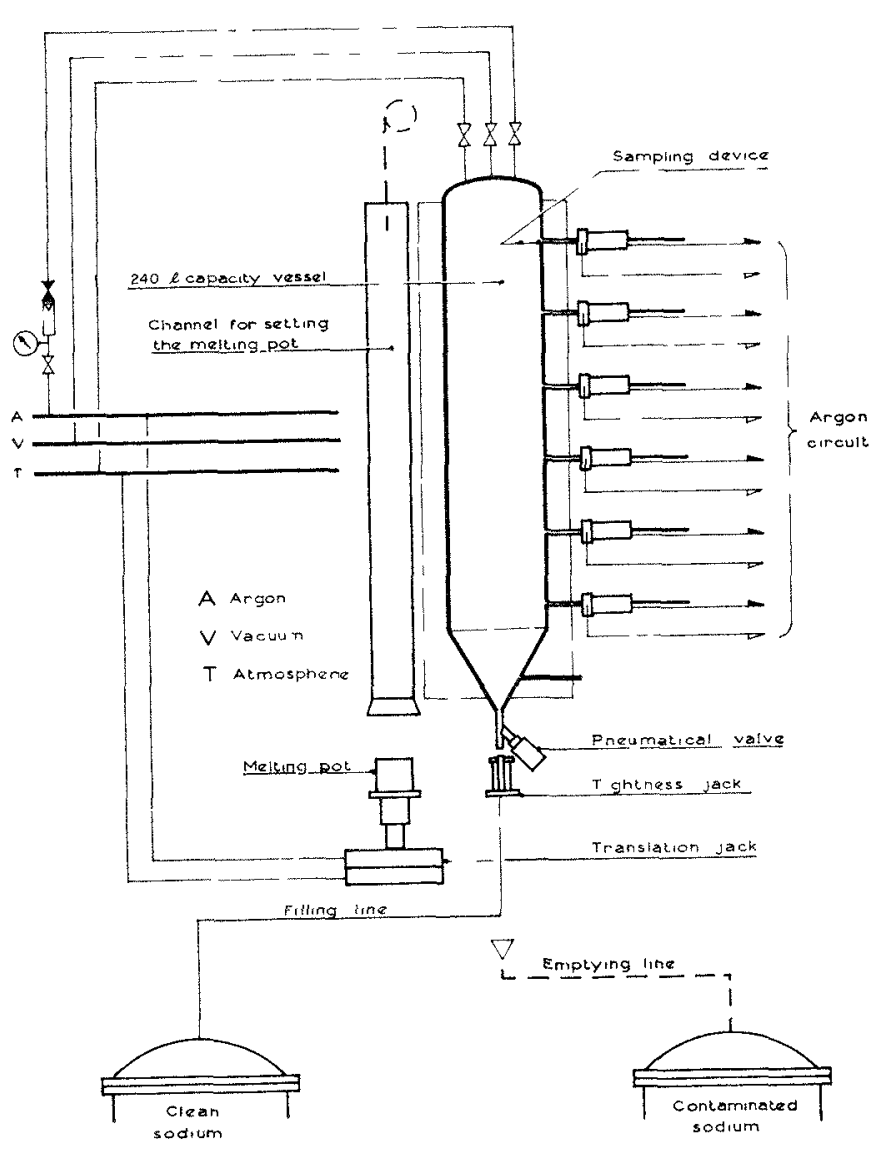

Frg. 6. Pirana, a Simplified Diagram

encouraging in this field. To sum up, this program is very much inspired by the one followed in waterreactor research, but it is clear that difficulties are much greater.

Preliminary work on studies with sodium started at the end of 1963. It has been dedicated to instrumentation and to the study of heating sources. Different ways have been proposed to obtain the necessary flux:

(a) A heating device using a high-frequency current generator of $500 \mathrm{kHz}$ has been tested. Already a heat flux of several hundred watts $/ \mathrm{cm}^{2}$ at the wall of a nickel tube of $4-\mathrm{mm}$ inner diameter and a length of $40 \mathrm{~cm}$ (see Fig. 4) has been reached.

(b) A heating pin able to dissipate by the Joule effect a flux of $2000 \mathrm{~W} / \mathrm{cm}^{2}$ is now under construction (see Fig. 5).

(c) Electronic bombardment is being studied.

\section{Physical Data for Ceramic Fuel in Liquid and Gaseous Phases}

Physical data about ceramic fuels outside the field of normal operating conditions are needed; however, no serious accident is foreseeable which would not involve meltdown and even vaporization of the fuel.
The lack of physical data hinders especially the correct evaluation of the feedback which results from the Doppler effect and shiftings of fuel under the impact of pressures of which it is the center.

1) Since the value of the Doppler coefficient in the liquid phase is not known, it has been taken as zero for accident calculations. Basic physics studies are to be undertaken.

2) The system formed by a partially vaporized core cannot be studied without a better knowledge of basic data: heat capacity, thermal conductivity, ete., even ignoring the part played by sodium under such conditions.

The possibility of using new experimental techniques for measurements of thermodynamic characteristics of uranium oxide at high temperatures is foreseen. Dynamic methods using heating by direct conduction or by laser impact in high-pressure molten systems are considered.

Whatever be the accuracy of the equation of state and of the basic data, it will remain difficult to describe mathematically the meltdown of a fuel subassembly owing to its complicated geometry. Experiments seem here almost unavoidable. The possibility of in-pile study of fuel meltdown should be contemplated. Nuclear heating seems indeed the only suitable way of achieving high-enough power densities to simulate correctly the meltdown phenomenon. The meltdown experiments which are taking place in TREAT reactor are being followed with interest, but it must be understood that our approach is somewhat different. We are not looking so much for power transients as for high power densities.

\section{Miscellaneous Studies}

Though the accurate description of an accidental sequence appears to be very difficult, a large variety of its consequences, such as involving contamination and fire, is fairly well-known. Thus it is wise to study the best way of dealing with these consequences independently of their origins. Indeed, if the reactor itself is presumed to be perfectly safe, many auxiliary and ancillary systems give rise to safety hazards that are not negligible.

\section{V.1. The Problem of the Containment Shell}

The problem of containment shells is highly explosive. The fight is hard between no-containment people and high-pressure-vapor-suppressor people. Our position is moderate.

(a) We are convineed that the primary shielding of the reactor can be designed in order to contain grossly all core accidents.

(b) It does follow necessarily that the strict requirements regarding containment shell tightness 


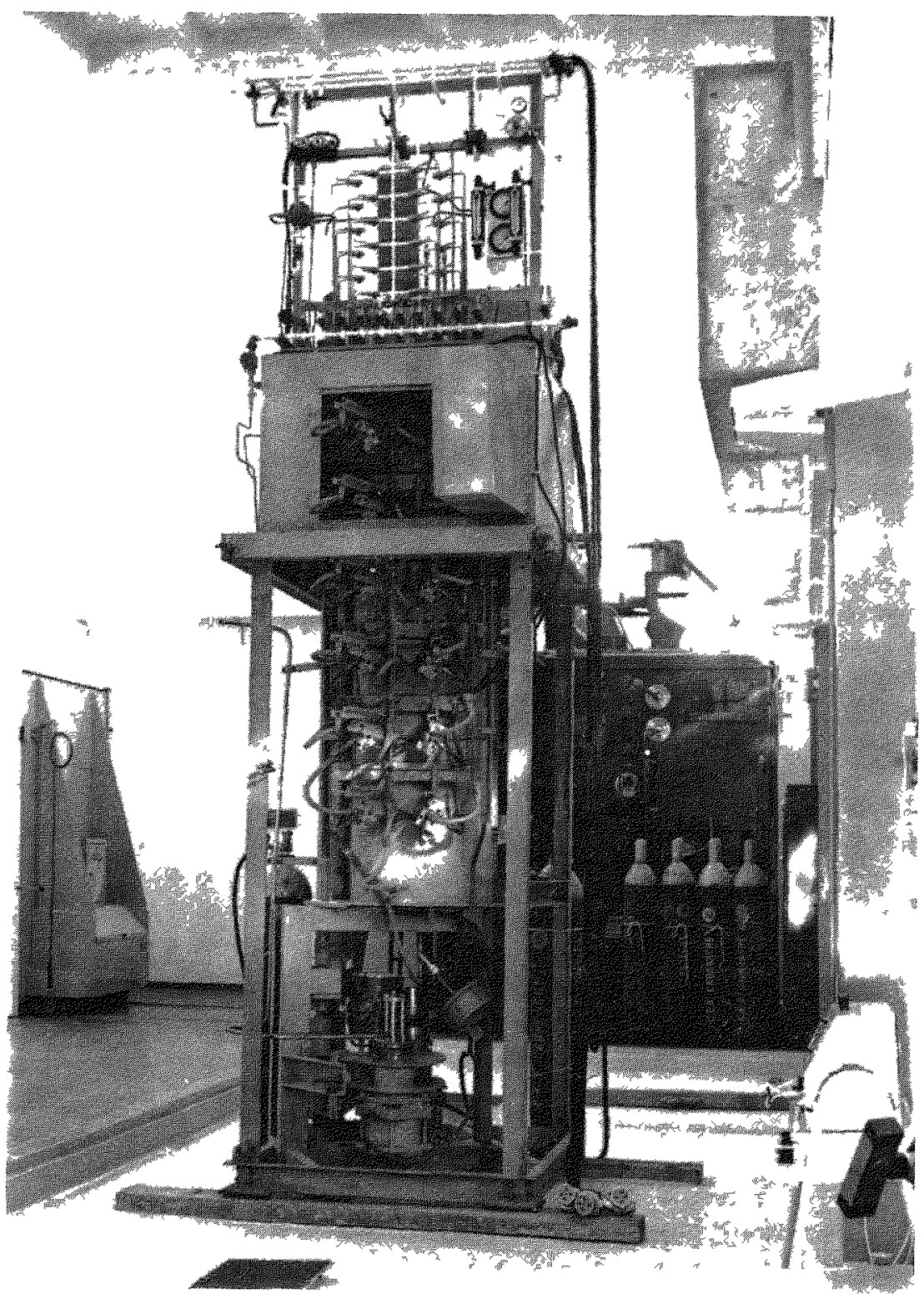

Fig. 7. General View of Pirana

should be dropped. Actually, accidents and minor incidents may entail a release of radioactivity which would be harmful, as, for instance, in meltdown accidents during fuel handling.

(c) With respect to point a) we no longer need to design containment shells to withstand dangerously large internal pressures arising from sodium fires.

(d) Does this mean that the containment shell has to be not resistant at all? In no case whatsoever! Indeed, the simple necessities of the construction: ovcrhead bridge crane, and shelter against snow and wind, lead to buildings which inherently offer some resistance to pressure. Furthermore, it is our experience that an appropriate tightness has a sense only if it can be periodically checked and the best inspec- tions consist in an overpressure at a level distinctly higher than the random background due to wind and temperature. We believe that the price supplement which is paid with respect to a classical building is very low, as soon as the overpressure required is low, e g., $50 \mathrm{~cm}$ of water. This solution is very close to the "control leakage" version, but it looks somewhat safer.

\section{V.2. Radiological Safety Studies-Pirana and Fanal experiments}

The decision to carry out experiments which would clarify the behavior of fission products in case of an accident with one of the reactors of the group was 


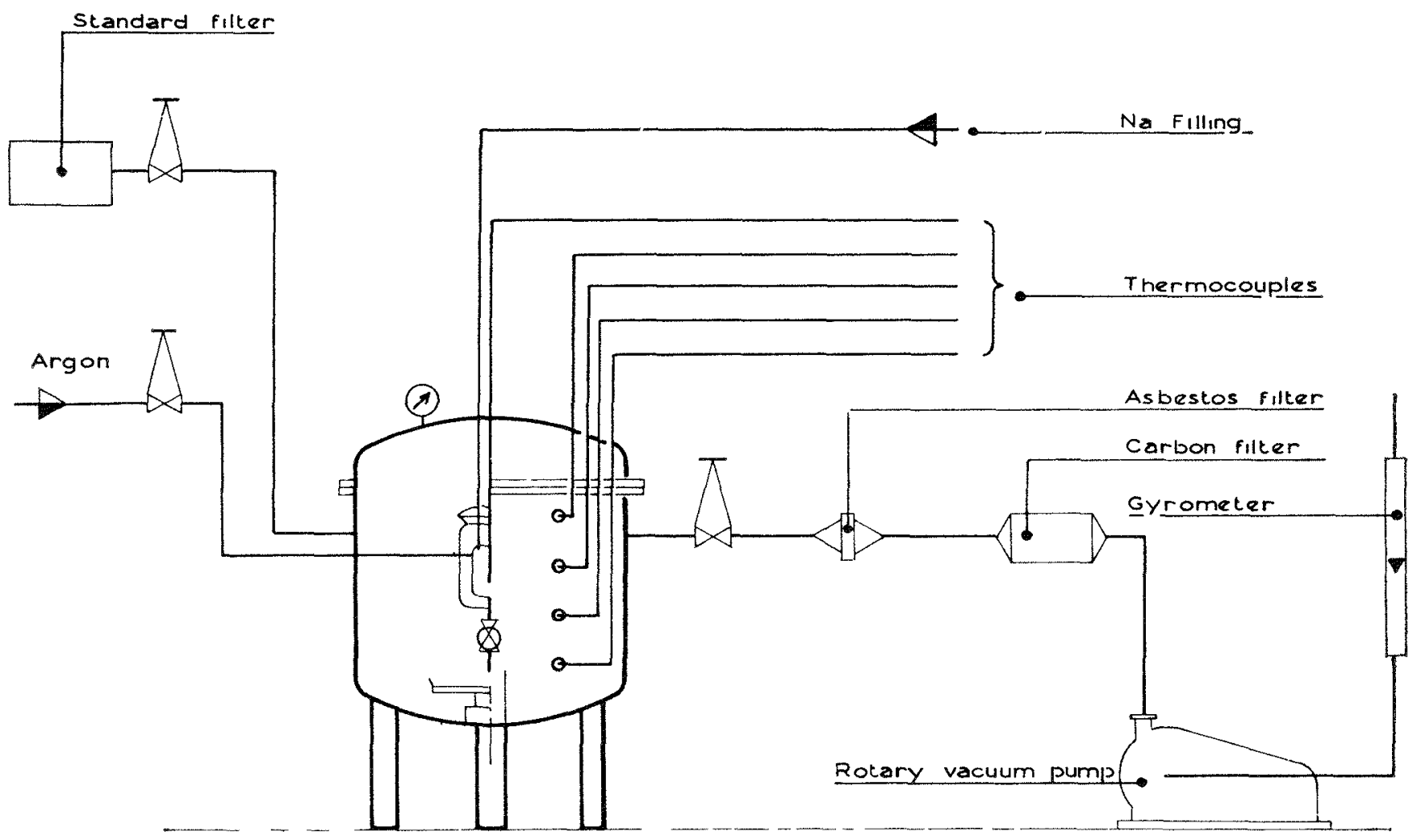

FIG 8 Fanal, a Simplified Dragram

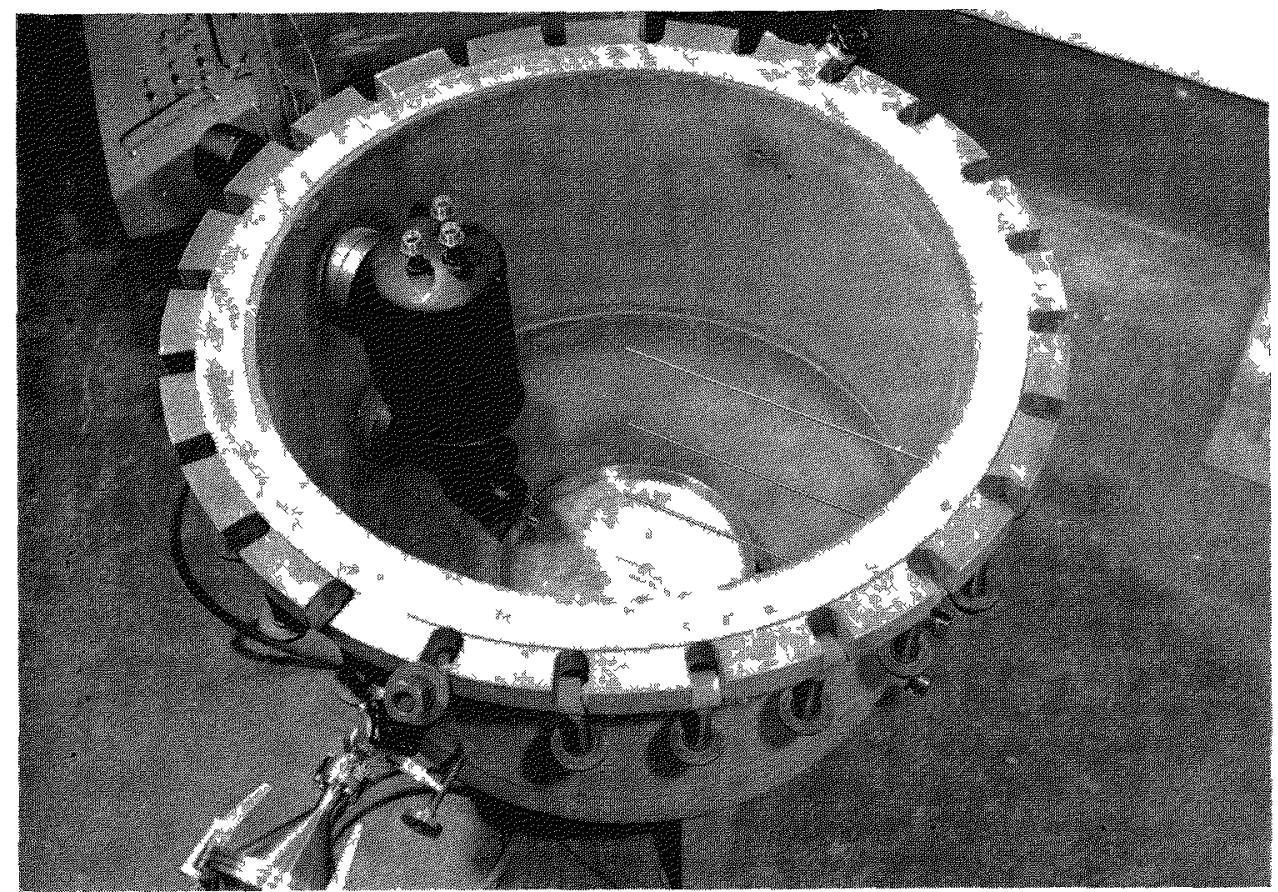

Fid 9 Vrew in the "Fanal" Vessel Showing the Sodum Meltdown Device 
E: Rupture device

$M$ : Safety membrane

P: Pressure capture device
T: Thermocouple

$V$ : Pneumatical valve

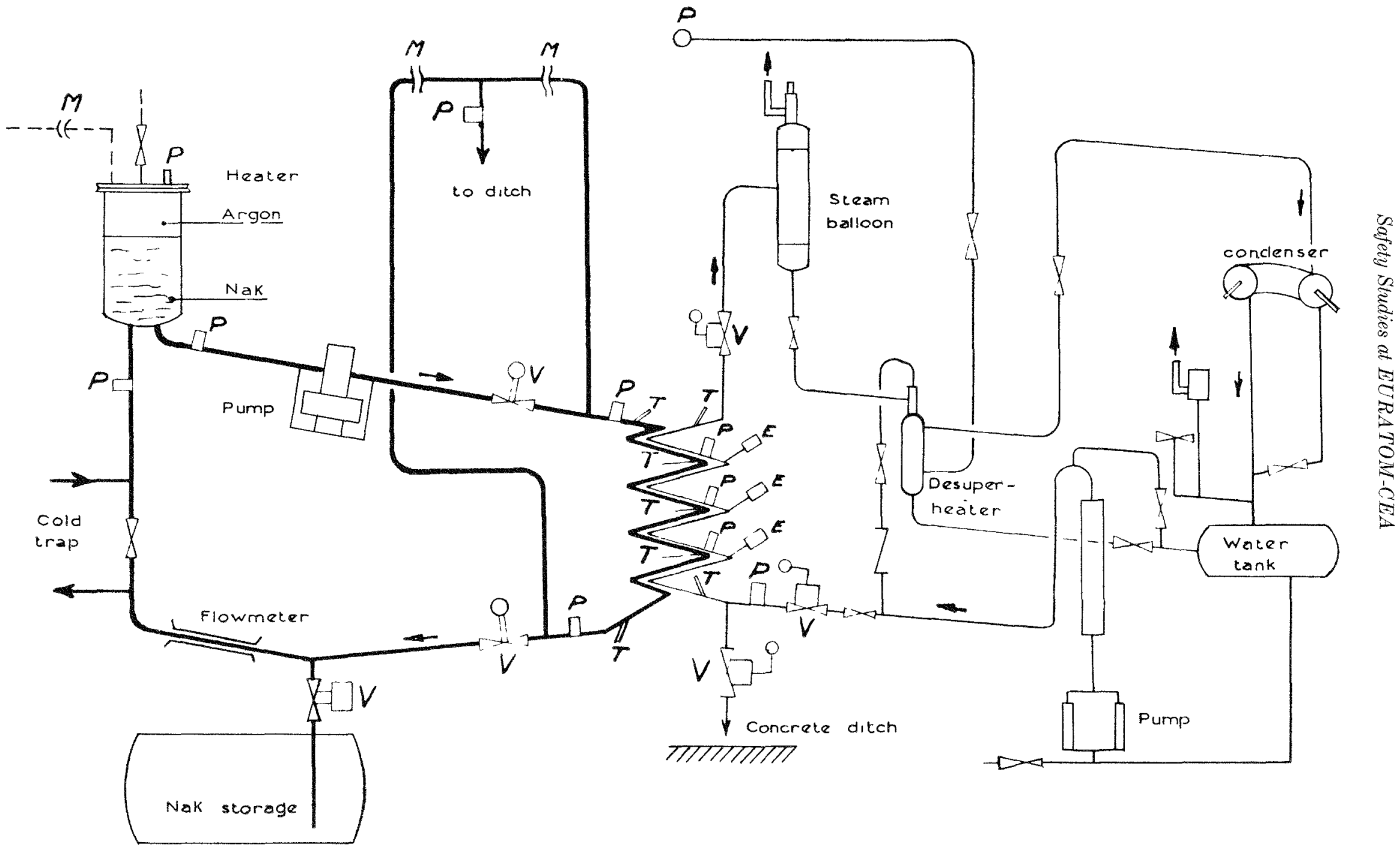

FIg. 10. NaK-Water Reaction Test Circuit $(50 \mathrm{~kW})$ 


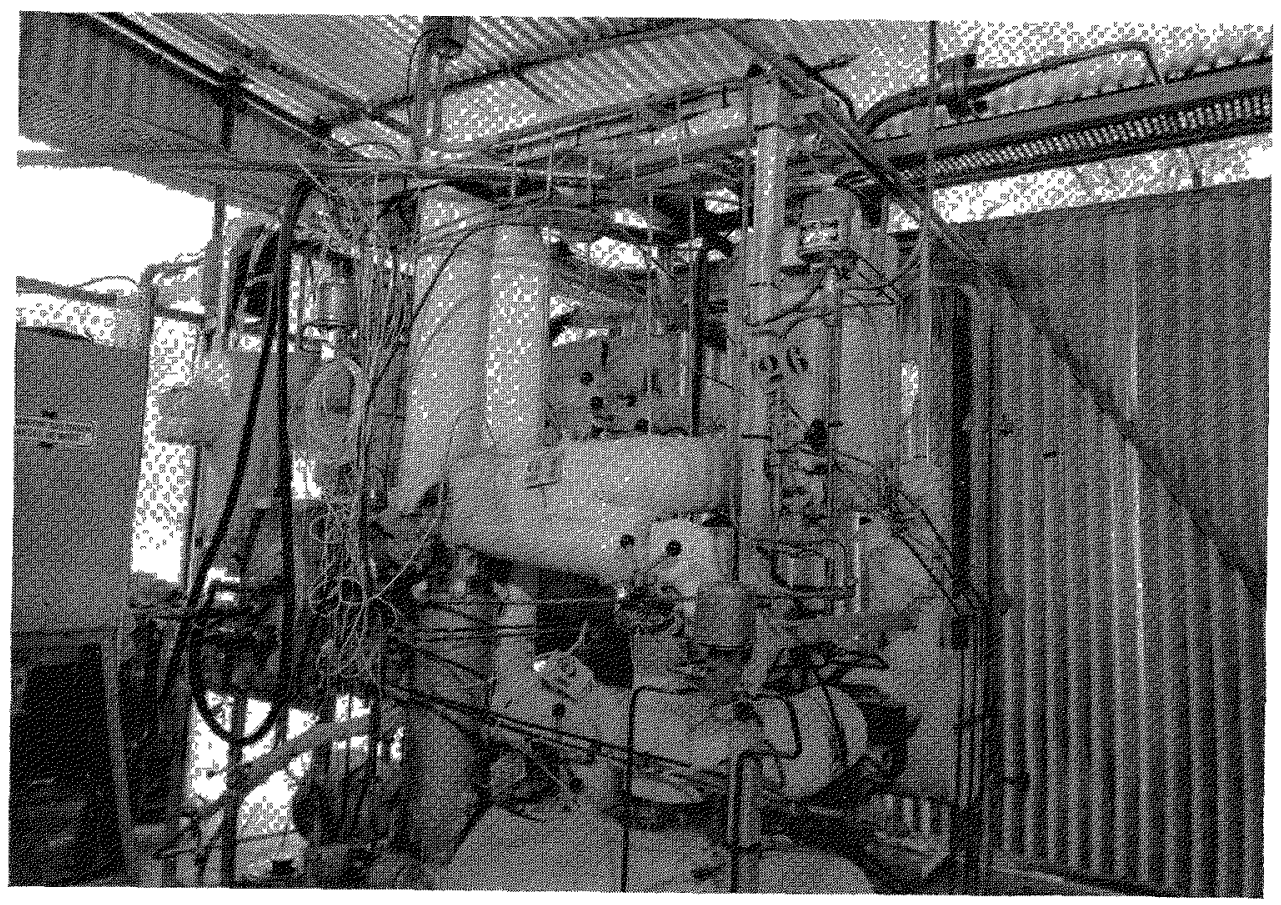

FIG. 11. General View of the Test Circuit for NaK-Water Reaction

taken at the end of 1963. Two series of studies are conducted.

(a) Pirana Tests (see Figures 6 and 7). These aim at knowing the nature and quantity of fission products which can pass through the sodium and escape into the upper inert gas blanket. An irradiated samplo of metallic uranium is melted at the bottom of a 240 liter vessel in direct contact with the sodium contained therein. The fuel sample is a hollow cylinder with an outer diameter of $10 \mathrm{~mm}$ and a height of $20 \mathrm{~mm}$; it is placed in a titanium melting pot and induction heated. On the vessel, devices to sample sodium are fixed at different levels for analysis. Fission gases, escaping above the free sodium level into the argon cover gas, are collected as well.

The tests already carried out with static sodium will be followed by circulating sodium experiments; the metallic uranium sample will also be replaced by a ceramic sample.

(b) Fanal Tests (see Figures 8 and 9). These aim at solving the problem of the escape of fission products of burning sodium, condensing of these fission products on cold surfaces, and the efficiency of the filtering.

The contaminated sodium is preheated and allowed to flow into a container where it is ignited. Sampling will take place inside the sodium, in the atmosphere, after the filters, and on the walls. Tests have been carried out so far only with uncontaminated sodium.
Sodium-Water or NaK-Water Reaction

Experiments have been conducted with single-wall steam-generators in order to check the efficiency of shutdown valves and pressure-relief devices. The test loop (see Figures 10 and 11) includes a single-tube, hair-pin, 50-kW once-through steam-generator. First tests were made with a NaK alloy because this fluid is now in use at the test station at Grand-Quevilly. ${ }^{(3)}$

A known steam or water leak in the NaK loop is created by an electric arc. At first, steam characteristics and hole cross section (about $10 \mathrm{~mm}^{2}$ ) are kept constant. Leak position in the generator is altered (the influence of the steam quality is thus studied). Further experiments will study the influence of geometrical parameters (type of generator, NaK cross section, group, etc...)

\section{Conclusion}

1. The proposed program does not intend to look into all the problems which arise from a safety review of the fast breeder conceptual design, but only to these which look to us most important. We expect that world-wide programs will be coordinated enough to cover the whole ficld.

2. The program is proposed for a four-year period.

3. Financial evaluation is under way. Two million dollars is not too bad a figure. 


\section{REFERENCHS}

1. H. A. Bethe and J. H. Tait, An Estimate of the Order of Magnitude of the Explosion When the ('ore of a Fast Reactor Collapses, UKAEA-RHM(56) 113.

2. Liquid Metal Fast Breeder Reactor Design Study (1000-MWe $\mathrm{UO}_{2}-\mathrm{PuO} \mathrm{O}_{2}$ fueled Plant), GFAP-4418 (Jan 1964).

3. CEA Report R-2520, Test Station Grand Quevilly.

\section{Discussion}

Mr. Stachura (AI) : I would like to comment on some of the items you have brought up. You had mentioned that the Doppler of the liquid phase is perhaps not established or that sometimes it was assumed to be zero. I think the normal procedure is to assume that the Doppler is simply temperature dependent, and it doesn't matter what the phase is. For example, in the GE studies if the fucl goes beyond melting, I believe that the code simply looks at the changes in $T$ and that $T(d k / d T)$ contribution goes in. Similarly in the Bethe-Tait-type analysis where the fuel gets up to critical temperatures and pressures, I believe the Doppler again, even though there exists a liquid or whatever phase you want to call it, is simply based on the temperature dependence. There might be some value in looking whether there is a Doppler effect on melting. If it really is simply temperature dependent, one would assume that there is none. Secondly, the problem of what happens to the fuel melting and reassembling I think is the general intention of the Bethe-Tait analysis; namely, that the fuel can reassemble at as rapid a rate as it would like. We have insertions as large as 100 or 200 dollars per second. The only mechanisms that then take place involve the equation of state to disassemble this critical mass and the Doppler effect. However, we have noticed in some recent TREAT studies another factor which could be of extreme inportance. In one case where we melted uranium carbide, we found that upon cladding failure an exposure of this molten uranium carbide to the liquid sodium generated large local sodium pressures which causes gross dispersion of the fuel material, so that this may be another mechanism for fuel motion. Presumably if melting occurred at the peak portions of the core, the reactivity effects would tend to be negative under those conditions.

Mr. Denielou: In answer to the first part of your question, I must say that the Doppler effect is assumed to be zero in accident studies as soon as we are in liquid phase.

Mr. Campise (AI): In mentioning containment philosophy you implied low-pressure containment. Does this mean that you are going to a large building that is leak tight or are you essentially ignoring a possible sodium fire in the containment building as one of your accidents?

Mr. Denielou: At present we believe that we can avoid a large escape of sodium from the primary containment. Thus we are not going to a very large building. You are right in saying that we assume we are not going to have what we call a "lake of flame" type of accident, in which the sodium burns and generates very high pressures.

Mr. Burton (Risley): Concerning the experiments in which you looked at the possibility of a propagation of a subassembly blockage by fission gas release, were you able to reach your confident position without doing experiments in sodium at the right temperature? And if so, what criterion of failure did you use?

Mr. Denielou: Are you asking whether or not we have performed in-pile experiments?

$M r$. Burton: The experiments could be out of pile, but did you do them, for example, in watcr as one possibility or did you do them at temperature?

Mr. Denielou: Yes, with the right pressure and temperature. The next step would be to make them in sodium, but $I$ think the latter might be avoided because the conclusion will be that a violent explosion of the pin is highly improbable by itself. If it's improbable by itself, it cannot propagate because of the difference in different situations in the pins.

$M r$. Burton: Another possibility is that you'll get heat transfer effects locally when you release the fission gas.

Mr. Denielou: Local loss of cooling may result. We believe that this will have a loss-of-coolant effect if it propagates. If it's a factor of 10, we hope that this will not have bad loss-of-coolant effect. Anyway, it will be included in the overall possible causes for coolant loss, which, I say, we don't see how we can avoid. One pin exploding will surely cause some local coolant defect, but many other causes can provoke the difficulty.

Mr. Wolfe (GE): Can you tell us what accident your containment is designed for?

Mr. Denielou: Not now, because our containment is not designed.

$M r$. Wolfe: Well, you mentioned studies of containment. How do you study containment if you don't know the accident or the criteria for the accident?

Mr. Denielou: We do not proceed in a very logical way. We believe that we will be able to demonstrate that the accident is contained within the primary circuit. Again, we believe that we will never be able to demonstrate that there is no fission product release or no small sodium fire. Thus we must have a tight 
building first and then one that does not need to withstand a very high pressure.

Mr. Wolfe: I didn't understand whether you, for instance, postulated the Bethe-Tait aceident or meltdown accident, or were going to design for it.

Mr. Denielou: No, I don't postulate it. I expostulate against the misuse of Bethe-Tait, which has people looking into the detail of every accident. We believe that some part of Bethe-Tait accidents can be contained, for instance, equivalent to $500 \mathrm{~kg}$ of $\mathrm{TNT}$; this can be safely contained within the primary circuit with some extra care.
Mr. Charak (ANL): What is the primary circuit? Is it piping or building?

Mr. Denielou: It is concrete and in the inside you've got the piping, but it is concrete which withstands the pressure.

Mr. Charak: There is no air?

Mr. Denielou: There is inert gas within the containment we are speaking about, but there is air in the building itself.

Mr. Charak: And you preclude fires in the containment but not in the building?

Mr. Denielou: Yes. 


\title{
Loss-of-Coolant Accidents in a Large Sodium-Cooled Fast Reactor
}

\author{
A. M. JUDD \\ L. K. Atomic Energy Authority \\ Winfrith, England \\ (PRESENTED BY E. IIICKS)
}

\section{INTRODUCTION}

This paper will explore the possibilities of analysing fast reactor accidents more realistically than has been attempted before.

The safety of a large sodium-cooled ceramic-fuelled fast reactor against overheating accidents depends on guarding against three potentially dangerous factors. Firstly, as in all fast reactors, the fuel is not arranged in its most reactive configuration, so that in an accident involving collapse of the core structure, the fuel could be rearranged to become prompt critical. Secondly, the sodium void coefficient of reactivity is positive over the central region of the core, so that it may be possible to achieve prompt criticality by losing coolant from part of the core, for example by local boiling, Thirdly, the fuel opcrates at very high temperature, which in the case of oxide may exceed $2000^{\circ} \mathrm{C}$, so that if the core does collapse, allowing hot fuel and coolant to come in contact, there is a possibility of explosive vapourisation.

In addition, safety may be affected by the details of the design of the specific reactor. For instance, the fuel cans may be pressurised by the buildup of fissionproduct gas so that if a can is broken, this gas is released into the coolant stream to cause ovcrheating and bursting of other cans.

During the course of its life the reactor may be subject to unscheduled perturbations of various degrees of severity, such as reductions or blockages of coolant flow, affecting all or part of the core, bursting of faulty pressurised fuel pins, or accidental reactivity additions. It is necessary to know whether such an incident can bring into play one of the more dangerous mochanisms mentioned above, and so turn into a major accident.

It is relatively simple to calculate the effects of a flow or reactivity perturbation if the integrity of the fuel is maintained, and there are no phase changes (i.e., the fuel or cans do not melt and the coolant does not boil). At the other end of the scale, it is possible to do explosion calculations to estimate the severity of a prompt-critical excursion when the whole of the core structure collapses. This paper attempts to fill the gap between these two types of calculations by following specific accidents as they develop beyond the point at which the coolant boils or the fuel collapses, and to predict in a realistic way the consequences of thesc accidents.

Work has been done on many of the individual mechanisms which are important in the development of such an accident. In this paper we try to gather these results together to present as coherent a picture as possible of the safety of the reactor, within the limitations of our method. Of course, we are unable to predict exactly what happens in every accident, but the exercise does enable us to see more clearly what are the important points in the design for the safety of the reactor, and also to see where our knowledge is most seriously lacking:

\section{SYNOPSIS}

\section{Summary of Method}

Basically the approach used here is to consider a series of fault conditions which might arise in a typical oxide-fuelled, sodium-cooled fast reactor. The accidents resulting from these faults are then analysed step-by-step, usually under some pessimistic assumptions, such as the failure of one or more reactor trip circuits.

There are, however, some mechanisms which are important for several of the accidents and require detailed consideration. Section three therefore is devoted to general discussions of certain aspects of failure, including:

coolant and fuel reactivity worth;

the behaviour of the coolant as it boils, and particularly the effects of nucleate boiling;

the release of fission-product gas from a burst fuel can.

In Section four these results are applied to the analysis of various accidents which are initiated by faults affecting either a single subassembly or the whole core. Because of the greater difficulty in detecting a single subassembly failure, these accidents are considered in greater detail. The initial faults are: 
TABLE 1. REACTOR MODEL

\begin{tabular}{|c|c|c|c|}
\hline & \multicolumn{3}{|c|}{ Dimensions (cm) } \\
\hline \multirow{8}{*}{$\begin{array}{l}\text { Core Height } \\
\text { Core Diameter } \\
\text { Diameter of Inner Enrich- } \\
\quad \text { ment Zone } \\
\text { Blanket Thickness: } \\
\text { Axial } \\
\text { Radial }\end{array}$} & \\
\hline & & $140 \mathrm{~cm}$ & \\
\hline & \multicolumn{3}{|c|}{$92 \mathrm{~cm}$} \\
\hline & \multirow{3}{*}{\multicolumn{3}{|c|}{$\begin{array}{l}23 \mathrm{~cm} \\
55 \mathrm{~cm}\end{array}$}} \\
\hline & & & \\
\hline & & & \\
\hline & \multicolumn{3}{|c|}{ Composition $(\mathrm{v} / \mathrm{o})$} \\
\hline & Fuel & $\begin{array}{c}\text { Stainless } \\
\text { Steel }\end{array}$ & Coolant \\
\hline Core and Axial Blankets & 36 & 21 & 43 \\
\hline Radial Blanket & 57 & 22 & 21 \\
\hline
\end{tabular}

Core Fuel is $\mathrm{UO}_{2} / \mathrm{PuO}_{2}$, Overall Density: $8.8 \mathrm{~g} / \mathrm{cm}^{3}$

Enrichment $[\mathrm{Pu} /(\mathrm{U}+\mathrm{Pu})]\left\{\begin{array}{l}\text { Inner Zone: } \\ \text { Outer Zone: } 20.4 \%\end{array}\right.$

Plutonium Isotopic Composition:

$\begin{array}{ll}239 & 80 \% \\ 240 & 16.8 \% \\ 241 & 2.8 \% \\ 242 & 0.4 \%\end{array}$

restriction of coolant flow in one subassembly;

sudden complete failure of coolant flow in one subassembly;

local coolant blockage affecting a small amount of the fuel in one subassembly.

We also consider whole-core accidents initiated by: coolant-circulating pump failure, and

sudden complete coolant flow failure occurring while the reactor is operating at power.

As a result of these analyses, certain problems stand out as being particularly important for the safety of the reactor, and these are discussed in Section five. They include the possibility of a single subassembly accident spreading to the whole core, and the time available for a trip mechanism to operate. Suggestions for future experimental and theoretical work are then made.

\section{Limitations of Method}

It is important to be quite clear as to exactly what can be accomplished by this approach. As a result of the limitations imposed by the use of the present method (which is to follow the development of specific accidents), we can expect to reach conclusions of the following types:

(i) We can place bounds on the severity of various transients (e.g., we can say that a certain accident cannot give more than a certain reactivity increase, or give rise to pressure pulses of more than a certain severity or duration).

(ii) We can pinpoint sensitive features of the design for safety (e.g., we may be able to show that if the rate of release of fission-product gas from a burst fuel can is prevented from exceeding certain value, failure of other cans is impossible).

(iii) We may be able to suggest areas where the present design is overcautious (e.g., we may be able to show that the margin between operating coolant temperature and can-failure temperature is unnecessarily wide).

(iv) We can gain increased confidence that no important dangers have been overlooked.

It is equally important to be clear as to what cannot be achieved. The two main limitations are:

(i) An accident is so complicated that we cannot hope to calculate exactly what happens. Instead, we have to concentrate on refining the limits we place on its severity.

(ii) We cannot prove that we have overlooked nothing, so there is always the possibility of a dangerous failure mechanism going unnoticed. Nevertheless, the more we study the problem, the greater is our confidence.

\section{PRELIMINARY CALCULATIONS}

In this section we discuss in general terms certain aspects of reactor failure to establish results for use in the analysis of specific accidents.

\section{Reactivity Worth of Coolant and Fuel}

\section{Reactor Model}

The reactor model which was used for reactivity calculations is described in outline in Table 1 . It is a typical mixed oxide-fuelled fast reactor producing 600 MIV (thermal), in the design of which no special steps have been taken to reduce the positive sodium void coefficient of reactivity. There are two radial fuel-enrichment zones to flatten the power distribution, so that the rating of the fuel at the inner edge of the outer zone is the same as that at the centre of the core.

The core is taken to consist of hexagonal subassemblies, each about $14 \mathrm{~cm}$ across flats. The inner and outer enrichment zones are of roughly equal volumes. Six subassembly positions in the outer part of the inner enrichment zone are occupied by control rods. For the purpose of this study, we ignore the differences in radius from the core axis between different subassemblies in one hexagonal row, so that all the subassemblies of a row are taken to have the same power rating and reactivity worths. The central seven subassemblies 
TABle 2. Renctivity Changes due to Loss of Sodrum from Single Subassembles

\begin{tabular}{|c|c|c|c|c|c|}
\hline Subassembly row & 1 & 2 & 3 & 4 & 5 \\
\hline Number of core subassemblies in row & 7 & 12 & 12 & 24 & 24 \\
\hline Power developed relative to central subassembly & 1.00 & 0.95 & 0.88 & 0.99 & 0.90 \\
\hline Reactivity worth of complete subassembly $(\Delta k, \%)$ & 0.87 & 0.78 & 0.68 & 0.81 & 0.55 \\
\hline $\begin{array}{l}\text { Reactivity change due to loss of sodium from core section of one } \\
\text { subassembly }(\%)\end{array}$ & 0.0198 & 0.0161 & 0.0092 & -0.0005 & -0.0199 \\
\hline $\begin{array}{l}\text { Reactivity change due to loss of sodium from one axial blanket } \\
\text { section }(\%)\end{array}$ & -0.0026 & -0.0024 & -0.0024 & -0.0023 & -0.0018 \\
\hline $\begin{array}{l}\text { Maximum positive reactivity due to loss of sodium from centre } \\
\text { part of subassembly }(\%)\end{array}$ & 0.0218 & 0.0182 & 0.0118 & 0.0041 & 0 \\
\hline
\end{tabular}

We take the delayed-neutron fraction to be $0.3 \%$

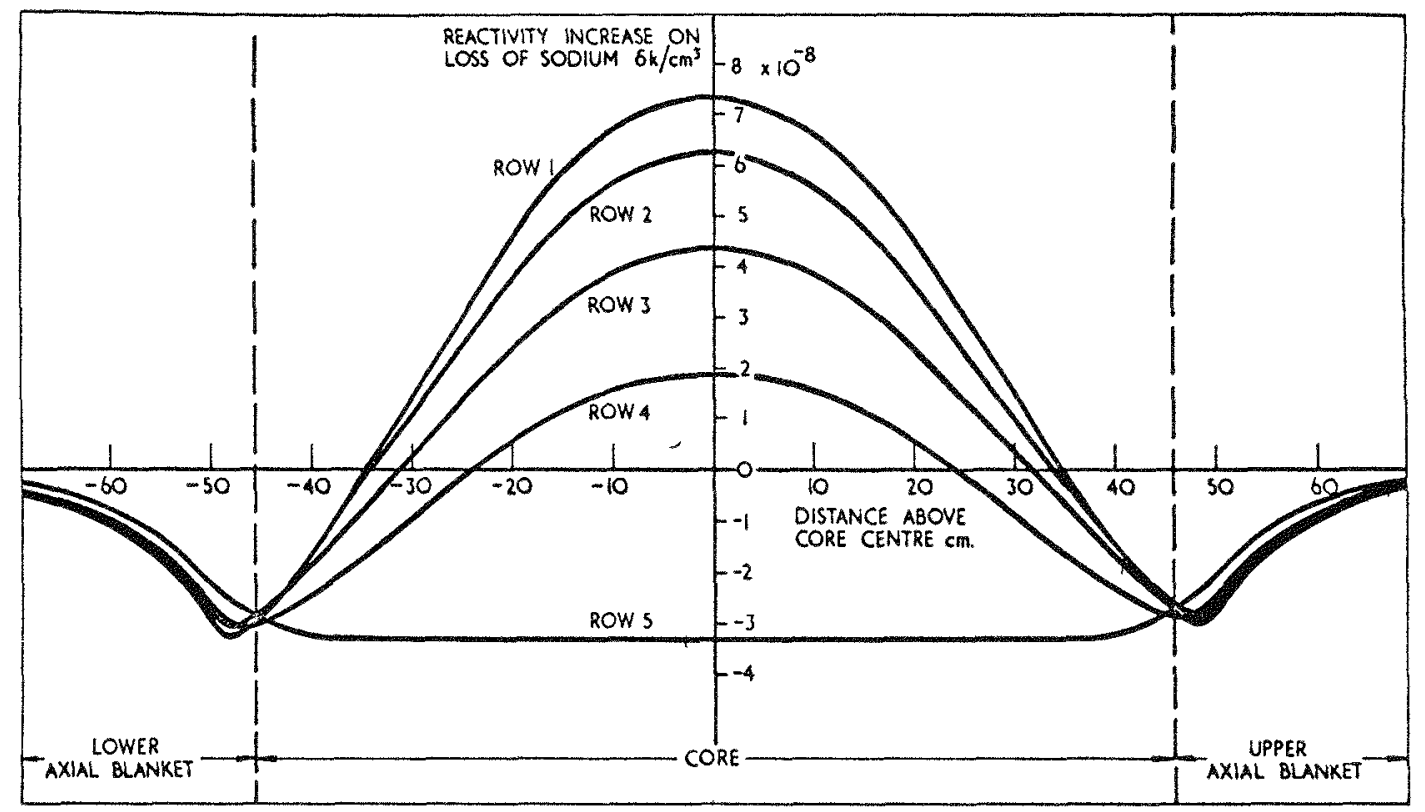

Fig. 1. Sodium Void Coefficient of Reactivity

are treated together as row 1 . The number of subassemblies in each row and their power rating are shown in Table 2.

\section{Sodium Void Coefficient}

The reactivity worth of loss of sodium was calculated by means of the CRAM diffusion-theory code ${ }^{(1)}$ with the FD 1 data set. ${ }^{(2)}$ Perturbation theory was used to find the worth of sodium at each mesh point, the mesh having been chosen to coincide with the rows of subassemblies. The results are given in Figure 1 , which shows the increase in reactivity due to the loss of $1 \mathrm{~cm}^{3}$ of sodium of density $0.833 \mathrm{~g} / \mathrm{cm}^{3}$ (correspond- ing to a temperature of $500^{\circ} \mathrm{C}$ ) from a point on the axis of a subassembly of each row, as a function of axial position. Table 2 also shows the reactivity increase due to loss of sodium from the core and axial blanket parts of one subassembly from each row, and also the maximum positive reactivity obtained by loss of sodium from the central part of a subassembly where the void coefficient is positive.

\section{Fuel Movement}

The reactivity effect of fuel movement in one subassembly has been estimated by first-order perturbation theory. If the fuel in a central subassembly melts, 


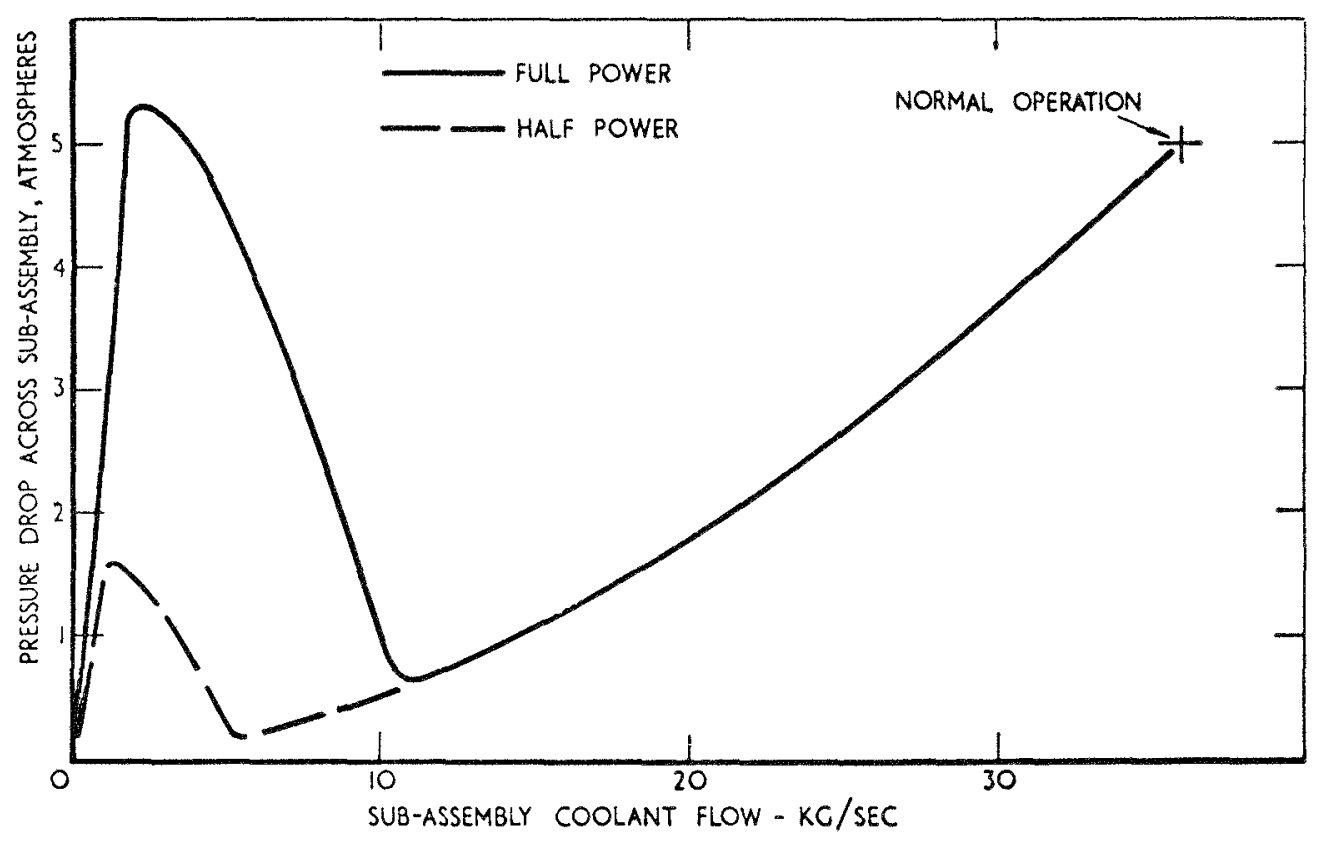

FIG. 2. Flow/Pressure Drop Characteristics for a Subassembly of Row 1

moves to the centre of the subassembly, and is compacted at its maximum theoretical density of 11.0 $\mathrm{g} / \mathrm{cm}^{3}$, there is a reactivity increase of $0.16 \%$, or about 56 cents, assuming that the axial blanket does not move. This estimate is not valid if the core undergoes a gross perturbation and can only be used for single subassembly accidents. For whole-core accidents in which the fuel in several subassemblies collapses, we have confined our attention to reactivity changes due to coolant loss, leaving a complete study for more detailed treatment.

\section{Doppler Effect}

The Doppler coefficient for the whole core is -4.6 $\times 10^{-6} \Delta k /{ }^{\circ} \mathrm{C}$ at a mean fuel temperature of $1000^{\circ} \mathrm{C}$, while the coefficient for heating row 1 alone is -0.72 $\times 10^{-6} \Delta k /{ }^{\circ} \mathrm{C}$. We assume the coefficient for heating the central subassembly is one-seventh of this, i.e., $-0.10 \times 10^{-6} \Delta \mathrm{k} /{ }^{\circ} \mathrm{C}$. The coefficient is taken to be inversely proportional to the mean fuel temperature.

\section{Coolant Behaviour}

\section{Steady-state Pressure Drop}

The relationship between the coolant flowrate in the central subassembly and the steady-state pressure drop across it is shown in Figure 2, taking the core inlet temperature to be $400^{\circ} \mathrm{C}$ and the temperature rise to be $200^{\circ} \mathrm{C}$. We have assumed that vapour is formed as soon as the sodium reaches its saturation temperature (the effects of superheating and nucleate boiling are discussed below).
While the coolant at the subassembly outlet is subcooled, the pressure drop is substantially independent of the heating rate, and decreases as the flow decreases and the outlet temperature rises. When the outlet temperature reaches the saturation temperature corresponding to the outlet pressure, boiling starts in the top of the subassembly. The large volume change on boiling gives high velocities in the boiling region, and a correspondingly large pressure drop due to friction and momentum increase, so that the pressure drop needed to maintain the flow increases rapidly as the flow decreases. The curves of Figure 2 were derived by the method described in detail by Stein, ${ }^{(3)}$ which is based on empirical correlations of two-phase flow data from experiments using water.

The effect of a partial blockage in the subassembly is to introduce an extra flow-dependent pressure drop which reduces the pressure drop available to maintain the flow in the manner shown in Figure 3. The reduced flow is now given by the intersection of the subassembly flow characteristic curve and the reduced pressure-drop curve (for example, $X$ in Figure 3 ). As the blockage increases, the flow decreases until a point $Y$ is reached. Here, boiling has started at the top of the subassembly in the axial blanket region.

The condition represented by $Y$ is, however, unstable, and a slight increase in the blockage gives a large change in flow as conditions jump to $Z$, where the flow is very small and most of the subassembly is filled with a mixture of liquid and vapour. This jump is known variously as a "flow excursion" or "choking." The nature of this flow excursion is not certain: it 


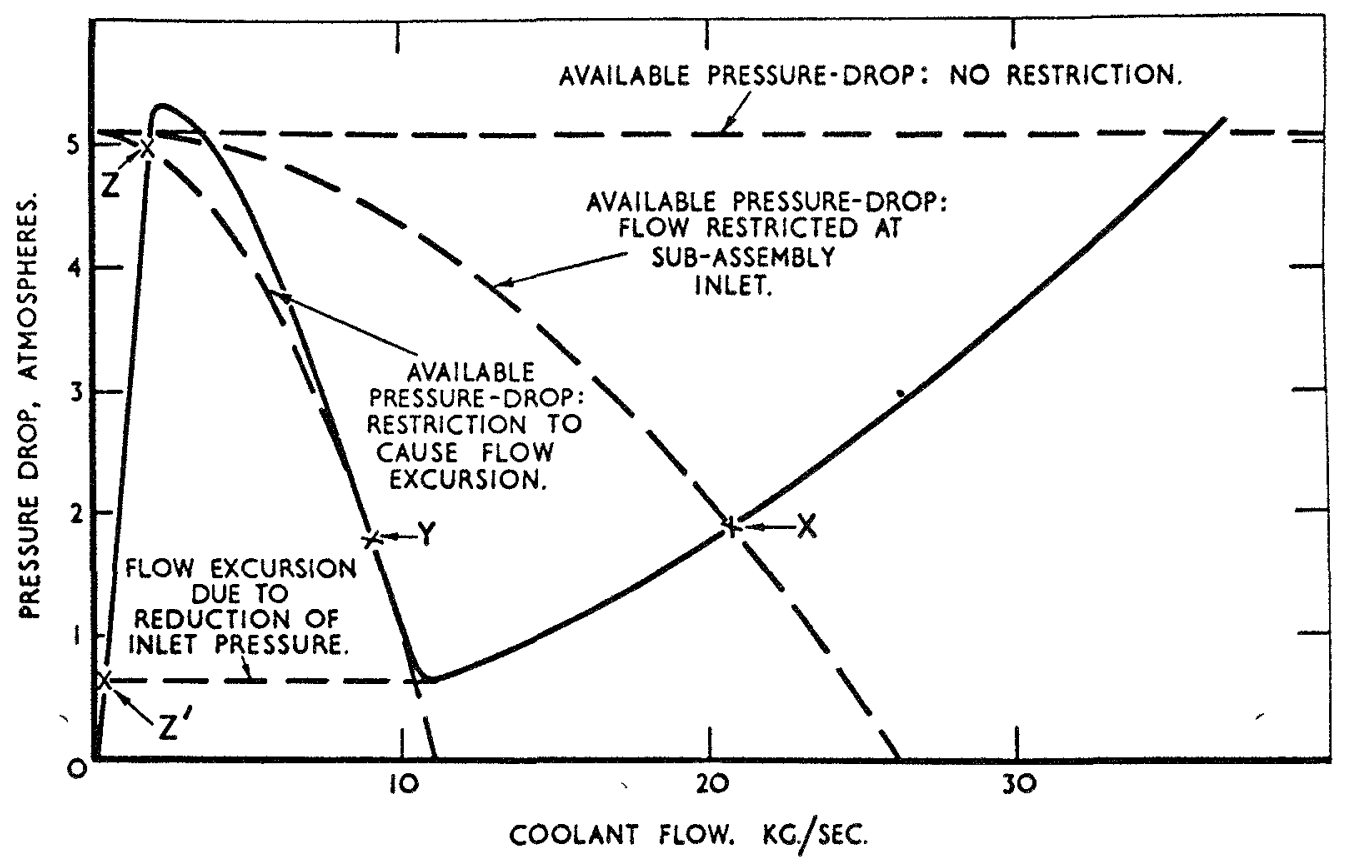

Fig. 3. Flow Excursions in a Subassembly of Row 1

cannot be instantaneous, as it is accompanied by a considerable rise in temperature of the fuel, and it may not be a smooth transition, especially if significant superheating of the liquid sodium takes place.

\section{Nucleate Boiling}

When a liquid boils on a heated surface, vapourisation is prevented by surface tension until the liquid temperature exceeds the saturation temperature corresponding to the ambient pressure. Vapour bubbles grow from nucleation sites, which may be envisaged as being small pits in the surface containing nuclei of trapped gas or vapour. Such a nucleus can only grow when the liquid temperature exceeds the saturation temperature by an amount (the superheat) depending on the tension in the curved surface of the trapped bubbles (and so on the radius of the site) and also on the ambient temperature. Water normally requires a superheat of $5-10^{\circ} \mathrm{C}$ when boiling on a normal enginecring surface, but there is experimental evidence to suggest that liquid metals require superheats as high as $400^{\circ} \mathrm{C}$ to initiate nucleation.

Figure 4 shows the superheat required to make a bubble grow as a function of the nucleation site radius for sodium at a pressure of $\mathbf{1 . 7} \mathrm{atm}$, at which the saturation temperature is $944^{\circ} \mathrm{C}$. It is based on the assumption that the surface tension falls linearly with temperature to zero at the critical temperature, which is taken as $2000^{\circ} \mathrm{C}$ for sodium. This value may be in error by $100^{\circ} \mathrm{C}$ or more, so that the high-temperature part of the curve for nucleation site radius less than $10^{-6} \mathrm{~cm}$ may be in error.

The actual superheat which occurs depends on the radius of the largest pits in the surface which are retaining bubbles of gas or vapour and so acting as nucleation sites. This, in turn, depends on the previous history of the surface and the liquid, and also on the cleanliness of the system. If the system is very clean, bubbles are retained only by the smaller pits, so that while water boils typically with a superheat of up to $10^{\circ} \mathrm{C}$, corresponding to a nucleation site radius of about $2 \times 10^{-4} \mathrm{~cm}$, superheats in excess of $100^{\circ} \mathrm{C}$ have been observed under very clean conditions, corresponding to radii of $10^{-5} \mathrm{~cm}$ or less. Also, if the system is particularly free from dissolved or adsorbed gas (as may be the case in a reactor after long periods of operation), few trapped gas bubbles will survive to form nucleation sites. Hoffman and Krakoviak ${ }^{(4)}$ report superheats in the range from 150 to $390^{\circ} \mathrm{C}$, depending on pressure, for potassium boiling in "as received" type 347 stainless steel tube. These results correspond to a site radius of about $4 \times 10^{-5} \mathrm{~cm}$. Thus it is likely that site radii in the range from $10^{-4}$ to $10^{-5}$ $\mathrm{cm}$ are appropriate for sodium boiling on normal engineering surfaces, corresponding to superheats of up to $300^{\circ} \mathrm{C}$. Moreover, making the system cleaner tends to increase the superheat.

The expansion of a bubble growing from a nucleation site is governed by the distribution of temperature and pressure in the moving liquid surrounding it. The problem cannot be solved analytically, and so far no 


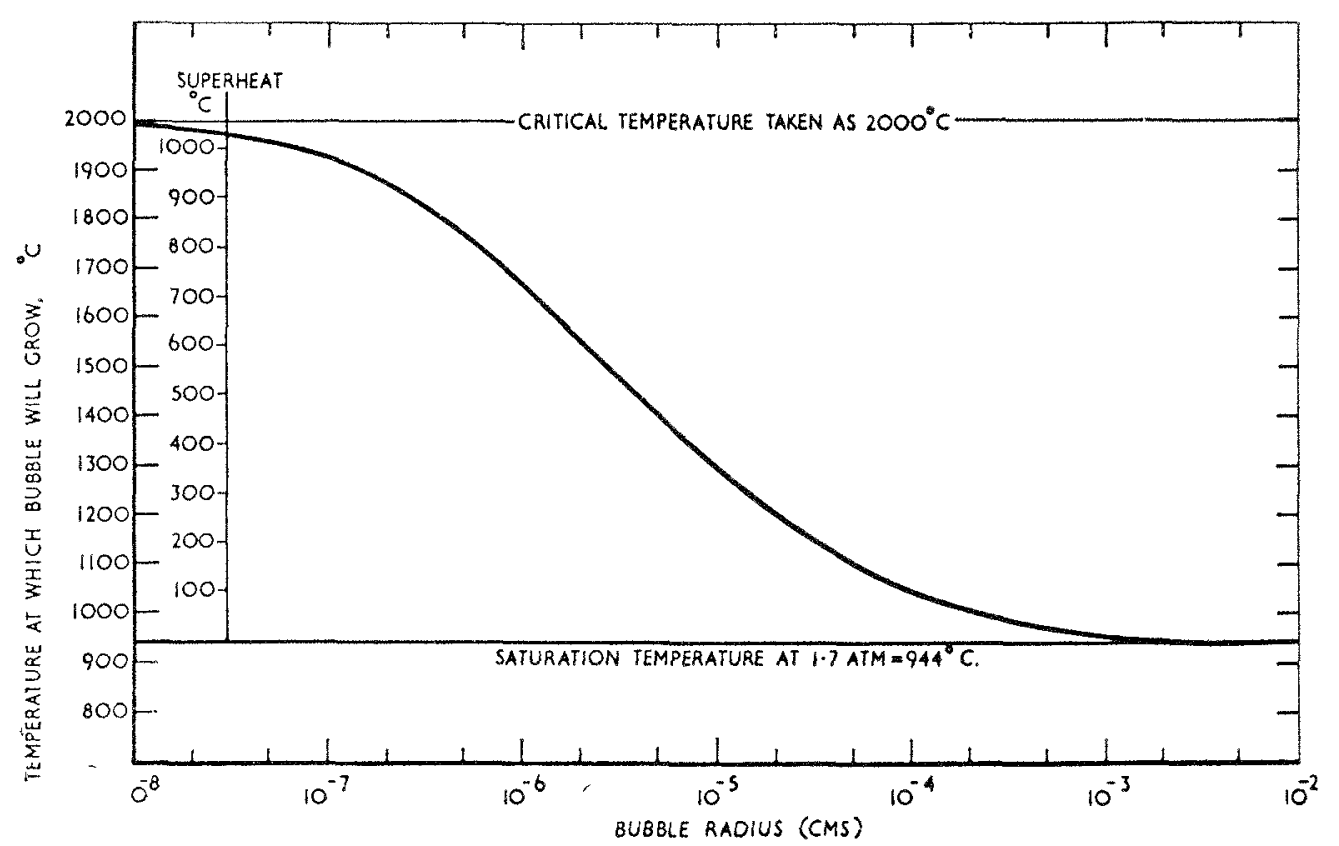

Frg. 4. Superheat and Nucleation Site Radius for Sodium Boiling at $1.7 \mathrm{Atm}$

accurate numerical solution has been attempted, but an approximate estimate of the pressure transicnt suggests that the pressure at a point $0.2 \mathrm{~cm}$ from the centre of an expanding bubble reaches a peak of about $10 \mathrm{~atm}$ in a pulse lasting for about $100 \mu \mathrm{s}$, if the nucleation site radius is $10^{-5} \mathrm{~cm}$ (i.e., the liquid sodium is superheated by $354^{\circ}$ to about $1300^{\circ} \mathrm{C}$ ). For a nucleation site radius of $10^{-6} \mathrm{~cm}$ (sodium at $1670^{\circ} \mathrm{C}$ ), the peak pressure is about $40 \mathrm{~atm}$, and the pulse lasts about $40 \mu$ s. These pressures are only rough estimates, but show that mechanical damage due to rapid expansion of bubbles in superheated liquid is unlikely.

\section{Coolant Ejection and Re-entry}

If the coolant flow in the central subassembly is suddenly stopped by complete blockage of the inlet, it is shown below that saturation is first reached just above the centre about 0.6 see after the flow stops. If the liquid sodium in the subassembly above this point is assumed to move as a rigid body, the forces acting on it and the rate at which it is ejected ean be found. The motion of the liquid-vapor interface is shown in Figure 5, assuming various amounts of superheating occur before vapour is produced. The top of the subassembly is voided in about 0.16 sec if there is no superheat. This is an oversimplified picture of what takes place, but it serves to indicate the time scale for coolant expulsion by boiling.

The liquid expelled from the top of the subassembly is followed by a stream of mixed vapour and liquid, possibly containing fission-product gas as well, which continues until most of the sodium has been lost from the subassembly. After this, the liquid sodium falls back into the subassembly and comes into contact again with the fuel which is now above the saturation temperature. Vapour is gencrated and the liquid blown out again. This proceses may be repeated in a cyclic manner, as observed for instance by Huebotter ${ }^{(5)}$ in experiments on a water model of part of the Enrico Fermi core. He reports a cyclic "chugging" motion with a period in the range from 0.2 to $0.5 \mathrm{sec}$, but does not give details of the pressures generated.

It is not clear how much sodium enters the subassembly in a "chugging" cycle of this type, but a rough estimate based on the correlation of limiting countercurrent liquid and gas flows due to Wallis ${ }^{(6)}$ and assuming coolant and fuel exchange heat through a $0.01-\mathrm{cm}$ vapour layer, suggests that the coolant falls back in to occupy about $30 \%$ of the core section of the subassembly before it is ejected again.

\section{Fuel-pin Behaviour}

\section{Temperature Distribution in the Fuel}

If the coolant flow in a subassembly is suddenly stopped by a blockage of the inlet, the temperature of the coolant and fuel rises. The temperature distribution in a single fuel pin and its associated coolant can be calculated analytically as a function of time after the flow stops. The variation of temperature in a fuel pin at the centre of the reactor is shown in Figure 6, assuming constant reactor power, for an 


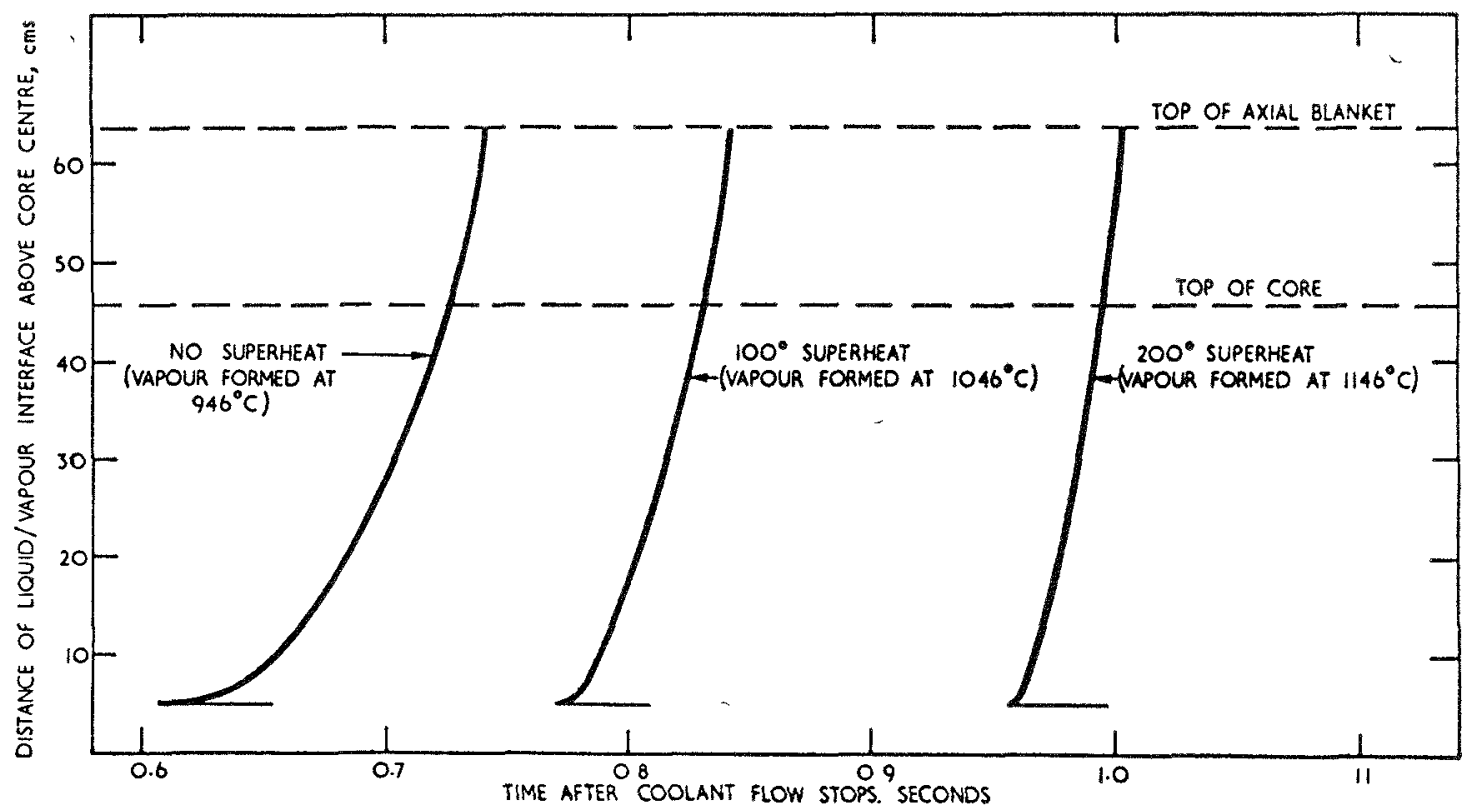

FIG. 5. Expulsion of Sodium from a Blocked Subassembly

oxide fuel pin of $0.25-\mathrm{cm}$ radius, clad in stainless steel $0.04 \mathrm{~cm}$. thick, with a fuel heat rating of $200 \mathrm{~W} / \mathrm{g}$. It will be seen that the coolant reaches its boiling point after 0.65 sec, and the can starts to melt after a further 0.51 sec.

\section{Explosive Vapourisation of the Coolant}

Interest in explosive release of energy by rapid vapourisation of the coolant as it comes into intimate contact with very hot fuel stems from the last of a series of transient tests with the SPERT-1 reactor, which are described by Miller et al. ${ }^{(7)}$ In this test, a light-water-moderated thermal reactor with highly enriched uranium-aluminum alloy fuel in the form of plates was subjected to a severe super-prompt-critical excursion. The nuclear excursion was terminated by expansion and boiling of the moderator, but $15 \mathrm{~ms}$ later an explosion occurred, giving pressures of $3-4000$ psi, destroying the reactor. The evidence suggests that the nuclear power was negligible when the explosion occurred.

It is suggested, although it is not certain, that the explosion was due to water coming in contact with molten fuel at a high temperature and in a very finely divided form. Heat was transferred very rapidly to the water, giving rise to high vapour pressures before the pressure could be relieved by expansion and the heat transfer rate reduced by the formation of a vapour blanket.

Until the SPERT results are adequately explained, it is impossible to say whether a similar explosion

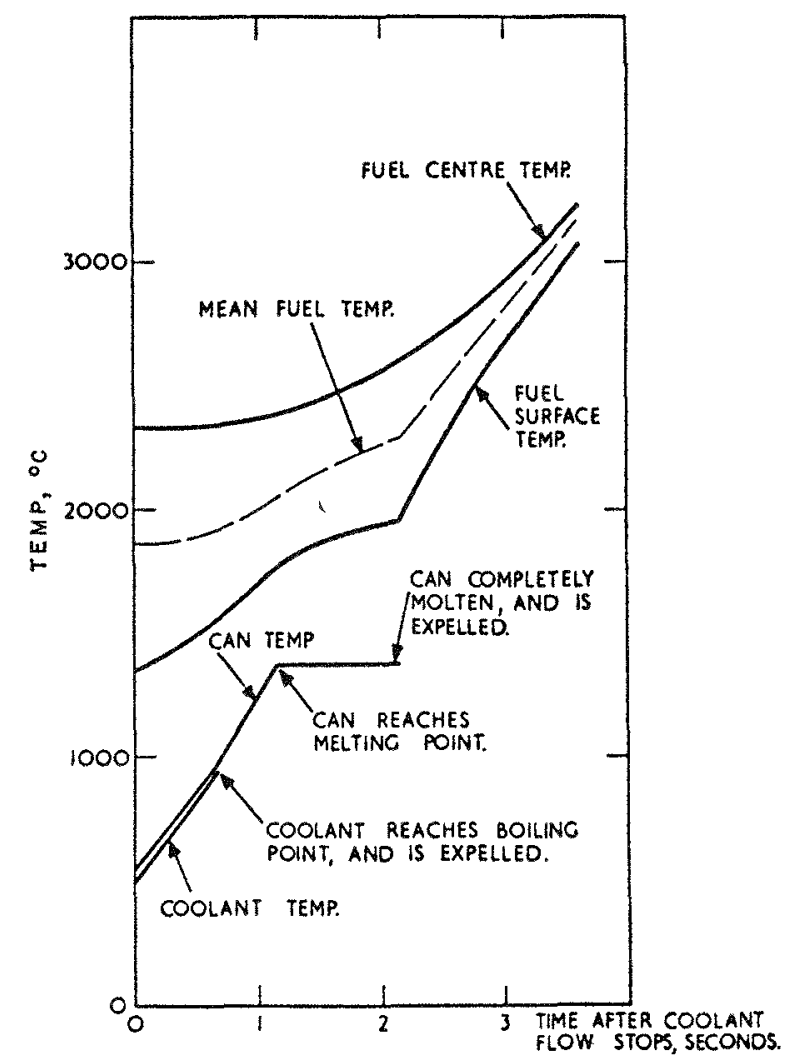

FIx. 6. Temperature Distribution in a Fuel Pin and Coolant after a Sudden Complete Flow Failure.

could take place in a different type of reactor. Nevertheless, even if the mechanism outlined above is not a full explanation of the SPERT results, it might in a 


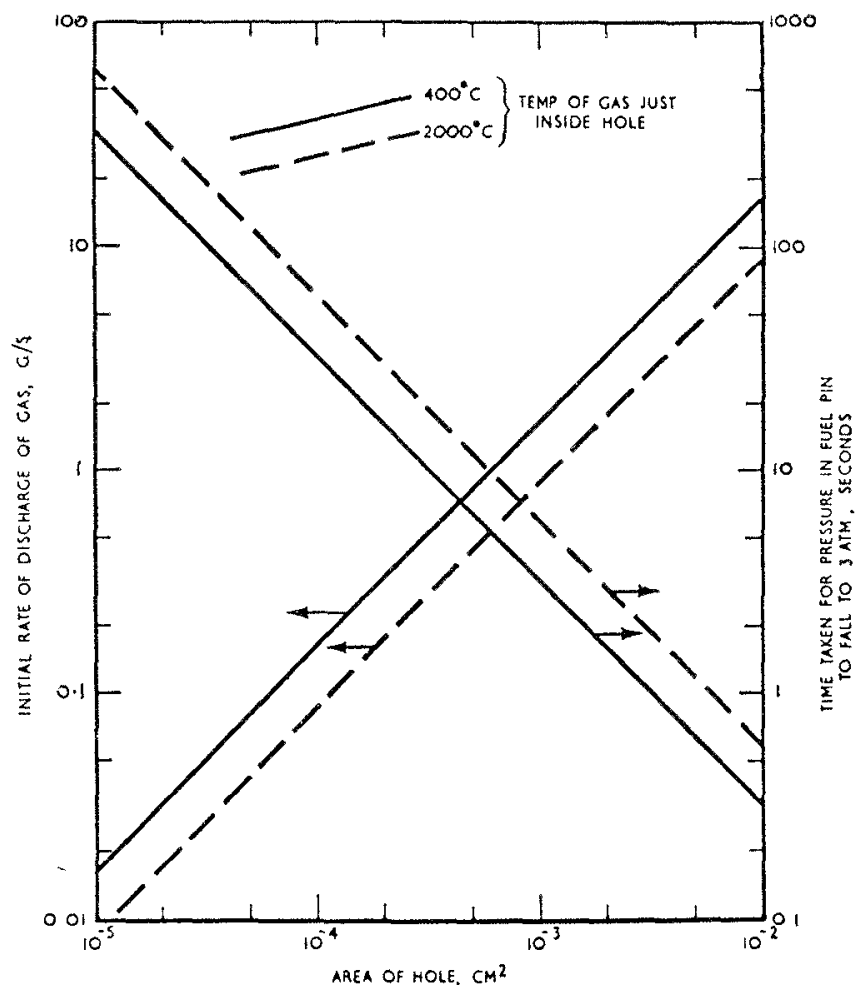

Fl(x. 7. Discharge of Fission-product Gas from Burst Fuel Pin

modified form give the rapid energy release in an oxide-fuelled fast reactor.

The situation envisaged is one where a subassembly, say, has been voided of coolant long enough for most of the fuel to reach a temperature near vapourisation, and also the fuel has broken into small pieces. The sodium then falls back in, becomes intimately mixed with the hot fuel, is heated very rapidly, and expands explosively. For this to happen, a large fraction of the fuel must be in a finely divided form, the sodiun must be mixed with it intimately and very rapidly in droplets which are small enough for their temperature to rise close to that of the fuel before they have time to expand and relieve the pressure, and mixing must take place in the right proportion to maximise the account of work available.

Further consideration of this process is beyond the scope of this paper; a more detailed study is presented by Hicks. ${ }^{(8)}$ It must be noted that although such an incident appears to be very unlikely, it cannot at this stage be ruled out as impossible for an explosion to occur in this manner, which could seriously distort the rest of the core and prevent shut-down of the reactor.

\section{Release of Fission-product Gas}

On irradiation to $10 \%$ burnup, each gram of fuel can release up to about $12 \mathrm{mg}$ of fission-product gas. If the fuel pin is pressurised, and a volume equal to the fuel volume is available to accommodate this gas at the sodium inlet temperature of $400^{\circ} \mathrm{C}$, it raises the pressure in the fucl pin to $45 \mathrm{~atm}$ (assuming it has the properties of xenon).

If for some reason the can is punctured, this gas escapes at a rate limited by sonic choking of the flow through the hole, until the pressure in the pin falls to about $3 \mathrm{~atm}$. Figure 7 shows the initial rate at which gas is released as a function of the area of the hole, and also the time taken for the pressure to fall to 3 atm, assuming the fuel in the can offers no resistance to the flow of gas from the storage space to the hole. Two values for the temperature of the gas as it reaches the hole, $400^{\circ} \mathrm{C}$ and $2000^{\circ} \mathrm{C}$, were taken, corresponding to the gas either taking up the maximum fuel temperature, or receiving no additional heat as it flows from the storage space.

\section{Cascade Failure}

It is possible that gas released from one broken pin might cause overheating of an adjacent pin, so that it bursts and releases more gas, causing failure of a third pin, and so on through the subassembly. If a fuel pin is completely blanketted by gas, so that it loses no heat, it takes the can at least 0.6 sec to reach the melting point of $1250^{\circ} \mathrm{C}$. Thus it is clear from Figure 7 that for a failure to spread in this way, bursting of the first pin must give a hole of area less than $5.4 \times$ $10^{-3} \mathrm{~cm}^{2}$ (at $400^{\circ} \mathrm{C}$ ) or of $10^{-2} \mathrm{~cm}^{2}$ (at $2000^{\circ} \mathrm{C}$ ) if the discharge is to last long enough to cause failure of another pin.

In addition to this upper limit on the size of the hole, it is possible to estimate a lower limit. It is difficult to see how the stream of gas can cause significant overheating of the adjacent pin if it occupies less than about $0.01 \mathrm{~cm}^{2}$ of the coolant flow area, which is equivalent to a stream of bubbles of about $0.1-\mathrm{cm}$ radius. This bubble stream cannot move more slowly than the bulk coolant velocity of about $1000 \mathrm{~cm} / \mathrm{sec}$, which implies that the minimum rate of release of gas to cause damage to an adjacent pin is about $0.04 \mathrm{~g} / \mathrm{sec}$. Figure 7 shows that this requires a hole of area greater than $2.5 \times 10^{-5} \mathrm{~cm}^{2}$ (at $400^{\circ} \mathrm{C}$ ) or of $4.7 \times$ $10^{-5} \mathrm{~cm}^{2}$ (at $2000^{\circ} \mathrm{C}$ ). The details of this argument may be open to criticism, but it is clear that some sort of lower limit can be arrived at in this way.

Thus, although we cannot rule out the possibility of a cascade failure of this type, it seems to be very unlikely. It needs all the fuel pins to fail, giving holes of which the areas fall in a narrow range, and it is inhibited by the fact that when one pin bursts, the jet of gas coming from it will be directed mainly towards the pins which have already failed. 


\section{ANALYSIS OF SPECIFIC ACCIDENTS}

\section{Single-subassembly Accidents}

Conditions to Cause Accidents

An overheating accident affecting initially a single subassembly can only be caused by a reduction of the coolant flow. To cover the range of possibilities we consider the following situations. First, we take the case of a reduction of the coolant flow due to a blockage at the subassembly inlet, which gradually increases in severity due to, say, accumulation of some sort of debris. Secondly, we consider sudden complete blockage of the coolant inlet, so that the flow stops instantaneously. The inlet would be designed to prevent this, but it does represent a worst possible flow failure. Thirdly, we have a local blockage, caused, for example, by accumulation of debris between the fuel pins, causing local flow reduction severe enough to make one or two pins fail.

In all cases we assume the accident happens to the central subassembly, as the reactivity changes due to fuel and coolant movement are most severe, and the power density highest, at the core centre.

\section{Partial Blockage of the Central Subassembly}

As the coolant flow is restricted, the subassembly outlet temperature rises until boiling starts in the axial blanket region. The bubbles of vapour released collapse violently in the subcooled sodium above the core. The flow in the subassembly in this situation is represented by the point $\mathrm{Y}$ in Figure 3.

Further increase of the blockage causes a flow excursion to $\mathrm{Z}$ in Figure 3, as explained above. The distribution of vapour and the temperature profile before and after the excursion are shown in Figure 8. The reactivity increase due to loss of sodium in the excursion is 5.5 cents. The nature of the transition is not certain, and it may not take place smoothly, especially if significant superheating of the liquid sodium takes place. As pointed out above, however, the pressures due to bubble growth in superheated sodium are unlikely to be severe enough to cause mechanical damage. There may be rapid reactivity fluctuations as the coolant is thrown about, but a maximum positive reactivity of 7.3 cents cannot be exceeded. The efforts of such reactivity changes on the reactor power on the time scale of the accident is negligible.

If the fuel pins are pressurised and near the end of their life, they may be weakencd by irradiation and fail due to the fission-product gas pressure before the sodium boils. The consequent release of fission-product gas may interrupt the coolant flow, but this is unlikely to have a significant effect. If the coolant flow is to

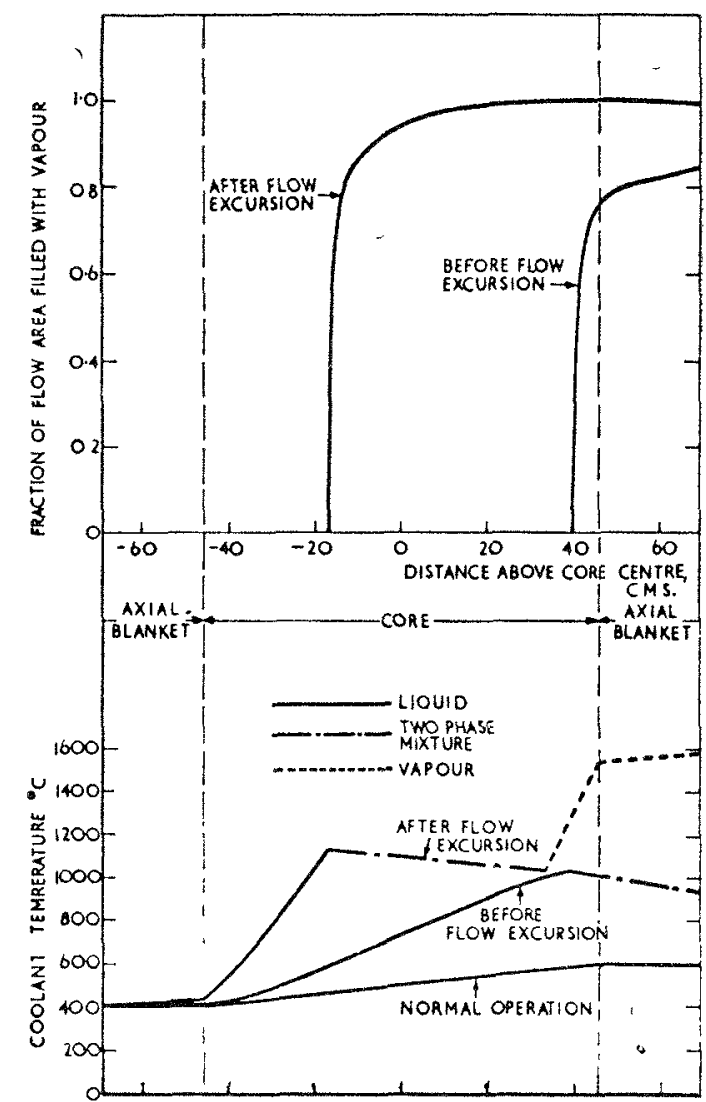

$F_{I G}$ 8. Vapour and Temperature Distribution in a Subassembly before and after a Flow Excursion.

be stopped, the gas must raise the pressure at the top of the subasscmbly above the inlet pressure, in which case the gas itself will be blown out of the subassembly at the sonic velocity of about $3 \times 10^{4} \mathrm{~cm} / \mathrm{sec}$. This would allow all the gas stored in the fuel pins to be discharged in less than $0.1 \mathrm{sec}$, so this is the maximum time for which the coolant flow can be interrupted. During this time the can surface temperature could only increase by $80^{\circ} \mathrm{C}$.

Within a second of the flow excursion taking place, the fuel cans at the top of the core will melt, as indicated in Figure 8, allowing the pins to collapse, and releasing any remaining fission-product gas. The reactivity change due to the Doppler effect up to the time the fuel pins collapse is about -0.5 cent. It is impossible to follow the progress of the accident in detail beyond this point. The maximum reactivity increase if all the fucl should collapse to the centre of the subassembly is 56 cents. It may be, however, that the debris of broken fuel and molten cans will be blown upwards by the sodium vapour stream to block the subassembly outlet. This, in turn, may cause expulsion of vapour downwards below the core.

If all the subassembly power goes into boiling so- 


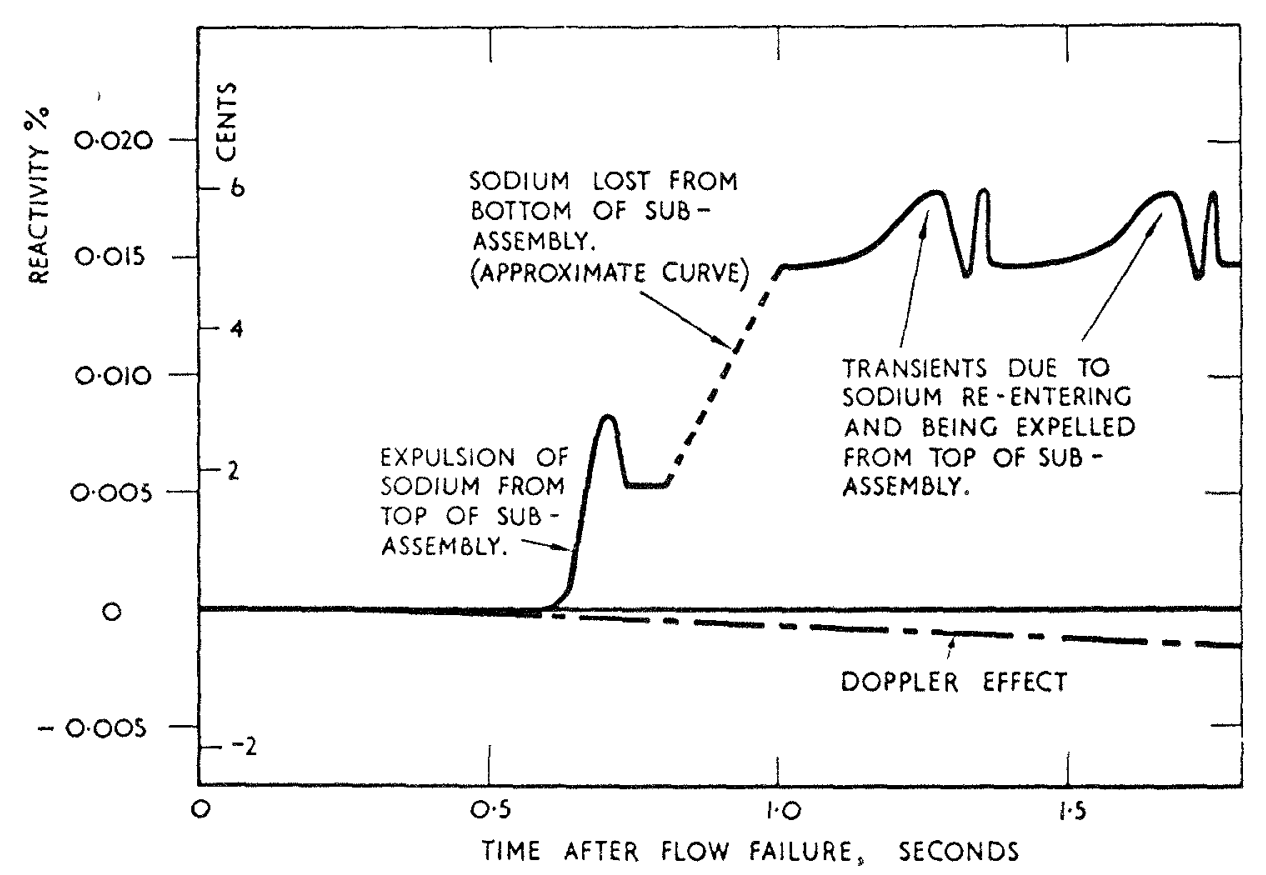

Fra. 9. Reactivity Changes due to Coolant Movement and Doppler Fffect on Sudden Complete Blockage of a Single Subassembly

dium, enough vapour is produced to fill the subassembly in $7 \mathrm{~ms}$. Such a large rate of vapour production can only continue for a few seconds, and a "chugging" motion, with liquid coolant entering the core region and being blown rapidly out again in a cyclic manner (as described above), must soon supervene.

If vapour gets below the core, there is a danger that it will enter other hitherto unaffected subassemblies, causing a positive reactivity transient, and maybe more melting. Against this, due to the high conductivity of sodium, any vapour entering the cold region below the core will condense quickly. It is not possible to analyse this situation without knowing how rapidly vapour can be generated in a "chugging" state. If vapour fills the six subassemblies adjacent to the one initially affected from the bottom upwards, the reactivity increases by a further 36 cents.

\section{Sudden Complete Blockage of the Central Subas- sembly}

Afer the coolant flow stops, the temperature in all the fuel pins except those nearest the wrapper rise, as shown in Figure 6. The coolant reaches saturation 0.6 sec after the blockage occurs, and the liquid sodium is expelled from the top of the subassembly in a further $0.16 \mathrm{sec}$. Loss of sodium from the top of a central subassembly gives a peak positive reactivity of 3.6 cents and a net increase (when the upper axial blanket is voided) of 2.4 cents.

After this, most of the rest of the coolant in the core region of the subassembly is ejected and then "chug- ging" takes place as described above, probably with a period of $0.2-0.5 \mathrm{sec}$ and with the coolant re-occupying about a third of the subassembly at most on each cycle. The maximum possible reactivity increase due to sodium loss from the centre part of the subassembly is 7.3 cents. The reactivity change due to the Doppler cffect up to the time the cans melt is -0.3 cent.

This cyclic motion continues until the fuel pins collapse when the cans melt about 1.2 see after the initial flow failure. The maxinum possible reactivity increase if the fuel melts and collapses to the centre of the subassembly is 56 cents. The stream of sodium vapour leaving the subassembly may, however, blow the fuel away from the core centre until it is stopped by the cooler axial blanket which does not collapse so readily.

Figure 9 shows the reactivity changes due to coolant movement and Doppler effect for this accident. It will be seen that these are small compared with the possible effect of fuel movement.

The subsequent course of the accident is similar to that of the partial-blockage accident described above. It is impossible for a high pressure to be maintained by reaction against material being ejected from the top of the subassembly for long enough to force vapour downwards against the inlet pressure, and so to get beneath the core and enter other subassemblies. Only if the top of the subassembly becomes blocked with fuel pin debris would this be possible.

\section{Local Flow Blockage}

In the case of blockage of a small number of the coolant flow channels, can failure may be caused either 
by coolant boiling or by bursting under fission-product gas pressure.

The possibility of the latter type of failure spreading to the other fuel pins purely by fission product gas blanketting is discussed above: it is only possible if the burst can releases gas at a rate which falls in a limited range. Cascade failure of a large number of pins is only possible if each succeeding pin releases gas at a correspondingly limited rate. Gross melting of the fuel pins in the subassembly can only result from such a cascade failure if the cans are deprived of coolant for about 0.6 sec. Although such a cascade failure appears to be very unlikely because it requires the pins to fail in a very restricted way, it cannnot be ruled out as impossible.

If the cans are not pressurised, or do not burst at low temperature, failure may be started if the initial blockage is large enough to cause the coolant to boil. If this causes the cans to melt, fuel at a temperature above $1400^{\circ} \mathrm{C}$ will be exposed, and vapour will be generated rapidly. It seems unlikely that this vapour will cause failure of other pins, however, as the bulk of the sodium flow in the subassembly is still at least $350^{\circ} \mathrm{C}$ below the saturation temperature, so that any vapour bubbles which leave the overheated channelsthat is, the channels affected by the blockage-will collapse very rapidly. If the blockage is so severe that vapour is generated fast enough to interrupt the whole subassembly coolant flow, "chugging" will take place, as described above.

\section{Whole-core Accidents}

\section{Conditions to Cause Accidents}

A whole-core overheating accident which is of some interest is that caused by failure of the electrical supply to the coolant-circulating pumps. We consider this accident below, assuming that the trip mechanisms do not function and the reactor power remains constant. Also, to represent a worst possible whole-core coolant aceident, we consider the case of the inlet pressure falling suddenly to zero, due, for example, to a sudden large brcach in the bottom of the reactor vessel.

A whole-core overheating accident can also be caused by an increase in reactor power. This could be due, for instance, to the insertion of reactivity at a steady rate by a faulty control rod mechanism, giving a slow, uncontrolled power increase. The consequences of this are very similar to those of the pump-failure accident, and we do not consider it in detail.

As explained above, we do not consider here the reactivity changes in whole-core accidents due to fuel movement, as these cannot be regarded as being given by small perturbations of the reactor. We confine our attention to the reactivity effects of coolant loss.
Coolant-pump Failure

If the coolant pumps fail and the reactor is not tripped, the coolant flow falls as the inlet pressure falls until the highest rated subassemblies reach a point corresponding to the minimum of the full-power curve in Figure 3. A flow excursion now takes place in these subassemblics, but as the inlet pressure is low, the flow after the excursion is very low, corresponding to the point $Z^{\prime}$ in Figure 3, and almost all the core and upper axial blanket are filled with vapour.

If we assume that the flow halves in 10 sec, in the manner indicated in Figure 10, we can find the time at which each row of subassemblies is subject to a flow excursion. This happens in the central subassembly after $17.2 \mathrm{sec}$, and so on as indicated in Figure 10, until the whole core and upper axial blanket are voided of sodium. We assume the flow decay characteristics are unaffected by the accumulation of vapor in the coolant circuit.

We estimate the reactivity effect of coolant vapourisation in a simple way as follows. We assume that a flow excursion involves loss of coolant from the top axial blanket region of the subassembly in $0.1 \mathrm{sec}$ and from the core region in a further $1.0 \mathrm{sec}$, and that the reactivity changes given by these stages are made as smooth ramps. Using now the reactivity values given in Table 2 , we can obtain the overall reactivity change due to coolant vapourisation. This is shown in Figure 10. It will be seen that after the flow excursion occurs in row 2, a peak reactivity of more than 50 cents is reached for half a second, but after the excursion in row 5 , the reactivity stays negative. It is important to note that the time scale for this accident is much longer than for any of the other accidents we have considered.

\section{Sudden Complete Whole-core Flow Failure}

The consequences of this accident for each individual subassembly are very similar to those of the sudden complete blockage discussed above. The coolant temperature rises as shown in Figure 6 and is expelled in the manner indicated in Figure 5, only in this case, as the inlet pressure is low, the coolant is expelled in both directions.

Figure 5 applies to the central subassembly, but we assume that it can be used for other subassemblies by increasing the time scale in inverse proportion to the power rating. Making this assumption and using the values of sodium void coefficient of Figure 1, we can find the reactivity transient due to sodium loss in this accident. This is shown in Figure 11. It will be seen that the reactivity reaches a positive peak of 70 cents and stays positive for less than 0.2 sec, after which it is strongly negative. 


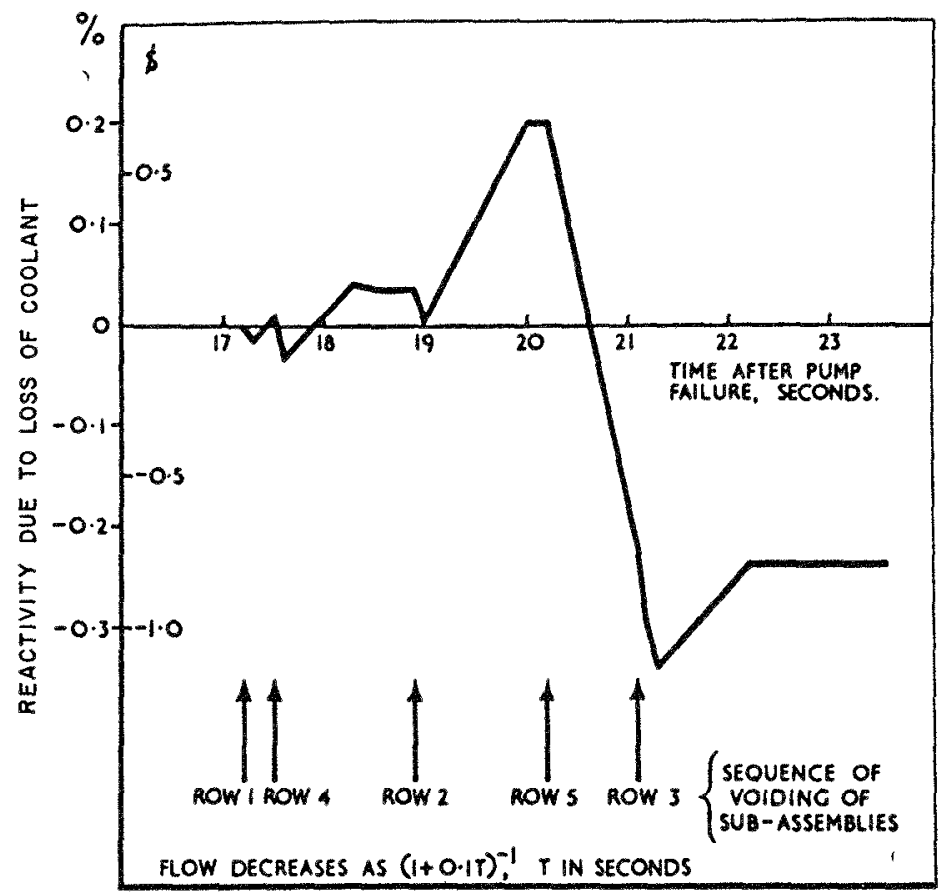

FIG. 10. Reactivity Changes due to Loss of Coolant in a Pump-Failure Accident

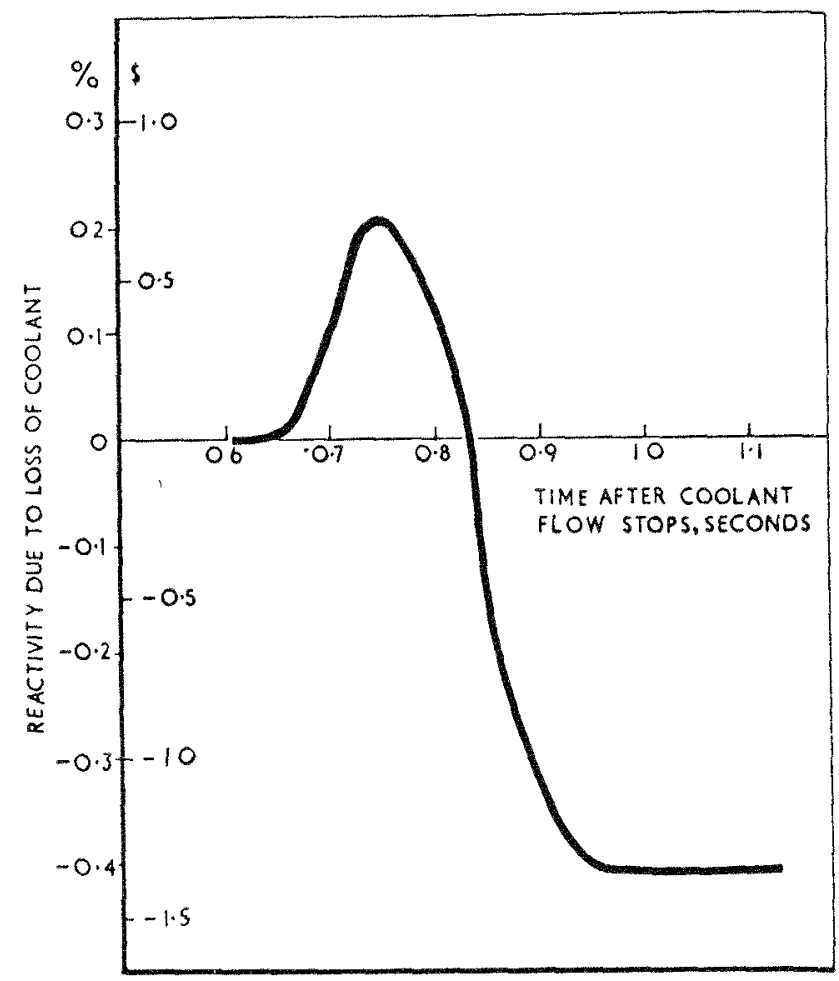

Fig. 11. Reactivity Changes due to Loss of Coolant after a Sudden Complete Flow Failure.

In this case, as can melting and collapse of the fuel cannot occur until $1.2 \mathrm{sec}$ after the flow failure, there is no possibility of reactivity loss due to fuel movement reducing the peak reactivity due to sodium loss.

\section{CONCLUSIONS}

\section{Positive Sodium Void Coefficient}

\section{Maximum Reactivity due to Loss of Sodium}

For the reactor described here, the maximum positive reactivity that can be reached by voiding sodium from the centre of one subassembly is 7 cents (see Table 2). Even allowing for inaccuracy in the data used in our calculation of the void coefficient, it is clear that loss of sodium will not in itself give a serious reactivity increase in an accident which is confined to a single subassembly.

The maximum positive reactivity that can be reached by voiding just the part of the whole core for which the void coefficient is positive, is nearly $\$ 2$. It might be suggested that for this reason such a reactor design is undesirable and should be altered to reduce this maximum reactivity. This may be economically undesirable, as making the sodium void coefficient more negative may impair the breeding propertics of the system and may also increase its engineering complexity.

Our analysis of specific accidents suggests, however, that it is not necessary to consider this maximum possible reactivity as a criterion for assessing the significance of a positive sodium void coefficient. The greatest positive reactivity due to sodium loss which can be reached in the more realistic, but still very pessimistic, accident conditions we have considered is $\$ 0.7$. We suggest that an analysis of this type gives a 
less restrictive, and yet still adequately cautious, criterion for assessing a positive sodium void coefficient. It also has the advantage of showing clearly the conditions necessary for such an accident to take place, so giving a better idea of its probability.

\section{Control of the Maximum Reactivity}

The severity of the reactivity addition in these accidents is reduced in two ways. Firstly, when boiling occurs, coolant is lost very rapidly from the whole length of a subassembly, so that the positive reactivity contribution from the centre is rapidly followed by a negative contribution from the ends. Secondly, the high power density in the outer enrichment zone of the core ensures that a negative effect due to voiding the outer subassemblies comes very soon after the positive effect from those near the core centre.

This may afford a useful means of controlling the effect of the positive sodium void coefficient, instead of altering the design of the reactor to reduce the coefficient and incurring economic penalties. If the power density in the outer zone is made such that in a whole-core accident boiling occurs first in the outer region of the core, the reactivity transient due to loss of sodium could be made to start with a negative change, and the positive reactivity peak reduced or eliminated.

It should be noted that these conclusions are based on the above analysis of whole-core accidents, which take no account of reactivity changes due to movement of coolint after the first expulsion from a subassembly or due to collapse of the fuel. We are not able to assess the reactivity effect of, for instance, the re-entry of sodium into the core, and our conclusions may be radically changed if this is found to be important.

\section{Pressurised Fuel Pins}

If the fuel pins are sealed to prevent the escape of fission-product gas, the increasing pressure in the pins and the decreasing strength of the cans under irradiation will cause the cans to burst before the melting point of steel is reached, and possibly even before the sodium boils. Only in the latter case, however, is there a possibility of a significant effect on the safety of the reactor, because if the coolant boils, the fuel will almost certainly collapse by melting if not bursting of the cans.

The possibility of the bursting of a single faulty pin causing cascade failure of all the pins in a subassembly has been discussed above. Although this cannot be dismissed as being impossible, it is very unlikely, as it depends on all the pins bursting in a restricted manner.

Apart from this, qualitatively the effects of an accident in which the cans burst and release fissionproduct gas rapidly are very similar to those of an accident in which the cans are intact until the coolant boils. In either case the subsequent course of the accident depends on the interruption of the coolant flow and the expulsion of coolant from the subassembly. The main difference is that failure occurs earlier if the cans burst below the coolant boiling point, so that, for example, a partial blockage of a single subassembly has to be less severe to cause a flow excursion, and in the case of the coolant-pump failure less time elapses before flow excursions occur in the highest rated subassemblies.

\section{Superheating of Liquid Sodium}

It is not clear whether liquid sodium can be superheated well above the saturation temperature in the manner described above in an operating reactor or not. If it can, there are two effects on the safety of the system. Firstly, the formation of vapour is delayed until a higher temperature is reached. This is an advantage, as for example it may enable a subassembly to survive a transient partial blockage of the coolant flow which otherwise would cause melting.

Secondly, when vapour is formed under conditions of high superheat, the bubbles will grow much more rapidly, which may give high pressure pulses and rapid reactivity changes. As pointed out above, the pressure transient due to an expanding bubble is unlikely to be severe enough, even for superheating to $1300^{\circ} \mathrm{C}$, to do significant mechanical damage.

In the accidents considered above, the possible rapid addition of reactivity is unimportant, as the reactor we have considered is always well below prompt critical while it is being subject to reactivity changes due to coolant movement, but in a reactor with a much more positive sodium void coefficient, it might be possible for the violent expulsion of superheated sodium from a subassembly to give a high rate of addition of reactivity at prompt critical, so increasing the energy yield of the consequent excursion.

\section{Effect of a Single-subassembly Accident on the Rest of the Core}

An accident which is initially confined to a single subassembly can affect other subassemblies by changes in reactivity, temperature, or pressure, or by interruption of the coolant flow.

The reactivity changes due to fuel and coolant movement in a single-subassembly accident cannot approach a dollar, so the resulting power transient will take place slowly. Damage to the rest of the reactor will be prevented by a high-power trip system.

Temperature changes in one subassembly are in- 
sulated from the rest of the core by two wrappers, the combined thickness of which would be about $0.5 \mathrm{~cm}$, cooled on one side by flowing sodium. If the fuel in the damaged subassembly is spread in a layer $0.2 \mathrm{~cm}$ thick on the inside of the wrapper, and the inside of this layer is at $3500^{\circ} \mathrm{C}$ (approximately the vapourisation tempcrature), it takes about 10 sec to melt through the two wrappers. It should be possible to shut down the reactor in a time of this order.

The pressures generated in expelling liquid coolant from a subassembly or in the growth of vapour bubbles in superheated coolant are too small to do significant mechanical damage. It might be possible, however, for an accident to spread to unaffected parts of the core by means of a pressure pulse produced by explosive vapourisation of the sodium as described above. This is outside the scope of this paper, but must be noted as a possibility.

If the outlet of a subassembly were blocked with molten fuel and all the available fission-product gas were ejected downwards, it would occupy a considerable volume below the core. It would be necessary to design the sodium inlets so that the volume of gas which could be released if all the fuel pins in one subassembly were broken would be trapped and not allowed to rise into adjacent subassemblies and cause overheating.

Under the worst possible conditions, sodium vapour could be generated so fast in a blocked subassembly that no trapping system could cope with it if it were expelled downwards. In this case, however, the vapour would condense very rapidly, and it seems unlikely that bubbles would last long enough to interrupt the sodium flow in other subassemblies.

\section{The Time Available to Trip the Reactor}

There is little difficulty in shutting down the reactor in a whole-core accident, as monitoring coolant temperature or flow or neutron flux, for example, afford several independent means of detection. Only in an accident as violent as a sudden complete flow failure is there any difficulty in shutting down quickly enough.

Detection of a single-subassembly accident may be much more difficult. It may be impossible to monitor the outlet temperature from every subassembly, and, in addition, in an accident involving complete blockage of the coolant flow the outlet temperature will not rise until the coolant is being expelled from the subassembly as it boils.

Even if it is possible to detect the onset of vapourisation it is unlikely that can melting and collapse of the fuel in the affected subassembly can be prevented, as this may follow within 0.6 sec. This is probably too short a time for a trip mechanism to act.
Provided it can be shown, however, that expulsion of vapour below the core cannot affect other subassemblies, there is no possibility of damage spreading to the rest of the core until the hot fuel melts through the wrappers in about 10 see. Thus, although gross damage to the affected subassembly cannot be prevented, it is possible to prevent major damage to the rest of the core.

Once again we have not considered the problem of preventing explosive vapourisation of the coolant, and this may present a more acute detection problem.

\section{Summary of Conclusions}

1. For the reactor considered here, the maximum positive reactivity due to loss of coolant actually reached in a whole-core accident is 70 cents, compared with a maximum of $\$ 2$ on voiding the centre part only of the core.

2. The method outlined here gives a realistic means of assessing the importance of a positive sodium void coefficient of reactivity.

3. The peak reactivity due to loss of sodium in a whole-core accident can be reduced by increasing the relative power density in the outer part of the core.

4. Cascade failure of all the fuel pins in a subassembly precipitated by release of fission-product gas from a single faulty pin is unlikely, but has not yet been shown to be impossible.

5. Apart from 4 above, the only effect of a pressurised fuel-pin design on the safety of the reactor is a possible reduction of the coolant temperature at which the fuel suffers damage.

6. Superheating of liquid sodium probably has little effect on the safety of the reactor, except in a design with a more positive sodium void coefficient, for which it might provide a means of increasing reactivity rapidly at prompt eritical.

7. Accidents can only spread from a single subassembly to the whole core by expulsion of vapour below the core (which seems to be very unlikely), by melting through the subassembly wrapper (which takes of the order of $10 \mathrm{sec}$ ), or by explosive vapourisation of the coolant.

8. In the event of a flow blockage in one subassembly, it is impossible to prevent collapse of the fuel in that subassembly, but it appears to be possible to prevent damage to the rest of the core.

As a result it seems that experimental and theom retical work should continue on:

the evaluation of the sodium void coefficient;

the possibility of explosive vapourisation of the coolant;

the possibility of cascade failure due to release of gas from a faulty fuel pin (see 4 above); 
the expansion and collapse of vapour bubbles in superheated and subcooled sodium (see 7 above).

\section{REFERENCES}

1. A. Hassitt, A Computer Programme to Solve the Multigroup Diffusion Equations, TRG Report 229 (R).

2. J. L. Rowlands and D. Wardleworth, A Nineteen Group Extension to the Yiftah, Okrent and Moldauer Cross-section Set, AEEW-M.472.

3. R. P. Stein, Studhes Related to Predicting Coolant Behaviour for Fast Reactor Safety, NDA 2147-8.

4. H. W. Hoffman and A. I. Krakoviak, Convective Bonling with Liquid Potassium, Proc. 1964 Heat Transfer and Fluid Mechanies Inst., Stanford Univ. Press (1964).

5. P. R. Huebotter, Study of Fast Reactor Meltdown Accidents Using Simulant Matemals, ADPA-155.

6. G. B. Wallis, Flooding Velocities for Air and Water in Vertical Tubes, AEEW-R123.

7. R. W. Miller, A. Sola, and R. K. MeCardell, Report of the SPERT-1 Destructive Test Programme on an Aluminum, Plate-type Water-moderated Reactor, 1DO-16883.

8. E. P. Hicks, Private communication.

\section{Discussion}

Mr. Noyes (AI) : I'd like to make a remark on the question of sodium superheat during boiling, which has been raised a number of times this morning. We have made quite a large number of experiments on the boiling of sodium, both with pool boiling and for a forcedconvection situation. We have never observed the degree of superheat which has been mentioned this morning. The most that we have ever observed is 20 $30^{\circ} \mathrm{F}$. This is not to say that such superheat is not possible, because it has been observed in experiments in other systems, but it is to say that it's not necessary, and we need to study, I think, mostly what causes the high degree of superheat and what can be done to prevent it.

Mr. Hicks: Even if the superheat were to occur, I'm far from sure that it would be a serious problem.

Mr. Zebroski (GE): I believe your calculations assume that the reactor continued running at full power after the channel blockage. In such a case you should be getting a rariety of signals from different sensors that something is happening. Have you evaluated the different sorts of signals you might get, such as temperature rise, noise, pressure changes, and so on? Do you have any judgment as to which of these might be the most effective in preventing going on to the later stages of the accident?

$M r$. Hicks: This is a very big question. It hasn't been considered from that point of view in this paper itself, but a lot of work is going on in other establishments and from other points of view to try and determine the answer to this type of question.

$M r$. Anderson (LASL) : Dr. Chernick mentioned his concern that designers were sacrificing economics or, frequently, brecding ratio, for safety. Frequently this is probably due to a desire to consider the worst accident without looking at its credibility. Do you think that your approach of trying to specify accidents without presupposition as to the severity could lead to downgrading of the safety criterion and therefore upgrading the economics of the system?

Mr. Hicks: You are almost asking a philosophical question. I think that one has to examine accidents without regard to their credibility to some extent, but I think at one stage one has simply got to make a considered judgment about credibility and its consequences.

Mr. Häfele (Karlsruhe): In bringing up the time scale of the sodium expulsion in your considerations, to what extent did you employ as an input the velocity of sound, or was that calculation independent of that consideration?

Mr. Hicks: It was independent of that; it was simply a Newtonian-type dynamical calculation of a column of liquid sodium being expelled as a rigid column by gas pressure.

$M r$. Häfele: Therefore, I assume that you did not look into the phenomena of shock short waves there.

Mr. Hicks: No. The time scale of 0.1 sec or more is very, very long in comparison with sonic velocities.

Mr. Häfele: No. 
0

0 


\section{Session II October 11, 1965}

\section{Fast Reactor Concepts}

Chairman: W. H. ZINN

Combustion Engineering

Secretary: D. K. Butler

Argonne National Laboratory 
0

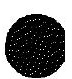




\title{
A Large Metal-fueled Fast Reactor Study Part I. Neutronies Calculations for a 1,000-MWe Plutonium-Uranium, Metal-fueled, Fast Breeder Reactor
}

\author{
D. K. Butler, W. B. Loewenstein, D. Meneghetti, and K. E. Phillips \\ Argonne National Laboratory \\ Argonne, Illinois
}

(PRESENTED BY D, MENEGHETTI)

\section{Introduction}

An evaluation study directed toward the design of a 1,000-MWe plutonium-uranium, metal-fueled, fast breeder reactor has been carried out at Argonne National Laboratory. (1) The effects of various design considerations as they relate to the physics, safety, engineering, and economic aspects of the system were evaluated. The study also served to assist in evaluating the relative merits of metal-versus ceramic-fueled fast breeders in this power range.

The general design evolved was that of a ring of six cylindrical core modules separated by and surrounded by fertile blanket. Each module is equivalent to about a 800-liter core loosely coupled to the neighboring modules. A simplified schematic is shown in Fig. 1.

The modular design reduces the magnitude of positive sodium roiding effects. To improve the negative Doppler coefficient and to effect core-module radial power flattening, a beryllium-containing region has been inserted at the core-blanket interfaces of the modules. To further improve the radial power flattening, the core modules have been radially two-zone enriched.

The inner and outer radial peripheries of the fertile blankets of the composite ring of the six modules are surrounded by beryllium regions. These are surrounded by the steel inner and outer radial reflectors. The external beryllium regions reduce fertile inventory and improve blanket breeding.

Use of fizzium alloy fuel (containing fission products) and low-capture alloy fuels, such as titanium or zirconium alloys, has been studied.

This paper describes results of some neutronics calculations for single-enriched and zoned-enriched modules carried out in support of this fast reactor study.

\section{Single-enrichment Module}

Calculations have been directed primarily toward understanding the neutronics of a module consisting of its core, blanket, and reflector regions. The effective thickness of a radial blanket associated with a module is dependent upon the radial direction from the module center. Furthermore, the overall outer beryllium region and steel reflector are not present in directions between neighboring modules. Cylindrical calculations for a module therefore included a range of radial blanket thicknesses with outer radial boundary conditions of zero current (without outer radial beryllium and reflector regions) and of zero flux (with outer radial beryllium and reflector regions).

Dimensions of the single-enrichment core module, associated blankets, beryllium regions, and reflectors are listed in Table 1. Atom densities of the materials in the various regions are listed in Table 2 . A modification of the Argonne 26-group-cross section set(2) was used. In the core these atomic densities represent: 45 $\mathrm{v} / \mathrm{o}$ fucl element, $40 \mathrm{v} / \mathrm{o}$ sodium, $9 \mathrm{v} / \mathrm{o}$ steel structure, and $6 \mathrm{v} / 0$ control mechanism. The fuel element composition is $30 \mathrm{v} / 0 \mathrm{Ti}-\mathrm{V}(20 \mathrm{w} / \mathrm{o} \mathrm{Ti}, 80 \mathrm{w} / \mathrm{o} \mathrm{V})$ clad and $70 \mathrm{v} / 0$ of $(\mathrm{U}+\mathrm{Pu}+4 \mathrm{w} / \mathrm{o}$ Fizzium + F.P. $)$. Using 5.5 heavy-atom percent burnup in the core as a basis, the fission product pairs are to represent an average of 2.75 percent heavy-atom burnup, i.e., $2.75 \%$ of the $\mathrm{U}+\mathrm{Pu}$ atoms are fission product pairs. The expanded $(\mathrm{U}+\mathrm{Pu}+$ Fizzium + F.P. $)$ density has been assumed to be $12 \mathrm{gm} / \mathrm{ec}$. The following atomic density ratios of plutonium isotopes to total plutonium atomic density have been assumed: 0.751 , $0.210,0.0295$, and 0.0085 for $\mathrm{Pu}^{239}, \mathrm{Pu}^{240}, \mathrm{Pu}^{241}$, and $\mathrm{Pu}^{242}$, respectively. The $6 \mathrm{v} / 0$ of control has been replaced by $4 \mathrm{v} / \mathrm{o}$ additional sodium and $2 \mathrm{v} / \mathrm{o}$ additional steel for most of the analyses to be described. This corresponds to the case of no poison control inserted in the core. The atom density of zirconium listed in Table 2 represents the addition in the analyses of zirconium to simulate the noncapture properties of of the listed fizzium capture material.

The radial beryllium-containing zone surrounding the module consists of $5 \mathrm{v} / 0$ sodium, $12 \mathrm{v} / \mathrm{o}$ steel, and $83 \mathrm{v} / \mathrm{o}$ beryllium.

The atomic densities of the inner radial blanket 
region represent $47 \mathrm{v} / 0$ fuel element, $10 \mathrm{v} / 0$ steel structure, and $43 \mathrm{v} / 0$ sodium. The $47 \mathrm{v} / 0$ fuel element consists of about $70 \% \mathrm{U}^{238}$ and $30 \mathrm{v} / \mathrm{o}$ titanium-vanadium cladding alloy. $2.4 \mathrm{w} / \mathrm{o}$ of the uranium fuel is $\mathrm{Pu}^{239}$, which represents the average fissile concentration at midlife.

The outer radial blanket region instead contains

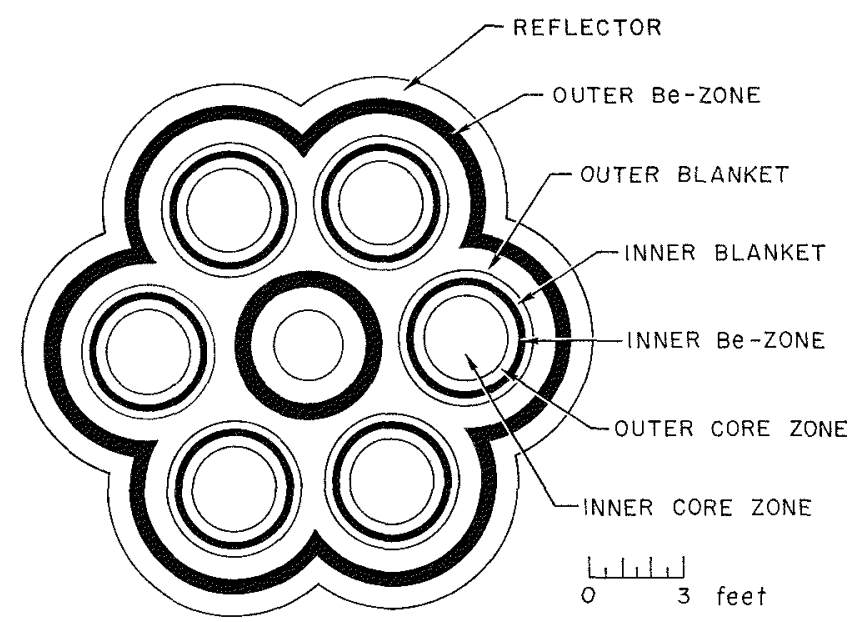

Frg. 1. Simplified Schematic of Horizontal Section of Reactor at Midplane of Core Modules.

TABle 1. Dimensions (cm) of Modvle Regions

Core- -840 liters; $92-\mathrm{cm}$ height, $53.9-\mathrm{cm}$ radius

\begin{tabular}{|c|c|c|}
\hline Inner Beryllium & & 5.3 \\
\hline & (Region 1 & 6.1 \\
\hline Radial Blankets & Region 2 & $\sim 17 \rightarrow 38$ \\
\hline Outer Beryllium & & 15 \\
\hline Radial Reflector & & 37 \\
\hline Axial Blanket & & 33 \\
\hline Axial Reflector & & 55 \\
\hline
\end{tabular}

TABle 2. Regonal Atomic Densities (1024 units) Used in the Calculations

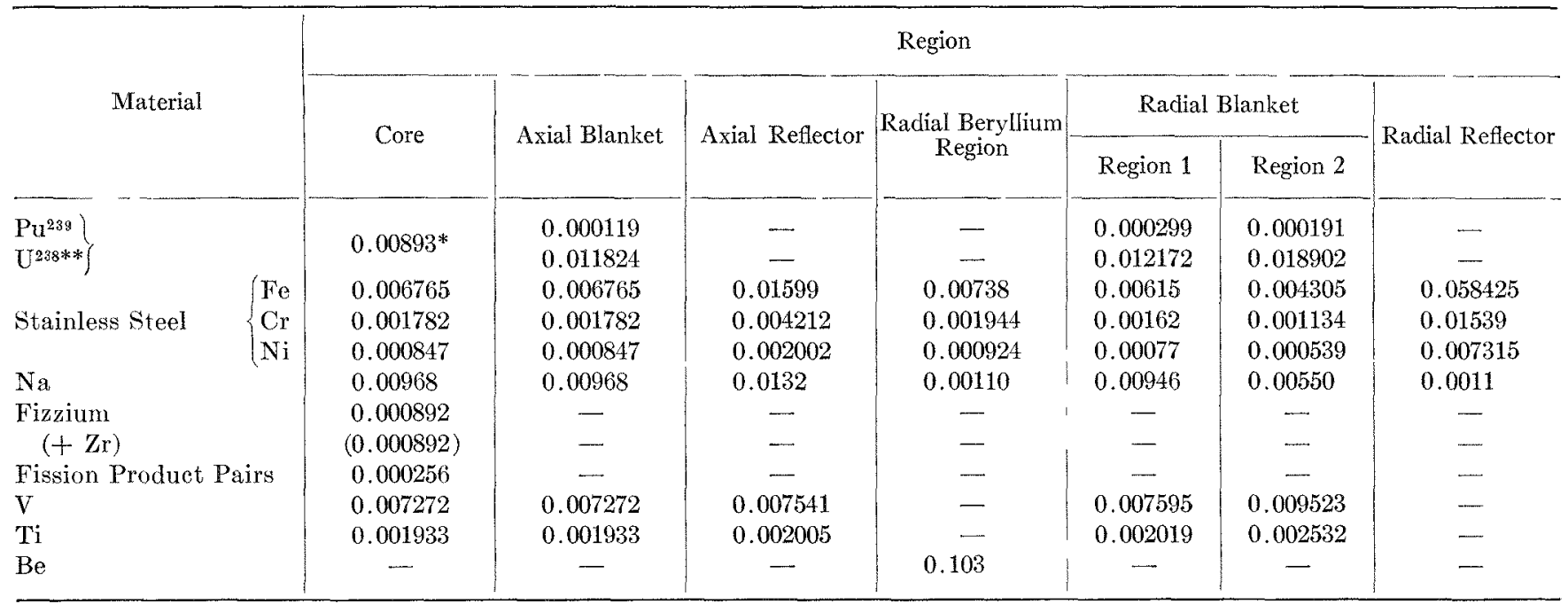

* For core, this quantity includes also the higher plutonium isotopes of masses 240,241 , and 242 , as well as $\mathrm{Pu}^{233}$ and $\mathrm{U}^{228}$.

** $1500^{\circ} \mathrm{K} \mathrm{U}^{238}$ cross-section parameters used for J)oppler calculations.
$68 \mathrm{r} / 0$ fuel element, $7 \mathrm{v} / 0$ steel structure, and $25 \mathrm{v} / 0$ sodium. The $68 \mathrm{v} / \mathrm{o}$ fuel element consists of about $74 \%$ $\mathrm{U}^{238}$ and $26 \%$ titanium-vanadium alloy. The average fissile concentration at midlife is represented by $\mathrm{Pu}^{239}$ present as one weight percent of the uranium fuel. The steel reflector is $95 \mathrm{v} / 0$ steel and $5 \mathrm{v} / 0$ sodium. The composition of the outer beryllium region is taken to be the same as that of the core-blanket interface region described previously.

The atomic densities in the axial blanket correspond to $45 \mathrm{v} / \mathrm{o}$ fuel element, $40 \mathrm{v} / 0$ sodium coolant, $9 \mathrm{v} / 0$ steel structural material, and $6 \mathrm{v} / \mathrm{o}$ for control movement. The latter has been represented by $4 \mathrm{v} / \mathrm{o}$ additional sodium and $2 \mathrm{v} / \mathrm{o}$ additional steel. The fuel element consists of about $70 \mathrm{v} / \mathrm{o} U^{238}$ and $30 \mathrm{v} / 0$ of the titanium-vanadium cladding alloy. One weight percent of the uranium fuel is assumed to be $\mathrm{Pu}^{239}$, which represents the average fissile concentration at midlife of the core. The expanded fuel density, i.e., $\left(\mathrm{U}^{238}+\mathrm{Pu}^{239}\right)$, is assumed to be $15 \mathrm{gm} / \mathrm{cc}$ in the blankets.

The atomic composition of the axial reflector corresponds to $60 \mathrm{v} / 0$ sodium, $26 \mathrm{v} / \mathrm{o}$ steel, and $14 \mathrm{v} / \mathrm{o}$ titanium-vanadium cladding alloy.

Corresponding quantities for the two-zoned enriched module are given in a subsequent section. Calculations were by one-dimensional diffusion theory. Calculated results for sodium void include considerations of the change in axial reflector savings upon removal of core sodium.

\section{Effects of Radial Beryllium-containing Regions and Variations of Radial Blanket Thickness}

The effects of the radial beryllium-containing regions at the core-blanket and/or blanket-reflector interfaces 
upon critical enrichment, breeding and core conversion ratio, sodium void effects, and Doppler effects of isolated modules are listed in Table 3.

The beryllium-containing region at the blanketreflector interface was assumed to have the identical composition as that listed for the core-blanket interface. The thickness, however, was $15 \mathrm{~cm}$ rather than $5.3 \mathrm{~cm}$.

The effects of the beryllium at the core-blanket interface is to augment the negative Doppler by greater than a factor of two.

Calculations show that the beryllium region can also augment the negative reactivity due to uniform voiding of core sodium.

The addition also of an outer beryllium region in the case of the thinner $(\sim 29 \mathrm{~cm})$ radial blanket results in a breeding ratio of 1.42 , which is comparable to 1.43 obtained with the thicker blanket.
The presence of the beryllium-containing region at the radial interface of core and blanket results in radial power flattening. This is evident in Fig. 2 , in which a comparison is made between the radial source distribution with and without the beryllium region. The spectral degradation resulting from the presence of the beryllium region results in preferential lowering of the spectrum in the radial peripheral region of the core. This is advantageous in that centrally located sodium voiding of the core does not diminish the peripherally weighted Doppler effect.

\section{Low-capture Alloy Fuel Compared with Fizzium- alloy Fuel}

The neutronics effects of the presence of fizzium in the fuel material of the core has been studied by substitution of an equal number of titanium atoms for the

Table 3. Efeects of Berylitum Regions and Blanket Thickness upon Isolated, Reflected Modelaes

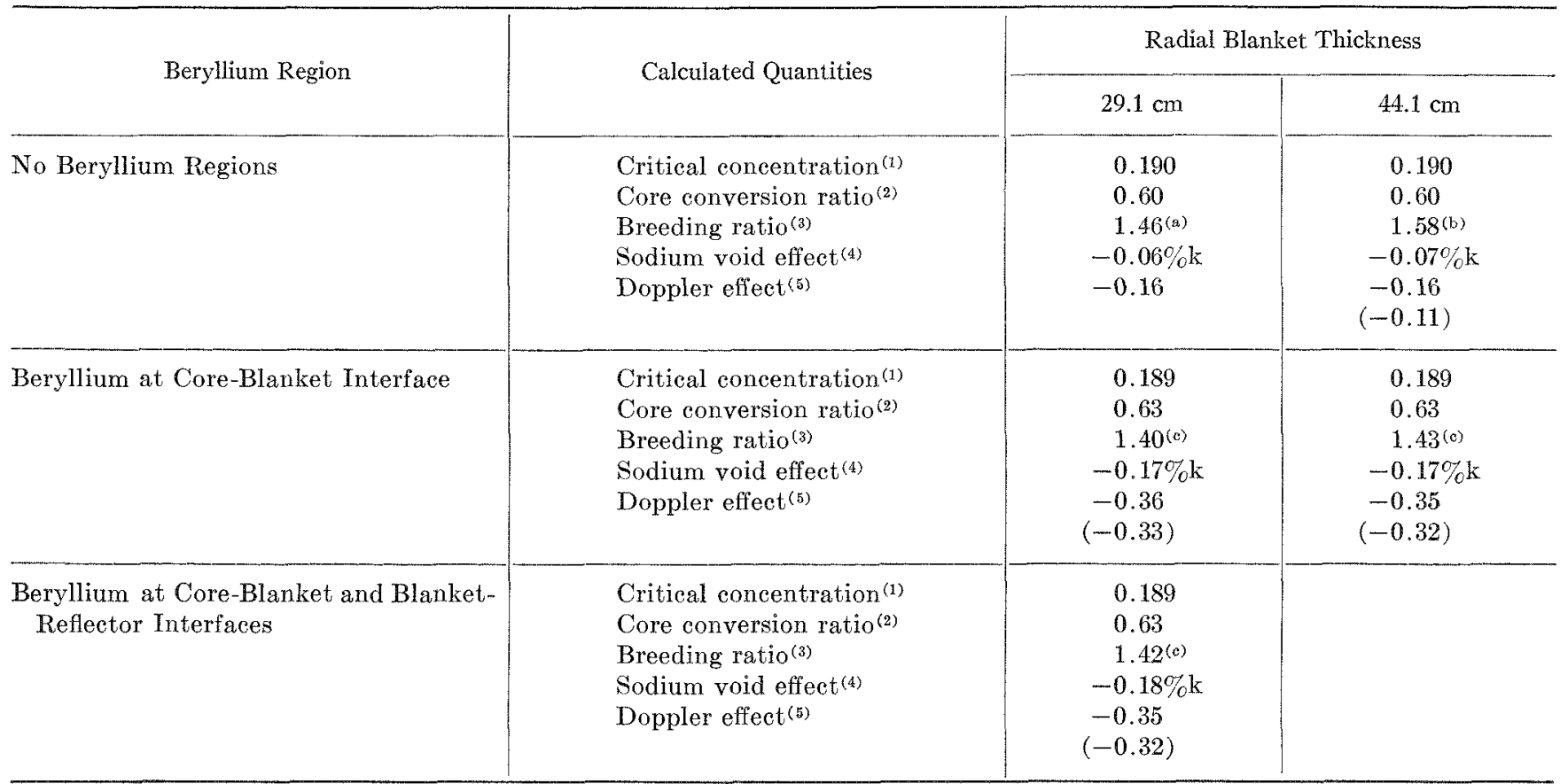

(a) Includes contributions of 0.33 from axial blankets.

(b) Includes contributions of 0.38 from axial blankets.

(c) Includes contributions of 0.31 from axial blankets.

(1) Critical concentration $=\frac{(\text { total } \mathrm{Pu})_{\text {in eore }}}{\left(\text { total } \mathrm{Pu}+\mathrm{U}^{238}\right)_{\text {in oore }}}$.

(2) Core conversion ratio $=\frac{\left(\text { captures in } \mathrm{U}^{238}, \mathrm{Pu}^{240} \text {, and } \mathrm{Pu}^{242}\right)_{\text {in core }}}{\left.\text { (absorptions in } \mathrm{Pu}^{239} \text { and in } \mathrm{Pu}^{241}\right)_{\text {in core }}}$.

(3) Breeding ratio $=\frac{\left(\text { captures in } \mathrm{U}^{228}, \mathrm{Pu}^{240} \text {, and } \mathrm{Pu}^{242}\right)_{\text {in core and blanket }}}{\left.\text { (absorptions in } \mathrm{Pu}^{239} \text { and in } \mathrm{Pu}^{241}\right)_{\text {in core and blanket }}}$.

(4) Reactivity resulting from a $40 \%$ reduction of core sodium.

(5) Based on reactivity resulting from $\mathrm{U}^{238}$ Doppler effect in core for temperature change from $750^{\circ} \mathrm{K}$ to $1500^{\circ} \mathrm{K}$ with constant sodium composition and (with $40 \%$ less sodium in core). The $\Delta k$ have been converted to $T(\partial k / \partial T)_{\text {Doppler }} 10^{2}$, assuming this quantity independent of temperature. 
fizzium atoms. This corresponds to an 8.9 a/o titanium concentration in the fuel meat.

Results of the calculations are given in Table 4 for systems in cylindrical geometry, having $\sim 29 \mathrm{~cm}$ of radial blanket, surrounded by $15 \mathrm{~cm}$ of beryllium-containing region, in turn surrounded by a $37-\mathrm{cm}$ reflector. The use of titanium rather than fizzium results in a breeding increase of about +0.06 .

By comparison with the sodium voiding results given in Table 3 the removal of fizzium results in a substantial improvement in the magnitude of the overall negative sodium void effect. It is noted, however, that the presence of beryllium at the core-blanket interface does not affect the sodium void reactivity as significantly with the titanium alloy fuel as with the fizzium alloy fuel.

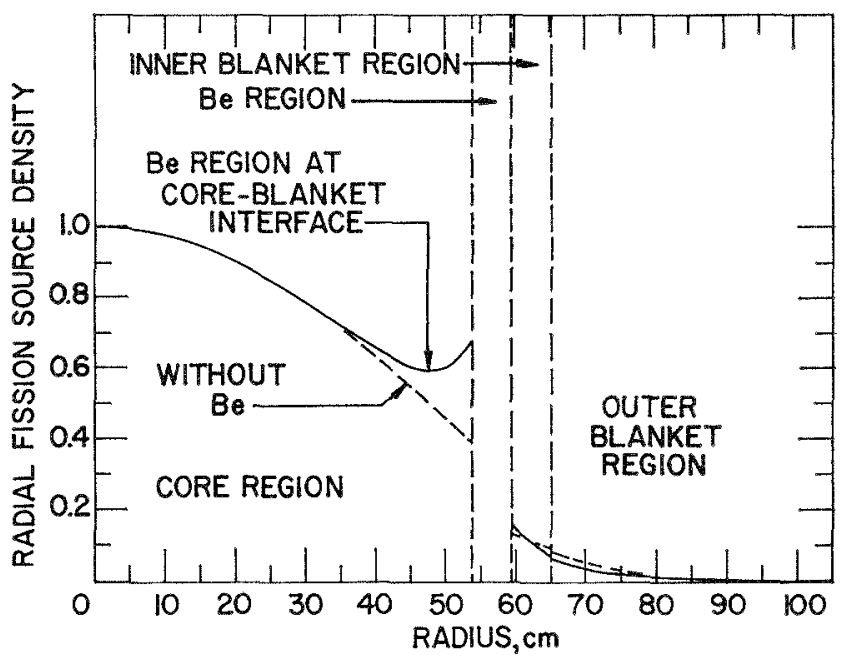

Frg. 2. Comparison of Radial Source Densities with and without Beryllium Region.

TABLE 4. Comparison of Titanivm AND Fizzium Alloy fuels

\begin{tabular}{l|c|c|c}
\hline \multicolumn{1}{c|}{ Beryllium Region } & $\begin{array}{c}\text { Calculated } \\
\text { Quantities* }\end{array}$ & $\begin{array}{c}\text { With } \\
\text { Titanium }\end{array}$ & $\begin{array}{c}\text { With } \\
\text { Fizzium }\end{array}$ \\
\cline { 2 - 3 } No Beryllium at Core- & $(1)$ & 0.182 & 0.190 \\
Blanket Interface; Be- & $(2)$ & 0.64 & 0.60 \\
ryllium at Blanket- & $(3)$ & $1.62^{(a)}$ & $1.56^{(a)}$ \\
Reflector Interface & $(4)$ & $-0.28 \% k$ & $-0.08 \% k$ \\
& $(5)$ & -0.23 & -0.16 \\
& & $(-0.17)$ & $(-0.12)$ \\
\hline Beryllium at Core-Blan- & $(1)$ & 0.179 & 0.189 \\
ket Interface and at & $(2)$ & 0.68 & 0.63 \\
Blanket-Reflector In- & $(3)$ & $1.49(\mathrm{~b})$ & $1.42^{(\mathrm{b})}$ \\
terface & $(4)$ & $-0.32 \% k$ & $-0.18 \%$ \\
& $(5)$ & -0.52 & -0.35 \\
& & $(-0.47)$ & $(-0.32)$ \\
\hline
\end{tabular}

* Identical footnotes as in Table 3.

(a) Includes contribution of 0.38 from axial blankets.

(b) Includes contribution of 0.31 from axial blankets.
TABle 5. Neutronics Chamacteristics Compared for Drfererent Fuei, Alloys*

\begin{tabular}{|c|c|c|c|c|c|c|}
\hline & \multicolumn{6}{|c|}{ Alloy } \\
\hline & \multirow{2}{*}{ None } & \multirow{2}{*}{$\begin{array}{c}\text { Fizzium, } \\
4 \mathrm{w} / \mathrm{o}\end{array}$} & \multicolumn{2}{|c|}{ Titanium } & \multirow{2}{*}{$\begin{array}{l}\text { Zir- } \\
\text { conium, } \\
22 \mathrm{a} / \mathrm{o}\end{array}$} & \multirow{2}{*}{$\begin{array}{c}\text { Carbon, } \\
1: 1\end{array}$} \\
\hline & & & $8.9 \mathrm{a} / \mathrm{o}$ & $30 \mathrm{a} / \mathrm{o}$ & & \\
\hline (1) & 0.16 & 0.18 & 0.17 & 0.17 & 0.17 & 0.17 \\
\hline (2) & 0.73 & 0.62 & 0.67 & 0.68 & 0.68 & 0.72 \\
\hline (3) & 1.67 & 1.57 & 1.64 & 1.61 & 1.62 & 1.54 \\
\hline (4) & -0.17 & -0.08 & -0.30 & -0.26 & -0.18 & -0.16 \\
\hline (5) & -0.16 & -0.12 & -0.17 & -0.20 & -0.17 & -0.35 \\
\hline
\end{tabular}

* Footnotes as in Table 3 (when applicable to spherical geometry).

Doppler reactivity effects are improved by removal of the fizzium. These values are approximately $40 \%$ more negative.

Relative results of employing various other fuel alloys are given in Table 5 , based on calculations in spherical geometry with surrounding blankets and reflectors corresponding to those of the previous cylindrical radial values. The core-blanket beryllium was not present. In these cases the density of $\mathrm{Pu}+\mathrm{U}$ atoms was held constant. For example, the $8.9 \mathrm{a} / 0$ titanium concentration in the fuel was increased to 30 a/o at the expense of void space. Analogously, the 8.9 a/o titanium was replaced by 22 a/o girconium. The last column gives the results of adding carbon atoms in 1:1 ratio to the heavy atoms.

\section{Intermodule Reactivity Coupling}

Because of the ring configuration, the intermodule coupling of a given module is dependent upon the radial direction. An estimate has been made of the intermodule reactivity coupling. Fizzium-alloy and titanium-alloy, single-enriched module cases were calculated without the outer beryllium and steel radial reflectors but with zero-current boundary conditions to simulate blanket regions between modules. A thickblanket $(38 \mathrm{~cm})$ case corresponds essentially to an uncoupled module. A thin-blanket $(23 \mathrm{~cm})$ case corresponds approximately to one-half the average blanket thickness separating the modules. Less than one-third of the radial blanket surrounding a module is positioned between core regions of neighboring modules.

One-third of the reactivity differences between the thick- and thin-blanket cases are given in Table 6 . Analogous values, similarly obtained, assuming 50\% less of the $\mathrm{Pu}^{239}$ in the radial blankets, are indicated in parentheses.

The reactivity coupling of a given module is seen to be quite small, especially when the inner core- 
TABLE 6. Estimated Miximum Reictivity Colpling of a Modtan to the Ring of Modules

\begin{tabular}{l|c|c}
\hline \multirow{2}{*}{ Beryllium Region } & \multicolumn{2}{|c}{ Fuel Alloy } \\
\cline { 2 - 3 } & Titanium & Fizzium \\
\hline None & $0.48 \%(0.40 \%)$ & $0.45 \%(0.38 \%)$ \\
\hline $\begin{array}{l}\text { At Core-Blanket Inter- } \\
\text { face }\end{array}$ & $0.15 \%(0.11 \%)$ & $0.13 \%(0.10 \%)$ \\
\hline
\end{tabular}

* With $50 \%$ less $\mathrm{Pu}^{239}$ in radial blankets.

blanket beryllium region surrounds each module. Fifty percent variation of $\mathrm{Pu}^{239}$ content in the radial blankets varies the reactivity coupling by about a $0.1 \% k$ increment, without the inner core-blanket beryllium regions present.

\section{Two-zoned Core Enrichments}

Use of two-zone enriched core modules together with a beryllium interface between core and radial blanket has been considered in order to improve power flattening. In the zoned-core case the module dimensions differ from those listed in Table 1. These dimensions are given in Table 7 .

The overall core volume in this case is thus about 784 liters. Analyses were by cylindrical one-dimensional geometry and zero-flux outer boundary conditions. The same axial reflector savings and variation of axial reflector savings used in the previous singlezoned-core cases also containing titanium fuel alloy were used.

In the two core zones the volume fractions are, respectively, 45.79 and $49.69 \mathrm{v} / \mathrm{o}$ fuel element, 39.42
TABle 7. Dimensions (cm) OF Module Regions

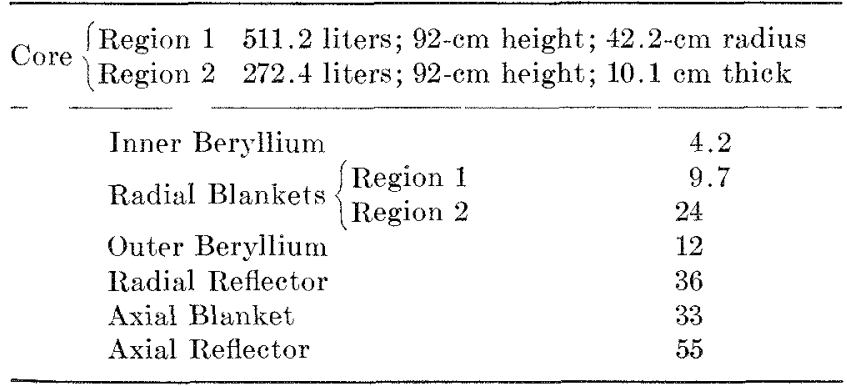

and 42.40 sodium, and 8.80 and 7.91 steel structure. The inner core zone in addition contains $6 \mathrm{v} / \mathrm{o}$ control made up of about $58 \mathrm{v} / \mathrm{o}$ sodium, $35 \mathrm{v} / 0$ steel, and an assumed $7 \mathrm{v} / 0 \quad B_{4}^{\text {nat }} \mathrm{C}$ to represent poison present during operation. The fuel-element composition is $31.3 \mathrm{v} / \mathrm{o} \mathrm{TiV}(20 \mathrm{w} / \mathrm{o}$ Ti and $80 \mathrm{w} / \mathrm{o} \mathrm{V})$ clad and 68.7 $\mathrm{v} / \mathrm{o}$ of $(\mathrm{U}+\mathrm{Pu}+\mathrm{Ti})$ alloy plus fission products pairs. If we take an average heavy atom burnup of $6 \%$, the assumed fission product pairs are taken to represent a time-averaged value in burnup cycle of $3 \%$ burnup, i.e., $3 \%$ of the $\mathrm{U}+\mathrm{Pu}$ atoms are fission product pairs. Expanded ( $\mathrm{U}+\mathrm{Pu}+\mathrm{Ti}+$ F.P.P. $)$ density has been retained at $12 \mathrm{gm} / \mathrm{cc}$. The fuel alloy has been assumed to contain about 8.9 a/o titanium.

Atom densities used in the calculations for the various regions are listed in Table 8 .

Results for the zoned core with and without the coreblanket beryllium region are given in Table 9 for three enrichment ratios in region 2, namely, $[\mathrm{Pu}$ / $(\mathrm{Pu}+\mathrm{U})]_{\mathrm{core}}=0.20,0.21$, and 0.22 . Criticality in all cases was by variation of $[\mathrm{Pu} /(\mathrm{Pu}+\mathrm{U})]_{\text {core }}$ in region 1.

TABLE 8. Regronal, Atom Densities (1024 units) Used in Zoned-cone Calcilations

\begin{tabular}{|c|c|c|c|c|c|c|c|c|}
\hline \multirow[b]{2}{*}{ Material } & & \multicolumn{7}{|c|}{ Region } \\
\hline & & \multicolumn{2}{|c|}{ Core } & $\begin{array}{c}\text { Radial Beryllium } \\
\text { Region }\end{array}$ & \multicolumn{2}{|c|}{ Radial Blanket } & $\begin{array}{c}\text { Outer Radial } \\
\text { Beryllium Region }\end{array}$ & Radial Reflector \\
\hline $\left.\begin{array}{l}\mathrm{Pu}^{239} \\
\mathrm{U}^{238}\end{array}\right\}$ & & $0.009088^{*}$ & $0.009862^{*}$ & - & $\begin{array}{l}0.000311 \\
0.012647\end{array}$ & $\begin{array}{l}0.000194 \\
0.019216\end{array}$ & - & - \\
\hline Stainless Steel & $\mathrm{Ni}$ & 0.000839 & 0.000609 & 0.001925 & 0.000609 & 0.000537 & 0.001078 & 0.007238 \\
\hline $\mathrm{Na}$ & & 0.009436 & 0.009328 & 0.002200 & 0.009328 & 0.005326 & 0.001320 & 0.001320 \\
\hline F. P. Pairs & & 0.000281 & 0.000305 & - & - & - & - & - \\
\hline V & & 0.00772 & 0.008378 & - & 0.008378 & 0.009527 & - & $一$ \\
\hline $\mathrm{Ti}$ & & 0.002961 & 0.003213 & 一 & 0.002052 & 0.002334 & - & - \\
\hline $\mathrm{Be}$ & & - & $-\cdots$ & 0.08034 & - & - & 0.09888 & - \\
\hline
\end{tabular}

* For core regions, these quantities include also the higher plutonium isotopes of masses 240,241 , and 242 , as well as $\mathrm{Pu}^{239}$ and $\mathrm{U}^{288}$. 
TABle 9. Results of Cylindrical Calculations of Two-zoned Core Modules

\begin{tabular}{|c|c|c|c|c|c|c|c|c|c|c|}
\hline \multirow{2}{*}{ Beryllium Region } & \multicolumn{2}{|c|}{$\left(\frac{P u}{P u+U}\right)_{\text {core }}$} & \multicolumn{2}{|c|}{$\left(\frac{\text { Fissile } \mathrm{PU}}{\mathrm{PU}+\mathrm{U}}\right)_{\text {eore }}$} & \multirow{2}{*}{$\begin{array}{c}\text { Core } \\
\text { Conversion } \\
\text { Ratio }\end{array}$} & \multirow{2}{*}{$\begin{array}{l}\text { Breeding } \\
\text { Ratio }\end{array}$} & \multirow{2}{*}{$\begin{array}{c}40 \% \mathrm{Na} \\
\text { Void in Core }\end{array}$} & \multicolumn{2}{|c|}{ Doppler ${ }^{(c)}$} & \multirow{2}{*}{$\begin{array}{l}\text { Source Peak } \\
\text { to Average at } \\
\text { Cylindrical } \\
\text { Midplane }\end{array}$} \\
\hline & Region 1 & Region 2 & Region 1 & Region 2 & & & & $\mathrm{Na}$ In & Na Out ${ }^{(d)}$ & \\
\hline $\begin{array}{l}\text { No Beryllium at Core- } \\
\text { Blanket Interface; } \\
\text { Beryllium at Blanket- } \\
\text { Reflector Interface }\end{array}$ & $\begin{array}{l}0.178 \\
0.176 \\
0.173\end{array}$ & $\begin{array}{l}0.200 \\
0.210 \\
0.220\end{array}$ & $\begin{array}{l}0.139 \\
0.137 \\
0.135\end{array}$ & $\begin{array}{l}0.156 \\
0.164 \\
0.172\end{array}$ & $\begin{array}{l}0.63 \\
0.63 \\
0.62\end{array}$ & $\begin{array}{l}1.63(1) \\
1.63 \\
1.63\end{array}$ & $\begin{array}{l}+0.02 \% \mathrm{k} \\
+0.06 \\
+0.10\end{array}$ & $\begin{array}{l}-0.21 \\
-0.21 \\
-0.21\end{array}$ & $\begin{array}{l}-0.16 \\
-0.16 \\
-0.16\end{array}$ & $\begin{array}{l}1.35 \\
1.32 \\
1.29\end{array}$ \\
\hline $\begin{array}{l}\text { Beryllium at Core-Blan- } \\
\text { ket Interface; Beryl- } \\
\text { lium at Blanket-Re- } \\
\text { flector Interface }\end{array}$ & $\begin{array}{l}0.176 \\
0.173 \\
0.170\end{array}$ & $\begin{array}{l}0.200 \\
0.210 \\
0.220\end{array}$ & $\begin{array}{l}0.137 \\
0.135 \\
0.132\end{array}$ & $\begin{array}{l}0.156 \\
0.164 \\
0.172\end{array}$ & $\begin{array}{l}0.66 \\
0.65 \\
0.65\end{array}$ & $\begin{array}{l}1.50^{(b)} \\
1.50 \\
1.50\end{array}$ & $\begin{array}{c}-0.05 \% \mathrm{k} \\
0.00 \\
+0.04\end{array}$ & $\begin{array}{l}-0.41 \\
-0.41 \\
-0.41\end{array}$ & $\begin{array}{l}-0.36 \\
-0.36 \\
-0.36\end{array}$ & $\begin{array}{l}1.25 \\
1.20 \\
1.16\end{array}$ \\
\hline
\end{tabular}

(a) Includes contribution of 0.38 from axial blankets.

(b) Includes contribution of 0.31 from axial blankets.

(c) $T(\partial k / \partial T)_{\text {Doppler }} 10^{2}$.

(d) For case of $40 \%$ less sodium in core.

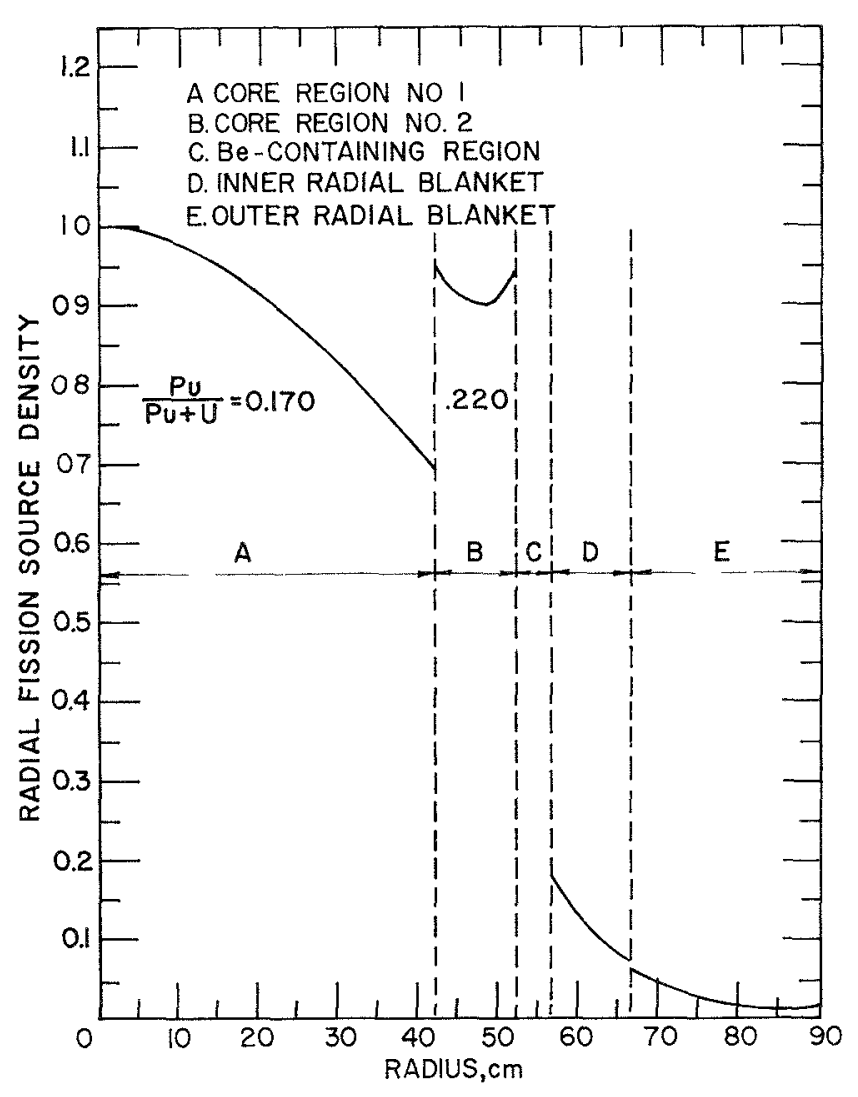

Fis. 3. Radial Source Density Distribution in a Zoned-core Module.

Comparisons of these two-zoned results with those of the analogous one-zoned titanium alloy-fueled cases in Table 4 show that the resulting breeding ratios are effectively unchanged even though in the central zone $\mathrm{B}_{4} \mathrm{C}$ is present in the case listed in Table 9. Doppler effects are not changed by the use of zone enrichment. The somewhat lesser optimistic Doppler in the two- zoned (relative to the one-zoned) beryllium interface case is instead largely due to the smaller effective thickness of this beryllium region. Zoned loading does, however, sizably decrease the previously very optimistic sodium void effect. This becomes more evident with increase in zone enrichment in the outer core. The combined effects of zoned loading and moderate-sized beryllium-containing region do, however, considerably improve the peak-to-average source value. Radial source distributions for one of the zoned cases containing the beryllium region is shown in Fig. 3 .

Integrated radial-dependent sodium removal from the two-zoned, beryllium-interfaced core having an outer core enrichment of $21 \%$ plutonium has also been calculated. Removals of $40 \%$ sodium internal to cylindrical radii $29.8 \mathrm{~cm}, 42.2 \mathrm{~cm}$, and $52.3 \mathrm{~cm}$ result in multiplication changes of $+0.24 \% k,+0.23 \% k$, and $0 \% k$, respectively (the latter value is for $40 \%$ sodium removal from the entire core).

\section{Equilibrium Fuel Cycle and Reactivity Loss from Burnup}

Two properties of the 1000-MWe system connected with long-term operation have been studied: the equilibrium fuel composition, and the change in reactivity to be expected between refuelings. The CYCLE code $^{(3)}$ was used. CYCLE is a one-dimensional code that calculates the equilibrium fuel composition for a specified burnup and refueling scheme. It also calculates the isotopic changes and changes in reactivity which must be made to maintain criticality over the cycle. Because a single dimension is assumed, the code in cylindrical geometry cannot take into account changes in the axial reflector savings that would occur in the actual system. On the other hand, if spherical geometry is used, the code assumes a uniform blanket 
all around. It is therefore necessary to estimate the characteristics of the proposed system on the basis of a series of problems with appropriately varied compositions and geometries.

In calculating the equilibrium composition, an additional restriction is that the core loading be uniform. Therefore, the properties of the reference design with the two enrichment zones were estimated subject to the assumption of a single enrichment with the same volume fraction of fucl, clad, structure, and coolant. It was also necessary to use a "single batch" core in which the whole core is reprocessed at once. To approximate the actual scheme proposed, it was assumed that one-third of the fission products and $2 \%$ of the heavy atoms corresponding to reprocessing losses were removed from the core fuel. The remainder was returned, combined with sufficient fresh $\mathrm{U}^{238}, \mathrm{Pu}^{239}$, and boron to restore the core to its initial uniform composition. The code adjusts the feed enrichment so that the system is critical at midcycle, but allows the reactivity to vary during the cycle as the composition changes with burnup.

Problems to determine equilibrium composition were run in spherical geometry with both the axial and radial blankets of the reference design. The equilibrium atom densities thus obtained were very close to those used in the reference design.

The code also computes the changes in isotopic composition in the blanket over the cycle. These were found to be in satisfactory agreement with those estimated for the reference design.

The reactivity change due to burnup during a cycle was determined in a separate calculation in which a desired burnup expressed as the fraction of fissions of the hcavy atoms during the cycle was specified. The burnup was taken to be $2 \%$ in the core. Like that of equilibrium composition, the reactivity change for the actual two-dimensional cylinder was estimated by means of a series of one-dimensional calculations. The reactivity change for the reference systems with two enrichment zones in cylindrical geometry was determined to be $2.6 \%$. In this calculation it was assumed that the height was constant, that is, there was no change in the axial direction. Calculations in spherical geometry analogous to those in which the equilibrium composition was determined showed that the correction for axial effects would be less than $0.2 \% \Delta k / k$.

The burnup calculations support the original assumptions on which the reference design was based, both as to equilibrium composition and the amount of reactivity which will be lost by burnup each cycle. The latter quantity also supports the estimate of average amount of boron which must be present for the purpose of the reactivity variation over a burnup cycle.

\section{Reactivity Coefficients and Dynamic Behavior of Modules}

Thermal expansion of the reactor modules can be divided into radial and axial components. Estimates of these components were made by calculating, in spherical geometry (with uniform core composition), the volume coefficient of expansion for the sphere and converting it into components of a cylinder, by means of the equation

$$
\frac{\Delta V}{V}=\frac{\Delta h}{h}+2\left(\frac{\Delta r}{r}\right)_{\text {es linder }} .
$$

Table 10 gives the results obtained in this way for various assumptions about the mechanism of the expansion.

Exploratory studies of the dynamic behavior of a single module of the 1000-MW system have been made for two kinds of transient conditions important to considerations of safety: ramp insertions of reactivity and loss of coolant flow. Calculations were performed with a modified version (RP351X) of the FORE ${ }^{(4)}$ program. This cylindrical-geometry code computes the temperature distribution as a function of time across an average and the hottest fuel pins under conditions of time-varying reactivity and, if desired, time-varying coolant velocity. The code includes feedback from Doppler effect and from expansion of fuel, clad, coolant, or structure.

Information was sought on the importance of the magnitude of the Doppler feedbacks and of fuel or structural expansion. Doppler coefficients appropriate for systems both with and without a beryllium region at the core-blanket interface were used. Thermal expansion coefficients corresponding to axial expansion of fuel meat and radial expansion due to the

TABlaE 10. Expansion Coffficiexts of Reactivity

$$
\left(\frac{\Delta k}{k}=\alpha \frac{\Delta h}{h}+\beta \frac{\Delta r}{r}\right) \text {. }
$$

\begin{tabular}{l|c|c}
\hline & $\alpha$ & $\beta$ \\
\hline $\begin{array}{l}\text { 1. Axial (Expanding Fuel, Displacing } \\
\text { Blanket) }\end{array}$ & -0.30 & \\
2. Axial (Contracting Fuel, Leaving \\
Clad, Coolant, and Structure in Gap) & -0.26 & \\
3. Radial (Contracting Core Radius, \\
Leaving Clad, Coolant, Structure in \\
$\begin{array}{l}\text { Gap) } \\
\text { 4. Radial (Contracting Core Radius, } \\
\text { Filling Gap with Inner Radial Blan- } \\
\text { ket) }\end{array}$ & -0.53 \\
\hline
\end{tabular}


stainless steel structure were also used. These were selected as possible sources of negative feedback for calculational purposes as examples. In practice this axial expansion of the fuel might be lowered by its confinement in the expanding Ti-V clad. In addition, availability of fuel expansion with the high-burnup fuel remains to be demonstrated. Samples of U-5 w/o Fs alloy, however, with burnup of about $1.3 \mathrm{a} / \mathrm{o}$ demonstrated essentially the same transient expansion in TREAT experiments as fresh fucl. ${ }^{(5)}$

The results shown in Table 11 indicate that the time before fuel melting may be increased $20 \%$ by the addition of a sufficiently thick region of beryllium. Fuel temperature coefficients of reactivity corresponding to axial expansion result in a $33 \%$ increase under the same conditions. For problems involving loss of coolant flow the input reactivity was held constant. The results indicate that a radial expansion coefficient of reactivity characteristic of stainless steel expansion contributes more feedback than the Doppler effect, even with the beryllium region present. The modular core has the feature (of importance for metal fuel because of the small Doppler effect available) of making possible significant expansion effects.

From the results it is seen that the prompt feedback available between operating temperature and fuel melting is not more than about $\$ 0.5$ from the combined effects of axial fuel expansion and Doppler effect. The Doppler effect is necessarily limited with metal fuel even with the addition of the thin, moderat.- ing beryllium annulus. This is primarily because there is only about a $20 \%$ rise in absolute temperature available between fuel opcrating and melting temperatures. For improved feedback, radial expansion introduced, for example, by a fuel cermet structure could provide an improved reactivity feedback mechanism.

In all of the studies of loss of coolant flow, initial boiling took place near the axial core boundary. Thus, initial sodium void reactivity would be negative. Initial coolant voiding in the ramp studies also took place above the core centerline, but not as close to the axial core extremity as with loss of flow. Generally speaking, the larger Doppler coefficient of reactivity obtained when a beryllium annulus was present seems to provide a time to melting that is consistent with times necessary to initiate protective devices for reactivity reduction. A small Doppler coefficient coupled with a reliable prompt axial expansion coefficient provides similar times to melting. In all studies it was assumed that the modules are relatively uncoupled. Detailed study of the interaction of a malfuction in one module and its influence upon a neighboring module is a problem requiring additional study.

Potential slumping of fuel pins within the clad presents an area of undetermined hazard. The initial, unirradiated, fuel-pin diameter is $0.15 \mathrm{in}$; t the inside clad diameter is $0.174 \mathrm{in}$. The large amount of effective void filled with bond sodium is required to accommodate irradiation growth. If effective slumping were to occur, the height of the fuel column in the clad could

TABle 11. Dynamic Behavior of Single Module

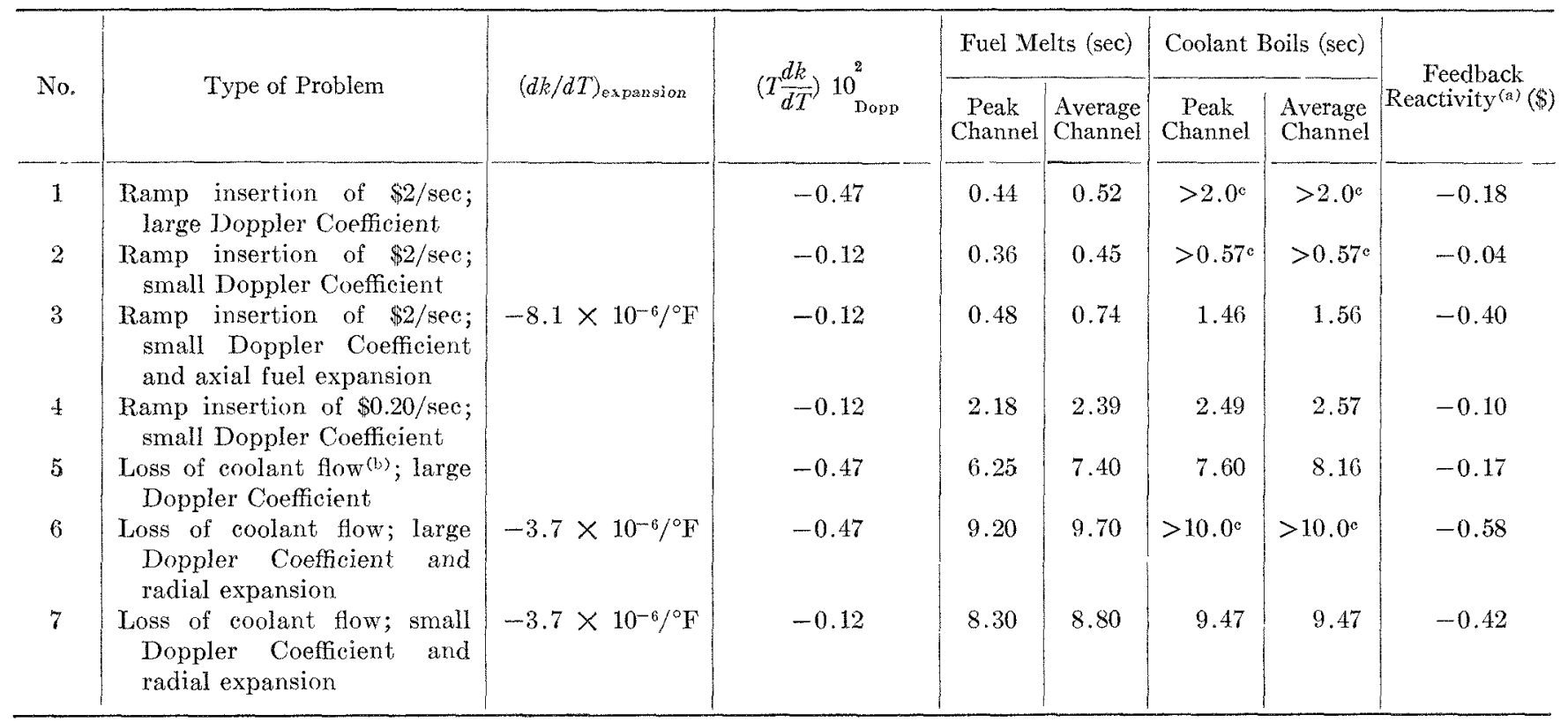

(a) Total feedback reactivity at time corresponding to peak channel fuel melting.

(b) Flow decays exponentially from $22 \mathrm{ft} / \mathrm{sec}$ for $13.2 \mathrm{sec}$ to a final velocity of $0.8 \mathrm{ft} / \mathrm{sec}$.

(e) Problem terminated at time shown before boiling occurred. 
be as much as $25 \%$ less than for the unirradiated pin. However, only freshly charged fuel is susceptible to efficient slumping, since it is expected that swelling to fill the radial gap within the clad will oceur when only partial burnup has been attained. Furthermore, one-third of the core is replaced at each cycle; thus, only one-third of the core would be available for efficient slumping for roughly half of the time between successive refuelings. It is believed that the reactivity increase would accrue gradually over a period of time, which would provide an opportunity for protective measures. If detailed design were to show that less void space is required in the fuel, the slumping reactivity would be correspondingly less. It is also possible to conceive of a metallic, segmented fuel element, consisting of individually supported segments within the clad, thus significantly reducing potential slumping reactivity. Such a design eliminates the (already unreliable) negative axial expansion coefficient of reactivity. This latter approach would, in this design concept, require both the beryllium annulus for enhanced Doppler reactivity as well as the fueled cermet for structural expansion reactivity.

\section{REFLRENCES}

1. L. E. Link et al., 1000-MWe Metal-fueled F'ast Breeder Reactor, ANL-7001 (to be published).

2. 1. M. O'shea, H. H. Hummel, W. B. Loewenstein, D. Okrent, and B. J. Toppel, Twenty-six Group Cross Sections, ANL-6858 (to be published).

3. B. J. Toppel, R. Avery, and (‘. J. Fischer, CYCLE and COST, Codes for Fast Reactor Fuel Cycle Analysis and Related Cost Evaluation, Trans. Am. Nuel. Soc. 5, No. 1, 92 (1962).

4. P. (ireebler, D. B. Sherer, and N. H. Walton, FORE, $A$ Computational Program for the Analysis of Fast Reactor Excursions, GEAP-4090 (Oct 1962).

5. C. E. Dickerman, L. E. Robinson, F. L. Willis, W. Stephany, and C. August, Status of Recent Meltdown Studies, Proc. International Conference on Safety, Fuels and Core Design in Large Fast Power Reactors (Oct 1965).

\section{Acknowledgments}

The authors wish to thank Dr. H. H. Hummel for his interest and helpful comments on various aspects of the study. Thanks are also due to Mr. K. A. Hub and Mr. J. T. Weills for numerous helpful discussions on engineering-related questions. 


\title{
A Large Metal-fueled Fast Reactor Study Part II. Core Design and Fuel Cyele
}

\author{
L. E. Link, J. E. Ayer, K. A. Hub, W. J. Mecha y, V. G. Trice, Jr., and J. T. Werlls \\ Argonne National Luboratory \\ Argonne, Illinois
}

(PRESENTED BY L. E. LINK)

\section{Introduction}

Four divisions of Argonne National Laboratory have participated in the preliminary study of a high-power, metal-fueled reactor system. The Metallurgy and Chemical Engineering Divisions have extrapolated their combined experience in remote fabrication and pyrochemical-processing experience to a large-scale fuel cycle facility for handling high-burnup core and blanket fuel. The Reactor Physics and Reactor Engineering Divisions have studied the neutronic performance and prepared the preliminary engineering design.

\section{Core Design}

The core is made up of six modules as shown in Fig. 1. The active zones of each module are surrounded by blanket regions which, in total, are edged inside and out by beryllium. The beryllium, in turn, is backed up by stainless steel. About one-third of the radial blanket is common to two modules. An 800-liter-core module with a length-to-diameter $(\mathrm{L} / \mathrm{D})$ ratio of 0.9 is consistent with a small sodium voiding reactivity effect. Six modules are required to generate heat for producing $1000 \mathrm{MW}(\mathrm{e})$.

The module features are reproduced to larger scale in Fig. 2. The 61 subassemblies of the core are divided into two zones of enrichment. The outer 24 subassemblies are at higher enrichment to improve power flattening. The outer zone subassemblies in combination with the inner radial blanket subassemblies have appropriately located beryllium to form the inner beryllium zone. The zone enrichments are adjusted to provide a radial maximum-to-average heat generation density in the core of 1.2. The inner enrichment zone contains the 13 control positions, a central position for regulating rod, and 2 concentric rings of positions for shim and safety rods.

The radial blanket is also in 2 zones. The 30 subassemblies adjacent to the core have smaller-diameter elements to match the requirement for higher heat removal.

The fuel elements are designed partially to accommodate and partially to constrain the tendency of me- tallic fuels to expand as burnup proceeds. The hoavy atom density of the fuel in its expanded form is 12 $\mathrm{g} / \mathrm{cm}^{3}$. The fuel elements are assembled into subassemblies as shown in Fig. 3. Tubular spacing of fuel elements is necessary to achieve acceptable pressure drop of the closely spaced fuel elements over their 87 -in. $(220-\mathrm{cm})$ length. The total subassembly length is 20.5 ft $(6.3 \mathrm{~m})$. The tops of the subassemblies extend above the level of the sodium so that refueling is performed with visual aid.

The characteristics and design performance of the fuel elements are indicated in Table 1 . A consistent approach has been applied to the design of a metal fuel element. The fuel is regarded as arbitrarily weak, and the fuel cladding is assumed to act as a tube pressurized according to the degree of burnup with an allowance for both the fission product gas volume and the temperature level. (1) The 87-in.-long fuel tube contains the uranium pin for the lower axial blanket, the fuel pin, upper uranium pin, and a plenum for displaced sodium and inert gas. The plenum height is set to provide the final volume of the inert gas without significant contribution to the wall stresses in the fuel tube. A plug is inserted above the upper blanket pin and restrained to allow only 2 in. (5-cm) of axial movement. Essentially, all of this movement is expected to be expansion of the fuel pin.

Two approximations of the burnup model were utilized in design to arrive at an average axial burnup of the fuel pin. An average burnup of 7.1 percent is consistent with the approximation of a uniform fission product gas pressure over fuel height producing the limiting stress condition at the top of the core where the coolant is hottest. A maximum of 8.3 percent is consistent with the approximation of a localized pressure against the cladding somewhat downstream of the core midplane, where a combination of sodium temperature rise and local burnup produces the limiting condition. The local burnup value is consistent with the average axial burnup approximation of 7.1 percent. The radial maximum-to-average power generation in the core reduces the burnup to 6 percent for the discharged fuel. The most recent irradiation data on these 


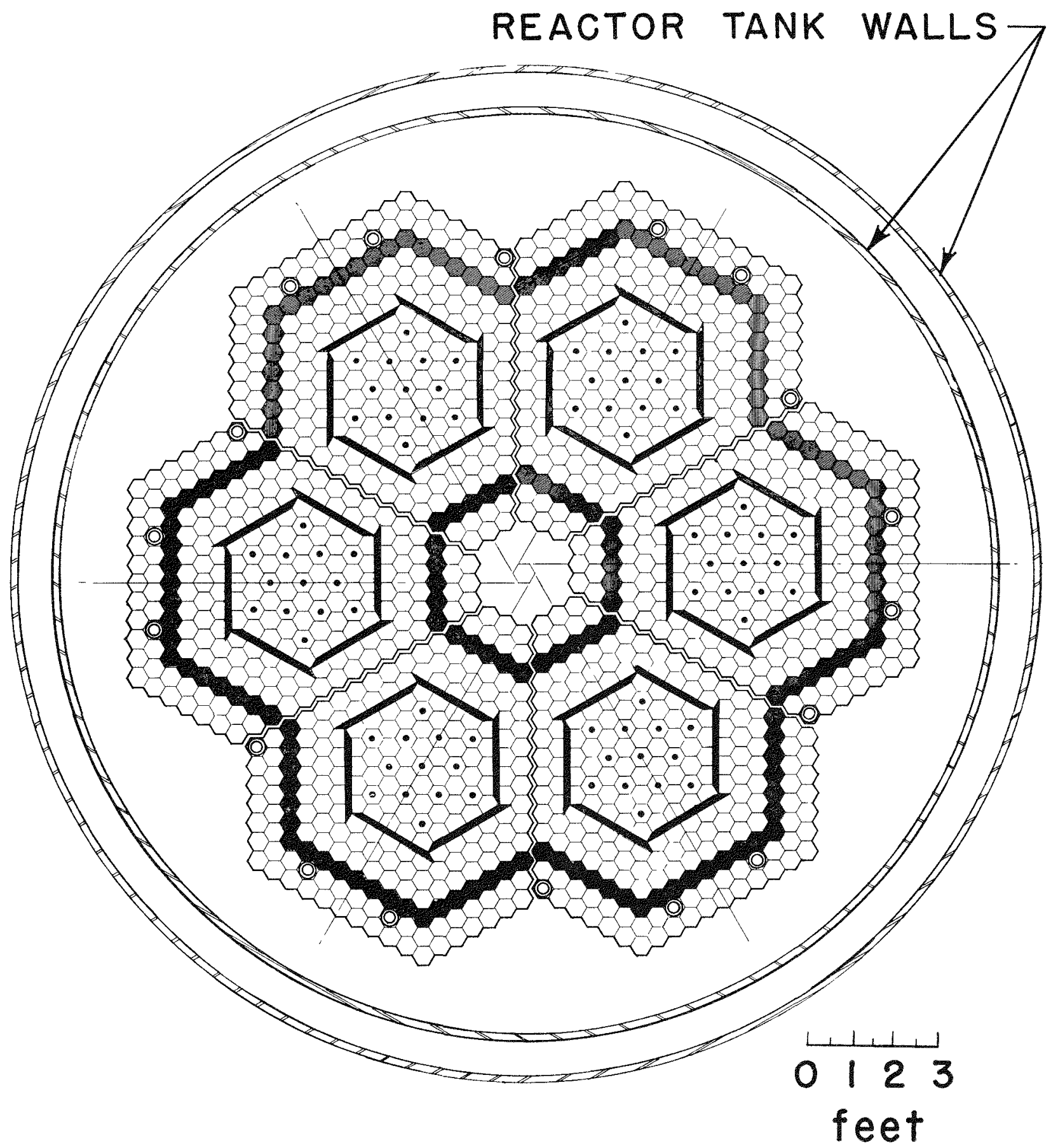

FIG. 1. Horizontal Section of Reactor at Midplane

types of metallic fuels lead to confidence that the reference design element has the capability to achieve an average burnup of at least 7 percent.

The design of the fuel element and the design of the subassembly are matched to the techniques and equipment determined to be suitable for remote manufacture of low-decontamination fuel. The $3-\mathrm{ft}(91-\mathrm{cm})$ long pin may be beyond the practical limit for casting in Vycor tubing, in which case two castings would be used. The difference in radial clearance between cast pin and fuel cladding is just large enough to give sodium bonding without prolonged vibration to ensure specified completeness of thermal contact. Fuel elements can be loaded into the hexagonal shroud tube in the manner of EBR-II manufacture to form the fuel subassembly.

The fuel subassemblies of a core module are locked radially by the differential expansion between a ti- 


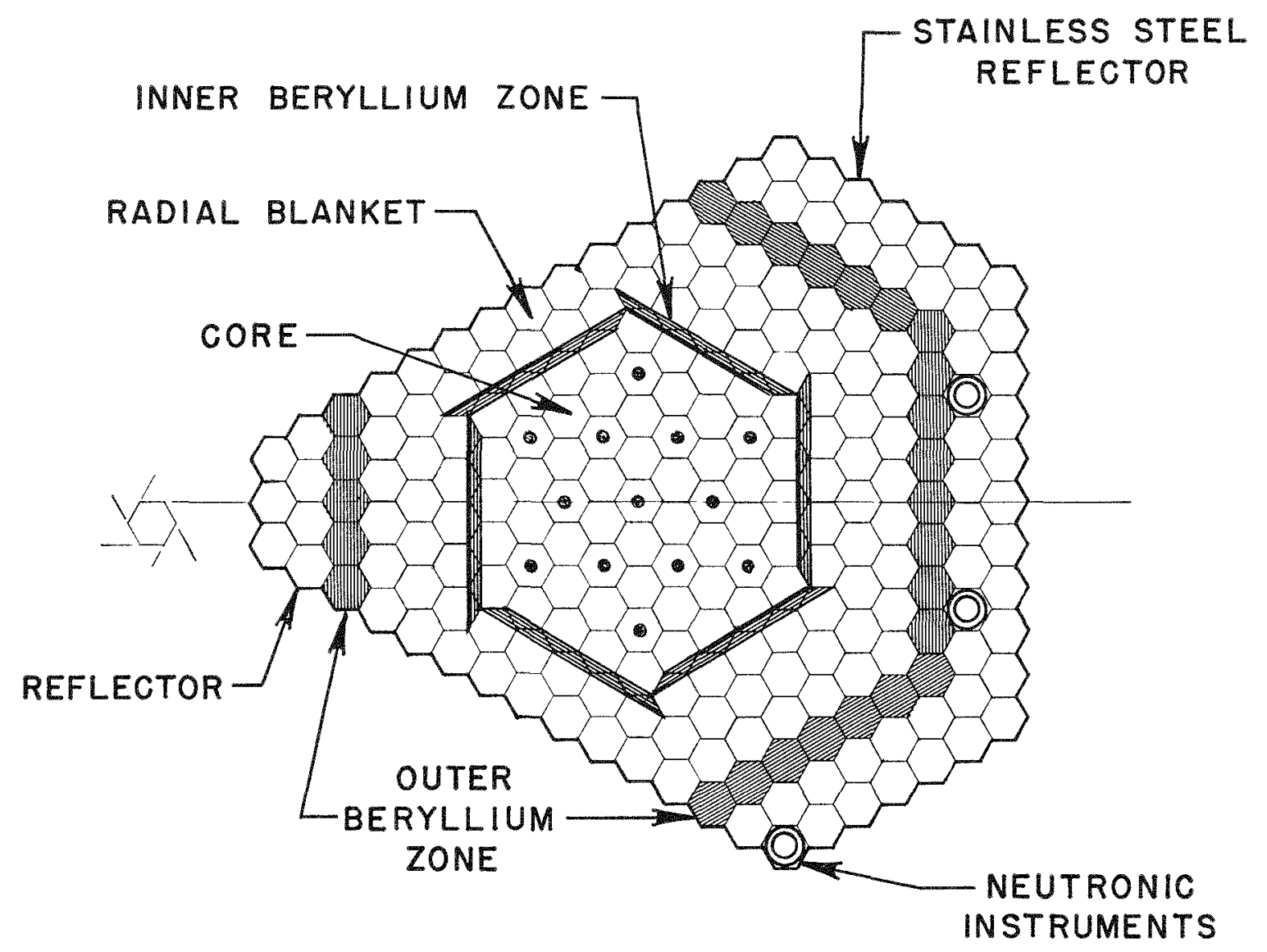

FItr. 2 Section of One Module

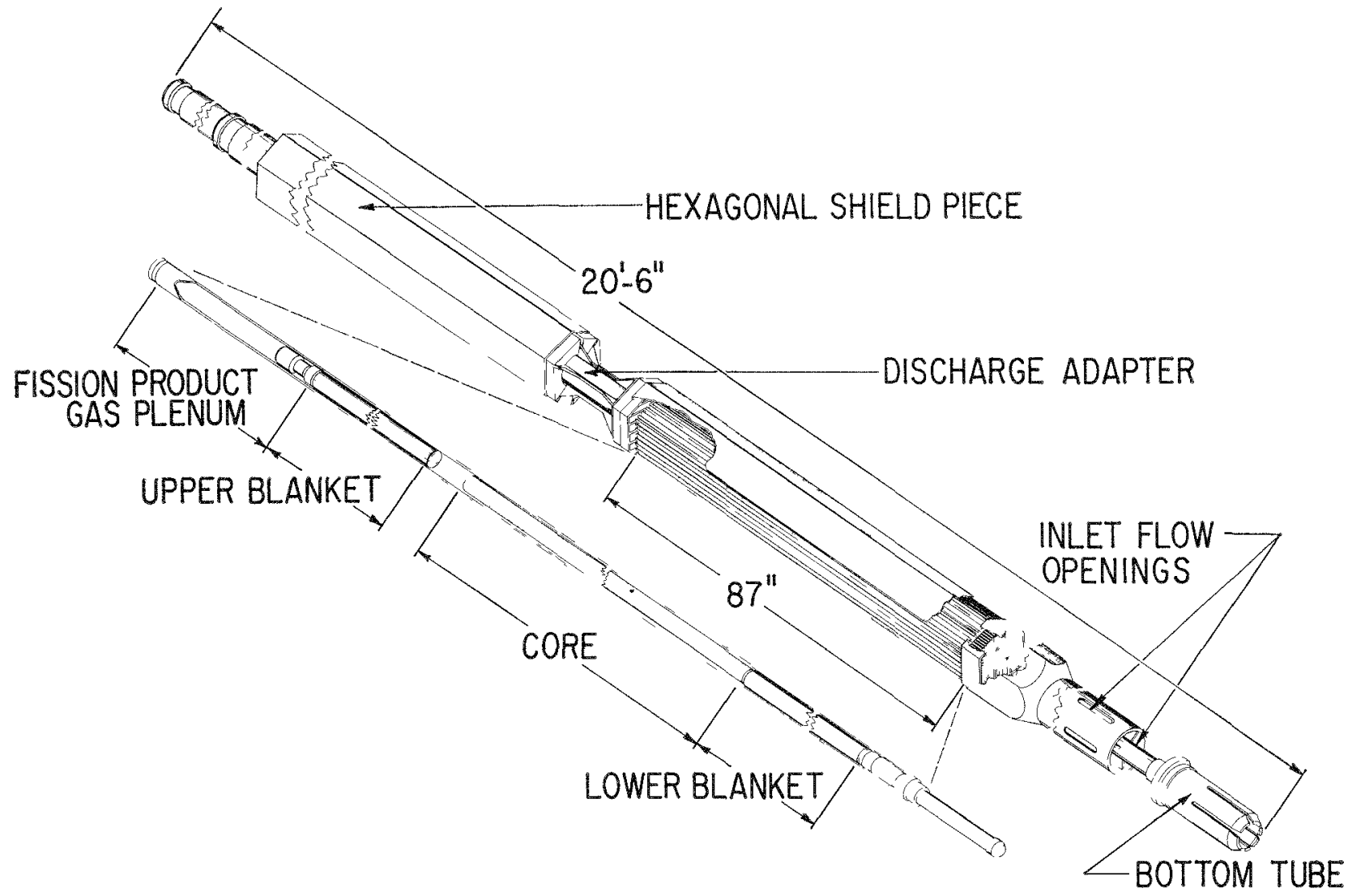

Fig. 3. Isometric View of Fuel Subassembly for 1000-MWe Metal Fuel Breeder Reactor Study 
tanium pivot plate and a stainless steel driver plate acting at two levels on the lower adapter of each subassembly. The radial clearances that are present among assemblies at the refueling temperature are removed before the approach to criticality by raising the temperature of the system to a point approaching the operating temperature.

\section{Reactor System}

Figure 4 is a schematic vertical section of the reactor and one of the six primary sodium loops. Two core modules are shown. Hot sodium exits from the subasscmbly hexagonal tubes just below the neutron-shield region and spreads out over the module area. Most of the flow is expected to be directly upward before radial flow to the six exit pipes. The shield fitted to the top of the reactor tank is rotated for refueling after control rod disengagement. A removable plug exposes the entire area module, including the outer beryllium, for subassembly replacement for relocation. It is proposed that a spent subassembly be lifted into a sodium-filled tube for transport to storage within the refueling cell.

Reactor characteristics are based on replacement of one-third of the core zone fuel in each module at each refueling period. One-sixth of the inner blanket zone and one-ninth of the outer blanket zone are replaced at each refueling cycle. The period of the refueling cycle is 206 days, with an annual plant load factor of $80 \%$ and $6 \%$ average burnup of the heavy atom content of the core fuel. The reactivity loss over such a cycle is about 3 percent $\Delta k / k$; roughly 10 percent of this is estimated to be due to axial expansion of the new fuel added at the beginning of each cycle.

The salient design information is summarized in Table 2 for the larger-scale features and reactor performance. The net electrical output is achieved by two turbine generator units matched to 1800-psig (122 atm) steam at $900^{\circ} \mathrm{F}\left(482^{\circ} \mathrm{C}\right)$ and $900^{\circ} \mathrm{F}\left(482^{\circ} \mathrm{C}\right)$ reheat.

\section{Fuel Cycle}

The vital function of a fuel cycle facility is to restore irradiated fuel to a form suitable for return to the reactor. For a fast reactor fuel the reprocessing requirements for restoration of the fuel are partial fission product removal and reconstruction of the heavy atom and inert alloying constituents.

Pyrochemical processing and remote fabrication are proposed for the 1000-MW (e) metal-fueled fast reactor. Pyrochemical processing is the generic name chosen by Argonne National Laboratory for a class of nonaqueous processes for metal, oxide, and carbide fuels that are currently under development. These processes employ liquid-phase reactions at tempera-
TABLE 1. Fuel Flement and Design Data

\begin{tabular}{|c|c|c|}
\hline \multicolumn{2}{|c|}{$\begin{array}{l}\text { Average Burnup in Discharged Fuel, \% } \\
\text { Fuel Element Composition, \% } \\
\text { Tanadium-20 w/o titanium cladding } \\
\text { Expanded U-Pu-9 a/o Ti Fuel }\end{array}$} & $\begin{array}{l}31 \\
69\end{array}$ \\
\hline & \multicolumn{2}{|c|}{ Fuel Pin Features: } \\
\hline & As Cast & Fxpanded \\
\hline $\begin{array}{l}\text { Density, } \mathrm{g} / \mathrm{cm}^{8} \\
\text { Diameter, in. (mm) } \\
\text { Length, in. (cm) }\end{array}$ & $\begin{array}{l}17.5 \\
0.150(3.8) \\
34(86)\end{array}$ & $\begin{array}{l}12 \\
0.174(4.4) \\
36(91)\end{array}$ \\
\hline $\begin{array}{l}\text { Fuel Tube } \\
\text { Outside Diameter, in. (mm) } \\
\text { Wall thickness, in. (mm) }\end{array}$ & & $\begin{array}{ll}0.210 & (5.3) \\
0.018 & (0.46)\end{array}$ \\
\hline $\begin{array}{l}\text { Fuel Enrichment as Charged } \\
\text { Inner Zone, } \mathrm{Pu}_{j} / \mathrm{Pu}+\mathrm{U}, \mathrm{w} / \\
\text { Outer Zone, } \mathrm{Pu}_{j} / \mathrm{Pu}+\mathrm{U} \text {, w }\end{array}$ & & $\begin{array}{l}13.5 \\
17.5\end{array}$ \\
\hline $\begin{array}{l}\text { Thermal Data } \\
\text { Average Linear Power, } \mathrm{kW} / \mathrm{ft} \\
\text { Hot Spot Cladding Temperat } \\
\text { Hot Spot Fuel Centerline } \\
{ }^{\circ} \mathrm{F}\left({ }^{\circ} \mathrm{C}\right)\end{array}$ & $\begin{array}{l}(\mathrm{W} / \mathrm{cm}) \\
\text { ure, }{ }^{\circ} \mathrm{F}\left({ }^{\circ} \mathrm{C}\right) \\
\text { Temperature, }\end{array}$ & $\begin{array}{r}6.6(217) \\
1126(608) \\
1439(782)\end{array}$ \\
\hline $\begin{array}{l}\text { Heat Flux, Btu/hr ft'2 } \\
\text { Maximum } \\
\text { Average }\end{array}$ & & $\begin{array}{l}6.9 \times 10^{5} \\
4.1 \times 10^{5}\end{array}$ \\
\hline
\end{tabular}

tures of 1200 to $2300^{\circ} \mathrm{F}\left(650\right.$ to $\left.1250^{\circ} \mathrm{C}\right)$ to effect adequate removal of fission products and the reconstruction of the core and blanket fuel discharged from fast reactors fueled with metal or ceramic fuel. Batch processes are used which employ oxidation-reductions in molten metal-fused salt systems to separate the fuel values from most of the fission products and then to effect the separation of uranium and plutonium. A pyrochemical process for metal fuels, melt refining, is employed in the EBR-II fuel cycle facility, and the remote fabrication procedures considered here are identical in principle to the EBR-II procedures. ${ }^{(2)}$

Comparative cost studies are currently being made at Argonne National Laboratory on various processing methods for fast reactor plutonium fuels. These studies indicate that a close-coupled pyrochemical processing plant has about half the cost of an aqueous processing plant of equal capacity processing highly burned and highly enriched plutonium-containing fuels. The lower cost of pyrochemical processing derives from the much lower cell-space requirement, the small volume, the convenient form of the wastes produced, and the shorter period of cooling required before processing.

A simplified flow chart of the fuel cycle proposed for metal fuel is shown in Fig. 5. The fuel cycle consists of three basic components: 1) breakdown of the fuel subassembly, 2) pyrochemical refining of the spent fuel, and 3 ) fabrication of the fuel subassembly. 


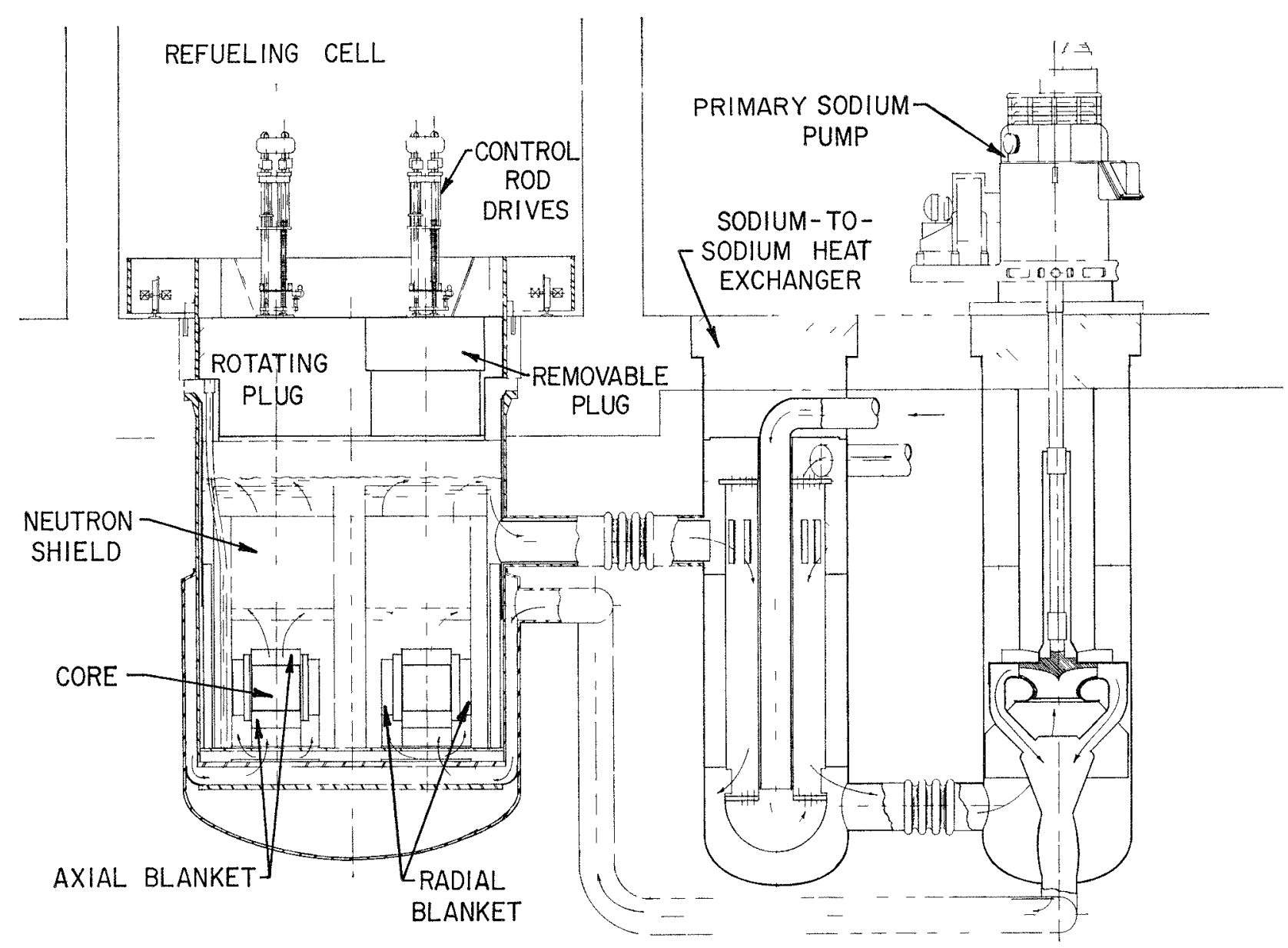

Fig. 4. Vertical Section of Reactor and One of Six Primary Sodium Loops

\section{Dismantling and Decanning}

The subassembly breakdown is a two-step operation involving dismantling and decanning; ${ }^{(4)}$ both of these are purely mechanical processes. The dismantling step is a process by which an expended fuel subassembly is stripped of its outer hardware. The exposed bundle of fuel rods is disassembled, and the rods are charged to a magazine which is then loaded into the decanning machine. The decanning machine removes the jacket from the fuel rods and reduces the unprotected fuel pin to short slugs. The fuel discharged from decanning is the input to pyrochemical refining.

\section{Processing}

The pyrochemical process is shown in Fig. 6. Six processing steps are required:

1. Core fuel is melted in a beryllia crucible and contacted with a fused salt. The rare earth, alkali metal, and alkaline earth fission products are extracted into the salt phase, which is discarded as waste. Volatile fission products are released to the offgas system.

2. Core fuel is chlorinated with cadmium chloride in a tungsten vessel. The noble metals remain in the metal phase, which is discarded as waste.

3. The salt from the chlorination step, which contains the uranium and plutonium from the core and axial blanket fuel, is charged to a stainless steel vessel. Uranium and plutonium chlorides from the core fuel are reduced by contacting with a solution of cadmium, zinc, and magnesium. The radial blanket fuel is dissolved. Alkali metal and alkaline earth fission products from the blanket fuel are extracted into the salt phase, which is discarded as waste. Volatile fission products and volatile chlorides of zirconium, niobium, and titanium are released. The rare earth and noble metal fission products in the blanket fuel are not removed.

4. The metal phase from step (3) is charged to a beryllia vessel and retorted to remove most of the cadmium and zinc. The uranium precipitates, and the plutonium remaining in solution in the supernatant is transferred to a scparate beryllia retort vessel.

5. The uranium product is retorted to separate solvent metal. Uranium is recovered as a metallic ingot. The solvent metal is recycled (to step 3 ). 
6. The plutonium product is retorted to separate solvent metal. Plutonium is recovered as a metallic ingot. The solvent metal is recycled (to step 3 ).

\section{Fabrication}

Recovered uranium and plutonium are alloyed with the required amount of titanium for feed for the fabrication of the core fuel. This process is a combination of four metallurgical and mechanical operations which convert the fuel alloy ingot into a core subassembly acceptable to the reactor. The various unit operations in the fabrication process are shown in Fig. 7. The blanket fuel is unalloyed uranium.

Fabrication starts with the conversion of a fuel alloy billet into right circular cylindrical castings. The procedure employed is a well-developed ${ }^{(5,6,7)}$ process called injection casting. The casting molds are stripped from the fuel pins and enter a waste stream. The fuel pins enter the mechanical phases of the operation, in which they are cropped to desired length and inspected. Inspection consists of scanning for gross surface de-

Table 2. General Reactor Features and Performance Data

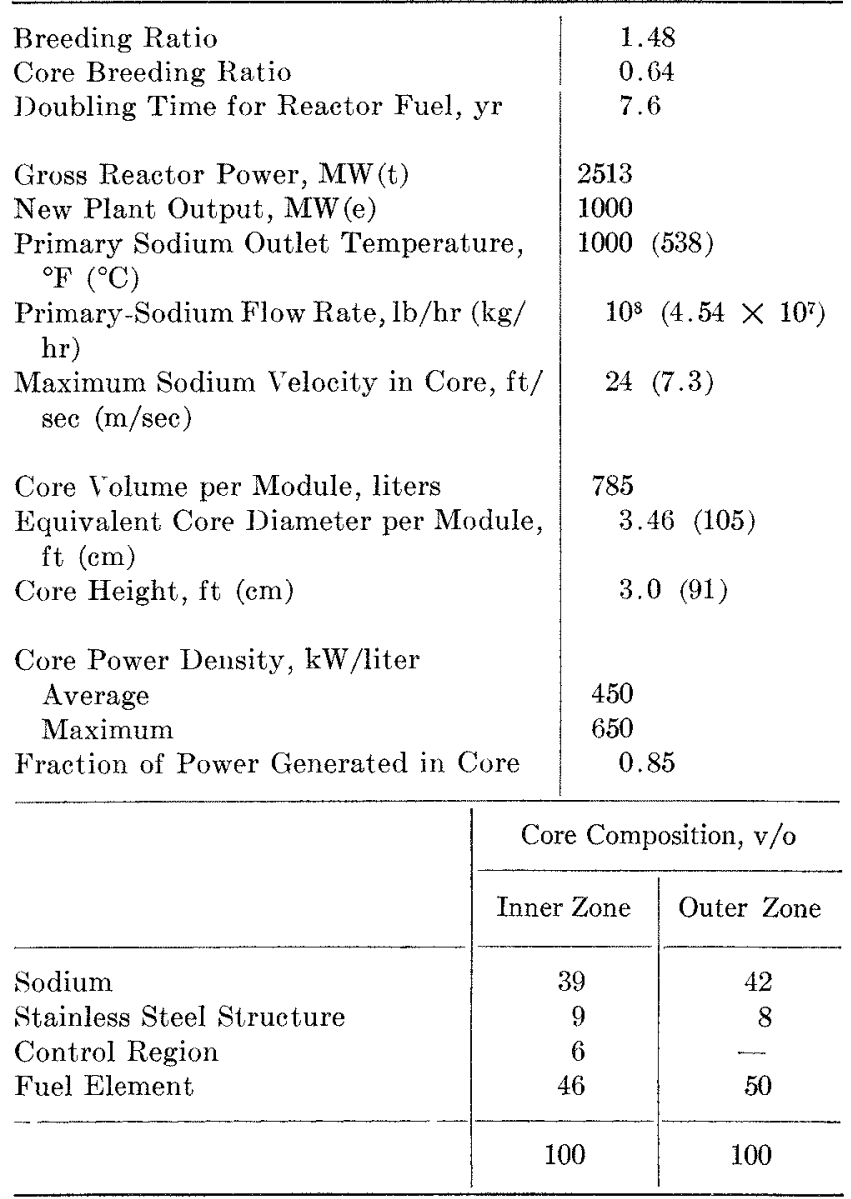

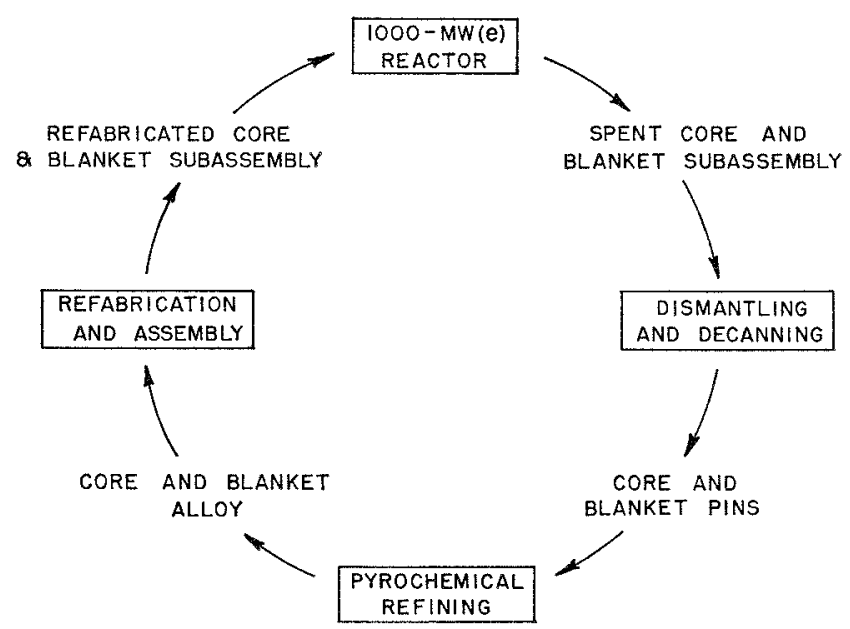

Fig. 5. Simplified Flow Chart of the Fuel Cycle

fects, weighing, and checking the diameter and length of the pin.

The assembly of fucl elements from fuel pins and jacket hardware is the second of the four basic fabrication operations. In this phase, sodium is loaded into preassembled and inspected jackets, which are introduced into the remote facility. Acceptable fuel pins from inspection are placed in the jackets containing sodium and bonded. Bonding consists of heating and impacting the jackets until a void-free annulus of sodium is achieved between the inside surface of the jacket and the fuel-pin perimeter. Following bonding, a restraint plug is inserted and held in place by a circumferential depression in the jacket. A top-end plug is then inserted into and fused to the jacket by welding.

The third broad fabrication operation is that of inspection of fuel elements. The end closure on each fuel element is inspected to insure leak-tight integrity. The process employed is a pressure technique developed for EBR-II application. ${ }^{(8)}$ Since the sodium annulus between the jacket and fuel is generous, no inspection for bond integrity is planned. If it were found necessary to inspect for sodium bond and reclaim non-bonded fuel elements, the technology to do this exists. ${ }^{(9)}$

The final operation in the fabrication scheme is the assembly of fuel elements into subassemblies acceptable for the reactor. In this operation fuel elements and tubular spacers are loadcd alternately, one row at a time, into a hexagonal array. The array is forced into a hexagonal shroud, and the protruding end of each fuel element is attached to a fuel-element support grid which is shouldered against the shroud. After all fuel rods have bcen attached to the grid, end fittings are mounted and welded to the hexagonal shroud. The completed fuel subassembly is gauged, inspected, and, if found acceptable, is ready for return to the reactor. 


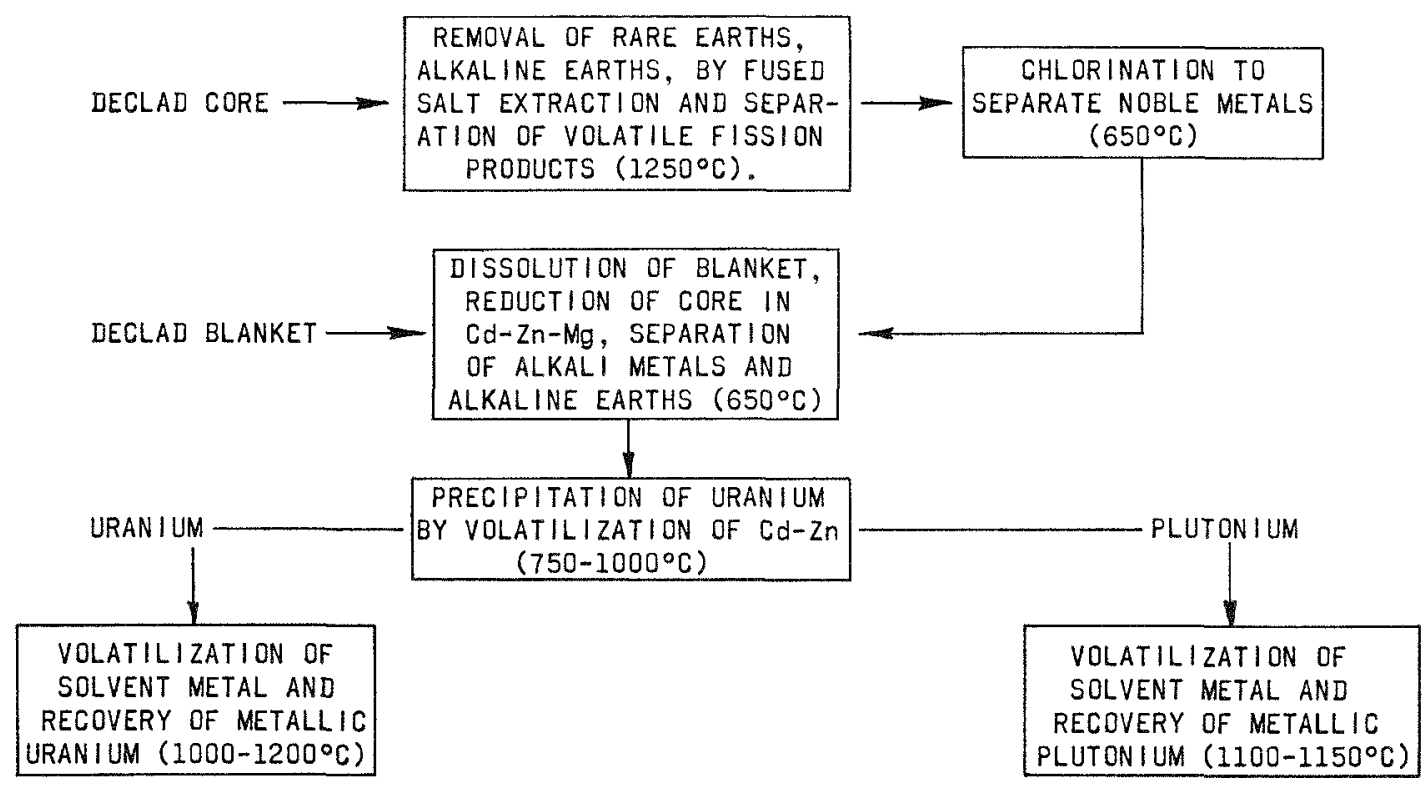

Fıa. 6. Principal Steps in Pyrochemical Processing

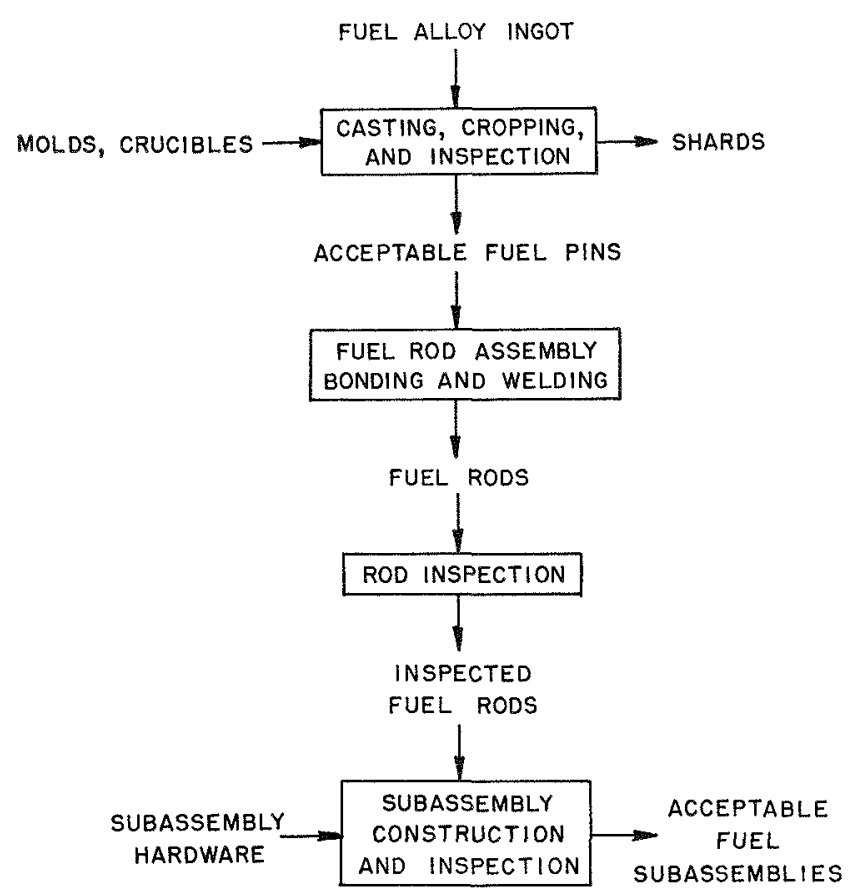

FIG. 7. Principal Steps in Fabrication

A conceptual sketch of the fuel cycle facility is presented in Fig. 8. Processing and fabrication operations are conducted remotely under an inert atmosphere in a heavily shielded annular cell which is $20 \mathrm{ft}(6 \mathrm{~m})$ wide and has an inner diameter of $74 \mathrm{ft}(22.5 \mathrm{~m})$. Fuel subassemblies enter and leave the cell via a tunnel. Operations in the cell are viewed directly through strategically placed windows and are controlled from a $64-\mathrm{ft}(19.5-\mathrm{m})$ diameter control room inside the annulus. Fabrication hardware is assembled and the fuel tubes loaded with sodium in a large glovebox which provides direct access to the cell. Supporting facilities are located along the outer perimeter of the facility and in the subcell area. Two wings are provided: one houses the administrative offices, lunchroom, and locker room; the other the warehouse, refabrication influents preparation facility, and the stockroom.

Costs

A cost estimate, based largely on EBR-II building and equipment costs, has been made for a fuel cycle facility for the Argonne Reference Metal Fueled Reactor. The plant capacity chosen is considered to be in the area of commercial interest. However, further economics can be realized for plants of larger scale since for most promising steps the criticality limitation on batch sizes has not been reached. An analysis of fuel-cycle economics indicated that for a single 1000MW (e) reactor, a two-shift-per-day, five-day-perweek plant operation is needed for the plant size chosen. This case has been termed Partial Capacity operation. The operation of the same plant on a schedule of three shifts per day, seven days per week, corresponding to an $80 \%$ plant factor, would afford the servicing of about three reactors of the characteristics of the reference metal fuel reactor. This case has been termed Full Capacity operation.

The estimated fuel cycle investment requirements for building and equipment appear in Table 3. For Partial Capacity operation the estimate is $\$ 11.4 \times 10^{6}$ and for Full Capacity operation $\$ 12.3 \times 10^{6}$. The cost difference is due to the added capacity for waste storage needed for a Full Capacity Plant. 
I. TUNNEL CONNECTING REACTOR AND FUEL CYCLE FACILITY

2. HOT MAINTENANCE SHOP

3. DECONTAMINATION CELL

4. ANALYTICAL LABORATORY

5. OUTER OPERATING ANNULUS

6. REFAB. INFLUENTS GLOVEBOX

7. ACCESS TUNNEL TO SUBCELL

8. WAREHOUSE

9. ADMIN. OFFICES, LUNCHROOM, AND LOCKER ROOM

10. PROCESSING ANNULUS

11. MAINTENANCE ANNULUS
12. INNER OPERATING ANNULUS

13. OFFICE SPACE

14. PROCESS CONTROL ROOM

15. SUBCELL

16. COLD RECEIVING DOCK

17. ELECTROMECHANICAL MANIPULATOR

18. BRIDGE CRANE

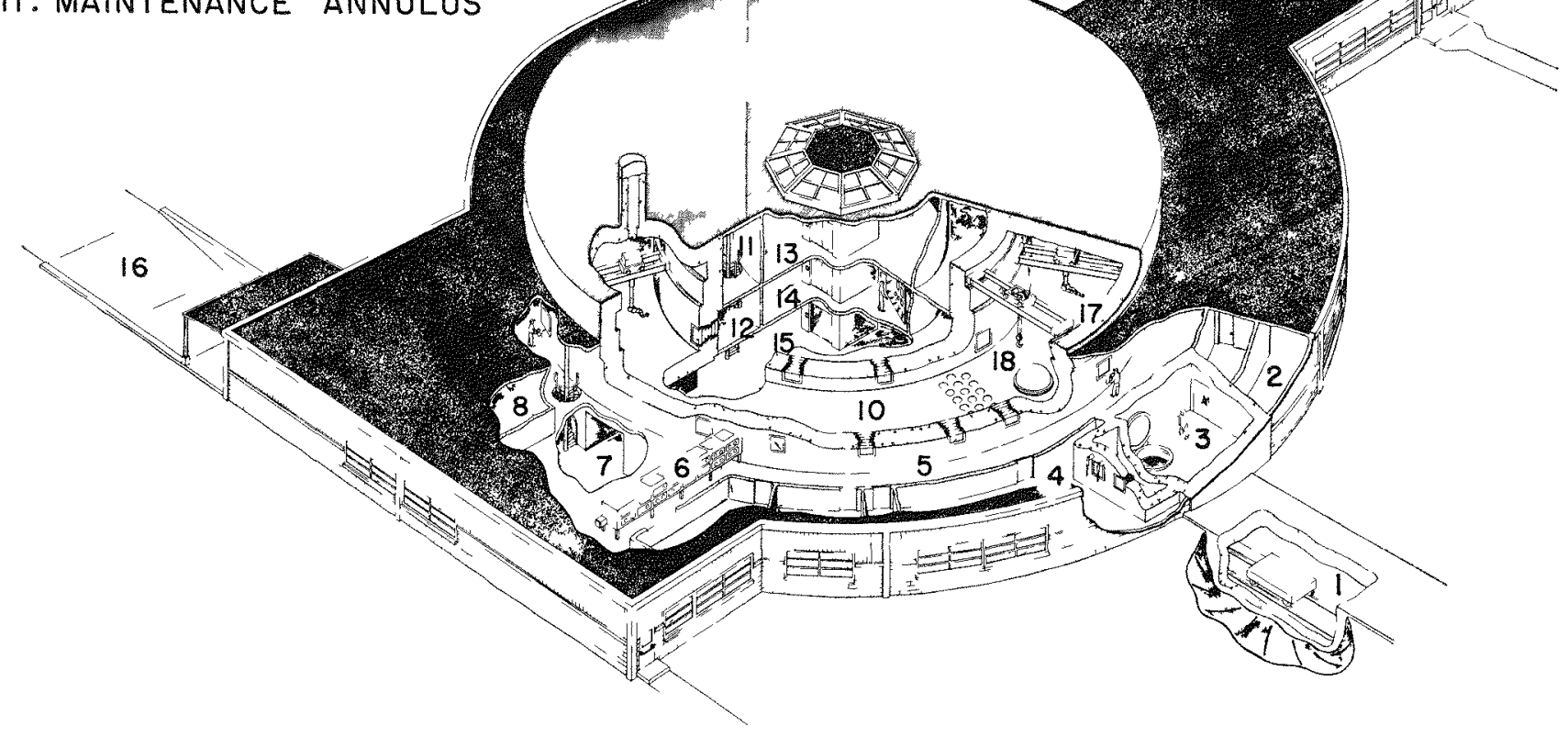

Fis 8 Fuel Cycle Faculity

Estimates of the annual charges for the fuel cycle facility are presented in Table 4 The charges are based on a $675 \%$ cost of money, a $13.0 \%$ return, and deprecration schedules of 30 years for bulding, 15 years for nonprocess equipment, and 5 years for process equipment. Allowances are included for construction overhead, startup expenses, and working capital. The

TABLE 3 SUMMARX OF FUEL CYCle INVES PMLAT REQT IRE mu vts for Reyerevce Metal fueled Reactor

\begin{tabular}{l|c|c}
\hline & \multicolumn{2}{|c}{$\begin{array}{c}\text { Investment in Buldring } \\
\text { and Equipment, } 10^{6} \$\end{array}$} \\
\hline $\begin{array}{c}\text { Partial Ca- } \\
\text { pacity* }\end{array}$ & $\begin{array}{c}\text { Full Ca- } \\
\text { pacity }\end{array}$ \\
\cline { 2 - 3 } $\begin{array}{l}\text { Processing and Refabrication } \\
\text { Waste Disposal }\end{array}$ & 102 & 102 \\
12 & 21 \\
\hline
\end{tabular}

* Annual throughput of $10800 \mathrm{~kg}$ Core Fuel and $23,600 \mathrm{~kg}$ Blanket Fuel

$\dagger$ Annual throughput of $28,400 \mathrm{~kg}$ Core Fuel and $62,100 \mathrm{~kg}$ Blanket Fuel
TABLE 4. Summary or A vual Charges for Fuel Crcle for Reflie $\backslash$ CE Metal FUel

\begin{tabular}{l|c|c}
\hline & \multicolumn{2}{|c|}{ Annual Charge, $10^{\circ} \$$} \\
\cline { 2 - 3 } & $\begin{array}{c}\text { Partal Ca- } \\
\text { pacity }\end{array}$ & $\begin{array}{c}\text { Full Ca- } \\
\text { pacity }\end{array}$ \\
\hline $\begin{array}{c}\text { Processing and Fabrication } \\
\text { a) Capital Charges and J)eprecia } \\
\text { tion }\end{array}$ & 259 & 299 \\
$\begin{array}{c}\text { b) Operating Expense } \\
\text { Waste Disposal } \\
\text { a) Capital Charges and Deprecia } \\
\text { tion }\end{array}$ & 024 & 011 \\
$\begin{array}{c}\text { b) Operating Expense } \\
\text { Total Annual Charge }\end{array}$ & 017 & 039 \\
\hline
\end{tabular}

* Annual throughput of $10800 \mathrm{~kg}$ Core Fuel and $23,600 \mathrm{~kg}$ Blanket Fuel

$\dagger$ Annual throughput of $28,400 \mathrm{~kg}$ Core Fuel and $62,100 \mathrm{~kg}$ Blanket Fuel. 
waste-disposal charge includes allowances for shipping and ultimate disposal offsite. The total annual charge is $\$ 7.1 \times 10^{6}$ for Partial Capacity operation and $\$ 14.4 \times 10^{6}$ for Full Capacity operation. This amounts to a total unit fuel cycle cost of $\$ 160$ per $\mathrm{kg}$ of uranium plus plutonium with plant operating at Full Capacity.

A fuel-cycle cost analysis has been made assuming the fuel-cycle facility operating at Full Capacity and using a plutonium value of $\$ 10$ per gram of $\mathrm{Pu}^{239}$ plus $\mathrm{Pu}^{241}$, private ownership of fuel, and $9 \%$ annual charge on working capital; the fuel-cycle cost is $0.9 \mathrm{mill} /$ $\mathrm{kWhr}$ at $6 \%$ average burnup of core fuel and 0.8 mill/kWhr at $7 \%$ average burnup. If these same rules are applied to two previous $1000-\mathrm{MW}$ (e) fast reactor studies, ${ }^{(10,11)}$ the fuel cycles represent $0.8 \mathrm{mill} / \mathrm{kWhr}$ for both. These latter studies presented fuel-cycle costs based on extrapolations of AEC reference charges for a hypothetical aqueous processing plant ${ }^{(12)}$ designed for enriched uranium, not high-plutonium, high-burnup fuels.

Based on recent Argonne fuel alloy development and test work, the metal fucls look quite promising. (13) The uranium-plutonium-titanium alloy used for the reference design is compatible with vanadium-alloy cladding. The uranium-plutonium-zirconium alloy is equally promising. In addition, when the zirconium content is $10 \mathrm{w} / 0$ or higher, the alloy appears compatible with stainless steel cladding at suitable operating temperatures; stainless steel has a significant cost advantage over the use of vanadium. Capsules of both fuel alloys are under test and as of September, 1965, have successfully gone beyond $4 \%$ burnup. Additional encouragement is found for metal fuels in irradiations of the less desirable uranium-plutonium-fissium alloy which has been irradiated beyond $6 \%$ burnup. Based on potential fuel-cycle cost, there appears to be no distinction, at present, between the mixed carbide, metal, or oxide fuel systems for fast reactors.

\section{BIBLTOGRAPHY AND REFERENCES}

1. L. R. Blake, Achieving High Burnup in Fast Reactors, Reac tor Science and Technology ( $J$. of Nuclear Energy, Parts A and B) 14, 31-48 (1961).

2. J. C. Hesson, M. J. Feldman, and L. Burris, Description and Proposed Operation of the Fuel Cycle Facility for the Second Experimental Breeder Reactor, AN1,-6605 (1963).

3. J. P. Simon and R. B. Wehrle, EBR-II Fuel Dismantling Equipment, 10th Hot Lab Proceedings, ANs, 99-110, November 1962

4. J. P. Simon and J. R. White, Mechanical Decanning of EBR-II Fuel Elements, 10th Hot Lab Proceedings, ANs, 91-98 (Nov. 1962).

5. A. B. Shuck, The Fabrication of Irradiated Reactor Fuels by Semiautomatic, Remote Controlled, Equipment, presented at the Nuclear Fngineering and Science Congress, 1957.

6. F. L. Yaggee, J. E. Ayer, and H. F. Jelinek, The Injection Casting of Uranium-Fissium Alloys as Applied to the
Fabrication of EBR-II Prototype Fuel Pins, Nuclear Metallurgy, Vol. IV, 51-62 (Nov. 1957).

7. H. F. Jelinek and G. M. Iverson, Equipment for the Remote Injection Casting of EBR-II Fuel, Nuclear Science and Engineering, 21, 405-411 (1962).

8. A. P. (xrunwald, Leak Testing of EBR-II Fuel Rods, Nuclear Science and Engineering, 21, 419-423 (1962).

9. T. C. Cameron and N. F. Hessler, Assembling Sodium Bonding, and Bond T'esting of EBR-II Fuel Rods, Nuclear Science and Engineering, 21, 424-431 (1962).

10. M. J. McNelly, Liquid Metal Fast Breeder Reactor Design Study (1000 $\mathrm{Mw}(e) \mathrm{UO}_{2}-\mathrm{PuO}_{2}$ Fueled Plant), GEAP-4418 (Jan 1964).

11. R. B. Steck, Liquid Metal Fast Breeder Reactor Design Study, WCAP-3251-1 (Jan. 1964).

12. Guide to Nuclear Power Cost Evaluation, TID-7025, Tol. 1-5 (March 1962).

13. Reactor Development Program Progress Report for August 1965, ANL-7090.

\section{Discussion}

Mr. Greebler (GE): Have you looked into the effects of the plutonium buildup in the two blankets, and what effect does this have on the coupling between the module of the sodium void effect and also on bypass sodium flow through the blankets?

$M r$. Meneghetti: These calculations have included the presence of some buildup in the blanket regions. However, the effect of the buildup on the coupling has not been studied. Perhaps Mr. Link can answer better the other part of your question.

Mr. Link: The same approach was taken in this particular study as was applied to EBR-II in that the fuel elements are orificed separately and must take the flow which comes about at the maximum heat generation at the end of the cycle, so they may slightly undercool at the beginning of cycle.

$M r$. Häfele (Karlsruhe): Upon what is that figure of $6 \%$ burnup based? A certain estimation or calculation, or do radiation experiments of that kind exist which lead to that figure?

Mr. Link: We have radiation experiments at the present time which have reached this figure under conditions that approach it.

Mr. Häfele: At what temperature was that?

Mr. Link: This particular one has a maximum temperature of $1400^{\circ} \mathrm{F}$ with $1100^{\circ} \mathrm{F}$ external or clad temperature.

Mr. Häfele: Did you have a look in capital cost potential of such a modular arrangement? Did you try to evaluate a capital cost aspect to some extent?

Mr. Link: No, we have not.

Mr. Wirtz (Karlsruhe): Why did you use 9 w/o titanium in your fuel?

Mr. Link: It gives a fair degree of radiation stability and raises the melting point of the alloy appreciably above that which we have experienced in the fizzium 
alloys in EBR-II. With this alloy we can operate at allowable temperatures higher by about $200^{\circ} \mathrm{C}$ than we have experienced with fizzium.

$M r$. Wirtz: What is the highest temperature in your fuel?

Mr. Link: A little over $1400^{\circ} \mathrm{F}$ for the internal temperature in the fuel.

$M r$.Zebroski (GE): What is the reactivity effect of the fuel column slumping by roughly $7 \mathrm{in}$., as I calculated, because of the large annulus that you have; the core length could decrease from $34 \mathrm{in}$. to about 27 or 28 in.? Secondly, does the doubling time of 7.6 years include the inventory in cooling and processing?

Mr. Link: Yes, it does. It includes the external investory and this is calculated on the so-called compound basis in which the assumption is made that the fuel on recovery is reinvested in some reactor system.

Mr. Meneghetti: With regard to the first question, this is an undetermined hazard at present. However, a large amount of effective void with bond sodium is required in order to accommodate a radiation growth. If effective slumping were to occur, the height of the fuel column in the clad could be as much as $25 \%$ less than for the unirradiated pin. Only freshly charged fuel is susceptible to efficient slumping, since it is expected that swelling to fill the radial gap within the clad will occur when only partial burnup has been obtained. Furthermore, only $1 / 3$ of the core is replaced at each cycle; thus, only $1 / 3$ of the core would be available for efficient slumping.

$M r$. Sherer (GE): Do you happen to know what the thickness of the hexagonal channel or subassembly wall is?

Mr. Link: About 80 mils on the hex ean arnund the 


\title{
Selected Optimization Studies in the Use of Carbide Fuel in Large Fast Breeder Reactors
}

\author{
G. A. Soren, A. H. Kazi, B. R. Then, A D) B. H. Cherriy \\ Unted Nuclear Corporation \\ White Plains, New York \\ (PIRESINTED BY G. A. SOFER)
}

\section{SELECTION OF (UPu)C FUEL-ROD DESIGN}

\subsection{Introduction}

Analyses have been performed in order to arrive at a (UPu)C fuel-rod design which will allow for currently expected fuel swelling at 100,000 MWD/MT and keep the maximum fuel temperature below $3500^{\circ} \mathrm{F}$ at a fuel rating of $30 \mathrm{~kW} / \mathrm{ft}$. Two principal methods can be considered to accommodate swelling. In the first, fuel-clad interference is permitted and the cladding is allowed to deform plastically. In the second, lower effective fuel density is assumed to absorb swelling. Both approaches to solve the problem of fuel swelling are being planned for current EBR-II irradiation tests. Fuel with high and low "effective" densities will be irradiated in Type 316 stainless steel clad to determine the best compromise between clad strain, fuel temperature, and effective fuel density. Only the results of such experimental data can guide the further design philosophy for ceramic fuel rods for high burnups.

If the fuel center temperature is to be kept below $3500^{\circ} \mathrm{F}$ at the beginning of life, a clad strain of $2.8 \%$ must be accommodated at the end of the 100,000$\mathrm{MWD} / \mathrm{T}$ life for a $\mathbf{9 5 \%}$ density fuel. At the present time, data on the effects of fast flux on the ductility of clad materials are lacking. The alternative approach of lower effective density of fuel to prevent excessive clad strain at end of life was therefore considered in this paper.

Helium is preferred to sodium as a thermal bond material for the following reasons:

1. Clad carburization is facilitated by the presence of sodium.

2. Fabrication costs are higher with sodium.

3. Uncontrolled presence of bubbles in the sodium can lead to excessive fuel temperatures.

4. Contact of sodium with fuel at the center of the pellet may cause excessive internal pressures.

Fission gas venting to the coolant is not taken as a near-term design objective.

Material properties and irradiation effects for the (UPu) C fuel were taken from data recently obtained by United Nuclear Corp. (1) The specific values of the properties used together with properties of the cladding, the filler gas, and the sodium coolant are listed in Table 1 . The gap conductance $\left(h_{q}\right)$ across the helium filler gas between the fuel and the clad was calculated based on the actual gap thickness between pellet and cladding, taking into account thermal expansion and swelling. When the fuel and clad are in contact, a maximum value of $h_{g}=2000 \mathrm{Btu} / \mathrm{hr}-\mathrm{ft}^{2}{ }^{\circ} \mathrm{F}$ was used.

A maximum volume increase of $18 \%$ at a burnup of $100,000 \mathrm{MWD}_{\mathrm{MT}}{ }^{(1)}$ is used and assumed to occur isotropically for fuel of $95 \%$ theoretical density based on irradiation swelling data recently determined. For fucl of less than $95 \%$ theoretical density, the internal voids are assuned to be effective in reducing the external dimensional increases of the fuel pellet. For a pellet with a density of $\rho / \rho T$, the external swelling is calculated as $0.18-\left[0.95-\left(\rho / \rho_{x}\right)\right]$.

The fuel conductivity is assumed to vary directly with the fuel density over the range investigated.

\subsection{Summary of Results}

\section{Fuel Density and Assembled Radial Gap Width}

The determination of the fuel density and assembled radial gap width for the reference fuel-rod design was 
TABLE 1. Materials Properties

\begin{tabular}{|c|c|c|c|}
\hline & \multicolumn{2}{|c|}{ Material } & \multirow{2}{*}{$\mathrm{Na}$} \\
\hline & (UPu)C & $316 \mathrm{SS}$ & \\
\hline Density, $\mathrm{g} / \mathrm{cm}^{3}$ & $\begin{array}{l}13.6 \\
(100 \% \text { theo- } \\
\text { retical) }\end{array}$ & 7.8 & 0.83 \\
\hline $\begin{array}{l}\text { Thermal conductivity, B'TU/ } \\
\text { hr-ft- }{ }^{\circ} \mathrm{F}\end{array}$ & 11.6 & 12.0 & 40 \\
\hline Specific heat, BTC $/ \mathrm{lb}-{ }^{\circ} \mathrm{F}$ & 0.062 & 0.134 & 0.3 \\
\hline $\begin{array}{l}\text { Coefficient of thermal expan- } \\
\text { sion, }{ }^{\circ} \mathbf{F}^{-1} \times 10^{6}\end{array}$ & 6.1 & 10.0 & \\
\hline
\end{tabular}

Conductivity of He filler gas $=0.18 \mathrm{BT} \mathrm{T} / \mathrm{hr}-\mathrm{ft}-{ }^{\circ} \mathrm{F}$.

conducted by means of a parametric survey with maximum fuel temperatures and end-of-life pellet-clad interference calculated as a function of effective density and assembled cold-gap thickness. Effective density is defined as the mass of fuel per unit volume inside the fuel rod, which includes the fuel pellet and the radial gap. Effective density is lower than the fuel pellet density by the ratio $\left(\frac{\text { pellet diameter }}{\text { clad inside diameter }}\right)^{2}$.

All calculations were made with the reference fuel-rod design of 0.265-in. diameter and with a clad thickness of $0.015 \mathrm{in}$.

The effects of the assembled radial gap and effective density on maximum fuel temperature are shown in Figure 1. The maximum fuel temperature, which occurs at the beginning of life, is strongly dependent upon the gap width because of the inverse relationship between gap conductance and gap width. Since the thermal conductivity of the fuel is quite high, the maximum fuel temperature is not strongly dependent on fuel density, although fuel conductivity is assumed to be directly proportional to fuel density.

Examination of Figure 1 reveals that an assembled gap width less than $\sim 0.0025 \mathrm{in}$. is required to keep the maximum fuel temperature below $3500^{\circ} \mathrm{F}$. Either of the densities shown would be acceptable.

However, as the assembled radial gap thickness is reduced, there is less room available to accommodate irradiation swelling of the fuel pellet, and the end-of-

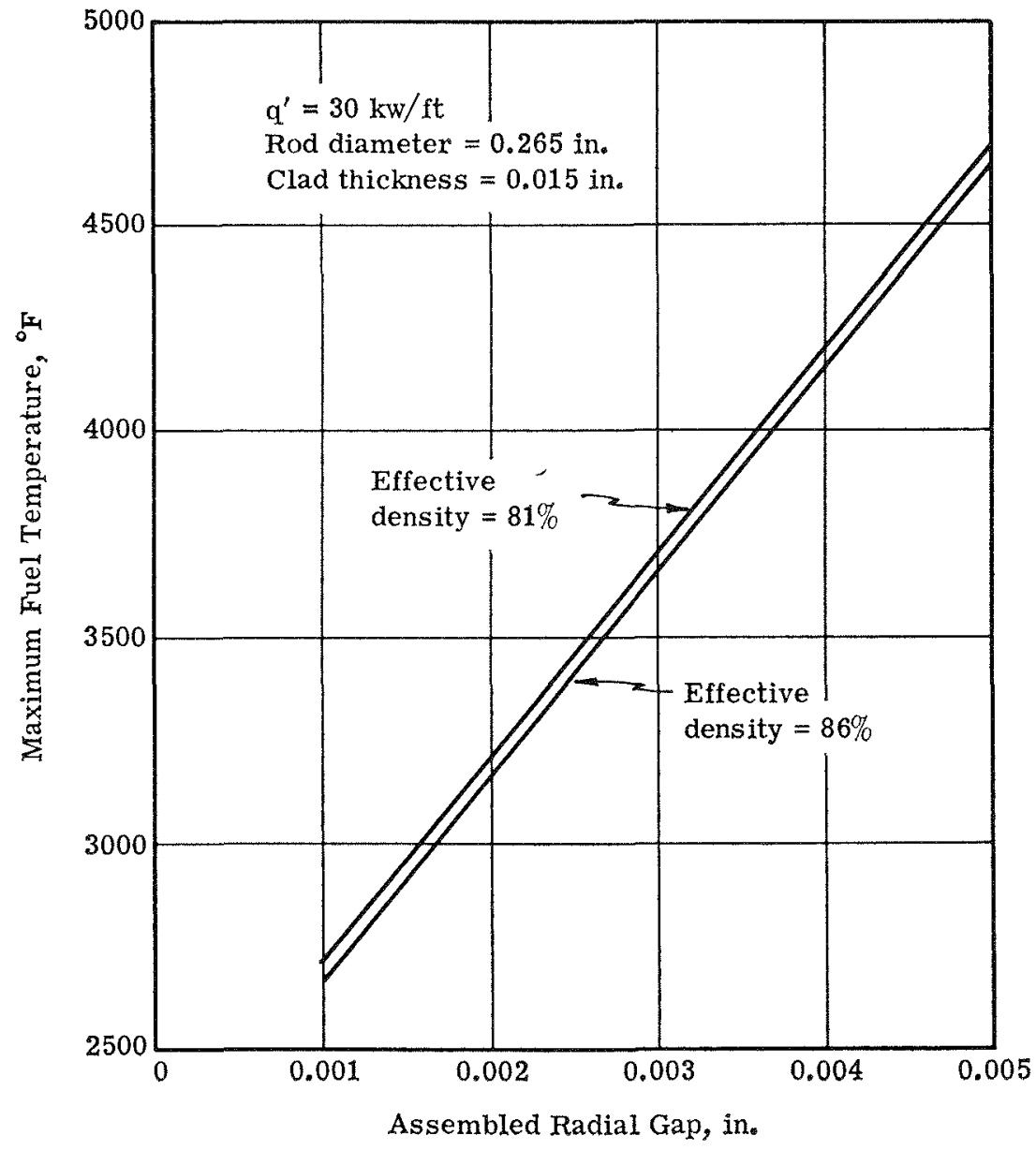

Fig. 1. Effect of Assembled Radial Crap on Maximum Fuel Temperature. 


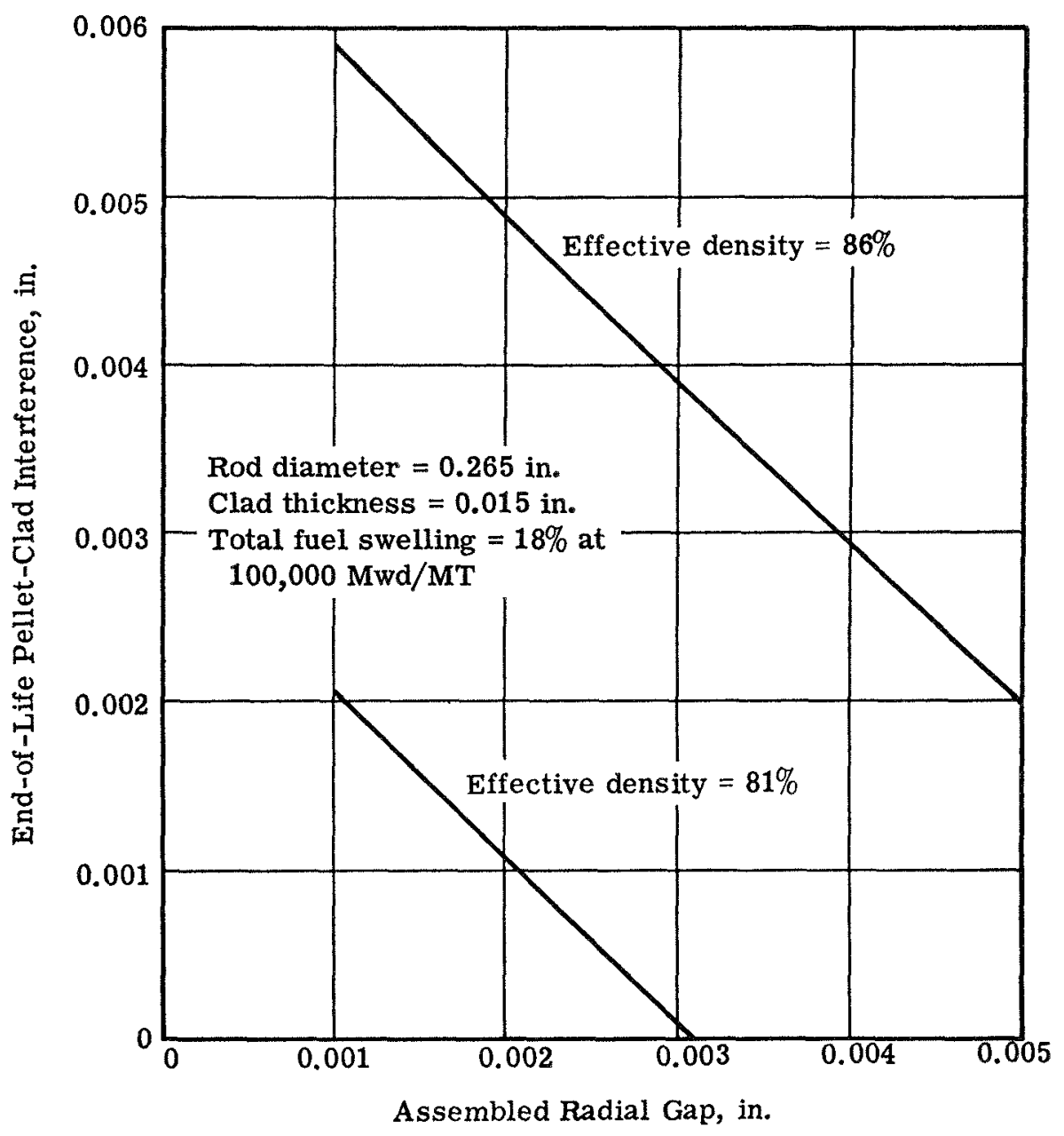

Fig. 2. Effect of Assembled Radial Gap on End-of-life Pellet-Clad Interference.

life pellet-clad interference increases, as shown in Figure 2. This interference is strongly dependent on effective density because of the swelling model discussed in Section 1.1. An assembled radial gap of greater than 0.003 in. is required to insure no pellet-clad interference at the end of life with the lower effective density $(81 \%)$. An effective density of $81 \%$ and an assembled radial gap of $0.002 \mathrm{in}$. were chosen to preserve acceptable nuclear characteristics. This corresponds to a pellet density of $83.7 \%$ theoretical.

\section{Reference Fuel-rod Characteristics}

The selected values of the assembled radial gap width and effective density for the reference fuel rod represent a compromise among the mutually conflicting requirements of breeding ratio, maximum fuel temperature, and pellet-clad interference. The nominal gap thickness of $0.002 \mathrm{in}$. and the effective density of $81 \%$, chosen for the reference fuel rod, result in a maximum fuel temperature of $3200^{\circ} \mathrm{F}$ and an end-of-life cold pellet-clad interference of 0.001 in. The corre-
Table 2. Reference Fuet-rod Characteristics

$\begin{array}{lc}\text { Rod outside diameter, in.: } & 0.265 \\ \text { Clad thickness, in.: } & 0.015 \\ \text { Rod inside diameter, in.: } & 0.235 \\ \text { Radial gap thickness, in.: } & 0.002 \\ \text { Fuel-pellet diameter, in.: } & 0.231 \\ \text { Effective density, \% theoretical: } & 80.9 \\ \text { Actual fuel density, \% theoretical: } & 83.7 \\ \text { Active core length, ft: } & 3.0\end{array}$

sponding hot pellet-clad interference at the end of life is $0.0005 \mathrm{in}$.

The characteristies of the reference fuel rod are listed in Table 2.

\section{CORE-BLANKET COMPOSITION AND CONFIGURATION}

\subsection{Introduction}

Analyses have been performed in order to assess the influence of $(\mathrm{UPu}) \mathrm{C}$ fuel density on reactor character- 
TABLE 3. Restlts of Composition-Conftguration Survey Calculations

\begin{tabular}{|c|c|c|c|c|c|c|c|c|c|c|c|c|c|c|}
\hline \multirow{2}{*}{$\begin{array}{l}\text { Case } \\
\text { No. }\end{array}$} & \multirow{2}{*}{ Reactor Type } & \multirow{2}{*}{$\mid \begin{array}{c}\text { Smeared } \\
(\mathrm{UPu}) \mathrm{C} \\
\text { Density, } \mathrm{g} / \mathrm{cm}^{2}\end{array}$} & \multirow{2}{*}{$\begin{array}{l}\text { Core Size, } \\
\mathrm{OD} \times \mathrm{H}, \mathrm{ft}\end{array}$} & \multirow{2}{*}{$\begin{array}{c}\text { Reactor } \\
\text { Size, OD } \times \\
\mathrm{H}, \mathrm{ft} \dagger\end{array}$} & \multirow{2}{*}{$\begin{array}{l}\text { Core } \\
\text { Volume, } \\
\text { liters }\end{array}$} & \multirow{2}{*}{$\begin{array}{l}\text { Specific } \\
\text { Power, } \\
\mathrm{kW} / \mathrm{kg} \\
\text { fissile }\end{array}$} & \multirow{2}{*}{$\begin{array}{c}\text { Inventory, } \\
\mathrm{kg}(49+ \\
4.1)\end{array}$} & \multirow{2}{*}{$\begin{array}{c}\text { Fissile } \\
\text { Enrichment } \\
49+41 / 40 \\
+28+49 \\
+41\end{array}$} & \multirow{2}{*}{$\begin{array}{c}\text { Core } \\
\text { Conver- } \\
\text { sion Ratio }\end{array}$} & \multirow{2}{*}{$\begin{array}{c}\text { Total } \\
\text { Breeding } \\
\text { Ratio }\end{array}$} & \multirow{2}{*}{$-T \frac{d k}{d t}$} & \multicolumn{2}{|c|}{$\begin{array}{c}\text { Na Void Effect, } \\
\% \Delta k / k\end{array}$} & \multirow{2}{*}{$\begin{array}{c}\text { Fraction } \\
\text { of Flux } \\
\text { below } \\
17 \mathrm{keV}\end{array}$} \\
\hline & & & & & & & & & & & & $\begin{array}{l}50 \% \\
\text { Core }\end{array}$ & $\begin{array}{l}100 \% \\
\text { Core }\end{array}$ & \\
\hline 1 & $\begin{array}{r}\text { Pancake, high- } \\
\text { density fuel }\end{array}$ & $\begin{array}{l}12.9(95 \% \\
\text { theo. })\end{array}$ & $10 \times 2$ & $12.5 \times 5$ & 4448 & 1200 & 1830 & 0.124 & 0.86 & 1.50 & 0.0045 & +0.621 & +1.25 & 0.29 \\
\hline 2 & $\begin{array}{l}\text { Pancake, medium- } \\
\text { density fuel }\end{array}$ & $11.0(81 \%)$ & $10 \times 2$ & $12.5 \times 5$ & 4448 & 1260 & 1749 & 0.139 & 0.75 & 1.44 & 0.004 & +0.687 & & 0.29 \\
\hline 3 & $\begin{array}{l}\text { Pancake, low-den- } \\
\text { sity fuel }\end{array}$ & $\begin{array}{c}9.03 \\
(66.5 \%)\end{array}$ & $10 \times 2$ & $12.5 \times 5$ & 4448 & 1320 & 1665 & 0.161 & 0.64 & 1.39 & 0.0035 & & & 0.29 \\
\hline 4 & $\begin{array}{l}7 \text { hexagonal core } \\
\text { modules }\end{array}$ & 11.0 & $\begin{array}{l}\text { Hexagonal module, } \\
3 \text { high } \times 1.7 \text { on } \\
\text { side }\end{array}$ & $18 \times 6$ & $635 \times 7$ & 770 & 2287 & 0.182 & 0.51 & 1.67 & 0.002 & & -1.33 & 0.24 \\
\hline 5 & $\begin{array}{l}3 \text { rhombic core } \\
\text { modules }\end{array}$ & 11.0 & $\begin{array}{l}4.5 \times 4.5 \times 3 \text { rhom- } \\
\text { bic module }\end{array}$ & $15 \times 6$ & $1483 \times 3$ & 1200 & 1836 & 0.146 & 0.70 & 1.65 & 0.003 & & -0.307 & 0.27 \\
\hline 6 & $\begin{array}{l}\text { Pancake, axial car- } \\
\text { bide blanket in } \\
\text { Case No. } 1\end{array}$ & 12.9 & $10 \times 2$ & $12.5 \times 5$ & 4418 & 1200 & 1830 & 0.124 & 0.84 & 1.60 & & & & \\
\hline 7 & $\begin{array}{l}\text { Pancake, thick } \\
\text { clad in Case No. } 1\end{array}$ & 12.9 & $10 \times 2$ & $12.5 \times 5$ & 4448 & 1180 & 1870 & 0.126 & 0.84 & 1.48 & & & & \\
\hline 8 & $\begin{array}{l}\text { Pancake, niobium } \\
\text { clad in Case No. } 1\end{array}$ & 12.9 & $10 \times 2$ & $12.5 \times 5$ & 4448 & 1060 & 2066 & 0.140 & 0.71 & 1.19 & $<0.001$ & & & 0.21 \\
\hline 9 & $\begin{array}{c}\text { Pancake, vanadium } \\
\text { clad in Case No. } 1\end{array}$ & 12.9 & $10 \times 2$ & $12.5 \times 5$ & 4448 & 1220 & 1800 & 0.122 & 0.86 & 1.50 & $\sim 0.005$ & & & 0.35 \\
\hline
\end{tabular}

* Core volume fractions are $27 \mathrm{v} / 0$ fuel, $14 \mathrm{v} / 0$ clad and structure, and $59 \mathrm{v} / 0$ sodium, except for Case No. 7 which contains $27 \mathrm{v} / 0$ fuel, $21 \mathrm{v} / 0$ clad and structure, and 52 $\mathrm{v} / \mathrm{o}$ sodium.

$\dagger$ Reactor height does not include fission gas reservoir. Axial blanket composition is same as core. Radial blanket composition is $55 \mathrm{v} / \mathrm{o}$ fuel, $20 \mathrm{v} / 0$ clad and structure, and $25 \mathrm{v} / 0$ sodium. Fuel is $\mathrm{UO}$ at $9.5 \mathrm{~g} / \mathrm{cm}^{3}$. 
TABLE 4. SPECTRAI INDICES rot CORE Region

\begin{tabular}{c|c|c|c|c}
\hline Case & $\sigma_{c}^{49} / \sigma_{f}^{49}$ & $\sigma_{c}^{28} / \sigma_{f}^{49}$ & $\sigma_{f}{ }^{10} / \sigma_{f}^{49}$ & $\sigma_{f}^{28} / \sigma_{f}^{49}$ \\
\hline 1 & 0.17 & 0.14 & 0.19 & 0.027 \\
2 & 0.17 & 0.14 & 0.19 & 0.027 \\
3 & 0.17 & 0.14 & 0.20 & 0.028 \\
4 & 0.14 & 0.13 & 0.22 & 0.033 \\
5 & 0.16 & 0.14 & 0.19 & 0.029 \\
8 & 0.13 & 0.13 & 022 & 0.031 \\
9 & 0.19 & 0.15 & 0.18 & 0.026 \\
\hline
\end{tabular}

istics in order to permit evaluation of the cost penalty associated with low-density fuel. Calculations have also been performed to determine the effect of a thick clad, as well as the use of niobium rather than stainless steel clad. Low-density fuel and restraint by thick (30 mils) clad provide two approaches to allow for expected fuel swelling at high burnup.

An investigation of reactor configurations was carried out to arrive at a reasonable compromise between economic parameters such as breeding ratio and inventory, on the one hand, and safety parameters, i.e., Doppler coefficient and sodium void reactivity offect, on the other hand.

The calculations were performed with the use of the following cross sections:
1. Fe, $\mathrm{Ni}, \mathrm{Cr}, \mathrm{O}, \mathrm{C}, \mathrm{Nb}$
16-group Hansen-Roach Set ${ }^{(2,3)}$
2. $U-238$
16-group Hansen-Roach as modi- fied by spielberg(:)

3. Pu-239, Pu-240

16-group Hansen-Roach, infinite dilute modified by self-shielding factors generated using methods of Graves. (5)

4. $\mathrm{Na}$

16-group Hansen-Roach with modification of the $\sigma_{c}$ in $2.85 \mathrm{keV}$ resonanre by data of Hibdon.(6)

5. V

16-group generated using $\mathrm{HRG}$ slowing-down calculation. ${ }^{(7)}$

Further details concerning these cross-section calculations are given in Reference (8).

Critical enrichment and reactivity were calculated by use of the $\mathrm{DS}_{2}$ approximation to the neutron transport equation by means of the DTF code. ${ }^{(9)}$ This code is a one-dimensional multigroup program for solving the neutron transport equation by the $S_{n}$ approximation.

Doppler coefficients were calculated by performing two $k$-eigenvalue calculations, one at $1000^{\circ} \mathrm{K}$ and one at $1500^{\circ} \mathrm{K}$, and determining $T(d k / d T)$ from these results as follows:

$$
T \frac{d k}{d T}=\text { Constant }=\frac{k(1500)-k(1000)}{\ln \frac{1500}{1000}} .
$$

For coolant void reactivity effects, two $k$-eigenvalue calculations were performed at operating temperature, one with full sodium density, and one with $50 \%$ or no sodium present, from which the void effect was calculated.

Breeding ratio and spectral indices were calculated directly from DTF output.

\subsection{Summary of Results}

The results of the various ealculations are summarized in Tables 3 and 4, and in Figure 3.

\section{Effect of Core Subdivision}

Comparing Cases 2, 5, and 4 of Table 3 , it is seen that as the core region is subdivided, critical loading and enrichment increase, and the sodium reactivity effect becomes more negative. These effects are due to increased core leakage and a harder neutron spectrum. The spectral hardening as well as increased enrichment results in a decreased Doppler coefficient.

The conversion ratio in the core decreases as core size is reduced, leading to more frequent refueling shutdowns. In the case of the modular cores, better use is made of the radial blanket, which can contain an appreciably larger fertile material volume fraction than is possible in the core and the axial blanket. (55 vs. $27 \mathrm{v} /$ o fuel).

It is seen that the three-core module reactor represents an attractive compromise between the pancake design (low total breeding ratio, low fissile fucl inventory, modest Doppler, positive sodium void effect) and the seven-core module arrangement (high total breeding ratio, high fissile fuel inventory, small Doppler, negative sodium void effect). The three-core modular arrangement also permits a relatively compact reactor configuration compared to a seven-core modular arrangement. For given core and blanket volumes, a four- or five-module arrangement is less compact.

\section{Effect of (UPu)C Fuel Density}

The effect of lower fuel density is seen mainly in an increase in enrichment and a reduction in overall breeding ratio, as seen in Table 3 and Figure 3 . The effect of fuel density on total breeding ratio is less intense than it is on core conversion ratio. It is seen from the data of Table 3 that a smeared fuel density as low as $\sim 80 \%$ theoretical yields a breeding ratio of 1.65 in the modular core. 


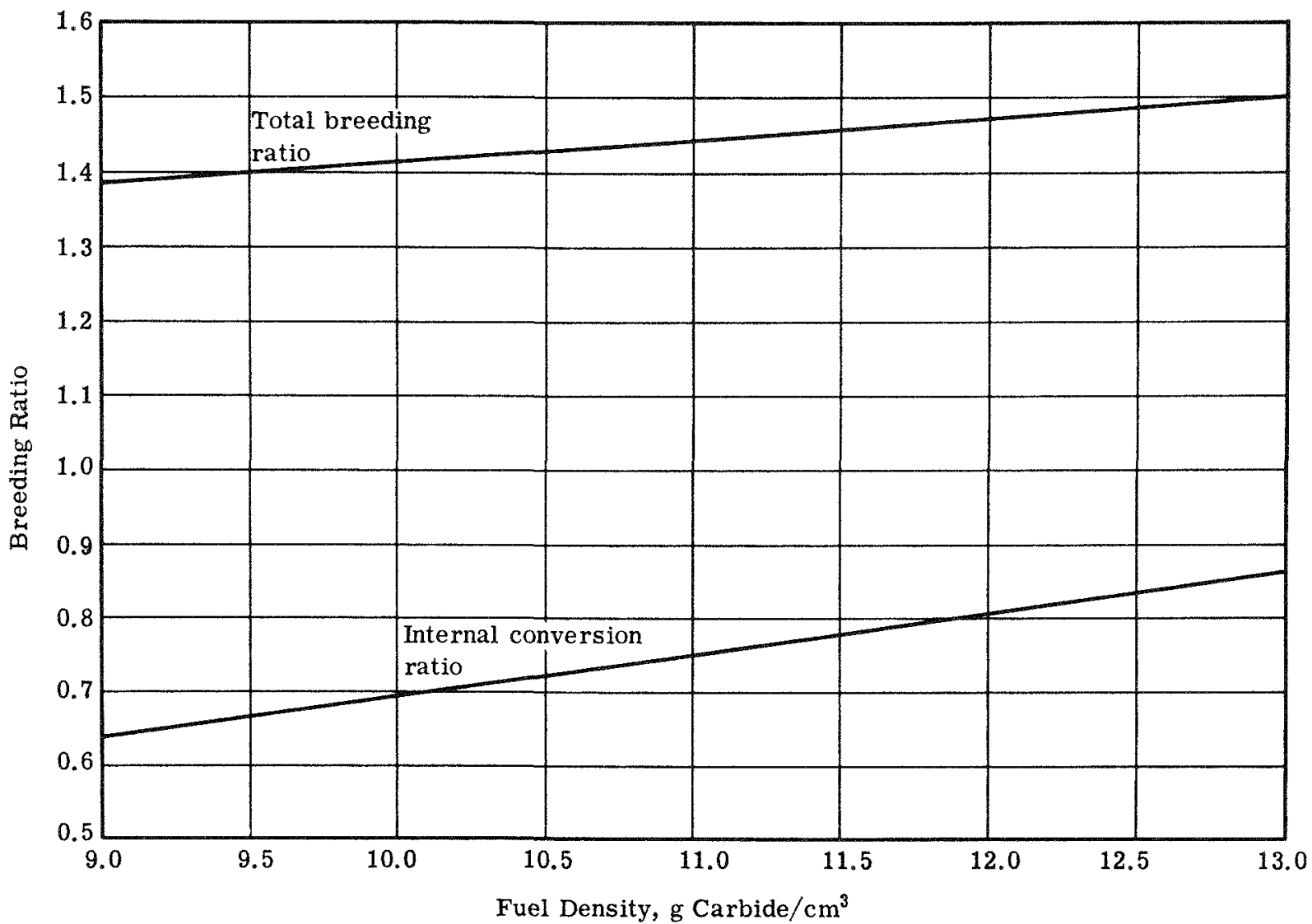

FIa. 3. Breeding Ratio vs Fuel Density for 1000-MW(e) (UPu)C Pancake Reactor.

\section{Effect of Thick Clad}

A calculation was performed to assess the effect of increasing the thickness of stainless steel clad from 15 to 30 mils. The stainless steel volume fractions corresponding to these two clad thicknesses are 6 and 13 $\mathrm{v} / \mathrm{o}$, respectively. Other structure accounts for $8 \mathrm{v} / \mathrm{o}$. It is seen from Table 3 (see Case 7) that the resultant penalty is small: an inerease of $50 \mathrm{~kg}$ in fissile inventory, and a reduction in breeding ratio of $\sim 0.02$.

\section{Effect of Niobium and Vanadium Clad}

Cases 8 and 9 in Table 3 show the effect of substituting niobium or vanadium clad for stainless steel. Although the substitution of niobium is not acceptable, that of vanadium is. There is an increase in Doppler coefficient when vanadium is used. These results are in agreement with a similar earlier analysis. ${ }^{(10)}$

The calculations reported to date on the effect of niobium, including the present calculation, make no allowance for self-shielding of the niobium resonances. Since the niobium resonances lie just above the important U-238 resonances, it is expected that proper treatment of the niobium resonances could appreci- ably alter the predicted effect of niobium on fast breeder performance.

\section{Effect of $\mathrm{UO}_{2}$ Versus $\mathrm{UC}$ Blanket}

Comparison of Cases 1 and 6 of Table 3 indicate the effect of substituting $\mathrm{UC}$ for the $\mathrm{UO}_{2}$ in the axial blanket of the pancake reactor. The core inventory and conversion ratio are essentially unchanged, while the total breeding ratio is increased by 0.1 . This is a significant increase; however, as discussed in Section 4.2 , it does not lead to a decrease in fuel-cycle costs due to the currently postulated higher fabrication costs of UC vs. $\mathrm{UO}_{2}$. Changeover to a carbide blanket is available at a relatively small cost penalty $(\sim .04$ mill $/ \mathrm{kWhr}(\mathrm{e})$ ) to raise the overall breeding ratio by $\sim 0.1$ should it be necessary to soften the spectrum in the core to enhance the Doppler coefficient.

\section{REACTIVITY TRANSIENTS}

\subsection{Introduction}

Response of (PuU) C to transients caused by reactivity insertion was examined to evaluate the effect of 

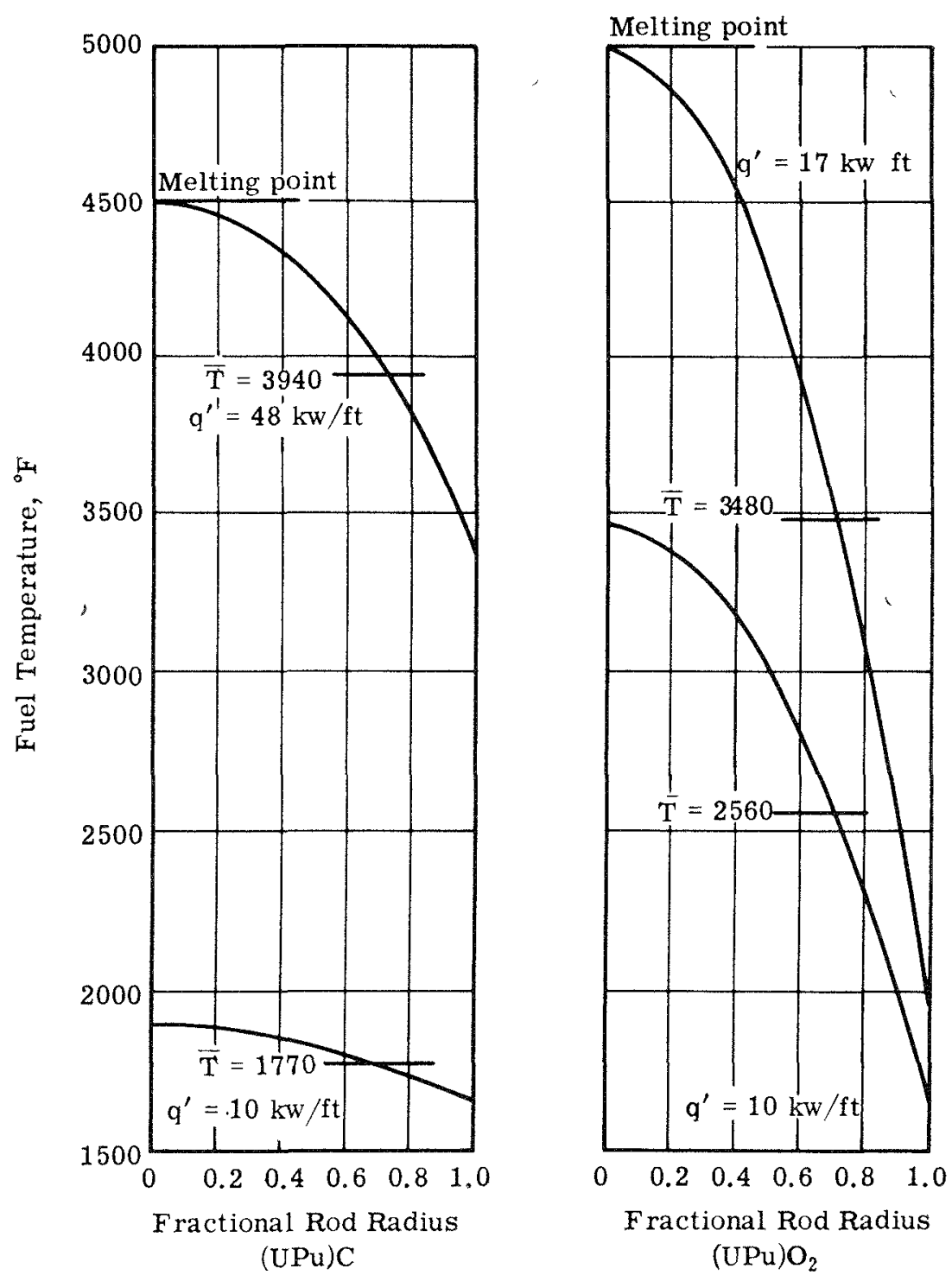

Fra. 4. Comparison of Carbide and Oxide Fuel Rods.

relatively low initial operating temperatures, and the effect of high conduetivity and thermal diffusivity of the fuel. This behavior was compared to $(\mathrm{PuU}) \mathrm{O}_{2}$ fuel under similar conditions of reactivity insertion to assess the differences in behavior between the two fuels.

The major contributor to this different response is the thermal conductivity, which is about $11.6 \mathrm{BTU} /$ hr-ft- ${ }^{\circ} \mathrm{F}$ for (UPu) C and about 1.5-2.0 BTU/hr-ft- ${ }^{\circ} \mathrm{F}$ for $(\mathrm{UPu}) \mathrm{O}_{2}$. Figure 4 illustrates the difference in steady-state temperatures between carbide and oxide fuel at $10 \mathrm{~kW} / \mathrm{ft}$, and the difference in maximum heat generation per unit length corresponding to incipient melting.

The difference in thermal conductivity is also reflected in a difference in thermal diffusivity, which is about $0.22 \mathrm{ft}^{2} / \mathrm{hr}$ for the carbide and $0.032 \mathrm{ft}^{2} / \mathrm{hr}$ for the oxide. This means that for the carbide the heat is released to the coolant much faster.

An investigation was made of the response of the reference carbide fuel rod described in Section 1 and a typical oxide-fueled core ${ }^{(11)}$ to transients produced by two reactivity insertions. A total reactivity of $\$ 1.40$, which is typical of the amount which might be inserted by the withdrawal of one group of control rods, was inserted at rates of $\$ 2.00 / \mathrm{sec}$ and $\$ 0.20 /$ sec. Table 5 shows the kinetics parameters for these problems which were run on the coupled kinetics-heat transfer code, SCARE, written at United Nuclear Corporation, which treats an individual fuel rod and its associated coolant. The Elrod lumped-parameter formulation $(12,13)$ is used as the transient heat transfer model for the radial conduction in the fuel rod. The set of simultaneous 
TABle 5. Parameters for Reactuvity Transients Calculations

Total Reactivity Insertion, $\$: 1.40$

Reactivity Insertion Rates, $\$ /$ see $: 2.00$ and 0.20

\begin{tabular}{|c|c|c|}
\hline & \multicolumn{2}{|c|}{ Fuel } \\
\hline & $(\mathrm{UPu}) \mathrm{C}$ & $(\mathrm{UPc}) \mathrm{O}_{2}$ \\
\hline Neutron Lifetime $l$, see $\times 10^{7}$ & 4.0 & 5.7 \\
\hline $\begin{array}{l}\text { Fffective Delayed-neutron Fraction } \\
\beta_{e f f}\end{array}$ & 0.0040 & 0.0032 \\
\hline $\begin{array}{l}\text { Woppler Coefficient Constant } \\
\qquad-T(d k / d t)\end{array}$ & 0.010 & 0.010 \\
\hline $\begin{array}{l}\text { Doppler Coefficient, at Initial Aver- } \\
\text { age Fuel Temperature, }{ }^{\circ} \mathrm{F}^{-1} \times 10^{6}\end{array}$ & -4.2 & -3.5 \\
\hline Initial Average Fuel Temperature, ${ }^{\circ} \mathrm{F}$ & 1940 & 2400 \\
\hline Average Power Rating, $\mathrm{kW} / \mathrm{ft}$ & 18.0 & 10.4 \\
\hline
\end{tabular}

first-order differential equations which are obtained by coupling the reactor kinetics equations and the heat, transfer equations with the temperature coefficients of reactivity are numerically solved by a modified RungeKutta finite-difference technique. ${ }^{(14)}$

\subsection{Summary of Results}

\section{Reactivity Transients in (UPu)C Fuel}

Figures 5 through 7 summarize the results of the reactivity transients analyses performed for (UPu)C fuel. In all cases, the total reactivity insertion was $\$ 1.40$, introduced at rates of $\$ 2.00 / \mathrm{sec}$ and $\$ 0.20 / \mathrm{sec}$. As seen from Figure 7, the maximum fuel temperature stays well below the melting point of $4500^{\circ} \mathrm{F}$. The sodium coolant does not boil.

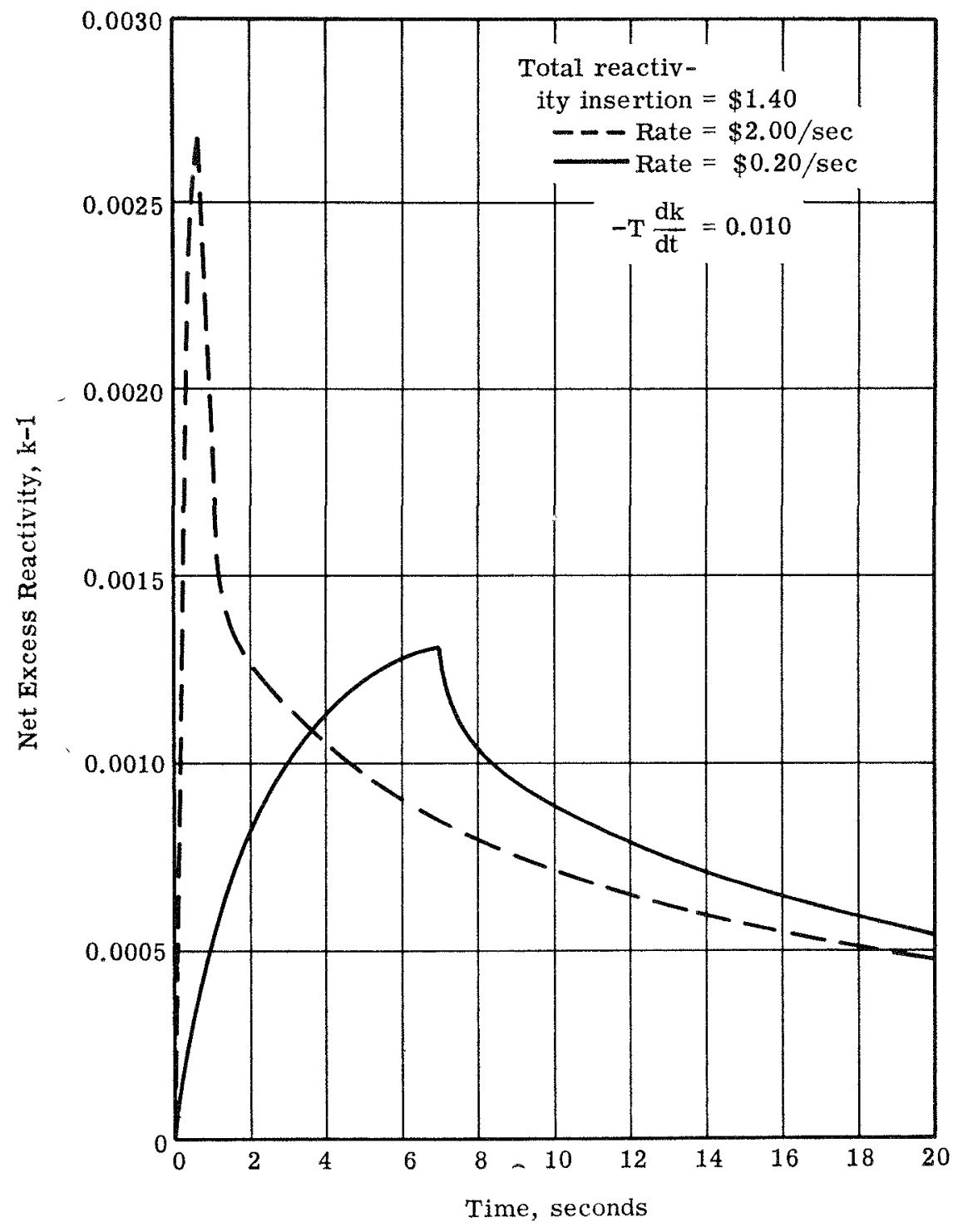

FIG. 5. Reactivity Insertion Accident-Net Excess Reactivity-Carbide Fuel. 


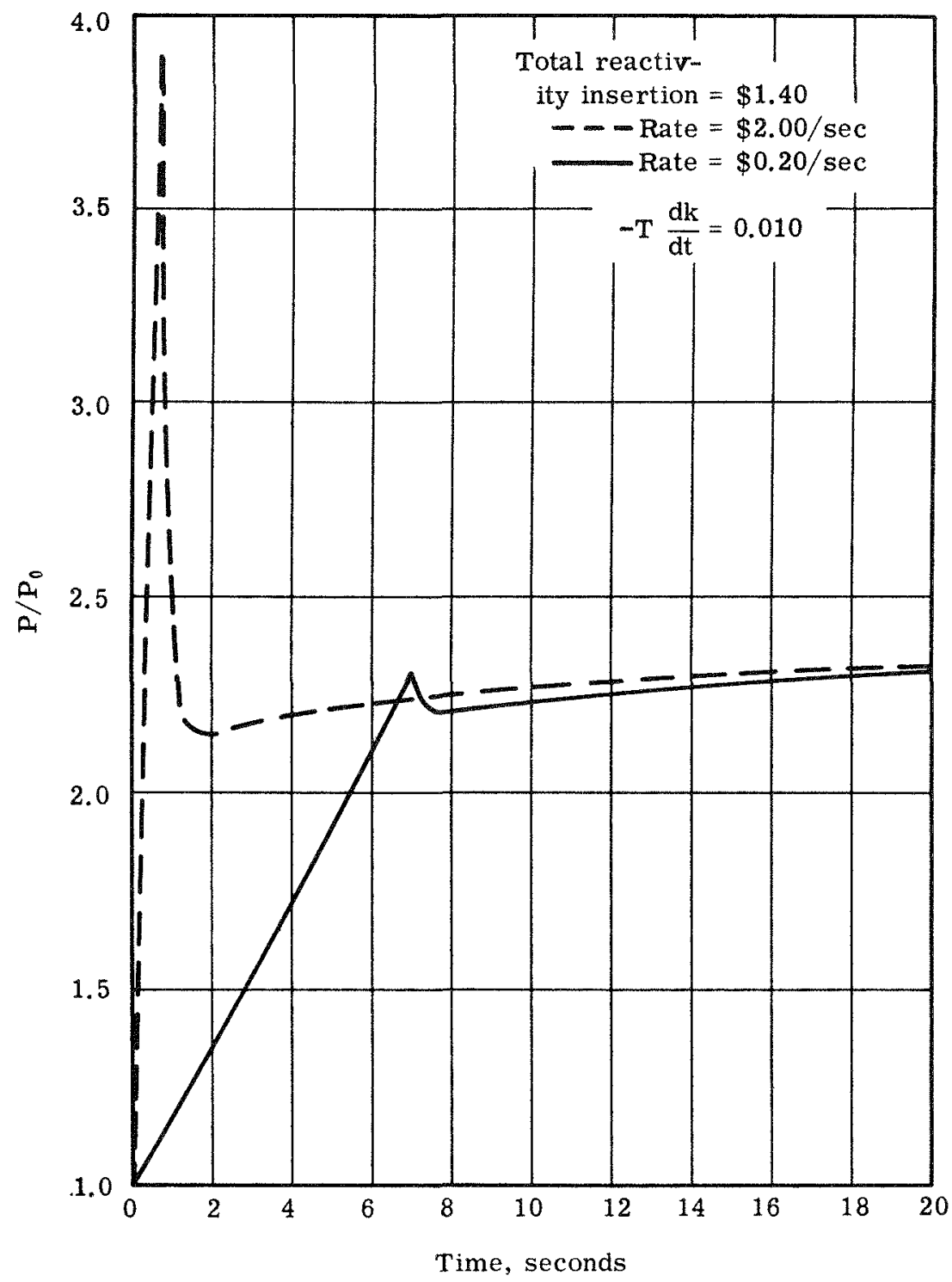

Fıg. 6. Reactivity Insertion Accident-Power Response-Carbide Fuel.

\section{Reactivity Transients in (UPu) $\mathrm{O}_{2}$ Fuel}

The results of reactivity transients analyses for (UPu) $\mathrm{O}_{2}$ fuel are summarized in Figures 8 through 10 . The reactivity insertion, reactivity insertion rate, and Doppler coefficient are the same as in the case of the carbide. From Figure 10 it is seen that the oxide melting point of $5000^{\circ} \mathrm{F}$ is reached in about one second for the fast transient and in about 5 sec for the slow transient. Again, as in the case of the carbide, the sodium coolant does not boil under these conditions.

Comparison between Transients in (UPu)C and $(U P u) O_{2} ;$ Conclusions

In general, for a given reactivity insertion, reactivity insertion rate, and Doppler coefficient, maximum fuel temperatures attained in a $(\mathrm{UPu}) \mathrm{C}$ fuel rod are sig- nificantly below those attained in a $(\mathrm{UPu}) \mathrm{O}_{2}$ fuel rod. This difference between carbide and oxide indicates that a lower Doppler coefficient may be tolerated for the carbide fuel. The results also indicate that a Doppler coefficient much less than $0.010[T(d k / d t)]$ will not be sufficient to override a total insertion of $\$ 1.40$ without resulting in fuel melting.

The effect of sodium void coefficient was factored in by repeating the above transient calculation with negative, zero, and positive void coefficients. The results indicated essentially no effect on fuel and eladding temperatures at $20 \mathrm{sec}$, since boiling did not take place in any of the cases considered. A negative void reactivity effect is considered particularly important for a carbide-fueled reactor because a larger amount of heat is deposited in the coolant in the earlier stages of the 


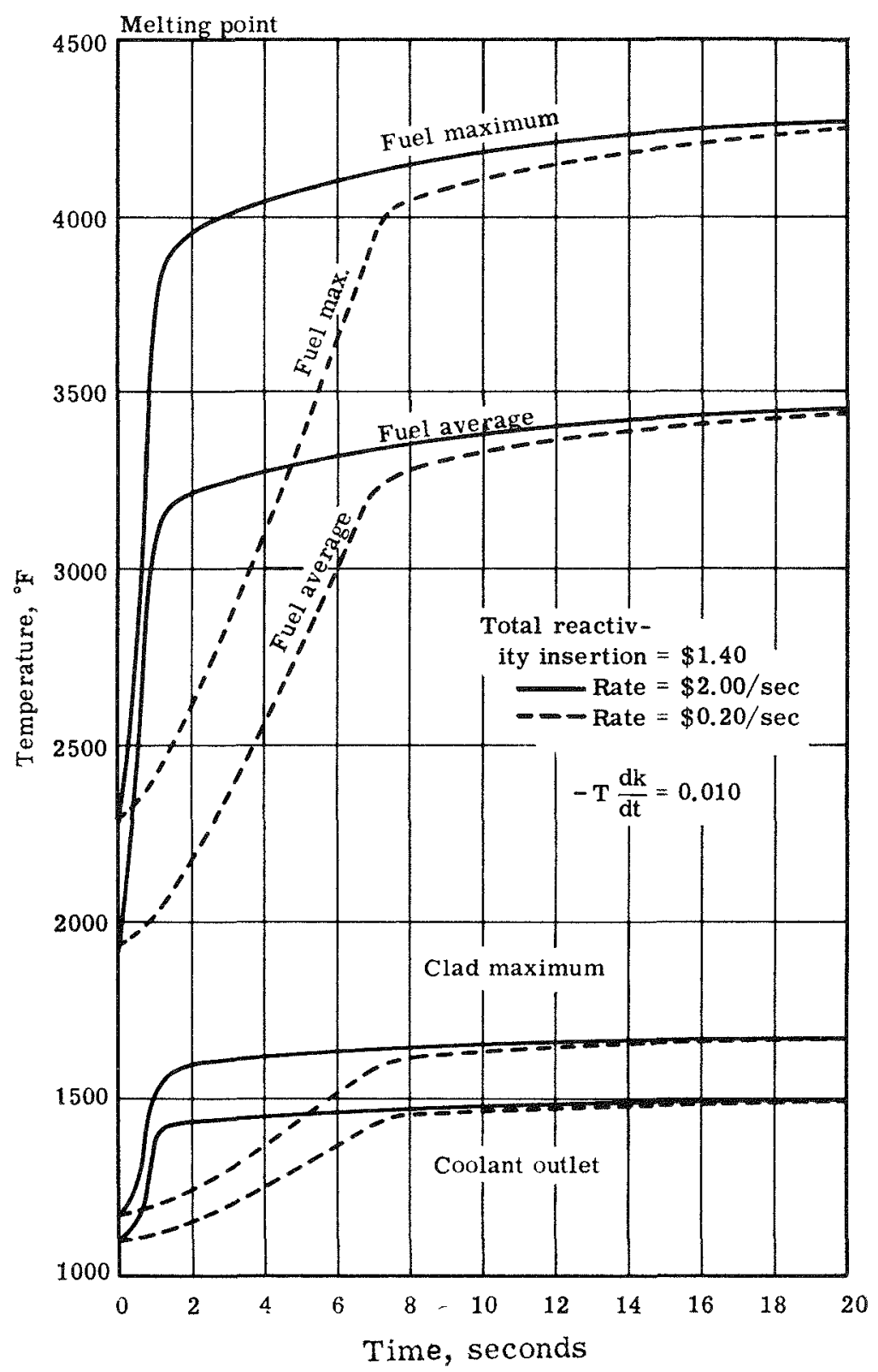

Fig. 7. Reactivity Insertion Accident-Temperatures in the Average Fuel Rod-Carbide Fuel.

transient, which could lead to boiling for large reactivity insertions (more than $\$ 1.40$ ).

\section{FUEL-CYCLE ANALYSIS}

\subsection{Introduction}

Fuel-cycle costs have been calculated in order to assess the economic effect of the following variations of design parameters:

1. the effect of reduced (UPu) C fuel density, in the range from $95 \%$ to $66.5 \%$ of theoretical density;

2. the effect of going from one single 1000-MWe pancake geometry core to a subdivided core consisting of three or seven core modules;

3. the effect of specific power $(\mathrm{kW} / \mathrm{kg}$ fissile at constant $\mathrm{kW} / \mathrm{ft}$ ) ;

4. the effect of plutonium value and working capital charges reflecting conditions under private fuel ownership;

5. the effect of burnup;

6. the effect of using a carbide rather than oxide axial blanket;

7. the effect of using a thick clad, which may be desirable to restrain fuel swelling, and other clad materials, such as niobium and vanadium. 


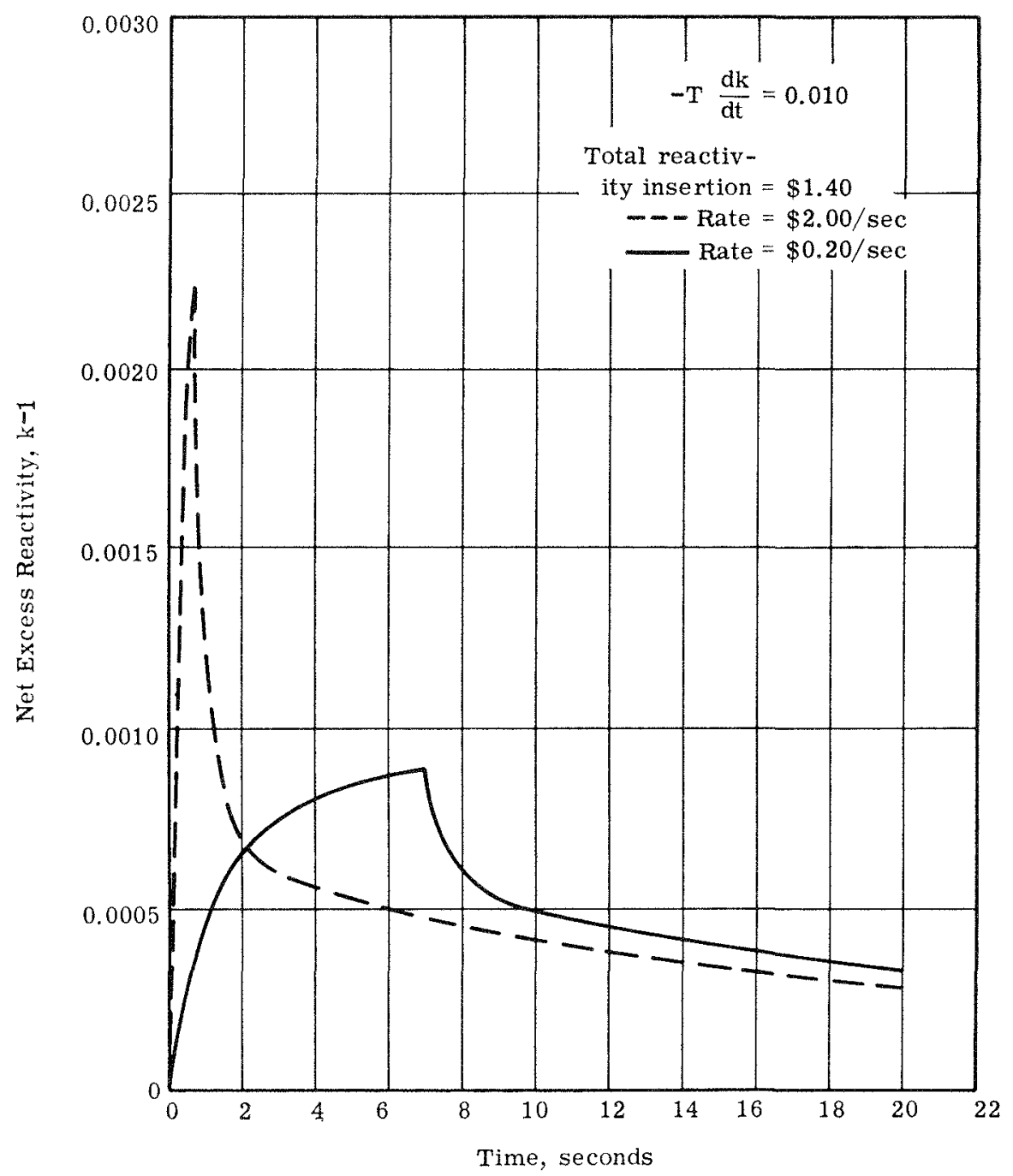

Fra. 8. Reactivity Insertion Accident-Net Excess Reactivity-Oxide Fuel.

A number of significant economic parameters, particularly relating to fabrication, processing, and shipping costs, can at this time be estimated only with considerable uncertainty. In Table 6 are listed the parameters used in the present study. These are present "best" estimates and are consistent with other recent analyses. ${ }^{(11,15,16)}$ Although the absolute magnitudes of fuel-cycle costs thus represent a projection with some uncertainty, the differences between the various cases, however, should reflect fairly reasonably the economic effects of design parameters in question.

\subsection{Summary of Results}

\section{Effect of Fuel Density}

Table 7 summarizes the fuel-cycle costs obtained for the 1000-MWe (UPu) C-fueled pancake reactor at fuel densities of 95,81 , and 66.5 percent of theoretical (sce
Table 3 , Cases 1,2 , and 3 ). In these reactors, both blankets are fueled with $\mathrm{UO}_{2}$, and the residence time of the radial blanket is twice that of the core and axial blanket. It is seen that going from $95 \%$ to $81 \%$ theoretical density increases fuel-cycle costs by a factor of 1.3 ; going from $95 \%$ to $66.5 \%$ increases costs by a factor of 1.65. Sixty-six and one-half per cent $(66.5 \%)$ is an unrealistically low density. At a density of about $80 \%$, fuel-cycle costs can be kept below $0.5 \mathrm{mill} / \mathrm{k}$ Whre for the pancake core geometry.

\section{Modular Versus Pancake Geometry}

The principal economic advantage of a modular versus a pancake core geometry is more efficient utilization of the radial blanket. The radial blanket may contain about twice the fuel volume fraction than the core, while the axial blanket must contain the same fuel volume fraction as the core so long as the core and axial 


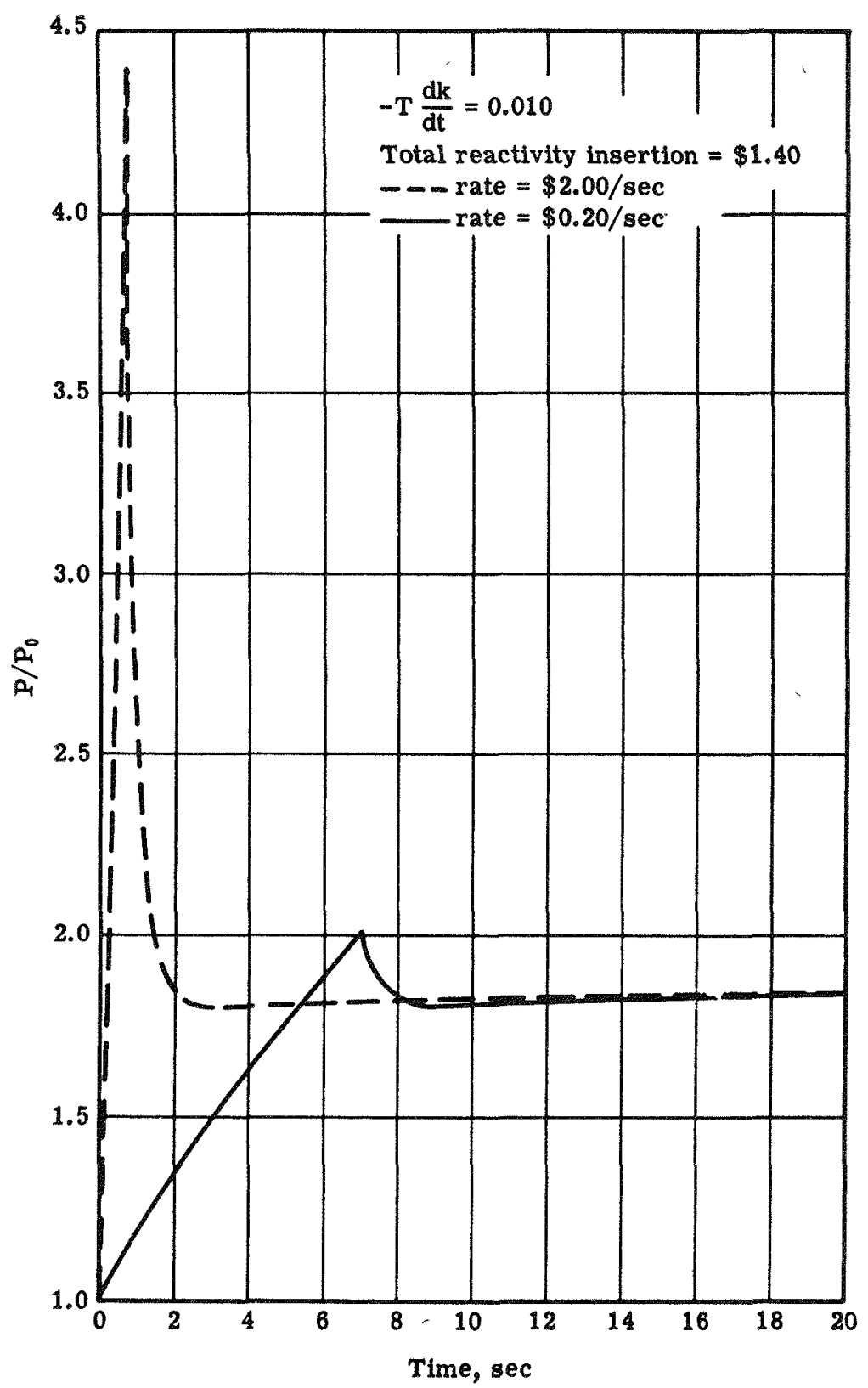

Fra. 9. Reactivity Insertion Accident Power Response-Oxide Fuel.

blanket form integral fuel assemblies. This results in a higher breeding ratio in the blanket for modular geometry.

A second advantage is that the radial blanket can have a longer residence time than the core. It does not have to be moved every time core fuel is changed. The axial blanket, on the other hand, generally has to be moved more frequently than is desirable, since it is integral with the core. This results in reduced radial blanket fabrication, reprocessing, and shipping costs per unit energy generated in the core, in the case of modular geometry.
A third advantage is that since the volume of a single core module is considerably smaller than the volume of a single core region reactor, the total breeding ratio is increased because of a somewhat harder spectrum.

There are two principal economic disadvantages with a modular core. First, the fissile fuel inventory is increased as core volume is decreased and, secondly, the core conversion ratio is reduced. Table 3 summarizes the physics data for a pancake, three-module, and seven-module reactor (Cases 2,5 and 4). The fuel inventories are 1749,1836 , and $2287 \mathrm{~kg}$ fissile plutonium, respectively; the core conversion ratios are $0.75,0.70$, 


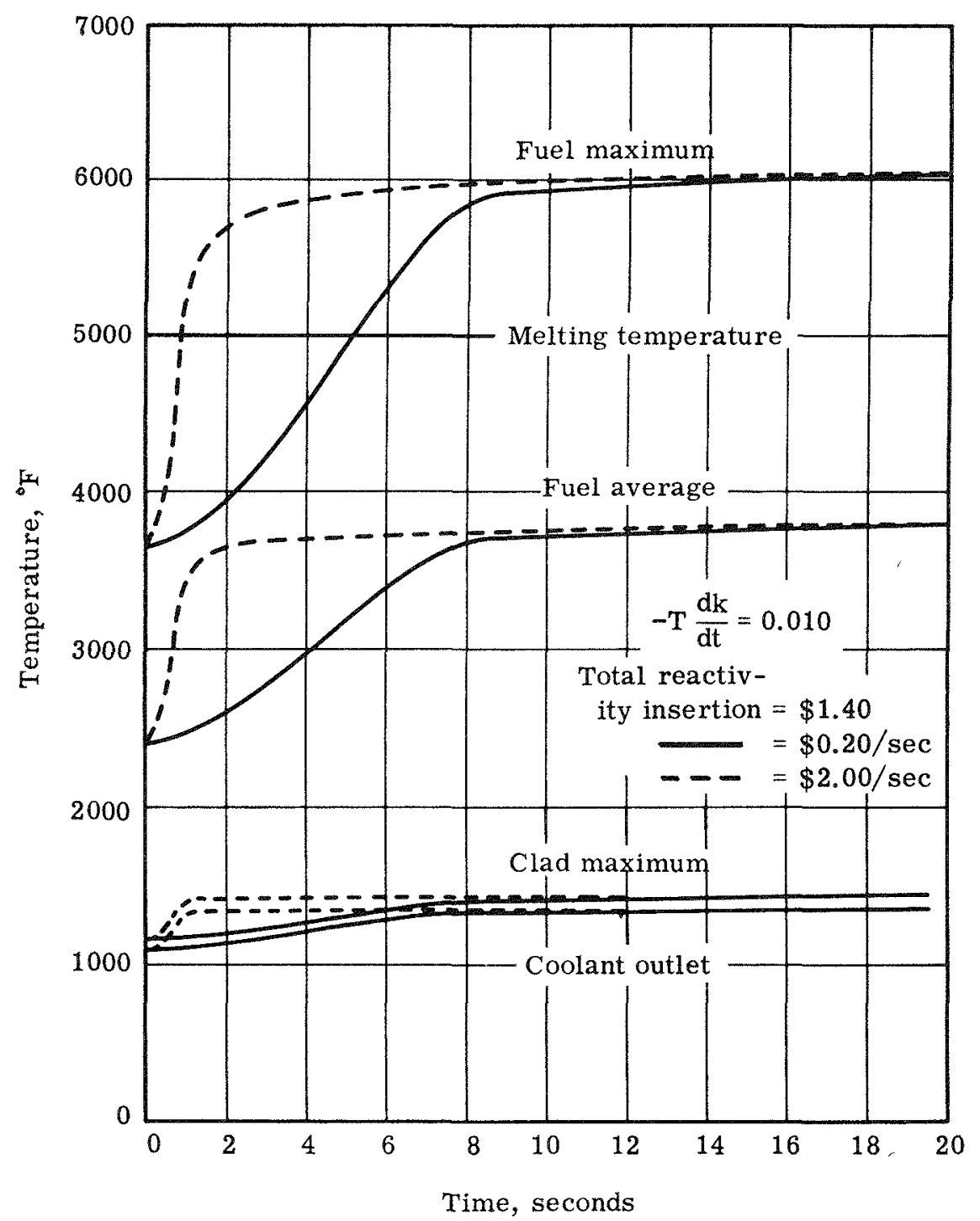

Fra. 10. Reactivity Insertion Accident--Temperatures in the Average Fuel Rod-Oxide Fuel.

and 0.51 , respectively; the total breeding ratios are $1.44,1.65$, and 1.67 , respectively.

Fuel-cycle costs for the three-module and the sevenmodule reactors are summarized in Table 8 . It is seen that the total fuel-cycle cost is estimated at 0.21 mill/ $\mathrm{kWhre}$, a reduction of more than $50 \%$ over the 0.44 mill/kWhre obtained for the pancake geometry. Comparing Case B of Table 7 and the data in Table 8, it is seen that for the modular case, core inventory costs have increased to 0.297 from $0.283 \mathrm{mill} / \mathrm{kWhre}$, and core burnup has increased to 0.291 from $0.242 \mathrm{mill} /$ kWhre. This is, however, more than offset by the increase of blanket breeding gain to -0.92 from -0.67 mill/kWhre.

As is also seen from Table 8 , the fuel inventory costs are beginning to hurt more in the case of the seven- module reactor, resulting in a $57 \%$ increase in fuelcycle cost over the three-module reactor. This fact, plus the fact that the seven-module reactor has a smaller Doppler coefficient (see Table 3), and larger volume of core plus blanket, makes it undesirable. The three-module core, on the other hand, has the advantages of low fuel-cycle cost, compactness, and negative core sodium void effect, and shows only a moderate decrease in Doppler coefficicnt.

\section{Effect of Specific Power}

For a given core composition and power output, increasing the specific power ( $\mathrm{kW} / \mathrm{kg}$ fissile) at constant linear power generation will decrease the core volume, increase inventory, decrease fuel-rod diameter, and change the breeding ratio. 
Figure 11 illustrates the effect of changing specific power on fuel-cycle costs for the three-module reference reactor. The data in this figure correspond to a constant maximum heat generation rate of $30 \mathrm{~kW} / \mathrm{ft}$, but variable fuel-rod size and variable core volume. There is some uncertainty on the variation of fabrication cost with fuel-rod diameter. In the range of interest, down to a diameter of about $0.2 \mathrm{in}$., it is expected that the fabrication cost will vary between costs proportional to (diameter) $)^{-1}$ and (diameter) ${ }^{-1 / 2}$. It is secn from Figure 11 that high specific power results in significant reduction of fuel-cycle costs. It appears that under conditions of high working-capital charges, such as are expected under private fuel ownership, one should push for as high specific power as is metallurgically feasible.

Unless fuel fabrication becomes actually impractical, fabrication costs are a secondary consideration. The calculations represented by Figure 11 neglect the effect of specific power on refueling interval, since no adequate information is available at this time on the period between refueling shutdowns. The core with higher specific power will have a lower core breeding ratio, and therefore more frequent refuelings, for a given amount of available control.

\section{Effect of Plutonium Value, Working-capital Charges, and Burnup}

Figure 12 illustrates the effect of fissile plutonium value on fuel-cycle costs. The modular core, having a higher breeding ratio than the pancake, shows a more pronounced cost variation with plutonium value. In the

TABLE 6. Parameters Used in Fuel-cycle Cost Axalysis [(UPu)C Reference Case, $81 \%$ Theoretical Density $]$

Core Fabrication

Core Processing

Blanket Fabrication (oxide)

Blanket Fabrication (carbide)

Blanket Processing (oxide)

Blanket Processing (carbide)

Core Shipping

Axial Blanket Shipping

Radial Blanket Shipping

Core Burnup

Thermal Efficiency

Load Factor

Plutonium Value

Working Capital Charge, Fuel Inventory

Working Capital Charge, Fabricated Fuel

Fuel Losses

$\$ 200 / \mathrm{kg} \mathrm{C}+\mathrm{Pu}^{*}$

$\$ 90 / \mathrm{kg} \mathbf{U}+\mathrm{Pu}$

$\$ 60 / \mathrm{kg} \mathrm{U}$

$\$ 100 / \mathrm{kg}$ U

$\$ 30 / \mathrm{kg} \mathrm{U}$

$\$ 50 / \mathrm{kg} \mathrm{U}$

$\$ 24 / \mathrm{kg} \mathrm{U}+\mathrm{Pu}$

$\$ 24 / \mathrm{kg} \mathrm{U}$

$\$ 12 / \mathrm{kg} \mathrm{U}$

$100,000 \mathrm{MWD} / \mathrm{MT}(\mathrm{U}+$

$$
\text { Pu) }
$$

$40 \%$

0.8

$\$ 10 / \mathrm{gm}$ fissile

$10 \%$ per annum

$10 \%$ per anwum

Included in above costs

* (For a 0.26 -in.-OD rod).

TABLF 7. Enfect of (UPu)C Fetel Dexstty

on Fuel cycle Costs

(1000-MWe Pancake Geometry Reactor, Costs in Mills/kWhre)

\begin{tabular}{l|c|c|c|c}
\hline Core & $\begin{array}{c}\text { Axial } \\
\text { Blanket }\end{array}$ & $\begin{array}{c}\text { Radial } \\
\text { Blanket }\end{array}$ & Sum \\
\hline
\end{tabular}

A. $95 \%$ Theoretical Density*

\begin{tabular}{l|r|r|r|r}
\hline Fabrication & 0.178 & 0.073 & 0.028 & 0.279 \\
Processing & 0.094 & 0.036 & 0.014 & 0.144 \\
Net Burnup (or Breed- & 0.133 & -0.445 & -0.162 & -0.474 \\
$\quad$ ing) & & & & \\
Fuel Invent ory & 0.297 & - & - & 0.297 \\
Shipping & 0.025 & 0.029 & 0.005 & 0.059 \\
Capitalization & 0.020 & 0.008 & 0.004 & 0.032 \\
& & & \\
& 0.75 & -0.30 & -0.11 & 0.34 \\
\hline
\end{tabular}

B. $81 \%$ Theoretical Density*

\begin{tabular}{|c|c|c|c|c|}
\hline Fabrication & 0.209 & 0.085 & 0.036 & 0.330 \\
\hline Processing & 0.094 & 0.042 & 0.018 & 0.154 \\
\hline $\begin{array}{l}\text { Net Burnup (or Breed- } \\
\text { ing) }\end{array}$ & 0.242 & -0.494 & -0.174 & -0.426 \\
\hline Fuel Inventory & 0.283 & - & $-\infty$ & 0.283 \\
\hline Shipping & 0.025 & 0.034 & 0.007 & 0.066 \\
\hline \multirow[t]{2}{*}{ Capitalization } & 0.020 & 0.008 & 0.004 & 0.032 \\
\hline & 0.87 & -0.32 & -0.11 & 0.44 \\
\hline
\end{tabular}

C. $66.5 \%$ Theoretical Density*

\begin{tabular}{l|c|r|r|r}
\hline Fabrication & 0.254 & 0.104 & 0.041 & 0.399 \\
Processing & 0.094 & 0.052 & 0.021 & 0.166 \\
Net Burnup (or Breed- & 0.356 & -0.544 & -0.198 & -0.386 \\
$\quad$ ing) & & & & \\
Fuel Inventory & 0.270 & - & - & 0.270 \\
Shipping & 0.025 & 0.042 & 0.008 & 0.075 \\
Capitalization & 0.020 & 0.008 & 0.004 & 0.032 \\
& 1.02 & -0.34 & -0.12 & 0.56 \\
\hline
\end{tabular}

* Smeared density with cold ID of fuel tube. Theoretical density taken as $13.6 \mathrm{~g}$ carbide/cc.

long term, plutonium value is expected to go up. This would favor the higher breeder.

The variation of fuel-cycle costs with working-capital charges and burnup is shown in Figures 13 and 14. Both variables have a strong influence on costs.

It is seen that an average burnup of 100,000 MWD/ $\mathrm{MT}$ is a reasonable design objective. Beyond this value, increased burnup begins to yield diminishing cost reductions.

It is seen that fuel-cycle costs are extremely sensitive to the average burnup achieved, especially for the modular core, for which a relatively high breeding ratio is predicted. The sensitivity of fuel-cycle cost to burnup at discharge becomes even stronger at plutonium values greater than $10 \mathrm{\$} / \mathrm{g}$ fissile. 
TABlE 8. FCel-Cycle Costrs of Three- ANd SevenMODUTE (UPu)C-FUELED 1000-MWe Reactors

[Costs in Mills/kWhre (UPu)C Fuel at $81 \%$ Theoretical Density]

\begin{tabular}{l|l|l|l}
\hline & Core & Blanket & Sum \\
\hline
\end{tabular}

A. Three-core Modules

\begin{tabular}{l|c|c|r}
\hline Fabrication & 0.209 & 0.112 & 0.321 \\
Processing & 0.094 & 0.056 & 0.150 \\
Burnup/Breeding & 0.291 & -0.917 & -0.626 \\
Inventory & 0.297 & - & 0.297 \\
Shipping & 0.025 & 0.011 & 0.036 \\
Capitalization & 0.020 & 0.015 & 0.035 \\
& 0.94 & -0.73 & 0.21 \\
\hline
\end{tabular}

B. Seven-core Modules

\begin{tabular}{l|c|c|r}
\hline Fabrication & 0.209 & 0.156 & 0.365 \\
Processing & 0.094 & 0.078 & 0.172 \\
Burnup/Breeding & 0.470 & -1.121 & -0.651 \\
Inventory & 0.370 & - & 0.370 \\
Shipping & 0.025 & 0.016 & 0.041 \\
Capitalization & 0.020 & 0.016 & 0.036 \\
& 1.19 & -0.86 & 0.33 \\
\hline
\end{tabular}

Carbide Versus Oxide Blanket

In the (UPu)C-fueled reactors under discussion, both the radial and axial blankets are taken as of $\mathrm{UO}_{2}$ rather than as of UC because of somewhat lower anticipated fabrication costs for $\mathrm{UO}_{2}$. In the case of the integral core-axial blanket-fuel assemblies, use is made of inert ceramic pellets to avoid interaction between oxide and carbide pellets at the core-blanket interface.

For the $95 \%$ theoretical density, (UPu) C-fueled pancake core, use of a UC axial blanket increases the total breeding ratio from 1.50 to 1.57 . Assuming UC fabrication costs of $\$ 100 / \mathrm{kg}$ and processing costs of $\$ 50 / \mathrm{kg}$, the result is a net fuel-cycle cost increase of $0.04 \mathrm{mill} / \mathrm{kWh}$ he over the oxide blanket.

\section{Effect of Clad Thickness and Material}

As shown in Table 3 , the effect of increasing the stainless steel-clad thickness from 15 to $30 \mathrm{mils}$ for the 95\% theoretical density, (UPu) C-fueled pancake reactor is to decrease the total breeding ratio by 0.02 , and increase enrichment by $0.2 \%$. The clad thickness is increased at the expense of sodium. The resultant change in fuel-cycle costs is less than 0.005 mill/

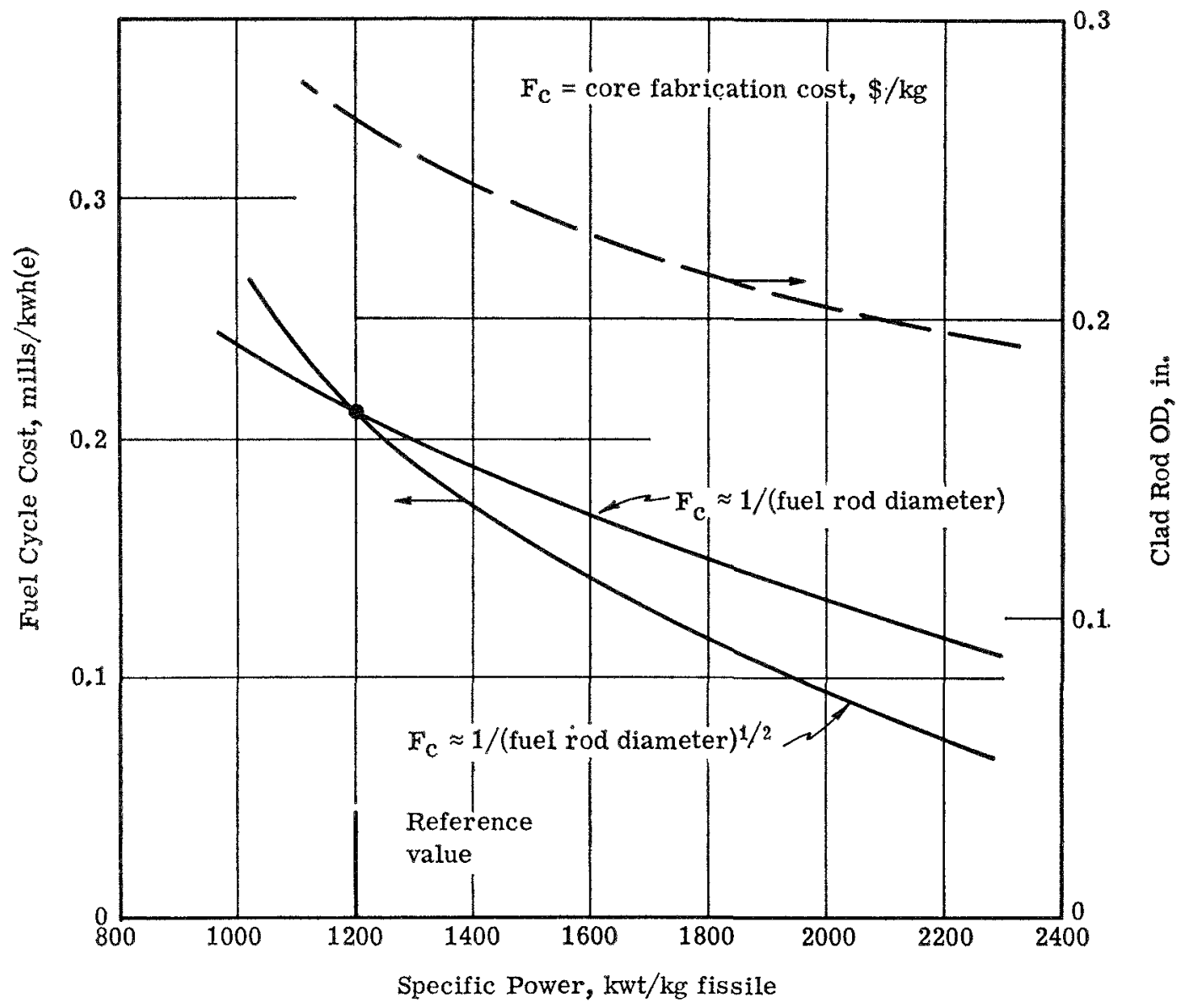

FIG. 11. Variation of Fuel-eycle Cost with Specific Power [333-MW(e) (UPu)C-fueled Core Module, Constant Linear Power at $30 \mathrm{~kW} / \mathrm{ft}$ max, Variable Core Volume and Enrichment]. 


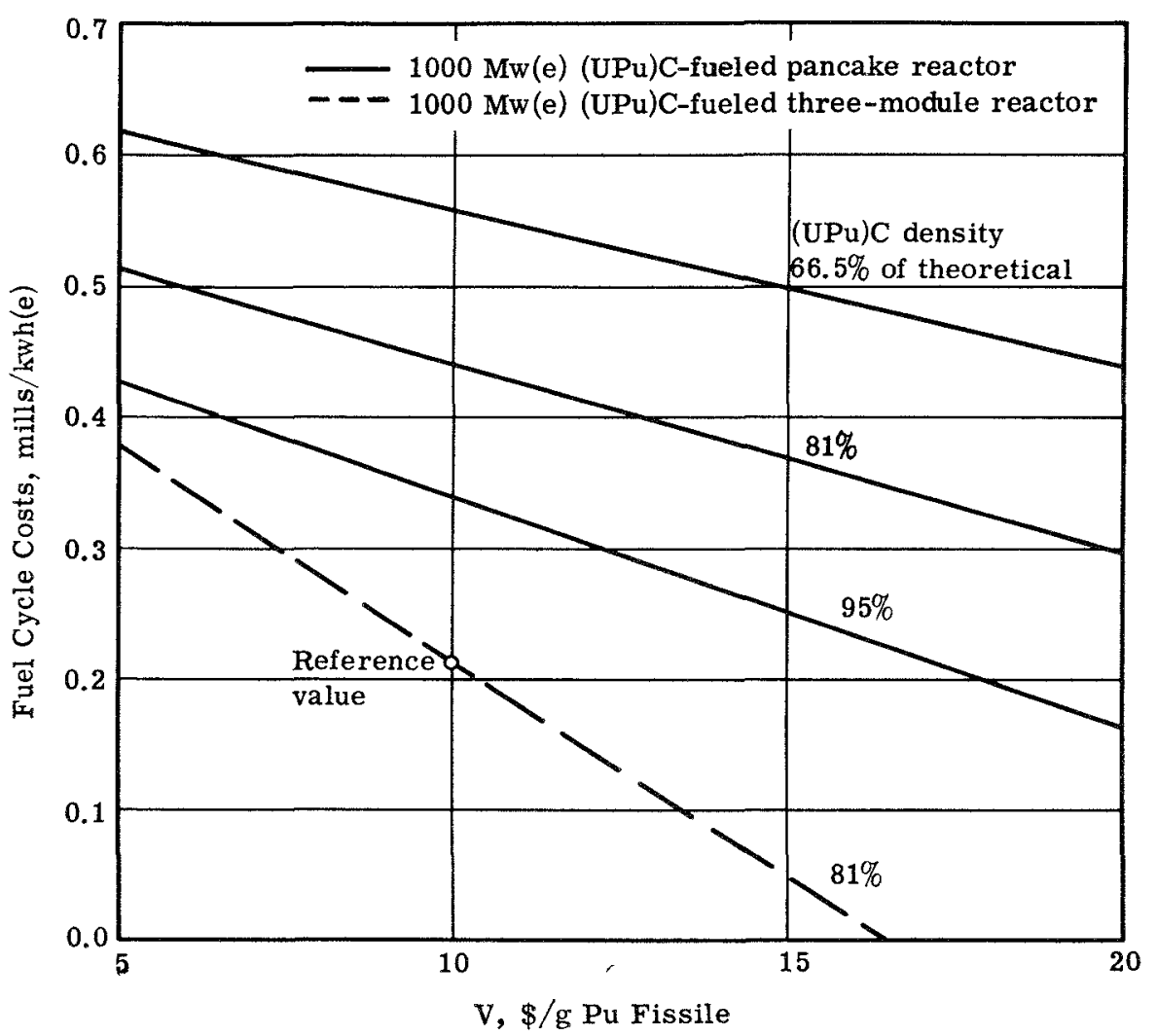

Fxa. 12. Variation of Fuel-cycle Costs with Plutonium Value as a Function of (UPu)C Fuel Density.

kWhre. Thus, a thicker stainless steel clad, which may be desirable to restrain fuel swelling or fission gas pressure, appears economically feasible.

Table 3 also presents data on the effect of using niobium clad: the breeding ratio is reduced appreciably, and the enrichment is significantly increased. This, plus considerations of safety and niobium cost, makes the use of niobium appear undesirable, as has also been shown in previous studies. ${ }^{(10)}$ However, as already noted above in Section 2 , the present treatment of the niobium cross sections is somewhat indaequate, and more refined calculations could yield different results.

The use of vanadium as a cladding material is satisfactory from a nuclear point of view, as is shown by Case 9 of Table 3. Costs due to the use of vanadium cladding cannot be calculated with any accuracy at this time since vanadium tubing is not available in commercial quantities. Again, the effect of vanadium is in agreement with previous work. ${ }^{(10)}$

\section{SUMMARY OF CONCEPTUAL REFERENCE DESIGN}

The characteristics of the $1000-\mathrm{MWe}$ (UPu)Cfueled conceptual reference design developed in the present study are summarized in Table 9. Figure 15 is a schematic sketch of the reactor arrangement, con- sisting of three rhombic-shaped 1500-liter core modules. Each module consists of fuel rods with $3-\mathrm{ft}$ core section, two 1.5-ft axial blanket regions, and a fission gas reservoir above the top axial blanket region. The core modules are separated by $3 \mathrm{ft}$ of radial blanket and stainless steel structure.

The present configuration was selected because of the following principal considerations:

1. The rhombic, 1500 -liter core modules represent a reasonable compromise in attempting to achieve attractive fuel inventory and breeding ratio while maintaining sutisfactory safety characteristics. The reactivity effect due to $100 \%$ loss of sodium from the core regions is negative. A good peak/average power ratio is also obtained without fuel zoning.

2 . The total breeding ratio is increased considerably over that of a single pancake reactor of equal core volume due to higher and more efficient radial blanket breeding. The result is a fuel-cycle cost of $0.21 \mathrm{mill} /$ $\mathrm{kWhre}$ versus $0.44 \mathrm{mill} / \mathrm{kWhre}$ for the pancake design.

3. Each of the three core modules is neutronically decoupled from the other core modules throughout core life by the presence of $3 \mathrm{ft}$ of radial blanket and steel structure between modules. The configuration is sufficiently compact to fit within an external blanket diameter of $\sim 15 \mathrm{ft}$. Decoupling the modules is expected to simplify reactor control and significantly reduces the 


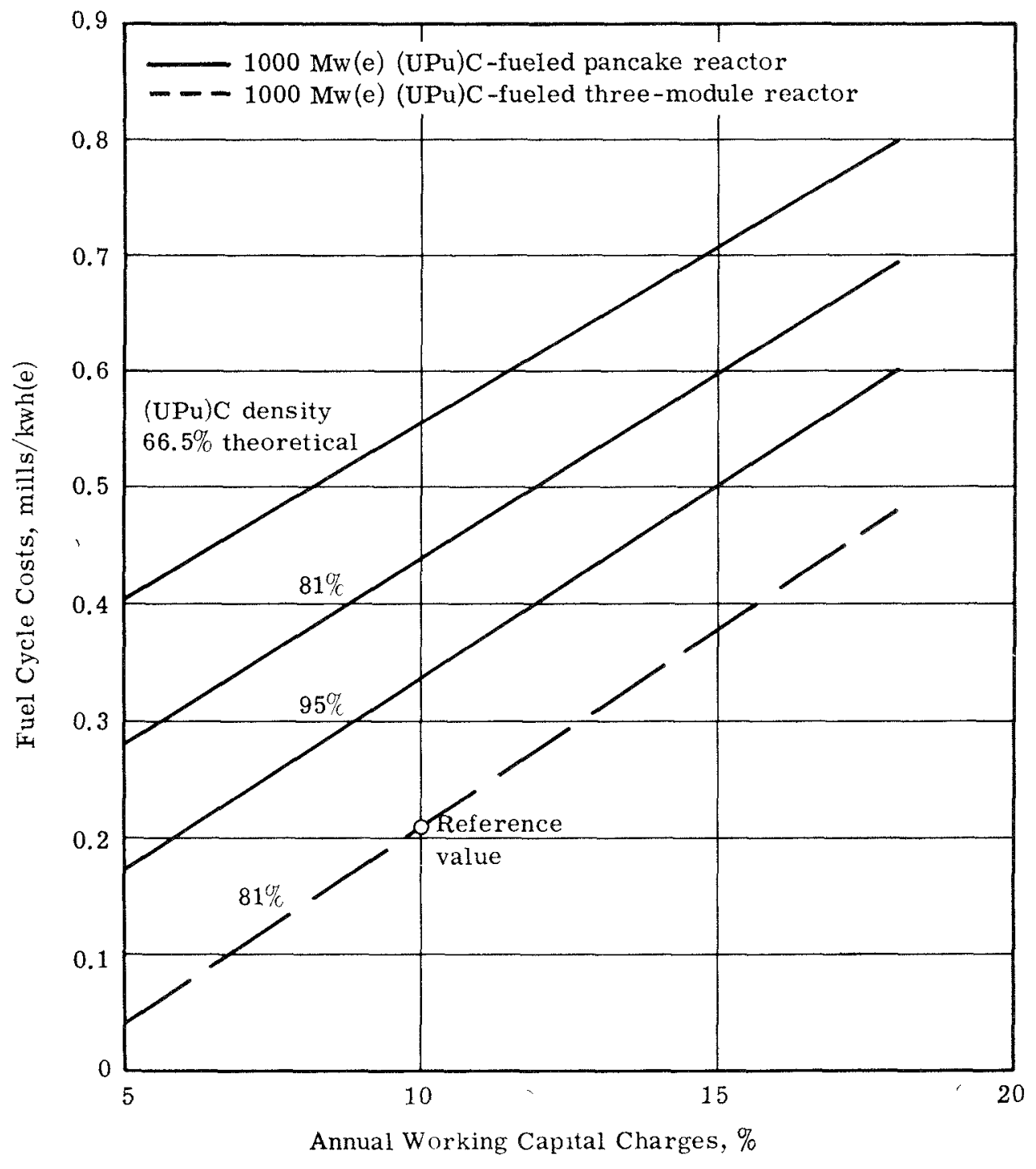

FIt. 13. Variation of Fuel-cycle Costs with Working Capital Charges as a Function of (UPu)C Fuel Density.

potential hazard of fuel meltdown. The presence of blanket between modules permits incorporation in the core of rigid structure midway between modules for protection against compaction of the entire fuel in the reactor. This structure could be extended above and below the core to provide for three separate flow loops. Such an arrangement would minimize the severity of flow blockage in a given fuel cluster over a pancake core supplied with flow from a single plenum.

4. A disadvantage of the modular core is a reduced Doppler coefficient. The Doppler coefficient for the present 1500 -liter module is about $25 \%$ smaller than for a 4500-liter pancake core. The significance of the Doppler coefficient has to be assessed for specific reactivity transients, and its magnitude is presently subject to considerable uncertainty. If it turns out that the available Doppler shutdown is inadequate, eren in the presence of favorable sodium void effects, the spectrum may have to be suitably softened. The modular core would continue to have a breeding ratio advantage over a pancake core with moderator such as $\mathrm{BeO}$ added to soften the spectrum. Furthermore, the initially high breeding ratio achievable with a modular carbide core, and possibly carbide blankets, should result in a breeding ratio in excess of 1.40 with a softened spectrum.

5. Core height in the present design is chosen as low as possible consistent with acceptable specific power and overall reactor diameter. The reference height of $3 \mathrm{ft}$ for the core and of $1 \frac{1 / 2}{\mathrm{ft}}$ for axial blankets results in an overall fuel-rod length of $6 \mathrm{ft}$ exclusive of fission gas volume. This is only $1 \mathrm{ft}$ longer than the pancake design.

As is discussed in Section 1, the reference fuel-rod 


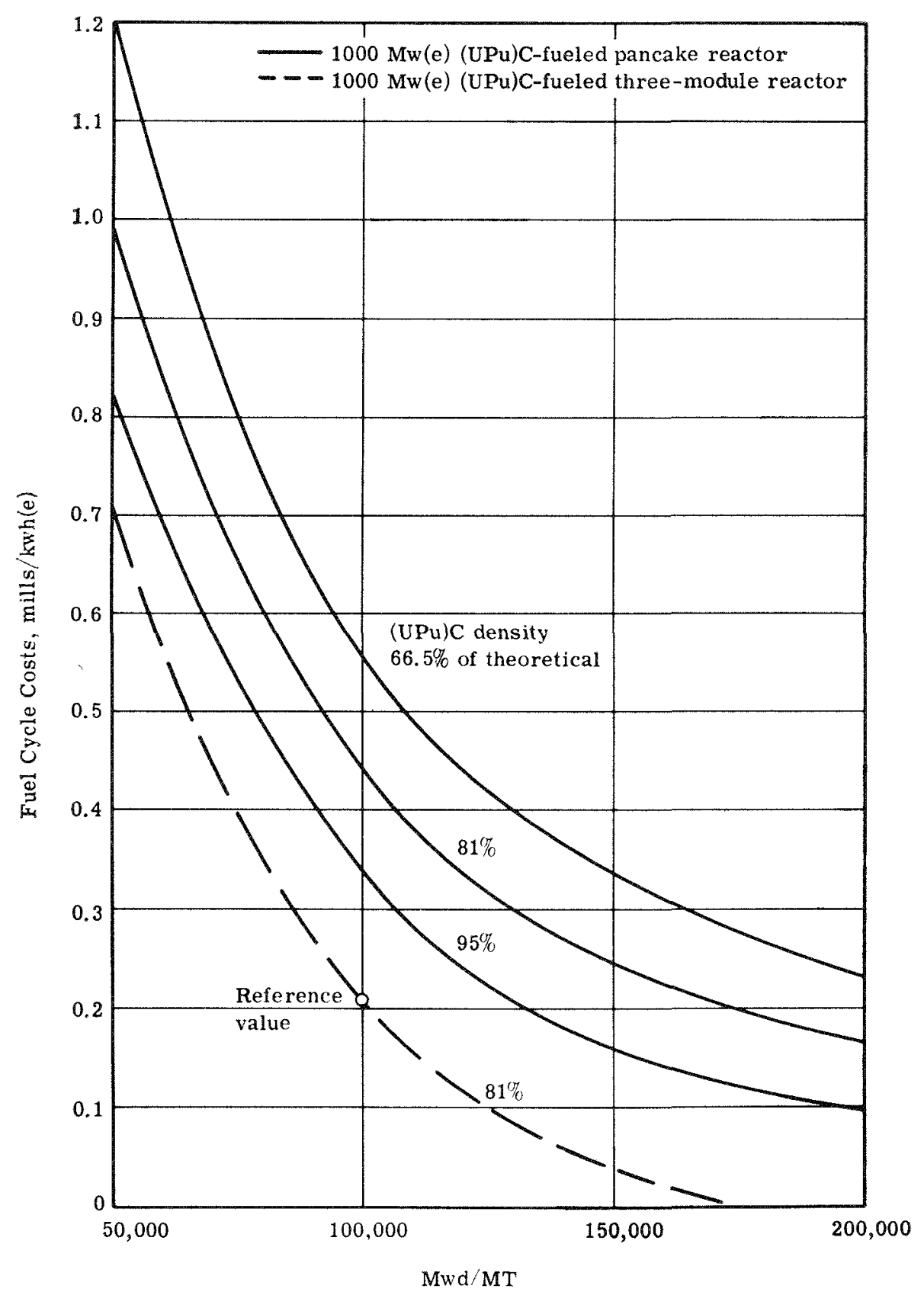

FIG. 14. Variation of Fuel-cycle Costs with Burnup as a Function of (UPu)C Fuel Density. 
TABLE 9. Characteristrcs of 1000-MWe (UPu)C Conceptual Reference Design

Reactor Power

Thermal Efficiency

Reactor Configuration

Reactor Size

Module Power

Fuel Inventory in 3 Modules

Enrichment, Fissile

Average Core Power Density

Average Specific Power

Core Conversion Ratio

Total Breeding Ratio

Doubling Time

Maximum Fuel Rating

Peak/Average Power Ratio

Coolant Inlet Temperature

Coolant Outlet Temperature

Maximum Fuel Temperature

Doppler Coefficient

Sodium Void Worth (100\% core module)

Burnup

Fuel Residence Time

Load Factor

Fuel-cycle Cost

Fuel Material

Fuel Density (smeared over cold elad ID)

Fuel-rod-clad OD

Clad Material

Clad Thickness

Thermal Bond Material

Cold Gap Width

Cold Pellet OD

Core Composition, v/o Fuel/ Sodium/Structure

Blanket Material

Blanket Thickness

Radial Blanket Composition,

v/o Fuel/Sodium/Structure

characteristics and smeared fuel density of $81 \%$ theoretical is based on presently postulated fuel swelling to $100,000 \mathrm{MWD} / \mathrm{MT}$ and the assumption that internal void will absorb this swelling. This is subject to verification through irradiation testing in a fast-flux environment. Present swelling data are based on thermal-flux irradiation tests. As has been shown in Section 4, increasing clad thickness from 0.015 to 0.030 in. will have a negligible effect on fuel-cycle costs. Thus, a thicker clad than the reference 0.015 in. may be used to restrain fuel swelling if it is verified that cladding embrittlement does not occur under fast-flux conditions. This might permit the use of smeared (UPu)C fuel densities closer to $95 \%$ of theoretical, with associated reduction in fuel-cycle costs (see Figure 12).

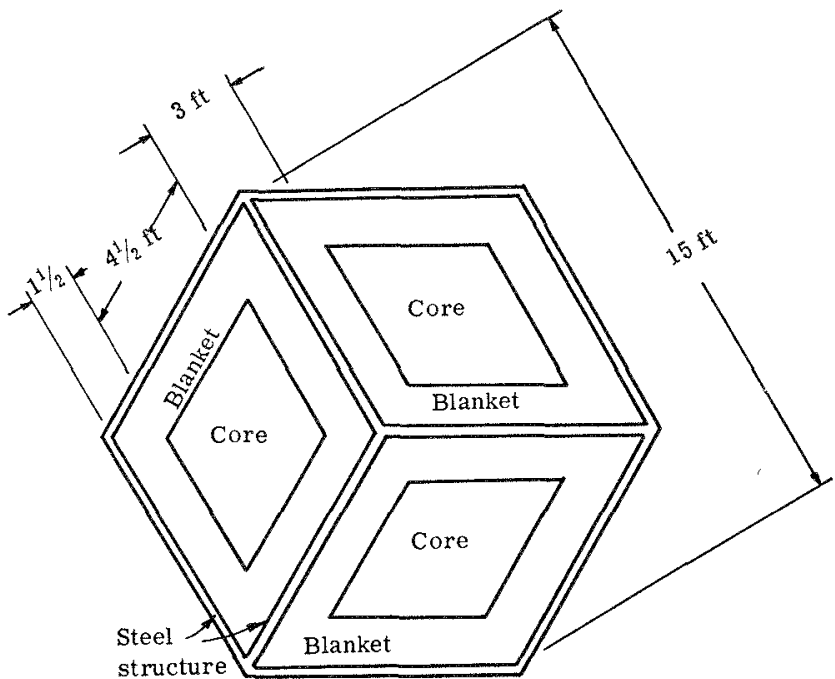

FIG. 15. Schematic Sketch of 1000-MW(e) Modular (UPu)C Reactor Configuration (Core Height $3 \mathrm{ft}$, Axial Blankets 1/2 ft Each, Fission Gas Reservoir).

It is evident that these possibilities must aw ait the results of extensive fast-flux irradiation testing.

\section{REFERENCES}

1. Strasser, A. A., et al., Uranium-Plutonium Carbide Fuels for F ast Breeder Reactors, ANS-100 (April 1965).

2. Connolly, Lucie D., Los Alamos Group-averaged Cross Sections, LAMS 2941 (Sept. 24, 1963).

3. Hansen, G. E., and Roach, W. H., Six and Sixteen Group Cross Sections for Fast and Intermediate Critical Assem. blies, LAMS-2543 (Dec 6, 1961).

4. Spielberg, D., DTK Cross-section Sets for $U-235$ and $U-298$ at 1000 and $1500^{\circ} \mathrm{K}$, UNC internal memo, Phys./Math2934 (April 1963).

5. Graves, C. C., General Methods and Graphs for Evaluation of Effective Cross Sections Used in Doppler Effect Calcula. tions for Fast Reactors, UNC-5064 (May 1964).

6. Hibdon, C. T., Phys. Rev. 118, 515 (1960).

7. Progress in Plutonium Utilization, HW-83601 (Dec 1964).

8. Cherry, B. H., Calculation of ANL Fast Reactor Intercomparison Problems, UNC-NDEO-527 (Sept 10, 1965).

9. Carlson, B. G., et al., DTF Users Manual, UNC Physics/ Math-3321 (Nov 1963).

10. Okrent, D., et al., Some Nuclear and Safety Considerations in the Design of Large $F$ ast Power Reactors, $\mathrm{P} / 267$, Geneva Conference (1964).

11. Liquid Metal Fast Breeder Reactor Design Study, GEAP4418 (1964).

12. Elrod, H. G., Jr., Improved Lumped Parameter Method for Transient Heat Conduction Calculations, J. Heat Transfer, 82(3), 181 (Aug 1960).

13. Graves, C. C., and Firstenberg, H., Evaluation of Approximate Methods for Predicting Transient Temperatures in $\mathrm{UO}_{2}$ and $\mathrm{UC}$ Fuel Elements, UNC-5063 (May 11, 1964).

14. Blue, L. R., and Hoffman, M., AIREK III, NAA Report AMTD-131 (Jan 1963).

15. Link, L. E., et al., Fuel Cycle Economics of Fast Reactors, $\mathrm{P} / 248$, Geneva Conference (1964).

16. Liquid Metal Fast Breeder Reactor Design Study, WCAP. $3251-1$ (1964). 


\section{Discussion}

Mr. Campise (AI): Was the Doppler coefficient you showed in your table the Doppler of the core only or was it the isothermal Doppler for the module?

Ir. Sofer: The Doppler for the module.

Mr. Campise: I find this strange because most of the modular studies have shown an increase in the Doppler coefficient by going to a blanketed module, like in the Argonne study a while back.

Mr. Sofer: The modules are neutronically separated so that really the modules behave as if they were cores by themselves. These cores are 1500 liters each, and the spectrum of a 1500-liter core is certainly harder than one of much larger volume. This is why the Doppler is low.

Mr. Dalle-Donne (Karlsruhe): Don't you think that the problem of the heat transfer coefficient between the fuel and the cladding is very important for carbides because of their very good thermal properties, particularly the thermal conductivity? In other words, you put something which has a very bad heat transfer coefficient in series with something which is very good. Should you not try to use sodium bonding?

$M r$. Sofer: We recognize this as being a definite problem. We're not ignoring it. The temperature drop across the gap is not negligible. However, there is this revealing aspect to it in that as the fuel temperature goes up, expansion will take place and tend to close the gap. Now the problem is: can we get it so that we don't overly strain the clad as a result of expansion and swelling? Sodium bonding is one way to do it, but we have problems with that, too.

Mr. Cobb (Nuclear Power Group, England) : In Fig. 1 you show that for a $5 \%$ change in carbide density you got about only a $2 \%$ change in the center fuel temperature. Could you say how much the actual change in conductivity was; presumable the gap-closing effect is coming in here?

$M r$. Sofer: I think I understand your question to be as follows: What is the change in conductivity of the fuel as we go from the pellet density of, say, $95 \%$ to $85 \%$ ? There are limited data on the subject, but we assumed in our calculations that the conductivity is proportional to the density.

Mr. Chernick (BNL): Have you assumed that the carbide fuel remains intact? I did notice some very large burnup figures, like a $100,000 \mathrm{MWd} / \mathrm{ton}$. What is going to happen when fission products get out and gases get mixed with the helium? This is a known problem with thermal reactors.

$M r$. Sofer: 'We really cannot be presumptuous on this matter. There have been all kinds of concern as to what happens really when carbide cracks. It has a nice conductivity as a solid pellet. What happens to that conductivity when it cracks? Radiation data we have obtained operating at $20 \mathrm{~kW} / \mathrm{ft}$ indicated that the conductivity doesn't drop appreciably. There was no grain growth and no sintering, which indicates that the conductivity somehow seems to maintain itself.

IIr. Chernick: I'm really more concerned about the conductivity of the gas, not the pellet itself. The small amount of the fission gases does seem to ruin the conductivity of helium.

Mr. Sofer: Yes, there have been data showing that the conductivity of the gas tends to drop when fission gas enters, but again we have to really go on the basis of experimental evidence.

Mr. Bradbury (Harwell): For what percentage of fission gas release from the fuel are you designing?

Mr. Sofer: At these burnups we would prefer to design for total release.

Mr. Kummerer (Karlsruhe): What is the reason that you consider mainly niobium cladding as a substitute for Type 316 stainless steel instead of the vanadiumtitanium alloy?

$M r$. Sofer: The reason I brought niobium into the picture was because the niobium behaves so well. People have always asked: "Well, now why can't you use niobium?" The answer is that, at least if the Hansen-Roach cross section for niobium is used, there is produced a rather detrimental effect on breeding ratio. We are now planning some pins with vanadium. One problem with vanadium is cost.

Mr. Kummerer: Is niobium material available in tubing?

Mr. Sofer: Niobium is costly, too. Of course, the preferred choice is stainless steel.

Mr. Bump (ANL): Do you have a reference for the conditions under which you observe the cracking in the stainless steel cladding?

$M r$. Sofer: The conditions were $100,000 \mathrm{MIWd}$ /ton of burnup, $20 \mathrm{~kW} / \mathrm{ft}$, temperatures of the pellet were up to about $2400^{\circ} \mathrm{F}$, and there was evidence of embrittlement of the cladding and, therefore, cracking of the cladding. We have been hopeful that the reason for this embrittlement was the presence of low amounts of boron in the cladding, which in a thermal flux may have resulted in helium precipitation which led to embrittlement. There is some evidence that small amounts of boron will lead to embrittlement in high-temperature radiation in thermal flux, supported by data from the British, but we will have to find out experimentally what happens in a fast spectrum.

$M r$. Bump: What is your attitude concerning the effect of the low smear density of the fuel with regards to safety, particularly slumping?

$M r$. Sofer: We are ignorant like everybody else is. 


\title{
A Re-examination of Design Factors for a 1000-MWe Fast Breeder Reactor
}

\author{
William S. Farmer axd David G. Strawion \\ Allis-Chalmers Manufacturing Company \\ 1 tomic Energy Division \\ Bethesda, Maryland \\ (PRESENTED BY D. ( . STRAWSON)
}

\section{Introduction}

The conflicting requirements eneountered in designing a large fast breeder reactor which will have good safety characteristies while maintaining attractive fuel-cycle costs and a low fuel doubling time were well demonstrated in four 1000-MWe Large Fast Breeder Design studies. ${ }^{(1-4)}$ Each of the organizations undertaking the studies worked independently under ground rules which, except for the type of fuel, were common to all. Each organization produced a design which represented a reasonable solution to the conflicting design requirements. These designs provide an opportunity for evaluating the merits of four different approaches to the design of a large fast breeder reactor.

\section{Major Conclusions of Studies}

We have reviewed the alternative approaches to the Allis-Chalmers annular core to see if they offer major advantages. Before proceeding, we feel that certain general conclusions can be drawn from the four design studies.

A major question prior to the studies was the feasibility of designing a fast reactor core capable of producing $1000 \mathrm{MWe}(2500 \mathrm{MWt})$; i.e., could such a core be designed to have reasonable safety characteristics and, if so, would the necessary design measures make the core unattractive in terms of breeding ratio or fuel-cycle economics? We believe this question of the feasibility of large breeder reactors was answered affirmatively by each of the studies. The Allis-Chalmers annular core, the General Electric "pancaked" cylinder, and the more conventional cylinder of Combustion Engineering are each a single core capable of producing $1000 \mathrm{MWe}$. The weakly coupled modules which make up the Westinghouse core may raise semantic arguments, but the Westinghouse concept accomplishes the production of 2500 MWt within a single space envelope and may also be thought of as a single core. Each of the cores exceeded the design criteria of a breeding ratio equal to 1.2 , and fuel-cycle costs were low for all designs.
Insofar as reasonable safety characteristics can be taken to mean a minimal reactivity gain on complete loss of core sodium, this was met by each of the design studies.

We believe that conclusions may also be drawn from the fuel-cycle costs shown in the design studies. The fucl-cycle costs reported in each study have been normalized to common fuel fabrication, shipping, and reprocessing procedures. ${ }^{(5)}$ Further normalization on the basis of eonsistent physies data has been performed ${ }^{(6)}$ to provide comparable values for the breeding ratio and hence the value of plutonium credit for each system. Fuel-cycle costs after these normalizations are generally low and range from a minimum of $0.43 \mathrm{mill} / \mathrm{kWh}$ to a maximum of $0.85 \mathrm{mill} / \mathrm{kW}^{\mathrm{T}} \mathrm{h}$. Demonstration of high burnups for the ceramic fuels through continuing research programs and the successful development of commercial-scale fabrication and processing procedures for mixed uranium-plutonium ceramics used in a sodium environment are, of course, prerequisites for these low fuel-cycle costs. However, achievement of fuel-cycle costs well under $1 \mathrm{mill} / \mathrm{kWh}$ seems a definite possibility and points to the strong economic attractiveness of large fast breeders. That such low fuel-cycle costs were achieved when the designs were based to a large extent on safety considerations and in general did not represent economic optimization even under assumed safety criteria, indicates room for further improvements in fuel-cycle costs.

The relative fuel-cycle costs shown in the studies also indicate that the particular core concept selected for the first-round large-size fast breeder will have a small effect on total power costs. For example, in the design studies in which four substantially different core concepts were presented, the fuel-cycle cost difference between the highest and lowest cost concept was approximately $0.4 \mathrm{mill} / \mathrm{kWh}$. Of this, approximately $0.2 \mathrm{mill} / \mathrm{kWh}$ could be attributed to the higher breeding gain and hence plutonium credit achieved through use of carbide rather than oxide fuel. The remaining $0.2-\mathrm{mill} / \mathrm{kWh}$ cost difference was due to differences in core configuration. On the basis of capital 
costs of $\$ 125 / \mathrm{kWe}$, total power costs of the order of 3.5 mills $/ \mathrm{kWh}$ have been estimated. (5) Thus, for these design studies, the effect of core concept as reflected in fuel-cycle costs amounts to only about $5 \%$ of the total power costs. Fuel-cycle cconomics is a major factor in the design of a large fast reactor core. However, due to the minor dependence of fuel-cycle costs on core concept, we think that proof of the attractiveness of fast reactors for large central station power production will be best accomplished if core design features of the first round of large fast reactors are selected with primary emphasis on attaining plant reliability and safety. Thus, simplicity of design, the inclusion of features which promote safety under both normal and abnormal operating conditions, and selection of design features to as large an extent as possible of the basis of proven technology should be main design considerations.

\section{Safety Considerations}

The basic core concept of each of the design studies was dictated to a large extent by the requirement for attaining satisfactory sodium voiding properties. Consequently, the designs do not achieve as high a plutonium specific power and internal conversion ratio or as low fuel-cycle costs and fuel doubling time as would be achieved with a cylindrical core having a ratio of length to diameter $(\mathrm{L} / \mathrm{D})$ near unity. However, we believe that the major emphasis given to sodium voiding properties was fully justified. Further improvements in fast reactor performance may come about through reductions in conservatism of the present designs resulting from better physics data and hence a greater certainty in calculated values of sodium and Doppler coefficients. Additional accident analysis and safety testing, particularly of the effects of transient partial sodium voiding, may also allow reductions in present conservative design criteria.

The specific eriteria which were applied to sodium voiding reactivity effects differed in the various studies. A design goal of our study was the attainment of zero or only small reactivity gain on complete loss of core sodium. The maximum reactivity gain resulting from sodium voiding, i.e., in central core regions only, was in excess of one dollar. In another of the design studies, design goals were to keep the maximum partial roiding reactivity effect under one dollar and obtain a negative effect for complete loss of core sodium. In the other two studies, reactivity gain due to complete core voiding and the maximum reactivity gain due to partial voiding were both in excess of one dollar. No answers as to what constitutes acceptable criteria are readily apparent. Hopefully, they will become apparent as detailed aceident studies of various reference designs are carried out.

For current design studies, however, we prefer to retain the criterion that core voiding shall result in zero or only small reactivity gain. This criterion does not eliminate all problems of sodium voiding, since maximum partial voiding reactivity effects may still be high. It at least, however, ensures that large cores have equivalent sodium voiding properties as small cores and minimizes the safety problems of expanding core sizes from those associated with a prototype to a full 1000-MWe design. Other minimum criteria applicable to more realistic cases of sodium voiding are (1) partial or complete loss of core sodium from a single fuel channel shall have a negligible reactivity effect and (2) progressive loss of sodium from the top of the core downward, including the effect of upper blanket (or reflector) voiding, shall at all times be negative.

Two of the design studies were based on use of oxide fuel; the other two were based on carbide. Present discussions of use of mixed oxides vs. carbides are primarily centered on (1) improvements possible through use of carbide in breeding ratio and power density versus (2) the advantage of oxide due to its more advanced technology. Although we believe the latter argument carries more weight at present, a clearcut choice of fuel material is not possible. It is worthwhile considering whether either fuel has a distinct safety advantage.

Present design of oxide fuel elements is based on operation of the fuel with the maximum central temperature just below the melting point. In present designs for carbide fuel, the maximum central temperature is well below the melting point. Thus, carbide fuel is eapable of greater Doppler compensation in going from operating temperatures to the melting point. This does not necessarily result, however, in a greater allowable reactivity insertion to the point of fuel failure for carbide cores. TREAT tests ${ }^{(7)}$ of unirradiated $\mathrm{UO}_{2}$ specimens have indicated that clad integrity could be maintained for transients when fuel temperatures reached $7000^{\circ} \mathrm{F}$. For full-power flow conditions and short-period transients corresponding to those of a fast reactor excursion, analytic studies ${ }^{(8)}$ have shown that high fuel vapor pressure due to fuel temperatures in excess of $6500^{\circ} \mathrm{F}$ may be the most likely mechanism for fuel failure in gas-bonded oxide fuel pins.

Sodium-bonded carbide fuel is presently receiving the greatest amount of attention. With this type of fuel, generation of high sodium rapor pressure in the bond may be a possible failure mechanism. Recent TREAT ${ }^{(9)}$ tests with sodium-bonded $\mathrm{C}^{\top} \mathrm{C}$ fuel re- 
TABIL 1. Calctiations for Anntlate Come

\begin{tabular}{l|c|c|c}
\hline Volume, liters & $\begin{array}{c}\text { Core } \\
\text { OD, }{ }^{*} \mathrm{ft}\end{array}$ & $\begin{array}{c}\text { Enrichment, } \\
\text { \%o (Fissile } \\
\mathrm{Pu} / \mathrm{U}+\mathrm{Pu})\end{array}$ & $\begin{array}{c}\text { Power Peak- } \\
\text { ing Factor, } \\
\text { Max/Avg }\end{array}$ \\
\hline 7820, Original Design & 16 & 17.95 & 1.52 \\
$\begin{array}{c}\text { Study } \\
7820, \text { Present Study }\end{array}$ & 16 & 17.82 & 1.52 \\
6470 (1) & 14 & 17.81 & 1.53 \\
4990 & 12 & 17.77 & 1.54 \\
\hline
\end{tabular}

* Ineluding 18 in. blanket.

sulted in high fuel dispersal throughout the test capsule for a transient in which the maximum measured temperature was believed to be $5700^{\circ} \mathrm{F}$. It was concluded for this test that local sodium pressures may have plovided the mechanism for fuel dispersal. Further testing work on transients is required for both oxides and carbides, particularly in defining the failure mechanism for sodium-bonded carbide during short-period transients. On the basis of present data it appears that sodium-bonded carbide fuel may fail at a lower temperature than gas-bonded oxide fuel. Therefore, the IOppler compensation to the point of fuel failure may not be any greater for carbide than oxide.

There is no clearcut advantage to oxide or carbide in sodium voiding reactivity effects. The positive spectral component of the void coefficient is higher for carbide due to the higher ratio of fertile to fissile atoms. This tends to be counteracted by the smaller core volume due to the higher power density possible through use of carbide, and hence greater magnitude of the negative leakage component of the void coefficient. Full exploitation of the high-power-density capability of carbide could yield an advantage to carbide but was not clearly demonstrated in the design studies.

The shorter time constant of carbide fuel (approximately $1 / 4$ that of oxide as given in two of the 1000 MWe design studies) may lead to disadvantages under some conditions. For example, following reactor scram and in the absence of rapid flow reduction, the core outlet temperature of the sodium will decrease at a rate determined primarily by the fuel-element time constant. Thus, thermal stresses in the hot leg of the primary circuit will be more severe for a carbidefueled core. In considering the possibility of partial sodium voiding near the center of the corc, a short time constant may also be a disadvantage. If reactivity is inserted when reactor power level and flow rate are both low, sodium boiling can be initiated at the core center, where high positive reactivity feedback is possible. Boiling at the core center is enhanced by a short time constant of the fuel.
It may be concluded from these considerations that safety characteristics of cores with oxide and carbide fuel will probably be somewhat different and will both have to be studied in greater detail. At present, neither fuel appears to offer a significant safety advantage over the other.

\section{Comparison of Core Geometries*}

The most significant difference among the four 1000 MWe studies was that of core geometry. In order to decrease the positive reactivity effect of removal of core sodium, the Allis-Chalmers annular core, the General Electric and Combustion Engineering pancaked cores, and the Westinghouse modular core each had one major core dimension shortened to promote core leakage. There is no core geometry which appears to represent a "best" design, since each has its own advantages and disadvantages. However, we have reviewed our original choice of an annular core in an attempt to decide if its disadvantages or if the advantages of the other designs indicated that some other choice would be preferable.

On the basis of parameters reported in the design studies, the annular concept seemed to have the major disadvantages of a high plutonium inventory and a large outside diameter for the core. Both of these tend to impose an economic penalty on the annular design. In other areas, it seemed approximately equivalent. Comparative calculations ${ }^{(6)}$ showed it to have the lowest reactivity effect on loss of core sodium, although both the modular core and annulus should be capable of achieving equivalent effects. Breeding ratios, when corrected for differences attributable to fuel material, were essentially the same for all designs. No significant difference in Doppler coefficient for unmoderated cores was demonstrated in the design studies, although the Doppler coefficient was substantially increased in one design by the addition of moderator. Thus, the possibility of achieving lower plutonium inventory in a core having smaller overall dimensions was the major incentive for investigating alternative concepts.

The parameters of plutonium inventory and total core size are strong functions of arerage core power density. In order to evaluate the effects of changing core power density, calculations for various sizes of annular cores were performed. In each, the height and thickness of the annulus were kept constant while the inner diameter was decreased. One-dimensional CRAM calculations were performed using the axial buckling of our original design study and twenty

\footnotetext{
* Physics analyses for this section were performed by John Lewellen, Allis-Chalmers Manufacturing Company, Atomic Energy Division.
} 
energy groups. The cross-section set was that of the original design study, except that the four groups of lowest energy were not used and nominal rather than specific composition-dependent values for heavy-element absorption cross sections were used. Fission product concentrations and plutonium isotropic ratios corresponded to a fuel burnup of $67,000 \mathrm{MWD} / \mathrm{T}$. Material volume fractions were those of the original study. For each core, a fissile material search was performed to determine critical loading, and power distributions were determined. The original annular core was recalculated, since a simpler cross section set and less detail in representing blanket plutonium distribution were used than for the original design study. Results are given in Table 1.

In going from the reference annular core to a 5000 -liter core, corresponding to a $55 \%$ increase in power density, equilibrium core enrichment decreased slightly to $99.7 \%$ of the reference. This indicates that, in the annular core, plutonium inventory is essentially in direct proportion to core volume, or inversely proportional to average power density. The power peaking factors also changed very slightly with core volume. Thus, plutonium inventory is also inversely proportional to peak power density in the core, a parameter important in defining thermal limitations of fuel-clement design. The fact that enrichment and powor distribution changed so slightly over the size range considered indicates that the annular core behaves as an infinitely long rectangular cylinder. Thus, other important parameters of the annular core, such as breeding ratio, Doppler coefficient, and sodium voiding reactivity effects, are expected to be relatively independent of core volume.

Using these characteristics of the annular core, we have performed some comparisons of plutonium inventory requirements and outer core dimension with alternative concepts of the design studies. These comparisons were performed under the assumption that cores are designed to the same peak power density. For oxide cores, this is equivalent to the same fuel-pin geometry. This is a rather rough basis of comparison, since for each design the fuel-pin geometry and power density are set by economic optimizations. However, we believe it to be a more equitable basis of comparison than assuming equal average core power densities, since differences in power peaking factors can require substantially different fuel designs in cores having the same average power density.

The original design studies showed a substantial advantage for the pancaked cores as regards plutonium inventory. Comparisons were made with the General Electric core, since it used the same fuel material and its sodium voiding effects were closer to
TaBle 2. Calcllatoxs for Modilat Core

\begin{tabular}{c|c|c|c|c|c}
\hline Module Size, liters & $\begin{array}{c}\text { Equiva- } \\
\text { lent Cy- } \\
\text { lindrical } \\
\mathrm{L} / \mathrm{D}\end{array}$ & $\begin{array}{c}\text { Fissile } \\
\text { Pu In- } \\
\text { ventory, } \\
\mathrm{kg}\end{array}$ & $\begin{array}{c}\text { Enrich- } \\
\text { ment, \% } \\
\text { Fissile } \\
\mathrm{Pu} / \mathrm{U} \\
+\mathrm{Pu})\end{array}$ & $\begin{array}{c}\text { Power } \\
\text { Peaking } \\
\text { Factor }\end{array}$ & $\begin{array}{c}\text { Electric } \\
\text { Power } \\
\text { per } \\
\text { Module, } \\
\text { MWe }\end{array}$ \\
\hline $\begin{array}{c}7820, \text { reference } \\
\text { annulus }\end{array}$ & - & 3033 & 17.8 & 1.52 & 1000 \\
500 & 1 & 242 & 22.3 & 1.72 & 57 \\
1000 & 1 & 396 & 18.2 & 1.86 & 105 \\
1500 & 1 & 539 & 16.5 & 1.95 & 150 \\
2000 & 1 & 676 & 15.5 & 2.01 & 195 \\
2500 & 1 & 810 & 14.9 & 2.06 & 237 \\
3500 & 1 & 1071 & 14.0 & 2.14 & 320 \\
1000 & 2 & 410 & 19.3 & 1.93 & 101 \\
3500 & 2 & 1136 & 14.9 & 2.23 & 307 \\
\hline
\end{tabular}

* Assuming same peak power density and core to blanket power split as for the 7,820 -liter reference annulus.

the annular design. This comparison was made on the basis of reported values of axial and radial power peaking factors, exclusive of hot spot factors. The annulus was assumed to have the same peak value of power per $\mathrm{kg}$ of fuel $(\mathrm{U}+\mathrm{Pu})$ as the pancake. (Power $/ \mathrm{kg}$ fuel was used instead of power density to eliminate differences attributable to fuel volume fraction and fuel density.) On this basis, the annular core inventory (initially fabricated) is substantially reduced but is still approximately $16 \%$ greater than the reference pancake. Removal of moderator from the reference pancake would decrease its inventory. However, the annular core was designed to give a lower core sodium voiding reactivity effect, and thickening the annular core to achieve equivalent sodium voiding effects would decrease its inventory. Further detailed studies would be required to determine the exact extent of inventory advantage and its effect on fuelcycle costs of the alternative designs. On the basis of this comparison, however, inventory requirements seem roughly equivalent.

Outer core diameters of the annulus and pancake were also compared on the basis of equivalent maximum specific powers. The diameters were not significantly different. However, the annular core had a slightly smaller diameter than the reference pancake, a different result than shown in the original design studies.

A direct comparison of the annular core with the modular core of the design studies is complicated by the fact that one design used carbide and the other used oxide fuel. To assess relative inventory requirements and core dimensions, we have performed calculations for a series of oxide-fueled spherical cores having the same material volume fractions as our reference annulus. For each core, one-dimensional CRAM 


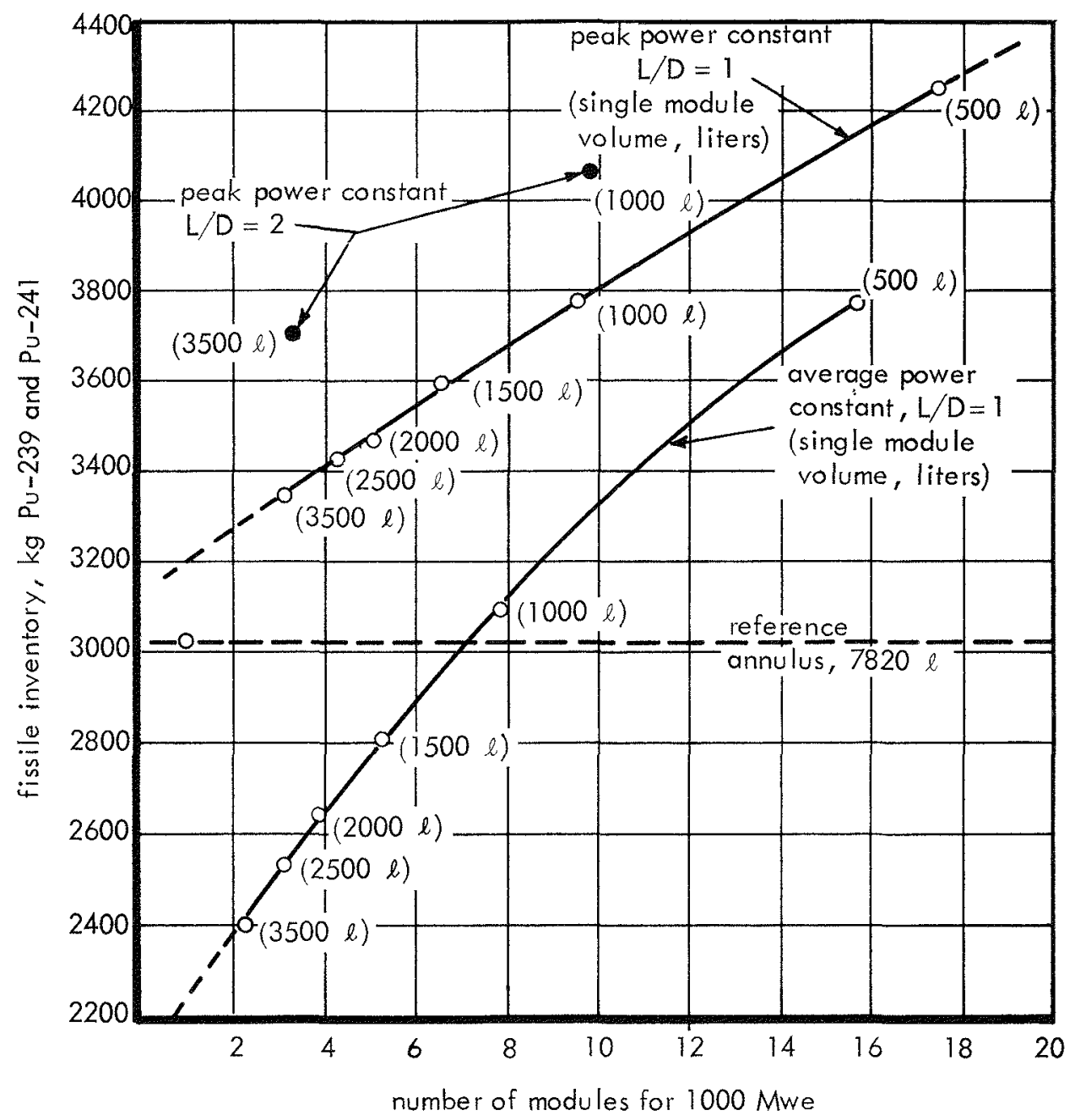

Fla. 1. Fissile Inventory Requirements for a 1000-MWe Modular Core.

calculations were performed to determine power distributions and eritical loading, with fission product concentration and plutonium isotopic ratios corresponding to $67,000 \mathrm{MWD} / \mathrm{MT}$. Effects of module coupling on inventory requirements were neglected. Spherical cores with volumes of 500, 1000, 1500, 2000, 2500 , and 3500 liters were computed. The spherical cores were used to represent a single cylindrical module of $L / D=1$. The effects of changing single-module length-to-diameter ratio were evaluated by calculations for cylinders of 1000 and 3500 liters having an $\mathrm{L} / \mathrm{D}=2$. Results are given in Table 2 and Figure 1 .

In Figure 1, total fissile inventory is plotted versus the number of core modules used to produce 1000 MWe (assuming the same core to blanket power split for each). Results for the modular cores are given in two curves. The upper curve corresponds to the assumption that the modular cores are designed to the same peak power density as the 7820-liter reference annulus, which we consider the proper basis of comparison. The second curre corresponds to the assump- tion that the modular and annular cores have the same average power density, or equal total core volumes. The dotted line represents fissile inventory for the annular core. These results show that, when designed to the same peak power density, a modular core will have a higher plutonium inventory than the annular core regardless of the number of core modules used. For cylinders having L/D greater than unity, the inventory advantage of the annular core is increased. The requirements of lower inventory for the annulus results from its low total power peaking factor relative to the modular cores. This permits a smaller total core volume for the same power production, which more than offsets the higher enrichment of the annulus when compared to modular cores made up of single modules of 1100 liters or greater. (For smaller-sized modules, the enrichment is higher than that of the annular core.)

We have also compared outer core diameters of annular and modular cores. The outer diameter of a modular core will depend upon the $\mathrm{L} / \mathrm{D}$ ratio of the 
individual modules, size and number of modules used, blanket thickness, and power density. We looked only at hexagonal cylinders with $\mathrm{L} / \mathrm{D}$ of 2 , employing the same blanket thickness as the Westinghouse 1000MWe design study modules, and with either 4 or 7 modules per core. Allowable power density was determined from our calculations for a spherical core. The outer core diameter of the reference annulus is $16 \mathrm{ft}$. A four-module core having the same peak power density would be $15.7 \mathrm{ft}$ in OD. A seven-module core of the same peak power density would be approximately $14.1 \mathrm{ft}$ in OD. If a 5000-liter annulus is used, corresponding to increasing the power density of the reference annulus, its outside diameter would be $12 \mathrm{ft}$. Four- and seven-module cores having the same peak power density as this smaller annulus would be 13.9 and $12.6 \mathrm{ft}$ in diameter, respectively. Although these results cannot give a definite answer as to whether a modular or annular core will have a greater diameter, they do indicate that neither design has a strong advantage as regards diameter.

From these comparisons of the annular core with the alternative geometries of the pancake and modular cores, we have concluded that the apparent disadvantages of high plutonium inventory and large outside diameter are not an inherent disadvantage of annular cores. On the basis of equivalent thermal design, there was a small inventory advantage to the reference pancake, although much less than that shown in the design studies. Our calculations with modular cores showed the annular core to have lower inventory requirements than a modular core, regardless of the number of modules used. No design showed a distinct advantage as regards core diameter.

\section{REFERENCES}

1. Large Fast Reactor Design Study, Allis-Chalmers Manufacturing Company, ACNP-64503 (1964).

2. Liquid Metal Fast Breeder Reactor Design Study, Combustion Engineering, Ine, CEND-200 (1964).

3. Liquid Metal Fast Breeder Reactor Design Study, General Electric Co., GEAP-4418 (1964).

4. Liquid Metal Fast Breeder Reactor Design Study, Westinghouse Electric Corp., WCAP-3251-1 (1964).

5. L. E. Link, C. J. Fischer, and E. L. Zebroski, Fuel Cycle Economics of Fast Reactors, Third Geneva Conference, $\mathrm{P} / 248$ (1964).

6. An Evaluation of Four Design Studies of A 1000 Wwe Ceramic Fueled Fast Breeder Reactor, COO-279 (Dec 1, 1964).

7. J. H. Field, Experimental Studies of Transient Effects in Fast Reactor Fuels, Series 1-- $\mathrm{CO}_{2}$ Irradiations, GEAP4130 (Nov 15, 1962).

8. R. B. Osborn and I). B. Sherer, Anatytical Studies of Transient Effects in Fast Reactor Fuels-1, GEAP-4058 (Aug 1962).

9. S. J. Stachura, R. N. Cordy, and M. Silberberg (Al), Uranium Carbide Transient-Heating Studies-Phase II, Trans. Am. Nucl. Soc. 8(1), 305 (June 1965).

\section{Discussion}

Mr. Gibson (AI): You indicated that the disadvantage of the modular core was the high inventory required, aside from considerations of plutonium availability; that's only a disadvantage if it economically is unattractive. Did you do any fuel-cycle studies of the modular versus the annular core?

Mr. Strawson: The original intent of our studies was to see if what appeared to be inventory disadvantage of the annular core was, in fact, a major disadvantage. We concluded from our studies that it was not. We have not carried through detailed studies of fuel-cycle cost. 


\title{
Application of the Vented Fuel Concept to a Sodium-cooled Power Breeder with $1000 \mathrm{MWe}^{*}$
}

\author{
D. SMint and W. Sommer \\ Institute for Reactor Development \\ Kernforschungszentrum, Karlsruhe, Germany
}

(PRISENTED BY D. SMIIT)

The principle of venting the fission gas of a fast ceramic reactor to its coolant has been well-known for some years. ${ }^{(1-2)}$ There is an impressive number of advantages:

1. No stress of the fuel cladding caused by fission gas pressure. This results in:

a. improved safety against canning rupture; $\dagger$

b. smaller wall thickness of cladding;

c. less structural material in the core, i.e., higher breeding ratio;

d. no need for the use of exotic canning materials.

2. More compact subassembly, since no fission gas plenum is necessary. This results in:

a. smaller pressure drop of coolant, smaller pumppower, and smaller wall thickness of subassembly boxes;

b. smaller height of reactor vessel, shielding, refueling equipment, and air locks.

3. No storage of high-pressure radioactive gas. This results in:

a. no sudden uncontrollable gas eruption during reactor operation in case of canning rupture or meltdown. This is of special importance in connection with the positive void coefficient;

b. no sudden uncontrollable gas eruption during the fuel handling and fuel reprocessing.

On the other hand, some sperial equipment is needed, which adds to the rapital costs of the station:

1. special plugs for the fuel rods;

2. a gas-purification plant with all exuipment (blowers, ('ooling aggregates, heat exchangers, etc.);

3. additional filtering equipment with high retention ability for halogens;

4. elaborate system for control of radioactivity;

5. special flanges and gaskets for high leak-tightness.

The most important item is the purification plant for the cover gas. But it should be kept in mind that we

\footnotetext{
* This paper has been prepared within the framework of the association EURATOM-Gesellschaft fur Kernforschung $\mathrm{mbH}$. in the field of fast breeder development.

$\uparrow$ This might not be true for fast excursions with sudden fuel melting and subsequent fission gas release from the oxide.
}

also save some costs for fission gas treatment at the reprocessing plant.

It is the purpose of this paper to give some answers to the following questions:

1. What are the principal design specifications for the gas-purifiration plant with respect to operation and safety of the reactor?

2. What are the numerical results for a reforence reactor?

3. What is the economical balance of the cited advantages and disadvantages of the "vented-toroolant" concept?

We are aware of the fact that many groups are working on this subject and probably have arrived at similar results. But very few of these have appeared in the literature so far. This contribution particularly is meant to stimulate the discussion on this very interesting matter.

\section{Specifications for the Gas-purification Plant}

Figure 1 shows the principal plant. Region I is the cover gas inside the reactor vessel, II the inside of the safety containment. Let a flow rate $g$ of fission gas enter region I; let $\dot{v}_{A}$ and $\dot{v}_{E}$ denote the flow rates to and from the purification plant, respectively. Let the leakage flow rate $L$ be from region $I$ to II, with flow rates $\dot{V}_{A}$ and $\dot{V}_{E}$ to and from the outer atmosphere. Normally $\dot{V}_{A}$ is given by the air-exchange rate of the containment volume, and $C_{A}$ is either given by the allowed artivity level inside the building or by the allowed activity output at the stack.

If the respective activity concentrations are $c_{g}, c_{E}$, $c_{A}, c_{L}, C_{E}$, and $C_{A}\left(\right.$ all in $\left.\mathrm{Ci} / \mathrm{cm}^{3}\right)$, we get the following balance:

$$
\begin{aligned}
& g c_{g}+\dot{v}_{E} c_{E}=\dot{v}_{A} c_{A}+L c_{L} ; \\
& L C_{L}+\dot{V}_{E} C_{E}=\dot{V}_{A} C_{A} .
\end{aligned}
$$

In general, $c_{E}=0 ; \dot{v}_{E}=\dot{v}_{A} ; c_{A}=c_{L} ;$ and $C_{E}=0$. For $L c_{L} \ll g c_{g}$, the result is

$$
\dot{v}_{A}=\frac{g c_{g}}{\dot{V}_{A} C_{A}} L
$$


the input of the purification plant. The leakage $L$, therefore, is the most important parameter for the size of the purification plant. There are several flanges for refueling purposes and control rod drives on the top of our proposed sodium-cooled reactor.

The problem is the definition of a feasible leak-tightness.

The cover gas is helium. The most extensive tests on this have been made in connection with the Dragon project..$^{(3-5)}$ The results have been converted to the cover-gas pressure of $1.2 \mathrm{~atm}$ of our reactor according to the Knudsen equation, in which the leakage is proportional to the pressure difference. The result is a leakage per unit length of

$$
1.5 \times 10^{-12}\left(\mathrm{~atm}_{-} \cdot \mathrm{cm}^{3} / \mathrm{sec}-\mathrm{cm}\right)
$$

or with a sealing length of $12,500 \mathrm{~cm}$, we get

$$
L=1.87 \times 10^{-8}\left(\mathrm{~atm}-\mathrm{cm}^{3} / \mathrm{sec}\right)
$$

for the total reactor top.

After determination of $g$ and $c_{g}$ the purification plant ('an be specified.

We feel it important to underline that these specifications are based on operational safety and not on the analysis of any major accident.

The results of an accident of the vented-1o-coolant and the vented-to-reservoir plant are the same, since in this case the destructions of a number of fuel cans in the latter is probable.

\section{Fission Gas Production and Numerical Results}

The 1000-IIWe reference reactor ${ }^{(6)}$ produces 0.775 $\left(\mathrm{cm}^{3} /\right.$ sec of the gaseous fission products bromine, iodine, xenon, and krypton, or approximately $1 \mathrm{~cm}^{3}$ per fuel rod, per day. We assume that these stay inside the fuel for a time $t_{C}=5$ days at the ambient pressure of $2 \mathrm{~atm}$. If it is possible to maintain higher gas pressures inside the rod, the retention time would rise proportionately, but it is extremely difficult to build up any overpressure at such a low flow rate.

During a time $l_{B}$ the gases pass the axial blanket, where $t_{B}$ is a function of pressure, temperature, and the free cross section.

If we take into account the production rate ${ }^{(7)}$ of the different isotopes produced directly by fission and the radioactive decay of their parents in the core zone, together with the results of Burris and Dillon, ${ }^{(8)}$ the activity $\left(\mathrm{Ci} / \mathrm{cm}^{3}\right)$ leaving the fuel rods ran be calculated. For a burnup of $100,000 \mathrm{\lambda IWd} / \mathrm{t}$ release fractions of $100 \%$ for xenon and krypton and of $50 \%$ for the halogens are assumed. The exit activity has been calculated for two cases:

a) the fission gas moves through the blanket as a rigid column;

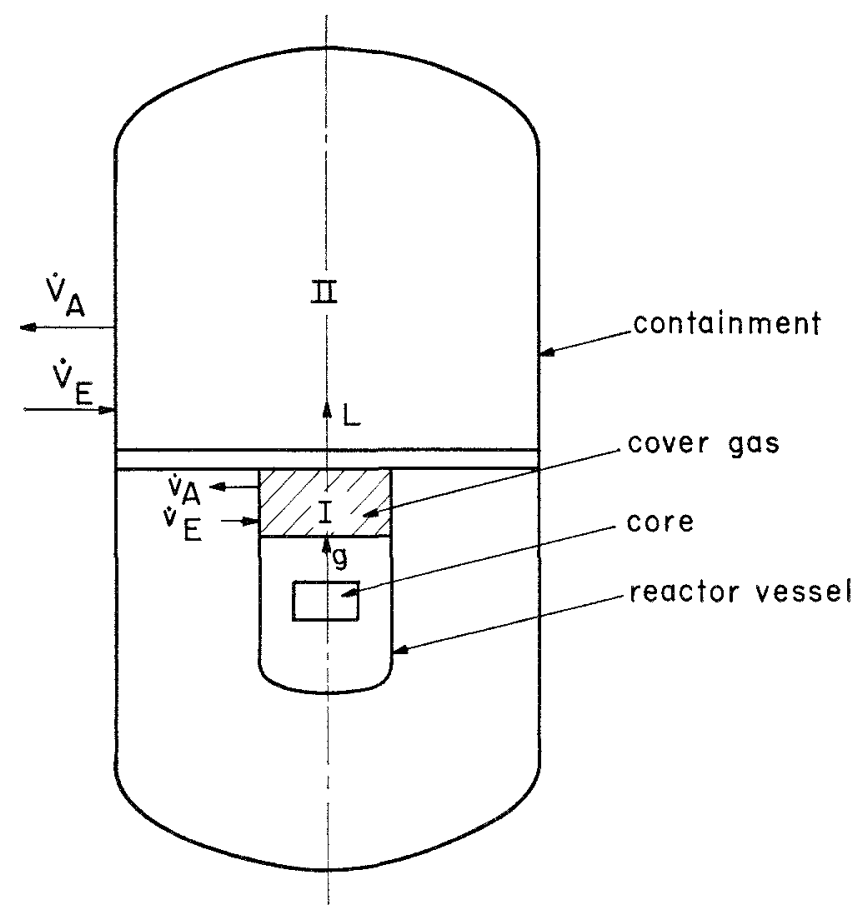

Frg. 1. Principal Reactor Layout. Basic Equations:

1. $g c_{y}+v_{E} c_{E}=\dot{v}_{A} c_{A}+L c_{L}$ for Region I

2. $L c_{L}+\dot{V}_{E} C_{E}=\dot{V}_{A} C_{A} \quad$ for Region II

3. $\quad \dot{v}_{A}=-\frac{y c_{G}}{\dot{V}_{A} C_{A}} L$

b) the gas entering the blanket zone always is completely mixed with the gas already there.

Tables 1 and 2 show the results for no mixing and complete mixing in the blanket, respectively.

Both I and $\mathrm{Br}$ will be chemically bound in sodium. We assume in accordance with $\mathrm{GE}^{(9)}$ that $0.02 \%$ of the iodine arriving at the coolant will be contained in the cover gas. The activity of $\mathrm{I}$ in $\mathrm{Na}$ is small compared with the activity of the $\mathrm{Na}$ itself. The activity of $\mathrm{Br}$ in the Na and the cover gas can be neglected.

If this be taken into areount, $c_{g}$ and $g$ can be evaluated, and by means of eq. (3) the input $\dot{v}_{\hat{A}}$ of the purification plant can be calculated. Figure 2 shows the results as a function of the fuel-element pressure for different assumptions for the process in blanket and plenum. It was assumed that

$$
\dot{V}_{A}=11 \mathrm{~m}^{3} / \mathrm{sec} ; \quad C_{A}=10^{-11} \mu \mathrm{Ci} / \mathrm{cm}^{3}
$$

(that is, one-tenth of maximum permissible concentration of unidentified mixture; however, without $\alpha$ emitters and without the $\beta$ emitters $\mathrm{Pb}^{210}, \mathrm{Ac}^{227}, \mathrm{Ra}^{228}$, and $\mathrm{Pu}^{241}$ ).

\section{Cost Evaluation}

A purification plant for $260 \mathrm{~m}^{3} / \mathrm{hr}$ helium has been planned in connection with Linde AG., Nuenchen, and 
TABLE 1. Exit Concentration OF Gaseots Fission Prodt cts (Ci/cmo Gas Prodtced in Core) as a Function of Frel Inside Presst re (No Plentm axd No Mixing in Blanket)

\begin{tabular}{l|c|c|c|c|c|c}
\hline \multirow{2}{*}{ Isotope } & Half-life & \multicolumn{5}{|c|}{ Fuel Inside Pressure (atm) } \\
\cline { 3 - 7 } & & 2 & 4 & 8 & 12 & 34 \\
\hline Kr-83m & $1.3 \mathrm{hr}$ & 0.004 & - & - & - & - \\
$\mathrm{Kr}-85 \mathrm{~m}$ & $4.4 \mathrm{hr}$ & 1.2 & 0.035 & 0.001 & - & - \\
$\mathrm{Kr}-85$ & $10.6 \mathrm{yr}$ & 0.0047 & 0.0046 & 0.0045 & 0.0044 & 0.0044 \\
$\mathrm{Kr}-87$ & $78.0 \mathrm{~min}$ & 0.0033 & - & - & - & - \\
$\mathrm{Kr}-88$ & $2.8 \mathrm{hr}$ & 0.9 & 0.0046 & - & - & - \\
$\mathrm{Kr}-89$ & $3.2 \mathrm{~min}$ & - & - & - & - & \\
& & & & & & \\
$\mathrm{I} 131$ & $8.1 \mathrm{~d}$ & 50.4 & 39.5 & 24.2 & 16.1 & 3.0 \\
$\mathrm{I}-132$ & $2.3 \mathrm{hr}$ & 0.9 & 0.0017 & - & - & - \\
I-133 & $21.0 \mathrm{hr}$ & 105.5 & 28.9 & 4.5 & 0.9 & - \\
$\mathrm{I}-134$ & $53.0 \mathrm{~min}$ & & - & & - & \\
I 135 & $6.7 \mathrm{hr}$ & 29.6 & 2.3 & 0.025 & - & - \\
& & & & & & \\
$\mathrm{Xe}-133$ & $5.3 \mathrm{~d}$ & 197.0 & 127.9 & 72.2 & 41.9 & 5.1 \\
$\mathrm{Xe}-135 \mathrm{~m}$ & $15.7 \mathrm{~min}$ & - & - & - & - & - \\
$\mathrm{Xe}-135$ & $9.2 \mathrm{hr}$ & 204.9 & 34.0 & 1.3 & 0.038 & - \\
$\mathrm{Xe}-138$ & $17.0 \mathrm{~min}$ & - & - & - & - & \\
\hline
\end{tabular}

TABLE 2. EXIT Concentration of Gaseous Fissiov Products (Ci/ $\mathrm{cm}^{3}$ (aAs Produced in Core) as a Function of Fued Inside Pressure (No Plinum and Complete Mixing in Bianket)

\begin{tabular}{|c|c|c|c|c|c|c|}
\hline \multirow{2}{*}{ Isotope } & \multirow{2}{*}{ Half-life } & \multicolumn{5}{|c|}{ Fuel Inside Pressure (atm) } \\
\hline & & 2 & 4 & 8 & 12 & 34 \\
\hline $\mathrm{Kr}-83 \mathrm{~m}$ & $1.3 \mathrm{hr}$ & 0.4 & 0.2 & - & - & - \\
\hline $\mathrm{Kr}-85 \mathrm{~m}$ & $4.4 \mathrm{hr}$ & 7.0 & 1.9 & 0.5 & 0.2 & 0.021 \\
\hline $\mathrm{Kr}_{\mathrm{r}-85}$ & $10.6 \mathrm{gr}$ & 0.0041 & 0.0046 & 0.0045 & 0.0044 & 0.0044 \\
\hline $\mathrm{Kr}-87$ & $78.0 \mathrm{~min}$ & 5.4 & 1.2 & 0.3 & 0.2 & 0.024 \\
\hline $\mathrm{Kr}-88$ & $2.8 \mathrm{hr}$ & 17.2 & 4.3 & 1.1 & 0.5 & 0.049 \\
\hline Kr -89 & $3.2 \mathrm{~min}$ & 0.4 & 0.2 & & - & 一 \\
\hline $\mathrm{I}-131$ & $8.1 d$ & 52.2 & 42.2 & 27.6 & 19.7 & 5.4 \\
\hline [-132 & $2.3 \mathrm{hr}$ & 36.5 & 8.4 & 2.3 & 1.1 & 0.1 \\
\hline I-133 & $21.0 \mathrm{hr}$ & 144.3 & 55.5 & 19.0 & 8.7 & 1.2 \\
\hline I-134 & $53.0 \mathrm{~min}$ & 13.7 & 3.5 & 0.9 & 0.035 & 0.005 \\
\hline I-135 & $6.7 \mathrm{hr}$ & 87.9 & 25.3 & 6.5 & 1.5 & 0.4 \\
\hline $\mathrm{Xe}-133$ & $5.3 \mathrm{~d}$ & 193.8 & 132.0 & 83.4 & 54.3 & 12.9 \\
\hline $\mathrm{Xe}-135 \mathrm{~m}$ & $15.7 \mathrm{~min}$ & 1.2 & 0.6 & 0.3 & 0.07 & - \\
\hline $\mathrm{Xe}-135$ & $9.2 \mathrm{hr}$ & 310.1 & 109.3 & 30.4 & 10.1 & 1.8 \\
\hline Xe-138 & $17.0 \mathrm{~min}$ & 7.9 & 1.9 & 0.5 & 0.2 & 0.024 \\
\hline
\end{tabular}

evaluated for a fuel inside pressure of $2 \mathrm{~atm}$, no plenum, and complete mixing. Xenon and krypton are removed from the cover gas by low-temperature adsorption. The plant will be relatively expensive because of the need for continuous operation and the large amount of decay heat. It needs $180 \mathrm{~kW}$ pumping power, $75 \mathrm{~m}^{3} / \mathrm{hr}$ coolant water, and $35 \mathrm{l} / \mathrm{hr}$ liquid nitrogen. Table 3 shows the heat production for different conditions.

Compared with the reference reactor with "venting to reservoir," the core height with venting to coolant is redured by $80 \mathrm{~cm}$. The most important changes are:

Pressure Drop, atm Cladding Thickness, $\mathrm{mm}$ Cladding Fraction of Fuel Eilement

Breeding Ratio

$\begin{array}{cc}\begin{array}{c}\text { Reference } \\ \text { Reactor }\end{array} & \begin{array}{c}\text { Vented to } \\ \text { Coolant }\end{array} \\ 4.25 & 3.20 \\ 0.35 & 0.15 \\ 0.25 & 0.096 \\ & \\ 1.42 & 1.59\end{array}$

Table 4 shows the resulting capital costs. The main contribution comes from the smaller fabrication cost of the shorter fuel elements with $\$ 205,000$ per core. For replacing one-third of the core per year, this is equivalent to $\$ 1.59 \times 10^{6}$ capital costs for a 15 -yr lifetime. The second largest contribution is the capitalized gain in breeding ratio (eapitalized for a load factor of 0.8 ).

The radial blanket is also considerably shorter. For 4 blankets during the reactor lifetime, this amount to $\$ 1.05 \times 10^{6}$. Smaller savings are gained by the reduction of pumping power.

Gas purification and storage at the reprocessing plant are not needed any more. The costs were evaluated in accordance with Alter. ${ }^{(10)}$ Finally, the reduced height of the core allows for some savings for the core vessel and its internals, such as supporting cylinders, insulation walls, reloading equipment, and thermal shielding. However, this contribution is small.

The last column at the right side of Table 4 shows the additional costs. The net saving amounts to $\$ 2.19 \times$ $10^{6}$ or $2.1 \%$ of the total plant cost.

\section{Preliminary Tests}

The important point is the development of a suitable plug. The diving-bell design of $\mathrm{GE}$ is well-known. ${ }^{(1)} \mathrm{It}$ promises a very reliable operation, but it nust be of considerable length and, therefore, adds to the pressure drop and to capital costs. We, therefore, thought it useful to study the behaviour of a very simple porous plug of sintered metal.

Two problems are of importance:

a) the ability to retain sodium from the fuel. In the case of a reactor shutdown and lowering of the fuel temperature, the outside overpressure of the sodium amounts to 1-2 atm.

b) the ability to stay porous for the fission gases.

In a simple apparatus the following plug materials have been tested: $\mathrm{Ni}, \mathrm{CrNi}$ 18/8, Fe, and CrNi 18/10, with pore sizes between 1 and $5 \mu$. Sodium leakage started at a pressure difference of about $1.5 \mathrm{~atm}$ for sodium temperatures of $500^{\circ} \mathrm{C}$, and at about $0.5 \mathrm{~atm}$ at $600^{\circ} \mathrm{C}$.

The first plugging experiments by artificial evaporation of iodine did not lead to a pressure buildup of the 


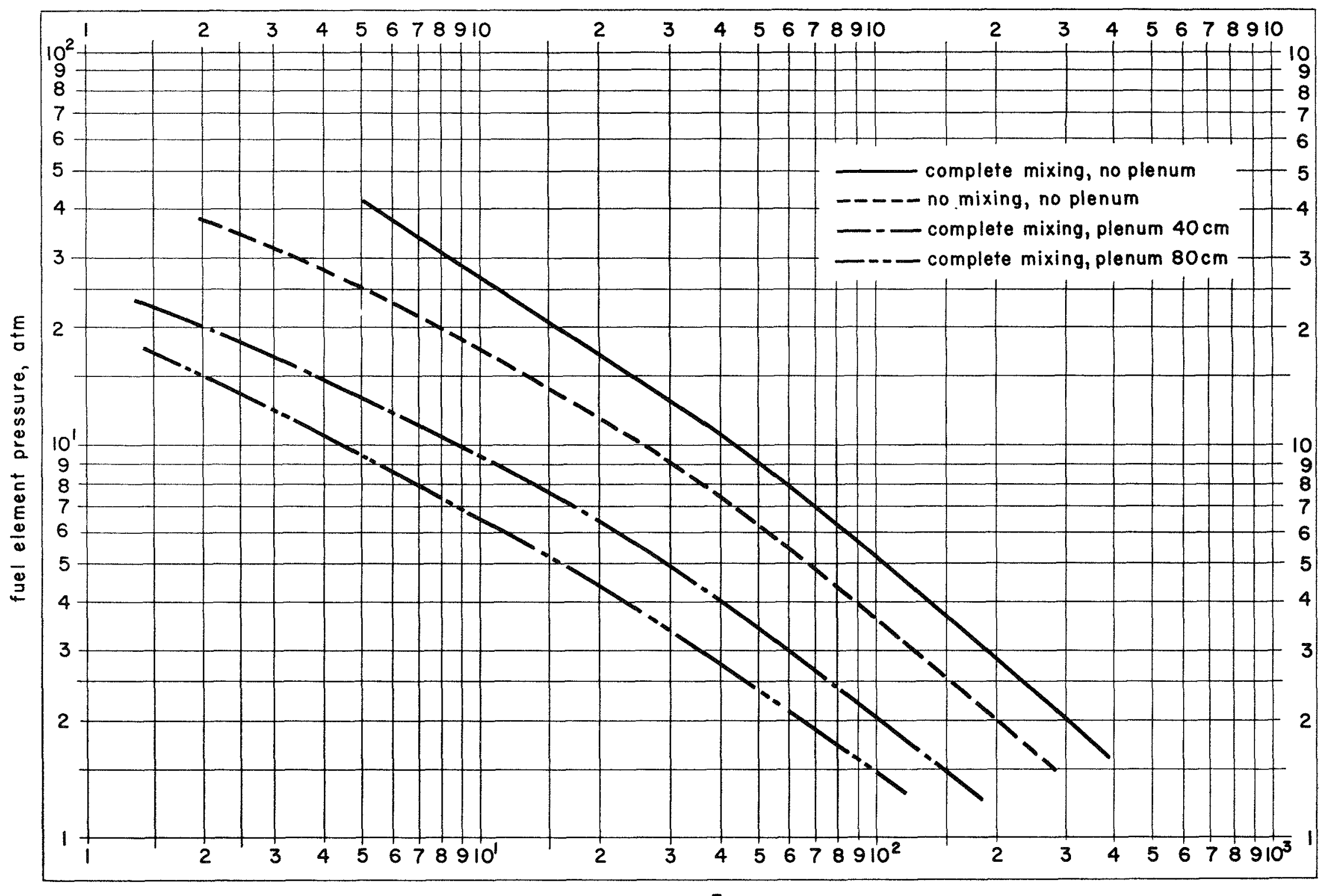

FIG. 2. Theoretical Input into Purification Plant. 
Table 3. Decay Heat to be Removed from the Adsorbers after $3000 \mathrm{Hr}$ of Operation Time for Different Mechanisms of Fission Gas Release (At 2-ATM Fuel Pressure)

\begin{tabular}{|c|c|c|c|c|}
\hline \multirow{2}{*}{ Release Mechanism } & \multicolumn{3}{|c|}{ Decay Heat of Isotopes (kW) } & \multirow{2}{*}{$\begin{array}{c}\text { Total Decay } \\
\text { Heat } \\
(\mathrm{kW})\end{array}$} \\
\hline & $\mathrm{Xe}-133$ & $\mathrm{Xe}-135$ & $\mathrm{Kr}-85$ & \\
\hline No Plenum; No Mixing & 280.0 & 57.5 & 0.15 & 337.7 \\
\hline $\begin{array}{l}\text { No Plenum; Complete } \\
\text { Mixing }\end{array}$ & 276.2 & 94.0 & 0.15 & 370.4 \\
\hline $\begin{array}{l}\text { Plenum }(40 \mathrm{~cm}) \text {; Com- } \\
\text { plete Mixing }\end{array}$ & 200.0 & 11.7 & 0.15 & 211.9 \\
\hline $\begin{array}{l}\text { Plenum }(80 \mathrm{~cm}) ; \mathrm{Com}- \\
\text { plete Mixing }\end{array}$ & 139.0 & 6.9 & 0.15 & 146.1 \\
\hline
\end{tabular}

TABle 4. Cost Balance for Application of a Fented-Fuel Concept

\begin{tabular}{l|c|c|c}
\hline \multicolumn{1}{c|}{ Savings } & $10^{6} \$$ & Additional Costs & $10^{6} \$$ \\
\hline Core Fabrication & 1.59 & Gas-purification Plant & 2.30 \\
Breeding Ratio & 1.58 & Operational Costs & 0.77 \\
Radial Blanket & 1.04 & & \\
Circulation Pumps & 0.50 & & \\
Gas Treatment at Re- & 0.34 & & \\
processing & & & \\
Reactor Vessel & 0.21 & & \\
\hline Total & 5.26 & & \\
\hline
\end{tabular}

gases. More tests are necessary for the evaluation of the sintered porous plug.

\section{Conclusions}

From this study the following conclusions can be drawn:

a) The venting of fission gases to the sodium coolant allows for savings of the order of $2 \%$ of the total plant cost, if compared to the reference reartor. In comparison to a more optimized reactor the savings are much smaller.

b) The main savings are from lower costs for core and radial blanket resulting from the elimination of the fission gas plenum and the reduction of cladding thickness, which causes a substantial rise in breeding ratio.

c) A low-temperature adsorption process seems to be the most suitable for the purification of the cover gas. The main problem is the decay heat of several hundred $\mathrm{kW}$, mainly from xenon.

d) The input specification of the purification plant comes from operational criteria. The unavoidable leakage into the containment building is the determining factor.

e) The most important advantage is the gain in safety. Sudden bursts of gas into the coolant and the consequences with a positive void coefficient are avoided. The transient behaviour needs further studies.

\section{LITERATURE}

1. F. J. Leit , Sodium-cooled Reactor Program, First Quarterly Report, October-December 1961 (GEAP-3888).

2. G. L. O'Neill, J. H. Iavies, and M. C. Johnson, Demonstration of Fission Gas Venting from Fast Oxide Reactor Fuels, ANS Annual Meeting, Philadelphia, Pa., 6/14$18 / 65$.

3. N. Pollit, The Leak-tightness of Demountable Flange Assemblies using Soft Iron Gaskets, Iragon Project Report 28 (April 1961).

4. D. Barnes and F. Wade, Leaktesting of the Test Vessel and Experimental Gaskets at Mannesmann, Hueckingen, Dragon Project Reports 23, 44, and 66 (1961).

5. D. Barnes and F. Wade, Leaktesting the Dragon Reactor Pressure Vessel, Dragon Project Report 154 (Jan 1963).

6. D. Sinidt and A. Muller, Referenzstudie für den $1000 \mathrm{MWe}$ natrium-gekühlten Schnellen Brutreaktor ( $\mathrm{Na}$ 1), KFK299 (1)ec 1964).

7. S. Kateoff, Fission-product Yields from $L$, Th and $P u$, Nucleonics, 18, No. 11 (Nov 1960).

8. L. Burris and I. G. Dillon, Estimation of Fission Product Spectra in IDischarged Fuel from Fast Reactors, ANL5743 (July 1957).

9. Personal communication.

10. H. W. Alter, A Fuel Reprocessing Plant for Fast Ceramic Reactors, GEAP-4028 (Feb 1962).

\section{Discussion}

Mr. Gueron (EURATOM, Brussels): In the economy found for the reprocessing plant, did you not assume (a) that you had an integrated reactor reprocessing plant, and (b) that you would have really $100 \%$ loss of fission gases? Are these assumptions perhaps not a little daring?

$M r$. Smidt: I think you are completely right. It was a point of this study to find out what, at the maximum, one can gain with venting the fission gas to coolant, and the maximum found is practically nothing. It becomes even worse if one takes into account some of these factors which might be too optimistic in this study. So, I agree with you.

Mr. Zebroski (GE): A very strong parameter which would influence the cost of the processing plant would be the delay time that you assume that the gas sustains within the fuel; if you can be clever enough, say to get 1200 hours delay time, you would issue only krypton-85 and have a negligible gas-purification-plant problem.

Mr. Smidt: That's completely true. The main cost comes from the decay heat. If you could build up a pressure of, say, 10 or $20 \mathrm{~atm}$ in your fuel rods, you would have much easier design of the purification plant. However, with a flow rate of one cubic centimeter a day, it is very difficult to get a reliable pressure in this order of magnitude.

$M r$. O'Neill (GE): You have chosen the Dragon data and the leakage rates obtained as an impossible situa- 
tion, whereas I think we would choose to develop a sealing system that could contain the cover gases.

Mr. Smidt: This would appear in the capital balance end as additional cost.

Mr. Wright (WAPD): The designing for a vented fuel system not only includes the purification problem, but possibly imposes burdens on maintenance and operation of the plant as well. On the other hand, if you look at the 100,000 MWd/ton burnup, one would have difficulty saying that these things are all going to get there without having one or several can ruptures and subsequent leakage. The real problem is not to compare a nonradioactive, nonfission product system to one having fission products, but maybe the difference in the quantities is what you have to compare. I don't think the difference is quite as great as you might guess.

Mr. Smidt: I would repeat again you need a purification plant in any case, but you could assume with no venting through the coolant you would have at least a longer decay time of the fission gases.

Mr. Okrent (ANL): I believe you indicated a safety advantage for venting, from the point of view that you would not be subject to rupture of cladding. How do you evaluate the possible safety disadvantage of a larger fission product burden in the sodium and the increased difficulty of detecting the failure of fuel?

$M r$. Smidt: You mean the activity of fission gases in the sodium?

Mr. Okrent: Yes.

Mr. Smidt: We haven't evaluated that. The question we put was whether we need the safety advantage that no sudden bursts of fission gases will be released under transient conditions. This has to be studied much more experimentally. The safety aspect of the picture isn't complete yet.
Mr. Häfele (Karlsruhe): Another advantage is the fact that the plenums at the entrance of the subassembly are no longer under strong pressures. With venting, the potential danger of sudden fission gas release from clad rupture is gone.

Mr. O'Nenll: With Dr. Smidt's permission I could help to answer Dr. Okrent's question, I think. There is a way to detect the difference between the fission products that escaped from the vented fuel and the failed fuel in that you look at the age of the fission products. We have some experimental data that says it takes 5 to 10 days for the gases to escape, so it is fairly easy to tell the difference.

Mr. Okrent: If you're looking for fission gases. But, it is a relatively slow detection system if fission gases have to get from the fuel through the coolant out to the cover gas. I was thinking in terms of a delayed-neutron detector, of the sort that is now on EBR-II, which, hopefully, might detect failure on a time scale of a minute or less. If you are running with vented fuel, is there a greater likelihood of having a reasonable amount of fissionable material in the sodium, and is there a greater background, so that the probability of detecting failure then is diminished?

$M r$. Jens (APDA): It seems that all fuels we have been talking about have not yet achieved the burnups that we're talking of, and that one of the real advantages of the vented fuel system might be the fact that you can reach the burnup you're talking about, whereas the rest of them can't. Is that burnup advantage at all worked into your numbers?

Mr. Smidt: No. We took a standard burnup, so to say, of $100,000 \mathrm{MWd} /$ ton. I agree with you; you have a point which might also be of value. 


\title{
Steam Cooling for Fast Reactors
}

\author{
R. A. Webb and D. C. Schluderberg \\ The Babcock \& Wilcox Company \\ Atomic Energy Division \\ Lynchburg, Virginia \\ (PRESENTED BY D. C. SCHLUDERBERG)
}

\section{Introduction}

Over the past six years, $B \& W$ has been engaged in the study and evolution of steam-cooled reactors. After looking at two thermal reactor designs, work was begun on a direct-cycle fast reactor incorporating a modern $3500-p s i, 1000^{\circ} \mathrm{F}$ steam cycle with $950^{\circ} \mathrm{F}$ reheat. The study of this reactor was jointly sponsored by the East Central Nuclear Group and B\&W; the results have been reported recently in papers presented at Detroit and Gatlinburg.

Since then, $B \& W$ has independently investigated the possibilities of cooling a direct-cycle fast reactor with steam at $1200 \mathrm{psi}$. The purpose of this paper is to describe some of the interesting and encouraging results obtained.

\section{Objectives of Study}

A core power of 2500 MWt was selected for the study in order to provide a net plant output in the neighborhood of $1000 \mathrm{MWe}$. The objectives of the study were to determine the following:

1. Can a 10-year doubling time be achieved?

2. What are the estimated fuel costs?

3. Does 1200-psi steam offer improved safety characteristics?

4. What is capital cost of a $1000-\mathrm{MWe}$ plant?

5. Can plant efficiency be improved?

\section{Breeding Requirements}

The first results of our study indicated that a doubling time under 10 years could be achieved. It was found that the required breeding ratio and specific power could be obtained with an equilibrium plutonium cycle based on a pancake core geometry and partial surface roughening.

The principal core design parameters are as follows:

1. Thermal power, $\mathrm{MW}$

2. Core diameter/core height, in.

$108 / 36$

3. Axial/radial blanket thickness, in.

$15 / 22$
4. Pin diameter/spacing, in.

$0.300 / 0.360$

5. Core volume, liters

5400

6. Fuel/Structure/Coolant/ Control Rod Fraction

$48.9 / 9.7 / 40.2 / 1.2$

Four-group radial lifetime calculations, using an equilibrium plutonium mixture of $64 \% \mathrm{Pu}-239,25 \%$ $\mathrm{Pu}-240,8 \% \mathrm{Pu}-241$, and $4 \% \mathrm{Pu}-242$, yielded the following information:

\section{Physics Parameters}

Core life, days of full power

Core irradiation, MWWD/Tonne

80,000

Fissile plutonium loading, $\mathrm{kg}$ metal

Total Pu + U loading, $\mathrm{kg}$ metal

22,450

Integrated overall breeding ratio

1.40

Integrated in-core breeding ratio

1.01

Doubling time, years

Specific power, $\mathrm{kW}$ thermal/gm fissile

9.0

plutonium in core

In these calculations, the three fast-group diffusiontheory coefficients were collapsed from a 68-group cross-section library. Resonance integrals were calculated for both fertile and fissile isotopes for all energy groups containing resonances.

Doppler broadening and Dancoff corrections were made in the resonance region. Thermal coefficients were generated by averaging thermal cross sections over a Wigner-Wilkins spectrum. Axial geometric buckling was calculated in the usual way for a cylinder and checked by axial criticality calculations. Fission products were treated on an infinitely dilute basis.

\section{Fuel Cost}

By means of the mass balance from these calculations, a fuel cost of $0.50 \mathrm{mill} / \mathrm{kWhr}$ was obtained. The components whereby this figure was derived are as follows:

Reprocessing, shipping, and fabrication

$\$ 8.48 \times 10^{6}$

Working capital charge at $10 \%$ interest

$8.05 \times 10^{6}$ 
Plutonium credit at $\$ 8861 / \mathrm{kg}$

( $2 \%$ loss)

Net core cost

$\mathrm{kWhr} /$ core

Fuel cost, mill/kWhr

If interest on the bred fuel during core life and cooling is left off, the cost falls to $0.42 \mathrm{mill} / \mathrm{kWhr}$. Further, if $80 \%$ of core and radial blanket cans are reused once, the savings in fabrication cost bring the fuel cost to $0.37 \mathrm{mill} / \mathrm{kWhr}$. These figures assume the entire radial blanket is reprocessed with each core. Some additional reduction should be possible with a better scheme for blanket management.

\section{Safety}

Modification of the plant design to increase capability with a positive voiding characteristic was considered. It was felt that a good start in this direction would be to find means to reduce the maximum credible rate of pressure loss from the system. This led to development of an integral arrangement of the Loeffler cycle (see Figure 1). Instead of enclosing only the reactor core and internals in a pressure vessel as shown here, we investigated the possibility of placing all principal components of the Loeffler cycle inside a reactor pressure vessel which is in turn enclosed in a highpressure close-fitting containment. This includes the reactor core and internals, desuperheating and steamseparating equipment, and the bypass coolant flow. The circulators are also enclosed in the pressure vessel, and these are driven by steam turbines which are mounted on the vessel and supplied steam directly from it.

How these components can be arranged in an actual reactor design is shown schematically in Figure 2. The turbines and circulators are mounted in the top head without interfering with refueling ports, which are not

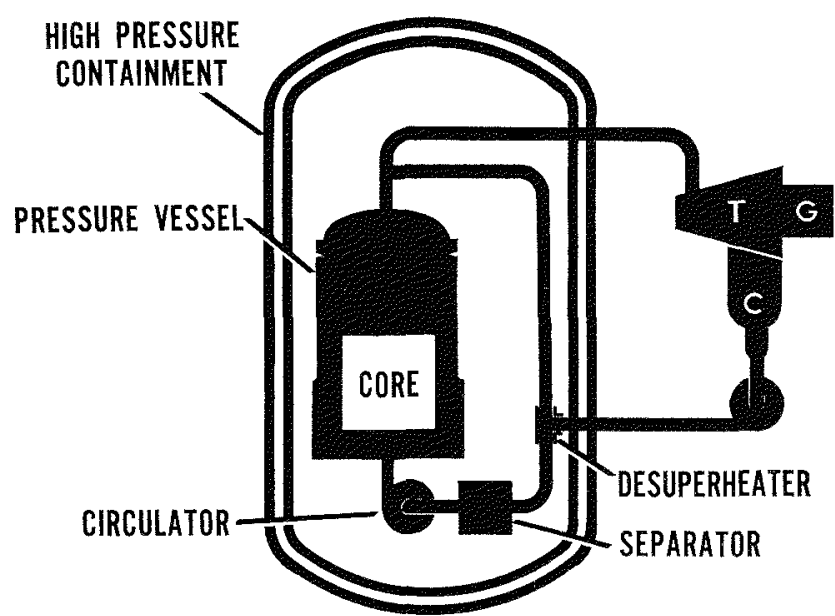

FIG. 1. Iritegral SCBR Flow Diagram.

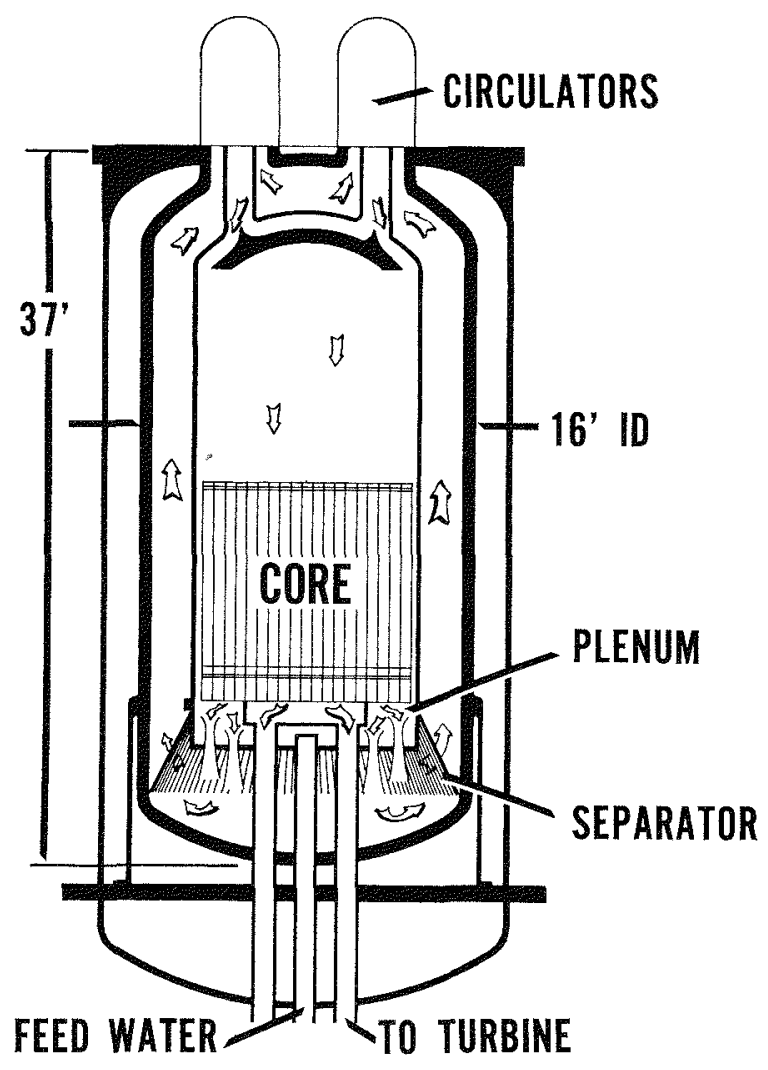

Fig. 2. Integral SCBR Schematic.

shown. Steam-desuperheating and scrubbing equipment are mounted below the core as shown. These consist of a multiplicity of spray-type desuperheaters followed by a steam-scrubber section such as shown.

By eliminating the relatively large external recirculating steam lines and using a close-fitting, fully pressurized containment, it becomes easier to control the rate of accidental coolant loss as well as to prevent core damage and provide dependable control rod action during such loss. These measures are further augmented by using four parallel turbine steam lines, each of which contains flow restrictors at the reactor vessel penetration.

Another advantage of the integral arrangement could be an inherently short constant for the time loop. This in combination with an excess of desuperheating spray may permit a significant automatic increase in steam generation rate as system pressure starts to fall during a coolant-loss accident. This would reduce the rate of pressure loss and thereby provide a longer time interval for control rod insertion.

If the reactivity change is negative with voiding, coolant loss would appear to involve only the problem of emergency cooling.

In this connection, we believe that improved emergency pumping characteristics are realized by mount- 


\section{CAPITAL $(14 \% \& 80 \%$ L.F.)}

FUEL
O.M. \& I

TOTAL MILS/KW. HR.
Fig. 3. Preliminary Economic Estimates for Integral sCBR.

ing the circulator-driving turbines within the highpressure containment and integral with the reactor vessel, and supplying them with steam directly from the vessel. Each circulator is also fitted with a pony motor to provide backup emergency pumping power and to drive the circulators during shutdown and startup operations.

\section{Capital Costs}

Another advantage of this design appears to be low capital costs. Preliminary estimates obtained by incremental adjustment of costs from the previous 3500-lb study indicate the integral 1200-psi plant would have a direct cost of $\$ 55.70 \times 10^{6}$. Adding $35 \%$ for indirect costs during construction and contingency, we get $\$ 75$ $\times 10^{6}$, which amounts to $\$ 80.00$ /installed $\mathrm{kW}$ based on a $940-\mathrm{MIW}$ net plant output. We belicve this low figure is due to basic simplicity of the integral arrangement plus the inherent low cost of a direct-cycle plant.

\section{Cost of Power Generation}

Figure 3 shows preliminary economic estimates in which the fuel and capital cost estimates have been combined with a $0.4-\mathrm{mill} / \mathrm{kWhr}$ charge for operation, maintenance, and insurance. This totals to 2.50 mills/ $\mathrm{kWh}$, so that the economic potential of this plant is indeed satisfactory.

\section{Plant Efficiency}

Turning now to plant efficiency, we found that the integral dosign materially reduced requirements for pumping power through elimination of external recirculating lines. If we take into account reasonable losses for flow restrictors, isolation valves, and headers, we find that cxternal recirculation would add about 75 psi to the circulator pumping head or $55 \mathrm{MW}$ to the pumping power requirement. This corresponds to about a 1.3 percentage point reduction in net plant efficiency, which we presently estimate to be about $37.5 \%$.

The fact that this is somewhat below the efficiency of modern reheat steam cycles encouraged us to look for ways to improve this value. In a Loeffler cycle, a major portion of the reactor outlet steam is recirculated to mix with feedwater which is added to maintain a mass balance in the system and provide reactor inlet steam at or near saturated conditions. The direct spray of water into this hot steam is an undesirable waste of availability as the temperature is degraded from 1050 to about $555^{\circ} \mathrm{F}$ without doing useful work.

This realization led to a reheat cycle designed to take advantage of the wasted availability (see Figure 4). A once-through boiler is placed between the core grid plate and desuperheaters to cool recirculated steam to about $660^{\circ} \mathrm{F}$. Feedwater enters the desuperheaters as before, but since the inlet stcam temperature is only $660^{\circ} \mathrm{F}$, most winds up in the bottom of the vessel as saturated water. This water is supplied to the boiler at 3800 psi through a booster feed pump. It is heated in the boiler in counterflow heat exchange to $950^{\circ} \mathrm{F}$ at 3500 psi, expanded in a high-pressure turbine, and returned to the reactor vessel. This turbine produces about 190 MW of power, and thereby increases net plant output about $100 \mathrm{MW}$ and efficiency about 4 percentage points.

The supercritical pressure boiler handles more heat than the reheater it replaces in the 3500-psi SCBR plant cycle. However, in spite of the increased heat load, the actual cost of the boiler may be lower, since the combinations of coolant flow, pressure, enthalpy rise, and specific volume appear more favorable for economical heat exchanger design.

Actually, this power cycle places the reactor between the high-pressure and intermediate-pressure turbines of a supercritical reheat cycle, thus permitting low-

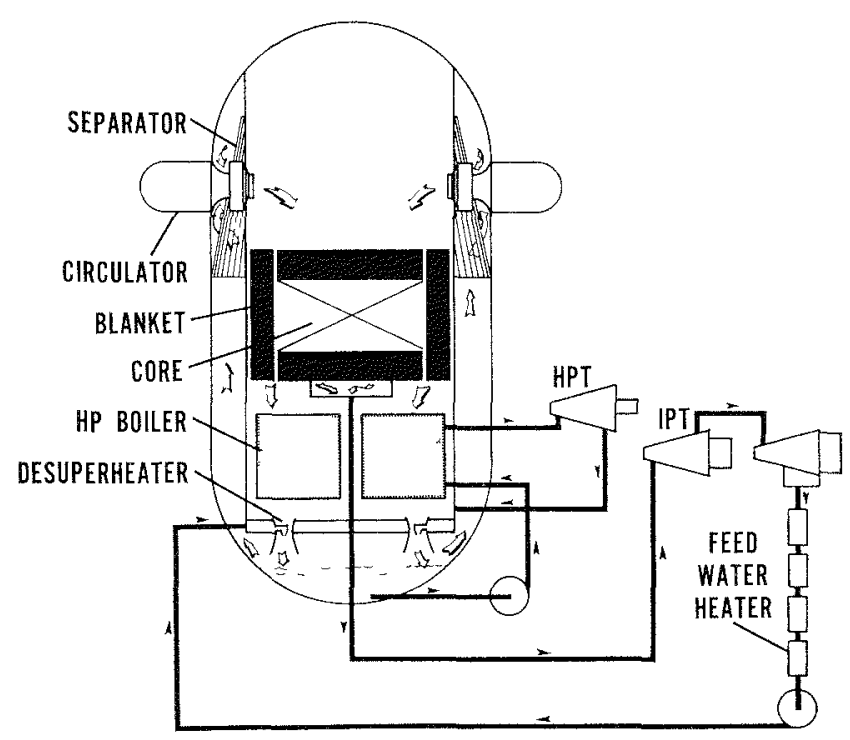

FIG. 4. Reheat Cycle Integral SCBR. 
pressure cooling of the reactor core and the resultant benefits of high breeding ratio. We hope it may constitute an advance in the art of applying a direct, highefficiency reheat steam cycle to a steam-cooled breeder reactor.

\section{Current Activity}

We are now studying methods to improve voiding characteristics, are continuing to develop plant design features to improve compatibility with a positive voiding $\Delta \mathrm{k}$, are investigating performance with other fuel cycles, and will continue investigation of the steam cycle just mentioned.

\section{Discussion}

Mr. Smidt (Karlsruhe): Am I right that in your first design you showed the integral design without the reheat cycle and that you have placed your Loeffler apparatus directly below the core? What are the problems of maintenance?

Mr. Schluderberg: Yes, we arranged the desuperheaters below the grid plate where the blanket bundles go in. Since there are no control rod drives in the blanket bundles, we can go through the holes in the grid plate and remove the nozzles and the venturis themselves. Then we have two arrangements: one where the scrubbers are below and one where the scrubbers are up, on the side where they can be gotten to more easily for maintenance.

Mr. Wall (GE) : Are the control rods top mounted or bottom mounted? How did you calculate the magnitude of the loss-of-coolant reactivity coefficient? What were your arrangements for emergency cooling?

$M r$. Schluderberg: The control rods themselves are pin rods that are located in the fuel-bundle can. One of the unique arrangements of the design, and why we can get down to a $9.7 \%$ structure fraction, was by taking the hexagonal spacer tube pieces (they're just short things braced together to form an array) and making the outer pins the whole length of the can, so that these become the can walls. We have put nine pin rods in each can, and they're actuated by a pressure command which is communicated through the grid plate; each pin is, of course, latched in place and requires a special pressure pulse to unlatch it before it can be driven out.

We have just started work on loss-of-coolant reactivity coefficient, and our results are quite preliminary. Perhaps Ralph Webb may say something about the methods we've used.

$M r$. Webb: The physics methods are the same as those which were given in the test cases which were reported in this morning's paper.
Mr. Fell (Winfrith): What is it about your present design that has given this big improvement in doubling time? Your previous study showed a breeding gain of 1.08 with supereritical steam. Have you reduced the ratio of hydrogen to plutonium in the core from your carlier design?

Mr. Schluderberg: Yes, we have reduced the ratio. The structure of the fuel fraction is about the same.

Mr. Fell: With low-pressure steam and the much bigger pin spacing there is a greater susceptibility to a flooding accident. Have you had to take special measures, in adding resonance poisons for example, to deal with this, and have thesc been reflected in your fuel cost?

Mr. Schluderberg: We have placed nine boron carbide pins around the holes in the fuel can. The others contain fuel pins themselves. Nine, of course, isn't the upper limit; we could add 18 or 27 , or as many as you wish, to get the amount of poisoned surface to combat the flooding problem. Of course, with equilibrium plutonium fuel it tends to be minimized compared to clean plutonium.

$M r$. Webb: We intend to hold down flooding by use of the control rods.

$M r$. Häfele (Karlsruhe): Were breeding considerations the basis for selecting 1200 psi as your operating pressure?

$M r$. Schluderberg: Yes. We also wanted to see if we could gain anything on the voiding problem. At supercritical pressure the average steam density in the core was about $61 / 2 \mathrm{lb} / \mathrm{cu} \mathrm{ft}$, and now it's down to 1.8 . Obviously this is a whole lot closer to a voided condition and you should get a lower value of $\Delta k$ with this.

Mr. Häfele: I would like to point out that according to our investigations there is a serious competition between breeding and safety. The voiding coefficient becomes better the higher the pressure, so that you are in a better position safety-wise. Therefore, our design pressure is higher than 1200 psi. We at Karlsruhe are following both sodium and steam in two parallel approaches. If we give tomorrow a panel paper on sodium and not on steam it is only for the reason that the reference design on steam will not be finished until January.

Mr. Thompson (MIT) : It appears that you may add water to the injector systems or you may add superheated steam. Would you explain in a little more detail from a safety viewpoint how you would go about controlling the possible injection and flooding or, on the other hand, the voiding in a rather dynamic system like this?

$M r$. Schluderberg: The analysis of the operation of the entire plant is in a preliminary state. We believe that the controls can be designed to prevent flooding 
the core due to an overflow of feedwater from the feedwater train as compared with the steam demand. If the pressure is falling in the system, if the coefficient is positive, and if the time constants are quick enough so that this is meaningful from the standpoint of how fast the pressure is falling, the temperature will start to rise. When it does, if there is enough excess spray, you will automatically generate more steam, which will tend to reduce the rate at which the pressure falls and give you more time to get your rods in. If the coefficient is negative instead of positive, then you would try to match the feedwater to the steam demand so that sometimes you would have slightly superheated steam. We ran some pretty extensive operational analysis calculations for the supercritical plant and found that it was indeed possible to design a system where the reactor would follow the load very well and protect itself against small mismatches in feedwater to steam flow. However, we have not gotten our computer methods modified to analyze the low-pressure system, so I can't say any more about it right now.

Mr. Dalle-Donne (Karlsruhe): I would like to ask if reducing pressure increased the cost of the turbines very much or not; secondly, what were the effects on the efficiency of the plant?

Mr. Schluderberg: Reducing the pressure would increase the cost, I think. The efficiency of the supercritical 3500 - $1 \mathrm{~b}$ plant, with $1050^{\circ} \mathrm{F}$ at the high pressure and $1000^{\circ} \mathrm{F}$ reheat, should be around $43 \%$. Without the reheat provision at $1200 \mathrm{lb}$ and with the lower pressure, I believe it would drop to about $381 / 2 \%$. Of course, you then tend to have a higher pumping power requirement because you don't have as good a coolant.

$M r$. Sofer (UNC): I'd like to make the following comment: Five years ago we studied this concept for the USAEC and pretty much agreed with the results that you mentioned here, namely, that there is a reduction in capital cost. However, there were two problems. One was that we had relatively high fuel inventory which at the time didn't show up in fuel-cycle costs because we were using $4.75 \%$ charges with private ownership; this is going to be higher. The second one was the void coefficient of the coolant. We had something like plus $\$ 20$ or thereabouts for voiding effect of the coolant from the 300-MW core (the core had an $\mathrm{L} / \mathrm{D}$ of one). I think you can do better by pancaking. With a $1000-\mathrm{MW}$ reactor I somehow feel that we have a long way to go to prevent the void coefficient from being positive, unless one goes to modular core or pancakes, double pancakes, triple pancakes, etc.

$M r$. Schluderberg: Certainly you have to run the leakage up to get that coefficient down. As far as the fuel costs and inventory, I don't think that you had a pancake arrangement, so that you couldn't cool it quite as efficiently as this. Also, as in the low-pressure gas systems, you have to go to surface roughening to get that little kick in thermal performance; otherwise the clad temperatures get too high for this specific power.

Mr. Häfele: I think that the early NDA study didn't have much plutonium-240 in it and that is the key for the help with void coefficients. 


\title{
Safety and Nuclear Considerations in the Design of the FTR*
}

\author{
F. R. Astley, D. R. de Halas, L. M. Finch, P. L. Hofmann, and W. W. Litthe, Jr. \\ Battelle Memorial Instıtute \\ Pacific Northwest Laboratory \\ Richland, Washington \\ (PRESENTED BY P. L. HOFMANN)
}

\section{Introduction}

A conceptual design study for a Fast Flux Test Facility (FFTF) was started by Battelle-Northwest in April, 1965. The study was directed toward the development of a conceptual design and a budget-stage cost estimate for a comprehensive irradiation testing facility to support fuel and material research and development necessary for fast breeder power reactors. The most important component of the FFTF is the Fast Test Reactor (FTR). This paper limits discussion to some of the nuclear and safety features of the FTR.

Although it appears obvious, the principal safety consideration is the provision for the test reactor itself. Although it is possible to perform some fast-neutron tests in power reactors, the design and operational optimizations for power generation are not necessarily compatible with testing. The FTR specifically provides for test-specimen handling, ranging of reactor operating conditions, wide-span reactivity control and adjustment, extensive data sensors and data logging, changing of experimental apparatus, detection of and operation with failed test specimens, and system decontamination. Each of these provisions will and should be part of the original reactor eapability.

Safety and nuclear considerations are closely related for fast reactors. This close relation is particularly true for a fast test reactor with its requirements for inherently great accessibility and flexibility for different experiments.

The design for the F"Th is not yet beyond the conceptual stage. Indeed, enough difficult problems have been identified to warrant continued intensive conceptual design. However, a preliminary conceptual design has been formulated. As presently conceived, the FTR will be sodium cooled and $\mathrm{PuO}_{2}$-stainless steel fueled with a 600 -liter core volume. Initial operation at 300 NWt is expected to produce a peak flux of $\sim 10^{16}$ $\mathrm{n} / \mathrm{cm}^{2}$-sec at the core center. Ultimate power operation (with corresponding flux increases) of the FTR is set at 400 MWt.

\footnotetext{
* Based on work performed under IT.S. Atomie Energy Commission Contract AT (45-1)-1830.
}

The current concept provides the facilities summarized in Table 1 for experimental irradiations in and near the core. The core concept is sufficiently general and versatile to permit substantial modification of kind, size, and number of test facilities.

To accommodate these facilities, the reactor core assembly is formed by tubular fuel subassemblies situated at the region of convergence of a skewed, hexagonal array of reactor tubes. The assembly is completed by an Inconel reflector closely surrounding the core (convergence) zone and by the various special-purpose components, such as safety and shim controls, test loop tubes, and flux-monitoring devices. The reactor tubesthe essential structural elements of the core - extend vertically above and below their region of convergence as an expanding array controlled by the geometry of the skewed system. The reactor tubes function (1) as containers to locate the fuel subassemblies in preferred positions in the core, (2) as coolant conduits to convey liquid sodium upward from a supply plenum past the fuel to discharge into the sodium pool in the reactor vessel, (3) as extended guides to permit remote chargedischarge operations from the top of the reactor, and (4) as isolation barriers for closed-loop tubes to prevent intermixing of primary reactor coolants with special coolants used for closed-loop experiments.

Radiation-damage effects leading to dimensional change or nonrecoverable loss of ductility in materials of construction were of concern in the FTR conceptual design. In the intense, high-energy FTR spectrum, both dislocations and hydrogen generation in nickel-bearing steels could lead to the loss of durtility. High operating temperatures would aggravate the effect. Therefore, major consideration was given to reducing the intensity of the damaging flux $(\mathrm{F}>0.1 \mathrm{MeV})$ incident on critical, nonremovable structural components. The design concept utilizes sufficient core shielding to reduce the flux intensities on the reactor vessel to levels tolerable for the design lifetime of the reactor. This shielding serves also as the neutron reflector.

Figure 1 shows a schematic of the FTR, including the prossure-containment vessel and the fuel-handling 
TABLE 1. FTR Fxperimental Facilities (also see Fig. 3)

\begin{tabular}{l|c|c}
\hline & Initial & Ultimate \\
\hline In-core Test Facilities & & \\
Closed-loop Fuel Tests, 6 in. & 2 & 4 \\
Closed-loop Fuel Tests, 4.5 in. & 2 & 2 \\
Packaged-loop Material Tests, 4.5 & 1 & 2 \\
in. & 2 & 2 \\
Open-loop Fuel Tests, 6 in. & 4 & 8 \\
Open-loop Fuel Tests, 4.5 in. & & \\
Reflector Test Facilities & 8 & 4 \\
Open-loop Material Tests & 0 & 4 \\
Packaged-loop Material Tests & 1 & 1 \\
Rapid Transit (Rabbit) Test & \\
\hline
\end{tabular}

machine. Figure 2 details the skewed-tube arrangement in the reactor. Figure 3 shows a cross section of the initial core arrangement, giving some indication of the complexity.

During the current development of the FTR conceptual design, a large number of parametric studies and safety analyses provided information for the selection of a reference core. It is clear that the optimum reactor size must be a compromise among safety of conducting fuel tests, scope of irradiation test problems to be investigated, number of tests to be conducted simultaneously, time available to do the testing, size of test subassemblies, and available capital and operating funds.

In nuclear design, four major objectives can be identified:

1. The FTR should have the ability of sustaining very high fast-flux levels, about $10^{16} \mathrm{n} / \mathrm{cm}^{2}$-ser above $0.1 \mathrm{MeV}$.

2. Ample control margins must be available to acconmodate low-reactivity test assemblies and to allow for substantial driver-fuel burnup.

3. Inherent safety features must be provided. Specifically, in-core loop design must be such that failure of test assemblies can be tolerated without damage to the reactor.

4. Sufficient alternative nuclear-design variants should be available so that changes in specific power and flux spectrum and the utilization of different driver fuels can be accomplished with relatively minor modifications, and without adverse effects on the safety features of the reactor.

\section{Driver Fuel, Flux Intensity, and Flux Spectra}

\section{A. Fissile-fuel Choices and Flux Level}

The average flux level $\phi$ in a fast reactor is given by

$$
\phi=C P / L \sigma_{f},
$$

where $P$ is the volumetric power density, $L$ the fissilefuel density, $\sigma_{f}$ the average microscopic fission cross section, and $C$ a conversion factor. Clearly, a high flux requires a high specific power $(\Lambda \mathrm{IW} / \mathrm{kg})$ and a low fission cross section. The high $\eta$ value of $\mathrm{Pu}^{239}$, compared with that of $\mathrm{U}^{235}$, results in relatively low fuel inventories and makes plutonium the preferred fuel for the FTR. These relations are illustrated in Figure 4 and 5 for a range of reactor sizes.

In Figure 4 the critical fuel density is shown as a function of core diameter for $\mathrm{PuO}_{2}$-SS and $\mathrm{UO}_{2}$-SS cermet cores. All calculations were made with a one-dimensional (radial) transport model with a buckling correction for axial leakage. The marked decreased in required critical inventories between the $\mathrm{Pu}$ and $\mathrm{U}^{235}$ cases is apparent. For a $600-\mathrm{l}$ core the $\mathrm{Pu} / \mathrm{U}$ inventory ratio is about 0.6 .

Figure 5 represents similar data in a somewhat different form. To obtain a large number of additional data points, a fundamental-mode model was used to compute the flux-to-power ratios for a wide range of fuel compositions. This ratio is shown as a function of the fundamental-mode radius $\left(R=\pi / \sqrt{B^{2}}\right)$.

Even though the control problem in a plutonium $(\beta \sim 0.0021)$ core is more difficult than in a $\mathrm{U}^{235}(\beta \sim$ 0.0064 ) fueled core, the flux advantage of the plutonium is so large that plutonium is presently considered to be the reference fuel for the FTR. For typical FTR core sizes, a one-third power density increase in the $\mathrm{U}^{235}$ core would be required to achieve the same flux level as that of the plutomium core; stated differently, for the same power density the $\mathrm{U}^{235}$ core would have a flux level $\sim 25 \%$ less than for the plutonium core. If the volumetric power density of the plutonium core is limiting, then the $U^{235}$ core volume would have to be nearly doubled to achieve the same flux level as that of the plutonium core. Hence, the incentives for adopting plutonium as the reference fissile material are high.

It should be mentioned that Figures 4 and $\tilde{5}$ are not completely consistent. The slight inconsistency is caused by the reflector moderation which, of course, cannot be incorporated in the fundamental-mode model.

\section{B. Core Size and Power Level}

The selection of core size and power level is not purely a problem of nuclear design. A variety of requirements enter into the selection process. Important among these are provision for adequate test space and required flux level.

To obtain the necessary relations among flux, power level, and core size, a series of static calculations were conducted for different cylindrical core volumes and length-to-diameter (L/D) ratios. Although the mechanical design called for a skewed core, the $4^{\circ}$ skew angle 


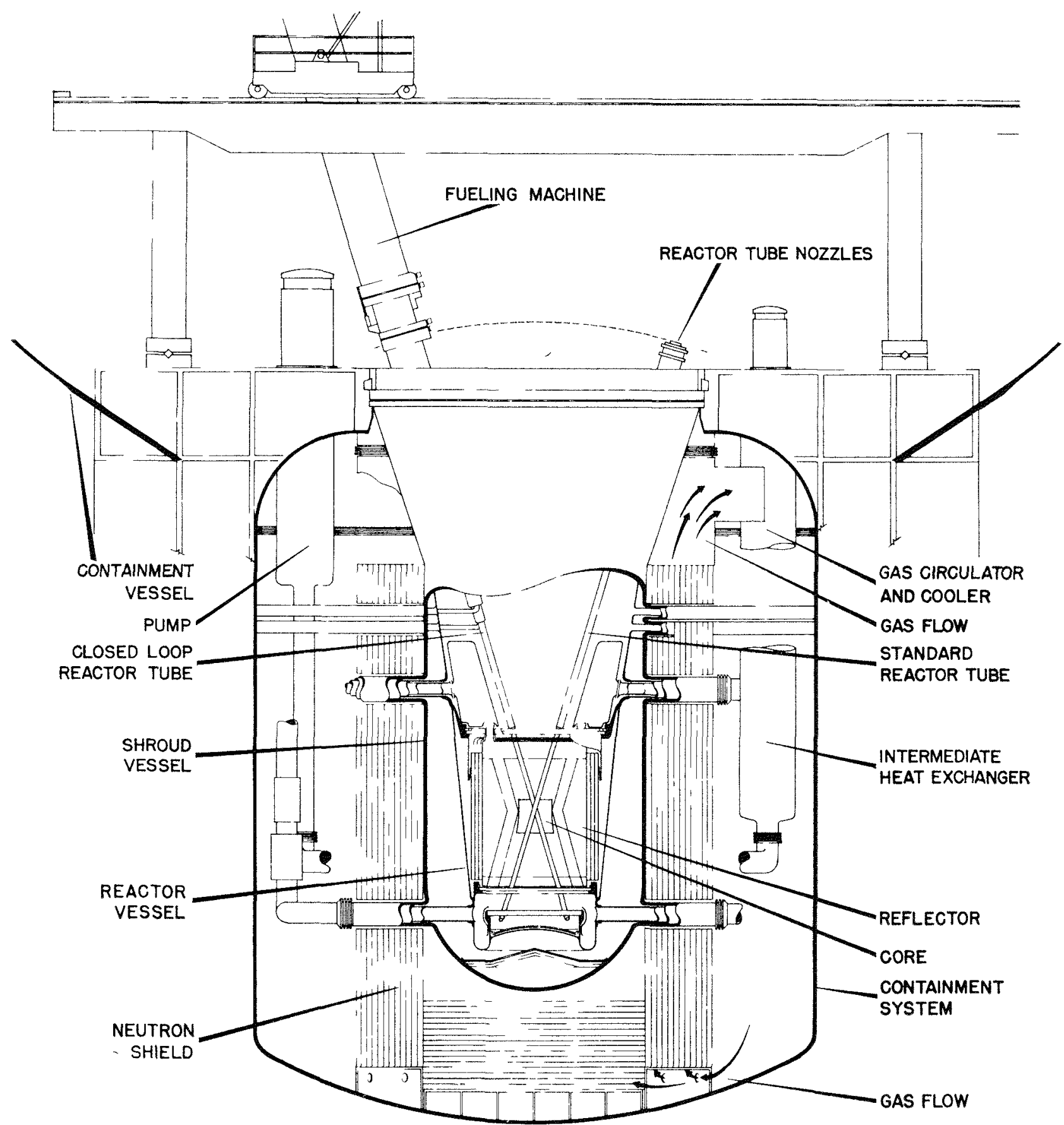

Fig 1. Reactor Section through FTR.

is so small that equivalent cylinders could be used to represent the cores adequately.

The calculations showed only a very slight dependence of fuel loading on L/D ratio. More importantly, as core volume is increased, the neutron leakage is reduced, and the volumetric fuel densities decrease from about $1 \mathrm{~kg} / \mathrm{l}$ for a 200 -liter core to about $1 / 2 \mathrm{~kg} / \mathrm{l}$ for an 800 liter core. Hence, for a constant flux level, while the required power increases as the volume of the core is made larger, this increase is not in direct proportion to core volume. Figure 6 illustrates these relations. Larger core volumes are gencrally advantageous, because they provide more test volume space. As a consequence of reduced fuel-centerline temperatures and temperature gradients, increases in system reliability, and hence core safety, can also be achicved. 


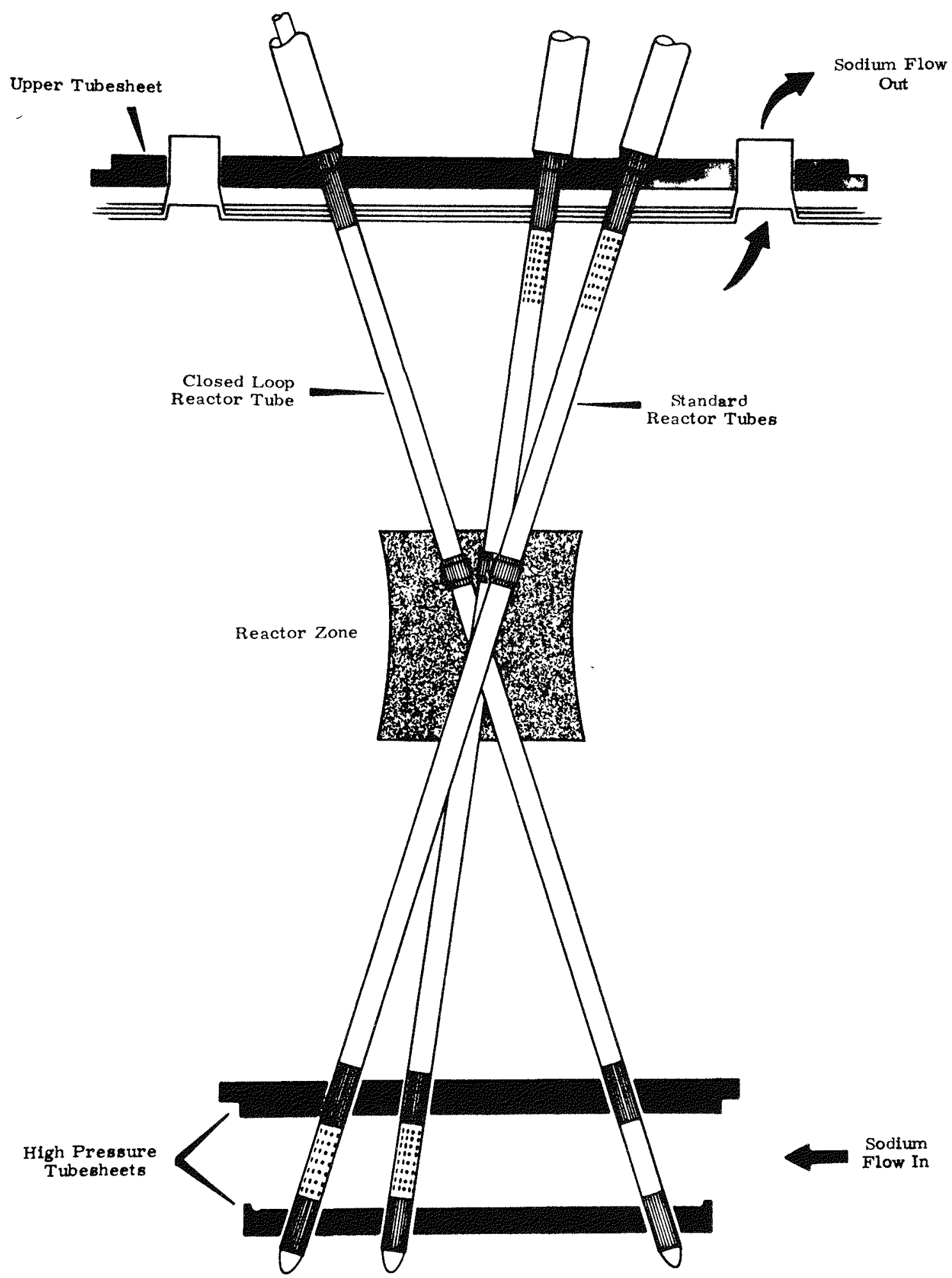

FIG. 2. Schematic of Tubed Core.

The present reference core has a $600-1$ volume. Cores of this size are still compact enough to result in rather hard neutron-flux spectra. The neutron-flux spectrum in the center of some typical FTR cores is shown in
Figure 7 for 5, 7, 9, and $11 \mathrm{v} / \mathrm{o} \mathrm{PuO}_{2}$ cores. As one would expect, the spectrum becomes harder as the fuel volume fraction is increased. This hardening causes the fissile fuel and structural material cross sections to decrease 

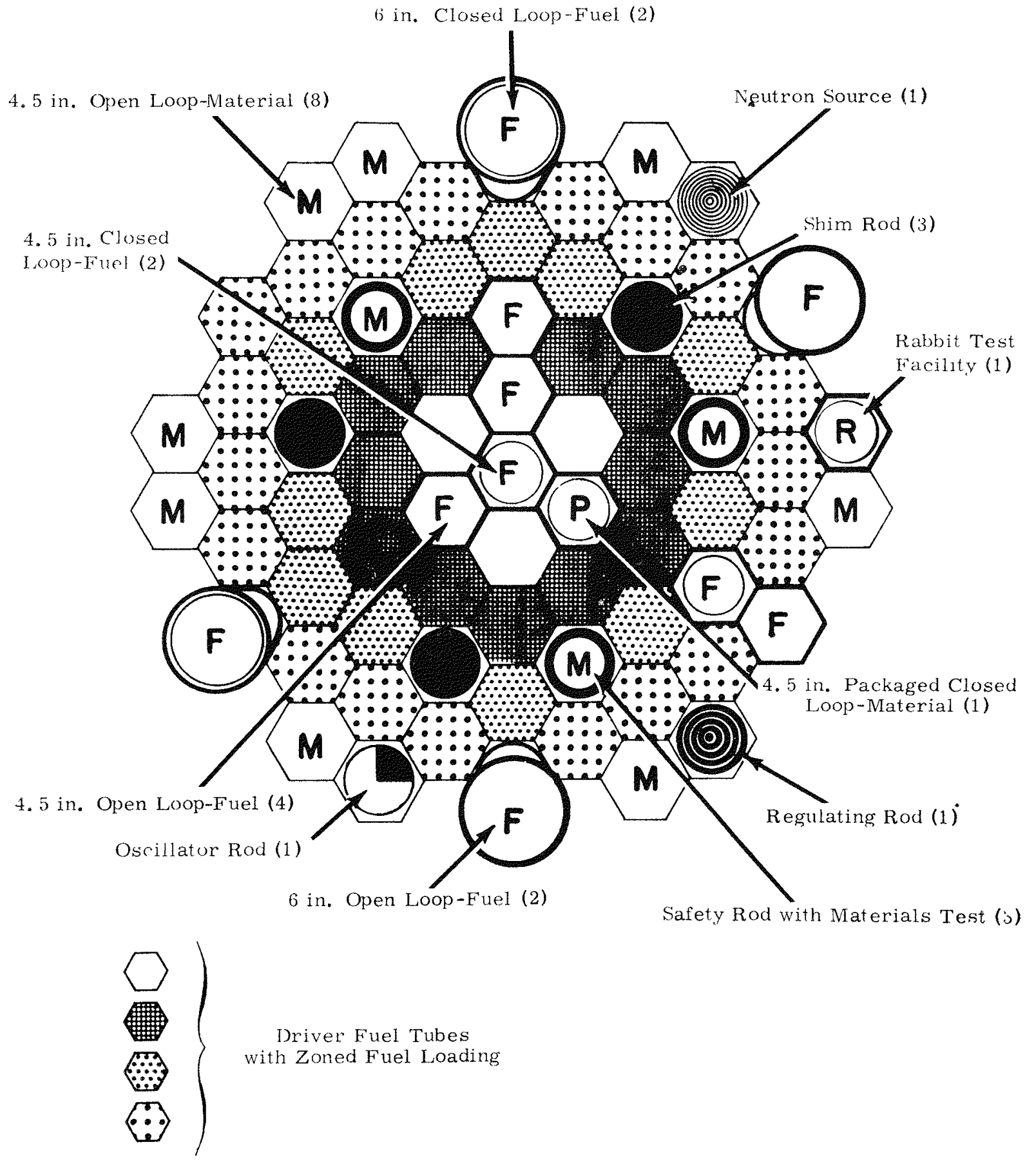

FIx. 3. Core Section, Initial Case.

and the fertile fuel cross section to increasc. For $5 \mathrm{v} / \mathrm{o}$ $\mathrm{PuO}_{2}$, more than 0.7 of the total flux is above $0.1 \mathrm{MeV}$; for $9 \mathrm{v} / \mathrm{O} \mathrm{PuO}_{2}, 0.8$ of the flux is over $0.1 \mathrm{MeV}$. Figure 8 compares the FTR spectrum with spectra from some typical fast breeder reactors.

The fuel is zoned in the present 600-1 reference core to flatten the power distribution. This zoning has only a very small effect on the spatial flux distribution. Some of the characteristics of this core are tabulated in Table 2.

\section{Control Statics}

Provision for adequate control of the FTR presents another important problem for nuclear design and safety. The FTR control requirements are much more stringent than for a typical breeder reactor. Require- 


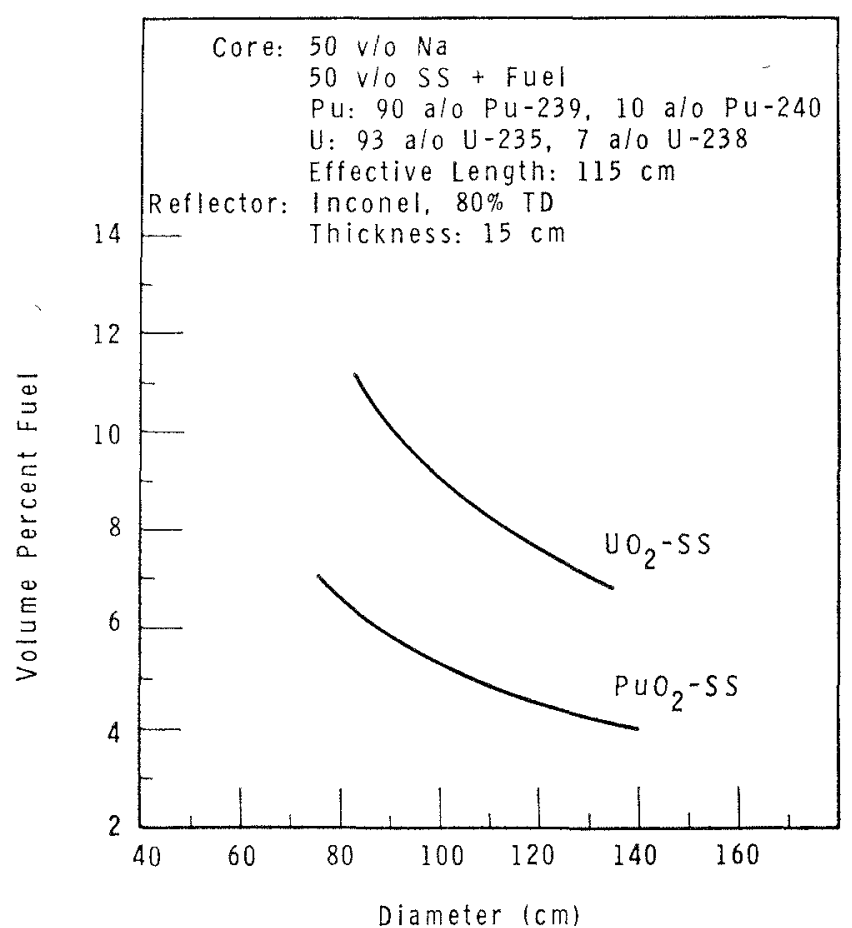

FI(x. 4. Fuel Loading vs Core Size for Cermet Cores.

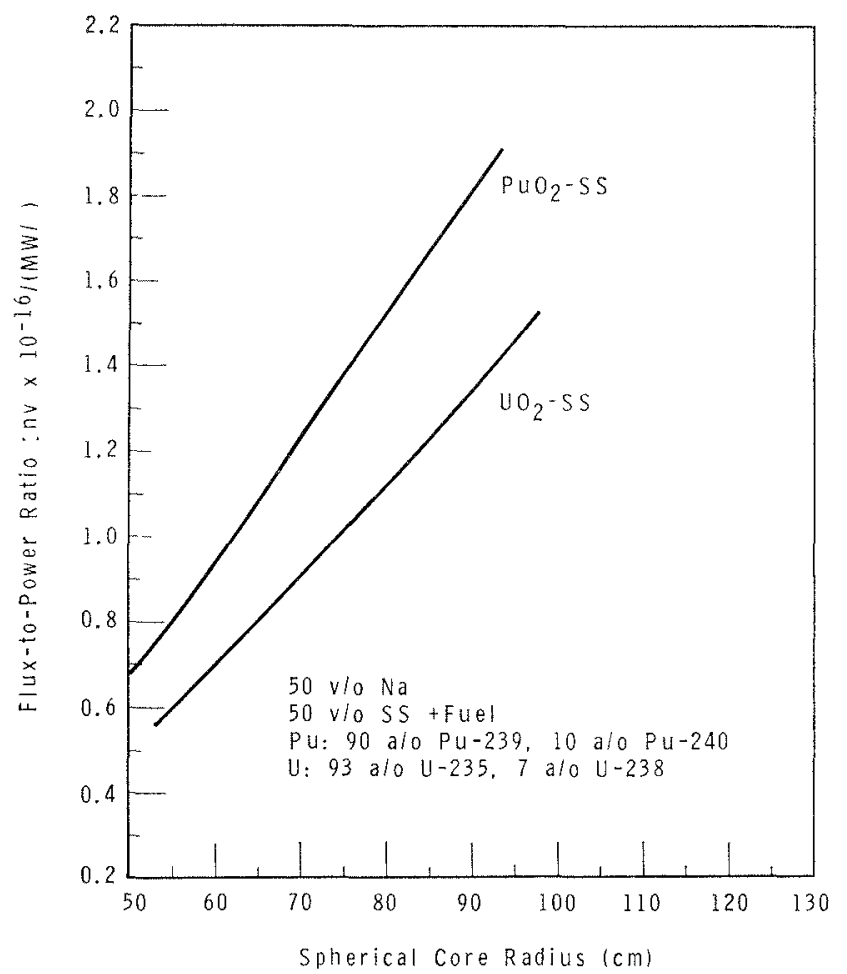

Fuc. 5. Flux-to-Power Ratio vs Reactor Size with Cermet, Cores.

ments as to burnup, temperature defect, and shutdown margin add to about $10 \% \Delta \mathrm{k}$. Additional control requirements would arise if low-reactivity test elements were to be accommodated. These latter requirements would probably have to be handled by rearrangement of driver fuel rather than by the merhanical system for reactor control.

Since the concentration of fertile fuel $\left(\mathrm{Pu}^{240}\right)$ is quite small and the buildup of fission products has a very small effect of reactivity, estimates of fuel life can be made fairly easily. If we consider only the burnup of the fissile nuclide,

$$
\delta k \sim\left\{\frac{\eta-1}{\eta}\right\} \frac{\delta M}{M}
$$

where $\delta k$ is the variation in reactivity, $\eta$ the effective eta of fuel, $M$ the mass of fissile fuel, and $\delta M$ the variation in fissile fuel mass. With an $(\eta-1) / \eta$ value of about 0.6 for a six-week operating cycle for FTR, the control margin required for burnup is of the order of $\sim 0.04 \Delta k$. This simple approach for the determination of burnup requirements has been checked with one-dimensional burnup calculations, and the agreement is quite satisfactory.

The FTR cermet cores have an overall negative temperature defect of $\sim 0.015 \Delta k$, made up of a sodium coolant and fuel expansion coefficient (see section IV).

Adding a shutdown margin of $0.045 \Delta k$ results in an overall control requirement of $10 \% \Delta k$.

\section{A. Reflector Control}

The possibility of reflector control was given serious consideration during the initial FTR conceptual design phases. Reflector control has the advantage of yiclding an uncluttered core design and of providing more test space in the core. It has the additional advantage of making it possible to utilize many relatively low-worth control elements in a fairly accessible area.

A series of rather detailed transport calculations were made to ascertain the margins for available reflector control. Idealized calculational models for rotating drums as well as for translating reflector control elements were formulated. A nickel/ $\mathrm{B}_{4} \mathrm{C}$ exchange was found to give the largest control swing.

As soon as practical engineering designs were considered in more detail, the control swings calculated for the fairly idealized models tended to decrease. This decrease arises because control elements must be kept reasonable in size, cannot be spaced too tightly around the core, and cannot be placed adjacent to the core.

For ITR core sizes, practically attainable reflector control margins seem to be about $\sim 0.05 \Delta k$. Compared to the $0.10 \Delta k$ requirements, these margins are inadequate, and hence reflector control was abandoned.

\section{B. In-core Rod Control}

To achieve an adequate reactivity swing in the FTR, in-core rod control has been adopted as the preferred 


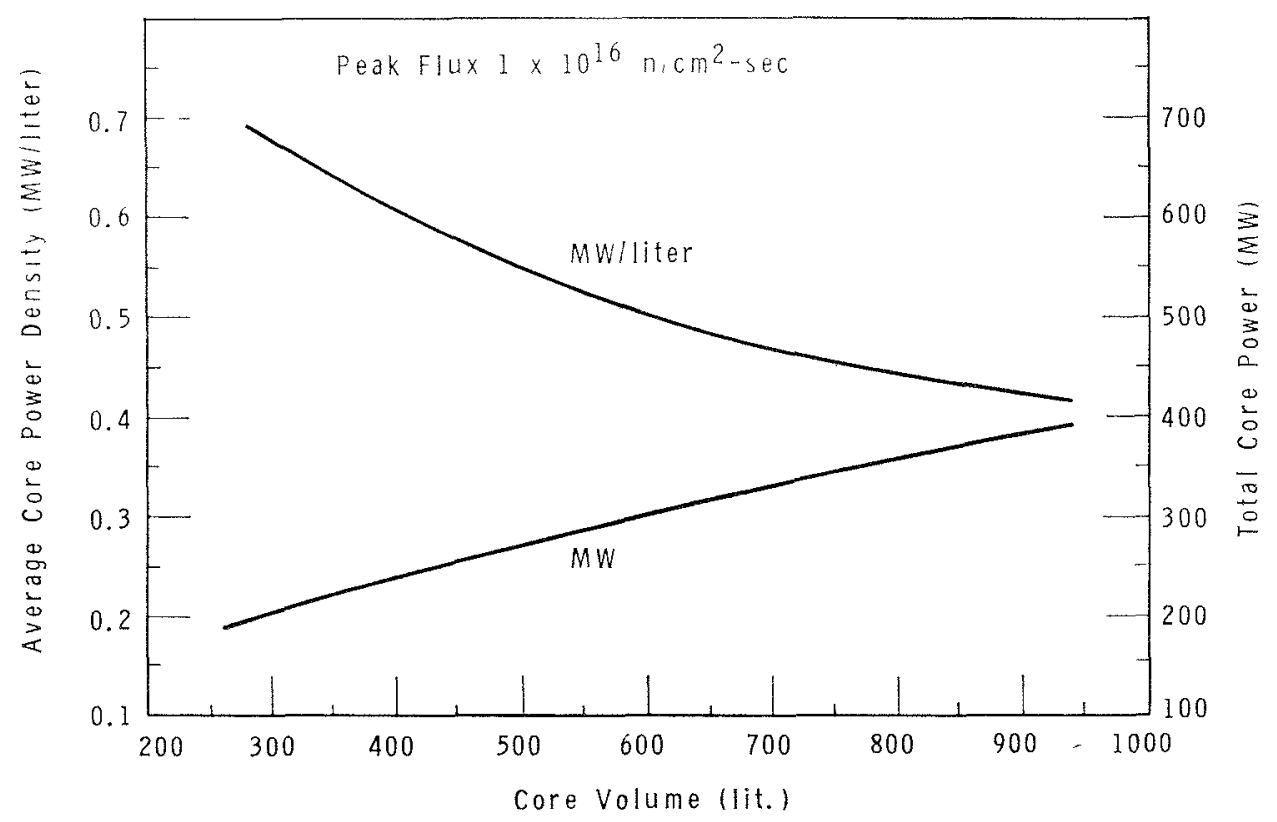

Fic. 6. Volume, Power, and Power-Density Relations.

control mode. In principle, one can use either fuel rods or poison rods for this purpose. Because movable fuel rods might present complex mechanical and cooling problems, major emphasis has been placed on the analysis of poison rods.

Even though the control rods will be placed in eccentric reactor positions, evaluation of central rod worth coupled with perturbation theory can be used to obtain estimates of off-center rod worths.

One-dimensional transport calculations were used to estimate the worth of centrally located $\mathrm{B}_{4} \mathrm{C}$ rods. In Figure 9 , the central rod worth as a function of $\mathrm{v} / \mathrm{o} \mathrm{B}_{4} \mathrm{C}$ is shown. A complete $\mathrm{Na} / \mathrm{B}_{4} \mathrm{C}$ interchange in the central zone causes a reactivity change of approximately 0.067 . This reactivity change is significantly greater than that raused by interchanging driver fuel $\left(7 \mathrm{v} / \mathrm{o} \mathrm{PuO}_{2}, 43\right.$ $\mathrm{v} / \mathrm{oSS}$, and $50 \mathrm{v} / \mathrm{o} \mathrm{Na}$ ) and sodium in the central region. For the fuel-sodium interchange, $\delta k \sim 0.018$.

To estimate the worths of off-center rods, perturbation theory can be used to obtain space-dependent reactivity coefficients of the component materials. The results of these calculations are shown in Figure 10.

The present design calls for three shim and three safety rods (plus one small power-regulating rod) with a reactivity worth of 2 to $2-1 / 2 \% \Delta k$ rod. This appears achievable with partially enriched boron carbide rods. Under normal operating conditions, the six control rods would provide the reactor with a very large shutdown margin. To guard against gross loading errors, it is presently proposed that a Nuclear Proof Test Facility (NPTF) be operated in conjunction with the FTR. This facility would simulate the reactor with the test ele- ments in it and would be used to preview new test elements before insertion in to the FTR.

\section{Temperature Coefficients and Reactor Kinetics}

\section{A. Temperature ('oefficients}

In the FTR cermet cores, the sodium coolant coefficient and the fuel expansion coefficient contribute to the overall negative temperature defect. The high-leakage FTR, in contrast with large breeder reactors, has a negative sodium void cocfficient because the effect of leakage variation predominates over the effect of spectrum variation. The effect of complete sodium voiding was calculated for a range of FTR core sizes and corresponding $\mathrm{PuO}_{2}$ fuel fractions. Sodium loss caused an apprecjable decrease for all cases considered. For instance, for an 80-cm-diameter core, containing initially $50 \mathrm{v} / \mathrm{o} \mathrm{Na}, 7 \mathrm{v} / \mathrm{o} \mathrm{PuO}_{2}$, and $43 \mathrm{v} / \mathrm{o}$ SS, complete sodium loss resulted in a reactivity decrease of $0.0925 \Delta k$. Furthermore, the spatial sodium worth is positive throughout the core.

It was also found that the effect of sodium removal is almost exactly a linear function of the amount removed. Hence, it is a fairly simple matter to translate the sodium void coefficients into sodium temperature coefficients. For the reference design this coefficient is about $-1 \phi /{ }^{\circ} \mathrm{K}$.

Another important temperature coefficient contributing to the overall safety of the core is the cermet fuel expansion coefficient. Both radial and axial core expansion coefficients were calculated. For typical FTR configurations, the axial fuel expansion coefficient is about $-0.5 \phi /{ }^{\circ} \mathrm{K}$. 


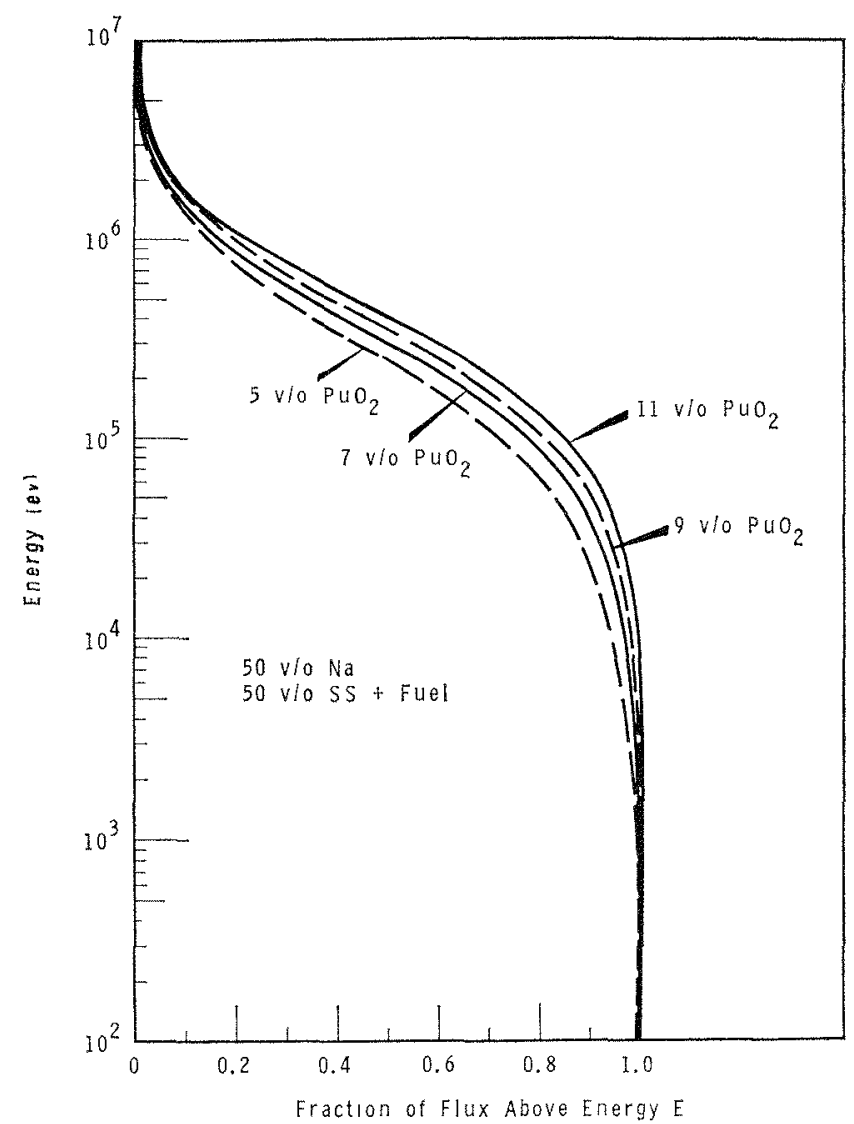

Fí. 7. Flux Spectra for Various Cermet Cores.

Based on the expansion coefficients shown above, the net temperature defect associated with sodium and fuel expansion is about $1.5 \% \Delta k$.

Since the FTR, as presently envisioned, is a fully enriched, hard-spectrum core, Doppler coefficients are expected to be small. The Doppler coefficients were calculated for a series of FTR configurations by means the Russian Cross Sections Set. Positive values of $T(d k / d T)$ from $\sim 6 \times 10^{-4}$ to $4 \times 10^{-4}$ were calculated for a range of $\mathrm{PuO}_{2} \mathrm{v} / 0$ from 5 to 9 . This is a rather small value, less by about a factor of 20 than the axial fuel expansion coefficient, and would therefore not appear to present a serious safety problem.

\section{B. Response to Ramp Reactivity Insertions}

The meltdown of a fuel subassembly in a central test zone could be considered a credible accident in the FFTF. This argument stems from the fact that test fuel is precisely that-test fuel-presumably with less reliable safety characteristics than driver fuel.

The reactor response to various fuel slumping rates (simulated by reactivity ramps) has been computed under highly idealized conditions. The objective was simply to show the approximate severity of a problem (fuel slumping) which is perhaps of more importance in a fuel test reactor than in a large breeder reactor.
The reactor power as a function of time is computed from the kinetic equations and the reactivity expression

$$
\rho(t)=\text { at }-b[T(t)-T(o)],
$$

where $t$ is the time, $a$ the rate of reactivity insertion, $b$ the reactivity shutdown coefficient, and $T$ the temperature of fuel. The time-dependent fuel temperature is obtained from the simple heat balance

$$
\frac{d T}{d t}=\frac{P}{C_{p}}-K T,
$$

where $C_{p}$ is the heat capacity and $K$ the reciprocal of the thermal time constant.

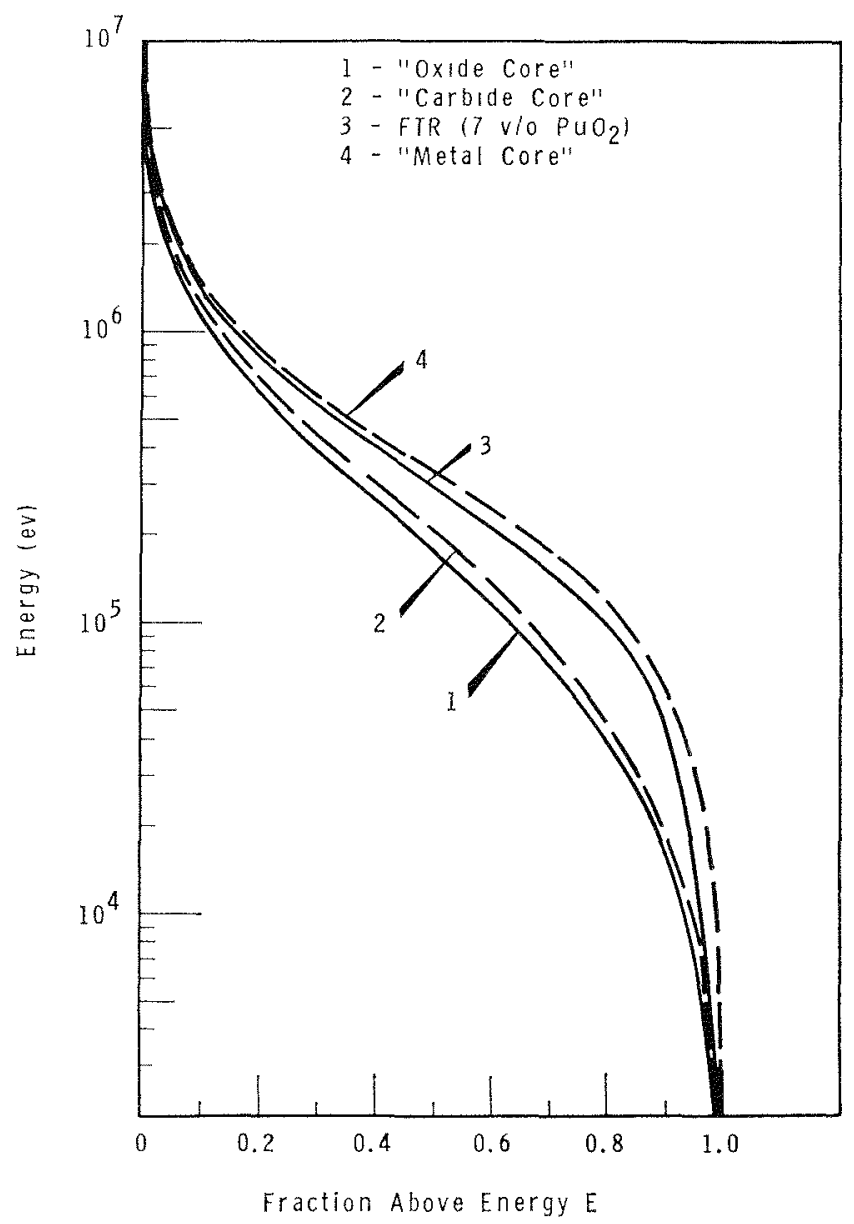

Fix. 8. Comparison of Typical Core Spectra.

\begin{tabular}{|c|c|c|c|c|}
\hline & \multicolumn{4}{|c|}{ Core Region } \\
\hline & $I$ & $I I$ & $I I I$ & $I V$ \\
\hline Number of Fuel Subassemblies & 3 & 10 & 11 & 21 \\
\hline $\begin{array}{l}\mathrm{PuO}_{2} \text { in Cermet }(70 \% \text { TD parti- } \\
\text { eles), v/o }\end{array}$ & 17.7 & 19.8 & 22.1 & 27.7 \\
\hline $\begin{array}{l}\mathrm{PuO}_{2} \text { in Cermet }(90 \% \text { TD parti- } \\
\text { cles), v/o }\end{array}$ & 13.7 & 15.4 & 17.2 & 21.6 \\
\hline$\phi(E>0.1 \mathrm{meV}) / \phi($ Total $)$ & 071 & 0.71 & 0.70 & 0.67 \\
\hline
\end{tabular}

TABLe 2. Zoned Reference Core 
The results are shown in Figures 11 for reactivity ramps from 1 to $\$ 50 /$ sec. A few general observations can be made from these results. First, the rate of reactivity insertion must be less than about $\$ 5 / \mathrm{sec}$ to avoid severe power excursions. The rate of reactivity insertion caused by a meltdown and gravity collapse of the upper one-fourth of a central fuel subassembly is estimated to be given by

$$
a(\$ / \mathrm{sec}) \sim 450[\delta M / M],
$$

where $\delta M / M$ is the fraction of fuel in the central subassembly. Therefore, to preclude a serious power excursion in this hypothetical accident, only about $1 \%$ of the fuel inventory should be placed in a central test region. Test elements of larger reactivity worth an be handled in off-center loops.

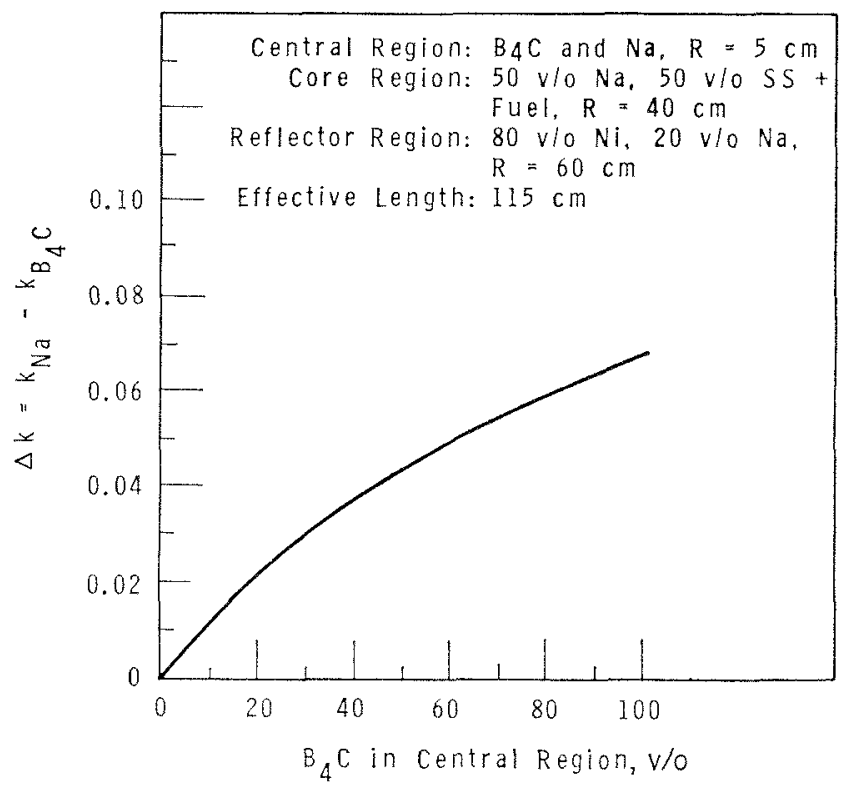

Fig. 9. Worth of Central $\mathrm{B}_{4} \mathrm{C}$ Rod.

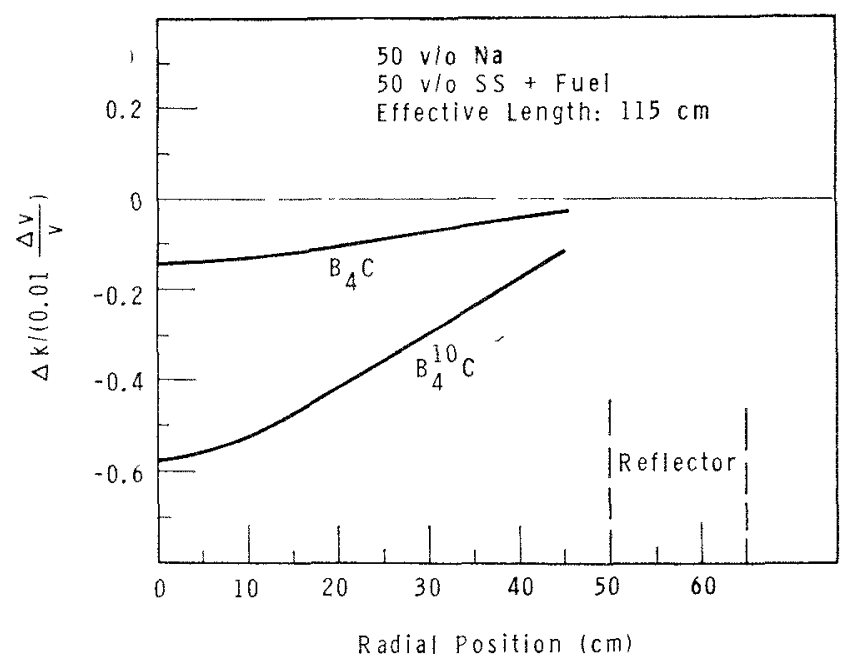

F1(x. 10. Radial Dependence of Poison Worth.

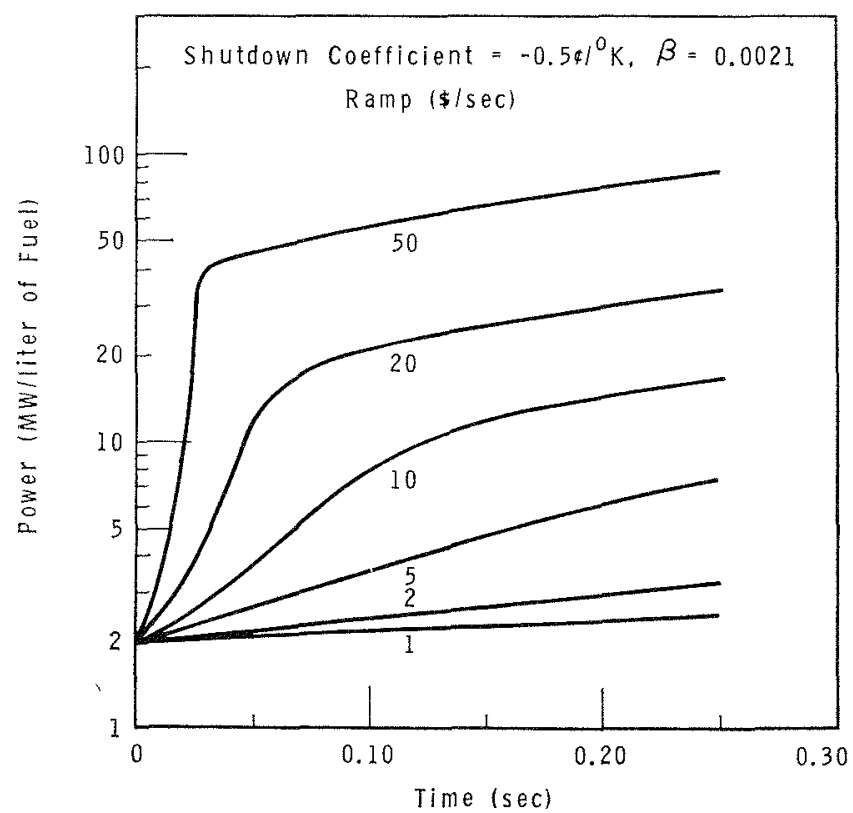

FIg. 11. Power Profiles for Various Reactivity Ramps.

\section{Nuclear Design Alternatives}

Two major alternatives of the nuclear design are receiving considerations:

1. alternative driver-fuel concepts;

2. possible use of $\mathrm{BeO}$ for modification of the core spectrum.

\section{A. Alternative Fuel Concepts}

Although $\mathrm{PuO}_{2}$-SS cermets are the preferred FTR driver fuel at the present time, alternative fueling concepts are being actively investigated. Chief among them are $\mathrm{PuO}_{2}-\mathrm{U}^{238} \mathrm{O}_{2}$ ceramics as driver fuel. The presence of $\mathrm{U}^{238}$ would increase the reactivity life of the core, would result in a net negative Doppler coefficient, and would increase the effective delayed-neutron fraction. Furthermore, it would offer the opportunity of attaining higher driver-fuel temperatures. On the other hand, the fuel expansion coefficient would be less than for the cermet, and the sodium coefficient might be adversely affected. The possibility of fuel rearrangement in the ceramic might also have undesirable effects on reactor safety, although it is believed that fuel relocation can be made negligible by proper design. In addition, the flux-topower ratio in the ceramic core is less than for the cermet core. Comparisons of fuel inventories and flux-to-power ratios for the cermet and ceramic cores are shown in Figures 12 and 13.

To examine the effect of $U^{238}$ on reactor lifetime, a zero-dimensional burnup code was used. The total fuel volume fraction (fertile and fissile) was held constant while the ratio of fertile to fissile fuel was varied. For cermet cores, containing large amounts of stainless steel 


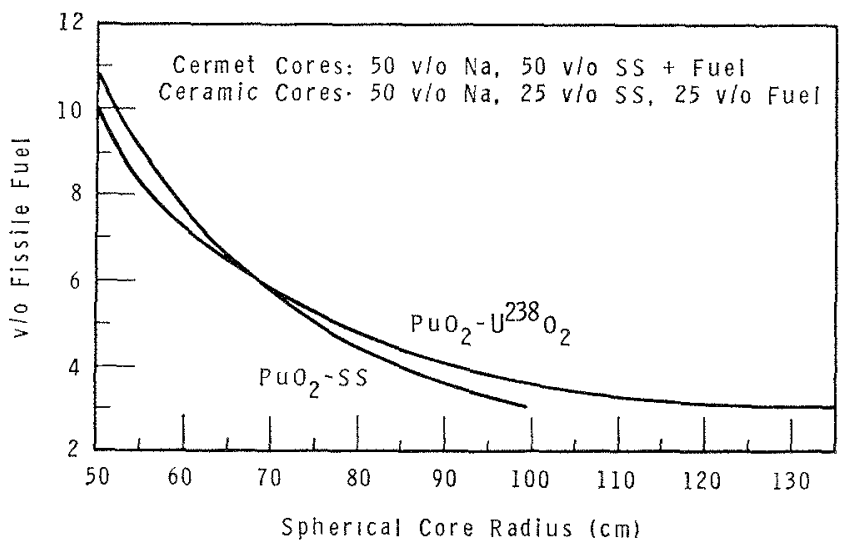

FI (i. 12. Fuel Loading vs Core size for Cermet and Ceramic Cores.

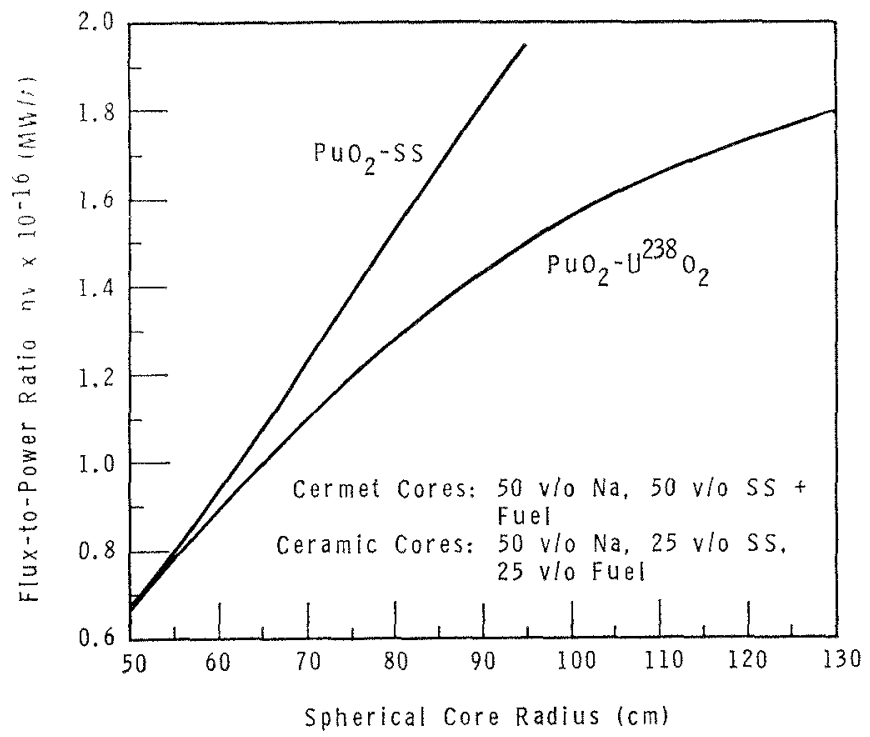

Fra. 13. Flux-to-Power Ratio vs Reactor Size for Cermet and Ceramic Cores.

$(\sim 40 \mathrm{v} / \mathrm{o} \mathrm{SS})$, the addition of $\mathrm{U}^{238}$ had very little effect on reactor life. If the stainless steel content can be decreased as in a ceramic core, some increases of reactivity lifetime can be achieved.

Although a cermet core does not offer any great potential for increase of reactivity lifetime by the use of $\mathrm{U}^{238}$, the addition of this resonance absorber may, nevertheless, be of interest in counteracting the effects of the positive plutonium Doppler coefficient. Calculations indicate that a fertile-to-fissile ratio of about $1 / 2$ would result in an overall zero Doppler coefficient for FTRtype cores. Although this addition of $\mathrm{U}^{238}$ is relatively modest, it might possibly have a detrimental effect on the physical endurance of the cermet.

\section{B. Use of BeO for Spectrum Softening}

For certain test situations, it may be desirable to soften the FTR neutron spectrum. Since beryllium is an excellent moderator, BeO could be used for this purpose. As $\mathrm{BeO}$ is added to the core, the required driverfuel inventory decreases and the specific power increases. For HTR core sizes, the replacement of $10 \mathrm{v} / \mathrm{o}$ SS by $10 \mathrm{v} / \mathrm{o} \mathrm{BeO}$ results in a fissile inventory reduction of about $30 \%$. Since the fission cross sections increase in the softer-spectrum core, this $30 \%$ reduction results in the overall flux increase of only about $10 \%$. However, the fast flux (that ahove 0.1 MeV) is redured in the $\mathrm{BeO}$ core.

A possible problem that could arise with the introduction of $\mathrm{BeO}$ into the core is the effect on the Doppler coefficient. Since the core is fully enriched, BeO tends to increase the positive Doppler coefficient of plutonium. This increase is a factor of about three for a core with $10 \mathrm{v} / \mathrm{O} \mathrm{BeO}$.

\section{Conclusion}

To summarize: a conceptual design for a Fast Flux Test Reactor has been formulated.

Steel cermets are presently considered to be the FTR reference fuel, because of their expected reliability and desirable safety features.

Plutonium is considered to be the reference fissile fucl, because it yields the highest flux achievable for a given power density.

In-core control with poison rods has been selected because it can supply the large control margins necessary for the operation of a test reactor.

The present design must be considered to be tentative. It is flexible enough so that many alternative approaches can be incorporated if the current investigations should show that this is necessary.

\section{APPENDIX: Methods and Cross Sections}

\section{A. Methods}

Three types of calculation routines have been used in the reactor statics studies. For broad-scale survey work, a simple fundamental-mode calculation has been used. This routine is part of the Battelle-Northwest code CRUNCH. ${ }^{(1)}$ The same point reactor formalism has also been applied in the calculations of driver-fuel burnup by means of the PYRE ${ }^{(2)}$ code.

For most of the design calculations, particularly those involving considerable geometric detail, one- and two-dimensional transport codes DTK and DDK of Los Alamos Scientific Laboratory were used.

For the reactor kinetics calculations the kinetic code KINETICS-I ${ }^{(3)}$ was used.

\section{B. Neutron Cross-section Sets}

In the earlier calculations, the 16-group HansenRoach fast reactor cross-section set ${ }^{(4)}$ was employed. In 
TABLE A-1. Effective Multiplication Constant* (Normalized to ANL set)

\begin{tabular}{|c|c|c|c|c|}
\hline \multirow{2}{*}{$\begin{array}{c}\text { Cross-section } \\
\text { Set }\end{array}$} & \multicolumn{4}{|c|}{$\mathrm{PuO}_{2}, \mathrm{v} / \mathrm{o}$} \\
\hline & 4 & 5 & 7 & 9 \\
\hline ANL-224 & 1.0 & 1.0 & 1.0 & 1.0 \\
\hline Hansen-Roach & 1.002488 & 0.9968790 & 0.9940673 & 0.9951723 \\
\hline YOM & 1.027956 & 1.028288 & 1.028095 & 1. 027783 \\
\hline
\end{tabular}

$* \mathrm{Na}=50 \mathrm{w} / \mathrm{o} ; \mathrm{SS}=(50-\mathrm{v} / \mathrm{o} \mathrm{Pu})$.

Table A-2. Material Buckling (B2) $\times 10^{-13} *$ $\left(k_{\mathrm{eft}} \mathrm{f}^{3}=1.00\right)$

\begin{tabular}{l|c|c|c|c}
\hline \multirow{2}{*}{$\begin{array}{c}\text { Cross-section } \\
\text { Set }\end{array}$} & \multicolumn{5}{|c}{$\mathrm{PuO}_{2}, \mathrm{v} / \mathrm{o}$} \\
\cline { 2 - 5 } & 4 & 5 & 7 & 9 \\
\hline ANL-224 & 1.393335 & 1.774857 & 2.522777 & 3.271783 \\
Hansen-Roach & 1.400043 & 1.764797 & 2.497220 & 3.245591 \\
YOM & 1.473527 & 1.870461 & 2.647579 & 3.426025 \\
\hline
\end{tabular}

$* \mathrm{Na}=50 \mathrm{v} / 0 ; \mathrm{SS}=(50-\mathrm{v} / \mathrm{oPu})$.

the later calculations-those more closely directed toward the Conceptual Design - the recent 22-group ANL fast cross-section set (ANL-224) was employed. For typical FTR sizes and compositions, these two sets gave very similar results. Some comparison calculations were also carried out with the original YOM set.

Table A-1 gives the fundamental-mode multiplication constants produced by the three sets for various fuel compositions. For each fuel composition, the critical material buckling from the ANL-224 set was employed. The variation in material buckling is shown in Table A-2.

\section{REFERENCES}

1. W. W. Little, Jx. and R. W. Hardie, CRUNCH-A Group Collapsing Code for Fast Reactor Analysis, BNWL-84 (April 29, 1965).

2. W. W. Little, Jr. and R. W. Hardie, PYRE-A Multigroup Burnup Code for Fast Reactors, BNWL-54 (April 1965).

3. W. W. Little, Jr., KINETICS-I-A Neutron Kinetics Code in FORTRAN IV, BNWL-111 (June 12, 1965).

4. G. E. Hansen et al., Los Alamos Group-averaged Cross Sections, LAMS-2941 (July 1963).

\section{Discussion}

Mr. Okrent (ANL): How much reactivity is available in the plutonium oxide-stainless steel cermet between operating temperature and failure point, wherever you deem this, as it heats up in the transient? If one is thinking in terms of a transient in which one relies on expansion of this fuel to terminate the rapid transient, how much reactivity is available before you have failed the fuel?
Mr. Hofmann: The peak temperatures at which the fuel runs is about $600^{\circ} \mathrm{C}$ and for the kind of ramp rate we have calculated the rise is about $100^{\circ} \mathrm{C}$. In other words, we would then be up to about $700^{\circ} \mathrm{C}$, and $700^{\circ} \mathrm{C}$ is well below melting of steel which is around $1400^{\circ} \mathrm{C}$.

$M r$. Okrent: As I recall, it's about $0.1 \% k$ for $100^{\circ}$ rise in fuel temperature. If one assumes that one can heat up $500^{\circ}$, let's say, that would be half a percent $\delta k$ to failure of the fuel.

Mr. Hofmann: But I'm not heating up 500.

Mr. Okrent: And you have a delayed fraction of $0.2 \%$. So that means that if you had a rapid reactivity insertion of about $0.7 \% \delta k$, you would have no more prompt negative feedback from the fuel, is that correct?

Mr. Hofmann: I don't really know what you are saying. The fuel is not expanding any more in the transients we studied.

$M r$. Okrent: I have a different transient in mind, one related to the maximum accident. How much reactivity would a fuel test in the central loop be worth? Is there a limit?

Mr. Hofmann: For the particular accident that we calculated, the rearrangement of such fuel if it were a coolant driver fuel, which would mean an extremely reactive element, the reactivity arrangement would bring about a dollar total. What I am saying is that an element may be worth 6 dollars. The particular melting model to which I have referred is a highly idealized model. The reactivity increase is about a dollar, and it occurs in a quarter second.

Mr. Okrent: This is our point of departure. It's not clear to me why a fuel element which is worth 6 dollars or more cannot, under the high forces due to a sodium bond or due to fission gas pressure, rapidly reassemble in the center of the core.

Mr. Hofmann: It doesn't have room. We assume, of course, that the fuel tubes did not fail. If I understand you correctly, what you would like me to do is take the top quarter and shove it into the center, and take the bottom quarter and shove it also into the center. This I think is physically possible in the sense that there is enough room to do this. So you might say we have analyzed the half maximum accident which you have just postulated, because we have only looked at the dropping of the top quarter into the center, which I think is very unrealistic.

Mr. Okrent: But you have dropped it under gravity. I'm also suggesting that a sodium bond or the fission gases may be considerably more rapid in achieving this assembly.

$M r$. Hofmann: The only thing I can say about that is that I don't know what will happen when this element melts. It's interesting to look at the experiments that Huebotter has done with lead and water. One of the 
conclusions that he derived from physics parameters is that he had not observed any rate faster than those induced by gravity. But I realize it is not a very strong answer to your question.

$M r$. Bradbury (Harwell): We have demonstrated in the case of $\mathrm{UO}_{2}$-stainless steel cermets that one of the main advantages to be gained from these fuels is that you can take advantage of the initial starting porosity, that is to say, with a high degree of restraint offered to the oxide particles. The fuel can be forced to swell in on itself and in this way you can delay the onset of swelling by changing the starting porosity of the fissile particles. In this connection, could you tell me what the starting density of the $\mathrm{Pu}-\mathrm{UO}_{2}$ would be in your fuel, and what target burnup you envisage?

Mr. Hofmann: I think that the densities of the ceramic states have been variously assumed to range between 70 and 90 of theoretical. I think we are aiming for about $15 \%$ total burnup over three cycles. The core would only burn up $5 \%$ during one cycle, but one cycle would be essentially $1 / 3$ of the total fuel like and $1 / 3$ of the fuel would be shuffled. 


\section{General Discussion}

Mr. Zinn (Combustion Engineering): I would like to start the general discussion by thanking all of the speakers for their efforts this afternoon.

Now, I will begin by directing a question to $\mathrm{Mr}$. Hofmann. You did mention that an experiment might melt down and add some reactivity, and you also said that this would increase the power in the reactor by about $50 \%$, but that it would not result in any serious consequences for the core itself. How about other experiments which might be in the reactor?

Mr. Hofmann (Battelle-Northwest): We have not looked at this particular question in detail. If these experiments are oxides as they might well be, the temperature would depend on what temperature the experiment is run at.

Mr. Zinn: Well, my question was a little bit of a leading one, because you have now started to answer it by limiting the experiments. $I$ am always impressed by the great costs of loops that are put into reactor cores, because they do involve separate cooling systems and so on. I think I would be especially impressed by the cost of putting loop experiments into fast reactors, so I want to ask some questions about the purpose of the loops. Are they to use coolants other than sodium?

Mr. Hofmann: The use of coolants other than sodium has been considered possible, but I don't.

Mr. Zinn: Such as gas, for instance.

Mr. IIofmann: Such as gas loops.

$M r$. Zinn: How about steam or water?

Mr. Hofmann: I have heard people talk about steam or water, but I takc a dim view of them at the moment.

$M r$. Zinn: In light of the fact that you have to, perhaps, think of limitations on all experiments because of the failure in one experiment triggering failures in others, which might in turn trigger the reactor into failure, and because you have to put limitations on loops, and because they cost a lot of money, I wonder if a simple high-power density reactor core without loops might not be a better facility for testing fuel.

Mr. Hofmann: I think you cannot test the great variety of fuel that you may want to test in fact.

$M r$. Zinn: Is the variety of fuel that we really have to consider for fast reactors so very great?

Mr. Hofmann: Do we know now what variety of fuels will be in 1980 ? I think at the moment some fuels may be considered to be the preferred fuels.

$M r$. Zinn: If the fast reactor business survives to 1980, hopefully, it won't matter whether this particular facility tests the fuel or not. In other words, if we haven't gotten some operating prototypes by 1980 , which are testing fucls, I wonder if we should be worrying about what happens here.

Mr. Hofmann: There is one other point. The fuels that might be tested might not only be fuels to be used in breeder reactors, but could possibly be fuels that might be used in special-purpose reactors.

Mr. Zinn: I think that is a valid point. We don't know what the special-purpose reactors will be except that we can be pretty sure that they will exist. I think our whole history shows that, so a general test facility might be very useful for that purpose. But I want to repeat that I am very impressed by the difficulty of adding experimental facilities of flexibility to a reactor that causes the size of a conference that we have here devoted to talking about safety. This is a different type of problem than we face in our general-purpose research reactor. It is a somewhat more difficult problem, and I think I would like to congratulate you and your colleagues for tackling this type of design.

$M r$. Zebroski (General Electric): Since the individual fuel channels are really all loops whether they are independent or not, and they are supported at rather great distances, one end from the other, do you have the possibility of an EBR-I type of reactivity effect due to bowing compaction?

Mr. Hofmann: We don't plan on that particular compaction as in EBR-I. In fact, friction pads are put between the tubes, and the tubes are essentially slightly forced out. We assume at the moment that our design can be made such that, in fact, we cannot get lateral motion which will result in the kind of compaction with which you seem to be concerned.

$M r$. Zebroski: With tolerances and differential temperature problems this is not an easy answer. The second half of the question is, if indeed the experimenters should find sympathy with Dr. Zinn's viewpoint that it is rather expensive to put their experiments in these loops, does the core have the flexibility to pcrmit conventional insertion of fuel between grid plates without the loop appurtenances?

Mr. Hofmann: There are about nine test positions located in the reflector. I would imagine that small pieces of fuel could be put into the reflector region.

Mr. Hennig (Battelle-Northwest): One of the advantages of the skewed-tube concept is that all of the positions in the reactor are, in essence, open loop positions. Each can be loaded with whatever kind of fuel one wishes to place inside, always within the envelope. 
Of particular advantage is the ability to monitor whatever tests are put in the open loops.

Mr. Zinn: The skewed-tube concept actually was very close to being used in the design of EBR-I. Alex Langsdorf of Argonne originated the design, but we gave it up because of the added expense of building it. It seemed to be an expensive way of arranging the core, but it had very many attractions, as it still has. Of course, we know it has been used in one reactor or two since then, but not fast reactors. It did have its origin in the fast reactor concept.

$M r$. Chernick (BNL): I have a question directed to either Webb or Schluderberg. It wasn't clear to me whether they had done a study of the transfer function of this reactor, in oscillations and reactivity and what not.

Mr. Webb (Babcock \& Wilcox): We have a sort of two-fold approach. We want our reactor to be safe, but at the same time we want the performance. I heard one comment that you could raise pressure and your voiding deficit would go down, which is true, but your performance will go down. We are trying to design the plant so that we can insert a time delay for the actual voiding to allow us time to get in our control rods. We have three or four pretty good ideas in the present design which we believe will allow this reactor to live with some positive coefficient. On the other hand, realizing we would like to get the coefficient negative, we have looked at adding solid moderators and we have also looked at changing geometry. We find that solid moderators help without too much detrimental effect on the performance. Although we have not completed a detailed plant analysis, it is in process now. As of now, the voiding deficit is about a little over $1 \%$, which we are sure we can reduce.

$M r$. Chernick: You say you haven't gone into any detailed calculations as to how you are going to control this reactor.

Mr. Webb: We have gone into it, we are in the middle of it, right now.

Mr. Chernick: You are convinced it is stable at operation.

Mr. Webb: Yes. 


\section{Session III}

\section{October 12, 1965}

\section{Panel on Safety of Large Fast Power Reactors}

Chairman: D. OKRENT Argonne National Laboratory

Secretaries: D. Meneghetti

D. A. Meneley

Argonne National I abooratory 
a

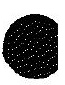




\title{
Contribution to Safety Problems Related to a 1000-MWe Fast Power Reactor Project
}

\author{
G. Denielou, R. Abuox, J. Fournier, J. Ladet, I. Puig, M. Robin, and C. P. Zaleski \\ EURATOM-CEA (Cadarache) \\ Bouches-du-Rhône, France \\ (PRESGNTED BY G. DENIELOU)
}

\section{Safety Philosophy}

Safety philosophies of different countries are strongly dependent on mentalities, laws, and on the technical know-how of each nation.

The French approach is rather empirical and pragmatic, being very much based on past expericnce. It is not easy to mold it in rigid patterns. Though a priori safety criteria are widely used, they are not fixed in a more detailed manner than possible at the present status of knowledge. They remain very often unwritten. At least formally, designers are given much freedom. They know only that they must be able to convince every scientifically and technically competent nonexpert of the validity of their hypotheses. It could be said that safety is achieved by the general agreement of learned people. The Reactor Safety Sub-Committee, whose authority is absolutely undisputed, embodics this agreement.

The cxample of the 1000-MWe reactor project which we shall discuss is a good illustration of what precedes. The authors are confident that they will be able to convince anybody of its good safety features. Actually, on a good many points they have already succeeded; on other points much research and development remains to be done. Such studies will no doubt lead to modifications of the values given hereafter. But it should be kept in mind that many other technical and economical reasons will also modify the project, not speaking of the lessons of Rapsodie and Phenix operating experience.

\section{Design Data for a 1000-MWe Reference Reactor}

II.1. The economic and technical trends for this fastneutron project are as follows:

1) The internal breeding ratio is to minimize longterm reactivity variations, i.e., is to be close to 1 . This leads to low enrichments which, in turn, give rise to a better use of fissile material.

2) Radial power flattening is to be achieved by the use of two concentric zones in a variable-enrichment core.
3) Core height is to be as low as is compatible with a technically sound pressure drop. (At constant breeding ratio the fuel volume fraction increases when the core height is reduced; see Fig. 1).

4) The time-dependent variation of the maximum/ mean specific power ratio is to be optimized. Thus, at the beginning of a fuel cycle specific power maximum in the external zone is higher than that in the inner one.

All these trends lead to minimize investment of fissile material.

II.2 The so-called 1000-G core, ${ }^{*}$ a description of which was presented at the Detroit Meeting in April $1965,{ }^{(1)}$ has been designed according to the abovementioned principles. Neutronic data pertaining to this core are given in Table 1.

\section{Safety and Dynamic Parameters}

\section{III.1. Temperature Coefficients of Reactivity}

The temperature coefficients of reactivity are given in Table 2. The following remarks may be made:

We assume that fuel subassemblies have been provided with buttons in the reactor midplane and that the core is tight for nominal working conditions.

The fraction of the temperature coefficient originating in the fuel dilatation has been calculated in two ways, according to whether the fuel is bound or not to its cladding. When the fuel is bound to its cladding (normally at high burnup), the dilatation is identical with that of its cladding; when the fuel is not bound to its cladding (at the beginning of irradiation), it dilates freely. The real coefficient is no doubt somewhere between these two bounds.

The sodium-dependent fraction of the temperature coefficient is practically zero for the reactor. It is itself the sum of two terms each corresponding to one zone of the reactor. The positive term is $+0.25 \cdot 10^{-5}$ $(\Delta k / k) /{ }^{\circ} \mathrm{C}$.

* This core differs essentially from the $1000 \mathrm{~A}$ core (presented at the Argonne Conference, October 1963) by a fuel volume fraction increase as foreseen by C. P. Zaleski at that time. 
Finally, the fraction dependent on the Doppler effect is $-0.6 \cdot 10^{-5}(\Delta k / k) /{ }^{\circ} \mathrm{C}$ at nominal conditions. The mean temperature weighted on the Doppler coefficient is then $1500^{\circ} \mathrm{K}[T(\partial k / \partial T)=0.09]$. We assume that the Doppler varies according to $T^{-3 / 2}$ in the solid phase and that it is zero in liquid and gaseous phases.

In this calculation the Hansen and Roach crosssection set was used. The coefficient depends very much on the cross-section set used. For instance, it is shown in Ref. 1 that, for a given reactor, the Russian cross-section set can give a $20 \%$ higher Doppler coefficient than the Hansen and Roach set.

\section{III.2. Power Coefficient}

The power coefficients are given in Table 3 . In order to calculate the power coefficient due to axial fuel expansion when the fuel is free in its cladding, we assumed that the expansion of a pellet was that corresponding to its own central temperature.

The Doppler effect provides the main contribution. to the power coefficient. The positive fraction of the sodium coefficient is about $0.01 \cdot 10^{-5}(\Delta k / k) / \mathrm{MW}$, that is, about $5 \%$ of the power coefficient due to the Doppler.

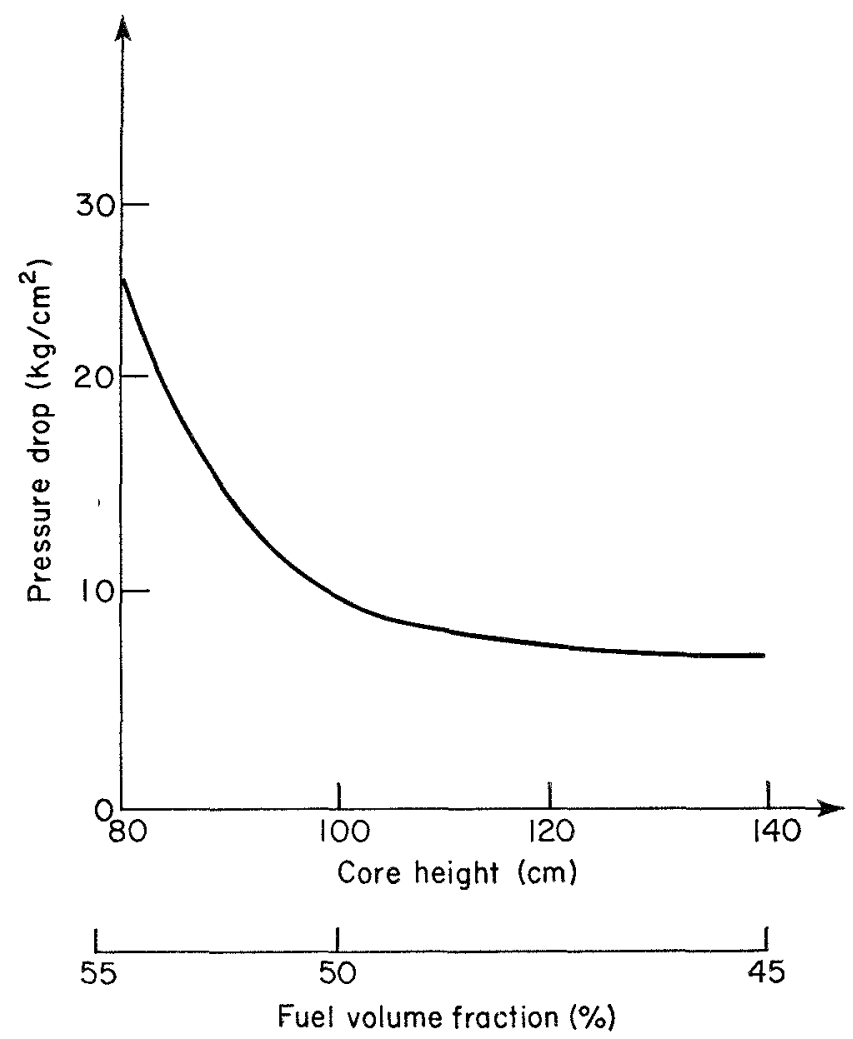

Fig. 1. Pressure Drop through the Core vs Core Height and Fuel Volume Fraction, the Associated Internal Breeding Ratio being Constant (0.93).
TABLE 1. Design Data for 1000-G Reactor Core

\begin{tabular}{l|c}
\hline Core Volume & 5200 liters \\
Number of Subassemblies & about 450 \\
Ratio of Internal Zone Volume & 0.54 \\
$\left(V_{1}\right)$ to Total Core Volume $(V)$ & $100 \mathrm{~cm}$ \\
Core Height & $2370 \mathrm{~kg}$ \\
$M_{c}$ (Fissile Plutonium) & $12.7 \%$ \\
Enrichment of Zone 1 & $18.2 \%$ \\
Enrichment of Zone 2 & 0.93 \\
Internal Breeding Ratio & $+0.14 \cdot 10^{-5}(\Delta k / k) /{ }^{\circ} \mathrm{C}$ \\
Sodium Coefficient in the Core & $-0.16 \cdot 10^{-5}(\Delta k / k) /{ }^{\circ} \mathrm{C}$ \\
Sodium Coefficient in the Blan- & \\
kets and Gap Transitions & $-0.02 \cdot 10^{-5}(\Delta k / k) /{ }^{\circ} \mathrm{C}$ \\
$\quad$ Total & $-0.6 \cdot 10^{-5}(\Delta k / k) /{ }^{\circ} \mathrm{C}$ at \\
Doppler Coefficient & $1500^{\circ} \mathrm{K}$ \\
$\beta_{\text {eif }}=330 \cdot 10^{-5}(\Delta k / k)$ & $1^{*}=3.6 \cdot 10^{-5} \mathrm{sec}$ \\
\hline
\end{tabular}

TABLI 2. Temperature Colfholents (IN tNits OF $\left.10^{-5}(\Delta k / k) /{ }^{\circ} \mathrm{C}\right)$ OF THE 1000 -G REACTOR

\begin{tabular}{|c|c|c|c|c|c|}
\hline & & & \multicolumn{3}{|c|}{ Total isothermal coefficient } \\
\hline & \multicolumn{2}{|c|}{ Free fuel } & \multicolumn{3}{|c|}{-1.5} \\
\hline & \multicolumn{2}{|c|}{ Bound fuel } & \multicolumn{3}{|c|}{-1.7} \\
\hline & $\begin{array}{c}\text { Core } \\
\text { including } \\
\text { absorb- } \\
\text { ing rods }\end{array}$ & $\begin{array}{c}\text { Gap } \\
\text { zones }\end{array}$ & $\begin{array}{c}\text { Axial } \\
\text { blankets }\end{array}$ & $\begin{array}{c}\text { Radial } \\
\text { blankets }\end{array}$ & Total \\
\hline Sodium Expansion & +0.14 & -0.10 & -0.01 & -0.05 & -0.02 \\
\hline Steel Expansion & +0.16 & -0.04 & +0.01 & -0.02 & +0.11 \\
\hline $\begin{array}{c}\text { Axial Expansion } \\
\text { of Free Fuel }\end{array}$ & -0.15 & & & & -0.15 \\
\hline $\begin{array}{c}\text { Axial Expansion } \\
\text { of a Bound Fuel }\end{array}$ & -0.33 & & & & -0.33 \\
\hline $\begin{array}{l}\text { Axial Expansion } \\
\text { of Fertile Ma- } \\
\text { terial }\end{array}$ & & & -0.01 & -0.00 & -0.01 \\
\hline \multirow[t]{2}{*}{ Doppler Effect* } & -0.60 & & & & -0.60 \\
\hline & \multicolumn{2}{|c|}{ Before tightening } & \multicolumn{3}{|c|}{ After tightening } \\
\hline Supporting Grid & \multirow{2}{*}{\multicolumn{2}{|c|}{$\begin{array}{c}-0.88 \\
0\end{array}$}} & \multirow{2}{*}{\multicolumn{3}{|c|}{$\begin{array}{l}-0.13 \\
-0.75\end{array}$}} \\
\hline $\begin{array}{l}\text { Effect of the But- } \\
\text { tons }\end{array}$ & & & & & \\
\hline Bowing & \multicolumn{2}{|c|}{0} & \multicolumn{3}{|c|}{+0.00} \\
\hline "Splaying" & \multicolumn{2}{|c|}{-0.02} & \multicolumn{3}{|c|}{0} \\
\hline
\end{tabular}

$*$ at $1500^{\circ} \mathrm{K}$.

\section{III.3. Integrated Negative Doppler Reactivity}

We define the fuel rupture temperature as a temperature for which the vapor pressure of the fuel is no longer negligible, that is, about $4000^{\circ} \mathrm{K}$.

We call "réserves Doppler" the integrated reactivities due to the Doppler effect between the nominal working point of the reactor and the point where the fuel rupture temperature at the fuel hot spot is reached. 
This "réserve" is about $\$ 1.1$, that is, $360 \cdot 10^{-5}$ $(\Delta k / k)$, in the case of an adiabatical temperature rise.

If we admit that the safety system operates, the Doppler-controlled step reactivity increase is

$$
[(360 / 2)+330] 10^{-5}=510 \cdot 10^{-5}(\Delta k / k),
$$

the dollar being taken at $330 \cdot 10^{-5}(\Delta / / k)$. The slow power rise which would lead to fuel vaporization at the hottest spot of the hot channel is $44 \%$ of the nominal power, that is, $1010 \mathrm{MW}$. It can be said that under the pessimistic assumption of a clad-bound fuel $250 \cdot 10^{-5}$ $(\Delta k / k)$ needs to be introduced in order to bring the fuel to its rupture point in the hot channel. Then, the clad hot spot will be about $790^{\circ} \mathrm{C}$. This means that in order to bring the fuel to its rupture point in the hot channel one needs:

$250 \cdot 10^{-5}(\Delta k / k)$ if one admits a safety-system failure;

$500 \cdot 10^{-5}(\Delta k / k)$ introduced at a very important insertion rate if the safety system is operating.

\section{III.4. Sodium Loss Reactivity}

Figure 2 shows the reactivity due to sodiun voidage beginning in the reactor midplane.

Figure 3 shows the reactivity due to sodium loss beginning in the upper axial blanket and propagating downwards.

\section{III.5. Reactivity Balance}

The reactivity necessary for reactor operation can be broken down as follows:

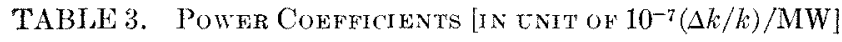
for Operation at Nominal Power

\begin{tabular}{|c|c|c|c|c|c|}
\hline & & & & \multicolumn{2}{|c|}{$\begin{array}{l}\text { Total power } \\
\text { coefficient }\end{array}$} \\
\hline & $\begin{array}{l}\text { Before } \\
\text { com- } \\
\text { pacting }\end{array}$ & \multicolumn{2}{|c|}{$\begin{array}{l}\text { Fuel free from } \\
\text { expansion } \\
\text { Bound fuel }\end{array}$} & \multicolumn{2}{|c|}{$\begin{array}{l}-26 \\
-20\end{array}$} \\
\hline & $\begin{array}{l}\text { After } \\
\text { com- } \\
\text { pacting }\end{array}$ & \multicolumn{2}{|c|}{$\begin{array}{l}\text { Fuel free from } \\
\text { expansion } \\
\text { Bound fuel }\end{array}$} & \multicolumn{2}{|c|}{$\begin{array}{l}-30 \\
-25\end{array}$} \\
\hline & Core & $\begin{array}{l}\text { Gap } \\
\text { Zones }\end{array}$ & $\begin{array}{l}\text { Axial } \\
\text { blan- } \\
\text { kets }\end{array}$ & $\begin{array}{l}\text { Radial } \\
\text { blan- } \\
\text { kets }\end{array}$ & Total \\
\hline Sodium Expansion & +0.7 & -0.7 & -0.0 & -0.0 & -0.0 \\
\hline Steel Expansion & +0.9 & -0.3 & -0.0 & -0.0 & +0.6 \\
\hline $\begin{array}{l}\text { Axial Expansion of } \\
\text { Free Fuel }\end{array}$ & -7.1 & & & & -7.1 \\
\hline $\begin{array}{l}\text { Axial Expansion of } \\
\text { Bound Fuel }\end{array}$ & -1.8 & & & & -1.8 \\
\hline $\begin{array}{c}\text { Axial Expansion of } \\
\text { Fertile Material }\end{array}$ & & & -0.0 & -0.0 & -0.0 \\
\hline Doppler Effect & -19 & & & & -19 \\
\hline Effect of the Buttons & -4.8 & & & & -4.8 \\
\hline
\end{tabular}

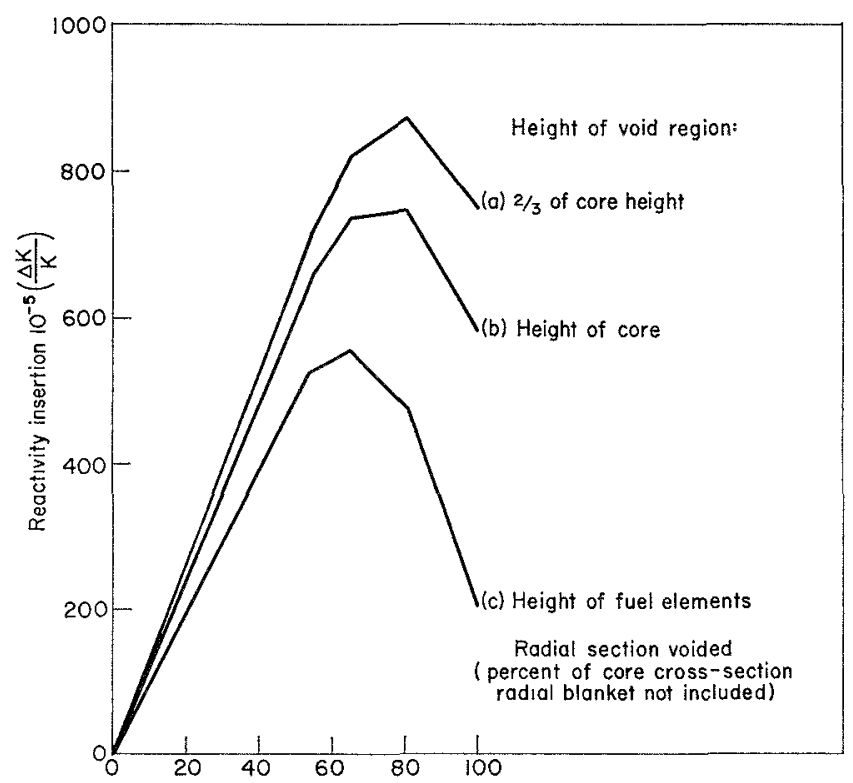

FIg. 2. Reactivity Effects Resulting from Sodium Loss Beginning at the Core Midplane.

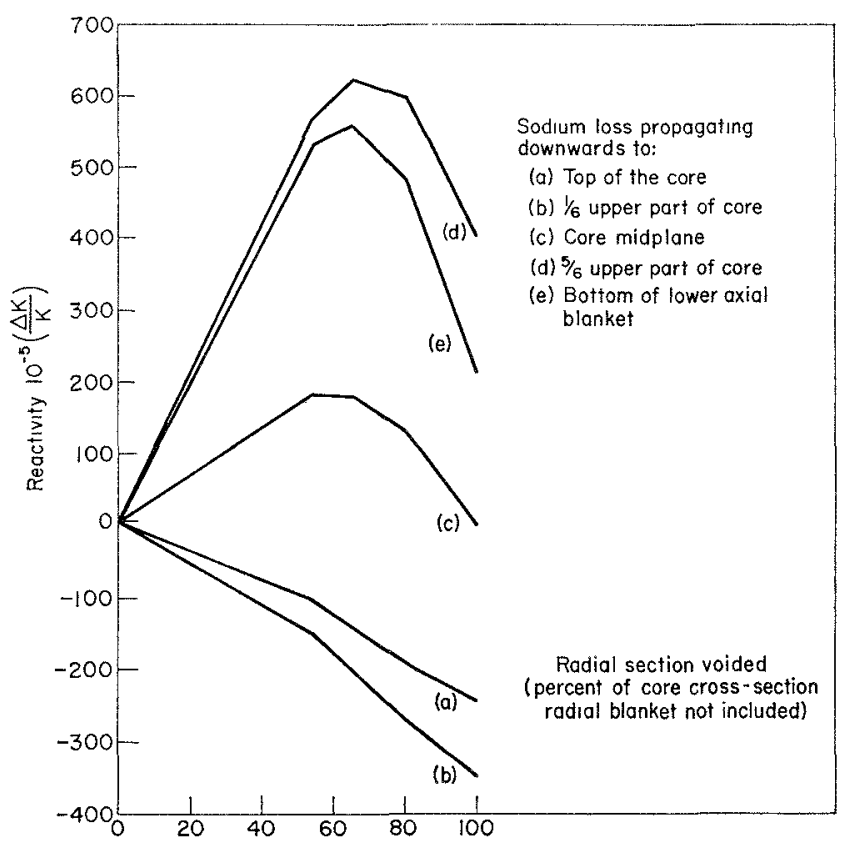

Fig. 3. Reactivity Effects Resulting from Sodium Loss Beginning in the Upper Axial Blanket.

at zero power from cold to hot reactor, $740 \cdot 10^{-5}$ $(\Delta k / k)$;

hot reactor, from zero power to nominal power, $1010 \cdot 10^{-5}(\Delta k / k)$;

fuel burnup during 6 months, $600 \cdot 10^{-5}(\Delta k / k)$;

control requirements, $140 \cdot 10^{-5}(\Delta k / k)$. 
The total reactivity requirements are thus $2500 \cdot 10^{-5}$ $(\Delta k / k)$, the total rod worth being $4800 \cdot 10^{-5}(\Delta k / k)$; the shutdown safety margin is thus $2300 \cdot 10^{-5}(\Delta k / k)$, about $\$ 7$.

\section{Safety Analysis}

Accidental sequences can be divided into three groups: those including necessarily such events which positively can be avoided by design; those including necessarily such events which cannot be avoided certainly and the consequences of which ean and must be limited by design; other accidents.

IV.1. The following accidents belong to the first group:

a. Reactor instabilities. Taking into account the strong Doppler coefficient, the experience gathered in this field since the days of EBR-I, and the proposed core structure, the stability of the reactor presents no safety problem. Space-dependent instability is not likely to occur.

b. Complete reactor vessel voidage. With or without a safety system, a complete voidage will necessarily lead to a generalized core meltdown. It must and can absolutely be avoided by design, whatever may be the consequences. (One has only to make sure that rupture of the reactor vessel cannot be the ultimate event of an accidental sequence.) The technical means are wellknown: piping entering from above, double-wall vessels, safety tanks, and even integrated circuits.

c. Total, generalized, and instantaneous flow stop. It is possible to ensure by design that there will never be a total loss of flow occurring at the same time in a great number of fuel subassemblies without having been noticed in due time for scramming. For this purpose, technical means are well-known: multiple loop systems, multiplying the number of independent electrical supplies, battery-supplied emergency motors, etc.

d. Simultaneous jamming of all safety rods.

e. Introduction of a significant quantity of slowingdown material into the reactor.

f. Loading accident (subassembly drop into a critical reactor).

g. Shim-rod ejection, the reactor being critical (though this ejection could be part of an accidental sequence; it cannot be the initial event).

IV.2. The following accidents belong to the second group:

a. Partial and instantaneous flow stop without immediate action of the safety system. Whatever might be the care taken, one cannot guarantee that there would neither be pump jamming nor loss of electrical power, although it seems that a coincidence of these two events is highly improbable.

b. Loading accident (subassembly drop, the reactor being suberitical).

IV.3. Unfortunately, some accidents cannot yet be included in the above-mentioned groups and are still being studied:

a. Generalized and total loss of flow. Are we able or not to assure that following an earthquake or any other act of God, the after-heat cooling of fission products will always be possible without core meltdown?

b. Complete plugging of a subassembly. Such a plugging will certainly melt down the subassembly. Unfortunately, it is difficult to incorporate this accident in the first group.

Is the propagation to other subassemblies impossible? We hope to be able to demonstrate that this event is not autocatalytic and that it will remain localized.

Nevertheless it is clear that we are still missing quantitative data on the physical characteristics of a $\mathrm{UO}_{2-}$ $\mathrm{PuO}_{2}$-stainless steel-sodium double- and even triplephase systems, or full-seale experiments substituting our lack of data.

\section{Conclusion}

\section{Maximum Hypothetical Accident}

In Irance we are not much familiar with the notion of a maximum hypothetical accident. To us the term seems somewhat self-contradictory. Does "hypothetical" mean that this accident never occurs or that it hopefully will never take place? In this case the word "hypothetical," although well-chosen, is not quite meaningful. Or does it mean that one is unable to conceive a sequence of events leading to such an accident? In this case it is difficult to understand what is its real sense. Actually, the only way which should enable us to use the word "accident" is: either it already occurred, or we are able to describe it in a credible way from the beginning to the end.

It seems more probable that "hypothetical" means here that we deal with an accidental sequence of which we do not know yet the initial event. In this case, which seems to us the only reasonable meaning, every possible step must be taken to get out of such an unfortunate situation. Actually, the accident is being studied. The worst accident we are studying now is surely the complete core meltdown following the two accidents mentioned in IV.3, namely, plugging of the fuel subassembly and complete loss of heat sink.

We believe that too much attention has been paid to a core disassembly following high-insertion reactivity 
and not enough to less explosive phenomena. In a way, core disassembly disregarding any possibility of fissile material reassembly is an easy means of terminating an accidental sequence. But what happens to a subcritical molten core? Is it also impossible to imagine slightly supercritical molten-down fissile assemblies giving rise to low-intensity repetitive nuclear excursions?

\section{REFERENCE}

1. C. P. Zaleski and D. Smidt, Fast Reactor Core Design and Safety Studies in France and the Federal Republic of Germany, Detroit Meeting, April 1965. 


\title{
The Karlsruhe Reference Design of a 1000-MWe Sodium-cooled Fast Breeder Reactor*
}

\author{
W. Häfele, D. Smidt, and K. Wirtz \\ Kernforschungszentrum \\ Karlsruhe, Germany \\ (PRESENTED BY W. HÄFELE)
}

\section{A. Introduction}

As has been described earlier, ${ }^{(1)}$ the present phase of the Karlsruhe Fast Breeder Project, which extends to 1967, is aimed towards the investigation of the feasibility of 1000-MWe fast breeder reactors. One principal coolant being considered is sodium. The purpose of the present Karlsruhe study, ${ }^{(2)}$ called Na-1, of a 1000 MWe sodium-cooled breeder reactor was to serve as a reference design. On the basis of this reference design, competition between breeding, safety, and economy is being explored as quantitatively as possible. In particular, the impact of safety on breeding and economy is being studied. A first set of results was presented by Smidt. ${ }^{(3)}$

The proper choice of a design basis arcident (DBA) and its impact on capital costs is being explored now and will serve as the basis of study Na-2. The forthcoming $\mathrm{Na}-2$ study will be a thorough safefy evaluation.

In this paper are described the present Na-1 design and its safety features with respect to incidents, the maximum hypothetical accident (MHA), and the DBA.

\section{B. A Description of the Karlsruhe Na-1 Reference Design}

A detailed description of the Na-1 design has been given. ${ }^{(2,}{ }^{4)}$ The descriptions should be considered as a background for this chapter. The table in the Appendix gives the most important data of the $\mathrm{Na}-1$ design.

\section{Choice of Fuel}

The fuel for $\mathrm{Ta}-1$ consists of mixed oxides of $\mathrm{UO}_{2}$ and $\mathrm{PuO}_{2}$. The design objective was to arrive at fuel-cycle costs below 1 mill/kWh. This can be met without difficulties by the use of the oxides. ${ }^{(5)}$ The advantages of using other types of fuel, such as the carbides, would lie in the areas of breeding, including the aspect of lowering fuel-cycle or capital costs. We will touch that point later. The choice for $\mathrm{UO}_{2}-\mathrm{PuO}_{2}$ as a fuel is

\footnotetext{
* This paper has been prepared within the framework of the association EURATOM-Gesellschaft für Kernforschung $\mathrm{mbH}$. in the field of fast breeder development.
}

also consistent with the Karlsruhe approach of looking for steam cooling, which excludes the use of the carbides. Another advantage is that the technology of $\mathrm{UO}_{2}$ as a fuel is available whereas that for UC is less developed and somewhat more difficult. All these have led to the use of $\mathrm{UO}_{2}-\mathrm{PuO}_{2}$ for the first large-seale fuel development at Karlsruhe.

One further argument for the oxides should be mentioned. Suppose that $\lambda$ is the heat conductivity of the fuel in the rod of diameter $R$ and $\alpha$ is the heat conductivity in the gap. Then the "effective" heat conductivity $\lambda_{\circ \text { ff }}$ between the center of fuel and the cladding is given by

$$
\lambda_{\mathrm{eff}}=\lambda \frac{2 \alpha R}{\alpha R+2 \lambda}
$$

If $\alpha R / 2 \lambda$ is small, then $\lambda_{\text {eff }} \rightarrow(\alpha R)(\alpha R / 2 \lambda)$. For a fast reactor fuel rod with carbide fuel, the value of $\lambda_{\text {eff }}$ is small for three reasons: 1) $R$ of a fast reactor rod is small (the situation is different for thermal reactors); 2) the problem of inner corrosion between fuel and clad is more serious for the carbides than for the oxides, and affects the value of $\alpha ; 3$ ) the high value of $\lambda$ in the case of the carbides emphasizes the difficulties of getting a comparable condurtivity $1 / 2 \alpha R$ in the gap.

\section{Choice of Cladding and Structural MLaterials}

In four recent 1000-MWe studies, austenitic stainless steel, with preference given to Types 316 and 304, has been chosen as cladding materials. In the Na-1 study we took Incoloy 800 as a reference point for advanced Incoloy types. On the other hand, stainless steel 16-13 (CrNi) may be used as an alternative for high creep strength and might possibly be used in the $\mathrm{Na}$-2 design. These two types of alloys seem to us to be more promising than Types 316 and 304. We expect these relatively low-nickel alloys to have, on the one hand, an acceptable corrosion behaviour with respect to mass transfer and, on the other, not to be harmful with respect to the neutronics. The neutronic evaluations with Incoloy 800, with its larger nickel content, showed that there is no great neutronic penalty for the use of such alloys. 
The sodium temperatures are lower than in the American studies. The mean sodium outlet temperature is $580^{\circ} \mathrm{C}$ (instead of $600-650^{\circ} \mathrm{C}$ as in the US studies). If the hot-channel factor is included, the maximum outlet temperature of sodium in our study is $630^{\circ} \mathrm{C}$. The mean inlet sodium temperature of the steam generator then amounts to $560^{\circ} \mathrm{C}$, thus leading to a steam temperature of $540^{\circ} \mathrm{C}$. The sodium outlet temperature of the steam generator was taken as $360^{\circ} \mathrm{C}$; the inlet temperature into the core will be $430^{\circ} \mathrm{C}$.

Having fixed these temperatures, one now can make a proper choice of the structural materials. The first choice is, of course, austenitic steel on the hot side of the loops; X8 $\mathrm{CrNi}+\mathrm{Nb} 1613$ is the only stainless steel which is allowed for high-temperature use in Germany. But for economic reasons we would prefer Type 304 or 316 and will discuss this question with our supervising authorities. We are also considering niobiumstabilized ferritic steel, 10 CrMoNbNi 910, advocated by the INTER-ATOM group. We cxpect further economic gains by use of this steel. Furthermore, we think it would be the most economic solution to use normal unstabilized ferritic steel 10 CrMo 910 for all cold parts of the loops with temperatures below $450^{\circ} \mathrm{C}$. Even the reactor vessel might be manufactured with this relatively cheap steel. Also this cheap ferritic steel may be used for the concentric in- and outlet tubes to the heat exchangers.

The most difficult part of the sodium circuits with respect to materials are surely the tubes of the steam generator for which we have no simple solution at hand. We think of a combination of normal ferritic steel for the evaporator section and of austenitic steel for the superheater part of the steam generator. (In this point the data of our study are misleading. Not an alternative but a combination of the austenitic and ferritic steel is intended.)

\section{The Core Design}

Originally a number of rather unconventional core designs were considered, for example, spherical fuel elements of canned oxide fuels. Ultimately, however, we arrived at a design with conventional pins in an hexagonal subassembly (see Fig. 1). The core has two zones of two enrichments optimized for power flattening and of the same volume. It also gives automatically a good Doppler coefficient $\left(7 \cdot 10^{-6}\right.$ at $\left.1400^{\circ} \mathrm{C}\right)$ because the center zone has the lower enrichment. The sodium void effect is acceptable. After considerable parametric studies, the height-to-diameter $(\mathrm{H} / \mathrm{D})$ ratio was chosen to be $1 / 3$ (it did not appear to be necessary to go to more extreme values or other extreme solutions in order to enhance leakage). A $\mathrm{H} / \mathrm{D}$ value of $1 / 3$ does not decrease the Doppler coefficient too strongly and also does not influence the fabrication costs of the fuel cycle too much. As will be pointed out below, a core of a low $\mathrm{H} / \mathrm{D}$ value may lead to somewhat lower values of the kinetic energy release in case of a major accident.

The fission gases are given to a gas plenum at the lower end of the core. Therefore we have a vented to plenum scheme (the aspects of a vented to coolant scheme have been evaluated by Sommer and Smidt $\left.{ }^{(6)}\right)$. There seem to be no major economical advantages but an improvement of safety.

The radial blanket has also two regions. In the outer region uranium metal is used in order to reduce the size of the radial blanket $(40 \mathrm{~cm})$, but because of the fuel cycle this appears not to be a good solution. This leads to the core arrangement shown in Fig. 1A.

The reason for having the plenum for the fission gases at the lower end is based on the lower sodium temperature. This leads to an $80-\mathrm{cm}$ length for the plenum, saving about $25 \mathrm{~cm}$. The height of the subassembly is thereby reduced, as is also the pressure drop of the sodium along the subassembly, therefore requiring somewhat less structural material. In addition, the thermal stresses in the subassembly are lower because the plenum does not participate in the displacement of the upper parts of the subassembly. The coolant fraction $(\alpha)$ in both core zones is $50 \%$. The principal argument for that choice was the rather low value of the pressure drop in the core $\left(4.25 \mathrm{~kg} / \mathrm{cm}^{2}\right)$ which, in turn, reduces the neressary volume fraction for the structural materials (to $10 \%$ ). The variation of the coolant fraction has already been discussed.(3)

The fuel subassemblies are fixed in a lower grid plate; the holddown is hydraulie, the direction of coolant flow upward. Among other reasons, the direction of natural convection and the pressure of the cover gas lead to the choice of upward flow.

Much attention was paid to the question of refueling (see Fig. 2A-C). There are three loading devices in the region above the core; each covers $1 / 3$ of the core and blanket area. Normal operation takes place under sodium and makes use of power steering. As long as everything is in order, quick loading and unloading is easily achieved. The principle of power steering immediately detects a mechanical failure. Now it is possible to lower the level of the sodium pool and to have optical access to the upper core surface by a periscope device. Lowering of the sodium level is possible because the sodium pool above the core is a mixing chamber. Presently $90 \%$ of the outcoming flow of the core is taken away from the outlet nozzles by thin vertical plates; only $10 \%$ of the flow reaches the outlet point directly. This decreases the effect of temperature shocks to the walls containing the sodium. Also the vertical plates act as walls in case the sodium level is lowered; 


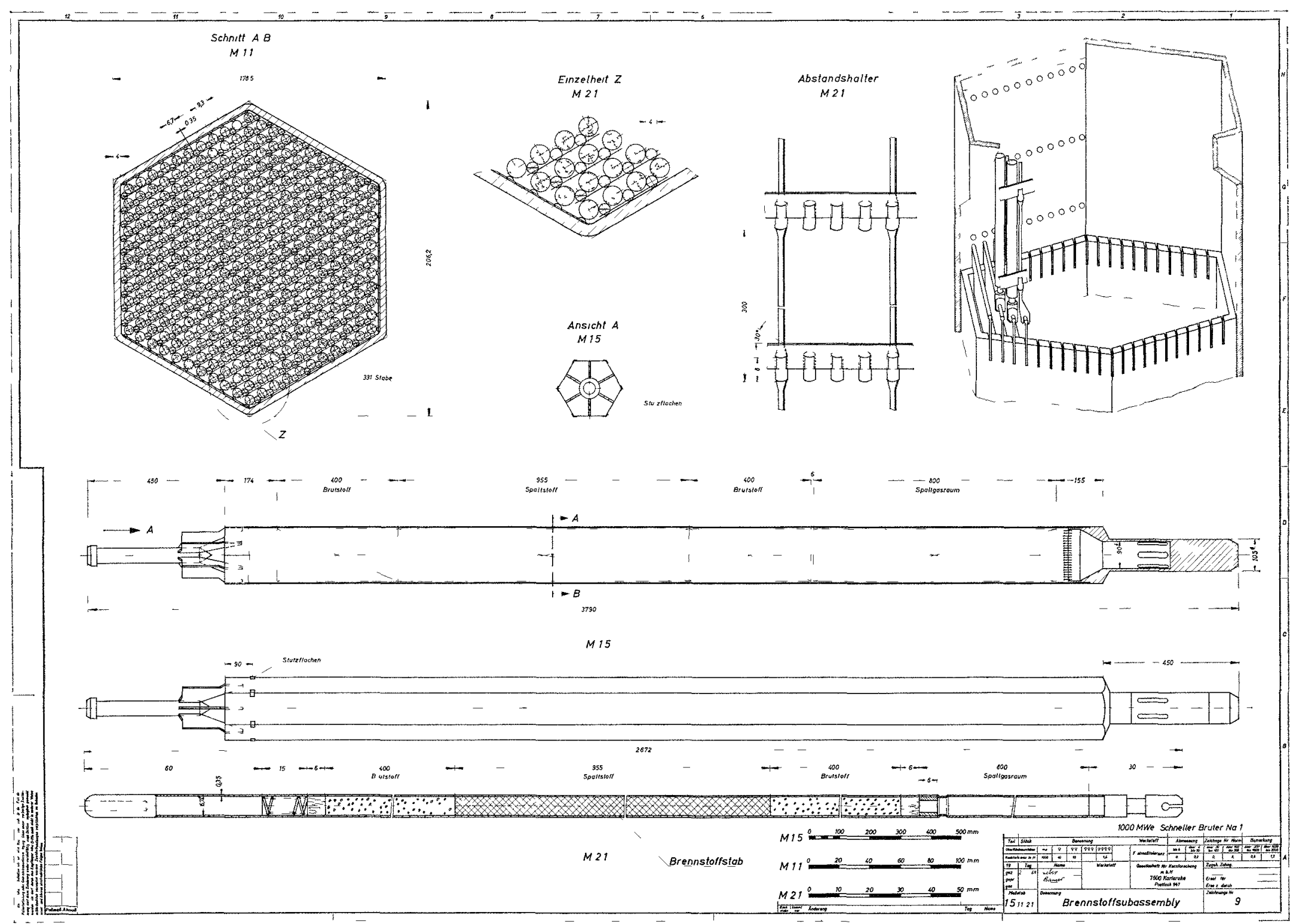

FIG. 1. Fuel Subassembly. 
in this case a sort of under-flow hot cell with optical access by a periscope device is established. The unloaded subassemblies are placed in transfer baskets at the outer perimeter of the radial blanket; from there they are taken away, after a cooling-off period of 100 days, by a rather simple device.

The use of three loading devices leads to very short interruptions for refueling. As long as more than one loading per year appears to be necessary, this may be of great importance for the question of availability. In our case we have a rating of $1 \mathrm{MWt} / \mathrm{kg}$ fissionable material and a three-batch loading scheme leading to refueling periods of 196 days. This is reasonable but not in coincidence with the yearly maintenance cycle; therefore very short interruptions for refueling are desirable.

It is probably more important to look for refueling periods of one year rather than for high ratings if they are associated with short refueling periods. The fuelcost benefit for such high ratings may be obtained only if a power refueling appears possible. This possibility should not be excluded for future reactor studies, however.

A refucling period of one year could increase the economic potential of the plant. Such a period can be obtained with high values of the excess reactivity. In most cases this would hurt breeding and economy except when the internal breeding ratio is somewhat larger than one.

In our case the excess reactivity for bridging a burnup of $1 / 3(100,000) \mathrm{MWd} /$ tonne is $2.4 \%$. This value is the sum of plutonium burnup $(0.75 \%)$ and fission product poisoning $(1.65 \%)$. The internal breeding ratio is 0.89 (inner zone 0.556 ; outer zone 0.334 ).

The nuclear core calculations were based on an equilibrium composition of plutonium isotopes. ${ }^{(7)}$ This equilibrium composition is such that only that amount of freshly bred plutonium from the blankets goes to the core which is necessary for making up the plutonium burnup in the core. In all probability this will not be the scheme for $\mathrm{Na}-2$. It is intended to have a mixed core-blanket aqueous reprocessing scheme instead. After one refueling period, $1 / \mathrm{n}$ of the core and $1 / 2 \mathrm{n}$ of the radial blanket would be mixed together in the process line. If the internal breeding ratio is in the neighbourhood of one, this leads to one unique plutonium isotope composition at all stages of the fuel cycle and there would be no plutonium holdup in the radial blanket.

4. The Design of the Primary Circuit (see Figures 3, 4, and 5)

The primary circuit is of the loop type and has a cover gas of argon. With a large reactor, a pool type

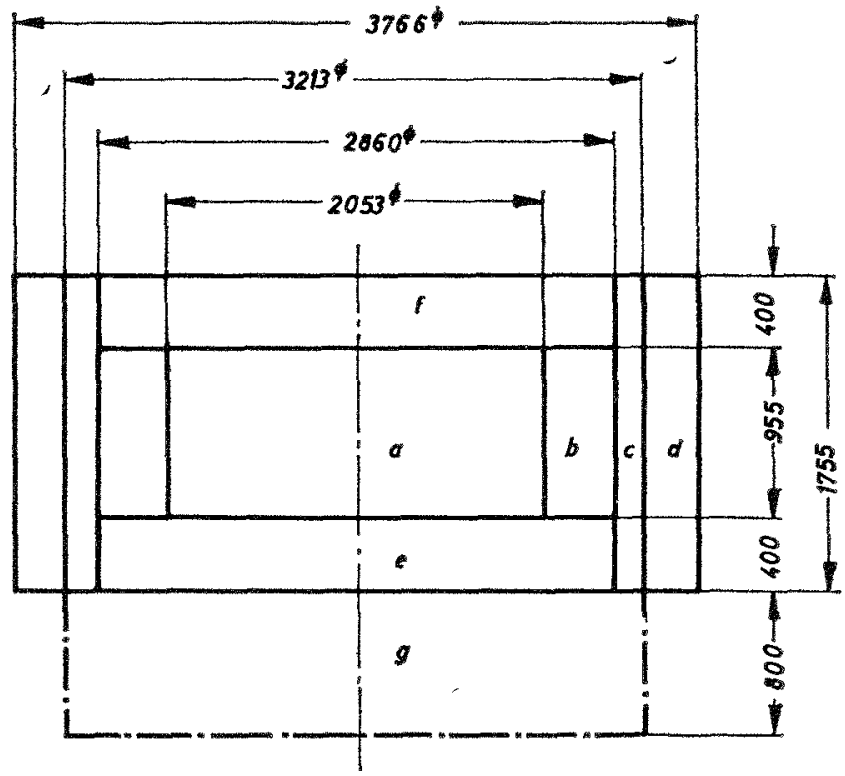

F1a. 1A. Division of Reactor Core into Zones. $a$, innere Spaltzone (Lone 1); $b$, aussere Spaltzone (Zone 2); $c$, innere Brutzone (Zone 3 ); $d$, aussere Brutzone (Zone 4); e, untere axiale Brutzone; $f$, obere axiale Brutzone; $g$, Spaltgesspeicher.

might lead to serious problems, such as unforseen flow patterns, thermal stresses in the structural plates for the pumps and the heat exchanger, radial expansion of the components in the pool with respect to the concrete shield plug, difficulties of having easy access for maintenance and for heavy instrumentation, and others. A solution of the loop type can be better overlooked and the design is more straightforward. But it is not excluded that ultimately the pool type is the right choice.

The loop type of solution for Na-1 provides two parallel circuits, that is, two intermediate heat exchangers are incorporated. Each circuit has two pumps in parallel, making a total of four. A higher number of heat exchangers does not seem advisable because this component should be a reliable one and a higher number of heat exchangers could perhaps even increase the failure probability. In the same sense, the use of four pumps is assumed to be optimum. By suspending the heat exchanger together with the pumps, radial expansion is provided, a good feature. All components of the primary circuit are in chambers of a concrete structure. The heavy motor drives for the sodium pumps are above the main floor, so that they are borne directly by the concrete structure; the pumps themselves are directly connected to the intermediate heat exchanger. The reactor tank itself (see Figures 6 and 7) has a double wall in order to guarantee that the sodium level is always above the reactor core. Convection from the reactor vessel to the intermediate heat 


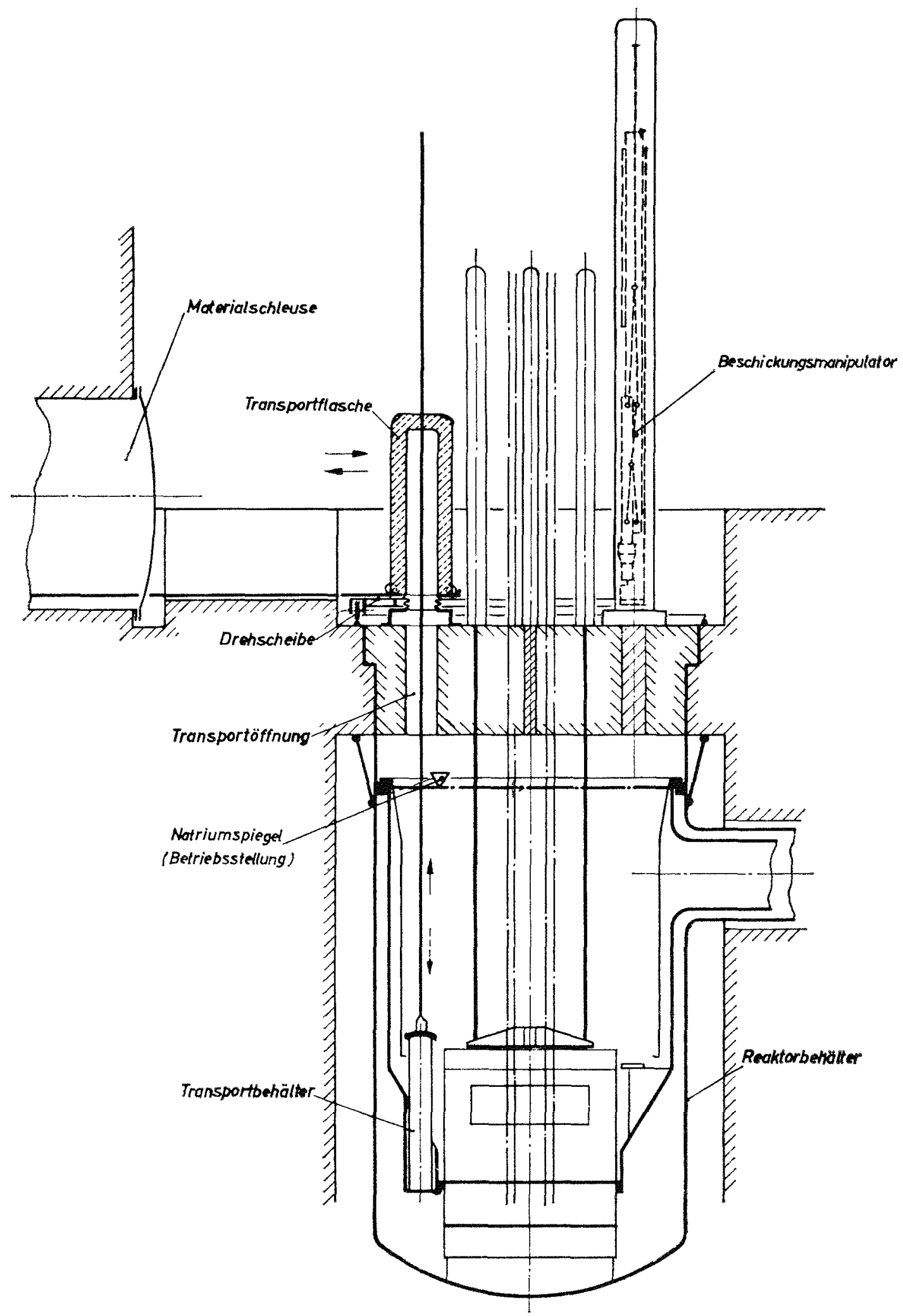

FIG. 2A. Transfer through Interlock of Fuel Transport Container into the Reactor Vessel. 


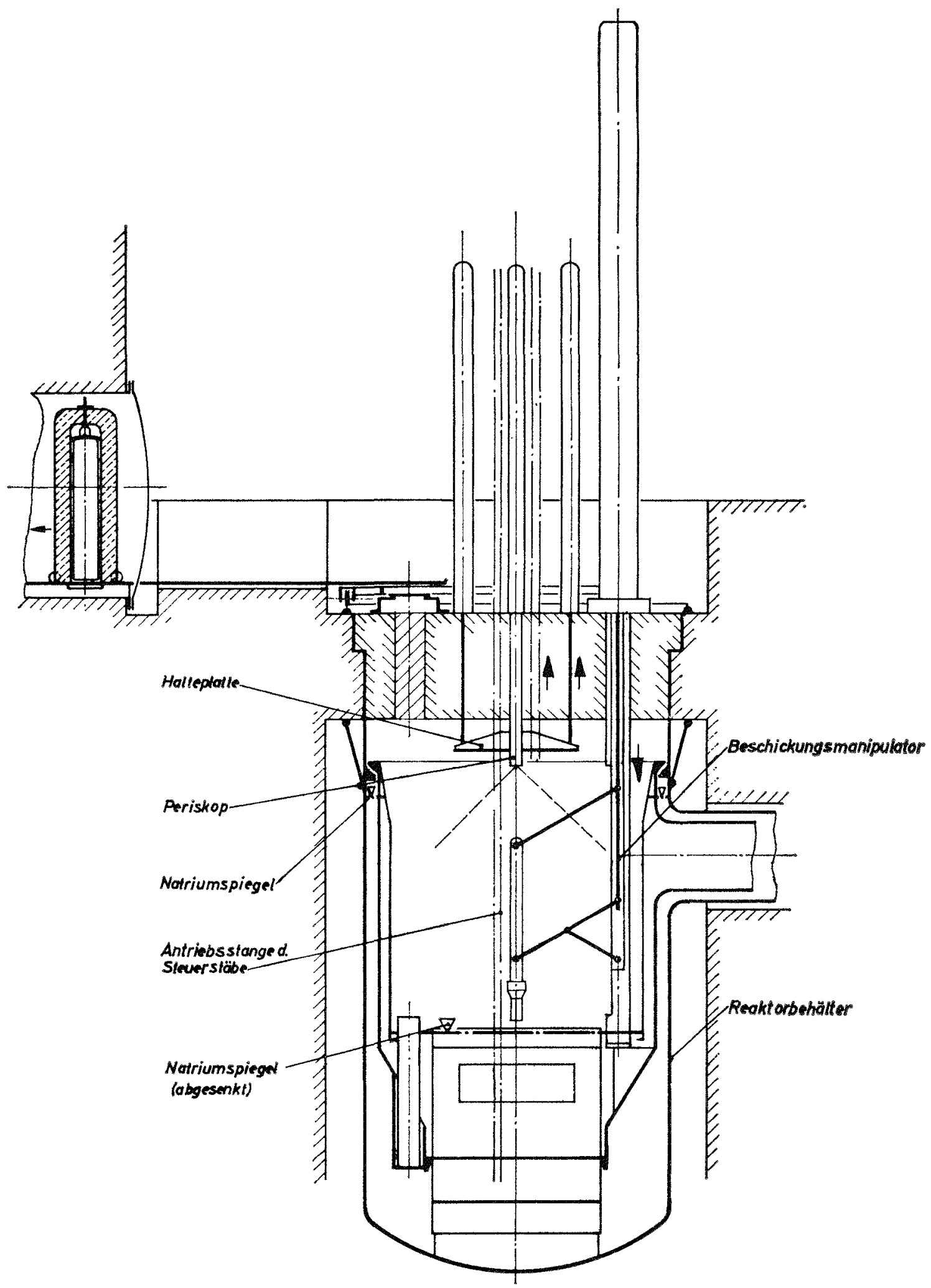

FIg. 2B. Preparation of the Reactor for Fuel-element Exchange. 


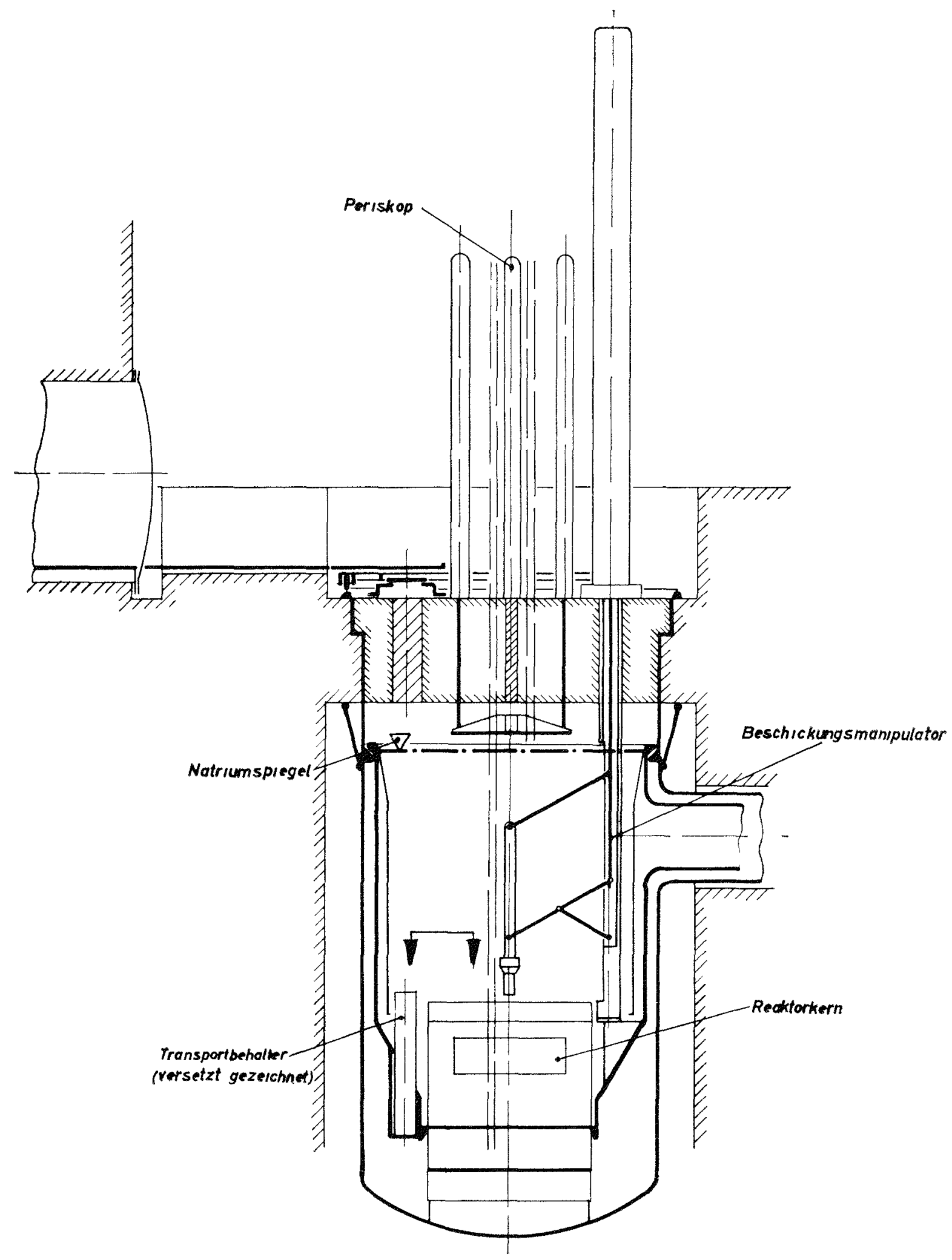

Fig 2C tuel element Exchange under Sodum 


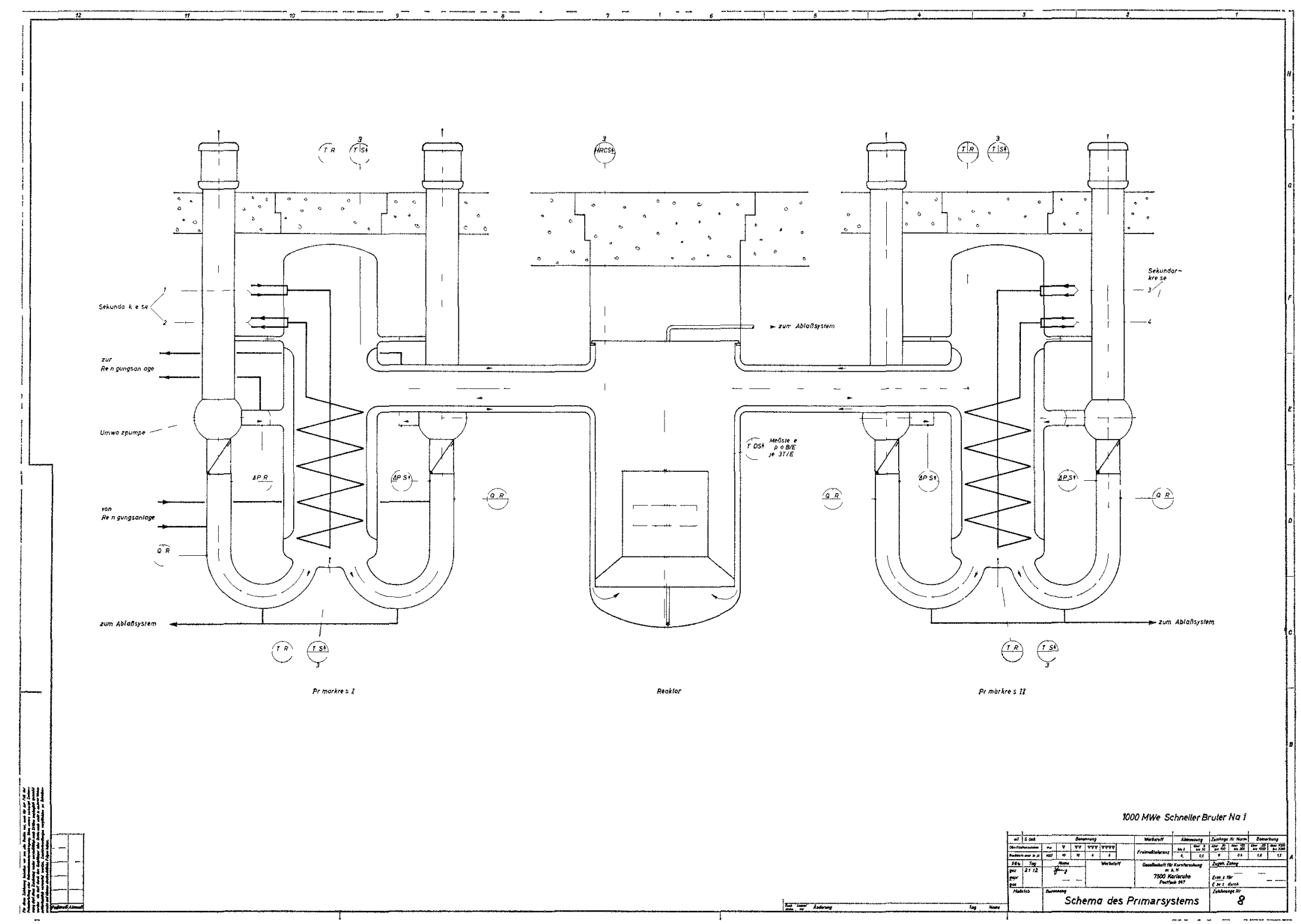




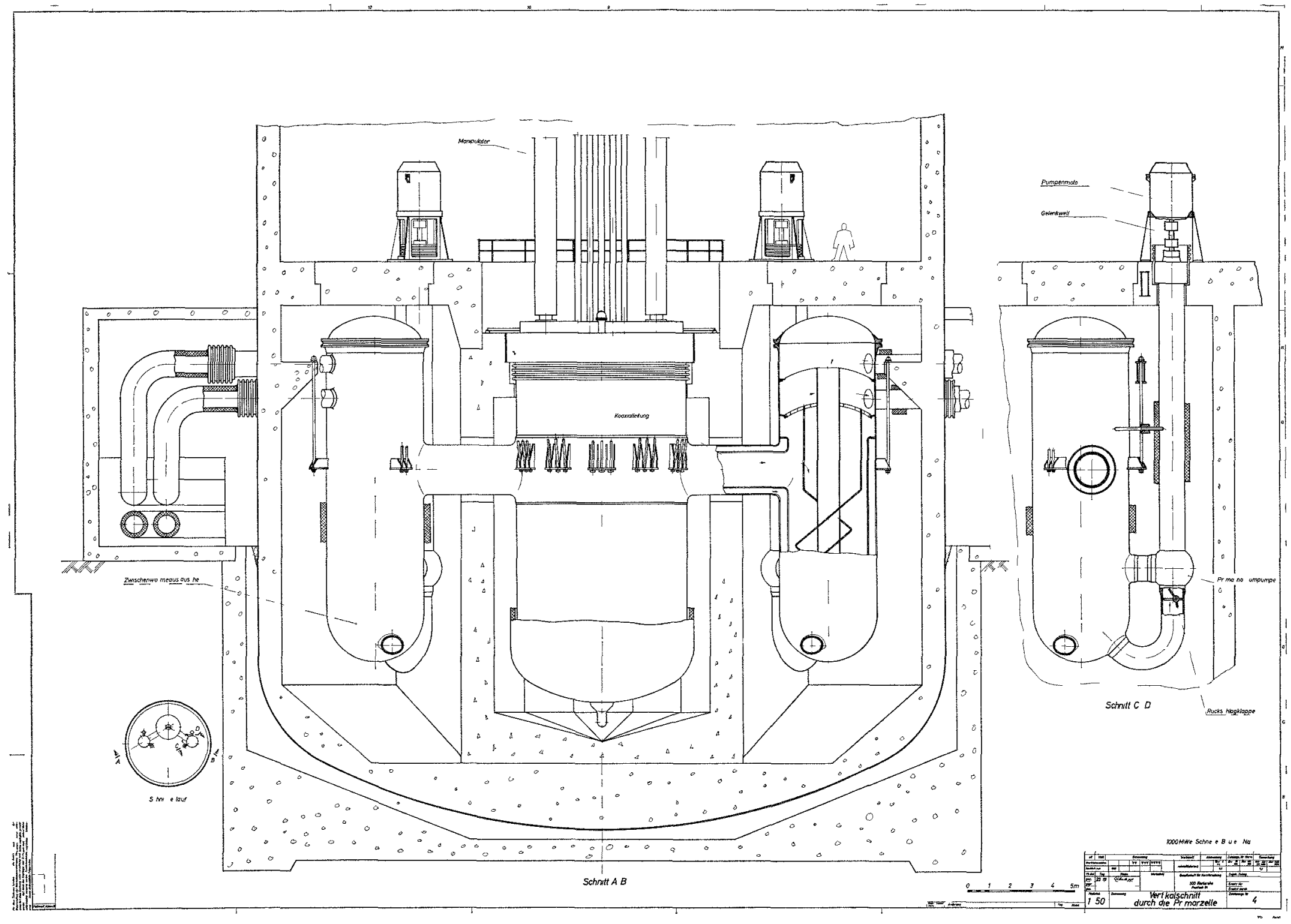

Fig 4 Vertical Section of the Primary Cell 


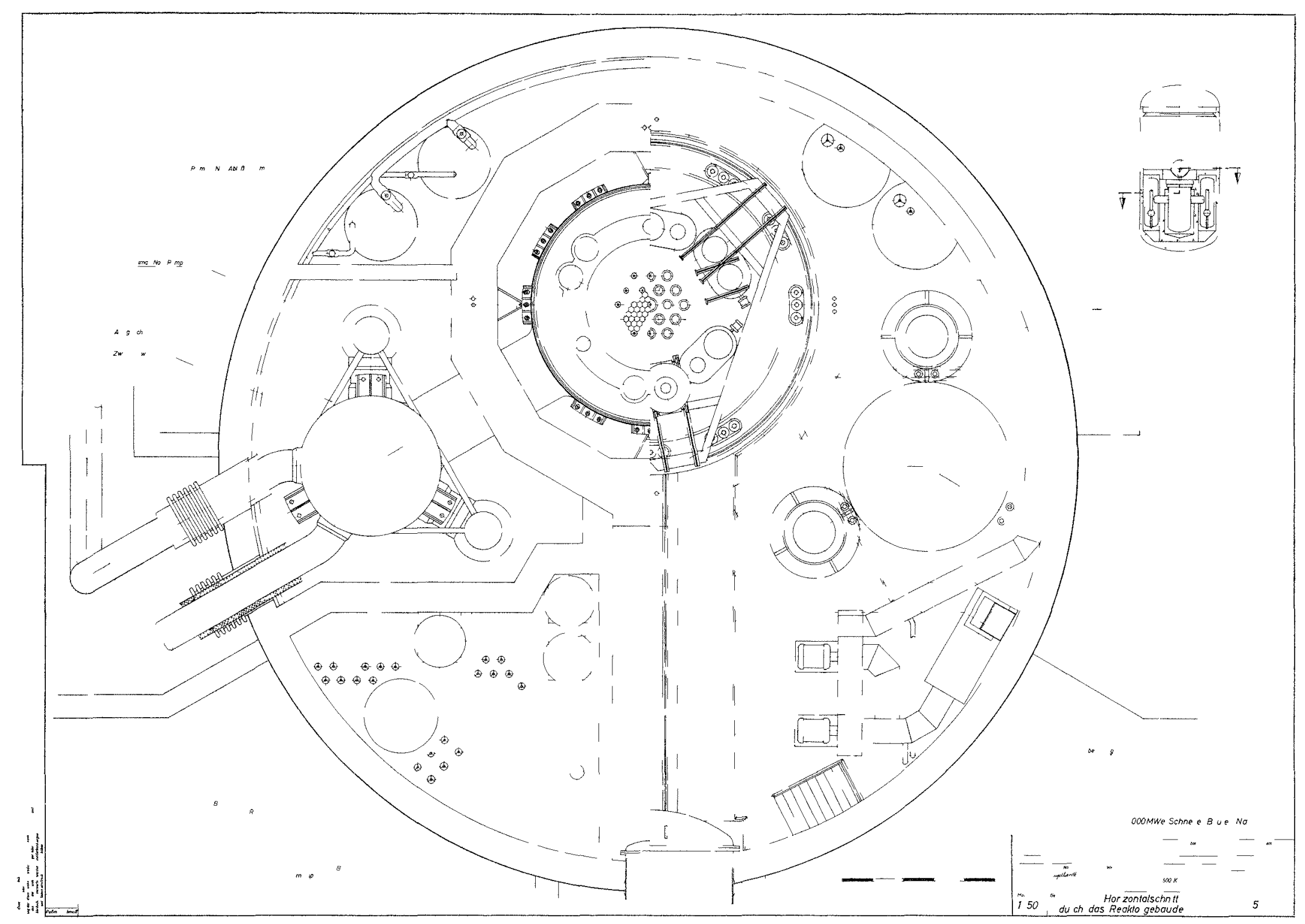

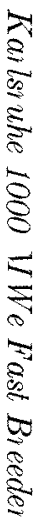

Hig 5 Horizontal Section of the Redetor Bulding 


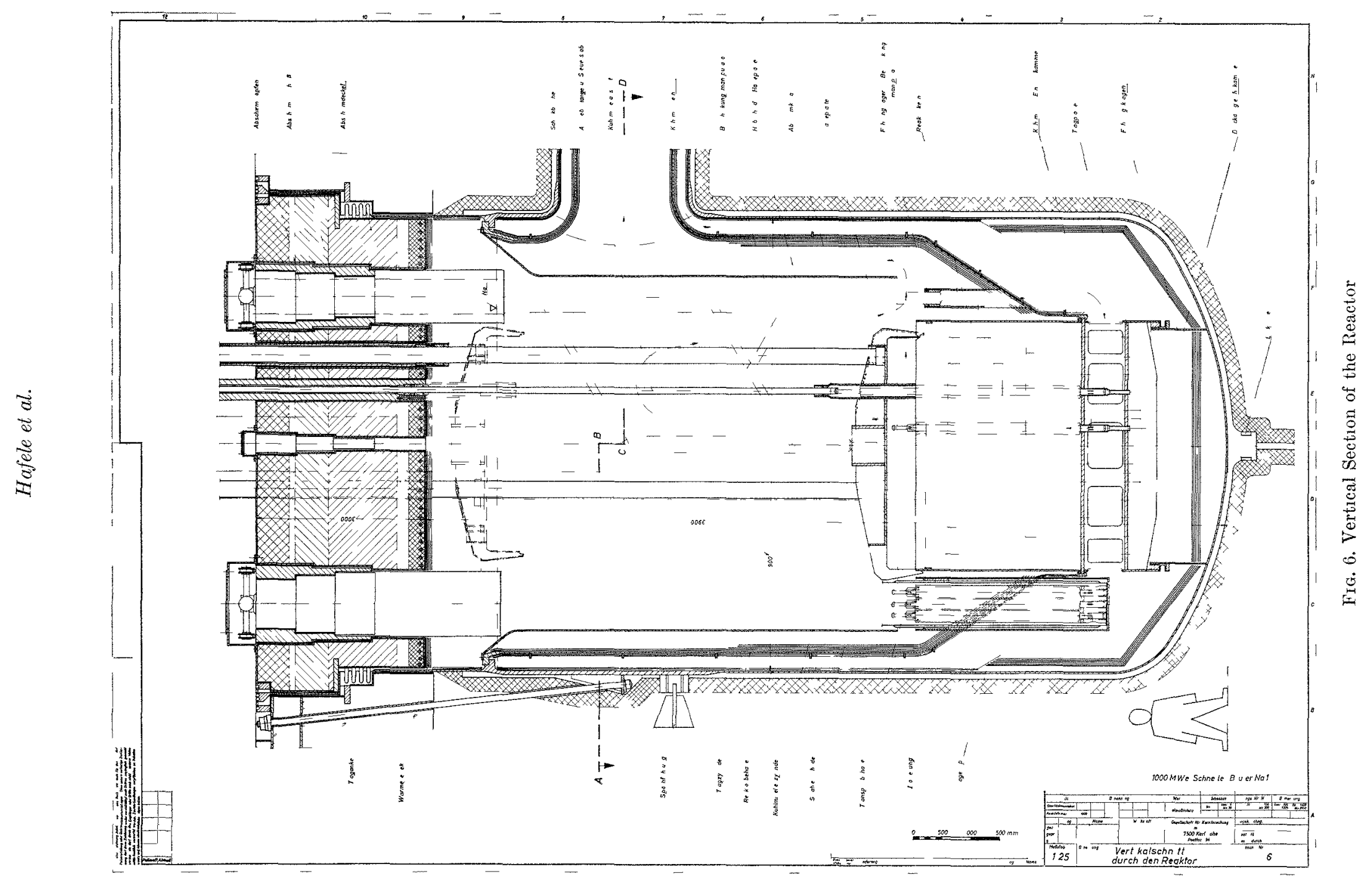




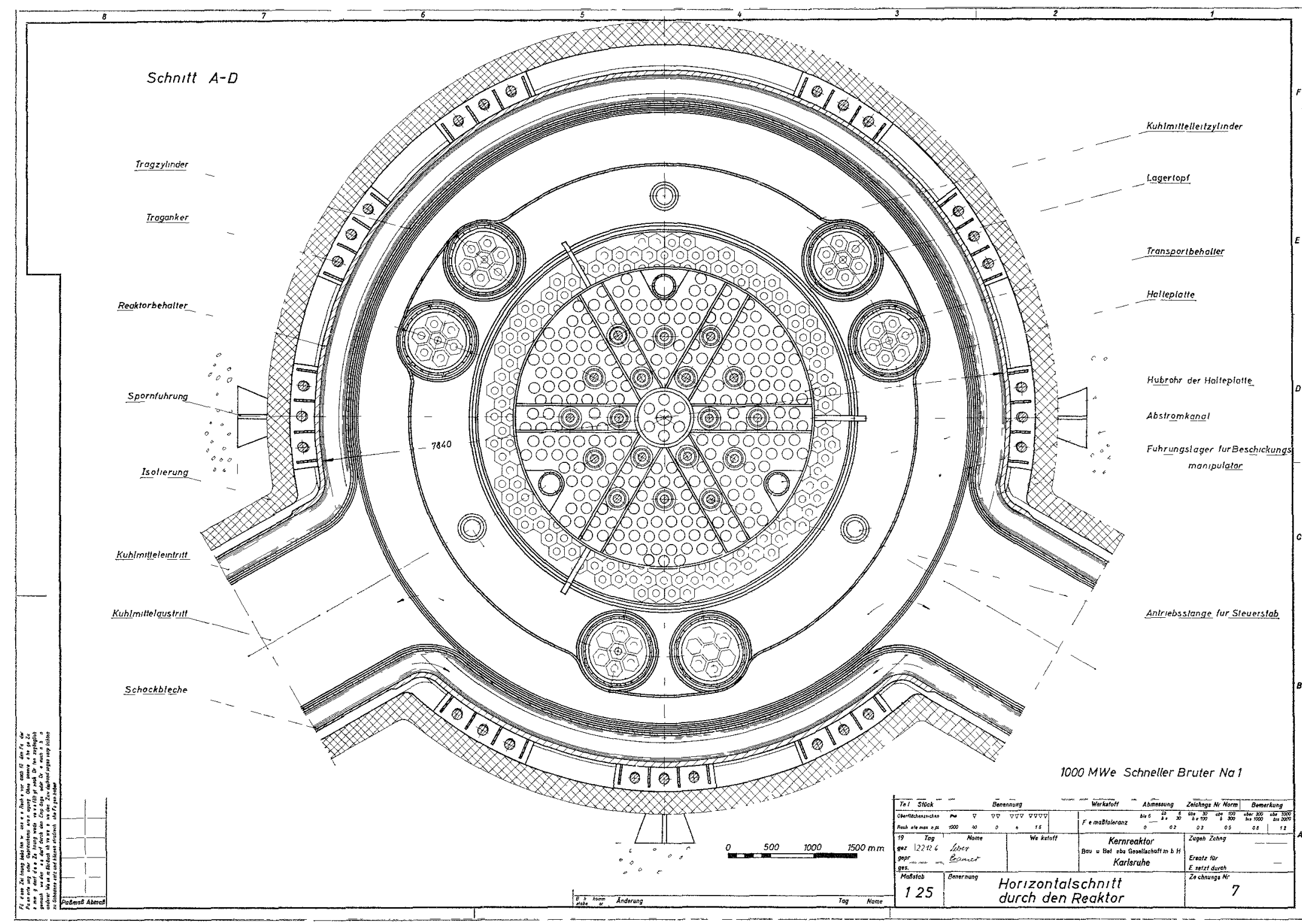

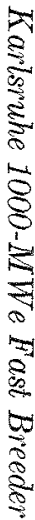

FIG. 7. Horizontal Section of the Reactor. 
exchanger provides a coolant flow large enough to pick up about $7 \%$ of the nominal heat production. As a first-order approximation, it was assumed that there will be no main primary pipe fajlure. Together with the cooling capability by natural convection this eliminates the necessity for an emergency cooling system. As we will see later on, the $\mathrm{Na}-1$ design does not make too pessimistic assumptions in order to study the capital cost potential of this kind of a reactor. The systems and safety analysis of $\mathrm{Na}-1$ may well lead to the requirement that there be an emergency cooling circuit for the coolant of the reactor vessel of the $\mathrm{Na}-2$ study.

All main pipes are coaxial. Therefore it is possible to have all outer pipe walls and all the containers at the same temperature, namely, that of the sodium entrance, $430^{\circ} \mathrm{C}$. On the other hand, this leads to great difficulties in having gate valves at the exit of the reactor vessel. With the intent of avoiding a main pipe break, no gate valves were provided in the Na-1 design. This is being subject to a careful re-evaluation for $\mathrm{Na}-2$.

The reactor tank is located eccentrically in the containment building in order to have a small diameter $(28 \mathrm{~m})$ for the reactor building.

The secondary circuit leaves the reactor building. Stcam generators and turbines are outside.

Advantage can be taken of the fact that there is a low pressure drop in the primary circuit $\left(5 \mathrm{~kg} / \mathrm{cm}^{2}\right)$. This, logether with the rather low constant overall temperature $\left(430^{\circ} \mathrm{C}\right)$ for vessels, containers, and the outer wall of the main pipes, leads to a possible use of perhaps a cheap ferritic material for these components. The 20-MWe KNK-reactor at Karlsruhe is designed along this line of arguments.

\section{The Neutronic Evaluation}

The neutronic evaluation was done in several steps. First, a parametric survey was made by calculating a large number of one-dimensional - one-core-zone reactor configurations. The results of these one-dimensional reactor calculations were checked against 8 twodimensional one-core-region reactors which fit thermodynamically in the scope of the design. The one-dimensional calculations at this stage were made with a slightly modified 16-group YOMI set; for the 8 two-

TABLE 1. SOdium VoId EFFects (One-dimensional Calculation by Using the ABN-Set)

\begin{tabular}{l|c|c|c|c}
\hline & Void $^{(1)}$ & Void $^{(1+2)}$ & Void (overall) & Void (max) \\
\hline \begin{tabular}{c|c}
$\Delta k$ \\
$\rho$
\end{tabular} & $\begin{array}{c}0.01032 \\
\$ 2.87\end{array}$ & $\begin{array}{c}0.00641 \\
\$ 1.78\end{array}$ & $\begin{array}{c}0.005445 \\
\$ 1.655\end{array}$ & $\begin{array}{c}0.011747 \\
\$ 3.27\end{array}$ \\
\hline
\end{tabular}

(1) and (2) referring to zones 1 and 2 ; $(\max )$ to $\mathrm{r}<118 \mathrm{~cm}$. dimensional calculations, a condensation into 5 groups was necessary for reasons of computer time. In the process of condensation, different weighting spectra were used for the core, and the axial and radial blankets. These spectra were obtained by one- and two-dimensional calculations. The results obtained after the condensation were cross checked and appeared to be satisfactory.

In the second step, optimization of the two-region core was accomplished. Again the 16-group YOM set in a slightly modified version was used. The optimization was done with respect to power flattening obtained by the use of proper enrichment in the two zones. This optimum appeared when the two core regions were of the same volume. These core dimensions and enrichments were used for the reference reactor. As mentioned before, the isotopic composition of plutonium was that of an equilibrium core with separate core-blanket management.

With these first data for the reference reactor at hand, a careful neutronics calculation for the reactor was made. The results were first calculated with a onedimensional code and then cross checked against a 5group two-dimensional $\mathrm{S}_{2}$ calculation. The agreement was quite good. Thereafter the calculation was repeated with the 26-group Russian set, because this set had all materials of interest available as self-shielded, 1emperature-dependent group constants, including those for the fission products. The details of the flux distribution, in partrcular in the radial blanket, were also considered.

The third step was to calculate the power and energy coefficients. The Doppler coefficient was calculated by means of the Karlsruhe code DCP (Doppler Coefficient by Perturbation), using as spectrum the results calculated with the Russian 26-group constants set $(A B N)$. The neutron lifetime and $\beta_{\text {eff }}$ were calculated. on the basis of the 16-group modified YOM set. The sodium void effect was calculated again, using a twodimensional code, "Twenty Grand," and 6 neutron groups coming from a condensation of the Russian 26 -group set. The results were compared with the corresponding one-dimensional results. The difference between the two maximal sodium void effects was less than $10 \%$ (compare Tables 1 and 2). One should note

TABLE 2. Sodrum VoId EFrects (Tho-dimensional Calculations with a 6-Group Set Coming FROM A CONDENSATION OF THE ABN SET)

\begin{tabular}{l|c|c|c|c}
\hline & Void $^{(1)}$ & Void $^{(1+2)}$ & Void (overall) & Void (max) \\
\cline { 2 - 4 }$\Delta k$ & $\begin{array}{c}0.00654 \\
\$ 1.8104\end{array}$ & $\begin{array}{c}0.00375 \\
\$ 1.037\end{array}$ & $\begin{array}{c}-000479 \\
\$-1.326\end{array}$ & $\begin{array}{c}0.01108 \\
\$ 3.066\end{array}$ \\
\hline
\end{tabular}

\footnotetext{
* See Table 1 footnotes.
} 
the difference in sign for the overall effect. This shows that calculational difficulties still exist.

Nuch attention was paid to proper calculation of burnup. The basis was a model for batch loading. Local groups of $n$ subassemblies were formed. Each subassembly of these local groups belonged to a different batch. Unloading of a subassembly occurs in the neighbourhood of $100,000 \mathrm{MWd} /$ tonne. If the number of batches is $n$, the average burnup in the local groups varies only for $100,000 / n$ between two successive refueling steps. A choice was made to have $n=3$ in the inner zone and the inner region of the outer zone, and $n=4$ in the outer region of the outer zone. This leads to a fuel lifetime of $3 \cdot 196 \mathrm{~d}$ and a reactivity change of $0.7 \%$ due to plutonium burnup; if fission products are included it will be $2.5 \%$. The choice of $n=3$ and $n=4$ in the outer zone of the core instead of $n=3$ in the whole core increased the average to maximum burnup by $6 \%$.

Finally, reactivity values were calculated on tho basis of a perturbation theory for the interchange of a subassembly of the outer with inner zone and for the sodium voiding of the central subassembly. The results are summarized in Table 3. Values have been published, ${ }^{(2)}$ but the calculation for the reference design is being improved continuously. New results have been calculated with the KFK 26-group set of constants. ${ }^{(9)}$ This set has the same group structure as the ABN set but starts from different microscopic data and uses a typical spectrum of a sodium-cooled $1000-\mathrm{MWe}$ reactor as a weighting spectrum. The main changes in the results are the following:

critical mass is increased by about $6 \%$;

the Doppler coefficient is decreased by about $25 \%$;

the maximum sodium void reactivity value increases from about $\$ 3$ to about $\$ 7$.

\section{The Accident Analysis}

The accident analysis for the Na-1 design is now under way. A first version has been prepared. ${ }^{(2)}$ First results are reported by Smidt et al. ${ }^{(3)}$

The basic approach for the accident analysis arose from an overall view as to the possibilities of accidents. We have four basic types of accidents:

a) loss of coolant flow;

b) accidental reactivity ramp insertions;

c) major breaks in the pipes containing sodium;

d) plugging of the cent ral (or an inner) subassembly.

a) The accident due to loss of coolant flow happens if all four pumps fail, as would occur if the regular power supply and that of the emergency drive fails. If, in addition, as a third coincidence, the safety system fails, the analysis indicates that one has a time interval of 80 sec before sodium begins to boil. After this
TABLE 3. The Effects of Flel Burnup for Various Numbers OF BatChes*

\begin{tabular}{|c|c|c|c|c|}
\hline \multirow{2}{*}{$\begin{array}{l}\text { Number of batches } \\
\text { in the inner and outer } \\
\text { zone of the core }\end{array}$} & \multicolumn{2}{|c|}{$\Delta k_{\text {(ff ff }}$} & \multirow{2}{*}{$\begin{array}{l}\text { Average to } \\
\text { maximum } \\
\text { burn-up }\end{array}$} & \multirow{2}{*}{$\begin{array}{l}\text { Time differ- } \\
\text { ence bet- } \\
\text { ween succes- } \\
\text { sive refueling } \\
\text { steps }\end{array}$} \\
\hline & $\begin{array}{c}\text { Without } \\
\text { fission } \\
\text { products }\end{array}$ & $\begin{array}{l}\text { Fission } \\
\text { products } \\
\text { included }\end{array}$ & & \\
\hline $\begin{array}{l}\text { Inner Zone } \\
\text { Outer Zone }\end{array}$ & $0.76^{\prime} / c$ & - & $79 \%$ & \\
\hline $\begin{array}{c}\text { Inner Zone } n=3 \\
\text { Outer Zone }{ }^{2}=3 \\
\text { Inner Region } \\
n=3 \\
\text { Outer Region } \\
n=4\end{array}$ & $0.74 \%$ & $2.5 \%$ & $85 \%$ & \\
\hline
\end{tabular}

* Calculation by using the ABN set.

** Averaged over the radius $r$.

time interval a sodium voiding accident is to be expected.

One can argue about the probability of a simultaneous mechanical failure. In that event only one further conditioned event, namely, that of the failure of the safety system, has to happen. But counting the simultaneous mechanical failure of all four pumps and, in particular, that of the safety system is a picture which is too rough, because these events are in themselves multiconditioned. In agreement with other assumptions concerning the mechanical design (see Sect. 4 above) we assume that there will be no such simultaneous mechanical failure. This leads to the conclusion that in the two conditioned cases of pump failure (regular and emergency drive failure) the safety system has to act within $80 \mathrm{sec}$.

b) Accidental insertions of reactivity can happen at the beginning of a sequence of events or as the consequence of sodium voiding or core compaction. Sodium voiding and core compaction lead to high values of the associated ramp rates $(10-100 \$ / \mathrm{sec})$, whereas reactivity insertions at the beginning of a sequence of events lead to low values of ramp rates. For example, the loading accident for a central subassembly gives $\$ 0.7$ in $350 \mathrm{msec}$, that is, $2 \$ / \mathrm{sec}$. Again, sodium voiding of the central subassembly leads to a change of $\$ 0.07$ in, say, 100 msec, resulting in a ramp rate of $0.7 \$ / \mathrm{sec}$. The size of an accidental reactivity insertion by a control rod runaway depends on the particular design, but will always be at ramp rates below $1 \$ /$ sec.

In the analysis of reactivity insertions the class of ramps between 0 and $20 \mathrm{~s} / \mathrm{sec}$ was considered. It is useful to make two distinctions. If a reactivity input is below $\$ 1$, the resulting excursion is limited as to reactivity value; it is largely the total reactivity value and not so much the ramp rate which determines the size of the excursion. If the reactivity is larger than $\$ 1$, 
it is in the region beyond $\$ 1$ the ramp rate and not the final reactivity value which determines the size of the excursion. The other distinction is that between low ramp rates leading to sodium boiling before fuel starts melting or vaporizing, and ramp rates leading to fuel melting or vaporization before sodium starts boilung. The limiting ramp rate for this distinction is a function of the reactor propertios, in particular of the Doppler coofficient and the average fuel temperature. In certain rases melting of the cladding instead of sodium boiling can be the relevant criterion.

The reactivity insertion which the reactor can accept without action of the safety system is 80.25 . These are rather weak insertions. For all other insertions stronger than that (reactivity value and ramp rate), scram by a safety system is required. Therefore the logical question is now: What kind of a safety system is required? The detailed studies of Smidt et al. ${ }^{(3)}$ give the answer for the Na-1 design in the form of a parametric study. The result is that for a delay time in the safety system of 50 msee and a realistic shutdown reactivity versus time characteristic having a $150 \mathrm{~s} / \mathrm{sec}$ ramp rate in its linear part and having a Doppler coefficient of $6 \times 10^{-6}$ at $1400^{\circ} \mathrm{K}$, a permitted accidental reactivity ramp of $10 \$ / \mathrm{sec}$ is allowed if the beginning of melting is the limit. The value of $150 \$ / \mathrm{sec}$ assumes, of course, that all shut-off rods are in action (10 rods at $\$ 1.5$ each). A delay time of 50 msec is a rather high value and means that more or less conventional shut-off systems can be applied. If the delay time would be 10 msec, about $14 \$ / \mathrm{sec}$ instead of $10 \mathrm{\$} / \mathrm{sec}$ could be handled. The influence of the Doppler coefficient is limited here. The reason is that its action reduces the net reactivity to values close to $\$ 1$ if the ramp insertion has led to reactivity values larger than $\$ 1$. Before prompt criticality is reached, the Doppler is of very small influence because the large periods of the power-level increase do not change the fuel temperature during that period. If $A=T(d k / d T)$ is the Doppler constant, $\Delta T$ the permissible fuel temperature interval in the case of an excursion, $T_{0}$ the fuel temperature at normal operation, and $r_{s}$ the accidental ramp rate, the time $t_{s}$ to fuel melting or vaporization is given by

$$
t_{s} \sim \frac{1+A \ln \left(1+\frac{\Delta T}{T_{0}}\right)}{r_{s}},
$$

Only if $A \ln \left[1+\left(\Delta T / T_{0}\right)\right]$ gives a significant contribution compared to unity does the Doppler have a stronger influence on the characteristics of the safety system. That is the case for carbide fuel at low rod power. For a 50-msec delay time, a Doppler coefficient of $4 \times 10^{-6}$ leads to $9.3 \$ / \mathrm{sec}$, a Doppler coefficient of $8.5 \times 10^{-6}$ to only $10.5 \$ /$ sec, permissible accidental ramp rates. The function of the Doppler coefficient is therefore to serve as an inherent safety mechanism against supercritical fast reactivity insertions and particularly to guarantee stability. The impact of the uncertainties in the nuclear calculation of the Doppler roefficient on the design of the safety system is limited.

One important conclusion to be drawn from these arguments is that an unconventional safety system. with very short delay times ( 1 msec or so) is required only in the case of accidental reactivity ramps much larger than, say, $20 \$ / \mathrm{sec}$. But it is difficult to think of such accidental ramp rates in the beginning of a sequence of events. Only sodium voiding of the core could give larger values, but according to arguments sodium voiding is only a consequence of accidents which are accompanied by a failure of the safety system. We therefore conclude that for the safety system a conventional version of it is sufficient. This differs from the earlier Karlsruhe thinking.

Whether an accidental ramp-reartivity insertion is simple or complex is a subject for a more detailed. safety discussion, but it is of no importance here in this context.

c) Major breaks of piping carrying sodium between the reactor vessel and the intermediate heat exchanger interrupt the circuit of natural convection for a primary circuit of the loop type. Such a break would require shut down and emergency cooling in the vessel.

According to the arguments of Sect. 4, such a major break of a pipe is excluded in the Na-1 study by definition, although it may be considered in the $\mathrm{Na}-2$ study. The reactor vessel itself has a double wall, and therefore a break is definitcly excluded.

d) Before considering an accident leading to a major sodium voiding of the core, the accidental plugging in the central subassembly shall be considered. If such a plugging takes place, the response of the neutron flux might be very small because of the low reactivity value of sodium voiding of the central subassembly. No reaction of the safety system therefore takes place, and the sodium in the central subassembly might boil. Smidt ${ }^{(3)}$ has emphasized that, e.g., in the case of the $\mathrm{Na}-1$ design at $28 \%$ of normal flow, boiling of the sodium occurs, but already at $22 \%$ of the normal flow unstable flow conditions may occur, thus keeping the coolant in the channel. This leads to the beginning of a more serious sequence of accidental events which will be discussed below. One can argue that boiling can perhaps be detected acoustically and therefore be used as an initiation of a scram signal. As long as the safety system works this kind of an accident might therefore be prevented.

But it is also possible that the sodium superheats and 
does not boil. (3) This can possibly not be detected by instrumentation. Thereafter sudden desuperheating may well lead to destruction of the central subassembly and its neighbours, thus leading to major core damage and ending in a serious core explosion. This will be discussed below. This difficulty of an undetectable plugging and superheating accident is an unresolved problem. In order to attack this problem much more has to be known on the mechanism of two-phase flow and superheat, both theoretically and experimentally. Fischer and Häfele ${ }^{(10)}$ and Friz ${ }^{(1)}$ have made a first step in this direction.

Along the same lines one also has to fear a break of the cladding at the fission gas plenum. This might propagate and result in a plugging of the coolant channel. This danger is somewhat emphasized in the Na-1 design, in which the fission gas plenum is at the lower end; putting it on the top of the fuel pin probably does not change the situation drastically.

We realize that all considerations of this chapter with the exception of the plugging and superheating accident do not lead to any larger accident in case the safety system works; in particular we realize that the size of the sodium void effect did not influence our considerations. The mechanism of the plugging and superheating accident therefore requires further thorough investigations. But the aceidents of types a-d lead to major damage in case of a simultaneous failure of the safety system. The sequence of events leading to such major damage all involve sodium voiding of major parts of the core. This leads to the maximum hypothetical accident.

\section{The Maximum Hypothetical Accident (MHA)}

Assume a reactivity ramp insertion as discussed under b) in the last section. The accidents discussed there have such a low value for rate of insertion that sodium boiling starts before fuel melting ends and vaporization of the fuel starts. In the case of the $\mathrm{Na}-1$ design, the limit for these reactivity ramps is $3 \$ /$ sec. We also assume that the safety system fails to react. Now it is not sufficient to assume that boiling begins at the upper end of the core where the sodium void effect is negative. According to the argument of Smidt, ${ }^{(3)}$ we have to assume unstable flow conditions. In this case voiding starts at the center of the core at the place of the largest positive void effect. If one wants to evaluate the ramp rate associated with sodium voiding, the situation is as follows:

The original calculation of the maximum integral void effect in the $\mathrm{Na}-1$ reference design gives a maximum value of $\$ 3$ for sodium voiding; the latest results indicate a higher value, elose to $\$ 7$. In the analysis of this accident the uncertainty in the nuclear calculation of the void effect appears for the first time to be of relevance. If one wants to continue to evaluate the maximum ramp rate, a second uncertainty appears, namely, that of the time scale for ejection of the sodium. Here the full scope of the two-phase flow of sodium appears. As Fischer and Häfele show, ${ }^{(10)}$ for low amounts of gas in the two-phase mixture one has to expect a rather low value of the velocity of sound (easily as low as $10 \mathrm{~m} / \mathrm{sec}$ ). This means that all kinds of shock-wave complications have to be expected in the pattern of flow. The time seale is probably uncertain to a much higher degree than the reactivity value of voiding. Both uncertainties combine if the ramp rate is calculated. Presently we bridge this difficulty by assuming simply a ramp rate of $100 \$ /$ sec.

After the original initiating excursion one finds that, in addition to the boiling of sodium, the fuel melts. The FORE analysis indicates that the hotter part of the fuel is already molten when the boiling starts. If the molten fuel touches the can, the heat resistance in the gap disappears and the temperature of the cladding is suddenly increased by about $500^{\circ} \mathrm{C}$. The immediately following formation of bubbles of sodium vapor is consistent with the above-mentioned second uncertainty of the time scale for sodium voiding. This formation of bubbles of sodium vapor means that there might be a sodium-steam explosion of the BORAX type.

After the melting of the can fuel, compaction inside and outside of the original fuel element could start. This would lead to another reactivity ramp. Both ramps, that of sodium voiding and that of fuel compaction, would add if the two involved mechanical processes would not interfere. They would lead to a nuclear excursion and subsequent explosion. Superimposed is the sodium-steam BORAX-type explosion.

We have not yet a clear jdea about the patterns of interactions of these two processes. Here is another open problem of great importance.

It is useful to realize the difference between this kind of an analysis and that for a small reactor with metallie fuel elements, like EFFBR or EBR-II. In the latter case we have a negative void effect. Any displacement of somewhat larger amounts of sodium shuts the reactor down; the two ramps involved there subtract and don't add as above. The metal fuel melts easily and drops to the bottom of the core, perhaps forming a second critical mass. This second critical mass is not located at the place of the first critical mass, that is, the core. The excursion in this second critical mass is known as a second excursion and constitutes the MHA. In our case, the accidental excursion occurs at the place of the original core and the BORAX type of sodium-steam explosion is superimposed; the ramps add and there is no time for a quiet meltdown. 
The plugging and superheating accident for the rentral subassembly can develop a similar final picture. Sudden desuperheat destroys the central subassembly and its neighbours. The probable interruption of the coolant flow there leads to boiling of sodium (or to supcrheat of sodium, which in turn would increase the area of subassembly destruction even further by a repetition of this mechanism). The ejection of sodium from a somewhat greater number of subassemblies leads now to a reactivity ramp of a relevant height and could initiate the scram rods. But here we have to assume that, even if the safety system works, the safety rods don't drop into the core because of mechani(al distortions there. Now the sequence of events develops as above.

The amount of buildup of kinetic energy in an excursion of a given ramp rate was calculated by $\mathrm{Böhm}^{(12)}$ and later by Küsters. They used a Bethe-Tait approach involving a spherical model for the supercritical mass. It is realized that extremely flat cores of, say, $\mathrm{H} / \mathrm{D}=$ $1 / 6$ may better be treated as a slab model, which should give smaller energy releases. This will be done.

In the Bethe-Tait type of ralculation there exists another uncertainty, namely, the uncertainty as to the equation of state for a realistic fuel, which is a mixture of $\mathrm{UO}_{2}-\mathrm{PuO}_{2}$ and fission products containing fission gases to a various degree.

At Karlsruhe originally an equation derived from the measurements of Ackermann ${ }^{(13)}$ were used. This led to a rather quick pressure buildup and limited the energy release nicely. A larger energy release is obtained by the use of the Wolfe-Meyer equation of state. ${ }^{(14)} \mathrm{A}$ safer approach is obtained by the use of the latter equation of state. The Doppler coefficient is included in the calculation. This results in a remarkable reduction of the energy release. The total energy release and that of kinetic energy for different values of the Doppler

TABLA 4. Energy Relaese for $\alpha_{R}=500 \$ / \mathrm{sec}$.

\begin{tabular}{c|c|c}
\hline Doppler Coefficient & $\Phi_{0}\left(n / \mathrm{cm}^{2} \mathrm{sec}\right)$ & $E(\mathrm{~kg} \mathrm{TNT})$ \\
\hline$-8 \times 10^{-6}$ & $10^{10}$ & 256 \\
$10^{14}$ & 48 \\
$10^{18}$ & 114 \\
$-4 \times 10^{-6}$ & $10^{10}$ & 539 \\
& $10^{14}$ & 84 \\
$-1 \times 10^{-6}$ & $10^{18}$ & 280 \\
& $10^{10}$ & 5474 \\
& $10^{14}$ & 2848 \\
0 & $10^{18}$ & 882 \\
& $10^{10}$ & 29814 \\
& $10^{14}$ & 18112 \\
$10^{18}$ & 8830 \\
\hline
\end{tabular}

TABLE 5. FNERGY RELEASE FOR $\alpha_{B}=100 \$ / \mathrm{sEC}$.

\begin{tabular}{c|c|c}
\hline Doppler Coefficient & $\Phi_{0}\left(n / \mathrm{cm}^{2}\right.$-sec $)$ & $E(\mathrm{~kg} \mathrm{TXT})$ \\
\hline$-8 \times 10^{-6}$ & $10^{10}$ & 34 \\
$10^{14}$ & $70^{18}$ & 17 \\
$-4 \times 10^{-6}$ & $10^{10}$ & 79 \\
& $10^{14}$ & 85 \\
$-1 \times 10^{-6}$ & $10^{18}$ & 85 \\
& $10^{10}$ & 43 \\
& $10^{14}$ & 284 \\
0 & $10^{18}$ & 239 \\
& $10^{10}$ & 6019 \\
& $10^{14}$ & 2196 \\
& $10^{18}$ & 2055 \\
\hline
\end{tabular}

coefficient at $2500^{\circ} \mathrm{K}$, the initial flux, and two ramp rates are given in Tables 4 and 5 .

The Na-1 reference design has a Doppler coefficient between $6 \times 10^{-6}$ and $8 \times 10^{-6}$ at $1400^{\circ} \mathrm{K}$; at $2500^{\circ} \mathrm{K}$ this leads to values of 3.3 and $4.5 \times 10^{-6}$, respectively. What to use for the correct value of the Doppler coefficient and the initial flux ronstitute principal difficulties in calculating the energy release. With a value of $4 \cdot 10^{-6}$ for the Doppler the energy release corresponds to that from $85 \mathrm{~kg}$ of TNT for $100 \$ / \mathrm{sec}$ and $\Phi_{0}=10^{14} \mathrm{n} / \mathrm{cm}^{2}$-sec, and at $500 \$ / \mathrm{sec}$ we have, for $\Phi_{0}=10^{10} \mathrm{n} / \mathrm{cm}^{2}$-sec an energy release corresponding to $539 \mathrm{~kg}$ TNT.

A major experimental effort is in preparation at Karlsruhe in order to investigate the equation of state experimentally.

\section{The Design Basis Accident (DBA) and the Design of the Containment}

About a year ago, when the Karlsruhe reference design, Na-1, neared its completion, the uncertainty of calculating the energy release in case of a MHA was larger than it appears today. So it was decided to accept in case of the Na-1 design the philosophy that the safety system does not fail. Reporting on the results of the third Geneva Conference, W. Häfele and P. Engelmann ${ }^{(15)}$ pointed out that this philosophy is accepted by many fast reactor groups. The mechanism of the plugging and superheating mechanism had not yet been detected, a fact which excluded the MHA as a DBA. The DBA is therefore a large sodium fire.

The steel container is designed to withstand a maximum pressure of $2.5 \mathrm{~atm}$. But it is highly improbable that this pressure builds up because only a fraction of the sodium can burn, for all the compartments containing the primary circuit have a nitrogen atmosphere; only in the case of a major explosion, which is 
excluded here, could all the sodium be burned. The diameter of the containment building (see Fig. 8) is only $28 \mathrm{~m}$, fixed by the size of the primary circuit. The height is determined by the installation and maintenance or repair procedures for the components of the primary circuit. At the inside of the steel containment a layer of concrete protects against temperature of a fire and mechanical damages.

The Na-1 reference design thus approaches the twocontainment solution of the SEFOR reactor. The DBA is therefore a nuclear explosion corresponding to that of $90 \mathrm{~kg}$ TNT. It will be contained in the compartments of the concrete structure of the primary loop. The second container, the steel shell in the case of SEFOR, shall withstand the pressure of an overall sodium fire. Therefore it will not be too difficult to extend the Na-1 reference design to a DBA of the SEFOR type. To exclude the MIHA as a DBA is therefore a less severe assumption. In the forthcoming weeks an effort will be made to evaluate the capital cost potential of such change of philosophy. Thereafter the basis for the DBA of the $\mathrm{Na}-2$ design will be selected.

9. Capital Costs of the Na-1 Reference Design, Its Areas of Greatest Potential for a Reduction, and Its Competition with Breeding and Safety.

One of the objectives of the Na-1 reference design was to get a rough idea of the costs and their distribution to use as a guide for further research and development.

The fuel costs are $0.3 \mathrm{DPf} / \mathrm{kWh}=0.75 \mathrm{mill} / \mathrm{kWh}$ at a price for plutonium of $10 \$ / \mathrm{g}$. This includes interest for inventory, as well as costs for refabrication, reprocessing, and the first core. It also includes the sale of bred material. The capital costs are $462.6 \times 10^{6} \mathrm{DM}=$ $\$ 116.0 \times 10^{6}$ or $\$ 116 / \mathrm{kWe}$. The $462.6 \times 10^{6} \mathrm{DM}$ are the sum of $262.6 \times 10^{6} \mathrm{DNI}$ for the conventional part $=$ $58 \%$ of the total capital costs. $200.0 \times 10^{6} \mathrm{DM}$ are assumed to be the costs for the reactor part, that is, $42 \%$ of the total sum. But the actual capital cost evaluation led to a value of only $116.96 \times 10^{6} \mathrm{DM} ; 83.05 \times$ $10^{6} \mathrm{DM}$ are considered to be a "contingency" due to general uncertainties in the area of costs for large components. If this "contingency" is excluded, the total sum is only $380.0 \times 10^{6} \mathrm{DM}=\$ 95 / \mathrm{kWe}$ with $68 \%$ for the conventional part and only $32 \%$ for the reactor part.

The amount of $116.95 \times 10^{6} \mathrm{DMI}=\$ 29 \times 10^{6}=$ $100 \%$ will serve as the reference value for comparisons. $\$ 11 \times 10^{6}$, or $38 \%$, is for the pumps; all the devices of the cooling circuits provide $\$ 19 \times 10^{6}$, or $65 \%$, of the total costs of the reactor part. The reactor itself gives only $\$ 5 \times 10^{6}$, that is, $17 \%$.

The reactor building should be in the order of $\$ 6 \times$ $10^{6}$.
A change from oxide to carbide fuel was considered. ${ }^{(3)}$ There are two ways to use the better properties of the carbides: It is possible to use the same rod power and to enjoy the higher breeding ratio, both internal and external, and the lower average temperature only, or to operate the reactor at higher rod power. Smidt's analysis indicates that in the second case, a higher cooling flux of sodium is required. This leads to higher pressure drops in the primary circuit and, therefore, to more expensive pumps and more structural material for the subassemblies, which in turn lowers the breeding ratio. The higher cost for the pumps nearly outbalances the gain in fuel-cycle costs if they are capitalized over a period of 15 years at $9.7 \%$. Fither way of use of the carbides instead of the oxides results in nearly the same cost reduction of about $20 \times 10^{6} \mathrm{DNI}=\$ 5 \times 10^{6}$.

The components of the coolant circuits are the area with the largest potential for possible cost reduction. In the Na-1 study we used cheap ferritic steel wherever it was possible. Up to now the cheap stainless steels of type 304 or 316 are forbidden for high-temperature use. If, however, their use were permitted, the thermal efficiency could be increased by perhaps $5 \%$. This could lead to a decrease of $0.2 \mathrm{mill} / \mathrm{kWh}$ at an $80 \%$ load factor. One should realize here, that already a decrease of about $0.3 \mathrm{mill} / \mathrm{kWh}$ is the incentive for the light water superheat development. The penalty for the change from the ferritic to the cheap stainless steel would amount to only $0.03 \mathrm{mill} / \mathrm{kWh}$. If, on the other hand, we continue to be forced to use a X8 Cr-Ni-Nb 16-13 type of stainless steel, gain and loss of costs may just cancel each other. The optimization of costs requires further attention.

Evaluation of the cost situation leads to the following conclusions:

a) The carbides have a cost advantage of over $5 \%$ when it is assumed that the fuel-cycle costs are capitalized over 15 years at $9.7 \%$. This advantage is large enough to be interesting, but it is not so large that the Karlsruhe group would follow a parallel development of both oxides and carbides. The development of the carbide fuel is a meaningful second step. The use of carbide also gives higher breeding and, with the same rod power as used for the oxides, the advantage of a higher inherent safety. Cost advantage, safety, and breeding all tend in the same direction.

b) Among the more direct reactor costs, those components required by the use of sodium have the largest potential cost. In particular, the pumps are very expensive so that a well-targeted development program for pumps is advisable. Also, the questions of materials to be used in the circuits and components containing sodium are of interest, especially in terms of the background of present engineering regulatory rules in 
Häfele et al.

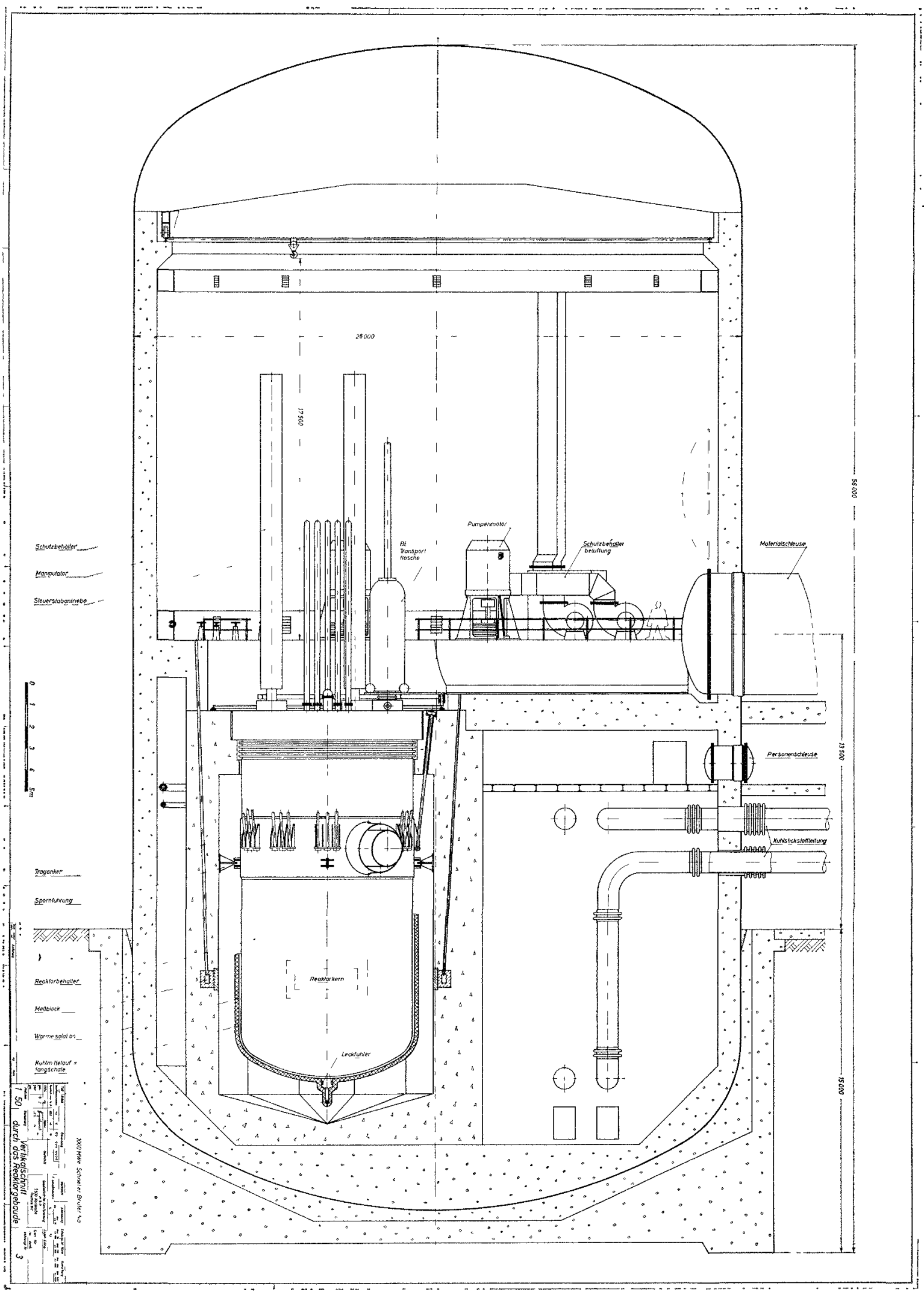

Fra. 8. Vertical Section of the Reactor Building. 
Germany. Serious consideration must be given to the use of cheap ferritic steel, particularly with respect to thermal efficiency.

c) The reactor building of the Na-1 design costs $6 \%$ of the whole plant. A more complicated building of the two-container principle able to withstand explosion of, say, $100-200 \mathrm{~kg} \mathrm{TNT}$, is being further investigated. It has been estimated that its use would increase the cost from 6 to 7 or $8 \%$ of the total. Therefore this does not result in a heavy competition between safety and economics.

d) The reactor itself involves $5 \%$ of the cost. Therefore any complication which appears to be necessary for safety or breeding again does not penalize the economies.

Generally one can say that the often-cited competition between economy, safety, and breeding does exist, but is not too heavy. In the case of fuel all three aspects are favourably affected by the use of the carbides.

\section{Conclusions}

1) A 1000-\IWe fast breeder reactor cooled by sodium appears technically feasible.

2) The safety properties of such a reactor do not constitute a major problem. Accidental reactivity insertions below $\$ 0.25$ can be accepted in the $\mathrm{Na}-1$ reference design without the action of the safety system. More severe reactivity insertions require the use of a rather conventional safety system (about a 50-msec delay time before mechanical movement starts).

3) The Doppler coefficient allows for the use of such a rather conventional safety system and guarantees overall stability. The actual size does not have too large an effect on the design characteristics. If the safety system works, the size of the sodium void effect does not have a large feedback on the design.

4) Within the realm of a working safety system, the recently detected plugging and superheating accident of a central subassembly requires further attention.

5) If the safety system can fail, major accidents are possible. Then the size of the sodium void effect is of great importance. Its positive sign leads to an addition of the ramp rates due to sodium voiding and fuel compaction. Both, the nuclear excursion and a sodiumsteam explosion superimpose. The Doppler coefficient decreases remarkably the release of kinetic energy.

6) Work should be done in the area of:

a) the nuclear evaluation of the sodium void effect;

b) two-phase flow of sodium;

c) sodium-steam explosion and the mechanism of core destruction;

d) a realistic equation of state for an oxide fuel.
7) The expected fucl-cycle cost for the Na-1 reference design is $0.7 \mathrm{mill} / \mathrm{kWh}$. The capital costs are $\$ 100 / \mathrm{kWh}$, the breeding ratio is about 1.4 ; the doubling time (of a population of fast breeder) is about 7 years. This is very satisfactory. ${ }^{(16)}$

8) The choice of fuel is oxide. Carbide could lead to a decrease of the order of $5 \%$ of the total costs (over 15 years). The development of a carbide fuel is therefore meaningful as a second fuel development. Carbide fuel cannot be used in a steam-cooled design.

9) The components of the cooling circuits make up nearly $65 \%$ of the cost of the more direct reactor part, the pumps alone providing $38 \%$. Careful development of cheap, large components because of the use of sodium is in that sense important. An optimization of cost between sodium outlet temperature (that is, thermal efficiency) and capital costs requires further attention.

10) The principle of large fast breeder reactors is to have fuel cycle costs below $1 \mathrm{mill} / \mathrm{kWh}$. Therefore, large-scale fuel testing to a burnup of $100,000 \mathrm{mWd}$ / tonne as a problem of long testing times is of great importance.

\section{APPENDIX}

\section{Data of the Karlsruhe Reference Design Na-1}

1. General Data

Reactor Type

Reactor Power

Thermal (MWt)

Electrical (MWe)

Fuel

Fertile Material

Coolant

Primary

Secondary

Tertiary

Total Breeding Ratio

Doubling Time ( $1 / 3$ out-of-pile

inventory)-for a Popula-

tion of Fast Breeders (yr)

Heterogeneous Fast
Breeder
2,500
1,000
$\mathrm{CO}_{2}$-PuO $\mathrm{O}_{2}$ at $87 \%$ of
theoretical density
$\mathrm{UO}_{2}$ and $\mathrm{U}$ metal
$\mathrm{Na}$
$\mathrm{Na}$
$\mathrm{H}_{2} \mathrm{O}$
1.385
7.2

\section{Reactor}

2.1 Reactor Core

2.1.1 Construction and Composition

$\begin{array}{lc}\text { Form } & \text { Upright Cylinder } \\ \text { Total Diameter (mm) } & 3,766 \\ \text { Total Height (mm) } & 1,755 \\ \text { Fuel Region } & \\ \quad \text { Diameter (mm) } & 2,860 \\ \text { Height (mm) } & 955 \\ \text { H/D Ratio } & 1 / 3 \\ \text { Thickness of Blanket: } & \\ \quad \text { Radial (mm) } & 400 \\ \text { Axial (mm) } & 400\end{array}$


Total Number of $Z_{0 \text { nes in Radial }}$ Direction (numbered from the Center outward):

in Fuel Region

in Radial Blanket Region

Total Number of Cells:

in Fuel Region

in Radial Blanket Region

Form of Cell

Width over Flats (mm)

Overall Length of Subassembly (mm)

Weight of Fuel Subassembly (kg)

Weight of $\mathrm{UO}_{2}$-Blanket Subassembly ( $\mathrm{kg}$ )

Weight of Metallic Uranium in Blanket Subassembly (kg)

Thickness of Subassembly Cladding ( $\mathrm{mm}$ )

Diameter of Fuel Pin ( $\mathrm{mm}$ )

Diameter of Blanket Pin (mm)

Cladding Thickness of Fuel Pin (mm)

Volume Ratio of Cladding to Fuel

Cladding Thickness of Blanket $\operatorname{Pin}(\mathrm{mm})$

Volume Ratio of Cladding to Fertile Material

Cladding Material

Number of Fuel Pins per Subassembly

Number of Blanket Pins per Subsembly

Fission Gas Plenum (below) (mm)

Maximum Fission Gas Pressure in Fuel Pin $\left(\mathrm{kg} / \mathrm{cm}^{2}\right)$

$\begin{array}{lcc}\text { Fuel Region } & \text { Zone 1 } & \text { Zone } 2 \\ \text { Outer Diameter (mm) } & 2,053 & 2,860 \\ \text { Number of Cells } & 118 & 111 \\ \text { Volume Fraction } & & \\ \quad \text { Coolant (v/o) } & 50.0 & 50.0 \\ \quad \text { Structural Material (v/o) } & 19.6 & 19.6 \\ \quad \text { Fissile Material (v/o) } & 3.2 & 4.1 \\ \quad \text { Fertile Material (v/o) } & 27.2 & 26.3 \\ \text { Number of Fuel Subassemblies } & 105 & 105 \\ \text { Number of Control Rod Subas- } & 12 & 6 \\ \quad \text { semblies } & & \\ \text { Fraction of Total Coolant per Fuel } & 0.465 & 0.465 \\ \quad \text { Cell } & & \\ \text { Fraction of Structural Material } & 0.121 & 0.121 \\ \quad \text { per Fuel Cell } & & \\ \quad & & \\ \text { Radial Blanket Region } & \text { Zone } 3 & \text { Zone } 4 \\ \text { Outer Diameter (mm) } & 3,213 & 3,766 \\ \text { Number of Cells } & 60 & 108 \\ \text { Number of Blanket Subassemblies } & 60 & 108 \\ \text { Volume Fraction } & & \\ \quad \text { Coolant (v/o) } & 29.8 & 29.8 \\ \text { Structural Material (v/o) } & 21.9 & 21.9 \\ \text { Fertile Material (v/o) } & 48.3 & 48.3\end{array}$

11.3

0.35

0.25

0.6

0.25

Incoloy 800

331

169

800

70
2.1.2 Thermodynamic Data

Thermal Power

Core (MWt)

Blanket Axial (MWt)

2,410

Blanket Radial (MWt)

Coolant Inlet Temperature $\left({ }^{\circ} \mathrm{C}\right)$

Max Outlet Temperature $\left({ }^{\circ} \mathrm{C}\right)$

Avg Outlet Temperature $\left({ }^{\circ} \mathrm{C}\right)$

Max Coolant Velocity (m/sec)

Pressure Loss

in Core $\left(\mathrm{kg} / \mathrm{cm}^{2}\right)$

in Axial Blanket $\left(\mathrm{kg} / \mathrm{cm}^{2}\right)$

in Fission Gas Plenum $\left(\mathrm{kg} / \mathrm{cm}^{2}\right)$

Max Fuel Temperature $\left({ }^{\circ} \mathrm{C}\right)$

Max Heat Flux $\left(W / \mathrm{cm}^{2}\right)$

Hot Channel Factors

Coolant Heating

Heat Transfer Coefficient

Rod Power

Hydraulic Dianeter in Fuel Region $(\mathrm{mm})$

Ratio of Avg to Max Power

Radial

Axial

0.82

0.80

$\begin{array}{lll}\text { Max Rod Power }(\mathrm{W} / \mathrm{cm}) & 566 & 539 \\ \text { Ratio of Avg to Max Power } & & \\ \text { Radial } & 0.897 & 0.745 \\ \text { Axial } & 0.80 & 0.80\end{array}$

Constants

Max Heat Transfer Coefficient between Cladding and Coolant $\left(\mathrm{W} / \mathrm{cm}^{2}{ }^{\circ} \mathrm{C}\right)$

Thermal Conductivity of Cladding (at $\left.600^{\circ} \mathrm{C}\right)\left(\mathrm{W} / \mathrm{cm}^{\circ}{ }^{\circ} \mathrm{C}\right)$

Heat Transfer Coefficient between Fuel and Cladding $\left(\mathrm{W} / \mathrm{cm}^{2}\right.$ ${ }^{\circ} \mathrm{C}$ )

Thermal Conductivity of Fuel $\left(\mathrm{W} / \mathrm{cm}-{ }^{\circ} \mathrm{C}\right)$

\subsubsection{Physics Data}

Critical Mass (Pu-239 + Pu-241) (kg)

Total Neutron Flux at Core Center $\left(n / \mathrm{cm}^{2}\right.$-sec $)$

Effective Neutron Lifetime (sec)

Effective Fraction of Delayed Neutrons

$(\%)$

(\$)

Worth of a Fuel Subassembly at Core Center, $\Delta k / k$

(\$)

Joppler Constant

$$
A_{\text {Dopp }}=T[(1 / k)(d k / d T)]
$$

Doppler Coefficient, $(1 / k)(d k / d T)$ $\left({ }^{\circ} \mathrm{K}^{-1}\right)$

Greatest Possible Reactivity Worth due to Partial Loss of sodium, $\Delta k / k$

(\$)

8.4

0.21

0.75

0.032

2,015

$10^{16}$

$3.38 \cdot 10^{-7}$

0.3548

1.0

$2.49 \cdot 10^{-3}$

0.7

$-1.19 \cdot 10^{-2}$

$-8.5 \cdot 10^{-6}$

0.01108 
Sodium Temperature Coeflicient $\left({ }^{\circ} \mathrm{C}^{-1}\right)$

Zone 1

Zone 2

Axial Blanket

Radial Blanket

Total

Fuel Composition:

$\mathrm{Pu}-239(\mathrm{a} / \mathrm{o})$

$\mathrm{Pu}-240(\mathrm{a} / \mathrm{o})$

$\mathrm{Pu}-241(\mathrm{a} / \mathrm{o})$

$\mathrm{Pu}-242(\mathrm{a} / \mathrm{o})$

U-238 (a/o)

Enrichment Fissionable

$$
\mathrm{Pu} /(\mathrm{Pu}+\mathrm{U})(\mathrm{a} / \mathrm{o})
$$

Atom Ratio of Fertile to Fissile Material

Volume Ratio of Fertile to Fissile Material

Composition Blanket Material

$\mathrm{U}-235(\mathrm{a} / \mathrm{o})$

$\mathrm{U}-238(\mathrm{a} / \mathrm{o})$

Core Volume (liter)

Rating

$\mathrm{Max}[\mathrm{MW} / \mathrm{kg}(\mathrm{Pu}-239+\mathrm{Pu}-$ 241)]

$\left[\left(\mathrm{kW} / \mathrm{cm}^{3}\left(\mathrm{PuO}_{2}+\mathrm{UO}_{2}\right)\right]\right.$

Avg $[\mathrm{MW} / \mathrm{kg}(\mathrm{Pu}-239+\mathrm{Pu}$ 241)]

Burnup

$\left[\mathrm{kW} / \mathrm{cm}^{3}\left(\mathrm{PuO}_{2}+\mathrm{VO}_{2}\right)\right]$

Max (Axial Average) [MWd/t$(\mathrm{U}+\mathrm{Pu})]$

$\operatorname{Avg}[\mathrm{MWd} / \mathrm{t}(\mathrm{U}+\mathrm{Pu})]$

Power Density

$\operatorname{Max}(\mathrm{MW} /$ liter)

Avg (MW/liter)

2.2 Reactor Vessel

Total Height ( $\mathrm{mm}$ )

Outer Diameter (mm)

Thickness of Wall (mm)

Core Support Grid Plate

Diameter (mm)

Height $(\mathrm{mm})$

Material

Eventually

Max Permissible Temperature of Containment $\left({ }^{\circ} \mathrm{C}\right)$

Max Permissible Pressure on Containment $\left(\mathrm{kg} / \mathrm{cm}^{2}\right)$
Breeding Ratio

$3.925 \cdot 10^{-6}$

0.556

0.334

0.254

0.241

1.385

Zone 1 Zone 2

$\begin{array}{rr}9.8726 & 12.9028 \\ 4.7013 & 6.1442 \\ 0.7835 & 1.0241 \\ 0.3134 & 0.4096 \\ 84.3292 & 79.5193 \\ & \\ 10.6561 & 13.9269 \\ 8.3843 & 6.1803 \\ & \\ 8.7110 & 6.4083\end{array}$

0.7

99.3

6,132

2.14

2.0

1.196

1.285

100,000

85,000

0.599

0.393

16,900

7,900

30

4,500

1,300

X8 Cr-Ni-Nb 16-13

$10 \mathrm{Cr}-\mathrm{Mo}-\mathrm{Ni}-\mathrm{Nb} \quad 9-10$

480

6

3. Primary Loop

3.1 Pipelines and Pumps

Coolant Volume $\left(\mathrm{m}^{3}\right)$

Activity of Coolant

$\mathrm{Na}-24\left(\mathrm{Ci} / \mathrm{cm}^{3}\right)$

$\mathrm{Na}-22\left(\mathrm{Ci} / \mathrm{cm}^{3}\right)$

Diameter of Pipe from Reactor to Intermediate Heat Exchanger (mm)

Coolant Velocity in Pipe $(\mathrm{m} / \mathrm{sec})$
$1.36 \cdot 10^{-1}$

1,700

3.5

Diameter of Coaxial Pipe from Intermediate Heat $F_{x}$ changer to Reactor

inside $(\mathrm{mm})$

outside (mm)

Coolant Velocity in Pipe $(\mathrm{m} / \mathrm{sec})$

Number of Pumps

Power Input of Tach Pump (kW)

Flow Rate through Each Pump ( $\mathrm{kg} / \mathrm{sec})$

Pressure Loss in Primary Loop $\left(\mathrm{kg} / \mathrm{cm}^{2}\right)$

Avg Coolant Circuit Time (sec)

1,900

2,200

7.7

4

2,940

3,296

5.6

62.4

\subsection{Intermediate Heat Exchanger}

Number of Exchangers

Type: Helical-tube Countercurrent Heat Hixchanger

Total Height (mm) $\quad 16,000$

Outer Diameter $(\mathrm{mm}) \quad 4,600$

Volume of Coolant

Primary $\left(\mathrm{m}^{3}\right)$

Secondary $\left(\mathrm{m}^{3}\right)$

Number of Exchanger Tubes

Diameter of Tubes ( $\mathrm{mm}$ )

Material

liventually

Surface Area per Heat Fxchanger $\left(\mathrm{m}^{2}\right)$

Avg Log Temperature Difference $\left({ }^{\circ} \mathrm{C}\right)$

Max Temperature Difference between Surfaces $\left({ }^{\circ} \mathrm{C}\right)$

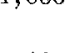

4,385

$20 \times 1.5$

X8 Cr-Ni-Nb 16-13

$10 \mathrm{Cr}-\mathrm{Mo}-\mathrm{Ni}-\mathrm{Nb} \quad 9-10$ 2,792

40

70

Primary Side

Coolant Flow Rate per Exchanger $(\mathrm{kg} / \mathrm{hr})$

Pressure Loss $\left(\mathrm{kg} / \mathrm{cm}^{2}\right)$

$23.73 \cdot 10^{6}$

Coolant Velocity between the Tubes $(\mathrm{m} / \mathrm{sec})$

Coolant Inlet Temperature $\left({ }^{\circ} \mathrm{C}\right)$

Coolant Outlet Temperature $\left({ }^{\circ} \mathrm{C}\right)$

0.43

1.6

580

430

Secondary Side

Coolant Flow Rate per Exchanger $(\mathrm{kg} / \mathrm{hr})$

Pressure Loss $\left(\mathrm{kg} / \mathrm{cm}^{2}\right)$

Coolant Velocity in the Tubes (m/sec)

Coolant Inlet Temperature $\left({ }^{\circ} \mathrm{C}\right)$

Coolant Outlet Temperature $\left({ }^{\circ} \mathrm{C}\right)$

$17.75 \cdot 10^{6}$

2.63

5.9

360

560

\section{REFERENCES}

1. W. Häfele et al., "The Karlsruhe Fast Breeder Project." III. International Conference on the Peaceful Uses of 1 tomic Energy, Geneva 1964, 28/P/539.

2. D. Smidt et al., Referenzstudie für den $1000 \mathrm{MWe}$ natriumgekühlten schnellen Brutreaktor (Na-1), KFK-299 (Dec 1964).

3. D. Smidt et al., see Paper in Session I of this Conference.

4. C. P. Zaleski et al., "Fast Reactor Core Design and Safety Studies in France and in the Federal Republic of Ger- 
many," National Topical Meeting on Fast Reactor Technology, Detroit, April 1965, ANS-100.

5. W. Hafele, Principles and Problems of the Development of a Fast Reactor Fuel Etement, Conference on Breeding, Economics and Safety in Large Fast Power Reactors, Argonne, October 1963, ANL-6792.

6. 1). Smidt and W. Sommer, Application of the Vented Fuel Concept to a Sodium-cooled Power Breeder with $1000 \mathrm{MWe}$, see paper in Session II of This Conference.

7. K. Ott and A. Jansen, "Long time Behaviour of Fast Breeders," IALA-Seminar on the Physics of Fast and Intermediate Reactors, Vienna, August 1961, SM-18/2.

8. K. Ott, K. Yiftah et al., A Comparison of Different Calculated Doppler Coefficients of Large Fast Breeder Reactors, ANL-6792 (Oct 1965).

9. K. H. Küsters and M. Metzenrotb, The Infuence of Some Important Group Constants on Integral Fast Reactor Quantities, See paper in Session VB of This Conference.

01. M. Fischer and W. Häfele, Shock Front Conditions in Two- phase Flow Including the Case of Desuperheating, See paper in Session VIIIB of This Conference.

11. G. Friz, Coolant Ejection Studies with 1 nalogy Experiments, See paper in Session VIIIB of This Conference.

12. H. Bohm, Parameter Dependence of After-meltdown Accidents, Conference on Breeding, Feonomics and Safety in. Large Fast Power Reactors, Argonne, October 1963, ANI -6792 .

13. R. I. Ackermann et al., High-temperature Thermodynamic Properties of $\mathrm{UO}_{2}$, J. Chem. Phys., 25, 1089 (1956).

14. B. Wolfe and R. A. Meyer, High-temperalure Equation of State of $\mathrm{CO}_{2}$, Trans. Amer. Nucl. Soc.. 7(1), (1964).

15. W. Hafele and P. Engelmann, Fast Reactor Programmes, Nuclear News, ANS, Vol. 7, No. 10 (1964).

16. H. Grümm et al., Demand for Vuclear Fuels and Costs of Different Reactor Types in Germany, Deutsches Atomforum e.V. and Kernforschungszentrum Karlsruhe, FORATOM-Kongress, Frankfurt, September 1965; Kernforschungszentrum Karlsruhe, KFK 366. 


\title{
Safety and Economic Characteristics of a 1000-MWe Fast Sodium-cooled Reactor Design*
}

\author{
K. P. Cohen and G. L. O'Neill \\ General Electric Company \\ Atlanced Products Operation \\ San Jose, California
}

(PRFELNTEI) BY K. P. COHEN)

\section{INTRODUCTION}

It is the purpose of this paper to present a 1965 concept of a 1000-MWe mixed plutonium-uranium oxide-fueled reactor, devoting particular attention to its safety characteristics. The approach has been to update safety criteria and redefine operating limits for fuel, cladding, and coolant in the light of present knowledge. Within these bounds we have selected a preliminary reference reactor core, coolant system, and containment design based on previous parametric studies. Once the design was established, hazard analyses were performed to predict the dynamic response of the plant to credible plant accidents. The hazards analyses were extended to include the more severe accidents which were deemed hypothetical because of

\footnotetext{
i * Work performed under Contract AT(04-3)-189, Project Agreement 10, between L. S. Atomic Energy Commission and General Electric Company.
}

the chain of improbable events required. A concept of reactor containment was then designed and an analysis made to prove its adequacy for even the hypothetical accident. Finally, the plant economics were determined, with emphasis on the effect of safety assumptions on the fuel-cycle costs. The possible effect on fuel-cycle costs of uncertainties in physics data and changes in limits of fuel performance were also determined.

\section{SUMMARY OF SAFETY CHARACTERISTICS}

\subsection{Safety Criteria}

Let us first discuss, for a moment, what safety criteria are and what use we shall make of them. Safoty criteria are principally useful in scoping studies and are ultimately superseded by detailed hazard analyses. But they serve to define problem areas and accident conditions which must be considered. With this appreciation, then, the criteria are as given below.

\section{Safety Criteria}

- 1. A negative power coefficient of reactivity is required under all credible circumstances. In addition, the local power coefficients of reactivity must be negative everywhere in the core to ensure stability of the power distribution.

2. In case of sodium loss from core and axial blankets, the blankets shall contribute sufficient negative reactivity so that the overall effect on reactivity will be zero or negative.

3. Core-design provisions shall prevent radial propagation from one subassembly to another of local formations of coolant voids.

4. The integrated negative Doppler reactivity from operating power level to the point of fuel rupture is required to be large compared to credible outside sources of rapid reactivity insertion.

5 . The performance of mechanical devices ultimately used to shut down the reactor shall be sufficiently rapid to avoid any damage, except minor local- 
ized fuel damage, following any credible combination of individual sources of reactivity addition.

6 . The design of the reactor, including its control, coolant, and refueling systems, shall be such as to limit all possible sources of reactivity addition to a magnitude of the order of that required to bring the reactor to its hot operating condition.

7. The estimated maximum potential explosive release of energy due to sodium voiding or core meltdown and compaction will not exceed the equivalent of $\sim 1000 \mathrm{lb}$ TNT.

These criteria are essentially identical to those used previously in GEAP-4418, (1) with the exception of criterion number seven.

\subsection{Significance of the Criteria}

1. The existence of negativo local power coefficients everywhere in the core assures stability of the power distribution as well as a net negative power coefficient under all credible circumstances.

2. For most cases, coolant loss will occur from the upper end of the subassemblies first. In general, then, with this criterion satisfied, coolant voiding will not produce consequential positive reactivity effects. Local coolant voiding could, in severe accidents, begin near the core midplane and produce a positive effect. This local condition would progress in a very short time over the entire subassembly (or subassemblies), and hence from a safety point of view, it is essential that the overall effect be small or negative. In effect, this criterion tends to limit actually expected voiding patterns way below the most reactive one.

3 . The third criterion is designed for minimization of gross coolant voiding during severe power excursions or local flow blockage when propagation could compound the accident. In effect, this is an admonition to the core designer to design the channels properly.

4. Criterion four is aimed at limiting the effect of rapid reactivity insertions; it sets, for example, the maximum reactivity in control rods.

5. Criterion five sets requirements of inherent negative feedbacks as well as requirements for mechanisms and instruments.

6. Criterion six establishes the indispensable reactivity change so that it can be used as a yardstick for measuring reactivity inputs from other sources. The cold-to-hot reactivity change measures the strength of normal fecdbacks. If the excess reactivity becomes very large, then insertion of a small fraction of it might overwhelm the normal reactivity feedbacks. For example, this criterion requires a limitation on excess reactivity in the core and hence may affect refueling times.

7. Criterion seven requires that reactor design limits the sodium voiding effect and sets a lower limit on the Doppler effect. Based on Bethe-Tait analysis, the criterion will be satisfied by a negative Doppler effect, $T(d k / d T)$, no weaker than -0.004 with sodium out of the reactor.

Once we have established the safety criteria and selected a reactor in accordance, we must decide which reactor accidents we will analyze.

\subsection{Classification of Accidents}

\subsubsection{Credible Accidents}

*...

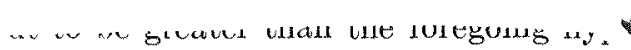
ical accident) to set an outer bound on explosive energy that the reactor can produce. A reactivity excursion was coupled with failure to seram and loss of flow so that sodium loss and meltdown occurred.

We have continued to consider the dynamic characteristics of fast reactors after loss of sodium as important. We have designed this reactor for control of the maximum pattern for inserting total void reactivity. This implies a certain pessimism about the ability to forccast the pattern of voiding. This is certainly the case at this time and may be so even after we have completed the necessary experimental programs on two-phase flow, etc., in sodium. Further, once you contemplate core voiding, no matter what the reactivity coefficient, you are facing a meltdown situation, and we have philosophical difficulties about taking the former seriously without the latter. So we also consider meltdown. This philosophy has led to a 
high-leakage core with addition of beryllia to improve the Doppler effect after loss of sodium.

We believe that loss of coolant, and hence core meltdown, can be made incredible and that eventually the designer will be released from these serious limitations. However, in the current work, we have tried to see how good a reactor can be designed even if you are forced to believe the unbelievable. This design is not a mere exercise because public acceptance of the first large fast reactors may well require designs which meet the consequence of the meltdown accident. In this context, then, we must strive to understand the implications of voiding in sodium-cooled reactors. The first reactor of this class will establish failure statistics of control drives and demonstrate independence of failure, thereby permiting subsequent assertion that failure to seram is impossible.

If you accept a loss-of-coolant accident, serious engineering attention to containment of a major explosion is necessary. In addition, problems of later reassembly of the core and the long-term removal of decay heat add cost and other complications. We have adopted a double containment system of a low-leakage concrete vault and a steel sphere because at this time this system is the most predictable and reliable. You will recognize that as the SEFOR containment concept. Perhaps eventually a low-pressure building with filtering capability may replace the low-leakage steel sphere.

\subsection{Design Implications}

Once the safety criteria are established, the designer is next faced with passing judgment on present uncertainties in the basic physics information and methodology. We have attempted to select a model which, while not an extreme of pessimism, can be expected to improve with time. The physies model differs from that of the previous design (GEAP-4418) by using lower fission and capture cross sections for $\mathrm{Pu}-239$ above 100 $\mathrm{keV}$, higher capture cross sections in iron and nickel, higher elastic energy-transfer cross sections for oxygen, and higher inelastic energy-transfer cross sections for $\mathrm{U}-238$. These result in a deterioration of the overall neutron economy.

We are aware that it is possible to achieve highleakage cores by using geometries, compositions, and arrangements other than the flat pancake which we used. Some of the alternative arrangements scem to show somewhat better fuel-cycle costs. However, the differences are within the error of physics methods. We believe that the comparative simplicity of the physical and mechanical design, as well as the dynamic analyses, more than justified continuation of this approach. Further, it was useful to have a design easily comparable with the previous one, so we could understand the trends caused by new safety criteria and new physies methods.

\section{SUMMARY OF DESIGN}

\subsection{Design Criteria}

The criteria used to select the core and coolant designs were developed with emphasis on safety and assurance of technical feasibility. The operating and transient design criteria mostly concern fuel performance.

\subsubsection{Operating Limits}

Fuel-operating limits have been selected to avoid axial fuel movement due to melting, and to prevent fuel-cladding mechanical interaction through control of the average fuel density (gap plus fuel pellet density).

1. The peak temperature limit requires that fresh fuel at the hot-spot-overpower condition shall be below the melting temperature.

2. The average fuel density has been selected so that differential fuel-cladding expansion is accommodated by an appropriate gap. At the fuel hot spot the differential expansion will require a minimum gap between the combination of the largest fuel-pellet diameter and the smallest cladding inside diameter. A statistical combination of these manufacturing tolerances is used to satisfy the design criteria.

In addition, a specified minimum value of the gap is required for fabrication. The combination of dimensional tolerances with the larger of either differential thermal expansion or the fabrication requirements determines the fuel-cladding gap. For the current design these criteria resulted in a smeared fuel density of $88 \%$. With the average $94 \%$ dense fuel, the nominal diametral gap is $0.008 \mathrm{in}$. The $94 \%$ is set by one of the transient limits as well as by consideration of ease of reproducible fabricability.

3. Cladding operating limits were established at $1250^{\circ} \mathrm{F}$, not including an overpower allowance. The value was selected on a conservative basis because of the apparent limit of $1300^{\circ} \mathrm{F}$ established by the Sodium Mass Transfer Program. ${ }^{(2)}$

We have adopted the vented-to-coolant concept for this reactor. In this fuel concept the limitations of cladding stress become secondary as long as fuelcladding mechanical interactions are minimized. Cladding strain is limited to a one percent diametral change at end of life. Creep criteria are of secondary importance. The one percent allowance for cladding strain should take care of the statistically rare combination of extreme tolerances plus fuel swelling. 
4. Corrosion must be limited to that amount which will leave a nominal thickness of 7 mils of unaffected cladding thickness at end of life. This is considered to be the minimum thickness capable of surviving the normal reactor environment. The corrosion allowance is based on the best available data at the hot-spot temperature (excluding overpower allowance), including a large safety factor.

5. Reactor sodium generally would be maintained at a plugging temperature near $250^{\circ} \mathrm{F}$ to minimize corrosion.

\subsubsection{Transient Limits}

These criteria are designed to guard against core damage as a result of credible accidents and abnormal operating conditions. They place further conditions on fuel design. They also serve to define the point beyond which core damage might be expected, a point of importance to hazard analyses.

1. The fuel transient limit has been selected so that no fuel can become completely molten, thereby putting a limit on the possible extent of fuel movement. The heat of fusion, which absorbs as much energy as a $1200^{\circ} \mathrm{F}$ tenperature rise, is important during a transient because it results in a thermal-arrest period during which the peak fuel temperature does not change significantly. The criterion is based on the assumption, for which we have some evidence, that during short transients fuel which is below melting or molten only in the center maintains its position within the fuel pin.

2. The average fuel-pellet density has been specified at $94 \%$ to minimize fuel-cladding interaction during a transient. During a transient which causes the centermost fuel to reach the molten condition, the volume change due to melting is thereby taken up within the fuel and, theoretically at least, does not exert a force on the cladding. This criterion requires that the percentage of porosity in the as-fabricated fuel be equal to about half the volume change on melting and that porosity in the semi-molten fuel be free to accommodate the fuel expansion. This criterion applies specifically to fresh fuel at reactor startup, where fuel hasn't. had time to redistribute radially.

3. The assumed transient failure point of the cladding and coolant has been selected conservatively at $1600^{\circ} \mathrm{F}$. Cladding ultimate strength is down by a factor of three from normal and is decreasing rapidly at this temperature.

\subsection{Reactor Design}

The reactor is a $1000-\mathrm{MWe}$ sodium-cooled, mixed plutonium-uranium oxide-fueled plant with a thermal rating of $2500 \mathrm{MW}$. Sodium at the reactor outlet tem- perature of $1100^{\circ} \mathrm{F}$ is used to produce steam at 3500 psia and $1000^{\circ} \mathrm{F}$. The tank-type style of reactor plant was maintained. The reactor coolant is circulated by four pumps and gives up its heat to secondary sodium coolant in six intermediate heat exchangers. The reactor is fueled with vented-to-coolant fuel which means that some $35 \%$ of the long-lived fission gases are normally in the argon cover gas.

\subsubsection{Core Description}

The core, blanket, and reflector arrangements are shown in Fig. 1. The core is a pancake 16 in. high with an equivalent diameter of $11.5 \mathrm{ft}$. Axial blankets, 18 in. thick, are placed above and below the core. Radially a moderating reflector, $7,3 \mathrm{in}$. thick, surrounds the core.

\subsubsection{Nuclear Design}

The core is divided into two radial zones of equal volumes. The same fissile enrichment is used in both zones, but the $\mathrm{BeO}$ content is varied from $10.5 \mathrm{v} / \mathrm{o}$ in the central zone to $3.6 \mathrm{v} / 0$ in the outer zone. The resulting power flattening is further enhanced by a $\mathrm{BeO}$ radial reflector which surrounds the core. A radial blanket outside the reflector was dropped as uneconomical.

The radial power profile is shown in Fig. 2. Because there is a higher volume fraction of fuel in the outer zone than in the central zone, the power density distribution (power/unit core volume) and the specific power distribution (power/unit fuel volume) are not the same. The midcycle radial peak to average power density is 1.22 , with the peak at the inner edge of zone 2 . The ratio of peak to average specific power is 1.23 , with the peak at the center of the core. The zone 1 and zone 2 power split is about $55 \%-45 \%$ and changes only slightly during operation. The thin core with 18-in. blankets results in a very flat axial power distribution in the core region. The peak-to-average ratio is 1.11 . The core-blanket power split at midcycle is $84 \%-16 \%$ and continuously shifts toward the blankets during operation.

A parametric study gave an early indication that larger fuel volume fractions than in our previous design were required to give acceptable neutron economy and that a core thinner than the previous 24-in.high reference core was required to produce a net negative reactivity effect with sodium voiding of core and axial blanket. The thin 16-in. core was selected, not only because it results in a negative total sodium loss reactivity effect, but also because it yields a relatively low maximum reactivity change $(\$ 1.60 \Delta k)$ due to voiding from the core center only. The spatial sodium voiding reactivity is shown in Fig. 3 .

The $\mathrm{BeO}$ incorporated in the core softens the 


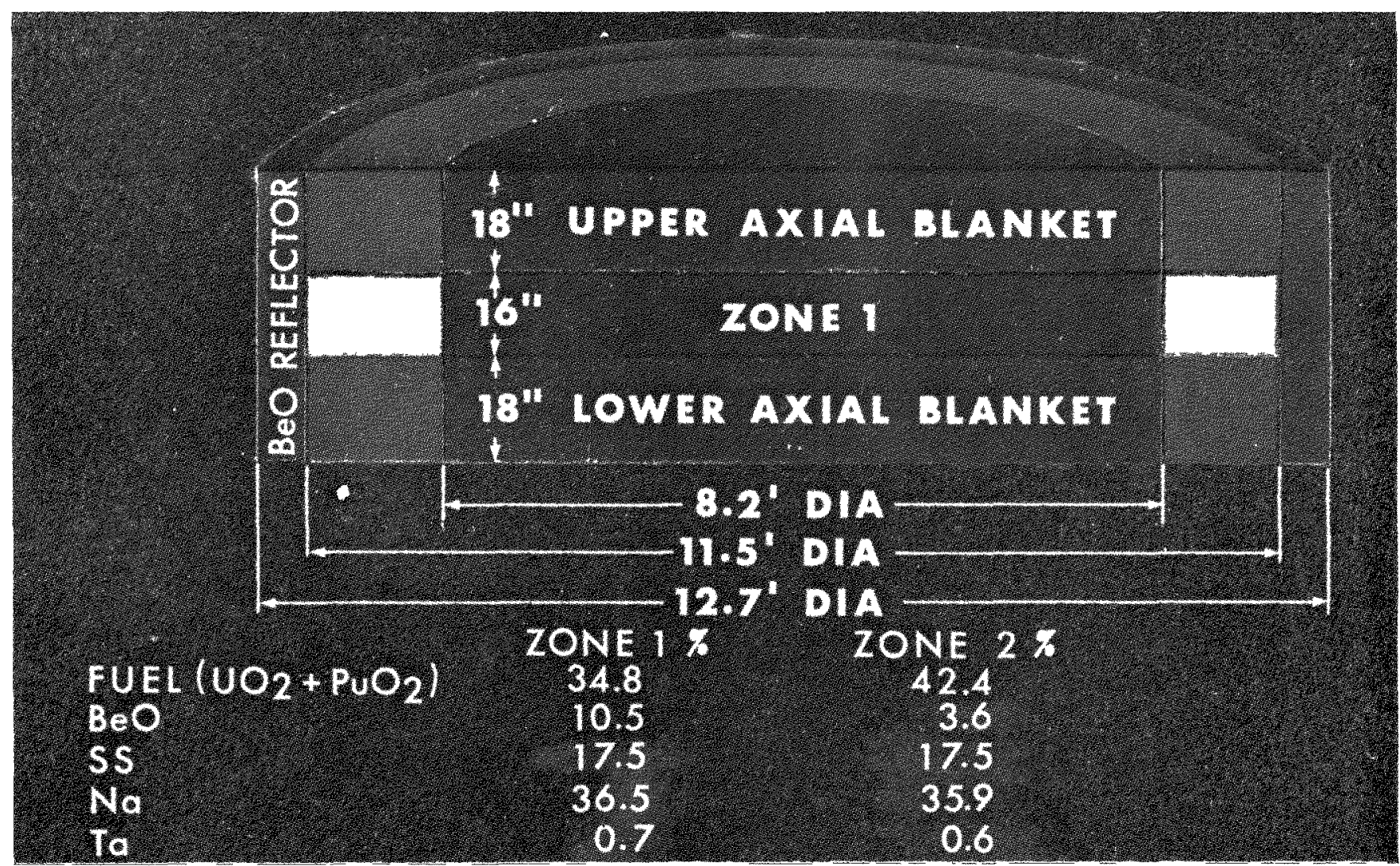

FIG 1 Core, Blanket and Reflector Arrangement

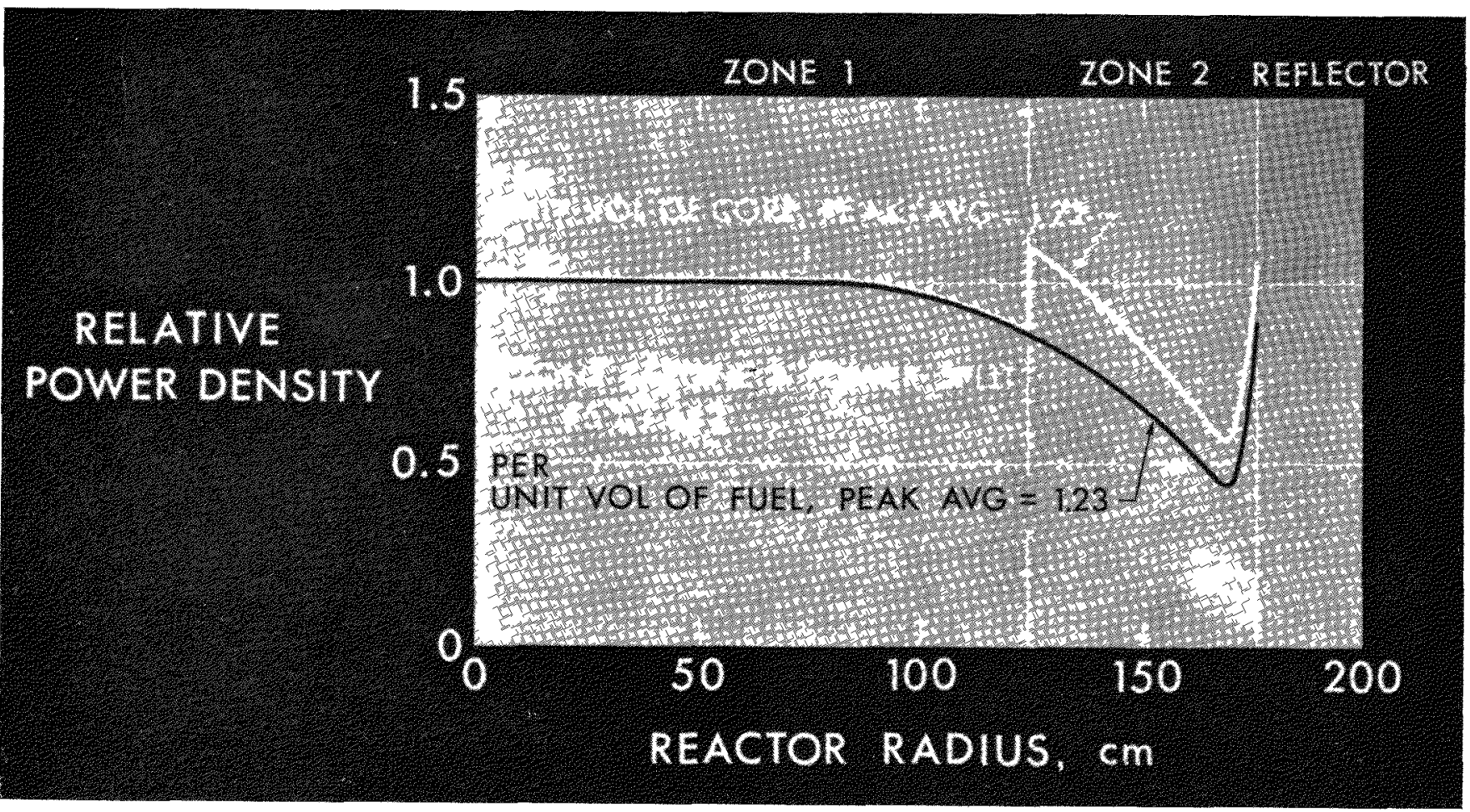

FIG 2 Radial Power Distribution at Mrdeycle 


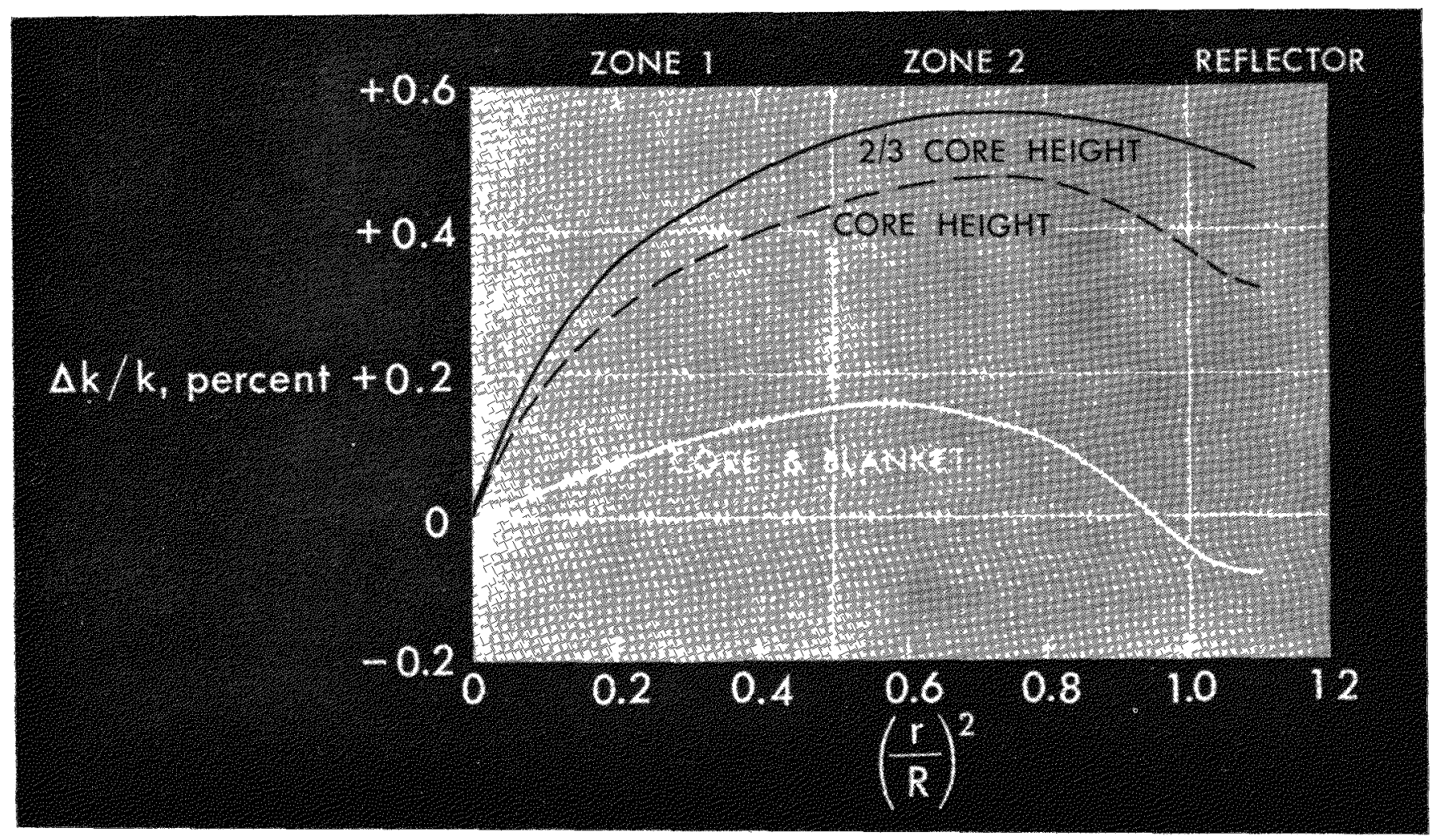

Fig 3 Reactivity Effects of Partial Sodium Volding

TABLE 1 Comparison with 1964 Design

\begin{tabular}{|c|c|c|c|}
\hline & GEAP-4418 & Currer & Design \\
\hline \multirow[t]{2}{*}{$\begin{array}{l}\text { Core Height } \\
\text { Core Diameter }\end{array}$} & \multirow[t]{2}{*}{$\begin{array}{l}24 \mathrm{in} \\
11.65 \mathrm{ft}\end{array}$} & \multicolumn{2}{|c|}{$\begin{array}{l}16 \mathrm{in.} \\
11.5 \mathrm{ft}\end{array}$} \\
\hline & & Zone 1 & Zone 2 \\
\hline$\%$ Fuel & 254 & 34.8 & 424 \\
\hline$\%$ Sodium & 479 & 36.5 & 359 \\
\hline$\%$ Steel & 188 & 17.5 & 17.5 \\
\hline$\% \mathrm{BeO}$ & 7.5 & 105 & 3.6 \\
\hline$\%$ Control & 0.40 & 0.7 & 0.6 \\
\hline \multicolumn{4}{|l|}{ Enrichment Supplied } \\
\hline Zone 1 & 15.7 & 17.9 & 17.9 \\
\hline Zone 2 & 18.0 & & \\
\hline Zone 3 & 20.2 & & \\
\hline
\end{tabular}

neutron spectrum sufficiently to provide a substantial (0.006) negative Doppler effect $[T(d k / d T)]$ even with all sodium removed. The $\mathrm{BeO}$, and particularly its concentration near the center of the reactor, also contributes to the low sodium loss effect mentioned previously. The high volume fraction of fuel results in reasonably good neutron economy and low inventory of fissile material; the breeding ratio is 1.18 with an internal conversion ratio of 0.63 in the core. At the midpoint of an equilibrium fuel cycle the average fissile enrichment in the core is $153 \%$. The total fissile inventory (Pu-239, Pu-241, U-235, and including fissile material in the blankets) at midcycle is 2470 $\mathrm{kg}$, resulting in an average specific power of 1010 $\mathrm{kW} / \mathrm{kg}$ fissile material.

3.2.2.1 Composition. Salient features of the core are given in Table 1 in comparison with the previous design. ${ }^{(1)}$

3.2.2.2 Reactivity and Power Coefficients. The main reactivity coefficients are shown in Table 2 compared to the previous design. ${ }^{(1)}$

The differences reflect core geometry and composition effects as well as different cross-section input and, in some instances, different calculational methods.

The overall power coefficients for the present design are given in Table 3 . The coefficients given are for the whole core and blanket, and reflect the effect of relatively small changes in power level about the normal operating condition. The net local power coefficient is negative everywhere in the core and thus safety criterion 1 is satisfied.

3.2.2.3 Control. The reactivity control requirements are shown in Table 4. The control description is shown in Table 5. The plan view of the core shown in Fig. 4 shows the control layout. The system consists of 64 topentry tantalum poison rods. Tantalum, clad in stainless steel, was selected because its cost over the life of 
the plant appears to be about half that of $\mathrm{B}_{4} \mathrm{C}$ and the rate of burnup is one-tenth that of $\mathrm{B}_{4} \mathrm{C}$. The higher thermal conductivity of tantalum, as well as its compatibility with stainless stcel to $1500^{\circ} \mathrm{F}$, were also important considerations.

The $3.7 \% \Delta k(\$ 10.6)$ worth of total swing was calculated for a six-month equilibrium fuel cycle at an $80 \%$ load factor. This reactivity change can be compensated by one-half of the control rods (shim rods). The remaining half of the control rods serve as scram rods and are withdrawn from the core during full power operation (maximum withdrawal rate $\sim 1 \phi$ / sec). The control rods are cooled in the main coolant stream by one percent of the total coolant flow. The rods would be designed with a hold-down provision so that they could not become missiles or be forced from the core.

For accident analyses, a control rod insertion rate was calculated from a one-g drop from $4 \mathrm{in}$. above the eore to 4 in. below the core. The rod displacement was found to be essentially a ramp with an effective delay of 0.2 sec with completion in 0.4 sec.

TABLE 2. CORE COMP IRISON - RE ACTIVITY AND Ni CLEAR P1R \METERS

\begin{tabular}{|c|c|c|}
\hline & GEAP'-4418 & Current Design. \\
\hline \multicolumn{3}{|l|}{ Doppler Effect $T(d h / d T)$} \\
\hline Sodium In & -0.01 & -0.0072 \\
\hline Sodium Out & -0.008 & -0.006 \\
\hline \multicolumn{3}{|l|}{ Sodium Void } \\
\hline Core and Axial Blanket & $\$-0.2$ & $\$-0.2$ \\
\hline Core Only & $\$+25$ & $\$+1.1$ \\
\hline Maximum & $\$+4.25$ & $\$+1.6$ \\
\hline \multicolumn{3}{|l|}{ Percent Fission below $9 \mathrm{keV}$} \\
\hline Zone 1 & 27 & 22 \\
\hline Zone 2 & 25 & 14 \\
\hline Zone 3 & 23 & \\
\hline \multicolumn{3}{|l|}{ Mean Fission Energy (keV) } \\
\hline Zone 1 & 110 & 115 \\
\hline Zone 2 & 128 & 208 \\
\hline Zone 3 & 139 & \\
\hline
\end{tabular}

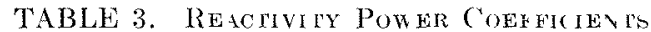
(At (sperating Temperatures)

\begin{tabular}{l|c}
\hline & $\Delta k / k$ per $\Delta P / P \times 10^{3}$ \\
Prompt & \\
Doppler Effect & -4.35 \\
Fuel Axial Expancion & -1.93 \\
Delayed & +0.02 \\
Sodium Density Reduction & +0.05 \\
Steel Axial Expansion & +0.00 \\
BeO Axial Expansion & -1.44 \\
Core Radial Expansion & \\
\hline
\end{tabular}

TABle 4. Control Requirements $(\$ 1=0.35 \% \Delta k)$

\begin{tabular}{l|c|c}
\hline $\begin{array}{l}\text { Shim Control } \\
\text { Burnup (Equilibrium Refueling }\end{array}$ & $\$ 10.6$ & $3.7 \%$ \\
$\begin{array}{l}\text { Cycle) } \\
\text { Seram Control } \\
\text { Cold to Operating at "Zero" }\end{array}$ & $\$ 1.4$ & $0.5 \%$ \\
$\quad \begin{array}{l}\text { Power } \\
\text { Zero to Full Power }\end{array}$ & 2.3 & 0.8 \\
$\begin{array}{l}\text { Reserve Control for Safety and } \\
\text { Shutdown Margin }\end{array}$ & 6.9 & 2.4 \\
\hline Total & $\$ 21.2$ & $7.4 \%$ \\
\hline
\end{tabular}

TABLE 5. CONTROL Description

\begin{tabular}{l|l}
\hline Control Material & Tantalum \\
Control Scram Worth & $3.7 \%(\$ 10.6)$ \\
Control Rod Diameter & $3.75 \mathrm{in}$. \\
Control Length & $24 \mathrm{in.}$ \\
Control Rod Drive Rate & $20 \mathrm{in.} / \mathrm{min}$ \\
Number of Seram Rods & 32 \\
Number of Burnup Control "Shim" Rods & 32 \\
Average Control Rod Worth & $0.12 \%(\$ 0.34)$ \\
"Effective" Control System Delay Time & $0.2 \mathrm{sec}$ \\
Total Scram Time & $0.4 \mathrm{sec}$ \\
\hline
\end{tabular}

\subsection{Fuel}

\subsubsection{Description of Fuel Pin}

The objective of the design is to relieve the pressure buildup due to fission gas without permitting ingress of sodium into the fucl while meeting the fuel design critcria. A single fuel pin is shown in Fig. 5. The fuel consists of oxide pellets stacked in stainless steel cladding. The total length of the pin is about $6 \frac{1}{4} \mathrm{ft}$ including $1 \frac{1}{4} \mathrm{ft}$ of core, $3 \mathrm{ft}$ of axial blanket, and 2 $\mathrm{ft}$ of vent. The fuel-pin description is given in Table 6 . A core loading consists of about 121,000 fuel pins.

The $\mathrm{BeO}$ moderator is similarly contained in stainless stcel cladding of the same size as the fuel. A core loading consists of about $22,000 \mathrm{BeO}$ pins.

A summary of other fuel pin specification details is given in Table 7. These fuel and moderator pins are arranged in two core zones and in two types of subassemblies as follows:

\begin{tabular}{|c|c|c|c|c|}
\hline Zone & No. & Kind & Fuel lins & BeO Pins \\
\hline \multirow[t]{2}{*}{1} & 34 & Full Subassembly & 420 & 126 \\
\hline & 107 & Corner Cutout & 376 & 114 \\
\hline \multirow[t]{2}{*}{2} & 53 & Full subassembly & 503 & 43 \\
\hline & 88 & Corner Cutout & 452 & 38 \\
\hline
\end{tabular}

The cladding material is to be fully annealed AISI type 316 stainless steel tubing with an ID of $0.245 \pm$ $0.002 \mathrm{in}$. and a wall thickness of $0.010 \pm 0.001 \mathrm{in}$.

The fuel design satisfied the design criteria in that 


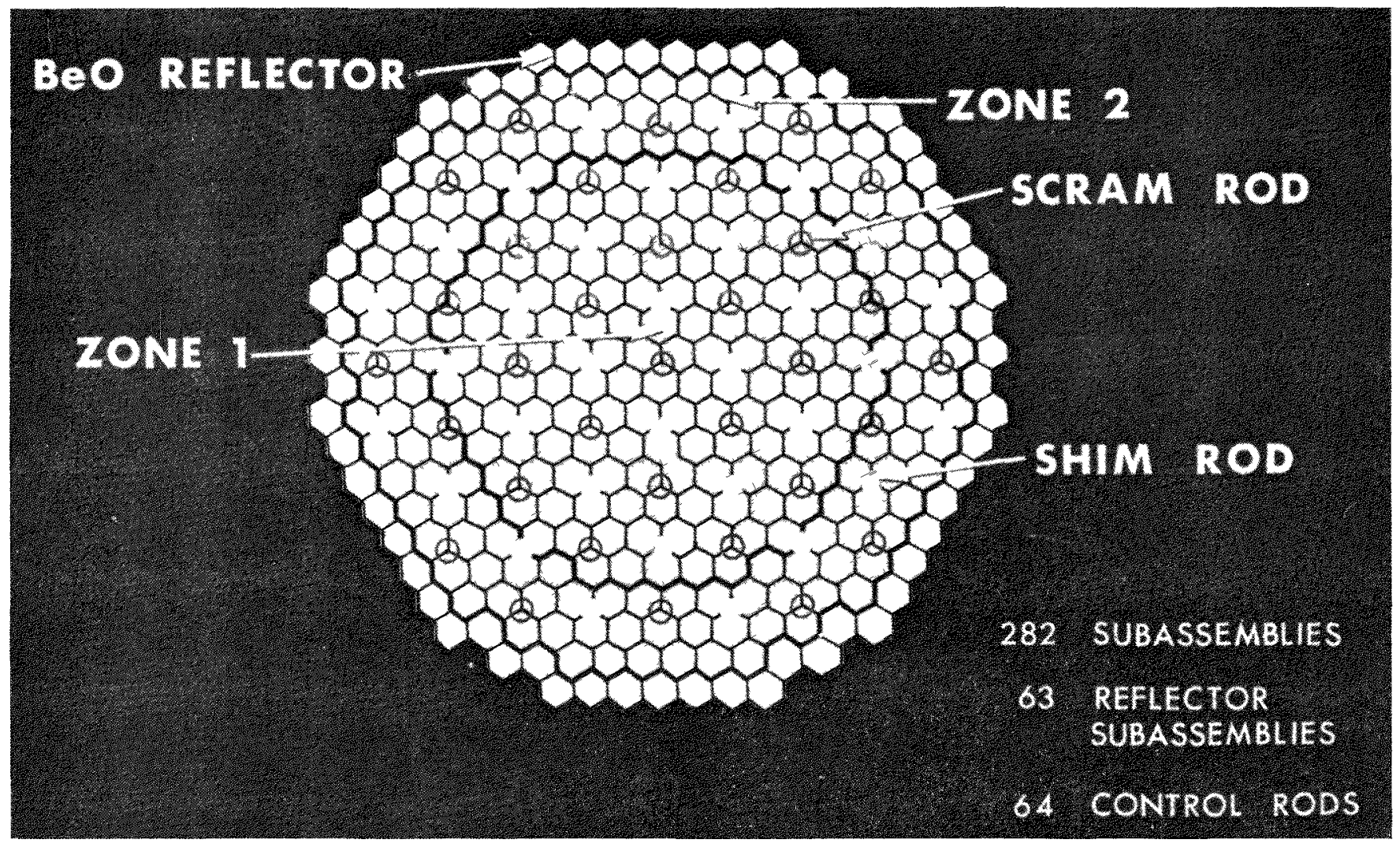

Fyx 4 Core Layout

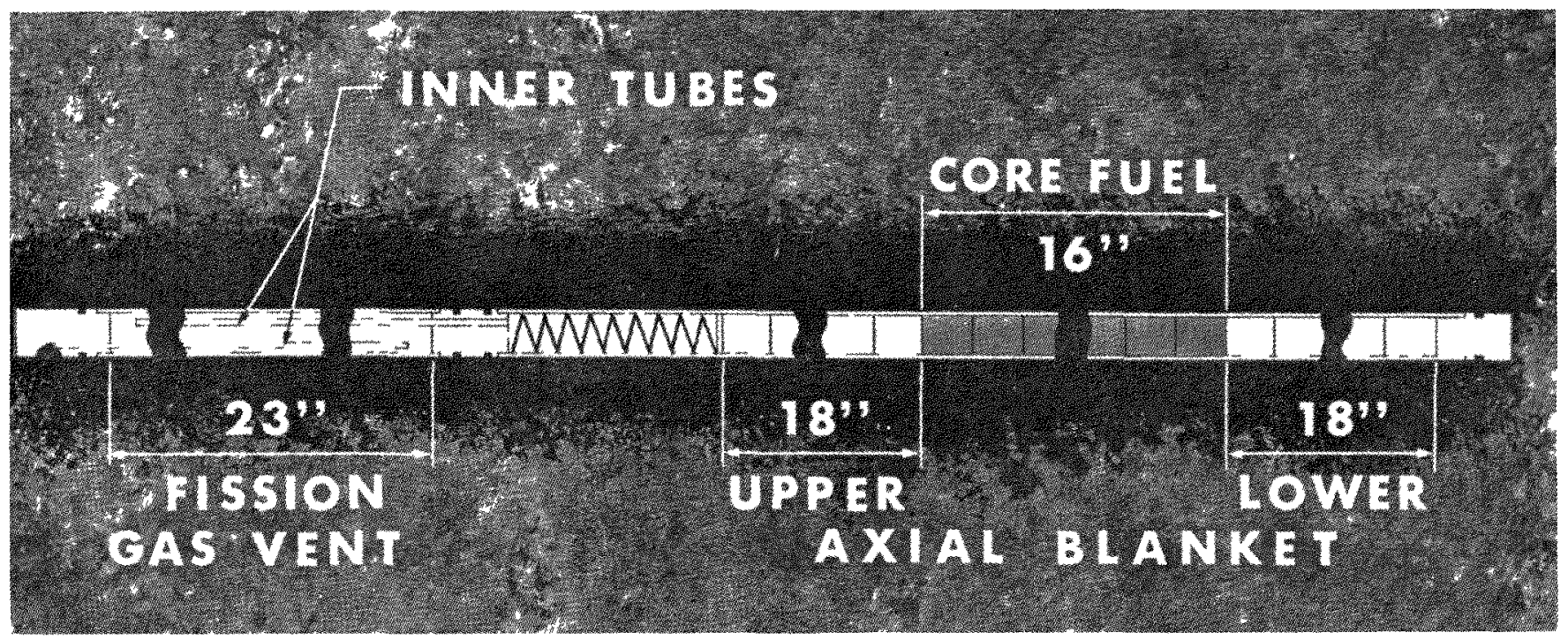

Fig 5 Fuel Pin

the density selected should prevent clad expansion duing the credible arcident tiansient The fuel-cladding gap assures against fuel interaction due to thermal expansion The worst case of differential fuel-cladding expansion is $36 \mathrm{mlls}$ The gap required for fabrication was assumed as 3 mils, so expansion is the controlling factor Fission products-induced 4nelling is accommodated by the low smeared denıty and large gap allowed for other reasons

The large tolerances specified are considered practical from the fabrication standpoint and do not restrict fuel performance. 
The vented fuel design with a "diving bell" vent has been used as the reference cladding design. The vent allows continuous release of long-lived fission product gases while retaining the significant fission products and fission product daughters. This low-pressure design removes the fission gas pressure stress from the cladding. The fuel rent device is described in a separate paper by G. L. O'Neill et al. (see paper in Session VIA).

Thermal stress is also insignificant, amounting to only 14,000 psi for 10-mil cladding at the hot spot. Thus, the vented-fuel cladding design is determined by the requirement of a reasonable end of life thickness.

The hot-spot corrosion rate based on the Sodium Mass Transfer Program is about 1 mil during the life of the fuel. If an uneertainty factor and an allowance for the ferrite layer which forms are applied, an effective maximum loss of 3 mils of cladding is predicted. It was next assumed that about 7 mils is an adequate end-of-life thickness, so 10 mils was selected as the asfabricated thickness.

\subsubsection{Performance of Fuel Pin}

Fuel performance is shown in Table 8 compared with the previous design. (1) The initial fissile loading is 2230 $\mathrm{kg}$ in core and $87 \mathrm{~kg}$ in blanket for a fissile specific power of $1079 \mathrm{~kW} / \mathrm{kg}$ fissile supplied.

The hot-spot linear power was determined in accordance with the criterion that no fucl should be higher than the melting temperature even at $10 \%$ overpower. The fuel at the peak linear power of $24.2 \mathrm{~kW} / \mathrm{ft}$ has a center temperature of $4928^{\circ} \mathrm{F}$ (the estimated melting point of the mixed oxide fuel). The ability of the fuel to operate under these conditions is dependent upon the formation of a central void in the hot-spot fuel. Because central voids are formed rapidly, low power operation for a few hours during startup with fresh fuel is required. This is normal practice and does not seem a handicap.

Credit for the center void is expressed as a geometric correction factor. In the current design a center void of nearly 56-mil diameter (assuming a gap conductivity of $1500 \mathrm{BTU} / \mathrm{hr}-\mathrm{ft}^{2}-{ }^{\circ} \mathrm{F}$ ) gives a $20 \%$ increase in allow-

TABLE 6. DESCRIPTION OF FUeI. PIN

\begin{tabular}{l|l}
\hline Fuel & $\mathrm{UO}_{2}-\mathrm{PuO}_{2}$ Sintered Pellets \\
Eurichment (Initial) & $17.9 \%$ fissile $\mathrm{Pu} /(\mathrm{Pu}+\mathrm{U})$ \\
Fuel-pellet Diameter, Cold & $0.237 \mathrm{in}$. \\
Cladding Material & AISI Type $316 \mathrm{SS}$ \\
OD $\times$ Thickness & $0.267 \times 0.010 \mathrm{in}$. \\
Pin Length & $77.25 \mathrm{in}$. \\
Fission Gas Vent Length & $24.0 \mathrm{in}$. \\
Ratio of Pitch to Pin Diameter & 1.13 \\
Pin Spacers & Spiral wire wrap \\
\hline
\end{tabular}

TABLE 7. Stmmary OF FUEL-CLAD SPECHICATIONS

\begin{tabular}{|c|c|}
\hline Fuel Composition & $\begin{array}{l}24.3 \% \mathrm{Pu} ; 17.88 \% \mathrm{Pu}-241+ \\
\mathrm{Pu}-239\end{array}$ \\
\hline Stoichiometry - Oxygen/Metal & $1.97-1.98$ \\
\hline Theoretical Density at STP & $11.09 \mathrm{gm} / \mathrm{ce}$ \\
\hline $\begin{array}{c}\text { Theoretical Density at Core } \\
\Lambda \vee g \text { Temp of } 2670^{\circ} \mathbf{F}\end{array}$ & $10.52 \mathrm{gm} / \mathrm{cc}$ \\
\hline Fuel-pellet Density & $94 \pm 2 \%$ theoretical \\
\hline Cold Smeared Jensity & $\begin{array}{l}9.75 \pm 0.32 \mathrm{gm} / \mathrm{cc} \\
87.9 \pm 3.3 \% \text { theoretical }\end{array}$ \\
\hline Hot Smeared Density & $\begin{array}{l}9.39 \pm 0.31 \mathrm{gm} / \mathrm{cc} \\
89.3 \pm 3.3 \% \text { theoretical }\end{array}$ \\
\hline Fuel Pellet OD & \\
\hline $\begin{array}{l}\text { Cold } \\
\text { llot }\end{array}$ & $\begin{array}{l}0.237 \pm 0.004 \mathrm{in} . \\
0.241 \pm 0.004 \mathrm{in} .\end{array}$ \\
\hline Clad ID & \\
\hline $\begin{array}{l}\text { Cold } \\
\text { IIot }\end{array}$ & $\begin{array}{l}0.2+5 \pm 0.002 \mathrm{in} . \\
0.2 \mathrm{k} \pm 0.002 \mathrm{in} .\end{array}$ \\
\hline $\begin{array}{l}\text { Fuel Clad Diametral Cap } \\
\text { (As Fabricated) } \\
\text { (Core Avg Temp) } \\
\text { (Iot Spot Temp) }\end{array}$ & $\begin{array}{r}8 \pm 4.5 \text { mils } \\
6 \pm 4.5 \text { mils } \\
4.5 \pm 4.5 \text { mils }\end{array}$ \\
\hline
\end{tabular}

TABLE 8. Performance OF Fuel PIN

\begin{tabular}{l|r|c}
\hline & Present & GEAP-4418 \\
\hline $\begin{array}{c}\text { Core Average Linear Power, } \\
\mathrm{kW} / \mathrm{ft}\end{array}$ & 12.4 & 10.4 \\
$\begin{array}{c}\text { Hot-spot Peak Linear Power, } \\
\mathrm{kW} / \mathrm{ft}\end{array}$ & 24.2 & 22.7 \\
$\begin{array}{c}\text { Core Average Specifie Poner, } \\
\mathrm{kW} / \mathrm{kg} \text { Mixed Oxide }\end{array}$ & 148.6 & 151 \\
$\begin{array}{c}\text { Fissile Specific Power, kW/kg } \\
\text { Fissile Material }\end{array}$ & 1079 & 1,000 \\
$\begin{array}{c}\text { Average Burnup, MWI)/T } \\
\text { Hot-spot Fuel Center Tem- } \\
\text { perature, }{ }^{\circ} \mathrm{F}\end{array}$ & 100,000 & 100,000 \\
Core Average Fuel Tempera- \\
ture, ${ }^{\circ} \mathrm{F}$
\end{tabular}

able linear power. This is possible when considering fuel densification alone and has often been observed experimentally.

Fuel conductivity was compiled from both out-ofpile and recent in-pile data. Data used was for $20 \%$ $\mathrm{PuO}_{2}-80 \% \quad \mathrm{CO}_{2}$ which was assumed appropriate for the current mixed-oxide composition $(24 \% \mathrm{Pu})$. The data used result in a total $\int_{0}^{t e} k d \theta$ to melting of 94 W/cm.

Other fuel performance details are given in Table 9. Peaking factors used in the design are given in Table 10.

\subsection{Subassembly}

A core subassembly (see Table 11) is shown schematically in Fig. 6. The core is comprised of 282 hexagonal channels which provide axial and lateral 
TABLE 9. Fene. Performance

\begin{tabular}{|c|c|c|c|}
\hline & $\begin{array}{c}\text { Core } \\
\text { (Average) }\end{array}$ & $\sim \underset{\text { Peak }}{\text { Nominal }}$ & Hot Spot \\
\hline $\mathrm{kW} / \mathrm{ft}$ & 12.4 & 20.0 & 24.2 \\
\hline $\begin{array}{l}\text { ID Heat Flux (BTU/hr- } \\
\left.\mathrm{ft}^{2}\right)\end{array}$ & 659,000 & $1,056,000$ & $1,280,000$ \\
\hline $\begin{array}{l}\text { OD Heat Flux (BTU/hr- } \\
\mathrm{ft}^{2} \text { ) }\end{array}$ & 610,500 & 977,000 & $1,180,000$ \\
\hline$T_{\mathrm{Na}}\left({ }^{\circ} \mathrm{F}\right)$ & 962 & 962 & 1,025 \\
\hline$\Delta T_{\mathrm{Na}}$ Film $\left({ }^{\circ} \mathrm{F}\right)$ & 30 & 49 & 59 \\
\hline$\Delta T_{\mathrm{clad}}\left({ }^{\circ} \mathrm{F}\right)$ & 42 & 67 & 86 \\
\hline$\Delta T_{\text {gap }}\left({ }^{\circ} \mathbf{F}\right)$ & 440 & 704 & 855 \\
\hline$T,\left({ }^{\circ} \mathbf{F}\right)$ & 1,474 & 1,782 & 2,025 \\
\hline $\int^{T_{s}}$ & & & \\
\hline $\int_{0} \quad k d T(\mathrm{~W} / \mathrm{em})$ & 35.0 & 37.5 & 41.3 \\
\hline $\begin{array}{l}\text { Sintering Dianeter } d_{1800^{\circ} \mathrm{F}} \\
\quad \text { (in.) }\end{array}$ & 0.140 & 0.178 & 0.220 \\
\hline Void Diameter $d_{o}$ (in.) & 0.034 & 0.044 & 0.056 \\
\hline $\begin{array}{l}l \text {, Center Void Correction } \\
\text { Factor }\end{array}$ & 0.92 & 0.89 & 0.83 \\
\hline $\int_{T_{s}}^{T_{\mathrm{c}}} k d T(\mathrm{~W} / \mathrm{cm})$ & 30.0 & 46.9 & 52.5 \\
\hline $\int_{0}^{T_{e} e} k d T(\mathrm{~W} / \mathrm{cm})$ & 65.0 & 81.4 & 93.8 \\
\hline$T_{c}\left({ }^{\circ} \mathrm{F}\right)$ & 3,866 & 4,595 & 4,928 \\
\hline
\end{tabular}

TABLE 10. PEIKING FaCtors

\begin{tabular}{l|l}
\hline Axial (Zone 1) & 1.10 \\
Radial (Beginning of Life) & 1.28 \\
Burnup & 1.12 \\
Overpow er Allowance & 1.10 \\
Hot-spot Cncertainty & 1.12 \\
& \\
Total Hot Spot & 1.38 \\
$\quad$ Total & 1.94 \\
Estimated $\gamma$-Heat Out-of-Fuel & $5 \%$
\end{tabular}

The fuel pins are held axially by two end grids whose wcbs are spaced to retain the fuel and $\mathrm{BeO}$ pins. The two end grids are joined by a center post which easily accommodates the differential radial and axial thermal expansions between the fuel pins and the post. The fuel and $\mathrm{BeO}$ pins are spaced by a spiral wrap on every pin. To take up the tolerances and maintain support, as well as for channeling the coolant past the fuel. Nose pieces, attached to the lower end of these channels, locate and support the subassemblies by mounting into the lower grid. The channel wall is designed to withstand a 40-psi differential pressure and requires a wall thickness of $0.139 \mathrm{in}$. Slotted channels permit this design by equalizing the pressure differential above the upper blanket. a tight pin array, the subasscmblies are subdivided into six bound groups of fuel pins which are held tight within the channel by springs.

The fuel and shield sections of the fuel are designed so the shield section may be removed before shipment. The shield rods are $2.2 \mathrm{in}$. in diameter and $13.5 \mathrm{ft}$ long.

\subsection{Core and Structure}

\subsubsection{Thermal Hydraulics}

Core thermal hydraulic values are summarized in Table 12. The coolant inlet temperature and temperature rise are $800^{\circ} \mathrm{F}$ and $300^{\circ} \mathrm{F}$, respectively, giving a bulk outlet temperature of $1100^{\circ} \mathrm{F}$, which is considered a practical upper limit at this time. These values are the same as in the previous design.

The temperature rise across the core was found with corrections for all forms of coolant dilution. In every subassembly the coolant flow is orificed to maintain the same maximum coolant exit temperature. The radial power gradient within the subassemblies contributes $8.4 \%$ dilution. Fuel burnup contributes $2.2 \%$ dilution. An estimated $1 \%$ of the coolant is required to cool control rods, and an estimated $5 \%$ was used to cool the radial reflector and thermal shield. The total dilution is about $17.7 \%$, calculated as follows:

$$
\text { Dilution }=-\frac{1+\text { power dilution }}{1-\text { flow dilution }} \text {. }
$$

The subassemblies are designed to reach a peak temperature rise of $300 \times 1.77=353^{\circ} \mathrm{F}$. The hot-channel factor of 1.29 results in a maximum temperature rise of $456^{\circ} \mathrm{F}$. The average core temperature rise is $319^{\circ} \mathrm{F}$.

The required coolant flow is $94.5 \times 10^{6} \mathrm{lb} / \mathrm{hr}$ of which $94 \%$ goes through the core subassemblics. The total effective coolant flow area is 3760 sq in., giving a core average coolant velocity of $18.3 \mathrm{ft} /$ sec.

A velocity peaking factor of 1.15 , derived from the radial power distribution, results in a peak velocity of $21.0 \mathrm{ft} / \mathrm{sec}$ in the inner subassembly of the outer

TABle 11. Stbissembly Description

\begin{tabular}{|c|c|c|}
\hline $\begin{array}{l}\text { Hexagonal Subassembly } \\
\text { Flats }\end{array}$ & Width across & $7.718 \mathrm{in}$. \\
\hline Channel Wall Thickness & & $0.139 \mathrm{in.}$ \\
\hline Interchaxnel Spacing & & $0.125 \mathrm{in}$. \\
\hline Lateral Pin Restraint & & spring \\
\hline Axial Pin Support & & end grids \\
\hline Fuel Pins/Full Hexagon & & \\
\hline Zone 1 & & 420 \\
\hline Zone 2 & & 503 \\
\hline BeO Pins/Full Hexagon & & \\
\hline Zone 1 & & 126 \\
\hline Zone 2 & & 43 \\
\hline Nose-piece Diameter & & $4.0 \mathrm{in.}$ \\
\hline
\end{tabular}




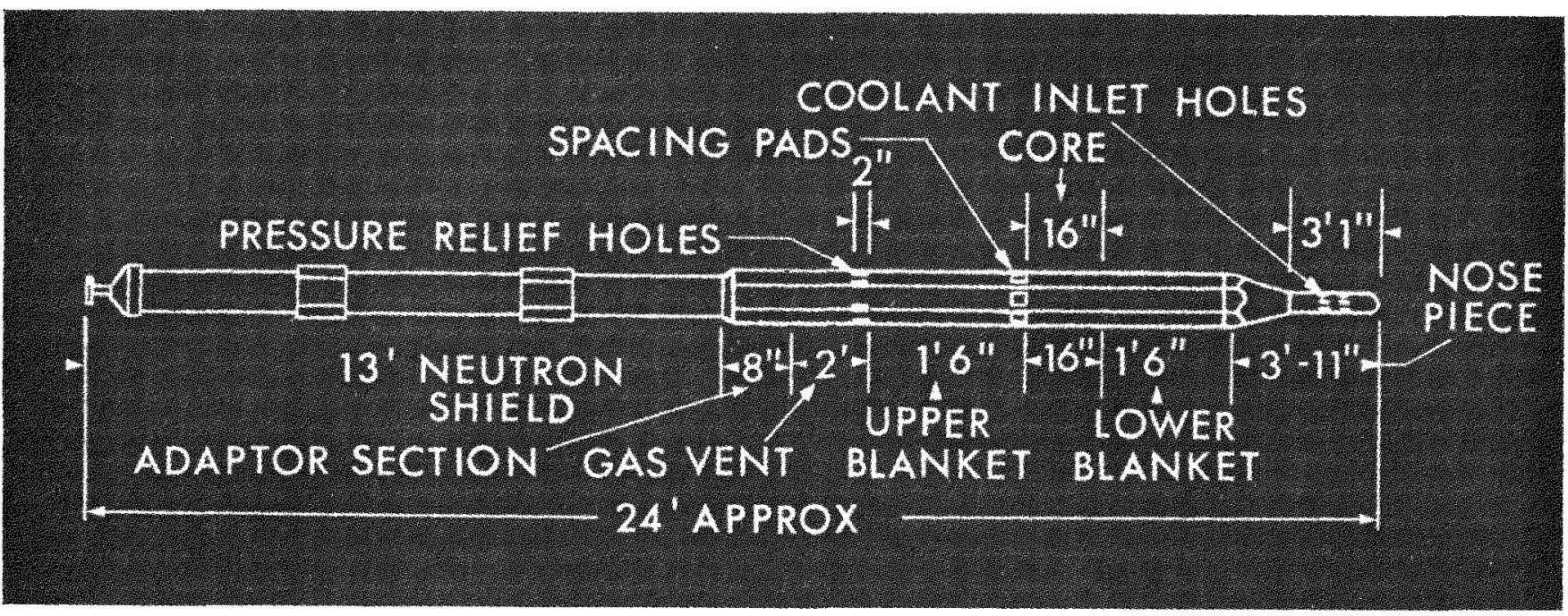

Fig. 6. Fuel Subassembly.

zone. These subassemblies are unorificed, thus setting the core pressure drop. The resultant pressure drop is 40.5 psi due to fuel pins, 16.5 psi due to the spiral wire wrap, and 6.4 psi due to entrance and exit losses. Pressure drop through the rest of the primary loop is 30.5 psi, giving a total pump head of 93.9 psi. Assuming a $75 \%$ efficient pump and motor, this requires $1.25 \%$ of the net plant output to drive the primary pumps.

\subsubsection{Core Support, Holddown, and Surrounding Struc- ture}

The 282 core subassemblies, 63 reflector subassemblies, and radial neutron shiclding are supported on the lower grid structure. This structure consists of two circular grid plates separated by $3 \mathrm{ft}$ of internal grid work.

Concentric holes in both plates accept the circular nose pieces of the subassemblies. The weight of the subasscmblies is supported at a conical seal face on the top grid plate. This grid structure is the coolant inlet plenum, coolant entering side holes in the nose pieces. Close fits of the nose pieces in the lower grid plate pro-

TABLE 12. THEHMAL HYoRAL hI'S

Inlet Temperature

Reactor Outlet Temperature Orifice Iesign Temperature IIot-channel Exit Temperature Reactor-coolant Flow Core Average Coolant Velocity Core Peak Coolant Velocity Estimated Worst Clad Corrosion Subassembly Pressure Drop Primary Loop Pressure Drop vides a hydraulic holddown, the area between the lower grid plate and the reactor vessel being opened to the primary tank.

The subassembly tightener grid is lowered over the top of the subassemblies and tightens the subassemblies laterally until the spacer pads at the core elevation are in contact. Cone-shaped guide-ins for all subassemblies and guide tubes lead to a simple cylindrical fit pulling the subassemblies together and aligning the control-rod guide tubes. Control-rod guide tubes protrude above this tightener grid, but the subassembly locations are provided with stops to prevent a subassembly from lifting out of the core.

Shiclding is provided between the reactor vessel and the moderating reflector subassemblies to attenuate the neutron flux on the vessel wall and to provide thermal shielding between the outlet sodium and the primary tank sodium.

\subsection{Reactor Tank and Coolant System}

The main sodium tank holds some $560,000 \mathrm{gal}$ of of sodium. During normal operation the tank sodium will be at the reactor inlet temperature of $800^{\circ} \mathrm{F}$. During refueling the temperature will be $350-400^{\circ} \mathrm{F}$. The tank is a vessel one inch thick, $52 \mathrm{ft}$ in diameter, and $47 \mathrm{ft}$ high. The tank structure would be built to withstand about 10 psig.

The reactor core structure is contained in an inner reactor pressure vessel which, along with all other components of the primary coolant system, is submerged in a large tank of sodium as shown in Fig. 7. Four 56,000-gpm, vertically mounted, centrifugal sodium pumps and six, counterflow, 15,000- $\mathrm{ft}^{2}$, intermediate heat exchangers provide the primary coolant system, and these are arranged in a circle around the 


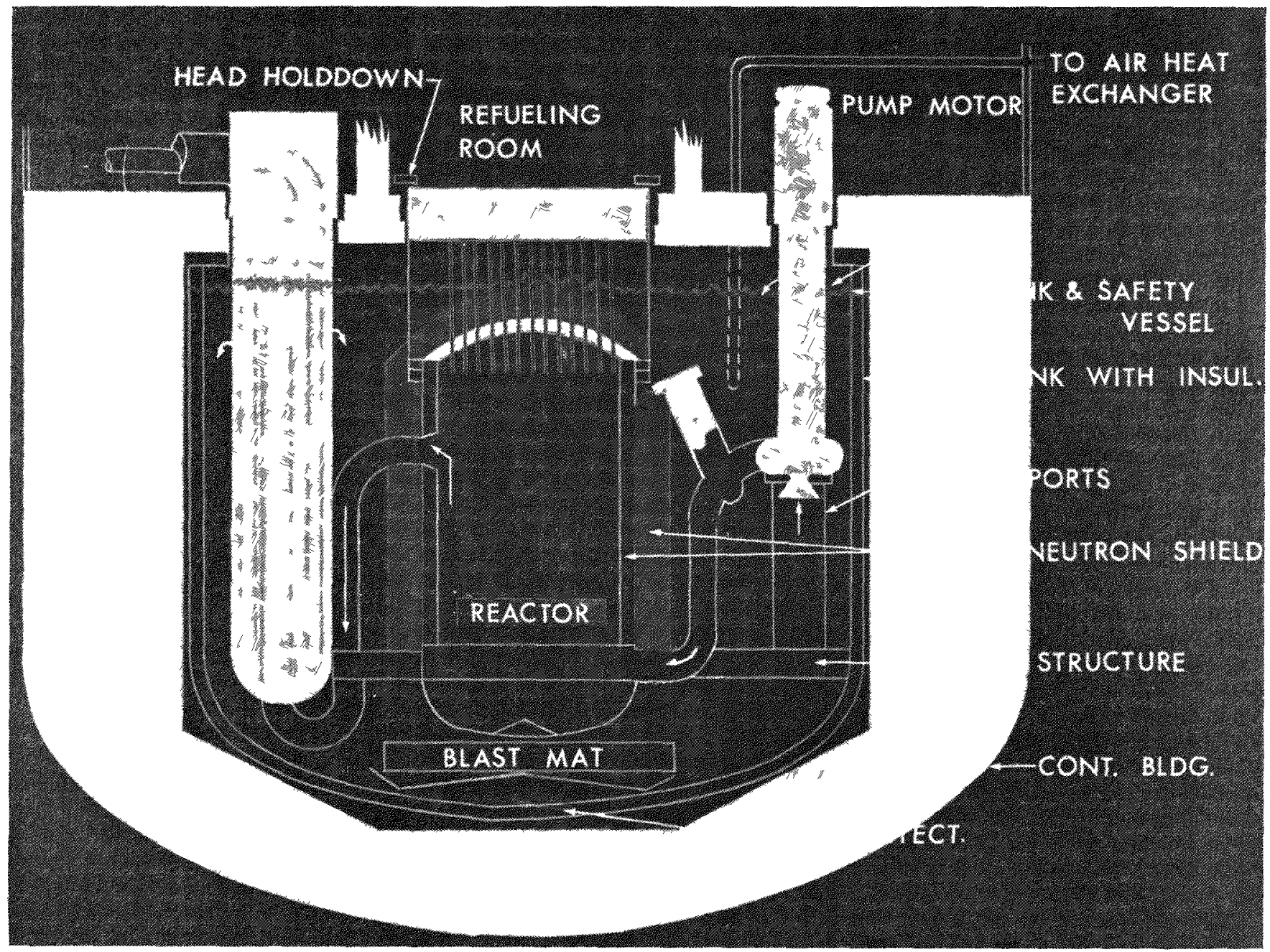

Tig 7 lertical Cross Section through Contanment Bulding

ressel The pumps take suction from the large tank and deliver sodium into the vessel through check valiex and $30-1 n$ pipes to an inlet plenum below the reactor From the plenum the flow is up through the core past the neutron shielding to an outlet region, then through 30-m lines to the six heat exchangers

This arlangement has been improved over that in the prei lou design by replacing the massive duct structure for the sodium with loops, and by supporting the reactor ressel and heat exchangers from the large tank wall instead of the top, which already must support the refueling cell The intermedate heat exchangers, pumps, check valves, and reactor head are removable Neutron shielding and any blast protectron are also contained mside the tank The blast shield surrounds the primary vessel, and stretch bolts would be used to hold the refueling "head" in place during dny accident

The sodium-purification units are also in the tank
The cold traps, plugging indieators, and pumps would be inserted through shreld plugs just like the pumps and heat exchangers Figure 8 shows an arrangement around the central reactor vessel

A safety ressel would be provided outside of the la1ge sodium tank, thereby assuring agamst loss of sodrum The space between the two vessels could then be monitored continuously

The satety vessel would be surrounded by a massive concrete structure which might possibly be lined to elminate any chance of ground water collecting A sump and removal system for water could also be provided

If one admits to core disassembly and meltdown accidents, as is postulated for this paper, the reactor tank must be designed to wathstand the impact resulting from such an accident, to prevent reassembly of the molten core, and to remove the decay heat

As previously mentioned, capability of pressure con- 


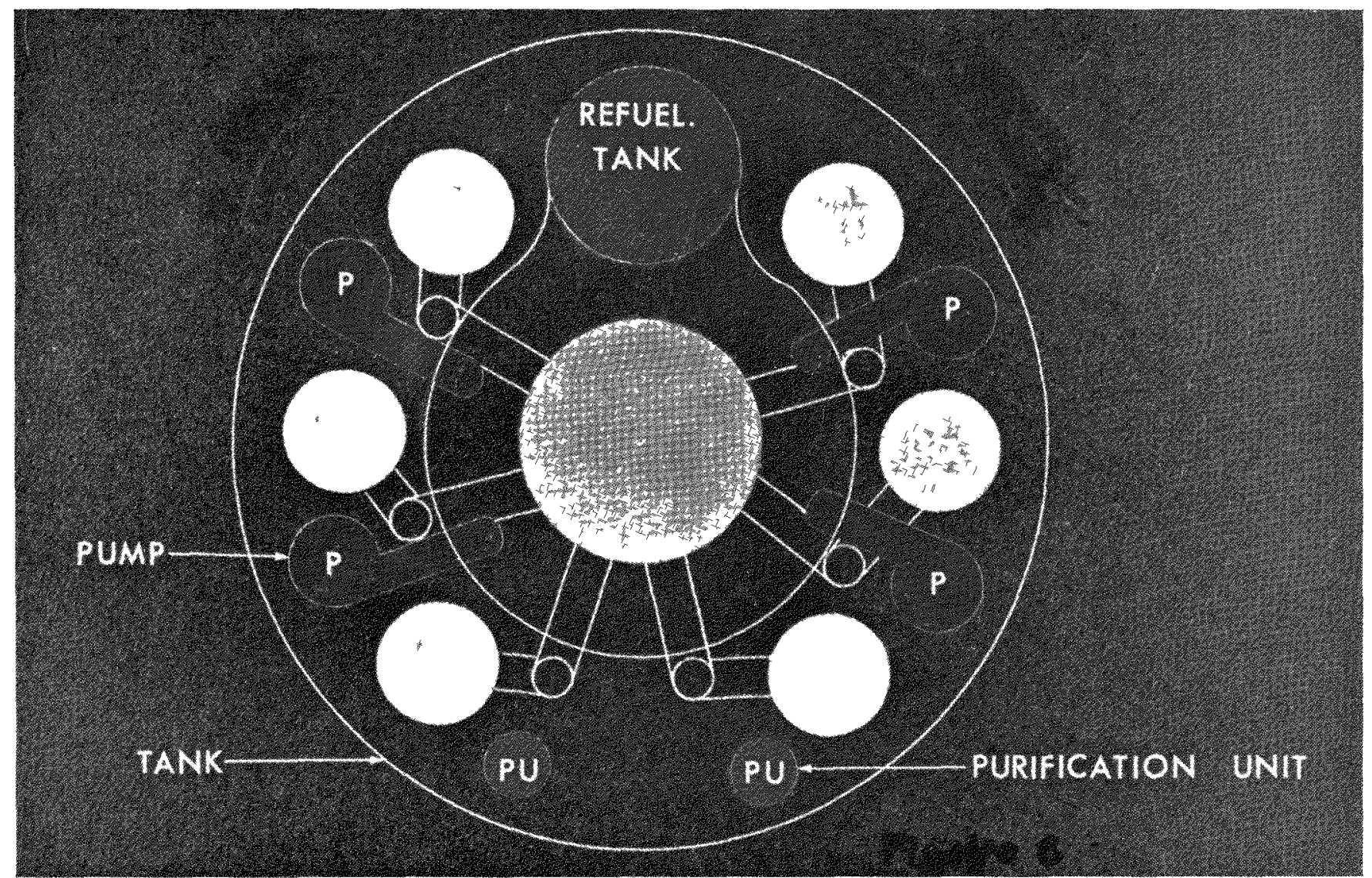

FIG 8 Plan $\bigvee$ lew of Reactor Tank.

taimment, a blast shield, and head holddown can be incorporated into the design. Further, core meltdown pans are shown which are protected by a blast mat.

The accident which destroys the core probably must be assumed to destroy the heat exchangers, so the continuous removal of decay heat could be a problem. It is proposed that until such design or analyses prove otherwise, a separate, blast-protected heat exchanger can be provided for removal of decay heat.

\section{SAFETY ANALYSIS}

\subsection{General Considerations}

This section describes the dynamic behavior of the reactor core under accident conditions, and evaluates the effectiveness of the control system and overall reactor design in meeting the safety criteria specified in Section 2.1 under accident conditions previously discussed in Section 2.3 .

Response of the reactor to credible accidents was analyzed by use of FORE, ${ }^{(3)}$ a digital computer code. The maximum hypothetical accidents wero analyzed by means of a modified version ${ }^{(4)}$ of the Bethe-Tait approach for analyzing core disassembly. Initial conditions for the disassembly calculations were obtained from FORE. Because of the difference in the Zone 1 and 2 compositions, a core-averaged composition was used to describe the core.

All calculated values of reactivity coefficients were conservatively modified by a factor of safety of 1.5 to account for uncertainties in reactivity coefficients. For credible accidents, a sodium-in Doppler effect of -0.0048 was used. In the case of the maximum hypothetical accidents, a sodium-out Doppler effect $[T(d k / d T)]$ of -0.004 , as well as the calculated value -0006 , was used. The normally prompt, negative coefficient from axial fuel expansion was assumed to be zero, more for consistency with past practice than from conviction. Other reactivity coefficients employed in the analyses were those from the radial and material expansions.

\subsection{Credible Accidents}

\subsubsection{Control Rod Ejection}

Between the time at which positive reactivity is inserted and control rods begin to move, the reactor 
behavior depends primarily on the Doppler effect to limit the severity of the accident. The full-power operating conditions correspond to the lowest Doppler feedback in normal operation because the Doppler effect is approximately inversely proportional to the absolute fuel temperature. Also, energy required for core failure is normally a minimum at full core power. Thus, the core is most vulnerable to reactivity insertions at full power. The ejection of the most reactive control rod was assumed at full power. Worth of the most reactive control rod is 60 cents. An instantaneous ejection of this rod was assumed.

The resulting maximum temperature rise during this extreme transient was less than one-fourth of the transient equivalent temperature rise of $1200^{\circ} \mathrm{F}$ for which the fucl has been designed. Only a negligible rise in cladding and coolant temperature occurred.

\subsubsection{Loss of Pumping Power}

Coolant coast-down resulting from an instantaneous loss of pumping power has been considered. A flow coast-down model was used, taking into consideration the inertia of the pump motors (without flywheels) and natural convective circulation of the sodium. It was assumed that the secondary system maintained the core inlet temperature at $800^{\circ} \mathrm{F}$. Scram was assumed to be initiated when pump power was lost.

Fuel temperatures were found to decrease alnost immediately. The maximum rise of coolant temperature during the entire coast-down period was less than $300^{\circ} \mathrm{F}$, leaving the hot-spot coolant temperature below the design maximum of $1600^{\circ} \mathrm{F}$.

\subsubsection{Local Flow Blockage}

Blockage of a single subassembly by debris or gas entrainment was also analyzed as a credible accident. Damage to the subassembly itself was not of major importance; rather, the sodium voiding effects and eventual local fuel slumping were of principal concern.

Both the voiding and slumping can produce positive reactivity additions. Accompanying the voiding can be a relatively large pressure pulse which has the potential of radial propagation unless design provisions are made against it.

Core calculations have shown that the combination of the most extreme voiding pattern and the complete melting and slumping of fuel from the upper half into the lower half of the core in one of the central subassemblies results in less than 40 cents of reactivity. Since this reactivity, and obviously the reactivity insertion rate, is less than that of the control rod ejection just discussed, this accident will be of no consequence to the rest of the core with respect to reactivity addition when followed by a normal scram.
There is a need for development of an adequate method for prevention of, or detection of, a local channel blockage.

Pressure within the channel due to the formation of sodium vapor was estimated to be of the order of 300 psi, based on sodium vapor pressure at the maximum temperature of the coolant. Extreme deformation of the hexagonal channel into a cylinder of equivalent volume and then ultimate rupture of this shape requires an innerchannel pressure of approximately $750 \mathrm{psi}$, if the channel material is near the boiling point of sodium. This, of course, assumes that the adjacent channels offer no restraint. Complete circular deformation of the channel would cause a maximum interference of approximately one-fourth inch with an adjacent channel. This interference would be localized and probably accommodated by local deformation in an adjacent channel without seriously disrupting coolant flow.

When this is considered as well as the fact that pressures of the order of $750 \mathrm{psi}$ would be required to cause such a phenomenon, radial propagation of a local blockage would not occur.

\subsubsection{Fuel-assembly Drop-in during Refueling}

The refueling accident was analyzed in terms of a series of pessimistic assumptions, e.g., a fuel assembly falling into the core under conditions where the reactor is just subcritical due to a combination of previous errors. This would require overenrichment of many subassemblies, combined with failure of the instruments to detect the condition. The model for reactivity addition during refueling was taken as an outer-zone subassembly inserted into the center of the core. The rate of insertion was taken as that due to free fall starting $20 \mathrm{ft}$ above the top of the core. The decelerating effect of the sodium was ignored. This resulted in a positive reactivity insertion of $\$ 1.70$ at approximately $\$ 46 / \mathrm{sec}$. It was assumed that the power is $10^{-9}$ times rated, and that the reactor coolant was at $400^{\circ} \mathrm{F}$.

This accident was analyzed under conditions of shutdown coolant flows of 10 and $15 \%$ of full flow and no provisions for mechanical shutdown. In the former case, core power began to level out near $850 \mathrm{MW}$, but the maximum temperature of the coolant in the hot channel exceeded the $1600^{\circ} \mathrm{F}$ criterion in $4.8 \mathrm{sec}$. With $15 \%$ full flow, core power approaches $1170 \mathrm{MW}$ with the main coolant temperature reaching $1600^{\circ} \mathrm{F}$ in approximately 10 sec. Fuel temperatures in both cases were well below the design maxima.

These results indicate that the core could survive this severe accident if some reserve control remained. Also, it is important to note that the rate of coolant flow has a significant effect on the time scale, that is, the time required to reach coolant temperature limits 
is lengthened when the flow is increased. This allows more time for the response of reserve control.

\subsection{Hypothetical Accidents}

As previously discussed, accidents in which the control system does not function are considered hypothetical. In such accidents the core will be destroyed, and the question of primary interest is the containability of the accompanying energy release.

Two types of accidents starting at full reactor power were considered: a rapid reactivity insertion leading to sodium voiding at full coolant flow, and an abrupt loss of flow followed by gross fuel melting and core compaction. (In the tank reactor model selected, a guillotine-type pipe break will not cause this accident.) In both accidents it was assumed scram did not occur, although there is a considerable difference in the two cases between the time the accident begins and disassembly occurs. It is conceivable that the accident due to rapid reactivity insertion occurs at times of the order of the delay time of the scram system, whereas the abrupt loss-of-flow accident requires several seconds or from ten to twenty times the scram time. In addition to the assumption of no scram, it was assumed that only Doppler and disassembly feedbacks are present.

In the case of the rapid reactivity insertion, reactivity was inserted at the rate of $\$ 2 /$ sec until excessive fuel vapor pressure was generated in the central channel, causing rupture of the fuel cladding. At this point it was postulated that the sodium was expelled from the core in the worst possible manner with an expulsion time of $0.1 \mathrm{sec}$, thereby producing a ramp insertion of $\$ 16 / \mathrm{sec}$. This caused a prompt-critical excursion which was terminated by disassembly. A center fuel temperature of $6500^{\circ} \mathrm{F}$ was chosen as the point at which vapor generation was sufficient to breach the cladding. The time required to reach this temperature was of the order of one second. Either flow blockage of several channels or ejection of several control rods must occur to produce a $\$ 2 /$ sec ramp over this time span.

For the abrupt loss-of-flow case it was assumed that either the most reactive control rod was ejected or local flow blockage occurred in a central channel, producing local sodium voiding and fuel compaction. After reaching the corresponding overpower condition, coolant flow was stopped, which initiates sodium voiding, and progressive radial melting and compaction of the fuel. It was assumed that as the fuel melts it compacts to $100 \%$ theoretical density under the influence of gravity.

Energy-release calculations were made with a modified version of the Bethe-Tait approach for

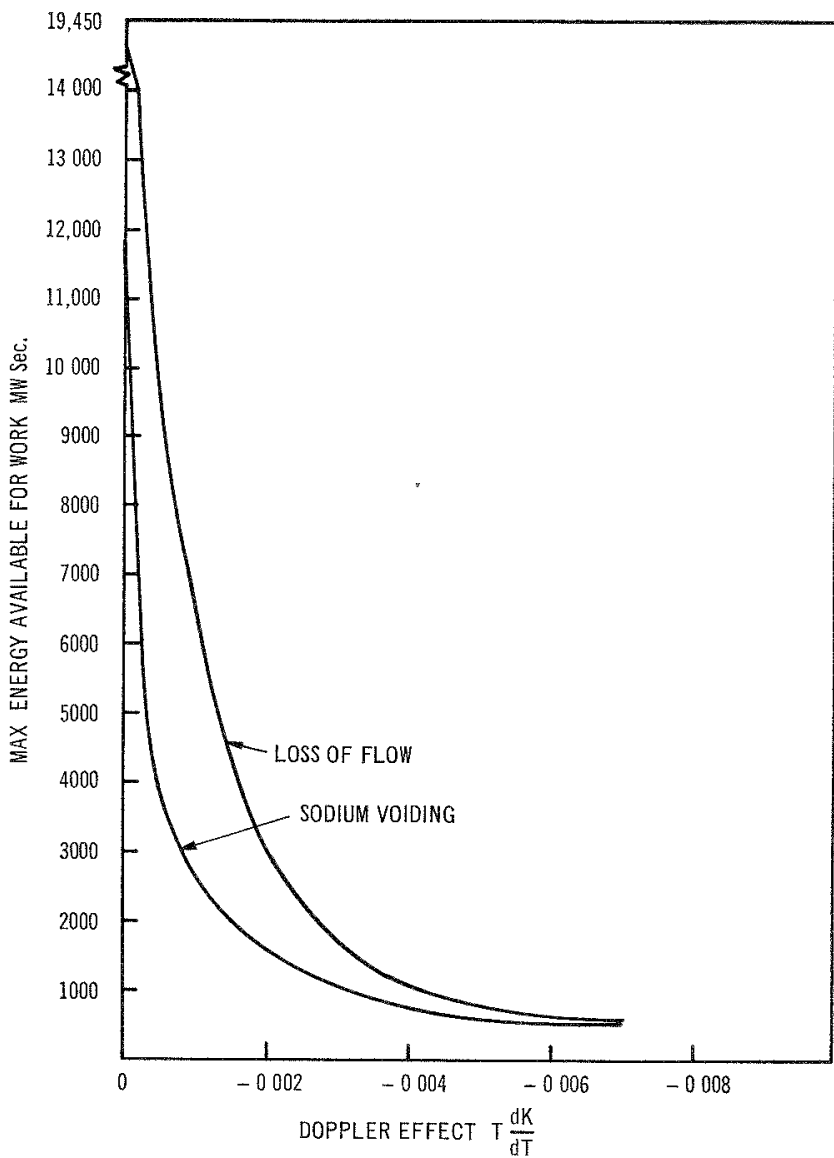

Fic. 9. Maximum Energy Avallable for Work vs Doppler Effect.

analyzing core disassembly. Energy distribution, precursor concentrations, core power, and reactivity obtained from FORE were used for initial conditions in the disassembly calculations. The equation of state for the fuel employed in the analysis was that of Meyer and Wolfe. ${ }^{(5)}$

The resulting energies available for work are shown in Fig. 9 for the two models as a function of Doppler effect. Figures 10 and 11 show the reactivity during these accidents.

In summary, the worst of these accidents (the loss of flow) produces 1030 and $640 \mathrm{MW}$-sec of available work, assuming isentropic expansions for $-[T(d k /$ $d T)]$ of 0.004 and 0.006 , respectively. If the results of the recent KIWI test ${ }^{(6)}$ are extrapolated for these core conditions, the available work is approximately 170 and $106 \mathrm{MW}$-sec, respectively.

\subsection{Containment}

A double-containment system has been provided in the current design because this system is the most predictable and reliable at this time. The containment features are shown in Figure 12. 

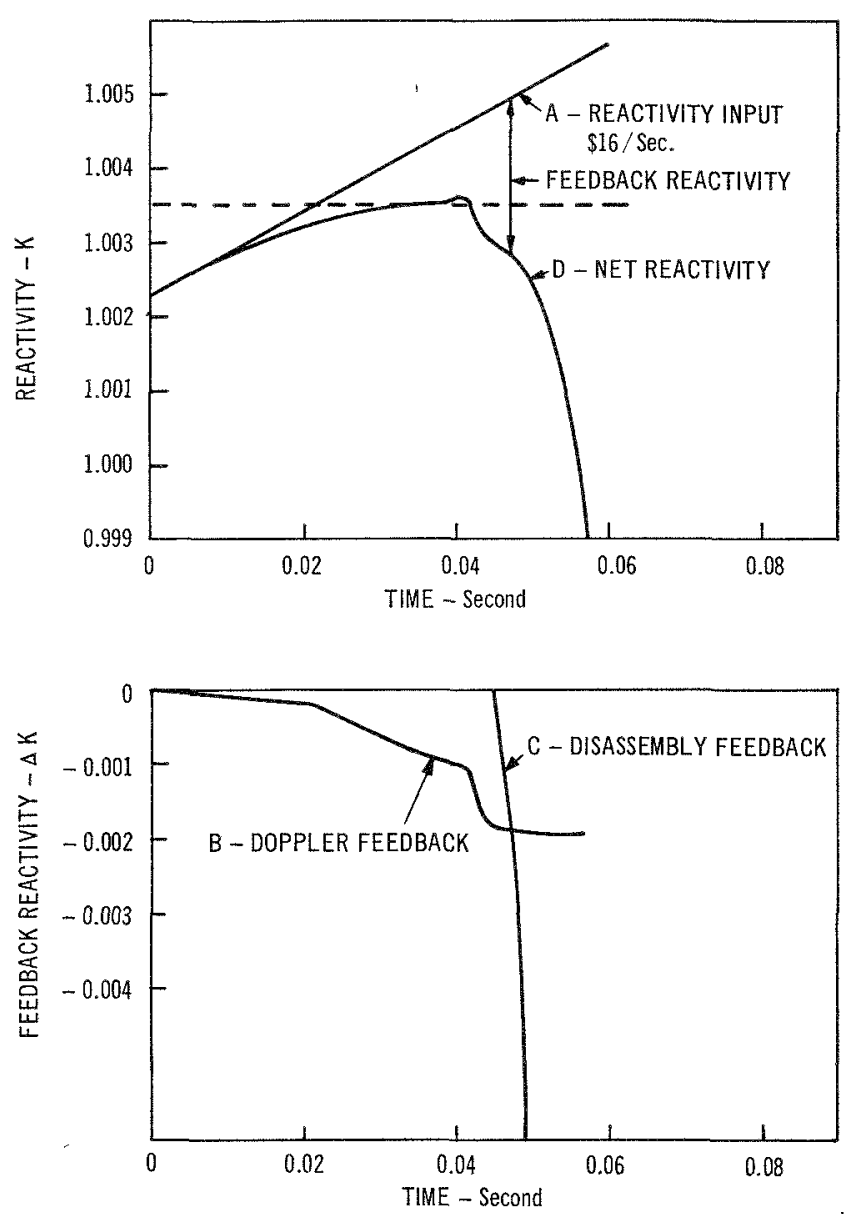

FIG. 10. Reactivity during Sodium Voiding Accident.

The outer container is a steel containment vessel which will contain 20 psig. The leakage rate for the sphere was assumed to be one percent of the contained volume over a 24 -hr period at 20 psig.

The inner container is the primary sodium tank. As previously stated, the tank is equipped with a safety vessel and placed in a massive concrete structure. The roof of the tank and all penetrations would be designed to withstand about 10-15 psig positive internal pressure and would normally be held at 0 to 2 psig. During refueling the pressure would be varied \pm 5 psi.

Because oxygen inleakage, and fission product and plutonium outleakage must be controlled, all floor penetrations and moving shafts would be equipped with seals designed for very low leakage at normal pressures of the cover gas. In order to test the adequacy of this design, these seals were assumed to leak $20 \mathrm{v} / 0$ of the cover gas per day at a differential. pressure of 10 psig. Containment of the maximum hypothetical accidents discussed in this paper was then considered and the radiological implications considered.
The large tank of sodium is considered capable of condensing the vapor ball resulting from the hypothetical burst before a significant contribution is made to the cover gas pressure by the vapor pressure of any core materials. One could design the system so that any such vapor ball would be expelled downward into the sodium tank. A transient pressure would be developed by a short-lived displacement of sodium, then a general heating of the vapor space due to the presence of high-temperature sodium. The total energy release in the accident would generate only a few degrees increase (averaged) in the tank (1688 MWsec $/^{\circ} \mathrm{F}$ rise). However, the decay heat can bring the tank contents to boiling in a few hours without cooling provisions.

The vapor space pressure (including the gas space in the interconnected gas surge tank), which developed as a result of increase of tank sodium temperature, was calculated on the basis that a 50-MW emergency cooler was used. The pressure resulting from the temperature pulse was used as input to a double-containment leakage program and the leakage rate computed.

The results if a 50-MW emergency cooler is used indicate a peak differential pressure of 9 psi between
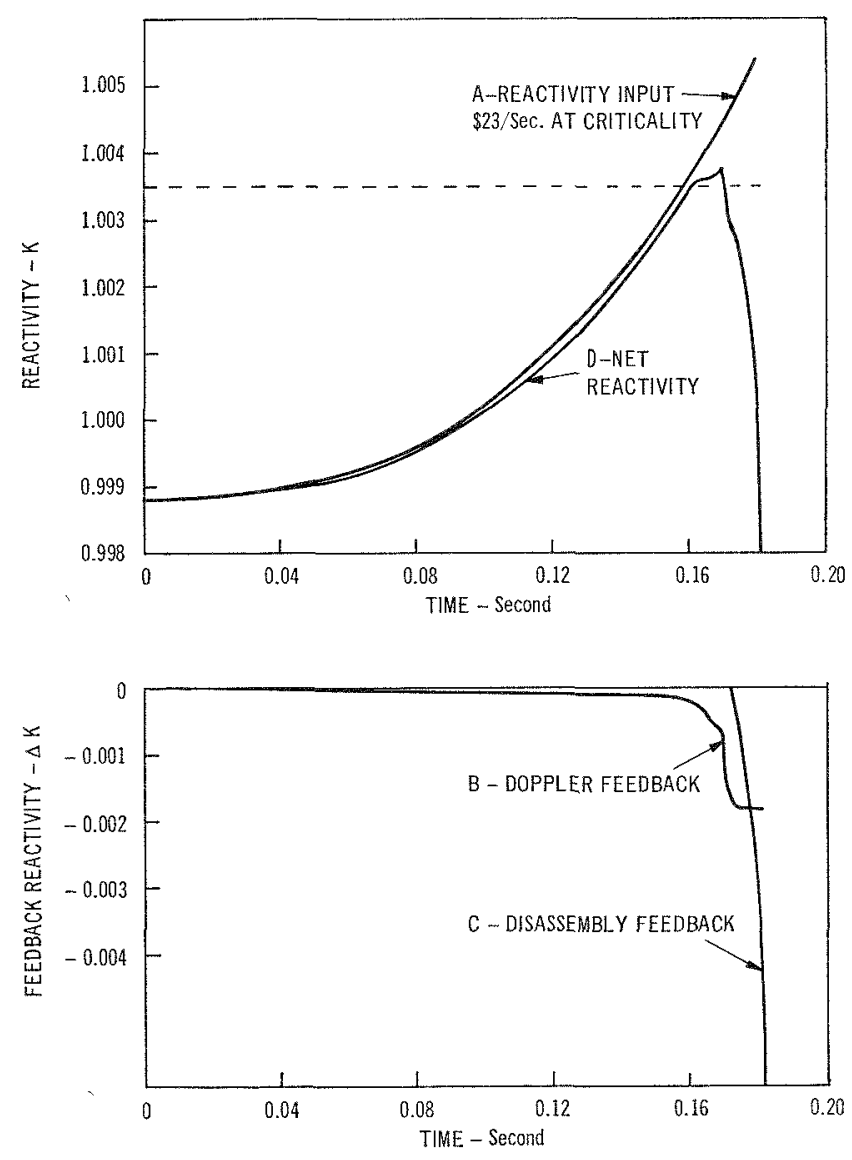

FIG. 11. Reactivity during Loss-of-Flow Accident. 


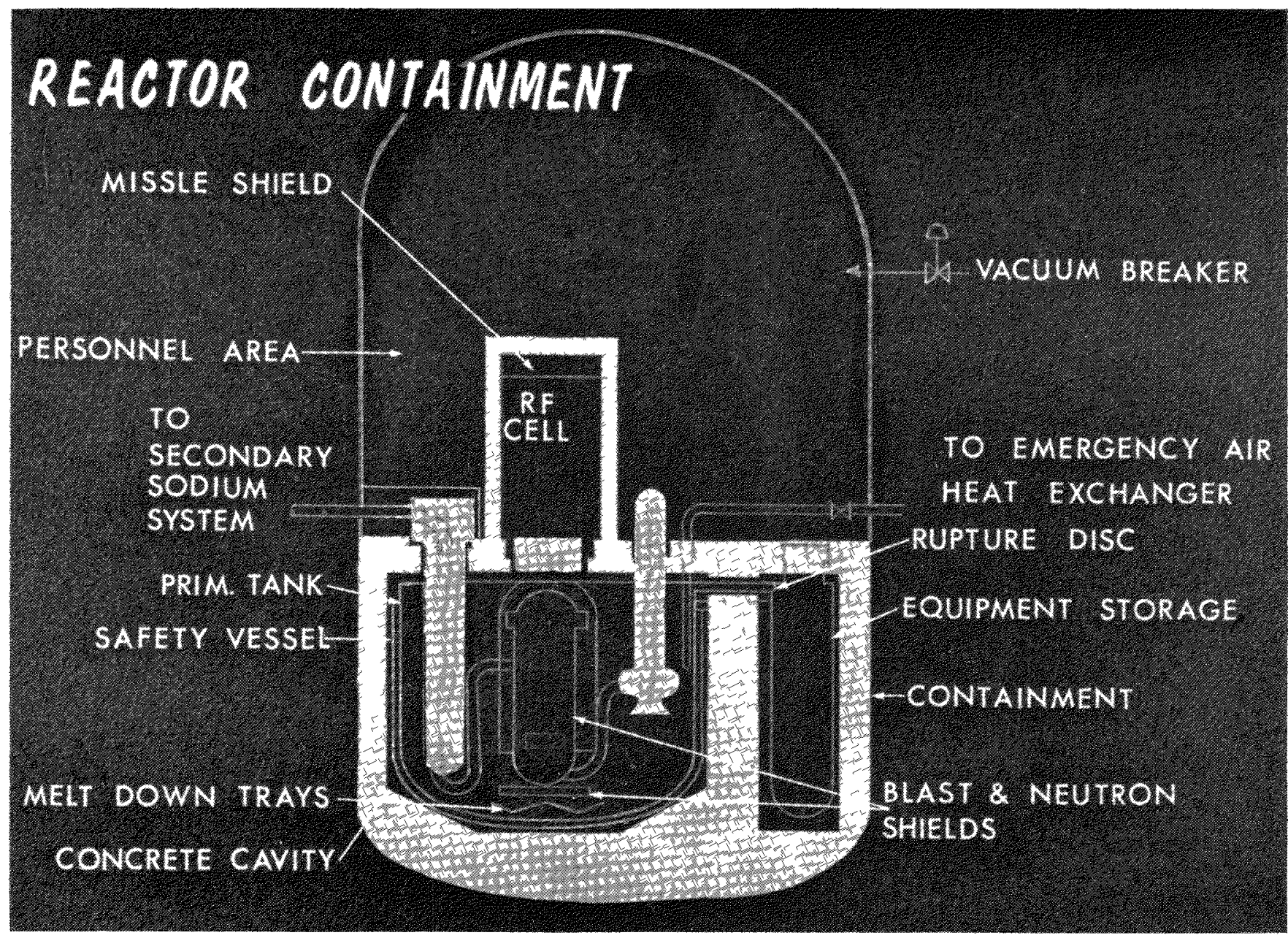

Fig. 12. Reactor Containment.

the inner and outer containments. The differential pressure between the outer containment sphere and a constant atmosphere increased to $2 \mathrm{in}$. of water after $10 \mathrm{hr}$, then fell to zero in about a week.

Plutonium and strontium leakage from this incident was calculated by assuming that a plutonium aerosol was present in the reactor vapor space. The plutonium concentration of the aerosol was taken as $0.264 \mathrm{gm} / \mathrm{m}^{3}$ of argon based on a specific gravity of about 8 . The resulting total leakage of plutonium to the atmosphere was $0.1 \mathrm{gm}$ over the week, assuming complete mixing in the outer containment. Strontium was assumed, conservatively, to escape in the same ratio as it exists with plutonium in the core (at 100,000 MWD/T). Some $0001 \mathrm{gm}$ of strontium would escape.

The release of about $0.1 \mathrm{gm}$ of plutonium and 0.001 $\mathrm{gm}$ strontium does not represent a serious radiological hazard in the context of a maximum accident result. Furthermore, the aerosol would settle and deposit on surfaces before it is released. In any event, calculations at a typical reactor site show these values to be at least one order of magnitude below acceptable accident limits.

Protection against sodium fires is provided by this design. Because the tank itself is designed to contain the hypothetical accident, the sodium is expected to be covered with an inert atmosphere throughout any incident. Small fires resulting from sodium escape from failed seals, etc., would be easily contained without pressurization of the outer containment, which would withstand approximately 20 psig in any event.

\section{ECONOMICS}

\subsection{Fuel-cycle Costs}

The fuel-cycle data important to cost calculations are given in Table 13. The refueling scheme is based on a six-month refueling cycle at $80 \%$ load factor. Note that the six-month refueling cycle is a limit imposed by safety criterion 6 . 
TABLE 13. FUEL-CYCle Data

Six-month Refueling Cycle, $80 \%$ Load Factor

Four-batch Loading in Zone 1, 5-batch in Zone 2

Average Fuel Burnup

Zone 1

Zone 2

Fissile Inventory at Midcycle

Core Fissile Enrichment at Mideycle

Total Breeding Ratio

Core Internal Conversion Ratio

Doubling Time at 30\% Out of Reactor Inventory

$115,700 \mathrm{MWD} / \mathrm{T}$ $105,500 \mathrm{MWD} / \mathrm{T}$ $2470 \mathrm{~kg}$ fissile $15.3 \%$

1.18

0.63

$13.4 \mathrm{yr}$

TABLE 14. FUEL-CYCle COST

\begin{tabular}{l|c}
\hline & Mills/kW-hr \\
\hline Fabrication & 0.343 \\
Inventory & 0.425 \\
Depletion & -0.197 \\
Recovery & 0.200 \\
First-core Capitalization & 0.101 \\
\hline \multicolumn{1}{c|}{ Total } & 0.872 \\
\hline
\end{tabular}

The initial fissile enrichment is $17.9 \%$. Fresh blanket material is depleted uranium of $0.3 \%$ U-235. The isotopic composition of the plutonium supplied to the core is calculated on the assumption that plutonium discharged from the core and blankets is mixed uniformly for recycling. The batch-refueling schemes yield nearly equal average burnup in the two zones with an overall average of about $110,000 \mathrm{MWD} / \mathrm{MT}$.

Fuel-cycle costs based on $10 \%$ inventory charges, $\$ 10 / \mathrm{gm}$ plutonium, shipping half-way across the USA, and $\$ 17,000 /$ day reprocessing charges per the AEC "standard" plant are given in Table 14.

\subsection{The Effect of Uncertainties in Nuclear Data}

The configuration and other important characteristics of a fast reactor core design are strongly influenced by the nuclear parameters of the fuel and other core materials. It is important therefore to have some assessment of the effect of uncertainties in the basic nuclear data.

An extensive survey was carried out to identify those uncertainties in nuclear data which were expected to be of significance from the point of view of fast reactor core design (see paper by Greebler et al., in Session I). In this presentation, the discussion will be restricted to the effects of only those changes in nuclear parameters which appear to be of greatest importance, and for which strong experimental evidence exists.

The nuclear design calculations for this $1000-\mathrm{MWe}$

Fast Ceramic Reactor used a conservative set of nuclear data. There is experimental evidence to suggest that calculations using these data predict:

1. a neutron spectrum which is too hard (quantitative information is given in the paper by Greebler et al.);

2. a positive Doppler contribution from $\mathrm{Pu}-239$ which is too large.

The significance of these predictions is shown in Table 15. If the nuclear data are adjusted in line with this experimental information, the design calculations show that at least $50 \%$ of the $\mathrm{BeO}$, which was included in the core to soften the neutron spectrum and to increase the Doppler coefficient, can be removed and still meet the Doppler and sodium-loss criteria of the reference core. The fissile inventory required decreases by about $3 \%$, and the breeding ratio increases from 1.18 to about 1.25 . This results in a decrease in the fuel-cycle cost of about $0.1 \mathrm{mill} / \mathrm{kW}$-hr. If one assumes a favorable combination of nuclear data within the limits of uncertainties reported by Greebler, and furthermore, if the safety criteria are relaxed to allow a calculated Doppler effect $[T(d k / d T)]$ of -0.004 (with sodium out), and a positive total sodium loss effect between $\$ 1$ and $\$ 2$, all of the $\mathrm{BeO}$ can be removed from the core. This leads to a $10 \%$ decrease in fissile inventory, a breeding ratio around 1.4 , and a fuel-cycle cost of $0.3 \mathrm{mill} / \mathrm{kW}$-hr less than that calculated for the reference core.

\subsection{Effect of Uncertainties on Current Fuel Per. formance}

Since construction of a prototype fast oxide-fueled reactor has begun in the USSR, and since immediate commitment of a prototype is under serious consideration in the UK, we thought it would be a useful exercise to design a 1000-MWe reactor as we would if we had to commit ourselves tomorrow morning. In this event we would have to design with additional safety factors to cover the inadequacies of our present information on fuel properties. We therefore assumed:

a. a $10 \%$ decrease in thermal conductivity of the fuel to allow for possible later unfavorable data;

b. a reduction in the fuel melting point of $250^{\circ} \mathrm{F}$, based on the possibility of noncongruent melting of mixed oxide;

c. an increase in overpower allowance from $110 \%$ to $120 \%$, to give added weight to errors in instrumentation;

d. no credit for central void formation in fuel;

e. an uncertainty factor of 1.2 for gap and sodium film coefficients.

These assumptions result in the core described in Table 16 when the core specific power is maintained approxi- 
TABLE 15. EFFECT OF UNCERT IINTIES IN NUCLEAR DA'T A

\begin{tabular}{|c|c|c|c|c|c|}
\hline & $\begin{array}{l}\text { Sodium-out } \\
\text { Doppler }\end{array}$ & $\begin{array}{l}\text { Total Loss of } \\
\text { Sodium }\end{array}$ & Percent Fissile & Breeding Ratio & $\begin{array}{l}\text { Fucl-cycle Cost or Cost } \\
\text { Improvement }\end{array}$ \\
\hline $\begin{array}{l}\text { Reference } \\
\text { Favorable Data, Remove } 50 \% \text { BeO } \\
\text { Ifighly Favorable Data, Remove } \\
\text { All BeO }\end{array}$ & $\begin{array}{l}-0.006 \\
-0.006 \\
-0.004\end{array}$ & $\begin{array}{c}\$-0.20 \\
0 \text { or slightly } \\
\$+1.60\end{array}$ & $\begin{array}{l}15.3 \\
15.0 \\
13.7\end{array}$ & $\begin{array}{l}1.18 \\
1.25 \\
1.40\end{array}$ & $\begin{array}{l}0.872 \mathrm{mill} / \mathrm{kW}-\mathrm{hr} \\
-0.1 \mathrm{mill} / \mathrm{kW}-\mathrm{hr} \\
-0.3 \mathrm{mill} / \mathrm{kW}-\mathrm{hr}\end{array}$ \\
\hline
\end{tabular}

TABle 16. Conservative Core Complaison

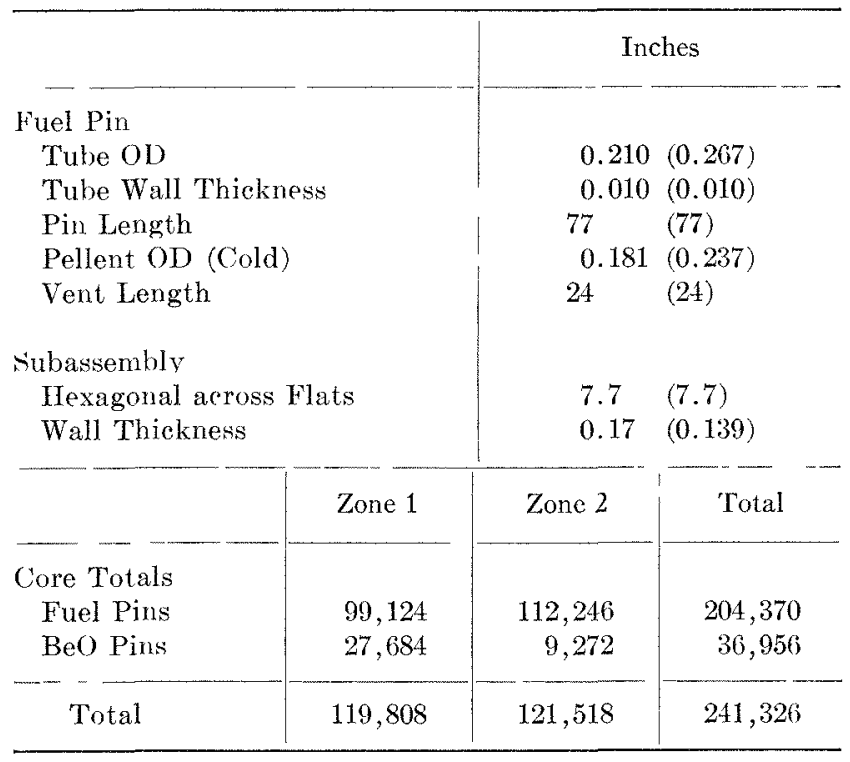

TABI. 17. "Conservatitue Curhent" Fuel Performance

Core Average Specific Power

Lot-spot Peak Linear Power

Core Average Specific Power

Fissile Specific Power

Hot-spot Fuel Center Temperature

Core Average Fuel Temperature

Average Burnup

mately constant. Values in parentheses are for the core discussed previously.

Fuel-pin performance was changed by these assumptions to that described in Table 17. By use of the same costing assumptions as before, the fuel-cycle costs for this core were calculated (see Table 18). These are a conservative limit on expected costs.

The incentive for the oxide-fueled fast reactor for immediate commitment is still considerable, even if less than we expect to attain at the conclusion of investigations presently underway. The attainment of high burnup remains an important assumption. Information supporting the 100,000-MWD/T figure used
TABle 18. "Conservitive Current" Fleicr'ycle Cosl's

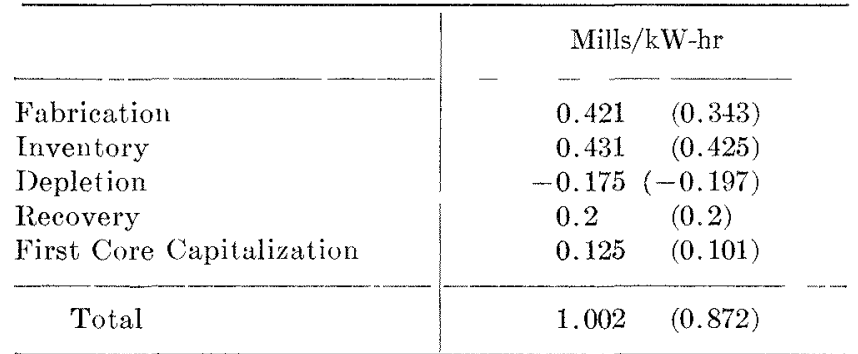

Again, the values in parentheses are those given previously.

TABIE 19. Construction Costs For 1000-MW Fisf Oxide Reictor (thousands of dollars)

\begin{tabular}{l|r}
\hline \multicolumn{1}{c|}{ Item } & \\
\hline Land & 220 \\
Site Improvements & 3,990 \\
Controls and Instrumentation & 2,970 \\
Turbine-Generator Building & 8,200 \\
Turbine-Generator Unit & 27,000 \\
Condenser for Circulating-water System & 7,500 \\
Accessory Electrical Iquipment & 8,750 \\
Feedwater System and Piping & 11,800 \\
\multicolumn{1}{c|}{ Subtotal "Non-nuclear" } & 70,430 \\
\hline Reactor Equipment & 16,100 \\
Cover-gas System & 850 \\
Primary Sodium Pumps and Drives & 3,280 \\
Intermediate Heat Exchangers & 4,150 \\
Secondary Sodium Pumps and Drives & 2,740 \\
Secondary Sodium Piping and Valves & 610 \\
Steam Generators & 19,800 \\
$\quad$ Subtotal "Nuclear Steam Supply" & $\$ 47,530$ \\
\hline Reactor Building & $\$ 7,100$ \\
\hline Total Plant Cost & $\$ 125,060$ \\
\hline
\end{tabular}

in the calculation is meager, and possibly a conservative present design would also back off to 50,000 or 75,000 MWD/T.

\subsection{Capital and Total Power Costs}

Capital and operating costs were not revised in this work. A similar plant was estimated in 1964 to cost 
$\$ 125 / \mathrm{kW}$, which is equivalent to $2.5 \mathrm{mills} / \mathrm{kW}-\mathrm{hr}$ at $80 \%$ plant factor and $14 \%$ per annum. These costs included all construction, overhead, and direct costs, as well as contingency and interest during construction. The estimate is repeated in Table 19 for completeness. Operating and maintenance costs were also estimated previously at an annual cost of $\$ 2,157,000$, which amounts to $0.34 \mathrm{mill} / \mathrm{kW}$-hr at $80 \%$ plant operating factor.

Thus, total power costs for this reactor are:

\begin{tabular}{lc} 
& mills $/ \mathrm{kW}-\mathrm{hr}$ \\
\cline { 2 - 2 } Capital & 2.5 \\
Operating & 0.31 \\
Fuel Cycle & $\overline{0.87}$ \\
\multicolumn{1}{c}{ Total } & $\overline{3.71}$
\end{tabular}

Thus, the incentive to derelop this reactor concept re- mains strong and essentially unchanged from the previous evaluation.

\section{REFERENCES}

1. M. J. MeNelly, Liquid Metal Fast Breeder Reactor Design Study (1000 .1WWe LO $\mathrm{O}_{2}-\mathrm{PuO}_{2}$ Fueled Plant), (x EAP-4418.

2. R. S. Young and R. W. Loekhart, (xeneral Hlectric Sodium Mass Transfer Program Contract AT (04-3)-189, P.A. 15 presented at ALC Sodium Components Development Program Information Meeting at Chicago, Illinois, $6 / 16 / 65$.

3, P. Greebler, 1). B. Sherer, and N. H. Walton, A Computational Program for the 1 nalysis of Fast Reactor Excursions -FORE, GHAP-4090 (Oet 1962).

4. B. Wolfe, N. Friedman, and R. Meyer, Meltdown Accidents in Large FCR's, GEAP-4009, to be published.

5. R. Meyer and B. Wolfe, High Temperature Equation of State of Uranium Dioxide, ANS Transactions, 7(1) (June 1964).

6. Private communication to B. Wolfe from W. Stratton. 


\title{
Conceptual Design and Preliminary Accident Analysis of a Sodium-cooled, Carbide-fueled, Large Modular Fast Reactor
}

\author{
J. H. Wright, W. F. Gunson, F. M. Heck, and R. S. Daleas \\ Westinghouse Electric Corporation \\ A tomic Power Division \\ Pittsburgh, Pennsylvania \\ (PRESENTEI) BY J. H. WRIGHT)
}

\section{Introduction}

This paper presents the conceptual design and some of the safety characteristics of a large, modular, sodium-cooled fast breeder reactor power plant. The reactor concept is essentially the same as that developed in reference (1) for the USAEC. Certain aspects of that design have been revised to produce a higherpower-density reactor ${ }^{(6)}$ which is more nearly consistent with private ownership of fuel and commercial utility practice.

One of the advantages of the modular concept is that by the addition of modules the thermal power rating can be extended, in units of a module, to any desired level. This flexibility in the concept removes all apparent size restrictions confronting fast reactor cores. The modular concept can easily be prototyped through the use of a single module for a demonstration plant. A second advantage is that the safety coefficients, particularly the sodium temperature and voiding, can be adjusted to any desired values through changing the dimensions, height, and diameter of the individual modules to obtain the necessary neutron leakage. In addition, the neutron coupling between modules can be made, within limits, to be any desired value through the freedom existing in adjusting the radial blanket thickness between modules.

The CEX (neutron-controlled expansion) fucl assemblies used in reference (1) were omitted from the core design as this supplementary axial-expansion safety device is not yet developed. A sodium-bonded carbide-fueled core may have a predictable expansion characteristic as the carbide fuels have physical properties, thermal conductivity, and expansion coefficients quite similar in values to those of metals.

To establish the expansion characteristics of carbide fuels, a study of the behavior of these fuels under irradiation, up to $100,000-\mathrm{MWD} / \mathrm{T}$ burnup, is required. If carbide fuels are shown not to have predictable expansion characteristics, then the stack of active fuel pellets can be divided among compartments and the fuel will move with the cladding. The clad is stainless steel whose expansion behavior is well known and should provide sodium-bonded carbidefueled cores with a dependable axial-expansion characteristic during transient conditions. The sodium bonding increases the fuel-clad conductance sufficiently to give a time constant less than 0.5 see for the heat being generated in the fuel to reach the clad. The clad thus has a response fast enough to be a major contributor in terminating a power excursion. Thus in any case adding the CEX safety device would improve the axial-expansion behavior of the core.

The sodium outlet temperature of the reactor has been lowered from $1200^{\circ} \mathrm{F}$ in reference (1) to $1100^{\circ} \mathrm{F}$. The $1100^{\circ} \mathrm{F}$ level is sufficiently high to achieve steam temperatures in the range from 950 to $1000^{\circ} \mathrm{F}$. This reduction in sodium outlet temperature permits a reduction in clad temperature while simultaneously increasing the reactor temperature rise and the total power. The increase in total power with the same fuel assembly reduced the inventory of fuel per unit of output. The lower temperature level reduces the requirements of the IHX and steam-generator materials. Concurrently, the pump size is reduced, the cost of heat exchanger apparatus decreased, and the reliability of the fuel elad is increased.

The number of control rods has been increased to reduce the hazard of a multiple accident culminating in control-rod expulsion, and to permit refueling on approximately an annual basis (instead of semi-annually) to improve the commercial acceptability of the plant.

Potential accidents to this reactor have been reviewed, and five events or chain of events have been chosen as representative of the more severe disturbances to which the system could be subjected. The response of the reactor to these disturbances has been determined by means of digital power-excursion computer programs. The analysis of these results indicates that the behavior of the reactor should not imperil the public or the operating staff, nor even result in economically severe damage to the plant. The 
most severe accident analyzed is a flow blockage to one fuel assembly. This is severe because of the difficulty in detecting such a small perturbation in the coolant flow until severe damage has been done to that one assembly. Even this case would not result in damage to the remainder of the core unless a large fraction of the safety rods refused to move in response to reactor over-power, over-temperature, and neutronlevel and neutron-period scram signals, a very improbable event.

\section{Reactor Description}

The reactor is a revision of the conceptual design developed in reference (1) for the USAEC. It consist of seven distinct regions or modules separated by a graphite reflecting region and coupled by the neutron flux. Each module contains a core region approximately 34 in. in diameter and $72 \mathrm{in}$, tall. Surrounding the cores are fertile, axial, and radial blankets. The core layout is illustrated in Fig. 1.

The thermal design of the core has been revised to increase the power density in order more nearly to optimize the fuel-cycle cost for private ownership of fuel, $10 \%$ annual charge, and commercial acceptability. In this revised design, ${ }^{(6)}$ the sodium outlet temperature is reduced from the $1200^{\circ} \mathrm{F}$ in reference (1) to $1100^{\circ} \mathrm{F}$. The increase in power density raises the combined thermal power rating of the seven modules from 2500 $\mathrm{MW}(\mathrm{t})^{(1)}$ to $3440 \mathrm{MW}(\mathrm{t})$, approximately $500 \mathrm{MW}(\mathrm{t})$ per module. The electrical output rating becomes 1375 MW (c), assuming a 40\% plant efficiency.

The reduction in coolant outlet temperature, while permitting a $30 \%$ increase in the specific power density, $\mathrm{kW} / \mathrm{kg}$ of fissile material, allows a reduction in the clad surface hot-spot temperature from $1400^{\circ} \mathrm{F}$ to $1300^{\circ} \mathrm{F}$, with a simultancous increase in the reactor coolant temperature rise from $220^{\circ} \mathrm{F}$ to $250^{\circ} \mathrm{F}$. The fuel-rod average and maximum linear powers are 15.6 $\mathrm{kW} / \mathrm{ft}$ and $38 \mathrm{~kW} / \mathrm{ft}$, respectively. The core average fuel temperature is approximately $1400^{\circ} \mathrm{F}$ whereas the maximum fuel centerline temperature is $2430^{\circ} \mathrm{F}$. The thermal calculations are based upon a fuel thermal conductivity at operating conditions of $10.5 \mathrm{BTC} / \mathrm{hr}$ $\mathrm{ft}-{ }^{\circ} \mathrm{F}$. The core pressure drop is approximately 100 psi. Table 1 summarizes the thermal and hydraulic characteristics of the core.

Each core consists of 37 fuel assembly positions; one (the center position) is occupied by a safety rod. Each of the 36 hexagonal fuel assemblies contains 127 fuel rods, of 0.300 -in. OD, on a triangular pitch with 0.426 -in. centers. The cladding is stainless steel, 10 mils thick. The fuel material is $90 \%$ dense, stabilized plutonium-uranium carbide. The fuel pellets are sodium bonded to the clad. The fuel rods are vented to the sodium coolant. The numerical data for the mechanical design are tabulated in Table 2. A more detailed description of the reactor mechanical design is given in Appendix A.

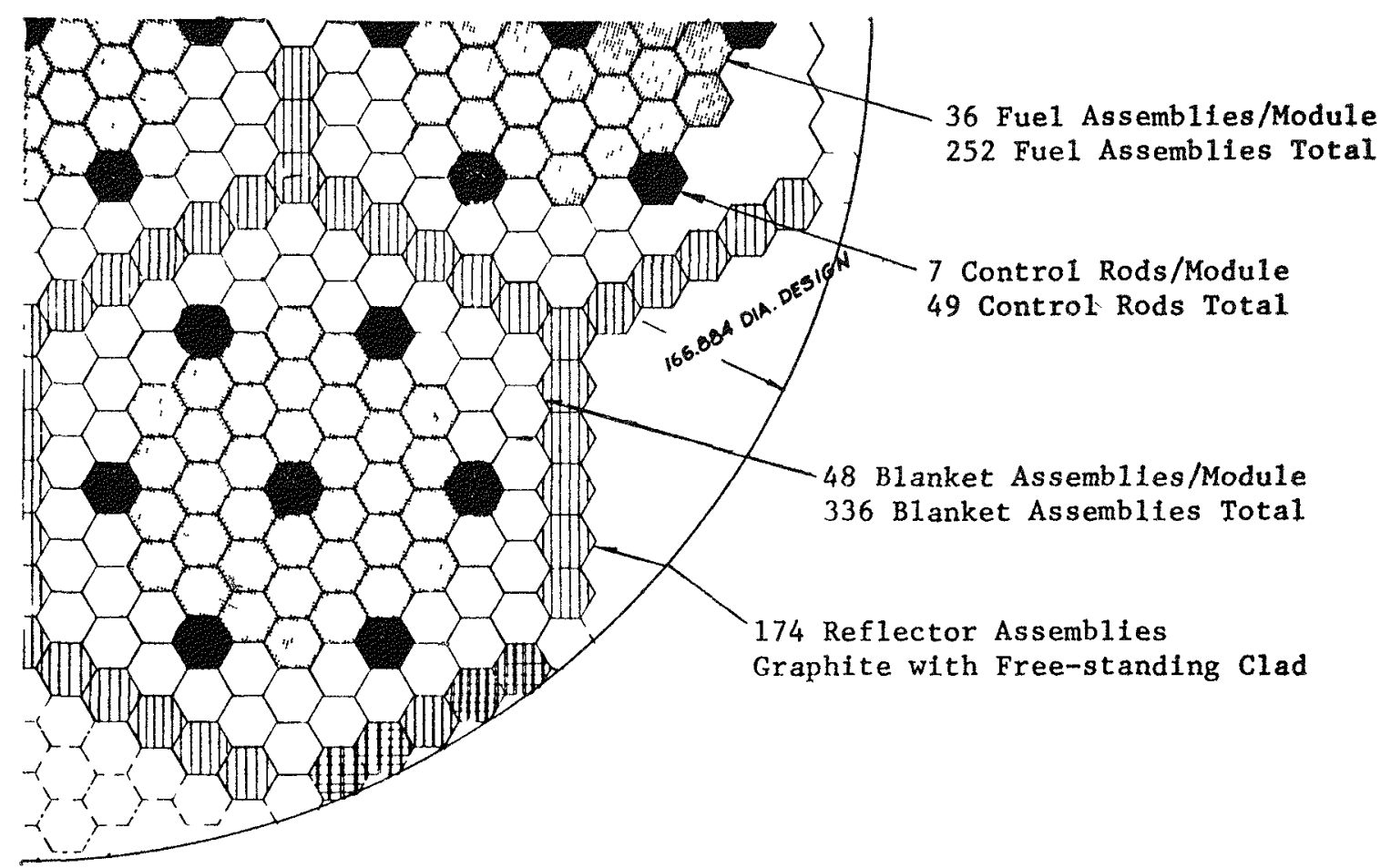

Fra. 1. One-quarter Reactor Core Secton. 
TABLE 1. RejCLOR Therm 4L Design D TT

\begin{tabular}{|c|c|}
\hline Power & 1 \\
\hline Total Thermal Output, $\mathrm{MW}(\mathrm{t})$ & 3440 \\
\hline Estinated Electrical Output, MW(e) & 1376 \\
\hline Thermal Output of Core per Module, MW(t) & $417^{*}$ \\
\hline $\begin{array}{l}\text { Thermal Output of Axial Blanket per Mod- } \\
\text { ule, MW }\end{array}$ & $11^{*}$ \\
\hline $\begin{array}{l}\text { Thermal Output of Radial Blanket per Mod- } \\
\text { ule, MW }\end{array}$ & $64^{*}$ \\
\hline Total Flow rate, $10^{6} \mathrm{Ib} / \mathrm{hr}$ & 20.7 \\
\hline Bypass Flow, Estimated at $10 \%$ & 2.1 \\
\hline Power Density, $\mathrm{kW} /$ liter of Core & 390 \\
\hline Specific Power, $\mathrm{kW} / \mathrm{kg}$ of Core Metal & 117.8 \\
\hline Average Linear Power, $\mathrm{kW} / \mathrm{ft}$ & 15.6 \\
\hline Maximum Linear Power, $\mathrm{kW} / \mathrm{ft}$ & 37.8 \\
\hline \multicolumn{2}{|l|}{ Heat Transfer } \\
\hline Total Heat Transfer Area, $\mathrm{ft}^{2}$ & 14,210 \\
\hline Average Heat Flux, $B t u / h r-f t^{2}$ & 678,000 \\
\hline Maximum Feat Flux, Btu/hr-ft $t^{2}$ & $1,640,000$ \\
\hline Total Flow Area, $\mathrm{ft}^{2}$ & 20.58 \\
\hline Average Coolant Velocitv, ft/sec & 30.3 \\
\hline Average Mass Velocity, $10^{6} \mathrm{lb} / \mathrm{ft}^{2}-\mathrm{hr}$ & 5.58 \\
\hline \multicolumn{2}{|l|}{ Temperatures, ${ }^{\circ} \mathrm{F}$ at Rated Power } \\
\hline Reactor Inlet & 850 \\
\hline Reactor Outlet & 1100 \\
\hline Average Channel Rise $(10 \%$ By pass $)$ & 275 \\
\hline Average Chamel Outlet & 1125 \\
\hline Ifot Channel Outlet & 1293 \\
\hline Maximum Clad (Outside) & 1326 \\
\hline Maximum Fuel Center & 2430 \\
\hline
\end{tabular}

* Time-averaged values (estimated).

The nuclear reactivity coefficients were calculated by means of improved methods developed since the issuance of reference (1). The new sodium-coefficient calculations are based on two-dimensional studies. The Doppler coefficient is based on resonance-integral calculations involving higher plutonium isotope and fission product effects than were used in reference (1). The Monte Carlo technique used in reference (1) has been improved and if used would give a Doppler coefficient $25 \%$ higher than the resonance-integral method. Table 3 summarizes the pertinent nuclear design data.

There are 49 control rods, 7 per module. The central rod in each module is a safety rod, and the six peripheral rods in the first row of radial blanket assemblies are used for power regulating and shim control. The total worth of the 49 rods is $6.9 \% \mathrm{k}$ against an estimated reactivity requirement of $6.3 \% k$ (see Table 3).

\section{Fuel-cycle Cost}

Fuel-cycle analyses of the Westinghouse modular fast breeder reactor designs have been placed on a more realistic basis than was directed by the USAEC during the four conceptual design studies. ${ }^{(1-4)}$ For example, a 10\% annual charge was assumed to apply on all working capital and inventory charges rather than the $4 \% / 4 \%$ directed by the Commission in 1963.

TABLE 2. RELCTOR MeChanell. Destan Dita

\begin{tabular}{|c|c|}
\hline \multicolumn{2}{|l|}{ Core Modules } \\
\hline Number of Modules & 7 \\
\hline Number of Fuel Assemblies/Module & 36 \\
\hline $\begin{array}{l}\text { Number of Blanket Ansemblies/Mod- } \\
\text { ule }\end{array}$ & 48 \\
\hline $\begin{array}{l}\text { Number of Reflector Assemblies/ } \\
\text { Module }\end{array}$ & 18 \\
\hline Number of Control Assemblies/Module & 7 \\
\hline \multicolumn{2}{|l|}{ Fupl Rods } \\
\hline Pellet Diameler, in. & 0.264 \\
\hline Total Fuel Meight, in. & 72.0 \\
\hline Clad Thickness, in. & 0.010 \\
\hline Clad Outside Diameter, in. & 0.300 \\
\hline Fuel Material & $(\mathrm{L}, \mathrm{Pu}) \mathrm{C}(\bmod )$ \\
\hline Fuel Material Density, c/c Theoretical & 90.0 \\
\hline Thermal Bond Material & $\mathrm{Na}$ \\
\hline Clad Material & $316 \mathrm{~L}$ \\
\hline \multicolumn{2}{|l|}{$\begin{array}{l}\text { Axial Blanket Rods (Integral whth Fuel } \\
\text { Rods) }\end{array}$} \\
\hline Blanket Iength each Lind, in. & 12.0 \\
\hline Blanket Material & $\mathrm{UC}$ \\
\hline $\begin{array}{l}\text { Blanket Material Density, } \% \text { Theo- } \\
\text { retical }\end{array}$ & 90 \\
\hline \multicolumn{2}{|l|}{ Fuel Assemblies } \\
\hline Type of Rod Array & triangular \\
\hline Fuel-rod Pitch, in. & 0.426 \\
\hline Type of Can & hexagonal \\
\hline Can Width across Flats, in. & 5.104 \\
\hline Fuel Rods per Assembly & 127 \\
\hline $\begin{array}{l}\text { Fuel Assembly Length (Approximate), } \\
\text { in. }\end{array}$ & 132 \\
\hline
\end{tabular}

TABLE 3. Nílear Design Data

\begin{tabular}{l|c}
\hline Equilibrium Cycle Enrichment, $\%$ & $\sim 15$ \\
Doppler Coefficient, $T(d k / d T)$ & -0.0034 \\
Axial Expansion, $k / \%$ Elongation & -0.0049 \\
Sodium Coefficient, $k /{ }^{\circ}$ F (Isothermal Average) & $\sim 0$ \\
Estimated Reactivity Requirements, $\% k:$ & \\
Cold to Hot & 1.3 \\
Fuel Depletion and Fission Products & 1.9 \\
Shutdown Margin (Minimum) & 3.0 \\
Load Following & 0.1 \\
$\quad$ Total & 6.3 \\
\hline Control Rod Worth, $\% k:$ & 2.0 \\
Control Rods, 7 in All, Total Worth $=$ & 4.9 \\
Peripheral Rods, 42 in All, Total Worth $=$ & 6.9 \\
\hline Total for Reactor & \\
\hline
\end{tabular}


TABLE 4. MODL 1 R-CORE FUEL-CYCLE COST B ISED ON Oyster Creek MElHod ${ }^{(8)}$

\begin{tabular}{|c|c|c|c|}
\hline & $\begin{array}{l}\Delta N L \text { Nor- } \\
\text { malization } \\
\text { (AEC Fuel } \\
\text { Ownership) }\end{array}$ & $\begin{array}{l}\text { AEC } \\
\text { Design }\end{array}$ & $\begin{array}{l}\text { HPD Design } \\
\text { (Today's } \\
\text { physics) }\end{array}$ \\
\hline $\begin{array}{l}\text { Working Capital } \\
\text { Fabrication } \\
\text { Processing } \\
\text { Plutonium } \\
\text { Credit(d) }\end{array}$ & $\begin{array}{c}0.39 \\
0.40 \\
0.21 \\
(0.39)\end{array}$ & $\begin{array}{l}0.88^{(\mathrm{c}, d, b)} \\
0.31 \\
0.27^{(\mathrm{e})} \\
(0.60)^{(a)}\end{array}$ & $\begin{array}{l}0.530 .63(c, d, f) \\
0.2-0.3 \\
0.25-0.28(c) \\
(0.45-0.5)\end{array}$ \\
\hline & 0.61 & 0.86 & $0.53-0.71$ \\
\hline
\end{tabular}

(a) Based on a breeding ratio of 1.57 as reported in WCAP. $3251-1$.

(b) Latest revised physics calculations gave a breeding ratio of 1.46 .

(c) Fuel (including bred material) fabrication, and processing at $10.4 \%$.

(d) Plutonium at $\$ 10 / \mathrm{gm}$ (fissile) as nitrate.

(e) Includes processing at $\$ 21,150 /$ day and NFs processing rates, shipping, and reprocessing losses at $1 \%$.

(f) Capacity factor at $88 \%$, typical of first fifteen years for Oyster Creek Plant. ${ }^{(8)}$

Further, estimates of the plutonium value have been carefully developed as a function of the commercial and technical status of the industry. ${ }^{(17)}$

The fuel-cycle costs based on private ownership of fuel and commercial utility practice are given in Table 4. The design reported in reference (1) and normalized by the Argonne National Laboratory ${ }^{(5)}$ is given in the first column. The fuel-cycle cost for this design based on present commercial-utility practice ${ }^{(16)}$ is given in the second column. The fuel-cycle costs for this high-power-density design is given in the third column. The reduction in the plutonium credit reflects a reassessment of the breeding gain obtained by the use of more sophisticated analytical tools and which was found to decrease by about $20 \%$.

This shifting of commercial-utility ground rules places strong emphasis upon obtaining higher specific power and results in the technical changes to the thermal design noted in reference (6). The more realistic appraisal of the commercial ground rules has led to an increase by a factor of 2 or 3 in the fuel-cycle cost over that reported in reference (1), which was based on AEC ground rules.

\section{Plant Description}

The reactor plant design utilizes separate components to maximize accessibility for maintenance. Three heat transfer loops are projected for this plant. The general arrangement of a loop is presented in Fig. 2 . This permits temporary operation at two-thirds ca- pacity with one component out of service. The entire primary system is doubly contained in an inert gas atmosphere to minimize the consequences of a primary sodium leak.

The plant layout stresses accessibility and ease of maintenance. These two factors contribute significantly to long-term plant safety. High accessibility permits thorough routine inspection and increases the probability of locating small or incipient problems before they become major problems or incidents. Ease of maintenance permits more routine maintenance to be accomplished and increases the probability that maintenance or repair work will be successful. Significant features of the plant design are discussed in more detail in Appendix B.

Each of the three loops consists of a circulating pump, the single intermediate heat exchanger, connecting double-walled piping, and instrumentation for operational control of the system. The pump suction is connected to the intermediate heat exchanger shell, while pump discharge is connected to the reactor vessel top inlet. The top discharge from the reactor vessel is connected to the intermediate heat exchanger. All system equipment that contains sodium is heattraced. All primary-system equipment is located inside the primary reactor containment. The environment around all primary-system equipment will be nitrogen or oxygen-depleted air to prevent sodium oxidation and radioactivity dispersal in the event of a leak.

The reactor vessel is of double-walled construction, consisting of a primary vessel and a secondary vessel, and is fabricated entirely from Type 304 stainless steel. The primary vessel provides containment for the reactor core and serves as the support member for the core and core barrel. The radial gap between the primary and secondary vessels is selected so that if drainage of sodium from the primary to the secondary vessel occurs, the core will remain submerged in sodium. During power operation this gap is filled with stagnant nitrogen. At shutdown, the sodium within the vessel is heated by circulating hot nitrogen between the two vessels.

The shield-plug thickness is $5 \mathrm{ft}$ and consists of the following materials listed from top to bottom: $4 \mathrm{in}$. of carbon steel; 12 in. of stacked $3 \mathrm{w} / \mathrm{o}$ boronated graphite block; a layer of carbon steel shielding (not a structural or pressure-containing component); a type 304 stainless steel structural member; $12 \mathrm{in}$. of boronated graphite block; a type 304 stainless steel structural and pressure-containing member; and $24 \mathrm{in}$. of blast-absorbing crushable material. The total minimum thickness of the four stecl plates is $111 / 2$ in. Nitrogen is provided for plug cooling in order to main- 


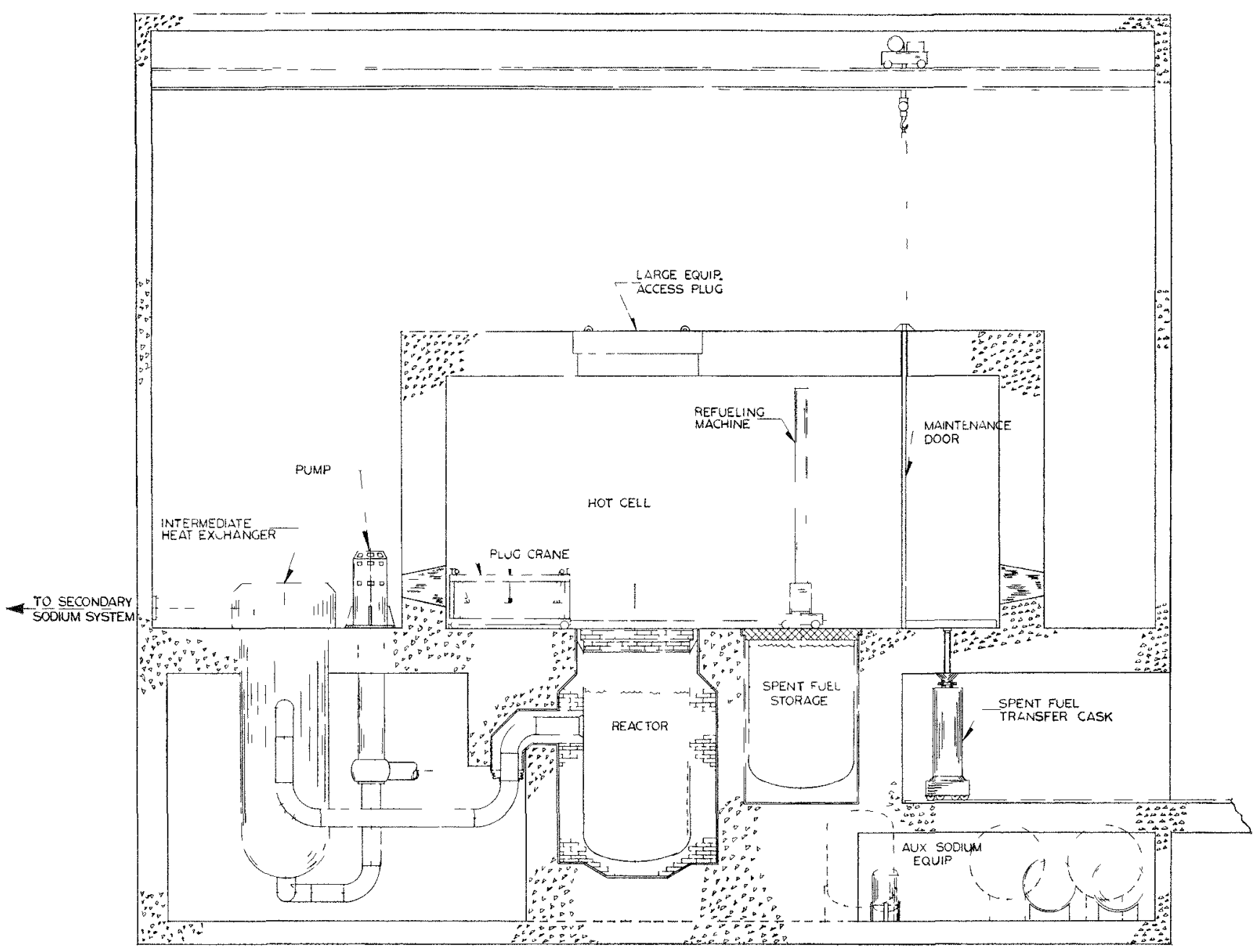

Fig. 2. Reactor Building Elevation.

tain the upper plug surface and seal at temperatures below $130^{\circ} \mathrm{F}$.

The control-rod drives, of a rack and pinion type, are located on the top of the upper plug and are completely enclosed by a gas-tight shell. Power, control, and gas-supply lines form helical coils which are suspended from the ceiling of the refueling cell. This arrangement makes it possible to remove the top plug from the reactor, hoist it up, and move the plug to its storage position without disconnecting any cables or gas-supply lines.

The three primary variable-speed pumps for circulating sodium are double suction, vertical, centrifugal units of the free surface type. All parts of the pumps in contact with sodium are stainless steel except the bearings and other special parts. Each pump is equipped with a smaller auxiliary motor connected to a separate power supply to insure adequate minimum sodium flow in the event of loss of power.

The intermediate (sodium to sodium) heat ex- changers are of the vertical shell-and-tube type with the primary sodium flowing through the shell side of the heat exchanger, entering and leaving through two diametrically opposed horizontal nozzles. Intermediate sodium flows through the tubes. The shell side contains a free sodium surface covered by argon gas to prevent oxidation and to minimize thermal stresses in the tube sheet. The argon space contains a sodiumoverflow line to accommodate volume surges.

The primary hot cell is a gas-tight structure enclosed by 6 -ft-thick concrete shield walls lined with stainless steel. Periscopes and lead glass windows are provided in the walls and ceiling for viewing critical operations. Provisions are made to heat the cell walls during refueling operations to prevent condensation of sodium vapor.

A shield door closes off one end of the cell, providing a maintenance area for the refueling machine. With the door closed air may be admitted to the maintenance area. 
The reactor is refucled by at remotely controlled refueling machine operating in the shielded, inert atmosphere cell. The refueling machine acconplishes all the fuel-handling operations, from the introduction of new fuel assomblies into the cell to the final loading of the spent fucl into the transfer cask. In addition to handling fuel, the refueling machine also removes and replaces reflector elements and control rods.

The refueling machine is a remotely controlled rectilinear crane that carries a vertical telescoping mast. Two cooling systems are provided with separate gas supplies. Automatic controls switch from one system to the other in the event of a system failure. The forced-cooling system must operate continuously during transfer of a spent core asscmbly. The design provides a method of checking the cooling system immediately after a fuel element is withdrawn from the sodium. If cooling gas is lost during a transfer, a fuel element could reach the boiling point of sodium in 5 min.

Spent fuel assemblies and control rods are stored in a sodium-filled tank located below the cell floor. Pipes extend through a top cover plate and shield down into a tank in which sodium coolant is circulated. This provides storage space for more than a full core load of fuel and blanket assemblies. Because sodium is used as the coolant, the storage pit can also be utilized for storing partially spent fuel during maintenance operations. In addition, the heat-rejection system and heat capacity of the spent-fuel storage pit are used to remove decay heat during shutdown from the reactor.

\section{Description of the Primary-system Safety Features}

The design of the Westinghouse Fast Brceder Reactor incorporates many design and safety features that will cnhance safe operation. Inherent safety features include a negligible sodium temperature coefficient and a negative Doppler coefficient of reactivity. The mechanical design is such that structural thermal expansion (radial and axial) contributes a negative feedback to the power coefficient.

Each module has one safety rod in the center. The seven of these together are worth $2 \%$ to the total reactor. Each module also has six identical peripheral control rods, three of which are used as safety rods and three as regulating rods. All 21 peripheral safety rods from the seven modules together are worth $2.45 \%$ to the total reactor, and all 21 peripheral control rods together are worth $2.45 \%$ to the total reactor. This provides a total shutdown margin in excess of $3 \%$ cold, and sufficient operating reactivity to permit an- nual refueling. One rod-drive power supply is provided for each module, which is capable of activating only one rod at a time. Thus the maximum number of rods, one rod in each module, which can be simultaneously actuated is seven. All rods can be scrammed simultaneously with a release time of $200 \mathrm{~ms}$ (similar to Fermi and EBR-II).

Positive reactivity due to partial voiding of sodium, although highly unlikely in the modular-core concept, can be controlled by rod scram action. The time between initiation of a power excursion and the beginning of sodium boiling is sufficient to permit the insertion of fast-acting control rods for all accidents studied. Further investigations of core voiding must be made to understand fully the interaction and time dependency of not only Doppler and sodium temperature coefficients, but also the dynamic behavior of sodium under abnormal conditions of boiling, twophase flow, expulsion, and voiding.

The reactor vessel is doubled-walled so that leaks in the reactor vessel cannot drain the core and leave it without cooling. The inlet and outlet nozzles to the reactor vessel are located above the core, so that leaks anywhere in the primary system cannot siphon the core dry. The primary piping is of double-wall construction with sodium-leak detectors located at the low points of the containment.

Blanket assemblies have smaller lower nozzles than fuel assemblies, so that fuel assemblies cannot inadvertently be inserted into blanket positions.

The shield plug is equipped with 2 ft of energyabsorbing material on the lower face to absorb partially the high-energy release in the event of the coremeltdown accident. In addition, strong holddown latches are located at the top of the plug to prevent the plug from becoming a missile.

The reactor protection system will detect potentially unsafe trends and conditions, and initiate corrective action. The reactor will scram automatically when an unsafe condition develops. The functions connected with the primary system which will automatically scram the reactor are listed in Table 5. Shutdown capability is provided by control rods that are spring assisted into the core. The reactivity shutdown margin is greater than $3.0 \%$ for the core with sodium.

A safety margin is provided by placing the reactor and its entire primary coolant system in reinforced concrete cells, which are backed up by a low-leakage steel containment shell. The two containment barriers will be designed to withstand conditions more severe than any that are expected to occur. The structural integrity of both containment barriers, reactor vessel, and piping will be provided by con- 
forming to all applicable design and structural codes, laws, and regulations of the appropriate regulating bodies.

The primary-system coolant pumps are equipped with flywheels attached to the rotor shaft of the drive motor. This provides inertia to the system to obtain a pump-coastdown characteristic giving $60 \%$ of full flow after 5 sec.

A coolant loss due to primary-system rupture would, by itself, cause no increase in pressure within the containment barriers as the reactor operates near atmospheric pressure, $15 \mathrm{psig}$, with temperatures at least $500^{\circ} \mathrm{F}$ below the boiling temperature of sodium at atmospheric pressures. Mechanisms that could increase the temperatures and pressures within the containment barriers are: (1) a nuclear power excursion giving a rapid release of energy, and (2) a chemical reaction of sodium with air or water vapor. The effect of a chemical reaction between sodium and air within the concrete cells is minimized by maintaining an inert atmosphere, nitrogen, within these cells. The mechanical design of the concrete cells provide protection in the improbable event of a nuclear power excursion.

\section{Accident Analyses}

A review of the reactor design yielded a list of the major accidents, credible and incredible, which can be postulated to occur to the reactor given the necessary conditions. Table $\mathrm{C}-1$ in Appendix $\mathrm{C}$ contains the resultant list along with preliminary engineering safeguards for controlling each event. The accidents are grouped into the following four categories:

1. fuel-handling mishaps;

2. start-up mishaps;

3. full-power mishaps;

4. sodium spills and fires.

By proper system design and the application of the suggested preliminary engineering safeguards, all the accidents listed can be controlled and prevented from leading to gross disassembly of the core. A sequence of events potentially leading to core meltdown and the resulting energy release is discussed later. A controlled accident may result in some degree of core damage which will not present a hazard to the personnel or to any part of the plant outside of the primary cooling system. During a controlled accident the control system functions properly and scrams the reactor. To obtain an uncontrolled accident an accumulation of mechanical failures, including failure to scram, with possible loss of administrative control are necessary.

Events initiating a power excursion do not occur in-

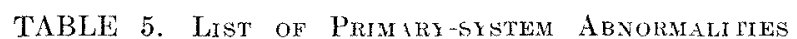
CALSING SCR AM AND/OK ALARM

1. Source flux level low (i)

2. Log count-rate level high

3. Log count-rate period short or detector voltage low ${ }^{(b)}$

4. $\log N$-period short or detector voltage low (b)

5. Power range above fixed level or detector voltage low ${ }^{(b)}$

6. Any control rod unlatched

7. Any control rod not "Full Down" (a)

8. Reactor inlet coolant flow low or rate of flow change high

9. Reactor outlet coolant temperature high or rate of change high

10. Primary pump power interuption

11. Primary system colant level low

12. Reactor cover gas pressure high

13. Cell isolation trip (access open)

14. Manual scram

15. Reactor "noise" due to boiling or voiding in a single fuel subassembly

16. Mismatched sodium outlet temperature from the various assemblies

(a) Effective only prior to reactor operation; bypass when control power is available.

(b) During startup, any two out of three chamels will cause seram and alarm.

stantaneously because all processes have a finite time dependence. Because of the time element associated with an accident, it is within engineering reality to control the worst accident, sodium voiding, by fastacting control rods capable of introducing several percent reactivity in a rather short time, i.e., less than $400 \mathrm{~ms}$ from release to full insertion. If a power excursion is terminated without gross fuel melting and collapse of the core, the accident will have been controlled and the large energy release associated with core compaction will have been prevented.

The characteristics of the proposed fast breeder reactor were analyzed for inherent stability and the consequences of several accidents selected from the first three categories in Table 5, which were considered most likely to result in core damage. The analyses which were performed concerned:

1. inherent stability;

2. dropping a fuel assembly into a just subcritical core;

3. expulsion at $100 \%$ thermal power of a regulating control rod;

4. loss of pump power at $100 \%$ thermal power;

5. pipe rupture at $100 \%$ power in one of three loops;

6. local flow blockage;

7. fuel slumping;

8. core meltdown.

The analyses of the above accidents were performed for an isolated core representing the center module 
TABLE 6. Renctivity COEfFicients for Modulak Core

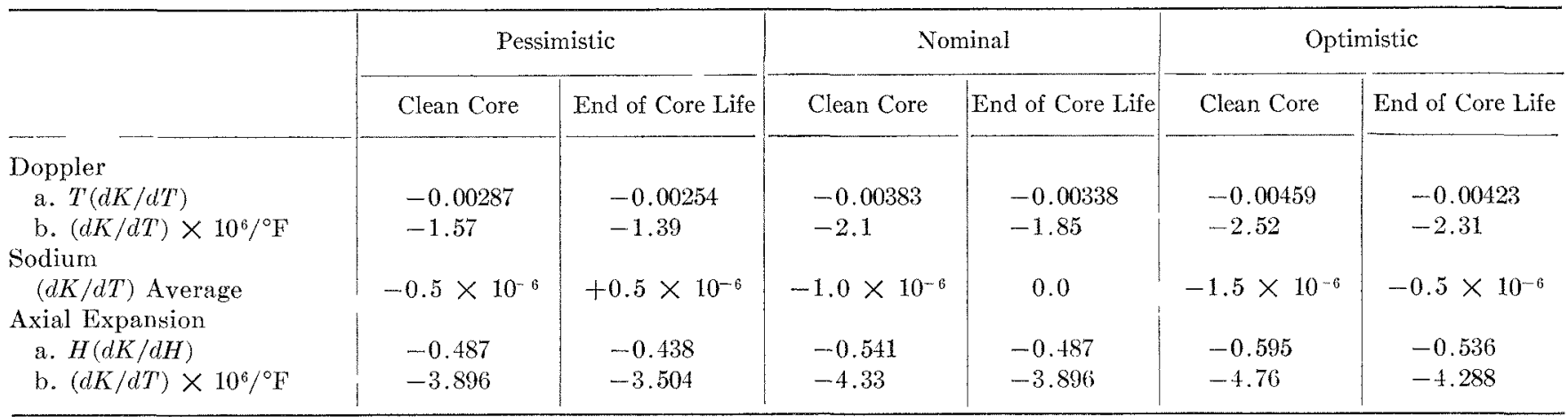

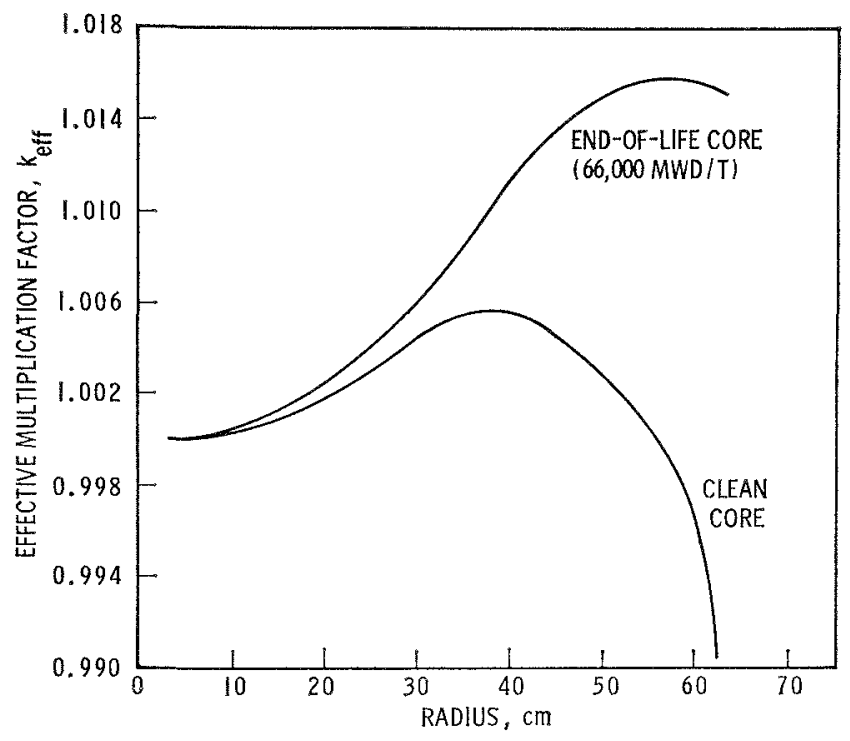

FIG. 3. Sodium Voiding $k_{\mathrm{tf}}$ as Function of Radius Voided.

of a multiple-coupled core system. A modular core designed on the basis of an isolated core would be conservative. In particular, the results of the analyses for dropping a fuel assembly and for expulsion of a control rod would be pessimistic for a coupled core system because of the neutronic cross-coupling effects. In a modular core there is information which indicates a reactivity insertion into one module is divided between all modules as some function of the cross coupling.

The first five analyses were performed with the WFORE digital computer code developed at Westinghouse. This code is similar to the FORE ${ }^{(9)}$ code. All reactivity feedbacks are responsive to temperature changes in the core. The Doppler effect varies inversely with fucl temperature. The core is divided into five axial segments. The sodium temperature coefficient is given a spatial distribution with core height. The axial expansion is a function, by an input control option, of the change in fuel or clad temperature.

The range of reactivity coefficients, Doppler, so- dium, and axial fuel expansion, for the clean core and the end-of-life core, 66,000-MWD/T burnup, used in the analyses are given in Table 6. All coefficients remain negative throughout core life. The effect of the change in isotopic composition of the fuel and fission products is to cause all coefficients to become less negative by a factor of 1.14 for Doppler, and 1.1 for axial fuel expansion. The optimistic and pessimistic values were obtained by applying estimated calculational error tolerances to the nominal values.

The reactivity added upon voiding the core is shown in Fig. 3. In the clean core, complete voiding removes approximately $1 \%$ in " $k$," whereas for the end-of-life core the effect of the fission products is to cause a positive reactivity addition of $1.54 \%$ in " $k$ " upon voiding. In the accidents analyzed, sodium voiding did not occur and thus voiding appears to be highly improbable in the modular core design.

\section{VI.1 Core Inherent Stability}

Local Inherent Stability. The local stability due to some local disturbance, such as flow starvation, was analyzed by use of the W-MABI computer code. The end-of-life core, 66,000-1MWD/T burnup, was selected for the analysis as it represents the worst condition. The core was voided out to a radius of $24.5 \mathrm{~cm}$, and the resultant power profiles are shown in Fig. 4. As the sodium density decreases in a local region, the power density in the affected region decreases. Any local disturbance thus does not propagate into a large local power excursion.

Overall Inherent Stability. The reactor response to a small reactivity insertion such as would be introduced at full power for a change in power level was studied to assess the overall inherent stability characteristies of the core. The reactivity insertion used was 10 cents in the form of a ramp of 10 cents per second. This insertion rate was dictated by the numerical stability of the computer code and represents a greater insertion ramp rate than would be designed into a con- 
trol system. The reactivity rate of insertion in a typical control system would be in the order of 0.05 to 0.10 cent per second.

The analyses were performed for a clean core and

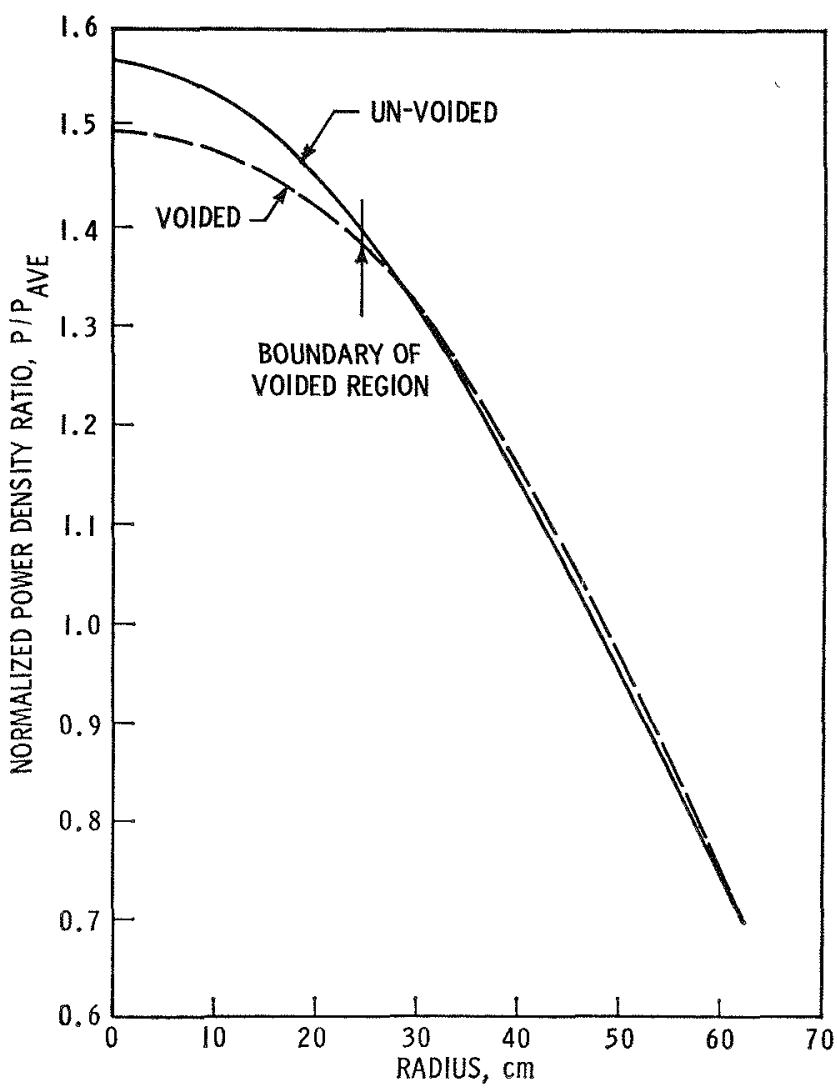

Fra. 4. Local Inberent Stability: End-of-Life Core Radial Power Profile. an end-of-life core. The fuel axial expansion coefficient was set equal to zero. The rise in power with time is shown in Fig. 5. In a clean core, the new equilibrium power is at approximately 101\% of full power, whereas with fission products the equilibrium power is $105 \%$ of full power. The new equilibrium power is reached at approximately 20 sec after the start of the reactivity insertion in the latter case. The fuel, clad, and coolant temperatures in the hot channel as a function of time are given in Table 7. The increases in temperature are $193^{\circ} \mathrm{F}$ for the fuel center, $74^{\circ} \mathrm{F}$ for the fuel surface, $68^{\circ} \mathrm{F}$ for the clad, and $56^{\circ} \mathrm{F}$ for the hot channel coolant outlet.

The results shown in Fig. 5 and Table 7 are optimistic, for the fast breeder being presented embodies variable-speed pumps in the primary system in order to hold a constant core temperature rise above $50 \%$ power. Except during the time lag for accelerating the pumps, the negative sodium temperature coefficient effect would be minimized. With this condition the equilibrium power would be higher than shown in Fig. 5.

\section{VI.2 Refueling Accident: Dropping Fuel Assembly into a Just Subcritical Core}

This accident assumes a loss of administrative control followed by a mechanical failure. Administrative control is lost during calculating the shutdown reactivity and the core is just subcritical with a center fuel assembly missing. Then, as the last fuel assembly is being lowered into the core, a mechanical failure causes the latch mechanism to release the fuel assembly, allowing it to fall into the core.

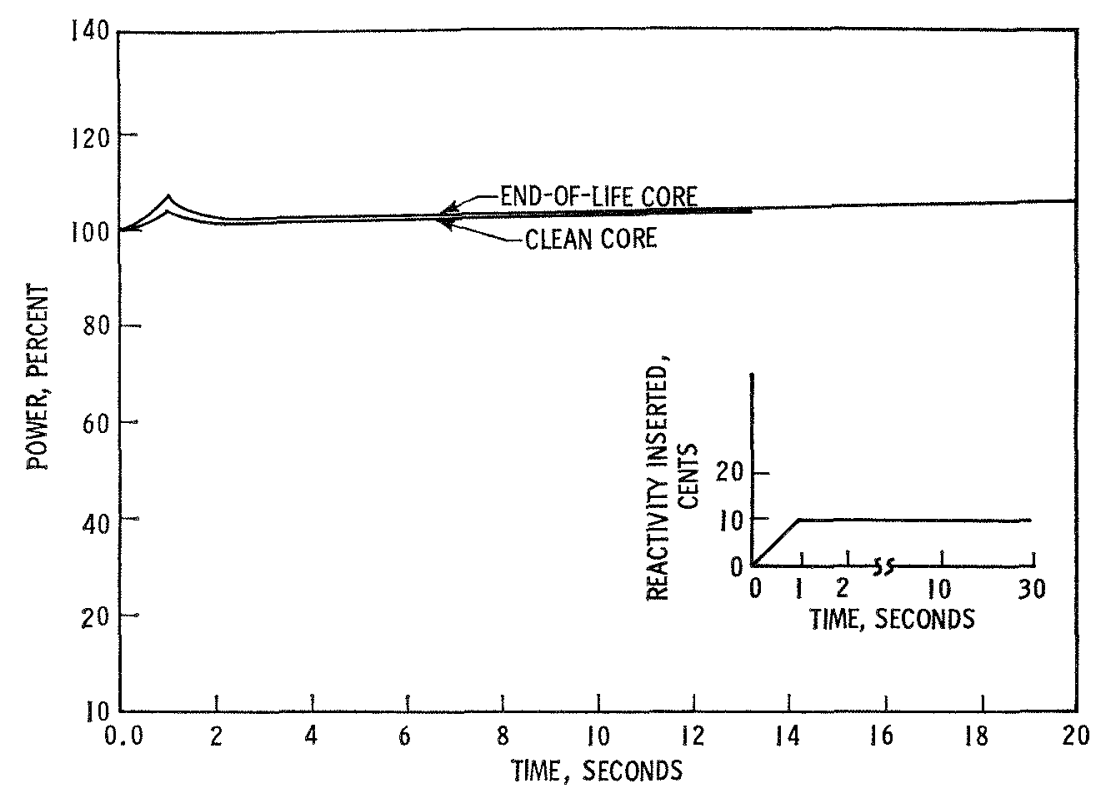

FIG. 5. Overall Inherent Stability. Reactor Power Response to a Change in Power Level. 
TABLf 7. Maximla Temperate res ( ${ }^{\circ} \mathrm{F}$ ) in Hol Channel FOR REACIVITY INSER FION OF 10k/SEC (TOTAL INSERTION 10ф) it Fula Power

\begin{tabular}{|c|c|c|c|c|c|c|c|c|}
\hline \multirow{2}{*}{$\begin{array}{l}\text { Time, } \\
\text { sec }\end{array}$} & \multicolumn{4}{|c|}{$\begin{array}{l}\text { Clean Core: Nominal } \\
\text { Sodium and Doppler } \\
\text { Reactivity Coefficients }\end{array}$} & \multicolumn{4}{|c|}{$\begin{array}{l}\text { Core End-of-Life: Nominal } \\
\text { Sodium and Doppler } \\
\text { Reactivity Coefficients }\end{array}$} \\
\hline & $\begin{array}{c}\text { Cool- } \\
\text { ant }\end{array}$ & Clad & $\begin{array}{l}\text { Fuel } \\
\text { Sur- } \\
\text { face }\end{array}$ & $\begin{array}{l}\text { Fuel } \\
\text { Center }\end{array}$ & $\begin{array}{c}\text { Cool- } \\
\text { ant }\end{array}$ & Clad & $\begin{array}{l}\text { Fuel } \\
\text { Sur- } \\
\text { face }\end{array}$ & $\begin{array}{c}\text { Fuel } \\
\text { Center }\end{array}$ \\
\hline 0.0 & 1175 & 1238 & 1274 & 1793 & 1175 & 1238 & 1274 & 1793 \\
\hline 1.03 & 1207 & 1274 & 1318 & 1917 & 1211 & 1279 & 1324 & 1929 \\
\hline 2.08 & 1218 & 1289 & 1331 & 1948 & 1226 & 1298 & 1342 & 1971 \\
\hline 3.1 & 1218 & 1290 & 1332 & 1919 & 1228 & 1301 & 1344 & 1977 \\
\hline 4.0 & 1218 & 1290 & 1382 & 1949 & 1228 & 1302 & 1345 & 1978 \\
\hline 5.0 & 1218 & 1290 & 1332 & 1949 & 1229 & 1303 & 1346 & 1980 \\
\hline 6.01 & 1218 & 1290 & 1332 & 1949 & 1229 & 1304 & 1347 & 1982 \\
\hline 7.02 & 1218 & 1291 & 1332 & 1949 & 1230 & 1304 & 1347 & 1983 \\
\hline 8.1 & 1218 & 1291 & 1332 & 1949 & 1230 & 1305 & 1348 & 1984 \\
\hline 9.1 & 1218 & 1291 & 1832 & 1949 & 1231 & 1305 & 1348 & 1985 \\
\hline 10.0 & 1218 & 1291 & 1332 & 1949 & 1231 & 1306 & 1348 & 1986 \\
\hline
\end{tabular}

The maximum worth of a fuel asscmbly is $75 \phi$. The fuel assembly is assumed to fall freely into the core. Under this assumption, 0.615 see is required for complete insertion, a 6 -ft travel. Including drag forces during the fall lengthens the insertion time to 0.7 sec. The reactivity addition is taken to be proportional to insertion instead of the usual "S"-shape distribution.

The core is assumed to be at a source and/or decay heat power level of $100 \mathrm{~W}$, a flow at $9 \%$ of full flow, and a coolant temperature of $400^{\circ} \mathrm{F}$. The cleancore pessimistic reactivity coefficient (see Table 6) were assumed for this analysis.

The rise in power as a function of time is shown in Fig. 6, and the hot-channel maximum temperatures for coolant outlet, clad, and fuel, are presented in Table 8. At 17 sec the power is $60 \mathrm{MW}$ and rising on a slope which appears to be approaching a maximum. The maximum fuel and coolant temperatures at 17 sec are $936^{\circ} \mathrm{F}$ and $887.3^{\circ} \mathrm{F}$, respectively.

\section{VI.3 Control-rod Expulsion at Full Power}

There are seven control rods in the isolated module. Four, each worth $1.2 \%$ in $k$, are safety rods and three, each worth 80 cents, are shim rods for load follow and burnup. In the analysis it was assumed that the rod to be expelled from the core is a shim rod in the fully inserted position. Expelling a control rod is a highly unlikely or even an incredible accident, as a sequence of events must happen. In expelling the control rod, it is assumed that the control-rod drive mechanism or the control-rod extension shaft breaks and that the full pressure drop in the core, $100 \mathrm{psi}$, acts on the control rod. Under the conditions assumed, the control rod will be expelled at an initial acceleration of $100 \mathrm{ft} / \mathrm{sec}^{2}(3 \mathrm{~g}$ 's), neglecting fluid drag forces. In the analysis, the initial acceleration rate was held constant for the full core height, $6 \mathrm{ft}$. The rod is fully out of the core in $0.35 \mathrm{sec}$.

The core was assumed to be at full power and flow. In the analyses the pessimistic reactivity values for both the clean core and the end-of-life core were used. The core power as a function of time is shown in Fig. 7 , and the maximum temperatures for coolant outlet, clad, and fuel in the hot channel are given in Table 9 . In both cases analyzed, the reactor power after the initial power spike approached an equilibrium power of $200 \%$ of full power for the clean core and of $212 \%$ of full power for the end-of-life core. The maximum coolant outlet and fuel center tempera-

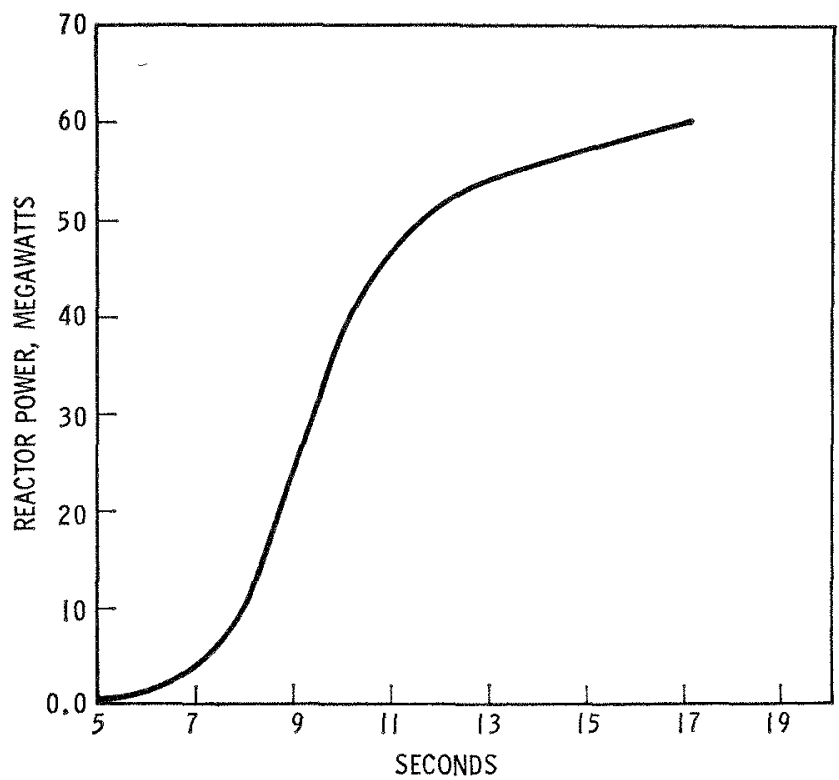

Fig. 6. Reactor Poner upon Dropping a Fuel Assembly Worth 75 Cents. Initial Power Is $100 \mathrm{~W}$, Initial Temperature Is $400^{\circ} \mathrm{F}$.

Table 8. Maximlim Temperatures ( ${ }^{\circ} \mathrm{F}$ ) in Hot Channis for Dropping Frel Assembly Accident. Assembly

Worte is 75 Cents. Accelleration is $32 \mathrm{FT} / \mathrm{sec}^{2}$. IntTl $1 \mathrm{~L}$ Power is $100 \mathrm{~W}$

\begin{tabular}{c|c|c|c|c}
\hline \multirow{2}{*}{ Time (sec) } & \multicolumn{3}{|c|}{$\begin{array}{c}\text { Clean Core: Pessimistic Sodium, Doppler, and } \\
\text { Expansion Reactivity Coefficients }\end{array}$} \\
\cline { 2 - 5 } & Coolant & Clad & $\begin{array}{c}\text { Fuel } \\
\text { Surface }\end{array}$ & $\begin{array}{c}\text { Fuel } \\
\text { Center }\end{array}$ \\
\hline & & & & \\
\hline 0.0 & 400.0 & 400.0 & 400.0 & 400.0 \\
5.0 & 400.2 & 400.2 & 400.2 & 400.5 \\
10.0 & 490.2 & 492.9 & 494.8 & 527.7 \\
15.0 & 816.6 & 819.6 & 821.5 & 867.7 \\
16.0 & 857.3 & 862.0 & 864.5 & 902.9 \\
17.0 & 887.3 & 893.2 & 896.3 & 936.0 \\
\hline
\end{tabular}


tures were $1565^{\circ} \mathrm{F}$ and $2933^{\circ} \mathrm{F}$, respectively, for the clean core, and $1610^{\circ} \mathrm{F}$ and $3046^{\circ} \mathrm{F}$ for the end-of-life core. Thus an isolated module can absorb this accident without the occurrence of even local core damage.

The results for control-rod expulsion are considered to be pessimistic for the modular systems. As previously mentioned, for a modular-core design existing information indicates a reactivity insertion into one module will be divided between all modules as some function of the cross coupling. Thus the module affected would feel only a fraction of the full reactivity insertion.

\section{VI.4 Loss of Primary-pump Power at Full Reactor Power}

The loss of primary-pump power is a credible accident for all nuclear reactors. Again, the total loss of all pump power is a highly unlikely or even an incredible accident as the pumps are supplied by more than one power supply. The safety and integrity of the core is strongly dependent on the flow-decay characteristics of the primary-system pump. Because of this importance, the primary pumps are assumed to be equipped with a high-inertia flywheel attached to the rotor of the drive motor to obtain the flowdecay characteristic curve given in Fig. 8.

The core conditions at the time of loss of electrical power to all three primary pumps were taken to be $100 \%$ power and $100 \%$ flow. The reactivity coefficients (Doppler, sodium, and axial expansion) used were the pessimistic values for both the clean core and the end-of-life core. As all the reactivity coefficients are negative, the decay in the reactor power (see Fig. 9) follows the pump flow but with a time delay. For example, at 5 sec in the clean core, the flow is $60 \%$ of full flow and the reactor power is $822.6 \%$ of full power. The maximum coolant outlet and fuel temperatures after $5 \mathrm{sec}$ were $1367^{\circ} \mathrm{F}$ and $1927^{\circ} \mathrm{F}$, respectively, for the clean core, and $1372^{\circ} \mathrm{F}$ and $1936^{\circ} \mathrm{F}$, respectively, for the end-of-life core (see Table 10).

These results, although performed for an isolated core, represent a modular-core design, for all modules in a loss-of-pumping-power accident would be affected simultaneously.

\section{VI.5 Rupture of Primary-coolant Piping in One of Three Loops}

The rupture of the primary-coolant piping can occur, but it is a highly unlikely accident. In the analysis of the pipe-rupture accident, it was assumed that the rupture occurs at the pump discharge in one of the three primary loops. The rupture was taken to be a clean break with the end of the pipe shifting in a manner so that the coolant discharged

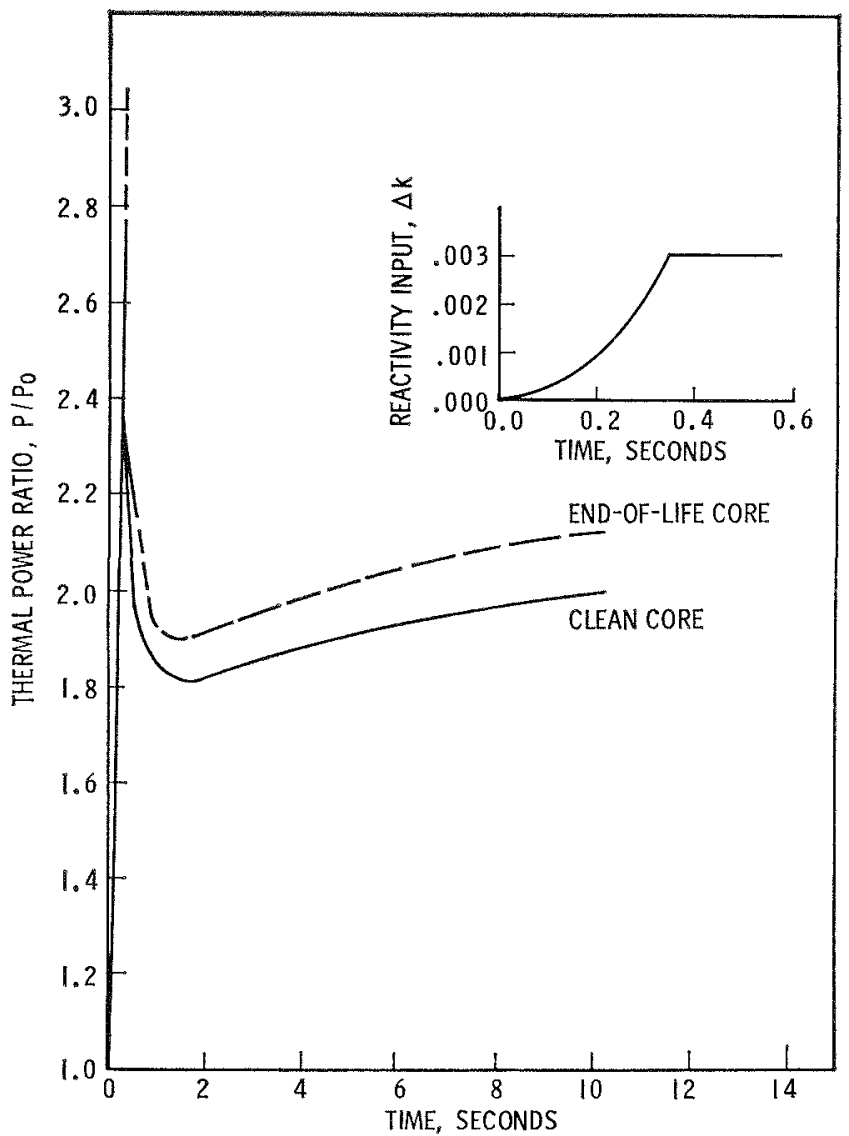

Fia. 7. Reactor Power for Expulsion of a Control Rod Worth 80 Cents at Full Power. Acceleration Rate of Expulsion Is $100 \mathrm{Ft} / \mathrm{Sec}^{2}$.

TABLe 9. Maximem Temperate res $\left({ }^{\circ} \mathrm{F}\right)$ in Hot Channel for Contror,-rod Expulsion Accident. RoD Worth Is 80 Cents. Acceleration of Expulston Is $100 \mathrm{FT} / \mathrm{sec}^{2}$. Initial Power Is Fill Power

\begin{tabular}{|c|c|c|c|c|c|c|c|c|}
\hline \multirow{2}{*}{$\begin{array}{l}\text { Time } \\
(\mathrm{sec})\end{array}$} & \multicolumn{4}{|c|}{$\begin{array}{l}\text { Clean Core: Pessimistic } \\
\text { Sodium, Doppler, and } \\
\text { ExpansionReactivity } \\
\text { Coefficients }\end{array}$} & \multicolumn{4}{|c|}{$\begin{array}{l}\text { Core End-of-Life: Pessimistic } \\
\text { Sodium, Doppler, and } \\
\text { Expansion Reactivity } \\
\text { Coefficients }\end{array}$} \\
\hline & $\begin{array}{c}\text { Cool- } \\
\text { ant }\end{array}$ & Clad & $\begin{array}{l}\text { Fuel } \\
\text { Sur- } \\
\text { face }\end{array}$ & $\begin{array}{c}\text { Fuel } \\
\text { Center }\end{array}$ & $\begin{array}{c}\text { Cool- } \\
\text { ant }\end{array}$ & Clad & $\begin{array}{l}\text { Fuel } \\
\text { Sur- } \\
\text { face }\end{array}$ & $\begin{array}{c}\text { Fuel } \\
\text { Center }\end{array}$ \\
\hline 0.0 & 1175 & 1200 & 1274 & 1793 & 1175 & 1238 & 1274 & 1793 \\
\hline 1.00 & 1458 & 1558 & 1650 & 2684 & 1479 & 1581 & 1678 & 2742 \\
\hline 2.09 & 1496 & 1619 & 1697 & 2766 & 1527 & 1655 & 1737 & 2845 \\
\hline 3.05 & 1510 & 1638 & 1715 & 2798 & 1545 & 1679 & 1760 & 2886 \\
\hline 4.02 & 1522 & 1653 & 1730 & 2828 & 1559 & 1696 & 1778 & 2919 \\
\hline 5.09 & 1533 & 1666 & 1744 & 2855 & 1572 & 1712 & 1795 & 2952 \\
\hline 6.06 & 1541 & 1676 & 1755 & 2875 & 1582 & 1725 & 1809 & 2978 \\
\hline 7.01 & 1548 & 1685 & 1764 & 2893 & 1590 & 1735 & 1819 & 2998 \\
\hline 8.00 & 1555 & 1692 & 1772 & 2908 & 1598 & 1744 & 1829 & 3015 \\
\hline 9.10 & 1561 & 1699 & 1780 & 2923 & 1605 & 1752 & 1838 & 3033 \\
\hline 10.0 & 1565 & 1705 & 1786 & 2933 & 1610 & 1759 & 1845 & 3046 \\
\hline
\end{tabular}




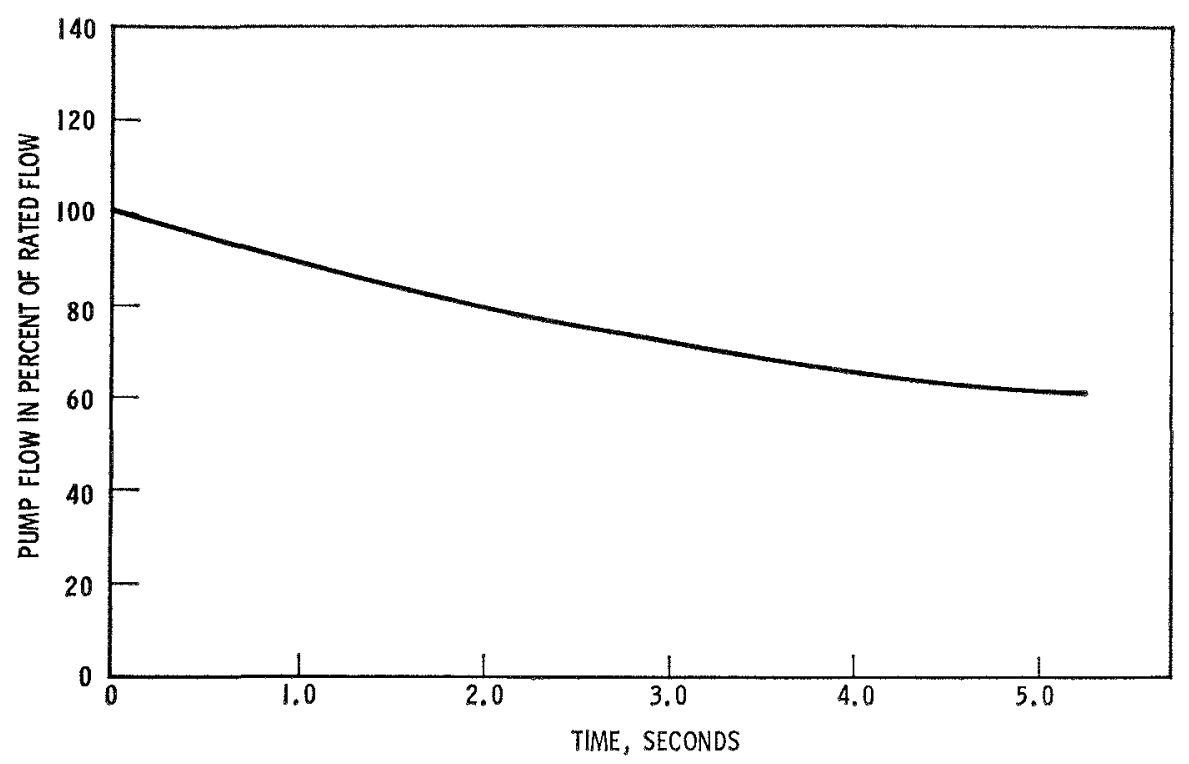

FIG. 8. Pump Flow during Coastdown, High-inertia Flywheel Attached to Rotor of Drive Motor.

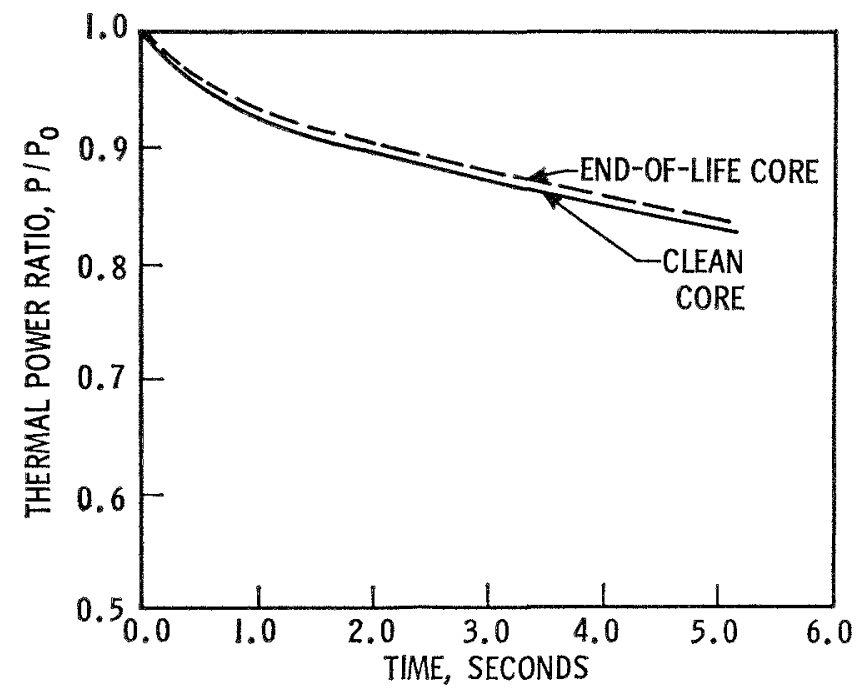

Fig. 9. Reactor Power for Loss of Primary-Pump Power Accident. Initial Power, $P_{0}$, Is 100\% Power. Initial Flow Is $100 \%$ Flow.

freely from both ends of the break into the pump cell. The pumps in the other two loops continued to operate with the flow being divided according to the pressure drop between the core and the ruptured. pipe. The pumps were assumed to lose suction at 3.1 sec once the level of the sodium pool above the core was lowered to the outlet nozzle, allowing the cover gas to enter the pump intake. The core flow with time is shown in Fig. 10 for the above assumptions.

The core conditions at the time of the pipe rupture were taken to be $100 \%$ power and $100 \%$ flow. The reactivity coefficients (Doppler, sodium, and axial fuel expansion) used in the analyses were the pessi- mistic values of Table 6 for both the clean core and the end-of-life core. As in the loss-of-primary-pumppower accident, the reactor power (see Fig. 11) follows the core flow but with a time lag. Up to the time, 3.1 sec, of losing pump suction, the maximum temperatures in the hot channel occurred between 2.0 and $2.6 \mathrm{sec}$ (see Table 11). The maximum coolant outlet and fuel center temperatures were $1661^{\circ} \mathrm{F}$ and $2018^{\circ} \mathrm{F}$, respectively, for the clean core, and $1694^{\circ} \mathrm{F}$ and $2066^{\circ} \mathrm{F}$, respectively, for the end-of-life core. The pressure at the core outlet was approximately 25 psia, giving a sodium boiling temperature of $1650^{\circ} \mathrm{F}$. This is a conservative boiling temperature as the liquid superheat required to initiate vapor formation is neglected. Thus, at worst, a very small amount of boiling may occur in the hot channel, but the reactor should be scrammed when boiling begins.

Table 10. Maximum Temperitures $\left({ }^{\circ} \mathrm{F}\right)$ in Hot Channer for Pump-cosstoown Accident

\begin{tabular}{|c|c|c|c|c|c|c|c|c|}
\hline \multirow{2}{*}{$\begin{array}{l}\text { Time } \\
\text { (sec) }\end{array}$} & \multicolumn{4}{|c|}{$\begin{array}{c}\text { Clean Core: Pessimistic } \\
\text { Sodium, Doppler, and } \\
\text { Expansion Reactivity } \\
\text { Coefficients }\end{array}$} & \multicolumn{4}{|c|}{$\begin{array}{l}\text { Core End-of-Life: Pessimistic } \\
\text { Sodium, Doppler, and } \\
\text { Expansion Reactivity } \\
\text { Coefficients }\end{array}$} \\
\hline & $\begin{array}{c}\text { Cool- } \\
\text { ant }\end{array}$ & Clad & $\begin{array}{l}\text { Fuel } \\
\text { Sur- } \\
\text { face }\end{array}$ & $\begin{array}{c}\text { Fuel } \\
\text { Center }\end{array}$ & $\begin{array}{c}\text { Cool- } \\
\text { ant }\end{array}$ & Clad & $\begin{array}{l}\text { Fuel } \\
\text { Sur- } \\
\text { face }\end{array}$ & $\begin{array}{l}\text { Fuel } \\
\text { Center }\end{array}$ \\
\hline 0.0 & 1175 & 1238 & 1274 & 1793 & 1175 & 1238 & 1274 & 1793 \\
\hline 1.10 & 1230 & 1289 & 1320 & 1921 & 1233 & 1292 & 1323 & 1925 \\
\hline 2.10 & 1271 & 1330 & 1359 & 1924 & 1271 & 1330 & 1359 & 1930 \\
\hline 3.00 & 1300 & 1358 & 1386 & 1921 & 1304 & 1362 & 1390 & 1928 \\
\hline 4.05 & 1336 & 1392 & 1419 & 1920 & 1341 & 1397 & 1425 & 1928 \\
\hline 5.00 & 1367 & 1422 & 1448 & 1927 & 1372 & 1427 & 1454 & 1936 \\
\hline
\end{tabular}




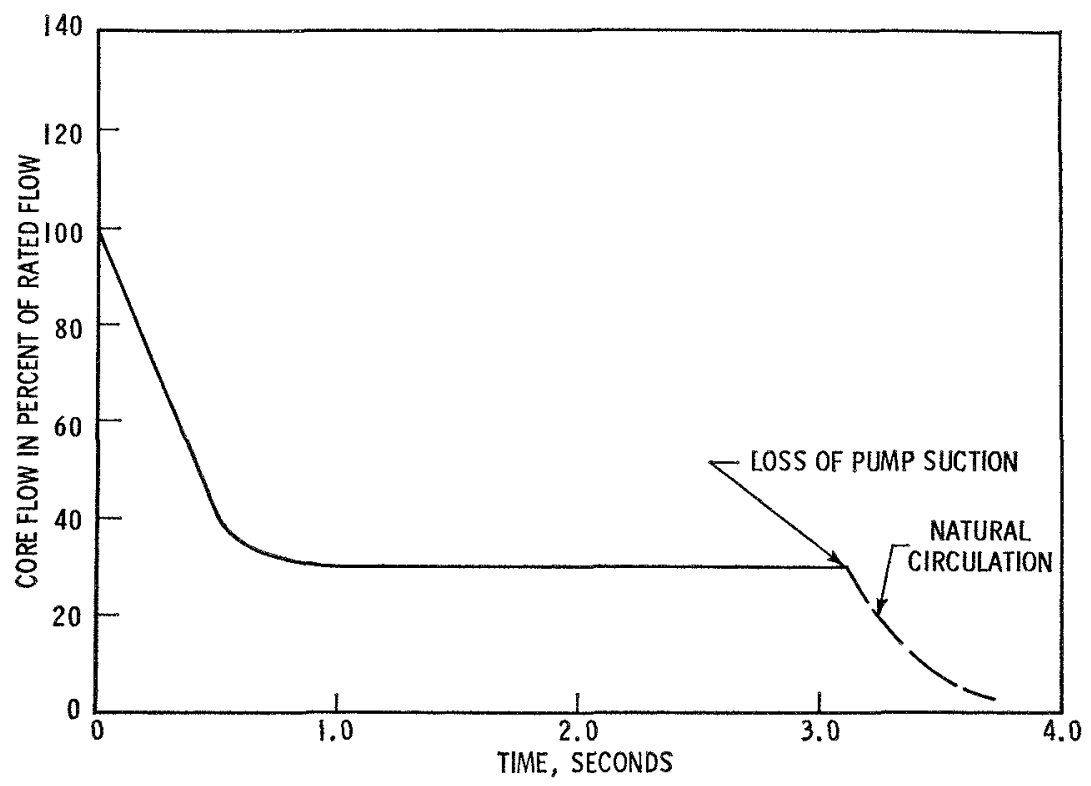

FIG. 10. Decay of Core Flow for Pipe-rupture Accident in One of Three Loops.

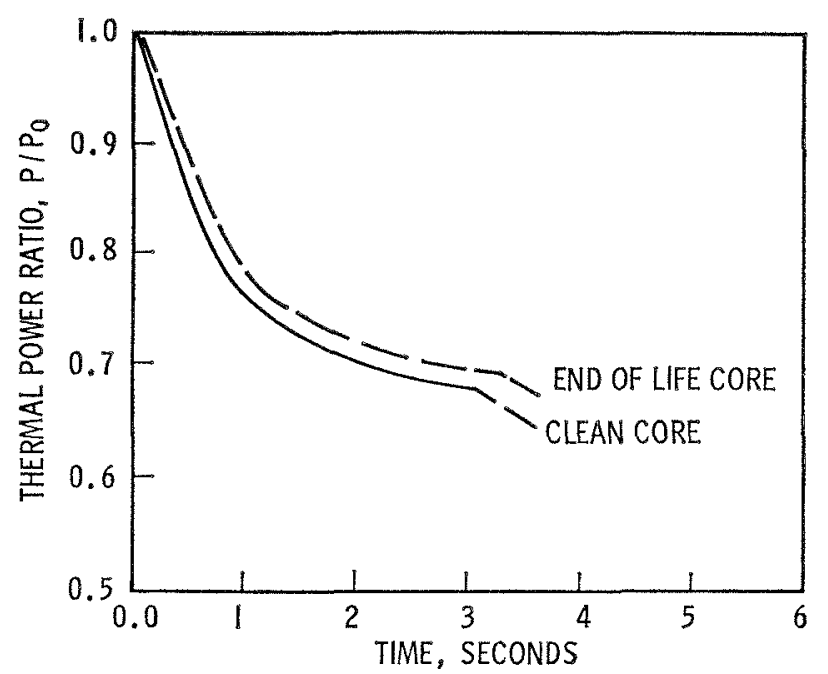

FIg. 11. Reactor Power for Primary-pipe-rupture Accident in One of Three Loops. Initial Power, $\mathrm{P}_{0}$, Is $100 \%$; Initial Flow Is $100 \%$.

\section{VI.6 Local Flow Blockages}

Coolant flow to a fuel assembly could be lost by blocking physically the inlet nozzle or by plugging with sodium oxide. If the inlet nozzle is blocked by buildup of sodium oxide, it is highly probable that the buildup would be uniform in all fuel assemblies rather than in a single fuel assembly. This would be manifested by a gradual increase in the core pressure drop, which would be detectable by differential-pressure monitors. Therefore, analysis for local loss of coolant flow is taken to be the instantaneous loss by mechanical blockage of flow to one fuel assembly, since the block- ing of more than one fuel assembly is a highly unlikely occurrence.

After losing flow to one fuel assembly, the temperature of the stagnant coolant rises until the boiling point is reached and voiding occurs. This takes place in approximately one second. The clad and fuel temperatures continue to increase, and clad melting occurs before the carbide fuel reaches its melting point, $4200^{\circ} \mathrm{F}$. If it may be assumed that the fuel stack remains as a column, the fuel melting temperature will not be reached until approximately 3.5 sec. Thus, as the clad melting occurs considerably before fuel melting, the fuel will remain in solid state rather than in a molten state when fuel compaction occurs in the blocked fuel assembly. The reactivity addition

TABLE 11. Maximum Temperatures $\left({ }^{\circ} \mathrm{F}\right)$ in Hot Channel for Pipe-rupture Accidents. Intthal Power is Full Power

\begin{tabular}{l|c|c|c|c|c|c|c|c}
\hline & \multicolumn{2}{|c|}{$\begin{array}{c}\text { Clean Core: Pessimistic } \\
\text { Sodium, Dopper, and } \\
\text { Expansion Reactivity } \\
\text { Cime }\end{array}$} & \multicolumn{3}{|c|}{$\begin{array}{c}\text { Cofficients } \\
\text { (sec) }\end{array}$} & $\begin{array}{c}\text { Core End-of-Life: Pessimistic } \\
\text { Sodium, Doppler, and } \\
\text { Expansion Reactivity } \\
\text { Coefficients }\end{array}$ \\
& $\begin{array}{c}\text { Cool- } \\
\text { ant }\end{array}$ & Clad & $\begin{array}{c}\text { Fuel } \\
\text { Sur- } \\
\text { face }\end{array}$ & $\begin{array}{c}\text { Fuel } \\
\text { Center }\end{array}$ & $\begin{array}{c}\text { Cool- } \\
\text { ant }\end{array}$ & Clad & $\begin{array}{c}\text { Fuel } \\
\text { Sur- } \\
\text { face }\end{array}$ & $\begin{array}{c}\text { Fuel } \\
\text { Center }\end{array}$ \\
\hline 0. & 1175 & 1238 & 1274 & 1793 & 1175 & 1238 & 1274 & 1793 \\
0.50 & 1334 & 1364 & 1398 & 1906 & 1344 & 1370 & 1407 & 1905 \\
1.02 & 1528 & 1544 & 1558 & 1903 & 1548 & 1560 & 1575 & 1925 \\
1.52 & 1633 & 1671 & 1680 & 1962 & 1655 & 1705 & 1693 & 2022 \\
2.07 & 1661 & 1699 & 1717 & 2004 & 1697 & 1757 & 1750 & 2059 \\
2.57 & 1640 & 1686 & 1712 & 2018 & 1694 & 1738 & 1734 & 2063 \\
3.05 & 1612 & 1680 & 1702 & 2016 & 1674 & 1723 & 1748 & 2066 \\
\hline
\end{tabular}


upon fuel compaction is less than one dollar. The reactor will, therefore, remain on a delayed power period, and a scram will prevent further melting of the core.

\section{VI.7 Fuel Slumping}

Fuel slumping and core collapse appear to be highly unlikely accidents in the modular core as fuel melting did not occur in any of the accidents analyzed. But if it may be assumed that by some unknown manner fuel melting does occur and the fuel slumps uniformly throughout the core within the clad, filling all the void apace and increasing its density from $90 \%$ to $95 \%$, the reactivity addition in the isolated module would be $\$ 18$. Then if the upper half of the core folds over into the bottom half, the total reactivity insertion including slumping within the core would be $\$ 75$.

\section{VI.8 Core Meltdown}

The energy release during core compaction was analyzed by a modified Bethe-Tait approach with Doppler feedback as used in the "WEAK EXPLOSION" code. The equation of state for PuC used in the analysis is given in reference 21 . The collapse time of the upper half of the core into the lower half, assuming free fall, was 0.3 see. The worth of such an incident was found to be about $\$ 75$, thus yielding a reactivity insertion rate of $\$ 250 / \mathrm{sec}$. The total energy released for the isolated module was approximately $2100 \mathrm{MW}$-sec.

\section{VI.9 Summary of Accident Analyses}

An isolated core of the same size as a module in the modular core has good safety characteristics. The accidents analyzed for an isolated core are sum-

TABLE 12. SUMM ARy OF ACCIDEN A AN Lyses

\begin{tabular}{|c|c|c|}
\hline Analyses Performed & Clean Core at Beginning of Life & $\begin{array}{l}\text { Core at End of Life }(66,000 \mathrm{MWD} / \mathrm{T}) \\
\text { with Fission Products }\end{array}$ \\
\hline $\begin{array}{l}\text { 1. Reactivity Coefficients } \\
\text { a. Doppler } \\
\text { b. Axial Expansion }\end{array}$ & Refarence (seo Table 5) & $\begin{array}{l}\text { Decreases by } 14 \% \\
\text { Decreases by } 10 \%\end{array}$ \\
\hline 2. Complete Core Voiding & Removes $1 \%$ in " $k$ " & Add $1.54 \%$ in " $k$ " during voiding \\
\hline $\begin{array}{l}\text { 3. Core Inherent Stability at } 100 \% \text { Power } \\
\text { and } 100 \% \text { Flow } \\
\text { a. Local Stability } \\
\text { b. Overall Stability to a Change in } \\
\text { Power Setting (10\% in one second) }\end{array}$ & $\begin{array}{l}\text { Good } \\
\text { Good-Reactor power increases by } 1 \% \text {. } \\
\text { Equilibrium power after } 10 \text { sec }\end{array}$ & $\begin{array}{l}\text { Good } \\
\text { Good-Poner increases by } 5 \% \text {. Equilib- } \\
\quad \text { rium after } 20 \mathrm{sec} \text {. }\end{array}$ \\
\hline $\begin{array}{l}\text { 4. Dropping of Fuel Assembly Worth } 75 \% \\
\text { into Just Suberitical Core. Initial } \\
\text { Power is } 100 \mathrm{~W} \text { and Flow is } 9 \% \text { of Full } \\
\text { Flow }\end{array}$ & $\begin{array}{l}\text { After } 17 \text { sec, power is } 60 \mathrm{MW} \text { and is ap- } \\
\text { proaching, a steady-state value }\end{array}$ & $\begin{array}{l}\text { Not applicable as fuel assemblies are } \\
\text { being removed at this condition }\end{array}$ \\
\hline $\begin{array}{l}\text { 5. Shim Control-rod Expulsion. Rod } \\
\text { Worth } 80 \mathrm{c} \text { at } 100 \mathrm{ft} / \mathrm{sec}^{2} .100 \% \text { reactor } \\
\text { power and flow }\end{array}$ & $\begin{array}{l}\text { Equilibrium power is } 200 \% \text { after } 10 \text { sec. } \\
\text { Sodium boiling and fuel melting do not } \\
\text { occur. Terminate excursion with over } \\
\text { power scram }\end{array}$ & $\begin{array}{l}\text { Equilibrium power is } 212 \% \text { after } 10 \text { sec. } \\
\text { Sodium boiling and fuel melting do not } \\
\text { occur. Terminate excursion with over- } \\
\text { power scram. }\end{array}$ \\
\hline $\begin{array}{l}\text { 6. Loss of Primary-pump Power at 100\% } \\
\text { Renctor Power and } 100 \% \text { Flow }\end{array}$ & $\begin{array}{l}\text { Reactor power decays as flow, but at a } \\
\text { lesser rate. Excursion can be terminated } \\
\text { by loss of pump power scram }\end{array}$ & $\begin{array}{l}\text { Reactor power decays as flow, but at a } \\
\text { lesser rate. Excursion can be terminated } \\
\text { by loss of pump power scram }\end{array}$ \\
\hline $\begin{array}{l}\text { 7. Local Flow Blockage of a Single Fuel } \\
\text { Assembly }\end{array}$ & $\begin{array}{l}\text { Sodium boiling occurs in } 1 \text { see. Clad melts } \\
\text { before fuel melts. A scram actuated by a } \\
\text { noize analyzer required to terminate } \\
\text { excursion before blocked assemblies } \\
\text { incur gross damage }\end{array}$ & $\begin{array}{l}\text { Sodium boiling occurs in one second. Clad } \\
\text { melts before fuel melts. A scram actu- } \\
\text { ated by a noise analyzer required to } \\
\text { terminate excursion before blocked } \\
\text { assemblies incur gross damage }\end{array}$ \\
\hline 8. Pipe Rupture & $\begin{array}{l}\text { Reactor power decays as flow, but at a } \\
\text { lesser rate. Excursion can be terminated } \\
\text { by low system pressure or low flow } \\
\text { scram }\end{array}$ & $\begin{array}{l}\text { Reactor power decays as flow, but at a } \\
\text { lesser rate. Excursion can be terminated } \\
\text { by low system pressure or low-flow } \\
\text { scram. }\end{array}$ \\
\hline
\end{tabular}


marized in Table 12. In each of the arcidents analyzed, the reactor power obtained, or was approaching, an equilibrium power below that required to cause even local core damage. The reactor could have been scrammed in each accident. Although the interaction of a multimodule was not analyzed in this study, it is reasonable to predict that it would have safety characteristics better than the single module analyzed because the neutronic cross-coupling effect divides among all modules any reactivity insertion occurring in one module. The results of the control-rod expulsion accident and the dropping of a fuel assembly for the single module are pessimistic compared to a multiple-module core. The other accidents, pipe rupture and loss of pump power, would essentially be identical for the modular core as all modules are affected simultaneously in the same manner. Of the accidents analyzed for the isolated core, the loss of flow to a single fuel assembly has the highest probability of leading to collapse and compaction of fucl. A local loss of flow is the most difficult accident to detect as the reactor power does not change before voiding occurs. A noise-analyzer instrumentation system for boiling would be one method of detecting this accident and initiating reactor scran before fuel compaction occurs (see Reference 20). If the loss of flow to a single fuel assembly propagates to complete core meltdown, a minimum of $8.5 \mathrm{sec}$ is available for scramming the reactor before the entire core is voided. The meltdown energy release calculated by the BetheTait method is approximately $2100 \mathrm{MW}$-sec for the isolated core.

The pipe-rupture and the loss-of-primary-pumppower accidents without scram could lead to core meltdown if the reactor was also on a fast period approaching $100 \%$ power prior to the accident. The power overshoot that would occur during these accidents would be sufficient to carry the hot-channel coolant outlet temperature above the boiling point. Because of the large superheat capabilities of sodium, this might not cause vapor formation in the hot channel. This approach to core meltdown requires a multiplicity of events to occur and therefore is highly unlikely to occur.

\section{Combination of Accidents Leading to Core or Fuel-assembly Meltdown}

The core-meltdown accident appears to be a highly unlikely accident in the modular-core concept. Of the major accidents selected and studied, the loss of flow at $100 \%$ power to a single fuel assembly in the end-oflife core without scram is the accident having the highest probability of leading to core meltdown. This accident is the most difficult to detect by means of the usual instrumentation, flow, pressure drop, coolant temperatures, ete., and would require a noise analyzer to initiate a signal upon boiling to scram the reactor.

The time history without scram of a core-meltdown accident resulting from the loss of flow to a single fuel assembly is shown in Fig. 12. After the fuel assembly is physically blocked at the inlet, the reactor power remains constant, raising all temperatures in the blocked assembly. The stagnant coolant in the blocked assembly begins to boil at the core centerline in $0.9 \mathrm{sec}$. The formation of vapor at the centerline may expel out of the top of the core the coolant above the center section. The coolant in the lower portion of the assembly is boiled off in 1.5 sec. Voiding of one fuel assembly inserts $0.02 \%$ in $k$. Fuel melting and collapse of the blocked fuel assembly begin after $3.5 \mathrm{sec}$, adding sufficient reactivity to raise the reactor power to approximately $270 \%$ of full power. The time of collapse is $0.3 \mathrm{sec}$, corresponding to free fall over a distance of $3 \mathrm{ft}$. After collapse of the single assembly, the fuel and coolant temperatures in the unblocked portion of the core are increased to the point of voiding the entire core after $8.5 \mathrm{sec}$. Voiding the entire end-of-life core adds $1.54 \%$ in " $k$ " to the system. The reactor power then rises on a prompt period, and fuel melting and collapse of the core begin after 10.6 sec. The energy release for the meltdown of the entire core is approximately $2100 \mathrm{MW}$-see.

The loss of flow to a single fuel assembly in a module of the modular core may not lead to core meltdown. In the modular core, the effectiveness of

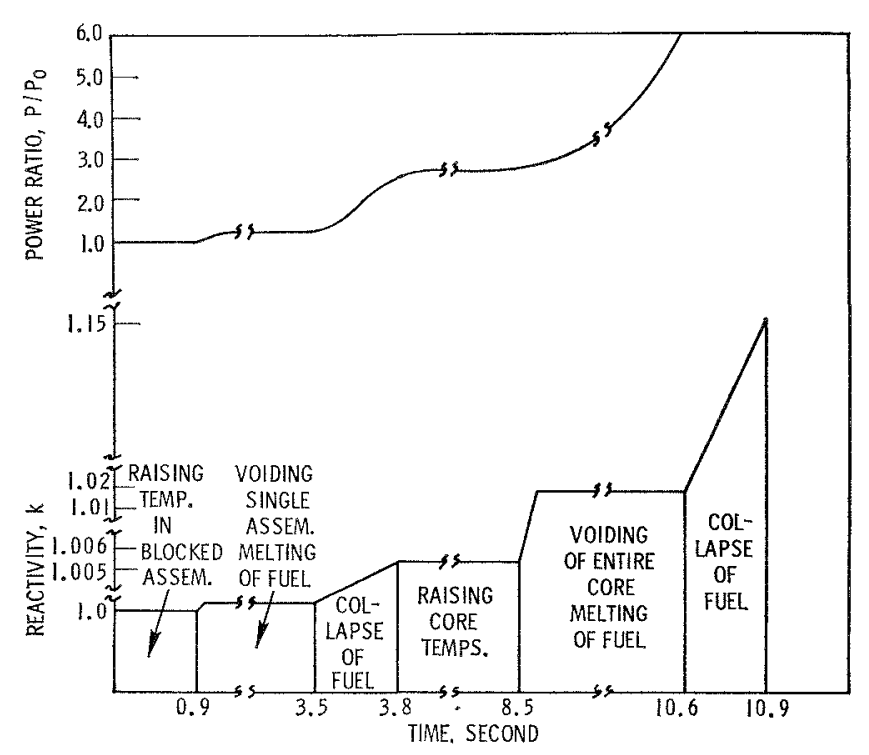

Fig. 12. Ifistogram of Core Meltdown Resulting from Loss of Hlow to a Single Fuel Assembly. 
the neutronic cross coupling in distributing among all modules a reactivity insertion in one module may be sufficient to confine the meltdown and fuel compaction to the fuel assembly which has lost flow. In addition, application of the controlled expansion concept (CEX) may provide sufficient negative feedback to override the reactivity worth of the collapsing of a single fuel assembly.

The simultaneous occurrence of an accident involving pipe rupture, the loss of primary pump power, or control-rod expulsion without scram when the reactor is already on the shortest controlled period approaching 100\% power would probably lead to core meltdown. In the analyses performed for these three accidents, the hot-channel coolant outlet temperature was approaching the boiling point, and the expected overshoot in power may be sufficient to give boiling and voiding beginning at the outlet of the core. Once the center fuel assemblies are voiding, fuel meltdown will follow in these fucl assemblies. In the modular core the control-rod expulsion accident may not insert sufficient reactivity in the module losing the control rod to lead to core meltdown because of the neutronic cross-coupling effects. The isolated core-pipe rupture and loss-of-primary-pumppower accidents represent the modular core behavior as all modules would be affected simultaneous in the same manner.

A spent fuel assembly dropped onto the floor of the hot cell during the refueling transfer process would meltdown from decay heat if cooling is not available. Fission products and radioactive material would be released to the hot cell atmosphere. The release of a large explosive force as in the core-meltdown accident would not occur, as the amount of material in a single fuel assembly is less than a critical mass.

If during refueling a spent assembly becomes jammed in the refueling machine with failure of all cooling systems, the decay heat will be sufficient to meltdown the fuel assembly. Again, fission products and radioactive material would be released to the refueling hot cell. A large explosive force would not follow the meltdown of the fuel assembly.

\section{Conclusions}

From the results of these preliminary accident analyses, it would appear that a fast breeder reactor designed with mixed uranium-plutonium carbide fuel in a multiple modular geometric array possesses inherently safe operating characteristics. Several key accidents were studied, all of which indicated inherent stability of the reactor core. In the course of this study, several added safety features were identified which could be readily designed into the overall reactor system, thereby providing further engineered safeguards for this fast breeder reactor. Admittedly, not all conceivable accidents were studied in this investigation, but a careful selection was made for study, on the basis of past experience, of incidents most likely to result in the worst accidents.

The high neutron-leakage characteristic of a modular core serves to provide a zero or slightly negative temperature coefficient while maintaining a good total breeding ratio by use of the interspersed blanket material between the modules. The large negative Doppler coefficient was a predominant accident-limiting mechanism in most of the accidents studied.

The accuracy with which the sodium, Doppler, and other temperature-dependent power coefficients are presently known leaves much to be desired, and, similarly, the adequacy with which the digital computer/ analog codes truly portray the actual mechanisms is also open to some question; however, the studies conducted would appear to represent a reasonable accurate "status-of-art" analysis.

It would appear from the results of these analyses that the particular core concept chosen may be overly conservative. Future cores could be considered having less strongly pronounced safoty advantages, liberalized in terms of higher specific powers and internal breeding ratios and lower neutron leakage, inventory requirements, doubling times, and overall fuel-cycle costs. In anticipation of improved designs of this sort, a more precise knowledge of the power coefficients and transient nature of fast breeder cores must be developed. In addition to a more precise knowledge of the Doppler and coolant coefficients, it is necessary to achieve a better understanding of the thermoand fluid dynamies as limited by sodium behavior during a prompt power burst.

\section{APPENDIX A}

\section{Mechanical Design of Reactor}

The reactor consists of seven hexagonal modules; each contains 37 fuel-assembly positions, one of which is occupied by a safety rod. Surrounding the fuel assemblies are 54 blanket assembly positions, of which six are occupied by control rods. Each module is surrounded by 36 reflector assemblies, each of which is shared by an adjacent module except on the outer 
periphery of the reactor. A onc-quarter core layout is shown in Fig. 1.

The reactor is contained within a double-walled reactor vessel with coolant nozzles located above the top of the fuel assemblies. The core support structure is attached to a core barrel suspended from the upper part of the inner reactor vessel. The reactor vessel is a composite plug comprised of blast-absorbing material on the bottom and radiation-absorbing material above.

Control-rod drive mechanisms are located on top of the plug. Access to the reactor for refueling or maintenance is obtained by uncoupling the control-roddrive lines, unlatching the plug from the vessel, hoisting the plug by means of a traveling hoist, and rolling it to one side.

The fuel assemblies are hexagonal, containing 127 rods. The rods are attached to an end fitting at the lower end. The end fitting in turn is attached to the can. The rods are laterally restrained by spring grids spaced uniformly up the can. The cans are terminated at the upper end by a lift fitting, and at the lower end by a mechanical latch similar to that described in Ref. 1. Bumper pads are provided at two levels on the exterior of the cans to take up clearance between cans.

The radial blanket assemblies are externally identical with fuel-assembly cans with latches of smaller diameter to prevent insertion of fuel-assembly cans into blanket positions.

The reflector-assembly cans are dimensionally similar to fuel-assembly cans. The reflector cans contain short lengths of graphite logs and do not depend upon the graphite for support. They are helium back filled and welded shut. The reflector cans have end fittings which socket into the core support structure at the bottom and into the reflector support grid at the top. The reflector support grid positions the refiector around each module and limits the lateral movement of fuel and blanket cans. It does not interfere with refueling.

The fuel rods are stacks of stabilized (U,Pu)C pellets contained in stainless steel clad. The stabilization provides compatibility with the clad by chemically tying up the offending free clement. The axial blankets are integral with the fuel rods. The fuel rods are sodium bonded and vented to the coolant.

The control-rod assembly consists of a hexagonal array of tubes occupying the center section of the modified fuel assembly. The absorber, $\mathrm{B}_{4} \mathrm{C}$ powder, bearing tubes are segmented, with a horizontal grid at about one-foot intervals, making a unit assembly of the tube array. The top grid from which all rods hang is provided with a vertical connecting member to the control-rod drive coupling. The radial spacer grids permit differential linear expansion of the individual rods to eliminate any bowing which might otherwise develop due to flux gradients around the control rod perimeter. Strips attached at the spacer grids provide running surfaces against the control rod guide tube.

The numerical data for the mechanical design are tabulated in Table 1.

\section{APPENDIX B}

\section{Plant Systems Description}

\section{Primary System}

The primary coolant system consists of three identical loops to transfer heat from the reactor core to the intermediate sodium system. The general arrangement of a loop is presented in Figures 2 and 13. Each loop consists of a circulating pump, the single intermediate heat exchanger, connecting double-walled piping, and instrumentation for operational control of the system. The pump suction is connected to the intermediate-heat-exchanger shell, whereas pump discharge is connected to the top inlet of the reactor vessel. The top discharge from the reactor vessel is connected to the intermediate heat exchanger.

All system equipment that contains sodium is heat-traced. All primary-system equipment is located inside the primary reactor containment. The environment around all primary-system equipment will be nitrogen or oxygen-depleted air to prevent sodium oxidation and radioactivity dispersal in the event of a leak.

\section{Reactor Vessel}

The reactor vessel (see Fig. 14) is of double-walled construction, consisting of a primary and a secondary vessel, and is fabricated entirely from Type 304 stainless steel. The primary vessel provides containment for the reactor core and serves at the support member for the core and core barrel. The upper end of the primary vessel is welded to the vessel support ring. The secondary vessel provides sodium containment in the unlikely event of a failure of the primary vessel. Three inches of stainless steel reflective insulation covers the outside of the secondary vessel.

The radial gap between the primary and secondary vessels is selected so that if drainage of sodium from the primary to the secondary vessel occurs, the core will remain submerged in sodium. During power operation this gap is filled with stagnant nitrogen. At 

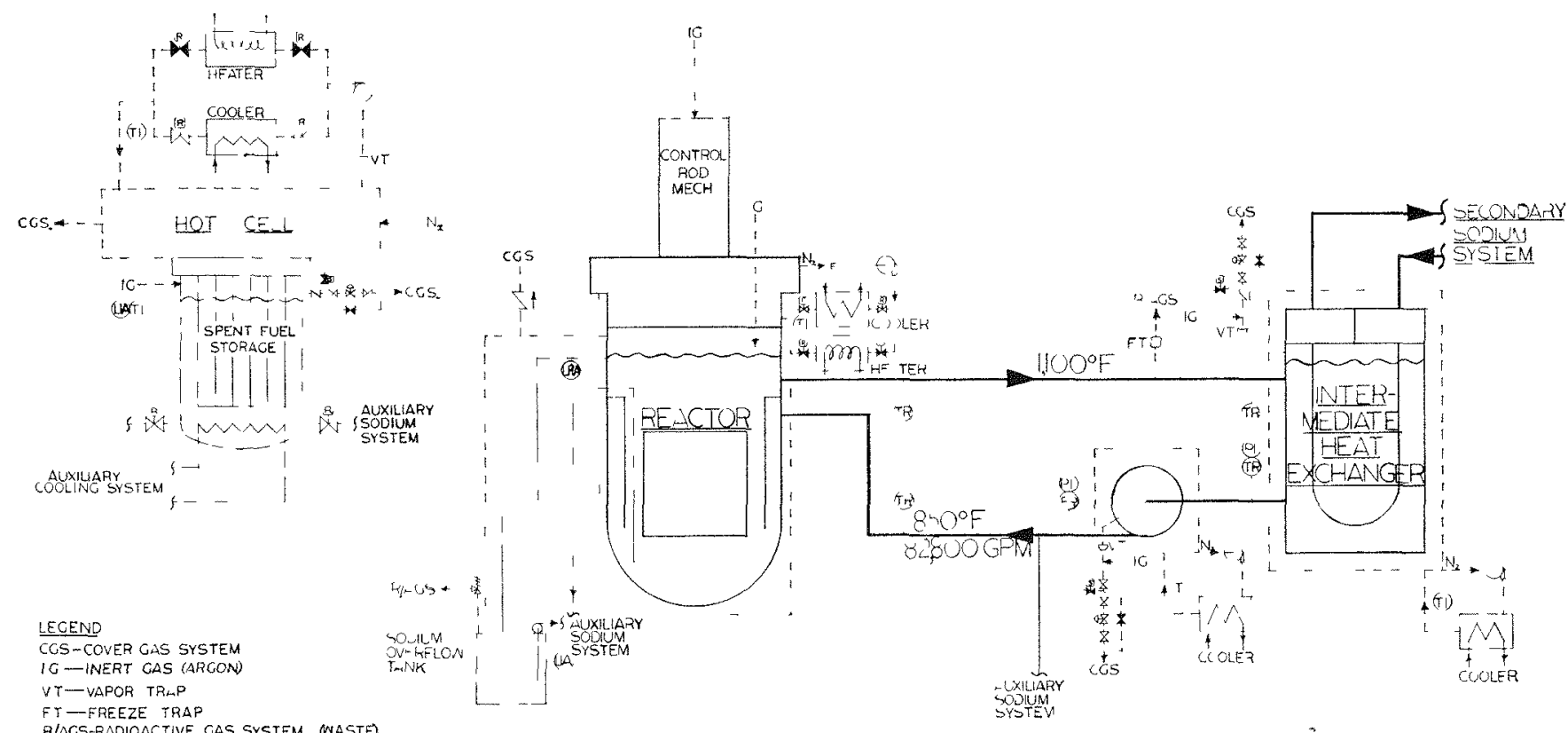

FI( . 13. One of Three Identical Loops.

shutdown, the sodium within the vessel is heated by circulating hot nitrogen between the two vessels.

\section{Shield Plug}

The shield plug has three major functions:

1. provides a full-diameter opening at the top of the vessel capable of containing the full design pressure;

2. provides shielding to attenuate neutron flux and gamma radiation;

3. provides support and alignment for control-rod drive mechanisms.

The total thickness of the plug shield is $5 \mathrm{ft}$. The plug shield consists of the following materials listed from top to bottom: 4 in. of carbon steel; 12 in. of stacked $3 \mathrm{w} / \mathrm{o}$ boronated graphite block; a layer of carbon steel shielding (not a structural or pressurecontaining component); a type 304 stainless steel structural member; 12 in. of boronated graphite block; a type 304 stainless steel structural and pressure-containing member; and 24 in. of blast-absorbing erushable material. The total minimum thickness of the four steel plates is $111 / 2$ in.

The two lower stainless steel plates, above the crushable material, provide the structural support for the plug assembly and the design pressure loading. The space between these two plates contains ribs or similar stiffening members to enhance the load-carrying capability of the assembly.

Reflective stainless steel insulation is attached to the bottom of the plug assembly as required to reduce the rate of heat transfer through the plug and to promote satisfactory temperature distributions throughout the upper region of the vessel and support structure.

Nitrogen is provided for plug cooling in order to maintain the upper plug surface and seal at temperatures below $130^{\circ} \mathrm{F}$.

\section{Closure of Shield Plug}

The vessel closure is sealed and secured in place during normal operating conditions (at a pressure of 15 psig) by a low-melting-point metal alloy. Cerroben, a lead, cadmium, tin and bismuth alloy, is typical of several possible seal materials. It has a density of $0.339 \mathrm{lb} / \mathrm{in}^{3}$ and a melting point of $158^{\circ} \mathrm{F}$. A shear strength of around 3500 psi makes it adequate for closure holddown at many times the normal operating pressure of 15 psig.

A rotating shear block closure provides a backup holddown and is capable of securing the plug at pressures of at least 150 psig. This mechanical closure is simple in design and will have the capability of being operated remotely by pneumatic cylinders mounted to the refueling-cell floor. Additional beams across the plug hold the plug in place in the event of a large release of explosive energy. Human access to the closure mechanism during plug removal is contemplated only under unusual conditions.

\section{Axial Thermal Shield and Meltdown Pan}

An axial thermal shield and meltdown pan assembly is supported at the bottom of the vessel below the core support structure. It consists of a 304 stain. 


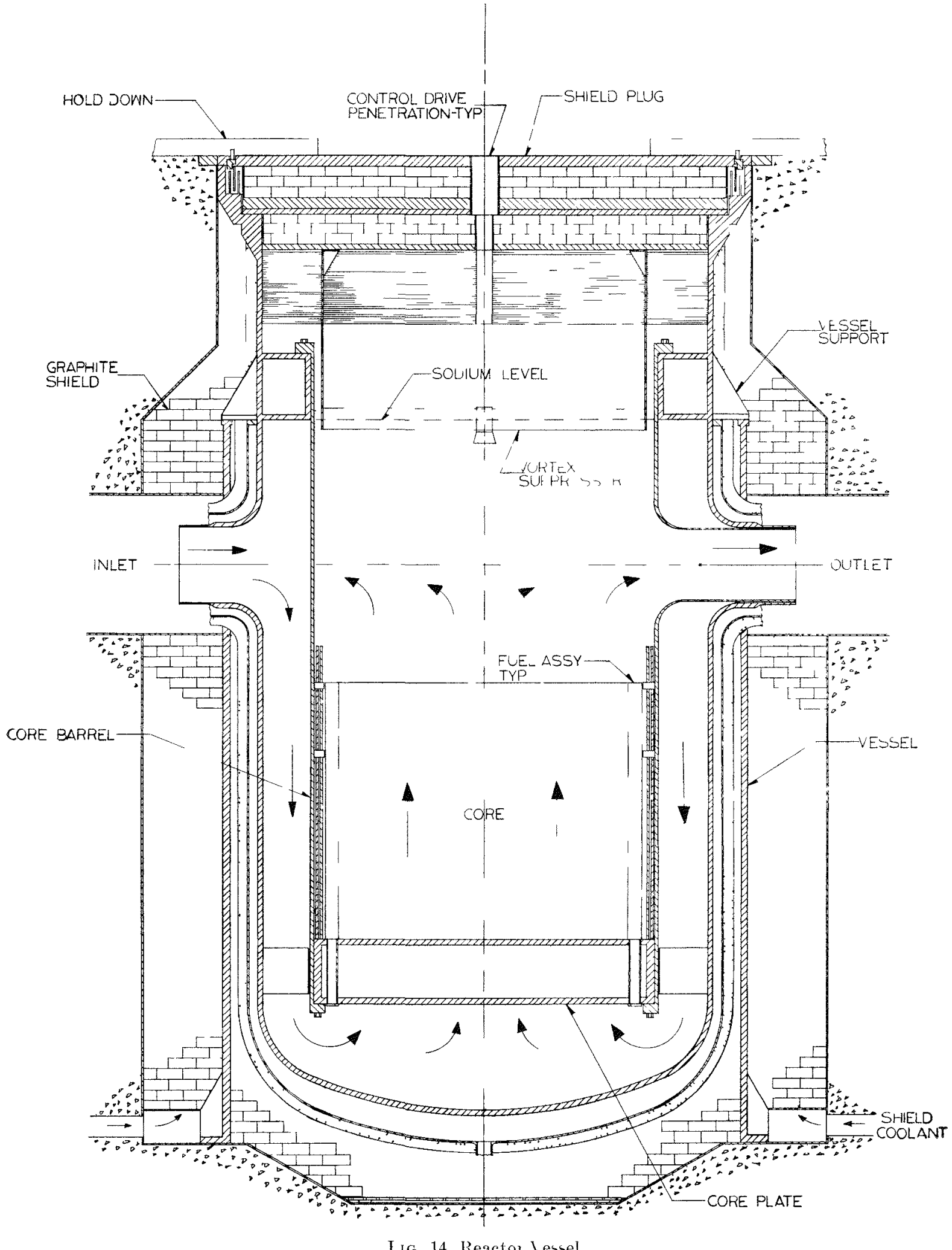

I IG 14 Reactor lessel 
less steel dished head with a conical piece welded to it. One-inch-diameter by 6-in.-length, $1.0 \mathrm{w} / \mathrm{O}$ natural boron-type 304 stainless steel rods are welded to the assembly.

\section{Control-rod Drive System}

The control-rod drives, of a rack-and-pinion type, are located on the top of the upper plug and are completely enclosed by the gas-tight shell. Power, control, and gas-supply lines form helical coils which are suspended from the ceiling of the refueling cell. This arrangement makes it possible to remove the top plug from the reactor and hoist it up and move the plug to its storage position without disconnecting any cables or gas-supply lines.

A positive pressure of inert gas is maintained in the containment shell around the drives to prevent contamination of the mechanisms by the cell atmosphere. Bellow seals similar to those used at Fermi and EBR-II isolate the control-rod drive components from the reactor cover gas. An inert gas pressure higher than the reactor cover gas pressure is maintained in the mechanism housing as further insurance against contamination.

The rack, which is attached to the control rod, is driven up and down by a pinion. As the rack moves up a spring is compressed. The motor torque is transmitted through a gear-reduction unit and an electromagnet cluteh to the pinion. Provision is made in the drive train for continuous indication of control-rod position.

A scram is initiated by cutting the power to the electromagnetic clutch, which allows the rack and control rod to fall freely. The spring in the rack housing provides an initial force to accelerate the control rod more rapidly at the beginning of a scram. A spring- or pneumatically-operated dash pot within the rack housing decelerates the control-rod train at the end of its fall.

The lower end of the rack assembly is attached to the control rod with a latch.

\section{Pumps}

The three variable-speed pumps for circulating primary-system sodium are double-suction, vertical centrifugal units of the free surface type, consisting of:

1. a pump drive motor mounted on a shield deck;

2. a pump volute located in the primary-sodiumsystem piping below the shield deck;

3. a pump impeller located in the volute and connected to the motor by a shaft;
4. a vertical column between the shield deck and the volute to contain the shaft.

The vertical column contains a sodium pool, a chamber for argon cover gas, gas-shaft seal, and other necessary pump components.

All parts of the pumps in contact with sodium are stainless steel except the bearings and other special parts. Each pump is equipped with a smaller auxiliary motor connected to a separate power supply to insure adequate minimum flow of sodium in the event of loss of power.

\section{Intermediate Heat Exchangers}

The intermediate (sodium to sodium) heat exchangers are of the vertical shell-and-tube type with the primary sodium on the shell side, entering and learing through two diametrically opposed horizontal nozzles. Intermediate sodium flows through the tubes. The shell side contains a free sodium surface covered by argon gas to prevent oxidation and to reduce tube-sheet thermal stresses. The argon space contains a sodium-overflow line to accommodate volume surges.

The thermal center of the heat exchanger is located above the center of the core to provide natural circulation of the coolant after a complete loss-of-power accident. The tube sheets and tube bundle may be removed from the shell for inspection or maintenance.

\section{Valves}

No loop-isolation or pump check valves are provided. Loop-isolation valves are omitted as power operation is not contemplated if one pump becomes inoperative. In this event, the plant would be shut down for the necessary repairs. By eliminating shutoff valves, system complexity is reduced, and plant economics are improved.

\section{Piping}

The primary piping is fabricated of ASTM A-358 Type 304 stainless steel. A wall thickness of $3 / 8$ in. was sclected for structural stability even though the piping code requires less than $1 / 4$ in.

All primary piping is enclosed by secondary containment piping to limit the loss of coolant in the event of a leak. Without a secondary shell surrounding the primary piping, a leak could drain the pumps and other sections of the primary system, preventing circulation of sodium to remove decay heat. The secondary-containment material in the reactor plenum is stainless steel and the rest is $11 / 4 \mathrm{Cr}, 1 / 2 \mathrm{Mo}$. Leak detectors are located at the low points of the containment. 


\section{Insulation and Heat Tracing}

All equipment that contains sodium is heat-traced to prevent sodium solidification. Selection of an ultimate heating system will be based on detailed engineering and economic analysis. The sodium piping and equipment are covered with insulation to reduce heat losses.

\section{Temperatures}

Temperature detectors in the hot and cold legs of each loop provide signals for control of the primary sodium system during startup, shutdown, and normal operation. The temperature signals are used by the reactor control and protection system for control of system temperature and are recorded in the main control room.

\section{Sodium Level}

The reactor vessel is equipped with a sodium-level detector. An alarm indicating low or high sodium level is sent to the reactor control and protection system to scram the reactor. Sodium level is recorded, and alarm indicators are located in the main control room.

\section{Flow of Primary Sodium}

Flow rates are measured in each cold leg of the primary sodium loop. A loop-flow signal in any loop actuates an alarm in the main control room. The low-flow alarm is sent to the reactor control and protection system, which initiates a low-flow scram if flow falls below a predetermined value.

\section{Cell Structures}

The primary-system hot cell is a gas-tight structure enclosed by 6 -ft-thick concrete shield walls lined with stainless steel. The floor and ceiling are also lined with stainless steel. Periscopes and lead glass windows are provided in the walls and ceiling for viewing critical operations. Provisions are made to heat the cell walls during refueling operations to prevent condensation of sodium vapor.

A shield door closes off one end of the cell, providing a maintenance area for the refueling machine. With the door closed, air may be admitted to the maintenance area. Air leakage to the rest of the cell is prevented by introducing inert gas between two sets of door seals at a pressure slightly higher than that in either cell area. Normally, this maintenance area will be used only when the reactor spent-fuel storage-pool plugs and the reactor top plug are in place in order to protect the sodium from possible air leakage. Maintenance inside the hot cell will be performed by personnel wearing breathing apparatus and protective clothing. The cell will not be supplied with air except for major maintenance when the sodium in the reactor vessel and storage pool must be covered or removed.

\section{Reactor Plug Crane}

The reactor plug crane is a remotely controlled bridge crane designed to lift the top plug from the reactor, move it aside for refueling, and replace it after refueling. Four separate hoists are provided to lift the plug, and a bridge drive is provided to move the crane along the rails.

The control-rod drives are lifted integrally with the plug after remote disconnection of the drive shafts. Electric cables connected to the drives have enough slack to allow the plug to be lifted and moved without disconnecting the cables.

The crane hoists are permanently connected to the plug top. During reactor operation, the crane is parked directly over the reactor. The lifting cables are rigged through sheaves over to the hoist mechanisms located on the end of the bridge in the equipment tunnel. The four separate hoists are driven by variable-speed DC motors, with speedadjustment circuits in the control system that allow the motors to be driven individually or balanced to function together. Each hoist drum has two separate drive units connected to the drum by electric clutches. Power is carried to the hoist and bridge drive through a feed rail in the ventilation corridor. Circuits are provided for each motor so that a complete dualdrive system is available for the hoists, bridge drive, and clutches.

When replacing the plug, the reactor plug crane is positioned over the reactor, by driving against fixed stops. Level-indicating instruments are located directly on the plug top, and readings can be checked through a wall periscope. Corrections to level the plug are made by driving the hoists individually.

\section{Fuel Handling}

The reactor is refueled by a remotely controlled refueling machine operating in the shielded, inertatmosphere cell. The refueling machine accomplishes all the fuel-handling operations, from the introduction of new fuel assemblies into the cell to the final loading of the spent fuel into the transfer cask. In addition to handling fuel, the refueling machine also removes and replaces reflector elements and control rods. All core components except control rods have lifting fixtures identical with those for the fuel.

The refueling machine is a remotely controlled 
TABle B-1. Design Pirameters of Refleling Michine

\begin{tabular}{|c|c|}
\hline Minimum Lift Capacity & $1000 \mathrm{lb}$ \\
\hline Nitrogen Cooling Flow & $3000 \mathrm{lb} / \mathrm{hr}$ at $200^{\circ} \mathrm{F}$ \\
\hline $\begin{array}{l}\text { Decay Heat of Hottest Fuel As- } \\
\text { sembly }\end{array}$ & $54 \mathrm{~kW}$ \\
\hline $\begin{array}{l}\text { Maximum Speed of Bridge } \\
\text { Travel }\end{array}$ & $20 \mathrm{ft} / \mathrm{min}$ \\
\hline $\begin{array}{l}\text { Maximum Speed of Trolley } \\
\text { Travel }\end{array}$ & $5 \mathrm{ft} / \mathrm{min}$ \\
\hline $\begin{array}{l}\text { Maximum Speed of Vertical } \\
\text { Mast }\end{array}$ & $20 \mathrm{ft} / \mathrm{min}$ \\
\hline Positioning Accurary, Linear & $\pm 1 / 32$ in. (mast extended) \\
\hline
\end{tabular}

rectilinear crane that carries a vertical telescoping mast. Fuel assemblies and control assemblies are transported with this machine between the reactor, storage pool, and entry and discharge ports. Inert-gas cooling with backup is provided for spent fuel elements during transfer.

The bridge and trolley are driven by variable-speed drive units powered and controlled from supply lines and control leads hung in festoons along the cell wall. The mast of the machine is made in telescoping sections with provision to prevent rotation. A gripper for engaging and lifting core components is attached to the bottom of the mast. In addition to the lifting function, the mast must be capable of exerting a downward force of at least $200 \mathrm{lb}$ in order to unlatch the fuel assembly from the support structure. The gripper mechanism is actuated with a pneumatic (nitrogen) cylinder located well above the sodium level. The gripper is designed so that it remains closed (latched) unless the pneumatic system is pressurized; even then it is not possible to open it if a weight exceeding $200 \mathrm{lb}$ is hanging from it.

The method of powering all refueling machine motions is through use of pneumatic (nitrogen) piston-type motors and pneumatic cylinders. All control systems and valves are located outside the cell. Reliability, simplicity, and environment were the major factors in selecting this type of power unit. Electric motors would require complex cooling systems to operate in the cell atmosphere.

Cables or auxiliary power drives are provided to move the refueling machine and unload the fuel into the storage pool in the event of a failure of the normal motive equipment. Spent core assemblies are cooled during transfer by flow of nitrogen gas through the mast assembly supplied from a hose trailed behind the machine.

Two cooling systems are provided with separate gas supplies. Automatic controls switch from one system to the other in the event of a system failure. The forced-cooling system must operate continuously dur- ing a transfer of a spent core assembly. The design provides a method of checking the cooling system immediately after a fuel element is withdrawn from the sodium. If cooling gas is lost during a transfer, a fuel element could reach the boiling point of sodium in 5 min.

The refueling machine is positioned at the desired location by aligning position markers attached to the machine with markers on the crane and trolley rails by means of optical equipment. An alternative system would use an electronic positioning system. Proper location is determined with an electrical position repeat-back system on both bridge and trolley which indicates the position of the vertical mast in relation to a fixed grid pattern. The electronic position-indication system would be backed up with the visual system described. The design parameters for the refueling machine are presented in Table B-1.

\section{Storage of Spent Fuel}

Spent fuel assemblies and control rods are stored in a sodium-filled tank located below the cell floor. Ports extend through a top cover plate and shield down into a tank in which sodium coolant is circulated. This provides storage space for more than a full core load of fuel and blanket assemblies. Because sodium is used as the coolant, the storage pit can also be utilized for storing partially spent fuel during maintenance operations. The heat-rejection system and heat capacity of the spent-fuel-storage pit are also used to remove decay heat from the reactor.

The shield plate and the height of sodium above the fuel provides sufficient gamma shielding in case the hot cell must be entered for maintenance. The storage-pool sodium is isolated from the cell atmosphere by seal plugs placed by the manipulator or refueling machine into the top guide sleeves of each storage position. An inert cover-gas blanket is maintained under the top plate at a pressure slightly below that in the refueling cell. This helps prevent contamination of the cell by fission gases released by the stored fuel. Decay heat is removed by circulating the sodium through an intermediate sodium-NaK heat exchange to maintain a temperature of $300^{\circ} \mathrm{F}$.

\section{Cover-gas System}

The cover-gas systems serve the general function of providing a protective inert atmosphere for the sodium coolant. In addition, the cover gas is used as a collection mechanism for fission product gases (released from the vented fuel), for pressure control, and for continuous purging of the control-rod drive mechanisms. 
Two argon cover-gas systems are used to carry out these functions. One of these is the cover-gas-supply system and the other is the cover-gas-purification system.

The supply system maintains an inert gas blanket in the reactor and in all piping, vessels, or equipment where a free surface of sodium exists. The system is designed to maintain the constant gas pressures required in the reactor, primary system, and the primary drain tank. Argon is also utilized as the displacement gas during draining, filling, and transferring sodium.

The purification system serves to remove gaseous fission products (relcased from vented fuel) from the argon cover gas from primary-system components. The gaseous fission products released to the cover gas are assumed to be xenon and krypton isotopes, as existing data indicate that the halogen fission gases released from the fuel react chemically with the coolant. The system is designed to handle the full flow of the reactor and the fuel-storage cover-gas volumes (where the fission gases are expected to concentrate) and also bleed flow from other primarysystem component cover-gas volumes where fission gases that become entrained in the sodium coolant may be released.

\section{Nitrogen System}

The purpose of the nitrogen system is to maintain an inert atmosphere in the hot cell, primary pump and pipe cells, heat exchanger cell, and maintenance cell to minimize the possibility of a fire with radioactive sodium. The system is also used to blanket areas where an inert atmosphere is desirable, such as the sodium melt stations and cyclone separators connected to the rupture dises from the steam generators. It also backs up compressed-air systems for pneumatic tools and instruments. In addition to its use for an inert atmosphere, the nitrogen is also used for cooling purposes.

\section{APPENIDIX C}

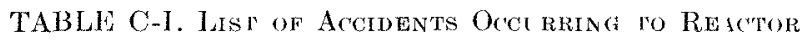

Accidents $\quad$ Feasibility $\mid$ Preliminary Engineering Safeguards

1. Improper removal of shield plug

2. Loss of power to fuel-handling machine

3. Failure of coolant system of fuel-handling machine

4. Jamming of spent fuel assembly partially removed from core

5. Structural failure of fuel-handling machine causing release of subassembly:

a. Drop into core

b. Dropped at some point before final storage

6. Loss of administrative control (not following procedures):

a. Coupled with mechanical failure (e.g., several control rods out of core and central assembly dropped in while refueling)

b. Independent of mechanical failure:

(1) Improper enrichment of fuel assembly

(2) Interehange of core and blanket assemblies
Credible

Credible

Credible

Credible

Credible

Credible

Srstem of checks and balances in any given operation to limit results after mechanical failure; make control rods, fuel assemblies, etc., with relatively low worths

Credible

Credible

Mechanisms and instrumentation insuring all control rods are disengaged and fully inserted into core

Have baekup power; make provisions for manual operation

Have temperature sensors to detect condition; auxiliary cooling system

Raise sodium level or use auxiliary inert gas cooling to prevent melting of rods and release of fission products

Keep core subcritical by an amount greater than the maximum worth of the fuel assembly during refueling; limit worth of assembly

Inert gas cooling

Production quality control

Assemblies have different mechanical characteristics or different size of inlet nozzles fitting core support plate 
TABLE C-I-Continued.

\begin{tabular}{|c|c|c|}
\hline Accidents & Feasibility & Preliminary Engineering Safeguards \\
\hline \multicolumn{3}{|c|}{ B. Startup Accidents } \\
\hline 2. Introduction of slug of cold sodium & Credible & $\begin{array}{l}\text { Design for low sodium worth; design with large inlet } \\
\text { plenum; loop-temperature measurements: seram } \\
\text { reactor }\end{array}$ \\
\hline 3. Introduction of moderator to core & Unlikely & $\begin{array}{l}\text { Do not have any hydrogenous material in the vicinity } \\
\text { of the primary system }\end{array}$ \\
\hline $\begin{array}{l}\text { 4. Bowing and resulting changes of equivalent core } \\
\text { radius }\end{array}$ & Credible & $\begin{array}{l}\text { Elements mechanically designed to force bowing to } \\
\text { be a negative addition of reactivity }\end{array}$ \\
\hline
\end{tabular}

\section{Full-power Mishaps}

\section{Failure of power}

a. Main power

b. Main power and emergency power

c. Main power, emergency power, and battery power

d. Main, emergency, and battery power-control rods fail to scram

2. Failure of pumps

a. Loss of primary flow

b. Loss of secondary flow

3. Loss of coolant

a. Gross failure of vessel

b. Pipe break in primary system

c. Leaks in intermediate heat exchanger

d. Leaks in sodium-steam generator

e. Local coolant blockage due to oxide buildup, mechanical blockage of flow passages, gross swelling of fuel elements, ete.

f. Pipe break in secondary system

g. Loss of coolant through boiling after large power excursion
Unlikely

Unlikely

Incredible

Ineredible

Unlikely

Credible

Unlikely

Unlikely

Credible

Credible

Credible

Unlikely

Credible
Have flywheels on pumps; emergency power; scram reactor. Each pump supplied by a separate power supply

Battery power; scram reactor

Scram reactor

Would lead to core meltdown

Loss-of-flow seram; auxiliary pump

Loss-of-flow scram; auxiliary pump

Double containment prevents uncovering of core; emergency sodium-injection system.

Auxiliary makeup-system leakage from secondary to primary; double-containment piping: leak detectors

Primary-system high-coolant-level seram

Have steam generator behind retaining wall so $\mathrm{Na}$ $\mathrm{H}_{2} \mathrm{O}$ reaction does not damage primary system; seram reactor

$\Delta P$ detectors to scram core; have cleanup systems to remove oxide; have screens in inlet pipes to stop debris from any failed components

Leak detectors scram reactor

Overpower scram of fast-acting control rods 
TABLE C-I.-Continued.

\begin{tabular}{l|c|c}
\hline \multicolumn{1}{c|}{ Accidents } & Feasibility & Preliminary Engineering Safegards \\
\hline 1. Control-rod expulsion & D. Unplanned Changes in Core Makeup \\
\hline 2. Fuel slumping within clad & Credible & $\begin{array}{l}\text { Limit worths of rods; have standby safety rods } \\
\text { carbide rather than oxide fuel to operate far from } \\
\text { fuel melting point. }\end{array}$ \\
$\begin{array}{ll}\text { 4. Control rod rails to scram } \\
\text { administrative control }\end{array}$ & $\begin{array}{l}\text { Control-rod mechanisms independent of each other; } \\
\text { have enough extra control-rod worth that one rod } \\
\text { could fail to scram with no consequences } \\
\text { Limit worth and rate of travel of rod; checks and } \\
\text { balances to minimize neglect of operating pro- } \\
\text { cedures }\end{array}$ \\
\hline
\end{tabular}

E. Sodium Spills and Fires

1. In and around reactor vessel

2. Elsewhere
Unlikely

Credible
Fliminate hydrogenous material; have emergency inert gas system for smothering purposes

Keep potential hazards (steam generators) in separate buildings or behind retaining walls; design containment to withstand theoretical maximum temperature and pressures generated in sodium fire in order to insure core safety

\section{BIBLIOGRAPHY}

1. Heck, F. M., et al., Liguid Metal Fast Breeder Reactor Design Study, WCAP-3251-1 (Jan 1964).

2. Klotz, C., et al., Large Fast Reactor Design Study, ANCP64503 (Jan 1964).

3. McNelly, M. J., et al., Liquid Metal Fast Breeder Reactor Design Study (1,000-MWe UO $\mathrm{O}_{2}-\mathrm{PuQ} \mathrm{Q}_{2}$-fueled Plant), GEAP-4418 (Jan 1964).

4. Visner, S., et al., Liquid Metal Fast Breeder Reactor Design Study, CEND-200 (Jan 1964).

5. An Evaluation of Four Design Studies of a 1,000-MWe Ceramic-fueled Fast Breeder Reactor, COO-279 (Dec 1964).

6. Gunson, W. E., High Power Density, Stainless Steel, Reference FBR Core Design, WCAP-2638 (July 1964).

7. Fuziger, J. H., et al., Resonance Integral Calculations for Evaluation of Doppler Coefficients-The RAPTURE Code, GEAP-3923 (June 1962).

8. Avery, R., Theory of Coupled Reactors, Proceedings of the Second United Nations International Conference on the Peaceful Uses of Atomic Energy, 12, P/1,858 (1958).

9. Grechler, P., et al., FORE, A Computational Program for the Analysis of Fast Reactor Excursions, GEAP-4090 (Oct 1962).

10. Schoeberle, D. F., Heestand, J., and Miller, L. B., A Method of Calculating Transient Temperatures in a Multiregion, Axisymmetric, Cylindrical Configuration, the ARGUS Program, 1089/RE248, Written in FORTRAN II, ANL6654 .

11. SEFOR Hazards and Safeguards Report.
12. Kato, W. Y., et al., Safety Analysis Report. Argonne Fast Critical Facility (ZPR-VI), ANL-6271.

13. Nicholsen, R. X., Methods for Determining the Energy Release in Hypothetical Reactor Meltdown Accidents (Dec 1962).

14. MeCarthy, W. J., et al., Studies of Nuclear Accidents in Fast Power Reactors, $\mathrm{P} / 2,165$, Proceedings of the Second International Conference on the Peaceful Uses of Atomic Energy (Geneva, 1958).

15. Okrent, D., et al., AX-1, A Computing Program for Coupled Neutronics-Hydrodynamics Calculations on the IBM-ro4 (1959).

16. Report on Economic Analysis for Oyster Creek Nuclear Electric Generating Station, Jersey Center Power and Light Company, Feb 17, 1964.

17. Smith, E. E., and Wright, J. H., Future Energy Needs and Nuclear Fuel Use, Atomies, May-June 1965.

18. Lieberman, J. A., et al., The Nuclear Safety Research and Development Program in the United States, Geneva Conference 1964 .

19. Okrent, D., et al., Some Nuclear and Safety Considerations in the Design of Large Fast Power Reactors, Geneva Conference 1964 .

20. Gunson, W. E., A Summary of the Safety Mechanisms of Fast Breeder Reactors, WCAP-2275.

21. Kubit, C. J., et al., High Temperature Thermodynamic Equation of State of Plutonium Carbide, Trans. ANS 8, No. 1 (June 1965).

22. Rajagopal, V., Reactor Noise Measurements on the Saxton Reactor, WCAP-2397 (Oct 1963). 


\title{
Safety Characteristics of Large Gas-cooled Fast Power Reactors*
}

\author{
Peter Fortescue, R. Shanstron, J. Bromo, J. M. Steen, A. Baxter, and H. Fenech (Consultant) $\dagger$ \\ General Atomic \\ San Diego, California
}

(PRESENTED BY P. FORTESCUE)

\section{INTRODUCTION}

Gas cooling for fast reactors was first studied seriously by General Atomic some four years ago. Initially, the main emphasis was on broadly based surveys aimed at assessing the general attractiveness of the idea, as judged not only by likely capital and fuel costs, but also by the operational, maintenance, and safety characteristics indicated.

This work involved investigation of a wide range of applications within the general field of economic production of electric power. Thus, the objective was more than just the design of a reactor to suit a set of arbitrary requirements, but was extended to include finding the particular applications most suited to the special characteristics that gas cooling provided.

By way of example, it was found that the system looked particularly favorable for very large mixed $\mathrm{Pu}-\mathrm{U}^{238}$ oxide- or carbide-fueled reactors of relatively low enrichment, and therefore having a high internal conversion ratio.

As a culmination of this phase of the work, an approximately 1000-MWt reference design was formulated as a basis for subsequent study including, in particular, much more specific investigation of safety aspects, which is the main concern of the present conference.

It is of particular interest to note that in the course of the studies of gas-cooled fast reactors so far completed, questions related to safety have, somewhat surprisingly, turned out to be primarily responsible for many of the attractive features of the system.

Insofar as early neglect of gases as contenders for fast reactor cooling was, as much as anything, due to worries about the safety of relying on a high-pressure coolant with a negligible capability of natural-convection cooling, this would indeed seem to represent an astonishing reorientation of thought.

It is therefore desirable at the outset to say some-

\footnotetext{
*Work performed under Contract AT(04-3)-167, Project Agreement 23, between U. S. Atomic Energy Commission and General Atomic.

$\uparrow$ Massachusetts Institute of Technology
}

thing about how these second thoughts on gas cooling could arise.

Fundamentally, the source of the major favorable claims made for the use of a gas as coolant for fast reactors stems from its very small interaction with the nuclear performance. Although this interaction with a liquid metal coolant like sodium is not at first glance a large one, consequences arise from it that can profoundly affect not only safety matters but, because of design compromises that this aspect entails, can also greatly modify the overall performance obtainable. This magnification of the possible consequences of small effects is characteristic of the whole nuclear business and arises from the fact that the satisfactory operation of any reactor vitally depends on the influence of second-order effects.

In the case of fast reactors which have to rely on differential thermal expansion and Doppler coefficients for their stability, and which characteristically have little heat capacity in their very high-power density cores, this leads to great importance being attached to even small reactivity changes that might arise, i.e., from local or complete expulsion of coolant.

At this time, one can make a fundamental point for gas cooling, namely, holes in a gas, hence, local voids at the core center (which are generally the worst worry), are impossible. As will be shown later, even the total loss of coolant (with $\mathrm{He}$ or $\mathrm{CO}_{2}$ ) leads to less than a dollar's increase in reactivity. To achieve a similar situation with a liquid coolant requires that the influence of the coolant on core neutron leakage terms be high enough to offset sufficiently the opposed effects on spectral-shift terms, or at least that a sufficiently high Doppler effects by retained to override the net deficiency. The trouble with putting things right this way is that in large systems performance can be scriously compromised and, furthermore, new safety difficulties can be created.

Any deliberate increase in core leakage drops the available internal conversion ratio, which in turn forces either employment of a high reactivity reserve for burnup, or much shortened refueling inter- 
vals. The former is fundamentally less safe (and wasteful of neutrons), and the latter is operationally undesirable and can, in fact, lead to a material increase in chances of involuntary down-time extension.

If deliberate spectrum softening must be resorted to in order to blanket the troubles with enough Doppler coefficient, then sacrifices of overall conversion ratio are involved which can be quite severe to a plutonium system.

That these factors are very real and important can perhaps be best illustrated by pointing out that overall conversion ratios as low as 1.2 , refueling requirements several times a year, and burnup-reactivity reserves of several dollars have been mentioned in recent published studies of large sodium systems. These figures may be compared with the combination of 1.5 overall conversion ratio, with no need for partial refueling at all for less than a dollar's reactivity swing that is contemplated for the equivalent gas-cooled systems. This low invested excess reactivity constitutes, in fact, a most important fundamental safety advantage which is reflected in the calculated consequences of a number of accident situations.

Although it is not suggested that all sodium systems will always have to live with the severity of the compromises implied by the figures just mentioned, they do illustrate the degree to which safety ground rules can influence the overall performance of fast reactors, and that this influence is far stronger in the case of liquid metal coolants than it is with gases such as $\mathrm{CO}_{2}$ or $\mathrm{He}$.

Before leaving the subject of coolant-nuclear interaction, a quite fundamental, but little appreciated, point in favor of gas coolants may be given, which turns out to be very important in the proper assessment of the significance of void coefficients. It is that whereas a liquid can be expelled from a reactor core very rapidly (by local boiling or gas entrainment), gases take much more time, for the entire primary circuit volume is involved in the associated time constants, and not just that of the relatively insignificant core contents. As a result, substantial coolant loss from the core region within the time normally required to detect this loss and to complete scram operations is impossible, barring a complete containment explosion. Thus, the reactivity worth of gas coolants is of much less significance than is the case with liquids, in addition to being of characteristically much lower magnitude.

In discussing only the nuclear consequences of coolant loss, we have thus far successfully avoided mentioning the main worry about gas cooling, which is concerned with the effect of a substantial loss in its heat transfer capabilities by a loss of pressure.
This is a point at which progressing development of gas-cooled thermal reactors has greatly modified earlier opinion by offering new techniques for reliable pressure containment, specifically, the notion of total containment of the primary circuit by a single, prestressed-concrete vessel. This completely changes ideas on containment integrity, fundamentally on account of the extreme degree of redundancy of the vessel structure, which limits local fault propagation, and because the strength members are outside the radiation- and temperature-influenced field.

It is indeed characteristic of this type of vessel to fail by local liner cracking, thus allowing a modest initial leak, followed by a measure of self-healing, to the extent that the loss of pressure leaves surplus force in the tensioning cables to close up the cracks in the system. Fracture of a main external cooling duct is, of course, eliminated as a case to be handled.

This type of containment has therefore been accepted as essential to the conception of the gascooled fast-reactor system, and the loss-of-coolant analysis with which we will deal more specifically later, has been directed toward an inquiry as to what rate of pressure loss is tolerable, without core damage, rather than toward looking at what postulated calamities might befall the integrity of the primary loop.

One further important general principle relating to the safety philosophy of the particular reactor system discussed here needs mention before passing to the more specific questions, and that concerns the means for dealing with steam or water flooding of the core, for fracture of a boiler tube is clearly a situation to be reckoned with.

Basically, the method adopted to ensure a continuous decrease in reactivity on water-steam introduction is to incorporate suitable resonance absorbers permanently in the fuel. Thanks to the normally hard spectrum, these have very little effect on normal operation, but progressively shut down the reactor as the spectrum is softened by water introduction. Having thus catered to water flooding, we gain the by no means insignificant side benefit of being able to flood the core deliberately to permit visible salvage operations, and to take care of longterm shutdown heat using only natural convection. The ability, in fact, to enlist the aid of regular faucet water when in trouble or when facing ultimate catastrophes must surely rank as a very reassuring feature.

Having thus briefly outlined some of the main safety aspects of the design philosophy of the system, we will now pass to the results of more specific analysis.

To clarify this, we will briefly describe the princi- 
TABLE 1. Detarled Specifications for Reference Design (Gadolinium resonance absorber)

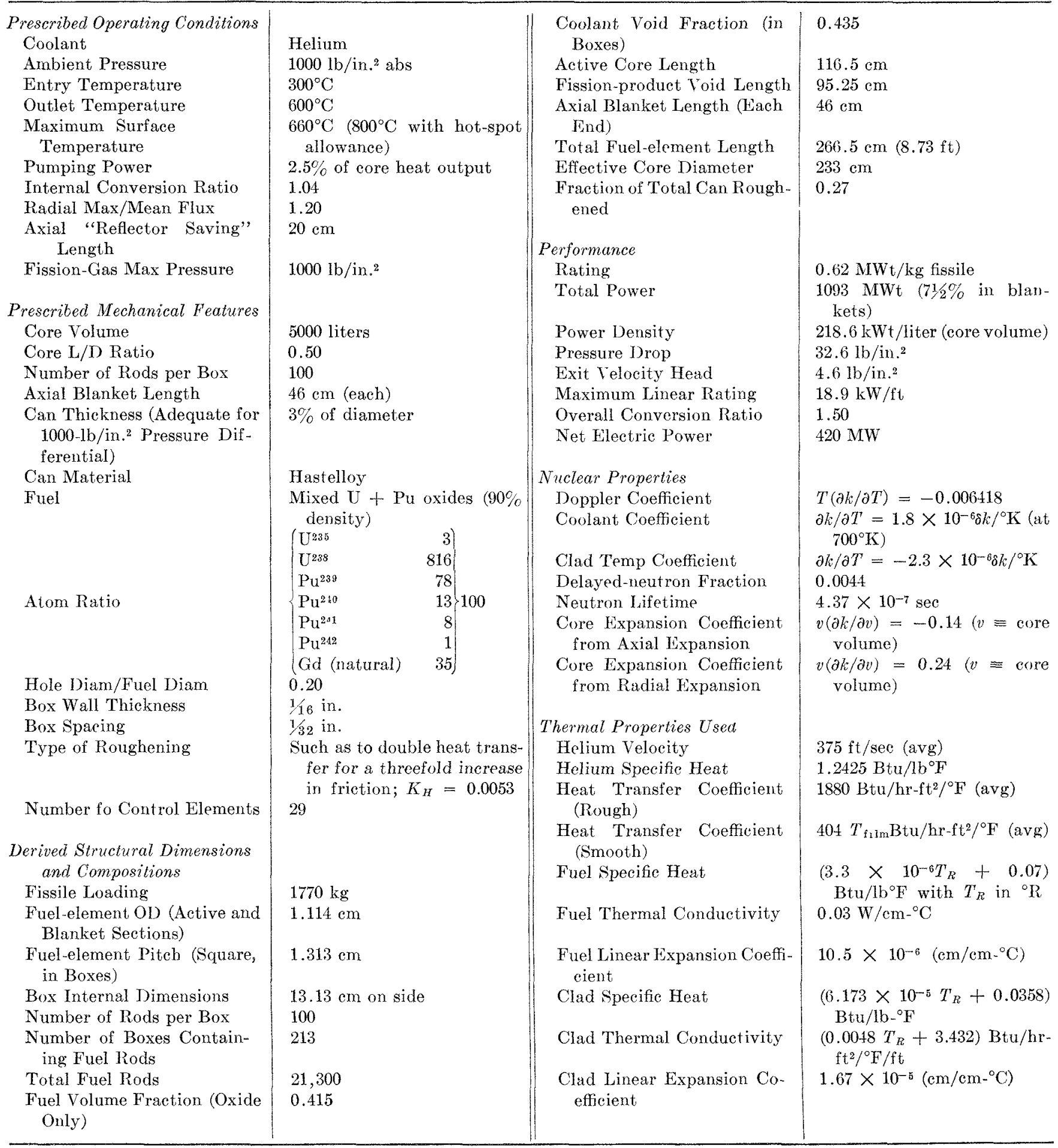


pal mechanical features of a reference design considered typical of the system, and to which the subsequent analysis specifically refers.

\section{REFERENCE DESIGN FEATURES}

Details of the reference design which formed the basis for the present work have been given in detail. (1) We will only mention enough of the leading features of this system to clarify what follows, particular emphasis being on items that concern safety. The leading dimensions and performance of the reference design are summarized in Table 1. A diagramatic representation of the core disposition is shown in Fig. 1.

As shown in Fig. 2, the entire reactor primary system-comprising a 5000-liter core with a surrounding blanket, circulators, steam generators, and associated main coolant ducting, as well as the control drive mechanisms-is housed in a simple, cylindrical, prestressed-concrete vessel. The vessel is illustrated as being housed in a concrete-lined shaft, the top face being at ground level, but this is not regarded in any way as a general necessity.
Coolant flow is downward through a $31 / 2$-ft-deep top grid plate (which is the only core constraint) to the core region, and then upward via multiple ducts, and through an internal concrete shield to the steamgeneration region located above. Apart from keeping the control mechanism and core-structure location planes close together and uniformly well cooled, this arrangement has the safety feature that in the event of meltdown, fuel would be expelled by both coolant flow and gravity in the direction of the hottest end of the system. Thus, not only would the melt not have to travel so far, but the possibility of subsequent freezing within the core is reduced greatly (a major source of concern in analysis of such accidents). The purpose of the internal conerete shield is to allow access, under shutdown or low-power conditions, to the steamgenerator tube banks and control drive mechanisms, and to reduce the activation of these components during high-power operation. Bottom loading is employed, primarily to avoid having to pass fuel elements through the top grid plate, which would have introduced considerable waste space between fuel

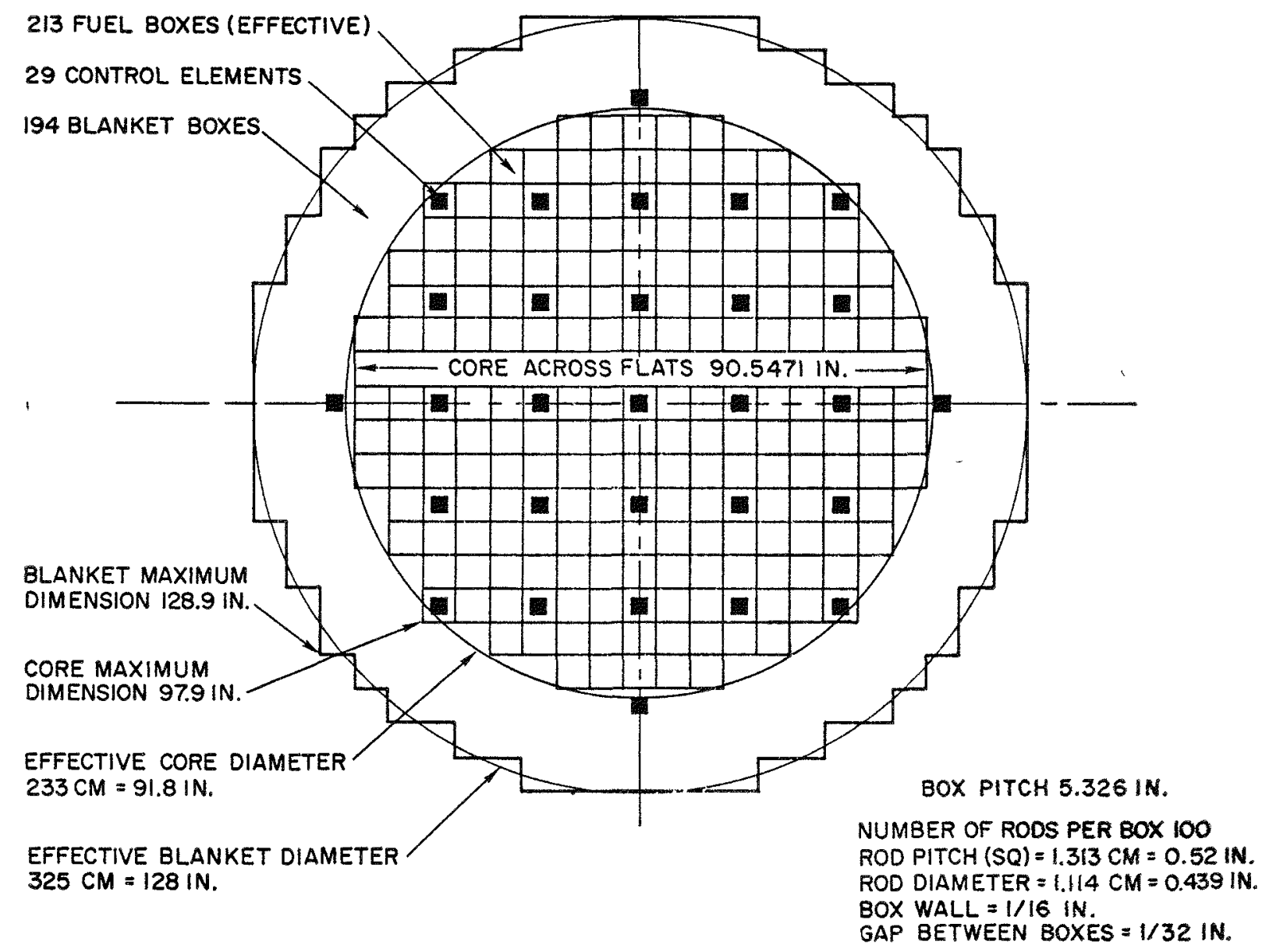

FIG. 1. Core Disposition. 

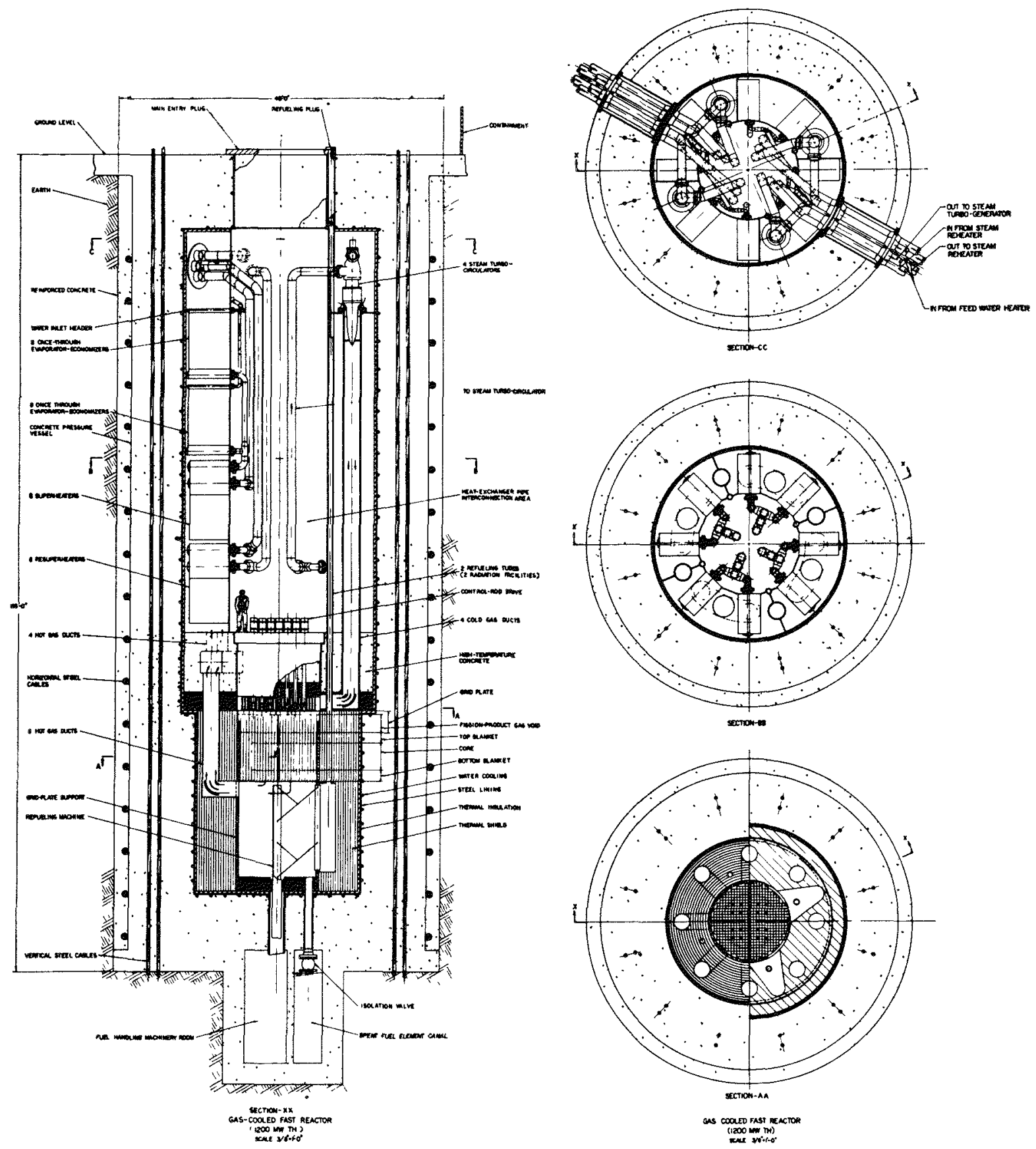

Frg. 2. Engineering Drawing of Primary System.

boxes, and which early analysis had shown to be particularly detrimental to performance. A removable internal fuel-transfer machine is employed; the only feature of this, however, that directly concerns the present safety aspects is that it allows dry reloading at atmospherie pressure, by incorporating a means for continuous cooling of the extracted fuel elements, derived from a tapping from the main circulator outlet. Although quite small local pumping power is involved (some $1 / 3 \mathrm{hp}$ ), without this, fuel limiting temperatures would be met after only about $3 \mathrm{~min}$ after removal from the core environment, even four days after shutdown.

Although dry reloading is thus catered for, performing the operation under water, after deliberate core flooding for the purpose, is being seriously considered as an alternative having much attraction. Notably there is completely eliminated the need for 
primary-circuit forced-cooling circulation during the operation, and generally there is provided a reassuring protection during the time of open access to the reactor vessel.

The steam generators comprise eight independent units, each of which incorporates once-through evaporator economizer, superheater, and re-superheater sections.

The re-superheater is used in this instance because an external steam-to-steam heat exchanger is proposed for reheat. This arrangement considerably reduces the bulk of the steam generator to be housed within the pressure vessel, as it avoids having to accommodate low-pressure steam there. In the present application, this reduction is useful because the core power density is so high in fast reactors that the vessel size is almost entirely determined by the steamgenerator bulk. Further advantages of this arrangement are that it keeps all steam pressures above that of the coolant and reduces necessary penetration sizes; both are factors of concern in limiting the maximum credible rate of coolant loss.

A further important safety consideration is the provision of a large free space below the core, into which, in the event of meltdown, the fuel could pass without the impediment of a lower grid plate. A conical splitter at the bottom of this space is incorporated to avoid reforming a critical assembly.

The circulators are driven by single-stage steam turbines, cach passing the full steam flow from a pair of boiler units. This arrangement has the merit of readily permitting design for $50 \%$ overspeed continuous operation under depressurized conditions.

This design concept was evolved for General Atomic's large HTGR's, where prototype development has been initiated on a machine that has much in common with the machine needed for the present concept. The circulators are located in the top of the main pressure vessel and are under coolant pressure internally and externally, their casings thus being relieved of sustained high pressures. Access is obtained by removal of a steel pressure dome and a concrete shield plug beneath, allowing complete removal of the circulator units.

The pressure vessel is of the prestressed-concrete type and is cylindrical in shape with flat cnds. The tension system proposed consists of a simple arrangement of separate axial and circumferential cables, capable of independent adjustment and continuous strain measurement.

The pressure vessel incorporates an inner steel lining faced with heat insulation, and immediately adjacent built-in water cooling coils.

\subsection{Fuel Elements}

The reference design is based on simple round-rod elements, incorporating core and axial blanket zones, together with a voided extension to limit fission-gaspressure buildup.

This approach is particularly well adapted to use with high-pressure coolants, since the fuel rods can be designed to limit fission-gas pressure (even with total release) to less than that of the coolant. Cnder these circumstances, the gas containment is obtained at no penalty in ean thickness, since the latter must be sufficient to deal with the possibility of pressure equalization resulting from small in-leaks of coolant, followed by depressurization.

The fuel pellets will be operated up to a maximum burnup of $100,000 \mathrm{MWd} /$ tonne. Taking a fission yield of stable rare gases of 0.2 atom per atom fissioned, and assuming all the gas to be released from the fucl to a space at the top of the fuel rod provided for its storage, the volume of reservoir required for 1000$\mathrm{lb} /$ in. $^{2}$ pressure at a storage temperature of $300^{\circ} \mathrm{C}$ (core inlet temperature) is approximately one-half the fuel volume for an axial max/mean power distribution of 1.3 .

\subsubsection{Fuel-rod Design}

The fuel rod for the reference design consists of a Hastelloy-X can, $\sim 0.44$-in. OD and 12.5 mils thick, sized to accommodate $46 \mathrm{in}$. of core and $18 \mathrm{in.}$ of blanket above and below the core. Continuous with this tubing is an extension, 37.5 in. long, which is used as a fission-product storage reservoir. This extension is swaged down to about $80 \%$ of the normal diameter to reduce pressure drop. The core and axial blanket pellets are $0.50 \mathrm{in}$. long and smaller than the internal diameter of the can by the amount necessary for ease of assembly; this amount depends on the tolerances in can diameter. The pellets have a small central hole, the prime purpose of which is to facilitate the free escape of fission gas to the end roid space in the event of a local melt, thus avoiding development of excessive local pressures. The fissionproduct storage reservoir is at the top (gas entry) end and is fitted with an end cap which attaches to an upper support in the fuel-element box. The lower end is capped, but not confined in the axial position. Approximatcly one-half the lower outlet of the active rod length carries surface roughening of the same type as used in the British AGR reactor.

\subsection{Fuel Boxes and Assembly}

The concept is based on bundles of fuel rods which can be separately orificed for coolant flow and which 

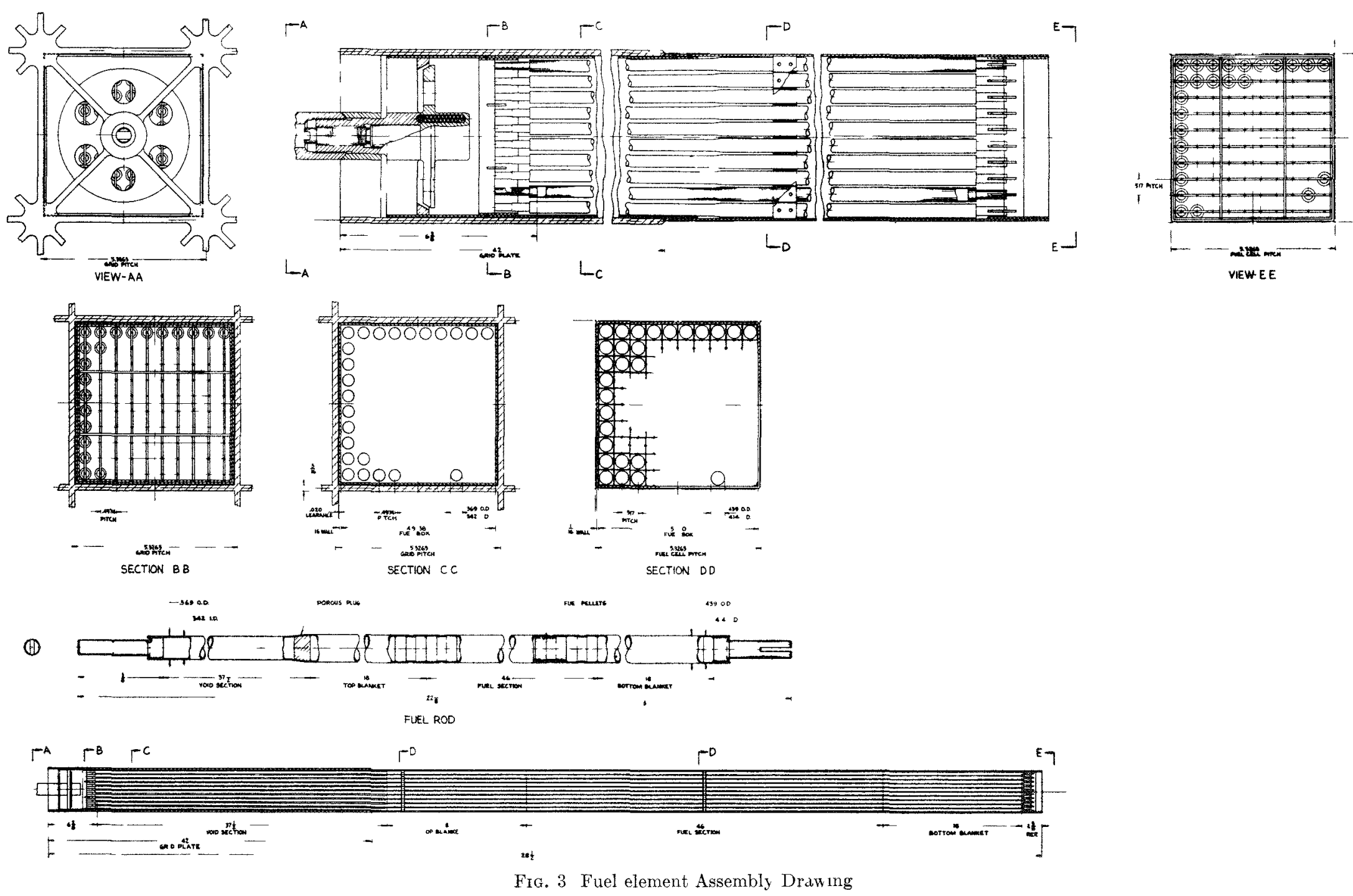
are contained in a box which acts as a support and flow barrier. Square-section fuel boxes are employed, the flat walls of which could have to withstand a pressure differential of the order of $30 \mathrm{lb} / \mathrm{in} .{ }^{2}$, the coolant pressure drop. A typical fuel-element assembly is shown in Fig. 3. The dimensional requirements dictated by nuclear, thermal, and hydrodynamic design are shown in Fig. 4.

To avoid the large pressure differential, the box interspaces are open at both ends, so that pressures are approximately equal inside and outside the fuel boxes along their whole length. This, of course, allows some coolant bypass, but the amount in our case is little over $1 \%$, owing to the close box spacing $\left(1 / 32^{-i n}\right.$. gaps) allowed by the bottom-loaded core design.

The fuel rods are attached to an internal support grid at the top of the fuel element. This support is in turn welded to the box structure inside the reduced box section. The top cap on the fuel rods is slotted to fit the support grid. This attachment is the only means of confining the rods in the longitudinal direction. The rest of the element is permitted to expand longitudinally under an increase in temperature and to slide within the spacing grids. This arrangement allows complete ejection of a partly melted fuel bundle under the combined influence of gravity and coolant pressure drop.

The fuel-element boxes are held in the grid plate by a nut which engages a threaded shaft attached to the top fitting of the box. The conical surface at the bottom of the nut and the beveled lower face of the grid plate provide, in effect, two cones located some 42 in. apart, which lock the fuel boxes in position and locate them with respect to the grid plate. The box retention nuts are operable from the top face of the internal shield plug by means of extension wrenches built into the latter.

\subsection{Control Rods and Drives}

Control is maintained by boron (in carbide form) in round steel cans operated in guide tubes located in specially adapted fuel boxes.

Drive is by direct rod-to-mechanisms located in the upper shielded compartment of the pressure containment.

These mechanisms employ a synchronous motoroperated ball screw for normal operation, with direct linear pneumatic actuation for scram, with hydraulic snubbing.

Followers are not used, since the downward extension of the rod is limited to the confines of the core by closed ends in the guide tubes, which retain the detached rods.

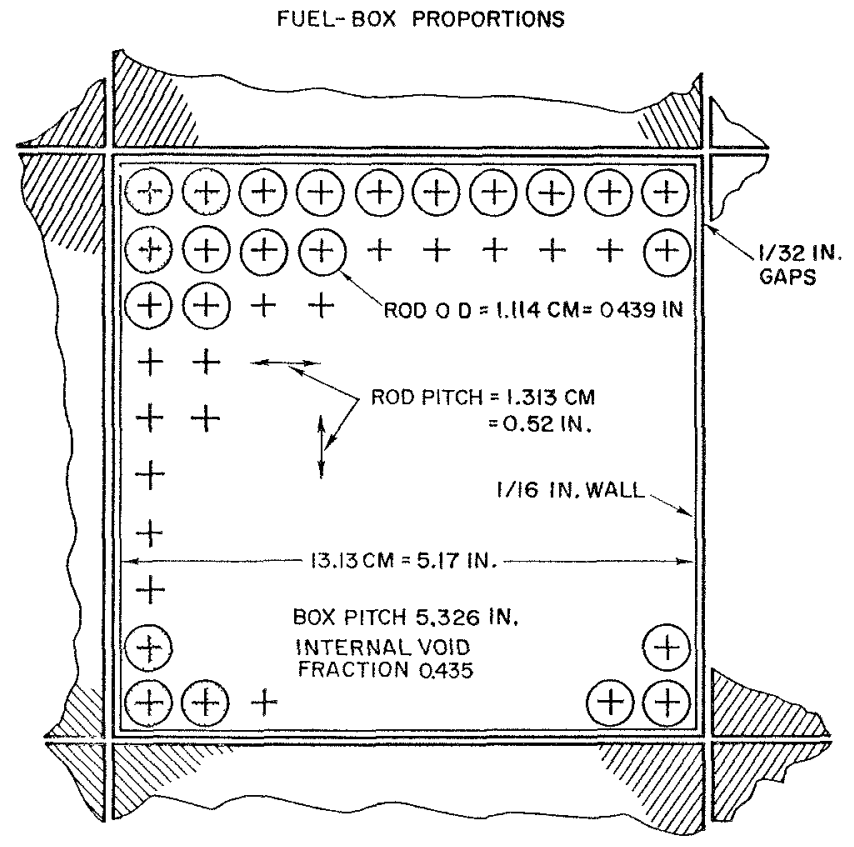

ACTIVE LENGTH $116.5 \mathrm{CM}=46 \mathrm{IN}$. END BLANKETS $46 \mathrm{CMEACH}=18.1 \mathrm{IN}$. VOID LENGTH $95.3 \mathrm{CM}=37.5 \mathrm{IN}$.

FIG. 4. Fuel-box Proportions.

\section{ANALYSIS OF SELECTED ACCIDENTS}

\subsection{Consequences of Coolant Loss}

Perhaps the most immediately obvious safety problem associated with use of a high-pressure gas as a reactor coolant is the possible effects of its inadvertent loss.

We are not here concerned with the credibility of the leak rates indicated, but are only interested in this context in what the core could stand without damage.

In the present concept, the situation is eased by the following design features:

1. integral prestressed-concrete containment, climinating external ducts or component casing failures as a source of rapid leak;

2. use of a core designed to allow water flooding to deal with long-term shutdown heat removal by natural convection;

3. use of multiple circulators (driven independently of external power supply) capable of $50 \%$ overspeed at low pressure;

4. availability of boiler sections as heat sinks already incorporated within the pressure vessel.

The problem to be dealt with quantitatively here is the estimation of the core-temperature history consequent of arbitrary coolant pressure leak rates, including modification of the results by application of different corrective measures-in particular, allowing 
circulator overspeed and the cutting out of feed heating after an appropriate interval.

\subsubsection{Pressure Deray}

Assuming isothermal initial reservoir conditions and neglecting viscous effects, it can be shown that the pressure in a container having a fixed-area leak decays exponentially to a point where the pressure ratio across the leak drops to a critical value (2.05 for helium). During this period, container pressure is given by

$$
P=P_{0} e^{-\left(t t_{c}\right)}
$$

where $P_{0}$ is the initial absolute pressure, $t$ the time, and $t_{r}$ a time constant, related (in the assumed ideal conditions) to the leak area $A$ and the initial container volume $V$ by

$$
t_{c}=\frac{V}{A}\left[\frac{M}{R T \gamma}\left(\frac{y+1}{2}\right)^{(y+1) /(y-1)}\right]^{1 / 2} .
$$

In the latter equation $M$ is the coolant molecular weight, $R$ the gas constant, $T$ the absolute initial temperature, and $\gamma$ the ratio of specific heats. For helium,

$$
t_{c}=0.302\left(\frac{V}{A \sqrt{T}}\right) \mathrm{sec},
$$

where $V$ is in litres, $A$ is in $\mathrm{cm}^{2}$, and $T$ is in ${ }^{\circ} \mathrm{K}$.

As a point of interest, the time constant for $\mathrm{CO}_{2}$ would be $\sim 3.5$ times larger, allowing even more time for corrective action after the same accident. After the critical pressure ratio across the leak is reached (which takes $31 / 2$ time constants), the relationships are much more complieated, but fortunately the flow equations can be integrated for the particular value of $\gamma$ for helium, yielding the relation

$$
\left(\frac{t^{\prime}}{t_{c}}\right)=\frac{5 \sqrt{3}}{16}\left[\frac{10}{3 \sqrt{3}}-\left({p_{R}}^{2 / 5}-1\right)^{1 / 2}\left({p_{R}}^{2 / 5}+2\right)\right]
$$

where $p_{R}$ is the ratio of container pressure to atmospheric pressure at time interval $t^{\prime}$, after the moment of reaching the critical pressure; $t_{c}$ is the same time constant as before.

Putting $p_{R}=1.0$ in the above equation, we find the rather surprising result that the time needed to equalize pressure is finite and equal to $(25 / 24)$ time constants after the critical point is reached, or approximately $4 \frac{1}{2}$ time constants after the initial leak starts.

Plotting the above functions has shown that, in practice, continuing the initial, simple, exponential pressure decay right down to atmospheric pressure, and thereafter keeping it constant, is a good enough approximation for analysis of the effect of coolant loss. This approximation was, in fact, used in computing what follows.

To preserve generality (and because total containment volume is not yet a settled quantity), it is preferable to work in terms of arbitrary time constants rather than in equivalent leak areas, but the relation between the two can be obtained in any particular case from Eq. (1). The containment shown in the illustrations of the reference design has an internal volume of some $30,000 \mathrm{ft}^{3}$, which would give a time constant of about 12 sec for an effective $1-\mathrm{ft}^{2}$ hole at the reactor intake. This gives some idea of the quantities involved.

It is emphasized that the hole area referred to here is an ideal one, approximated only by a carefully designed nozzle. In practice, the same area, spread out in the form of long cracks, for instance, would have many times less flow rate owing to the various effects that have been ignored. Even a single unobstructed near-circular orifice would only permit about half this flow rate because of flow-distribution irregularity.

In calculating core temperatures during and subsequent to coolant leakage, further assumptions are necessary concerning the heat rejection (via the boiler) during the period. The assumption made is that reactor inlet temperature remains constant for any one case. Owing to the close approach temperature that an economic steam system would require, this assumption is considered justified. The effect of feed-heating removal is taken to be an immediate reduction of reactor gas inlet temperature by approximately $350^{\circ} \mathrm{F}$ from its normal value.

\subsubsection{Power after Shutdown}

The mathematical model used for afterheat generation following reactor shutdown is

$$
P=A t^{-B},
$$

where $P$ is the percent of initial operating power, $t$ is time in seconds, and the constants $A$ and $B$ change over different time intervals in accordance with the following:

\begin{tabular}{ccc}
$\begin{array}{c}\text { Time Interval } \\
\text { (sec) }\end{array}$ & $A$ & $B$ \\
\hline 1.0 to 10 & 12.05 & \\
10 to 150 & 15.31 & 0.0639 \\
150 to $4 \times 10^{6}$ & 26.02 & 0.1807 \\
$4 \times 10^{6}$ to $2 \times 10^{8}$ & 53.18 & 0.3834
\end{tabular}




\subsubsection{Scram Time Delay}

From the leak rates considered, the behavior of the system is relatively insensitive to time delay before the reactor is shut down. The time delay used was 0.5 sec, which is pessimistic considering the magnitude of the initiating phenomena.

\subsubsection{Normal Corrective Measures}

The steam-driven turbine circulators are capable of $50 \%$ overspeed under reduced pressure conditions and represent a readily available means for improving coolant flow. A conservative 0.5 -min time delay for this action is used in the analysis. Another normally available means of improving the cooling is the reduction of core inlet temperature, which may be accomplished by bypassing the feedwater heaters in the steam plant. For the purpose of this study, it is assumed that the inlet temperature can be reduced by $\sim 350^{\circ} \mathrm{F}$ in the 0.5 -min time delay.

\subsubsection{Heat Transfer Analysis}

The heat transfer under transient conditions was calculated by means of the RAT code, which permits the subdivision of the fuel element under consideration into many pieces, both radially and axially, and follows the changes in temperature in all pieces during the course of the transient. The code can be used for a variable heat transfer condition along the fuel element, and the rough and smooth sections of the reactor element were properly considered.

\subsubsection{Results of Analysis}

The most important effect of a loss-of-coolant accident is the rise in cladding temperature, since the cladding must be kept appreciably below the molting point to prevent a failure. The reference cladding material is Hastelloy- $X$, which has a melting point of $2350^{\circ} \mathrm{F}$. Figure 5 shows a history of the maximum cladding temperature with a pressure decay constant of $0.5 \mathrm{~min}$ for the case of (1) no corrective action, (2) a reduction of inlet temperature by $\sim 350^{\circ}$ F, (3) an overspeed of $50 \%$, and (4) both (2) and (3). The full corrective action of (4) limits the temperature to a maximum of $1976^{\circ} \mathrm{F}$, which is just about equal to the acceptable limit. It should be noted that the low thermal capacity of the fuel element yields an immediate and rapid drop in the cladding temperature after the reactor is scrammed. It is interesting to note here that the core heat capacity is, in fact, so low that the stored heat in the fuel really constitutes no problem - it is all dumped to coolant long before pressure could fall far and while there is still a good heat transfer. It is the nuclear afterheat which constitutes the essential difficulty, but the effects of this are sufficiently slow in building up as to allow a quite reasonable delay in effecting preventive measures.

This situation can be reckoned as one useful byproduct of having, for other reasons, to employ so large a pressure vessel volume in relation to that of the core alone.

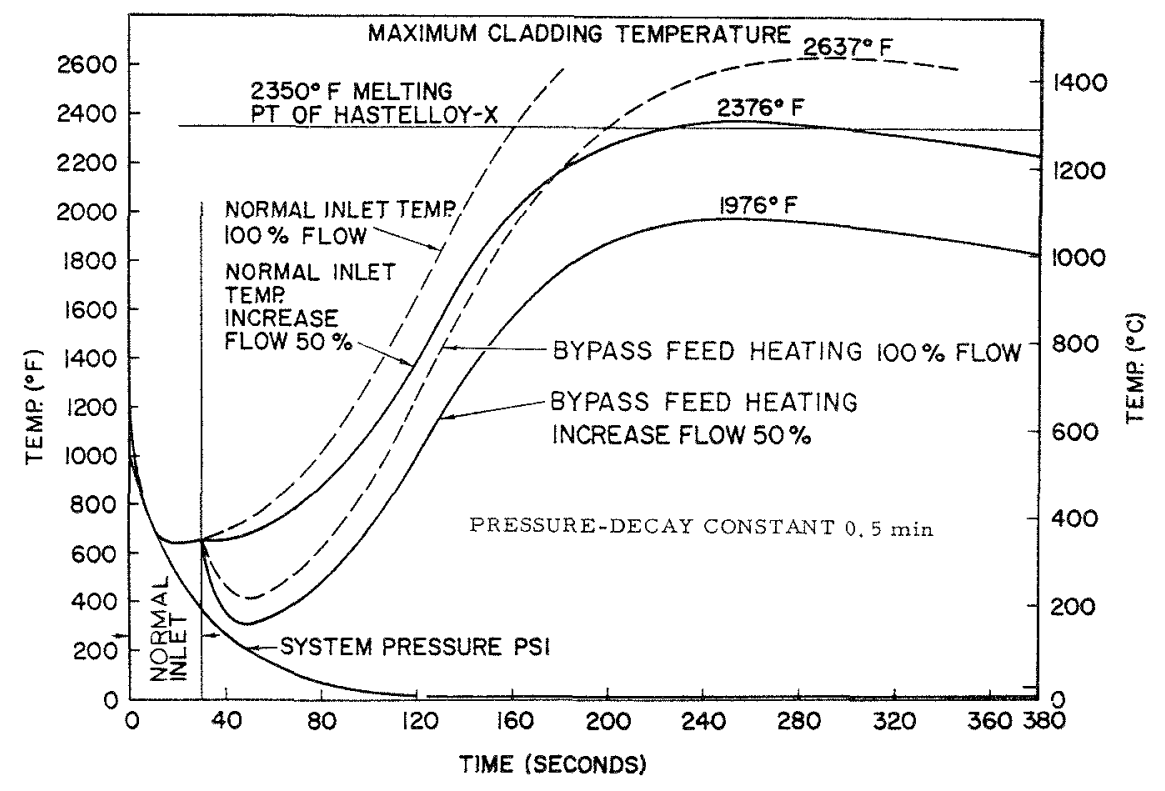

Frg. 5. Maximum Cladding Temperature Histories for Various Combinations of Corrective Actions on Inlet Temperature and Circulator Speed. 


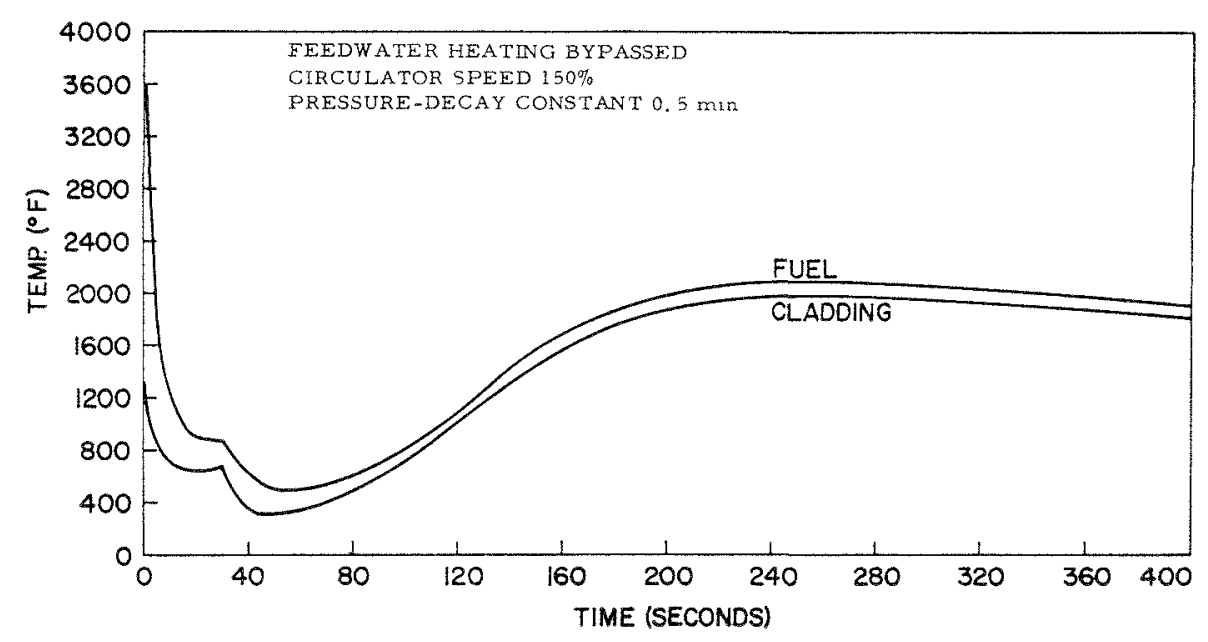

FIG.6. Maximum Fuel and Maxiumm Cladding Temperatures as a Function of Time.

Figures 6 through 9 show the temperatures at other points in the fuel element for case (4). Figure 10 shows that a leak of twice this rate would not lead to a melting of the cladding. If the only corrective action taken is the overspeed of the circulators, the maximum cladding temperature is as shown in Fig. 11 for various leak rates. This figure shows that without this corrective measure, the same temperatures would be reached for a leak rate of $25 \%$ of the magnitude considered in the detailed study.

Thus, it would appear that with scram initiated within one-half second of the start of trouble, it is possible to deal with a leak that would halve the pressure in as short a time as about 20 sec with the normal cooling arrangements, and without can failure.

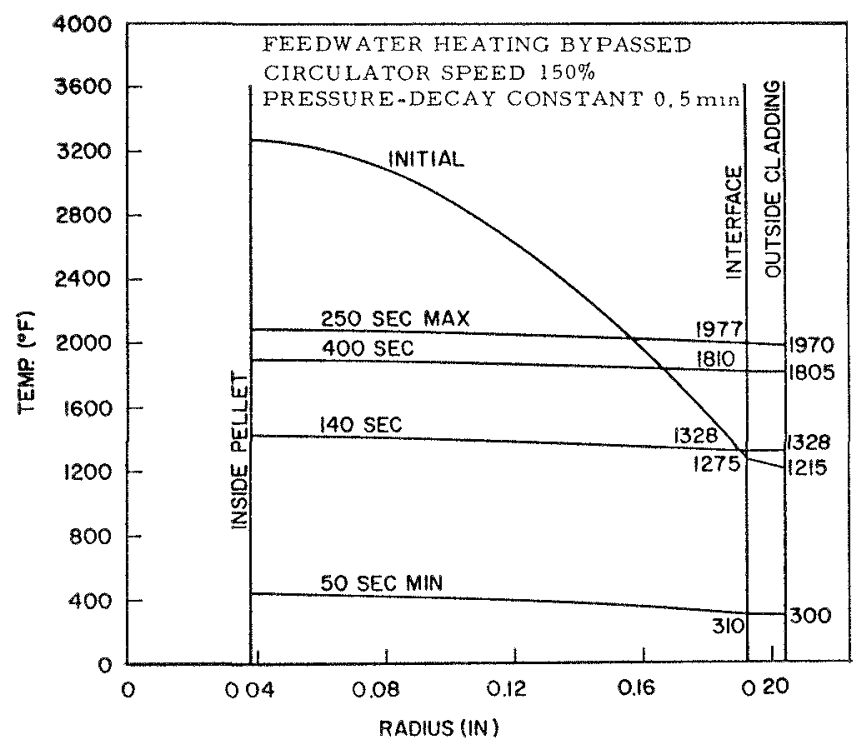

Fig. 7. Radial Temperature Profiles at Point of Maximum Cladding Temperature for Various Times after Beginning of Accident.

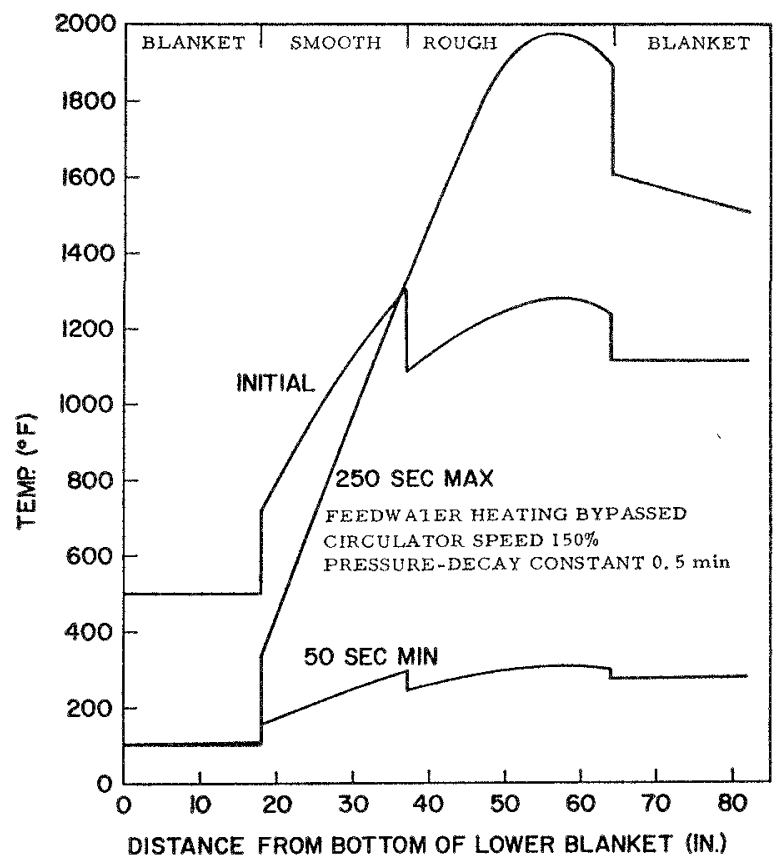

FIg. 8. Maximum Cladding Temperature Axial Profiles at Various Times after Beginning of Accident.

This requires the remedial measures of cutting off the feed-water heating within a half minute, and an overspeed allowance on the blowers. The power demand of the latter is quite low. The ambient pressure would be nearly atmospheric by the time the speed increase is required.

\subsection{Reactivity Changes Caused by Introduction of Water into the Core}

Several possible accident or operational situations require analysis of the reactivity change consequent to the introduction of water or steam into the coolant channels of a GCFR: first, the rupture of a steam 
generator tube; second, the availability and physical properties of water make it a logical choice for emergency coolant should the mass flow rate of helium be severely reduced; and third, the performance of refueling operations with a completely flooded core is being seriously considered, either as an emergency substitute for dry refueling or as a standard operational procedure.

In connection with these measures, the reactivity change due to water entry has been studied for all

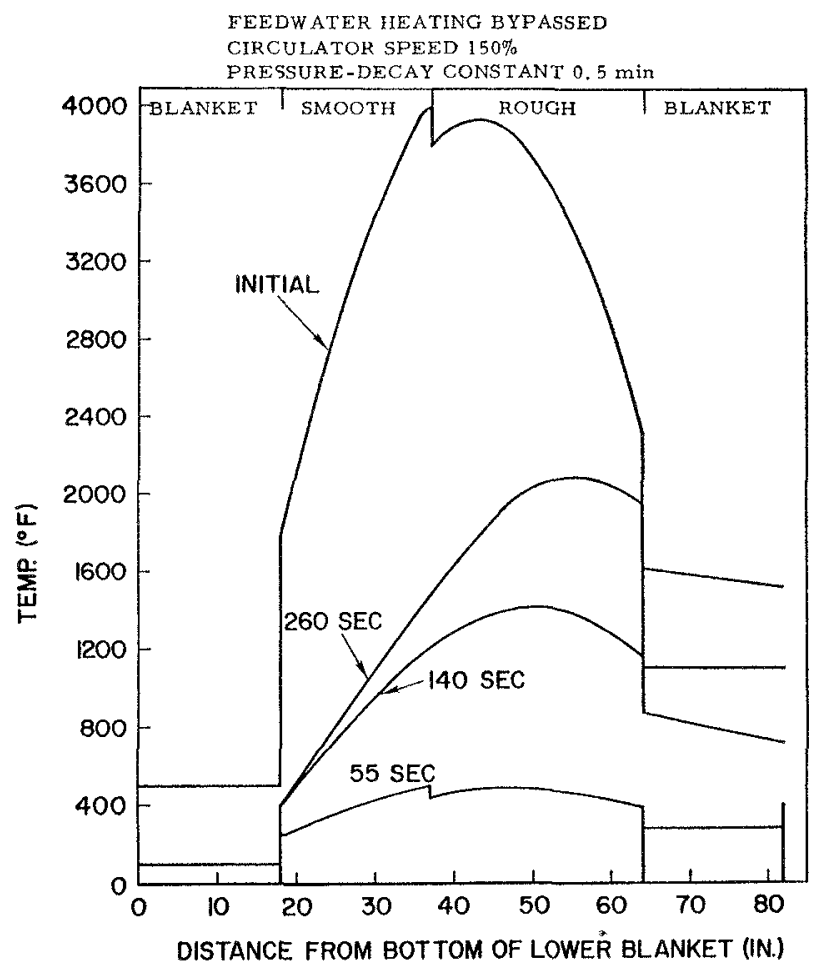

FIc. 9. Maximum Fuel Temperature Axial Profiles at Various Times after Beginning of Accident. possible concentrations of water in the coolant channels and these results are summarized in Fig. 12 . The top curve is for GCFR fuel with no additional parasitic absorber and shows the important characteristics that small amounts of water give negative reactivity insertions. However, the reactivity becomes positive after $10 \%$ flooding and increases to $11 \%$ in reactivity at the fully flooded condition (for the case of unpoisoned fuel, unpoisoned water). In all calculations, it was assumed that the flooding was with cold water and that all control rods were out.

In reality, a boiler-tube rupture would introduce steam rather than cold water into the core. In Fig. 12 , this accident would correspond to a very small percentage of the flooded condition. For example, an upper boundary on the steam density is $2.25 \mathrm{lb} / \mathrm{ft}^{3}$, corresponding to the saturation point for $1000 \mathrm{psi}$. This maximum steam density corresponds to a $3.6 \%$ flooded condition in Fig. 12, well within the negativereactivity area.

Therefore, in the event of ruptured steam-generator tubes, the reactor would inherently shut down for any quantity of steam entry and without the incorporation of additional absorbent material in the core.

However, situations of emergency core cooling with watcr or underwater refueling require either the introduction of additional poison or an ensured scram of a large number of control rods (critical volumes are small with $50 \%$ water and $10 \%$ enriched fuel). As shown in Fig. 12, if approximately $3 \times 10^{20}$ atoms of gadolinium are incorporated per cubic centimeter of core volume, the multiplication factor then decreases for any degree of water content. This concentration corresponds to approximately one atom of gadolinium for every three fissile atoms.

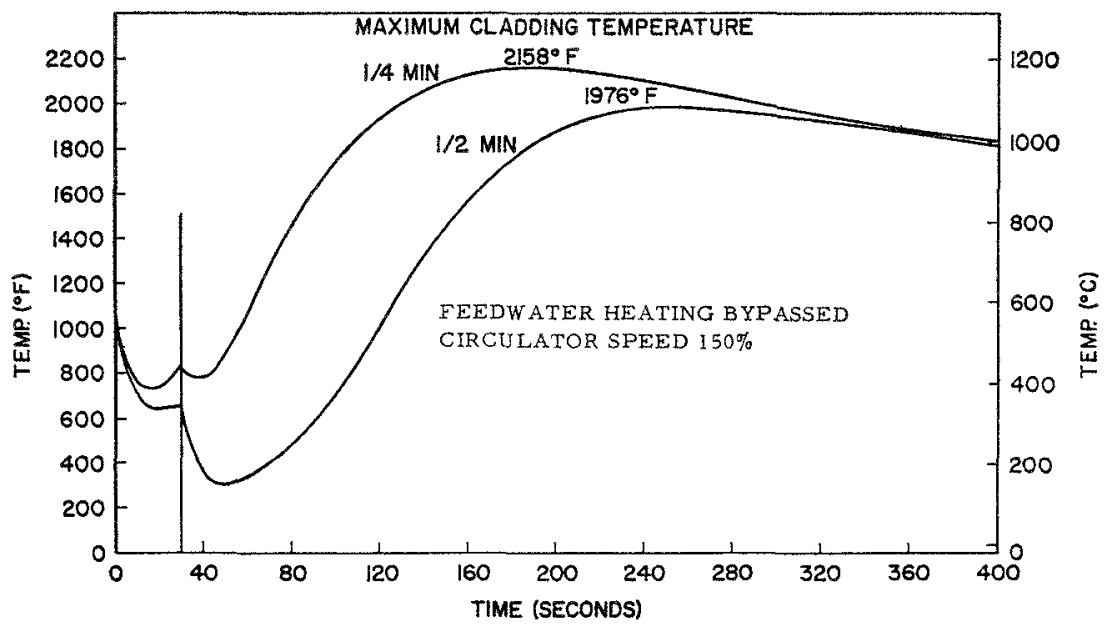

Frg. 10. Maximum Cladding Temperature Histories for Various Pressure-decay Constants. 


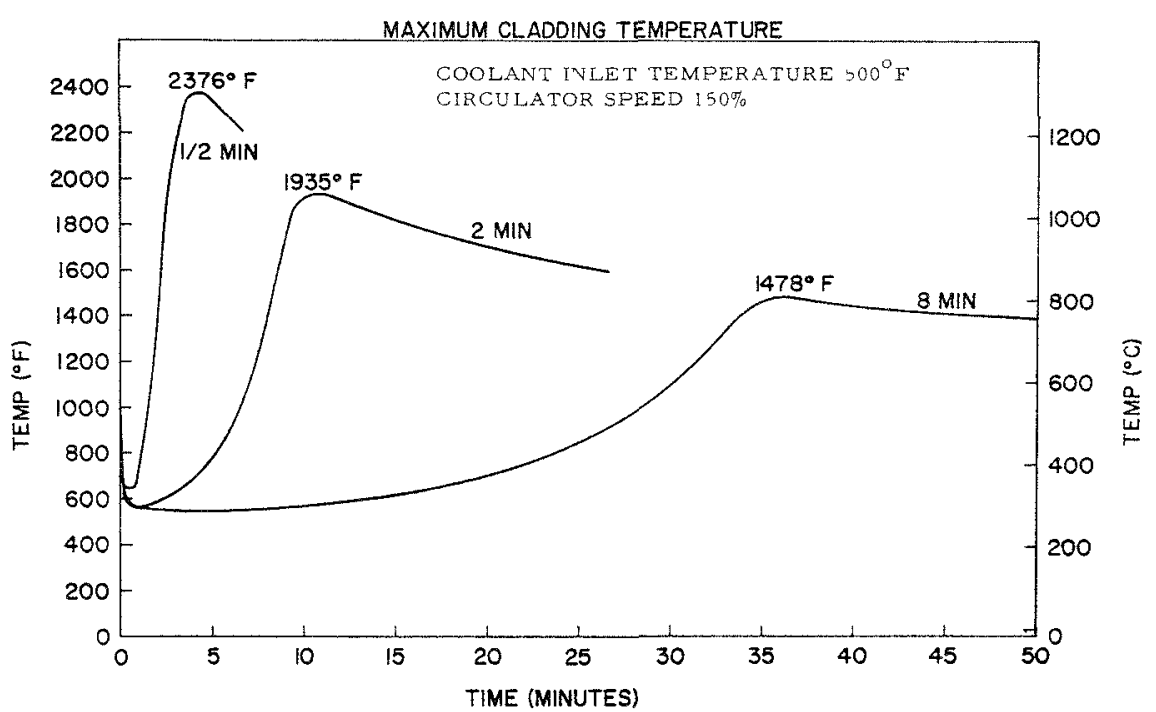

Fia. 11. Maximum Cladding Temperature Histories for I arious Pressure decay Constants.

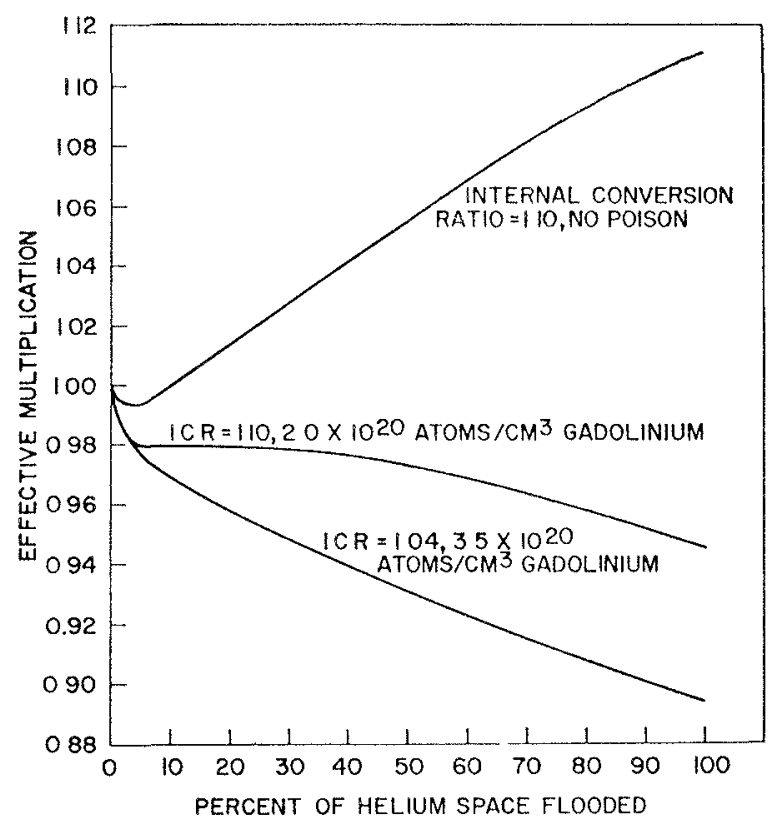

Fit. 12. Fiffective Multiplication as a Function of Percent of Helium space Flooded.

We are considering incorporating this quantity of gadolinium, in oxide form, in the fuel itself (which was the assumption for the calculations of Fig. 12), since it appears that the loss of conversion ratio is small (approximately 0.05), and the measure enables the use of unpoisoned water for flooding. The alternative possibility of incorporating poisons in the water is also being considered, either as a substitute for or as a supplement to fuel poisoning (which would decrease or eliminate any loss in conversion ratio at operating condition).
In summary, this analysis was of a generally conservative nature, in that no scram was assumed, all poison was included in the fuel material, relatively low $(13 \%)$ concentrations of $\mathrm{Pu}^{240}$ were assumed for the reference fuel (the poison requirements are sensitive to this value), and a single, albeit effective, element was allowed as poison material. It can be concluded that the reactivity can indeed be caused to decrease for any degree of water flooding in a GCFR with a minimal penalty to the neutron economy at operating conditions.

\subsection{Analysis of Control-rod Withdrawal}

\subsubsection{Analytical Methods Employed}

The analysis of various step and ramp transients was carried out with the use of a modified version of the BLOOST code, consisting of a group of kinetics subroutines which solve the lumped-parameter kinetics equations coupled with a two-dimensional heattransfer calculation for a fuel element and associated coolant channel. Six groups of delayed neutrons are included. The heat-transfer routines provide the local and average values of cladding, fuel, and coolant temperatures which are used to compute feedback to the kinetics subroutines. The reactivity contribution due to local fucl temperature change may be weighted pointwise by an arbitrary weighting function so as to give the properly averaged Doppler reactivity. In the cases considered in this report, the product of the flux and adjoint flux was used as the weighting function.

A detailed temperature map of the element provided at each time step makes possible observation of hot spots, local melting, ete. Provision is made for 
arbitrary reactivity insertion and for scrams triggered by preset power levels after a specified delay time.

\subsubsection{Rod Withdrawal from Hot Operating Conditions}

3.3.2.1. Description of the Accident. The reactor is considered to be at hot operating conditions when the maximum fuel temperature in the hottest rod is approximately $2200^{\circ} \mathrm{C}$ and maximum elad temperature in the hottest rod is approximately $680^{\circ} \mathrm{C}$. One dollar of excess reactivity is available and is held down by three control rods, with each rod holding down approximately $0.0014 \delta k$ (less than one dollar of reactivity is required for burnup). It is presumed that one rod is withdrawn at the rate of $10 \mathrm{in.} / \mathrm{min}$. The scram is assumed to occur at $120 \%$ of full power.

3.3.2.2. Results of the Accident. Figures 13 and 14 show the changes of tempcrature and reactivity as a function of time during the course of the accident. The reactivity insertion is almost linear. As may be observed from Fig. 13, the feedback reactivity from Doppler and core-expansion effects follows the accident reactivity closely, and the two effects are in a quasi-equilibrium state after about 10 sec.

In Fig. 14, the temperature is plotted as a function of time. There is a temperature rise at the maximum temperature point in the hottest element of $320^{\circ} \mathrm{C}$. The peak temperature of $2520^{\circ} \mathrm{C}$ is below the melting point of the oxide fuel. The average fuel temperature in the hottest element also shows a small rise in temperature, in this ease only $160^{\circ} \mathrm{C}$. The cladding temperature increases to a maximum of $760^{\circ} \mathrm{C}$ on the inner surface of the can, which is well within the temperature limitation of Hastelloy-X cladding material.

It should be noted that the time needed to reach $120 \%$ of full power, at which point the scram is initiated, is almost 65 see, with the maximum period being $130 \mathrm{sec}$. It is unlikely that an excursion of this nature would be permitted to proceed this far under normal operating conditions.

In summary, control-rod withdrawal from the hot operating condition causes no damage, even if the accident is allowed to proceed until power has reached $120 \%$ of the normal value. The resulting temperature rises are more sensitive to the fractional overpower setting for the scram signal than to the rate of rod withdrawal, owing to the relatively long periods involved which allow the temperature to come into equilibrium with the power level.

\subsubsection{Rod Withdrawal from Cold Critical Condition}

3.3.3.1. Description of the Accident. In this case, the reactor was assumed to be operating at $1 \mathrm{kWt}$ and at a uniform temperature of $300^{\circ} \mathrm{C}$. Excess re-

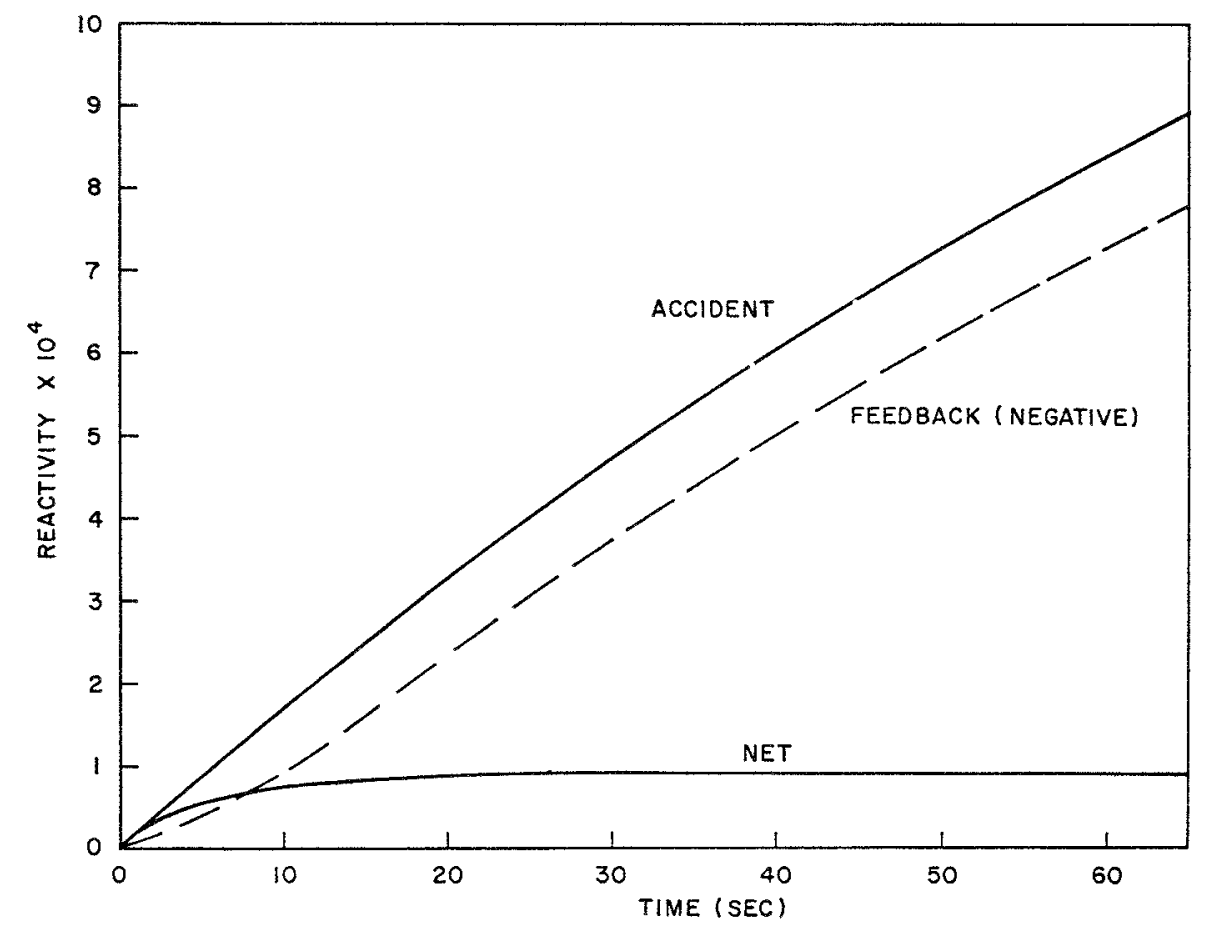

FIG. 13. Feedback and Net Reactivity as a Function of Time Up to the Beginning of Scram for Rod Withdrawal from Hot Operating Conditions. 


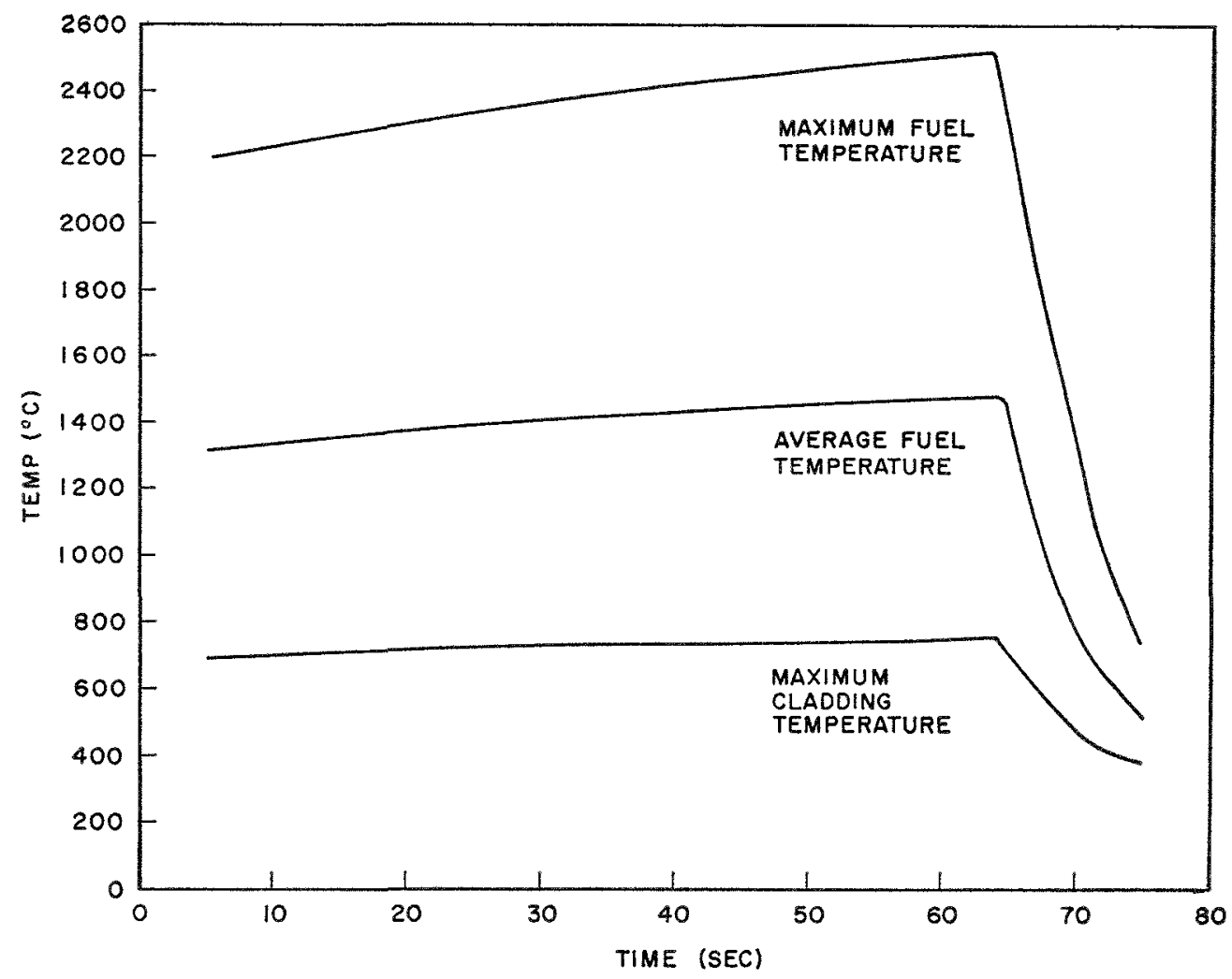

FIG. 14. Maximum Fuel Temperature, Average Fuel Temperature, and Maximum Cladding Temperature as a Function of Time for Rod Withdrawal from Hot Operating Conditions.

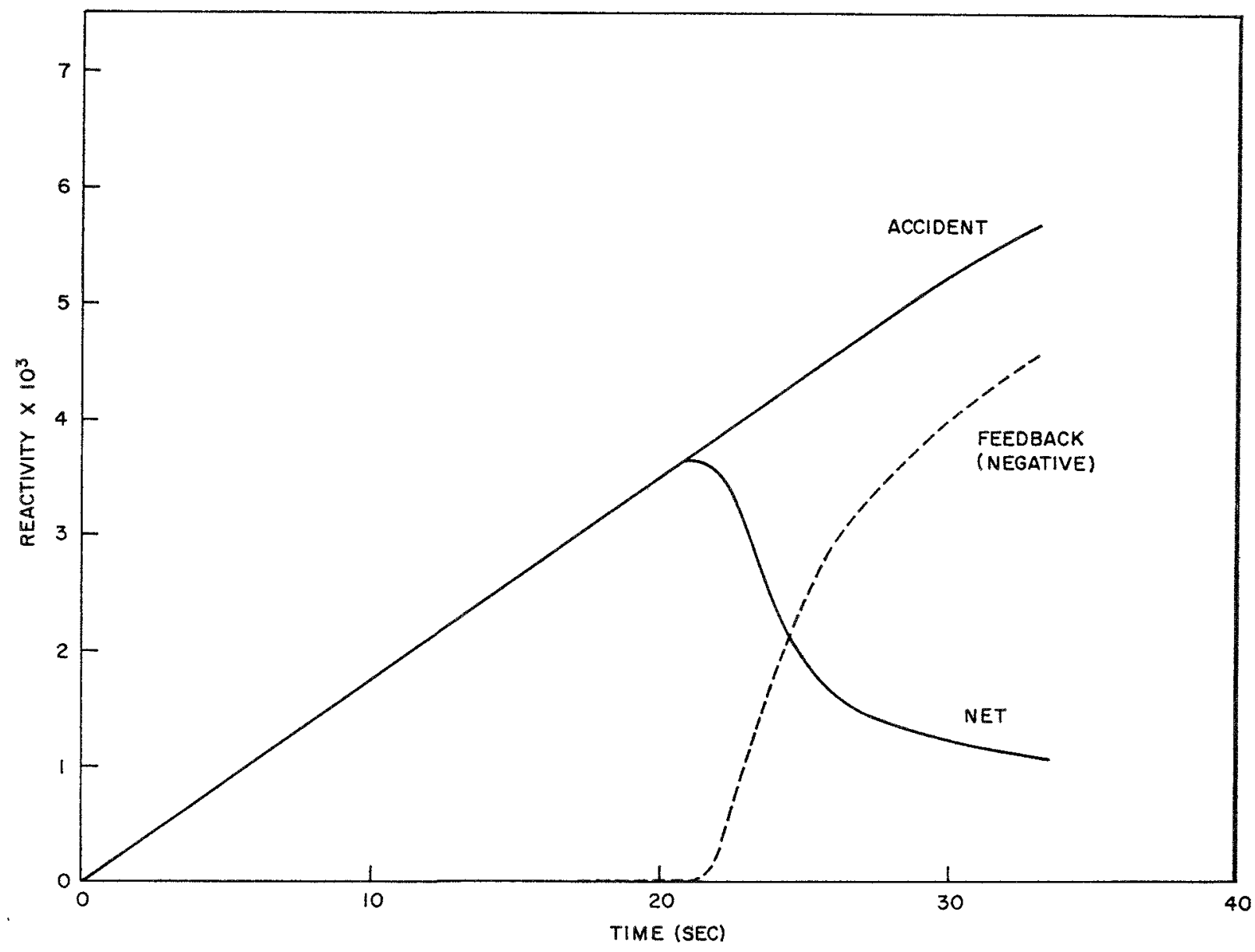

FIG. 15. Accident, Feedback, and Net Reactivity as a Function of Time for Cold Startup Accident. 
activity in the core included one dollar's burnup reactivity plus approximately two dollar's burnup reactivity in the cold to hot swing. This reactivity was being held down by 11 control rods, each inserted to the same position as were the 3 control rods in the rod-withdrawal accident. To initiate the accident, the extreme assumption is made that all 11 rods are simultaneously withdrawn at a rate of 10 in./min. Various scram possibilities are considered.

3.3.3.2. Results of the Study. Figures 15 through 17 describe the course of the accident. In Figure 15, the reactivity due to rod withdrawal as well as the feedback and net reactivities are shown as a function of time. As may be observed, there is no effective temperature feedback until after about 21 sec have elapsed. At this point, the rods have inserted almost one dollar and the feedback reactivity increases rapidly, then leveling off to become parallel to the accident curve. The net reactivity consequently decreases as the feedback increasess and levels off to a constant value of approximately 0.001. This is con- siderably higher than that of Fig. 13, since the insertion rate assumed for the cold startup case is eleven times the rate for the single rod withdrawal accident.

The power profile is shown in Fig. 16. The initial power overshoot is small and is soon compensated for by the temperature feedbacks. The power then increases at a rate of approximately $15 \mathrm{MWt} / \mathrm{sec}$.

Figure 17 shows the peak temperatures in the fuel and cladding as functions of time in this accident. It is not until about 21 sec have elapsed that any change is noted. After this time, the temperatures increase rapidly. The peak temperatures achieved in the course of this accident depend very strongly on the time at which scram is initiated. If the scram is tripped during the period passing through a preset value as low as even one second, or by $50 \%$ power, the accident will be terminated prior to any sensible temperature change in the core.

A scram at $70 \%$ power would limit the fuel temperature to $1260^{\circ} \mathrm{C}$ and the cladding temperature to $500^{\circ} \mathrm{C}$. Even if scram occurred only after $120 \%$ of

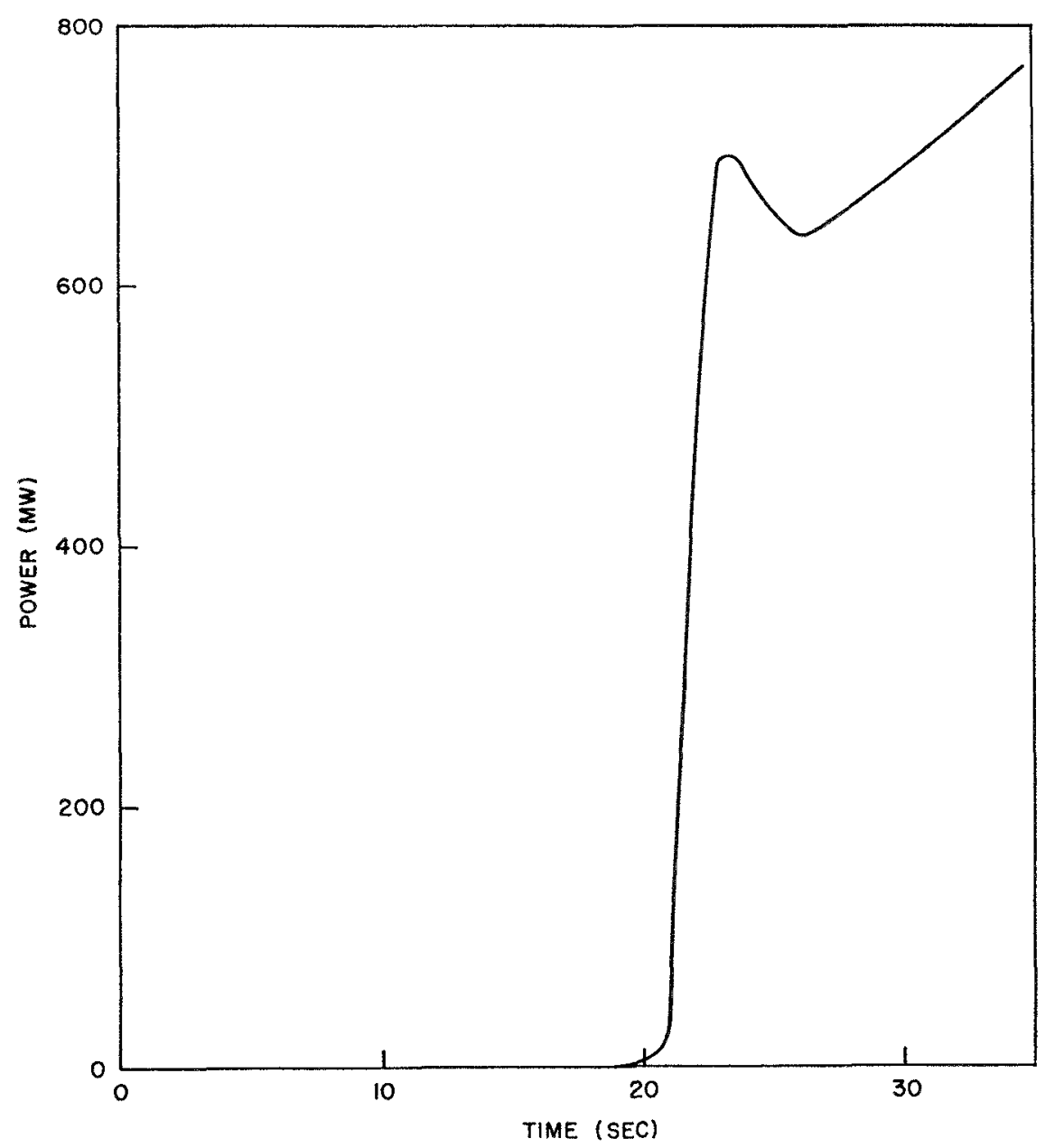

FIG. 16. Reactor Power as a Function of Time for Cold Startup Accident. 


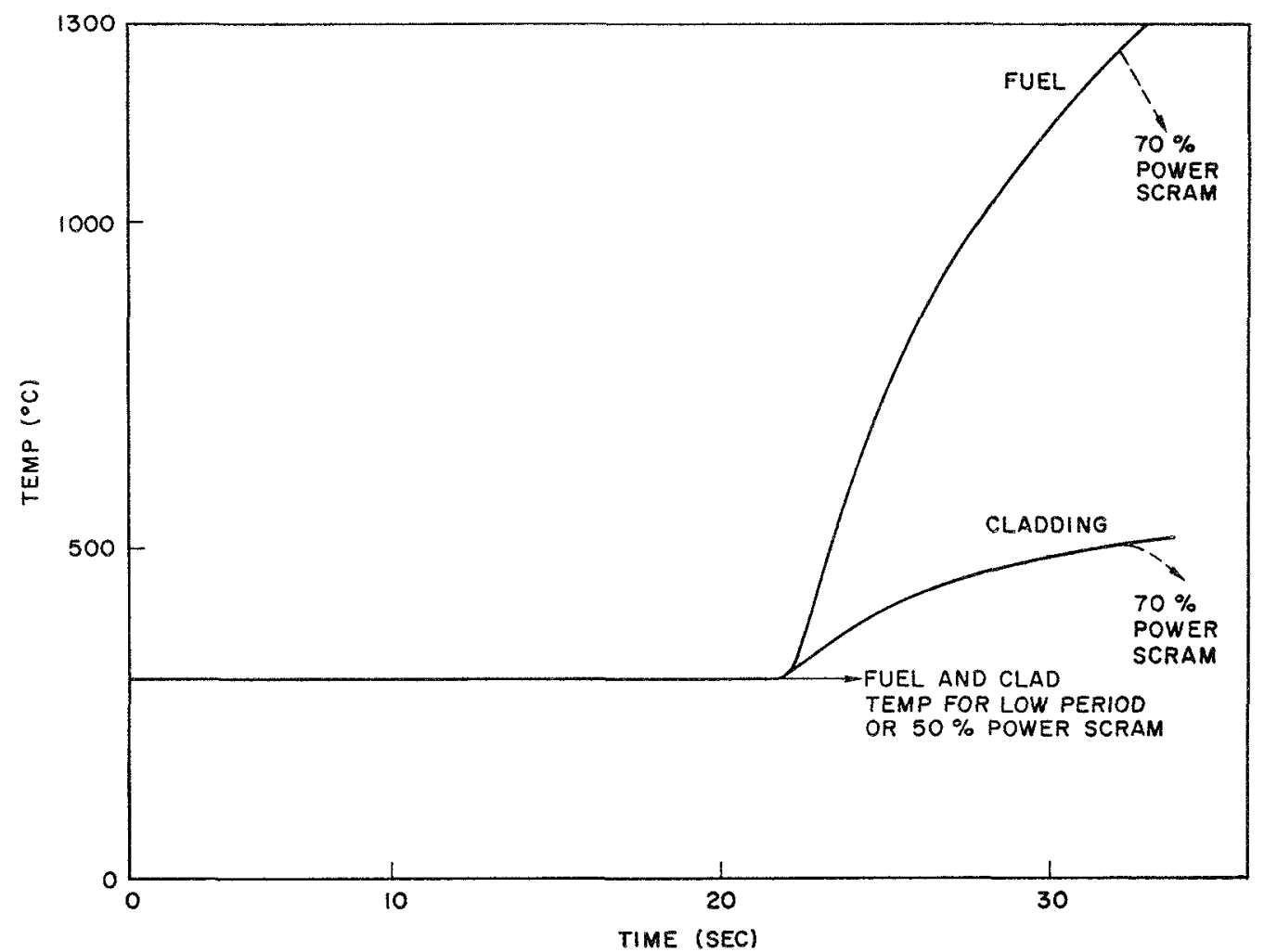

FIg. 17. Maximum Fuel and Cladding Temperature as a Function of Time for Cold Startup Accident.

full power, temperatures would remain below fuel melting points (approximately the same temperatures as those of Fig. 14).

In summary, the cold-startup accident considered here does not appear to involve consequences as sovere as those yielded by rod withdrawal from hot operating conditions. The two accidents ultimately become similar and are not damaging to the core or any of its members.

\subsection{Fuel-assembly Insertion at Cold Critical}

The worth of the central fuel assembly has been determined to be approximately $0.4 \% \delta k$ when added to an otherwise fully loaded core. Assuming that the fuel-loading machine is restricted to an insertion rate of $10 \mathrm{in.} / \mathrm{min}$, then the reactivity insertion rate due to the insertion of even the central fuel element is less than that considered in the cold-startup accident. Fuel and clad temperatures for this case are clearly less than those obtained in the previous analysis of the cold-startup accident.

\section{STABILITY ANALYSIS}

An important part of the safety considerations for the GCFR has been the study of the inherent stability of the system as a function of such important parameters as power level and temperature-feedback mechanisms. By inherent stability we mean stability without external control. By stability we mean, loosely, that the reactor is insensitive to small perturbations in its power level, temperatures, or the physical constants of the system. Thus after any perturbation it will remain close to or return to an equilibrium state.

\subsection{Description of the Calculation Model}

The usual space-independent reactor kinetics equations in the form

$$
\begin{aligned}
& \frac{d n}{d t}=\frac{\rho\left(n, T_{j, k} t\right)-\beta}{\Lambda} n(t)+\sum_{i=1}^{6} \lambda_{i} C_{i}(t) \\
& \frac{d C_{i}}{d t}=\frac{\beta_{i}}{\Lambda} n(t)-\lambda_{i} C_{i}(t) \quad(i=1, \cdots 6)
\end{aligned}
$$

are assumed to be sufficient.

An average fuel element and its cladding material are considered to be divided into a number of axial and radial regions. The average temperatures of these regions are assumed to be given approximately by the relationship

$$
\begin{aligned}
C_{j, k} \frac{d T_{j, k}}{d t}=f_{j, k} n(t) & +h_{j, k-1}\left[T_{j, k-1}(t)-T_{j, k}(t)\right] \\
& -h_{j, k}\left[T_{j, k}(t)-T_{j, k+1}(t)\right] \\
& +g_{j-1, k}\left[T_{j-1, k}(t)-T_{j, k}(t)\right] \\
& -g_{j, k}\left[T_{j, k}(t)-T_{j, k+1}(t)\right]
\end{aligned}
$$


The outer radial regions correspond to the coolant channel, in which the temperature is assumed to be given by

$$
\begin{aligned}
& C_{j, n}\left[\frac{d T_{j, n}}{d t}+\frac{v}{2 l}\left(T_{j+1}(t)-T_{\jmath-1}(t)\right)\right] \\
&=h_{\jmath, n-1}\left[T_{\jmath, n-1}(t)-T_{\jmath, n}(t)\right] .
\end{aligned}
$$

Appropriate boundary conditions are derived from specification of inlet coolant temperature and zero temperature gradient at the fuel centerline. Equations (1) through (4) are then rewritten in the conventional manner to give the state variables in terms of perturbations from the hot-critical equilibrium point, and the $\rho(t) n(t)$ term in Eq. (1) is linearized.

The reactivity feedback was assumed to be local from fuel, cladding, and coolant temperatures as given by

$$
\rho(l)=\sum_{k} \sum_{j} W_{j, k} \delta T_{j, k}(t),
$$

where the term $W_{j, k}$ includes the reactivity coefficient and the appropriate spatial weighting function.

The resulting set of differential equations are then of the form

$$
\frac{d \bar{x}}{d t}=A \bar{x},
$$

where $A$ is a matrix with constant coefficients and $x$ is the vector

$\bar{x}=\left\{\delta n(t), \delta C_{1}(t), \cdots, \delta C_{6}(t), \delta T_{1,1}(t), \cdots, \delta T_{m, n}(t)\right\}$.

A number of different and more or less restrictive stability criteria could be cited as background for this study. Typical of these is the following criterion from Bellman:(2) "If all solutions of Eq. (6) approach zero as $t \rightarrow \infty$, the same holds for solutions of

$$
\frac{d \bar{x}}{d t}=[A+B(t)] x
$$

provided that

$$
\|B(t)\| \leq c_{1}, \quad \text { for } t \geq t_{0},
$$

where $c_{1}$ is a constant that depends on $A$. " That is, the system is asymptotically stable against the perturbation $B(t)$. For other types of perturbations other criteria may of course be preferable.

A computer routine called GASA was written to evaluate the roots of the matrix $A$ of (6), check for the sign of the real parts, and hence determine the system stability. In addition, the routine evaluates the transfer function relating any of the variables to an external reactivity perturbation or any other state variable.

For this study the GCER fuel element was divided into one to five axial regions and three radial regions (fuel, cladding, and coolant). Axial heat transfer was neglected, since the fuel is in pelletized form.

Three reactivity temperature mechanisms were included in this model: the Doppler effect in the fuel, the effect due to axial cxpansion in the cladding, and the effect of changing density with temperature in the coolant. These temperature coefficients were considered to have the major effect on the dynamic behavior of the system.

The Doppler reactivity effect is inversely proportional to the absolute temperature and thus is nonlinear. However, for small perturbations and considering the other approximations of the model, a linear variation of reactivity feedback with temperature due to the fuel was assumed to be adequate. A flux-adjoint flux weighting of the Doppler feedback contribution from each axial zone was employed.

The important reactivity effect in the cladding was assumed to be axial expansion, which lowers the macroscopic cross sections in the region of expansion by decreasing the density. This core expansion is assumed to take place instantaneously with temperature changes in the cladding and is assumed to be independent of fuel temperature. A uniform weighting of the cladding temperature in each axial region was used to determine the reactivity contribution of that region. The main reactivity feedback mechanism in the coolant is a combination of leakage and moderation changes due to changes in density. It was assumed that the time constant for core bowing effects was long relative to coolant density effects and therefore the negative bowing feedback term was neglected. The coolant axial weighting function was calculated from the GAZE criticality code for hot operating conditions.

\subsection{Results of GCFR Stability Analysis}

The stability regions for the reference design GCFR were mapped as a function of the temperature-feedback coefficients by means of the GASA code. Results were obtained for values of the Doppler coefficient ranging from $10^{-8}$ to $10^{-5} /{ }^{\circ} \mathrm{C}$, for values of the coolant coefficient from $+10^{-7} /{ }^{\circ} \mathrm{C}$ to $+10^{-4} /{ }^{\circ} \mathrm{C}$, and for three values of the cladding coefficient, namely, 0 , $-4.2 \times 10^{-7} /{ }^{\circ} \mathrm{C}$, and $-1.0 \times 10^{-6} /{ }^{\circ} \mathrm{C}$. These values more than adequately cover the range of variation in the coefficients possible for this design.

The stability regions were calculated for assumptions of one, three, and five axial zones. It was found that three are sufficient. The resulting stability map is shown in Fig. 18 for the three-zone case. The region 


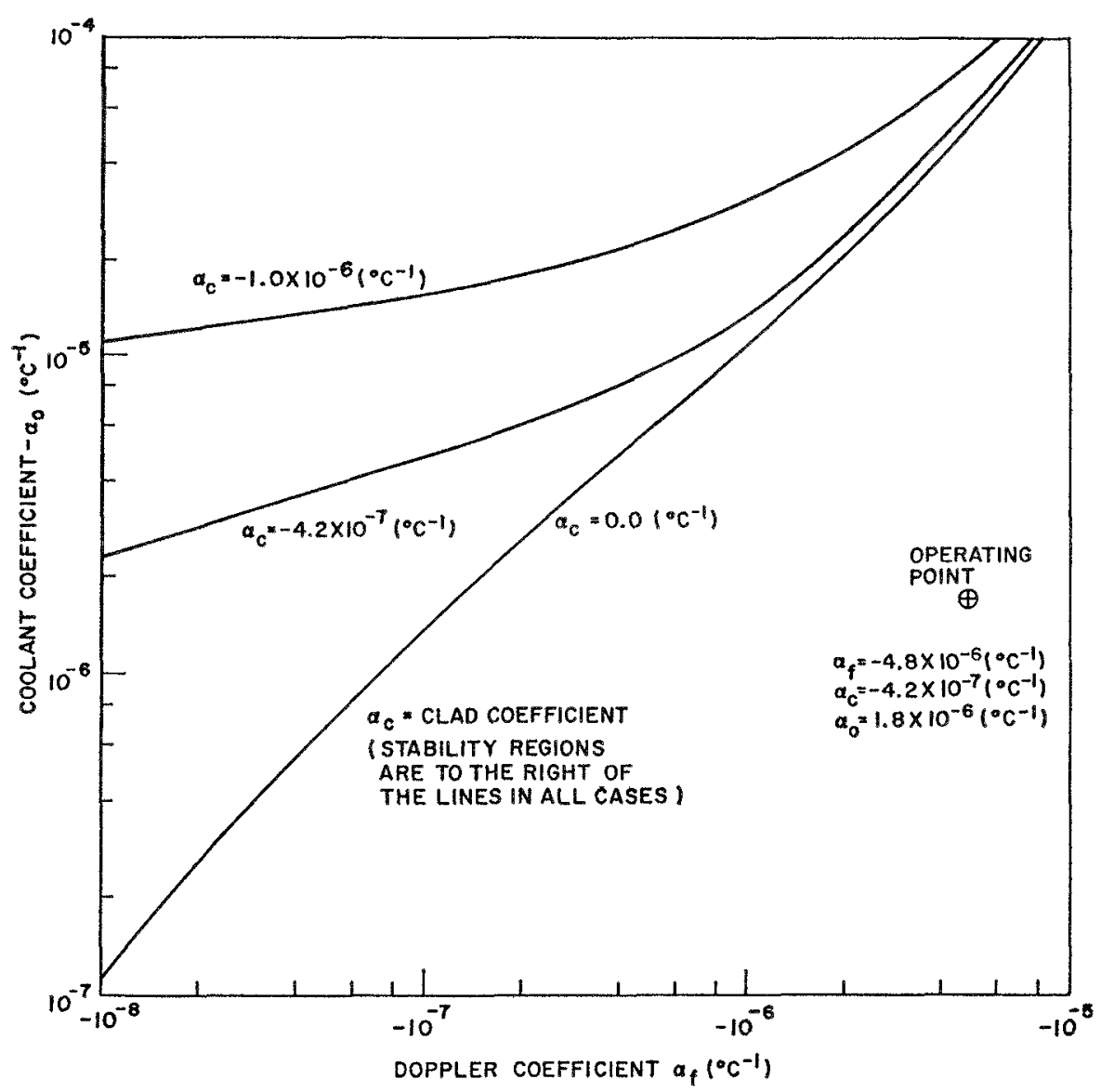

FIG. 18. Stability Map for GCFR.

of stability lies to the right of the curves, and it can be seen that the operating point is well within the stable region.

Since the roots of the matrix A are evaluated numerically to determine the stability of the system, the value of the parameters at which the real part of the largest root changes signs is not determined exactly, but only within certain limits. These limits are determined by the number of points at which the roots are evaluated and the accuracy with which the roots can be evaluated by the numerical procedure. The uncertainty associated with the curves given in Fig. 18 is about $8 \%$ of the value of the fuel and coolant temperature coefficients at each point.

The stability boundary curves for $\alpha_{c}=0.0,-4.2$ $\times 10^{-7}$, and $-1.0 \times 10^{-6}\left({ }^{\circ} \mathrm{C}\right)$ come very close together for values of the Doppler and coolant coefficients greater than $8 \times 10^{-6}\left({ }^{\circ} \mathrm{C}\right)-1$. This indicates that the cladding coefficient has little effect on the size of the stability region when these coefficients are large.

\subsection{Conclusions}

The results of this stability study indicate that the GCFR system is stable for the design operating parameters. Moreover, the results show that the coolant coefficient would have to be made more positive by at least an order of magnitude and the Doppler coefficient reduced by an order of magnitude for the system to become unstable.

\section{MAXIMUM ENERGY RELEASE STUDIES}

Certain characteristics of fast reactors, such as the sensitivity of reactivity to reductions in the dimensions of the core, have focused attention on the possibility of severe accidents which would lead to an appreciable amount of energy being released with termination after vaporization of all or part of the fuel in the core. All fast reactors which have been built in the United States, or under consideration, have been subjected to such accident analysis, and a survey of the work in this area is given in Ref. (3). Analysis of severe accidents in the Fermi Fast Breeder 
Reactor and the EBR-II were made for highly enriched metal-alloy fuel which gives a relatively hard spectrum and almost no Doppler coefficient. The SEFOR analysis relates to uranium oxide fuel in a relatively low-power reactor. All previous studies have been made for reactors with sodium coolant.

The intent of this work is to make use of the present information and calculational methods available to examine the case for the GCFR, with the purpose of understanding the differences in going to large cores cooled with gas, producing relatively high powers, and with strong Doppler coefficients.

\subsection{Causes of Severe Accidents}

The sudden release of damaging quantities of energy requires the insertion of somewhat more than a dollar in reactivity in time periods of the order of a hundredth of a second or less. In past reactor safeguards studies, such accidents have been postulated on the hypothetical events of core collapse, fuel melting followed by core compaction, and in the case of SEFOR, a lengthy chain of events ending with the rapid insertion of reactivity with an experimental transient device. The ensuing power surges in these cases were sufficient to cause pressure buildup, which then expanded the core and ended the excursions.

Before attempting any meaningful analysis, it is therefore necessary to examine any possible circumstances which might cause a prompt excursion in GCFR and estimate the rate of reactivity insertion at prompt criticality involved in this initiating accident.

\subsubsection{Possible Reactivity Accidents Other Than Core Rearrangement}

In the present design, the operating core has less than one dollar $(0.0044 \delta k / k)$ of excess reactivity for burnup. All the control rods are operated from the top of the core, thus eliminating a fallout. The coolant flow is downward, eliminating possible expulsion from the pressure drop. The worth of each rod is also less than one dollar. The central fuel assembly (containing 100 pins) is worth only one dollar and, moreover, cannot fall into the core since the fuel is fed from the bottom.

The friction forces due to the gas flow would cause some deflection of the grid plate. It has been calculated that this would increase the core volume by $0.04 \%$ and, therefore, would be worth only $8 \times 10^{-5} \delta k / k$, or two cents in reactivity.

Complete loss of helium coolant is calculated to increase reactivity by thirty cents. This amount of reactivity is insufficient to cause a severe accident; moreover, it is impossible suddenly to void the GCFR core due to the relatively large surrounding gas volume, the prestressed-concrete containment, and the limitations on the cross-sectional area of individual penetrations to the vessel. Indeed, these characteristics of gas coolant appear to offer intrinsic and significant safety improvements over liquid metals for large systems.

\subsubsection{Core Collapse}

Since the core is suspended from the grid plate, a major dislocation of the plate, possibly due to an earthquake or outside explosion, might cause the core to fall toward the bottom of the refueling space. Were the entire core to fall on a flat surface, it would then be compacted. Calculations indicated that large rates of reactivity insertion, of the order of $\$ 100 /$ sec, would be attained from such an accident.

Even though this accident is highly improbable, it is believed that special preventative measures should be taken. Therefore, we have incorporated into the GCFR design a splitting cone in the space below the core.

The central void caused by the splitting cone is sufficient to cause a net negative reactivity insertion and, therefore, to eliminate this accident possibility.

\subsubsection{Core Meltdown}

In previous analyses of metal-fueled reactors, it has been hypothesized that an accident situation of this type might result in central melting of fuel and subsequent refreezing in the lower core regions, followed by a gravity drop of upper fuel from which large reactivity insertions can be obtained. In the case of GCFR, the safety situation would appear to be considerably improved.

The use of prestressed-concrete containment, redundancy of circulators directly driven by steam with the possibility of $50 \%$ overspeed, as well as providing for emergency water flooding have previously been discussed for prevention of loss-of-coolant or loss-of-flow accidents. Nevertheless, should all these measures fail and the central fuel melt, the reactivity can be expected to decrease for the GCFR. The reason is as follows.

Contrary to the metal-fueled case, it could be expected that cladding adjacent to molten $\mathrm{UO}_{2}$ fuel would promptly melt, thus leaving a very weak support structure for the fuel pins. Since there is no lower support plate in the GCFR fuel assemblies, the bottom half of the fuel elements would fall out of the core and shut down the accident situation. It is important that the initial downward motion of the 
melted fuel is in the direction of decreasing reactivity worth (since the melt would occur at or below the center line).

In summary, at this stage of our investigations, we have not isolated any accidental situation which would lead to the order of reactivity insertions of $\$ 100 /$ sec in the current GCFR design. The study has, however, led to the specification of a splitting cone below the core to eliminate the possibility of sudden compaction of a critical mass of falling fuel.

Despite the lack of a justifying accident situation, we have proceeded to analyze the energy release which would result from arbitrarily large reactivity insertion rates.

\subsection{Methods of Analysis of a Severe Accident}

The various analytic methods developed to study this type of accident are offsprings of the original analysis of Bethe and Tait. (4) A comprehensive survey of these methods was made by Nicholson ${ }^{(5)}$ and by W. J. MeCarthy and D. Okrent. ${ }^{(6)}$ The main disadvantage of the analytic solution is that it does not allow for the Doppler correction, which can be very significant in large cores. ${ }^{(5,7)}$ A second drawback of the analytic solution is that during a severe accident, only the central portion of the core behaves as a homogeneous mixture. The outer part of the core does not initially experience any pressure surge and should be considered as heterogeneous.

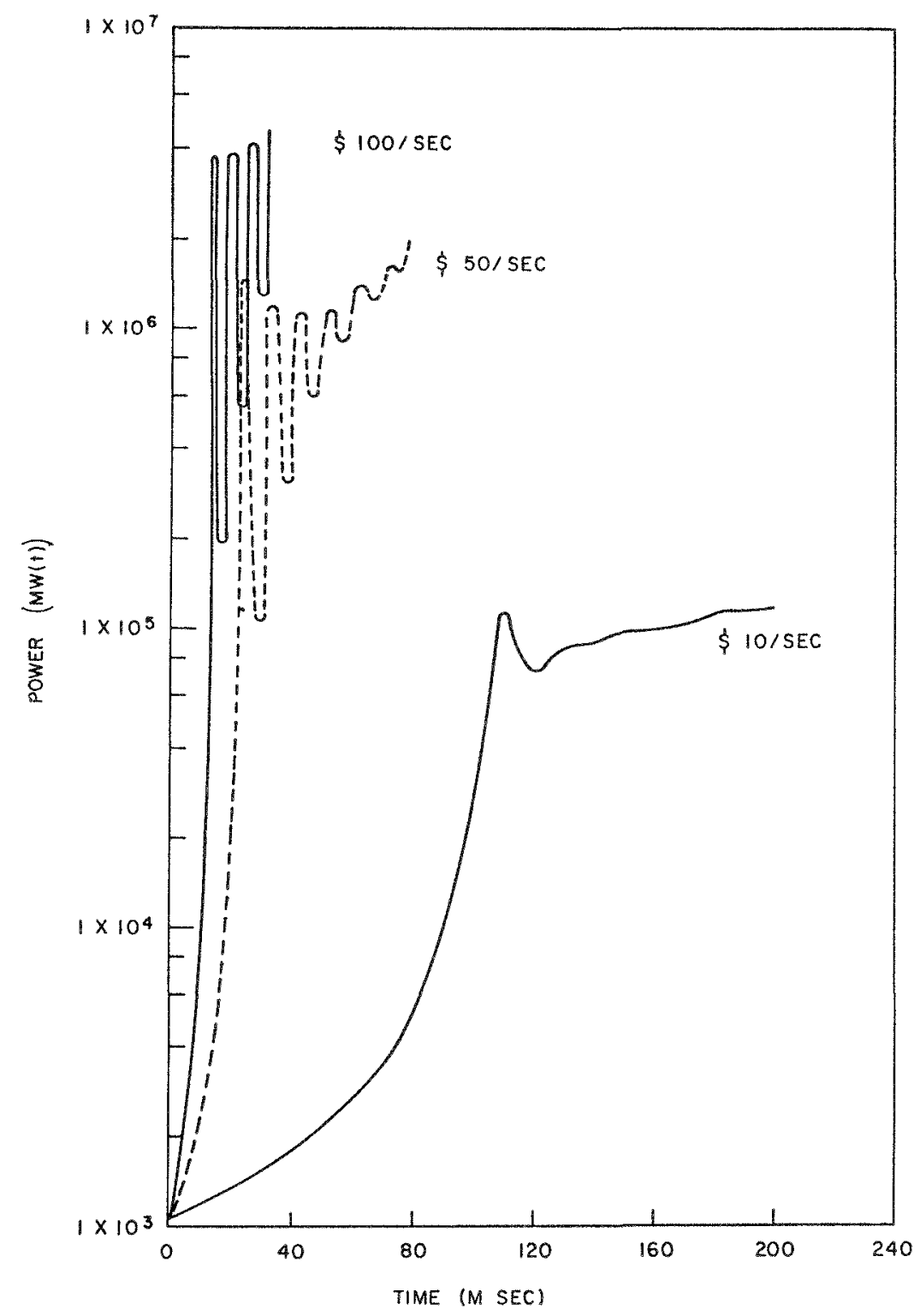

FIG. 19. Power as a Function of Time for Different Reactivity Ramps, Assuming No Disassembly. 


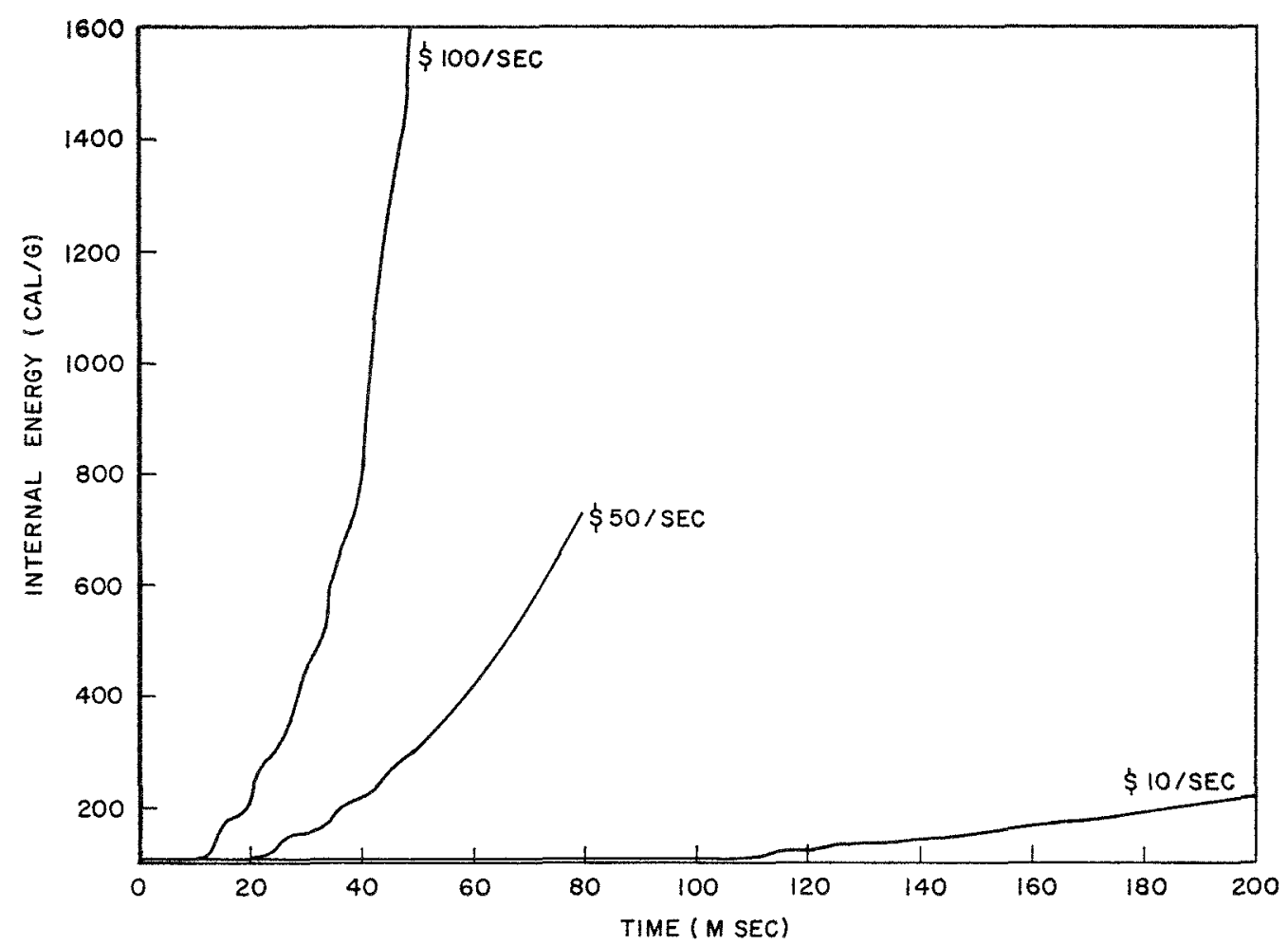

FIG. 20. Average Internal Energy of Fuel as a Function of Time, Assuming No Disassembly.

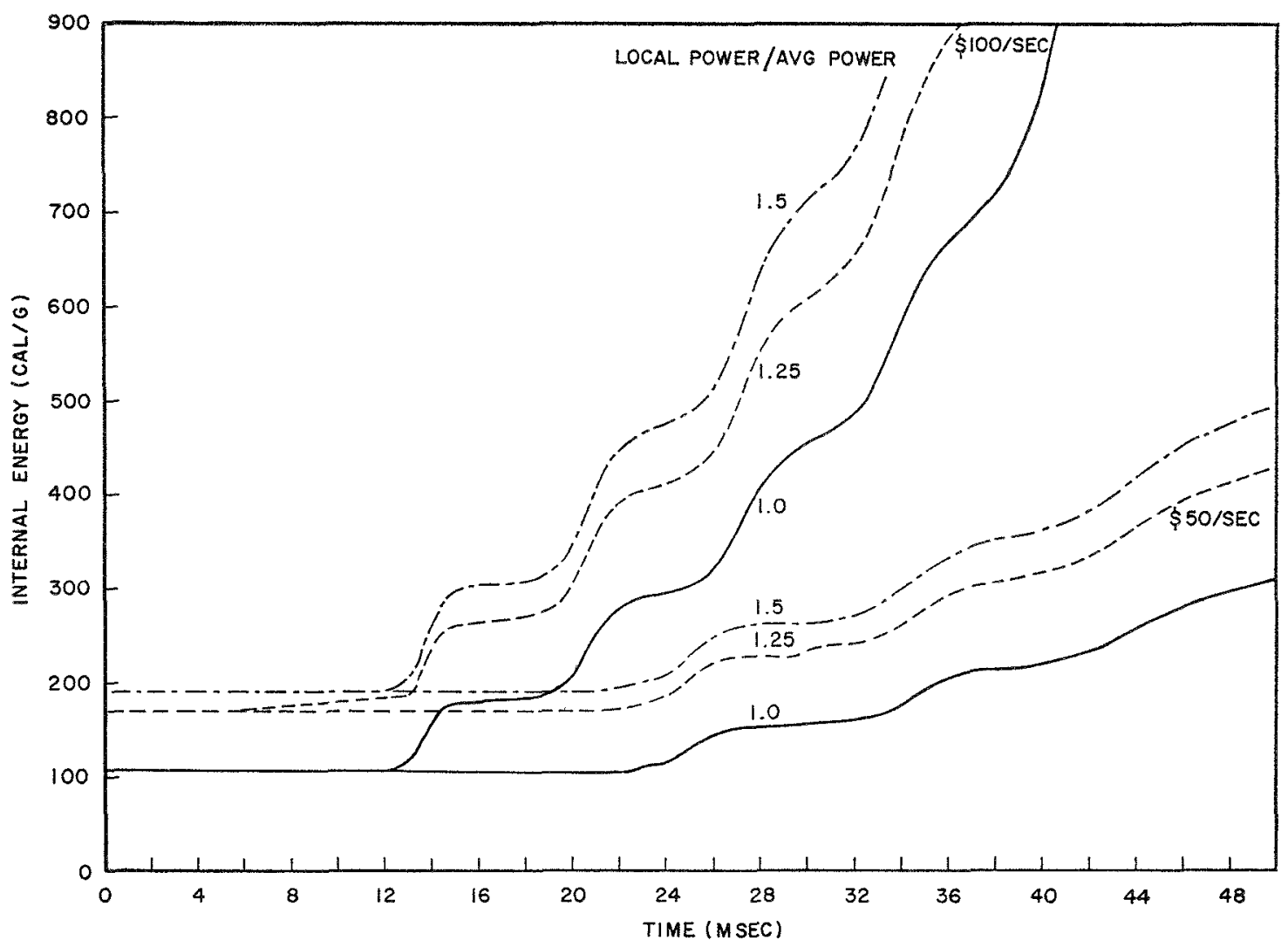

FIG. 21. Internal Energy in Different Fuel Regions as a Function of Time. 


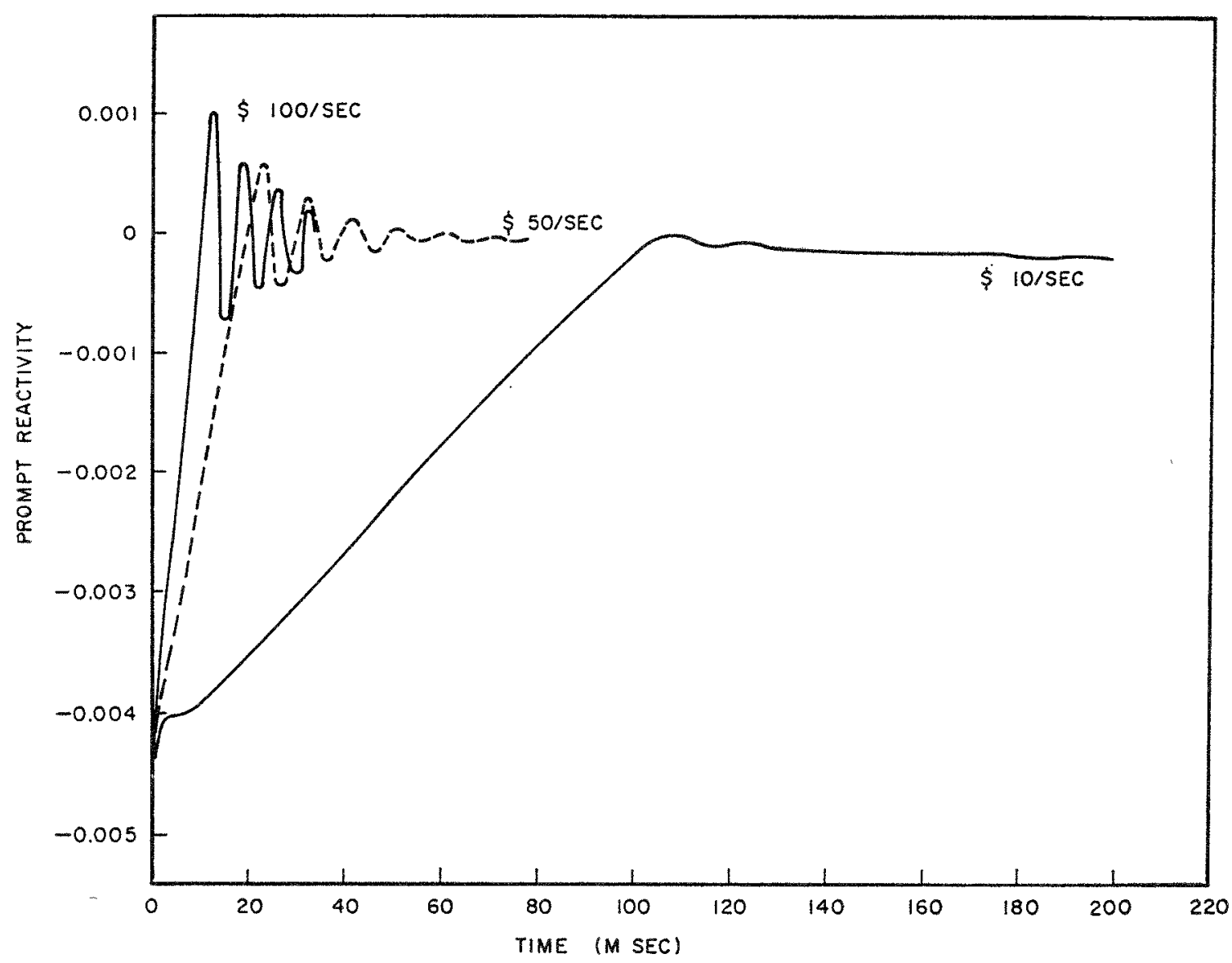

FIG. 22. Prompt Reactivity as a Function of Time.

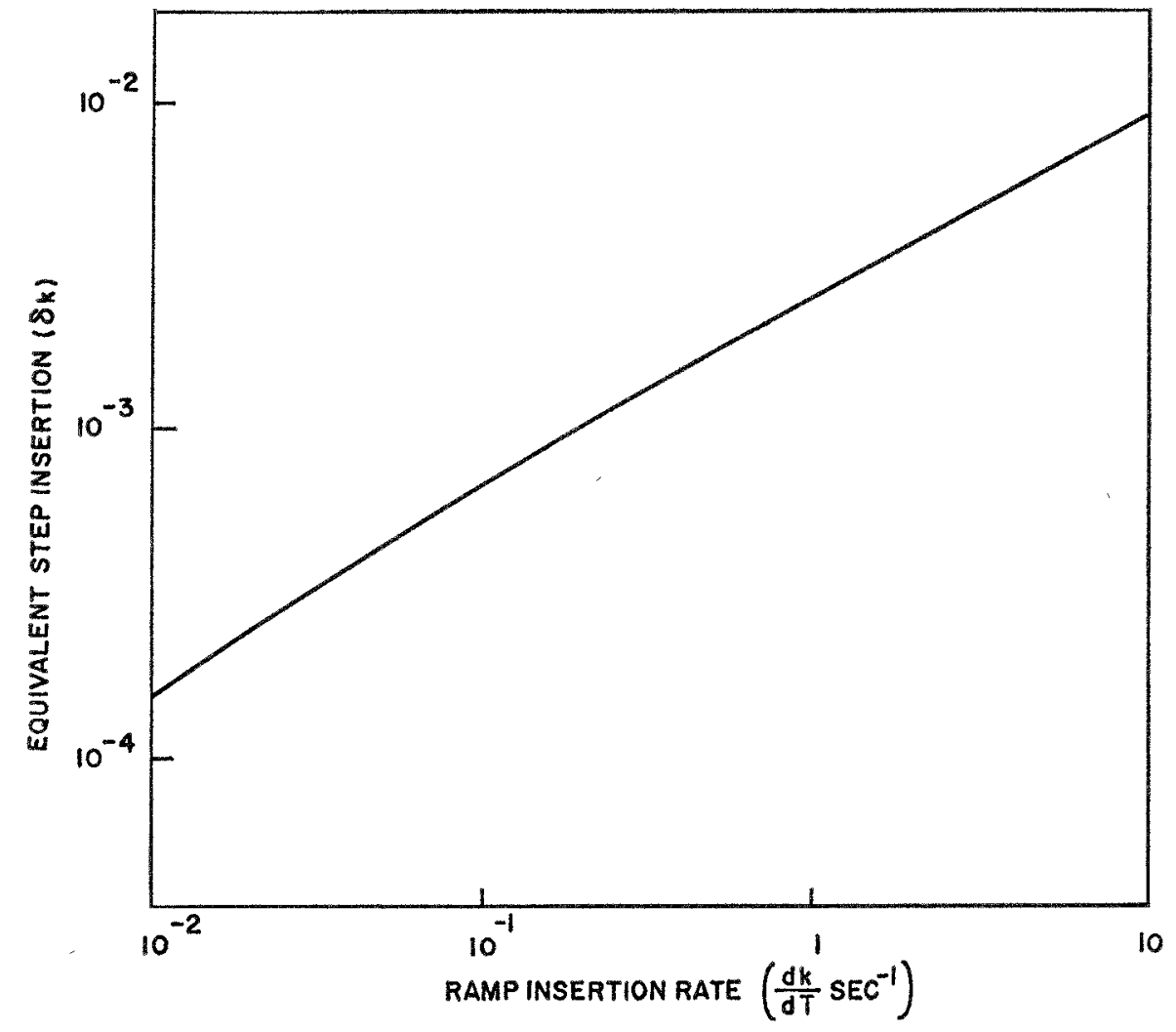

FIG. 23. Equivalent Step Reactivity as a Function of Ramp Insertion Rate. 
In addition, we had available two machine-calculational tools, the AX-IT code ${ }^{(6)}$ and the BLOOST code. ${ }^{(8)}$ AX-IT was modified to include a Doppler contribution to reactivity. Although it could be used either for ramp insertions of reactivity (simulated by imposing inward velocities on the various spherical layers), it was found to be more accurate and to use less computer time to specify step insertions of reactivity. The step insertion, which was equivalent to the desired ramp rate, was obtained from the BLOOST code. BLOOST allows consideration of delayed neutrons, axial and radial temperature distributions in the fuel element, and spatial weighting of the Doppler reactivity contribution. AX-IT considers only prompt neutrons and spherical geometry, but per- forms hydrodynamic calculations in addition to neutronic calculations. Pressure changes are calculated from the equation of state, energy input, and material density; acceleration of spherical shells is calculated from the pressure gradients, and reactivity from onegroup transport theory.

\subsubsection{Results for Ramp Insertions, Assuming No Dissassembly}

Figure 19 shows the power as a function of time for the reference design GCFR, as calculated from BLOOST, for ramp reactivity insertions of $\$ 10 / \mathrm{sec}$, $\$ 50 /$ sec, and $\$ 100 /$ sec. The oscillatory behavior is due to the Doppler feedback (the cladding reactivity coefficient was included but was negligible due to the

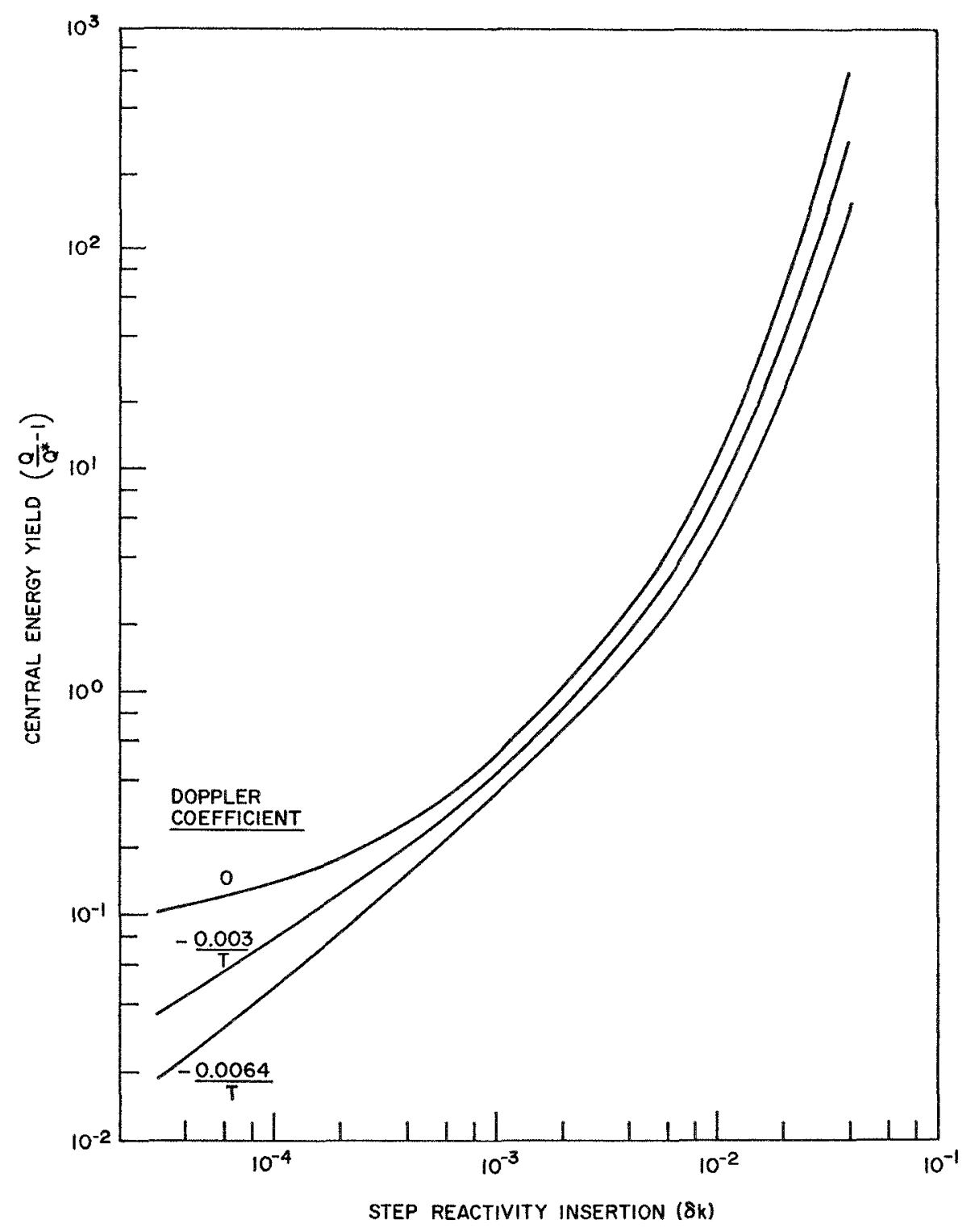

FIG. 24. Relative Energy Yield for Hottest Fuel as a Function of Step Reactivity Insertion. 
prompt and, therefore, adiabatic nature of the energy release). Peaks of later power pulses can be higher or lower than the initial peaks, depending on the balance between the $1 / T$ dependence of the Doppler coefficient, the axial weighting of the coefficient, and the ramp-insertion rate. The oscillations are damped by delayed-neutron effects.

The results are only physically meaningful up to the energy threshold at which significant pressures are obtained, since the outward velocity would then rapidly shut down the reaction.

Figure 20 shows the average internal energy of the fuel as a function of time for the three cases considered, and Fig. 21 shows the distribution of fuel at different internal energy levels for the $\$ 100$ / sec and $\$ 50 /$ sec cases. It was estimated from the equation-ofstate data for $\mathrm{UO}_{2}-\mathrm{PuO}_{2}$ that the threshold energy for pressure generation, $Q^{*}$, is approximately 300 $\mathrm{cal} / \mathrm{g} \cdot{ }^{(9)}$

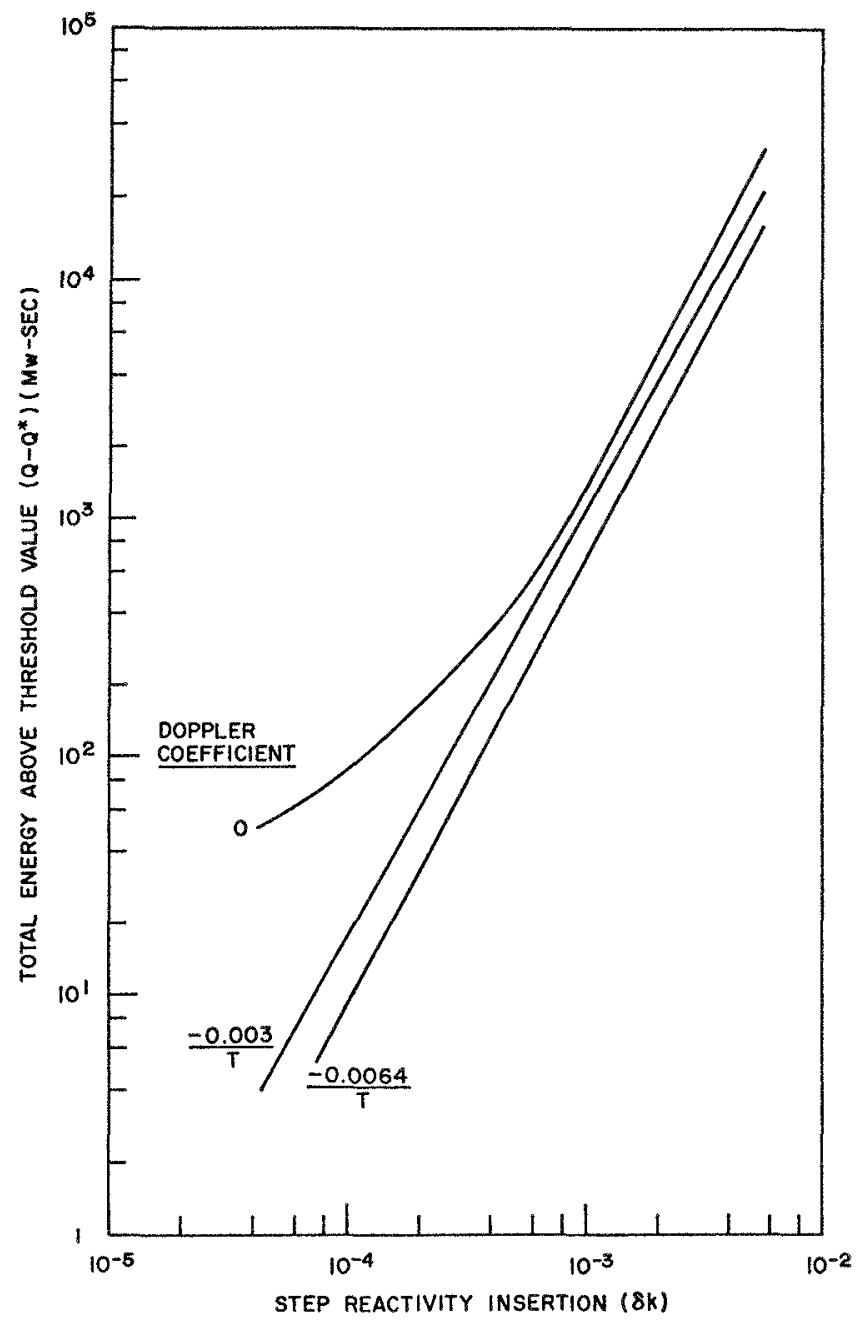

FIG. 25. Total Energy above Threshold Value as a Funetion of Step Reactivity Insertion.
It is seen that this level would be reached on the first pulse of a $\$ 100 /$ sec accident, and the second pulse for a $\$ 50 / \mathrm{sec}$ ramp. As will be shown later from the AX-IT calculations, the cnergy release would be terminated in less than a millisecond after reaching the threshold point.

It can be seen from Fig. 21 that a relatively small fraction of the fuel will be vaporized and that this fraction will, in general, decrease as the maximumto-average power ratio increases. This indicates that the energy release in accidents of this type is less than proportional to reactor power, since the maximum-to-average power ratio can be expected to increase for larger cores.

Figure 22 shows the prompt reactivity as a function of time for the different cases. Results from these cases are further plotted in Fig. 23 and can be used to correlate the AX-IT energy releases with the results for proper insertion rate. The prompt reac-

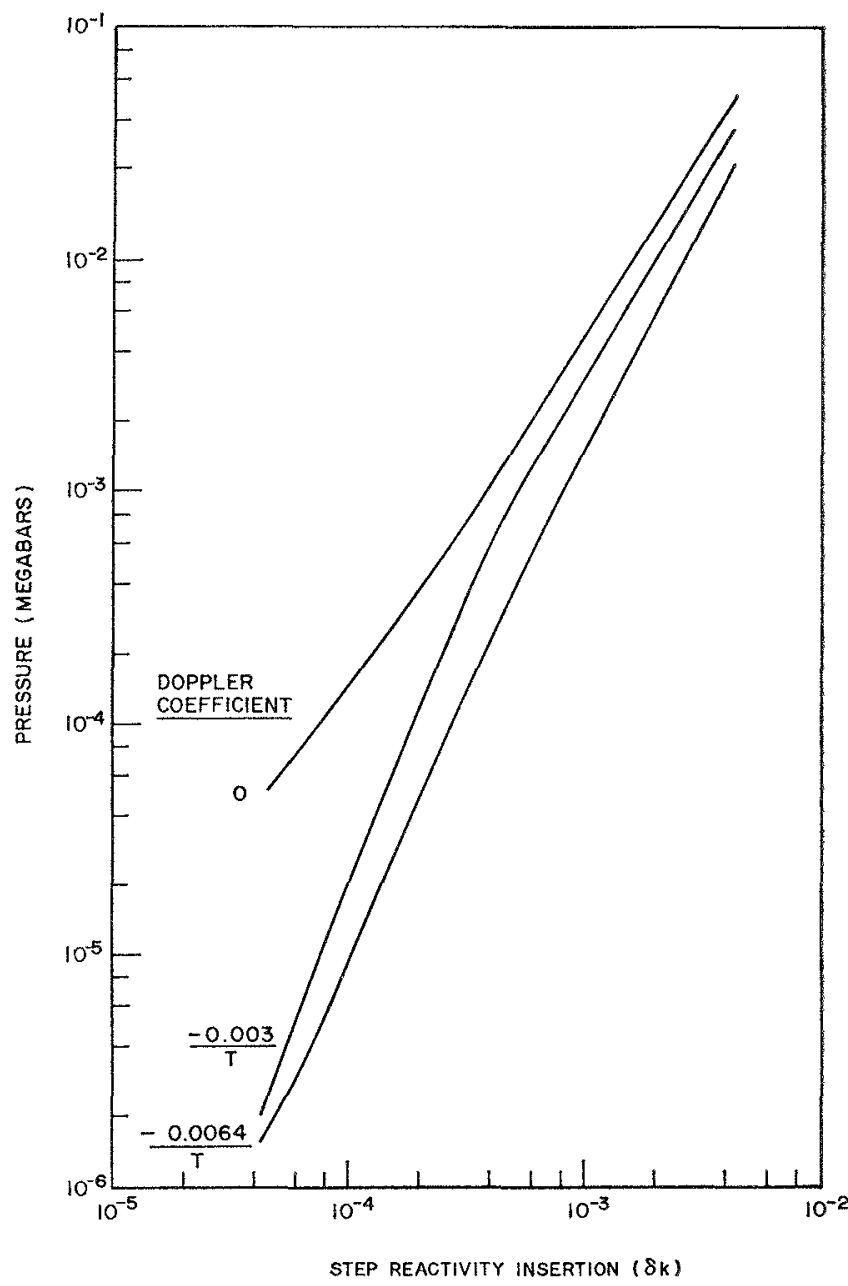

FIG. 26. Maximum Pressure as Function of Step Reactivity Insertion. 


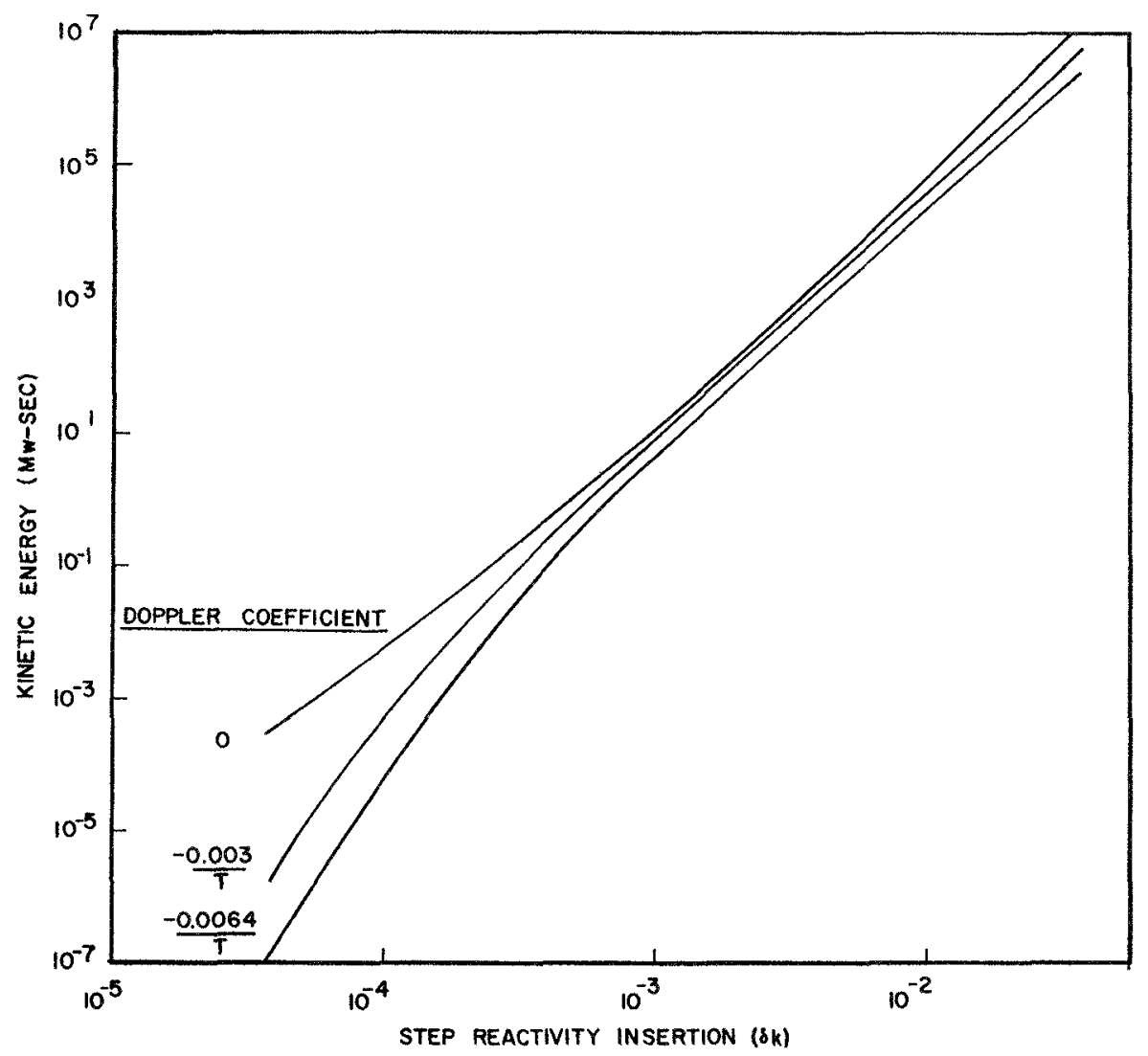

FIG. 27. Kinetic Energy as a Function of Step Reactivity Insertion.

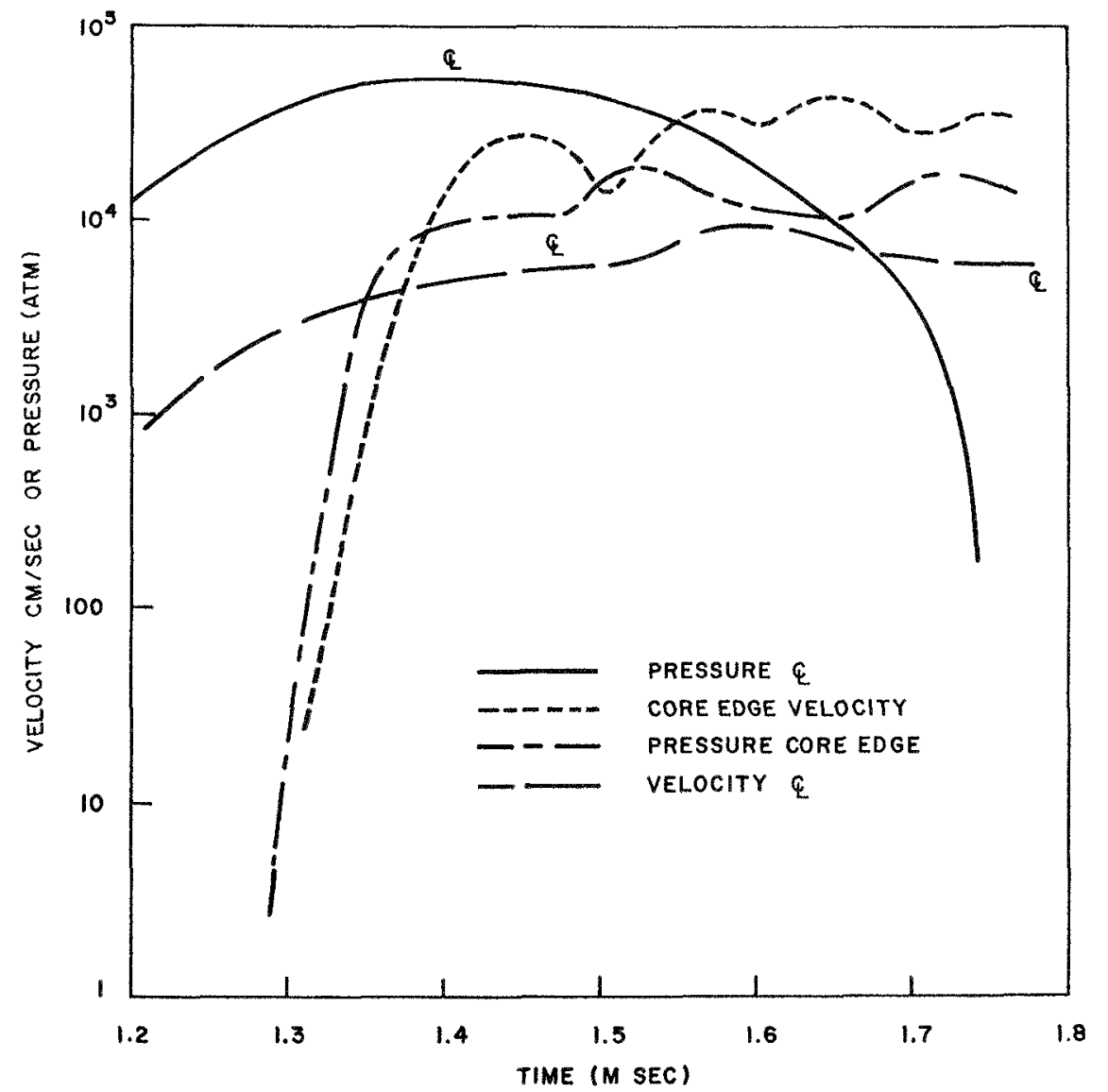

Fig. 28. Local Pressures and Velocities as Functions of Time for $\$ 600 / \operatorname{Sec} \operatorname{Ramp}\left(\delta k_{p}=4.4 \times 10^{-3}\right)$. 
tivities involved are sufficiently close to zero so that delayed neutrons should not be neglected.

\subsubsection{Correlation of Ramp with Equivalent Step Insertion of Reactivity}

In Fig. 23 is presented the relationship between ramp and step reactivity insertions which were used for the AX-IT code. The equivalent step reactivity corresponds to the ramp insertion (from BLOOST code) at the peak power of the first pulse. Since one dollar is $0.0044 \delta k$ for GCFR, Fig. 22 shows that a $\$ 50 /$ sec insertion rate is equivalent to a step insertion of $10^{-3}$ in reactivity and that the step insertion is roughly proportional to the square root of the rampinsertion rate.

\subsubsection{Results of Calculations for Energy Release}

Results from the AX-IT code are plotted in Figs. 24 through 30 . The information includes results for

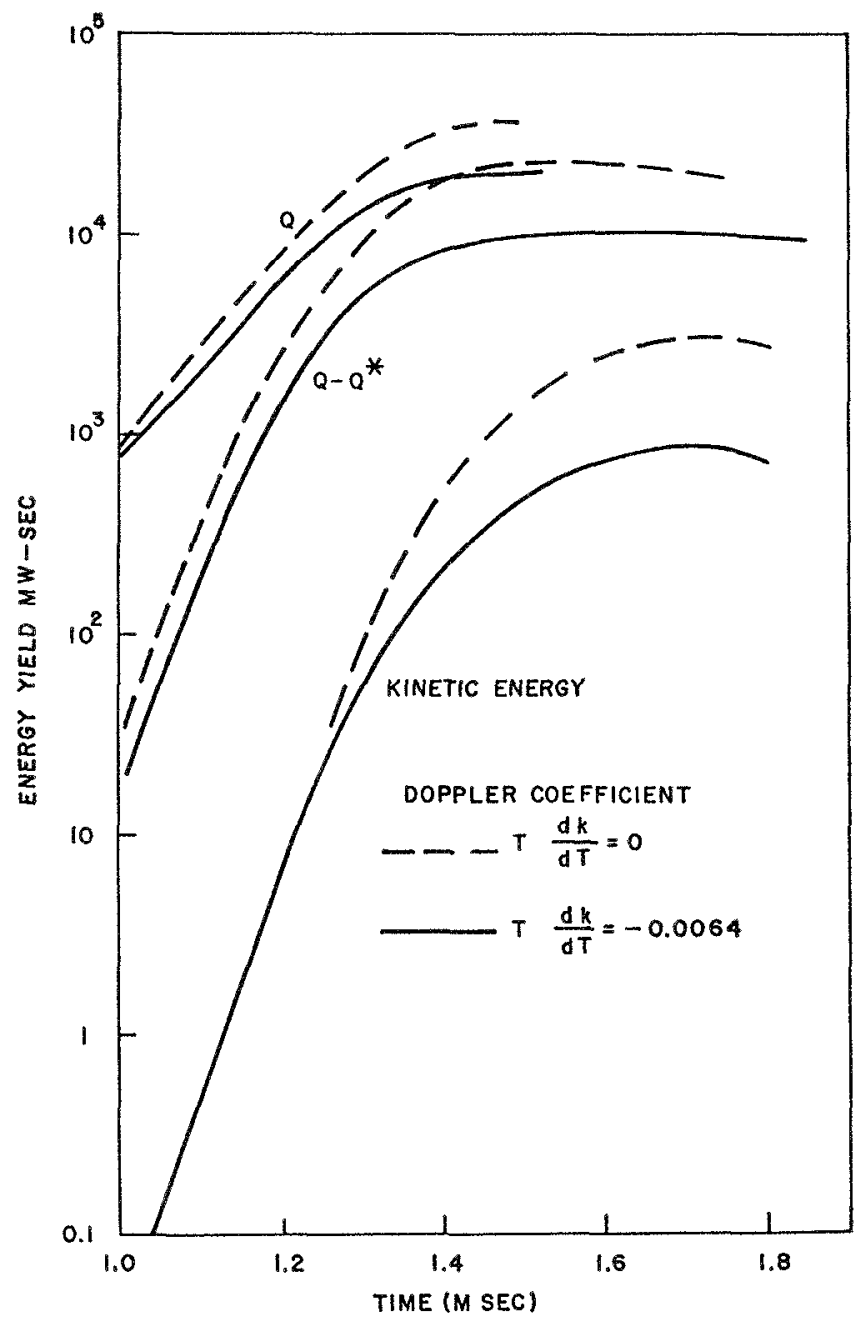

Fra. 29. Energy Yields as a Function of Time for Step Insertion of $4.4 \times 10^{-3}$ in Reactivity. energy release, maximum pressures, and kinetic energies for different step insertions of reactivity.

The work available from such an accident is bounded by the internal energy of the fuel above the threshold value, $Q-Q^{*}$. This function is plotted in Fig. 25. It is seen that a $\$ 50 /$ sec insertion rate would result in a $\left(Q-Q^{*}\right)$ of approximately 700 $M W$-sec. This function $\left(Q-Q^{*}\right)$ is roughly proportional to the square of the step reactivity insertion, or roughly proportional to the ramp insertion rate.

Approximate curves for the maximum pressure attained in the accident are given in Fig. 26. A value of the order of $1000 \mathrm{~atm}$ is observed for a $\$ 50 / \mathrm{sec}$ insertion rate; the maximum local pressure is also approximately proportional to the ramp insertion rate.

The pressures shown in Fig. 26 are local values, and the shock wave should be reduced considerably by the surrounding massive, and relatively porous, unmelted fuel and blanket material. Furthermore, the very large space available between the core and the reactor vessel (the latter sized by the boiler dimensions) allows room for the insertion of large quantities of porous material to extend this effect and further protect the vessel from impulsive pressure leaks. This effect was, however, not taken into account for this study.

The kinetic energy of the core and blanket materials was obtained from the calculation for the treatment of the outer regions as homogeneous mixtures. These values are shown in Fig. 27 and are another measure of the energy available for damage. For a $\$ 50 /$ sec ramp, the kinetic energy is only $10 \mathrm{MW}$-sec, since this function drops off sharply as reactivity insertion is reduced. It is roughly proportional to the square of the ramp insertion rate.

In Fig. 28 are plotted the pressures and velocities of the core center and core edge for very severe accident conditions, corresponding to a reactivity ramp of $\$ 600 /$ sec.

In Figs. 29 and 30 the total cnergy pulse $Q$, the upper bound on available work, $Q-Q^{*}$, and the kinetic energy are plotted as functions of time for step insertions of $4.4 \times 10^{-3}$ and $4.4 \times 10^{-4}$ in reactivity, corresponding to ramp rates of $\$ 600 / \mathrm{sec}$ and $\$ 6 / \mathrm{sec}$. It is seen that the energy pulse is terminated in the order of milliseconds or less in all cases.

\subsection{Conclusions}

The calculations of energy release presented here are of a survey nature intended to outline important design points and to lend understanding to the functional dependence of the variables, rather than to arrive at definite design values. 


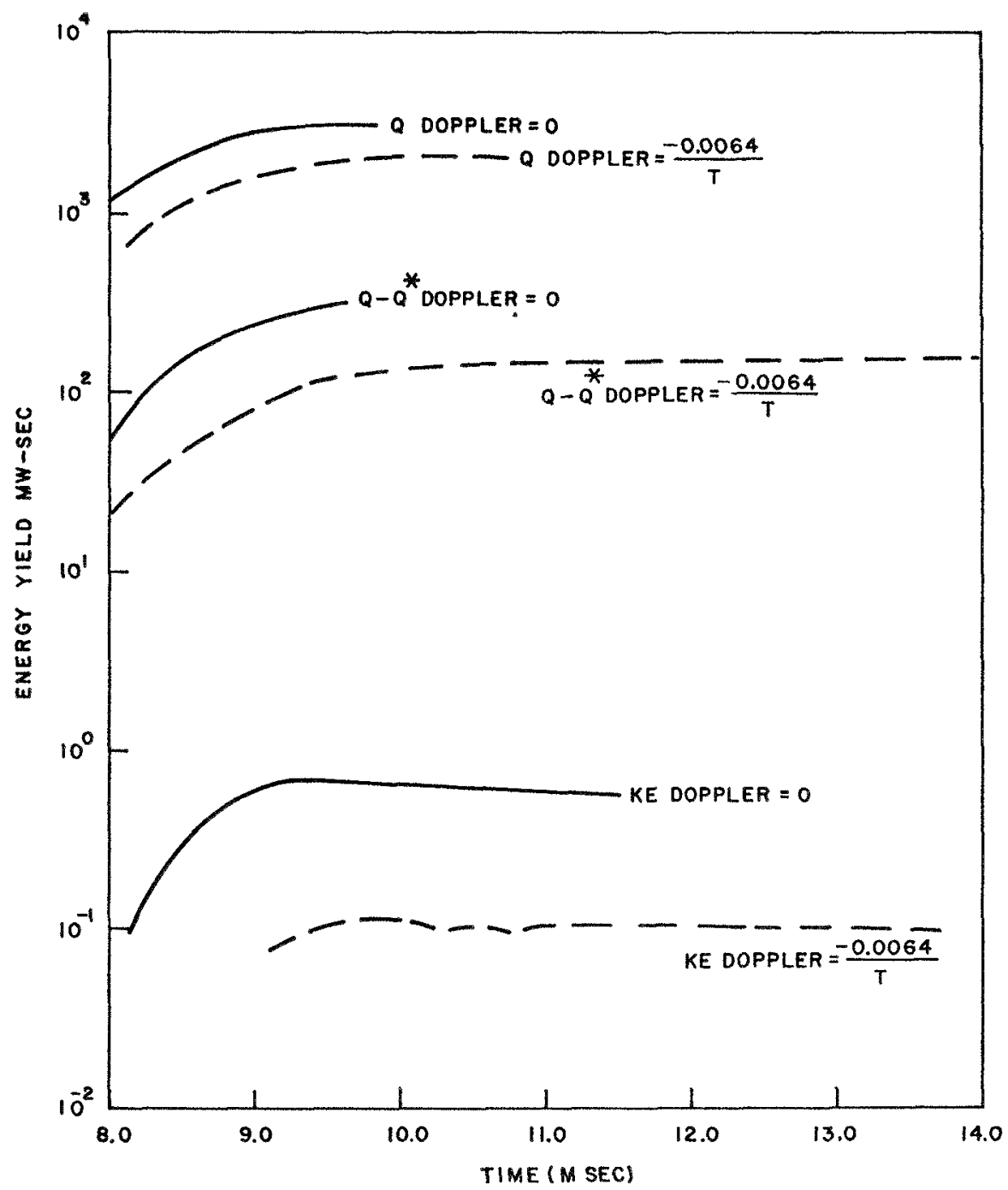

Fig. 30. Energy Yields as a Function of Time for Step Insertion of $4.4 \times 10^{-4}$ in Reactivity.

Several important characteristics have been discovered.

1. The specific mechanical design configuration of a reactor is of great importance in eliminating potential causes of severe accidents, e.g., top support of fuel, withdrawal of control rods upward against the coolant flow direction, provision of splitting cones below the reactor core, and a stiff grid plate.

2. The minimal reactivity worth of gas as compared with liquid metal is a distinct advantage for the GCFR.

3. The Doppler coefficient in large ceramic reactors is of considerable importance, in that many reactivity accidents can be terminated by this mechanism alone, without disassembly or undue core melting.

4. The quantized nature of the energy pulses allows time for disassembly between pulses. The energy release is proportional to the fraction of fuel which is vaporized during a single pulse, so that the maximumto-average specific power for the fuel is an important design variable. On the other hand, it would not be desirable that the power be overly shaped so as to peak near the core boundaries, since an initial accident could then lead to core compaction, rather than disassembly.

5. The unmolten, compressible material surrounding the vaporized region should prove of great effectiveness as a blast shield.

6. The substitution of gas for liquid-metal coolant should also be beneficial in reducing the effective speed of sound in the core structure, i.e., the pressure wave should be much less without tamping by liquid metal.

7. The concrete pressure vessel should prove an effective blast shield due to its large wall inertia.

8. The low fuel enrichment that goes with design 
for high internal conversion ratio is valuable from the safety point of view, in increasing the minimum critical value, i.e., reducing the inherent number of critical measures in the system.
9. No accident situations have yet been discovered for the GCFR which would be of the severity required to result in such energy releases of the orders considered in this section of the study.

\section{APPENDIX A}

\section{Reactivity Coefficients}

\section{A.1. Doppler Coefficient}

For this study, the Doppler coefficient due to the broadening of $U^{238}$ resonances was evaluated by the Nordheim integral method for well-spaced resonances. The effect of some of the approximations in this method were checked with the GAROL code, which includes the effects of resonance overlap and downscatter from all nuclides. Although the more accurate calculation showed that there was considerable variation in the collision density as a function of energy, the overall Doppler coefficient changed by only approximately $5 \%$. This effect, which tends to increase the magnitude of the Doppler coefficient, is somewhat balanced by the opposite effect of $\mathrm{Pu}^{239}$. The coefficient as evaluated shows a $1 / T$ dependence and is given by the equation

$$
\frac{\delta \mathrm{k}}{\delta T}=\frac{-0.006418}{T} .
$$

The hot-to-cold swing from $293^{\circ} \mathrm{K}$ to $1341^{\circ} \mathrm{K}$ is 0.009755 , or $\$ 2.27$.

\section{A.2. Helium Density Coefficient}

The helium density coefficient was evaluated from one-dimensional diffusion-theory codes and from modified zero-dimensional burnup studies. The results

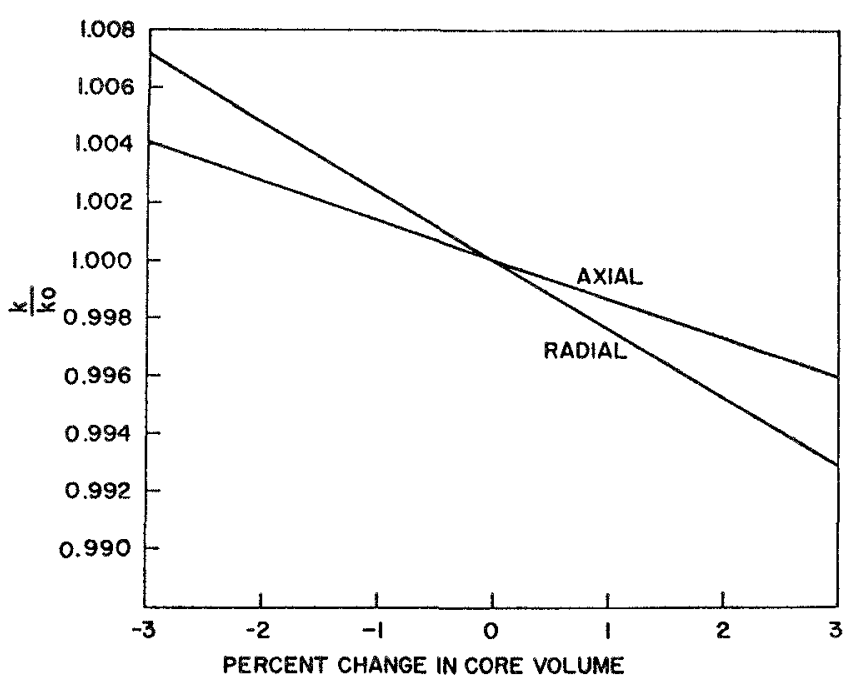

FIG. 31. Criticality Factor as a Function of Change in Core Volume. showed that complete loss of helium at beginning of life would lead to an increase in reactivity of some 30 cents. The burnup studies showed that this amount would be reduced somewhat during the lifetime of the core.

The helium temperature coefficient is evaluated by assuming that the reactivity change is essentially linear with the coolant density. If the perfect-gas laws are utilized, the coefficient can be evaluated as a function of temperature, giving

$$
\frac{\partial k}{\partial T}=\alpha \frac{T_{0}}{T^{2}}
$$

where $\alpha$ is 30 cents or 0.00129 in multiplication.

\section{A.3. Core Expansion}

The change in reactivity as a function of change in core volume is shown in Fig. 31. The evaluation is a result of one-dimensional diffusion-theory calculations. From the slope of the curve, it may be determined that the reactivity changes by $0.0014 \delta /$ per percent change in volume in the axial direction and by 0.0024 $\delta k$ per percent change in volume in the radial direction.

To determine the temperature coefficient of reactivity due to expansion, it remains only to determine the expansion coefficients in the radial and axial directions. In the axial direction, the assumption was made that elongation of the core due to temperature effects was due to the axial expansion of the cladding alone. This leads to the expression

$$
\frac{\delta k}{\delta T_{\mathrm{clad}}}=-2.3 \times 10^{-6} \delta k /{ }^{\circ} \mathrm{C}
$$

and yields a cold-to-hot swing of $-\$ 0.31$.

Evaluation of the temperature coefficient of expansion in the radial direction is somewhat more involved. The assumption is made that the bowing effect is a function only of the difference between the temperature of the inner box wall and that of the outer wall. The temperature gradient across the fuel pins is neglected. The reason for this assumption is as follows. Although the box structure is quite rigid, the pins are long, thin rods which are quite flexible in comparison, and the stress that would be required to keep the pins straight is small compared with the stress required to bend the box.

It is further assumed that the box walls, with their large area exposed to coolant flow, are directly re- 


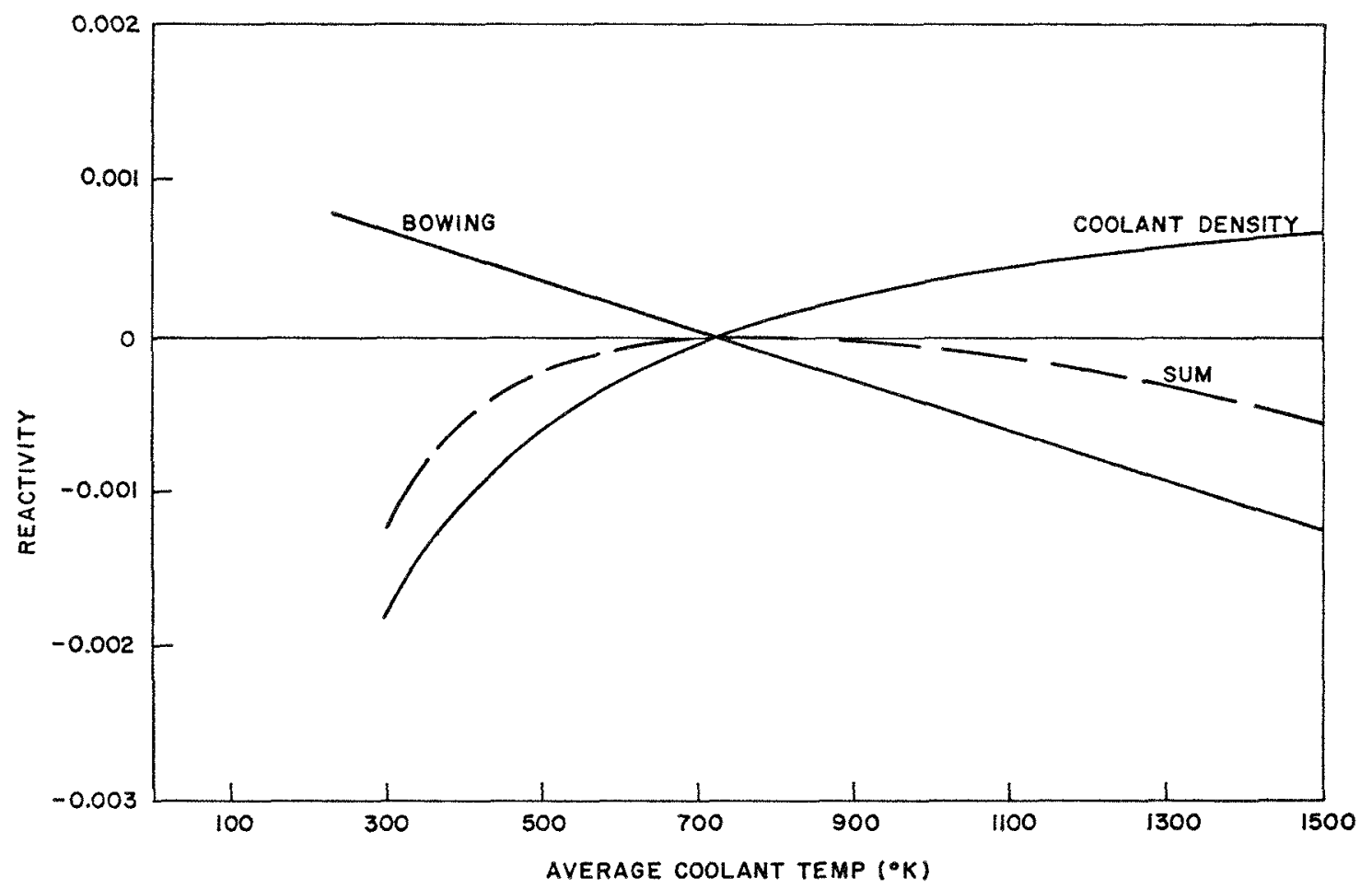

Frg. 32. Reactivity Feedback due to Coolant Density Change and Bowing as a Function of Coolant Temperature.

sponsive to the coolant temperature. The temperature difference between the inner and outer box walls is derived from the assumptions that the coolant does not mix as it travels downward through the boxes and that the radial peaking of the power profile results in a similar peaking in the temperature rise of the coolant as it passes through the box. These lead to the expression

$$
\delta k / \delta T=-1.6 \times 10^{-6} \delta k /{ }^{\circ} \mathrm{C},
$$

where $\delta T$ refers to the change in the average coolant temperature.

In Fig. 32 are plotted the reactivity due to bowing-induced radial expansion and that due to helium density, both as a function of average coolant temperature. Their sum is also plotted. It is of interest to note that the sum indicates that the reactivity contribution from the sum of these two effects is always negative or zero.

It is recognized that in a fast transient situation

TABLE 2. Delayed-NeUtron Groups

\begin{tabular}{c|l|l}
\hline Group No. & Fractional $\beta$ & $\lambda\left(\mathrm{sec}^{-1}\right)$ \\
\hline 1 & 0.0001 & $1.29 \times 10^{-2}$ \\
2 & 0.000867 & $3.11 \times 10^{-2}$ \\
3 & 0.000808 & $1.34 \times 10^{-1}$ \\
4 & 0.001612 & $3.31 \times 10^{-1}$ \\
5 & 0.000761 & 1.26 \\
6 & 0.000121 & 3.21 \\
\hline
\end{tabular}

the negative effect of box bowing would be somewhat delayed compared with the coolant temperature rise, which in turn is delayed relative to the fuel temperature rise. In the analyses of this study, fuel assembly bowing was neglected in Section 4 for stability analysis and Section 5 for fast transients, and was assumed to be in equilibrium with the coolant temperature for the slow transients of Section 3.

\section{A.4 Grid-plate Bowing}

Gricl-plate bowing has been calculated by determining the amount of spreading of the core that would result from the coolant drag forces pulling on the grid plate and deflecting it in a concave manner. This effect has been shown to be worth about 2 cents in negative reactivity at full flow and has been neglected in all calculations.

\section{A.5. Reactor Properties}

The neutron lifetime of $4.37 \times 10^{-7}$ sec was evaluated by averaging $1 / v$ over the reactor spectrum and then using the relationship

$$
l=\left\langle\frac{1}{v}\right\rangle \frac{1}{\left\langle\nu \Sigma_{f}\right\rangle} .
$$

The fraction of neutrons in each delayed group and the decay constant for each of these groups are given in Table 2, where contributions from fissions in all nuclides are included. The average delayedneutron fraction gives a value of 0.0044 for the dollar. 


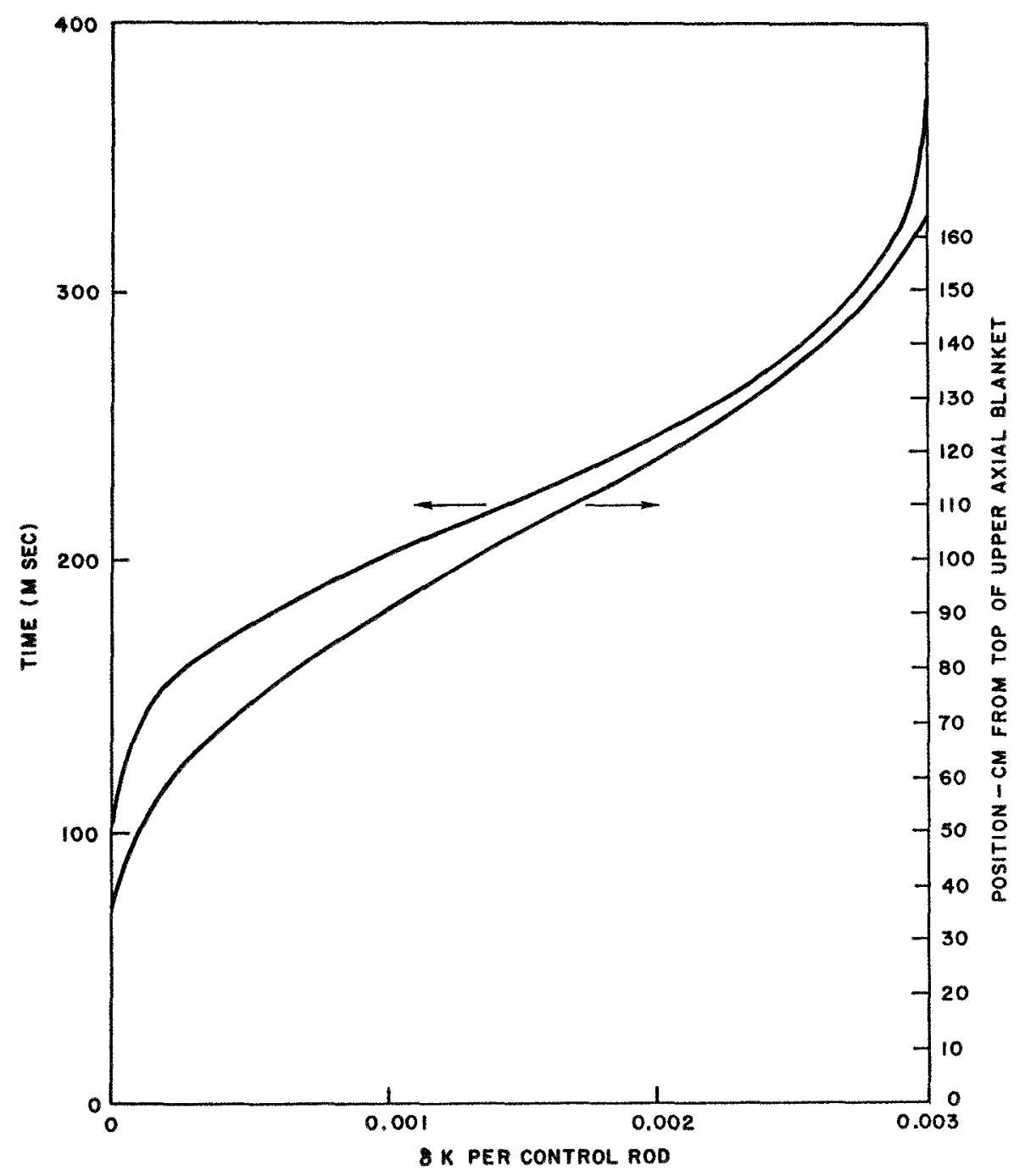

Fig. 33. Reactivity Inserted per Control Rod as a Function of Time after Scram and Position.

\section{A.6. Control Rods}

The 29 control rods are required to hold down approximately $9 \%$ in reactivity. This results in an average control-rod worth of approximately $0.3 \%$, or 70 cents per rod. The $9 \%$ is made up of approximately $1 \%$ in cold-to-hot swing and about 1 dollar in excess reactivity for burnup. The remainder is the shutdown margin. The withdrawal rate is set at $10 \mathrm{in} / \mathrm{min}$. The scram rate is set by assuming an initial acceleration of $5 \mathrm{~g}$ and a symmetrical velocity position diagram. Figure 33 also shows the scram reactivity insertion per rod as a function of time.

\section{REFERENCES}

1. A Study of a Gas-cooled Fast Breeder Reactor - Initial Study - Core Design Analysis and System Development Program, Final Summary Report, GA-5537 (April 15, 1964).

2. Bellman, Richard, Stability Theory of Differential Equa- tions, MeGraw-Hill Book Company, Inc., New York (1953).

3. Thompson, J. J., and J. G. Beckerley (eds.), The Technology of Nuclear Reactor Safety, Massachusetts Institute of Technology Press, Cambridge (1964).

4. Bethe, H., and J. H. Tait, An Estimate of the Order of Magnitude of the Explosion When the Core of a Fast Reactor Collapses, UKAEA Report RHM-56/113 (1956).

5. Nicholson, R. B., Methods for Determining the Energy Release in Hypothetical Reactor Meltdown Accidents, APDA-150 (Dec. 1962); Ph.D. Thesis, University of Michigan, December, 1961.

6. Okrent, D., and W. J. McCarthy, $A X-I T-A$ Computing Program for Coupled Neutronics-Hydrodynamics Calculations on the IBM-704, ANL-5977 (May 1969).

7. Wolfe, B., N. Friedman, and I). Riley, Influence of the Doppler Effect on the Meltdown Accident, GEAP 4420 (Nov. 18. (1963).

8. Merrill, M., BLOOST-5: A Combined Reactor Kinetics-Heat Transfer Code for the IBM-7044: Preliminary Description, GAMD-6644 (Aug 17, 1965).

9. Fenech, H., Energy Release Calculations, unpublished. 


\title{
Session IV October 12, 1965
}

\section{Discussion of Papers Among'Panel}

\author{
Chairman: D. OKRENT \\ Argonne National Laboratory \\ Secretaries: D. Neneghetti \\ D. A. Meneley \\ Argonne National Laboratory
}

Panel Members:

M. Benedict, Massachusetts Institute of Technology

K. P. Cohen, General Electric

G. P. Denielou, CEA

P. Fortescue, General Atomic

W. Häfele, Karlsruhe

D. Okrent, Argonne

L. Silverman, Harvard University

T. J. Thompson, Massachusetts Institute of Technology

J. H. Wright, Westinghouse

W. H. Zinn, Combustion Engineering 
- 
Mr. Okrent: Good afternoon gentlemen. I should like to introduce the independent members of the Panel. On my immediate right we have Dr. Leslie Silverman of Harvard University, and a former chairman of the Advisory Committee on Reactor Safeguards. Next to him we have Dr. Manson Benedict of Massachusetts Institute of Technology, a former member of the Advisory Committee on Reactor Safeguards and a member of the General Advisory Committee. Next is Dr. W. H. Zinn, Vice-President of Combustion Engineering and the first Director of Argonne National Laboratory, and finally we have Dr. T. J. Thompson of MIT and a former chairman of the Advisory Committee on Reactor Safcguards. At the other end of the table we have the five reactor designers. Since this panel is in itself an experiment, we will attempt a trial procedure which we may modify as we go along. We will start by asking the independent members to develop questions of interest. We will expect all the various reactor designers to chime in as they think it appropriate, and in particular, to answer questions that may be put to them. Dr. Silverman is nearest to me. Would you care to start?

Mr. Silverman: I might say I don't qualify as a fast reactor man. I am quite interested to know something about the relative costs of these units in terms of containment. We are always harassed by people who have regarded the Reactor Safeguards Committee as the Reactor Prevention Committee. Added safeguards must be placed upon the reactor to make it qualify in terms of the prevention of any undue risk to the health and safety of the public. The cost of containment and the cost of engineered safeguards fit this category. In addition to that, we are also concerned with the effectiveness of these devices and their reliability. So I think a good general question to all the speakers would be "Can they develop anything along this line on the cost of these 'paper reactors' at the present time?" And, can they give us some feeling for the accident problem as they see it? One of the healthy things I heard this morning was the consideration of something that was probably long overdue, and that is to call the accident we worry about the design basis accident instead of the maximum credible. Everybody has trouble with the word "credible;" and the next thing they are concerned about is what's "maximum credible." Other applicants come in and talk about the hypothetical accident or, in some cases, a maximum credible operating accident. So I think terminology here is another problem, and I would like to encourage the use of design basis acci- dent as being more realistic, because we hope that none of these accidents ever take place. I would also like to hear the panel members develop some information on backup shutdown devices, and would like to see some more information presented on the large sodium fire, which may go along with the accidents that seem to be considered in the design. That is all that I had at the moment, Mr. Chairman.

$M r$. Okrent: I wonder if any of the reactor designers would care to comment on costs of containment and engineered safeguards relative to overall plant costs.

Mr. Häfele: I should say that our cost evaluation of the Sodium-I design indicates that the overall cost of the plant is $\$ 116,000,000$, equal to $462,000,000$ Deutsche Marks (DM). Of these $462,000,000$ DM, $200,000,000$ are assumed to be the cost for the reactor parts. That is $42 \%$ of the total plant. Of the $200,000,000$ DMI for the reactor parts, however, $83,000,000$ DM were considered to be sort of a contingency. That is more than $40 \%$ of the reactor parts, because you really are not sure how expensive the large sodium components will finally be. Therefore the basis for calculation in the reactor part is $116,000,000 \mathrm{DM}$, or $32 \%$ of the overall sum. Of this, $24,000,000 \mathrm{DM}$ goes to the reactor building and construction, including the container. That is a 6 to $7 \%$ item, if you take the whole overall price of $460,000,000$ DM.

Mr. Okrent: As I understand it then, the containment part is a 6 or $7 \%$ item.

Mr. Häfele: Yes. In our evaluation it comes out to be $6 \%$ of the whole cost. In another containment philosophy we have pursucd, it would increase to perhaps $8 \%$. Our design is already close to a two-shell containment, having, so to say, the inner compartment a steel and concrete structure. We believe that an increase from 6 to $8 \%$ would be sufficient to cover the case where one insists on having the double-containment facility. Our conclusion is that this is not too heavy competition between safety and economy. The burden might much more be on the side of operation and maintenance by having this tightness in the inner part of the reactor building.

Mr. Okrent: Dr. Cohen, did you have a comment?

Mr. Cohen: Yes. I wanted to say that it is really very difficult to isolate the containment cost from the other costs associated with safety, and even to separate the costs associated with safety from the costs associated with just radiation. The entire construction of a nuclear plant has unusual requirements compared to let's say building a ship, as regards tightness, weld inspection, etc. We have many times tried to evalu- 
ate the containment costs. Depending upon the types of assumptions made, one gets that anywhere that 2 to $20 \%$ of the whole plant cost could be attributed to containment.

Mr. Fortescue: Speaking on containment, the gascooled system has special problems of its own, which differentiate it considerably from the sodium system. Of course, the essential difference is the proposal to use prestressed concrete for the primary containment. In this respect, it is different even from the steamcooled systems, which are not readily adaptable to that type of containment, primarily on account of problems with thermal insulation. However, having adopted the idea of using prestressed concrete for primary containment, a different kind of philosophy is used in determining the second containment. In normal practice, if you can use such a word in this business, prestressed concrete vessels are used without secondary containment. They are so used in Europe with natural uranium and thermal systems. It seems to me the essential point is this. If one accepts the characteristic of the prestressed-concrete containment, that by virtue of its redundancy and the fact that the tensile members are outside the temperature and irradiation field, and so forth, catastrophic failure is to be ruled out. If you accept this philosophy, the source of concern is a modest rate of leak. What dictates the need for a secondary containment is whether or not, and to what extent, you assume a number of failed fuel elements to occur at the same time as this leak, because the only reason for wanting to contain the reactor is to prevent egress of the fission products. The philosophy which we have adopted is that we would provide, not a hermetically sealed secondary containment, i.e., one capable of a positive pressure, but a form of containment which is not pressurized and which is normally vented through filters. In effect, this involves the philosophy that you are accepting a degree of leak which is considered maximum credible to the system of gas, and furthermore assuming rather arbitrarily that, say, 1 or $2 \%$ of the fuel elements are, in fact, discharging their fission products into the confinement. It is not at all difficult to deal with that. It would be very difficult to deal with a leak plus all of the fission products.

Mr. Okrent: Are you not indeed in trouble if you have only a confinement system and a catastrophic rupture of your pressure vessel results? You then have nothing to eatch the noble gases.

Mr. Fortescue: That is right. Indeed that remark applies to any use of primary containment with no secondary containment, so I don't think we would go that far. What we say is, we would have the delay and filtering system. To get some idea of the order of magnitude of attenuation which you can get with a practical filter, we believe you could dump up to, say, 10-20 megacuries of activity into such a containment and keep within site boundary restrictions, meaning, in particular, 25-R whole-body dose at, site boundary.

Mr. Okrent: Would this be with a tight building or a vented building?

Mr. Fortescue: Vented building with trapping as far as you can go. Of course, the noble gases go right through, in fact, most of this trouble is the noble gases. But the cost of the nonsealed containment would be very much less than that of total (pressurized) containment.

Mr. Denielou: I would first concur with the opinion of Karl Cohen. I think it is so very difficult to distinguish between what is due to safety considerations and what is due only to radiation. Then, I would make a distinction between tightness and withstanding pressure. In our experience, if you design a building for withstanding only snow, winds, and so forth, it doesn't cost very much to have it tight. You can rely on very simple devices, such as siphon type of things to have the wires out, and so on. The tightness, in my opinion, is very cheap, but pressure costs a lot.

Mr. Wright: You know, it is easy to look at something that costs $\$ 120,000,000$ and say well, so what if there is another $\$ 6$ or $\$ 8,000,000$ associated with containment. But, for the companies represented in this room that have been fighting this reactor battle on the commercial front at least, that is a huge amount of money. Our first competition has been and will remain the coal industry, after which internal competition in the nuclear industry is the problem. There have been far more coal power stations built in the last year than nuclear power plants. And I. think that this will continue for some time; therefore, I do not view lightly an extra $\$ 6$ or $\$ 8,000,000$. I would concur that the cost of a fully pressurized containment system with shielding, around such a reactor as we are talking about, would probably not exceed $\$ 8,000,000$. I might even guess it as $\$ 5$ to $\$ 8,000,000$. Concerning the sodium fire, the philosophy which we employ, and which I guess is the same as the Germans use, is that of a primary containment system which might be likened unto a prestressed concrete vessol, except that its normal operation is at zero pressure. This would seem to provide a lot of safety margin. But at the same time, I would have difficulty at this moment to buy a prestressed concrete vessel for any reactor, without extra containment. And certainly the case of radioactive sodium fires requires considerable attention with regard to containment. I'm afraid I would be inclined 
to feel that you have to consider this $\$ 5$ to $\$ 8,000,000$ for a while yet.

Mr. Silverman: Part of the cost of containment, as I see it, is inherent in the design. You want to keep inert gas in the primary containment because you have sodium in your system. It is pretty hard to operate without inert gas under those circumstances. I brought the sodium fire into the picture because this is considered one of the "after" problems; as somebody stated, if one could do away with containment, then the sodium fire would be perhaps the controlling accident.

Mr. Fortescue: It does seem to me that the question which very strongly influences the cost of secondary containment is the matter of how seriously one takes this maximum impossible accident, i.e., the BetheTait explosive type of reaction. We all do our calculations in figures in terms of tons of TNT yield and then, it seems to me, we all stop at that point. Do we or don't we have to make a secondary containment that really does contain that? In other words, if you have got to take the pressure which results from the dumping of that energy into heat energy, and also the burning of any sodium, which presumably goes along with it, with the assumption that all of the fission products not just a few of them, are in there, which scems to go along with the big bang, it seems to me you have a mighty expensive containment. So I am wondering how seriously this has been reflected in the cost of their containment? Or is it that the calculations stop with the big bang?

Mr. Okrent: I believe the idea of the two-containment system is that even in the presence of such an explosive event, there is no fire, because it is confined within an inert atmosphere.

Mr. Fortescue: But not all systems envisage necessarily an inert atmosphere in the secondary containment.

Mr. Okrent: No, this is within the primary containment, where the sodium is contained.

Mr. Fortescue: I am not thinking of the primary containment rupturing in a big way. I am thinking of a subsequent gentle escape of high pressure, which must be considered to be in the primary containment, as a result of the heating of the gas that is there. Eventually it sort of fizzles out into the secondary containment and then you are stuck with the problem. Are you going to deal with that pressure or not?

Mr. Okrent: I suppose the answer depends on what one assumes as the leak rate. If you rupture your primary containment, then $I$ think you do have many things leaking into whatever you have outside. If it is truly a primary containment, you don't; it is a minor leakage. Could I ask Dr. Silverman if he wants to pursue the question of engineered safeguards at this time?

Mr. Silverman: I think probably I have taken up enough time, I will let somebody else take over.

Mr. Okrent: Dr. Benedict would you care to raise a point?

Mr. Benedict: Well, there are two somewhat related points that $I$ would be glad to have comments on from the reactor designers. One has to do with the penalty which is paid or the compromises which are made when an attempt is made to design a sodiumcooled fast reactor such that the increase in reactivity on loss of sodium doesn't lead to any serious consequences. I think the steps which Dr. Cohen described, which have led to his company's design of a reactor in which the increase in reactivity on loss of sodium is down to a tolerable level, certainly are very valuable. They give us valuable insight into what can be done to make this reactivity increase acceptable, and also what penalty we have to pay in order to make it as low as it has been made. As I see it, the penalties are first that the breeding gain is much lower than would be possible if we did not soften the spectrum and increase the leakage; second, the internal conversion ratio is substantially lower. I think, Dr. Cohen, you are down somewhere around 0.7 , or thereabout.

Mr. Cohen: That is right.

$M r$. Benedict: Which in turn gives two other disadvantages to the reactor, of which one is the rather high swing in reactivity between fresh fuel and fuel when it is time to replace the fuel. I think you had something like $\$ 7$ worth of reactivity change, did you not?

Mr. Cohen: No, I think it is about $\$ 10$.

Mr. Benedict: $\$ 10$, and the need for rather frequent refueling. I note that if the internal conversion ratio can be kept close to unity, or as was the case with the gas-cooled reactor, is above unity, there is practically no reactivity swing during fuel lifetime, and the intervals between refuelings can be much higher. I wonder, as a matter of judgment, whether the panel members believe that we have gained more than we have lost in introducing this substantial reactivity change during burnup in place of a higher reactivity change in case sodium voiding occurs. The other point that I noted is that there is a very substantial difference in the amount of reactivity increase which takes place on loss of coolant in the several designs that were discussed today; I am sure that the audience recognizes that there is both a difference in numbers here and a difference in design philosophy. If I have three of the numbers correct, in case of the Karlsruhe design presently the maximum increase in reactivity which can 
result in the most unfavorable loss of sodium coolant was something like $\$ 7$. In the case of the General Electric design, because of steps which have been taken to soften the spectrum and otherwise make this effect smaller, the number is down to about $\$ 1.60$. Finally, in reading the General Atomic paper, I note that the increase in reactivity resulting from a complete loss of helium coolant from the $1000-\mathrm{Ib} / \mathrm{in}^{2}{ }^{2}$ pressure down to atmospheric was some $\$ 0.30$. These of course represent very significant differences, provided one has to be concerned about a loss of a substantial fraction of the coolant in each of these cases. It becomes very important to consider how likely it is, particularly for the design which would have an increase in reactivity of $\$ 7$ on a most unfavorable loss of coolant, whether this much coolant, or any appreciable fraction of this much coolant, could be lost, and how quickly this loss could take place.

Now, as was emphasized earlier in the Conference, the sodium coolant has the very undesirable characteristic of being prone to very substantial superheat. One of Dr. Rosenow's students at MIT has found that unless rather special precautions are taken to prevent superheating, superheat to the extent of $50^{\circ} \mathrm{F}$ can easily be obtained before any boiling occurs at all. A simple estimate indicates that if we had sodium initially at, say, $1500^{\circ} \mathrm{F}$ or at its boiling point at atmospheric pressure, and $50^{\circ} \mathrm{F}$ superheat were to take place followed by flashing, some $50 \%$ of the volume initially occupied by the liquid sodium would now be occupied by sodium vapor. This could mean that if there were a substantial fraction of the reactor in which the sodium was superheated to the extent of $50^{\circ} \mathrm{F}$, say by blockage of one or more fuel assemblies, almost instantaneously $50 \%$ of this volume would become sodium void. Thus it becomes very important in a sodium-cooled reactor which has as large a sodium void coefficient as $\$ 7$, for instance, to do everything possible to prevent sodium superheat. Reliance cannot be put on acoustic or other means to determine when superheating is likely to take place, because this is almost an instantaneous event. One has to be assured in the first place that the coolant flow is maintained and that superheating is not occurring. Thus unless sodium superheat can be prevented, measures to keep the reactivity increase to a tolerable level in case the sodium flashes are essential. Otherwise some other coolant, such as steam or gas which does not have this problem associated with it, becomes a safer cooling medium. I would like to have the panel develop these two points further. First, perhaps we could ask Dr. Cohen his view as to the relative balance between the gains and losses which the GE design has in going to a low internal conversion ratio and a substantial reactivity swing during burnup, in order to keep the reactivity increase on sodium voiding down to a low level.

Mr. Cohen: I believe you have in part answered this yourself in your second question. We have, in fact, taken quite seriously the possibility of sodium flashing, and this is the reason we have striven so hard to reduce the reactivity effect. You quite correctly pointed out that we didn't get this free, even in the matter of safety from other types of accidents. And this is one of the reasons why we have another special criterion to deal with: the burnup reactivity swing, or at least to try to limit it. We are not very happy about the compromise that taking the void to be credible has forced us to make. We have, however, believed that we ought at least to look the devil in the eye and see how bad it gets, so we have some idea of the limit of the price that we may have to pay. I thoroughly subscribe to the point of view of Dr. Häfele, which is that we must study this problem of superheat and understand it. I have some feeling that the kind of egg-crate structures which are characteristic of the core are going to be fairly resistant to even a considerable shock wave. This is just the kind of structure to build to absorb shocks. Unless the shock from superheating is much severer than we think. By this structure we hope to localize the shock.

Mr. Häfele: If I may, I would also like to reply to your two points. First, I had the impression that you implied that the total breeding would change in the case of a very pancaked core, i.e., a high-leakage core. If one makes a comparison by the same assumptions, usually the total breeding ratio is somewhat increased, provided the blanket is thick enough, because the spectrum is somewhat hardened. Only the partition between external breeding and internal breeding is heavily changed. Indeed, since the internal breeding ratio goes down considerably, we at Karlsruhe believe that a point not yet brought to the full attention of the design people is the question of availability and maintenance cycle. We tried to evaluate this quantitatively (by Rheinisch-Westfälische Elektrizitätgesellschaft) and they said that it will be of very great importance to have a yearly maintenance cycle, but they couldn't evaluate it in the form of figures. Therefore it is difficult, or up to now impossible, to get the numbers, but we do believe that a yearly maintenance cycle, and therefore a fuel cycle of one year, is of great importance when one can quantitatively evaluate the necessity of having such a high availability. One would be also in a position to evaluate the penalty of not having it, or to weigh it against the safety difficulties.

My second comment concerns the figure of $\$ 7$ for 
the sodium void effect. First, the design basis for this Sodium-1 reactor was a sodium void coefficient of $\$ 3$. We calculated the $\$ 7$ figure only recently, after having introduced our own 26-group set. Here I should stress the point that even if we assume the same microscopic input and only use different weighting spectra, the value varies widely; the value of $\$ 7$ is the maximum value calculated. Really we do not know what the figure is. This uncertainty is underlined if you calculate the sodium void coefficient first with two-dimensional methods, and then with one-dimensional methods. In this case, the overall effect comes out with a different sign. All this reflects a situation in which one has to say we don't know what the effect really is. If the value were really $\$ 7$, then probably it would be necessary to change the core design as I mentioned in my oral presentation. I agree with you that the necessity of changing the core design is indeed emphasized by the supcrheat aspects. We have tried to stress this point and we are working vigorously on understanding this phenomenon. On the other hand, I do come back to the point that it is of great impact and importance only if you accept the fact that the safety system can fail.

$M r$. Benedict: I am afraid I don't exactly understand that point of view. Suppose a substantial volume of superheated sodium can be built up in the core through some undetected reduction in flow, and this sodium then suddenly flashes. This can occur much too rapidly for the safety system to compensate it, and one could even have a prompt reactivity excursion.

Mr. Häfele: Yes, assuming that it is not possible to detect the plugging by instrumentation.

$M r$. Okrent: Could I ask whether you both mean the same thing though? I believe Dr. Benedict had in mind more than one subassembly, in order to get a significant amount of reactivity added.

Mr. Benedict: One has to have a sufficient volume to give more than $\$ 1$ worth, or else the control system can presumably compensate for it.

Mr. Okrent: I don't belicve that a single subassembly is likely to contribute a dollar, even if it is completely voided.

Mr. Häfele: I implied that already propagation has taken place. Avoiding propagation would be a measure against it, but it would be a heavy penalty for breeding, because the boxes of the subassemblies must be strong in that case. I agree with Dr. Okrent that plugging of a central subassembly gives you only 7 to 10 cents, an amount automatically controlled by the control system.

Mr. Denielou: In reply to Dr. Benedict's question, I think the comparison between the safety advantages of putting in beryllium and the economic advantages of using it is difficult to make. But the disadvantages are more certain than the advantages. When I look at the sodium voidage figure, I have, with or without beryllium, $\$ 1.6$ or $\$ 1.8$ for a sodium voidage beginning: in the upper axial blanket, and maybe $\$ 2$ or $\$ 3$ if it begins in the central planes. But because of the uncertainties already mentioned, within $\$ 1.6$, $\$ 2$, and $\$ 3$, it doesn't seem to matter so much. On the contrary, the disadvantages to the doubling time from softening the spectrum seem very clear.

Mr. Cohen: May I respond to that? I would like first to suggest that when one compares beryllium in and beryllium out, one should probably make sure that one is doing the same calculation in the same way. It is very difficult to compare calculations done at two different sites, as we saw earlier in this meeting.

I do believe there is a considerable difference between, say, $\$ 3$ and $\$ 1.60$. If I have $\$ 3, \mathrm{I}$ don't have to get the very very worst pattern to be super prompt critical. Consequently, the probability of getting something bad is larger. If I have just barely enough maximum sodium void reactivity to get me into trouble, I have to have a perfectly planned mishap in order to get into trouble. So I think there is considerable difference between smaller and larger amounts of the void reactivity.

Mr. Benedict: There is one thought that occurred to me upon hearing Dr. Cohen's paper this morning. I was wondering whether in view of the very substantial reduction in breeding gain which the introduction of beryllia into the plutonium-fueled reactor cvidently brings about, whether your system might not have a higher breeding gain if it were using the thorium- $U^{233}$ cycle rather than the plutonium-uranium cycle. Have you given any consideration to that?

Mr. Cohen: We have given some consideration to it, but our point of view is that we ought to exhaust the possibilities of the $\mathrm{U}-\mathrm{Pu}$ system, including the sodium, before we go to another.

Mr. Zinn: I make this comment that we are making progress of sorts. At the conference two years ago, we had the sodium void problem before us in full measure, for the first time, and it gave everyone a great deal of concern. Between then and now, rather ingeniously, people have invented a way of getting the whole core voided by getting simple blockage of one fuel channel. So now I have two questions that are both directed towards what I would consider to be engineered safeguards. What is being thought of by the designers as measures to inhibit the consequences of flow blockage in one channel, and inhibit the propagation of this cffect through the core? For instance, has consideration been given to stronger and differ- 
ently shaped cans? I notice they are all hexagonal. Has one thought of ways of getting the coolant to the fuel pins through more than one opening or orifice? I suspect that we may wish to use some engineered safeguards to mitigate this effect which was pointed up rather dramatically this morning.

The second question involves the observation that the boundary line between the hypothetical maximum accident and the credible one scems to be the place where the control rods function, and I said control rods deliberately, because $\mathrm{I}$ belicve all the designs terminate an unpleasant event by the movement of control rods, which I assume is always a movement of fuel or absorbers in the core. This means that the mechanical arrangements for making this movement are concentrated in one area, always on top of the reactor, a restricted area, and there is no other backup. There isn't redundancy for this control-rod system. My second question is: has there been thought of a redundant system or a backup system.

Mr. Denielou: To the first question, I think that the current design will be able to avoid the propagation of the melting in a single fuel subassembly. The only point is we are not able to demonstrate it to learned people. So before trying to avoid it, we prefer studying it; it costs money. Concerning the second point, we do not anticipate any other backup system. There is a feeling of defiance against all these fuseable systems, and so on, in France, more or less. We prefer having one single reliable system to a multiplicity of systems.

Mr. Fortescue: I think there is a measure of redundancy built into control rods, insofar as you don't need all of them to go in to shut the reactor down in any particular case. For instance, in the design I was giving this morning, only eleven out of the 27 control rods would be sufficient to shut the reactor down to room-temperature conditions. So there is a measure of redundancy in all control rods insofar that you don't need them all to go in. Beyond that, the only other measure that has occurred to us is to make quite sure that the design of the control rod is such that there is a limited rate of withdrawal. This would mean nonreversible mechanisms. It would seem to be sort of a primary goal in any control drive system used for fast reactors, meaning a serew or some such device which cannot possibly exceed a certain speed, and driven by synchronized motors or something like that.

Mr. Häfele: With respect to that question of a redundant safety system, I would like to say the following: As long as a logical pattern on which the safety system is designed is defined, then the safety system is always redundant. The problem is whether that logieal definition covers all possible events and cir- cumstances. Therefore the question goes back to the very first question, to define the scheme of a failure. Because it might easily be that one common reason for failure for all rods has been overlooked and then despite your logical layout of the safety system, no redundancy exists in that system any more.

$M r$. Cohen: I would like to say that these are very good points that Dr. Zinn has brought up. On redundant systems, I think that we haven't done enough on them. There is the redundancy, of course, in the fact that all rods do not have to work. The point that all of these rods are localized in one place is, of course, a matter for concern. One should do more thinking.

Mr. Zinn: Might I interject a remark? What brought it to my mind was the statement by Dr. Benedict about getting a rapid pressure pulse in the reactor due to vaporization of the superheated sodium. And I am reminded of the fact that in the SL-1 accident, every single control rod drive was seized in its channel by a water hammer which acted on all of them in the same way, making all of them inoperative. The pressure pulse actually made all the rods imbed firmly in the reactor structure. They couldn't have moved. Therefore, I even question whether identical design of control rods should be used-whether one shouldn't put some variation into the design so that the same simple accident couldn't catch all of them.

Mr. Thompson: Just by chance, I had a question in the same general area. It seemed to me that one of the problems is the reliability that one can place on any one of the systems. I happen to have a very good mechanical engineer that works with me. Every time $I$ ask him to make something that is really failsafe, he tells me to do it electronically. I also have a very able electronics expert that works with me, and he says that if you want to do it really reliably, do it with some sort of a mechanical interlinkage. The problem really is that experience has not to date shown us that we can indeed design a perfectly reliable control system. I know of a recent instance in which all of the control rods in one particular reactor were seized in such a way that they could not be moved, bccause there was an attack on the seals that attached the control rods to the rest of the mechanism. It happened that this reactor was shut down at the time, but the same situation occurring in the reactor in operation might prevent totally all control rods from going into the reactor. I know of at least two or three instances in which the scram system for a reactor was either totally or partially disabled while in operation for longer or shorter periods. I have to say in all honesty that a part of our scram system at MIT went out for a fraction of a week; we don't 
know how much of a fraction. We check it every Monday before startup, and it went out because a relay shorted from primary to secondary. This is a sealed-type relay. I have talked to many electronics people and none of them have ever seen this happen before.

In one major reactor in the United States, the scram control system was totally inoperative, in that it could not have been scrammed, for a period of time as long as the total month. There was no way that this reactor could have been scrammed during that period. These things do happen. Experience says that they do and therefore all of us must anticipate such failures and believe that they may happen. There are many control authorities including those in Canada and those in a number of laboratories in the United States that have asserted within the last two to three years that it is impossible to build a fail-safe system. If this is indeed true, then one has to think a good deal about putting all your eggs in one basket and assuming that the control system will always work perfectly. It appears likely that an unusually heavy burden is being placed on the operability of a single control system. It seems to me that it is particularly important that there be no common cause which could prevent all the rods from going in. For instance, at least in one case involving one control rod, sodium vapor going through a bellows seal in a fast reactor froze that rod so that it was inoperable. You can imagine that in some reactors this might be particularly bad, say in a reactor where there is only a very small change in reactivity with lifetime, so that the control rods might actually remain almost motionless for long periods of time. If they did, they might indeed freeze in some such manner.

Mr. Denielou: We would move them from time to time.

$M r$. Thompson: But then you have a rod-exercise program. In fact, I have yet to see the perfect control-rod design. I would like to distinguish also between two things. One is redundancy, which has been mentioned several times. Redundaney is a repetitive application or use in parallel, so to speak, of the same sort of mechanism or system, for instance, three power-level trip circuits with at least two required to be operative at all times. But there is another principle that is also important. That is diversity. Very often you do not foresee some accident that may occur which will, in fact, immobilize your entire control system or any sets of control, systems of identical design. It is, therefore, worthwhile for the reactor designer to attempt the challenge of trying to work diversity into fast reactor control systems. I would like to hear all of the five reactor designers involved here answer three or four questions. One, do you believe it credible that there could be a major meltdown, and perhaps a reassembly, in the event that a control rod seram failed to work? In other words, what are the consequences of a failure to scram? As a corollary, if the rods fail to work, will boiling then result in a major accident? Two, do you believe that you can design a completely reliable control system? Three, do you have any ideas for a backup system? I think that has been pretty much answered. I think nobody does at the moment. Question number four is brought to mind by something that Denielou said. He said, I believe, it is a little schizophrenic to ask a man to design a system not to boil, and then ask him to make an analysis of what happens when it boils. In a way he is right. But in another way, this is exactly the thing the man should do, because he should really know the consequences of exceeding the boiling point. If anybody should know it, it should be the man who designs the plant, unless it is the man who will eventually operate the plant. If the designer realizes what will happen when the boiling point is exceeded, then he may indeed worry about whether the boiling point will be reached, and he will know what sort of risks he is taking. A reactor designer must not only explore the normal behavior of his reactor, but he must know what happens as he goes groping into the darkness of the regions beyond normal operation. He must know whether he is going to reach a cliff and fall off and be killed, or he must know whether it is a nice gentle slope that will gradually lead him down onto, perhaps, some lower plane. It would appear that one could get into a situation with a large reactor in which there was nothing one could do except to sit there and watch it happen. If this is indeed true, when such reactors are really designed, then $I$ think the situation is indeed precarious. Therefore the fourth question I would like to ask the panelists is: How close do you really feel you can come to the boiling point of sodium in your reactor in a safe way?

Mr. Okrent: The first question is what happens to a reactor in an accident if indeed there is no scram. Mr. Wright?

Mr. Wright: I think I answered the question in the presentation, namely, that if we get flow blockage of one channel, a mechanical scram is absolutely required, or we progress through reactivity insertion to a single assembly meltdown which in turn gives a power-level rise to boiling, which in turn gives total core meltdown and criticality. I think the answer is that we envisage no way other than engineered safeguards to overcome that problem.

Mr. Häfele: I am not satisfied with the answer, because an engineering safeguard is just a prolongation 
of the safety system. Therefore, I think it is not really the answer to what happens if the safety system fails.

$M r$. Wright: I think you presumed that the safety system would fail, didn't you, Mr. Thompson?

Mr. Thompson: Yes, if you say it wouldn't scram, either because of electronic reasons or because the rods were all frozen in place, or something like that.

Mr. Häfele: And your answer is engineered safeguards?

Mr. Wright: No, if the use of control rods is thrown out, then I see no way of stopping it.

Mr. Okrent: Is this true of all the sodium-cooled designs? Dr. Cohen, could your design take blockage and voiding in single subassembly and ride it out, as it were?

Mr. Cohen: A single subassembly, certainly. I think the basic question which Dr. Thompson has asked is: can your reactor get into trouble in an emergency situation if you fail to scram? Say, for example, that power is lost to the pumps; the pumping drops and seram does not occur, the reactor keeping at power. Although some reliance may be placed in the structural coefficient, and so forth, the answer is basically yes, we are going to get into trouble.

Mr. Fortescue: Although most of the reasons so far proposed for things going wrong seem to be connected with sodium, obviously, we still have the same problem with the gas-cooled system, namcly, that one must suppose it is a possibility that you cannot scram any of the rods; but to imagine that would require a common cause. I think it is beyond credibility that coincidence or separate kinds of things wrong with the different ones should occur at the same time. Thus it is likely that a common cause causes the jamming of the rods. Further, one has to look to see whether this common cause comes at the particularly unfortunate moment when you need to seram. If it is a question of a common cause of jamming and you have time to make matters right, in other words, you are allowed to run the reactor for a while while you think, there are various means possible to shut down the gas-cooled system other than the control rods. One, of course, common to any reactor, would be a rather extreme one, namely, to unload the fuel elements, which could be done provided you didn't care what happens to one if you drop it. I mean it would be difficult to arrange a tidy removal, while the reactor is running (MCCH LACGHTER). In general, we certainly endorse the philosophy that diversity in the types of drive should be introduced. Although it is casy to introduce diversity into the drive mechanism, I think that is not really the trouble. What must be done is to introduce diversity into what is inside the core, a diversity which reacts in a different way to a com- mon cause. That is the essential thing. All one can do is to acknowledge that it is a good thing in principle. I must remark that the kind of common cause capable and so violent as to jam simultaneously all the elements would presumably be one which justified pretty drastic remedial measures, even though these damage the core.

Mr. Thompson: I want to point out again that you don't have to jam the rods. I repeat again one major reactor worked for a large fraction of a month with its entire scram system electronically inoperative. Regardless of the signals given, no scram would occur. Therefore, mechanical ability was insufficient in this case. This is the sort of thing that can lie in wait for the unwary. It is not necessary that this sort of failure happen at the instant another causal failure or perturbation of the system occurs. The inoperative system need only wait for something else to happen, something that required scram.

Mr. Fortescue: I think there are some sort of ameliorating things one can do in the design, for instance, adoption of a solid drive. One would not use a cable drive for a fast reactor. A solid connection between the two with a substantial machine capable of overcoming minor friction could be worked into the design to mitigate this.

Mr. Okrent: I believe in the case mentioned that would not have helped. I wonder if we can go on to the last of Dr. Thompson's questions concerning how close one can come to boiling. Is there a big margin or a small margin to boiling in one's design?

Mr. Denielou: I would prefer answering the first question, if I may. If the control system fails to operate, we can imagine incidents which will destroy the reactor. But $I$ think it is almost the same for any type of reactor.

Mr. Cohen: There were some other questions which I don't think were answered. One was: can you design a perfect safety system? I think that question answers itself. Then there was the statement that nobody has any ideas for backup. I deny that. For example, we have ideas for backup which would function despite binding. There could exist a thimble in the reactor and material or poison falls in the thimble. Of course, there is a need for a great many better ideas, because obviously this one has got some practical difficulties.

Mr. Wright: With regard to the same type of a thing, certainly there are other backup systems that are wilder and less conventional, but I would like to mention two points here. One is that the whole future concept of this reactor may take on a different form with time. Instead of building a nice little plant that we can justify putting next door to some other nice plant built at Ravenswood, maybe this is the time to 
address ourselves to problems of truly highly reliable equipinent, which is placed underground at some considerable distance. Naybe 15 or 20 years from now, reactor systems particularly of this type, might be located $100 \mathrm{ft}$ below grade, hopefully with components which worked for an extended period of time without maintenance. Then maybe what we would be risking is the reactor system, as opposed to any particular safety problems. This approach might make feasible the going to a less safe reactor than any of those you have heard of today. Back to Dr. Benedict's question, in which he spoke of the economic incentives for moving away from a safe reactor. I think there are tremendous economic incentives. For example, with a simple equidimensional right circular cylinder for the core, there is a very high internal breeding ratio, above 1 . The total inventory requirements to run the reactor are dropped by as much as a third. The reactivity requirements for lifetime are zero. At the same time it doesn't have the kind of sodium void characteristies that we would like, but I am not sure that this is really an ultimate constraint. A gain can be made on the fuel cycle that is of the order of four-tenths of a mil or more. In addition to that, doubling time is dropped down to 6 or 7 years. Whether or not for this performance we simply have to have a whole new concept of reactors and how to deal with their safety, I don't know.

Concerning a backup scheme, sodium cores have enough pressure drop that they would be lifted except for the holddown derices. One scheme that can be thought of is a system that has no bottom core-support plate. It has basically a system of channels at the top into which the fuel assemblies are drawn from the bottom. They are pulled up into this position. They are held there by some kind of fusible wire. Having enough weight attached to the bottom, you could either pull it in two or pull the whole assembly out if it overheated. Such backups are certainly possible, if we look at the space programs and other far-out things. I think we can reach quite far in terms of having additional safety systems. I bet we could get a dozen from the audience right now.

Mr. Denielou: That is a French patent.

Mr. Wright: Oh-I am sorry. (LAUGHTER)

$M r$. Okrent: One thing that has been said at times in the past is that the nuclear accident that occurs from the worst sodium expulsion is no worse, in general, than the accident calculated if the core melts. Since the core melts when the sodium leaves, it hardly matters whether the coefficient was positive or negative. I would like to ask the sodium reactor designers whether they think this way or not.

Mr. Denielou: Yes.
$M r$. Cohen: I would say no for a short answer. We don't know very much about what the meltdown accident is going to be, and though one can indeed make ealculations about it, one would be naive to really believe them. The accidents due to voiding are one thing, and the accident from melting another. I don't take the point of view that necessarily one is worse than the other: it could well be either way.

Mr. Okrent: I guess to use a phrase that was suggested before, if you took a design basis meltdown accident, and a design basis sodium voiding accident, would you have any different requirements for your primary containment system?

Mr. Cohen: That just depends on the void coefficients that you assume in your void accident, and on the maximum to average power ratio, and consequently the rate of assembly, in your meltdown accident. There isn't any one answer.

Mr. Häfele: Tentatively I would say there is a difference, because if sodium starts boiling first and fuel evaporates thereafter, both the ramp rates of the fuel compaction and of sodium boiling add. That means you add the ramp rates, and you can easily get 200 or 300 , even more, dollars per second, which has a strong impact on the energy release in that excursion. If only fuel vaporization occurred, then a completely different ramp rate may be meaningful in leading to an energy release. I would even stress the point a little bit further. One has to recognize the fact that perhaps even no nuclear excursion occurs at all, except the one which vaporizes the fuel, which has to be a really strong excursion. That means no larger excursion would take place, just a plain sodium vapor explosion takes place. These are two completely different mechanisms, and therefore we do envisage a situation in which the energy release is remarkedly different. We have a feeling at Karlsruhe that one should repeat a BORAX-type experiment of the old days. It was very illuminating and many results were concluded from it.

Mr. Silverman: Mr. Wright raised a point which I think is very important, namely, that of locating a reactor underground. We already know what we can do underground with proper attention to geology, faults, and things of this type to avoid venting. Optimum economic design could provide the incentive aspects to go underground and point out the benefits to be gained. I am not saying you should design the maximum unsafe reactor. What $I$ am pointing out is that if you design a reactor for the best technology and economic yields, then the thing to do is to look at it in a location that is underground and see whether or not you have a better safety position for such a reactor as an incentive. 
Mr. Zinn: When you say underground, how far underground? Does this mean below a layer of rock which is impervious, or does this mean just out of sight?

Mr. Silverman: Based on what Edward Teller says you can calculate what the energy yield is of the given explosion and decide on the depth. I don't remember how many kilotons for how many feet, but he has a formula that fits nicely.

$M r$. Zinn: But if we believe what we saw this morning that everyone comes up with $1000 \mathrm{~kg}$ or less TNT, then we really don't have an explosion-containment problem; it is a gas-containment problem. Is that any better underground than above, where you can perhaps find leaks that exist?

Mr. Silverman: It is better underground in my opinion, because we are already doing it underground. We have underground gas-storage chambers all over the country now.

Mr. Zinn: A fairly leaky business, isn't it?

Mr. Silverman: No, it isn't too bad. I can assure you that the economies of it are such that the leaks are negligible and that the actual amounts that are leaking so far can be considered acceptable by any standards we have for health and safety.

Mr. Zinn: I know something about this. When they were trying to locate the first such large underground storage in this area, we provided them with a radioactive tracer so that they could go around and find all the wells that had been abandoned which were leaking. This had to be done over a period of a year and a half. There were a lot of these wells to find and plug up. I don't believe they found them all to this day, in terms of the kind of leaks we are considering here. I wonder if it is really so that these things are tight in terms of the quantities of radioactivity that we are dealing with in these accident conditions.

$M r$. Silverman: A number of tests of this type are being carried out with radioactivity to investigate the possibility for underground storage of rad-wastes from normal processing. Of course, there are different geological formations everywhere. If you get underneath the water table, there is no question but that you have a capping phenomena that seals it off. $I$ am not able to pick out specific areas, but where studies have been made and the wells have been drilled, good locations have been obtained. You don't have to just put it down without any kind of a ceiling. You can certainly provide the kind of sealant they are providing for liquefied natural gas and things of that kind. They actually freeze a layer of mud around the location they are dealing with.

Mr. Häfele: I am very astonished to hear that you want to bring reactors underground. I read in all articles, journals, and technical journals and newspapers that the USAEC wants to bring reactors downtown into the big cities. And I think that is the right path, and undoubtedly it will happen with some reactors. If fast reactors cannot compete with that trend, they won't succeed at all. In Karlsruhe we won't follow that trend.

Mr. Silverman: A point that might be raised here is that such reactors may be suitable downtown in big cities if they are 500 to $1000 \mathrm{ft}$ below the city. We are unable to say now that these are so safe you can do it on the basis of the information available.

$M r$. Fortescue: I agree with Häfele and am against putting reactors underground. The essential point is the desire to contain fission products. I don't see the leak-tight standards associated with containment that are remotely connected with shallow underground stuff. It is quite a different thing thing from going down in a salt mine. This we are not prepared to do, I hope.

Mr. Denielou: May I strongly concur. We are not that desperate.

Mr. Wright: I rather think the point was lost somewhere down the line. The point was that it might be possible to design a less-safe reactor having far better economics. On that basis, the overall economics might be superior by running a less safe reactor below grade. Now, certainly, I suspect that we will be in the battle trying to locate one in the middle of cities, just like everyone else. But the reactor that we try to put there will be very profoundly safe. I am just saying that there might be a trade-off in these things. We are not saying that all reactors go underground.

Mr. Cohen: Might I make a facetious remark, namely, that as soon as you put the reactor underground, you are going to put a hatchway for people to walk down. And, when you go to review the safety of this reactor, since these are engineered safeguards, the hatches will be assumed to be left open.

$M r$. Okrent: I believe Dr. Silverman had a more detailed, technical question concerning backup engineered safeguards. I wonder if we could look at what the gas-cooled reactor has to offer in the way of emergency heat removal in the event primary systems just don't work. What kind of emergency heat removal is there in the event that the core melts down to the bottom of the vessel, and so forth?

Mr. Fortescue: We do propose to have emergency cooling other than by the steam-turbine circulation system. This is primarily apart from safety. From the pure measure of convenience in commissioning the plant, it would be necessary to do this. Certainly one has to take into account the possibility of melt- 
down of a single fuel element. This could arise from failure of a single pin which was self-propagating within the fuel cell. Because of that, we certainly would have to have the other backup safety, namely, the containment around the vessel, not a pressurized containment, but a leaking one. It is not essential with a prestressed concrete vessel, but it is a backup because you have to reckon on some radioactivity in the primary circuit. We have redundancy in the four circulators, each operated by a separate boiler. Still, there is one possible common cause for them all stopping at once, either (a) total absence of boiler feedwater, or (b) a main turbine casing going, which would preclude the passage of steam. Because of that, we want an emergency-shutdown blower, which, fortunately, doesn't require a great deal of power. There are also minor backups to keep the lining of the pressure vessel cooled to avoid damaging the concrete. This is done by a redundant system having two or more independent water eircuits. This is not a matter of vital consequence for immediate safety, because the damage done to concrete is not of a catastrophic nature. It just might put the reactor vessel out of commission. I think the system doesn't differ from that for sodium here. I don't see any great difference in the fact that this happens to be gas-cooled rather than sodium-cooled.

Mr. Okrent: Is there any problem in the unlikely event of the core melting down onto your separatora problem of the decay heat supplying more energy than you find convenient to take away? What I have in mind is that Dr. Cohen mentioned that he felt that the large pool of sodium represents a big adrantage in keeping things cool after the design-basis accident. At the moment I don't see any reason why gas-cooled reactors are less likely to require a designbasis accident. Therefore, I am asking: what do you do after this design accident?

Mr. Fortescue: I should have mentioned that the splitting cone contains poison material as well to accentuate the effect of the splitting. But I think one assumes that when it is down there, it is presumably partly melted and certainly things would get pretty hot down there. But I don't really see that this endangers the pressure vessel. In the long term you flood the reactor with water eventually.

Mr. Häfele: Could I ask Dr. Fortescue what is the design-basis accident in your design?

$M r$. Fortescue: There are a number of safety-oriented things which have caused headaches. If you are asking which one caused the worst headache, it is the loss of coolant. The Ioss-of-coolant accident caused us to reject everything but a prestressed concrete vessel, which is a limitation on design. The worst acci- dent that we envisage is the meltdown of the core which, of course, means that you have got fission products there as well. We add to this a maximum credible leak from the vessel, which we hope is small enough to be dealt with by the filter system.

Mr. Okrent: Are there comments on the question of the credibility of failure of the vessel? It seems to me this is a key part of the armor and everything seems to be hinged on this. I think it might be worth exploring this point for a minute.

$M r$. Zinn: I have a question that has always concerned me. Are there any openings that have to be sealed with gaskets, steel covers, and so on in the concrete vessel? How are they given a similar integrity to the concrete and its stressing cable?

Mr. Fortescue: In general, the penetration usually consists of a fairly thick-walled tube, a metal tube, which passes through the concrete wall and is welded to the inner skin forming part of it. In general, that tube has in itself on its outer surface rings which interlock with the concrete, so that the main thrust on any end cap is taken and transmitted to the concrete and not presented to the welded joint between the relatively thin surfaces of the inner skin. In general, the biggest main openings that we envisage are maybe 5 $\mathrm{ft}$ in diameter. They are to get access to the blowers and so forth. Now a plug there would consist in fact of a steel disc, if you like, of sufficiently thick strength to withstand the pressure. That steel dise is held into the steel tube by split rings around the outside to take the thrust. You back that up with seal welding to take the small leaks. That is roughly the way things are sealed up.

$M r$. Zinn: In view of that type of sealing, is the concrete vessel really that much different from what would be a well-designed steel containment vessel?

Mr. Fortescue: Oh, I think it is. The essential point about the concrete vessel is its extreme redundancy. The concrete vessel as a whole is an extremely redundant structure. In other words, its strength comes from a multiplicity of separate cables and no means of propagation of failure from one cable to the next. I don't quite see how one can postulate that the individual openings offer a safety problem comparable to the possibility of, say, fracturing a duct. Nobody supposes a steel vessel would blow up-and I am not talking about fast reactors here, I am talking about steel vessels, in general. But one does have to suppose that a main duct fractures, and this is indeed a possibility because there are thermal stresses of considerable magnitude in ducts. The fracture of a main duct is the thing to avoid. This is avoided with a prestressed concrete vessel, and I don't see that the fracture of a large thick dise is the thing one need contemplate. 
Mr. Zinn: I think one must distinguish between two cases. For a sodium fast reactor, the containment building has to contain a pressure which presumably can be calculated in advance and designed for, and have a leakage rate which is specified and small enough, which is frequently determined by the inventory of fission products in the whole core. In the gas-cooled reactor exactly the same situation exists. It seems to me that in both cases it is the leakage from the containment that determines the difficulty or the expense of building it, not so much the ability to contain the pressure.

$M r$. Fortescue: If you are talking about the rate of leakage from the primary system, one of the points where the totally enclosed system scores is that there are indeed a very much less number of penetrations. They may be big but the total number of penetrations is very small, because all of the equipment is internal. It is true you have got to take steam pipes out, but they are of much smaller diameter. One of the points you have to ensure is that there can't be a leak if you break a steam pipe at such a point that a hole is left in the ressel where the steam pipe was. This has to be gotten around by design, and a redundancy is built in around such penetrations, a redundancy which does involve bellows as one possibility.

Mr. Silverman: Can your steam boiler break or blow up inside that containment?

Mr. Fortescue: (LAUGITER) It is assumed, in the calculations of the strength of the vessel, that the steam boiler blows up. It is assumed tube fractures result. Insofar as one has no pressure shell around the boiler, there is another area in which one has gained.

$M r$. Okrent: I recall a talk at a recent special ANS mecting on concrete vessels in which a German expert, in response to a question I raised, said that a catastrophic failure of a prestressed concrete pressure vessel could occur in two ways. One would be a shear mode of failure, the other around enclosures. If that was the feeling of one expert, I wonder how one can design on the basis of incredibility of a catastrophic failure.

$M r$. Fortescue: This depends a lot on the design of the individual vessel. I think the first French prestressed concrete vessel had a dish end. The end was not prestressed-it was dished concrete. It was conceivable in such a design that you could blow the whole end out. But if you are prestressing the ends, the sides, and everything else, you took away the concrete; it would look just like a bird cage. It is just a complete tangle of wires erisscrossing in every direction, and it is the very epitome of redundancy. Incidentally, some people are used to seeing what happens to concrete in patios, and so forth, and they may get the impression that it is an unpredictable material. As a matter of fact, it is surprisingly not so. At GA they have recently completed a destructive test on a concrete model; that darn thing failed within one percent of the pressure at which they said it would. Now that is nothing but a coincidence, but it is amazing-LAUGHTER-how close you can get with a bit of luck.

Mr. Benedict: I would like to return to Dr. Häfele's comment that in the case of sodium-cooled fast reactors it would be highly desirable to have some type of excursion test which led ultimately to one of such severity that the reactor was destroyed, like the BORAX experiments. It seems to me that in dealing with some of the questions that arise in overheating of sodium in reactors, notably the unpredictability of the degree of superheating and the unpredictability of the character of the two-phase flow that would result upon boiling, no amount of careful pre-experiment analysis can hope to anticipate exactly what will happen in the reactor. A great deal of very fine engineering and nuclear analy sis has been made for the expected performance of these fast reactors, and I am inclined to attach rather more confidence in the results. I think that we know some of the nuclear coefficients better than we think we do. But I am very skeptical about our ability to predict the detailed character of the combined sodium boiling in core and subsequent fuel meltdown when we have to attempt to predict how two or three different phases of materials will interact in a few milliseconds or a few tenths of a second. I don't think we can do that, and I think that we do have to have tests to destruction of a typical, or more than one typical, sodium-cooled fast reactor before we can really be on confident ground.

Mr. Häfele: When I used the term BORAX I was not eager to destroy a reactor. Rather, I wished to make the point that in particular the mechanism of explosion of sodium vapor should be investigated.

Mr. Benedict: I didn't mean to imply that only destructive experiments should be made. I believe that the philosophy which guided the first BORAX experiment, which was to carry out transient experiments of progressively increasing severity, understanding as much as we could from each succeeding experiment, and perhaps ultimately leading to a destructive test, was what was needed in the case of sodium-cooled reactors also. Don't you think so, Dr. Zinn?

$M r$. Zinn: Yes, although I recognize the problem is pretty difficult. The sort of experiment you have to do with a sodium-cooled reactor is much more costly than the experiments that have been done with water-cooled reactors.

Mr. Häfele: If I recall properly, by the end of the fifties there was a series of papers investigating the 
mechanism of steam explosion in case of water. A parallel action should be necessary to some extent at least in case of sodium vapor.

$M r$. Cohen: Let me say that we are not going to do this experiment in SEFOR.

Mr. Zinn: I want to lower the intellectual level of the discussion and ask the speakers of this morning what they think of the problem of persuading the utility companies to accept these reactors from the point of view of maintenance, and maintenance in connection with much more minor accidents. I might mention some of them. We would start out with the melting of a single fuel channel, which might be possible in all designs. (It might be possible even during the time of fuel transfer, but even simpler things might be matters of concern.) What if someone drops some objects into a sodium-cooled reactor. How difficult is it to satisfy oneself that this won't be the cause of the melting of a fuel channel? With what we heard this morning, perhaps this is a more serious problem in connection with propagation. How about problems of jammed loading mechanisms and problems of jammed control rods, even just from the point of view of lost operating time and the difficulty of maintenance? Is this likely to be a handicap to the fast reactors? That is really the question.

Mr. Häfele: I think it is an important point, and I tried to bring out this morning during my report, that we did pay a great deal of attention to that point. The loading and unloading schemes were designed in such a way that regular loading would be done by power steering, not by a blind mechanism, but by a feeling mechanism under sodium. The Sodium-I design is such that if something goes wrong the sodium level can be lowered. Optical access is then available and therefore repair or adjustment work would not be too difficult. Further, we tripled the number of loading and unloading mechanisms, all to decrease the probability of failure and to increase the question of availability.

Mr. Fortescue: The one thing that comes up more and more from utilities is the question "can the fast reactor ever be a kilowatt factory in a commercial proposition"? We find much more expression of concern from the utilities as to whether it can be operated by other than by a flock of geniuses. Is it likely to be a system in which some slight fault causes shutdown for a couple of weeks? Things like that worry them far more than the explosions we have been talking about, and I think somewhat justifiably because this is real and the explosions are hypothetical. Indeed, these are the very motives which make us look at gas cooling in the first place. We didn't look at gas cooling to try and make it inherently safer, or to make it a better job at all.
Fundamentally, we looked at it first of all to see whether this approach was one which went in the direction of operational ease of maintenance. For instance, the sort of thing which is extremely attractive to an operator is the thought that he can do repairs to the reactor visually, rather than groping underneath an invisible blanket by remote control. The prospect of being able to flood the reactor with water deliberately is regarded as an extremely attractive thing from the point of view of putting things right. The absence of refueling at frequent intervals was received with major enthusiasm, perhaps beyond what it deserves. The accent on keeping high availability is very great. One of the things that does worry the utilities is the thought that they would forever be down for a couple of days for something that wouldn't hurt fossil fuel at all. Perhaps a similar accident could be put right in ten minutes in a fossil-fueled plant.

$M r$. Okrent: I have a feeling looking at your allenclosed design that there might be trouble with maintenance of your systems, the control-rod drives and the like. Should you get a reasonable number of fuel-element failures, as anyone may expect, what happens to the general activity for maintenance within the pressure vessel? Do you indeed have a simple system to maintain?

$M r$. Fortescue: Maintenance of what you might call routine machinery, like blowers and eirculators, seems to worry utilities a lot less than maintenance of the black magic part inside the reactor. The specific question is: how do you deal with the maintenance of totally enclosed system? The answer is that the operation is always done when you are depressurized, and furthermore when you have atmospheric air present instead of helium. Maintenance is not supposed to require remote control at all, because the measures taken to allow this are (a) that the entire compartment is fully shielded to the extent that you can work in there, and (b) there is an overpressure put into that area, so there is a deliberate leak of clean helium out of the section into the rest of the reactor, which is passed through a filtered system. This provides a big time factor to shut things down if activity is detected early. But there are measures taken to make sure there is no plate-out on the outside of the reactor. Now there well may be some plate-out on the inside of things, if the reason why you opened up is a melted fuel element or something. The general position is that when it comes to activity on the inside of the boiler tube or something like that, you have to face up to decontamination. But it is a very much different thing than contaminating the outside of something. In general, the acceptance of 
the difficulty of getting at an enclosed system generally seems to be that it outweighs the disadvantage. In particular, the ability to see what you are doing in the core region is what is considered so attractive.

$M r$. Zinn: I would like to ask the people who have vented fuel whether this gives any problems for maintenance? Do they expect any problems?

Mr. Cohen: There will be a paper on this tomorrow. The answer is that this was one of the first things looked at. Based on experimental results, it does not seem at this time to be a major concern. Concerning the utilities, I think it is fair to say that they are concerned about sodium-cooled reactors, as I imagine they would be concerned about any other reactor type which has had the somewhat checkered history of our sodium reactors. They are, however, people who are very much interested in the basic promise of the system, and I think that they are prepared to consider it honestly. Let me remark that I think that we are now a very highly technological society, even more so than we were, say, 15 years ago, and continually improving. I feel that the methods of reliability analysis, the improved instrumentation, and other electronic techniques which are coming along, including the feedback mechanisms which Dr. Häfele described, are a very good answer to some of the pitfalls that some of our complicated mechanisms have run into. I believe that handling sodium is a problem that a sophisticated society will be able to solve.

Mr. Wright: I agree that there is a lot of very deep concern in the country today. I think it is going to take a lot of demonstration and a lot of getting a hold of this thing called reliability to overcome it.

$M r$. Silverman: I would like to ask if it is just the reactor type, or the fact that some utilities have tried mercury boilers and combined this with a heat exchange mechanism. Their experience has been somewhat dubious, so that they are not apt to jump into another sodium system that has more complications.

Mr. Wright: I think that is a negligible part of the problem, if a part.

Mr. Silverman: Well, you may or may not recall that two or three utilities had mercury boilers. The experiences they had with the mercury boilers was such that-they couldn't get rid of them fast enough, as far as experience goes. So liquid metal systems have a bad reputation in the utilities.

$M r$. Cohen: I would remark that the people who stayed along with the mercury boilers recently came in for windfall profits when they sold the mercury. (LAUGHTER.)
Mr. Thompson: I would like to change the subject a bit. It seems to me that the design of a fast reactor with a 1000-MW(e) capacity may not, today, be quite realistic. We listened to Dr. Okrent's figures and I agree with Professor Benedict that these are too divergent; if those of you who have made such calculations get together and talk a little, and compare your results with appropriate definitive experiments, this will be clarified. But experience with water reactors have shown that things have gone a little more slowly, that unforeseen problems and challenges have arisen at each step of the growth. These challenges include components problems which you haven't even mentioned here at all. Materials problems have been discussed considerably, as well as reactivity problems, control problems, and so on. The development of the water reactor was aided enormously by the Naval program and even to some extent by the production reactor program in this country. I think there is no question but that water reactors were accelerated a good ten years by the fact that the Navy wanted these very badly. The fast power reactor program in this country has been very nicely and firmly launched right here at Argonne. One of the finest efforts was directed by Dr. Zinn. The Enrico Fermi Reactor has helped immensely. But this program has not had the benefit of the very large expenditure of funds available as had the Naval program. In fact, last night we heard that the total investment in the fast reactor program in this country to date is about comparable to the cost of a single very high-energy accelerator. If I were to guess, I would venture that in the long run the funds put into the fast reactors will bear more fruit in terms of benefit to mankind than they will for the accelerators, even though I still believe that there should be continuing efforts in both directions.

I think it is perhaps unfortunate that people started out to aim at the goal of a 1000-MW (e) plant immediately. Such a plant is as large as the biggest of the proposed water reactors. The reactors that are really on the final design drawing boards now are for about half that size. I would also caution that there aren't a huge number of utilities that could usefully use a 1000-MW (e) plant, at least right now. The fraction of the local load that should be borne by one plant is open to question. It seems to me that one must crawl before he can walk, and walk before he can run. Hence, it seems to me that too strong a selling pitch at this time for a $1000-\mathrm{MW}$ plant may, in fact, be a bad thing for the fast reactor industry. The Congress or perhaps even to some extent the AEC will believe that these very large plants can 
be built tomorrow. If the fast reactor design groups of the world do that and then do not build them, the public and the groups with funds may become discouraged and say "it is just another boondoggle." They will start to withdraw funds prematurely. I think this might be even worse than slow and steady progress. It secms to me then that the thing one should do in the next round is to try for a prototype which will work, which will be safe, and which pays less attention to economics.

We have talked very little about the engincering problems. I cannot help but remember that in $\mathbf{1 9 5 5}$ everybody then was talking about "prosaic" boiling and pressurized water reactors. But it seems to me that the water reactor technologists have been very busy for ten years trying to get some of these prosaic reactors to run correctly and properly with minimum down time. I therefore say that although I believe in a strong fast reactor program, there are going to be similar discouraging periods for fast reactors. I think we ought to prepare funding groups like Congress and others for the fact that there will be periods of darkness before the dawn.

The 1000-MW studies should be used to delineate quite carefully what the problems are or may be. Much of this is being done. Yet there is always a temptation in paper studies to minimize the problems and come out optimistically, especially under economic competition from other types of reactors and from other bidders for development contracts. The responsibility of building a real reactor will force one to face reality very quickly. With these thoughts in mind I would like to ask each of the participants here, if they were to try to design a reactor of this type tomorrow, what are the four or five principal problems? What are the experiments, the things you would like to have answered, that you feel are essential in order to make a plant of the type you propose work, and work safely?

Mr. Häfele: I think that this comment must be answered from the side of the designers. We gave a great deal of thought as to whether the target of $1000 \mathrm{MW}$ (e) would be correct or not, and I think it is correct for two reasons. First of all, it is that large because we have to identify the area where the problems are, and they are the bigger the larger the reactor is. There is competition between economy, safety, and breeding, which is a triangle, and the triangle is the larger, the bigger the power output is. Therefore, the problems become the clearer the bigger the reactor is. In order to do that and to follow that, it is reasonable to investigate 1000-MW (e) reactors. That is one point.

The second point is that all of us have to remind ourselves that we are shooting for a flying target. At the earliest the reactor will be ready by 1980 . At that time, even in Germany, plans will be for the 1000- or 1200-MW (e) size. Arriving at a reactor which is, by nature, limited say to $600 \mathrm{MW}$ or so, wouldn't make sense. We don't say that we will build a 1000MW(e) reactor tomorrow, but we do say that after we understand its problems, we will be ready to commit a prototype of $300 \mathrm{MW}$ (e) tomorrow. There is a great big difference, and I think the procedure is logical and reasonable. If you ask what is necessary, what do you want to have, in order to commit a reactor, then I think we also can give at least the main answers. First of all, I would say one should see the big sodium components in operation, safely and cheaply. Secondly, one should have a fuel element able to give 100,000 MW days/ton, but not only with one pin rather than with a high number of pins irradiated in fast flux. Thirdly, one should have had a plutonium critical to resolve these final questions of sodium void. These are the three main areas to our judgment. This is somewhat less difficult for the commitment of a prototype of an intermediate size, but the problems to be attacked should be typical for the larger size.

$M r$. Denielou: May I give the answer to the same subject? I think I would place, first, the fuel element having 100,000 MW days/ton; secondly to be sure that there is no propagation mechanism for the melting of the core from one point; thirdly, to have the most reliable steam generators.

Mr. Wright: Although safety and fuel are important and exciting present problems, there are ways around them. We feel that the obtaining of reliable components at anything like a reasonable cost is the very major problem which stands in the way for a long period of time. The system must work as it is designed to work. I don't believe that we are very close to that in the sodium system. I am sure that each of us here should give every attention we possibly can, including a prayer at night occasionally, that the SEFOR thing runs, because that thing has got to run. If that thing doesn't run well, we are all going to be in lots of trouble I believe. In terms of what has to be done, I think carbide fuel is an eventual fuel, but to start out with I would certainly choose an oxide core. I suspect that it would be loaded down with beryllium-beryllia or graphite, so that it could be made safe. I believe that it can be made safe. The potential may not be particularly striking, but that is another economic problem. I would suspect that 50,000 MW days/ton is enough to carry the thing along to where is would be very in- 
teresting and very attractive. It is not at all mandatory until you get into, perhaps, the third core to go on to higher burnups. The basic problem is the old unglamorous problem of hardware, and how to glue it together so that it really works.

$M r$. Cohen: I agree with the last three speakers. There is no question but that we need a great deal of work and information in lots of areas: physics, fuel, safety, sodium technology, sodium components, major equipment, and instruments. Major components are a major headache. And, of course, SEFOR has to work. I think it is certainly a good idea to take a look at where you are going, and to look at what a 1000-MW plant looks like to be sure you are directing your development program in the proper way. I don't think there is any question about anybody pretending to commit himself to a $1000-\mathrm{MW}$ plant tomorrow morning. I also agree that a smaller plant presents a considerably easier problem.

Mr. Fortescue: I think without a doubt that the development of the fuel is the essential part of the problem. The essential difference between this and the thermal reactors, to my mind, is the economic need for very high burnups in fast reactors, which poses problems not even looked at on thermal reactors. If we are going to achieve $10 \%$ burnup, this is certainly going to mean some metallurgical development of no mean order, because it is extremely difficult to achieve these high burnups in the face of a phenomenon like embrittlement. If you are going to have to pass the fuel cans through the plastic-flow region many times during the course of the reactor, there is no doubt that quite a considerable development will have to be acquired to work up to anything like this economic $10 \%$ burnup. But fortunately, one has some time to do that because the first thing to do is to get the plant right-the $10 \%$ burnup can follow, I think. Of course, in the gas-cooled reactor field we have our other special problems, namely, the prestressed concrete vessel, because if we are going to tie one to the other like that, right there you have got a development problem. Worse than that in a way, you have got an acceptance problem as well. It is difficult to see how this can be other than time-consuming. On the question of size, our impression from talking to the utilities is that 500 MW and upward are just what they want; in fact, it is extremely difficult to sell anything less. I would almost go so far as to say they are more interested in the ability to make it big than the ability to make it economical at the moment. I think possibly one factor in this is that for a utility to accept a reactor at all means sort of practically reorganizing themselves. An entirely dif- ferent kind of staff might be required, and so forth. If you are going to accept that perturbation, you might as well justify it by a large-scale perturbation of your profits and revenue.

Mr. Häfele: May I address a question to the panelists? How large, in your judgment, is the credibility of a major sodium pipe break? Is it incredible or is it credible?

$M r$. Silverman: It is probably time to take that break now, but I think it is credible.

Mr. Denielou: May I reply to a question we managed to escape. We were asked at what distance from boiling are we going to operate? Let me give the answer now very briefly. We are limited by the cladding temperature of $650^{\circ} \mathrm{C}$ at the present time. This sets up a limitation and we have not been thinking of going much further.

Mr. Thompson: I should note that members of any committees are speaking here as individuals and their opinions are their own and do not reflect committee opinions. Just to show that this is indeed true, I would say on my own part that the credibility of a major failure of pipe carrying sodium is in my opinion pretty well down the list. I would say that there are many, many other things that are more likely to happen.

Mr. Okrent: I think I will try to get questions from the floor now.

Mr. Zebroski (GE): I have a combination of a question and a comment on the one really substantial new accident that has developed at this conference, that is, the superheating and sodium voiding accident, and I think Dr. Benedict, as a chemical engineer, might want to invent with me a solution for this kind of problem. I think this solution can go along two lines. You can provide an exceptionally hot surface somewhere within the element which would attain sufficient superheat and boil earlier, or you could provide a venturi, which would provide a localized low-pressure region and a lower saturation temperature, or both. This would prevent a coherent wave front of sudden voiding following on the superheating of the entire channel cross section, as postulated in Dr. Benedict's calculation.

I would then observe that most reactor designers spend a great deal of time trying to "disinvent" these solutions to the problem, because we have hotspot temperatures of the order of $1275^{\circ} \mathrm{F}$ corresponding to an exit bulk temperature of $1100^{\circ} \mathrm{F}$ after considerable efforts to minimize hot spots. For a single pin it is easy to discover and calculate peripheral temperature variations greater than $50^{\circ} \mathrm{F}$. So I wonder if when we do these tests in a multi-rod 
channel configuration (rather than an idealized, thermally symmetrical, single-channel experiment) if it is really likely that we will have a substantial volume of superheated sodium involved. Dr. Benedict suggested this proviso-whether we would have a substantial rolume that would superheat and create a large region of voiding instantaneously.

Mr. Benedict: Well, admittedly there is a wide variation in metal surface temperatures, even in the best designed reactor. But it seems to me that the kind of thing which could lead to a rather rapid and rather extensive superheating of sodium would be flow stoppage, which $I$ think is in a class of a credible maloperation. This could occur in such a way that even though there were local points of higher metal temperatures, a substantial volume of sodium could be heated above the boiling point before the hottest point triggered the phase change that can be beneficial if it occurs with just a limited number of BTUs of superheat stored up, and can be very destructive if a substantial number of BTUs of heat are stored up. In line with your search for an invention which would make this a less unfortunate instance, I haven't any particular substance to suggest, but if we could find something which had a limited solubility in sodium and appeared in the vapor phase so that the sodium fizzed a little bit in flowing through the reactor, I think we might have means for catalyzing the information of the second phase almost at every point in the reactor.

Mr. Voice: Fission gases from vented fuel would do this automatically.

$M r$. Schluderberg (B\&W): Dr. Benedict, may I direct this question to you? Do you believe that in view of all the questions about sodium and other coolants that have been raised today, a coolant can be picked at this time upon which to base the national program? Isn't it possible that maybe we are rejecting a stone now that may become the cornerstone later?

Mr. Benedict: I was going to ask Dr. Häfele that question. I know that you would be interested in his answer to it too. What is the status of the Karlsruhe investigation of steam cooling for fast reactors? What pressures and temperatures of steam does he think would be most economic and most feasible as a third possibility among these fast reactor coolants?

Mr. Häfele: I think the kind of difficulty Schluderberg deseribes will continue. It will continue to be difficult to choose between different coolants. Therefore, at Karlsruhe we are more or less decided to go ahead with two different coolants, sodium and steam. We no longer are trying for a decision between these two coolants-rather, we have made the decision to do both in the prototype stage.

Mr. Denielou: Don't you think that the decision about a choice of coolants is going to be taken by the United Kingdom and Soviet Union?

Mr. Häfele: We make our decision by ourselves.

Mr. Cohen: Maybe I should inject a note of levity into this serious conversation by remarking it is often noticed that when people divorce, they end up marrying the same kind of person as the second wife or second husband. What I'm about to suggest is that perhaps all of these problens about sodium are here because we really have studied a great deal about it and know a great deal about it. Now I myself am very much interested in other coolants, and I expect to be continually looking at other reactors forever. I am never going to give up on them. But I think one should be awfully careful about saying "Okay, we have come to some difficulties here, or maybe even a little thorny patch, and so let's just go over on the other side of the hill where the grass is greener." I am afraid that when you start working around there in the same depth you will find some other and different problems. One thing true is that any program to be carried through needs continuity, it needs money, and it needs a lot of people working on it in a dedicated way. I wonder whether you can do a great many programs having these requirements simultaneously without soon finding you can't do any?

Mr. Fortescue: I agree wholeheartedly with Häfele that we should be looking at two coolants, but the only trouble is that I differ on the two coolants. I think we should be looking at sodium and helium as the coolants. I would genuinely like to hear from the steam people what fundamentally they see in steam.

Mr. Häfele: I think there is a big difference between a gas-cooled reactor and a steam-cooled reactor, because a steam-cooled reactor does have a good thermodynamic heat transfer property and you can operate at $650^{\circ} \mathrm{C}$ cladding temperature. At these temperatures you do have rather good creep strengths, even with somewhat ordinary metals, say stainless steel 1613 or so, or Inconel, which is available today. You don't have to rely on the principle of a collapsed cladding. Whereas applying $\mathrm{CO}_{2}$ or helium, you would have to apply the principle of collapsed cladding material. And I don't think that you can rely on that principle for a burnup of $100,000 \mathrm{MWD} /$ ton because of all the swelling of fuel inside. That is one big point.

The second big point is that steam cooling might become a natural evolution from present water technology, in particular boiling water reactor technology. 
By the same token, it solves the problem of superheat; therefore, it is very natural to consider steam. The steam pressures also should be dictated by the conditions of today's power stations, which use 2,200 psi. Then you can evolve from all this technology.

The third point is that even now I am not convinced that the capital cost of a gas-cooled design is lower than that of the steam-cooled. I would say on the contrary. I admit that in the case of the gas cooling you do have the advantage of the better internal breeding ratio. But this should be balanced against the question of economy, reliability, and experience in the steam field. In doing this, we do arrive at steam and not at $\mathrm{CO}_{2}$ or helium.

$M r$. Fortescue: On the question of the steam being a better coolant, it is a better coolant than helium if it is operated near saturation at the intake to the reactor, or if you go to supercritical pressures. But the essential point in my mind is this-does this do you any good? The point is that in any steam system one wishes to eliminate the boiler. There is a potential capital gain there. But the moment you do this, you arcept a new limitation, namely, you must operate the reactor at the same pressure that you choose for the external plant. Now the external plant wants a very high pressure to keep the capital costs down, so the reactor gets given the high pressure. But then this excellence as a coolant disappears completely, because at such pressures the helium would be more than good enough as the coolant. The point is: it seems to me unnecessary to overdo the goodness of a coolant. At 1,000 psi, helium is, in fact, capable of giving you 38 to $40 \%$ overall plant efficiency without exceeding the $650^{\circ} \mathrm{C}$ can temperature which Häfele mentioned. It seems to me that the real issue is not that at all. It is a question of safety. With the steam system you are admittedly still left with the compromises which the gas was trying to free you from in the sodium system, the proof of which is in the lower conversion ratio that goes with the steam. Not only do you have to accept the lower conversion ratio, but you have to accept 4 or 5 dollars worth of reactivity tied up in the coolant. This seems to be typical. Furthermore, you have not got yourself freedom from a void coefficient, so in fact you are again comprising the core shape to achieve adequate void coefficients. I don't honestly believe that the steam has sufficiently removed itself from the problems of the sodium system to justify a small increase in its heat transfer properties which is useless to you if you don't retain the boiler.

Mr. Häfele: I disagree. In the paper by Dr. DalleDonne, to be presented tomorrow, there is a thorough evaluation of the thermodynamic properties in the primary circuit. On the basis of this paper steam is better even if you increase the pressure of helium. You don't have the freedom to do that because it is rather easy to have blowers at $180 \mathrm{~atm}$ in the case of steam, but I don't know of any large-scale helium blowers for that pressure. That is just the reason why we have chosen steam, not to have to jump into a new technology; what you propose is virtually a new technology.

Your other argument was that the safety problem would be more difficult. With steam cooling at 180 atmospheres we will have an insignificant positive, or if necessary, also a negative voiding coefficient, provided that there is some plutonium-240 present. The details of this will be described in the paper by Dr. Ott. Therefore, it is not true that there is a large holdup of positive reactivity in the coolant. On the contrary. By the same token we improve the efficiency. Therefore, we see a number of reasons to choose steam, and I cannot agree with your conclusions.

Mr. Okrent: May 1 ask one small question? I thought that Dr. Fortescue said something about not exceeding $650^{\circ} \mathrm{C}$, but in Table $\mathrm{I}$ of his paper, it mentions $800^{\circ} \mathrm{C}$ with a hot-spot allowance.

$M r$. Fortescue: Oh, that is the hot-spot allowance. $650^{\circ} \mathrm{C}$ is the design maximum. It is just the basis of stating it. That goes along with an $800^{\circ} \mathrm{C}$ hot spot.

Mr. Häfele: I should repeat my first argument: One cannot work with the principle of a collapsed cladding for a burnup of 100,000 megawatt days per ton.

Mr. Fortescue: I think this answer about having secured an adequate coolant void coefficient with the steam has been done at the expense of conversion ratio. That is my point. I think you can make any reactor okay as long as you compromise in some particular direction. Concerning the other point-are we talking about a system with an isolated primary system, or are we talking about what I would call an open system in which you take the steam down to the turbine?

Mr. Häfele: Open primary system, but with a lot of inventions which will be patented and published thereafter.

Mr. Fortescue: I think that is the whole point. It is a question of contrived safety and fundamental safety which is at issue here. For instance, we believe that total enclosement of the primary circuit is an essential for any pressurized fast reactor. That is accepting a limitation. But not only can you not have the prestressed concrete construction with the steam, but what do you do when you break a main 
steam pipe down to the turbines? It isn 't just the reactivity increase; you have still got to get the heat out.

Wr. Häfele: I am ready to defend our steam design in three to four months on that basis. Publicly.

$M r$. Katman (B\&W): We've been quoting a lot of void deficits and density deficits throughout the entire conference. Looking at the intercomparison would lead me to believe that we really don't know what we are talking about when we are quoting all of these void deficits and density deficits. There was a 2 to $3 \%$ spread in the numbers calculated by all the groups. When you talk about a $2 \%$ positive coefficient or a $1 \%$ positive and you can have a $3 \%$ or a $2 \%$ error, then it really doesn't mean too much; you might be positive or you might be negative. Realistically, do we know what we are talking about? I wonder if it wouldn't be a good thing at this point to say all right, now we've got a lot of paper studies; let's go out and measure a few of these density deficits and see if we have meaningful numbers. Maybe in a year or two we can go at it again. I would like to know some of the plus or minuses on the calculated density deficits and void coefficients that the various panel members expect. They must be of the order of $100 \%$ of the number.

Mr. Okrent: I believe estimates have been given for some of the uncertainties. Perhaps one or two of the speakers would care to indicate whether their designs would be vitally changed if there were, say, a 1 or $2 \%$ difference, by that $I$ mean if the total amount of reactivity gained by sodium voiding were increased by $1 \% \Delta k$ from what they now calculate.

$M r$. Cohen: What we tried to do, of course, was to begin with the fact that we didn't know exactly how to calculate these coefficients, and consequently, to make allowances in the design for this. In our safety analyscs, as I pointed out this morning, we divided most of our favorable feedback coefficients by 1.5 in order to take care of this sort of variation. I think we all agree that it would be fine to measure these things; on the other hand, when you do a design you are obliged to believe and act consequentially on your numbers, because this in itself is training for what you're going to do when you finally have good numbers.

Mr. Gast (ANL): I want to belabor this void coefficient a little more, not for itself but to lead into something else. The panel, I think, are all agreed that $\$ 7.00$ was too much. One member of the panel thought that $\$ 3.00$ was too much, but that $\$ 1.60$ was all right. If I can put my own interpretation on what I have heard, I believe that in a particular design, some limit, say $\mathrm{X}$, is chosen for void effect which is acceptable on the basis that if you do have an accident and the safety system doesn't operate, then the consequences of this accident will exceed some limits of tolerance if the value of the void coefficient is above $\mathrm{X}$. It isn't at all clear what these limits of tolerance are. I want to recall also that all of the speakers mentioned almost in the same breath a thousand pounds of TNT as the consequence of a meltdown accident and seem to imply that all of the designs were quite capable of enduring this kind of thing. I wonder if the panel has any comments or viewpoints on what kind of tolerable limits one could set and then use these to determine in some sort of a logical way what is an acceptable void effect in a particular design.

Mr. Häfele: I think there is a basic difficulty in order to do this. Reactivity worths are one question, but these excursions are ramp rate limited. One has to know the time scale. Mr. Fischer and I evaluated the boundaries for the time scale for a given reactivity burst, and there is an uncertainty of the same order. There is even a third difficulty, namely, the equation of state. You are referring to the fact that everybody is saying one thousand pounds of TNT. In our panel contribution, we said it varies between 50 and $500 \mathrm{~kg}$, and only the upper limit coincides with a thousand pounds of TNT. If a different equation of state is used, for example, that of Ackerman, much smaller values come out. All three uncertainties have to be resolved before one can give an upper limit for the reactivity worth of the sodium void.

Mr. Greebler (GE): There has been much emphasis on the virtues of a high internal breeding ratio, and we certainly agree with the advantages that were cited. It might be worthwhile pointing out that there are some disadvantages also. For example, with our reactor, which has an internal breeding ratio of 0.7 forced on us by the sodium void problem, the fuel which has reached $100,000 \mathrm{MWD} / \mathrm{t}$ burnup is loafing along at about $80 \%$ of the power density of the clean fuel in the reactor. Since you gentlemen all agree that the ability of the fuel to achieve long lifetime at high specific power is a vital feasibility question, it would seem that this is a potential advantage for the lower internal breeding ratio. On the other hand, the 1.1 internal breeding ratio which eliminates the need for burnup control would impose the peak power on the fuel element which is at the highest burnup. I wonder what comments you might have on that.

$M r$. Fortescue: There is one little point. One of the side benefits of a high internal conversion ratio is that you can flatten power better because the power 
Mr. Bartels remarked that we will build a prototype and after some time we will build another. My experience with the competitive system in this country has persuaded me that reactors are not going to come one at a time in the next round.

$M r$. Wright: We believe that the technology can fall into line so that enginecring could start on a prototype job in 1969. This will give us a chance to get some results out of SEFOR and assume there will be sufficient program in certain of the other areas. Let's look at the thing this way. In France they have one designer, one builder, one customer. In the Naval program we had maybe a couple of designers, one customer. In Russia there is one designer and one customer, and the UK follows this line pretty well. The reason that we have good successful operating reactors today is twofold. Certainly the Naval program helped tremendously as regards time scale, but basically there was a profit motive here for private industry to invest a good chunk of their money to go into the thing and take the risks. And there are still risks being taken on the contracts being issued in the last three or four months. They are willing enough to go in and take the risks to make the thing move. Now the real problem is: is the fast breeder development program in this country going to become an academic institution? If so, I assure you, the power will never be competitive out of it unless it is on a $3 \%$ public power system. In order for fast breeder power to ever get competitive in the United States, we are going to have to have two or more industrial groups capable of competing on it, and this is the only way we are going to get there.

$M r$. Wensch (AEC Washington): I'd like to return to the fast reactor Spert-type facility. Some years ago we proposed such a facility to the fast reactor community and received no endorsement that it was needed. Unfortunately, we already had the money and were ready to go with what I considered a program which had been formulated rather carefully and given the greatest amount of consideration. In view of the remarks made this afternoon, Mr. Chairman, I wonder if we couldn't receive recommendations in more detail from Dr. Benedict, Dr. Zinn, Mr. Thompson, and Dr. Häfele.

Mr. Okrent: Were you asking that they try to define this experiment now before the close of the session?

Mr. Wensch: No, but let's not fool ourselves. Building the facility may not be as difficult as really delineating the program. We went through this some years ago, and if the need is here $I$ think we have lost some years. The preparation of a program is very important.

Mr. Okrent: Perhaps I might offer a comment first. I think, in reference to the earlier consideration of such an experiment, had it been done at that time it would have completely missed this particular phenomenon, since it had not been identified. At this time, I think it is more likely that one can define experiments that will be pertinent to large sodium reactors than it was then.

$M r$. Wright: Which is a reason for the 1000-MIW studies perhaps.

Mr. Okrent: And for discussions of this kind. If any of the gentlemen on the panel would care to try to outline this experiment in more detail now, we'd be happy to have them do it. If not, could we leave it at saying that more will be done about this.

Mr. Bump (ANL): I'd like to remind the conference that in the proceedings of the Argonne conference of two years ago, a facility such as we are talking about was described in some detail by APDA. I remember that they quoted 50 million dollars for the facility. I don't know if this is the same one Dr. Wenseh is talking about or not, but if you add another 50 million to operating the facility and performing the experiments, you get an idea of what is involved in this. I might also add that we have been thinking of doing at least small experiments in a loop in the FARET which would presumably be uscful in this area.

Mr. Okrent: I believe the paper at the 1963 conference dealt with something related to the problem of fuel meltdown, and the thought in that particular paper was that one would like to melt of the order of 10 or $15 \%$ of the core in a single experiment without melting the rest. I don't believe that particular program had the problem of propagation of an accident in mind, although conceivably the facility for the job might not be very much different. The cost also might not be much different.

Mr. Stachura (AI): I'd like to bring to the attention of the audience some experiments which I mentioned yesterday, and which I think have a very direct bearing on what we have been talking about. There seems to be a great deal of talk about superheating sodium coolant, about a lack of understanding of meltdown behavior, about fuel compaction, as well as a whole range of meltdown and boiling problems in fast reactors. We have done some experiments in TREAT, and I think there are several qualifications that are worth making here. For one, the heating rates that people calculate for large fast excursions due to reactivity insertions that are at all realistic--control-rod ejection, this type of thing-are not at all so fast that the TREAT power profiles cannot produce the type of thermal behavior of the fuel that these calculations predict. In fact, in one 
experiment in $\mathrm{CC}$ was heated with about 250 BTU's per cubic inch within one second, which corresponds to an adiabatic temperature rise of about 7000 . $8000^{\circ} \mathrm{F}$. The fuel melted, sagged in the cladding, sat there while the sodium bond appeared to have gotten out of the way. The sample was sitting in stagnant coolant, such as exists in a blocked flow condition. The coolant remained at about $1200^{\circ} \mathrm{F}$ for about another second, and then began to heat rapidly as the fuel contacted the cladding and improved the heat transfer. Finally, upon failure of the cladding, the fuel was thrown, blown randomly, spread throughout the capsule in a very dispersed way by virtue of the fact that cladding failure resulted in contact of this $4000^{\circ} \mathrm{F}$ fuel with the cold coolant. It most likely radiated to the coolant and produced large local pressures which then produced a dispersion mechanism. I think this dispersion mechanism ought to be considered as a possible means of obtaining negative reactivity effects when fuel is moved from central portions of the melting reactor into regions where the worth is less. One of the significant things is that it is very difficult, when looking at real reactor accidents, to see where the sodium coolant will obtain the superheat at the kind of heating rates that we see and with the kind of reactor cladding materials that we have. It looks as if tremendous heating rates, that are just not obtained in real accidents, must be necessary. Superheats certainly are measured when somebody does a basic experiment with a thermocouple at the metal surface and looks at the fact that a certain energy is required to create the bubble surface. In some cases, superheats of 30 or 50 degrees may have been obtained. I don't know if anybody has measured the very large superheats that are sometimes quoted. Dick Noyes at Atomies International has done a large number of boiling experiments in which he has not seen amounts of superheat larger than 30 degrees. We may be able to use TREAT to establish the importance of this problem. We may be able also to use other heating techniques before we build a large facility to study a large reactor boiling experiment. I think that there certainly is a need to fill the gap in information about meltdown and about transient boiling. But $\mathrm{I}$ think there are a lot of experiments, which have not been funded, that are more in this line and that don't require very large expenditures for large facilities.

Mr. Dalle-Donne (Karlsruhe) : I think I can answer some of the questions in regard to sodium superheat. In some experiments at Oak Ridge with potassium superheats of almost $200^{\circ} \mathrm{F}$ were measured. Later, because theoretical calculations indicated that sodium should have greater degree of superheat, experiments were done with sodium; these are not yet published, but the sodium superheat was so high that they could not boil sodium. The temperature was so high that they could not follow it. So I think the question of superheat is very debatable. There are very different experimental data-I think because this phenomenon depends on causes which are not completely known.

Mr. Segal (APDA): I think it is worth pointing out again that, whatever the uncertainties, at least two countries do feel themselves ready to commence construction of a large fast reactor, and furthermore in the Soviet case, they don't appear to be worried about containment at all.

Mr. Häfele: As an answer, 1 would like to refer to a paper of Engelmann and mine at the end of the third Geneva conference, where just that point was outlined and explained.

Mr. Wright: I think those of us who have had an opportunity to meet with the Russians at the Geneva conference and in Russia have found that the problem of containment and of reactor design philosophy, safety wise, is a little bit different there from what it is here.

Mr. Thompson: It may be wise to end on a positive note. We should remember that we are today talking in a restricted community of technical people. all involved in one way or another in fast reactors. We are now getting to the part of the work where we really begin to understand the problems. Contrary to the earlier impression that I may have left, I do feel that the 1000-MW studies reported indeed add quite a bit to our understanding in outlining the problem, and I think they were important. I think we should recognize that the next plants won't be those 1000 MW (e) plants, and we shouldn't mislead the general public to believe they will be. But I think we all believe in fast reactors, that they are absolutely essential, that we are coming to grips at last with the problems, and that we should try as rapidly as possible to obtain answers to those various problems.

Mr. Cohen: I would merely like to remak on the question of the experimental proof of the propagation problem. I would like to say again that I believe we should do experiments, but this doesn't mean that we should immediately do them in a reactor. We ought to begin with out-of-pile loops and see whether we really have a problem of reactor dimensions or not. For what it's worth, my guess is we don't.

Mr. Okrent: Are there any other comments on any subject? If not, thank you gentlemen. The meeting is adjourned. 


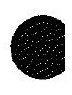

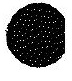




\title{
Session V-A \\ October 13, 1965
}

\section{Fuels and Fuel Behavior}

\author{
Chairman: M. Benedict \\ Massachusetts Institute of Technology
}

Secretary: O. L. Kruger

Argonne National Laboratory 


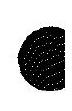




\title{
Metal Fuels for Fast Reactors
}

\author{
R. E. Macherey, L. R. Kelman, and J. H. Kittel \\ Argonne Vational Laboratory \\ Argonne, Illinois
}

(PRESENTED BY R. E. MACHEREY)

\section{Introduction}

The successful attainment of 5.7 a/o burnup at a maximum jacket surface temperature of $620^{\circ} \mathrm{C}$ and of $8.7 \mathrm{a} / \mathrm{o}$ burnup at a maximum jacket temperature of $545^{\circ} \mathrm{C}$ are discussed in this paper; also discussed are what is being done to further this burnup, to improve its reliability, and to reduce its cost. Uranium-plutonium alloys have properties which make them attractive as fuels for fast power breeder reactors: (a) high fertile-fissile atom density, (b) high thermal conductivity, (c) large reliable thermal expansion, and (d) excellent resistance to sodium corrosion. In addition, uranium-plutonium alloys are: (a) relatively easy to prepare (no synthesis problems are involved), (b) casily controlled in composition and impurity level (no stoichiometry problems are present), (c) amenable to pyrorefining, and (d) readily fabricated and remotely refabricated. Cranium-plutonium alloys have some disadrantages, such as: (a) relatively low melting temperature, (b) incompatibility with some otherwise attractive jacket materials, and (c) low irradiation-swelling temperature.

The advantages of metallic uranium-plutonium fuel are substantial enough to warrant a vigorous exploitation. The weak features of this alloy fuel are open to attack via: (a) alloy development and heat treatment, (b) development of superior jacket materials, and (c) application of mechanical restraint for suppression of swelling.

The alloy fuels of primary interest in this paper are uranium-plutonium-fizzium, uranium-plutoniumtitanium, and uranium-plutonium-zirconium. Jacket materials associated with these fuels are $\mathrm{V}-\mathrm{Ti}$ and other refractory alloys, Type 304 stainless stecl, and other high-strength iron-base alloys. The fuel design used exclusively in this work consists of an uraniumplutonium alloy fuel pin, jacketed in a loose fitting tube closed with appropriate end fittings. The annular space between fuel and jacket is sodium filled for heat transfer purposes.

\section{Summary}

In the area of irradiation performance, burnup levels to $5.7 \mathrm{a} / \mathrm{o}$ have been reached in prototype fuel rods of $\mathrm{C}-15 \mathrm{w} / \mathrm{o} \mathrm{Pu}-10 \mathrm{w} / \mathrm{o} \mathrm{Fz}$ alloy jacketed in $\mathrm{V}$ $20 \mathrm{w} / \mathrm{o} \mathrm{Ti}$ alloy tubing. Irradiation was performed at significant temperatures: $620^{\circ} \mathrm{C}$ maximum at the jacket surface and $720^{\circ} \mathrm{C}$ maximum at the fuel center. A higher burnup of $8.7 \mathrm{a} / \mathrm{o}$ has been achieved in the same fuel alloy jacketed in $\mathrm{Nb}-1 \mathrm{w} / \mathrm{o} \mathrm{Zr}$ alloy tubing but at lower temperatures: $545^{\circ} \mathrm{C}$ maximum at the jacket surface and $625^{\circ} \mathrm{C}$ maximum at the fuel center.

Newer irradiations are being conducted with more advanced metallic fuels. A fuel $\operatorname{rod}$ of U-15 w/o Pu-10 w/o Ti alloy jacketed in V-20 w/o Ti alloy has reached 4.6 a/o burnup at temperatures of $790^{\circ} \mathrm{C}$ maximum at the fuel center and of $570^{\circ} \mathrm{C}$ maximum at the jacket surface. Another alloy, U-18.5 w/o Pu-14.1 $\mathrm{w} / \mathrm{O} \mathrm{Zr}$, also jacketed in $\mathrm{V}-20 \mathrm{w} / \mathrm{O} \mathrm{Zr}$ alloy, has achieved 4.2 a/o burnup at even higher temperatures: $820^{\circ} \mathrm{C}$ maximum at the fuel center and $600^{\circ} \mathrm{C}$ maximum at the jacket surface. These fuel rods will receive further irradiation.

In the area of alloy development, significant progress has been made in the improvement of melting temperature and in the suppression of phase transformation. Titanium additions of $6.5 \mathrm{w} / \mathrm{o}$ to U-15 w/o $\mathrm{Pu}$ alloy raise the solidus temperature some $300^{\circ} \mathrm{C}$ above the $820^{\circ} \mathrm{C}$ solidus for $\mathrm{U}-15 \mathrm{w} / \mathrm{O} \mathrm{Pu}-10 \mathrm{w} / \mathrm{o} \mathrm{Fz}$ alloy. The addition of $10 \mathrm{w} / \mathrm{o} \mathrm{Zr}$ to U-15 w/o Pu also raises the solidus temperature the same amount. This increase in melting temperature of the fuel can be used directly in increasing power density, improving reliability, or increasing fuel diameter. Raising of the melting temperature may also raise swelling temperatures of the fuel.

The prospect of suppressing phase transformations in $\mathrm{U}-\mathrm{Pu}$ alloys within the operating temperature range of the fuel appears promising in the U-Pu-Ti alloy system. In the binary U-Ti system, a compound $\mathrm{U}_{2} \mathrm{Ti}$ is formed which undergoes no phase change below $900^{\circ} \mathrm{C}$, at which temperature the compound decomposes into a gamma U-Ti phase. Indications are that as much as half of the uranium can be replaced by plutonium in this compound, but at the expense of transformation.

The most exciting development is the discovery that the higher $\mathrm{U}-\mathrm{Pu}-\mathrm{Zr}$ alloys are compatible with Type 
304 stainless steel and form no molten reaction products to temperatures above $800^{\circ} \mathrm{C}$, some $200^{\circ} \mathrm{C}$ above the temperature at which inolten phases form in the $U$ $\mathrm{Pu}-\mathrm{Fz}$ versus Type 304 stainless steel. This development opens a whole new area of jacket combinations, with great potential from both performance and economic standpoints.

In the sections which follow, details of irradiation performance, alloy development and compatibility, and jacket corrosion resistance for U-Pu fuel alloy and jacket systems are discussed. Nothing will be reported on fuel, jacket, and fuel-element fabrication, as these segments of fast reactor fuel-element program are well developed. ${ }^{(1,2)}$

The fuel systems of importance are:

a) U-Pu-Fr fuel- $\mathrm{V}$ alloy jacket;

b) U-Pu-Ti fuel-V alloy jacket;

c) U-Pu-Zr fuel--stainless steel and V alloy jacket.

Other refractory alloy jackets, such as $\mathrm{Nb}-1 \mathrm{w} / \mathrm{o} \mathrm{Zr}$ alloy, have been used for proof of principle largely because of availability.

\section{Irradiation Performance}

Uranium-plutonium-fizzium alloys have by themselves very low resistance to irradiation swelling. Breakaway swelling for these alloys, in general, occurs around $370^{\circ} \mathrm{C}$ for low burnup. ${ }^{(3)}$ Alloy modification within the scope of fizzium additions have not proven

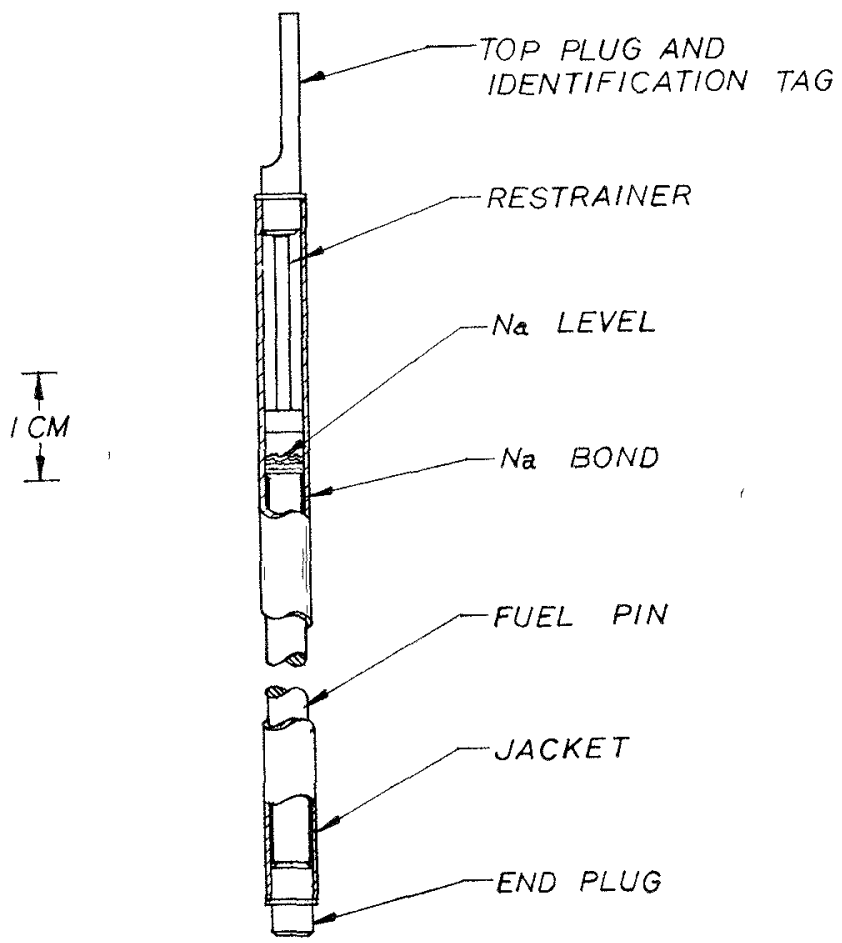

FIg. 1. Cutaway Drawing of CP-5 Irradiation Specimen Showing Components. effective in improving resistance to irradiation swelling. Alloys which fall outside the fizzium range may show improved swelling resistance through increased melting temperature and suppression of phase transformation. These are subjects of current investigation.

A positive approach has been taken by Argonne National Laboratory for the control of swelling in U-Pu alloy fuels which does not depend upon developing a cure for the swelling phenomenon itself. The approach is confinement of swelling by jacket restraint. ${ }^{(4)}$ A jacket must possess adequate strength, ductility, compatibility, corrosion resistance, and irradiation stability to perform this function.

The irradiation of metallic $\mathrm{U}-\mathrm{Pu}$ fast reactor fuels has been carried out with prototype EBR-II fuel rods having reference cross-sectional dimensions but scaled down in length by a factor of seven. A large part of this irradiation work has been carried out in temperature-monitored and -controlled capsules positioned within the tubular fuel of the CP-5 reactor, where an appreciable fast flux exists. ${ }^{(5)} \mathrm{A}$ sketch of a typical irradiation specimen is shown in Fig. 1. Other irradiations have been with full-length fuel rods in temperature-controlled capsules in the MTR reactor. Irradiations have been initiated in EBR-II on encapsulated fuel rods.

\section{A. High-burnup Specimens}

The high-burnup experiment eited previously was designed to explore the effectiveness of jacket wall thickness and plutonium content on the restraint of fuel swelling. Two fuel alloys were used in this experiment, $\mathrm{U}-15 \mathrm{w} / \mathrm{0} \mathrm{Pu}-10 \mathrm{w} / \mathrm{o} \mathrm{Fz}$ and $\mathrm{U}-10 \mathrm{w} / \mathrm{o}$ $\mathrm{Pu}-10 \mathrm{w} / \mathrm{o} \mathrm{Fz}$, all fuel pins being $0.144 \mathrm{in}$. in diameter and 2 in. long. The uranium component of these alloys was enriched $7.7 \%$ in the $15 \mathrm{w} / \mathrm{o} \mathrm{Pu}$ alloy and $15.5 \%$ in the $10 \mathrm{w} / \mathrm{o} \mathrm{Pu}$ alloy. The jackets were $\mathrm{Nb}-1 \mathrm{w} / \mathrm{o} \mathrm{Zr}$ alloy tubing, $0.156 \mathrm{in}$. in ID and 0.015 , 0.020 , and 0.025 in. in wall thicknesses. Sodium was used in the annuli between fuel and jacket as a heat transfer medium. The six specimens were irradiated in a single capsule for 29 months in CP-5. A calculated burnup of $8.7 \mathrm{a} / 0$ was achieved in the higher plutonium alloys by the end of September 1965. Irradiation was carried out at maximum temperatures of $625^{\circ} \mathrm{C}$ for the central fuel and $545^{\circ} \mathrm{C}$ for the jacket surface. Time-averaged temperatures were, however, lower, being $495^{\circ} \mathrm{C}$ for that central fuel and $430^{\circ} \mathrm{C}$ for the jacket surface. Design parameters and irradiation conditions for this capsule are summarized in Table 1.

As shown in Fig. 2, five of the six pins survived this high burnup without defect or deformation. The jacket of one fucl pin suffered a local fracture at 
TABLE 1 CP-5 InR LDITTON Data Fon CAPSLle No CP $33^{* * *}$

\begin{tabular}{|c|c|c|c|c|c|c|c|c|}
\hline \multirow{2}{*}{ Spec No } & \multirow{2}{*}{ I uel Comp, w/o } & \multirow{2}{*}{$\begin{array}{c}\text { Jacket Comp, } \\
\text { w/o }\end{array}$} & \multicolumn{2}{|c|}{ Jacket Dimensions } & \multirow{2}{*}{ Vold Vol, \% } & \multicolumn{2}{|c|}{ Estimated Burnup* } & \multirow{2}{*}{$\begin{array}{l}\text { Calculated } \\
\text { Burnup, a/o }\end{array}$} \\
\hline & & & $\mathrm{ID}$, in & Wall, in & & $\mathrm{a} / \mathrm{OU}+\mathrm{Pu}$ & $\underset{10^{-21}}{\mathrm{f} / \mathrm{cm}^{3} \times}$ & \\
\hline C $115 \mathrm{~A}$ & $\mathrm{U}-10 \mathrm{Pu}-10 \mathrm{~F} z$ & $\mathrm{Nb} 1 \mathrm{Zr}$ & 0156 & 0015 & 222 & 71 & 28 & 50 \\
\hline $\mathrm{C} 116 \mathrm{~B}$ & $\mathrm{U}-10 \mathrm{Pu} 10 \mathrm{Fz}$ & $\mathrm{Nb} 1 \mathrm{Zr}$ & 0156 & 0020 & 232 & 71 & 28 & 59 \\
\hline C $119 \mathrm{~A}$ & $\mathrm{U} 15 \mathrm{Pu} 10 \mathrm{Fr}$ & $\mathrm{Nb} 1 \mathrm{Zr}$ & 0156 & 0015 & 233 & 87 & 33 & $53 \mathrm{~F}^{* *}$ \\
\hline $\mathrm{C} 118 \mathrm{C}$ & $\mathrm{U} 15 \mathrm{Pu} 10 \mathrm{~F} z$ & $\mathrm{Nb} 1 \mathrm{Zr}$ & 0156 & 0025 & 238 & 87 & 33 & 94 \\
\hline $\mathrm{C}-117 \mathrm{~A}$ & $\mathrm{U} 15 \mathrm{Pu} 10 \mathrm{~F}$ & Nb $1 Z x$ & $0 \quad 156$ & 0015 & 298 & 87 & 33 & 72 \\
\hline C $120 \mathrm{C}$ & $\mathrm{U} 10 \mathrm{Pu} 10 \mathrm{~F}$ & $\mathrm{Nb} 1 \mathrm{Zr}$ & 0156 & 0025 & 242 & 71 & 28 & 67 \\
\hline
\end{tabular}

* As of September 30,1965

** Indicates jacket fallure

*** Maxmum temperature at the jacket surface $w a n 55^{\circ} \mathrm{C}$ and at the fuel center $625^{\circ} \mathrm{C}$.
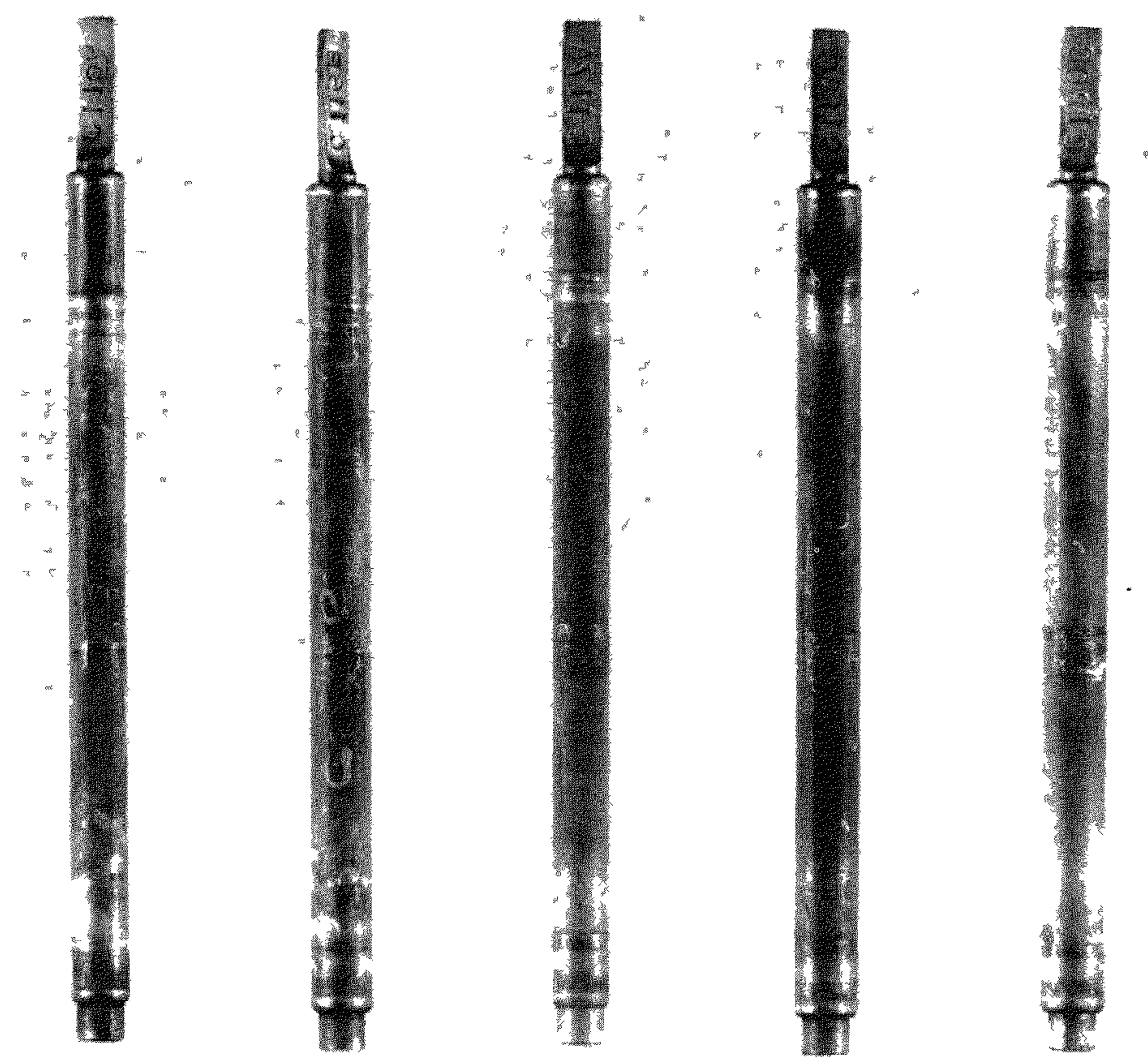

Specimen No.

C-115A

C-116B

C-117A

C- $118 \mathrm{C}$

C-120C

Composition, w/0 U-10Pu-10Fz

Burnup, a/o 7.1 7.1 8.7

$\mathrm{U}-15 \mathrm{Pu}-10 \mathrm{Fz}$

8.7

$\mathrm{U}-10 \mathrm{Pu}-10 \mathrm{Fz}$

Fig. 2. Appearance of U-Pu-F 4 Allor, Jacketed an Nb 1 w/o Zr Alloy after Irradiation to High Burnup 


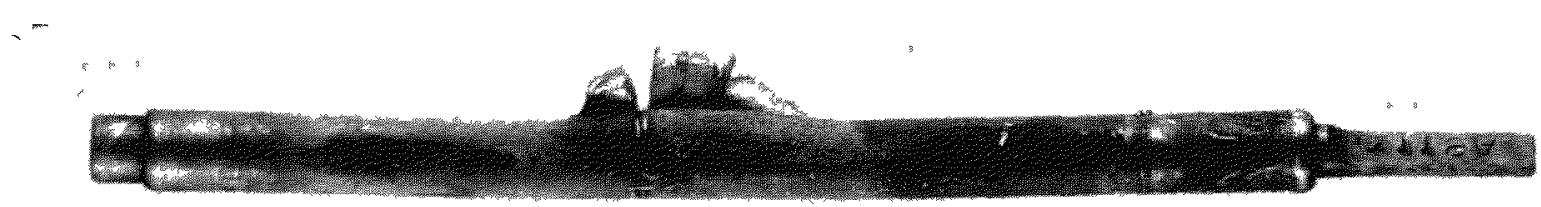

Fia. 3. U-15 w/o Pu-10 w/o Fz Alloy Specimen Jacketed in Nb-1 w/o Zr Alloy Showing Jacket Rupture after 8.7 a/o Burnup

some indicated burnup level between $5.0 \mathrm{a} / \mathrm{o}$ when last neutron radiographed and $8.7 \mathrm{a} / \mathrm{o}$ when examined. The fuel in the failed rod had extruded from the jacket, as shown in Fig. 3, assuming the shape of the jacket opening. This specimen was jacketed in stress-relieved, 0.015-in.-wall tubing. The two specimens jacketed in annealed tubing of the same wall thickness survived without significant change in external dimensions. Success of the specimen jacketed in annealed tubing might be attributed to higher ductility and perhaps higher creep strength. The range in jacket wall thickness was used to study its effect in attaining high burnup. These experiments indicate, however, that a wall thickness of $0.025 \mathrm{in}$. is not required for the burnup levels reached here.

This experiment has demonstrated, in principle, the effectiveness of a strong jacket upon restraint of fuel swelling to attractive burnup in spite of the fact that $\mathrm{X} b-1 \mathrm{w} / \mathrm{o} \mathrm{Zr}$ alloy is not presently the preferred jacket. This level of burnup in an U-Pu alloy is the highest ever reported for this type of fuel.

For purposes of comparison, $8.7 \mathrm{a} / \mathrm{o}$ burnup in the uranium-plutonium-fizzium alloys used in this experiment produced a fission density of $3.3 \times 10^{22}$ fissions/ $\mathrm{cm}^{3}$. This fission density is equivalent to over 100,000 $\mathrm{MWd} / \mathrm{T}$ burnup in dense mixed oxide fuel, and to $80,000 \mathrm{MWd} / \mathrm{T}$ burnup in mixed carbide fuel.

More recent developments have yielded jacket materials, based upon vanadium, which have features more attractive than those for niobium alloys. Among these features are: (a) better nuclcar properties, (b) superior compatibility with fuel, (c) higher mechanical properties, (d) good fabricability, and (e) improved corrosion resistance to sodium.

Irradiation experiments involving $\mathrm{V}-20 \mathrm{w} / \mathrm{o} \mathrm{Ti}$ alloy jackets were started in September 1964 upon availability of tubing from ANL alloy and fabrication development. A burnup level of $5.7 \mathrm{a} / \mathrm{o}$ was reached in a group of six U-15 w/o Pu-10 w/o Fz alloy fuel rods at the end of September 1965. All specimens were jacketed in tubing of 0.016 -in. wall thickness having varying inside diameters ranging from 0.157 to $0.169 \mathrm{in}$. All fuel used was $0.144 \mathrm{in}$. in diameter and $2 \mathrm{in}$. long. The varying gaps between fuel and jacket (filled with $\mathrm{NaK}$ ) coupled with varying allowable axial expansion of the fuel (clearance between fucl and top restrainer) were established in these specimens so that jacket failure calculated by formulae developed by Foote ${ }^{(6)}$ would occur at approximately $3.1 \mathrm{a} / \mathrm{o}$ burnup. Details of these design parameters and irradiation conditions for these specimens are shown in Table 2. Irradiations of these specimens were carricd out at substantially higher temperatures, $620^{\circ} \mathrm{C}$ maximum at the surface and $720^{\circ} \mathrm{C}$ maximum for the center temperature (timeaveraged temperatures about $70^{\circ} \mathrm{C}$ lower), than the previously discussed high-burnup specimens. Upon removal from the reactor at $5.7 \mathrm{a} / \mathrm{o}$ burnup, the capsule was radiographed by neutron techniques developed at ANL. (7) Four of the six fuel rods showed evidence of jacket deformation. These specimens as listed in Table 2 (in Capsule No. CP-40) include those having the largest and those having the smallest annuli between fuel and jacket. The significant feature of these irradiations is the fact that two of the specimens certainly far exceeded, and the others probably exceeded, the calculated achievable burnup.

Another capsule of fuel specimens has been irradiated to $4.0 \mathrm{a} / \mathrm{o}$ burnup at a maximum surface temperature of $630^{\circ} \mathrm{C}$ and a maximum fuel center temperature of $740^{\circ} \mathrm{C}$ (time-averaged temperatures about $75^{\circ} \mathrm{C}$ lower). Plutonium content was varied in these specimens as well as the gap between fuel and jacket, the gap between fuel and upper restrainer, and the void space above the bond. Both U-15 w/o Pu-10 $\mathrm{w} / \mathrm{o} \mathrm{Fz}$ and $\mathrm{U}-10 \mathrm{w} / \mathrm{o} \mathrm{Pu}-10 \mathrm{w} / \mathrm{o} \mathrm{Fz}$ alloy fuels, 0.144 in. in diameter and $2 \mathrm{in}$. long, were used. The annuli between fuel and jacket in these rods varied from approximately 0.006 to 0.012 in. Fuel jackets of $\mathrm{V}-20 \mathrm{w} / \mathrm{o} \mathrm{Ti}$ were used, all having 0.016 -in. wall thicknesses. The design of these specimens was such that the calculated burnup at failure would be 6.0 a/o. Design and operating data for the specimens, all in Capsule No. CP-41, are given in Table 2. Neutron radiography of the capsule at 4.0 a/o burnup has indicated jacket deformation in one rod, a 10 w/o Pu alloy.

The burnup already reached in many of these irradiation experiments are at levels attractive for fast reactor fuel systems projected for large-scale plants. The conditions under which these experiments are being conducted, in water-cooled thermal reactors, are 
TABIE 2. CP-5 Capsule IrR IDition Data for V-20 w/o Ti Jackets of 0.016-in. Wall Thickness

\begin{tabular}{|c|c|c|c|c|c|c|c|c|c|c|c|}
\hline \multirow{2}{*}{ Capsule No. } & \multirow{2}{*}{$\begin{array}{l}\text { Specimen } \\
\text { No. }\end{array}$} & \multirow{2}{*}{ Fuel Comp, w/o } & \multirow{2}{*}{$\begin{array}{l}\text { Jacket } \\
\text { ID, in. }\end{array}$} & \multirow{2}{*}{$\begin{array}{l}\text { Restrainer } \\
\text { above } \\
\text { Fuels, in. }\end{array}$} & \multirow{2}{*}{$\begin{array}{l}\text { Void } \\
\text { Vol, } \%\end{array}$} & \multicolumn{2}{|c|}{ Max Temp, ${ }^{\circ} \mathrm{C}$} & \multicolumn{2}{|c|}{ Estimated Burnup* } & \multirow{2}{*}{\multicolumn{2}{|c|}{$\begin{array}{l}\text { Calculated J } \\
\text { Burnup, } \\
\text { a/o }\end{array}$}} \\
\hline & & & & & & $\begin{array}{c}\text { Jacket } \\
\text { Surface }\end{array}$ & $\begin{array}{l}\text { Fuel } \\
\text { Center }\end{array}$ & $\underset{\mathrm{Pu}}{\mathrm{a} \mathrm{o}}+$ & $\mathrm{f} / \mathrm{cm}_{10^{-21}} \times$ & & \\
\hline \multirow{4}{*}{$\mathrm{CP}-40$} & NI & $\mathrm{U}-15 \mathrm{Pu}-10 \mathrm{Fz}$ & 0.157 & 0.001 & 23.5 & 620 & 720 & 5.7 & 2.2 & 3.1 & $\mathrm{~F}^{* *}$ \\
\hline & $\mathrm{N} 2$ & & 0.157 & 0.010 & 23.6 & 620 & 720 & 5.7 & 2.2 & 3.1 & \\
\hline & N3 & & 0.165 & 0.010 & 26.5 & 620 & 720 & 5.7 & 2.2 & 3.1 & $\mathrm{~F}$ \\
\hline & N6 & & 0.169 & 0010 & 27.5 & 620 & 720 & 5.7 & 2.2 & 3.1 & $\mathrm{~F}$ \\
\hline \multirow[t]{4}{*}{$\mathrm{CP}-41$} & N7 & $\mathrm{U}-15 \mathrm{Pu}-10 \mathrm{Fz}$ & 0.165 & 0.001 & 41.1 & 630 & 740 & 4.0 & 1.5 & 6 & \\
\hline & N8 & & 0.161 & 0.010 & 39.9 & 630 & 740 & 4.0 & 1.5 & 6 & \\
\hline & N9 & & 0157 & 0.018 & 36.7 & 630 & 740 & 4.0 & 1.5 & 6 & \\
\hline & $\mathrm{N} 10$ & $\mathrm{U}-10 \mathrm{Pu}-10 \mathrm{Fz}$ & 0.165 & 0.001 & 41.1 & 630 & 740 & 4.0 & 1.5 & 6 & $\mathrm{~F}$ \\
\hline
\end{tabular}

* As of September 30, 1965 .

** Indicates possible jacket failure.

in some instances more severe than those anticipated in a sodium-cooled fast reactor. During the course of irradiation, the specimens are subjected to many and violent thermal cycles, occasioned by reactor scrams and scheduled shutdowns. The number and magnitude of thermal cycles the fuel would receive in a sodiumcooled fast reactor would be substantially less. The severity of irradiation damage to the jacket material is probably substantially less in CP-5 reactor on a comparable temperature level because of the much lower dose sustained for equivalent burnup.

\section{B. Advanced-fuel Irradiations}

One characteristic common to most of the failures experienced with $\mathrm{U}-\mathrm{Pu}-\mathrm{Fz}$ alloys is the presence of what appears to be once-molten fuel. Whether this apparent melting occurred before or after jacket rupture cannot be determined from these experiments. In either case, however, increasing melting temperature of the fuel would be helpful.

The development work with advanced fuel is based, in part, upon the desire to increase the melting temperature of U-Pu alloy fuels.

Two alloying elements have been selected from those that raise the melting temperature of the binary $\mathrm{C}-\mathrm{Pu}$ alloys. The two more potent elements and the least objcctionable from the physics standpoint are titanium and zirconium. Irradiation experiments have been initiated to determine the irradiation stability of fuel rods designed according to calculations to reach about 10 a/o burnup. To obtain a high burnup rate (being limited by the heat transfer capability of the capsule) the capsule contains only a single fuel rod. One capsule contains an $\mathrm{U}-15 \mathrm{w} / \mathrm{o} \mathrm{Pu}-10 \mathrm{w} / \mathrm{o}$ Ti alloy fuel pin jacketed and NaK bonded in 0.015-in.-wall, V-20 w/o Ti alloy tubing. The other capsule is loaded with one rod of L-18.5 w/o Pu-14.1 w/o $\mathrm{Zr}$ alloy also jacketed in $\mathrm{V}-20 \mathrm{w} / \mathrm{o} \mathrm{Ti}$ alloy tubing. The fuel pins are $0.144 \mathrm{in}$. in diameter and $4 \mathrm{in}$. long. The inside diameter of the $\mathrm{V}-\mathrm{Ti}$ alloy jacket is $0.173 \mathrm{in}$. for the $\mathrm{U}-\mathrm{Pu}-\mathrm{Ti}$ fuel and $0.175 \mathrm{in}$. for the U-Pu-Zr fuel, leaving annuli of 0.0145 and $0.0155 \mathrm{in}$., respectively.

These fuel rods have been irradiated at a substantial rate of about $0.7 \mathrm{a} / 0$ per month in CP-5. As shown in Table 3, the U-Pu-Ti alloy rod had reached $4.6 \mathrm{a} / \mathrm{o}$ burnup at the end of August; the $\mathrm{U}-\mathrm{Pu}-\mathrm{Zr}$ alloy rod had reached $4.2 \mathrm{a} / \mathrm{o}$ burnup. Ir-

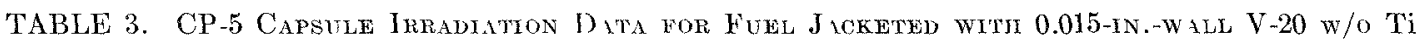

\begin{tabular}{|c|c|c|c|c|c|c|c|c|c|c|}
\hline \multirow{2}{*}{$\begin{array}{c}\text { Capsule } \\
\text { No. }\end{array}$} & \multirow{2}{*}{$\begin{array}{c}\text { Spec. } \\
\text { No. }\end{array}$} & \multirow{2}{*}{ Fuel Comp, w/o } & \multirow{2}{*}{$\begin{array}{l}\text { Jacket } \\
\text { ID, in. }\end{array}$} & \multirow{2}{*}{$\begin{array}{l}\text { Restrainer } \\
\text { above Fuel, } \\
\text { in. }\end{array}$} & \multirow{2}{*}{$\begin{array}{c}\text { Void Vol, } \\
\%\end{array}$} & \multicolumn{2}{|c|}{$\operatorname{Max} \operatorname{Temp},{ }^{\circ} \mathrm{C}$} & \multicolumn{2}{|c|}{ Estimated Burnup* } & \multirow{2}{*}{$\begin{array}{c}\text { Calculated } \\
\text { Burnup, } \\
\text { a/o }\end{array}$} \\
\hline & & & & & & $\begin{array}{l}\text { Jacket } \\
\text { Surface }\end{array}$ & $\begin{array}{c}\text { Fuel } \\
\text { Center }\end{array}$ & $\begin{array}{c}a / o \\
\mathrm{U}+\mathrm{Pu}\end{array}$ & $\underset{10^{-21}}{\mathrm{f} / \mathrm{cm}^{3}} \times$ & \\
\hline CP -44 & N14 & U-15-Pu-10Ti & 0.173 & 0.59 & 64 & 570 & 790 & 4.6 & 1.7 & 9.6 \\
\hline$C P-45$ & N15 & $\mathrm{U}-18.5 \mathrm{Pu}-14.1 \mathrm{Zr}$ & 0.175 & 0.59 & 73 & 600 & 820 & 4.2 & 1.4 & 10.0 \\
\hline
\end{tabular}

* As of September 30, 1965. 
radiation temperatures are substantial, being $570^{\circ} \mathrm{C}$ maximum at the surface and $790^{\circ} \mathrm{C}$ maximum at the fuel center for the $\mathrm{U}-\mathrm{Pu}-\mathrm{Ti}$ alloy, and $600^{\circ} \mathrm{C}$ maximum at the surface and $820^{\circ} \mathrm{C}$ maximum at the fuel center for $\mathrm{C}-\mathrm{Pu}-\mathrm{Zr}$.

Neutron radiographs of both capsules after CP-5 shutdown indicates that the jackets are intact on both fuel rods. The U-Pu-Ti alloy rod, however, has clongated about $15 \%$ to the restrainer, indicating the need for a restrainer to control fuel length. The U-Pu-Zr fuel rod has developed two narrow bands of density reduction, one near the top of the rod and the other somewhat below the center, with some increase in length. The significance of these areas is not known.

The burnup levels reached in these fuels on first attempts are very encouraging, particularly since irradiation temperatures are high for metallic fuels. Both of these capsules were to be returned to the reactor for further irradiation.

\section{Fuel-alloy Development}

\section{A. Elevating Melting Temperature}

The initial objectives of the uranium-plutonium alloy development are simple and straightforward: (a) to increase the solidus temperature about $300^{\circ} \mathrm{C}$ abore that for $\mathrm{U}-\mathrm{Pu}-\mathrm{Fz}$ alloys, and (b) to suppress phase transformations within the operating temperature range of the fuel. The melting temperature has direct effect upon the thermal performance of a fuel since melting for all intent and purpose limits the

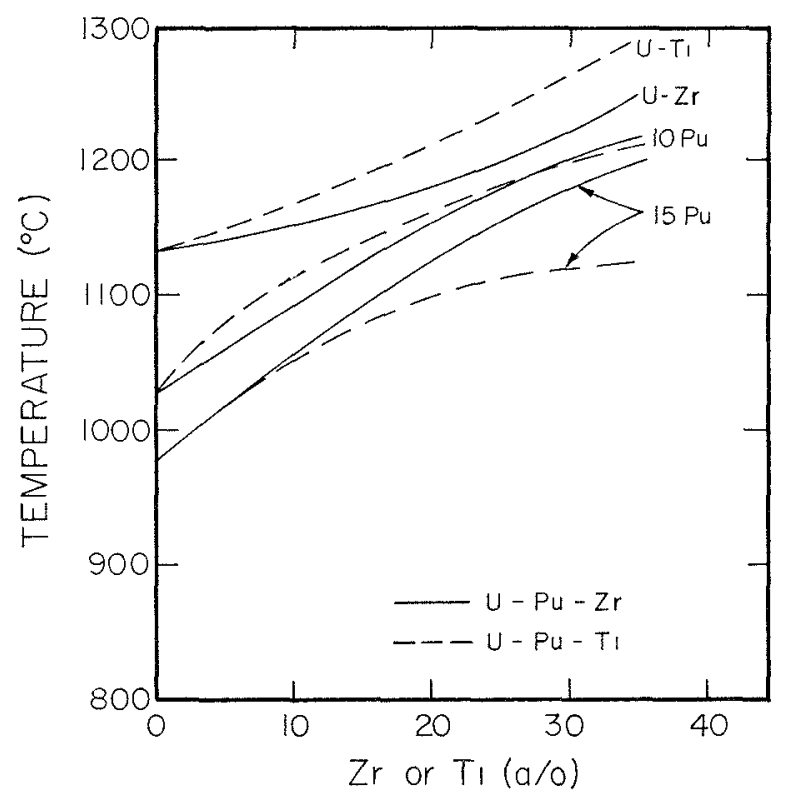

FIG. 4. Effect of Zireonium and Titanium on Solidus of U-Pu Alloys. top temperature at which the fuel can be used. The higher the tempcrature, the higher the power rating for a given fuel and fuel geometry. Melting temperature could also indirectly effect the swelling characteristic of the fuel.

The $\mathrm{U}-\mathrm{Pu}-\mathrm{Fz}$ alloys were taken to be the equilibrium alloys containing fission product elements not removed by the melt-refining process ${ }^{(8)}$ now being used to pyrorefine a similar U-Fz alloy for EBR-II. The alloy designated $10 \mathrm{w} / \mathrm{o} \mathrm{Fz}$ contains $2.80 \mathrm{w} / \mathrm{o}$ $\mathrm{Zr}, 2.75 \mathrm{w} / \mathrm{o} \mathrm{Mo}, 2.97 \mathrm{w} / \mathrm{o} \mathrm{Ru}, 0.50 \mathrm{w} / \mathrm{o} \mathrm{Rh}$, and 1.00 $\mathrm{w} / \mathrm{o} \mathrm{Pd}$ in the synthetic form. Technetium will be present in the fission-produced alloy. It has been replaced by equivalent amounts of molybdenum and ruthenium in the synthetic alloy. Severe restrictions have been placed upon the operating conditions under which the fizzium fuels can be used because of its low solidus temperature, $820^{\circ} \mathrm{C}$ for $\mathrm{U}-20 \mathrm{w} / \mathrm{o} \quad \mathrm{Pu}-10$ $\mathrm{w} / \mathrm{o} \mathrm{Fz}, 865^{\circ} \mathrm{C}$ for $\mathrm{U}-15 \mathrm{w} / \mathrm{o} \mathrm{Pu}-10 \mathrm{w} / \mathrm{o} \mathrm{Fz}$, and $910^{\circ} \mathrm{C}$ for $\mathrm{C}^{\prime}-10 \mathrm{w} / \mathrm{o} \mathrm{Pu}-10 \mathrm{w} / 0 \mathrm{Fz}$.

A number of elements were known or suspected to increase the melting temperature of $\mathrm{U}-\mathrm{Pu}$ alloys. These include molybdenum, niobium, scandium, titanium, yttrium, and zirconium. Ternary alloys of each of these dements with uranium and plutonium were prepared and screened for their effectiveness in increasing the solidus temperature. Of this group, zirconium and titanium were found to be most effective. It is fortuitous that these elements are the least undesirable from a physics standpoint.

The effect of zirconium and titanium additions upon the solidus temperature of U-Pu alloy is shown in Figure 4. It is evident that $\mathrm{U}-\mathrm{Pu}-\mathrm{Zr}$ and $\mathrm{U}-\mathrm{Pu}-\mathrm{Ti}$ alloys have much higher solidus and liquidus temperatures than do equivalent $\mathrm{U}-\mathrm{Pu}-\mathrm{Fz}$ alloys, as is shown in Fig. 5.

In comparison, an $\mathrm{U}-10 \mathrm{w} / \mathrm{o} \mathrm{Pu}-10 \mathrm{w} / \mathrm{o} \mathrm{Zr}$ alloy starts to melt at approximately $1150^{\circ} \mathrm{C}$, whereas an U-10 w/o Pu-10 w/o Fz alloy begins to melt at $910^{\circ} \mathrm{C}$. This increase in solidus temperature can be put to good use in the application of this fuel.

\section{B. Suppression of Phase Transformation}

Phase transformations directly affect the thermal stability of a fuel system because abrupt dimensional (density) changes that take place upon transformation contribute to performance limitations. Phase transformations could also markedly increase the swelling rate of fucl. For these and other reasons phase transformations are to be avoided or at least made as sluggish as possible.

Phase transformation can be suppressed by adding alloying elements which slow the rate of transformation sufficiently to prevent their taking place within 


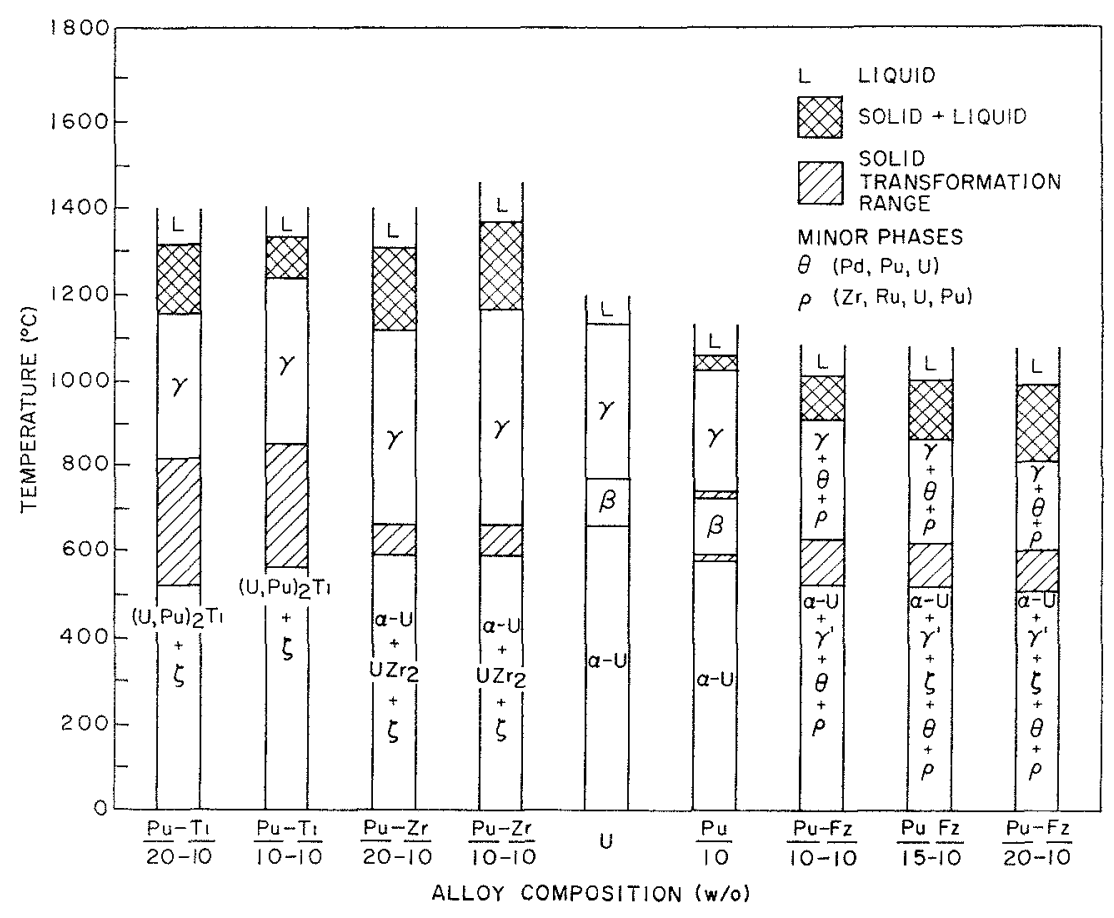

Fig. 5. Schematic Comparison of Phase Fields of Some U-Pu-based Alloys.

the life of the fuel. Although some elements retard transformation, none have been found which are sufficiently potent to be effective in temperature ranges of interest in reactor operation. Molybdenum and the fissium elements are examples of those which retard transformation.

Another method of suppressing phase transformation is by alloying into a phase field which displays no transformation in the temperature ranges of interest. This stable form is the one of interest. The $\mathrm{U}-\mathrm{Ti}$ alloy has this feature of interest in a useful composition range. In this alloy system, there is a compound, $\mathrm{U}_{2} \mathrm{Ti}$, having a hexagonal structure which is stable up to about $900^{\circ} \mathrm{C}$. Above this temperature this compound transforms into a gamma-phase uranium. The $\mathrm{U}_{2}$ Ti compound exists over a narrow composition range. Plutonium can be substituted for about one-half of the uranium in this compound, ${ }^{(9)}$ but at the sacrifice of lowering the upper temperature in the range for which the compound exists. Alloys containing less titanium than the compound amount consists of $(\mathrm{U}, \mathrm{Pu})_{2} \mathrm{Ti}$ and $\mathrm{U}-\mathrm{Pu}$ alpha phase and a small amount of zeta phase.

\section{Physical and Mechanical Properties}

The most pertinent properties have been determined for $\mathrm{C}-\mathrm{Pu}-\mathrm{Zr}$ and $\mathrm{C}-\mathrm{Pu}-\mathrm{Ti}$ alloys of interest. The major influence on properties by additions of zirconium and titanium is the increase in melting temperature of these alloys over that of the binary
$\mathrm{U}-\mathrm{Pu}$, as has been discussed above. The microstructure of these ternary alloys, of course, is much simpler than that for $\mathrm{U}-\mathrm{Pu}-\mathrm{F} z$ alloys.

Thermal cycling of U-Pu-Zr and U-Pu-Ti alloys shows these alloys to be very stable to this treatment. Only very small density and dimensional changes occur after 100 cycles between $450^{\circ} \mathrm{C}$ and $700^{\circ} \mathrm{C}$ at a transfer rate of $10 \mathrm{~min}$. This cycle was designed to simulate normal reactor operation and not a scram, however. Density and dimensional changes are slightly less than those measured for $\mathrm{U}-\mathrm{Pu}-\mathrm{Fz}$ alloys and substantially lower than the changes developed in unalloyed uranium and the binary $\mathrm{U}-10 \mathrm{w} / \mathrm{o} \mathrm{Pu}$ alloy.

Based on preliminary data, the thermal conductivities of equivalent $\mathrm{U}-\mathrm{Pu}-\mathrm{Zr}$ and $\mathrm{U}-\mathrm{Pu}-\mathrm{Fz}$ alloys appear to be similar. Alloys of higher zirconium content have somewhat lower conductivities. As was expected, the $(\mathrm{U}, \mathrm{Pu})_{2} \mathrm{Ti}$ compound and the U-10 $\mathrm{w} / \mathrm{o} \mathrm{Pu}-8 \mathrm{w} / \mathrm{o}$ $\mathrm{Ti}$ alloy have the lowest conductivity of the metal fuels measured.

Mechanical properties of $\mathrm{U}-\mathrm{Pu}-\mathrm{Zr}$ and $\mathrm{U}-\mathrm{Pu}-\mathrm{Ti}$ alloys have been found to compare with those of U-Pu-Fz. Uranium-plutonium-base alloys are weak at elevated temperatures, having ultimate tensile strengths of 2 to $7 \mathrm{~kg}-\mathrm{mm}^{-2}$ between 600 and $700^{\circ} \mathrm{C}$. Room-temperature properties, however, can be high and can vary widely depending upon the alloy and its history.

Creep strengths of U-Pu-Ti and U-Pu-Zr alloys 
TABle 4. Creep in U-Pu-base Alloys (Time in Mintemes to Atrain 1\% Stratn)

\begin{tabular}{c|c|c|c|c|c|c|c}
\hline \multirow{2}{*}{$\begin{array}{c}\text { Stress } \\
\left(\mathrm{kg}-\mathrm{mm}^{-2}\right)\end{array}$} & \multicolumn{2}{|c|}{$\mathrm{U}-15 \mathrm{w} / \mathrm{o}$ Pu-10 w/o Ti } & \multicolumn{2}{|c|}{$\mathrm{U}-18.5$ w/o Pu-14 w/o Zr } \\
\cline { 2 - 7 } & $600^{\circ} \mathrm{C}$ & $650^{\circ} \mathrm{C}$ & $700^{\circ} \mathrm{C}$ & $600^{\circ} \mathrm{C}$ & $675^{\circ} \mathrm{C}$ & $700^{\circ} \mathrm{C}$ \\
\hline 0.28 & - & 210 & 64 & - & 94 & 70 \\
0.42 & 170 & 35 & 30 & $3.8 \times 10^{4}$ & 27 & 32 \\
0.56 & 120 & 30 & 17 & $3.7 \times 10^{4}$ & 13 & 12 \\
\hline
\end{tabular}

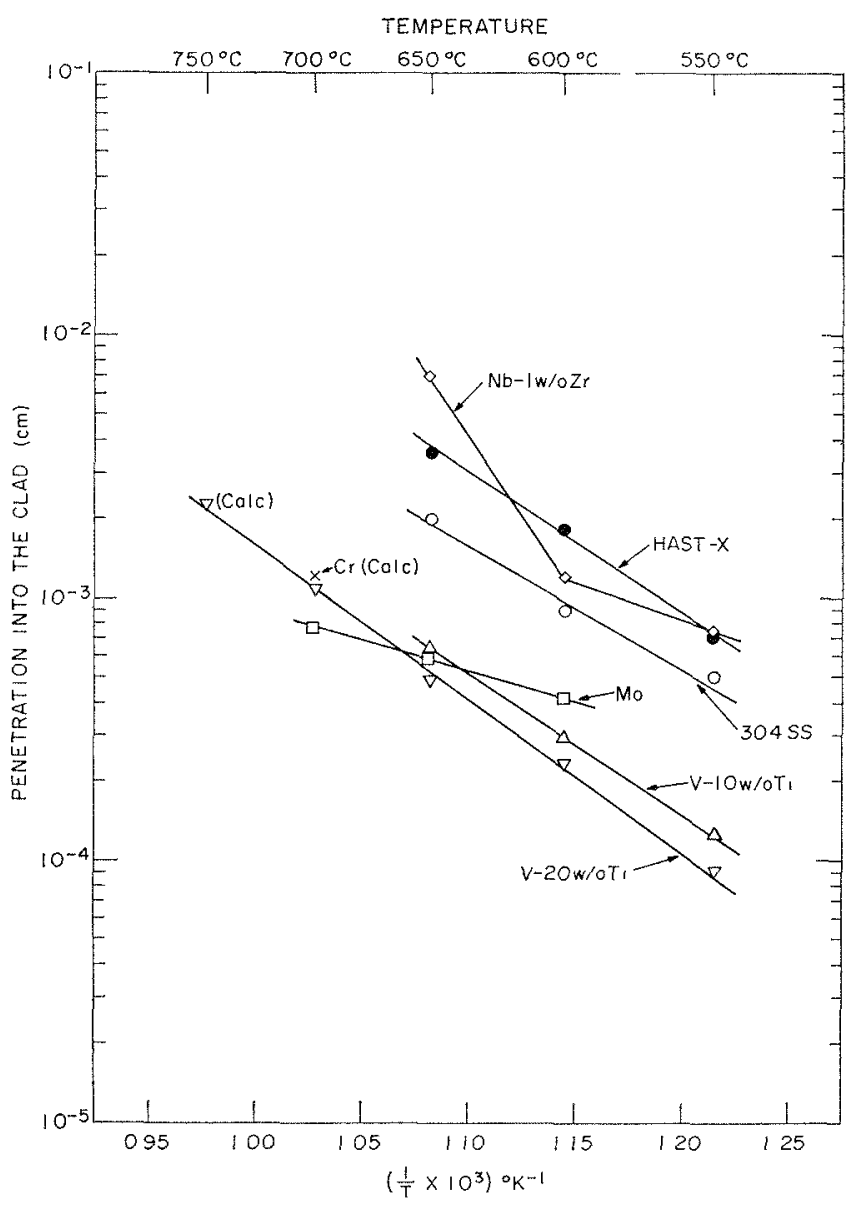

FIG. 6, Diffusion Penetration of U-10 w/o Pu-10 w/o Fz into Prospective Claddings at a Constant Time of 42 Days.

are very low above $600^{\circ} \mathrm{C}$, in the temperature range of most interest in reactor operation. These low creep rates are indicated in Table 4 . The abrupt change in rate experienced by $\mathrm{U}-18.5 \mathrm{w} / \mathrm{o} \mathrm{Pu}-14.0$ w/o $\mathrm{Zr}$ alloy between 600 and $675^{\circ} \mathrm{C}$ can be associated with a phase transformation which takes place in this temperature range. These low-level mechanical properties can be used to advantage if enhanced performance can be realized from the weak fuelstrong jacket concept. The fuel in a weak condition can conform to the will of the jacket more readily than can a strong fuel.

\section{Compatibility of Fuel and Jacket}

Compatibility between fuel and jacket is a very important, if not a limiting, factor in the development of high-performance, high-burnup fuel systems. Extensive work has been done at ANL to determine and to understand interactions between fuel and jacket materials appropriate for metallic $\mathrm{U}-\mathrm{Pu}$ fuel systems. Extensive investigation has been made with $\mathrm{U}-\mathrm{Pu}-\mathrm{Fz}$ fuels, employing diffusion couples with various jacket materials in the temperature range from 550 to $750^{\circ} \mathrm{C} .{ }^{(10,11)}$ Interpenetration between fuel and jacket has been determined prineipally by metallographic means, with checks made by microprobe analysis.

As shown in Fig. 6, V-Ti alloys, molybdenum, and chromium are most compatible with U-10 w/o Pu-10 w/o Fz fuel of the materials studied. Type 304 stainless stcel and Hastelloy-X are considerably poorer in behavior, with $\mathrm{Nb}-1 \mathrm{w} / \mathrm{o} \mathrm{Zr}$ alloy being the worst. All these materials follow the parabolic rate law except $\mathrm{Nb}-1 \mathrm{w} / \mathrm{o} \mathrm{Zr}$ and $\mathrm{Nb}$, which follow a linear behavior.

For all intents and purposes, iron- and nickel-base alloys must be excluded from reactor use with the fizzium alloy fuels because of the low melting temperature of the reaction products. For example, molten phases developed in Type 304 stainless steel versus $\mathrm{U}-\mathrm{Pu}-\mathrm{Fz}$ and $\mathrm{Hastelloy}-\mathrm{X}$ versus $\mathrm{U}-\mathrm{Pu}-\mathrm{Fz}$ at temperatures between 650 and $675^{\circ} \mathrm{C}$. This temperature is much too close to reactor operating temperatures contemplated to be tolerated. Extrapolation of the penetration data obtained to longer periods yields the one-year penetration levels shown in Table 5 . These extrapolations show $\mathrm{V}$ and V-Ti alloys to be superior to all others except chromium, for which limited data are available.

Study of the penetration data indicates that approximate rates may be predicted from examination of the binary phase diagrams of the major elements

TABle 5. Penetration of U-Pu-Fz Alaloys into Pótenta a Cladding Materi dro at Variotis Temperatures lextrapoL $\triangle T E D$ TO ONE YE1K $\left(\mathrm{cm} \times 10^{4}\right)$

\begin{tabular}{|c|c|c|c|c|c|}
\hline Cladding/Fuel (w/o) & $550^{\circ} \mathrm{C}$ & $600^{\circ} \mathrm{C}$ & $650^{\circ} \mathrm{C}$ & $700^{\circ} \mathrm{C}$ & $750^{\circ} \mathrm{C}$ \\
\hline $\mathrm{V}-20 \mathrm{Ti} / \mathrm{U}-10 \mathrm{Pu}-10 \mathrm{~F} z$ & $3^{*}$ & 6 & 15 & 30 & $75^{*}$ \\
\hline $\mathrm{V}-20 \mathrm{Ti} / \mathrm{U}-15 \mathrm{Pu}-10 \mathrm{~F} z$ & & $6^{*}$ & 15 & $50^{*}$ & $100^{*}$ \\
\hline $\mathrm{V}-10 \mathrm{Ti} / \mathrm{C}-\mathrm{Pu}-10 \mathrm{Fz}$ & 4 & 10 & 20 & & \\
\hline $\mathrm{Cr} / \mathrm{U}-10 \mathrm{Pu}-10 \mathrm{~F} z$ & & & & $30^{*}$ & \\
\hline $\mathrm{Mo} / \mathrm{U}-10 \mathrm{Pu}-10 \mathrm{Fz}$ & & 10 & 15 & 25 & \\
\hline $\mathrm{Mo} / \mathrm{U}-15 \mathrm{Pu}-10 \mathrm{Fz}$ & & $15^{*}$ & $25^{*}$ & $35^{*}$ & \\
\hline $304 \mathrm{SS} / \mathrm{U}-10 \mathrm{Pu}-10 \mathrm{Fz}$ & 15 & 25 & 60 & & \\
\hline $304 \mathrm{SS} / \mathrm{U}-15 \mathrm{Pu}-10 \mathrm{Fz}$ & & & $100^{*}$ & & \\
\hline Ifast-X/U-10Pu-10Fz & 25 & 50 & 110 & & \\
\hline IIast-X/U-15Pu-10Fz & & & $190^{*}$ & & \\
\hline $\mathrm{Nb} / \mathrm{U}-10 \mathrm{Pu}-10 \mathrm{Fz}$ & 20 & 45 & 700 & & \\
\hline $\mathrm{Nb} / \mathrm{U}-20 \mathrm{Pu}-10 \mathrm{Fz}$ & 30 & 75 & 1300 & & \\
\hline $\mathrm{Nb}-1 \mathrm{Zr} / \mathrm{U}-10 \mathrm{Pu}-10 \mathrm{Fz}$ & 25 & 35 & 600 & & \\
\hline
\end{tabular}

* Extrapolated from a single point. 
in the fuel and jackets. Some general indications are that phase systems which have limited solubility, particularly on the jacket side of the system, and form no intermetallic compounds have good compatibility. Conversely, fuel-jacket systems which have extensive solid solubility or which form intermetallic compounds appear to have poorer compatibility.

As was stated earlier, the most exciting feature of the advanced fuel work is the discovery of relatively high compatibility temperatures of U-Pu-Zr alloys with Type 304 stainless steel. Whereas the U-10 w/o Pu-10 w/o Fz alloy forms a molten phase with stainless steel at temperatures slightly above $650^{\circ} \mathrm{C}$, zirconium alloys having even higher plutonium contents do not form a molten phase with
Type 304 stainless steel until a temperature of $850^{\circ} \mathrm{C}$ is approached. This discovery opens a whole new area of otherwise very desirable jacket materials for consideration of use with these alloy fuels.

In addition to raising the temperature at which reaction products between fuel and jacket melt, these zirconium alloys show very low penetration rates into stainless steel at normal operating temperatures $\left(600-700^{\circ} \mathrm{C}\right)$. For example, test results at $650^{\circ} \mathrm{C}$ predict less than $10 \mu$ penetration by U-18.5 w/o $\mathrm{Pu}-14.0 \mathrm{w} / \mathrm{o} \mathrm{Zr}$ alloy into Type 304 stainless steel in one year. This penetration rate increases slowly with temperature, but is still relatively low at $800^{\circ} \mathrm{C}$ (less than $100 \mu$ ) in one year. These changes in rate with temperature are shown metallographically in Fig. 7. Changes in fuel structure in the diffusion

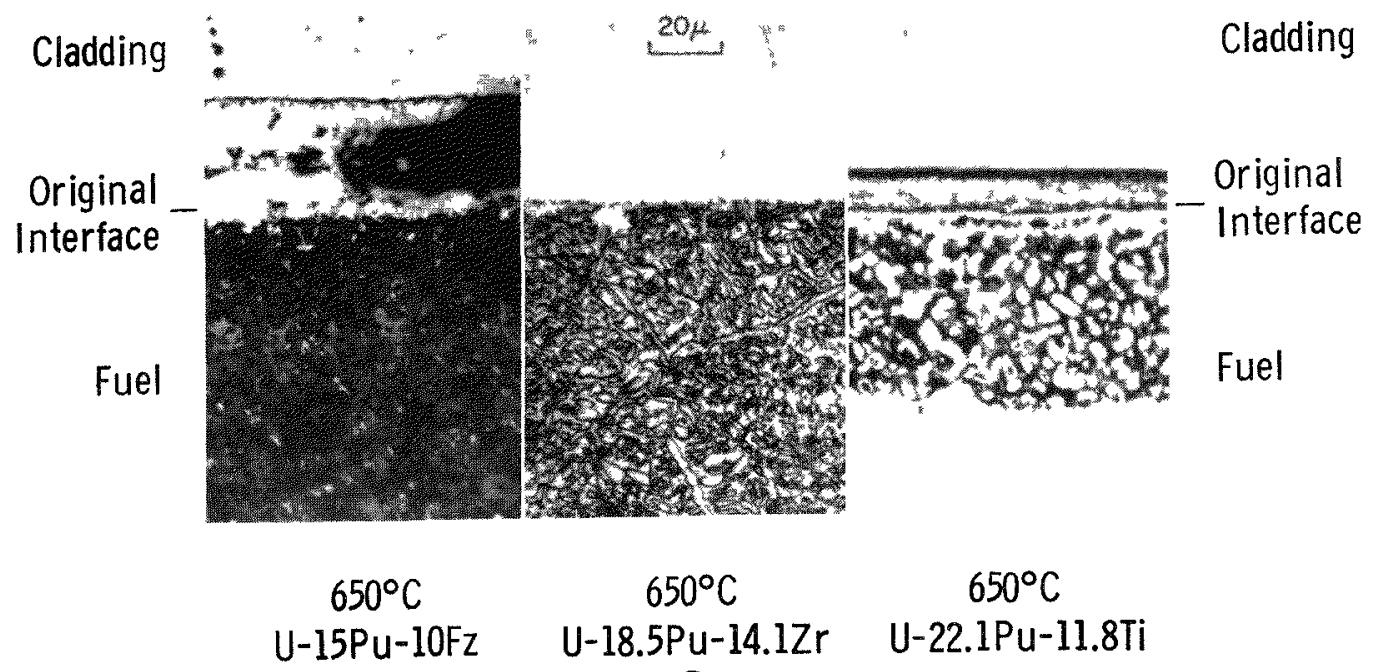

(a)

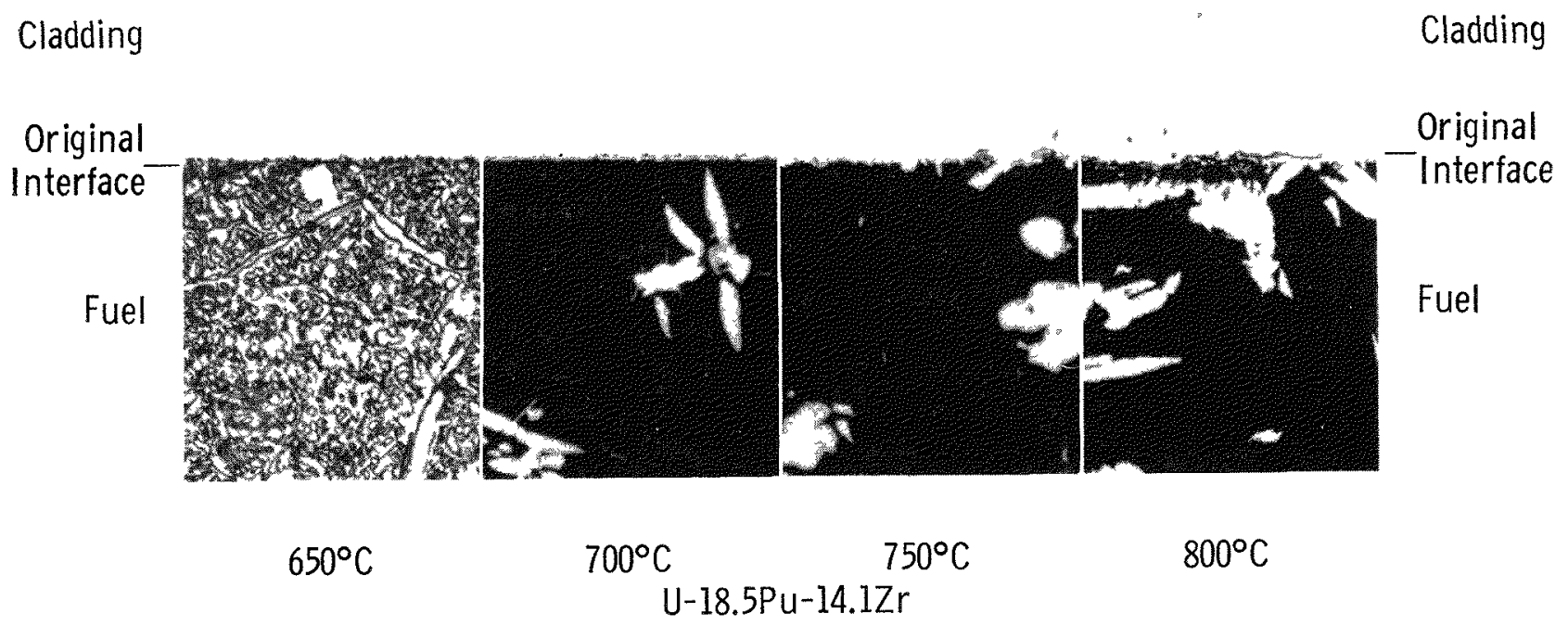

(b)

Fra. 7. Compatibility of $30+\mathrm{SS}$ with U-Pu-based Fuels (compositions in w/o) after 7 Days at Temperature. 
TABLE 6. Penetration of U-Pu-Zr Fuel into 304 SS, H istelloy-X, ind Incoloy-800 Cladding

\begin{tabular}{|c|c|c|c|c|c|c|c|c|c|c|}
\hline \multirow{3}{*}{ Cladding } & \multirow{3}{*}{ Temp $\left({ }^{\circ} \mathrm{C}\right)$} & \multicolumn{9}{|c|}{ Penetration $\left(\mathrm{cm} \times 10^{4}\right)$} \\
\hline & & \multicolumn{3}{|c|}{ U-16.6 w/o Pu-6.3 w/o Zr } & \multicolumn{3}{|c|}{$\mathrm{U}-15 \mathrm{w} / \mathrm{o} \mathrm{Pu}-10 \mathrm{w} / \mathrm{o} \mathrm{Zr}$} & \multicolumn{3}{|c|}{$\mathrm{U}-18.5 \mathrm{w} / \mathrm{o} \mathrm{Pu}-14.1 \mathrm{w} / \mathrm{o} \mathrm{Zr}$} \\
\hline & & 7 Days & 17 Days & 42 Days & 7 Days & 17 Days & 42 Days & 7 Days & 17 Days & 42 Days \\
\hline $304 \mathrm{SS}$ & $\begin{array}{l}650 \\
700 \\
750 \\
800 \\
850\end{array}$ & Melted ${ }^{*}$ & 8 & & 4 & 2 & & $\begin{array}{c}<1 \\
1 \\
2 \\
9 \\
\text { Melted }\end{array}$ & 2 & $\begin{array}{r}6 \\
17\end{array}$ \\
\hline Hastelloy-X & $\begin{array}{l}700 \\
750 \\
800\end{array}$ & Melted ${ }^{*}$ & 50 & & Melted* & 10 & & Melted* & & $\begin{array}{c}12 \\
\text { Melted* }\end{array}$ \\
\hline Incoloy- 800 & $\begin{array}{l}750 \\
800\end{array}$ & & & & $\begin{array}{c}16 \\
\text { Melted* }\end{array}$ & & 38 & & & \\
\hline
\end{tabular}

* Melting of the reaction products and accelerated penetration occurred.

couples at higher temperature can be attributed to the fuel being held in the gamma phase.

Compatibility of U-Pu-Zr alloys with some alloys of higher strength have been studied. Hastelloy-X and Incoloy-800 are inferior in both rate of penetration and temperature of melting when compared with Type 304 stainless steel. Data for these systems are given in Table 6 . The conclusions to be drawn from results with these couples are: (a) penetration rate decreases and melting temperature increases with increasing zirconium content in the fuel; (b) higher nickel content in the jacket material decreases compatibility.

A number of alloys of higher strength are now under study to determine what levels of nickel, and also of cobalt, can be tolerated while maintaining compatibility: Haynes $\$ 56,16-25-6,16-15-6$, and $\mathrm{N}-155$. Early results with $\mathrm{N}-155$, which contains 20 $\mathrm{w} / \mathrm{o} \mathrm{Ni}$ and $20 \mathrm{w} / \mathrm{o} \mathrm{Co}$, indicate satisfactory compatibility at $750^{\circ} \mathrm{C}$.

The mechanism that leads to the excellent compatibility between U-Pu-Zr alloys and iron-base alloys is under investigation. Further work is needed to correlate this effect with observation made by others ${ }^{(12)}$ which show complex diffusion paths are taken in some ternary systems. Expcriments are under way with a simplified U-Zr versus Fe system. Melting has been prevented to $800^{\circ} \mathrm{C}$ in uranium alloys containing greater than $9.7 \mathrm{w} / 0 \mathrm{Zr}$. Indications are that the addition of zirconium avoids regions having molten phases in the binary U-Fe system and that improvement in compatibility is indeed due to the zirconium.

\section{Development of Vanadium Alloys}

Vanadium-base alloys possess many qualities required of a good jacket material for metallic U-Pu fuels. They have acceptable nuclear properties, have excellent compatibility with $\mathrm{U}-\mathrm{Pu}$ alloy fuels, have good strength, and can be fabricated into high-quality tubing without resorting to exotic methods. The only point in question (aside from verifying its ability to restrain high swelling $\mathrm{U}-\mathrm{Pu}$ alloy fuels) is its resistance to corrosion by sodium.

The corrosion behavior of vanadium and vanadiumbase alloys in sodium at $650^{\circ} \mathrm{C}$ is very sensitive to the oxygen content of the alkali metal (other contaminants may also effect corrosion rates). Variation in the oxygen content of the corroding sodium from 50 ppm to hot-gettered metal, believed to be under $5 \mathrm{ppm}$ oxygen, changes the corrosion behavior of V-Ti alloys from a linear weight loss at the highoxygen end to parabolic weight gains in hot-gettered sodium.

The corrosion data for V-20 w/o Ti alloy establishing the parabolic rate are plotted in Fig. 8. These low rates have been substantiated by metallographic examination and hardness traverses made on sections through test samples. After $323 \mathrm{hr}$ the surface oxide phase was less than $1 \mu$ thick. X-ray diffraction pattern indicated the oxide to be single-phase $\mathrm{VO}_{0} 9$ with titanium present. Total contamination in the metal evaluated by hardness traverse indicated oxygen penetration to be less than $25 \mu$.

A parabolic rate constant was determined from the corrosion data and found to be $2.763\left(\mathrm{mg} / \mathrm{cm}^{2}\right)^{2} \mathrm{hr}^{-1}$.

The corrosion resistance of $\mathrm{V}-\mathrm{Ti}$ alloys is not as good as originally believed; however, the performance of $\mathrm{V}-20 \mathrm{w} / \mathrm{o} \mathrm{Ti}$ alloy in low-oxygen sodium holds promise, particularly in view of purity levels indicated in the primary sodium systems in EBR-II and Fermi, and in the $\mathrm{NaK}$ system at the Dounreay Fast Reactor. 


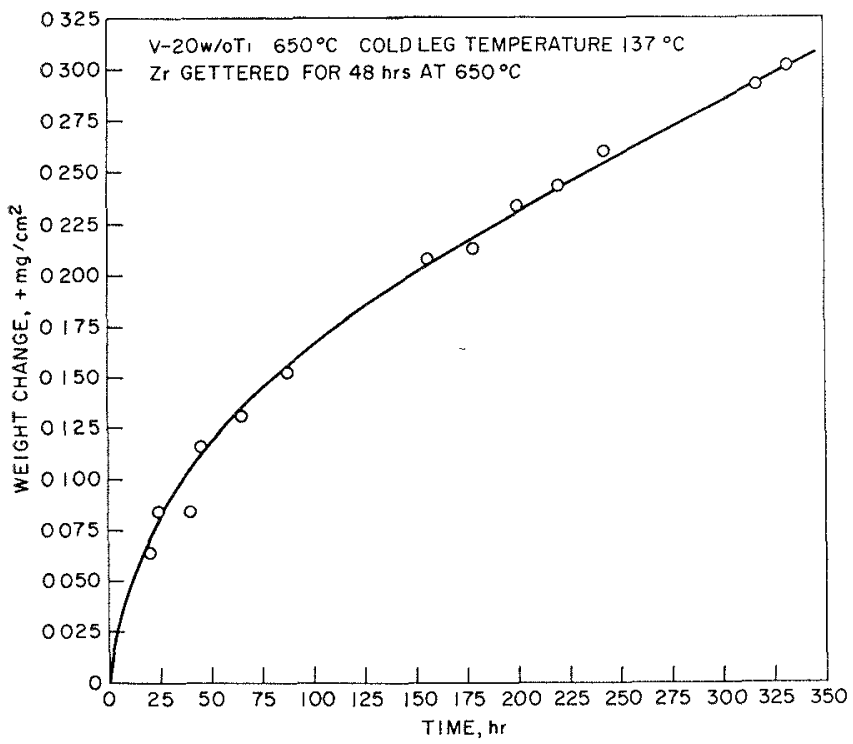

Frg. 8. Weight Change of $\mathrm{V}$-Ti Alloy in Hot-gettered Sodium.

Accelerated screening tests in sodium containing oxygen levels of about $50 \mathrm{ppm}$ indicate improved resistance of other alloys. On a comparative basis, higher titanium alloys up to $50 \mathrm{a} / \mathrm{o} \mathrm{Ti}$ and some ternary V-Ti-Cr alloys exhibit lower corrosion rates than $\mathrm{V}-20 \mathrm{w} / \mathrm{o}$ Ti alloys.

Metallurgical structure has also been found to affect corrosion rate. Annealed V-Ti alloys (for one hour at $900^{\circ} \mathrm{C}$ ) have shown significantly lower total penetration than the same material in the coldworked condition. Alloy composition and heat treatment are two areas which will be exploited.

\section{Conclusions}

The effectiveness of refractory alloy jackets in restraining high-swelling U-Pu alloy fuels has been demonstrated to high burnup levels of $8.7 \mathrm{a} / \mathrm{o}$. This achievement in itsolf is substantial, but is even more impressive when compared with burnup levels achievable only a short time ago. Two years ago burnup levels of $1.6 \mathrm{a} / \mathrm{o}$ could not be exceeded in U-20 w/o Pu-10 w/o Fz alloys. From that time to the present a fivefold increase in burnup level has been achieved through: (a) modification of design, principally increased wall thickness, increased void space to accept sodium displaced from the bond, and increased void space into which fuel can swell, and (b) substantial improvement in quality of the jacket tubing, mostly resulting from ANL developments in tubing fabrication.

The burnup levels already successfully achieved in metal fuels are considered adequate for economic competition as fuels for the fast reactor. ${ }^{(13)}$ This burnup level, however, must be duplicated in a truly fast environment and repeated to develop confidence.
The prospects that metallic fuel specimens now in preparation will exceed burnups of $10 \mathrm{a} / \mathrm{o}$ are very good by virtue of the effectiveness of zirconium and titanium in raising the melting temperature of uranium-plutonium alloys. The compatibility of U-Pu$\mathrm{Zr}$ alloys with stainless steel is a further promising development since it can effectively improve the economics and safety of metallic fuel systems, and may improve performance.

\section{REFERENCES}

1. A. B. Shuck and J. E. Ayer, Engineering Considerations for Remote Refabrication of EBR-II Fuel Elements, Nucl. Sci. Eng., 12, 398-404 (1962).

2. W. R. Burt, Jr., W. C. Kramer, R. 1). MeGowan, F, J. Karasek, and R. M. Mayfield, Consolidation and Fabrication Techniques for Vanadium-20 w/o Titanium (TV20), ANL-6928 (Feb 1965).

3. J. A. Horak, J. H. Kittel, and R. J. Dunworth, The Effects of Irradiation on Uranium-Plutomium-Fissium Fuel Alloys, ANL-6429 (1962).

4. W. N. Beck, J. H. Kittel, and R. J. Fousek, Irradiations of U-20Pu-10F's Alloy Fuel Rods, ANL-6750 (1965).

5. W. N. Beck and R. J. Fousek, Instrumented Temperaturecontrolled Capsules for Irradiations in the CP-5 Reactor, ANL-6554 (1962).

6. W. N. Beck, W. F. Murphy, J. H. Kittel, and F. G. Foote, Irradiation Behavior of Plutonium Alloy Fuels for Fast Reactors, to be presented at the Third International Conference on Plutonium, London, 22-26 November 1965.

7. H. Berger and W. N. Beck, Neutron Radiographic Inspection of Radioactive Irradiated k'uel Specimens, Nucl. Sci. Eng., 15, 411-414 (1963).

8. J. C. Hesson, M. J. Feldman, and L. Burris, Description and Proposed Operation of the Fuel Cycle Facility for the Second Experimental Breeder Reactor (EBR-II), ANL-6605 (April 1963).

9. Quarterly Status Report on Advanced Reactor T'echnology (ART) for Period Ending January 31, 1965, LA-3244-MS, p. 31 .

10. C. M. Walter, Interdiffusion between Uranium-5 w/o Fissium Alloy and Type 304 Stainless Steel, ANL-6816 (March 1964).

11. C. M. Walter and J. A. Lahti, Compatibility of U-Pu-Fz Fuel Alloy with Potential Cladding Materials, ANL-7051 (to be published).

12. J. S. Kirkaldy and L. C. Brown, Diffusion Behavior in Ternary, Multi-phase Systems, Canadian Met. Quarterly, 2(1), 89 (1963).

13. L. E. Link, J. E. Ayer, K. A. Hub, W. J. Mecham, I. G. Trice, Jr., and J. T. Weills, A Large Metal-fueled F'ast Reactor Study. Part II: Core Design and Fuel Cycle, see paper in Session II of this Conference.

\section{Discussion}

Mr. Weinberg (ORNL): I wonder if from your experiments you have any way of telling whether the cladding materials undergo the high-temperature embrittlement which is on the minds of so many people, such as the helium-induced embrittlement?

Mr. Macherey: The experiments conducted so far 
have been in a thermal environment. Although this is not really indicative of the exposures the material will get in fast environment, at these burnup levels and dose rates the materials appear to have appreciable ductility at the end of exposure. We don't have definitive experiments at this point which would indicate the change in properties of the material.

Mr. Weinberg: You say this because the cans did not split?

Mr. Macherey: Yes.

Mr. Weinberg: Even though they were under a fair amount of stress?

Mr. Macherey: Yes.

Mr. Weinberg: Do you have any idea of the boron contents of your cladding?

Mr. Macherey: No. These experiments were run with refractory metal jackets principally, and not many of these have been analyzed prior to this point.

Mr. Weinberg: Perhaps I could turn the question around and ask: do you understand why your jackets don't show this high-temperature embrittlement?

Mr. Macherey: No; I wouldn't care to guess.

$M r$. Kummerer (Karlsruhe): What was the maximum or average power in the pins? Also, what was the basis for your calculations for burnup in relation to swelling?

Mr. Macherey: The burnup calculation relates the pressure developed by gaseous fission products upon the jacket under conditions where no restraint is assumed from the material itself. By means of the ideal-gas equation $P V=n R T$ the amount of fission gas generated in the fuel was correlated with the void volume available initially in the pin. As the fission gases form, the fuel expands and applies pressure on the jacket. The resulting pressure is a function of the amount of gas produced and the amount of space available in the fuel.

$M r$. Kummerer: For this calculation, you assume given strength of the material in this temperature?

Mr. Macherey: Yes, around 30,000 psi or perhaps a little higher for $\mathrm{Nb}-1 \mathrm{w} / \mathrm{o} \mathrm{Zr}$ and a little bit less for the V-Ti jackets.

Mr. Kummerer: I would be interested in your answer about the power.

Mr. Machery: In the high-burnup specimen at the low temperature, the power density was equivalent to, or say, calculated to $7.6 \mathrm{~kW} / \mathrm{ft}$ for the titanium specimens and to $12.8 \mathrm{~kW} / \mathrm{ft}$ for the zirconium alloy.

$M r$. Campise (AI) : In discussing the corrosion resistance of $\mathrm{V}-20 \mathrm{w} / \mathrm{o}$ Ti you mention an oxide buildup on the surface. I was wondering whether in the presence of a high-intensity fast-neutron flux this surface protection that you are getting would be affected or stripped off by high-energy neutrons and therefore increase the corrosion rate of the clad.

Mr. Macherey: It is possible, but I think that most definitive experiments aimed at improving or accelerating reaction rates haven't been particularly fruitful. Stress in the jacket could certainly affect this, as well as fission fragments. We do have experiments underway in EBR-II to determine effects of neutron environment on this corrosion rate. 


\title{
Compatibility of Modified Carbide Fuels with Stainless Steel
}

\author{
K. R. Jorbax, R. J. Herbst, and R. (․ Palit \\ Westinghouse Electric Corporation \\ Atomic Power Division \\ Forest Hills, Pennsylvania
}

(PRESENTED BY R. J. HERBST)

\section{Introduction}

The monocarbides of uranium and plutonium are potential reactor fuels. The high density of heavy metal atoms in the monocarbides recommends use of a mixture of the two in fast breeder reactors (FBR), especially since the high thermal conductivity of these compounds and their admixtures permits operation at high power density. To utilize this latter advantage fully, fucl-element designs incorporating carbide materials include a liquid-metal thermal bond, o.g. Na or NaK, at the fuel-clad interface.

A factor limiting the utility of carbide fuels is incompatibility with stainless steel clad materials. Control of the carbon content in contemporary procedures used to prepare uranium, plutonium, or mixed uranium-plutonium monocarbide is limited to \pm 0.1 w/o. Since uranium and carbon hare negligible solubility in the monocarbide, ${ }^{(1)}$ fuels fabricated from nominally stoichiometric compositions invariably include a secondary phase. The secondary phase, free metal in the case of less than stoichiometric carbon contents (hypostoichiometric) and higher carbides (e.g., $\mathrm{U}_{2} \mathrm{C}_{3}, \mathrm{Pu}_{2} \mathrm{C}_{3}$, or $\mathrm{UC}_{2}$ ) in the ease of greater than stoichiometric carbon contents (hyperstoichiometric), contributes to incompatibility with stainless steels. The incompatibility is exaggerated in liquidmetal-bonder systems wherein the liquid metal may act as a vehicle to accelerate reaction.

The free metal present in hypostoichiometric compositions may react with iron-base-alloy cladding. materials. The product of this reaction with uranium metal is a eutectic composition melting at $727^{\circ} \mathrm{C} .{ }^{(2)}$ If plutonium metal is present, the eutectic composition may melt as low as $425^{\circ} \mathrm{C} .^{(3)}$ The presence of a eutcetic melt will greatly accelerate the rate of subsequent reaction and contribute to clad failure. Accordingly, the free metal present in hypostoichiometric compositions is dangerous or, at best, limiting.

The mechanism of clad interaction with hyperstoichiometric compositions involves decarburization of the fuel and subsequent carburization of the clad alloy. ${ }^{(4)}$ The resulting embrittlement and reduction in the ductility of the clad material will contribute to premature clad failure. A liquid-metal thermal bond will accelerate the progress of these reactions. Liquid sodium will decarburize the higher carbides in hyperstoichiometric compositions and the carbon will precipitate in the clad alloy. The rate of this reaction is prohibitive above $540^{\circ} \mathrm{C}$.(5) An oxygen-contaminated thermal bond will further accelerate the progress of carburization, even in nearly stoichiometric compositions, through oxidation of the carbide.

Considerable experimental effort has been expended in attempts to synthesize single-phase monocarbides. In the case of uranium monocarbide, UC, these studies have sought to expand the range of carbon contents over which the single phase can be obtained. Alloying additions which themselves form stable monocarbides over a range of carbon contents, e.g., zirconium carbide, have been made to UC. ${ }^{(6)}$ No system has been identified that does not result in prohibitive dilution of the fission-fertile atom density.

The Westinghouse Atomic Power Division has postulated a significant departure from the usual goal of a single-phase fuel to improve the compatibility of carbide fucls with stainless steel.(7) Elemental or compound modifying additions may be made to offstoichiometric compositions to react or combine with the normally occurring sccondary phases. The resultant polyphase composition contains secondary phases more stable and refractory than the uranium, plutonium, or mixed uranium-plutonium metals or their higher carbides. The required addition should satisfy the following eriteria:

a. The addition should effectively combine with the secondary phases initially present, forming a stable compound that precludes formation of lowmelting eutecties with the cladding constituents.

b. The phases present in the final modified composition should have high melting points.

c. The final secondary phases must be compatible with the monocarbide.

d. Uranium and plutonium should be mutually sol- 


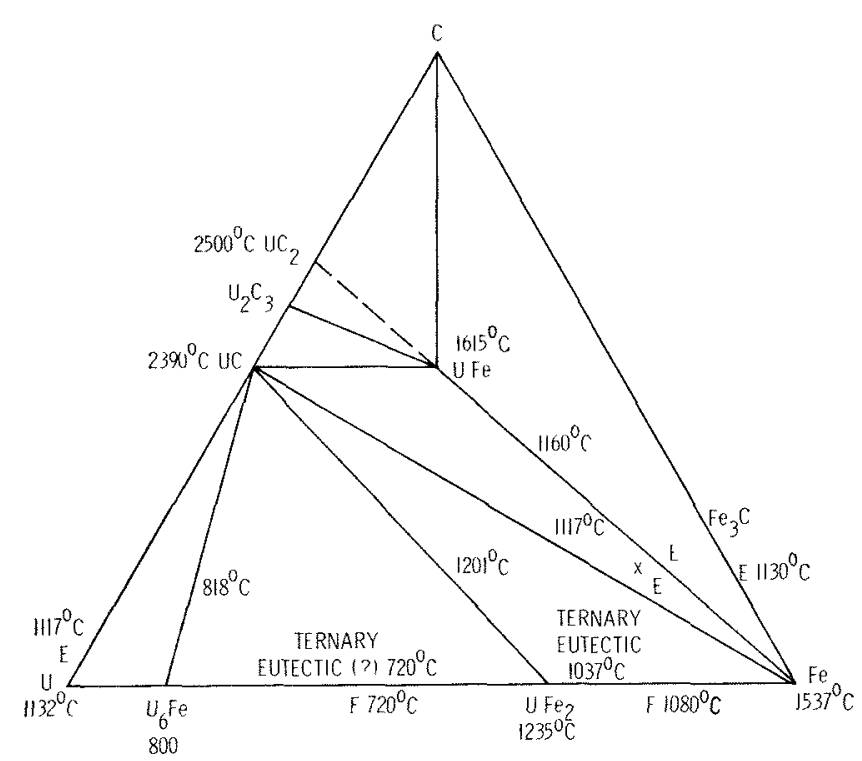

Fra. 1. Tie Lines in the U-C.Fe Srstem.

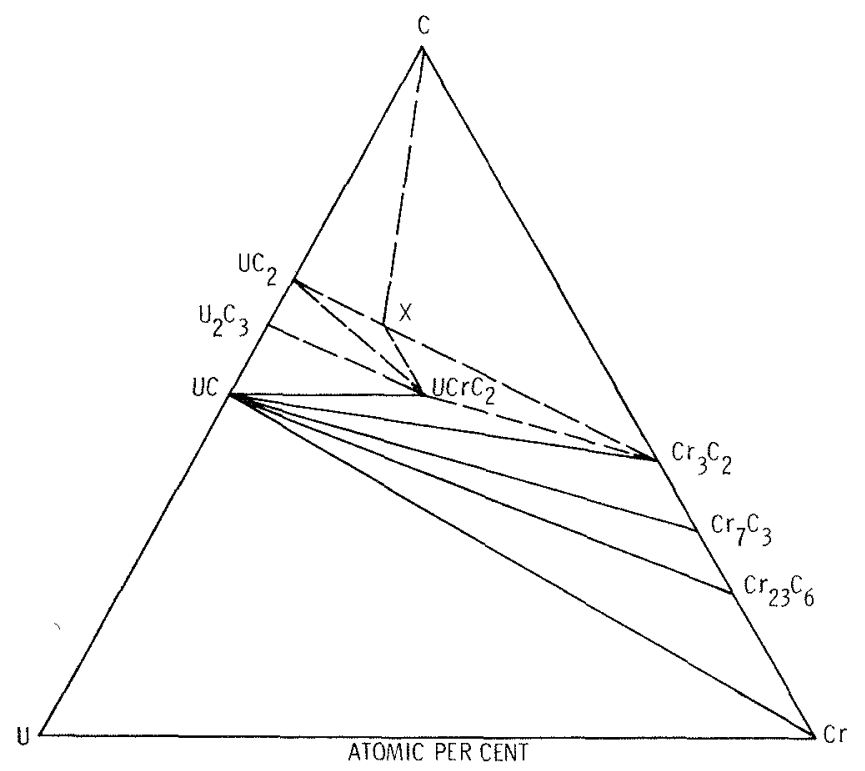

Frr. 2. Tie Lines in the IT C.Cr System. ${ }^{(9)}$

uble in the final phases so that low-melting uraniumcompound/plutoniun-compound euterties cannot form.

e. The amount of modifying addition required should be minimal.

Any compound present in the final composition should also have a low neutron absorption cross section and good irradiation stability.

Review of pertinent phase equilibria has revealed several ternary ranges of composition in $\mathrm{U}-\mathrm{C}-\mathrm{X}$ systems. A significant feature of these ranges is the thrce-phase equilibrium that can be achieved with variable carbon content. Promising addition materials satisfying the criteria cited above include iron, chromium, and the compounds $\mathrm{UFe}_{2}$ and $\mathrm{Cr}_{23} \mathrm{C}_{6}$.
Tie lines in the ternary systems $\mathrm{U}-\mathrm{C}-\mathrm{Fe}$ and $\mathrm{U}-\mathrm{C}-\mathrm{Cr}$ are shown in Figures 1 and 2. ${ }^{(8,9)}$ The particular areas of interest in these diagrams are shown in greater detail in Figures 3 and 4 . It is supposed that sufficient analogy exists between these areas and corresponding areas of interest, over narrow compositional ranges, in the $\mathrm{Pu}-\mathrm{C}-\mathrm{Fe}$ and $\mathrm{Pu}-\mathrm{C}-\mathrm{Cr}$ systems so that qualitative conclusions reached from study of the uranium systems will be applicable to the quasiternary (, $\mathrm{Pu})-\mathrm{C}-\mathrm{X}$ systems.

By reference to Figures 3 and 4, it can be shown that small additions of $\mathrm{Fe}$ or $\mathrm{Cr}_{23} \mathrm{C}_{6}$ to initially hypostoichiometric $\mathrm{UC}$ can be used to bring the total composition into the $\mathrm{CC}-\mathrm{Fe}-\mathrm{CF} e_{2}$ or $\mathrm{UC}-\mathrm{Cr}-\mathrm{Cr}_{23} \mathrm{C}_{6}$ composition triangles, respectively. The phase compositions of equilibrium mixtures in these triangles will contain only these phases.

With specific reference to the iron modification and Fig. 3. the lowest-melting eutectic in the subject composition triangle, $\mathrm{CC}-\mathrm{Fe}_{\mathrm{C}} \mathrm{UFe}_{2}$, is a ternary melting at $1037^{\circ} \mathrm{C}^{(8)}$ This temperature is considerably greater than the previously cited limiting eutectic $\left(\sim 720^{\circ}\right.$ () relating to reaction of the unnodified composition with iron-base-alloy-clad materials. (Harwell investigators have reported an analogous quasiternary eutectic melting at $1043^{\circ} \mathrm{C}$ in the $\mathrm{U}_{0.7} \mathrm{Pu}_{0.3} \mathrm{C}-\mathrm{Fe}$ system. ${ }^{\left({ }^{8}\right)}$ ) The ternary eutectic temperature is considerably greater than the maximum fuel surface temperature $\left(825^{\circ} \mathrm{C}\right)$ predicted for an advanced reactor design. Since no evidence of decarburization of stoichiometric $\mathrm{CC}$ has been reported and no other carbides are present in the modified equilibrium composition, clad carburization is not anticipated. Accordingly, this fuel composition and stainless steel cladding can be expected to be highly compatible under operating FBR conditions.

An analogous argument can be constructed for $\mathrm{Cr}_{23} \mathrm{C}_{6}$ modifying additions to $\mathrm{UC}_{1-\mathrm{x}}$ compositions by reference to $\mathrm{Fig}$. 4 . In this instance the secondary phases are $\mathrm{Cr}$ and $\mathrm{Cr}_{23} \mathrm{C}_{6}$. A ternary eutectic melting at $1315^{\circ} \mathrm{C}$ in the $\mathrm{C}^{\top} \mathrm{C}-\mathrm{Cr}_{-}-\mathrm{Cr}_{23} \mathrm{C}_{6}$ region of the $\mathrm{C}-\mathrm{C}-\mathrm{Cr}$ system has been identified. ${ }^{(9)}$ Thermodynamic calculations indicate that decarburization of $\mathrm{Cr}_{23} \mathrm{C}_{7}$ in liquid soctium will not proceed at typical fast reactor operating temperatures.

Again, by reference to Figures 3 and 4 , a scheme for modifying initially hyperstoichiometric compositions to the ame phase equilibrium with additions of $\mathrm{CFe}_{2}$ and $\mathrm{Cr}$ can be deduced.

An advantage of chromium- over iron-modified compositions may also be hypothesized. Since none of the secondary phases in the chromium-modified fuels is a fissile phase, good irradiation performance 


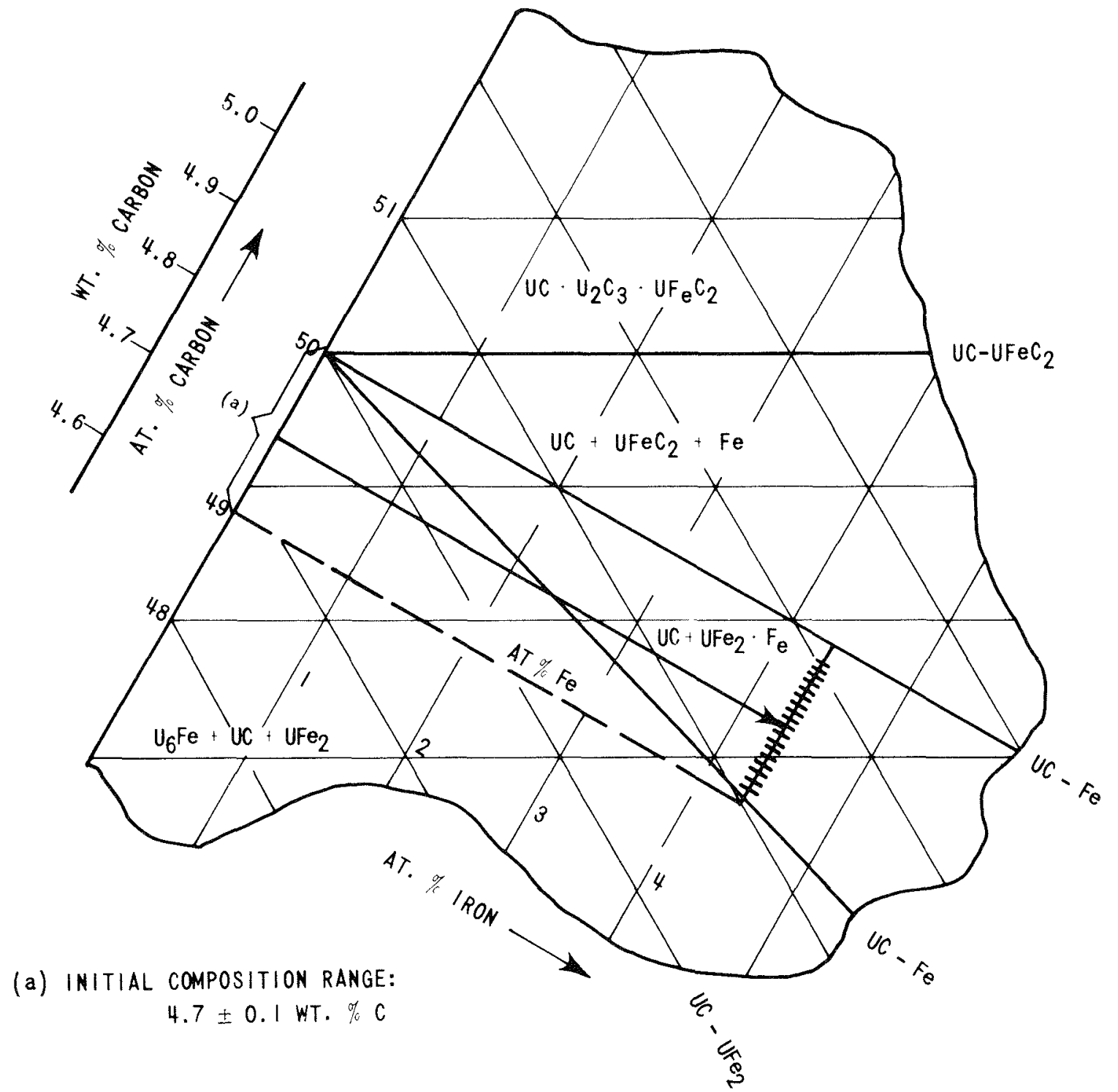

FIG. 3. Enlarged Area of the U-C-Fe System Showing How Iron Additions Mar Be Made to Initially Hyposteichiometric Uranium Carbide to Modify the Final Phase Composition.

comparable to that of the stoichiometric monocarbide is expected.

Figure 5 shows the dilution effects and relative volume of secondary phases introduced by the modifying additions as a function of the initial carbon content of uranium monocarbide. In each instance these effects are minimal and tolerable.

An experimental program is currently in progress at WAPD with the objective of (a) demonstrating that off-stoichiometric uranium monocarbide can be modified to the phase compositions cited above by the addition of $\mathrm{Fe}, \mathrm{Cr}_{23} \mathrm{C}_{6}, \mathrm{UFe}_{2}$, or $\mathrm{Cr}$ as indicated, and (b) to prove that the so-modified carbide compositions possess the hypothesized improved compatibility with stainless steels.

\section{Experimental Procedures}

\section{A. Materials}

Finely divided $(<44 \quad \mu)$ hypostoichiometric, depleted uranium carbide powders were obtained from the Vitro Laboratories. The powders were prepared by milling the product of a high-intensity arc process. ${ }^{(10)}$ Two powders (WAPD-1 and -2) varying in effective carbon content were obtained. The results of the vendor's chemical and spectrographic analyses are given in Table 1. Compositions with carbon contents between WAPD-1 and -2 were prepared by mechanically mixing weighed fractions of WAPD-1 and $\mathbf{- 2}$ in an agate mortar and pestle. 


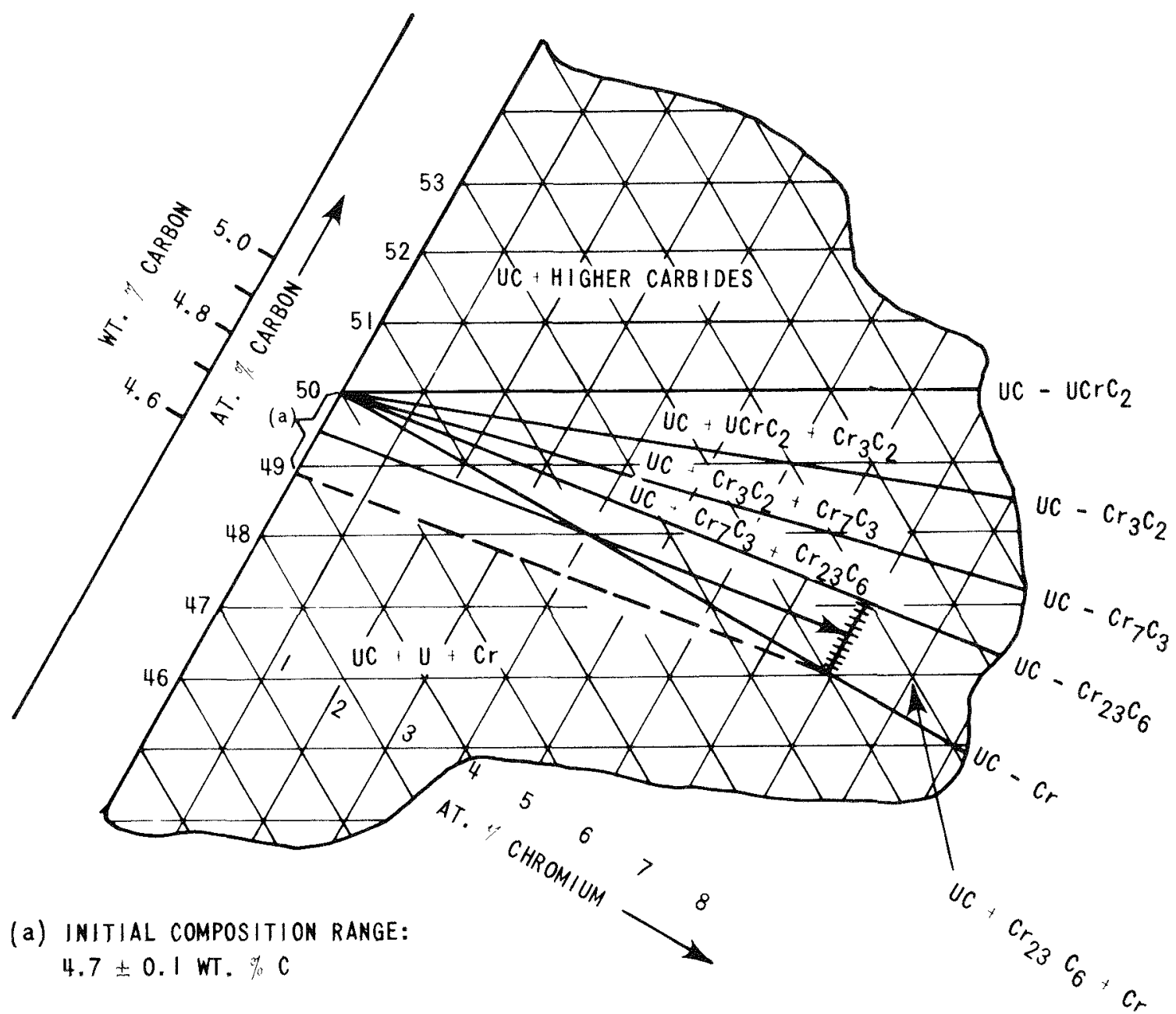

FIG. 4. Enlarged Area of the U-C-Cr System Showing How $\mathrm{Cr}_{23} \mathrm{C}_{6}$ Additions May Be Made to Initially Hypostoichiometric Uranium Carbide to Modify the Final Phase Composition.

Iron ${ }^{(a)}$ and chromium carbide $\left(\mathrm{Cr}_{23} \mathrm{C}_{6}\right)^{(b)}$ were prepared as powders with less than a $44 \mu$ particle size. Other elements specifically sought but not found or present in quantities below the limits of detection of the method used include:

$\mathrm{Ag}, \mathrm{Al}, \mathrm{Ar}, \mathrm{Au}, \mathrm{B}, \mathrm{Ba}, \mathrm{Be}, \mathrm{Bi}, \mathrm{Ca}, \mathrm{Cd}, \mathrm{Co}, \mathrm{Cr}, \mathrm{Ga}$, $\mathrm{Ge}, \mathrm{Hf}$, In, Li, Mg, Mn, Na, Nb, Ni, Os, Pb, Pt, Rh, Ru, Sb, Si, Sn, Sr, Ta, Te, Tl, Ti, V, W, Zn, Zr.

\section{B. Pellet Fabrication}

Pellet fabrication was carried out in a modified, inert gas weld box, continuously purged with dry argon. The oxygen and moisture contents of the box effuent were continuously monitored. Fabrication procedures were terminated when the level of either of these constituents in the effluent exceeded $40 \mathrm{ppm}$. A contaminated box atmosphere was removed by evacu-

(a)Atomergic Metals Co., 1001 Franklin Ave., Garden City, N. J.

(b) Consolidated Astronautics, Inc., 41-45 Crescent St., Long Island $1, N . Y$.

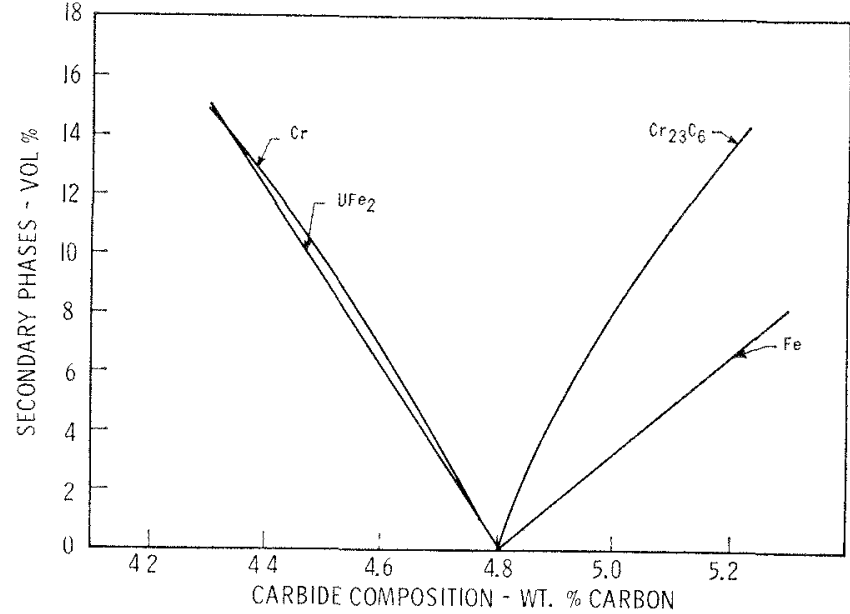

FIG. 5. Tolume of Secondary Phases Contributed by Iron and Chromium Modifying Additions to Initially Hypostoichio. metric Uranium Monocarbides.

ation and repeatedly backfilling with clean argon before fabrication procedures were reinitiated. 
It was recognized that incomplete removal of forming aids during sintering would contribute significant

TABle 1. Chemical and Spectrographic Analyses of Uranium Carbide Powders Used for Fabrication and Compatibility Studies

\begin{tabular}{l|r|r}
\hline Element & WAPD-1 & WAPD-2 \\
\hline C, w/o & 4.20 & 4.54 \\
O, ppm & 2240 & 1905 \\
N, ppm & $<100$ & $<100$ \\
Fe,ppm & 30 & 30 \\
Mo, ppm & 300 & 300 \\
Cu, ppm & 10 & 10 \\
\hline
\end{tabular}

carbon to the final pellet composition. Therefore, pellets were fabricated from the as-received powders without binder or lubricating additions.

The pellets were cold pressed on a laboratory press at variable pressures in a carbide-lined die. The forming pressure used was adequate to impart a degree of mechanical strength to the compact sufficient to resist disintegration during subsequent handling, but not so great as to produce laminations or other recognizable defects in the sintered pellet.

Sintering was done in vacuum or argon in an alumina $\left(\mathrm{Al}_{2} \mathrm{O}_{3}\right)$ retort in a molybdenum wire resistance furnace.

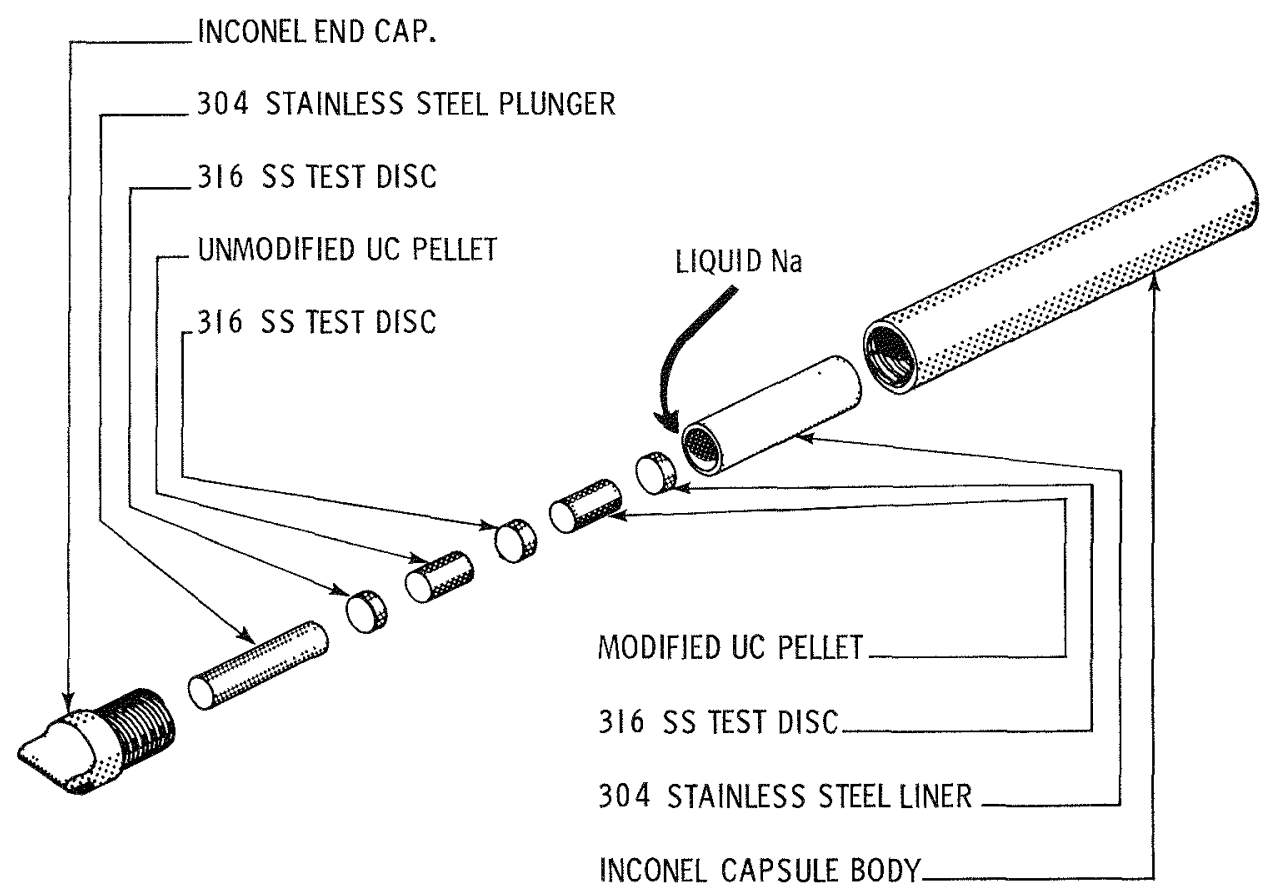

Frg. 6. Test Capsule Used to Ascertain the Compatibility of Monocarbide Compositions with 316 Stainless Steel.

TABle 2. Procedures for Metaliographic Identification of Secondary Phases in Hypostoichiometric and Modified Uranium Carbide Compositions

\begin{tabular}{|c|c|c|}
\hline ANTICIPATED PHASES & METALLOGRAPHIC PROCEOURFS & INTERPRETATION \\
\hline (a) $\cup \mathbb{I N} \cup C$ & $\begin{array}{l}\text { (1) CHEMICAL ETCH. HNO } 3 \text { : HAC. } \\
\mathrm{H}_{2} \mathrm{O}(1: 1) \text { 1). } 8 \text { SECS (MAX.) }\end{array}$ & $\begin{array}{l}\text { UC: STAINS TO A VARIETY OF COLORS } \\
\text { AND SHADES. U HIGH REFIECTANCE, } \\
\text { WHITE MATRIX AREA }\end{array}$ \\
\hline (b) $\mathrm{U}, \mathrm{UFe}_{2}, \mathrm{Fe} \mathrm{IN} \mathrm{UC}$ & $\begin{array}{l}\text { (1) CHEMICAL ETCH } \mathrm{HNO}_{3} \text { : HAC. } \\
\mathrm{H}_{2} \mathrm{O} \text { (1:I:1).t- } 8 \text { SECS. (MAX.) } \\
\text { (2) OXIDIZE AT } 50^{\circ} \mathrm{C} \text { FOR } 17 \text { HRS. }\end{array}$ & $\begin{array}{l}\text { UC STAINS TO A VARIETY OF COLORS } \\
\text { AND SHADES. U OXIDIZES TO RFD- } \\
\text { BROWN. UFE2. UNALTERED, HIGH } \\
\text { REFLECTANCE PHASE. Fe. ATIACKED, } \\
\text { BY ETCHANT. . B BLACK IN EUTECTIC } \\
\text { STRUCTURES. }\end{array}$ \\
\hline (c) $\mathrm{Cr}, \mathrm{Cr}_{23} \mathrm{C}_{6} \mathrm{IN} \mathrm{UC}$ & $\begin{array}{l}\text { (1) ELECTROLYTIC ETCH. } \\
\text { V-5-O VOLTS. ELECTROLYTE } \\
\text { OXALIC ACID (25 GMS) IN } \\
\text { METHYL ALCHOHOL }(100 \mathrm{ml})\end{array}$ & $\begin{array}{l}\text { UC. STAINS TO A VARIETY OF COLORS } \\
\text { AND SHADES. } \mathrm{Cr}_{23} \mathrm{C}_{6} \text { ATTACKED, } \\
\text { REDUCED REFLECTANCE, MATRIX } \\
\text { AREA. Cr. UNATTACKED, HIGHER } \\
\text { REFLECIANCE PHASE. }\end{array}$ \\
\hline
\end{tabular}




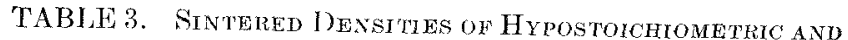
Monhied Uranitim Monocarbide Compositions

\begin{tabular}{|c|c|c|c|c|}
\hline COMPOSITION & $\begin{array}{c}\text { SINIERING } \\
\text { TEMPERATURE }\end{array}$ & ATMOSPHERE ${ }^{(a)}$ & TIME & $\begin{array}{l}\text { DENSITY } \\
\text { gms /an }{ }^{3}\end{array}$ \\
\hline$u c_{i-x}$ & $1150^{\circ} \mathrm{C}$ & v & 2 & 10.00 \\
\hline$U C_{1-x}$ & $1300^{\circ} \mathrm{C}$ & v & 2 & 10.15 \\
\hline$U \mathrm{C}_{1-x}+\mathrm{Fe}$ & $1300^{\circ} \mathrm{C}$ & v & 2 & 11.84 \\
\hline $\mathrm{UC}_{\mathrm{I}-\mathrm{x}}+\mathrm{Fe}$ & $1300^{\circ} \mathrm{C}$ & V & 3 & 11.45 \\
\hline$u C_{1-x}$ & $1425^{\circ} \mathrm{C}$ & A & 4 & 13.0 \\
\hline$U C_{1-x}+F e$ & $1425^{\circ} \mathrm{C}$ & A & 4 & 12. \\
\hline $\mathrm{UC}_{1-x}+\mathrm{Cr}_{23} \mathrm{C}_{6}$ & $1425^{\circ} \mathrm{C}$ & A & 4 & 11.0 \\
\hline$u c_{1-x}+C_{23}-c_{6}$ & $1450^{\circ} \mathrm{C}$ & A & 4 & 12.3 \\
\hline
\end{tabular}

(d)-V-VACUUM A-ARGON

\section{Compatibility Tests}

Details of the test capsule assembly used for compatibility studies are given in Fig. 6. The difference in thermal expansion between the Inconel outer capsule and the stainless steel plunger served to load the pellet-disc couple and insure intimacy of contact at the test temperature. The sodium-filled test capsules were assembled by inserting the stainless steel liner and then adding sodium. A test disc, a modified pellet, a second test disc, an unmodified pellet, a third test disc, and the plunger followed in that order. The end caps were finger tightened and then welded closed. The furnaces used for compatibility testing accommodated two capsules of this design. Normally one sodium-filled and one argon-filled capsule constituted the test complement for each furnace.

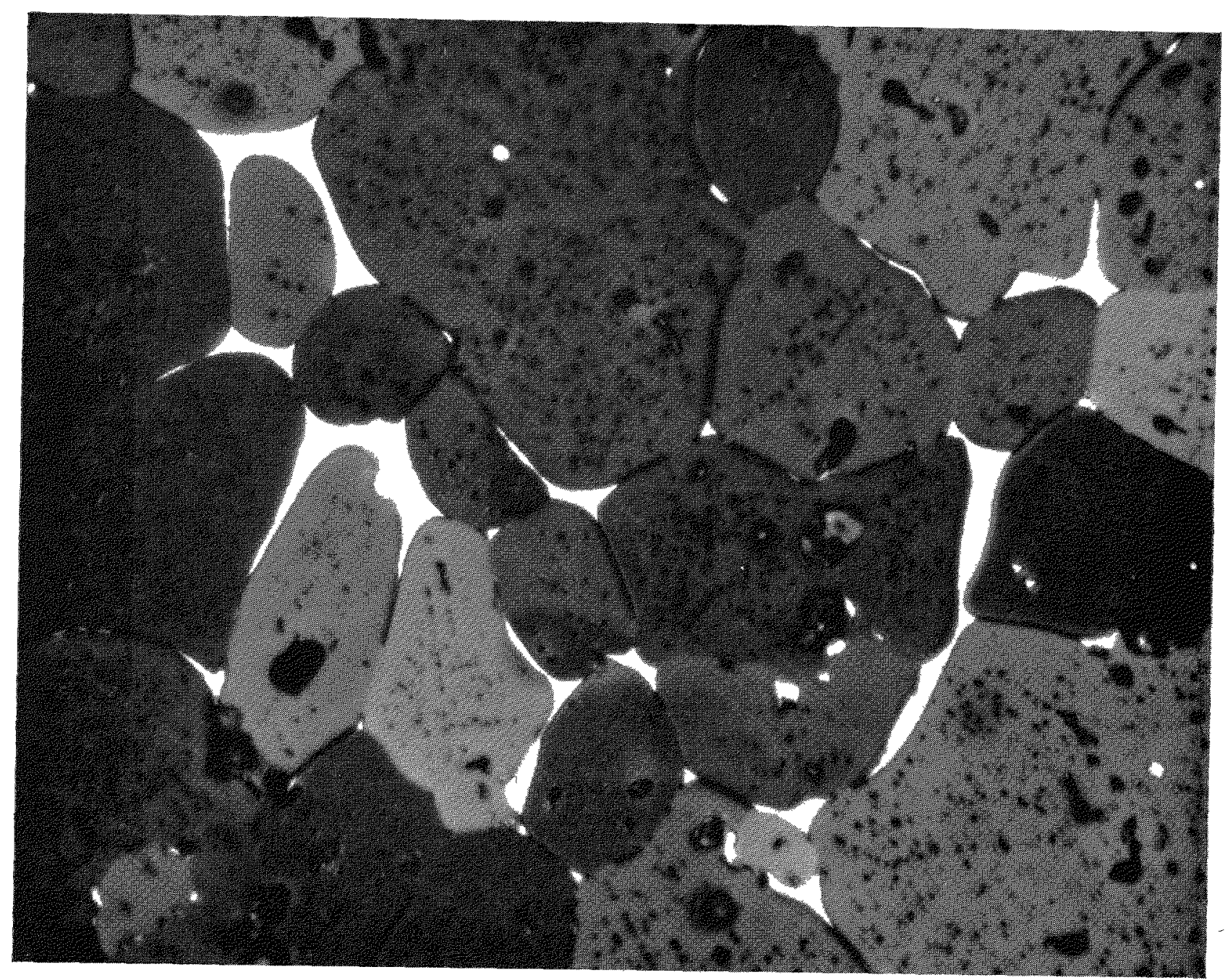

Fic. 7. Microstructure of Sintered, Hypostoichiometric Uranium Monocarbide. White Phase is Uranium Metal. (Specimen Sintered in Argon at $1450^{\circ} \mathrm{C}$ for 4 Hr. Density: $13.2 \mathrm{gm} / \mathrm{cm}^{3}$.) 


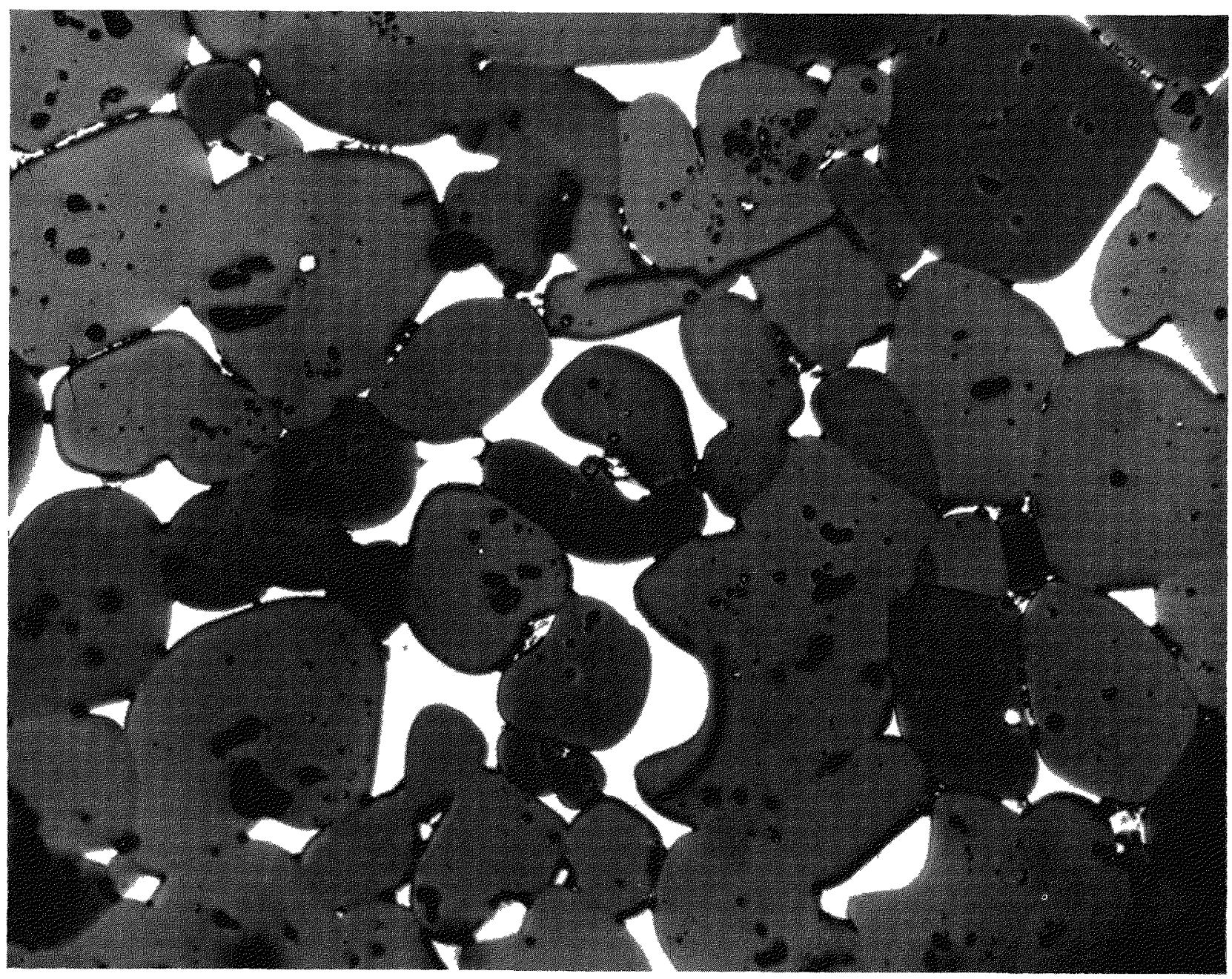

Fig. 8. Microstructure of Sintered Iron-Modified Uranium Monocarbide. Secondary Phases are UFe 2 (White) and LFe ${ }_{2}$-Fe Eutectic. (Specimen Sintered in Vacuum at $1300^{\circ} \mathrm{C}$ for $2 \mathrm{Hr}$. Density: $11.0 \mathrm{gm} / \mathrm{cm}^{3}$.)

\section{Analytical Procedures}

Sintered pellets were dimensioned and weighed for density determinations.

The sintered pellets were also examined metallographically. The compatibility test couples (pellets and discs) were also examined by metallographic techniques. Procedures employed to prepare metallographic sections and identify significant phases in the various microstructures are given in Table 2 .

$\mathrm{X}$-ray diffraction studies were also performed on sintered pellets to identify the important secondary phases. Greatest success was realized by this method in the case of chromium carbide $\left(\mathrm{Cr}_{23} \mathrm{C}_{6}\right)$ modified compositions. The secondary phases in these compositions were concentrated by leaching away the uranium carbide (UC) with an acid wash. The finely divided residue, enriched in the secondary phases, was studied with a Debye-Scherrer, 114.6-mm camera, and $\mathrm{Cu} \mathbf{K}_{\alpha}$ radiation.

\section{Results and Discussion}

\section{A. Pellet Fabrication}

Specimen history is important to the interpretation of compatibility test results. Accordingly, a discussion of the specimen-preparation procedures is required. Initial sintering experiments were done in vacuum. The results of these studies were inconsistent and aggravated by repeated failure of the ceramic retort in which the sintering was conducted. Test speciments sintered in argon gas produced reliable data, and most of the results discussed herein were obtained with specimens sintered in this atmosphere.

The hypostoichiometric and modified compositions sinter via a liquid-phase mechanism. The minimum 


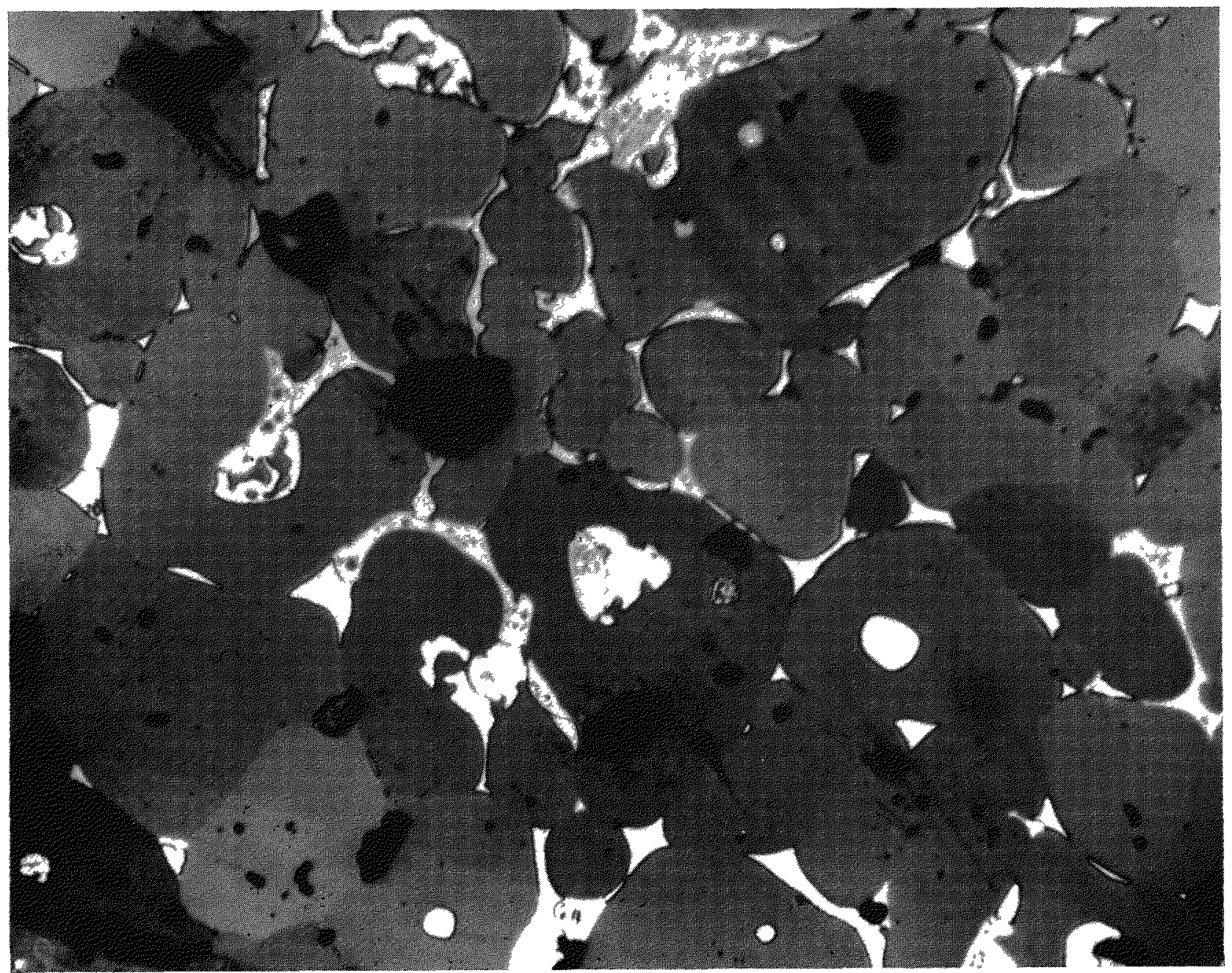

FIG. 9. Microstructure of Sintered, $\mathrm{Cr}_{23} \mathrm{C}_{6}$-Modified Uranium Monocarbide. Secondary Phases Are Cr (Ifigher Reflectance) and $\mathrm{Cr}_{23} \mathrm{C}_{6}$. (Specimen Sintered in Argon at $1450^{\circ} \mathrm{C}$ for $4 \mathrm{Hr}$. Density: $12.4 \mathrm{gm} / \mathrm{cm}^{3}$.)

sintering temperature was defined by the first-melting constituents among the reactants. The first-melting constituent (barring significant solid-state reaction at lower temperatures) is uranium.

Test specimens sintered at $1150^{\circ} \mathrm{C}$, i.e., slightly above the uranium melting point, did not densify appreciably. It was concluded, after several sintering trials, that adequate densification concomitant with development of the desired phase equilibria could be accomplished at $1300^{\circ} \mathrm{C}$ and $1450^{\circ} \mathrm{C}$ in the iron- and $\mathrm{Cr}_{23} \mathrm{C}_{6}$-modified compositions, respectively. The densification of several compositions sintered under variable conditions is summarized in Table 3 . Figures 7, 8, and 9 are photomicrographs showing the microstructure of the hypostoichiometric, iron-modified and $\mathrm{Cr}_{23} \mathrm{C}_{6}$-modified compositions after heat treatment for $4 \mathrm{hr}$ in argon. There was no metallographic evidence of free uranium metal in any of the sintered, modified compositions.

An experiment was conducted to ascertain the effect of variable carbon content on the sintering behavior of powder compacts to which a constant quantity of modifying addition was made. An amount of $\mathrm{Cr}_{23} \mathrm{C}_{6}$ was added which was adequate to modify fully the least stoichiometric composition. The resultant densification and microstructures of the modified and unmodified compositions are compared in Figures 10, 11, and 12. The degree of densification among the compositions was almost equal. The anticipated phases were observed in approximately the relative amounts expected for each composition. It was concluded that densification of variable compositions was not difficult to control over a range of carbon contents attainable by contemporary synthe- 


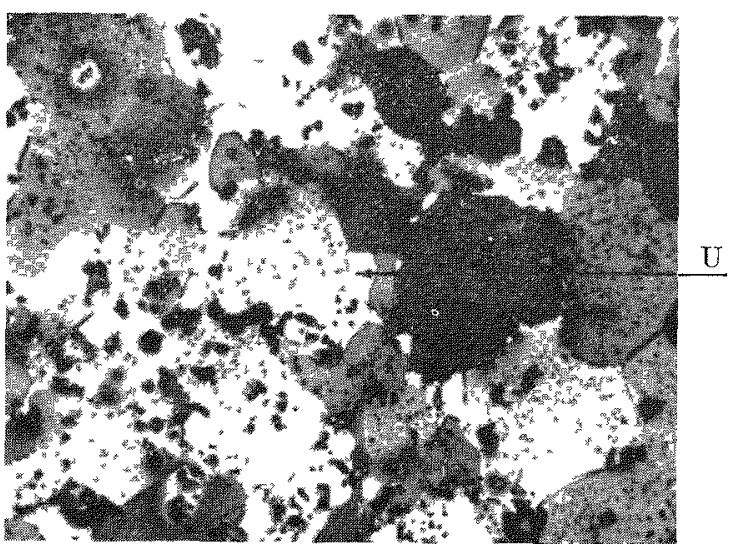

(a)

$450 \times$

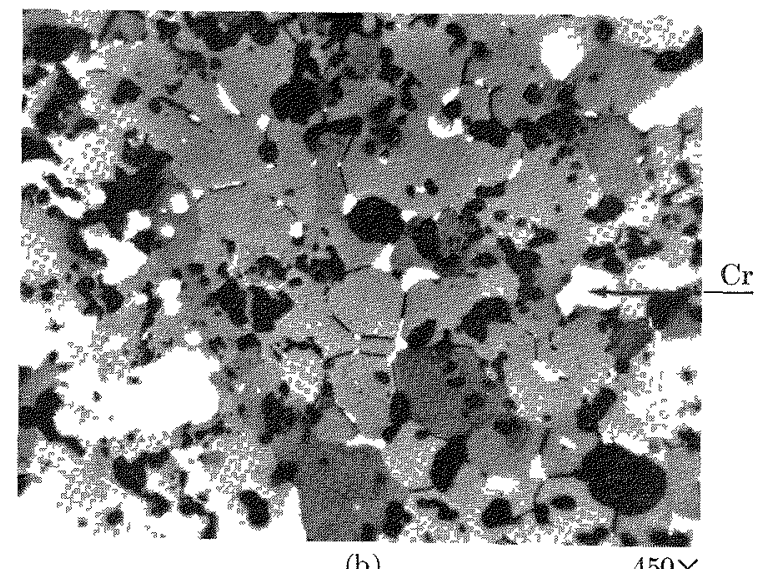

FIG. 10. Microstructure of (a) Iypostoichiometric Uranium Monocarbide (Approximate Composition, $\mathrm{CC}_{0} 9_{0}$ ) and (b) $\mathrm{Cr}_{23} \mathrm{C}_{\mathrm{B}^{-}}$ modified $\mathrm{UC}_{0} 95$.

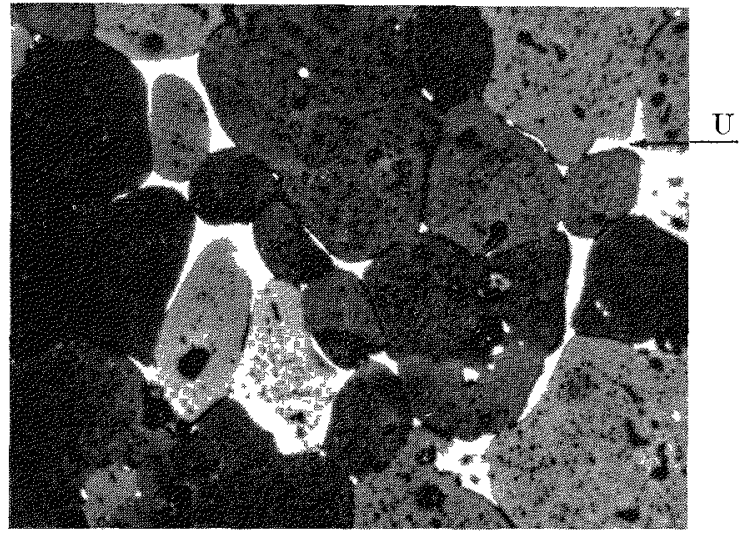

(a)

$390 \times$

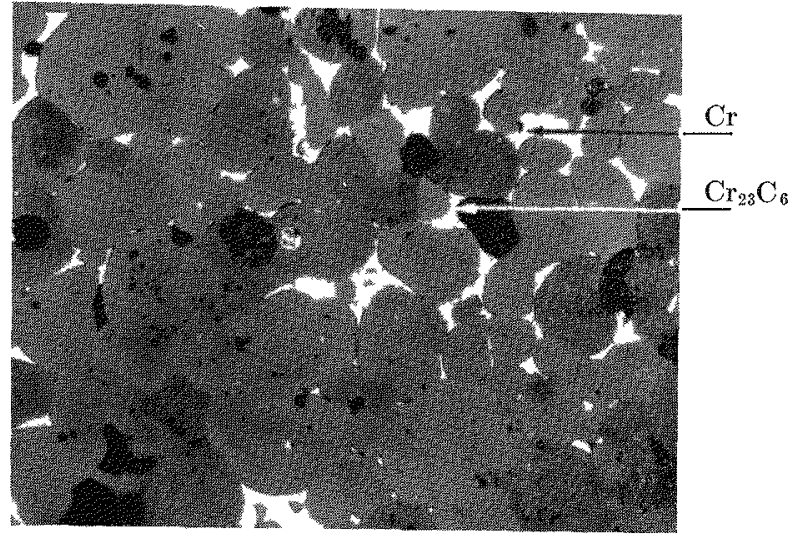

(b)

$390 \times$

FIg. 11. Microstructure of (a) Hypostoichiometric Uranium Carbide (Approximate Composition: $\mathrm{UC}_{097}$ ) and (b) $\mathrm{Cr}_{23} \mathrm{C}_{67}$ modified $\mathrm{UC}_{0} 87$. Note Two-phase Nature of Matrix regions.

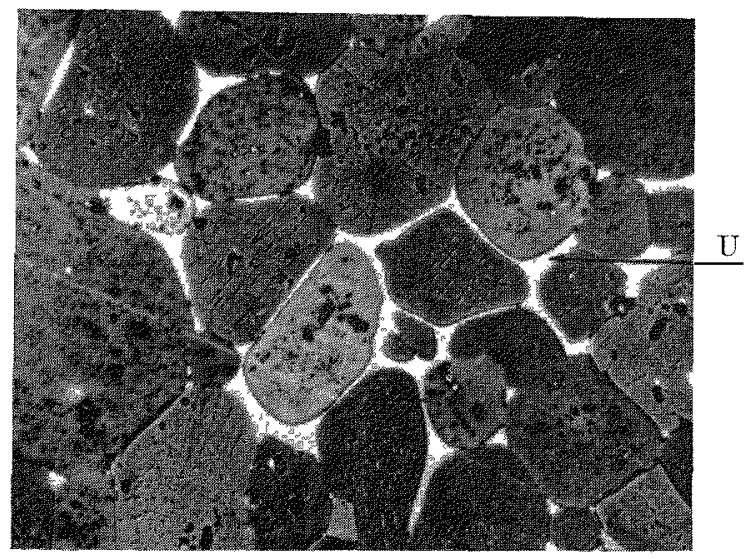

(a)

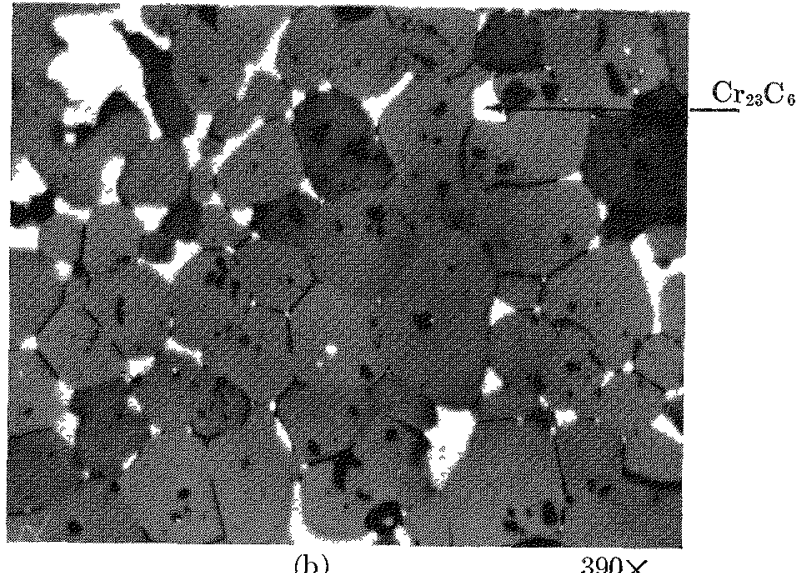

(b)

$390 \times$

FIa. 12. Microstructure of (a) Hypostoichiometric Uranium Monocarbide (Approximate Composition: $\mathrm{UC}_{0.99}$ ) and (b) $\mathrm{Cr}_{23} \mathrm{C}_{6}$ modified $\mathrm{UC}_{0} 99$. 
TABle 4. Stmmary of Modified Chrbide Compatibility Tests Completed or in Progress at WaPD

\begin{tabular}{|c|c|c|c|c|c|c|c|c|}
\hline \multirow{2}{*}{$\begin{array}{l}\text { TEST } \\
\text { NO. }\end{array}$} & \multirow{2}{*}{$\begin{array}{l}\text { CAPSULE } \\
\text { DESIGNATION }\end{array}$} & \multirow{2}{*}{$\begin{array}{l}\text { TEMPERATURE } \\
{ }_{0}^{\mathrm{C}} \mathrm{C}\end{array}$} & \multirow{2}{*}{$\begin{array}{l}\text { TIME } \\
\text { HRS }\end{array}$} & \multicolumn{2}{|c|}{ ENVIRONMENT } & \multirow{2}{*}{$\begin{array}{l}\text { TEST PELLET } \\
\text { COMPOSITION }\end{array}$} & \multicolumn{2}{|c|}{ OBSERVATIONS } \\
\hline & & & & $\mathrm{Na}$ & $\mathrm{A}$ & & UNMODIFIED PELLET & MODIFIED PELLET \\
\hline 0 & A & 815 & 24 & & $x$ & $U C_{1-X}+F e$ & $\begin{array}{l}\text { REACTION, DEPTH OF REACTION } \\
\text { ZONE IN } 316 \text { TEST DISC: } 150 \mu\end{array}$ & NO REACTION \\
\hline 00 & B & 815 & 72 & & $x$ & $U C_{1-X}+F e$ & $\begin{array}{l}\text { REACTION, DEPTH OF REACTION } \\
\text { ZONE IN } 316 \text { TEST DISC: } 110 \mu\end{array}$ & NO REACTION \\
\hline \multirow{2}{*}{1} & C & \multirow{2}{*}{790} & \multirow{2}{*}{500} & \multirow[t]{2}{*}{$x$} & & $U C_{1-x}+F e$ & $\begin{array}{l}\text { REACTION, DEPTH OF REACTION } \\
\text { ZONE IN } 316 \text { TEST DISC: } 175 \mu\end{array}$ & $\begin{array}{l}\text { NO REACTION, MECHANICAL } \\
\text { ADHERENCE OF PELLET TO } \\
\text { TEST DISC. }\end{array}$ \\
\hline & D & & & & & $\mathrm{UC}_{1}-\mathrm{X}+\mathrm{Fe}$ & $\begin{array}{l}\text { REACTION, DEPTH OF REACTION } \\
\text { ZONE IN } 316 \text { TEST DISC: } 150 \mu\end{array}$ & $\begin{array}{l}\text { NO REACTION, MECHANICAL } \\
\text { ADHERENCE OF PELLET TO } \\
\text { IEST DISC. }\end{array}$ \\
\hline \multirow{2}{*}{2} & E & \multirow{2}{*}{730} & \multirow{2}{*}{1000} & \multirow[t]{2}{*}{$x$} & \multirow[b]{2}{*}{$x$} & $U C_{1-X}+F e$ & NO REACTION & NO REACIION \\
\hline & $F$ & & & & & $U c_{1-x}+F e$ & NO REACTION & NO REACTION \\
\hline 3 & - & 760 & 700 & $x$ & & 一 & $\begin{array}{l}\mathrm{Cr}_{23} \mathrm{C}_{6} \text { POWDER IN Na IN A THIN } \\
\text { STEEL TUBE. NO CARBURIZATION }\end{array}$ & $\begin{array}{l}\text { WALLED }(330 \mu) \text { STAINLESS } \\
\text { OF THE STAINLESS STEEL }\end{array}$ \\
\hline \multirow{2}{*}{4} & G & \multirow{2}{*}{815} & \multirow{2}{*}{500} & $x$ & & $U \mathrm{C}_{1-\mathrm{X}}+\mathrm{Cr}_{23} \mathrm{C}_{6}$ & \multirow{2}{*}{\multicolumn{2}{|c|}{$\begin{array}{l}316 \text { TEST DISCS. SHOWED EVIDENCE OF CARBURIZATION } \\
\text { FROM REACTION WITH BOTH MODIFIED AND UNMODIFIED } \\
\text { COMPOSITIONS INDEPENDENT OF THE ENVIRONMENT. }\end{array}$}} \\
\hline & $\mathrm{H}$ & & & & $x$ & $U \mathrm{CC}_{1-\mathrm{X}}+\mathrm{Cr}_{23} \mathrm{C}_{6}$ & & \\
\hline \multirow{2}{*}{5} & I & \multirow{2}{*}{830} & \multirow{2}{*}{1200} & $x$ & & $U C_{1-X}+F e$ & \multicolumn{2}{|c|}{$\begin{array}{l}\text { TEST CAPSULE IN TEST APPARATUS UPSIDE DOWN. NO Na } \\
\text { IN THE VICINITY OF THE TEST COUPLE. NO REACTION. } \\
\end{array}$} \\
\hline & J & & & & & $U C_{1-x}+F e$ & TEST CAPSULE LEAKED. NO REAC & CTION \\
\hline \multirow{2}{*}{6} & K & \multirow{2}{*}{730} & \multirow{2}{*}{ - } & $x$ & & $U C_{1-X}+F e$ & IN PROGRESS & IN PROGRESS \\
\hline & $\mathrm{L}$ & & & & $x$ & $U C_{1-X}+F e$ & IN PROGRESS & IN PROGRESS \\
\hline \multirow{2}{*}{7} & M & \multirow{2}{*}{830} & \multirow{2}{*}{200} & $x$ & & $U \mathrm{C}_{1-\mathrm{X}}+\mathrm{Cr}_{23} \mathrm{C}_{6}$ & NONE & NONE \\
\hline & $N$ & & & $x$ & & $\mathrm{UC}_{1-\mathrm{X}}+\mathrm{Cr}_{23} \mathrm{C}_{6}$ & NONE & NONE \\
\hline \multirow{2}{*}{8} & 0 & \multirow{2}{*}{730} & \multirow{2}{*}{ - } & $x$ & & $u c_{1-x}+\mathrm{Cr}_{23} \mathrm{C}_{6}$ & IN PROGRESS & IN PROGRESS \\
\hline & $P$ & & & $x$ & & $\mathrm{UC}_{1-\mathrm{X}}+\mathrm{Cr}_{23} \mathrm{C}_{6}$ & IN PROGRESS & IN PROGRESS \\
\hline
\end{tabular}

sis procedures. Complete phase modification was also easily realized in every instance of compositional variation.

\section{B. Compatibility Experiments}

Compatibility studies completed or still in progress are summarized in Table 4. Most of this work has been concerned with the iron-modified composition. Figure 13a shows the surface of a 316 stainless steel disc tested in contact with an iron-modified uranium carbide pellet at $790^{\circ} \mathrm{C}$ for $500 \mathrm{hr}$. Figure $13 \mathrm{~b}$ shows a typical cross section through the same disc. There is no evidence of reaction between the disc and the modified carbide pellet.

The surface of a 316 stainless steel disc tested in contact with an unmodified uranium carbide pellet under the same conditions is shown in Figures 14a and $14 \mathrm{~b}$. Significant reaction with the pellet is evidenced by the extensive area of pitting. Figure $14 \mathrm{~b}$ shows the penetration of the reaction zone $(\sim 150 \mu)$ at a cross section taken through the reacted area. Metallographic examinations of the fuel pellet indicated that the reaction involved metal throughout the pellet volume and was not limited to surface concentrations.

Although reaction was evidenced in nearly every instance where the unmodified composition was tested, the extent of the reaction was not a well-behaved function of the test variables, i.e., time and temperature. Accordingly, while failure by the hypothesized reaction mechanism may be concluded to be a certainty in a stainless clad-hypostoichiometric monocarbide system, the kinetics of the failure are not readily predicted.

The absence of evidence of a reaction at the lowest test temperature $\left(\sim 730^{\circ} \mathrm{C}\right)$ suggests that the reaction with the clad is accelerated in the presence of a liquid phase. This temperature very nearly approxi- 
mates the melting point $\left(725^{\circ} \mathrm{C}\right)$ of the lowest-melting iron-uranium eutectic. This eutectic occurs at approximately $35 \mathrm{a} / \mathrm{o}$ iron, and compositions containing greater than $14 \mathrm{a} / \mathrm{o}$ iron will form a liquid phase at this temperature. At higher temperatures a liquid phase will form at a composition containing significantly less iron (e.g., 25 a/o at $816^{\circ} \mathrm{C}$ ).

Compatibility tests with chromium carbide-modified compositions have only recently begun. Test 3 was run to demonstrate the stability of the $\mathrm{Cr}_{23} \mathrm{C}_{6}$ and its resistance to decarburization in sodium. Finely divided $\mathrm{Cr}_{23} \mathrm{C}_{6}$ and liquid sodium were added to the capsule liner and held at $760^{\circ} \mathrm{C}$ for $700 \mathrm{hr}$. There was no evidence of carburization of the liner. Oxygen contamination of the sodium or a leak in the capsule may have contributed to the progress of the carburi-

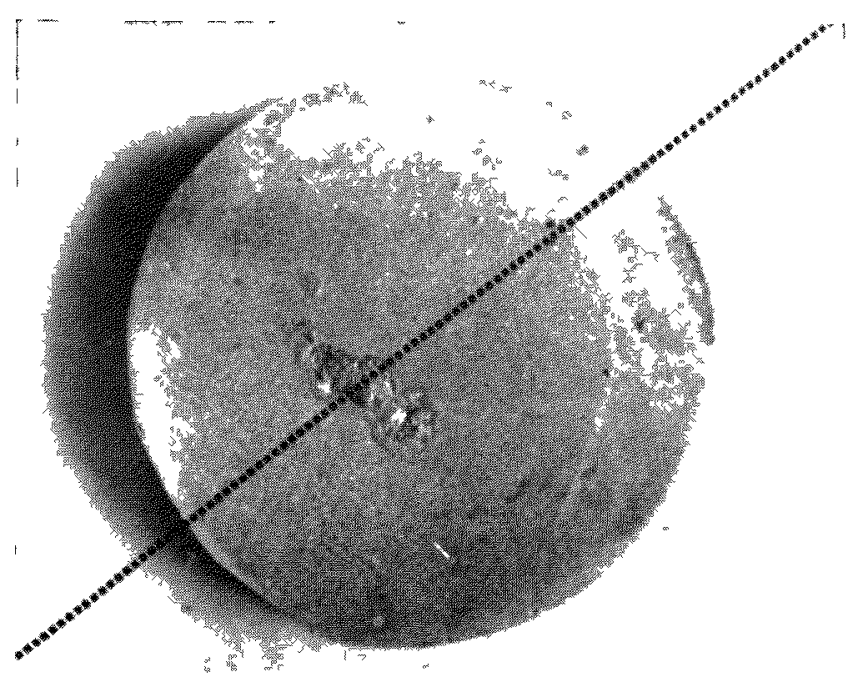

(a)

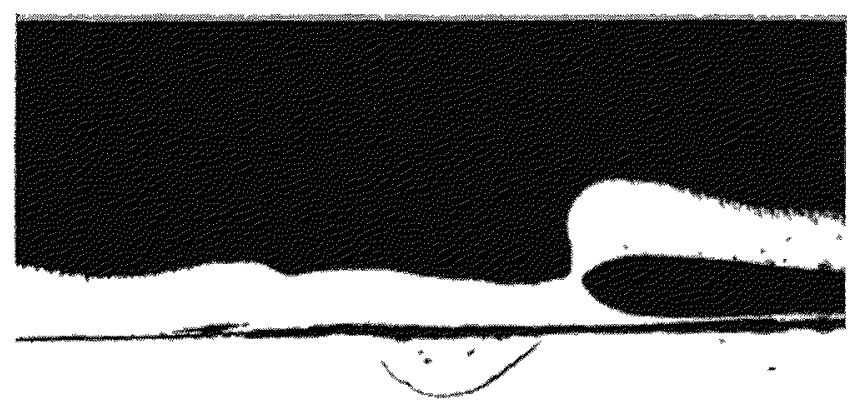

(b)

$37.5 \times$

Frg. 13. (a) 316 Stainless Steel Disc Annealed for $500 \mathrm{Hr}$ at $790^{\circ} \mathrm{C}$ in Contact with Unmodified, Hypostoichiometric Uranium Carbide in Sodium; (b) Transverse Section through the Test Dise Shown in (a) Taken Along the Line Indicated. Note depth of reaction zone, $175 \mu$.

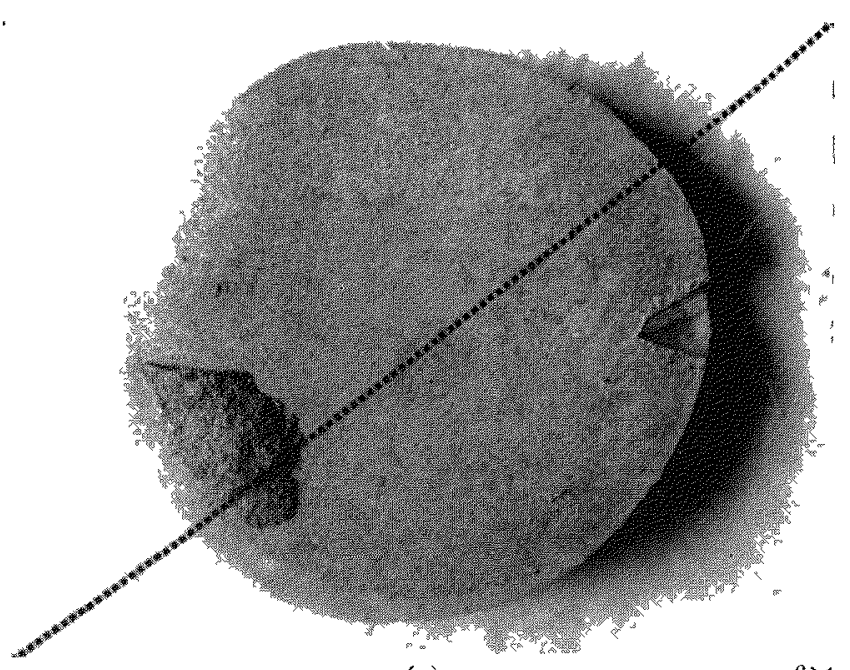

(a)

$6 \times$

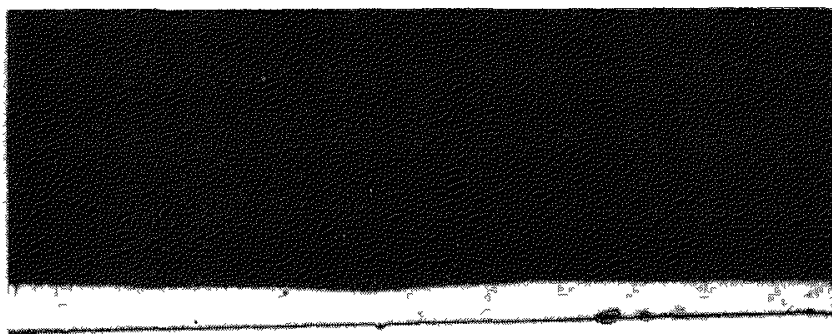

(b)

$37.5 \times$

Frg. 14. (a) 316 Stainless Steel Irse Annealed for $500 \mathrm{Hr}$ at $790^{\circ} \mathrm{C}$ in Contact with Iron-modified, Hypostoichometric Uranium Monocarbide in Sodum; (b) Transverse Section through the Test Iise Shown in (a) Taken Along the Line Indicated. Note absence of reaction. [Notch on right hand side of (a) was for identification. Artifact at left is mechanically adhered carbide.]

zation noted in Tests 4 and 7 . These tests are being repeated.

\section{Summary}

The compatibility of liquid metal-bonded, nonstoichiometric, mixed monocarbide (U,Pu)C fast breeder reactor fuels with a leading candidate clad material, stainless steel, is poor. A compositional innovation designed to improve the poor compatibility of this material combination has been defined. This innovation involves the addition of controlled amounts of a modifying element or compound to off-stoichiometric compositions. The polyphase composition re- 
sulting from heat treatment includes no low-melting eutectic formers or unstable higher carbides.

Fxperimental study of iron and chromium carbide $\left(\mathrm{Cr}_{23} \mathrm{C}_{6}\right)$ modified, initially hypostoichiometric, uranium monocarbide $\left(\mathrm{CC}_{1-\mathrm{x}}\right)$ compositions has been initiated. Modified compositions were successfully pelletized by cold pressing and sintering. Sintering in these compositions proceeds via a liquid-phase mechanism at relatively low temperatures. Development of the requisite phase equilibrium also occurs in short times at the same temperatures.

Improved compatibility was experimentally demonstrated in argon and liquid metal environments between contact-bonded, iron-modified, initially hypostoichiometric uranium monocarbide and 316 stainless steel. Initial experiments with gas- and sodiumbonded $\mathrm{Cr}_{23} \mathrm{C}_{6}$-modified $\mathrm{UC}_{1-\mathrm{X}}$ are inconclusive and continuing.

\section{IREFERENCES}

1. Chubb, W., and Dickerson, R. F., Research on Uranium Carbides at Battelle Memorial Institute, 4th Uranium Carbide Meeting, East Hartford, Connecticut, May 20-21, 1963.

2. A. N. Holden, Physical Metallurgy of Uranium, AddisonWesley Publishing Co., Inc., Reading, Mass. (1958).

3. The Metal Plutonium, Ed., A. S. Coffinberry and W. N. Miner, Univ, of Chicago Press, Chicago, Ill (1961).

4. P. E. Elkins, Compatibility of Uranium Carbide Fuels with Cladding Materials, NAA-SR-7502 (1964).

5. B. A. Webb, Carburization of Austenitic Stainless Steel by Uranium Carbides in Sodum Systems, NAA-SR-6246 (May 1963).

6. E. Rudy and F. Benesovsky, Contribution to the Study of the Stabitity of Uranium Decarbide and the Carbon Stable Regions in the Partial Systems of UC with ZrC, HfC, NbC and TaC, Monatsh Chem., 94, 202-204.

7. WCAP-3251-1, Liquid Metal Fast Breeder Reactor Design Study (Jan. 1961).

8. Nichols, J. L., and Marples, J. A. C., An Investigation of the $U-F e-C$ and $P u F e-C$ Ternary Phase Duagrams with Some Observations on the U-Pu-Fe Quarternary. Paper A17 Presented at Harwell Symposium on Carbides in Nuclear Energy, November 1963.

9. Briggs, G., Dutta, S. K., and White, J., Phase Relationships in the systems $U-C r-C, U-N i-C$ and $U-F e-C r C$, Paper A16 Presented at Harwell Symposium on Carbides in Nuclear Energy, November 1963.

10. Gibson, J. O., and Weidman, R., Chemical Synthesis Via the High Intensity Arc Process, Chem. Eng. Prog., 59(a), $53-56$ (1963).

\section{Discussion}

Mr. Siegel (AI): I gather that the formation of the alloys is by powder metallurgy techniques. Can they be made directly from a single arc melting process?

Mr. Herbst: We haven't considered the problem at any length. There appears to be difficulty with plutonium in that it exhibits a variable volatility. We thus directed our efforts at a practical scheme for fabricating the material, liquid-phase sintering at a reasonably low temperature.

Mr. Siegel: Do you know whether that process is likely to add significantly to the fabrication cost?

Mr. IIerbst: We believe that this represents a cost reduction effectively from a fabrication standpoint. Our experience with oxide, for example, has been by powder metallurgical procedures.

$M r$. Siegel: With hypostoichiometric UC in contact with stainless steel we have observed basically no practical incompatibility up to $1600^{\circ} \mathrm{F}$, with what amounts to a sodium-bonded couple.

Mr. Stahl (UNC): You say you have liquid-phase sintering. Have you investigated at all the stability of the fuel under long-time high-temperature treatments? What happens to your second phase?

Mr. Herbst: No, we haven't carried out this kind of investigation. The composition is obviously tailored to the Westinghouse Fast Breeder Reactor design. Maximum operating temperatures of the Westinghouse system will include a finite volume of liquid phase at operating temperatures, and this is considered a disadvantage. The chromium addition leads to a higher melting point and will get us over this, so that we should have no liquid phase under reactor operating conditions.

Mr. Stahl: Have you investigated all the diffusion layers between the fuel and cladding so that you don't have to worry about stoichiometry?

Mr. Herbst: No, our whole program is based upon the modifying additions of secondary phases. We haven't initiated any work in the area of diffusion barriers, for example.

Mr. Walter (ANL): Have you irradiated any of this material?

Mr. Herbst: No irradiation experiments have been conducted yet. We do have an irradiation experiment proposed for both iron- and chromium-modified compositions for EBR-II.

Mrr. Walter: What is your feeling on the effect of changes of stoichiometry in the carbide due to formation of fission product carbides and things like this? This may throw your phase relations completely off and change compatibility. Also, even though you are forming higher-melting material, it looks like it is mostly in grain boundaries rather than in $\mathrm{C}-\mathrm{Pu}$ alloy. The melting point is still considerably lower than that of carbide. One would think there might be some problems with just irradiation damage in the fuel in comparison to a normal hyperstoichiometric carbide.

Mr. Herbst: We believe, for example, that in the case of chromium-modified carbide we have no fissile 
secondary phases in grain-boundary regions. Thus we anticipate improved compatibility or improved irradiation performance comparable to the stoichiometric composition, which has been demonstrated to be good in other irradiation studies. The dilution or effective modification of the composition of the carbide under irradiation has been considered, but the answer escapes me at the moment.

Mr. Kummerer (Karlsruhe): You have carried out this investigation with pure carbide fuel. We are more interested in the mixed carbide fuel, for which the compatibility may be quite different than for pure carbide. To what extent do you consider these results as a simulation for the real mixed carbide fuels?

$M r$. Herbst: Our feeling particularly with regard to the iron modification is that it is a pretty good mockup, based primarily on the Harwell work, which has included the quasiternary $\mathrm{U}_{0.7}$ and $\mathrm{Pu}_{0.3}$. The phase relations derived from these studies are not significantly different from what has been reported for UC-Fe. 


\title{
Mixed-carbide Fuels for Fast Reactors
}

\author{
L. A. Nentark, J. E. Ayer, (). L. lirgeer, T. W. Lathiler, axi C. M. Whlter \\ Argonne Tational Laboratory \\ Argonne, Illinois
}

(PRLSENTED BY L. A. NITMARK)

\section{Introduction}

Carbide fuels have long been recognized for their potential superiority over metal and oxide fuels. This potential is bascd on superior irradiation behavior over metals and better thermal conductivity, higher density, and lower release of fission gases than in the case of oxides. The realization of this potential, however, has been hampered by the problem of cladding compatibility. It is toward the solution of this problem that much of the work on carbides is directed.

For the purpose of defining carbide compositions of interest as fast reactor fuels, the term "hypostoichiometric" will be used in this paper to designate alloys that contain metal in equilibrium with the monocarbide, ( $\mathrm{C}, \mathrm{Pu}) \mathrm{C}$ phase. The term "hyperstoichiometric" will refer to compositions with monocarbide in equilibrium with higher carbon phases, (U,Pu) ${ }_{2} \mathrm{C}_{3}$ and $(\mathrm{U}, \mathrm{Pu}) \mathrm{C}_{2}$. Oxygen has been shown to have substitutional solubility with $\left(\mathrm{U}_{0.85} \mathrm{Pu}_{0.15}\right) \mathrm{C}$ to the extent of $40 \mathrm{a} / \mathrm{o}$ of the carbon content, ${ }^{(1)}$ and nitrogen forms a complete substitutional solid solution. (2) As a result, single-phase monocarbide is produced when the nonmetal-atom $\mathrm{C}+\mathrm{O}+\mathrm{N}$ content totals $50 \mathrm{a} / \mathrm{o}$. A hyperstoichiometric amount of $\mathrm{C}+\mathrm{O}+\mathrm{N}$ apparently produces a microstructure with monocarbide in equilibrium with higher carbide, but it is also conceivable that an oxide phase could also be present in threephase equilibrium.

It appears that the most desirable microstructure is stoichiometric or hyperstoichiometric. ${ }^{(3)}$ The questionable irradiation behavior of a free metal phase coupled with its possible eutectic reaction with the cladding precludes the use of hypostoichiometric carbide with prosent technology. At present, hyperstoichiometric carbide must be used with a helium contact bond between itself and the cladding because a sodium bond will transfer carbon to the cladding. The contact bond, however, will create higher fuel temperatures than the sodium bond. The higher temperature with its deleterious effects is cited as the principal disadvantage of helium contact bonding and, hence, of hyperstoichiometric fuel. Nevertheless, until proven conclusively that hyperstoichiometric carbide has a significant temperature limitation, this composition is considered a better choice than hypostoichiometric. Stoichiometric carbide suffers from none of the aforesaid disadvantages. Its major drawback is the inability to produce it in large-scale quantities because of its narrow range of carbon content.

The carbide development program of the Metallurgy Division at Argonne National Laboratory has concentrated on making and studying stoichiometric or slightly hyperstoichiometric mixed carbides with a uranium-plutonium ratio of $4: 1$. This paper will discuss some of the results of this program.

\section{Synthesis}

The techniques for synthesis of the mixed carbides have, in general, been extensions of processes used for the preparation of uranium monocarbide. Four techniques that have been used extensively for this purpose are: (1) high-temperature fusion of metal-carbon or oxide-carbon mixtures, either under arc or by a plasma, (2) carbothermic reduction of the oxides, (3) raction-sintering of mixtures of uranium-plutonium hydrides with carbon or mixtures of higher uranium-plutonium carbides, and (4) gas-metal reactions. Most of these methods for the synthesis of mixed carbides have been investigated on a laboratory-scale at Argonne. A fifth method, that of direct sintering of mixtures of uranium-plutonium carbides, has also been tried. The advantages and disadvantages of each process depend primarily on the desired composition of the fuel and its final physical form: sintered pellets, cast slugs, or powder with particle sizes that are needed for vibratory compaction.

Arc-melting(t) and carbothermic reduction of the mix ${ }^{2}$ oxides ${ }^{(5)}$ were the first methods used at Argonne to prepare cast and pressed-and-sintered pellets for early irradiation tests. ${ }^{(6)}$ More recently the arc-melting process has been used to synthesize material for sintering of pellets and vibratory compaction. This process was used because homogeneous material with reproducible carbon composition and low oxygen content was desired as a sintering material for preparation of pellets. Previous work had shown that arc- 
melting produces such a product on a laboratory scale.

Buttons of a $\left(\mathrm{U}_{0}{ }_{8} \mathrm{Pu}_{02}\right) \mathrm{C}$ composition weighing about $50 \mathrm{gm}$ were prepared by fusion of the elements with a tungsten-tipped electrode. The homogenized buttons were always very close to stoichiometric composition, and they usually contained less than 0.03 w/o oxygen plus nitrogen. The melts were kept small to avoid appreciable contamination from the tungsten electrode. The time in the liquid state was kept short to avoid detectable plutonium losses. This material was then crushed and sintered into pellets by a procedure described later.

Buttons of approximately $100-\mathrm{gm}$ weight were also made by the above procedure with the exception that a carbon-tipped electrode was user instear of tungsten. As expected, the compositions of the first few melts were hyperstoichiometric because of the high affinity of plutonium for carbon. The carbon content of the starting materials was then adjusted, and a significant reduction in the carbon content of the homogenized button resulted. This procedure, however, does not appear promising for production of large quantities of material with reproducible compositions because of the difficulty in controlling the carbon content.

\section{Fabrication}

A parallel study is being made of two concepts of fuel elements. One concept uses pressed-and-sintered pellets contact bonded to the jacket, and the other uses powder vibratorily compacted in fuel tubes. Although the pellet-making process is relatively simple, the large number of pellets required to fuel and refuel a reactor suggest a need for a bulk-processing technique. Vibratory compaction ${ }^{(7,8,9)}$ is considered a likely competitor for the pellet process.

\section{Sintered Pellets}

Are-fused buttons of $\left(\mathrm{U}_{0.8} \mathrm{Pu}_{02}\right) \mathrm{C}$ were crushed to powder that was screened to various particle sizes in gloveboxes that contained an inert atmosphere of nitrogen with impurities of oxygen and water vapor of about 0.005 and $0.01 \mathrm{w} / 0$, respectively. The powder was coated with $0.5 \mathrm{w} / \mathrm{o}$ Carbowax binder and pressed into pellets at a pressure of about $21 \mathrm{~kg} / \mathrm{mm}^{2}$.

Powders with high oxygen contents were undesirable for fabrication because they produced dimensional variations in the form of an hour-glass shape in the sintered pellets. The lowest attainable oxygen content was considered best for preparation of pellets with uniform diameters over their entire length. High oxygen contents also produced wide variations in phases from the center to the surface of pellets. Loss of car-

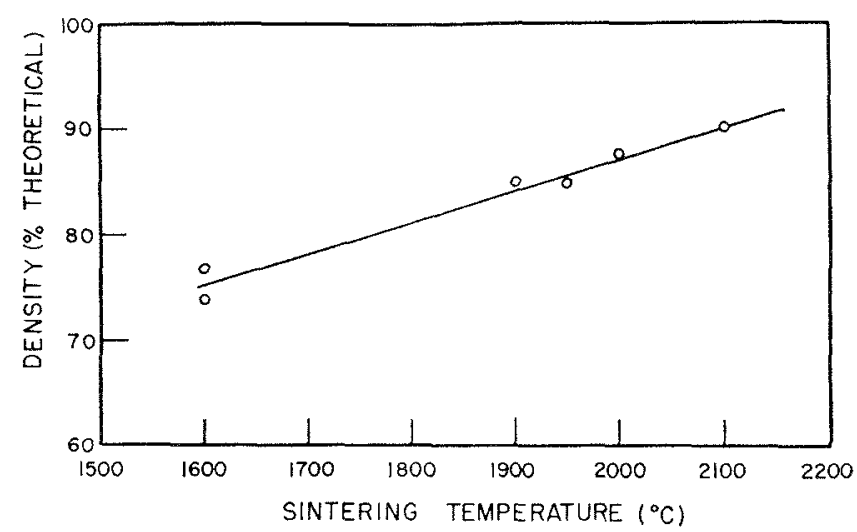

FIG. 1. The Change in Density with Sintering Temperature for $\left(\mathrm{U}_{0.8}, \mathrm{Pu}_{0.2}\right) \mathrm{C}$ Pellets Prepared from $<44 \mu$ Powder and Fired in Argon.

bon at the surface through the CO reaction resulted in a hypostoichiometric composition at the surface and hyperstoichiometric composition in the center of a pellet.

The changes in density of the pellets were investigated as a function of particle size and sintering temperature. Preliminary work showed that pellets from the solid-solution material lost plutonium when fired under vacuum at temperatures of $1600^{\circ} \mathrm{C}$ and above for $2 \mathrm{hr}$. To reduce plutonium raporization, pellets were fired in a flowing, high-purity argon gas at a pressure slightly above atmospheric. No plutonium loss was detected, and all subsequent studies were earried out under these conditions.

Pellets prepared from powder with a particle size of about $10 \mu$ attained densities of 80 to $85 \%$ of theoretical when sintered under argon for $2 \mathrm{hr}$ at $1600^{\circ} \mathrm{C}$. Densities of $90 \%$ of theoretical were obtained when the sintering temperature was increased to $2000^{\circ} \mathrm{C}$ for $2 \mathrm{hr}$. The fine particle size of the powder caused oxygen pickup to the extent of 1 to 2 w/o. Carbon contents of the pellets were low, which indicated loss by reaction to form $\mathrm{CO}$, and values ranged from 3.2 to $3.9 \mathrm{w} / 0$ carbon. In comparison, pellets prepared from powder with a particle size of about $40 \mu$ and sintered at $1600^{\circ} \mathrm{C}$ had lower densities (about $76 \%$ of theoretical) but the oxygen content was usually less than $0.15 \mathrm{w} / \mathrm{o}$ and the amount of carbon was always about the same as that present in the arcmelted material. Because of this problem of severe oxygen contamination of fine powders, all further work was conducted with powder with a particle size of about $40 \mu$.

The changes in density of pellets sintered at increasing temperatures for $2 \mathrm{hr}$ are shown in Fig. 1. Pellets of $90 \%$ of theoretical density were obtained by sintering at $2100^{\circ} \mathrm{C}$. Metallographic and X-ray examination showed that only the solid-solution (U, 


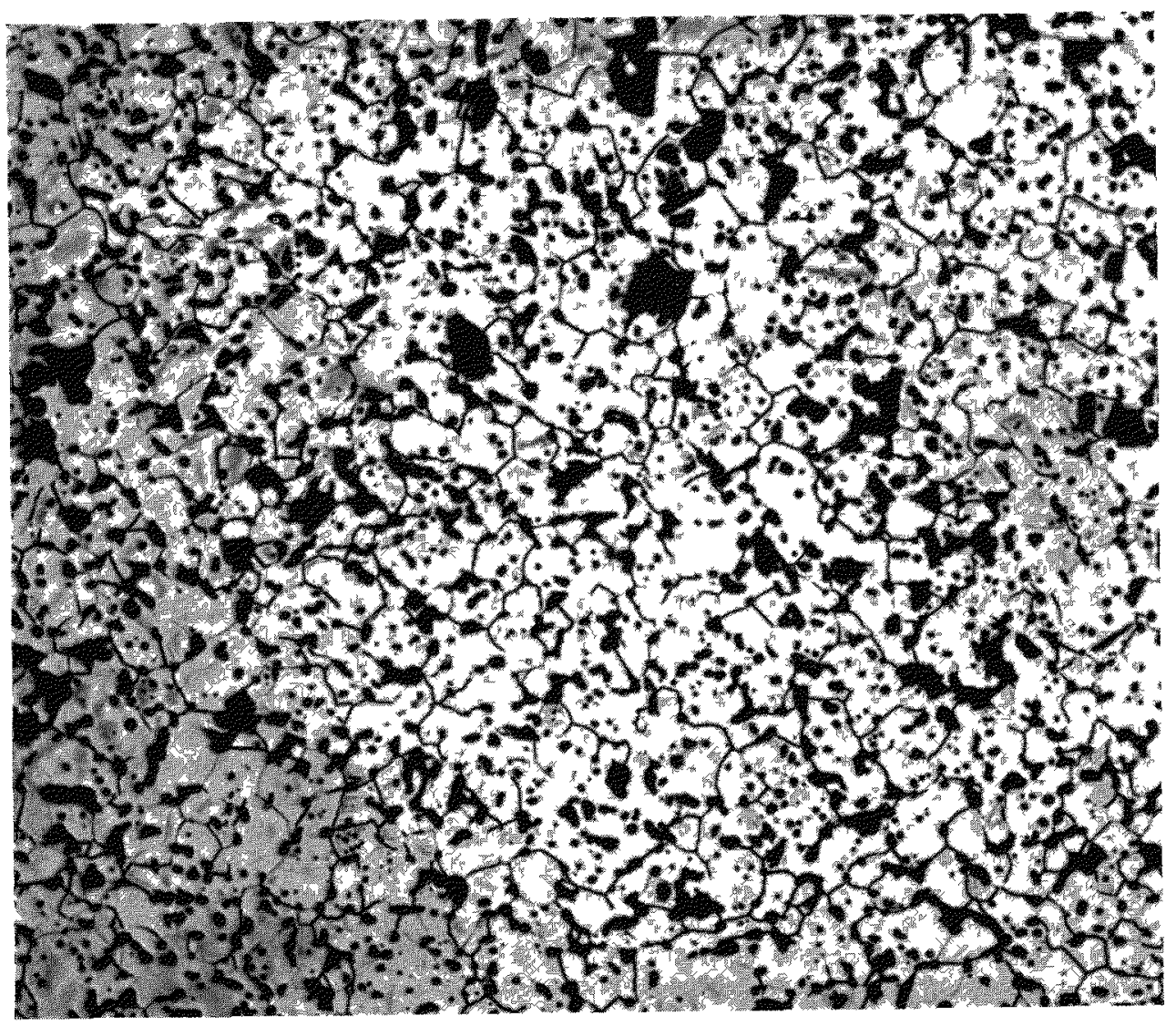

Fir 2 Mrrostructure of $\left(\mathrm{U}_{0}{ }_{8} \mathrm{Pu}_{0}\right)$ C Made by Arc melting and Then Sintered $(\times 200)$

$\mathrm{Pu}) \mathrm{C}$ phase was present in these pellet, There wa no detectable loss of plutonum from sintoring under argon at this temperature

The above procedure was used to make pellets 642 $\mathrm{mm}$ in diameter by 12 to $15 \mathrm{~mm}$ long for irradiation studies in MTR, teat fuel elements for KBR-II, and compatibility studien Densities of about $85 \%$ of theoretical were desued for the irradiation expen iment From the curve of $F i g$ it can be seen that a density of $85 \%$ of theoretical 15 obtamed after sintering at about $1900^{\circ} \mathrm{C}$ for $2 \mathrm{hr}$ The shinkage of these pellets was controlled to $\pm 005 \mathrm{~mm}$ so that they could be loaded into fuel jackets without grinding to sze Some of the pellets for the MTR irradiations were made with axial holes to accommodate ther mocouples Every effort was made to prepare single-phase ( $\mathrm{T}^{\top} \mathrm{s}$ $\mathrm{Pu}_{0}$ ) C that was well-characterized thiough chemical analyss and metallographic exammation Chemical analysis showed the pellets contaned 464 to $472 \mathrm{w} / \mathrm{o}$ carbon, 011 to $012 \mathrm{w} / \mathrm{o}$ oxygen, and 0014 to 0020 w/o nitrogen The mierostructure of a typical pellet is shown in Fig 2

\section{Vibratory Compaction}

Two u ibratorily compacted uanum-plutonum carbide systems are being studied at Argonne colid- colution powder, and mixed powders of $\mathrm{CC}$ and $\mathrm{PuC}$ The colid-solution ponder is made in the same way as the feed for suntered pellets The latter procedure (the IC and PuC can be handled separately until the mixing step), although simplified, is made at the cost of a significantly reduced Doppler effect These factors will have to be considered in the final analysis of this system

Small, 50-mm-long fuel specimens of physically mxed $\mathrm{IC}^{\mathrm{C}}-20 \mathrm{w} / \mathrm{o}$ PuC powders have been made for capsule-irradiation studies (10) Three size fractions of powder were blended and then vibrated in toto The coarce $(20 / 40$ mesh $)$ and fine ( -325 mesh) fractions were $\mathrm{CC}$, and the mudle $(120 / 200 \mathrm{mech})$ fraction was $\mathrm{PuC}$ The PuC was made at Argonne by the reaction of elemental plutonium and carbon (11) The UC was purchased commercially The average packing efficrency in the specimens was $80 \%$ The small specimen -1ze and short compaction times minimized component separation which is common in a pre-blended. cystem It was realized that this would not be the rase with full-size fuel rods

A basic study of particle packing in systems of spheres ${ }^{(12)}$ provided the mathematical relationships necesary to predict the prmary bunary, and tenary packing efficiences in full-scale rods This work has 


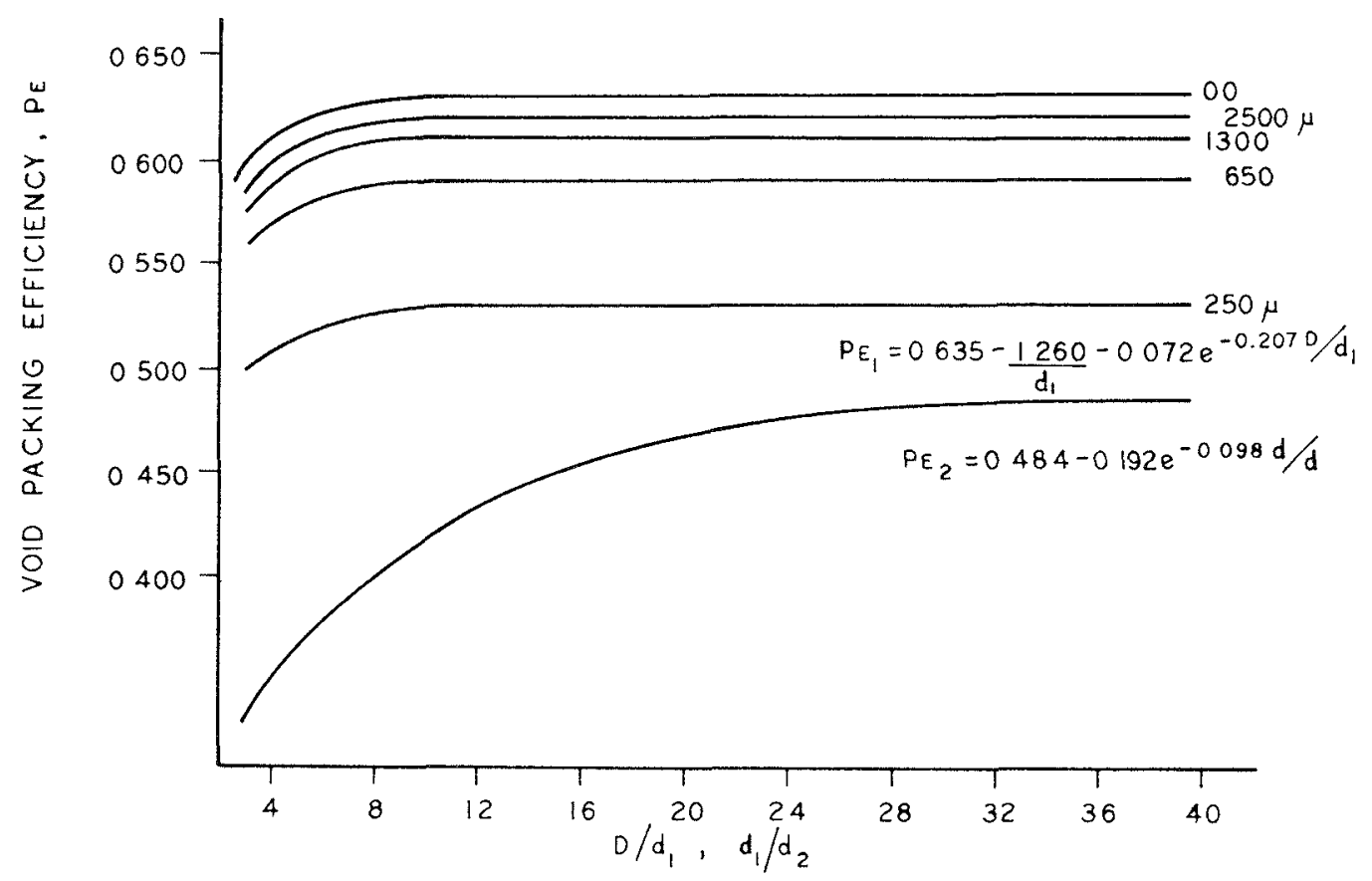

Fig. 3. Void Packing Efficiency vs Ratio of Diameter of Container to First Fraction, First Fraction to Second Fraction, etc.

since been extended to include the packing characteristics of irregularly shaped particles. ${ }^{(13)}$ The information gleaned from these studies has been applied to the loading of a series of uranium carbide-plutonium carbide fuel specimens for irradiation in EBR-II.(14) These specimens, the first full-scale vibratory-compacted rods made at Argonne, were made by an infiltration technique. (15)

Eleven mixed carbide rods containing $80 \mathrm{w} / 0$ uranium carbide and $20 \mathrm{w} / \mathrm{o}$ plutonium carbide and five solid-solution uranium-plutonium carbide rods were made by the new technique. The density of the mixed carbide system was specified at $80 \%$ of theoretical. Since the distribution and concentration of $\mathrm{PuC}$ in the solid solution with $\mathrm{CC}$ was uniform at $20 \mathrm{w} / \mathrm{O}$ $\mathrm{PuC}$, the only requirement on the solid-solution fuel body was uniform density. In the case of the solidsolution specimens, as high a density as might be practically attained was allowed.

Before loading any of the jackets with the two carbide materials, it was necessary to determine the quantities and sizes of grains to be loaded. The determination was based upon the results of newly developed information on particle packing. (13) Experiments with malleable, nonfissile materials compacted into rigid tubes indicated that the maximum packing fraction for a two-component system of angular particles in a small-diameter, $6.5-\mathrm{mm}$ tube would be about $80 \%$. Since the particle density of the carbides was about $97.5 \%$ of theoretical, such a packing fraction would yield a fuel density of only $78 \%$. An obvious solution to the problem of raising the packing density would, in a model system, be the use of three components. However, in the past it was found that efforts to produce uniform density in ternary systems of angular particles were singularly unsuccessful. An alternative would be to use the popular procedure of blending three components and pouring them into the jackets while vibrating. ${ }^{(7,8)}$ This solution was rejected because of nonuniform density and particle distribution resulting from the rapid settling of the finest fraction and subsequent levitation of the coarser particles.

The procedures to yield an acceptable product involved a compromise between the infiltration technique and the blending approach. A three-component system was derised in which settling and levitation were prevented and the infiltration of the finest fraction came about with little difficulty. To accomplish this, a blend of two size components was made. The coarse size was chosen to yield the maximum packing efficiency of angular particles in a $6.5-\mathrm{mm}$ tube according to the equation ${ }^{(13)}$

$$
P e_{1}=0.635-\frac{1.260}{d_{1}}-0.072 e^{-0207 D / d_{1}},
$$

where $P e_{1}$ is the packing efficiency of the coarse component, $d_{1}$ is the particle dimension in microns, and $D$ is the inside diameter of the tube, $6500 \mu$. The relationship between packing efficiency and particle size is shown in Fig. 3, which was derived from experimentation with angular particles.

The optimum size for the coarse fraction, $d_{1}$, was found to be $1200 \mu$. The determination was made by 


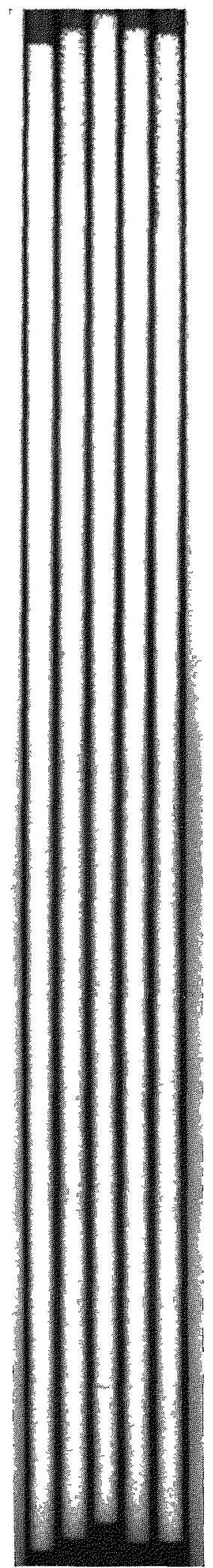

FIG. 4. Cramma-radiographs of Vipac UC-20 w/o PuC Showing Top Areas Depleted of Fines. taking the derivative of $P e_{1}$ with respect to $d_{1}$ in the above equation, setting the derivative equal to zero, and solving for $d_{1}$. The maximum packing efficiency of the coarse component in this system was $58.6 \%$.

In order to increase the packing fraction of the first component to about $66 \%$, a second component was added. The size of the second component was selected such that it would be small enough to fit into the large voids in the coarse-component matrix and large enough so that it could not migrate through the paths between large voids. The second-component size was determined to be $600 \mu$, one-half the coarse-component size.

Several runs were made in which angular blastcleaning grit was used ac a stand-in for fissile materials. The two-component first fraction was blended, added to a sample jacket, held in place by a "thimble," (12) and infiltrated with -325 mesh powder. The model runs yielded dencitics of about $82 \%$ in which $20 \%$ of the material loaded was -325 mesh. The -325 mesh iraction was a substitute for $\mathrm{PuC}$ in the mixed carbide system.

The ten specimens of mixed UC and PuC, if nellbehaved, should have been homogeneous fuel columns of $80 \%$ of theoretical density. Radiography of the actual fuel columns showed void regions at the tube wall near the restrainer. The voids were indicative of low density resulting from the absence of the fine fraction at the top of the column. This condition was confirmed by gamma radiography, as shown in Fig. 4 .

The low density at the top of the fucl column was caused by the deviation of the carbide system from the model. The most significant difference between the theory and practice was that the model system was made up of angularly shaped particles of malleable material, while the carbides were highly brittle. The effect of the brittle nature of the fuel was to provide a wider range of particle size during settling of the matrix than was introduced into the jacket as feed. Experience gained with some rejected specimens showed that $20 \%$ of a carbide fraction could be reduced to a smaller size during the loading of the matrix material alone. Since the loading of the -325 mesh fraction took as much as $8 \mathrm{hr}$, a considerable attrition could be expected. Although one would not expect the generation of additional void volume by particle cleavage, a similar effect does exist.

It was previously reported that the packing efficiency of approximately tetragonal shapes may be increased by rounding the edges of the particles. (13) The increase in packing efficiency of milled particles is brought about by at least three conditions: (1) the 
rounding of edges reduces the surface-to-volume ratio (the particle approaches spherical geometry), (2) the voids in the matrix have rounded walls that reduce resistance to the entrance of the fine fraction, and (3) extremely fine powders that result from attrition add another size fraction to the particle size distribution. Since the extent to which the carbides are affected by abrasion is not known, it is difficult to predict the degree of this effect.

Five specimens of solid-solution (U,Pu)C were assembled in which maximum uniform density was desired. Maximum density was achieved by adding the -325 mesh fraction until the rod would accept no more. The roid packing efficiency reached in these specimens was $86.7 \pm 1.6 \%$, which yields a theroretical density of $84.4 \pm 1.5 \%$ for the $97.5 \%$ dense particles. Gamma and autoradiography of these specimens showed uniform density over the entire length of the fuel column. Uniform distribution of plutonium, inherent in solid-solution feed materials, was confirmed by autoradiography.

The loading of the solid-solution rods gave information on the quantity of -325 mesh material which could be added to the blended matrix of coarse particles.

One rod containing mixed carbide was made on the basis of this information. The -325 mesh fraction, made up of $18.4 \% \mathrm{UC}$ and $81.6 \% \mathrm{PuC}$, was added until no additional material could be infiltrated into the $\mathrm{UC}$ matrix. The finished fuel rod showed no voids in the gamma radiograph. The total packing fraction was $86.0 \%$, the theoretical density was $83.8 \%$, and the plutonium carbide content was $19.3 \%$.

The determinations of plutonium distribution and uniformity of density by the techniques employed were qualitative, at best. Therefore, the densities achieved are "smear" densities and indications of homogeneity are relative only. Investigations are now under way to evaluate systems for the determination of particle distribution in vibratory-compacted bodies. When such capability is realized, quality control of compacted fuel columns will become possible. At present a fuel rod is completely assembled before being inspected for fuel distribution and density. Capability of examination in a glovebox before closure welding of the fuel rod will permit adjustment of the loading and guarantee final assembly of an acceptable product.

\section{Compatibility}

The initial phase of the Argonne compatibility studies on (U,Pu)C consisted of surveying the interaction of a number of cladding materials, including refractory metals, and iron- and nickel-base alloys with stoichiometric, single-phase (U,Pu)C over the temperature range from 650 to $1100^{\circ} \mathrm{C}$. This portion of the program is concerned with contact bonding in the absence of sodium and represents the extreme contact conditions found in a helium contact-bonded fuel element. The emphasis has been placed on ironand nickel-base claddings at 650 to $800^{\circ} \mathrm{C}$. Later portions of the program will investigate the effect of stoichiometry, stabilizing additions, sodium environment, and irradiation.

The $(\mathrm{U}, \mathrm{Pu}) \mathrm{C}$ was prepared by sintering pellets pressed from crushed, arc-melted stock as described in the preceding section. The $\mathrm{U} / \mathrm{Pu}$ ratio was $4: 1$, and a typical chemical analysis showed a carbon content of $4.74 \mathrm{w} / \mathrm{o}$, with oxygen and nitrogen impurities of 0.09 and $0.03 \mathrm{w} / \mathrm{o}$, respectively. Examination of the pellets showed single-phase monocarbide of approximately $86 \%$ of theoretical density.

Each compatibility assembly consisted of a pellet of the carbide fuel between two disks of cladding material held in contact with a molybdenum frame. In addition, a third metal disk was added to the assembly to ensure that the fuel-cladding assembly had a sufficiently greater thermal expansion than that of its molybdenum holder to maintain continued contact between fuel and cladding at elevated temperatures.

Approximately $0.8 \mathrm{~mm}$ was ground off each ond of the carbide pollets to reduce the chance of surfaceimpurity phases. The fuel-cladding contact surfaces were then polished flat by use of $4 / 0$ emery paper on a glass plate and a special specimen holder. Immediately after polishing the specimens were assembled and pressure applied by a serew in one end of the molybdenum holder. The assembly was then placed in a capsule, evacuated, backfilled with onethird atmosphere of helium, and sealed. Stainless steel capsules were used for the $800^{\circ} \mathrm{C}$ heat treatments; quartz capsules were used for the heat treatments at higher temperature. Equivalent results were obtained in each capsule material.

The results of the compatibility tests made to date should be considered as a preliminary survey. The extent of carbide reaction with the various cladding materials are summarized in Table 1 and are discussed briefly below. Some representative reactions with these materials are illustrated in Fig. 5.

Type 304 Stainless Steel-The results show no reaction of single-phase $(\mathrm{U}, \mathrm{Pu}) \mathrm{C}$ with Type 304 stainless steel at $800^{\circ} \mathrm{C}$ for 42 days. At $1100^{\circ} \mathrm{C}$, heavy grain-boundary penetration was noted, with eventual melting occurring between 17 and 42 days. 


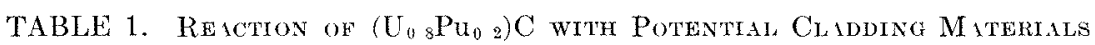

\begin{tabular}{|c|c|c|c|c|c|c|}
\hline \multirow{2}{*}{ Material } & \multirow{2}{*}{ Temp $\left({ }^{\circ} \mathrm{C}\right)$} & \multicolumn{3}{|c|}{ Reaction Zone Width $(\mu)$} & \multirow{2}{*}{$\begin{array}{l}\text { Direction of } \\
\text { Reaction Zone }\end{array}$} & \multirow{2}{*}{ Remarks } \\
\hline & & 7 Days & 17 Days & 42 Days & & \\
\hline $\mathrm{V}-20 \mathrm{w} / \mathrm{o} \mathrm{Ti}$ & $\begin{array}{r}800 \\
950 \\
1100\end{array}$ & $\begin{array}{l}\text { - } \\
160(320)^{*} \\
750(>1300)^{*}\end{array}$ & $100(270)^{*}$ & $\begin{array}{c}350(700)^{*} \\
>1300\end{array}$ & $\begin{array}{l}\text { Cladding } \\
\text { Cladding } \\
\text { Cladding }\end{array}$ & $\begin{array}{l}\text { Nonuniform continuous band, } \\
\text { poorly defined due to reac- } \\
\text { tion along stringers }\end{array}$ \\
\hline Niobium & $\begin{array}{r}950 \\
1100\end{array}$ & $\begin{array}{l}2 \\
8\end{array}$ & - & - & $\begin{array}{l}\text { Cladding } \\
\text { Cladding }\end{array}$ & $\begin{array}{l}\text { Uniform continuous band } \\
\text { Uniform continuous band }\end{array}$ \\
\hline Molybdenum & 1100 & 6 & - & - & Cladding & Uniform continuous band \\
\hline Tantalum & 1100 & 4 & - & - & Cladding & Uniform continuous band \\
\hline Chromium & 1100 & $\mathrm{Nil}$ & - & - & - & - \\
\hline Tungsten & 1100 & $\mathrm{Nil}$ & - & - & - & - \\
\hline
\end{tabular}

* Values in parentheses indicate extent of reaction along stringers.

Hastelloy-X-At $800^{\circ} \mathrm{C}$, slight reaction $(8 \mu$ at 42 days) was noted with Hastelloy-X at the interface. At $950^{\circ} \mathrm{C}$, the reaction zone is shown to be elearly in the direction of the fuel.

Vanadium-20 w/o Titanium-This alloy appeared to react quite extensively with $(\mathrm{U}, \mathrm{Pu}) \mathrm{C}$ at $800^{\circ} \mathrm{C}$. The reaction zone was rather irregular and appeared to follow stringers (particles aligned in the extrusion direction) observed in as-received vanadium-titanium material.

Vanadium-No reaction was noted at $950^{\circ} \mathrm{C}$ up to 17 days, but a few small grain-boundary penetrations were observed after 42 days. This second phase was present in much greater amounts at $1100^{\circ} \mathrm{C}$.

Refractory Metals ( $T a, W . M o, N b, C r)$-Tantalum, niobium, and molybdenum showed slight reaction (4-8 $\mu$ ) at $1100^{\circ} \mathrm{C}$ for 7 days; no evidence of reaction was observed with chromium and tungsten under these conditions.

The program of compatibility studies also includes new concepts in claddings and in fuel composition. These are described below.

Cladding Materials-Stainless steel, because of its low cost, good compatibility with sodium, and ease of fabrication, will continue to receive attention even though its strength is significantly lowered at temperatures above $600^{\circ} \mathrm{C}$. Increased nickel content, although imparting greater strength to iron-nickel-base alloys, appears also to lessen the compatibility of these alloys with carbide fuels. High nickel alloys, such as Inconel or Nimonic, have been reported ${ }^{(16,17)}$ as incompatible with carbide fuels. Hastelloy-X $(47 \% \mathrm{Ni})$ has shown some reaction at $800^{\circ} \mathrm{C}$ with stoichiometric material. Because of these factors, the investigation is being expanded to include high strength iron-nickel-chromium and iron-nickel-chromium-cobalt alloys with relatively low nickel contents $(13-25 \%)$. These alloys represent an interesting range of composition while having a significant increase of strength over stainless steel at $700^{\circ} \mathrm{C}$. The compositions of the potential cladding materials receiving immediate attention are listed in $\mathrm{Ta}-$ ble 2. Three of these alloys contain substantial amounts of cobalt or manganese, the influence of which is unknown with regard to compatibility with uraniumplutonium carbides or to resistance to corrosion by sodium.

Effects of Carbide Stoichiometry-Although all material considerations favor the use of stoichiometric monocarbide, its permissible range of carbon content is so narrow that large-scale production is not eco- 


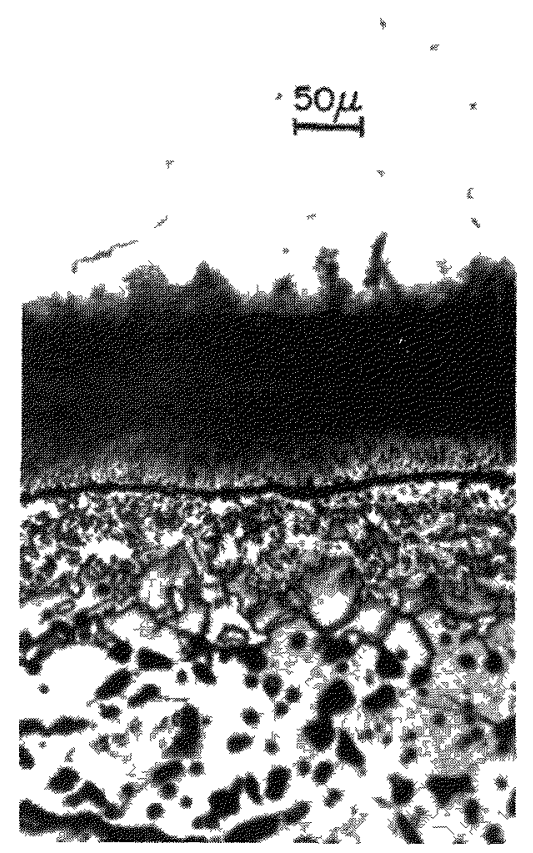

Hastelloy $\mathrm{X}$

$7 \mathrm{Ddr}$, at $950^{\circ} \mathrm{C}$

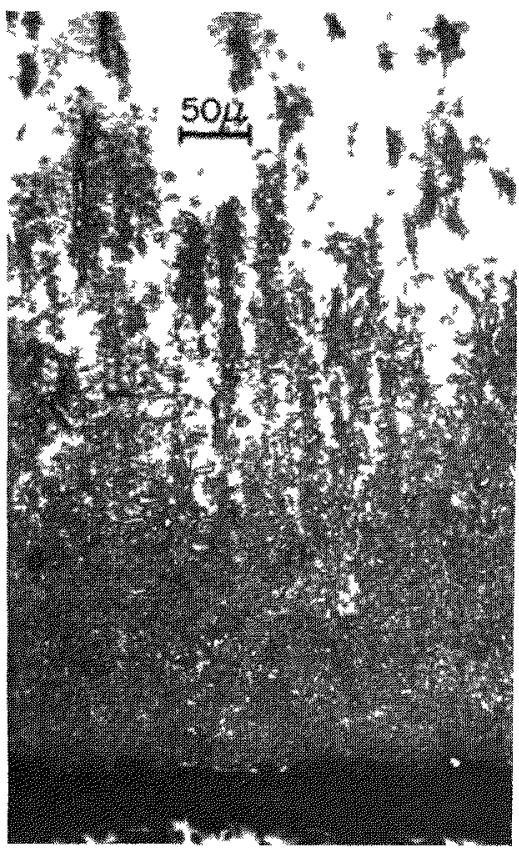

Vanadium $20 \mathrm{w} / 0$ Titanium $42 \mathrm{IJ}$ irs at $950^{\circ} \mathrm{C}$

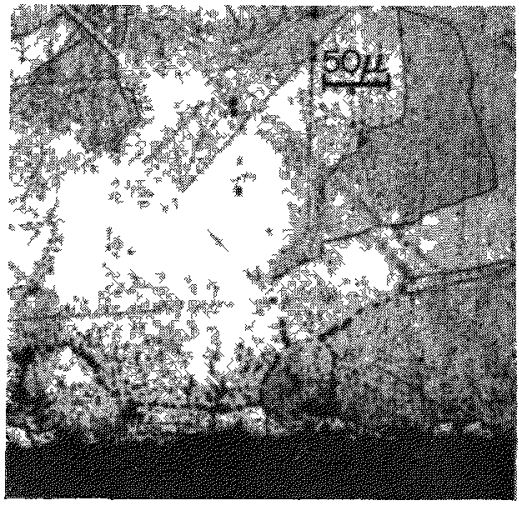

304 Stamless steel $7 \mathrm{Day}$ at $1100^{\circ} \mathrm{C}$

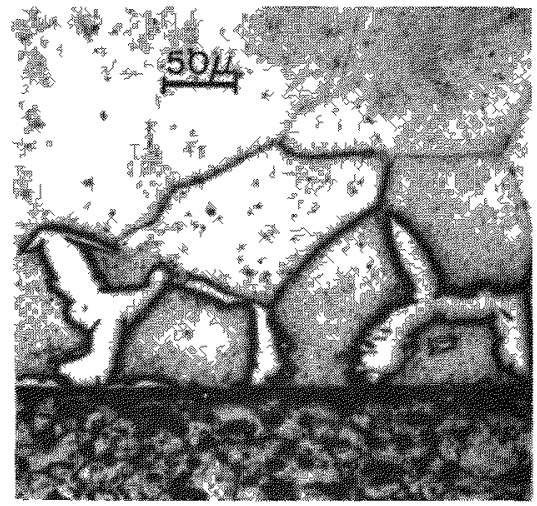

I nnadium

7 )dys at $1100^{\circ} \mathrm{C}$

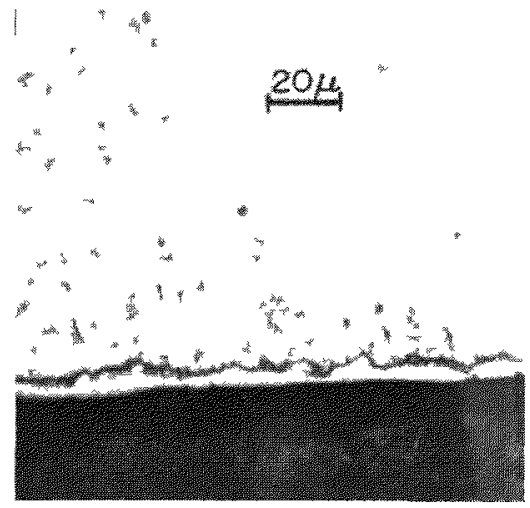

Niobium

7 Dars at $1100^{\circ} \mathrm{C}$

Fic 5 Reaction of Sungle phase ( $\left.\mathbf{C}_{0}{ }_{4} \mathrm{Pu}_{02}\right) \mathrm{C}$ (Bottom) with Valous Matenals

nomically feasble at this time Hyperstowchometric carbide in helium contact-bonded systems appears to be the next chorce Although stanless steels are 1eported to be compatible with slightly hyperstorchiometric (U,Pu)C at $816^{\circ} \mathrm{C}$ for $4000 \mathrm{hr}$, the compatibility of hyperstorchometric $(\mathrm{T}, \mathrm{Pu}) \mathrm{C}$ and newer potentral cladding materials is yet to be determined

Addations to Carbude Fuels-The lower thermodynamic atability of the carbide phases $(\mathrm{U}, \mathrm{Pu})_{2} \mathrm{C}_{3}$ and $(\mathrm{U}, \mathrm{Pu}) \mathrm{C}_{2}$ in comparison to $(\mathrm{C}, \mathrm{Pu}) \mathrm{C}$ can present compatibility problems in hyperstorchiometric carbide fuels because of their tendency to transfer carbon to the cladding, especially in the presence of a sodrum bond The carbon that is transferred either forms a cladding carbide at the interface or diffuses into the cladding and degrades its mechanical propertree, notably ductility It 1 s therefore desirable to eliminate these higher carbides This might be accomplished in two ways (1) the addition of an element which would reduce $(\mathrm{C}, \mathrm{Pu})_{2} \mathrm{C}_{3}$ and $(\mathrm{C}, \mathrm{Pu}) \mathrm{C}_{2}$, and form a compound that would give the fuel greate 1 ovcrall thermodyname stability, and (2) the addition of an element that would form a very stable carbide existing as a ingle-phase compound over a relatively wide composition range In the latter method it is hoped that the fuel pellet would consist of either uranium-plutonium monocarbide plus a metal carbide with a wide range of stolchiometry or 
TABle 2. Potential Cladding Materials for $\left(\mathrm{U}_{9} \mathrm{Pu}\right) \mathrm{C}$

\begin{tabular}{|c|c|c|c|c|c|c|c|c|c|c|c|}
\hline \multirow{2}{*}{ Alloy } & \multicolumn{11}{|c|}{ Composition $(w / o)$} \\
\hline & $\mathrm{Fe}$ & $\mathrm{Ni}$ & $\mathrm{Cr}$ & $\mathrm{Co}$ & $\mathrm{Mn}$ & Mo & $\mathrm{Nb}+\mathrm{Ta}$ & $W$ & C & $\mathrm{N}$ & $\mathrm{Si}$ \\
\hline Hastelloy-X & 18 & 48 & 22 & 1.5 & 1 & 9 & - & 0.6 & 0.10 & 一 & 1 \\
\hline $16-25-6$ & 50 & 25 & 16 & - & 1.3 & 6 & - & - & 0.08 & 0.15 & 0.70 \\
\hline $16-15-6$ & 55 & 15 & 16 & - & 7.5 & 6 & - & - & 0.07 & 0.35 & 0.50 \\
\hline
\end{tabular}

TABLE 3. SUMM IRY OH VIPAC CIRBIDE IRR UDITIONS

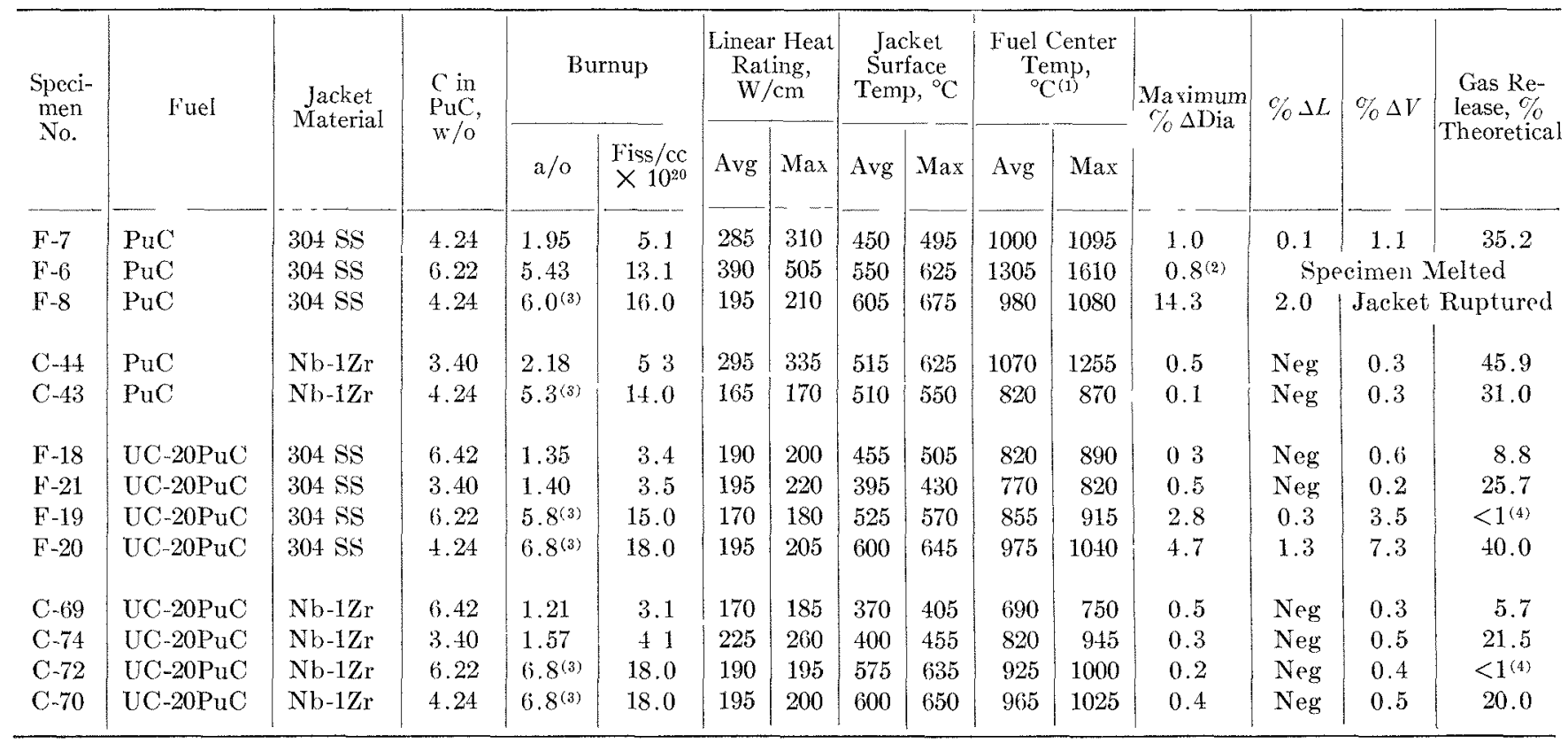

(1) Calculated assuming a fuel-jecket interface conductance of $0.136 \mathrm{cal} / \mathrm{sec}-\mathrm{cm}^{2}{ }^{\circ} \mathrm{C}\left(1000 \mathrm{Btu} / \mathrm{hr}-\mathrm{ft}^{2}{ }^{\circ} \mathrm{F}\right)$.

(2) Measured on remaining pieces.

(3) Burnup estimated from heat-generation rates.

(4) Based only on xenon release.

of a uranium-plutonium-metal monocarbide with a wider range of stoichiometry than that of (U,Pu)C. Titanium and zirconium additions are being tried by the French. ${ }^{(18)}$

Since these additions will be made to the hyperstoichiometric powders before sintering of the pellets, phase studies of the fired pellets should indicate the feasibility of these methods before compatibility tests are run. Preparations are now being made for these phase studies.

\section{Irradiation}

The irradiation program has as its objective the investigation of fuel behavior with respect to swelling, fission-gas release, micro- and macro-structural changes, and cladding compatibility. Both pellet fuel and vibratorily compacted fuel are being studied.
So far, the emphasis has been on vibratorily compacted physical mixtures of UC-20 w/o PuC powders. A few vibratorily compacted $\mathrm{PuC}$ powder specimens have also been irradiated to obtain information on the single-carbide system. The specimen fabrication has been discussed previously under "Vibratory Compaction." These specimens were $70 \mathrm{~mm}$ overall with a 50-mm-long fuel section and a small, 13-mm gas plenum (20\% of the fuel volume) above the fuel. The fuel diameters were 3.96 or $6.53 \mathrm{~mm}$. The jackets were either $\mathrm{Nb}-1 \mathrm{w} / \mathrm{o} \mathrm{Zr}$ or 304 stainless steel, 0.23 or $0.30 \mathrm{~mm}$ in thickness. The carbon content of the UC was $4.8 \mathrm{w} / \mathrm{o}$; that of the $\mathrm{PuC}$ ranged from 3.4 to 6.4 w/o. Free plutonium was present in the low-carbon batches and sesquicarbide was evident in the highcarbon batches. The uranium was not enriched since the irradiations were to be made in thermal reactors. 

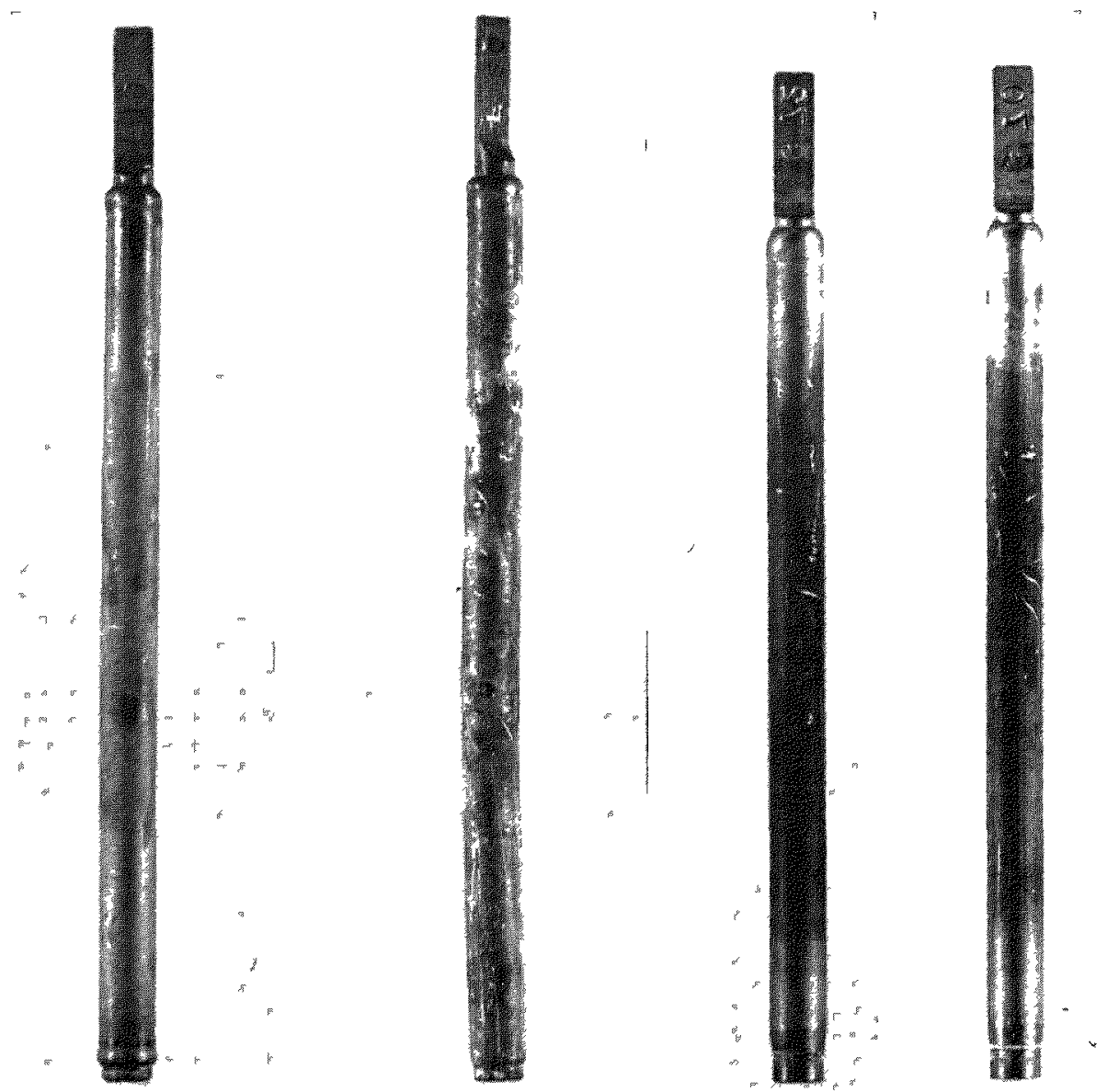

Fig. 6. From Left, UC-20 w/o PuC Specmens F 19 (304 SS), F-20 (304 SS), C 72 (Nb-1 w/o Zr), and C-70 (Nb-1 w/o Zr) after Irradiation $(13 \times)$.

The $\mathrm{PuC}$ was then the only component undergoing significant fissioning, and the results are characteristic of the PuC powder and not the UC.

The irradiation program for the vibratorily compacted specimens called for nominal burnup levels of 2,5 , and $10 \mathrm{a} / 0$ at jacket surface temperatures of 600 , 800 , and $1000^{\circ} \mathrm{C}$. The irradiations are done in the $\mathrm{CP}-$ 5 and MTR reactors in instrumented capsules. The MTR capsules are also temperature-controlled, but at the sacrifice of high linear heat ratings. Irradiations have been completed at the 2 and 5 a/o burnup levels at $600^{\circ} \mathrm{C}$, although the $2 \mathrm{a} / 0$ specimens did not all quite achieve this burnup or temperature. Specimens for the $10 \mathrm{a} / \mathrm{o}$ level at $600^{\circ} \mathrm{C}$ and $800^{\circ} \mathrm{C}$ are now in MTR, and 2 and 5 a/o, $1000^{\circ} \mathrm{C}$ capsules are being prepared.

The irradiation data and postirradiation examination results on specimens that have been examined are given in Table 3 . All specimens had $0.23-\mathrm{mm}$-thrck jackets and a fuel diameter of $396 \mathrm{~mm}$. Burnups ranged from 12 to $6.8 \mathrm{a} / \mathrm{o}\left(3.1 \times 10^{20}\right.$ to $18 \times 10^{20}$ fiss $/ \mathrm{cc}$ ) with average surface temperatures between $370^{\circ} \mathrm{C}$ and $605^{\circ} \mathrm{C}$. Maximum linear heat ratings were between 170 and $505 \mathrm{~W} / \mathrm{cm}(5.2$ and $15.4 \mathrm{~kW} / \mathrm{ft}$, respectively). The burnups were determined from $\mathrm{Te}^{99}$ analyses unless noted otherwise. The heat-generation rates were in turn calculated from the burnups The higher burnup specimens of $\mathrm{UC}-\mathrm{PuC}$ and $\mathrm{PuC}$ are shown in Figures 6 and 7 , respectively.

Inspection of the data indicated the following:

1. No significant diameter, length, or volume changes occurred when $\mathrm{Nb}-1 \mathrm{w} / \mathrm{o} \mathrm{Zr}$ was the jacket material for $\mathrm{CC}-20 \mathrm{w} / \mathrm{o} \mathrm{PuC}$. This was independent of fuel material, carbon content, burnup, heat rating, or temperature.

2. Changes in diameter, length, and volume occurred on stainless steel jackets with UC-20 w/o PuC. Primary dependence appeared to be on burnup, with secondary dependence on temperature and heat rating

3. Stainless steel jackets with $\mathrm{PuC}$ had diameter increases apparently dependent on burnup.

4. Fission-gas release was dependent on the carbon content of the PuC. 

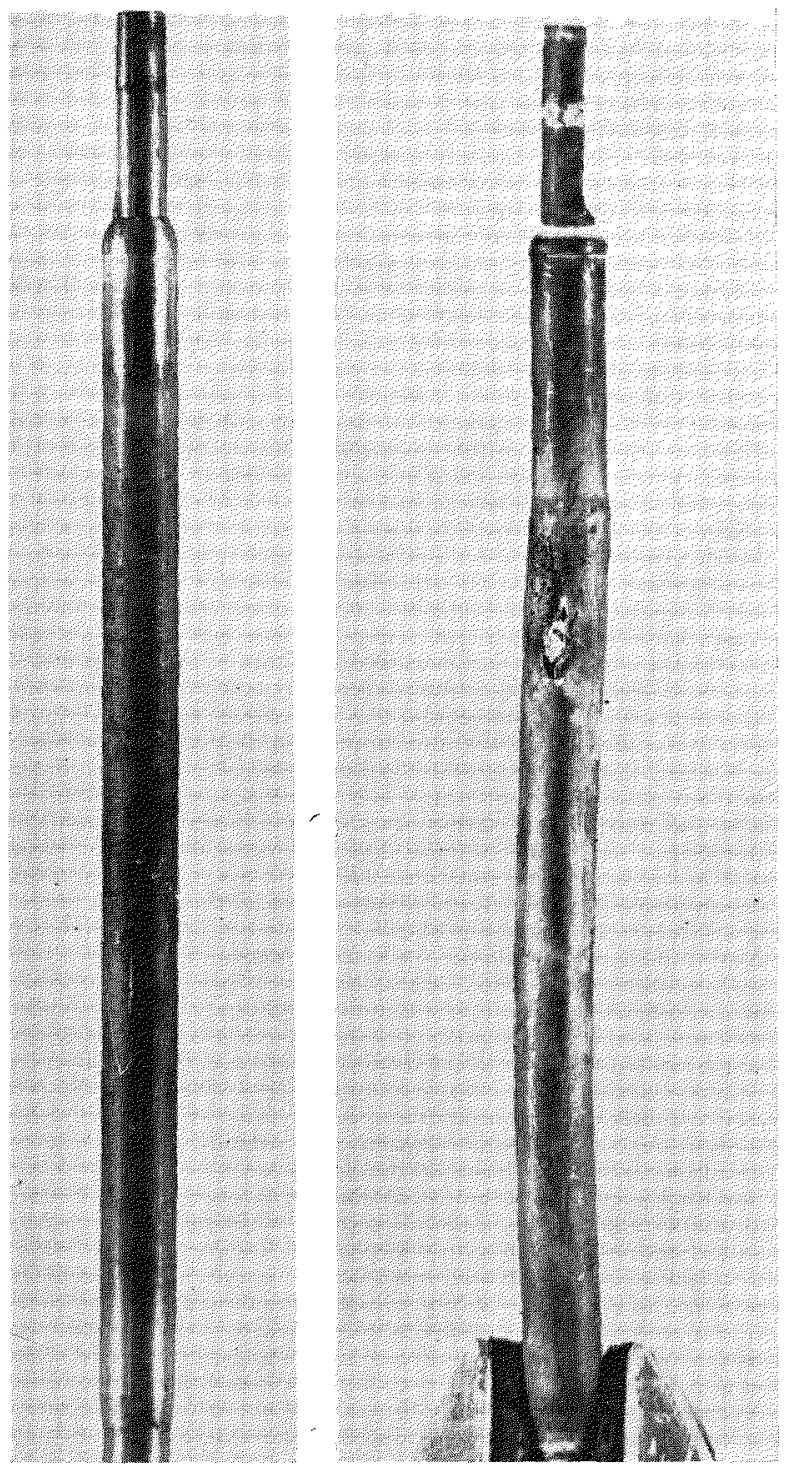

FIG. 7. Specimens C-43 (Left) and F-8 (Right), PuC in $\mathrm{Nb}-1 \mathrm{w} / \mathrm{O} \mathrm{Zr}$ and 304 Stainless Steel, Respectively, after Irradiation $(1.5 \times)$.

All mixed-carbide specimens were intact. Two stainless-jacketed $\mathrm{PuC}$ specimens failed from different causes. Specimen F-6 melted in a large area of the fuel section, as shown in Fig. 8. The calculated center temperature of $1610^{\circ} \mathrm{C}$ in only $40^{\circ}$ below the melting point of $\mathrm{PuC}$. Because of the nature of the assumptions, the calculation can be off enough to make up the difference. Preliminary metallography indicated the fuel melted and reacted with the jacket. It is not known when during the irradiation this occurred, because the maximum thermocouple reading indicated a jacket surface temperature of only $625^{\circ} \mathrm{C}$.

The jacket of specimen F-8, shown in Fig. 7, apparently ruptured because of high internal gas pressure. The failure is typical of those observed in tube- burst tests. From the gas collected from specimen $\mathrm{C}-43$, it was estimated that the maximum cladding stress might have been $4.5 \mathrm{~kg} / \mathrm{mm}^{2}$. The specimen also had a double bow, which is evident in Fig. 7. Bowing was also evident to a lesser degree of specimen F-20 (shown in Fig. 6). These specimens have not been metallographically examined as yet, but the fuel just inside the split of specimen F-8 appears to be massive and not particulate.

Only cursory metallography of the low-burnup specimens has been done. Other than evidence of melted fuel in the remains of specimen F-6, no gross fuel changes have been noted. In-pile sintering of UC and $\mathrm{PuC}$ has so far been found only to a small degree.

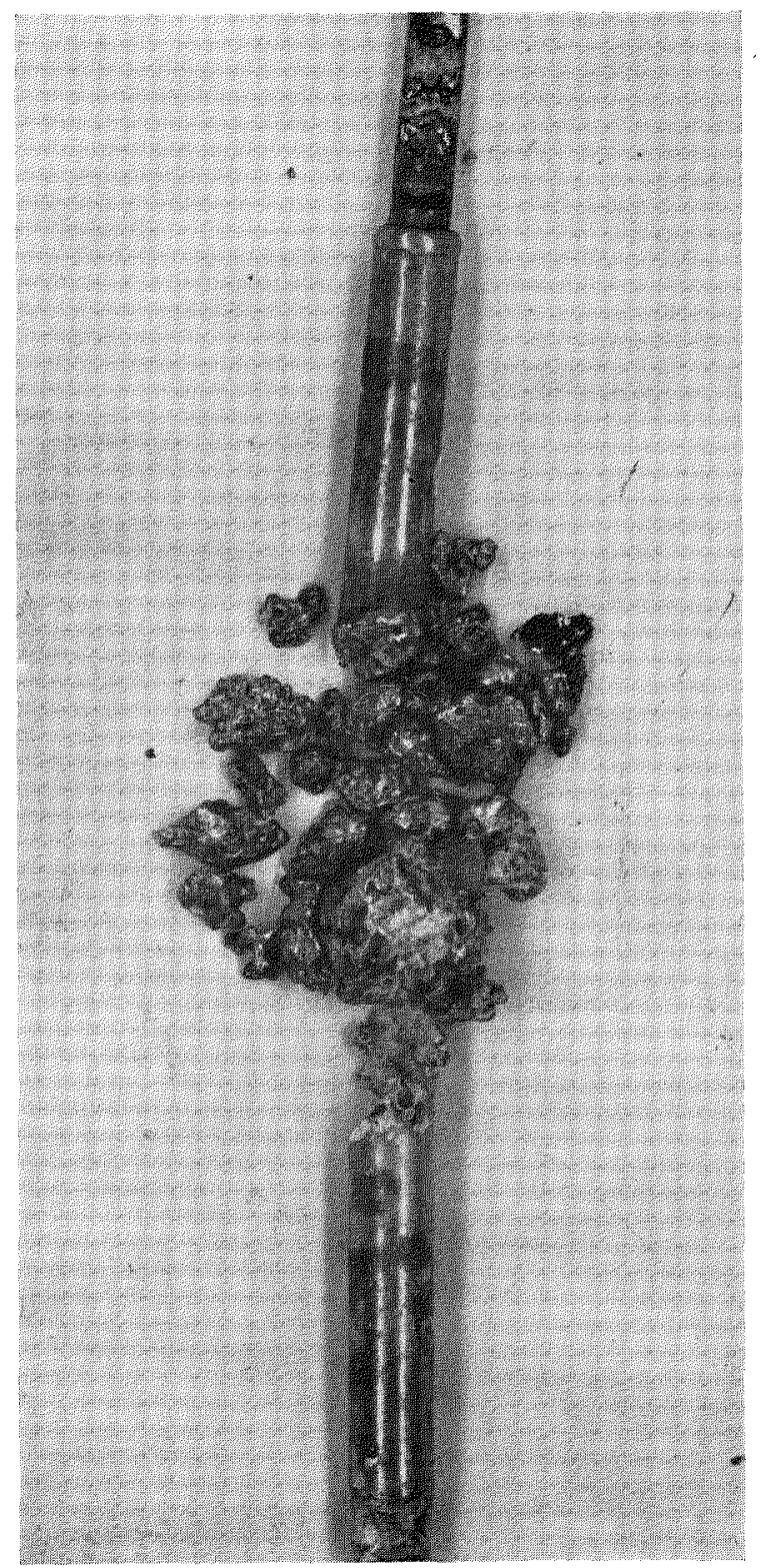

FIG. 8. Specimen F-6 (PuC in 304 Stainless Steel) Shown Reassembled after Meltdown during Irradiation (1.5X). 
Figure 9 shows a small $\mathrm{PuC}$ particle to which smaller UC particles have become attached. Extensive sintering was found in specimen $\mathrm{C}-44$, which was all $\mathrm{PuC}$. It is expected that sintering of $\mathrm{UC}$ and $\mathrm{PuC}$ will be evident on a larger scale in the higher-temperature specimens. Other than the melted specimen, the only fuel-jacket reaction noted was a thin intermittent layer on the fuel side of the fuel-cladding interface in $\mathrm{PuC}$ specimen $\mathrm{C}-44$. The layer is as yet unidentified, but may be free plutonium metal from the hypostoichiometric carbide.

The fission-gas results are indicative of the effect of a free metal phase. Hypostoichiometric carbide released significantly more gas then the hyperstoichiometric. The quantities of gas released are commensurate with the irradiation temperatures and are not considered excessive.

As a logical step in the irradiation program, the vibratorily compacted fuels are being tested in fullscale fuel rods in EBR-II, where they will be in a fast flux and have heat ratings of interest for direct reactor application. This phase of the program now includes solid-solution powders and also pressed-and-sintered pellets. The fabrication of all these fuel specimens has been previously discussed in this paper.

Three vibratorily compacted fuel rods are now under irradiation in EBR-II at heat ratings of 650 to $790 \mathrm{~W} / \mathrm{cm}(20$ to $24 \mathrm{~kW} / \mathrm{ft})$ and jacket surface temperatures of about $600^{\circ} \mathrm{C}$. Jacket materials are 304 stainless steel, Hastelloy-X, and Nb-1 w/o Z $\mathrm{r}$. Cladding thicknesses have been increased to $0.30 \mathrm{~mm}$ for Nb-1 w/o Zr, $0.38 \mathrm{~mm}$ for Hastelloy-X, and 0.51 $\mathrm{mm}$ for stainless steel. These rods will be removed after $5 \mathrm{a} / \mathrm{o}$ burnup. Other specimens will be irradiated to 2 and $10 \mathrm{a} / \mathrm{o}$, as well as $5 \mathrm{a} / \mathrm{o}$.

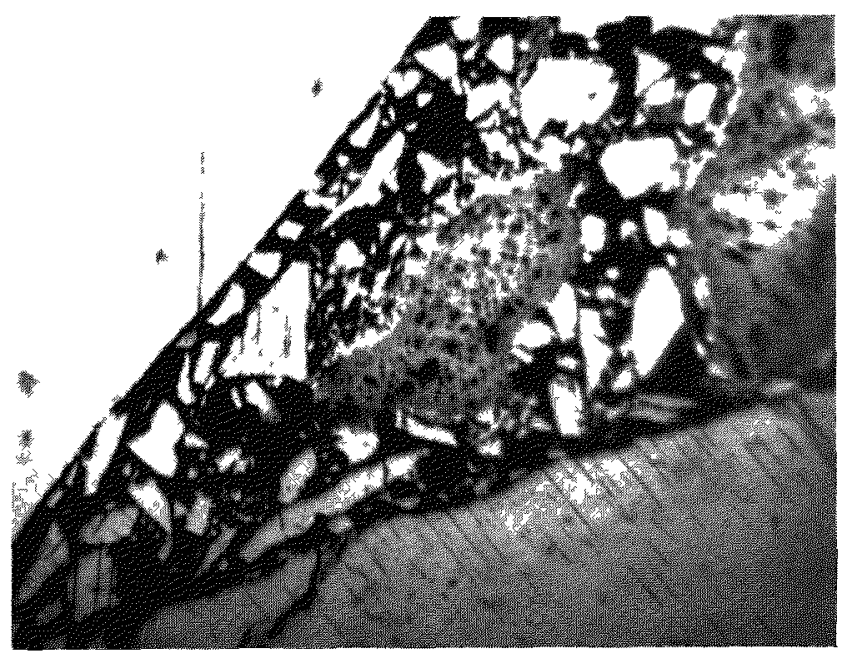

FIG. 9. PuC Particle with Adhering UC Particles in Specimen C-74 $(\times 400)$.

\section{Conclusions}

The present difficulty in making large quantities of stoichiometric $(\mathrm{C}, \mathrm{Pu}) \mathrm{C}$ leaves slightly hyperstoichiometric carbide as the favored composition for fuels with today's technology. Techniques have been demonstrated for producing high-quality pressed-and-sintered pellets and vibratorily compacted fuel columns. Although compatibility tests have shown stainless steel to be an acceptable jacket material from this standpoint, there is still a need for making nonstoichiometrie fuel more compatible with the jacket and for finding stronger jacket materials. The promising irradiation results on vibratorily compacted carbide indicate that this system is worthy of consideration for future reactors.

\section{Acknowledgment}

The authors wish to acknowledge the endeavors of many of their coworkers which made this paper possible.

\section{REFERENCES}

1. N. H. Brett, E. A. Harper, H. J. Hedger, and J. S. Pottinger, The Substitutional Solubulity in Oxygen in $U$-C, $\left(U_{0}{ }_{85} P u_{0}{ }_{15}\right)-C$ and $P u C$, Carbides in Nuclear Energy, Vol. I, Macmillan Company, London (1964) p. 162.

2. F. Anselin, G. Dean, R. Lorenzelli, and R. Pascard, $O n$ the System $(U, P u)(C N O)$ as Applied to Sintered Carbides, Carbonitrides, and Oxycarbides, ibid., p. 113.

3. A. Strasser, C. Wheelock, and L. A. Neimark, C'raniumPlutonium Carbide Fuels for Fast Breeder Reactors, Proceedings of the ANS Meeting on Fast Reactor Technology, ANS-100, Detroit, April 1965.

4. O. L. Kruger, Preparation and Some Properties of Arc-cast Plutonium Monocarbide, J. Nucl. Mat. 7(2), 142 (1962).

5. R. C. Lied and (x. D. White, Preliminary Studies in the System Uc-PuC, J. Am. Cer. Soc., 45(4), 149 (1962).

6. J. H. Kittel et al., Preliminary Irradiations of $P u C$ and ${ }^{\top} C^{\prime}-P u C$, ANL-6678 (1963).

7. J. J. Hauth, Vibrationally Compacted Ceramic Fuels, HW-67777 (1961).

8. S. W. Porembka, C. B. Boyer, and J. J. Hauth, Fabrication of $\mathrm{UO}_{2}$ fruel Rods by Vibrational Compaction and Pressure Bonding, BMI-1637 (1963).

9. J. J. Hauth, Canadian Patent 653,597 (Dec 1960).

10. Annual Progress Report for 1964, Metallurgy Division, Argonne National Laboratory, ANL-7000, p. 41.

11. W. (․ Tope, Production of Plutonium Monocarbide by Reaction of Elemental Plutonium and Carbon, ANI 46969 , to be published.

12. J. W. Ayer and F. E. Soppet, Vibratory Compaction, I: Compaction of Spherical Shapes, J. Am. Cer. Soc., 48(4), 180-183 (1965).

13. J. F. Ayer and F. E. Soppet, Vibratory Compaction. II: Compaction of Angular Shapes, to be published.

14. J. E. Ayer et al., Nuclear-fuel-element Loading by Vibratory Compaction, I'ranium-Plutonium Carbide Specimens for EBR-II Irradiation, ANL-7075, to be published.

15. J. E. Ayer and F. E. Soppet, U.S. Patent Application S.N. $358,389-5-30,416$. 
16. 1). Stahl and A. Strasser, Properties of Solid Solution Uranium-Plutonium Carbides, Carbides in Nuclear Energy, Vol. I, Macmillan Company, London (1964).

17. L. Russell et al,, Monocarbides as Reactor Fuels, P/154, Third Geneva Conference on the Peaceful Uses of Atomic Wnergy (1964).

18. R. Boucher and P. Barthélémy, Etudes D'Alliages a Base de Plutonium, to be presented at The Institute of Metals' Third International Conference on Plutonium, November, 1965.

\section{Discussion}

$M r$. Persiani (ANL): There have been two statements made about the mass transfer between fuel and clad. Mr. Neimark resorts to a gas-bonded system and thus, he says, carbon transfer is avoided or minimized; Mr. Siegel stated that he observed no transfer of metal to the clad with sodium bonding. I was wondering if Mr. Siegel might comment on any observation as to the carburization of the clad in the presence of a sodium bond.

Mr. Seigel (AI): We have observed carburization of the cladding with hyper materials. This occurs fairly rapidly. The comment I made earlier was that up to $1600^{\circ} \mathrm{F}$ we did not observe significant reaction between hypo material and stainless cladding to form low-melting eutectics.

$M r$. Stachura (AI): You had excluded the sodiumbonded hypostoichiometric carbide on the basis of the possible free uranium-stainless reaction and on the basis of the questionable nature of having a fissioning material as the uranium phase in the grain boundaries. Is there firm experimental evidence that indicates that these are indeed serious problems?

Mr. Neimark: It would be difficult to so conclude from the work that we have done ourselves, but I think that it has been shown by the British that there is a significant amount of swelling attributed to a metal phase in grain boundaries. This was pointed out in a paper recently at the Detroit ANS meeting that, in a temperature range and at power densities necessary for fast reactors, this would be a considerably larger problem than it is in the thermal reactors for which a metal phase is now acceptable. I belicve there is significant evidence to this effect.

Mr. Runnalls (AECL): It is possible to calculate from the available thermodynamic information that, as one irradiates plutonium carbide, the fission products require more carbon then ean be provided by $\mathrm{PuC}$ and, therefore, one of the things that one might expect in a long-irradiated fuel is the possibility that plutonium metal could be freed. Perhaps it is more important than considerations of compatibility with the sheet (clad) to find out whether or not the plutonium in a liquid phase flows by gravity towards the bottom of the fuel, that is, relocates itself. Do you have any experimental evidence from your irradiations of $\mathrm{PuC}$ which would seem to settle this question as to whether or not plutonium segregates towards the bottom of the element?

Mr. Neimark: We have just cursorily examined by metallography a few specimens. The high-burnup ones have not even been sectioned for examination. The problem you mention about needing an excess amount of carbon to take care of both the initial metal phases that are present when you load the rod and also those that are formed as fission products has been considered; it is what may be considered a secondary reason for going to a hyperstoichiometric fuel to begin with, to get some excess carbon in there. We will look for plutonium segregation, but have not seen it as yet.

Mr. Kittel (ANL): A question came up a few minutes ago about whether we had evidence that would lead us to prefer hyper mixed carbide over hypo material. Certainly fission-gas release is much higher in the hypo material as compared to the hyper.

Mr. Skavdahl (Battelle-Northwest) : I am interested in your view of the Doppler effect and the rate of response of the Doppler effect in physically mixed $\mathrm{PuC}-\mathrm{CC}$. What relative response rate do you anticipate between, say, solid-solution carbide versus the physical mixture where the $\mathrm{PuC}$ is a separate pure phase?

Mr. Neimark: I cannot answer your question.

Mr. Benedict (MITT): It seems to me that one might anticipate a slight delay in the Doppler effect in the fortile material, but it would only be a few milliseconds at most. Why is it thought that there is any reduction in the overall effect when a new temperature is established?

Mr. Neimark: The uranium and plutonium atoms are separated by a finite amount, a greater amount than would be found in a solid-solution material, so there would be a time lag due to heat transfer.

$M r$. Benedict: Yes, I can see that. But the end result, I would think, once the fuel had reached its new temperature distribution, would be to give about the same amount of reactivity change, would it not?

Mr. Persiani: The Doppler coefficient might be divided into two parts: the delay component and the prompt component. I think the prompt effect is somewhat compromised. The delayed component is somewhat increased when the fissile and fertile material is separated, but I don't think you compromise the whole Doppler effect.

Mr. Kummerer (Karlsruhe): One takes carbide fuel in order to have an importantly increased thermal conductivity. I wonder whether it is consistent to use 
this carbide fuel in powder compaction form because every powder has a bad thermal conductivity?

Mr. Neimark: The increased thermal conductivity of the carbide allows you to use vibratory compaction, which is a desirable process. I would not make the same statement for an oxide.

$M r$. Skavdahl: I am interested in your negative approach on the oxide, and I would like to hear some comment upon that.

Mr. Neimark: It was not a negative approach on the oxide; it was in defense of the carbide.
Mr. Gibson (AI): I note your comments on the hypo based upon the swelling problems of the hypo with the thermal bond; surely the swelling isn't such a serious problem. It would seem the principal disadvantage that you attribute to the hypo would be mostly restricted to the release of fission gases. Is that right?

Mr. Neimark: This was brought out; yes, it is one point. It is a major contributing factor why hyper would be considered better. 


\title{
An Account of Some Work on the Safety Aspects of Carbide Fast Reactor Fuels
}

\author{
B. T. Bradblry, L. E. Russell, and M. B. Waldron \\ Metallurgy Division \\ Atomic Energy Research Establishment \\ Harwell, United Kingdom
}

(PRESENTED BY B. T. BRADBURY)

\section{Introduction}

\subsection{The Choice of Fuel for a Fast Reactor}

Ceramic materials are becoming increasingly important as fast reactor fuels. Such fuels should be capable of achieving a high burnup in the region of $100,000 \mathrm{MWD} /$ te to be economically attractive.

A current design for fast react or fuel element allows for gas release by voidage. ${ }^{(1,2)}$ This means that a plenum space is included, and the volume of this space is calculated to allow for the effects of combined pressure stresses and thermal stresses. Such designs take account of the fact that irradiation results obtained with oxide fuels, at the heat ratings and to the burnups relevant to fast-reactor operation, have indicated that a large percentage of the fission gases is released from the fuel. Thus, in a fast reactor where the fuel is highly rated, i.e., $\sim 200 \mathrm{~W} / \mathrm{g}$ fuel, even with a small-diameter oxide pellet, the poor thermal conductivity of this fuel gives rise to high centre temperatures of the fuel, and this in turn leads 10 high percentages of gas release.

Carbide fuel offers two main advantages over oxide: it has a higher fissile density and a higher thermal conductivity. Thus, a carbide-fuelled fast reactor could have a more compact core, giving rise to a smaller inventory. The higher fissile density of carbide, and the fact that its structure comprises one nommetallic atom per molecule compared with two for oxide, leads to better breeding characteristics. Improved performance may be experted to result from its higher thermal conductivity, but the question of how to take advantage of the relatively high thermal conductivity compared with oxide is still oper.

One possible way is to design to the same parameters as for oxide fuel, so that low temperatures at the fuel centre are obtained with the possibility of low release of fission gas. A considerable amount of information on the irradiation behaviour of U-C (up to 30,000 $\mathrm{MWD} / \mathrm{te})^{(3,4)}$ and (UPu)-C (up to $113,000 \mathrm{MWD} /$ te) ${ }^{(5,6)}$ has been reported by a number of workers. Their main conclusions are as follows:
(1) There are no important differences in irradiation behaviour between UC and (UPu)C.

(2) Ilypostoichiometric material, containing free metal, shows a high rate of swelling and large gas releases.

(3) Single-phase and hyperstoichiometric material is dimensionally stable to $50,000 \mathrm{MWD} /$ te if subjected to some restraint. The swelling rate observed corresponds to about $1.6 \%$ dencity decrease per $1 \%$ burnup at the highest burnups.

(4) $\mathrm{Up}$ to $30,000 \mathrm{MWD} / \mathrm{te}$ for $\mathrm{UC}$ and 40,000 MWD/te for $(\mathrm{UPu}) \mathrm{C}$ and at moderate centre temperatures, fission gas release, with some exceptions, is low. At burnups of $\sim 100,000$ MWD/te and fuel temperatures of $\sim 1200^{\circ} \mathrm{C}$, Strasser ${ }^{(6)}$ has recently reported appreciable gas release $(\sim 30 \%)$ from mixed carbide.

(5) Examination of mixed carbides irradiated up to $113,000 \mathrm{MWD} / \mathrm{Te}$ has not revealed any evidence for gross changes in the carbon-metal ratio or for the separation of solid fission products into a discrete phase.

(6) Replica and thin-film electron microscopy have shown evidence for gas bubble formation in LC. ${ }^{(7,8)}$

In current work at Harwell, a gas-retentive carbide fuel is being examined, with the object of attaining a basic understanding of the behaviour of the fission gases in UC and (UPu)C in relation to their effects on gas release and $\rightarrow$ welling. The firs 1 part of the programme comprises six experiments which are designed to compare the irradiation behaviour of sintered and are-cast fuel of composition $\left(\mathrm{U}_{0}{ }_{85} \mathrm{Pu}_{015}\right) \mathrm{C}$. Burnup levels be1ween 40,000 and 100,000 \IWD/1e are being arhieved. lour of the experiments are being irradiated under fast reactor conditions and the remaining two at high latings in a thermal flux. Irradiations have been com pleted for three experiments, and some of the postirradiation results are reported here. In all experiments, the carbide was prepared from arc-melted stork and the oxygen content of the fuel was generally $₹ 0.2 \mathrm{w} / \mathrm{o}$. 
An attractive way of preparing monocarbides is by the carbothermic reduction of oxides. The second part of the irradiation programme, which is now getting underway, is designed 10 study the irradiation behaviour of such carbides at high burnup as a function of oxygen content. Oxycarbides, defined here as monocarbides containing $\widetilde{>} 0.3 \mathrm{w} / 0$ oxygen, (an be prepared at lower temperatures or in higher $\mathrm{CO}$ pressures than monocarbides. These materials have obvious interest as reactor fuels provided that there is no significant deterioration in those properties which determine fuel behaviour under irradiation. Thus, in support of the irradiation work on these materials, uranium-plutonium oxycarbides prepared by the carbothermic reduction process have been pressed and sintered into pellets containing $\sim 0.5 \mathrm{w} / \mathrm{O}$ oxygen, and used in tests which rompared their high-temperature stability with that of uranium oxycarbides. The compratibility of (UPu) carbides with various cladding materials has also been studied.

In summary, three aspects of fuel and cladding behaviour of importance to the safety of an operating fuel element are under investigation:

(1) the compatibility of (UPu)-C and U-C with 3161 stainlens steel cladding material under both gas-bonded and sodium-bonded conditions. The compatibility of ( $\mathrm{CPu}$-C with a number of nickel-based alloys has been investigated;

(2) the gas release and swelling behaviour of ( $\mathrm{TPu})-\mathrm{C}$ of varying composition, using sintered, arc-cast, and powder fuel. Irradiations in both thermal and fast fluxes and being carried out;

(3) with the growing interest in plutonium-bearing oxycurbides, some initial experiments have been completed which give some indication of the stability of these fuels at high temperatures.

The experimental results so far obtained are described and discussed in this paper.

\section{High-burnup Miniature Fuel-element Irradia- tion}

\subsection{Introduction}

As stated previously, one of the major objectives of the irradiation programme is to study the effect of fabrication and composition variables on the irradiation behaviour of $\left(\mathrm{C}_{0.85} \mathrm{Pu}_{0.15}\right)$-C. In one experiment, a sample has recently been irradiated in the Dounreay Fast Reactor (DFR) to a maximum fuel burnup of $5.2 \%$. The results obtained from this experiment are described here.

\subsection{Fabrication of the Fuel}

Sintered Fuel. This fuel was prepared by crushing and milling arc-melted carbide, which was formed into compacts at pressures up to $20-40 \mathrm{tsi}$. The pellets were sintered in flowing argon at $1550^{\circ} \mathrm{C}$ for $4 \mathrm{hr}$. Densities and analyses for $\mathrm{Pu}, \mathrm{C}, \mathrm{O}$, and $\mathrm{N}$ were carried out on selerted pellets.

Pre-irradiation details of the fuel are given in Table 1, and Fig. 1 shows typical fuel microstructures.

Powder Specimens. Two powder specimens were irradiated. These were prepared by crushing arc-melted fuel to $-30+200$ mesh $(500-74 \mu)$, and tapping this into the irradiation cans so that it occupied $60 \%$ of the internal volume of the cans.

Arc-cast F'uel. The experimental technique which was used for the preparation of the irradiation specimens has been described elsewhere. ${ }^{(9)}$ A 600-g skull was used, and precautions were taken to ensure uniform composition. Casting was carried out by the Durville method, using massive copper moulds. A typical preirradiation microstructure of the arc-east fuel is shown in Fig. 2.

\subsection{Irradiation Details}

Figure 3 shows the general assembly of the fuel cans, and Table 1 gives the irradiation parameters for each specimen. Eleven specimens, each 1 in. long, were irradiated in 0.030-in.-thick Nimonic 90 alloy cans which were lined with tantalum, fabricated from co-extruded tubing. The ends of the cans were closed with Nimonic 90 end caps, which were seal welded into the can. A tantalum liner was also provided for the end caps integrally with tantalum spacers, the latter serving to position the specimens longitudinally in the can. Nimonic 90 cans were chosen to provide strong cans 10 counteract the effect of irradiation, but subsequently it was demonstraied that nickel-base alloys undergo considerable irradiation embrittlement. Since there was some doubt concerning the compatibility of (CPu)-C with Nimonic alloys at $800^{\circ} \mathrm{C}$ and above, the tantalum liners were incorporated to prevent reaction between the fuel and can, and thus ensure the maximum rhance of achieving the target burmups without fuel-can interaction.

It was, however, also suspected that some incompatibility might exist between tantalum and Nimonic $90 \mathrm{in}$ view of the information available on the $\mathrm{Ni}-\mathrm{Ta}$ phase diagram. ${ }^{(10)}$ The specimens were a very close diametral fit in the cans, the radial clearance being about $0.0003 \mathrm{in}$, and the cans were filled with reactorgrade helium. Ifeat removal from the specimen cans was through close fitting matrix blocks of steel, selected for their thermal conductivities in relation to the required temperature differences across them. These blocks were in turn a good fit in the outer sheath, so that a solid metal path of controlled thermal resistance 
TABle 1. Fast Reactor Experimente-Specimen Detalls

\begin{tabular}{|c|c|c|c|c|c|c|c|c|c|c|c|c|c|c|c|c|}
\hline \multirow{2}{*}{$\begin{array}{c}\text { Can } \\
\text { Number } \\
\text { and Fuel } \\
\text { Type }\end{array}$} & \multirow{2}{*}{$\begin{array}{l}\text { Specimen } \\
\text { Number }\end{array}$} & \multirow{2}{*}{$\begin{array}{l}\text { Specimen } \\
\text { Length } \\
\text { and } \\
\text { Diameter } \\
\text { (in.) }\end{array}$} & \multicolumn{5}{|c|}{ Specimen Composition (w/o) } & \multirow{2}{*}{\begin{tabular}{|} 
Equivalent \\
Carbon \\
Content \\
$(w / 0)$
\end{tabular}} & \multirow{2}{*}{ Microstructure } & \multicolumn{2}{|c|}{ Density $\left(\mathrm{g} / \mathrm{cm}^{3}\right)$} & \multirow{2}{*}{$\begin{array}{l}\text { Specimen } \\
\text { Rating } \\
(\mathrm{W} / \mathrm{g})\end{array}$} & \multirow{2}{*}{$\begin{array}{c}\text { Estimated } \\
\text { Surface } \\
\text { Temp } \\
\left({ }^{\circ} \mathrm{C}\right)\end{array}$} & \multirow{2}{*}{$\begin{array}{c}\text { Estimated } \\
\text { Centre } \\
\text { Temp } \\
\left({ }^{\circ} \mathrm{C}\right)\end{array}$} & \multirow{2}{*}{$\begin{array}{l}\text { Estimated } \\
\text { Burnup } \\
\text { (\% heavy } \\
\text { atoms) }\end{array}$} & \multirow{2}{*}{$\begin{array}{c}\text { Fstimated } \\
\text { Fission Gas } \\
\text { Release } \\
(\%)\end{array}$} \\
\hline & & & C & $\mathrm{O}$ & $\mathrm{N}$ & $\mathrm{Pu}$ & $\mathrm{U}$ & & & $\begin{array}{l}\text { Sintered } \\
\text { Bulk }\end{array}$ & $\begin{array}{l}\text { Theo- } \\
\text { retical }\end{array}$ & & & & & \\
\hline $\begin{array}{c}1 \\
\text { Sintered }\end{array}$ & 8139 & $\begin{array}{l}1.024 \\
0.32\end{array}$ & 4.76 & 0.24 & 0.04 & 13.0 & 82.0 & 4.97 & $\mathrm{MC}+\mathrm{M}_{2} \mathrm{C}_{3}$ & 12.9 & 13.4 & 144 & 1040 & 1610 & 3.95 & - \\
\hline $\begin{array}{c}2 \\
\text { Sintered }\end{array}$ & 8140 & $\begin{array}{l}1.018 \\
0.29\end{array}$ & 5.32 & 0.07 & 0.02 & 12.6 & 81.99 & 5.38 & $\mathrm{MC}+\mathrm{M}_{2} \mathrm{C}_{3}$ & 12.8 & 13.3 & 162 & 940 & 1440 & 4.45 & - \\
\hline $\begin{array}{c}3 \\
\text { Sintered }\end{array}$ & 8141 & $\begin{array}{l}1.015 \\
0.27\end{array}$ & 4.73 & 0.09 & 0.07 & 13.2 & 81.90 & 4.86 & $\mathrm{MC}+\mathrm{MI}_{2} \mathrm{C}_{3}$ & 13.0 & 13.4 & 180 & 950 & 1460 & 4.95 & - \\
\hline $\begin{array}{c}4 \\
\text { Sintered }\end{array}$ & 8142 & $\begin{array}{l}1.013 \\
0.26\end{array}$ & 5.32 & 0.07 & 0.02 & 12.6 & 81.99 & 5.38 & $\mathrm{MC}+\mathrm{M}_{2} \mathrm{C}_{3}$ & 12.8 & 13.3 & 190 & 930 & 1430 & 5.20 & - \\
\hline $\begin{array}{c}5 \\
\text { Are-cast }\end{array}$ & 8143 & $\begin{array}{l}1.068 \\
0.25\end{array}$ & 4.76 & 一 & - & - & - & - & $\begin{array}{l}\mathrm{MC}+\text { trace of } \\
\text { free metal }\end{array}$ & - & - & 199 & 940 & 1420 & 5.40 & - \\
\hline $\begin{array}{c}6 \\
\text { Sintered }\end{array}$ & 8144 & $\begin{array}{l}1.024 \\
0.25\end{array}$ & 4.76 & 0.24 & 0.04 & 13.0 & 82.0 & 4.97 & $\mathrm{MC}+\mathrm{M}_{2} \mathrm{C}_{3}$ & 12.9 & 13.4 & 201 & 950 & 1430 & 5.50 & - \\
\hline $\begin{array}{c}7 \\
\text { Powder }\end{array}$ & 8145 & $\begin{array}{l}1.023 \\
0.25\end{array}$ & 5.32 & 0.07 & 0.02 & 12.6 & 82.0 & 5.39 & - & $60 \%$ T.D. & 13.3 & 199 & 800 & 1270 & 5.40 & $0.2-0.5$ \\
\hline $\begin{array}{c}8 \\
\text { Powder }\end{array}$ & 8146 & $\begin{array}{l}1.016 \\
0.26\end{array}$ & 5.32 & 0.07 & 0.02 & 12.6 & 82.0 & 5.39 & - & $60 \%$ T.D. & 13.3 & 190 & 800 & 1250 & 5.20 & $1.25-2.5$ \\
\hline $\begin{array}{c}9 \\
\text { Arc-cast }\end{array}$ & 8147 & $\begin{array}{l}1.017 \\
0.27\end{array}$ & 4.75 & - & - & 14.2 & - & - & $\begin{array}{l}\mathrm{MC}+\text { trace of } \\
\text { free metal }\end{array}$ & 13.36 & 13.6 & 180 & 930 & 1410 & 4.95 & - \\
\hline $\begin{array}{c}10 \\
\text { Sintered }\end{array}$ & 8148 & $\begin{array}{l}1.022 \\
0.29\end{array}$ & 4.00 & 0.07 & 0.10 & 14.3 & 81.53 & 4.14 & $\begin{array}{l}\text { MC in metal } \\
\text { matrix }\end{array}$ & 13.5 & 14.2 & 162 & 990 & 1530 & 4.45 & - \\
\hline $\begin{array}{c}11 \\
\text { Arc-cast }\end{array}$ & 8149 & $\begin{array}{l}1.016 \\
0.32\end{array}$ & 4.80 & - & - & - & - & - & $\begin{array}{l}M C+\text { trace of } \\
\text { free metal }\end{array}$ & 13.54 & 13.6 & 144 & 1020 & 1610 & 3.95 & - \\
\hline
\end{tabular}




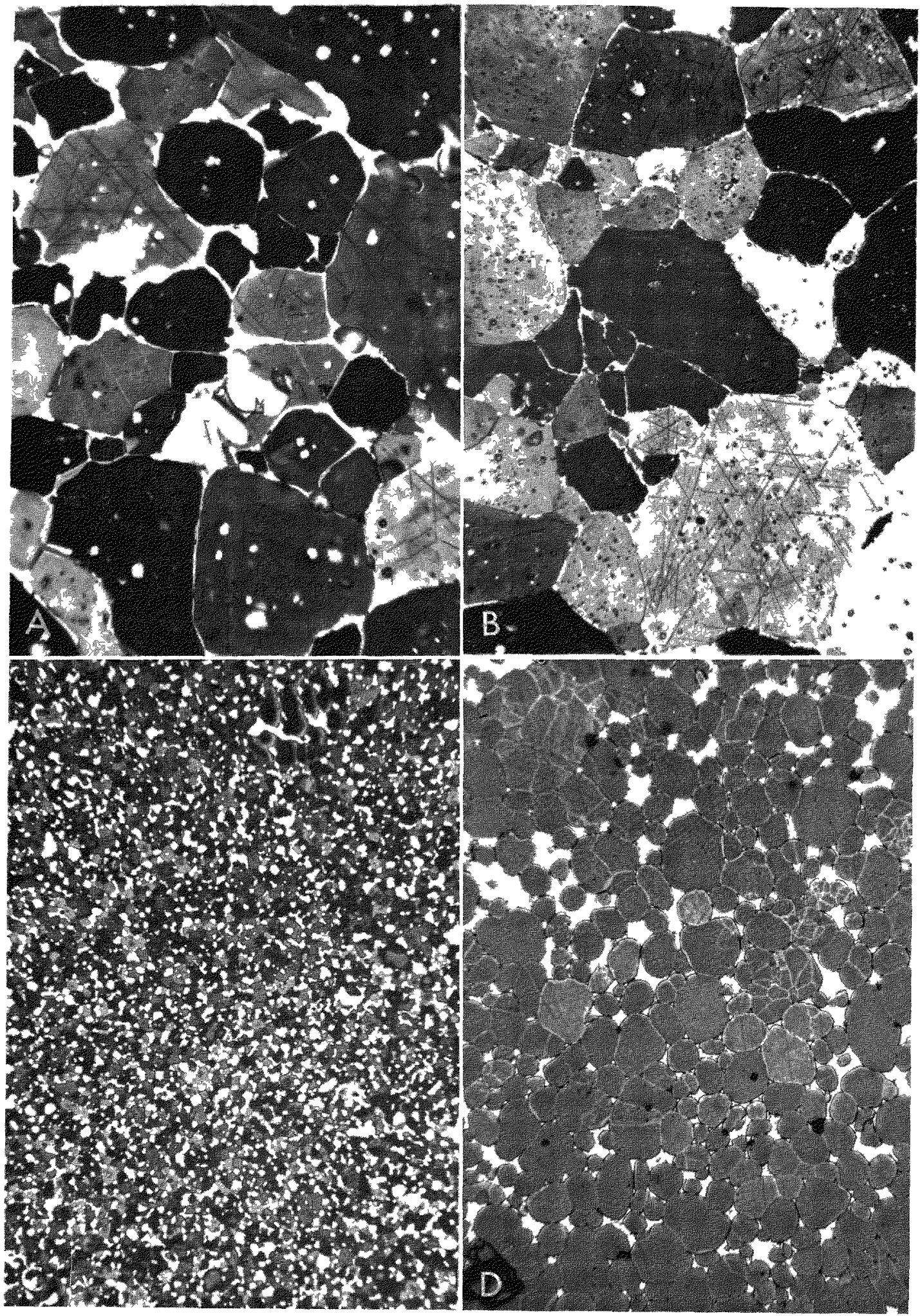

Fig 1 Pie irradiation Mic rostructures of Sintered ( $\mathrm{U}_{8}$ Pug $) \mathrm{C}$ (a) Typic ll of Cans 1 and 6 (MC $+\mathrm{MI} \mathrm{C}_{3}$ ) (X235) (b) Typical of Can $3\left(\mathrm{MC}+\mathrm{M}_{2} \mathrm{C}_{3}\right)$ ( $\left.\times 94\right)$ (c) Trpical of Cans 2 and $4\left(\mathrm{MC}+\mathrm{M}_{2} \mathrm{C}_{3}\right)(\times 235)$ (d) Trpical of Can 10 (MC in Vetal Matrix) $(\times 940)$ 


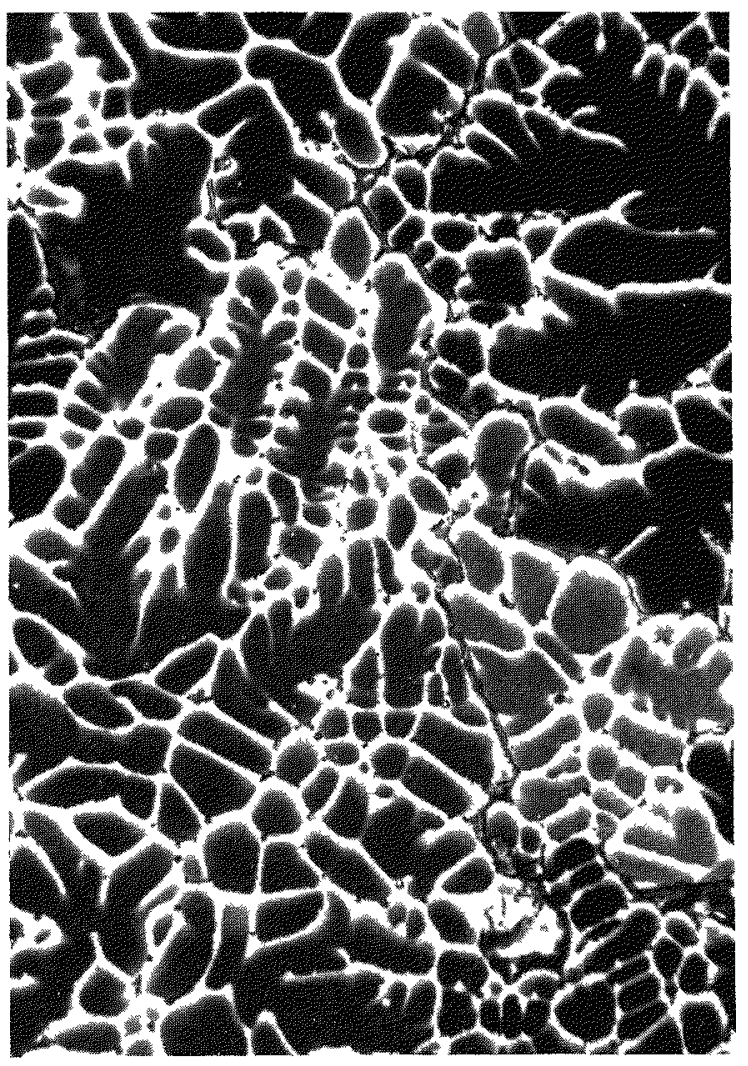

Fig. 2. Pre-irradiation Microstructure of Are-cast (U $\mathrm{U}_{05-}$

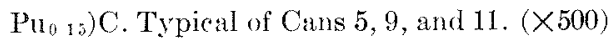

arried the heat from the specimens to the reactor roolant.

Because of the marked longitudinal variation in the reactor flux, the specimen diameters were varied from $0.30 \mathrm{in}$. at either end of the rig to $0.26 \mathrm{in}$, at the centre, so that the linear ratings of each specimen were roughly constant. In the period prior to insertion of the rig into the DIRR, the operating power was increased from 30 to $58 \mathrm{NW}$, and this could only be partially arcommodated by moving the rig to a different position in the core. In fact, as a result of this, the operating conditions for the rig were far more severe than those for which it was designed, and this has aused many failures in the Nimonic 90 cans. These will be discussed later.

\subsection{Postirradiation Examination}

Radiography and Macroscopic Eramination. Radiography showed that four cans (samples 4, 6, 10, and 11) had failed very severely; in all but one case (can 11), one end cap had fractured from the can body. Can 10, which contained sintered hypostoichiometric carbide, was a particularly serious failure. Gross swelling of the fuel and the matrix block (see Fig. 4) was found. This is not surprising in view of the large proportion of free metal in this specimen, as shown in the pre-irradiation structure in Fig. 1D. Can 11 was extracted from its matrix block, by machining down the mild steel, to investigate the general condition of the fuel can. The fuel can was found to be badly cracked both circumferentially and along its length, and eventually fell into two pieces during handling (see Fig. 5). At this stage, it was decided to carry out any further investigations with the fuel cans still in position in the matrix blocks; seven cans remained to be examined, two of which (cans 7 and 8 containing powder fuel) were known to be intact.

Release of Fission Gas. The remaining seven cans were punctured to determine the levels of gas released from the fuel. Gas samples were obtained only from cans 7 and 8 , suggesting that the ot her 5 cans containing solid fuel had failed. Table 1 gives the estimated gas releases from cans 7 and 8 , which were very low.

Metallography of the Fuel. Individual fuel cans were recovered from their matrix blocks, and selected pellets (or slices of fuel in the case of the arc-ast material) and sheathing were extracted from near the centre and ends of each fuel element. The sheathed pellets or slices of fuel were then prepared for metallography.

The powder fuel in cans 7 and 8 was first exposed by slitting the cladding at one end, near to the tantalum end shim. The fuel was found to have densified and wa: no longer a fine powder. Fxamination of thin slices takcn from the end and centre of the element revealed that the powder had sintered during irradiation to form a porous mass. This effert was most pronounced at the rentre of the fuel sections, whereas in the circumferential regions the original particle size persisted (see Fig. 6).

Fxamination of pellets from all the sintered elements indieated that, with the exception of Can 10, there was little change in the microstructure, other than cracking of the fuel. Can 10, which contained hypostoichiometric fuel, was found to be severely distorted (as shown in Fig. 4 previously). This dimensional (hange is believed to be raused by the free metal present in this fuel, although postirradiation mirroscopy indicated that the metal phase had migrated during irradiation (see Fig. 7).

The three elements containing arc-cast fuel have also been examined. In all cases, the fuel had not undergone any serious changes, although there was a marked increase in the amount of grain-interior porosity. There is no doubt due to the agglomeration of fission gascs into resolvable gas-filled voids.

Can 11 containing arc-cast fuel exhibited a serious circumferential crack in the fuel ("an and subsequently fell into two picees during handling. The smaller half was sectioned transversely and longitudinally. The transverse section is shown in Fig. 8 and the longitudinal 


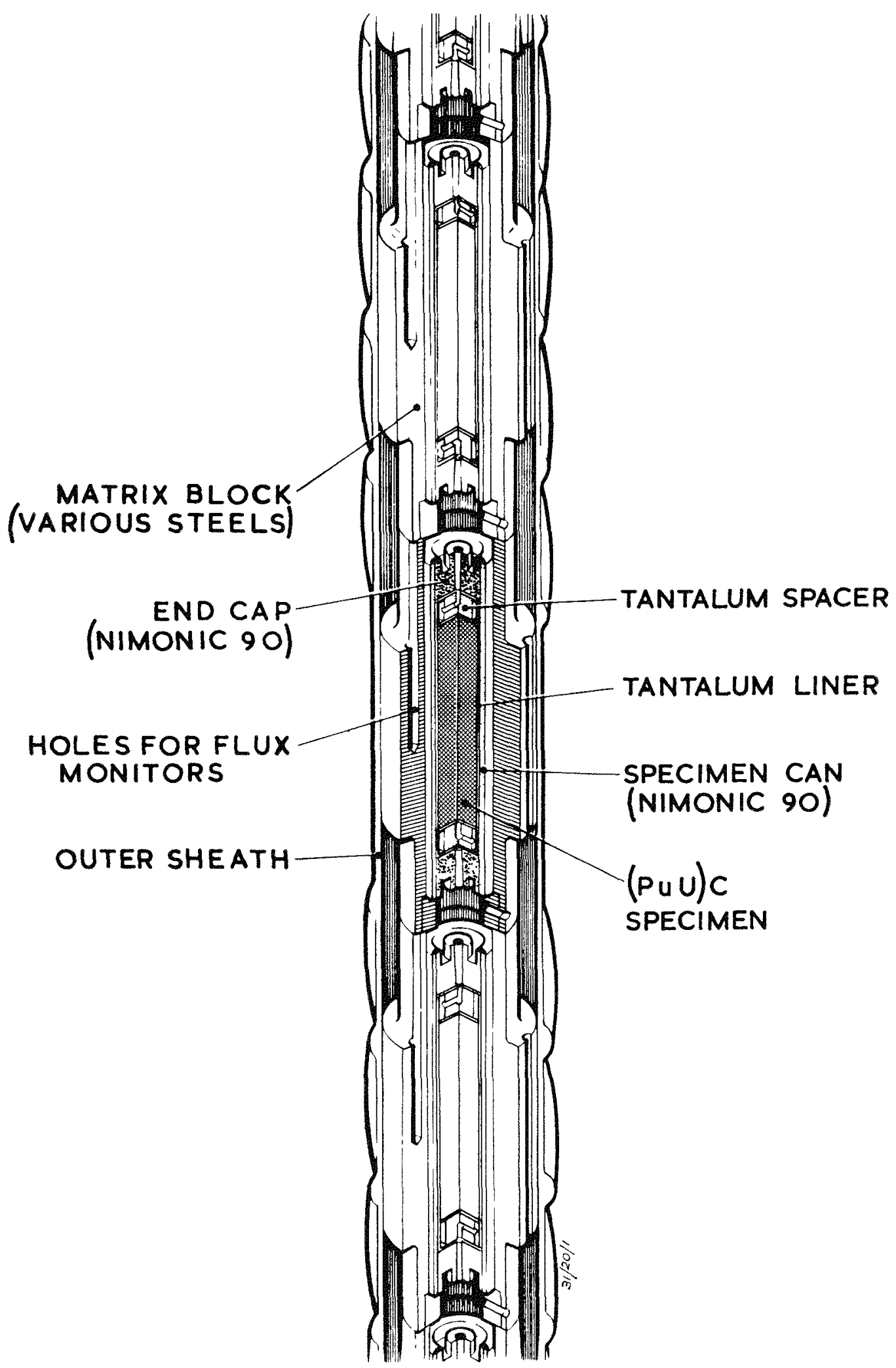

Fia. 3. General Assembly of Irradiation Fuel Cans. 


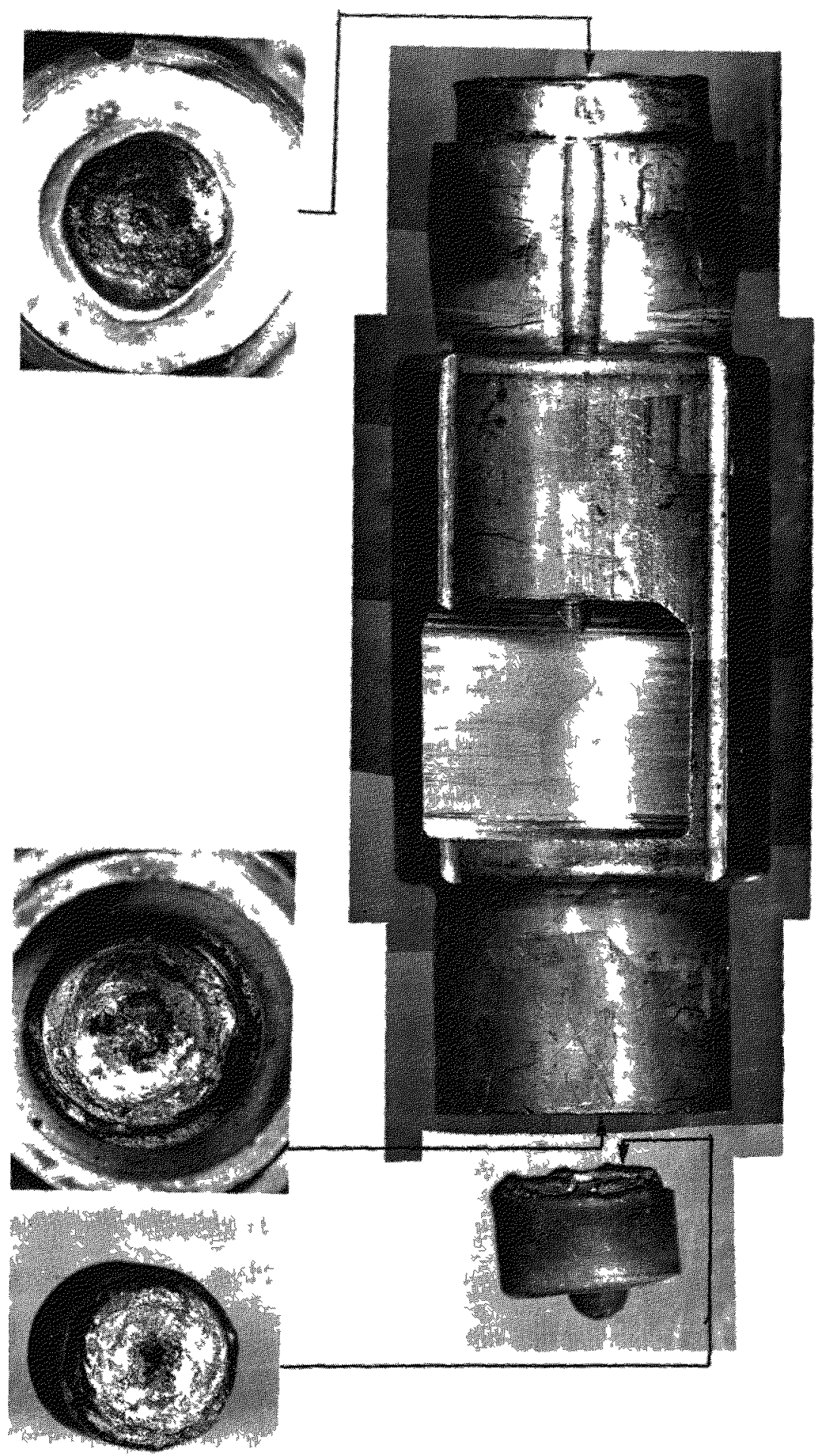

Fra 4 Matrix Block ( ont tinung Can 10 Showing Distortion due to Swelling of the Hypostorchrometrie Fuel 

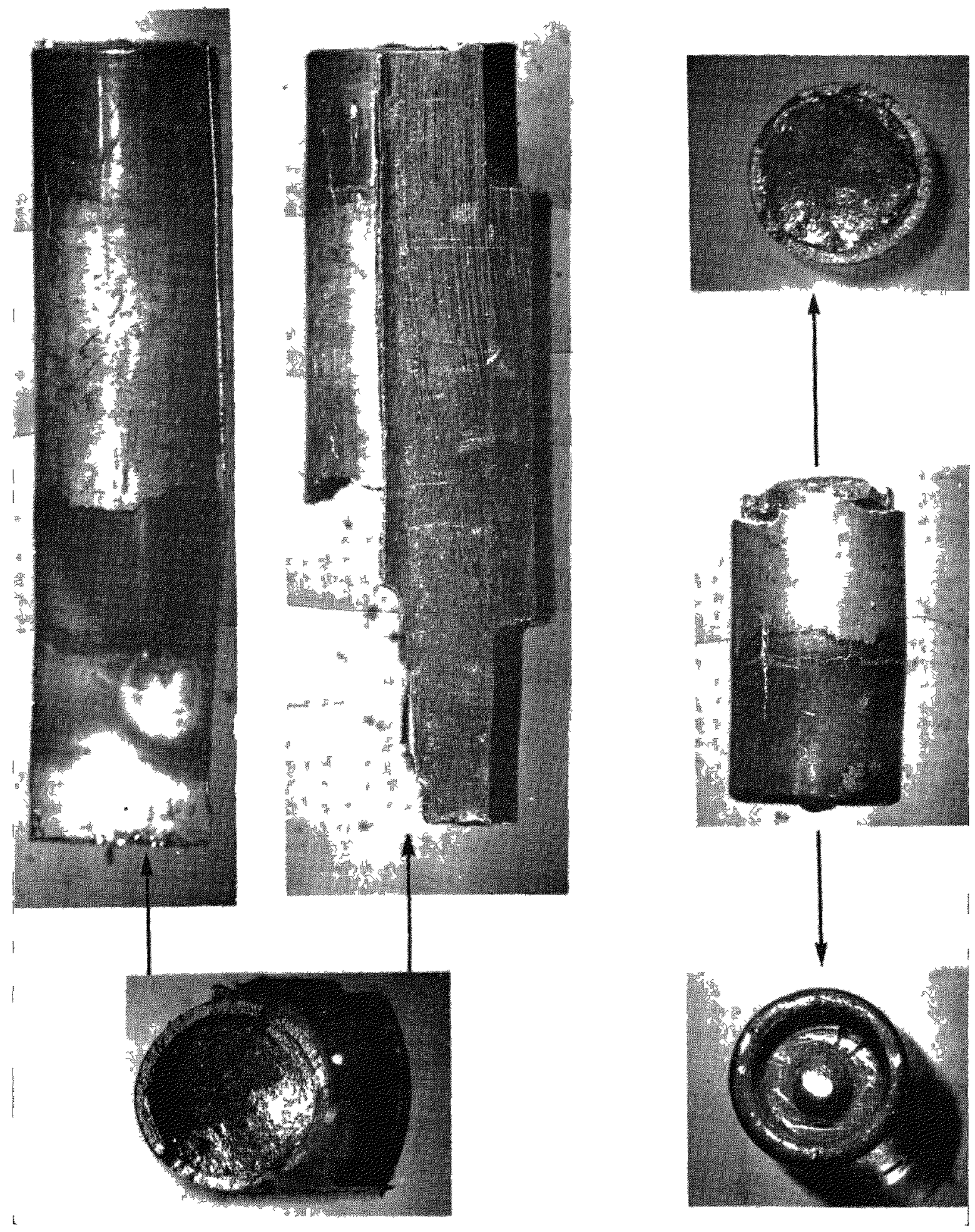

Hia 5 Fallure of Can 11 due to Serrous Circumferential Crach

section in I ig 9 I rom $\mathrm{I}$ ig 8 it appear, that little change, other than cracking, had occurred in the fuel, the columnar grain structure which was evident in the circumferental regions of the fuel before irradiation persisted 1 or the hotter regrons of the fuel, there is some evidence in I ig 9 for red tion between the hot fuel and the dantalum end $-p a c e r$, and betw een the tantalum spacer and the \monic 90 end (ap I Igure 10 illustrates 


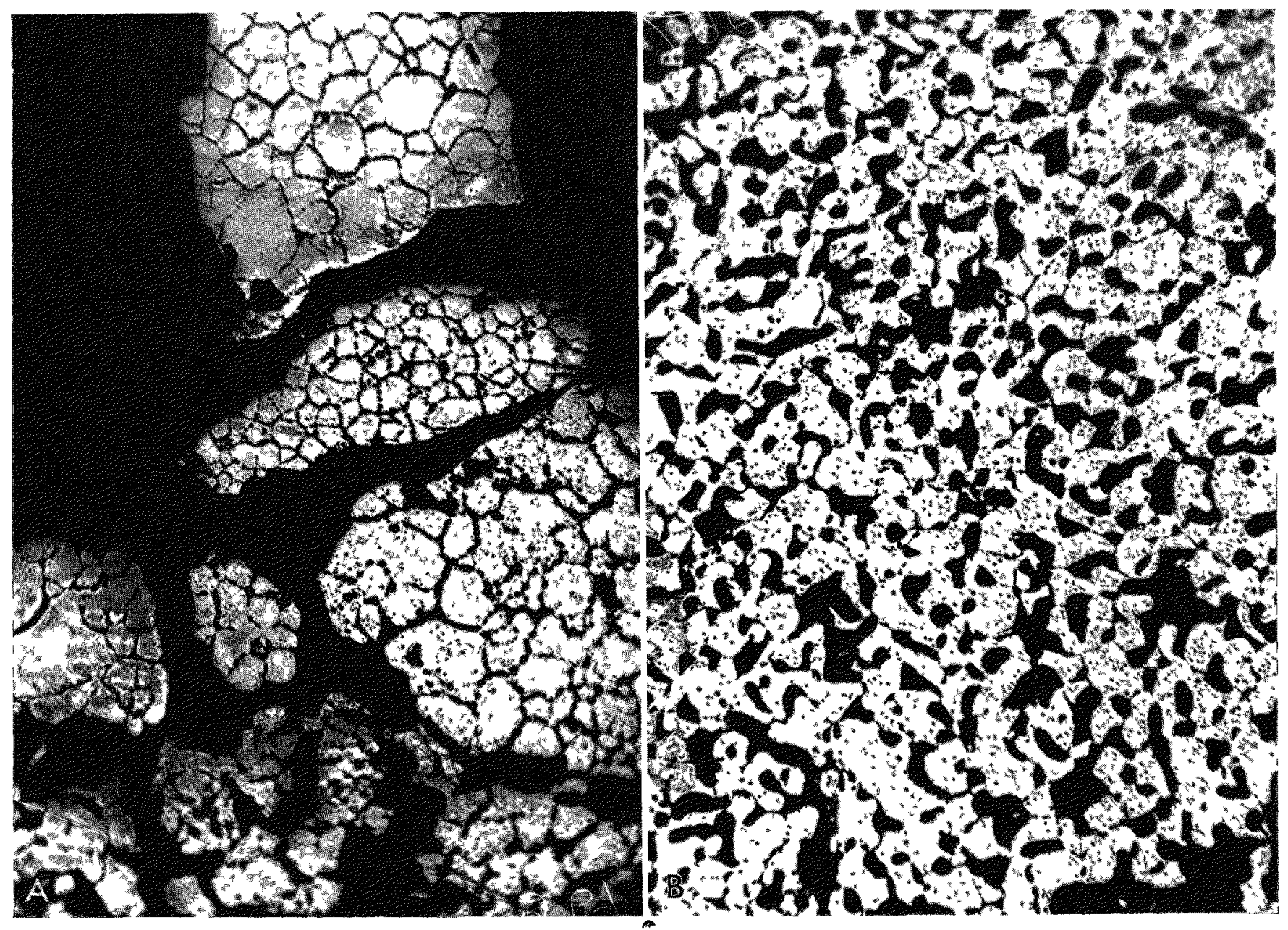

Fig 6 Miciostructures Showing Partial Densification of the Powder Fuel in Can 7 ( $\times 153)$

the high concentration of gran interior porosity in the fuel structure

\section{The ('ompatzbuluty of Vmonec 90 and T'antalum}

As stated before, Nimonic 90 clddding was selected to provide strong cans, and the tantalum interlayer was incorporated because the compatibility of ( $\mathrm{CPu}) \mathrm{C}$ with Nimone alloys at temperatures $>800^{\circ} \mathrm{C}$ was doubtful. However, it was also surpected that come incompatibility might exist between tantalum and Nimonc 90 Because of these doubt, a senes of laboratory compatibility tests(11) were started on Nimonic 90 and tantalum very soon after insertion of the rig into the reartor

Since there was a possibility that nic hel might diffuse right through the tantalum and reart with the carbide, couples consistmg of $\mathrm{\lambda monc} 90$ and (arbide separated by $0.006 \mathrm{~m}$ thrck tantalum heet were mantanned in contact by differentral expansion on a capsule of the hind hown in Iig 11. In this way, any incompatibility between the tantalum Nimonic diffurion products and the carbide could also be noted if penetration of the tantalum occured. Initially, three test temperatures were used 760,830 , and $900^{\circ} \mathrm{C}$, to enable approximate inter and extrapolation of the results to be made However, during the course of the tests, it became clear that the actual in radiation tests were iunning at higher temperatures than origmally envisaged, in an attempl to produce $1000^{\circ} \mathrm{C}$ data, couples already I unnng at lower temperatures were rerun at the higher temperature and the time at the lower temperature converted to an equivalent time at $1000^{\circ} \mathrm{C}$, using the actuvation energy derived from the low temperalure runs

The heat treated couples were examined by metal lography and electron-microprobe analysis No reaction was obscrved at $760^{\circ} \mathrm{C}$ The results for the higher temperatures are plotted in Fig. 12, the derived diffusion coefferents and activation energy are given in Table 2 The glowth appeared to follow the expected parabolic law initially, but then slowed down, although the zones were slightly niegular and the plotted data, which 
relate to the maximum penetration depth into the tantalum, are therefore somewhat uncertain, the effect seems to be genuine. This has complicated the interpretation, and the pairs of parabolas shown at 830 and $900^{\circ} \mathrm{C}$ in Fig. 12 relate, firstly, to the best fit to the initial points $(<120$ days $)$ and, secondly, to all the points on the basis of minimum deviations. It is doubtful if a simple Arrhenius plot is applicable in the present case, and it is not surprising therefore that the parabolas fitted to the derived points at $1000^{\circ} \mathrm{C}$ give values for the diffusion coefficient which are rather greater than the calculated diffusion coefficient from the data at Iower temperatures.

Microseopic examination revealed that for the Nimonic side of the couple, depletion of $\mathrm{Ni}_{3} \mathrm{Al}$ and $\mathrm{Cr}_{23} \mathrm{C}_{6}$ occurred in the surface layers (see $\mathrm{Fig}$. 13) whilst on the tantalum side a narrow, and probably isotropic, surface zone was followed by a wider anisotropic zone (see Fig. 14). The compositions of these zones have not been fully determined, but microprobe analysis indirated that cobalt is present in large amounts in the anisotropic zone which also contains nickel to an ap-

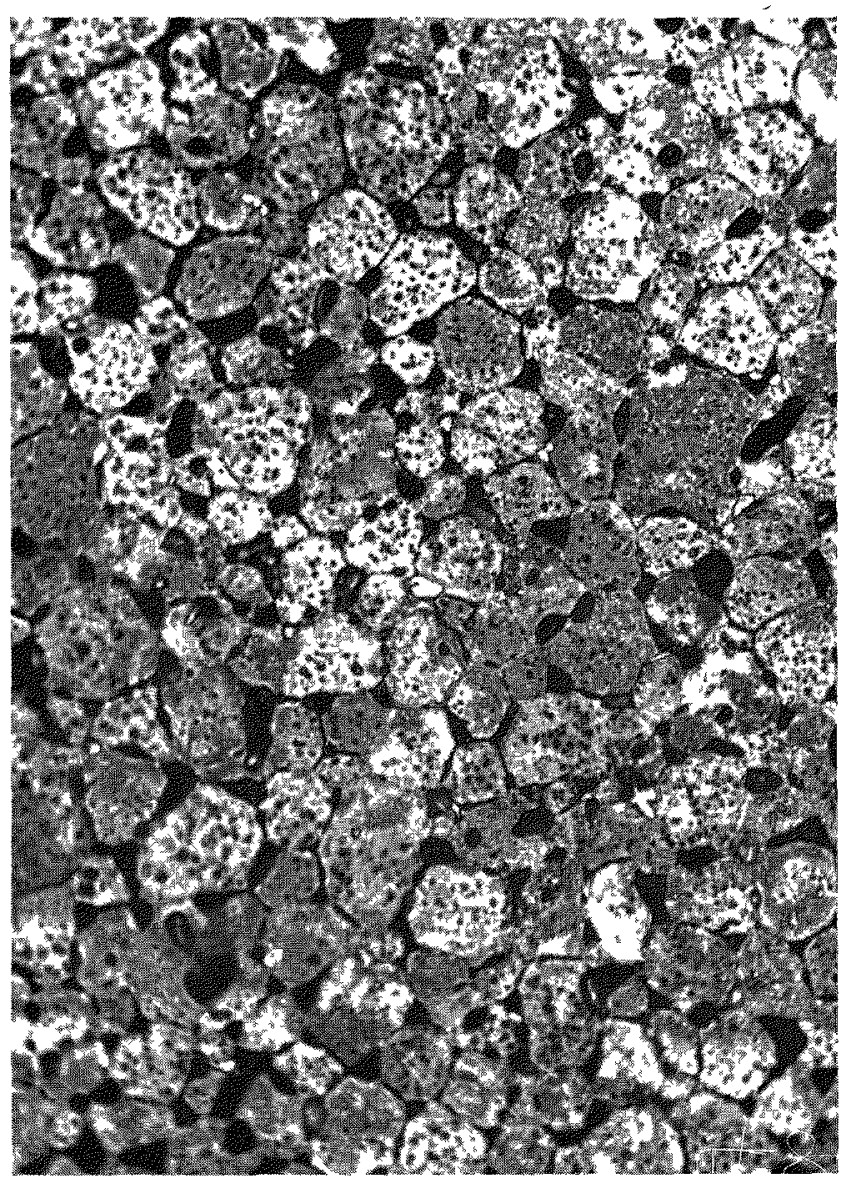

FIg. 7. Postirradiation Microstructure of Metal-rich Fuel in Can 10.

Note absence of free metal compared with pre-irradiation structure. $(\times 342)$ preciable level. Chromium is also prosent, but the $\mathrm{Cr} / \mathrm{Ni}$ ratio appears to be lower than in the Nimonic. No data were obtained for aluminium or titanium.

In view of the depletion of $\mathrm{Cr}_{23} \mathrm{C}_{6}$, it is tempting to suggest that the narrow surface zone is lantalum earbide; the formation of such a zone might explain the gradual departure of the zone depth below the value expected from a parabolie growth law. (There is, however, no confirmatory evidence of the componition of this zone.) Such a theory might also acrount for the relatively slow diffusion of Ximonic 90 into tantalum compared to the reported behaviour of pure nickel.

In spite of the uncertainties in interpretation, the experiment clearly demonstrated that there was no danger of the 0.006 -in. tantalum foil being penetrated by the Nimonic during the carbide-irradiation experiments deseribed herein. Even at $1000^{\circ} \mathrm{C}$, the time required to penetrate the tantalum would be about 1 year. In fact, postirradiation metallographie examination of sections from all eleven cans revealed that the depth of penetration was very slight in all cases.

\subsection{The Cause of Radial Cracking in ('an 11}

Postirradiation examination of the fuel cans from this rig has revealed a variety of can failures, and the cause of failure in all cases is being studied in some detail. Only can 11 has been considered so far. Looking sperifically at this can, two main modes of failure were apparent:

(1) complete circumferential fracture of the Nimonic can (see Fig. 5) which may have been caused by fuel swelling and axial tensile failure;

(2) a number of radial cracks in the can walls, which seem to propagate inwards from the outer surface. Some of these cracks do not propagate entirely across the wall thickness, but terminate at varying distances across the sertion (see Fig. 8). This mode of failure is due to thermal stressing and is discussed in more detail below.

The can was designed to have a wall thickness such as to minimise the stresser arising from the radial transmission of heat across the wall, and from the containment of an internal pressure resulting from release of fission gas.

The initial stress system was predominantly thermal, and it was well within the capabilities of the unirradiated clad material to withstand these stresses. Indeed, a substantial allowance had been made for any deterioration in the mat erial strength with neutron irradiation.

As stated previously, the operating conditions for the rig were far more severe than anticipated, and thermal stresses in the can wall were excessive. In fact, the can was originally designed to operate at $612^{\circ} \mathrm{C}$, but actually achieved a temperature of $870^{\circ} \mathrm{C}$, and 


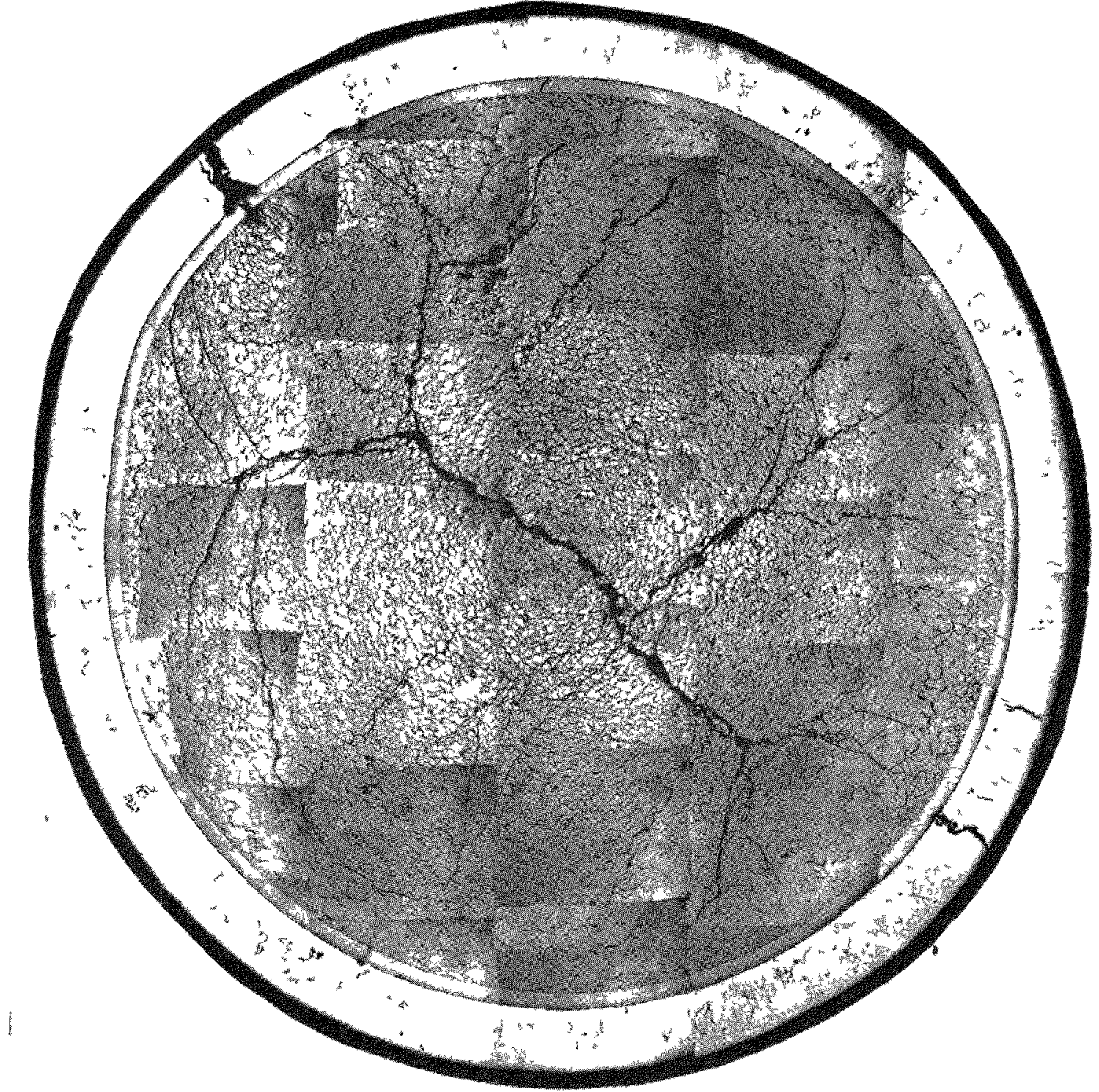

Fif 8 Transverse hection of Aic east I uel in Can 11 Vote farlures m Nimonic 90 can (X14)

the can was subjected to a maximum tensile thermal stress of 8.3 th At this highel temperature, the theimal stress exceed the 100 hr rupture stres for the Imome 90 cladding material, and it is theretore reatonable to suppose that the cracks observed in the can wall were mitrated within the first week of 1 radiation, when the mechancal propentes of the clad matenal would have been relatively unaffected by radiation damage (the neutron dose rate was $\sim 5 \times 10^{18}$ neutrons $/ \mathrm{cm}^{2} /$ hour)

As the cracks propagated 1hrough the wall, the effective thrckness of ean matelal ahead of oarh crack was reduced If we ignore stres concentrations at the (rack lip), an approximation for the changing stress pattern ran be obtaned by calculating the stresses in successively $\rightarrow$ maller ran wall thickness.

The thermal stresses in the can wall can be obtamed using an equation derived by Tmoshenko ${ }^{(2)}$

$$
\begin{aligned}
& \left(\sigma_{t}\right)_{r=\mathrm{r}_{2}}=\frac{E \alpha \Delta T}{2(1-\mu) \log \frac{\gamma_{2}}{\gamma_{1}}} \\
& \left(1-\frac{2 r_{1}^{2}}{r_{2}^{2}-r_{1}^{2}} \log _{e} \frac{r_{2}}{r_{1}}\right),
\end{aligned}
$$




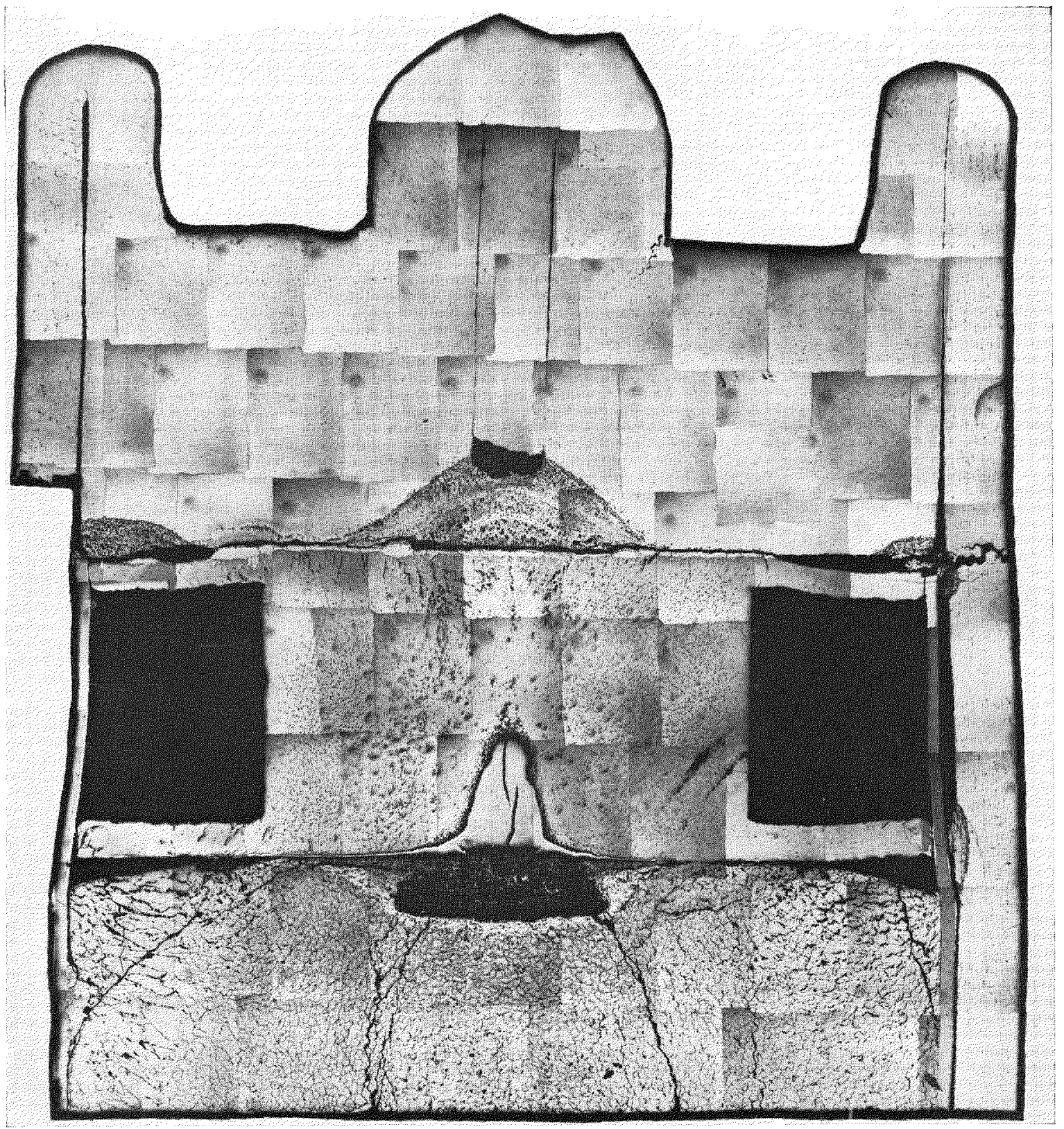

Fig. 9. Longitudinal Section of Fuel, End Spacer, and End Cap in Can 11.

Note regions of reaction between fuel and spacer, and between end cap and spacer. $(\times 16)$

where $\sigma_{t}$ is the thermal stress, $r_{1}$ is the can inner radius, $r_{2}$ the can outer radius, $E$ is Young's modulus, $\alpha$ is the coefficient of thermal expansion, $\mu$ is Poisson's ratio, and $T$ is the temperature drop across the can wall.

Poisson's ratio can be determined from $E$ and the rigidity modulus $G$ by means of the expression

$$
\mu=\frac{E}{2 G}-1 \text {. }
$$

The thermal stress values obtained from these calcula- tions are as follows:

\begin{tabular}{cc}
$\begin{array}{c}\text { Thermal Stress in Outer } \\
\text { Fibres of Can Wall or at } \\
\text { the Tips of Cracks (tsi) }\end{array}$ & $\begin{array}{c}\text { Crack Propagation as } \\
\text { \% of Wall Thickness } \\
(\%)\end{array}$ \\
\hline 8.3 & 0 \\
6.05 & 31 \\
2.60 & 69
\end{tabular}

Figure 15 is a plot of the rupture and proof stresses for Nimonic 90, in which the calculated values shown above have been superimposed. The calculated values 


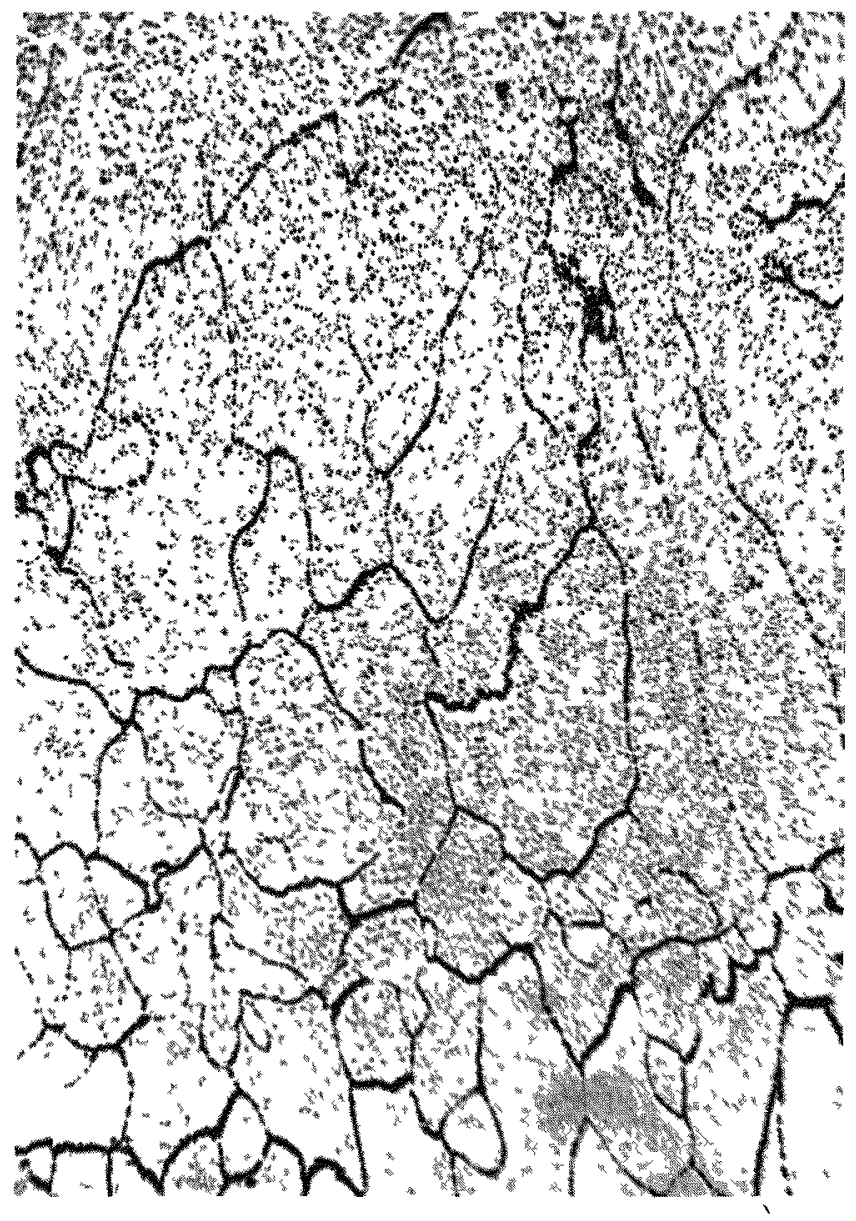

Fic 10 (arim mterion Poiosity in Aic east Fuel (Can 11) $(\times 150)$

in Fig 15 confum the belief that fallure in can 11, by radial craching in the can wall, would have occurred duing the first week of in radiation. Calculations smilar to those presented above are at present being made for the other ten ran in thrs wiadiation astembly

\section{Compatibility Studies of $\left(\mathbf{U}_{085} \mathbf{P u}_{01}\right) \cdot \mathrm{C}$}

\section{Introduction}

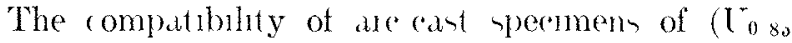
$\left.\mathrm{Pu}_{15}\right) \mathrm{C}$ with 316L, stanles steel and rextam higher nuchel alloys has been studed at temperatures up to $900^{\circ} \mathrm{C}$ In addition, a limited number of tests have been canried out for both $\mathrm{C}^{\circ} \mathrm{C}$ and $(\mathrm{UPu}) \mathrm{C}$ with $316 \mathrm{~L}$ steel in the presence of a codsum bond. The dry compatibility tests were undertaken with couplen saled in welded, thick wall $\mathrm{A}$ monone $80 \mathrm{\Lambda}$ (ans using expansion rod of 347 steel to ensure that rontact was mantaned between the comtituent of the couples at operating temperatures. A case of hnown incompatibility was used to lest the cans at the maximum temperature employed, $900^{\circ} \mathrm{C}$, to ensure that creep of the Nimonic
801 wan not caung low of contart In the sodiun teste, canc of 316L steel were used, in wheh was plared the aribide surounded by a sodium miterlayer, the mner surfare of the can arted as the specimen

In the dry tests, evidence has been sought for pousble tranfer of earbon to the steel and also for plutonum or uranum trancfer, with aroociated diffusion and compound formation.

An upper limat to the temperature at whath the materials can be compatsble is provided by the formation of hiquid phanes. Thus, for hypostorehiometre $(\mathrm{CPu}) \mathrm{C}$, a plutonum rich metal phase is present which an form a eutectic with nichel at temperatures of less than $500^{\circ} \mathrm{C}$ With storchometrie and hyperstorehi metulc ( $\mathrm{CPu}) \mathrm{C}$, eutertice orrur between the mono carbide and the transition netals. For $\mathrm{CC} F e$, the eutectic temperature is $1113^{\circ} \mathrm{C}^{(13)}$ and is lowered by addition of chromium or nichel, ${ }^{(14)}$ the relevant value for $316 \mathrm{~L}$ stanlem steel being $1003^{\circ} \mathrm{C}$. Surprisngly, the value for $\left(\mathrm{C}_{0}{ }_{85} \mathrm{Pu}_{015}\right) \mathrm{C}$ with $316 \mathrm{~L}$ is slightly higher, being $1029^{\circ} \mathrm{C}$.

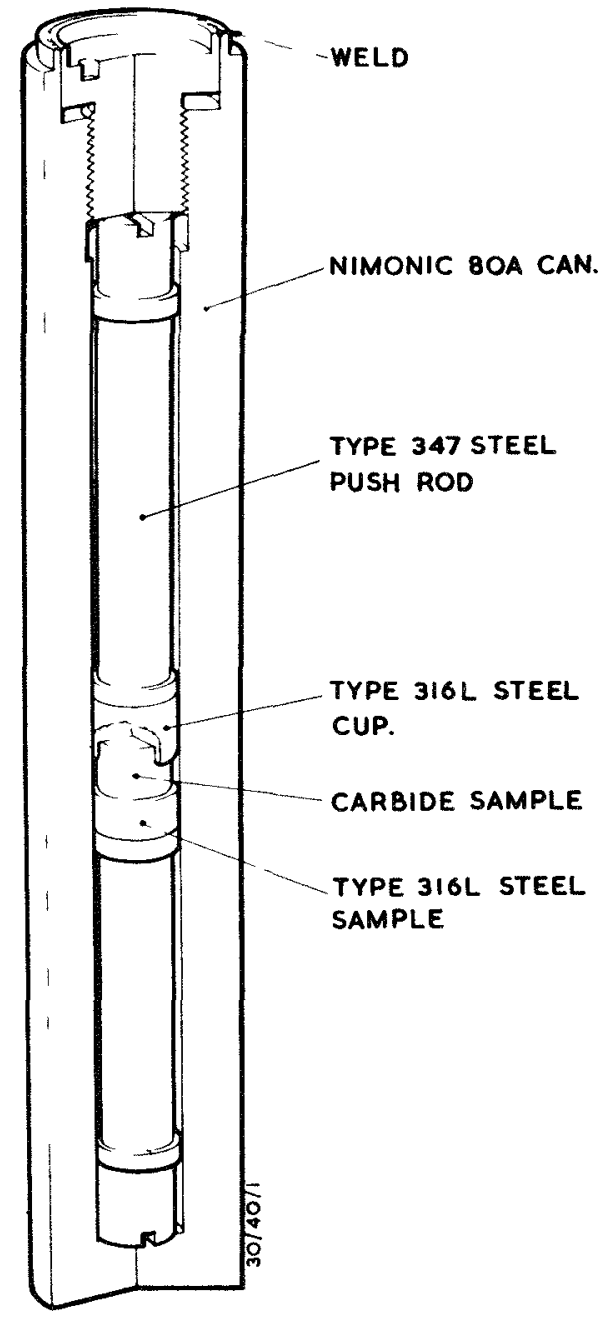

Fig 11 General Assembly of Compatıbility Capsule 


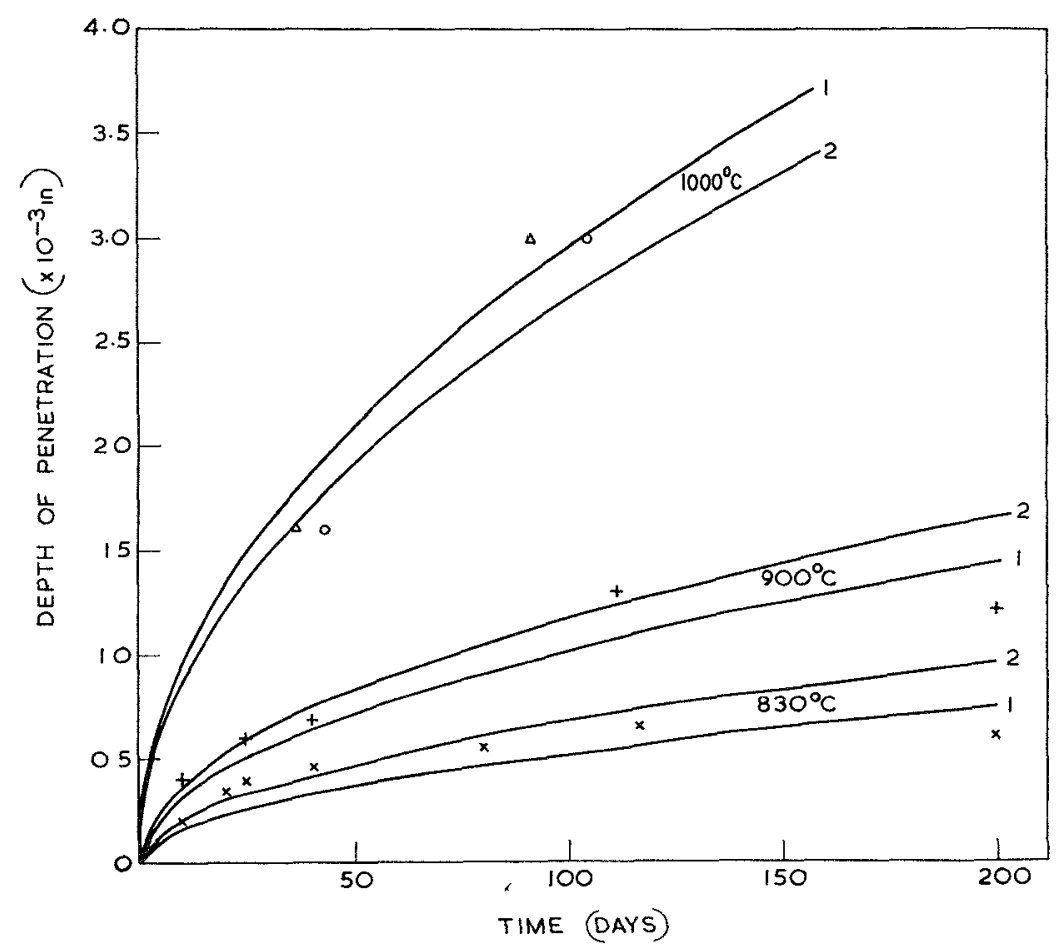

PENETRATION OF NIMONIC 90 INTO TANTALUM .

Fig. 12. Penetration of Nimonic 90 into Tantalum.

Curves 1 : best fit to experimental points up to 50 days; Curves 2 : best fit to experimental points up to 200 days.

\section{$3.2(U P u)-C / 316 L$ Compatibility}

Compatibility studies with $316 \mathrm{~L}$ steels are complirated by the variable response to heat treatments of steels from different manufacturers. In the present work, vacuum melted steels supplied by Samuel Fox, Firth-Vickers, and Telcon, together with an air melted Willan steel, have been studied at various times. The as-received steels, after ageing at $6.50-900^{\circ} \mathrm{C}$, all showed a surface layer of cold-work-induced precipitation, the precipitates being mainly carbide with some $\sigma$ phase (see Fig. 16). The recommended solution treatment of $20 \mathrm{~min}$ at $1050^{\circ} \mathrm{C}^{(15)}$ eliminated cold-work effects in the Telcon and Firth Vickers stcels, but not in that from Samuel Fox; indeed, extending the heat treatment times to $24 \mathrm{hr}$ and the temperature to $1100^{\circ} \mathrm{C}$ failed to remove the effect completely in the latter steel.

Details of the heat-treatment conditions for the compatibility couples are given in Table 3 . Transverse sections through the interface zone, at points where (arbide had stuck to the steel, did not show any reaction products in either the steel or the carbide (see Fig. 17), nor was there any evidence of depletion of $\mathrm{MC}_{2}$ or $\mathrm{II}_{2} \mathrm{C}_{3}$ phases in the hyperstoichiometric carbides.

Some localised carburisation was noticed at points of poor contact, but the zone depth was slight and showed no increase with time or temperature. There is
TABLE 2. Diffesion Data for Penetration of Nimonic 90 into Tantalum

\begin{tabular}{|c|c|c|c|c|}
\hline \multirow{2}{*}{ Temperature $\left({ }^{\circ} \mathrm{C}\right)$} & \multicolumn{2}{|c|}{$\mathrm{D}\left(\mathrm{cm}^{2} / \mathrm{sec}\right) \times 10^{-7}$} & \multicolumn{2}{|c|}{$\begin{array}{l}\text { Activation Energy } \\
\text { (kcal) }\end{array}$} \\
\hline & Curves 1 & Curves 2 & Curves 1 & Curves 2 \\
\hline 830 & 2.0 & 3.4 & \multirow{3}{*}{48.9} & \multirow{3}{*}{40.5} \\
\hline 900 & 7.7 & 10.3 & & \\
\hline 1000 & $40-68^{*}$ & $39.6-65.5^{*}$ & & \\
\hline
\end{tabular}

* First figure is value derived from data at lower temperature; second figure from $1000^{\circ} \mathrm{C}$ parabolas.

evidence that this is not an effect due to cold working; it could be due either to gas-phase carburising asso(riated with the presence of hydrogen in the carbides, as suggested by Batey, ${ }^{(16)}$ or to surface diffusion. On the available evidence it is believed that gas-phase carburising is the most probable mechanism. (This phenomenon is discussed in detail in a paper by French and Hodkin to be presented at the Third International Conference on Plutonium, London, November 1965.)

Autoradiographic studies confirmed that no diffusion of plutonium into the steel had occurred, which may bo taken as further evidence of the essential compatibility of stoichiometric and hyperstoichiometric $\left(\mathrm{U}_{0.85}\right.$ $\mathrm{Pu}_{0.16}$ - $\mathrm{C}$ with $316 \mathrm{~L}$ steel at temperatures up to $900^{\circ} \mathrm{C}$. 


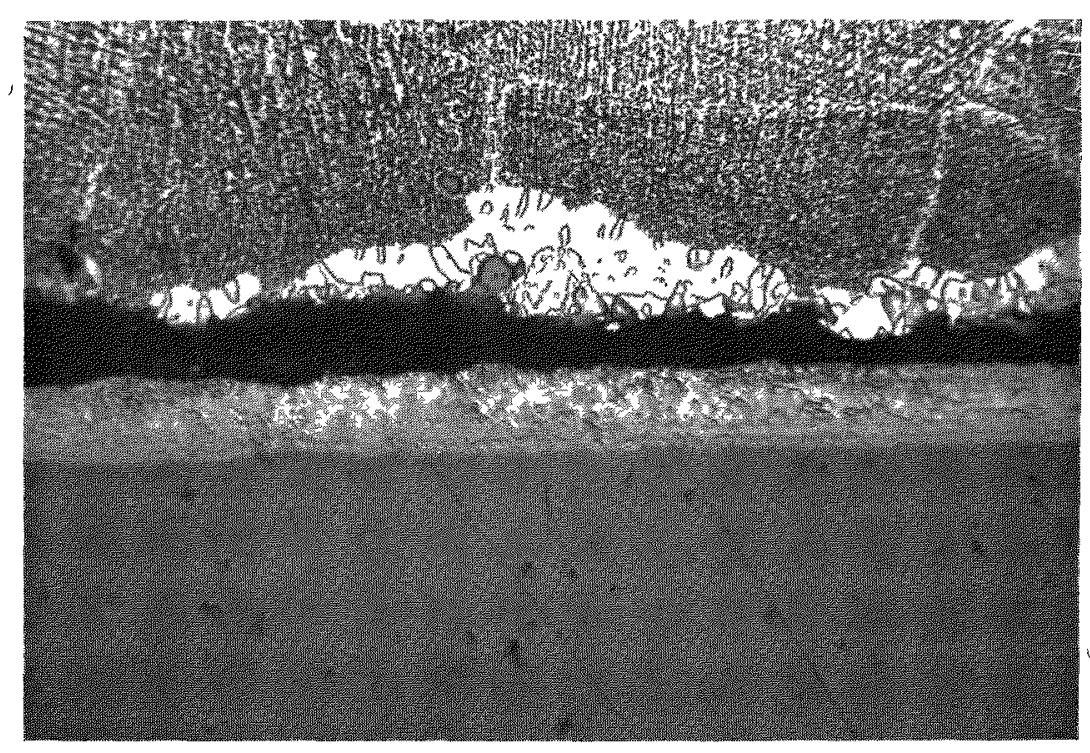

Ifa 13. Ta-Nimonic 90 Interface after Heatmg for 117 Jays at $830^{\circ} \mathrm{C}$. Show ty pical reaction zone on Nimonle 90 side $(\times 750)$

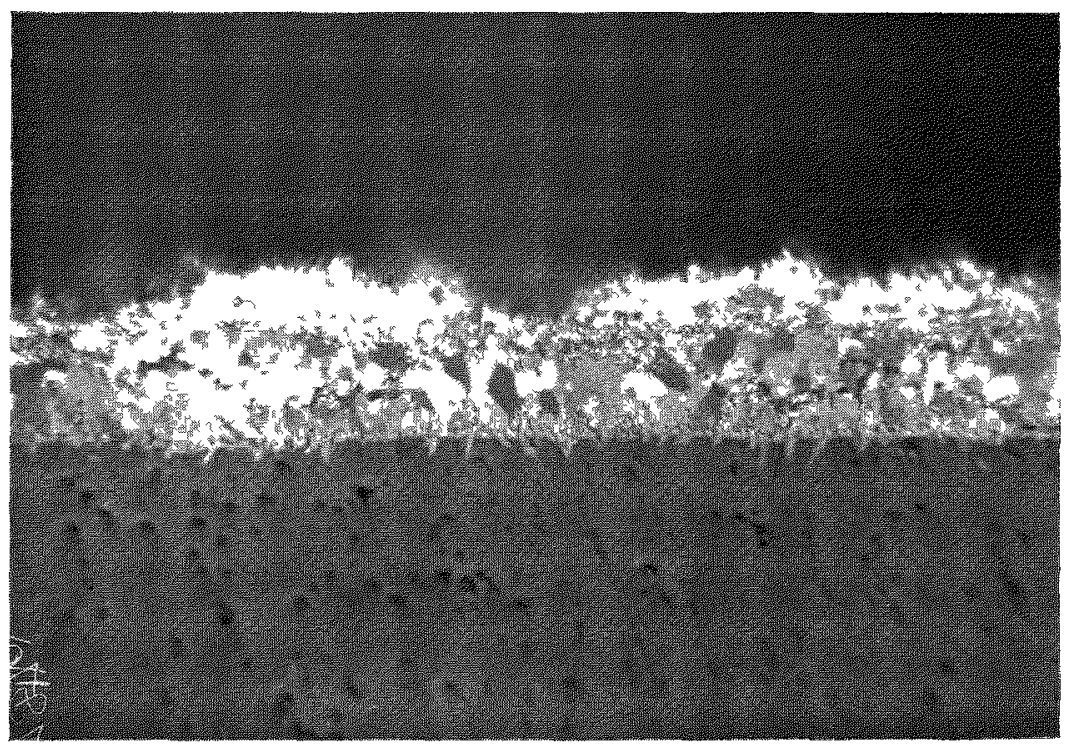

FId. 14. Ta-Nimonic 90 Interface after Ileating for 117 Days at $830^{\circ} \mathrm{C}$. Shows typical reaction zone on Ta Side $(\times 750)$

3.3 The Behaviour of $U-C / 316 L$ and $(U P u)-C / 316 L$ in the Presence of Sodzum

In view of the carburisation of stainless steel by hyperstoichiometric U-C in the presence of sodium as reported by Webb, ${ }^{(17)}$ an attempt has been made to compare the behaviour of $(\mathrm{UPu})-\mathrm{C}$ containing $\mathrm{MC}_{2}$ needles plus a grain-boundary precipitate of the $\mathrm{M}_{2} \mathrm{C}_{3}$ phase with U-C of a similar carbon content but with all excess carbon present as $\mathrm{UC}_{2}$.

For periods of up to 3 months at temperatures up to $800^{\circ} \mathrm{C}$, depletion of the $\mathrm{MC}_{2}$ phase occurred in both materials, but with no depletion of $\mathrm{MI}_{2} \mathrm{C}_{3}$ in the mixed rarbide (see Figs. 18 and 19). Fine globular precipitates were seen on slip planes in the stainless steel, together with coarser precipitation in the surface layer (see Fig. 20). The zone depth is plotted in Fig. 21; the exact carbon equivalence of each alloy is unknown, but undoubtedly they are all hyperstoichiometric, oxygen and nitrogen contents totalling upwards of $500 \mathrm{ppm}$. In general, the rate of carburisation was less for (UPu)-C than for U-C for the same carbon content, presumably due to the smaller amount of $\mathrm{MC}_{2}$ present in the mixed carbide.

Metallographic examination of the lowest carbon 
content quoted for the mixed carbide, namely, 4.79 $\mathrm{w} / \mathrm{o} \mathrm{C}$, suggests a composition equivalent of about 4.9 w/o C. Detertable carbon transfer can thus occur in a material which is only slightly hyperstoichiometrie, provided that the excess carbon is present to some extent as $\mathrm{MC}_{2}$. It should be noted that the structure of the second phase in sintered material is very different from that in arc-cast, and further work is needed to

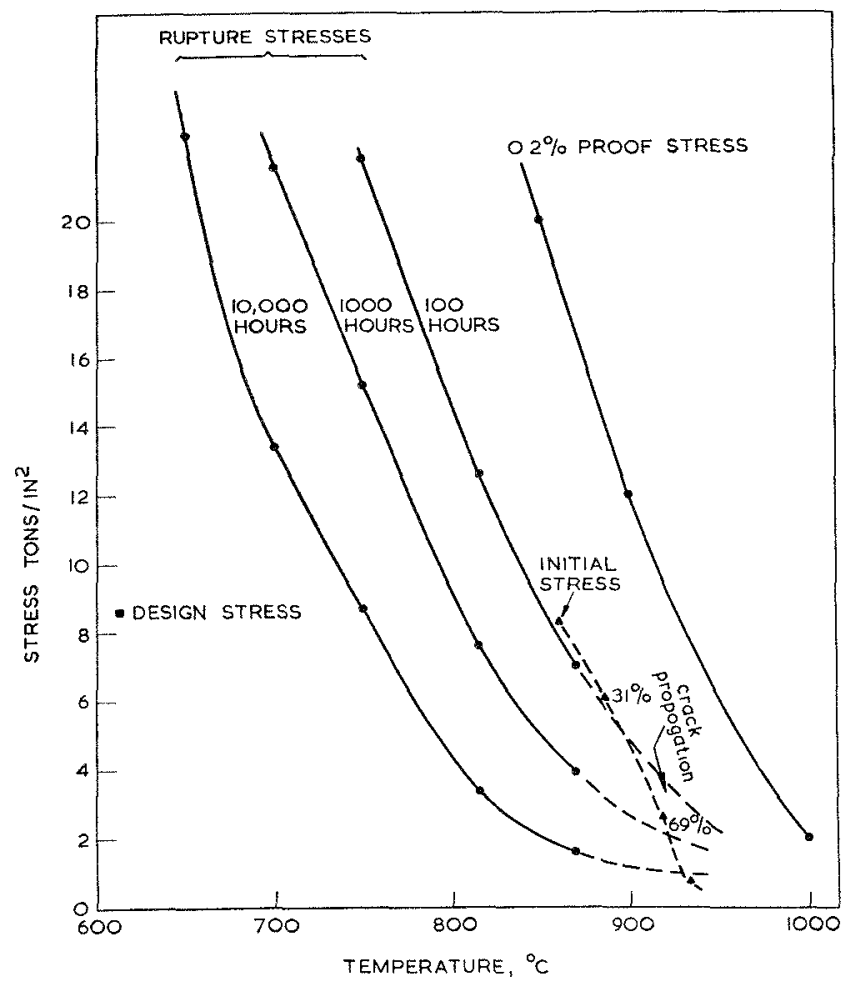

Fig. 15. Relationship between the Thermal Stresses and Material Strength in Can 11.
Table 3. Maximum Heat Treatment Times for (UPu)-C/316L Couples

\begin{tabular}{|c|c|c|c|}
\hline Steel & $\begin{array}{l}\text { Carbon } \\
\text { Content of } \\
\text { (UPu)-C } \\
\text { (wt \%) }\end{array}$ & $\begin{array}{c}\text { Heat } \\
\text { Treatment } \\
\text { Temperature } \\
\left({ }^{\circ} \mathrm{C}\right)\end{array}$ & $\begin{array}{l}\text { Time } \\
\text { (days) }\end{array}$ \\
\hline Willan (Air-melted) & $\begin{array}{r}4.72 \\
4.72 \\
488 \\
488 \\
488 \\
5.06\end{array}$ & $\begin{array}{l}800 \\
900 \\
800 \\
850 \\
900 \\
800\end{array}$ & $\begin{array}{l}28 \\
28 \\
56 \\
28 \\
28 \\
86\end{array}$ \\
\hline $\begin{array}{l}\text { Samuel Fox and Firth } \\
\text { rickers (Both Vac- } \\
\text { uum-melted) }\end{array}$ & $\begin{array}{l}4.88 \\
4.88 \\
4.82\end{array}$ & $\begin{array}{l}650 \\
800 \\
900\end{array}$ & $\begin{array}{l}56 \\
56 \\
56\end{array}$ \\
\hline
\end{tabular}

determine the influence of such differences on compatibility in the presenee of sodium.

\subsection{Compatibitity of (UPu)-C with Nickel-based Alloys}

Hyperstoichiometric mixed carbide reacted with nickel within 1 week at 800 or $900^{\circ} \mathrm{C}$. The reaction products consisted of free carbon and (UPu)-Ni compounds. The depth of the reaction zone was $0.003 \mathrm{in}$. at $800^{\circ} \mathrm{C}$ after a heat treatment for 1 week.

The nickel alloy EPE7 (36.7 Ni. $18.1 \mathrm{Cr}, 4.7$ Mo, 2.5 $\mathrm{Ti}$ and $\mathrm{Al}, 0.17 \mathrm{Si}$, with $\mathrm{Fe}$ to balance) showed a fine globular (arbide presipitate after 14 days at $800^{\circ} \mathrm{C}$, accompanied by depletion of the $\mathrm{IIC}_{2}$ phase in the surface of the carbide.

Nimonic $80 \mathrm{~A}$ showed localised reaction after 56 days at $650^{\circ} \mathrm{C}$, again accompanied by depletion of the $\mathrm{MC}_{2}$ in the carbide. At temperatures of $800900^{\circ} \mathrm{C}$, severe local pitting of the Nimonic occurred, and a

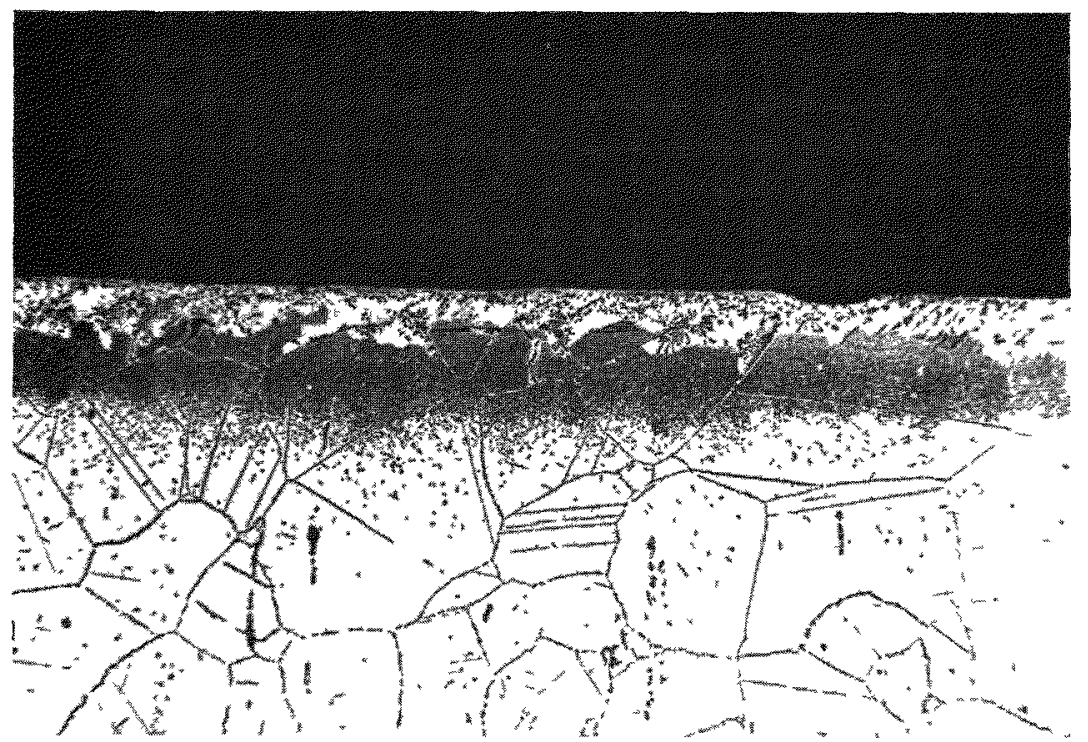

FIG. 16. Typical Precipitation in the Cold-worked Jayer of 316L Stainless Steel. $(\times 250)$ 


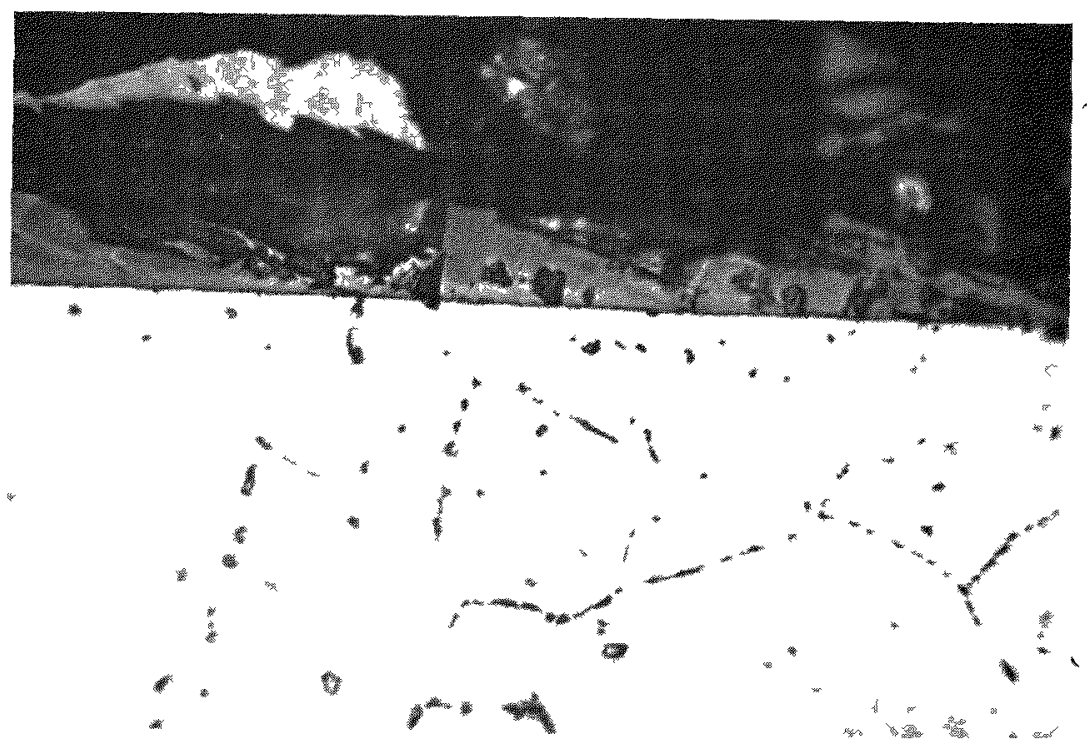

FIG 17 Interface of (UPu) $488 \mathrm{w} / 0 \mathrm{C} / 316 \mathrm{I}$, stimless Steel after Heating for 56 I)ays at $900^{\circ} \mathrm{C}$ No Reaction (X750).

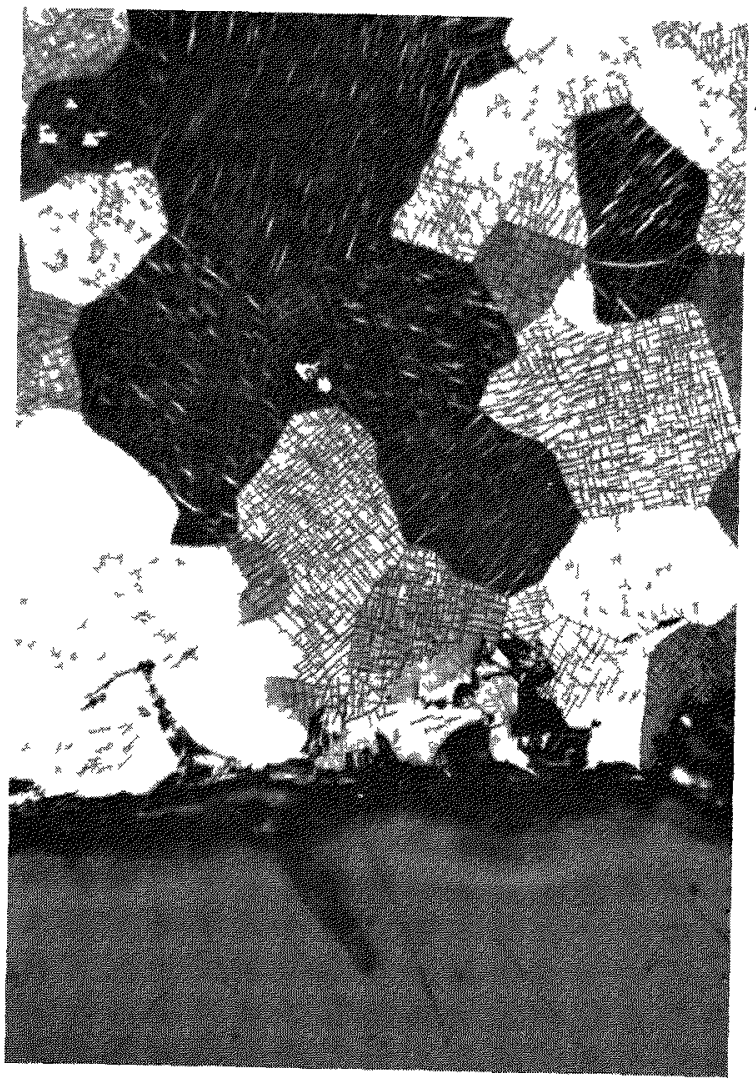

Fra. 18 Interface of $U 489 \mathrm{w} / \mathrm{O} \mathrm{C} / \mathrm{Na} / 316 \mathrm{~L}$ after 28 1)ays at $700^{\circ} \mathrm{C}$

Shows Ty pical Depletion of $\mathrm{MC}_{2}$ Phase from Carbide Surface $(\times 250)$

rontinuour reiction zone was observed (see Fig 22) that reached a depth of $00016 \mathrm{~m}$ after 28 days at $900^{\circ} \mathrm{C}$.

The behaviour of such clads with (UPu) C appears to be worst at intermediate mekel contents, this may

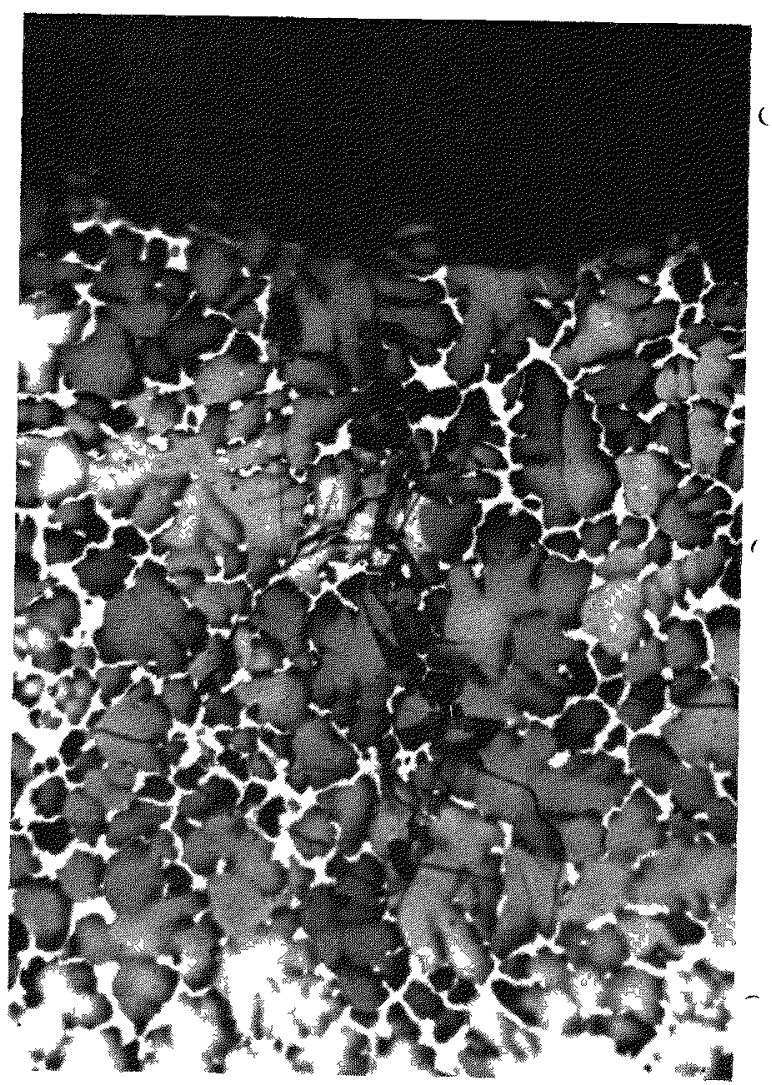

FIG 19 Inteifdce of (UPu) $522 \mathrm{w} / 0$ C/Na/316L after 84 Days at $800^{\circ} \mathrm{C}$

Shows Typieal Depletion of $\mathrm{MC}_{2}$ Phase from Carbide Sur face $\mathrm{No}_{0}$ Depletion of $\mathrm{M}_{2} \mathrm{C}_{3}$ Phase $(\times 250)$

be compared with the behaviour with UC, which improves steadily as the michel content of the clad is rased $\left({ }^{18}\right)$ and with $\mathrm{PuC}$ which worsens with rising nickel content (19) 


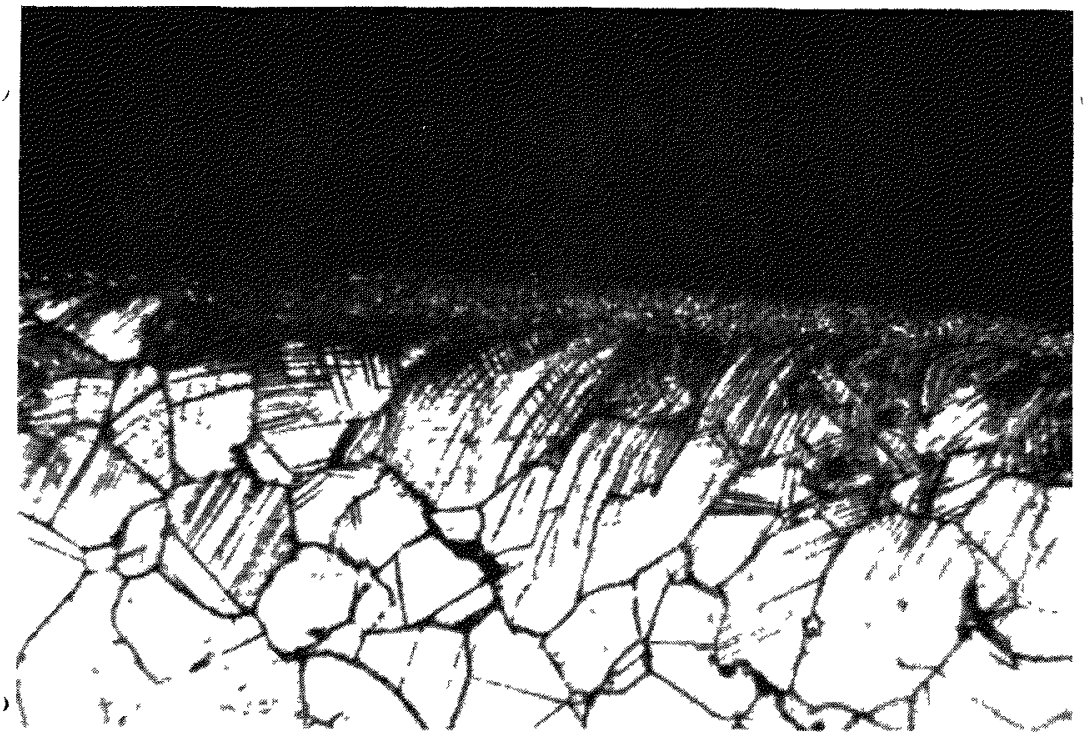

Frg. 20. Interface of $\mathrm{U}-4.89 \mathrm{w} / \mathrm{O} \mathrm{C} / \mathrm{Na} / 316 \mathrm{~L}$ after 84 days at $700^{\circ} \mathrm{C}$. Shows typical carburisation of steel. $(\times 1000)$

\section{The Stability of Oxycarbides at High Tempera- tures}

\subsection{Introduction}

Oxycarbides are known to be unstable at very high temperatures, and this property is used to reduce the oxygen content of carbides to low levels by melting. However, the temperatures encountered in operating carbide fuel pellets are likely to be well below $2000^{\circ} \mathrm{C}$ (at least in the case of a 0.25 -in.-dia pellet operating at $200 \mathrm{~W} / \mathrm{g}$ ), and thus it is of interest to determine the conditions under which decomposition of oxycarbides is likely to occur.

The pressure-temperature-composition curves in Fig. 23 indicate that at $1700^{\circ} \mathrm{C}$, uranium oxycarbides containing more than $10 \mathrm{~m} / \mathrm{o}$ UO will tend to decompose by loss of $\mathrm{CO}$ to yield uranium and oxycurbides of lower oxygen content when the $C()$ pressure falls below $10^{-2} \mathrm{~mm}$. Whilst this relationship has not been precisely determined, the general behaviour is support ed by reports from various workers. Anselin et al., ${ }^{(20)}$ for example, state that whoreas $\mathrm{T}^{\top} \mathrm{C}$ containing $<$.) $\mathrm{m} / \mathrm{o} \mathrm{UO} \mathrm{CO}_{2}$ is stable in vacuum at $1800^{\circ} \mathrm{C}$, decomposition of oxycarbides containing $>15 \mathrm{~m} / \mathrm{o} \mathrm{CO}$ oceurs in a vacuum of $10^{-5} \mathrm{~mm} \mathrm{Hg}$ at $1600^{\circ} \mathrm{C}$.

\subsection{Recent Experimental Results}

At Harwell, Roberts and Regann ${ }^{(21)}$ have studied the behaviour of 1-3-g pellets of $\mathrm{UC}_{1-x} \mathrm{O}_{x}$ and $(\mathrm{CPu})-$ $\mathrm{C}_{1-x} \mathrm{O}_{\mathrm{x}}$ containing $0.5 \mathrm{w} / \mathrm{o}$ oxygen during heat treatment in tantalum containers in vacuum or argon, at temperatures between $1600^{\circ} \mathrm{C}$ and $1800^{\circ} \mathrm{C}$. The tests were performed in separate furnaces, $A$ for $\mathrm{UC}_{1-\mathrm{x}} \mathrm{O}_{\mathrm{x}}$ and $\mathrm{B}$ for $(\mathrm{UPu}) \mathrm{C}_{1-\mathrm{x}} \mathrm{O}_{\mathrm{x}}$, and the single cross check which was carried out with a uranium oxycarbide containing $0.4 \mathrm{w} / \mathrm{o}$ oxygen yielded different weight losses, which were $25 \%$ in $\mathrm{A}$ and $12.8 \%$ in B after $65 \mathrm{hr}$ at $1800^{\circ} \mathrm{C}$. The ultimate pressure attained in both furnaces was : $\times 10^{-6} \mathrm{~mm}$, but the geometries of the furnaces were different, and this could well account for the observed discrepancy. Nevertheless, although the absolute values of results obtained may not be in close agreement, the trends which emerge from these tests, which are described below, are believed to be real.

Thus, the rate of weight loss from these oxycarbides when heated at $1800^{\circ} \mathrm{C}$ in varuum is initially high, but falls with increase of heat-treatment time. Increasing the amount of plutonium over the range 10-25\% of $(\mathrm{CPu}) \mathrm{C}_{1-1} \mathrm{O}_{\mathrm{x}}$ for a constant initial oxygen content of $0.4-0.5 \%$ appears to increase the stability quite markedly, so that the weight loss after $6.5 \mathrm{hr}$ for $\left(\mathrm{U}_{0.75^{-}}\right.$ $\left.\mathrm{Pu}_{0.25}\right) \mathrm{C}_{1-\mathrm{x}}\left(\mathrm{O}_{\mathrm{x}}\right.$ is 0.16 of that for $\left(\mathrm{C}_{0.85} \mathrm{Pu}_{0.15}\right) \mathrm{C}_{1-\mathrm{x}} \mathrm{O}_{\mathrm{x}}$, and in $0.4-0.08$ of that for $\mathrm{LC}_{1-\mathrm{x}} \mathrm{O}$. This is illustrated in Fig. 24. As shown in Fig. 25, a considerable decrease in the weight loss is found when the heat-treatment temperature is lowered to $1600^{\circ} \mathrm{C}$, the relative decrease being greater for the sperimens containing plutonium.

An investigation into composition changes taking place during heat treatment revealed that the period of greatest weight lowis corresponds to the period during which the oxygen content is decreasing most rapidly (see Fig. 26). The $\left(\mathrm{C}_{0}{ }_{85} \mathrm{Pu}_{0.15}\right) \mathrm{C}_{1-\mathrm{x}} \mathrm{O}_{\mathrm{x}}$ specimens showed the same general behaviour as $\mathrm{I}^{r} \mathrm{C}_{1-\mathrm{x}} \mathrm{O}_{\mathrm{x}}$, but the final much higher. This observation provides further evi- 

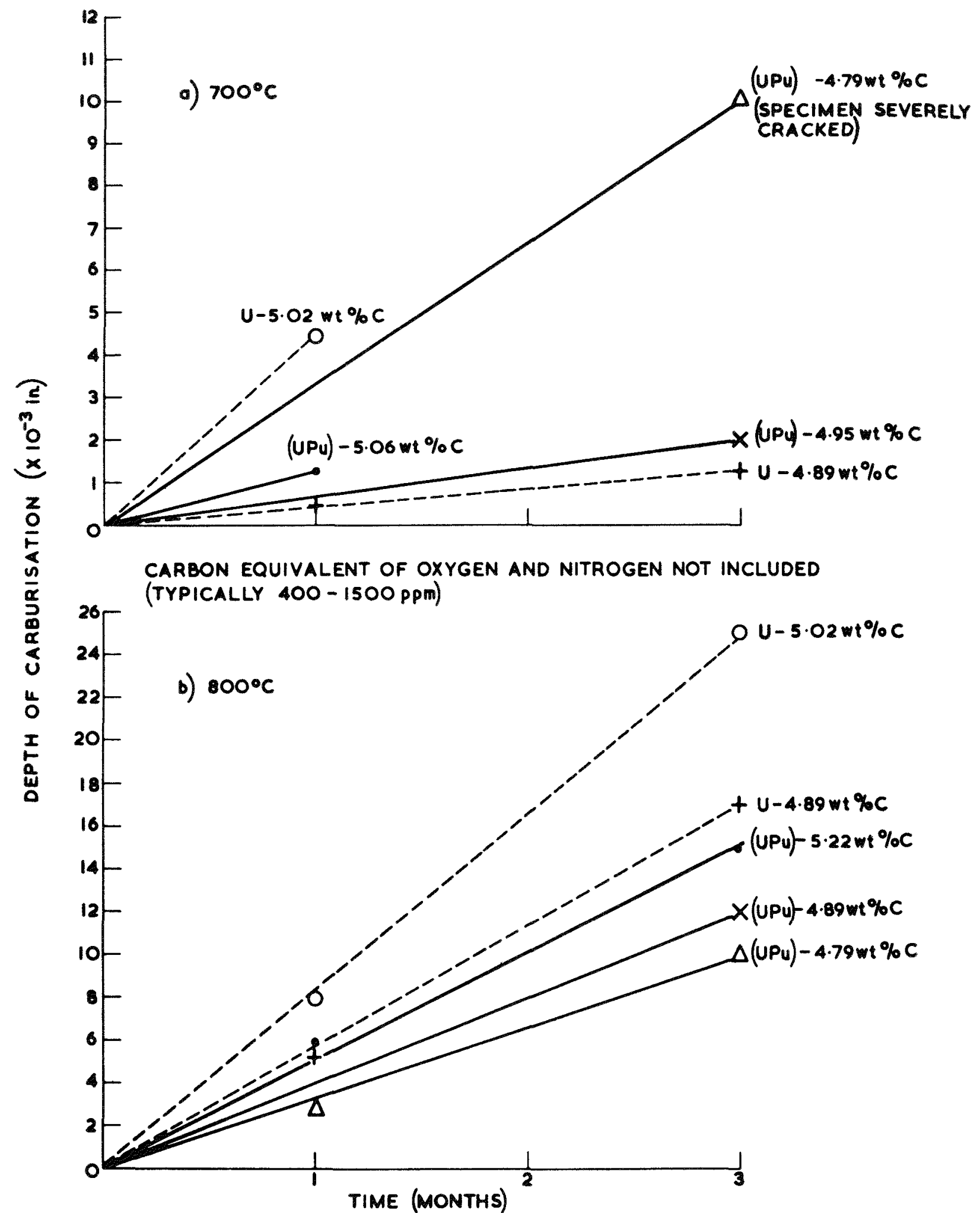

Fig. 21. Curves Illustrating the Rate of Carburisation of Steel. (Sodium Compatibility 'Tests.)

dence for the increased stability of plutonium-rontaining oxycarbides as compared with uranium oxycarbides. The period over which most of the oxygen was lost also corresponds to the period over which plutonium
Josses were highest. This suggest s that the principal mechanism for plutonium loss may involve the volatilisation of either $\mathrm{PuO}$ or an oxygen-rich plutonium oxycarbide. Losses of plutonium during the reaction of 


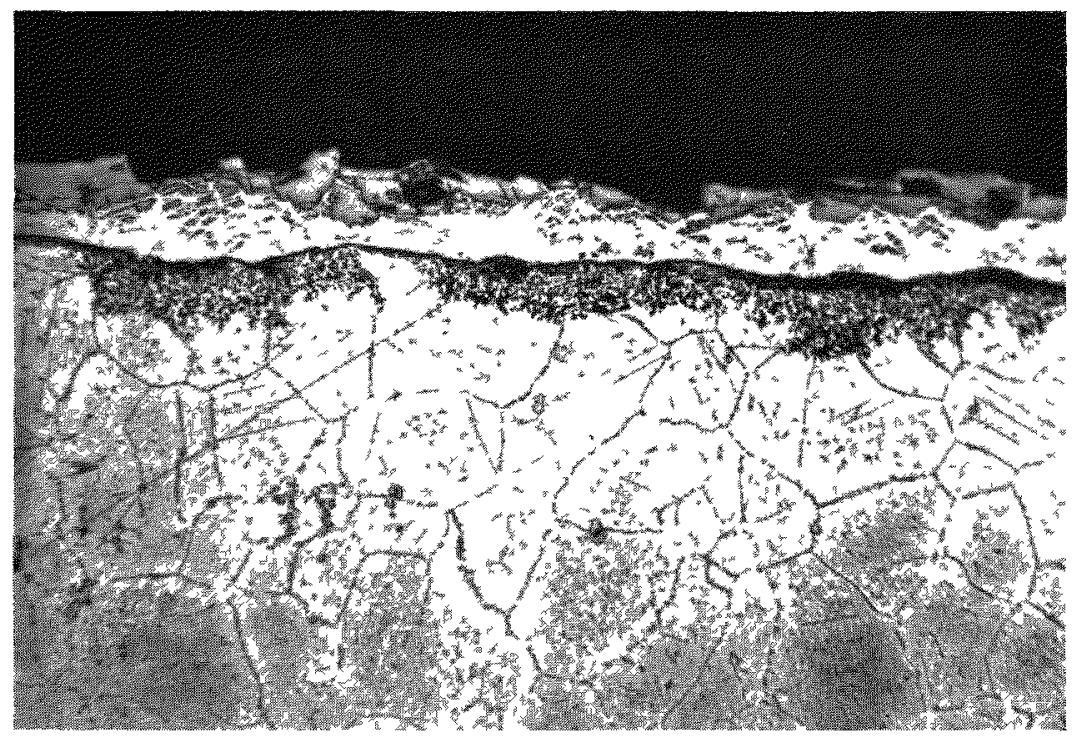

FIG. 22. Interface of (UPu) $-4.89 \mathrm{w} / \mathrm{o} \mathrm{C} / \mathrm{Nimone} 80 \mathrm{~A}$ after 28 Days at $800^{\circ} \mathrm{C}$. Typical reaction product in carbide and localised pitting in Nimonic $80 \mathrm{~A}$. ( $\times 750)$

oxides with carbon in vacuum have also been reported by Horspool et al, ${ }^{(22)}$ and others.

There is an initial decrease in carbon content as $\mathrm{CO}$ is lost, but thereafter the carbon content show a tendency to increase, particularly after long heat treatments at high temperatures. The microstructures of heat-treated pellets tend to be single phase at their centres and show evidence of free metal near their outer surfaces (see Fig. 27). These microstructures support the results of chemical analyses which show that the equivalent carbon content, i.e., w/o C + $(12 / 16) \mathrm{w} / \mathrm{o} \mathrm{O}$, has remained essentially constant during heat treatment, and that the decrease in oxygen content has been balanced by an increase in carbon content.

The dependence of the stability of oxycarbides on oxygen content has not been studied in any great detail, but one recent experıment carried out in vacuum at $1800^{\circ} \mathrm{C}$ showed that, whereas $\left(\mathrm{U}_{0}{ }_{85} \mathrm{Pu}_{015}\right) \mathrm{C}_{1-\mathrm{x}} \mathrm{O}_{\mathrm{x}}$ containing $0.49 \mathrm{w} / 0$ oxygen lost $7.8 \mathrm{w} / \mathrm{o}$ after $6.5 \mathrm{hr}$, a sample of similar composition but containing 0.10 $\mathrm{w} / \mathrm{o}$ oxygen lost only $0.95 \mathrm{w} / \mathrm{o}$ over the same period. Calculations based on the Langmuir evaporation equation indicate that the weight loss from uranium oxy "arbides is greater than that anticipated from oxygen-free $\mathrm{UC}$ by vapourisation of uranium. However, application of the same equation to $(\mathrm{U}, \mathrm{Pu}) \mathrm{C}$ indicates that the weight loss may be lower in oxycarbides than in oxygen-free material, but in 1 his case the values for the vapour pressure of uranium over $(\mathrm{CPu}) \mathrm{C}$, as measured by Stahl and Strasser, are 10-100 times higher than the values used for the vapour pressure of uranium over UC.

Since the conditions inside an operating fuel can are very different from those existing inside a vacuum furnace at $1800^{\circ} \mathrm{C}$, most of the above-mentioned results will tend to give a pessmistic impression of the in-pile stability of oxycarbides. With a fuel centre temperature of $1100-1300^{\circ} \mathrm{C}$, and a fuel surface temperature of $\sim 900^{\circ} \mathrm{C}$, very little decomposition would occur, even if the atmosphere in the fuel can was maintained at $10^{-6} \mathrm{~mm} \mathrm{CO}$ or if the fuel element was of a vented design.

The tendency to decompose during operation will be further reduced by the presence of other gases, such as helium filler gas, and xenon and krypton fission gases, and by the rapidly established equilibrium $\mathrm{CO}$ pressure, unless some fuel-element components act as getters for the CO. To investigate the influence of another gas on the suppression of volatility, a test was performed in which a sample of $\left(\mathrm{U}_{0.85} \mathrm{Pu}_{015}\right) \mathrm{C}_{1-\mathrm{x}}$ containing $0.49 \%$ oxygen was heated for $20 \mathrm{hr}$ at $1700^{\circ} \mathrm{C}$ in $1 \mathrm{~atm}$ of argon. Lnder these conditions there was no detectable change in composition, and the weight loss was $0.47 \%$. When a similar pellet was heated in a helium pressure of $0.3 \mathrm{~atm}$ inside a sealed tantalum can, one end of which was maintained at $1600^{\circ} \mathrm{C}$ for $65 \mathrm{hr}$, and the other maintained at $\sim 800^{\circ} \mathrm{C}$, the pellet which was situated at the hot end lost only $0.11 \mathrm{w} / \mathrm{o}$, whereas a pellet of similar composition lost $0.51 \mathrm{w} / \mathrm{o}$ when heated for the same time at $1600^{\circ} \mathrm{C}$ in vacuum.

\section{Discussion}

The work described in this paper is mainly relevant to the behaviour of individual fuel elements in a fast reactor. It must, however, be appreciated that the work has wider implications, in that changes within a single 


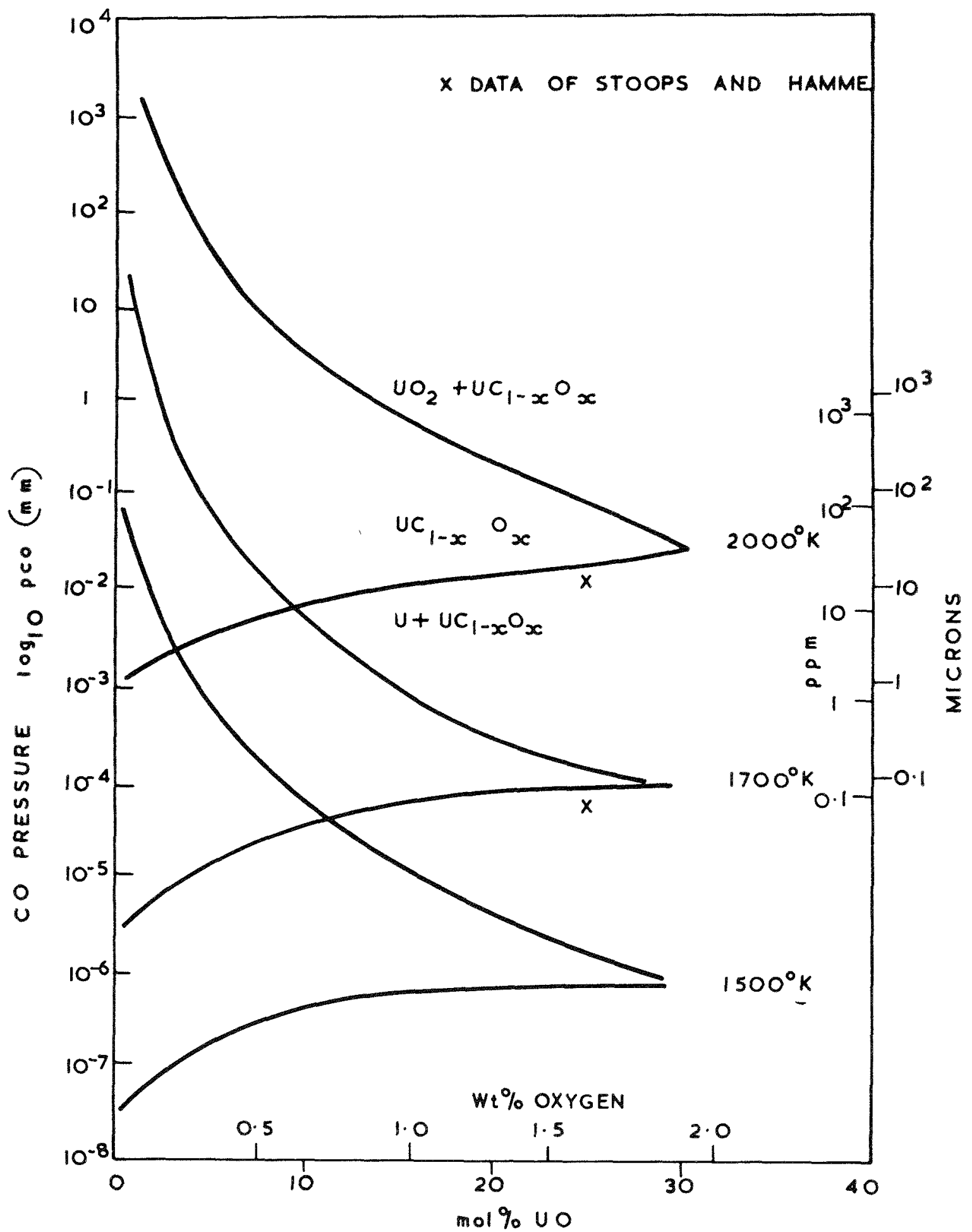

Fig. 23. CO Pressures in Equilibrium with $\mathrm{UC}_{1-\mathrm{x}} \mathrm{O}_{\mathrm{x}}$.

fuel element may influence the behaviour of other fuel elements in a cluster or subassembly, and thereby affect the behaviour of the react or core itself. Examples of such interactions are axial redistribution of plutonium within the fuel element and geometrical changes in the fuel element caused by fuel swelling. Such effects could perturb the coolant flow through the narrow passages between the fuel elements and cause a progressive change in other fuel elements comprising a subassembly. A consideration of the consequences of the deterioration 


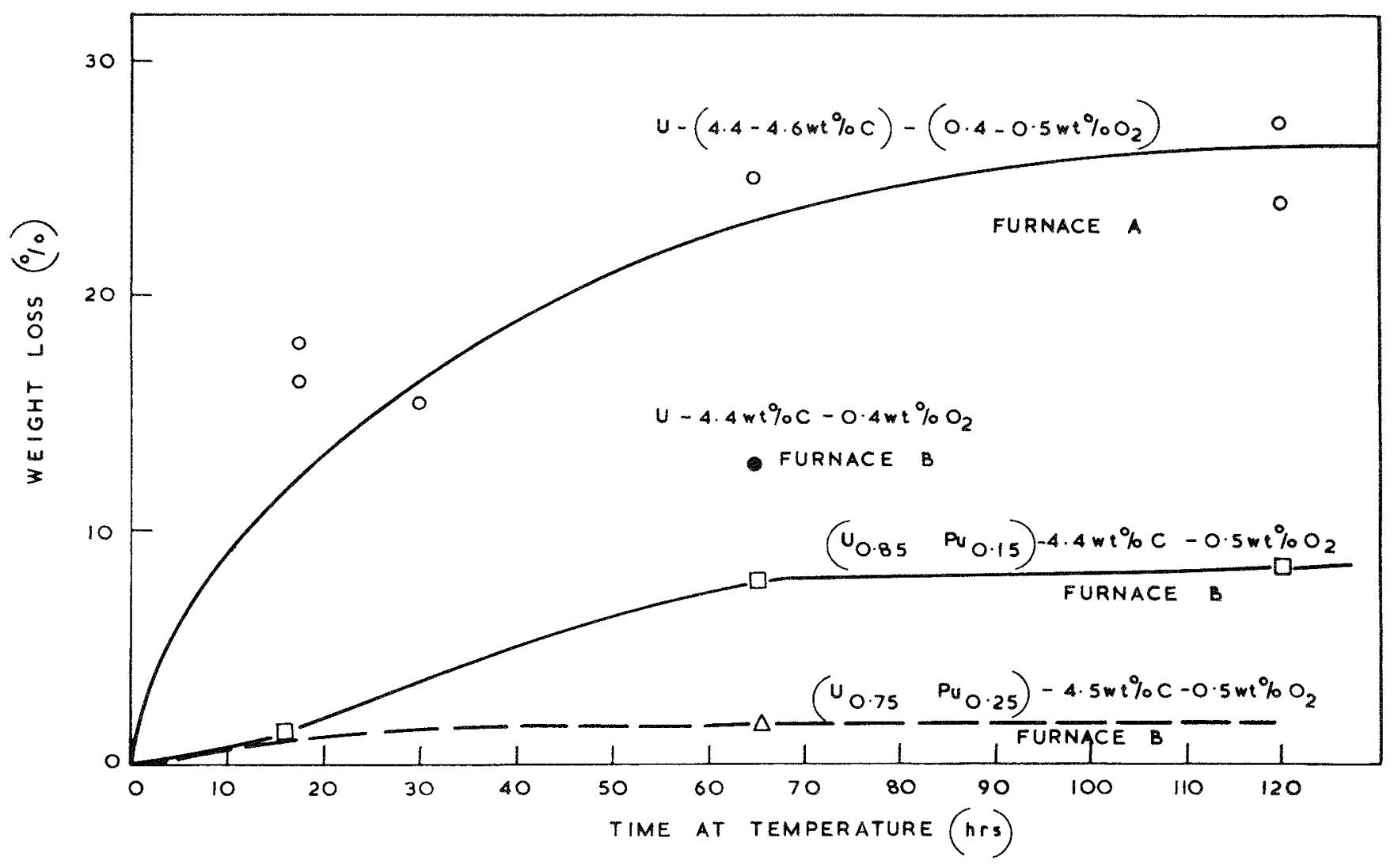

Fig. 24. Weight Loss in Vacuum at $1800^{\circ} \mathrm{C}$ from Uranium-and Uranium-Plutonium-Oxycarbides.

of a fuel element are outside the scope of this paper, in which it is assumed that the criteria for safety of the fuel element are that it should remain intact and geometrically stable throughout its life, and that no major redistribution of the fuel or its constituents should oceur.

In a fast reactor, in which the fucl is required to go to high burnup, the production and behaviour of the gaseous and nongaseous fission products will probably determine the useful life of the fuel. Thus, even in oxide fuel, for which centre temperatures are high and a large proportion of the fission gases is released, those fission products which are retained in the cooler regions of the fuel may eventually cause gross fuel swelling.

The fact that carbide seems to be a gas-retentive fuel means that it is very important to underst and the behaviour of the retained gases and solids, especially as they may increase fuel swelling and limit the burnup attainable. For any fissile material, there is a practical limit to the concentration of foreign atoms, e.g., fission product aloms, which it can accommodate and remain dimensionally stable. This limit will depend upon fuel rating and temperature, but even in the lowertemperature regions of the fuel, swelling and gas release will eventually occur. At Marwell, the irradiation work on carbides is concerned with the behaviour of these fuels under conditions where fission product gas release may be kept at a minimum level $(<10 \%$ of the gases produced). This requirement dictates that the fuel centre temperature should not exceed $1500-$ $1600^{\circ} \mathrm{C}$, which is the temperature at which diffusion release becomes significant. Fortunately, the thermal conductivity of (UPu)-C is sufficiently high to fix fuel centre temperatures of 0.25 -in.-diameter pellets operating at $\sim 200 \mathrm{~W} / \mathrm{gm}$ at about $1100-1300^{\circ} \mathrm{C}$. This may give a reasonable margin of safety sufficient to permit short-term involuntary increases in reactor power, but more work is required to demonstrate this fact. The two powder specimens irradiated to $\sim 5.2 \%$ burnup were found to give low gas-releasc values, and it seems reasonable to conclude from this that the gas releases from the remaining nine elements containing solid fuel were also low. In this connection, Strasser(6) has reported that at centre temperatures of $1200^{\circ} \mathrm{C}$, gas releases from $\left(\mathrm{C}_{0.80} \mathrm{Pu}_{0.20}\right)-\mathrm{C}$ are low $(<1.5 \%)$ up to $40,000 \mathrm{XWD} / \mathrm{te}$, but become appreciable $(\sim 30 \%)$ at $100,000 \mathrm{NWD} / \mathrm{te}$.

More recently at Harwell irradiation of $\left(\mathrm{C}_{0.85^{-}}\right.$ $\left.\mathrm{Pu}_{0.15}\right)$-C to burnups of $90,000 \mathrm{NWD} /$ te at fuel centre temperatures of $\sim 850^{\circ} \mathrm{C}$ has given gas releases of $<1 \%{ }^{(23)}$

In the light of these results, and on the basis of the 


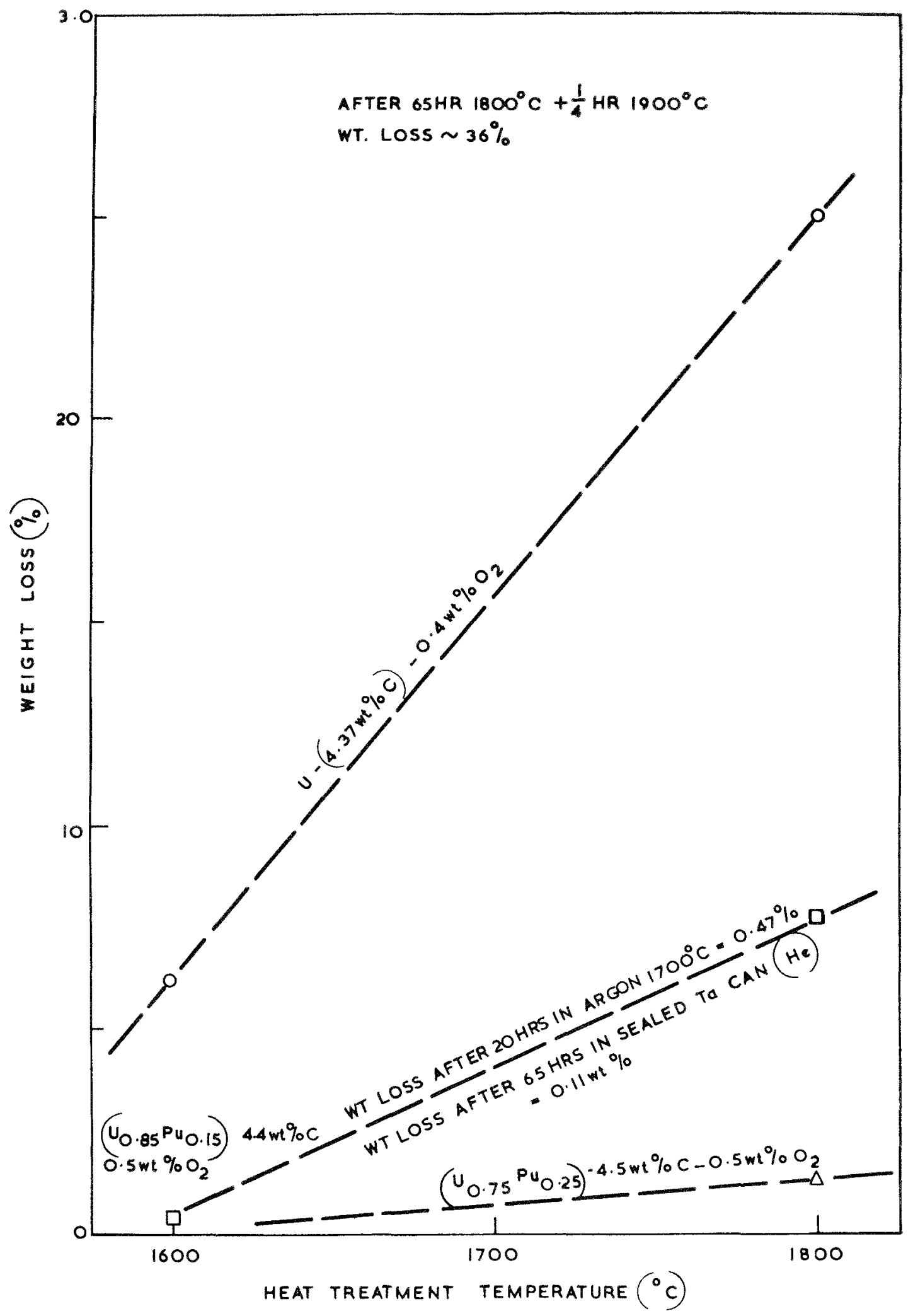

FIg. 25. Weight Loss from Oxycarbides after Heat Treatment for $65 \mathrm{Hr}$ at Various Temperatures. 


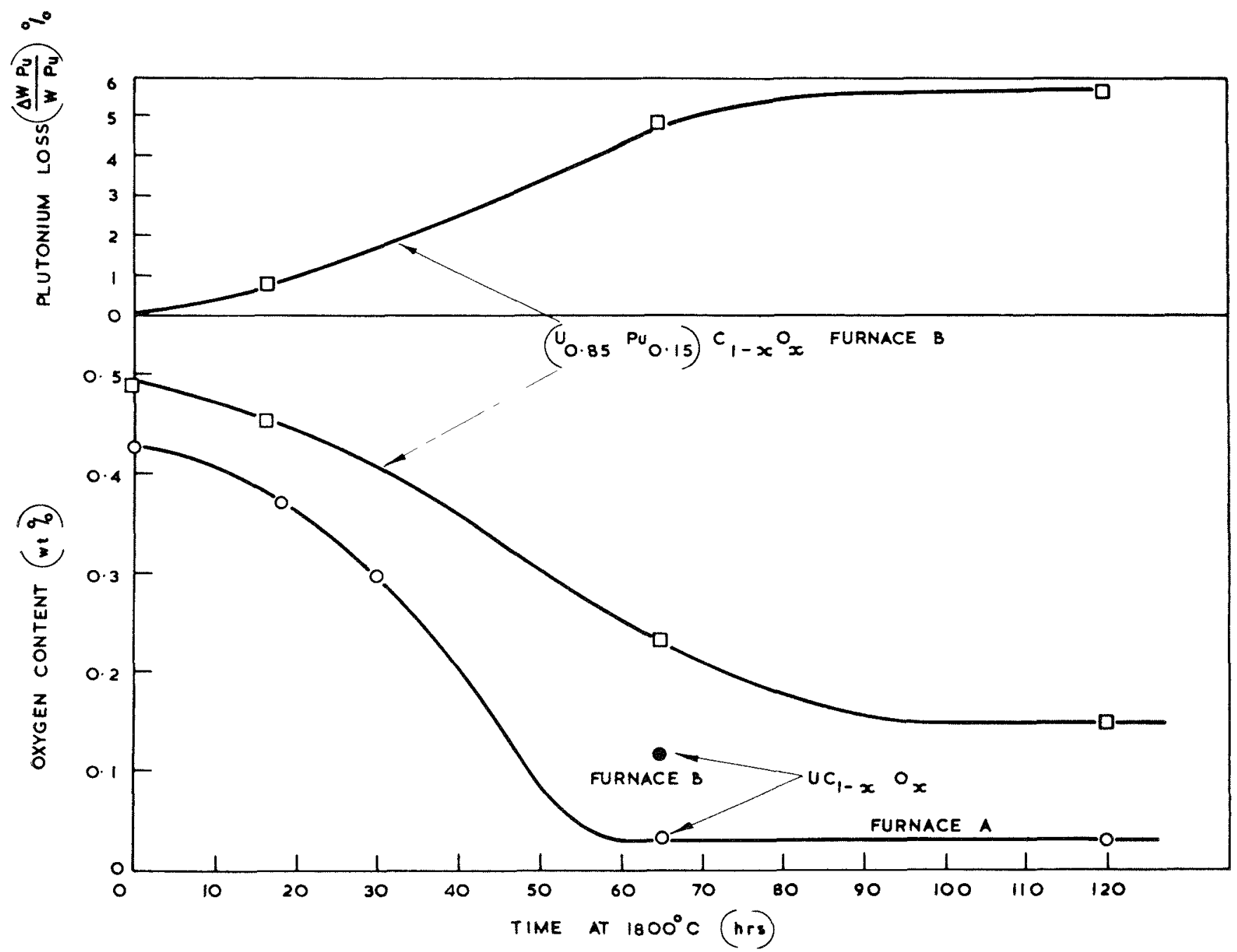

Fic. 26. Change in Oxygen and Plutonium Contents after IIeat Treatment at $1800^{\circ} \mathrm{C}$ in Vacuum.

preceding arguments which imply an enhanced swelling rate for a fission product-retentive fuel, possible means of accommodating fuel welling must be sought. Several ways can be considered:

(1) Strengthen the an by increasing its wall thickness, and thereby rest rain the swolling fuel. This method may affect the neutron physics of the system; in any rase, high thermal strenses in the can may preclude it as a uneful technique.

(2) L'se a stronger clad, avoiding neutron-absorbing matcrials such as tantalum and molybdenum. The favoured rladding materials are type 316 or 304 stainless steel, although in a thermal flux their duetilitien derrease markedly above $600^{\circ} \mathrm{C}$ and exposures of $10^{20}$ nvt. This cmbrittlement is believed to be caused by the agglomeration of helium or lithium atoms, which are produced by the transmutation of boron. The effect of neutron irradiation on various cladding materials is considered in detail elsewhere. ${ }^{(24)}$

(3) Develop a fuel composition which can accommodate swelling when subjected to sufficient restraint. Preliminary data suggest that oxyearbides containing $\sim 1 \mathrm{w} / \mathrm{o}$ oxygen may be ronsiderably more plastic than monocarbide at $\sim 1000^{\circ} \mathrm{C}$, and this may have important ronsecquences in this ronnection.

(1) Develop the rodium-bonded element concept to areommodate either fission produet release or fuel swelling due to fission produet retention. Because of the strong possibility of carbon transfer from hyperst oichiometric "arbide, this method neecsitates very elose control on fuel stoi(hiometry.

(5) Control the movement and growth of fission gas bubbles by making minor additions to the carbide. 


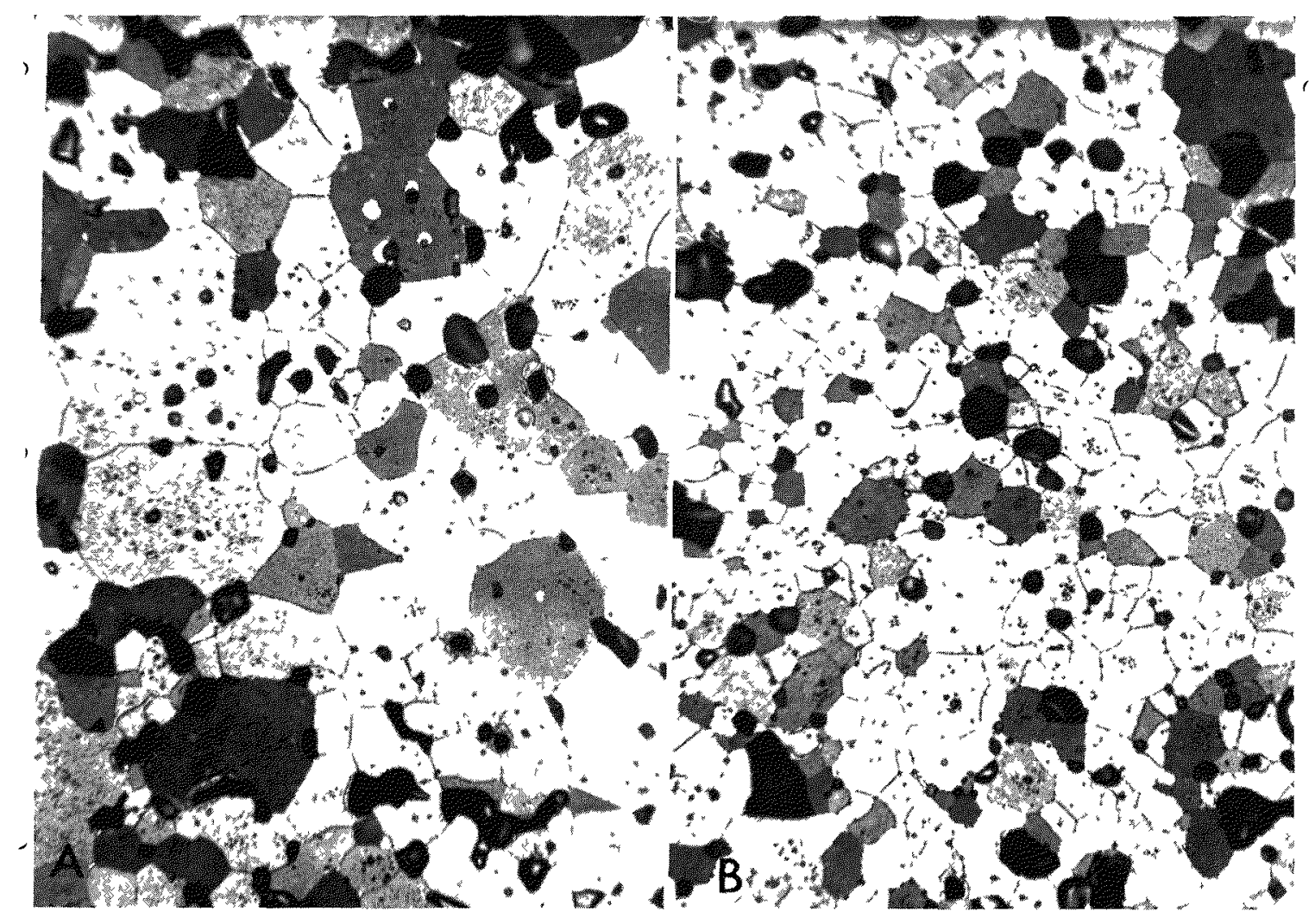

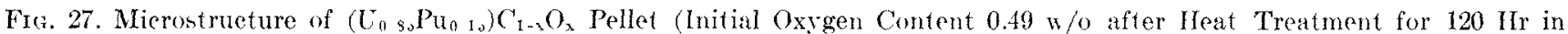
Vacuum at $1800^{\circ} \mathrm{C}$. (a) Surface Showng Metal (White). (b) (entre. (X250)

Because of the improved plasticity of oxycarbide, and also for the reasons stated earlier in the paper, the high-burnup irradiation behaviour of this fuel is being studied at Harwell. Most of the concepts described above will be examined in this programme.

A further aspect of the safety of a fuel element concerns the compatibility of fuel with clad both under normal operating conditions and under fault conditions leading to power transients. From the work described in this paper and other published information, it is (lear that no compatibility problems arise between stoirhiometric carbide and stainless steel at probable operating temperatures in the absence of a sodium bond, the slight localised carburisation seen at points of poor contact being of no practical ignificance. It would seem that short-term excursions of the clad in excess of $900^{\circ} \mathrm{C}$ can also be tolerated, but that the formation of liquid phases above about $1000^{\circ} \mathrm{C}$ sets an absolute limit to any permiscible excursion lasting more than a few seconds. With hypostoichiometric carbides, the existence of low-melting phases with nickel above $500^{\circ} \mathrm{C}$ makes the rompatibility of this material somewhat uncertain. The matcrial used in this work in some instances contained slight traces of free uranium without there being evidence of incompati- bility, but no testing of marked hypostoichiometric material in dry contact with stainless steel has so far been reported in the literature.

The presence of a sodium bond radically alters the compatibility of hyperstoichiometric (arbides with stainless steel. Sinizer ${ }^{(25)}$ established that serious carburisation of steel cladding occurred when excess rarbon was present as dicarbide, and Webb has shown ${ }^{(17)}$ that this effect is reduced if the sescuic arbide is present rather than diearbide. Similar behaviour was reported by Batey et al. ${ }^{(16)}$ for mixed arbides in whirh the seeond phase is predominantly sesquicarbide. In the present work, the proportion of second phase was small compared with that in other invertigations, such as might be expected with a fairly rigid specification of carbon rontent in nominally stoichiometric fuel. These preliminary results suggest that for such material carburiation may be kept to a low level if dicarbide can be eliminated from the microstrueture, but that otherwice it in a serious problem. Further work is needed to establinh the precise bchaviour of sesquicarbide, particularly if present in a different morphology, as in sintered material where it is not normally found in a continuous form. The influence of oxygen content on 
compatibility through its effect on the occurrence of second phases is also worth further study.

The use of tantalum/Nimonic 90 co-extruded tubing as a cladding in the experiments described here may have wider application if high-strength nickel-bearing alloys become of importance for constraint of swelling. This work suggests that the reaction between tantalum and nickel, confined as it largely is to the tantalum component, may be kept within arceptable limits for applications to fuel elements.

As far as the concept of a moderate-cent re-temperature, gas-retentive carbide fuel is concerned, the experiments reported here on the behaviour of carbide fuels at $1800^{\circ} \mathrm{C}$ are, in fact, tests of these materials under conditions which are extremely unlikely to arise. If they did, the conditions would be of very brief duration and not at all comparable to treatments of the order of $100 \mathrm{hr}$ which were used here.

The monocarbides of uranium and uranium-plutonium have proved to be remarkably stable as far as composition changes during irradiation are concerned. Also, Strasser ${ }^{(6)}$ has shown that (UPu)-oxycarbides containing up to $0.4 \mathrm{w} / \mathrm{o}$ oxygen undergo no microstructural ehanges and that the amounts of $\mathrm{MC}$ and $\mathrm{II}_{2} \mathrm{C}_{3}$ phasen present in these materials remain sensibly constant to $10 \mathrm{a} / \mathrm{o}$ burnup at centre temperatures of $1100-1.500^{\circ} \mathrm{C}$. Further, there is no evidence for the formation of metallic phases.

There is, however, a report of a significant deterioration of uranium oxycarbide during irradiation at a temperature of $1000^{\circ} \mathrm{C}$, which suggests that under the influence of irradiation, decomposition of the oxycarbide can occur some $800^{\circ} \mathrm{C}$ lower than it would have done under normal conditions. In fact, Accary and Delmas ${ }^{(26)}$ report that this decomposition process eventually led to the formation of metallic uranium by the loss of $\mathrm{CO}$. The subsequent substantial swelling which occurred is believed to have resulted from the precipitation and growth of gas bubbles in the free metal, and could limit the value of oxycarbides as fuels. As mentioned earlier, a further problem would arise from the presence of hot uranium or uranium-plutonium metal in contact with the canning material.

Further work is urgently needed to study the effect of irradiation on the stability of (UPu)-oxycarbides. Until this work has been performed, the results of Strasser and those reported here indicate that, provided the temperature of the fucl is kept at the levels required to give low gas-release rates, the fuels should be stable both with regard to the redistribution of plutonium and to composition changes resulting from the loss of $\mathrm{CO}$.

\section{Acknowledgments}

The authors wish to thank all their colleagues in the Netallurgy and Researeh Reartors Divisions at Harwell whose efforts have made this paper possible. In particular, they thank MI. J. D. L. Harrison, Mr. W. G. Roberts, MIr. J. W. Isaacs, Mr. J. M. North, and Mr. B. Riley for fabrication of the fuel, Mr. M. W. George for designing and commissioning the irradiation rig, Mr. $J$. Sheldon for the careful preparation and assembly of the irradiation cans, and Mr. D. Hodkin for the compatibility experiments.

They also extend their thanks to Mr. D. Gilling who was responsible for the gas-sampling and metallographic exanination of the fuel pins, and to the personnel of the Active Handling Laboratories in Metallurgy Division, particularly to Mr. J. A. E. Olden, MIr. G. Comley, Mr. L. Thorn, Mr. E. Fidler, and Mr. E. Gerard.

\section{REFERENCES}

1. K. M. Horst and B. A. Hutchins, GEAP-3880 (1962).

2. E. L. Zebroski, J. P. Mustelier, and C. Caldwell, Fast Reactor Technology, ANS-100 (1965), p. 110.

3. B. T. Bradbury, J. E. Cole, B. R. T. Frost, and J. D. B. Lambert, ('arbides in Nuclear Energy, 2, p. 880, Macmillan \& Co., London (1964).

4. J. Crane and E. Gordon, Carbides in Nuclear Energy, 2, p. 896, Macmillan \& Co., London (1964).

5. A. Strasser, ANS-6792 (Dec 1963), p. 297.

6. A. Strasser, C. Wheelock, and L. Neimark, Fast Reactor Technology, ANS-100 (1965), p. 126.

7. B. T. Bradbury, unpublished work at AERL, 1965.

8. B. L. Eyre, unpublished work at ALRE, 1965.

9. B. R. T. Frost, P. G. Mardon, and L. E. Russell, American Nuclear Society Proceedings on Plutonium as a Power Reactor Fuel, HW-75007, Sec. 4.1 (1962).

10. J. H. Kittel, ANL-4937.

11. I). J. Hodkin and P. G. Mardon, work at AERE to be pub lished, 1965.

12. S. Timoshenko and J. N. Goodier, Theory of Elasticity, MeGraw-Hill Book Co., Ine., New York (1951).

13. G. Briggs, J. Barton, and J. White, Powder Metallurgy in the Nuclear Age, (Metallwerk Plansee, Reuthe, Tyrol, 1962), p. 249.

14. J. L. Nichols and J. A. C. Marples, Carbides in Nuclear Energy, Macmillan \& Co., London (1964), p. 246.

15. Firth-Viekers Data Sheet, FV 555.

16. W. Batey, K. Bolton, D. M. Donaldson, and C. Yates, Carbides in Nuclear Energy, p. 392, Macmillan \& Co., London (1964).

17. B. A. Webb, NAA-SR-6246 (1963).

18. J. A. Christensen, HW-72031 (1963).

19. J). J. Hodkin, unpublished work at AERE, 1965.

20. F. Anselin, G. Dean, R. Lorenzelli, and R. Pascard, Carbides in Nuclear Energy, 1, p. 113, Macmillan \& Co., London (1964).

21. W. (x. Roberts and M. C. Regan, unpublished work at AERE, 1965.

22. J. M. Horspool, J. A. S. Mowat, and S. Pickles, Carbides in Nuclear Energy, 2, p. 572, Macmillan \& Co., London (1964).

23. B. T. Bradbury, J. E. Cole, and M. Allen, unpublished work at AERE, 1965 .

24. D. R. Harries et al., A/CONF/28/P/162, Geneva, 1964.

25. I). J. Sinizer, B. A. Webb, and S. Berger, NAA-SR-7248 (1962). 
26. A. Accary and R. Delmas, A/CONF/28/P/59, Cieneva, 1964.

\section{Discussion}

Mr. Zebroski (GE): I'd like to repeat the question which has been asked in two different forms already, which is, do you have some feeling of as to how you might accommodate, in the case of stoichiometric material, the shift in stoichiometry which you can calculate with burnup? I think this shift unfortunately might go either way, because even though the fission products become in excess of the carbon, the binding energies on the average, or for some of them I should say, may be lower than the heavy metal that they displace. Thus you might have a problem of coping with excess carbon locally as well as with excess metal.

Mr. Bradbury: Unfortunately we haven't got the neressary thermodynamic data to say which way things will go. I think we need to measure or to plot $R T \log \mathrm{p}$ against $1 / T$ and compare this for the various solid fission products which are produced with, say, the cladding. This is one thing we must do in the immediate future. One other thing is that, looking at Dr. Strasser's work at Cnited Nuclear, the metallography appears to me to be completely different from that of the oxide; if you take U or U-Pu oxide in excess of $4 \%$ burnup, say, we start to see segregation of second phases which we know are solid fission products, in what form is beside the point at the moment. In the case of carbide we see nothing like this, so that the solution of the fission products in carbides relative to those of oxides is completely different. I think we ought to try to find something more about this. 


\title{
Clad Compatibility with Mixed Oxide Fuel and Sodium
}

\author{
F. A. Comprelli, F. J. Hetzler, and T. A. Latritzen \\ General Electric Company \\ Advanced Products Operation \\ San Jose, Calufornia
}

(PRESENTED BY F. A. COMPRELLI)

\section{INTRODUCTION}

Compatibility is a term frequently used by materials technologists to describe the chemical stability of materials in close contact. As a design criterion, compatibility is of importance since it is one of the factors which determines the selection and useful lifetime of materials in the reactor system.

Although high metal temperatures are found in many areas of a sodium-cooled fast reactor, the highest are associated with the fuel cladding. It is there that diffusion processes and chemical reactions are most likely to be limiting.

The need for system compatibility is clearly demonstrated by considering the possible effects of reactions between the clad material and its enviroment. As reactions occur between the fuel and cladding, new phases may form at the interface or penetrate locally, e.g., along grain boundaries. These, in turn, can affect the useable strength or ductility of the cladding, with consequent increased frequency or severity of fuel-element failures.

Reactions between the cladding and sodium coolant may result in either general (uniform) or localized attack on the outer clad surface, as well as changes in alloy chemistry. Thus the properties of the cladding can be modified both by changes in the effective loadbearing cross section, and by changes in composition and microstructure.

The maintenance of clad integrity under service conditions for fast reactor fuel-element lifetimes of 2 to $4 \mathrm{yr}$ is the primary objective in the screening, selection, and optimization of a fucl-cladding material. Modifications in the structural characteristics of the cladding, at the fucl-clad or clad-coolant interfaces, can be used to give sensitive indications of the relative merit of different materials prior to mounting large-scale proof-test programs.

It is the purpose of this paper to present several observations of fuel-clad and clad-coolant reactions, to analyze some of the factors which influence them, and to evaluate the effect of such reactions on the expected performance characteristics of cladding materials, especially for applications with oxide fuel.

\section{REACTIONS AT CLAD-COOLANT INTER- FACE}

\section{A. Structural Observation}

The hot-zone environment of a dynamic sodiumcooled system promotes specific changes in the surface composition of system materials. A leaching process, in which various alloy constituents are selectively removed from the alloy surface by the liquid sodium and taken into solution, strongly influences the ironchromium-nickel ratios at the surface of the stainless steel. The reverse process, in which impurity elcments in the sodium are deposited on and absorbed into the stainless steel surface, is also commonly observed. The carburization of stainless steel in the hot zone is a well-documented ${ }^{(1-6)}$ example of this effect. Studies at General Electric have been made to determine the magnitude and nature of the localized phenomena at FCR conditions and their general effect on mechanical properties. ${ }^{(7)}$

\section{A.1. Elemental Leaching}

The process of elcmental leaching has been dcscribed by Epstein ${ }^{(8)}$ and Mottley ${ }^{(9)}$ as a combination of dissolution and chemical reaction mechanisms which remove alloy constituents at various rates. $\mathrm{Ob}-$ servations ${ }^{(1)}$ made on type 316 stainless steel samples in $1200^{\circ} \mathrm{F}$ pumped sodium loops have verified this process. Analyses of sample surfaces by electron microprobe have shown ${ }^{(10)}$ iron-chromium-nickel ratios which are notably different from the original compositions (see Fig. 1). This change in surface composition produces a visible surface layer. If the layer continues to develop and the depletion of alloy constituents persists beyond a specific minimum threshold level, the layer becomes ferritic.

Austenitic type 316 stainless steel has been tested in reactor-grade sodium (calcium controlled to less than $50 \mathrm{ppm}$ ) under the loop conditions given in Table 1. The depth of the layer under these conditions is dependent on several variables, especially oxide content and specimen temperature. ${ }^{(1)}$ For the oxide levels of 12 and $50 \mathrm{ppm}$, average depths of 

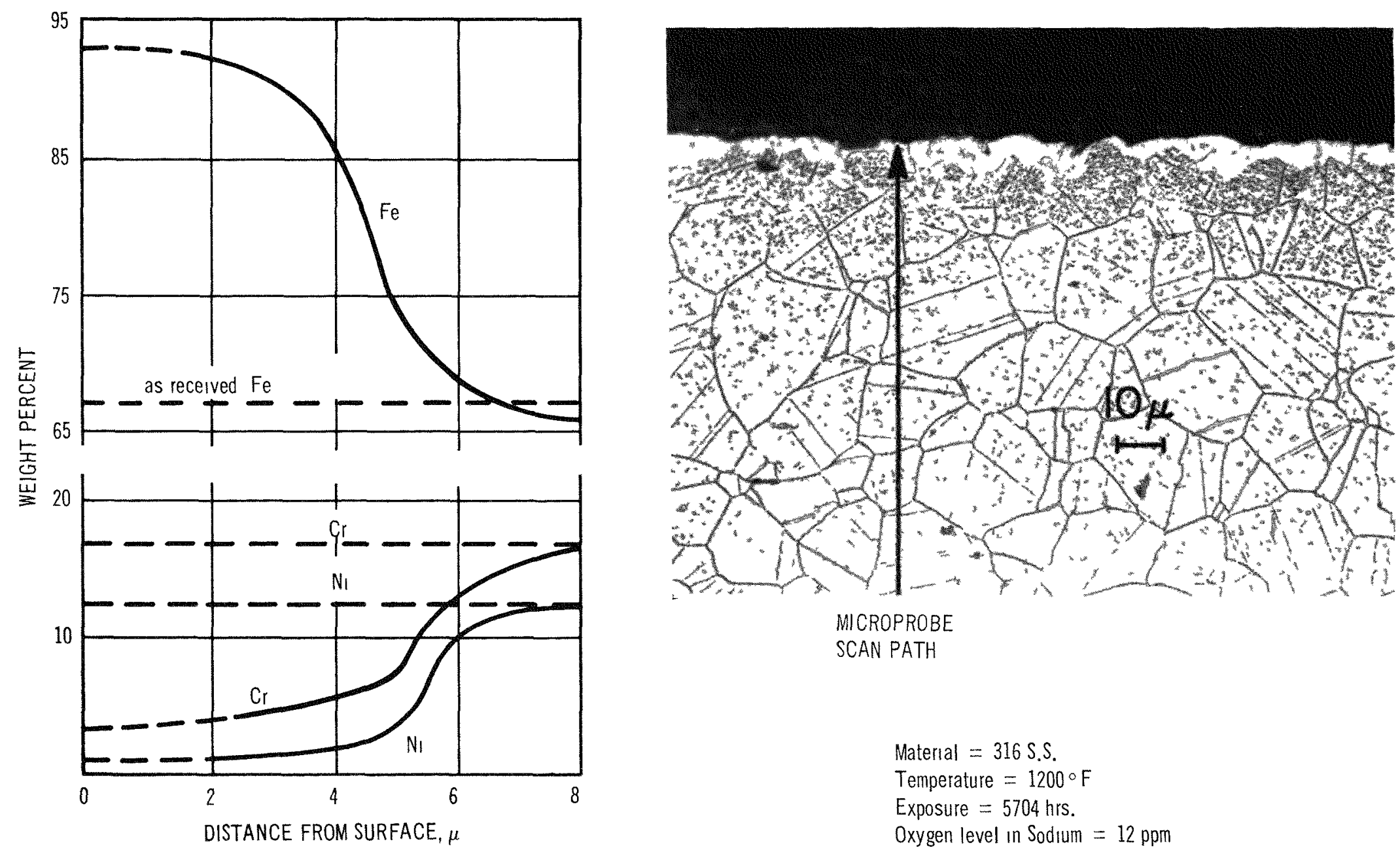

FIs 1 Trpical Structural and Compositional Changes in Trpe 316 Stanless steel Associated with Llemental Leaching in Sodum

Material $=316$ S.S.

Temperature $=1200^{\circ} \mathrm{F}$

Exposure $=5704$ hrs.

Oxygen level in Sodium $=12 \mathrm{ppm}$ 
TABLE 1. Ravge of LoOp CondtTon Tested

\begin{tabular}{|c|c|c|c|c|c|c|}
\hline \multirow{2}{*}{$\begin{array}{l}\text { Sodium Velocity } \\
\text { fps }\end{array}$} & \multirow{2}{*}{ Max Temp, ${ }^{\circ} \mathrm{F}$} & \multirow{2}{*}{$\Delta t,{ }^{\circ} \mathrm{F}$} & \multirow{2}{*}{ Oxide Contents, ppm } & \multicolumn{2}{|c|}{ Material Combinations } & \multirow{2}{*}{ Time, hr } \\
\hline & & & & Hot Leg & Cold Leg & \\
\hline
\end{tabular}

TABLE 2. Feret fic layer Forvation In Type 316 Stainless STEel afrer Exposure for $2800 \mathrm{Hr}$

\begin{tabular}{c|c|c}
\hline \multirow{2}{*}{ Temperature, ${ }^{\circ} \mathrm{F}$} & \multicolumn{2}{|c}{ Average Depth after $2800 \mathrm{Hr}$, mil } \\
\cline { 2 - 3 } & $12 \mathrm{ppm} \mathrm{Na}_{2} \mathrm{O}$ & $50 \mathrm{ppm} \mathrm{Na}_{2} \mathrm{O}$ \\
\hline 1000 & Trace & Trace \\
1100 & Trace & 0.1 \\
1200 & 0.2 & 03 \\
1300 & 0.3 & 08 \\
\hline
\end{tabular}

the ferritic layer in Type 316 stainless steel after $2800 \mathrm{hr}$ at various test temperatures are listed in Table 2.

Analysis of the test data indicated that the ferritic layer reached a limiting depth under a specific set of conditions within $1000 \mathrm{hr}$. The quasi-equilibrium state was maintained over a test period of at least $5000 \mathrm{hr}$ unless the environmental conditions were altered.

An additional factor affecting layer formation is cold work. Figure 2 shows a cold-rolled sample exposed for $400 \mathrm{hr}$ in $1300^{\circ} \mathrm{F}$ sodium at an oxide concentration of $50 \mathrm{ppm}$. The unusually rapid layer formation and growth is probably caused by increased diffusion rates associated with severe lattice deformation. (11) The number of lattice vacancies would be greatly increased, allowing the elemental alloy constituents to diffuse more rapidly to the liquid metalsolid interface. A similar acceleration of layer growth should also occur if the test coupon is subjected to an applied stress. ${ }^{(12)}$

The direct influence of the surface layer on material properties is effectively minor. Small amounts of surface ferrite are subject to rusting if air is allowed to enter the system during shut-down periods. Rust particles might flake off during subsequent reactor operation and restrict small flow passages. Control of the reactor cover gas during operational shut downs or liquid soidum coolant filtration could rectify the problem.

Because of the volume change accompanying the alloy depletion and structural transformation to the body-centered cubic lattice from the denser face-centered cubic structure, the ferritic layer contains numerous structural defects. In this regard, the surface

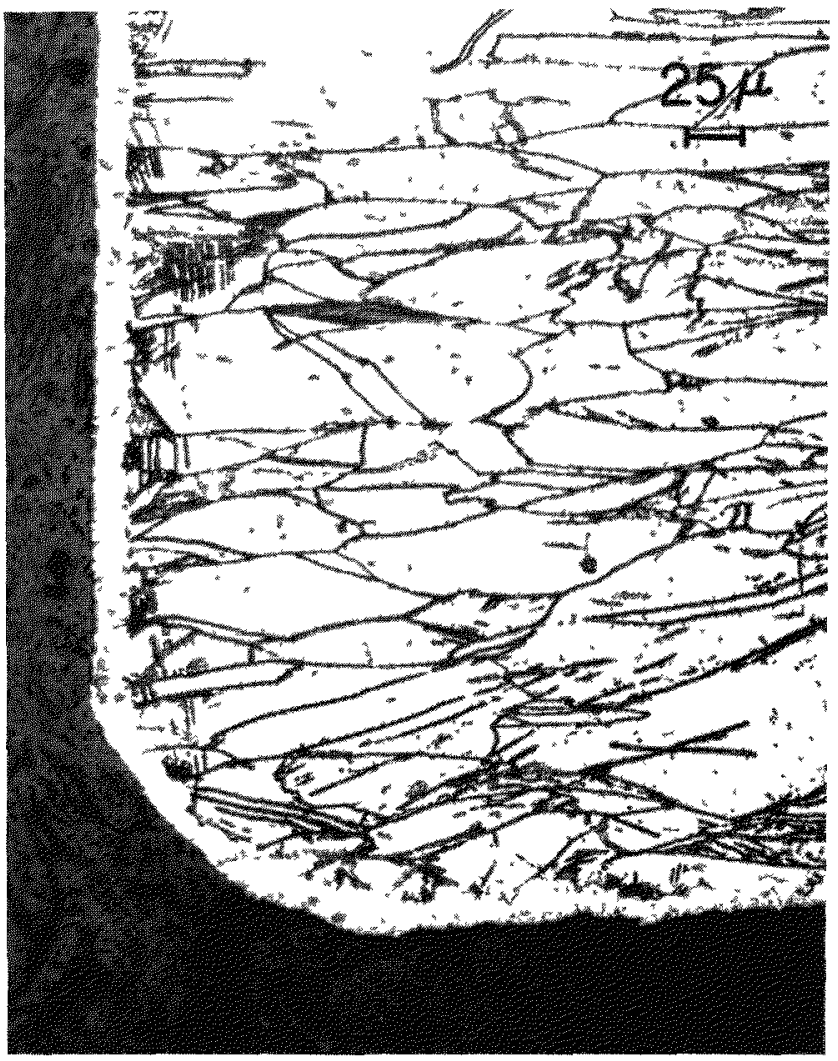

FIg. 2. Ferritic Laver Formation on Cold-worked Coupon after 400 Hours in Sodium at $1330^{\circ} \mathrm{F}$.

layer may act as a nucleation site for damage mechanisms. The layer often extends along grain boundaries to penetration depths greater than the average bulk penetration. We may speculate that such penetrations may act as notehes to promote thermal fatigue and act as stress concentration sites during strain cycling. Although the austenitic stainless steels are not particularly notch sensitive in their annealed state, they may become increasingly so with irradiation due to severe embrittlement. Differences in thermal expansion characteristics of the ferritic layer $\left(6.5 \times 10^{-6} \mathrm{in} . / \mathrm{in} .{ }^{\circ} \mathrm{F}\right)$ to that of the austenitic matrix $\left(10.6 \times 10^{-6}\right.$ in. $\left./ \mathrm{in} .{ }^{\circ} \mathrm{F}\right)$ also suggest a possible mechanism for initiating localized structural damage. 


\section{A.2. Elemental Absorption}

The absorption of carbon by stainless steel has been the subject of several investigations. ${ }^{(2-4,7)}$ At temperatures above $1000^{\circ} \mathrm{F}$, austenitic stainless steel has been shown to carburize readily if carbon is present in the sodium stream. Carbon has been found in as-received sodium as graphite particles which have sloughed off from electrodes in the electrochemical process of sodium production. Since carbon is slightly soluble in sodium, the graphite probably dissolves slowly and either forms as yet unidentified compounds or enters into an "active" state which readily engages in the carburization of stainless steel. Other carbon sources stem from organic materials which may accidentally be introduced into the sodium and from cover gas containing carbon monoxide or dioxide. The availability of a major carburization source is inherent if bimetallic systems are considered which combine austenitic and unstabilized ferritic steels. The ferritic alloy $21 / 4$ Cr-1 Mo has been observed ${ }^{1,2}$, 4, 6) to donate "active" carbon to the sodium coolant until the carbon content in the ferritic alloy is reduced below analytically detectable limits. Carburization of the austenitic material in this case is dependent on the total quantity of unstabilized ferritic alloy in the system. Carbide stabilizers such as niobium and titanium have proven effective in reducing or eliminating this problem, as was demonstrated with $5 \mathrm{Cr}-1 / 2 \mathrm{Mo}^{-T i(1,2)}$ and the German alloy $10 \mathrm{Cr} \mathrm{Mo} \mathrm{Nb} 910,{ }^{(4)}$ a niobium-stabilized 21/4 Cr1 Mo ferritic.

Prior work at General Electric on general carbon movement has been reported by Plumlee. ${ }^{(2)}$ This report will cover more recent work comparing the carburization of types $304,316 \mathrm{~L}, 316$, and 347 stainless steels and Incoloy 800 at $1050^{\circ} \mathrm{F}$ in an isothermal pumped sodium loop. Tubular cladding samples were exposed for periods up to $700 \mathrm{hr}$ in carbon-contaminated sodium. The source of carbon contamination was a coil of carburized plain carbon steel placed in the sodium stream. Thin $(\sim 0.001$ in. $)$ foils of type 304 stainless steel were also suspended in the sodium stream so that a more accurate estimate of the surface carbon composition of the thicker tubular samples could be obtained. The tubes were subsequently burst-tested at room temperature, metallographically examined, and analyzed for gross carbon content. The carburization results (see Fig. 3) were similar for types $304,316 \mathrm{~L}$, and 316 , whereas type 347 showed pick-up response. Incoloy-800, with its higher nickel content, showed significantly lower rates of carburization than did the other alloys. (The lower carbon levels at $700 \mathrm{hr}$ were associated with exhaustion of the carbon source.) In the room-tem- perature burst tests (see Fig. 4), Incoloy 800 exhibited the maximum increase in strengths, with the $\mathbf{3 0 0}$ series alloys showing slightly smaller gains.

Analysis of the combined results shows a general relationship between carbon pickup, room-temperature burst strength, and the distribution of the absorbed carbon in the microstructure. The highernickel alloy Incoloy 800 showed the least amount of carbon pickup, but the largest increase in hoop strength. The absorbed carbon in Incoloy 800 (see Fig. 5) is heavily concentrated at the surface, the area of maximum fiber stress, thereby magnifying the strengthening effect of the carbon. This concentration of carbon also had a minimal effect on the fracture strain of Incoloy 800, as shown in Table 3. This small reduction in ductility is associated with the small percentage of the cross-sectional area affected by the carbon.

The 300 series of stainless steel alloys absorbed larger quantities of carbon, but these were distributed to greater depths (see Fig. 6) than in Incoloy 800. Therefore, a larger percentage of the cross-sectional area was affected, resulting in lower fracture strains and smaller increases in burst strength.

Other trace impurity elements, such as hydrogen and nitrogen, are commonly present in sodium systems and may cause effects similar to those associated with carbon. Only isolated instances of this type have been reported, and there has not yet been a requirement for a systematic effort to evaluate the importance of such effects.

\section{B. Localized Corrosion}

General corrosion or mass-transfer phenomena have been the subject of intensive investigations. In general, these studies have served to point out the significance of several factors:

(1) For temperatures of $1200^{\circ} \mathrm{F}$ or lower and oxygen levels $\leq 10 \mathrm{ppm}$, general corrosion* per se of austenitic stainless steels does not appear to be of sufficient magnitude to warrant concern for the system integrity for anticipated lifetimes of the order of 20 yr or more.

(2) The overall performance of the test material appears to be dependent on: its location within the system, the test temperature, the temperature distribution, the coolant chemistry, and the system geometry and flow characteristics.

(3) The test parameters which have exerted a strong influence on the alloy behavior in test loops

\footnotetext{
* Jefined as a uniform reaction between a material and the corrosive environment, resulting in a reaction product which does not contribute to the structural integrity of the material.
} 


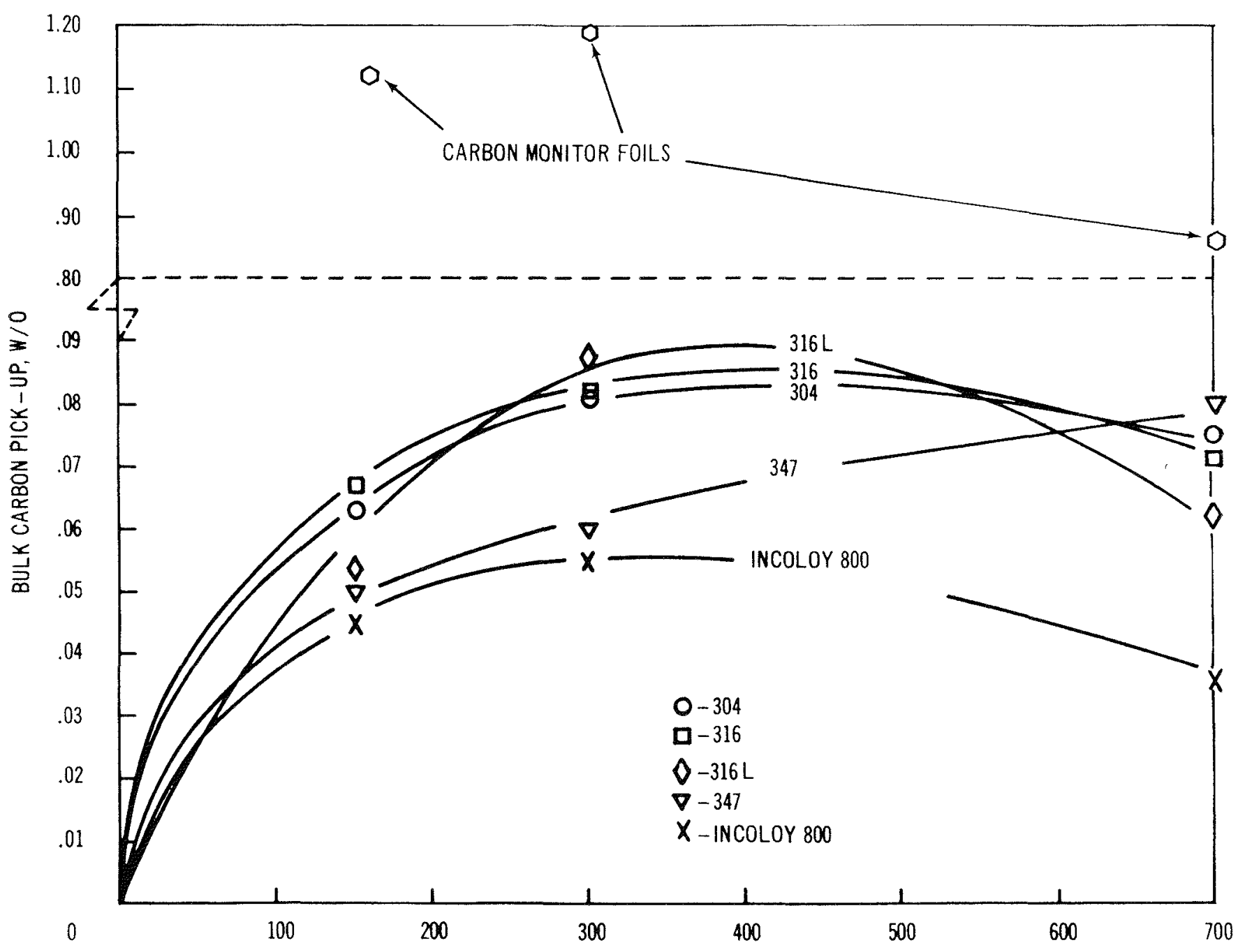

EXPOSURE TIME, HOURS

Fia. 3. Increase in Carbon Level of Selected Austenitic Alloys after Exposure to Contaminated Sodium.

have often been representative of operating extremes or casualty conditions in a large plant.

Recent observations have added another factor which is common in other systems, but which has not been systematically explored in sodium, namely, localized attack. Localized effects can influence the performance of an alloy in the sodium environment in two ways: (a) structural modifications associated with the selective elemental leaching and surface impurity concentrations (discussed previously), and (b) localized corrosion effects occurring under conditions for which general corrosion is small.

Recent examinations of the piping from the sodium mass-transfer test loops have revealed the occurrence of localized intergranular penetrations in addition to general corrosion effects. This attack was first observed in an austenitic stainless steel type 316 which operated at $1200^{\circ} \mathrm{F}$ (maximum) for approximately $30,000 \mathrm{hr}$. The grain-boundary penetrations, to a maximum depth of $0.007 \mathrm{in}$., were invariably found only in the piping between the last hot-leg test section and the first cooler (neither of which exhibited localized attack). The extent of penetration observed would be of little concern in thick-walled regions of a sodium system (unless also subjected to strain cycling). For thin members, such as fuel cladding, such penetration would define a scrious operating limit. There is, accordingly, considerable interest in determining the nature of the attack and the parameters which control or influence it.

\section{B.1. Experimental Observations}

A section of loop piping from the attacked region was examined metallographically and revealed ex- 


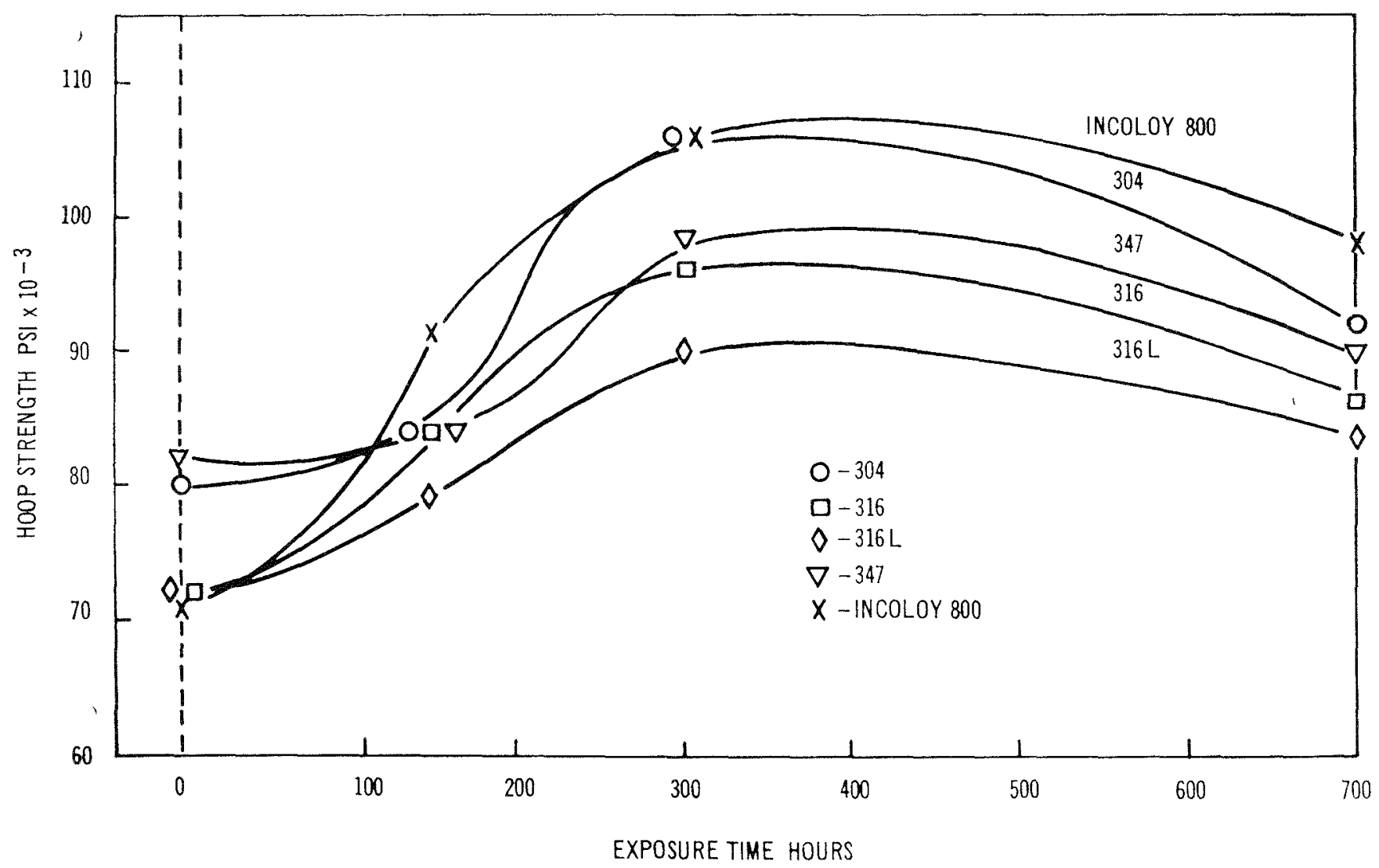

FIa 4 Hoop Strength of Selected Austenitic Alloys after Exposure to Carbon-contammated Sodium.

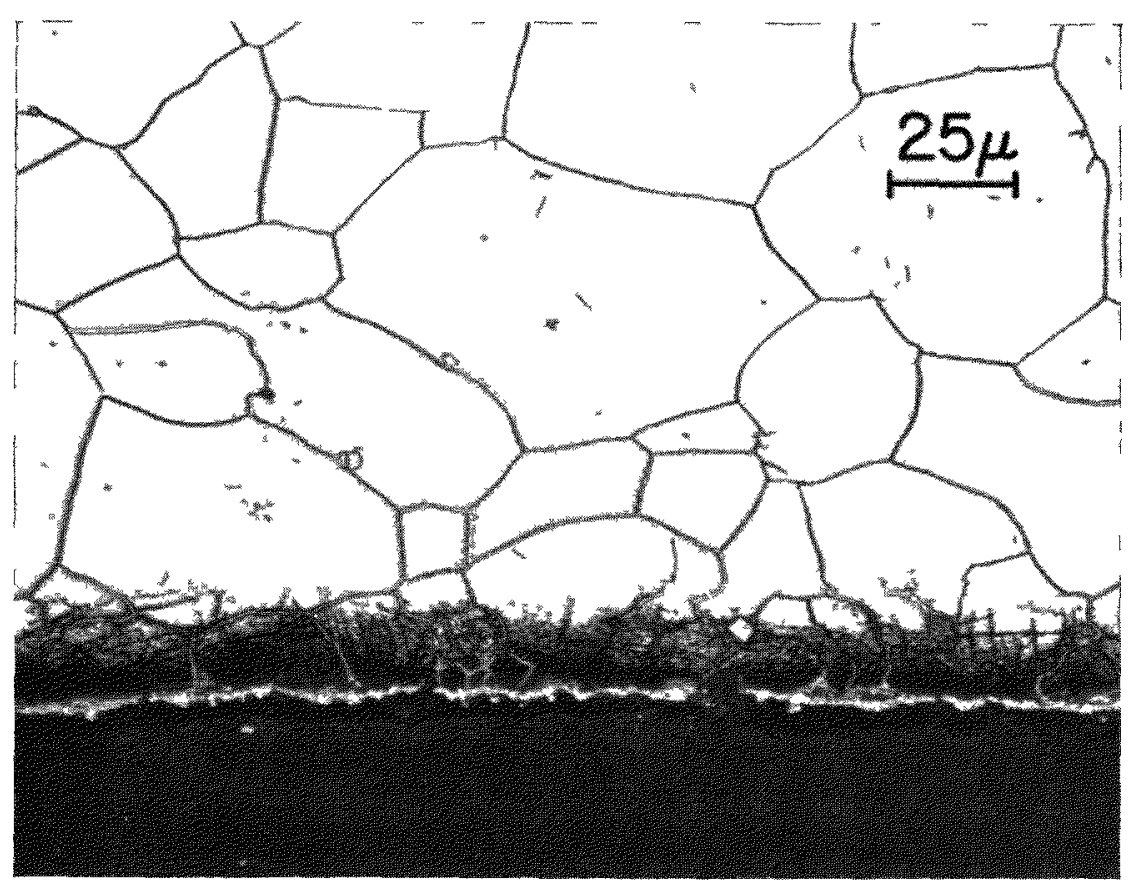

FIG. 5. Carburized Surface of Incoloy 800 after 700 Hours in Carbon-contaminated Sodium at $1050^{\circ}$ F. 
tensive intergranular penetration (see Fig. 7). Since the attack was characteristically intergranular, the differences in topology and precipitate morphology between the attacked and unattacked grain boundaries were examined. This was accomplished by fracturing a section of the pipe at liquid nitrogen temperature to produce a predominantly brittle (intergranular) rupture in the unattacked bulk of the pipe section.

A fractographic analysis, utilizing both two-step
TABLE 3. Fracture Strain of Test Alloys after 300 me Exposure

\begin{tabular}{c|c|c}
\hline \multirow{2}{*}{ Alloy } & \multicolumn{2}{|c}{ Strain } \\
\cline { 2 - 3 } & Pre-exposure, \% & Post-exposure, \% \\
\hline Incoloy 800 & 17 & 14 \\
Type 304 & 40 & 17 \\
Type 316 & 38 & 19 \\
Type 347 & 25 & 9 \\
\hline
\end{tabular}

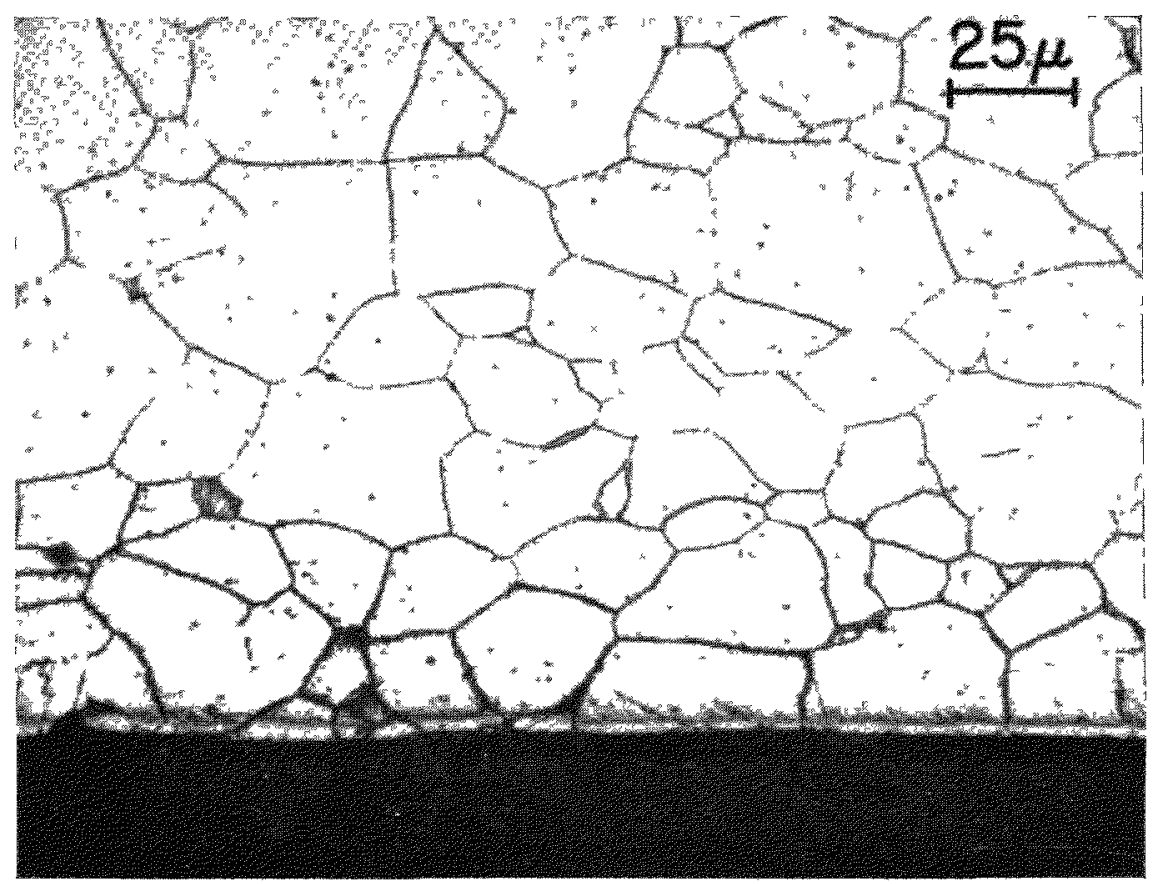

Fig. 6. Carburized Surface of Type 316 Stainless Steel after 700 Hours in Carbon-contaminated Sodium at $1050^{\circ}$ F.

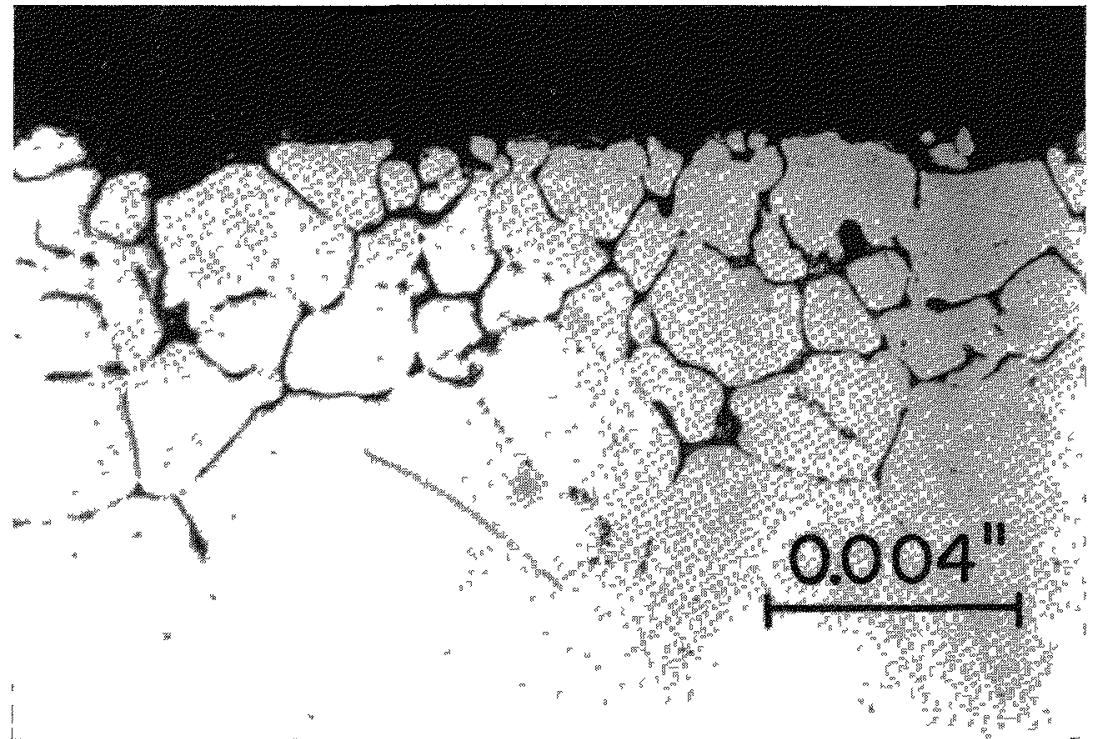

FIG. 7. Intergranular Penetration in Type 316 Stainless Steel. 
carbon and carbon-extraction replicas, was carried out and yielded the following observations

(a) All replicas of the fracture surface away from the attacked area (see Figures 8 and 9 ) were typical of intergranular fracture and showed severe sensitization in the form of continuous sheets of minute, angular partıcles whıch were identıfied by clectron diffraction as $\mathrm{M}_{23} \mathrm{C}_{6}$

(b) Examination of the replicas of the attacked intergranular surfaces (see F1g 10) revealed 'steps' or "serrations" which were not present on the original unexposed surface and are typical of a heavily etched sulface This suggests that the corrosive environment preferentially attacked and exposed certain crystallographic facets of the grains

(c) The distribution of carbides on the attacked surfaces was substantially different than on the unattacked surfaces The presence of large angular carbides indicated that the carbide particles themselves were not chemically attacked The smaller carbide partıcles were piobably dislodged from the surface during the fracture process after the carbidematilx bonding had been weakened and disıupted by the corrosive attack

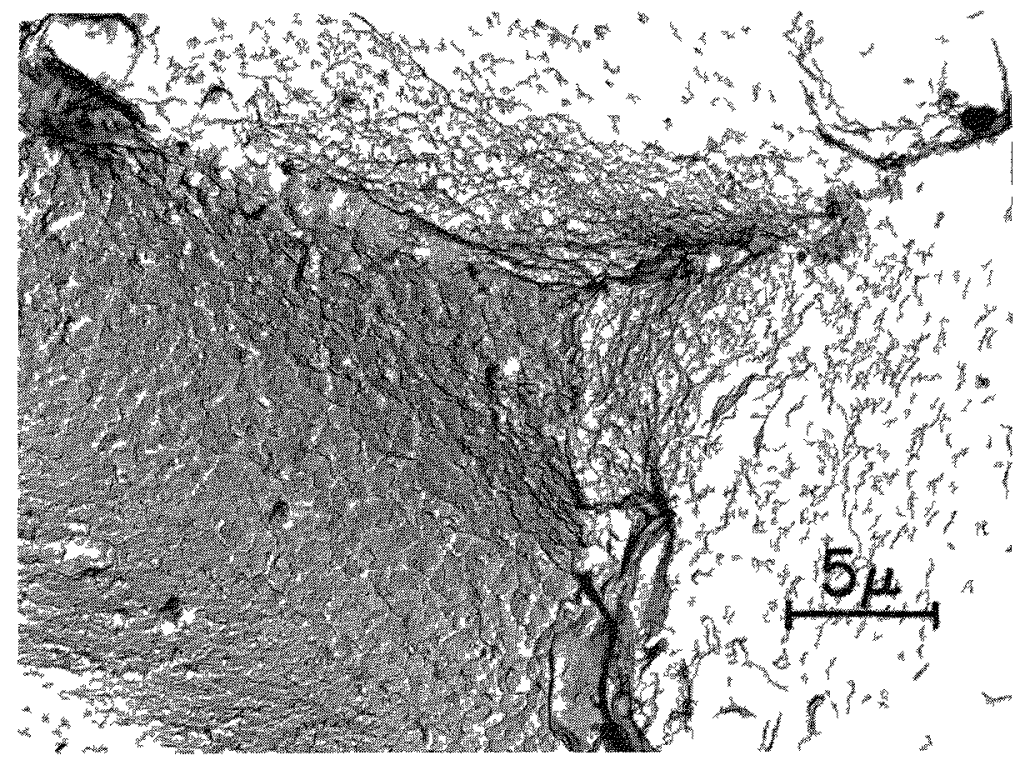

Fig 8 Transmission Electron Micrograph of Plastic carbon Replica of Intergranular Fracture Surface Away from Attacked Area, Showng Complete Gram boundar Coverage by Precipitation

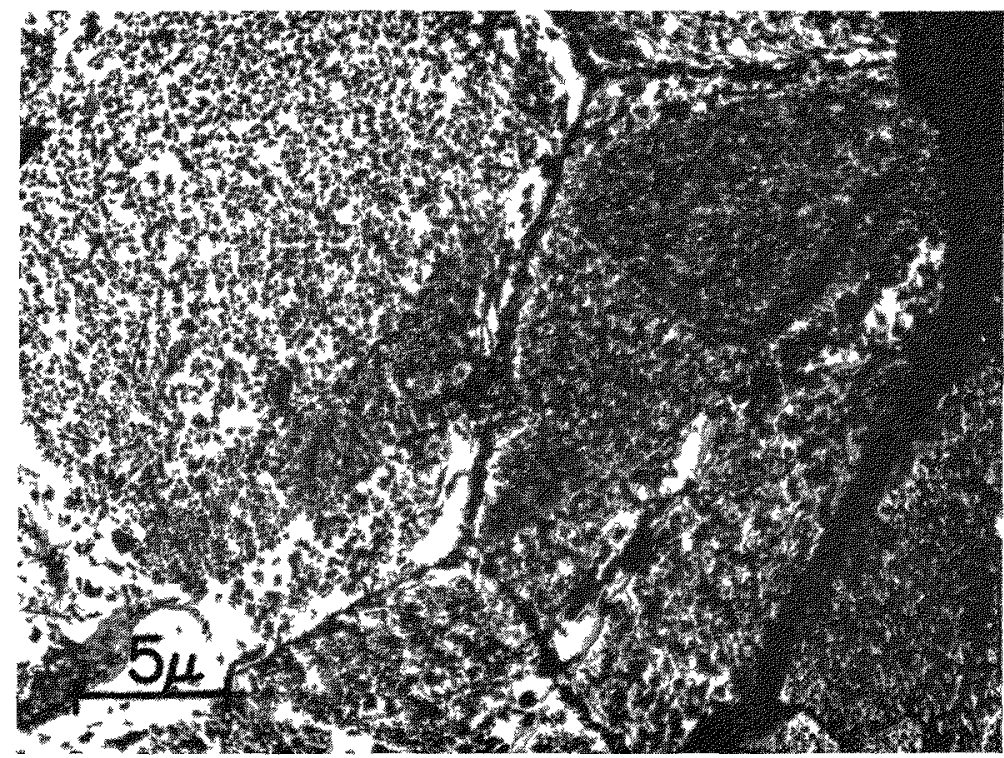

Fig 9 Transmission blectron Micrograph of Carbon extraction Replica of Intergranular Fracture Surface Anaj from Attacked Area, Showing Complete Sensitization 
(d) No positive evidence of any corrosion reaction product in the grain boundary could be established Particles of different cizes and shapes were observed (see Tigures 11 and 12) and examined by electron diffraction, but no positive identification could be made

\section{B 2 Pertinent Considerations}

Several facets of the observed penctration are smimiar in appearance and character to data reported from bdsic corrosion studies ${ }^{13}$ 14) and applied problem areas such as failure analysis (15) In a theoretical work ${ }^{(16)}$ on the structural mechanisms associated with intergranula corrosion, two enterna were proposed for this mode of attack

(1) the existence of a continuous structural path along which the chemical redctivity is high relative to the surrounding matrix,

(2) the cxistence of a normal stress across the path of high chcmical iedctivity

B 21 Structural (onsiderations The most obvious -tructural conı1deration is that of chromium depletion in the allor lattree adjacent to the carbide precipitate, providing a path of reduced corrosion resistance Experimental measurcments of the attack rate suggcat a typical value of $17 \mathrm{mils} / \mathrm{yl}^{(5)}$ determined over

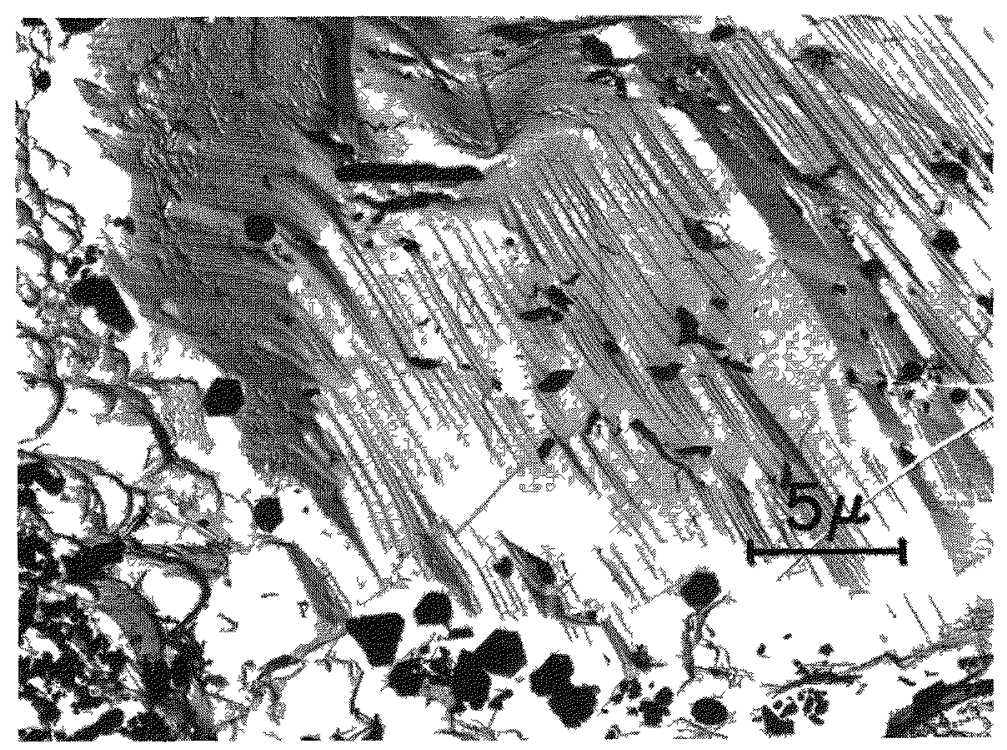

Fig 10 Transmisson I lectron Micrograph of Carhon extraction Replic $a$ of Attacked Gram boundary Surface.

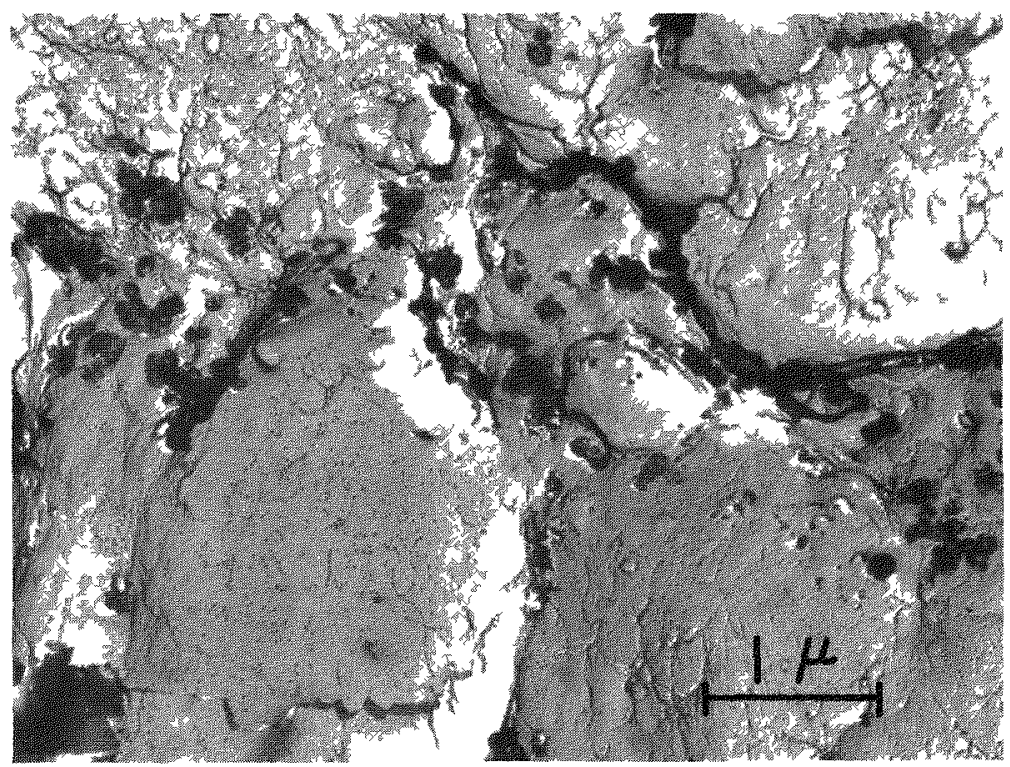

Fig 11 Transmission Electron Microgr zph of Aggregate of Płntic'cs Removed from the Attacked Gian boundary Surfaces and Thought to be Cor rosion. Products 


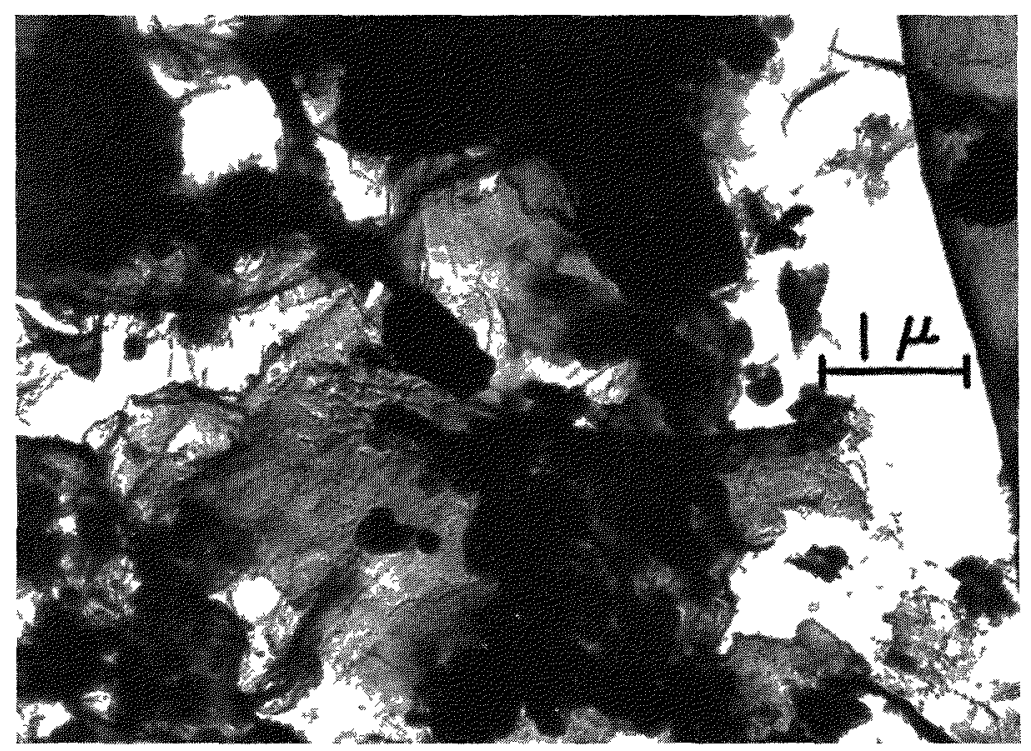

Fig. 12. Transmission Electron Micrograph of Polycrystalline Particles Removed from the Attacked Grain-boundary Surfaces and Thought to be Corrosion Products.

a period of $3 \mathrm{yr}$. Although an initial depletion of chromium may exist at the metal-carbide interface, the diffusion of chromium orer long periods of time will certainly tend to reduce any concentration gradients [assuming a finite supply of insoluble carbon available for formation of $\left(\mathrm{Fe},(\mathrm{Cr})_{23} \mathrm{C}_{46}\right]$.

Electron microprobe studies of various alloy systems ${ }^{(17,18)}$ have indicated that, within the resolution capabilities of this technique, the region adjacent to an intergranular solute-rich precipitate was not solute depleted, especially at time and temperature conditions under which solute diffusion was significant $\left(T>1 / 2 T_{m}\right)$.

Several studies have been reported which discuss the morphology of carbides in austenitic steels and its influence on corrosion recistance. Stickler and Vinckier ${ }^{(19)}$ reported a direct correspondence between corrosion behavior and the morphology of the grainboundary carbide. Their data indicated that poor corrosion resistance was associated with sheets of connected small particles, and good corrosion resistance with isolated larger particles. The morphology and distribution of the precipitate were strongly dependent upon time and temperature.

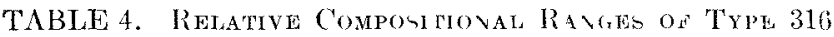
Matrix a $\backslash$ ) Compley ('akbine $\mathrm{ML}_{3} \mathrm{C}_{6}$

\begin{tabular}{c|c|c}
\hline Element & Matrix, w/o & $\mathrm{M}_{23} \mathrm{C}_{6}, \mathrm{w} / \mathrm{o}$ \\
\hline $\mathrm{Cr}$ & $16-18$ & $60-70$ \\
$\mathrm{Fe}$ & $68-70$ & $15-20$ \\
$\mathrm{Ni}$ & 1012 & 46 \\
$\mathrm{Mo}$ & 23 & $10-15$ \\
\hline
\end{tabular}

Corroborating data were presented by Bendure et al. (20) in a study of the carbide-precipitation characteristics of austenitic stainless steels. Their data also showed that susceptibility to intergranular attack was much more pronounced after sensitization at $1250^{\circ} \mathrm{F}$ (sheets of interconnected small particles) than after similar exposure at $1500^{\circ} \mathrm{F}$ (large, widely spaced precipitates).

Further work by Stickler and Vinckier ${ }^{(21)}$ indicated that corrosion attack of sensitized austenitic stainless steel in the Strauss $\left(\mathrm{CuSO}_{4}-\mathrm{H}_{2} \mathrm{SO}_{4}\right)$ solution proceeded by a dissolution of the matrix along a narrow region adjacent to the intergranular carbide particles, which remained essentially unattacked. These experimental data suggosted that an electrochemical process was operative between the noble carbide and the less-noble matrix. However, exposure of the same steel in the Huey solution $\left(65 \% \mathrm{HNO}_{3}\right)$ resulted in intergranular corrosion which advanced by atttacking and dissolying the grain-boundary carbides. Therefore, it is evident that the corrosive environment is as important a factor in determining the corrosion mechanism as the structural characteristics of the steel.

The principal cation in the complex carbide $\mathrm{M}_{23} \mathrm{C}_{6}$ is $\mathrm{Cr}$, but small amounts of $\mathrm{Fe}, \mathrm{Ni}$, and $\mathrm{Mo}$ can be taken into solution. When compared with the nominal composition of the 316 alloy matrix (see Table $4^{(22)}$ ), the complex carbide is rich in alloying constituents which would make it more noble than the adjacent matrix.

The conclusions reached in these experimental studies indicated that intergranular corrosion of a sensi- 
tized austenitic stainless steel was associated with an electrochemical reaction between the noble carbide and less-noble matrix, and was strongly dependent upon the morphology and distribution of the intergranular precipitates and the chemical nature of the corrosive environment.

B.2.2 Considerations of Applied Stress. Since the observed attack appears to be a selective and localized phenomenon, many variables within the material structure may assume importance, such as orientation effects, coherent precipitates, stacking faults, and other dislocation barriers. Therefore, relatively low macrostresses may induce locally high microstresses to initiate and propagate cracking.

As postulated by Robertson and Tetelman, ${ }^{(16)}$ a stress normal to the chemically reactive path is required to satisfy fracture criteria. Stress also provides strain energy at the metal-carbide interface, increasing the chemical reactivity therc. A certain degree of strain energy is also present due to the lattice mismatch at the metal-carbide interface. The alloy matrix has a face-centered cubic structure with a lattice parameter of $3.584 \AA$, while the carbide has a modified face-centered cubic structure with a lattice parameter of $10.64 \AA$, approximately 3 times that of the matrix.

The opportunity for substantial stresses to develop in the loop piping was present during loop startup. During the initial stages of the preheat cycle, a circumferential temperature gradient of $260^{\circ} \mathrm{F}$ (maximum) was impressed upon the $1 / 2$-in. Schedule 40 loop piping. A thermal-stress analysis revealed that the average stress on the ID surface of the piping was of the order of 35,000 psi. As the preheat time increased, the temperature of the piping approached an equilibrium level of $700^{\circ} \mathrm{F}$, reducing the thermal stresses to an insignificant level. During loop operation at $1200^{\circ} \mathrm{F}$, the temperature differential across the piping wall was approximately $10-15^{\circ} \mathrm{F}$, producing minor thermal stresses.

B.2.3 Considerations of Corrosive Environment. A study of the procedures of loop operation indicated that during loop shutdown, the internal piping surfaces, coated with a residual sodium film, were subjected to limited atmospheric contamination. Under these conditions the sodium film quickly reacts to form $\mathrm{Na}_{2} \mathrm{O}$ and $\mathrm{NaOH}$. The combination of the highly corrosive film, the high thermal stresses, and increasing temperature during the preheat cycle presented an environmental condition favorable for corrosive attack.

Attack of the type observed in the loops had been noticed previously ${ }^{(23)}$ under two slightly different sets of circumstances: (a) When a sodium system has sprung a leak, and sodium has dripped out, solidified on the outside of the steel, and subsequently undergone atmospheric oxidation, inspection of the leak region frequently discloses intergranular attack.

(b) When corrosion tabs of steel samples have been withdrawn from a sodium system and stored in a supposedly inert atmosphere without washing or dissolving away the surface film of sodium, air in-leakage in time produces a white layer of $\mathrm{Na}_{2} \mathrm{O}$ on the sample, beneath which intergranular penetration is observed upon subsequent examination.

Several experimenters ${ }^{(13-15)}$ have studied the corrosion of stainless steels in caustic solutions at temperatures up to $400^{\circ} \mathrm{C}$. Their data show that caustic solutions will produce intergranular corrosion in both unsensitized and stabilized austenitic stainless steels. The magnitude of the attack in caustic solutions was dependent upon and increased with increasing solution concentration, applied stress, and test temperature.

Snowden ${ }^{(14)}$ also pointed out several other interesting aspects of caustic corrosion which have direct application to the analysis of the present problem. His data showed a difference in the appearance of the attack as a function of solution strength. Oversaturated solutions tended to produce cracks more slowly, and with a broader and stubbier appearance, than the more dilute solutions, which usually produced a finebranched crack. Further tests with $\mathrm{NaOH}$ at high concentrations near its melting point revealed mixed inter- and transgranular cracking.

Snowden also evaluated the effect of low $(0.03 \%)$ and higher $(0.07-0.11 \%)$ carbon levels in stainless steels under similar corrosive conditions. The low-carbon alloy exhibited transgranular cracking while the higher-carbon alloys revealed intergranular cracking. This difference in behavior was attributed to grainboundary segregation of the carbon atoms. This observation is in direct agreement with the later results of several workers ${ }^{(19-21)}$ who showed the direct correlation between corrosion behavior and the morphology of the grain-boundary carbide.

\section{B.3 Discussion}

Review of the published data indicates that the observed loop penetrations are very similar in character to those observed in basic corrosion studies and applied problems such as failure analysis. These data show that intergranular attack can occur in unstressed metal beneath a layer of $\mathrm{Na}_{2} \mathrm{O}$ at room temperature and also in dilute to medium-strength solutions of $\mathrm{NaOH}$ at temperatures of $250-300^{\circ} \mathrm{C}$ in stressed coupons. Comparison of these and comple- 
mentary data with the observed attack suggests: (1) that the intergranular penetration of the loop piping was associated with the presence of $\mathrm{Na}_{2} \mathrm{O}$ and $\mathrm{NaOH}$, (2) progressed through an electrochemical-mechanical mechanism, and (3) occurred during the preheat cycle of loop startup. The first two suggestions are based upon the close similarity of published data and metallurgical observations of the loop piping. The same type of cracking has been reliably reproduced under controlled environmental conditions similar to those which can be reasonably postulated as present on the internal surfaces of the piping. The third suggestion is based upon an examination of loop-operating procedures. Only during loop startup can substantial thermal stresses and a concentrated caustic environment be present. When the flowing sodium is introduced into the system, the pipe walls become essentially isothermal, and any $\mathrm{Na}_{2} \mathrm{O}-\mathrm{NaOH}$ layer on the wall surface is rapidly washed away or taken into solution. Therefore, progression of the attack during high-tcmperature operation is unlikely. It would then appear that the penetration occurred in steps with each shutdown-startup period representing an incremental advance.

\section{FUEL-CLAD INTERFACE REAC'TIONS}

Modifications in the structural characteristics of the cladding may occur by a number of possible fuel-clad, clad-coolant, or irradiation-induced reactions, most of which tend to reduce the effective hightemperature strength of the cladding, either by chemical thinning or embrittlement.

The work reported here is a compilation of recent observations from several irradiation tests in which reactions at the fucl-clad interface were observed.

\section{A. Examination of a High-burnup Fuel Pin}

\section{A.1. General}

A test capsule containing ( $\mathrm{Pu}, \mathrm{C}) \mathrm{O}_{2}$ fuel was fabricated to evaluate the in-pile swelling behavior of mixed oxide fuels under typical fast reactor operating

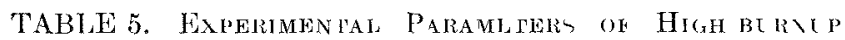
FT EL PIV

\begin{tabular}{|c|c|}
\hline $\mathrm{Pu}\left(\mathbf{P u}_{\mathrm{u}}+\mathrm{I}^{\top}\right)$ Ratio & 203 \\
\hline Storkhometry $(0 / \mathrm{M})$ & 204 \\
\hline Pellet Density (Avg) & $96 \% /$ Theoretioal \\
\hline Smeared I)ensity (Avg Cold) & $92 \%$ theoretical \\
\hline Cladding & 347 s4 \\
\hline Clad Thickness & $15 \mathrm{mils}$ \\
\hline Diametral (xap (Avg) & $19 \mathrm{muls}$ \\
\hline Environment & $\mathrm{NaK}$ \\
\hline Neutron Flux & $9 \times 10^{1} \cdot \mathrm{nV}$ \\
\hline Burnup $(\mathrm{MWl}) / \mathrm{T})$ & 70,000 \\
\hline Clad OI) Temperature $\left({ }^{\circ} \mathbf{F}\right)$ & 1,000 \\
\hline
\end{tabular}

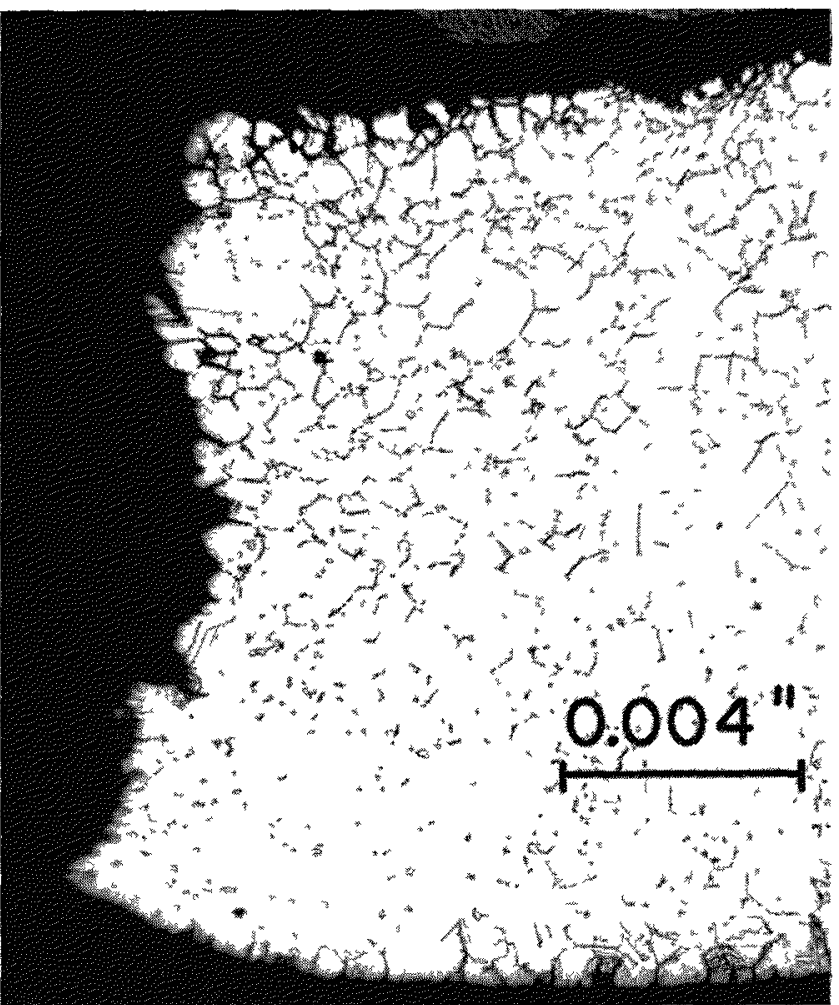

FIx. 13 Fanlure in Type 347 stamless siteel Cladding.

conditions. The fabrication and irradiation parameters are contained in Table 5.

Postirradiation examination ${ }^{(24)}$ of this pin revealed a longitudinal clad rupture immediately adjacent to a spacing wire attached to the outer surface of the cladding. Clad failure was attributed to a reduction in clad yreld strength due to local overheating in this region. Overheating is believed to have been initiated by incomplete sodium bonding of the test capsule, or growth of a gas bubble in the NaK annulus. Metallographic examination of the failed region revcaled considerable necking at the OD surface (see Fig. 13), suggestrve of ductile fracture. The remainder of the cladding faled intergranularly.

Metallography of the ID surface of the cladding near the fallure showed a region consisting of heavy gram-boundary precipitation extending to a depth of approximately $3 \mathrm{mlls}$. Although this surface was highly irregular due to the breakaway of precipitateenreloped surface grains (see Fig. 14), no gross chemical attack was observed at any point along the fuelclad interface. The OI) surface of the cladding showed no abnormal exposure effects.

\section{A.2 Analysis of Precipitates}

Precipitates were extracted from four regions along the clad cross section: (1) the ID region of high- 
density grain-boundary precipitation, (2) the region adjacent to and including the OD surface, (3) the central portion, and (4) the complete radial clad cross section. Electron-microseopic examination of the extraction replicas showed that the OD surface and central region of the cladding contained a random distribution of massive globular precipitates (see Fig. 15) characteristic of those found in a stabilized stainless steel. The ID surface, however, exhibited a continuous grain-boundary array of thin angular precipitates (see Fig. 16) as well as the globular forms.

Analysis of the bulk-extracted precipitates by $\mathrm{X}$-ray (Debye-Scherrer) diffraction revealed only one discrete phase: a face-centered cubic structure with a lattice parameter of $4.434 \pm 0.005 \AA$. This value agrees fairly well with that for $\mathrm{NbC}$, but suggests nonstoichiometry. Pearson ${ }^{(25)}$ reports that a lattice parameter of this value would match a composition of approximately $\mathrm{NbC}_{0.72}$. The possibility exists, however, that the phase contains nitrogen in solution, corresponding to a composition $\mathrm{Nb}(\mathrm{C}, \mathrm{N})$ which contains approximately $30 \mathrm{~m} / \mathrm{o}$ nitrogen. The fact that the primary constituent of the phase is niobium was substantiated by X-ray fluorescence analysis of the extracted precipitates, which identi-

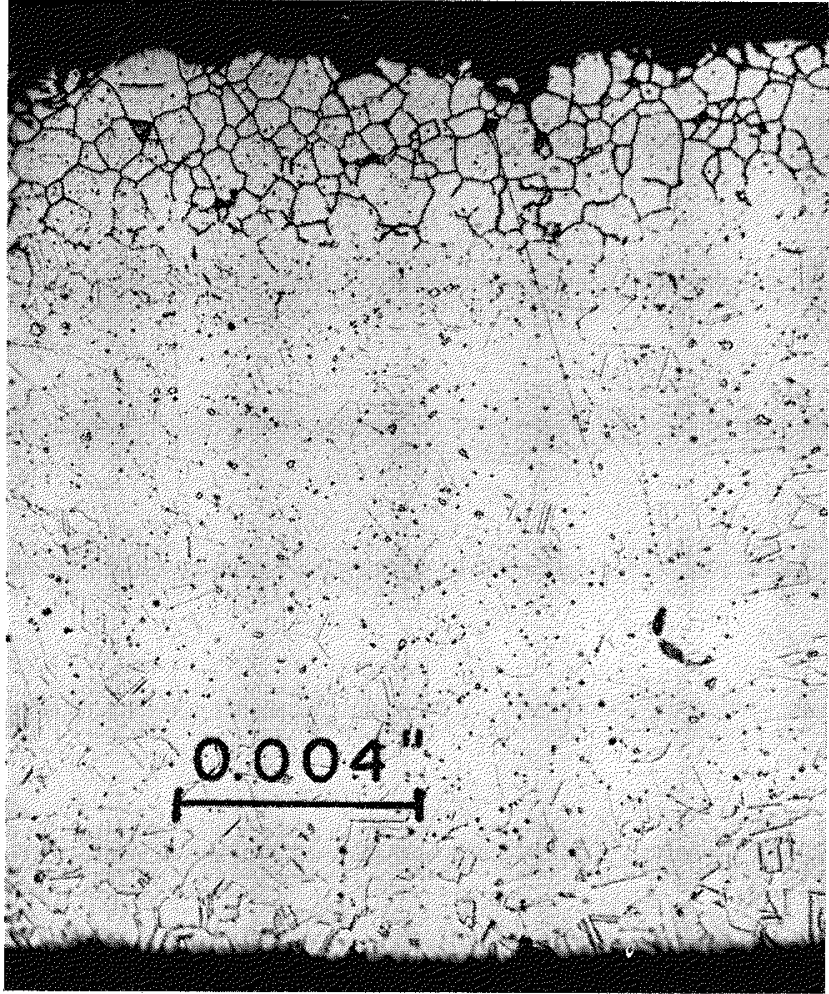

FIG. 14. Grain-boundary Precipitation, II) Surface of Type 347 Stainless Steel Cladding.

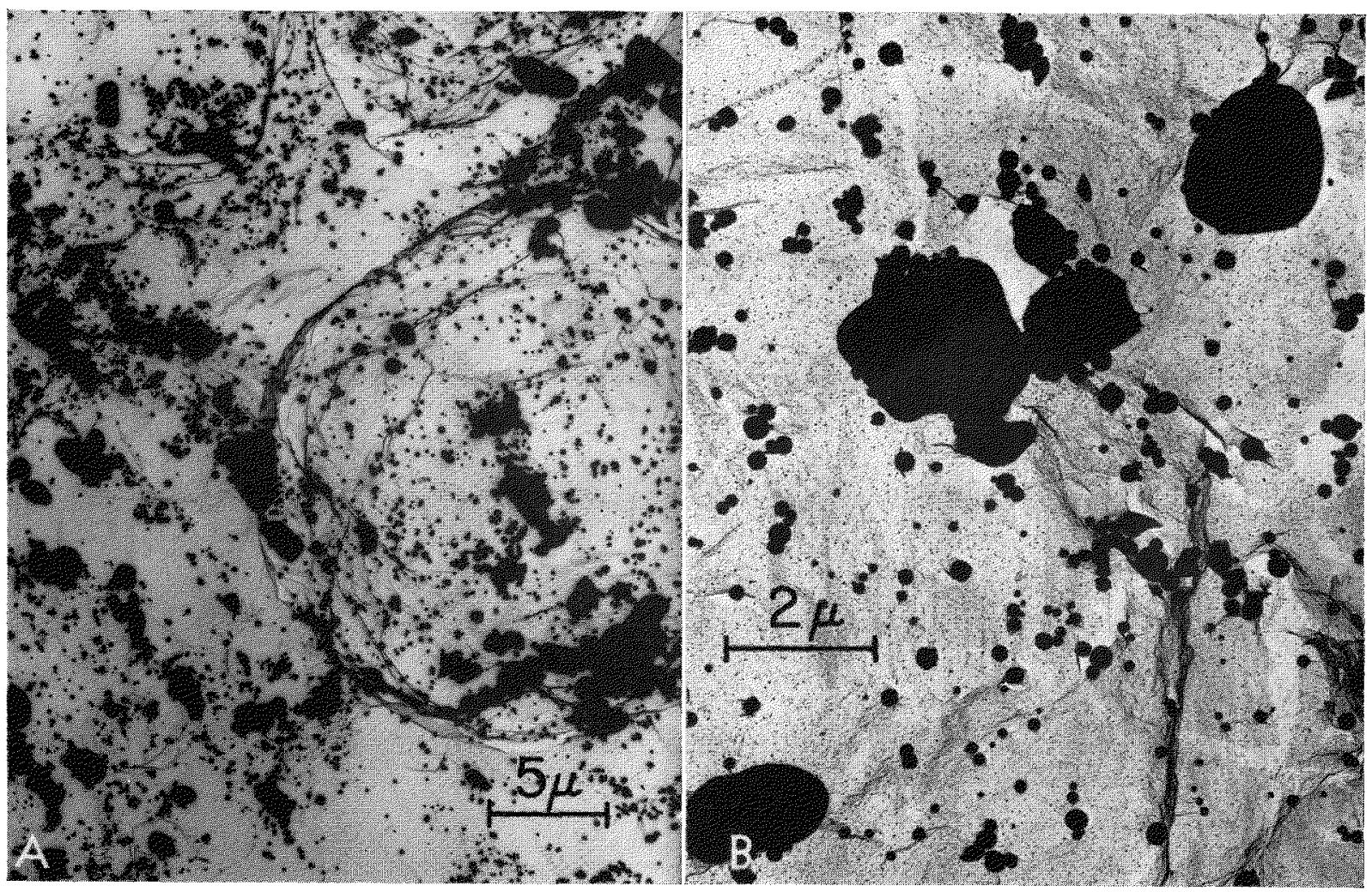

FIg. 15. Transmission Electron Micrographs of Carbon-extraction Replica Showing Carbides Characteristic of Midradial Region of Cladding. 


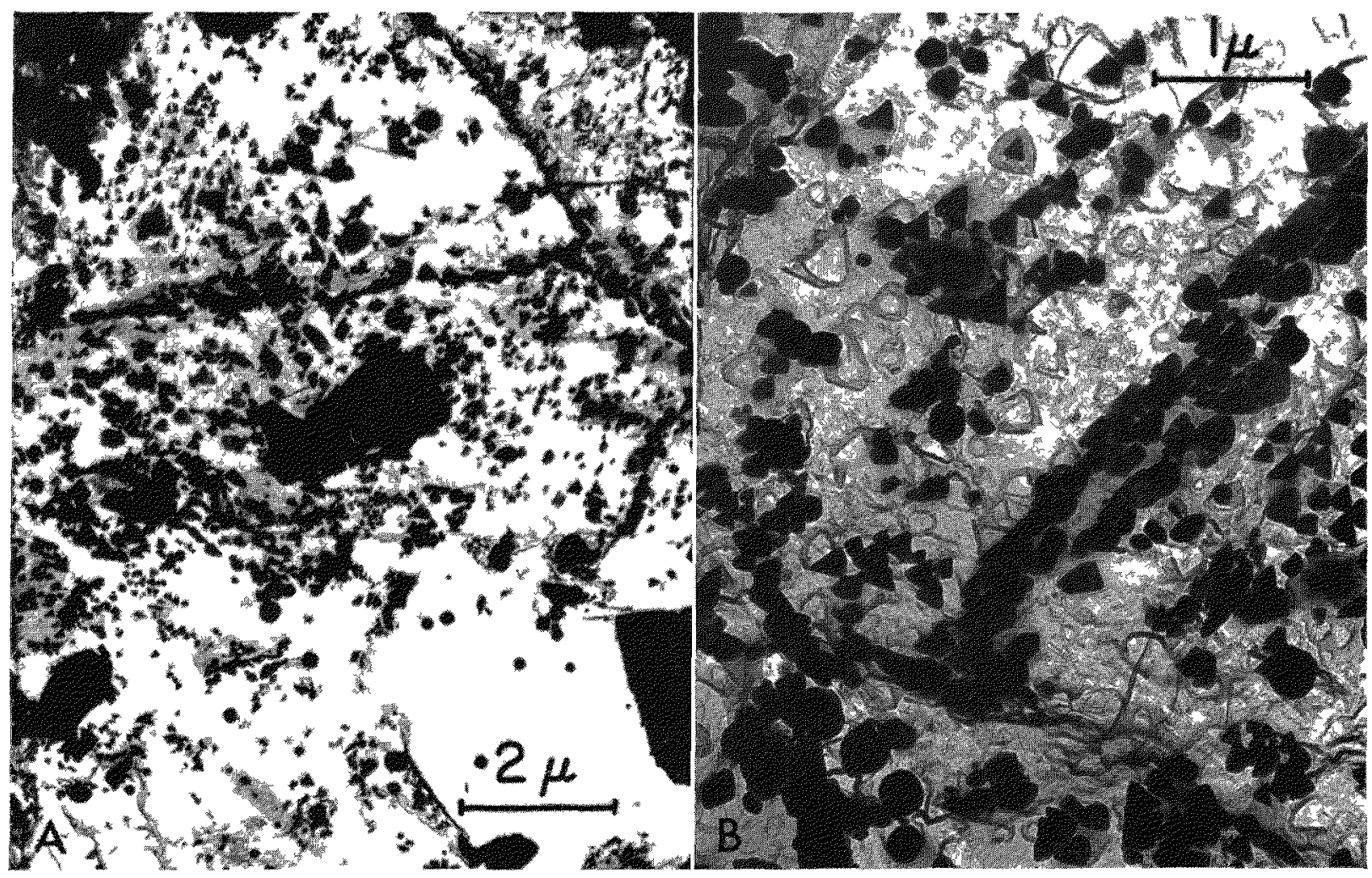

Hig 16 Tranımisuion Electron Micrographs of Carbon extraction Replica Showing Carbides (Characteristic of ID Region of Cladding

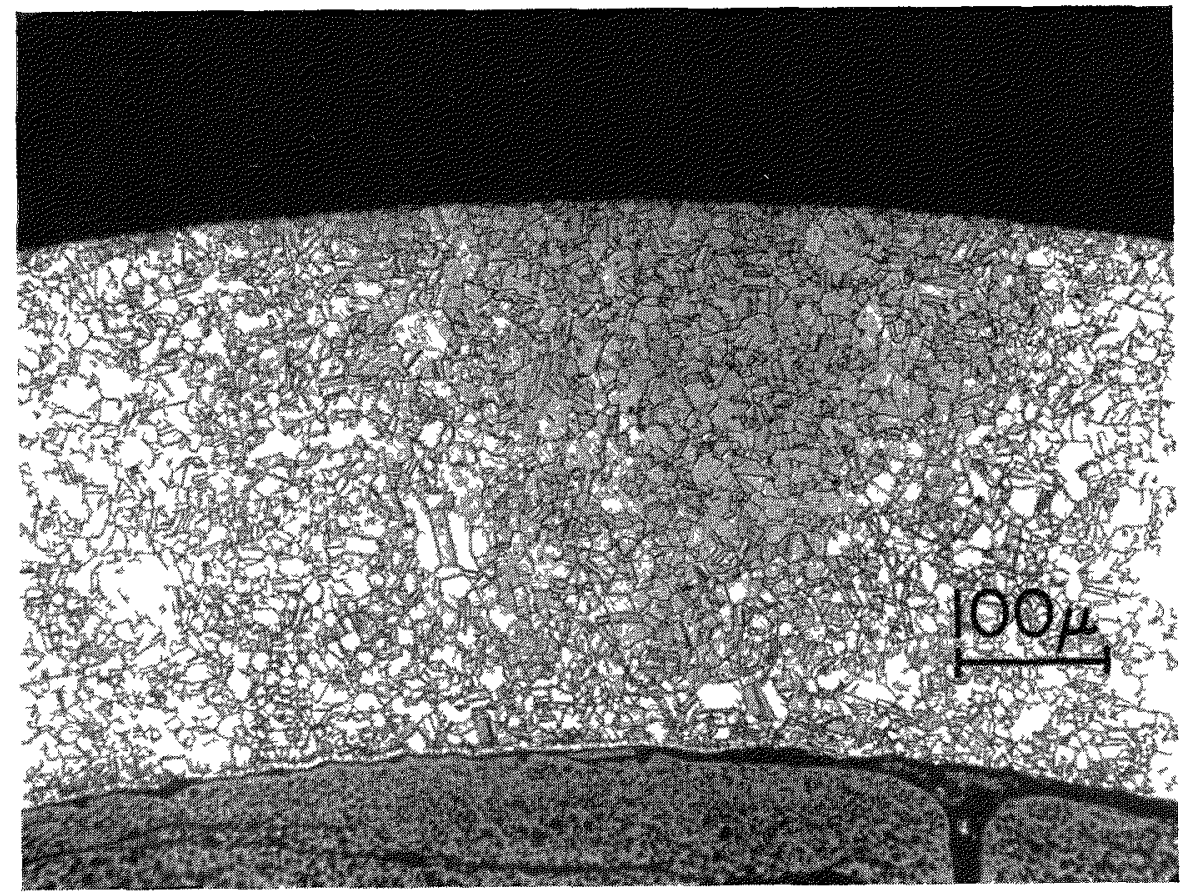

Frc 17 Fuel clad Interface Reaction after a Bunup of 77,400 MW1)/T 
fied nrobrum as the only malor element present $A$ weak trace of 1 on was aloo detected (X-1 ay fluorecence does not detect carbon or nitrogen).

Since the X-ray diffraction of the bulk extraction failed to show the presence of a second precipitate, an electron-diffraction analysis of the two precip1tates observed in the carbon-extraction replica from the clad ID surface was performed Although the large precipitates were too thick for electron transmission, the thin angular particles yolded good diffraction patterns, the spot spacings of which corre- spond to the eompound $\mathrm{II}_{-3} \mathrm{C}_{6}$, sinee thrs compound could not be remolved by X-ray diffraction of the precupitaten remoled by bulk extraction, it is reasonable to assume that a much larger volume of niobuum-base particles than $\mathrm{M}_{23} \mathrm{C}_{4}$ partieles was present in the fuel cladding $A$ subscquent $X$-ray diffraction analysis of an extraction icpliea of the ID surface revealed both phases

It was, therefore, coneluded that the majority of the $\mathrm{M}_{23} \mathrm{C}_{6}$ precipitate oceus in the narrow zone at the inside surface of the cladding and constitutes

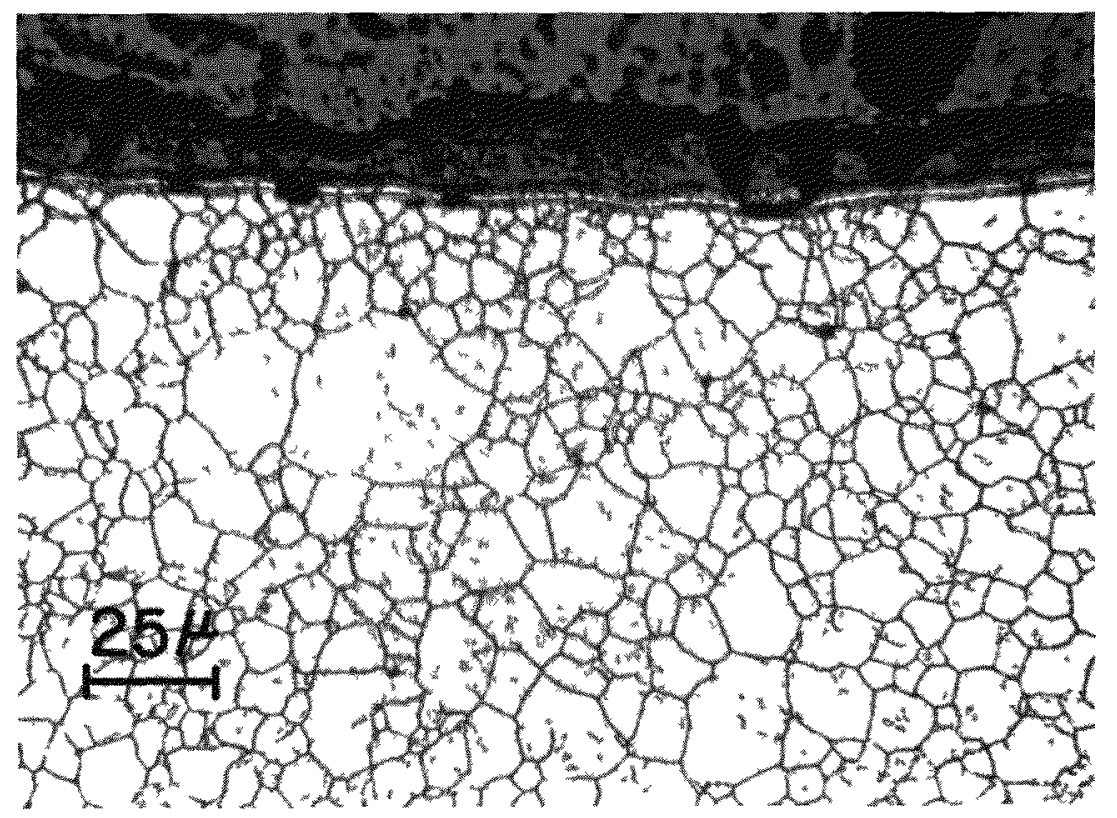

Fig 18 Reaction at Fuel clad Interface of a $\left(\mathbf{P u ~}^{\top}\right) \mathrm{O}_{2}$ Pin Irradiated to a Burnup of 28,000 MWD/T.
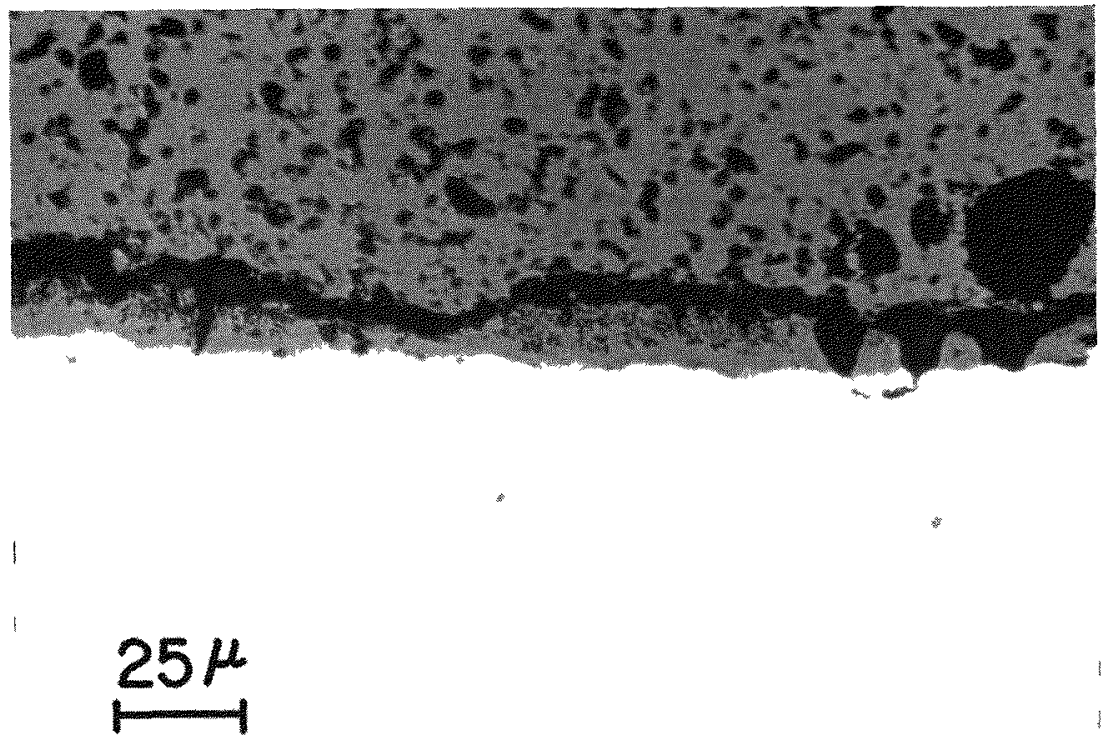

TiG 19 As polished Appearance of Fuel clad Reaction Shown in Figure 18. 

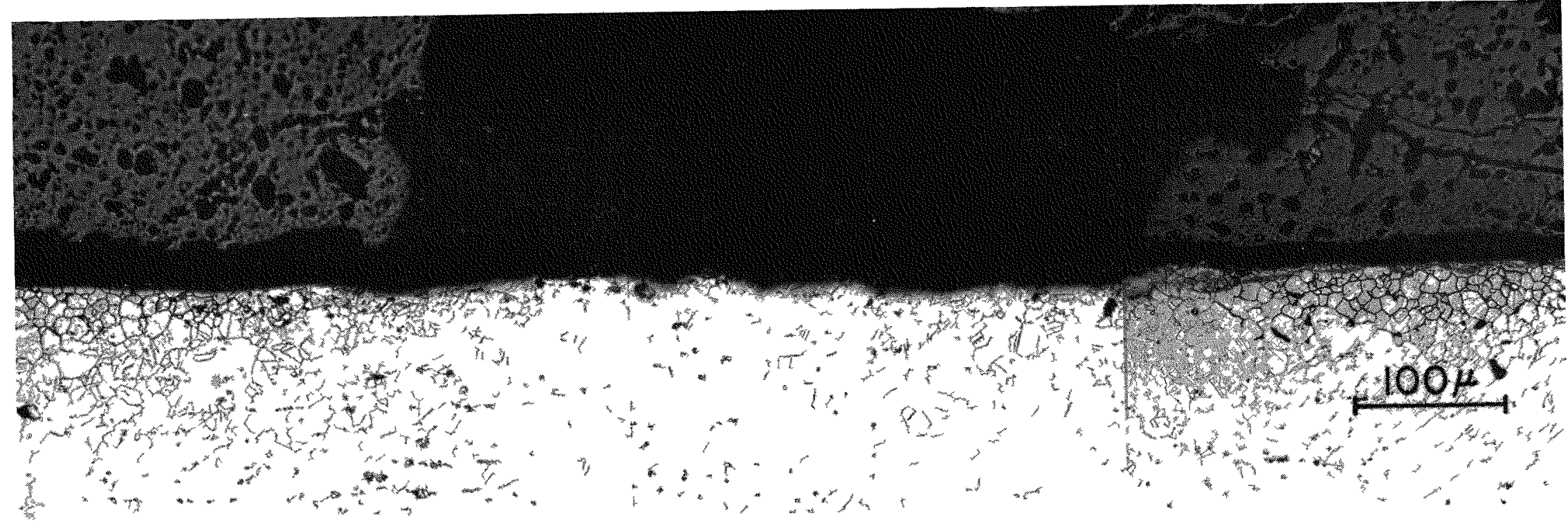

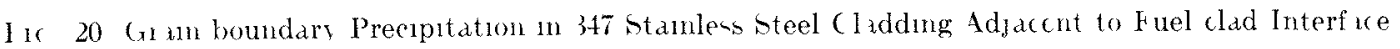


the heavy grain-boundary precipitate phase observed in optical metallography.

Precipitation effects of this type are essentially those which would be expected due to the thermal exposure alone, without regard to neutron exposure or compatibility considerations. However, the overall sharacteristies of the precipitation, including the well-defined boundary, suggest that the mixed oxide fuel is exerting an influence on the diffusion rates of alloy constituents and, therefore, the rate and characteristics of structural transformations.

The observation of a preferential buildup of carbides at or near the ID surface of the cladding suggests that a carbon gradient exists in the material. However, a microhardness survey, prompted by the fact that an increase in carbon content in most steels is reflected in increased hardness, failed to reveal any substantial gradient across the cladding.

\section{B. General Observations of Fuel-clad Interface Re* actions}

The clad-fuel interfaces of a series of fuel pins irradiated to burnups up to $99,000 \mathrm{MWD} / \mathrm{T}$ were examined by optical microscopy. The results indicated that, for exposures as high as $99,000 \mathrm{MWD} / \mathrm{T}$ and maximum ID clad surface temperatures of $1260^{\circ} \mathrm{F}$, little or no general reactions occurred between fuel and cladding in either pellet-filled, 347 stainless steel pins or swaged-orer 304 stainless steel pins. In several instances, however, localized reactions were observed in a number of the 347 stainless steel pins containing pelleted fuel, although none of the pins failed.

A section of a pin $(\mathrm{V}-2-\mathrm{P})$, which sustained a burnup of $77,400 \mathrm{MWD} / \mathrm{T}$, shows a thin surface layer $(6$ to $7 \mu)$ on the clad ID in a region of intimate fuel-cladding contact (see Fig. 17). (The maximum clad ID temperature of this pin was $1180^{\circ} \mathrm{F}$.)

A duplex layer of possible fuel-clad reaction product is evident on the transverse cross section of a (Pu-U) $\mathrm{O}_{2}$ pin (VIII-2-P) (see Fig. 18), which had been irradiated to a burnup of $28,000 \mathrm{MWD} / \mathrm{T}$ and attained a maximum clad ID temperature of $1220^{\circ} \mathrm{F}$. The layer had a thickness of approximately $14 \mu$ and was contiguous with the cladding, but had the gray as-polished appearance of nonporous fuel (see Fig. 19). A thin, 4- $\mu$ uniform layer with well-defined interfaces, sandwiched between the gray phase and the bulk of the cladding, was revealed by etching. The grain structure of this layer could not be fully resolved by optical metallography although the grainboundary network of the adjacent undisturbed cladding appeared to be continuous across the layer.

Grain-boundary prccipitation along the ID surface of the cladding, similar in appearance to that noted on the previously described high-burnup capsule, was observed in a number of pins. However, such precipitation was generally restricted to regions where the fuel-clad gap was nonexistent or sufficiently narrow to assure that intimate contact existed at the test conditions (see Fig. 20).

The mechanisms responsible for the localized reactions observed in this investigation have not been firmly established. A number of possible reaction mechanisms ${ }^{(26)}$ may be operative at the fuel-clad interface, the most likely of which are (1) transport of such impurities in the fuel as water, carbon compounds, or halogens to the reaction interface, (2) vapor transport of oxide, iodide, or other volatile fission products or their daughters to the interface, (3) selective oxidation of some clad alloy constituents, and (4) fission product recoil from fuel surface entering clad surface with disruption of the protective film.

\section{CONCLUSIONS}

The following conclusions are offered:

(1) Localized corrosion which exhibited characteristics similar to caustic corrosion has been observed in a pumped sodium system. The attack was associated with intermittent atmospherie contamination of sodium-coated interior surfaces and with large thermal stresses present during startup.

(2) Compatibility studies of mixed oxide fuel and austenitic stainless steels showed general interactions of less than 1 mil, and localized grain-boundary disturbances up to 3 mils. These reactions were not observed in all fuel pins, nor were they observed uniformly along the fuel-clad interface in any one pin.

(3) Application of electron microscopy and microprobe techniques resulted in specific improvements in the quality and accuracy of diagnoses of such reaction and structural effects, and afford promise of a firmer basis for screening and performance analysis of candidate cladding alloys.

\section{ACKNOWLEDGMENTS}

The authors are grateful to the Atomic Energy Commission for permission to publish this work, to E. L. Zcbroski for his many helpful suggestions, and to C. F. Wolff for the electron microscopy.

\section{REFERENCES}

1. F. J. Hetzler and R. S. Young, Sodium Mass Transfer: II. Screening Test Data and Inalysis, GEAP-3726 (June 1962).

2. I). E. Plumlee. Studies of Carbon Movement in High Temerature Sodium Loops, AEC Corrosion Symposium, Augusta, Ga., April 27, 1965. 
3. D. I. Simizen and E. N. Pearson, Relationship of Carburizing Potential to Operating Temperature Limitations in SRE, NAA-SR-MEMO-7804 (Nov 1962).

4. J. Motz, Die Untersuchung eines warmfesten ferritischen Stahles auf seine Verwendbarkeit in Natriumanlagen bis zu Temperaturen von $605^{\circ} \mathrm{C}$, INTERATOM, Bensberg, Germany, Sept. 1963.

5. R. S. Young and R. W. Lockhart, Information MeetingAEC Sodium Components Development Program, Chicago, Illinois, June 1965.

6. Effect of High Temperature Sodizm on Austenitic and Ferritic Steels, Physical Properties of Materials, MSAR 64-81 (July 1964).

7. F. J. Hetzler and T. A. Lauritzen, The Effect of Carboncontaminated Sodium on Austenitic Stainless Steel, ANS Transactions, 8(No. 2), Nov. 1965.

8. I. F. Epstein, Statc and Dynamic Corrosion and Mass Transfer in Liquid Metal Systems, Chem. Eng. Prog. Sym., Ser. 53, No. 20 (1957); Proc. Conf. Peaceful Uses of Atomic Energy, Vol. 9, p. 311, New York (1956).

9. J. 1). Mottley, Sodium Mass Transfer: VIII Corrosion of Stainless Steel in Isothermal Regions of a Flowing Sodium System, GEAP-4313 (Feb 1964).

10. E. G. Brush, Partially Selective Corrosion of Type-316 Stainless Steel in High-temperature Sodium, ANS Transactions, 8, No. 2 (Nov 1965).

11. A. H. Cottrell, Vacancies and Other Point Defects in Wetals and Alloys, The Institute of Metals (1958), p. 1.

12. D. McLean, ibid., p. 159.

13. J. N. Wanklyn and D. Jones, The Corrosion of A ustemtic Stainless Steels under Heat Transfer in High-temperature Water, J. Nuclear Materials, 1 (No, 2), 154 (July 1959).

14. P. P. Snowden, Stress Corrosion of Austenitic Stainless Steel by High-temperature Solutions and Contaminated Sieam, J. Iron and Steel Institute, 194, 181 (Feb 1960).

15. G. F. Rieger and E. A. Lees, General Electric APED, report in preparation.

16. W D. Robertson and A. S. Tetelman, "A Unified Structural Mechanism for Intergranular and Transgranular Corrosion Cracking," Strengthening Mechanisms in Soldds, American Society for Metals, 1962.

17. B. E. Hopkinson and K. Cx. Carroll, Chromium Distribution around Grain Boundary Carbides Found in A L stenitic Stainless Steel, Nature, 184, 1479 (1959).

18. J. B. Clark, Electron Probe Test of Two Theories of Denuded Zone Formution in Aged Alloys, Aeta Met., 12, 1197 (1964).

19. R. Stickler and A. Tinckier, Morphology of Grain-boundary Carbides and Its Influence on Intergranular Corrosion of 304 Stainless Steel, Trans. ASM, 54, 362 (1961).

20. J. R. Bendure et al., Quantity and Form of Carbides in Austenitic and Precipitation Hardening Stainless Steels, Trans. AIME, 221, 1032 (1961).

21. R. Stickler and A. I inckier, Electron Wicroscopy Investiga tion of the Intergranular Corrosion Fracture Surfaces in a Sensitized Austenitic Stainless Steel, Corrosion Science, 3, 1 (1963).

22. F. A. Comprelli and J. E. Lewis, Microstructural Evaluation of Superheat Cladding Materials, (GEAP-4751 (Jan 1965).

23. L. F. Epstein, General Electric-Tallecitos, personal communication (July 1965).

24. E. L. Zebroski, W. E. Baily, and D. P. Hines, Iradiation Behavior of Hyper- and Hypostoichiometric PlutoniaUrania Fuel at $700,00 \mathrm{MWD} / \mathrm{T}$, (HEAP-4897 (March 1965).
25. W. B. Pearson, A Handbook of Lattice Spacings and Structures of Metals and Alloys, Pergamon Press (1958).

26. E. L. Zebroski, J. P. Mustelier, and C. Caldwell, Oxide Fuels for Fast Reactors, Presented at the ANS National Topical Meeting on Fast Reactor Technology, Detroit, Michigan, April 1965.

\section{Discussion}

Mr. Kummerer (Karlsruhe): My question is related directly to the fuel-clad interaction; I want to know whether you could find any change of stoichiometry of the fuel directly on the surface.

Mr. Comprelli: We did not look directly for that in our observations.

Mr. Mustelier (CEA, France): What do you think of the compatibility between stainless steel and mixed oxide in the presence of a sodium bond, for example in the case of a rupture of cladding?

Mr. Comprelli: We did not carry out a direct experiment on this particular item. I assume that you are referring to a fuel element to which has been intentionally added sodium and sodium does not enter due to rupture. Is that correct?

Mr. Mustelier: I think the result is the same. I just want to know if you happen to see transfer of fission products or an attack when sodium is placed between $\mathrm{UO}_{2}-\mathrm{PuO}_{2}$ and stainless steel.

Mr. Comprelli: If I understand you correctly, you refer to fission products leaving the fuel, entering sodium, and then acting as a corrosion medium with respect to the cladding. We have not analyzed this, but it will be in our present program. We have taken a cursory look at the effect of sodium-fuel reaction products on the cladding. Our present results, which are preliminary, indicate that there is a minor reaction but nothing of eatastrophic measure.

Mr. Okrent (ANL): Would you expect any severe compatibility problems if you were running oxides at higher ratings and higher burnups?

Mr. Comprelli: The observations reported today are pretty representative of the general trend which we have observed in our fuel pins, and we have irradiated these pins up to $99,000 \mathrm{MWD} / \mathrm{T}$. The magnitude of the effects, as we have seen them, do not indicate that this problem is insurmountable. We have had interface temperatures of approximately $1250^{\circ} \mathrm{F}$, and the magnitudes of the reactions under those conditions were not substantially larger than those observed under the slightly lower temperatures. However, I do expect a slight increase with increasing temperature.

Mr. Okrent: How much margin do you think you have between satisfactory operation and rapid deterioration? In other words, does one have to really 
have a very good handle on the compatibility question?

Mr. Comprelli: I don't believe I'm prepared to say we have a real good handle on it, based on the observations we've made to date. $\mathrm{W}^{\top} \mathrm{e}$ 've made essentially cursory examinations of the pins we have available.

Mr. Stahl (UNC): In one of your micrographs there was no gap between your fuel and clad. Did you start out with a reasonable cold clad gap? Did you have any failures because of swelling?

Mr. Comprelli: We do not have any failures that can be attributed directly to swelling. In the high- burnup pin, to which I believe you are referring, the cold diametral gap was approximately 2 mils.

$M r$. Kummerer: In one of your slides, sodium had an oxygen content of $12 \mathrm{ppm}$. Did you determine the oxygen content before or after the irradiation experiments?

$M r$. Comprelli: The oxygen level to which you refer was associated with the microprobe trace across the 316 sample. That was not an irradiation experiment; it was a loop experiment. The oxygen level was obtained through determination of the plugging temperature. 


\title{
Effect of Stoichiometry on the Properties of Mixed Oxide U-Pu Fuel
}

\author{
E. L. Zebroski, W. L. Lyon, and W. E. Baily \\ General Electric Company \\ Advanced Products Operation \\ San Jose, California
}

(PRESENTED BY E. L. ZEBROSKI)

\section{Introduction}

Conventional oxide fuels for present economic power reactors typically employ essentially stoichiometric fuel, $\mathrm{UO}_{2+x}$, where $x$ is positive and lies in a range up to 0.02 . This has proven satisfactory for relatively low-burnup applications $(10,000$ to $25,000 \mathrm{MWD} / \mathrm{T})$ in which stoichiometry changes with burnup are small.

Fast reactor fuels of the type $(\mathrm{U}, \mathrm{Pu}) \mathrm{O}_{x}$ are required to attain average burnup of 75,000 to $100,000 \mathrm{MWD} / \mathrm{T}$ to be of economic interest, and local peak burnups up to $130,000 \mathrm{MWD} / \mathrm{T}$. The choice of preferred stoichiometry for fast spectrum power reactor application may prove to be appreciably different from the practice for present power reactors for several reasons:

(1) A wider range of stoichiometry and properties is conveniently attainable; for $\left(\mathrm{Pu}_{0.2} \mathrm{U}_{0.8}\right) \mathrm{O}_{2+x}$, the value of $x$ can be readily adjusted to lie in the range $x=$ \pm 0.03 .

(2) The change in composition and properties with burnup is more significant (final composition is up to $\sim 25 \mathrm{a} / \mathrm{o}$ fission products relative to uranium plus plutonium).

(3) The average operating temperature of the fuel is significantly higher (because of higher coolant temperature, higher heat fluxes, and higher specific power).

This paper is intended to cover the status of some fuel preparation and properties data, as well as observations of fuel performance, which lead to a tentative preference for a stoichiometry of 1.97 to 1.98 for mixed U-Pu oxide fuel.

\section{Preparation and Homogeneity of Mixed Oxide Solid-solution Fuel}

The preparation of a solid solution of $\mathrm{PuO}_{2}$ and $\mathrm{CO}_{2}$ can be accomplished by either coprecipitation or by efficient physical mixing of powders of $\mathrm{PuO}_{2}$ and $\mathrm{UO}_{2}$ followed by sintering or by pressing and sintering. Coprecipitation is at present the reference method since it provides a high degree of assurance of formation of a homogeneous, single-phase solid solution. Coprecipitation is accomplished by the simultaneous precipitation of ammonium diuranate and plutonium hydroxide with ammonium hydroxide from nitrate solution. Subsequent steps include filtration, drying, and reduction. The flow diagram for this operation is shown in Fig. 1.

Physically mixed powders can also be used to produce a near-homogeneous solid solution (after sintering) by careful control of starting materials, particle size, and mixing procedures to insure against agglomeration of the powders during mixing. Departure from ideal process conditions results in localized high concentrations of $\mathrm{PuO}_{2}$ and/or $\mathrm{UO}_{2}$ which are difficult to guard against by conventional inspection methods. Electron-beam microprobe analysis affords the most sensitive measure of local homogeneity available at present.

Microprobe analyses (conducted with the cooperation of NUMEC and the Mound Laboratory) were performed with physically mixed and sintered powders and coprecipitated mixed oxides to establish the degree of homogeneity attainable. (1) These results are summarized in Table 1 and Fig. 2. The slight departure from unity in maximum to minimum ratio noted in Table 1 for coprecipitated fuel may be within instrumental error. The physically mixed powders generally have readily detectable inhomogeneities.

The feasibility of attaining nearly equivalent homogencity by the mixed-powder route is shown by the sample represented in linc 2 of Table 1 . With sufficient experience in process control, and the development of convenient inspection methods to give statistical assurance of homogeneity, this may become another acceptable route for fast reactor fuel fabrication.

The samples in the lower three lines of Table 1 represent different degrees of inhomogeneity with mixed powders. For the samples which are not sintered, significant deterioration of the prompt Doppler would have to be accepted. This increases the calculated energy release for high-ramp-rate reactivity-insertion accidents, and consequently increases the difficulty and cost of reactor containment. Sintering and homogenization which may occur in situ in the hotter portions of operating fuel does not significantly alleviate the safety problem of small-scale inhomogeneity. The $1 / T$ de- 

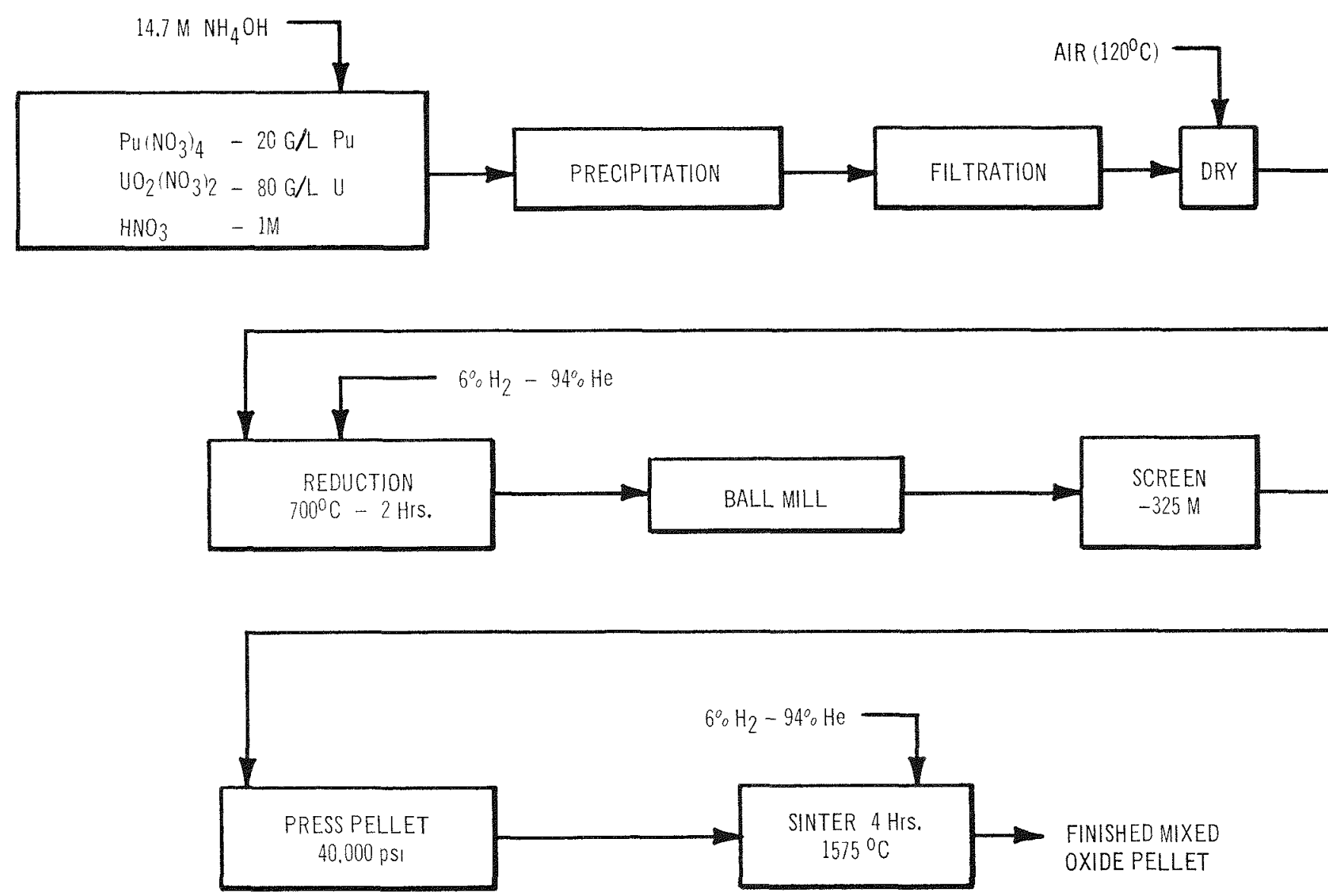

Frr, 1. Coprecipitation Flow Diagram.

pendence of the Doppler coefficient implies that a large part of the useable Doppler $\Delta k$ in a reactor transient must come from the colder regions of the fuel, which may not be rapidly homogenized by thermal diffusion. A much smaller loss of Doppler $\Delta k$ is calculated for large-scale inhomogeneities (limited radial concentration gradients) as treated in another paper at this Symposium. ${ }^{(2)}$

\section{Control and Measurement of Stoichiometry}

Mixed oxide of uranium and plutonium, $(\mathrm{U}, \mathrm{Pu}) \mathrm{O}_{2}$, can be conveniently fabricated with direct control of stoichiometry over a range of oxygen-to-metal ratios from 1.97 to 2.00 . These are obtainable during sintering of $(\mathrm{Pu}, \mathrm{U}) \mathrm{O}_{2}$ fuel by control of the moisture content of the sintering-furnace atmosphere. By addition of water vapor to the helium- $6 \%$ hydrogen furnace gas to a level of about $1 \%$ at room temperature, stoichiometric mixed oxide is obtained. If helium-hydrogen gas is passed through magnesium perchlorate drying columns before entering the sintering furnace, fuel with oxygento-metal ratio of 1.97 is obtained. Analyses have shown that constant $\mathrm{O} / \mathrm{M}$ ratio is obtained within a given
TABILE 1. SMaLla-SCALE INHONOGENEI TY OF MuXed OXIDE Fleh

\begin{tabular}{c|c|c|c|c}
\hline Type of Fuel & $\begin{array}{c}\text { Agglomer- } \\
\text { ation } \\
\text { Regions, } \mu\end{array}$ & $\begin{array}{c}\text { Mean } \\
\mathrm{PuO}_{2} \\
\text { Conc, w/o }\end{array}$ & $\begin{array}{c}\text { Max/Min } \\
\text { Plutonium } \\
\text { Content }\end{array}$ & $\begin{array}{c}\text { Max/Avg } \\
\text { Plutonium } \\
\text { Content }\end{array}$ \\
\hline $\begin{array}{c}\text { Coprecipitated, } \\
\text { Dry Sintering }\end{array}$ & $<1^{*}$ & 15.0 & 1.08 & 1.04 \\
$\begin{array}{c}\text { Physically Mixed, } \\
\text { Dry Sintered }\end{array}$ & $<1^{*}$ & 15.0 & 1.26 & 1.08 \\
$\begin{array}{l}\text { Physically Mixed, } \\
\text { Wet Sintered }\end{array}$ & $\sim 5^{*}$ & 15.0 & 1.92 & 1.30 \\
$\begin{array}{l}\text { Ball Milled for 4 } \\
\text { Hr and Im- } \\
\text { pacted }\end{array}$ & $20-40^{* *}$ & 20.0 & 2.69 & 1.74 \\
Ball Milled for 64 \\
$\begin{array}{l}\text { Hr and Im- } \\
\text { pacted }\end{array}$ & $20-30^{* *}$ & 20.0 & 1.93 & 1.44 \\
\hline
\end{tabular}

* Analysis with 1- $\mu$-dia spot.

**Analysis with 20 - $\mu$-dia spot. (3)

pellet and throughout a batch of pellets sintered at one time, provided that an even temperature is maintained across the charge.

A still wider range of stoichiometry is available by auxiliary process steps. Hyperstoichiometric mixed 


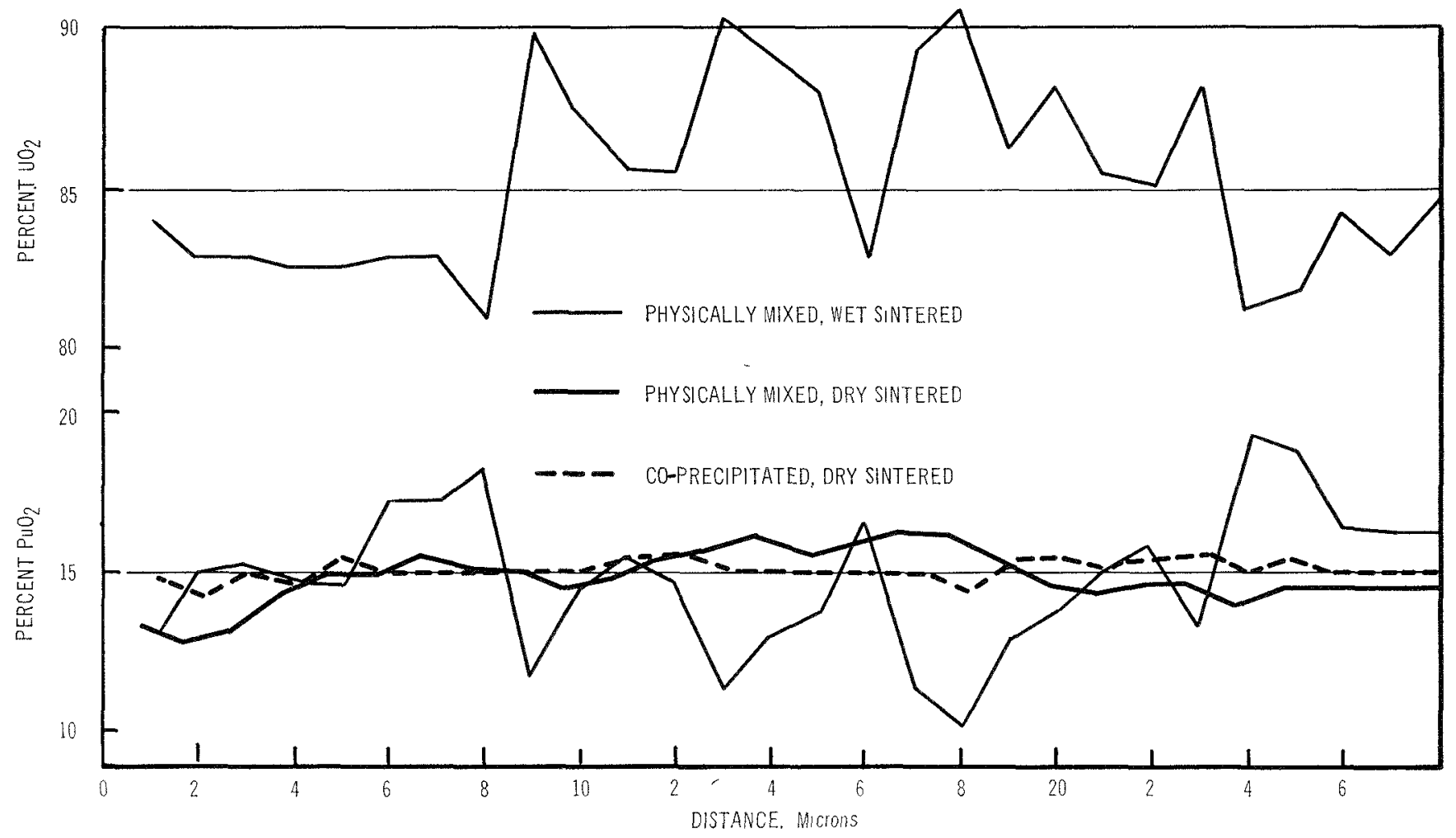

FIG. 2. Plutonium Distribution in $\mathrm{UO}_{2}-15 \mathrm{w} / 0 \mathrm{PuO}_{2}$ Fuels.

oxide is prepared by the controlled oxidation in air at $400^{\circ} \mathrm{C}$ of initially stoichiometric specimens. The batch of pellets or powder is heated until a prescribed weight increase has been obtained, and then soaked in pure argon at $700^{\circ} \mathrm{C}$ to obtain a uniform distribution of the excess oxygen. Pellets with tailored $\mathrm{O} / \mathrm{M}$ levels of 2.010 to 2.060 have been made for property measurements and irradiation tests.

The analysis for $O / M$ ratio uses the thermogravimetric procedure based upon oxidation followed by reduction in hydrogen at $700^{\circ} \mathrm{C} \cdot{ }^{(4)} \mathrm{A}$ macro technique with precision of \pm 0.001 is used for whole pellets or powder samples of 1 to $10 \mathrm{gm}$. A microthermogravimetric apparatus has been developed for samples as small as $2 \mathrm{mg}$. Local shielding makes possible the handling of highly irradiated fuel samples in the latter apparatus. Attempts to measure $\mathrm{O} / \mathrm{M}$ of irradiated samples to date have been hampered by the extreme sensitivity of the measurement to traces of organic materials used in mounting and retrieving irradiated specimens.

The redistribution of oxygen in fuel operating with typical large thermal gradients can be roughly predicted by extrapolation to higher temperatures of the U-Pu-O thermodynamic data of Markin and Bones.*

* Unpublished work, Harwell, 1964.
For an effective oxygen partial pressure of $10^{-9} \mathrm{~atm}$, fuel of the initial composition $(0.2 \mathrm{Pu}-0.8 \mathrm{U}) \mathrm{O}_{2.00}$ is estimated to reach an $\mathrm{O} / \mathrm{M}$ of about 1.91 at the center $\left(\sim 2500^{\circ} \mathrm{C}\right)$, an $\mathrm{O} / \mathrm{M}$ of 2.00 at $70 \%$ radius $\left(\sim 1900^{\circ} \mathrm{C}\right)$, and an $\mathrm{O} / \mathrm{M}$ of $\sim 2.005$ at the fuel surface $\left(\sim 1300^{\circ} \mathrm{C}\right)$.

\section{Nonstoichiometry in Mixed Oxides of Pluto- nium and Uranium}

Nonstoichiometric mixed oxides are eharacterized as compounds which exist as a single phase over a range of chemical compositions. More specifically, they can be defined as a solid solution of binary oxides, $\left(\mathrm{M}_{a}\right.$, $\left.\mathrm{M}_{1-a}\right) \mathrm{O}_{x}$, where $a$ and $x$ are variable. The term "nonstoichiometric" is sometimes used loosely, since in many cases departures from stoichiometry are accompanied by the formation of a new phase or phases which are not readily detected by chemical analysis and which may be interpreted as a possible defect structure. An example of this is the $\mathrm{UO}_{2-x}$ system. At room temperature a stoichiometry of 1.97 actually refers to a two-phase mixture of very closely stoichiometric $\mathrm{UO}_{2.00}$ and a second phase of uranium metal. Under equilibrium conditions the $\mathrm{UO}_{2-x}$ structure exists only at high temperatures. At higher temperatures, the $\mathrm{UO}_{2}$ system does exhibit a true departure from stoichiometry, and at $1400^{\circ} \mathrm{C}$ the defect structure $\mathrm{UO}_{2+x}$ (where $x$ may be positive or negative) exists between 1.98 and 
approximately 2.20. A more detailed discussion of some of the effects of this transformation on physical properties is given in Sec. V. Departures from stoichiometry exist at oxygen sites which are vacant for the $2-x$ condition. In the $2+x$ condition additional oxygen atoms are located in interstitial sites which readily accommodate the extra oxygen in a stable structure.

In the mixed oxide system of $\mathrm{PuO}_{2} \mathrm{UO}_{2}$ there exists a unique combination of an oxide $\left(\mathrm{PuO}_{2}\right)$ whose highest oxide state is (IV) and of an $\left(\mathrm{UO}_{2}\right)$ oxide which has a maximum oxide state of (VI). In the other direction, $\mathrm{PuO}_{2}$ exhibits a true nonstoichiometric defect structure down to an oxygen-to-metal ratio of approximately 1.98 and below that is a two-phase structure of $\alpha-\mathrm{Pu}_{2} \mathrm{O}_{3}$ and $\mathrm{PuO}_{1.98} .{ }^{(5)}$ Excess oxygen added to $\mathrm{UO}_{2}$ exists as either a defect structure or as a two-phase structure of $\mathrm{UO}_{2}$ and $\mathrm{U}_{4} \mathrm{O}_{9}$, depending on the temperature and rate of cooling.

Both $\mathrm{UO}_{2}$ and $\mathrm{PuO}_{2}$ exhibit fluorite-type facecentered cubic crystal structures which have no known allotropic modifications up to their melting point and have a $\mathrm{U} / \mathrm{Pu}$ ionic-radius ratio of 1.04 . These observations along with $\mathrm{X}$-ray diffraction data on the mixed oxides have confirmed that a complete series of solid solutions exist across the phase diagram, which is single phase for all stoichiometric compositions. Figure 3 shows room-temperature $\mathrm{X}$-ray diffraction results which indicate an almost linear change in cell size of the solid solution of $\mathrm{PuO}_{2}$ and $\mathrm{UO}_{2}$ with composition. ${ }^{(6)}$ Per Vegard's law this confirms that complete solid solubility exists in this system. This has also been verified by several other investigators. ${ }^{(7,8)}$

A series of solid solutions exists between various stoichiometries in specific compositions of $\left(\mathrm{Pu}_{a} \mathrm{C}_{1-a}\right) \mathrm{O}_{2}$. Compositions of $\left(\mathrm{Pu}_{0.2} \mathrm{U}_{0.8}\right) \mathrm{O}_{2+x}$ have been studied between stoichiometries of 1.975 and 2.01, and have been found to exhibit a single-phase structure with a linear decreasing cell size with increasing oxygen content. ${ }^{(9)}$ This is shown in Figure 4 and also in Table 2. A similar correlation has also been established by Dean for 30 and $50 \mathrm{w} / \mathrm{o} \mathrm{PuO}_{2}$ mixed oxides. ${ }^{(10)}$ Trace quantities of alpha- $\mathrm{Pu}_{2} \mathrm{O}_{3}$ have been found in various compositions and stoichiometries of $\mathrm{PuO}_{2}-\mathrm{UO}_{2}$ solid solutions including hyperstoichiometric compositions. This effect is not well understood, but may reflect slowness in attainment of equilibrium distribution. The contraction of the unit-cell size with increasing oxygen content is similar to that noted in the single-phase $\mathrm{UO}_{2+x}$ structure in which the cell size varies almost linearly with increasing oxygen from $5.472 \AA$ for $x=2.00$ to $5.450 \AA$ for $x=$

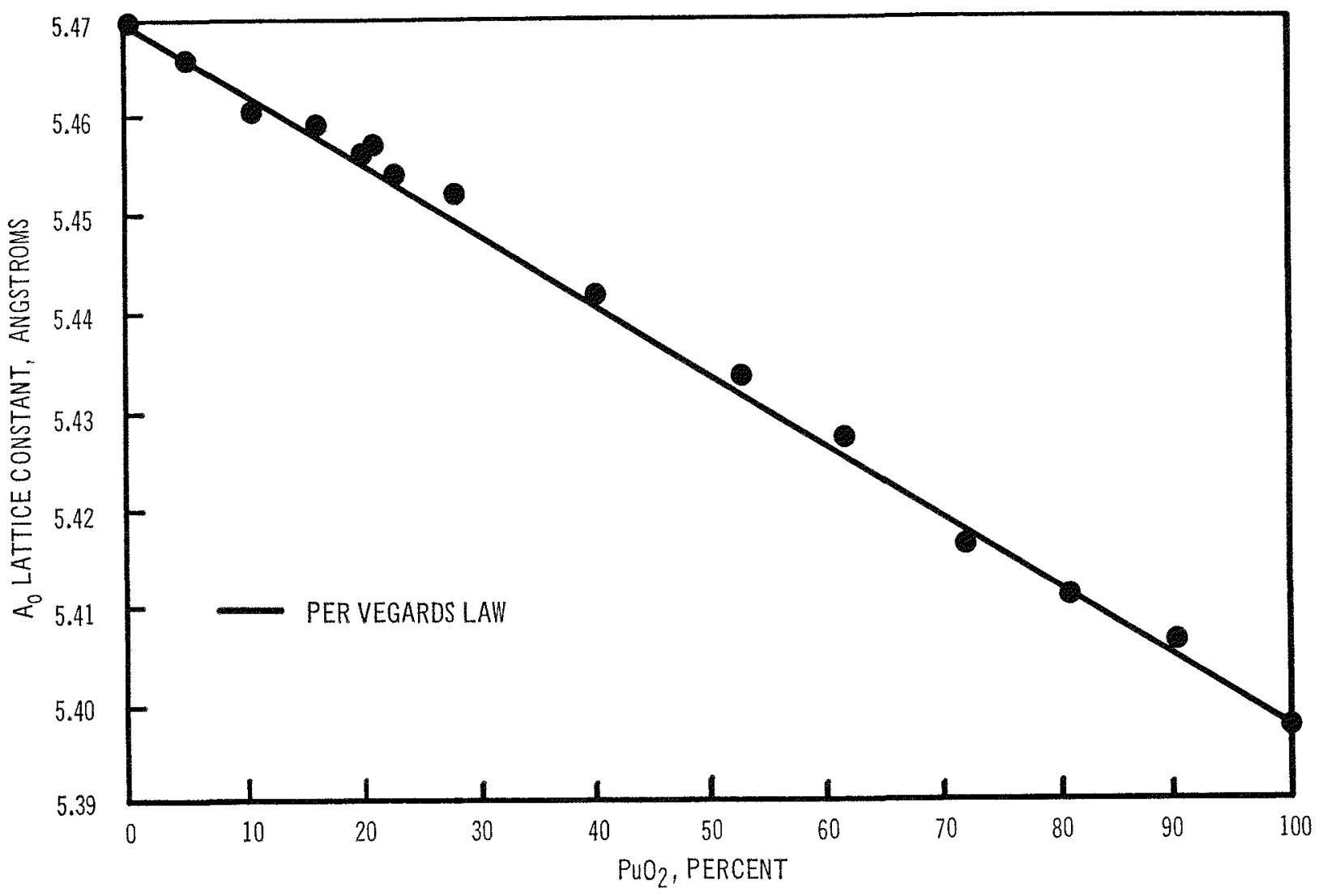

Fra. 3. Measured Change in Lattice Parameter with Composition in the $\mathrm{UO}_{2}-\mathrm{PuO}_{2}$ System. 


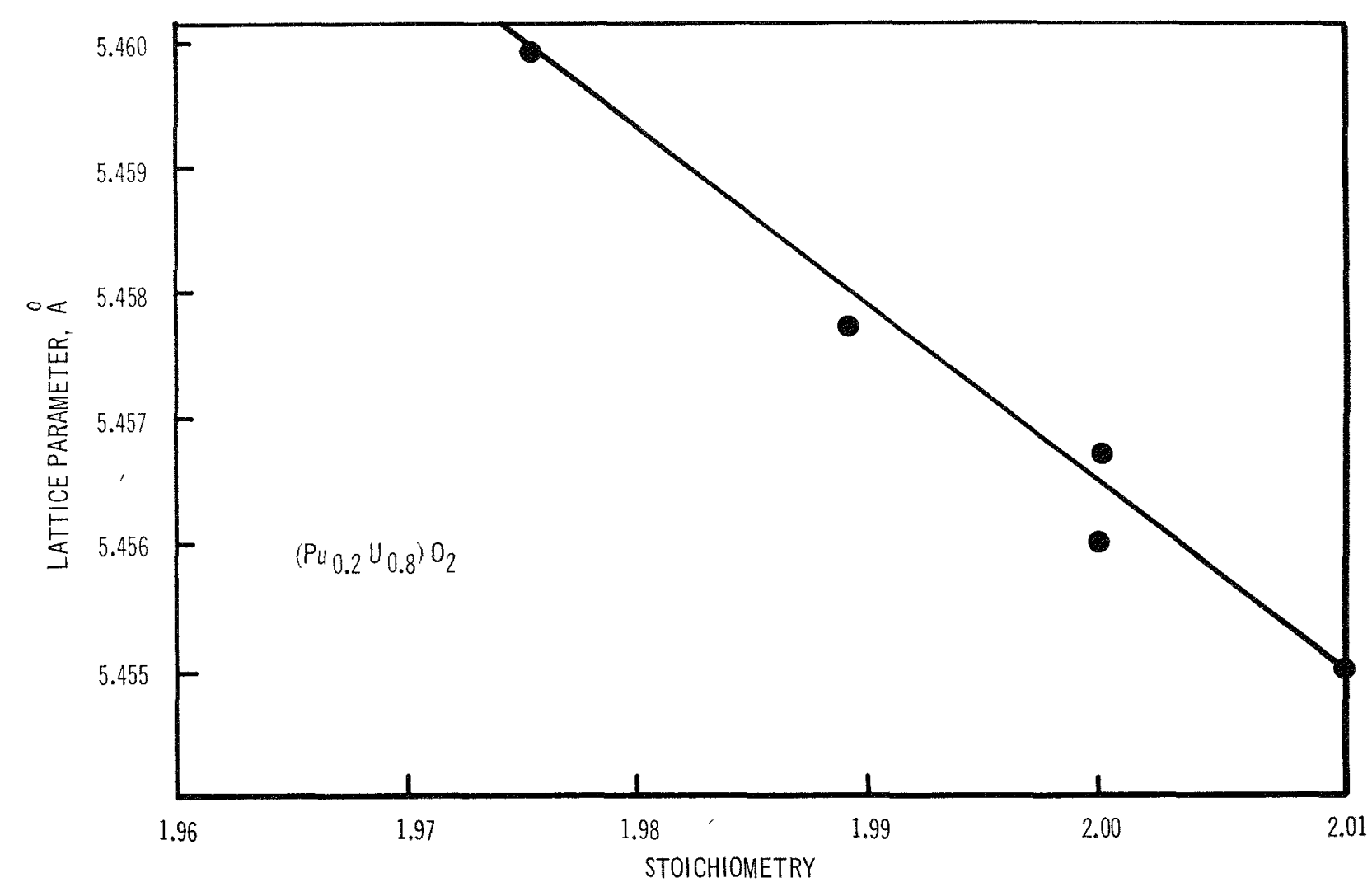

Fug. 4. Relationship of Cnit Cell Size with Stoichiometry in $\left(\mathrm{Pu}_{0.2} \mathrm{U}_{0} 8\right)_{2}$ Specimens.

'Table: 2. Change in La'trice Parameters and Second Phases in Mixed Oxide wrth Varying Oxygen Content

\begin{tabular}{|c|c|c|c|c|c|}
\hline $\mathrm{O} / \mathrm{M}$, Chemical & $\begin{array}{c}\mathrm{PuO}_{2}, \text { Chemical, } \\
\text { w/o }\end{array}$ & $\underset{\text { Xray }}{\mathrm{Pu}(\mathrm{Pu}}+\mathrm{U})$ & $\begin{array}{l}\text { Lattice Parameter of } \\
\text { Solid-solution Phase, }\end{array}$ & Trace Phases & $\begin{array}{c}\text { Relative Amount of } \\
\text { Trace Phases }\end{array}$ \\
\hline 2.010 & 0.200 & 0.2037 & $5.455 \pm 0.001$ & Alpha $\mathrm{Pu}_{2} \mathrm{O}_{3} *$ & Weak minor \\
\hline 2.000 & 0.200 & 0.2036 & $5.4567 \pm 0.0002$ & Alpha $\mathrm{Pu}_{2} \mathrm{O}_{3}{ }^{*}$ & Weak minor \\
\hline 1.989 & 0.20 (nominal) & 0.1873 & $5.4577 \pm 0.0002$ & Alpha $\mathrm{Pu}_{2} \mathrm{O}_{3}{ }^{*}$ & Tery weak \\
\hline 1.976 & 0.200 & 0.2016 & $5.460 \pm 0.007$ & Alpha $\mathrm{Pu}_{2} \mathrm{O}_{3}$ * & Weak \\
\hline
\end{tabular}

$* \mathrm{Pu}_{2} \mathrm{O}_{3}-\mathrm{Pu}_{4} \mathrm{O}_{7}$-Identity of these phases is based on a measured lattice parameter of the second phase at 11.03 to $11.01 \AA$. The structure of alpha- $\mathrm{Pu}_{2} \mathrm{O}_{3}$ was indexed by Zachariasen and was shown to be a defect structure that can contain additional oxygen atoms in the range $\mathrm{Pu}_{2} \mathrm{O}_{3}$ to $\mathrm{Pu}_{4} \mathrm{O}_{7}$. (11, 12) Ellinger in a separate study found that the lattice parameter of alpha- $\mathrm{Pu} \mathrm{O}_{3} \mathrm{O}_{3}$ could vary between $11.00 \pm 0.02$ to $11.04 \pm 0.02 \AA$ for oxygen-rich and oxygen-deficient st ructures, respectively.(13)

2.18. This variation, however, is a function of the cooling rate since the equilibrium structure for $\mathrm{UO}_{2+x}$ is $\mathrm{C}_{4} \mathrm{O}_{9}+\mathrm{I}^{\top} \mathrm{O}_{2}$ (two phase). It should be noted that whenever $\mathrm{U}_{4} \mathrm{O}_{9}$ is observed the corresponding $\mathrm{CO}_{2}$ cell size is that for $\mathrm{UO}_{2.00} .^{(14)}$ Such a correlation in the hyperstoichiometric mixed oxide system has not been found at this time.

Metallographic examination of mixed oxides as a function of stoichiometry have shown no apparent correlation of second phase with stoichiometry. Figure 5 shows the microstructure of $\mathrm{CO}_{2}$ at 1.98 stoichiometry with its characteristic $\mathrm{UO}_{2}$ plus free uranium. Precipitates of free uranium have been noted in specimens of 1.99 and lower stoichiometry. Metallographic examina- tions of mixed oxides in the neighborhood of $20 \mathrm{w} / 0$ $\mathrm{PuO}_{2}$ have indicated that the mixed oxide exhibits less distinctive etching behavior than $\mathrm{UO}_{2}$.

The structure of hyperstoichiometric mixed oxide has been found commonly to exhibit microcracks dispersed throughout the specimen. These cracks are apparently associated with the fabrication technique at the stage of controlled oxidation of either 1.98 or 2.00 mixed oxide to 2.05 . The cracks presumably occur due to the density decrease on oxidation. Figure 6 shows an electron micrograph of mixed oxide fuel showing the striated microcontour substructure normally associated with coprecipitated mixed oxide. ${ }^{(15)}$ This substructure is apparently formed during the coprecipita- 


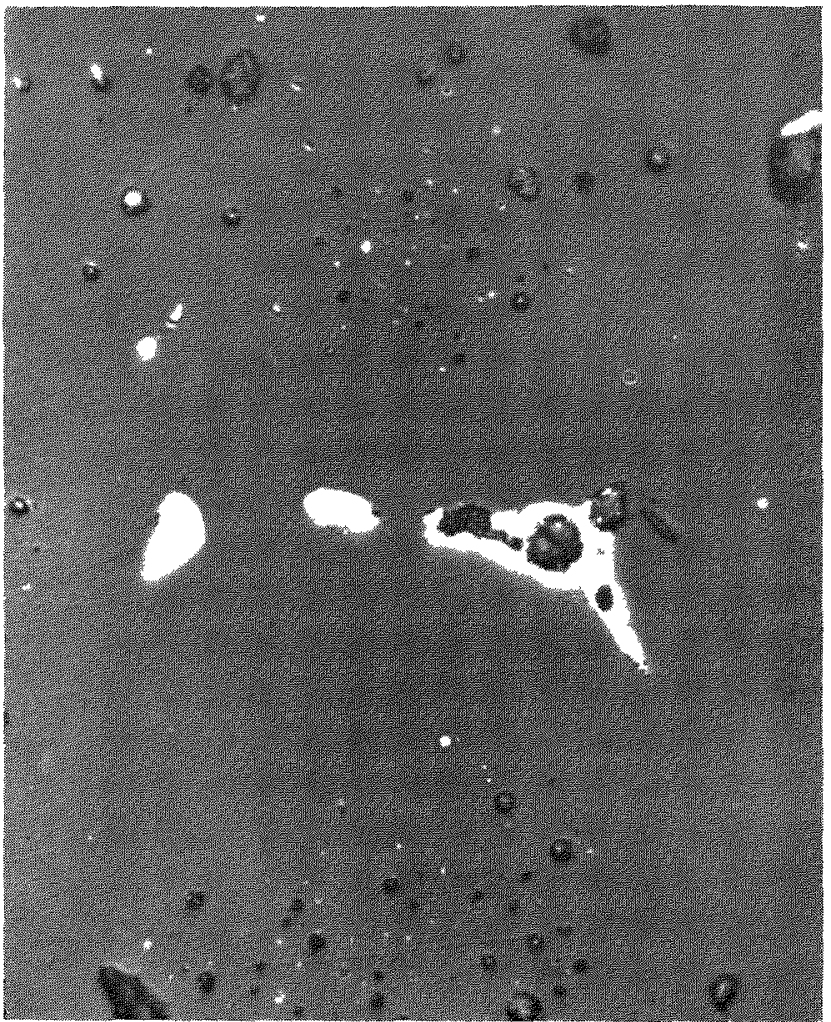

$465 \times$

FIG. 5. Microstructure of Tacuum Reduced $\mathrm{UO}_{2-1}$.

tion operation and probably represents a periodic series of either coherent changes in composition or orientation within each grain.

\section{Effect of Stoichiometry on Some Properties of Mixed Oxides and $\mathrm{UO}_{2}$}

\section{A. Thermal Conductivity of Hypostoichiometric $\mathrm{CO}_{2}$}

The adverse effect of excess oxygen on the conductivity and irradiation behavior of $\mathrm{CO}_{2}$ was demonstrated earlier by Robertson in a series of in-pile experiments. ${ }^{(16)}$ These measurements showed that excess oxygen in $\mathrm{UO}_{2}$ seriously decreases its average thermal conductivity (as defined by postirradiation microstructure). Out-ofpile measurements of thermal conductivity have been made of the effect of stoichiometry on the thermal conductivity of $\mathrm{CO}_{2-x}$ and mixed oxide samples under the FCR program. ${ }^{(17)}$ The purposes of these experiments were to establish information on the phase change of $\mathrm{CO}_{2}+\mathrm{T}$ to $\mathrm{CO}_{2-x}$ in hypostoichiometric $\mathrm{UO}_{2}$ and how this phase change might affect thermal conductivity, as well as to determine whether analogous effects were present in the mixed U-Pu oxide system.

Figure 7 shows the change in $\mathrm{UO}_{2}$ thermal conductivity as a function temperature for three different stoichiometries. The results from this study showed that the $\mathrm{CO}_{197}$ had a slightly higher thermal conductivity than $\mathrm{UO}_{1.98}$ in the temperature range from 800 to $1400^{\circ} \mathrm{C}$, and both of these hypostoichiometric compositions exhibited $30 \%$ higher conductivity than $\mathrm{UO}_{200}$ in this temperature range. All hypostoichiometric specimens tested exhibited a threshold temperature at which the conductivity rapidly decreased to a value close to that for $\mathrm{CO}_{200}$. This threshold temperature was found to be characteristic for a given stoichiometry and was found to be present on repeated cycling through this range. The thermal conductivity of stoichiometric $\mathrm{UO}_{2}$ was found 10 agree well with values obtained by some recent investigations. ${ }^{(18,}$ 19) The increase in thermal conductivity postulated or observed by some investigators at temperatures above $1600^{\circ} \mathrm{C}$ was not observed in these experiments. This may te due to the facts that the highest temperature observed was $1800^{\circ} \mathrm{C}$ and that $\Delta T$ used in those measurements was relatively large $\left(\sim 1000^{\circ} \mathrm{C}\right)$ to simulate fuel behavior.

It is hypothesized that the break in the conductivity eurves of $\mathrm{UO}_{198}$ and $\mathrm{CO}_{197}$ is associated with the transformation temperature of $\mathrm{C}^{\top} \mathrm{O}_{20}+\mathrm{I}^{2}$ to the defect structure $\mathrm{UO}_{2} \ldots$. Figure 8 shows the partial $\mathrm{UO}_{2-x}$ phase

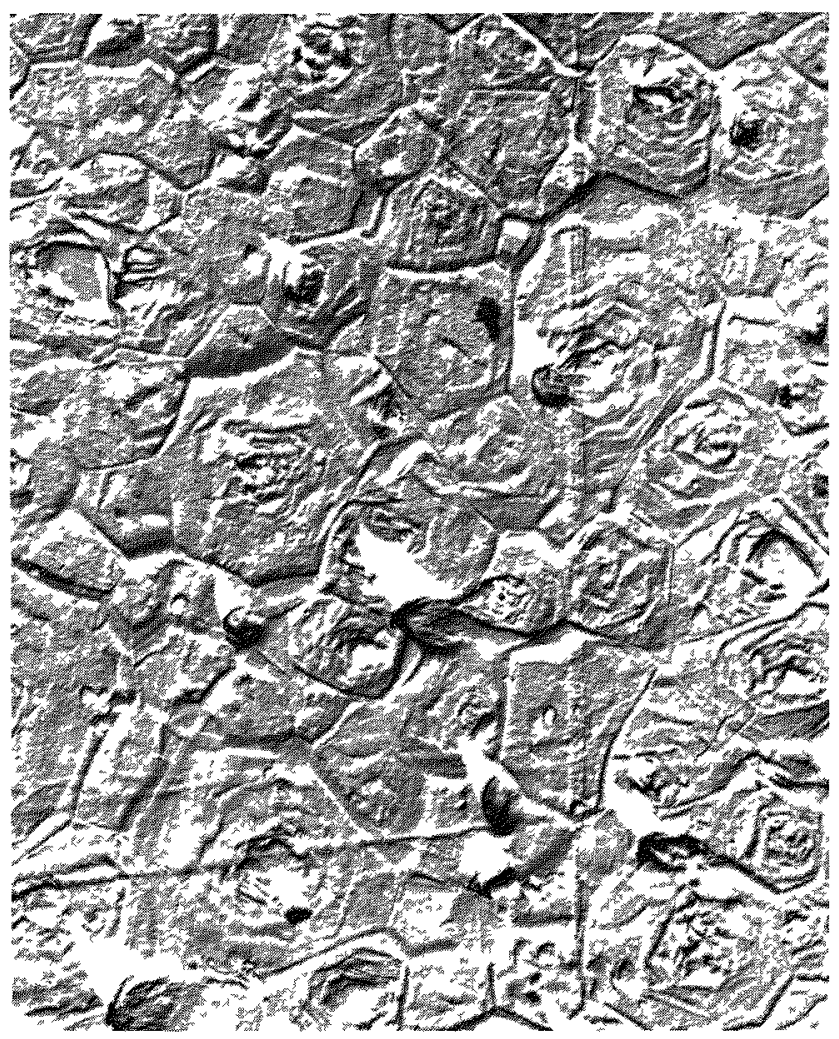

$6100 \times$

Fla. 6. Electron Micrograph of $\left(\mathrm{Pu}_{0} \mathrm{U}_{0} \mathrm{U}_{8}\right) \mathrm{O}_{2}$ Showing Sub structure within the Gran. 


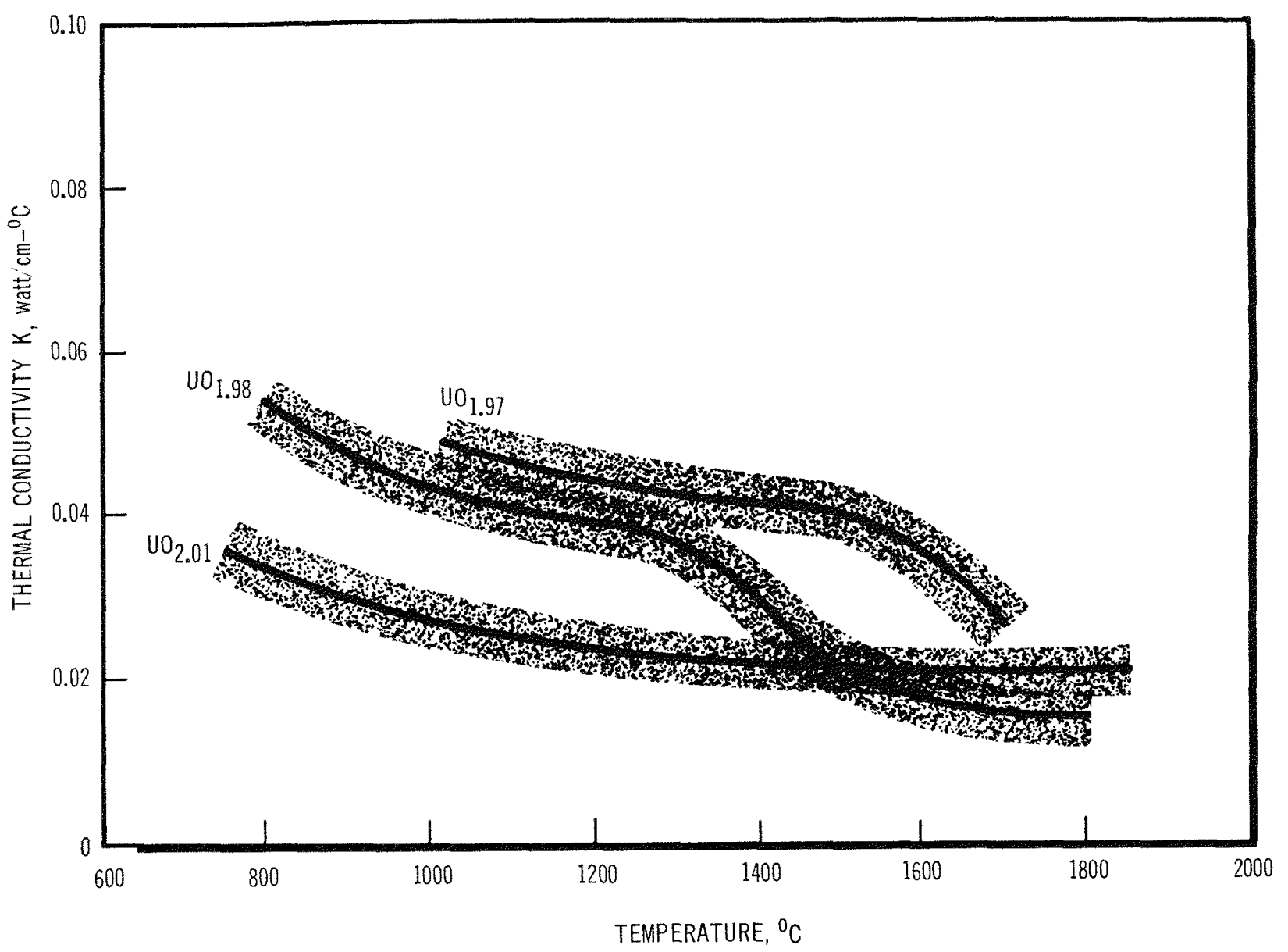

Fic. 7. Thermal Conductivity of Both Stoichiometric and Irypostoichiometric UO $\mathrm{O}_{2}$ as a Funetion of Temperature.

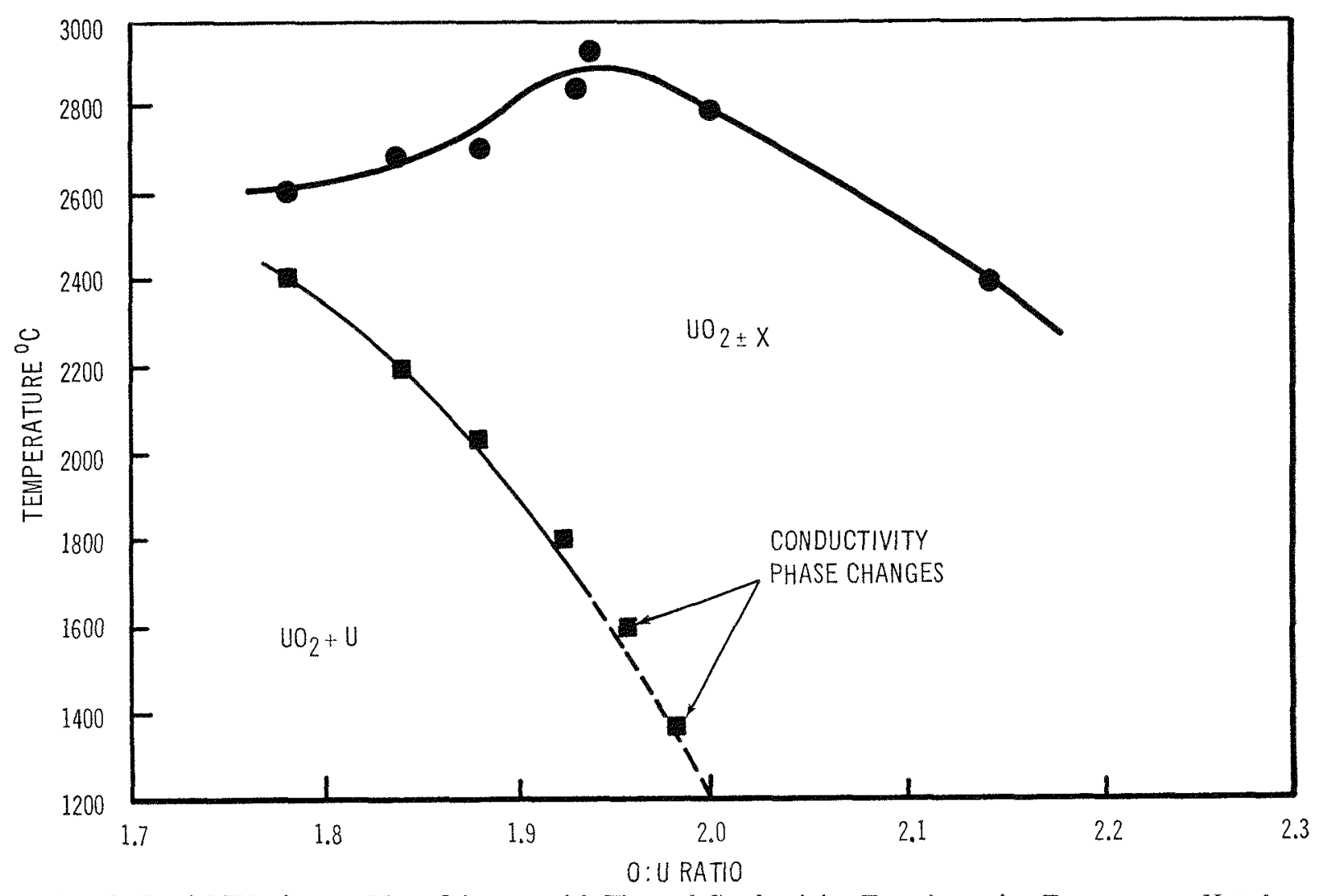

FIG. 8. Partial $\mathrm{CO}_{2}$-Oxygen Phase Diagram with Thermal Conductivity Transformation Temperatures Noted. 
diagram with the observed threshold temperatures proposed by Bates. ${ }^{(20)}$ By extrapolating the transformation lines, the change in slopes in the conductivity data can be associated with the transformation temperature of the process $\left(\mathrm{UO}_{2.0}+\mathrm{U}\right)$ to $\mathrm{UO}_{2-x}$.

The higher thermal conductivities of the $\mathrm{UO}_{1.98}$ and $\mathrm{UO}_{1.97}$ specimens below $1400^{\circ} \mathrm{C}$ is apparently associated with the presence of free uranium in the $\mathrm{UO}_{2}$ matrix. Higher thermal conductivity of $\mathrm{UO}_{2-x}$ material has also been qualitatively observed by Notley at temperatures below $1400^{\circ} \mathrm{C}$.(21) Thermal conductivity changes caused by a noncontinuous dispersed phase based on a modified Maxwell-Eucken relationship ${ }^{(22)}$ indicated that the high thermal conductivity of the $\mathrm{UO}_{2.0}+\mathrm{U}$ region is not caused by just the increased thermal conductivity of free uranium. One possible cause for this higher thermal conductivity is that the presence of free uranium in the $\mathrm{UO}_{2}$ matrix provides an excess of free electrons, which would result in an additional contribution to the conductivity function.

\section{B. Mixed Oxides}

The effect of stoichiometry on the thermal conductivity of mixed oxide was found to be significantly different than that measured for $\mathrm{UO}_{2}$. Figure 9 shows the measured thermal conductivities of $\left(\mathrm{Pu}_{0.2} \mathrm{U}_{0.8}\right) \mathrm{O}_{2}$ specimens with stoichiometries of $2.04,2.00$, and $1.98{ }^{(23)}$ These results showed that the $\mathrm{UO}_{2}+\mathrm{U}$ to $\mathrm{UO}_{2-x}$ transformation temperature is displaced in the hypostoichiometric direction with the addition of $\mathrm{PuO}_{2}$. This has been verified by both metallographic and X-ray diffraction data (see Sec. II), which show that $\left(\mathrm{Pu}_{0.2} \mathrm{U}_{0.8}\right) \mathrm{O}_{2}$ is single phase between 1.975 and 2.01 stoichiometry. Out-of-pile measurements on specimens with a stoichiometry of 2.05 are considered atypical since the hyperstoichiometric pellets were found to have microcracks dispersed throughout their volume. They exhibited lower conductivity than the 1.98 material to about $1200-1400^{\circ} \mathrm{C}$. Rising conductivity at higher temperatures can be attributed to either heating of microcracks as plasticity develops, or to the dissolution of a second phase. In general, the hyperstoichiometric specimens exhibited a lower thermal conductivity than either the stoichiometric or hypostoichiometric fuel.

\section{Melling Point}

Melting points for the mixed oxide system have been measured by both $\mathrm{V}$-filament technique with milligram samples and thermal-arrest measurements with massive

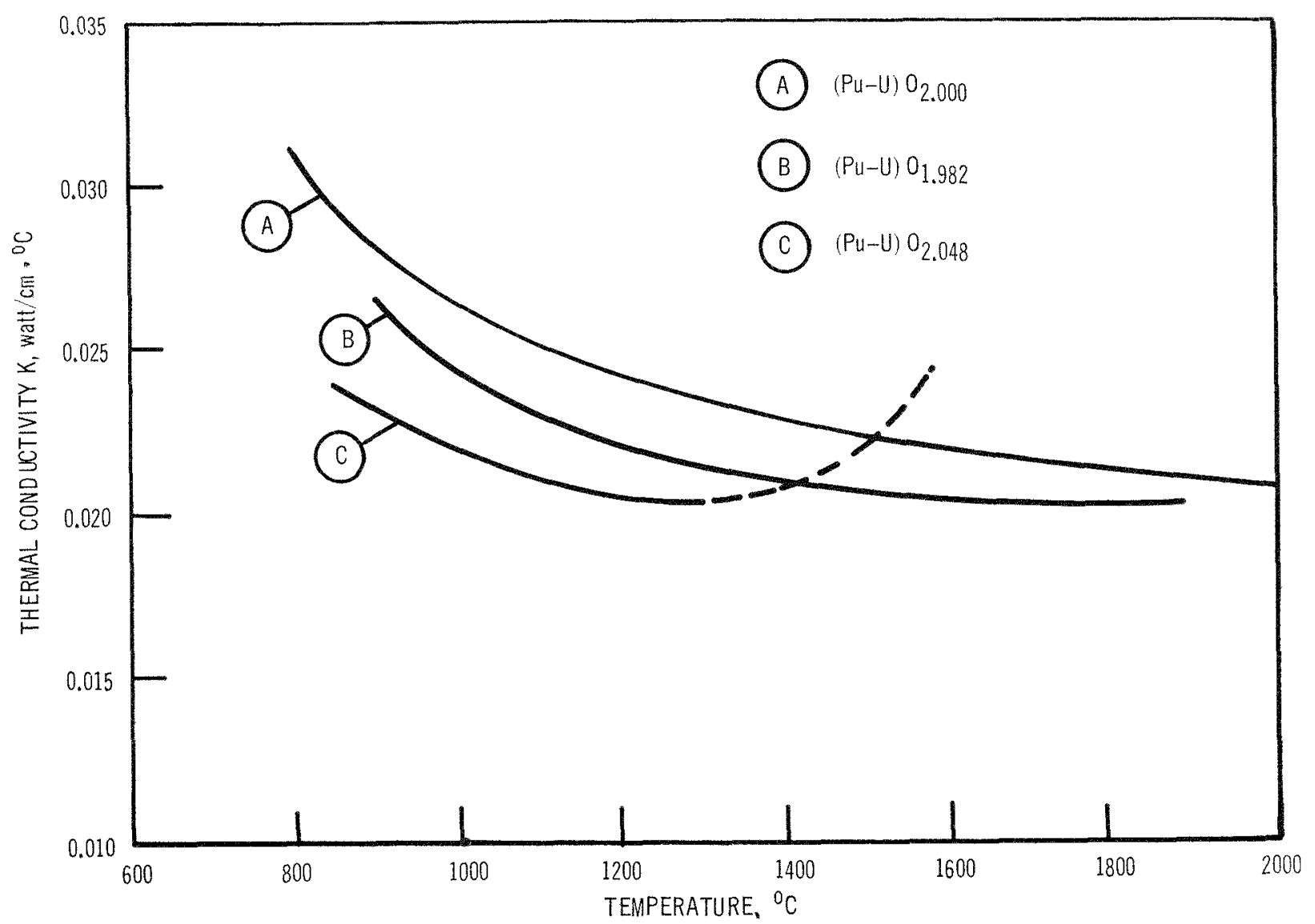

Fig. 9. Thermal Conductivity of Both Hypo- and Stoichiometric $\left(\mathrm{Pu}_{0.2} \mathrm{U}_{0}{ }_{8}\right) \mathrm{O}_{2+\mathrm{x}}$ as a Function of Temperature. 
samples. Early measurements of the $\mathrm{PuO}_{2}-\mathrm{UO}_{2}$ system by the $V$-filament method indicated an anomalous maximum in the melting point at approximately 10 $\mathrm{W} / \mathrm{O} \mathrm{PuO}{ }_{2}{ }^{(24,25)}$

Recent V-filament melting-point measurements on the mixed oxide system under the FCR program also showed an anomalous maximum, but at $5 \mathrm{w} / \mathrm{o} \mathrm{PuO}_{2}$ rather than at the earlicr noted value for $10 \mathrm{w} / \mathrm{o}$ $\mathrm{PuO}_{2}$, ${ }^{(26)}$ Thermal arrest-melting measurements of the $\mathrm{PuO}_{2}-\mathrm{UO}_{2}$ system indicate that with adequate control of sample composition during the measurement this maximum is not observed.

Figure 10 shows the proposed liquidus for the $\mathrm{PuO}_{2}$ $\mathrm{CO}_{2}$ system as measured by thermal-arrest techniques. ${ }^{(27)}$ These measurements indicated a simple binary phase diagram with complete miscibility for the $\mathrm{PuO}_{2}-\mathrm{UO}_{2}$ system. Thermal-arrest measurements at all compositions did not indicate any significant difference between the solidus and liquidus. On this basis it is reasonable to expect that the $\mathrm{PuO}_{2}-\mathrm{UO}_{2}$ system will exhibit a very narrow two-phase field which is consistent with other metal oxide systems, such as $\mathrm{UO}_{2}-\mathrm{ZrO}_{2}, \mathrm{UO}_{2}-\mathrm{ThO}_{2}, \mathrm{Al}_{2} \mathrm{O}_{3}-\mathrm{Cr}_{2} \mathrm{O}_{3}, \mathrm{CeO}_{2}-\mathrm{ZrO}_{2}$, and $\mathrm{ThO}_{2}-\mathrm{ZrO}_{2}$, all of which exhibit a narrow liquid-solid band, approximately 50 to $125^{\circ} \mathrm{C}$ wide.

The melting point of mixed oxides has been shown to be dependent on the stoichiometry. Figure 11 shows both tungsten $V$-filament and thermal-arrest measurements for $\left(\mathrm{Pu}_{0.2} \mathrm{U}_{0.8}\right) \mathrm{O}_{2 \pm x}$ specimens. Results from both techniques agree very well and show that the hypostoichiometric melting temperature is significantly higher than the stoichiometric composition. A similar relationship has also been observed for the $\mathrm{UO}_{2-x}$ system when a $150^{\circ} \mathrm{C}$ higher melting point was observed for $\mathrm{UO}_{198} \cdot{ }^{(28)}$

The maximum noted in $V$-filament molting measurements at approximately 5 to $10 \mathrm{w} / \mathrm{o} \mathrm{PuO}_{2}$ is believed due to a greater rate of loss of oxygen at high temperatures, giving rise to a $(\mathrm{Pu}, \mathrm{U}) \mathrm{O}_{2-x}$ solid-solution compound that forms before melting. This new material then melts at the hypostoichiometric value and gives an apparent maximum in the melting data for stoichiometric specimens. The absence of a maximum in enclosed capsules used for the thermal arrest implies that with massive samples there is little or no oxygen loss.

\section{Electrical Properties of Mixed Oxides at Low T'm- peratures}

Electrical properties of both $\mathrm{PuO}_{2}$ and mixtures of $\mathrm{PuO}_{2}$ and $\mathrm{UO}_{2}$ have been used by a number of investigators to establish phase boundaries and transformation temperatures. Figure 12 shows summary plots of the electrical-property studies. ${ }^{(9)}$ Thermoelectric measure-

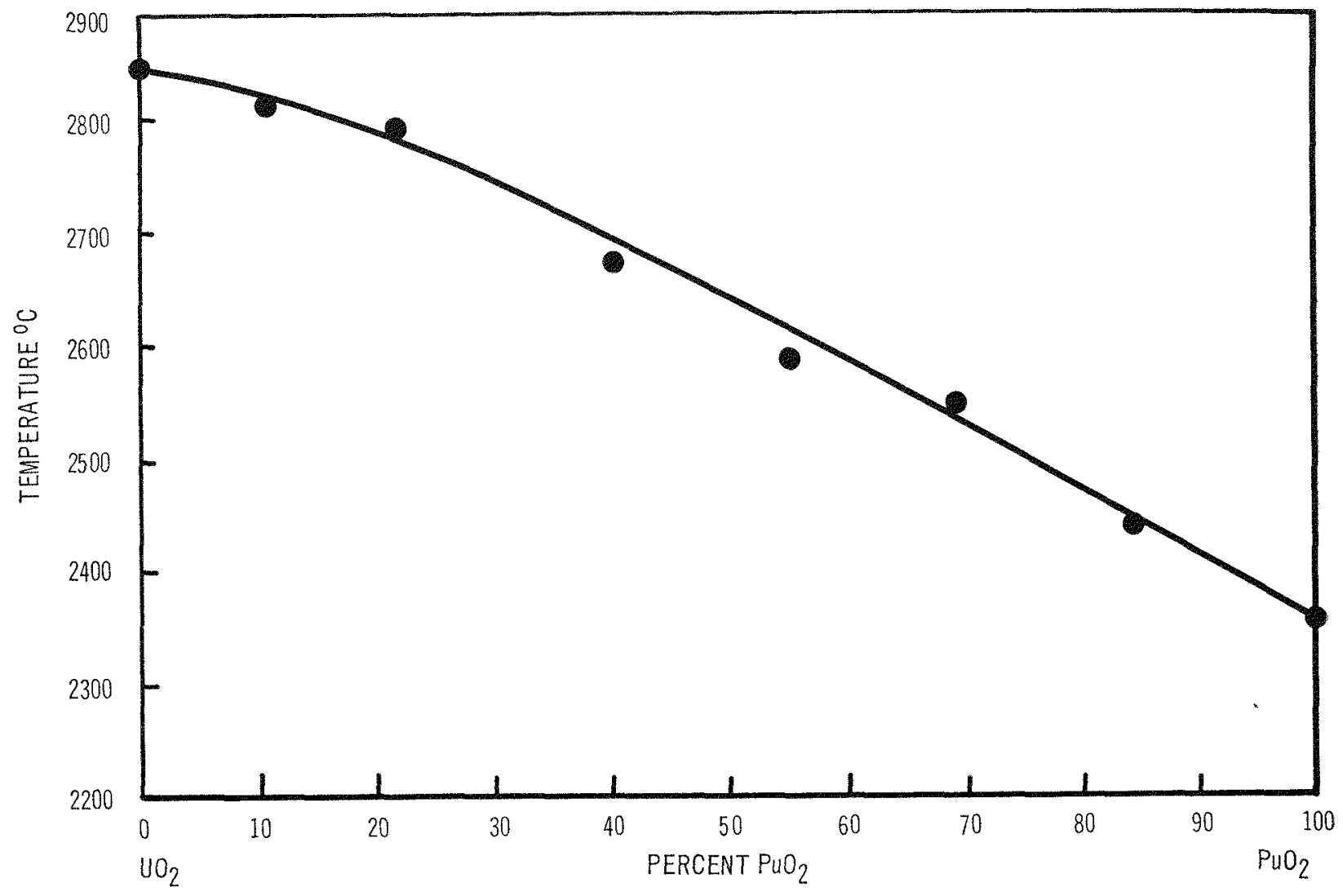

Fic. 10. Liquidus of the $\mathrm{PuO}_{2}-\mathrm{UO}_{2}$ System. 


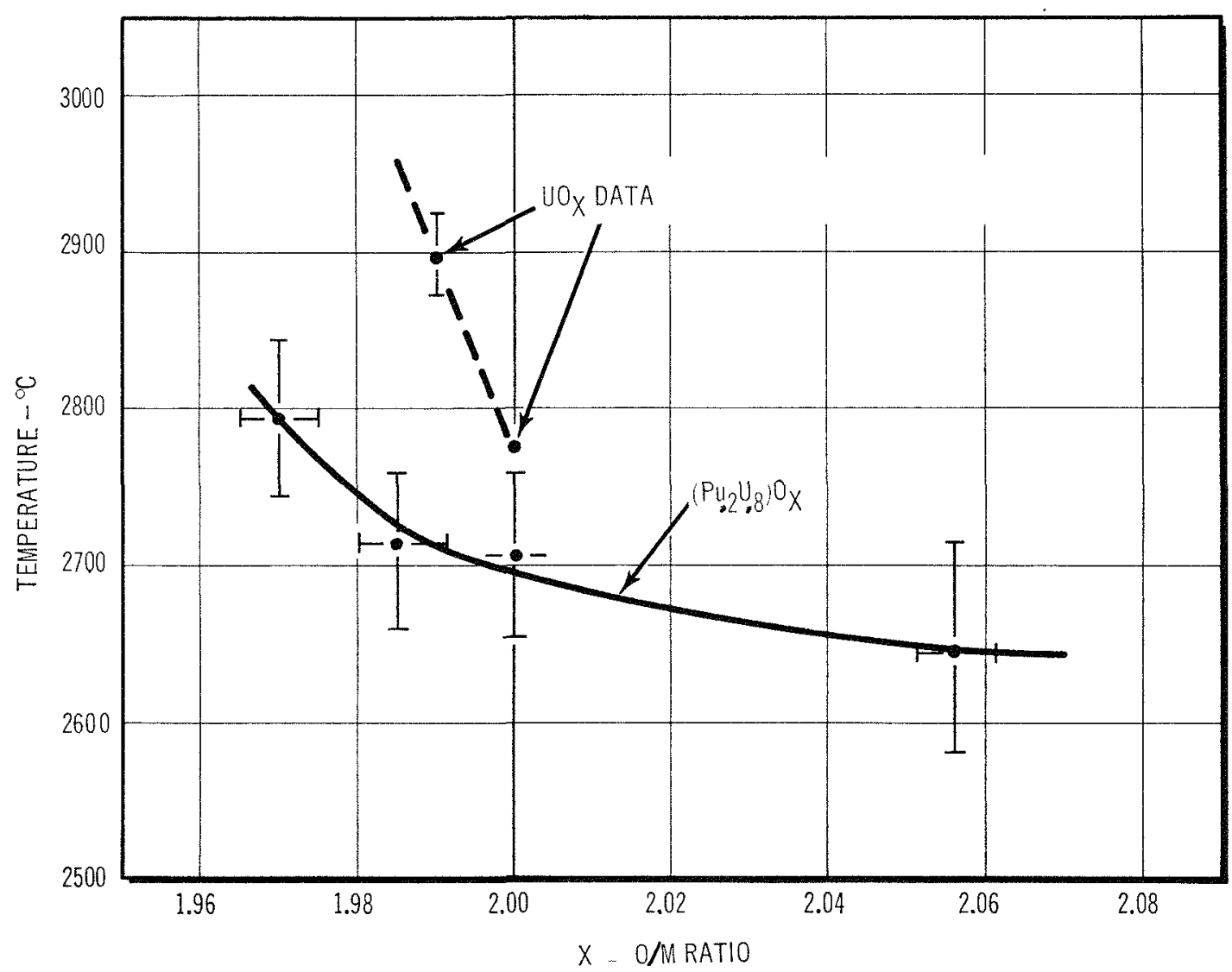

Fic. 11. Effect of Stoichiometry on the Melting Point of $\left(\mathrm{Pu}_{0.2} \mathrm{U}_{0.8}\right) \mathrm{O}_{2}$ Fuel.

ments of the $\mathrm{PuO}_{2}-\mathrm{UO}_{2}$ system show an almost linear decrease in the Scebeck coefficient from $\mathrm{LO}_{2}$ to $\mathrm{PuO}_{2}$. A gradual decrease from $\mathrm{p}$ - to n-type conduction was noted with increased $\mathrm{PuO}_{2}$ content.

Electrical resistivity and thermoelectric measurements were found to give values quite sensitive to stoichiometry. Both propertics increased with decreasing stoichiometry in a manner similar to $\mathrm{CO}_{2}$. The electrical resistivity was found to increase from a value of $4 \times 10^{3} \mathrm{ohm}$-cm for $\left(\mathrm{Pu}_{0.2} \mathrm{U}_{0.8}\right) \mathrm{O}_{1.975}$, suggesting that the electrically neutral compositions in mixed oxides is closer to 1.975 than 2.00 in mixed oxide. (A similar situation was reported by Duncan ${ }^{(28)}$ for the $\mathrm{UO}_{2-x}$ system, which showed that a minimum charge carrier concentration exists at $\sim 1.99 \mathrm{O} / \mathrm{U}$ ratio rather than at 2.00.)

\section{In-pile Behavior of Stoichiometric and Hypo- stoichiometric Mixed Oxides}

\section{A. Short-time Tests}

Both hypo- and stoichiometric $\left(\mathrm{Pu}_{0.2} \mathrm{U}_{0.8}\right) \mathrm{O}_{2}$ specimens have been irradiated under gross melting conditions to establish the difference in the $\int_{T_{s}}^{T_{m}} K d \theta$ of these fuels. This was accomplished by irradiating a hydraulic rabbit capsule in the MTR containing both 1.98 and 2.00 stoichiometry fuel in separate compartments to establish the relative amounts of melting in each fuel section. The stoichiometric specimen exhibited significantly larger radius of melting than the hypostoichiometric fuel. ${ }^{(29)}$ Figure 13 shows the irradiated microstructure of the two fuel specimens. (The central void in these specimens is a shrinkage void and is not necessarily related to the maximum temperature.)

Table 3 lists the capsule test parameters and results. Significantly more melting was noted in the stoichiometric sample and the integral to melting, from surface to center, was $64 \mathrm{~W} / \mathrm{cm}$ and $46 \mathrm{~W} / \mathrm{cm}$ for the samples of stoichiometry 1.98 and 2.00, respectively. Volume change on melting was measured by providing minimum free volume in the pin so that fucl expansion in melting would cause yielding of the cladding. The observed volume change was between 8 and $12 \%$, based on clad expansion. The results from this experiment indicated that hypostoichiometric fuel can operate at significantly higher power level than stoichiometric fuel, based on center melting as a limit. Part of this difference is due to the higher melting point (about $100^{\circ} \mathrm{C}$ ) of the hypostoichiometric fuel. An additional contribution to the higher integral to melting implies 

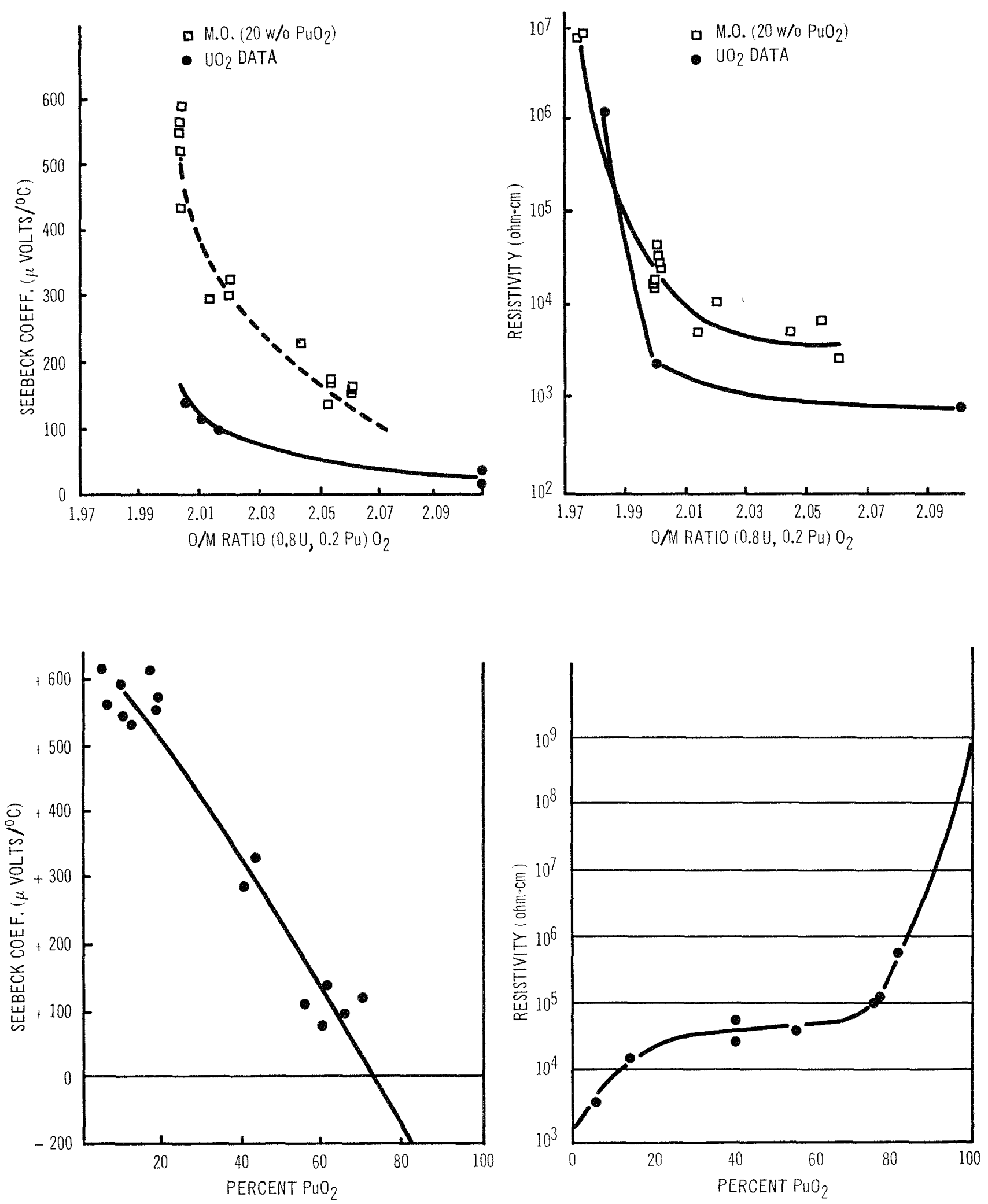

Fig. 12. Electrical Properties of Mixtures of $\mathrm{PuO}_{2}$ and $\mathrm{UO}_{2}$. 


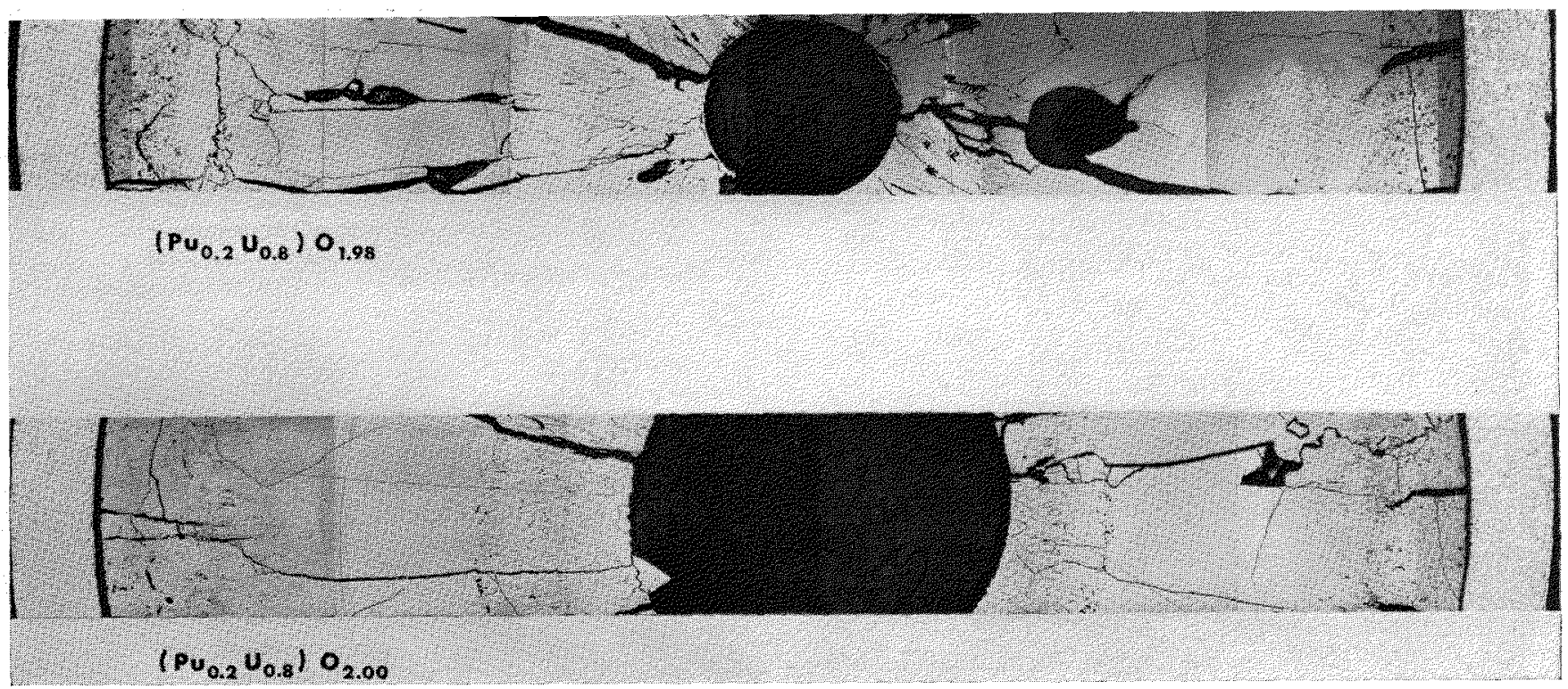

FIG. 13. Microstructure of Hypo- and Stoichiometric Fuel Irradiated at Identical Power Levels.

TABLE 3. Smeared In-pile Thermal Conductivity Results From Hypo- and SToichiometric $\left(\mathrm{Pu}_{0.2} \mathrm{U}_{0.8}\right) \mathrm{O}_{2}$

\begin{tabular}{|c|c|c|}
\hline & $\left(\mathrm{Pu}_{0.2} \mathrm{U}_{0.8}\right) \mathrm{O}_{1.98}$ & $\left(\mathrm{Pu}_{0.2} \mathrm{U}_{0.8}\right) \mathrm{O}_{2.08}$ \\
\hline \multicolumn{3}{|l|}{ Fuel-operating Parameters } \\
\hline $\begin{array}{l}\text { 1. Composition, w/o } \\
\mathrm{PuO}_{2}\end{array}$ & 20 & 20 \\
\hline 2. Stoichiometry & 1.98 & 2.00 \\
\hline $\begin{array}{l}\text { 3. Density, \% Theoreti- } \\
\text { cal }\end{array}$ & 94.5 & 94.5 \\
\hline 4. Backfill Gas & Argon & Argon \\
\hline 5. Pellet Diameter, in. & 0.218 & 0.218 \\
\hline $\begin{array}{l}\text { 6. Diametrical Gap } \\
(\text { Avg), in. }\end{array}$ & 0.002 & 0.002 \\
\hline $\begin{array}{l}\text { 7. } \Phi_{c} / \Phi_{s}, \text { Self-shielding } \\
\text { Factor }\end{array}$ & 0.38 & 0.38 \\
\hline 8. Time in Pile, hr & 5 & 5 \\
\hline \multicolumn{3}{|l|}{ Test Results } \\
\hline 1. Fuel Pin Power, $\mathrm{kW} / \mathrm{ft}$ & $46.6 \pm 5$ & $46.6 \pm 5$ \\
\hline $2 . \int_{T_{s}}^{\alpha_{s}} K d \theta, \mathrm{W} / \mathrm{cm}$ & $94.4 \pm 10$ & $94.4 \pm 10$ \\
\hline $3 . \int_{T_{s}}^{T_{m}} K d \theta, \mathrm{W} / \mathrm{cm}$ & $64 \pm 7$ & $46 \pm 5$ \\
\hline 4. Molten diameter, in. & 0.160 & 0.175 \\
\hline
\end{tabular}

that the high-temperature conductivity of hypostoichiometric fuel is slightly greater than stoichiometric fuel.

\section{B. High-burnup Stability}

Both hyper- and hypostoichiometric mixed oxides have been irradiated under identical power and thermalcycling conditions to an average peak burnup of 70,000 MWD/Tm. ${ }^{(30)}$ Table 4 lists the in-pile test parameters of the experiment.
TABLE 4. IN-PILE Test Parmaeters

\begin{tabular}{l|c|c}
\hline \multicolumn{1}{c|}{ Pin Number } & E2B-1 & E2B-2 \\
\cline { 2 - 3 } Stoichiometry & 2.04 & 1.97 \\
Fuel length, in. & 8 & 10 \\
Diametral gap, mil & 1.8 & 1.8 \\
Fuel diameter, in. & 0.150 & 0.150 \\
Peak power, $\mathrm{kW} / \mathrm{ft}$ & $8.0 \pm 1.0$ & $18.0 \pm 1.0$ \\
Peak $\int_{0}^{T_{0}} K d \theta, \mathrm{W} / \mathrm{cm}$ & $\sim 1000$ & $\sim 1000$ \\
Fuel surface temperature, ${ }^{\circ} \mathrm{C}$ & $\sim 2600$ & $\sim 2600$ \\
Peak fuel-center temperature, ${ }^{\circ} \mathrm{C}$ & & \\
\hline
\end{tabular}

The results from this experiment indicated no systematic difference between the performance of the fuels at a burnup of $70,000 \mathrm{MWD} / \mathrm{T}$. The only microstructural difference noted was that the hyperstoichiometric fuel had a larger central void, suggesting that the irradiated hyperstoichiometric fuel might have a lower thermal conductivity (or higher volatility) than the hypostoichiometric fuel. This is shown in Fig. 14. Metallic fission products were noted dispersed throughout the high-temperature region $\left(1800-2600^{\circ} \mathrm{C}\right)$ in both fuel specimens, and no correlation of the volume of the insoluble phases could be made with stoichiometry. Similar metallic fission products have been previously reported for high-burnup $\mathrm{UO}_{2}$ and $\mathrm{UO}_{2}-\mathrm{ZrO}_{2}$ fuel. ${ }^{(31)}$

\section{Summary and Conelusions}

The reference fabrication method for solid-solution mixed oxide fuel is coprecipitation, pressing, and sintering in hydrogen. Homogeneity with respect to the 


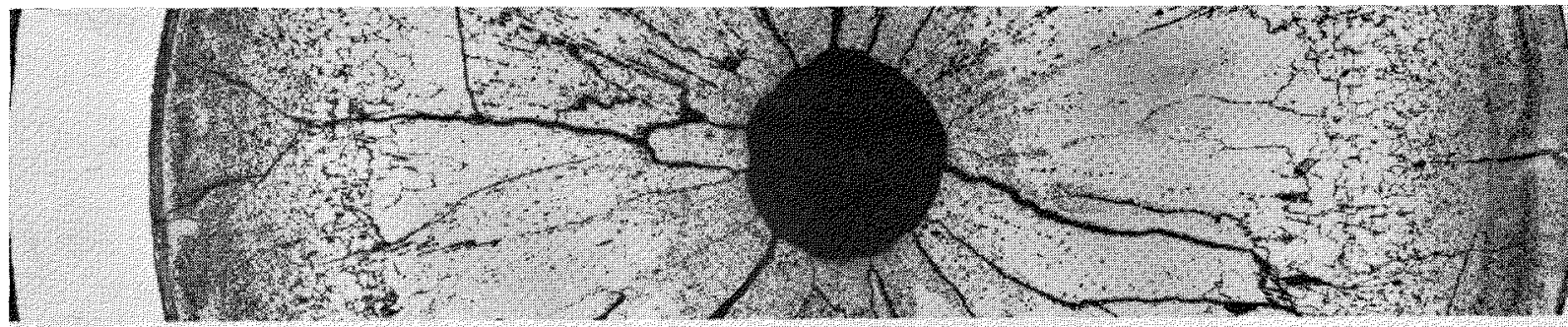

HYPO-STOICHIOMETRIC

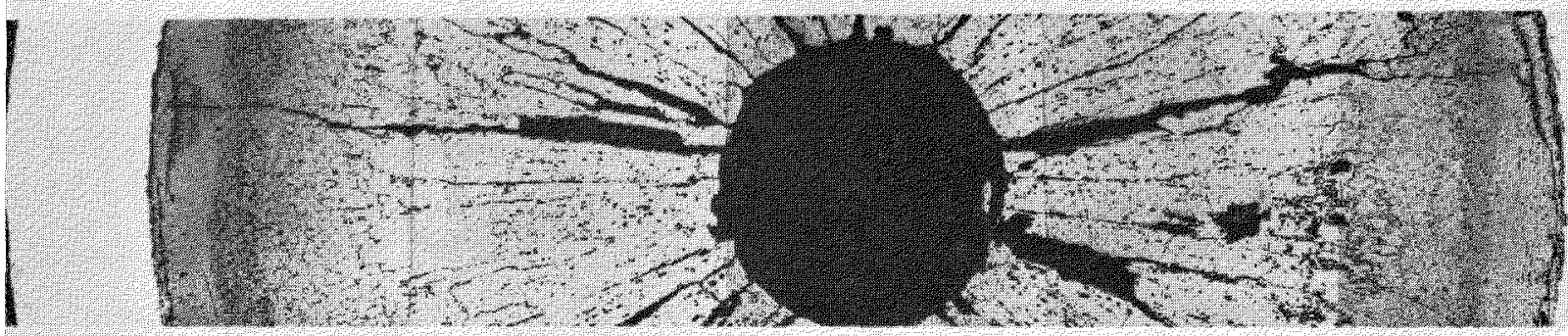

HYPER - STOICHIOMETRIC

$70,000 \mathrm{MWD} / \mathrm{T}$

FIG. 14. Microstructure of Hyper- and Hypostoichiometric Mixed Oxide at 70,000 MWD/T.

micron scale of measurement is readily attained by this process.

Mixing of $\mathrm{UO}_{2}$ and $\mathrm{PuO}_{2}$ powders also shows promise of attaining near-equivalent homogeneity (and 1.97 stoichiometry) after sintering, provided that agglomeration is avoided in the mixing process. Mixtures with agglomerates present imply significant loss of prompt Doppler coefficient.

There is a preliminary basis for preference of stoichiometry of 1.97 to 1.98 for mixed oxide fuel. The most significant properties are thermal conductivity, clad and sodium compatibility, melting point, gas retention, volume expansion (with temperature, with fission products, and on melting), and creep rates under stress. Most of these properties have some dependence on hoth stoichiometry and on burnup.

The usual stoichiometry for $\mathrm{UO}_{2}$ fuels is 2.00 . Sufficient information has been developed to rule out the hyperstoichiometric $\mathrm{UO}_{2}$ compositions based on their poorer thermal conductivity. Hypostoichiometric $\mathrm{UO}_{2}$ on the other hand, shows very definite potential based on a slightly higher thermal conductivity and melting point. One reason for the relative lack of information on hyperstoichiometric $\mathrm{UO}_{2}$ is the difficulty of fabrication.

With mixed oxides of $\mathrm{PuO}_{2}$ and $\mathrm{UO}_{2}$, hyperstoichiometric compositions are conveniently prepared. Outof-pile thermal conductivity data show differences at low temperatures, but closely similar conductivity at
1200 to $1600^{\circ} \mathrm{C}$ for varying stoichiometry. In-pile smeared thermal conductivity data covering a range up to central melting at $2750^{\circ} \mathrm{C}$ indicate approximately $20 \%$ higher conductivity for 1.97 material when compared with 2.00 . The higher melting point of the hypostoichiometric composition is also an advantage. Preliminary results suggest that the overall advantage of 1.97 material in conductivity and/or melting point persists to high burnup. Hypostoichiometric mixed oxide fuel has also better compatibility with sodium at high temperature than either the 2.00 or 2.05 compositions.

Unequivocal selection of stoichiometry is not yet possible since this will involve evaluation and weighting of the relative importance of properties such as those listed above, some of which are not yet accurately known except as approximate limits.

\section{REFERENCES}

1. T. Lauritzen, GEAP-4931, and forthcoming NUMEC Quarterly Report, July-September 1965, NUMEC-3524-8.

2. P. Novak et al., Plutonium and Fission Products Redistribution in Mixed Oxide Fuel, see paper in Session V-A of this conference.

3. S. Goldsmith et al., A Physically Mixed and Impacted $\mathrm{UO}_{2}-\mathrm{PuO}_{2}$ Fast Reactor Fuel, ANs Trans., 7, No. 2 (Nov 1964).

4. W. L. Lyon, The Measurement of Oxygen to Metal Ratio ir Solid Solutions of Uranium and Plutonium Dioxides, GEAP_4271 (May 1963). 
5. T. D. Chikalla and C. E. McNeilly, The Plutonium-Oxygen System, HW-74802 (Sept 1962).

6. Fast Ceramic Reactor Development Program, Tenth Quarterly Report, January-March 1964, GEAP-4601.

7. T. D. Chikalla, Sintering Studies on the System $\mathrm{UO}_{2}-\mathrm{PuO} \mathrm{O}_{2}$. Part I-Solid Solution Formation, HW-60276 (April 27, 1959).

8. F. H. Ellinger and R. N. Mulford, $\mathrm{UO}_{2}-\mathrm{PuO}_{2}$ Solid Solutions, J. Am. Chem. Soc., 80, 2023 (1958).

9. W. E. Baily and W. L. Lyon, Electrical Properties of the Plutonium-Uranium Mixed Oxide System, GEAP-4675 (Sept 1965).

10. G. Dean, Etude des Oxydes de Plutonium et de Leaur Solubilite dans l'Oxyde d'Uranum $\mathrm{UO}_{2}$, to be presented at the 3rd Intnl. Conference on Plutonium, November 1965, London, England.

11. W. H. Zachariasen, Crystal Structure Studies of Oxides of Plutonium, AECD-1787.

12. W. H. Zachariasen, Metallurgical Progress Report, CN-2069 (Aug 1964), p. 35.

13. F. H. Ellinger, "A Review of the Intermetallic Compounds of Plutonium," The Metal Plutonium, The University of Chicago Press (1961), pp. 281-308.

14. Private communication from W. Cummings, GE-Tallecitos X-ray Diffraction Group.

15. W. E. Baily, C. N. Craig, and U. Wolff, Observations on Microstructural Changes with Burnup in Mixed-oxide Fuels, to be presented as ANS Winter Meeting, Washtion, 1965.

16. J. A. L. Robertson, "Some Consequence of Excess Oxygen in $\mathrm{US}_{2}$," Symposium on Radiation Effects in Refractory Fuel Compounds, ASTM Special Technical Publication No. 306 (March 1962).

17. F. J. Hetzler and E. L. Zebroski, Thermal Conductivity of Stoichometric and Hypostoichiometric Uranium Oxide and High Temperatures, ANS Trans., 7, No. 2 (Nov 1964).

18. A. D. Feith, The Thermal Conductivity of $\mathrm{UO}_{2}$ to $2500^{\circ} \mathrm{C}$, J. Nuc. Matls., 16, 231-2 (June 1965).

19. T. G. Godfrey, W. Fulkerson, T. G. Kollie, J. P. Moore, and D. L. MeElroy, Thermal Conductivity of Uranium Dioxide from -57 to $1100^{\circ} \mathrm{C}$ by a Radial Heat Flow Technique, J. Am. Cer. Soc., 48, No.6 (June 1965).

20. J. L. Bates, "Formation of Substoichiometric $\mathrm{UO}_{2}$," in Ceramics Research and Development Operation Quarterly Report Oct.-Dec., 1964, HW-81603.

21. J. E. May, M. J. F. Notley, R. L. Stoute, and J. A. L. Robertson, Observations on the Thermal Conductivity of Uranium Oxide, Trans. ANS, 5, No. 2 (Nov 1962).

22. W. D. Kingery, Introduction to Ceramics, John Wiley \& Sons, Inc., New York (1960).

23. F. J. Hetzler, F. F. Lannin, K. J. Perry, and E. L. Zebroski, Thermal Conductivity of $20: 80(P u, U) \mathrm{O}_{2}$, Trans. ANS, 8, No. 1 (June 1965).

24. S. W. Pijanowski and L. S. DeLuca, Melting Points in the System $\mathrm{PuO}_{2}-\mathrm{CO}_{2}$, KAPL-1957 (April 1960).

25. T. D. Chikalla, The Liquidus for the System $\mathrm{UO}_{2}-\mathrm{PuO}_{2}$, HW-69832 (June 1961).

26. Fast Ceramic Reactor Development Program, Eleventh Quarterly Report, April-June 1964, GEAP-4640.

27. W. L. Lyon and W. E. Baily, The Melting Point of Plutonia-Urania Fuels, to be presented at ANS Winter Meeting, Washington 1965.

28. R. N. Duncan and H. M. Ferrari, Preparation and Proper- ties of Hypostoichiometric Uranium Dioxide, Trans. ANS, 6, No. 1 (June 1963).

29. W. E. Baily and C. N. Craig, Relative In-pile Thermal Conductivity of Stoichiometric and Hypostoichiometric Mixed Oxides of Plutonium and Uranium, to be presented in Winter ANS Meeting, Washington, 1965.

30. E. L. Zebroski, W. E. Baily, and D. P. Hines, Irradiation Behavior of Hyper- and Hypostoichiometric PlutoniaUrania Fuel at 70,000 MWD/T, GEAP-4897 (March 1965).

31. M. Bleiberg, R. M. Berman and B. Lustman, Effects of High Burnups on Oxide Ceramic Fuels, WAPI)-T-1455 (March 1962).

\section{Discussion}

Mr. Wirtz (Karlsruhe): We have observed that stoichiometric $\mathrm{UO}_{2}$ after a fairly short time in a temperature gradient at high temperature changes its stoichiometry by a few percent. We have also observed that thermal conductivity is a function of stoichiometry, as you have already said. So after a short while, every fuel will have adapted to that gradient of stoichiometry. Does this mean something in your consideration?

$M r$. Zebroski: No, I think this was fully anticipated. This was the thing that I was giving the calculation on. There is no question that the phenomenon occurs. The question is to what extent it occurs. This also explains why a simple attempt to construct in-pile peak operating temperature curves from out-of-pile temperature data, particularly low temperature with low thermal gradient, is doomed to failure. I think it is more complicated, but the complexities are the kind I think we can handle now.

Mr. Kummerer (Karlsruhe): You have proposed stoichiometry of 1.97 to 1.98 as a reference phase. As I understand it, that means stoichiometry during the production of the material, because I think that the stoichiometry does change markedly with a different temperature of the fuel. Is this assumption right?

Mr. Zebroski: Yes. The stoichiometry of an initial fabricated 1.98 material will vary from as low as 1.91 at the center to 2.006 at the edge for an effective oxygen partial pressure of $10^{-11} \mathrm{~atm}$.

Mr. Kummerer: You mentioned that the wet sintering process leads to a rather high inhomogeneity over a distance of 20 to 40 microns. What were the conditions of this wet sintering, and what is your definition of inhomogeneity?

Mr. Zebroski: We have simply characterized inhomogeneity by peak to minimum ratio concentration and by effective wavelength of the inhomogeneity as determined by the microprobe. This tends to understate homogeneity, because if the inhomogeneity has a dimension of 1 micron and the analytical method has a resolution of 3 microns, you will not see the extremes 
in concentration and you have to make allowance for this.

Mr. Bradbury (Harwell): In the case of uranium oxide we have observed microsegregation of the fission products to form grain-boundary inclusions. Have you observed similar effects from high-burnup mixed oxide?

Mr. Zebroski: As far as I know, every fuel element we've ever looked at always shows this effect above perhaps 30,000 to $40,000 \mathrm{MWD} / \mathrm{T}$; you always see the second phase.

Mr. Bradbury: Have you examined the constituents of these inclusions at all?

$M r$. Zebroski: I think Bates' work is still the most definitive on this.

$M r$. Skavdahl (Battelle-Northwest): I am interested as to how you analyzed our results to get a 20- to 40micron inhomogeneity. You reported a 20-micron spot on the probe. We went down to a $1 / 2$-micron spot. Our particle size analysis and statistical analysis of the data gave an average particle size of 2 microns and a maximum particle size of about 10 or 12 microns.

$M r$. Zebroski: I think this comes back to the definition of inhomogeneity, which is an area of controversy at least three years old now, and I would guess three of six years more will be needed before it is resolved. I would like to observe again that in the resolution between safety and economics, the economic gain to permitting gross inhomogeneity is very trivial. There are good procedures available for getting homogencity; why defend inhomogeneity so desperately? 


\title{
Comment
}

\author{
R. E. SkavdahL \\ Battelle Memorial Institute \\ Pacific Northwest Laboratory \\ Richland, Washington
}

I. would like to present some recent phase-equilibria data on the plutonium-oxygen and uranium-oxygen binary systems obtained at Battelle-Northwest.

Several substoichiometric plutonium oxide samples, varying in $\mathrm{O} / \mathrm{Pu}$ atomic ratio from 1.92 to about 1.6 , were examined by differential thermal analysis and were found to exhibit (during cooling) a broad exothermic peak at about $250^{\circ} \mathrm{C}$. This corresponds quite closely to the eutectoid reaction at $300^{\circ} \mathrm{C}$ shown in the plutonium-oxygen phase diagram which we published in 1963 . The lower limit of $\mathrm{O} / \mathrm{Pu}$ ratio is given as "approximately" 1.6 , since oxidation of the samples at such low stoichiometry made it difficult to determine the precise stoichiometry existing at the time of the observed reaction.

A small, extremely sharp exothermic reaction on heating was observed at about $875^{\circ} \mathrm{C}$ for a sample which, before heating, had an $\mathrm{O} / \mathrm{Pu}$ ratio of 1.55 , and after cooling, an $\mathrm{O} / \mathrm{Pu}$ ratio of 1.62 . Once again, sample oxidation proved to be a problem. However, the observed reaction agrees very well with the allotropic transformation of $\alpha-\mathrm{Pu}_{2} \mathrm{O}_{3}$ to $\alpha^{\prime}-\mathrm{Pu}_{2} \mathrm{O}_{3}$ shown in our phase diagram to occur at $800^{\circ} \mathrm{C}$ between $\mathrm{O} / \mathrm{Pu} 1.50$ and 1.62. The differential thermal analyses were performed by C. E. McNeilly.

High-temperature reflection electron micrographs were taken by Daniel, Chikalla, and Bauer during the course of heating a sample of $\mathrm{PuO}_{1.8}$. The smooth polished surface of the specimen began to coarsen at about $700^{\circ} \mathrm{C}$, which is consistent with the transformation to the single-phase $\mathrm{PuO}_{2-\mathrm{x}}$ predicted by the phase diagram. No further changes were noted until a temperature of $1000^{\circ} \mathrm{C}$ was reached. After cooling to room temperature, the sample surface was marked by a series of heavy ridges similar to the lamellae typical of substoichiometric plutonium oxide and which we normally take to result from the eutectoid reaction at $300^{\circ} \mathrm{C}$.

Increased confidence in the location of the substoichiometrie liquidus line in the uranium-oxygen system has resulted from additional melting-point data by Bates and Horn. Seven data points have been added, as shown in Fig. 1. Each point represents approximately 15 separate melting-point determinations.

A capsule containing uranium oxide with $\mathrm{O} / \mathrm{U}$ between 1.93 and 1.98 was irradiated for one cycle in the MTR. At the heat-generation conditions $(23 \mathrm{~kW} / \mathrm{ft})$ of the irradiation, melting to $\sim 40 \%$ of the fuel radius and columnar grain growth to $\sim 72 \%$ of the fuel radius would be expected in stoichiometric $\mathrm{UO}_{2}$. However, the substoichiometric material exhibited melting to only about $20 \%$ of the radius and columnar grain growth to about $70 \%$. This is further evidence of the improved thermal conductivity of substoichiometric oxide fuel.

\section{Discussion}

Mr. Okrent (ANL): This really relates to a previous question raised by Dr. Wirtz. Since the stoichiometry in fact varies with radial fission during an actual irradiation, what does it mean when you say had the stoichiometry been 2.00 you would have gotten $40 \%$ melting during irradiation and in fact you got $20 \%$ melting? It is not clear to me how one makes the specification that he wishes 1.97 ; this must mean that he expects some different gradients under actual operation with 1.97 than were it 1.92 or 2.02. I would appreciate having both of these points elaborated.

$M r$. Skavdahl: The observation was an empirical one, based on what we had previously observed in stoichiometric $\mathrm{UO}_{2}$ experiments at these power-generation conditions. With $\mathrm{UO}_{2}$ we would have expected to have observed about $40 \%$ melting along the radius. However, we started with a lower stoichiometry, again in a gross sense, and we got less melting for the same power generation. I would not care to draw a plot of melting radius or thermal conductivity versus stoichiometry at various positions. I am not able to do that myself.

$M r$. Kelman (ANL) : I'm confused about the reference to liquidus temperatures and melting point with no reference to solidus temperatures; what is it that you are referring to? 


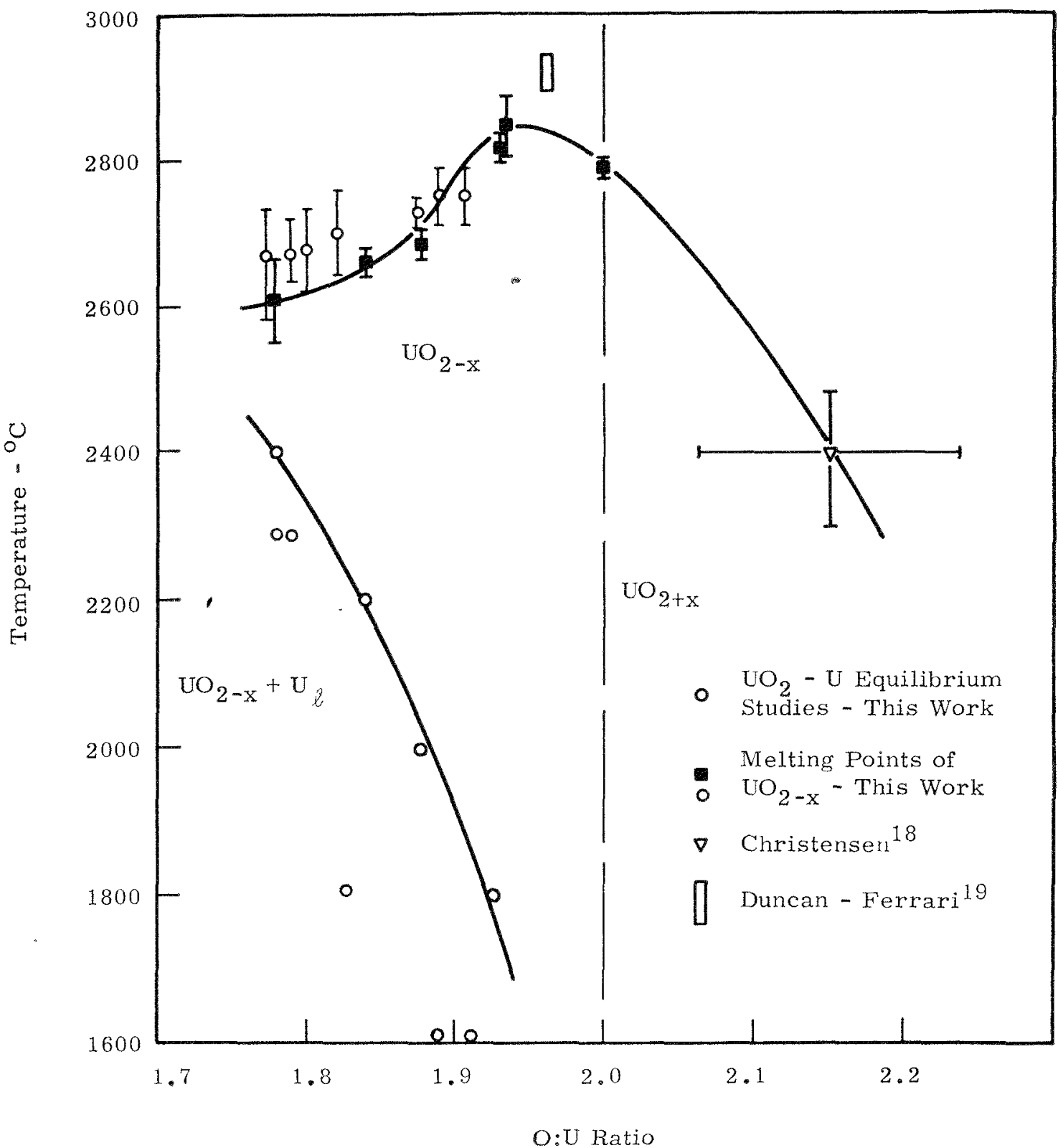

FIG. 1. Tentative Oxygen-Uranium Phase Diagram.

Mr. Skavdahl: The melting points were those observed.

Mr. Kelman: But is this supposed to be congruent melting? Or do you have a solidus and liquidus temperature?

Mr. Skavdahl: It is congruent melting for a single phase.

Mr. Zebroski (GE): I think the question is that you can't just have a single line. With a massive sample you get a thermal arrest, but it is a tiny one. Thus the solidus and the liquidus lines are separated by no more than 50 to $100^{\circ}$, and this is in accord with many other binaries like urania-ceria, urania-thoria, urania-zirconia, and so on, which also have very narrow differences of the order of from as little as 25 to 100 degrees. I think it just becomes experimental technique when it is that small.

Mr. Skavdahl: I think the error band is actually almost bigger than the difference in the temperature between the solidus and liquidus.

Mr. Okrent: I wonder if Dr. Zebroski would give more of the reasons for the 1.97 in view of the fact that he does have 1.97 at only one radius in the actual operations.

Mr. Zebroski: Well, I think Dr. Skavdahl really gave a very good answer to that empirically. You 
have an average value and then you swing about the tie line. It works out from the volume relationships that the mid-volume point, which is about $70 \%$ of the radius thermodynamically, stays about the original stoichiometry. The bulk of the fuel at that point rises very slightly to just about stoichiometric, and a very small volume fraction of the fuel, I think of the order of $5 \%$, reaches the extreme low limit of stoichiometry that I mentioned.

Mr. Okrent: I assume that there may be other properties that deteriorate which we don't yet under- stand, such as swelling. This may turn out to be worse in the cooler portions.

Mr. Zebroski: This has already been observed. Nobody has done experiments in which he says stoichiometry shall stay level. This has been with us always.

Mr. Okrent: I agree. What I am wondering is, will everything be optimum at the 1.97 or only some properties?

Mr. Zebroski: No, this is only a present choice. As of right now, the choice looks like a reasonable compromise with the many properties you have. 


\title{
Plutonium and Fission Product Redistribution in Mixed Oxide Fuels
}

\author{
P. E. Novak, T. A. Lauritzen, R. Protsik, J. H. Davies, axd E. L. Zebroski \\ General Electric Company \\ Advanced Products Operation \\ San Jose, California \\ (PRESENTED BY P E. NOVAK)
}

\section{Introduction}

The distribution of plutonium and fission products in mixed oxide fuels is important to the safety and life of fast reactor systems. The interest in plutonium distribution stems from the importance of the negative Doppler coefficient to reactor safety and its dependence on fuel homogeneity. Separation of the plutonium from the uranium reduces the average temperature rise in $\mathrm{U}-238$ relative to the case of complete homogeneity and thus reduces the negative Doppler coefficient. Fission product concentration gradients do not materially influence the Doppler physics of a fast reactor system because of the relatively small amount of heat liberated by neutron capture in fission products. However, the concentrations of chemically reactive species, such as cesium and its precursor, iodine, near the fuel-clad interface may adversely affect the performance of the cladding material.

Several physical and chemical processes are potentially available to produce plutonium and fission product concentration gradients in operating mixed oxide fuels. The most likely mechanisms are:

(1) zone refining by solidus-liquidus separation (change of state);

(2) zone refining by solid-state pore sweeping in a thermal gradient;

(3) differential mass transport of plutonium-rich phases by vaporization and deposition;

(4) solid-state diffusion-induced separation in a thermal gradient (Soret effect);

(5) electrorefining by an emf produced in a thermal gradient;

(6) selective chemical reactions in fuel operating in contact with sodium.

In addition, fission product daughters with gaseous precursors typically show extensive redistribution within the fuel by diffusion and flow of the gases.

It is the purpose of this paper to explore some of these separating or de-mixing phenomena which might occur and to assess both their magnitude and consequences to the operation of fast reactor systems.

\section{Previous Observations of Plutonium and Fis- sion Produet Concentration Gradients in Oxide Fuel}

Many investigators have observed migration of volatile and gaseous fission products in $\mathrm{UO}_{2}$, thoria, and to a limited extent in mixed oxides. However, there is still a wide variation in the patterns reported, presumably because of the difficulty of determining or controlling all of the parameters which can affect the distribution of fission products in the fuel. One fairly consistent pattern is the concentration of $\mathrm{Cs}-137$ at the cooler periphery of the fuel. ${ }^{(1-4)}$ Another is the tendency for slight enrichment (10 to $20 \%$ of average value) of refractory fission products, such as $(\mathrm{Ce}, \mathrm{Pr})-$ 144 , at the fuel center.

There is little agreement on plutonium relocation. Both the 1963 work of Ruiz ${ }^{(5)}$ on the FCR program and the later work on $\mathrm{UO}_{2}$ by Bates ${ }^{(1)}$ indicated slight increases in plutonium concentrations near the center of mixed oxide and $\mathrm{UO}_{2}$ fuel rods, whereas Bailey and Chikalla(2) reported insignificant migration of plutonium.

Lewis ${ }^{(4)}$ reported that the measured plutonium concentration in irradiated $\mathrm{UO}_{2}$ varied less than $\pm 5 \%$, with the exception of two unexplained low-plutonium data points at the midradius of one pellet rod. Figures 1 and 2 show some of these observations.

In much of this work it is not clear whether the trends reported are significantly larger than the possible sampling and analytical uncertainties. However, there does appear to be agreement that the plutonium concentration generally follows the refractory (Cr,Pr)-144 fission product concentration. Interpretation of some of these experimental results is complicated by the large self-shielding corrections that must be made to correct for the preferential surface burnup of plutonium, and for both thermal and resonance capture by $\mathrm{U}-238$ in a thermal reactor spectrum. 
Plutonium and Fission Product Redistribution in Mixed Oxide Fuels

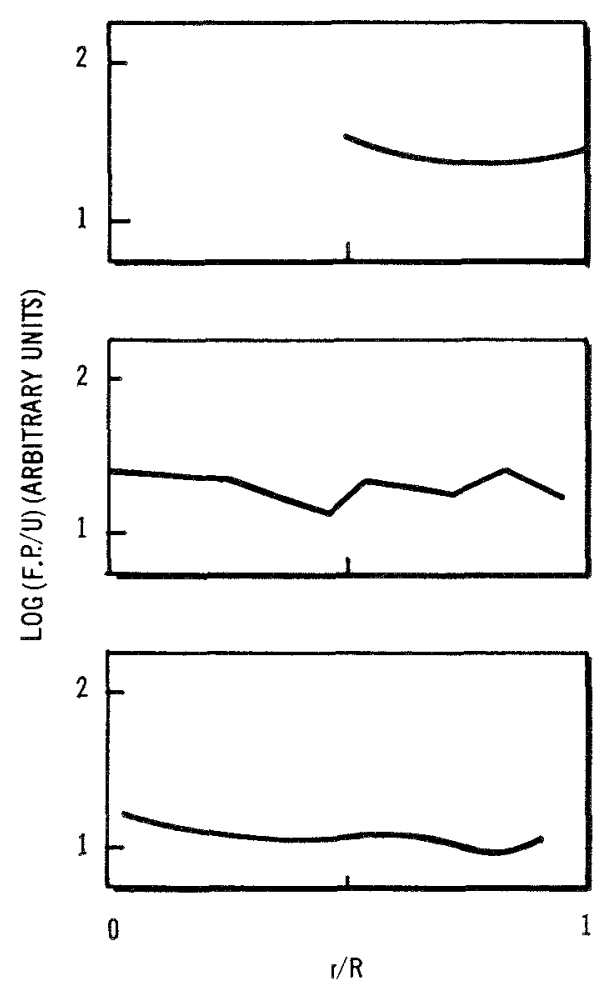

a) (Ce, Pr)-144 DISTRIBUTION

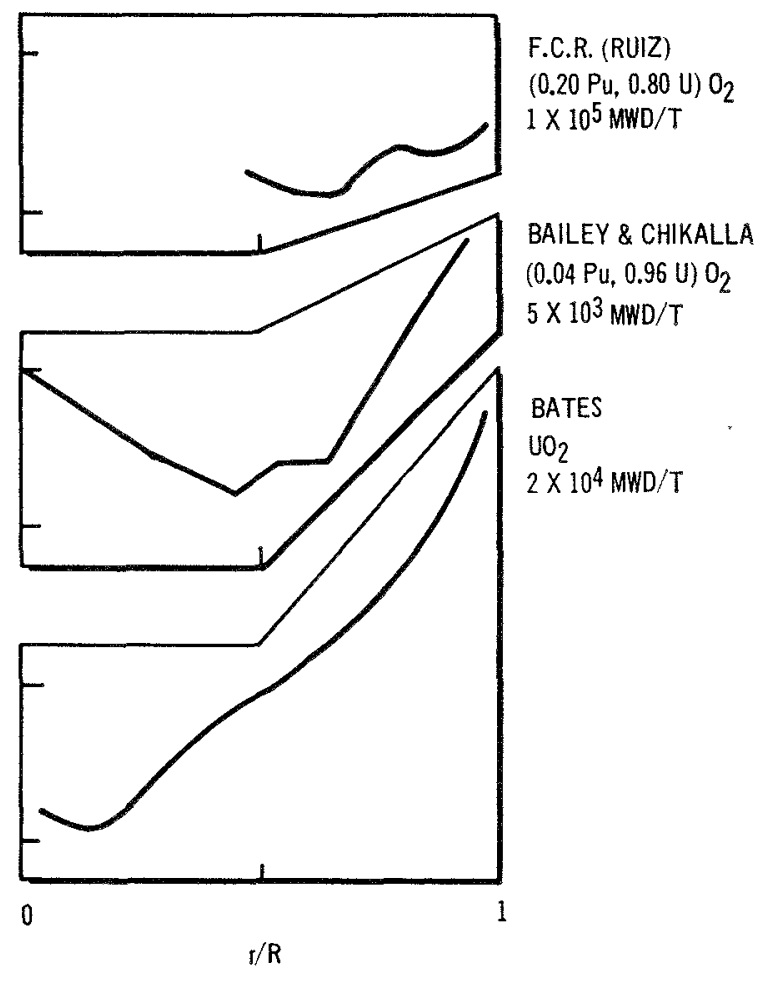

b) Cs-137 DISTRIBUTION

Frg. 1. Previously Observed Fission Product Distribution in Oxide Fuel.

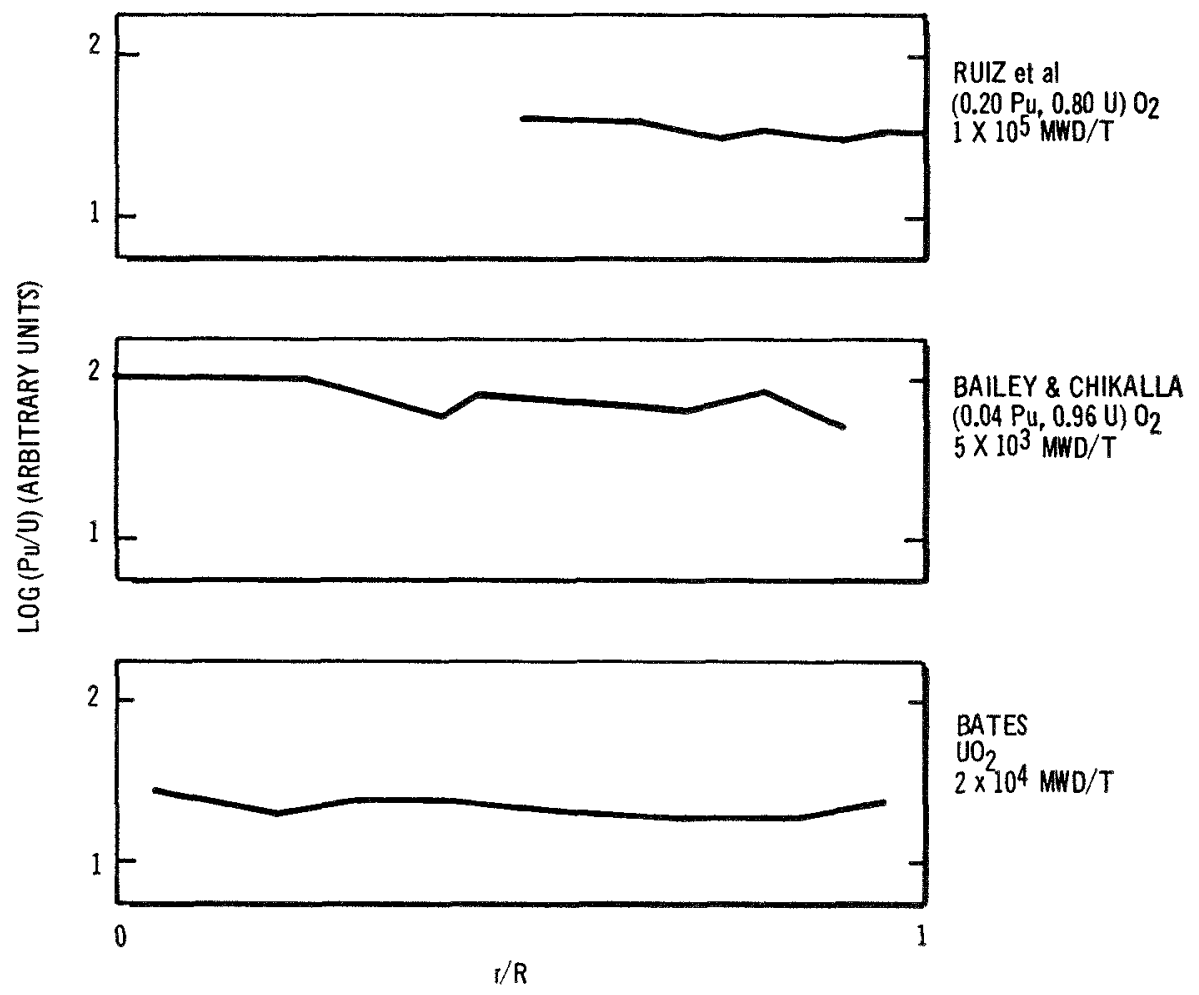

FIG. 2. Previously Observed Plutonium Distribution in Oxide Fuel. 


\section{Recent Measurements of Plutonium and Fis- sion Product Concentration Gradients in Mixed Oxide Fuel}

Recent measurements were made both in-pile and out-of-pile to determme plutonium migration rates quantitatively. Davies and Novak ${ }^{(\varepsilon)}$ extended the work of Schmitz and Lindner ${ }^{(7)}$ from 1500 to $2400^{\circ} \mathrm{C}$, and measured the self-diffusion coefficients of $\mathrm{Pu}-242$ and U-233 in $\mathrm{UO}_{2}$ to be $3.4 \times 10^{-10}$ and $2.6 \times 10^{-10}$ $\mathrm{cm}^{2} /$ sec, respectively, at $2400^{\circ} \mathrm{C}$, indicating plutonium to be slightly more mobile than uranium in the solid state.

An in-pile experiment was then performed to evaluate the effect of stoichiometry on plutonium and fission product distribution after irradiation and to evaluate possible separation effects. Figure 3 is a montage which correlates the distribution of (1) alpha activity, (2) beta-gamma activity, and (3) specimen microstructure for 0.25 -in.-diameter, $20 \%$ plutonium$80 \%$ uranium mixed oxide specimens of three different initial stoichiometries irradiated to $5000 \mathrm{MWD} / \mathrm{T}$.

Striking variations in structures and distribution of both fission products and plutonium are evident. However, the tracer-spiked regions intended to measure plutonium diffusion coefficients at several different temperatures were lost due to accidental gas blanketing of portions of the $\mathrm{NaK}$ bond external to the fuclpin cladding. This caused clad failure. Thus these specimens do not represent normal full-power operation; rather, they are indicative of possible effects under extreme operating conditions. This experiment has been repeated under more normal conditions; the results are presently being analyzed.

The microstructures of the hyperstoichiometric $(\mathrm{O} / \mathrm{M}=2.05)$ and stoichiometric $(\mathrm{O} / \mathrm{M}=2.00)$ fuels are shown in Fig. 4. They consist of three discrete rings about a central shrinkage void. The ring immediately adjacent to the void has vestiges of dendritic grains, probably formed either by vapor transport or by solidification of a molten two-component system. The former hypothesis agrees with observations reported at Hanford; ${ }^{(8)}$ the latter agrees with work done at Chalk River. ${ }^{(9)}$ The middle ring in both specimens consists of short, thick columnar grains, the innermost portions of which are essentially free of voids. The remainder of the fuel contains long, thin columnar grains extending out to the periphery.

The hypostoichiometric $(\mathrm{O} / \mathrm{M}=1.98)$ pin contains only one structural zone, consisting of long, thin columnar grains extending from the void to the periphery. No discrete pore-sweeping boundary is evident, and substantial clad deformation was observed in the overheated zone, suggesting that this specimen experienced a somewhat higher local temperature than the other two. The entire cross section may have undergone melting.

The alpha autoradiographs for this specimen (see Fig. 3) show a higher alpha activity (plutonium concentration) in the fuel bordering the central shrinkage void, and along the pellet periphery, with a relative depletion in the midradial region. The width of the inner high-alpha-actrvity zone $(\sim 20$ mils $)$ corresponds to the width of the dendritic layer in the hyperstoichiometric and stoichiometric pins. The zone width in the hypostoichiometric pin is of similar magnitude, although its interfaces do not correlate with a discrete fuel structure. Progressing outward, the gradwal reduction in alpha activity in the region of the midradius is followed by a stcep actirity rise as the pellet periphery is approached. This is particularly cvident in the hypostoichiometric pin, which shows a well-defined peripheral band of high alpha activity from which radial needles of similar high activity

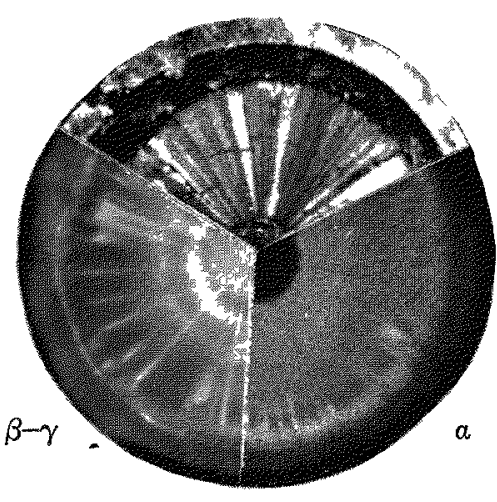

$0 / \mathrm{M}=1.98$

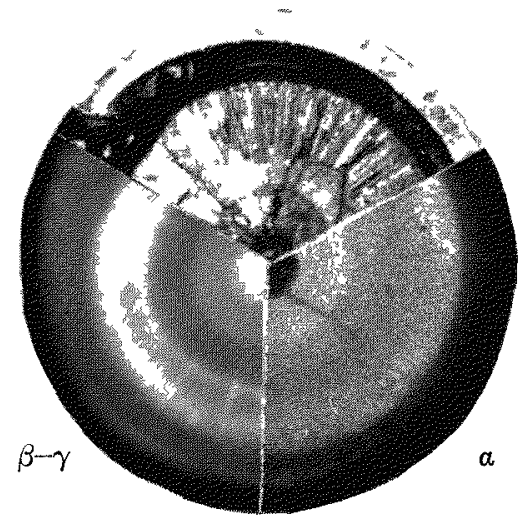

$0 / \mathrm{M}=2.00$

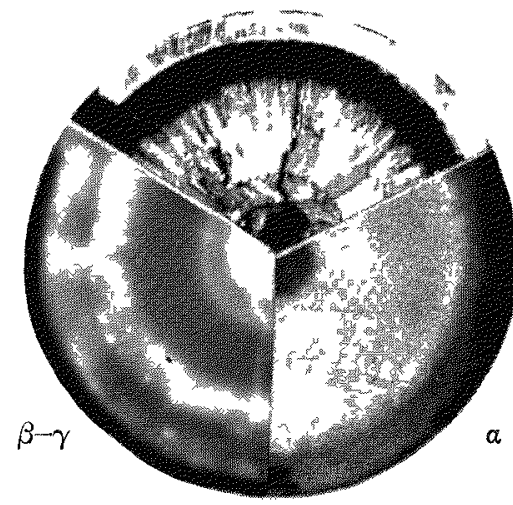

$0 / \mathrm{AI}=2.05$

Fig. 3. Plutonum and Fission Product Distribution in Mixed Oxide Fuel. 


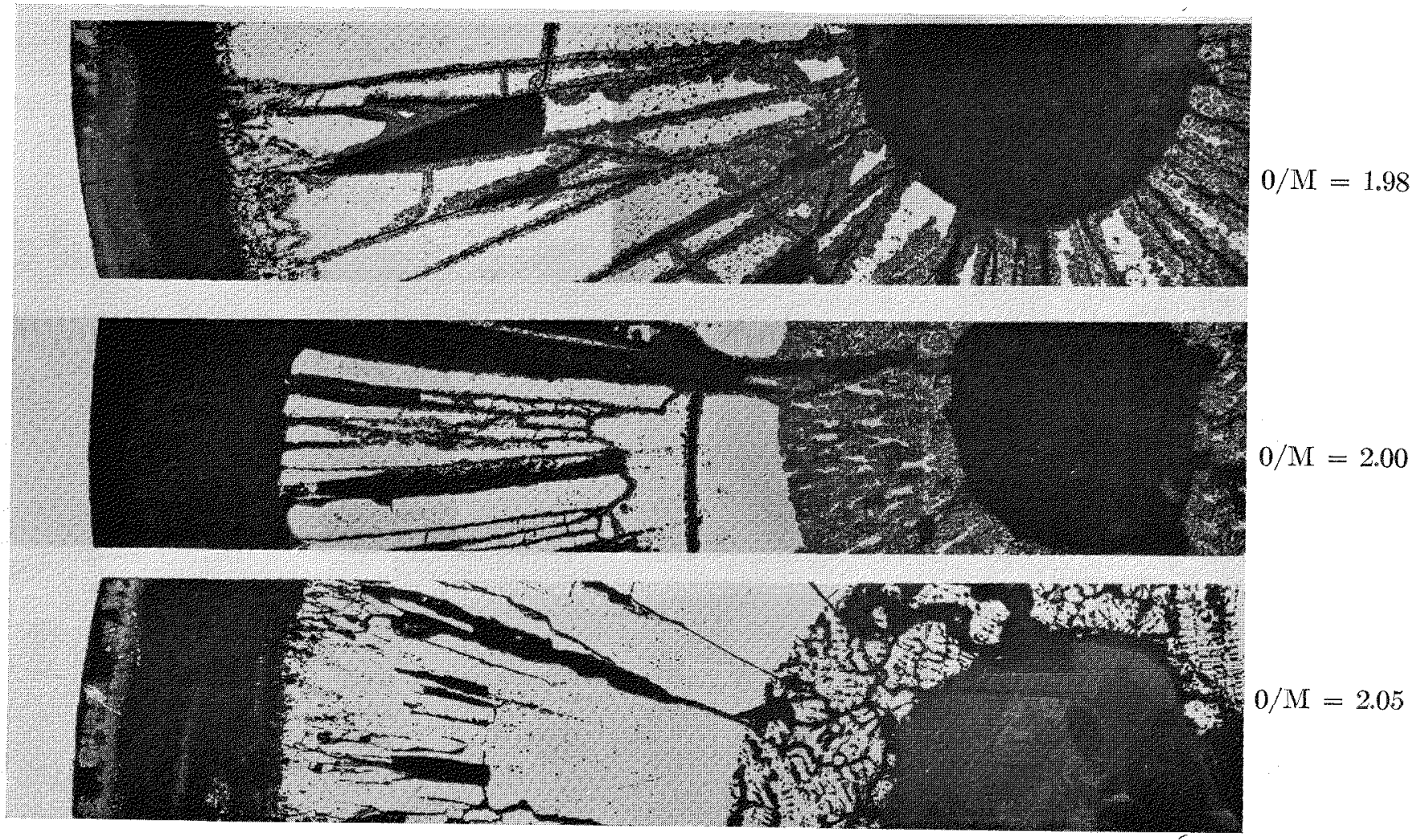

Frg. 4. Mixed Oxide Fuel Structure.

extend toward the thermal center. The spaces between the needles exhibit low alpha activity suggestive of plutonium depletion. In addition, the fuel-clad gap, which contains an apparent NaK-fuel reaction product, also appears depleted in alpha activity. The gradual activity changes in the midradial region and at the periphery bear no direct correlation with the columnar grain structure observed in these regions, nor is there any observable activity change in the region where pore sweeping ceases.

Beta-gamma autoradiographs, representative of the same three fuel surfaces, show a closer correlation with grain structure. Again the hyperstoichiometrie and stoichiometric pins show similar distribution. The midradial band is essentially void of beta-gamma activity, while the periphery of the fuel contains the highest beta-gamma activity. The dendritic region exhibits somewhat lower activity. Beta-gamma activity distribution in the hypostoichiometric pin is concentrated primarily in a thick zone surrounding the central void and along the pellet periphery. Thin fingers of high activity were observed radially throughout the entire fuel cross section.

Figure 5 shows the plutonium concentration as a function of position along the radii of the stoichiometric and hypostoichiometric specimens. It also shows plutonium distribution in a very-low-burnup mixed oxide specimen that sustained gross central melting during a 10-min irradiation. Note that melting in the unfailed fuel pin did not cause the degree of segregation observed in the higher-burnup, failed fuel specimens that operated in contact with sodium. There appears to have been no axial plutonium redistribution.

The solid lines shown in Fig. 5 represent ultrasonically drilled 0.015 - and 0.030 -in.-diameter microcore samples from the high-burnup specimens and $\mathrm{X}$-ray fluorescence analysis on the low-burnup specimen. In the X-ray fluorescence analysis a $0.040 \times$ 0.080-in. elliptical probe was used. The dashed line in the hypostoichiometric specimen represents the analysis of two loosely held fuel particles removed from the highly alpha-active, needle-like zone and periphery of the specimen with adhesive backed tape.

Mass-spectrometric analyses of sample solutions showed that the concentration of plutonium across the fuel sections correlated closely with the relative alphaactivity profile shown in the autoradiographs.

The concentration of both plutonium and fission products into two general areas of the fuel indicates that separation occurred by at least two discrete mechanisms. Enrichment around the central shrink- 


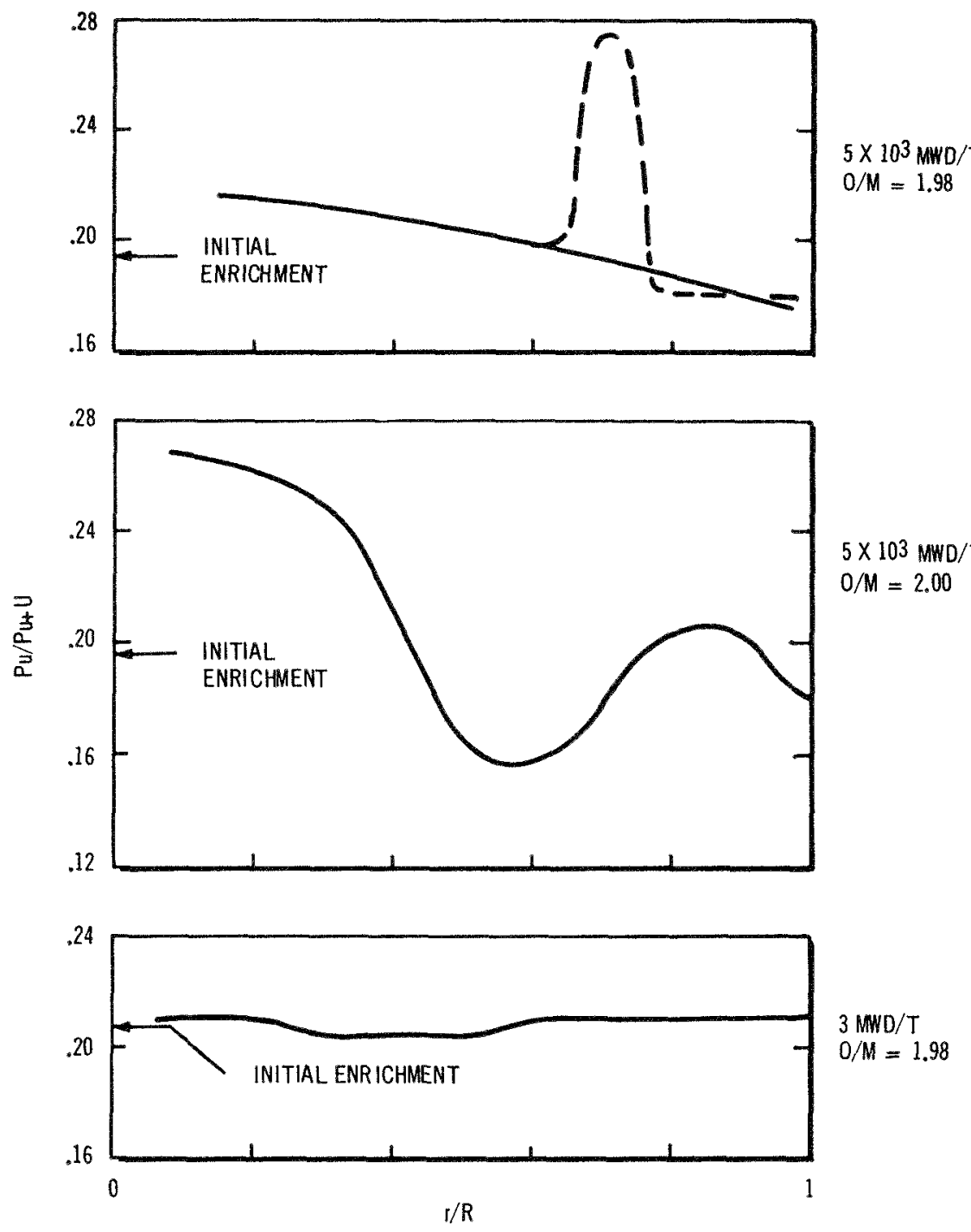

Fia. 5. Observed Plutonium Distribution in Mixed Oxide.

age void with evidence of gross fuel melting suggests the preferential separation of plutonium upon the solidification of molten mixed oxide. Zone refining may contribute to the central enrichment by preferentially depleting the midradial region. Sodium reaction with the failed fuel probably accounts for the narrow concentration and depletion bands at the periphery.

In the case of fission products, the same mechanisms that separated plutonium can separate refractory fission products. However, the additional process of "distillation" is available to concentrate volatile fission products and their precursors at the periphery of the fuel.

\section{Consequences of Plutonium Redistribution on the Calculated Doppler}

The consequences of various patterns of plutonium redistribution on Doppler reactivity were calculated for a large, sodium-cooled fast reactor fueled with mixed oxide. Assumptions used in these calculations include: no heat conduction during a transient, no Doppler saturation, no effect on neutron spectrum by plutonium redistribution, negligible spatial neutronflux depression, no radial fuel densification, no axial migration, and the final fuel temperature is a function of initial temperature and fissionable isotope concentration. The reactor analyzed had a 2-ft-high by 11.7-ft-diameter pancake core with a ratio of 0.092:0.005, respectively. The plutonium content of the mixed oxide fuel was $24 \%$, with $13.65 \% \mathrm{Pu}-239$ and $7.67 \% \mathrm{Pu}-240$, the balance being higher isotopes of plutonium. The calculational transients terminated at an average fuel temperature of $2000^{\circ} \mathrm{K}$.

The two basic calculational models for plutonium segregation are shown in Fig. 6. The parabolic distribution corresponds to the core-sampled hypostoichiometric specimen; the parabolic distribution without 


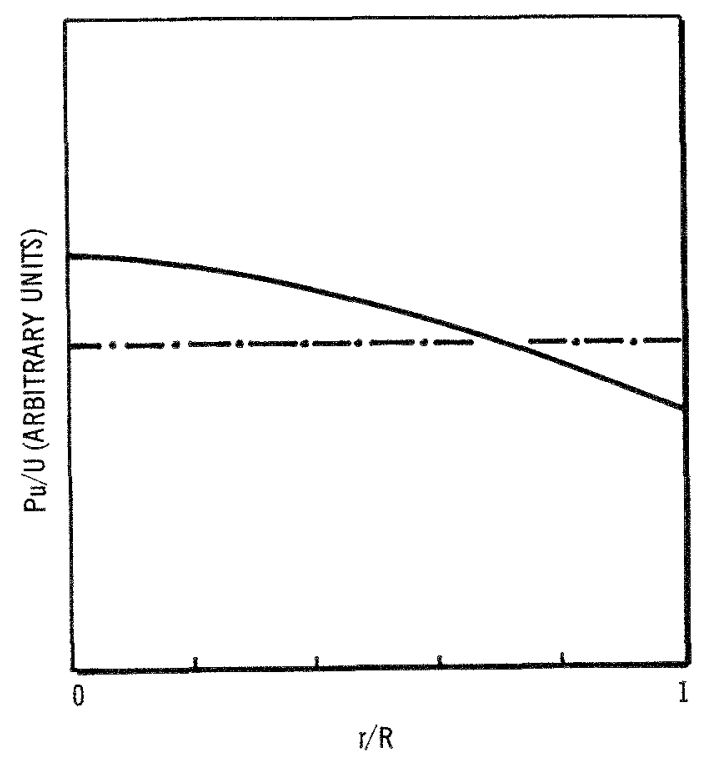

a) PARABOLIC PU DISTRIBUTION

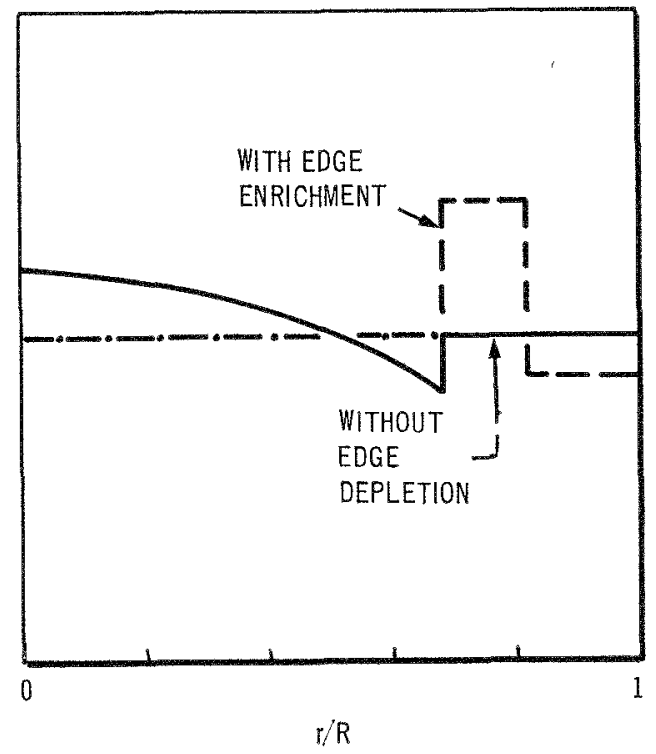

b) PARABOLIC PU DISTRIBUTION (MODIFIED)

Fıg. 6. Calculational Plutonium Distribution Models.

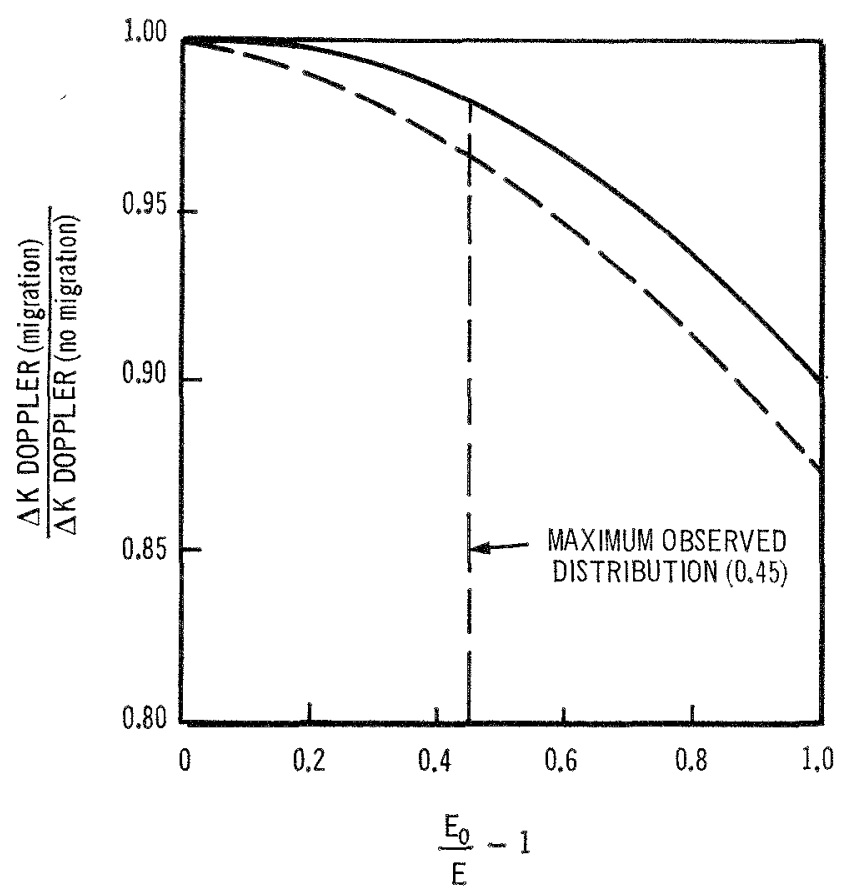

a) PARABOLIC PU DISTRIBUTION

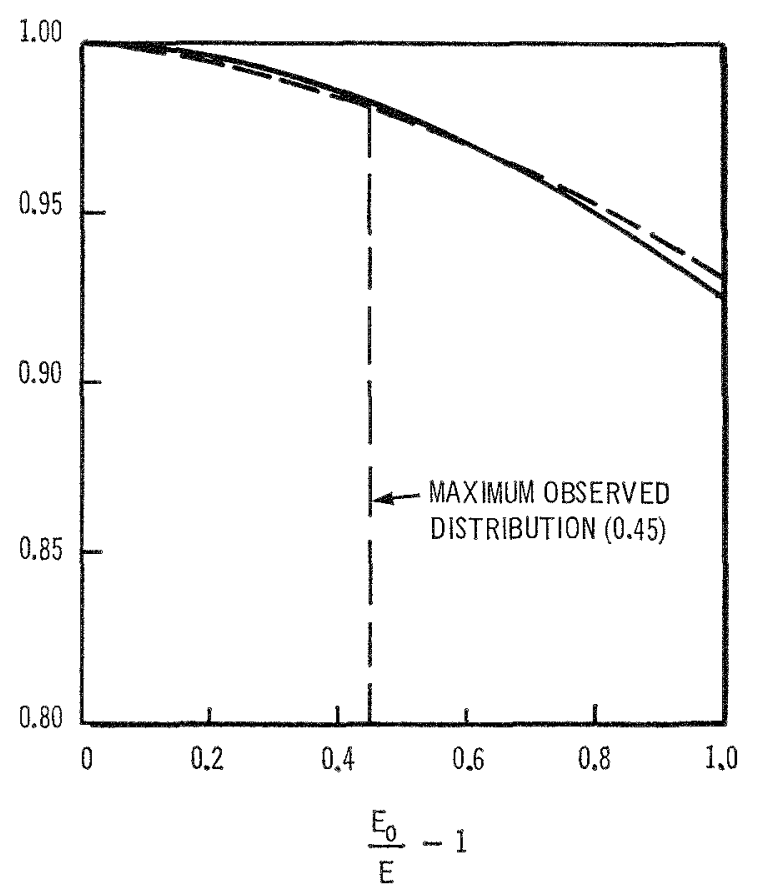

b) PARABOLIC PU DISTRIBUTION WITHOUT EDGE DEPLETION

$$
\begin{array}{ll}
- & 750-2000 \% \\
- & 1450-2000 \%
\end{array}
$$

Fig 7. Effect of Plutonium Migration on the Doppler Coefficient of Reactivity. 
TABle 1. Calcclated Doppler Reactivity Cefange due to Observed Puutonium Redistribution

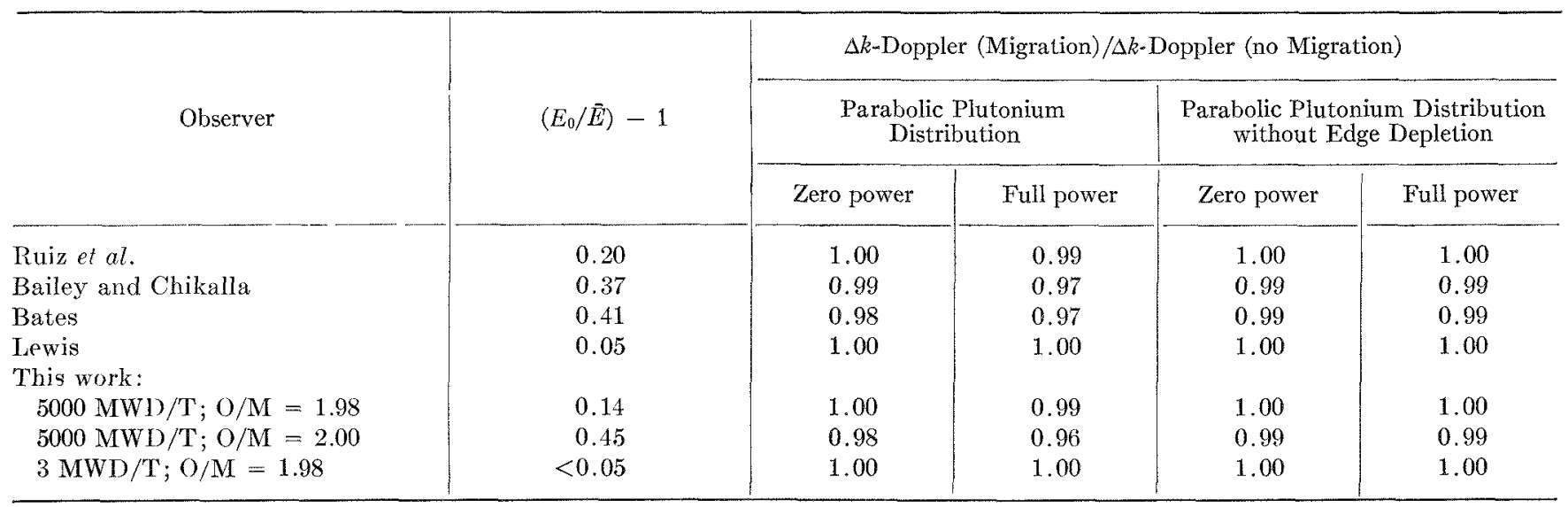

edge depletion corresponds to the other specimens discussed here. The dashed line represents the edge enrichment noted in the loosely held particulate samples from the hypostoichiometric specimen.

Figure 7 exhibits the effect of plutonium redistribution on Doppler reactivity $\Delta k$-Doppler (migration) $/ \Delta k$-Doppler (no migration) as a function of central $\left(E_{0}\right)$ and average $(\bar{E})$ fuel enrichment for each of the above models.

Table 1 shows the calculated Doppler reactivity change for zero power and full power for each of the observed instances of plutonium segregation, both for the parabolic plutonium distribution and for the parabolic distribution without edge depletion. In the fullpower calculation an initial parabolic temperature profile in the fuel rod was assumed. The assumption of the parabolic plutonium distribution with edge enrichment increases the Doppler degradation by approximately $1 \%$ compared to the parabolic distribution without edge depletion. Note that in the cases of unfailed mixed oxide fuel (Ruiz et al., and Bailey and Chikalla) the Doppler is reduced a maximum of $3 \%$. The use of Bates' observations for these calculations is open to serious question because his plutonium distribution data is a measure of plutonium buildup in $\mathrm{UO}_{2}$ fuel in a thermal-neutron spectrum rather than separation of an initial two-component system. However, applying such data to a mixed oxide fuel would correspond to a $3 \%$ decrease in Doppler. The differences in calculated Doppler change for the two high-burnup specimens of this work correspond primarily to the differences in detailed thermal history during gas blanketing and $\mathrm{NaK}$ attack on the fuel after failure. These vary from 0 to $4 \%$ and represent extreme conditions.

\section{Conclusions}

The following conclusions are drawn from the above analyses:
(1) Plutonium redistribution has been observed in high-performance mixed oxide fuel. The central region tends to become enriched in plutonium with a corresponding depletion in the midradius.

(2) The maximum effect of the segregation observed in this report is to reduce the calculated Doppler shutdown reactivity by $4 \%$.

(3) Refractory fission products tend to follow the observed distribution of plutonium while volatile species concentrate in the cooler peripheral regions of the fuel.

\section{REFERENCES}

1. J. L. Bates, $F$ ission Products Distribution in Irradiated $\mathrm{UO}_{2}$, Trans. ANS, 7, 389 (1964).

2. W. J. Bailey and T. D. Chikalla, Irradiation of UraniumPlutonium Oxide, HW-SA-3129 (Nov 8, 1963).

3. Sodium Cooled Reactors Program, Fast Ceramic Reactor Development Program, Fourth Quarterly Report, JulySeptember, 196:, GEAP-4080.

4. W. B. Lewis et al., Fission-gas Behaviour in $\mathrm{UO}_{2}$ Fuel, Third United Nations International Conference on the Peaceful Uses of Atomic Energy, A/Conf. 28/p/19, May, 1964.

5. C. P. Ruiz et al., The Transport of Plutonium in $\mathrm{PuO}_{2} / \mathrm{CO}_{2}$ Fuels: I: Radial Distribution of Plutonium in Highly Irradiated FCR Fuel, Trans. ANS, 6 (1963).

6. J. H. Davies and P. E. Novak, Diffusion of Plutonuum and Uranium in Oxide Fuel at $2400^{\circ} \mathrm{C}$, ibid., 7, 393 (1964).

7. F. Schmitz and R. Lindner, Diffusion of Plutonium in $\mathrm{UO}_{2}$, Sonderdruck and Radiochimica. Acta, 1, 218-220 (1963).

8. Novel Ceramic Fuel Fabrication Processes, HW-64629 (April $15,1960)$.

9. J. A. L. Robertson, High Temperature Properties of Ceramic Fuels: Their Significance and Measurement, Trans. ANS, 5, No. 1 (1962).

\section{Discussion}

Mr. Wirtz (Karlsruhe): I missed in your presentation any indication that temperature may play a part in the segregation of plutonium. It is well possible that the segregation is very strongly dependent on 


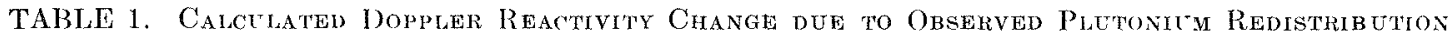

\begin{tabular}{|c|c|c|c|c|c|}
\hline \multirow{3}{*}{ Observer } & \multirow{3}{*}{$\left(E_{0} / \bar{E}\right)-1$} & \multicolumn{4}{|c|}{$\Delta k=$ Doppler (Migration) $/ \Delta k$-Doppler (no Migration) } \\
\hline & & \multicolumn{2}{|c|}{$\begin{array}{l}\text { Parabolic Plutonium } \\
\text { Distribution }\end{array}$} & \multicolumn{2}{|c|}{$\begin{array}{l}\text { Parabolic Plutonium Distribution } \\
\text { without Edge Depletion }\end{array}$} \\
\hline & & Zero power & Full power & Zero power & Full power \\
\hline Ruiz et al. & 0.20 & 1.00 & 0.99 & 1.00 & 1.00 \\
\hline Bailey and Chikalla & 0.37 & 0.99 & 0.97 & 0.99 & 0.99 \\
\hline Bates & 0.41 & 0.98 & 0.97 & 0.99 & 0.99 \\
\hline Lewis & 0.05 & 1.00 & 1.00 & 1.00 & 1.00 \\
\hline \multicolumn{6}{|l|}{ This work: } \\
\hline $5000 \mathrm{MWI} / \mathrm{T} ; \mathrm{O} / \mathrm{M}=1.98$ & 0.14 & 1.00 & 0.99 & 1.00 & 1.00 \\
\hline $5000 \mathrm{MWD} / \mathrm{T} ; \mathrm{O} / \mathrm{M}=200$ & 0.45 & 0.98 & 0.96 & 0.99 & 0.99 \\
\hline $3 \mathrm{MWD} / \mathrm{T} ; \mathrm{O} / \mathrm{M}=1.98$ & $<0.05$ & 1.00 & 1.00 & 1.00 & 1.00 \\
\hline
\end{tabular}

edge depletion corresponds to the other specimens discussed here. The dashed line represents the edge enrichment noted in the loosely held particulate samples from the hypostoichiometric specimen.

Figure 7 exhibits the effect of plutonium redistribution on 10oppler reactivity $\Delta k$-Doppler (migration) $/ \Delta k$-Doppler (no migration) as a function of central $\left(E_{0}\right)$ and average $(\bar{E})$ fucl enrichment for each of the above models.

Table 1 shows the calculated Doppler reactivity change for zero power and full power for each of the observed instances of plutonium segregation, both for the parabolic plutonium distribution and for the parabolic distribution without edge depletion. In the fullpower calculation an initial parabolic temperature profile in the fuel rod was assumed. The assumption of the parabolic plutonium distribution with edge enrichment increases the Doppler degradation by approximately $1 \%$ compared to the parabolic distribution without edge depletion. Note that in the cases of unfailed mixed oxide fuel (Ruiz et al., and Bailey and Chikalla) the Doppler is reduced a maximum of $3 \%$. The use of Bates' observations for these calculations is open to serious question because his plutonium distribution data is a measure of plutonium buildup in $\mathrm{UO}_{2}$ fuel in a thermal-neutron spectrum rather than separation of an initial two-component system. However, applying such data to a mixed oxide fuel would correspond to a $3 \%$ decrease in Doppler. The differences in calculated Doppler change for the two high-burnup specimens of this work correspond primarily to the differences in detailed thermal history during gas blanketing and NaK attack on the fuel after failure. These vary from 0 to $4 \%$ and represent extreme conditions.

\section{Conclusions}

The following conclusions are drawn from the above analyses:
(1) Plutonium redistribution has been observed in high-performance mixed oxide fuel. The central region tends to become enriched in plutonium with a corresponding depletion in the midradius.

(2) The maximum effect of the segregation observed in this report is to reduce the calculated Doppler shutdown reactivity by $4 \%$.

(3) Refractory fission products tend to follow the observed distribution of plutonium while volatile species concentrate in the cooler peripheral regions of the fuel.

\section{REFERENCES}

1. J. 1. Bates, Fission Products Distrubution in Irradiated $\mathrm{UO}_{2}$, Trans. ANS, 7, 389 (1964).

2. W. J. Bailey and T. 1). Chikalla, Irradiation of Tranium Plutonium Oxide, HW-SA-3129 (Nov 8, 1963).

3. Sodium Cooled Reactors Program, Fast Ceramic Reactor Development Program, Fourth Quarterly Report, JulySeptember, 1962, GEAP-4080.

4. W. B. Lewis et al., Fission-gas Behaviour in $\mathrm{HO}_{2} \mathrm{Fuel}$, Third United Nations International Conference on the Peaceful I'ses of Atomic Energy, A/Conf. 28/p/19, May, 1964 .

5. C. P. Ruiz et al., The Transport of Plutonium in $\mathrm{PuO}_{2} / \mathrm{UO}_{2}$ Fuels: I: Radial Distribution of Plutonium in Highly Irradiated FCR Fuel, Trans. ANS, 6 (1963).

6. J. H. Javies and P. E. Novak, Diffusion of Plutonum and Uranium in Oxide $F$ uel at $2400^{\circ} \mathrm{C}$, ibud., 7, 393 (1964).

7. $\mathrm{F}$. Schmitz and R. Lindner, Diffuszon of Plutonizm in $\mathrm{C}^{\top} \mathrm{O}_{2}$, Sonderdruck and Radiochimica Acta, 1, 218-220 (1963).

8. Novel Ceramic Fuel Fabrication Processes, HW-64629 (April $15,1960)$.

9. J. A. I. Robertson, High Temperature Properties of Ceramic Fuels: Their Significance and Measurement, Trans. ANS, 5, No. 1 (1962)

\section{Discussion}

Mr. Wirta (Karlsruhe): I missed in your presentation any indication that temperature may play a part in the segregation of plutonium. It is well possible that the segregation is very strongly dependent on 
temperature and may even sometimes change sign with change of temperature. Have you observed such effects?

Mr. Novak: Our data apply to high-performance fuels, for which $\int k d \theta$ is over $80-85$. The original FCR data are for a high-performance pin running at maybe $17 \mathrm{~kW} / \mathrm{ft}$ linear power; Bailey and Chikalla's experiments also ran about there. I would suspect that in a low-temperature pin you would not sce these gross magnitudes; in fact, for a pin that sustained gross center melting on short burnup in a 10minute irradiation there was very little segregation.

Mr. Persiani (ANL): Would you care to comment on the migration properties as a function of the fabrication technique when you talk about solidsolution fuels or mechanical mixed fuels, and also as a function of temperature?

Mr. Novak: I believe that all the mixed oxide pins that were discussed were certainly the FCR ones which were coprecipitated mixed oxides. The $\mathrm{UO}_{2}$ work by Bates was a high-burnup situation.

Mr. Persiani: Would you expect a different behavior if you had a physical homogeneous mixture?

Mr. Novak: I don't know. We have not studied this particular case. There would probably be different effects on the Doppler.

Mr. Okrent: Your study of the change in the Doppler effect indicated that this wasn't very large. I wonder if you had the same sort of buildup of plutonium in the center of the fuel and you went on to the unlikely accident where you were supercritical and needed a certain amount of Doppler effect prior to fuel failure. In this case, would the amount of energy the reactor could absorb prior to fuel failure be markedly influeneed by the fact that you now had an appreciable concentration of plutonium near the center of the pin and could reach vaporization, for example, earlier in the transient?

Mr. Novak: Yes, with the plutonium near the center in a fast transient it would heat up higher. The plutonium would melt. This particular effect was not taken into account in this calculation.

Mr. Campise (AI): Were these irradiations done in a thermal reactor?

Mr. Novak: Yes.

Mr. Campise: What kind of surface-to-center flux depression did you have, and what effect would the increased capture rate in $U^{238}$ have on the plutonium distribution in the pins?

Mr. Novak: The center-to-surface flux depression, as I recall, was of the order of about a half. In these lower burnup cases, namely, $5000 \mathrm{MWI} / \mathrm{T}$, for which we did the microsampling, the burnout of plutonium or burnup of plutonium at the edge would be virtually compensated within limits of any of our measurements by the thermal and resonance capture of $\mathrm{U}^{238}$. 


\title{
Microanalytical Studies of Micro- and Macro-segregation in Oxide Fuels*
}

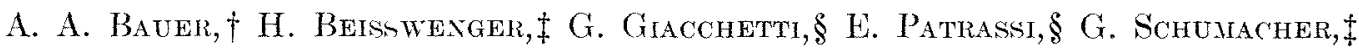 \\ AND R. Theisex $\$$ \\ Instutute for 1 pplied Reactor Physics \\ Kernforschungszentrum, Karlsruhe, Germany
}

(PRLSLNTED BY K. WIRTZ)

\section{Introduction}

For the evaluation and development of fast reactor oxide fuels, techniques applicable to the study of segregation in the fuel are required. From the viewpoint of nuclear safety, metallic-ion segregation and quantitative data on the ion mass transfer in a thermal gradient are of particular inportance. Evaluation of preparation and fabrication procedures, as they affect fucl homogeneity, is desired. Because thermal, mechanical, and chemical properties vary remarkably with stojehiometry, techniques capable of quantitatively detecting oxygen segregation, which can seriously affect fuel performance, also are required.

Electron-microprobe techniques, if adequately adapted for high-sensitivity analysis in the presence of radioactive material, are uniquely suited to the study of short-range segregation. Quantitative elementary microanalysis is effective for the elements boron to plutonium (atomic number $Z$ from 5 to 94 ), and highly localised point analysis of microvolumes ranging from $1 \mu^{3}$ to integrated surface scannings of $(300)(300)(1)$ $\mu^{3}$ with a detection limit of about $0.01 \%$.

A segregation of solid fission products as a consequence of temperature distribution in oxide fuels during irradiation has been previously observed. ${ }^{(1,2)}$

The present study was condurted to investigate the segregation of $\mathrm{CeO}_{2}$ in $\mathrm{UO}_{2}$ annealed in a temperature gradient. The $\mathrm{CeO}_{2}$ was intended to serve as a substitute for $\mathrm{PuO}_{2}$, as well as being a representative of solid fission products. The results obtained are preliminary in nature, but they demonstrate the value of the technique in studying the segregation problem.

\footnotetext{
* This paper has been prepared within the framework of the association EURATOM Gesellschaft fur Kernforsehung mbH. in the field of fast breeder development.

$\dagger$ U. S. Atomic Energy Commission, delegated to the Karlsruhe Fast Breeder Project.

† Institute for Neutron Physics and Reactor Technology, Karlsruhe.

\$EURATOM, Brussels, delegated to the Karlsruhe Fast Breeder Project.
}

\section{Outline of Experimental Procedure and Neces - sary Adaptations for Microanalysis of Ir- radiated Specimens}

For unirradiated plutonium-containing targets, only minor changes in conventional electron probe microprobe analysis have to be introduced. A vacuum evaporated thin layer of about $80 \AA$ nickel, plated simultaneously on the surface of the metallographically mounted and polished surface of the specimen and the ralibration standards, meets all the following requirements. Satisfactory retention of fugitive activity, increase of thermal and electrical conductivity of ceramic materials, perfect adherenco to the specimen surface, sufficient optical transmission for microscopical obcervation of structure and localisation of the analysis and, above all, insignificant cffect on the $\mathrm{X}$-ray excitation and emission efficiency.

For microanalysis of irradiated materials, the presence of an intense source of gamma radiation necessitates radiobiological protection for the operator. The electron-optical bench, including the specimen stage and the associated electronic units of a commercial CAMECA D $46^{(3)}$ instrument, are shown in Fig. 1. A 10 -cm-thick protection wall of lead, surrounding the specimen chamber, the remote specimen-introduction system, and the drives for the specimen stage and spectrometer scan are shown in Fig. 2.

For microanalysis of unirradiated material or general servicing, the lead shield can be hydropneumatically lifted upwards. The upper part of the electron-optical bench is screened by a 7-cm-thick Denal $(D=18)$ plate placed above the objective lens and pierced to leave only passage to the electron beam. Below the specimen stage a combined Denal-lead plate protects the lower part of the bench with the vacuum pumping units. The use of a new type of X-ray spectrometer, ${ }^{(3)}$ with a constant take-off angle of the emergent $X$ rays, independent of their wavelengths, facilitates enormously the correct channeling of the characteristic $\mathrm{X}$ rays and the elimination of direct or scattered gamma radiation. 


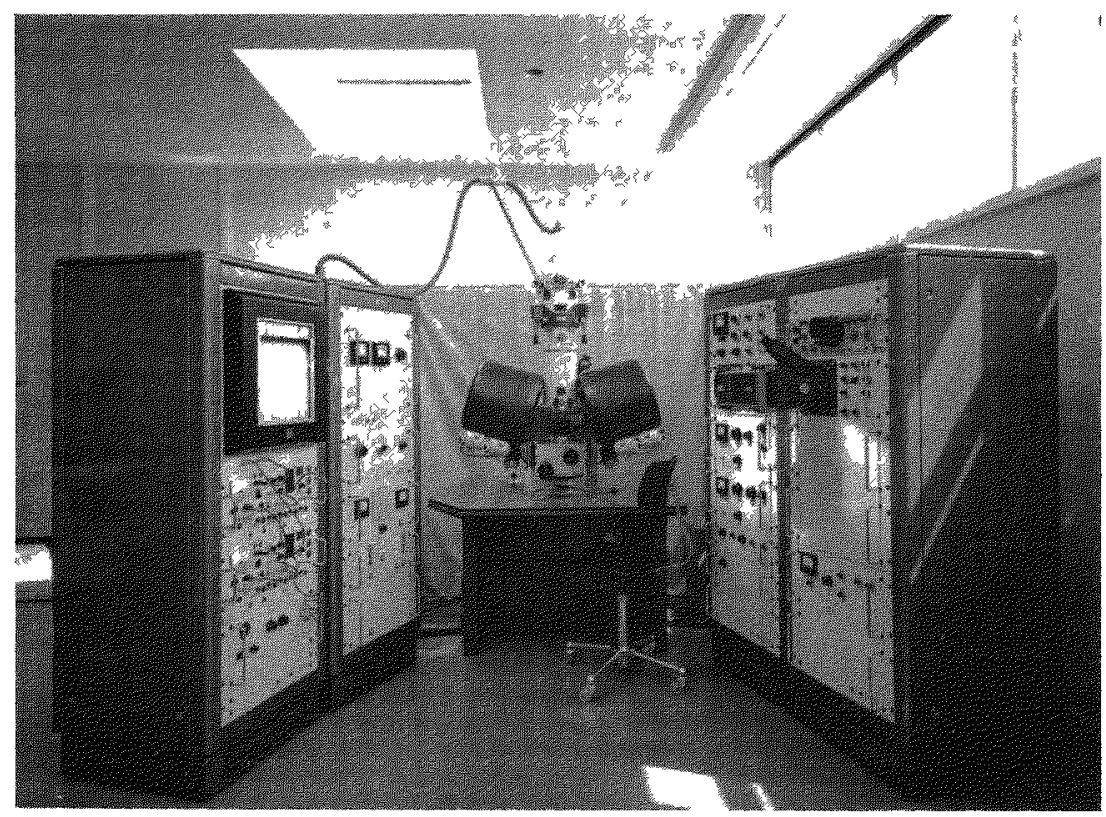

FIG. 1. Commercial CAMECA Mirroanalyzer and Associated Electrone Units.

A picreed eylindrical collimator (of DENAL) is placed immediately after the X-ray entry window of the spectrometer, leaving passage only for the $\mathrm{X}$ ray in the direction of the monochromator. A second collimator, consisting of a DENAL tube, is placed before the window of the detector and is automatically oriented in the direction of the analysed radiation.

The correlation of the sensilivity of analysis, $\Delta C / C$, with the total counting interval $\Delta t$, in seconds, required for one analytic determination, as influenced by the mean counting rate $r_{c}$ (in I/sec) and the peak to background ratio $\mathrm{P} / \mathrm{B}$ (both to be determined experimentally on the specimen) is shown in the nomogram of Fig. 3 and corresponds to the statistically obtained sensitivity formula ${ }^{(4)}$ for microprobe analysis:

$\frac{\Delta C}{C}=\frac{2 \sqrt{r_{c}}}{\sqrt{\Delta^{t}}\left(r_{o}-r_{B}\right)} ; \quad P / B=r_{c} / r_{B} ;$

$$
y=r_{c}\left(\frac{\Delta c}{c}\right)^{2} .
$$

As a practical example the dashed lines in Fig. 3 show how it is possible to determine the necessary counting interval from the required sensitivity. The gamma activity of irradiated material noticeably decreases the peak-to-background ratio, even for correctly shielded instruments employing pulse-height discrimination analysis. The diagram in the upper right corner of Fig. 3 shows the experimentally determined variation of the $P / B$ ratio with sample activity; it is strictly applicable only for the experinental conditions and the specific shielding of the instrument used. The reduction of the $P / B$ ratio for microanalysis of irradiated materials consequently requires an increased counting interval. However, the formation rate of the well-known carbon contamination spot at the point of impact of the electron beam limits the counting interval in conventional microanalysis to about 50 to 100 sec.

Consequently the use of a decontamination device (such as an argon stream directed through a massspectrometer vacuum leak and a nonmagnetic capillary onto the point of impact of the electrons, ${ }^{(4)}$ which permits arbitrary long counting time intervals) is required. For the determinations of uranium, both the $\mathrm{UL}_{\alpha 1}$ and the $\mathrm{U}_{\beta}$ lines and the $\mathrm{Ce} \mathrm{L}_{\alpha 1}$ line were used. For the determination of local variation of oxygen an empirically calibrated fixed-charge procedure, described by Heinrich, ${ }^{(5)}$ was used.

\section{Materials and Thermal Diffusion Treatment}

$\mathrm{UO}_{2} \cdot \mathrm{CeO}_{2}$ pellets, $8 \mathrm{~mm}$ in diameter and $10 \mathrm{~mm}$ long; were produced by NUKELI, Germany, either by mechanical mixing of powders or by coprecipitation followed by sintering in a hydrogen atmosphere at temperatures ranging from $1500^{\circ} \mathrm{C}$ to $1650^{\circ} \mathrm{C}$ for $2 \mathrm{hr}$. The samples were submitted to thermal diffusion for up to $600 \mathrm{hr}$ and temperature gradients ranging up to $750^{\circ} \mathrm{C} / \mathrm{cm}$, developed by placing a specimen on a watercooled pedestal, the other end being heated in a refractory metal furnace.

For some special studies of microsegregation in the high-temperature range, pellets with a central hole 


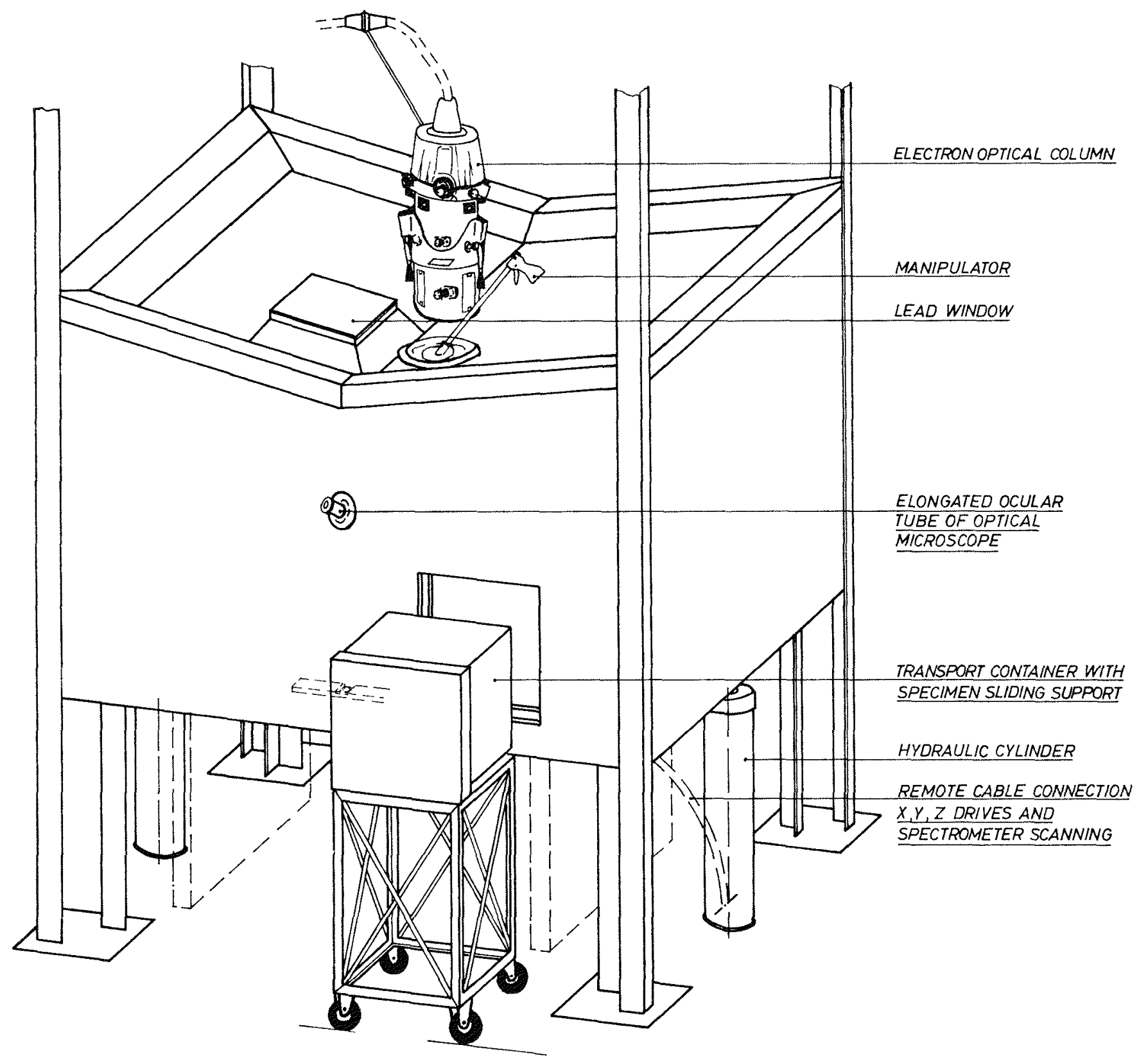

Fig 2. Flectron Microanalyzer for Irradiated Materials.

were radially heated by a tungsten rod and thermal gradients up to $5000^{\circ} \mathrm{C} / \mathrm{cm}$ were reached.

After the thermal treatment, the pellets were sectioned longitudinally by a diamond wheel, and one surface metallographically prepared and submitted for microanalysis. Both macrosegregation and microsegregation were studied by quantitative electron-microprobe analysis. ${ }^{(4)}$ For the macrosegregation studies, mean cerium concentrations were obtained by quantitative scanning of surface regions of $50 \times 300 \mu$, accomplished either by mechanical scanning, in which case the specimen is translated under a static electron probe, or by use of the CAMIECA semi-electronic scanning device. This technique was necessary to decrease the scatter of statistical point analysis, caused by local inhomogeneities. Conventional point-by-point analysis was used to study microsegregation, a consequence of the interaction of small $\mathrm{CeO}_{2}$ inclusions within the matrix.

\section{Experimental Results}

Metallographic inspection and quantitative microanalysis demonstrated that the as-sintered material consisted of an apparently homogeneous (U,Ce) $\mathrm{O}_{2}$ matrix containing inclusions of pure $\mathrm{CeO}_{2}$. These inclusions, with varying diarneters up to $100 \mu$, were 


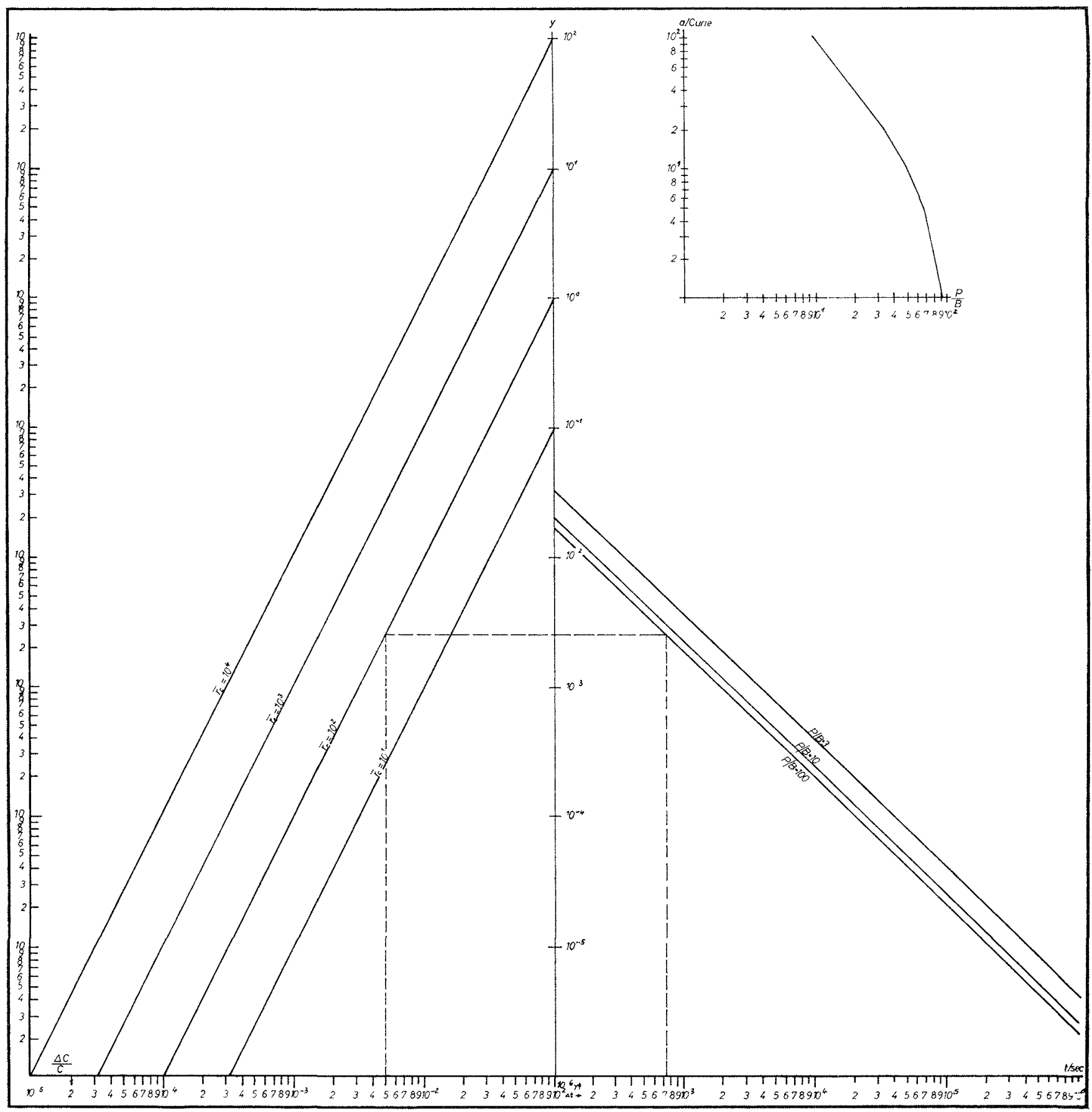

Fig. 3. Nomogram for Correlation of Required Sensitivity, Counting Interval, Counting Rate, Peak to Background Ratio, and Sample Activity.

randomly distributed and frequent in pellets sintered from mechanically mixed powders, smaller and only rarely observed in coprecipitated material.

\subsection{Macrosegregation after Thermal Diffusion}

The typical effect of a high thermal gradient on cerium mass transfer in sintered pellets of coprecipitated $\mathrm{UO}_{2}-\mathrm{CeO}_{2}$ is shown in Figures 4 and 5 . Identical behaviour was observed in mechanically mixed fuels, as is seen by the cerium concentration profile along the temperature axis, shown in Fig. 6.

The essential effects of annealing in a temperature gradient are the migration of cerium to regions of higher temperatures and preferential Ioss by evaporation at the highest temperature.

Three distinct zones can be identified: 
In a first zone, up to temperatures of $1450^{\circ} \mathrm{C}$, no variation of the original concentration was observable, because of low diffusion rate. In the temperature range above $1450^{\circ} \mathrm{C}$, thermal diffusion results in preferential cerium migration towards regions of higher temperatures.

At temperatures at which equiaxed grain growth occurs (about $1900^{\circ} \mathrm{C}$ ), the cerium concentration decreases, because of preferential loss by evaporation of cerium at the high-temperature end of the specimen. At these temperatures an inversion of the Soret superposition may occur. However it is difficult at this stage to separate thermal diffusivity effects from those due to preferential evaporation. The position and the amplitude of the minimum and the maxinum are time dependent, moving to lower temperatures with increased time, while simultaneously the depth of the minimum increases. When a crack occurs across the specimen, it acts as a diffusion barrier, as illustrated in Fig. 6.

\subsection{Microsegregation}

The mechanism responsible for the kineties of macrosegregation can be qualitatively studied by the micro- analytical determination of the $\mathrm{CeO}_{2}$ inclusion, the (U,Ce) $\mathrm{O}_{2}$ matrix, and their interaction.

In the region up to $1450^{\circ} \mathrm{C}$ no interaction between the $\mathrm{CeO}_{2}$ particles and the matrix occurred, as can be seen in Fig. 7, which shows a surface scanning inage in the $\mathrm{Ce} \mathrm{L}_{\alpha 1}$ line and a linear scanning line across the interface of the inclusion and matrix, where the $\mathrm{CeO}_{2}$ concentration drops immediately to the matrix concentration. The hollow spherical aspert of the $\mathrm{CeO}_{2}$ inclusions is probably due to a lack of plasticity of the pure $\mathrm{CeO}_{2}$ powders during the pelletising step.

In the temperature range from $1450^{\circ} \mathrm{C}$ up to $1900^{\circ} \mathrm{C}$ the diffusion mechanisms have been evaluated from the depth $\mathrm{X}$ of the interdiffusion layer between the matrix. By linear Ce-scannings along a line of equithermal flux, the chemical diffusion coefficients can be determined. ${ }^{(6)}$ The effect of a positive thermal gradient $+(d T / d x)$ is measured by scanning the Ce-concentration profile against the direction of the thermal flux (towards the higher-temperature region); the influence of a negative thermal gradient, $-(d T / d x)$, is measured in the opposite direction.

Typical linear scanning curves in the three direc tions are compared in Fig. 8, and the results are sum

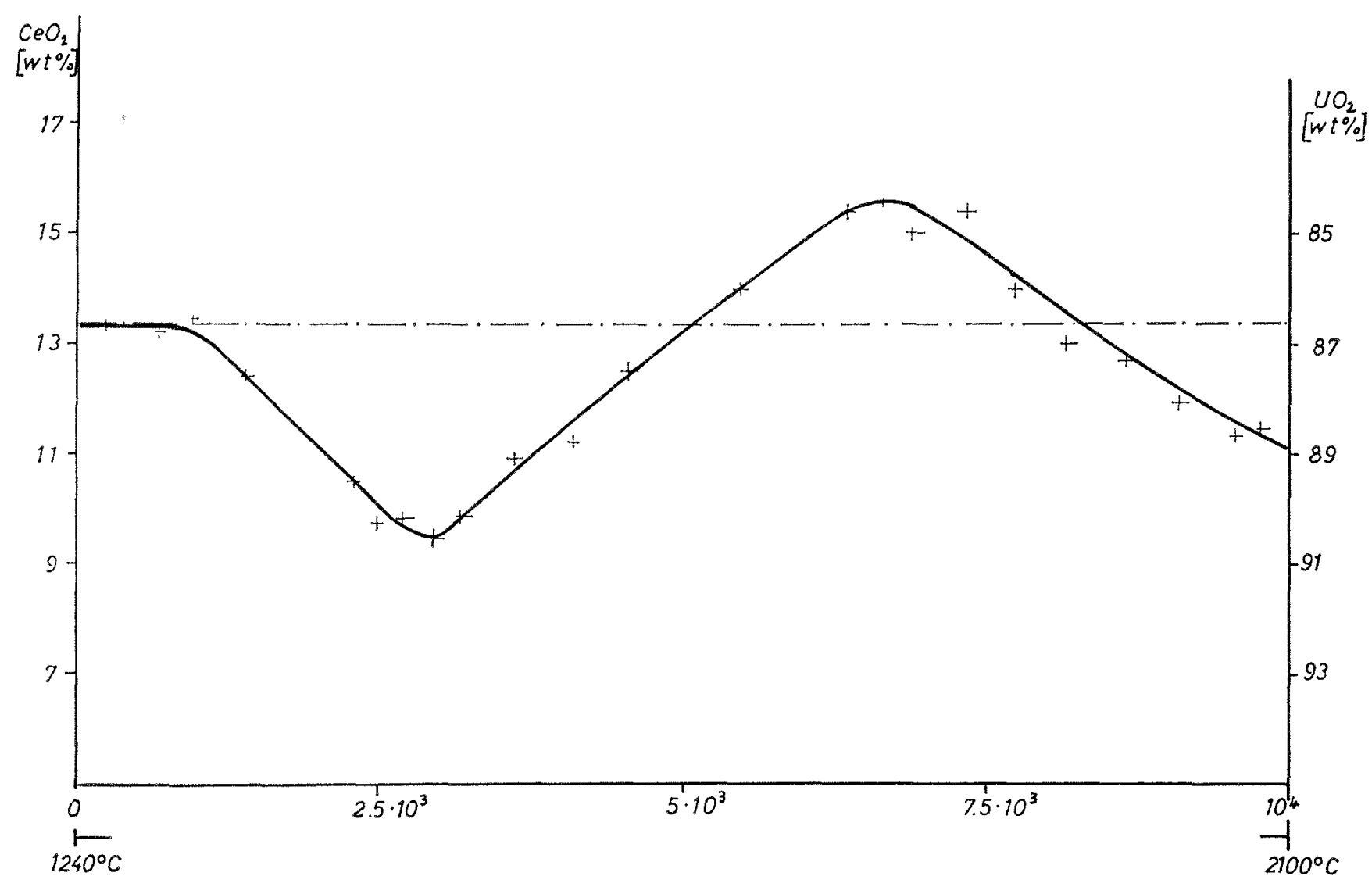

FIG. 4. Effect of Thermal Gradient $\left[(d T / d x)=860^{\circ} \mathrm{C} / \mathrm{cm}\right]$ on Cerium Segregation in Coprecipitated $\mathrm{UO}_{2}-\mathrm{CeO}_{2}(20 \mathrm{~m} / \mathrm{o}) . \mathrm{Diffusion}$ Annealing at $2100^{\circ} \mathrm{C}-1240^{\circ} \mathrm{C}$ for $185 \mathrm{Hr}$. 


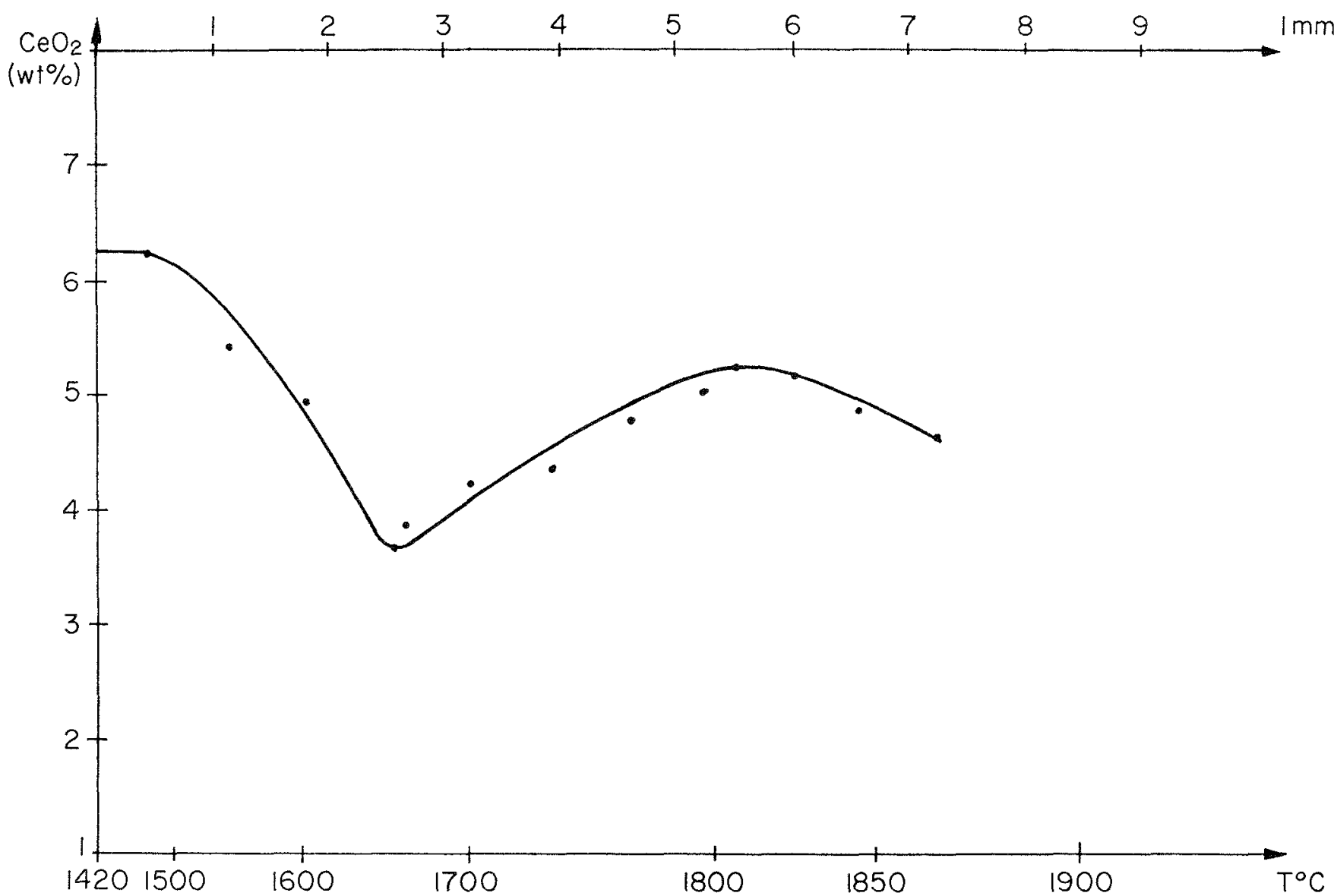

FIG. 5. Effect of Thermal Gradient $\left[(d T / d x)=562.5^{\circ} \mathrm{C} / \mathrm{cm}\right]$ on Cerium Segregation in Coprecipitated $\mathrm{UO}_{2}$-CeO2 $(6 \mathrm{w} / \mathrm{o}) . \mathrm{Diffu}-$ sion Annealing at $1870^{\circ} \mathrm{C}-1420^{\circ} \mathrm{C}$ for $600 \mathrm{Hr}$.

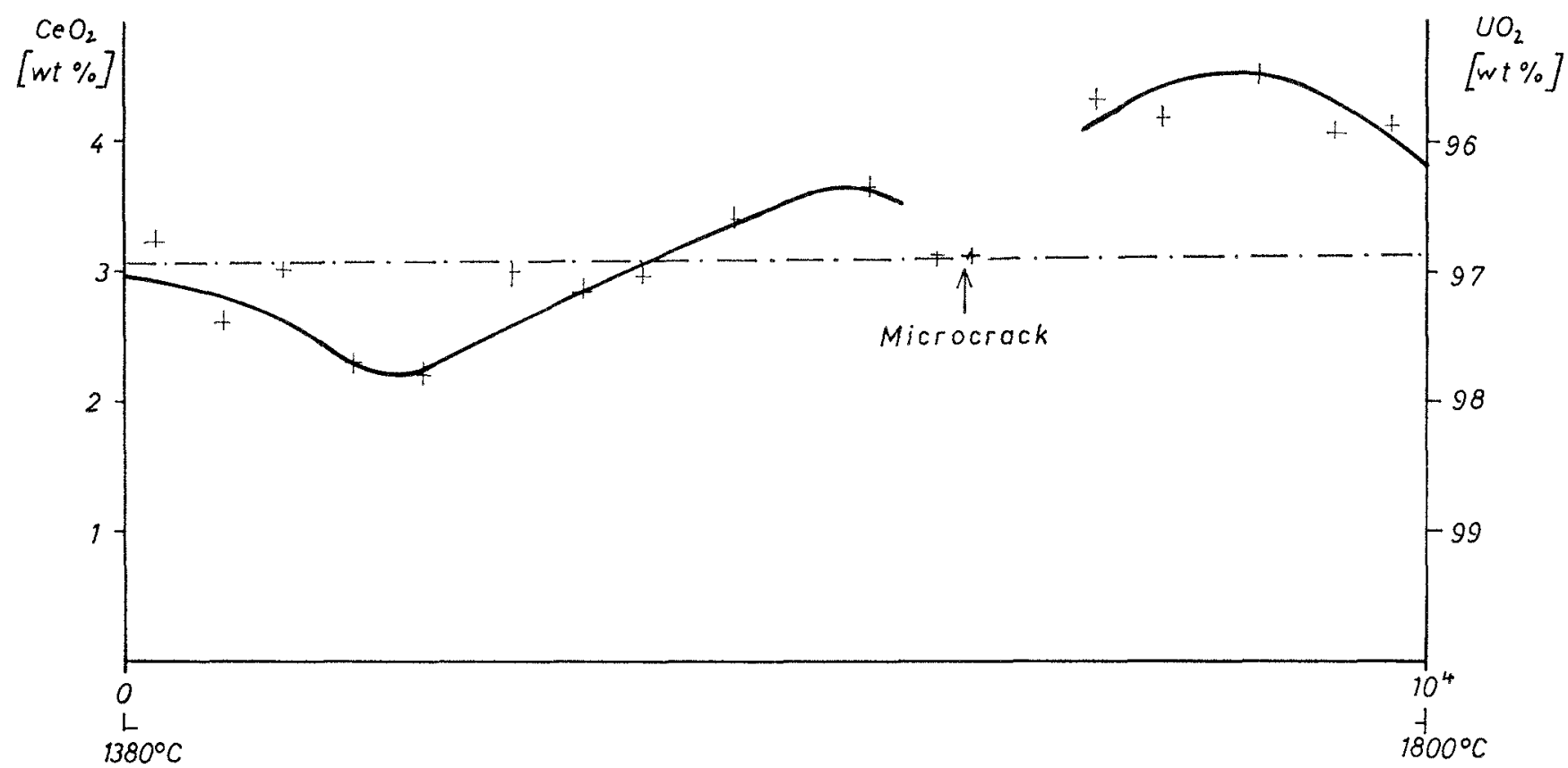

FIG. 6. Effect of Thermal Ciradient $\left[(d T / d x)=420^{\circ} \mathrm{C} / \mathrm{cm}\right]$ on Cerium Segregation in Wet, Mechanically $\mathrm{Mixed} \mathrm{LO}_{2}-\mathrm{CeO} \mathrm{C}_{2}(3 \mathrm{w} / \mathrm{o})$. Annealing at $1800^{\circ} \mathrm{C}-1380^{\circ} \mathrm{C}$ for $40 \mathrm{Hr}$. 

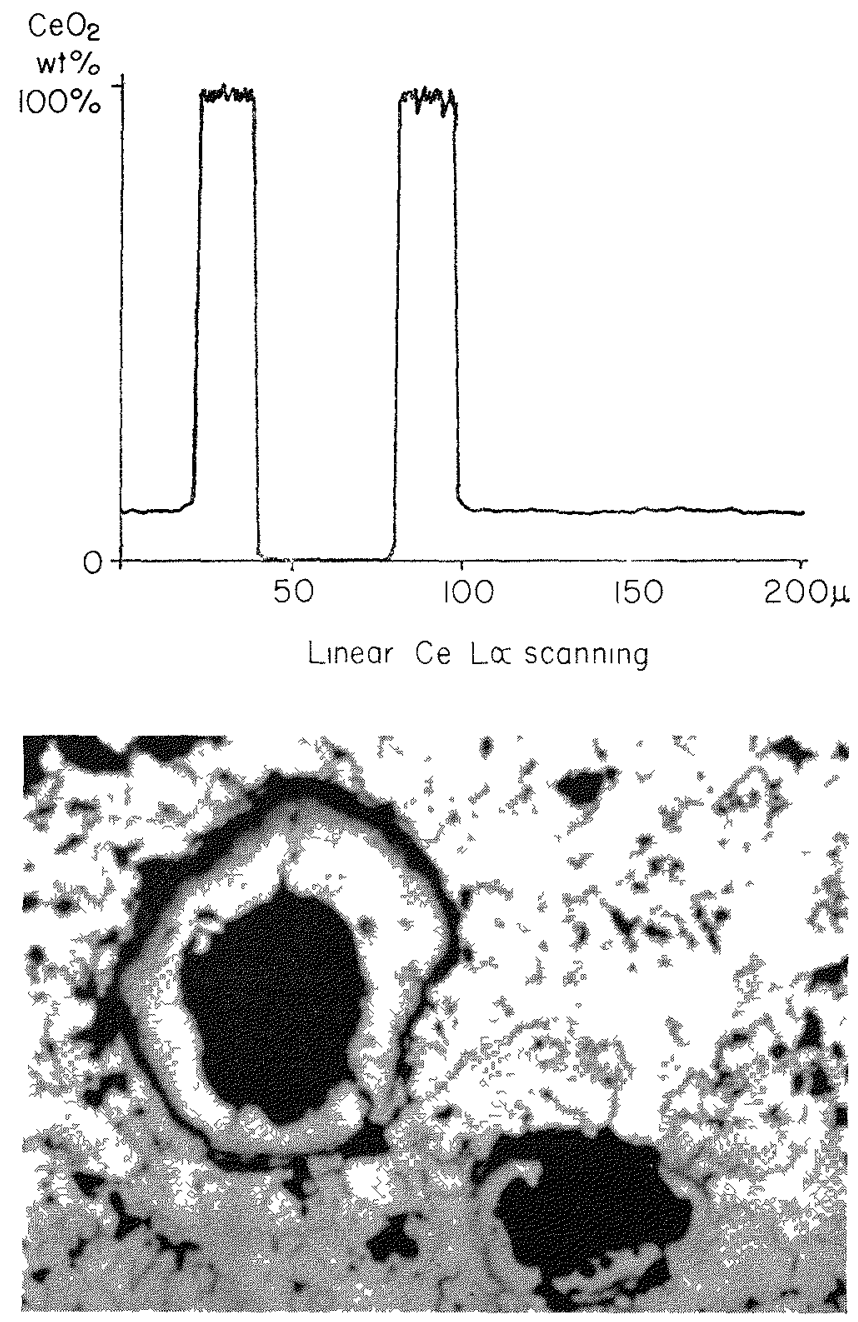

$\mathrm{F}_{1 \mathrm{i}} 7$ Effect of a Thermal (izadent on $\mathrm{CeO}_{2}$ Inclusuon in $\mathrm{UO}_{2}-\mathrm{CeO}_{2}$ at $1400^{\circ} \mathrm{C}$.

marized in lig. 9. The direction of enhanced diffusion noted around the inclusions is in exact agreement with the direction of diffusion in a thermal gradient observed on a macroseale, described in Sect. 4.1. In the temperature range above $1900^{\circ} \mathrm{C}$, the $\mathrm{CeO}_{2}$ concentration decreases with increasing temperature, as evaporation appears to become a predominant mechanism. This is clearly seen in the absorbed electron image of Fig. 10 (the brightness of the image is proportional to the mean. atomic number of the specimen), where around the small equiaxed grains a cerium dcpleted zone is seen. High-sensitivity statistical analysis gives evidence of a decrease of cerium and oxygen from the center to the grain boundary of the crystals. The steepest cerium gradient is observed in the direction of the higher temperature $[+(d T / d x)]$. The grain-boundary zone of the columnar grains is clearly hypostoichiometric. The formation and growth of the columnar grains by vapor transport can be inferred by observation of the cerium and oxygen gradients within the grains.

For the initual long annealing periods, the $\mathrm{CeO}_{2}$ inclustons dissolved completely in the solid solution at higher temperature. Recently, short-tme diffusion treatments were performed on axially heated pellets involving a very high thermal gradient, and inclusions were still partially observed at temperatures up to $2100^{\circ} \mathrm{C}$. Porosity and a brittle interdiffusion layer prohibited a cuantitative analysis, however, in comparison with the diffusion studies in the second temperature zone; an inversion of the relative interpenetration depth with the thermal gradient, $d T / d x$, was observed.

In all specimens examined to date, no metallic uranium inclusions have been observed, the metallic inclusions seen in the electron picture of Fig. 11 being precipitates of the refractory metal used in the heating plates. Analysis of the heating plates showed an absorption of evaporated $\mathrm{CeO}_{2}$ and, to a minor extent, of $\mathrm{UO}_{2}$.

\section{Discussion}

Two significant mechanisms for the migration of $\mathrm{CeO}_{2}$ in $\mathrm{UO}_{2}$ have been identıfied in these studies: thermal diffusion and vapor transport. Thermal diffusion of $\mathrm{CeO}_{2}$ in the direction of increasing temperature predommates at lower temperatures; above about $1900^{\circ} \mathrm{C}$ preferential evaporation of $\mathrm{CeO}_{2}$ from the high-temperature end of the specmen and vapor transport, leading to a segregation on a microscale, predominates. The maximum in $\mathrm{CeO}_{2}$ concentration observed near the lower temperature limit of the columnar-grain region is consistent with the increased activity of soldd fission products noted at the same location in irradiated oxide fuels. ${ }^{(2)}$ In view of the chemical similarities between the lanthanide and actinide metals, a similar segregation pattern for $\mathrm{PuO}_{2} \mathrm{In}_{\mathrm{UO}}$ might be expected.

On the basis of the relative sintering behaviour of $\mathrm{CeO}_{2}$ and $\mathrm{PuO}_{2}$ containing $\mathrm{UO}_{2}$ fucl, more rapid plutonium diffusion is indicated.

From the concentration curves of the type shown in Figures 4-6, the temperature dependence of the chemical and thermal diffusion coefficients, and of the Soret effect, can be calculated by analog and digital computing methods. Such calculations are now in progress and will be published when completed. ${ }^{(7)}$

In order to provide an estimate of the Soret effect, it is assumed that after a diffusion gradient treatment of $600 \mathrm{hr}$ an almost steady state has been reached. At this point the chenical and thermal diffusion driving forces are balanced and the atomic flux $J$ becomes zero. Thus,

$$
J=-D \rho \frac{d c}{d x}+D^{\prime} \rho c(1-c) \frac{d T}{d x}=0
$$


where

$D=$ chemical diffusion coefficient;

$D^{\prime}=$ thermal diffusion coefficient;

$c=$ weight fraction;

$T=$ temperature, in ${ }^{\circ} \mathrm{K}$;

$x$ = distance.

The Soret coefficient is

$$
S_{T}=\frac{D_{T}^{\prime}}{D T}=\frac{d c / d x}{c(1-c) \frac{d T}{d x}} .
$$

From the $\mathrm{CeO}_{2}$ concentration profile in Fig. 5 a coefficient

$$
S_{T}=2.3 \times 10^{-3}\left(\text { per }^{\circ} \mathrm{K}\right)
$$

is estimated at $1750^{\circ} \mathrm{C}$.

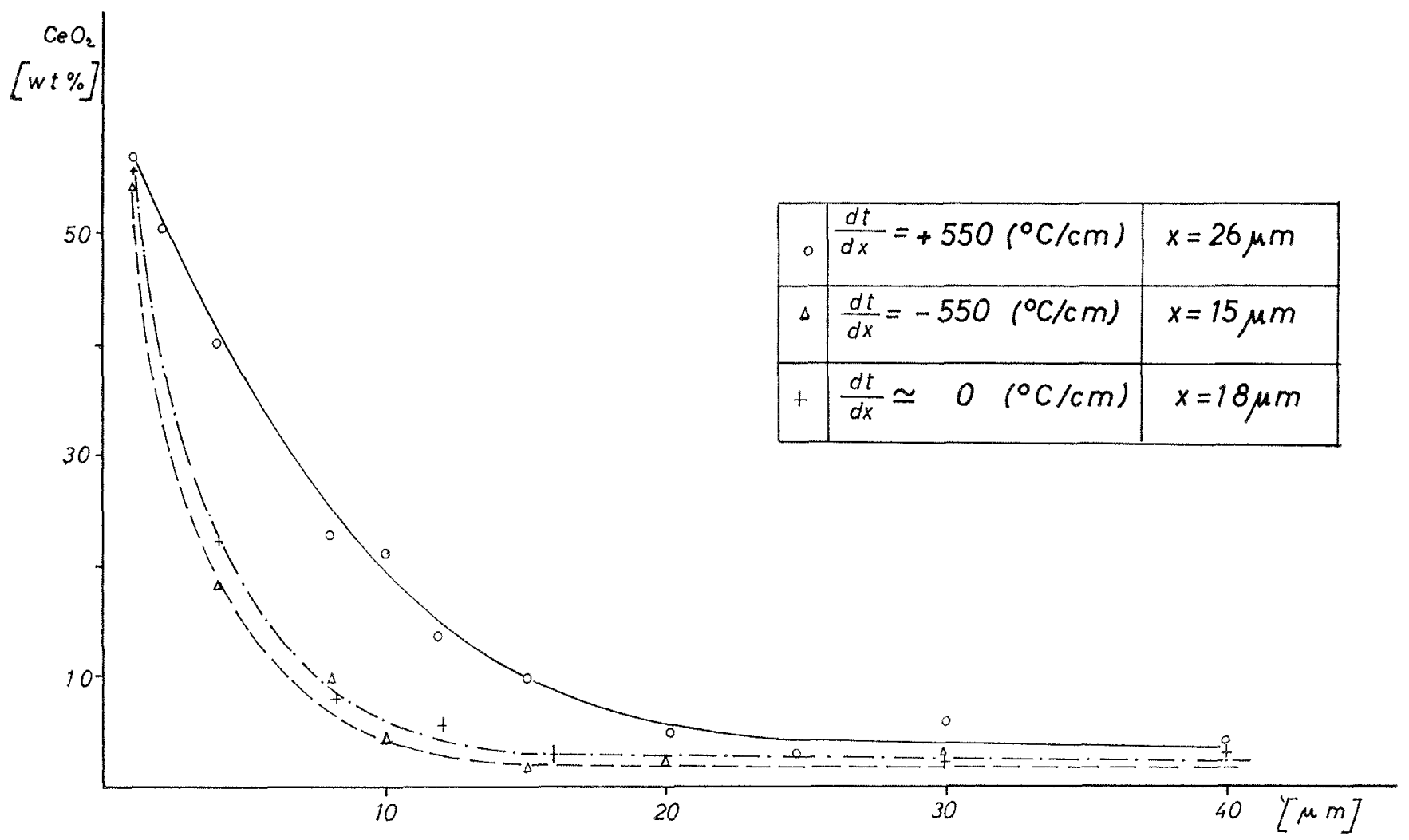

FIG. 8. Influence of Thermal Gradient on Mass Transport in $\mathrm{UO}_{2}-\mathrm{CeO}_{2}$ Pellets (No. 43) at $1600^{\circ} \mathrm{C}$. Mechanically Mixed $\mathrm{UO}_{2-}$

\begin{tabular}{|c|c|c|c|c|c|}
\hline Specimen No. & Fabrication Method & $\begin{array}{c}\text { Temperature } \\
\text { Gradient, } \Delta T / \mathrm{cm}\end{array}$ & $\begin{array}{l}\text { Temperature of } \\
\text { Inclusion, }{ }^{\circ} \mathrm{C}\end{array}$ & $\underset{\mathrm{sec}}{\text { Annealing Period, }}$ & $\begin{array}{c}\text { Penetration, } \\
\mathrm{cm}^{-4}\end{array}$ \\
\hline 27 & Mechanically mixed & $\begin{array}{r}+550 \\
-550 \\
0\end{array}$ & 1750 & $5.04 \times 10^{1}$ & $\begin{array}{l}26 \\
15 \\
18\end{array}$ \\
\hline 43 & Mechanically mixed & $\begin{array}{r}+420 \\
-420 \\
0\end{array}$ & 1600 & $1.44 \times 10^{5}$ & $\begin{array}{l}32 \\
20 \\
23\end{array}$ \\
\hline 57 & Coprecipitated & $\begin{array}{r}+750 \\
-750 \\
0\end{array}$ & 1570 & $4.18 \times 10^{5}$ & $\begin{array}{l}55 \\
32 \\
38\end{array}$ \\
\hline 100 & $\begin{array}{l}\text { Coarse; mechanically } \\
\text { mixed }\end{array}$ & $\begin{array}{r}+5000 \\
-5000 \\
0\end{array}$ & 2000 & $2.16 \times 10^{4}$ & To be analysed \\
\hline
\end{tabular}
$\mathrm{CeO}_{2}(4 \mathrm{w} / \mathrm{o})$.

Fr. 9. Influence of Temperature Gradient on Microsegregation 


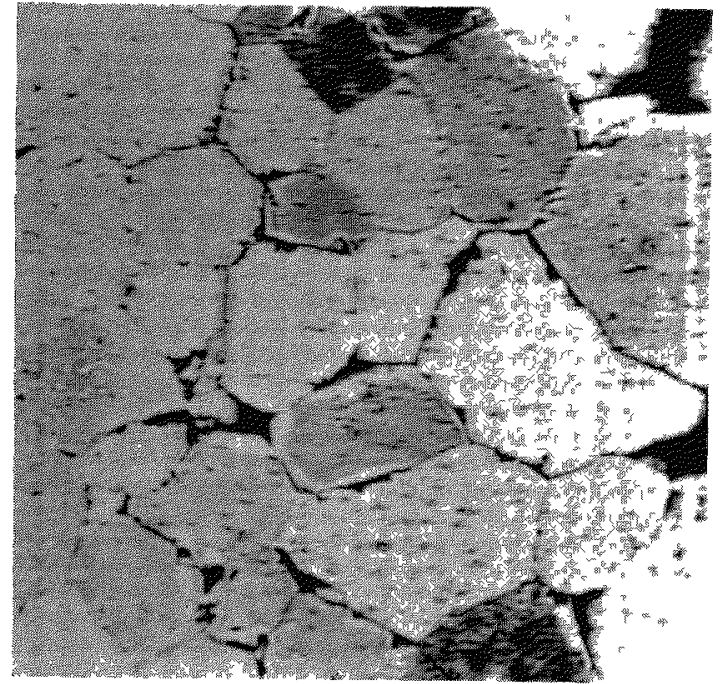

Fia. 10. Absorbed Electron Image Showing ('erium Depletion at Gram Boundary of Equaxed Grams, $200 \mu \times 200 \mu$.

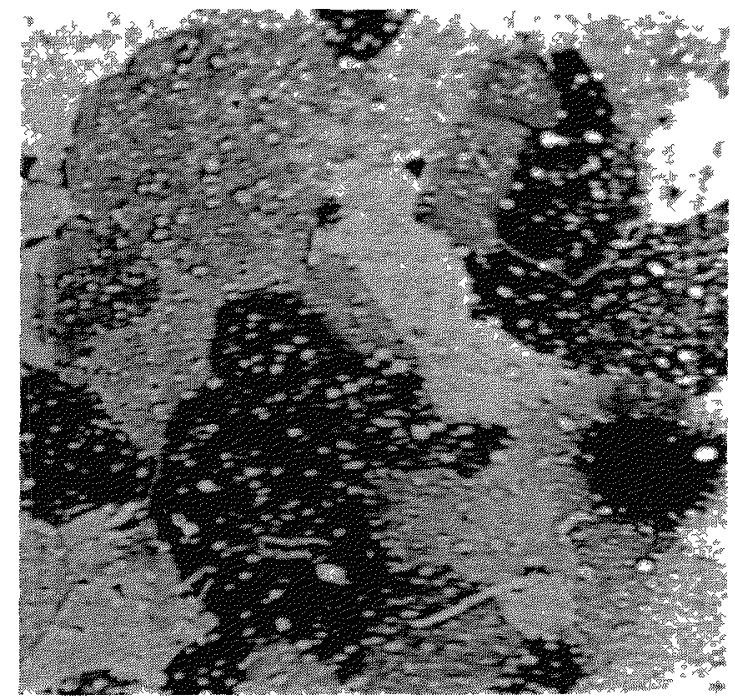

Fia. 11 Absorbed Electron Image Showing Refractory Metal Inclusions at Temperatures of $2050^{\circ} \mathrm{C}$ in (U,Ce) $\mathrm{O}_{2}$ (Sample No. 57), $200 \mu \times 200 \mu$

Furthermore, in view of the importance of the problem of plutonium relocation, and its effect on power distribution and reactivity in Fast Reactors, it is planned to extend these studics to both unirradiated and irradiated plutonium containing $\mathrm{UO}_{2}$ fuel. It is anticipated that the analysis of specimens with up to 100-Ci artivity will be possible with the shielded microprobe unit described in this paper.

\section{Acknowledgment}

The authors wish to express their sincere thanks to Prof. Dr. K. Wirtz for encouraging the work on thermal diffusion and to Prof. Häfele for stimulating the microprobe work.

\section{REFERENCES}

1. Bates, J. L., I.A.E.A. Symposium on Thermodynamics, Vienna (1965).

2. Rider, B. F., Ruiz, C. P., Peterson, J. P., and Luke, P. S. GEAP-4278 (1963).

3. Cameca, S. A, Courbevoi (S. et O.) France, Technical Notice (1964).

4. Theisen, R., Quantitative Electron Mucroanalysis, Springer Yerlag, New York (1965).

5. Heinrich, K., II Intern. Conf. X-ray Microanalysis, Paris (1965).

6. Ciacchetti, G., and Theisen, R., unpublished.

7. Beisswenger, H. et al., to be published.

\section{Discussion}

Mr. Zebroski (GE): We published some calculations of the Soret constant some years ago. The results were very disappointing; the constant was not a constant. The calculations involved a good many thermodynamic quantities which have not been measured and which probably will not be measured in the near future because of the difficulty in making these measurements in high temperature. I think this is a convenient way of correlating the data, but $I$ believe it will not be useful for making predictions.

Mr. Wirtz: I do not agree entirely. Indeed you must find out what thermodynamic values are behind this picture and you know that for the Soret coefficient

$$
\begin{gathered}
J=D \frac{\partial c}{\partial x}+D^{\prime} c(1-c) \frac{d T}{d x}=0 \\
-\frac{D^{\prime}}{D}=\frac{1}{c} \frac{d c}{d T^{T}}=S=-\frac{Q}{k T^{\prime 2}}=2.3 \times 10^{3} /{ }^{\circ} \mathrm{C} \text { at } 1750^{\circ} \mathrm{C},
\end{gathered}
$$

whore $Q$ is measured at one of the temperatures. It is also temperature dependent, but I am quite sure that for a certain mixture of uranium and plutonium oxide this value $Q$ is unique. It should be measured. It will vary with temperature and composition of the specimens but it is a physical constant. I think it would be of much more value to find out those physical constants instead of presenting to us measurements of different probes and different reactors and the different irradiation conditions and at different temperatures, and never find out what these physical values are.

Mr. Paul (NUMEC): If this sort of analysis does eventually prove to be of some value, the same sort of attack or approach might be of some value in examining the thermal conductivity of the various types of fuels, since the same principles of irreversible thermodynamics should apply. 
Mr. Wirtz: No, no, definitely not.

Mr. Paul: Part of the conductivity would be due to the transport of the various materials through the fuel.

$M r$. Wirtz: Only a very small amount of (fuel) heat conductivity would arise from this effect.

Mr. Zebroski: I think that we should mention that we have seen no segregation at all. What we observed were just concentration gradients, which you might call de facto segregation. But with the five or six different mechanisms operative and particularly when you get a wavy concentration gradient in the actual thermogradient situation, I think it is very bold indeed to ascribe this entirely to the Soret effect. In fact, I think that Dr. Theisen did suggest two other mechanisms operated in the same sample.

$M r$. Wirtz: I agree entirely. 


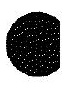




\title{
Session V-B \\ October 13, 1965
}

\section{Mostly Reactivity Coefficients}

\author{
Chairman: R. Avery \\ Argonne National Laboratory
}

Secretary: B. J. Toppel

Argonne National Laboratory 
0

0 


\title{
Recent Investigations of Fast Reactor Reactivity Coefficients
}

\author{
H. H. Hummel, R. N. Hwang, and K. Phillips \\ Argonne National Laboratory \\ Argonne, Illinois
}

(PRESENTED BY H. H. HUMMEL)

\section{Equivalence between Homogeneous and Heter- ogeneous Resonance Integrals in Cylindrical Geometry}

Although the equivalence relation between homogeneous and heterogeneous resonance integrals ${ }^{(1)}$ has proven very useful, it seemed desirable to determine whether it can be made sufficiently accurate for use in. multigroup cross-section-generation codes.

A key assumption of the theory is that the flux in each region can be assumed spatially flat. Another important assumption is that the probability $P_{0}(E)$ that a uniformly distributed source of neutrons of energy $E$ will escape from an isolated rod without suffering a collision is given by the rational approximation

$$
P_{0}(E)=\frac{1}{1+\left(\Sigma_{0}(E) / \Sigma_{e 0}\right)},
$$

where $\Sigma_{0}(E)$ is the macroscopic total cross section of the rod, and

$$
\Sigma_{e 0}=S_{0} / 4 V_{0}=1 / 2 R_{0} .
$$

Here $S, V$, and $R$ are the surface, volume, and radius, respectively, of the rod; subscripts 0 and 1 refer to fuel and moderator regions, respectively. The rational approximation is not extremely accurate, and an improvement suggested by Levine ${ }^{(2)}$ is to replace Eq. (1) by

$$
P_{0}(E)=\frac{1}{1+\left(\Sigma_{0}(E) / \Sigma_{e 0} a\right)}
$$

where $a$ is a constant, assumed to be the same for every resonance.

If one regards $a$ as a function of $E$, as will be done for both $a$ and $\Sigma_{0}$ in the following, clearly the rational approximation can be made exact, but it is then no longer useful for determining an equivalence relationship. It was suggested by Otter ${ }^{(3)}$ that a mean value $\vec{a}$ be defined for each resonance by, in effect, integrating over the resonance by breaking it up into intervals, in each of which Eq. (2) is applied with appropriate constant values of parameters. However, it was found by
Otter that Levine's assumption with a constant value of $\bar{a}$ of 1.35 for all resonances gave quite good results.

For a lattice in which Dancoff interaction must be taken into account, if an equivalence relation is to be obtained, the escape probability for the rod in the lattice, $P_{0}^{*}$, must again be cast in the rational form

$$
P_{0}^{*}=\frac{1}{1+\left(\Sigma_{0} / \Sigma_{e 0}^{*}\right)} .
$$

An approximate relationship between $P_{0}$ and $P_{0}^{*}$,

$$
\frac{1}{P_{0}^{*}}=\frac{1}{P_{0}}+\frac{\Sigma_{0} C}{\Sigma_{e 0}(1-C)},
$$

has been developed ${ }^{(4,6)}$ by use of the assumption that paths in successive fuel pin cells are uncorrelated. When, as suggested by Levine, ${ }^{(2)}$ the correction factor $a$ is introduced for the lattice case also, one obtains from Eqs. (3), (4), and (5),

$$
\Sigma_{e 0}^{*}=\Sigma_{e 0}(1-C) \frac{a}{1+(a-1) C} .
$$

The factor $a$ is still that for $P_{0}$ and appears here because of the relation Eq. (5) between $P_{0}$ and $P_{0}^{*}$.

The equivalent $\sigma_{p}$, the effective reactor scattering cross section in barns per absorber atom to be used in a homogeneous resonance integral calculation, is then

$$
\sigma_{p}=\sigma_{p 0}+\frac{\Sigma_{e 0}^{*}}{N_{0}}
$$

where $\sigma_{p 0}$ is the fuel-rod scattering cross section per absorber atom in barns and $N_{0}$ is the concentration of absorber atoms in the fuel in atoms/ce $\times 10^{-24}$.

The accuracy of Eq. (6) was checked by calculations kindly performed by E. Pennington, who used his collision probability code. (7) This code uses the flat-flux approximation and an isotropic cell-boundary condition. The calculations were carried out for monoenergetic neutrons for a 0.25 -in. pin with steel clad and sodium coolant typical of a fast power breeder. Various values of the relevant cross sections were assumed to cover the range of escape probabilities of interest. Equation (6) was found to be accurate to about $1 \%$ for $a$ defined by the correct isolated-rod escape proba- 
bility and with $C$ obtained from the calculation with Pennington's code. It was found also that clad and coolant regions could be homogenized in the calculation of $C$ with good accuracy, a result previously obtained by Pennington ${ }^{(8)}$ for water latices. The discrepancy in $\gamma=1-C$ noted in this case between the two- and three-region cells did not exceed $1 \%$.

An approximate method of calculating the Dancoff factor, proposed by Bell, ${ }^{(5)}$ is based on the equation

$$
\gamma_{B}=1-C_{B}=\frac{1}{1+\left(\Sigma_{e 1} / \Sigma_{1}\right)},
$$

where $\Sigma_{e 1}$ in this case is

$$
\Sigma_{e \mathbf{I}}=\frac{S_{0}}{4 V_{1}}=\frac{R_{0}}{2\left(R_{1}^{2}-R_{0}^{2}\right)}
$$

and $R_{1}$ is the outer radius of the moderator (or in this case, homogenized clad and coolant). It has been found by Leslie, Hill, and Jonsson ${ }^{(9)}$ that $\gamma$ does indeed depend to a good approximation only on $\Sigma_{1} / \Sigma_{e 1}$, although $\mathrm{Eq}$. (8) gives values about $12 \%$ too low at large values of $\Sigma_{1} / \Sigma_{e 1}$, in the range from 2 to 3 . Calculations of $\gamma$ were carried out in Ref. 8 with a collision-probability cell calculation with isotropic return quite similar to Pennington's for a square cell with $V_{1} / V_{0}=1$. Calculations were also carried out in Ref. 9 for this same cell with a Monte Carlo calculation, with the result that the collision-probability results were about $3 \%$ too high at large $\Sigma_{1} / \Sigma_{e 1}$. Values of $\gamma$ calculated by Penning$\operatorname{ton}^{(8)}$ for a series of different water-moderated lattices, as well as those discussed in the present paper, all fall very close, within 1 or $2 \%$ in most cases, to the curve of $\gamma$ versus $\Sigma_{1} / \Sigma_{e 1}$ for the escape-probability calculation of Ref. 9, which is reproduced in Fig. 1. The points

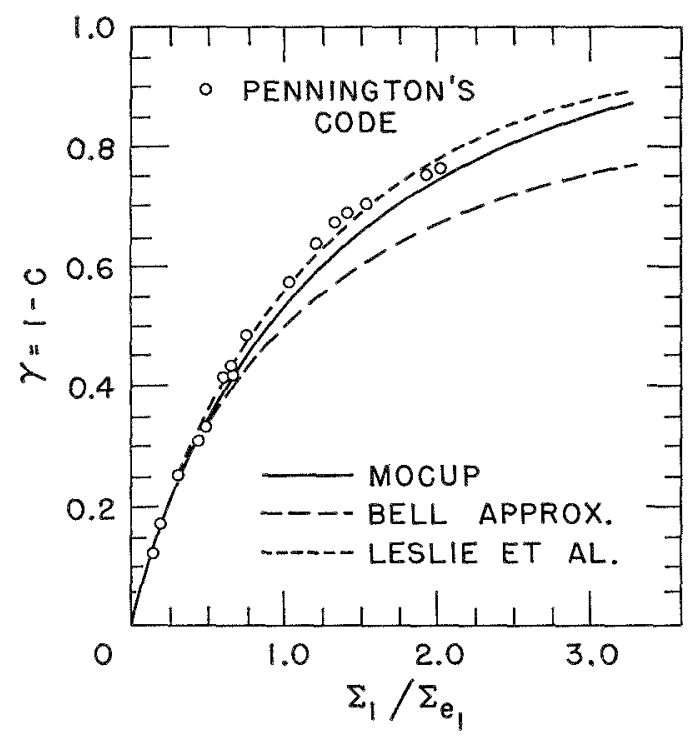

FIG. 1. Values of the Dancoff Factor for Various Approximations. obtained by Pennington's code are also plotted. If $\gamma$ really does depend only on $\Sigma_{1} / \Sigma_{e 1}$, then it seems one would do just as well by making an accurate determination of this universal curve and using an empirical fit. Whether this is really so perhaps requires further exploration. In any event, it is suggested that for the time being a modified Bell approximation be obtained by using a fit to the Monte Carlo curve of Ref. 9 for $\gamma$ versus $\Sigma_{1} / \Sigma_{e 1}$. A simple fit, which is $2 \%$ low at $\Sigma_{1} / \Sigma_{e 1}=3.0$ and closer at smaller values, is

$$
\gamma=\gamma_{B}+\gamma_{B}^{4}\left(1-\gamma_{B}\right)
$$

In using Eq. (6), one is still faced with the problem of what mean value $\bar{a}$ to use. It would be possible to make $\vec{a}$ a function of $C$. This may not be worthwhile, however, since for $C$ nearly unity the factor on the far right side of Eq. (6) is nearly unity regardless of $\bar{a}$; for small $C$, the case of an isolated rod is approached.

An investigation of the appropriate value of $\vec{a}$ was made by use of the code of Kier. ${ }^{(10)}$ By means of this code the Boltzmann equation is solved in the resonance region for a two-region cylindrical cell with up to three resonant isotopes allowed in the fuel region. Isotropic return is assumed at the cell boundary. Since a comparison was made with resonance integral calculations made in the narrow-resonance approximation and without interference scattering, Kier's code was modified to be consistent with these assumptions. The effect of spatial flux variation was still present, however.

The calculations were performed for an oxide fuel pin with stainless steel clad and sodium coolant, the latter two being assumed homogenized. Cell dimensions and compositions were chosen (11) according to the specifications for the comparison calculations proposed for this conference by Okrent. Fuel and cell outer radii were $0.318 \mathrm{~cm}$ and $0.505 \mathrm{~cm}$, respectively.

Calculations were done for a series of U-238 resonances between 1.0 and $1.3 \mathrm{keV}$, using the Firk, Lynn, and Moxon parameters. Resonances with neutron widths loss than $0.01 \mathrm{eV}$ werc excluded because of the weakness of the self-shielding of such resonances. Values of $\bar{a}$ for $C=0$, the isolated-rod case, are given in Table 1, and are seen to lie between 1.19 and 1.53, except for one value at 1.59. The former two values of $a$ seem to be reasonable lower and upper limits according to the curve of $a$ versus optical chord length in the fuel presented by Otter. ${ }^{\left({ }^{3}\right)}$ The precision of the determination of $\bar{a}$ at small neutron widths is not very high.

Calculations were also made with Dancoff interaction present and varied by varying the assumed sodium scattering cross section. Calculations were made for $\sigma_{s}$ of steel assumed constant at $10 \mathrm{~b}$ and $\sigma_{s}$ of sodium assumed to be $10,20,30,60$, and $100 \mathrm{~b}$, corresponding to values of $C$ of $0.758,0.663,0.584,0.494$, and 0.276 , 
TABLE 1. ISOLATED-ROD VALUES OF $\bar{a}$

\begin{tabular}{c|c|c}
\hline $\begin{array}{c}\text { Resonance Energy, } \\
\text { keV }\end{array}$ & $\Gamma_{n}, \mathrm{eV}$ & $\bar{a}$ \\
\hline 1.328 & 0.010 & 1.50 \\
1.281 & 0.027 & 1.53 \\
1.276 & 0.025 & 1.46 \\
1.255 & 0.236 & 1.21 \\
1.221 & 0.014 & 1.53 \\
1.204 & 0.057 & 1.42 \\
1.186 & 0.030 & 1.44 \\
1.178 & 0.043 & 1.41 \\
1.149 & 0.274 & 1.19 \\
1.130 & 0.010 & 1.59 \\
1.061 & 0.051 & 1.39 \\
1.029 & 0.019 & 1.53 \\
\hline
\end{tabular}

respectively. It was found that for $\sigma_{s}$ of 10 and $20 \mathrm{~b}$, the equivalent $\sigma_{p}$ obtained by the relations given here were accurate to 1 or $2 \mathrm{~b}$ out of totals of 48 and $56 \mathrm{~b}$, respectively, using $\bar{a}=1.35$. At smaller values of $C$, however, greater disparities appeared. Results at $\sigma_{s}=$ 30 and $100 \mathrm{~b}$ are shown in Figs. 2 and 3, with inferred $\sigma_{p}$ values for individual resonances plotted against neutron width. It is found in these cases that $\sigma_{p}$ is slightly larger than expected and indeed at small neutron widths is larger than would correspond to a possible value of $\bar{a}$. Not shown are results for $\sigma_{s}=60 \mathrm{~b}$. In this case apparent $\sigma_{p}$ values, which were expected to be about 70 to $76 \mathrm{~b}$, were about $13 \mathrm{~b}$, a slightly larger discrepancy than in the cases for 30 and $100 \mathrm{~b}$. It is not obvious why this should be, and it may be due to an undetected error. In any event, the errors in the equivalence relations indicated here are of small consequence for fast reactor calculations.

Comparisons were made at $300^{\circ} \mathrm{K}$ and at $750^{\circ} \mathrm{K}$. At the higher temperature the inferred $\sigma_{p}$ was usually slightly larger than at the lower one, the difference being 1 to $2 \mathrm{~b}$. This indicates that the use of the equivalence relations leads to an underestimation of the Doppler effect by several percent, which is not a significant error.

While the results here indicate that equivalence theory is satisfactory for U-238 resonances in the energy range considered, it is still possible that significant errors could occur at lower energy, where resonances are stronger and the flat-flux approximation is less realistic.

\section{Effect of Parameter Variation on Sodium Void Effect}

We consider the effect of variations in the fission cross section of $\mathrm{Pu}^{239}$ and the capture cross section of $\mathrm{U}^{238}$ from the reference values used in the comparison calculations done for this Conference, ${ }^{(11)}$ referred to as the Set 232 values and given in Table 2. These cross sections were identical with those of Set $224^{(12)}$ above $25 \mathrm{keV}$.

$\sigma_{f}$ of $P u^{239}$

We have found that, particularly for a ceramicfueled system, the value of $\sigma_{f}$ of $\mathrm{Pu}^{239}$ at quite low energies, in the range 1 to $3 \mathrm{keV}$, can be significant for the sodium void effect. Although there is not much flux at such low energies, the energy variation of the adjoint function can be significantly affected at higher energies by events occurring in the range from 1 to 3 $\mathrm{keV}$ because of the elastic-transfer coupling term in the adjoint function. This is a significant source of uncertainty because of the large uncertainty in $\sigma_{f}$ of $\mathrm{Pu}^{239}$ in this energy range. We have considered an alternative set of values to those of Set 232, based on data presented by Hemmendinger ${ }^{(13)}$ and based on Los Alamos bomb test measurements (see Table 2). Since these data are

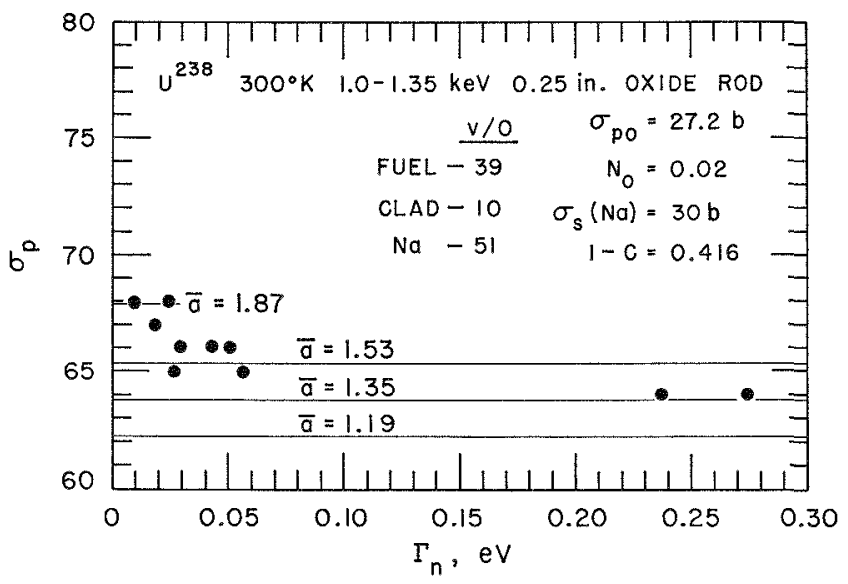

Fia. 2. Values of $\sigma_{p}$ as a Function of $\Gamma_{n}$ of the Resonance (for $\sigma_{s}$ of Sodium Equal to $30 \mathrm{~b}$ ).

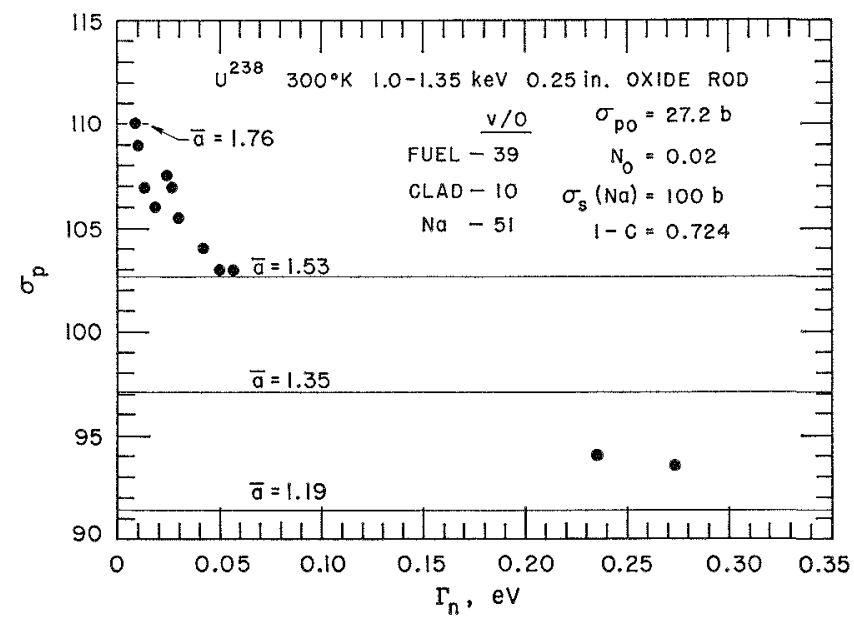

FIG. 3. Values of $\sigma_{p}$ as a Function of $\Gamma_{n}$ of the Resonance (for $\sigma_{s}$ of Sodium Equal to $100 \mathrm{~b}$ ). 
TABLE 2. Cross-section Variations Studied

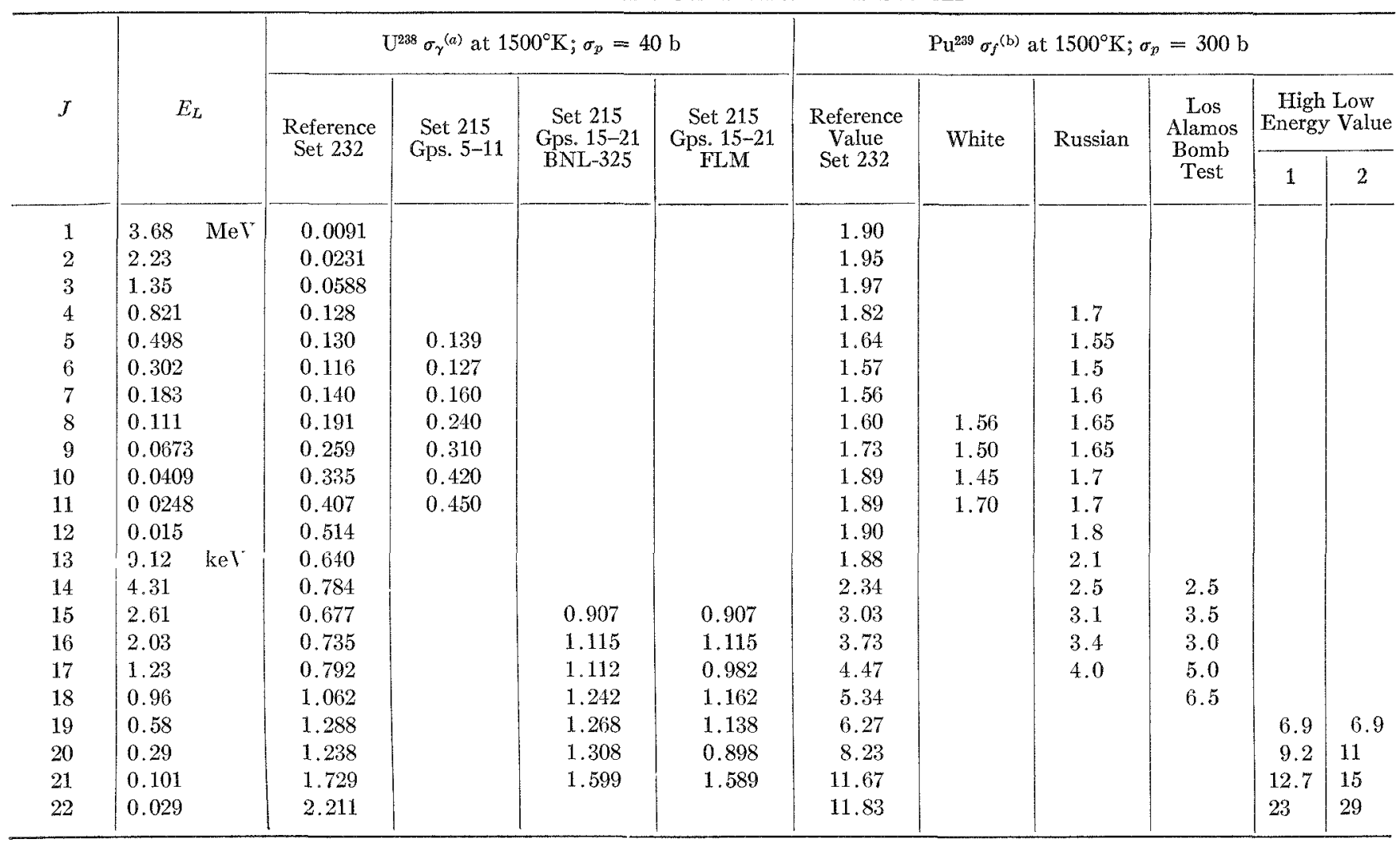

(a) Calculated values include flux correction factor.

(b) $\alpha$ was kept constant in $\sigma_{f}$ variations.

preliminary and since resonance parameters are not available, the values inferred here are not to be taken too seriously. The question of self-shielding has been ignored here, but should be small for $\mathrm{Pu}^{239}$ in the energy range above $1 \mathrm{keV}$. The Los Alamos values are generally higher than those for Set 232 except in the case of Group 16 by from 2.0 to $2.6 \mathrm{keV}$.

At still lower energies, below $1 \mathrm{keV}$, the measured cross section as shown in BNL-325 ${ }^{(14)}$ suggests that the Set 232 values of $\sigma_{j}$ for $\mathrm{Pu}^{239}$, which were based on a statistical distribution of parameters above Group 22, may be too low. Calculations with parameters recently obtained from Harwell(15) give a value of around $14-15 \mathrm{~b}$ at $1500^{\circ} \mathrm{K}$ and of $\sigma_{p}=300 \mathrm{~b}$ for Group 21. The $\sigma_{f}$ cross sections for $\mathrm{Pu}^{239}$ in the Bondarenko book ${ }^{(16)}$ are also higher. We have therefore tried two alternative sets of higher low-energy values. The Bondarenko values appear to fall in between these, but a careful interpolation with respect to energy, temperature, and $\sigma_{p}$ has not been done.

Another variation made in $\sigma_{f}$ of $\mathrm{Pu}^{239}$ corresponds to recent data of $\mathrm{P}$. White, ${ }^{(17)}$ which indicate considerably lower values in the energy range from 25 to 200 $\mathrm{keV}$ than were used in Set 232.
Still another variation is the use of Russian data ${ }^{(18)}$ for the $\sigma_{f}$ of $\mathrm{Pu}^{23 \dot{s}}$. These values are not greatly different from those of Set 232 , but the statistical uncertainty is rather large.

$\sigma_{\gamma}$ of $U^{233}$

The values assumed for Set 232 (and for Set 224) are lower than most of the data in BNL-325 in the energy range $25-300 \mathrm{keV}$ because of a desire to give some weight to the data of Moxon. ${ }^{(19)}$ The effect of use of values from set 215 , used in calculations presented at the October, 1963 conference at Argonne, ${ }^{(20)}$ was studied. Cross sections in this set are higher than those of Set 232 in the energy region $25-800 \mathrm{keV}$ (Groups 5-11) and are close to the curve in BNL-325. The Set 215 cross sections are also higher below $4 \mathrm{keV}$, as a consequence of using the BNL-325 parameters and unresolved region parameters corresponding to them, whereas the Set 232 values use the new Columbia resonance parameters. ${ }^{(21)}$ This was also studied as a variation for Groups 15-21. Another variation was to use the Set 215 cross sections for Groups 15-21, but with the Firk, Lynn, and Moxon parameters ${ }^{(22)}$ for Groups 17-21, below $2.0 \mathrm{keV}$. 


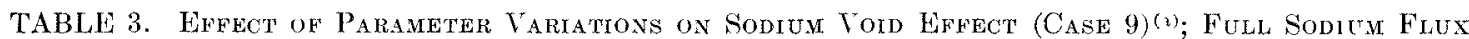

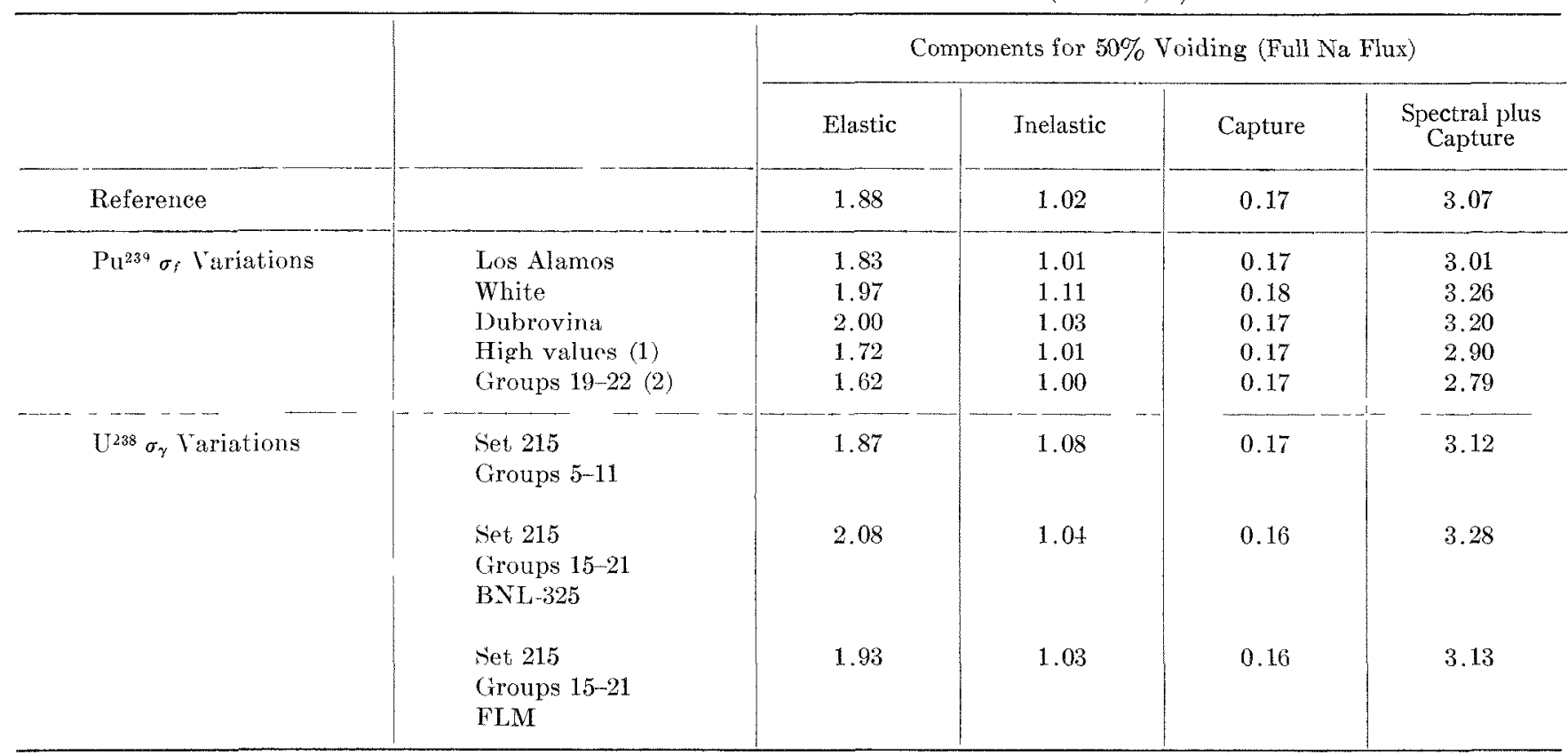

(a) Atoms/ce $\times 10^{-21}: \mathrm{Na}: 0.0123 ; \mathrm{Pu}^{239}: 0.0009 ; \mathrm{U}^{238}: 0.0063 ; \mathrm{Pu}_{\mathbf{u}^{240}}: 0.00045 ;$ Stainless Steel : $0.011 ;$ Oxygen: $0.0144 ;$ Fission Product Pairs: 0.00036 .

\section{Results of Parameter Variation (see Table 3)}

$\sigma_{f}$ for $P u^{239}$. The use of the low-energy cross sections inferred from the Los Alamos bomb test experiment reduced the positive spectral component slightly. This was caused by the fact that the varied cross section was mainly above the original one. This resulted in a reduction in the energy variation of the adjoint function. The higher values at still lower energy have also resulted in less positive void effects for the same reason.

The use of the new White data, corresponding to a lower fission cross section in the range from 25 to 200 $\mathrm{keV}$, has resulted in a more positive effect as expected. The data of Dubrovina and Shigin, which also lie generally below the Set 232 values, also give more positive results because of a more rapid variation of the adjoint function.

$\sigma_{\gamma}$ for $U^{238}$. Although none of the variations had a very important effect, the use of the higher cross sections at lower energy causes the void effect to be more positive, because the energy variation of the adjoint function becomes more rapid. This effect is less pronounced with the Firk, Lynn, and Moxon cross sections, as they give lower cross sections than do the BNL-325 parameters.

\section{Effect of Presence of $\mathbf{P u}^{241}$ on Sodium Void Effect}

It is expected that the substitution of $\mathrm{Pu}^{241}$ for $\mathrm{Pu}^{239}$ will result in a more negative sodium void effect for
Tablia 4. Spectral Plus Capture $\delta 7$ for $50 \%$ Sodium Vording

10\% of $P u^{239}$ Replaced with $P u^{2 a 1} ; F^{x}$ ull Sodium Flux

\begin{tabular}{c|c|c|c}
\hline \multirow{2}{*}{$\begin{array}{c}\text { Fertile to Fissile } \\
\text { Ratio }\end{array}$} & Case & \multicolumn{2}{|c|}{$\% \delta k$} \\
\cline { 2 - 3 } & & Original & $\mathrm{Pu}^{241}$ Present \\
\hline 9.5 & 8 & 4.02 & 3.20 \\
7.5 & 9 & 3.07 & 2.41 \\
5.5 & 10 & 2.07 & 1.51 \\
\hline
\end{tabular}

the same ratio of fertile to fissile atoms because the $\mathrm{Pu}^{241}$ fission cross section varies more rapidly with energy than that of $\mathrm{Pu}^{239}$. This has been studied for Cases 8, 9, and 10 of the comparison calculations for this Meeting. ${ }^{(11)}$ These are oxide-fueled compositions with ratios of fertile to fissile atoms as indicated in Table 4. The composition is otherwise as indicated in Table 3, the total fuel atoms/cc remaining the same. Ten percent of the $\mathrm{Pu}^{239}$ was replaced with $\mathrm{Pu}^{241}$, the $\mathrm{Pu}^{241}$ cross sections being given in Ref. 11. Fuel-cycle studies indicate that equilibrium $\mathrm{Pu}^{241}$ contents are generally in the range from 7 to $10 \%$ of the $\mathrm{Pu}^{239}$ content.

The results, given in Table 4, show that, as expected, the void effect is considerably less positive with $\mathrm{Pu}^{241}$ present. Note that this comparison is for a fixed fertile to fissile ratio and takes no account of a possible change in critical enrichment for a given reactor size as a result of the substitution. 


\section{Nonlinearity in Spectral Component as a Function of Sodium Content}

It has been found that nonlinearity is due primarily to variations in the energy dependence of the adjoint function, and hardly at all to variation of effective sodium cross section or flux, except for events occurring in the vicinity of the $2.85-\mathrm{keV}$ sodium resonance. Nonlinearities of the adjoint function tend to be associated with local variations in energy of $\eta$, the fission neutrons produced per neutron captured. When strong moderation is present, these variations are smoothed out and do not cause corresponding variations in the adjoint function. In the limit of no moderation, the adjoint function becomes equal to $\eta$, aside from the effect of leakage. As sodium is removed and moderation is

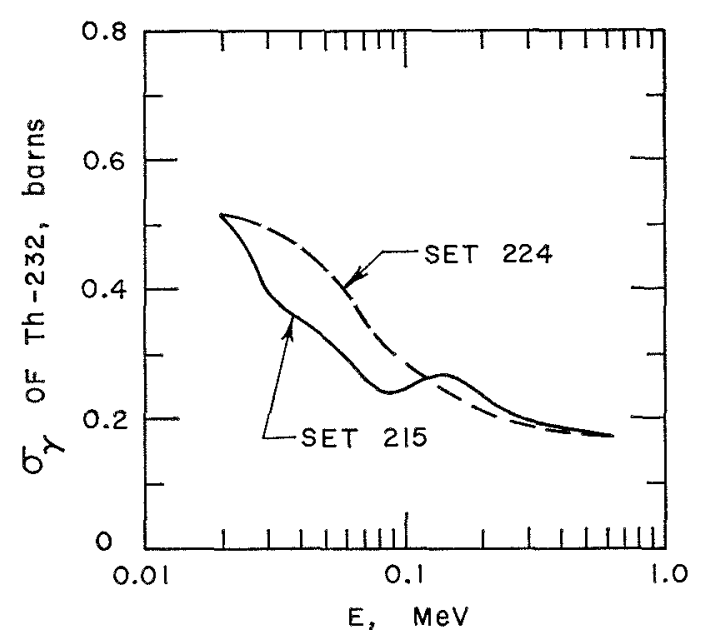

FIg. 4. Assumed Energy Variation of $\sigma_{\gamma}$ of Th-232 for Crosssection Sets 215 and 224 . reduced, therefore, any local variations in $\eta$ tend to appear in the adjoint function. This is not very marked with oxide systems, as strong moderation is still present. With a metal system rather strong effects can occur.

The example which is given in the following is admittedly a rather extreme one in that the ratio of sodium to fuel is rather high. It is taken from a report to be published by Singh and Hummel ${ }^{(23)}$ on reactivity coefficients in $\mathrm{U}^{233}{ }^{-} \mathrm{Th}^{232}$-fueled systems. Results were obtained from the use cross-section Sets 215 and 224 . The essential difference between the two for the purpose of the present discussion is in the assumed energy variation of the $T^{232}$ (apture cross section (see Fig. 4). The basis for the eurve for Set 224 is reported in Ref. 12 . The lower curve, for Set 215, favors data of Moxon. ${ }^{(19)}$ As a result of the dip in the cross section below $0.1 \mathrm{MeV}$, the adjoint function for the metal-fueled case treated by Set 215 shows a rise just below $0.1 \mathrm{MeV}$, which is barely perceptible for the full sodium case (see Fig. 5) and quite strong for the no-sodium case (see Fig. 6). This effect is suppressed for the Set 215 oxide case, and not present at all for the Set 224 metal case. The consequences for the elastic removal component of the sodium void effect may be seen in Table 5. Although the variations in the adjoint function which occur in the region of greatest flux amount to only a few percent, they correspond to large changes in the sodium void effect. The results in Table 5 were obtained by perturbation theory and correspond to $50 \%$ sodium voiding using the indicated flux. The nonlinearity for the Set 215 case is very strong; that in the Set 224 metal case is much less. Peculiarity in the behavior of the void effect in the oxide cases is due mostly to events occur-

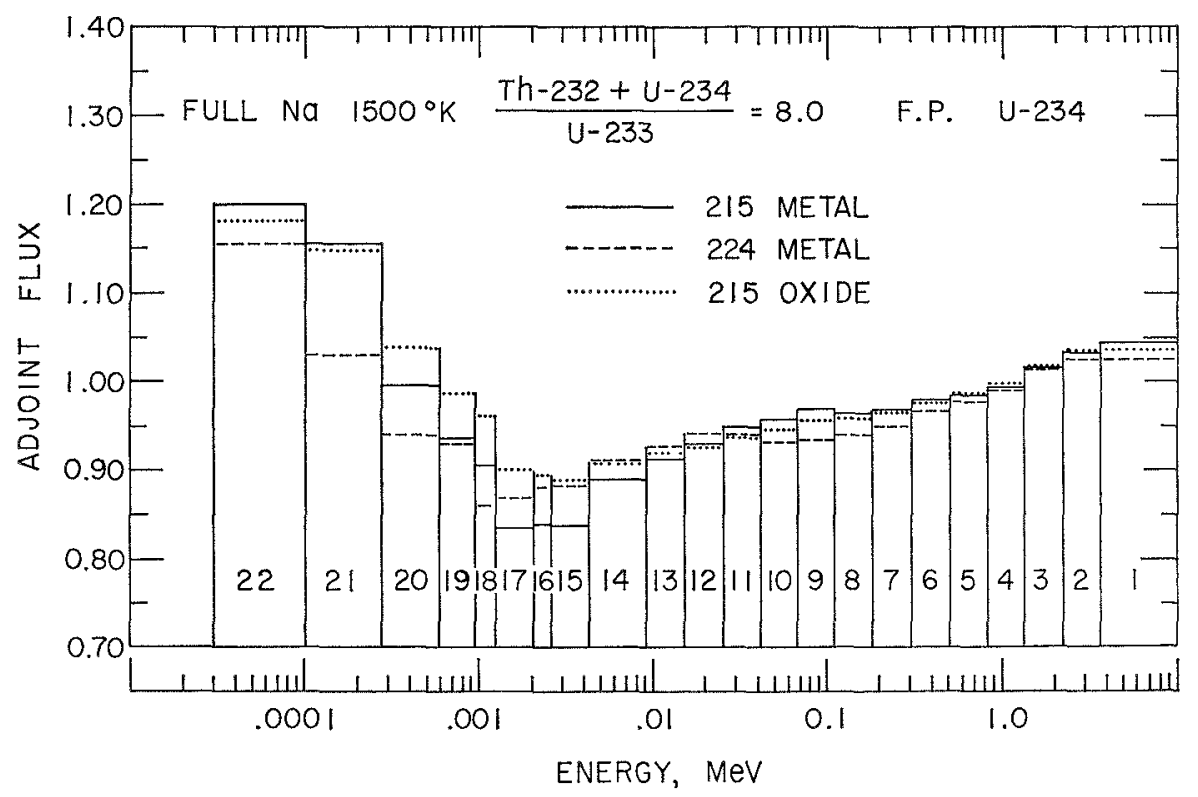

Frg. 5. Adjoint Function as a Function of Energy in a $\mathrm{U}^{233}$-Th System; Fuel Sodium. 


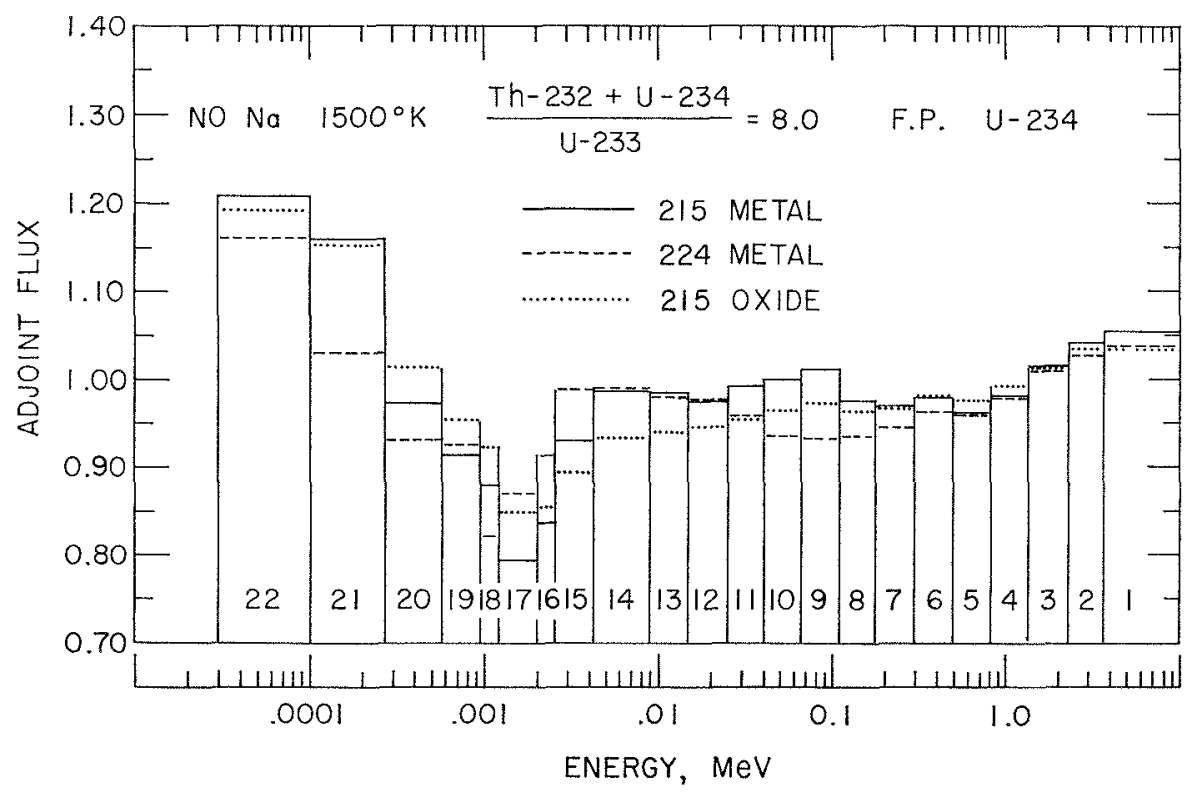

FIG. 6. Adjoint Function as a Function of Energy in a U233-Th System; No Sodium.

TABLE 5. Elastic Removal Component for 50\% VOIDING, \% $\Delta k$

$$
\frac{T h^{232}+U^{234}}{U^{233}}=8.0 ; 70 \mathrm{v} / 0 \mathrm{Na} ; 1500^{\circ} \mathrm{K}
$$

\begin{tabular}{|c|c|c|c|c|}
\hline $\begin{array}{l}\text { Flux Used, } \% \\
\text { Sodium }\end{array}$ & 100 & 50 & 10 & 0 \\
\hline \multicolumn{5}{|l|}{ Set 215} \\
\hline Metal & 1.05 & 0.85 & $\begin{array}{c}0.03 \\
(0.01)^{(4)}\end{array}$ & $\begin{array}{l}-0.26 \\
(0.23)^{(a)}\end{array}$ \\
\hline Oxide & 0.47 & $\begin{array}{l}1.15 \\
(0.04)\end{array}$ & $\begin{array}{l}2.18 \\
(0.47)^{(\omega)}\end{array}$ & $\begin{array}{c}3.71 \\
(2.62)^{(a)}\end{array}$ \\
\hline \multicolumn{5}{|l|}{ Set 224} \\
\hline Metal & 1.09 & 1.08 & & $\begin{array}{l}0.99 \\
(0.41)^{\langle a\rangle}\end{array}$ \\
\hline Oxide & 1.13 & 1.74 & & $\begin{array}{l}5.14 \\
(3.52)^{(a)}\end{array}$ \\
\hline
\end{tabular}

(a) Contribution from Group $15(2.8-4.0 \mathrm{kel})$.

ring in the vicinity of the $2.85-\mathrm{keV}$ sodium resonance. The contribution to $\delta \mathrm{k}$ in this region is indicated in parentheses in Table 5. The large effect at zero sodium flux is due to a failure in perturbation theory, since the unperturbed flux is being multiplied by a large sodium transfer cross section. As sodium is introduced, a flux depression appears in the vicinity of the resonance and the effect is quickly reduced.

The results given here suggest that interesting effects may be observed in some cases by looking for nonlinearities in sodium void effect in different systems for various degrees of moderation. Useful information about the energy variation of both the $\mathrm{Th}^{232}$ and the
$\mathrm{U}^{239}$ capture cross sections could conceivably be obtained.

\section{Acknowledgment}

The authors wish to thank E. Pennington for performing some of the calculations with his collision probability code, and P. Kier for assistance in the use and modification of his code for integration of the Boltzmann equation.

\section{REFERENCES}

1. J. Chernick and R. Vernon, Some Refinements in the Calculation of Resonance Integrals, Nucl. Sci. Eng, 4, 649 (1958).

2. M. M. Levine, Resonance Integral Calculations for U-238 Lattices, Nucl. Sei. Eng. 16, 271 (1963).

3. J. M. Otter, Escape Probability Approximations in Lumped Absorbers, NAA-SR-9744 (1964).

4. W. Rothenstein, Collision Probabilities and Resonance Integrals for Lattices, Nuel. Sci. Eng. 7, 1962 (1960).

5. G. I. Bell, A Simple Treaiment for Effective Resonance Integrals in Dense Lattices, Nucl. Sci. Eng. 5, 138 (1959).

6. L. W. Nordheim, The Theory of Resonance Absorption, Proc. Symposium Appl. Math. XI, 58 (1961).

7. E. Pennington, Collision Probabilities in Cylindrical Lattices, Nucl. Sci. Eng. 19, 215 (1964).

8. E. Pennington, "Hi-C Uniform Lattice Calculations," in Reactor Physics Division Annual Report, ANL-7110 (1965).

9. D. C. Leslie, J. (4. Hill, and A. Jonsson, Improvements to the Theory of Resonance Escape in Heterogeneous Fuel. Nucl. Sci. Eng. 22, 78 (1965).

10. P. H. Kier, A Method of Calculating Mutual Shielding between Resonance of Different Nuclides, Trans. Am. Nuc. Soc. 8, No. 1, 286 (1965).

11. H. H. Hummel, K. E. Phillips, and D. M. O'Shea, Calculations of Intercomparison Problems for ANL Fast Reactor Meeting, to be published. 
12. D. M. O'Shea, H. H. Hummel, W. B. Loewenstein, D. Okrent, and B. J. Toppel, Twenty-six-group Cross Sections, ANL-6858, to be published.

13. A. Hemmendinger, Cross-section Measurements Made with Neutrons from a Nuclear Detonation, Physics Today 18, No. 8, 17-23 (Aug 1965).

14. Neutron Cross Sections, BNL-325 (July 1, 1955).

15. C. A. Uttley, "Total Neutron Cross Section Measure. ments," Nuclear Physics Division Progress Report, $7 / 1 / 64$ to 2/28/65, AERE-PR/NP 8, (1965).

16. L. P. Abagyan, N. O. Bozazyants, I. I. Bondarenko, and M. N. Nikolaev, Group Constants of Fast and Intermediate Neutrons for the Designing of Nuclear Reactors, Physical Power Engineering Inst., State Committee for Use of Atomic Energy, USSR (1962).

17. P. H. White, J. G. Hodgkinson, and G. J. Wall, Measurement of Fission Cross Sections for Neutrons of Energies in the Range 40-500 keV, SM 60/14, IAEA Symposium on the Physics and Chemistry of Fission, Salzburg, Austria, 22-26 March, 1965.

18. S. M. Dubrovina and $T$. A. Shigin, Bulletin of the Information Center on Nuclear Data (First Issue), State Com- mittee on the Utilization of Atomic Energy of the USSR, Atomizdat (1964).

19. A. T. G. Ferguson and N. J. Pattenden, Neutron Crosssection Measurements at Harwell in Support of the U.K. Fast Reactor Programme, Proc. of the Conf. on Breeding, Fconomics, and Safety in Large Fast Power Reactors, ANL 6792 (1963), pp. 11-13.

20. H. H. Hummel, K. Phillips, and A. L. Rago, Calculations of Sodium Void Reactivity Effect for Large Fast Oxide Reactors in Spherical and Slab Geometry, Proc. of the Conf. on Breeding, Economics, and Safety in Large Fast Power Reactors, ANL-6792 (1963), pp. 65-74.

21. J. Garg, J. Rainwater, J. S. Petersen, and W. W. Havens, Jr., Neutron Resonance Spectroscopy. III. Th-238 and U-288, Phys. Rev. 134, 5B, B985-B1009 (1965).

22. F. W. K. Firk, J. E. Lynn, and M. C. Moxon, Resonance Parameters of the Neutron Cross Section of U-238, Nucl. Phys. 41, 614 (1963).

23. H. H. Hummel and R. S. Singh, Parametric Studies of the Reactivity Coefficients for Large U-233-Th-fueled Fast Reactors, ANL-6930, to be published. 


\title{
A Comment on the Comparison of Some Sodium Void Coefficients with Experimental Data
}

\author{
A. Travelui \\ Argonne National Laboratory \\ Argonne, Illinois
}

The difficulties which are encountered in the evaluation of the sodium void coefficient in fast reactors may be considered to belong, in general, to one of two different categories. The first includes all the problems involved in the determination of the correct spatial behavior of the real and adjoint fluxes in the reactor, which often requires complex calculations to take into account transport effects, inhomogeneities, anisotropy, irregular geometry, and two- or three-dimensional effects. The second category includes the determination of a suitable set of cross sections to be used in the (alculations. The combination of all these sources of uncertainty may cause a considerable inaccuracy in the calculations, and the disagreement which may be found among the values calculated by different authors for the sodium void coefficient of similar reactors has been put well in evidence by Okrent. (1)

One logical procedure intended to improve the accuracy of the calculations is to face separately the different causes of inaccuracy, and it is with this consideration in mind that a set of calculations has been made on the variation of the sodium void coefficient when different cross-section sets are used. The purpose of these calculations was to compare the results with experimental data in such a way that a criterion could be at hand, however modest, to assign a figure of merit to each cross-section set.

The reactor considered in the calculations was chosen to be similar in shape and composition to Assembly $45 \mathrm{~A}$ of $\mathrm{ZPR}-3 .^{(2)}$ It is sufficient here to say that it is a zoned reactor, cylindrical in shape, and that it consists of a reflector, of a driver, of a filter, and of a central zone. The composition of the central zone is such that for every atom of plutonium-239 there are, in the central zone, approximately six atoms of uranium-238, eight atoms of sodium, four atoms of graphite, and twelve atoms of stainless steel. Three different sets of cross sections were used in the calculations. The first and the second set were, respectively, the Argonne Set 224 and the Argonne Set 232.(3) The third set was the Argonne Set 224 with a modification introduced by D. K. Butler to include the White cross sections for plutonium-239 and for uranium-235; we shall refer to this set as to the Set $22 t+W$.

The parameter which was calculated with each set of cross sections, and compared with the experimental data, was the value of the central sodium worth. This value was chosen for the comparison, rather than the value of a distributed sodium worth, because at the center of the reactor the leakage component of the sodium worth vanishes, and this decreases the effect of the uncertainty involved in the determination of the spatial behavior of the fluxes.

The value of the central sodium worth in a critical reactor is given, in perturbation theory and in a concise matrix notation, by the following expression:

$$
\begin{aligned}
\text { Sodium Worth }=\frac{\left\langle\phi^{+}\left|\sigma_{s}^{\mathrm{Na}}-\sigma_{s o}^{\mathrm{Na}}-\sigma_{c}^{\mathrm{Na}}\right| \phi\right\rangle_{0}}{\left\langle\phi^{+} \mid \chi\right\rangle_{0}\left\langle\nu \Sigma_{f} \mid \phi\right\rangle_{0}} \\
\cdot \frac{\left\langle\phi^{+} \mid \chi\right\rangle_{0}\left\langle\nu \Sigma_{f} \mid \phi\right\rangle_{0}}{\int_{V}\left\langle\phi^{+} \mid \chi\right\rangle\left\langle\nu \Sigma_{f} \mid \phi\right\rangle d \mathbf{r}} C,
\end{aligned}
$$

where $\left\langle\phi^{+}\right|$and $|\phi\rangle$ are, respectively, the adjoint and the real fluxes; $\left[\sigma_{s}^{\mathrm{Na}}\right],\left[\sigma_{s o}^{\mathrm{Na}}\right]$, and $\left[\sigma_{r}^{\mathrm{Na}}\right]$ are the total microscopic scattering cross sections, the microscopic scattering kernel, and the microscopic capture cross section, of sodium; $\langle\nu|,| \chi\rangle,\left[\Sigma_{f}\right]$, and $V$ are the average number of neutrons produced in a fission, the fission spectrum, the macroscopic fission cross section of the core, and the volume of the core. The subscript 0 refers to quantities evaluated at the center of the reactor, and $C$ is equal to the product of the number of atoms which are present in a kilogram of sodium times the number of inhours per unit excess reactivity.

If the central zone is large enough for the fundamental mode to be cstablished at the center of the reactor, the first factor on the right hand side of $\mathrm{Eq}$. (1) may be considered very close to the result of a fundamental mode calculation, and it is almost independent on the accuracy with which the spatial dependence of $\left\langle\phi^{+}\right|$ and $|\phi\rangle$ is evaluated. The second factor, on the other hand, does not depend of the material whose worth is being calculated. Thus, the calculated ratio of the central worths of two different materials should be 
TABLF, 1. MATERIAL, Worths in Assembly 45A

\begin{tabular}{|c|c|c|c|c|}
\hline & Set 224 & Set 232 & $\begin{array}{l}\text { Set } 224 \\
+W\end{array}$ & Experimental \\
\hline \multicolumn{5}{|l|}{$\begin{array}{c}\text { Central Worths } \\
(\mathrm{Ih} / \mathrm{kg})\end{array}$} \\
\hline $\mathrm{Na}$ & -4.76 & -5.96 & -5.89 & $-4.7 \pm 0.4$ \\
\hline Pu Fuel & 188.1 & 199.2 & 174.1 & $176 \pm 1.0$ \\
\hline Depleted $\mathbf{U}$ & -8.47 & -9.35 & -8.66 & $-7.6 \pm 0.1$ \\
\hline \multicolumn{5}{|l|}{ Central. Worth } \\
\hline $\mathrm{Na} / \mathrm{Pu}$ Fuel & 0.0253 & 0.0299 & 0.0338 & $0.0267 \pm 0.0024$ \\
\hline $\mathrm{Na} /$ Depleted $\mathrm{U}$ & 0.562 & 0.637 & 0.681 & $0.618 \pm 0.061$ \\
\hline$--\ldots-\ldots-$ & 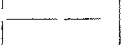 & & $-\cdots$ & $-\cdots--$ \\
\hline $\begin{array}{l}\text { Na loid Coeffi- } \\
\text { cient* }\end{array}$ & 0.601 & 0.717 & 0.805 & $(0.662)$ \\
\hline
\end{tabular}

* Fundamental Mode Calculations (\% $\Delta k / k$ for $50 \%$ sodium removal).

almost independent of the spatial variation of the fluxes, and its comparison with the experimental data should provide a more significant indication of the reliability of the cross-section set which is being employed.

The calculations were performed by perturbation theory in MACH-1, a one-dimensional diffusion code developed by D. A. Meneley. In view of the previous considerations, it was believed that the limitations of diffusion theory would not affect the results for the worth ratios to any appreciable extent. Table 1 shows the calculated results for the worths of sodium, of plutonium fuel $\left(95.4 \% \mathrm{Pu}^{239}\right.$ and $\left.4.6 \% \mathrm{Pu}^{240}\right)$, and of depleted uranium $\left(99.794 \% \mathrm{U}^{238}\right.$ and $\left.0.206 \% \mathrm{C}^{235}\right)$, with their ratios, and the corresponding experimental values. ${ }^{(4)}$

The agreement between calculated and experimental values is remarkably good with all the cross-section sets, both for the absolute values of the worths and for their ratios. From the comparison of the worth ratios we may conclude that the experimental results fall in between the results that have been calculated with the Argonne (ross-section Sets 224 and 232, which rate almost equally well, with Set $224+\mathrm{W}$ coming up last. It should be noted, however, that all three cross-section sets come through the test quite satisfactorily, and, in effect, all almost within the experimental error. Thus, the result of the calculations should be considered an indication of the relative worth of the different crosssection sets, rather than a final conclusion.

The last line of Table 1 gives the results of a fundamental-mode calculation of the sodium void coefficient for a $50 \%$ sodium removal throughout the core, as obtained from the evaluation of the first factor on the right-hand side of Eq. (1), and, by interpolation, what may be considered as an experimental result for a fundamental-mode sodium void coefficient. This result would be very close to the spectral-plus-capture components for uniform voiding of a reflected reactor with a core composition equal to the composition of the central zone, since the core size would be large and the effect of the reflector on the core spectrum would not be important. These values are given for orientation purposes, and show that the reactivity differences between theory and experiment are not as serious as one might have feared, considering the uncertainties in relevant parameters.

\section{REFERENCES}

1. D. Okrent, "Intercomparison of Caleulations", See paper in Session I of This Conference.

2. (i. J. Fischer, D. A. Meneley, R. N. Hwang, E. F. Groh, and C. E. Till, Measurement and Analysis of Doppler Effect in Plutonium fueled Fast Reactor Assemblies, see Paper in Session VIB of This Conference.

3. H. H. Hummel, R. N. Hwang, and K. Phillips, Recent Investigations of Fast Reactor ("vefficients, see Paper in Session VB of This Conference.

4. Argonne National Laboratory Reactor Development Program Progress Report, ANI 7003 (1965).

\section{Discussion}

Mr. Küsters (Karlsruhe): I would like to make a comment. I agree that the uncertainties in the microscopic cross sections for the sodium void effect may be not so severe as the uncertainty in the calculational methods. I remind you of the comment Dr. Häfele gave yesterday in the Panel Discussion. He said that we have found that in using or in calculating the sodium void for a two-dimensional reactor with one-dimensional calculations with suitable buckling, we got a positive effect; when we made two-dimensional calculations, we got a negative effect. The difference was about $\$ 3$. I would say that the sodium void is much more sensitive to the calculational methods than to the cross sections.

Mr. Hummel (ANL): I think that if you look at the spectral or leakage components separately, the spectral component is probably not overly sensitive to the details of the geometry. This is what really does the damage. Admittedly the leakage component gives you trouble, but this is something I think we can certainly work up to. I think it would be helpful to separate these two parts of the void effect when you discuss the problem. 


\title{
The Influence of Some Important Group Constants on Integral Fast Reactor Quantities*
}

\author{
H. Küsters axd M. Metzenkoth \\ Institute of Neutron Physics and Reactor Engineenng \\ Kernforschungszentrum, Karlsruhe, Germany
}

(PRESENTEI BY H. KÜSTERS)

\section{Introduction}

It is well known that for large fast reactors the calculation of critical mass and safety coefficients is possible only to an unsatisfactory degree of accuracy. One of the main reasons comes from the fact that the group constants used in the multigroup calculations are uncertain. The objects of this paper are to find the importance of some uncertaintics and the basis for further improvements in the calculational methods for dilute fast reactors. For this purpose it is useful to distinguish between two sources of uncertainties in group constants: those arising from the weighting spectrum and those from uncertainties in the microscopie data.

In the first part of this paper the effect of spectral fine structure with respect to resonance self-shielding and the influence of the macroscopic weighting spectrum is discussed. It is investigated how reactivity coefficients like the Doppler coefficient (DC), sodium density coefficients, or breeding ratios, important for economical considerations, are influenced by different weighting spec$1 \mathrm{ra}$, which are used in various sets of group constants.

In the second part the effect of some uncertain microscopic data with respect to the calculation of dilute fast reactors is investigated. The aim is to have a better knowledge of the importance of these group constants in fast reactor calculations. Some of these effects are separated during the preparation of the first Karlsruhe group-constant set KFK 26-10, which is briefly described here.

The method of calculating the DC used here is based on perturbation theory. ${ }^{(1)}$ Sodium void or loss is calculated in two criticality calculations. The resonance selfshielding is treated in narrow resonance approximation and is determined in advance of each reactor calculation. This is the procedure described in the Russian group-constant set. ${ }^{(3)}$

* This paper has been prepared within the framework of the association EURATOM Gesellschaft fur Kernforschung mbH. in the field of fast breeder development.

\section{Methodical Questions}

\section{The EIfect of Resonance Self-shielding}

The theoretical investigation of fast reactors was first made for relatively small assemblies with hard spectra. A large number of these facilities have been calculated with good results in critical masses with the unshielded group-constant set "YOMI" given by S. Yiftah, D. Okrent, and P. A. Moldauer and by S. Yiftah, MI. Sieger ("YONI-64"). (2)

For dilute fast reactors there is an appreciable amount of neutrons below $10 \mathrm{keV}$ and the effect of resonance self-shielding should be remarkable. Nevertheless, the YOM set gives good results in criticality for these large reactors (see Table 6 below, for instance).

To have a better understanding of the shielding effect on neutron multiplication, we made a series of fundamental-mode ealculations for reactors of different sizes and compositions with the shielded and unshielded Russian " $\mathrm{AB} N$ " set of I. I. Bondarenko et al. (3) The result is shown in Fig. 1. The ordinate is the difference of the shielded $\left(k_{e f f, s}\right)$ and unshielded calculations. All reactors are made critical with buckling iteration so that $k_{\mathrm{eff}, s}=$ 1 always. The abscissa is the escape probability of neutrons for the system, defined by $P=1-\left(k_{\mathrm{eff}, s} / k_{\infty, s}\right)$.

For large fast reactors of about 7000-liter core volume there is a loss in reactivity of about $6 \%$ if the calculation is made with unshielded cross sections. This reactivity loss is mainly due to a decrease in $k_{\infty}$. For small cores with high escape probability, the neutron balance is made up mainly of leaking neutrons and fissioning neutrons. In these cores with hard spectra only the resonances of structural materials like iron and nickel contribute to the shielding effect. However, these resonances are almost scattering resonances, and therefore the use of unshielded scattering cross sections results in increasing transport cross sections (higher by a factor of 2 near the iron resonance at $40 \mathrm{keV}$ ) and in lowering of leakage. The fundamental-mode calculation yields a gain of about $8 \%$ in reactivity for the core composition 


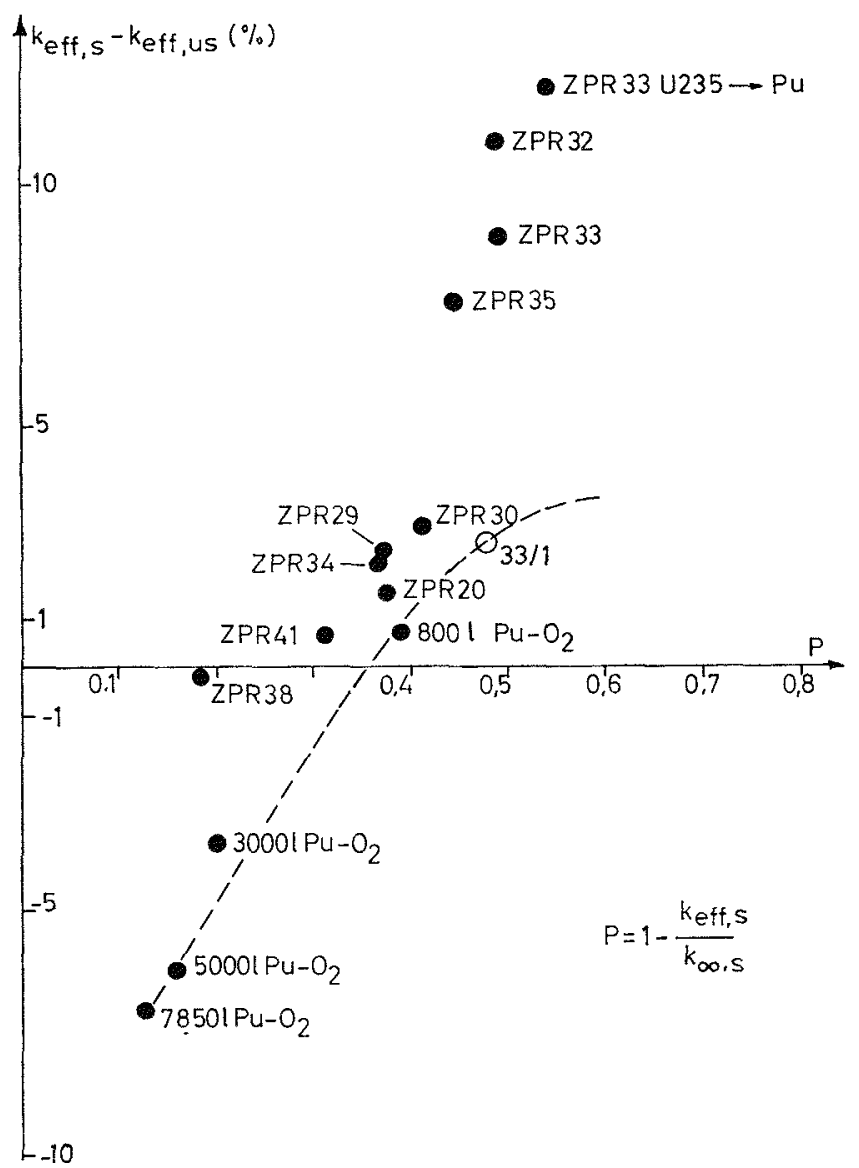

Fic. 1. The Effect of Resonance Self-shielding for Some Reactors.

$k_{\mathrm{eff}, \mathrm{s}}$ is the multiplication factor for the shielded calculations $\left(k_{\mathrm{eff}, \mathrm{s}}=1\right), k_{\mathrm{eff}}$, us for the unshielded calculations. $P$ is the escape probability of neutrons for the system.

of the ZPR III/33 assembly. Naturally, the effect is overestimated by this calculation, but the general trend should be correct. A one-dimensional check for ZPR $\mathrm{III} / 33$ gives $+2.7 \%$ reactivity gain. For the large cores the fundamental-mode calculations give very good results. The dashed line in Fig. 1 gives the probable tendency.

In a similar way as does the multiplication constant, the resonance self-shielding affects the neutron lifetimes: an increase for small and a decrease of about $30 \%$ for large fast reactors, if no shielding corrections are made. The contribution to the average lifetime from below 10 $\mathrm{keV}$ is about $40 \%$. Spectra for large reactors calculated with shielding corrections are increased in the lowenergy range, and any DC ealculation made with "unshielded spectra" is meaningless (see ref. 1).

It is assumed that the complex problem of resonance self-shielding may be treated with an accuracy of about $20 \%$. Therefore, the uncertainty caused by the effect of resonance shielding is about $6 \%$ for the determination of neutron lifetimes and about $1 \%$ in the multiplication constant. This situation makes it very clear that continued attention should be paid to this problem. Especially the resonance data should be more accurate. Also, the proper shielding of the transport cross section has to be studied. This is important for the scattering resonances of the structural materials, where the narrowresonance approximation or even a fine-group representation as used in ELMIIOE are not the suitable methods.

\section{The Influence of the Weighting Spectrum}

Nany of the group-constant sets in use for the calculation of large fast reactors contain about 30 groups up to $10 \mathrm{MeV}$. The use of such a group number with most compulers gives a reasonable machine time.

As a basis for our investigation, we chose the boundaries of the Russian ABN set. The question of whether such a group width is sufficiently small with respect to the weighting spectrum was first investigated by calculating group constants for the weighting spectra, $1 / E$, YOMI-weighting flux (see ref. 2) and a spectrum (KFK) of a sodium-cooled, 1,000- IIWe reactor. The microscopic data were taken from the Karlsruhe Nuclear Data File KEDAK. ${ }^{(4)}$

As a typical example the group constants for fission of $\mathrm{Pu}^{239}$ are shown in Fig. 2. There are no essential differences in the most interesting energy region for fast reactors. Only in the region of resolved resonances below 100 $\mathrm{eV}$ are there discrepancies up to $50 \%$. Here only few resonances are located in one group, and therefore the position of group boundaries and the shape of the weighting spectra are important. From this it follows directly that the high and relatively broad resonances of sodium and the structural materials acquire special interest. These scattering resonances enter into the diffusion calculation besides the determination of resonance shielding only in the elastic-removal and transport cross sections.

In this paper we will discuss only the effect of the weighting spectrum on the elastic-removal group constants. A detailed investigation about the transport cross section, especially in connertion with the average scattering cosine, is under way.

Neutrons are removed from a group by elastic scattering with an isotope of mass $A$ only out of the degradation interval to the extent $E_{L} / \alpha$, where $E_{L}$ is the lower group boundary and $\alpha=\left(\frac{A-1}{A+1}\right)^{2}$. Since $\alpha$ is near unity for isotopes in a sodium-cooled fast reactor $(\alpha=$ 0.78 for $\left.\mathrm{O}^{16}\right)$, the degradation interval is mostly small compared with the width of the group. As a first step we took the coarse group structure of the $A B N$ set and investigated only the degradation interval with the appropriate shielding in the narrow-resonance approxima- 


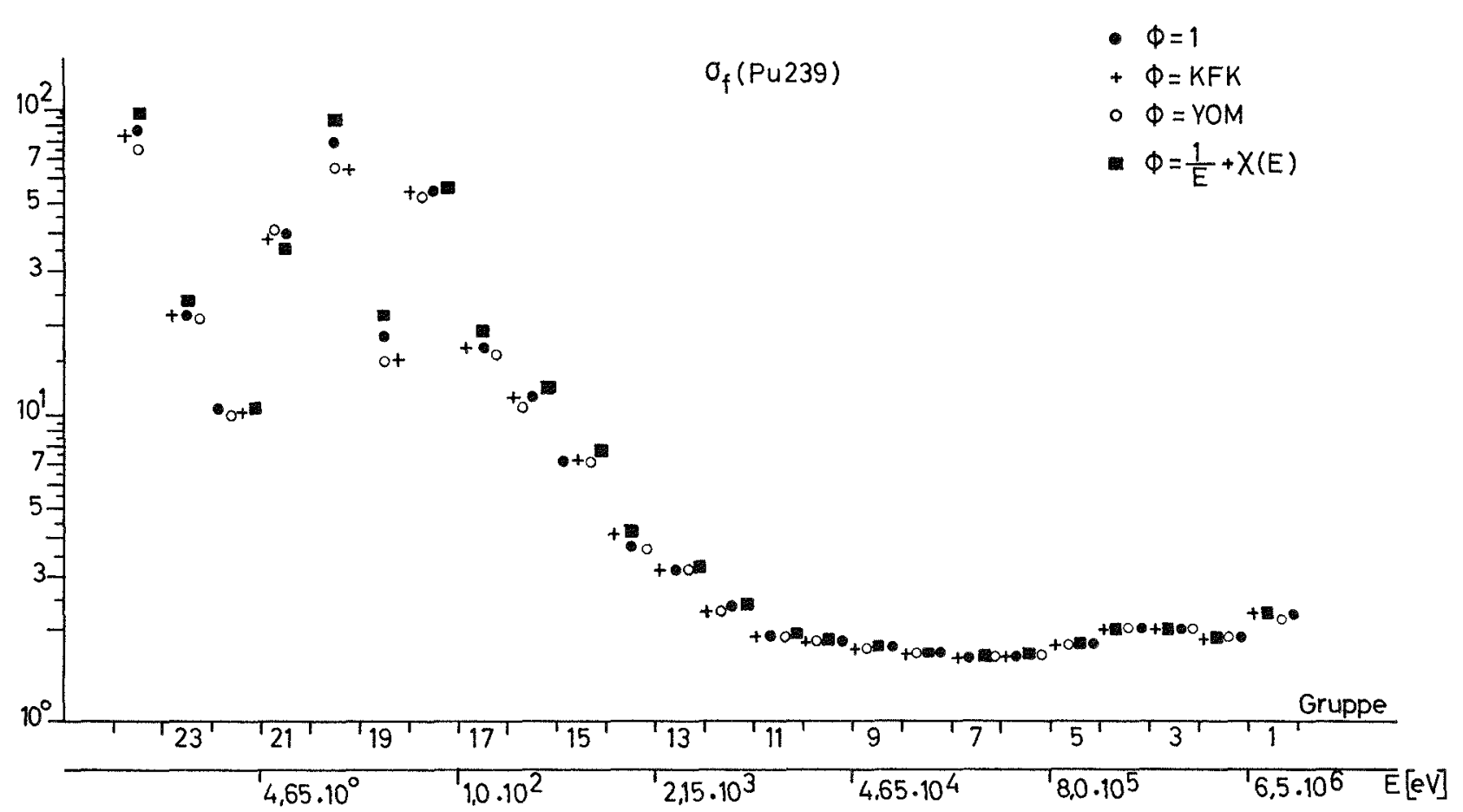

FIg. 2. $\sigma_{f}^{i}$ for $\mathbf{P u}^{239}$ in ABN Group Boundaries for Different Weighting Spectra.

tion. The anisotropic scattcring in the center-of-mass system for high energies is considered as an isotropic scattering with a pseudo-material by averaging the scattering cosine of each material over the degradation interval to which an energy loss, chararterizing the pseudo-material, is connected. For constant scattering cross section and fixed average spectrum in a group, a de(reasing weighting spectrum with increasing energy will give higher elastic-removal cross sections than increasing spectra. In reactors with oxide or carbide fuel the effect may be severe.

In order to study the effect of different weighting spectra, we replaced in the ABN set only the elastic-removal group constants, because other group constants are not sensitive to the weighting and therefore remain unchanged. With KEDAK data we calculated the removal constants for the 9 materials $\mathrm{C}, \mathrm{O}, \mathrm{Na}, \mathrm{Cr}, \mathrm{Fe}$, $\mathrm{Ni}, \mathrm{U}^{235}, \mathrm{U}^{238}$, and $\mathrm{Pu}^{239}$. In Fig. 3 are shown the YOM and KFK weighting spectra in lethargy representation, in Fig. 4 the corresponding elastic-removal group constants for $\mathrm{O}^{16}$.

The differences are remarkable and should have a great influence on integral reactor quantities. For a dilute fast test reactor we calculated Doppler coefficients $\left[(1 / k)(d k / d T)\right.$ related $\left.10900^{\circ} \mathrm{K}\right]$, the loss of $40 \%$ sodium from the reactor core, neutron lifetimes, and breeding ratios (see Table 1 ). The core composition is as follows: $\mathrm{Cr}, 2.56 \mathrm{v} / \mathrm{o} ; \mathrm{Fe}, 9.52 \mathrm{v} / \mathrm{o} ; \mathrm{Na}, 55.9 \mathrm{v} / \mathrm{o}$; $\mathrm{Ni}, 0.9 \mathrm{v} / 0 ; \mathrm{O}, 15 \mathrm{v} / \mathrm{o} ; \mathrm{Pu}^{239}, 1.5 \mathrm{v} / \mathrm{o} ; \mathrm{U}^{238}, 13.5 \mathrm{v} / \mathrm{o}$.

The group sets $\mathrm{K} 1$ and $\mathrm{K} 2$ were based on the same microscopic data and are different only in the weighting spectra (different elastic-removal constants). The sets $\mathrm{K} 1$ and $\mathrm{ABN}$ have the same weighting speetrum (1/E+ fission spectrum) in the first three groups, but K1 elastic-removal group constants were calculated with KEDAK data. As compared with ABN or YOM, the different cross sections and procedure in calculating the clastic moderation gave $2 \%$ difference in the multiplication constant, and $0.3 \%$ for the loss of $40 \%$ sodium (using the same group set for the original and voided reactor, but with new determination of the shielding factors for removal of neutrons). The Doppler coefficient decreased by about $16 \%$. The difference in neutron multiplication arose from differences in the removal cross sections above $30 \mathrm{keV}$ only. The breeding ratios are practically not influenced. It should be noted here that the breeding ratios and DC belong to the reactors which are made critical by variation of particle number densities of $\mathrm{Pu}^{239}$ and $\mathrm{U}^{238}\left(\mathrm{~N}_{9}+\mathrm{N}_{8}=\right.$ const.). The YOM weighting in $\mathrm{K} 2$ gave an increase in $k_{\text {eff }}$ by about $0.6 \%$ compared with $\mathrm{K} 1$, but the $\mathrm{DC}$ was lowered by a factor of about $t$ wo. This results from the spectral shift to higher energies because below $100 \mathrm{keV}$ the YOM weighting gives lower elastic removal than $1 / E$-weighting. Therefore we have less neutrons in the resonance region. The lifetime data reproduced the spectral shifts and showed the same tendency as the DC variation.

The KFK set is a completely new group set in which all microscopic data were taken from the KEDAK file. This set is briefly described later on; we compare the 


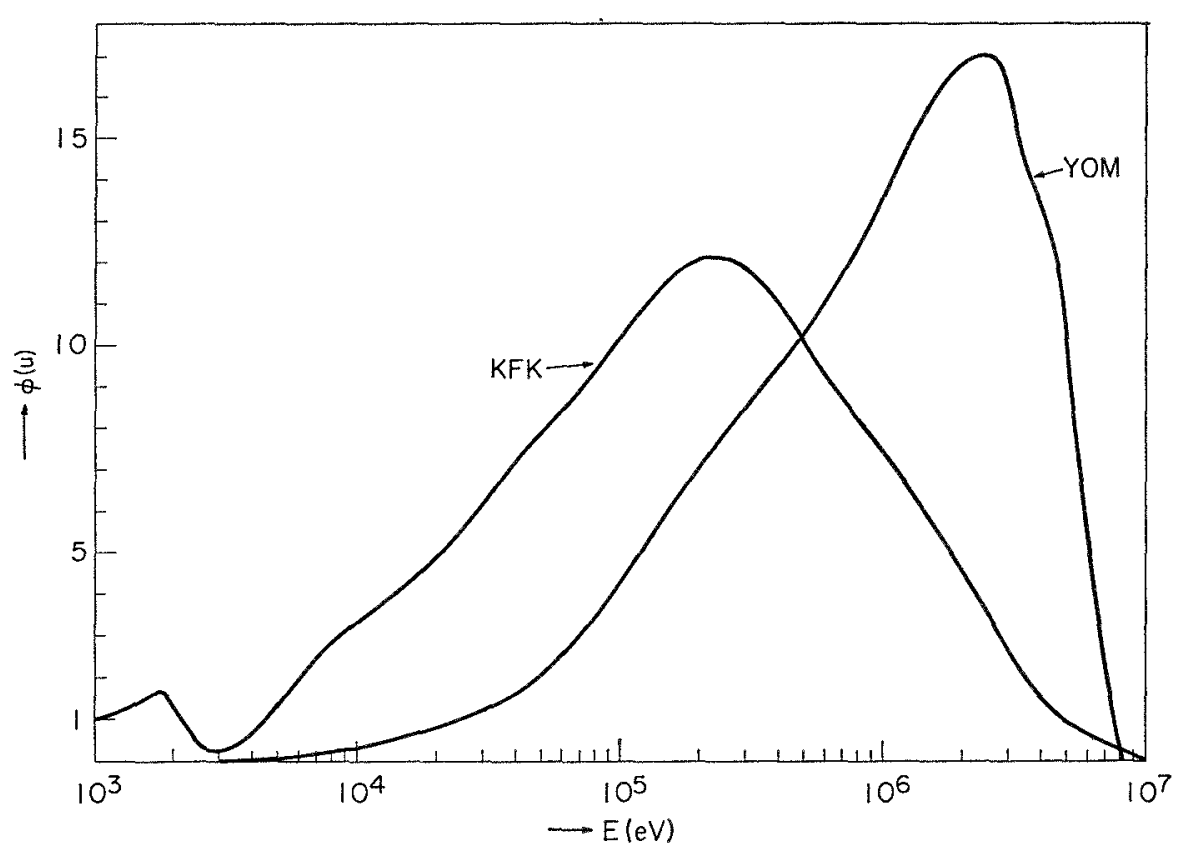

FIG. 3. Weighting Spectra Used for Elastic-removal Compilation.

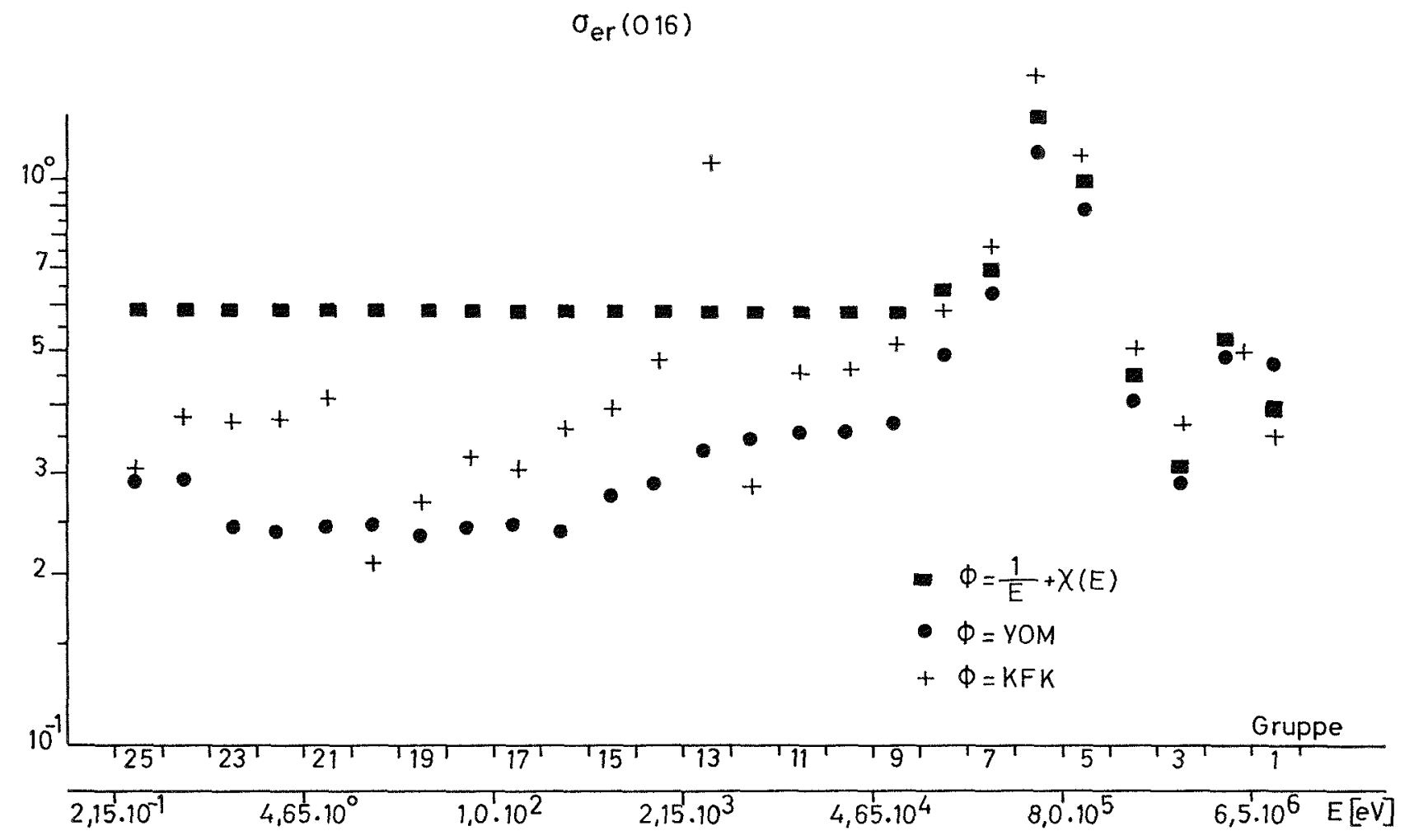

FIx. 4. Elastic-removal Group Constants for the Weighting Spectra Shown in Fig. 3.

KFK and other sets in Table 6 below. We see that despite great differences in microscopic data the breeding ratios are nearly unchanged. The sodium coefficients for the various Karlsruhe sets are not very different. The increase of $0.6 \%$ compared with $A B N$ results partly $(0.3 \%)$ from the cross sections used (ABN-K1).
The other $0.3 \%$ is due to the weighting spectrum. Figures 3 and 4 show that in the range from 0 to $100 \mathrm{keV}$ the elastic-removal constants are smaller in KFK than with $1 / E$-weighting; above $100 \mathrm{keV}$ these constants are larger so as to result in a spectral shift to lower energies. However, one has to be careful in speaking about a 
TaBle 1. The Results for a 7850-liter Core of a Plutonium Oxide Breeder with Different Group Sets

\begin{tabular}{|c|c|c|c|c|c|c|c|}
\hline Group Set* & $\begin{array}{l}\text { Critical Mass, } \\
\qquad \mathrm{kg} \mathrm{Pu}{ }^{239}\end{array}$ & $\begin{array}{c}\Delta k(\%) \text { for } \\
\mathrm{M}=2242 \mathrm{~kg}\end{array}$ & $\begin{array}{c}\Delta \%(\%) \text { for } 40 \% \\
\text { Sodium Loss }\end{array}$ & $\begin{array}{l}\text { Doppler Coeffi- } \\
\text { cient }\left(\times 10^{5}\right)\end{array}$ & $\begin{array}{l}\text { Lifetime } \\
\left(\times 10^{7} \mathrm{sec}\right)\end{array}$ & $\underset{\text { Ratio }}{\text { Internal Breeding }}$ & $\begin{array}{c}\text { Breeding } \\
\text { Ratio }\end{array}$ \\
\hline$A B N$ & 2242 & 0 & 0.52 & -1.88 & 6.37 & 1.04 & 1.43 \\
\hline $\mathrm{K} 1$ & 2340 & -2.15 & 0.83 & -1.56 & 5.95 & 1.06 & 1.44 \\
\hline $\mathrm{K} 2$ & 2296 & -1.48 & 1.17 & -0.81 & 4.82 & 1.10 & 1.52 \\
\hline
\end{tabular}

* K1: $\sigma_{e r}$ with 1/E-weighting (KFK-data). K2: $\sigma_{e r}$ with YOM-weighting flux. KFK 26-10: 1,000-MWe spectrum; all group constants are KFK data.

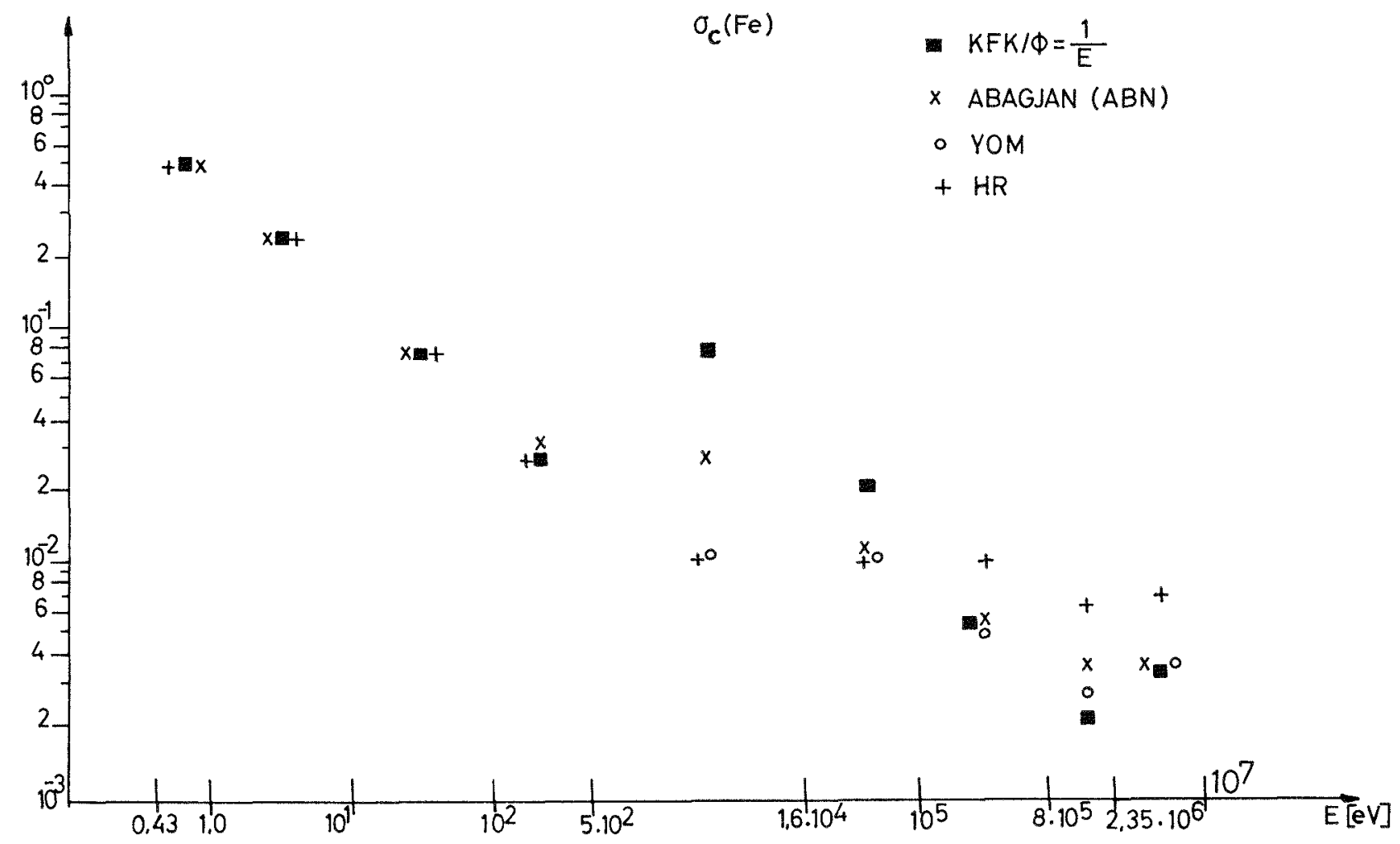

FIG. 5. Comparison of Four Group-constant Sets for the Capture Constants of Fe.

"shift" of the spectrum, because below $10 \mathrm{keV}$ there are less neutrons than with $1 / E$-weighting, more neutrons naturally than with YOM-flux. The DC is therefore smaller than for $1 / E$-weighting and for $\mathrm{ABN}(20 \%)$.

If one calculates the spectrum of a reactor without sodium with a group set that includes the dip at the 3 -keV sodium resonance in its weighting spectrum, one finds also a clear dip in the calculated spectrum at the sodium resonance. This dip mainly results from the elastic-removal group constants of the light elements, all of which have a peak below and a minimum above the resonance. We removed the "psuedo-dip" and used this spectrum as a weighting for new elastic-removal cross sections to build a new group set, KFK 26-11, which was used for calculating total coolant loss. We calculated the total loss, $\Delta h$, and obtained

$$
\begin{aligned}
\Delta k_{\text {eff }}(\text { KFK 26-10) } & =0.029 \quad \text { (same group set) } \\
k_{\text {eff }}(\text { KFK 26-10) } & -k_{\text {eff }}(\text { KFK 26-11) }=0.025 .
\end{aligned}
$$

The loss $\Delta k$ is decreased by about $0.5 \%$ if one takes the corresponding system group sets. It should be noted that the spectra should have been converged if such small changes are expected.

We see that the weighting spectrum has an important influence on integral fast reactor quantities by the process of elastic moderation.

In a second step we are investigating whether the method of calculating the elastic moderation, briefly described above, is sufficient. An additional comparison with ELMOE calculations on an IBM 7090 computer was prepared. This code cannot be used on the Karlsruhe IBM 7074 because of its small storage capacity. 


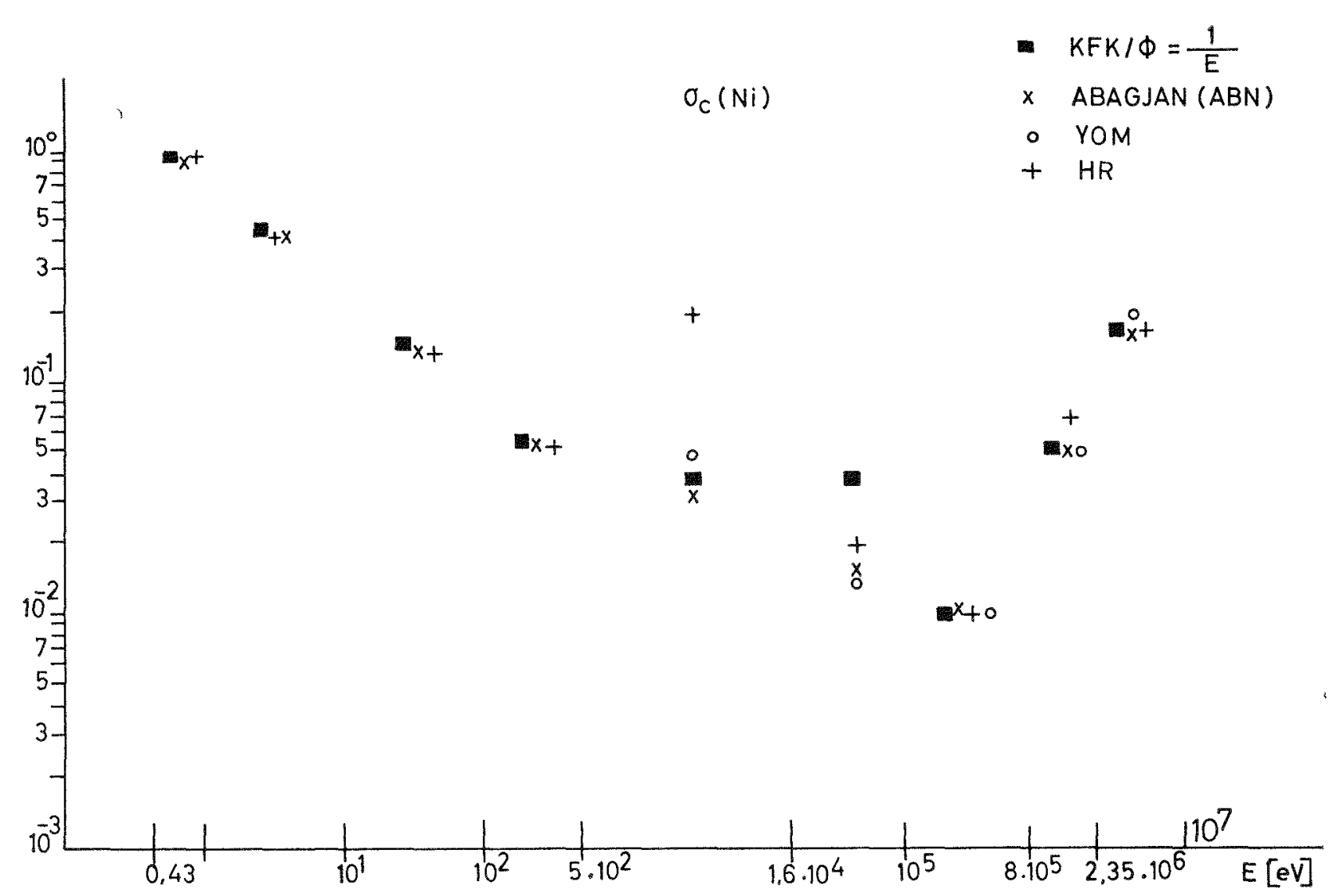

FIG. 6. Comparison of Four Group-constant Sets for the Capture Constants of $\mathrm{Ni}_{\text {. }}$

We can draw a further conclusion from the results discussed above. Multizoned cores and blankets should not be calculated by using one group set prepared with a core or an average reactor spectrum as a weighting only. To be more accurate, one needs at least two group sets, one for core materials and one for blanket materials.

\section{The Karlsruhe Group Set KFK 26-10}

Most of the discussion on changes in neutron multiplication arising from differences in microscopic data is done in chapter IV by comparing the results of the Karlsruhe group set KFK 26-10 with those of the ABN set. Therefore it is necessary to give a short description of the characteristics of the KFK set.

\section{Comparison of Microscopic Data}

This part is mainly based on a comparison of group constants. ${ }^{(5)}$ In Figures 5,6 , and 7 the capture data of several authors for $\mathrm{Fe}, \mathrm{Ni}$, and $\mathrm{U}^{238}$ are compared. The boundaries of the 9 groups are shown as intersections on the abscissa. They are chosen in such a way as to coincide optimally with the boundaries of the different sets. The group constants HR of Hansen and Roach, ${ }^{(6)}$
YOM, and $A B N$ are condensed into these groups by means of the corresponding weighting fluxes. The Karlsruhe data KFK are taken from the KEDAK file and are revised data. ${ }^{(7)} 1 / \mathrm{E}$-weighting was used in order to provide a direct comparison with $\mathrm{ABN}^{\top}$ and $\mathrm{HR}$.

For $\mathrm{Fe}^{56}$ large discrepancies exist for the capture group constants in the region above $500 \mathrm{eV}$. The KFK values, which are based on recent Harwell and Oak Ridge measurements, ${ }^{(7)}$ are higher by a factor of about 2 than the $A B N$ data up to $100 \mathrm{keV}$. YOM and $\mathrm{HR}$ deviate from KFK by a factor of about 10 between 0.2 and $1.6 \mathrm{keV}$. For higher energies there is better agreement.

The situation is only a little better for $\mathrm{Ni}$. The KFK resonance capture cross sections are based on capture widths estimated from the thermal isotopic capture cross sections. ${ }^{(7)}$ These data are higher by a factor of about 2 than the other data between 0.5 and $1.6 \mathrm{keV}$.

For U23s in Fig. 7 the agreement in the capture constants is good. The KFK data above $130 \mathrm{keV}$ are based on recent Harwell measurements.

In Fig. 8 the inelastic cross section of $\mathrm{Pu}^{239}$ is shown. The abscissa now is the lethargy $u$. Below $2 \mathrm{MeV}$ the data of the $A N L^{(8)}$ and $A B N$ sets are different by a factor of about 10; KFK values coincide with YOM 


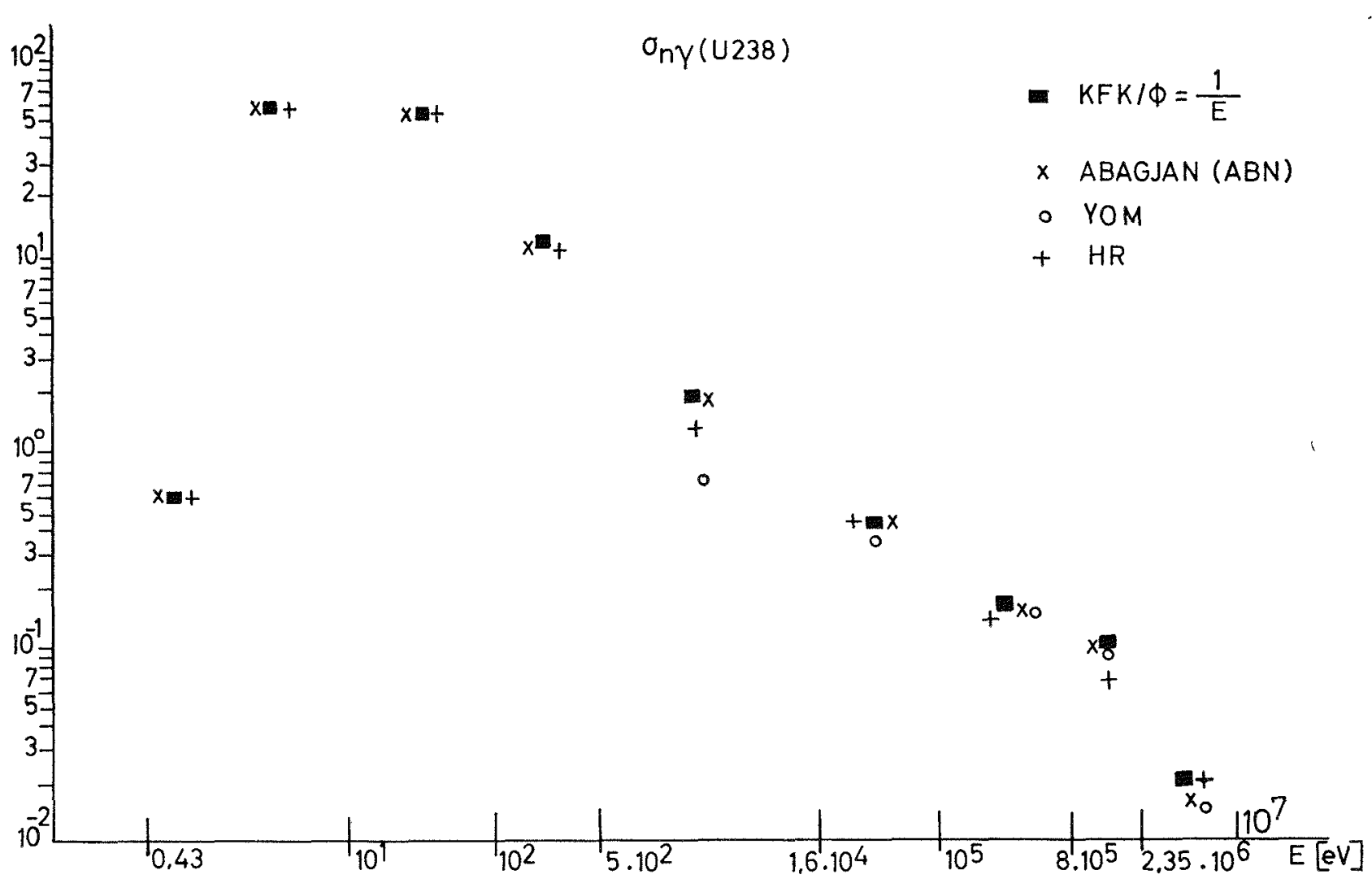

Fr(x. 7. Comparison of Four Group-constant Sets for the Capture Constants of U238.

data, which are lower by a factor of about 2 compared with $A B N$.

For $\mathrm{Pu}^{239} 1$ he KFK data for fission are lower by about $20 \%$ (recent Russian and British measurements) between 10 and $50 \mathrm{keV}$ than $\mathrm{ABN}$. The $\alpha$-value remained unchanged in this region.

\section{Preparation of the KFK Set}

The group set was prepared with the Karlsruhe MIIGROS-system, which combines several codes. For a prescribed weighting spectrum, the unshielded group constants were calculated from data in the KEIDAK file. Elastic-removal constants were determined as described in chapter II.

The KEDAK file now contains data only for the materials $\mathrm{H}, \mathrm{He}, \mathrm{C}, \mathrm{O}^{16}, \mathrm{Na}, \mathrm{Cr}, \mathrm{Fe}, \mathrm{Ni}, \mathrm{Mo}$, U235, U2238, and $\mathrm{Pu}^{239}$. In order to have a very complete set, we took the $A B N$ set as a basis and replaced the unshielded group constants of the nine materials by KFK data. Calculations for the lest reactor in chapter II were therefore made with KFK constants only. The spatially averaged spectrum of the Karlsruhe Na-1 sodiumcooled reference design reactor was used as a weighting. Since the different codes in the block for calculating resonance shielding in MIIGROS had not yet been fully combined, and because calculations mostly showed good agreement with the Russian shielding factors, we chose as a first step the Russian ones with only slight modifications for the removal values. We have 26 groups; the group boundaries are given in the appendix. Using the same microscopic data and calculational methods, exchanging only the spectrum, we then obtained the set KFK 26-11, which was used for the calculation of total coolant loss.

\section{Microscopic Uncertainties}

Naturally, it is always possible to check the used data by comparing integral data with those measured in critical facilities. Yet, all these results have to be interpreted very carefully, and it is not always possible to come to a conclusion about microscopic data.

Therefore, we thought it might be helpful to see how the uncertainties work in large fast reactors. At first we made a calculation concerning the sodium resonance. Up to the last conference at Antwerp in July 1965, the peak value of the sodium resonance at $3 \mathrm{keV}$ had been uncertain by about $30 \%$. We tried to find the action of this resonance by removing the peak in the K-1 set and calculating coolant void and DC with the new set K1R. Table 2 gives the results.

As expected, there was almost no effect on the void effect for sodium, but the DC decreased by about $20 \%$. 


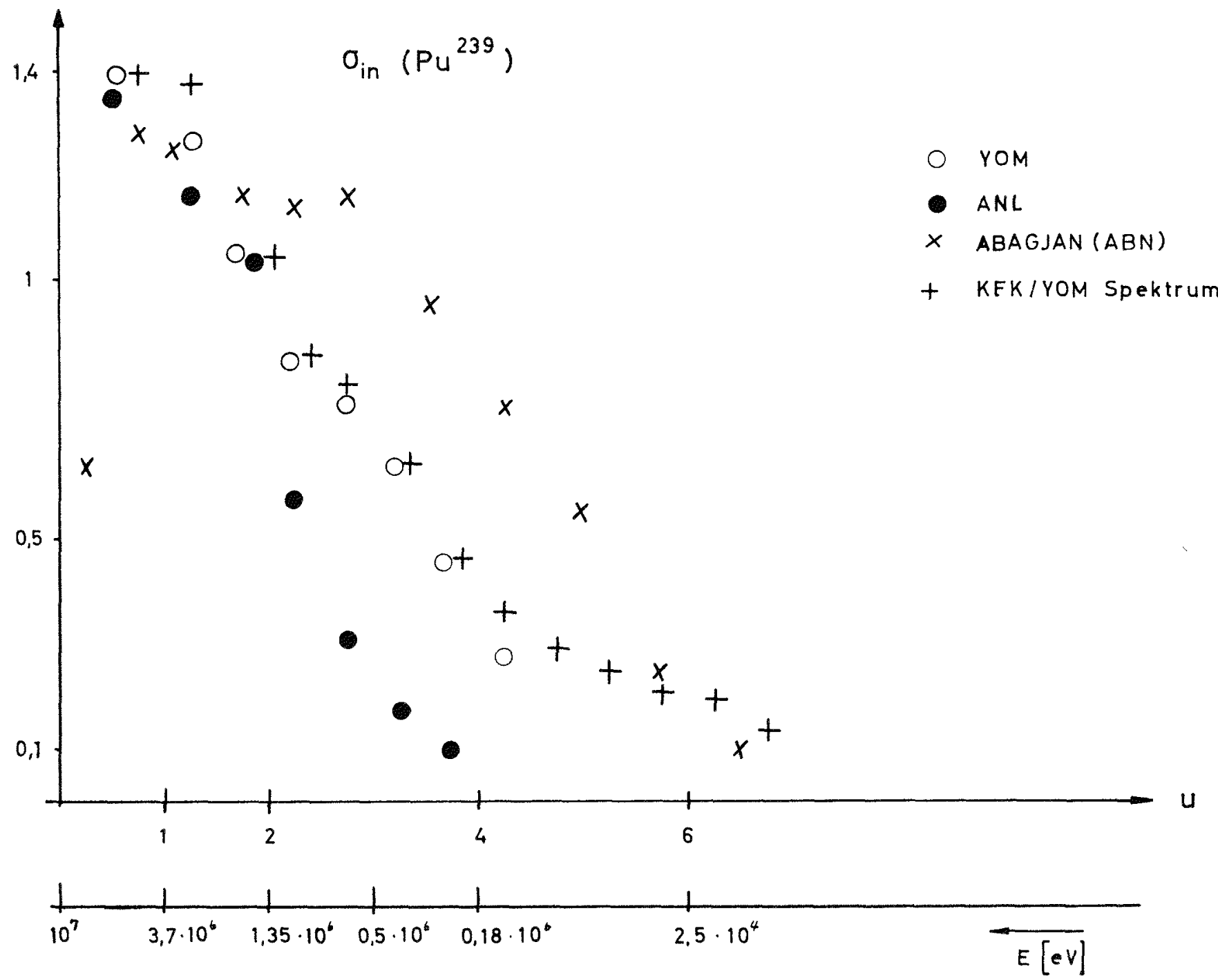

Fig. 8. The Group Constants for Inelastic Scattering in $\mathrm{Pu}^{239}$.

TABLE 2. Comparison OF SODICM VOLD AND DC, Calcilatige with axd without hodum Resonance

\begin{tabular}{l|c|c|c}
\hline \multirow{2}{*}{ Set } & $\begin{array}{c}\Delta k(\%) \text { for } 40 \% \\
\text { Sodium Loss }\end{array}$ & $\begin{array}{c}\text { Doppler Co- } \\
\text { efficient }\left(\times 10^{5}\right)\end{array}$ & $\begin{array}{c}\text { Lifetime } \\
\left(\times 10^{7} \mathrm{sec}\right)\end{array}$ \\
\hline K1 & 0.83 & -1.69 & 6.21 \\
K1R & 0.89 & -1.34 & 5.81 \\
\hline
\end{tabular}

An uncertainty of about $30 \%$ in height therefore may have less than $7 \%$ influence on $D C$. It should be noted that these results are valid for weighting spectra without any resonance dip. The problems previously discussed are equally significant, because the weighting flux changes the elastic moderation of all light materials.

The realistic influence on criticality of the discrepancies shown in Figures 5 through 9 were estimated from calculations done with $A B N$ and KFK set.

Table 3 gives the average group constants for fission and capture, condensed from the 26-group $\mathrm{ABN}$ and KFK sets, using the same 1,000-MWe spectrum for condensation. The mentioned differences for $\mathrm{Fe}, \mathrm{Ni}$, and $\mathrm{Pu}$ are reproduced; the average capture value of $\mathrm{U}^{238}$ is smaller in KFK by about $5 \%$. It should be mentioned that the $\mathrm{O}^{16}$ capture is overestimated by about $40 \%$, so that the difference with respect to $A B N$ is even larger. The microscopic cross sections for the higher isotopes of plutonium were taken to be the same in the KFK and $A B N^{\top}$ sets.

Table $t$ shows the results of calculations for a spherical model of the following core composition $(\mathrm{Na} 1): \mathrm{Cr}$, $4.6 \mathrm{v} / \mathrm{o} ; \mathrm{Na}, 49.8 \mathrm{v} / \mathrm{o} ; \mathrm{O}, 14 \mathrm{v} / \mathrm{o} ; \mathrm{Pu}^{240}, 0.7 \mathrm{v} / \mathrm{o} ; \mathrm{Pu}^{242}$, $0.05 \mathrm{v} / \mathrm{o} ; \mathrm{Fe}, 8.8 \mathrm{v} / 0 ; \mathrm{Ni}, 5.6 \mathrm{v} / \mathrm{o} ; \mathrm{Pu}^{239}, 1.4 \mathrm{v} / \mathrm{o} ; \mathrm{Pu}^{241}$, $0.1 \mathrm{v} / 0 ; \mathrm{U}^{238}, 11.7 \mathrm{v} / 0$. The reaction rates in Table 4 are normalized to make the total production of neutrons in the core unity. Both reactors are made critical. The KFK column shows that the capture in iron contributes 
about $2 \%$ to the balance, that in niekel about $2.5 \%$. In other words, without any capture in iron or nickel about $2 \%$ change in criticality can be expected. If one now compares $A B N$ with $\mathrm{KFK}$ values, the increase in the iron capture cross section by a factor of 2 results in an additional contribution in absorption rate per neutron born in the core of less than $1 \%$, for nickel of less than $1 / 2 \%$. The increased capture probability in iron from 1 to $2 \%$ is partially due to the softening of the spectra by KFK data.

It is remarkable that the lowering of the $\mathrm{Pu}^{239}$ fission cross section between 10 and $50 \mathrm{keV}$ is completely compensated in this reactor by the higher spectrum in this region, so that the fission rates are nearly the same. This is clearly an effect of riticality. $\mathrm{U}^{238}$ has about $2.5 \%$ increase in capture rates, which is also due to spectral effects.

Because the reactor is made critical with both sets by radial iteration, the leakage compensates the higher absorption rates in $\mathrm{KHK}$. The total breeding ratio with KFK is nearly unchanged compared with $A B N$, but the internal breeding is increased by about $6.5 \%$. More-

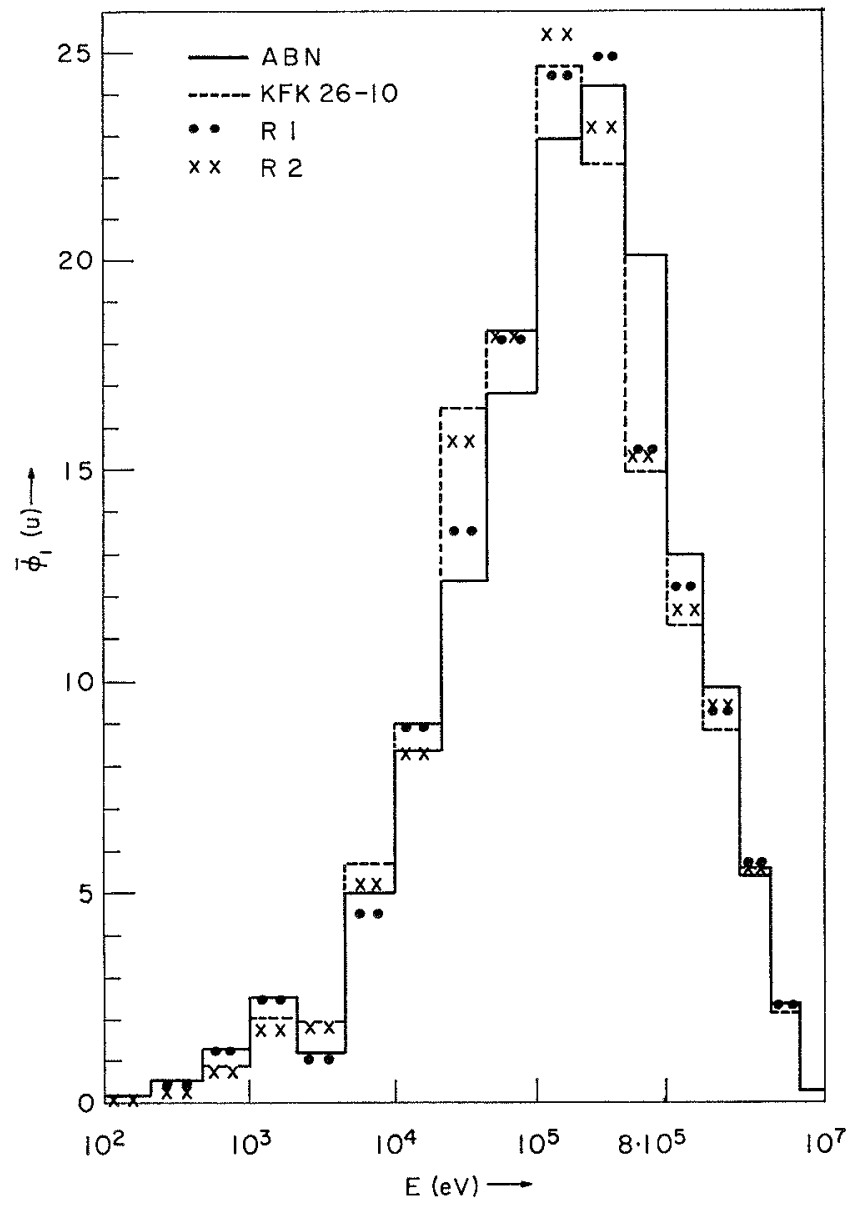

FIG. 9. Suecessive Fundamental-mode Spectra during KFK 26-10 Preparation.
TABle 3. Comparison of Average Microscoplc ('ross SECTIONS IN A 1,000-MWe SPECTRUM (COVDENSED FRoM 26 Groups) FOR ABN AND KFK DATA

\begin{tabular}{l|c|c|c|c|c|c}
\hline \multirow{2}{*}{ Element } & \multicolumn{2}{|c|}{$\bar{\sigma}_{f}$} & \multicolumn{2}{|c|}{$\bar{\nu} \sigma_{f}$} & \multicolumn{2}{|c}{$\bar{\sigma}_{c}$} \\
\cline { 3 - 6 } & $\mathrm{ABN}$ & $\mathrm{KFK}$ & $\mathrm{ABN}$ & $\mathrm{KFK}$ & $\mathrm{ABN}$ & $\mathrm{KFK}$ \\
\hline $\mathrm{Cr}$ & - & - & & & $9.86 \cdot 10^{-3}$ & $9.12 \cdot 10^{-3}$ \\
$\mathrm{Fe}$ & - & - & & & $9.66 \cdot 10^{-3}$ & $1.97 \cdot 10^{-2}$ \\
$\mathrm{Na}$ & & & & - & $2.46 \cdot 10^{-3}$ & $2.77 \cdot 10^{-3}$ \\
$\mathrm{Ni}$ & - & -- & & & $2.33 \cdot 10^{-2}$ & $2.85 \cdot 10^{-2}$ \\
$\mathrm{O}^{16}$ & & - & - & - & $1.03 \cdot 10^{-3}$ & $9.0 \cdot 10^{-1}$ \\
$\mathrm{Pu}^{239}$ & 2.110 & 1.986 & 6.150 & 5.790 & $5.91 \cdot 10^{-1}$ & $5.06 \cdot 10^{-1}$ \\
$\mathrm{U}^{238}$ & 0.040 & 0.039 & 0.112 & 0.113 & $1.31 \cdot 10^{-1}$ & $4.10 \cdot 10^{-1}$ \\
\hline
\end{tabular}

TABLE 1. Comparison of Fission and Absorption Rate; FOR ABN A $\backslash$ D KFK SET

\begin{tabular}{|c|c|c|c|c|}
\hline \multirow{2}{*}{ Element } & \multicolumn{2}{|c|}{$R_{f}(\%)$} & \multicolumn{2}{|c|}{$R_{A}(\%)$} \\
\hline & $\mathrm{ABN}$ & KFK & $\Lambda B N$ & $\mathrm{KFK}$ \\
\hline $\mathrm{Cr}$ & - & - & 0.57 & 0.60 \\
\hline $\mathrm{Fe}$ & - & & 0.95 & 2.07 \\
\hline $\mathrm{Na}$ & - & -- & 0.30 & 0.35 \\
\hline $\mathrm{Ni}$ & & & 1.98 & 2.41 \\
\hline$O$ & $\rightarrow$ & - & 0.25 & 0.22 \\
\hline $\mathrm{Pu}^{239}$ & 24.84 & 24.78 & 31.55 & 31.02 \\
\hline $\mathrm{Pu}^{240}$ & 1.94 & 1.79 & 6.61 & 6.87 \\
\hline$P u^{241}$ & 2.90 & 3.30 & 345 & 3.84 \\
\hline $\mathrm{Pu}^{242}$ & 0.09 & 0.09 & 0.29 & 0.30 \\
\hline $\mathrm{C}^{238}$ & 1.31 & 4.05 & 35.79 & 38.08 \\
\hline- & ---- & - - & - & - \\
\hline Sum & 34.14 & 34.01 & 81.78 & 85.76 \\
\hline Leakage & & & 18.22 & 14.24 \\
\hline Total & & & 100.00 & 100.00 \\
\hline
\end{tabular}

TABLE 5. SEPara IION OF DISCREPANCTES BETWEEN THE ABN and KFK Sets duri $\backslash$ G Preparation or KFK 26-10

\begin{tabular}{l|c|c|c|c}
\hline & $\mathrm{ABN}$ & $\mathrm{R} 1$ & $\mathrm{R} 2$ & $\mathrm{KKK}$ \\
\hline$k_{\mathrm{efi}}$ & 1.0038 & 0.9953 & 0.9823 & 0.9701 \\
$\frac{k_{\mathrm{eff}}-k_{\mathrm{eff}}^{\mathrm{ABN}}}{k_{\mathrm{eff}}^{\mathrm{ABN}}}(\%)$ & 0 & -0.85 & -2.15 & -3.37 \\
Lifetime $\left(\times 10^{7} \mathrm{sec}\right)$ & 3.76 & 3.79 & 3.48 & 3.70 \\
$k_{\infty}$ & 1.266 & 1.246 & 1.234 & 1.221 \\
\hline
\end{tabular}

over, one can plainly see that a change in the average $\nu$-values for $\mathrm{Pu}^{240}$ of $15 \%$ (as is true between YOMI and $A B N$ ) gives a change in the balance by about $1 \%$, which is also the difference in the multiplication. The differences in the higher plutonium rates are due to spectral effects. 
TABLE 6. COMParison OF Results OBtarved from Different Grolp sets for a 5,000-li l'er Pletonita (OXIDE BREEDER

\begin{tabular}{l|c|c|c|c|c}
\hline \multicolumn{1}{c|}{ Set $^{*}$} & $\begin{array}{c}\text { Critical } \\
\text { Mass } \\
(\mathrm{kg} \\
\left.\mathrm{Pu}^{239}\right)\end{array}$ & $\begin{array}{c}\text { Breeding } \\
\text { Ratio }\end{array}$ & $\begin{array}{c}\text { Internal } \\
\text { Breding } \\
\text { Ratio }\end{array}$ & $\begin{array}{c}\Delta k(\%) \\
\text { for 40\% } \\
\text { Sodium } \\
\text { Loss }\end{array}$ & $\begin{array}{c}\text { Fraction } \\
\text { of Fissions } \\
\text { below } \\
9.1 \mathrm{keV}\end{array}$ \\
\hline ANI. (26) & 1514 & 1.34 & 0.89 & +0.25 & 0.17 \\
YOM (16) & 1536 & 1.35 & 0.92 & +1.22 & 0.11 \\
HR SH (16) & 1567 & 1.43 & 0.94 & -0.23 & - \\
HR UNSH & 1721 & 1.51 & 1.02 & +1.22 & \\
ABN (26) & 1523 & 1.37 & 0.93 & +0.13 & 0.178 \\
KEK (26) & 1643 & 1.375 & 0.938 & +0.69 & 0.185 \\
\hline
\end{tabular}

* I alue in parentheses gives number of groups.

TABLE 7. COMPARISON OF RESILTS FROM DIFFERENT (XROUP SETS FOR ZPR III/39

\begin{tabular}{|c|c|c|c|c|}
\hline ZPR III/39 & $\mathrm{ABN}$ & YOM & KFK & Expt. \\
\hline$k_{\mathrm{cff}}$ & 1.0000 & 0.9839 & 0.9928 & 1.0 \\
\hline $\begin{array}{l}\text { Critical Mass (kg } \\
\left.\mathrm{U}^{235}\right)\end{array}$ & 388 & 422 & 403 & 426.5 \\
\hline $\begin{array}{c}\text { Deviation from } \\
M_{\text {erp }}(/ /)\end{array}$ & -9.0 & -1.0 & -5.5 & - \\
\hline$\sigma_{f} \mathrm{U}^{233} / \sigma_{f} \mathrm{U}^{255}$ & 0.0488 & 0.052 & 0.0483 & 0.0475 \\
\hline$\sigma_{f} \mathrm{Pu}^{239} / \sigma_{f} \mathrm{~L}^{235}$ & 1.1560 & 1.230 & 1.183 & 1.180 \\
\hline
\end{tabular}

During preparation we separated the single effects of the KlK set with fundamental-mode calculations. The results are shown in Table 5 and in Fig. 9. We started with the ABN set and successively replaced values for the nine materials mentioned above. The shielding fuc- tors remained the same except for the elastic-removal cross sections. In a first step we replaced $\mathrm{O}^{16}$ and $C$ (spectrum R1 in Fig. 9). For a typical oxide breeder the replacement of oxygen results in a loss in multiplication of $1 \%$. This is almost exclusively due to the different description of elastic moderation (above $0.4 \mathrm{MeV}$ ). The peak of the spectrum is shifted remarkably. In the next step we exchanged $\mathrm{Na}, \mathrm{Cr}, \mathrm{Fe}$, and $\mathrm{Ni}$. The resulting spectrum is R2, and the decrease in multiplication is about $1 \%$ also, caused by the increased capture in iron and nickel. Finally, values for $\mathrm{U}^{235}, \mathrm{U}^{238}$, and $\mathrm{Pu}^{239}$ were replaced, resulting in a further lowering in multiplication (fission of $\mathrm{Pu}^{239}$ and increased absorption in $\mathrm{U}^{238}$ by spect ral shift).

In Table 6 the KFK-set is compared with group sets already mentioned. Integral data were calculated for a 5,000 -liter fast oxide reactor, which is described in the Geneva paper P/267 (1964) by D. Okrent et al.

Moreover, a comparison was made with the measured data of the spherical assembly ZPR III/39. In Table 7 we find better agreement, for the critical mass than given by use of $\mathrm{ABN}$; the KFK spectral indices are in a better agreement with experiment than are the YOM and $A B N$ results. These two comparisons very clearly show the difficult situation in obtaining final conclusions about used sets.

\section{Acknowledgment}

The authors appreciate many helpful discussions with R. Froelich, K. Ott, and J. J. Schmidt. We are obliged for the assistance of H. Bachmann, B. Ortmann, and D. Woll in preparing the different group sets.

\section{Appendix}

Group Bocndaries for the ABN and KFK Sets

\begin{tabular}{|c|c|c|c|c|c|}
\hline Group & $E_{n}$ & $\Delta u$ & Group & $E_{n}$ & $\Delta u$ \\
\hline 1 & $6.5-10.5 \mathrm{MeV}^{+}$ & 0.48 & 14 & $1.0-2.15 \mathrm{keV}^{-}$ & 0.77 \\
\hline 2 & $4.0-6.5 \mathrm{Mel}$ & 0.48 & 15 & $465-1000 \mathrm{el}$ & 0.6 \\
\hline 4 & $1.4-2.5 \mathrm{Mel}^{\prime}$ & 0.57 & 17 & $100-215 \mathrm{el}^{\prime}$ & 0.77 \\
\hline 5 & $0.8-1.4 \mathrm{Mel}$ & 0.57 & 18 & $46.5-100 \mathrm{eV}$ & 0.77 \\
\hline 6 & $0.4-0.8 \mathrm{Mel}$ & 0.69 & 19 & $21.5-16.5 \mathrm{el}$ & 0.77 \\
\hline 9 & $46.5-100 \mathrm{kel}$ & 0.77 & 22 & $2.15-4.65 \mathrm{eV}$ & 0.77 \\
\hline 10 & $21.5-46.5 \mathrm{keV}$ & 0.77 & 23 & $1.0-2.15 \mathrm{eV}$ & 0.77 \\
\hline 11 & $10.0-21.5 \mathrm{keV}$ & 0.77 & 24 & $0.465-1.0 \mathrm{el}$ & 0.77 \\
\hline 12 & $4.65-10.0 \mathrm{keV}$ & 0.77 & 25 & $0.215-0.465 \mathrm{eV}^{\mathrm{Y}}$ & 0.77 \\
\hline 13 & $2.15-4.65 \mathrm{kel}$ & 0.77 & $T$ & $0.0252 \mathrm{eV}$ & \\
\hline
\end{tabular}

\section{REFERENCES}

1. R. Froelieh and K. Ott, Nuel. Sci. Eng. 22, 487 (1965); IR. Froelich, K. Ott, (*. Shaviv, and S. Yiftah, see paper in Session VB presented at This Conference.

2. S. Yiftah, D. Okrent, and P. A. Moldauer, Fast Reactor Cross
Sections, Pergamon Press, New York (1960); see also S. Yiftah and M. Sieger, Israel Report IA-980.

3. L. P. Abagjan, N. O. Bazazjane, I. I. Bondarenko, and M. N. Nicola'ev, Translation KFK-tr-144.

4. U. Moller, J. J. Sehmidt, and H. Stittgen, KED.1K The 
Karlsruhe Nuclear Data-File, Internal Karlsruhex Reports.

5. H. Küsters, K. Ott, and J. J. Schmidt, Comparison of Different Group Constant Sets, to be published.

6. G. E. Hansen and W. Roach, LAMS-2543 (1961).

7. J. J. Schmidt, KFK-120 (EANDC-F-35 u), Vol. 1, to be published.

8. D. M. O'shea, H. H. Hummel, W. B. Loewenstein, and I). Okrent, 26-group Cross Sections, Trans. Am. Nucl. Soc. 7, 2 (Nov 64). See also R. Avery et al., (Geneva Conference $1964, \mathrm{P} / 259$

\section{Discussion}

Mr. Yiftah (Israel AEC): In Fig. 2 are shown four different weighting spectra. Were the same macroscopic data used with different weighting spectra? Was the same number of groups used in all the sets? Was a typical 1000-MW(e) flux used as a weighting flux for the calculation?

$M r$. Küsters: The answer to the three questions is yes. Except for the ABN set all the same microscopic cross sections were used. The groups were the same, as was the group structure. We placed the nine materials in the Russian group-constants set to have a very complete one. Therefore, we left the group structure totally the same.

Mr. Chernick (BNL): Since you have indicated that self-shielding effects are important, could you give more details? Was this a homogeneous calculation?

Mr. Küsters: Yes.

Mr. Chernick: This is also the case for sodium loss; you didn't go to complete sodium loss, but still even that would be homogeneous.

Mr. Küsters: Yes, still homogeneous.

$M r$. Chernick: Has anybody checked the Russian selfshielding factor for homogeneous systems just to see how adequate they are?

Mr. Küsters: Yes, we did and found that for plutonium and uranium there was a discrepancy with our own calculations of less than $5 \%$ for the fissile and fertile materials. Additional discrepancies were found for the structural materials.

Mr. Chernick: How about the streaming effect? There seems to be a great deal of uncertainty in the spectrum which causes this. In addition, it seems to me that, in view of the importance of the voiding problem as indicated yesterday by the Panel, here again heterogeneity cffects become important, especially for the pancake cores. For realism one would have to take this into account.

Mr. Küsters: Yes. We are now taking into account the heterogeneity in the self-shielding effect by the rational approximation of Wigner as the first step.

$M r$. Ott (Karlsruhe): I would like to reply to your question about the streaming effect. We made a study about the influence of the streaming effect on the sodium void effect, and we found, at least for the usual fast reactor shapes, it is really small, because there are about 100,000 pins. In other words, the reactor is in itself approximately homogeneous and large streaming effects cannot oceur.

Mr. Perry (ORNL): Do I understand correctly that you conclude the KFK set is the one best suited for your purposes?

Mr. Küsters: Yes.

Mr. Perry: Was this the set on which the comparison calculations were based?

Mr. Küsters: Yes.

Mr. Hummel (ANL): Our experience with ELiIOE has been that it seems to be possible to defino effective cross sections that are applicable to a fairly wide variety of reactors. So I would expect the procedure that the speaker indicated to be fairly successful. On the slides the speaker indicated some results with an ANL-26 group set, results which were rather negative. I wish to warn people that there is one material in this cross section set that has a big error in the plutonium fission cross section. I think that on some listings it was designated material 2 at $300^{\circ}$ and sigma $p$ of 300 . The apparent fission cross section about 2 or 3 kilovolts was way too big, and this would lead to much too negative a sodium void effect. This might have been the one you used in that comparison. That material should be thrown out.

Mr. Küsters: I took this figure from the Geneva paper.

Mr. Hummel: I think that those figures came from the erroneous cross section. 


\title{
Some Calculations of Fast Reactor Doppler and Sodium Loss Coefficients with Particular Reference to the Effects of Core Lattice Structure and Radial Fuel Temperature Distribution
}

\author{
J. Cond, C. Durston, and Mrss W. V. Wright \\ Atomic Energy Establishment \\ Winfrith, United Kingdom \\ (PRESHNTED BY J. COI)I)
}

\section{Iniroduction}

This paper gives the results of calculations concerning two physies problems of dilute fast power reactors: (1) the effects of core heterogeneity in the main Doppler region of the spectrum; and (2) the treatment of resonance capture in a fuel rod with a radial temperature distribution.

For current reactor designs the neutron mean free path is usually several times greater than the cell dimensions over nost of the spectrum, and heterogeneity effects are unlikely to be very important. At low energies, however, the mean free path will be very small near resonance peaks, and heterogeneity effects might become more significant. In particular, the stronger self-shielding of resonances in lumped fuel, as compared with the homogenized cell, will influence the inultigroup cross sections and their Doppler change with temperature.

The heterogeneity calculations described in Section 2 refer to a particular unreflected reactor lattice. The reproduction constant $k_{\text {eff }}$ as well as the Doppler and sodium loss coefficients are compared with the results for a homogeneous system of the same size and material composition. The treatment of heterogeneity is limited to the energy range below $15 \mathrm{keV}$. The calculations are a particular application of a numerical method for treating the resonance-energy region which has been developed at Winfrith. ${ }^{(1)}$

A different problem requiring consideration of the lattice cell structure is the ealculation of resonance capture in a fuel rod having a radial temperature distribution. A common approximation is to use the mean temperature. However, some Monte Carlo calculations for a fast reactor lattice ${ }^{(2)}$ cast doubt on this procedure, and further study of the problem was considered necessary. In Section 3 are given the results of calculations, also by the Monte Carlo method, for a hypothetical cell containing a uranium-238 dioxide rod, which is subdivided into three radial regions at different temperatures. The resonance eapture within a limited energy range is compared with results for uniform rod temperatures.

\section{Comparison of Doppler and Sodium Loss Co- efficients for Homogeneous and Heterogeneous Systems}

\section{(a) Method of Calculation}

The aim of the investigation was to study the effects of core heterogeneity in the spectral region below 15 $\mathrm{keV}$, which gives rise to most of the Doppler coefficient in typical dilute fast reactors.

The calculations refer to an unreflected reactor containing $39.3 \mathrm{v} / \mathrm{o} \mathrm{Pu}^{239}-\mathrm{U}^{238}$ dioxide fuel $(\mathrm{U} / \mathrm{Pu}=$ $5), 50.7 \mathrm{v} / 0$ sodium and $10 \mathrm{v} / 0$ steel. A square lattice cell was assumed of side 0.354 in., containing a central fuel rod $0.28 \mathrm{in}$. in diameter. The steel and sodium were assumed to be uniformly mixed in the outer cell region. The specifications are similar to Case No. 4 of reference 3 , but a two-region square cell was chosen for convenience of calculation. The geometrical buckling was fixed at $15.4 \mathrm{~m}^{-2}$, giving a system close to critical size.

The reproduction constant $k_{\text {eff }}$ was calculated for different fuel temperatures and sodium densities by assumption of the standard fundamental-mode solution in multigroup diffusion theory. A 33-group set of cross sections was used, covering the energy range from $10 \mathrm{MeV}$ to $0.4 \mathrm{eV}$ in half lethargy units, apart from the top group, which was of unit width. Above $15 \mathrm{keV}$ and below $37 \mathrm{eV}$ the group cross sections were taken from the current Winfrith data set for dilute fast reactor calculations (FD2), and were uncorrected for heterogeneity or Doppler broadening. Between 15 $\mathrm{keV}$ and $37 \mathrm{eV}$ the group cross sections were calculated by a numerical method for treating the resonance region, ${ }^{(1)}$ using two STRETCH codes, GENEX and SDR.

The purpose of GENEX is to compute a library of Doppler-broadened cross sections for the fissile and 
fertile isotopes below about $20 \mathrm{keV}$. Resolved resonance widths and spacings are used where available, while in the unresolved region they are generated by sampling from assumed statistical distributions. The cross sections are computed over a suitably chosen energy mesh which is fine enough to represent the variation through individual resonances, namely, about $10^{5}$ points between thermal energies and 20 $\mathrm{keV}$.

The SDR code uses GENEX cross sections in a numerical solution of the Boltzmann transport equation for a lattice cell, starting with a neutron source at $20 \mathrm{keV}$. The $2.85-\mathrm{keV}$ sodium resonance cross section was represented analytically by a series of straightline segments. The code treats the spatial variation of flux across the cell by the method of collision probabilities. These functions are computed at each energy mesh point by a separate routine, using for the present cell geometry the method of reference 4. An editing code, SDRED, finally processes the computed flux spectrum and cross sections to give reaction rates and group cross sections averaged over preseribed energy ranges. For the present application, macroscopic group cross sections were computed for the cell, which included averaging over the two-region spatial flux distribution as well as over energy.

Sets of 12-group cross sections between $15 \mathrm{keV}$ and $37 \mathrm{eV}$ were obtained in this way for fuel temperatures 300 and $900^{\circ} \mathrm{K}$, and for zero, 50 , and $100 \%$ sodium density. Similar sets were also derived for the comparative case of the homogenized lattice cell.

\section{(b) Results}

The calculated values of $k_{\text {eff }}$, the average Doppler coefficient between 300 and $900^{\circ} \mathrm{K}$, and the reactivity change on complete loss of sodium at $300^{\circ} \mathrm{K}$ are shown in Table 1. The contributions to the sodium loss coefficient from terms other than the leakage are also included. As explained above, heterogeneity is allowed for only in the resonance region below $15 \mathrm{keV}$, and the lattice is treated as homogeneous above this energy.

It will be seen that the predicted effects of heterogeneity are small. Thus, $k_{\text {eff }}$ is increased by only $0.07 \%$. A large change in this quantity was not to be expected, however, since only about one-sixth of the total reactivity comes from below $15 \mathrm{keV}$. The calculations of Storrer et $a l .^{(5)}$ for a somewhat similar system suggested that $k_{\text {eff }}$ might be increased by a further $0.1 \%$ from heterogeneity effects above $15 \mathrm{keV}$, when allowance is made for the larger diameter of the fuel rod considered by those authors.

The magnitude of the negative Doppler coefficient
TABLE 1. Tre Effect of Heterogeneity Below $15 \mathrm{keT}$ on $k_{\text {eff }}$, THe Doppler Coefficient, and rhe 100\% Sodium Loss CoffFictent

\begin{tabular}{|c|c|c|}
\hline \multirow{2}{*}{ Parameter } & \multicolumn{2}{|c|}{ System } \\
\hline & $\begin{array}{l}\text { Homogeneous } \\
\text { Lattice }\end{array}$ & $\begin{array}{l}\text { Heterogeneous } \\
\text { Lattice }\end{array}$ \\
\hline$k_{\mathrm{eff}}\left(300^{\circ} \mathrm{K}\right)$ & 1.0000 & 1.0007 \\
\hline $\begin{array}{l}\text { Doppler Coefficient }\left(10^{-5} \text { per }\right. \\
\left.{ }^{\circ} \mathrm{C}\right)\end{array}$ & -1.07 & -1.10 \\
\hline $\begin{array}{r}\text { Sodium Loss Coefficient }(\%) \\
d k / k)\end{array}$ & -9.45 & -9.51 \\
\hline $\begin{array}{l}\text { Sodium Loss Coefficient Ex- } \\
\text { cluding Leakage Term (\% } \\
d k / k)\end{array}$ & +2.23 & +2.17 \\
\hline
\end{tabular}

is about $3 \%$ greater in the heterogeneous case. A perturbation-theory analysis of the results showed that this figure is the balance of several small competing terms. The main contribution arises from the increased Doppler change in the absorption cross section for the energy groups near the $2.85-\mathrm{keV}$ sodium resonance. The small change in the Doppler coefficient is consistent with the conclusion of Greebler and Goldman $^{(6)}$ that heterogeneity in large fast ceramic reactors would have a small effect on $\mathrm{Pu}^{239}$ and $\mathrm{U}^{238}$ multigroup cross sections.

The results in Table 1 show that the effect of heterogeneity on the sodium loss coefficient is also small. The overall coefficient is slightly more negative, mainly because of a $3 \%$ decrease in the positive nonleakage component. This component includes the effects of spectral hardening and the stronger selfshielding of fission and capture resonances which occur when sodium is removed. The reduction of $3 \%$ due to heterogeneity comes in roughly equal parts from the spectral hardening and resonance self-shielding terms.

The calculations in Table 1 were based upon Hibdon's data for the $2.85-\mathrm{keV}$ sodium resonance. A similar set of calculations was also performed using alternative data due to Lynn, Firk, and Moxon (Harwell). The peak total cross sections are, respectively, about 630 and $370 \mathrm{~b}$, but the cross section below the resonance is higher in the latter data. The change from Hibdon to Lynn et al. data gave rise to only small differences in the results. For both the homogeneous and heterogeneous cases, $k_{\text {efr }}$ was reduced by $0.1 \%$, and the magnitude of the Doppler coefficient by $1 \%$. The main effect was to increase the positive nonleakage component of the sodium loss coefficient by about $5 \%$, e.g., the figure of $2.23 \% d k / k$ shown in Table 1 for the homogeneous case was increased to $2.35 \%$ $d k / k$, yielding a slightly less negative overall sodium 
TABle 2. U238 Resonance Capture and Its Doppler Change from $300^{\circ} \mathrm{K}$, for Uniform Temperatures OF 300,600, AND $900^{\circ} \mathrm{K}$, AND A RADIAL

Temperature Distribution With Mean Value $600^{\circ} \mathrm{K}$

\begin{tabular}{|c|c|c|c|}
\hline $\begin{array}{c}\text { Energy } \\
\text { Range } \\
(\mathrm{keV})\end{array}$ & $\begin{array}{c}\text { Temperature } \\
\left({ }^{\circ} \mathrm{K}\right)\end{array}$ & $\mathrm{U}^{238}$ Capture & Doppler Change \\
\hline $1.6-2.1$ & $\begin{array}{c}300 \\
600 \\
900 \\
\text { Distribution }\end{array}$ & $\begin{array}{l}0.085 \pm 0.001 \\
0.096 \pm 0.001 \\
0.104 \pm 0.001 \\
0.096 \pm 0.001\end{array}$ & $\begin{array}{l}0.011 \pm 0.0007 \\
0.019 \pm 0.0007 \\
0.011 \pm 00007\end{array}$ \\
\hline $0.8-1.6$ & $\begin{array}{c}300 \\
600 \\
900 \\
\text { Distribution }\end{array}$ & $\begin{array}{l}0.248 \pm 0.002 \\
0.278 \pm 0.003 \\
0.302 \pm 0.003 \\
0.277 \pm 0.003\end{array}$ & $\begin{array}{l}\text { - } \\
0.030 \pm 0.0025 \\
0.054 \pm 0.0025 \\
0.029 \pm 0.0025\end{array}$ \\
\hline
\end{tabular}

loss coefficient, $-9.33 \%$ as against the quoted $-9.45 \%$ $d k / k$ with Hibdon data.

It should be emphasized that the small effects of heterogeneity obtained above refer to a typical lattice in a power reactor. Considerably more important heterogeneity corrections have been indicated by experimental and theroretical work on fast zero-energy assemblies such as ZPR-III and ZEBRA. There the fissile, fertile, coolant, and structural materials are often present in the form of separate plates, giving a larger cell size than in a power reactor. In the latter system a considerable degree of homogeneity is already achieved through the use of mixed oxide or carbide fuel pins.

\section{Uranium-238 Resonance Capture for a Nonunim form Radial Temperature Distribution}

\section{(a) Method of Calculation}

The calculations refer to a square lattice composed of a $U^{238}$ dioxide rod surrounded by sodium. The rod diameter was chosen to be 0.20 in. and the cell width $0.30 \mathrm{in}$. The assumed isotopic concentrations in units of $10^{24}$ atoms per ec were $U^{238}: 0.020$; oxygen: 0.040 in the rod; sodium: 0.022 in the outer cell region. A hypothetical temperature distribution was defined by subdividing the rod into three concentric radial regions of equal cross-sectional area. The central region was at $900^{\circ} \mathrm{K}$, the intermediate region at $600^{\circ} \mathrm{K}$, and the surface region at $300^{\circ} \mathrm{K}$.

The $\mathrm{U}^{238}$ resonance eapture of neutrons slowing down below a prescribed source energy was calculated by a Monte Carlo code MOCUP for the IBM 7090 computer. ${ }^{(7)}$ Reflective boundary conditions were imposed at the outer cell surface, so that effectively the results refer to a lattice array rather than to an isolated cell. The library of $\mathrm{U}^{238}$ resonance cross sections obtained by the GENEX code (see Section 2) was used with MOCUP.

Calculations were made separately for two energy ranges, 2.1 to $1.6 \mathrm{keV}$, and 1.6 to $0.8 \mathrm{keV}$, which lie in the main Doppler region. The numbers of neutron histories followed were 16,384 and 6,044, respectively, yielding about the same percentage standard error for the calculated $U^{238}$ capture in each range.

Comparative calculations were also made for uniform rod temperatures of 300,600 , and $900^{\circ} \mathrm{K}$. Runs at different temperatures were correlated by choosing the same source neutrons and the same sequence of random numbers used for tracking, in order to reduce the standard error on the Doppler change in capture.

\section{(b) Results}

The calculated $U^{238}$ capture, and its Doppler change relative to a uniform rod temperature of $300^{\circ} \mathrm{K}$, are shown in Table 2. The capture is normalized to one source neutron entering the top of each energy range. Results are included for uniform rod temperatures of 300,600 , and $900^{\circ} \mathrm{K}$, as well as for the assumed 3region radial temperature distribution.

The standard error of the Doppler changes is less than might be expected from the quoted error for the capture, by about $30 \%$ for the lower energy range and about $50 \%$ for the higher. These reductions are a consequence of the correlation of MOCUP runs explained above.

The volume-weighted mean of the assumed temperature distribution is $600^{\circ} \mathrm{K}$. It will be seen from Table 2 that the results for the case of the temperature distribution are in good agreement with those for a uniform temperature equal to this value. One may conclude that the present work gives support to the common approximation of replacing a radial distribution of fuel-rod temperature by the mean value when calculating resonance absorption in fast reactors. The statistical uncertainty in the results does not, however, exclude the possibility of errors of order $\pm 10 \%$ in Doppler coefficients calculated by this rule. Such errors may not be considered excessive compared to those due to current imperfections in the methods and data used for calculations, or to uncertainties in predicted temperature conditions in power reactors.

Blässer and Matthes ${ }^{(2)}$ performed Monte Carlo calculations for a lattice of 0.125 -in.-diameter rods of $10 \%$ enriched uranium, cooled with sodium. A parabolic radial temperature distribution was assumed, and the energy range 1 to $10 \mathrm{keV}$ was investigated. 
The quantitics calculated were the partial Doppler coefficients with respect to increments in the rod surface and centre temperatures. The ratio of these coefficients over a range of temperature conditions was shown to vary between about 2.5 and 4 , in contrast to the value of unity expected if the mean-temperature rule were correct.

A direct comparison with these results could not be made in the present investigation since cross-section data were not available at a sufficient number of temperature values. However, the problem considered corresponds roughly to the determination of the centre-temperature Doppler coefficient as defined by Blässer and Matthes.

The agreement with the mean-temperature rule in the present work suggested that it may thus be the behaviour of the surface rather than of the centre temperature coefficient which is responsible for the results obtained by these authors. Collins ${ }^{(8)}$ has investigated the problem of capture in a bare slab of $\mathrm{C}^{238}$ with a parabolic temperature distribution, assuming normally incident neutrons. For a range of slab widths and temperature conditions he found that the ratio of the surface to centre temperature cocfficients of capture departed only slightly from the value given by the mean-temperature rule. The present work, together with Collins' results, suggest that the use of this rule should be an adequate approximation in Doppler calculations for the type of fast reactor considered here.

To conclude, we show in Table 3 the radial distribution of $\mathrm{U}^{238}$ capture in the rod, for uniform temperatures and for the temperature distribution. The energy range is 0.8 to $1.6 \mathrm{keV}$, but similar trends were also found for the higher range. Standard errors are not quoted individually, but lay within the limits \pm 0.0005 to \pm 0.0025 .

For uniform temperatures the capture distribution is nearly flat, although with a tendency to rise slightly towards the rod surface. For the temperature distribution, however, the capture decreases radially outwards.

Comparison of the results suggests that the capture at any point in the rod is determined primarily by the local temperature and is relatively insensitive to the temperature elsewhere. For instance, in the case of the temperature distribution, the capture $(0.083)$ in the surface region at $300^{\circ} \mathrm{K}$ is close to the value $(0.086)$ when the rod is at a uniform temperature of $300^{\circ} \mathrm{K}$, and likewise for the other two regions. This suggests that a suitable analytical approach to the problem would be to evaluate the resonance capture as a function of uniform rod temperature, and then
TABLE 3. Radial Distribution of U238 Resonaxce

Capture Between 0.8 and $1.6 \mathrm{keV}$, for Timiform Rod Temperatures aNd a Radial Temperature Instribetion

\begin{tabular}{c|c|c|c}
\hline \multirow{2}{*}{$\begin{array}{c}\text { Temperature } \\
\left({ }^{\circ} \mathrm{K}\right)\end{array}$} & \multicolumn{3}{|c}{ Rod Region } \\
\cline { 2 - 4 } & Central & Intermediate & Surface \\
\hline 300 & 0.080 & 0.081 & 0.086 \\
600 & 0.092 & 0.091 & 0.095 \\
900 & 0.099 & 0.099 & 0.103 \\
Distribution & 0.103 & 0.090 & 0.083 \\
& $\left(900^{\circ} \mathrm{K}\right)$ & $\left(600^{\circ} \mathrm{K}\right)$ & $\left(300^{\circ} \mathrm{K}\right)$ \\
\hline
\end{tabular}

average over the actual radial temperature distribution.

\section{Conclusions}

1. The reactivity of a typical dilute fast power reactor core, and the Doppler and sodium loss coefficients, are found to be only slightly influenced by heterogeneity effects in the resonance energy region below $15 \mathrm{keV}$. The use of Lynn, Firk, and Moxon's data for the $2.85-\mathrm{keV}$ sodium resonance in place of Hibdon's data also gives rise to only small changes in the results.

2. The $\mathrm{U}^{238}$ resonance capture in a fuel rod having a particular radial temperature distribution agrees closely with the results for the uniform mean temperature over the energy range from 0.8 to $2.1 \mathrm{keV}$ which was investigated. The statistical crror in the results, due to use of the Monte Carlo method, does not, however, preclude the possibility of $\pm 10 \%$ errors in Doppler coefficients calculated assuming the mean fuel temperature.

\section{REFERENCES}

1. Brissenden, R. J., and Durston, C., The Calculation of Neutron Spectra in the Doppler Region, Conference on the Application of Computing Methods to Reactor Problems, American Nuclear Society Meeting, May 1965, at the Argonne National Itaboratory, CSA, ANL-7050.

2. Blässer, G., and Matthes, W., Recent Investigations Concerning the Influence of Non-uniform Temperature Distribution in Doppler Effect Calculations, Euratom Report EUR $401 \mathrm{e}(1964)$.

3. D. Okrent, Specification of Calculations for Intercomparison to be made at the Conference on Safety, Fuels and Core Design in Large Fast Reactors, Argonne National Laboratory, Oct 1965.

4. Brissenden, R. J., A Formula for the Escape Probability of a Rod in a Uniform Lattice, AEEW-R.282 (1963).

5. Storrer, F., Khairallah, A., Cadilhac, M., and Benoist, P., Heterogeneity Calculation for Fast Reactors by a Perturbation Method, American Nuclear Society Meeting, San Francisco, Nov. 30/Dec. 3., 1964.

6. Greebler, P., and Goldman, E., Doppler Calculations for 
Large Ceramic Fast heactors: Effects of Improved Methods and Recent Cross Section Information, GEAP-4092 (1962).

7. Bannister, (X., Basher, J. C., and Pull, I. C., A.E.E., Winfrith, to be published.

8. Collins, P. J., Resonance Absorption of Neutrons in a Slab with a Parabolic Temperature Variation, AEEW-M.580 (1965).

\section{Discussion}

Mr. Ott (Karlsruhe): In your heterogeneity study, you showed remarkably small effects, and the heterogeneity value for $k_{\text {eff }}$ was larger than in the homogeneous case. How shall one understand this?

$M r$. Codd: I think that arises from the change in the resonance group cross sections with self-shielding. Self-shielding is stronger in the heterogeneous case.

Mr. Ott: In calculating the Doppler coefficients, in which you consider the temperature distribution over the reactor, have you also considered the temperature distribution of the additional energy release?

$M r$. Codd: No, we have not extended the investigation outside this simple temperature variation across the fucl pin.

$M r$. Ott: I meant that the cross temperature distribution over the reactor influences your calculations of the Doppler coefficient. A second influence will come from the space distribution of the additional energy release. The two effects are different.

Mr. Codd: This is limited to the case of uniform temperature conditions across the core.

$M r$. Ott: Do you know of the early investigation of Paul Greebler about the influence of the microscopic temperature distribution over the rod?

Mr. Codd: Yes.

$M r$. Ott: Grcebler derived that the deviation of, say, an effective temperature from the average temperature should be expected to be of the order of some percent.

Mr. Codd: Yes, we were aware of that work and it is discussed in this paper. I think we wanted to do a little more because Greebler's considerations particularly refer to conditions at the center and in the extreme wings of the resonances; in our calculations we integrate over the whole resonance. In general, our results support Dr. Greebler's conclusions; however, the results indicate an even smaller effect due to the temperature distribution, because we have taken into account conditions in the middle parts of the resonance as well.

Mr. Engelder (Babcock \& Wilcox): In support of your calculations which indicate the temperature distribution within the fuel pin is relatively unimportant, I would like to comment that Dr. Pettis at our laboratory has measured the resonance integral of thorium oxide under both conditions of a temperature distribution such as you have shown and under an average temperature through the fuel pin. He found essentially no difference in the resonance absorption between those two cases. This, of course, is in a $1 / E$ spectrum. The measurement is for thorium rather than uranium, but I think from considerations of the Debye temperature you would expect this effect to be more serious in thorium than in uranium. We were not able to create exactly a parabolic temperature distribution across the pin. It did have a temperature profile much like the one you have drawn on the blackboard, but it didn't quite have the same temperature gradient at the surface of the pin where you would expect the effect to be most serious. Nonetheless, based on these measurements, there should be no very strong effect of the temperature distribution through the pin.

$M r$. Codd: Thank you very much; is this work to be published?

Mr. Engelder: It has been published as a B\&W report within the last three months.

$M r$. Yiftah (Israel AEC): I just wanted to remark that Mr. Shaviv and I looked into the same problem of core heterogeneity using the Monte Carlo technique and our results corroborate yours. The core heterogeneity had only a very slight influence on the results.

Mr. Wall (GE): I have three questions and one comment regarding the heterogeneity effect. Would you describe the spatial cell which you are weighting and the size of the fuel pin? Secondly, what kind of spectrum were you weighting this over? You were looking below $15 \mathrm{keV}$, so what was the magnitude of the contribution with different uranium resonances? Thirdly, what is the relative effect of the sodium resonance and the uranium resonances on the heterogeneity effects you showed? The comment I would like to make is that in the experiments we have done at the MSTA at Vallecitos we have looked at the heterogeneity effect and have seen a very small effect. There was no sodium present in the system.

$M r$. Codd: We used a square of 0.35 -in. OD and a fuel rod of 0.280 -in. diameter. The volume proportion of fuel was $40 \%$ and I would say it was Case No. 4 specified by Dr. Okrent. We chose a square cell because we happened to have a collision-probability routine for this particular geometry. As regards your second question, we calculated our spectrum. This is part of the function of the Code SDR, which solves the Boltzmann transport equation in the resonance region. So we had to assume no spectrum. This was a slowing-down spectrum with depths at all the resonances.

Mr. Wall: The point of my question was the relative weight as regards $U^{238}$. We have done some meas- 
urements, to be reported on this afternoon, which indicate that there was more weighting at the $1-\mathrm{keV}$ $\mathrm{U}^{238}$ resonance than we expected from our calculations. Therefore, the relative weighting of the different resonances was somewhat important.

$M r$. Codd: We have applied no extraneous weighting to resonances. The calculation integrates right down through cnergy taking each resonance as the neutrons come to it. In this energy region there are the resolved resonance parameters, and we don't have to make any separate assumptions about weighting.

Mr. Dunenfeld (Allison Division, GMC): We are very much interested in the Doppler effect for transient conditions. Under those conditions the temperature profile within the fuel can be inverted from the sort you showed. What would the effect of that be on the heterogeneity effect?

Mr. Codd: We have not actually done that case although we have thought of doing it. Judging from the results we have, we think that the capture in any particular region depends mainly on the local temperature conditions rather than upon the particular variation of the temperature over the whole rod. I would think that in the inverted temperature conditions that one would gain a good agreement or approximation using the mean temperature. 


\title{
Results of Doppler Coefficient Calculations for Fast Reactors and Comparison of Different Methods*
}

\author{
PART I BY \\ R. Froelich and K. OtT \\ Institute of Neutron Physics and Reactor Engineering \\ Kernforschungszentrum, Karlsruhe, Germany \\ PART II $\dagger$ BI \\ G. Shaviv and S. Yiftah \\ Department of Nuclear Science, \\ Israel Institute of Technology \\ and Israel $A E C$ \\ Haifa, Israel \\ (PRESENTED BY K. OTT)
}

PART I

\section{Introduction}

The results of Doppler coefficient calculations of three different origins are compared and analysed. The aim of this analysis is to find out the special assumptions which are responsible for obvious discrepancies. This paper gives preliminary results. The three groups of results are calculated from

$(\alpha)$ the Karlsruhe nuclear data file ${ }^{(1)}$ using the Karlsruhe code $\mathrm{DCP}^{(2)}$ for calculation of Doppler coefficients from temperature derivatives of self-shielded cross sections;

( $\beta$ ) a Russian self-shielded group-constants set $\mathrm{ABN} ;{ }^{(3)}$

$(\gamma)$ a $\mathrm{YOM}^{(4)}$ group-constants set with corrections for the temperature dependence and for lowenergy neutrons. These last results are presented in Part II of this paper, by Shaviv and Yiftah.

As a result of such an analysis one should expect a separation of all the discrepancies in three classes:

a first class (C1) coming from the use of different resonance parameters;

a second class (C2) coming from the spectrum, mainly caused by the use of different sets of group constants;

a third class (C3) coming from the use of different

* This paper has been prepared within the framework of the association EURATOM-Gesellschaft für Kernforschung mbH. in the field of fast breeder development.

$\dagger$ This work is partly supported by the Kernforschungszen trum Karlsruhe-EURATOM Association on Fast Reactors. methods for the calculation of the Doppler reactivity effect.

For such an analysis the full knowledge of the nuclear data used by the two other groups $[(\beta)$ and $(\gamma)]$ in form of a data file as required for the Karlsruhe code DCP is necessary. Unfortunately, this has not been possible to manage up to now. Therefore, this paper contains only part of the comparison which we are carrying out. Beside the comparison of the final results as they were originally calculated, the temperature dependence of the Doppler coefficients is carefully investigated. Furthermore, the three different ways which are in common use for the calculation of Doppler coefficients are discussed and compared. These are the perturbation calculations of $d k / d T$ and $\Delta k$, and successive $k$ calculation.

These comparisons and discussions will show some discrepancies and uncertainties. A summary of these will be given in section. VI.

\section{Survey of the Methods of Calculation Used}

The results compared in this paper were calculated by two (and sometimes three) different methods which are in common use:

1. the usual perturbation calculation of the Doppler coefficient $d k / d T$ based on the temperature derivatives of the effective cross sections, $d \Sigma / d T$. One part of the Karlsruhe results, subsequently marked " $\alpha$," was calculated in this way (DCP).

2. The calculation of a Doppler $\Delta k$ by means of 
two multigroup calculations using effective cross sections which differ by an amount $\Delta \Sigma$ corresponding to a finite temperature interval $\Delta T$. The second part of the Karlsruhe results and all the Israelic results marked " $\beta$ " and " $\gamma$," respectively, were obtained by this method.

For the comparison of the results evidently a temperature law is necessary. This may cause some uncertainties to enter into the comparison.

3. Sometimes a third method was used for the calculation of Doppler $\Delta k$-values, namely, the determination of $\Delta k$ from $\Delta \Sigma$ by means of first-order perturbation theory. However, in this method it is unclear at which temperature the neutron spectrum shall be called "unperturbed," because the spectrum may change remarkably by shifting the temperature from one end to the other of the interval under consideration.

These difficulties do not arise in the first and second methods, where it is possible to choose the proper temperature or spatial temperature distribution in the effective cross sections for the multigroup calculation of the neutron flux.

( $\alpha$ In the Karlsruhe Doppler calculation code DCP, the handling of the resonance cross sections is based essentially on the paper of Nicholson ${ }^{(5)}$ with some improvements concerning the approximation of the $\psi$-function and the mutual shielding of resonances of the same resonance series. ${ }^{(2)}$ The resonance data are listed in Ref. (1). The neutron flux and adjoint-flux spectrum in most cases were calculated from the Russian set of group constants, $A B N,{ }^{(3)}$ and in a few cases with the Karlsruhe set of group constants, KFK 26-10. ${ }^{(6)}$

$(\beta)$ The Russian set of group constants ${ }^{(3)}$ (26 groups) is available for three temperatures. It may be interpolated for other temperature values. By means of successive multigroup calculations the Doppler $\Delta k$ were determined.

$(\gamma)$ The Doppler $\Delta k$ calculations of the Israeli group are described in Part II of this paper.

\section{Comparison of Doppler $\Delta k$ Values and Doppler Coefficients}

For comparison we have chosen in the first part of this paper two sequences of reactors with decreasing core volume. The first sequence investigated in more detail is identical with the first four cases proposed for intercomparison at this conference. They differ only* in the ratios of fertile to fissile material: $y=$ $\mathrm{U}^{238} / \mathrm{Pu}^{239}=11,9,7$, and 5. They are treated in the

* Concentrations in $10^{24}$ atoms $/ \mathrm{cm}^{3} ; \mathrm{Na}: 0.0123$; stainless steel : 0.0011 ; oxygen: $0.0144 ; \mathrm{Pu}^{239}+\mathrm{U}^{238}: 0.0072$.
TABLE $1 . \Delta k$ BETWEN $300^{\circ} \mathrm{K}$ A $\backslash 1900^{\circ} \mathrm{K}$

\begin{tabular}{|c|c|c|c|}
\hline$y=\frac{\mathrm{U}^{238}}{\mathrm{Pu}^{259}}$ & $\begin{array}{c}-\Delta k_{\alpha} \\
\text { (Karlsruhe) } \\
\text { from } d k / d T \text { - } \\
\text { Calculation } \\
\text { and } 1 / T^{x} \text { Law }\end{array}$ & $\begin{array}{l}-\Delta k_{\beta}(\mathrm{ABN}) \\
\text { Successive } \\
k \text { Calculation }\end{array}$ & $\frac{\Delta k_{\beta}-\Delta k_{\alpha}}{\Delta k_{\alpha}}(\%)$ \\
\hline 11 & 0.0258 & 0.0237 & -8.2 \\
\hline 9 & 0.0195 & 0.0178 & -9 \\
\hline 7 & 0.0132 & 0.0120 & -9.1 \\
\hline 5 & 0.00746 & 0.00659 & -11.6 \\
\hline
\end{tabular}

TABIE 2. $\Delta k$ BE FWEEN $900^{\circ} \mathrm{K}$ AND $2,100^{\circ} \mathrm{K}$

\begin{tabular}{|c|c|c|c|c|}
\hline$y=$ & $\frac{\mathrm{U}^{238}}{\mathrm{Pu}^{239}}$ & $\begin{array}{c}-\Delta k_{\alpha} \\
\text { (Karlsruhe) } \\
\text { from } d k / d T- \\
\text { Calculation } \\
\text { and } 1 / T^{x} \text { Law }\end{array}$ & $\begin{array}{c}-\Delta k_{\beta}(\mathrm{ABN}) \\
\text { Successive } \\
k \text { Calculation }\end{array}$ & $\frac{\Delta k_{\beta}-\Delta k_{\alpha}}{\Delta k_{\alpha}}(\%)$ \\
\hline & 11 & 0.0190 & 0.0190 & 0 \\
\hline & 9 & 0.0141 & 0.0145 & +2.9 \\
\hline & 7 & 0.00939 & 0.00983 & +4.7 \\
\hline & 5 & 0.00513 & 0.00559 & +9 \\
\hline
\end{tabular}

zero-dimensional approximation using $\mathrm{B}^{2}$ to go critical. The second sequence consists of the $\mathrm{Pu}^{239}$-oxide-fueled, blanketed spheres (case $\mathrm{Pu}$ "A") of Part II of this paper. The core volumes in this sequence are 800 , 1,500 , and 2,500 liters, respectively.

First comparison: The same neutron spectra, different resonance data, different methods of calculation.

In Table 1 the Doppler reactivity changes between 300 and $900^{\circ} \mathrm{K}$ are compared for the different ratios $(y)$ of fertile to fissile material. The $\Delta k$ values of column 2 were determined from the Doppler coefficients calculated by means of the Karlsruhe Doppler perturbation code DCP using the temperature law $a / T^{x}$ fitted at 300 and $900^{\circ} \mathrm{K}$. The spectra were calculated by means of the $A B N$ group-constants set. The values in column 3 result from two successive multigroup calculations at 300 and $900^{\circ} \mathrm{K}$, respectively.

In Table 2 the same values corresponding to the temperature interval from 900 to $2,100^{\circ} \mathrm{K}$ are listed.

It should be emphasized that all the pairs of $\Delta k$ compared in Tables 1 and 2 contain the same neutron spectrum, so that the discrepancies actually refer to the resonance data (C1) and the method of handling these data (C3). It is evident from Table 1 that between 300 and $900^{\circ} \mathrm{K}$ the Karlsruhe DCP values are about $9 \%$ higher than the values calculated from $A B N$ and that this difference is nearly independent of reactor size. Table 2 shows discrepancies of the opposite sign in the interval from 900 to $2,100^{\circ} \mathrm{K}$, strongly increasing with decreasing reactor size.

This follows from the first comparison: Using the same neutron spectra, we obtained from different 
data and different calculational methods Doppler $\Delta k$ values which are in agreement up to $\pm 10 \%$. The remaining discrepancies have a trend from which one may suggest that the method of handling the resonance data may be more responsible for the differences than the resonance data themselves are.

Second comparison: Different neutron spectra, identical resonance data, identical method of calculation. Results are given in. Table 3.

From the second comparison we see that: The Doppler coefficients calculated with the ABN spectrum are about $15-20 \%$ larger than those obtained with the KFK-26-10 spectrum, showing the large influence of the neutron spectrum on the Doppler coefficient. This may also explain the still larger discrepancios shown in Table 3.

These differences in the spectra are discussed by H. Küsters and M. Metzenroth. ${ }^{(6)}$

Third comparison: Diffcrent spectra, different resonance data, different methods of calculation.

The comparison of the values in Table 4 shows remarkably large differences in the Doppler coefficients in cases where spectra, resonance data, and methods of calculation are all different ('f. Part II of this paper). In the brackets of the last column are given the values calculated from the ABN set of group constants, showing relatively small differences from $A_{\alpha}$ because

TABLE 3. COMPARISON OF $d k_{\alpha} / d T$ (900 $\left.\mathrm{K}\right)$ DETERMINED by Perterbation Method DCP with the Same $d \Sigma / d T$ 's and DIFFeren? SPEC'Tra Calcetlated WITH IMHFERENT (MROUP-CONSTANT SETS

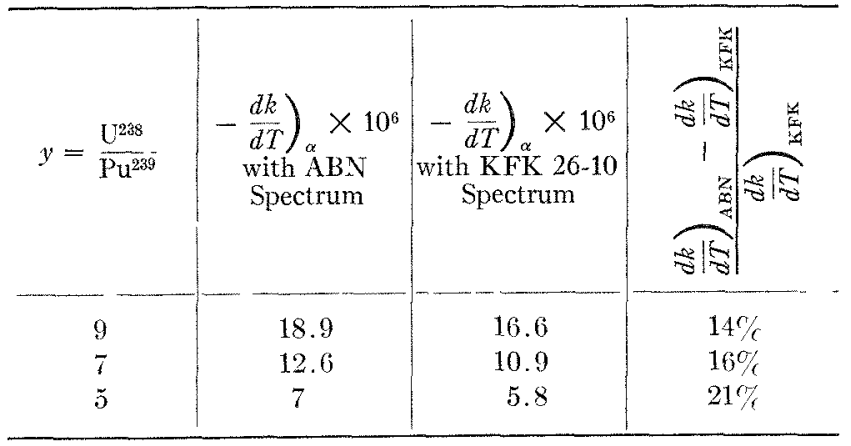

TABLF 4. Comparison of Rent(ed Dopplar Coefficients $\left[A(T)=T^{*}(d k / d T)\right]$ AT $900^{\circ} \mathrm{K}$ FOR THE Pu ${ }^{239}$ O2-Flteled BLANKE'led SPHERES OF ANL-6212 (See Part II of this paper)

\begin{tabular}{c|c|c}
\hline $\begin{array}{c}\text { Core Volume, } \\
\text { liters }\end{array}$ & $\begin{array}{c}-A_{\gamma} \text { (Israel) from } \\
\text { Successive } \\
k \text { Calculation } \\
\text { and } T^{-x} \text { Law }\end{array}$ & $\begin{array}{c}-A_{\alpha} \text { (Karlsruhe) from } \\
\text { Direct } T \frac{d k}{d T} \\
\text { Calculation (DCP) }\end{array}$ \\
\hline 800 & 0.00126 & $0.0084(0.00334)$ \\
1,500 & 0.00364 & $0.0061(0.00580)$ \\
2,500 & 0.00536 & $0.0087(0.00815)$ \\
\hline
\end{tabular}

both results are calculated with the same neutron spectra,

\section{Comparison of the Temperature Law for $d k / d T$}

The previous comparisons suggest a detailed investigation be made of the temperature dependence of the Doppler coefficient, $d k / d T$. lirst, we split the largest part of the temperature dependence, namely, a factor $1 / T,{ }^{(7)}$ and consider the quantity

$$
A(T)=T(d k / d T),
$$

which will be called "reduced Doppler coefficient" in the following. As a generalization of the 1/ $T$ law we use

$$
d k / d T \simeq a / T^{x}
$$

which was used in Section III to link $d k / d T$ with $\Delta k$.

For the determination of $a$ and $x$ of equation (2) one needs two values of $\Delta k$ or $d k / d T$. If only one value is available, one has to make an assumption about $a$ or $x$. Usually one puts $x=1$. From our Doppler coefficient calculations we computed three values for $d k /$ $d T$, so that one can determine special approximations for the intervals from 300 to $900^{\circ} \mathrm{K}$ and from 900 to $2,100^{\circ} \mathrm{K}$. In Figures 1 and 2 these approximations are compared for the four "zero-dimensional" reference reactors which were discussed above. It can be seen that the use of eq. (2) instead of just $1 / T$ in these cases improves the Doppler coefficient by less than $5 \%$. The main result from Figures 1 and 2 is that the temperature dependence of the reduced Doppler coefficient is remarkably different for the calculational procedures $\alpha$ and $\beta$.

Another investigation of the temperature dependence is given in Fig. 3, in which the exponent $x$ is plotted for increasing core volume characterized by $y$. Also in this plot the results of procedures $(\alpha)$ and $(\beta)$ show an opposite behaviour. With decreasing $y$ the neutron spectrum is shifted to higher energies, and one should expect from this an increasing value of $x$, representing the trend from the $T^{-1 / 2}$ law ${ }^{(s)}$ of thermal reactors to a $T^{-3 / 2}$ law $^{(5)}$ for very hard neutron spectra. The results of procedure $(\alpha)$ indicate this trend, whereas these of procedure $(\beta)$ show the opposite trend. Unfortunately, we could not use the results of Part II of this paper for a further comparison of this kind, because in these sequences there is no unique trend in $x$ as a function of core volume.

In the preceding analysis we used $x$ as a quantity independent of temperature. If this assumption were accurate, one could use the $1 / T^{x}$ law also for extrapolation to higher temperatures which may be useful for excursion calculations. Figure 4 shows the two values of $x$ determined from the three Doppler coefficients $(\alpha)$ 
mentioned above. We plotted only the results for the reactor with $y=9$. For all the other reactors we got quite similar values. We conclude from this that for the purpose of extrapolation the use of the $1 / T^{x}$ law is not an essential improvement compared with the use of the $1 / T$ law. However, since $x$ sensitively depends on the trends of the Doppler coefficients, the analysis of $x$ is a useful tool to check results for their internal consistency.

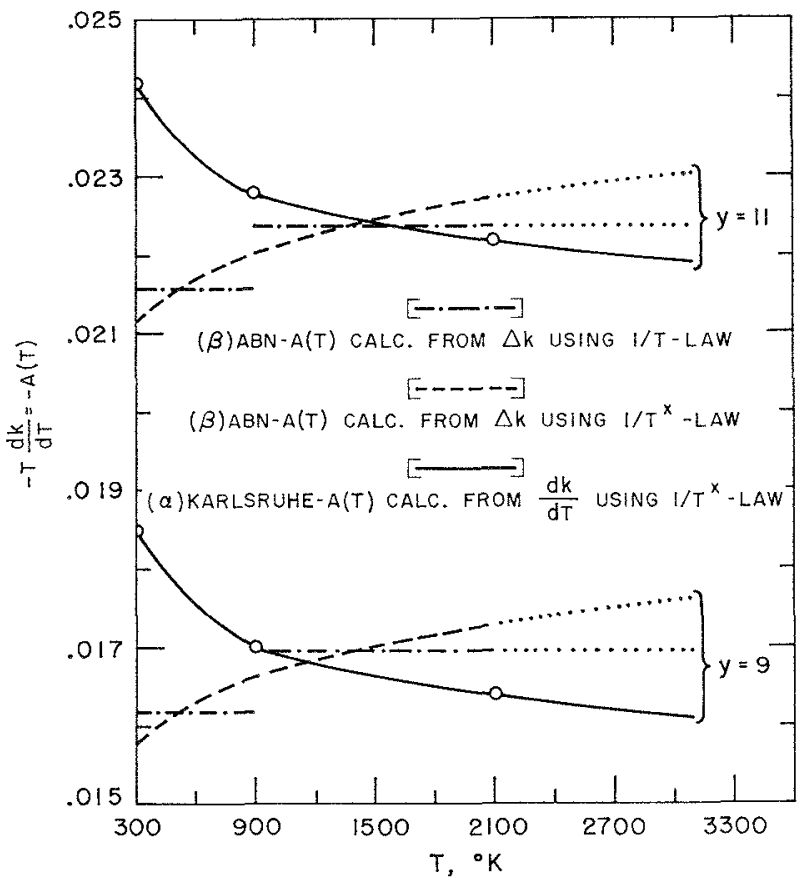

Fia. 1. Comparison of Reduced I oppler Coefficients Caleulated by Different Methods for $y=11$ and 9 .

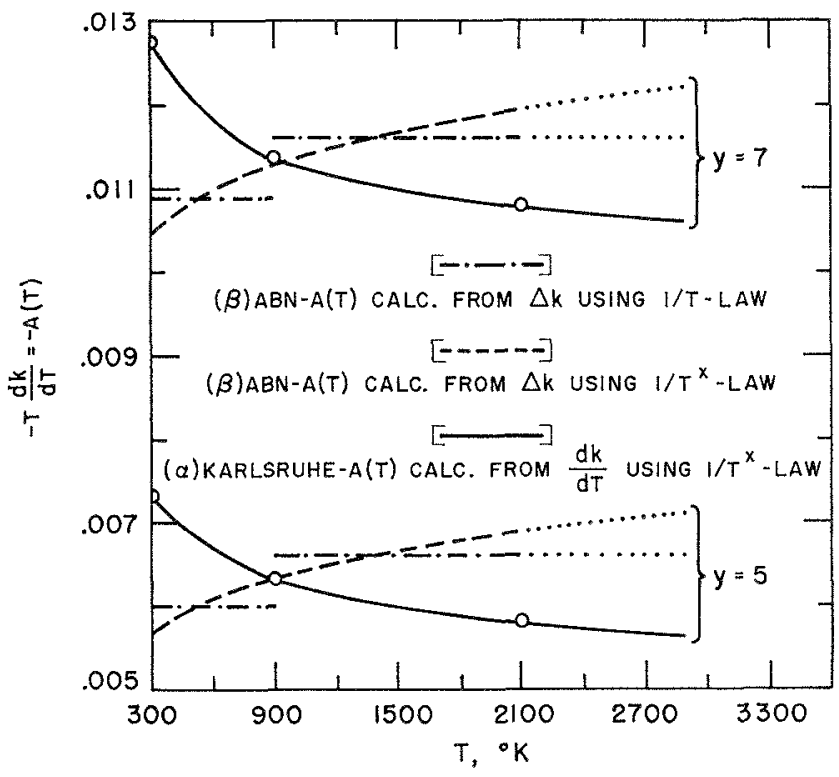

Fig. 2. Comparison of Reduced I oppler Coefficients Calculated by Different Methods for $y=7$ and 5 .

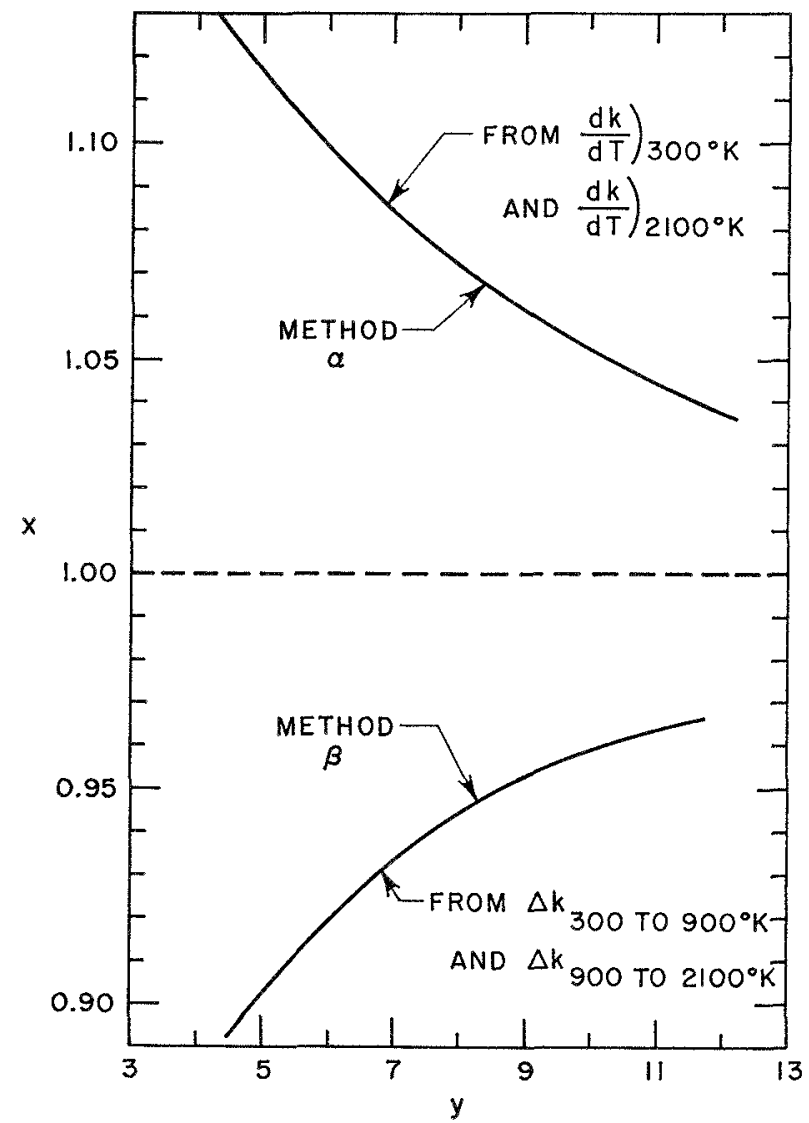

Fic. 3. Lxponent $x$ of the Law $d k / d^{\prime} T^{\prime} \sim 1 / T^{x}$ as Function of $y^{*}$

\section{Short Investigation of $\Delta k$ Calculation by Means of First-order Perturbation Theory from $\Delta \Sigma$}

The third method, ${ }^{(7,9)}$ mentioned in section II, namely, the use of first-order perturbation theory for the calculation of $\Delta k$ from $\Delta \Sigma$, involves an uncertainty in the choice of the "unperturbed" spectrum. To investigate the sensitivity with respect to the choice of the unperturbed spectrum we calculated $\Delta k$ from $\Delta \Sigma$ values corresponding to temperature intervals from 300 to $900^{\circ} \mathrm{K}$ and from 900 to $2,100^{\circ} \mathrm{K}$. The results are listed in Tables 5 and 6 , and are compared with the exact values of $\Delta k$ calculated from successive $k$ calculations. These tables show that for the temperature interval considered here the $\Delta k_{\text {pert }}$ values are up to $10 \%$ too high if the spectrum corresponding to the lower end of the temperature interval is used or up to $10 \%$ too low for the spectrum of the higher end of the interval. This suggests a comparison of the average value of both with the correct $\Delta k$. These average values, which are listed in the next-to-last columus of Tables 5 and 6 , show deviations from the correct values of only 0 to $3 \%$.

Clearly, all these deviations have to decrease if the temperature interval is decreased. 


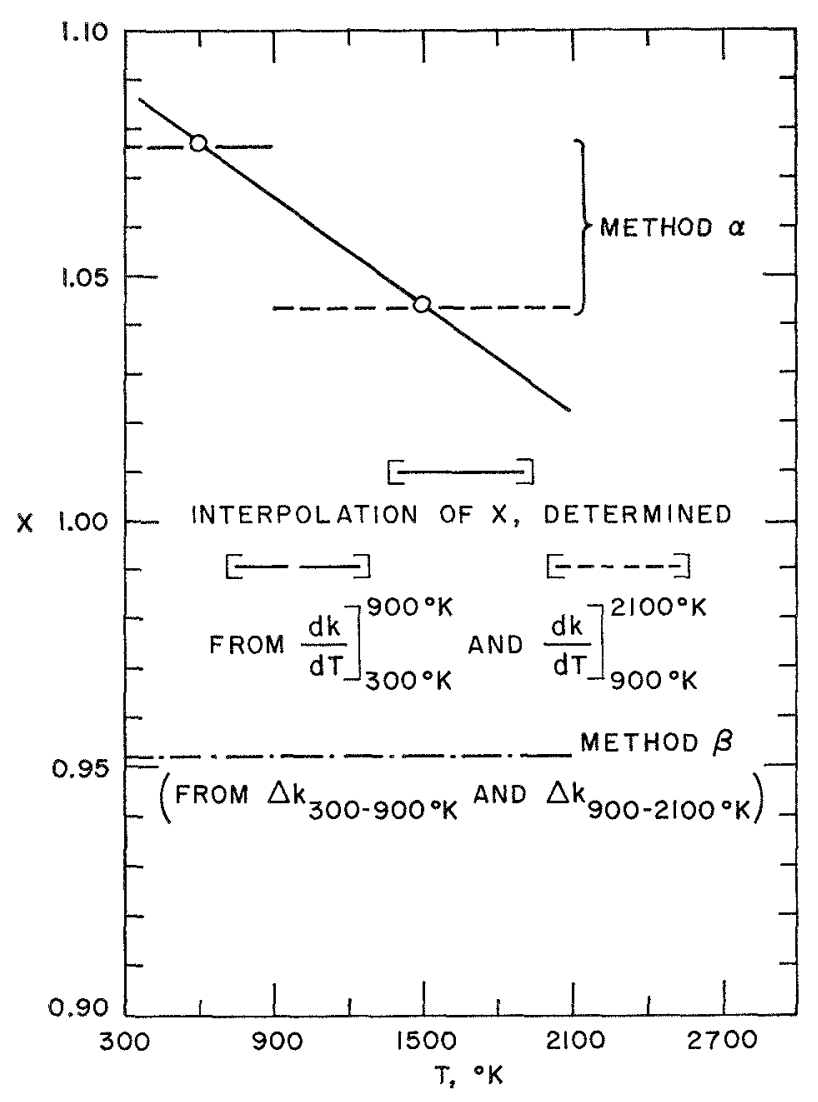

FJG. 4. Exponent $x$ of the Law $d k / d T \sim 1 / T^{x}$ as Function of $T$.

\section{Final Discussion}

The aim of this investigation was to find out the real roots of discrepancies in calculated Doppler reactivity results which usually appear if one compares Doppler coefficients of different origins. In the introduction these roots were discussed and subdivided into three classes: C1 to C3. In this paper three groups of results $[(\alpha)$, $(\beta)$, and $(\gamma)]$ are compared. The investigation is not completed up to now. The first results are qualitatively the following:

C1: The influence of the use of different resonance data on calculated Doppler reactivity results seems fairly small. From former investigations ${ }^{(1,9)}$ it follows that one has to expect not essentially more than $10 \%$ variation. A draft comparison with the results of Shaviv and Yiftah shows still lower differences.

$\mathrm{C} 2$ : The influence of the neutron spectrum used for the calculation of the Doppler reartivity effect is very strong. Tables 3 and 4 show discrepancies of more than $20 \%$ coming from the use of different spectra calculated from different sets of group constants. A further effect which may lead to different spectra is the use of an incorrect reference temperature, which may happen if a group-constant set is available only for a few temperatures. The errors coming from this are small if the temperature used is not too far from the real one (compare Tables 5 and 6). Also, the use of dyuamic spectra instead of static ones influences the Doppler reactivity effect only by small amounts. (In a typical example, for an excursion caused by $\$ 1.5$ step reactivity the Doppler coefficient was decreased by less than $7 \%$.)

C3: The reactivity calculated from $\Delta \Sigma$ by successive $\mathrm{k}$ calculation and the determination of $d k / d T$ from $d \Sigma / d T$ by means of perturbation theory are straight forward, and $\Delta k$ (by successive $k$ calculations) agrees with $\int_{\Delta T}(d k / d T) d T$ from perturbation theory, if $\Delta \Sigma$ corresponds strictly to $d \mathbf{\Sigma} / d T$. Therefore methodical differences can only rome from the use of different methods for the calculation of $\Delta \Sigma$ or $d \Sigma / d T$ out of the resonance data.

Tables 1 and 2 show that the values of different Doppler $\Delta k$ calculations agree fairly well if the same neutron spectrum is used. However, the temperature dependence disagrees in two respects (see Fig. 1 to 3). The reduced Doppler coefficient $T(d / / d T)$ decreases with temperature in the Karlsruhe $(\alpha)$ result and increases in the IBN results. Furthermore, the exponent $x$ in the temperature law $1 / T^{x}$ decreases with decreasing core volume in the $A B N$ results, whereas the Karlsruhe results show the opposite trend. We do not think that this comes from

TABLE 5. $\Delta k$ BE TWEEN $300^{\circ} \mathrm{K}$ AND $900^{\circ} \mathrm{K}$

\begin{tabular}{|c|c|c|c|c|}
\hline$y=\frac{\mathrm{U}^{238}}{\mathrm{Pu}^{239}}$ & $-\Delta k_{300}^{300} 900$ & $-\Delta k_{300-900}^{900}$ & $-\Delta k_{300-900}^{\overline{300-900}}$ & $\begin{array}{c}-\Delta k \\
\text { (Successive } \\
k \\
\text { Calculation) }\end{array}$ \\
\hline 11 & 0.0247 & 0.0216 & 0.0232 & 0.0237 \\
\hline 9 & 00187 & 0.0163 & 0.0176 & 0.0178 \\
\hline 7 & $\begin{array}{lll}0 & 0128\end{array}$ & 0.0112 & 0.0120 & 0.0120 \\
\hline 5 & 0.00715 & 0.00629 & 0.00672 & 0.0659 \\
\hline
\end{tabular}

$\Delta k_{300-900}^{300}$ calculated from $\Delta \Sigma_{300-900}$ and spectra at $300^{\circ} \mathrm{K}$. $\Delta k_{300-900}^{900}$ calculated from $\Delta \Sigma_{300-900}$ and spectra at $900^{\circ} \mathrm{K}$. $\Delta k_{300-900}^{300-900}=1 / 2\left(\Delta k_{300-900}^{300}+\Delta k_{300-900}^{900}\right)$.

TABLE 6. $\Delta k$ BETWEEN $900^{\circ} \mathrm{K}$ A $\ 2,100^{\circ} \mathrm{K}$

\begin{tabular}{c|c|c|c|c}
\hline$y-\frac{\mathrm{U}^{238}}{\mathrm{Pu}^{239}}$ & $-\Delta k_{900-2,100}^{900}$ & $-\Delta k_{900-2,100}^{2,100}$ & $-\Delta k_{900-2,100}$ & $\begin{array}{c}-\Delta k \\
\text { (Successive } \\
k \\
\text { Calculation) }\end{array}$ \\
\hline 11 & 0.0207 & 0.0182 & 0.0194 & 0.0190 \\
9 & 0.0157 & 0.0138 & 00147 & 00144 \\
7 & 0.0107 & 0.00948 & 0.0101 & 0.00983 \\
5 & 0.00607 & 00545 & 0.00576 & 0.00559 \\
\hline
\end{tabular}

$\Delta k_{900-2,100}^{900}$ calculated from $\Delta \Sigma_{900-2,100}$ and spectra at $900^{\circ} \mathrm{K}$. $\Delta k_{900}^{2,100}$, 100 calculated from $\Delta \Sigma_{910-2,100}$ and spectra at $2,100^{\circ} \mathrm{K}$. $\Delta k_{900-2,100}^{900-2,100}=J / 2\left(\Delta k_{900-2,100}^{900}+\Delta k_{900-2,100}^{2,100}\right)$. 
different resonance data. We guess that it is caused by different handling of the resonance data.

Further investigations will hopefully clear this dis- crepancy. Besides these, further improvements in the calculated Doppler coefficients depend upon improvement of the neutron spectrum.

\section{PART II}

\section{Introduction}

Recently six design studies of a 1000-NWe fast plutonium breeder were published in the CSA, France, and Germany. ${ }^{(10-15)}$ The cores of the designed 1000 MWe reactors, intended to be in operation in the seventies, differ in geometry and type of fuel, and have volumes in the range from 3500 to 7500 liters. Smaller reactors, however, in the range of 150-300 MWe and core volumes of 1000-1500 liters will be built and operated in various countries before undertaking the 1000-MWe unit.

As a matter of fact, the USSR has already started construction of the biggest fast reactor to date, BN-350, a 1000-MWt reactor to be used for power production and desalination of the Caspian Sea water near Schevchenko. This reactor, which has a core volume of 1870 liters, is to be completed and begin operation in 1968-69.

It was thought to be of interest, therefore, to study in detail one of the main safety mechanisms in fast reactors, the Doppler effect, for systems with core volumes in the range of $1000-2500$ liters. The results of ealculations of the change in neutron multiplication factor due to Doppler effect which were obtained in different ways are compared. In all cases the isothermal Doppler effect was calculated for a uniform rise in temperature over the whole core.

The comparison made here relates only to the possible different ways to calculate $\Delta k$ caused by $\Delta \Sigma$ or $d k / d T$ caused by $d \Sigma / d T$, and the relation between these quantities.

\section{Description of the Calculated Systems}

The calculated systems were first described by Yiftah and Okrent.(16) Details are given in Table 7. All systems are spherically symmetric. Three plutonium compositions were assumed and are denoted by $\mathrm{Pu}$ "A," $\mathrm{Pu}$ "B," and $\mathrm{Pu}$ "C." $\mathrm{Pu}$ "A" is pure $\mathrm{Pu}^{239}$. $\mathrm{Pu}$ "B" corresponds to the isotopic composition of plutonium after one cycle of irradiation in a thermal reactor fueled with enriched uranium (in this case the Yankee reactor fueled by oxide fuel and cooled by pressurized water). $\mathrm{Pu}$ "C" corresponds to the plutonium obtained after several fucl cycles in thermal reactors with high burnup. This is the plutonium isotopic composition obtained at equilibrium. The reason. for choosing these special isotopic compositions for plutonium is that they represent and embrace the possible isotopic composition which will be obtained from various origins.

The core volume of the calculated systems ranges from 800 to 2500 liters. Three chemical compositions of the fuel were assumed: metal, oxide, and carbide fuel. This assumption seems to cover the possible fuel composition of the fast breeder reactors.

\section{Methods of Calculation of $\Delta \Sigma$}

A full description of the methods for the calculation of $\Delta \Sigma$ can be found in reference (17). A short summary for the sake of orientation is given here. The method

TABLE 7. COMPOSITION AND STRECIURE OF Calculated systems

Core Volume: 800,1500 , and 2500 liters

Core Volume Fractions: Fuel and Fertile Isotopes, 0.25 Struetural Materials, 0.25 Coolant, 0.50

\begin{tabular}{c|c}
\hline Fuel & Density $(\mathrm{gm} / \mathrm{cc})$ \\
\hline${\mathrm{Pu}-\mathrm{U}^{238}}^{238}$ & 19 \\
$\mathrm{PuO}_{2}-\mathrm{UO}_{2}$ & 8.4 \\
$\mathrm{PuC}-\mathrm{UC}^{-}$ & 11.39 \\
\hline
\end{tabular}

Plutonium Isotopic Composition

\begin{tabular}{c|c|c|c|c}
\hline & $\mathrm{Pu}^{239}$ & $\mathrm{Pu}^{245}$ & $\mathrm{Pu}^{241}$ & $\mathrm{Pu}^{242}$ \\
\hline $\begin{array}{c}\text { Type (Notation Only for } \\
\text { this Work) }\end{array}$ & \multicolumn{4}{|c|}{ Per Cent } \\
A & 100 & 0 & 0 & 0 \\
B & 74.7 & 10.2 & $12 . t$ & 2.7 \\
C & 40 & 10 & 25 & 25 \\
\hline
\end{tabular}

Coolant: Liquid Sodium; Density: $0.84 \mathrm{gm} / \mathrm{cc}$.

Blanket: Thickness of $45 \mathrm{~cm}$.

Blanket Volume Fraction

U238 (Metal), 0.6

$\mathrm{Na}, 0.2$

Fe, 0.2

Reflector: Thickness of $30 \mathrm{rm}$.

Reflector Volume Fraction

$\mathrm{Fe}, 0.6$

$\mathrm{Na}, 0.4$ 
used for the calculation of $\Delta \Sigma$ changed with the energy range involved and with the type of isotope. At very high energies, e.g., above $6.5 \mathrm{keV}$ for $\mathrm{Pu}^{239}, 1 \mathrm{keV}$ for $\mathrm{Pu}^{241}$, and about $30 \mathrm{keV}$ for $\mathrm{U}^{238}$, and high temperatures, the method $\mathrm{A}$ of Nicholson ${ }^{(5)}$ was used. The two basic assumptions of this method are:

(1) At very high energies the resonances overlap strongly.

(2) The asymptotic expression for the $\psi$ function can be used.

In the case of fissile isotopes at medium energies assumption (1) was replaced by the assumption:

(3) The total contribution of the resonant isotopes to the cross section is small.

In practical cases this assumption can be valid at lower energies than those at which (1) is valid. Although at high energies and temperatures (2) can be justified, this is not the case at low energies; hence, when assumption (3) was used, no use was made of (2). Because the second method consumes more computing time, it was replaced by the first method for energies above that at which both methods gave practically the same result (to within an accuracy of $1-3 \%)$.

Method B of Nicholson was used for fissile isotopes at very low energies and for fertile isotopes at low energies.

The effects of heterogeneity, interaction between resonances of the fertile and fissile isotopes, large sodium resonances on the effective cross section, etc., were treated by the use of the Monte Carlo method.
Complete tables of all the resonance parameters used in the calculation can be found in ref. (17). The basic cross-section set used was the 16-group cross-section. set of Yiftah, Okrent, and Moldauer. ${ }^{(4)}$ The changes in the effective group cross sections for a specific system were obtained by averaging the changes in effective cross section obtained with the mothods mentioned above, over the approximated neutron flux of the system. Examples of neutron fluxes are giver in Fig. 5. In many cases the change in effective cross section decreases rapidly with energy; hence averaging is of importance. The flux histogram obtained for each system from the eriticality ealculation at $300^{\circ} \mathrm{K}$ with the multigroup model was approximated by a smooth curve which was later used for averaging the changes in effective cross section.

The 16-group cross-section set used extends from 10 $\mathrm{MeV}$ down to $0.5 \mathrm{keV}$. However, a non-nogligible contribution to the total Doppler effect comes from below this energy. Since the 16th energy group is the last energy group in this cross-section set, it contains all the neutrons with energy below $2.1 \mathrm{keV}$, including those neutrons that in reality are scattered to lower energies than $0.5 \mathrm{keV}$. Since in this cross-section set there is no energy group below $0.5 \mathrm{keV}$, all the neutrons with energy below $0.5 \mathrm{keV}$ remain in the 16th group. The overestimation of neutrons in this energy range is expressed in the maximum found in the case of large oxide-fueled reactor for the lowest-energy group (see Fig. 5). The procedure used was to assume that the 16th energy group extends down to $0.1 \mathrm{keV}$. The

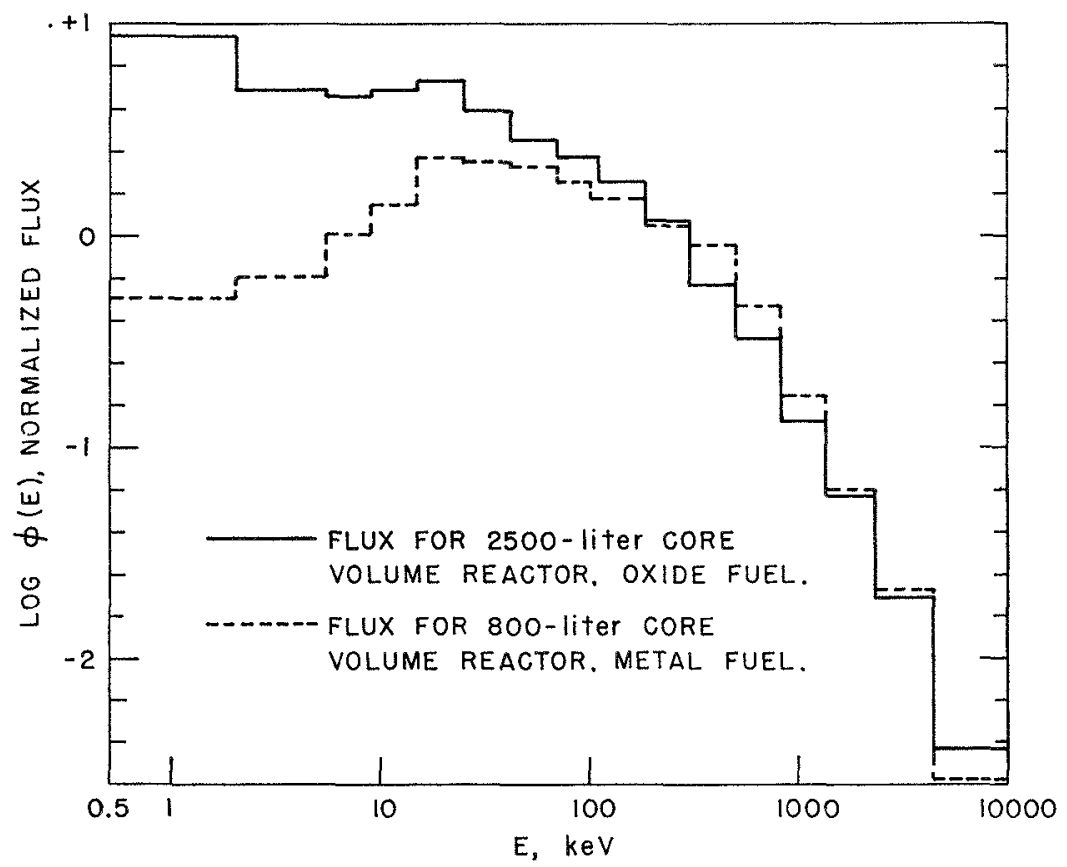

FıG. 5. Spectra for Oxide-fueled Reactors of Two Different Sizes (2500 Liters and 800 Liters). 
TABle 8. Results of the Calculition of the Doppler Efeet $-\Delta k \times 10^{4}$

\begin{tabular}{|c|c|c|c|c|c|c|c|}
\hline \multirow{2}{*}{$\begin{array}{l}\text { Core Volume } \\
\text { (liters) }\end{array}$} & \multirow{2}{*}{$\begin{array}{l}\text { Fucl } \\
\text { Type }\end{array}$} & \multicolumn{2}{|c|}{$P_{u}$ "A" } & \multicolumn{2}{|c|}{$\mathrm{Pu}$ "B" } & \multicolumn{2}{|c|}{$\mathrm{Pu}$ " C $"$} \\
\hline & & $\Delta k\left(300-900^{\circ} \mathrm{K}\right)$ & $\Delta k\left(300-1500^{\circ} \mathrm{K}\right)$ & $\Delta k\left(300-900^{\circ} \mathrm{K}\right)$ & $\Delta k\left(300-1500^{\circ} \mathrm{K}\right)$ & $\Delta k\left(300-900^{\circ} \mathrm{K}\right)$ & $\Delta k\left(300-1500^{\circ} \mathrm{K}\right)$ \\
\hline \multirow[t]{3}{*}{800} & Metal & 14.7 & 20.0 & 15.9 & 21.8 & 16.7 & 23.0 \\
\hline & Oxide & 12.4 & 19.2 & 13.8 & 20.5 & 19.4 & 27.4 \\
\hline & Carbide & 24.1 & 33.8 & 29.6 & 40.9 & 38.5 & 48.2 \\
\hline \multirow[t]{3}{*}{1500} & Metal & 19.7 & 26.7 & 21.2 & 29.3 & 22.4 & 30.9 \\
\hline & Oxide & 41.8 & 60.0 & 46.7 & 65.7 & 61.1 & 85.3 \\
\hline & Carbide & 39.7 & 57.4 & 46.8 & 63.8 & 59.3 & 79.0 \\
\hline \multirow[t]{3}{*}{2500} & Metal & 24.2 & 328 & 25.6 & 35.3 & 27.3 & 37.4 \\
\hline & Oxide & 63.3 & 89.8 & 81.2 & 100 & 86.3 & 129 \\
\hline & Carbide & 54.1 & 75.3 & 68.7 & $86 . t$ & 76.2 & 102 \\
\hline
\end{tabular}

change in the effective group cross section for this group was obtained by averaging the change in the effective cross section over the region $0.1-2.1 \mathrm{keV}$. The cross section used for the criticality calculation. was not ehanged. The total number of neutrons below $0.5 \mathrm{keV}$ is small and does not affect appreciably the criticality calculations. The averaging flux was assumed to be $\phi(E)|\propto| E^{1 / 2}$.

The procedure adopted here is not very accurate, and the calculations were repeated with additional energy groups in this energy range. It is estimated that the possible error introduced in the group effective cross section for this group by wrong averaging flux and neutron-flux overestimation for this energy is $15-20 \%$.

\section{Methods of Calculation of $\Delta k$}

The calculation of the change in neutron multiplication factor $k$ due to temperature rise was carried out by a one-dimensional diffusion approximation.

Two ways were tried: the sucressive $k$ method and perturbation. In the first method the procedure is as follows: the neutron multiplication factor of the system at $300^{\circ} \mathrm{K}$ is found by the use of standard cross-section tables, in this case the YOM cross-section set. After convergence is reached, the changes in effective cross section corresponding to a given temperature rise are added to the cold cross-section set and the new $k$ is sought. This procedure is repeated till the highest temperature is reached. The convergence criterion (the ratio between the differenee in neutron source in the $i$ th and $(i-1)^{\prime}$ th iteration, and the noutron source in the $i$ th iteration) was $10^{-6}$. The convergence is usually rapid, and only 4- 5 iterations are needed. Table 8 summarizes the results for $\Delta k\left(300-900^{\circ} \mathrm{K}\right)$ and $\Delta k\left(300-1500^{\circ} \mathrm{K}\right)$.

In the second method, first-order perturbation was used. $\Lambda$ comparison of the results of both methods has shown that in all cases the result obtained by perturba-
TABLE 9. Comparion OF Grotp Ficxes aT TWO TEMPERATLRES

The fluxes are normalized to unity: $\sum_{g=1}^{16} \phi_{g}=1$

\begin{tabular}{c|c|c|c|c}
\hline $\begin{array}{c}\text { Energy } \\
\text { Range, } \\
\text { keV }\end{array}$ & $\begin{array}{c}\text { 2500-liter Core Volume; } \\
\text { Oxide Fuel. Pu A. }\end{array}$ & $\begin{array}{l}\text { 800-liter Core Volume; } \\
\text { Metal Fuel. Pu A. }\end{array}$ \\
\cline { 2 - 5 } & $\phi_{g}\left(300^{\circ} \mathrm{K}\right)$ & $\phi_{g}\left(1500^{\circ} \mathrm{K}\right)$ & $\phi_{g}\left(300^{\circ} \mathrm{K}\right)$ & $\phi_{g}\left(1500^{\circ} \mathrm{K}\right)$ \\
\hline $0.5-2.1$ & 0.0139 & 0.00926 & 0.000809 & 0.000476 \\
$2.1-5.5$ & 0.0156 & 0.0153 & 0.00217 & 0.00192 \\
$5.5-9.1$ & 0.0165 & 00164 & 0.00355 & 0.00326 \\
$9.1-25$ & 0.0277 & 0.0277 & 0.00820 & 000767 \\
$15-25$ & 0.0525 & 0.0525 & 0.0232 & 0.0220 \\
$25-10.7$ & 0.0617 & 0.0619 & 0.0354 & 0.0339 \\
$40.7-67$ & 0.0744 & 0.0748 & 0.0556 & 0.0535 \\
$67-110$ & 0.0999 & 0.1005 & 0.0773 & 0.0745 \\
110180 & 0.1245 & 0.1253 & 0.1299 & 0.1253 \\
$180-300$ & 0.1339 & 0.1347 & 0.1581 & 0.1525 \\
$300-500$ & 0.1220 & 0.1227 & 0.1777 & 0.1715 \\
$500-825$ & 0.1024 & 0.1030 & 0.1451 & 0.1400 \\
8251350 & 0.0682 & 0.0685 & 0.0868 & 0.0837 \\
$1350-2225$ & 0.0485 & 0.0488 & 0.0521 & 0.0502 \\
$2225-3668$ & 0.0264 & 0.0265 & 0.0294 & 0.0284 \\
$3668-10000$ & 0.0120 & 0.0121 & 0.0148 & 0.0142 \\
\hline
\end{tabular}

tion theory is greater by about $20 \%$ than that obtained by the successive $k$ method. Such a great difference between the first-order approximation and the exact calculation is surprising because the percentage change in $\Delta \Sigma$ is small, less than $5 \%$ at most. When all $\Delta \Sigma$ were halved, the difference in percent between the results obtained by the two methods did not change. This fact shows that the difference is of a systematic nature inherent with the first-order perturbation calculation. In Table 9 the group fluxes at two temperatures are compared. It can be seen that in the center of a core having a volume of 2500 liters and oxide fuel the change in neutron spectrum may be of the order of $20 \%$ at the very low-energy part. Hence, such a change in neutron spectrum over the whole core can cause such a difference between the successive $k$ method in which 
TABLE 10. Comparison OF Reselits of $-\Delta /\left(300-900^{\circ} \mathrm{K}\right) \times$ $10^{4}$ Obtained in Karlskuhe and Israel

\begin{tabular}{c|c|c|c}
\hline \multirow{2}{*}{$\begin{array}{c}\text { Core Volume } \\
\text { (liters) }\end{array}$} & $\begin{array}{c}\text { Fuel Type } \\
\text { Pu "A" }\end{array}$ & \multicolumn{2}{|c|}{$-\Delta k\left(300-900^{\circ} \mathrm{K}\right) \times 10^{4}$} \\
\cline { 3 - 4 } & Metal & 14.7 & 24.8 \\
\hline 800 & Oxide & 12.4 & 37.4 \\
& Carbide & 24.1 & 51.7 \\
\hline 1500 & Oxrael & Karlsruhe \\
\hline 2500 & Oxide & 42 & 67 \\
\hline-- & Oxide & 63 & 95.7 \\
\hline
\end{tabular}

the exact flux is used and the first-order perturbation method in which the flux at $300^{\circ} \mathrm{K}$ is used.

\section{Comparison of Results}

The comparison between the results obtained in Israel and the results obtained in Karlsruhe was made by assuming the law $d k / d T=\mathrm{A} / T$ for the change of neutron multiplication factor with temperature.

Table 10 summarizes the results obtained in Karlsruhe and Israel for the same systems. The results obtained at Karlsruhe are systematically greater than those obtained in Israel. A thorough comparison of the methods and data used has shown that practically the results obtained for $\Delta \Sigma$ or $d \Sigma / d T$ are the same. It is estimated that the cumulative error in these figures (error introduced by the various approximation in the calculation of $\Delta z$ and $\Delta k$ ) are of the order of $1 \%$.

Under these conditions it follows that the difference between the results is caused by the use of different basic data. It is known ${ }^{(18,19)}$ that the YOM crosssection set yields a harder neutron spectrum than that obtained by the use of the cross-section set of Abagjan et al. ${ }^{(18)}$ This will result in a smaller number of neutrons at low energies; hence, the Doppler effect which comes into play at low energies will have less neutrons to act on.

\section{REFERENCES}

1. J. J. Schmidt, EANDC-E-46 L (1963); R. Froelich, K. Ott, and J. J. Schmidt, ANL-6792, pp. 765-773 (1963).

2. DCP (Doppler Coefficient Calculation by Perturbation Theory), Code description in preparation. Theory is based on: R. Froelich, K. Ott, and J. J. Schmidt, ANL6792, pp. 777-788 (1963); R. Froelich and K. Ott, NSI-22, pp. 487-488 (1965); R. Froelich, KFK-367 (1965).

3. L. P. Abagjan, N. O. Bazazjane, I. I. Bondarenko, and M. N. Nikolaev, Translation: KFK-tr-144.

4. S. Yiftah, D. Okrent, and P. A. Moldauer, Fast Reactor Cross Sections, Pergamon Press, New York (1960).

5. R. B. Nicholson, Doppler Effect in Fast Reactors, APDA139 (1960).

6. H. Kusters and M. Metzenroth, see paper presented at this Session of This Conference.
7. B. A. Hutehins, and P. Greebler, Seminar on Physics of Fast and Intermediate Reactors, Vienna (1961).

8. L. Dresner, Resonance Absorption in Nuclear Reactors, Pergamon Press, New York (1960).

9. H. H. Hummel and A. L. Rago, ANL -6792, pp. 747-761 (1963).

10. McNelly, M. J. (Project Engineer), Liquzd Metal Fast Breeder Reactor Design Study, GFAP-1418 (1964).

11. Klotz, C. (Project Management), Large Fast Reactor Design Study, ACNP-64503 (1964).

12. Heck, F. M. (Project Engineer), Laquid Metal Fast Breeder Reactor Design Study, WCAP-3251-1 (1964).

13. Visner, S. (Project Manager), Liquid Metal Fast Breeder Reactor Design Study, CWND-200 (1964).

14. I endryes, (x., Zaleski, C. P., et al., Litudes Preliminaires Conduit a un Concept de Reacteur a Neutrons Rapides de 1000 HWc, CEA-R 2554 (EUR. $2235 \mathrm{f}$ ).

15. Smidt, D., Muller, A., et al., Natrumgekuhlten Sehnellen Brutreaktor ( $\mathrm{Nal}$ ), PSB-Bericht 122 (Gesellschaft fur Kernforsthung m.b.h. Karlsruhe), (1964).

16. Yiftah, S. and Okrent, 1)., Some Physics Calculations on the Performance of Large Fast Breeder Power Reactors, ANL-6212 (1960).

17. Szwarcbaum, G. (Shaviv), and S. Yiftah, ('alculation of the Doppter Effect in Fast Reactors, IA-1039 (1965).

18. Sere Ref. 4, p. 120.

19. Hutchins, B. A., A remark made at the meeting between the Israeli and the Karlsruhe groups, June 3, 1965.

20. L. P. Abagjan et al.. Teutron Group Constants for Fast and Intermediate Reactor Calculation, Moscow (1964). (translated in Reference 3)

\section{Discussion.}

Mr. Till (ANL): We have just recently completed a series of measurements with ZPR-6 on the temperature dependence of the Doppler effect from 300 to $1100^{\circ} \mathrm{K}$; this was for a 2600 -liter carbide core. The temperature dependence of the $U^{238}$ Doppler effect seems to be almost exactly as $1 / T$ between those temperatures, to within 2 or $3 \%$.

Mr. Hummel (ANL): I would like to go a little more deeply into the discrepancy between the Karlsruhe results and those given by Yiftah. It seems to me that a discrepancy of this magnitude could be due only to a large disparity in the number of low-energy neutrons. It certainly is not due to differences in the resonance parameters of $\mathrm{U}^{238}$ or anything like that. When you talked about spectrum, is what you were referring to that the effect of elastic removal cross sections at low energy was determined to see how many neutrons were slowed down to below $25 \mathrm{keV}$ or something similar?

Mr. Ott: Yes.

$M r$. Ancarani (ANL): The dependence of $x$ as shown by you seems to be related to the $y$ value. Were the compositions of the reactors the same as far as coolant and structure material are concerned, or was there a change?

Mr. Ott: No, only the enrichment was changed. All of the ingredients of the reactor were the same. 


\title{
Effect of the Fluctuations in Collision Density on Fast-reactor Doppler Effect Calculations
}

\author{
R. N. Hwang \\ Argonne National Laboratory \\ Argonne, Illinois
}

\section{Introduction}

Because of its simplicity, the narrow-resonance (NR) approximation has been most widely used in studies of the Doppler effect for large fast reactors. In this approximation, the neutron collision density is taken to be constant in lethargy and temperature not only over a particular resonance, but also over a lethargy interval containing many resonances. Although the applicability of the NR approximation that greatly simplifies the Doppler calculation has been gratifying, it is important to know, however, whether or not the approximation introduces errors.

Even though all resonances in the energy region of interest for the fast reactor studies are nearly always narrow compared to the maximum increment in lethargy per collision, the collision density, strictly speaking, is lethargy and temperature dependent. Since it acts as a negative or positive source, a resonance will introduce localized transients in collision density, which are sometimes referred to as Placzek oscillations. To make the problem even more complicated, the fluctuation in collision density caused by resonances of one substance may affect the resonance integrals of other substances in the mixture. Hence, the resonance integral of a particular resonance depends, to some extent, on the resonance scattering and absorbing characteristics of resonances of the same and different nuclides at higher energies. Since the Doppler effect is accumulative from resonance to resonance over a rather broad energy span $(\sim 300 \mathrm{eV}$ to $10 \mathrm{keV})$, these temperaturedependent and nonasyn.ptotic contributions in the collision density may play a role in the Doppler studies.

The primary purpose of this paper is to investigate the behaviors of the fluctuations in collision density and their influence on the Doppler calculations for a large fast reactor. The present work is restricted to the case of an infinite homogeneous medium with composition typical of a dilute fast reactor. Three special topics concerning the Doppler theory have been investigated:

(1) effect of Placzek oscillations on the reaction rate of individual resonances;

(2) effect of incomplete recovery in neutron collision density between resonances on the group reac- tion rates and the effective cross section of a single resonant material in the mixture of heavy scatterers;

(3) a more general approach to the problem of resonance overlap between two different nuclides.

Investigations were carried out both analytically and numerically. The first and second topics were intended to illustrate the nature of the problem; the third topic is of great practical importance. An approximate method which accounts for the fluctuations in the collision density has been developed in order to treat the problem of resonance overlap in the region where resonances are only partially resolved for the fertile isotopes and completely unresolved for the fissile isotopes. It was found that the errors introduced by the fluctuation in the collision density are generally small when the total Doppler effect of the system is considered, even though the NR approximation may become unreliable in the lower end of the Doppler region.

\section{Physical and Mathematical Aspects of the Slowing-down Problem}

With no lack of generality, the well-known slowingdown equation for an infinite homogeneous mixture of $N$ nuclides with a monoenergetic source of unit strength can be described by a Volterra integral equation of the second kind, as pointed out by Corngold. ${ }^{(1)}$ Since, for practical purposes, the energy region of interest is well below the source encrgy, an arbitrary lower lethargy limit $u_{1}$ is chosen so that the collision density below $u_{1}$ is assumed to be uniform and unperturbed by the resonance structure. Hence, Corngold's equation can be rewritten into the following form:

$$
\begin{gathered}
F(u)=\bar{\xi} P(u)-\int_{0}^{u} K\left(u, u^{\prime}\right) F^{\prime}\left(u^{\prime}\right) d u^{\prime} \\
\cong 1-\int_{u_{1}}^{u} K\left(u, u^{\prime}\right) F\left(u^{\prime}\right) d u^{\prime} ; \\
u>u_{1}>>>0,
\end{gathered}
$$

where $F(u)$ is the collision density normalized to its asymptotic value $1 / \bar{\xi}$, with $\bar{\xi}$ the average lethargy 
increment per collision for the mixture, and the kernel $K\left(u, u^{\prime}\right)$ is to be defined. $P(u)$ is the Placzek function $^{(1,2)}$ for the nonabsorptive mixture which assumes its asymptotic value $1 / \bar{\xi}$ far from the source energy.

It is quite clear from Eq. (1) that the perturbation in $F(u)$ caused by resonances depends entirely on the characteristics of $K\left(u, u^{\prime}\right)$. This kernel $K\left(u, u^{\prime}\right)$, given in Ref. 1, ean be rewritten into the following form:

$$
\begin{aligned}
K(u, u)= & \sum_{1}^{N} G_{N}\left(u-u^{\prime}\right) g_{N}\left(u^{\prime}\right) \\
= & P\left(u-u^{\prime}\right) \sum_{1}^{h} \frac{\Sigma_{R h}\left(u^{\prime}\right)}{\Sigma_{t}\left(u^{\prime}\right)} \\
& -\sum_{1}^{h} G_{h}\left(u, u^{\prime}\right) \frac{\Sigma_{R S h}\left(u^{\prime}\right)}{\Sigma_{t}\left(u^{\prime}\right)},
\end{aligned}
$$

with

$$
g_{N}\left(u^{\prime}\right)=\frac{\Sigma_{S N}(0)}{\Sigma_{t}(0)}-\frac{\Sigma_{S N}(0)+\Sigma_{R S N}\left(u^{\prime}\right)}{\Sigma_{t}\left(u^{\prime}\right)},
$$

where $h$ designates resonance absorbers only, $\Sigma_{R h}\left(u^{\prime}\right)$ and $\Sigma_{R s h}\left(u^{\prime}\right)$ total macroscopic resonance cross section and resonance scattering cross section of resonance absorber $h$, respectively, and $\Sigma_{t}\left(u^{\prime}\right)$ total macroscopic cross section of the mixture:

$$
\Sigma_{t}(U)=\Sigma_{i}(0)+\sum_{i}^{h} \Sigma_{R h}(u)
$$

The function $P\left(u-u^{\prime}\right)$ is the Placzek function ${ }^{(2)}$ for the mixture, defined as

$$
P(X)=\sum_{1}^{N} \frac{\Sigma_{S N}(0)}{\Sigma_{t}(0)} G_{N}(X),
$$

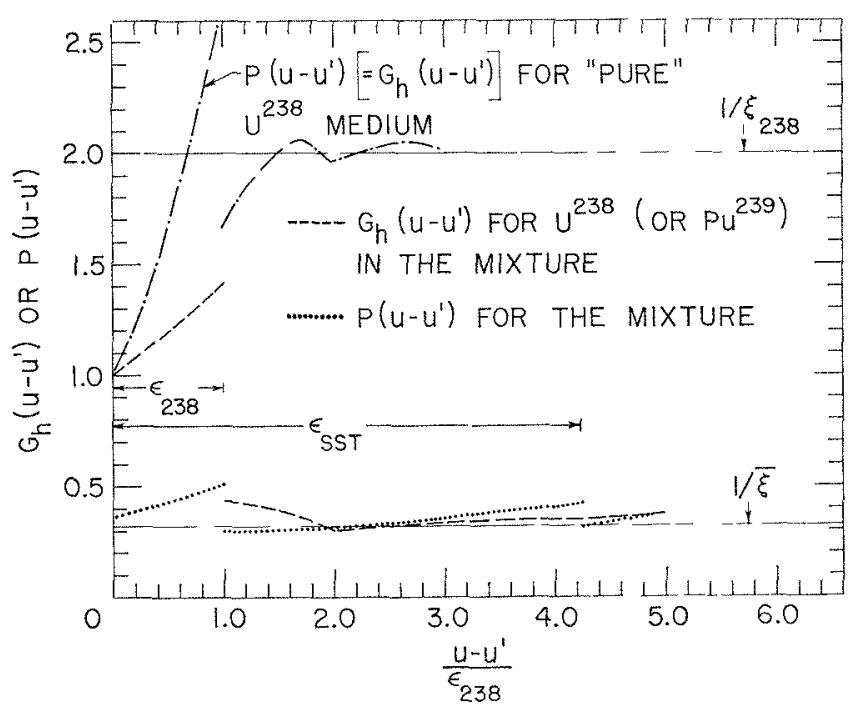

FIG. 1. Variations of Functions $P\left(u-u^{\prime}\right)$ and $G_{h}\left(u-u^{\prime}\right)$. and the Green's function $G_{N}\left(u-u^{\prime}\right)$ introduced by Corngold is the solution of the following equation:

$$
\begin{aligned}
G_{N}(u)= & {\left[\sum_{r=1}^{N} \frac{\Sigma_{S r}(0)}{\Sigma_{t}(0)}\right.} \\
& \left.\cdot \int_{u-\varepsilon_{r}}^{u} d u^{\prime} \frac{e^{u^{\prime}-u}}{1-\alpha_{r}} G_{N}\left(u^{\prime}\right)\right]+S_{N}(u),
\end{aligned}
$$

with

$$
\begin{aligned}
S_{N}(u) & =\frac{e^{-u}}{1-\alpha_{N}} \text { for } 0<u<\varepsilon_{N} \\
& =0 \quad \text { for } \quad u>\varepsilon_{N}^{+} ; \\
\alpha_{N} & =\left(\frac{A_{N}-1}{A_{N}+1}\right)^{2} ; \\
\varepsilon_{N} & =\begin{array}{l}
\text { maximum increment in lethargy per colli- } \\
\text { sion for nuclide } N ;
\end{array} \\
A_{N}= & \text { atomic mass for nuclide } N .
\end{aligned}
$$

Note that neither $G_{N}(X)$ nor $P(X)$ depends on the resonance properties of the nuclides in the mixture. $G_{N}\left(u-u^{\prime}\right)$ can be pictured as the kernel describing neutrons whose first collision is with nuclide $N$, as pointed out by Corngold.

\section{A. Characteristics of the Kernel $G_{h}\left(u-u^{\prime}\right)$ and $P\left(u-u^{\prime}\right)$ in a Typical Fast System}

Consider an infinite homogeneous medium with two heavy absorbers with approximately the same atomic weight, presumably fertile and fissile isotopes, mixed with a number of scatterers. Let the subscripts $h(=1$ and 2$)$ and $m(1,2, \cdots, N-h)$ designate the heavy absorbers and background scatterers, respectively. Note that $\varepsilon_{h}=\varepsilon_{1} \cong \varepsilon_{2}$. It is generally true for a large fast system that $\varepsilon_{m} \gg \varepsilon_{k}$. The smallest $\varepsilon_{m}$ for such system is that of stainless steel, which is more than 4 times larger than $\varepsilon_{h}$. By using the same techniques given by Placzek ${ }^{(2)}$ and directly differentiating Eq. (5), one obtains a first-order inhomogeneous differential equation for $G_{N}(u)$, which has a solution of the following form:

$$
\begin{aligned}
& G_{N}(u)=-e^{(Q-1) u} \int d u^{\prime} \sum_{r=1}^{N} \frac{\alpha_{r}}{1-\alpha_{r}} \frac{\Sigma_{S r}(0)}{\Sigma_{t}(0)} \\
& \cdot G_{N}\left(u^{\prime}-\varepsilon_{r}\right) e^{(Q-1) u}+c e^{(Q-1) u} \text { for } u \geqslant 0,
\end{aligned}
$$

where the constant $c$ is to be determined by appropriate initial and boundary conditions, and

$$
Q=\sum_{1}^{N} \overline{1} \frac{1}{-\alpha_{N}} \frac{\Sigma_{S N}(0)}{\Sigma_{t}(0)}
$$

By stepwise integration, Eq. (6) can be solved quite 
readily at least for the first few intervals. The results are given in Appendix 1.

Figure 1 shows the variations of $G_{h}\left(u-u^{\prime}\right)$ and $P\left(u-u^{\prime}\right)$ normalized to $\left(1-\alpha_{h}\right)$ versus $\left(u-u^{\prime}\right)$ for a system typical of a dilute fast reactor composition given in Table 1. As expected, $P\left(u-u^{\prime}\right)$ is relatively flat and insensitive to $\left(u-u^{\prime}\right)$, due to the competition in neutron scattering among all scatterers in the mixture. On the other hand, $G_{h}\left(u-u^{\prime}\right)$, which is the same for fertile and fissile isotopes with approximately identical $\left(1-\alpha_{h}\right)$ values, is significantly higher than $P\left(u-u^{\prime}\right)$ in the first collision interval of $0 \leqslant u-u^{\prime} \leq$ $\overline{\varepsilon_{h}}$ as a sharp discontinuity occurs at $u-u^{\prime}=\varepsilon_{h}$. It is interesting to note that $G_{h}\left(u-u^{\prime}\right)$ has only one jump at $u-u^{\prime}=\varepsilon_{h}$, whereas the jumps for $P\left(u-u^{\prime}\right)$ occur at all $u-u^{\prime}=\varepsilon_{N}$. The second jump in $P\left(u-u^{\prime}\right)$ which appears in Fig. 1 occurs where $u-u^{\prime}$ is equal to the maximum increment in lethargy per collision of stainless steel. It is quite clear from Fig. 1 that $G_{h}\left(u-u^{\prime}\right)$ and $P\left(u-u^{\prime}\right)$ become almost identical for $u-u^{\prime}>\sim 2 \varepsilon_{k}$ as both funetions rapidly approach their common asymptotic value $1 / \bar{\xi}$. It is not difficult to see from Eq. (1), Eq. (2), and Fig. 1 that there is a sudden rise in $F(u)$ beyond its asymptotic value near a resonance whose neutron width is large.

In the limiting case, if there is only one type of nuclide in the mixture (i.e., $N=h=1), G_{k}\left(u-u^{\prime}\right.$ ) and $P\left(u-u^{\prime}\right)$ become identical as $Q=1 /\left(1-\alpha_{h}\right)$. The dotted line shows $G_{h}\left(u-u^{\prime}\right)$ for a "pure" $\mathrm{U}^{238}$ medium.

\section{B. General Solution}

In any finite lethargy region $u_{1} \leq u^{\prime} \leq u \leq u_{2}$ far away from the source, $K\left(u, u^{\prime}\right)$ is well defined and bounded everywhere in the triangle to the right of the $45^{\circ}$ line as shown in Fig. 2. So long as $K\left(u-u^{\prime}\right)$ hac only a finite number of discontinuities which are regularly distributed, ${ }^{(3,4)} \mathrm{Eq}$. (1) has a unique continuous solution represented by the usual Neumann series:

$$
\begin{gathered}
F(u)=1-\int_{u_{1}}^{u} K\left(u, u^{\prime}\right) d u^{\prime}+\int_{u_{1}}^{u} K\left(u, u^{\prime}\right) d u^{\prime} \\
\cdot \int_{u_{1}}^{u} K\left(u, u^{\prime \prime}\right) d u^{\prime \prime}-\ldots \\
+(-1)^{n} \int_{u_{1}}^{u} K\left(u, u^{\prime}\right) d u^{\prime} \\
\cdots \int_{u_{1}}^{u} K\left(u^{(n-1)} u^{(n)}\right) d u^{(n)}
\end{gathered}
$$

provided that the series converges uniformly in $u_{1} \leq$ $u \leq u_{2}$. Since both $P\left(u-u^{\prime}\right)$ and $G_{h}\left(u-u^{\prime}\right)$ are

\begin{tabular}{|c|c|c|c|c|c|}
\hline & \multicolumn{5}{|c|}{ Material } \\
\hline & $U^{2888}$ & $\mathrm{Pu}^{239}$ & SS & $\mathrm{Na}$ & $\mathrm{O}$ \\
\hline Atoms/ce & 0.00637 & 0.00098 & 0.01355 & $\begin{array}{lll}0 & 01144\end{array}$ & $\begin{array}{lll}0 & 01568\end{array}$ \\
\hline$\sigma_{s}(0), b$ & 10 & 10 & 10 & 3 & 3.5 \\
\hline $1-\alpha_{N}$ & 001667 & 70.01667 & 0.0689 & 0.1597 & 0.2215 \\
\hline
\end{tabular}
bounded, and the reaction rates in the interval $u_{2}-u_{1}$
TABLE 1. ATOM DENSITY FOR THE FAST REACTOR UNDER STIDY

$Q=22.931$

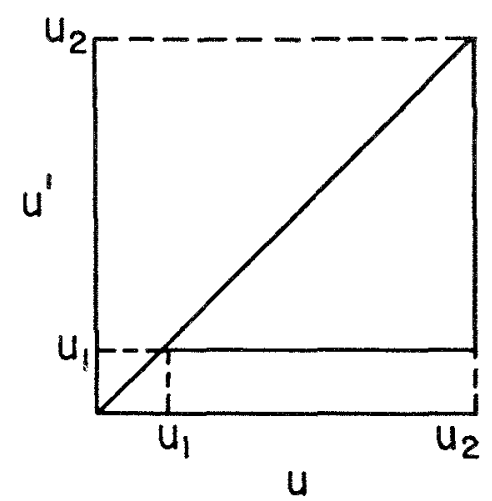

FIG. 2. The Region where $F(u)$ is Defined.

must be finite, it can be shown quite readily that the $n$ th-order term in Eq. (8), $F_{n}(u)$, is convergent for

$$
\left|F_{n}(u)\right|<\frac{M^{n}}{n !} ; \quad \begin{aligned}
& u_{1} \leqslant u \leqslant u_{2} \\
& n=0,1,2, \cdots,
\end{aligned}
$$

where $M$ is a positive and finite constant. Consequently, the series in Eq. (8) converges absolutely and uniformly in the interval $u_{1} \leq u \leq u_{2}$.

Fven though Eq. (8), in general, cannot be described explicitly by a simple analytical function of the closed form, it will lead to the following approximations under rertain conditions:

(1) Wigner's approximation; ${ }^{(1,5,13)}$

(2) Spinney's approximation ${ }^{(6)}$ for narrow and "isolated" resonances;

(3) first-order approximation for the relatively highencrgy region where $F(u)$ can be described adequately by its zero and first-order terms.

Wigner's approximation does not account for the Placzek oscillations characterized by the nonuniform part of the kernel $G_{N}\left(u-u^{\prime}\right)$. Spinney's approximation and the first-order approximation, howeves, are believed to be very useful in the Doppler effect studies for fast reactors and will be further discussed.

\section{Placzek Oscillations due to "Isolated" Res- onances}

In contrast to the Wigner's approximation, the nonasymptotic behaviors of $F(u)$ near a resonance can 
be estimated rigorously if 1 ) the extent of the resonance under consideration is small compared to the maximum lethargy increment per collision and 2) $F(u$ ) recovers between resonances, or, in other words, resonances are considered as "isolated." Under these conditions, the kernel $G_{N}\left(u-u^{\prime}\right)$ in Eq. (8) can be replaced by its value in the first collision interval $0<u-u^{\prime} \leq \varepsilon_{h}$ as given by $\mathrm{Eq}$. (3A) and $\mathrm{Eq}$. (6A). By substituting Eq. (3A) and Eq. (6A) of Appendix 1 into Eq. (8) and changing the order of integration, the collision density over a particular resonance under consideration becomes

$$
\begin{aligned}
f(u)^{\prime}=1 & -\int_{u_{1}}^{u} e^{(Q-1)\left(u-u^{\prime}\right)} \sum_{1}^{N} \frac{g_{N}\left(u^{\prime}\right)}{1-\alpha_{N}} d u^{\prime} \\
& +\int_{u_{1}}^{u} d u^{\prime \prime} e^{(Q-1)\left(u-u^{\prime \prime}\right)} \sum_{1}^{N} \frac{g_{N}\left(u^{\prime \prime}\right)}{1-\alpha_{N}} \\
& \cdot \int_{u^{\prime \prime}}^{u} \sum_{1}^{N} \frac{g_{N}\left(u^{\prime}\right)}{1-\alpha_{N}} d u^{\prime} \cdots \\
=1- & \int_{u_{1}}^{u} e^{(Q-1)\left(u-u^{\prime}\right)} \sum_{0}^{n} \frac{(-1)^{n}}{(n+1) !} \\
& \cdot\left(\sum_{1}^{N} \frac{g_{N}\left(u^{\prime}\right)}{1-\alpha_{n}}\right)\left[\sum_{1}^{N} \int_{u_{\mathrm{I}}}^{u} \frac{g_{\mathrm{V}}\left(u^{\prime \prime}\right)}{1-\alpha_{N}} d u^{\prime \prime}\right]^{n} .
\end{aligned}
$$

Equation (9) can also be obtained by an alternative approach in which the method of the resolvent kemal ${ }^{(4)}$ is used for solving $\mathrm{Eq}$. (1). It is interesting to note that Eq. (9) becomes a simple analytical function of the closed form if $e^{(Q-1)\left(u-u^{*}\right)} \approx 1$. The latter condition is valid so long as the extent of the resonance is much smaller than $\varepsilon_{h}$. Hence, over an isolated narrow resonance, one has

$$
\begin{aligned}
F(u)= & \exp -\left[\int_{u_{1}}^{u} \sum_{1}^{N} \frac{g_{N}\left(u^{\prime}\right)}{1-\alpha_{N}} d u^{\prime}\right] \\
= & \exp \left\{-\int_{u_{1}}^{u}\left[Q \frac{\Sigma_{R h}\left(u^{\prime}\right)}{\Sigma_{t}\left(u^{\prime}\right)}\right.\right. \\
& \left.\left.-\frac{1}{1-\alpha_{h}} \frac{\Sigma_{R S h}\left(u^{\prime}\right)}{\Sigma_{t}\left(u^{\prime}\right)}\right] d u^{\prime}\right\},
\end{aligned}
$$

which is exactly identical with the gencralized form of Spinney's approximation ${ }^{(6)}$ obtained by an entirely different approach.

The "moderator effect" is included in Eq. (10). The presence of heavy scatterers in the mixture tends to reduce the sudden rise in $F(u)$ near the resonance, as clearly indicated in $\mathrm{Eq} .(10)$. Fquation (10) becomes identical with the form given in Ref. 6 if the moderator effect is ignored, i.e.,

$$
Q \approx \frac{1}{1-\alpha_{h}} \frac{\Sigma_{s h}(0)}{\Sigma_{t}(0)} .
$$

Under conditions that Eq. (10) is valid, the resonance integral for an isolated resonance can be writter as

$$
\begin{aligned}
\mathrm{RI}=(\mathrm{RI})_{\mathrm{NR}}\{1 & +\sum_{1}^{m}\left[\left(\frac{\Gamma_{n} / \Gamma}{1-\alpha_{n}}-Q\right)\right. \\
& \left.\left.\cdot \frac{\Gamma}{\sigma_{p} \Gamma_{x}}(\mathrm{RI})_{\mathrm{NR}}\right]^{m} /(m+1) !\right\},
\end{aligned}
$$

where $\Gamma_{n}, \Gamma_{2}$, and $\Gamma$ are neutron width, capture (or fission) width, and total width, respectively, and $(\mathrm{RI})_{\mathrm{NR}}$ is the resonance integral obtained by the narrow-resonance approximation.

Equations (10) and (11) can be used as a powerful tool in determining the effect of Placzek oscillation in the Doppler region where resonances are always narrow.

It is apparent from Eq. (11) that, for an isolated resonance, the NR approximation underestimates or overestimates the resonance integral depending on whether $\Gamma_{n} / \Gamma>$ or $<\left(1-\alpha_{h}\right) Q$. For compositions typical of a large fast reactor with high concentrations of both stainless steel and sodium, $Q$ is of the order of 20 . Hence, $Q\left(1-\alpha_{h}\right)$ is of the order of $1 / 3$. Table 2 gives some representative values of resonance integrals of $\mathrm{U}^{238}$ for the fast system described in Table 1 as obtained by NR approximation, Spinney's approximation, and the exact calculation of MISH-MASH* Code, ${ }^{(7)}$ respectively. The NR approximation based on uniform $F(u)$ appears to be reasonably accurate insofar as the magnitude of $\mathrm{RI}$ is concerned. Errors ranging from about $10 \%$ in $\delta(\mathrm{RI})$ due to temperature were observed for all scattering resonances with large $\Gamma_{n}$, whereas there is virtually no error for resonances with small $\Gamma_{n}$.

Unlike the situation with $\mathrm{U}^{238}$, resonances of $\mathrm{Pu}^{239}$, in general, do not produce drastic perturbation in the localized $F(u)$ due to the relatively small $\Gamma_{n} / \Gamma$ ratio and low concentration. Calculations of the statistically averaged fission and capture rates for $\mathrm{Pu}^{239}$ by means of Fq. (11) have been made. No noticeable effect was observed. In order to illustrate the problem of the Placzek oscillation further, calculations of the example given by Codd and Collins ${ }^{(8)}$ were repeated by using both MISH-MASH Code and an analytical method taking only the zero-order and first-order term in Eq. (8). The results are given in Fig. 3 together with the effect of a $\mathrm{Pu}^{239}$ resonance, obtained by use of the average parameters given in Ref, 8 . The dotted curve shows the case in which the neutron width was increased five times; no significant effect is observed.

\section{Effect of Incomplete Recovery in $F(u)$ be- tween Resonances for a Single Resonant Sub- stance in the Mixture}

\section{A. $U^{238}$ Resonances Alone}

The Placzek oscillations in $F^{*}(u)$ are somewhat different when a group of resonances are considered. The

\footnotetext{
* Homogeneous version of RIFF-RAFF Code (Ref. 7).
} 
Table 2. Representative Values of Resonance Integrals and Their Doppler Change by Varous Methods

\begin{tabular}{|c|c|c|c|c|c|c|c|}
\hline \multirow{2}{*}{$\begin{array}{c}\text { Resonance } \\
\text { Energy, keV }\end{array}$} & \multirow{2}{*}{$\Gamma_{n}$} & \multicolumn{2}{|c|}{ NR Approx. } & \multicolumn{2}{|c|}{ Spinney's Appros. } & \multicolumn{2}{|c|}{ Exact (MISH-MASH) } \\
\hline & & $\underset{500^{\circ} \mathrm{K}}{\mathrm{RI}}$ & $\begin{array}{c}\delta(\mathrm{RI}) \\
1100-500^{\circ} \mathrm{K}\end{array}$ & $\underset{500^{\circ} \mathrm{K}}{\mathrm{RI}}$ & $\begin{array}{c}\delta(\mathrm{RT}) \\
1100-500^{\circ} \mathrm{K}\end{array}$ & $\underset{500^{\circ} \mathrm{K}}{\mathrm{RI}}$ & $\begin{array}{c}\delta(\mathrm{RI}) \\
1100-500^{\circ} \mathrm{K}\end{array}$ \\
\hline 1.802 & 0.67 & 0.006090 & 0.000615 & 0.006281 & 0.0071 & 0.006227 & 0.000707 \\
\hline 1.710 & 0.046 & 0.012965 & 0.001847 & 0.013048 & 0.001873 & 0.013079 & 0.00189 \\
\hline 1. 428 & 0.020 & 0.015390 & 0.001574 & 0.015392 & 0.001598 & 0.015470 & 0.001599 \\
\hline 1.1490 & 0.274 & 0.013318 & 0.002183 & 0.014060 & 0.002442 & 0.013964 & 0.002472 \\
\hline 1.0610 & 0.051 & 0.024092 & 0.004865 & 0.024426 & 0.005014 & 0.02440 & 0.005020 \\
\hline 0.9970 & 035 & 0.015067 & 0.001833 & 0.016290 & 0002149 & 0.016090 & 0.002190 \\
\hline 0.985 & 0.001 & 0.003887 & 0.000490 & 0.003884 & 0.000490 & 0.003910 & 0.00049 \\
\hline 0.7930 & 0.0024 & 0.012540 & 0.000480 & 0.012507 & 0.000477 & 0.012587 & 0.000480 \\
\hline 0.6970 & 0.057 & 0.038274 & 0.00900 & 0.039258 & 0.009518 & 0.039157 & 0.009594 \\
\hline
\end{tabular}

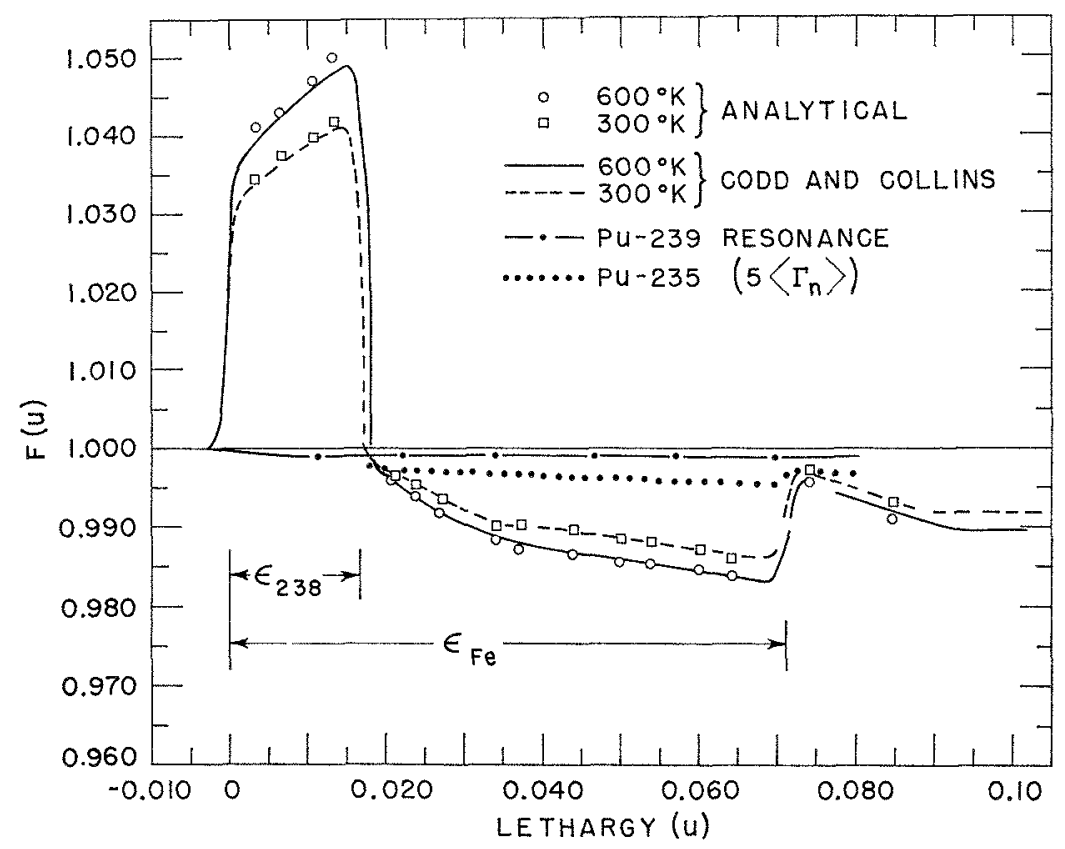

FIG. 3. Codd and Collins Problem.

localized increase in $F(u)$ due to the resonance scattering of a particular resonance may be partially or completely balanced out by the resonance absorptions of resonances at higher energy. Figures 4 through 6 show some interesting results in $F(u)$ due to $\mathrm{U}^{238}$ alone as obtained by MISH-MASH Code in different energy intervals. For energy intervals around or below about $1 \mathrm{keV}$, where the maximum lethargy increment per collision, $\varepsilon_{h}$, is equal to or smaller than the average spacing of $\mathrm{U}^{238}, F(u)$ tends to drift below the asymptotic value as $u$ increases with the exception of few localized rises caused by the resonances with extremely large neutron width. The true group reaction rates in these intervals will be smaller than those obtained by the NR approximation.
On the other hand, for energy intervals beyond about $1.4 \mathrm{keV}$, where $\varepsilon_{b}$ becomes larger than the average spacing between $\mathrm{U}^{238}$ resonances, $F(u)$ fluctuates about the asymptotic value with increasingly smaller amplitude at the higher energy interval. Hence, it is not surprising that the group reaction rates obtained by the NR approximation become nearly identical with those obtained by exact calculations beyond $\sim 1.4 \mathrm{keV}$ as the nonasymptotic contribution due to individual resonances nearly washes out statistically, as indicated in Table 3. Table 3 also gives the effective cross section for each energy interval as obtained by the two methods.

The NR approximation tends to underestimate $\delta \tilde{\sigma}_{\gamma}$ slightly in the last two groups, while it overestimates $\delta \tilde{\sigma}_{\gamma}$ slightly in the higher-energy groups. Errors in both 
the effective cross section and its Doppler change $\delta \tilde{\sigma}_{\gamma}$ appear to be unimportant.

\section{B. $P u^{239}$ Resonances Alone}

Unlike the case for $\mathrm{U}^{238}$ resonances, resonances of fissile isotopes in general exhibit a somewhat different behavior in the Doppler region. Since the absorption of a particular $\mathrm{Pu}^{239}$ resonance is nearly always more significant than the corresponding resonance scattering

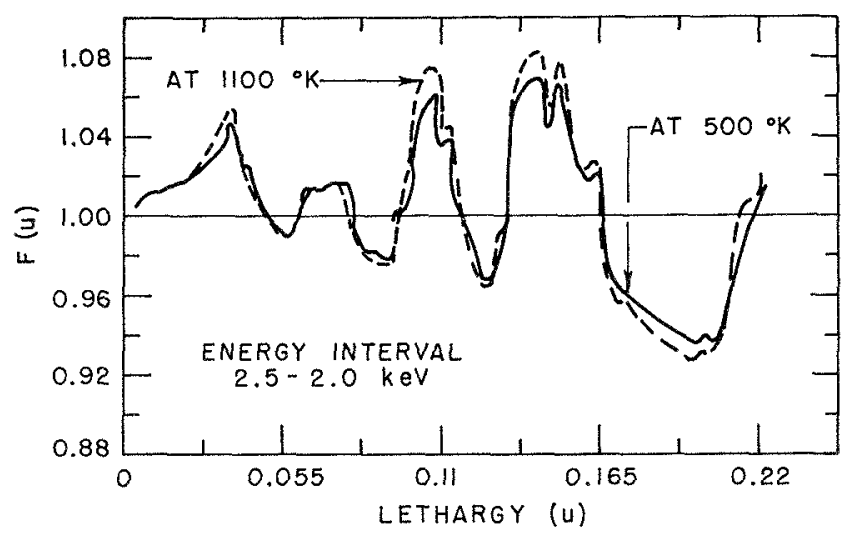

FIQ. 4 . Variation of $F^{\prime}(u)$ between 2.5 and $2 \mathrm{keV}\left(\mathbf{C}^{238}\right.$ Resonances Only).

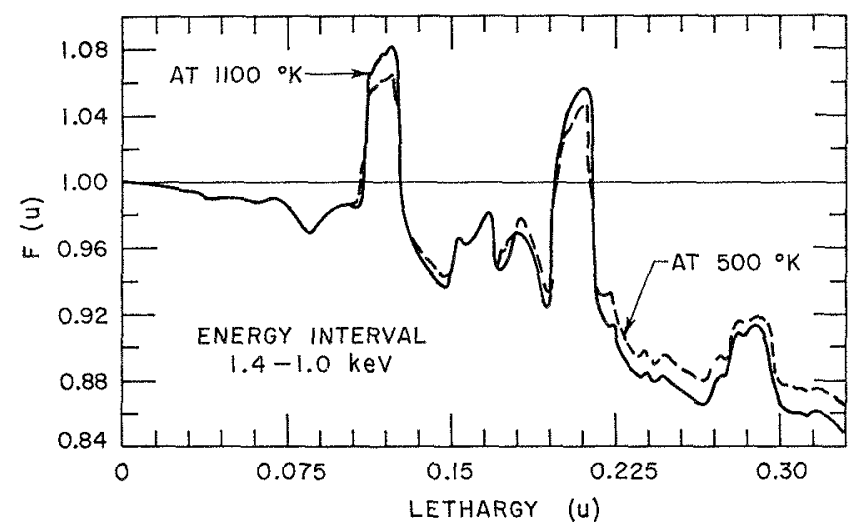

Fig. 5. Variations of $F^{\prime}(u)$ between 1.4 and $1.0 \mathrm{keV}$ (U238 Resonances Only).

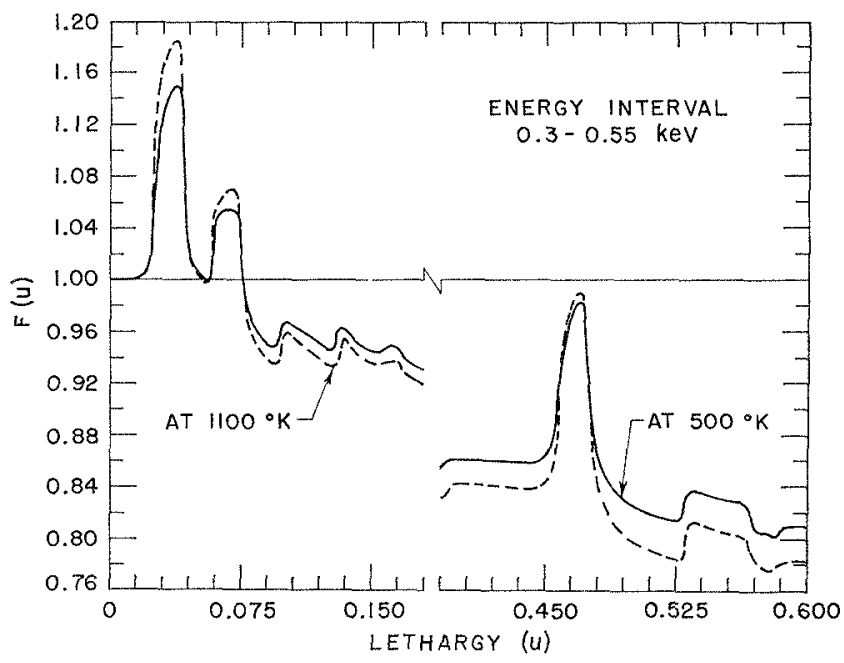

$F_{t G}, 6$. Variation of $F(u)$ between 0.55 and $0.3 \mathrm{kel}^{r}\left(\mathrm{U}^{238}\right.$ Resonances Only).

on the statistical basis, as indicated by the results of calculation using $\mathrm{Eq}$. (11), the localized collision density is practically unaffected by the existence of a particular $\mathrm{Pu}^{239}$ resonance. Instead, $F(u)$ beyond the first collision interval $\mathcal{E}_{2}$ from a given resonance will be depleted due to resonance absorption. By referring to Eq. (2) and Fig. 3 , it is quite clear that

$$
\begin{aligned}
K\left(u, u^{\prime}\right) & =P\left(u-u^{\prime}\right) \frac{\Sigma_{a 2}\left(u^{\prime}\right)}{\Sigma_{t}\left(u^{\prime}\right)}+\frac{\Sigma_{R S 2}\left(u^{\prime}\right)}{\Sigma_{t}\left(u^{\prime}\right)} \\
& \cdot\left[P\left(u-u^{\prime}\right)-G_{2}\left(u-u^{\prime}\right)\right] \\
\approx & P\left(u-u^{\prime}\right) \frac{\Sigma_{a 2}\left(u^{\prime}\right)}{\Sigma_{t}\left(u^{\prime}\right)} \\
& \approx \frac{1}{\bar{\xi}} \frac{\Sigma_{a 2}\left(u^{\prime}\right)}{\Sigma_{t}\left(u^{\prime}\right)}
\end{aligned}
$$

as long as $\Sigma_{a 2}\left(u^{\prime}\right)>>>\Sigma_{R S_{2}}\left(u^{\prime}\right)$. The substitution of Fq. (12) into Eq. (8) gives the well-known Wigner's approximation $^{(1,5)}$

\begin{tabular}{|c|c|c|c|c|c|c|c|c|}
\hline \multirow{2}{*}{ Energy Interval, $\mathrm{keV}$} & \multicolumn{4}{|c|}{ NR Approximation } & \multicolumn{4}{|c|}{$\begin{array}{c}\text { Exact Calculation } \\
\text { (MISH-MASH CODE) }\end{array}$} \\
\hline & $\tilde{R}_{\gamma}$ at $500^{\circ} \mathrm{K}$ & $\begin{array}{l}\delta \tilde{R}_{\gamma} 700- \\
1100^{\circ} \mathrm{K}\end{array}$ & $\tilde{\sigma}$ at $500^{\circ} \mathrm{K}$ & $\begin{array}{l}\delta \tilde{\sigma}_{\gamma} 500- \\
1100^{\circ} \mathrm{K}\end{array}$ & $R_{\gamma}$ at $500^{\circ} \mathrm{K}$ & $\begin{array}{l}\delta \tilde{R}_{\gamma} 500- \\
1100^{\circ} \mathrm{K}\end{array}$ & $\tilde{\sigma}_{\gamma}$ at $500^{\circ} \mathrm{K}$ & $\begin{array}{l}\delta \tilde{\sigma}_{\gamma} 500- \\
1100^{\circ} \mathrm{K}\end{array}$ \\
\hline $2.5-2.0$ & 0.71161 & 0.07873 & 0.7501 & 0.09012 & 0.70269 & 0.07818 & 0.0749 & 0.08995 \\
\hline $2.0-1.4$ & 0.73080 & 0.1093 & 0.7998 & 0.13035 & 0.74450 & 0.10787 & 0.8086 & 0.12856 \\
\hline $1.4-1.0$ & 0.9895 & 0.1240 & 1.0513 & 0.14275 & 0.97695 & 0.10928 & 1.0885 & 0.13723 \\
\hline $1.0-0.55$ & 0.96510 & 0.1638 & 1.0454 & 0.19659 & 0.91621 & 0.14081 & 1.0753 & 0.20206 \\
\hline $0.55-0.30$ & 0.75019 & 0.11250 & 0.7782 & 0.12290 & 0.74683 & 0.10465 & 0.8641 & 0.14335 \\
\hline
\end{tabular}

$$
F(u)=\exp \left[-\frac{1}{\xi} \int_{u_{1}}^{u} \frac{\Sigma_{a 2}\left(u^{\prime}\right)}{\Sigma_{t}\left(u^{\prime}\right)} d u^{\prime}\right]
$$

Table 3. Efefective Cross sections and Group Reaction Rates for Resolved U238 Resonances*

* Fink's ${ }^{(1)}$ parameters were used beyond $1802 \mathrm{eV}$ and Columbia Parameters ${ }^{(12)}$ were used for higher energies 
Equation (13) is a relatively smooth function of $u$ within a reasonably small lethargy interval $u_{2}-u_{1}$. As far as the effective group cross section is concerned, the NR approximation is believed to be quite good in the sense that the depletion in the reaction rates due to resonance absorption within the group nearly cancels out the corresponding depletion in the group flux when Fq. (13) is used. To illustrate this point further, calculations have been made with use of the preliminary values of $\mathrm{Pu}^{239}$ parameters by Uttley ${ }^{(9)}$ between 100 $\mathrm{eV}$ and $300 \mathrm{cV}$ and by MISH-MLASH Code with results given in Fig. 7 and Table 4 . It is interesting to note that $F(u)$ given in Fig. 7 can be approximated fairly accurately by use of Eq. (13).

\section{First-order Approximations and Group Reac- tion Rates for Overlapping Resonances in the Doppler Region}

For convenience, let

$$
F(u)=1+T(u),
$$

where $T^{\prime}(u)$ represents the nonuniform contributions to $F(u)$ given by the higher-order terms in Eq. (8). Since Eq. (8) is a convergent series within each finite lethargy group considered in multigroup calculations, all higher-order terms can be pictured as $n$ th-order perturbations caused by resonances to the otherwise uniform $F(u)$ of zero order. In particular, for energy region beyond $\sim 300 \mathrm{eV}$ and reasonably small group width, the first-order term is believed to be the only significant contributor to $T(u)$ as other higher-order terms are expected to become negligible. For an infinite homogeneous medium consisting of two resonant isotopes and any number of backgroup scatterers, the first-order approximation gives

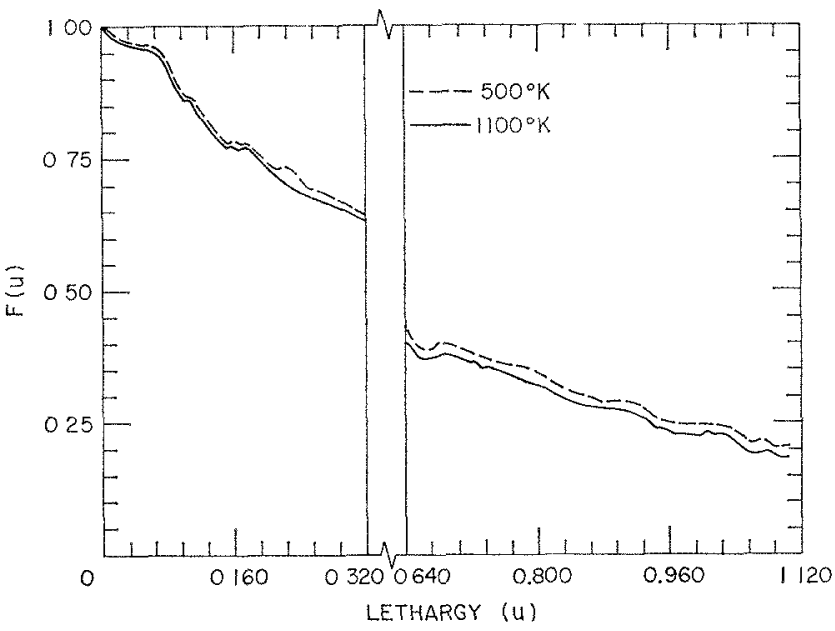

FIG. 7. Variation of $F(u)$ between 0.3 and $0.1 \mathrm{keV}\left(\mathrm{Pu}^{293}\right.$ Resonances Only).
TABLE 4. Efrective Cross Sectioss for Resolyeu $\mathrm{Pu}^{239}$ Resonances $(300-100 \mathrm{eV})$

\begin{tabular}{|c|c|c|c|c|}
\hline $\begin{array}{c}\text { Energy } \\
\text { Interval, eV }\end{array}$ & $\tilde{\sigma}_{f}$ at $500^{\circ} \mathrm{K}$ & $\begin{array}{l}\delta \tilde{\sigma}_{f} \text { at } 1100 \\
-500^{\circ} \mathrm{K}\end{array}$ & $\tilde{\sigma}_{\gamma}$ at $500^{\circ} \mathrm{K}$ & $\begin{array}{c}\delta \tilde{\sigma}_{\gamma} \text { at } 1100 \\
-500^{\circ} \mathrm{K}\end{array}$ \\
\hline \multicolumn{5}{|c|}{ a) NR Approximation. } \\
\hline $300-100$ & 14.2846 & 0.7461 & 9.3887 & 0.8059 \\
\hline $300-200$ & 12.2455 & 0.6186 & 9.5169 & 0.7314 \\
\hline
\end{tabular}

b) Exact Calculation (MISH-MAsH)

\begin{tabular}{l|l|l|l|l}
\hline 300100 & 13.9882 & 06457 & 93530 & 0.7432 \\
$300-200$ & 13.0891 & 0.6438 & 9.6364 & 0.7452 \\
\hline
\end{tabular}

$$
\begin{aligned}
T(u) \cong-\sum_{1}^{2} \int_{u_{1}}^{u} d u & {\left[P\left(u-u^{\prime}\right) \frac{\Sigma_{R h}\left(u^{\prime}\right)}{\Sigma_{t}\left(u^{\prime}\right)}\right.} \\
& \left.-G_{h}\left(u-u^{\prime}\right) \frac{\Sigma_{R S h}\left(u^{\prime}\right)}{\Sigma_{t}\left(u^{\prime}\right)}\right] \\
= & T_{1}^{(1)}(u)+T_{2}^{(1)}(u)+C_{12}(u),
\end{aligned}
$$

where $T^{(1)}(u)$ is the first-order perturbation caused by the resonances of nuclide 1 alone as if the resonances of nuclide 2 did not exist, while $T_{2}^{(1)}(u)$ is the first-order perturbation due to resonances of nuclide 2 alone. $C_{12}(u)$ is a second-order "interfering" term which is composed of second-order terms such as

$$
\int_{u_{1}}^{u} \frac{\Sigma_{R 1}\left(u^{\prime}\right) \cdot \Sigma_{R S 2}\left(u^{\prime}\right)}{\left.\Sigma_{t}(0)+\Sigma_{R 1}\left(u^{\prime}\right)\right] \Sigma_{t}\left(u^{\prime}\right)} d u^{\prime} \text {, ete. }
$$

Under the condition that the fluctuations caused by the fertile and the fissile isotopes individually are adequately described by their first-order terms $T_{1}^{(1)}(u)$ and $T_{2}^{(1)}(u)$, respectively, the quantity $C_{12}$ will be ignored for reasonably small group width. $T_{1}^{(1)}(u)+$ $T_{2}^{(1)}(u) \gg C_{32}$ is believed to be true for all cases considered in this work.

\section{A. Effective Cross Sectron and Group Reaction Rate}

The effective cross section of process $x$ for a particular nuclide $h$ within energy interval $E_{1}-E_{2}$ (corresponding to the lethargy interval $u_{2}-u_{1}$ ) is defined as

$$
\tilde{\sigma}_{2 h}=\frac{\int_{u_{1}}^{u_{2}} d u \frac{\sigma_{x h}(u) F(u)}{\Sigma_{t}(u)}}{\int_{u_{1}}^{u_{2}} d u \frac{F(u)}{\Sigma_{t}(u)}}=\frac{\tilde{R}_{x h}}{f^{*}},
$$

where $\tilde{R}_{x h}$ is the group reaction rate of process $x$ for nuclide $h$ weighted by the asymptotic group flux $\left(u_{2}-u_{1}\right) / \sigma_{P h}$ and $f^{*}$ is referred to as the flux correction fartor. Here

$$
\sigma_{P h}=\frac{\Sigma_{i}(0)}{N_{h}}
$$

and $N_{h}$ is atom density of nuclide $h$. 
Assume that the separation between any resonance $k$ of nuclide 2 at $E_{k}$ with respect to a particular resonance $i$ of nuclide 1 at $E_{\imath}$ is described by a random distribution since there is no correlation between resoonances of 1 and 2 . Using the identity

$$
\begin{aligned}
\frac{\sigma_{x 2}(u)}{\Sigma_{t}(u)} & =\frac{\sigma_{x 2}(u)}{\Sigma_{t}(0)+\Sigma_{R 2}(u)} \\
& -\left[\frac{\sigma_{x 2}(u) \Sigma_{R 1}(u)}{\left[\Sigma_{t}(0)+\Sigma_{R 2}(u)\right]\left[\Sigma_{t}(0)+\Sigma_{R 1}(u)\right]} \cdots\right]
\end{aligned}
$$

and substituting Eq. (15) into Eq. (16), one has for unresolved resonances

$$
\begin{aligned}
& \tilde{R}_{x 2} \cong \sum_{\substack{\text { sppn } \\
\text { states }}}\left\{\frac{\sigma_{P 2}}{\left\langle S_{2}\right\rangle}\left\langle\int_{\Delta E} d E \frac{\sigma_{x 2}(E)}{\Sigma_{t}(0)+\Sigma_{R 2}(E)}\right\rangle\right. \\
& {\left[1-\frac{\left\langle\int_{\Delta E} \frac{d E \Sigma_{R 1}(E)}{\Sigma_{t}(0)+\Sigma_{R 1}(E)}\right\rangle}{\left\langle S_{1}\right\rangle}\right.} \\
& \left.+\frac{E_{2}+E_{1}^{r}}{2\left\langle S_{1}\right\rangle}\left\langle\int_{\Delta u} d u T_{1}^{(1)}(u)\right\rangle\right] \\
& +\frac{\sigma_{P 2}\left(E_{2}+E_{1}\right)}{2\left\langle S_{2}\right\rangle} \\
& \text { - } \left.\left\langle\int_{\Delta u} \frac{\sigma_{x 2}(u) T_{2}^{(1)}(u)}{\Sigma_{t}(0)+\Sigma_{R 2}(u)} d u\right\rangle\right\}
\end{aligned}
$$

where \langle\rangle is the standard symbol for the statistical averaging over distribution functions of resonance width and, if necessary, over spacings as well. Since $\left(u_{2}-u_{1}\right) \cong 2\left(E_{1}-E_{2}\right) /\left(E_{1}+E_{2}\right)$ for small group width under consideration, the symbol $\left(E_{1}+E_{2}\right) /$ $(2\langle s\rangle) \cdot\langle\rangle$ can be replaced by

$$
\frac{1}{u_{2}-u_{1}} \int_{u_{1}}^{u_{2}}
$$

wherever the resolved resonances are considered. Eq. (18) is identical with the usual overlap approximation based on constant $F(u)$ if $T_{1}^{(1)}(u)$ and $T_{2}^{(1)}(u)$ are ignored.

The reaction rate for $U^{238}, \widetilde{R}_{\gamma 1}$, can be obtained by interchanging the subscripts 1 and 2 in Eq. (18). Similarly, the flux correction factor $f^{*}$ can be obtained by the same procedure:

$$
\begin{aligned}
f^{*} \cong f+\frac{1}{u_{2}-u_{1}} \sum_{1}^{2} & {\left[\int_{\Delta u} d u T_{h}^{(1)}(u)\right.} \\
& \left.+\int_{\Delta u} d u \frac{\Sigma_{R h}(u) T_{h}^{(1)}(u)}{\Sigma_{t}(0)+\Sigma_{R h}(u)}\right],
\end{aligned}
$$

where $f$, the flux correction factor when $F(u)$ is taken to constant, is defined in Ref. 10.

The last term in Eq. (18) and Eq. (19) is, in general, rather difficult to evaluate unless the resonances are resolved. For resolved $U^{238}$ resonances, this quantity is just the difference between the reaction rates obtained by the NR approximation and the exact calculation using MIISH-MASH Code when $\mathrm{Pu}^{239}$ resonances were ignored. From the values given in Table 3 , it is believed that the quantity

$$
\frac{\sigma_{P 1}}{u_{2}-u_{1}} \int_{u_{1}}^{u_{2}} \frac{\Sigma_{\gamma 1}(u) T_{1}^{(1)}(u) d u}{\Sigma_{t}(0)+\Sigma_{R 1}(u)}
$$

is negligible compared to the zero-order term in $\mathrm{Eq}$. (18) and $\mathrm{Eq}$. (19) in the high-energy region beyond about $1.4 \mathrm{keV}$. For $\mathrm{Pu}^{239}$, the latter quantity can be estimated only on the basis of its strong absorbing characteristics and of Eq. (13) discussed in Section IV. Since $T_{2}^{(1)}(u)$ is essentially lethargy independent in the interval where a given resonance is considered, the quantity

$$
\left\langle\int \frac{\Sigma_{r_{2}}(u) T_{2}^{(1)}(u)}{\Sigma_{t}(0)+\Sigma_{R 2}(u)} d u\right\rangle
$$

can be approximated by

$$
\begin{aligned}
& \left\langle\int \frac{\Sigma_{x 2}(u) T_{2}^{(1)}(u)}{\Sigma_{t}(0)+\Sigma_{R 2}(u)} d u\right\rangle \\
& \cong \bar{T}_{2}^{(1)}(u) \\
& \left\langle\int \frac{\Sigma_{x 2}(u) d u}{\Sigma_{t}(0)+\Sigma_{R 2}(u)}\right\rangle,
\end{aligned}
$$

where $\overline{T_{2}^{(1)}(u)}$ is taken to be the average neutron loss within a given group, $u_{2}-u_{1}$ containing many resonances, i.e.,

$$
\begin{aligned}
\overline{T_{2}^{(1)}(u)} & \cong \frac{1}{2 \bar{\xi}} \int_{u_{1}}^{u_{2}} \frac{\Sigma_{\gamma 2}(u)+\Sigma_{f 2}(u)}{\Sigma_{t}(0)+\Sigma_{R 2}(u)} d u \\
& =\frac{E_{1}-E_{2}}{2 \bar{\xi} E_{0}} \frac{\left\langle\left(\Gamma_{f}+\Gamma_{\gamma}\right) J_{2}\right\rangle}{\langle S\rangle_{2}} .
\end{aligned}
$$

Here $E_{0}$ is the midpoint between $E_{1}$ and $E_{2}$, and $J_{2}$ is the usual $J$ function for nuclide 2 . It is interesting to note that the quantity

$$
\frac{\sigma_{P h}\left(E_{1}-E_{2}\right)}{2\left\langle S_{h}\right\rangle}\left\langle\int \frac{\sigma_{x h}(u) T_{h}^{(1)}(u) d u}{\Sigma_{i}(0)+\Sigma_{R h}(u)}\right\rangle
$$

for both $\mathrm{U}^{238}$ and $\mathrm{Pu}^{239}$ becomes negligible in the higherenergy region beyond about $1.4 \mathrm{keV}$, so that the effective cross section can be approximated by

$$
\tilde{\Sigma}_{x h} \cong \frac{\sum \frac{\sigma_{P h}}{\left\langle S_{h}\right\rangle}\left\langle\int_{\Delta E} d E \frac{\Sigma_{x h}(E)}{\Sigma_{t}(0)+\Sigma_{R h}(E)}\right\rangle}{1-\frac{1}{\left\langle S_{h h}\right\rangle}\left\langle\int_{\Delta E} \frac{\Sigma_{R h}(E) d E}{\Sigma_{t}(0)+\Sigma_{R h}(E)}\right\rangle}+.
$$

The quantity $\left\langle\int_{\Delta u} d u T_{k}^{(1)}(u)\right\rangle$ represents an additional overlap correction term, and this quantity may become of the same order of magnitude as the usual overlap term in the NR approximation, ${ }^{(8,10)}$ especially in the energy region below about $1.4 \mathrm{keV}$. It will be shown that 
$\left\langle\int d u T_{h}^{(1)}(u)\right\rangle$ is related to $J$ functions of the NR approximation in a rather simple way.

\section{B. Evaluation of $\left\langle\int_{\Delta u} d u T_{1}^{(1)}(u) d u\right\rangle$}

From the discussions given in Section II, it is clear that

$$
G_{N}\left(u-u^{\prime}\right) \cong 1 / \bar{\xi}
$$

for all $2 \varepsilon_{n}^{+} \leqslant u-u^{\prime}$. Using Eq. (23), and Eqs. (3A), $(4 \mathrm{~A}),(6 \mathrm{~A})$, and $(7 \mathrm{~A})$ of Appendix $\mathrm{I}$, and changing the order of integration, one has Eq. (24) (see foot of page). These constants depend only on the relative concentrations and the scattering properties characterized by

TABLE 5. $1 /\left(u_{2}-u_{1}\right) \int d u T_{1}(u)$ FOR U238 IN THE Resolved and UnResolved Region

\begin{tabular}{c|c|c|c|c}
\hline \multirow{2}{*}{$\begin{array}{c}\text { Energy } \\
\text { Interval, keV }\end{array}$} & $\begin{array}{c}\text { Exact Calculation* } \\
\text { (MISH-MASH) }\end{array}$ & $\begin{array}{c}\text { First-order Approximation } \\
\mathrm{Eq}-\mathrm{X}\end{array}$ \\
\cline { 3 - 5 } & $500^{\circ} \mathrm{K}$ & $1100^{\circ} \mathrm{K}$ & $500^{\circ} \mathrm{K}$ & $1100^{\circ} \mathrm{K}$ \\
\hline $1.5-9.0$ & Unresolved Region & +0.01307 & +0.01520 \\
$9.0-6.0$ & & $\begin{array}{l}+0.01266 \\
+0.0001885\end{array}$ & +0.002384 \\
$6.0-4.0$ & & & -0.00097 & +0.00121 \\
$4.0-2.5$ & & & +0.01034 & +0.01373 \\
$2.5-2.0$ & & & \\
& & & & \\
$2.0-1.4$ & +0.0036 & +0.0049 & +0.0041 & +0.0046 \\
$1.4-1.0$ & -0.0550 & -0.0584 & -0.0540 & -0.0582 \\
$1.0-0.55$ & -0.0769 & -0.0889 & -0.0840 & -0.0962 \\
$0.55-0.30$ & -0.1018 & -0.1147 & -0.1023 & -0.1175 \\
\hline
\end{tabular}

* Firk's parameters. atomic weights of each nuclide in the mixture. All integrals in $\mathrm{Eq}$. (2) can be obtained quite readily from any resonance integral code using the NR approximation. For unresolved resonances, Eq. (24) becomes

$$
\begin{aligned}
& \frac{1}{u_{2}-u_{1}} \int_{u_{1}}^{u_{2}} T_{h}^{(1)}(u) d u \\
& \cong \frac{-1}{\left\langle S_{h}\right\rangle}\left\{\left\langle\left(Q \Gamma_{h}-\frac{\Gamma_{n h}}{1-\alpha_{n}}\right) J_{h}\right\rangle\left(A_{1}+A_{2}\right)\right. \\
& \quad-A_{3}\left\langle\left(\frac{\Sigma_{S h}(0)}{\Sigma_{i}(0)} \Gamma_{h}-\frac{\Gamma_{n h}}{1-\alpha_{n}}\right) J_{h}\right\rangle \\
& \left.\quad+\ln \frac{\alpha_{n}^{2} E_{k}}{E_{2}}\left\langle\left(\Gamma_{f h}+\Gamma_{\gamma h}\right) J_{h}\right\rangle\right\},
\end{aligned}
$$

where $u_{2}-u_{1}>>>\varepsilon_{k}$ and $J_{h}$ is the usual $J$ function for nuclide $h$ defined in the Ref. 13 .

Table 5 shows some results of exact calculations of

$$
\frac{1}{u_{2}-u_{1}} \int_{u_{1}}^{u_{2}} d u T_{1}^{(1)}(u)
$$

in the resolved region of $\mathrm{U}^{238}$ using MISH-MASH Code as compared to the approximation using Eq. (24), together with calculations for unresolved region using Eq. (25). Excellent agreement between the exact calculation and the approximation implies that $T_{1}(u)$ is adequately described by its first-order term $T_{1}^{(1)}(u)$. It is interesting to note that

$$
\frac{1}{u_{2}-u_{1}} \int_{u_{1}}^{u_{2}} d u T_{1}^{(1)}(u)
$$

becomes small beyond about $1.4 \mathrm{keV}$ as the effect of

$$
\begin{aligned}
\frac{1}{u_{2}-u_{1}} \int_{u_{1}}^{u_{2}} T_{h}^{(1)}(u) d u= & -\frac{1}{u_{2}-u_{1}}\left\{A_{1} \int_{u_{1}}^{u_{2}} \frac{\left[Q \Sigma_{R h}\left(u^{\prime}\right)-\frac{1}{1-\alpha_{h}} \Sigma_{R S h}\left(u^{\prime}\right)\right]}{\Sigma_{t}(0)+\Sigma_{R h}\left(u^{\prime}\right)} d u^{\prime}\right. \\
& +A_{2} \int_{u_{1}}^{u_{2}-\varepsilon_{h}} d u^{\prime} \frac{\left[Q \Sigma_{R h}\left(u^{\prime}\right)-\frac{1}{1-\alpha_{h}} \Sigma_{R S h}\left(u^{\prime}\right)\right]}{\Sigma_{t}(0)+\Sigma_{R h}} \\
& -A_{3} \int_{u_{1}}^{u_{2}-\varepsilon_{h}} d u^{\prime} \frac{\left[\left(\sum_{1}^{2} \frac{\Sigma_{S h}(0)}{\Sigma_{t}(0)}\right) \Sigma_{R h}\left(u^{\prime}\right)-\frac{1}{1-\alpha_{h}} \Sigma_{R S h}\left(u^{\prime}\right)\right]}{\Sigma_{i}(0)+\Sigma_{R h}\left(u^{\prime}\right)} \\
& \left.+\ln \frac{\alpha_{h}^{2} L_{k}^{\prime}}{E_{2}^{\prime}} \int_{u_{1}}^{u_{2}-2 \varepsilon_{h}} \frac{\Sigma_{a h}\left(u^{\prime}\right)}{\Sigma_{t}(0)+\Sigma_{R h}\left(u^{\prime}\right)} d u^{\prime}\right\},
\end{aligned}
$$

where

$$
\begin{aligned}
& A_{1}=\frac{e^{(Q 1) \varepsilon_{h}}-1}{Q-1} ; \\
& A_{2}=\frac{\alpha_{h}\left(\alpha_{h} e^{Q \varepsilon_{h}}-1\right)}{Q-1}\left[e^{Q \varepsilon_{h}}+\frac{\left(\sum_{1}^{2} \Sigma_{S h}(0) / \Sigma_{t}(0)\right)}{(Q-1)\left(1-\alpha_{h}\right)}\right]-\frac{\alpha_{h}^{2} \varepsilon_{h}\left(\sum_{1}^{2} \frac{\Sigma_{S h}(0)}{\Sigma_{i}(0)}\right) e^{Q \varepsilon_{h}}}{(Q-1)\left(1-\alpha_{h}\right)} \\
& A_{3}=\frac{\alpha_{h}\left(\alpha_{h} e^{Q \varepsilon_{h}}-1\right)}{\left(1-\alpha_{h}\right)(Q-1)} ; \\
& E_{k}=\text { resonance energy for the } k^{\text {th }} \text { resonance within } u_{2}-u_{1} .
\end{aligned}
$$


the fluctuations in $F(u)$ introduced by resonances of nuclide 1 on nuclide 2 nearly washes out statistically. For energy regions below $1.4 \mathrm{keV}$, however, the latter quantity is about the same order of magnitude as the overlap correction term obtained by NR approximation.

\section{Results of Calculations and Discussions}

The quantity $\int T_{h}^{(1)}(u) d u$ in Eq. (18) represents an additional overlap correction term when the reaction rates of $\mathrm{U}^{238}$ and $\mathrm{Pu}^{239}$ are considered. The presence of this quantity together with the last terms in Eq. (18) will affect the relative magnitudes of the reaction rates in the system. The effect of these additional terms on the total Doppler effect of the system and on the effective cross sections of individual isotopes will be discussed separately in order as follows:

For fast reactors which have a spectrum soft enough to produce a large Doppler effect to be significant for safety, the overall Doppler effect of the system is expected to depend almost entirely on the relative reaction rates of fissile and fertile jsotopes, as discussed in References 10 and 14. Hence, the significance of the nonuniform terms in $F(u)$ depends on whether their presence will affect the ratios $\hat{R}_{\gamma 2} / \hat{R}_{f 2}$ and $\hat{R}_{\gamma 1} / \hat{R}_{f 2}$ at various temperatures. Note that

$$
\frac{\tilde{\sigma}_{\gamma 2}}{\tilde{\sigma}_{f 2}}=\frac{\tilde{R}_{\gamma 2}}{\tilde{R}_{f 2}}=\frac{\int \frac{\Sigma_{\gamma 2}(E)}{\Sigma_{t}(0)+\Sigma_{R 2}(E)} d E^{\prime}}{\int \frac{\Sigma_{f 2}(E)}{\Sigma_{t}(0)+\Sigma_{R 2}(E)} d E}
$$

is affected neither by the presence of nonuniform terms when Eq. (18) and Eq. (20) are used, nor by the correction terms due to interaction between different nuclides when the usual NR approximation is used.

The question is then whether $R_{\gamma 1} / R_{f 2}$ will be affected.
Table 6 shows the quantities $\nu \tilde{\Sigma}_{j 2} /\left(\tilde{\Sigma}_{f 2}+\tilde{\Sigma}_{\gamma 2}+\tilde{\Sigma}_{\gamma 1}\right)$ at $500^{\circ} \mathrm{K}$, and the Doppler change

$$
\delta\left[\nu \tilde{\Sigma}_{f 2} /\left(\tilde{\Sigma}_{f 2}+\tilde{\Sigma}_{\gamma 2}+\tilde{\Sigma}_{\gamma 1}\right)\right]
$$

between 1100 and $500^{\circ} \mathrm{K}$ obtained by various methods at various energy intervals. The NR approximation with overlap correction seems to overestimate the Doppler change slightly at high energy intervals, and underestimate values slightly below about $1 \mathrm{keV}$, as compared to the case where the nonuniform contributions are included. Nevertheless, the results indicate that NR approximation is fairly accurate for practical purpose.

Table 7 gives the effective cross section and the change due to temperature obtained by two different methods in which resonance interactions were taken into account. The magnitudes of $\tilde{\sigma}_{x}$ appears to be almost unaffected by the presences of the nonuniform terms in the collision density, as expected, whereas discrepancies of a few percent in $\delta \tilde{\sigma}_{x}$ were observed for both $\mathrm{U}^{238}$ and $\mathrm{Pu}^{239}$. Since the discrepancies in $\delta \tilde{\sigma}_{\gamma 1}, \delta \tilde{\sigma}_{\gamma 2}$, and $\delta \tilde{\sigma}_{f 2}$ are small and oriented in the same direction, the result also indicates that the NR approximation is fairly accurate for practical purpose when the total Doppler effect of the system is considered.

It is important to realize, however, that all results given in Tables 6 and 7 were based on the assumption that the separation between any particular resonance of $\mathrm{Pu}^{239}$ with respect to a given resonance of $\mathrm{U}^{238}$ has a random distribution. In reality, the reaction rates of $\mathrm{Pu}^{239}$ depend to a great extent on the actual location of each $\mathrm{Pu}^{239}$ resonance with respect to $\mathrm{U}^{238}$ resonances, as indicated by the recent work of Kelber and Kier. ${ }^{(15)}$ Hence, the random distribution may not give an accurate estimation of $\widetilde{R}_{\gamma 2} / \widetilde{R}_{f 2}$ and $\widetilde{R}_{\gamma 1} / \tilde{R}_{f 2}$. As far as the total Doppler effect of a particular group is concerned,

\begin{tabular}{|c|c|c|c|c|c|c|}
\hline \multirow[b]{2}{*}{$\underset{\mathrm{keV}}{\text { Energy }}$} & \multicolumn{2}{|c|}{$\begin{array}{l}\text { NR Approximation } \\
\text { (no interaction) }\end{array}$} & \multicolumn{2}{|c|}{$\begin{array}{l}\text { RN Approximation } \\
\text { (with interaction) }\end{array}$} & \multicolumn{2}{|c|}{$\begin{array}{l}\text { First-order Approximation } \\
\text { [interaction and fluctuation in } F(u)]\end{array}$} \\
\hline & $\frac{\nu \tilde{\Sigma}_{f 2}}{\tilde{\Sigma}_{f 2}+\tilde{\Sigma}_{\gamma 2}+\tilde{\Sigma}_{\gamma 1}}$ & $\delta\left(\begin{array}{c}\frac{\nu \tilde{\Sigma}_{f 2}}{\tilde{\Sigma}_{f 2}+\tilde{\Sigma}_{\gamma^{2}}+\tilde{\Sigma}_{\gamma 1}} \\
500-1100^{\circ} \mathrm{K}\end{array}\right)$ & $\begin{array}{c}\frac{\nu \tilde{\Sigma}_{f^{2}}}{\tilde{\Sigma}_{f^{2}}+\tilde{\Sigma}_{\gamma^{2}}+\tilde{\Sigma}_{\gamma 1}} \\
\text { at } 500^{\circ} \mathrm{K}\end{array}$ & $\delta\left(\frac{\nu \tilde{\Sigma}_{f 2}}{\tilde{\Sigma}_{f 2}+\tilde{\Sigma}_{\gamma^{2}}+\tilde{\Sigma}_{\gamma 1}}\right)$ & $\begin{array}{c}\frac{\nu \tilde{\Sigma}_{f 2}}{\tilde{\Sigma}_{f 2}+\tilde{\Sigma}_{\gamma^{2}}+\tilde{\Sigma}_{\gamma 1}} \\
\text { at } 500^{\circ} \mathrm{K}\end{array}$ & $\left(\begin{array}{c}\left(\frac{\nu \tilde{\Sigma}_{f 2}}{\tilde{\Sigma}_{f 2}+\tilde{\Sigma}_{\gamma 2}+\tilde{\Sigma}_{\gamma 1}}\right) \\
500-1100^{\circ} \mathrm{K}\end{array}\right.$ \\
\hline $\begin{array}{c}15-9.0 \\
9.0-4.0 \\
9.0-6.0 \\
6.0-4.0 \\
4.0-2.8 \\
2.8-2.0 \\
2.0-1.4 \\
1.4-1.0 \\
1.0-0.55 \\
0.55-0.30\end{array}$ & $\begin{array}{l}0.9909 \\
0.9722 \\
0.9750 \\
0.9676 \\
0.9719 \\
0.9971 \\
1.0360 \\
0.9824 \\
1.0875 \\
1.2915\end{array}$ & $\begin{array}{l}-0.0198 \\
-0.0288 \\
-0.0267 \\
-0.0334 \\
-0.0394 \\
-0.0445 \\
-0.0545 \\
-0.0428 \\
-0.0548 \\
-0.0363\end{array}$ & $\begin{array}{l}0.9671 \\
0.9463 \\
0.9495 \\
0.9414 \\
0.9460 \\
0.9725 \\
1.0097 \\
0.9714 \\
1.0718 \\
1.2977\end{array}$ & $\begin{array}{l}-0.0222 \\
-0.0325 \\
-0.0300 \\
-0.0377 \\
-0.0446 \\
-0.0538 \\
-0.0598 \\
-0.0466 \\
-0.0612 \\
-0.0375\end{array}$ & $\begin{array}{l}0.9748 \\
0.9337 \\
0.9569 \\
0.9434 \\
0.9477 \\
0.9819 \\
1.0169 \\
0.9570 \\
1.0733 \\
1.2818\end{array}$ & $\begin{array}{l}-0.0212 \\
-0.0339 \\
-0.0286 \\
-0.0367 \\
-0.0437 \\
-0.0490 \\
-0.0603 \\
-0.0436 \\
-0.0630 \\
-0.0420\end{array}$ \\
\hline
\end{tabular}

TABLE $6 . \quad \eta$ and $\delta \eta$ at Various Energy Intervals by Different Methods 
TABLF 7. EFzective Cross Sections $\left(500-1100^{\circ} \mathrm{K}\right)$

\begin{tabular}{|c|c|c|c|c|c|c|c|c|}
\hline Energy Interval (keV) & \multicolumn{7}{|c|}{ a) $\mathrm{Pu}^{23 \theta^{*}}$} & dation) \\
\hline $15-9.0$ & 1.2007 & 0.00355 & 0.6573 & 0.00189 & 1.2017 & 0.003277 & 0.6579 & 0.001735 \\
\hline $9.0-4.0$ & 1.8040 & 0.00978 & 1.0147 & 0.00542 & 1.8279 & 0.008452 & 1.0282 & 0004672 \\
\hline $4.0-2.8$ & 2.7187 & 0.02719 & 1.5722 & 0.01586 & 2.7231 & 0.024493 & 1.5748 & 0.013401 \\
\hline $2.8-2.4$ & 3.4864 & 0.05056 & 2.0504 & 0.03047 & 3.4980 & 0.045594 & 2.0572 & 0.027557 \\
\hline $2.0-1.4$ & 4.1013 & 0.07580 & 2.4382 & 0.04676 & 4.1077 & 0.0703348 & 2.4420 & 0043518 \\
\hline $1.4-1.0$ & 4.9777 & 0.12184 & 2.9964 & 0.07741 & 5.0190 & 0.1016004 & 3.0213 & 0065246 \\
\hline $1.0-0.55$ & 6.2564 & 0.21209 & 3.8180 & 0.13987 & 6.3552 & 0.1867786 & 3.8783 & 0.124549 \\
\hline $0.55-0.30$ & 8.3160 & 0.41194 & 5.1450 & 0.28603 & 90783 & 0.4487196 & 5.6160 & 0311630 \\
\hline
\end{tabular}

$$
\text { b) } \mathrm{U} 238^{* * *}
$$

\begin{tabular}{|c|c|c|c|c|}
\hline \multirow{2}{*}{ Ënergy Interval (keV) } & \multicolumn{2}{|c|}{ NR Approximation (no fluctuation) } & \multicolumn{2}{|c|}{ First Order Approximation (with fluctuation) } \\
\hline & $\tilde{\sigma}_{\gamma}$ at $500^{\circ} \mathrm{K}$ & $\delta \tilde{\sigma}_{\gamma}$ & $\tilde{\sigma}_{\gamma}$ at $500^{\circ} \mathrm{K}$ & $\hat{\delta} \tilde{\sigma}_{\gamma}$ \\
\hline $15-9.0$ & 0.2661 & 0.01384 & 0.2619 & 0.12944 \\
\hline $9.0-4.0$ & 0.4139 & 0.03265 & 0.4310 & 0.03498 \\
\hline $9.0-6.0$ & 0.3752 & 0.02702 & 0.3693 & 0.02528 \\
\hline $6.0-4.0$ & 0.4931 & 0.04583 & 0.4917 & 0.04421 \\
\hline $4.0-2.8$ & 0.6176 & 0.07008 & 0.6162 & 0.06793 \\
\hline $2.8-2.0$ & 0.7420 & 0.10535 & 0.7291 & 0.09366 \\
\hline $2.0-1.4$ & 0.7998 & 0.13035 & 0.7882 & 0.12846 \\
\hline $1.4-1.0$ & 1.0513 & 0.14275 & 1.0947 & 013511 \\
\hline $1.0-0.55$ & 1.0454 & 0.19659 & 1.0580 & 0.19848 \\
\hline 0.550 .30 & 0.7782 & 0.12290 & 0.8881 & 0.15072 \\
\hline
\end{tabular}

${ }^{*} y=1$ dist ribution for neutron width and $\nu=2$ for fission width; $\left\langle\Gamma_{f}\right\rangle=0.099 \mathrm{el} ;$ strength functions $=10^{-4}$.

** Fink et al. parameters below $1.8 \mathrm{keV}$.

$\nu=1 ;$ strength function $=10^{-4}$ for unresolved resonances.

however, the NR approximation and the assumption of random separation probably will not lead to significant errors in the energy group beyond about $1.4 \mathrm{keV}$ for the following reasons:

1. The localized variation in the collision density becomes relatively iusensitive to the exact location of a particular resonance in the high-energy region, where the maximum energy loss per collision becomes large compared to the average spacing of each heavy nuclide.

2. $\mathrm{U}^{238}$ is the principal contributor to the total Doppler effect of the group. The relative location of a particular $\mathrm{Pu}^{239}$ resonance with respect to a $\mathrm{U}^{238}$ resonance is not likely to affect the ratio $\tilde{\Sigma}_{\gamma 1} / \tilde{\Sigma}_{f 2}$ eignificantly.

3. The presence of many resonances within a given group provides better statistics.

On the other hand, for lower-energy groups in which $\mathrm{Pu}^{239}$ resonances are significant, the error introduced by the usual NR approximation may become much greater than those inferred by Tables 6 and 7 . table 8. Calculations of Capture-to-fission Piatios Using Resolved Parameters for BOTH $\mathrm{Pu}^{239}$ AND U238

\begin{tabular}{|c|c|c|c|c|}
\hline & \multicolumn{2}{|c|}{ NR Approximation } & \multicolumn{2}{|c|}{ Exact Calculation } \\
\hline & $\begin{array}{l}\text { Without } \\
\text { Inter- } \\
\text { action }\end{array}$ & $\begin{array}{l}\text { With } \\
\text { Inter- } \\
\text { action }\end{array}$ & $\begin{array}{l}\text { Without } \\
\text { Inter- } \\
\text { action }\end{array}$ & $\begin{array}{l}\text { With } \\
\text { Inter- } \\
\text { action }\end{array}$ \\
\hline $\begin{array}{l}\tilde{\Sigma}_{\gamma 2} / \tilde{\Sigma}_{f 2} \text { at } 500^{\circ} \mathrm{K} \\
\delta\left(\tilde{\Sigma}_{\gamma 2} / \tilde{\Sigma}_{j 2}\right), 500-1100^{\circ} \mathrm{K}\end{array}$ & $\begin{array}{l}0.6572 \\
0.0209\end{array}$ & $\begin{array}{l}0.6572 \\
0.0209\end{array}$ & $\begin{array}{l}0.6686 \\
0.0212\end{array}$ & $\begin{array}{l}0.6939 \\
0.0286\end{array}$ \\
\hline
\end{tabular}

Table 8 shows some results of NR approximation and the exact calculations using resolved parameters $^{(9,12)}$ for both $\mathrm{Pu}^{239}$ and $\mathrm{U}^{238}$ between 300 and 100 $\mathrm{eV}$. The results also indicate that, contrary to Eq. (26), the $\alpha$-value $\tilde{\Sigma}_{\gamma 2} / \tilde{\Sigma}_{f 2}$ and its Doppler change are affected by the resonance interaction. For this reason, the statistical treatment may not be adequate in the low-energy groups when the resonance interaction is considered. 


\section{Conclusion}

In conclusion, the results of this study can be summarized as follows:

\section{On the Resonance Integral of Individual Resonances}

In the energy region of interest for Doppler studies of a large fast reactor, the effect of Placzek oscillation, which ean be estimated accurately by Spinney's approximation, depends strongly on the relative magnitude of the neutron width and absorption width of the resonance. For $\mathrm{U}^{238}$ the $\mathrm{NR}$ approximation tends to underestimate the Doppler change in the resonance integral by about $10 \%$ for cases where $\Gamma_{n}>>>\Gamma_{\gamma}$, although it introduces practically no error for cases where $\Gamma_{n}$ is comparable or less than $\Gamma_{\gamma}$. For $\mathrm{Pu}^{239}$ resonances the NR approximation is believed to be accurate.

2. On the Effective Cross Section of a Single Resonant Absorber in the Mixture

For $\mathrm{U}^{238}$ small discrepancies in $\tilde{\sigma}_{\gamma}$ and $\delta \tilde{\sigma}_{\gamma}$ obtained by the NR approximation and the exact calculation were observed in the low-energy groups. Neither the effective cross section nor the group reaction rate will be affected beyond about $1.4 \mathrm{keV}$, for the collision density begins to oscillate about its asymptotic value. For $\mathrm{Pu}^{239}$, the effective cross section is nearly unaffected by the depletion in the collision density within the group so long as the group width is reasonably small. The NR approximation is believed to be accurate.

\section{On the Total Doppler Effect when Resonance Inter- actions are Considered}

The NR approximation appears to overestimate the Doppler effect slightly in all high-energy groups beyond about $1 \mathrm{keV}$, whereas it underestimates the Doppler effect in the low-energy groups. In general, the NR approximation is believed to be fairly accurate for practical purposes as far as the overall Doppler effect of a large fast reactor is concerned. The statistical treatment used in this work, however, may introduce noticeable errors in the lower-energy groups for which the Doppler effect within a given group depends to a great extent on the actual locations of $\mathrm{Pu}^{239}$ resonances with respect to given $\mathrm{U}^{238}$ resonances.

\section{Acknowledgment}

The author is indebted to Dr. P. H. Kier for providing the MISH-MASH Code prior to its publication. Discussions with Dr. H. H. Hummel have been very helpful on this subject.

\section{REFERENCES}

1. N. Corngold, Slowing Down of Neutrons in Infinite Homogenous Media, Proc. Phys. Soc. (London), A70 (1957).

2. G. Placzek, On the Theory of the Slowing Down of Neutrons in Heavy Substances, Phys. Rev. 69 (1946).

3. G. C. Evans, Volterra's Integral Equation of the Second Kind with Discontinuous Kernel, Trans. Amer. Math. Soc., 1, 393-413; 12, 429-472 (1911).

4. F. Smithies, Integral Equations, Cambridge University Press, (1958).

5. E. P. Wigner, E. Creutz, H. Jupnik, and T. Snyder, J. Appl. Phys. 26, 260 (1955).

6. K. T. Spinney, Resonance Absorption in Homogeneous Mixture, BNL-433 (1956).

7. P. H. Kier, RIFF-RAFF, A Program for Computation of Resonance Integral in a Two-region Cell, ANL-7033.

8. J. Codd and P. Collins, Proc. Conf. Breeding Economics and Safety in Large Fast Reactors, ANL-6792 (1963), pp. 711-726.

9. C. A. Vttley, Physics Division Progress Report, AERE$\operatorname{Pr} / \mathrm{NPS}$ (1964).

10. R. N. Hwang, Doppler Effect Calculations with Interference Corrections, Nucl, Sci. Eng. 21, 4, 523 (1965).

11. F. W. Fink, J. E. Lynn, and M. C. Moxon, Nucl. Phys. 41, $614(1963)$.

12. J. B. Garg, J. Rainwater, J. S. Petersen, and W. W. Havens, Jr., Neutron Resonance Spectroscopy III. Th-232 and $U$-298, Phys. Rev. 134 (1964).

13. L. I)resner, Resonance Absorption in Nuclear Reactors, Pergamon Press (1960).

14. H. H. Hummel and R. N. Hwang, Effect of Resonance Correction to Group Flux in Fast Reactor Doppler Effect Calculation, Nuel. Sci. Eng. 23, 98 (1965).

15. C. N. Kelber and P. H. Kier, On Group Cross Sections of Fissile Nuclides in the Unresolved Energy Range, Tran. Am. Nucl. Soc. (Washington Meeting).

\section{Appendix I}

The constant $C$ in Eq. (6) can be determined by the initial condition

$$
G_{N}\left(0^{+}\right)=\frac{1}{1-\alpha_{N^{*}}}
$$

and the boundary condition

$$
G_{N}\left(n \varepsilon_{n}^{+}\right)=\lim _{\delta \rightarrow 0} G_{N}\left(n \varepsilon_{N}+\delta\right),
$$

where $G_{N}(X)$ is defined by Eq. (5) and (6). By stepwise integrations, one has
(1) for heavy absorbers of approximately the same atomic weight,

$$
\begin{gathered}
G_{h}(X)=\frac{e^{(Q-1) X}}{1-\alpha_{h}} \text { when } 0 \leqslant X \leqslant \overline{\varepsilon_{h}} ; \\
=\frac{e^{(Q-1) X}}{1-\alpha_{h}}\left[1-\left(1-\frac{\varepsilon_{h}-X}{1-\alpha_{h}}\right.\right. \\
\left.\left.\cdot \sum_{i}^{2} \frac{\Sigma_{s h}(0)}{\Sigma_{t}(0)}\right) e^{--Q \varepsilon_{h}}\right] \text { when } \\
\varepsilon_{h}^{+} \leq X \leq 2 \varepsilon_{h}^{-}
\end{gathered}
$$




$$
\begin{aligned}
& =\frac{e^{(Q-1) X}}{1-\alpha_{h}}\left[1-e^{-Q \varepsilon_{h}} \frac{\varepsilon_{h} e^{-Q \varepsilon_{h}}}{1-\alpha_{h}} \sum_{1}^{2} \frac{\Sigma_{s h}(0)}{\Sigma_{t}(0)}\right] \\
& \quad-\frac{\alpha_{h} e^{(Q-1)\left(X-\varepsilon_{h}\right)}}{\left(1-\alpha_{h}\right)^{2}} \cdot\left(\sum_{1}^{2} \frac{\Sigma_{s h}(0)}{\Sigma_{t}(0)}\right) \\
& \cdot\left[( X - 2 \varepsilon _ { h } ) \left(1-e^{-Q \varepsilon_{h}}\right.\right. \\
& \left.+\frac{\varepsilon_{h} e^{Q \varepsilon_{h}}}{1-\alpha_{h}} \sum_{1}^{2} \frac{\Sigma_{s}(0)}{\Sigma_{t}(0)}\right) \\
& -\frac{\left(X-\varepsilon_{h}\right)^{2}-\varepsilon_{h}^{2}}{2\left(1-\alpha_{h}\right)} \\
& \left.\cdot\left(\sum_{1}^{2} \frac{\Sigma_{s h}(0)}{\Sigma_{t}(0)}\right) e^{-Q \varepsilon_{h}}\right] \text { when } 2 \varepsilon_{h}^{+} \\
& \leqslant X \leqslant 3 \varepsilon_{h}^{-} ;
\end{aligned}
$$

etc.

(2) for moderators in the mixture,

$$
\begin{aligned}
& G_{m}(X)= \frac{e^{(Q-1) X}}{1-\alpha_{m}} \text { when } 0 \leqslant X \leqslant \overline{\varepsilon_{h}} ; \\
&=\frac{e^{(Q-1) X}}{1-\alpha_{m}}\left[1+\frac{\varepsilon_{h}-X}{1-\alpha_{h}}\right. \\
&\left.\cdot\left(\sum_{1}^{2} \frac{\Sigma_{s h}(0)}{\Sigma_{t}(0)}\right) e^{-Q \varepsilon_{h}}\right] \text { when } \\
& \cdot \varepsilon_{h}^{+} \leqslant X \leqslant 2 \varepsilon_{h}^{-} ; \\
&= \frac{e^{(Q-1) X}}{1-\alpha_{m}}\left[1-\frac{\varepsilon_{h} e^{-Q \varepsilon_{h}}}{1-\alpha_{h}}\right. \\
&\left.\cdot\left(\sum_{1}^{2} \frac{\Sigma_{s h}(0)}{\Sigma_{b}(0)}\right)\right] \\
&-\left(\frac{\alpha_{h}}{1-\alpha_{h}} \sum_{1}^{2} \frac{\Sigma_{s h}(0)}{\Sigma_{t}(0)}\right) \\
& \cdot \frac{e^{(Q-1)\left(X-\varepsilon_{h}\right)}}{1-\alpha_{m}}\left[\left(X-2 \varepsilon_{h}\right)\right. \\
&\left.\cdot\left(1+\frac{\varepsilon_{h} e^{-Q \varepsilon_{h}}}{1-\alpha_{h}} \sum_{i}^{2} \frac{\Sigma_{s h}(0)}{\Sigma_{t}(0)}\right)\right] \\
&-\frac{\left(X-\varepsilon_{h}\right)^{2}-\varepsilon_{h}^{2}}{2\left(1-\alpha_{h}\right)}
\end{aligned}
$$

$$
\begin{aligned}
& \cdot\left(\sum_{1}^{2} \frac{\Sigma_{s h}(0)}{\Sigma_{i}(0)}\right) e^{-a \varepsilon_{h}} \text { when } \\
& 2 \varepsilon_{h}^{+}<X<3 \varepsilon_{h}^{-} .
\end{aligned}
$$

\section{Discussion}

Mr. Chernick (BNL): I think you showed that at low energies it does make a difference just what the resonances are. At the energies of interest to fast reactors, that is, in the koV range, many resonances are smeared together into big blocks and lumps. What do you hope to do to resolve this into real resonances and to try to get at the real effect of interactions?

$M r$. Hwang: We need more parameters to solve that problem. In my opinion, we should not push our argument too far down as regards energy, that is, below 1 $\mathrm{keV}$.

Mr. Chernick: It is a little work, but could you get a set of random resonances that will be reasonable and will still fit what has been seen on, say, the BNL crosssection curves?

Mr. Hwang: This is a problem at which Dr. Kelber and Dr. Kier at Argonne are looking. I think I will pass that question to Dr. Kelber.

Mr. Kelber (ANL): We have attempted to verify a conjecture of Brissenden's which has been referred to earlier this morning, namely, that one can compute the effects of the unresolved resonances by simply sampling from the distribution and producing a set of parameters which obey all the known statistical laws. We have not attempted to do what Brissenden has suggested, that is, use forced sampling in those regions where we have broad resolution measurements. What we have directed our attention to is the variance in the results obtained by use of a number of different samples. I think it would be premature at this time to draw any broad conclusions. We have been able to show that the variance observed for a particular example is large, but for many cases of practical calculation is tolerable. This is the best we can do with the present structure. If this problem is to be resolved further, we have to introduce such schemes as forced sampling to take into account broad resolution measurements. This should remarkably reduce the variance because these measurements are in general available in just the regions of highest statistical worth. 


\title{
Comparison of Monte Carlo and Resonance Integral Methods in the Determination of Doppler Effects in Fast Reactors
}

\author{
J. F. Olhoeft, H. E. Kirug \\ Westinghouse Electric Corporation \\ A tome Power Division \\ Pitksurgh, Pennsylvania \\ AND \\ R. N. HWancr \\ Argonne National Laboratory \\ Argonne, Illinois
}

(PRESHNTEL) BY J. E. OLHOEFT)

\section{Introduction}

This paper describes a comparison of the calculations by the Monte Carlo method and resonance integral techniques, of Doppler effect in fast reactors. These comparisons have been based on a common set of ground rules which have been selected to minimize the effect of uncertainties in nonfuel cross sections. In this way discrepancies in results can be attributed to the ditferences in the treatments of the Doppler-broadened ross sections and the slowing-down models.

With the Monte Carlo method the physical behavior of neutrons in a reactor is simulated on a digital computer. Neutrons can be introduced into the reactor system, distributed in energy according to the fission spectrum, and followed in spatial propagation and in energy degradation as they experience scattering collisions with various nuclei until absorption occurs. The reactor geometry can be duplicated to a high degree of detail. The collection of statistical data as the neutrons slow down can provide information of capture rates, fission rates, scattering rates, flux distribution and spectrum; also values of derivatives of absorption rates with respect to some parameter (i.e., temperature) can be determined. In principle, Monte Carlo can be as accurate as the theoretiral models describing nicroscopic interactions between neutrons and nuclei. The resonances of the light elements as well as heavy elements could be included in a Monte Carlo algorithm. In practice, because of computer size linitations and economics of running times, the resultant Monte Carlo model usually represents a departure from a true physical mockup while retaining the features important to the application under consideration. Furthermore, like physical experiments, Monte Carlo "experiments" have stat istiral uncertainties inherent in the results due to the probabilistic nature of the calculation.

Resonance integral theory has been the standard method of calculating the Doppler coefficient in fast reactors. ${ }^{(1-10)}$ Because of the rapidly varying flux spectrum over the energy range important to the Doppler roefficient, the gross slowing-down problem is generally treated by multigroup techniques, using Doppler-broadened, self-shiclded cross sections obtained from the resonance-integral calculations. The purpose of this paper is to make a comparison between this method and Nonte Carlo.

\section{Monte Carlo Method}

The Monte Carlo calculations were performed with the REPTTITIOUS 3 code, which simulates the neutron slowing down and the cell geometry exactly. The original Monte Carlo code REP developed by Richtmyer et al..$^{(11-13)}$ permits only a single fertile resonance absorber, generally U-238. In order to study resonance interference effects between isotopes, this code was extended, as REPHTITIOUS 3, to include a second resonance isotope which may have resonance characteristics of a fissile material, for example, $\mathrm{Pu}-239$.

In the case of the fertile resonances, the REP treatment is retained intact; the Doppler-broadened cross sections are based on the Breit-Wigner single-level formula and are calculated for each neutron energy as needed in the Monte Carlo chains from tabulations of the Doppler-broadening functions $\psi$ and $\chi$. Above 1 $\mathrm{keV}$, parameters for the unresolved resonances are taken as the proper average except for $\Gamma_{n}^{0}$, which is selected from the Porter-Thomas distribution.

Due to storage limitations in the computer core and 
the neressity of preserving reasonable execution time, certain approximations are made in regards to the second absorber. These relate to the explicit representation of the Doppler-broadened resolved and unresolved resonances for a fissile isotope in a Monte Carlo calculation. For the fissile absorber it is assumed that the Doppler-broadened cross sections may be represented by a sum of Gaussian-shaped pseudo-broadened functions each of which has the correct peak and integral value for the resonance it represents (details are given in Appendix A). Thus, the resonance parameters are broadened rather than the line-shape function. This parameter broadening is done for both resolved and statistically determined unresolved resonances. For the unresolved resonances, sets of resonance parameters and level spacings are selected from appropriate chisquared distributions and then broadened. In order to obtain statistical averaging, a new set is selected by REPETITIOUS 3 after every $n$ histories ( $n$ is arbitrary).

For a given neutron energy in the Monte Carlo slowing-down process, two neighboring fertule resonances and generally 20 neighboring unresolved fissile resonances are considered in determining the respective (ross sections. Below $53 \mathrm{eV}$ all resolved fissile resonances are included. In this way interference effects between resonances of the two different species and the overlap of the same species are taken into account. Further details of the REPETITIOUS 3 code are described in Appendix A.

\section{Resonance Integral-Multigroup Method}

In order to determine the Doppler effect on the same basis as in the Monte Carlo method, the resonanceintegral technique is coupled with multigroup analysis.

The details of the resonance-integral method used in this study have been reported by Hwang ${ }^{(14,15)}$ and will only be summarized here. The narrow-resonance approximation is used to determine the effective cross section of a given process exhibiting resonance phenomena. The total collision density is assumed to be constant in energy over a narrow bandwidth. The total macroscopic cross section in this instance includes the effective background scattering cross section and the total resonance cross section of each isotope present. In all cases the Breit-Wigner single-level formula is assumed and the Doppler broadening is accounted for by the usual $\psi$ function. Interference between potential and resonance scattering is neglected; however, interference or overlap between resonances of the same or different species is included as discussed in the following paragraph.

As a result of the analyses reported in references 14 and 15 , the effective cross section for a resonance absorber in a fast reactor spectrum can be described as the effective cross section determined by the isolated resonance approximation minus a correction term. The correction term embodies the effects of selfoverlap of resonances of the same species and interference interactions of resonances of different species. This correction term can be evaluated by series expansion. For neutron energies below about $25 \mathrm{keV}$ the resonances of the fertile isotope are assumed isolated, since its average separation is, in general, greater than its Doppler width. Therefore, the interference of only the nearest fertile resonance upon a set of fissile resonances is concidered. However, self-overlap of many fissile resonances is included. The treatment for the unresolved resonances is performed by statistical averaging over the appropriate $\chi^{2}$ distribution.

The Doppler effect on reaction rates is determined by employing the temperature-dependent effective cross sections of the resonance absorbers in calculations based on multigroup diffusion theory. The changes in multiplication constant and changes in reaction rates with temperature are observed. The lattice cell is simulated by homogenization of materials and cross sections; heterogeneity effects are accounted for by modification of the background seattering cross sections appearing in the resonance-integral calculations. Separate multigroup calculations are performed for each temperature, rather than employing perturbation theory, in order to also account for changes in multigroup flux spectra with temperature.

\section{Geometry, Composition, and Cross-section Data}

The physical model selected for this study is an infinite array of semi-infinite, cylindrical fuel rods in a hexagonal lattice cooled by sodium. Figure 1 represents a typical cell. The fuel pellet, $15 \%$ enriched $\mathrm{U}^{238}-\mathrm{Pu}^{239}$ monocarbide, is sodium bonded to the stainless steel cladding. The geometry of the cell is given in Table 1,

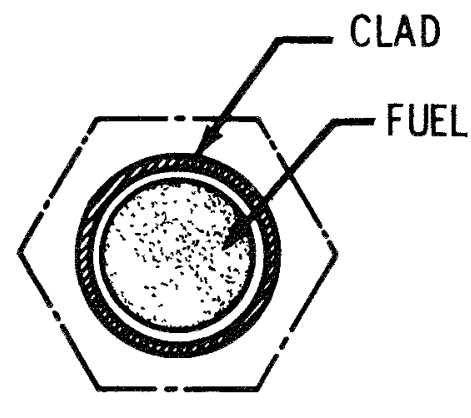

Frg. 1. Typical Cell.

The unshaded areas represent sodium lattice. 
'TABLe 1. La itice Cela Geometry

\begin{tabular}{|c|c|}
\hline $\begin{array}{l}\text { Fuel Pellet } \\
\text { Cladding }\end{array}$ & $\begin{array}{l}0.268 \text {-in. OD } ; 0.3403-\mathrm{cm} \text { radius } \\
\begin{cases}0.280-\mathrm{in} . \mathrm{II}) ; 0.3558-\mathrm{cm} \text { radius } \\
0.300-\mathrm{in} . \text { OD } ; 0.3810-\mathrm{cm} \text { radius. }\end{cases} \end{array}$ \\
\hline $\begin{array}{l}\text { Hexagonal Piteh } \\
\text { Cell Area }\end{array}$ & $\begin{array}{ll}0.426 \mathrm{in} . & 1.0820-\mathrm{cm} \text { pitch } \\
& 1.0139 \mathrm{~cm}^{2}\end{array}$ \\
\hline
\end{tabular}

TABLE 2. Composition

\begin{tabular}{l|l|l|l}
\hline \multirow{2}{*}{ Nuclide } & $\begin{array}{l}\text { Volume } \\
\text { Fraction }\end{array}$ & \multicolumn{2}{|c}{ Number Density, atoms/b-cm } \\
\cline { 3 - 4 } & & In Lattice & $\begin{array}{c}\text { Homogenized } \\
\text { Over Cell }\end{array}$ \\
\hline $\mathrm{U}^{238}$ & 0.35883 & 0.02499 & 0.0089675 \\
$\mathrm{Pu}$ & 0.35883 & 0.00441 & 0.0015825 \\
$\mathrm{C}$ & 0.35883 & 0.02940 & 0.01055 \\
$\mathrm{Na}$ & 0.58364 & 0.02160 & 0.01261 \\
$\mathrm{SS} \mathrm{304}$ & 0.05753 & 0.08520 & 0.00490 \\
\hline
\end{tabular}

* SS 304 composition: $10.4 \mathrm{a} / \mathrm{O} \mathrm{Ni}, 19.7 \mathrm{a} / \mathrm{o} \mathrm{Cr}, 69.9 \mathrm{a} / \mathrm{o} \mathrm{Fe}$.

the composition in Table 2, and the cross-section data in Table 3.

$U^{238}$

Resolved resonances: tabulation in Appendix B.

Unresolved resonances: $1.0-30 \mathrm{keV} ; l=0$ neutrons only.

$g=1.0$

$\bar{D}=18.5 \mathrm{eV}$

$\bar{S}_{0}=0.95 \times 10^{-4} \mathrm{eV}^{-1 / 2}$;

$\bar{\Gamma}_{\gamma}=24.6 \mathrm{mV}$ (constant);

$\bar{\Gamma}_{n}^{0}=1.76 \times 10^{-3} \mathrm{eV}^{1 / 2}$ (Porter-Thomas distribution).

$P u^{239}$

I. Resonance Treatment

Resolved resonances: tabulation in Appendix B.

Unresolved resonances: $53 \mathrm{oV}$ to $5.5 \mathrm{keV} ; l=0$ neutrons only.

$\vec{g}=0.5$;

$\bar{D}=2.9 \mathrm{eV}(\nu=8)$;

$\bar{S}_{0}=1.2 \times 10^{-4}$;

$\bar{\Gamma}_{\gamma}=37.51 \mathrm{mV}$ (constant);

$\bar{\Gamma}_{f}=100.92 \mathrm{mV}(\nu=1)$;

$\widetilde{\Gamma}_{n}^{0}=0.70 \times 10^{-3} \mathrm{eV}^{1 / 2} \quad$ (Porter-Thomas distribution)

Above unresolved resonances: $5.5-30 \mathrm{keV}$;

$\sigma_{f}=2.5 \mathrm{~b}$ (constant); $\quad \alpha=0.45$;

$\sigma_{a}=3.625 \mathrm{~b}$ (constant).

II. " $1 / \mathrm{v}$ " treatment

$\sigma_{f}=222 \mathrm{~b}$ at $1 \mathrm{eV}$;

$\sigma_{a}=342 \mathrm{~b}$ at $1 \mathrm{eV}$.

\section{Execution of Problem}

Three uniform fuel temperatures were selected to determine the isothermal Doppler effect: 293.6, 888, and $1482.4^{\circ} \mathrm{K}$.

\section{A. Monte Carlo Procedure}

There is provision in the REPETITIOUS 3 code to initiate histories from a source that simulates the asymptotic slowing-down density in a flux spectrum constant in lethargy and in space. For this application the histories are started by neutrons crossing the 30 keV energy boundary. Several thousand histories were followed from birth to death. The geometry routine duplicates Figure 1 exactly.

The following cases were run, each consisting of 1024 histories:

I. $\mathrm{Pu}^{239}$ treated as a resonance absorber below 5.5 $\mathrm{keV}: 16$ cases at $293.6^{\circ} \mathrm{K} ; 20$ cases at $888^{\circ} \mathrm{K}$; 16 cases at $1482.4^{\circ} \mathrm{K}$.

II. $\mathrm{Pu}^{239}$ treated as a " $1 / \mathrm{v}$ " absorber over the full energy range: 10 cases at $293.6^{\circ} \mathrm{K}$ and 10 cases at $888^{\circ} \mathrm{K}$

Spectral and regionwise reaction rates are determined in the REPETITIOUS 3 calculation. By performing the Nonte Carlo computer runs for each temperature as sets of distinct cases, statistical information could be generated. The reaction rates for each case are average values for 1024 histories. The standard deviations reported in Section 6 are based on the assumption that this set of averages for each temperature follows a normal distribution.

The total reaction rates for $\mathrm{U}^{238}$ capture, $\mathrm{Pu}^{239}$ fission and capture, and nonfuel absorption are also available for subcases and therefore further tests of variances were made as well as normality checks based on the methods of Shapiro and Wilk. ${ }^{(16,17)}$

The second set of cases $\left(\mathrm{Pu}^{229}\right.$ as " $1 / \mathrm{v}$ ") were selected to provide a simplified model for comparing Monte Carlo and resonance-integral results. The objective was to eliminate the uncertainties involved in the pseudobroadening of $\mathrm{Pu}^{239}$ and to compare the methods when only $\mathrm{U}^{238}$ resonances are present.

\section{B. Resonance Integral-Multigroup Procedure}

The effective cross sections for the resonance nuclides were dotermined for each temperature by the method described in section 3 . The values are tabulated in

TABLE 3. Cross-Section Data

\begin{tabular}{l|c|c|c|c}
\hline Nuclide & $\begin{array}{c}\text { Mass } \\
\text { Number }\end{array}$ & $\xi$ & $\sigma_{s}, \mathrm{~b}^{*}$ & $\sigma_{a}(1 \mathrm{eV}), \mathrm{b}$ \\
\hline $\mathrm{U}^{238}$ & 238 & 0.00814 & 10.7 & - \\
$\mathrm{Pu}^{239}$ & 239 & 0.00820 & 10.7 & - \\
$\mathrm{C}$ & 12 & 0.15785 & 4.7 & 0.0007 \\
$\mathrm{Na}$ & 23 & 0.08450 & 3.2 & 0.0804 \\
$\mathrm{SS} 304$ & 54.73 & 0.03610 & 10.53 & 0.9483 \\
\hline
\end{tabular}

* $\sigma_{s}$ is constant with energy. $\sigma_{a}$ is " $1 / \mathrm{v}$ " with energy except as noted in text. 
Appendix $\mathrm{C}$ for the energy range from $30 \mathrm{eV}$ to $30 \mathrm{keV}$. Two sets of cross sections are given for $\mathrm{U}^{2.8}$ : one for the presence of $\mathrm{Pu}^{239}$ resonances and the second for the " $1 / \mathrm{v}$ " treatment of $\mathrm{Pu}^{239}$. The main reason for the different values between the two sets is due to the correction factor for the integrated flux. As discussed in reference 15 , since the same energy-dependent factor applies to both fertile and fissile isotopes, it can generally be neglected in the case of the mixture of $\mathrm{U}^{238}$ and $\mathrm{Pu}^{239}$ resonances without affecting the Doppler effect.

Since the primary concern in this study is that part of the energy spectrum below $30 \mathrm{keV}$, this low-energy tail was divided into a relatively fine structure for slowing-down calculations. Above $40.7 \mathrm{keV}$ the YOM(18) cross sections were used with modifications to update the fuel data and to account for the elastic scattering resonances of the light elements. ${ }^{(19-21)}$ Below $40.7 \mathrm{keV}$ several sets of elastic removal and " $1 / \mathrm{v}$ " absorption and fission cross sections were ealculated theoretically for various functional forms of flux shape. The assumed fluxes covered variations from $\mathrm{d} E / E$ to $E \mathrm{~d} E$. The initial multigroup structure was similar to that given in Appendix $\mathrm{C}$ except that groups above $0.3 \mathrm{keV}$ with lethargy widths equal to 0.5 or larger were divided in half. The corresponding $\mathrm{U}^{238}$ and $\mathrm{Pu}^{239}$ (ross sections for $888^{\circ} \mathrm{K}$ were obtained by interpolation assuming a smooth energy dependence. Several iterations were passed through in comparing assujed flux shapes to the resultant nultigroup fluxes calculated by using elastic removal and " $1 / \mathrm{v}$ " cross sections corresponding to the assumed flux shapes, until convergence was obtained. The generated flux spectra are presented in Appendix D.

Once convergence was obtained for $888^{\circ} \mathrm{K}$, the multigroup libraries were compacted to the energy structure

TABLE 4. Reaction Rates and Dopplen LifEcts $\leq 30 \mathrm{keV}$

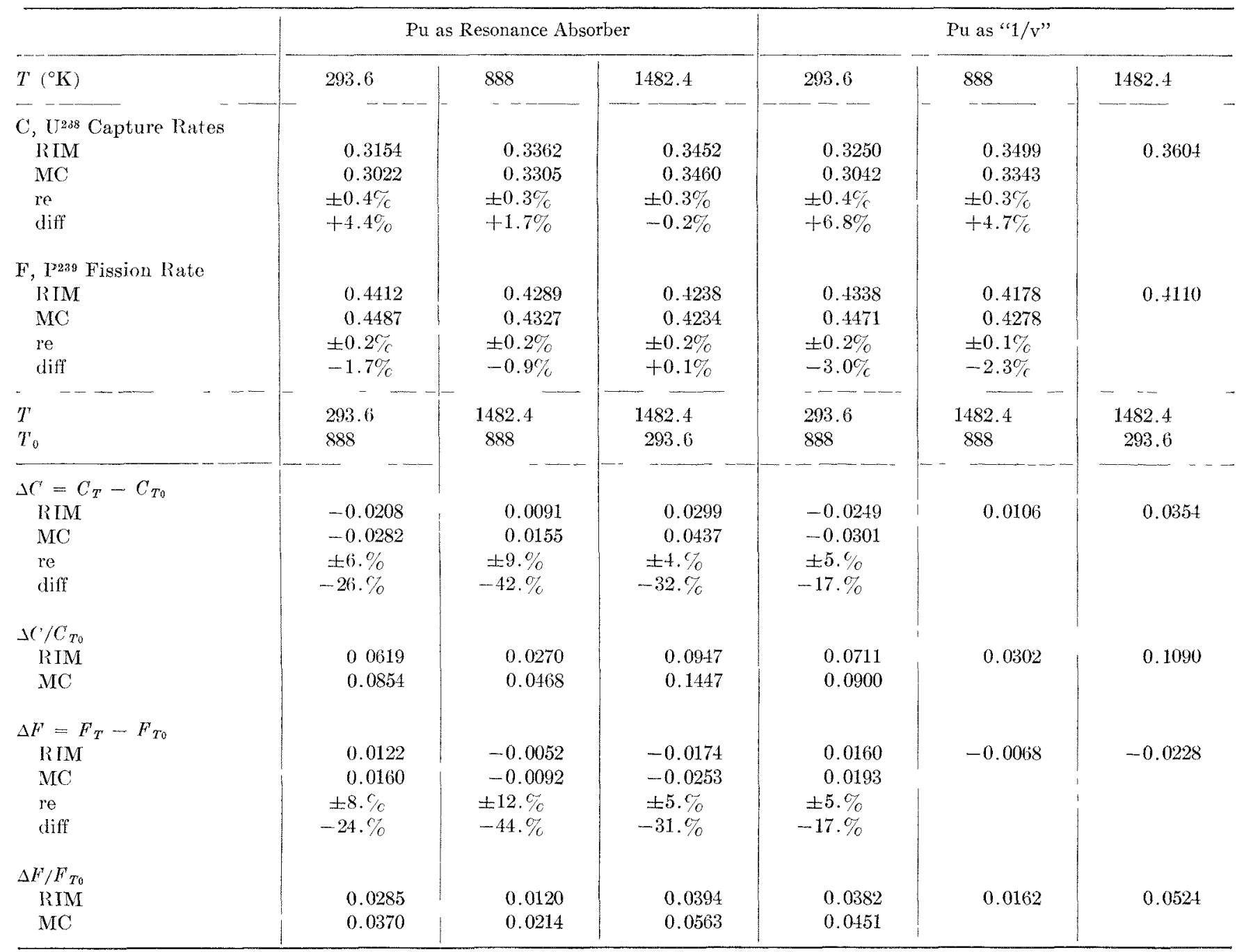

Note: The minus sign in the reported differences indicates that the absolute values of RIM are less than MC. 
given in Appendix C. One library, identified as RH7, was formed for the resonance treatment of $\mathrm{Pu}^{239}$, and the other, identified as RH9, was formed for the " $1 / \mathrm{v}$ " treatment of $\mathrm{Pu}^{239}$. By means of these two libraries and the cross sections tabulated in Appendix C, further multigroup calculations were performed to determine the Doppler effect. As a test of the sensitivity of the results to the low-energy libraries, both $\mathrm{RH7}$ and RH9 libraries were used for the resonance and " $1 / \mathrm{v}$ " treatments of $\mathrm{P}^{2} \mathrm{u}^{239}$; no differences were distinguishable in the resultant total Doppler effects.

\section{Results}

In the Monte Carlo results the reaction rates in each material and region are presented as a fraction such that the total of reaction rates sums to one. In other words, the reaction rate in a particular material is also the probability that a neutron be absorbed in that material. This normalization is constant for all temperatures and arises because each neutron entered inte the system as a source at $30 \mathrm{keV}$ must be absorbed as it slows down. Fission rates are not handled any differently than capture rates in that no new secondary neutrons are injected into the Monte Carlo system if a fission oreurs, but the fact is nerely recorded.

By detcmining reaction rates at cach temperature with multigroup analysis as an eigenvalue problem using temperature-dependent cross sections from the resonance-integral calculation, the same normalization as in Monte Carlo is possible. In fact, the results of the multigroup analysis also indicate that the total absorption rate below $30 \mathrm{keV}$ also remains constant with temperature, although not equal to one. This occurs because of the source renormalization that takes place in the eigenvalue problem and as a consequence of the fact that the absorption rate above $30 \mathrm{keV}$ does not change with temperature. In the presentation of the results from the resonance integral-nultigroup calculation, the reaction rates below $30 \mathrm{keV}$ are normalized to the total absorption rate below ep $\mathrm{keV}$.

In Table 4 the capture rates in $U^{238}$ and the fission rates in $\mathrm{Pu}^{239}$ are presented for each temperature for the MC (Monte Carlo) and RIM (resonance integralmultigroup analysis) results. These represent the reaction rates summed over all energy groups below $30 \mathrm{keV}$. Also the changes and relative changes in reaction rates with temperature are also given. The reference temperature is generally $888^{\circ} \mathrm{K}$. The discrepancies between the RIII and $M C$ results are reported relative to the $M C$ results so that they can be compared with the relative error (re) from the statistical fluctuations of the $\mathrm{MC}$ calculations. The re are based on $1 \sigma$ standard deviation.

TABLE 5. COMParison OF $\alpha$ AND $\eta$

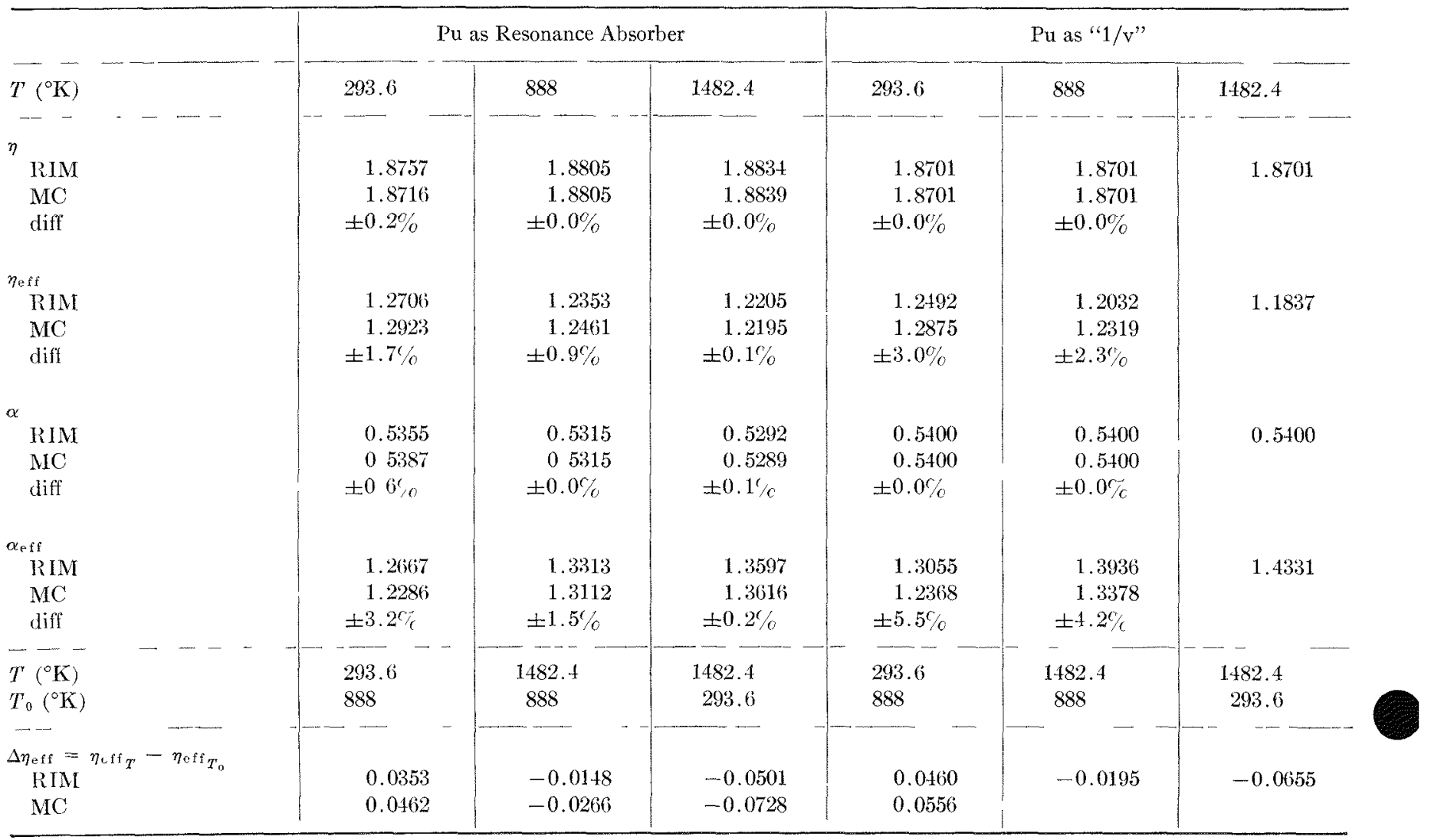


A minus sign attached to the difference (diff) in the Table indicates that the magnitudes of the RIM results are less than those of the $\mathrm{MC}$ results.

In Table 5 the comparative values of $\alpha$ (the ratio of $\mathrm{Pu}^{239}$ capture to fission), $\alpha_{\text {eff }}$ (ratio of total captures in all materials to $\mathrm{Pu}^{239}$ fissions), and $\eta$ (neutrons emitted from fission per $\mathrm{Pu}^{239}$ absorption), and $\eta_{\text {eff }}$ (the value of $k_{\infty}$ for the neutrons below $30 \mathrm{keV}$ ) are presented for each temperature.

In Tables 6-8, the partial spectrum comparisons are made. The energy range below $30 \mathrm{keV}$ is also broken into subranges with break points at 5.5 and $1.0 \mathrm{keV}$. The partial reaction rates are presented to make a comparison over three energy bands. Between 5.5 and $30 \mathrm{keV} \mathrm{U}^{238}$ is treated as unresolved resonances and $\mathrm{Pu}^{239}$ is treated as constant or as " $1 / \mathrm{v}$." Between

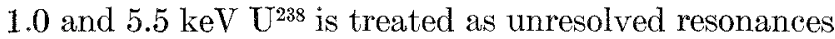

and $\mathrm{Pu}^{239}$ as unresolved resonances or " $1 / \mathrm{v}$." Below $1.0 \mathrm{keV} \mathrm{U} \mathrm{U}^{238}$ is treated as resolved resonances and $\mathrm{Pu}^{239}$ as unresolved (with the exception below $53 \mathrm{eV}$ where $\mathrm{Pu}^{239}$ resonances are resolved) or " $1 / \mathrm{v}$." The contribution of the lowest energy range to the Doppler effect is quite small; therefore the results are renormalized to make the comparison. The results are renormalized as if the total absorption below the reference energy, 30, 5.5, and $1.0 \mathrm{keV}$, respectively, is 1.0. This is done for the lower-energy ranges as an attempt to isolate each energy range and remove the effects of uncertainties above the reference energy.

In Figures 2 and 3 the spectra for the $\mathrm{Pu}^{239}$ fission rates at $888^{\circ} \mathrm{K}$ are given for the two cases: $\mathrm{Pu}^{239}$ as " $1 / \mathrm{v}$ " and as a resonance absorber. The Doppler-effect spectra are presented in Figures 4 and 5 for the two cases. The reference temperature is $293.6^{\circ} \mathrm{K}$ for the

TABLE 6. U238 Partial Spectral Compartson

\begin{tabular}{|c|c|c|c|c|c|c|c|c|c|}
\hline \multirow{2}{*}{$\bar{T}\left({ }^{\circ} \mathbf{K}\right)$} & \multicolumn{3}{|c|}{$5.5-30 \mathrm{keV}$} & \multicolumn{3}{|c|}{$1.0-5.5 \mathrm{keV}$} & \multicolumn{3}{|c|}{$0.03-1.0 \mathrm{keV}$} \\
\hline & 293.6 & 888 & 1482.4 & 293.6 & 888 & 1482.4 & 293.6 & 888 & 1482.4 \\
\hline \multicolumn{10}{|c|}{$\begin{array}{l}\text { C, U238 Capture Rate } \\
\text { Pu as Resonance Absorber }\end{array}$} \\
\hline RIM & 0.1508 & 0.1595 & 0.1630 & 0.3436 & 0.3799 & 0.3961 & 0.3387 & 0.3819 & 0.4047 \\
\hline $\mathrm{MC}$ & 0.1519 & 0.1656 & 0.1714 & 0.3097 & 0.3581 & 0.3846 & 0.2998 & 0.3380 & 0.3681 \\
\hline re & $\pm 0.4 \%$ & $\pm 0.3 \%$ & $\pm 0.3 \%$ & $\pm 0.7 \%$ & $\pm 0.5 \%$ & $\pm 0.5 \%$ & $\pm 0.9 \%$ & $\pm 1.2 \%$ & $\pm 1.0 \%$ \\
\hline & $-0.7 \%$ & $-3.7 \%$ & $-4.9 \%$ & $+11 . \%$ & $+6.1 \%$ & $+3.0 \%$ & $+13 . \%$ & $+13 . \%$ & $+10 . \%$ \\
\hline \multicolumn{10}{|c|}{ Pu as " $1 / v "$} \\
\hline RIM & 0.1736 & 0.1845 & 0.1889 & 0.3099 & 0.3467 & 0.3630 & 0.2957 & 0.3500 & 0.3774 \\
\hline $\mathrm{MC}$ & 0.1660 & 0.1813 & & 0.2716 & 0.3116 & & 0.2563 & 0.3003 & \\
\hline re & $\pm 0.4 \%$ & $\pm 0.4 \%$ & & $\pm 0.7 \%$ & $\pm 0.7 \%$ & & $\pm 1.5 \%$ & $\pm 1.2 \%$ & \\
\hline & $+4.6 \%$ & $+1.7 \%$ & & $+14 . \%$ & $+11 . \%$ & & $+15 . \%$ & $+17 . \%$ & \\
\hline $\begin{array}{l}T\left({ }^{\circ} \mathrm{K}\right) \\
T_{0}\left({ }^{\circ} \mathrm{K}\right)\end{array}$ & $\begin{array}{l}293.6 \\
888\end{array}$ & $\begin{array}{c}1482.4 \\
888\end{array}$ & $\begin{array}{r}1482.4 \\
293.6\end{array}$ & $\begin{array}{l}293.6 \\
888\end{array}$ & $\begin{array}{c}1482.4 \\
888\end{array}$ & 1482.4 & 293.6 & 1482.4 & $\begin{array}{r}1482.4 \\
202.6\end{array}$ \\
\hline \multirow{2}{*}{\multicolumn{10}{|c|}{$\Delta C=C_{T}-C_{T_{0}}$}} \\
\hline & & & & & & & & & \\
\hline RIM & -0.0086 & 0.0035 & 0.0122 & -0.0363 & 0.0162 & 0.0525 & -0.0432 & 0.0229 & 0.0660 \\
\hline $\mathrm{MC}$ & -0.0137 & 0.0059 & 0.0196 & -0.0484 & 0.0265 & 0.0749 & -0.0382 & 0.0302 & 0.0683 \\
\hline re & $\pm 6 . \%$ & $\pm 12 . \%$ & $\pm 4 . \%$ & $\pm 6 . \%$ & $\pm 10 . \%$ & $\pm 4 . \%$ & $\pm 13 . \%$ & $\pm 18 . \%$ & $\pm 6 . \%$ \\
\hline diff & $-37 . \%$ & $-40 . \%$ & $-38 . \%$ & $-25 . \%$ & $-39 . \%$ & $-30 . \%$ & $+13 . \%$ & $-24 . \%$ & $-3 . \%$ \\
\hline \multicolumn{10}{|l|}{$\Delta C / C_{T_{0}}$} \\
\hline R IM & 0.0542 & 0.0222 & 0.0808 & 0.0956 & 0.0426 & 0.1529 & 0.1131 & 0.0599 & 0.1950 \\
\hline $\mathrm{MC}$ & 0.0827 & 0.0354 & 0.1287 & 0.1352 & 0.0739 & 0.2418 & 0.1129 & 0.0892 & 0.2277 \\
\hline \multirow{2}{*}{\multicolumn{10}{|c|}{$\begin{array}{l}\Delta C=C_{T}-C_{T_{0}} \\
\text { Pu as " } 1 / \mathrm{v} "\end{array}$}} \\
\hline & & & & & & & & & \\
\hline RIM & -0.0109 & 0.0044 & 0.0153 & -0.0368 & 0.0163 & 0.0531 & -0.0543 & 0.0274 & 0.0818 \\
\hline $\mathrm{MC}$ & -0.0154 & & & -0.0400 & & & -0.0440 & & \\
\hline re & $\pm 7 . \%$ & & & $\pm 7 . \%$ & & & $\pm 12 . \%$ & & \\
\hline diff & $-29 . \%$ & & & $-8 . \%$ & & & $+24 . \%$ & & \\
\hline \multicolumn{10}{|l|}{$\Delta C / C_{T_{0}}^{\gamma}$} \\
\hline RIM & 0.0589 & 0.0240 & 0.0880 & 0.1060 & 0.0471 & 0.1713 & 0.1552 & 0.0783 & 0.2765 \\
\hline$M C$ & 0.0848 & & & 0.1285 & & & 0.1465 & & \\
\hline
\end{tabular}


TABLE 7. Pu ${ }^{239}$ Partial Spectral Comparison

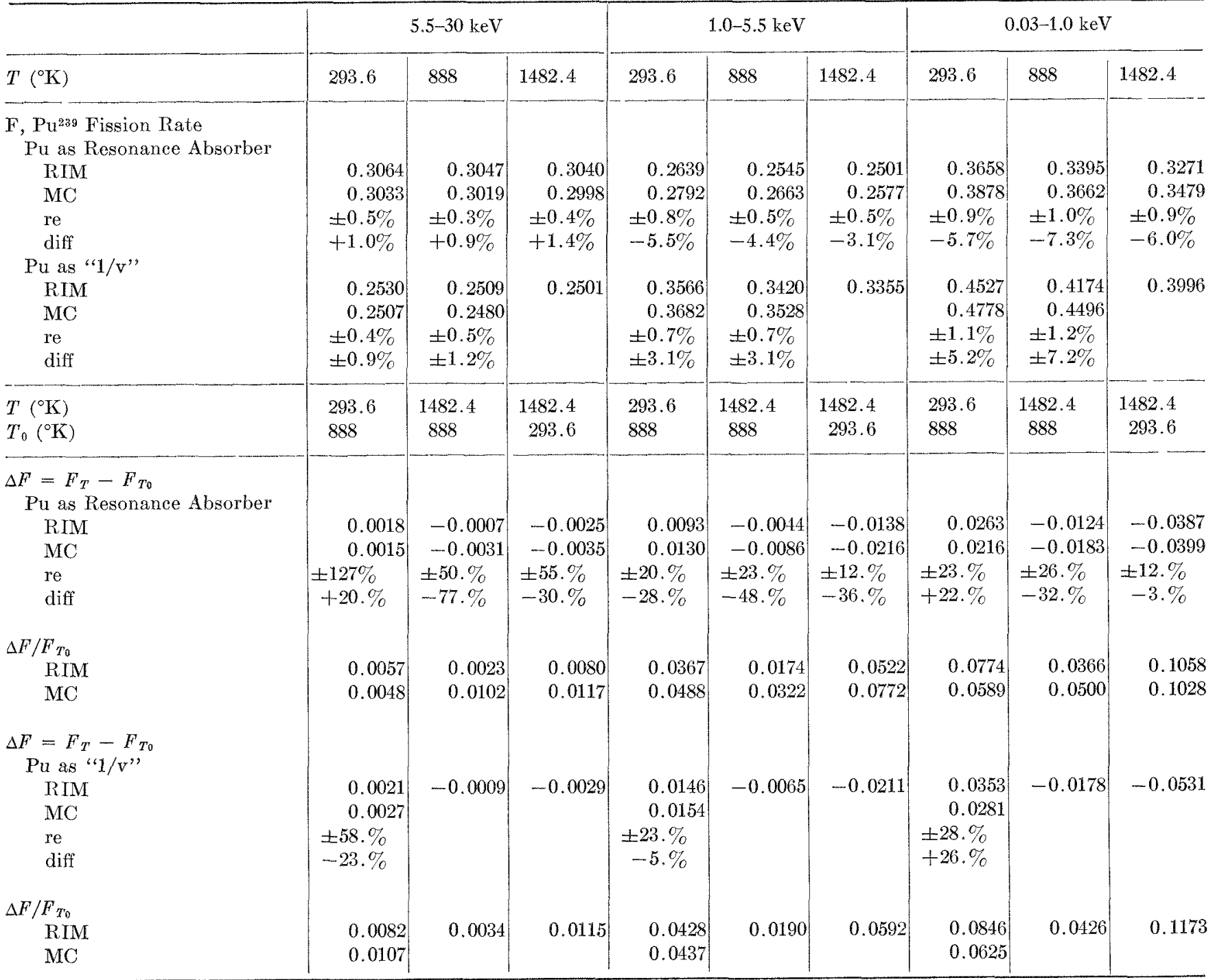

latter two figures. The error flags on the $\mathrm{MC}$ results are shown for one standard deviation.

\section{Discussion of Results and Conclusions}

The study is not complete but from the results presented, the following observations are made.

\section{Total Spectrum $\leq 30 \mathrm{keV}$}

Agreement between the MC and RIM methods in determining the $\mathrm{U}^{238}$ capture rates and $\mathrm{Pu}^{239}$ fission rates are quite good; only a discrepancy of a few per cent is noted. A trend seems to be that agreement improves with increasing temperature. For $\mathrm{U}^{238}$ capture rates the RIM method predicts the larger values, and for $\mathrm{Pu}^{2.9}$ fission rate the $\mathrm{MC}$ method gives the larger values. The agreement in both reaction rates is gen- erally better when $\mathrm{Pu}^{239}$ is treated as a resonance absorber than in the simplified " $1 / \mathrm{v}$ " cases.

However, for the Doppler-effect determination, agreement is better in the " $1 / \mathrm{v}$ " cases. In fact, for the temperature changes involving the $1482.4^{\circ} \mathrm{K}$ temperature in the $\mathrm{Pu}^{229}$ resonance treatment, rather large disagreements are noted, as large as $40 \%$ for a temperature change from 293.6 to $1482.4^{\circ} \mathrm{K}$. The Doppler coefficient in an infinite reactor is approximately proportional to $\Delta F / F$; therefore differences in $\Delta F / F$ tend to induce the same uncertainties in the Doppler coefficient.

Table 5 indicates good agreement between the two methods in the calculation of $\alpha$ and $\eta$ for $\mathrm{Pu}^{239}$, the diserepancy being only a fraction of a per cent. However, generally greater discrepancies occur in the determination of $\alpha_{\text {eff }}$ and $\eta_{\text {eff }}$ (partial $k_{\infty}$ ), except for 
Table 8. Partial Spectral Comparisov of $\alpha$ a yD $\eta$

\begin{tabular}{|c|c|c|c|c|c|c|c|c|c|}
\hline \multirow[b]{2}{*}{$T\left({ }^{\circ} \mathbf{K}\right)$} & \multicolumn{3}{|c|}{$5.5-30 \mathrm{keV}$} & \multicolumn{3}{|c|}{$1.0-5.5 \mathrm{keV}$} & \multicolumn{3}{|c|}{$0.03-1.0 \mathrm{keV}$} \\
\hline & 293.6 & 888 & 1482.4 & 293.6 & 888 & 1482.4 & 293.6 & 888 & 1482.4 \\
\hline$m-\cdots-\cdots-\cdots$ & -- & $-\cdots$ & $-\cdots$ & $-\cdots-\cdots$ & $-\cdots$ & $\cdots$ & $-\cdots$ & $-\infty-\infty$ & -- \\
\hline \multicolumn{10}{|l|}{${ }^{\eta}$ Pu as Resonance Absorber } \\
\hline RIM & 1.9862 & 1.9862 & 1.9862 & 1.6788 & 1.6778 & 1.6787 & $1.616+$ & 1.6049 & 1.6038 \\
\hline $\mathrm{MC}$ & 1.9862 & 1.9862 & 1.9862 & 1.6867 & 1.6908 & 1.6913 & 1.6167 & 1.6135 & 1.6057 \\
\hline \multicolumn{10}{|l|}{$\mathrm{Pu}$ as " $1 / \mathrm{v}$ " } \\
\hline RIM & 1.8701 & 1.8701 & 1.8701 & 1.8701 & 18701 & 1.8701 & 1.8701 & 1.8701 & 1.8701 \\
\hline $\mathrm{MC}$ & 1.8701 & 1.8701 & & 1.8701 & 1.8701 & & 1.8701 & 1.8701 & \\
\hline \multirow{2}{*}{\multicolumn{10}{|c|}{$\eta_{\mathrm{off}}$}} \\
\hline \multicolumn{8}{|l|}{ Pu as Resonance Absorber } & & \\
\hline RIM & 1.4730 & 1.4504 & 1.4414 & 0.9461 & 0.8902 & 0.8661 & 10528 & 0.9783 & 0.9416 \\
\hline $\mathrm{MC}$ & 1.4642 & 1.4322 & 1.4157 & 1.0137 & 0.9372 & 0.8944 & 1.1167 & 1.0546 & 1.0018 \\
\hline \multicolumn{10}{|l|}{ Pu as " $1 / \mathrm{v} "$} \\
\hline RIM & 1.2845 & 1.2575 & 1.2458 & 1.1876 & 1.1206 & 1.0917 & 1.3035 & 1.2030 & 1.1509 \\
\hline $\mathrm{MC}$ & 1.2982 & 1.2590 & & 1.2554 & 1.1803 & & 1.3759 & 1.2950 & \\
\hline \multicolumn{10}{|l|}{$\alpha$} \\
\hline \multicolumn{10}{|l|}{ Pu as Resonance Absorber } \\
\hline $\mathrm{RIM}$ & 0.4500 & 04500 & 0.4500 & 0.7155 & 0.7165 & 0.7156 & 0.7817 & 0.7945 & 0.7958 \\
\hline MC & 04500 & 04500 & 0.4500 & 0.7075 & 0.7034 & 0.7028 & 0.7814 & 0.7849 & 0.7936 \\
\hline \multicolumn{10}{|l|}{ Pu as " $1 / \mathrm{v} "$} \\
\hline RIM & 0.5400 & 0.5400 & 0.5400 & 0.5400 & 0.5400 & 0.5400 & 0.5400 & 0.5400 & 0.5400 \\
\hline $\mathrm{MC}$ & 0.5400 & 0.5400 & & 05400 & 0.5400 & & 0.5400 & 0.5400 & \\
\hline \multicolumn{10}{|l|}{$\alpha_{\varepsilon f f}$} \\
\hline \multicolumn{10}{|l|}{ Pu as Resonance $A b s o r b e r$} \\
\hline RIM & 0.9544 & 0.9855 & 0.9983 & 2.0436 & 2.2348 & 2.3251 & 1.7337 & 19452 & 2.0571 \\
\hline $\mathrm{MC}$ & 0.9631 & 1.0108 & 1.0343 & 18412 & 2.0731 & 2.2201 & 1.5790 & 1.7308 & 1.8747 \\
\hline \multicolumn{10}{|l|}{ Pu as " $1 / \mathrm{v}$ " } \\
\hline RIM & 1.2426 & 1.2913 & 1.3117 & 1.4252 & 15698 & 1.6380 & 1.2088 & 1.3957 & 1.5024 \\
\hline $\mathrm{MC}$ & 1.2185 & 1.2875 & & 1.2940 & 1.4400 & & 1.0931 & 12240 & \\
\hline
\end{tabular}

$1482.4^{\circ} \mathrm{K}$, where the agreement is within $0.2 \%$. This suggests that the relative capture and fission rates are in very good agreement with each other but that the absolute reaction rates relative to the complete system are generally in error by a few per cent. The latter is in agreement with the observations made in the first paragraph. In fact, the discrepancies noted between RIMI and $\mathrm{MC}$ in $\alpha_{\text {eff }}$ and $\eta_{\text {eff }}$ are due to the discrepancies in $\mathrm{Pu}^{239}$ fission rates since the total absorption rate below $30 \mathrm{keV}$ has been normalized to 1.0. Likewise the re in the MC statisties is the same for $\alpha_{\text {eff }}$ and $\eta_{\text {eff }}$ as those given in Table 4 for the fission. rates. Therefore the MIC re and diecrepancies for the change in $\alpha_{\text {eff }}$ and $\eta_{\text {eff }}$ with temperature are the same as reported in Table 4 for $\Delta F$.

\section{Partial Spectrum}

Examination of Tables 6-8 for the partial reaction rates over 3 different energy ranges shows that the contribution to the discrepancies discussed in the previ- ous paragraphs can be partially synthesized. The discrepancies between the two methods appear to be larger for the partial reaction rates below $5.5 \mathrm{keV}$ than above, and also there is a change in sign. For the highest energy range the agreement gets better with a decrease in temperature, which is opposite to the effect noted above for the total reaction rates. The "plus" and "minus" discrepancies above and below $5.5 \mathrm{keV}$ tend to cancel each other at $1482.4^{\circ} \mathrm{K}$. As mentioned in Scetion 6 , the results for the two lower energy ranges have been renormalized so that the total absorption below the maximum energy of the specified range is 1.0 ; therefore the three energy ranges do not contribute equally to the total reaction rate.

For the changes in partial reaction rates with temperature, the MIC statisties become substantially worse due to dividing the results into three different energy ranges. The only statistically significant difference for the Doppler effect on the partial fission rate is noted for the energy range from 1.0 to $5.5 \mathrm{keV}$ when $\mathrm{Pu}^{239}$ is 


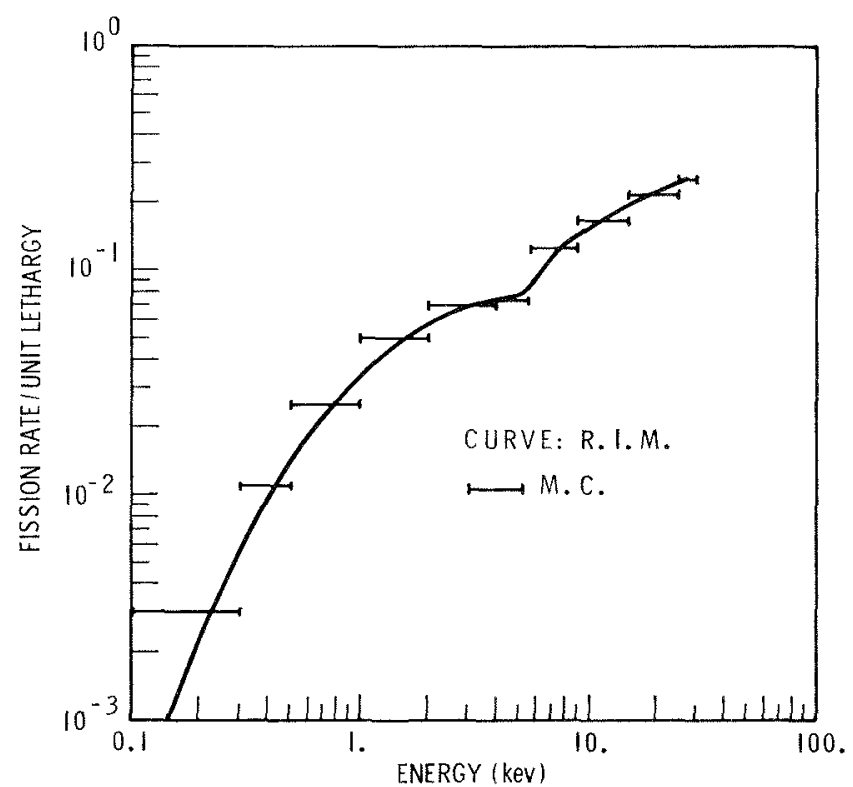

Fic. 2. Fission Rate Spectrum below $30 \mathrm{keT}$ at $888^{\circ} \mathrm{K}$; $\mathrm{Pu}^{239}$ Treated as " $1 / \mathrm{v}$ ".

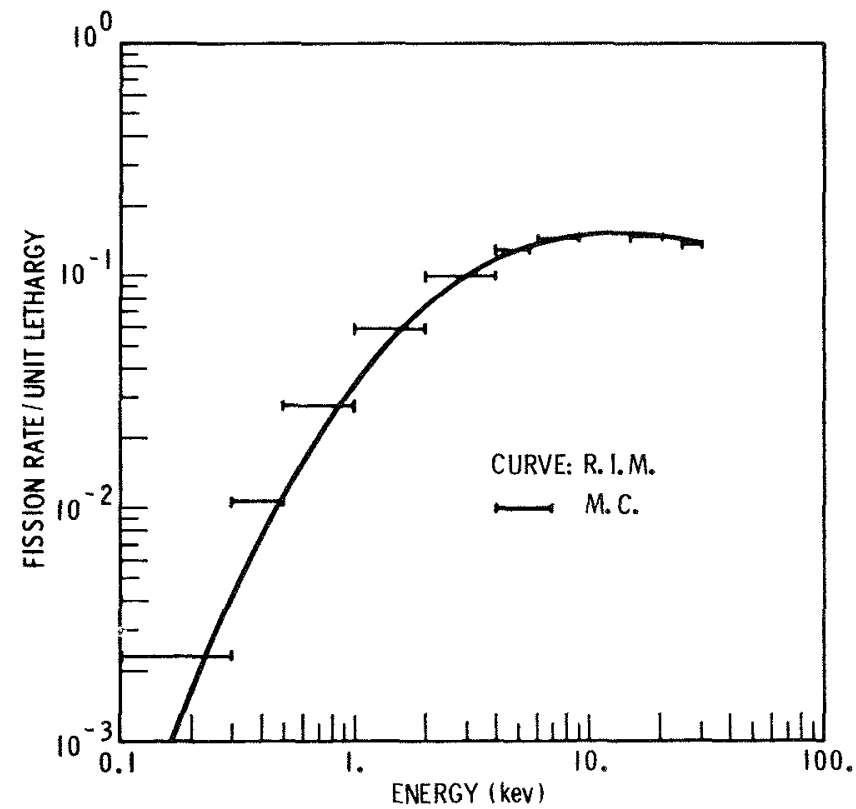

FI(x. 3. Fission Rate Spectrum below $30 \mathrm{keV}$ at $888^{\circ} \mathrm{K}$; $\mathrm{Pu}^{239}$ Treated as Resonance Absorber.

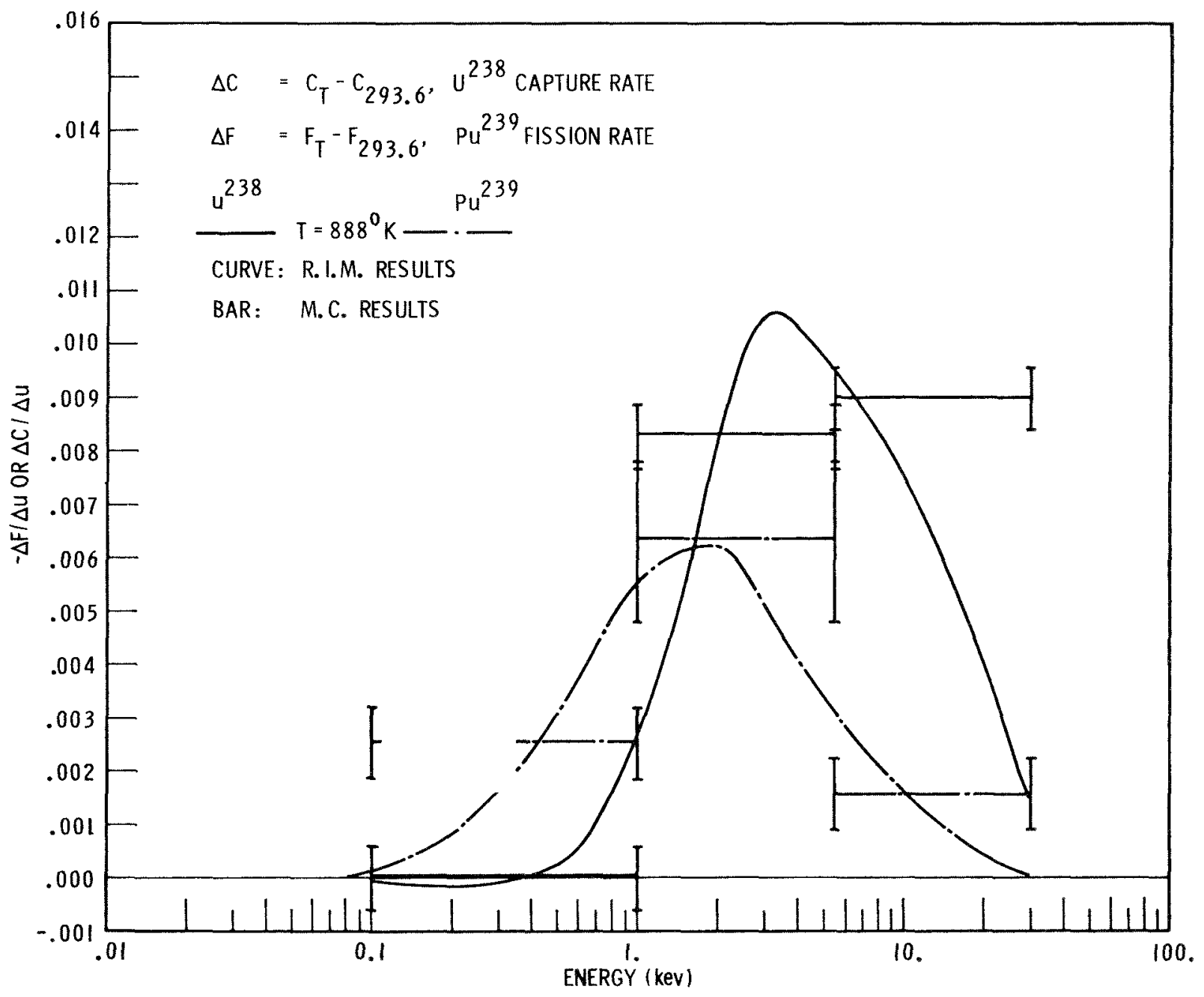

Fig. 4. Doppler Hffect below $30 \mathrm{keV}$ for $\mathrm{Pu}^{239}$ Treated as " $1 / \mathrm{v}$ ". 


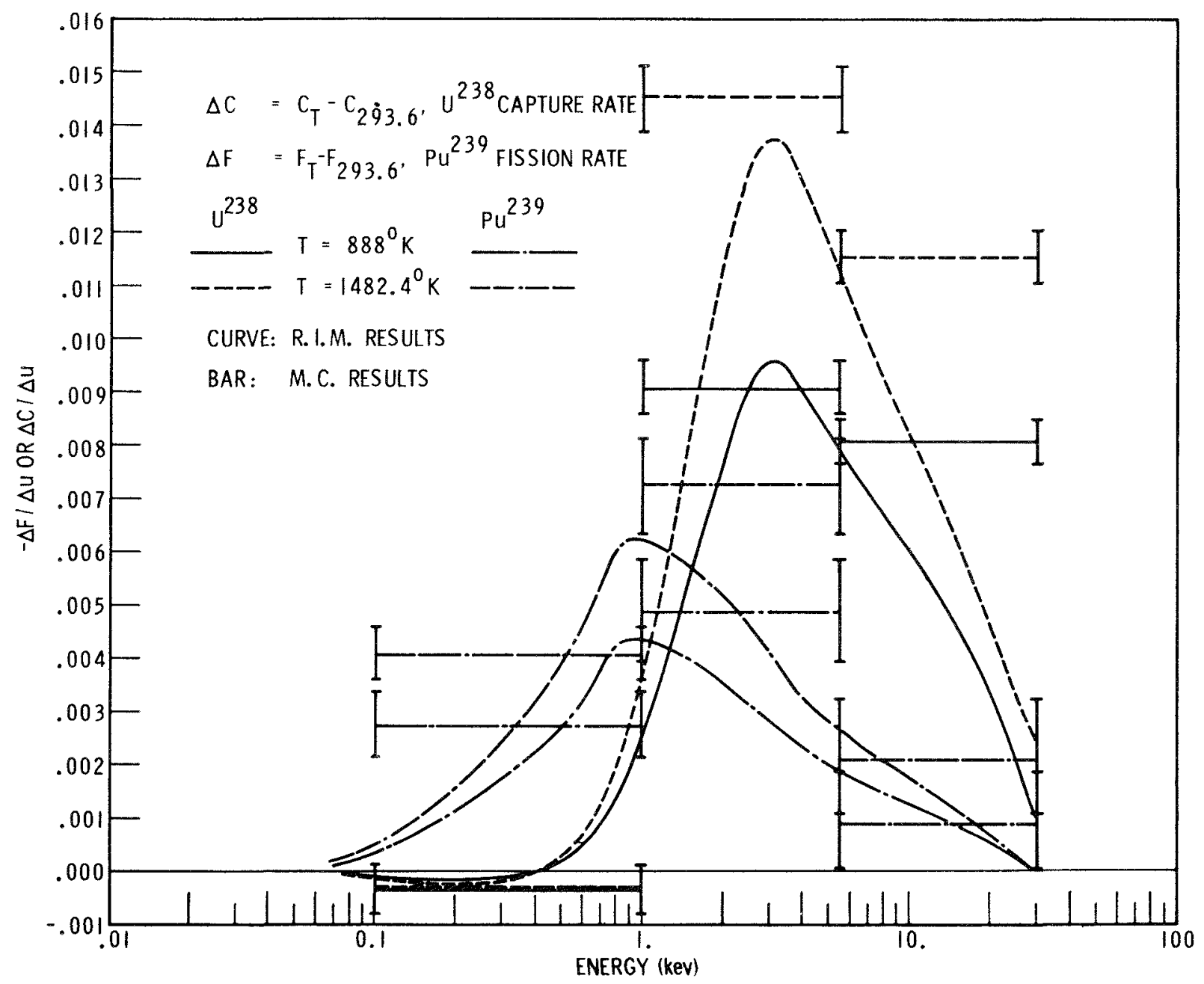

Fig. 5. Doppler Effect below $30 \mathrm{keV}$ for $\mathrm{Pu}^{239}$ Treated as Resonance Absorber.

treated as a resonance absorber. For the Doppler effect on the partial $\mathrm{U}^{238}$ capture rate, the greatest significant difference between the two methods appears generally for the energy range above $5.5 \mathrm{keV}$, the discrepancics being larger than $30 \%$, with the $\mathrm{MC}$ method predicting a larger Doppler effect. It is noted that in this energy range the differences can be attributed only to the $\mathrm{U}^{238}$ resonance treatments or the slowing-down treatments since plutonium cross sections are either constant or " $1 / \mathrm{v} . "$

Figures 2-5 further illustrate the spectral results for reaction rates and Doppler effect. There is no renormalization below $5.5 \mathrm{keV}$ as discussed in the presentation of Tables 6-8. Figures 2 and 3 show good agreement in the fission rate spectra generated by $\mathrm{MC}$ and RIMI. The RIM results exhibit a faster attenuation of reaction rates as the neutrons slow down.

In conclusion, there is generally good agreement in reaction rates considering the rather different assumptions made in the two methods. However, because of the small changes in reaction rates with temperature, the reasonably small discrepancies in reaction rates produce quite large differences in Doppler effect. The results indicate that uncertainties in the Doppler effect and therefore in the Doppler coefficient are large enough to justify further sophistication in methods, both for $\mathrm{MC}$ and for RIMI.

\section{Acknowledgments}

It is a pleasure to acknowledge Dr. R. A. Dannels for his development and write-up of REPETITIOUS 3, Drs. H. H. Hummel and G. H. Minton for their valuable discussions, Messrs. E. L. Hill and R. P. Price for their very able assistance in computer programming and computations, and Miss Roseann Litzinger for her fine typing.

\section{REFERENCES}

1. G. Goertzel, H. Feshbach, H. Hurwitz, G. Roe, and H. Yamanchi, Estimation of Doppler Effect in Intermediate and Fast Neutron Reactors, Proc. Int. Conf. on PUAE, Geneva, $5 \mathrm{P} / 613(1955)$. 
2. A. M. Lane, J. F. Lynn, and J. S. Story, An Estimation of the Doppler Effect in Fast Neutron Reactors, AERE T/M 137 (1956).

3. H. A. Bethe, On the Doppler Effect in Fast Reactors, API)A119 (1957).

4. R. B. Nicholson, The Doppler Effect in Fast Neutron Reactors, API)A-136 (1960).

5. P. Greebler and E. Goldman, Doppler Calculations for Large Fast Ceramic Reactors-Effects of Improved Methods and Recent Cross Section Information, (xEAP-4092 (1)ec 1962).

6. P. (ireebler, "Recent Improvements in Calculations of Doppler and Sodium Reactivity Effects for Large Fast Reactors," Proc. of Conf. on Breeding, Economics and Safety in Large Fast Power Reactors, ANL-6792 (Oct 1963) 51.

7. H. H. Hummel and A. L. Rago, "Effect of Parameter Variation in Doppler-Effeet Calculations," "bid., 747.

8. J. Codd and P. J. Collins, "Some Calculations Concerning the Influence of Resonance Overlapping on the Doppler Effect in a Dilute Fast Reactor," ibid., 711.

9. R. Froelich, K. Ott, and J. J. Schmidt, "Dependence of Fast Reactor Doppler Coefficients on Nuclear I)ata Uncertainties," ibid., 765.

10. J. Codd, J. E. Beardwood, 1). C. Leslie, and H. M. Sumner, Studies of Resonance Absorption and the Doppler Phenomenon for Fast and Thermal Reactors, Proc. 3rd Int. Conf. on PUAE, Geneva, P/172 (1964).

11. R. D. Richtmyer, Resonance Capture Calculations for Lattices by the Monte Carlo Method, BNL-433 (Sept 1956).

12. R. I. Richtmyer, Monte Carlo Methods, Am. Math. Soc. Meeting, April 24, 1959.

13. R. D. Richtmyer, R. Van Norton, and A. Wolfe, Monte Carlo Calculations of Resonance Capture in Reactor
Lattices, Proc. 2nd Int. Conf. on PUAE, Geneva, 16 (1958) 180.

14. R. N. Hwang, "An Improved Method of Doppler-effect Calculation for Fissile Materials in the Intermediate Fnergy Region," Proc. of Conf. on Breeding, Economics and Safely in Large Fast Power Reactors, ANL-6792 (Oct 1963), 727 .

15. R. N. Hwang, Doppler Effect Calculations with Interference Corrections, Nucl. Sci. Eng., 21523 (1965).

16. G. L. Burrows, and D. B. Macmillan, Confidence Limits for Monte Carlo Calculations, Nucl. Sci. Eng. 22, 384 (July 1965).

17. S. Shapiro and M. B. Wilk, An Analysis of Variance Test for Normality (Complete Samples), ATL, G. E. Report No. 64GL140 (Sept 3, 1964) (also submitted for publication to Buometrika).

18. S. Yiftah, D. Okrent, and P. A. Moldauer, Fast Reactor Cross Sections, Pergamon Press (1960).

19. F. M. Heck, Liquid Metal Fast Breeder Reactor Design Study, WCAP-3251 (Jan 1964).

20. M. G. Bhide and H. Hummel, Calculations of the Doppler Coefficient of Large Ceramic-fueled Fast Reactors, ANI6601 (1962).

21. A. L. Rago and H. H. Hummel, ELMOE: An IBM-704 Program Treating Elastic Scattering Resonances in Fast Reactors, ANL 6805 (Jan 1964).

22. F. L. Fillmore, ARES-1, A Resonance Integral Code, Trans. Am. Nucl. Soe., 5 (No 1), 57 (1962) and AMTD-130 (May 1962), NAA Program Deseription.

23. D. J. Hughes and R. B. Schwartz, Neutron Cross Sections, BNL-325, 2nd Ed. (1958).

24. J. S. Fraser and R. B. Schwartz, Nucl. Phys., 30, 269 (1962).

25. L. M. Bollinger, R. E. Coté, and G. E. Thomas, "The Slow Neutron Cross Sections of PIutonium-239,"' Proc. 2nd Int. Conf. on PUAE, Geneva, 15127 (1958).

\section{Appendix A}

\section{The REPETITIOUs 3 Code}

REPETITIOUS 3 is a multiregion, cell-geometry, slowing-down, Monte Carlo code which permits inclusion of one primary isotope having strong isolated resonances characteristic of $\mathrm{U}^{238}$ or other fertile material, and of another secondary (lesser amount present) isotope which may have resonance characteristics of fissile materials. Thus, the competition which occurs when two resonance absorbers are present is included in the REPETITIOUS calculation. Because of limitations in the core storage available with the IBM 7094, and in order to obtain reasonable computation times, the methods for accounting for Doppler broadening in the two absorbers are somewhat different; greater accuracy is obtained in the calculation for $\mathrm{U}^{238}$. Effects arising from overlap of resonances in a single absorber or from competition between the resonances of the two materials are limited to the accuracy inherent in a BreitWigner single-level representation for the resonances and, for the second material, in the approximations discussed below.
The relative probabilities of interactions of various kinds at a given point in the calculation are determined by the cross sections of the individual materials for various proresses. The cross sections are determined as follows:

\section{1) Resonance Absorber No. 1 (e.g., $U^{238}$ )}

It is assumed that, in addition to a background $1 / \mathrm{v}$ component to the cross section, only the two adjacent resonances (i.e., the first resonance having the energy of its maximum greater than $E$ and the first resonance having the energy of its maximum less than $E$ ) contribute to the cross section at energy $E$. It has been found that such a treatment is quite adequate for $\mathrm{U}^{238}$, in which the average level spacing is of the order of 18 $\mathrm{eV}$.

For energies less than a designated energy $E_{R}$ (which separates the resolved from the unresolved resonances), the cross sections are given by

$$
\sigma_{a}(E)=\frac{\text { const }}{\sqrt{E}}+\left[\sqrt{\frac{E}{E_{0}}} \psi(x, \theta) \sigma_{0} \frac{\Gamma_{\gamma}}{\Gamma}\right]_{1}
$$




$$
\begin{aligned}
+ & {\left[\sqrt{\frac{E}{E_{0}}} \psi(x, \theta) \sigma_{0} \frac{\Gamma_{\gamma}}{\Gamma}\right]_{2} } \\
\sigma_{s}=\sigma_{\mathrm{pot}} & +\left[\psi(x, \theta) \sigma_{0} \frac{\Gamma_{n}}{\Gamma}+\sqrt{\sigma_{\mathrm{pot}} \sigma_{0}} \frac{\Gamma_{n}}{\Gamma} \chi(x, \theta)\right]_{1} \\
+ & {\left[\psi(x, \theta) \sigma_{0} \frac{\Gamma_{n}}{\Gamma}+\sqrt{\sigma_{\mathrm{pot}} \sigma_{0}} \frac{\Gamma_{n}}{\Gamma} \chi(x, \theta)\right]_{2}, }
\end{aligned}
$$

where:

$$
\begin{aligned}
x= & 2\left(E_{-}-E_{0}\right) / \mathrm{I} ; \\
\sigma_{0}= & 4 \pi \lambda_{0}^{2} g\left(\Gamma_{n} / \Gamma\right) \\
\theta= & 4 E_{0} k T / A \mathrm{~T}^{2} ; \\
\lambda_{0}= & \text { the wavelength of the neutron in the center-of- } \\
& \text { mass systen at energy } E_{0} ; \\
k= & \text { Boltzmann's constant; } \\
T= & \text { the absolute temperature; } \\
A= & \text { ratio of nuclear mass to neutron mass; } \\
E_{0}= & \text { the energy of the neutron at the maximum of } \\
& \text { the appropriate resonance; } \\
E= & \text { the energy of the neutron at which the cross } \\
& \text { section is calculated; } \\
\Gamma= & \text { the total resonance half-width; } \\
\Gamma_{\gamma}= & \text { the resonance half-width for neutron capture; } \\
\Gamma_{n}= & \text { the resonance hall-width for neutron scatter- } \\
& \text { ing; } \\
g= & \text { the statistical spin factor for the nuclide and } \\
& \text { level involved. }
\end{aligned}
$$

Here Doppler broadening is explicitly contained in the development of the $\psi$ and $\chi$ functions through the parameter $\theta$. Tables of $\psi$ and $x$ are contained in the code.

In the unresolved region $\left(E>E_{R}\right)$, resonances are determined statistically. The energies corresponding to the maxima for the resonances are fixed with uniform spacing corresponding to the average level spacing determined from data on resolved resonances. For each resonance, the value of $\Gamma_{\gamma}$ is constant, and the value of $\Gamma_{n}$ is determined on the basis of a random selection from a Porter-Thomas distribution. The same formulas used for the determination of the values for the eross sections of resolved resonances are applicable to those of the unresolved resonances.

\section{2) Resonance Absorber No. $2\left(e . g ., P u^{239}\right)$}

For this material, an approxination was made in the treatment of Doppler broadening. It is assumed that the broadened shape is a Guassian having the correct area and peak value for the cross section. This assumption eliminates the necessity for calculation of the $\psi$ and $\chi$ functions at each energy $E$ for which the cross section is required.
The cross sections for the resolved region are given by

$$
\begin{aligned}
& \sigma_{c}=\sum_{\substack{u 11 \\
\text { resolved }}}\left[\sqrt{\frac{E}{E_{0}}} \sigma_{0} \psi(0, \theta) \frac{\Gamma_{\gamma}}{\Gamma} \frac{1}{1+x^{\prime 2}}\right] ; \\
& \sigma_{f}=\sum_{\substack{\text { al1 } \\
\text { resolved }}}\left[\sqrt{\frac{E}{E_{0}}} \sigma_{0} \psi(0, \theta) \frac{\Gamma_{f}}{\Gamma} \frac{1}{1+x^{\prime 2}}\right] ; \\
& \sigma_{s}=\sigma_{\text {pot }}+\sum_{\substack{a 11 \\
\text { resolved }}}\left[\sigma_{0} \psi(0, \theta) \frac{\Gamma_{n}}{\Gamma} \frac{1}{1+x^{\prime 2}}\right],
\end{aligned}
$$

where

$$
x^{\prime}=\frac{2\left(E-E_{0}\right) \psi(0, \theta)}{\Gamma} .
$$

Above the region of resolved resonances, statistical resonances are assumed. For each such resonance, $\Gamma_{\gamma}$ is taken to be constant, and $\Gamma_{f}, \Gamma_{n}$, and $D$ (energy spacing between resonances) are drawn from $\chi$-squared distributions with degrees of freedom selected to obtain a good fit to the corresponding distributions determined from experimental data for the resolved resonances. The $g$ values for the statistical resonances may be determined by a random-number sampling such that the frequency of values matches that of resolved resonances. The cross sections at energy $E$ are then calculated as for the resolved energy range except that the sum is limited to twenty resonances: ten above and ten. below the energy $E$. The actual distribution in energy and values of parameters for the statistical resonances are read from a library tape at the beginning of the calculation, and a different set is read periodically after a sperified number of histories. Above the statistical region, the cross sections for the second resonance absorber are given by

$$
\begin{aligned}
\sigma_{j} & =\frac{\text { constant }}{\sqrt{\bar{E}}} \text { or constant; } \\
\sigma_{\gamma} & =\alpha \sigma_{f} ; \\
\sigma_{s} & =\sigma_{p}=\sigma_{\text {potentidl }} .
\end{aligned}
$$

Values for $\sigma_{p}, \alpha$, and the constant in $\sigma_{f}$ are input quantities.

\section{3) Nonresonance Materials}

The cross sections for nonresonance materials are obtained at all energies as follows:

$$
\begin{gathered}
\sigma_{a}=\frac{\text { constant }}{\sqrt{E}} ; \\
\sigma_{s}=\sigma_{p}=\sigma_{\text {potential }} .
\end{gathered}
$$

Values for $\sigma_{p}$ and the constant are provided for each material. 
Appendix $B$

REsONAVCE PaIRAMETHRs (23-20)

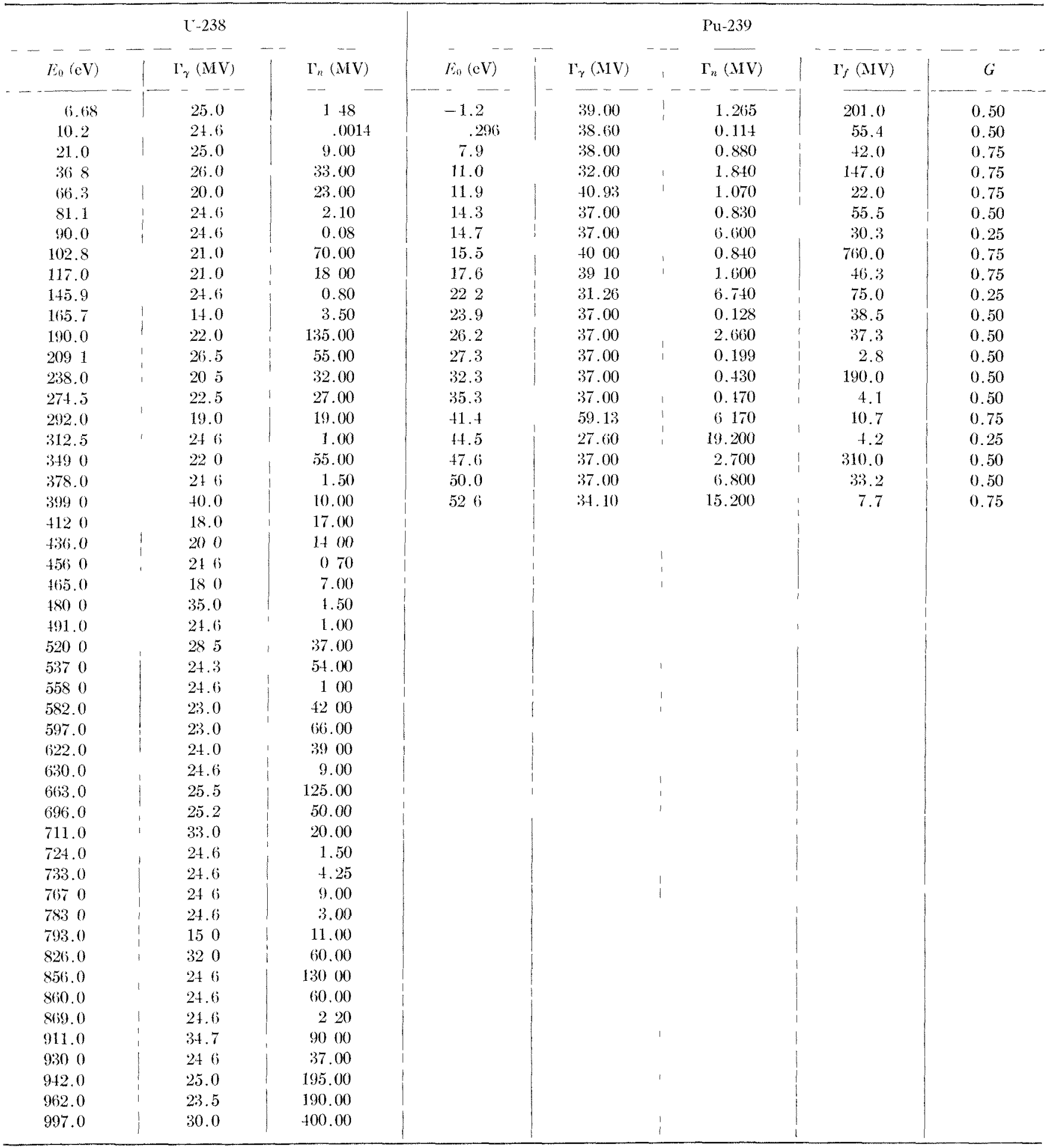




\section{Appendix C}

RI L-238 CROSS SECTIONS

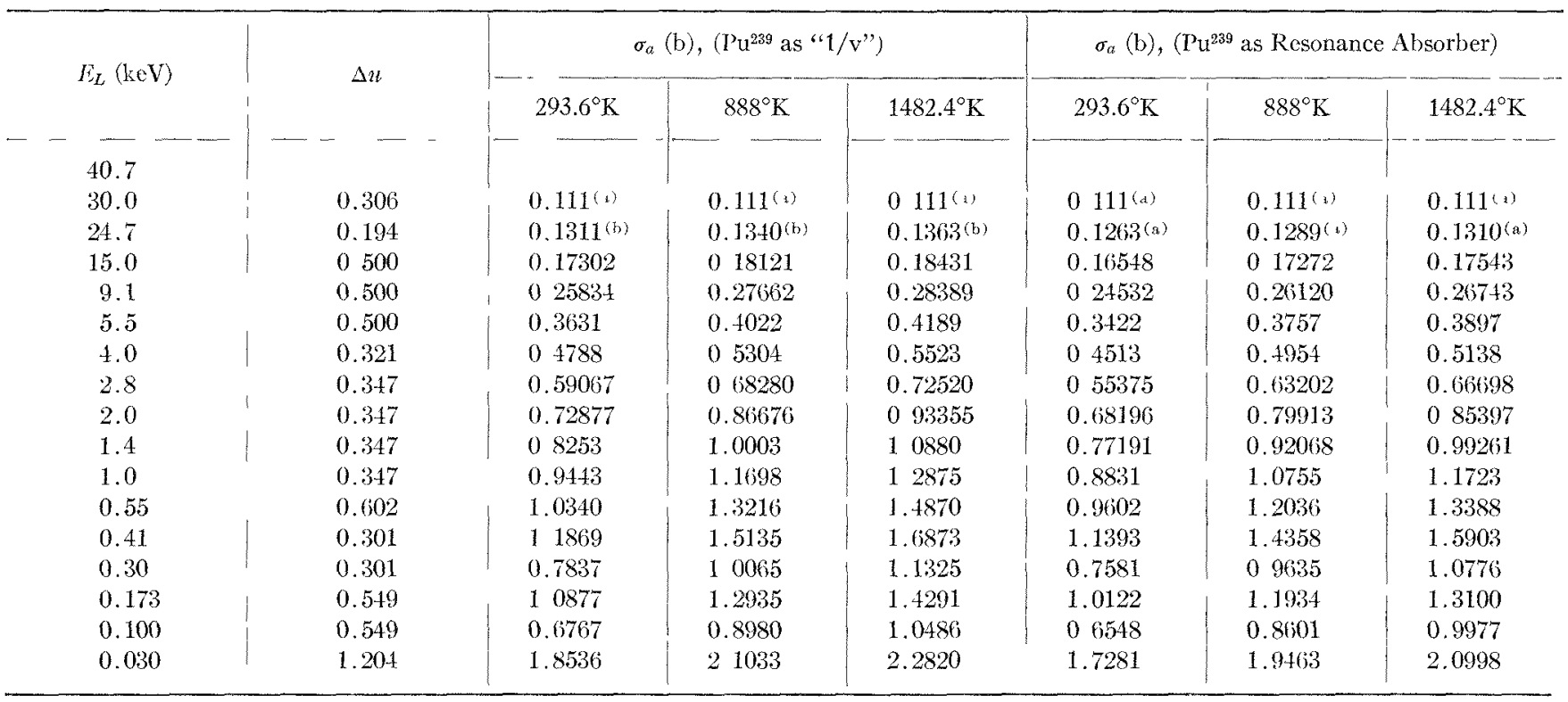

(1) Results from ARES calculations (22)

(b) ARES results modified by extrapolated values of Hwang's "f" factor. (15)

Note: Scattering cross section per Y ${ }^{238}$ atom, $\sigma_{p}$, is 50.1 barns.

RI Pu 239 Cross Sections

\begin{tabular}{|c|c|c|c|c|c|c|c|c|c|c|c|c|c|}
\hline \multirow{2}{*}{$E_{L_{L}}(\mathrm{keV})$} & \multirow{2}{*}{$u$} & \multicolumn{3}{|c|}{$\sigma /(b)$} & \multicolumn{3}{|c|}{$\sigma_{\gamma}(\mathrm{b})$} & \multicolumn{3}{|c|}{$\sigma_{a}(b)$} & \multicolumn{3}{|c|}{$\nu \sigma_{f}(\mathrm{~b})$} \\
\hline & & $293.6^{\circ} \mathrm{K}$ & $888^{\circ} \mathrm{K}$ & $1482.4^{\circ} \mathrm{K}$ & $293.6^{\circ} \mathrm{K}$ & $888^{\circ} \mathrm{K}$ & $1482.4^{\circ} \mathrm{K}$ & $293.6^{\circ} \mathrm{K}$ & $888^{\circ} \mathrm{K}$ & $1482.4^{\circ} \mathrm{K}$ & $293.6^{\circ} \mathrm{K}$ & $888^{\circ} \mathrm{K}$ & $1482.4^{\circ} \mathrm{K}$ \\
\hline 40.7 & & & & & & & & & & & & & \\
\hline 30.0 & 0.306 & 2.50 & 2.50 & 250 & 1.125 & 1125 & 1125 & 3.625 & 3.625 & 3.625 & 7.20 & 7.20 & 7.20 \\
\hline 24.7 & 0.194 & 2.50 & 2.50 & 2.50 & 1.125 & 1.125 & 1.125 & 3.625 & 3.625 & 3.625 & 7.20 & 7.20 & 7.20 \\
\hline 15.0 & 0.500 & 2.50 & 2.50 & 2.50 & 1.125 & 1.125 & 1125 & 3.625 & 3.625 & 3.625 & 7.20 & 7.20 & 7.20 \\
\hline 9.1 & 0.500 & 2.50 & 2.50 & 2.50 & 1.125 & 1125 & 1125 & & 3625 & & 7.20 & 7.20 & 7.20 \\
\hline 5.5 & 0.500 & 2.50 & 2.50 & 250 & 1.125 & 1.125 & 1.125 & 5 & 3.625 & 3 & 7. & 7.20 & 7.20 \\
\hline 4.0 & 1 & & 1.874 & & 1.2 & 1.264 & 1.2 & & 3.138 & 3. & & & \\
\hline 2.8 & & 2.3 & 2.3459 & & 1.6 & 1.6 & 1.6 & & 4.0 & 00 & & 6.7563 & 551 \\
\hline 2.0 & 0.347 & 2.9756 & 3.0047 & 3.0082 & 2.1611 & 2.1852 & 2.1878 & 5.1367 & 5.1900 & 5.1960 & 8.5697 & 8.6536 & 8.6636 \\
\hline 1.4 & 0.347 & 3.4824 & 3.5333 & 3.5428 & 2.5777 & 26218 & 2.6294 & 6.0602 & 6.1551 & 6.1722 & $\begin{array}{ll}10 & 0294\end{array}$ & 10.1760 & 10.2031 \\
\hline 1,0 & 0.347 & 4.1948 & 4.2881 & 4.3114 & 3.1737 & 3.2590 & 3.2790 & 7.3685 & 7.5471 & 7.5904 & 12.0809 & 12.3198 & 12.4168 \\
\hline 0.55 & 0.602 & 5.1731 & 5.3403 & 5.3901 & 4.0076 & 4.1728 & 4.2193 & 9.1807 & 9.5131 & 9.6091 & 14.8985 & 15.3801 & 15.5235 \\
\hline 0.71 & 0.301 & 6.2737 & 6.6423 & 6.7992 & 4.9723 & 5.3567 & 5.5080 & 11.2460 & 11.9990 & 12.3072 & 18.0683 & 19.1298 & 19.5817 \\
\hline 0.30 & 0.301 & 7.7265 & 8.1918 & 8.3899 & 6.1238 & 6.6063 & 67965 & 13.8503 & 14.7981 & 15.1864 & 22.2523 & 23.5924 & 24.1629 \\
\hline 0.173 & & 8.3343 & 9.0023 & & & 7.4289 & 77740 & 14.9849 & 16.4312 & 17.0943 & 24.0028 & 25.9266 & 26.8425 \\
\hline 0.100 & 0.549 & 9.9704 & 10.7527 & 11.1297 & 7.9562 & 8.8733 & 9.2832 & 17.9266 & 19.6260 & 20.4129 & & 30.9678 & 32.0535 \\
\hline 0.030 & 1.204 & 12.9568 & 13.9465 & 14.5534 & 9.8878 & 11.3186 & 12.1052 & 22.8446 & 25.2651 & 26.6586 & 37.3156 & 40.1659 & 41.9138 \\
\hline
\end{tabular}

Note: Scattering cross section per $\mathrm{Pu}^{239}$ atom, $\sigma_{p}$, is 283.9 barns, $p=2.88$ below $40.7 \mathrm{keV}$. 


\section{Appendix D}

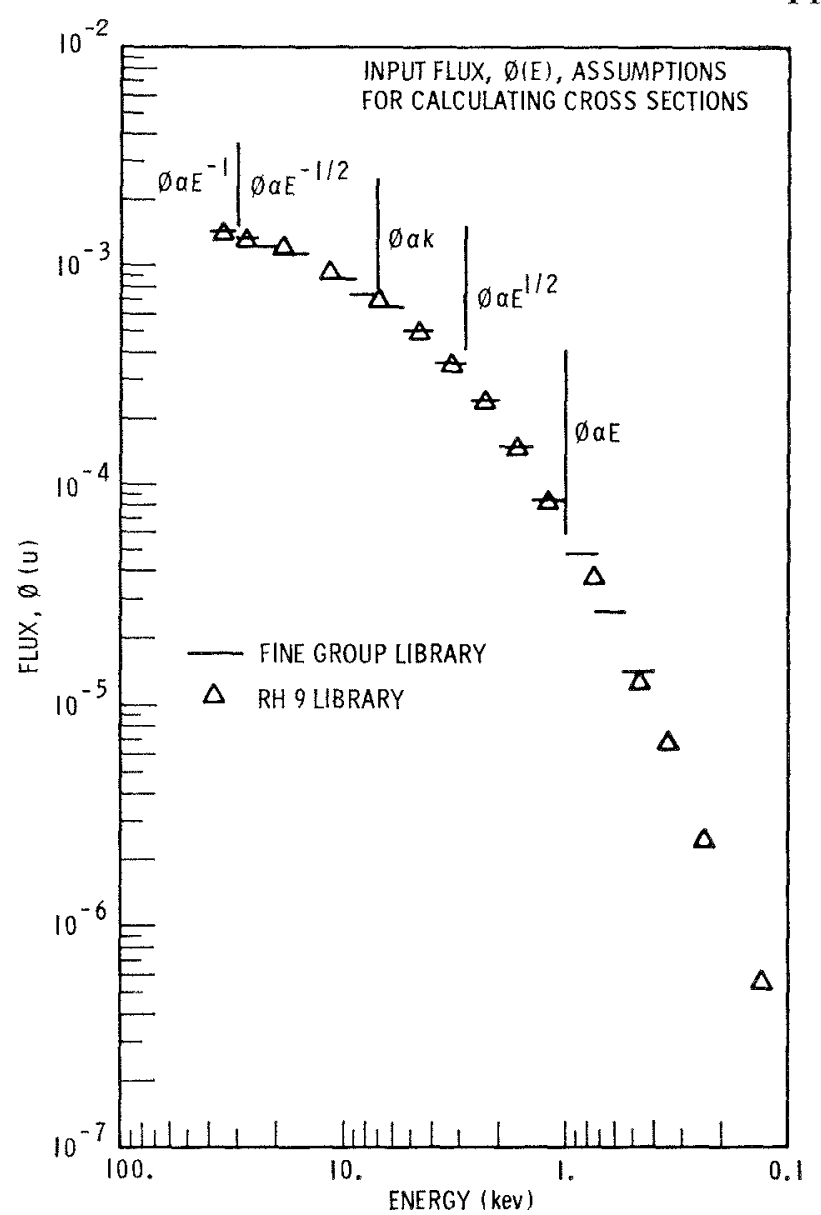

FIG. D.1. Flux spectrum at $888^{\circ} \mathrm{K}$ of $\mathrm{Pu}^{239}$ Treated as " $1 / \mathrm{v} "$.

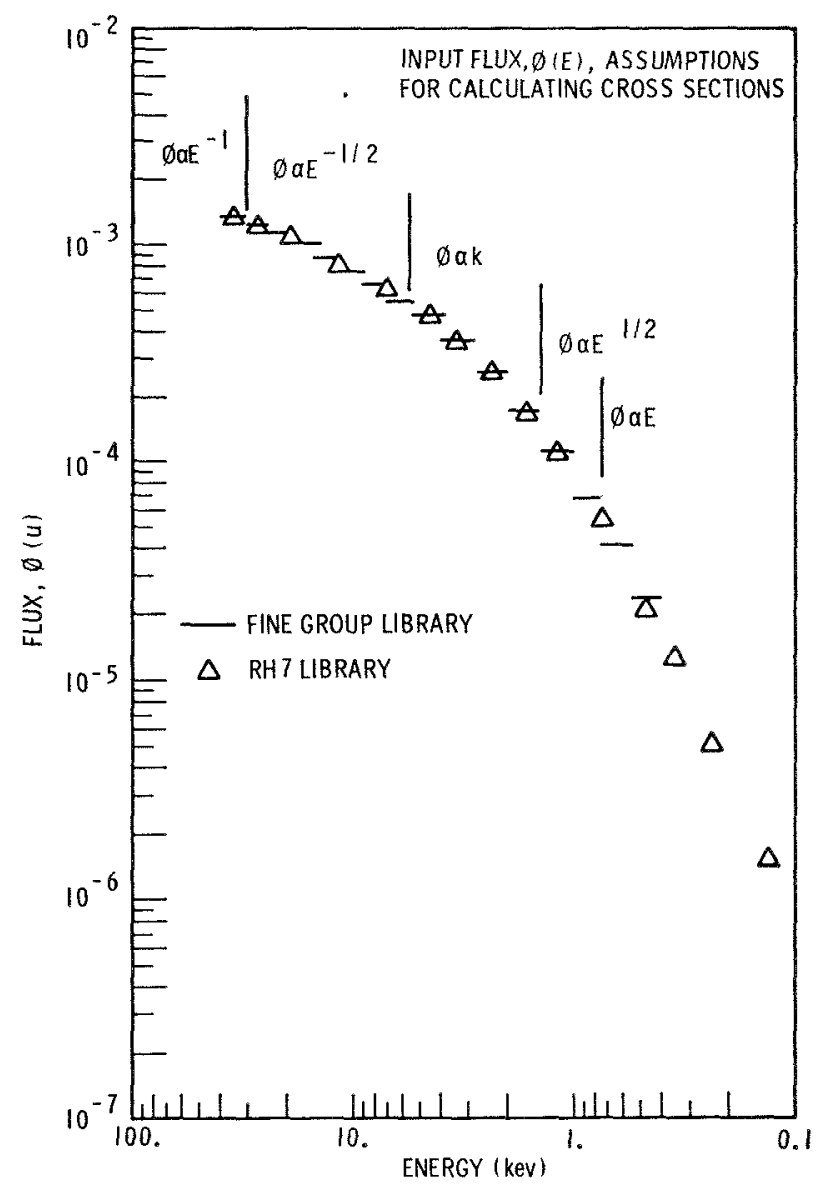

Fic. D.2. Flux Spectrum at $888^{\circ} \mathrm{K}$ of $\mathrm{Pu}^{239}$ Treated as Resonance Absorber.

\section{Discussion}

Mr. Yiftah (Israel AEC): I have two questions. You mentioned using Monte Carlo to calculate the Doppler effect and the reaction rate, rather than the reactivity. My first question is, why? Secondly, since Monte Carlo has been used at Westinghouse for calculating the total Doppler effect for typical big fast reactors, as mentioned in your $1000-\mathrm{MW}$ study, the question is how do these calculations compare with other methods, such as perturbation or successive $k$ as far as numerical results, computing time, ete? The main point of my second question is, of course, do you consider the Monte Carlo technique useful for calculating the Doppler effect of fast reactors?

$M r$. Olhoeft: I think it is useful. I will answer your last question first. At the present stage of technology it is expensive and therefore one cannot use it for a complete design study. You have to look at typical cases, but $I$ think it is very useful for making comparisons between the Monte Carlo results and some of the other mothods. As computers become faster and faster, computer economies will become much more favorable for computational times; then I think Monte Carlos will actually see some greater application. The reason why the Doppler effect was compared rather than the Doppler coefficient is that what we tried to do here was to make the comparison as basic as possible in the respect that we wanted to remove any uncertainties at the higher energy of the spectrum and also any uncertainties of reactor size which would have an effect on leakage. Certainly the Doppler coefficient is sensitive to the reactor, whereas what we were trying to do here was to compare methods for energies below $30 \mathrm{keV}$. The eigenvalue calculation compares much more directly with the Monte Carlo. Because of the fact that the total absorptions below $30 \mathrm{keV}$ can be normalized to one, if you do a perturbation analysis, you cannot do this. So this seemed to be a natural way to do it.

Mr. Levine (BNL): You mentioned that the Doppler 
broadening of the $\mathrm{Pu}^{239}$ resonances is not accomplished in quite the same way as that of the $\mathrm{U}^{238}$. Do you know how much discrepancy might be caused by that?

$M r$. Olhoeft: We have investigated this somewhat. If one uses the approximation that is built into the code, one can use this as an approximation for the $\psi$ function. Then if you introduce this into the $J$ function for calculating by the resonance-integral method, you may compare these results with, for example, the $J$ calculated by a more exact method. For the case of plutonium where we are quite dilute, the approximation seems to be quite good as regards the reaction rate. We only looked at a fairly typical set of resonances. We used the average resonance parameters. But at about $2 \mathrm{keV}$ there is about a $2 \%$ difference in reaction rates. In the Doppler effect, there is a larger difference, as I recall of the order of $20 \%$. But the approximate method actually underestimates the change in the Doppler effect of $\mathrm{Pu}^{239}$. Of course, in this particular study, the $\mathrm{U}^{238}$ is what contributes the largest effect to the total Doppler effect, so that $20 \%$ is probably reduced to more like a 4 or $5 \%$ effect in this study. This would tend to bring closer agreement in the results.

Mr. Codd (Winfrith): Could you please repeat the standard deviation of your Monte Carlo results with the Doppler coefficient?

Mr. Olhoeft: I did not report the absolute numbers. What I reported was a relative error which would be the ratio of the standard deviation to the quantity that it was referring to. In terms of the reaction rates, we had a relative error of about $0.4 \%$. This was for roughly 10,000 histories when plutonium was introduced as $1 / \mathrm{v}$ material, and between 16 , and 20,000 histories when plutonium was introduced as a resonance $a b-$ sorber. The actual standard deviations will be reported in the proceedings.

Mr. Codd: Yes. I wasn't clear how the discrepancies you obtained between the two methods were related to the standard deviation of your Monte Carlo. That was the point of my question.

Mr. Olhoeft: I see. Well, it is certainly related, but I believe that in most of the cases the discrepancies were outside of the statistical limits. For instance, in the $1 / \mathrm{v}$ case we had a difference between the two methods of $17 \%$; the Doppler effect of $\mathrm{Pu}^{239}$ and the $3 \sigma$ standard deviations was actually about $15 \%$, so that it was outside of the limits even if we go so far as to say a $3 \sigma$ standard deviation. 


\title{
Transient Temperature Distributions in Fast Reactor Fuels with Widely Varying Thermal Diffusivity
}

\author{
C. K. Saxathanan, J. C. Carter, and R. O. Brittan \\ Argonne National Laboratory \\ Argonne, Illinois \\ (PRESENTED BY C. K. SANATHANAN)
}

\section{Introduction}

The limitations on the thermal performance of reactor fuels, both statically and dynamically, are governed by the thermal diffusivity of the fuel material. Estimates of the peak central temperatures are necessary to determine limiting power densities for normal operation and of physical behavior in abnormal rircumstances. In addition, complete temperature distributions are necessary to determine coupling between heat generation and cooling, both statically and dynamically.

It has been possible to meet this problem in the past because fuel materials have had high thermal conductivity and because fuels have had operating temperatures limited by low melting points. When the conductivity is high, its variation with temperature low, and thermal gradients small, the equation for heat conduction can be greatly simplified. Errors in assuming "'onstant" values for the thermal diffusivity are insignificant and can be ignored.

Now, however, $\mathrm{CO}_{2}$ fuels have been proposed for large fast reactors. ${ }^{(1,2)}$ In ralculating temperature distributions for these fuels, large errors will ensue if simplified methods are employed. $\mathrm{CO}_{2}$ has very low thermal conductivity which decreases with temperature to a minimum, then increases sharply. It is desirable to operate a reactor fueled with $\mathrm{UO}_{2}$ with renterline temperatures approaching the melting point in order to realize maximum power densities. The large center-to-edge tempcrature ratios result in conductivities on both sides of the minimum, with thermal radiation strongly influencing heat flow.

In addition, $\mathrm{UO}_{2}$ fuels are subjected to transient heating experiments in. TREAT which may result in rapid temperature rises to well above the melting point. For these tests it is necessary to predict the temperature so that the burst intensity can be properly planned. This is true especially if the damage threshold is approached cautiously in a series of shots. The unreliability of temperature measurements in the $\mathrm{UO}_{2}$ make extrapolations to the next test in a series useless.

Because of these design and test requirements, a study has been made in which new calculational methods for determining transient temperature distributions in $\mathrm{UO}_{2}$ fuel elements have been devised and determination of transient temperature and heat flow behavior undertaken.

\section{Significance of Variable Conductivity}

The effect of neglecting variation of thermal conductivity and its gradient can be shown by analysis of the usual steady-state heat-conduction equation for a flat plate:

$$
\frac{d}{d x}\left[K \frac{d T}{d x}\right]+q=0
$$

with boundary conditions

$$
\begin{array}{r}
d T /\left.d x\right|_{x=0}=0 ; \\
-K d T /\left.d x\right|_{x=l}=q l,
\end{array}
$$

where $T$ is temperature, $x$ is the space coordinate, $q$ is volumetric heat-generation rate, $l$ is the plate halfthickness, and $K$ is thermal conductivity.

Equation (1) may be rewritten as

$$
K \frac{d^{2} T}{d x^{2}}+\frac{d K}{d T}\left(\frac{d T}{d x}\right)^{2}+q=0 .
$$

When dealing with materials in which variation of $K$ with $T$ is insignificant, and the thermal gradient $d T / d x$ is small, the quantity in parentheses in Eq. (4) is neglected. ${ }^{(3-7)}$ However, this simplification would lead to significant error if the material were $\mathrm{UO}_{2}$.

From reported measurements, ${ }^{(8-26)}$ the thermal conductivity of $\mathrm{UO}_{2}$ is small at low temperatures and decreases significantly as temperature increases. Then, as thermal radiation becomes significant in transferring heat, the conductivity begins to increase sharply. The term expressing conductivity as a function of temperature in the range below that where radiation is significant can be taken as

$$
K=A /(B+T),
$$

where $A$ and $B$ are constants and have been assigned the values $A=42$ and $B=400$ on the basis of experimental observation. ${ }^{(16)}$ 
Through the use of Eq. (5), Eq. (4) has been solved for $T(x)$ exactly and with the term in parentheses neglected. The comparative results are displayed in Fig. 1 for $q=1000 \mathrm{~W} / \mathrm{cc}$ and a coolant temperat ure of $500^{\circ} \mathrm{K}$. The center-plane temperature yielded by the simplified solution is seen to be $50 \%$ higher than that given by the exact solution.

This is a simple steady-state problem for illustrative purposes, since temperatures reached are in the range in which thermal radiation is significant. With the radiation component of $\mathrm{K}$ increasing nearly as the cube of the temperature, the thermal gradient of $\mathrm{K}$ takes on greater significance in the central region of the plate. However, the example chosen dramatically illustrates the errors which may be introduced by neglecting the temperature and conductivity gradients in calculating temperature distributions in low-conductivity materials like $\mathrm{CO}_{2}$. For example, this feature makes the ARGUS $\operatorname{code}^{(4)}$ in its present form unsuitable for use in calculation of temperature distributions in $\mathrm{UO}_{2}$ elements (especially those to be tested in TRHAT).

\section{Thermal Diffusivity of $\mathrm{UO}_{2}$}

Before going into the study of transient effects, some discussion of the particular make-up of thermal diffusivity of $\mathrm{YO}_{2}$ is in order. The diffusivity is defined as the ratio of thermal conductivity to heat capacity (which in turn is the product of specific heat and density). Diffusivity is the key parameter signifying the space-time-dependent behavior of heat flow and temperature. In many materials it varies little with temperature and is frequently considered constant in making calculations. However, in a low-conductivity material like $\mathrm{CO}_{2}$ it is dominated by $K(T)$ and varies significantly.

With respect to $K(T)$, the predominant mechanisms of heat transfer in ceramics are generally recognized to be phonon conduction and photon conduction (internal radiation).

At low temperatures, phonon conduction is dominant; its effect, in most cases, diminishes with temperature. On the other hand, photon ronduction becomes increasingly significant at higher temperatures. In materials such as $\mathrm{CO}_{2}$, photon conduction is so significant at higher temperatures (say above $1800^{\circ} \mathrm{K}$ ) that it causes the overall conductivity of these materials to increase with temperature. This has been observed by several investigators. ${ }^{14,24)}$ The phonon-conduction componont of the thermal conductivity is usually expressed as in Eq. $(.5):(16,25)$

$$
K_{\text {phon }}=A /(B+T) \text {. }
$$

The photon-conduction component is considered to be $e^{(26)}$

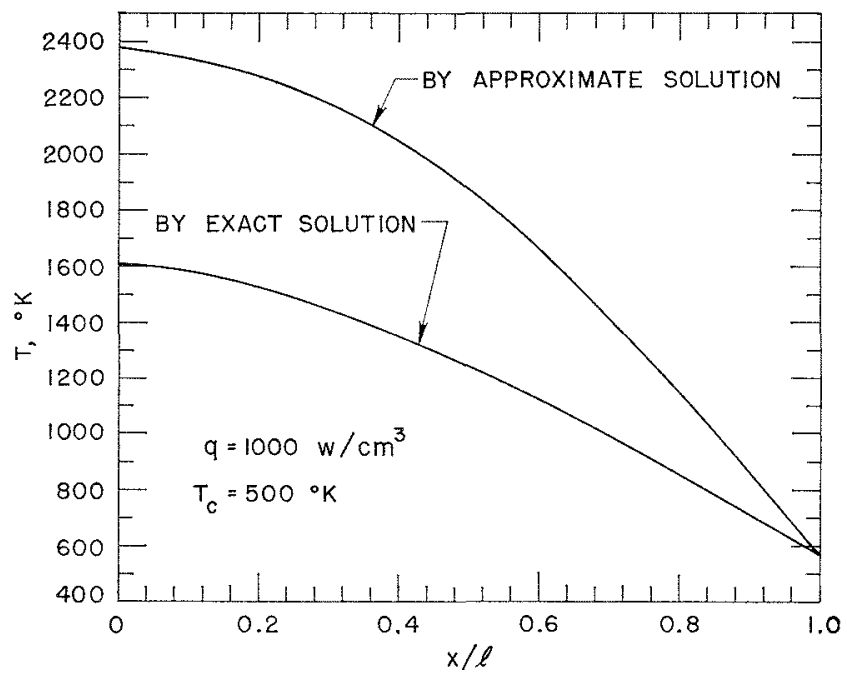

Fic. 1. Comparison of the Approximate with the Exact Temperature Distribution in a $\mathrm{UO}_{2}$ Plate.

$$
K_{\text {phot }}=\left(16 n^{2} \sigma / 3 \beta_{r}\right) T^{3},
$$

where $n$ is the index of refraction, $\sigma$ the Stefan-Boltzmann constant, and $\beta_{2}$ the inverse mean free path of radiation. It has been found ${ }^{(26)}$ that $\beta_{r}$ is essentially proportional to $T$ in crystalline $\mathrm{CO}_{2}$. The temperature dependence of $\beta_{r}$ is much more complex in nonerystalline $\mathrm{UO}_{2}$ and is not fully understood. The total effective thermal conductivity is evidently the sum of (5a) and (6):

$$
K(T)=[A /(B+T)]+\left(16 n^{2} \sigma / 3 \beta_{3}\right) T^{3} .
$$

The values of $A$ and $B$, and the coefficient of $T^{3}$ may be obtained from experimental data. However, to facilitate the calculations which follow it was found more convenient to express $K(T)$ as a third-degree power series:

$$
K=K_{0}+K_{1} T+K_{2} T^{2}+K_{3} T^{3} .
$$

This allows determination of the coefficients $K_{0}$, $K_{1}, \cdots$ either from $\mathrm{Eq} .(7)$ or directly from an experimental plot of $K$ versus $T$ by a curve-fitting procedure $^{(28)}$ which leads to errors of less than $1 / 2 \%$. Use of the series expression has distinct computational advantages over the form of $\mathrm{E}(\mathrm{l}$. (7) and facilitates extension of the computational methods to other ceramic materials.

After a careful study of the recent publications on the conductivity of $\mathrm{CO}_{2}$ (both experimental and theoretical), ${ }^{(3,8,10,14,18-26)}$ it was found that the variation of conductivity with temperature can be given in a certain range of values shown by curves $K_{2}, K_{3}$, and $K_{4}$ in Fig. 2. In this figure, curve $K_{1}$ is obtained by assuming the unrealistic condition of no radiative transfer in the medium and hence represents the value 


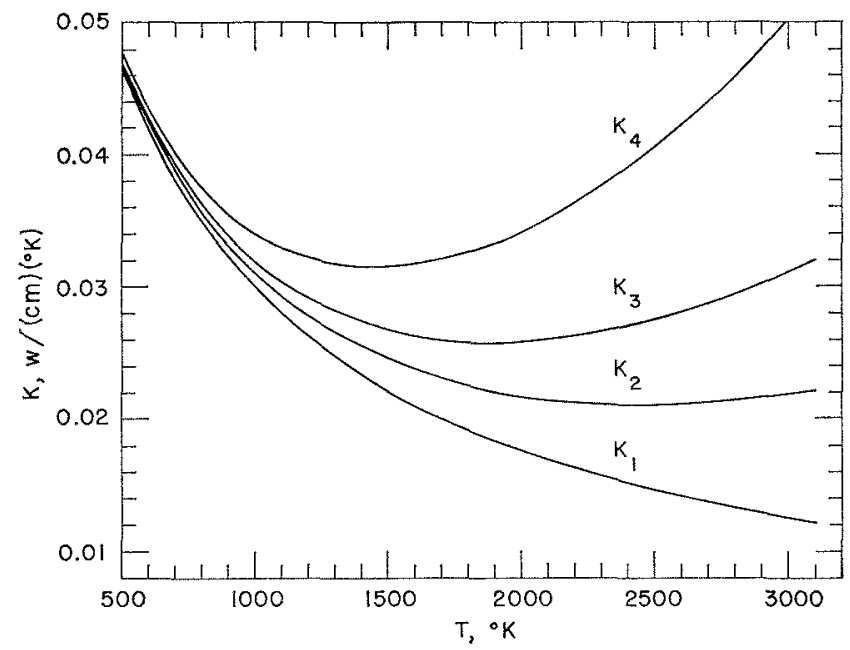

FIG. 2. Conductivity of $\mathrm{UO}_{2}$ vs Temperature.

used earlier in the simple steady-state problem as given by $\mathrm{Eq}$. (5) with values of $A$ and $B$ from Ref. 16, that is,

$$
K=42 /(400+T) .
$$

The other component of the diffusivity (heat capacity) also varies with temperature. ${ }^{(3)}$ Although variation is not pronounced, the variation is taken into account in determining the transient behavior of the temperature. Depending on the porosity, irradiation level, and stoichiometry, the volumetric heat capacity may increase or decrease with temperature. It appears that this variation is linear and within $\pm 8 \%$ in the temperature range from 500 to $3000^{\circ} \mathrm{K}$. Hence it will be necessary to express the product of specific heat $c(T)$ and density $\rho(T)$ as

$$
c(T) \rho(T)=g_{0}+g_{1} T,
$$

where

$$
\begin{aligned}
& g_{0}=3.31 \mathrm{~W} \text {-sec } / \mathrm{cc} ; \\
& g_{1}= \pm 1.27 \times 10^{-4} \mathrm{~W} \text {-sec } / \mathrm{cc} /{ }^{\circ} \mathrm{K} .
\end{aligned}
$$

These elements of the thermal diffusivity have a profound effect on the transient heat-transfer processes within the $\mathrm{UO}_{2}$ medium. It is this effect that is examined in the study to follow.

\section{Transient Thermal Characteristics of $\mathrm{UO}_{2}$}

A critical evaluation of the transient performance of a reactor system involves the simultaneous solution of a complex system of differential equations describing heat transfer in the fuel element and the coolant, and the neutron kinetics in the reactor.

This particular study is directed towards analyzing the transient thermal characteristies of $\mathrm{UO}_{2}$ as the first step in the evaluation without reference to any specific reactor system. Later it is planned to extend the studies to a system represented by a $\mathrm{UO}_{2}$-fueled, high-performance fast reactor for the purpose of understanding the influence of the thermal characteristics on the dynamics of the system. At present, indications are that the future system study will employ an analogdigital hybrid computer. For this portion of the study, the analog computer is best suited because of the parametric nature of the study and the time dependence. A brief account of this initial phase is presented here.

Although the fuel elements in the fast reactor of interest will probably be cylindrical, flat-plate geometry is used here, with no cladding to simplify the space dependence. In addition, the temperature of the coolant and the heat transfer coefficient at the fuel-coolant interface are assumed to be constant. (As the next step in the study, these constraints will be removed and the computational scheme will be extended to include cylindrical geometry.)

Cnder these constraints, the time-dependent conservation of energy in one-dimensional space is expressed as

$$
\frac{\partial}{\partial x}\left[K(T) \frac{\partial T}{\partial x}\right]+q=c(T) \rho(T) \frac{\partial T}{\partial t} .
$$

The boundary conditions chosen are

$$
\begin{aligned}
\partial T /\left.\partial x\right|_{x=0} & =0 ; \\
-K(T) \partial T /\left.\partial x\right|_{x=l} & =h\left(T_{l}-T_{c}\right),
\end{aligned}
$$

where $x$ and $t$ are the independent space and time coordinates, respectively, $l$ is the plate half-thickness, $q$ is the volumetric heat-generation rate, $h$ is the heat transfer coefficient at the fuel-coolant interface, $T_{l}$ is the fuel boundary temperature, and $T_{c}$ is the coolant

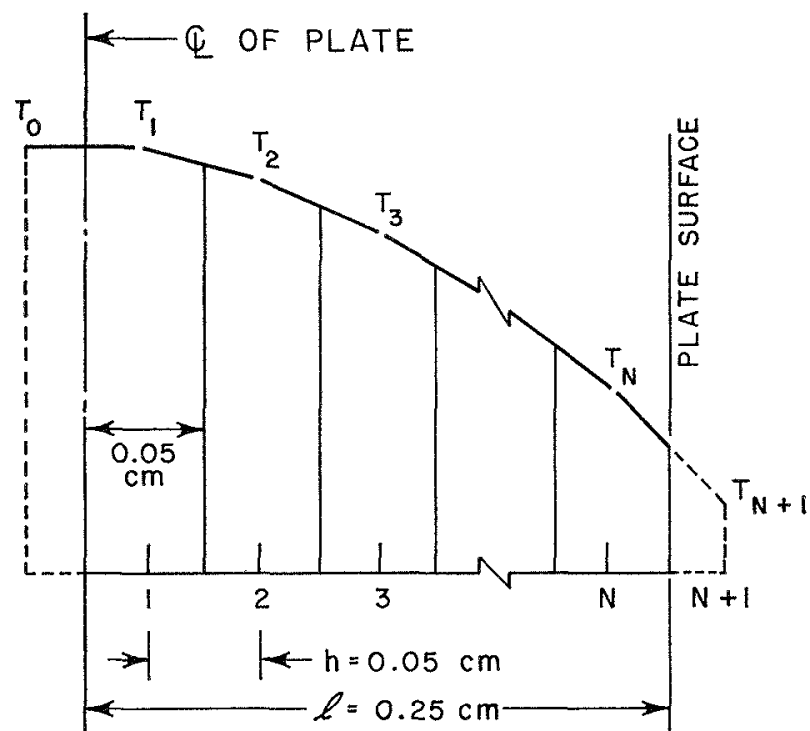

FIG. 3. Sectionalization of Space for Analog Computation. 
temperature. $K(T)$ is given by $\mathrm{Eq} .(8)$, and $c(T) \rho(T)$ is given by Eq. (10).

It is assumed that the material is isotropic and that the volumetric heat-generation rate is uniform, so that the temperature distribution is taken to be symmetrical with respect to the plate midplane.

The half-thickness is divided into $\mathrm{N}$ sections of width $\mathrm{h}$ as shown in Fig. 3. The temperature distribution between centers of any two consecutive sections is assumed to be linear. This enables one to integrate Eq. (11) within each of these sections, thereby leaving only $T_{0}, T_{1}, T_{2}, \cdots T_{N}, T_{N+1}$ as the unknown functions of time. Both $T_{0}$ and $T_{N+1}$ are outside the boundaries. This method of solution of the heat-conduction equation is found to require the minimum number of sections. Several other differential-equation formulations were investigated before arriving at this conclusion.

Now consider any section $i$ and integrate Eq. (11) in this section:

$$
\begin{aligned}
K_{i, i+1} \frac{\left(T_{i+1}-T_{i}\right)}{h}- & K_{i-1, i} \frac{\left(T_{i}-T_{i-1}\right)}{h} \\
& +q h=\int_{(\imath-1) h}^{i h} c \rho \frac{\partial}{\partial t} T d x,
\end{aligned}
$$

where $K_{i, 2+1}$ is the conductivity at the boundary of section $i$ and $i+1$, and is evaluated at

$$
T=1 / 2\left(T_{\imath}+T_{\imath+1}\right) .
$$

To show the influence of variable ronductivity alone, $c \rho$ is assumed constant. The added influence of variable

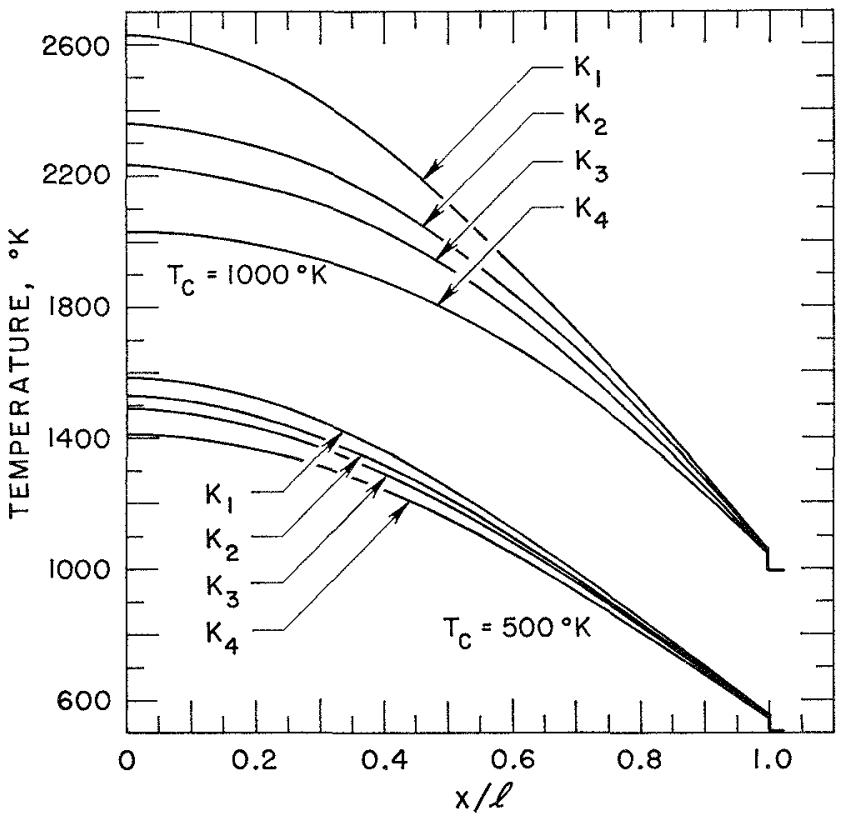

FIG. 4. The Effect of Radiative Transfer upon Temperature Distribution in a Plate of $\mathrm{UO}_{2}$.

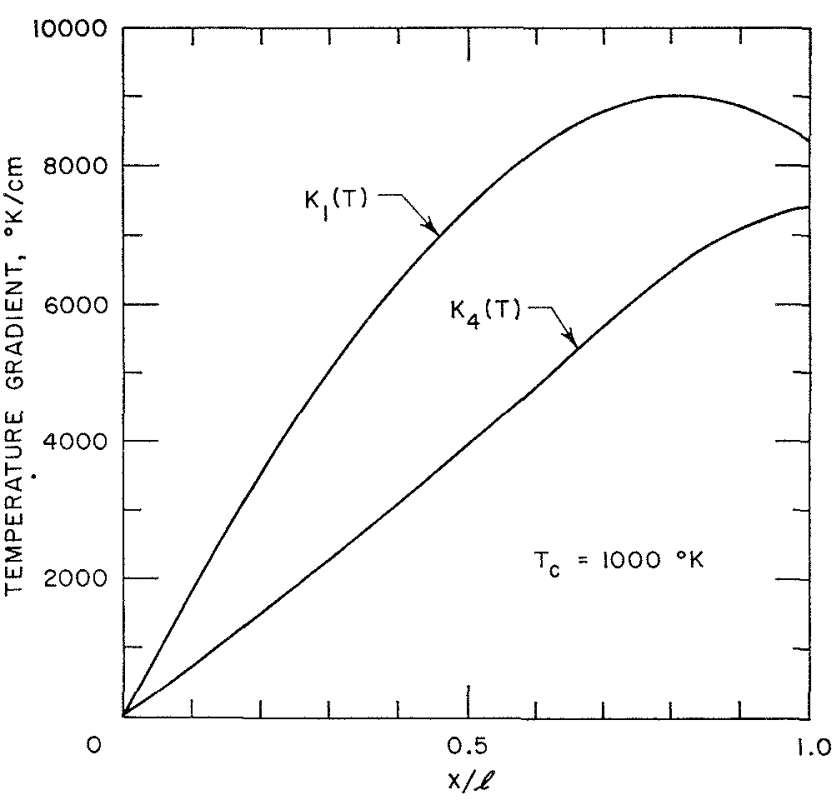

FIG. 5. The Effect of Radiative Transfer upon Temperature Gradient.

heat capacity is studied separately. Hence two sets of equations must be developed.

If $c \rho$ is not a function of $T$, the right-hand side of Eq. (14) may be expressed as

$$
\begin{aligned}
c \rho \frac{\partial}{\partial t} \int_{(i-1) h}^{i h} T d x & \\
= & c \rho \frac{\partial}{\partial t}\left[\int_{0}^{h / 2}\left(T_{i}+\frac{T_{i-1}-T_{i}}{h} x\right) d x\right. \\
& \left.\left.\quad+\int_{0}^{h / 2}\left(T_{i}-\frac{T_{i}-T_{i+1}}{h} x\right) d x\right] \cdot\right\}
\end{aligned}
$$

Because of the symmetry about the center plane,

$$
T_{0}=T_{1} \quad \text { (first boundary condition). }
$$

The outer boundary temperature is given by

$$
T_{l}=\frac{T_{N}+T_{N+1}}{2}
$$

Application of the second boundary condition yields

$$
-K_{N, N+1}\left(\frac{T_{N+1}-T_{N}}{h}\right)=h\left(T_{l}-T_{c}\right) .
$$

Thus, one may write $N$ equations of the form of $\mathrm{Eq}$. (14), for $i=1,2, \cdots N$. The boundary conditions give two more equations. These $N+2$ equations are used to solve for the $N+2$ unknown temperatures $T_{0}, T_{1}, \cdots, T_{N+1}$.

However, if $c \rho$ is assumed to vary linearly with respect to $T$, as given by Eq. (10), the right-hand side of Eq. (14) may be integrated to yield 


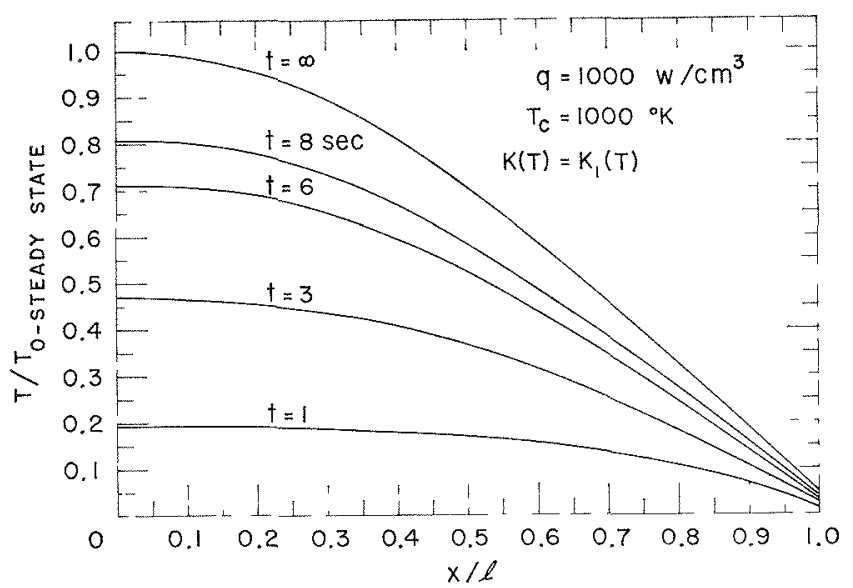

FI(x. 6. Distrubution of Temperature Resulting from a step Input of Heat with $K(T)=K_{1}\left(T^{\prime}\right)$.

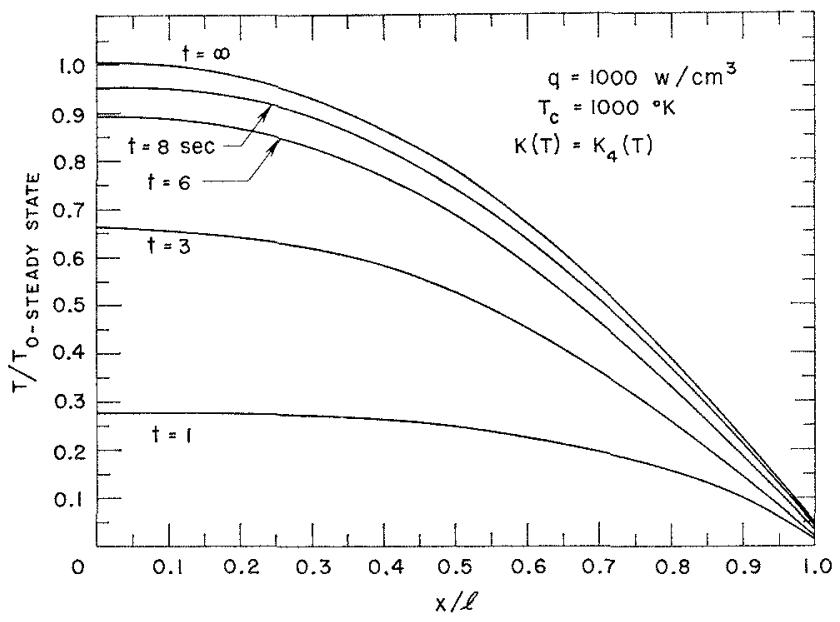

Frc. 7. Distribution of Temperature Resulting from a Step Input of Heat with $K(T)=K_{4}(T)$.

$$
\left.\begin{array}{l}
\int_{(i-1) h}^{\imath h} c(T) \rho(T) \frac{\partial}{\partial t} T d x \\
=\int_{(\imath-1) h}^{\imath h}\left(g_{0}+g_{1} T\right) \frac{\partial T}{\partial t} d x \\
\quad=h g_{0} \frac{\partial}{\partial t}\left[1 / 8 T_{\imath-1}+3 / 4 T_{\imath}+1 / 8 T_{i+1}\right] \\
\quad+1 / 6 h g_{1} \frac{\partial}{\partial t}\left[1 / 4 T_{\imath-1}^{2}+\frac{11}{2} T_{1}^{2}+1 / 4 T_{\imath+1}^{2}\right] .
\end{array}\right\}
$$

It is noted that the above method of solution of Eq. (11) has the distinct advantage that there are no memory requirements in the analog computer to deal with the nonlinearity of the equation and the boundary conditions. Further, the method is equally applicable to cylindrical geometry and is found to require the minimum number of spatial subdivisions to achieve a certain desired accuracy in the solution.

Since an exact analytic solution of Eq. (11) is avail- able for the steady-state case, ${ }^{(29)}$ it was compared with the analog solution for various numbers $N$ of subdivisions. It was found that $N=5$ is adequate for this study, since the error for this case is less than $1 / 2 \%$. For this example, $K(T)$ was taken from curve $K_{4}$ of Fig. 2; other quantities used were $q=1000 \mathrm{~W} / \mathrm{cc}$, $l=0.25 \mathrm{~cm}, T_{c}=500^{\circ} \mathrm{K}, h=6.67 \mathrm{~W} / \mathrm{cm}^{2} /{ }^{\circ} \mathrm{K}$, and $c \rho$ was held constant but given by Eq. (10) with $g_{1}=0$. It is noted that the computed curve touches the theoretical curve in each subsection and that the small crror is always $\geq 0$.

The following significant results of the present study include the effect of internal radiation upon the steadystate and transient distribution of the temperature, its gradient within the medium, and the effect of $c \rho$ being a function of temperature.

The steady-state distribution of temperature for each of the curves of conductivity versus temperature in Fig. 2 is shown in Fig. 4. This figure clearly shows that photon conduction has a significant influence upon the steady-state temperature distribution.

Radiative transfer also has a sizable effect upon the temperature gradient in the $\mathrm{CO}_{2}$ plate (which incidentally determines the thermal stress in the material). This is illustrated in Fig. 5. The effect of increasing the radiative transfer upon the transient response is illustrated in Figs. 6 and 7 in which has been considered the time-dependent temperature distribution resulting from a step increase in the internal heat-generation

TABLE 1. TIME Constants (SE()

\begin{tabular}{c|c|c}
\hline Conductivity & $T_{r}=500^{\circ} \mathrm{K}$ & $T_{c}=1000^{\circ} \mathrm{K}$ \\
\hline$K_{1}(T)$ & 3.40 & 4.80 \\
$K_{2}(T)$ & 3.25 & 4.00 \\
$K_{3}(T)$ & 2.95 & 3.45 \\
$K_{4}(T)$ & 2.60 & 2.85 \\
\hline
\end{tabular}

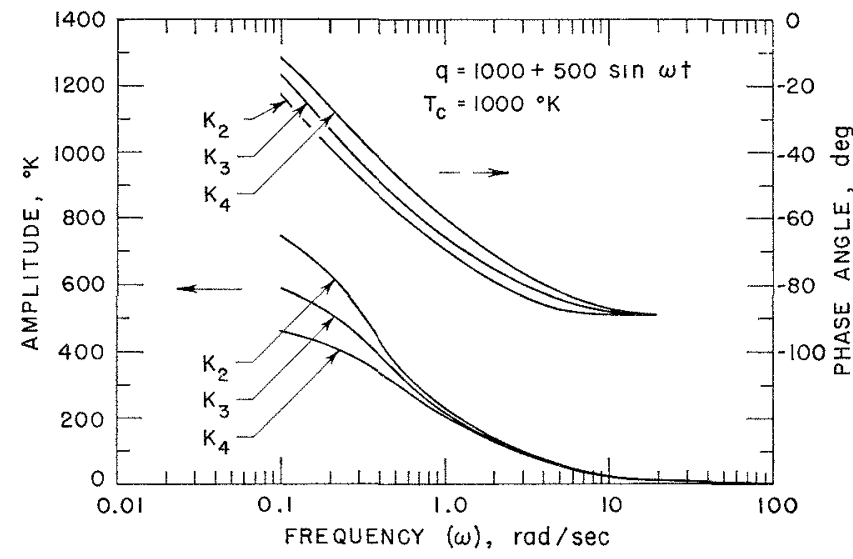

Frc. 8. Frequency Response of the Center-plane Temperatures. 
rate of $1000 \mathrm{~W} / \mathrm{cc}$ for $T_{c}=1000^{\circ} \mathrm{K}$. Each figure is normalized to the corresponding steady-state centerplane temperature rise. $K_{1}(T)$ and $K_{4}(T)$ from Fig. 2 were used. The relative speed with which the temperature distributions attain their steady states may be observed from these figures.

The familiar "iime constant" used in connection with linear systems may also be chosen as a measure to indicate the speed of response. This "time constant" is defined as the time taken by the response to reach $[1-(1 / e)]$ of its final value for a step increase of the forcing function. The "time constants" associated with the center-plane temperature responses corresponding to the various conductivity curves of Fig. 2 for coolant temperatures of 500 and $1000^{\circ} \mathrm{K}$, respectively, are obtained for a step increase in the volumetric heatgeneration rate of $1000 \mathrm{~W} /$ ce (see Table 1 ).

The effect of internal radiation on the transient behavior may also be illustrated by considering the frequency response. This consists of observing the amplitude and phase delay of oscillations of the centerplane temperature for a variation of the volumetric heat-generation rate $q$ of the form

$$
q=q_{0}+A \sin \omega t,
$$

where $q_{0}$ is a certain steady value.

The amplitudes and phase responses of the center plane temperatures are given in Fig. 8 for a coolant temperature of $1000^{\circ} \mathrm{K}$. It is apparent that the internal radiation effects are more pronounced at higher temperatures and for lower frequencies.

It is also clear that the attentuation of the amplitude of the response is quite rapid with increasing frequency. This means that high-frequency fluctuations in the heat-generation rate encountered in reactor operations have little disturbing effert upon the $\mathrm{UO}_{2}$ fuel temperature for frequencies of practical interest, * even though its thermal conductivity (and consequently its ability to diffuse heat) is considerably smaller than the metallic fuels.

A linear variation of $c \rho$ with respect to $T$ is considered next. $c \rho$ is chosen as in $\mathrm{Eq}$. (10), and the integration is carried out with the use of Eq. (18). $q$ is taken as 1000 $\mathrm{W} / \mathrm{ce}$ (applied as a step function), $T_{c}=1000^{\circ} \mathrm{K}$, and the conductivity is given by curve $K_{4}$ of Fig. 2. Figure 9 gives the response of the center-plane temperature for the + and - values of $g_{1}$. Note that the "time constant" is 3.1 see for the positive value of $g$, and 2.3 sec for the negative value of $g_{1}$.

In conclusion it is stressed that internal radiation has a significant effect upon both the steady-state and

\footnotetext{
* The "cutoff" frequency for $\mathrm{UO}_{2}$ is about 15 times less than that of metallic uranium.
}

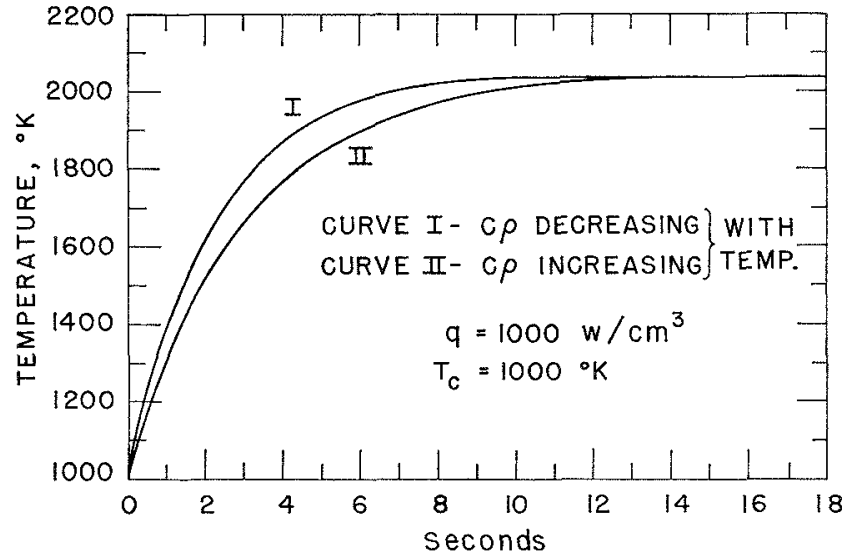

FIa. 9. The Effect of $c \rho$ upon the Response.

transient thermal response of $\mathrm{UO}_{2}$, which in turn affects the reartor design involving use of this material. The recent experimental determinations of thermal conductivity indicate that there is a substantial contribution from internal radiation. However, at present there is disagreement in the quantitative functional relationship of conductivity with temperature. The range of experimental functional relationships shown in Fig. 2 for $\mathrm{UO}_{2}$ covers a wide variation of its properties, such as oxygen content, porosity, and structure. An experimental determination of the variation of conductivity with temperature corresponding to the exact form of $\mathrm{CO}_{2}$ used in any design is, therefore, essential to a realistic evaluation of the physical behavior of that specific reactor design.

\section{REFERENCES}

1. In Evaluation of Four Design Studies of a 1000-1/We Ceramic-pueled Fast Breeder Reactor, COO-279 (Dec. 1, 1964).

2. W. R. Simmons, P. J. Persiani, and T. R. Bump, Fast Reactor Test Facility (FARET) and Initial Experimental Program, ANS National Topical Meeting on Fast Reactor Technology, Detroit, Michigan, April 26-28, 1965.

3. W. 1. Kingery, A. M. Ross, and J. Belle, "Thermal Conductivity," in Uranium Dooxide: Properties and Vuclear Ipplications (J. Belle, ed.) LSAEC, Washington, D. C., (1961).

4. D. F. Schoeberle, J. Heestand, and L. B. Miller, A Method of Calculating Transient Temperatures in a Iultiregion, 1xysymmetric, Cylindrical Configuration. The ARGUS Program, 10891/RE248, Written in Fortran II, ANI,6654 (1963).

5. J. Heestand, I). F. Schoeberle, and I. B. Miller, The Calculation of Transient Temperature Distributions in a Solid Cylinarical Pin, Cooled on the Surface, ANL-6237 (1960).

6. P. Greebler, D. B. Sherer, and N. H. Walton, FORE-A Computational Program for the Analysis of Fast Reactor Excursions, GEAP-4090 (1962).

7. R. J. Wagner, Heat-1, A One-dimensional Time Dependent or Steady State Heat Conduction Code for the IBM-650, IDO16867 (1963). 
8. W. D. Kingery et al., Thermal Conductivity, X. Data for Several Pure Oxide Materials Corrected to Zero Porosity, J. Amer. Ceram. Soc., 37, 108 (1954).

9. J. C. Jamieson and A. W. Lawson, High Temperature Heat Conductivity of Some Metal Oxides, J. Appl. Phys., 29, 1313 (1958).

10. A. D. Feith, Thermal Conductivity of $\mathrm{CO}_{2}$ by a Radial Heat Flow Method, TID-21668, General Electric Co., Cincin-nati.

11. J. L. Bates, Thermal Conductivity of $\mathrm{UO}_{2}$ Improves at High Temperatures, Nucleonics, 19, No. 6, 83 (1961).

12. W. D. Kingery and M. C. McQuarrie, Thermal Conductivity: 1, Cancepts of Measurement and Factors Affecting Thermal Conductivity of Ceramic Materials, J. Amer. Ceram. Soc., 37, 67 (1954).

13. M. F. Lyons et al., $\mathrm{UO}_{2}$ Pellet Thermal Conductivity from Irradiations with Central Melting, Trans. Am. Nucl. Soc., 7, No. 1, 106 (1964).

14. A. D. Feith, The Thermal Conductivity of $\mathrm{UO}_{2}$ up to $2500^{\circ} \mathrm{C}$, J. Nuclear Materials, 16, 231 (1965).

15. J. C. Hedge and I. B. Fieldhouse, Measurement of Thermal Conductivity of Uranium Oxide, AECU-3381 (Sept. 20, 1956).

16. R. Scott, Thermal Conductivity of $\mathrm{UO}_{2}$, AERE-M/R-2526 (March 1958).

17. T. J. Pashos et al., Irradiation Behavior of Ceramic Fuels, Proc. 1964 Geneva Conference, Paper No. A-CONF.28, $\mathrm{P} / 240$.

18. Power Reactor Technology, 6, No. 3, 12 (1963).

19. J. E. May et al., Observations on the Thermal Conductivity of Uranium Dioxide, Trans. Am. Nucl. Soc., 5, No. 2, 473 (1962).

20. J. L. Daniel, J. Matolich, Jr., and H. W. Deem, Thermal Conductivity of $\mathrm{UO}_{2}, \mathrm{HW}-69945$ (Sept. 1962).

21. 1). W. Lee and W. D. Kingery, Radiation Energy Transfer and Thermal Conductivity of Ceramic Oxides, J. Amer. Ceram. Soc., 43, 594 (1960).

22. J. Lambert Bates, Thermal Conductivity of $\mathrm{UO}_{2}$ Improves at High Temperatures, Nucleonics, 19, No. 6, 83 (1961).

23. 1). H. Whitmore, Excitation Processes in Ceramics and Anomalous Increase in Thermal Conductivity at Elevated Temperalures, J. Appl. Phys., 31, 1109 (1960).

24. R. Tiskanta and R. J. Grosh, "Heat Transfer in Thermal Radiation Absorbing and Scattering Media," International Developments in Heat Transfer, Part IV, p. 820, ASMF, New York (1961).

25. R. D. Reiswig, Thermal Conductivity of $\mathrm{UO}_{2}$ to $2100^{\circ} \mathrm{C}, \mathrm{J}$. Am. Ceram. Soc., 44, 48 (1961).

26. R. Yiskanta, Influence of Internal Thermal Radiation on Heat Transfer in $\mathrm{UO}_{2}$ Fuel Elements, Nucl. Sci. Eng. 21, 13 (1965).

27. S. Chandrasekhar, "Radiative Transfer", (I)over Publications, Inc., New York, 1960).

28. B. Garbow, A General Program for Least Square Polynomial Fit (FORTRAN II), ANME 209, ANL Code Center (1961).

29. H. S. Carslaw and J. C. Jaeger, Conduction of Heat in Series, Clarendon Press, Oxford (1959), p. 89.

\section{Discussion}

Mr. Fell (Winfrith): In your first graph, which presented a fairly marked difference in center temperature between the results with a constant $K$ and the results with varying $K$, what value of $K$ did you use for the constant value? Was it in fact the low-temperature value or was it that associated with the mean temperature of the fuel?

Mr. Sanathanan: I think you have misunderstood what I said. I said that there was a great difference between the exact and the approximate solutions of the heat conduction equation. This was because the approximate solution was obtained by neglecting the term containing $d K / d T$ in the equation. This approximation is good in materials where $K$ does not vary appreciably with temperature. But for $K(T)=42 /$ $(400+T)$, such an approximation is erroneous.

Mr. Lellouche (BNL): How many zones did you use in your analysis?

Mr. Sanathanan: We used 5 zones, and the errors were of the order of $1 \%$ or less.

Mr. Lellouche: When you say you used five, did you try ten or fifteen to start with?

Mr. Sanathanan: We first tried to solve the equation by writing it as a difference equation and considering 10 zones. We found that the errors were of the order of $20 \%$. We used up the entire analog facility at this point. It was this disappointing result that lead us to the new method of solution. First, we tried to solve the equation with 3 zones; and the errors were only of the order of $4 \%$. When we increased the number of zones to five, our errors were within $1 \%$. We did not go for more zones. The errors that 1 have been referring to are for the steady state distribution of temperature.

Mr. Lellouche: Few-zone techniques, while they very well mockup steady-state calculations of this sort, very often are far wrong in predicting the transient phenomena. This is one point. Secondly, why not go to a digital instead of an analog computer? You could then use one or two hundred points and get a relatively exact solution. The multipliers in your analog, being mechanical or electrical, are often wrong when small multiplications are involved. Then perhaps the problem must be rescaled in the middle of it.

Mr. Sanathanan: This is a historical question - the conflict between the use of analog and digital. As far as I am concerned, it doesn't make any difference; if any computer can solve the problem to the accuracies that you are expecting to solve, I think it is well and good. The analog computer had a particular advantage for our kind of parametric study. In this problem we had no difficulty with scaling.

Let me answer your previous question. The approximation in our few-zone technique is that the temperature varies linearly between mid-points of the zones. The temperature at the mid-points are kept completely unrestricted. Therefore, during transients or steady state, if the temperature distribution is not very ir- 
regular in the material, the present few-zone technique is quite adequate.

$M r . C o b b$ (Nuclear Power Group, England): In your curves of the various conductivities that you showed, it seemed to me you had a rather general fit to various conductivity values. My understanding was that the irradiation term didn't come in until much higher temperatures, something over $2000^{\circ} \mathrm{K}$. It seems to me that the differences between the $K_{1}$ and $K_{4}$ values was due almost really to differences in mean conductivity levels, rather than just to irradiation effect at high temperatures. Secondly, I notice you have only solved in slab geometry; what about the case of cylindrical geometry which is what the pin designs represent?

$M r$. Sanathanan: The method we used is very general. There is no difference in the analog simulation. Both cylindrical and slab amount to the same pro- graming, identical except that the pot settings are different.

To answer your first question, it is certainly obvious that when you increase the radiation contribution, the mean value of the conductivity also is going to increase with temperature. We are interested in temperature ranges from 2000 to $3000^{\circ} \mathrm{K}$. We are not planning to devote our attention to temperature ranges with which we are very familiar in the case of metallic fuels, that is, around $1500^{\circ} \mathrm{K}$.

Mr. Cobb: My point was that you have in fact fitted the curves to a cubic equation. I don't see at the low temperatures where you can say whether it is radiation or just differences in the basic data.

$M r$ Sanathanan: At low temperatures, say up to $1500^{\circ} \mathrm{K}$, you don't have to worry about radiation at all; the contribution will be less than $5 \%$ or so. 


\title{
Transient Temperature Distribution in Fuel Elements
}

\author{
J. E. Godts axd E. H. Restell, Jr. \\ Westinghouse Electric Corporation \\ A tomic Power Division \\ Pittsburgh, Pennsylvania
}

(PRESENTED BY E. RESTELLI)

\section{Introduction}

From a reactor systems analysis point of view, (alculation of fuel rod and cermet (ceramic-metallic expansion controlling rod) ${ }^{*}$ temperatures must provide accurate feedback to the remainder of the plant model. It must also be concise enough to minimize the amount of analog equipment or digital computing time required.

The representation for the problem is usually a onedimensional sectionalized nodal model, as shown in Figure 1, with the following defining equation:

$$
\begin{aligned}
\left(\rho C_{p}\right)_{\imath} T_{\imath}=R_{\imath+1}\left(T_{\imath+1}\right. & \left.-T_{\imath}\right) \\
& -R_{\imath, \imath-1}\left(T_{\imath}-T_{\imath-1}\right)+Q_{\imath},
\end{aligned}
$$

where the heat-transfer resistances are defined from appropriate steady-state profiles or by the heattransfer properties of the fuel element. The disadvantage of employing such a nodal approach is a possible inaccuracy when only a few sections are used. Furthermore, computed temperatures are limited to the centers of selected sections, and accurate information about the temperature gradient is not given directly, although the latter is important in determining stresses on the rod or cermet. Another disadvantage arises from the need to determine the value at each node selected in order to obtain averages. These values may fluctuate widely during transients because of the spatial distribution within the nodal regions.

Alternatives to the nodal approach involve finding transform solutions, in which spatial modes, $\psi_{n}(z)$, describe the spatial dependency of the solution, as given by

$$
T(z, t)=\sum a_{n}(t) \psi_{n}(z)
$$

* In the Westinghouse fast breeder reactor design, the fuel rods are assembled into two bundles, in line with each other. Each such bundle is one-half of the core height. The bundles are connected by central cermet rods, fabricated by powder metallurgy from a mixture of fuel, ceramic, and metal, that serve as continuous structural members through the core. Thermal expansion of the cermet rods thus results in a net reactivity decrease for the core.
An advantage of the transform-solution method is that the temperature and its derivative may be given directly at any point on the profile. In fast reactors especially, due to the high power densities, fuel and clad motion (by thermal expansion) exert a determining effect on stability and control, and thus have a strong bearing on power distribution. The system analyst is interested in the temperature distributions arising in the fuel, clad, and controlling device (such as the cermet rod), as well as their average temperatures. These average temperatures provide elements for evaluating the fuel Doppler feedback, clad-expansion feedback, controlling CEX feedback, etc. In calculating average temperatures by modal analysis, the higher the rank of the modes, the smaller their influence due to their wavelike spatial behavior. As a result, average temperature calculations require only a few terms to attain sufficient accuracy for transient temperature representation.

For the above reasons, temperature computation by finite transformation was applied to analyses of the Westinghouse Fast Breeder Reactor design utilizing the CEX (Controlled Cermet Expansion) concept. Initial work in this area employed separate transformations for the fuel and clad. ${ }^{(1,2)}$

Use of finite transform techniques (with kernels possessing analytical finite discontinuities) in treating temperature distributions in multircgion problems has been discussed by Kaplan ${ }^{(3)}$ and was applied to an analysis of a nuclear fuel rod. ${ }^{(4)}$

This paper describes a transform solution for temperature distributions within a cladded fuel rod. Discontinuous kernels and mixed geometry are used with finality and completeness $†$ preserved in the transformation. By considering the problem in mixed geometries, there is considerable simplification of the transcendental equation that yields the eigenvalues of the transformation. Also included are quantitative checks with nodal method solutions and an analysis of several TREAT experiments, in which the fast periods considered are typical of those which may be expected in a fast reactor.

$\dagger$ Finality and completeness-see reference 5, paragraph 1. 


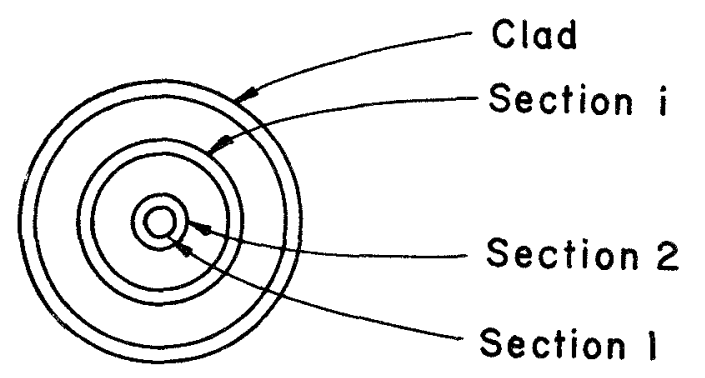

Fig. 1. Section through Fuel rod Nodal Model.

\section{Summary of Finite Transform Solution for Cylindrical Fuel, Rectangular Clad Repre- sentation}

Since the cladding is thin relative to the radius of the fuel rod, steady-state considerations show that slab representation of the clad gives negligible error. ${ }^{(6)}$ The problem considered is shown in Figure 2.

With the substitutions*

$$
\begin{aligned}
& u_{f}=T_{j}^{*}-T_{\text {avg cool }}^{*} ; \\
& u_{c}=T_{c}^{*}-T_{\mathrm{av} z \cos }^{*},
\end{aligned}
$$

where ${ }^{*}$ denotes the deviation from steady state, the appropriate conduction equations for the fuel and clad become

$$
\begin{aligned}
\frac{1}{z_{f}} \frac{\partial}{\partial z_{f}}\left(z_{f} k_{f} \frac{\partial u_{f}}{\partial z_{f}}\right)+Q\left(z_{f}, t\right)-\rho_{f} C_{p f} & \frac{\partial T_{\mathrm{avg} \text { cool }}^{*}}{\partial t} \\
& =\rho_{f} C_{p f} \frac{\partial u_{f}}{\partial t}
\end{aligned}
$$

for $0 \leq z_{f} \leq a$, and

$$
\frac{\partial}{\partial z_{c}}\left(k_{c} \frac{\partial}{\partial z_{c}} u_{c}\right)-\rho_{c} C_{p c} \frac{\partial T_{\alpha v g \text { cool }}^{*}}{\partial t}=\rho_{c} C_{p c} \frac{\partial u_{c}}{\partial t}
$$

for $0 \leq z_{c} \leq l$, where $Q\left(z_{c}, t\right)=0$. Boundary conditions for the problem are:

$$
\begin{aligned}
& \nabla u_{f}=0 \\
& \text { (a) } z_{f}=0 \text {; } \\
& k_{f} \nabla u_{f}=k_{c}^{\prime} \nabla u_{c} \\
& \left.-k_{j} \nabla u_{f}=h_{f o}\left(u_{f}-u_{c}\right)\right\} \\
& \text { (a) } z_{f}=a ; \quad z_{\iota}=0 \text {; } \\
& -k_{c} \nabla u_{c}=h_{c w}^{\prime} u_{c} \\
& \text { (a) } z_{c}=l \text {, }
\end{aligned}
$$

where $k_{c}^{\prime}=k_{c}[(a+1 / 2 l) / a]$ and $h_{c w}^{\prime}=h_{c w}[(a+l) / a]$ are the appropriate modifications to account for slab representation of the clad. The transform solution is as

* Convergence of series solutions using the dependent variables defined in (3) has been checked ${ }^{(6)}$ and found to be more rapid than series solutions for the variable $T_{f}^{*}$ when there are variations in $T_{\text {arg cool }}^{*}$. given in (2):

$$
u(z, t)=\sum_{n} a_{n}(t) \psi_{n}(z),
$$

where $a_{n}(t)$ is given by the time bchavior of the transformed variable divided by the normalizing function and where the transform kernel $\psi_{n}(z)$ is the composite function

$$
\psi_{n}(z)=\delta_{f \imath} \psi_{n f}\left(z_{f}\right)+\delta_{c \imath} \psi_{n c}\left(z_{c}\right) ; \quad(i=f, c) .
$$

$\psi_{n f}\left(z_{f}\right)$ and $\psi_{n c}\left(z_{c}\right)$ are given by

$$
\frac{1}{z_{f}} \frac{d}{d z_{j}}\left(z_{f} k_{f} \frac{d}{d z_{j}} \psi_{n f}\left(z_{f}\right)\right)+\lambda_{n}^{2}\left(\rho_{f}\left({ }_{p j}\right) \psi_{n f}\left(z_{j}\right)=0\right.
$$

and

$$
\frac{d}{d z_{c}}\left(k_{c} \frac{d}{d z_{c}} \psi_{n c}\left(z_{c}\right)\right)+\lambda_{n}^{2}\left(\rho_{c} C_{p e}\right) \psi_{n c}\left(z_{c}\right)=0,
$$

which are the eigenfunction equations from (4) and $(5)$, respectively. $\psi_{n f}\left(z_{f}\right)$ and $\psi_{n c}\left(z_{c}\right)$ also satisfy the boundary conditions given in $(6)$.

The unique transformed equation is found by multiplying equation (4) by $\psi_{n f}$ and integrating over the fuel region, and similarly multiplying equation (5) by $\psi_{n c}$ and integrating over the clad domain and summing the results. The domain elements are $2 \pi z_{f} d z_{f}=d \mathbb{R}_{f}$ for the fuel and $2 \pi\left(a+\frac{1}{2} l\right) d z_{c}=d \mathbb{R}_{c}$ for the clad, since rectangular coordinates are used for the clad. Performance of these operations yields

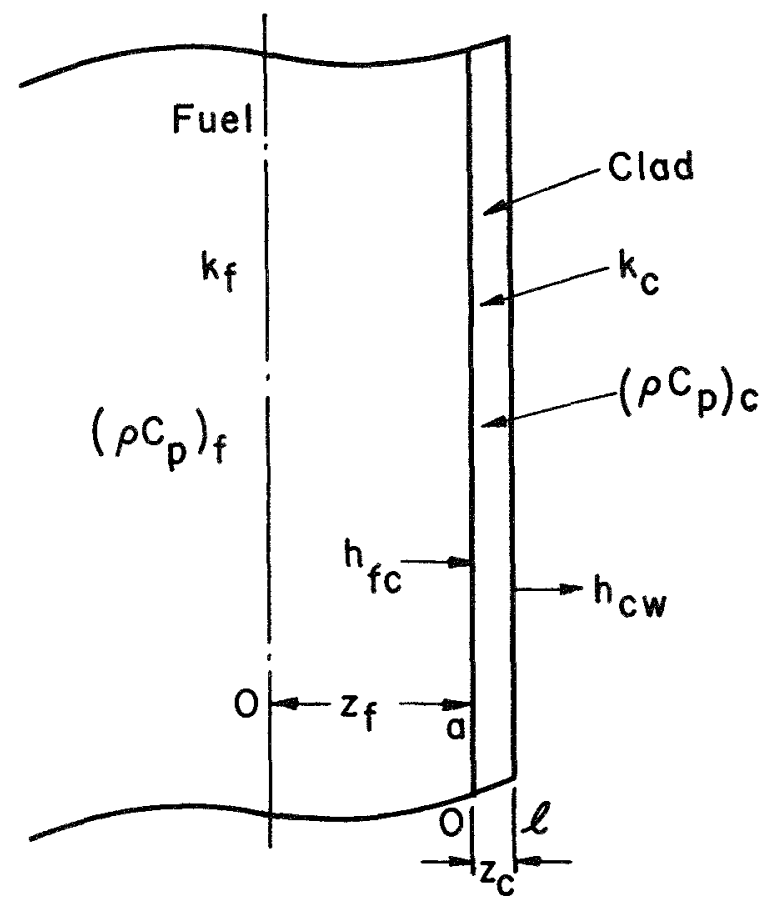

FIg. 2. Fuel-rod Nodal Model. 


$$
\begin{array}{r}
\int_{o}^{a} \frac{1}{z_{f}} \frac{\partial}{\partial z_{f}} z_{f} k_{f} \frac{\partial u_{f}}{\partial z_{f}} \psi_{n f} d \mathbb{R}_{f}+\int_{0}^{l} \frac{\partial}{\partial z_{c}} k_{c} \frac{\partial u_{c}}{\partial z_{c}} \psi_{n c} d R_{c} \\
-\int_{o}^{a}\left[Q\left(z_{f}, t\right)-\rho_{f} C_{p f} \frac{d T_{\mathrm{avg} \text { cool }}^{*}}{d t}\right] \psi_{n f} d \mathbb{R}_{f} \\
-\int_{o}^{l} \rho_{c} C_{p c} \frac{d T_{\mathrm{avg} \text { cool }}^{*}}{d t} \psi_{n c} d R_{c} \\
=\int_{o}^{a} \rho_{f} C_{p f} \psi_{n f} \frac{\partial u_{f}}{\partial t} d \mathbb{R}_{f}+\int_{o}^{l} \rho_{c} C_{p c} \psi_{n o} \frac{\partial u_{c}}{\partial t} d R_{R_{c}} .
\end{array}
$$

Define the transformed variable as

$$
\int_{0}^{a} \rho_{f} C_{p j} \psi_{n j} u_{f} d R_{f}+\int_{0}^{l} \rho_{c} C_{p c} \psi_{n c} u_{c} d R_{c}=\bar{u}_{n} .
$$

With this and with

$$
\begin{aligned}
& Q\left(z_{f}, t\right)=Q\left(z_{f}\right) Q(t), \\
& \int_{0}^{a} Q\left(z_{f}\right) \psi_{n f} d \mathbb{R}_{f}=Q_{n},
\end{aligned}
$$

and

$$
\int_{0}^{a} \rho_{f} C_{p f} \psi_{n f} d R_{f}+\int_{0}^{l} \rho_{c} C_{p c} \psi_{n c} d R_{c}=G_{n},
$$

(11) becomes

$$
\begin{aligned}
\int_{0}^{a} \frac{1}{z_{f}} \frac{d}{d z_{f}} z_{f} k_{f} \frac{d u_{f}}{d z_{f}} \psi_{n f}\left(z_{f}\right) d R_{f} \\
+\int_{0}^{l} \frac{d}{d z_{c}} k_{c} \frac{d u_{c}}{d z_{c}} \psi_{n c}\left(z_{c}\right) d R_{c} \\
+Q_{n} Q(t)-G_{n} \frac{d T_{\text {avg cool }}^{*}}{d t}=\frac{d \bar{u}_{n}}{d t} .
\end{aligned}
$$

A double integration by parts of the above integral terms interchanges $\psi_{n j}$ with $u_{f}$ and $\psi_{n c}$ with $u_{c}$. Then, using (9) and (10), and the transform definition, the time behavior of the transformed variable is given by

$$
-\lambda_{n}^{2} \bar{u}_{n}+Q_{n} Q(t)-G_{n} \frac{d T_{a v g \text { cool }}^{*}}{d t}=\frac{d \bar{u}_{n}}{d t} .
$$

Since $\bar{u}_{n}(t)$ is defined only by the $n^{\text {th }}$ mode, the transform possesses the property of finality.

Using the orthogonality property for $\psi_{n}\left(z_{\imath}\right)$ (see reference 7 ), the series solution for $u\left(z_{2}, t\right)$ becomes

$$
u\left(z_{i}, t\right)=\sum_{n} \frac{\bar{u}_{n}\left[\delta_{f_{2}} \psi_{n f}\left(z_{f}\right)+\delta_{c 2} \psi_{n c}\left(z_{c}\right)\right]}{N_{n}},
$$

where

$$
\begin{aligned}
N_{n}=\int_{0}^{a} \rho_{f} C_{p j} \psi_{n f}^{2}\left(z_{f}\right) d R_{f} & \\
& +\int_{0}^{l} \rho_{c} C_{p c} \psi_{n c}^{2}\left(z_{v}\right) d \boldsymbol{R}_{c} .
\end{aligned}
$$

From (18), the equations expressing the average temperatures in the fuel and the clad are given by

$$
\begin{array}{r}
u_{i_{d \mathrm{Y}}}\left(z_{i}, t\right)=\sum_{n} \frac{\bar{u}_{n}}{N_{n} \mathbb{R}_{\imath}} \int_{\mathbb{R}_{\imath}} \psi_{n i} d \mathbb{R}_{i} ; \\
\mathbb{R}_{i}=\int_{\mathbb{R}_{i}} d \mathbb{R}_{i} .
\end{array}
$$

\section{Solution Eigenvalues}

The solution of (9) and (10), subject to be boundary conditions of (16), yjelds the functions $\psi_{n f}\left(z_{f}\right), \psi_{n c}\left(z_{c}\right)$, and the values of $\lambda_{n}^{2}$, thus satisfying the problem. With the aid of the first three conditions listed in (6), for constant thermal conductivity, we obtain

$$
\begin{gathered}
\psi_{n f}\left(z_{j}\right)=A_{n f} J_{0}\left(p_{n f} z_{j}\right) ; \\
\psi_{n c}\left(z_{c}\right)=-\frac{p_{n f} k_{f}}{p_{n c} k_{c}} J_{1}\left(p_{n j} a\right) \sin \left(p_{n c} z_{c}\right)+ \\
\left\{J_{0}\left(p_{n f} a\right)-\frac{p_{n f} k_{f}}{h_{f c}} J_{1}\left(p_{n f} a\right)\right\} \cos \left(p_{n c} z_{c}\right),
\end{gathered}
$$

where

$$
p_{n i}=\sqrt{\frac{\rho_{2} C_{p i}}{k_{\imath}}} \lambda_{n} ; \quad i=c, f .
$$

By means of (22) and the final condition of (6), the transcendental equation for the solution eigenvalues with $\mathbf{u}$ as the root is

$$
\frac{b(\tan (\mu \mathfrak{u})+\nu \mathfrak{u})}{\nu \mathfrak{u} \tan (\mu \mathfrak{u})-1}+\frac{J_{0}(\mathfrak{u})}{J_{1}(\mathfrak{u})}-\xi \mathbf{u}=f(\mathfrak{u})=0,
$$

where

$$
\left.\begin{array}{l}
b=\sqrt{\frac{\rho_{f} C_{p f} k_{f}}{\rho_{c} C_{p c} k_{c}}} ; \quad \gamma=\frac{p_{n f}}{p_{n c}}=b \frac{k_{c}}{k_{f}} ; \\
\mu=\frac{l}{a \gamma} ; \quad \nu=\frac{k_{c}}{a \gamma h_{c w}} ; \quad \xi=\frac{b k_{c}}{h_{f c} a \gamma}
\end{array}\right\},
$$

and $\mathfrak{u}=a \gamma p_{n c}=a p_{n f}$, where (23) depends on the five parameters listed above, which are defined by the heat transfer properties of the fuel rod. A typical plot of the function, whose roots may be readily evaluated by a digital computer, is given in Fig. 3 .

With the fuel-rod parameters specified, and the eigenvalues and eigenfunctions determined from (21), (22) and (23), plus knowing the form of $Q(t)$ and $d T_{\mathrm{avg} \mathrm{cool} /}^{*} d t$, transient temperature profiles may be developed by (18).

\section{Sample Calculations}

The transform solution outlined above was applied to several transient test cases (power steps, ramp, and impulses) for parameters representing both thermal and 


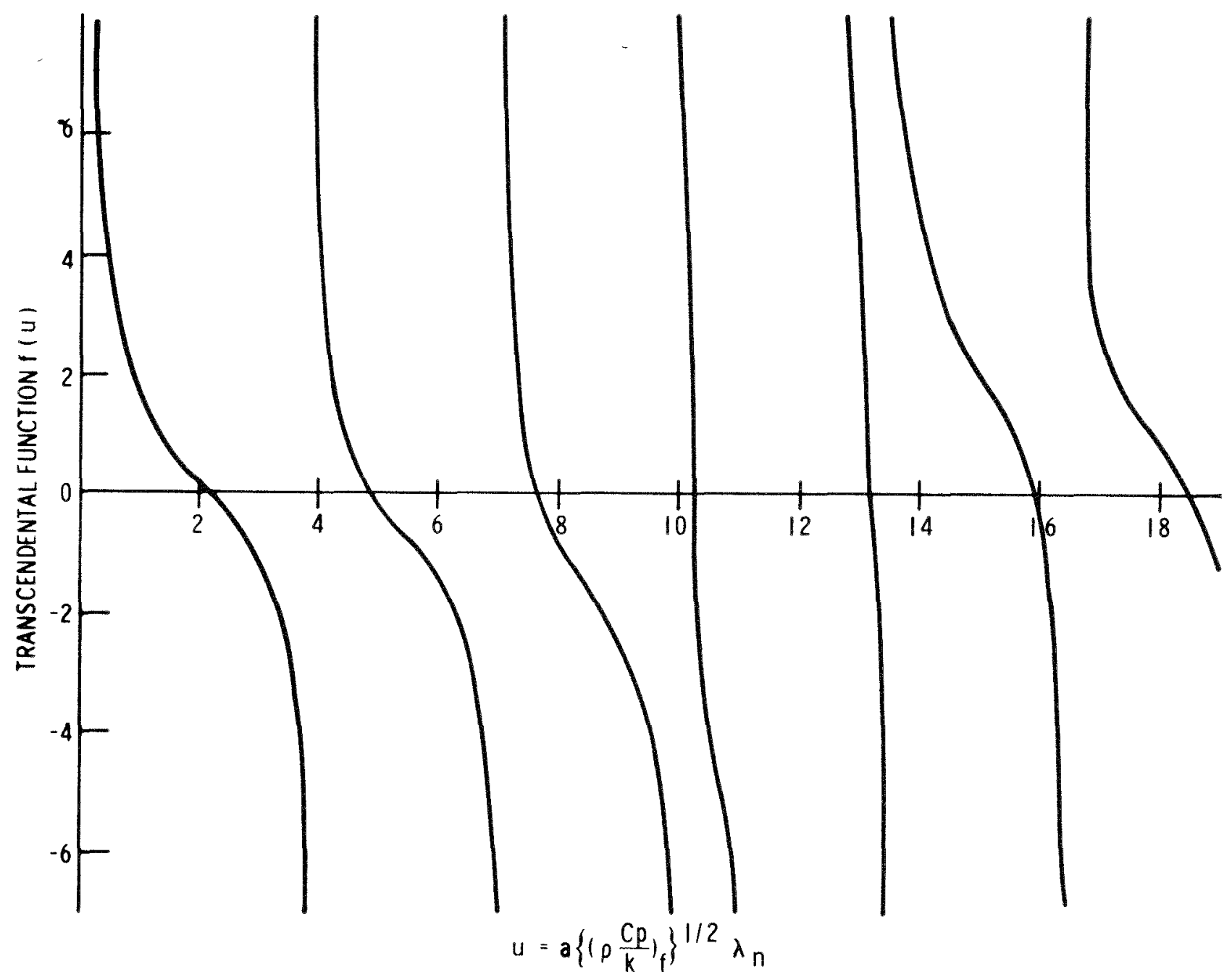

FIG. 3. Solution for Transcendental Function.

fast reactor fuel rods. As a quantitative check of the method, Fig. 4 shows the 3-mode transient profile for a $10 \%$ step in reactor power (using thermal rod parameters) as compared to the "more precise" solution using seven modes and checked using a 5-node calculation. The consistency between these solutions for this "mild" transient serves as a check on the modal method of solution. Rapid convergence of the computed average temperatures, as given by the transformation method for this transient, is illustrated in Figures 5 and 6 . Convergence of the average fuel temperature is very rapid, since the modes beyond the first oscillate spatially throughout the fuel, and their integral effect becomes progressively smaller as the order increases. Convergence of the average clad temperature is slower, however, since for this problem, the modes of lower order are of the same sign. The physical reason for this behavior is that the response of the clad is faster than that of the fuel, and hence requires more modes for an accurate description.

A direct comparison between the modal and nodal methods of comparable complexity is given in Fig. 7 for 3-mode and 3-node solutions. A. 7-mode solution is considered as the "more precise" answer. Close observation of the figure shows the modal solution is more accurate. This comparison indicates that the modal method offers an improvement over a nodal solution, especially when only a few terms are involved, and thus merits further consideration.

As a qualitative check, transient profiles were determined by finite transformation for TREAT experiments CEN 65 and CEN $67^{(8)}$ (see Figures 8, 9, and 10). For these transients, a nonuniform heat-generation profile having a $17 \%$ drop from the outer edge to the center of the pellet was used. The input to the pellet was approximated from the initial period of the reac- 


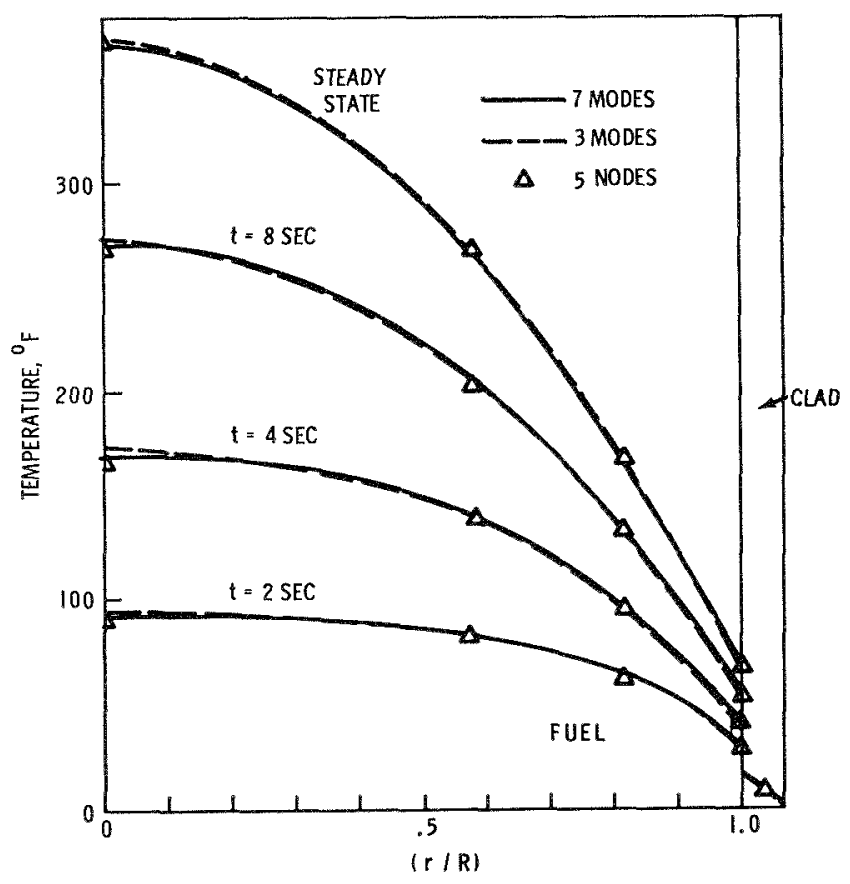

Fxa. 4. Transient Profile for $10 \%$ Step in Power at $T=0$.

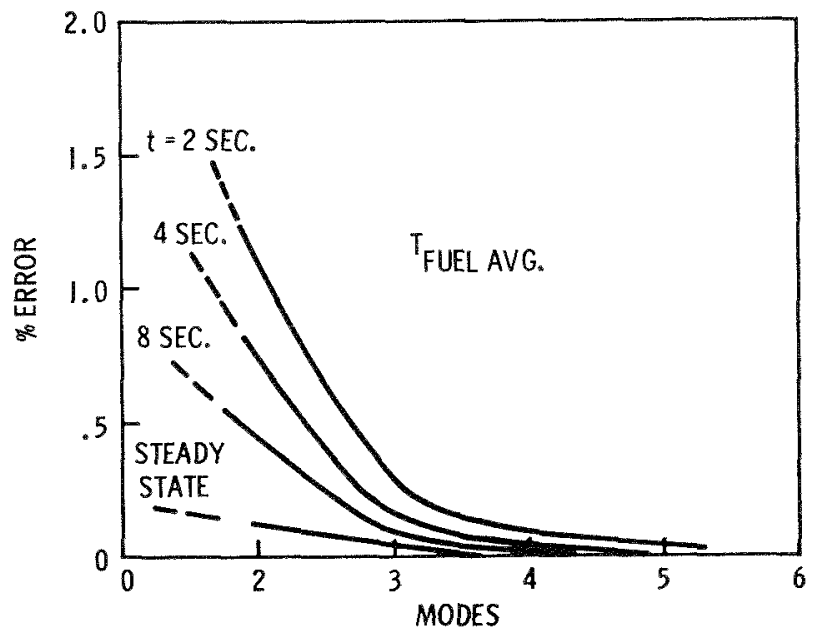

Fig. 5. Modal Solution Average Values for Fuel Temperature.

tor, maximum reactor power, and the total energy imparted to the sample, and was considered to follow the form of the transient behavior calculated for the TREAT reactor. ${ }^{(9)}$ Energies imparted to the samples were 244 and $240 \mathrm{cal} / \mathrm{gm}$, respectively.

Calculations for CEN 65 showed that the sample would reach fuel melting first at $(r / R)$ equal to approximately 0.67. For test CEN 67, heat transfer from the sample was insufficient to produce fuel melting. Considering the greater probability of clad failure when molten fuel is formed in the vicinity of the clad, the calculations show that clad under test CEN 65 would rupture, while that in test CEN 67 should remain intact. These conclusions are consistent with the experimental results.

\section{Conclusions}

Finite transformation represents a classical method of solution for temperature distributions in solids. Complexity of the analysis of the nuclear fuel rod by finite transformation may be reduced considerably, especially in the transcendental equation defining the eigenvalues of the problem, by considering a problem in discontinuous (two) geometries-cylindrical fuel

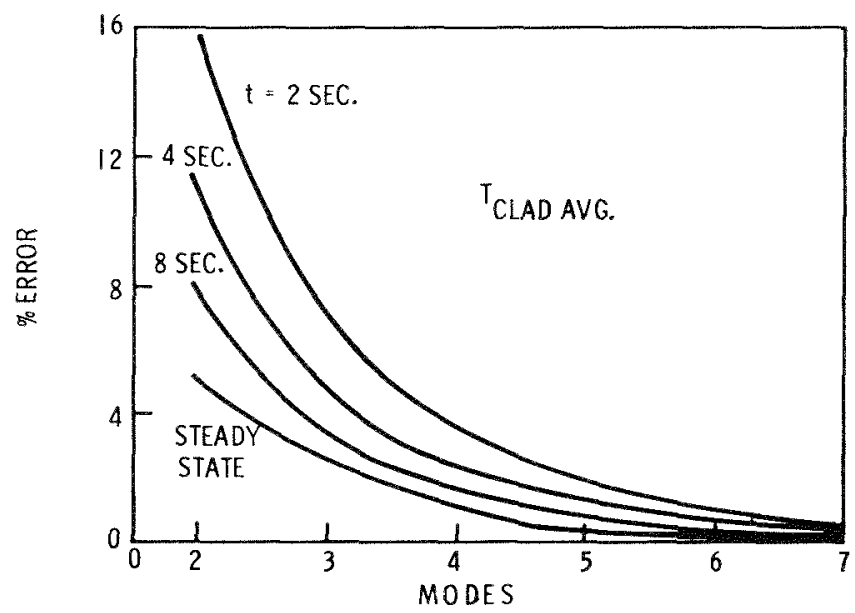

Fıa. 6. Modal Solution Average Talues for Clad Tempera ture.

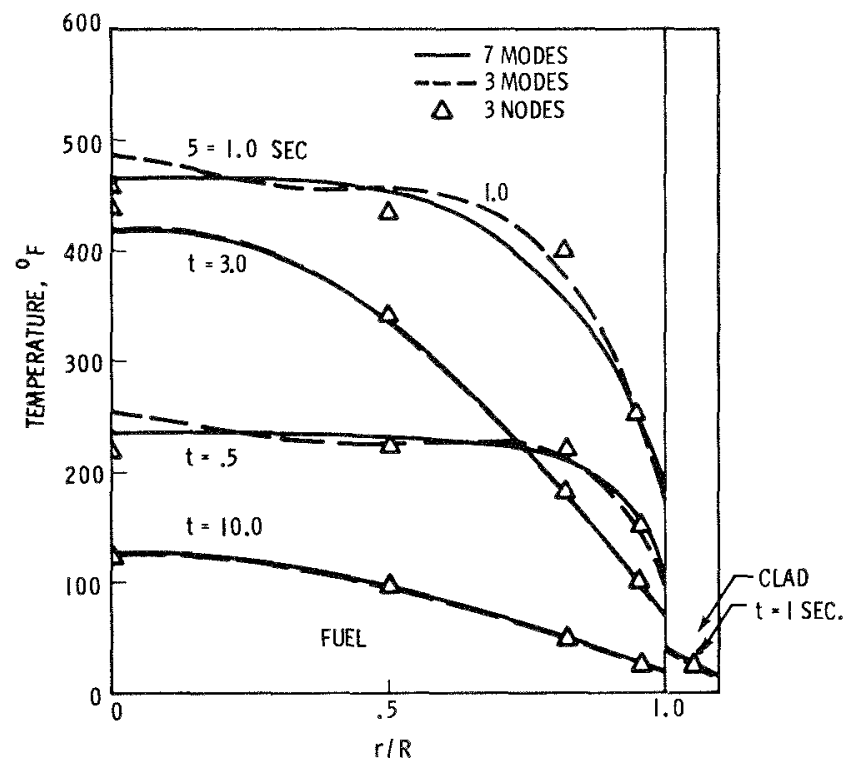

FIG. 7. Modal and Nodal Solutions for a 1-sec Full-power Pulse. 


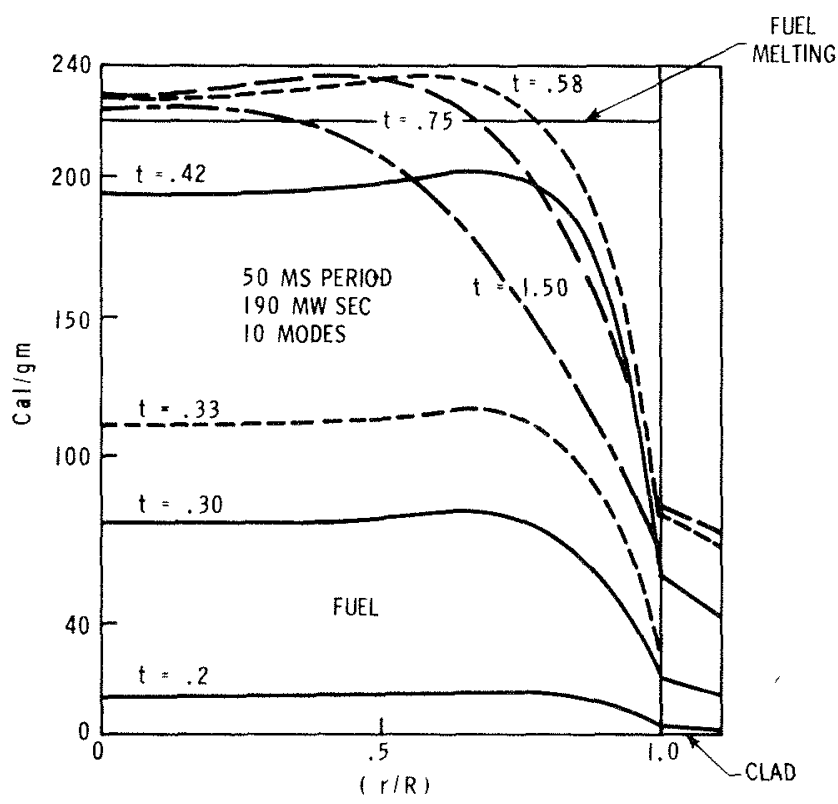

FIg. 8. Energy Release in ThEAT Experiment CEN 65 as a Function of Radius.

with slab-geometry clad. From the sample calculations performed, the method of modal analysis appears comparable to nodal models of the same complexity for "mild" or slow transients, and for fast transients is considered to be an improvement, especially when only a few modes or a few sections are involved. Furthermore, the modal method is analytical and now ready for further detailed improvements that will add to its versatility and accuracy. Nevertheless, further merits or disadvantages of finite transformation for represent-

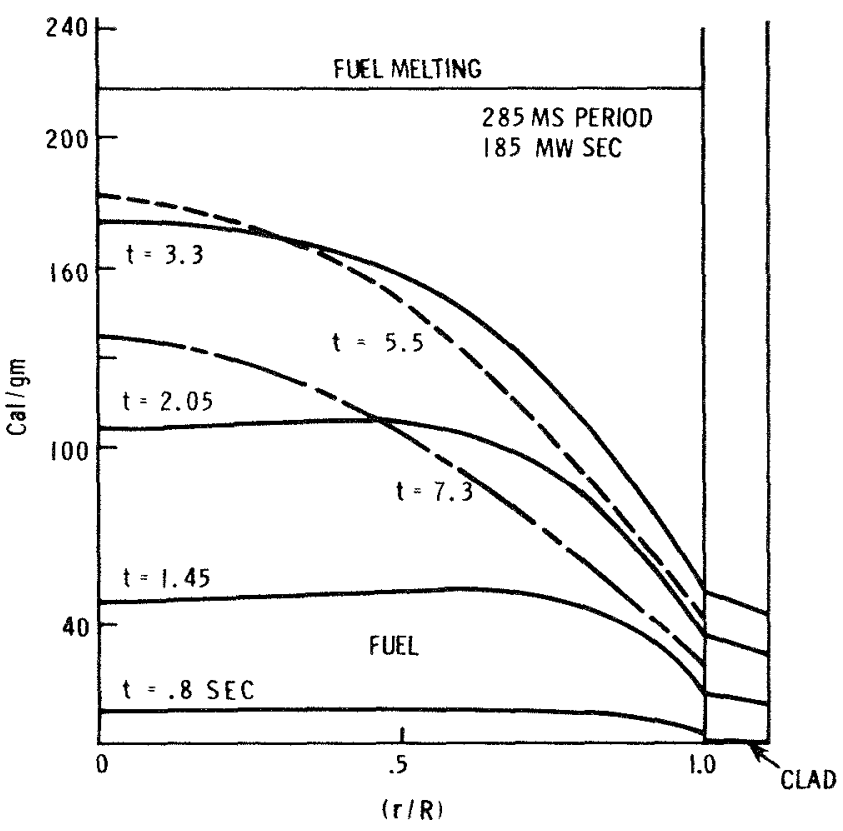

FIG. 10. Energy Reletse in TREAT kxperiment CHN 67 as a Function of Radius.

ing fuel-rod temperatures (an be perceived only by further application and cherks of the method.

It is stressed that the above approach represents only a step forward to achieving more accurate modal solutions. The inclusion of variable properties with temperature is the next goal in the advancement of the method. The nonlinear problem that arises from considering properties as a function of average temperature may be treated directly by the above method, using a stepwise procedure in time.

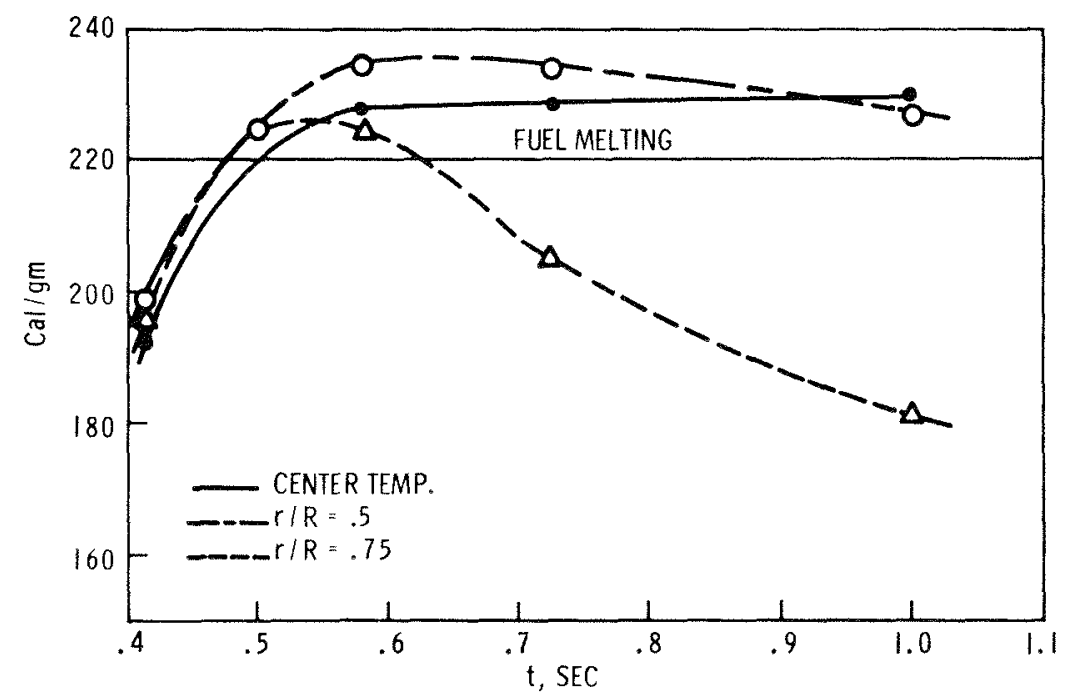

Fig. 9. Energy Release in TREAT Experiment CEN 65 as a Funetion of Time. 


\section{Glossary of Terms}

Sub f

denotes fuel

Sub $c$

denotes clad

Sub avg cool

denotes average coolant

Symbol Location

$a$

$a_{n}(t)$

$b$

$f(\mathbf{u})$

$h_{j c}$

$h_{c w}$

$h_{c w}^{\prime}$

$k_{i}$
$k_{i}^{\prime}$

$l$

$p_{n i}$

$u_{i}, u(z, t)$

$\bar{u}_{n}$

$\mathfrak{u}$

$z_{i}$

$A_{n f}$

$C_{p i}$

$G_{n}$

$J_{n}(\mathfrak{u})$

$N_{n}$

$Q_{n}$

$Q_{i}, Q\left(z_{i}, t\right)$

$Q(t)$

$Q\left(z_{f}\right)$

$R_{i},_{i+1}$

$R_{i}$

$T_{i}$

$\gamma$

$\delta_{f i}$

$\lambda_{n}$

$\mu$

$\nu$

$\xi$

$\rho$

$\psi_{n}, \psi_{n}(z)$

$\psi_{n i}\left(z_{i}\right)$

$\partial / \partial z_{i}$

\section{$\nabla$}

$(-)$

(*)
Fig. 2

Fig. 2

Fig. 2

Fig. 2

Fig. 2

Fig. 2
Description

Fuel-pellet radius

Coefficient of series solution

Coefficient ratio of fuel or clad

Eigenvalue function

Fuel-to-clad film coefficient

Clad-to-coolant film coefficient

Modified $h_{c w}$

Thermal conductivity

Modified clad conductivity

Clad thickness

Eigenfunction of region $i$

Relative temperature of region $i$

Transformed relative temperature

Root of the eigenvalue transcendental equation

Independent variable of region $i$

Kernel coefficient

Specific heat of region $i$

Notation

Bessel function of first kind of order $n$ and argument $\mathbf{u}$

Transform normalizing function

Notation

Volumetric heat generation in region $i$

Volumetric power time function

Spatial power distribution

Nodal-model heat-transfer resistance

Region $i$ domain

Temperature of region $i$

Eigenvalue ratio

Kronecker delta symbol

Eigenvalue of order $n$

Fuel, clad coefficient ratio

Clad dimensionless coefficient

Clad dimensionless coefficient

Specific mass

Figenfunction of order $n$

Eigenfunction in region $i$

Partial derivative with respect to coordinate $z_{i}$

Denotes gradient

Denotes transformed variable

Denotes deviation from steady state

Dimension Symbols

$\begin{array}{ll}\text { H } & \text { Energy } \\ \text { L } & \text { Length } \\ \text { II } & \text { Mass } \\ \text { T } & \text { Time } \\ \Theta & \text { Temperature }\end{array}$

Dimensions

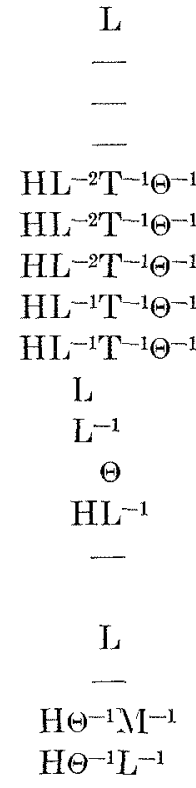

$\mathrm{H} \Theta \mathrm{H}^{-1} \mathrm{~L}^{-1}$

$L^{2}$

$\mathrm{HL}^{-3} \mathrm{~T}^{-1}$

$\mathrm{HL}^{-3 \mathrm{~T}^{-1}}$

$H L^{-2} \mathrm{~T}^{-1} \Theta^{-1}$

$\mathrm{L}^{2}$

$\Theta$

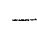

$\mathrm{T}^{-1}$

$\mathrm{NIL}-3$ 


\section{REFERENCES}

1. Liquid Metal Fast Breeder Reactor Design Study, WCAP. 3251-1 (Jan. 1964).

2. Batenburg, A., and Godts, J. E., On the Simulation of Fuel Element Temperatures Based on Finite Transforms, WCAP2261 (May 1963).

3. Kaplan, S., Extensions and Applications of the Method of Finite Transforms, Ph.D. thesis, University of Pittsburgh, 1960 .

4. Matsch, L. A., Transient One-dimensional Temperature Distribution, M. S. thesis, University of Pittsburgh, 1960.

5. Sommerfeld, A., Partial Differential Equations, Academic Press, Inc., New York (1949).

6. Godts, J. E., Simulation of the Temperature Distribution in a Clad, WCAP 4406 (April 1964).

7. Restelli, E. F., and Godts, J. E., Transient Temperature Distribution in Fuel Elements, Westinghouse Atomic Power Division, May 1965.

8. Liimatainen, R. C., Ivins, R. O., Deerwester, M. F. and Testa, F. J., Studies of Metal-Water Reactions at High Temperatures, ANL-6250 (Jan. 1962).

9. Okrent, I)., Dickerman, C. E., Gasidlo, J., O'Shea, D. M., and Schoeberle, D. F., The Reactor Kineties of the Tran sient Reactor T'est Facility, ANL-6174 (Sept. 1960).

\section{Discussion}

$M r$. Cobb (Nuclear Power Group, England): In some of the temperature profiles you showed there was actually a dip at the center of the pin. What was the reason for that; was it a flux depression?

Mr. Restelli: No. I forgot to mention that in the TREAT experiments there was nonuniform heat generation. In ANL $-6250^{(8)}$ there is a calculation that indicates a $17 \%$ rise in power across the fuel pin, and this was included in the calculation.

$M r$. Cobb: Otherwise the conductivity and specific heat were assumed constant across the pin?

Mr. Restelli: Yes.

$M r$ Lellouche (BNL): You stated that the mathematical technique you used permitted you to get a single equation for each mode.

Mr. Restelli: Yes, time-dependent.

Mr. Lellouche: But when you use a different type of mode in the clad, the amplitude of the mode would no longer be a single function of a single interior mode, but a sum of the amplitudes of the interior. They wouldn't separate, would they?

Mr. Restelli: They will separate, yes. It is possible to obtain a match, via the boundary conditions, of first mode in the fuel with first mode in the clad.

$M r$. Lellouche: Then did you use a time-dependent or a time-independent boundary condition?

Mr. Restelli: It doesn't matter. We matched thermal fluxes and film coefficients.

$M r$. Godts (WAPD): We used, in fact, a series of dis- continuous functions. When we speak of a mode, that mode describes the properties within the fuel element as well as in the clad. That means that the transform equation will be representative of the behavior of the fuel and the clad at the same time. If we have a time variation of the properties, like the film coefficient between fuel and clad, we must have a new series of eigenfunctions. If we use a digital computer, these eigenfunctions can be easily tabulated, offering a very easy possibility to solve those problems.

$M r$. Lellouche: In a full-feedback model in which you try to mock up the dynamics, I no longer think that this would be a valuable technique as opposed to a straight numerical multipoint analysis because of the nonlinearities that arise.

Mr. Godts: This might be true although there is something working for us. We found out for the same complexity, that means, the same number of modes and nodes, analog computing requires slightly less equipment; I suppose that with digital techniques we would have slightly shorter time except naybe for certain specific nonlinearitics.

Mr. Fell (Winfrith): It seemed to me from your graphs that in all cases you are using essentially a linear temperature fall through the clad, which I think is obviously the right thing to do. Although you might use many modes in the fuel, there is no sense in ever using more than one mode in the clad. Now, if you have many modes in the fuel and are sticking to one mode in the clad, it should effectively come out the same if you ean put your boundary conditions as a single boundary condition on the surface of the fuel. So the mathematies turns out really to be no different from doing effective mode shapes over the fuel alone.

Mr. Restelli: I don't think I agree with that. I did plot all the drops across the clad linearly and didn't bother to calculate the intermediate points from the calculation. However, the effect of the higher modes in the clad had quite a bit of influence on the solution. In fart, since the clad is thin, it has a fast response, and it really requires more modes than the fuel for an accurate representation.

Mr. Fell: I am essentially saying that you can always treat the clad as if it were simply a conductor in parallel with the heat-sink term. In fact, this will fail to be true only if you have to take into account modes which put a node in the middle of the clad.

$M r$. Restelli: In other words, just add a node to the outer edge of the solution, is what you are saying. Well, yes, that would be one way of treating the problem. I think I like the modal approach through the clad just as well. 


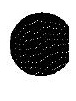

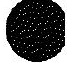




\title{
Session VI-A \\ October 13, 1965
}

\section{Fuels and Fuel Behavior}

\author{
Chairman: S. LAwroski \\ Argonne National Laboratory
}

Secretary: H. Savage

Argonne National Laboratory 
0

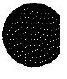




\title{
Fast Reactor Safety Considerations Related to Fuel Macrostructure
}

\author{
R. E. Petelison and S. Goldsmith \\ Battelle Memorial Institute \\ Pacific Northwest Laboratory \\ Richland, Washington \\ (PRESHNTED BY R. E. PETERSON)
}

\section{Introduction}

Inherent safety in fast reactor core design has continued to receive substantial attention as evidenced by the abundance of work reported on the Doppler effect. ${ }^{(1,2)}$ Controlled fuel expansion also has been suggested as an alternative or augmenting mechanism for limiting fast power reactor excursions. ${ }^{(3)}$ In each case fuel design plays a dominant role in achieving maximum safety characteristics for a given reactor design.

Fuels of the ceramic type, such as oxides, carbides, nitrides, and sulphides, possess some measure of inherent safety by virtue of the negative Doppler effect associated with the fertile fuel isotopes. Metallic or cermet fuels offer similar protection because of a relatively large negative reactivity expansion characteristic. It is often assumed in the analysis of transient reactor behavior that both of these mechanisms provide instantaneous negative reactivity feedback in proportion to the energy generated. The validity of this assumption depends upon the rapidity with which the temperature of the bulk fuel rises relative to the energy generated. If the fuel has a completely homogeneous structure or if the excursion is in the subprompt critical range, then instantaneous feedback is an adequate assumption. However, if some small amount of time is required for heat transfer to take place from fissile components of the fuel, such that the bulk fuel temperature lags the energy generation, then the assumption of instantaneous feedback for very fast excursions can be substantially in error. Further, in the case of an expanding fuel, inertia limits the response following a temperature rise in addition to thermal time delays encountered. It is reasonable to conclude that these considerations assume importance only in the range of supcrprompt accidents, and it is in this regime that the present work applies.

The transient response of the fuel to a very fast burst, whether it be entirely thermal or thermoelastic, depends upon such macroscopic properties as density, homogeneity, thermal conductivity, specific heat, and elastic modulus. Investigations have been undertaken to assess the importance of homogeneity to prompt Doppler feedback in mixed oxide fucls. ${ }^{(4,5)}$ However, no evaluations of this kind have been published for other ceramic fuels or for cermets. Further, it is of interest to investigate the importance of other macroscopic fuel properties to inherent safety as well. This paper summarizes recent work relating these properties to fast reactor safety when the reactor undergoes a prompt burst accident.

\section{Transient Heat Transfer in Inhomogeneous Fuels}

Transient heat transfer within the bulk fuel is a consideration when the fissile component is not homogeneously mixed with the bulk fuel. Inhomogeneities in fuel can be due to the fuel design, method of manufacture, or may develop under certain conditions of irradiation.

Some examples of inhomogeneous structure are shown in Fig. 1. An indication of the wide range in dispersion of fissile material in different fuel types is apparent.

Three mechanisms by which heat is transported within the fuel are conduction, radiation, and recoil of fission fragments. In selecting a physical model for analysis of these processes, the fuel is assumed to be composed of spherical particles of fissile material uniformly dispersed in the bulk fuel, as shown in Fig. 2. Although the choice of this model is somewhat arbitrary, the results are relatively insensitive to exact geometry.

The diffusion equation governing the heat-conduction process in this model is

$$
\nabla^{2} u(r, l)=\frac{1}{K} \frac{\partial u}{\partial t}(r, l),
$$

where $u$ is the temperature at point $r$ and time $t$, and $K$ the thermal diffusivity.

Solutions have been obtained ${ }^{(4)}$ for the temperature rise $\Delta T_{1}$ of the fissile particle and that of the surrounding fuel, $\Delta T_{2}$, of the forms

$$
\Delta T_{1}=A \psi+B_{1} \sum_{n=1}^{\infty} g_{n}
$$



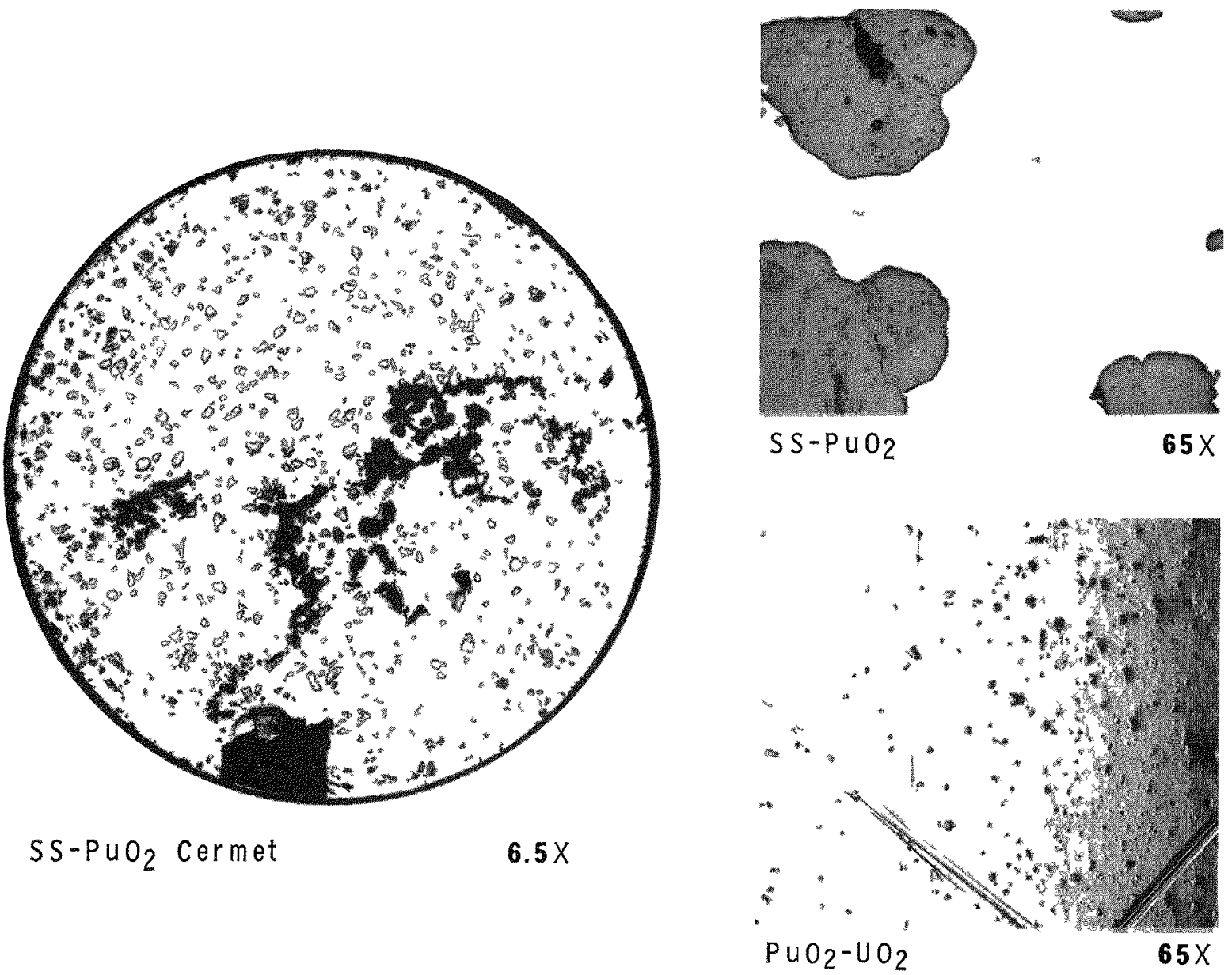

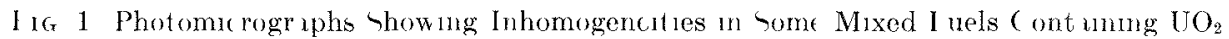

and

$$
\Delta T_{2}=1 \psi-B_{2} \sum_{n=1}^{\infty} q
$$

where the firnt $t \in 1 \mathrm{~m}$ in each case is the heat gentiation and the second term reprenents the sum of all the modes of heat conduction into the fucl The $g_{n}$ are goterned by time constants relited to the fuel composition as follows

$$
\tau_{n}=\frac{d_{1}}{4 K} \frac{1}{L^{7 / 3} \lambda_{n}^{2}},
$$

where $d$ is the ficsile particle dianeter, $E^{r}$ the enuch ment rolume fraction, $\lambda_{r}$ the eigenv alue, and

$$
K=\text { therm } \lambda \text { d diffusirity }=k / \rho c_{p}
$$

Here $h$ is the thermal conducturity, $\rho$ the fucl density, and $c_{p}$ the fuel specific heat

Thus, the fuel parametex affecting the time delay are thermal diffusivity (involving thermal conduc tivity, density, and specific heat), particle size, and enuchment Values for theimal propertes of some

selected fuel typen are hown in Table 1 near normal operating fuel temperatues

It is intercsting to note that the carbides and mitrides bave the most favolable tiansient heat transfer (har acteristus The diffurrity of a abides cxceeds cren that of stanless steel bec uuse of the higher specific heat of the litter Oxides and sulphides, on the other hand, are the le wh farolable because of then low conductivity Tme constants for fundamental mode heat tiansfer are hown on $1 \mathrm{gg} 3$ as a function of par ticle diameter and enuchment Since a large fraction of the heat transfers in thrs mode, neglect of the terms of higher order does not introduce large error

In the case of heat deposted in the bulk fuel by fission fragment iccorl fiom tissile particles, the proress is effectively instantaneour However, the amount of heat transferred by this merhamsm depends upon fissile particle size and separation, 1 e, homogeneity. The mean iange of these fiagments is from about 9 to $12 \mu$ in ceramic type fuels Analyses have been canied out which show that for very small particles, of approximately $1 \mu$ drameter, (loce to $90 \%$ of the energy 
escapes in this manner; in the limit of very large particles $(>100 \mu)$, the fraction approaches $33 \% .^{(5)}$ In any case, if fissile particles are separated by no more than about twice the mean range, substantial instantaneous heating of the bulk fuel is obtained. As a point of reference the particle separation distance for several particle sizes and fuel enrichments is shown in Table 2, for which a uniform distribution has been assumed.

One can also add to the heat from fission-fragment recoil, the threshold fission heating in $\mathrm{C}-238$ or Th-232 if the bulk fuel is a fissile-fertile mixture. This fraction is near $12 \%$ in a 1500 -liter carbide core with U-238, but is only about $4 \%$ with thorium fuel, beeanse of its lower fission cross section and higher fast fission threshold. Radiation undoubtedly supplements the other heat-transport mechanisms, although its net effect is uncertain because of photon scattering at grain boundarics and because of increasing opacity to infrared wavelengths with increasing fuel temperature. Some conservatism is retained by neglect of this component in the present work.

\section{Thermal Expansion Kinetics}

For a fuel in which inherent safety is derived from a negative reactivity coefficient of thermal expansion, inertial effects become important in a fast burst in

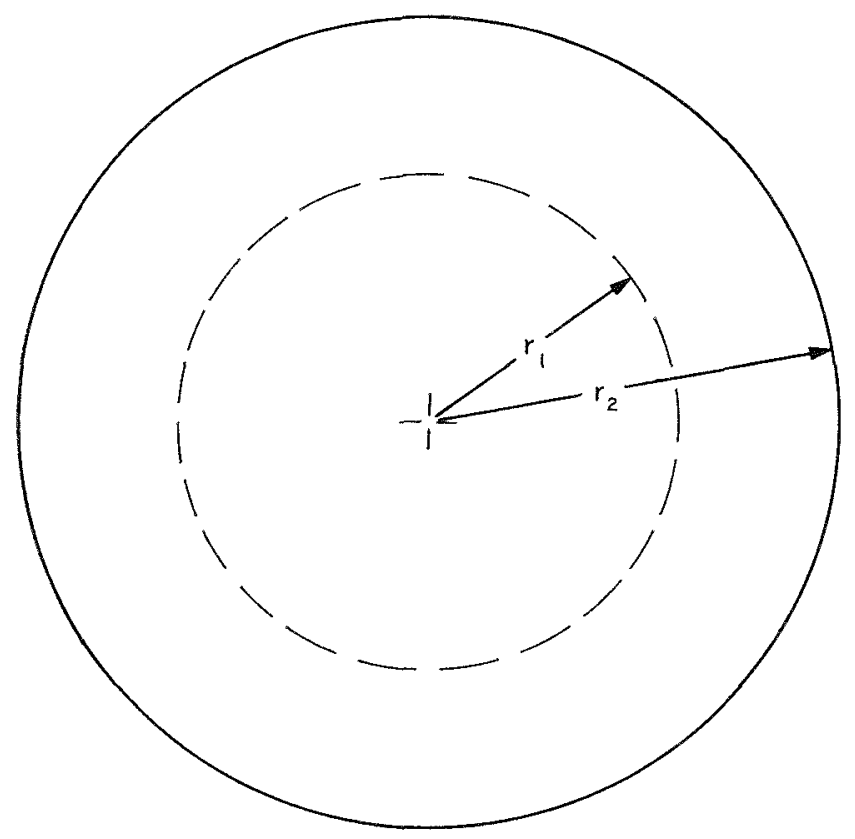

Fl(x. 2. Physical Model.

$$
\begin{aligned}
\nabla^{2} u & =\frac{1}{K} \frac{\partial u}{\partial t} \\
\tau_{\mathrm{J}} & =-\frac{1}{\lambda_{\mathrm{J}}^{2} K}
\end{aligned}
$$

\begin{tabular}{|c|c|c|c|c|c|}
\hline $\begin{array}{c}\text { Fuel } \\
\text { Material }\end{array}$ & $\begin{array}{c}\text { Theoreti- } \\
\text { cal } \\
\text { Density } \\
\left(\mathrm{g} / \mathrm{cm}^{3}\right)\end{array}$ & $\begin{array}{c}\text { Thermal } \\
\text { Conductiv- } \\
\text { ity (cal/ } \\
\mathrm{cm}-\mathrm{sec}-{ }^{\circ} \mathrm{C}\end{array} \mid$ & $\begin{array}{c}\text { Specific } \\
\text { Heat } \\
\text { (cal/ } \\
\left.\text { (cal } / \mathrm{g}-{ }^{\circ} \mathrm{C}\right)\end{array}$ & $\begin{array}{c}\text { Thermal } \\
\text { Diffusiv- } \\
\text { i1y } \\
\left(\mathrm{cm}^{2} / \mathrm{sec}\right)\end{array}$ & $\begin{array}{l}\text { Melting } \\
\text { Point } \\
\left({ }^{\circ} \mathrm{C}\right)\end{array}$ \\
\hline $\mathrm{UO}_{2}$ & 10.97 & 0.008 & 0.078 & 0.009 & 2750 \\
\hline $\mathrm{PuO}_{2}$ & 11.47 & (a) & (d) & 0.009 & 2280 \\
\hline ITC & 13.63 & 0.06 & 0.045 & 0.098 & 2350 \\
\hline $\mathrm{PuC}$ & 13.6 & (a) & (d) & 0.098 & 1850 \\
\hline IUN & 14.32 & 0.05 & 0.077 & 0.045 & 2885 \\
\hline PuN & 14.23 & (a) & 0.061 & 0.058 & 2750 \\
\hline Us & 10.87 & 0.004 & 0.055 & 0.007 & 2462 \\
\hline Pus & 10.60 & (a) & $(d)$ & 0.007 & 2400 \\
\hline $\mathrm{ThO}_{2}$ & 10.00 & 0.0078 & 0.073 & 0.01 & 3250 \\
\hline $\begin{array}{l}\text { Stainless } \\
\text { Steel }\end{array}$ & 7.9 & 0.05 & 0.12 & 0.05 & 1400 \\
\hline
\end{tabular}

TABLE 1. Thermal Properties or Fuel Materials

(a) Value not available; assumed the same as uranium compound.

addition to the thermal delay considered above. The problem in expansion reactivity feedback has been investigated in comnection with the design of fast pulsed reactors. ${ }^{(6)}$ The time required for expansion feedback to become effective is governed by the time required for the elastic wave to propagate throughout the fuel. In the case of a fuel rod in which the wavelength of the induced stress wave is long relative to the transverse dimension, the wave equation yielding the displarement is

$$
\frac{\partial^{2} y(x, t)}{\partial x^{2}} \frac{1}{v^{2}}-\frac{\partial^{2} y(x, t)}{\partial t^{2}}=\beta \frac{\partial T(x, t)}{\partial x},
$$

where

$$
\begin{aligned}
y & =\text { the longitudinal displacement at point } x \\
& \text { and time } t ; \\
\beta & =\text { coefficient of linear expansion; } \\
T(x, t) & =\text { temperature distribution funetion; } \\
w & =\text { wave velocity }=\sqrt{M / \rho} \\
I & =\text { Young's nodulus; } \\
\rho & =\text { mass density. }
\end{aligned}
$$

The solution to this equation for stress-free ends is readily obtained for a generalized temperature function. ${ }^{(7)}$ Soodak has shown that for a temperature $T=T_{0}^{\alpha t} \cos B x$, the linear expansion coefficient $\beta$ is reduced by an inertial factor such that ${ }^{(8)}$

$$
\beta_{\mathrm{eff}}=\frac{\beta}{1+\left(\alpha^{2} / \omega^{2}\right)},
$$

where

$$
\begin{aligned}
& \alpha=\text { inverse reactor period; } \\
& \omega=\text { natural rod frequency }=\pi v / 2 L ; \\
& L=\text { rod length; } \\
& v=\text { wave velocity. }
\end{aligned}
$$




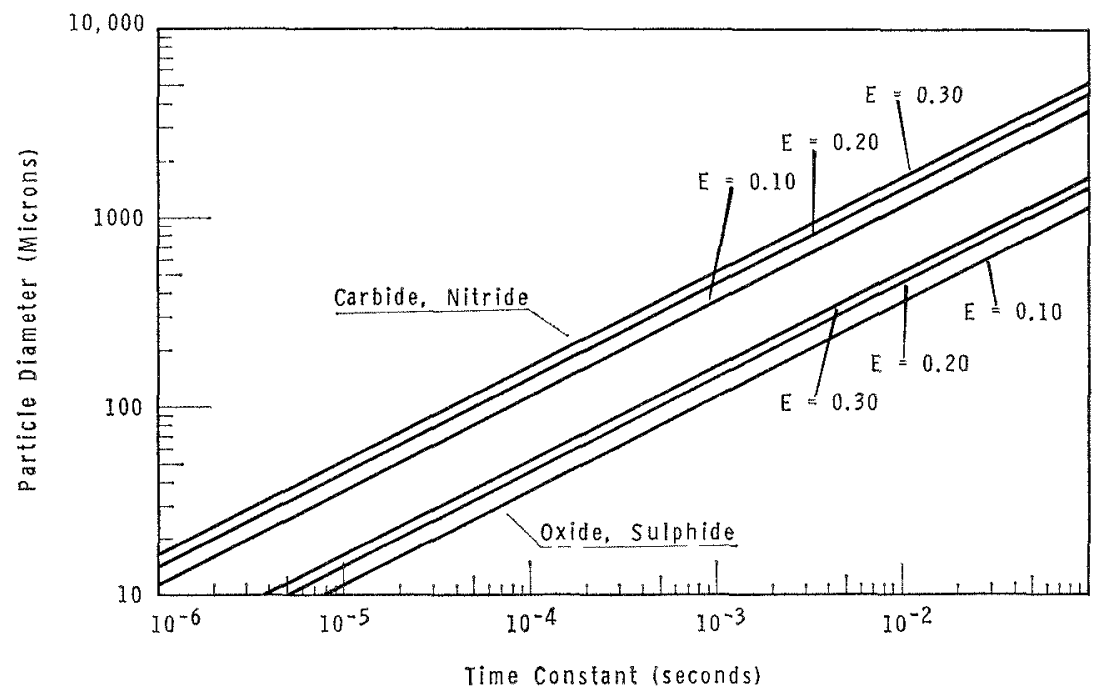

Fra. 3. Time Constants for Fundamental-mode Heat Transfer $\tau_{\nu}=d_{1}^{2} / 4 K E^{1 / 2} \lambda_{\nu}^{2}$.

TABLE 2. Particlue Separation Distance

\begin{tabular}{c|c|c|c}
\hline \multirow{2}{*}{$\begin{array}{c}(\mu) \\
\text { Particle Diameter }\end{array}$} & \multicolumn{3}{|c}{ Enrichment Volume Fraction } \\
\cline { 2 - 4 } & 0.10 & 0.20 & 0.30 \\
\hline 10 & 11.5 & 7.1 & $\begin{array}{c}4.9 \\
100\end{array}$ \\
115 & 71 & 49 \\
1000 & 1150 & 710 & 490 \\
\hline
\end{tabular}

Thus, the importance of inertia to the speed of response depends upon the ratio of the inverse period to the natural frequency of the fuel rod. Properties affecting fuel expansion are shown in Table 3 for several cermet fuels. Expansion coefficients reported here are mean values between 20 and $1000^{\circ} \mathrm{C}$.

It is evident that the wave velocity in all the above cermets is near $4000 \mathrm{~m} / \mathrm{sec}$ if the elastic modulus is not strongly affected by composition. There appear to be little data available on this latter property, however. It should be noted, that if the effective density of a rod is increased by attaching to it additional nonexpanding mass, the response is slowed substantially from that indicated by the calculated wave velocities in Table 3.

A further point of interest is that during measurements on the expansion coefficient of SS-PuO ${ }_{2}$ cermets at Battelle-Northwest, a certain amount of stress relieving was observed during the first temperature cycle of the specimen. Although this does not affect the expansion coefficient or reactor safety, it does indicate the desirability to anneal the fuel before use to avoid a reactivity penalty from initial lengthening of the fuel.

\section{Energy Release in Fast Bursts}

To permit a comparison of the importance of thermal and inertial delays and to relate these to fuel design rriteria, an estimate of the energy release under assumed accidental conditions is desirable. Since only a relative comparison is necessary, an exponential-burst approximation is adopted, that is, the reactor power is assumed to rise exponentially on a constant period until the peak of the excursion is reached. At this point the reactivity feedback mechanisms become instantaneously effective and reduce the total reactivity to zero. The energy release to the peak of the burst is given by

$$
A_{p}=A \int_{0}^{t_{p}} e^{\alpha t} d t=\frac{A}{\alpha}\left(e^{\alpha t_{p}}-1\right) \approx \frac{A}{\alpha} e^{\alpha t_{p}},
$$

where

$$
\begin{aligned}
A & =\text { initial power } \\
\alpha & =\text { prompt reactor period; } \\
t_{p} & =\text { elapsed time to peak of burst. }
\end{aligned}
$$

In general, the reactivity balance at the peak of the burst is

\begin{tabular}{|c|c|c|c|c|}
\hline Cermet Type & $\begin{array}{l}\text { Density } \\
\left(\mathrm{g} / \mathrm{cm}^{3}\right)\end{array}$ & $\begin{array}{c}\text { Elastic } \\
\text { Modulus } \\
\text { (dynes/ } \\
\left.\mathrm{cm}^{2}\right)^{(a)}\end{array}$ & $\begin{array}{c}\text { Mean } \\
\text { Expansion } \\
\text { Coeffi- } \\
\text { cient }(\mathrm{cm} / \\
\mathrm{cm}-{ }^{\circ} \mathrm{C} \times \\
\left.10^{6}\right)\end{array}$ & $\begin{array}{c}\text { Wave } \\
\text { Velocity } \\
\text { (cm/sec } \\
\left.\times 10^{-5}\right)\end{array}$ \\
\hline $\mathrm{SS}-24.5 \mathrm{v} / \mathrm{O} \mathrm{UO}_{2}$ & 8.7 & $1.38 \times 10^{12}$ & 19.5 & 3.98 \\
\hline $\mathrm{SS}-50 \mathrm{v} / \mathrm{O} \mathrm{UO}_{2}$ & 9.4 & $1.38 \times 10^{12}$ & 18.8 & 3.84 \\
\hline$S \mathrm{SS}-63.5 \mathrm{v} / \mathrm{O} \mathrm{UO}_{2}$ & 9.7 & $1.38 \times 10^{12}$ & 18.1 & 3.77 \\
\hline $\mathrm{SS}-20 \mathrm{v} / 0 \mathrm{PuO}_{2}$ & 8.6 & $1.38 \times 10^{12}$ & 17.5 & 4.0 \\
\hline
\end{tabular}

$$
\Delta k+\gamma_{1} \Delta T_{1}=\left(\gamma_{2}+\lambda\right) \Delta T_{2}
$$

Table 3. Expansion Properties of Cermets

(a) Exact values not available; assumed same as stainless steel. 
where

$\Delta k=$ excess prompt reartivity;

$\gamma_{1}=$ Doppler coefficient of fissile fuel;

$\gamma_{2}=$ Doppler coefficient of fertile fuel;

$\lambda=$ reactivity coefficient of expansion;

$\Delta T_{1}=$ temperature rise of fissile fuel particle;

$\Delta T_{2}=$ temperature rise of surrounding fuel.

By means of equations (2), (3), and (6), the energy release to the peak of the burst in terms of the thermal and inertial delay factors and reactivity roefficients may be obtained, recalling that in (2) and (3) the first term is identical with equation (7).

It is convenient to evaluate the ratio of the energy release at the peak of the burst to that obtained if the feedback were instantancous. This quantity, which will be referred to as the energy release ratio, is as follows:
The significance of a positive Doppler coefficient in fuel of inhomogeneous structure is rather striking, as shown by the large increase in relative energy release with increasing time dolay and/or decreasing period. Direct heating of the fuel, either by fission fragment recoils or threshold fission, aids substantially in holding the energy release down. Thus, from a fuel design standpoint it is important to keep the thermal time constant below the shortest credible period anticipated in a power excursion. Referring to the time constants shown in Fig. 3, carbides and nitrides offer an advantage over oxides and sulphides in this regard.

In the second type of fuel investigated, that in which expansion is the principal shutdown mechanism, the energy release ratio as a function of inverse period was evaluated for a range of thermal time constants from

$$
\frac{Q_{p}^{\prime}}{Q_{p}}=\frac{\gamma_{2}+\lambda-\gamma_{1}}{\gamma_{2}\left[1-\frac{B_{2} \alpha}{A} \sum_{n=1}^{\infty} g_{n}\right]+\frac{\lambda}{1+\alpha^{2} / \omega^{2}}\left[1-\frac{B_{2} \alpha}{A} \sum_{n=1}^{\infty} g_{n}\right]-\gamma_{1}\left[1+\frac{B_{1} \alpha}{A} \sum_{n=1}^{\infty} g_{n}\right]}
$$

where all terms have been defined previously except for $B_{1}, B_{2}$, and the $g_{n}$, which are given by

$$
\begin{aligned}
& B_{1}=\frac{6}{\rho C_{p}} \frac{\left(q_{01}-q_{02}\right)}{E} ; \\
& B_{2}=\frac{6}{\rho C_{p}} \frac{\left(q_{01}-q_{02}\right)}{1-E}
\end{aligned}
$$

with

$E=$ enrichment volume fraction;

$q_{01}=$ the initial power density in fuel particle;

$q_{02}=$ the initial power density in surrounding fuel; and for the exponential-burst approximation,

$$
g_{n} \approx \frac{a_{n} \tau_{n}}{1+\alpha \tau_{n}}
$$

where

$$
a_{n}=\frac{\left[\sin \left(\frac{\lambda_{n} r_{1}}{r_{2}}-\frac{\lambda_{n} r_{1}}{r_{2}}\right) \cos \left(\frac{\lambda_{n} r_{1}}{r_{2}}\right)\right]^{2}}{\left(\lambda_{n}^{2}-\sin ^{2} \lambda_{n}\right) \lambda_{n}^{2}} .
$$

The energy release ratio as given by equation (9) has been evaluated for two different fuel types. The first, typical of a mixture of plutonium and uranium compounds, incorporates positive and negative Doppler but no expansion. The second, characterizing a cermet, takes account of positive Doppler and negative expansion. Both are for an assumed enrichment of about $20 \mathrm{v} / \mathrm{o}$. In Figure 4, results of the first calculation are shown. The energy release ratio is given as a function of the ratio of the fundamental-mode time constant to the prompt period. Parameters of each curve are the ratio of positive to negative Doppler coefficients, $\gamma_{1} / \gamma_{2}$, and the fraction of the energy appearing directly in the bulk fuel, $\varepsilon$.
0 to $1 \mathrm{~ms}$ and a ratio of positive Doppler to negative expansion coefficients of 0 and $10 \%$. The results obtaincd are shown in Fig. 5 for a fuel rod $200 \mathrm{~cm}$ in length. The curves for $\gamma_{1}=0$ represent only the inertial delay, which is seen to become important when the reactor period falls below about $1 \mathrm{~ms}$ in this case. The effect of thermal delay is, of course, most pronounced when positive Doppler feed-back exists. However, inclusion of a small amount of fertile material may be used to introduce sufficient negative Doppler to neutralize this. Nevertholess, the inertial factor

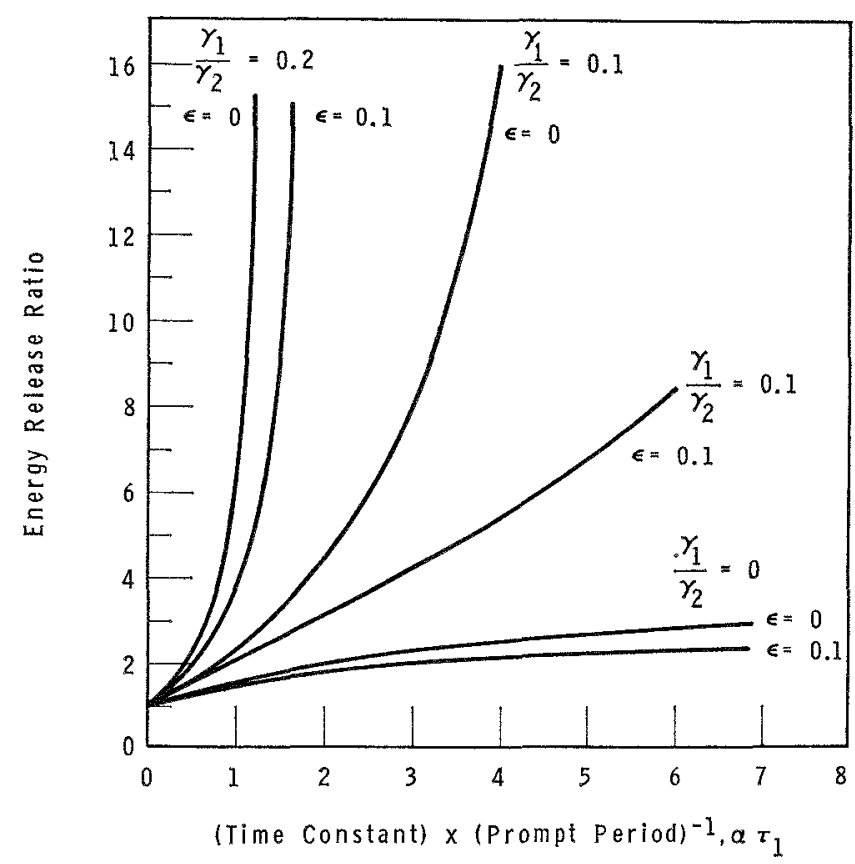

FIG. 4. Energy Release Ratio as a Function of Time Constant/Prompt Period Ratio. 


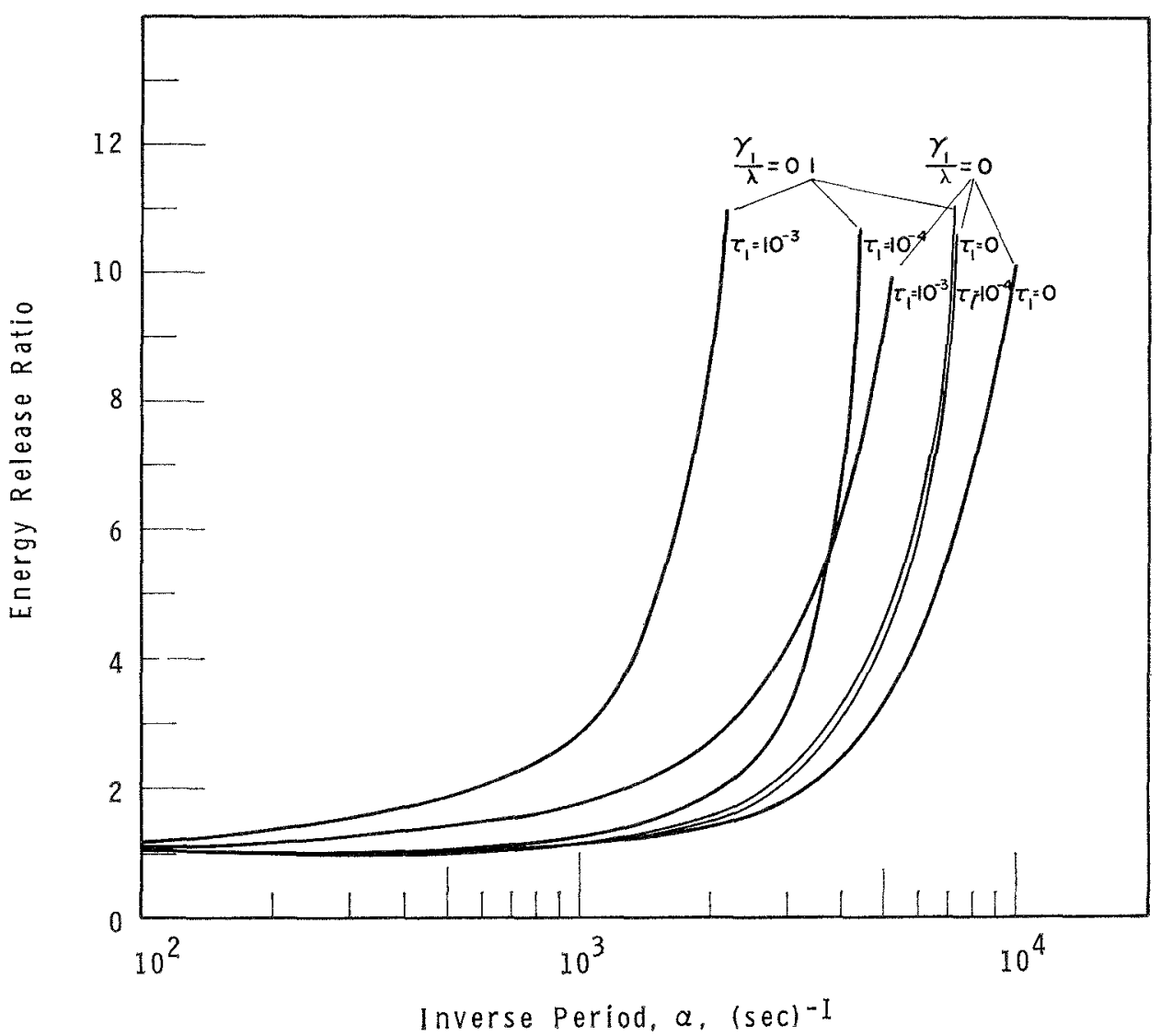

Fig. 5. Energy Release Ratio vs Inverse Period in Cermet Fuel. Length $=200 \mathrm{~cm}$.

remains limiting in fuel of this type. Direct heating by fission-fragment recoils is probably not nearly as effective in cermets as in ceramic fuels because of the shorter range $(\sim 2.5 \mu$ in stainless steel). Some advantage is also gained by incorporating a fuel having a high diffusivity, such as carbide or nitride, in the steel matrix. Reducing the fuel length has the largest effect in reducing the inertial factor. In the present case, reducing the length by a factor of two decreases by about the same factor the period at which a sharp rise in the energy release ratio takes place.

There are several defects in the present calculations which perhaps should be mentioned before attempting to interpret these results. First, the exponential-burst model is known to be in error near the peak of an excursion. However, since we are calculating only ratios of energy release rather than absolute magnitudes, less sensitivity to burst shape is expected. Second, reactivity coefficients that are linear with temperature were used for convenience. This approximation will more strongly affect those "ases in which large temperature changes take place in one fuel component. Finally, no changes in state are assumed for the fuel, again affecting energy release for the more severe accidents. It is believed that in spite of the preceding limitations, the periods and/or time constants for which the energy release rises above that of instantaneous feedback response are reliably predicted.

\section{Conclusions and Recommendations}

It is evident from the preceding analyses that thermal and elastic properties related to fuel macrostructure can be of utmost importance in fast reactor power excursions in the prompt-critical range. A certain amount of inhomogeneity in fast reactor fucls may be tolerated, depending upon the shortest credible reactor period one postulates, the amount of positive reactivity feedback, and the amount of direct heating by fission fragment recoils or threshold fission. With positive Doppler feedback the energy release is very sensitive to the ratio of the time constant to the period. Thus in a large fast oxide breeder, for example, 20- $\mathrm{PuO}_{2}$ particles would appear adequate to assure inherent safety for reactor periods down to about $0.1 \mathrm{~ms}$. Carbides and nitrides would permit an increase in particle size by about a factor of three for the same range of periods.

For ceramis fuels coprecipitation of the components will yield excellent homogeneity in fuel manufacture. Similar results have been obtained by physically 
mixing $\mathrm{UO}_{2}-\mathrm{PuO}_{2}$ powders and dry ball-milling. ${ }^{(9)}$ While the possibility exists that gross inhomogeneities in the fuel mixture may occur, the probability of such an occurrence is considered to be very small. When proper techniques and mixing times are employed, physically mixing $\mathrm{UO}_{2}$ and $\mathrm{PuO}_{2}$ powder has consistently yielded a uniform mixture. Once a uniform mixture of $\mathrm{UO}_{2}-\mathrm{PuO}_{2}$ is obtained, the mixture can be consolidated (by pneumatic impaction or sintering) to maintain a uniform distribution of the two materials during subsequent processing. To guard against the possibility of $\mathrm{PuO}_{2}$ segregation, the fabricated fuel pins can be subjected to a gamma-scanning test. This test for nonuniformity of $\mathrm{PuO}_{2}$ distribution is simple, rapid, and inexpensive, and can be applied to each fabricated fuel pin.

Cermet fuels will provide adequate inherent safety by fuel expansion providing the inverse reactor period remains smaller than the natural vibration frequency of the fuel rod. The energy release climbs very rapidly beyond this point, establishing a threshold beyond which the response is too slow to be effective. The effect of a thermal delay in heating of the bulk fuel is to move this threshold towards longer periods. In the example presented in this study the threshold occurs for periods between 0.1 and $1 \mathrm{~ms}$, depending on the choice of the amount of positive Doppler feedback and time delay. It is concluded that cermet fuels must be critically examined if employed in a reactor in which a prompt accident is credible.

Finally, the question should be answered whether fuel designed and manufactured to provide nuclear safety continues to retain those properties throughout long residence in a reactor. Observed structural changes in physically mixed $\mathrm{UO}_{2}-\mathrm{PuO}_{2}$ indicate that in the vicinity of $1700^{\circ} \mathrm{C}$ and higher, extensive homogenization occurs. Similar homogenization probably occurs in carbides and nitrides considered here as well. Of particular concern would be any strong tendency for migration and reconcentration of the fissile component with exposure. Investigations of segregation mechanisms have been carried out for oxides and to a lesser extent for carbides. ${ }^{(10,11)}$ Results so far appear encouraging. Whether or not the other properties change appreciably with long exposure can not be determined without additional measurements on irradiated fuel.

\section{REFERENCES}

1. Proceedings of the Conference on Breeding Economics and Safety in Large Fast Power Reactors, ANL-6792 (Dec. 1963).

2. Proceedings of the Conference on Safety, Fuets and Core Design in Large Fast Power Reactors, Oct. 11-14, 1965; to be published.

3. R. B. Steck et al., Liquid Metal Fast Breeder Design Study, WCAP-32511 (Jan. 1964).
4. A. Fraude, Untersuchungen über die Wirkung von zwei Temperaturkoeffizienten der Reaktivität, die aus der Entmischung eines ursprünglich homogenen Brennstoffes resultieren, Report INR-NR 59/63, Kernreaktor Bau und Be1 riebgesellschaft mbh., Karlsruhe (Oct. 1963).

5. R. E. Peterson, Neutron Kinetics Problems Associated with Mixed Oxide Fuels, HW-81259 (July 1964).

6. (․ Breidenbach et al., Preliminary Design of the ORNL Fast Burst Reactor, NAD-2136-1 (1960).

7. J. E. Michaels, Thermally Induced Elastic Wave Propagation in Slender Bars, Proceedings of the Third U. S. National Congress of Applied Mechanies, 1958.

8. H. Soodak, Expansion Feedback in Fast Pulsed Reactors, Transactions of the American Nuclear Soriety, 5, No. 1 (June 1962).

9. S. Goodsmith et al., A Physically Mixed and Impacted $\mathrm{CO}_{2-}$ $\mathrm{PuO}_{2}$ Fast Reactor $\mathrm{Fuel}$, Transactions of the American Nuclear Society, 7, No. 2 (Nov. 1964).

10. E. L. Zebroski, J. P. Mustelier, and C. Caldwell, Oxide Fuels For Fast Reactors, Fast Reactor Technology, National Topical Meeting, ANS-100, April, 1965. See also P. Novak et al., Paper in Session VA of This Conference.

11. C. D. Bingham and K. M. Broom, A Comparison of Burning Data from Uranium Carbide Development Programs with Consequences of High Temperature Operations, Trans. ANS, 8, No. 1 (June 1965).

\section{Discussion}

$M r$. Lawroski (ANL): In regard to your remarks about the situation with respect to cermets, up to what loading of the ceramic in the cermet does this apply?

$M r$. Peterson: In the particular case this was a 20 v/o cermet.

Mr. Kummerer (Karlsruhe): Concerning the reactivity feedback coming out from the thermal expansion, the inertia plays a role and the inertia is connected with the density of material. Is this influence coming from the inertia linear or nonlinear with the density?

$M r$. Peterson: The wave velocity is equal to the square root of the modulus of elasticity divided by the density. Thus it is a square-root dependence.

Mr. Okrent (ANL): Up to what temperature would the stainless steel cermet be temperature-independent, and where would its expansion properties markedly be affected by unusual effects?

Mr. Peterson: The effect depends upon the modulus of elasticity. I would expect less response at higher temperatures due to a decrease in the modulus.

$M r$. Sregel (AI): I notice you used the modulus for stainless steel in the absence of a value for the cermet. Doesn't one have to be rather careful in these rather heterogeneous materials if the two wave velocities are significantly different, as they possibly might be?

Mr. Peterson: Yes.

Mr. Siegel: I think stainless steel probably has the higher wave velocity.

Mr. Peterson: I would have to defer that to someone who has investigated the physical properties of $\mathrm{UO}_{2}$ to some greater extent than I have. 


\title{
Molten Plutonium Alloys as Fast Reactor Fuels
}

\author{
L. D. KIRKBRIDE \\ Los Alamos Scientific Laboratory \\ Los Alamos, New Mexico
}

\begin{abstract}
A Bstract
Molten plutonium alloys are being investigated by LASL as fuels for fast reactors. LAMPRE-I, a 1-1IW reactor experiment, utilized a $\mathrm{Pu}-\mathrm{Fe}$ alloy of $16 \mathrm{~g}$ $\mathrm{Pu} / \mathrm{ce}$ and demonstrated the feasibility of using molten alloys as reactor fuel. Current effort is being directed toward developing the less corrosive $\mathrm{Pu}-\mathrm{Co}-\mathrm{Ce}$ alloy system which will be employed in the IAASL Molten Plutonium Burnup Experiment $\left(\mathrm{MPBF}_{\mathrm{f}}\right)$. Results of current research and development regarding the $\mathrm{Pu}-\mathrm{Co}-\mathrm{Ce}$ fuel system are summarized.
\end{abstract}

\section{Introduction}

Molten metal alloys for utilization as fuel in fast reactors have several attractive properties, such as: capability of release of the bulk (approaching 100\%) of volatile firsion products; a large thermal expansion coefficient $\left(\sim 90 \times 10^{-6}(\Delta \mathrm{v} / \mathrm{v}) /{ }^{\circ} \mathrm{C}\right)$; the potential for simplified fuel handling and low fabrication costs; and the elimination of fuel slump as an accident consideration.

LAMPRE-I, a $1-\mathrm{MWt}$ reactor experiment, employed $\mathrm{Pu}-\mathrm{Fe}$ fuel of $16 \mathrm{~g} \mathrm{Pu} / \mathrm{cc}$ and demonstrated, in a small system, the feasibility of using a molten alloy as a fast reactor fuel. However, $\mathrm{Pu}-\mathrm{Fe}$ fuel is not a suitable fuel for large power reactors $(\sim 1000$ MWe) because of its high plutonium density (and consequently low allowable specific power).

An alternative fuel alloy, $\mathrm{Pu}-\mathrm{Co}-\mathrm{Ce}$, will be utilized in the Molten Plutonium Burnup Experiment (MPBE), ${ }^{(2)}$ which is presently being designed. This fuel alloy offers two major advantages over Pu-Fe: (1) the plutonium concentration is variable, and over the entire range of alloys from 0 to $13 \mathrm{~g} \mathrm{Pu} / \mathrm{cc}$ the melting point is less than $450^{\circ} \mathrm{C}$ (see Fig. 1); (2) the fuel is far less corrosive to the selected containment materials than is $\mathrm{Pu}-\mathrm{Fe}$ at equivalent test conditions.

Coupled with the advantages of molten alloys listed above, three areas are of concern. The first, and most obvious, is the potential for corrosion of the container and loss of the fuel. Present experience indicates that tantalum or Ta-W alloys are the most likly container materials. The development effort which has been expended on this facet has resulted in improvements to the extent that reliable long-torm fuel containment may be anticipated.

The second problem area is that $\mathrm{Pu}-\mathrm{Co}-\mathrm{Ce}$ fuels exhibit large volumetric expansions during freczing of from $1-3 \%$ for plutonium concentrations from $0-8$ g Pu/ce (see Fig. 2). This situation poses a potential operational nuisance in that the core may have to be maintained in a molten condition or under controlled conditions if mechanical damage to the container is to be avoided.

The third area is associated with the formation of a $\mathrm{Ta}-\mathrm{Co}$ intermetallic reaction layer which may be damaged during irradiation and cause undesirable mass-transport effects. Preliminary evidence obtained by radiotracer experiments in the absence of irradiation indicate that no major difficulties will be presented by this phenomenon.

The following sections will summarize the major results of current research on the various aspects of the problem of development of molten alloys and define areas which require in-pile evaluation.

\section{Corrosion Behavior}

One of the most important aspects of developing a molten-alloy fuel system is demonstrating the capability of containing the molten metal for extended periods of time. It was established early in the LAMPRE program that the corrosion-failure mechanism involved with liquid plutonium alloys contained in refractory metals was that of intergranular penetration (IGP) of the plutonium through the grain boundaries of the container. ${ }^{(3)}$ It was also realized, although somewhat later, that corrosion behavior as measured by length of time before a fuel element showed detectable plutonium on the surface was statistical in nature, with a ratio of shortest observed lifetime to longest of $<0.1$. Figure 3 shows the fraction failed versus test time for $\mathrm{Pu}-\mathrm{Fe}$ and $\mathrm{Pu}-\mathrm{Co}-\mathrm{Ce}$ under all test conditions up to $850^{\circ} \mathrm{C}$, plotted according to the Weibull distribution.

Because the time interval required to obtain meaningful corrosion data at temperatures of interest in reactor applications $\left(500-750^{\circ} \mathrm{C}\right)$ is in the thousands of hours, test equipment designed to obtain lifetime 
data at temperatures up to $1150^{\circ} \mathrm{C}$ has been recently employed. Figure 4 shows mean lifetime data obtained at elevated temperatures in vacuum $(\sim 1 \times$ $10^{-7}$ Torr) for both plutonium and $\mathrm{Pu}-\mathrm{Fe}$ eutectic alloy. It is significant that for the $\mathrm{Pu}-\mathrm{Fe}$ fuel, the high-temperature and the lifetime data obtained for a sodium environment may be represented by the same straight line, which lends support to the validity of extrapolating high-temperature data to obtain corrosion-behavior information at lower temperatures.

Tests to determine the lifetime of $\mathrm{Pu}-\mathrm{Co}-\mathrm{Ce}$ fuels of interest to the MPBE are in progress. Corrosion tests have encompassed fuel concentrations from 5-8 g $\mathrm{Pu} / \mathrm{ec}$ in contact with tantalum and Ta-W alloys (up to $10 \mathrm{w} / \mathrm{o} \mathrm{W}$ ) and at temperatures up to $1100^{\circ} \mathrm{C}$. Since there have been few failures associated with tests carried out at $850^{\circ} \mathrm{C}$ and below, no definite conclusion can be drawn concerning expected lifetime of this fuel system. However, several generalizations can be drawn from data available:

a. When all information from $\mathrm{Pu}-\mathrm{Co}-\mathrm{Ce}$ alloy corrosion tests (that is, all combinations of fuel composition, container material, and test temperature) have been pooled, it is observed that of 135 tests 27 have resulted in failures, of which 26 have been in welds.

b. The test result indicate that container walls are not penetrated by $\mathrm{Pu}-\mathrm{Co}-\mathrm{Ce}$ (in dramatic contrast to $\mathrm{Pu}-\mathrm{Fe}$ fuel) but that only welds are subject to penetration, up to the maximum observation time to date, $7500 \mathrm{hr}$ at $850^{\circ} \mathrm{C}$ (see Fig. 3).

c. When welds are removed from consideration, there is an indication that significant corrosion of

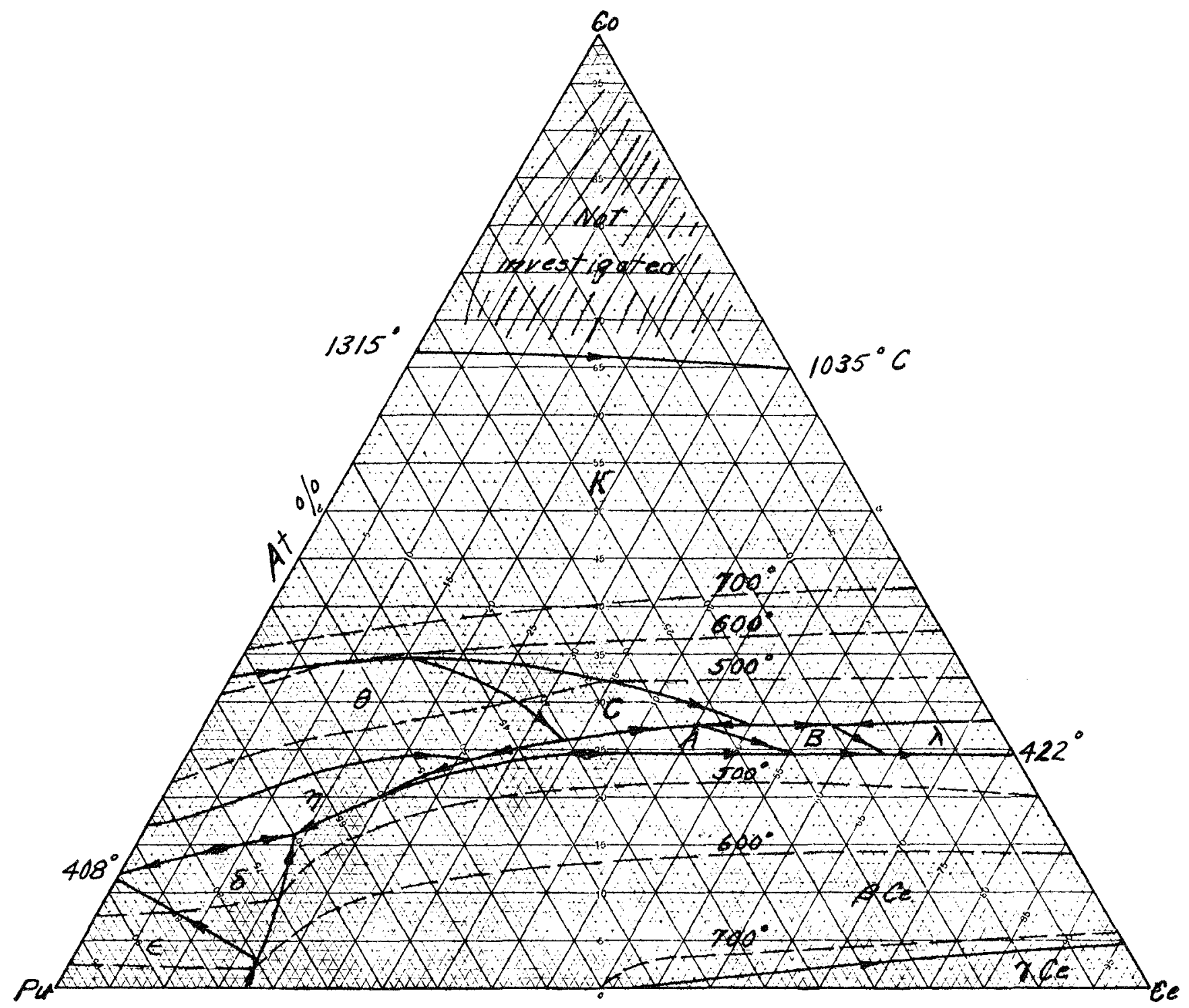

FIG. 1. Liquidus Projection of the System Plutonium-Cobalt-Cerium. 


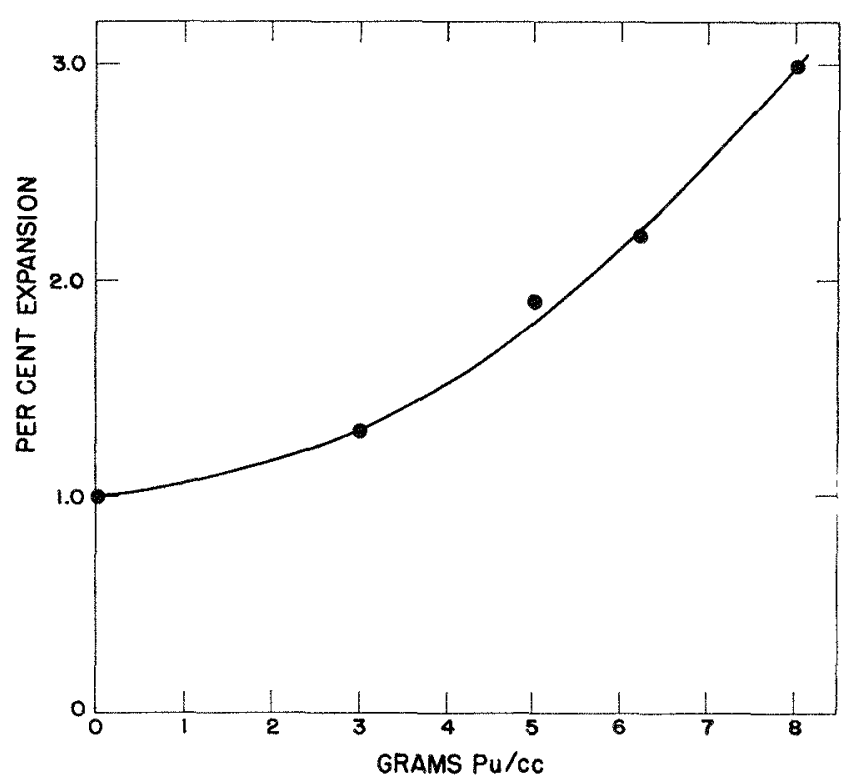

Fic. 2. Volumetric Expansion during Freezing of $\mathrm{Pu}-\mathrm{Co}-\mathrm{Ce}$ Alloys as a Function of Plutonium Concentration.

tantalum or Ta-W alloys will not occur at temperatures up to $850^{\circ} \mathrm{C}$.

d. The lifetime prior to failure of $\mathrm{Pu}-\mathrm{Co}-\mathrm{Ce}$ alloys contained in tantalum or Ta-W alloys will be significantly better than for Pu-Fe alloy (see Fig. 3).

It has been found that a carburized layer on the internal surface of the container or the diffusion of earbon into the tantalum grain boundaries is exceptionally effective in preventing plutonium penetration with the highly corrosive $\mathrm{Pu}-\mathrm{Fe}$ fucl. Evidence also indicates the latter treatment to be effective in prevention of plutonium penetration of welds by $\mathrm{Pu}-\mathrm{Co}$ -
Ce fuel. For example, when carbon was allowed to diffuse into the tantalum grain boundaries from the external surface of the container in a run with Pu-Fe fuel, the tantalum capsule did not fail after $380 \mathrm{hr}$ at $1100^{\circ} \mathrm{C}$, whereas without the carburization treatment the lifetime is less than $48 \mathrm{hr}$ (see Fig. 4). Cnder similar conditions, two tests with $8 \mathrm{~g} \mathrm{Pu} / \mathrm{cc} \mathrm{Pu}-\mathrm{Co}-\mathrm{Ce}$ fuel did not exhibit failure after $700 \mathrm{hr}$ at $1100^{\circ} \mathrm{C}$, and one test lasted $1500 \mathrm{hr}$ without failure (see Fig. 4). All tests were discontinued before failure was observed. It is anticipated that exploitation of the pronounced deterrent effect on plutonium penetration of carbon in the tantalum grain boundaries will eliminate IGP in a reactor system.

It should be pointed out that in all the corrosion work mentioned in this paper a "failure" due to IGP is defined as detectable plutonium (by alpha counting) on the surface of the container following a given test. In general, the failures obtained with $\mathrm{Pu}-\mathrm{Co}-\mathrm{Ce}$ fuels have been of the order of $<10,000 \mathrm{cpm}$ or $<10^{-7}$ $g$ plutonium on the outer surface of the containers. It is obvious that the presence of plutonium on the surface of a capsule of the magnitude described will not constitute a "failure" in the sense of being of operational importance in a reactor. No evidence at this time indicates that a "failure" of $\sim 10^{-7} \mathrm{~g}$ plutonium will ever progress to a magnitude that will allow liquid fuel to flow from the containers into the sodium coolant. In fact, some evidence exists that in certain instances so-called "failures" may become nonfailures if the test is allowed to continue after initial detection of plutonium. Table 1 shows data obtained on two test capsules that are under current investigation. Fur-

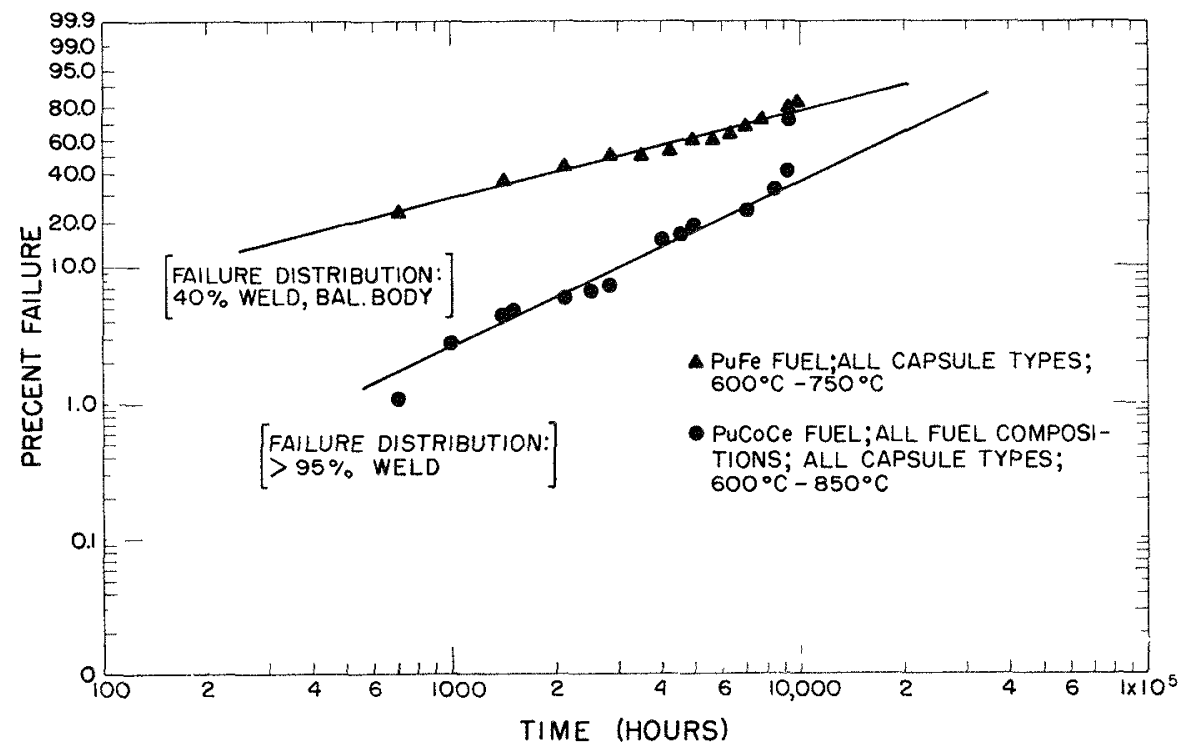

Frg. 3. Failure Analysis of PuFe and Pu-Co-Ce Fuels. (Weibull Distribution Plot.) 


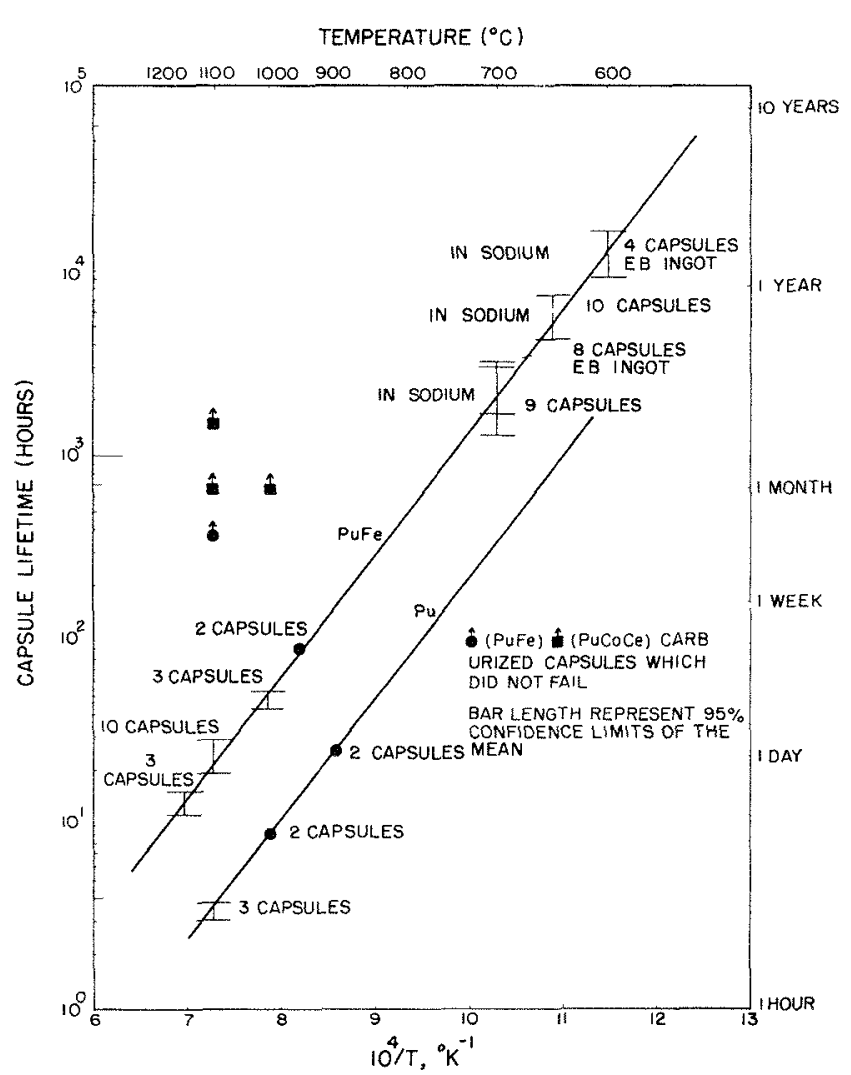

$F_{I G} 4$ Mean Lifetime of Plutonum and Plutonum Alloys Contamed in Unalloyed Tantalum Note the effect of external carburization of the tantalum on lifetime of $\mathrm{PuFe}$ and $\mathrm{Pu}$ Co Ce alloys

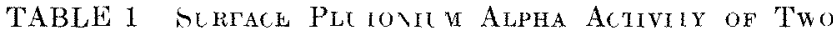
Possible Capsute Fathe res:

\begin{tabular}{|c|c|c|c|c|c|}
\hline I est Condition & $\begin{array}{l}\text { Test } \\
\text { Time } \\
\text { (hr) }\end{array}$ & $\begin{array}{c}\text { After } \\
\text { Pre acid } \\
\text { Wash, } \\
\text { cpm }\end{array}$ & $\begin{array}{c}\text { After } \\
\text { 1st Acid } \\
\text { Wash, }{ }^{t} \\
\text { cpm }\end{array}$ & $\begin{array}{l}\text { After } \\
\text { 2nd } \\
\text { Acid } \\
\text { Wash, } \\
\text { cpm }\end{array}$ & $\begin{array}{c}\text { After } \\
\text { 3rd } \\
\text { Acid } \\
\text { Wash, } \\
\text { cpm }\end{array}$ \\
\hline Ta Contaner, $8 \mathrm{~g}$ & 1500 & 2000 & 250 & - & - \\
\hline $\mathrm{Pu} / \mathrm{cc} \mathrm{Pu} \mathrm{CoCe}$, & 2500 & 1200 & 200 & 100 & \\
\hline $850^{\circ} \mathrm{C}$ & 3500 & None & - & - & - \\
\hline Ta $10 \mathrm{~W}$ Contamer, $8 \mathrm{~g}$ & 2500 & 200 & None & - & - \\
\hline $\mathrm{Pu} / \mathrm{cc} \quad \mathrm{Pu} \mathrm{Co} \mathrm{Ce}$, & 3500 & 10,000 & 1900 & 500 & 500 \\
\hline $850^{\circ} \mathrm{C}$ & 4500 & 2000 & 1100 & 500 & 300 \\
\hline
\end{tabular}

a Falure defined as $\geq 500 \mathrm{cpm}$ after 3 acid washes

b Acrd wash used was HF. $\backslash_{\backslash} \mathrm{O}_{3}: \mathrm{H}_{2} \mathrm{SO}_{4}$ in $2: 2: 5$ volume ratio for 5 sec

ther studies are in progress concerning the conditions which govein the size of plutonium leaks.

\section{Mass Transfer}

The transition metals $\mathrm{Fe}$, $\mathrm{Co}$, and $\mathrm{N}_{1}$ form intermetallic compounds with refractory metals $\mathrm{Nb}$, Ta, and $W$ This intermetallic compound formation has been observed when both $\mathrm{Pu}-\mathrm{Fe}^{(4)}$ and $\mathrm{Pu}-\mathrm{Co}-\mathrm{Ce}^{(5)}$ fuek are in contact with tantalum or it alloys Figure 5 show a reaction ldyer formed between 62 g Pu/ce Pu-Co-Ce fuel and tantalum the formation of this layer presents two potential problems with respect to long-term behavior at high temperatures.

First, sinee these compounds, TaCo or $\mathrm{Ta}_{2} \mathrm{Co}$, have lower tantalum activities than does pure tantalum, It mught be possible to "stablish a "short crrcuit" pitting corrosion of spallation if the intermetallie should occur The pitting would be due to dissolution of bare tantalum followed by precipitation of tantalum as the intermotallie compound at an immediately adjacent sink

Direct spallation of the TaCo layer has not been observed in any test run to date, although in some cases the precipitated crystal (-ee Fig 4) have been dislodged from the surface However, in no case has

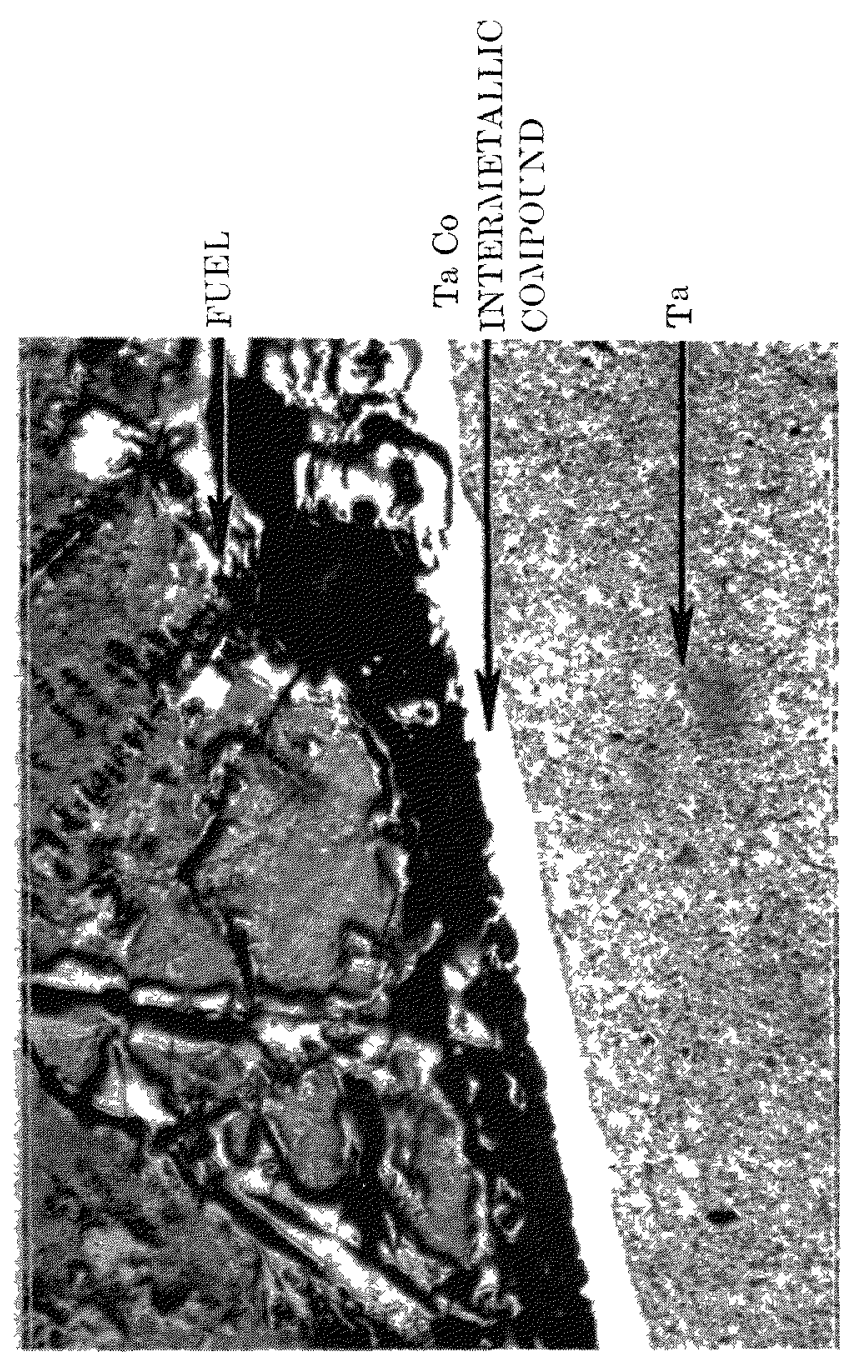

Fra 5 TaCo Layer Formed on Tantalum by $62 \mathrm{~g}$ Pu/ce Fuel 
the diffusion layer been separated from the tantalum substrate.

No pitting corrosion due to preferential TaCo crystal removal has been observed. However, tests at $1100^{\circ} \mathrm{C}$ with $\mathrm{Pu}-\mathrm{Fe}$ fuel (which forms the similar reaction layer $\mathrm{TaFe}$ ) have definitely indicated pitting

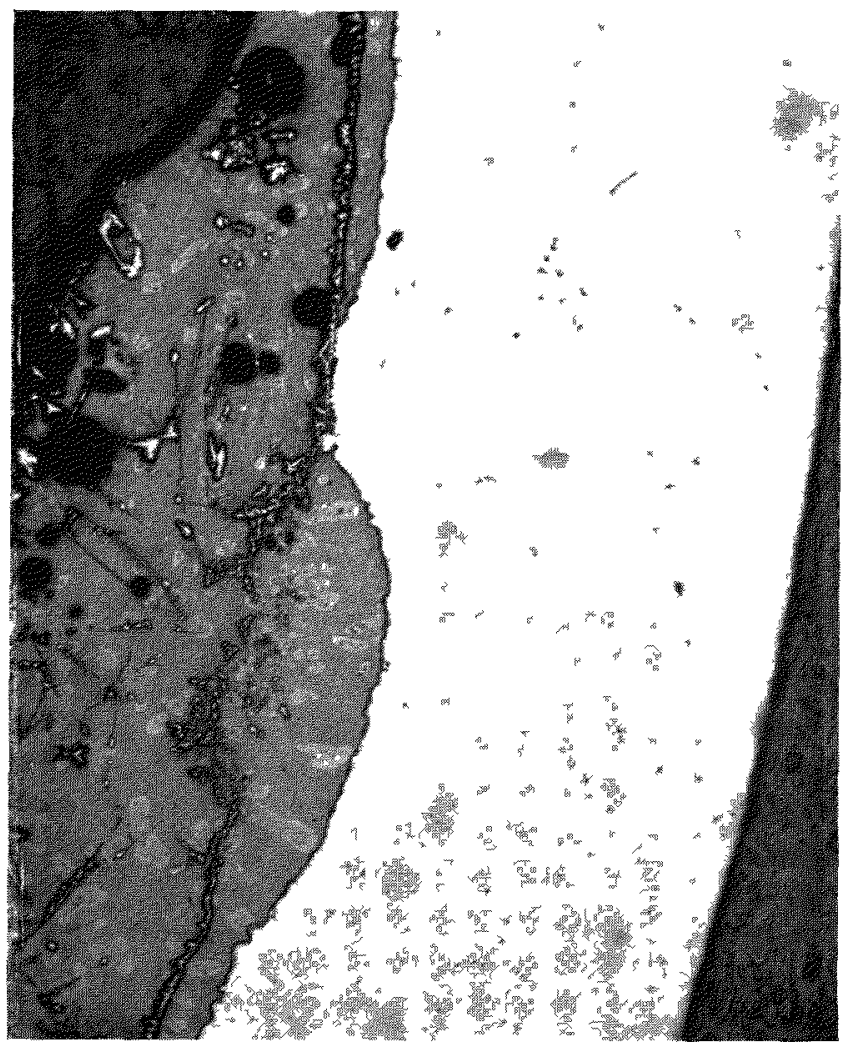

Fic 6 Pitting Corrosion of Tantalum 1 Contact with PuFe Alloy for $16 \mathrm{hr}$ at $1100^{\circ} \mathrm{C}$ Pit has occurred where Ta Fe mtermetallie compound spalled off the tantalum wall

TABLE 2 EXperimen ial, Condition for Mass Tratsi har

\begin{tabular}{|c|c|c|c|}
\hline Run No & $\begin{array}{c}\text { Pu-Co Ce } \\
\text { l uel Tested } \\
\text { g Pu cc }\end{array} \mid$ & $\begin{array}{l}\text { Type of Actuve Tab and } \\
\text { Capsule }\end{array}$ & $\begin{array}{l}\text { Temperature } \\
\text { Conditions, }{ }^{\circ} \mathrm{C}\end{array}$ \\
\hline 3 & 5 & $\begin{array}{l}\text { Ta capsule, annealed Ta } \\
\text { tab at bottom }\end{array}$ & $700^{\approx}$ to $610^{\mathrm{b}}$ \\
\hline 4 & 5 & $\begin{array}{l}\text { Ta capsule, highly worked } \\
\text { tab at bottom }\end{array}$ & $700^{\circ} t, 610^{\mathrm{b}}$ \\
\hline 5 & 5 & $\begin{array}{l}\text { Carbided Ta capsule, an } \\
\text { nealed uncarbided Ta } \\
\text { tab at bottom }\end{array}$ & $700^{\circ}$ to $610^{\mathrm{b}}$ \\
\hline 6 & 8 & $\begin{array}{l}\text { Ta capsule, anvealed Ta } \\
\text { tab at bottom }\end{array}$ & $750^{a}$ to $650^{b}$ \\
\hline 7 & 8 & $\begin{array}{l}\text { Ta capsule, annealed } \mathrm{Ta} \\
\text { rung in wall at gas/liq } \\
\text { quid interface }\end{array}$ & $750^{\circ}$ to $650^{\circ}$ \\
\hline
\end{tabular}

a Temperature at bottom of capsule

b Temperature at fuel/gas interface table 3 Overall Ratis of Mass Trangfer

\begin{tabular}{|c|c|c|c|}
\hline Run No & $\begin{array}{l}\text { Average Rate, } \\
\text { mils/year }\end{array}$ & $\begin{array}{c}\text { Maximum Rate, } \\
\text { mils/year }\end{array}$ & Time of Run, hr \\
\hline 3 & 0006 & 0014 & 3300 \\
\hline 4 & 0025 & 0038 & 3100 \\
\hline 5 & 0038 & 0104 & 2000 \\
\hline 6 & 0185 & 095 & 720 \\
\hline 7 & 055 & 620 & $\begin{array}{c}384 \\
\text { (In progress) }\end{array}$ \\
\hline Run No & \multicolumn{3}{|c|}{ Comments } \\
\hline 3 & \multicolumn{3}{|c|}{$\begin{array}{l}\text { No } \mathrm{Ta}_{2} \mathrm{Co} \text { formed on the capsule walls } \mathrm{Ta}_{2} \mathrm{Co} \\
\text { precipitated at gas/liquid interface Large } \\
\text { quantities of } \mathrm{Ta}_{2} \mathrm{Co} \text { formed in the zone } \\
\text { between the tab and the bottom end cap } \\
\text { of the capsule }\end{array}$} \\
\hline 4 & \multicolumn{3}{|c|}{$\begin{array}{l}\text { The run was similar to Run } 3 \text { except the mass } \\
\text { transfer rates were higher by a factor of } 3 \text { to } \\
4\end{array}$} \\
\hline 5 & \multicolumn{3}{|c|}{ 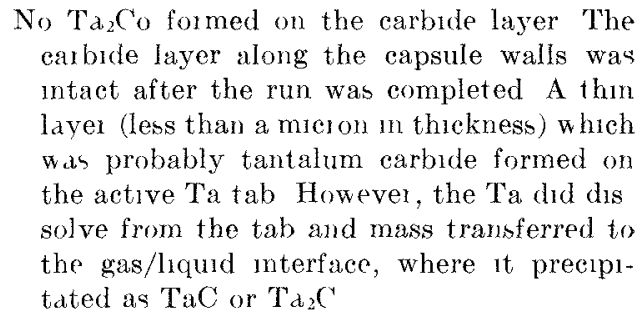 } \\
\hline 6 & \multicolumn{3}{|c|}{$\begin{array}{l}\text { Ta } a_{2} \text { o ciystals piecipitated only at the gas/ } \\
\text { liquid interface No Ta } a_{2} \mathrm{Co} \text { formed on the } \\
\text { walls of the capsule The overall rate of } \\
\text { mass transfer appeared to be controlled by } \\
\text { the rate of precipitation at the interface } \\
\text { Periodically, showers of crystals would form } \\
\text { at the interface, and dung these periods the } \\
\text { mass transfer from the tab would markedly } \\
\text { Increase }\end{array}$} \\
\hline 7 & \multicolumn{3}{|c|}{$\begin{array}{l}\text { A very large quantity of Ta dissolved from the } \\
\text { ring mserted during the first } 11_{2} \text { days } \\
\text { Then, no more transfel was noted Probably } \\
\mathrm{Ta}_{2} \mathrm{Co} \text { cisstals preempitated on the Ta sur- } \\
\text { faces on and around the ring insert and pre } \\
\text { vented further mass transfer The formation } \\
\text { of the lay el and its structure wll be checked } \\
\text { by post mortem analy sis of the capsule }\end{array}$} \\
\hline
\end{tabular}

and subsequent IGP through the unprotected container wall in the pit (see Fig. 6 ).

Since the TaCo intermetallic compound acts as a precipitation sink for tantalum in solution in the fuel, mass transfer of tantalum is expected because of the free energy differences in different parts of the capsule arising from temperature gradients. Thermal-gradient mass transfer of tantalum has been demonstrated by tracer techniques. Tables 2 and 3 summarize the available data Mass-transfer rates of the magnitudes cited should present little difficulty, providing pitting is not initiated.

The second potential problem posed by the forma- 
Visual Film Color Observation
Gold Color

Stops in Section $4 \quad$ Blue Color
Internal Surface

Appears to be Clean, Shiny Tantalum

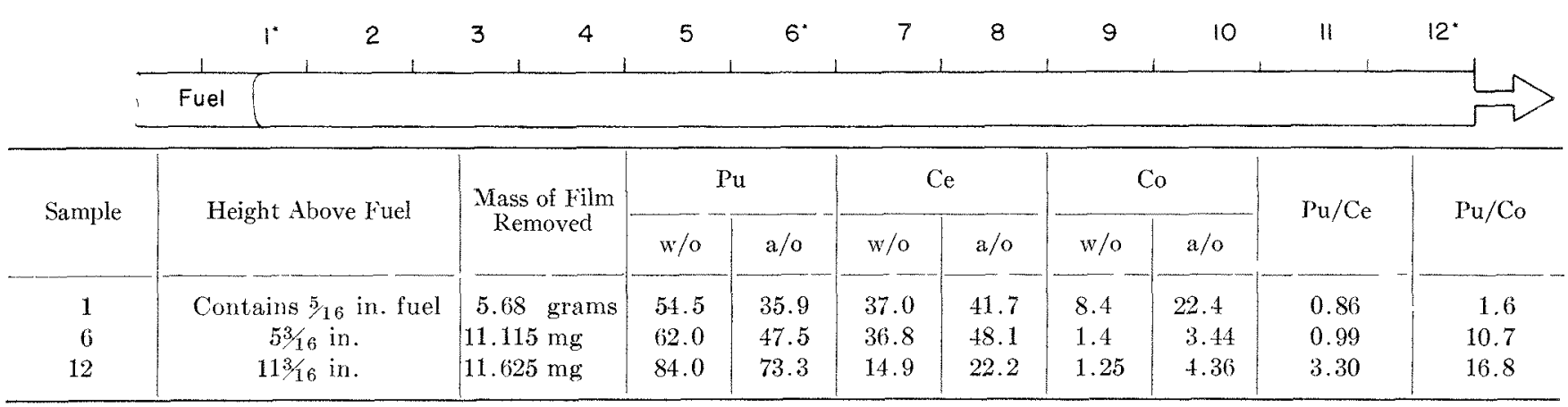

All sections 1 in. in length.

* = Samples analyzed.

Frg. 7. Composition Variation of the Pu-Co Ce Film that Climbed the Tantalum Alloy Capsule Wall during Testing at $700^{\circ} \mathrm{C}$ for $500 \mathrm{Hr}$. Note plutonium enrichment as cobalt concentration decreases due to Ta-Co intermetallic compound formation.

tion of the intermetallic compound is that moltenplutonium alloys all exhibit a "climb" phenomenon on metal walls. This phenomenon is manifest by a $<1-3-\mu$-thick fuel layer climbing on tantalum to heights of $5 \mathrm{in}$. or more above the meniscus. As the fuel climbs, the cobalt in the alloy forms a TaCo or $\mathrm{Ta}_{2} \mathrm{Co}$ intermetallic compound; consequently the plutonium content of the fuel becomes enriched, thereby giving the fuel increased tendency for intergranular penetration of the tantalum wall.

Figure 7 shows the analysis of the fuel film on a capsule wall after exposure for $500 \mathrm{hr}$ at $700^{\circ} \mathrm{C}$. Plutonium was observed on the external surface of this capsule by alpha counting in the region of sections 11 and 12 , even though no visible plutonium was observed on the internal surfaces of these sections. Leaching of the internal surface with $\mathrm{HCl}$ gave results as shown. The enrichment of plutonium is caused by the depletion of cobalt via intermetallic formation up to approximately $5.5 \mathrm{in}$. above the fuel level. Since about a $10^{\circ} \mathrm{C}$ temperature gradient exists along this length of capsule (top $\sim 10^{\circ}$ cooler than the fuel level), further enrichment in plutonium is caused by sliding along isotherms of the phase diagram (see Fig. 1), caused by precipitation of $\beta \mathrm{Ce}$ at approximately $700^{\circ} \mathrm{C}$ and $4.5 \mathrm{a} / \mathrm{o}$ Co.

Although plutonium was found by chemical analysis in the section 10, the mass of plutonium involved was extremely small, i.e., $11.625 \mathrm{mg}$. Consequently, it is not expected that a leak in this area would cause macroscopic amounts of fuel to be lost from the capsule. It is known that the presence of a $\mathrm{Ta}$-Co or other intermetallic compound prevents diffusion of plutonium in tantalum grain boundaries. Consequently, the method that would prevent. IGP is to prevent the fuel film from forming $\mathrm{TaCo}$ as it climbs.
Preliminary work indicates that the presence of a $\mathrm{Ta}_{x} \mathrm{C}$ layer on the container surface completely prevents Ta-Co intermetallic layer formation and may be self-healing. The carbide layer is established by bulk diffusion of carbon into the tantalum and is not subject to spallation in thin layers (i.e., $<5 \mu$ ), although spallation of a portion of the layer (at the $\mathrm{TaC}-\mathrm{Ta}_{2} \mathrm{C}$ interface) has been observed with thick layers (i.e., 10-15 $\mu$ ). Run 5 (sce Table 3 ) indicates that although no $\mathrm{Ta}_{2} \mathrm{Co}$ formed as a sink for the active tantalum in the carburized-container run, the dissolved tantalum apparently precipitated as $\mathrm{Ta}_{x} \mathrm{C}$ and transferred at a rate approximately 4 times that for the uncarburized system. However, the rate observed, i.e., $0.04 \mathrm{mil} / \mathrm{yr}$, is considered not to be significant from a practical standpoint.

The presence of a carburized surface has been shown to enhance the IGP resistance of tantalum as well as to prevent $\mathrm{Ta}_{2} \mathrm{Co}$ intermetallic compound formation. Therefore, because of the uncertainty associated with the tantalum mass-transport phenomenon and pitting corrosion, a carbide layer has been specified for the reference capsule of the MPBE core, until and unless it can be proven by further experimentation that such a layer is not necessary.

\section{Mechanical Properties of the Fuel Container}

The mechanical properties of the container, as well as its resistance to corrosion, are important considerations in selecting a suitable container material. Tungsten additions to tantalum have pronounced effects on hardness, ultimate and yield strengths, and total per cent elongation. Increasing tungsten content of the alloy rapidly increases ultimate and yield strengths, and moderately lowers percentage elongation. Figure 8 shows yield strengths and ultimate ten- 


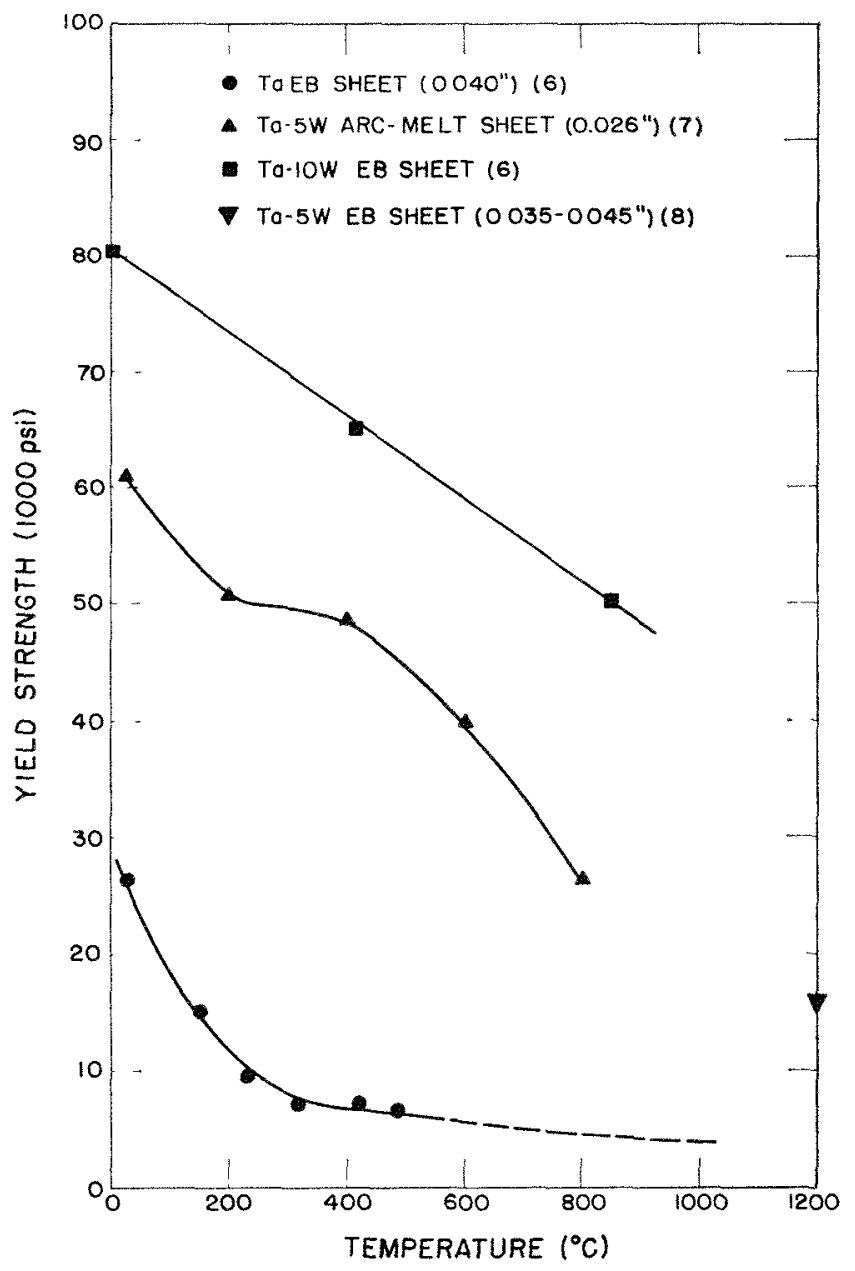

FIG. 8. Elevated-temperature Yield Strength Properties for $\mathrm{Ta}, \mathrm{Ta}-5 \mathrm{w} / 0 \mathrm{~W}$, and $\mathrm{Ta}-10 \mathrm{w} / 0 \mathrm{~W}$ sheet.

sile strengths for Ta, Ta- $5 \mathrm{w} / \mathrm{o} \mathrm{W}$, and Ta- $10 \mathrm{w} / \mathrm{o} \mathrm{W}$ alloys. The high temperature yield strength of $\mathrm{Ta}$ is markedly improved by $\mathrm{W}$ additions. It is seen that for temperatures of interest for fast reactor applications $\left(500-750^{\circ} \mathrm{C}\right)$ the yield strength of $\mathrm{Ta}-5 \mathrm{w} / \mathrm{o} \mathrm{W}$ is 5-7 times that for tantalum. Consequently, since container strength is important for preventing capsule distortion in the event of inadvertent freezing of the fuel, and since tungsten additions to tantalum may be beneficial to corrosion performance, the material selected for use in the MPBE is Ta-5 w/o W.

\section{Irradiation Studies}

The effect of irradiation on the mechanical properties of Ta-W alloys is virtually unknown. Roomtemperature tensile tests on tantalum irradiated to $\sim 9.4 \times 10^{19}$ nvt (fast) indicates an increase in yield strength of greater than $200 \%$ and an elongation decrease of $\sim 80 \%{ }^{(9)}$ New data on the effect of irradiation on the room-temperature tensile properties of tantalum and Ta-10 w/o W have been recently re- ported. ${ }^{(10)}$ However, information concerning the quality of the test material is inadequate, and the data are of little value in predicting the behavior of $\mathrm{Ta}-5 \mathrm{w} / \mathrm{O} \mathrm{W}$ during irradiation.

Body-centered-cubic materials show virtually complete recovery of mechanical properties at $\sim 0.35$ $T / T_{m}$ (ratio of absolute test temperature to absolute melting temperature). (11) Based on irradiation data on the high-temperature mechanical properties of $\mathrm{W}$, $\mathrm{Mo}$, and $\mathrm{Nb}$, it is expected that Ta-5 $\mathrm{W} / \mathrm{O} \mathrm{W}$ will demonstrate an irradiation-induced increase of yield strength and a reduction of total elongation since the temperature range of the container in the reactor is between 0.235 and $0.30 \mathrm{~T} / \mathrm{T}_{\mathrm{m}}$. The magnitude of these changes of mechanical properties after fastneutron irradiation is under investigation.

The effect of fission product recoil damage on both $\mathrm{Ta}(W)-\mathrm{Co}$ reaction layers and $\mathrm{Ta}(W)-\mathrm{C}$ layers is being studied by irradiation testing in the OWR, a 5-MW thermal-flux research reactor at LASL. Because of the fission density profile obtained in such a test, the tantalum wall actually is exposed to a total fission fragment generation density that is 5-6 times the average for the fuel element. Consequently, such testing is a way of intensifying the fission recoil into the container surface and obtaining large equivalent surface effects of burnup relative to a fast reactor environment which has a flat fission density across the fuel diameter.

The first of these experiments (OWREX-1) designed to investigate wall effects has been completed. The experimental fuel element consisted of an 8 -in.long capsule containing $\sim 2.2$ in. of $6.2-\mathrm{g} \mathrm{Pu} / \mathrm{cc} \mathrm{Pu}-$ Co-Ce fuel $[38.5$ a/o $(58.0 \mathrm{w} / \mathrm{o}) \mathrm{Pu}, 24.6 \mathrm{a} / \mathrm{o}(9.0$ w/o) Co, balance Ce]. The container was an unalloyed, deep-drawn tantalum capsule. Fuel heat was removed by a naturally convecting sodium-coolant system with the bottom of the fuel maintained at $\sim 470^{\circ} \mathrm{C}$ and the top at $\sim 600^{\circ} \mathrm{C}$.

The average burnup of this capsule was calculated to be $2.5 \times 10^{19}$ fissions $/$ ce or $0.15 \mathrm{a} / \mathrm{o} \mathrm{Pu}$ burnup. Radiochemical burnup analysis has not yet been completed. The fission density at the wall was calculated to be $1.25 \times 10^{20}$ fission/ec. Figure 9 shows a postirradiation radiograph of this capsule. Significant features shown in the radiograph are the exceptionally clean meniscus and the presence of fission-gas bubbles along the capsule wall (similar to those observed in LAMPRE-I fuel capsules). Netallographic examination showed no wall effects other than those expected from an equivalent thermal treatment out-of-reactor (e.g., $\mathrm{Ta}_{2} \mathrm{Co}$ being formed at the meniscus-wall interface).

The second capsule (OWREX-2), identical to 


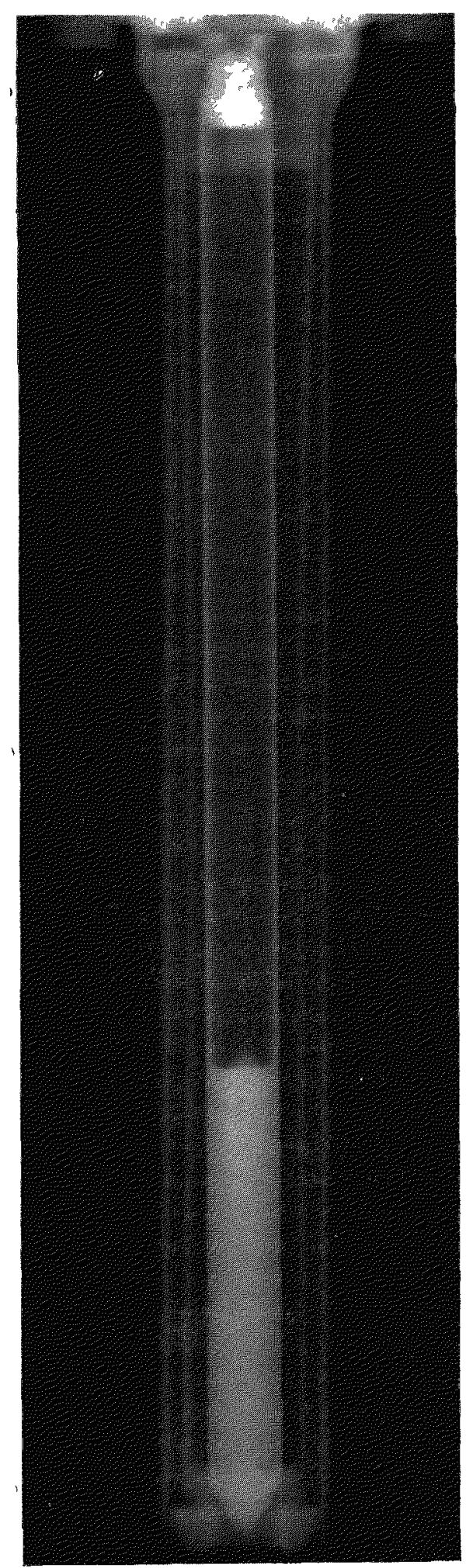

OWREX-1, was irradiated to $\sim 1 \mathrm{a} / \mathrm{O} \mathrm{Pu}$ burnup, or $1.6 \times 10^{20}$ fissions $/ \mathrm{cc}$, with a fission density at the container wall equivalent to $6 \mathrm{a} / \mathrm{o} \mathrm{Pu}$ burnup. A postirradiation radiograph of this capsule is shown in Fig. 10. The radiograph shows this capsule to be substantially the same as OWREX-1. Since postirradiation examination of this capsule is still in progress, no conclusions ean be drawn regarding wall effects. However, although fuel enrichment is expected

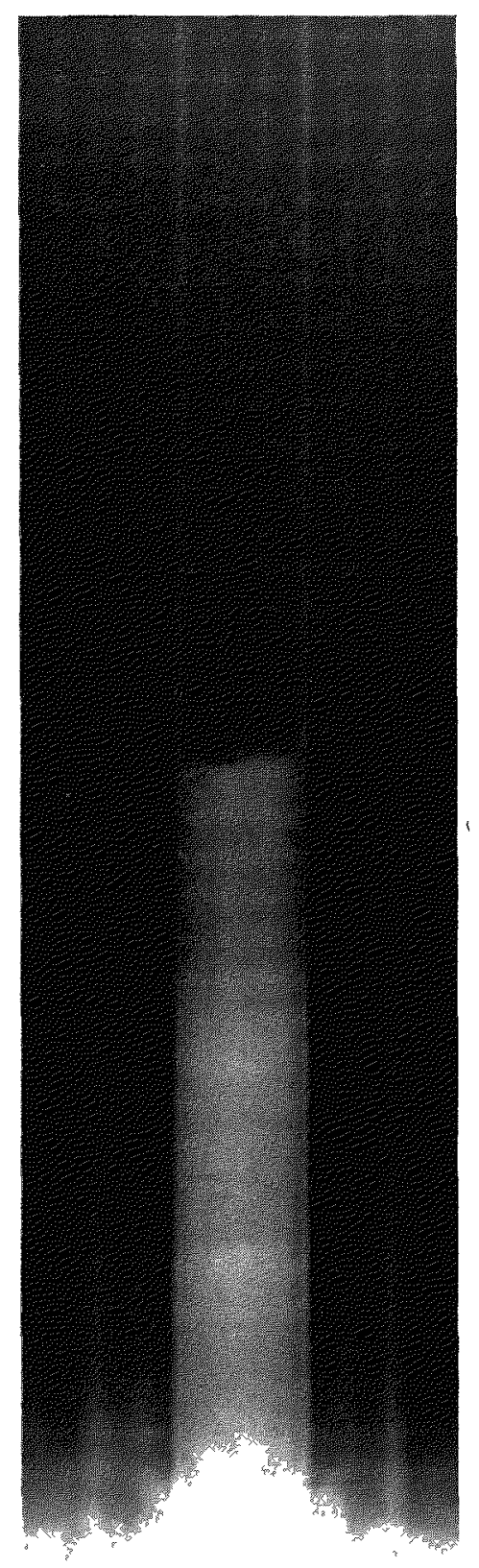

Fig. 9. Postirradiation Radiograph of OWREX-1 Irradiation Capsule. Fuel Composition $6.2 \mathrm{~g} \mathrm{Pu} / \mathrm{cc}$; Average Burnup; $\sim 0.15 \mathrm{a} / \mathrm{o} \mathrm{Pu}$.
Fig. 10. Postirradiation Radiograph of Fueled Region of OWREX-2 Irradiation Capsule. Fuel Composition $6.2 \mathrm{~g} \mathrm{Pu} / \mathrm{ce}$; Average Burnup; $0.9 \mathrm{a} / \mathrm{o} \mathrm{Pu}$. 
in the gas-phase portion of the capsule by "fuel climb," the capsule was "swipe elean" with respect to plutonium contamination, indicating that plutonium did not exhibit intergranular penetration of the capsule during the test $(\sim 2400 \mathrm{hr}$ at temperature).

A further significant point is that these two capsules are loaded with the same amount of fuel and that both are the same height (to within a few mils) after irradiation. This result indicates that fission-gas release reached a steady state within the 2 -week operational period of OIVREX-1 and that volume pereent of bubbles within the fuel remained constant after an initial transient condition.

Two experiments are being inserted in the OWR that are designed to determine the irradiation effect on $\mathrm{Ta}-5 \mathrm{w} / \mathrm{O} \mathrm{W}$ containers with and without a carbide coating. It is anticipated these capsules will be run to at least $3 \%$ average plutonium burnup.

\section{Conclusions}

Our studies of potential core materials for fast reactors may be summarized as follows. Molten PuCo-Ce fucl alloys appear to be much less corrosive than the Pu-Fe eutectic alloy used in LAMPRE. Ta-5 w/o W alloy, solected as a core container, has higher yicld strength by at least a factor of 5 than tantalum in the unirradiated condition, and it is expected that this increased strength will minimize or eliminate capsule distortion if the fuel is frozen. Although several major questions regarding fucl irradiation performance still must be answered (e.g., the effect of high fission products/ce on the chemical behavior of the fuel, the rate of fission-gas release at high power densities, etc.), Pu-Co-Ce alloys offer considerable potential as a practical fast reactor fuel.

\section{REFERENCES}

1. Hannum, W. H., and Kirkbride, L. 1)., The Molten Plu tonium Burnup Experiment, La-3384-MS, in publication.

2. Hannum, W. H., Nuclear Design of MPBE. See paper in Session VII B of This Conference.

3. Bidwell, R. M., Ferguson, W. E., Burwell, C. C., Hammond, R. P., Davidson, K. V., Wycoff, W. R., and Anderson, R. W., The Development of High Purity Tantalum and Alloys for Liquid Plutonium Containment in LAMPRE I. Nucl. Sci. Eng., 14, 109-122 (1962).

4. LAMPRE Operations Report, in preparation.

5. Litton, F. B., and Biery, J. C., Reaction of Tantalum with Wolten Cernum-Cobalt Alloys, to be published.

6. Schmidt, F. F., and Ogden, H. R., Engineering Propertzes of Tantalum and Tantalum Alloys, I)MIC-189 (Sept 13, 1963).

7. Brasier, R. I., Unpublished data, Los Alamos Scientific Laboratory.

8. Schmidt, F. F., Klopp, W. D., Maykuth, D. J., Ogden, H. R., and Jaffee, R. I., Investigation of the Properties of Tantalum and Its Alloys, WADD-TR 61-106 (May 1961).
9. Franklin, C. K., Stahl, 1), Shober, F. R., and Dickerson, R. F., Effects of Imadiation on the Mechanical Properties of Tantalum, BMI-1476 (Nov 18, 1960).

10. Claudson, T. T., and Pessl, H. J., Irradiation Effects on High Temperature Reactor Structural Materials, BNWL-23 (Feb 1965).

11. Moteff, J., Radiation Damage in Body centered Cubic Metals and Illoys, GE-TM-6e-92 (Aug 1965).

\section{Discussion}

Mr. Lauroski (ANL): From the inception of this program I recall that you had problems in acquiring tantalum of the right purity and tantalum technology. It is obvious that over the years you have made considerable progress as reported here in the ability to keep these molten alloys for long durations. Would you care to comment as to what have been some of the major improvements in the tantalum technology over and above the additions of tungsten that you mention?

Mr. Kirkbride: We have succeeded in getting the tantalum too pure. I think this is clear from the effect of carbon on the penetration. This is the old problem of establishing a maxinum in your specifications on impurities and not minima, and I believe that we've got to a point now where we are actually too pure. We are routinely getting seamless drawings of tantalum tubing with 100 ppm total impurities, not just interstitials, but total inpurities. This is just too pure, and now we are having to go back and add carbon in a selective manner. (Of course, the cost of tantalum has come down considerably, and routinely tantalum tubing to meet the best of tubing specifications can be obtained for $\$ 30-50 / \mathrm{ft}$.

Mr. Lawroski: How about the matter of welding?

Mr. Kirkbride: Again welding is a problem. The problem is again one of purity. We are not introducing any contamination normally in our welds, even are welds; TIG welds are coming out much softer than the base material, maybe 80 or $70 \mathrm{dph}$, for instance.

Mr. Kummerer (Karlsruhe): As regards cobaltcontaining fuel, how do you consider the drawback of the high cobalt activity in the light of the reprocessing difficulties?

Mr. Kirkbride: Since it's a fast reactor we don't particularly get high cobalt activity, not as you would in a thermal reactor. Of course, in the thermal irradiations the capsules come out very warm. But for instance in LAMPRE, the stainless steels with cobalt in them don't become too active. I think it would cause a problem, just because we do have 10 w/o cobalt in the fuel, but I don't think this is an overriding economic consideration. 
Mr. Kummerer: But in fast flux are not the cobalt cross sections comparatively higher than those of the other materials?

Mr. Kirkbride: Yes. You pay a penalty; there must be a balance between penalties and advantages.

$M r$. Lawroski: Have you studied the addition of the uranium to these alloys?

Mr. Kirlabride: Yes. We tried for a considerable number of years to find a low-melting internal breeding fuel, a uranium-plutonium system. It turns out that the uranium-plutonium-manganese system is about the lowest melting, at $738^{\circ} \mathrm{C}$. This is a pretty large price to pay in that the coolant temperature is too high if you are going to make this a molten system. Besides that, the uranium-plutonium-manganese system is really a universal solvent. It will dissolve almost anything, so we have given that up as a bad cause.

Mr. Blumenthal (ANL): To what extent will the fission gases fill the melt and restore a kind of a uniformity of the melt?

Mr. Kirkbride: In a fast system one obviously has convection because the temperature gradient that's induced across the capsule, and also the longitudinal gradient in the capsule actually causes quite a convection. However, in LAMPRE. which had a flat fission density across the fuel, we did only see bubbles along the walls. In this particular case, the longitudinal temperature gradient and the power density tend to counteract one another, and I am not sure that we are getting any convection in our irradiations in the thermal flux. However, it is quite obvious that there must be some kind of convection as bubbles break from the wall and rise. But I don't anticipate that the fission gas, in itself, is going to cause any appreciable stirring; it is going to be a thermalconvection stirring. Fission gases are growing too slowly to make much of a contribution at all.

Mr. Lawroski: In our pyrochemical studies for reprocessing we don't require the long durations for which you would have to provide a solution if you wanted to incorporate substantial amounts of uranium along with plutonium and magnesium.

Mr. Kirkbride: That's a good point. We were successful in containing this alloy for several thousand hours, but that is about it.

$M r$. Zegler (ANL): Is there any concern about the homogeneity of these liquids at temperature?

$M r$. Kirkbride: As far as we have been able to determine, they are homogeneous; there are no twophase, two-liquid regions in the system; there are no solid phases outside, except perhaps precipitated fission products. We see no indication whatsoever that these matcrials are not totally $100 \%$ homogeneous, as you would expect. Even though there is quite a density mismatch between components, once you get the atoms mixed, they are mixed, However, solidification can cause segregation. If that occurs, there may be considerable difficulty in getting the liquid homogeneous again, because then it can only become homogeneous by diffusion process. When we make these alloys initially, they are stirred and melted, cast, recast, and they come out very homogeneous.

Mr. Lauroski: This morning we heard considerable progress reported concerning the irradiation capability in carbide and metallic type fuels, and just now we have learned about the molten metal fuel. But we have not heard very much about any change in situation concerning irradiation for the oxide fuels. It was back in 1961, I believe, when it was first reported that oxide had been taken up to about 100,000 MWd/T, although I must admit I did not attend all the presentations. I have not heard if there has been any further progress in the irradiation of oxide fuels. There are, of course, a considerable amount reported in a better understanding of some of the factors involved with oxide. I take this opportunity to mention it now, so that those of you who have been working on oxide fuel will be prepared to make some comment on this point at the end of the session. 


\title{
The Application of Experimental Data from TREAT Meltdown Studies to Reactor Accident Analysis
}

\author{
S. J. Stachura and M. Silberberg \\ Atomics International \\ North American Aviation \\ Canoga Park, California \\ (PRESHNTED BY S. J. STACHURA)
}

During a recent study ${ }^{(1)}$ of the coupled-reactor concept at Atomies International, it was necessary to describe the behavior of the core during very large reactivity insertions under various conditions of protective action. Revisions were made to the digital code AIREK, (3) which was then used to treat the neutron kinetics together with an approximate thermal model of the core during reactor excursions. Because the reference core was fueled with mixed oxides and was operated at relatively high specific power levels, the magnitude of tolerable insertions was usually determined by gross fuel melting. The possible course of events subsequent to fuel melting and the manner in which changes would occur in the distribution of materials within the core was interpreted from recent $\mathrm{AI}$ experiments ${ }^{(2)}$ in TREAT in which ceramicfuel meltdown in sodium had been studied.

This effort represents an initial attempt to place the models for maximum accidents on a more physically realistic basis. It should also be noted that if the mechanisms of fuel dispersion as described in this study can be more completely characterized and generalized, the dependence on the Bethe-Tait criterion can be reduced.

\section{A. Pertinent Information from TREAT Studies}

To date, the AI TREAT experiments have included examination of the transient heating behavior of relatively large-diameter $(0.85$-in. $)$, hypostoichiometric, uranium carbide fuel, sodium bonded to 10 and 20-mil-wall stainless-steel tubing in a stagnant sodium environment. These TREAT transients have produced melting of the central portions of the fuel and, in one case, included gross melting of the fuel, temperature levels of about $6000^{\circ} \mathrm{F}$, and substantial redistribution of materials within the test capsule. It is this latter case which has generated experimental evidence regarding the possible course of meltdown, and the results of that experiment will now be reviewed briefly.

The capsule design is shown in Figure 1. Figure 2 presents the thermal and pressure behavior during the transient, and Table 1 describes the various events which are taking place. Figure 3 shows $\mathrm{X}$ radiographs of the capsule prior to irradiation and following the experiment. Figure 4 is a photograph of the capsule after vertical sectioning.

For present purposes, the gross dispersion of fuel throughout the capsule is most notable. During capsule disassembly, it was observed that the sodium was not present at the bottom of the graphite crucible, but rather was frozen on the surfaces of the capsule as were substantial portions of the fuel and cladding. Some of the fuel was present in two or three larger pieces, but these together probably comprised less than half of the original fuel slug.

As a result of these observations and analysis of the thermal and pressure data, the following conclusions were drawn:

1) The rapid transfer of thermal energy from molten fuel and clad to coolant produced large local pressures of sodium vapor which caused the observed gross dispersal of the fuel mixture. This phenomenon should also be observed for oxide meltdown in sodium, since a large part of the energy exchange is likely to take place by radiation. The application of the present results to oxide fuel assumes energy transfer similar to the carbide behavior.

2) The fuel appears to have possessed considerable momentum since it was forced through fairly small openings in the system. On this basis, it is assumed that in reactor situations the disassembly can occur over distances of a few feet, and the approximate models of fuel motion incorporated this assumption.

3) The sodium bond generated rather modest pressures prior to cladding failure, and it appears that either film boiling occurred in the bond space (with liquid sodium adhering to the cladding) or that the bond region was completely voided.

4) As a result of the above observation of pressure and thermal behavior, it is believed that the presence 


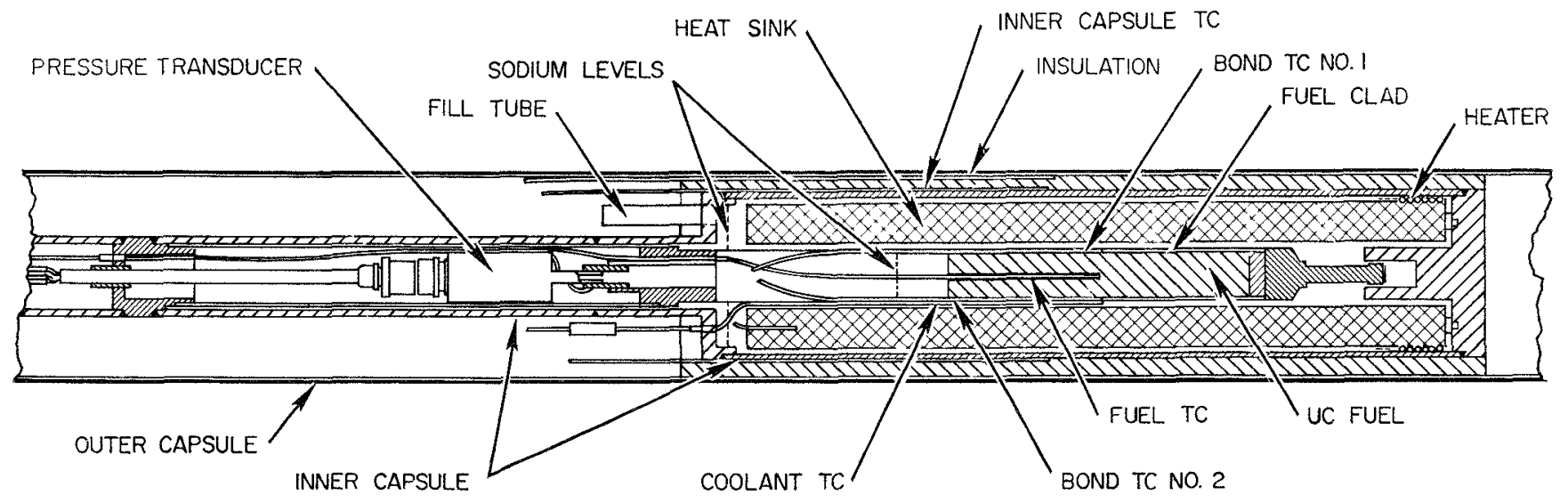

Frg. 1. TREAT UC Capsule Assembly.

of the sodium bond did not play a major role in the meltdown process.

5. There was a considerable time lag (approximately $1 \mathrm{sec}$ ) following completion of the burst and prior to cladding failure. Such a lag could be associated with the time required for the surface temperatures of the ${ }^{2} \mathrm{C}$ to reach melting, the time (probably minimal) required for collapse of the fuel in the clad, and the time to melt or react with the clad.

6) The time lag for a gas-bonded pin would be expected to be somewhat shorter, because the temperatures of the fuel surface are higher and rise more rapidly than in the case of a liquid-metal-bonded pin.

It should be noted that in the capsule experiment the energy generation within the fuel had ceased long before fuel motion began, so that the motion in a large core with residual (or high) power levels would be likely to be more extensive.

\section{B. Considerations in Describing Meltdown for a Large Core}

Fuel melting in a large core could occur in a variety of ways and for a number of reasons; loss of coolant, channel blockage, inadvertent high enrichment, of the fuel or increased system (or local) reactivity are all possible causes of fuel melting. The extrapolation from the simple conditions of the experiment to the complex situations which may arise in reactor operation cannot be made on any rigorous basis.

However, there is one case which is amenable to description, namely, the condition in which symmetric axial and radial flux gradients exist within the core and in which the temperature distribution in the fuel resembles the flux distribution (based on average fuel temperature). Under these conditions, a reactivity transient which produces no gross changes in the flux shape of the core would be likely to produce molten fuel in the center of the core, and the region of molten fuel would assume some approximately cylindrical shape which expands both axially and radially. It is for this condition that the results of the TREAT meltdown study were applied.

The reactivity effects associated with two types of fuel motion were calculated, and these effects were incoporated into the AIREK kineties code. This modified code will now be described, followed by a description of the method of ealculating reactivity changes associated with fuel motion, and the results for the coupled core design will then be presented.

\section{AIREK 3C Description}

The digital reactor kinetics code AIREK $3 \mathrm{C}^{(1,3)}$ was employed for the analyses of both moderate and severe accidents of the coupled core. This code treated four channels: the maximum and average rod in the fast region, and the average rod in each of the radial blankets. There were three axial nodes per channel. The heat transfer and flow could be varied from channel to channel.

In the transient condition, the neutron econony is time, energy, and space dependent. AIREK 3C treated the time dependence correctly, included the effects of delayed neutrons, and neglected changes in the energy spectrum and flux shape. Doppler and void reactivity feedbacks are about one order of magnitude more important than coolant and structural temperature coefficients or expansion coefficients. Consequently, for accidents in which important changes in configuration did not oceur, Doppler and void were the only feedbacks considered. Fucl melting and coolant vaporization were incorporated into the analysis, while temperature dependence of thermal properties and heat capacities associated with clad and structures were neglected. Axial, radial, and local nonrandom peaking factors were employed in determining the power and temperature levels of the maxi- 


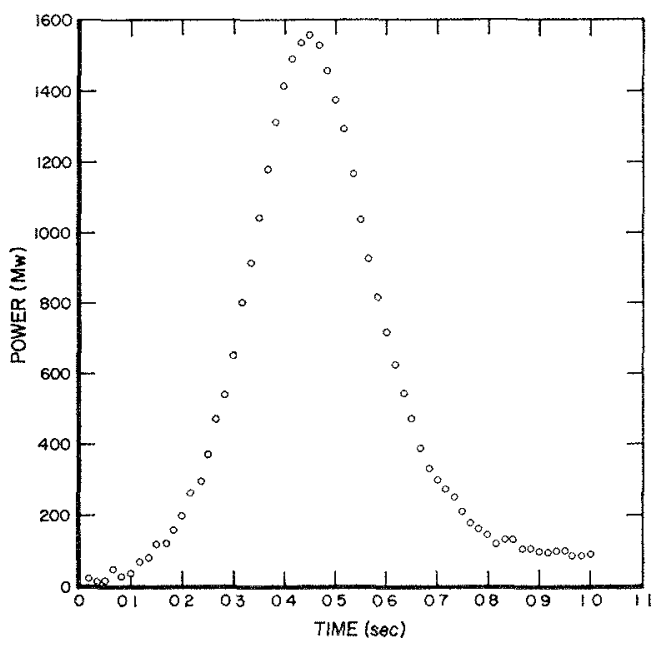

a. Reactor Power
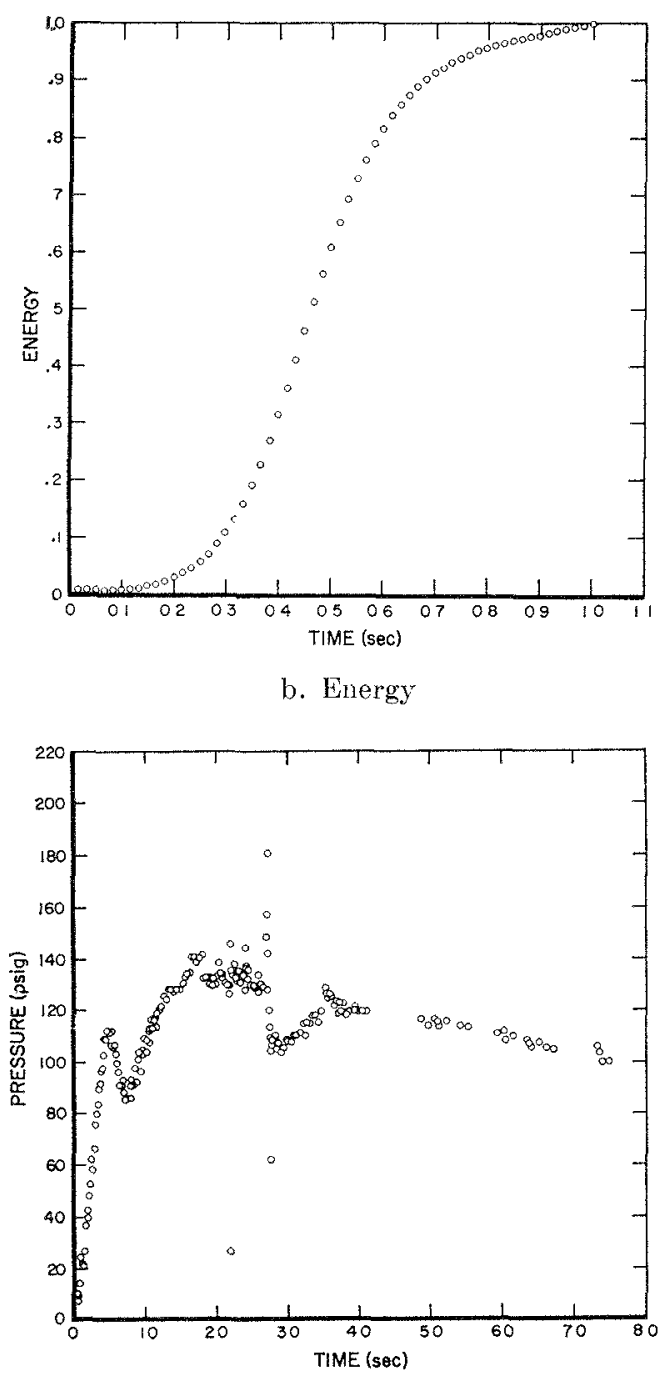

c. Fuel-Section Pressure

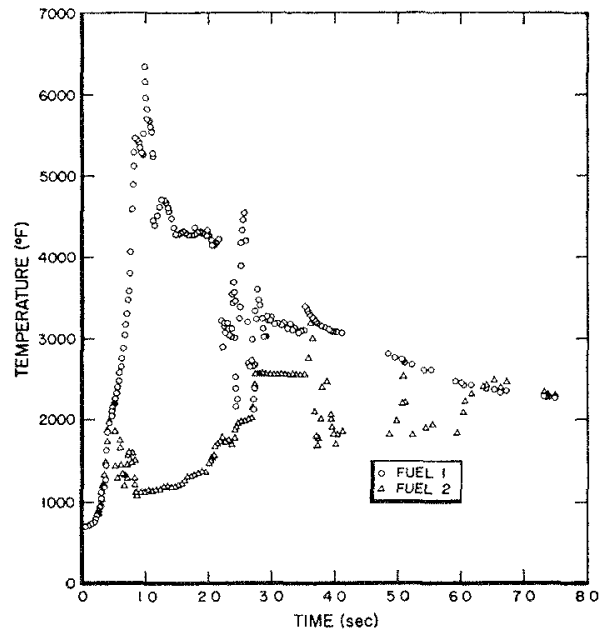

d. Fuel Temperatures

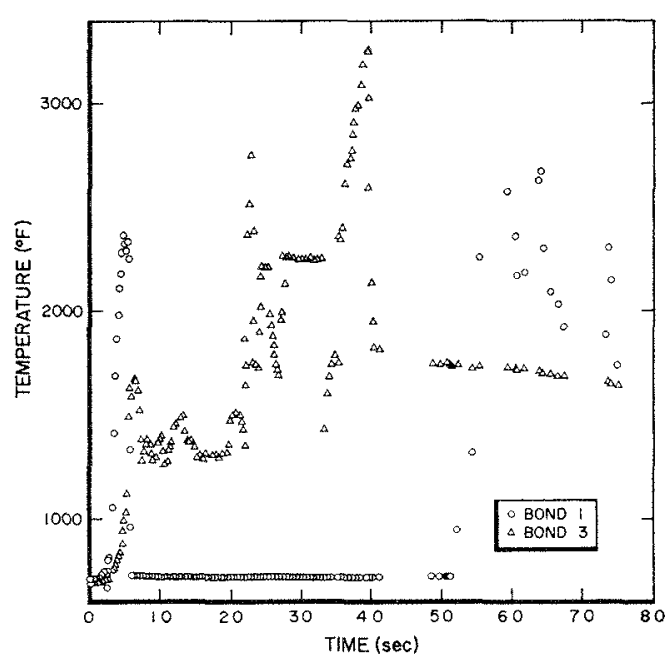

e. Bond Temperatures

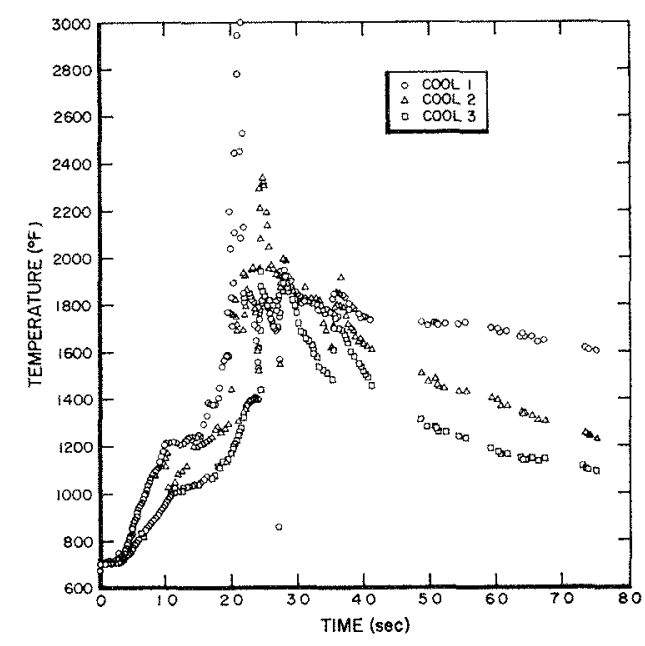

f. Coolant Temperatures

Fig. 2. Data from TREAT Capsule Experiment. 
mum channel. An overall fuel-coolant heat transfer coefficient was employed. Inlet coolant temperature and coolant flow rates were not varied during a nonboiling transient. In order to simulate pump-loss or pipe-rupture accidents, flow was initially set at some fraction of full flow. Flow was also changed to some new value when boiling occurred to simulate a flow reduction due to two-phase fluid pressure drop.

\section{Results of Calculations for Coupled Core}

Calculations were performed using a 15-group diffusion code (CAESAR) to determine the changes in reactivity in a coupled core module associated with voiding from 1 to $10 \%$ of the center of the core or fast region. Two calculational models were used to approximate the successive effects of fuel redistribution within a module:

1) cylindrical-cylinders of material of appropriate size removed from core;

2) spherical-spherical void growth in center of core with uniform redistribution of this material into remainder of core.

The composition and geometry of the reference module are given in Table 2 and Figure 5. The results of these calculations are presented in Figure 6.

TABLE 1. A\ALYSIS OF TREAT DA'TA

\begin{tabular}{|c|c|}
\hline Time (sec) & Description \\
\hline 0.45 & $\begin{array}{l}\text { About } 50 \% \text { of total energy released. Central } \\
\text { fuel temperature was } \sim 2200^{\circ} \mathrm{F} \text {. }\end{array}$ \\
\hline 0.5 to 0.6 & $\begin{array}{l}\text { Bond TC's farl (note decrease in readings to } \\
1500^{\circ} \mathrm{F} \text { ). Small pulses (few psi-not seen } \\
\text { on data printout) on fuel-section pressure } \\
\text { transducer-fluctuations ceased from } 0.8 \\
\text { to } 1.7 \text { sec. }\end{array}$ \\
\hline 07 & $\begin{array}{l}>90 \% \text { of energy released. Central fuel tem } \\
\text { perature read } \sim 4000^{\circ} \mathrm{F} .\end{array}$ \\
\hline 1.0 & $\begin{array}{l}\text { Burst complete. Central fuel temperature } \\
\text { read } \sim 5200^{\circ} \mathrm{F} \text {. For } \sim 06 \text { sec, coolant tem- } \\
\text { peratures level at } 1200-1300^{\circ} \mathrm{F} \text { (these events } \\
\text { are associated with time to melt edge } \\
\text { of fuel, sag into clad, and heat and melt } \\
\text { or react with clad). }\end{array}$ \\
\hline 1.7 to 2.0 & $\begin{array}{l}\text { Central fuel temperature reading dropped } \\
1000^{\circ} \mathrm{F} \text {. Bond temperature readings fluctu- } \\
\text { ated severely. Coolant temperatures fluctu- } \\
\text { ated severely. Pressure pulses up to } \sim 100 \\
\text { psi occurred several times at } \sim 0.2 \text {-sec in- } \\
\text { tervals (associated with failure of cladding } \\
\text { and motion of molten fuel and clad into } \\
\text { stagnant coolant, rapid energy transfer to } \\
\text { coolant with subsequent large local coolant } \\
\text { pressures producing fuel dispersion). }\end{array}$ \\
\hline
\end{tabular}
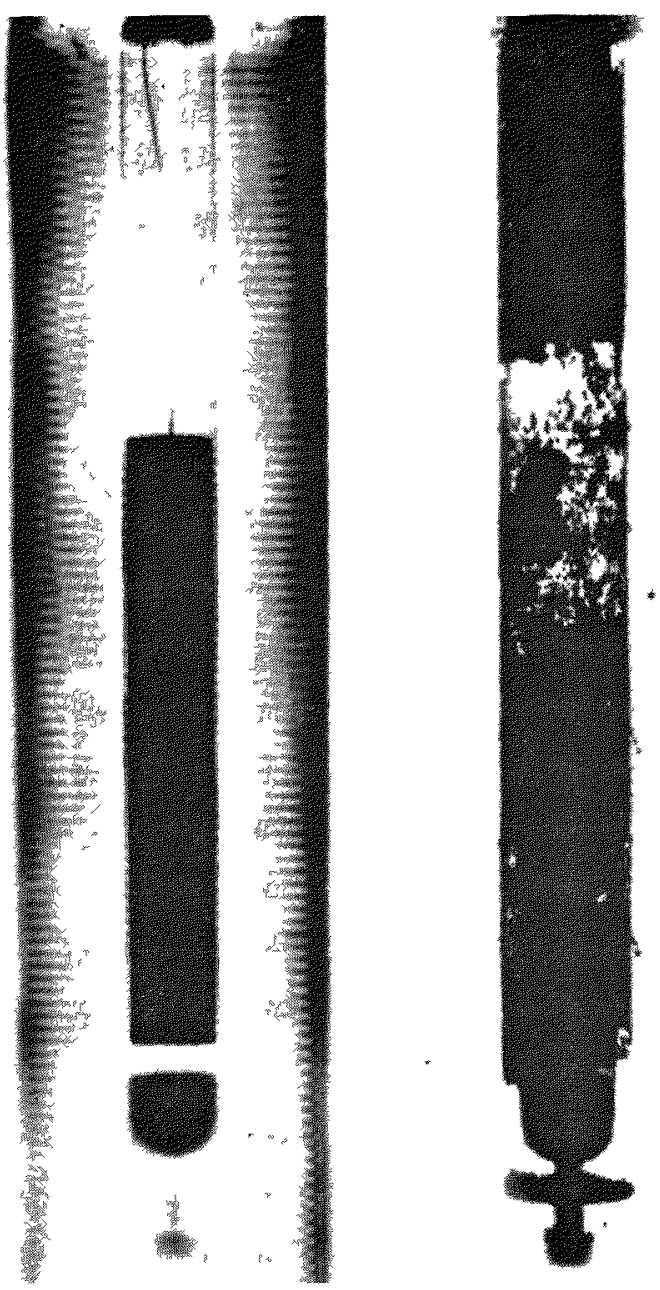

Fia. 3. X Radiographs of Capsule 1 before and after Irradiation.

To demonstrate the incorporation of fuel redistribution into the AIREK code, the reference coupled core design will be used. Consideration is given to an accident in which the control rods are ejected at a rapid rate and in which protective action does not occur so that residual power levels produce eventual melting of the gas-bonded oxide fuel in the peaked regions of the core. Based on the temperature distribution within the core and the rate of temperature rise of the fuel, the rate at which melting propagates from the center of the core was estimated. From the more conservative calculation of associated reactivity effects for the spherical model, the rate of change in reactivity due to displacement of fuel from the center of the core to the outer regions was determined. At the point of gross fuel melting, the reactivity effect was triggered. In the case under consideration, the redistribution resulted in rather large negative reactivity effects, so that propagation of meltdown would not proceed more than a few percent, at which point the core would be 


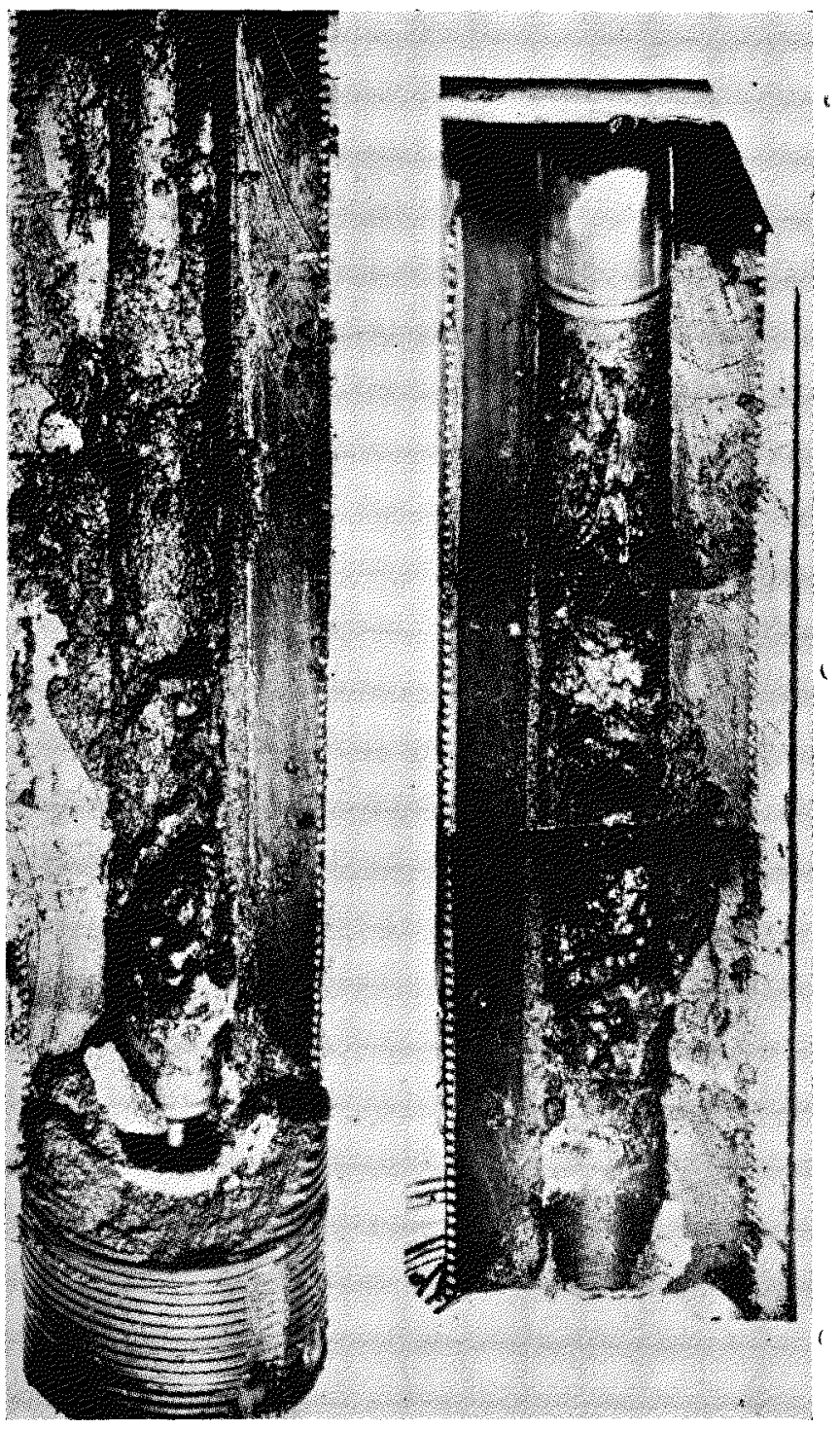

FIG. 4. Disassembled Capsule after Irradiation.

TABle 2. Composition ( $/$ o o of Reference Couplad Core Module

\begin{tabular}{l|r|r|r}
\hline & Core & $\begin{array}{c}\text { Inner } \\
\text { Blanket }\end{array}$ & $\begin{array}{c}\text { Outer } \\
\text { Blanket }\end{array}$ \\
\hline U-238 & 10.2 & 22.4 & 22.6 \\
Pu-239,-240,-241,-242 & 5.2 & 0.4 & 0.2 \\
Oxygen & 32.9 & 45.8 & 45.8 \\
Iron & 28.8 & 22.8 & 22.8 \\
Sodium & 21.9 & 8.6 & 8.6 \\
Fission Products (modera- & 1.0 & & \\
$\quad$ tor is 100\% C) & & & \\
\hline
\end{tabular}

quite subcritical. The results of the analysis are presented in Figure 7 . The model could be improved by a better understanding of the role of the coolant, the effects on the structures, and the functioning of safety and protective mechanisms (emergency cooling systems, etc.).

\section{E. Discussion of Parameters which Influence Melt- down Behavior}

Heating rate will exert an extremely important influence on the course of meltdown, since rapid heating rates will produce high fuel temperatures and relatively minor heat transfer to other materials in the core, whereas slow heating rates could produce coolant vaporization, cladding melting, or failure prior to gross fuel melting. Thus, the initial temperature distribution and the rate of heating during the excursion will play a major role in the course of events.

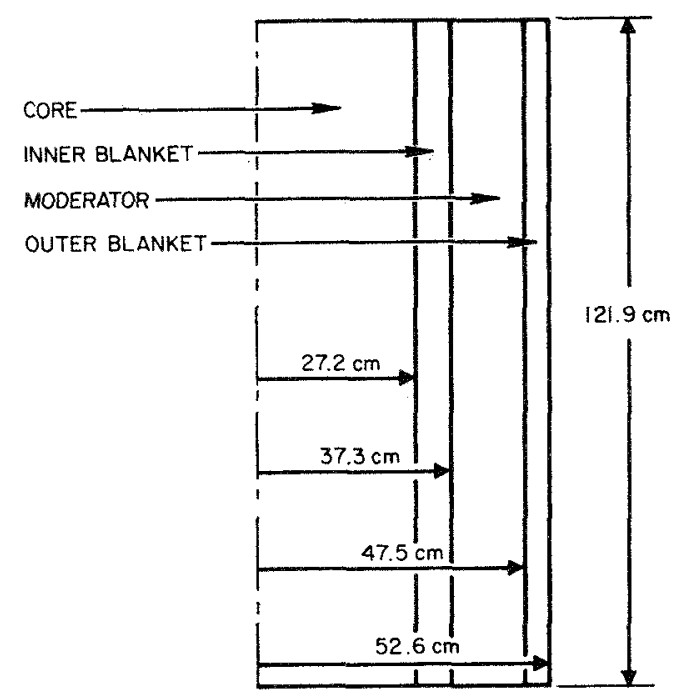

a) CYLINDRICAL

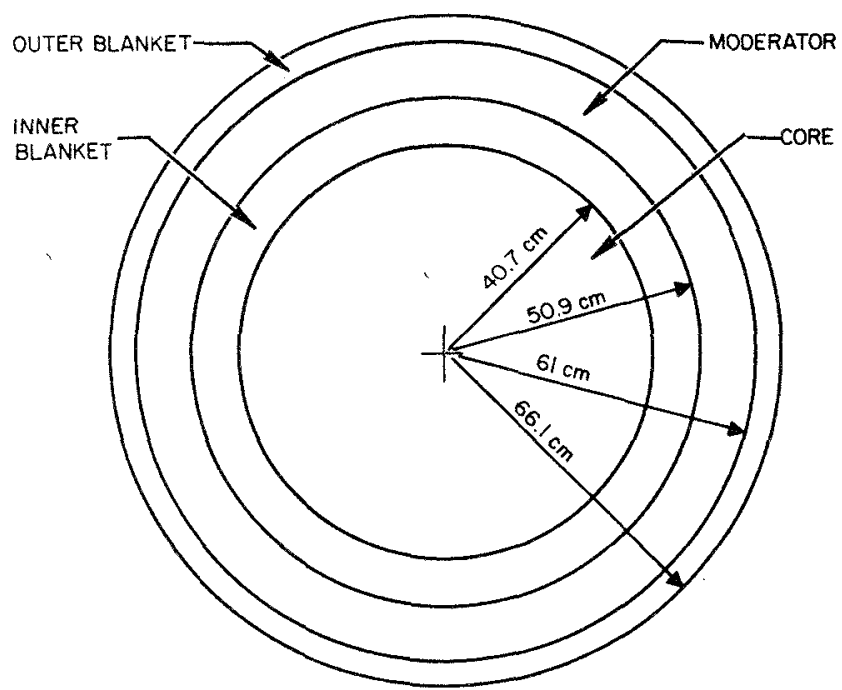

b) SPHERICAL

Fig. 5, Geometry of Reference Coupled Reactor Module. 


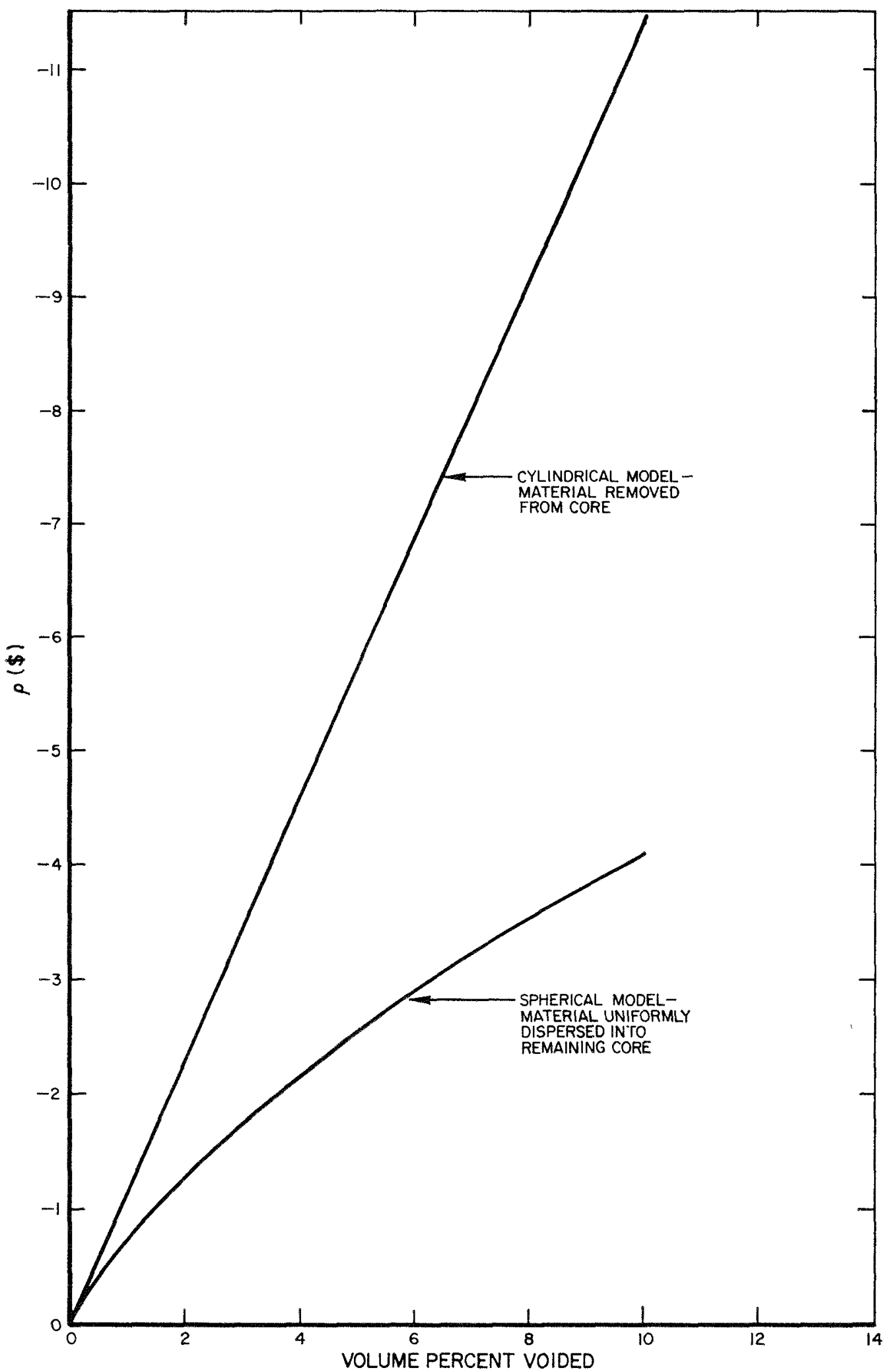

FIG. 6. Results of Coupled Reactor Reactivity Calculations for Fuel Motion. 

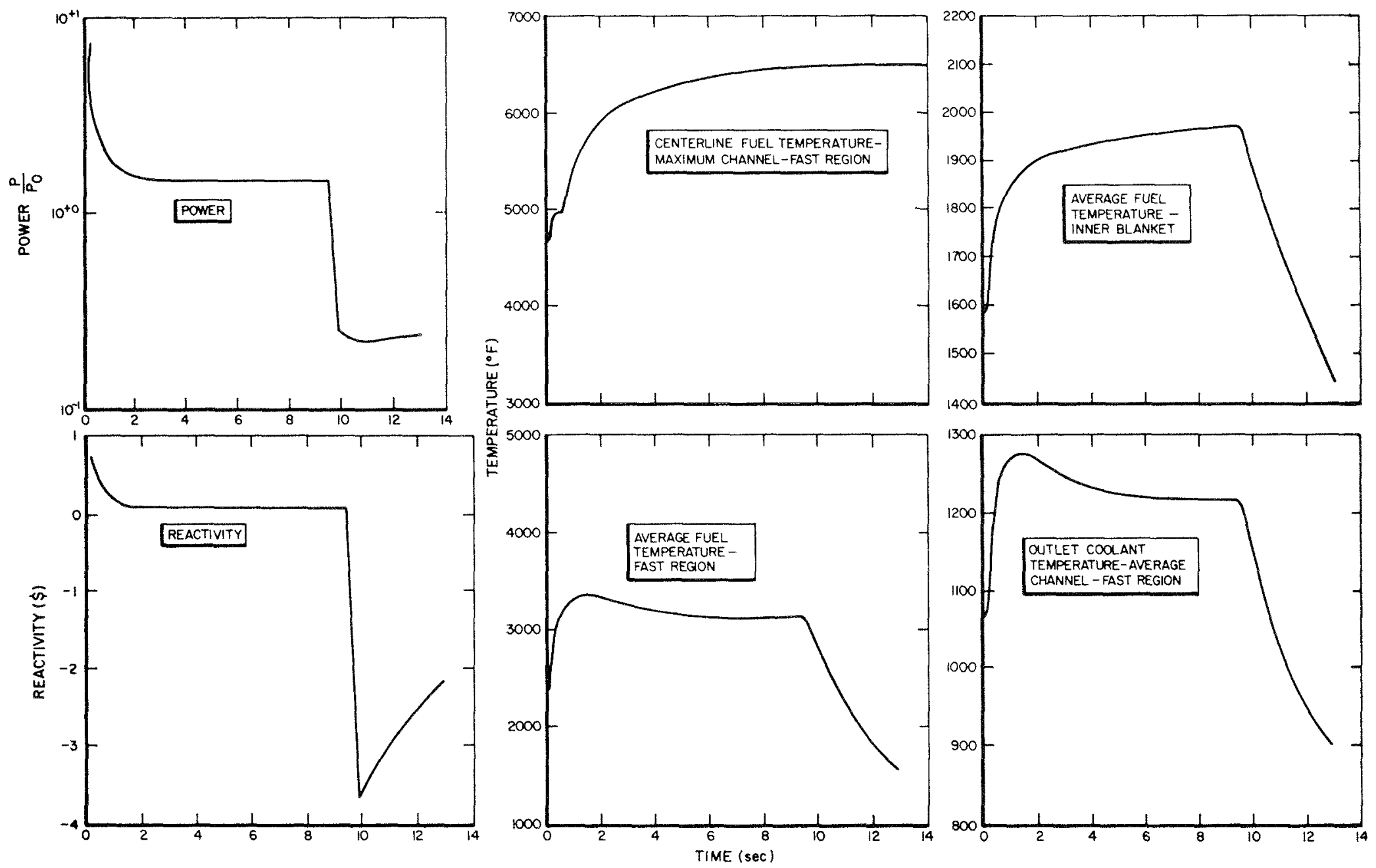

Frg. 7. Results of AIREK Analysis of Coupled Reactor Control Rod Ejection Accident with Disassembly Model. 
Similarly, the fucl-rod design, including fuel and clad material, type of bond, use of vented elements, and geometry, will also influence the sequence of events.

The interesting part of the TREAT study was the minor role of the bond and the time lag associated with cladding penetration. These results imply that heating rates which produce melting in the millisccond-to-second time interval might not produce very different results provided that maximum temperatures do not rise above the boiling point of the fuel. This contention should be checked by TREAT data on ceramic-fuel meltdown at slower and faster heating rates. Quite clearly, the extent of heating plays a major role, and it appears that for failure of ceramicfuel elements, sufficient energy must be deposited in the fuel to produce fairly complete melting of the fuel slug. Energy levels which produce fuel temperatures well above the fuel boiling point (at the pressure in the fucl rod) are likely to result in different consequences than those observed.

Since the failure observed was a result of slow cladding penetration by molten fuel rather than clad rupture, quite different behavior could occur in highly pressurized fuel rods. However, vented fuel elements would be expected to behave in a fashion similar to that observed in the experiment.

The core design, coolant flow, and pressure distribution will affect the course of material redistribution; however, the most important parameter is the temperature distribution in the core, since it will produce appreciable differences in the rate of propagation of molten regions of the core following melting of the hottest portions.

Finally, further work on the melting process in ceramic fuels is required to study the consequence of long-term fuel irradiation, such as the foaming which has been observed to occur upon meltdown of highly irradiated metallic fuel

\section{REFERENCES}

1. 1). T. Eggen and H. A. Morewitz, Feasibility Study of a 1000-WWe Sodium-cooled Fast Reactor, NAA-SR-11378, Vols. I and III.

2. S. J. Stachura, R. N. Cordy, and M. Silberberg, Uranium Carbide Transient Heating Studies-Phase II, American Nuclear Society Transactions, meeting held June 21-25, 1965 at Gatlinburg, Tenn.

3. L. R. Blue and M. Hoffman, AIREK Generalized Program for the Numerical Solution of Space Independent Reactor Kinetues Equations, NAA-SR-Memo 9197.

\section{Discussion}

Mr. Zebroski (GE): I think you know about the results of a long series of similar TREAT tests with oxide fuel capsules which have been extensively published. Many of these went well into the melting range and one into the high boiling range, that is, the $\mathrm{UO}_{2}$ boiled and reached a pressure of 800 psi. Yet in none of these did we have any explosive kind of behavior in the sodium bond, nor disruption of the capsule nor even of the fuel cladding. Do you think that this might be related to the much shorter time constant of release of heat of the carbide fuel?

Mr. Stachura: The case of the oxide fuel is somewhat different from the bonded case because the gas gap produces a rather large temperature difference from the edge of the fucl to the cladding. The cladding is kept very cold throughout the course of the burst. Although central temperatures well above melting, as high as $7000^{\circ} \mathrm{F}$, I believe, were reached in some cases, at no time did the edge of the fuel ever reach these temperatures. In specimen $\mathrm{C}-2-\mathrm{C}$ the edge of the fuel was molten, but the melting of the fuel at the edge did not cause penetration of the clad. It may be possible that the edge was at the melting point and the slight energy of fusion to melt it partially may have been transferred to the cladding which was still cool because of being in contact with the stagnant liquid coolant. There really wasn't sufficient energy available to lead to penetration of the cladding. When the cladding transfers heat to the stagnant coolants, which transfers heat to the heat sink, it takes quite a considerable amount of energy to reach the threshold at which the cladding will fail. It is difficult to understand how energy may continually be pumped into the oxide pin to very high values and never lead to failure of the element. I think there is going to be a point at which failure will ultimately occur, and it just has not been attained.

Mr. Zebroski: I wonder if the difference might not be characterized by a difference in the peak instantaneous heat flux prior to failure. I think the oxide 
rods never went much above a typical full-power heat flux or perhaps a full-power peak heat flux of perhaps around a million to a million and a half. I would suspect that with the shorter time constant in the sodium bond you probably ran heat fluxes of the order of 3 to 5 million.

Mr. Stachura: We did until the point at which the coolant temperature remained fairly constant. At that point, the boiling of the bond probably reduees the heat transfer quite considerably. By means of analog thermal models we have found that at the time the bond reaches the boiling temperature, there is imposed the resistance of sodium vapor or something corresponding to a film boiling coefficient. We rather accurately predicted thermal behavior; in fact, in the case of the large burst, we allowed the fuel to sag into the clad, thereby reducing by half the conductivity of fuel. The temperature rise took from $11 / 2$ to $2 \mathrm{sec}$; this was reproduced in this analysis, and finally the cladding reached melting temperatures.

Mr. Zebroski: Do you know the peak heat flux?

$M r$. Stachura: During the early part of the burst when the sodiun bond is still at $1800^{\circ} \mathrm{F}$, I imagine it is several million; during the last part of the burst, it must go down considerably.

Mr. Dalle-Donne (Karlsruhe): I don't think that from your results you can deduce that you will never have a large superheat in a sodium-cooled reactor. I don't think that the main effect is due to the smoothness of the surfaces, because unfortunately sodium, if it is in forced convection, after a certain time fills the biggest holes in the wall, if you like, so only the smallest holes remain active. This means a very high superheat.

Mr. Stachura: Yes, the experiments of Rohsenow and Marto, I think, show that there was even a hysteresis effect. But after boiling started at more modest superheats and proceeded for a while, then in order to sustain boiling at these sites rather large superheats were required. I think this definitely is related, however, to the size of the activation sites. They even found that it was related to the nature of the materials from which they were boiling. I will certainly agree that we cannot say we were really not measuring surface temperature. It was not a result that was designed to detect exactly the superheat. Quite clearly you have to have some superheat to form the vapor bubbles. The main point was to show that here was a ease in which heat was transferred to the sodium to create the bubbles. I'm not sure whether the forced convection is as effective in eliminating the inert gases from the site as are the boiling processes themselves when the liquid is collapsing at these sites.
There may be some problems associated with making inactive sites that, let's say, are initially active. However, I would think that we could roughen cladding surfaces to avoid any real serious superheat problem.

Mr. Dalle-Donne: That method was tried, but it did not reduce the superheat very much. One thing which could be effective would be the presence of an impurity in sodium that modified the surface tension; maybe that is the reason that you didn't find any superheat.

Mr. Noyes (AI): On the question of superheat, I think there is one factor that we haven't mentioned in this discussion, namely, surface heat flux. Rohsenow and Marto's experiment showed, among other thing:, that the superheat phenomenon was heat-flux-dependent, and the Oak Ridge experiments were mostly done at low heat flux. At high heat fluxes, the transition is something around 1 or $2 \times 10^{5}$ heat flux. Now in the reactor and in these experiments, the heat fluxes were of the order of $10^{6}$. You're much less likely to experience superheating under these high heat fluxes than you would expect under the low heat fluxes at which the Oak Ridge experiments were done. Here is a factor which needs to be kept in mind in the consideration of superheat questions.

Mr. Stachura: Was their system closed so that the gradient through the sodium could have been small enough that inert gas pressure could have actually resulted in some pressurization of the system to also inhibit boiling formation of bubbles?

Mr. Dalle-Donne: I think the important factor here is not heat flux. It is the gradient of temperature perpendicular to the wall that is important, because from that depends how the bubble can grow. I think the smaller this gradient is, the easier it is to superheat. You can always think of an accident in a sodium-cooled reactor in which the temperature of the sodium in a channel or in a subassembly is practically 
three-phase mixtures, etc. I don't think any energetic reactions were involved. I believe the pressure pulses were due primarily to the large local temperatures of the sodium that were generated upon passage of energy from the fuel to the sodium. These local pres- sures transmitted the pulse, which was damped quite a bit since it had to go through some vapor space; nevertheless they were the cause for these pulses. The only materials in the capsules were fuel, stainless steel, tantalum, and thermocouple materials. 


\title{
Mixed Oxide Fuel Movement at Various Power Levels and in Transients*
}

\author{
S. A. Rabin, W. E. Baily, J. E. Haxison, tai) F. W. Knight \\ General Electric Company \\ I dvanced Products Operatzon \\ San Jose, Calfornia \\ (PREATENTEI) BY S. A. RABIN)
}

\section{Introduction}

A fast reactor fuel typically is not deployed in its most reactive configuration. Consequently, fast reactor cores are subject to possible positive reactivity changes due to fuel movement, ${ }^{(1)}$ the sensitivity being greater in small, high-leakage cores. Movement of fuel could also affect its exposure lifetime. In this paper we will summarize our experimental observations on the movements of mixed oxidet fuel within the cladding in various fuel-tenperature regimes as they relate to (a) fuel-cladding interactions and (b) reactivity changes.

\section{Fuel-cladding Mechanical Interactions}

The basic question of interest from the standpoint of the operating life of a fuel element is whether the differential expansion of the fucl relative to that of its cladding will generate fuel-cladding mechanical interactions which, in the extreme, could lead to distortion or failure of the cladding. It is normal to provide sufficient as-fabricated void, in particular a diametral gap, such that the differential thermal expansion is accommodated within the fuel and stresses are not introduced in the cladding. Were movement of the fuel to alter this initial design condition, a potential for stressing the cladding could develop.

In-pile experiments in which the fuel density, radial gap and/or axial gap have been varied indicate that the differential thermal expansion can be accommodated within the range of values of interest in an FCR. For example, mixed oxide fuel pins irradiated for short term in EBR-II at power levels up to 16 $\mathrm{kW} / \mathrm{ft}$ exhibited no cladding distortion. (2) This dimensional stability is exhibited despite thermal-stress fracture of the fuel due to temperature gradients, which is typical in oxide fuels. The consequences of fuel fracture appear to be alleriated by the facts that: (a) the size into which oxide fuel fragments is lim-

* This work was performed under USAEC Contract AT (04-3)-189, Project Agreement No. 10.

$\dagger$ Mixed oxide as used in this paper refers to a solid solution of $\mathrm{PuO}_{2}$ and $\mathrm{UO}_{2}$, a typical composition being $20 \mathrm{w} / \mathrm{o} \mathrm{PuO}_{2}$ and $80 \mathrm{w} / \mathrm{O} \mathrm{TO}_{2}$. ited, ${ }^{(3-5)}$ and (b) crack healing occurs during heating of the fuel. In support of these observations, power cycling of oxide fuel pins which employed a selfsupporting elardling has revealed no evidence of strain cycling. ${ }^{(3,6.7)}$ I) ata have been reported ${ }^{(8)}$ which suggests that mixed oxide fuel exhibits better resistance to thermal fracture than $\mathrm{CO}_{2}$. We have irradiated the two materials under identical conditions and found no significant difference in the fracture behavior (see Fig. 1).

Radial migration of fuel away from the center is commonly obscrved at central fuel temperatures above about $1800^{\circ} \mathrm{C}$, but below melting. A central void forms in a matter of minutes, effectively reducing the central temperature. The primary transport mechanism appears to be vaporization from the hot side of a pore and condensation on the cold side. The columnar grains and central void in the inner half of the fast-flux-irradiated, mixed oxide fuel in Fig. 2 are the result of this type of movement.

Mixed-oxide irradiations at equivalent power levels of the same $\mathrm{Pu} / \mathrm{C}$ ratio but different stoichiometries have revealed (9) less redistribution in fuel with a slight oxygen deficiency as compared to the fucl with excess oxygen. This is evident from the relative size of the central void (see Fig. 3). In addition, irradiation of an instrumented capsule containing stoichiometric $\mathrm{CO}_{2}$ and hypostoichiometric $(\mathrm{O} / \mathrm{M}=1.97)$ mixed oxide pins in tandem at equal rod power indicates (10) that radial migration in the $\mathrm{PuO}_{2}-\mathrm{UO}_{2}$ is less despite operation at somewhat higher temperatures (see Fig. $4)$.

Radial relocation of the fuel does not appear to influence fuel-cladding interactions in the steady state. The accessibility of the void to the fuel and plasticity of the mixed oxide are believed to be contributing fac tor to the absence of cladding strain despite the radial inovement. Circumferential restraint on the fuel column by the cladding and by the outer, cooler fuel regions is substantial even under FCR conditions. The plastic portion of the fucl can flow and occupy the available void volume rather than stretch the cladding. 


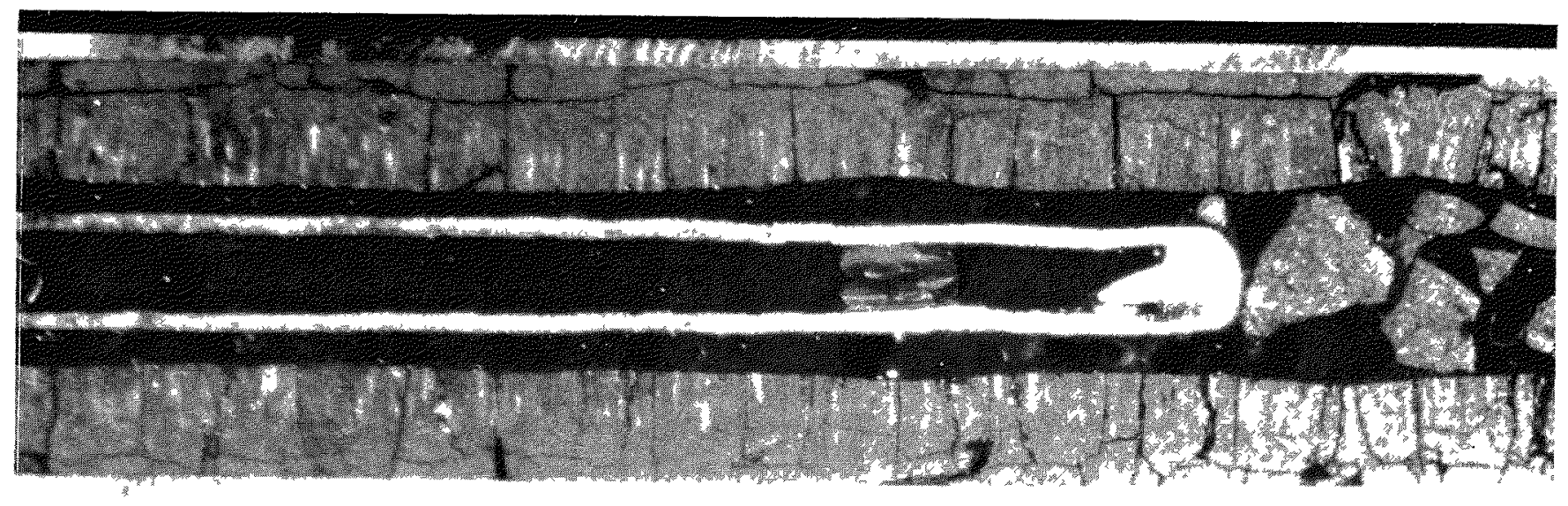

MIXED OXIDE

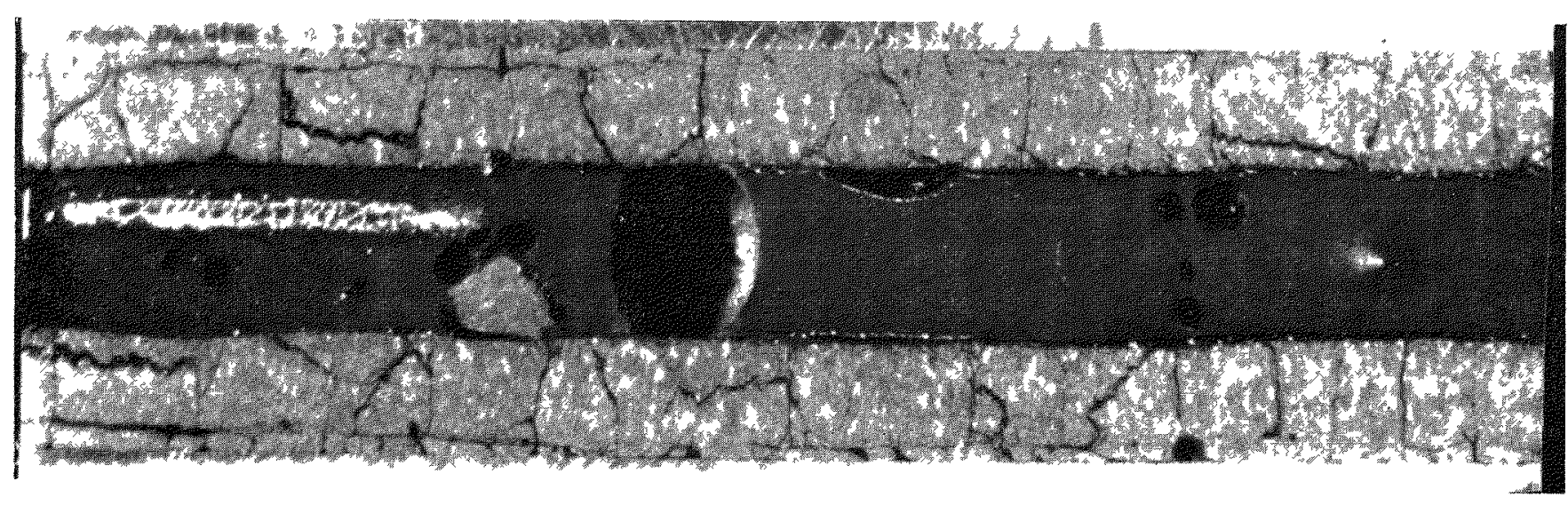

$\mathrm{UO}_{2}$

Fick. 1. Similarity of Fracture Pattern in Irradiated Mixed Oxide and U( $)_{2}$.

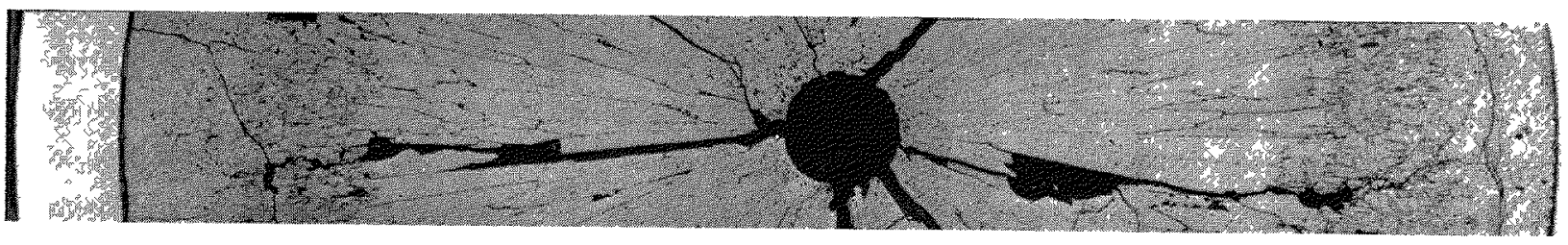

$100 \times$

FIG. 2. Diametral Composite of Mixed Oxide Irradiated in a Fast Flux for Short Term at $15 \mathrm{~kW} / \mathrm{ft}$.

Another source of fuel expansion at lower powers and high burnups is fuel swelling due to the combined expansion effects of solid fission product agglomeration and retained fission gas pressure. The reported swelling rates for $\mathrm{UO}_{2}$ and $\mathrm{PuO}_{2}$ cover a broad range, viz., 0.6 to $1.7 \% \Delta V$ per a/o burnup. ${ }^{(11,12)}$ The same studies indicated that the swelling rate is inversely related to the original void volume and that an irradiation-induced plasticity ocrurs. Thus one can speculate that fuel swelling could be accommodated by provision of appropriate void within the fuel pin.
In investigation of fuel swelling, measurable fissiongas porosity (see Fig. 5) was observed ${ }^{(9)}$ in the cooler portion of a high-burnup $(70,000 \mathrm{MWD} / \mathrm{MT})$, highsmear-density ( $92 \%$ of theoretical density), mixed oxide fuel pin. The power level in this section was approximately $10 \mathrm{~kW} / \mathrm{ft}$, and the porosity occupied about $15 \%$ of the cross section. Additional evidence of fuel movement in this region was the disappearance of the as-fabricated radial gap. Nevertheless, the diameter in this region of the fuel pin exhibited only a slight increase, which suggests that the swell- 


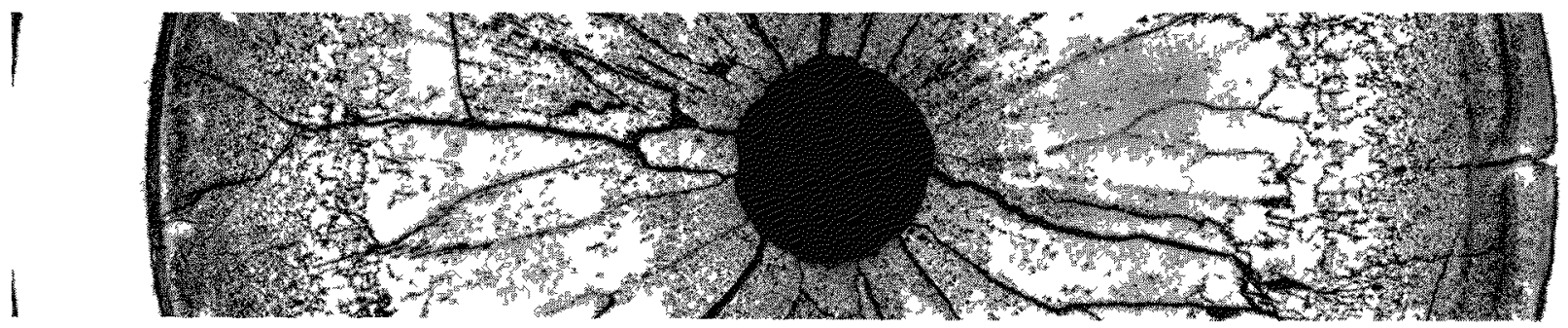

HYPO STOICHIONETRIC
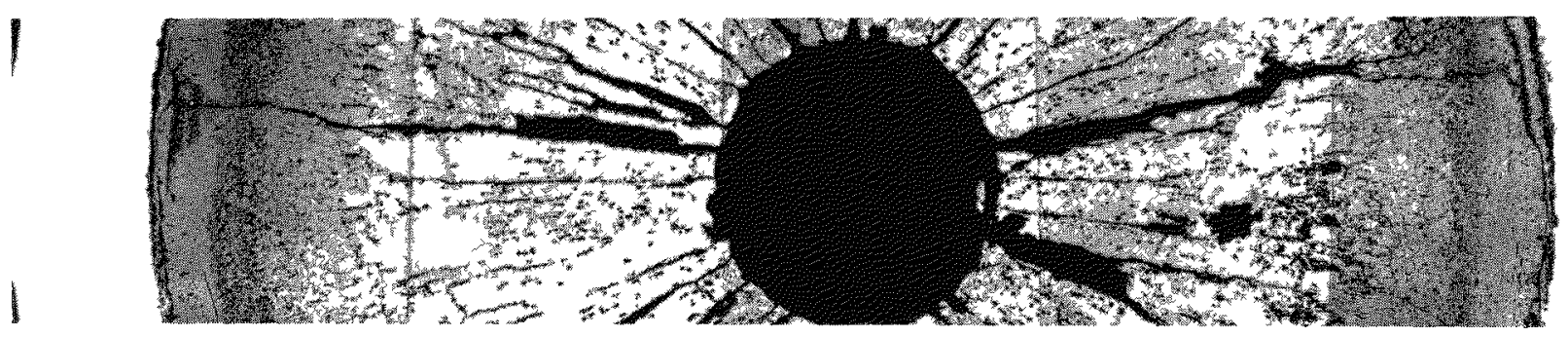

HYPER STOICHIO VETRIC

$70,000 \mathrm{VWW} / \mathrm{T}$

TIr 3 Cffect of store hrometry on Radial Tuel Movement in Mixed Oxide Furl
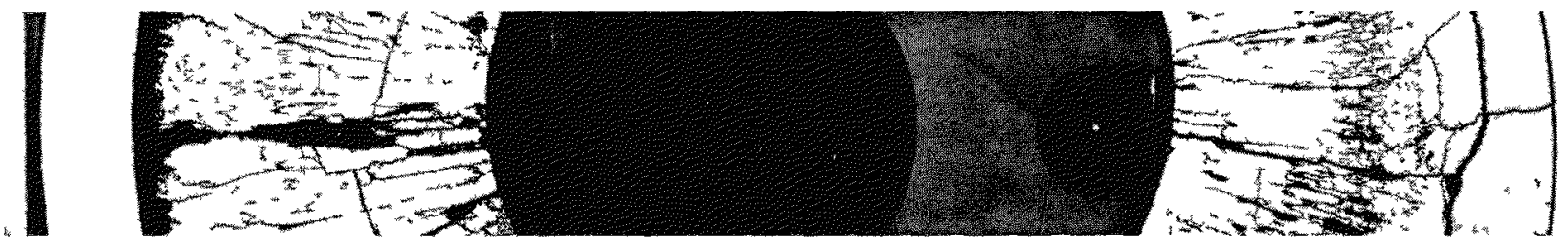

AS ETCHED

$20 \mathrm{~W} / \mathrm{O} \mathrm{PuO} 280 \mathrm{~W} / \mathrm{O} \mathrm{UO}_{2}(\mathrm{o} / \mathrm{m} 197)$
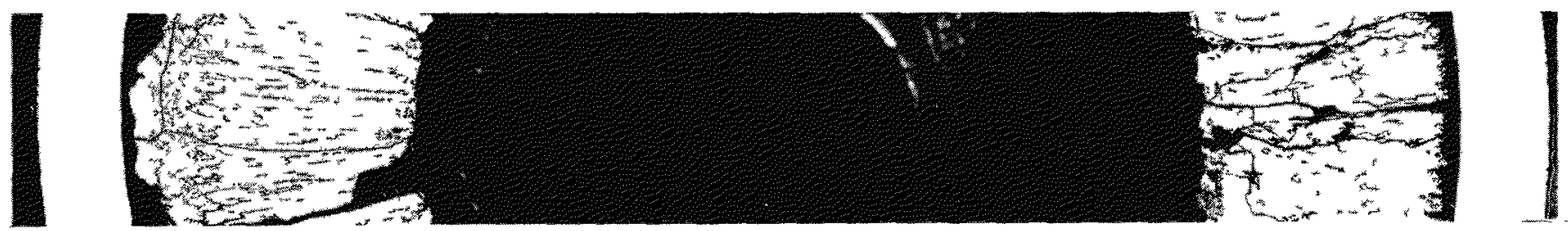

AS ETCHED

$\mathrm{UO}_{2}(\mathrm{o} / \mathrm{m} 2.00)$

$50 \times$

Fis 4 Comparison of Radial Migration in UO and Mixed Oxide Irradiated at Equal Rod Powers

ing was largely accommodated in this high-density fuel pin More precise experiments to evaluate the extent of swclling and its accommodition under representative FCR conditions are in progress, both in fast and thermal peetrd

When the mixed oxide fucl is oprated above the solidus temperature, an additional expansion is contributed by the change in rolume on melting Ind1rect in-prle medsurements(10 13) of the mixed oxide indicate that the molten rolumetric expansion is of the order of 8 to $12 \%$, which is comparable to the 7 to $10 \% \Delta V$ experieneed by $\mathrm{IO}_{2}$ A significant result flom steddy-state high-power tosts(13) is the demonstration that the molten expansion per se can be accommodited in dxidlly unrestramed, shoit fuel segment if sufherent vold space is avalable This same response has been displayed during transicnt nrad1ations in wheh up to $75 \%$ of the fuel volume became molten very rapidly (14)

Fuel morement and fission-gas generation during steddy-state iriadiation may indrectly bear upon the subsequent transient tuel performance Transient testing of a non-pre-mradiated and a pre-mrradiated $(7900$ MWI/AT at high power) specmen under dupl1- 
cate conditions, during which three-quarters of the fuel was molten, revealed that eladding deformation occurred only in the latter pin. ${ }^{(15)}$ It was surmised that fission gases released during the transient were trapped in the fuel, could not escape into the gas plenum, and thus exerted an appreciable pressure against the cladding. Further transient testing of fuel pre-irradiated to higher burnups is in progress. It should be noted that pre-irradiating and transient irradiating in a thermal flux represents a more severe

\section{HIGH BLRNLP}

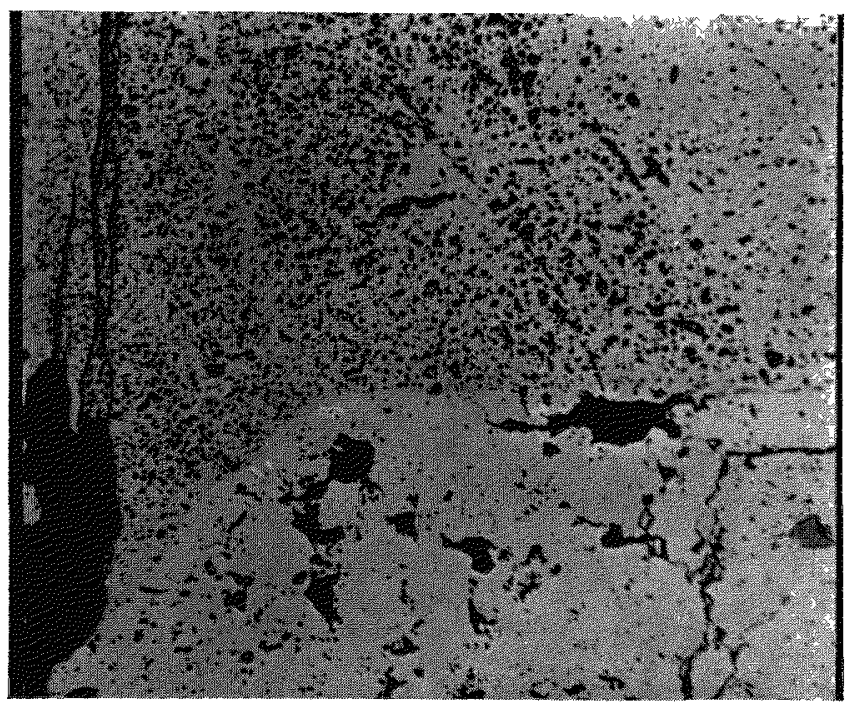

As Polished

$250 \times$

Fig. 5. Fission-gas Bubbles in High-burnup Mixed Oxide Fuel. test of the fuel than irradiation in a fast-flux environment due to the flux-depression effects in the thermal spectrum.

\section{Reactivy Effects}

Potential sources of $\Delta k$ due to fuel movement in a fast reactor include:

1. subassembly bowing;

2. Pu-U separation;

3. thermal expansion of the fuel column;

4. axial redistribution of fuel.

subassembly bowing is beyond the scope of this paper. Differential migration of plutonium and its effect on the Doppler coefficient are covered in a separate paper. ${ }^{(16)}$ In a large reactor the negative reactivity effect from thermal expansion is relatively small due to the inherently low neutron leakage. Furthermore, the axial expansion of fuel under irradiation is not fully predictable. A conservative approach thus takes no credit for this mode of fuel movement.

We will confine our attention to the item of fucl redistribution. Reactivity insertion rates due to fuel redistribution would be of concern in the event that both the magnitude of the movement toward the reactor midplane were large and such movement were rapid. A generalized assessment of the effects of fuel transport on reactor physics and control is not practical, principally because the consequences are a fun:tion of the specific reactor design (core size, shape, etc.). For example, a comparative analysis (17) of four ceramic-fueled reactor concepts indicates that the reactivity effects from axial translation of fuel differ notably and would be minimized by a pancake-

TABle 1. Axial Isistribution of Irradiated Mixed Oxide Ft el

\begin{tabular}{|c|c|c|c|c|c|c|c|c|}
\hline \multirow{2}{*}{$\begin{array}{l}\text { Specimen } \\
\text { Number }\end{array}$} & \multirow{2}{*}{$\begin{array}{l}\text { Fuel Length } \\
\text { (in.) }\end{array}$} & \multirow{2}{*}{$\begin{array}{l}\text { Smear Density } \\
(\% \text { Theoretical })\end{array}$} & \multirow{2}{*}{$\begin{array}{l}\text { Peak Rating } \\
\qquad(\mathrm{kW} / \mathrm{ft})\end{array}$} & \multirow{2}{*}{$\begin{array}{l}\text { Burnup } \\
(\mathrm{MWD} / \mathrm{T})\end{array}$} & \multirow{2}{*}{$\begin{array}{l}\text { Max Molien } \\
\text { Area }(\%)\end{array}$} & \multicolumn{3}{|c|}{$\begin{array}{l}\text { Postirradiation Smear Density } \\
(\% \text { Theoretical })\end{array}$} \\
\hline & & & & & & $\begin{array}{l}\text { Top } \\
\text { Segment }\end{array}$ & Middle Segment & $\begin{array}{l}\text { Botton } \\
\text { Segment }\end{array}$ \\
\hline FIE & 14.2 & 87 & 15 & 2,000 & 0 & & No change & \\
\hline $\mathrm{B} 4 \mathrm{~A}$ & 5.1 & 93 & 23 & 16,000 & 0 & & No change & \\
\hline $\mathrm{B} 6 \mathrm{~A}$ & 2.2 & 93 & 28 & Nil & 5 & & No change & \\
\hline $\mathrm{C} 3 \mathrm{~A}$ & 6.0 & 93 & 29 & 9,000 & 8 & 89 & 94 & $96^{(\mathrm{d})}$ \\
\hline $\mathrm{B} 6 \mathrm{~B}$ & 2.2 & 93 & 32 & Nil & 11 & 91 & 94 & 96 \\
\hline $\mathrm{C} 2 \mathrm{~B}$ & 6.0 & 91 & $\operatorname{Tran}(b)$ & Nil & 75 & 85 & 90 & $96^{(d)}$ \\
\hline $\mathrm{C} 3 \mathrm{~B}$ & 6.0 & 93 & 25 & 7,900 & & & Indetermined & \\
\hline & & & $\operatorname{Tran}(b)$ & Nil & 75 & 92 & 89 & $95^{(d)}$ \\
\hline
\end{tabular}

(a) Annular pellets (95\% theoretical density).

(b) Tran = Transient irradiated

(c) Metallographic preparation may have missed coring.

(d) Some fuel cast into hollow insulator pellet at end of fuel column. 


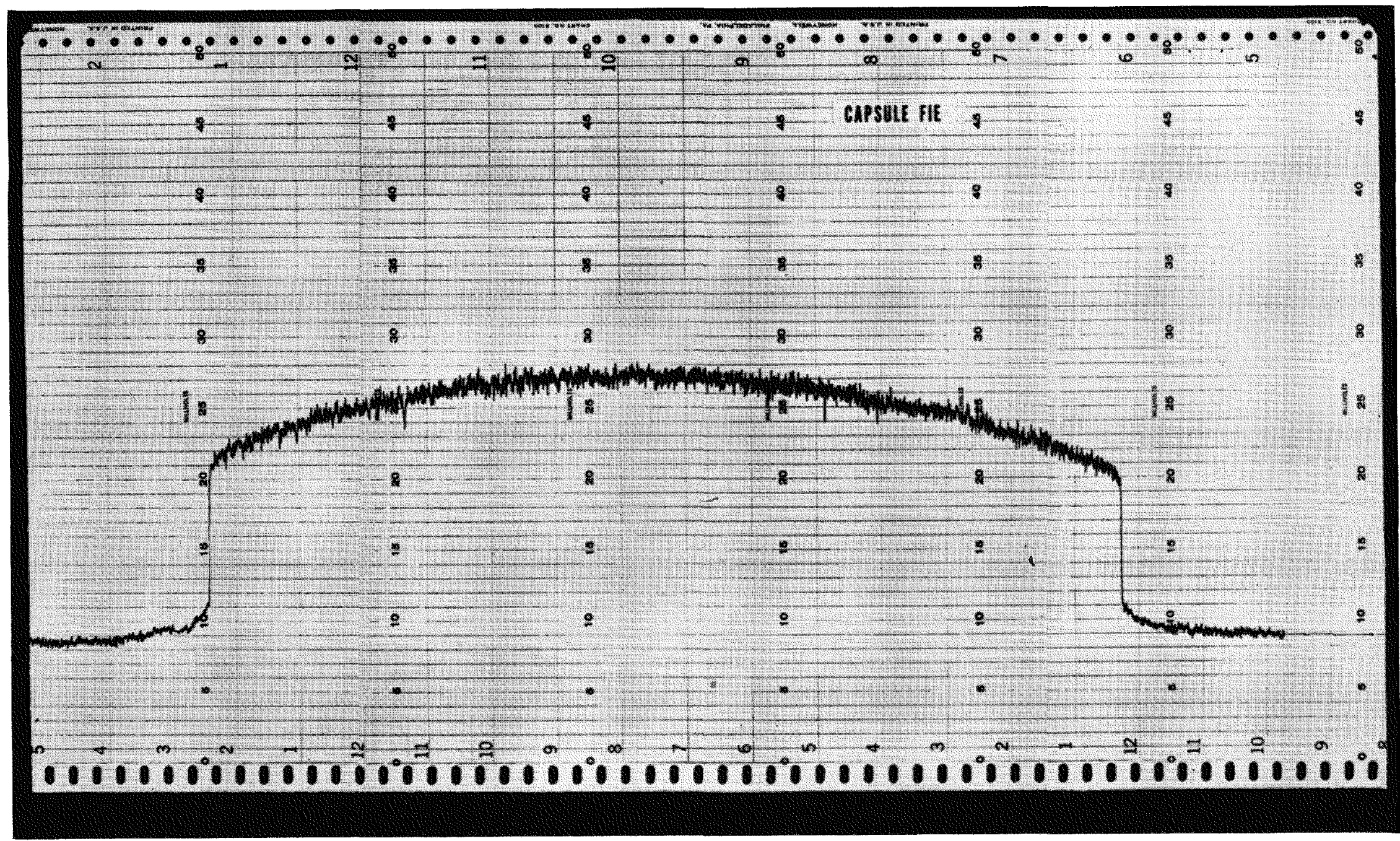

FIG. 6. Gross Gamma Scan of Reference FCR Fuel Irradiated in EBR-II Illustrating Axial Stability of Mixed Oxide. 

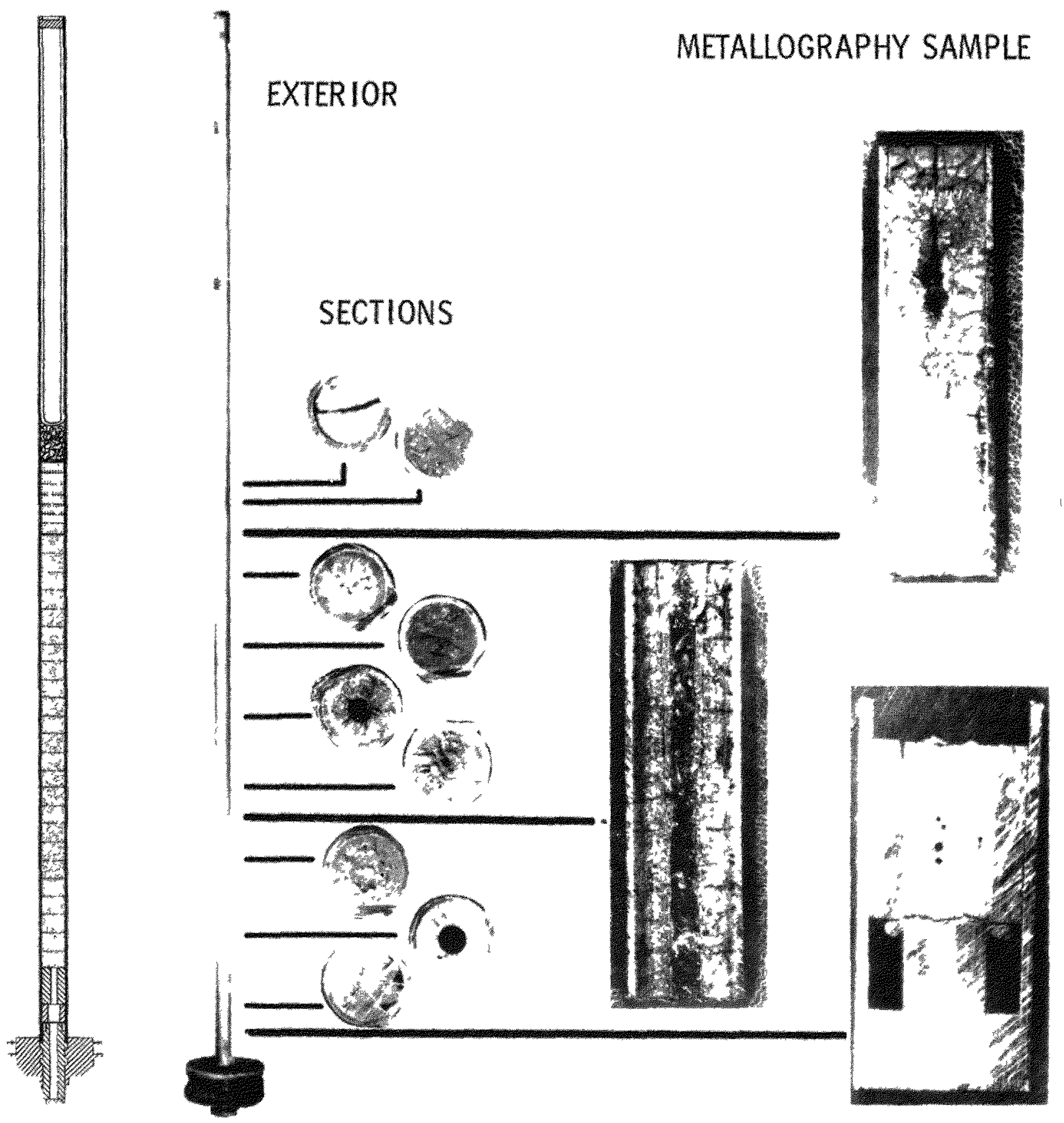

Fig 7 Upward Transport of Pre irrdiated Mixed Oxide Fuel Subsequently Melted during a Transient Test.

shaped core. Theoretical consequences concerning the relative importance of fuel movement in a hypothetical accident are discussed elsewhere; ${ }^{(18)}$ we will stress the emprrical observations made to date on the mixed oxide.

Numerous specmens 1rradiated under a variety of conditions have been examined for axial fuel movement. Gross gamma scans were performed to determine whether the radioactivity profile departs from the integrated flux curve. Multrchannel analyses plus single-channel scans for volatile and nonvolatile nuclides were uned to distinguish variations due to fuol redistribution $v_{s}$. those due to fission product migration. After the fuel pin was sectioned transversely, the fuel length was dirided into segment and the density estmuated from the roid distribution in each segment Typical results from these exammations are summarized in Table 1. 


\section{ESTIMATED PEAK FUEL TEMP: $5000 \pm 500^{\circ} \mathrm{F}$}

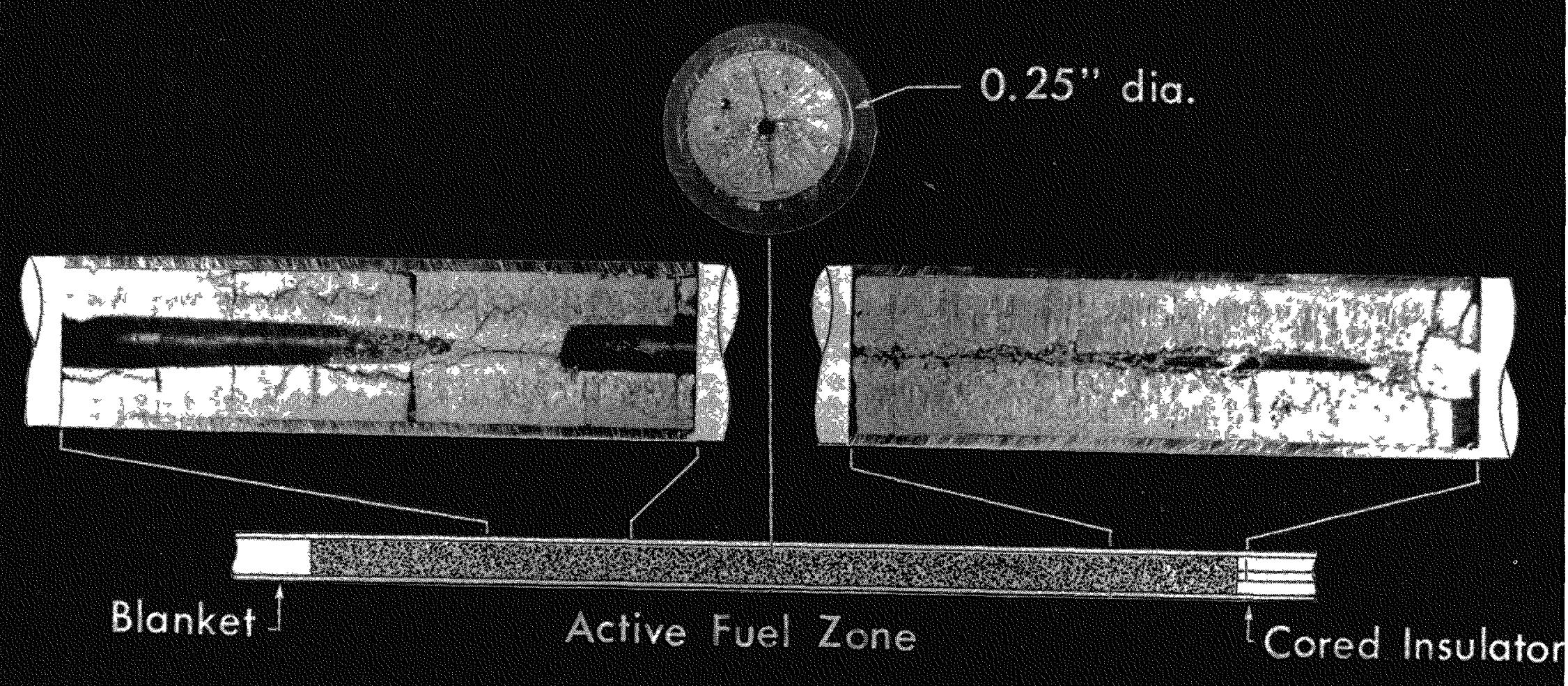

Fig 8 Solidification Pattern of Mixed Oxide Fuel Following Transient Irradiation 
As shown in the tabulation, no evidence has been observed in the FCR program of any significant axial redistribution in elements where the mixed oxide fuel has operated below center melting. Similar conclusions relative to axial fuel movement may be drawn for $\mathrm{CO}_{2}$ operated under BWR conditions. ${ }^{(19)}$ The axial stability of reference FCR fuel irradiated in a fast flux at $15 \mathrm{~kW} / \mathrm{ft}$ can further be inferred from Fig. 6 , which shows that the activity curve matches the flux profile and is quite smooth. Contrary to some postulations, fragments of fuel have not been observed to fall into the central void when one has formed.

When the linear power is sufficiently high to cause fuel melting, fluid flow becomes a potential source of fuel redistribution. Axial relocation of the mixed oxide has occurred in most pins in which a portion of the fuel was operated in the molten regime. Table 1 summarizes the results in terms of axial density change.

Liquefaction does not necessarily result in slumping, i.e., downward movement. Gravity is not the only force to which molten fuel is subject. The ultimate disposition of the once-molten fuel in the pin is subject to variation depending upon its condition prior to melting and can be affected by at least five phenomena:

a. volume expansion on melting;

b. slumping of molten fuel by gravity;

c. boiling or gas evolution from the liquid;

d. expulsion of liquid through cracks;

c. shrinkage on freezing.

Items a, c, and d can lead to expansion of the fuel.

Upward transport of pre-irradiated mixed oxide fuel subsequently melted during a transient is illustrated in Fig. 7. The upward movement is attributed to pressure from the sudden release of internal fission gas and perhaps vaporization of the fuel. This tendency of the fuel to separate axially during a power surge would be helpful, of course, in limiting an accidental excursion. A similar phenomenon has been noted in transient tests of sodium-bonded uranium carbide fuel in which vaporization of the bond generated high local pressures and caused fuel relocation of this nature. ${ }^{(20)}$

The downward morement of once-molten fuel follows an irregular pattern. It appears that the central portion of the fuel pin is completely filled with molten fuel at the time the maximum fuel temperature occurs, and that much of the downward movement occurs during solidification; this is evidenced by the conical shrinkage cavity which forms (see Fig. 8), in much the same manner as in a casting poured into a mold without an external reservoir of liquid.

In the current $1000-1 I W$ e design ${ }^{(21)}$ fucl design limits have been selected to preclude axial fuel movement due to melting, i.e., all fuel is kept below
TABLE 2. SI MUARY OF FAPERIMEATAL WOHK ON MOVEMEAT of Mided Oxine Fuel

1. Differential fuel-cladding thermal expansion can be accommodated under representative $\mathrm{FCR}$ conditions.

2. Radial movement occurs, as in $\mathrm{CO}_{2}$, but introduces no objectionable fuel-cladding interactions.

3. High-burnup swelling tests in thermal and fast fluxes are in progress.

4. No evidence of significant axial redistribution exists in mixed oxide operated below center meltmg.

5. Both upward and downward movement of fuel are noted as a result of transient melting.

6. Molten expansion in an overpower condition can be accommodated in short fuel segments provided sufficient void volume is available.

incipient melting in the steady state and no fuel rises above the liquidus during a transient.

\section{Conclusions}

The principle conclusions relative to experimental observations on movement of mixed oxide fuel are summarized in Table 2.

\section{REFERENCES}

1. F. J. Leitz, Significant Factors in the Evaluation of Fast Reactor Fuel Performance, ANS Meeting on Fast Reactor Technology, Detroit, Michigan, April 26-28, 1965, ANS-100.

2. Sodium Cooled Reactors, Fast Ceramic Reactor Development Program, Fifteenth Quarterly Report, May-July 1965, GEAP-4931.

3. J. D. Eichenberg et al., Effects of Irradiation on Bulk Uranium Oxide, WAPI)-183 (Oct 1957).

4. B. Wedell, 1 Theoretical Evaluation of Crack Formation in $\mathrm{CO}_{2}$ Fuel Pellets and Its Effect upon DOR-Reactor Fuel Element Design, R ISO Report * 52 (July 1962).

5. A. S. Bain, Cracking and Bulk Movement in Irradiated Uranium Oxide Fuel Elements, AECL-1827 (Sept 1963).

6. W. R. Martin and J. R. Weir, Dimensional Behavior of the Expermental Gas-cooled Reactor Fuel Element at Elevated Temperatures, ORNL-3103 (July 19, 1961).

7. Southwest Experiment Fast Oxide Reactor Developmental Program, Fourth Quarterly Report, February-April 1965, GEAP-4846 (May 1965).

8. L. E. J. Roberts et al., The Behavior of $\mathrm{UO}_{2}$ and of $(U$, $\mathrm{Pu}_{2} \mathrm{O}_{2}$ Fuel Materials under Irradiation, A/CONF. 28/P/155, 3rd. Intern. Conf. on Peaceful Uses of Atomic Energy, Geneva, 1964.

9. E. L. Zebroski, W. E. Baily, and I). P. Hines, Irradiation Behavior of Hyper- and Hypo-Stoichiometric PlutoniaUrania Fuel at 700,000 MWD/T, GEAP-4897 (March 1965).

10. W. E. Baily and C. N. Craig, Relative In-pile Thermal Conductivity of Stoichiometric and Hypostoichiometric Mixed Oxides of Plutonia and Urania, Trans. ANS, 8 (Nov 1965).

11. E. L. Zebroski, J. P. Mustelier, and C. Caldwell, Oxide Fuels for Fast Reactors, ANS Meeting on Fast Reactor Technology, Detroit, Michigan, April 26-28, 1965, ANS 100 .

12. J. B. Ainscough, The Iradiation of Sintered Uranium 
Oxide to High Burn-up, Nuclear Engineering 8, 272-277 (Aug 1963).

13. S. A. Rabin, F. W. Knight, and G. L. O'Neill, High Power Operation of $\mathrm{PuO}_{2}-\mathrm{CO}_{2}$ Fast Reactor Fuel, GEAP-4873 (1965).

14. J. E. Hanson, J. H. Field, and S. A. Rabin, Experimental Studies of Transient Effects in Fast Reactor Fuels. Series $\mathrm{II--Mixed} \mathrm{Oxide}\left(\mathrm{PuO}_{2}-\mathrm{UO}_{2}\right)$ Irradiations, GEAP-4804 (June 1965).

15. J. F. Hanson, Response of Low Burnup-Irradiated MixedOxide $\left(\mathrm{PuO}_{2}-\mathrm{UO}_{2}\right)$ Fuel to Transient Overpower, Trans. ANS 8 (Nov 1965).

16. P. E. Novak et al., Plutonium and Fission Products Redistrabution in Mixed Oxide Fuels, see paper in Session VA of This Conference.

17. P. R. Huebotter et al., Topical Report on the Evaluation of EOCR as a Fast Fuel Test Facility, APDA-169 (June $25,1965)$.

18. D. B. Sherer, An Analysis of Fast Reactor Transient Response and Safety in Selected Accidents, see paper in Session I of This Conference.

19. C. N. Spalaris, private communication, (GE-APHI).

20. S. J. Stachura, R. N. Cordy, and M. Silberberg, Uranium Carbide Transient Heating Studies-Phase II, Trans. ANS 8, 1 (June 1965).

21. K. P. Cohen and (x. L. O'Neill, Safety and Economic ('haracteristics of a 1000-MWe Fast Sodium-cooled Reactor Design, see paper in Session III of This Conference.

\section{Discussion}

Mr. Okrent (ANL): You said that the smear density is about $87 \%$ for the oxide. I recall that the Russians, who have had a reasonable amount of experience with oxide, at Detroit said that they would go down to about $75 \%$ or $80 \%$ in order to get high burnup. So I find an implication that to achieve 100,000 $\mathrm{MWV} / \mathrm{T}$, there is some feeling that lower densities than your $87 \%$ are needed. Would you care to comment to this point?

Mr. Rabin: I don't have all the details on the Russian work, although I know they have looked at $\mathrm{CO}_{2}$ and $\mathrm{PuO}_{2}$ individually. I'm not completely familiar on what basis they have come up with their design; were they extrapolating $\mathrm{PuO}_{2}$ data or just how did this come about? The reported swelling rates would not indicate that a low density is needed.

Mr. Okrent: Have you achieved $100,000 \mathrm{MWd} / \mathrm{T}$ or more with an $87 \%$ density fuel?

Mr. Rabin: No.

$M r$. Okrent: What I am trying to see is: at $87 \%$ have you built in a compromise for safety which you will give up later? In order to get $100,000 \mathrm{MWd} / \mathrm{T}$, will you indeed have to go to $80 \%$ density or $75 \%$, as the Russians have suggested?

Mr. Rabin: Well ...

Mr. Okrent: You mentioned the bright side of how fuel might move in an oxide, mainly first that it doesn't eseape the jacket during the transient, at least you haven't been able to make it do this, and sec- ondly, you think it will move from the center in both directions. But I think one can equally well visualize that fission gas might be primarily released near the bottom, where more is trapped in the fuel since this is a colder region. The molten part of the fuel might be more towards the top. You might have a general motion all in one direction, perhaps upward, but with a net compaction of many pins, adding reactivity. Is it clear that fuel always moves in such a way as to lose reactivity?

Mr. Rabin: No. First, the profile would be symmetrical so it will be presumably as cold at the top as at the bottom.

Mr. Okrent: Which profile? There are various ways of getting to the melting point. You may have an overpower incident or a partial loss of flow.

Mr. Rabin: My remarks were not intended to eonvey the impression that it will always be favorable. In general, the presumption has been that it will always be unfavorable. The point that I was trying to make was this is not necessarily so. In other words, it has been frequently assumed that compaction will occur. These results show that this is not necessarily so. We are not at this time intending to use this upward movement as a design basis for inclusion in our safety factors.

Mr. Stachura (AI): In capsule C-2-B was there not a cladding failure?

Mr. Rabin: No, that was $\mathrm{C}-2-\mathrm{C}$, which ran at higher power than C-2-B.

Mr. Stachura: Did C-2-C produce a penetration of the cladding?

Mr. Rabin: Yes.

Mr. Bump (ANL): You say that you are designing so that during the transient the fuel does not melt. What transients are these at which the fuel does not melt? A second related question is this: General Electric apparently does not use the statistical-type approach in combining the hot channel factors or uncertainty factors. This puts you at a disadvantage for otherwise you could presumably go to higher power without melting.

Mr. Rabin: I would like to refer the answer to this question to Mr. Sherer or Mr. O'Neill.

$M r$. Sherer (GE): We do use the statistical approach for those factors when we believe it is proper to do so. We don't believe it is applicable to those factors that are of a systematic nature; unless you have a large number of these uncertainties, we don't believe that you could properly apply a statistical approach. We do apply it to fabrication tolerances for example.

Mr. Bump: What transients will not put the fuel above melting?

$M r$. O'Neill (GE): We design for the normal re- 
actor operating transients, for example, the overpower, the normal power overshoot, and that sort of transient, to stay below melting, but we do not consider it in our accident transients, of course.

Mr. Kummerer (Karlsruhe): My question is not related directly to the fuel movement but to the cladding behavior in this transient test. Did you ob- serve any longitudinal strain onto the cladding of the specimens which were pre-irradiated before the transient test?

Mr. Rabin: The particular specimen was not measured. A specimen pre-irradiated under comparable conditions which was examined and not transient tested showed no axial expansion. 


\title{
Status of Recent Meltdown Studies
}

\author{
C. E. Dickerman, L. E. Robinson, F. L. Willis, W. Stephaxy, and C. August \\ Argonne National Laboratory \\ Argonne, Illinois
}

(PRESENTED BY C. E. DICKERMAN)

\section{Introduction}

Analysis of the consequences of a "meltdown-type" incident in a fast reactor requires knowledge of the phenomena governing the movement of core materials -coolant, as well as fuel and structure-resulting from abnormal operating conditions. Information needed includes modes of fuel failure and the conditions which produce the various types of failure, types and velocities of fuel movement, and coolant effects. Pre-failure movements of coolant or fuel may also be involved. Given these data, then, and a hypothetical accident, the next step consists of determining types of movements, the various velocities, degrees of coherence of movement over the core, and phasing these results into kinetics calculations. At this stage, there are various possibilities: the incident may be shown to have terminated; secondary movements may be found to be possible, requiring additional analysis; or shutdown due to internal pressures may have to be calculated. Even in the case of the most widely studied system, EBR-II, meltdown-related data are incomplete. It is the purpose of this paper to review the current status of meltdown studies, cmphasizing recent work. Because separate papers relate results obtained by Atomics International and General Electric experimenters, this report will be restricted to the Argonne National Laboratory program.

\section{Failure Phase}

\section{A. Coherence of Failure Study}

At the last October Fast Reactor Conference, we gave a preliminary report(1) on calculations of the coherence in fuel failure for hypothetical accidents in an EBR-II-like reactor, based on the experimental data then available for the Mark-I fuel. Except for a particularly incredible case, degree of coherence was sufficiently large to play a significant role in limiting possible rates of reactivity addition resulting from failure. (2) Those calculations can now be shown to have a wider range of validity than could have been demonstrated then.

(1) Effects of Prior Irradiation. Although localized, preliminary cladding failures have been observed, massive failure and gross fuel movement still appear (for burnups in the range of interest of EBR-II Mark 1) to be characterized by the same thresholds, delays, and mechanisms as unirradiated samples. ${ }^{(3)}$ One later sample run just past the failure threshold, with axially shaped power profile, showed failure at the hottest region and some fuel movement from ends to the center. Expulsion of fuel from the cladding was not complete.

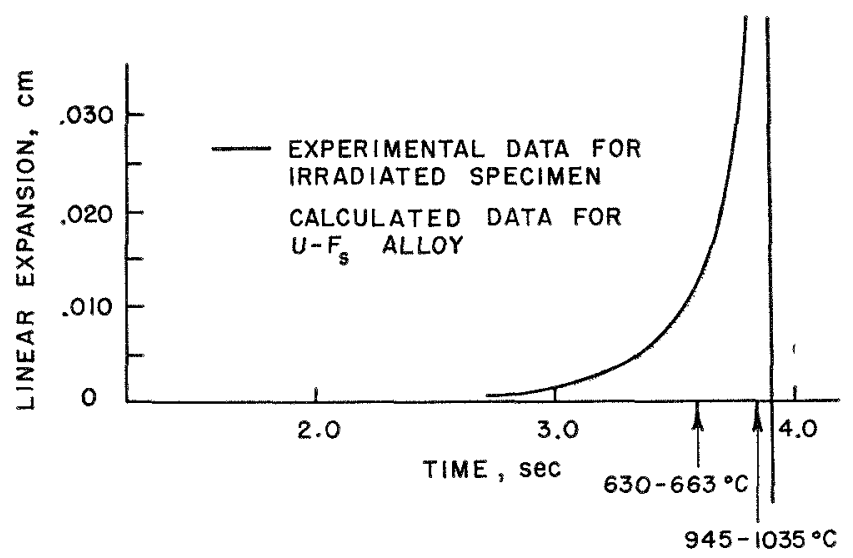

FIG. 1. Comparison of Experimental Linear Expansion of Irradiated Sample with Expansion Calculated Using Data for Unirradiated U-5 w/o Fs Alloy. Sample Heating Rate: $1000^{\circ} \mathrm{C} /$ sec.

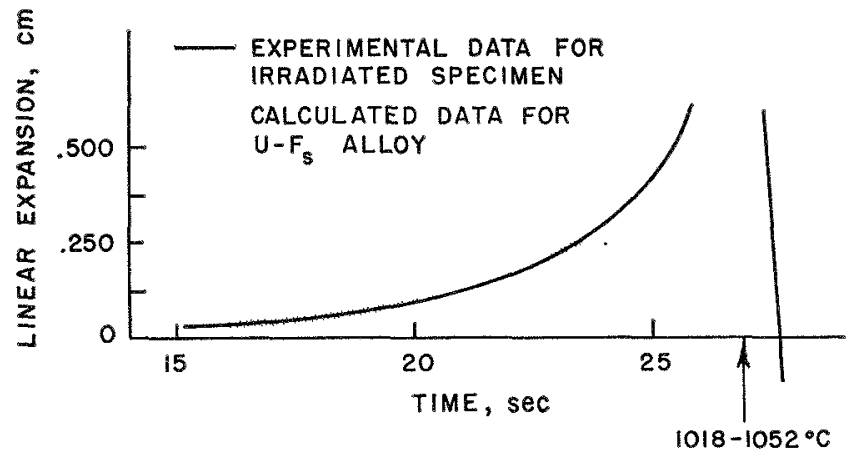

FIG. 2. Comparison of Experimental Linear Expansion of Irradiated Sample with Expansion Calculated Using Data for Unirradiated U-5 w/o Fs Alloy. Sample Heating Rate: $80^{\circ} \mathrm{C}$ / sec. 

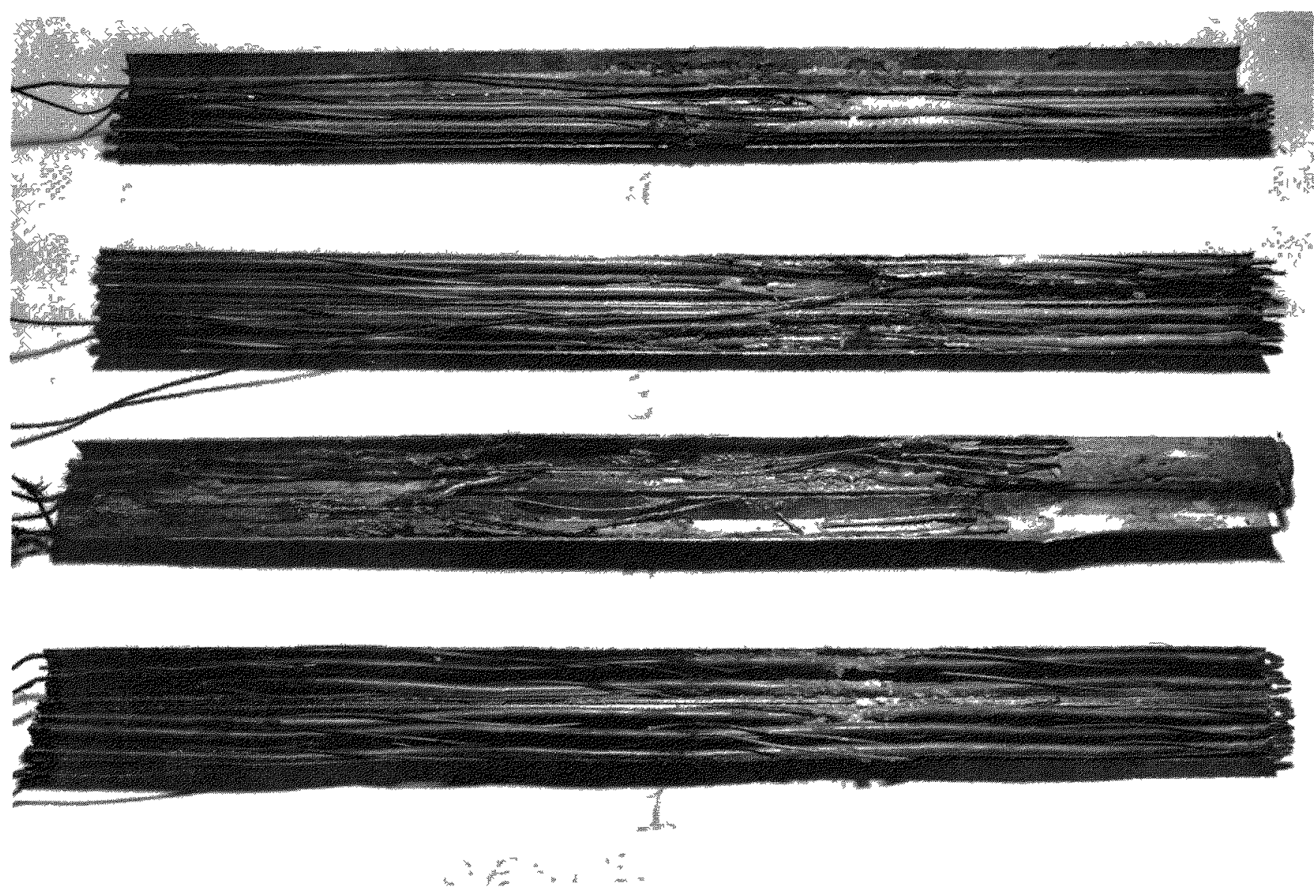

Fig. 3. Post experiment Condition of 7 pin Clustels of EBR-II Pins, Run in Ineri Gas.

For short transients in the time scale of TREAT excursions, excessive swelling of EBR-II Mark-I fuel alloy appears to occur at temperatures well above the normal operating range. ${ }^{(4)}$ In Fig. 1 are compared measured expansions of unrestrained irradiated EBRII Mark-I fuel alloys with those expected on the basis of data for unirradiated material given similar heat treatment. Massive swelling is associated with the melting range of $1000-1080^{\circ} \mathrm{C}$, in the middle of which the sample collapsed. ${ }^{(4)}$ The specimen was heated at the rate of approximately $1000^{\circ} \mathrm{C} / \mathrm{sec}$.

Because of calibration uncertainties (thermocouples could not be attached directly to the sample; hence temperatures were calculated from reactor transient data, power calibrations, and enthalpy data), a band is shown for the calculated expansion. Figure 2 is a similar comparison for a sample heated at about $80^{\circ} \mathrm{C} / \mathrm{sec}$. Both samples had burnup of about $1.3 \mathrm{a} / \mathrm{o}$.

(2) Capabilities of Subassembly Containment. Some subassembly containment is necessary to prevent excessive propagation of failure. Figure 3 shows four small clusters, opened up after being run in the absence of sodium coolant, in subassembly mockups of one-mm-thick 304 stainless steel, and with a shaped axial power profile. Three were run to nild failure, and one (second from bottom) to extensive failure. In the last case, failure appears to have occurred in the hottest region, with expulsion of fuel there, and subsequent movement of molten fuel-cladding eutectic to the subassembly bottom, where it solidified without attacking the cladding. This result is quite consistent with out-of-pile penetration studies. ${ }^{(5)}$

(3) Effects of Coolant. Results from recent loop experiments with single pins in the center of a ring of six dummy pins $^{(6)}$ are consistent with thresholds and failure mechanisms discovered earlier in tests with single pins in dry and stagnant sodium capsules, and used in the calculations of coherence of failure. ${ }^{(2)}$

\section{B. Mechanisms of Failure}

Competition between cladding failure due to internal pressure and dissolution by molten fuel has been reviewed for the sodium-bonded EBR-II fuel pin. ${ }^{(1)}$ Details of the cladding dissolution must be 


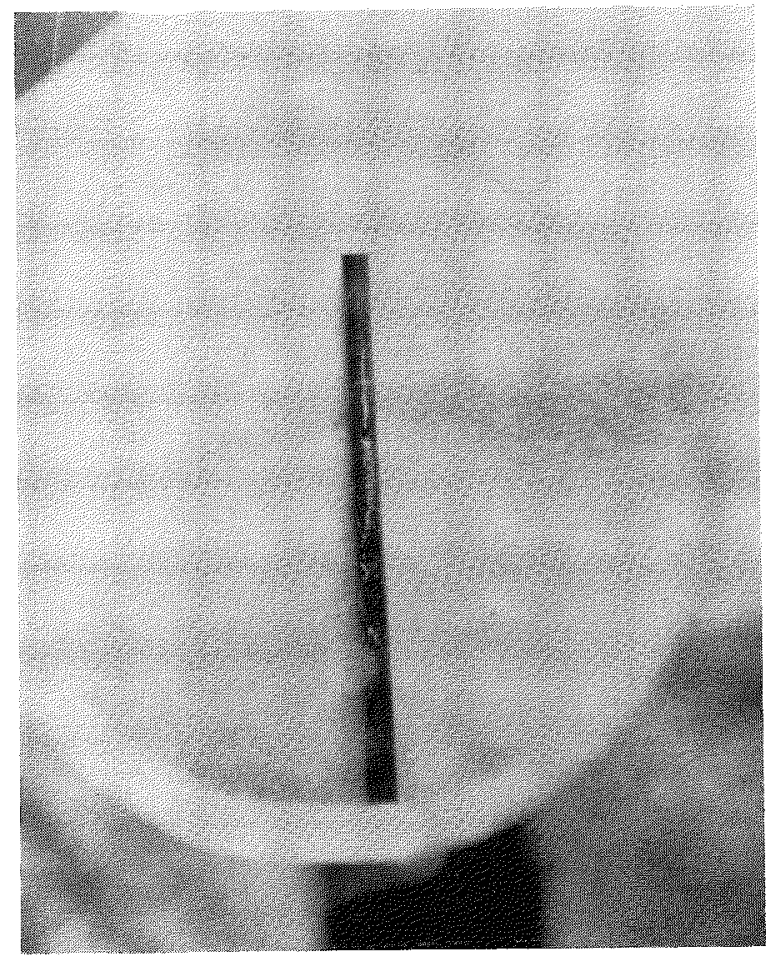

Ftg. 4. Solidified Fuel-Cladding Eutectic in Surface Crack of EBR-II Fuel Alloy of Epecimen Carried Almost to Failure in Integral Sodium Loop. cladding, following exposure in sodium just short of the failure threshold, ${ }^{(6)}$ in a TREAT package sodium loop. ${ }^{(7)}$ A surface crack, filled with uranium-steel eutectic, is clearly visible. This phenomenon of propagation of molten material through a cracked surface shell may also be important for brittle ceramics.

Thermal expansion may also be a source of failure. Figure 5 shows a $\mathrm{Nb}$ alloy-clad UC pin which ruptured in a TREAT transient as a result of resistance to axial expansion: the ceramic fuel fragmented and, acting like a hydraulic medium, transmitted the axial loading into a radial loading in the central region of the cladding, producing a pressure failure of the niobium tubing. Because of the nature of the pressure loading, nearly all of the fuel remained inside the cladding after the cladding rupture.

\section{Movement Phase}

As illustrated in Fig. 3, fuel movement can be a multistage process, in which the initial movement associated with failure is followed by secondary motion (s).

The role of internal pressure in producing fuel movement upon failure has been surveyed over a range of conditions. However, the metallurgical conditions, geometry, and temperature distributions of

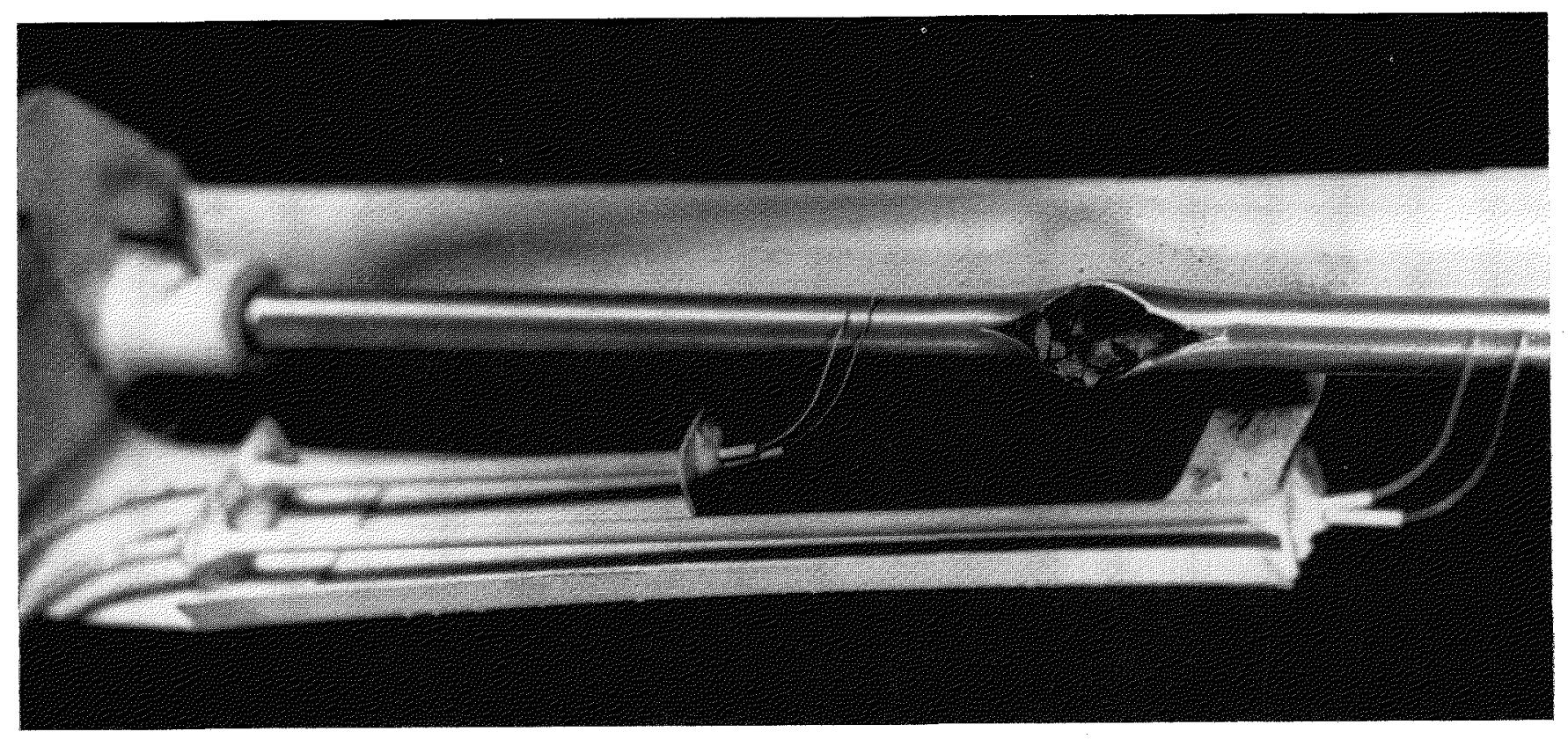

Fig. 5. Appearance of Nb Alloy-clad UC Element after Failure Produced by Transient Mechanical Loading of Ceramic Fuel.

specified, however, since actual attack of the cladding may result from high-temperature molten material moving from the interior via small cracks in the cooler fuel surface. This is demonstrated in Fig. 4, which shows an EBR-II fuel pin after removal of the fuel pins at the time of failure must be known if this phenomenon is to be analyzed properly.

As an example, Fig. 6 is an enlargement of a recent metallographic photo of uranium "fibers" in the thorium matrix of an EBR-II-type Th-U fuel pin which 


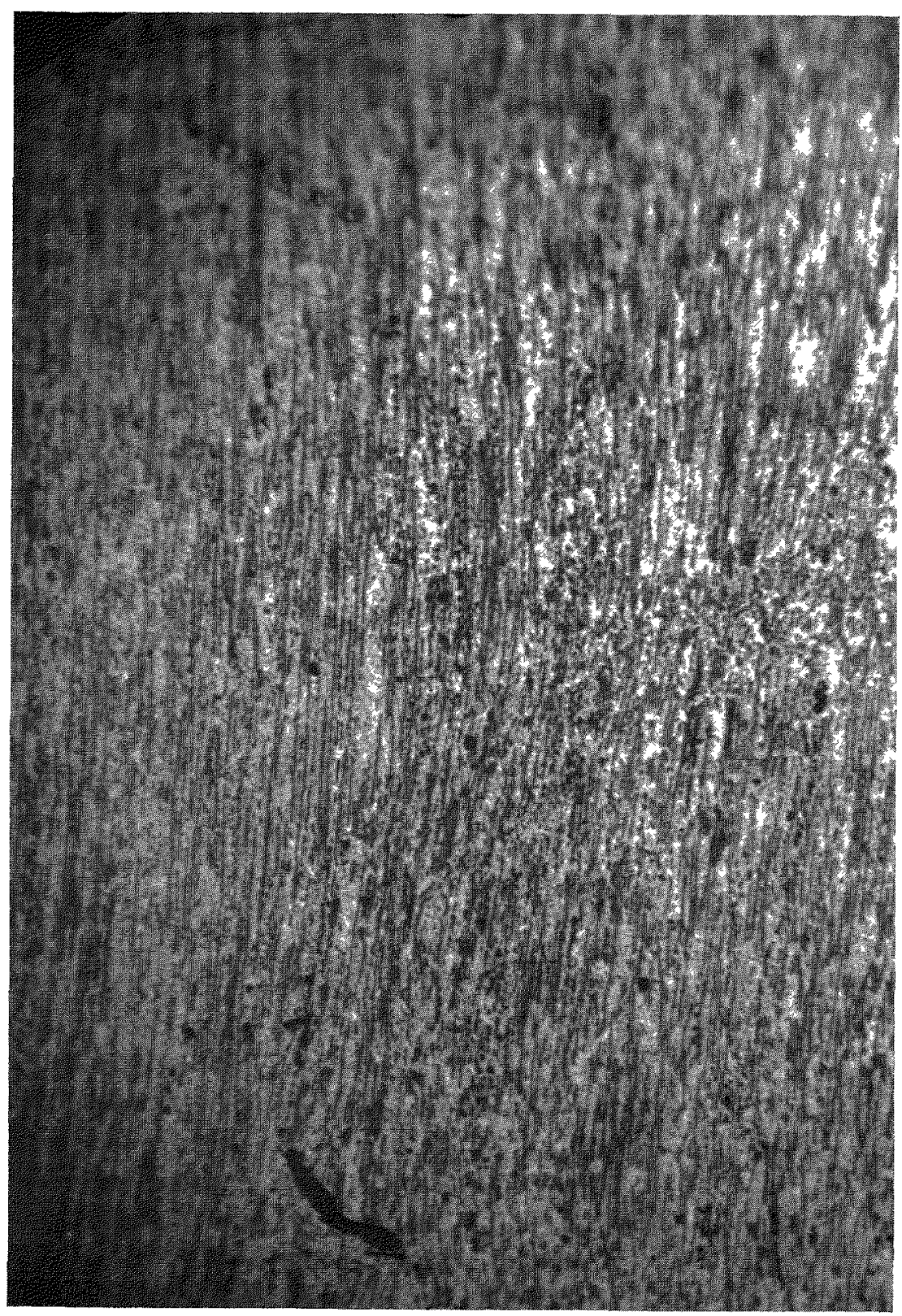

Fig. 6. Fiber Structure of Th U Fuel Pin after TREAT Transient with Maximum Cladding Temperature of $1360^{\circ} \mathrm{C}$.

was run in a TREAT transient. Although maximum cladding temperatures were $\sim 200^{\circ} \mathrm{C}$ above the solidus temperature and the uranium phase of the alloy was molten, the material retained the fibrous structure typical of its fabrication by extrusion. ${ }^{(8)}$ This relative metallurgical stability at high temperatures explains why samples of this fucl type did not display fuel movement resulting from pressure of bond sodium when the steel cladding failed. ${ }^{(9)}$ Because of the competing phenomena involved, prior irradiation and a sodium coolant may change this behavior significantly.

Effects of temperature distributions are illustrated in Fig. 7, which was used in the analyses of a melt- down experiment with an EBR-II-type pin run to extensive failure in a TREAT sodium loop. ${ }^{(6)} \mathrm{Calcu}-$ lated clarding bursting pressure-calculated pressure differential required to burst the tubing plus ambient pressure in the loop-is shown as a function of cladding temperature. In this nonisothermal case, "cladding temperature" is taken to be that of the central node point in the cladding region. Also shown is the internal pressure due to argon cover gas inside the cladding and vapor pressure of the sodium bond, displayed as a function of center cladding temperatures, for the transient temperatures calculated up to time of peak power using the ARGUS code. ${ }^{(10)}$ The 


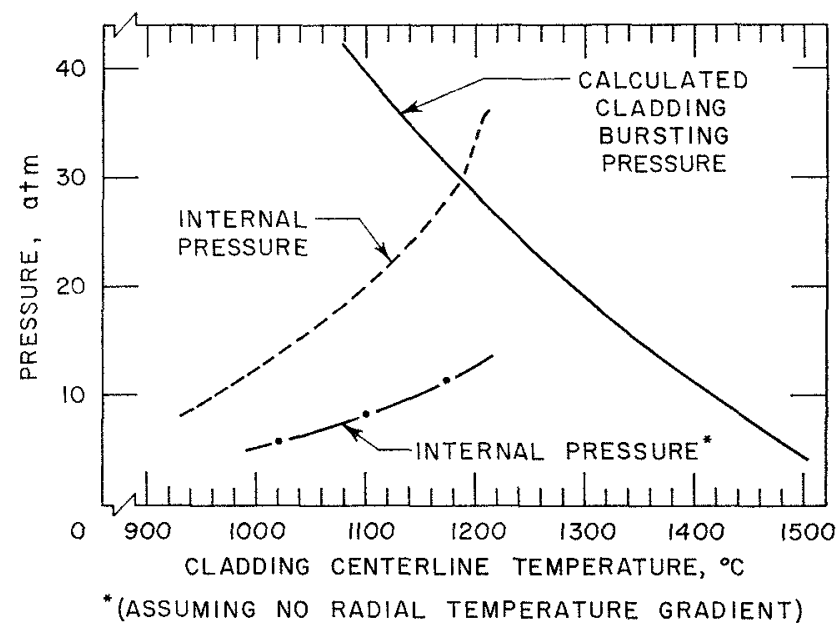

Fig 7 Calculated Internal Pressures and Cladding Burst ing Pressures of EBR II PIn in Sodium Tr 855

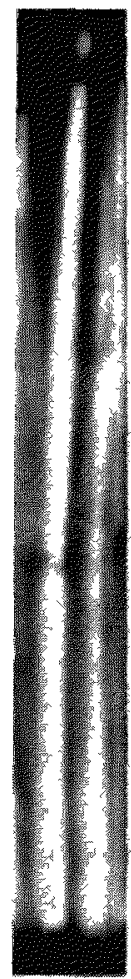

A

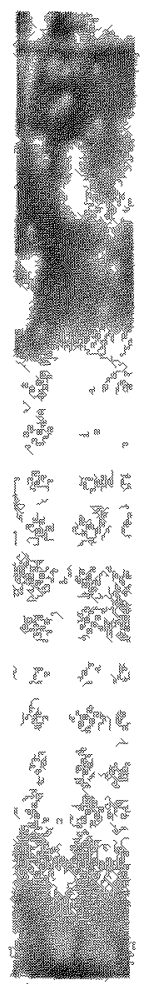

B

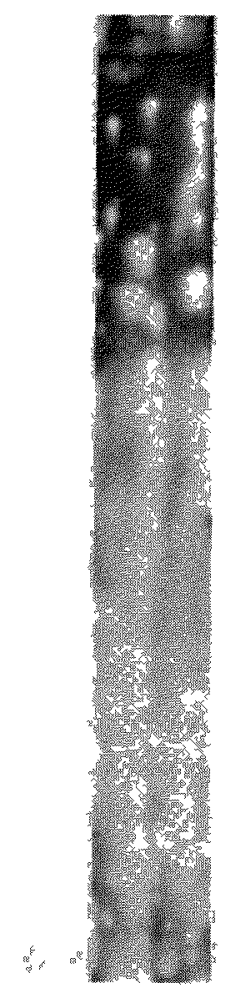

C

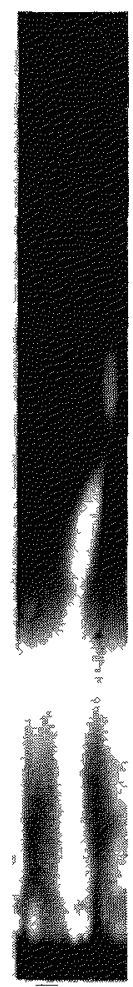

$\mathrm{D}$
Fig 8 still Frames showing High temperature Fallure of Ta clad $\mathrm{UO}_{2}$ Sample

analyses led to a prediction of a cladding failure due to internal gas pressure at about 187 sec. Experimentally, a sharp pressure spike of about $3 \mathrm{~atm}$ was recorded at the inlet to the test section at $1.86 \mathrm{sec}$, and the sample postmortem showed extensive failure along the length of the pin, with pressure rather than cladding dissolution as the dominant mode of failure.
By contrast, internal pressures calculated under the assumption that the bond sodium vapor pressure was that of sodium at the cladding temperatures (i.e., no radial temperature gradient) were much lower.

Although fuel movement due to gas pressure inside the cladding is often considered to be a unique characteristic of sodium-bonded metallic fuels, it can be realized for gas-bonded ceramic pins. Figure 8 shows still prints taken from the color motion picture film record of a high-temperature fallure of a Ta-clad $\mathrm{UO}_{2}$ specimen run in an mert gas environment. ${ }^{(11)}$ The figure traces the course of the test from the heating of the cladding through fallure and expulsion of molten oxide. Meltdown fragment velocities measured from the films ranged from 106 to $290 \mathrm{~cm} / \mathrm{sec}$.

The secondary movements are not well-characterized yet, particularly with regard to the effects of coolant. These effects depend, among other things, on the relative timing of failure and coolant expulsion. Thus, it is possible to predict that coolant boiling could cause fuel dispersal, or that, conversely, the coolant could be expelled prior to farlure and then rush back in and compact fuel material. There is evidence that secondary movements may not be as important in the case of irradiated metal fuel, because of the large volume change observed upon meltdown of metallic fuel. ${ }^{(12)}$ Frothing phenomena may also be significant for irradiated ceramic fuel; this point has yet to be established experimentally.

\section{Plans}

It is planned to expand the experimental program with metallic uranium fuel to include studies of the effects of clustering fuel pins in a sodium environment, with relatively realistic axial and radial power profiles. Behavior of irradiated specimens in sodium and in chusters is also to be investigated.

A stock of irradiated oxide pins is being accumulated for experiments with inert gas and sodium environments. Effects of sodium bonding and environment on unirradiated oxide pins will also be studied The program of experiments on carbide specimens will be expanded as more samples become available. Work on multicomponent fuels (like the Th-U matrix) will continue, in pace with their development.

As the behavior of the uranium fuels becomes better characterized, it will become appropriate to phase in study of plutonium fuels. Procurement has been initiated on a range of promising plutonium fuel samples-both ceramic and metal.

\section{Acknowledgments}

It is a pleasure to acknowledge the contributions of R. Brittan, for helpful discussions and guidance; E. 
Sowa, J. Heap, and G. Golden, for development of experimental facilities; J. Boland, TREAT Manager, and his efficient reactor staff.

\section{REFERENCES}

1. C. Dickerman, E. Sowa, L. Robinson, and D. Gopinath, Meltdown Studies, Proc. Conf. on Breeding, Economy and Safety in Large Fast Power Reactors, October 7-10, 1963, ANL-6792 (1963).

2. D. V. Gopinath and C. E. Dickerman, Calculations of Coherence of Failure for Hypothetical Meltdown Accidents in an EBR-II-like Reactor, ANL-6844 (1964).

3. C. E. Dickerman and L. E. Robinson, Photographic Experiments on Meltdown of Irradiated Melallic Uranium Fuel Pins, Trans. Am. Nucl. Soc. 7, No. 1, 137 (1964).

4. C. F. Dickerman and L. F. Robinson, TREAT Experiments to Provide Data for Fast Reactor Safety Kinetics Calculations, Proc. Symposium on Reactor Kinetics and Control, to be published.

5. C. M. Walter and L. R. Kelman, Penetration Rate Studies of Slainless Steel by Molten Uranium and UraniumFissium Alloy, J. Nuc. Mat. 6, 281 (1962).

6. C. E. Dickerman, L. E. Robinson, W. Stephany, and F. L. Willis, Fast Reactor Safety Experiments on EBR-II Sam. ples in Flowing Sodium Environment, Trans. Amer. Nucl. Soc. 8, No. 2, to be published.

7. E. S. Sowa and J. C. Heap, The Development of a Small Integral Sodium Loop for In-pile Fuel Failure Studies in the Presence of Flowing Sodium, Trans. Amer. Nuel. Soc. 8, No. 2, to be published.

8. B. Blumenthal, private communication.

9. C. E. Dickerman, L. E. Robinson, and R. Stewart, Fast Reactor Safety Experiments on EBR-II-type ThoriumUranium Fuel, Trans. Amer. Nucl. Soc. 8, No. 1, 309 (1965).

10. D. F. Schoeberle, L. B. Miller, and J. Heestand, A Method of Calculating Transient Temperatures in a Multiregion, Axisymmetric Cylindrical Configuration, the ARGUS Program, 1089 RP248, Written in Fortran II, ANL-6654 (1963).

11. C. E. Dickerman, I. E. Robinson, E. S. Sowa, and S. B. Skladzien, Studies of Fast Reactor Fuel Element Behavior under Transient Heating to Failure. II. In-pile Experiments on $\mathrm{UO}_{2}$ Samples in the Absence of Coolant, ANL-6845 (1965).

12. J. H. Monaweck, C. E. Dickerman, and E. S. Sowa, Behavior of Irradiated Metallic Fuel Elements Exposed to Nuclear Excursions in TREAT, Trans. Amer. Nucl. Soc. 6, No. 2, 374 (1963). 


\title{
Safety and Economic Aspects of Vented Fuel in Sodium-cooled Reactors*
}

\author{
G. L. O'Neill, F. W. Kxight, H. J. Leitz, and D. B. Sherer \\ General Electric Company \\ A dvanced Products Operation \\ San Jose, California
}

(PRESENTEI) BY G. L. O'NEILL)

\section{Introduction}

One of the major advantages of fast ceramic reactors over other power reactor systems is their potential for achieving low fucl-cycle cost. Fulfillment of this potential requires high fuel performance as wcll as the good neutron economy inherent in fast reactors. In particular, high burnup and high power density are required in order to minimize the fabrication, recovery, and fuel-inventory components of fuel-cycle costs.

\section{Fission-gas Accommodation}

The release of considerable fractions of generated fission gas from high-burnup, high-power-density FCR fuel is well-known. Thus, the accommodation of these fission gases is a major design problem for the FCR fuel and system. Various ways for accommodating fission gases in fuel pins are shown in Table 1. The broad objective of all these designs is to relieve the pressure buildup due to fission gas without permitting release of daughter activity or ingress of sodium into the fucl-blanket region. If the gas is retained in the core region, pressure buildup inside the fuel element roquires restraining cladding thicknesses which are generally precluded by considerations of both thermal stress and core composition. Alternatively, very low-density fuel, which allows space for fission gas, also leads to a poorer neutron economy.

If the gas is transferred out of the core region, as appears necessary, it may be retained in a plenum chamber integral with the fuel cladding, but remote from the core, or it may be released into the coolant and cover gas of the primary system. Three leading concents are shown schematically in Figure 1.

The vented-to-plenum concept, basically an extension of fucl cladding into a region above or below the core, appears the most attractive pressure-vessel design. The concept offers the potential for removal of the pressure stress from the cladding as well as a

\footnotetext{
* Work performed under Contract AT (04-3)-189, Project Agreement 10, between the U. S. Atomic Energy Commission and General Electric Company.
}

potential improvement of behavior of fuel-pin failure, especially as regards gas blanketing and autocatalytic failure of adjacent pins.

The restraining clad appears the least likely, due to high thermal stresses and poor neutron economy.

The diving-bell design was picked as the simplest pressure-relieved example, since it does not extend to cover gas nor does it rely on moring parts and special material properties. The vent is shown schematically, in greater detail, in Figure 2. The volume within the bell, and thus the length of the vent, is determined by the criterion that sodium cannot be forced into the fuel region on shutdown.

The potential disadvantages of rented-to-coolant systems might include additional requirements as regards shielding, cover-gas systems, fucl-handling system, and maintenance due to the release of the fission product gases and subsequent buildup of daughter activity.

Most of these disadvantages ean be neutralized by retention of the fission gases inside the fuel until the fission gases decay and the fission-gas daughters (Cs, $\mathrm{Sr}, \mathrm{Ba}$, and $\mathrm{La}$ ) are deposited inside the fuel pin. If one examines the fission product chains, we find that all potentially troublesome daughter products can be eliminated by an adequate delay time within the fuel.

\section{Vented-to-coolant Test}

A preliminary demonstration test of vented-to-coolant fuel has been successfully performed and previously reported.(2) This test was designed to identify the fission products released, to measure their release fraction, and to determine the delay time experienced by the gases prior to their escape.

The test fuel rod was comprised of $95 \%$ dense $(90 \%$ overall average including the fuel-cladding gap), 20 $\mathrm{w} / \mathrm{O} \mathrm{PuO}_{2}-80 \mathrm{w} / \mathrm{o} \mathrm{UO}$ pelleted fuel, $0.25 \mathrm{in}$. in diameter by 5 in. long with a 2 -in.-long $\mathrm{UO}_{2}$ blanket. The fuel operated at linear powers of 20 to $24 \mathrm{~kW} / \mathrm{ft}$ in a thermal flux which approached conditions for central melting. Total exposure was $16,500 \mathrm{MWD} / \mathrm{T}$. 
Results of this experiment are given in Table 2. Release of the long-lived and stable fission gases agreed elosely with a calculated release of $40-50 \%$ for these operating conditions.

In Table 2 note the dependence of gaseous release fraction on half-life. By plotting these data as in Fig. 3, with the release fraction normalized to that of stable gase's plotted against half-life on semilogarithmic paper, the slope gives the hold-up time. The holdup time in this test turned out to be near 5 days. This explains the lack of daughter activity that escaped into the simulated coolant and cover gas.

The only fission-gas daughter released in significant quantity was Cs-133, the daughter of 5.3-day $\mathrm{Xe}-133$. It turned out that neutron activation of Cs-133 to Cs-134 produced the majority of activity present outside the fuel, excluding that due to $\mathrm{Na}-24$. A summary of this test is given below:

1. Some $44 \%$ of the noble fission gas was released, consistent with the $40-50 \%$ predicted by calculation.

2. The effective gas-delay time was estimated to be about 5 days based on isotopic analysis of krypton and xenon released.

3 . The predominant activity in the sodium, after the decay of $\mathrm{Na-24}$, was $\mathrm{Cs}-134$, an activation product of $\mathrm{C}-133$, which in turn is a daughter of $\mathrm{Xe}-133$.

4. The release fractions of all other nuclides (including fissile) (Sr-89, Sr-90, Y-91, Zr-95, Ru-103, I-131, Cs-137, Ba-140, and Ce-144) were at or near the detection limit in the range from $10^{-6}$ to $10^{-10}$.

5. No deleterious effects to the fuel were observed due to possible exposure to sodium vapor. Traces of sodium were obscrved at the top of the "blanket," which may have been responsible for retention of radio-iodine in this test.

Thus, the fissile elements and the nongaseous fission products and daughter elements do not distill out of the fuel (and blanket), and the gaseous fission products are sufficiently delayed in their release so that Cs-134 seems the major concern to vented-to-coolant system design.

We are continuing this work to back up these conclusions and to date have found nothing to refute them.

TABLE 1. FISSTON-GAS ACCOMMODATION

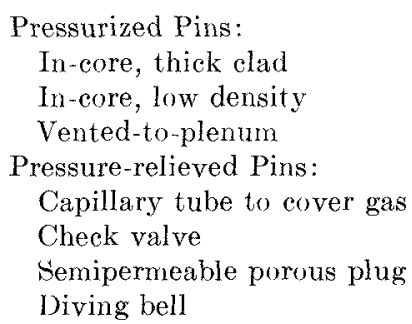

\section{Implications to FCR Design}

A technical and economic analysis ${ }^{(1)}$ has been carried out comparing a large reactor plant using ventedto-coolant fuel with one having vented-to-plenum fuel. As a basis for comparison, a 1000-MWe FCR design with a submerged tank-type primary system was utilized and the test results described above extrapolated. This system is described in another paper. ${ }^{3}$ Figure 4 is a schematic view of this type of system. The cover-gas system, shielding, fuel-handling, maintenance and decontamination, pumping power, reactor vessel, reactor building, fuel costs, and safety features were considered. Cost incentives or penalites for vented-to-coolant as compared to vented-to-plenum fuel were identified and their magnitudes were estimated. For purposes of the comparison, one percent failed fuel was assumed present in either case, a basis which presumably favors the vented-to-plenum case in view of its greater failure potential due to the pressure stress.

Before such estimates were possible, questions such as (a) the effects of Cs-134 activity, (b) the covergas activity, (c) the gaseous decay tank system design, etc., were analyzed. Examples of the type of analysis performed are given below.

The rented-to-coolant cover-gas activity is illustrated in Table 3 as a function of fuel-pin holdup time. Note the total activity in the system at 5 days, the basis of this analysis.

The Cs-134 activity in the system, assuming 100\% of the released Cs-133 found its way into the sodium coolant, is shown in Fig. 5. Since this activity is within an order of magnitude of the unavoidable Na-24 activity, the problem should be a second-order one. Note also the effect of one percent fuel failures. The Cs-137 activity from one percent failures is near the Na-24 level, i.e., the plant must be designed for about this level of activity in any event. Thus, no plant design changes were chargeable to vented-tocoolant in this regard.

\section{a. Economic Analyses}

Without giving the details of each analysis, let us skip to the results of the economic analysis. The reader is referred to GEAP-4770 ${ }^{(1)}$ for these details. As a result of these analyses, it appears that where changes in capital costs can be identified, they are small, and no significant net penalty or gain is apparent (see Table 4). It does seem that there is a small net gain, however. Accordingly, this capital cost analysis provides no basis for choice between the designs. Likewise cost differences resulting from fuel handling, shipping, pumping power-right down the list-were also found to be relatively small. 


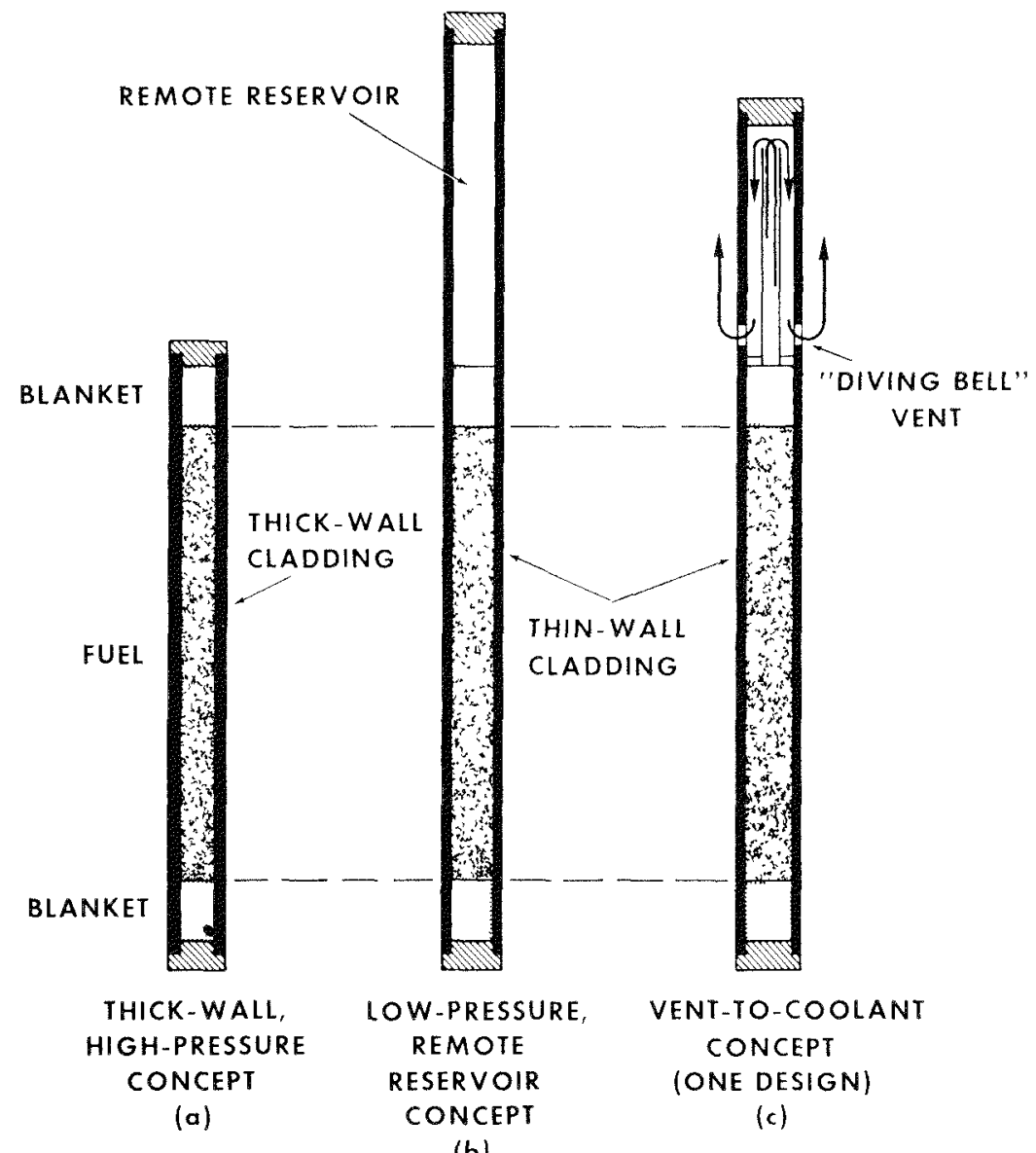

(b)

Fra. 1. Schematie of Fission-gas Aceommodation Concepts.

Should vented-to-coolant fuel result in a gain in burnup, the cost advantage is about $0.01 \mathrm{mill} / \mathrm{kW}-\mathrm{hr}$ per 10,000 MWD/T difference. It turns out that the significant differences lie in the area of safety.

\section{b. Safety Considerations}

Consider further the question of safety. When the cladding need not perform as a pressure vessel, less demands need be placed on the fuel-cladding properties, which are subject to possible deterioration over the life of the fuel in the fast flux and sodium environment. Therefore, the vented-to-coolant fuel may be expected to be less subject to fuel-pin failures due to this cause, although a quantitative prediction of the improvement is not possible.

Secondly, the probability of failure propagation should be reduced by a low internal pressure. The possibility that fission gas under high pressure could be injected into the coolant stream through a minor defect, causing vapor blanketing of adjacent pins and possibly choking off of coolant flow is effectively eliminated. An elementary analysis of this situation showed that other fuel pins, surrounding a vented-to- plenum pin which failed, would probably be gas blanketed long enough for cladding failure.

Although some preventative fuel-pin designs come to mind, such that an impedance is placed between the gas plenum and fuel, there will always be a larger gas inventory available for injection into the coolant from vented-to-plenum designs. In this sense the vented-to-coolant design is again superior.

Even if vented-to-coolant designs do not decrease the incidence of failures, i.e., failures turn out to be a function of something other than pressure, a decrease in damage potential is apparent from a failure for any reason. The reduced pressure of the contained fission gas could make many failures less dramatic. For example, solid or molten fuel is less subject to expulsion through the break-threatening blockage of a fuel channel or sodium voiding.

Additionally, some second-order safety characteristics are apparent during fuel handling and shipping. Handling of the unpressurized, irradiated fuel is obviously less hazardous than handling of pressurized ones. Canning during shipment of vented-to- 
coolant seems straightforward and probably required, in any event, for defective fuel.

A possible disadvantage of vented fuel, which was considered, was the concern that fission-gas bubbles might increase the tendency for sodium voiding in the core. Sodium velocities in the primary tank were found low enough to allow adequate de-entrainment of the gas. The gas remaining in the coolant was calculated to be $<0.001 \%$ of the total produced at $100,000 \mathrm{MWD} / \mathrm{T}$, which should be sufficient to guarantee that bubbles do not coagulate and elog a fuel channel. These calculations indicated that a bubble large enough to travel $>0.5 \mathrm{ft} / \mathrm{sec}$ vertically will be released in the low-velocity, baffled region provided in the sodium tank. This corresponds to a bubble size of about 0.02 in., whereas the spacing between fuel pins is several times this value. Thus, even if a bubble did not escape, it would probably pass through the core unnoticed, and could even serve to eliminate superheat by providing nucleation sites on a continuous basis.

\section{c. Detection System for Failed Fuel}

In reviewing the possible systems for detection of fuel failure in a sodium-cooled reactor with vented-

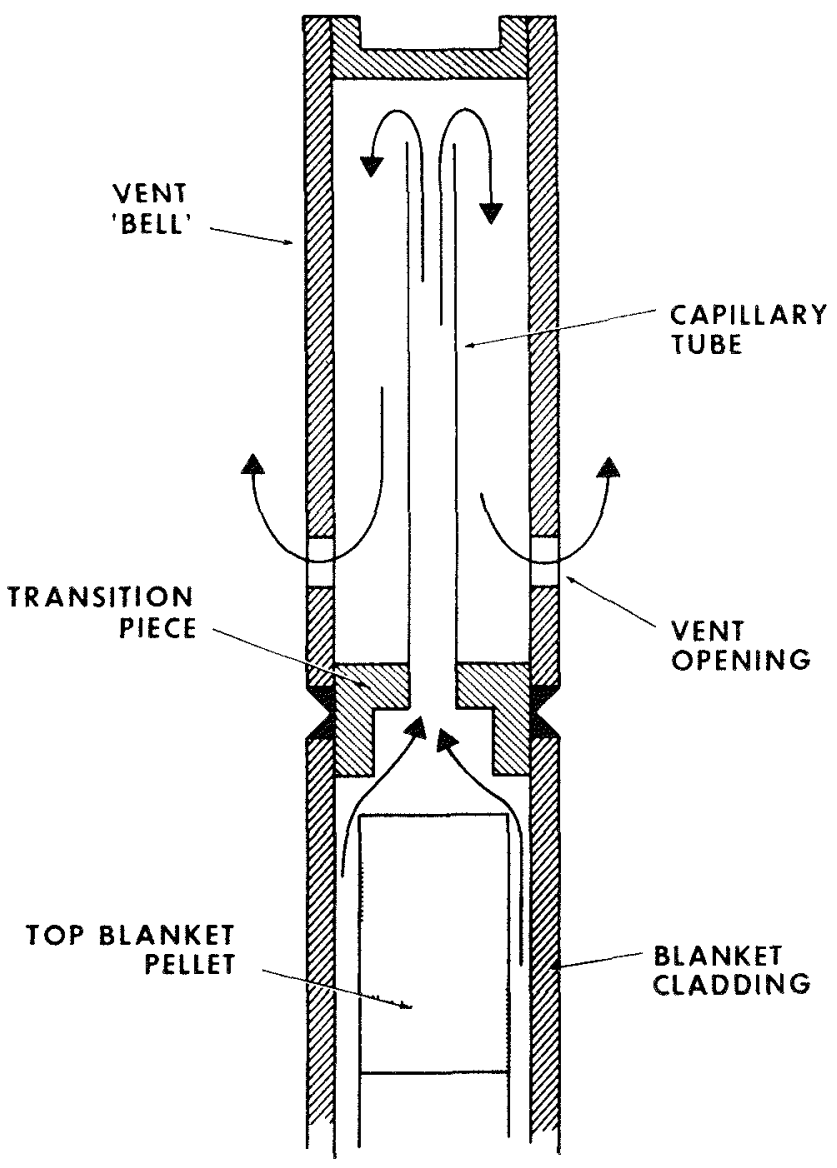

Fra. 2. "Diving Bell" Vent Device.
Table 2. Fission Products Released to CoOlant or Cover Gas

\begin{tabular}{l|c|c}
\hline \multicolumn{1}{c|}{ Nuclide } & Half-life & $\begin{array}{l}\text { Release } \\
\text { Fraction }\end{array}$ \\
\hline Gases : & & \\
Kr-85 & $10.6 \mathrm{yr}$ & 0.44 \\
Xe-131m & $12 \mathrm{~d}$ & 0.30 \\
Xe-133 & $5.3 \mathrm{~d}$ & 0.27 \\
Xe-133m & $2.3 \mathrm{~d}$ & 0.05 \\
Xe-135 & $9.2 \mathrm{~h}$ & 0.0003 \\
Solids: & & \\
Ru-103 & $40 \mathrm{~d}$ & $4 \times 10^{-10}$ \\
I-131 & $8 \mathrm{~d}$ & $1 \times 10^{-8}$ \\
Cs-137 & $29 \mathrm{yr}$ & $1 \times 10^{-6}$ \\
Other (Sr, Y, Zr, Ba, Ce) & & $10^{-6}$ to $10^{-8}$ \\
\hline
\end{tabular}

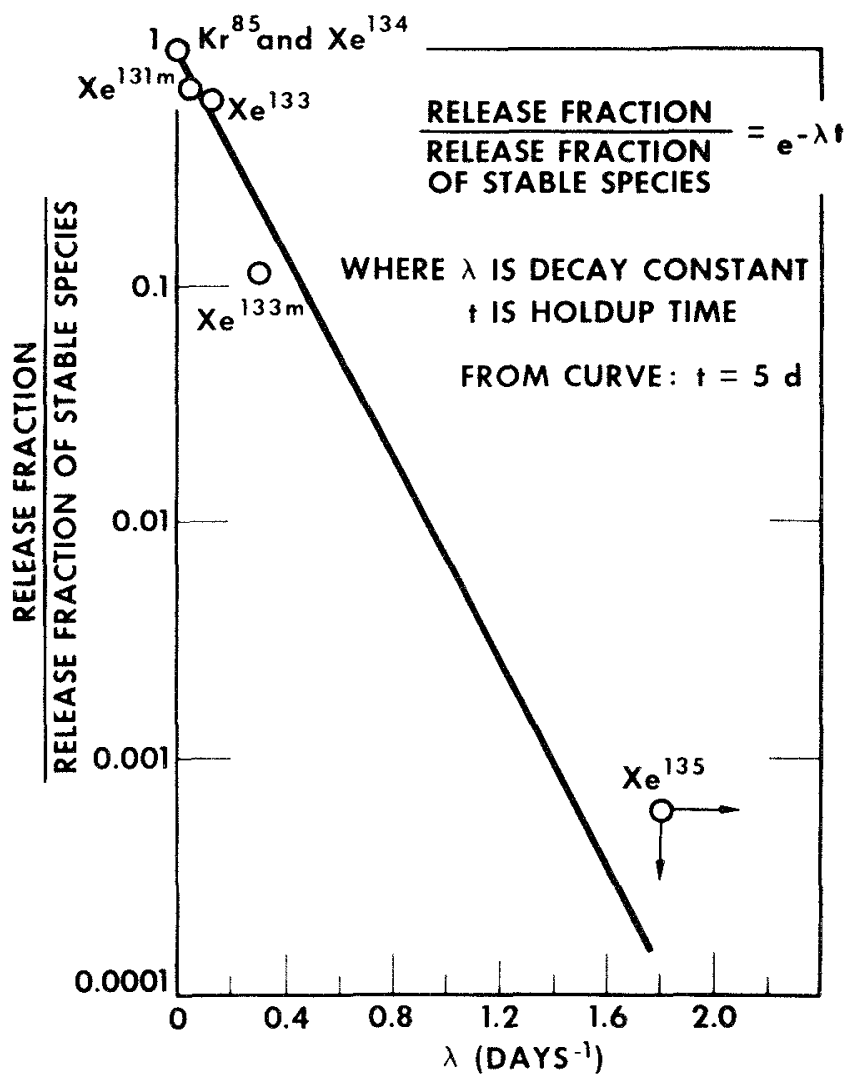

Frg. 3. Fuel-pin Holdup Time.

to-coolant fuel, the two main objectives of leak detection and leak location must be kept in mind. Several methods have been suggested, and it is not our purpose here to review the candidates. However, all rely on sampling of either the reactor coolant or cover gas, and then analyzing the sample for material or activity which would indicate the presence of defective fuel.

Most available methods are applicable to the detection of failed fuel. Discrimination between previously 


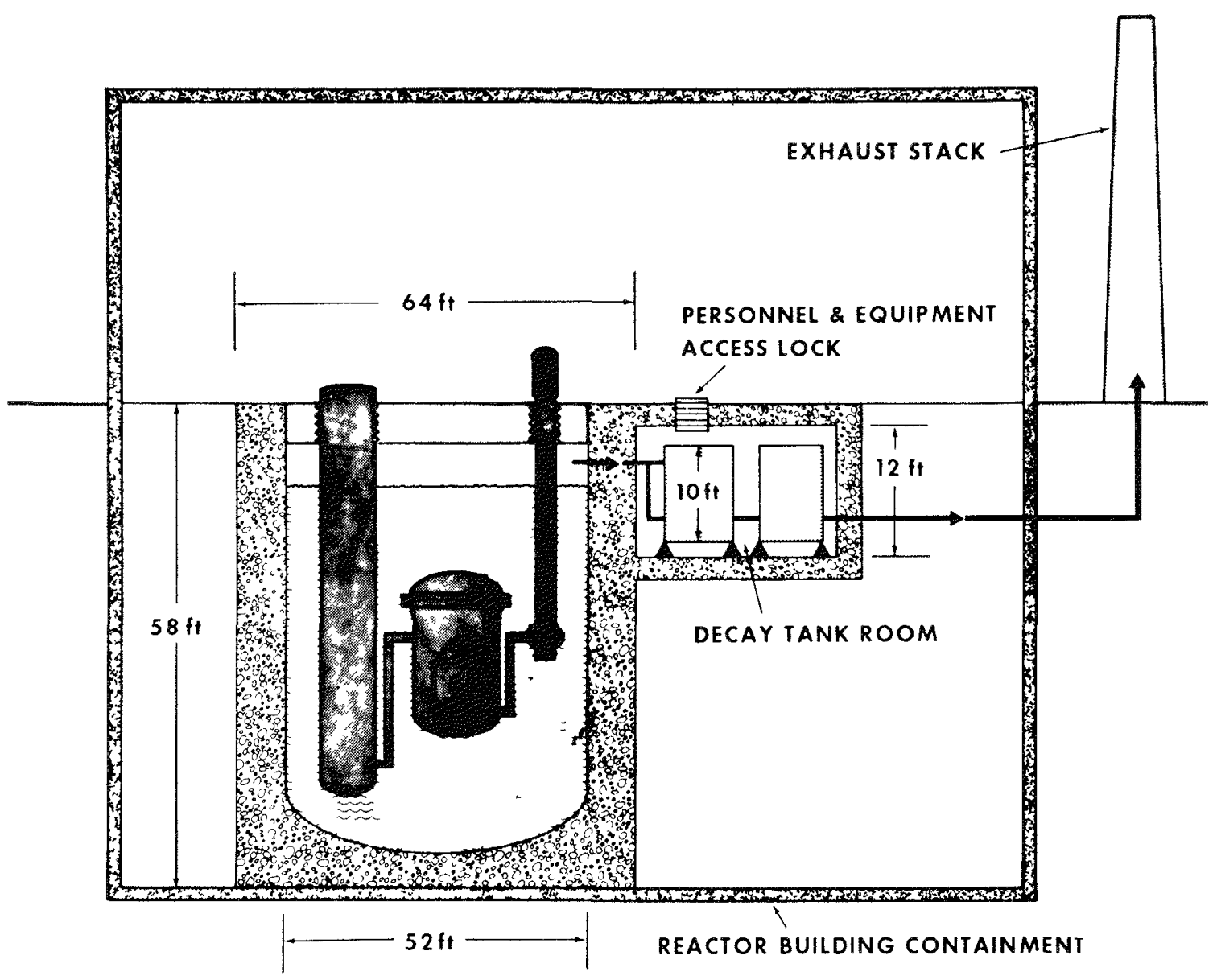

Fic 4. Tank and Cover-gas System.

released activity and incremental fresh activity will be a problem which must be solved in either ventedto-coolant or vented-to-plenum fuel systems because leakers probably will always be present in the core. Discrimination between aged (vented) fission gases and fresh (failed) fission gases seems possible in principle, and delayed-neutron detectors should also continue to work. The delayed-neutron emitters present in a vented fuel system will signal fucl failure just as in a nonvented fuel system because the shortlived halides do not reach the coolant in either design except through defects.

Also, experimental evidence indicates that the heavy elements do not leave vented fuel except through defects, so no interference with activity monitors or with operation and maintenance is inferred. In fact, the frequency of fuel-pin failures should be decreased by application of vented fuel so the amount of both delayed-neutron emitters and fissile material in the coolant should be decreased.

Location of failed fuel, if it can be done, is a much more difficult problem. If the origin of the activity is to be sufficiently localized, it probably requires sodium sampling from each subassembly, regardless of fuel design. The difference between vented-to-coolant and vented-to-plenum fuel would be only that entrained fission gases could not be used as indicators of failure for the vented-to-coolant fuel.

\section{Conclusions}

In summary, the vented-to-coolant fuel has been shown to be a primary contender as a ceramic fuel for a fast reactor. In some respects the vented design is superior to nonvented designs.

In the area of economics, vented-to-coolant designs show no significant increase in costs and possibly a small net reduction.

The value of improved propagative-failure behavior of vented fuel pins is difficult to assess, but appears beneficial and important. However, the longterm capability for withstanding the sodium and sodium vapor environment remains to be demonstrated.

Finally, an important advantage is that the vented- 
TABle 3. Equilibium Cover-gas Activity (Ci) for Variods Hold-up Trmes* for Vented-to-coolant Fuel

\begin{tabular}{|c|c|c|c|c|c|c|c|c|c|c|}
\hline ISOTOPE & $11 / 2$ & 0 & $10 \mathrm{~m}$ & $1 \mathrm{~h}$ & $5 \mathrm{~h}$ & $15 \mathrm{~h}$ & Id & $5 d$ & $20 d$ & $60 d$ \\
\hline$K_{r-89}$ & $3.2 \mathrm{~m}$ & $3.54 \times 10^{7}$ & $4.06 \times 10^{6}$ & $3.54 \times 10^{1}$ & & & & & & \\
\hline$x_{e-137}$ & $3.8 \mathrm{~m}$ & $4.52 \times 10^{7}$ & $7.18 \times 10^{6}$ & $6.8 \times 10^{2}$ & & & & & & \\
\hline $\mathrm{Xe}-135 \mathrm{~m}$ & $15 \mathrm{~m}$ & $1.38 \times 10^{7}$ & $8.84 \times 10^{6}$ & $8.6 \times 10^{2}$ & $1.38 \times 10^{-1}$ & & & & & \\
\hline$X e-138$ & $17 \mathrm{~m}$ & $4.20 \times 10^{7}$ & $2.82 \times 10^{7}$ & $3.77 \times 10^{6}$ & $2.1 \times 10^{2}$ & & & & & \\
\hline$K_{r-87}$ & $1.3 \mathrm{~h}$ & $2.08 \times 10^{7}$ & $1.91 \times 10^{7}$ & $1.22 \times 10^{7}$ & $1.04 \times 10^{6}$ & $7.3 \times 10^{3}$ & $5.72 \times 10^{1}$ & & & \\
\hline $\mathrm{Kr}-83 \mathrm{~m}$ & $1.86 \mathrm{~h}$ & $3.70 \times 10^{6}$ & $3.45 \times 10^{6}$ & $2.56 \times 10^{6}$ & $5.55 \times 10^{4}$ & $1.45 \times 10^{4}$ & $1.37 \times 10^{2}$ & & & \\
\hline$K_{r-88}$ & $2.8 \mathrm{~h}$ & $2.84 \times 10^{7}$ & $2.73 \times 10^{7}$ & $2.22 \times 10^{7}$ & $8.5 \times 10^{6}$ & $7.1 \times 10^{5}$ & $7.4 \times 10^{4}$ & & & \\
\hline $\mathrm{Kr}-85 \mathrm{~m}$ & $4.4 \mathrm{~m}$ & $1.15 \times 10^{7}$ & $1.12 \times 10^{7}$ & $9.8 \times 10^{6}$ & $5.2 \times 10^{6}$ & $1.06 \times 10^{6}$ & $2.63 \times 10^{5}$ & & & \\
\hline$x e-135$ & $9.2 \mathrm{~h}$ & $4.90 \times 10^{7}$ & $4.75 \times 10^{7}$ & $4.52 \times 10^{7}$ & $3.34 \times 10^{7}$ & $1.45 \times 10^{7}$ & $8.04 \times 10^{6}$ & $5.23 \times 10^{3}$ & & \\
\hline$x e-133 m$ & $2.3 d$ & $1.30 \times 10^{6}$ & $1.30 \times 10^{6}$ & $1.28 \times 10^{6}$ & $1.22 \times 10^{6}$ & $1.08 \times 10^{6}$ & $9.62 \times 10^{5}$ & $2.86 \times 10^{5}$ & $3.25 \times 10^{3}$ & \\
\hline$x e-133$ & $5.3 \mathrm{~d}$ & $5.32 \times 10^{7}$ & $5.32 \times 10^{7}$ & $5.32 \times 10^{7}$ & $5.15 \times 10^{7}$ & $4.9 \times 10^{7}$ & $4.66 \times 10^{7}$ & $2.76 \times 10^{7}$ & $3.72 \times 10^{6}$ & $2.12 \times 10^{4}$ \\
\hline $\mathrm{Xe}-131 \mathrm{~m}$ & $12 d$ & $1.54 \times 10^{5}$ & $1.54 \times 10^{5}$ & $1.54 \times 10^{5}$ & $1.54 \times 10^{5}$ & $1.48 \times 10^{5}$ & $1.45 \times 10^{5}$ & $1.15 \times 10^{5}$ & $5.4 \times 10^{4}$ & $9.8 \times 10^{3}$ \\
\hline$K_{r-85}$ & $10.6 y$ & $7.5 \times 10^{2}$ & $7.5 \times 10^{2}$ & $7.5 \times 10^{2}$ & $7.5 \times 10^{2}$ & $7.5 \times 10^{2}$ & $7.5 \times 10^{2}$ & $7.5 \times 10^{2}$ & $7.5 \times 10^{2}$ & $7.5 \times 10^{2}$ \\
\hline TOTAL & & $2.76 \times 10^{8}$ & $2.11 \times 10^{8}$ & $1.51 \times 10^{8}$ & $1.01 \times 10^{8}$ & $6.67 \times 10^{7}$ & $5.6 \times 10^{7}$ & $2.8 \times 10^{7}$ & $3.79 \times 10^{6}$ & $2.67 \times 10^{4}$ \\
\hline
\end{tabular}

-ASSUME 35\% RELEASE FRACTION FROM THE FUEL

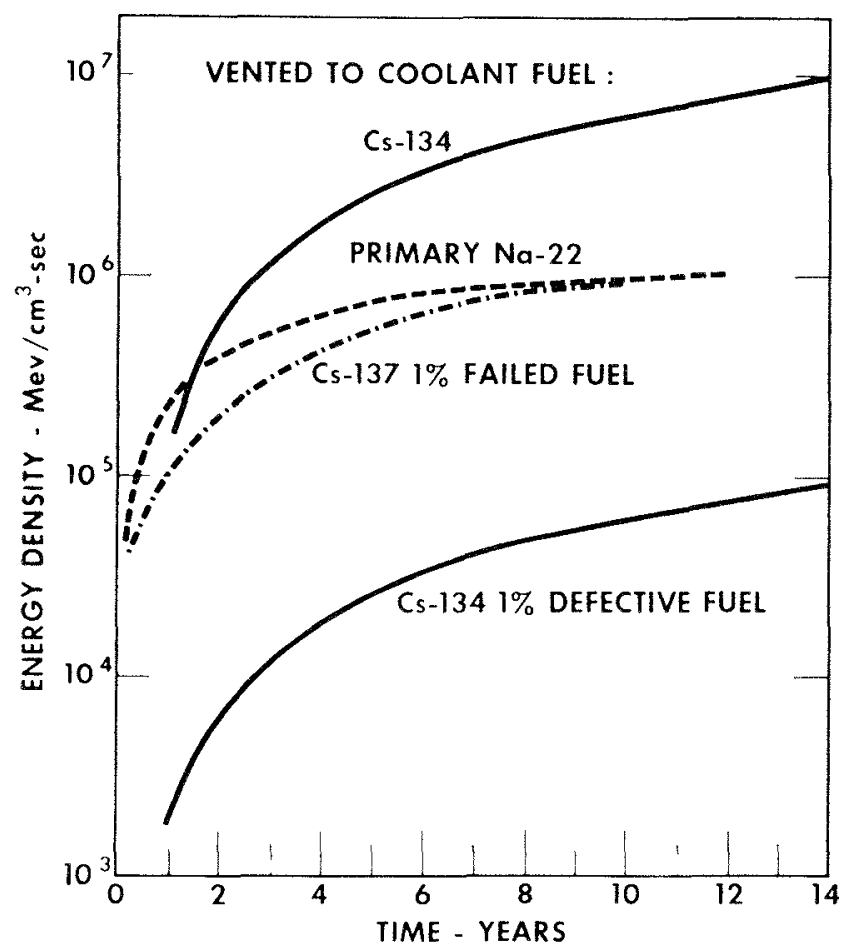

Frg. 5. Activity in the Coolant System.
Table 4. Technical and Economic Comparison

Basis:

1000-MWe, FCR, tank-type reactor, demonstration test results.

Conclusion:

Slight capital cost advantage to vented-to-coolant fuel.

Slight handling and shipping advantage to vented-to-plenum fuel.

Slight fuel-performance advantage to vented-to-coolant fuel

Slight pumping-power advantage to vented-to-coolant fuel.

slight decontamination and maintenance advantage to vented-to-plenum fuel.

to-coolant concept places less pressure-stress resistance on cladding properties in the fast flux and sodium environment.

\section{REFERHNCES}

1. J. J)uffy, J. Gilbertson, F. Knight, and (:. O'Neill, A Tech nical and Economic Evaluation of Vented Fuel for Sodiumcooled Fast Ceramic Reactors, GE.1P-47\%o.

2. G. L. O'Neill et al., Demonstration of Fission Gas Venting from Fast Oxide Reactor Fuels, ANS Annual Meeting, Philadelphia, Pa. 6/14-18/65. 
3. K. Cohen and G. O'Neill, Safety and Economic ('haracteristucs of a 1000-MWe l'ast Soduum-cooled Reactor Design, see paper in Session III of this Conference.

\section{Discussion}

Mr. Campise (AI): I think there is one obvious economic incentive to vented elements that has been overlooked thus far. It is not at all obvious that the plenum design is going to make the grade as far as being able to provide us reliable $100,000-\mathrm{MWd} / \mathrm{T}$ performance, whereas the vented concept just may increase the probablility that if one looks at fuelcycle costs you will find maybe $0.3-0.5 \mathrm{mill} / \mathrm{kWh}$, and this indeed is an economical ineentive.

Mr. O'Neill: Yes, we looked at the fuel costs and fuel-performance advantages, and I certainly agree with you that there is one. We find, however, that it is more like $0.01 \mathrm{mill} / \mathrm{kWh}$ per $10,000 \mathrm{MWd}$ per ton difference. I agree with you that this is a potential benefit of venting to coolant, and I strongly believe that the vented-to-coolant design will have less failure potential than the vented-to-plenum design. Of course, this is part of the reason why we have selected it for the design which Dr. Cohen discussed yesterday.

Mr. Mall (LASL): What is the fate of the cesium once it gets into the sodium?

Mr. O'Neill: The best I have seen are the papers on BR-5 by the Russians. We don't really have good data on the fate of the fission products after they get into the coolant, although we are addressing ourselves to that problem. The Russians seem to report a deposition in the vapor phase, which was out of proportion with the amount of sodium there is, as if there was some sort of preferential deposition of cesium, which we find very interesting.

$\mathrm{Mr}$. Kummerer (Karlsruhe): Under normal operation conditions, I find this proposal a very attractive one. But for transient or overpower condition the delay time for the fission gases in this fuel goes down to about zero and, as we can pick up from your table, the activity of the material is raised by some orders of magnitude. What is your comment?

Mr. O'Neill: I suspect you mean normal transients. The particular test data that I showed were the result of some 20 startups and shutdowns, including startup and shutdown transients for example. We did not find any effect due to this although we were looking for one. For example, when we took the capsule out of the reactor we rushed it to the hot cell and got immediate data to look for short-lived fission products; we did not find any. I don't think there will be enough transients in the steady-state operation of the reactor to cause a normal steady-state problem; I should hope not.
Mr. Kummerer: Excuse me, I must correct mysolf. I did not mean a normal transient; I meant mainly an overpowered condition coming out from a little accident or so. Then you have to some extent a swift release of all the fission gases or most of the fission gases to the sodium. The sodium activity may increase by a factor of 10 or 100 , and this is a problem as regards the safety record of this reactor.

Mr. O'Neill: I don't really see what that has to do with the difference betwreen vented-to-coolant and any other kind of fuel.

Mr. Kunkel (AI): With regard to the question on cesium that was asked, we have some data that indicate that the cesium is preferentially deposited in the cold trap. We will talk more about this tomorrow.

Mr. Runnalls (AECL): I understand from Mr. Comprelli's presentation that there is expected to be a fuel-clad interaction which might be as thick as about 3 mils, and there is also likely to be a ferritic layer produced on the outside of the reference 316 SS, which is perhaps 3 mils thick. In addition, if I remember the numbers correctly, there is some evidence of intergranular sodium penetration to about 7 mils. Now, Dr. Cohen yesterday indicated that the reference design is 10-mil $316 \mathrm{SS}$. How do you hope to avoid venting the fission gas through the sheet?

Mr. O'Neill: Dr. Zebroski, would you like to answer that?

Mr. Zebroski (GE): I think Mr. Comprelli mentioned that these phenomena on both the inside and outside of the cladding are statistically very rare; you don't see them on every pin, and you see them only in occasional spots in the pins where they are found. I think the assumption that you simultaneously have maximum penetration on the outer surface and the maximum penetration on the inner surface facing each other is one of the cases where we will use the statistical approach and say that's rare enough to neglect.

$M r$. O'Neill: Iet me tell you now how we would design today; it is the way that Dr. Cohen described it yesterday. We assume there is a $100 \%$ safety factor in the mass-transfer data which we have today, including the ferrite layer, and it came to, I believe, something around 3.5 mils. We then decided what the minimum end-of-life cladding thickness should be, selected 7 mils, but made the cladding 10 mils thick. I might point out that it is not particularly significant to the vented-fuel cause to have 10 mils of cladding as compared to 15. It's again one of those insignificant economic gains. It is beneficial, however.

$M r$. Okrent (ANL): Is it clear that one can design to rent and at the same time not get sodium back in or not flood under the various conditions of starting up 
reactors and having temperatures somewhat different from what one designed for, etc.? Are you very optimistic that this is a solved problem?

Mr. O'Neill: Actually, we have only the single test, which was very successful; we are very proud of it. We are continuing the work.

Mr. Okrent: It used to appear that the oxide is likely to achieve high burnup by releasing its fission product gases, thereby not swelling like metals and carbides. It was on this basis that it gained the reputation as a $100,000-\mathrm{MWd} / \mathrm{T}$ fuel. Now, however, there seems to be some question as to whether the released fission product gases can be held within a fuel element that goes to high burnup. Secondly, one is quite concerned about the sudden release of these fission product gases and their possible adverse effects on safety. And, hence, serious looks are being given to a new design concept whereby the fission product gases get into the sodium. Am I correct? Is there a strong reservation concerning the overall desirability and likelihood of success for the vented-to-plenum oxide concept at burnups of $100,000 \mathrm{MWd} / \mathrm{T}$ or more?

Mr. O'Neill: I don't mean to degrade the ventedto-plenum concept by advocating vented-to-coolant. I certainly believe that we can design vented-to-plenum fuel pins that can make it to $100,000 \mathrm{MWd} / \mathrm{T}$. We have no evidence to indicate that we ean't. However, we have precious little evidence to indicate that we can, too.

Mr. Okrent: I believe you said you're concerned about the escape of the released fission product gases, and I think the conference has been concerned about the consequences of boiling which might follow this escape.

Mr. O'Veill: I also indicated that we had several ideas of how we might put impedance between the plenum and the failure and help the problem along for the vented-to-plenum design, as well. We haven't set to work on that problem, if it is a problem. We're just in the process of analyzing it, and we have quite an improvement in our technique to make a right comparison. If it is a problem I am sure we will come up with a design of each type that can handle the problem.

Mr. Kelman (ANL): Can you tell me how much allowance you make in your design for the swelling of the fuel due to retained fission gases and solid fission products?

Mr. O'Neill: We do not make a direct allowance for swelling in our fuel design at this time. We designed the fuel in the way I described as I started the paper, namely, by combination of the fabrication tolerances at the hot spot with the overpower. In the cooler region of the core, where the swelling is likely to be the biggest problem, there will be a gap and porosity existing in the fuel, which should accommodate quite a bit of swelling. There are no data existing on the swelling of mixed oxide fuel pins that you can use to set down a criterion that would be based on swelling alone. We will be ready and willing to change our criteria when such data come along.

Mr. Morrison (Battelle-Columbus): Concerning the vented-to-coolant concept, it seems to me that the principle of operation involves sorption of the fission products on the surface, either of your capillary or your diving bell. I wonder if you could envision at high burnup the possibility of saturating the selective surface sites for sorption and cutting down your delay time for the release.

Mr. O'Neill: Yes. Not only that, the cesium deposits in the blanket on the way to the venting devices, I'm sure. In fact, we analyzed this experiment destructively and found that the delay time had occurred for the most part before it ever got out of the fuel and blanket region. But to get to your question: does it cause a change or decrease in the delay time? I really doubt that, because it's a diffusion phenomenon for the most part.

Mr. Morrison: It seems to me it's a diffusion phenomenon to get it there, but it's a sorption phenomenon once it stays there. If you may envision a saturation of the surface sites or if you get cnough fission products plated out there, you might get an increase in temperature in this region and get a desorption.

Mr. O'Neill: Yes, I guess I should point out that a complementary part of this vented-to-fuel development program is a comprehensive study of the potential for fission product deposition (we call it plugging) in the blanket region. We have seen some slight evidence that it occurs. We haven't seen any evidence that it is a real serious problem.

Mr. Zebroski: May I add to your answer on the swelling. As I mentioned in the paper this morning, we have observed swelling in the colder regions of the fuel, but it's very difficult to distinguish this from the redistribution of the porosity because it is of the same order of magnitude as the fabrication tolerances that you can ordinarily achieve. We see porosity whose total volume is of the same order as the 1-mil gap on the outside, so the reason that we have to give an indeterminate answer is simply that this is a very hard quantity to measure quantitatively. We do have fairly strong indications that it is small. We have in progress a series of four experiments, effectively dilatometer experiments, which are specifically designed for this purpose. The fuel has no place to go; it starts out at very high density with extremely high restraint, and we expect to be able to measure con- 
tinuously, that is, nondestructively, the expansion of the fuel up to about $150,000 \mathrm{MWd} / \mathrm{T}$ at about four different temperatures. We will get this low-temperature effect and hopefully find a threshold temperature at which gas release reduces the swelling rate. The old Bettis data which give swelling rates for thin wafer fuel at fairly low temperature, I think, give an upper limit to the probable effect that we would design for.

\section{General Discussion}

Mr. Lauroski (ANL): I would like to thank the speakers for their excellent presentations and the audience for the stimulating comments and questions that they have provided. The meeting is now open for gencral questions.

Mr. Stachura (AI): There was one comment which Dr. Dickerman made which I thought I agreed with at first, but on reffection, I find I disagree. This was the comment about the fact that while fuel could melt into sodium and become dispersed, in similar fashion a channel could void and the sodium could fall into it and compact the fuel. At first I thought this might fall into the category where you do have a voidingfirst condition. In the case of ceramic fuel, however, suppose the coolant did come back down into contact with, let's say, molten fucl. Since it hasn't solidified into cooler portions and is mobile, the vapor pressures would be much larger than any hydrodynamic pressures of simple sodium coming back into the core by gravity. I think in that case we would still have an effective dispersion effect, although as I mentioned earlier a previously voided channel requires an experiment where we look at meltdown into a voided region,

Mr. Lawroski: I would like to ask now for someone to comment on the question: what are some of the modes of failures that have been reported, as least in part, of oxide fuels that have been planned to go to rather high irradiations, but failed? Obviously some of the comments that we have had in connection with rented fuels here raise this question as well.

$M r$. Leitz (CE): Perhaps you have reference to some of the failures that we have experienced in capsule tests which have been conducted since the first series of 40 successful tests. We have had a few failures with these irradiations. One, I believe, was associated with an overambitious rod power that we exposed a pin to, prior to planned transient irradiation. As a result of exposures, I think in excess of $30 \mathrm{~kW} / \mathrm{ft}$, and end flux peaking, we lost the end of the pin. The other failures have been attributable to the difficulties associated with sodium bonding fuel capsules for these irradiation tests; the development of gas bubbles in the bonds, not associated particularly with fission gases, but introduced in the course of fabrication and unfortunately not removed thereafter, led to cladding burnout. I believe the French had some similar un- fortunate experience and possibly others who have tried high-performance fuel tests with sodium bonds.

$M r$. Lawroski: Have you had failures that you can't account for in this way, failures that have occurred prior to when you would have expected it, based on the early irradiations that went to $100,000 \mathrm{MWd}$ ?

Mr, Leitz: I don't recall any.

Mr. Dickerman (ANL) : I would like to point out that if the coolant rushes back in, and comes into contact with high-temperature fuel, the pressures will be generated at that location; presumably the majority of the fuel will be nearer the center of the core, so you do have a mechanism whereby pressure can be generated in a place that will drive the fuel, for example, towards the center of the core. I believe this is the sort of thing which has been used to describe one of the GE irradiations in TREAT.

Mr. Leitz: For one of the irradiations in TREAT in which there was a failure, reconstruction suggests that the pressures which were generated in the coolant tended to force the material back into the region inside the cladding.

Mr. Rabin (GE): The aluminum heat sink which was around the pin filled the central core such that when we examined it, we found that the aluminum heat sink had solidified inside the central core and was combined with some of the $\mathrm{NaK}$ that was in the annulus around the pin; evidently the differential pressure pushed it in. This was a non-preirradiated transient specimen.

Mr. Stachura: I believe that the GE capsule was pressurized on the outside to about $10 \mathrm{~atm}$. I think the failure that did occur was that the oxide did penetrate the clad, but apparently the dispersion that we noticed was not observed. Perhaps the energy was insufficient, so that the contact of fuel with the cladding was enough to bring the temperatures closer to failure temperatures, yet not get the pressures much above $10 \mathrm{~atm}$. However, I will certainly agree that the motion of molten fuels into regions other than the peak flux regions could produce pressures that may drive it again randomly. We need to look at some experiments in which there is a longer range of travel and resolidification of the fuel in the core.

Mr. Boland (ANL): I would like to urge caution in interpreting the single-pin experiments in TREAT too literally. We have yet to run clusters of fuel ele- 
ments and look to see what happens. Do we get the significant sodium pressures that we think might be possible due to superheating, with an appreciable mass loading of the subassembly caused by the effect of accelerating what would normally be a sodium head on any power reactor system? I think these singlepin experiments, although interesting, might lead us down the wrong path if we put too much faith in them.

Mr. Kummerer (Karlsruhe): It is clear that if we consider the maximum hypothetical or credible accident, then for both the vented and nonvented fuel concepts, the accident is the same. If the reactor explodes there is no difference. But there are different stages of safety considerations. If we have only some overpower with a nonvented fuel, then we have no accident; we have only an irregularity. But if we have a rented fuel then we have an accident, because the activity of the sodium goes up by a factor of 100 or so, and we need to shut down the reactor to clean the sodium, etc.

Mr. Campise (AI): I think one point that has to be considered is that when we finally get around to engincering a sodium-cooled fast reactor which is designed for vented elements, more than likely there will be auxiliary systems to scrub the sodium as well as to handle the normal venting of fission gases. We have demonstrated in the past that the normal traps in a primary system will trap solid fission products, and the gaseous fission products will be handled by the auxiliary system. It is highly probable that in an overpower situation most of this material will be trapped in the system employed in the design of the vented element reactor.

Mr. Lawroski: How much evidence do you have of this, other than empirieal?

Mr. Campise: As I recall, we dumped something like $10,000 \mathrm{Ci}$ of fission products into the $\mathrm{SRE}$, which were predominantly removed in cold traps.

Mr. Kunkel (AI): Naybe I could shed a little light on this. We did find that during the accident at the SRE, when there was a considerable quantity of fission product activity dumped into the sodium, that the level of the activity decreased markedly over what one would anticipate due to the normal radioactive decay, and that the majority of this material was deposited in the cold trap in the piping and in other areas of the system.

Mr. Lauroski: This was of the metallic fuel, was it not?

$M r$. Cohen (GE) : I would like to say that those of us who are working with oxide fuel are not satisfied with the amount of evidence which we have in support of these fuels. I don't mean that we are having bad results. What's happening now is that we are getting results few and far between. If a conclusion of this discussion is that we should redouble our efforts to have in-fast-reactor-flux irradiation of oxide fuels and perhaps a bundle, which would not have the misleading results that you get from sodium bonding in EBR-II, for example, I think we would be a lot further along in satisfying the chairman and other people that we have enough evidence.

Mr. Zebroski (GE): I would like to try to also give a speculative comment on Dr. Kummerer's question of the gas release in a transient. I think you have to understand that the instantaneous inventory in the fuel is relatively small, and that in addition to the total rise and absolute temperature of the fuel volume plus the delay time there still is some plenum. The total rise in absolute temperature is less than a factor of two. Thus, on the average, the instantaneous dumpout in the limited class of transients which you must be talking about (that is, the ones which are big enough to be considered an incident, and yet small enough so that you don't damage the fuel), doesn't lead to melting or damaging of the fuel. If you consider this limited class of accidents, you wouldn't be dumping kilocuries into the coolant, only smaller quantities and probably of the same order as the steady-state quantity would be involved.

Mr. Wensch (AEC Washington): It was quite a few years ago that we initiated the work on the rented-fuel-element program. I think I would like to state the objectives of that program so we can see if we have fulfilled the mission. The first objective was to determine the effect of leakers and know what the effect would be in terms of short-range and long-range consequences. If the results indicated that the consequences were tolerable, then we would go to vented fuel elements. It seems to me that great caution nust be exercised in considering vented fuel elements for any fast reactor in the future in order to assure ourselves that we can live with any long-term damage which could result to the primary system. I see no evidence that a program has been laid out to the Commission or to this audience which showed that these end objectives will be reached at the time of, say, the planned prototype for either the Germans or ourselves.

Mr. Lauroski: We must bear in mind that the Russians and BR-5 were able to deal with a large amount of what amounts to vented fuel and clean up the reactor and enable themselves to reload it with another fuel.

Mr. Cohen: I would like to repeat to Dr. Wensch the assurance that I gave him earlier that he will see that program. 
0

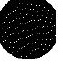




\section{Session VI-B \\ October 13, 1965}

\section{Mostly Reactivity Coefficients}

Chairman: F. W. Thalgotr

Argonne National Laboratory

Secretary: R. A. Lewis

Argonne National Laboratory 
0

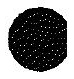




\title{
Doppler Coefficient Measurements in Zebra Core 5
}

\author{
A. R. Baker and R. C. Whegler \\ A tomec Energy Establishment \\ Winfrth Heath, Unted Kingdom \\ (PRESENTED BY R. C. WHEELER)
}

\section{Introduction}

In order to measure the Doppler coefficient associated with a zone of fissile material, the ZEBRA reactor was modified in October 1964 so as to accommodate a central hot loop. A series of measurements was completed during the following ten months with various assemblies of fissile and nonfissile pins within the loop, with neutron spectra approximating to that expected in a typiral power fast reactor. The core (Core 5 in the Zebra series) was arranged in two regions, an outer concentrated region of $\mathrm{U}^{285}$ and graphite 'driving' an inner region of more dilute mixed plutonium and natural uranium oxide. The inner region included the test zone (inside the loop) and a cold buffer zone, whose composition matched that of the test zone in the case of the four main assemblies of mixed oxides. In these assemblies it was found that the measured Doppler coefficients were slightly more negative than was predicted by the calculations. Other measurements indicated an apparent small negative coefficient for plutonium (i.e., in the absence of uranium) and a small negative coefficient for steel.

The loop had the following advantages over Doppler experiments with small fuel samples:

(a) the amount of fuel was large enough to give significant reactivity changes;

(b) the heated zone was nearly large enough to establish its own neutron spectrum (i.e., even without the matched buffer zone);

(c) it was possible to include correct reactor constituents including sodium;

(d) the effect of the zone boundary was comparatively small.

\section{Details of the Loop}

The main features of the loop are shown in Fig. 1. Inside the reactor, the loop consisted of an insulating structure made up of four square concentric steel tubes, which occupied the central 26 by 26 -cm hole in the core lattice structure, a hole normally filled by 2.5 fuel sheaths. Inside this structure a 16 by 16 array of pins, supported on a pin base plate symmetrically about the core centre-plane, could be accommodated on a square pitch of 1.13 ( $\mathrm{m}$ (see Fig. 2). The temperature of the pins was controlled by circulating nitrogen gas through the gaps between the pins. In addition, an electrical heating tape of $6-\mathrm{kW}$ capacity was wound round the innermost steel wall and was operated in such a manner as to achicve an approximately constant radial temperature distribution in the pin zone. The space between the first and third steel walls of the insulating structure, and between the third and fourth steel walls, was filled with Santocell insulant (mainly low-density $\mathrm{SiO}_{2}$ ). In the space between the second and third steel walls, a flow of air at the ambient temperature of the buffer zone was blown upwards by means of a second circulator in the so-called 'guard-ring' circuit. The two insulating walls and the flow of air in the guard-ring circuit together restricted the hoat flow from the inner test region to the outer core to a maximum of about $2 \pi \mathrm{W}$.

The walls of the inner loop were supported by means of four trunnions which connected to the outer sieel wall at the core centre-plane. Because the temperature variations of the outer wall were small (e.g., less than $1^{\circ} \mathrm{C}$ when the loop temperatures were cycled), the vertical movements of the trunnions due to thermal expansions were negligible. This particular method of supporting the loop was chosen to minimise the reactivity effects of thermal expansion and to simplify their calculations (see below). The loop structure was shown to expand as expected by a series of tests using Invar pushrods and external dial gauges.

Outside the biological shielding of the reactor were situated the heaters, cooler, and circulators associated with the three circuits of the experiment. The main circuit heater (40 kW) and cooler (a nitrogen-to-containment-air heat exchanger of about the same capacity) were operated by an automatic control system such that any desired pin zone temperature in the range from 100 to $550^{\circ} \mathrm{C}$ could be reached and maintained. The temperatures in the loop were monitored by resistance thermometers situated in the gas stream, and also at six positions inside three temperature probes which occupied positions in the assembly of pins. A temperature change over the complete range could be accomplished in about $2 \mathrm{hr}$. Another, smaller circulator and 


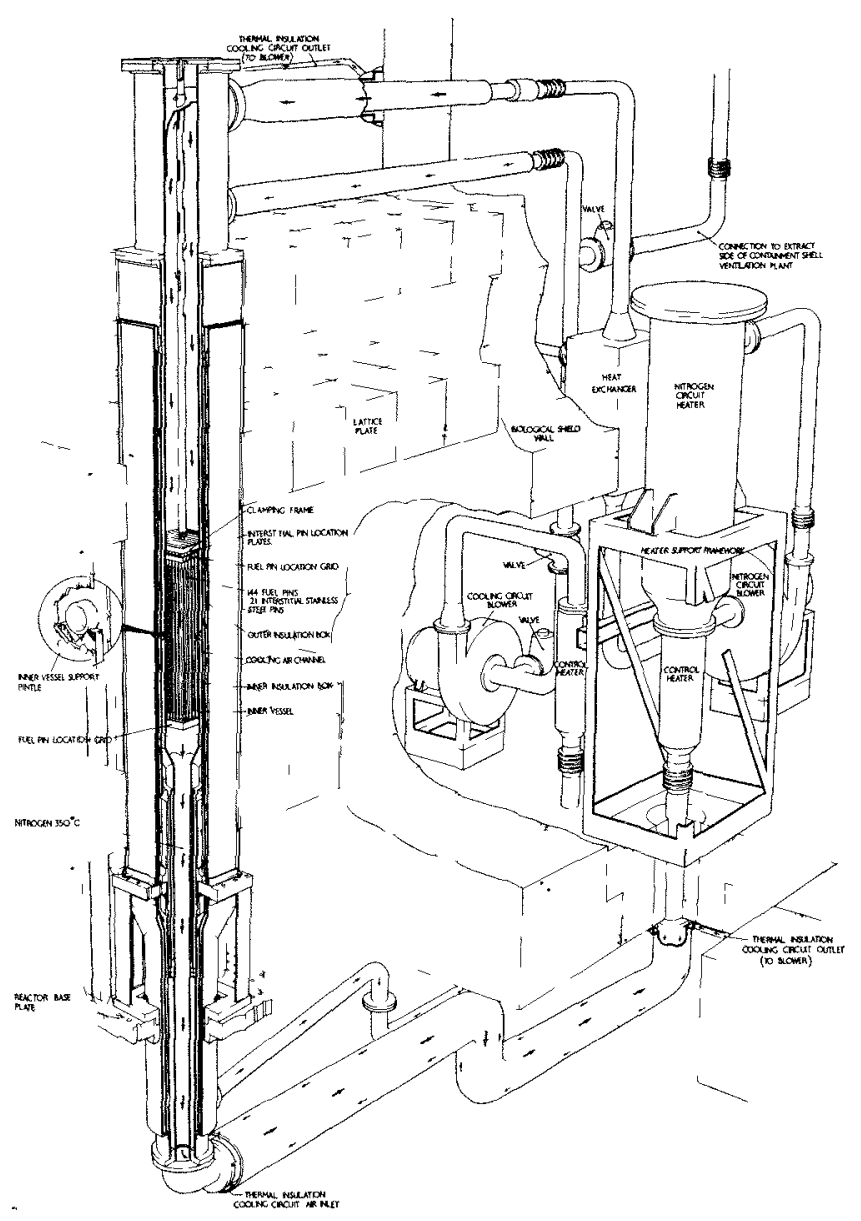

heater were associated with the guard-ring circuit; a third blower was associated with heat-scavenge circuits which were arranged to remove heat eseaping from the lagged pipes of the main circuit.

A small proportion of the main circuit was bypassed through a filter paper in a subsidiary loop, and an $\alpha$ seintillation counter was fixed close to the filter so that an automatic warning of the release of $\mathrm{PuO}_{2}$ particles (i.e., in the event of fuel-pin failure) would have been received in the control room. Other alarms were assoriated with high-temperature sensors in the test zone (and in the outer zones), with low gas flows in the three cir('uits, and with sodium leakage. Two detectors were used for observing leakage of sodium. One, at the lowest point of the main circuit, measured the resistance of a gas gap that would have been submerged in sodium in the event of any considerable leak. The other, situated further downstream, measured the attenuation of a beam from a sodium vapour lamp in passing across the pipe of the main circuit.

\section{The Pins Used in the Loop}

Three types of fuel pins and four types of nonfissile pins were used in the various arrangements in the test zone (see Table 1). The fuel pins (Fig. 3) were canned in 0.25 -mm-thick stainless steel tubes, of $11-\mathrm{mm}$ outside diameter. The top and bottom end pieces were

Fig. 1. Zebra Doppler Experiment.

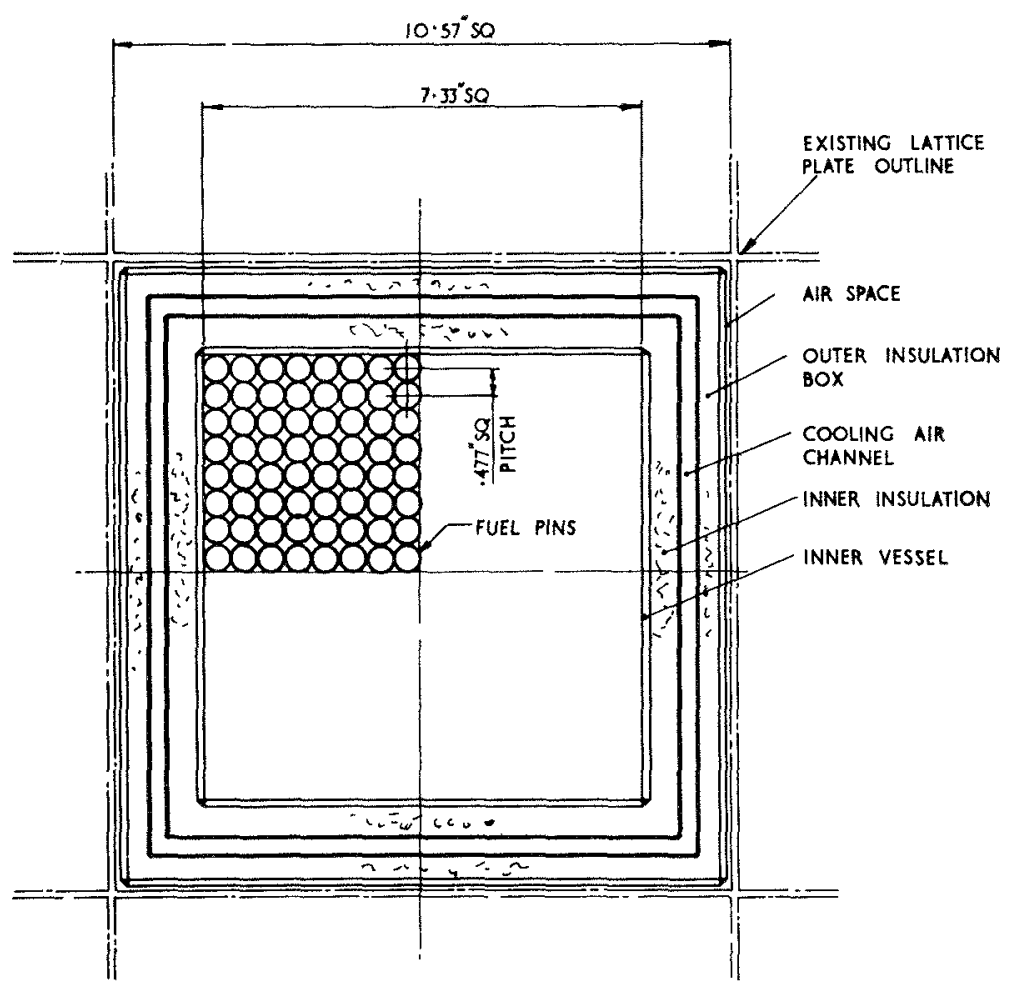

FIG. 2. Sectional Plan $\backslash$ iew through Doppler Loop. 
Table 1. Ni mbers of Pins Loaded in Tesi Zone

\begin{tabular}{|c|c|c|c|c|c|c|c|c|c|}
\hline $\begin{array}{l}\text { Assem- } \\
\text { bly }\end{array}$ & A & B & $\mathrm{D}$ & $\mathrm{Fe}$ & Void & $\mathrm{Na}$ & $\mathrm{Al}$ & $\begin{array}{c}\text { Tem- } \\
\text { per- } \\
\text { ature } \\
\text { Probes }\end{array}$ & $\begin{array}{l}\text { Buffer Zone } \\
\text { Composition }\end{array}$ \\
\hline A & 128 & $\ldots$ & - & 128 & - & - & - & - & Matched A \\
\hline B & - & 128 & - & 125 & - & - & - & 3 & “ \\
\hline $\mathrm{C}$ & 一 & 128 & - & - & 125 & - & - & 3 & Matched D \\
\hline $\mathrm{D}$ & 128 & 一 & - & - & 125 & - & 一 & 3 & " \\
\hline $\mathbf{E}$ & - & - & - & 128 & 125 & -- & - & 3 & “ \\
\hline $\mathrm{F}$ & -- & - & - & -1 & - & - & - & 3 & “ \\
\hline$G$ & - & - & - & - & - & - & - & 3 & Matched H \\
\hline $\mathrm{H}$ & 128 & - & - & - & - & 125 & 一 & 3 & " \\
\hline I & - & - & - & - & - & - & - & 3 & Matched J \\
\hline$J$ & 96 & -1 & - & 32 & - & 125 & - & 3 & " \\
\hline$J(1)$ & 54 & - & 一 & 18 & 50 & 125 & - & 3 & “ \\
\hline $\mathrm{J}(2)$ & 24 & - & - & 8 & 96 & 125 & - & 3 & “ \\
\hline$J(3)$ & 6 & - & - & 2 & 120 & 125 & $\cdots$ & 3 & “ \\
\hline$J(4)$ & - & - & - & - & 128 & 125 & 一 & 3 & " \\
\hline $\mathrm{K}$ & - & - & 16 & 112 & - & 125 & - & 3 & ، \\
\hline $\mathrm{L}$ & - & - & - & 253 & & - & - & 3 & $"$ \\
\hline $\mathrm{M}$ & - & - & - & 128 & 125 & - & - & 3 & " \\
\hline $\mathrm{N}$ & 125 & $\cdots$ & - & 128 & - & - & - & 3 & " \\
\hline $\mathrm{O}$ & - & - & - & - & 一 & - & 253 & 3 & "t \\
\hline
\end{tabular}

welded to the tubes, both edge and seam welds being used at each end. The fucl was in the form of sintered pellets of $10-\mathrm{mm}$ diameter and 6 - or $10-\mathrm{mm}$ height. Above each fuel column (69 cm high) was a nimonic spring which ensured contact between the faces of the pellets during thermal cycling. Each fuel element was thermally cycled after manufacture and then tested for leak tightness before use in the loop. The three fuel types were as follows:

Type A pins each containing $555 \mathrm{~g}$ of $16 \% \mathrm{PuO}_{2}$ and $84 \%$ depleted $\mathrm{UO}_{2}$ (mixture by weight);

Type $\mathrm{B}$ pins each containing $427 \mathrm{~g}$ with the same

$\mathrm{PuO}_{2}$ content as in type $\mathrm{A}$, the balance made up of boron steel and steel;

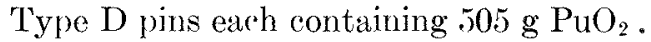

The ' $A$ ' pins were specified to represent the composition of a typical power fast reactor fuel (but not with the typical diameter of a power-producing fuel pin). The ' $B$ ' and ' $D$ ' pins were designed to help separate the contribution of plutonium to the Doppler effert. The ' $D$ ' pins were filled, like the ' $A$ ' pins, with a column of homogeneous pellets. However, the ' $B$ ' pins were loaded with three types of pellets in a regular sequence, as follows:

first pellet: $100 \% \mathrm{PuO}_{2} ; 3.2$-g mass and 6 -mm height; second pellet: boron steel; $8.7-\mathrm{g}$ mass and $14-\mathrm{mm}$ height;

third pellet: steel; 3.7-g mass and 5-mm height;

The total length was $25 \mathrm{~mm}$. Each 'B' pin contained 27 such cells of 3 pellets.

The steel was designed to achieve approximately the same total scattering cross section per plutonium atom as in the ' $\mathrm{A}$ ' pins, and the boron content (see Table 2) was chosen so as to match the neutron spectra of the two assemblies with ' $\mathrm{B}$ ' pins to the corresponding assemblies with ' $A$ ' pins. Calculations show that the spectra were in fact comparable (see Table 7 below).

\section{The Core Arrangement Outside the Loop}

The arrangement of the outer core zones is shown in Figures 4 and $\tilde{j}$, and some dimensions are given in Table 3. Immediately outside the insulator zone of the loop, a buffer zone ( $16 \mathrm{~cm}$, or $2 \frac{1}{2} \mathrm{scattering}$ mean free paths thick) was loaded with standard Zebra fuel sheaths and fucl pieces. The composition (but not the heterogeneity) of this zone (see Table 2) was made close to that of the test zone in the cases of assemblies $\mathrm{A}, \mathrm{D}, \mathrm{H}$, and $\mathrm{J}$, the mixed oxide assemblies investigated. (Carbon was used to mockup part of the oxygen content.)

Thus the neutron spectra in the pin zones of these

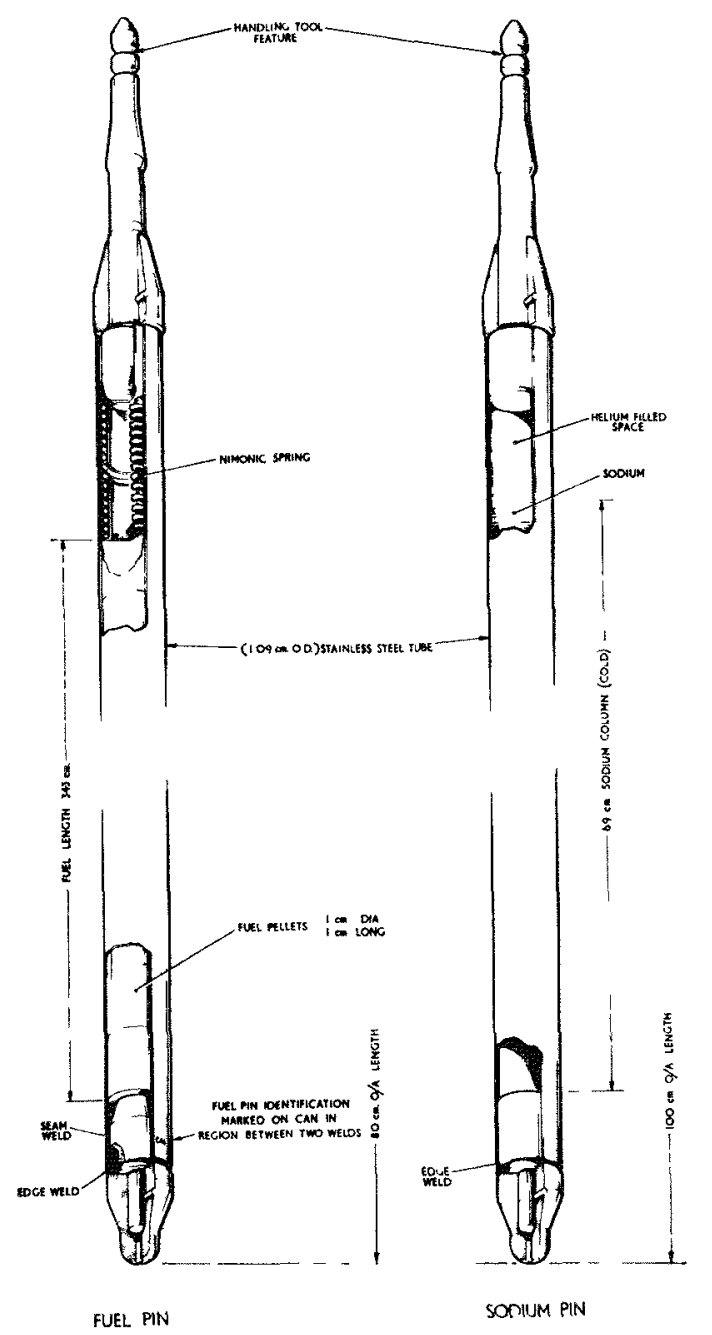

FIG. 3. Schematic of Pins Used in Tests. 
TABLE 2. ZoNe Compositions (atoms/ $\mathrm{cm}^{3} \times 10^{-22}$ )

\begin{tabular}{|c|c|c|c|c|c|c|c|c|c|c|}
\hline Zone & $\mathrm{C}^{235}$ & $\mathrm{C}^{238}$ & $\mathrm{Pu}^{239}$ & $\mathrm{Pu}^{240}$ & $\mathrm{O}$ & $\mathrm{C}$ & $\begin{array}{c}\mathrm{Fe}+\mathrm{Cr} \\
+\mathrm{Ni}\end{array}$ & Natural B & $\mathrm{Na}$ & $\mathrm{Al}$ \\
\hline Driver & 0.431 & 0.239 & - & - & $\ldots$ & 5.591 & 0.540 & 一 & $-\infty$ & $\cdots$ \\
\hline Buffer A, B & 0.004 & 0.597 & 0.114 & 0006 & 1.203 & 0.440 & 3.360 & - & 一 & 0.325 \\
\hline Test A (and N) & 0.003 & 0.583 & 0.105 & 0.006 & 1.461 & - & 3.42 & - & - & - \\
\hline Test B & - & - & 0.101 & 0.006 & 0.214 & - & 5.45 & 0.253 & 一 & - \\
\hline $\operatorname{Test} 1)$ & 0.003 & 0.583 & 0.105 & 0.006 & 1.461 & - & 0.570 & 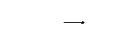 & - & - \\
\hline Test $\mathrm{E}(\operatorname{ard} \mathrm{M})$ & - & - & - & & - & & 3.420 & 一 & - & 一 \\
\hline Buffer $G, H$ & 0.004 & 0.556 & 0.108 & 0.006 & 1.120 & 0.410 & 0.974 & - & 0.668 & 0.421 \\
\hline Test $\mathrm{H}$ & 0.003 & 0.583 & 0.105 & 0.006 & 1.461 & 一 & 0.570 & - & 0.838 & - \\
\hline Buffer I to $O$ & 0.003 & 0.415 & 0.080 & 0.004 & 0.836 & .305 & 1.655 & - & 0.754 & - \\
\hline Test J & 0.002 & 0.437 & 0.079 & 0.004 & 1.096 & & 1.282 & - & 0.838 & $\cdots$ \\
\hline Insulator & & - & 一 & & 0.086 & 一 & 1.965 & 一 & - & 0.05 \\
\hline
\end{tabular}

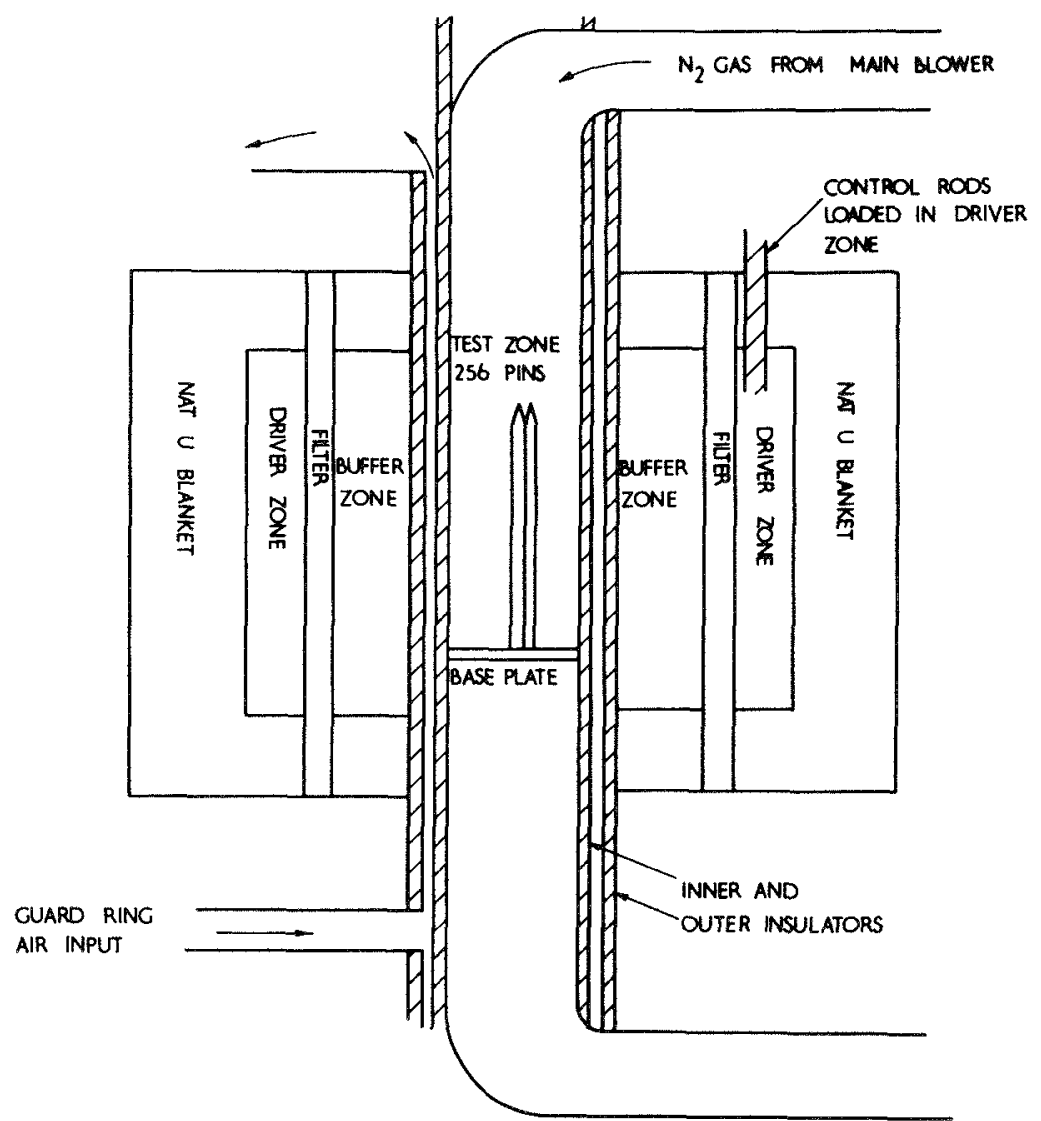

Fit. 4. Schematic Diagram of Core 5 (Not to Scale).

assemblies were close to those which would apply in infinite zones of the same compositions. The same matehing of compositions of zones did not apply to assemblies $\mathrm{B}, \mathrm{C}$, and $\mathrm{K}$, because here the purpose was to separate the plutonium Dopplor contribution in spectra appropriate to assemblies A, D, and J, respectively, and therefore the buffer compositions of the latter were retained (see Table 1). Outside the buffer 


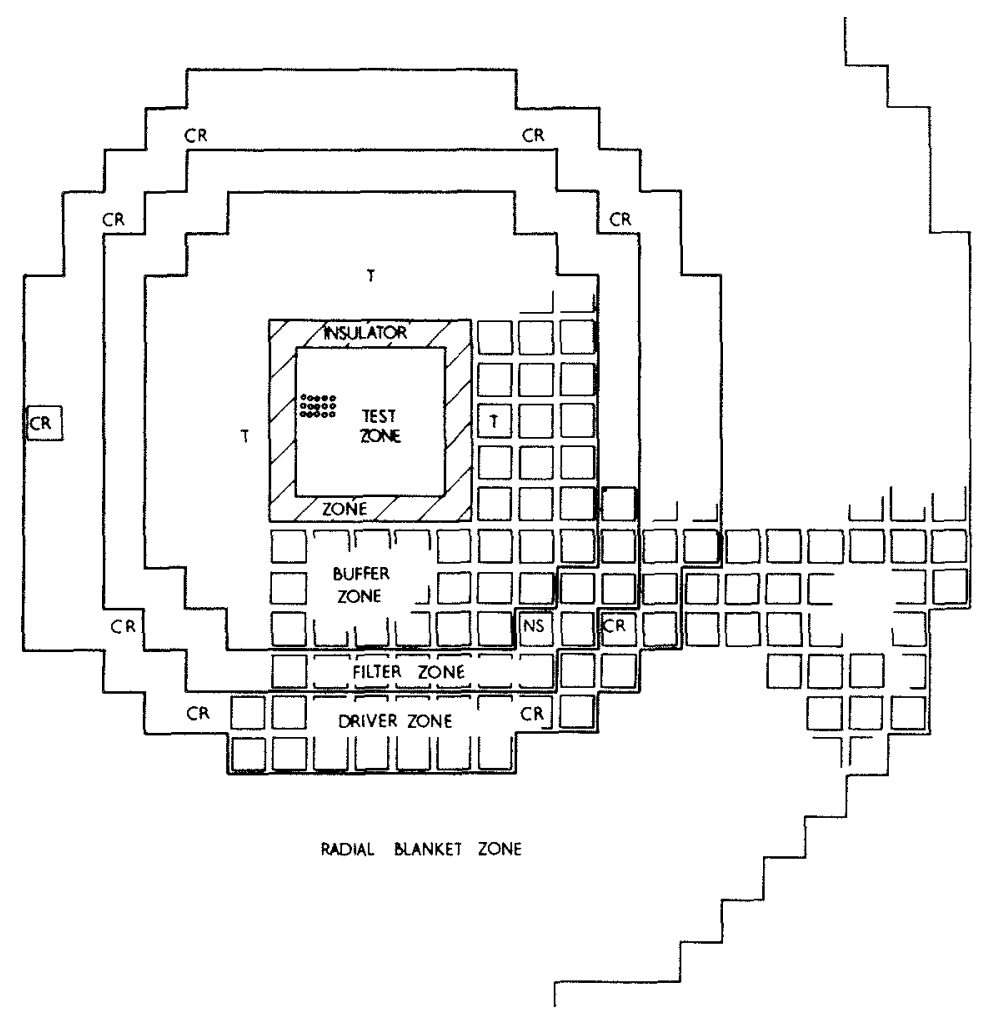

T. TEMPERATURE ELEMENT

CR. CONTROLL ROD POSITION

NS * NEUTRON SOURCE MECHUNISM

FIG. 5. Core-5A Loading Plan

zone were loaded, in order, a filter zone, it driver zone, and a radial blanket zone. The filter zone consisted of approximately one row of fuel sheaths loaded with iron plates. The object of the filter zone was to use the inelastic scattering and absorption of the iron to reduce the proportion of high-energy and very low-energy neutrons, respectively, coming from the driver zone, and so obtain a better spectrum match between it and the buffer zone. The driver zone consisted of $93 \% \mathrm{U}^{235}$ plates, $37 \% \mathrm{U}^{235}$ plates, and graphite plates. The control rods, which had a fuel section and a $\mathrm{B}^{10}$ section, were loaded in this zone. Outside the driver zone (and above and below the driver and buffer zones) was a $30-\mathrm{cm}$ thick natural uranium blanket.

A series of subsidiary experiments with chambers, which measured the reaction rate ratio $\mathrm{B}^{10}(n, \alpha) /$ $\mathrm{U}^{235}(n, f)$, indicated that the spectrum radially across the assembly was constant from the centre to near the outer boundary of the buffer. Similar measurements in the vertical direction through the centre showed only small variations along the height of the pins.

\section{Experimental Details}

The reactivity at each test zone temperature was measured by the movements of a calibrated control rod at a constant reactor power (usually about $5 \mathrm{~W}$ ). The calibration was carried out by the reactor-period
Table 3. Zone Boundaries Used in Cylindrical Modela or Core and Fisbrle Loadings

\begin{tabular}{l|c|c|cc}
\hline \multicolumn{1}{c|}{ Zone } & $\begin{array}{c}\text { Outer Radius } \\
(\mathrm{cm})\end{array}$ & $\begin{array}{r}\text { Height of Zone } \\
(\mathrm{cm})\end{array}$ & $\begin{array}{r}\text { Fissile Content } \\
(\mathrm{kg})\end{array}$ \\
\hline Test & 10.75 & 68.6 & 9.5 & $\mathrm{Pu}^{239(\mathrm{a})}$ \\
Insulation & 15.35 & 81.3 & - & \\
Buffer & 31.9 & 505 & 108 & $\mathrm{Pu}^{239(\mathrm{a})}$ \\
Filter & 38.2 & 50.5 & - & \\
Driver & $48.0^{a}$ & 50.5 & 426 & $\mathrm{U}^{235(\mathrm{a})}$ \\
Radial Blanket & 78.1 & 81.3 & - & \\
\hline
\end{tabular}

(a) Assembly 5A.

method, using calculated values of the total effective delayed-neutron fraction (0.0058 in 'A'). Typically, reactivity changes could be measured to an accuracy of about $7 \times 10^{-7}$. The reactivity changes had to be corrected for the effect of thermal expansions of the pins and structure (see below), and for background-reactivity drift. Reactivity drift occurred because of nuclear heating and because of heat loss from the loop to the outer core. The rate of temperature increase in the driver elements at the core centre-plane when the power was $5 \mathrm{~W}$ was about $0.0 .5^{\circ} \mathrm{C} / \mathrm{hr}$, and this gave rise to a linear reactivity drift of about $-2 \times 10^{-6} / \mathrm{hr}$. The method of measuring the reactivity of the test zone at a temperature $\mathrm{T}$ in the presence of drift was to balance 


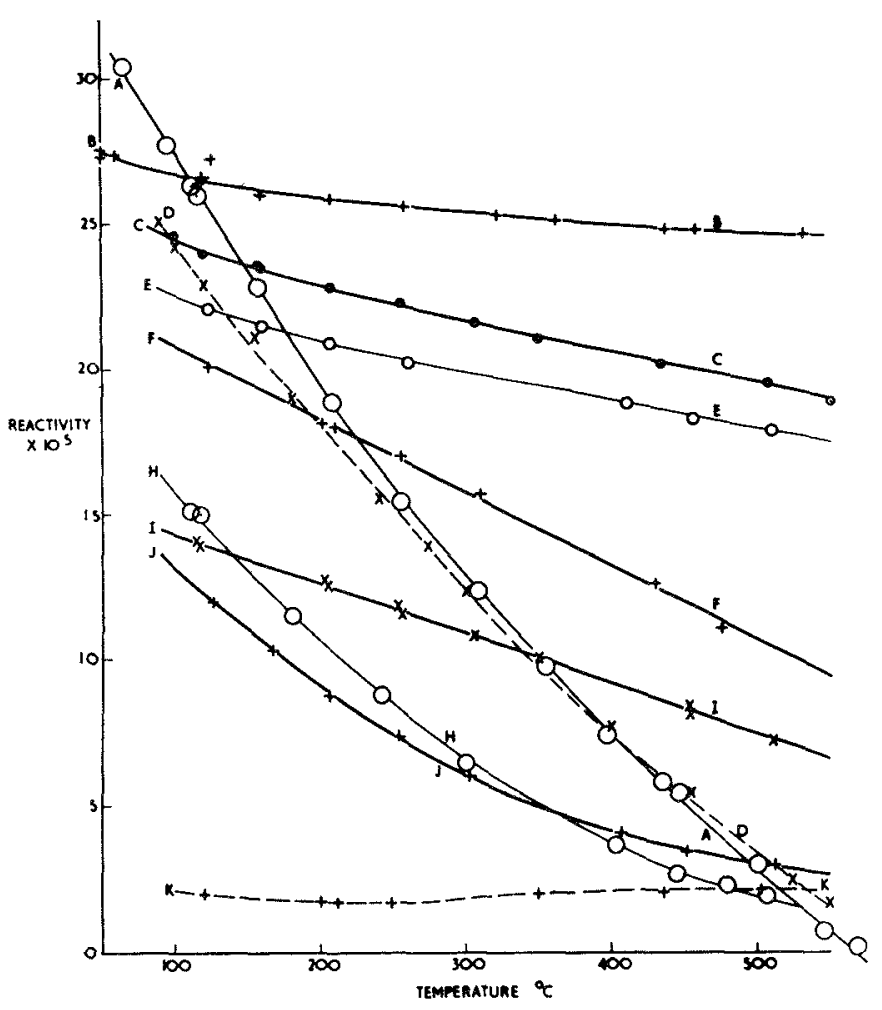

Fig. 6. Measured Temperature Coefficients (Uncorrected).

the reactor sucecssively at $350, \mathrm{~T}, 3.50^{\circ} \mathrm{C}$, and to interpolate linearly with time between the $350^{\circ} \mathrm{C}$ balances so as to obtain the effective background reactivity at the time of the balance point at $\mathrm{T}$. Thus reactivity changes were all related for convenience to one test-zone temperature $\left(350^{\circ} \mathrm{C}\right)$. The temperature coefficients measured for each assembly are shown in Fig. 6, and (after correction for expansion effects) in Fig. 8.

Heat flow from the test zone to the buffer zone varied with test-zone temperature. In a typical low-temperature cycle (e.g., $350-120-350^{\circ} \mathrm{C}$ ) the temperature inside an inner-row buffer element measured by a resistance thermometer at the core centre-plane varied by about $0.1^{\circ} \mathrm{C}$, and in a typical high-temperature cycle (e.g., $350-550-350$ ) by up to $0.6^{\circ} \mathrm{C}$. (The time response of the inner buffer to test-zone (hanges was about $1 \mathrm{hr}$.) Calculations showed that these temperature changes made negligible corrections to the measured reactivity changes. Subsidiary experiments were carried out to check this by rumning the guard-ring cireuit at a series of different temperatures, it being shown that the results were insensitive to buffer zone changes of this order.

The mean temperature in the test zone could be measured by means of six resistance thermometers to an accuracy of about $0.5^{\circ} \mathrm{C}$. The temperature variations along the length of the temperature probes in contact with the pins never varied by more than about $2^{\circ} \mathrm{C}$ when the system was balanced, even at $5.50^{\circ} \mathrm{C}$. Varia- tions radially across the pin zone were also of the order of $2^{\circ} \mathrm{C}$.

\section{Allowance for Pin Expansions}

The temperature coefficient $C_{1}$ associated with the thermal expansion of the column of material in a can (for constant pin base-plate position) was allowed for by use of the expression

$$
C_{1}=\alpha N\left[-W+l_{1}\left(\frac{d W}{d l}\right)_{1}\right]
$$

where $\alpha$ is the linear thermal expansion coefficient, $N$ is the number of pins loaded of the type being considered, $W$ is the reactivity worth of the column of material at the reactor ambient temperature $\left(50^{\circ} \mathrm{C}\right), l_{1}$ is the length of the column, and $(d W / d l)_{1}$ is the reartivity worth per unit length at the top of the column. The expression was evaluated for each type of pin in turn.

Published values of $\alpha$ were used for steel, sodium (i.e., liquid sodium in steel), and aluminum; but for the pellets of the A, B, and D pins, $\alpha$ was measured in the laboratory by use of an apparatus with silica push rods and dial gauges. Values of $W$ and $d W / d l$ were measured in the reactor in each assembly. It was found that, in general, $W$ for fissile pins was nearly constant across the test zone, and it was sufficiently accurate to measure it in one or two positions only. For nonfissile pins, for which the radial variations were more important, values of $W$ were frequently obtained by averaging the effect obtained with 9 or 16 pins distributed over the zone.

Table 4 gives typical values in assembly $\mathbf{J}$. The accuracies of the estimates were, however, dependent mainly on the values of $(d W / d l)_{1}$, which were obtained in two ways. Measurements of small-sample reactivity worth were obtained for the important materials at a series of heights through the centre of the core, and these were used to deduce $d W / d l$. Secondly, for the case of sodium, steel, and aluminum, the reactivity worths of special pins with an increased length of material (2.5, 5, and 7.5 ('m extra for sodium; $2.5 \mathrm{~cm}$ extra for steel and aluminum) were measured. By comparing the reactivity worths of these special pins with the worths (W) of the normal pins, values of $d W / d l$ were again obtained. The two methods were in agreement in the majority of assomblies to within the errors. An additional refinement in the case of some steel pins involved increasing the length by $2.9 \mathrm{~cm}$ and at the same time rutting a flat onto the side of the pin such that the total mass was equal to the mass of a normal steel pin. The special pin was thus a reasonable geometric model of a normal pin at $400^{\circ} \mathrm{C}$, and the difference in reartivity worths between the pins was then directly related to the temperature coefficient $C_{1}$ for steel pins. 
TABLE 4. Example of Input Data for Axial Expansion Corrections (Assembly J)

\begin{tabular}{|c|c|c|c|c|c|c|}
\hline & 'A' Fuel & & Steel & Steel Can & Sodium & Units \\
\hline $\begin{array}{l}\alpha \\
l_{1} \\
l_{3} \\
W \\
d W / d l \\
d^{2} W / d l^{2} \\
d w / d l\end{array}$ & $\begin{array}{c}10.3 \\
68.6 \\
34.3 \\
+6.9 \pm 0.1 \\
+7.3 \pm 1 \\
-\end{array}$ & & $\begin{array}{c}18.5 \\
72.4 \\
34.3 \\
-0.87 \pm 0.04 \\
+2.7 \pm 0.3 \\
+2.0 \pm 0.2 \\
+2.7 \pm 0.3\end{array}$ & $\begin{array}{c}220 \\
68.6^{(\mathrm{a})} \\
34.3 \\
+0.135 \pm 0.004 \\
+2 \pm 0.2 \\
-\end{array}$ & $\begin{array}{c}18.5 \\
72.4 \\
34.3 \\
-0.09 \pm 0.01 \\
+0.3 \pm 0.03 \\
-\end{array}$ & $\begin{array}{c}10^{-6}{ }^{\circ} \mathrm{C} \\
\mathrm{cm} \\
\mathrm{cm} \\
\times 10^{-5} \\
\times 10^{-7} \mathrm{~cm} \\
\times 10^{-8} / \mathrm{cm}^{2} \\
\times 10^{-9} / \mathrm{g} / \mathrm{cm}\end{array}$ \\
\hline & & $\begin{array}{r}l_{2}: \\
R: \\
M: \\
\\
W_{2}: \\
d W_{2} / d R:\end{array}$ & $\begin{aligned} & 36.1 \mathrm{~cm} \text { (bas } \\
& 39.3 \mathrm{~cm}(\mathrm{tol} \\
&: \quad 10.9 \mathrm{~cm} \\
& 2.4 \times 10^{3} \mathrm{~g} \\
& 12.4 \mathrm{~g} \text { (bott } \\
& 17.8 \mathrm{~g} \text { (top } \\
& \quad 1.25 \pm 0.2 \\
&: \quad 2.8 \pm 1.5\end{aligned}$ & $\begin{array}{l}\text { late, bottom end fit } \\
\text { ad fittings) } \\
\text { (base-plate) } \\
\text { end fitting per pin) } \\
\text { fitting per pin) } \\
\times 10^{-4} \\
\times 10^{-5} / \mathrm{cm}\end{array}$ & & \\
\hline
\end{tabular}

(a) i.e., at ambient.

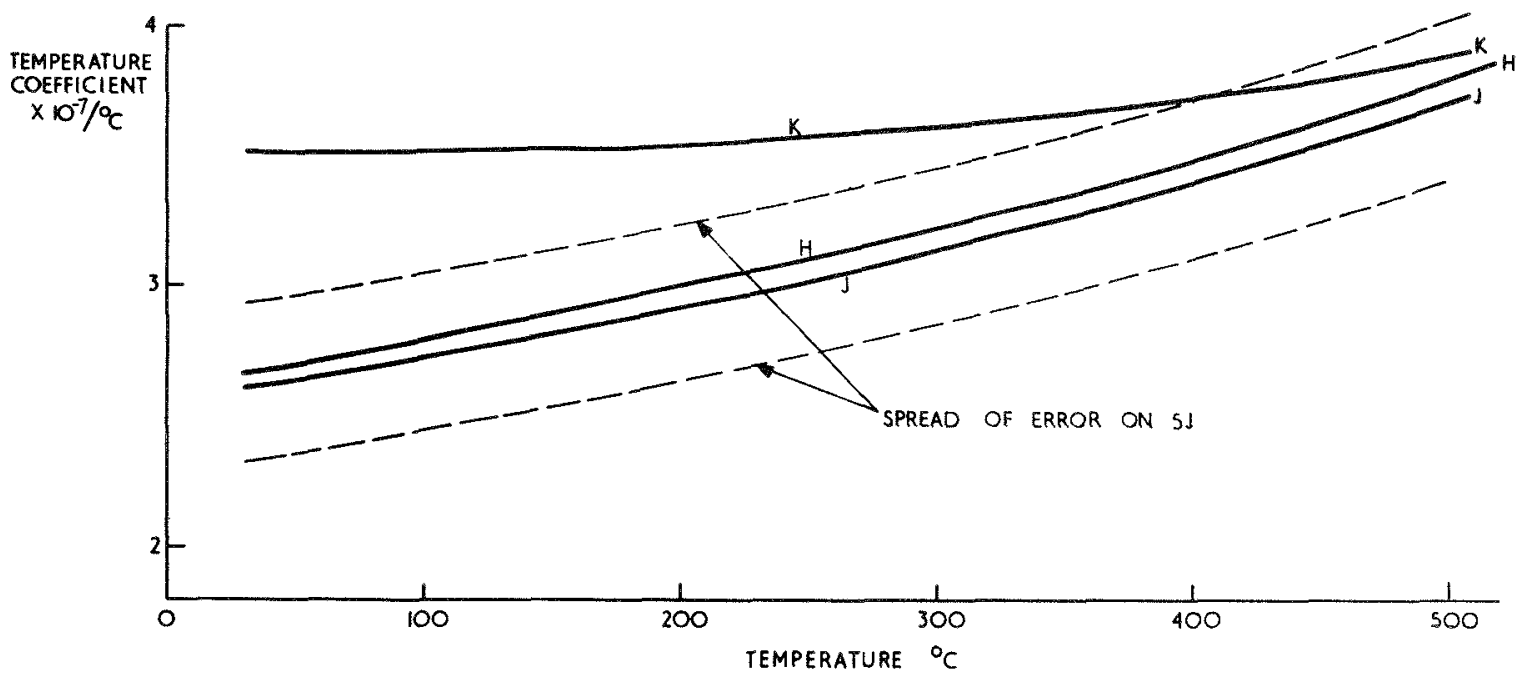

FIG. 7. Estimated Correction of Temperature Coefficient for Assemblies with Sodium Pins.

Measurements with small samples indicated that $d W / d l$ and hence $C_{1}$ were constant for expansions over the range of $50-550^{\circ} \mathrm{C}$ for fuel, steel, and aluminum. But for sodium, a column of which expanded by about $7.5 \mathrm{~cm}$ over this temperature range, this was not true, and $C_{1}$ was evaluated as a function of temperature (see Fig. 7). The temperature coefficients after correction for expansion effects are shown in Fig. 8.

The reactivity effect of the steel tubes of the cans was evaluated in the same way as for the solid steel pins. The effects of the top and bottom end pieces $\left(C_{2}\right)$, and of the steel pin base-plate $\left(C_{3}\right)$ were each evaluated by means of the expression

$$
C_{2} \text { or } C_{3}=\alpha l_{2} M(d w / d l)_{2},
$$

where $\alpha$ is the linear expansion coefficient for steel, $l_{2}$ is the distance of the end fitting, or steel plate, from the core centre-plane, $M$ is the mass of steel, and $(d w / d l)_{2}$ is the gradient of the reactivity worth of steel per $\mathrm{g}$ (each part of the steel structure expanded away from the core centre-plane because of the trunnions holding the inner loop at this position were connected to the cold outer structure).

The movement of the base-plate also contributed a reactivity effect by moving the pins, an effect not included in expression (1). This was evaluated for each type of pin by the expression

$$
C_{4}=\alpha l_{3}\left[(d W / d l)_{l_{3}}-(d W / d l)_{l_{1}-l_{3}}\right]
$$

where $\alpha$ and $(d W / d l)$ are as above, $l_{3}$ is the distance of the bottom of the column of material from the core centre-plane, and $l_{1}-l_{3}$ is the distance of the top of 


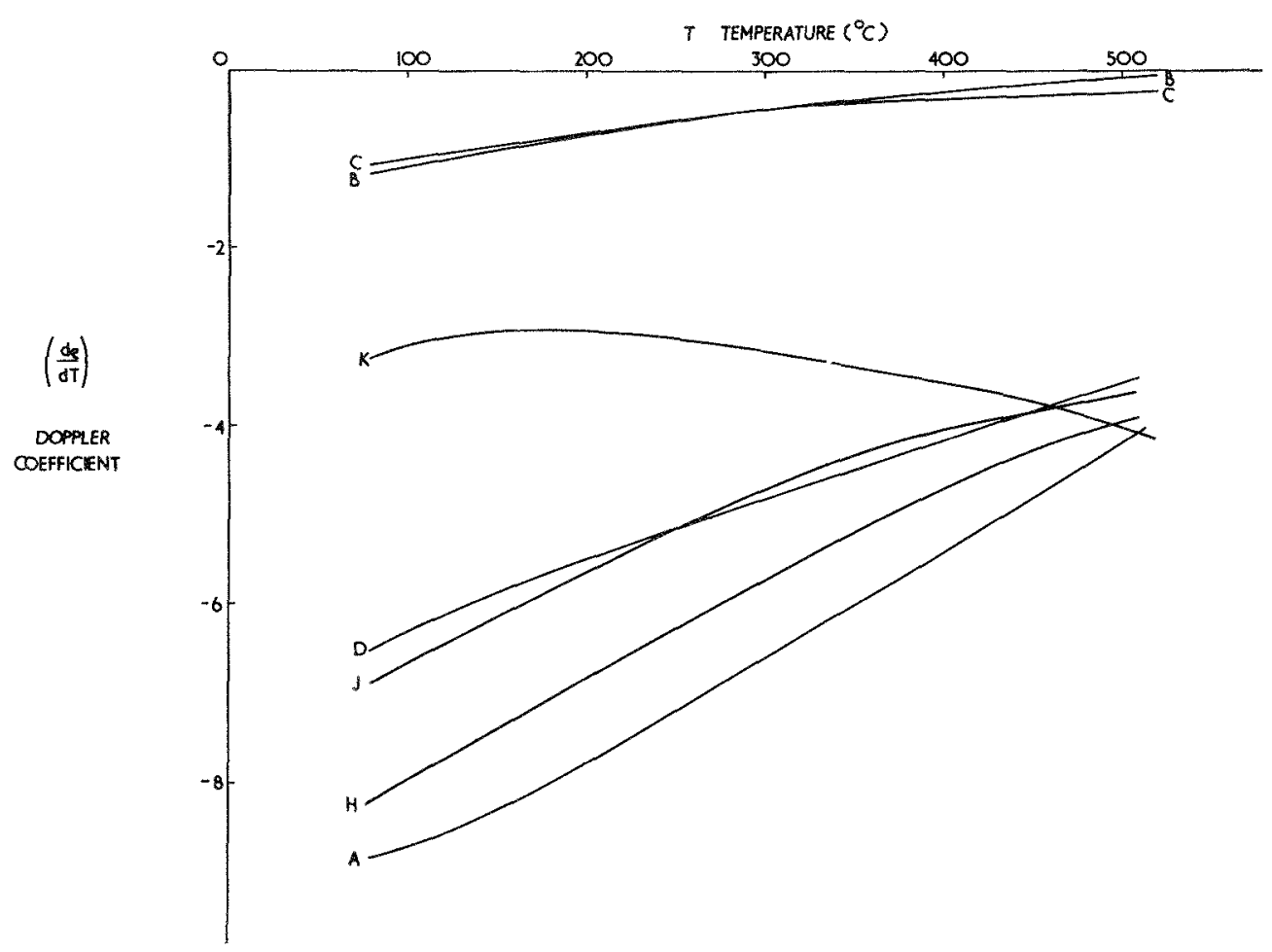

FIG. 8. Measured Doppler Coefficients as a Function of Temperature.

the column from the core centre-plane. For fuel pins where the column was symmetrical about the centreplane, $C_{4}$ was zero. For steel and aluminum pins (and for a sodium column near ambient) the columns were nearly symmetric about the centre-plane, and the expression (3) could be simplified to

$$
C_{4}=\alpha l_{3}\left(l_{1}-2 l_{3}\right)\left(\frac{d^{2} W}{d l^{2}}\right)_{l_{3}}
$$

In general the values of $C_{4}$ were much less than those found for $C_{1}, C_{2}$, and $C_{3}$.

The expansion effect of the inner walls of the loop in contact with the pins was allowed for by use of the expressions

$$
\begin{aligned}
& C_{5}=-\alpha k_{1} W_{2} ; \\
& C_{6}=-\alpha k_{2} R\left(d W_{2} / d r\right)_{R},
\end{aligned}
$$

where $C_{5}$ and $C_{6}$ represent the axial and radial contributions, respectively. Here $W_{2}$ is the worth of a long steel sample $(0.32$ by 17.5 by $150 \mathrm{~cm})$ which was measured close to the inner wall of the loop (of 0.34-cm thickness), with the lower edge at the core centre-plane.

A scaling factor $k_{1}$ was found which allowed for the difference in mass of the sample and the innermost wall of the loop, and allowed also for the change in geometry of the walls in the region beneath the pin base-plate. A device for moving the steel sample radially enabled values for $(d W / d r)$ to be measured. The effective radius of the test zone is given by $R$, and $k_{2}$ is a sealing factor similar to $k_{1}$. A small correction term was also applied to both $C_{5}$ and $C_{6}$ to allow for the expansion of the second steel wall, which had a mass about one-half of the first wall, and ran at about one-quarter of the temperature rise of the first wall.

Originally, it had been supposed that because the core had been designed so that both the spectrum and flux were approximately constant radially across the loop and inner buffer, the contribution $C_{6}$ due to radial movement of the walls of the loop would be small or negligible. In fact it was found that $C_{6}$ was an important correction term. Several experiments were carried out to understand the mechanism of this reactivity change, which is now believed to be due to changes in the number of neutrons streaming up the loop. Accurate determination of the magnitude of $C_{6}$ proved to be difficult, and except for the sodium expansion term, this effect was the largest source of error in the corrections and thus in the Doppler coefficients.

Small coefficients were also estimated for the expansion effects of the Santocell insulant and of the nitrogen gas. The reactivity worth of a large sample $(4 \mathrm{~kg})$ of the former was measured in the pin zone, and a reactivity coefficient estimated assuming the effective thermal expansion coefficient to be that of steel. The coefficient associated with the nitrogen was estimated from meas- 
Doppler Coefficients in Zebra Core 5

TABLE 5. Breakdown OF FXPansion Corrections $\left(\times 10^{-7} /{ }^{\circ} \mathrm{C}\right)$

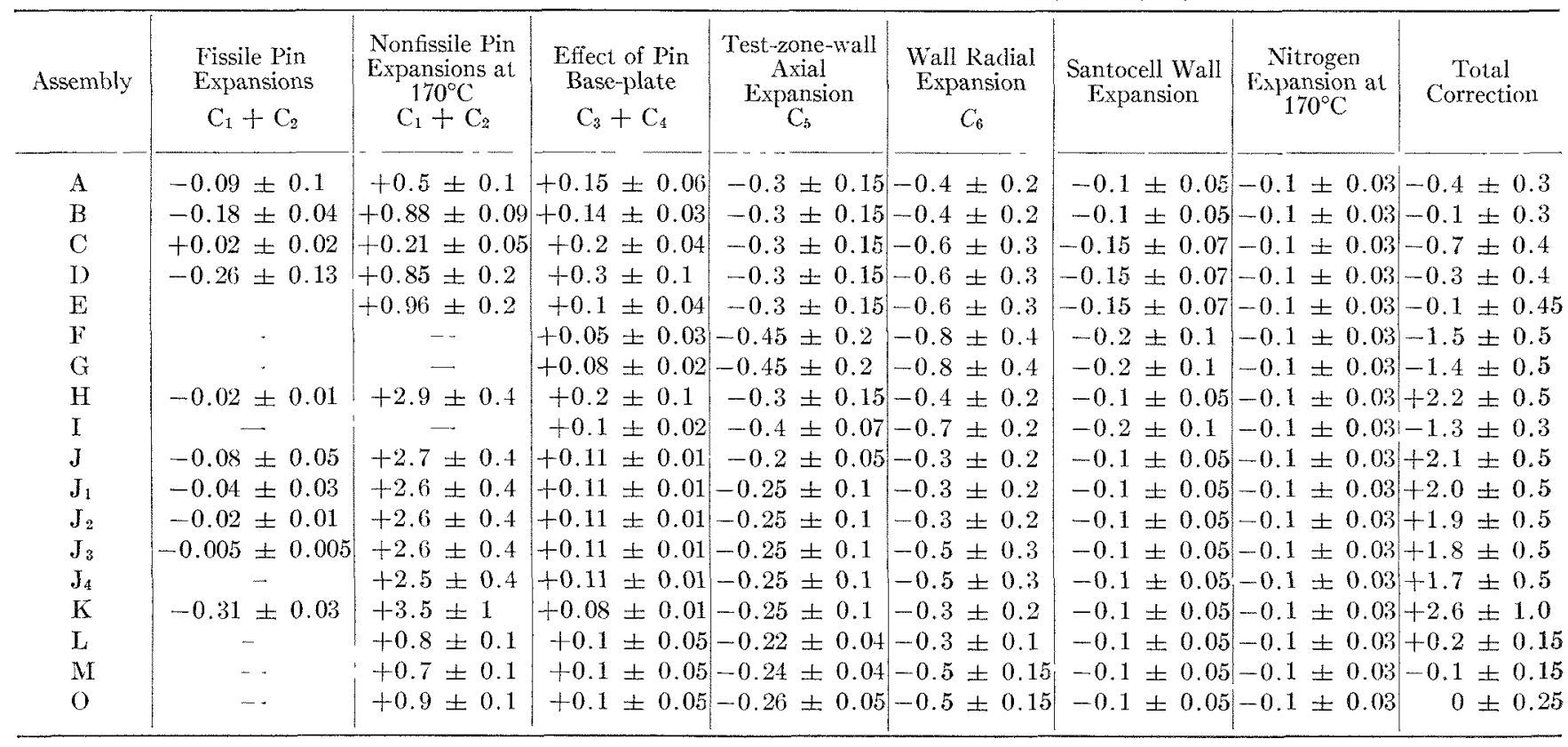

urements at varying loop pressure at constant temperature, and also from a series of reactivity worth measurements with a $\mathrm{N}_{\mathrm{aN}} \mathrm{N}_{3}$ sample.

These corrections are summarised in Table .).

\section{Effect of Varying Test-zone Area}

A number of variants of assembly J were loaded in order to determine whether the measured Doppler roefficients were dependent on the width of the low density insulating zone between the buffer and test. zones. The sodium pins were left in the alternate positions of $J$, but the numbers of fuel and steel pins were replaced in stages by void pins, two rows at a time, starting with the outer rows first. At each stage until the final one, there was an inner pin region of reduced area whose composition was the same as in J. Finally, in $\mathrm{J}_{4}$, the test zone contained only sodium and void pins. After correction for expansion effects, the Doppler coefficient was found to be an approximately linear function of the mass of fuel in the zone (see Fig. 9 below). It was concluded that the width of the gap between the test and buffer zones was not important.

\section{Discussion of Results}

The measured temperature coefficients together with the corrected Doppler coefficients are shown in Table 6. The corrections are relatively small for the cases of the two assemblies with $\mathrm{U}^{238}$ and without sodium $(\Lambda$ and $\mathrm{D})$, but they are important for all other assemblies. In particular, there is a large expansion correction which is necessary for each assembly containing sodium, which arises because of the high thermal expansion coefficient of sodium. In each case the error of the Doppler coefficient is attributed mainly to the errors inherent in the derivation of the corrections, rather than to the errors associated with the measurements of the temperature coefficients (see Fig. 6).

The results with empty cores ( $F, G$, and I) show small residual negative coefficients which are only slightly larger than the errors. It is concluded that the method of deriving the corrections for structure expansions is at least approximately correct. It is, however, possible that there are unexplained expansion mechanisms in the loop (not accounted for above) whose effects are of the order of $-0.5 \times 10^{-7} /{ }^{\circ} \mathrm{C}$, and this must be taken into account when discussing the results of the fueled assemblies. (A comprehensive search was made for fucl-element movements and temperature rises in the buffer and driver zones, but nothing was found which might account for such a coefficient.) With the test zonc loaded mainly with sodium $[\mathrm{J}(4)]$ or aluminum $(O)$, barely significant, small residual negative coefficients were observed. With steel, however, the residual coefficients become more significantly negative ( $\mathrm{F}, \mathrm{L}$, and $\mathrm{M}$ ), and the results show some evidence of a small negative Doppler coefficient for steel. (These residual coefficients are plotted as a function of steel loading in Fig. 10).

The positive coefficients calculated for the plutonium assemblies $B, C$, and $K$ were not observed. The evidence in $\mathrm{B}$ and $\mathrm{C}$ is for a near-zero or even a slightly negative plutonium contribution. The result from $\mathrm{K}$ is anomalous and shows a significant negative effect, even allowing for the large errors associated with the sodium 


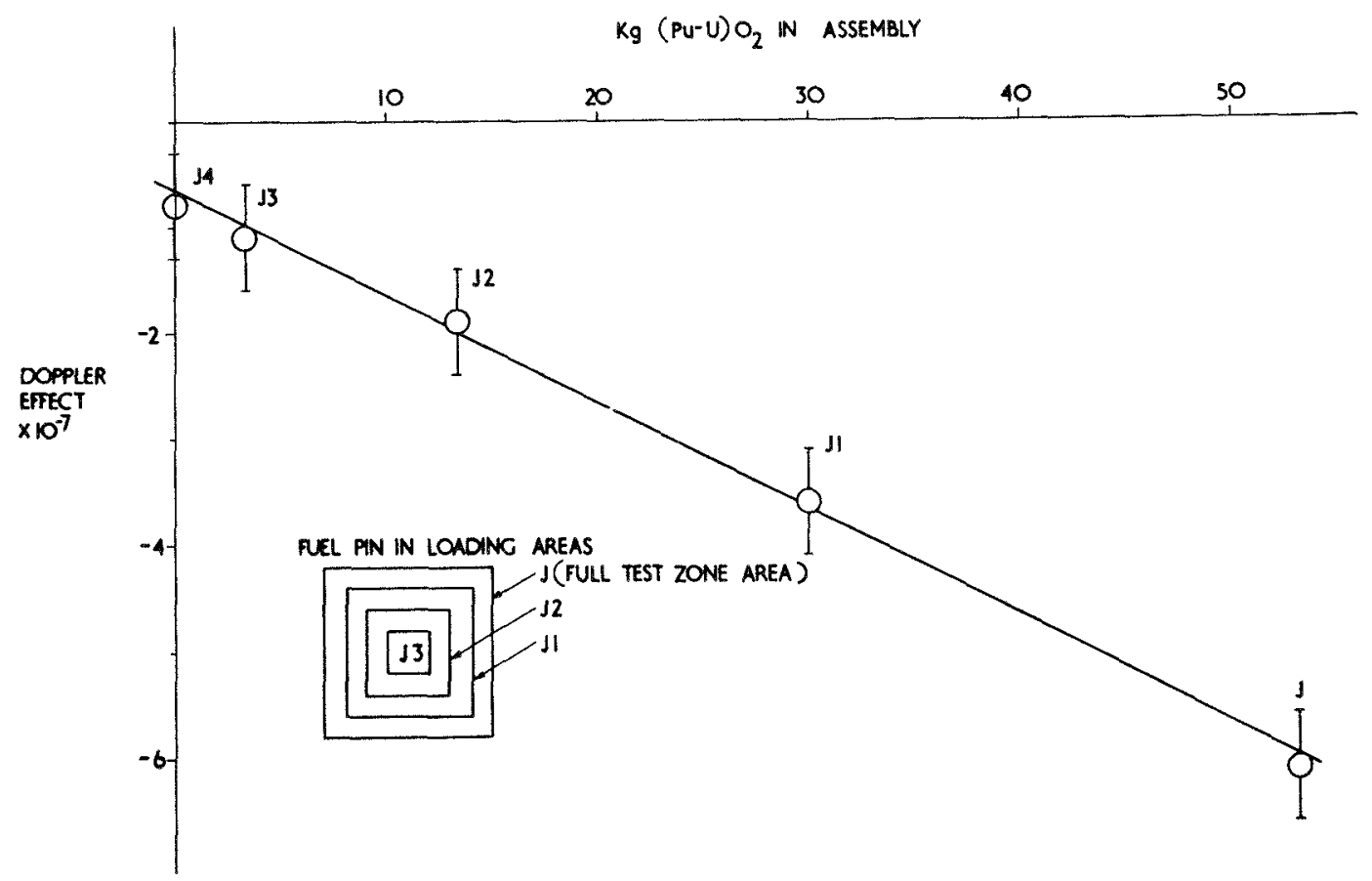

FIG. 9. Dependence of Doppler Effect on Test Zone Area.

TABLE 6. COMParison OF Results $\left(\times 10^{-7} /{ }^{\circ} \mathrm{C}\right)$

\begin{tabular}{l|c|c|c|c|c|c|c|}
\hline Assembly & Brief Description of & $\begin{array}{c}\text { Measured } \\
\text { Temperature } \\
\text { Assembly } \\
\text { (Uncorrient at 170 }\end{array}$ & $\begin{array}{c}\text { Corrected } \\
\text { Temperature } \\
\text { Coefficient } \\
\text { (Doppler Eftect) }\end{array}$ & $\begin{array}{c}\text { Hansen and } \\
\text { Roach }\end{array}$ & FD1 & FD2 \\
\hline
\end{tabular}

(1) Assemblies with Mixed Oxide (A) Pins $\left(16 \% \mathrm{PuO}_{2}, 84 \% \mathrm{U}^{8} \mathrm{O}_{2}\right)$

\begin{tabular}{|c|c|c|c|c|c|c|}
\hline $\begin{array}{l}A \\
D \\
H \\
J\end{array}$ & $\begin{array}{cl}\text { A pins } & +\mathrm{Fe} \\
، & + \text { Void } \\
" & +\mathrm{Na} \\
& +\mathrm{Na}+\mathrm{Fe}\end{array}$ & $\begin{array}{l}-7.7 \pm 0.07 \\
-6.0 \pm 0.1 \\
-5.3 \pm 0.1 \\
-4.0 \pm 0.1\end{array}$ & $\begin{array}{l}-8.1 \pm 0.3 \\
-5.7 \pm 0.4 \\
-7.5 \pm 0.6 \\
-6.1 \pm 0.5\end{array}$ & $\begin{array}{l}-6.3 \\
-5.0 \\
-5.7 \\
-4.5\end{array}$ & $\begin{array}{l}-3.5 \\
-2.8 \\
-4.3 \\
-3.7\end{array}$ & $\begin{array}{l}-6.6 \\
-4.6 \\
-6.3 \\
-5.0\end{array}$ \\
\hline \multicolumn{7}{|c|}{ (2) Assemblies with $\mathrm{PuO}_{2}$ without $\mathrm{U}^{238}$} \\
\hline $\begin{array}{l}\mathrm{B} \\
\mathrm{C} \\
\mathrm{K}\end{array}$ & $\begin{aligned} \mathrm{B} \text { pins } & +\mathrm{Fe} \\
6 & +\mathrm{Void} \\
\mathrm{D} \text { pins } & +\mathrm{Na}+\mathrm{Fe}\end{aligned}$ & $\begin{array}{l}-0.9 \pm 0.1 \\
-1.5 \pm 0.1 \\
-0.3 \pm 0.05\end{array}$ & $\begin{array}{l}-0.8 \pm 0.3 \\
-0.8 \pm 0.4 \\
-2.9 \pm 1.0\end{array}$ & $\begin{array}{l}+1.5 \\
+1.1 \\
+1.4\end{array}$ & $\begin{array}{l}+1.5 \\
+1.0 \\
+\end{array}$ & $\begin{array}{l}+2.8 \\
+1.8 \\
+3.0\end{array}$ \\
\hline \multicolumn{7}{|c|}{ (3) Assemblies with Nonfissile Pins Only } \\
\hline $\begin{array}{l}\mathrm{E} \\
\mathrm{L} \\
\mathrm{M} \\
\mathrm{J}(4) \\
\mathrm{O}\end{array}$ & $\begin{array}{llll}\text { half loading of } & \mathrm{Fe} \\
\text { full } & \text { " } & \text { " } \\
\text { half } & \text { " } & \text { " } \\
\text { half } & \text { " } & \mathrm{Na} \\
\text { full } & \text { " } & \mathrm{Al}\end{array}$ & $\begin{array}{l}-1.4 \pm 0.05 \\
-1.5 \pm 0.1 \\
-1.1 \pm 0.1 \\
+1.2 \pm 0.1 \\
-0.3 \pm 0.1\end{array}$ & $\begin{array}{l}-1.3 \pm 0.5 \\
-1.7 \pm 0.2 \\
-1.0 \pm 0.2 \\
-0.5 \pm 0.5 \\
-0.3 \pm 0.3\end{array}$ & $\begin{array}{l}0 \\
0 \\
0 \\
0 \\
0\end{array}$ & $\begin{array}{l}0 \\
0 \\
0 \\
0 \\
0\end{array}$ & $\begin{array}{l}0 \\
0 \\
0 \\
0 \\
0\end{array}$ \\
\hline \multicolumn{7}{|c|}{ (4) Assemblies without Pins } \\
\hline $\begin{array}{l}F \\
G \\
I\end{array}$ & - & $\begin{array}{r}-2.35 \pm 0.05 \\
-2.3 \pm 0.05 \\
-1.75 \pm 0.05\end{array}$ & $\begin{array}{l}-0.8 \pm 0.6 \\
-0.9 \pm 0.6 \\
-0.4 \pm 0.3\end{array}$ & $\begin{array}{l}0 \\
0 \\
0\end{array}$ & $\begin{array}{l}0 \\
0 \\
0\end{array}$ & $\begin{array}{l}0 \\
0 \\
0\end{array}$ \\
\hline
\end{tabular}


expansions. The reason for this result is not yet under stood.

Three calculations are included in the comparison of

table 7. Calcllated Central Neutron Spectra ${ }^{(a)}$

\begin{tabular}{c|r|r|r|r|r|r|r|r}
\hline \multirow{2}{*}{ Group } & \multirow{2}{*}{$E_{L}, \mathrm{keV}$} & \multicolumn{7}{|c}{ Assembly } \\
\cline { 3 - 9 } & & $\mathrm{A}$ & $\mathrm{B}$ & $\mathrm{C}$ & $\mathrm{D}$ & $\mathrm{H}$ & $\mathrm{J}$ & $\mathrm{K}$ \\
\hline 1 & 3000 & 279 & 281 & 364 & 353 & 338 & 294 & 299 \\
2 & 1400 & 727 & 745 & 938 & 896 & 847 & 760 & 775 \\
3 & 900 & 599 & 688 & 742 & 645 & 586 & 566 & 633 \\
4 & 400 & 1809 & 1943 & 1961 & 1857 & 1671 & 1645 & 1707 \\
5 & 100 & 3312 & 3280 & 3182 & 3248 & 3244 & 3265 & 3191 \\
6 & 17 & 2184 & 2125 & 1962 & 2031 & 2136 & 2195 & 2119 \\
7 & 3 & 741 & 648 & 623 & 695 & 795 & 828 & 776 \\
8 & 0.55 & 284 & 238 & 199 & 234 & 316 & 357 & 376 \\
9 & 0.10 & 55 & 41 & 26 & 36 & 58 & 76 & 90 \\
10 & 0.03 & 10 & 10 & 4 & 4 & 8 & 13 & 33 \\
$11-16$ & - & 0 & 0 & 0 & 0 & 0 & 0 & 1 \\
\hline Totals & & 10000 & 10000 & 10000 & 10000 & 10000 & 10000 & 10000 \\
\hline
\end{tabular}

(a) Using Hansen and Roach cross-section data (LAMS 2543) and the CRAM diffusion-theory programme. results. These are based, respectively, on fluxes and adjoint fluxes calculated using the Hansen and Roach set; the FD1 set, which is a Winfrith extension to the YOMI set; and the FD2 set, which is based on the UKAEA nuclear-data library. The calculations were carried out in one dimension by use of the CRAM diffusion-theory programme. The method of calculating the Doppler coefficient using perturbation theory and allowing for the heterogeneity of the pins was described by Smith and Rowlands, ${ }^{(1)}$ and more details are given in another paper at this conference. ${ }^{(2)}$

The calculations were carried out for the range 300 $600^{\circ} \mathrm{K}$. In order to compare them with experiment, the Doppler coefficients are quoted in Table 6 at $170^{\circ} \mathrm{C}$, this being the weighted mean temperature for this range if it is assumed that the Doppler coefficients are inversely proportional to temperature. The three calculations tend to underestimate the negative coefficients moasured for the mixed oxide assemblies $\mathrm{A}, \mathrm{D}, \mathrm{H}$, and $\mathrm{J}$ by between $15 \%$ and $50 \%$. However, the results show that the method of estimating the $\mathrm{U}^{238}$ contribution to the Doppler effect is soundly based.

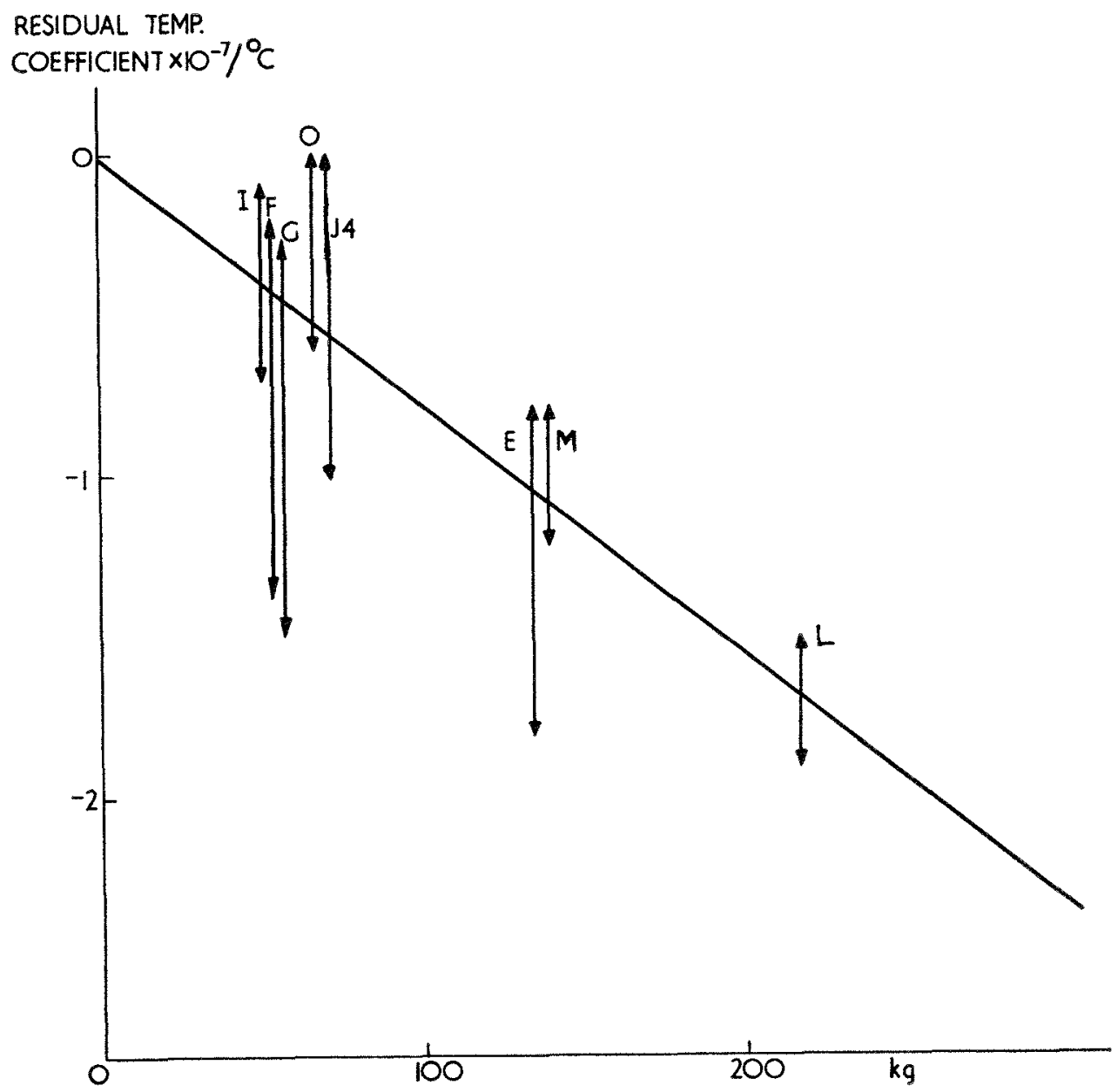

FIG. 10. Residual Temperature Coefficient as a Function of Mass of Heated Steel in Pin Region. 


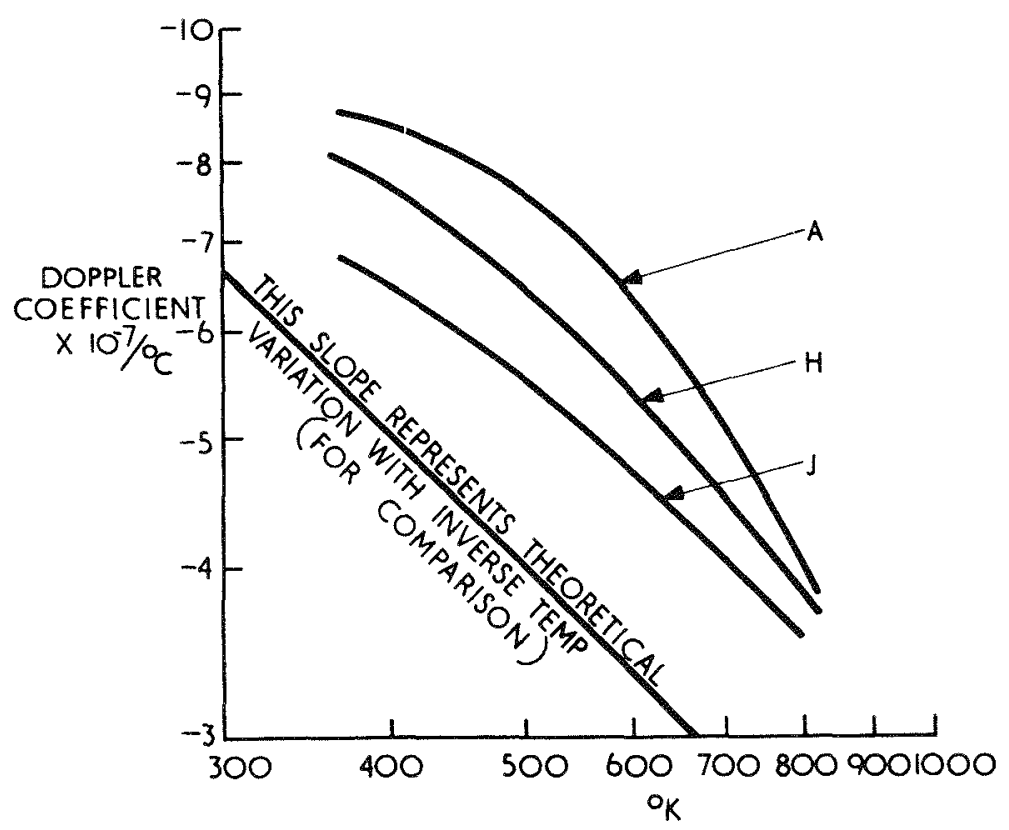

FI*. 11. Temperature Dependence of Measured Doppler Coefficients.

The results also confirm that the Doppler effect varies roughly with inverse temperature, which is the usual assumption made when extrapolating the effect up to the temperature range of a power reactor. The Doppler cocfficients when plotted logarithmically against the absolute temperature (see Fig. 11) can be seen to vary approximately as $T$ to the power ' $a$ ' $\left(T^{a}\right)$, where for

assembly $\mathrm{A}, \quad a=-1.0 \pm 0.2$;

for assembly $\mathrm{H}, \quad a=-1.0 \pm 0.1$

and for assembly $\mathrm{J}, a=-0.9 \pm 0.1$

over the range of temperature ronsidered.

\section{Acknowledgments}

The authors would like to thank all members of the Zebra team who operated the reactor or assisted with the experiments; and, in particular, Mr. R. MI. Absalom who participated in the experimental work of the Doppler experiment; Mr. C. R. Patterson who performed the computer calculations; Mr. G. Ingram who performed the perturbation experiments upon which the expansion corrections are based; and Mr. A. S. Belcher who assisted with the processing of the data. They would also like to thank Mr. J. Codd and Mr. J. L. Rowlands for advice on the theory of the Doppler effect, and Mr. G. Gunnill and his team who were responsible for the engineering design of the loop.

\section{REFERENCES}

1. D. C. G. Smith and J. L. Rowlands, The calculation of reacivity and temperature changes following a disturbance in reactor conditions, Paper presented at ANS meeting on Fast Reactor Technology, April 1965.

2. J. L. Rowlands and D. Wardleworth, The interpretation of Doppler and sodium reactivity worth measurements in zero power reactors, see next paper.

\section{Discussion}

Mr. Kato (ANL): Do you plan to continue this series of heated-zone experiments in which you might vary the surrounding core compositions and also use different, test elements?

Mr. Wheeler: We haven't any definite plans at the moment, but we can put this loop back at fairly short notice. Although we are not doing any loop experiments in the present assembly, we may very well do so in the future.

Mr. Engelmann (Karlsruhe): It seems to me that you introduce quite an uncertainty due to the expansion of sodium. Did you even consider the use of sodium in a compound? We are planning such experiments at Karlsruhe, and we were thinking of using a lead-sodium alloy which has a higher melting point and a relatively low expansion at these temperatures.

Mr. Wheeler: It is quite true that we lost a lot of accuracy in the experiments with sodium, and we might well consider something as you suggest for another occasion. But the advantages of having sodium in was, I think, that we showed there was nothing special about the presence of sodium which made the calculations worse.

Mr. Yiftah (Israel AEC): Has any attempt been made to analyze the negatively measured and positively 
ralculated Doppler effect of plutonium, a phenomenon somewhat similar to the negative Doppler effect of uranium-23j measured at Atomies International a couple of years ago?

Mr. Wheeler: I think possibly Mr. Codd may be able to say something about this in the next paper.

IMr. Pitterle (APDA): George Collin at Los Alamos has been advocating a slightly different distribution of resonance parameters for $\mathrm{Pu}^{239}$. His belief is that the $J=0$ sequence is a sequence of very broad resonances with average fission width between 1 or perhaps $2 \mathrm{eV}$, and the $J=1$ sequence for $S$ waves being much smaller fission widths as well as following the $\nu=1$ distribution of fission widths. We have made some Doppler calculations for plutonium under these conditions, and we find our results less by about a factor of 2 than what we calculated for the test cases of Okrent. The $\alpha$ values are very strongly dependent in this case because $80 \%$ or more of the Doppler effect is from the $J=1$ sequence. If these were high, as some people indicated, although trying to fit parameters to these $\alpha$ values does not seem reasonable, it could well be negative.

$M$. Fischer (ANL): I would like to ask what fission widths were used for the $J=1$ and 0 states.

Mr. Pitterle: We have tried a number of different combinations. We assume the average fission width for the $J=0$ sequence to be about $1 \mathrm{eV}$. Then we attempt to fit group-dependent $\sigma_{f}$ and $\alpha$ to obtain both the neutron widths and the fission width for the $J=1$ sequence. At the moment the values for $\sigma_{f}$ and $\alpha$ that we are using are the UK values above a keV and the Schmidt values below. The energy range is from 10
keV to $100 \mathrm{eV}$ with a values varying uniformly from about 0.5 to 0.75 over this energy range. This fitting procedure yields $J=1$ fission widths varying from 0.06 to $0.09 \mathrm{eV}$ and strength eunctions varying from 0.8 to $1 \times 10^{-4}$. We have also carried out another case which is in agreement with the feelings of George Cowan and Ilans Bethe. That is to assume that most of the lowenergy resonances that have been resolved are $J=1$ resonances and that many of the very broad $J=0$ resonances have not been resolved. If we take the recommended values in the recent $\mathrm{BNL}$ supplement and throw out the one plutonium resonance which has a very broad width, we obtain an average fission width of about 70 millivolts for the $J=1$ sequence. We then assumed a strength function of $1 \times 10^{-4}$ and extrapolated the low-energy fission width of 70 millivolts exponentially with an increase of a factor of e in 100 $\mathrm{keV}$. This procedure yields a Doppler coefficient within less than $20 \%$ of that obtained by the fitting procedure, although the parameters and resulting $\sigma_{f}$ and $\alpha$ values are significantly different. The $J=0$ sequence is dominantly a fission contribution, and this contribution is sensitive to the strength function. An increase in the strength function requires a smaller fission width for the $J=1$ sequence to obtain a given $\alpha$ value. It is significant that the contribution of the $J=0$ sequence to the Doppler effect is not negligible. For example, at $300^{\circ} \mathrm{K}$ this sequence contributes about $10 \%$ of the Doppler effect, while at $1500^{\circ} \mathrm{K}$ it contributes about $20 \%$ of the Doppler effect. This is berause the Doppler coefficient of this sequence does not decrease as rapidly with temperature as the $J=1$ sequence. 


\title{
The Interpretation of Doppler and Sodium Reactivity Worth Measurements in Zero Power Reactors, with Particular Reference to the Zebra Doppler Loop Measurements
}

\author{
J. L. Rowlands and D. Wardleworth \\ Atomic Energy Authority \\ Winfrith, United Kingdom \\ (PRHSENTED BY J. CODD)
}

\section{Introduction}

The kinetic behaviour of large sodium-cooled fast reactors is dominated by the Doppler and sodium reactivity coefficients. Unfortunately, the accurate calculation of these coefficients is one of the most difficult problems in the neutron physics of fast reactors, and many uncertainties in both the data and the methods of calculation remain, in particular, in the methods of interpreting measurements in zero-power reactors.

In order to reduce the uncertainty in the Doppler effect, a series of measurements was made recently in the Zebra Zero Power Reactor by heating the central part of the core to $550^{\circ} \mathrm{C}$. (1) The extrapolation of the results obtained from this experiment involves the treatment of the heterogeneity of the test zone and the effect of its finite size. The methods and approximations used in analysing the results are discussed below.

The nuclear data for plutonium are still of less than the desired accuracy, and in this paper a study is made of the effect on the Doppler coefficient from $\mathrm{Pu}^{239}$ of the present uncertainties in the data from $0.1 \mathrm{keV}$ to $10 \mathrm{keV}$, and, in particular, in the resonance parameter data in the unresolved resonance region.

Useful experimental data on the sodium coefficient has been reported from a series of experiments with ZPR-VI Assemblies 2 and 3. Calculations have been made to allow a comparison with these measurements using the standard methods of calculation, and some of the approximations involved in these methods are examined.

\section{Interpretation of Doppler Effect Measurements}

\subsection{The Effect of Data Uncertainties on the Doppler Coefficient of $P u^{239}$}

Recent measurements at Harwell ${ }^{(2)}$ and Saclay ${ }^{(3)}$ of $\mathrm{Pu}^{239}$ cross sections in the resonance region have shown the existence of very broad resonances, with widths of up to about $6 \mathrm{eV}$. The present measurements cover only part of the resonance region from which the Doppler effect arises in fast reactors, and the way in which the resonance data are to be extrapolated into the unresolved region is uncertain. However, it has been thought worthwhile to make Doppler coefficient calculations for $\mathrm{Pu}^{239}$ on the basis of the recent Harwell measurements to see how broad resonances affect the coefficient.

Uttley $^{(2)}$ has made a single-level analysis of the resonances in the total cross section of $\mathrm{Pu}^{239}$ from 100 to $300 \mathrm{eV}$ and has obtained values of the resonance energy, the total width $\Gamma, \sigma_{0} \mathrm{I}$, and $g \Gamma_{n}$ (where $g$ is the statistical spin factor and $\sigma_{0}$ the peak cross section). He also finds a background cross section of $3.6 \mathrm{~b}$ in the interval from 100 to $200 \mathrm{eV}$, and of $7 \mathrm{~b}$ in the interval from 200 to $300 \mathrm{eV}$, which is unaccounted for in the single-level analysis of the data and which it is presumed corresponds to very broad levels.

Calculations have been made using Uttley's resonance parameters by assuming the resonances to form a single sequence with $g=1 / 2$. A constant capture width of $\Gamma_{c}=40 \mathrm{meV}$ was assumed, which is the mean obtained by Story ${ }^{(4)}$ from an analysis of Bollinger's data. ${ }^{(5)}$ For extrapolation of the fission width distribution above $300 \mathrm{eV}$ the resonances were divided into six approximately equal groups and the mean value of $\Gamma_{f}$ for each group found and rounded off. The six values of $\mathrm{T}_{f}$ used were $2, \tilde{\mathrm{n}} 20,300,100,45,25$, and $10 \mathrm{meV}$, the mean value of $\Gamma_{f}$ being $500 \mathrm{meV}$. In each energy group in the unresolved region the cross section was represented by the 24 resonances formed by taking these six values of $\Gamma_{f}$ and four values of $\Gamma_{n}$ (sampled from a Porter-Thomas distribution), calculated at the mid-lethargy of the group. The strength function assumed was $2.3 \times 10^{-4}$ (twice the usual value, because of the assumption of a single resonance sequence with $g=1 / 2$ ). The background cross section was allowed for by increasing the fission cross section calculated from the resonance parameters by one third. (The mean value of the observed background, $5 \mathrm{~b}$, is about a quarter of the mean fission cross section.) The potential scattering cross section was taken to be $10.3 \mathrm{~b}$.

Effective group cross sections have been calculated 
for seven half-lethargy groups spanning the range from $101 \mathrm{eV}$ to $3.36 \mathrm{keV}$ by means of the code ERIC $2,{ }^{(6)}$ which treats the resonances as isolated. The infinitedilution fission and capture cross sections and the cross sections at $300^{\circ} \mathrm{K}$ and $600^{\circ} \mathrm{K}$ for environment background cross sections of 10,100 and $400 \mathrm{~b}$ have been calculated and are given in Tables 1 and 2 .

The infinite-dilution cross sections are given in Table 1. The fission cross section in group 16 is about twice the value recommended in the evaluation of Douglas and Barry, ${ }^{(7)}$ but the value in group 22 is not incon-

TABle 1. $\mathrm{Pu}^{239}$ Cross Sections Calculated From the Assumed Resonance Parameters (Infinite Dilutron VALUES)

\begin{tabular}{c|c|r|r|r|r}
\hline Group & $\begin{array}{c}\text { Lower } \\
\text { Energy, } \mathrm{eV}\end{array}$ & $\sigma_{f}, \mathrm{~b}$ & $\sigma_{c}, \mathrm{~b}$ & $\alpha$ & \multicolumn{1}{|c|}{$\sigma_{a}, \mathrm{~b}$} \\
\cline { 2 - 4 } & 3360 & & & & \\
\hline 16 & 2040 & 5.47 & 1.80 & 0.329 & 7.27 \\
17 & 1230 & 7.39 & 2.56 & 0.346 & 9.95 \\
18 & 748 & 9.93 & 3.60 & 0.363 & 13.53 \\
19 & 454 & 13.24 & 5.00 & 0.378 & 18.24 \\
20 & 275 & 17.61 & 7.30 & 0.415 & 24.91 \\
21 & 167 & 18.96 & 7.95 & 0.419 & 26.91 \\
22 & 101 & 24.97 & 12.61 & 0.505 & 37.58 \\
\hline
\end{tabular}

sistent with current data. The values of $\alpha$ in all groups are about $30-40 \%$ lower than the values recommended by Froelich, Ott, and Schmidt. ${ }^{(8)}$ Uttley $^{(9)}$ has made an analysis based on preliminary results of James' $\mathrm{Pu}^{239}$ fission cross section and his own total-cross-section measurements which gives an $\alpha$ of about 1.2 in the range from $600 \mathrm{eV}$ to $2 \mathrm{keV}$. This corresponds with a dip in the mean fission cross section, and so the compound elastic scattering is uncertain. The total absorption cross section is not inconsistent with Uttley's estimates when a curve which varies monotonically with energy is drawn through his mean values in $100-\mathrm{eV}$ and $1-\mathrm{keV}$ intervals. (The average values in some intervals deviate by about a factor of two from this monotonic curve.) Neglecting the constant background fission cross section would increase $\alpha$ by a factor $4 / 3$ and so make it more consistent with the data of Froelich, Ott, and Schmidt.

In Table 2 the fission and capture cross sections at $300^{\circ} \mathrm{K}$ and the difference between the fission and capture cross sections at $600^{\circ} \mathrm{K}$ and $300^{\circ} \mathrm{K}, \delta \sigma_{f}$ and $\delta \sigma_{c}$, are tabulated. The values are given for three $\sigma_{B}$ values (the environment background cross section per atom of the resonant absorber ) : $\sigma_{B}=400 \mathrm{~b}$, a value which is typical of a dilute fast reactor; $\sigma_{B}=100 \mathrm{~b}$, and $\sigma_{B}=10 \mathrm{~b}$, a value appropriate to a sample of $\mathrm{Pu}^{239}$ metal of about $1-\mathrm{cm}$

TABLe 2. Pu ${ }^{239}$ Cross Sections at $300^{\circ} \mathrm{K}$ and the Difference between Cross Sections at $600^{\circ} \mathrm{K}$ and $300^{\circ} \mathrm{K}$

\begin{tabular}{l|c|c|c|c|c|c|c|c|c|c|c}
\hline Group & $\sigma_{f}, \mathrm{~b}$ & $\sigma_{c}, \mathrm{~b}$ & $\alpha$ & $\sigma_{a}, \mathrm{~b}$ & $\delta \sigma_{f}, \mathrm{~b}$ & $\delta \sigma_{c}, \mathrm{~b}$ & $\alpha^{\prime}$ & $\alpha^{\prime} / \alpha$ \\
\hline
\end{tabular}

C. $\sigma_{B}=10 \mathrm{~b}$

\begin{tabular}{|c|c|c|c|c|c|c|c|c|}
\hline 16 & 4.71 & 1.32 & 0.28 & 0.26 & 0.15 & 0.60 & 2.14 & 0.25 \\
\hline 17 & 4.99 & 1.63 & 0.27 & 0.37 & 0.23 & 0.63 & 2.29 & 0.28 \\
\hline 18 & 7.52 & 1.93 & 0.26 & 0.49 & 0.32 & 0.65 & 2.53 & 0.31 \\
\hline 19 & 9.38 & 2.23 & 0.24 & 0.59 & 0.39 & 0.66 & 2.79 & 0.34 \\
\hline 21 & 12.16 & 2.76 & 0.23 & 0.75 & 0.54 & 0.72 & 3.15 & 0.40 \\
\hline 22 & 14.65 & 3.44 & 0.24 & 0.75 & 0.55 & 0.74 & 3.13 & 0.40 \\
\hline
\end{tabular}


TABLE 3. RATHO OF THE U238 CAPTURE AND B ${ }^{10}$ ABsorption Cross Sections to the Pu ${ }^{239}$ Absorption Cross SECTION AT $300^{\circ} \mathrm{K}$ FOR $\sigma_{B}=400 \mathrm{~b}$

\begin{tabular}{c|c|c|c|c}
\hline Group & $\sigma_{c}^{\mathrm{U} 238}(\mathrm{a}), \mathrm{b}$ & $\sigma_{a}^{\mathrm{B} 10}, \mathrm{~b}$ & $\sigma_{c}^{\mathrm{U} 238} / \sigma_{a}^{\mathrm{Pu}^{239}}$ & $\sigma_{a}^{\mathrm{B} 10} / \sigma_{a}^{\mathrm{Pu}^{239}}$ \\
\cline { 1 - 2 } & 0.6 & 12.2 & 0.084 & 1.7 \\
\cline { 1 - 2 } 16 & 0.9 & 15.7 & 0.094 & 1.6 \\
18 & 1.0 & 20.3 & 0.078 & 1.6 \\
20 & 1.0 & 33.0 & 0.046 & 1.5 \\
21 & 1.9 & 42.3 & 0.081 & 1.8 \\
22 & 1.7 & 54.3 & 0.057 & 1.8 \\
\hline
\end{tabular}

(a) These values are the shielded values appropriate to a dilute fast reactor.

radius. Also tabulated are the values of $\alpha$ and $\alpha^{\prime}=$ $\delta \sigma_{c} / \delta \sigma_{f}$. For a narrow distribution of fission widths $\alpha^{\prime} / \alpha$ is close to unity, increasing as the distribution of fission widths increases relative to the distribution of capture widths (here assumed to be constant). For the distribution of fission widths used in these calculations $\alpha^{\prime} / \alpha$ lies approximately in the range from 2 to 3 . Even if the constant background fission were neglected, $\alpha^{\prime} / \alpha$ would lie in the range from 1.5 to 2.3 .

\subsection{Compositions in which the Doppler Coefficient of $P u^{239}$ Might be Negative}

Some guidance about the compositions of $\mathrm{Pu}^{239}$ which might have a negative Doppler effect can be obtained by the following simple considerations. The Doppler effect for $\mathrm{Pu}^{239}$ is proportional to

$$
\begin{aligned}
\nu \delta \sigma_{f}-\left(\delta \sigma_{f}+\right. & \left.\delta \sigma_{c}\right) \phi^{*} \\
& =\nu \delta \sigma_{f}\left[1-\left(1+\alpha^{\prime}\right)\left(\phi^{*} / \nu\right)\right],
\end{aligned}
$$

where $\phi^{*}$ is the importance of low-energy neutrons relative to the importance of fission neutrons. This importance is the probability of a neutron causing fission, times $\nu$. If we neglect the variation of $\phi^{*}$ with energy at low energies, this is

$$
\begin{aligned}
\phi^{*}=\frac{N_{\mathrm{g}} \nu \sigma_{f}}{N_{9}\left(\sigma_{f}+\sigma_{c}\right)+\Sigma a,} & \text { rem }+B^{2} D \\
& =\frac{\nu}{(1+\alpha)(1+Y)},
\end{aligned}
$$

where

$$
Y=\frac{\Sigma a, \text { rem }+B^{2} D}{N_{9}\left(\sigma_{f}+\sigma_{c}\right)}=\frac{\sigma_{\mathrm{rem}}}{\sigma_{f}+\sigma_{c}}
$$

is the ratio of the absorption in the remaining constituents plus the leakage, to the absorption in $\mathrm{Pu}^{239}$ (in the resonance region).

The Doppler effect is therefore proportional to

$$
(1+Y)-\frac{1+\alpha^{\prime}}{1+\alpha}=Y-\frac{\alpha^{\prime}-\alpha}{1+\alpha}=Y-F .
$$

The quantity $F=\left(\alpha^{\prime}-\alpha\right) /(1+\alpha)$ is also tabulated in Table 2. The Doppler coefficient is negative when $F$ is larger than $Y$ (or, alternatively, when $\alpha^{\prime} / \alpha$ is larger than $\left.\sigma_{\mathrm{rem}} / \sigma_{c}\right)$.

If the present representation of the resonances gives a correct estimate of $F$, then the Doppler coefficient of $\mathrm{Pu}^{239}$ will be negative when the ratio of the leakage plus absorption in other constituents to the absorption in $\mathrm{Pu}^{239}$ is less than about 0.35 , on the average, through the Doppler energy region (100 to $2,000 \mathrm{eV}$ ).

In Table 3 the $\mathrm{U}^{238}$ capture cross section (shielded appropriately to a dilute fast reactor) and the $\mathrm{B}^{10}$ absorption cross section are given. Also given are the ratios of these to the calculated $\mathrm{Pu}^{239}$ absorption cross section. For a core in which the leakage and absorption in the Doppler region in isotopes other than $\mathrm{Pu}^{239}$ and $\mathrm{U}^{238}$ are small, the $\mathrm{Pu}^{239}$ Doppler coefficient would be zero for a ratio of $\mathrm{U}^{238}$ to $\mathrm{Pu}^{239}$ of about 5 , according to the above analysis. This is a typical value for a large dilute fast reactor. It would be negative for smaller ratios of $\mathrm{U}^{238}$ to $\mathrm{Pu}^{239}$ and positive for larger values. In the Zebra Doppler Loop U-PuO 2 pins (A), the ratio is about 6 , and so the above analysis would be expected to give a small positive $\mathrm{Pu}^{239}$ Doppler coefficient for these. The Zebra Doppler Loop Pins (B) contain a ratio of $\mathrm{B}^{10}$ to $\mathrm{Pu}^{239}$ of 0.5 , and so these would be expected to have a positive $\mathrm{Pu}^{239}$ coefficient. The $\mathrm{PuO}_{2}$ pins in Core $5 \mathrm{~K}$ are diluted with steel and sodium, and so the $\mathrm{Pu}^{239}$ coefficient would be expected to be negative.

The variation of neutron importance with energy also contributes to the Doppler effect; a neutron importance which increases towards lower energies makes a positive contribution to the Doppler coefficient because the increase in the absorption cross section with temperature moves the mean absorption to a higher neutron cnergy. An a value which increases towards lower energies tends to make the Doppler coefficient slightly more negative than the above simple treatment would suggest, but the variation of $\alpha$ with energy in the Doppler region is uncertain.

It seems possible for the Doppler effect of a small sample of $\mathrm{Pu}^{239}$ to be positive in a core which has a negative $\mathrm{Pu}^{239}$ coefficient. This is because $\alpha^{\prime}$ is smaller for the small value of $\sigma_{B}$ appropriate to a sample of metal or oxide. The $F$ value calculated from the $\alpha^{\prime}$ value corresponding to $\sigma_{B}=10 \mathrm{~b}$ and the $\alpha$ value corresponding to $\sigma_{B}=400 \mathrm{~b}$ is about 0.23 , compared with the value of 0.35 obtained with the $\alpha^{\prime}$ for $\sigma_{B}=$ $400 \mathrm{~b}$. However, the sample may perturb the neutron importance, and first-flight collisions in the sample may increase the importance of fission neutrons.

Since the extrapolation of Uttley's resonance data is inconsistent with estimates of $\alpha$, the sign of the $\mathrm{Pu}^{239}$ Doppler coefficient in fast reactors must be regarded 
as uncertain. If the distribution of fission widths is approximately the same as that assumed here, the ratio $\alpha^{\prime} / \alpha$ would remain approximately the same and $F$ would increase with increase in $\alpha$, leading to less positive $\mathrm{Pu}^{239}$ Doppler coefficients. If, however, the higher values of $\alpha$ correspond to a reduction in the constant background fission and the number of broad resonances, F will decrease.

\subsection{I'u Doppler Coefficients in a Sodium-cooled Reactor and in the Doppler loop}

These cross sections have been used in reactivity calculations for a large sodium-cooled fast reactor and for two of the Zebra Doppler Loop compositions. The $\mathrm{Pu}^{239}$ Doppler coefficient obtained for the large fast reactor was $+7 \times 10^{-7} \delta \mathrm{k} /{ }^{\circ} \mathrm{C}$ (the mean value for the range from $300^{\circ} \mathrm{K}$ to $600^{\circ} \mathrm{K}$ ) compared with the value $+7 \times 10^{-6} \mathrm{\delta} /{ }^{\circ} \mathrm{C}$ obtained using a mean fission width of $\Gamma_{f}=78 \mathrm{meV}$ and a narrow distribution. The reactor was a two-zone core with a $\mathrm{U}^{-238} / \mathrm{Pu}^{239}$ ratio of $6.03 \mathrm{in}$ the inner core, and of 4.21 in the outer core, the plutonium containing $17 \% \mathrm{Pu}^{240}$

For the I-Pu oxide pins in the Zebra Doppler Loop the absorption in other isotopes, relative to that in $\mathrm{Pu}^{239}$, is least in Core $5 \mathrm{D}$, which contains no diluent pins. The mean $\mathrm{Pu}^{239}$ coefficient obtained using tho cross sections in Table $2 \mathrm{~A}\left(\sigma_{B}=400 \mathrm{~b}\right)$ was $2.4 \times$ $10^{-8} \delta \mathrm{K} /{ }^{\circ} \mathrm{C}$ compared with the value $12 \times 10^{-8} \delta \mathrm{k} /{ }^{\circ} \mathrm{C}$ obtained using the narrow fission width distribution and $\sigma_{s} \simeq 200 \mathrm{~b}$, and the value $-5.8 \times 10^{-8} \delta \mathrm{k} /{ }^{\circ} \mathrm{C}$ obtained for the $\mathrm{U}^{238}$ coefficient. Assuming a $1 / \sigma_{s}$ dependence for the $\mathrm{Pu}^{239}$ coefficient in this range $\left(\sigma_{s}=\right.$ $\sigma_{B}+\sigma_{p}$, and $\sigma_{p}=10.3 \mathrm{~b}$ ) gives for a $\sigma_{s}$ value of $200 \mathrm{~b}$, $5 \times 10^{-8} \delta \mathrm{k} /{ }^{\circ} \mathrm{C}$ for the cross sections in Table 2 , compared with $12 \times 10^{-8} \delta k^{\circ} \mathrm{C}$ for the narrow width distribution.

For the plutonium pins, the value was also $2.4 \times$ $10^{-8} \delta k /{ }^{\circ} \mathrm{C}$ (for $\sigma_{B}=400 \mathrm{~b}$ ) compared with the value $18 \times 10^{-8} \delta \mathrm{k} /{ }^{\circ} \mathrm{C}$ (for $\sigma_{s} \simeq 200 \mathrm{~b}$ ) for the narrow fission width distribution. Allowing for the differences in $\sigma_{s}$ this is a reduction to one quarter.

\subsection{The Effect of Heterogeneity and Sample Size on the Doppler Effect}

For the interpretation of the Zebra Doppler Loop experiment, and of measurements of the Doppler effect of small samples, the effects of heterogeneity and sample size have been allowed for by using effective cross sections for equivalent homogeneous media. To obtain this equivalence the spacemaveraged flux in the homogeneous region containing the heated resonant absorber must be expressed in the form

$$
\begin{aligned}
\bar{\phi}_{0}(E) & =1 /\left[S+\aleph_{0}(E)\right] \\
& =1 / N\left[\sigma_{s}+\sigma_{\text {ess }}(E)\right],
\end{aligned}
$$

where $\Sigma_{0}(E)$ is the total macroscopic cross section in this region, $S$ is a constant, $N$ is the atomic density of the resonant absorber in the region, $\sigma_{s}$ the effective barkground cross section, and $\sigma_{\text {res }}$ the resonant aross section.

The effective rross section for the resonant absorber averaged over the energy dependence of the flux in the region is then the same as that for an infinite homogeneous medium which has a macroscopic cross section (for scattering out of the resonances) larger than that of the matcrial in the region by an amount $S$. Creebler and Goldman ${ }^{(10)}$ have called this increase in the effective background cross section, $N \sigma_{\text {tseape }}$.

Two methods have been used to obtain $\sigma_{\text {seape. }}$. The first is to obtain the constant $S$ from a rational fit to the collision probability which is appropriate to a cross section in the wings of a resonance. In doing this the methods of Bell ${ }^{(1)}$ and Rothenstein ${ }^{(12)}$ are followed. The second is to obtain the mean flux in the matcrial for two values of the resonant cross section and to assume a linear variation of the reciprocal of the flux. These methods are not as accurate as those of Otter ${ }^{(13)}$ and Kelber, ${ }^{(14)}$ who average the parameters through resonances, but they are expected to give values of $\sigma_{\text {esoape }}$ which are accurate to about $\pm .50 \%$. The main uncertainty probably arises from approximating the complex geometry by two uniform homogencous regions.

The effert of the heterogeneity on the group-averaged flux has not been treated in the Zebra calculations, the effertive cross section for the resonant region being taken to be the value appropriate to the cell-averaged flux.

\subsection{Derivation of $\sigma_{\text {eseape }}$ for an Isolated Specimen and a Regular Lattice}

In the case of a two-homogeneous-region problem, where region 0 contains the resonant absorber and region 1 contains nonresonant material, the mean flux in region 0 can be written in the form (2.4) if the collision probability $P_{00}$ can be written in the form

$$
1 /\left(1-P_{00}\right)=1+K \Sigma_{0}
$$

where $K$ is approximately independent of $\Sigma_{0}$. (Here $P_{i j}$ is the probability that a neutron originating in region $i$ will make its next collision in region $j$.) It then follows that $\sigma_{\text {escape }}=1 / N K$. It is well known that $P_{00}$ can be approximated in this form for isolated regions of resonant material and for regular lattices. For an isolated region $K=C l_{0}$ where $C$ is of the order of unity, $l_{0}$ being the mean chord in the region $4 V_{0} / S$, and so 
concerned, the insulation to a large extent isolates the test zone and buffer region spertra. At the top and the bottom of the zone the insulation will be greater and so it is a reasonable approximation to take $\sigma_{\text {seap }} \simeq$ $1 / l_{z} N$. This expression has been derived for a homogeneous zone. In the heterogeneous case, the effect of the finite zone size is to increase $G$ (the probability that a neutron leaving the fuel region will make its next collision in the moderator without first entering a fuel region ).

The probability of a neutron entering a fuel cell is reduced by a constant amount (on average), depending on the size of the zone. This reduction can be treated by increasing the effective size of the fuel cell, by increasing $l_{1} \Sigma_{1}$ to that the correct value for $\sigma_{\epsilon \text { scape }}$ is obtained in the limit as $l_{0}$ and $l_{1} \rightarrow 0$ :

$$
\begin{aligned}
\sigma_{\text {t scape }} & =\frac{l_{1} \Sigma_{1}+\left(l_{0} / l_{z} f\right)}{N_{0} l_{0}\left[1+E\left(l_{1} \Sigma_{1}\right)+\left(l_{0} / l_{z} f\right)\right]} \\
& =1+E\left(l_{1} \Sigma_{1}+N_{0} l_{0} \sigma_{z}\right)
\end{aligned}
$$

where $f$ is the fuel volume fraction and $\sigma_{z}=1 / N_{0} f l_{s}$.

The fuel pins in the test zone have a hoight of $(8.56$ ( $\mathrm{m}$ and the cross section is a square of width $18.2 \mathrm{~cm}$; thus $l_{z}=16 \mathrm{~cm}$. The finite size of the test zone is equivalent in the calculation of the effective background cross section $\sigma_{s}$, to increasing the mean macroscopic (ross section by $1 / l_{z}=0.0625 \mathrm{~cm}^{-1}$. This is $40 \%$ of the mean macroscopic cross section rorresponding to the U-Pu oxide pins (Set A) and $20 \%$ of the value for the Pu pins (Set B) (in the absence of diluent pins).

\subsection{Values of the Effective Background ('ross Section, $\sigma_{s}$, for $U^{238}$ and Pu ${ }^{239}$ in the Zebra Doppler Loop}

We may summarize the results of the previous two sections. Approximate values for the effective background cross sections appropriate for computing the resonance shielding factors and Doppler coefficients for $\mathrm{U}^{238}$ and $\mathrm{Pu}^{239}$ in the Zebra Doppler Loop are given in Table 7. In deriving these, the background cross sections have been assumed to be independent of energy,

'TABle 7. Approximate Valies of $\sigma_{q}$, the EFfective Background Cross Section (in barns)

\begin{tabular}{l|c|c|c|c|c}
\hline \multicolumn{1}{c|}{ Pins } & Component & $\begin{array}{c}\text { Homoge- } \\
\text { neous }\end{array}$ & $\begin{array}{c}\text { Heteroge- } \\
\text { neous }\end{array}$ & $\begin{array}{c}\text { Finite } \\
\text { Zone Size }\end{array}$ & Total \\
\hline $\mathrm{A}$ & $\mathrm{U}^{238}$ & 26.6 & 26.3 & 9.5 & 36 \\
$\mathrm{~A}+\mathrm{SS}$ & $\mathrm{Pu}^{239}$ & 148 & 147 & 51 & 198 \\
& $\mathrm{U}^{238}$ & 858 & 61.1 & 65 & 68 \\
$\mathrm{~B}$ & $\mathrm{Pu}^{239}$ & 477 & 339 & 35 & 374 \\
$\mathrm{~B}+\mathrm{SS}$ & $\mathrm{Pu}^{239}$ & 297 & 162 & 29 & 191 \\
& $\mathrm{Pu}^{239}$ & 637 & 220 & 19 & 239 \\
\hline
\end{tabular}

a cross section of 11 being assumed for steel atoms and of $3.7 \mathrm{~b}$ for oxygen, with a potential scattering cross section of $10 \mathrm{~b}$ for uranium and plutonium isotopes.

A single, chergy-independent value cannot be given for the "ase of sodium diluent pin because of the 3 -keV sodium resonance. The effect of heterogeneity and zone size are largest for $\mathrm{L}^{238}$ in the case of the $\mathrm{T}^{\top}-\mathrm{P}_{\mathrm{u}}$ pins in the absenere of diluent pins, when $\sigma_{s}$ is about $35 \%$ higher than the homogencous value.

The effects of heterogeneity and zone size are largest for $\mathrm{Pu}^{239}$ in the case of the plutonium pins with the steel diluent pins, when the $\sigma$, value is only $37 \%$ of the homogeneou value and has a large uncertainty because of the simplifications made in the calculation of the heterogeneity effect. The $P u^{239}$ Doppler cocfficient is about thrice the homogeneous value, with an unrertainty of a factor of about two arising because of the simplifications made in treating the heterogeneity.

\subsection{The Doppler Effect of a Small Specimen in a Core Containing the Same Resonant Absorber}

1 way of obtaining a value for $\sigma$, in this case is to ralculate the mean flux in the specimen, $\bar{\sigma}_{0}$, corresponding to the resonant absorber cross section between resonanees, $\phi_{n}$, and for a value within a resonance, $\phi$, and to assume a linear variation of $1 / \bar{\phi}_{0}$ with the resonant absorber cross scrtion between these two values. This gives

$$
\sigma_{\varsigma}=\frac{\phi_{r}-\sigma_{r}}{\phi_{n}-\phi_{r}}
$$

$\sigma /$, being the value of the resonant cross section for which $\phi_{r}$ was calculated.

Taking the flux in the specimen at energies between resonances to be the same as that in the core and assuming the narrow-resonance approximation gives

$$
\sigma_{\text {escape }}=1 / C_{0} l_{0} N
$$

for a specimen in a core containing no resonant absorber. The value of $C_{0}$ is the one corresponding to the specimen ross section within the resonance.

As an example of the estimation of $\sigma_{s}$ for a specimen in a homogeneous core containing the same resonant absorber as the specimen, a $\mathrm{U}^{23 b}$ metal specimen of density $N_{0}$ is considered in a homogeneous core containing $\mathrm{U}^{238}$ at density $N_{1}$. The remaining core constituents are assumed to be nonresonant and to have a macroscopic cross section $\Sigma_{m}$. The mean flux in the specimen is given by

$$
\Sigma_{0} \phi_{0}=S_{0} P_{00}+S_{1} P_{01}
$$

where $S_{0}$ is the neutron source density in the specimen and $S_{1}$ the source density in the core (these soure 
densities being assumed to be spatially ('onstant). Eliminating $P_{01}$ by using the reciprocity relationship

$$
\Sigma_{1} P_{01}=\Sigma_{0}\left(1-P_{00}\right)
$$

and writing

$$
\Sigma_{0}=N_{0}\left(\sigma_{p}+\sigma_{\mathrm{rss}}\right)
$$

and

$$
\Sigma_{1}=\Sigma_{m}+N_{1}\left(\sigma_{p}+\sigma_{\mathrm{res}}\right),
$$

where $\sigma_{p}$ is the potential scattering rross section of $\mathrm{U}^{238}$ and $\sigma_{\text {res }}$ the resonant cross section of $\mathrm{U}^{238}$, the mean flux becomes

$$
\bar{\sigma}_{0}=\frac{S_{0} P_{00}}{N_{0}\left(\sigma_{p}+\sigma_{\mathrm{res}}\right)}+\frac{S_{1}\left(1-P_{00}\right)}{\Sigma_{m}+\frac{N_{1}\left(\sigma_{p}+\sigma_{\mathrm{res}}\right)}{N_{0}}} .
$$

If the source densities in the resonances are taken to be the values corresponding to scattering from between resonances by a uniform flux in core and specimen, then

$$
S_{0}=N_{0} \sigma_{p} \phi_{n} ; \quad S_{1}=\left(\Sigma_{m}+N_{1} \sigma_{p}\right) \phi_{n}
$$

and

$$
\frac{\phi_{0}}{\phi_{n}}=\frac{P_{010}}{1+\frac{\sigma_{\text {res }}}{\sigma_{p}}}+\frac{1-P_{000}}{1+\frac{\sigma_{\text {res }}}{\sigma_{m}^{*}}}
$$

where

$$
\sigma_{m}^{*}=\frac{\Sigma_{m}}{N_{1}}+\sigma_{p} .
$$

Taking the values $\sigma_{p}=10 \mathrm{~b}$ and $\sigma_{m}^{*}=40 \mathrm{~b}$, a $\mathrm{U}^{238}$ density in the sperimen of $N_{0}=0.05 \times 10^{-24}$ atoms/ $\mathrm{cm}^{3}$, and a cylindrical rod of $1-\mathrm{cm}$ diameter gives $\sigma_{s}=17 \mathrm{~b}$ (for $\sigma_{r}=40 \mathrm{~b}$ ). The value given by this method for a $\mathrm{C}^{238}$,pecimen in a core which does not contain $\mathrm{U}^{238}$ is $\sigma_{s}=36 \mathrm{~b}$. The presence of the $\mathrm{U}^{238}$ in the core has a significant effect on the effective background cross section for $\mathrm{C}^{238}$ in the specimen.

Calculations of the $\mathrm{Pu}^{239}$ coefficient based on Uttley's resonance measurements in the energy range from 100 to $300 \mathrm{eV}$ still prediet positive values for the U-PuO, pins and the $\mathrm{Pu}$-boron pins, but the values are redured to a third or a quarter of the values given in Table 8 . It is expected that these data will predict a negative $\mathrm{Pu}^{239}$ coefficient for core $5 \mathrm{~K}$, which contained no $\mathrm{L}^{\mathrm{T} 338}$ or boron.

Calculations have also been made with use of the Hansen and Roach cross-section set ${ }^{(21)}$ and the FD1 set. Some calculations were made with a two-dimensional model, and the heterogeneous values of $\sigma_{s}$ were used in the Hansen and Roach calculations. These are

\begin{tabular}{|c|c|c|c|c|c|c|}
\hline A & A pins & $+\mathrm{Fe}$ & $-8.1 \pm 0.3$ & -8.3 & +1.7 & -6.6 \\
\hline I) & “ & + Void & $-5.7 \pm 0.4$ & -5.8 & +1.2 & -4.6 \\
\hline $\mathrm{H}$ & “" & $+\mathrm{Na}$ & $-7.5 \pm 0.6$ & -8.7 & +2.4 & -6.3 \\
\hline$J$ & "6 & $+\mathrm{Na}+\mathrm{Fe}$ & $-6.1 \pm 0.5$ & -6.5 & +1.5 & -5.0 \\
\hline
\end{tabular}
reported in the paper by Baker and Wheeler. ${ }^{(1)}$
TABLiti 8. Comparison of Measured and Calculated DOppler Coeffichents

\begin{tabular}{c|c|c|c|c|}
\hline $\begin{array}{c}\text { Assem- } \\
\text { bly }\end{array}$ & Composition & Measured & \multicolumn{2}{|c}{ Calculated } \\
\hline
\end{tabular}

(1) Assemblies with Mixed Oxide Pins: $16 \% \mathrm{PuO}_{2}$, and $84 \% \mathrm{UO}_{2}$ (Set A)

(2) Assemblies with $\mathrm{PuO}_{2}$ without $\mathrm{U}^{\mathrm{T} 238}$ (Sets $\mathrm{B}$ and $\mathrm{D}$ )

\begin{tabular}{l|l|l|r|r}
\hline $\mathrm{B}$ & $\mathrm{B}$ pins $+\mathrm{Fe}$ & $-0.8 \pm 0.3$ & +2.8 & +2.8 \\
$\mathrm{C}$ & " + Void & $-0.8 \pm 0.4$ & +1.8 & +1.8 \\
$\mathrm{~K}$ & $\mathrm{D}$ pins $+\mathrm{Na}+\mathrm{Fe}$ & $-2.9 \pm 0.8$ & +3.0 & +3.0 \\
\hline
\end{tabular}

2.10 Comparison of Doppler ('oefficients Calculated Ising the FDQ Set with the Values Measured in the Zebra Doppler Loop

Calculations have been made with the HD2 crosssection set ${ }^{(20)}$ for a homogeneous model of the Zebra Doppler Loop. Diffusion-thcory calculations were made for an infinite eylinder with an axial buckling, and the calculated Doppler coefficient was reduced by $15 \%$ to allow for the length of the fuel pins being less than the height of the core. Heterogeneity and test-zone size were allowed for by multiplying the calculated Doppler coefficients by a factor. This factor was the inverse ratio of the $\sigma_{s}$ values for the homogeneous and heterogeneous models for the $\mathrm{Pu}^{239}$ coefficient and the change was one-fifth of the percentage difference between these $\sigma_{s}$ values for the $\mathrm{I}^{238}$ coefficient. The resonance data used in the Doppler calculations were those described in the report on the FD1 set. ${ }^{(22)}$ The coefficient is the average value for the range from $300^{\circ} \mathrm{K}$ to $600^{\circ} \mathrm{K}$.

The $\mathrm{Pu}^{239}$ coefficient was calculated to be positive, whereas the measured value is estimated to be negative. If the $\mathrm{Pu}^{239}$ coefficient had been taken to be zero for the U-Pu oxide pins, the ealculated coefficient would be closer to the measured value, being a slight overestimate, whereas the calculated net coefficient is an underestimate.

\section{The Sodium Coefficient}

\subsection{Introduction}

The methods used at present in the UKAEA at Winfrith for the calculation of sodium loss and temperature coefficients for fast reactor design studies are 
based on diffusion theory and the multigroup (rosssection set FD2. ${ }^{(20)}$ The basic nuclear data used for the FD2 compilation are largely those in the UKAEA Nuclear Data Files, ${ }^{(28)}$ with the following two main. exceptions:

1. The sodium cross sections and the elastic removal cross sections for the main light diluents have been evaluated by means of the ANL progran ELMOE. ${ }^{(26)}$

2. Heavy-nuclide self-shielding factors are evalu-

TABLE 9. Composition of ZPR-VI Assembly 2

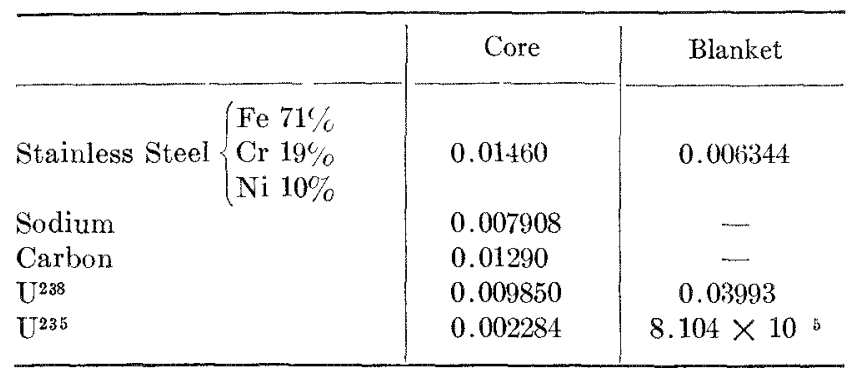

ated for each mixture by methods that, were used for the cross-section set FD1. ${ }^{(22)}$

The first systematic set of measurements of the sodium coefficient, in a zero-power facility were made in ZPR-VI. This section of the paper is a description of a series of calculations performed to check the bases of the Winfrith methods against the experimental measurements made in ZPR-VI Assembly 2.

\subsection{The ZPR-VI Assembly \& Sodium Coeflcient Meas- urements -Two-dimensional $(R Z)$ Calculations}

ZPR-VI Assembly 2 was a cylindrical, U ${ }^{235}$-fuelled assembly, with a core 36 in. in both height and diameter. The composition is given in Table 9; sodium is one of the main diluents.

Measurements of the sodium coefficient were made by removing sodium from various regions of the core and determining the ensuing reactivity change. A description of the core and of the experiments can be found in the ANL progress reports for 1964 (the sodium

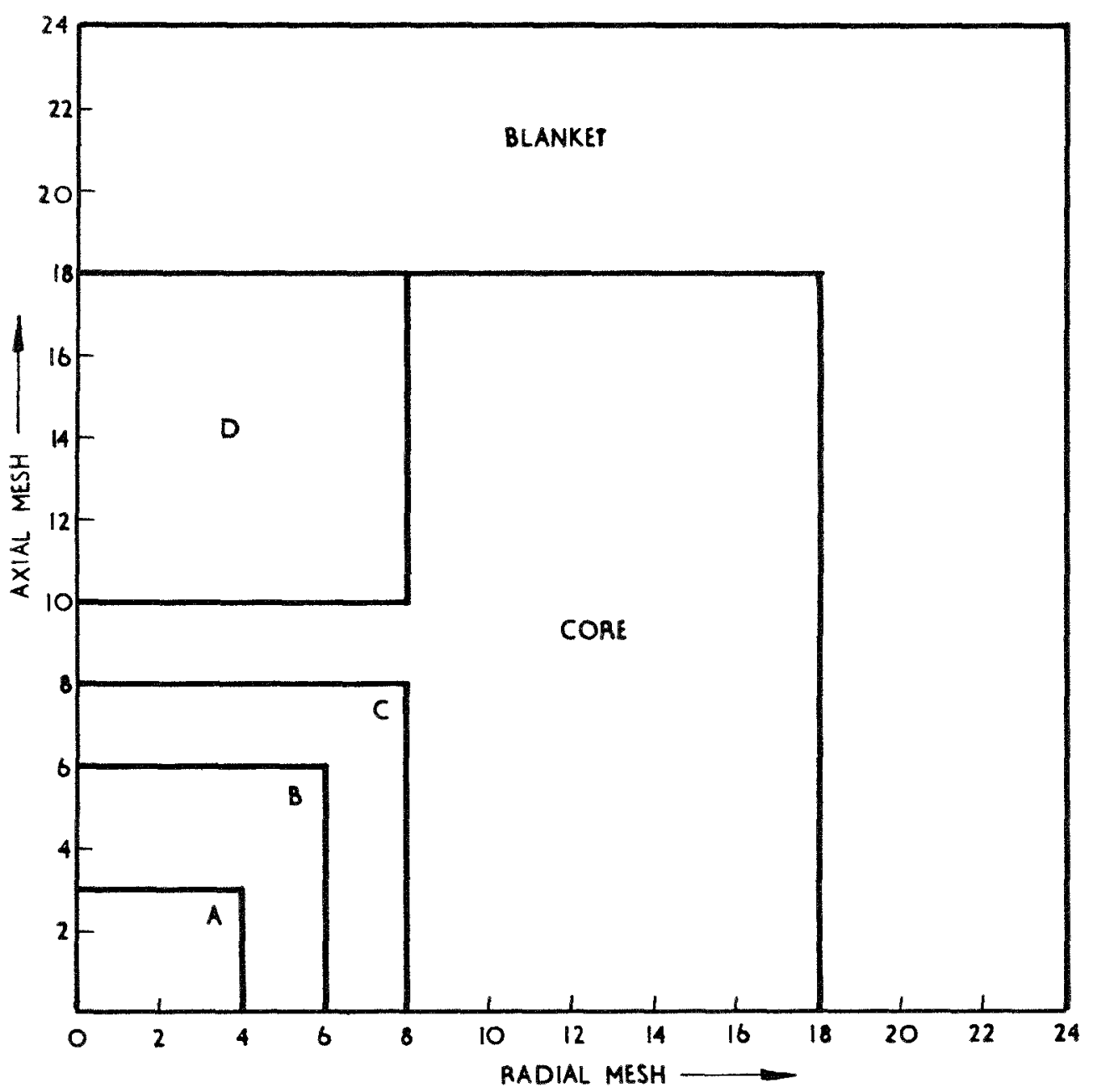

FIG. 1. Layout of Zones A, B, C, and D. 
coefficient measurements are reported in ANL-6904 ${ }^{(23)}$ ) and in a paper by Grifoni et al. ${ }^{(24)}$ Some modifications of the experimental results are made in this latter paper, which also reports one-dimensional slab and cylindrical diffusion-theory calculations.

Because of the importance of the sodium coefficient in fast reactor design and the suspected large error in the calculation of this paraneter, it is of obvious value to check the present calculation methods and crosssection data against this set of experiments. Twodimensional (RZ) diffusion-theory calculations (using the program CRAII $\left.{ }^{(25)}\right)$ were performed, using the Winfrith data sets FD1 and FD2, to obtain a normal and adjoint flux. The sodium coefficients for the experimental configurations were calculated by use of firstorder perturbation theory.

The layout of the experimental regions and their relationship to the calculation model is shown in Figs. 1,2 , and 3 . The nomenclature for the regions is that of ANL-6904, not that of Grifoni et al. The mesh spacing is given in Table 10; an even mesh was used between the points in the table. The adequacy of the mesh was checked by a series of 3 -group calculations, doubling and redoubling the standard $24 \times 24$ mesh.

Table 11 compares the coefficients from the $24 \times 24$, $48 \times 48$, and $96 \times 96$ calculations. The largest change in decreasing the mesh spacing by a fartor of 4 is 0.02 $\times 10^{-4} \delta k / k$ per $\mathrm{kg}$. In the standard calculation the first 24 groups of FD1 and FD2 were used, that is, the energy representation ceased at $37 \mathrm{eV}$, which is certainly adequate in this case. The normal flux at the centre in the 24th group is smaller by about six orders of magnitude than the peak flux in any group. A homogeneous model was used.

In FD1 and FD2 the sodium cross sections are defined as the change in system macroscopic cross sections per atom of sodium removed. This change was evaluated by use of ELMOE, and therefore the changes in the group cross sections for the light nuclides on removal of sodium are included. The sodium cross sections in the FD2 set were evaluated for a typical plutoniumfuelled fast power reactor and are therefore not entirely

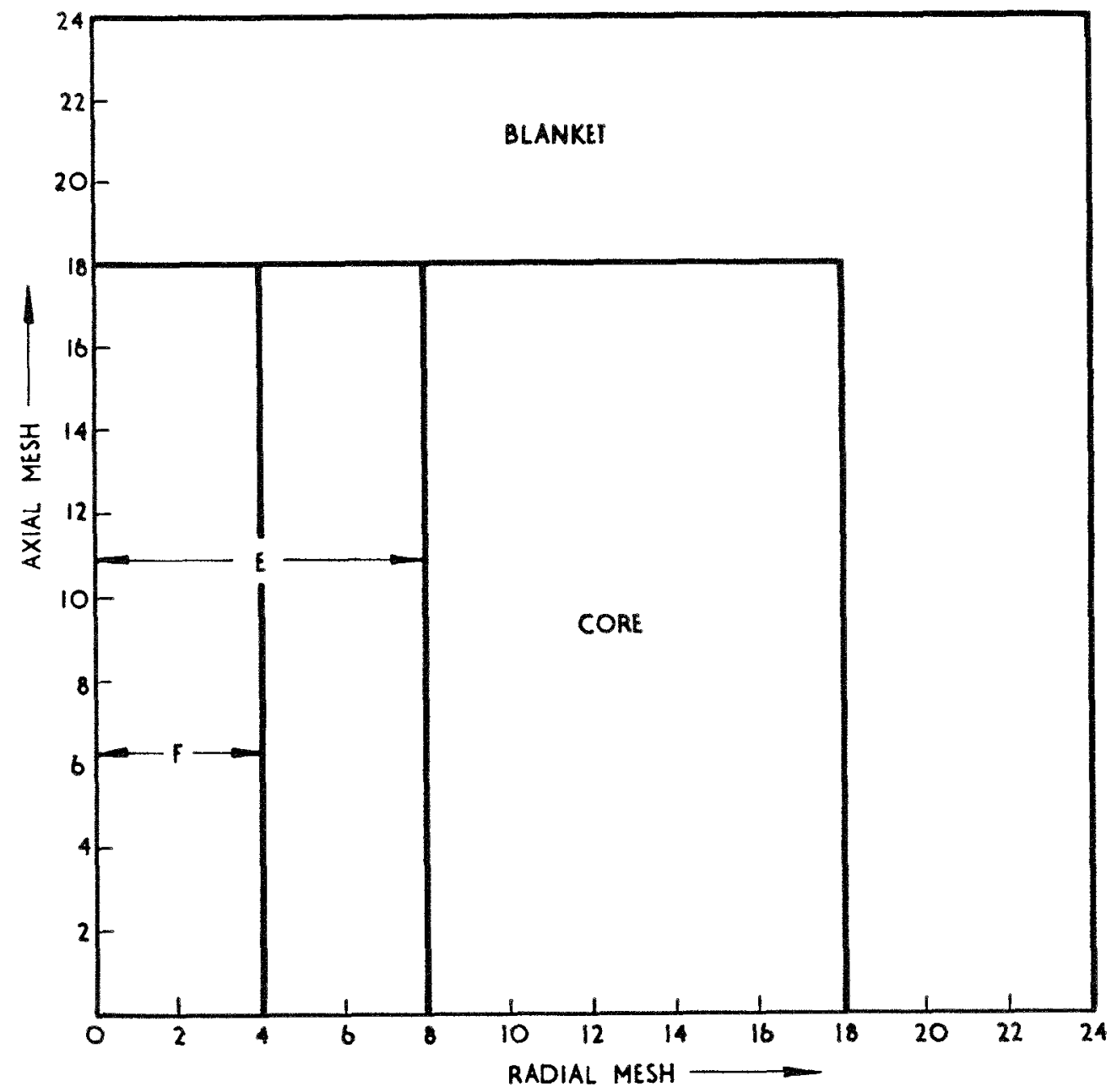

FIG. 2. Layout of Zones $E$ and F. 


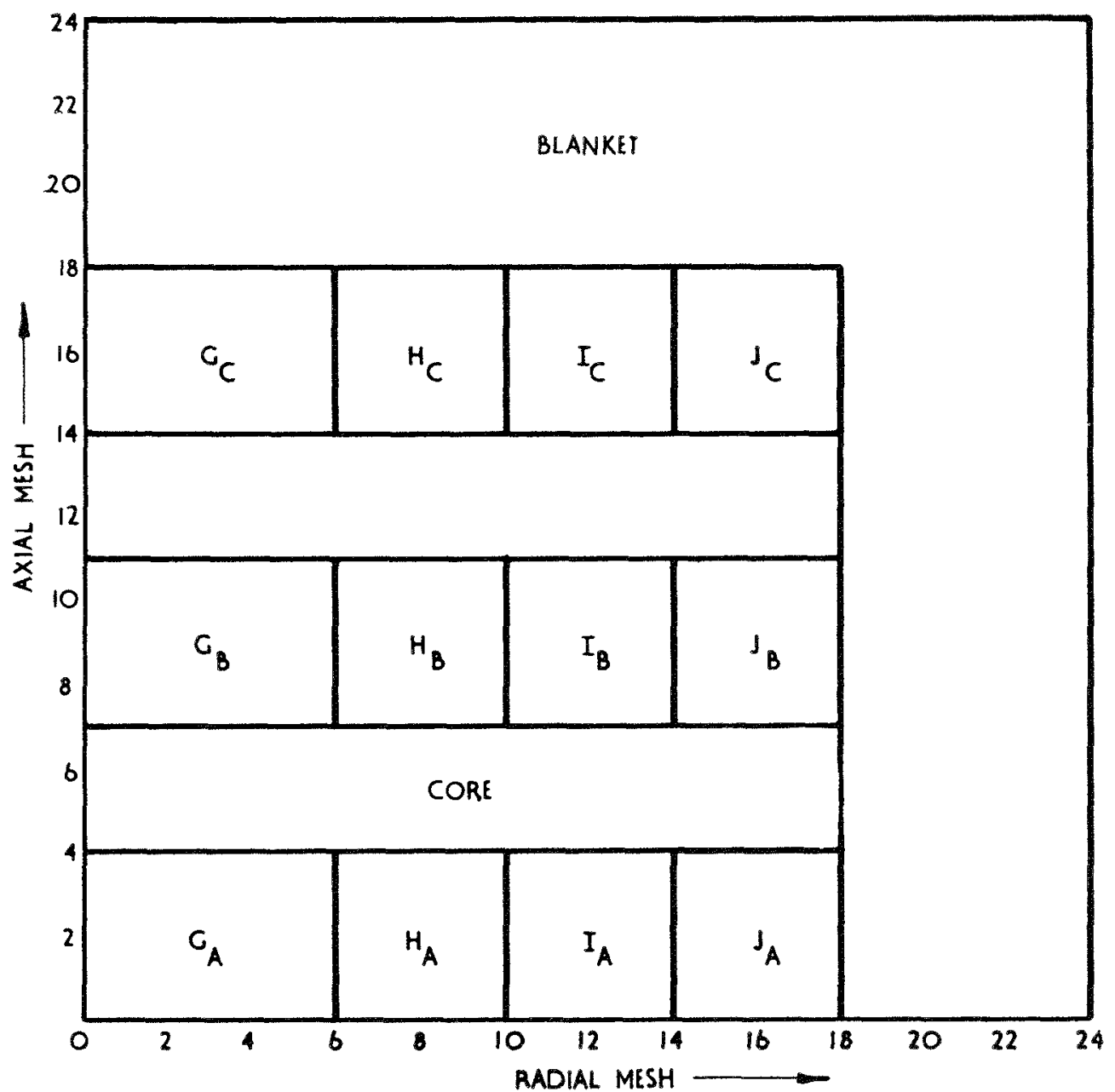

FIG. 3. Layout of Zones G through J.

TABLE 10 . THE $24 \times 24$ Mesh

\begin{tabular}{r|r|r|r}
\hline \multicolumn{2}{c|}{ Radial } & \multicolumn{2}{|c}{ Axial } \\
\hline Mesh Point & Radius (cm) & Mesh Point & $\begin{array}{c}\text { Height above } \\
\text { Centre-line (cm) }\end{array}$ \\
\hline 4 & 9.15 & 18 & 45.72 \\
6 & 14.03 & 24 & 75.72 \\
8 & 19.76 & & \\
10 & 24.70 & & \\
14 & 34.80 & & \\
18 & 46.15 & & \\
24 & 76.15 & & \\
\hline
\end{tabular}

applicable to this assembly. The corresponding cross sections for this assembly have been evaluated but have not yet been used in a two-dimensional calculation.

The FD1 and FD2 libraries in CRAM include a facility for automatic heavy-nuclide self-shielding according to a homogeneous model. The change in the
TABLE 11. The Effect of Degreased Mesh Spacing: 3 Groups $\left(\delta k / k\right.$ per $\left.\mathrm{kg} \times 10^{4}\right)$

\begin{tabular}{c|r|r|r}
\hline & $24 \times 24$ & $48 \times 48$ & $96 \times 96$ \\
\hline$A$ & -0.08 & -0.07 & -0.07 \\
$B$ & 0.05 & 0.07 & 0.07 \\
$C$ & 0.13 & 0.14 & 0.14 \\
$D$ & 0.89 & 0.90 & 0.90 \\
$E$ & 0.55 & 0.56 & 0.56 \\
$F$ & 0.44 & 0.46 & 0.46 \\
$G_{A}$ & 0.01 & 0.02 & 0.02 \\
$G_{B}$ & 0.46 & 0.48 & 0.48 \\
$G_{C}$ & 1.06 & 1.06 & 1.06 \\
$H_{A}$ & 0.38 & 0.40 & 0.40 \\
$H_{B}$ & 0.65 & 0.66 & 0.66 \\
$H_{C}$ & 0.97 & 0.97 & 0.97 \\
$I_{A}$ & 0.82 & 0.83 & 0.83 \\
$I_{B}$ & 0.85 & 0.86 & 0.86 \\
$I_{C}$ & 0.83 & 0.83 & 0.83 \\
$J_{A}$ & 1.16 & 1.16 & 1.16 \\
$J_{B}$ & 0.98 & 0.98 & 0.98 \\
$J_{C}$ & 0.68 & 0.68 & 0.68 \\
\hline
\end{tabular}


TABLE 12. The Tho-dmanstonal (RZ) Restuts $\left(\delta(1 / k)\right.$ per $\left.\mathrm{kg} \times 10^{4}\right)$

\begin{tabular}{l|l|l|l}
\hline & FD1 & FD2 & Experiment \\
\hline$A$ & 0.33 & 0.46 & $0.36 \pm 0.07$ \\
$B$ & 0.48 & 0.61 & $0 \pm 6 \pm 0.02$ \\
$C$ & 0.67 & 0.80 & $0.66 \pm 0.02$ \\
$D$ & 1.31 & 1.42 & $1.41 \pm 0.02$ \\
$E$ & 0.97 & 1.09 & $1.09 \pm 0.02$ \\
$F^{*}$ & 0.86 & 0.98 & 1.00 \\
$G_{A}$ & 0.43 & 0.56 & $0.43 \pm 0.06$ \\
$G_{B}$ & 0.90 & 1.03 & $0.92 \pm 0.06$ \\
$G_{C}$ & 1.44 & 154 & $1.72 \pm 0.06$ \\
$H_{A}$ & 0.84 & 0.97 & $1.02 \pm 0.06$ \\
$H_{B}$ & 1.07 & 1.20 & $0.98 \pm 0.06$ \\
$H_{C}$ & 1.30 & 1.37 & $1.26 \pm 0.06$ \\
$I_{A}$ & 1.26 & 1.39 & $1.37 \pm 0.04$ \\
$I_{B}$ & 1.22 & 1.32 & $1.27 \pm 0.04$ \\
$I_{C}$ & 1.07 & 1.11 & $1.19 \pm 0.04$ \\
$J_{A}$ & 1.50 & 1.59 & $1.65 \pm 0.06$ \\
$J_{B}$ & 1.24 & 1.30 & $1.21 \pm 0.06$ \\
$J_{C}$ & 0.82 & 0.83 & $0.77 \pm 0.03$ \\
\hline
\end{tabular}

TABLE 13. IJSCREpancy betw enen Caldellation AND FXPERIMENT $(\%)$

\begin{tabular}{l|r|r|r|r|r|r}
\hline & \multicolumn{2}{|c|}{$A$} & \multicolumn{2}{c|}{$B$} & \multicolumn{2}{c|}{$C$} \\
\hline & FD1 & FD2 & FD1 & FD2 & FD1 & FD2 \\
\hline$G$ & 0 & +29 & -3 & +12 & -17 & -12 \\
$H$ & -22 & -5 & -10 & +22 & +3 & +9 \\
$I$ & -9 & +2 & -4 & +4 & -11 & -7 \\
$J$ & -10 & -4 & +3 & +7 & +7 & +7 \\
\hline
\end{tabular}

uranium cross sections with removal of sodium evaluated in this way was included in the perturbation calculations. No heterogeneity correction was made, and this will therefore not be a very aceurate estimate of the effect.

The calculated sodium coefficients for FD1 and FD2 are compared with experiment in Table 12. The coefficients are $\delta(1 / k)$ per kilogram, and the experimental values were derived from the reported values, which were in $\mathrm{Ih} / \mathrm{kg}$, using the reported relation $4 \tilde{\jmath} 1$ $\mathrm{Ih}=1 \% \delta k / k$. Fxperimental uncel tainties were reported only for regions $A, B, C, D$, and $E$. The experimental errors for the other regions were guessed as 1 inhour for reactivity changes greater than $20 \mathrm{Ih}, 1 / 2 \mathrm{Ih}$ for reactivity changes less than $20 \mathrm{Ih}$. They are in the table only to give an estimate of the kind of uncertainty that is thought to exist, and it is arknowledged that they might be disputed.

A brief description of the layout of the regions is as follows: $A, B$, and $C$ were central cylinders, with a length-to-diametcr ratio $(\mathrm{L} / \mathrm{D})$ of about one, increas- ing in size alphabetically from 1 to $C ; D$ was a cylinder at the top of the core; $E$ and $F$ were cylinders of different diameter, decreasing alphabetically, from the top of the core to the centreline; the regions $G$ to $J$ were cylinders $(G)$ and rings $(I I$ to $J$ ) mapping the sodium coefficient through the core, going out from the centre alphabetically from $G$ to $J$, and upwards from the centre alphabetically in the subscript from $A$ to $C$; $G_{A}$ is therefore a central cylinder and $J_{C}$ a ring at a top corner.

Table 13 is in the form of a map of the percentage discrepancy between each of the cross-section sets and the experimental measurements, laid out as a quarter axial section through the core for regions $G_{A}$ to $J_{C}$. Column $C$ and row $J$ are next to the blanket and row $H$ contains the control rods.

\subsection{One-dimensional (Spherical) Calculations}

A number of one-dimensional calculations were also performed in spherical geometry. Their aims were to check in a limited way:

1. the adequacy of first-order perturbation theory;

2. the effect of sodium data from ELMIOE derived sperifically for ZPR-VI Assembly 2.

The regions $A, B$ and ${ }^{\gamma}$ at the centre of the core lend themselves to a simple spherical model. The sodium coefficient was calculated:

1. directly, as the difference between two $k$ calculations using CRAMI

2. by first-order perturbation theory.

The calculations used FD1, FD2, and FD2*, where $\mathrm{FD}^{*}$ contains light-nuclide cross sections from ELAIOE derived for this assembly. The results are in Table 14 as $\delta k / k$. The results indicate that:

1. the difference between direct calculation and

TABLF 14. The One-Drmensional Results $\left(\delta k / k \times 10^{4}\right)$

\begin{tabular}{c|c|c|c|c}
\hline & Experiment & Direct 1d. & 1st-order 1d. & 1st-order 2d. \\
\hline F D1 & & & & \\
A & 0.44 & 0.44 & 0.446 & 0.401 \\
$B$ & 2.62 & 3.01 & 3.08 & 2.72 \\
C $^{Y}$ & 9.98 & 10.13 & 10.61 & 10.06 \\
& & & & \\
FI2 & & & & \\
A & 0.44 & 0.57 & 0.596 & 0.552 \\
$B$ & 2.62 & 3.52 & 3.84 & 3.45 \\
( & 9.98 & 11.40 & 12.60 & 12.06 \\
& & & & \\
FI)2* & & & & \\
$A$ & 0.44 & 0.46 & 0.486 & \\
$B$ & 2.62 & 3.04 & 3.34 & \\
$C$ & 9.98 & 10.30 & 11.45 & \\
\hline
\end{tabular}


TABLE 15. SODILM Coeffichent Components FOR REGIONS $A, B$, AND $C$

\begin{tabular}{c|r|r|r|r}
\hline & \multicolumn{2}{|c|}{ FD1 } & \multicolumn{2}{c}{ FD2 } \\
\cline { 2 - 4 } Leakage & \multicolumn{2}{|c|}{$1 \mathrm{~d}$} & $2 \mathrm{~d}$ & \multicolumn{2}{c}{$1 \mathrm{~d}$} & $2 \mathrm{~d}$ \\
\hline$A$ & & & & \\
$B$ & 0.113 & 0.108 & 0.121 & 0.116 \\
$C$ & 0.301 & 0.276 & 0.322 & 0295 \\
& 0.503 & 0.496 & 0.538 & 0.530 \\
Moderation & & & & \\
$A$ & & & & \\
$B$ & 0.496 & 0.455 & 0.758 & 0.719 \\
$C$ & 0.471 & 0.420 & 0.722 & 0.661 \\
& 0.404 & 0.369 & 0.623 & 0.585 \\
Absorption & & & & \\
$A$ & & & & \\
$B$ & -0.087 & -0.083 & -0.172 & -0.165 \\
$C$ & -0.083 & -0.079 & -0.164 & -0.156 \\
& -0.073 & -0.071 & -0.143 & -0.140 \\
Self-Shielding & & & & \\
$A$ & -0.153 & -0.149 & -0.214 & -0.215 \\
$B$ & -0.147 & -0.139 & -0.204 & -0.192 \\
$C$ & -0.129 & -0.124 & -0.179 & -0.172 \\
\hline
\end{tabular}

first-order perturbation theory is small, but not negligible for the larger reactivity changes. The results here are somewhat ambiguous. For case $C$, FD1 shows a $5 \%$ difference whereas FD2 and FD2* show a difference of $11 \%$;

2. $\mathrm{FD}^{*}$ improves the agreement with experiment over FD2.

Table 15 lists the sodium coefficient components for FD1 and FD2 in $1 \mathrm{~d}$ and $2 \mathrm{~d}$. One point to note in this table is that the self-shielding term (that part of the coefficient resulting from the change in heavynuclide self-shielding with loss of sodium) is of the same order as the absorption term (due to the loss of the capture in sodium).

\subsection{An Interpretation of the Calculations for ZPR-VI Assembly 2}

The shape, in the energy dimension, of the adjoint function in a uranium-fuelled system is significantly different from that in a plutonium-fuelled system. Sodium coefficient measurements in uranium-tuelled systems, such as ZPR-VI Assembly 2, are therefore of no

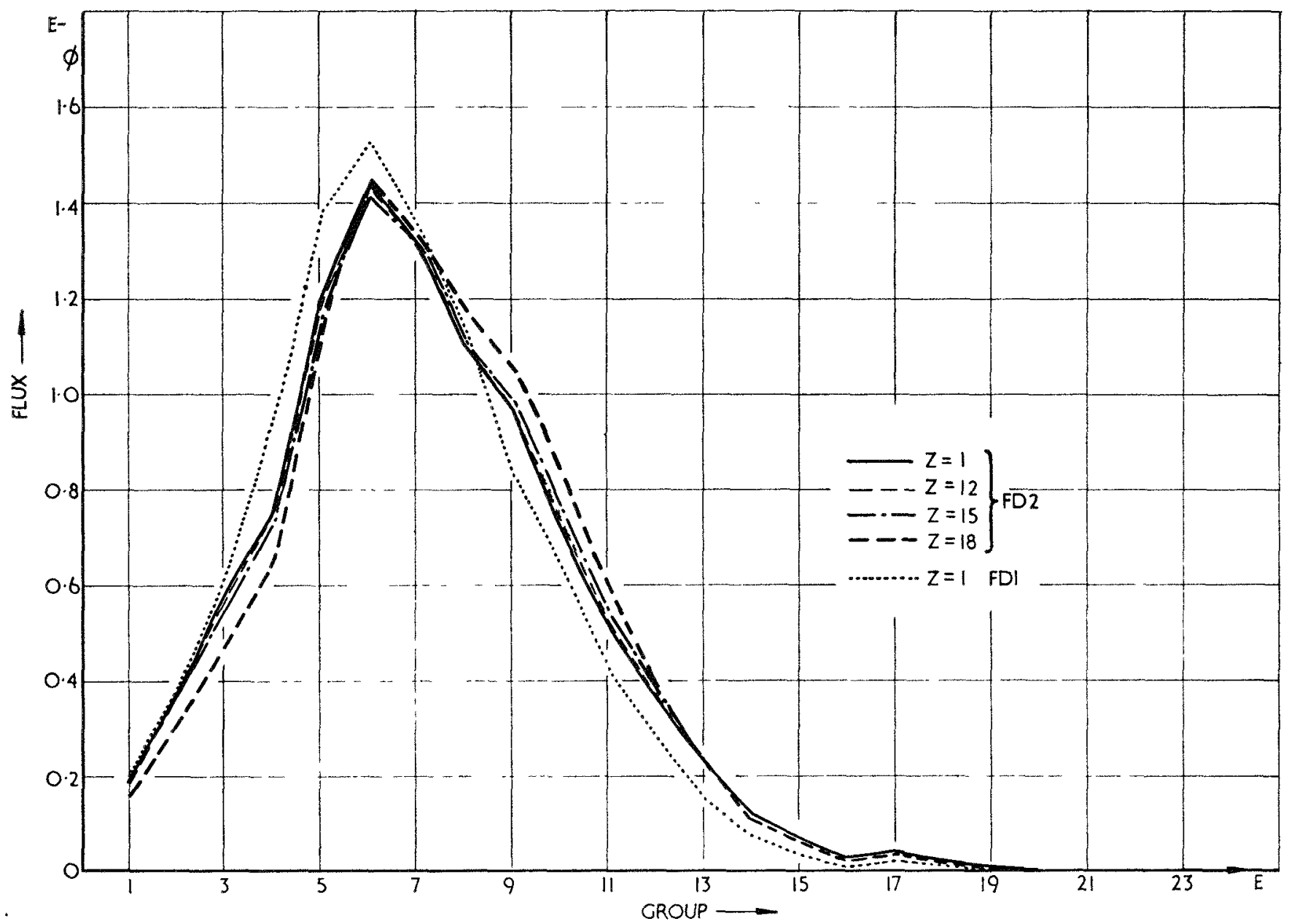

FIG. 4. The Normal Spectrum along the Central Axis. 
direct relevance to plutonium-fuelled reactor design in so far as the moderation term is concerned. (The moderation term is that part of the sodium coefficient which derives from the change in moderation with loss of sodium.) An accurate calculation of the leakage term (which derives from the change in leakage with loss of sodium) depends on an aceurate calculation of the normal flux spectrum, of the spatial variation of the flux, and on an accurate knowledge of the transport cross sections for sodium and for the system. The spectrum will depend strongly on the amount and type of moderator in the system rather than on the fuel, and the diluent composition of ZPR-VI Assembly 2 is to some extent typical of most sodium-cooled reactors at present under consideration. In ZPR-VI Assembly 2 the main contributor to the system transport eross section is $\mathrm{U}^{238}$. This again is a typical situation. The accuracy with which the leakage component is being calculated here is, therefore, of immediate relevance to plutonium-fuelled as well as uranium-fuelled reactor design.
The discrepancy map (see Table 13) indicates an overestimate of the sodium coefficient for all excepting the outer regions by FD2. It is of some interest to see whether these discrepancies can be explained by assuming a constant error in the moderation term. If we assume that the spatial variation of the flux is being calculated correctly, whether the error in a given term of the coefficient is constant will depend on whether the spectrum is constant over the core. Figures 4 and 5 show the variation of the spectra along the central axis. The spcctra are plotted at the centre and towards the edge. It can be seen that the normal spectrum varies hardly at all, and the adjoint does not change until the experimental region $G_{C}$ is reached. This stability of the spectra is reflected in the groupby-group breakdown of the terms shown in Figs. 6 and 7. In Fig. 6 the fraction of the total leakage term from each group is plotted for the most extreme cases, $G_{A}$ at the centre and $J_{C}$ at the top corner. There is very little change. In Fig. 7 the group breakdown of the moderation term is plotted (normalised to the group 1

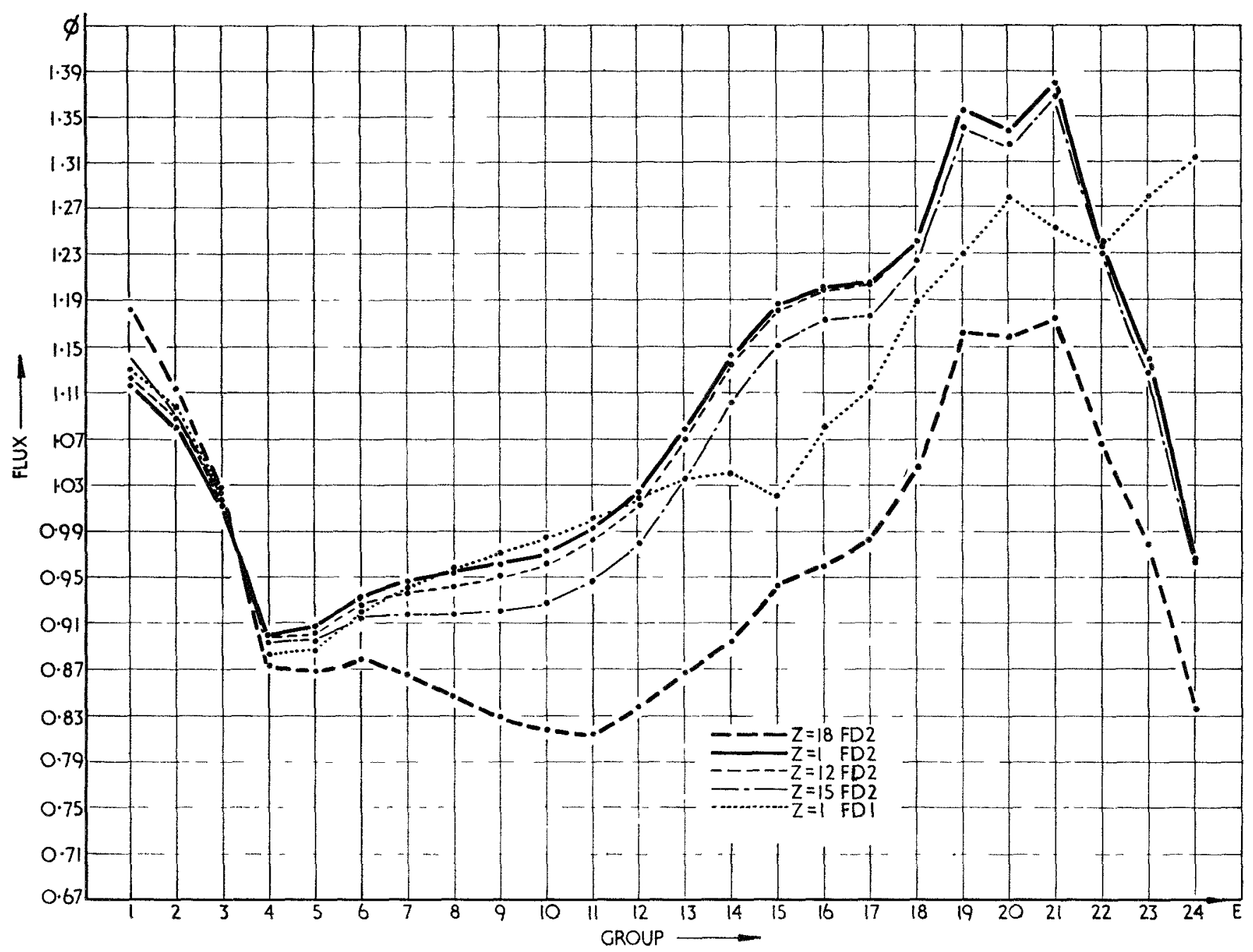

F1G. 5. The Adjoint Spectrum along the Central Axis. 


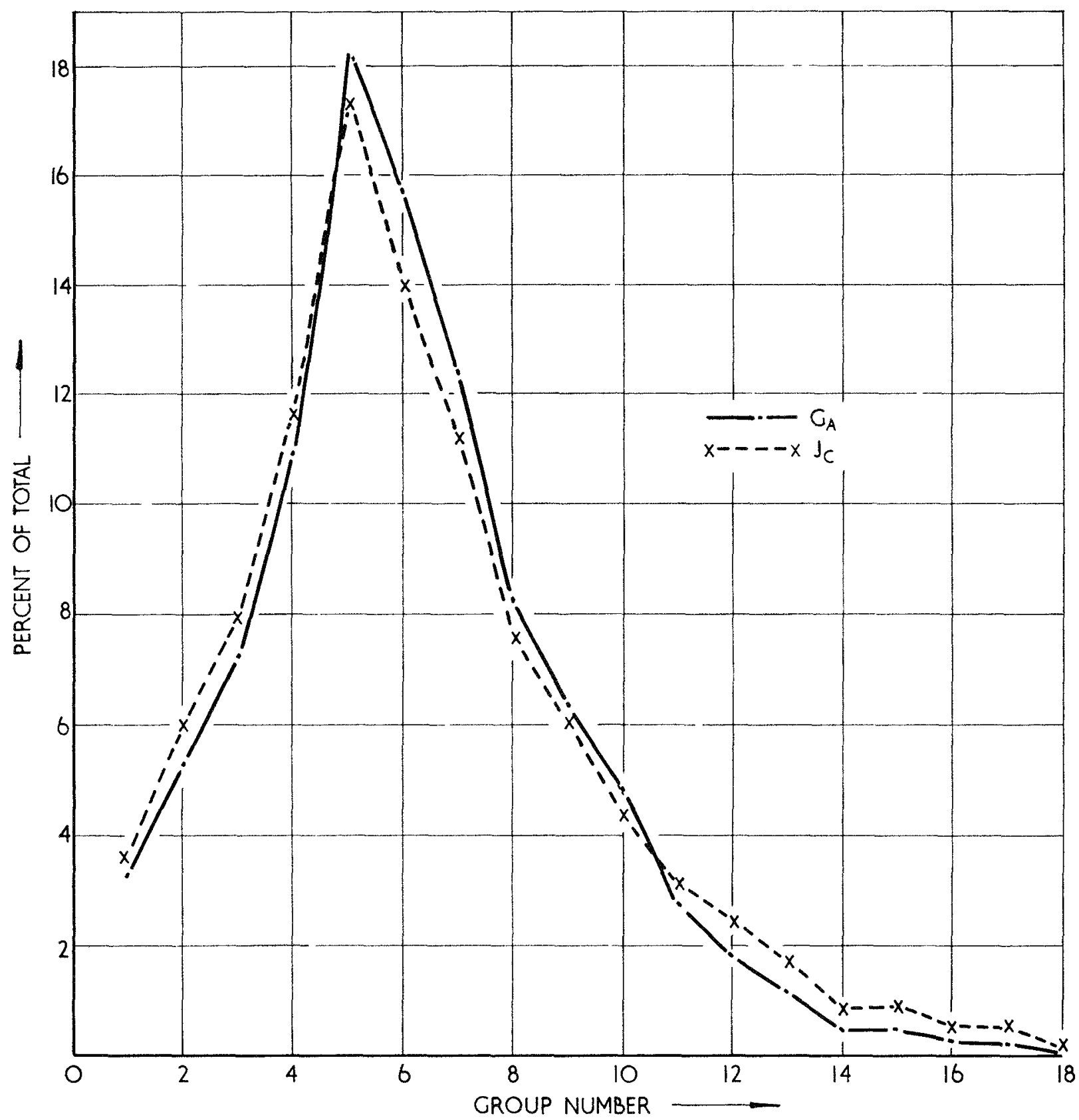

FI(x. 6. Fraetion of Total Leakage Component (FI)2).

contribution) for $G_{A}, G_{B}$, and $G_{C}$. The graphs for $G_{A}$ and $G_{B}$ are very similar. There should therefore be some constant discrepancy for the terms separately away from the core-blanket interface.

Table 16 shows the ratios between the terms for those regions away from the core edge. The self-shiclding, absorption, and moderation terms depend on the spatial variation of the flux in a similar way. The leakage term depends on the spatial variation of the current. Any errors in the calculation of the former three terms cannot easily be separated, and they can most simply be considered together.

In Table 17 the effect of reducing the calculated sum of the moderation, absorption and self-shielding terms by $40 \%$ is shown. The agreement with the experimental measurements is considerably improved except for the regions $H_{A}$ and $H_{B}$. These regions contain the control rods, which may introduce some significant perturbation to the spectrum and therefore introduce an effect not included in the calculation. That 


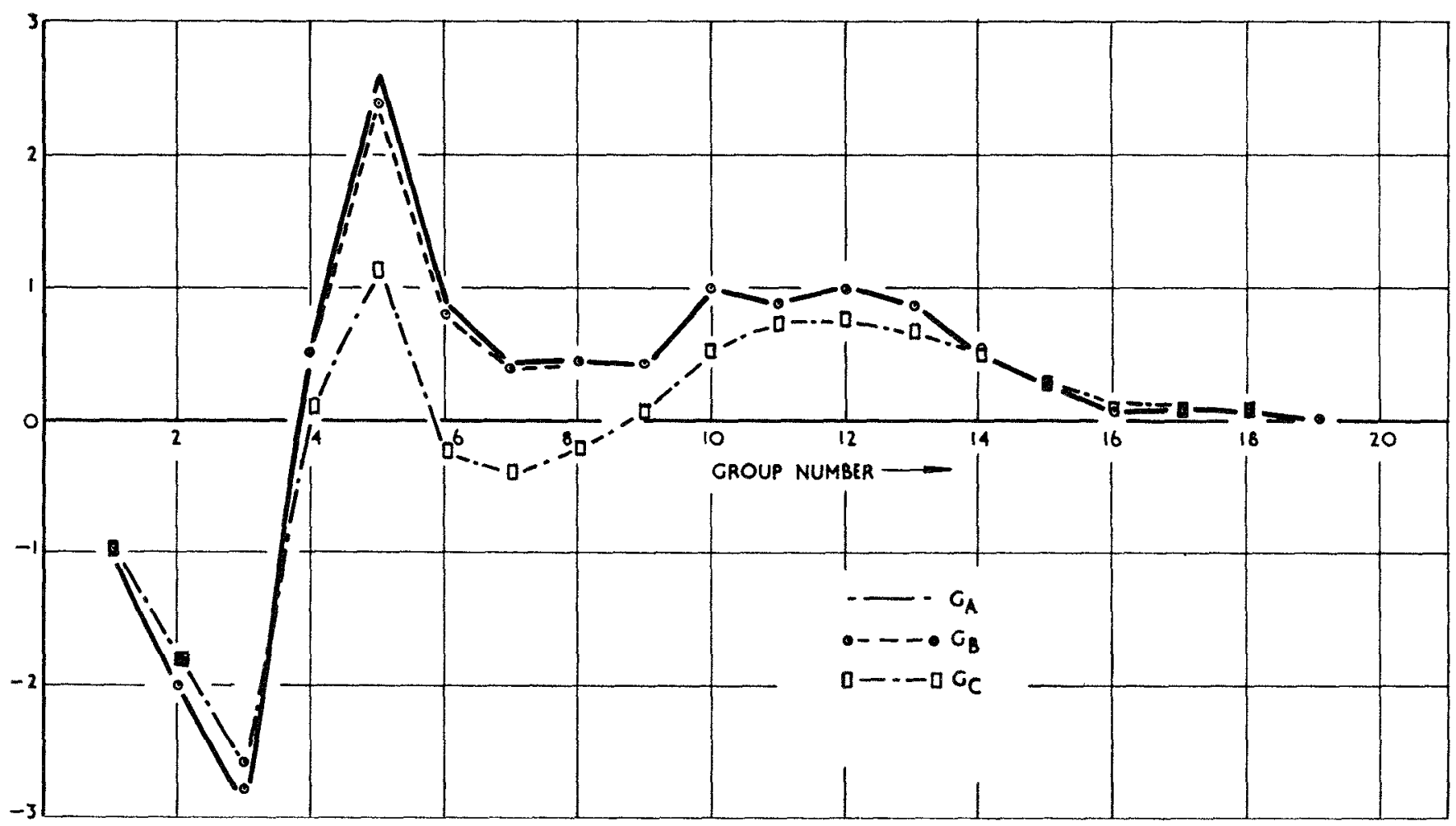

FIG. 7. The Moderation Component (FD2) (Normalised to Group 1 Contribution = -1).

TABLE 16. Ratro BeTWeEN Terms (FD2)

\begin{tabular}{l|c|c|c}
\hline & Self-shielding & Moderation & Leakage \\
\cline { 3 - 4 } & Absorption & Absorption & Moderation \\
\hline$A$ & 1.30 & -4.4 & 0.2 \\
$B$ & 1.23 & -4.2 & 0.4 \\
$C$ & 1.22 & -4.2 & 0.9 \\
$G_{A}$ & 1.22 & -4.2 & 0.4 \\
$G_{B}$ & 1.20 & -4.0 & 1.8 \\
$H_{A}$ & 1.23 & -4.1 & 1.4 \\
$H_{B}$ & 1.22 & -3.8 & 3.0 \\
$I_{A}$ & 1.22 & -3.3 & 4.7 \\
$I_{B}$ & 1.22 & -3.1 & 6.9 \\
\hline
\end{tabular}

there is something atypical about the coefficients in the cylindrical shell $H$ can be seen from the distortion in the discrepancy map (see Table 13) in this shell. Also in Table 12 it can be seen that the measured coefficient in $H_{A}$ is greater than that in $H_{B}$, whereas the calculated coefficient is significantly Iess. This seems to indicate that the simple homogeneous model assumed is inadequate for these regions.

\section{Conclusions}

Calculations of the $\mathrm{Pu}^{239}$ Doppler coefficient based on an extrapolation of the resonance parameters obtained from measurements of the $\mathrm{Pu}^{239}$ total cross
Table 17. Modified Calculation Results (FI)

\begin{tabular}{l|c|c|c|c|c|c}
\hline & Leakage & $\begin{array}{c}\text { Other } \\
\text { Terms }\end{array}$ & $\begin{array}{c}\text { Modified } \\
\text { Other } \\
\text { Terms }\end{array}$ & Total & $\begin{array}{c}\text { Modified } \\
\text { Total }\end{array}$ & Experiment \\
\hline$A$ & 0.12 & 0.34 & 0.20 & 0.46 & 0.32 & $0.36 \pm 0.07$ \\
$B$ & 0.29 & 0.31 & 0.19 & 0.60 & 0.48 & $0.46 \pm 0.02$ \\
$C$ & 0.53 & 0.27 & 0.16 & 0.80 & 0.69 & $0.66 \pm 0.02$ \\
$G_{A}$ & 0.24 & 0.32 & 0.19 & 0.56 & 0.43 & $0.43 \pm 0.06$ \\
$G_{B}$ & 0.83 & 0.20 & 0.12 & 1.03 & 0.95 & $0.92 \pm 0.06$ \\
$H_{A}$ & 0.74 & 0.23 & 0.14 & 0.97 & 0.88 & $1.02 \pm 0.06$ \\
$H_{B}$ & 1.05 & 0.15 & 0.09 & 1.20 & 1.14 & $0.98 \pm 0.06$ \\
$I_{A}$ & 1.30 & 0.09 & 0.05 & 1.39 & 1.35 & $1.37 \pm 0.04$ \\
$I_{B}$ & 1.26 & 0.06 & 0.04 & 1.32 & 1.30 & $1.27 \pm 0.04$ \\
\hline
\end{tabular}

section made recently at Harwell result in smaller values than those calculated previously. The measurements showed the existence of some very broad resonances. $\mathrm{A} \mathrm{Pu}^{239}$ Doppler coefficient smaller by an order of magnitude than the value obtained using a narrow fission width distribution has been obtained for a large dilute fast reactor, and negative values could be expected in cores with less absorption relative to the $\mathrm{Pu}^{239}$ absorption in the resonance region (for example, with a $\mathrm{U}^{238} / \mathrm{Pu}^{239}$ ratio of less than about 5). However, the $\alpha$ values obtained in this extrapolation are about $35 \%$ smaller than the usually accepted values, and so calculations of the $\mathrm{I}^{2} \mathrm{u}^{239}$ coefficient based on currently 
available resonance data must be regarded as uncertain, even in sign.

The approximate methods used to treat the heterogeneity of the Zebra Doppler test zone, and its finite size, have been described. The unit cell is a complicated one; the simple treatment is expected to give the heterogeneity corrections only to an accuracy of about $\pm 50 \%$. The heterogeneity correction to the $\mathrm{Pu}^{239}$ coefficient is a factor of about three for the plutonium pins when there are diluent pins present. Methods of allowing for the sample size of a small Doppler sample in a core which contains the same material as the sample are also described.

The Doppler coefficients calculated using the FD2 set and the narrow fission width resonance data for $\mathrm{Pu}^{239}$ give positive values for the $\mathrm{Pu}^{239}$ coefficients in the Zebra Doppler Loop, whereas the measured values are negative. The recent $\mathrm{Pu}^{239}$ data would lead to values smaller by a factor of 3 or 4 and probably to a negative value in core $5 \mathrm{~K}$. The $\mathrm{U}^{238}$ coefficients are in good agreement with the Doppler coefficients measured for the mixed $\mathrm{U}-\mathrm{P} u$ oxide pins when the $\mathrm{Pu}^{239}$ coefficients are taken to be zero.

Calculations of sodium reactivity worth have been made to compare with the measurements made in ZPR-VI Assembly 2. The calculations were for a homogeneous model, and used diffusion theory and first order perturbation theory.

The results suggest that the FD2 cross-section set overestimates the sum of the moderation, self-shielding and absorption components of the sodium coefficient in ZPR-VI Assembly 2, but there is no evidence of a systematic error in the calculation of the leakage component. Reducing the sum of moderation, self-shielding, and absorption components by $40 \%$ brings the calculated values into reasonable agreement with the measured values.

A possible explanation of the discrepancy between calculation and experiment may be the inadequary of the homogeneous model. Neasurements with ZPR-VI Assembly $2^{(29)}$ and Assembly $3^{(30)}$ indicate large heterogeneity effects. These could introduce the kind of constant discrepancy found. If this is so, a discrepancy of a similar magnitude would not be expected in an assembly with a different lattice structure.

\section{Acknowledgment}

The Authors would like to thank Mr. E. Begg for making available the results of his calculations of the Zebra Doppler Loop Doppler coefficicnts using the FD1 and FD2 cross-section sets and Miss Linda Grimshaw for help with the computations.

\section{Addendum}

Changes in the values of Tables 12 through 17, and in some of the conclusions, were made by the authors since the time of the Conference.

\section{REFERENCES}

1. A. R. Baker and R. C. Wheeler, Doppler coefficient measurements in Zebra Core 5 , see preceding paper.

2. C. A. Uttley, Single level analysis of the resonances in the total cross section of $P u^{239}$ from $100 \mathrm{eV}$ to $300 \mathrm{eV}$. International Conference on the Study of Nuclear Structure with Neutrons, Antwerp (July 1965).

3. J. Blons, H. Derrien, A. Michaudon, and P. Ribon, Study of resonance parameters of $P u^{239}$, International Conference on the Study of Nuclear Structure with Neutrons, Antwerp (July 1965).

4. J. S. Story, internal document, A.E.E., Winfrith (1963).

5. L. M. Bollinger, R. E. Coté, and G. E. Thomas, Proc. 2nd Geneva Conference, 15, 127 (1958).

6. Mrs. H. M. Sumner, ERIC 2, a Fortran programme to calculate resonance integrals and effective capture and fission. cross sections, AEEW-R323 (1964).

7. A. C. Douglas and J. F. Barry, Neutron Cross Sections of $P u^{239}$ in the Energy Range $1 \mathrm{keV}$ to $15 \mathrm{MeV}$, AWRE 0-79/64 (Jan 1965).

8. R. Froelich, K. Ott, and J. J. Schmidt, Dependence of Fast Reactor Doppler Effects on Nuclear-data Uncertainties, Proceedings of the Conference on Breeding, Economics and Safety in Large Fast Power Reactor, ANL-6792 (Oct 1963), p. 765 .

9. C. A. Uttley, private communication, AERE Harwell, 1965.

10. P. Greebler and E. Goldman, Doppler Calculations for Large Fast Ceramic Reactors, GEAP-4092 (1)ec 1962).

11. G. I. Bell, Theory of Effective Cross Sections, LA-2322 (Oct 1959).

12. W. Rothenstein, Collision Probabilities and Resonance Integrals for Lattices, Nucl. Sci. Eng., 7, 162 (1960).

13. J. M. Otter, Escape Probability Approximations in Lumped Resonance Absorbers, NAA-SR-9744 (Aug 1964).

14. C. N. Kelber, Improved Rational Escape Probabilities in Lumped Absorbers, Nucl. Sci. Eng., 22, 244 (1965).

15. D. C. Leslie, J. G. Hill, and A. Jonsson, Improvements to the Theory of Resonance Escape in Heterogeneous Fuel. 1. Regular Arrays of Fuel Rods. Nucl. Sci. Eng., 22, 78 (1965).

16. Y. Fukai, Nucl. Sci. Eng., 9, 370 (1961),

17. M. M. Levine, Resonance Integral Calculations for $U^{238}$ Lattices, Nucl. Sci. Eng., 16, 271 (1963).

18. Y. Fukai, First-fight Collision Probability in Moderatorcylindrical Fuel System, J. Nuel. Energy, 17, 115 (1963).

19. F. Storrer, A. Khairallah, and J. Ozeroff, Measurements of the Doppler Coefficient in Large F'ast Power Reactors using a Fast Critical Assembly and an Experimental Fast Reactor, Proceedings of the Conference on Breeding, Economics and Safety in Large Fast Power Reactors, ANL-6792 (Oct 1963).

20. R. W. Smith, The FID Cross-section Set, to be published, A.E.E., Winfrith.

21. G. E. Hansen and W. H. Roach, Six-and Sixteen-group Cross Sections for Fast and Intermediate Critical Assemblies, I,AMS-2543 (1961).

22. J. L. Rowlands and D. Wardleworth, A Nineleen-group 
Extension to the Yiftah, Okrent, and Moldauer Crosssection Sel, AFEW-M 472 (1965).

23. Argonne National Laboratory, Reactor Development Program Progress Report for May 1964, ANL-6904.

24. S. Grifoni, M. Nozawa, and W. Y. Kato, Sodium Void Coefficient Measurements in a Large Carbide Fast Assembly, Trans. ANS, 7, No. 2, p. 250 (Nov 1964).

25. A. Hassitt, A Computer Program to Solve the Multigroup Diffusion Equations, TRG Report 229 (R) (March 1962).

26. A. L. Rago and H. H. Hummel, ELMOE: An IBM-ro4 Program Treating Elastic Scattering Resonances in Fast

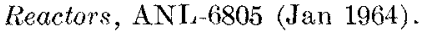

27. S. Yiftah, D. Okrent, and P. Moldauer, F'ast Reactor Cross Sections, Pergamon Press (1960).

28. Susan M. Miller and K. Parker, List of Data Files A vailable at 15th A pril 1965, AWRE Report 0-55/65 (July 1965).

29. G. K. Rusch and F. H. Helm, private communication.

30. Reactor Development Program Progress Report for June 1964 , ANL-6912.

\section{Discussion}

Mr. Greebler (GE): Did I understand you to say that the $25 \%$ and $40 \%$ adjustment of the two components was done empirically as a recipe to get better agreement between the measured and calculated results, or was it based on some specific factors in the calculation in the cross sections and so on?

$M r$. Codd: It has no theoretical justification; on the other hand, the leakage terms are more important in the outer regions of the reactor, and that gives a good idea of the $25 \%$. Likewise, at the center the moderation is relatively more important. They are not just guesses.

Mr. Peterson (Battelle-Northwest): I understood that you made a $15 \%$ correction to the one-dimensional diffusion calculations for the fact that the heated region was not completely filling the axial length. Could you explain how you arrived at the $15 \%$ ?

Mr. Codd: I think all I can say there is that this was based on various two-dimensional studies in which one tried to simulate the geometry and compensation of the regions above the loop more accurately. That is all.

Mr. Segal (APDA): I would just like to ask if your calculations of the sodium worth in Fermi, also dominated by the leakage effect, have shown a similar discrepancy. I would like to remind you that when I did similar calculations I found a $25 \%$ discrepancy but in the opposite direction of the leakage term. I am of the opinion that the discrepancies are due to data principally and to hope for $25 \%$ in heterogeneity effects is perhaps too optimistic.

Mr. Codd: You referred to Fermi calculations. I don't think the authors would feel able to say very much about Fermi work at the present without the comparisons with Fermi data.

Mr. Nims (APDA): I noticed in your map of the calculated sodium worth that in the central axial position the use of FD2 overestimated the worth by about $18 \%$, which is inconsistent with the author's statement about underestimating by the order of $1 \%$.

Mr. Codd: That correction factor of $40 \%$ refers only to the $\mathrm{FD} 2$ data set, and therefore the reduction in the moderation component would make that $18 \%$ come close to zero. One would require different corrections for the other data-set values where the sign of the error was opposite.

Mr. Chernick (BNL): You say Uttley and Company are serious about these low values of $\alpha$ for plutonium. Is this simply ad hoc for the moment?

Mr. Codd: I doubt if Dr. Uttley knows about these low values; our calculations are very preliminary.

Mr. Chernick: But he took a big leap forward in assuming that these resonances were all fission. Is that your understanding of what he has done about the broad resonances?

Mr. Codd: That is what he has done, I think, on the basis of Lynn's recent theory. The low $\alpha$ values are purely the responsibility of the authors, no one else.

Mr. Engelmann (Karlsruhe): In the conference here two years ago, there was a paper of Storrer on the effect of a hot sample in a cold surrounding. In your loop, of course, the center was very much larger; have you tried to calculate the correction due to the effect of the cold reactor and hot sample?

$M r$. Codd: That is discussed in the paper. But in between the main reactor and the loop, there is an insulating zone, and the author suggests, in fact, that this essentially disconnects the two regions insofar as the resonance steps in the flux are concerned.

Mr. Engelmann: If this is the case, then the Doppler effect as you calculated it should not agree with the Doppler effect which you measured, because in your calculation, you assume that the reactor is homogeneous and at the same temperature. I got it that this surrounding layer between the loop and the core zone did, in the resonance region, decouple the cold reactor and heated center. Is this correct?

Mr. Codd: Yes.

Mr. Engelmann: Then in your calculation the Doppler effect is calculated for the whole reactor.

Mr. Codd: No, only for the heated loop region.

Mr. Pitterle (APDA): Just a comment on those $\alpha$ values which they had for this broad sequence. As I have mentioned before, I find that the $\alpha$ values are very sensitive to the $\nu$ values assumed for the fission distribution. If one neglects this, assuming certain sets of parameters, one very easily gets very low $\alpha$ values; but if one assumes a $\nu=1$ distribution for the $j=0$ sequence, for instance, this drops the average $\left.\Gamma_{f}(\Gamma) / \Gamma\right)$ term for the infinite-dilution cross section, and this 
begins to raise the $\alpha$ value. I think it's significant as well for the $j=0$ sequence where we had been assuming a $\nu$ of 5 or 10 . And here again, it's surprising how much difference this makes in the $\alpha$ value.

$M r$. Codd: Thank you. The authors in fact don't assume a theoretical law for the fission width distribution. They group Uttley's parameters into six groups, each having a representative mean width, and assume that this can be extrapolated and that these groups apply to all energies. 


\title{
Accuracy of Power Reactor Void Coefficients Extrapolated from Measurements in Critical Assemblies
}

\author{
T. C. Engelder, N. L. Snidow, and T. S. Katmay \\ The Babcock \& Wilcox Company \\ Lynchburg, Virginia
}

(PRESENTED BY T. C. ENGELDER)

\section{INTRODUCTION}

It is generally agreed that we cannot undertake construction of a large, fast power reactor for commercial use until large-scale critical experiments have been conducted to confirm the reliability of crucial design calculations for such a reactor. Although complete and unambiguous information could be obtained if the critical assembly mocked up the reference power reactor design in every detail, a more flexible and less expensive approach is preferable. In this paper we consider how accurately the crucial parameters for nuclear design can be derived from what might be termed a "practical" critical assembly that may differ from the reference power reactor in such respects as size, temperature, and isotopic composition of plutonium. The parameter used to illustrate the approach, the coolant void coefficient, was selected because of its great importance and particular sensitivity to these differences. (A similar approach could be taken for other important nuclear parameters, such as the Doppler coefficient and the breeding gain.)

The reference power reactor is the MARK-XV design for a 1000-MWe supercritical, steam-cooled, fast breeder reactor (SCBR). ${ }^{(1)}$ The annular core is $18.8 \mathrm{in}$. thick by $62.5 \mathrm{in}$. high, and has a volume of approximately 4560 liters; the annulus is blanketed on the inside, outside, and at the top end. The fuel is a mixture of plutonium and depleted uranium oxide in nickel-based alloy tubes approximately $1 / 4 \mathrm{in}$. in diameter, and the coolant is supercritical steam. Under operating conditions, the average fuel temperature is $2485^{\circ} \mathrm{F}$, and the average steam density is $6.6 \mathrm{lb} / \mathrm{ft}^{3}\left(3500 \mathrm{psi}, 850^{\circ} \mathrm{F}\right)$. At the beginning of core life, the isotopic composition of plutonium is an "equilibrium mixture" that contains $68 \% \mathrm{Pu}-239,28 \% \mathrm{Pu}-240$, and $4 \% \mathrm{Pu}-241$. The atomic ratio of fissionable isotopes (Pu-239 $+\mathrm{Pu}-241)$ to total plutonium plus uranium is approximately 0.16 , and the critical mass is approximately $2700 \mathrm{~kg}$ plutonium. Core volume fractions are $43 \%$ fuel, $40 \%$ coolant, $8 \%$ cladding, $5 \%$ structure, and $4 \% \mathrm{~B}_{4} \mathrm{C}$ (enriched) control rods.
It is apparent from this description of the reference power reactor that a critical assembly would be considerably simplified if absolute duplication of the reference reactor were not required. Relaxation of the design requirements in at least four major areas is worthy of consideration:

1. Size: Decrease the size to reduce the cost of materials, facility, and containment.

2. Temperature: Eliminate the high cost and complexity of a hot critical facility.

3. Plutonium composition: Utilize available plutonium, which is low in $\mathrm{Pu}-240$ content.

4. Heterogeneity: Consider using cheaper fuel forms. The following sections evaluate the effects of these four variables on the void coefficient and on the correction required for its extrapolation from that measured in simpler critical assemblies to the value for the reference power reactor.

\section{TECHNICAL APPROACH}

The void coefficient $\Delta k_{v}$ is defined by the equation $\Delta k_{v} \equiv k_{0}-k_{100}$, where $k_{100}$ is the value of $k_{\text {eff }}$ with the supercritical steam coolant present under normal operating conditions, and $k_{0}$ is the equivalent value when the coolant is completely removed from the core and blanket. The void coefficient for the reference power reactor (REF) can then be written

$$
\begin{aligned}
\left(k_{0}-k_{100}\right)^{\mathrm{REF}}= & \left(k_{0}-k_{100}\right)^{\mathrm{CA}}+\left(k_{100}^{\mathrm{CA}}-k_{100}^{\mathrm{REF}}\right) \\
& -\left(k_{0}^{\mathrm{CA}}-k_{0}^{\mathrm{REF}}\right) \\
= & \left(k_{0}-k_{100}\right)^{\mathrm{CA}}+\mathrm{CF}_{\text {total }},
\end{aligned}
$$

where the superseript CA refers to the critical assembly.

We assume that the void coefficient is measured directly in the critical assembly, and we are not concerned here with the details of this measurement or its accuracy. To obtain the desired void coefficient for the reference power reactor, a correction factor (CF) must be added to the measured value to account for the differences between the critical assembly and the reference design (such as size, temperature, and composi- 
tion). The correction factor (which is zero for the trivial case in which the critical assembly is identical with the reference reactor) must be derived from auxiliary experiments and/or theoretical calculations. However, the virtue of the stratagem expressed in eq. $2-1$ is that the term most difficult to compute (because it involves a large spectrum change) is measured directly, whereas each term in the correction factor involves much smaller

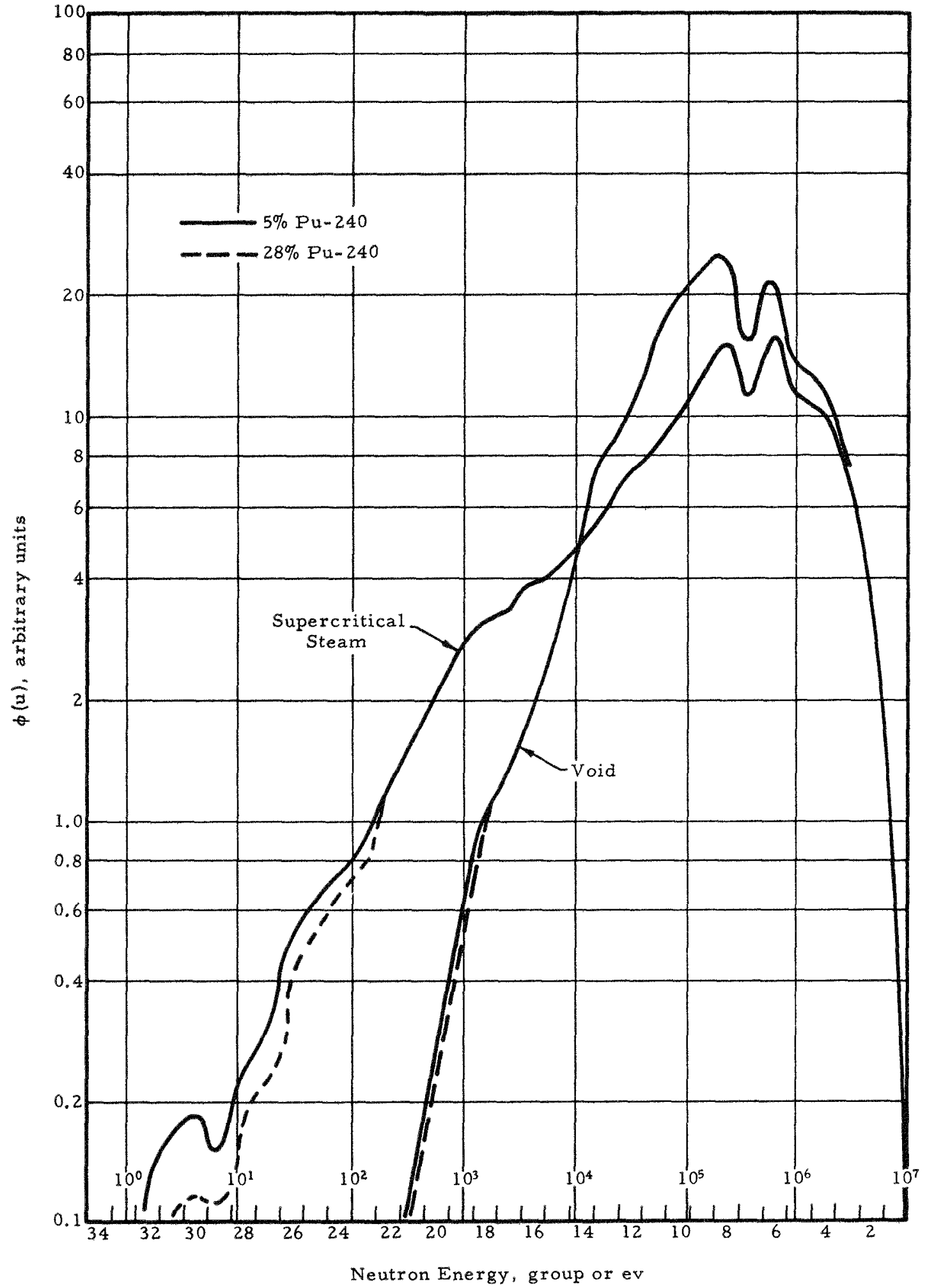

FIG. 1. Neutron Spectrum in the Reference Power Reactor. 
spectral changes and should be computable with less error.

This point is illustrated in Figures 1 and 2. The neutron spectrum at the center of the reference power reactor at room temperature is given in Fig. 1; the spectrum when readily available plutonium $(5 \% \mathrm{Pu}-240)$ is substituted for "equilibrium" plutonium (28\% $\mathrm{Pu}-240)$ is shown for comparison. The spectral differences, given as flux ratios in Fig. 2, show the difficulty of computing the void coefficient directly, which is caused by the large spectral change on voiding (median fission energy in- rreases from about $50 \mathrm{keV}$ to $100 \mathrm{keV}$ ). On the other hand, in this example, the spectral differences caused by a change in the isotopic composition of plutonium are much smaller, so the correction factor is easier to compute. Similar conclusions are reached when spectral changes caused by critical-assembly size or temperature are compared.

In the following analysis, the magnitude of the correction factor is obtained by comparing calculated values of $\left(k_{0}-k_{100}\right)^{\mathrm{RIF}}$ and $\left(k_{0}-k_{100}\right)^{\mathrm{CA}}$, and the accuracy with which we can determine this correction factor is

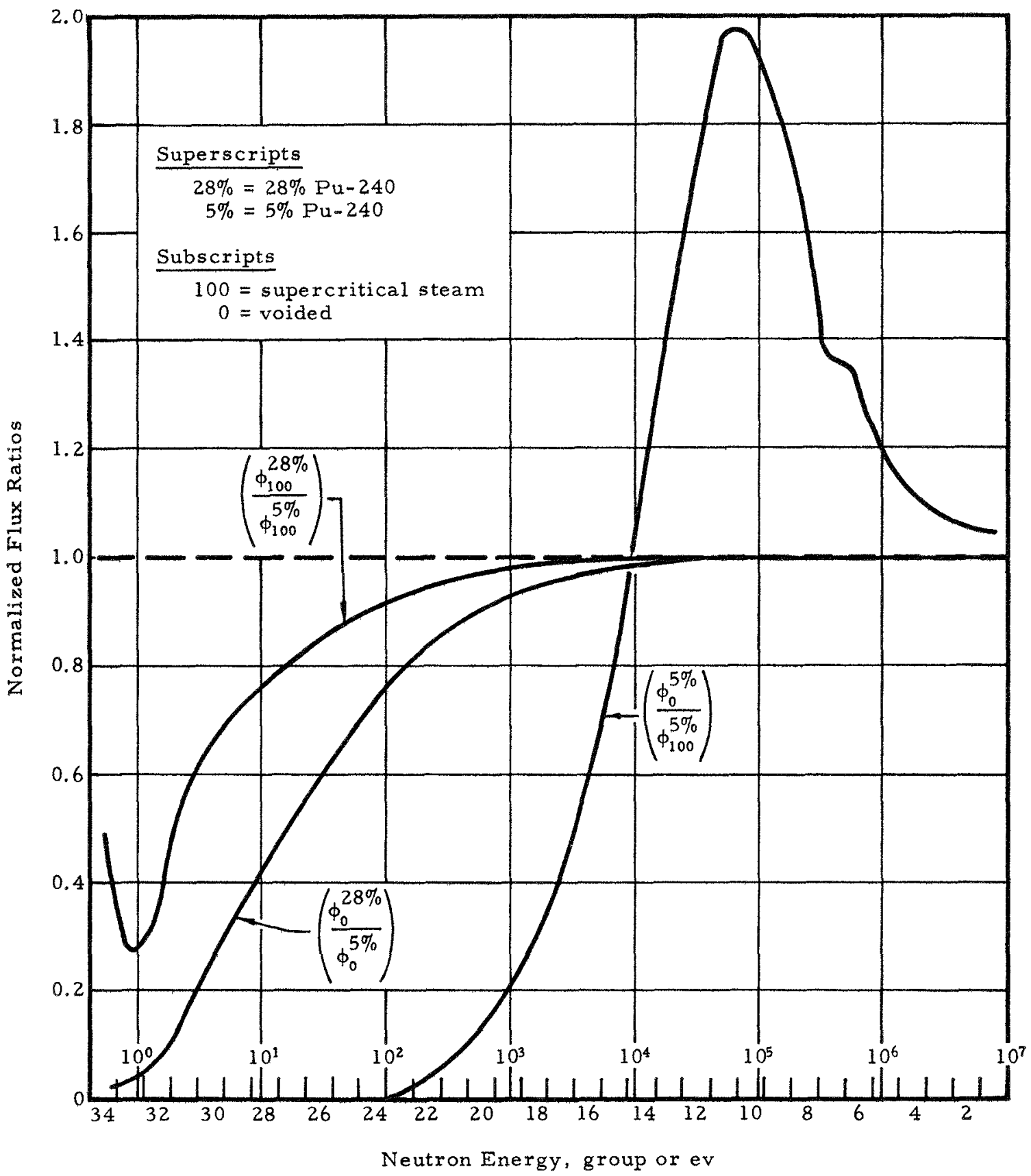

FIG. 2. Effect of Coolant Density and Pu-240 Content on Spectrum. 
estimated in various ways. The results represent the uncertainty in the void coefficient of the reference power reactor when it is extrapolated from measurements in a simpler critical assembly.

With few exceptions (noted in the text), we used the general analytical method given by the WANDA code to obtain $k_{\mathrm{ef}}$ and the four-group fluxes from numerical solutions of the one-dimensional, few-group neutrondiffusion equations. Four-group, flux-weighted coefficients were generated by means of the RIP, MUPT-5, and KATE-1 codes in combination with the 68 - or 34group B\&W standard cross-section library. RIP calculates the resonance integral of those elements in which the cross sections are shielded. Shielding and Doppler broadening of the neutron resonances were computed by use of the simple equivalence re]ations that lead to the NR or NRIA approximations. This corresponds to the use of the Adler-Nordheim method (e.g., GAM-1), except that Wigner's rational approximation of the escape probability is introduced, yielding a single expression that incorporates both the volume- and surfaceabsorption 1erms. The Dancoff factor was calculated by Sauer's method. MLVT-5 cmploys a Fourier transform. to calculate the flux-weighted, few-group constants for the three fast groups in the Pl diffusion approximation. The thermal-neutron spectrum was computed according to the Wigner-Wilkins theory, and average thermal group constants were obtained from the KATE-1 subroutine.

When the four-group representation is used, the energy bounds are

Group 1: $10 \mathrm{MeV}$ to $0.3 \mathrm{MeV}$

Group 2: $0.3 \mathrm{MeV}$ to $5 \mathrm{keV}^{r}$

Group $3: 5 \mathrm{keV}$ to $0.4 \mathrm{eV}$

Group 4:0.4 eV to 0 .

TABLE 1. EFFEC OF SHAPE AND SIZE ON THE VOID COEFFICIENT

\begin{tabular}{|c|c|c|c|c|}
\hline $\begin{array}{l}\text { Critical } \\
\text { Assembly } \\
\text { Size }\end{array}$ & $\begin{array}{l}\text { Fuel Tem- } \\
\text { perature, }\end{array}$ & $\begin{array}{c}\mathrm{Pu}-240 \\
\text { Concen- } \\
\text { tration, } \%\end{array}$ & $k_{0}-k_{100}$ & $\begin{array}{c}\text { Correction } \\
\text { Factor } \\
C F_{\text {size }}\end{array}$ \\
\hline Annulus & 20 & 28 & -0.0373 & \\
\hline$\infty / 1$ Slab & 20 & 28 & -0.0366 & $<0.001$ \\
\hline 5/1 Slab & 20 & 28 & -0.0379 & $<0.001$ \\
\hline $3 / 1$ Slab & 20 & 28 & -0.0404 & +0.003 \\
\hline 1/1 Slab & 20 & 28 & -0.0524 & +0.015 \\
\hline Annulus & 20 & 5 & -0.0638 & - \\
\hline $5 / 1$ Slab & 20 & 5 & -0.0634 & $<0.001$ \\
\hline Annulus & 1363 & 28 & -0.0157 & - \\
\hline 5/1 Slab & 1363 & 28 & -0.0170 & +0.001 \\
\hline Annulus & 1363 & 5 & -0.0405 & . \\
\hline 5/1 Slab & 1363 & 5 & -0.0411 & $<0.001$ \\
\hline
\end{tabular}

Although more sophisticated analytical techniques are arailable (and have been used to obtain a better absolute value of the void coefficient for reactor design studies), we are concerned here with changes in the void coefficient rather than with absolute values, so the simplified treatment should be adequate for error analysis.

\section{EFFECT OF SHAPE ANI SIZE}

The reduction of material costs, particularly for plutonium fuel, is the major incentive for decreasing the size of the critical assembly. Equipment and containment costs would also be substantially reduced by decreased plutonium inventory requirements.

A reasonable choice for the shape of the critical assembly is a slab that matches, in thickness, the annular core of the reference reactor. This shape is preferable to others (e.g., cylindrical) because it closely reproduces the reference spatial variation of the neutron flux and spectrum in the transverse direction. Since the core of the reference reactor is so thin in one dimension that asymptotic conditions exist in only about half of the core volume, the nonasymptotic region is of major importance and should be accurately simulated in the critical assembly. The slab core is provided with blankets against both faces, which are identical to the outer blanket of the reference reactor, and with nickel reflectors against the short sides. In order to avoid complex corrections for differences in axial leakage, the dimensions and composition in the axial direction are identical to those of the reference reactor.

Table 1 shows calculations for the reference reactor shape and size (annular core) and for slabs varying in breadth (expressed as the ratio of breadth to thickness); in each case the concentration of plutonium is adjusted slightly to normalize to $k_{0}=1$. The shape and size effect may be expressed analytically in the manner of eq. $2-1$ :

$$
\begin{aligned}
\left(k_{0}-k_{100}\right)^{\mathrm{ann}}= & \left(k_{0}-k_{100}\right)^{\mathrm{slab}}\left(k_{100}^{\mathrm{slab}}-k_{100}^{\mathrm{ann}}\right) \\
& -\left(k_{0}^{\mathrm{slab}}-k_{0}^{\mathrm{ann}}\right) \\
= & \left(k_{0}-k_{100}\right)^{\mathrm{slab}}+\mathrm{C} F_{\mathrm{szze}} .
\end{aligned}
$$

As experted, leakage increases as the slab size decreases, making the void coefficient more negative. However, the change is small, and the void coefficient for the reference reactor shape and size should be attainable with good arcuracy from measurements with $5 / 1$ or $3 / 1$ slabs.

The last three sets of calculations in Table 1 show the effect of fuel temperature and isotopic composition of plutonium on the size correction. The void coefficient itself changes appreciably, so these effects must be carefully considered (see below). However, the difference between the void coefficient for the reference reactor annulus and that for the slab remains small, indicating 
that the size correction is quite insensitive to the fuel temperature and isotopic composition in the range considered.

The reliability of these one-dimensional calculations was assessed by re-doing an extreme case (3/1 slab) in two dimensions using the PDQ code. The resulting void coefficient was within 0.001 of the one-dimensional case.

Although the correction factor for size is very small if the void coefficient is measured, for example, in a $5 / 1$ slab critical assembly, it does not necessarily follow that the correction factor can be calculated with that accuracy. For example, if $k_{\infty}^{*}$ is defined as the ratio of productions to absorptions in the center of the core, and the nonleakage probability $P_{N L}^{*}$ is defined as the ratio of $k_{\text {eff }}$ to $k_{\infty}^{*}$, the difference in these parameters between the reference annulus and the $5 / 1$ slab is approximately $3 \%$. The change in the void coefficient is very small because the changes in $k_{\infty}^{*}$ and $P_{N L}^{*}$ are in opposite directions and strongly cancel. This type of procedure is not useful for error analysis, however, because $k_{\infty}$ and $\mathrm{P}_{\mathrm{XI}}$ are not strictly separable in these high-leakage cores.

The approach used here is to calculate the sensitivity of $\mathrm{CF}_{\mathrm{szz}}$ to changes in cross sections in the manner deseribed more fully below (for the cross-section changes listed in Table 8, the changes in $\mathrm{CF}_{\mathrm{s} z \mathrm{z}}$ are generally much smaller than the reported values for $\mathrm{CF}_{\text {comp }}$ and are usually of the opposite sign). The only larger effects result from parameters that particularly influence leakage (specifically, inelastic scattering in U-238 and clastic scattering in oxygen), and in these cases the $\mathrm{CF}_{41 z e}$ uncertainty is only about 0.001 .

In summary, the uncertainty in the reference reactor void coefficient (accounting for differences in shape and size) is approximately \pm 0.001 if the void (oefficient is measured in a $5 / 1$ slab critical assembly. Further, the accuracy could possibly be improved, making the case for a $3 / 1$ slab critical assembly more attractive, by effecting some separation of $k_{\infty}$ and $\mathrm{P}_{\mathrm{N} \alpha}$ in supplementary experiments. Such experiments, involving small changes in the slab dimensions compensated by changes in the hydrogen density, may permit a more accurate correction for the small differences in $k_{\infty}$ and $\mathrm{P}_{\mathrm{NL}}$ between the slab critical assembly and the reference annular core.

\section{EFFECT OF TEMPERATURE}

The eritical experiments would be much simpler if they were performed at room temperature, thereby avoiding the complexity and cost associated with the type of hot critical facility required to match the operating temperature of the reference reactor. As would be experted, any parameter (such as temperature or pressure) that changes the coolant density will have a large
TABLE 2. EFFect of Fi el Temperatere ON THe Vold Coefficient

\begin{tabular}{|c|c|c|c|c|c|c|}
\hline $\begin{array}{c}\text { Critical } \\
\text { Assembly } \\
\text { Size }\end{array}$ & $\begin{array}{c}\mathrm{Pu}-240 \\
\mathrm{Conc} \\
\%\end{array}$ & $\begin{array}{l}\text { luel } \\
\text { Temp, } \\
{ }^{\circ} \mathrm{C}\end{array}$ & $k_{0}-k_{100}$ & $\begin{array}{c}\text { Correc- } \\
\text { tion } \\
\text { Factor, } \\
C F_{\text {temp }}\end{array}$ & $\begin{array}{c}k_{100}^{\text {cold }}- \\
k_{100}^{\text {hot }}\end{array}$ & $k_{0}^{k_{0}^{\text {cold }}-}$ \\
\hline $\begin{array}{l}\text { Annulus } \\
\text { Annulus }\end{array}$ & $\begin{array}{l}28 \\
28\end{array}$ & $\begin{array}{r}20 \\
1363\end{array}$ & $\left.\begin{array}{l}-0.0354 \\
-0.0148\end{array}\right\}$ & +0.021 & +0.026 & +0.005 \\
\hline $\begin{array}{l}5 / 1 \text { slab } \\
5 / 1 \text { slab }\end{array}$ & $\begin{array}{l}28 \\
28\end{array}$ & $\begin{array}{r}20 \\
1363\end{array}$ & $\left.\begin{array}{c}-0.0380 \\
-0.0182\end{array}\right\}$ & +0.020 & +0.024 & +0.004 \\
\hline $\begin{array}{l}5 / 1 \mathrm{slab} \\
5 / 1 \mathrm{Slab}\end{array}$ & $\begin{array}{l}5 \\
5\end{array}$ & $\begin{array}{r}20 \\
1363\end{array}$ & $\left.\begin{array}{l}-0.0639 \\
-0.0427\end{array}\right\}$ & +0.021 & $+0.02 t$ & +0.005 \\
\hline
\end{tabular}

effert on the void coefficient. However, the supercritical steam can be simulated in room-temperature experiments simply by using expanded plastic materials whose hydrogen density is the same as that in the reference power reactor at operating temperature and pressure.

The effert of the fuel temperature on the void coefficient is given in Table 2; expressed analytically,

$$
\begin{aligned}
\left(k_{0}-k_{100}\right)^{\text {hot }}= & \left(k_{0}-k_{100}\right)^{\text {cold }} \\
& +\left(k_{100}^{\text {eold }}-k_{100}^{\text {hot }}\right) \\
& -\left(k_{0}^{\text {cold }}-k_{0}^{\text {hot }}\right) \\
= & \left(k_{0}-k_{100}\right)^{\text {cold }}+\mathrm{CF}_{\text {temp }} .
\end{aligned}
$$

In calculating the reactivity change from cold to hot, $k_{0}$ was not renormalized to unity, so these void coefficients differ from those given in Table 1 . The results indicate that the correction factor to be applied to room-temperature measurements of the void coefficient is (at least to the first order) relatively insensitive to the shape and size of the critical assembly and to its plutonium isotopic composition.

The temperature correction is so large that it is doubtful that it can be calculated accurately enough from basic data. For example, estimates of the uncertainty in fast reactor Doppler calculations range from $\pm 20 \%$ to higher values, depending on the core composition and initial assumptions. Even if the optimistic value is assumed, the resulting uncertainty in the temperature correction $( \pm 0.005)$ is substantial.

Although we can expect improvements in the calculation of Doppler coefficients, for the purposes of this study, it is probably more realistic to assume that the Doppler coefficient is measured in the critical assembly, rather than to rely on calculations alone for the temperature correction. The magnitude of the temperature defect in the SCBR is shown in the last two columns of Table 2; it is five times larger under operating conditions than when the reactor is voided because of the much larger component of low-energy flux (see Fig. 1). The 
corresponding mean core Doppler cocfficients are $19 \times$ $10^{-6} \Delta k / k-^{\circ} \mathrm{C}$ and $3.7 \times 10^{-6} \Delta k / k^{-}{ }^{\circ} \mathrm{C}$.

Doppler measurements can be made in critical assemblies by several techniques (e.g., see G. J. Fischer ${ }^{(2)}$ ) 10 an accuracy of perhaps \pm 10 to $20 \%$. Furthermore, it seems reasonable to expect that the accuracy can and soon will be improved to \pm 5 to $10 \%$ as a result of current investigations of systematic errors (expansion effects, influence of heated-region size on incident spectrum, perturbation by heater), and improvements in experimental terhniques (suppression of reactor drift, increased sensitivity, extension to higher temperature). If such measurements are made in the critical assembly, as a function of position so that the spatial variation of the neutron spertrum is properly weighted, and with and without coolant present, we should be able to obtain $C F_{\text {trmp }}$ to an aceuracy of \pm 0.0013 to \pm 0.0026 .

For reasons discussed in the next section, it is preferable to fuel the critical assembly with readily available plutonium (5\% Pu-240) rather than with "equilibrium" plutonium (28\% P'u-240). Nlthough the calculations indicate that the temperature correction is approximately the same (within 0.001) for both types of fuel, such calculations are not very reliable for the three-component systems under consideration (U-238, Pu-239, $\mathrm{Pu}-240$ ) berause of large uncertainties in resonance cross sections, resonance interference, and overlap efferts. Therefore, if the critical assembly is fueled with plutonium that contains $5 \% \mathrm{Pu}-240$, the Doppler measurements should be made with both $5 \% \mathrm{Pu}-240$ and $28 \%$ $\mathrm{Pu}-240$ fuel samples to provide supplementary information on the $\mathrm{Pu}-240$ contribution and its interactions with the U-238 and Pu-239.

The neutron spectra in assemblics fueled with $5 \%$ and $28 \% \mathrm{Pu}-240$ material differ by less than $10 \%$ above about $100 \mathrm{eV}$ (and by no more than $20 \%$ at lower energies); the changes with temperature in the two spectra agree within $2 \%$ at all energies. Hence the correction for spectral differences is small and within an energy range where the cross sections are well known, and the

TABLE 3. EFFec THE TOID COEFFICIENT

\begin{tabular}{|c|c|c|c|c|}
\hline $\begin{array}{c}\text { Critiual } \\
\text { Assembly } \\
\text { Size }\end{array}$ & $\begin{array}{l}\text { Fuel Tem- } \\
\text { perature, } \\
{ }^{\circ} \mathrm{C}\end{array}$ & $\begin{array}{c}\text { P-240 Con- } \\
\text { centration, } \\
\%\end{array}$ & $k_{0}-k_{100}$ & $\begin{array}{c}\text { Correction } \\
\text { Factor } \\
C F_{\text {comp }}\end{array}$ \\
\hline $\begin{array}{l}5 / 1 \text { Slab } \\
5 / 1 \text { Slab }\end{array}$ & $\begin{array}{l}20 \\
20\end{array}$ & $\begin{array}{r}28 \\
5\end{array}$ & $\begin{array}{r}-0.0379 \\
-00634\end{array}$ & +0.026 \\
\hline $\begin{array}{l}\text { Annulus } \\
\text { Annulus }\end{array}$ & $\begin{array}{l}20 \\
20\end{array}$ & $\begin{array}{r}28 \\
5\end{array}$ & $\begin{array}{l}-0.0373 \\
-0.0638\end{array}$ & +0.026 \\
\hline $\begin{array}{l}\text { Annulus } \\
\text { Annulus }\end{array}$ & $\begin{array}{l}1363 \\
1363\end{array}$ & $\begin{array}{r}28 \\
5\end{array}$ & $\begin{array}{r}-0.0157 \\
-0.0405\end{array}$ & +0025 \\
\hline
\end{tabular}

Pu-239 and Pu-240 contributions should be small fractions of the U-238 contributions. Therefore, if the Doppler calculations are normalized to the experimental results in a critical assembly fueled with plutonium that contains $5 \% \mathrm{Pu}-240$, we should be able to extrapolate to the "equilibrium" plutonium case with good accuracy.

\section{EFFECT OF FUEL COMPOSITION}

The fuel for the reference power reactor is an "equilibrium" plutonium mixture that contains $28 \% \mathrm{Pu}-240$. It is probably unrealistic to assume that the required quantity of high-Pu-240 material (500 to $1000 \mathrm{~kg}$ ) is readily available for the critical experiments. The use of $\mathrm{Pu}-240$ also imposes some practical (although surmountable) difficulties in such areas as health physies, neutron background, and heat generation. Therefore, we must consider whether these experiments will provide useful results if they are fueled with readily available, low-Pu-240 plutonium; we nust carefully estimate the accuracy with which the void coefficient measured in such a critical assembly can be corrected for the difference in $\mathrm{Pu}-240$ concentration.

An alternative solution to this problem may be mentioned in passing: to simulate the Pu-240 with a more commonly available material, such as indium. Indium roughly approximates the capture cross section of $\mathrm{Pu}$ 240 over the entire energy range, within about $\pm 50 \%$; its fission cross section is zero. Calculations indicate that the equilibrium plutonium neutron spectrum can be matched fairly well by adding indium to pure $\mathrm{Pu}-239$; the resulting void coefficients arc the same, but it is difficult to specify the exart indium concentration because of large uncertainties in the Pu-240 and indium cross sections.

$\mathrm{Pu}-240$ self-shields, and some credit can be taken for the tendency toward saturation. However, the Pu-240 concentration must be at least $20 \%$ to obtain a resonance integral that is within $10 \%$ of the value for the $28 \%$ reference concentration $(1050 \mathrm{~b})$. In order to obtain a conservative limit, we assume in this analysis that the plutonium contains only $5 \% \mathrm{Pu}-240$; this material should be available in reasonable quantitien.

Using the same technical approach as before,

$$
\begin{aligned}
\left(k-k_{100}\right)^{28 \%}= & \left(k_{0}-k_{100}\right)^{5 \%} \\
& +\left(k_{100}^{5 \%}-k_{100}^{28 \%}\right) \\
& \quad-\left(k_{0}^{5 \%}-k_{0}^{28 \%}\right) \\
= & \left(k_{0}-k_{100}\right)^{5 \%}+\mathrm{CF}_{\text {comp }},
\end{aligned}
$$

where the superscripts refer to the Pu-240 isotopic concentration. The results, summarized in Table 3 , show that the correction (for plutonium isotopic composition) 
to the void coefficient measured in a critical assembly fueled with $5 \% \mathrm{Pu}-240$ plutonium is independent ( $\leqq 0.001)$ of critical assembly shape, size, or temperature. However, the void coefficient itself is strongly dependent on the plutonium isotopic composition; hence the source of this dependency must be carefully considered in order to estimate the accuracy with which this large correction factor can be determined.

\subsection{Effect of Pu-240}

Table 4 lists the fraction of $\mathrm{Pu}-240$ captures and fissions in a room-temperature, $5 / 1$ slab critical assembly fueled with plutonium that contains 5 or $28 \% \mathrm{Pu}-240$; the energy distribution of the $\mathrm{Pu}-240$ captures for these cases is plotted in Figure 3. Fortunately, a large fraction of the captures occurs at low energies where the cross section is known quite accurately; under operating conditions, with $5 \% \mathrm{Pu}-240$ fuel, roughly $1 / 3$ of the captures occur in the large resonance at $1.05 \mathrm{eV}, 1 / 3$ occur in the ten resolved resonances up to $119 \mathrm{eV}$, and only $1 / 3$ occur at higher energies. Since the cross section is known much more accurately below $167 \mathrm{eV}$ than above, this energy is a convenient break point in evaluating the uncertainty in the correction factor for $\mathrm{Pu}-240$ concentration.

\subsubsection{Analysis below $167 \mathrm{eV}$}

Using as extreme limits the uncertainties in the resolved resonance parameters reported in BNL-325, the correction factor in eq. 5-1 was recomputed by the standard 34-group method. The difference between the two calculations represents a conservative estimate of the uncertainty in the correction factor. A similar calculation was performed for the smooth (nonresonant) part of the capture cross section between 0.4 and $167 \mathrm{eV}$, assuming $a \pm 10 \%$ uncertainty in this value, which is consistent with available data. The resulting contributions to the uncertainty in the correction factor, summarized in Table 5 , are quite small.

We must also consider the error that may be contributed by the theoretical model used to compute the self-shielding in these resonances. As a guide, two other resonance-integral programs were compared with the RIP program used in the standard calculation; in all three models isolated resonances were assumed. RIP uses the Wigner rational approximation for the escape probability and the equivalence theorem to form an expression for the resonance integral; the use of the NR or NRIA approximation for each resonance must be specified. ${ }^{(3)}$ The Cohen-Goldstein intermediate absorption theory is used for ERIC-2. ${ }^{(4)} \mathrm{It}$ is an improvement over RIP in that it does not require a choice between NR and NRIA. GANIT utilizes the Nordheim integral treatment to obtain a numerical solution to the two-region, infinite-medium, slowing-down equations with an asym-
TABLE 4. Nectron Balance in Criticat Assembly

\begin{tabular}{l|c|c|c|c}
\hline \multirow{2}{*}{ Process } & \multicolumn{2}{|c|}{$5 \%$ Pu-240 } & \multicolumn{2}{c}{$28 \% \mathrm{Pu}-240$} \\
\cline { 2 - 5 } & 0 & 100 & 0 & 100 \\
\hline Pu-240 Captures & 0.0035 & 0.0154 & 0.0232 & 0.0598 \\
Pu-240 Fissions & 0.0033 & 0.0027 & 0.0223 & 0.0183 \\
Total Captures & 0.2867 & 0.3662 & 0.2914 & 0.3813 \\
Total Fissions & 0.3260 & 0.3591 & 0.3239 & 0.3484 \\
Total Leakage & 0.3873 & 0.2747 & 0.3847 & 0.2703 \\
\hline \multicolumn{1}{c|}{ Total } & 1.0000 & 1.0000 & 1.0000 & 1.0000 \\
\hline
\end{tabular}

metric Doppler-broadened Breit-Wigner resonance shape; ${ }^{(5)}$ thus a rigorous expression for the integral is formed. GANIT is an improved and faster version of ZUT. The results for the case with coolant present are compared in Table 6 for several microgroups in the 34 group scheme. It is evident that the effect of a model change is no greater than that caused by uncertainties in the resonance parameters themsclves, with a slight exception for the case of the $1.05-\mathrm{eV}$ resonance. The contribution to the uncertainty in the correction factor (see Table 5) is still negligible.

The remaining uncertainty in the region below 167 $\mathrm{eV}$ is that due to the alculation of the incident neutron spectrum. To avoid the complex task of estimating this error analytically, it is assumed that the spectrum will be measured by resonance foil-activation techniques which are useful in this energy range. If the calculated spectrum is forced to fit the measured value within $\pm 10 \%$, the contribution to the uncertainty in the correction factor is very small (see Table 5).

\subsubsection{Analysis above $167 \mathrm{eV}$}

Unfortunately, the differential capture cross section of $\mathrm{Pu}-240$ has not been measured above $167 \mathrm{eV}$. In many cross-section libraries, including that at $\mathrm{B \& W}$, it is assumed that the capture cross section is 1.75 times that of U-238; this ratio has been derived from statistical considerations. ${ }^{(6)}$ At Argonne National Laboratory ${ }^{(7)}$ a ratio of 1.4 above $2.5 \mathrm{keV}$ is used and the cross section computed at lower energies, using an s-wave strength function of $2.0 \times 10^{-4}$, and $\Gamma_{\gamma}$ and $\bar{D}$ in the resolved range from $B N L-325$. To provide another point for comparison, ERIC-2 was run to compute microgroup cross sections from $167 \mathrm{eV}$ to $15.03 \mathrm{keV}$, using the same inputs. The new cross sections were $38 \%$ lower in the lowest microgroup and $18 \%$ higher in the highest, varying smoothly over this range. However, it is impossible to make a realistic estimate of the uncertainty in the $\mathrm{Pu}-240$ capture cross section without experimental evidence. As an illustration of the seriousness of this deficiency, the uncertainty in the correction to the void 


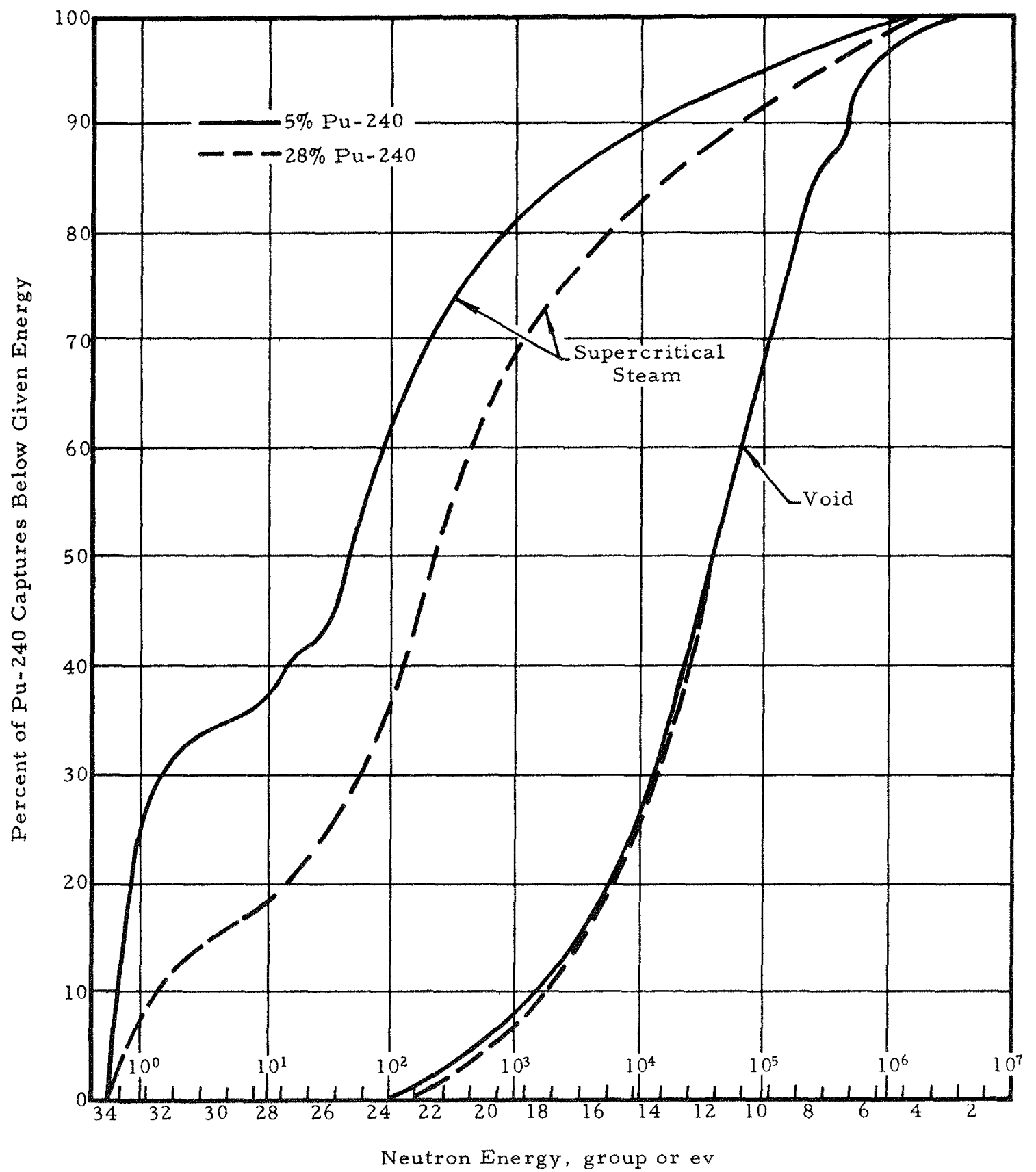

Fic. 3. Hhergy I)istrubution of Pu-240 Captures.

roefficient, assuming a $50 \%$ uncertainty in the capture cross section of $\mathrm{Pu}-240$ above $167 \mathrm{eV}$, is approximately $\pm 0.00 \% 5$.

Therefore, it in worth considering at this point what additional data we can obtain from these critical experiments (beyond the void coefficient) to reduce the dependency of the correction fartor for plutonium isotopic composition on Pu-240 cross-sectional uncertainties.

The details of these auxiliary experiments are discussed in the next sertion, but the conclusions are pre- sented here. The following approach is suggested: (1) measure the total Pu-240 capture integral in the critical ansembly by means of reactivity techniques; (2) calculate the contribution from 0.4 to $167 \mathrm{eV}$; (3) derive the integral above $167 \mathrm{eV}$ by difference. Table 7 shows the results for the two types of fuel and their respective spectra. The uncertainty in the calculated integral to $167 \mathrm{eV}$ was obtained by weighting the individual sourees of error (disrussed previously) by the number of raptures. The uncertainty in the measured total integral 
TABLE 5. UVCer Fal Correcion die to $\mathrm{Pu} 240$

\begin{tabular}{|c|c|c|c|c|}
\hline \multirow{2}{*}{ Source } & \multirow{2}{*}{$\begin{array}{l}\text { Uncer- } \\
\text { tainty, } \\
\qquad \%\end{array}$} & \multicolumn{3}{|c|}{ Uncertainty in } \\
\hline & & $k_{100}^{5 \%}-k_{100}^{28 \%}$ & $k_{0}^{5 \%}-k_{0}^{28 \%}$ & $C F_{\text {corrup }}$ \\
\hline \multicolumn{5}{|l|}{$\begin{array}{l}\text { Captures below } 167 \\
\text { el' }\end{array}$} \\
\hline 1.05-el Resonance & 5 & 0.0001 & 00000 & 0.0001 \\
\hline $\begin{array}{c}\text { Next } 10 \text { Reso } \\
\text { nances }\end{array}$ & 17 & 0.0008 & 0.0000 & 0.0008 \\
\hline Smooth Capture & 10 & 0.0007 & 0.0000 & 0.0007 \\
\hline Shielding Model & 17 & 0.0007 & 0.0000 & 0.0007 \\
\hline Spectrum Model & 10 & 0.0011 & 0.0000 & 00011 \\
\hline \multicolumn{5}{|l|}{$\begin{array}{l}\text { Captures above } 167 \\
\text { el }\end{array}$} \\
\hline $\begin{array}{l}\text { With Single-zone } \\
\text { Expt }\end{array}$ & 35 & 0.0098 & 00059 & 0.0039 \\
\hline $\begin{array}{l}\text { With Two zone } \\
\text { Expt }\end{array}$ & 17 & 0.0047 & 0.0029 & 0.0018 \\
\hline Measurement of $\bar{\sigma}_{j}$ & 9 & 0.0024 & 0.0027 & -00003 \\
\hline Measurement of $\bar{\nu}$ & 7 & 0.0031 & 0.0036 & -00005 \\
\hline Total Uncertainty & & & & \\
\hline $\begin{array}{l}\text { With Single-zone } \\
\text { Expt }\end{array}$ & - & - & - & 0.0043 \\
\hline $\begin{array}{l}\text { With Two-zone } \\
\text { Expt }\end{array}$ & - & - & - & 0.0025 \\
\hline
\end{tabular}

includes correction for Pu-240 fissions and for captures and fissions in the accompanying Pu-239. The uncertainty in the derived integral above $167 \mathrm{eV}$ assumes that the other two errors are random.

If the total capture integral is measured in a critical assembly fueled with plutonium that contains $5 \% \mathrm{Pu}-$ 240 , the integral above $167 \mathrm{eV}$ can be derived to about $\pm 3.5 \%$. If more arcurate results are required, a twozone experiment can be performed. In the 1 wo-zone experiment, the critical assembly contains a small central zone fueled with $28 \% \mathrm{Pu}-240$ material and large enough to achieve a neutron spectrum characteristic of a large single-zone assembly fueled entirely with $28 \%$ P'u-240 plutonium. If the total capture integral is measured in this type of experinent, the integral above $167 \mathrm{eV}$ can be derived much more aceurately, because the spectrum is harder, and a much larger fraction of the measured total capture ocrurs in the energy range of interest $(>167 \mathrm{eV})$.

TABLH 7. Pu-240 CAPTU RE INTEgRals

\begin{tabular}{|c|c|c|c|c|}
\hline \multirow{3}{*}{ Identification } & \multicolumn{2}{|c|}{ Total Captures } & \multicolumn{2}{|c|}{$\begin{array}{l}\text { Uncertainty } \\
\qquad( \pm \%)\end{array}$} \\
\hline & - & $\longrightarrow$ & - & \\
\hline & $\begin{array}{l}5 \% \\
P \mathrm{u}-240\end{array}$ & $\begin{array}{l}28 \% \\
\text { Pu-240 }\end{array}$ & $\begin{array}{l}5 \% \\
\mathrm{Pu}-240\end{array}$ & $\begin{array}{l}28 \% \\
\mathrm{Pu}-240\end{array}$ \\
\hline 1.05-el Resonance & 0.0028 & 00020 & - & - \\
\hline $\begin{array}{l}\text { Next } 10 \text { Resolved Reso- } \\
\text { nances }\end{array}$ & 0.0033 & 00083 & - & -- \\
\hline $\begin{array}{l}\text { Smooth Capture, } 0.4167 \\
\mathrm{eY}^{\circ} \\
\rho^{167}\end{array}$ & 0.0030 & 0.0147 & & - \\
\hline $\int_{\substack{0.4 \\
\text { lated } \\
\int^{10^{7}}}} \sigma_{c}(E) \phi(E) d E$-Calcu- & 0.0100 & 0.0250 & 16 & 15 \\
\hline $\begin{array}{l}\int_{04} \sigma_{\iota}(E) \phi(E) d E-\text { Meits- } \\
\text { ured } \\
f^{10^{7}}\end{array}$ & 0.0154 & 0.0598 & 7 & 8 \\
\hline $\begin{array}{l}\left.\int_{167} \sigma_{e}\left(E^{\prime}\right) \phi\left(E^{\top}\right) d E-1\right) \mathrm{e}- \\
\text { rived }\end{array}$ & 0.0054 & 0.0348 & 35 & 17 \\
\hline
\end{tabular}

table 6. Comparison of Low-energy Pu-240 Resonanee-integral Calculations

\begin{tabular}{|c|c|c|c|c|c|c|c|c|c|}
\hline \multirow{2}{*}{\multicolumn{2}{|c|}{ Microgroup }} & \multirow{2}{*}{$\begin{array}{l}\text { Low-energy } \\
\text { Limit, } \mathrm{eV}\end{array}$} & \multicolumn{3}{|c|}{ Group Resonance Integral } & \multicolumn{4}{|c|}{ Group Capture Cross Section } \\
\hline & & & RIP & ERIC-2 & GANIT & RIP & $\operatorname{RIP}^{(\mathrm{a})}$ & ERTC-2 & GANIT \\
\hline \multicolumn{10}{|c|}{ Plutonium Containing $5 \%$ Pu-240 } \\
\hline & 33 & \multirow{5}{*}{$\begin{array}{c}0.638 \\
13.71 \\
37.27 \\
61.44 \\
101.3\end{array}$} & +64.02 & 184.86 & (b) & 1208 & \multirow{5}{*}{$\begin{array}{r}1249 \\
31 \\
28 \\
22 \\
20\end{array}$} & 1250 & \multirow{5}{*}{$\begin{array}{l}\text { (b) } \\
30 \\
28 \\
22 \\
21\end{array}$} \\
\hline & 27 & & 7.17 & 7.26 & 7.44 & 29 & & 30 & \\
\hline & 25 & & 8.21 & 8.80 & 9.12 & 26 & & 27 & \\
\hline & 24 & & 9.25 & 10.28 & 10.76 & 19 & & 21 & \\
\hline & 23 & & 4.92 & 5.48 & 5.79 & 19 & & 20 & \\
\hline \multicolumn{10}{|c|}{ Plutonium Containing $28 \% \mathrm{Pu}-240$} \\
\hline & 33 & 0.638 & 1195.0 & 1274.0 & (b) & 2671 & 2775 & 2829 & (b) \\
\hline & 27 & 13.71 & 15.6 & 15.7 & 15.9 & 46 & 49 & 46 & 47 \\
\hline & 25 & 37.27 & 19.4 & 20.0 & 20.5 & 48 & 53 & 49 & 51 \\
\hline & 24 & 61.44 & 22.1 & 23.0 & 23.7 & 44 & 54 & 46 & 47 \\
\hline & 23 & 101.3 & 12.2 & 12.5 & 12.9 & 33 & 38 & 34 & 35 \\
\hline
\end{tabular}

(a) RIP using extreme values of resonance parameters.

(b) Not run; should fall between RIP and RIP.(a) 
No additional error is encountered in deriving the effective cross section from these integral values, because the neutron spectrum above $167 \mathrm{eV}$ is the same (within the arcuracy of the integral value) for all cases appearing in the correction factor (sec Fig. 2). Of course, measurements should be made with both 5 and $28 \%$ $\mathrm{Pu}-240$ samples, beeause the resonance shielding differs somewhat in this energy range. Using eq. 5-1, the uncertainty in the correction to the void coefficient due to uncertainties in the $\mathrm{Pu}-240$ capture cross section above $167 \mathrm{eV}$ was computed; the results are included in the summary in Table $\tilde{J}$.

\subsubsection{A uxiliary Experiments}

In addition to the void roefficient itself, other measurements may be taken in the critieal assembly to provide supplementary information on the Pu-240 cross sections and to reduce the uncertainty in the void coefficient correction for $\mathrm{Pu}-240$ concentration.

The total fission integral of Pu-240 can be measured relative to $\mathrm{Pu}-239$ (to about $\pm 2 \%$ ) by activation or counting techniques, e.g., Kirn. ${ }^{\left({ }^{(}\right)} \mathrm{l}^{\mathrm{P} u-239}$ is the preferred normalizer since its fiscion cross section varies less with energy than does that of U-233 or U-235. If we use a conservative estimate of the uncertainty in computing $\tilde{\sigma}_{f}^{239}$, the total fission integral

$$
\int_{0}^{\infty} \sigma_{f}^{240}\left(E_{i}\right) \phi(E) d E
$$

can be obtained to an accuracy of about $\pm 9 \%$ from measurements in the critical assembly under voided and supercritical steam conditions. It is not necessary to subdivide the fission integral into discrete energy ranges, nor to make the measurement in a spectrum characteristic of $28 \% \mathrm{Pu}-240$ fuel, because the fission integral differs by less than $1 \%$ in the two spectra. As summarized in Table 5, the effect of a $9 \%$ uncertainty in $\bar{\sigma}_{f}^{240}$ on the correction to the void coefficient is negligible.

Data on the total capture integral and $\bar{\nu}$ can be derived from reactivity measurements in the critical assembly, e.g., Ref. 9, which are proportional to $(\vec{v}-$ 1) $\bar{\sigma}_{f}-\bar{\sigma}_{c}^{*}$, where $\bar{\sigma}_{c}^{*}$ includes a small scattering correction that can be obtained experimentally. If such measurements are made in the harder, spectrum-voided critical assembly, to emphasize $(\bar{\nu}-1) \bar{\sigma}_{f}$ over $\bar{\sigma}_{c}^{*}, \bar{\nu}^{240}$ can be obtained to about \pm 0.2 using the previously determined value of $\bar{\sigma}_{f}^{240}$ and a very conservative estimate of $\bar{\sigma}_{c}^{240}$. As in the case of $\bar{\sigma}_{f}^{240}, \bar{\nu}^{240}$ is essentially the same in a spectrum characteristic of $28 \% \mathrm{Pu}-240$ fuel, and (as summarized in Table 5) this uncertainty has a negligible effect on the correction to the void coefficient.

Information on $\bar{\sigma}_{c}^{240}$ can be obtained from the same type of reactivity measurement if the spectrum is softer to allow captures to predominate over fissions. Whereas the reactivity is about 2.5 times more sensitive to ( $\vec{\nu}-$ 1) $\bar{\sigma}_{f}$ under voided conditions, it is about 2.j) times more sensitive to $\bar{\sigma}_{\theta}$ when the supercritical steam is prosent. By performing precise reactivity comparisons with plutonium samples that contain varying concentrations of $\mathrm{Pu}-240$, the total capture integral

$$
\int_{0}^{\infty} \sigma_{c}^{240}(E) \phi_{100}(E) d E
$$

may be obtained to an accurary of about $\pm 7 \%$. The following sources of error were considered in making this estimate: (1) accuracy of reactivity measurements; (2) corrections for fissions and captures in $\mathrm{Pu}-239$; (3) correction for fissions in Pu-240 as obtained from the above measurements in the voided spectrum; (4) calculation of the $\mathrm{Pu}-239$ normalization.

As stated previously, the capture integral above 167 $\mathrm{eV}$ can be much more accurately derived if the reactivity measurements are made in a spectrum characteristic of $28 \%$ Pu-240 fuel. Because of the large quantity of this material required to fuel the entire critical assembly, and the resulting cost and availability problems, the two-zone approach may be considered. With plutonium that contains $5 \% \mathrm{Pu}-240$ in the outer zone and $28 \% \mathrm{Pu}-240$ in the inner zone, calculations indicate that the inner zone needs a radius of only about $16 \mathrm{~cm}$ for the neutron spertrum to be asymptotic (to within $\pm 2 \%$ ) over a $2.54-\mathrm{cm}$ radius. The total quantity of the $28 \% \mathrm{Pu}-240$ plutonium is only $20 \mathrm{~kg}$, a very substantial reduction from the 750 to $1150 \mathrm{~kg}$ that would be required to fuel the entire critical assembly. The adjointspectrum match has not yet been examined, so the actual required quantity of $28 \% \mathrm{Pu}-240$ material may be somewhat larger.

\subsubsection{Summary of Pu-24O Corrections}

The corrections to the void coefficient for the difference in P'u-240 concentration between a critical assembly that uses $5 \% \mathrm{Pu}-240$ fuel and the reference power reartor, which contains $28 \% \mathrm{Pu}-240$ fuel, are collected and summarized in Table 5.

\subsection{Effect of Other Cross Sections}

Because of the large magnitude of the correction factor for the difference in fuel composition, it is necessary to consider how much error is introduced in this calculation by uncertainties in other nuclear cross sections and parameters. Recalling eq. 5-1, the sensitivity of the correction factor to changes in cross sections is given by the net change in four criticality calculations:

$$
\mathrm{CF}_{\text {comp }}=\left(k_{100}^{5 \%}-k_{100}^{28 \%}\right)-\left(k_{0}^{50 \%}-k_{0}^{28 \%}\right) .
$$

Therefore, although there may be a relatively large un- 
rertainty in computing any one value of $k_{\text {eff }}$, we would expect a strong cancellation of errors in computing differences in $k_{\text {eff }}$ and much less uncertainty in the final value of $\mathrm{CF}_{\text {comp }}$.

The uncertainties to be applied to the cross sections could be obtained from an evaluation of experimental mirroscopic cross-section data. However, one of the primary purposes of this (or any other) (ritical experiment program is to obtain integral data that can be used to improve the reliability of the set cross section. It is obvious, then, that the proper assessment of the error in the calculated correction factor requires some prior assumption as to the eventual accuracy of the integral data; it is equally obvious that the degree of success will not be known until the experiments are completed and their results are analyzed. However, in order to proceed at this time, the following assumption will be made: $k_{\text {efi }}$ will be measured in the critical experiments as a function of hydrogen density, from void to supereritical steam density, and these data will be matched to within $\pm 2 \%$ over the entire range by plausible adjustments in the cross sections. The calculations could be forced to fit the measurements exartly by arbitrary changes in the cross sections, but this does not rule out the possibility that errors may then be revealed in the calculation of the correction factor.

Table 8 shows the results of a four-macrogroup condensation that has the energy limits stated in Section 2. The fourth column represents the change in a particular cross section required to produce a $2 \%$ change in either $k_{0}$ or $k_{100}$, and the last three columns are the changes that occur in the correction factor (eq. 5-2) when the cross section is increased by that amount. Group 4 is not shown because in all cases the changes required in thermal cross sections to cause a $2 \%$ change in $k_{\text {eff }}$ are greater than $200 \%$, which is clearly outside reasonable bounds for experimental errors. The change in the capture eross sections for the cladding materials is the difference between the $B \& W$ and $\mathrm{ANL}$ and ORNI, libraries, and is principally in group 2. The change in the inelastic scattering matrix represents the difference between the Westinghouse MUFT-5 library and GAM-1, which is used in the B\&W library. As expected, the uncertainties in the correction factor are fairly small, even though $k_{\mathrm{eff}}$ itself changes by $2 \%$, because the spectral and compositional differences between pairs of terms are small.

The reason for deriving the uncertainty in the correction factor separately for each isotope, process, and energy range on the assumption that all of the uncertainty caused by a $2 \%$ mismatch in $k_{\text {ef }}$ is concentrated there rather than randomly, is to avoid the problem of deciding how to compound individual errors. From this point of view, the maximum uncertainty in $\mathrm{CF}_{\text {comp }}$ is the largest number in the last column of Table 8 , i.e., 0.004. Those cases for which the change in $\mathrm{CF}_{\text {comp }}$ is small, and those requiring an unrealistically large crosssection change can be disregarded. Of the remaining cases, no combination of compensating simultaneous cross-section changes could be found that changed $\mathrm{CF}_{\text {comp }}$ by more than 0.004 , subject to the constraint that $k_{0}$ or $k_{100}$ did not change by more than $2 \%$.

TABle 8. UnCertaixty in Votd Coefficient Correctron due to Other Cross Sections

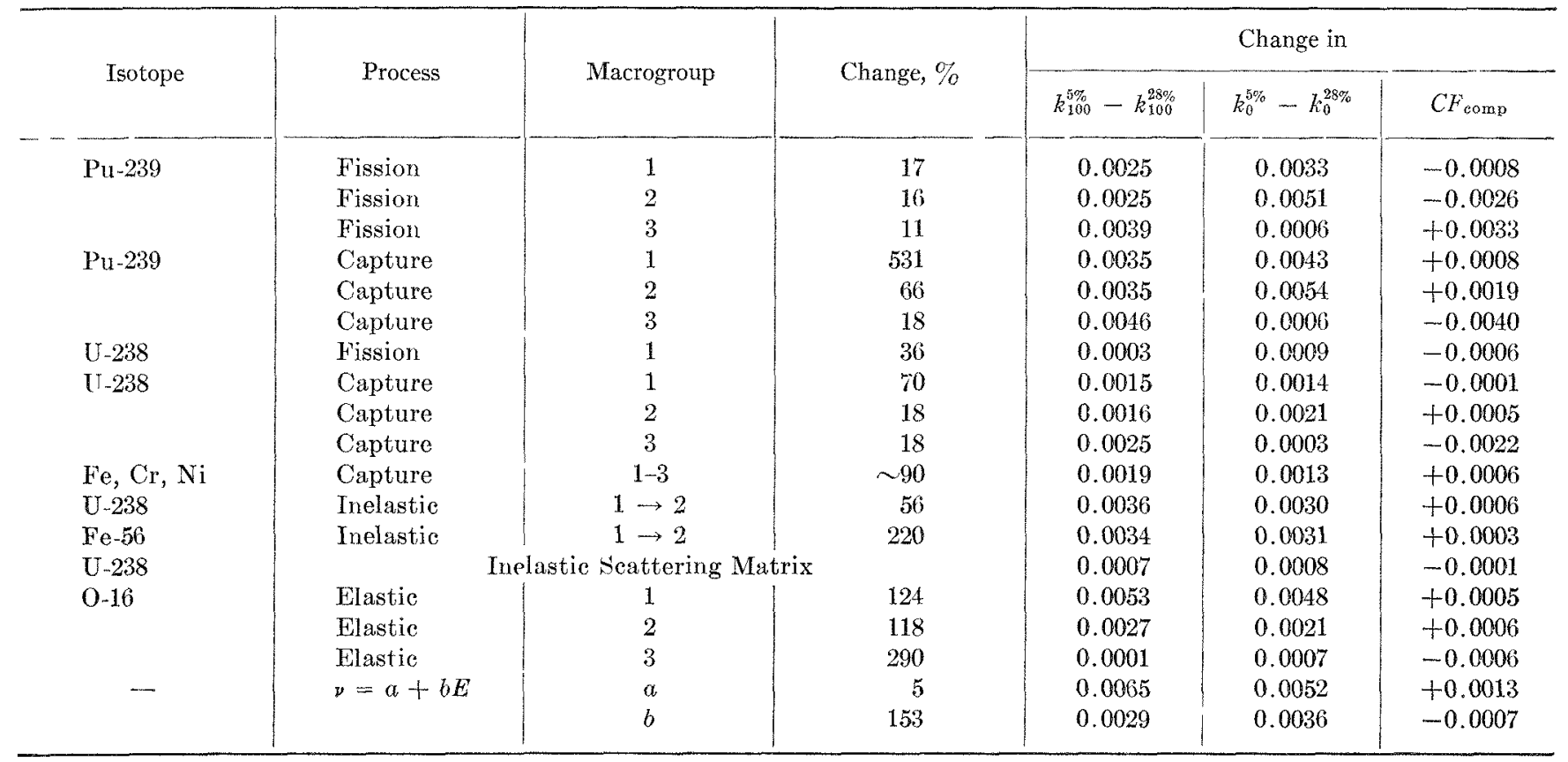




\section{EFFECT OF HETEROGENEITY}

Although the local heterogeneities of the reference power reactor could be reproduced in exact detail in the critical assembly, the experiments would be simplified if some freedom could be allowed in this area without seriously compromising the aceuracy of the roid roefficient.

The use of expanded plastics to simulate the low hydrogen density of supereritical steam at operating ronditions introduces foreign elements, e.g., carbon, and some mismatch in the $\mathrm{O} / \mathrm{H}$ ratio. On the basis of comparative calculations between supercritical steam and expanded polyethylene, in which the hydrogen density was matched exactly, $k_{100}$ changed only by 0.0008 ; therefore, the effect on the correction factor to the void coefficient should be entirely negligible. Iifferences in hydrogen bonding are negligible in these systems.

Because of the particular sensitivity of the SCBR to resonance effects, we prefer to use a fucl form in the critical assembly that approximates, at least to the first order, that of the reference power reactor, i.e., $\mathrm{Pu}-\mathrm{C}$ oxide in metal tubes. However, the cost and handling problems of fuel pins would be substantially reduced if the diameter could be increased over the $0.230 \mathrm{~min}$. reference design. Calculations were performed with 0.5-and 0.75-in.-diameter pins, taking into account the change in Dancoff factor and self-shielding; the correction factors to the void coefficient were found to be 0.0017 and 0.0030 , respectively. If the correction factor could be computed to an arcuracy of $\pm .50 \%$, the

TABLE 9. Summary of Corrections to the VOIJ COEFFICIENT

\begin{tabular}{|c|c|c|c|}
\hline Source & $k_{0}-k_{100}$ & $\begin{array}{l}\text { Estimated } \\
\text { Cncer- } \\
\text { tainty }\end{array}$ & Comments \\
\hline $\begin{array}{l}\text { Measurement in Criti- } \\
\text { cal Assembly }\end{array}$ & -0.063 & $<0.001$ & Assumed \\
\hline $\begin{array}{l}\text { Correction for Shape } \\
\text { and Size }\end{array}$ & $<0.001$ & 0.001 & $\begin{array}{c}\text { Use } 5 / 1 \text { Slab } \\
\text { Critical As- } \\
\text { sembly }\end{array}$ \\
\hline $\begin{array}{l}\text { Correction for Tem- } \\
\text { perature }\end{array}$ & +0.021 & $\begin{array}{l}0.0013 \\
\text { to } \\
0.0026\end{array}$ & $\begin{array}{l}\text { Measure Dop- } \\
\text { pler to } \pm 5 \text { or } \\
10 \%\end{array}$ \\
\hline $\begin{array}{l}\text { Correction for Fuel } \\
\text { Composition } \\
\mathrm{Pu}-240\end{array}$ & +0.026 & 0.0025 & $\begin{array}{c}\text { Use Two-zone } \\
\text { Experiment }\end{array}$ \\
\hline Other Isotopes & & $\begin{array}{l}0.0020 \\
\text { to } \\
0.0040\end{array}$ & $\begin{array}{l}\text { Match } k_{\mathrm{eff}} \text { to } \\
\quad \pm 1 \text { or } 2 \%\end{array}$ \\
\hline $\begin{array}{c}\text { Value beduced for } \\
\text { Operating SCBR }\end{array}$ & -0.016 & $\begin{array}{l}0.004 \\
\text { to } \\
0.005\end{array}$ & $\begin{array}{l}\text { Errors Added } \\
\text { Randomly }\end{array}$ \\
\hline
\end{tabular}

use of 0.5-in.diameter pins would appear to be an attractive alternate because it would reduce the number of pins required by more than a fartor of four. The incentive justifies a more detailed evaluation of this possibility.

The metal-and alloy-plated types of fuel commonly used to permit major composition changes in other fast rritical assemblies have not been considered here because they are more expensive, and the addition of oxygen is a problem. Before such fuel forms could be considered for SCBR experiments, the accuracy of the void coefficient correction for heterogeneity would have to be studied in detail, not only because the correction is expected to be large, but also because it must be determined with absolute aceuracy $( \pm 0.001$ to 0.002$)$.

The reference power reactor has a number of control rods approximately $1 \frac{1}{4} \mathrm{in}$. in diameter located on approximately 6 -in. centers. Since the control rods and holes affect the void cocficient, the void roefficient must be measured in the critical assembly with and without control rods, as well as in the clean, uniform lattice. Although this is a measured rather than a calculated heterogeneity correction, it must be shown that the void roefficient in a lab ritical assembly is the same as that in the annular reference design, with control rods present in both ases. Two-dimensional diffusion calculations were performed with the PDQ code, using the same routine for computing group constants that was described earlier for the case of the clean, uniform lattice. With the control rods in, the difference between the void coefficient of the critical assembly and that of the reference reactor was only 0.0005 ; with the control rods removed, and the rod guides and holes remaining in the core, the difference was only 0.0006 . Thus, the correction factor is negligible for this source of heterogeneity.

\section{SUMMARY AND CONCLUSIONS}

The major differences between what may be considered a "practical" "ritical assembly and the operating reference SCBR reactor are summarized in Table 9 . The magnitude of the correction factors and the estimated accuracy of the extrapolation of the void coefficient, as measured in a critical assembly, to that for the reference power reactor are shown for one of several possible sets of assumptions.

In this comparison, the critical assembly is assumed to be a $5 / 1$ slab, rather than the full-size referencedesign annular core. The measurements are taken at room temperature, and the extrapolation to the operating temperature is derived, from Doppler measurements in the critical assembly, to an accuracy of either \pm 5 or $10 \%$. The critical assembly is fueled with readily 
available, 5\% $\mathrm{Pu}-240$ plutonium. To correct to the reference design "equilibrium" plutonium $(28 \% \mathrm{Pu}$ 240), Pu-240 cross sections are derived from integral measurements in a two-zone assembly $(\sim 20 \mathrm{~kg}$ highPu-240 material), and the basis for the uncertainty in the correction due to other cross sections is a match of the experimental $k_{\text {ef }}$ to \pm 1 or $2 \%$. The size and shape of the reference design fuel element are used to avoid any heterogeneity correction.

If the void coefficient is required to an accuracy of better than \pm 0.004 to 0.005 , it is evident that the entire critical assembly will have to be fueled with high-Pu-240 plutonium, since the correction for fuel composition has the greatest uncertainty. It should be unneressary to go as high as $28 \% \mathrm{Pu}-240$, however. From a rough estimate, it appears that the use of 15 to $20 \%$ Pu-240 material should improve the accuracy by about a factor of two and make this source of uncertainty consistent with the optimistic value for the uncertainty in the temperature correction. The resulting void coefficient accuracy would then be about \pm 0.002 . Short of a full temperature mockup, it does not seem realistic to expect accuracies much better than about \pm 0.002 .

If accuracy in the range from \pm 0.004 to 0.005 can be tolerated, further simplifications can be considered. The critical assembly could be a $3 / 1$ slab rather than a $5 / 1$ slab, thereby saving an additional $40 \%$ in fuel and material costs, and the diameter of the fuel pins could be doubled, decreasing the number of fuel pins by a factor of four. If optimistic values are assumed for the accuracy of temperature and fuel-composition corrections, then the overall accuracy of the void coefficient would be about \pm 0.004 . However, if the temperature correction is limited to $10 \%$ accuracy and the critical-experiment data are matched only to $\pm 2 \%$ in $k_{\text {eff }}$, the overall accuracy for the void coefficient would decrease to about \pm 0.006 .

The following conclusions, although strictly applicable only to the SCBR, should be considered when designing a critical assembly to measure the void coefficient for large fast power reactors:

1. The size of the assembly can be reduced substantially without introducing errors that are larger than those from other sources. For example, the core volume and critical mass of the 3,1 slab ritical assembly are only about $1 / 4$ as large as the reference reactor volume and mass.

2. The correction for temperature is quite important and is highly dependent on accurate Doppler measurements. Although Doppler measurements for kinetics and safety information are needed only to perhaps $\pm 20 \%$ accuracy, techniques must be developed to the point where \pm 5 to $10 \%$ accuracy can be guaranteed when the void coefficient is considered.

3. The largest correction is that for plutonium isotopic composition, and unless 750 to $1150 \mathrm{~kg}$ of higherPu-240 material is made available and used for these experiments, it represents the principal limitation on the accuracy of measurements of the void coefficient. The uncertainty in this correction will be reduced as the accuracy of the microscopic cross-section data for $\mathrm{Pu}-240$ and the other common isotopes is improved.

4. Heterogeneity corrections can be avoided by using the reference-reactor fuel forms. However, the use of other fuel forms would impose a heavy burden on the heterogeneity correction, and the arcuracy of the void coefficient could well be limited by the accuracy of such calculations.

\section{REFERENCES}

1. Edlund, M. C., MacMillan, J. H., and Schluderberg, I). C., Cooling Fast Breeders with Steam, Nucleonics, 23, No. 5, p. 60 (May 1965).

2. Fischer, G. J., et al., A.TS Transactions, Vol. 7, No. 2, p. 252 (Nov 1964).

3. Roy, I). H., RIP: A General Purpose Resonance Integral Program for the Philco-2000, BAW-TM-383 (1963).

4. Sumner, H. M., A Fortran Program to Calculate Resonance Integrals and from Them Effective Capture and Fission Cross Sections, AEEW-R-393 (1964).

5. Nordheim, I. W., 1 Program of Research and Calculation of Resonance Absorption, GA-25.87 (1961).

6. Douglas, A. C., Neutron Cross Sections of Pu-240 in the Energy Range $1 \mathrm{keV}$ to $15 \mathrm{MeV}, \mathrm{AWRE}-0.91 / 64$ (1965).

7. Hummel, H. H. (ANL) to Engelder, T. C. $(B \& W)$, private communication, March 1965.

8. Kirn, F. S., Symposium on Neutron Dosimetry, Vol. 2, p. 497, International Atomic Energy Agency, 1963.

9. Smith, R. D., and Sanders, J. E., Second UN International Conference on the Peaceful Uses of Atomic Energy Geneva, Proceedings, 12, 3 (1958). 


\title{
Sodium-void Coefficients
}

\author{
W. Y. Kato, G. K. Rusch, and F. H. Helm \\ Argomne National Laboratory \\ Argonne, Illinois
}

(PRESENTED BY W. Y. KATO)

An understanding of the sodium-void coefficient for large fast sodium-cooled power reactors is of paramount importance from safety considerations. Considerable effort is being devoted both experimentally and theoretically to further the understanding of this effect by Argonne National Laboratory. In the past, the sodium-void effect has been measured on a number of assemblies constructed on ZPR-3.(1,2) During the past two years, there have been two large carbide assemblies which have been thoroughly studied for the sodium coefficient on ZPR- $6 .^{(3,4)}$ This paper is a status report on the work with the ZPR-6 facility.

The first assembly studied was a 610 -liter uranium carbide core which had a length-to-diameter ratio of approximately unity. The second core was a flat, pancake type of core whose volume was about 950 liters

TABLE 1. CORE 1) IMENSTONS OF ZPR-6 $(\imath)$

\begin{tabular}{l|c|c}
\hline & \multicolumn{2}{|c}{ Assembly No. } \\
\cline { 2 - 3 } & 2 & 3 \\
\hline Volume (liters) & 610 & 950 \\
Length (cm) & 91.4 & 50.8 \\
Diameter (cm) & 92 & 154.5 \\
L/D & 1 & $1 / 3$ \\
Critical Mass (kg) & 544 & 850 \\
\hline
\end{tabular}

(a) Both cores had $30-\mathrm{cm}$-thick axial and radial blankets.

TABLE 2. Compostion for Assemblies 2 AND 3 of ZPR-6

\begin{tabular}{|c|c|c|c|c|}
\hline & \multicolumn{2}{|c|}{ Core } & \multicolumn{2}{|c|}{ Blanket } \\
\hline & $\begin{array}{c}\text { Atom } \\
\text { Density, } \times \\
10^{24} \mathrm{~cm}^{-3}\end{array}$ & $\begin{array}{c}\text { YOM } \\
\text { Volume } \\
\text { Fraction }(a)\end{array}$ & $\begin{array}{c}\text { Atom Density, } \\
\times 10^{24} \mathrm{~cm}^{-3}\end{array}$ & $\begin{array}{l}\text { YOM } \\
\text { Volume } \\
\text { Fraction }\end{array}$ \\
\hline U-235 & 0.0022859 & 0.04760 & $8.104 \times 10^{-5}$ & 0.001688 \\
\hline $\mathrm{U}-238$ & 0.009853 & 0.2052 & 0.03993 & 0.8318 \\
\hline $\mathrm{C}$ & 0.01290 & 0.1540 & & \\
\hline $\mathrm{Na}$ & 0.007910 & 0.3595 & & \\
\hline SS-304 & 0.01475 & 0.17506 & 0.006003 & 0.07122 \\
\hline
\end{tabular}

(a) Corrected volume fractions for use with the macroscopic cross sections by Yiftah, Okrent, and Moldauer, Fast Reactor Cross Sections, Pergamon Press. with a length-to-diameter ratio of $\sim 1: 3$. A comparison of the dimensions of the two cores is given in Table 1. Both Assemblies No. 2 and 3 had the identical composition as given in Table 2 .

A typical drawer loading is shown in Fig. 1. The drawer was $2 \mathrm{in}$. in width and $2 \mathrm{in}$. in height. The depleted uranium and graphite were $\sim 1 / 8$ in. in thickness, the fuel plates $1 / 16 \mathrm{in}$. in thickness, and the sodium $1 / 4$ in. in thickness. The sodium-void measurements were performed by three separate runs, in each of which critical positions of the rods were established at a given power level. First, a reference run establishing the critical rod positions for a normal core configuration was made. Second, a run with sodium voided in the region of interest was made. Sodium cans were replaced by stainless steel frames which contained, with an accuracy of about $10 \%$, the same amount of stainless steel as the cans. Third, a run was made in which the worth of stainless steel was measured. This was necessary in order to correct for the difference in stainless steel between the sodium cans and the frames. In general, all results were found to be reproducible to between 0.5 and $1 \mathrm{Ih}$.

In both cores, a thorough mapping of the sodiumvoid coefficient as a function of location has been accomplished. With Assembly No. 3 a second type of measurement, concerning the effects of material arrangement and heterogeneity on the sodium-void coefficient, was carried out. The latter measurements were expected to be helpful in interpreting the sodiumvoid mapping results.

The mapping of the sodium-void coefficient was accomplished by obtaining sodium versus void measurements in central regions with different diameters, in regions along the core axis, in cylindrical rings, and finally in a sector comprising $1 / 8$ of the total of the core. Outlines of some of the regions are shown in Figs. 2 to 4; measured results are given in Tables 3, 4 , and 5 . Figure 2 shows examples of the regions which were voided in the core for both assemblies. Table 3 gives a comparison of the sodium-void coefficients which were obtained in the central regions in Assemblies 2 and 3 . Tables 4 and 5 give the sodium-void co- 


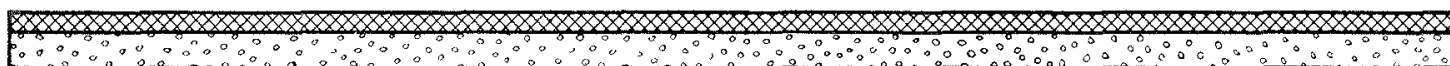
6 13

$\begin{array}{llllllllllll} & 1 & 1 & \mid & \mid & \mid & \mid & \mid & \mid & \mid & 1 \\ 0 & 1 & 2 & 3 & 4 & \begin{array}{c}5 \\ \text { inch }\end{array} & 6 & 7 & 8 & 9 & 10\end{array}$

\section{QDIIS ENRICHED $u^{235}$ \\ DEPLETED U ${ }^{238}$

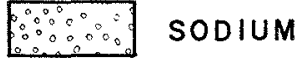

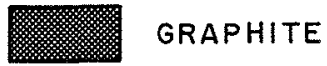

\section{STAINLESS STEEL}

FIa. 1. Typical Drawer Loading for Assembly No. 3.
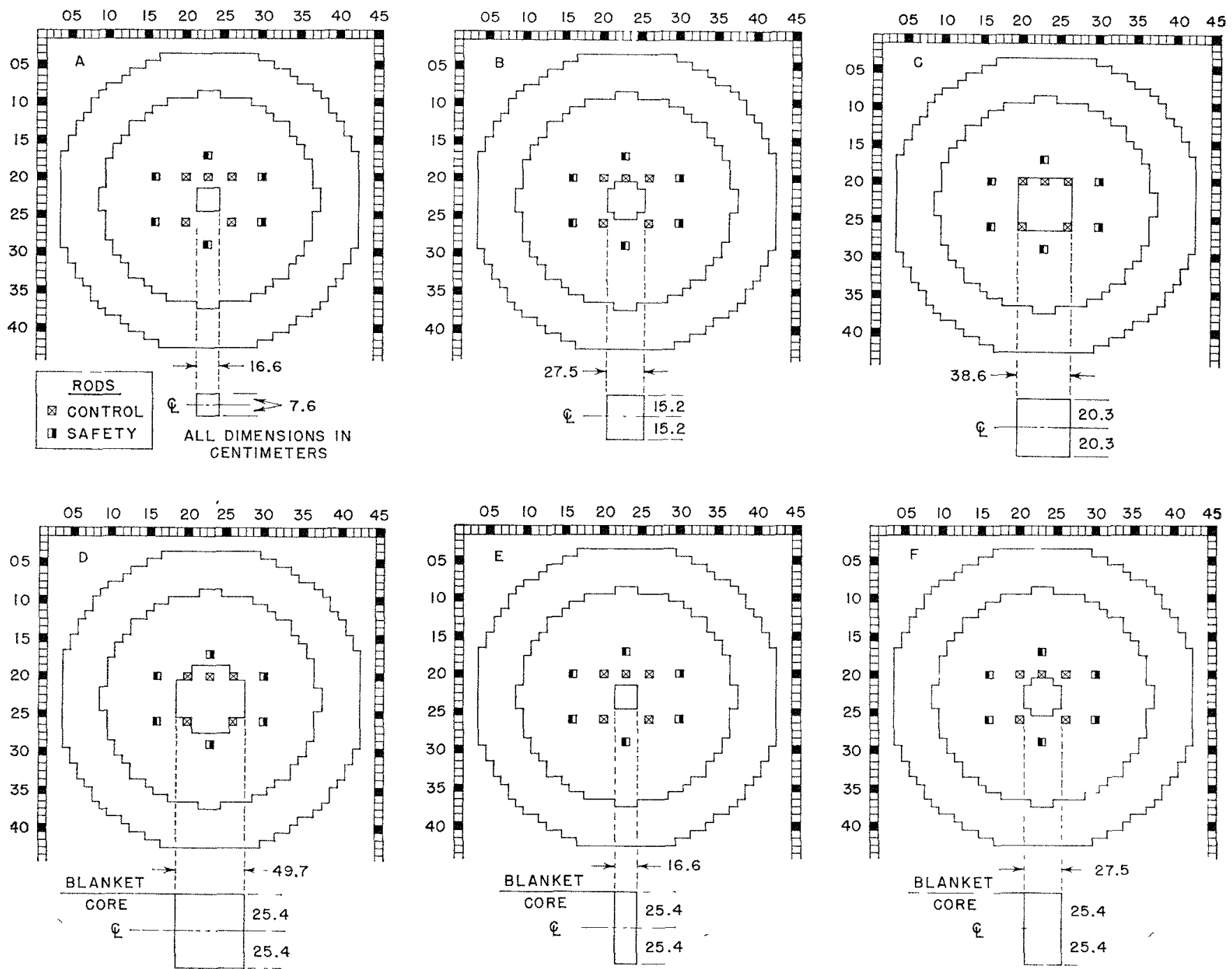

FIG. 2. Central Regions. 
efficients of axial and cylindrical ring regions for both assemblies. These regions correspond to those shown in Figs. 3 and 4.

Experiments to study the heterogeneity effect were done in a central region of Askembly No. 3 isee Fig. 2C) with a 28-cm diameter which contained 21 drawers in earh half of the reactor. Axially, the void extended either $30.5 \mathrm{~cm}$ or $50.8 \mathrm{~cm}$, which constituted
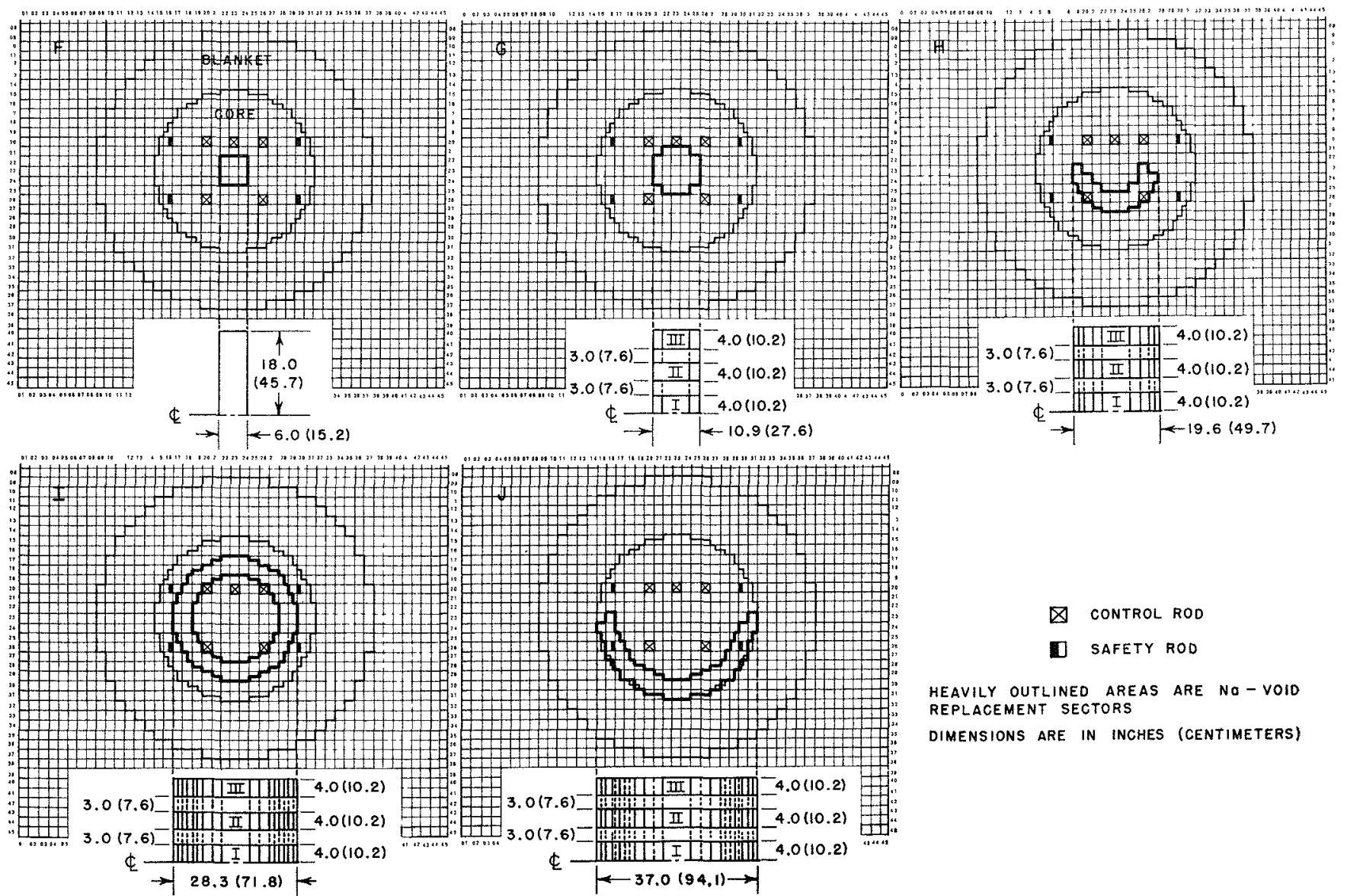

HEAVILY OUTLINED AREAS ARE NO - VOID REPLACEMENT SEGTORS

DIMENSIONS ARE IN INGHES (CENTIMETERS)

FIG. 3. Axial and Cylindrical Ring Regions for Assembly No. 2.
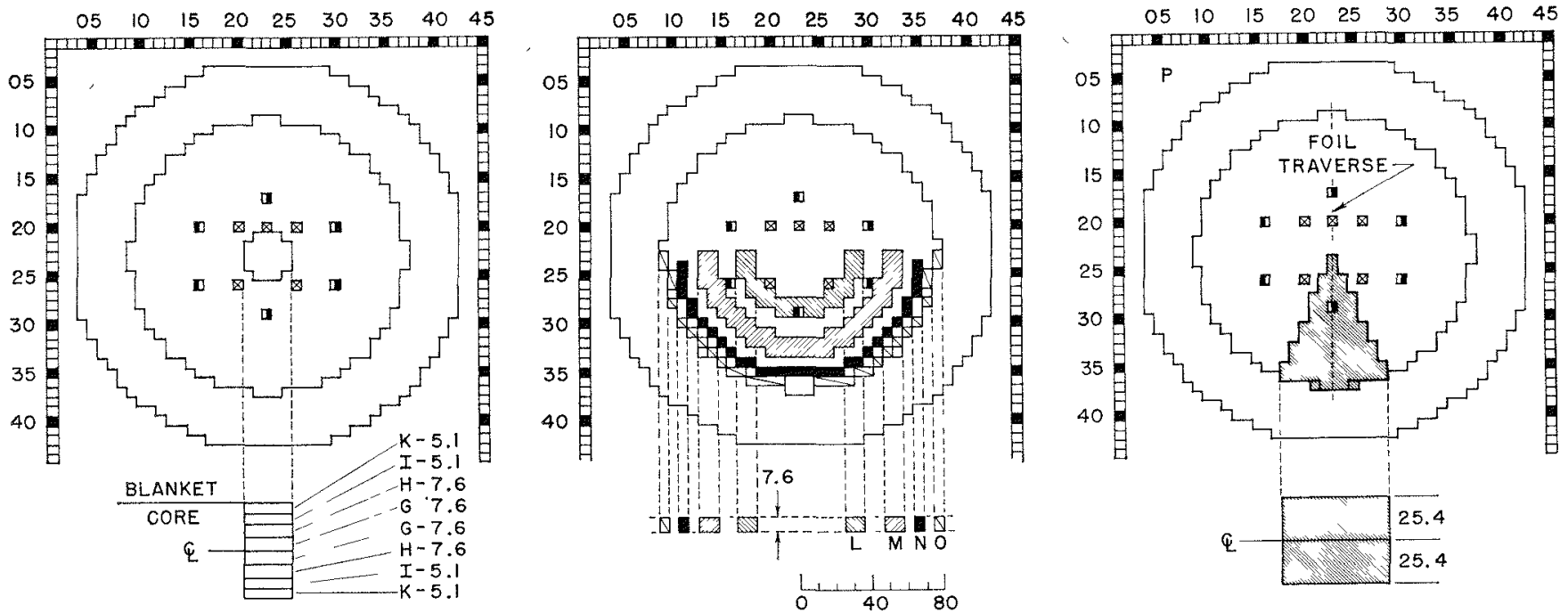

FrG. 4. Axial and Cylindrical Ring Regions for Assembly No. 3. 
TABLE 3. Sodra Coefricints in Central Region

\begin{tabular}{c|c|c|c|c}
\hline Region & $\begin{array}{c}\text { Length, } \\
\mathrm{cm}\end{array}$ & $\begin{array}{c}\text { Diameter, } \\
\mathrm{cm}\end{array}$ & $\begin{array}{c}\text { Assembly } \\
2, \mathrm{Ih} / \mathrm{kg}\end{array}$ & $\begin{array}{c}\text { Assembly } \\
3, \mathrm{Ih} / \mathrm{kg}\end{array}$ \\
\hline $\mathrm{A}$ & 15.2 & 18.7 & -1.6 & -- \\
$\mathrm{B}$ & 30.5 & 28.5 & -2.1 & -1.31 \\
$\mathrm{C}$ & 40.6 & 40.2 & -2.99 & - \\
& 40.6 & 41.3 & - & -2.29 \\
H. & 50.8 & 18.7 & - & -3.54 \\
F & 508 & 28.5 & - & -3.48 \\
D) & 50.8 & 49.8 & - & -3.23 \\
& & & & - \\
\hline
\end{tabular}

the entire length of the core. Measurements were made with:

1. different materials placed next to the sodium;

2. different widths of sodium columns;

3. partial voids in a 1 -in.-wide sodium column;

4. discontinuous or staggered roid in the normal drawer loading.

Typical examples of the effects of material arrangement and heterogeneity are shown in Tables 6 and 7 . In Tables 6 through 8 , the alphabetic notation represents the materials as follows:

D: depleted uranium

$\mathrm{N}$ : sodium

C: carbon

E: enriched uranium.

The letters which are italicized indicate those columns which were voided.

In Table 6 are given results when all of the sodium in the three types of drawers were replaced by void. The first line of Table 6 represents the way in which a normal drawer was loaded. The second and third lines represent modified patterns in which, in the former case, the sodium is predominantly adjacent to the enriched uranium and graphite. In the latter case, the sodium is predominantly next to $\mathrm{C}^{228}$. It can be seen there is as much as about $20 \%$ variation between the highest and lowest value for the sodium coefficient.

Table 7 gives results from a series of measurements in which the drawers were severely modified to determine the effect of a particular material adjacent to the sodium can. The first line shows the case in which only the sodium adjacent to the depleted uranium was voided. The second line shows the measurement when only the sodium next to the graphite was replaced. The third case represents the situation when all of the sodium was removed. Again, it can be seen that there are large variations of the sodium coefficient which are dependent on the material arrangement.
TABLE 4. Somum-vord Coefficents of Axial Regrons

\begin{tabular}{|c|c|c|c|c|c|c|c|}
\hline \multicolumn{4}{|c|}{ Assembly 2 (see Fig. 3) } & \multicolumn{4}{|c|}{ Assembly 3 (see Fig. 4) } \\
\hline \multirow{2}{*}{ Region } & \multicolumn{2}{|c|}{$\begin{array}{l}\text { Axial } \\
\text { Boundaries }\end{array}$} & \multirow{2}{*}{ Ih $/ \mathrm{kg}$} & \multirow{2}{*}{ Region } & \multicolumn{2}{|c|}{$\begin{array}{l}\text { Axial } \\
\text { Boundaries }\end{array}$} & \multirow{2}{*}{$\mathrm{Ih} / \mathrm{kg}$} \\
\hline & Inner & Outer & & & Inner & Outer & \\
\hline (xI & 0 & 10.16 & -1.9 & $G$ & 0 & 7.62 & -0.18 \\
\hline (XII & 17.78 & 27.94 & -4.0 & $H$ & 7.62 & 15.24 & -2.33 \\
\hline \multirow[t]{2}{*}{ (XIII } & 35.56 & 45.72 & -7.5 & I & 15.24 & 20.32 & -5.0 \\
\hline & & & & $K$ & 20.32 & 25.4 & -5.8 \\
\hline
\end{tabular}

TABLE 5. SODI'M-VOID COEFFICENTS OF Cylindrical Rings

\begin{tabular}{|c|c|c|c|c|c|c|c|}
\hline \multicolumn{4}{|c|}{ Assembly 2 (see Fig. 3 ) } & \multicolumn{4}{|c|}{ Assembly 3 (see Fig. 4) } \\
\hline \multirow{2}{*}{ Ring } & \multicolumn{2}{|c|}{ Radius } & \multirow{2}{*}{$\mathrm{Ih} / \mathrm{kg}$} & \multirow{2}{*}{ Ring } & \multicolumn{2}{|c|}{ Radius } & \multirow{2}{*}{$\mathrm{Ih} / \mathrm{kg}$} \\
\hline & Inner & Outer & & & Inner & Outer & \\
\hline $\mathrm{HI}$ & 14.6 & 249 & -4.4 & $\mathrm{~L}$ & 25.9 & 36.5 & -1.06 \\
\hline II & 25.1 & 35.4 & -5.96 & $\mathrm{M}$ & 46.3 & 58.2 & -2.16 \\
\hline \multirow[t]{2}{*}{$J I$} & 35.5 & 46.2 & -7.26 & $\mathrm{~N}$ & 65.1 & 70.0 & -2.25 \\
\hline & & & & 0 & 2.7 & 77.9 & -2.13 \\
\hline
\end{tabular}

TABLE 6. HETEROGENEITY EFFECTS FOR I OHD LENGTH of $30.5 \mathrm{cM}$

\begin{tabular}{|c|c|}
\hline Drawer Pattern & $\mathrm{Ih} / \mathrm{kg}$ \\
\hline I) $N \times C E D N N C D N N E C D N N$ & -1.31 \\
\hline$N N C D E N N C D D N V E D C N N$ & -1.48 \\
\hline NNCEDNNIDCDNNCEDNN & -1.25 \\
\hline Average & -1.35 \\
\hline
\end{tabular}

TABLE 7. Heterogeneity Efrects

\begin{tabular}{|c|c|c|}
\hline \multirow{3}{*}{ Pattern } & \multicolumn{2}{|c|}{ Void Length } \\
\hline & $30.5 \mathrm{~cm}$ & $50.8 \mathrm{~cm}$ \\
\hline & $\mathrm{Ih} / \mathrm{kg}$ & $\mathrm{Ih} / \mathrm{kg}$ \\
\hline 1) NNCECNNCI $N N D E D N N$ & -1.20 & -3.10 \\
\hline I) NNCEC $N N C D N N D E D N N$ & -1.97 & -4.06 \\
\hline 1) $N N C E C N N C D N N D \mathrm{ED} N N$ & -1.34 & -3.59 \\
\hline Average & -1.50 & -3.58 \\
\hline
\end{tabular}

Further measurements were made with sodium columns of varying thickness and with different configurations of partial voids in sodium columns of a constant thickness of $1 \mathrm{in.}$. In each case the remaining core materials were rearranged to approximate a homogeneous mixture. The results given in Table 8 show 
Table 8. Heterogenhity Effects due to Variable Thickness of SOdicm Coltuns

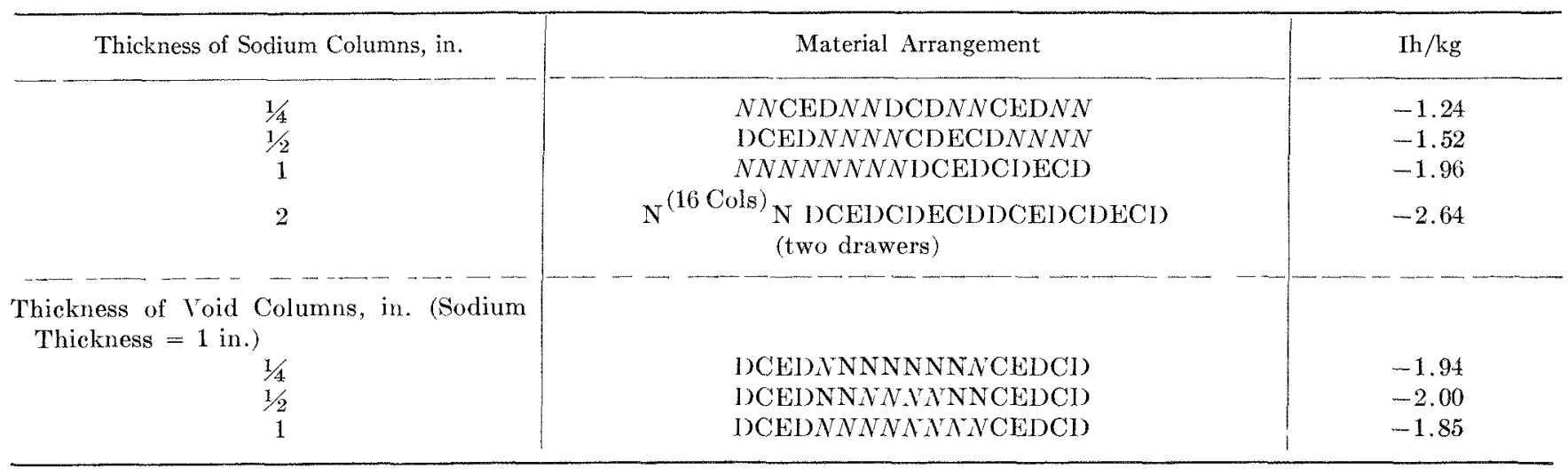

that the sodium-void coefficient changed considerably when the thickness of the sodium columns was varied, but was essentially the same when the thickness of the sodium column was fixed and only the voiding pattern was changed.

To date only simple one-dimensional diffusion-type sodium-void calculations have been made for comparison with the experimental results. Agreement between experiment and theory has only been quantitative.

From looking at these heterogeneity experimental results, it would appear necessary to use two-dimensional transport calculations which properly take into account the material arrangement in the drawers. Otherwise an agreement between experimental and theoretical results better than about $10 \%$ must be considered fortuitous. It would appear evident that for larger dilute cores, where the spectrum is more degraded, it is even more important to take into account the heterogeneity effects.

\section{REFERENCES}

1. P. I. Amundson et al., Critical Studies of Uranium-Steel and Uranium-Steel-Soduum Fast Reactor Cores (ZPR-3 Assemblies 32 and 33), ANL-6690 (Feb 1963).

2. J. M. Gasidlo et al., Doppler Effect Measurements, Sodium Void Coefficients, and Critical Studies of a 5000-liter Fast Power Breeder Reactor by the Two-zone Method (ZPR-3 Assembly 43), ANL-6838 (May 1964).

3. G. K. Rusch, E. R. Bohme, L. R. Dates, S. Grifoni, W. Y. Kato, G. W. Main, H. H. Meister, M. Nozawa, and R. L. Stover, "Investigations of a 600-liter Uranium Carbide Core (ZPR-6 Assembly No. 2)," Reactor Physics Division Annual Report, July 1, 1963 to June 30, 1964, ANL 7010, p. 91, (January 1965).

4. F. H. Helm, W. Y. Kato, G. W. Main, and G. K. Rusch, "Physics Parameters and Sodium Toid Coefficients," Reactor Physics Division Annual Report, ANL-7110 (to be published). 


\title{
Measurement and Analysis of Doppler Effect in Plutonium-fueled Fast Reactor Assemblies
}

\author{
G. J. Fischer, D. A. Meneley, R. N. Hwang, E. F. Groh, and C. E. Till \\ Argonne National Laboratory \\ Argonne, Illinois \\ (PRESENTED) BY G. J. FISCHER)
}

\section{Introduction}

The measurements described in this paper were made with the ZPR-3 fast critical assembly of the Argonne National Laboratory, Zone-loading procedures were used in Assembly $45 \mathrm{~A}$ to produce an allfast assembly which contained in its central zone a mockup of a large fast power breeder using plutonium-239, uranium-238 monocarbide fuel, sodium coolant, and stainless steel clad and structure. The center zone of Assembly 45 differed from that of $45 \mathrm{~A}$ in the replacement of $40 \%$ of the sodium cans by graphite. This change gave a second neutron-energy spectrum having more neutrons in the region which is the source of the Doppler effect reactivity changes. Figure 1 shows the geometrical arrangement of this loading in ZPR-3; Table 1 gives the outer radii and atomic compositions of the zones.

An unusually small central zone was designed for this assembly in order to reduce reactor-drift problems related to decay heat of the plutonium and to reduce the neutron-source problem. The real and adjoint spectral match at the position of the Doppler measurement deviated by a few percent at most from the single-region spectrum for neutrons in the energy internal that contributes to the Doppler effect.

\section{Equipment and Procedure}

A schematic diagram of the apparatus to measure the Ioppler effect as used in ZPR-3 is shown in Fig. 2. A drive unit moved the Doppler equipment back and forth, exchanging the Doppler elements, at one of two temperatures, and reference material at the center of the assembly. A fine, calibrated control rod maintained the power of the reactor essentially constant throughout the Doppler measurement. If there were no secondary events occurring at the same time, the change in the reactivity worth of the Doppler elements on heating would be due to the Doppler effect alone. An important part of the Argonne Doppler program consists of designing against and estimating the magnitude of these secondary effects.

The construction of the basic Doppler element is shown by the photograph of Fig. 3. Pellets of appropriate isotopes, in the form of oxides at 0.70 theoretical density, were pressed into cylinders, $0.5003 \mathrm{in}$. in diameter and $0.500 \mathrm{in}$. in length, and fired. Twelve' of these pellets were used to produce an element 6 in. long.

Two such Doppler elements were mounted in line, symmetrically about the axial center of the reactor in which they were to be used, as indicated in Fig. 2; the separation between them was $12.1 \mathrm{~cm}$. One end of each Doppler element was fixed in position in such manner that both elements expanded in the same direction when heated. This design caused the material of one element to move in the direction of a slightly increasing flux gradient, and the material of the other clement to move into a correspondingly decreasing flux gradient. A first-order cancellation of flux-gradient expansion effects occurred as a result. Theoretical calculations by two-dimensional transport theory were used to evaluatc the importance of residual expansion effects, which were significant for the fissile isotopes only.

Table 2 lists the kinds of Doppler elements which were prepared in this manner and gives their isotopic composition. In addition, elements of similar design but having diameters of $0.73 \mathrm{in}$. and $1.004 \mathrm{in}$. have been fabricated and filled with natural uranium dioxide.

\section{Results and Conclusions}

The experimental results obtained using Doppler elements made up of $\mathrm{U}-238$ and $\mathrm{U}-238$ mixed with $\mathrm{Pu}-239$ in a ratio of seven to one are shown in Table 3 ; results of theoretical analysis of these measurements are also included in the table. The following section will discuss both experimental and theoretical uncertainties of the numbers reported in this and the next table.

It can be seen that there is relatively satisfactory agreement between experiment and theory for the cases of Table 3 . There is much less theoretical uncertainty, arising mainly from uncertainties in basic nuclear data, for $\mathrm{U}-238$ than for the cases of fissile 


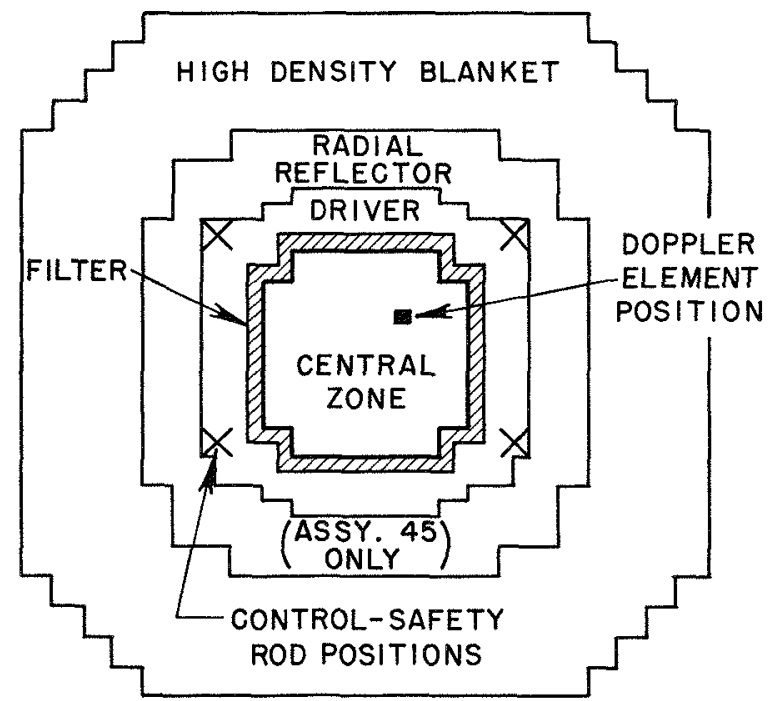

Fut. 1. Cross-sectional V'iew of ZPR 3 Assemblies 45 and $45 \mathrm{~A}$

isotopes. The results for $\mathrm{C}^{\top}-238$ indicate a significant reactivity loss of $-16 \times 10^{-6} \Delta k / k$ per $\mathrm{kg}$ for the temperature range used in the power breeder spectrum of Assembly 45A. For the mixed 7:1 isotopes, the reactivity loss of $-13.6 \times 10^{-6} \Delta k / k$ per $\mathrm{kg}$ is within $3 \%$ of being proportional to the relative density of U-238 in this Doppler element compared to that of the $\mathrm{C}-238$ element, indicating a very small positive plutonium-239 Doppler contribution.

The consequence of spectral softening can be scen by comparing the U-238 result for Assembly 45A to that for Assembly 45. A factor of almost two in Doppler response occurs. In all cases the theoretical procedures tend to underpredict the U-238 Doppler re- sponse. This means that if the relatively well-known U-238 Doppler cross sections were perfectly accounted for, the calculations must underestimate the flux in the energy interval of the Doppler effect.

The measurement with the empty or dummy "Doppler elements" indicates a lack of serious instrumental or other non-nuclear reactivity effects.

The theoretical estimates of the Doppler reactivity changes were based upon perturbation-theory calculations using multigroup cross sections appropriate for the experiment conditions. Radial values of the real and adjoint multigroup fluxes were obtained by means of eylindrical one-dimensional diffusion-theory calculations. The axial dependence of these fluxes was estimated from results of nine-group two-dimensional transport calculations, while the normalization of the perturbation integral was taken from a twodimensional transport calculation of plutonium worth at the core center. This worth calculation checked

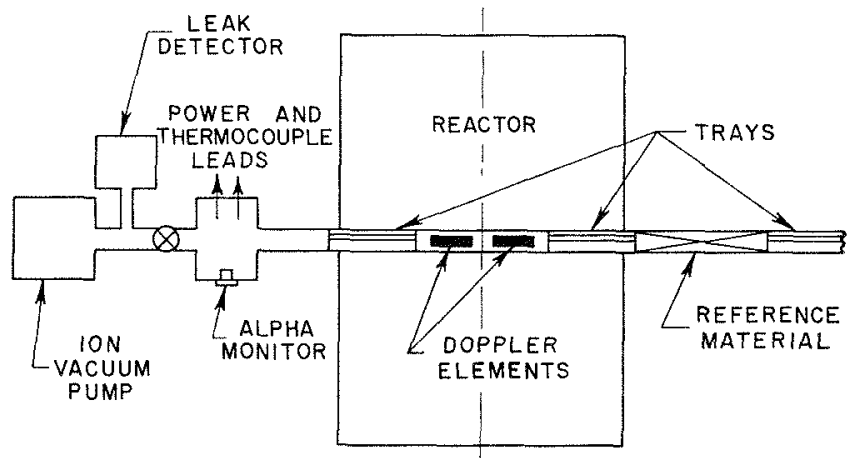

Fig. 2. Schematic Diagram of Apparatus to Measure the Doppler Lffect in ZPR-3. Dimensions have been distorted.

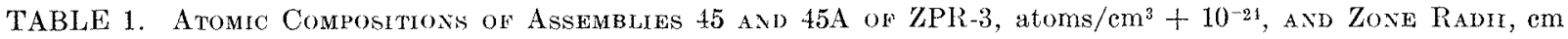

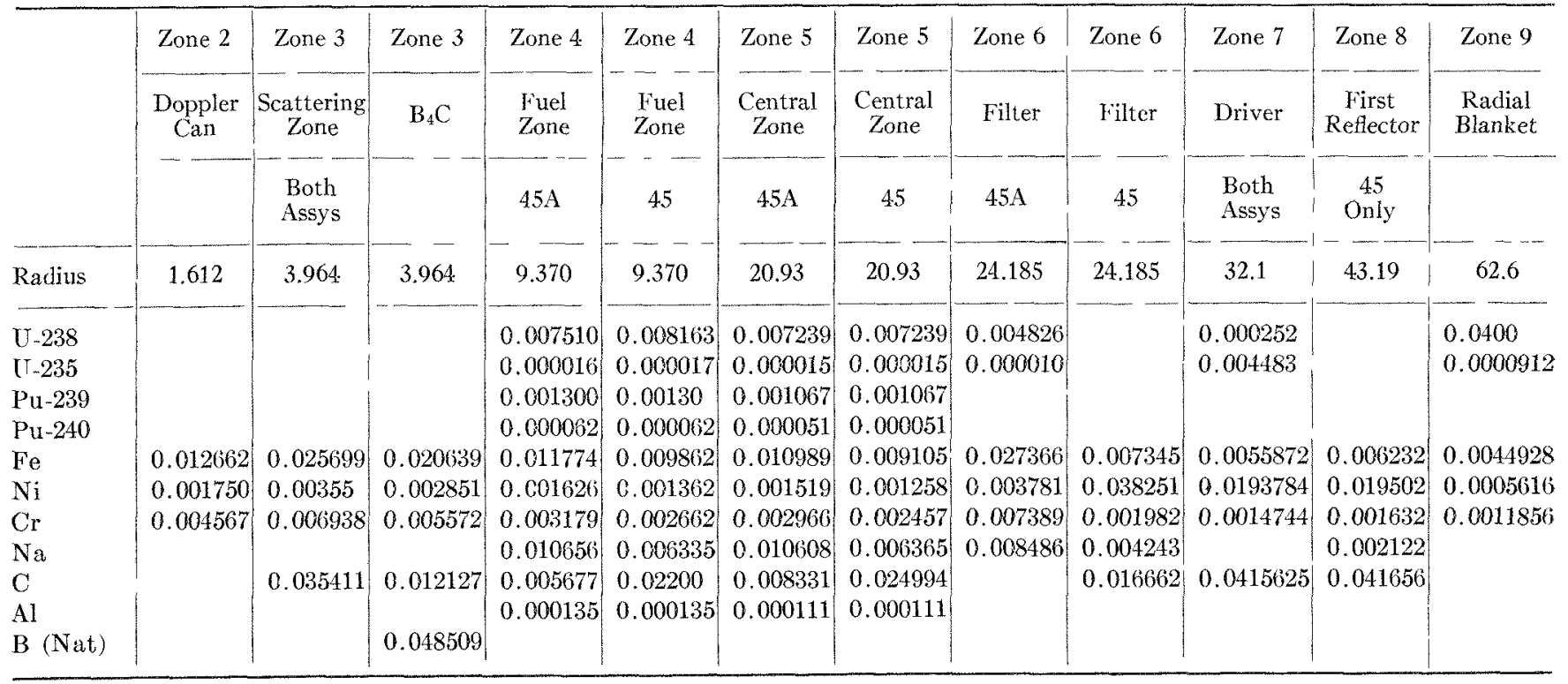




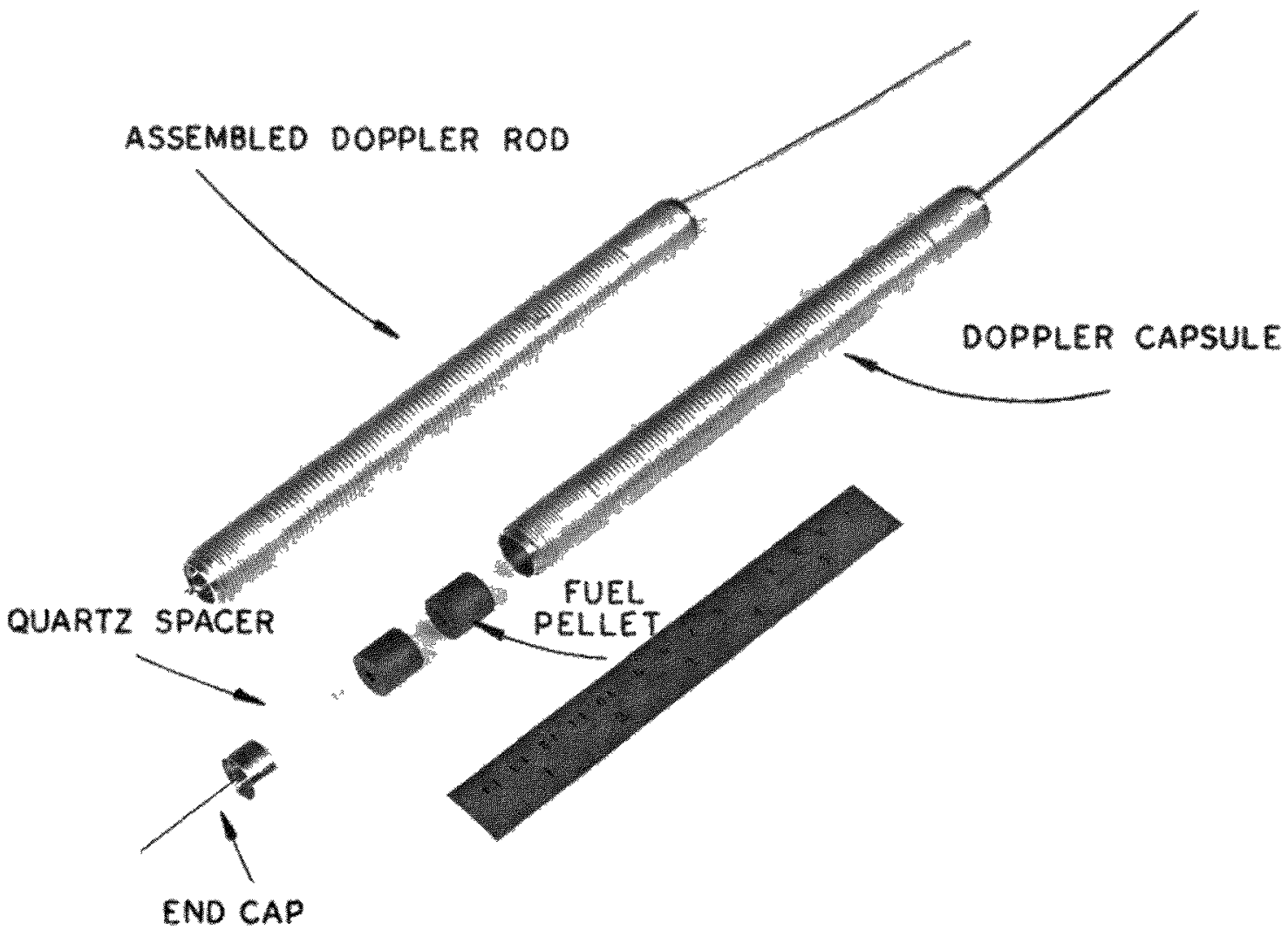

Fig. 3. Basic Doppler Element.

Extra windings of the sheathed heater wire at each end provided a flat axial temperature distribution. Three thermocouples along the length of every element measured this distribution. The jacket was a high-nickel alloy

well against the experimentaly measured worth of plutonium at the same position in the reactor, giving $-185 \mathrm{In} / \mathrm{kg}$ theoretically versus $-176 \pm 4 \mathrm{In} / \mathrm{kg}$ experimentally. The radial flux dependence given by diffusion theory also compared well with that of a similar one-dimensional transport calculation for the case of a plutonium Doppler element at the center of Assembly 45A. A two-dimensional, nine-group transport calculation of one of the Doppler reactivity changes agreed with the perturbation calculation of the same case to within a few percent.

Table 4 contains the results of measurements made with Pu-239 and, for completeness, experimental Doppler results for $[-235$ obtained by J. Gasidlo and $P$. Amundson in the same assembly. (1) These fissile isotope results lead to some very interesting observations which, certainly, must be tested by further experimental work. The correction for the expansion effect has been evaluated with some eare, so that the experimental results, as corrected for expansion effects, will be compared to the theoretical results.

The experimental results for $\mathrm{Pu}-239$ show remark-
TABLE 2. Atomic Densi ties of Doppler Elament, atoms $/ \mathrm{cm}^{3} \times 10^{-24}$, A $500^{\circ} \mathrm{K}$ Effective length $=15.28 \mathrm{~cm}$ Effectuve radius $=0.6366 \mathrm{~cm}$

\begin{tabular}{|c|c|c|c|c|c|}
\hline \multirow{2}{*}{ Isotope } & \multicolumn{5}{|c|}{ Type Element } \\
\hline & $\mathrm{Pu}-239$ & $\begin{array}{c}\mathrm{Pu}-239 \\
\mathrm{U}-238\end{array}$ & $\mathrm{U}-238$ & $\begin{array}{l}\mathrm{U}-238 \\
(1 \text { in. })\end{array}$ & $\mathrm{U}-235$ \\
\hline Pu-239 & 001471 & 0.001528 & - & - & - \\
\hline Pu 240 & 0.00038 & 0.000123 & - & - & - \\
\hline $\mathrm{U}^{\top}-238$ & $一$ & 0.013267 & 001560 & 0.01732 & 0.000421 \\
\hline U 235 & - & 0.000095 & 000011 & 0.000124 & 0.01535 \\
\hline 0 & 0.03016 & 0.030118 & 0.03142 & 0.03488 & 003155 \\
\hline
\end{tabular}

ably small, positive Doppler reactivity responses. This holds true even for the much softer spectrum of Assembly 45, where there should be many more neutrons in the region of the Doppler effect.

It can be seen from Table 4 that as the neutron energy spectrum hardens in proceeding from Assembly 45 to Assembly $45 \mathrm{~A}$ and then to Assembly $45 \mathrm{~A}$ with $\mathrm{B}_{4} \mathrm{C}$ about the Doppler element, agreement be- 
TABLE 3. Doppler Reactivity Changes for U-238 and for U $238+$ Pu-239 IN $7 / 1$ Ratio $\left(\Delta T=500\right.$ To $\left.1100^{\circ} \mathrm{K}\right)$

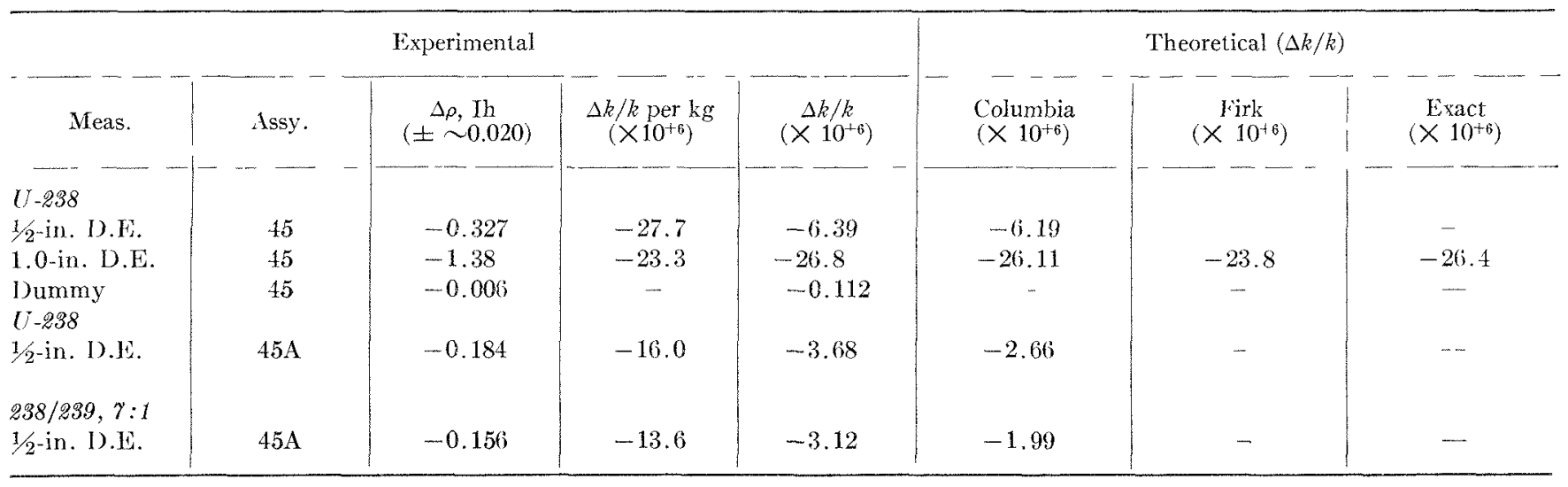

TABLE 4. Doppler Rhachulty Changhs for Pu-239 and U-235 $\left(\Delta T=500\right.$ To $\left.1100^{\circ} \mathrm{K}\right)$

\begin{tabular}{|c|c|c|c|c|c|c|}
\hline \multirow{2}{*}{ Measured $^{(a)}$} & \multicolumn{3}{|c|}{ Experimental } & \multirow{2}{*}{$\begin{array}{c}\text { Expansion } \\
\text { Correction, } \Delta k / k \\
\left(\times 10^{+6}\right)\end{array}$} & \multirow{2}{*}{$\begin{array}{l}\text { Corrected } \\
\Delta k / k \text { expt } \\
\left(\times 10^{+6}\right)\end{array}$} & \multirow{2}{*}{$\begin{array}{c}\Delta k / k \text { theory } \\
\left(\times 10^{-6}\right)\end{array}$} \\
\hline & $( \pm \stackrel{\Delta \rho, \mathrm{Th}}{\sim 0.020)}$ & $\begin{array}{c}\Delta k / k \\
\left(\times 10^{+6}\right)\end{array}$ & $\begin{array}{c}\Delta k / k \text { per kg } \\
\left(\times 10^{+6}\right)\end{array}$ & & & \\
\hline \multicolumn{7}{|l|}{$P U-239$} \\
\hline First Measurement & -0.060 & -1.21 & -5.36 & - & . & - \\
\hline 3 Wks Later & -0.049 & -0.99 & -4.39 & - & -- & - \\
\hline Homogeneous & -0.034 & -0.68 & -3.02 & +1.35 & +0.67 & - \\
\hline In $\mathrm{B}_{4} \mathrm{C}$ & +0.042 & +0.83 & +3.70 & +0.90 & +1.73 & +2.33 \\
\hline 1 Element & -0.044 & - & 一 & - & -- & - \\
\hline Assy. 45 & -0.024 & -0.56 & -2.49 & +1.63 & +1.07 & +12.95 \\
\hline$U-235$ & & & & & & \\
\hline
\end{tabular}

(a) Assembly 45A, except as indicated.

tween theory and experiment improves remarkably. This behavior may be related to uncertainties in the lower-energy Doppler cross sections for plutonium239 , discussed in the next section. The experimental Doppler reactivity change for $\mathrm{C}-235$ is calculated much better. 'That the basic cross-section information, in particular the value of $\alpha$, is relatively better known for $\mathrm{U}-235$ tends to support the $\mathrm{U}-238$ evidence that the flux in the Doppler region may be reasonably well calculated.

The case labeled "Homogeneous" represents the experimental difference resulting from replacing the "scattering zone" and "fuel zone" by a completely uniform core region. One sees that the Dancoff correction is not large. In the "1 element" case, that one of the two Doppler elements which expands into a decreasing flux gradient was heated while the other was not. The difference between this reactivity change and half of the two-element response is a measure of the compensation occurring, as explained in Section II, when the two elements expand in the same direction and into opposite flux gradients.

The U-235 measurement was done with the same apparatus except for the U-235 Doppler elements, which were fabricated in the same manner as the plutonium elements. The procedure of the measurement was also the same. Experiments are currently being performed by one of us (CET) with Doppler elements constrained to prevent axial expansion, constrained to prevent both radial and axial expansion, and having expansion properties very much like those used in the experiment reported here, in another assembly on ZPR-6.

The results obtained in these measurements indicate that the positive Doppler reactivity contribution of plutonium-239 in the fuel, and in the neutron-energy spectrum of a large power breeder reactor, is small in magnitude compared to the strong negative Doppler effect of U-238. Further experiments should be performed to verify and refine these measure- 
ments as well as to extend their range. It should also be remembered that these results are for a "clean" fast reactor. The presence of fission products and of $\mathrm{Pu}-240$, which builds up with fuel recycle, would reduce the magnitude of the results seen here. ${ }^{(2)}$

\section{Experimental and Theoretical Uncertainties}

The principal source of experimental uncertainty was reactor drift during the experimental data taking operation. Because the central zone of the assembly was plutonium-fueled, there was a significant amount of heat to be removed by drawing air through the zone. The procedure led in turn to a drift sensitivity related to the temperature of the reactor cell air as it entered the matrix. Control of these parameters improved continuously during the course of the experiments and has been worked on in anticipation of future work. Another source of difficulty which was observed during the course of measurements reported here and corrected is related to the cooling air, which removed about $35 \mathrm{~W}$ of heat per Doppler element at $1100^{\circ} \mathrm{K}$. A cover over the Doppler element drive tube now forces the air to move through the dummy vacuum manifold tube, preventing direct contact with the matrix. The reactivity signal due to the fissile isotopes was unnecessarily small. Time limitations did not allow for preparation and employment of several pairs of Doppler elements to thereby multiply the signal, as was possible with the equipment available.

The expansion correction is a serious problem which is being treated by further design. The corrections used in this report were calculated by 2-dimensional (2-D) transport theory. Both 5- and 9-group cross sections were produced for the 2-D code for each reactor region by a flux-weighting routine working from a 25-group cross-section set related to the set 224 reported elsewhere. (3) Only a very slight reactivity difference was seen when the expansion effect was calculated when nine instead of five groups were used and when the convergence criterion was tightened by a factor of two.

A relatively large and probably quite conservative overall experimental uncertainty of $\pm 0.40 \times 10^{-6}$ $\Delta k / k$ has been assigned to all data of Tables 3 and 4 . Improved data-taking procedures currently being used for Doppler measurements with ZPR-6 (CET) are producing results with uncertainties smaller by approximately an order of magnitude than this.

The theoretical calculations have separate difficulties of their own. A continuous problem to all fast reactor calculations is whether the calculational procedure produces the correct flux magnitude in the tail of the fast reactor neutron-energy spectrum, where the entire Doppler effect occurs. ${ }^{(4)}$ For the calculations
TABle 5. Doppler Change in Effective Cross Sections For URanum-238 for 1.0-in.-Diameter Rod $\left(500-1100^{\circ} \mathrm{K}\right)$

\begin{tabular}{c|l|c|c}
\hline \multirow{2}{*}{$\begin{array}{c}\text { Energy Interval, } \\
\mathrm{keV}\end{array}$} & \multicolumn{2}{|c|}{ NR Approximation } & $\begin{array}{c}\text { Exact } \\
\text { (RIFF-RAFF) }\end{array}$ \\
\cline { 2 - 4 } & $\begin{array}{c}\text { Columbia } \\
\text { Parameters } \\
\delta \tilde{\sigma}_{\gamma}\end{array}$ & $\begin{array}{c}\text { Firk's } \\
\text { Parameters } \\
\delta \tilde{\sigma}_{\gamma}\end{array}$ & $\begin{array}{c}\text { Firk's } \\
\text { Parameters } \\
\delta \tilde{\sigma}_{\gamma}\end{array}$ \\
\hline $4.0-2.8$ & 0.04764 & & \\
-2.0 & 0.06962 & \multicolumn{2}{|c|}{ Unresolved Region } \\
-1.4 & 0.08608 & 0.13035 & 0.13367 \\
-1.0 & 0.13215 & 0.13976 & 0.14111 \\
-0.55 & 0.19323 & 0.19212 & 0.20029 \\
-0.30 & 0.21485 & 0.12612 & 0.14168 \\
-0.10 & 0.34438 (IA) & 0.23037 & 0.32911 \\
-0.030 & 0.2920 (IA) & 0.18940 & 0.37472 \\
\hline
\end{tabular}

TABle 6. Doppler Change in EfFective Cross Sections FOR Plutonium-239 (500-1100K)

\begin{tabular}{c|c|c|c|c}
\hline \multirow{2}{*}{$\begin{array}{c}\text { Energy Interval, } \\
\mathrm{keV}\end{array}$} & \multicolumn{2}{|c|}{ Schmidt Parameters } & \multicolumn{2}{|c}{ Hummel Parameters } \\
\cline { 2 - 5 } & $\delta \tilde{\sigma}_{f}$ & $\delta \tilde{\sigma}_{\gamma}$ & $\delta \tilde{\sigma}_{f}$ & $\delta \tilde{\sigma}_{\gamma}$ \\
\hline $15.0-9.1$ & 0.0095 & 0.0043 & 0.0102 & 0.0053 \\
-4.0 & 0.0286 & 0.0173 & 0.0284 & 0.0155 \\
-2.8 & 0.0722 & 0.0412 & 0.0753 & 0.0429 \\
-2.0 & 0.1238 & 0.0757 & 0.1337 & 0.0773 \\
-1.4 & 0.1708 & 0.1165 & 0.1882 & 0.1158 \\
-1.0 & 0.2519 & 0.1876 & 0.2849 & 0.1808 \\
-0.55 & 0.3840 & 0.3119 & 0.4445 & 0.2905 \\
-0.30 & 0.6534 & 0.5846 & 0.7680 & 0.5275 \\
-0.10 & 0.8853 & 0.8715 & 1.0524 & 0.7624 \\
-0.03 & 0.9423 & 1.0591 & 1.1498 & 0.9006 \\
\hline
\end{tabular}

reported here most regional cross sections were produced by use of the ELMOE code(5) with radial leakage buckling corrections being added. Other cross sections were prepared from the new $\mathrm{ANL}_{4}$ code $\mathrm{MC}^{2}$, which produces multigroup cross sections from a basic library containing very detailed nuclear data.

The reaction cross sections for the Doppler effect for the analyses of the experiment were produced by one of the authors $(\mathrm{RH})$. The remainder of the discussion will center on this procedure and on uncertainties in the Doppler cross section.

The Doppler cross sections reported in Tables 5 and 6 were calculated using the usual narrow-resonance approximation, the assumption of a flat neutron source, flat flux, and Wigner's rational approximation applied to calculate the escape probability for the Doppler element, which then leads to the well-known equivalence relation. It is therefore possible to write the effective cross section into the form appropriate to a homogeneous medium by introducing an equivalent potential scattering cross section. 
The evaluation of the effective cross section has been discussed extensively for eases with and without resonance overlap. ${ }^{(6-8)}$ Overlap corrections were included in the Doppler reaction cross sections used here. A diseussion of some of the possible limitations of the use of assumptions leading to the simplified form of the effective cross section is given later in this paper.

The best available Doppler-region nuclear parameters are those for U-238. For this study the recent Columbia University parameters ${ }^{(9)}$ which extend the resolved region to $4 \mathrm{keV}$ were used. This covers a substantial portion of interest in these calculations. For comparison, the data of Firk, Lynn, and Moxon ${ }^{(10)}$ have also been used. The resultant Doppler changes in cross sections for $\mathrm{L}^{\top}-238$ are shown in Table 5. Because resonance parameters exist for energies beyond those for which the above procedure might be in doubt, a more exact ealculation was made by means of RIFF-RAFF.(11) This code takes into account lethargy, space, and temperature dependence of the source, and by use of correct escape probabilities, calculates both the resonance integrals and the perturbed group flux. Results of use of this code are included in Table 5. They show that the exact method gives essentially the same cross sections in regions for which there is significant flux.

Our lack of adequate nuclear data for the fissile isotopes in the Doppler neutron energy region could account for a major part of the disagreement with experiment. Because almost all resonances are not resolved in the Doppler region, we must rely on a statistical treatment involving an extrapolation from resolved resonances at low energies. The Doppler effect of the fissile isotope, which is determined by the competition between fission and capture, is very sensitive to the value of $\alpha$ and even more so to the value of the ratio $\delta \tilde{\sigma}_{f} / \delta \tilde{\sigma}_{\gamma}$. These ratios in turn depend strongly on the fission width distribution function and also on the average fission width.

For the calculation of $\mathrm{Pu}-239$ Doppler effect in this paper a $v=1$ (Porter-Thomas) distribution for the fission width and average fission widths of 0.04 $\mathrm{eV}$ and $0.156 \mathrm{eV}$ for spins $J=0$ and $J=1$, respectively, as suggested by Schmidt, (12) were uscd. Slightly higher values of the fission widths were used beyond $5 \mathrm{keV}$ to obtain better agreement with measured $\alpha$ values at high energy. For comparison, calculations were also made using the spin-independent $\Gamma_{f}$ of 0.099 $\mathrm{eV}$ and $\mathrm{a} v=2$ (random) distribution which has been widely used in the past. ${ }^{(2)}$ For U-235 the $\chi$-squared distribution of $y=2$ was used. The average fission width $\Gamma_{f}$ was varied about the usual value of 0.120 $\mathrm{eV}$ in such a way that the resulting average $\alpha$ valuc would agree approximately with the measured values at various energies as given in Ref. 13 .

Table 6 gives the group-dependent change in cross sections obtained by use of these two sets of assumptions.

Cnlike the case for ${ }^{2}-238$, the use of the flat-flux assumption appears to be dubious for a rod composed of high concentration of a fissile isotope below $1 \mathrm{keV}$ bccause significant flux depression must occur in this region. Therefore, narrow-resonance calculations based on a flat-flux assumption may not be satisfactory. This seems to be indicated by the experimental results which agree relatively well with theory when the lower-energy neutrons have been removed in the $\mathrm{B}_{4} \mathrm{C}$ experiment. Whether the discrepancies between theory and experiment for the soft-spectral case are due to uncertainties in knowledge of resonance parameters or the validity of the oversimplified method, or both, is not yet certain.

It seems very evident that much more theoretical and experimental work must be done with the fissile isotopes in varying fast reactor spectra.

\section{Acknowledgment}

J. Gasidlo and P. Amundson were responsible for supervision of the ZPR-3 reactor while these measurements were being made. We are most happy to acknowledge their capable help.

A number of shop men in Building D-20, and, in particular, Clare Kotora and the machinists of the D-316 shop, are to be thanked for their help. W. Hills helped greatly during various stages of the experiment.

D. White did most of the work of producing the ceramic pellets of the Doppler elements, while J. Handwerk, A. Shuck, J. Ayer, and A. Hins, among others, were exceptionally cooperative and helpful in planning and executing the ceramics work.

\section{REFHRENCES}

1. J. Gasidlo andP. Amundson, Nuel. Sei. Eng., 23, 392-3 (1965)

2. H. H. Hummel and A. I. Rago, Effect of Parameter Variation in Doppler Effert Calculations, ANL-6792, p. 747 (1963).

3. I). M. O'Shea et al., Twenty-six-group Cross Sections, ANL-6858 (to be published).

4. I). Meneghetti and J. R. White, Calculations of ZZPR-3 Fast Assemblies using Twenty-six Group Cross Section Set, Trans. Amer. Nucl. Sei., 7, 237 (1964).

5. A. L. Rago and H. H. Hummel, Elmoe: An IBM-704 Program Treating Elastic Scattering Resonances in Fast Reactors, ANL 6805 (1964).

6. R. N. Hwang, Nuel. Sci. Eng., 21, 523-535 (1964).

7. R. B. Nicholson, The Doppler Effect in Fast Veutron Reac tors, APDA-139 (1960). 
8. P. Greebler et al., Calculation of Doppler Coefficient and Other Safety Parameters for a Large Fast Oxide Reactor, GLAP-3646 (1961).

9. J. B. Garg, J. Rainwater, J. S. Peterson, and W. W. Havens, Jr., Phys. Rev., 134B, 985-1009 (1964). (1964)

10. F. W. Firk, J. E. Lynn and M. C. Moxon, Nuel. Phys., 41, $61+(1963)$

11. P. H. Kier, 1 Method of Calculating Mutual Shielding be- tween Resonances of Different Nuclides, Trans. Am. Nucl. Soc., 8, No. 1 (1965).

12. J. J. Schmidt, AEC-HNEA Seminar on Evaluation of Neutron Cross Section Data (1965).

13. G. desaussure, L. W. Weston, R. Gwin, J. E. Russell, and R. W. Hockenburg, Measurement of $\alpha$, the Ratio of the Neutron Capture Cross Section to the Fission Cross Section, for $U$-235 in the Energy Region from $3.25 \mathrm{eV}$ to $3.25 \mathrm{keV}$, ORNL-3738 (1965). 


\title{
Recent Doppler Measurements with ZPR-6
}

\author{
C. E. Till, R. A. Lewis, and E. F. Gron \\ Argonne National Laboratory \\ Argonne, Illinois
}

(PRESENTED BY C. E. THLL)

I want to report briefly on the current series of Doppler measurements with ZPR-6 that are just now being finished. These measurements follow chronologically the set described by Dr. Fischer in the last paper. We have not had time to analyze the results in any depth or for any detailed comparison with theory, but we believe that some of the results are of sufficient interest that they should be presented to this conference. The data have been reduced and analyzed to, I think, final form.

The equipment used for the current ineasurements is similar in part to that used in the measurements described in the last paper. We have made, however, two significant changes. First, by improving the datagathering techniques-and this largely is in providing a servo-controlled regulating rod for the reactivity measurement, fully automated with the oscillator system-we have improved the precision of the measurements by better than an order of magnitude. This, in turn, has allowed us to trace out the temperature dependence of the Doppler effect, something that was not feasible in the previous series, while still retaining precisions at each temperature better than the previous results by a factor of about ten. Secondly, in this series we have taken a direct approach to the problem of separating expansion effects from the true Doppler effect by designing three different sample types, each identical to the others except that in one type the sample material is allowed to expand freely; in the second type the axial expansion is constrained, and in the third type expansion is constrained both axially and radially. By comparing the results obtained from use of these otherwise identical samples, the expansion effects are shown directly. We believe, in fact, that one of the significant advantages of the heated-sample technique is the control one has over experimental conditions and, in particular, a potential control over the expansion problem.

The measurements were done in a zoned version of a $2600-$ liter carbide core. The core contained $42 \mathrm{v} / 0$ sodium, with a fuel isotopic ratio of $7: 1 \mathrm{U}^{238}$ to $\mathrm{C}^{235}$.

One of the first things of concern was the possible effect on the Doppler effect in the heated sample of resonances in material around the sample, which is the same as the sample material, but unheated-the so-called "hot-sample, cold-reactor" effect. We compared the results of two measurements with the same sample in two slightly different environments. The first measurement was done in the normal reactor environment. In the second, a zone of material about a mean free path or so thick around the sample had the $\tau^{\text {T238 }}$ removed and replaced by molybdenum-the quantity of molybdenum selected so that the calculated Doppler energy fluxes and adjoints were held constant for the two cases. The idea was to form an isolation zone around the sample without perturbing the gross fluxes or adjoints in the Doppler energy region. How successful we were in this matching was tested experimentally by measuring the worth of boron in each environment. The two boron worths checked to fraction of one percent.

Figure 1 gives the results of measurements with the $7: 1$ sample, having an isotopic ratio the same as the reactor itself. The upper curve shows the result with the sample in the isolation zone and the lower curve that with the sample in the reactor environment. One can see that there is probably a statistically significant, but small, effect at lower temperatures, but no very significant effect overall is noted.

Figure 2 shows the results of three separate experiments. The upper curve gives the $\mathrm{U}^{23}$ Doppler effect, the curve containing experimental results from two determinations about ten weeks apart. In the intervening time, the core and sample arrangement had been changed around several times. They were then returned to the original configuration and the measurement redone. The result gives us considerable confidence in our reproducibility, for as is apparent in the figure, all points fell on the same curre. I might say in passing that the shape of the curve fits a $1 / T$ dependence within a few percent. The lower curve is the result for the same sample but with a $1 / 2$-in. natural boron carbide filter installed around the element to illustrate that the measured effect is, in fact, due to low-energy neutrons. The calculated reduction in 
the Doppler energy fluxes and adjoints caused by the boron was such that the calculated Doppler effect was reduced to about $20 \%$ of its previous value and the measurement agrees nicely.

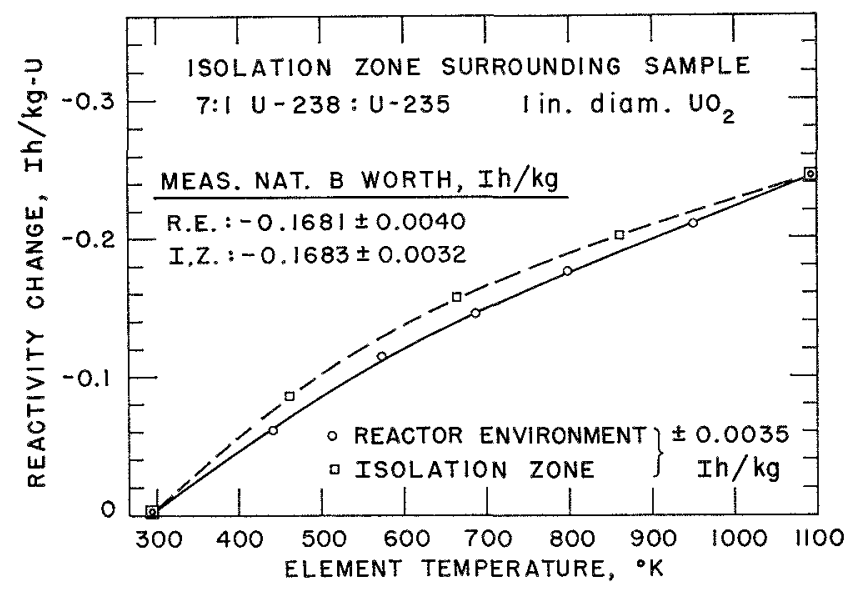

Frg. 1. Reactivity Change as a Function of Temperature with an Isolation Zone Surrounding Sample.

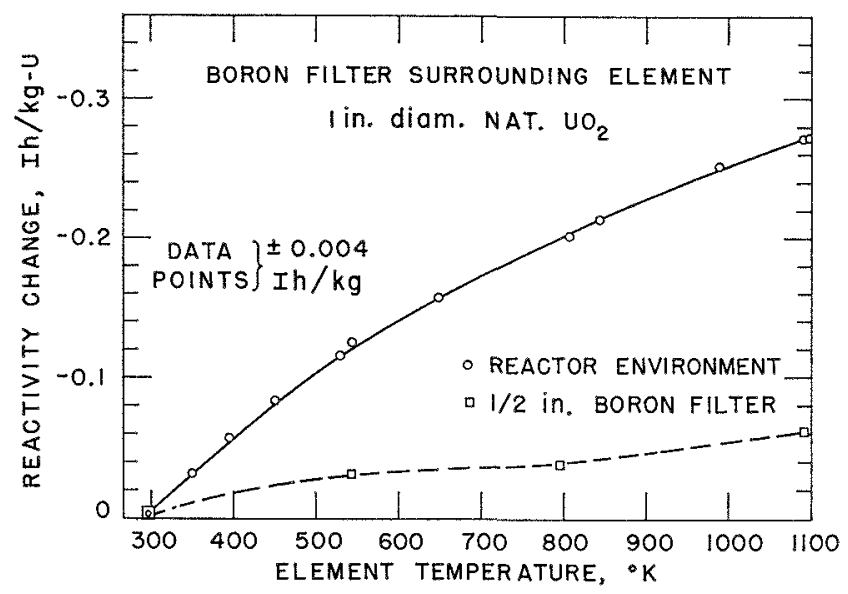

FIa. 2. Reactivity Change, with a Boron Filter Surrounding the Doppler Element, as a Function of Temperature.

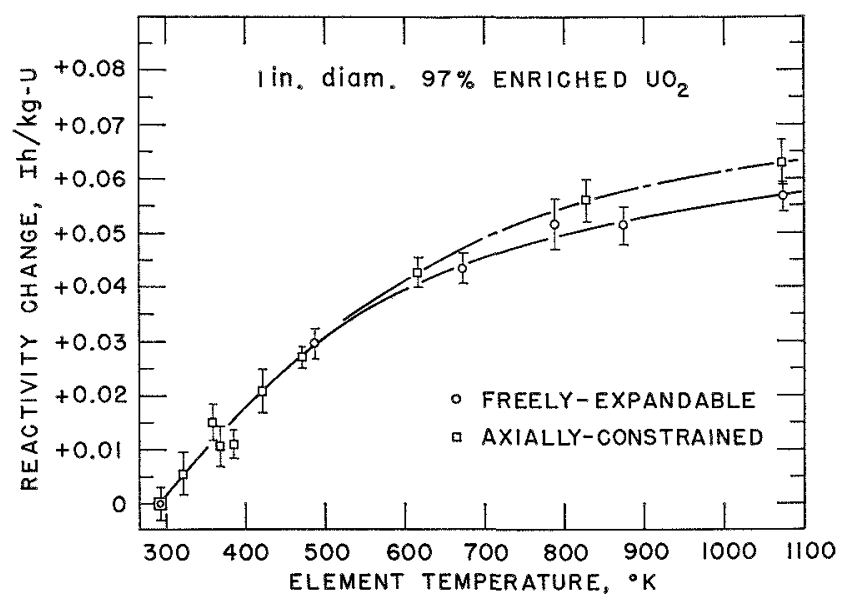

FIG. 3. Reactivity Changes with Freely Expanding and Axially Constrained Samples as a Function of Temperature.

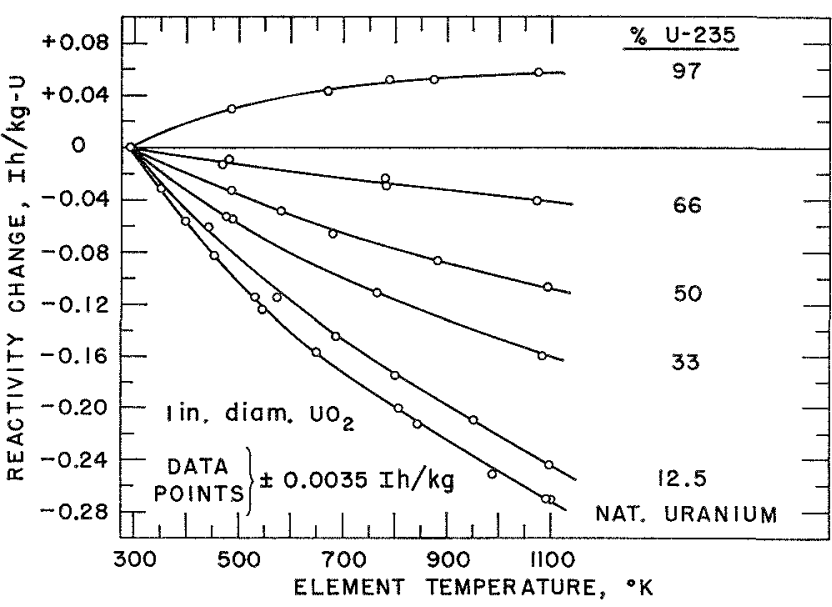

FIG. 4. Reactivity Changes for Elements of Various Isotopie Ratios of $\mathrm{U}^{235}$ to $\mathrm{D}^{236}$ as a Function of Temperature.

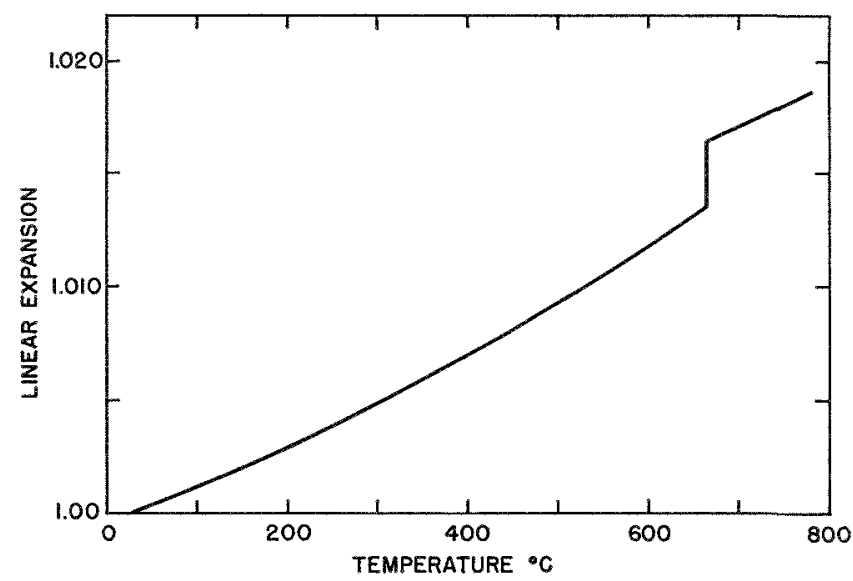

FıG. 5. Linear Thermal Expansion of Uranium Metal.

Figure 3 compares the results for the $\mathrm{U}^{235}$ oxide freely expanding element and for a similar element in which no axial expansion was allowed. An expansion effect is apparent, but it is not large. The $U^{235}$ Doppler effect thus is shown to be positive in these spectra and, comparing its magnitude with the $\mathrm{U}^{238}$ Doppler effect given in Fig. 2 , to be about $1 / 3$ the magnitude at low temperatures, decreasing to about $1 / 5$ at $1100^{\circ} \mathrm{K}$. The shape, therefore, is such that the $\mathrm{U}^{235}$ Doppler effect appears to fall off more rapidly than the approximate $1 / T$ dependence found for $U^{238}$.

Figure 4 summarizes data for samples with varying isotopic ratios of $U^{238}$ to $U^{235}$. In general, the Doppler effect of the mixtures seems to be about that given by pro-rating, simply on the basis of the amounts present in each mixture, the experimental results of the two component parts.

Finally, in Figures 5 and 6 are shown the results of a measurement that gives strong confirmation of the essentially positive nature of the $U^{235}$ Doppler 


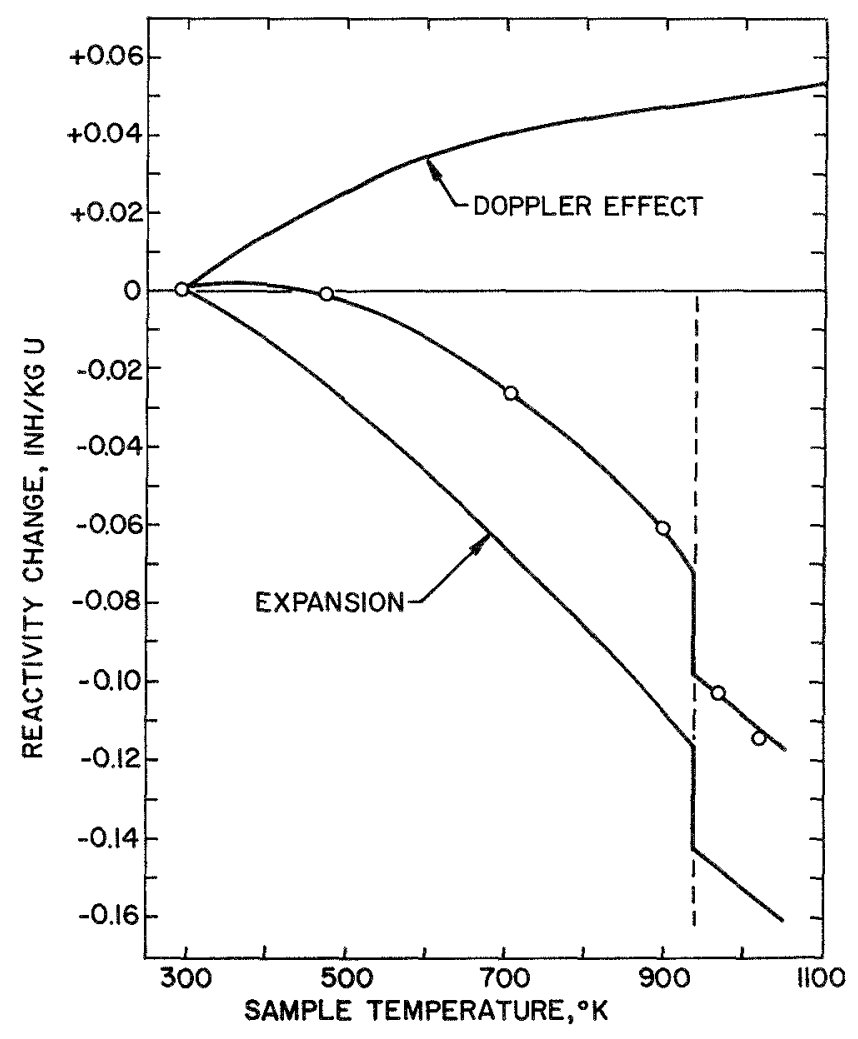

Fra. 6. Reactivity Changes of Uaso Metal Samples as a "Function of Temperature.

effect in these spectra, and also gives quantitative indication of the reason why negative effects have been observed in the past on heating $U^{235}$ metal. The measurement consisted simply of a Doppler measurement of a $U^{235}$ metal sample in the normal manner and taking advantage of the volume change in the $\alpha-\beta$ phase change of the metal to calibrate the reactivity effect of expansion. Figure 5 simply shows the expansion of as-cast uranium metal on heating, with the discontinuity at the phase change.

In Fig. 6 the circles indicate the measured reactivity effect on heating. The lower curve shows the reactivity effect of expansion as given by Fig. 5, calibrated in reactivity units by the experimentally observed reactivity change through the phase change. The upper curve is the experimentally observed $\mathrm{U}^{235}$ Doppler effect taken from the $\mathrm{U}^{235}$ oxide nonexpanding element result shown in Fig. 3. Thus both upper and lower curves are based on experiment.

The middle curve is the sum of the upper and lower curves. The agreement with the observed result is, of course, remarkable. However, even if the agreement were worsened considerably, the data would still be convincing, for the odd shape of the observed reactivity curve, much different than any other result we obtained, can only be explained on the basis of a positive Doppler effect falling off rapidly with increasing temperature combined with strong negative expansion effect increasing in a discontinuous manner through the phase change.

\section{Discussion}

Mr. Pitterle (APDA): I wonder if you could comment on how thick the nickel heating element around the sample is?

$M r$. Till: 50 mils.

Mr. Pitterle: I wonder if you are planning to pursue this zone around the sample any further. I don't believe the question here is the hot sample, cold reactor effect, but what is the flux incident on the sample. And here, I think, very often the question comes out what is the effect of the heterogeneity of the core? I wondered if you plan any experiments of two types-one, where you might vary the sample size of this corc around it, and sce how much of the fine structure is coming from the core and how much might be from the sample; secondly, whether you might be considering varying the plates immediately around the sample, perhaps to try and take one extreme of homogeneity versus another to see what types of effect this has. I would like to emphasize this because I think the interpretation in terms of the Doppler effect of a reactor is much clearer when you can put the homogeneous zones around the sample, provided your heating-element thickness is so small as not to perturb the fine structure.

Mr. Till: We have, in fact, done experiments in which the immediate environment around the sample has been changed. The nomal reactor environment around the sample was built out of small pieces selected so that we did have a much more homogeneous arrangement of materials than is true throughout the remainder of the reactor. We then removed that more homogeneous zone and replaced it with material arrayed in the same way as the rest of the reactor, and we got no significant difference. As for the other point about interpretation of the hot sample in a cold reactor, the calculations I have done indicated that one doesn't expect much effect from the interaction between the cold resonances and the hot-sample resonances as long as the temperature change is high enough that the sharp dip in the flux from the cold resonance doesn't really bear on the resonance broadening taking place out in the wings. Certainly the region around the center line of the cold resonance gives the sort of sharp dip that isn't very important as soon as the $\Delta T$ becomes large.

Mr. Chernick (BNL): One always worries about these small-sample experiments with very small reactivity exchange. Is there any chance that you might 
take a bundle of elements and repeat the measurements?

Mr. Till: We are planning just such a measurement with essentially a cluster. We have no plans at the moment of going on to a large heated zone, but we have started design of a cluster experiment.

Mr. Küsters (Karlsruhe): The results giving the negative plutonium Doppler coefficients seem to be the same as the British have obtained. Only the correction for expansion seems to be different. I wonder if Dr. Codd or Dr. Fischer would say the only correction one has to do to have a positive coefficient is to put the right correction on the expansion coefficient?

Mr. Fischer (ANL): I think that in these series of measurements we have obtained some very interosting clues in that the plutonium Doppler coefficient is much smaller than we had earlier expected it to be, but I don't think we can say that we have the ultimate experiment here. 


\title{
Recent Results of Doppler Measurements in Fast-neutron Spectra
}

\author{
S. G. Carpenter, L. A. Mountford, T. H. Springer, and R. J. Tuttlet \\ Atomics International \\ Canoga Park, California \\ (PRESENTED BY S. G. CARPENTER)
}

I would like to discuss briefly the status of the Fast Spectrum Doppler Measurements program at Atomics International and to outline the areas of study in which future work will be carried out. It should be pointed out that some of the results to be shown have already been discussed in recent technical meetings; consequently, a full paper will not be given. However, since the last ANS meeting, measurements have been carried out on some new materials, the addition of which brings the extent of material coverage to encompass $\mathrm{W}$, $\mathrm{Ta}, \mathrm{Nb}$, and $\mathrm{Mo}$, in metal form, Th as metal and oxide, and $\mathrm{U}^{238}$ and $\mathrm{U}^{235}$ as metal, oxide, and carbide forms. The range of spectra have extended from $200-\mathrm{keV}$ median fission energy (mfe) systems down to approximately $0.01-\mathrm{eV}$ mfe. Our measurements were made in a manner very similar to the technique discussed in the last paper, i.e., small samples in a multiregion reactor. The samples were generally $4 \mathrm{in}$. long and $0.438 \mathrm{in}$. in diameter.

The scale of median fission energy in Fig. 1 is a very rough indication, taken from calculations, of the energies at which the various experiments were made. These results apply to $\mathrm{U}^{235}$ metal, and the $\Delta \rho$ were determined over the temperature interval from 20 to $600^{\circ} \mathrm{C}$. The experimental measurements of the total temperature cocfficient of reactivity are shown by the square symbols and the dashed line. The expansion coefficient of reactivity is represented by the circular symbol and the dotted line. The former values are the results which were reported, at one-mfe value, as a negative Doppler coefficient for $U^{235}$ at the meeting two years ago. Since that time, we have done careful expansion-effect measurements by five independent methods, including the use of an alpha-beta phase change and a sample whose density has been artificially reduced to approximately $85 \%$ of theoretical, in order to find the effect of expansion of the samples in these cores.

When we make an algebraic subtraction point by point, the points represented by the triangles in Fig. 1 result. A rough curve drawn through these points is shown as the solid line. This curve now represents our estimate of the Doppler effect in the
$\mathrm{U}^{235}$ material, roughly as a function of median fission energy. These measurements were done in some cases with polyethylene regions around the sample to reduce the energy of the neutron spectrum and to give us an indication of those lower-energy cores which might be of interest for further experiments.

Our main conclusion from this work is that the Doppler effect in fast cores is positive for $U^{235}$. It is about half the effect that we calculate and curves over a little faster than our calculation would predict. It does go negative someplace, probably in the low$\mathrm{keV}$ region and then continues negative below that point. Similarly, the expansion coefficient goes through zero, going from negative to positive, as one decreases the mfe from $200 \mathrm{keV}$ to the low-eV region.

Figure 2 shows the results from a typical calculation. The curves are similar to those shown by Till, in the last paper, of the total measured effect, the expansion effect, and then the subtraction giving the Doppler effect for the sample as a function of energy. The measured total reactivity as a function of temperature will vary from a curve which starts out in the negative direction and continues in this direction with constant slope for a hard spectrum to a curve similar to Fig. 2, where it starts out in the negative direction, goes through zero, and then becomes positive for a soft spectrum.

Among the Doppler problems which are currently under study is one concerned with fitting the measured points to various temperature-dependent functions and extrapolating the results to very high temperatures. In Fig. 3, the measured change in the reactivity with respect to temperature $T$ has been compared with plots of $T^{-\gamma}$. The two particular curves plotted in this case are for $\gamma=1 / 2$ and 1 , made by a least-squares fit. Our results for $\mathrm{U}^{238}$, shown in Fig. 4 , in general show that the exponent $\gamma$ is slightly less than 1 for the hard cores and becomes smaller as we go down to the low-energy cores. For the fast cores, the fit is a little harder to make because the smaller signal makes more difficult the distinguishing among the several curves.

Our immediate plans for the future are to use 


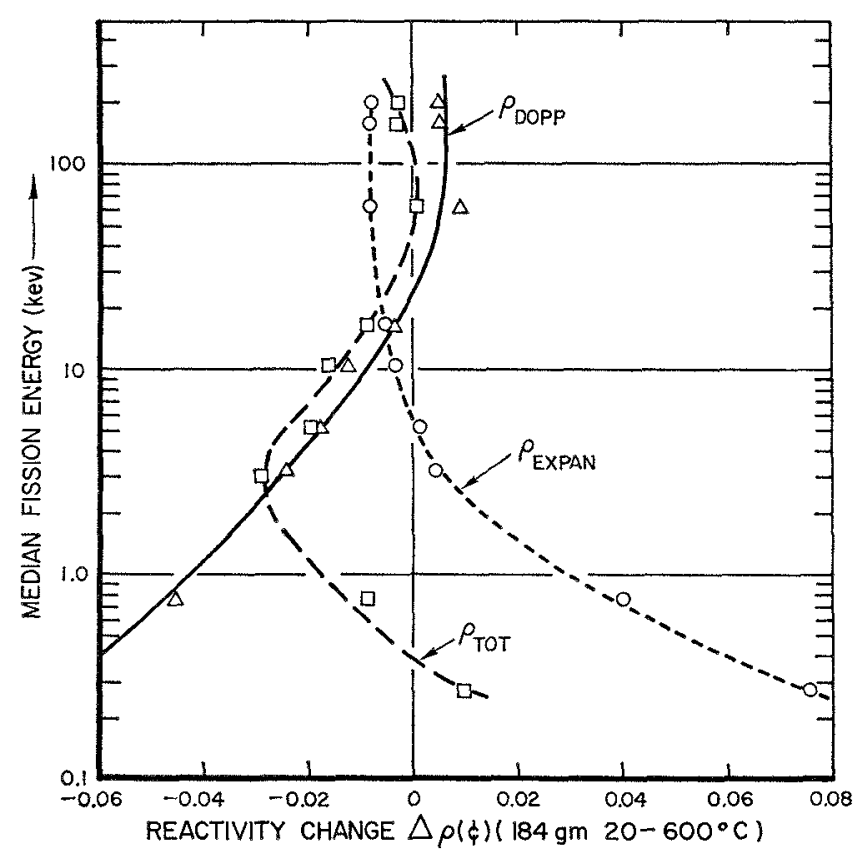

Fig. 1. Variation of Reactivity of $\mathrm{U}^{235}$ due to Temperature and Expansion, and Derived Doppler Reactivity for Different Calculated Median Fission Energies.

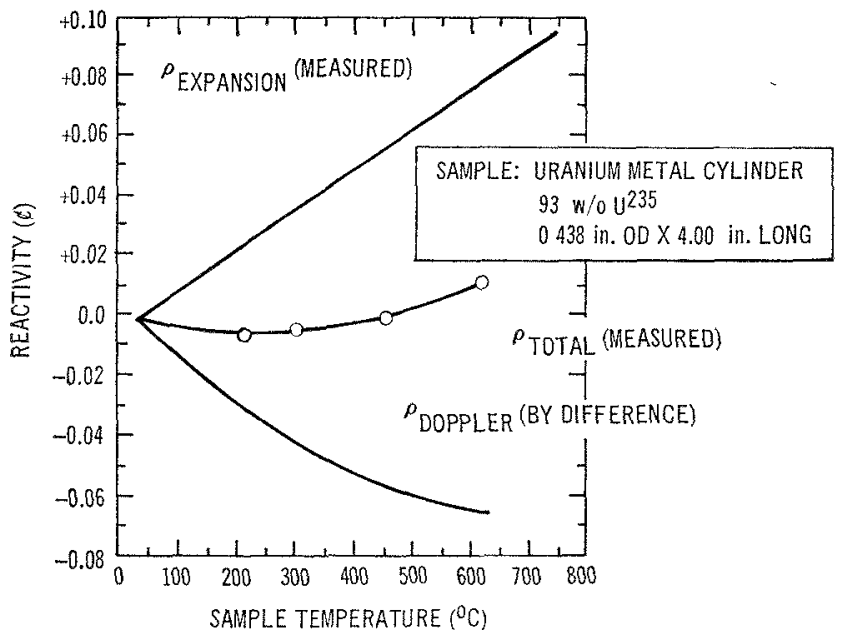

FIG. 2. Reactivity Worth vs Temperature for $U^{235}$.

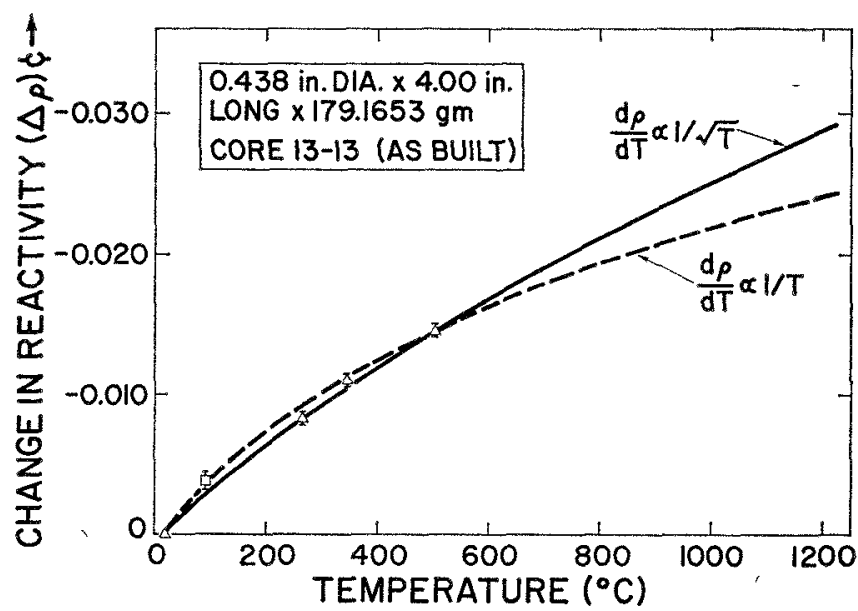

FIG. 3. Temperature Coefficient of $U^{238}$ Metal.

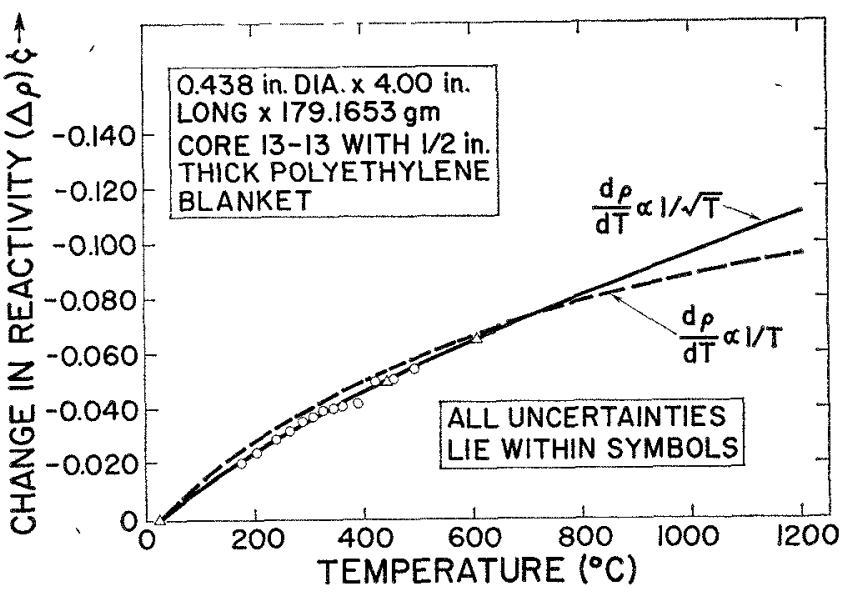

FIG. 4. Temperature Coefficient of $\mathrm{U}^{238}$ Metal.

plutonium, which we now have on order, in different isotopic compositions, including a small sample of almost pure plutonium-241. We plan to go to temperatures of the order of $2000^{\circ} \mathrm{C}$ and are just in the process of starting measurements in which the reactivity is determined as a function of temperature from room temperature down to 40 or $50^{\circ} \mathrm{K}$. Particular attention is being paid to the effects of the Debye temperature. 


\title{
Doppler Effect in $\mathbf{U}^{238}$ Capture and $\mathbf{U}^{225}$ Fission in a Fast-neutron Spectrum*
}

\author{
G. R. Pflasterer, Jr., R. Sher, $†$ W. A. Mayer, $\ddagger$ and F. G. Warzek \\ General Electric Company \\ Vallecutos A tomic Laboratory \\ Pleasanton, Calfornia \\ (PRESENTEI) BY (․ R PFLASTERER, JR)
}

\section{INTRODUCTION}

The Doppler effect which is so important to the inherent safety of fast reactors has been investigated in the fast-neutron spectrum of the Nixed Spectrum Critical Assembly (ISCA). Reference (1) describes the reactor and other experimental results obtained in this program, and reference (2) describes the physics design of the MSCA. This study includes both reactivity and activation determinations of the U-238 Doppler effect. The activation measurements, the subject of this paper, are believed to be the first to be performed in a fast spectrum.

\section{CONCLUSIONS AND RECOMMENDATIONS}

The activation Doppler measurement is an extremely worthwhile complement to the more familiar reactivity measurement; it permits a direct evaluation of the ability to predict the change in U-238 capture. Further, the activation technique provides the only opportunity to separate the effects of cross section and fine flux spectrum components of the gross Doppler effect. The measurement technique requires careful control of foil position and counting procedures, since the signal is in the 1 to $2 \%$ range. Nevertheless, experience has shown that reproducible measurements can be performed to \pm 0.002 or approximately $10 \%$ of the magnitude of the observable Doppler effect.

The activation measurements have indicated a significant underprediction of the Doppler effect. This discrepancy is believed to be at least partly due to the calculated neutron spectrum being substantially too hard. This conclusion is borne out by measurements of neutron spectrum and $1 / v$ poison worth. These results, coupled with the generally good agreement obtained between predicted and measured Doppler reactivity efferts in similar fast spectra, indicate that present

\footnotetext{
* Work sponsored by Atomic Energy Commission under Contract AT (04-3)-189, P A. 13.

† Stanford University, Consultant to Vallecitos Atomic Laboratory.

$\ddagger$ A.E.G., Frankfurt, Kernenergieanlage.
}

cross sections must lead to compensating errors. For example, an overprediction of the change in U-238 capture and an underprediction of the "importance" of this change due to significant errors in predicting gross spectra could explain present discrepancies. Future work in this area should be closely coupled with neutron-spectrum determinations.

Additional work is clearly needed to resolve scveral unanswered questions. First, what is the energy distribution of Doppler events in typical fast spectra? This can be determined by a series of measurements with varying foil thicknesses and known fuel buffers between the foil and fuel environment. Second, what is the flux energy spectra? This can be determined by proton-reroil and time-of-flight spectrum measurements over the energy range from $50 \mathrm{eV}$ to $1 \mathrm{MeV}$. Finally, through a comparison to reactivity measurements in known spectra remaining compensating errors could be evaluated and hopefully eliminated.

\section{MEASUREMENT ME'THOD}

\subsection{Comparison of Activation and Reactivity Techniques}

Two basic methods of measuring the Doppler effect are suitable for application in zero-power (critical) assemblies. ${ }^{(3)}$

In the activation method a sanple of either fissile or fertile material is irradiated at an elevated temperature and its induced activity compared to that of a cold sample which was irradiated under the same conditions.

In the reactivity method a sample is heated and the resulting change in reactivity measured. The measurement may be cither static or oscillatory. Further, the oscillator measurements may involve either thermal or mechanical oscillation.

Each measurement method measures different components of the Doppler effect. The two techniques are, therefore, complementary rather than duplicative in their approach. The reactivity measurement, to first order, determines exactly the reactivity effect per gram at the selected location of the reactor that would oceur 
in a reactor power excursion, whercas the activation measurement determines the exact change in neutron capture or fission in a reference spectra. Attention will now be focused on the activation technique which was the method used in this study.

Considering an infinite homogeneous medium at uniform temperature, the reaction rate in volume $v$, per unit energy, at energy $E$ is

$$
R=\int_{\imath} \varphi \sigma d v=v \varphi \sigma .
$$

Upon taking the derivative with respect to temperature we obtain

$$
\frac{d R}{d T}=v\left(\varphi \frac{d \sigma}{d T}+\sigma \frac{d \varphi}{d T}\right) .
$$

This expression illustrates the relative contributions, to first order, of a change in cross section of the heated sample and of a change in the spectrun impingent upon the sample. For very small samples (foils) almost all reactions in the foil are due to neutrons whose last collision was in either surrounding fuel or structural material. Under these circumstances only the first term on the right-hand side (RHS) of Eq. (2) is important. On the other hand, if the heated samples are large, or if a heated buffer zone is present immediately surrounding the sample, the second term on the RHS of Equation (2) becomes important. This term may be either positive or negative. For the present measurements only the first term was determined.

\subsection{Experimental Technique}

Two identical furnaces loaded with uranium metal foils are placed in symmetric positions within the fast core of the MISCA. Figure 1 shows a cutaway view of the sperial fuel bundle and furnace in the foil-holder region. Figure 2 shows the foil-placement detail. Three concentric tubes are used in the furnace construction to provide thermal insulation and paths for gas flow. The furnace assembly fits into a special fast core fuel bundle from which the central nine fuel rods have been removed, leaving the outer sixteen rods in place. Cooling air is introduced into the fuel channel at the lower end and flows upward along the outside of the furnace and then down through the outer furnace annulus. An inert cover gas (argon) is maintained in the innermost tube to prevent oxidation of the heated uranium foils. The sample foil holder is aluminum with heater wire wrapped on the lower portion. The furnace design minimizes neutron scattering between the foil and the surrounding fuel to assure a resonance flux spectrum at the foil location representative of the fuel environment. However, for selected experiments an 0.15-in.-thick nickel sleeve was inserted between the foil and the inner

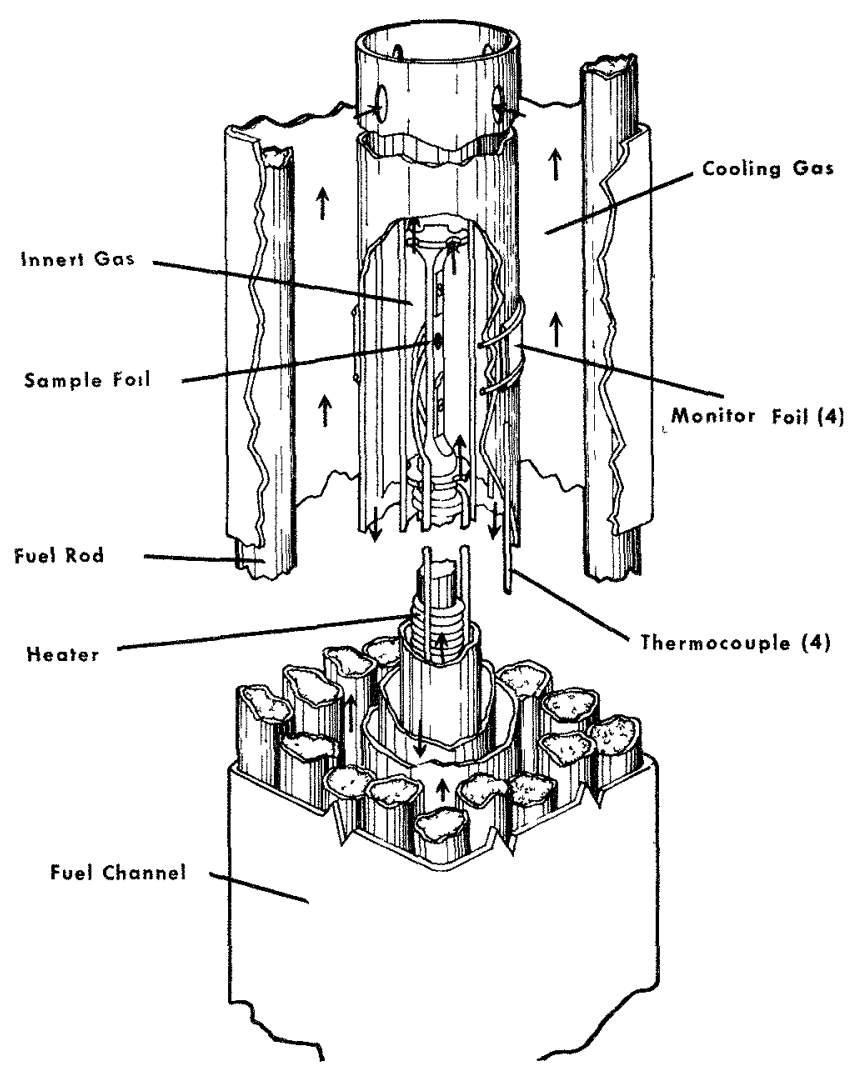

Fia. 1. Furnace for Doppler Effect.

furnace tube to increase intentionally this non-fuel scattering and, hence, provide a basis for experimentally extrapolating to zero scattering.

Two thermocouples are used at the sample-foil location with a third located at the center of the coiled heater wire. There are also two thermocouples on the outer furnace tube at the foil elevation. Four enriched U-235 flux-monitored foils are mounted on the outer furnace tube at the same elevation as the heated foil. These foils permit determination of small asymmetries in the gross flux distribution between the two furnace locations. Great care was taken in the design and testing of the furnace components to assure a negligible effect due to thermal expansion of the structural components.

One-half-inch-diameter uranium metal foils of both $93 \%$ and natural enrichment are utilized. For most of the experiments the foils are all 3 mils thick; however, some of the experiments utilized 0.5 -mil-thick natural uranium metal foils. The foils for a given irradiation are matched closely in weight to eliminate any differences in gamma self-absorption.

One natural and one enriched uranium metal foil are loaded into the foil holder which, in turn, is loaded into the furnace assembly. Two 1-mil-thick buffer foils of aluminum are used to separate the uranium foils and to prevent transfer of fission products between them. Two 


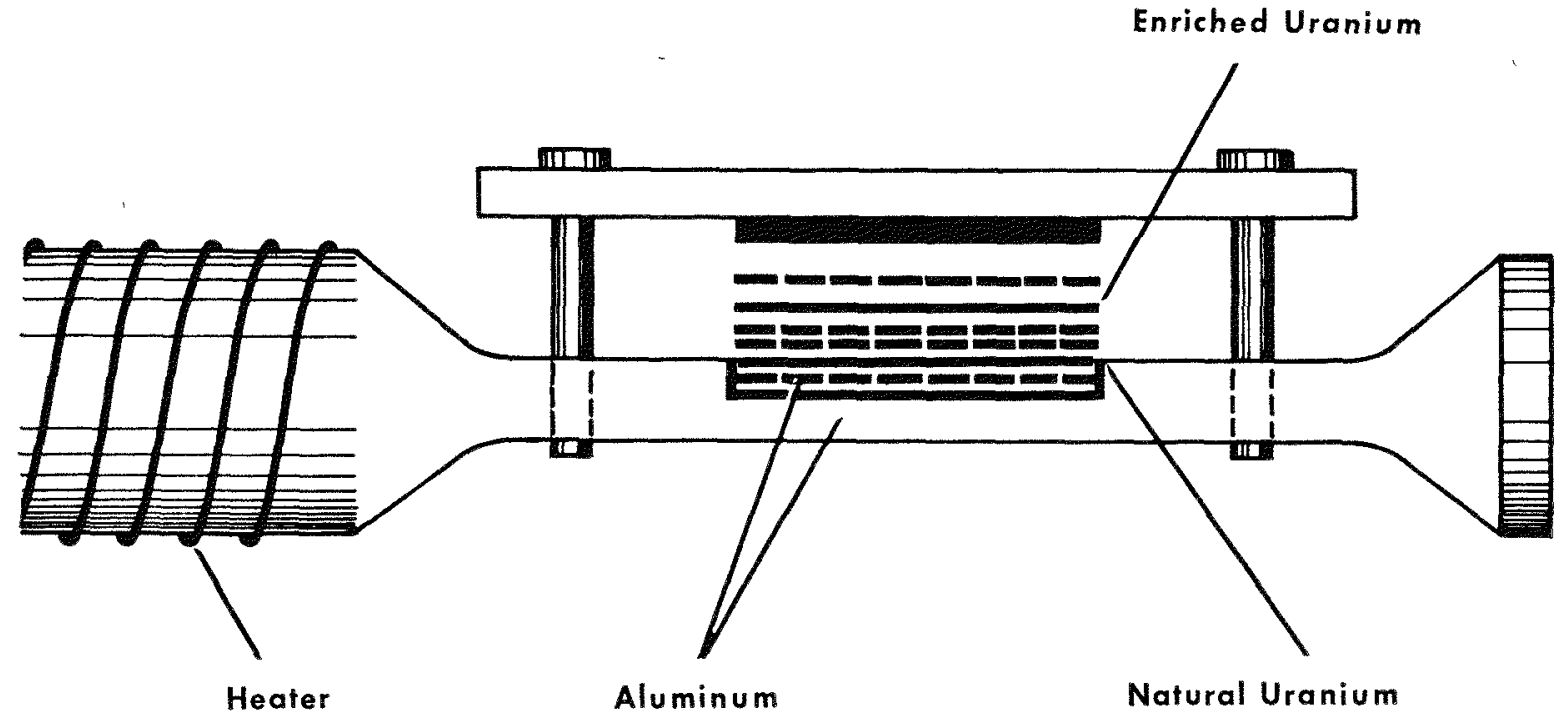

FIG. 2. Foil-holder Furnace Assembly.

other aluminum foils are used to prevent fission product contamination of the foil holder. Four enriched U-235 foils are installed in the flux-monitor locations on the outer furnace tube. The entire furnace assembly is then loaded into the appropriate fuel bundle position in the MSCA fast core.

After approximately $2 \mathrm{hr}$ of argon purging, one of the two furnaces is heated to the desired temperature. Approximately $45 \mathrm{~min}$ is required to reach $800^{\circ} \mathrm{F}$ starting from room temperature. Once the desired temperature is obtained, the reartor is brought to power. To induce the desired foil activity it is necessary to irradiate at approximately $1000 \mathrm{~W}$ for one hour; after completion of irradiation the reactor is shut down, the furnace allowed to cool, and the short-lived fission products permitted to decay. After approximately $15 \mathrm{hr}$ the foils are removed and their counting is initiated.

The irradiated foils are gamma counted with a $3 \times$ 3 -in. sodium iodide (T1) scintillation crystal. The U-238 capture is inferred by observing the $106 \mathrm{keV}, 56.3-\mathrm{hr}-$ half-life activity due to Np-239. The fission product activity on the other hand is determined by observing the gross gamma activity between 500 to $800 \mathrm{keV}$, which has approximately a $24-h r$ half-life at one day after formation. The seintillation pulses observed by the photomultiplier are amplified and analyzed by a 256-channel pulse-height analyzer. Two unirradiated foils, one natural and one enriched, are used to determine the residual background activity. The uranium metal foils from each irradiation are counted approximately once every hour over the two days following the irradiation. The counts obtained from the natural uranium foils in the $100-\mathrm{keV}$ region are corrected for the contribution of fission product gammas, utilizing the measured gross fission product gamma activity in the 400 - to $800-\mathrm{keV}$ region as a measure of total fission product activity present.

\subsection{Analytic Model}

It is convenient to analyze the Doppler effect observed in the heated foil in terms of a three-region system: the foil, the surrounding non-fuel material, and the fuel environment. The following assumptions are then made:

1. A scattering collision with fuel or non-fuel material results in a neutron energy change large compared to the resonance width (NR approximation).

2 . The resonances are widely separated so that the flux outside a resonance is constant in lethargy and space.

3 . Resonance scattering ean be neglected.

4. Cross sections are independent of space within a given region.

Using the methods outlined by Reynolds, ${ }^{(2)}$ it follows that

$$
\begin{aligned}
I_{1}=(1 & \left.-T_{0}\right) \int_{\mathrm{RES}} \sigma_{a, 1} \frac{\Sigma_{p, 1}+S}{\Sigma_{t, 1}+S} d E \\
& +T_{0} \int_{\mathrm{RES}} \sigma_{a, 1} \frac{\Sigma_{p, 3}}{\Sigma_{t, 3}} d E \\
& +T_{0} \int_{\mathrm{RES}} \sigma_{a, 1} \frac{\Sigma_{p, 1}}{S+\Sigma_{t, 1}}-\left[1-\frac{\Sigma_{p, 3} \Sigma_{t, 1}}{\Sigma_{p, 1} \Sigma_{t, 3}}\right] d E,
\end{aligned}
$$

where

$T_{0}=$ the transmission probability across the nonfuel region between the foil and the fuel;

$\sigma_{a, \mathbf{1}}=$ microscopic absorption cross section of the foil at temperature $T_{\mathbf{1}}$; 


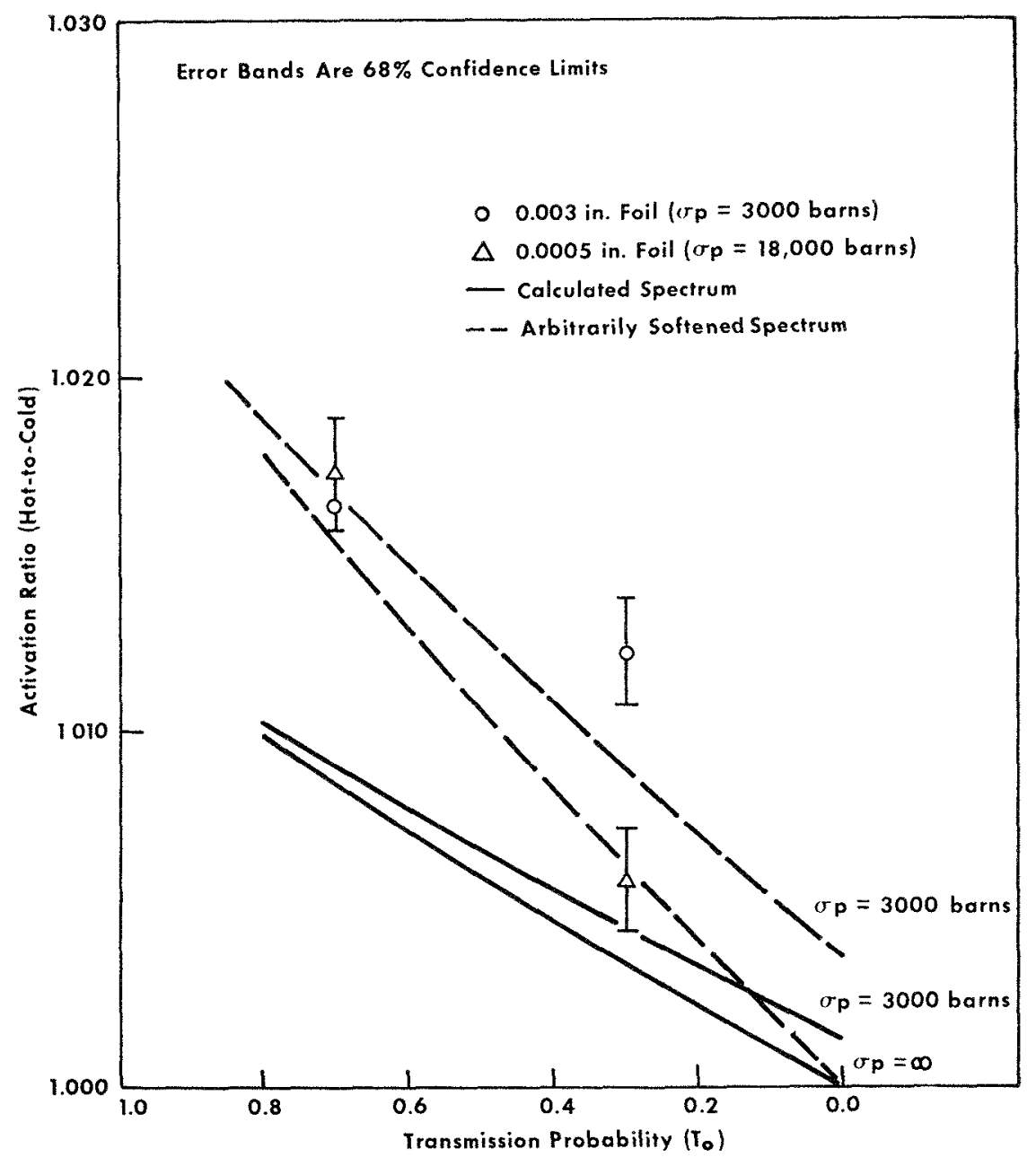

FIG. 3. Doppler-activation Results for $780 \pm 20^{\circ} \mathrm{F}$ Foil Temperature $5.5 \mathrm{In}$. from Core Center for 23.1-23.6 w/o Enrichment.

$\Sigma_{p}=$ the macroscopic partial scattering cross section;

$S=$ reciprocal foil thickness $\left[S /\left(S+\Sigma_{t, 1}\right)\right.$ is used for the foil escape probability];

$\Sigma_{t}=$ the macroscopic total cross section with subscript 1 referring to the foil region and subseript 3 referring to the fuel region.

In most practical cases the third integral may be neglected, giving

$$
\begin{aligned}
I_{1}=\left(1-T_{0}\right) \int_{\operatorname{RES}} \sigma_{\alpha, 1} \frac{\Sigma_{p, 1}+S}{\Sigma_{t, 1}+S} d E & \\
& \quad+T_{0} \int_{\text {RES }} \sigma_{\alpha, 1} \frac{\Sigma_{p, 3}}{\Sigma_{t, 3}} d E .
\end{aligned}
$$

The gross neutron-energy spectrum is obtained from a 24-group diffusion-theory calculation. The resonance integrals are evaluated over specific energy intervals in the form

$$
I=\int_{\mathrm{RES}} \sigma_{a}\left(T_{1}\right)\left[\frac{\Sigma_{p}+S}{\Sigma_{t}\left(T_{3}\right)+S}\right] d L^{\prime}
$$

where $T_{1}$ and $T_{3}$ can be equal or unequal temperatures. Resolved resonance parameters are used up to $1 \mathrm{keV}$, and a statistical model used for resonances above $1 \mathrm{keV}$. Doppler-broadened cross sections were obtained by use of the RAPTURE Code ${ }^{(j)}$ which has been modified by the addition of the PITFORK option to calculate integrals of the type shown in Eq. 5. The transmission probability $T_{0}$ for the intermediate region between the foil and fuel is determined by a collision probability method reported by Reynolds. ${ }^{(4)}$

Note that this expression for the foil resonance integral is independent of the foil thickness in the linit of $T_{0}=1$. Dependence on the foil thickness should be present at this limit, and its absence is the result of neglecting an apparently small term in the resonanceintegral expression. For this reason, the predicted values in Fig. 3 are not shown at the value $T_{0}=1$. 


\section{RESULTS}

\subsection{Comparisons with Theory}

The results, expressed as the nommalized ratio of the heated-foil activity to the unheated-foil activity, may be compared to the ratio of effective resonance integrals over narrow energy intervals (see Eq. 3) weighted by the calculated multigroup flux spectrum. Several measurement conditions were utilized in the program as indicated in Table 1. The foil thickness was varied to evaluate the effect of changes in the potential sattering rross sertion. The nickel slecve was inserted to determine the effect of varying $T_{0}$, the transmission probability of the region between the foil and surrounding fuel. The potential scattering cross sections were 3000 and $18,000 \mathrm{~b}$, respertively, for the 3 - and 0.5 -mil foil thicknesses. The transmission probabilities were 0.3 and 0.7 , respectively, with and without the nickel sleeve in plare.

The measurements at 5.5 in., with and without the nickel slceve present, are compared with predictions on Fig. 3. Here, the activation ratio (hot to cold) is plotted as a function of $T_{0}$ of the non-fuel region. Calculated rurves are shown for potential seattering cross sections corresponding to both the 3 - and 0.5 -mil foils.

Two important conclusions can be drawn immediately. First, the ralculated values for the actual 23.1 w/o U-235 enrichment lie considerably below the measured points, indicating that the spectrum is likely to be softer than predicted. To evaluate this possibility further, the calculated spectrum was arbitrarily softened to yield agreement with the measurements. This agreement was obtained by using a fictitious enrichment of $16 \mathrm{w} / \mathrm{O} \mathrm{U}-235$. The degree of spectrum change required to bring experiment and theory into agreement can be assessed by the fact that the median fission energy decreased from 200 to $1.50 \mathrm{keV}$ and the fraction of fissions below $9.1 \mathrm{keV}$ increased from 8.0 to $12.4 \%$, respectively. Second, the 3 -mil foil exhibits a very strong self-shielding effect, namely, the weak depend-

TABLE 1. Resllts of Armvatron Meastrements

\begin{tabular}{c|c|c|c|c|c}
\hline $\begin{array}{c}\text { Distance } \\
\text { from }\end{array}$ & $\begin{array}{c}\text { Temper- } \\
\text { Fast-core } \\
\text { Center } \\
\text { (in.) }\end{array}$ & $\begin{array}{c}\text { Foil } \\
\text { Thick- } \\
\text { ness } \\
\text { (in.) }\end{array}$ & $\begin{array}{c}\text { Nickel } \\
\text { Sleeve } \\
\text { around } \\
\text { Foil }\end{array}$ & $\begin{array}{c}\text { Measure- } \\
\text { ment } \\
\text { Repeti- } \\
\text { tions }\end{array}$ & $\begin{array}{c}\left(A_{H} / A_{C} \text { ) for }\right. \\
\text { U-238 }\end{array}$ \\
-5.5 & $-780 \pm 20$ & 0.003 & No & 14 & $1.016 \pm 0.001$ \\
5.5 & $780 \pm 20$ & 0.003 & Yes & 4 & $1.012 \pm 0.002$ \\
5.5 & $780 \pm 20$ & 0.0005 & No & 4 & $1.017 \pm 0.002$ \\
5.5 & $780 \pm 20$ & 0.0005 & Yes & 4 & $1.096 \pm 0.002$ \\
12.2 & $780 \pm 20$ & 0.003 & No & \pm & $1.019 \pm 0.002$ \\
12.2 & $780 \pm 20$ & 0.003 & Yes & 4 & $1.017 \pm 0.002$ \\
5.5 & $440 \pm 20$ & 0.003 & No & 2 & $1.0098 \pm 0.003$ \\
\hline
\end{tabular}

ence upon the presonce of the nickel sleeve. The data for the 0.5 -mil foil, on the other hand, indicate a stronger dependence on the presence of the nickel sleeve and, hence, less self-sbielding. Both conclusions are further substantiated by the data for the 3 -mil foil at $12.2 \mathrm{in}$. Table 1 summarizes all the measured values.

\subsection{Measurement Errors}

It is important to consider random and systematic errors in the activation ratio measurement so that realistic error bounds can be established. The random errors considered here are:

1. determination of gamma activity of the uranium foil;

2. determination of uranium weighe in the foils;

3. overall random error in the measurement of the activation ratio of foils irradiated at the same temperature.

The systematic errors which have been ronsidered are:

1. errors in the flux correction due to relative motion of the furnaces or thermal expansion effects in the heated furnace;

2. asymmetry in the neutron spectrum causing different Doppler effects at the two supposedly symmetric furnace locations;

3. effect of the $106-\mathrm{keV}$ gamma-pulse-height spectrum width used in determining the hot-to-cold No-239 activation ratio;

4. effect of the wait time after irradiation at which the activation ratio is determined;

5. effect of fission product artivity on the determination of the Np-239 artivity;

6. self-absorption of gamma radiation in the foils.

\subsubsection{Random Errors}

4.2.1.1 Determination of $N p$-999 Activity. The heated and unheated foils were in the sequence $A-B-B-A$, and the activation ratio determined by the ratio of the sun of the respective artivities. This sequence required about 10 to $15 \mathrm{~min}$ and was repeated many times for one set of foils so as to accumulate about $10^{6}$ counts in the $106-\mathrm{keV}$ peak for each foil. The error in the activation ratio as determined from a single A-B-B-A sequence is $(2 / N)^{1 / 2}$, where $N$ is the total counts arcumulated for one foil in the sequence. A plot of the cumulative distribution function obtained from 16 surh rounting sequences showed the data to be nomally distributed. About $10^{\circ}$ ccunts were accumulated on each foil for the determination of each ratio value.

Thus, the standard error of the activation ratio samples due to counting statisties alone was about 0.003 . The measured standard deviation of the 16 samples was 0.0037 , indicating that the counting error is dominant. The vast majority of data obtained showed the same 
result, so that it is believed that errors due to positioning the foils on the crystal and variation of pulse-heightanalysis equipment are negligible.

4.2.1.2 Determination of Foil Weights. When rombining the results of activation ratios determined from irradiation of several sets of foils, the errors in uranium weights nust also be considered.

The foils used in a given irradiation were first cleaned and then weighed repeatedly. The random error of weighing appeared to be about $0.1 \%$. The foils were also weighed after irradiation, and no signifieant weight gain or loss was noted. The 0.003-in.-thick foils weighed approximately $0.15 \mathrm{~g}$; the random weight error was about $0.00015 \mathrm{~g}$. For most of the weighings, a balance was used with a vernier scale marked in 0.0001 -g increments. Weighings were repeated two or three times, re-zeroing the balance between weighings. Repeatability of 0.0001 or 0.0002 was obtained in the weighings. The 0.0005-in.-thick natural uranium foils were weighed with a balance whose vernier scale could be read to $0.00001 \mathrm{~g}$. These weighings also appeared to have a random error of about $0.1 \%$. Some of the 0.003 -in.-thick natural uranium foils were also weighed with this more sensitive balance. It is estimated that the actual random error in the uranium content of the foils was approximately $0.1 \%$ of the foil weight when surface oxidation and moisture are considered.

4.2.1.3 Overall Random Error in the Measurement. The hot-to-cold activation ratio corrected for the neutron flux difference at the two furnaces can be expressed for error estimation purposes as

$$
R=\frac{N_{H}}{M_{k I}} \frac{M_{c}}{N_{c}} R_{\varphi}
$$

where

$$
\begin{aligned}
N= & \text { Total Np-239 counts accumulated for one foil } \\
& \text { (subscript } H \text { indicates heated foil, } c \text { an un- } \\
& \text { heated foil); } \\
M= & \text { Foil weight; } \\
R \varphi= & \text { Flux-correction ratio. }
\end{aligned}
$$

The error in $R$ can be written as

$$
\frac{\sigma^{2}(R)}{R^{2}}=\frac{2 \sigma^{2}(N)}{N^{2}}+\frac{5}{2} \frac{\sigma^{2}(M)}{M^{2}}+\frac{1}{2} \frac{\sigma^{2}(F)}{F^{2}},
$$

where

$$
\begin{aligned}
N= & \text { Total Np-239 counts accumulaied for one } \\
& \text { natural uranium foil } \\
F= & \text { Total fission product counts accumulated for } \\
& \text { one flux-monitor foil } \\
M= & \text { Foil weight. }
\end{aligned}
$$

Since the ratio $R$ is nearly 1.0 , we have

$$
\sigma(R)=\left[2 \frac{\sigma^{2}(N)}{N^{2}}+\frac{5}{2} \frac{\sigma^{2}(M)}{M^{2}}+\frac{1}{2} \frac{\sigma^{2}(F)}{F^{2}}\right]^{1 / 2} .
$$

In most cases, the count statistics yield

$$
\begin{aligned}
\frac{\sigma^{2}(N)}{N^{2}} & =\frac{1}{N} \\
& =10^{-6} \quad \text { (Natural uranium-foil counts) }
\end{aligned}
$$

and

$$
\begin{aligned}
\frac{\sigma^{2}(F)}{F^{2}} & =\frac{1}{F} \\
& =10^{-6} \quad \text { (Flux-monitor-foil counts). }
\end{aligned}
$$

The weight error is estimated to be $0.1 \%$, so

$$
\frac{\sigma^{2}(M)}{M^{2}}=10^{-6} \text {. }
$$

Thus, the population standard error $\sigma(R)$ is

$$
\sigma(R)=\sqrt{5} \times \overline{10}^{-6}=0.0022 .
$$

A total of nine separate irradiations were made over a period of one year with both furnaces at the same temperature. In two of the nine cases, the furnaces were both at $800^{\circ} \mathrm{F}$; in the other seven, they were both at room temperature. The cumulative probability distribution for the nine ratios shows the data to be normally distributed. The data were treated in exactly the same manner as the other heated-foil cases, including flux correction. The average value and standard error of the nine sample values are

$$
R\left(T_{1}=T_{2}\right)=1.0000 \pm 0.0023 .
$$

The ratio is expressed as the activation ratio of Furnace $\# 1 /$ Furnace \#2. It is thus demonstrated that the actual population standard deviation is essentially equal to the expected value.

A total of 14 data points were obtained from separate irradiations at the same conditions over a period of one year:

Foil size $-0.003 \mathrm{in}$. thick by $0.5 \mathrm{in}$. in diameter;

Foil location- 5.5 in. from center of fast core;

Heated foil temperature $-780 \pm 20^{\circ} \mathrm{F}$.

Four of the irradiations were made with a fast-core U-235 enrichment of $23.6 \mathrm{w} / \mathrm{o}$ and with $40 \%$ of the reactor power in the fast core. The other 10 irradiations were made with a fast-core enrichment of $23.1 \mathrm{w} / 0$, and with $30 \%$ of the reactor power in the fast core. Since no calculation shows that an enrichment change from 23.6 to $23.1 \mathrm{w} / \mathrm{o}$ has a significant effect on the measurement, all 14 points are considered to have been obtained under the same conditions. The cunulative probability distribution of the 14 points shows the data to be normally distributed. The average value and standard error of the 14 sample values are

$$
R=1.0164 \pm 0.0022 .
$$


Again, the population standard deviation of these 14 samples is essentially that predicted from consideration of the basic measurement errors.

The data obtained during the period $9 / 29 / 64$ to $10 / 2 / 64$ (shown in Table 3 below) appear to be in general lower than the other data points. Since these were rather early measurenents and the careful routine of foil weighing, activation, and counting was not as uniformly established, these four points have been given a weighting of 0.25 compared to the remaining ten data points.

By use of the $T$-statistic it is possible to establish the range of the true mean about the sample mean as

$$
\bar{X}-\frac{\sigma}{\sqrt{n}} T_{p}<m<\bar{X}+\frac{\sigma}{\sqrt{n}} T_{p} .
$$

This relation is read as follows: "Given a sample of size $n$ with mean $\bar{X}$, we are $\mathrm{P} \%$ certain that the true mean lies within $\pm(\sigma / \sqrt{n}) T_{p}$ about the sample mean." $T_{p}$ is the value of the $T$-statistic for probability $P$ with $n-1$ degrees of freedom. Assuming $\sigma=0.0025$, we obtain the results in Table 2 for various sample sizes. Assuming the Doppler activation difference to be $1.6 \%$ (Doppler ratio of 1.016), Table 2 shows that a sample of size 4 should give a random error of \pm 10 to $25 \%$ in the measurement, depending on the desired confidence level.

\subsubsection{Systematic Errors}

The effects of foil thickness and furnace structural material on the measurement were investigated experimentally and discussed earlier in this paper. It was felt that those effects were fundamental to the interpretation of the measurement and deserved special investigation. Other possible systematic errors have also been investigated and are the subject of this Section.

4.2.2.1 Effects of Thermal Expansion and Relative Motion. The radial displacement of the heated sample foil relative to the outer flux-monitor foils was measured in a prototype furnace. Holes were drilled through the furnace tube walls at the foil locations. Nicrometer dial gauges with 0.0005 -in. resolution were used to measure the change in position of the inner foil holder relative to the outer tube. The total relative displacement at a sample foil temperature of $750^{\circ} \mathrm{F}$ was less than $0.010 \mathrm{in}$.

Table 2. Range of True Mean abovt Sample Mean

\begin{tabular}{c|c|c|c}
\hline & \multicolumn{3}{|c}{ Probability } \\
\hline & $95 \%$ & $80 \%$ & $68 \%$ \\
\hline 2 & \pm 00224 & \pm 0.0054 & \pm 0.0030 \\
4 & \pm 0.0040 & \pm 0.0020 & \pm 0.0014 \\
7 & \pm 0.0023 & \pm 0.0014 & \pm 0.0011 \\
14 & \pm 0.0015 & \pm 0.0009 & \pm 0.0007 \\
\hline
\end{tabular}

TABLE 3. EFfect of Interchanging Heated-foll Position At 5.5-1n. Symmetric Locations

\begin{tabular}{c|c|c|c}
\hline Irradiation Date & $\begin{array}{c}\text { *1 Hot/ } \\
\text { Cold }\end{array}$ & Irradiation Date & $\begin{array}{c}\text { *2 Hot/ } \\
\text { Cold }\end{array}$ \\
\hline $6 / 12 / 64$ & 1.0173 & $6 / 18 / 64$ & 1.0159 \\
$6 / 18 / 64$ & 1.0188 & $6 / 30 / 64$ & 1.0161 \\
$9 / 29 / 64$ & 1.0151 & $9 / 30 / 64$ & 1.0107 \\
$10 / 1 / 64$ & 1.0142 & $10 / 2 / 64$ & 1.0138 \\
$4 / 21 / 65$ & 1.0137 & $4 / 22 / 64$ & 1.0175 \\
$6 / 27 / 65$ & 1.0160 & $6 / 26 / 65$ & 1.0171 \\
$7 / 14 / 65$ & 1.0173 & $7 / 15 / 65$ & 1.0170 \\
\hline Average = $1.0160 \pm 0.0019$ & Average = $1.0154 \pm 0.0024$ \\
\hline
\end{tabular}

The activity data obtained from the four monitor foils showed the following radial flux gradients at various core locations:

\begin{tabular}{cc}
$\begin{array}{c}\text { Distance from Fast Core } \\
\text { Center, in. }\end{array}$ & $\begin{array}{c}\text { Radial Flux Gradient at } \\
\text { Furnace, \%/in. }\end{array}$ \\
\hline 5.5 & 1.5 \\
12.2 & 3.3
\end{tabular}

Thus, a displacement of $0.010 \mathrm{in}$. along the line of maximum gradient yields an activation difference of about $0.015 \%$ at the 5.5 -in. location and of $0.03 \%$ at the 12.2 in. location. This would contribute a 1 to $2 \%$ error in a Doppler activation difference of $1.6 \%$ (Doppler ratio of 1.016).

Since the fuel bundles in the core have about a 0.1 -in. freedom of motion in the radial direction, about $0.1 \%$ to $0.6 \%$ flux correction is possible. This is the range of flux correction observed experimentally.

The axial expansion of the sample foil holder was measured to be $0.062 \mathrm{in}$. relative to the outer furnace tube when the sample foil temperature was raised from $72^{\circ} \mathrm{F}$ to $750^{\circ} \mathrm{F}$. This value compares favorably with an estimated expansion of $0.08 \mathrm{in}$. In each measurement the heated sample holder was shimmed down $0.0625 \mathrm{in}$. so that it would expand into the same position as the unheated foil holder. Activity measurements with enriched uranium flux-monitor foils showed an axial gradient of $0.6 \% /$ in., so that small uncertainties in the relative position are negligible with respect to the Doppler ratio of the order of 1.016 .

4.2.2.2 Asymmetry of the Doppler Effect at the Two Furnace Locations. The 14 Doppler ratio values obtained at 5.5 in. with the 3 -mil foils were obtained by heating the foil in both symmetric locations in the fast core. Table 3 compares the average values obtained for 7 measurements with the heated foil in each location. The error shown in Table 3 is the standard deviation of the seven samples. Evidently there is no measurable significant effect of interchanging the position of the heated foil at the 5.5-in. location. The standard deviation of 
each of the seven sets of measurements is approximately that which is expected considering the basic measurement errors.

4.2.2.3 Effect of the Pulse-height spectrum Width Used to Determine the Np-239 Actiration Ratio. Usually, the 106 -keV gamma activity of $\mathrm{Np}$-239 was determined by using a 45 -keV energy width about the peak, that is, $106 \pm 23 \mathrm{keV}$. The effect on the Doppler ratio of varying this energy width was studied by using the data of two separate irradiations. Figure 4 shows the variation obtained.

The error bands shown for the data in Fig. 4 reflect the error due to counting statisties. There appears to be a trend established by the results of the two separate irradiations, such that the average change in activation ratio with energy width is $-2.3 \times 10^{-5} / \mathrm{keV}$, that is, as the energy width is narrowed, the hot-to-cold ratio increases. However, this observed effect is well within the linit of the statistical crror of counting, so that it is difficult to establish such an effect definitely.

The value of the activation ratio obtained by extrupolating these curves to zero encrgy width may or may not be the true value, since it is possible to either under-correct or over-correct the data in trying to obtain the true $\mathrm{Np}-239$ activity. At any rate, we can say that the apparent error is small and within the random error of the measurement.

4.2.2.4 Effect of Time after Irradiation at Which Activity is Measured. The Np-239 activity was measured at times from 12 to $100 \mathrm{hr}$ after the irradiation. Examination of the hot-to-cold artivation ratios thus obtained showed no trend with time.

4.2.2.5 Effect of Fission Product Activity on the $N p$-239 Activation Measurement. In the fast-neutron spectrum characteristic of the MSCA, the U-235 and U-238 fission product activity in the $106-\mathrm{keV} \gamma$-ray

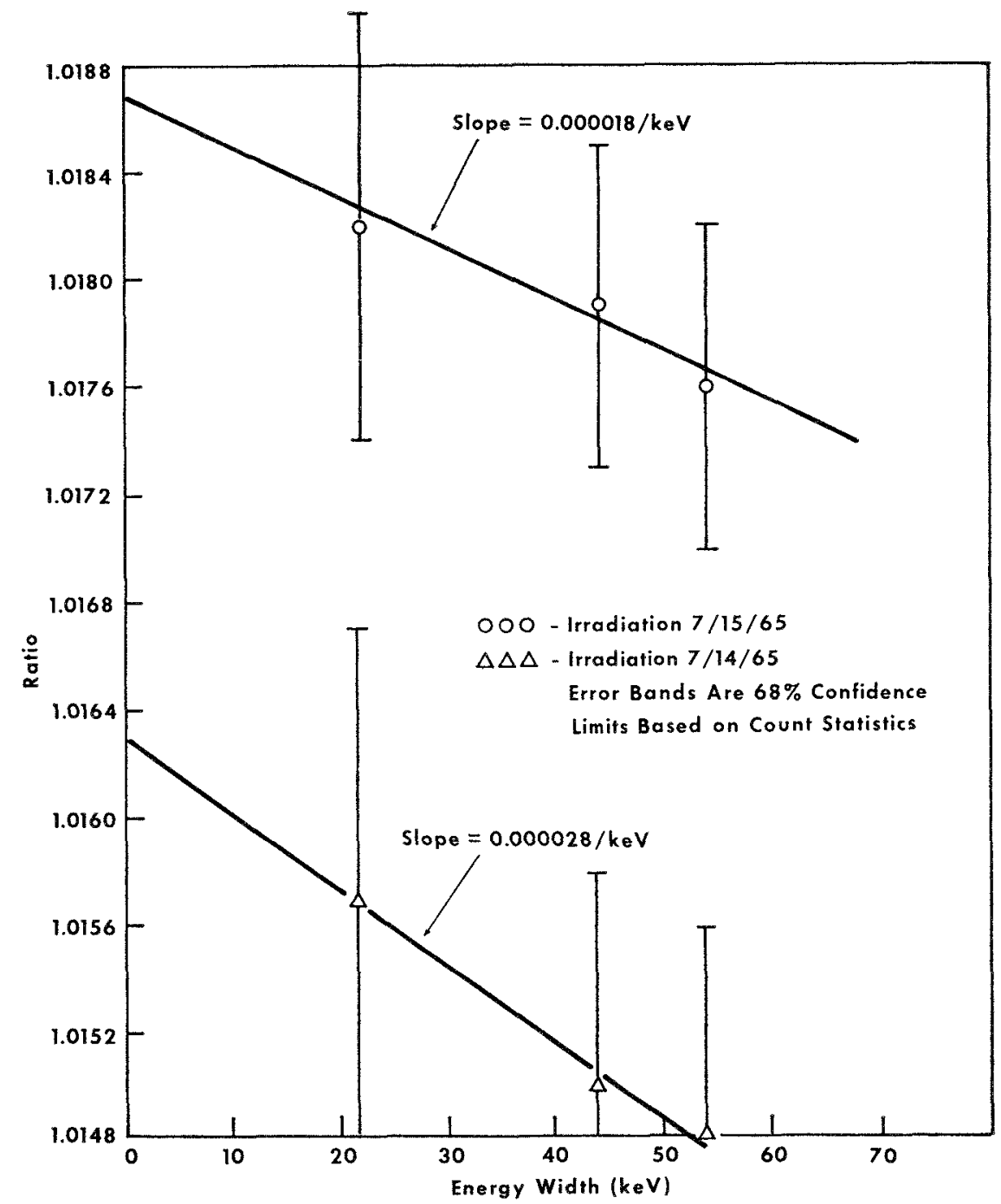

FIG. 4. Np-239 Activation Ratio vs Energy Width Used for 106-keV Peak. 
peak of Np-239 is believed to contribute negligible error to the measurement. By measuring the gamma activity in both natural and enriched uranium foils in the 106-keV (Nj-239) and 400-to-800-keV (fission product) energy regions, it is possible to obtain an estimate of the fission product aretivity in the $106-\mathrm{keV}$ energy region. The effect of this experimentally determined correction was to raise the hot-to-cold $\mathrm{N}_{\mathrm{p}} \mathrm{p}-239$ activation ratio by approximately 0.0005 , that is, if the ratio without correction is 1.0170 , then after the fission product correction it is 1.0175 .

4.2.2.6 Effect of Self-absorption of Gamma Activity in the Foils. The distribution of Np-239 in the foil will be nonuniform berause of the foil self-shielding effect. Thus, the Np-239 may be distributed somewhat more uniformly in the heated foil than in the unheated one. The self-absorption of the gamma activity would then result in a lower measured artivity for the heated foil. This difference in gamma self-absorption due to spatial distribution of the Xp-239 was investigated analytically, Assuming the Doppler line has a rectangular shape, we can derive an approximate expression for the measured gamma artivity. For the $1.5 \%$ change in activation due to the Doppler effect, it was determined that the ditference in gamma self-absorption can be neglected. This is not necessarily true for cases in which the Doppler effect is large and spatial self-shickling is appreciable.

\section{REFERENCES}

1. Mixed Spectrum Superheat Critical Experiments-Coupled Core, GEAP 4680 (Oct 1965).

2. A. B. Reynolds, Physics Design of the Mixed Spectrum Critical Assembly, GEAP-4320 (Aug 1963).

3. F. Storrer, A. Khairallah, and J. Ozeroff, Theoretical Aspects of the Doppler Coefficient in a Critical A ssembly, ANI 46792 , p. 823 (I)ec 1963).
4. A. B. Reynolds, Resonance Integral for the Doppler ITeasure ment in Fast Reactors by the Fonl Actrvation Technique, Nucl. sci. Fng, 22, 1, 487 (1965).

5. J. H. Ferziger et al., Resonance Integral ('alculations for Evaluation of Doppler Coeffcients The RAPTlKE Code, (iEAP 3923 (1962).

\section{Discussion}

1hr. Darey (ANL): In your counting at $106 \mathrm{kcr}$, I understand you have a contribution of a few percent due to fission products, both from U-235 and U-238. I wonder, in the case of heating the foil, whether or not some evaporation of fission produrts could oecur? Unless you correct for this effect, would it not appear as an apparent Doppler effect?

Mr. Pflasterer: First of all, I neglected to say that the U-235 Doppler measurenent, which wo also measured but for which we did not show any results, turned out within the error of our measurement to be essentially zero; that is to say, the cold-cold value of the ratio was one and the hot-cold ratio was also one within the $0.2 \%$ crror. To measure this Doppler effect in U-235 fission, we used a 500 - to $800-\mathrm{keV}$ gamma spectrum. If the fission products were driven off by raising the temperature, the gamma-spectra energy would be of the order of 0.8 or higher $\mathrm{MeV}$. The effect, insofar as it concerns the 500 - to $800-\mathrm{keV}$ region, is essentially zero or at least below the resolution of the measurement. We also have not detected any variation in the correction that we apply, that is, we have not detected any variation of this correction as a function of either foil thickness or time of radiation. We did make some irradiations that lasted one hour and some that lasted three hours, so that the boil-off time might be different in those cases. We believe that all the indications are that the effect is quite small and within the resolution of the experiment. 


\title{
Transient Flux Distributions by Discontinuous Modal Synthesis
}

\author{
J. E. GovTs \\ Westinghouse Electric Corporation \\ A tomic Power Division \\ Pittsburgh, Pennsylvania \\ ANi) \\ A. S. Wexnsteiv \\ Department of Mechanical Engzneerng \\ Carnegie Institute of Technology \\ Pittsburgh, Pennsylvania
}

(PIRESENTED BY J. E. GOITS)

\section{Introduction}

The neutron-flux distribution in a core transicnt is, without doubt, one of the most investigated phenomena in nuclear technology. The literature survey in reference (1) indicates the extent of domestic and foreign attempts to perfect existing solutions.

Figure 1 shows schematically the convergence of two separate systems of study towards a common goal of description of the transient flux. Although the compromise forces a loss in precision in description of the flux distribution and in plant-feedback description, it does bring a useful tool within reach of the nuclear engineer and systems analyst.

The neutron kinetic problem, hence the systemanalysis and control-design problems, are more critical in fast reactor plants than in thermal reactor plants. There are several reassons for this, among which are.

the larger power swings due to a lower fraction of delayed-neutron and shorter average lifetime;

the greater importance of fuel motion resulting from temperature changes due to the higher power densities and mean free path-core dimension ratio; the effect of a positive coolant coefficient that must be compensated for by the moderator density rhanges, as in some thermal reactors, ${ }^{*}$ to ascertain spatial stability.

Furthermore, the control-rod circuits require faster and more precise operation; hence, knowledge of flux distribution during reactor transients is of paramount importance to the reactor designer.

In systems analysis, a complete plant representation is usually considered, with the core divided into (at most) three regions. The resulting kinetic calculations must thus be adapted to few-region models. Furthermore, analyses of such models are usually porformed

* The study presented in this paper must be extended to a complete two-neutron-group model to enable an adequate representation of this effect. by analog computation. Available deseriptions of the transient flux are unsuitable for these complete-system analyses because of their complexity or inadaptability to analog simulation. For this reason, a modal-synthesis method which is attractive for few-region problems and is applicable to analog or digital computation was considered as most appropriate.

In the modal synthesis used, the overall modes across the reactor are taken an a composite of the region modes, with the interface conditions of continuous flux and current being satisfied. In turn, the region-wise modes satisfy the buckling equation for each respective region, giving rise to "intrinsie" or region-wise buckling. Defined in this fashion, the overall modes are functions with finite discontinuities in their derivatives at the region interfaces.

This paper presents the $1 / 2$-group kinetics model considered for a systems study of the Westinghouse Fast Breeder Reactor. ${ }^{(2)}$ The slow-flux group is averaged over the core and represented as a point, while the modal synthesis is used to describe the spatial behavior for the fast-neutron group.

Attention is focused on the modal-analysis problem, with a brief description of the averaging process proposed for two-dimensional analysis. This topic opens the way for further investigation and elearly shows the strong points of the process for analog-computer complexes having repetitive compressed time units. Elements of the analog analyses necessary to implement the transform solution are discussed, and an example of application via digital procedures is briefly described.

\section{The Reactor Equations}

The equations used are derived from diffusion theory and are the familiar two-group diffusion equations with $m$ delayed-neutron groups. It has been observed that for the fast reactor considered, the slow-neutron flux defined by energies below the $5-\mathrm{keV}$ level, has a rela- 

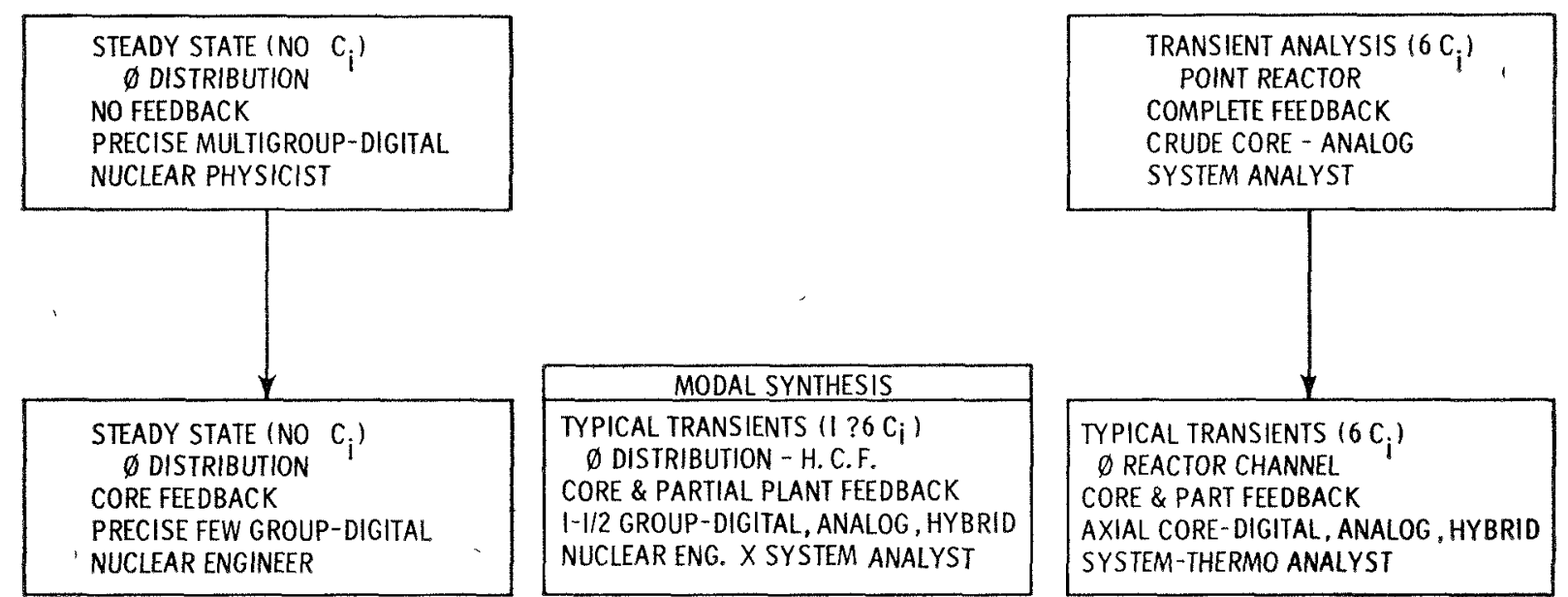

FIG. 1. Convergence of the Studies towards the Modal Synthesis Model.

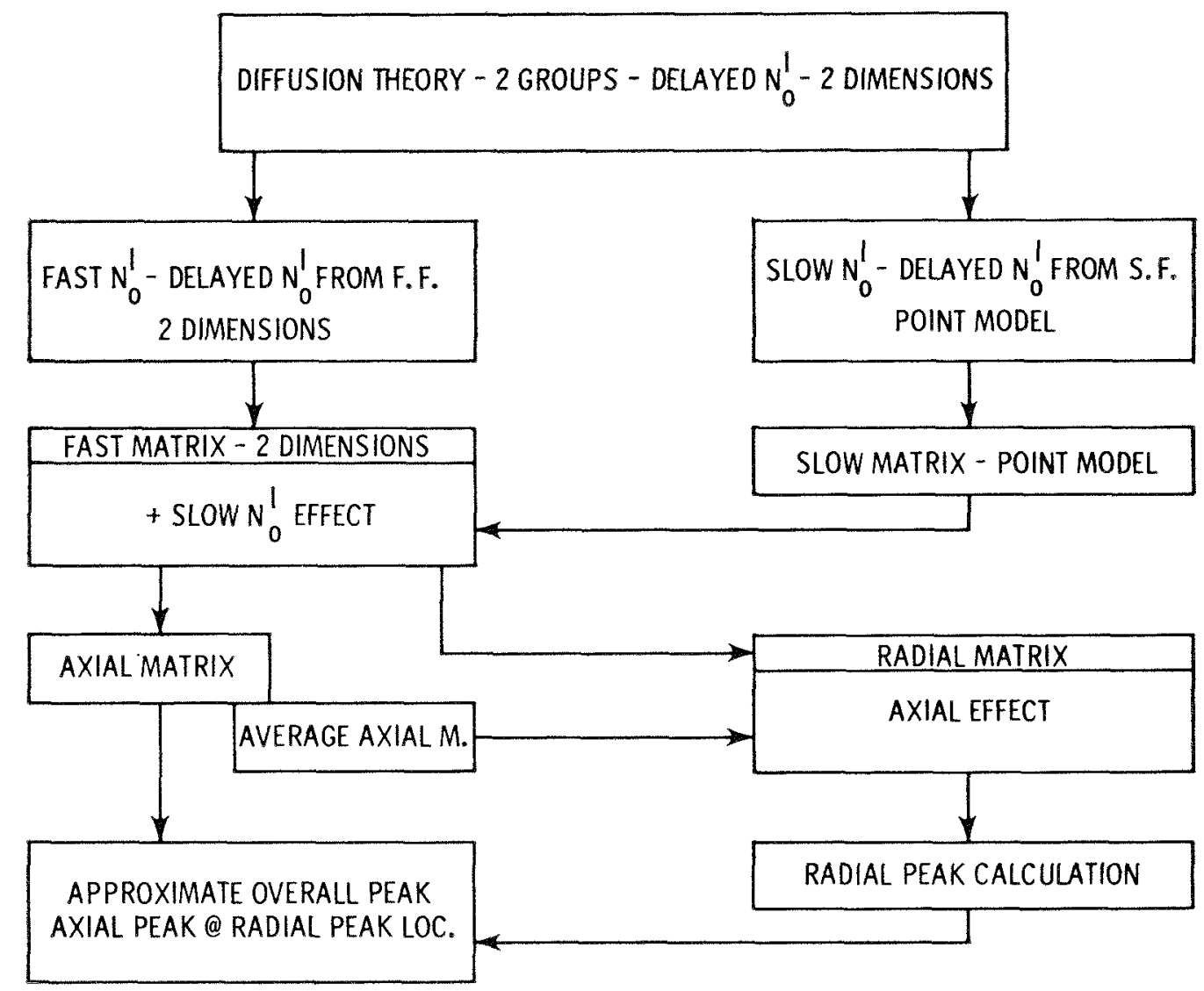

Fig. 2. The Flow Pattem of the Reactor Equation.

tively flat distribution and an effectiveness of only i) to $10 \% .{ }^{(2)}$ The slow flux may thus be approximated by a point representation without sacrificing overall accuracy. In order to separate the entire slow-neutron flux effect, the delayed-neutron group is divided into two parts, those arising from fast fission and those arising from slow fission. The line of thinking is shown in the upper section of Figure 2.
The neutron kinetic equations may then be written in a matrix form, with the dependent variable expressed as a column matrix of flux dimension containing the flux and the precursor densities multiplied by the average velocity of the neutron-flux group. This gives the following set of equations:

$$
\begin{aligned}
l_{f} \phi_{f}+\underline{l}_{f} \underline{\phi}_{s}+\underline{S}_{f} & =\dot{\phi}_{f} ; \\
\underline{l}_{s} \underline{\phi}_{s}+\underline{S}_{s} & =\underline{\dot{\phi}}_{s},
\end{aligned}
$$


with

$$
\begin{aligned}
& \underline{L}_{f}=\left[\begin{array}{crcc}
{\left[\nabla D_{f} \nabla-\left(\Sigma_{A}^{f}+\Sigma_{R}^{f}\right)+(1-\beta) \nu_{f} \Sigma_{f}^{f}\right] v_{f}} & \lambda_{1} & \cdots & \lambda_{m} \\
\beta_{f 1}^{\prime} v_{f} & -\lambda_{1} & \cdots & 0 \\
\cdot & \cdot & \cdots & \cdot \\
\cdot & \cdot & \cdots & \cdot \\
\cdot & \cdot & \cdots & \cdot \\
\beta_{j m}^{\prime} v_{f} & 0 & \cdots & -\lambda_{m}
\end{array}\right] ; \\
& l_{f}=\left[\begin{array}{cccc}
(1-\beta) \nu_{s} \Sigma_{f}^{s} v_{f} & \lambda_{1} & \cdots & \lambda_{m} \\
0 & 0 & \cdots & 0 \\
. & \cdot & \cdots & \cdot \\
. & \cdot & \cdots & . \\
. & \cdot & \cdots & . \\
0 & 0 & \cdots & 0
\end{array}\right] \\
& \underline{l}_{s}=\left[\begin{array}{cccc}
\left(\frac{S}{\alpha V}-\Sigma_{A_{\mathrm{al}} \mathrm{g}}^{s}\right) v_{s} & 0 & \cdots & 0 \\
\beta_{s 1}^{\prime} v_{s} & -\lambda_{1} & \cdots & 0 \\
\cdot & \cdot & \cdots & \cdot \\
\cdot & \cdot & \cdots & \cdot \\
\beta_{s m}^{\prime} v_{s} & 0 & \cdots & -\lambda_{m}
\end{array}\right] \\
& \underline{\phi}_{f}=\left[\begin{array}{c}
\Phi_{f} \\
C_{f 1} v_{f} \\
\vdots \\
C_{f m} v_{f}
\end{array}\right] ; \quad \underline{\phi}_{s}=\left[\begin{array}{c}
\Phi_{s} \\
C_{s 1} v_{s} \\
\vdots \\
C_{s m} v_{s}
\end{array}\right] ; \quad \underline{S}_{f}=\left[\begin{array}{c}
S_{f} v_{f} \\
0 \\
\vdots \\
0
\end{array}\right] ; \quad S_{s}=\left[\begin{array}{c}
\left(\not \Sigma_{R}{ }^{f} \Phi_{f}+S_{s}\right)_{\text {avg }} v_{s} \\
0 \\
\vdots \\
0
\end{array}\right] .
\end{aligned}
$$

All the factors of equations (1) and (2) are dimensionally defined. Artificial sources can be included through the terms $S_{f}$ or $S_{s}$. The subscript avg means that a core average must be evaluated. No weighting function is specified but, if judged necessary, may be included.

Equation (2) can be directly solved:

$$
\underline{\phi}_{s}=e^{\underline{l}_{s} t}\left(\underline{\phi}_{s o}+\int_{0}^{t} e^{-\underline{l}_{s} t} \underline{S}_{s} d t\right),
$$

where $\phi_{s o}$ is the slow-neutron flux matrix at time $t=0$. This is a column matrix, consisting of the initial average slow-neutron flux and initial average precursor densities due to slow-neutron fissions. (The precursor densities are multiplied by the slow-neutron average velocities as in $\phi_{s}$.) The problem then resolves to a time and space solution of (1), for which the modal analysis is used as described in the following.

\section{The Kernel and the Transform Equations}

Equation (1) may be solved by the use of a transform method. A one-dimensional problem is first considered. The transform kernel is defined by

$$
L_{f} \underline{\psi}_{n j}=-\chi_{n j} \underline{\psi}_{n j}
$$

(with appropriate boundary conditions), giving the modal solution

$$
\phi_{f}=\sum_{n} \sum_{j} a_{n j}(t) \underline{\psi}_{n j}
$$

with

$$
\underline{\psi}_{n \jmath}=\left[\begin{array}{c}
\psi_{n \jmath} \\
\xi_{(n \jmath) 1} \\
\vdots \\
\xi_{(n \jmath) m}
\end{array}\right] .
$$

The double subscript is due to the presence of the precursors. For a given flux kernel shape defined by the subscript $n$, all of the precursor kernel distribution shapes are determined, but not their relative amplitudes. The $m+1$ solutions with the different relative amplitudes are necessary in order to satisfy the $m+1$ initial distributions of the flux and precursors.

An adjoint kernel equation is formed by

$$
\underline{L}_{j}^{T} \underline{\psi}_{n j}^{*}=-\lambda_{n j}^{2} \underline{\psi}_{n j}^{*},
$$

with identical boundary conditions as for (3). The eigenvalues $X_{n j}^{2}$ are evidently the same for equations (4) and (5) as can be easily demonstrated, ${ }^{(2,3)}$ and the functions $\underline{\psi}_{n j}$ and $\underline{\psi}_{n j}^{*}$ present the property of biorthogonality (on two indices).

By definition, the transform equation is

$$
\int_{\text {core }} \underline{\phi}^{T} \underline{\psi}_{n j}^{*} d(\text { core })=\bar{\phi}_{n j},
$$

which is a scalar variable. 
We make the replacement

$$
a_{n j}(t)=\frac{\bar{\phi}_{n j}(t)}{N_{n j}}
$$

in (1) with $N_{n}$, defined by

$$
\int_{\text {core }} \underline{\psi}_{n_{3}}^{T_{j}} \underline{\psi}_{n_{j}}^{*} d(\text { core })=N_{n_{j}} \text {. }
$$

By taking the transpose of (1), multiplying by $\underline{\psi}_{n j}^{*}$, and integrating over the core using (3), (6), and the biorthogonality property, we obtain the following:

$$
-\chi_{n \jmath}^{2} \bar{\phi}_{n \jmath}+\bar{S}=\overline{\dot{\phi}}_{n j} .
$$

The flux matrix solution is given by solving (9) and introducing the result in (7) and (4):

$$
\begin{aligned}
& \underline{\phi}=\sum_{n}^{\infty} \sum_{\jmath}^{m+1} \\
& \frac{\exp \left(-\chi_{n \jmath}^{2} t\right)\left[\bar{\phi}_{0(n)\rangle}+\int_{0}^{t} \bar{S}(t) \exp \left(\chi_{n j}^{2} t\right) d t\right]_{\Psi_{n \jmath}}}{N_{n j}}
\end{aligned}
$$

where $\bar{\phi}_{0\left(n_{3}\right)}$ is given by the transformation of the initial $(t=0)$ flux distribution matrix.

\section{The Multiregion, Multidimensional Reactor}

As was indicated in the Introduction, the preceding transformation may be applied to multiregion problems ${ }^{(1,3)}$ such as multicore flux deseription. The constants defining the flux descriptions are related by the boundary conditions between each region, such as flux rontinuity and current continuity. If the kernels are ronsidered as functions satisfying the diffusion matrix $L$ and the boundary conditions between each region, as well as the outer boundary conditions, the transformed dependent variable has the same properties. It follows, then, that the flux description in each region is a function of the material buckling of that region; since the buckling changes from region to region, the function expressing the flux is discontinuous (analyti(ally speaking). An important consideration is that the first mode describes the steady-state asymptotic condition, whereas the higher modes describe the departure of that condition. This property largely decreases the number of terms necessary to obtain an acceptable description of the transient flux for systems analysis of a nuclear plant.

For a multidimensional analysis, by appropriate decomposition of the terms of the first-row, first-column element of the $L_{f}$ matrix, the matrix can be decomposed into as many components as there are dimen- sions to be considered. Thus, the first row and first column of each romponent would contain the derivatives of one dimension. As an example, for a decomposition performed along the $x$ (axial) and $r$ (radial) dimensions of a reactor (the line of thoughts can be followed in Fig. 2), $L_{f}=L_{x}+L_{r}$, where $L_{x x}$ contains only derivatives of the form $\partial / \partial x$ and $L_{r}$ only derivatives of the form $\partial / \partial r$. The terms of the second to the $m+1$ columns are left with the $L_{r r}$ matrix; henee the $L_{x}$ matrix has only its first column different from zero. A spatial transformation may be applied to the $x$ coordinate, and the resulting transformed equation is only a function of the coordinate $r$. This equation can be solved either as a one-dimensional problem, or further transformed and solved by a second modal synt hesis.

The transformed variable may now be averaged in the $x$ direction. The resulting equation contains only the r-independent variable. This approach enables approximation of the peak flux value by the following proecss:

1. The peak of the average ${ }^{*}$ in the $r$ direction is first found by "normal processes."

2. It is assumed that the overall peak occurs at the same value of $r$ as the peak of the flux averaged in $x$, i.e., $f(r)$. Consequently, a detailed modal synthesis is performed at that value of $r$ in the $x$ direction, and the peak value is retained as an approximated value of the ovrrall peak.

This problem was adapted for analog-computer simulation studies which are considered as follows.

\section{The Analog-computer Simulation ${ }^{(1,3)}$}

The analog-computer study of transient-flux descriptions by finite transformation as given by equation (10) consisted of the following:

finding the eigenvalues and eigenfunctions for the respective modes and orders;

obtaining solution of the flux equation with discontimuous spatial coefficients in the steady state;

solving boundary-value problems for the transicnt flux.

The first items above define the elements necessary for the axial transformation; the two last items give the solution in the radial coordinate for the average values in a steady state and during a transient.

From a computer programming point of view, the first two items constitute a simple iteration problem; the third is one of iteration and memory.

Figure 3 shows the machine search for a first eigenvalue by iteration. As may be seen, the iteration continues until $\lambda_{n}^{2}$ satisfies the eondition of zero flux at the

\footnotetext{
* Actually the peak of $\int_{x \times \text { ia } 1} \phi(r, x) d x / \int_{\text {axia }} d x=f(r)$.
} 


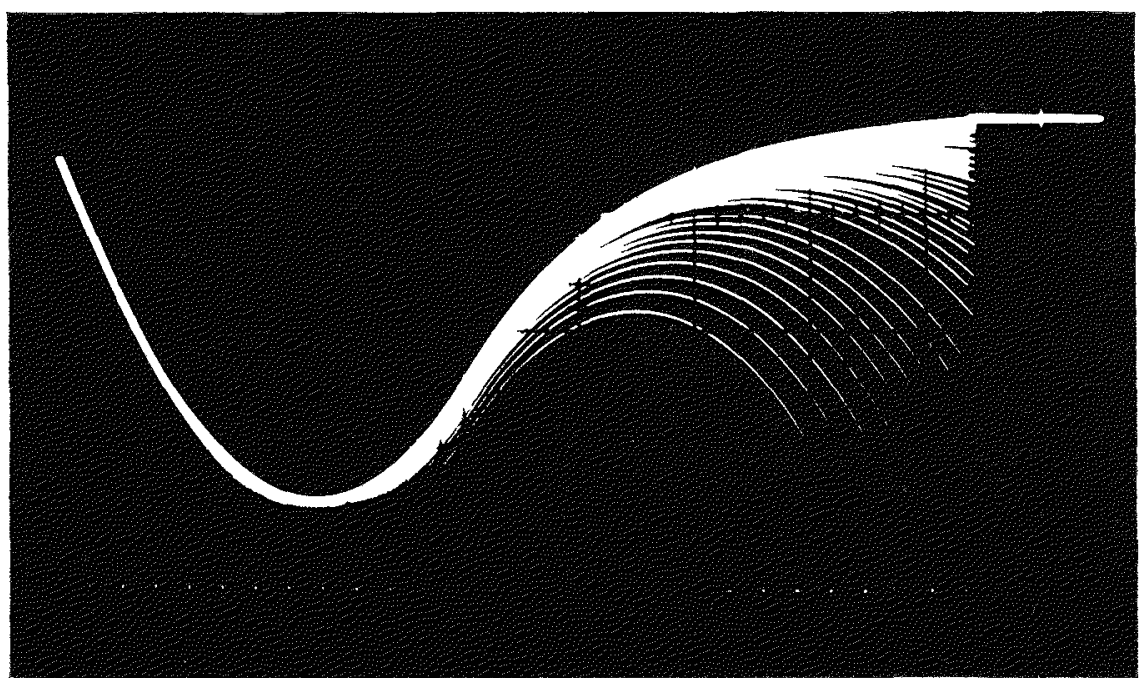

FIG 3. Boundary-value Search for Fixed Initial Tangent Search of the Eigenvalue

reactor boundary. Figure 4 shows the computed eigenfunction together with its first and second derivatives. A typical rod-insertion process in a critical core is shown in Figure 5 , with the dotted line showing the extent of the rod insertion. In Figures 3,4 , and 5 , the spatial coordinate is horizontal; the dependent variablo is vertical, directed downwards.

A block diagram of a complete space-1ime simulation of a nuclear plant with a core divided into two regions is given in Fig. 6. This simulation makes use of a repetitive and a real-time analog computer linked together and controlled by logic intervention. All sections of this simulation have been tested successfully. However, the present memory capacity of the equipment constitutes the limiting feature in coupling the repetitive core calculations with the remainder of the plant data.

A simple digital check of the transtorm method was also performed. In this check, a slab reactor consiting of two regions, with one flux group (thermal) and one group of delayed neutrons, was considered. The matrix $L$ was updated step-wise in time so that fuel-temperature feedback could be taken into account; hence the solution modes were changed with time. Transients were computed for absorption cross section decreased in the center, rodded region, which was considered as $1 / 38$ and $1 / 8$ of the core volume. Worst-condition profiles (largest deviation from steady state) were checked by a steady-state calculation through use of the adiabatic assumption. ${ }^{(3)}$

\section{Conclusion}

Although the transient-flux distribution can be calculated by different existing methods, the technique outlined above is advantageous and adaptable to

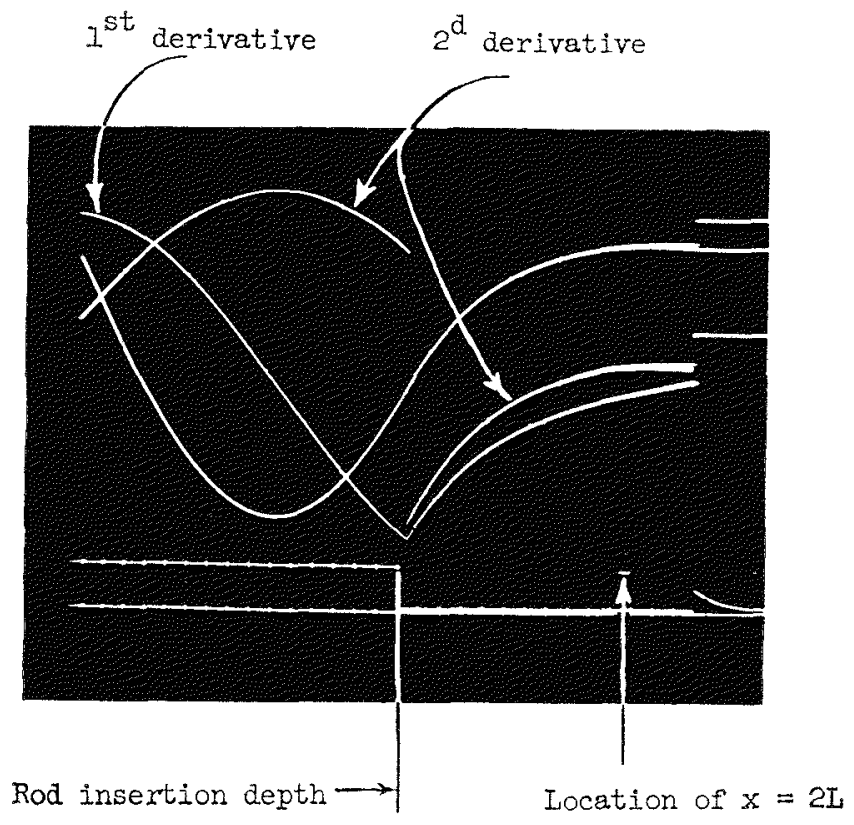

FIG. 4. The Eigenfunction Solution and Its Derivative.

systems analyses. It is, in effect, a compromise between elaborate multigroup calculations and simple point or adiabatic representations.

The 1/2-group behavior of the Westinghouse Fast Breeder Reactor evolves primarily to a time and space solution for the fast-neutron group. All elements of the modal analyses necessary to perform this task have been investigated via analog simulation, using repetitive compressed time units with adequate logic and by a simple digital check.

The merits and disadvantages of modal analysis for design and analysis of fast reactors will become apparent only through further investigation. 


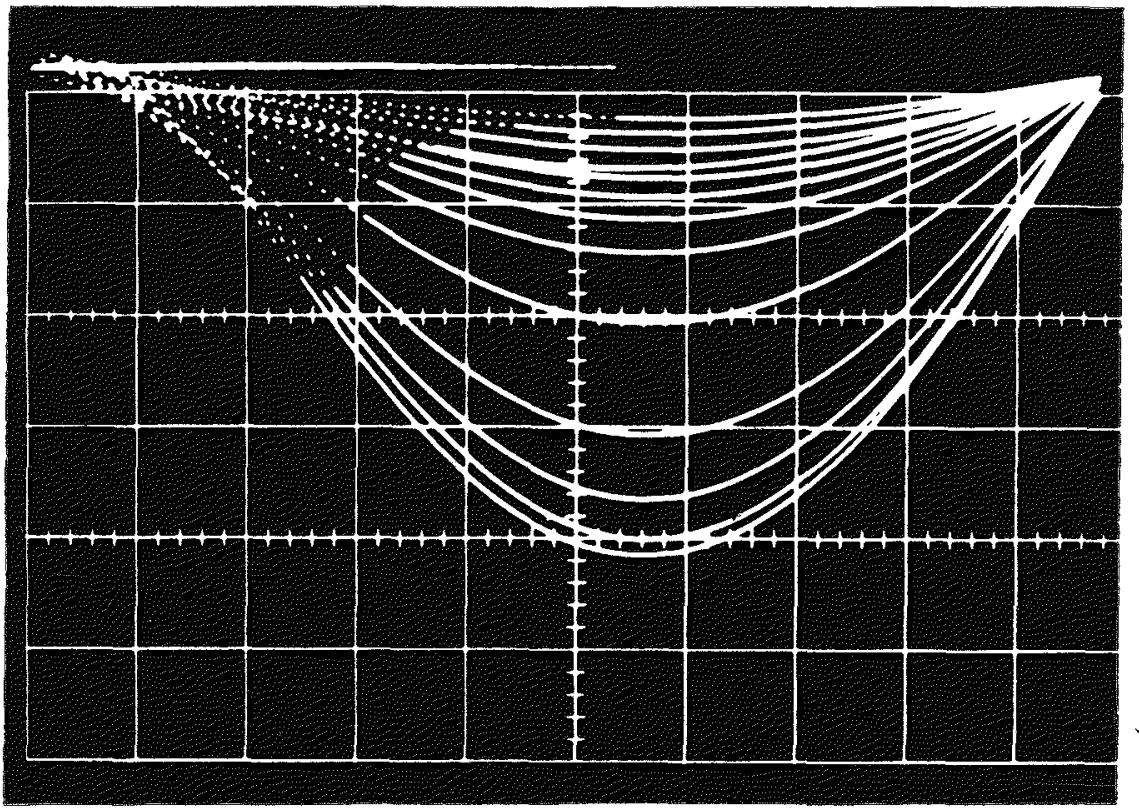

Fıg. 5. Rod-insertion Process in an Initially Critical Core (Rod Inserted from the Left).

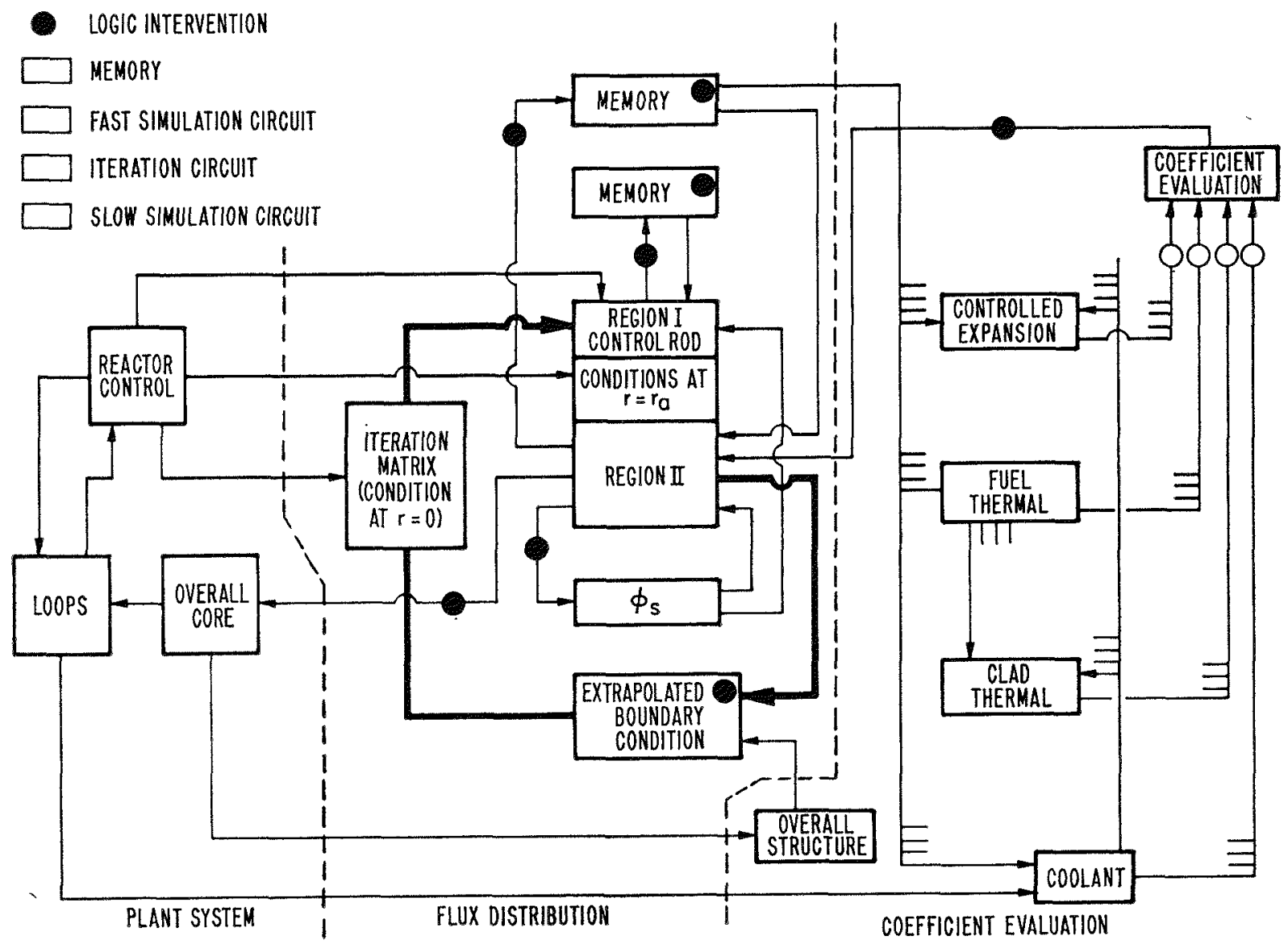

Frg. 6. Analog Simulation of a Fast Reactor Plant. 
The above approach is an initial step to bring transient-flux-distribution solutions within the scope of complete systems analysis. It is anticipated that subsequent improvements in equipment and technique will permit complete solution by analog methods and that the above kinetic treatment will be incorporated into an overall plant digital code.

\section{Acknowledgment}

The authors extend their thanks to Dr. E. F. Restelli, Jr. for his helpful suggestions during the study and the review of this paper.

\section{REFERENCES}

1. Godts, J. E., Analog Analysis of Transient Veutron Flux by Discontinuous Synthesis, Ph.l). thesis, Camegie Institute of Technology, 1965 (Also WCAP-2828, Westinghouse Atomic Power Division, July 1965).
2. Liquid Metal Fast Breeder Reactor Design Study, WCAP3251-1 (Jan 1964).

3. Godts, J. E., and Restelli, E. F., Jr., Transient Flux Distributions by Discontinuous Modal Synthesis, WCAP-2755 (July 1965).

\section{BIBLIOURAPHY}

Radkowsky, A., Naval Reactor Physics Handbook, Volume I, U. S. Government Printing Office (1964).

Kaplan, S., Henry, A. F., Margolis, S. G., and Taylor, J. J., Space-time Reactor Dynamics, Third Cnited Nations International Conference on the Peaceful Uses of Atomic Energy, A/Conf 28/P/271, May 1964.

Kaplan, S., Extensions and Applications of the Method of Finite Transforms, University of Pittsburgh, Ph.D. thesis, 1960.

Kaplan, S., Some New Methods of Flux Synthesis, Nucl. Sei. Eng., 13, 22-31 (1962).

Henry, A. F., The Application of Inhour llodes to the Description of Nonseparable Reactor Transients, Nucl. Sci. Fing., 20, 338-351 (1964).

Dougherty, 1). E., and Shen, C. N., The Space-time Neutron Kinetic Equations Obtained by Semi-direct Variational Methods, Nucl. Sci. Eng., 13, 141-148 (1962). 


\title{
Criteria for the Density of Monitoring Points in Large Reactors
}

\author{
Paul F. Gast \\ Argonne National Laboratory \\ Argonne, Illinois
}

\section{Summary}

In large fast reactors of modular core design, each module is very nearly critical independently. In this situation, small changes in the nuclear properties of individual modules can lead to large power imbalances, and each module must be monitored as if it were a separate reactor. Corresponding control capability is required.

In large, nonmodular cores, the density of monitoring points requires additional criteria for its determination. Monitoring results are used to estimate the local power at all points in the reactor. The maximum tolerable deviation between such estimated power and the actual power is one of the required criteria. When the largest expected deviation in local nuclear properties is chosen as a second criterion, the density of monitoring locations can be fixed. An illustrative description is given of the application of these criteria to a situation involving both radial and angular variations in the local $k$.

A general theory of reactor monitoring applicable to problems of this type is outlined in the final section.

\section{Introduction}

In the operation of nuclear reactors it is necessary not only that the total power remain within design limits, but also that the local power in various portions of the reactor remain within design limits at those locations. The power, or some quantity closely related to it, is usually observed at a finite number of locations in the reactor. The power at all other points is estimated by use of reactor theory based upon the known or estimated composition of the reactor.

This estimation process becomes expecially significant in large reactors, both because small variations in the reactor properties can produce disproportionately large power shifts and because the large size may provide greater scope for local variations in composition, temperature, and positioning of the reactor components.

In this paper we consider the effect that these possible variations have upon the problem of monitoring the performance of a reactor in a quasi-steady state. Kinetic situations are not considered, but the arguments here used appear capable of extension to include variations in time.

\section{Description of the Criteria}

In large fast reactors of modular design, each module characteristically consists of a core surrounded by a blanket and possibly some reflecting material. A number of these modules are assembled closely to form the complete reactor. The modules are loosely coupled neutronically, and each module would be nearly critical in an isolated condition. In such a situation, small deviations in the nuclear properties of individual modules could produce large variations in the flux and power distributions in the total reactor. It is clear that a suitable monitoring process must be earried out for each individual module and that it must be possible to use the results in the operation of a control system of similar distribution. Only in this way can a desirable power distribution be maintained.

In large reactors of nonmodular design a similar situation exists. Portions of the core would be nearly critical in isolation and are weakly coupled to other portions. The maintaining of a balanced power distribution will require similar monitoring and control facilities. However, there is no naturally occurring module which can be used as a basis for design of the monitoring system. Any portion of the large core could be regarded as a core surrounded by a high-quality reflector and nearly critical by itself. Some additional criteria must therefore be introduced in order to determine the number and spacing of the monitoring points.

In many reactors a large number of temperature monitors are placed in the outlet coolant stream near the outlets of individual channels for the combined purpose of monitoring power and local coolant flow. It might therefore seem that the monitoring question has been answered by providing an abundance of points. However, independent monitoring of local power is still required in order to separate power variations from flow variations. Also, an abundance of data requires a reduction process which recreates the problem on a new level.

The criteria for the selection of monitoring points here proposed are related to the uncertainties attached to the interpolation or extrapolation of the measured values to obtain values of the quantity of interest at nonmonitored points. Some uncertainties exist with 
respect to temperatures, compositions, and positions of materials in local regions of a reactor, and these produce corresponding uncertainties in the local neutronic properties. Normally, some upper and lower bounds can be placed on these uncertainties but, within these bounds, local properties may vary from place to place in such a manner that overall criticality is maintained.

Local increases in the reproduction factor are compensated by local increases in power, which in turn lead to increases in net neutron leakage away from that region, thus reestablishing a steady state.

When the power is measured at a number of monitoring points in a reactor, the expected power at all other points can be determined based upon the normal or expected properties of the reactor medium. The reactor design will provide for the effects of such a power distribution when all of the measured values are within the design limits. The design will also provide for some local excesses above the expected values.

Thus the two considerations used in establishing monitoring locations are: first, the expected deviations in the local properties of the reactor medium away from their normal values; second, the maximum undeterted increases in power above expected levels which the design can tolerate. Since, in gencral, these power uncertainties increase when the distance between monitoring points increases, these considerations may be used to determine the monitor spacing.

In applying these considerations to an actual reactor design, detailed calculations would probably be carried out by multigroup methods to determine the power uncertainties from the property uncertainties. It should be noted, however, that existing monitoring devices provide a signal proportional to a weighted sum over the energy groups. Thus, the full power of a multigroup calculation is lost in the absence of a detector able to produce values of the flux in individual energy groups. Such detectors are not beyond the bounds of possibility, ${ }^{(1)}$ and further exploration of the concepts here presented may indicate its usefulness for applications to power reactors.

For an exploratory study of the concept here presented we have chosen to avoid the complexities of multigroup calculations in the belief that a one-group representation of the quantity determined by the monitoring devices would illustrate the main features of the method.

Two cases have been studied: a large cylindrical (pancake) reactor treated as a bare core, and the same core treated as perfectly reflected so that a flat power distribution is its normal condition. Figure 1 shows the undetected power deviations as percentages of peak power that result from local leakage increases as per-

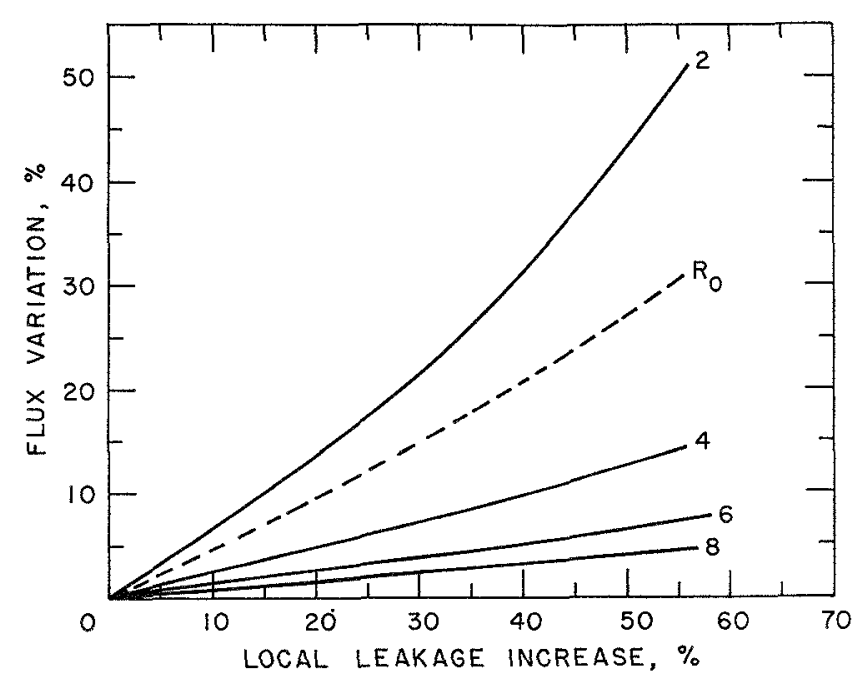

FIG. 1 Flux 1 ariation in Bare Reactor.

cent of normal radial leakage for various detector arrangements. The solid lines indicate flux variations resulting from angular variations of the reactor properties with monitors equally spaced on a single ring at about 0.5 to 0.6 of the reactor radius. The curves were calculated by use of a variational method described in the Appendix. Radial variations for the same conditions are indicated by the dashed $R_{0}$ curve.

Addition of a detector at the center of the reartor will strongly reduce the radial variations. The amount of the reduction is dependent both upon the maximum local increases and decreases of reactivity to be expected. However, some general approximate arguments indicate that, with a central detector, radial variations will again exceed the angular ones when more than about eight monitors are used on the ring. For patterns containing more than nine detectors, the locations should be approximately equally spaced over the reactor. Methods for treating this type of problem are outlined in the next section.

Results for the perfectly reflected core with a normal flat power distribution are shown in Fig. 2. In the previous case, the requirement that the flux vanish on the outer extrapolated surface provided an additional monitoring surface, at no extra charge. Loss of this constraint in the present case is reflected in the much larger variations escaping detertion by a given monitoring pattern. As with the first case, addition of a central monitor would reduce the radial variations, but beyond the octant monitoring pattern, a single ring is not the best distribution. The curves with higher numbers of monitors have been included only for the purpose of indicating the trend of improvement for a ring configuration. 


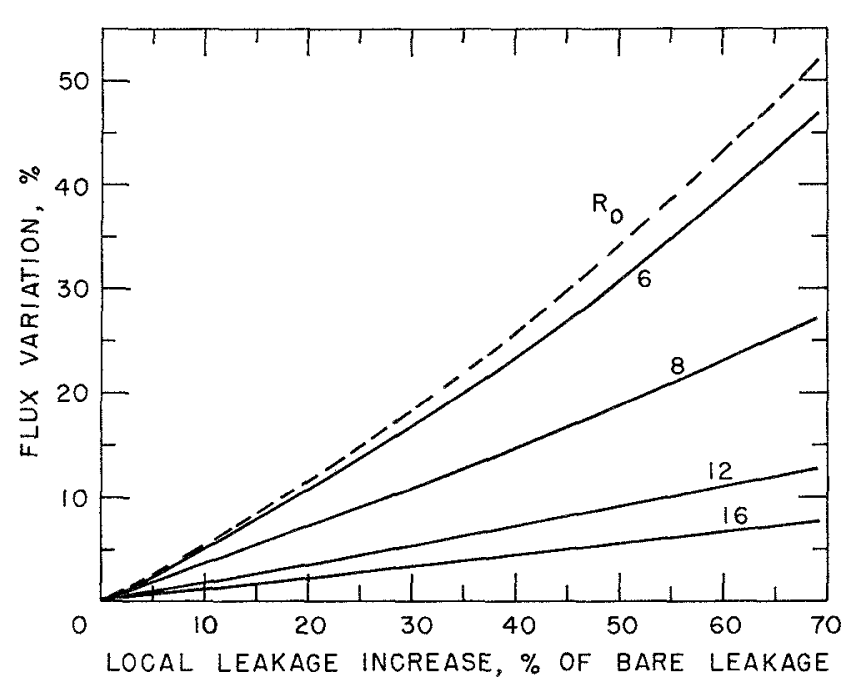

FIg. 2. Flux Variation in Flat Reactor.

\section{A More General Monitoring Theory}

A number of methods exist for fitting calculated reactor flux or power distributions to values measured at a selected number of points in a reactor. From the standpoint of design and use of monitoring systems, it is an advantage to have the measured values appear explicitly in a linear manner in the fitted expression. Such a method is available and can be applied in general to the type of problems we are considering. ${ }^{(2)}$

If, for example, the flux is measured at a number of locations $r_{2}$ in a reactor to obtain the values $\phi_{2}$, then the flux throughout the reactor can be obtained from the expression

$$
\phi(r)=\sum_{i} \frac{\phi_{i}}{\psi_{i}\left(\underline{r}_{i}\right)} \psi_{i}(r)
$$

where the $\psi_{2}$ are a specier of Green's functions, different from, but related to, the ordinary Green's functions and having the following properties:
They are solutions of the equation satisfied by the flux:

$$
H_{\phi}=0 .
$$

They satisfy the boundary conditions satisfied by the flux on the external real boundaries of the reactor and

$$
\psi_{i}\left(\underline{r}_{3}\right)=0 \text { for } \jmath \neq i .
$$

These conditions allow the $\psi_{2}$ to be constructed, usually by machine computation. If there is more than one monitoring point, the function $\psi_{\diamond}$ will have a positive singularity at $r_{\imath}$. Choice of a cutoff radius about $P_{2}$ is arbitrary and may be chosen differently for the different points as a means of assigning different weights to the measurements.

For our purposes, $H$ is to be regarded as consisting of a normal part $H_{0}$ and a part representing the maximum expected deviations in the reactor properties, $\delta H$. ('orrespondingly, $\delta \psi_{2}$ will be generated which can be used to determine $\delta \phi(r)$ between the mcasured points.

The smoothness of the $\phi(r)$ constructed from Eq. (1) with a given set of measurements is an indication of the accuracy with which Eq. (2) represents the artual reactor situation. Lack of a good fit will be evidenced by discontinuities in the gradient of $\phi$ at the points $\underline{r}_{2}$. Adjustments in $H$ would then be required to obtain smoothness. The adjustments will in general not be uniquely determined, and some $\delta H$ will remain to contribute a $\delta \phi$ for areas between the monitoring points.

It is not beyond the realm of possibility that such computations could be continuously performed by romputer in the monitoring of an operating reartor and the results displayed for the guidance of the operators.

The general theory also appears capable of extension to kinetic situations though the inclusion of the time variable.

\section{Appendix}

The radial variations in flux were computed by use of a variational method described by Becker, ${ }^{(3)}$ which applies the least-squares method to the error in satisfying the flux equation. The functional to be minimized is

$$
\varepsilon^{2}=\int\left(\nabla^{2} \phi+B^{2} \phi\right)^{2} d V
$$

The problem is one in which alternate sectors of the reactor have values of $\mathrm{B}^{2}$ above and below the value $\mathrm{B}_{0}{ }^{2}$ required for criticality of a homogeneous reactor of the same size in such a way that rriticality is maintained. We are here concerned only with the sectors having increased buckling, since these will be the higher-power regions. Each sector is $\pi / p$ in angular width and the trial function taken for $\phi$ is

$$
\phi=J_{0}\left(\alpha_{01} r\right)+A J_{p}\left(\alpha_{p 1} r\right) \cos p \hat{\theta},
$$

where $\alpha_{2 j}$ is the $j$ th root of $J_{2}$. Inserting (4) in (3) and carrying out the variational process with respect to the parameter $A$, we obtain

$$
A=\frac{4}{\pi} \frac{\left(B^{2}-\alpha_{01}^{2}\right)}{\left(\alpha_{p 1}^{2}-B^{2}\right)} \frac{\int_{0}^{1} J_{0}\left(\alpha_{01} r^{r}\right) J_{p}\left(\alpha_{p 1} r\right) r d r}{\int_{0}^{1} J_{2}^{p}\left(\alpha_{p 1} r^{r}\right) r d r^{r}} .
$$

For the normally flat reactor the 1rial function was 


$$
\phi=1+A J_{p}\left(\beta_{p l} r\right) \cos p \theta,
$$

where $\beta_{p 1}$ is the first maximum of $J_{p}$. This resulted in

$$
\Lambda=\frac{4}{\pi} \frac{B^{2}}{\beta_{p 1}^{2}-B^{2}} \frac{\int_{0}^{1} J_{p}\left(\beta_{p 1} r^{2}\right) r d r}{\int_{0}^{1} J_{2}^{p}\left(\beta_{p 1^{r}}\right) r d r} .
$$

Thanks are due to Dr. H. C. Thareher for computation of the Bessel function integrals.

\section{REFERENCES}

1. Reactor Development Program Progress Report for August 1965, ANL-7090, pp. 18-19.

2. P. F. (Xast, A General Reactor Monitoring Theory, to be published.

3. M. Becker. The Principles and Ipptications of Variational Wethods, M.I.T. Press (1964).

\section{Discussion}

IIr. Thompson (MIIT): I wonder if you've tried to apply this model to any thermal reactors, first, and second, have you thought about extending this to locations of control rods?

Mr. Gast: Yes, as a matter of fact, although I put this in as a paper for a Fast Reactor Symposium, it was really sparked by some problems that had occurred at Hanford over a number of years. I think it might be simpler to apply to thermal reartors in the sense that in most of them one could do this adequately with at most two-group ralculations; I am not sure this would be the case with fast reactors. If there is a control rod near a monitor, one has to include that in his operator deseription in order to get any kind of a proper solution to the problem. It docs, I think, raise the question of whether it might not be worthwhile to attach some special computers to the reactor control room which might develop this kind of information in a continuous way for operators.

Mr. Godts (WAPD): Do you feel that this would be applicable to finding the transient-flux distribution with the operational limits?

Mr. Gast: In a formal sense, yes. One can include the time variable and go through the whole thing again. Thic becomes much more complicated if you are thinking of transients in which you may have boiling and that kind of thing. It gets to be very diffieult.

Mr. Godts: I was thinking of the inclusion of an on-line computer.

Mr. Gast: That might possibly be helpful.

Mr. Burton (Risley): This does seem to be a very useful technique for the thermal reactor. But in the present state of our knowledge of the fast reactor, as we have seen throughout the conference, what is worrying us is the local blockage. Do you see any prospect for improving your resolution to do this?

Mr. Gast: I think that is a problem that is somewhat beyond the formalism that is included here.

Mr. Burton: Yes, but you're liable to do something formally by considering, for example, the extent that the sodium boiling of voiding would reproduce on these monitors. It might be possible.

Mr. Gast: I think that might be possible. I think, however, that a direct approach, such as monitoring temperatures or looking directly for hydraulic effects, might be better if you are looking for blorked tubes.

Mrr. Burton: Well, in engineering terms, this is what we are finding particularly difficult. One really wants to achieve a minimum number of monitors, and this is one way of integrating the effect. It does have merit, in principle. 


\section{General Discussion}

Mr. Chernick (BNL): I have a question I first raised two years ago. At that time I wondered whether it might not be better in the long run, in getting Doppler coefficients, to set up an expensive facility, with good heating sources and everything that goes with it, as they have done for thermal reactors. I would like to get the opinion of the experimental people as to whether they are satisfied with what has been done and whether it is perhaps not too late to still ask for an expensive attack on this problem.

$M r$. Greebler (GE): I think the SEFOR program is an answer.

$M r$. Chernick: That's what I was told last time.

$M r$. Fischer (ANL): I don't think you would want a more expensive answer than SEFOR. But there are other measurements, which you have seen even today. The question is what do you want to do.

$M r$. Chernick: SEFOR is an answer in an integral way.

Mr. Fischer: I think they are trying in one integral way to make this kind of a measurement. But I think Wheeler, this morning, gave you a first British pass at the kind of a thing you are asking for. And Till has mentioned that he and Lewis are planning a small clustered-pin experiment.

Mr. Chernick: Is this going to give us the answer? With the kind of facility I am talking about we would have some certainty within some given time of having the answer, not only to the U-238 Doppler effect, which is fairly well pinned down already, but also to the question of the U-235 and plutonium Doppler effects.

Mr. Fischer: The fact that the Doppler effect for $\mathrm{U}-238$ is negative, large and pretty well known is the big thing that you need to know. I think we have to go on now asking-as Till will in ZPR-6 in the near future-what happens to the Doppler coefficient when you remove sodium? I think we should build ZPPR as rapidly as possible to find out what the change in the Doppler coefficient is when you put in things like plutonium-240, for which you really can't expect to have good cross sections in three to five years. You want to do the dirty plutonium Doppler experiments. You'd like to do something about fission products. I think you can do all of these things with the criticals rather nicely. SEFOR will corer only one specific composition.

Mr. Greebler: I was just going to say something about the temperature effect. It was mentioned that the Pu-239 effect may be near zero at room temperature, but may come up appreciably because of the influence of Doppler worth on the resonances. It is important that we be able to cover the full range of temperatures in a reactor at full power and perhaps under transient conditions above power. We may see some surprises, particularly in regard to the $\mathrm{Pu}-239$ effect in that area.

Mr. Fischer: In this area of the working criticals, both at AI and at Argonne, we are reaching very rapidly to significantly higher temperatures. I think this will show up pretty nicely.

Mr. Thompson (MIT): Speaking as a naive experimentalist, it appears to me that perhaps both approaches may be required. The small-sample technique allows you to make corrections for such things as cxpansion, and the in-out type of measurement allows you always to zero back in a way which I think will be found to be quite difficult with the large integral experiments of the SEFOR type. With the SEFOR you are very likely to be actually measuring a combination of Doppler plus expansion and overall core expansion plus individual rod expansion. Therefore I think in the long run you will need both.

$M r$. Chernick: One other thing that hasn't been mentioned is the question of spectrum effects. You may know what the delta signal is, but without such a facility it is going to be extremely difficult to determine spectrum effects on the Doppler.

Mr. Till (ANL): I think that the results that I showed this afternoon would indicate that, although the signal for the small-sample experiments is small, we have techniques that can measure the signal to the sort of accuracies that we need. Further, the smallsample technique does allow us a good way of separating these Doppler and expansion effects, and I think this is a significant point.

Mr. Zartman (USAEC, Washington): It seems to me that one has to be very careful with these integral expcriments in being able to differentiate, just as has been mentioned by others, between the various effects that you sum up in these large integral experiments. I would like to see, however, one thing in the other experiments taken into greater consideration than I think anybody has, and that is the spectrum in which the measurements are being made. I don't think, or at least I am not convinced, that the actual spectrum in which these measurements are made is known well enough and compared or taken into consideration, in 
the comparison between the results of the different people that have been reporting. Isn't this one thing that ought to receive a much greater attention than it has in the past? I am not too much concerned that we won't be able to interpret or measure the small signals. I think there has been a good deal of improvement along this line. There is a need for further ingenuity in the preparation of the samples and in attempts to separate expansion effects from true reactivity changes of the material. I would like to hear a little more about the spectrum aspect.

Mr. Fischer: I think we all agree with you that spectrum is a very important quantity in trying to analyze what we claim to have measured. For years at Argonne on our assemblies we have been trying various devices to measure spectrum. I would say the best news about spectra is essentially a bulletin out of ZPR-6. Ed Bennett has been able to measure down into the kilovolt region, which is a tremendous achievement. Recently at San Francisco, he reported a comparison between measurement and calculation for the current $4 \mathrm{Z}$ assembly of ZPR-6. Since then he has worked on the thermal source reactor in a Snell block and has been able to see a small undetected experimental difficulty he hadn't known about at San Francisco. When he made a correction to the numbers he reported at San Francisco, there was surprisingly good agreement from his results of the analysis of the spectrum, done by a proton-recoil technique, and the spectrum we calculated using ELMOE corrected set 224. The disagreement, which was almost a factor two in the lowestenergy region, is now very largely removed with Bennett's latest correction. The results are surprisingly good. This I think is our best hope for spectrum. There is some sandwich work with lithium detectors in Idaho, probably the next best thing currently going on in spectra.

$M r$. Greebler: I thought that the time-of-flight technique provided the best method of measuring the spectrum. Does anybody have a feeling as to how good the advanced proton-recoil methods compare with the time-of-flight procedures? Further, is there need for time-of-flight equipment with critical assemblies?

Mr. Thalgott (ANL): It seems to me that the time-of-flight technique works best for the low-energy flux, and there may be some difficult problems at the higher energies in the few-kilovolt range. I think that it will be necessary for us to pursue these things, as the British have been doing, and also at Karlsruhe. The second point-concerning the spectrum. I think we must be able to reconcile calculation with experiment as far as the spectrum is concerned if we are to calculate the Doppler effect in a reactor. However, the measurements in eritical experiments are made in the spectrum that is associated with that composition, and the calculations are either right or wrong. The neutrons behave themselves in the critical as they should, and the measurements should then reflect the proper spectrum for this mixture of materials. Two years ago I agreed with Chernick that an elaborate facility might very well be necessary, even though there is some difficulty with respect to measurements in such a facility that I don't quite know about. The measurements in critical experiments hadn't been made and $I$ thought they might turn out to be extremely difficult. The measurements reported today seem to be reasonably consistent, and it is gratifying to see that such measurements can be made. I still am not sure that this answers the whole problem, but I have much more confidence now than I did two years ago.

$M r$. Chernick: We have to be careful how we interpret any type of experiment, including large-scale experiments. I am impressed with what was done at Hanford on measuring Dopplers in their PCTR and what was done at Winfrith in measuring assemblies where the fucl elements had been heated with gas and so on. So there is some precedent in thermal reactors indicating the flexibility and the high probablility of being able to get good data from such a measurement. There is one thing you mentioned I agree on-the time-of-flight method. This is an extremely good tool for going up at least to $10^{4} \mathrm{eV}$. I am impressed with what the Russians have already done and reported at the Geneva conference with their IBR reactor with various filters-iron, stainless steel, and things like that. They seem to get extremely great detail in the spectrum in this energy range. Beyond $10^{4} \mathrm{eV}$ the resolution gets very bad. I think that new techniques are going to be needed to determine the spectrum in that range. But for the extremely important range from about 100 to $10^{4} \mathrm{eV} \mathrm{I}$ think that good time-of-flight equipment is very useful. 


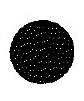

0 


\section{Session VII-A \\ October 14, 1965}

\section{Maximum Accident and Containment}

Chairman: W. J. McCARTHY, JR.

Power Reactor Development Corporation.

Secretary: G J. Fischer

Argonne National Laboratory 


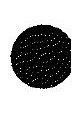




\title{
A Critical Review of the Properties of Materials at the High Temperatures and Pressures Significant for Fast Reactor Safety
}

\author{
David) Millerer \\ Argonne National Laboratory \\ Argonne, Jllinois
}

The equation of state plays an important role in calculations of the course of a hypothetical fast reactor excursion, for it serves as the link between the neutronic reactions and the dynamic behavior of a core which leads to ultimate shutdown. Determination of the energy appearing as kinetic energy provides insight into the potential damage which might result from such an excursion. The principal relations necessary for such calculations are the pressure as a function of energy and volume for hydrodynamies caleulations, which give the time variation of geometry (including purely elastic deformations such as thermal expansion), and the temperature as a function of energy and volume, to give the proper temperature dependence of nuclear rross sections (with special emphasis on the Doppler (coefficient).

Several techniques for determining these relations have been used, and will be critically reviewed and rompared. In addition, some future directions will be indicated.

Gnergy releases estimated for hypothetical nuclear arcidents could lead to temperatures and pressures beyond those possible with static experiments and present technology. Resort must therefore be made to theory and correlation for the estimation of these physical properties at extreme conditions.

The equation of state as applied to fast reactors has been reviewed briefly by Okrent. ${ }^{(1,2)}$ Effort has been roncentrated on the application of the law of corresponding states ${ }^{(3-7)}$ and on empirical relations which were fit to reactor excursion data.

It should be noted that the energy to heat uranium from room temperature to the boiling point is $0.65 \times$ $10^{10} \mathrm{ergs} / \mathrm{gm}$, whereas the heat of vaporization at the boiling point is about $1.7 \times 10^{10} \mathrm{erg} / \mathrm{gm} .^{(8)}$

\section{Corresponding States}

The well-known law of rorresponding states ${ }^{(7)}$ implies that substances are characterized by their thermodynamic eritical properties: ritical temperature $T_{c}$, critical density $\rho_{c}$, (or critical volume $V_{c}$ ), and critical pressure $P_{c}$, and that materials at the same reduced temperature, pressure, and volume will have similar behavior.
This approximate relation has been used by several investigators ${ }^{(3-5)}$ to first estimate the critical properties of reactor fuel materials and to then use generalized correlations $^{(7)}$ to predict their equation of state.

Elrod $^{(5,6)}$ has used empirical information on lowboiling-point nommetallic liquids to estimate $T_{c}=$ $14,200^{\circ} \mathrm{K}$ and $P_{c}=5,130 \mathrm{~atm}$ for uranium. Brout ${ }^{(3)}$ accepted his estimate of $T_{r}$ but used the empirical relations $V_{c} / V_{\text {solid }}=3.11$ and $P_{c} V_{c} / R T_{c}=0.3$ for many substances to obtain $V_{c}=40 \mathrm{~cm}^{3} / \mathrm{mol}$ and $P_{c}=8650 \mathrm{~atm}$.

Gross ${ }^{(9)}$ has recently suggested application of the entropy of vaporization vs reduced temperature (with mereury as the only well-measured metal) as an alternative method of estimating critical temperature. The application of the law of "rectilinear diameters" was also suggested, and the two methods lead to consistent agreement for all but the transition metals, such as $\mathrm{Fe}$, $\mathrm{Co}$, and $\mathrm{Ni}$. The latter technique will be discussed below.

Since the first edition of their text, which was used by Brout and others, Hougen and Watson ${ }^{(7)}$ found it necessary to use a third parameter, the critical compressibility $Z_{c}=(p V / R T)_{c}$, to correlate accurately the thermophysical properties of ordinary substances. The range of their correlations is for $0.24<Z_{c}<0.30$, which corresponds to most of the inert gases and organic materials upon which the correlations are based.

Table 1 shows that many materials, including mercury (the only heavy metal for which experimental data exist to the critical point) do not fall in this range and would not be expected to agree well with the correlations.

Empirical rules for estimating ritical properties (annot be relied upon. One such rule states that the critical temperature is 1.5 times the absolute boiling temperature $T_{b}$. For mercury, bismuth chloride, and cesium $T_{c} / T_{b}$ is $2.7 .5,1.66$ and 2.0 , respectively. Similar rules for volumetric behavior which have been used ${ }^{(10)}$ are equally unreliable.

Brout, in applying the corresponding-states correlation, comments that the agreement is not good for argon gas at high pressures, although this is one of the 
materials on which the correlation is based and which has a $Z_{c}$ in the range of the correlation. In his consideration of heat capacity, Brout rejects a correspondingstate principle based on argon and neon because they are unlike uranium, although he is willing to acespt the generalized correlations which are not bared on any metallic fluids.

For the materials $\left(\mathrm{CO}_{2}, \mathrm{CH}_{4}, \mathrm{NII}_{3}\right.$, ete $)$ on which the generalized correlation used by Brout is based, $T_{c}$ is so low that ionization is negligible. Calculations ${ }^{(11)}$ from the Langmuir-Saha equation show, as was rationalized by Nicholson, ${ }^{(12)}$ that ionization is not important until 3 or 4 times the critical temperature. Morris ${ }^{(13)}$ has considered the effects of jonization in an attempt to shore up the law of corresponding states for metals. His approach is to provide a modification to the Grüneisen equation

$$
P=\rho \gamma(\rho)\left[E(\rho, T)-E_{0}^{\gamma}(\rho)\right],
$$

where

$P$ is the pressure;

$\rho$ is the density;

$E$ is the internal energy;

$E_{0}$ is the Cirüneisen energy parameter;

$\gamma$ is the Crüneisen ratio $\alpha / \rho c_{p} \kappa_{s}$;

$\alpha$ is the volumetric thermal expansion coefficient, $-(1 / V)\left(\partial \mathrm{I}^{\mathrm{r}} / \partial T\right)_{p}$;

$c_{p}$ is the constant pressure heat capacity;

$\kappa_{s}$ in the adiabatio compressibility $(1 / V)(\partial V / \partial P)_{s}$, by considering the effect of the electrons on pressure and heat rapacity.

In a study of experimental data on shock compression of reduced-density uranium Skidmore and Morris ${ }^{(8)}$ used the law of corresponding states without modification.

Although the law of corresponding states appears to be inapplicable for high accuracy it should be useful as a guide until more satisfactory estimation procedures ran be tested.

As a temporary expedient to explore the temperature, pressure, and density ranges of interest, the densitytemperature relation for the saturated liquid-vapor region has been estimated (up to the critical point) for some materials of reactor interest by the "rectilinear law" method. ${ }^{(9)}$ This method assumes that one-half

TABle 1. Meast Red Crithan, Propertes

\begin{tabular}{l|c|c|c|c}
\hline & $T_{c},{ }^{\circ} \mathrm{K}$ & $\rho_{\varepsilon}, \mathrm{g} / \mathrm{cm}^{3}$ & $P_{c}, \operatorname{atm}$ & $Z_{c}=\frac{P_{c} M}{R \bar{T}_{c} \rho_{c}}$ \\
\cline { 2 - 5 } & $1733 \pm 50,4.70 \pm 0.20$ & $1587 \pm 50$ & 0477 \\
Mercury & $1178 \pm 5$ & $1.210 \pm 0.006$ & 118.1 & 0.318 \\
BiCl $_{3}$ & 547.9 & 0.237 & 47.7 & 0.19 \\
Acetonitrile & 38.3 & 0.0151 & 16.3 & 1.37 \\
Deuterium & & & \\
\hline
\end{tabular}

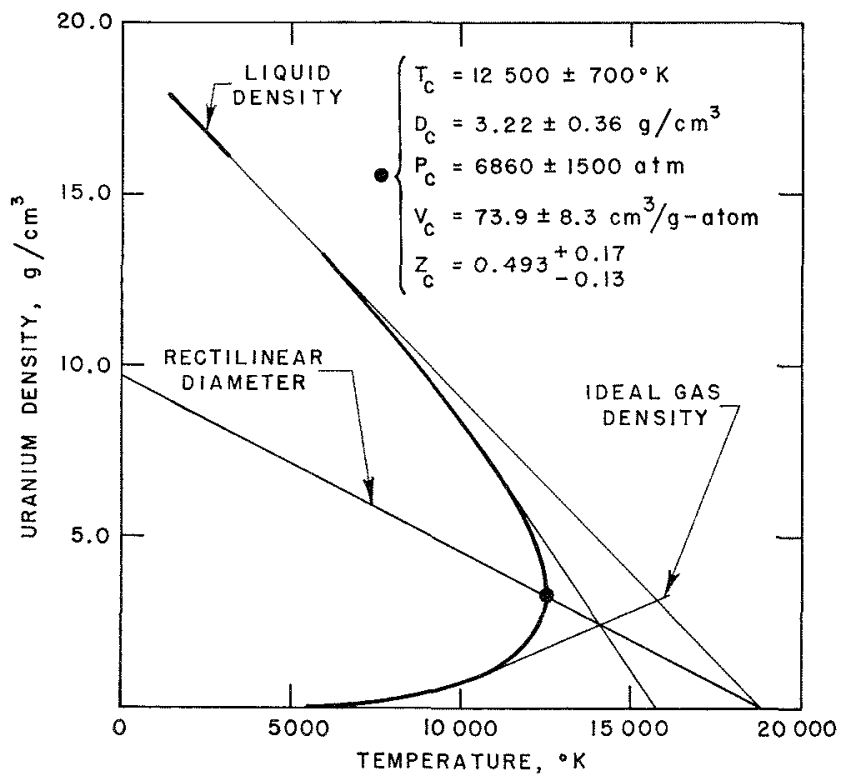

Fir. 1. Density vs Temperature for Ciranium

the sum of the liquid and vapor densitien is linear up to the rritical point. This empirical law has been substantiated for quantum fluids, such as hydrogen and helium; polar liquids, such as ammonia and hydrogen fluoride; a fused salt, $\mathrm{BiCl}_{3}$, and metals, such as mercury, resium, rubidium and sodium, ${ }^{(14)}$ and many ordinary fluids.

By the use of data for the density of the liquid, as a function of temperature along the saturation curve, and estimates of the vapor density from a vapor pressure curve or its extrapolation, the coexistence curves can be estimated. The terhnique is illustrated in Fig. 1 for uranium. The constants estimated for other reactor materials are given in Table 2. Care must be taken in the use of these estimates since they require extrapolation of experimental data far beyond the region of measurement. This is particularly true for vapor pressures.

1 parameter study has been made ${ }^{(15)}$ to investigate the effects of uncertainties in experimental data on the propertics of $\mathrm{CO}_{2}$. Rectilinear-law curves were constructed for several vapor pressure equations, eritical compressibilities, coefficients of expansion of the liquid, and heat of fusion. The results tend to cluster in a fairly narrow range around the value obtained for the "best" values chosen for the needed data. The results are presented in Table 3 .

Figure 2 shows the vapor pressure eurves of $\mathrm{UO}_{2}$ used for the calculations. The code AIT is used for the Ackerman ${ }^{(16)}$ low-temperature, $A H T$ for Ackerman ${ }^{(16)}$ high-temperature, $\mathrm{O}$ for Ohse, ${ }^{(17)}$ and $\mathrm{B} M \mathrm{II}^{(18)}$ for an equation reported by Battelle Memorial Institute. An estimated heat of fusion of $3.3 \mathrm{kcal} / \mathrm{mol}$ at the melting 
TABLF 2. Es fim a ned Cratical Properties of Reactor Materials

\begin{tabular}{l|c|c|c|c}
\hline Material & $\begin{array}{c}\text { Critical Temperature } \\
T_{c},{ }^{\circ} \mathrm{K}\end{array}$ & Critical Density $\rho_{c}, \mathrm{~g} / \mathrm{cm}^{3}$ & Critical Pressure $P_{c}$, atm & $\begin{array}{c}\text { Critical Compressibility } \\
Z_{c}=\left(P_{c} / R T_{c}\right)\left(M / \rho_{c}\right)\end{array}$ \\
\hline Uranium & $12,500 \pm 700$ & $3.22 \pm 0.36$ & $6860 \pm 1500$ & $0.493 \pm 0.15$ \\
Plutonium & $10,000 \pm 500$ & $1.414 \pm 0.380$ & $1800 \pm 350$ & $0.371 \pm 0.020$ \\
UO $\mathrm{O}_{2}$ & 9,115 & 1.59 & 1230 & 0.27 \\
Iron & 7,000 & 1.22 & 3,060 & 0.245 \\
Sodium & 2,780 & 0.17 & 440 & 0.285 \\
Beryllium & 8,600 & 0.435 & 14,000 & 0.411 \\
\hline
\end{tabular}

(a) Assumed.

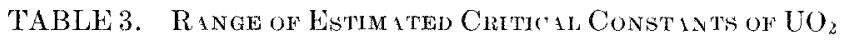

\begin{tabular}{l|r|r|r}
\hline & \multicolumn{1}{|c|}{$T_{c},{ }^{\circ} \mathrm{K}$} & $P_{c}, \mathrm{~atm}$ & $\rho_{c}, \mathrm{gm} / \mathrm{cm}^{3}$ \\
\hline High Temperature & 12,780 & 493 & 0.127 \\
Low Temperature & 4,910 & 1037 & 3.47 \\
Most Probable & 9,115 & 1230 & 1.59 \\
\hline
\end{tabular}

point is included. The effect of the choice of vapor pressure equation is shown in Fig. 3 with the "most probable" heat of fusion of $3.3 \mathrm{kcal} / \mathrm{mol}$, liquid expansion coefficient of $1.05 \times 10^{-4 \circ} \mathrm{K}$ and critical compressibility of 0.27 used. The critical temperatures found range from $5100^{\circ} \mathrm{K}$ for the AHT to 9100 for the A.LT vapor pressure curves.

In Figure 4 is demonstrated the effect of varying the critical compressibilty from 0.20 to 0.40 with the AHT vapor pressure relation and the "most probable" constants. Critical temperatures estimated are about $5 \%$ lower and $7 \%$ higher for $Z_{c}=0.20$ and 0.40 , respectively, as compared to $Z_{c}=0.27$.

The choice of the value for the latent heat of fusion is seen in Fig. 5 to make a major change in the estimated critical temperature. The values used represent several different correlations of heat of fusion.

In Fig. 6 the effect of the slope of the curve of liquid density vs temperature is investigated, using the error limits of Christiansen's ${ }^{(19)}$ measurements (which rovered a very small temperature range compared to those considered here). The net change is less than $\pm 2 \%$, well within other errors in applying the rectilineardiameter method.

An additional technique which can be used is comparison of the entropy of vaporization ${ }^{(9)}$ vs. reduced temperature using a known material as a standard. This is illustrated in Fig. 7 for some common substances. Here the entropy of vaporization is calculated from the heat of vaporization and temperature or by use of the rectilinear-law curves and the Clapeyron equation. Figure 8 shows the effect of the choice of vapor pressure equation with the choice of "best" constants. Some confidence is gained in the choice of the ALT curve and the "best" constants since the entropy curve

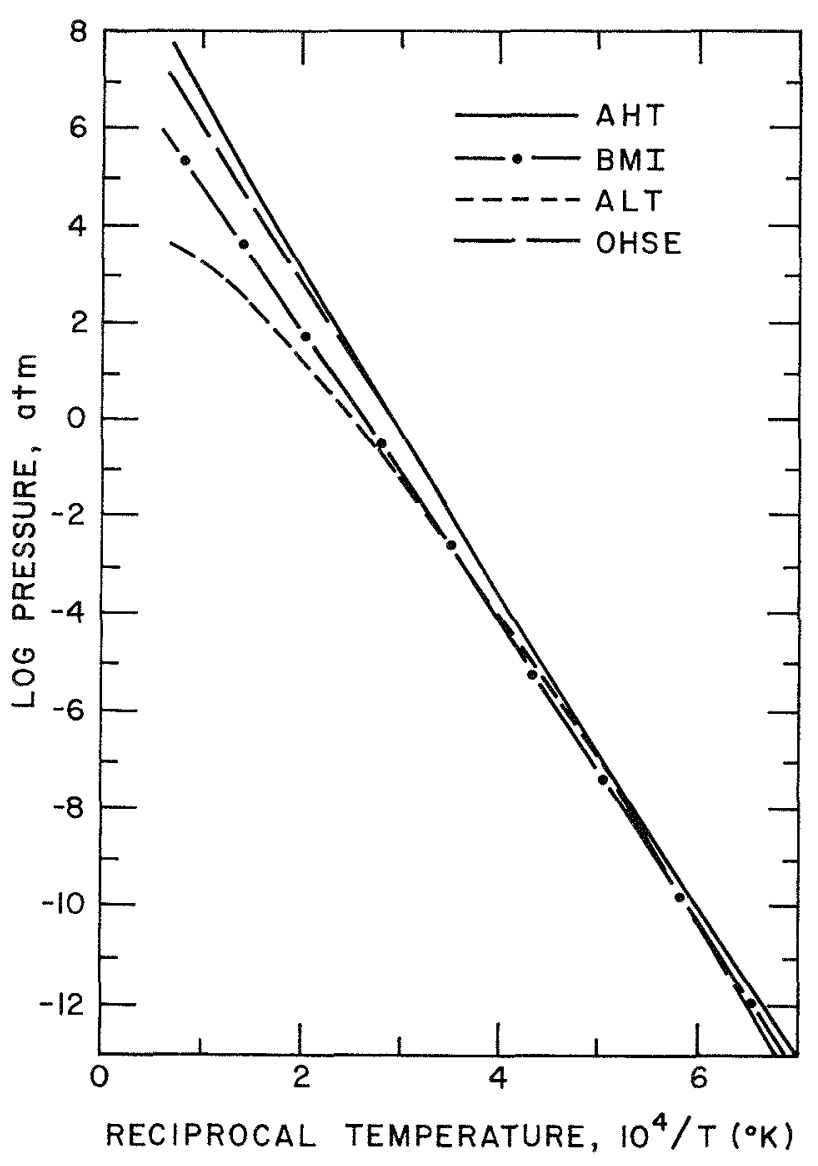

FIG. 2. Vapor Pressure of $\mathrm{UO}_{2}$

derived from them falls between the curves of mercury and the common substances. This may be due to coineidence; any additional experimental data would help justify these choices. Agreement with the results of Meyer and Wolfe ${ }^{(20)}$ is within the accuracy of their assumptions. The results of the variation of parameters used in this study shows care must be used in applying this method.

In general, critical temperatures derived from the entropy of vaporization for metals using mercury as the "stand-in" agree well with those from the rectilinear-law plots except for the transition metals Fe, 


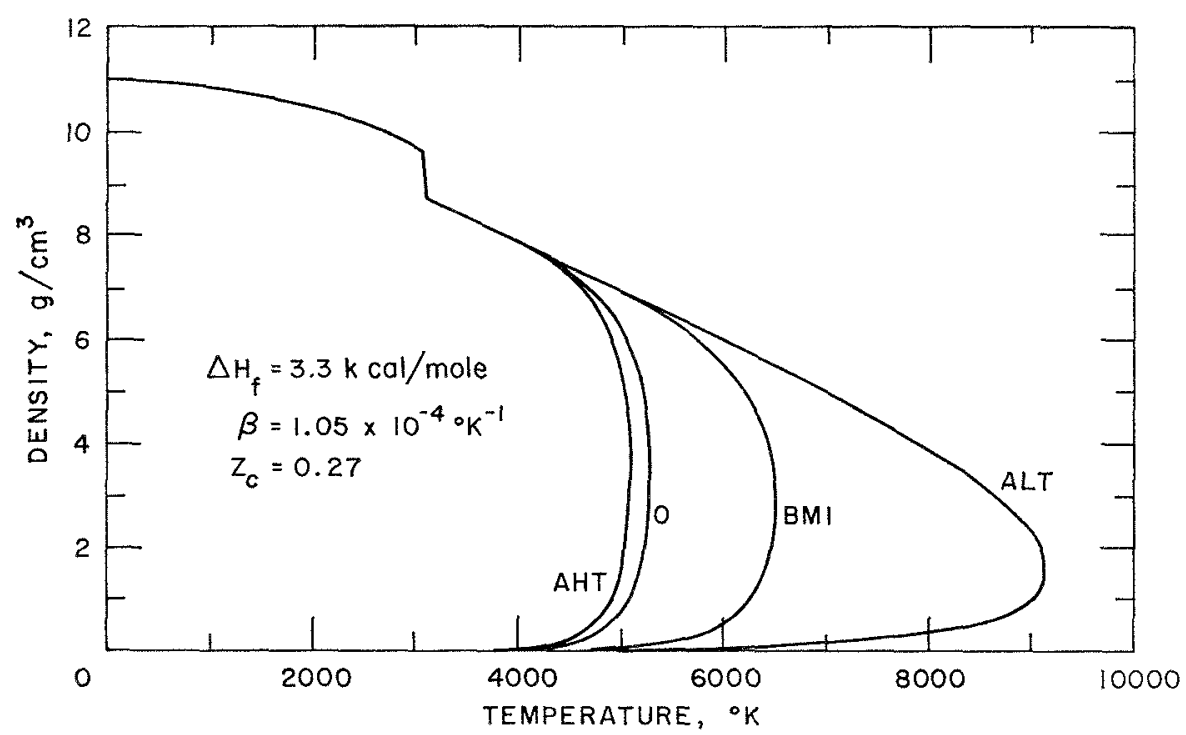

Fig. 3. Effect of Vapor Pressure for $\mathrm{CO}_{2}$.

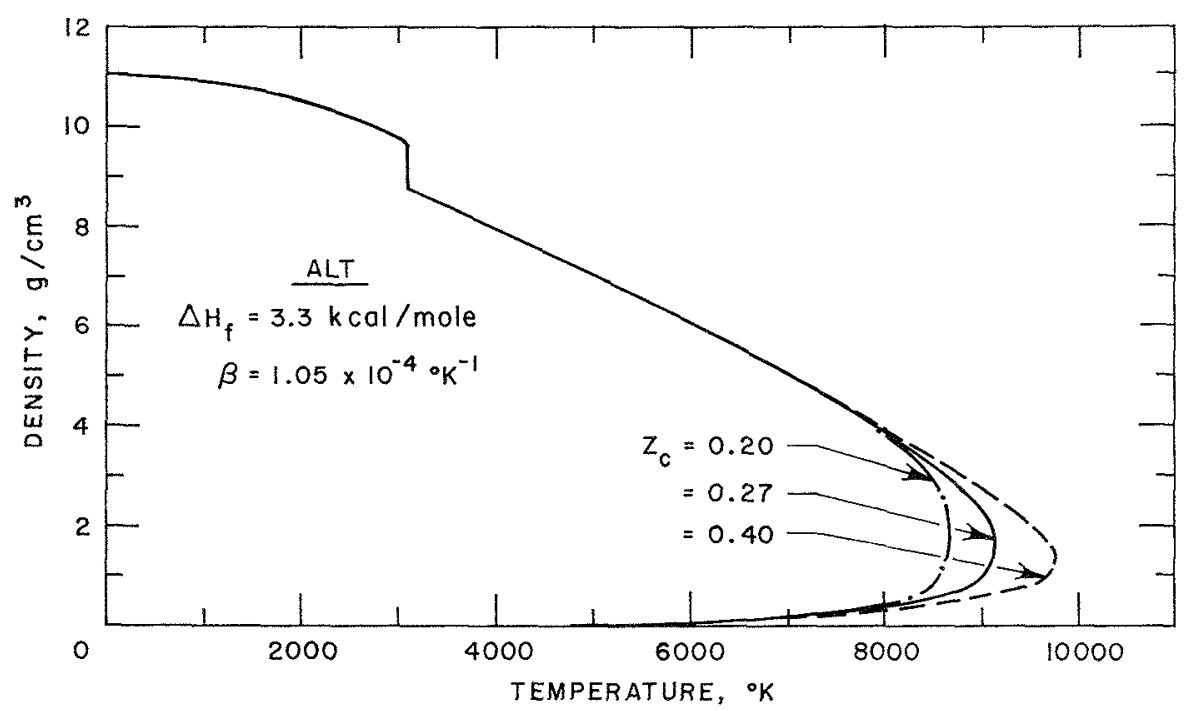

FIt. 4. Effect of Critical Compressibility for $\mathrm{CO}_{2}$.

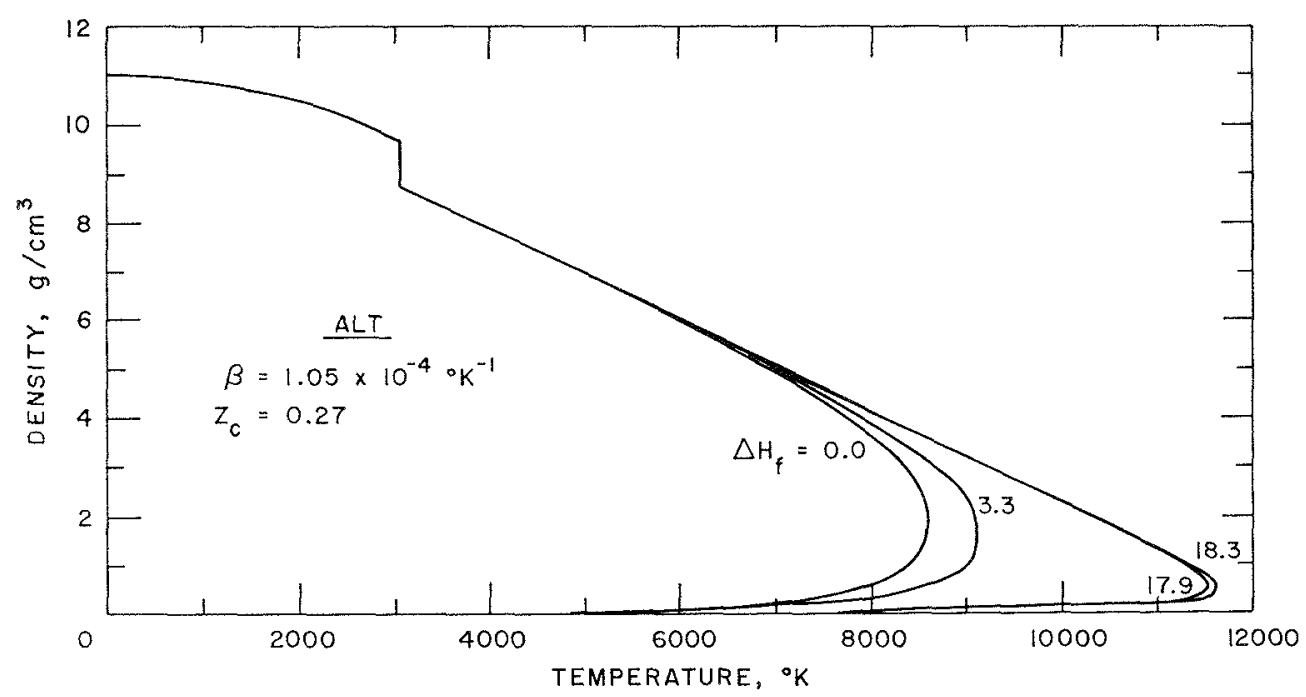

Fug. 5. Effect of Heat of Fusion for $\mathrm{UO}_{2}$. 


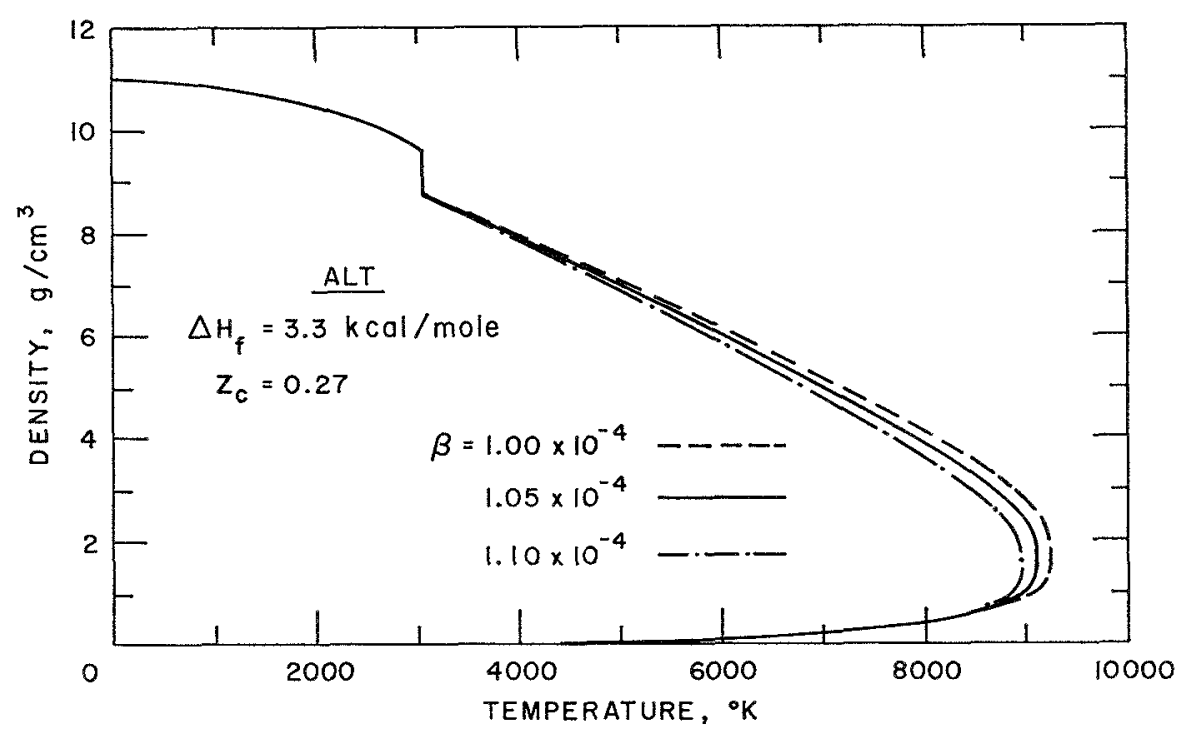

Fig. 6. Effect of Liquid Expansion Coefficient for $\mathrm{UO}_{2}$.

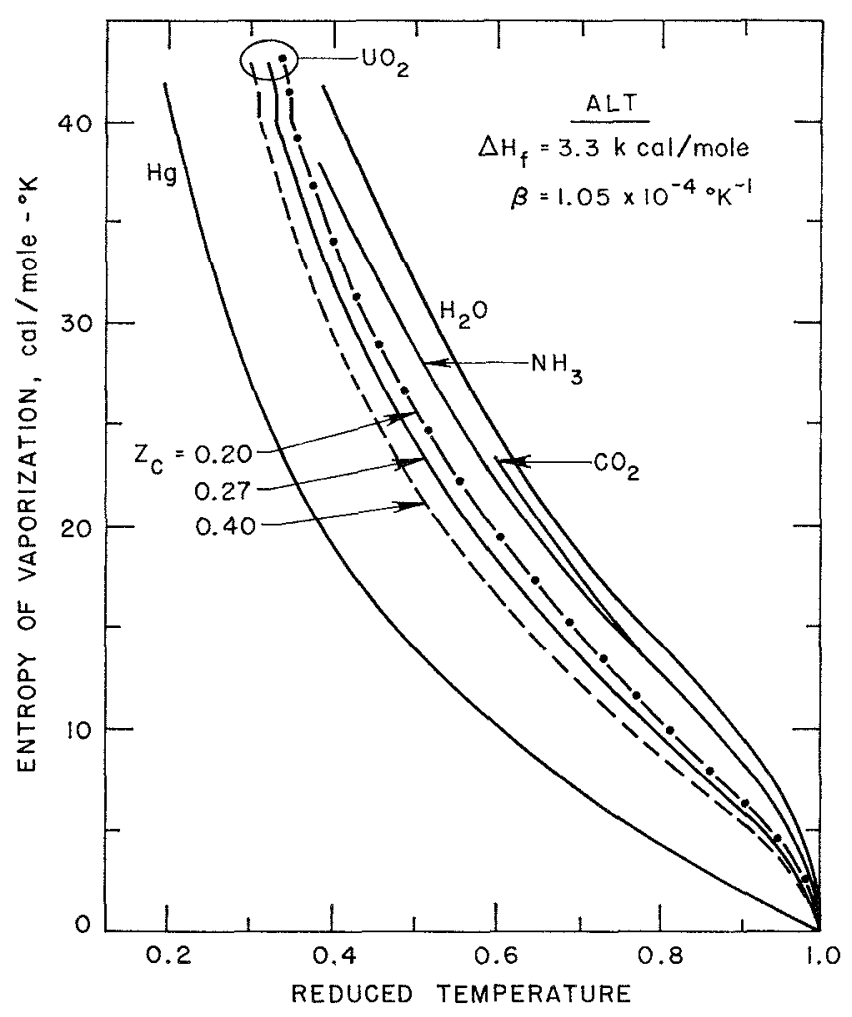

FIG. 7. Effect of Material and of Critical Compressibility for $\mathrm{UO}_{2}$.

Co, and Ni. The disagreement is explained on the basis of the d-band electrons and the rectilinear-law estimate is favored.

\section{Vapor Pressure}

The vapor pressures of reactor fuel materials are important in determining the equilibrium pressure as a

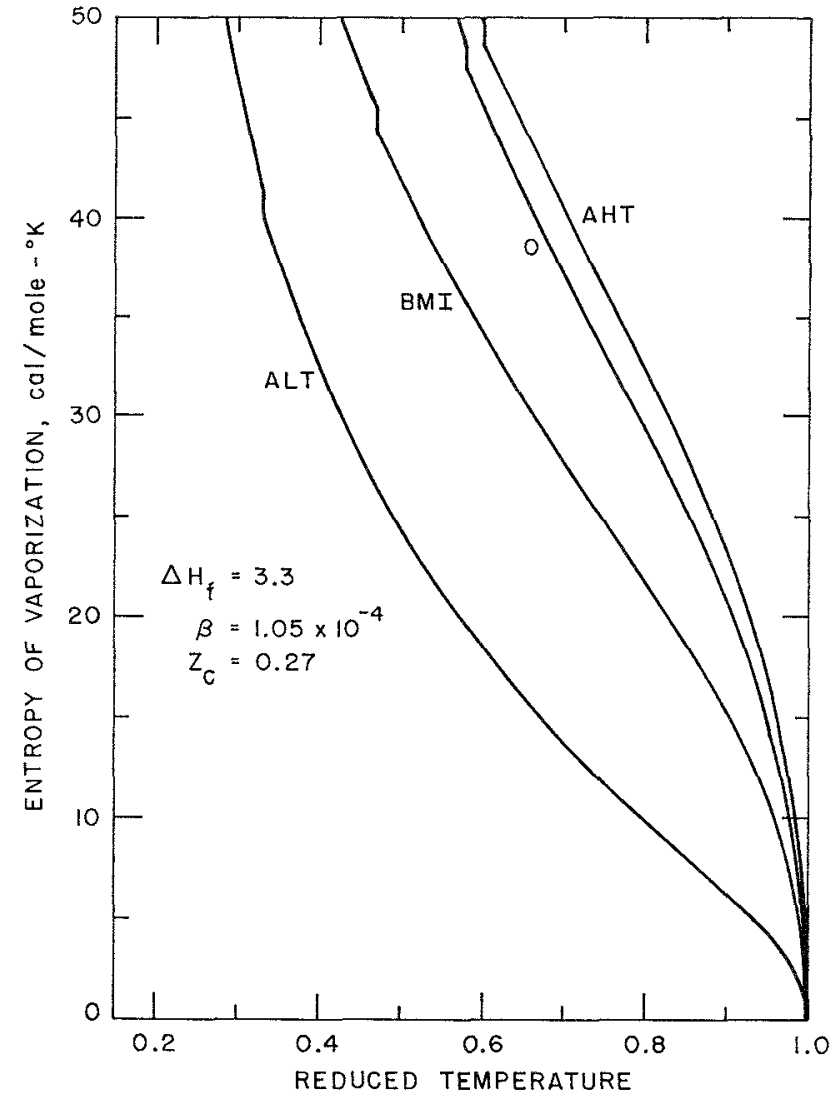

Fix. 8. Effect of Vapor Pressure Curve for $\mathrm{UO}_{2}$.

function of temperature below the thermodynamic critical point. Since evaporation is a rate-limited process it is possible that a phase transition would not occur in a sufficiently rapid transient.

A selection of vapor pressure equations for substances of interest as reactor fuels is given in Table 4 . In gen- 
eral, these equations were obtained by fitting experimental data taken by effusion, transpiration, or massspectrometric methods at pressures below $1 \mathrm{~mm} \mathrm{Hg}$. They are therefore sensitive to the details of stoichiometry, vacuum, and the materials of containment used for the experiment. For reactor safety calculations, these equations must often be extrapolated six to nine orders of magnitude higher in pressure than experimentally measured. Such extrapolations are often reasonably accurate for ordinary substances, but at higher temperatures the effects of dissociation, excited states, and ionization would be expected to complicate the behavior for the reactor fuel materials.

The first vapor pressure equation for $\mathrm{PuC}$ in the table was developed by Palmfreyman and Potter ${ }^{(30)}$ from results of a transpiration experiment and is based on five experimental determinations of vapor pressure at four temperatures with only $65^{\circ} \mathrm{C}$ in range. These are shown in Figure 9. As the maximum weight loss in these experiments was $14 \mathrm{mg}$, the presence of $5 \mathrm{ppm}$ oxygen in the argon carrier gas could have led to significant error, since at the temperatures of the experiment $\mathrm{PuO}_{2}$ is more volatile than PuC.

The heat of vaporization (which is related to the slope of the curve) from this work is out of line with values for other artinide carbides and with the results of recent measurements at Los Alamos, ${ }^{(31)}$ and therefore nust be suspect. This work ${ }^{(30)}$ was used, without critical examination, as the basis for the estimation of equationof-state information by Kubit ${ }^{(10)}$ along with a questionable rule for estimating critical volumes.

Critical temperatures and pressures estimated by Kubit are sufficiently low as to be within experimental reach in steady or transient measurements. Such experiments are under consideration by the author and C. E. Dickerman.

The lack of agreement by several investigators study -

TABLE 4. Vapor Pressithes of Re ictor Fuel Materials

\begin{tabular}{|c|c|c|c|}
\hline Material & Reference & $\log P(\operatorname{atm})$ & Phase \\
\hline $\mathrm{L}$ & 21 & $5.702-(23,300 / T)$ & liquid \\
\hline$\left[\mathrm{IO}_{2}\right.$ & 16 & $\begin{array}{r}22.805-(33,115 / T) \\
-4.026 \log T\end{array}$ & solid \\
\hline $\mathrm{UC}$ & 22 & $7.219-(32,910 / T)^{\{(\alpha)}$ & solid \\
\hline UN & 23 & $\begin{aligned} 8.913- & (29,510 / T) \\
+ & \left(5.27 \times 10^{-18} T^{5}\right)^{(6)}\end{aligned}$ & solid \\
\hline Us & 24 & $7.666-(29,970 / T)$ & solid \\
\hline $\mathrm{Pu}$ & 25,26 & $5.014-(17,587 / T)$ & liquid \\
\hline $\mathrm{PuO}_{2}$ & $27,28,29$ & $8.129-(27,900 / T)$ & solid \\
\hline$P_{u C}$ & 30 & $22.3-(51,000 / T)$ & solid \\
\hline $\mathrm{PuC}(2)$ & 31 & $5.096-(18,561 / T)$ & solid \\
\hline
\end{tabular}

(a) Total pressure above LC.

(b) Decomposition pressure of nitrogen above UN.

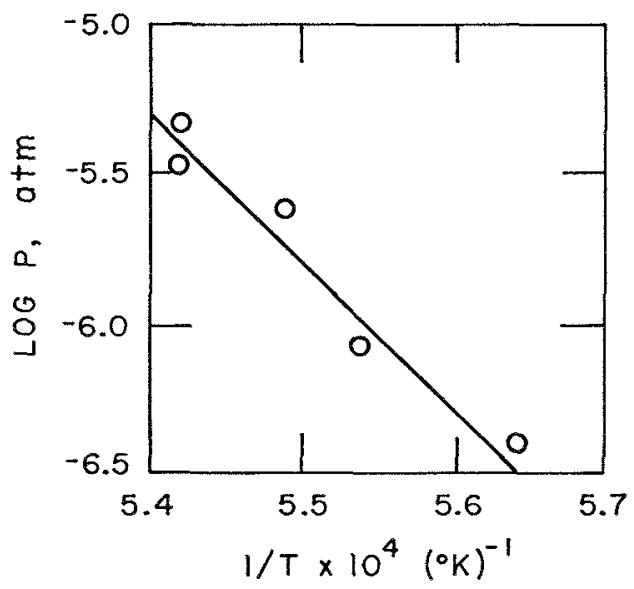

Fig. 9. Vapor Pressure of PuC.

ing the same substance, $\mathrm{T}^{\top} \mathrm{O}_{2},{ }^{(16-18)}$ is indicative of the importance of careful technique, the necessity for characterization of the sample, and the residual gases in high-vacuum systems in order to obtain relations which can be extrapolated over wide temperature ranges reliably. In this most widely studied reactor fuel material the effects of stoichiometry are marked. Both hypoand hyperstoichiometric $\mathrm{CO}_{2}$ have higher vapor pressures and tend to vaporize to the stoichiometric material under equilibrium conditions. ${ }^{(32)}$ Recent experiments at Argonne ${ }^{\text {(32) }}$ and elsewhere tend to confirm Ackerman's ${ }^{(16)}$ low-temperature equation.

\section{Equations of State}

The Grüneisen equation ${ }^{(33,34)}$ which relates energy, density, and pressure can be written in the form.

$$
P(\rho, T)-P_{k}(\rho)=\rho \gamma(\rho)\left[E(\rho, T)-E_{k}(\rho)\right],
$$

where

$$
\begin{aligned}
& P=\text { pressure, dyne } / \mathrm{cm}^{2} \\
& T=\text { temperature, }{ }^{\circ} \mathrm{K} ; \\
& \rho=\text { density, } \mathrm{g} / \mathrm{cm}^{3} ; \\
& \gamma \rho=\text { Grüneisen ratio; } \\
& E=\text { specific internal energy, } \mathrm{erg} / \mathrm{gm}
\end{aligned}
$$

and $P_{k}$ and $E_{k}$ are the pressure and specific intermal energy as functions only of density at $0^{\circ} \mathrm{K}$, and are related by

$$
P_{k}=\rho^{2} \frac{\partial E_{k}}{\partial \rho}
$$

Now if Eq. (1) is rearranged and $Q^{*}$ is defined by

$\gamma(\rho)\left[E_{k}(\rho)-\frac{P_{h}(\rho)}{\rho \gamma(\rho)}\right]=E(\rho, T)+Q^{*}(\gamma-1)$,

then 


$$
\begin{aligned}
Q^{*}=\frac{\gamma}{\gamma-1} & E_{h}(\rho)-\frac{P_{h}(\rho)}{\rho(\gamma-1)}-\frac{E(\rho, T)}{\gamma-1} \\
& =\frac{\gamma}{\gamma-1} E_{k}-\frac{\rho}{\gamma-1} \frac{\partial E_{k}}{\partial \rho}-\frac{E}{\gamma-1} .
\end{aligned}
$$

Equation 1 may be written in the form of the BetheTait "threshold equation of state" (35)

$$
P(\rho, T)=\rho[\gamma-1]\left[E(\rho, T)-Q^{*}\right],
$$

where $P=0$ for $E<Q^{*}$.

It can be seem that, in general, $Q^{*}$ is not a constant in spite of its utility in providing a physical feeling for the buildup of pressure when the energy is greater than $Q^{*}$.

The limiting value of the Grüneisen coefficient for a monatomic substance at sufficiently high temperatures is that of an ideal gas:

$$
\operatorname{Lim}_{T \rightarrow \infty} \gamma=\frac{R}{C_{v}}=\frac{2}{3}
$$

Consideration of this limiting lower bound indicates that $\gamma-1$ could be negative in the threshold equation of state, and pressure would become negative for increasing encrgy and temperature. Caution should be exereised in using that relation other than as a curve fit over limited ranges of energy and density.

In the first formulation of the computer code $\mathrm{AX}-1^{(36)}$ the equation

$$
P=a+b T+c \rho
$$

was used along with the caloric equation

$$
\frac{\partial E}{\partial T}=C_{v}=A_{c v}+B_{c v} T
$$

with the constants recommended by Stratton for uranium:

$$
\begin{aligned}
& a=0.545649 \text { megabar } \\
& b=278.46 / \mathrm{keV} ; \\
& c=0.02873 \mathrm{~cm}^{3} / \mathrm{gm} ; \\
& A_{c v}=12.163 \mathrm{~cm}^{2} / \mu \mathrm{sec}^{2}-\mathrm{keV} \\
& B_{c v}=5.78 \times 10^{3} \mathrm{~cm}^{2} / \mu \mathrm{sec}^{2}-\mathrm{keV}
\end{aligned}
$$

for tempcratures measured in kilovolts $\left(1.16 \times 10^{7 \circ} \mathrm{K}\right)$ and pressures measured in megabars $\left(0.9869 \times 10^{6}\right.$ atm). Jankus ${ }^{(37)}$ has shown the thermodynamic inconsistency of Equations 6 and 7 by means of thermodynamic identities. The constants $a, b$, and $c$ are also inconsistent at the critical point.

Equation (6) can be compared with Brout's work in reduced form or directly. This is shown in Fig. 10 and 11. The van der Waals equation, which generally gives good agreement above the critiral point, is also shown for comparison. It can be seen that Stratton's equation is at variance with Brout's calculations and the van der Waals equation, which show reasonable agreement. If the results of Brout's calculations and Stratton's equation are plotted on an absolute basis, normalized to a common point as in Fig. 11, the disagreement persists but is not as marked. Also shown is the linearenergy-threshold model used in the Bethe-Tait calculation with a value of the Grüneisen ratio taken as 2 .

Constancy of the Grüneisen ratio has also often been assumed. Only Nicholson ${ }^{(12)}$ has attempted to include the effect of density, and he assumes a linear relation between this ratio and density. It can be seen from. H'ig. 12 that the slope and values chosen by Nicholson do not agree with extrapolated values of $\gamma$ from experimental shock compression data of Persson and Persson. ${ }^{(38)}$ The effects of reduced density are presently under consideration and these will be reported later.

\section{Shock Equation of State}

Generation of extremely high pressures by shock compression provides important information on the equation of state ${ }^{(39,40)}$ ('ogent to reactor safety. The basic equation necessary to interpret the measurements is the conservation of mass across the shock wave:

$\rho_{0} C_{s}=\rho_{1}\left(L_{s}^{r}-L_{p}{ }_{p}\right)$

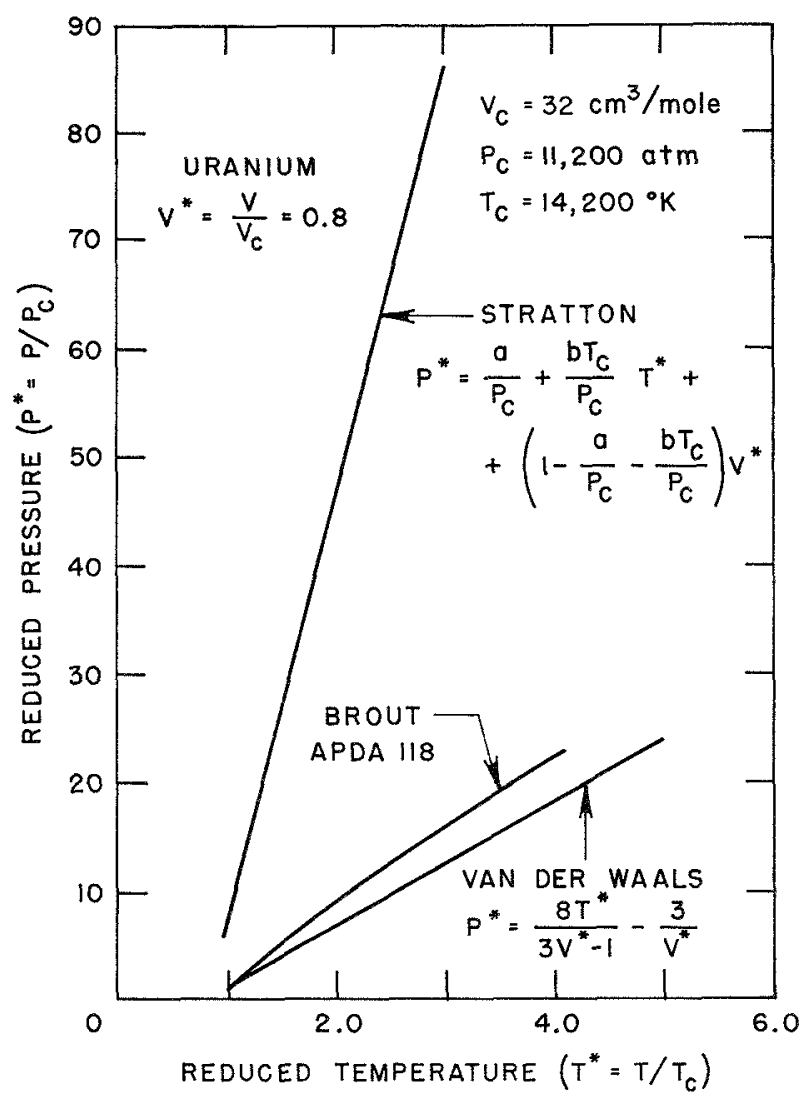

Fig. 10. Estimate of Reduced Pressure of Uranium as a Function of Reduced Temperature. 


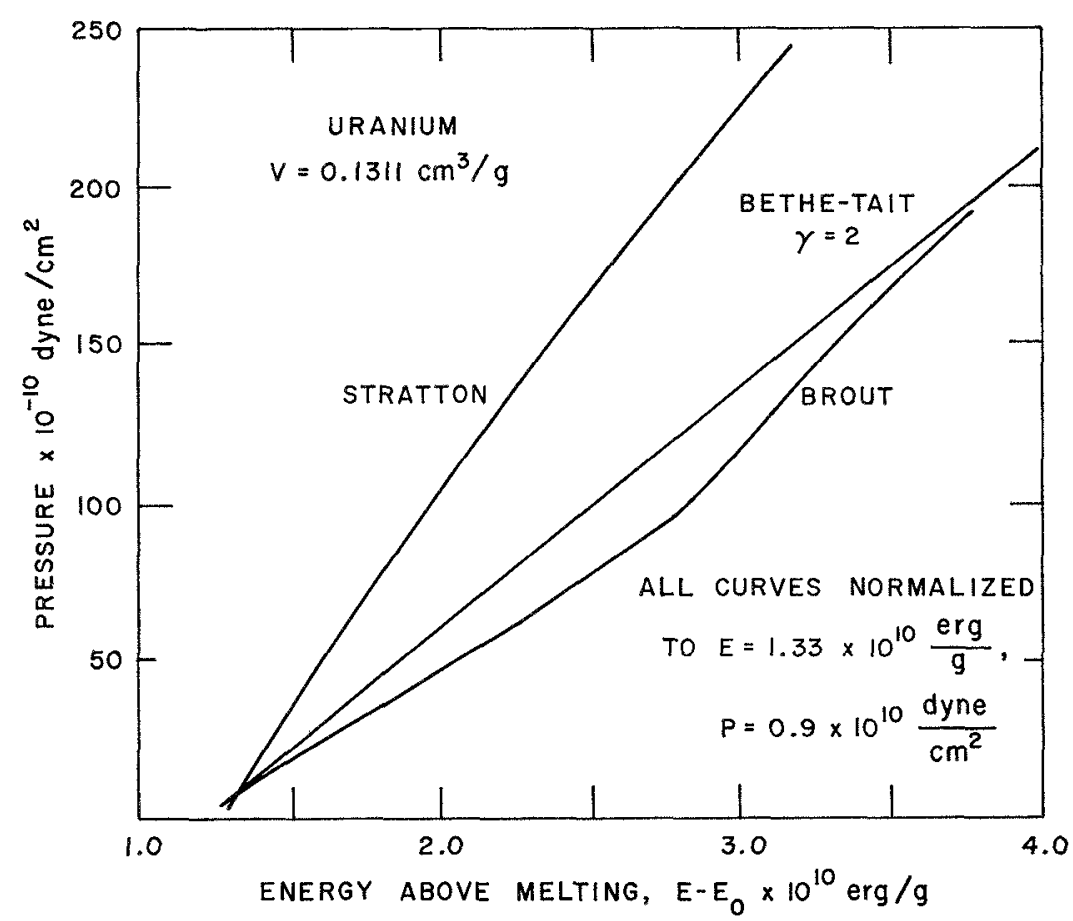

Fra. 11. Estimates of the Pressure of Uranium above the Melting Point.

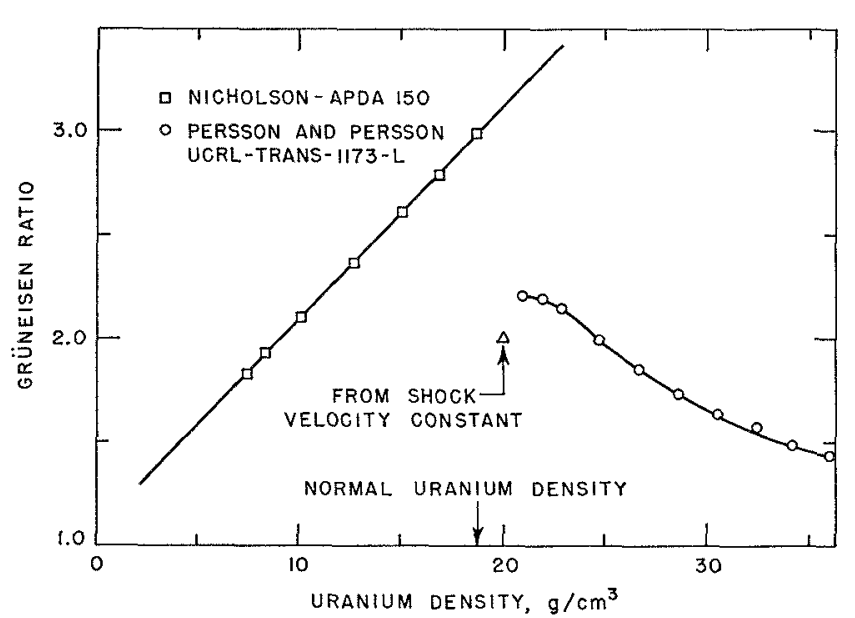

Frg. 12. The Grüneisen Ratio for Uranium as a Function of Density.

where

$$
\begin{aligned}
\rho_{0} & =\text { density before shock; } \\
\rho_{1} & =\text { density after shock; } \\
U_{p} & =\text { particle velocity; } \\
U_{s} & =\text { shock velocity. }
\end{aligned}
$$

The velocities are usually measured by photographic techniques or by closing of electrical circuits. The law of conservation of momentum is similarly given by

$$
P_{0}-P_{1}=\rho_{0} U_{s} U_{p},
$$

where $P_{0}$ and $P_{1}$ are the pressure before and after the shock, respectively. Conservation of energy across the shock leads to

$$
P_{1} U_{p}=1 / 2 \rho_{0} U_{s} U_{p}^{2}+\rho_{0} U_{s}\left(E_{1}-E_{0}\right) .
$$

These can be combined to give the Rankine-Hugoniot equation :

$$
E_{1}-E_{0}=1 / 2\left(V_{0}-V_{1}\right)\left(P_{1}+P_{0}\right),
$$

where $V=1 / \rho$ is the volume.

Experimental measurements in regions where no phase transitions occur usually provide a linear relation ${ }^{(39,40)}$ between shork and particle velocity:

$$
C_{s}=C+S U_{p} .
$$

This holds for experiments with high-density $C^{\Upsilon(8,38)}$ and is approximately true for low-density material, ${ }^{(8)}$ although the investigators fitted a different form of equation to the results from porous $U$. The data for uranium are shown in Fig. 13. When these data are combined with Eqs. 8 and 9, an expression is obtained for the pressure-density behavior on the RankineHugoniot curve or shock-Hugoniot, as it is called:

$$
P_{H}=C^{2} \rho_{0}\left(1-\frac{\rho_{0}}{\rho}\right) /\left[1-S\left(1-\frac{\rho_{0}}{\rho}\right)\right]^{2} \text {. }
$$

Although $C$ and $S$ are constants for fitting the data, in the limit of zero pressure, $C$ is the velocity of sound, and experimental data are in good agreement with this conclusion. It can also be shown that $S$ is related to the Grüneisen ratio $\gamma$ by

$$
S=\frac{\gamma+1}{2} .
$$

Here the agreement is not so good. 
As a first approximation for porous materials, $S_{\text {porous }}=S_{\text {solid }}$, whercas $C_{\text {porous }}=C_{\text {solid }}\left(\rho_{\text {porous }} /\right.$ $\left.\rho_{\text {solid }}\right)^{2}$. This approximation was found to work for iron as well as uranium.

Since

$$
\gamma=\frac{\alpha}{\rho \kappa_{s} c_{p}}=\frac{\alpha}{\rho \kappa_{T} c_{v}}
$$

where

$\alpha=$ volumetric expansion coefficient, $(1 / V)(\partial V /$ $\partial T)_{p}$;

$\kappa_{s, T}=$ adiabatic or isothermal compressibility, $-(1 / V)\left(\partial V / \partial P^{\prime}\right)_{s, T}$;

$c_{p, v}=$ specific heat at constant pressure or volume, $(\partial E / \partial T)_{p, v}$,

the compressibilities are related, as may be obtained thermodynamically, by

$$
\kappa_{T}=\kappa_{s}+\frac{T \alpha^{2}}{\rho c_{p}} .
$$

Compresibilities are available from high pressure compression or velocity of sound measurements where $C_{s}=\sqrt{1 / \kappa_{s} \rho}$ or can be estimated from elastic properties. If $K$ is the bulk modulus of compressibility, $E$ is

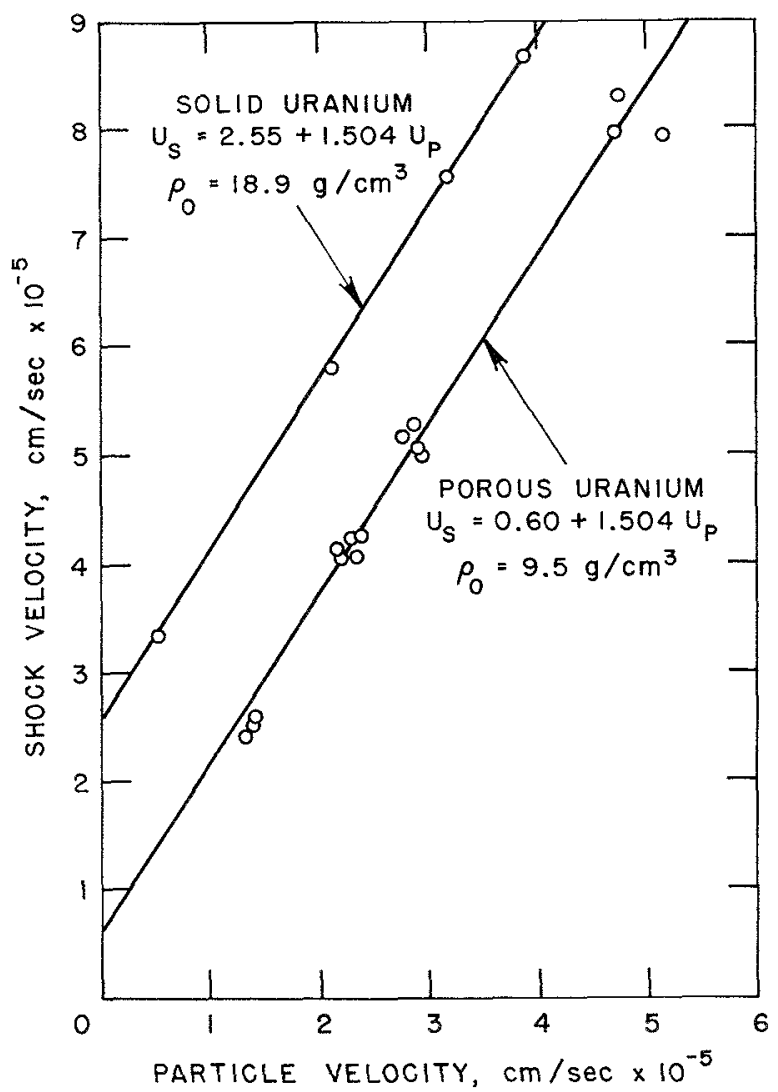

Fug. 13. Data of skidmore and Morris for Shock and Particle Velocities.
TABLE 5. Shock-velocity Constints for Materlals OF REACTOR INTEREST

\begin{tabular}{l|r|l|l|l}
\hline Material & $\rho, \mathrm{gm} / \mathrm{cm}^{3}$ & $\gamma$ & $\begin{array}{c}C,(\mathrm{~cm} / \mathrm{sec}) \\
\times 10^{-5}\end{array}$ & $S$ \\
\hline $\mathrm{U}$ & 19.04 & 1.91 & 2.55 & 1.504 \\
$\mathrm{UO} 2$ & 10.97 & 1.88 & 2.37 & 1.438 \\
$\mathrm{UC}$ & 13.61 & 2.11 & 2.08 & 1.555 \\
$\mathrm{CN}$ & 14.32 & 0.915 & 2.80 & 0.858 \\
$\mathrm{Pu}$ & 19.79 & 2.56 & 1.543 & 1.780 \\
$\mathrm{Al}$ & 2.79 & 2.18 & 5.25 & 1.39 \\
$\mathrm{Be}$ & 1.85 & 1.16 & 7.98 & 1.09 \\
$\mathrm{Fe}$ & 7.84 & 1.60 & 3.80 & 1.58 \\
$\mathrm{Mo}$ & 10.20 & 1.58 & 5.16 & 1.24 \\
$\mathrm{Na}$ & 0.97 & 1.17 & 2.563 & 1.50 \\
$\mathrm{Nb}$ & 8.60 & - & 4.45 & 1.21 \\
$\mathrm{Th}$ & 11.68 & 1.26 & 2.13 & 1.28 \\
$\mathrm{Ti}$ & 4.51 & 1.10 & 4.78 & 1.09 \\
$\mathrm{~V}$ & 6.10 & 1.29 & 5.11 & 1.21 \\
$\mathrm{~W}$ & 19.71 & 1.54 & 4.00 & 1.27 \\
$\mathrm{Zr}$ & 6.49 & - & 3.77 & 0.93 \\
\hline
\end{tabular}

Young's modulus of elasticity, and $\nu$ is Poisson's ratio, then

$$
\kappa_{T}=1 / K=3(1-2 \nu) / E .
$$

The Grüneisen ratio can be calculated from its definition if the other parameters are available, as they generally are. This leads to a good estimate of the shock equation of state. Estimated values of $C$ and $S$ for reactor marials are given in Table 5 .

The energy can be obtained from the relation

$$
\begin{aligned}
& E_{H}=E_{k}+\frac{C^{2}}{2}\left\{\left[1-\left(\rho_{0} / \rho\right)\right] /\right. \\
& {\left[1-S\left(1-\left(\rho_{0} / \rho\right)\right]\right\}^{2}=E_{h}+\frac{P_{H}\left[1-\left(\rho_{0} / \rho\right)\right]}{2 \rho_{0}} .}
\end{aligned}
$$

All that is necessary to estimate the energy in shock compression is an equation to calculate $P_{k}$ or $E_{k}$ since they are related. Very accurate measurements of sonic velocity as a function of pressure ${ }^{(41)}$ or estimations from statistical theories of the atom ${ }^{(42,53)}$ can be used to provide an interpolation equation for $P_{k}$.

This estimation ${ }^{(44)}$ is shown in Fig. 14. Here are plotted the simple Thomas-Fermi (TF), ${ }^{(42)}$ ThomasFermi-Dirac (TFD), ${ }^{(42)}$ and Thomas-Fermi with correlation correction (TFC) ${ }^{43}$ models. The points plotted are calculated values. It can be seen that knowing the pressure from shock experiments in the region from 0.1 to 0.5 megabar and using the statistical theories as asymptotic limits, one can fit an equation to a smooth rurve fit to these. The form for such a curve was suggested by Kormer ${ }^{(45)}$ and used by Altschuler. ${ }^{(46)}$

An equation fitted to similar curves for uranium is referred to in Ref. 38 : 


$$
p_{k}=1.08\left[\left(\rho / \rho_{0}\right)-1\right]+3.88\left[\left(\rho / \rho_{0}\right)-1\right]^{2.30} .
$$

This equation was integrated numerically from Eq. 2 to find $E_{k}$ and $E_{H}$ from Eq. 17. With $P_{k}$ and/or $E_{k}$ one can ealculate the pressure and energy for any density and temperature from ${ }^{(4 t)}$

$$
\begin{aligned}
P^{\prime}(\rho, T)= & P_{k}(\rho)+\gamma(\rho) c_{v} \rho^{\prime} T \\
& +1 / 4 \rho_{0} \beta_{0}\left(\rho / \rho_{0}\right)^{1 / 2} T^{2} \\
E(\rho, T)= & E_{k}(\rho)+c_{r} T+\left[1 / 2 \beta_{0} T^{2} /\left(\rho / \rho_{0}\right)^{1 / 2}\right],
\end{aligned}
$$

where $\beta_{0}$ is the electronic contribution to the heat capacity and is measured at very low temperatures. Alternatively, a Debye model ${ }^{(46)}$ (an be used, and the equations become

$$
\begin{aligned}
& P(\rho, T)=P_{k}(\rho)+\gamma(\rho) \rho \frac{3 R T}{A} D\left(\frac{\theta}{T}\right) \\
&+\frac{\gamma_{\theta}}{2} \beta(\rho) \rho T^{2} ; \\
& E(\rho, T)=E_{k}^{\prime}(\rho)+\frac{3 R T}{A} D\left(\frac{\theta}{T}\right)+\frac{\beta(\rho)}{2} T^{2},
\end{aligned}
$$

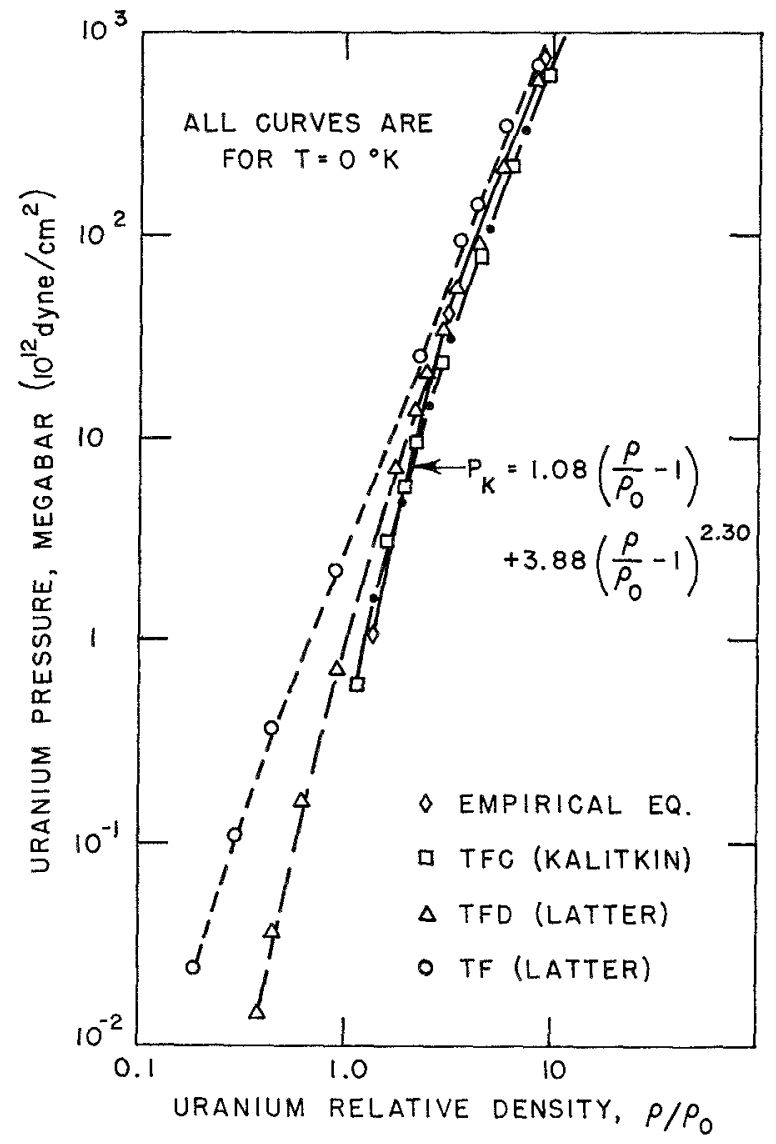

FIG. 14. Estimates of Uranium Pressures.

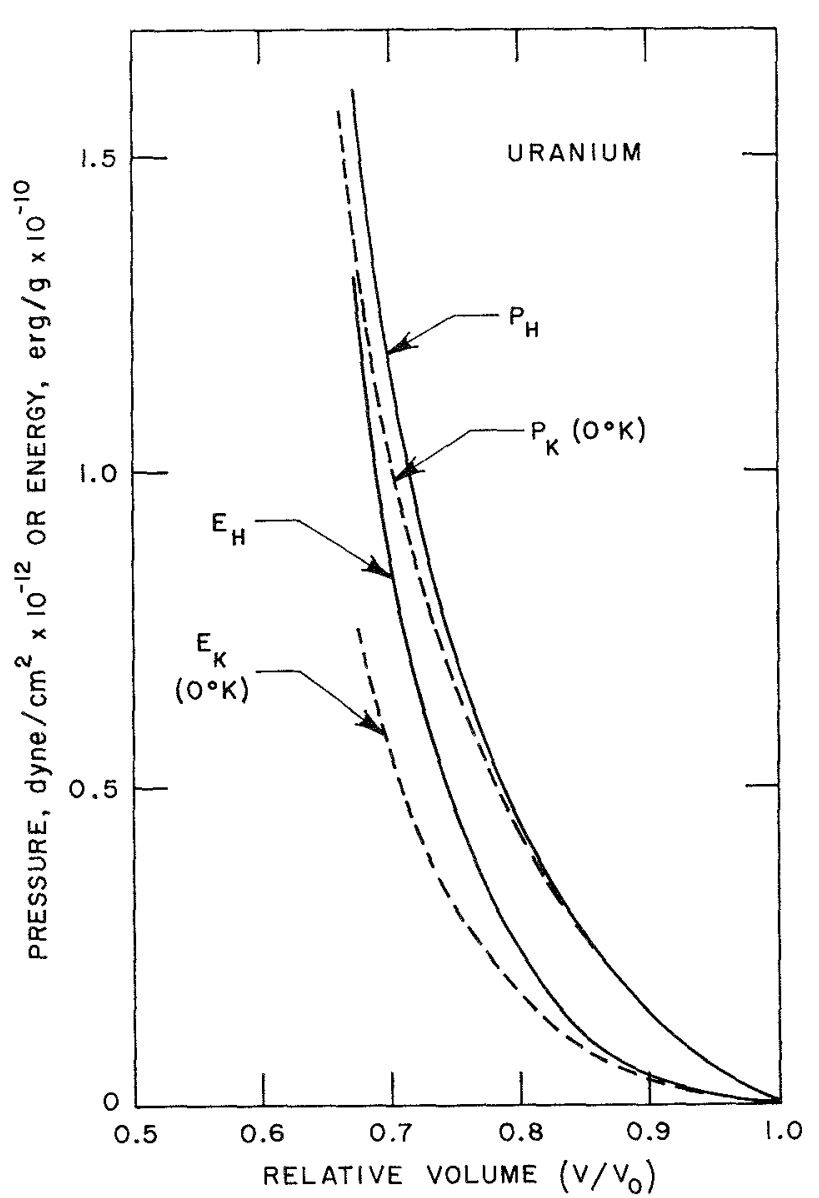

F19. 15. The Effect of Temperature on Pressure and Energy as a Function of Compression for Uranium.

where

$$
A=\text { atomic weight; }
$$

$D(\theta / T)=$ Debye function;

$\theta=$ Debye temperature;

$\beta(\rho)=\beta_{0}\left(\rho_{0} / \rho\right)^{\gamma e}=$ approximate density dependence of the electronic heat capacity

$\gamma_{e}=$ electronic Grüneisen ratio (which varies from 0.5 to $2 / 3$ for free electrons)

The results of calculations for uranium are shown in Figs. 1.5 and 16. Here the values ${ }^{(38)} 1.04 \times 10^{6} \mathrm{erg} /$ $\mathrm{gm}-{ }^{\circ} \mathrm{K}$ and $170 \mathrm{erg} / \mathrm{gm}-{ }^{\circ} \mathrm{K}$ were used for $c_{v}$ and $\beta_{0}$. In Fig. 17, the pressure and temperature along the shock Hugoniot as well as the cold compressive pressure are plotted vs energy. It can be seen that the pressure is approximately parabolic in energy while the temperature is approximately linear with energy.

The results of these calculations can be compared to the equations of state used for fast reactor calculations as compiled in the recent publication of Kazi and Cherry $^{(48)}$ and shown in Fig. 17. 


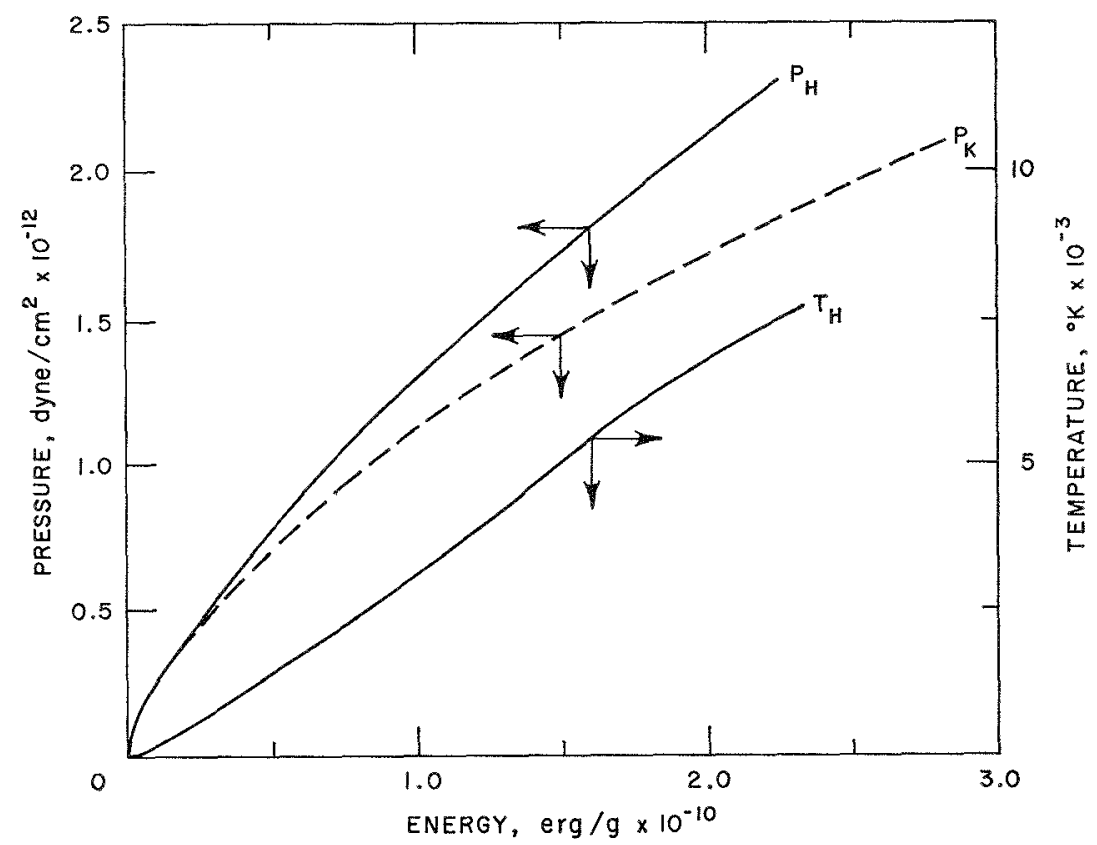

FIG. 16. Pressure and Temperature as a Function of Internal Energy for Uranium.

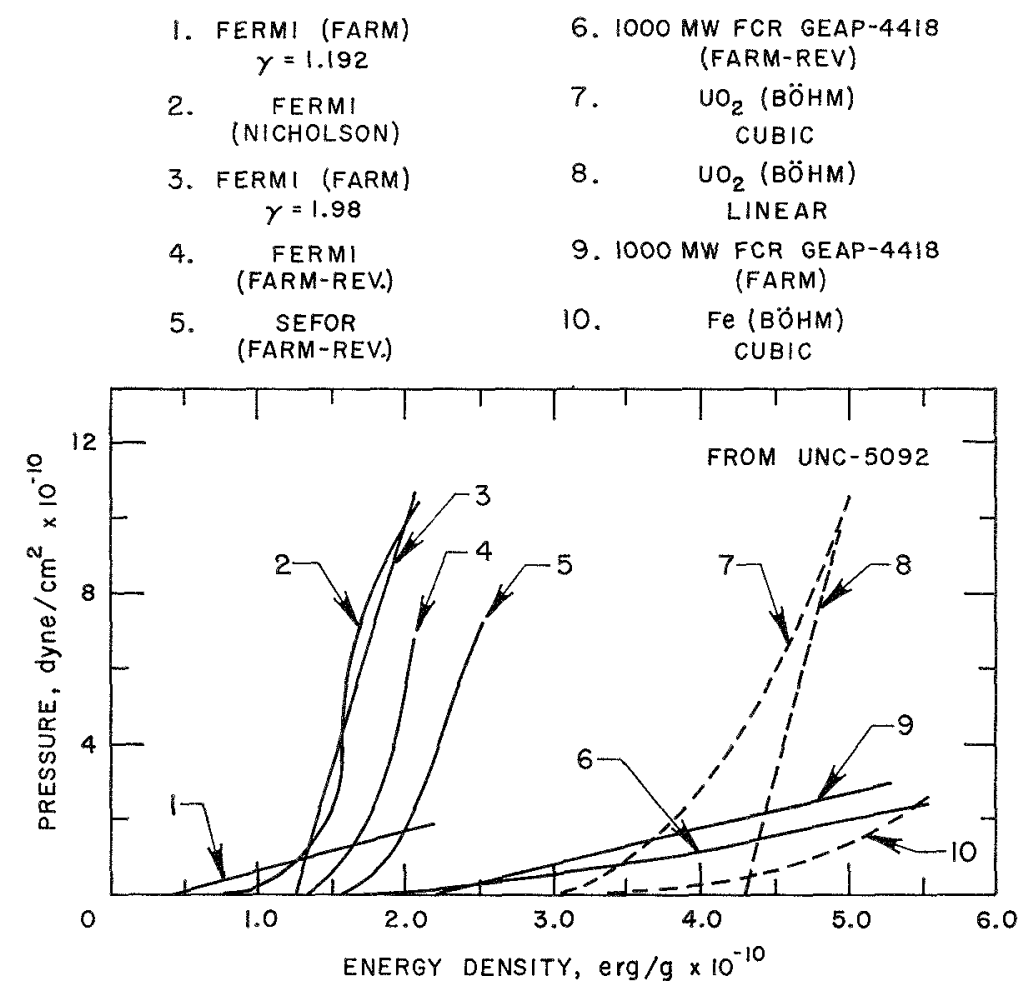

FIG. 17. Equations of State Which Have Been Used for Calculation of Hypothetical Reactor Accidents.

\section{BIBLIOGRAPHY}

1. W. J. McCarthy, Jr., and D. Okrent, "Fast Reactor Kinetics," Technology of Nuclear Reactor Safety, Vol. I, 530-607, Fd. by T. J. Thompson and J. G. Beckerley, MIT Press, 1964.
2. D. Okrent, A Look at Fast Reactor Safety, Nuclear Safety 6, No. 4, 317-342 (1965).

3. R. H. Brout, Equation of State and Heat Content of Uranium, APDA-118 (Feb 20, 1957).

4. II. Elrod, A Method for the Estimation of Critical Temperatures and Pressures, NDA-14-121 (Feb 2, 1956). 
5. H. Elrod, Estimation of the Critical Temperature and Pressure of Uranium, NDA-14-122 (Feb 1956).

6. H. Elrod, Revisions of Estimated Saturation Properties of Uranium, NDA-14-139 (1957).

7. O. A. Hougen, K. M. Watson, and R. A. Ragatz, Chemical Process Principles. Vol. II, Thermodynamics, 2nd Ed., John Wiley (1959).

8. I. C. Skidmore and E. Morris, Experimental Equations of State Data for Uranium and Its Interpretation in the Critical Region, Thermodynamics of Nuclear Materials, International Atomic Energy Agency, p. 173, Vienna, 1962 (STI/PUB/58); see also E. Morris, The Equation of State of Uranium in the Critical Region, AWRE-0-61/62, 1962.

9. A. V. Grosse, The Temperature Range of Liquid Metals and an Estimate of Their Critical Constants, J. Inorg. Nucl. Chem., 22, 23 (1961).

10. C. J. Kubit, R. G. Palm, and W. Gunson, High Temperature Thermodynamic Equation of State of Plutonium Carbide, Trans. Amer. Nucl. Soc. 8, 29 (1965).

11. D. Miller (unpublished work). See Reactor Development Program Progress Report, ANL-7017 (Feb 1965).

12. R. B. Nicholson, Methods for Determining the Energy Release in Hypothetical Reactor Meltdown Accidents, APDA150 (Dec 1962), or Nucl. Sci. Eng. 18, 207 (1964).

13. E. Morris, The Equation of State of Copper and Other Metals near their Critical Points, AWRE 0-36/64.

14. I. G. Dillon, Critical Temperature and Densities of Alkali Metals, ANL-7025 (Aug 1965).

15. R. D. Johnson and D. Miller (unpublished work), Reactor Physies Division, Argonne National Laboratory (1965).

16. R. J. Ackerman, P. W. Gilles, and R. J. Thorn, High Temperature Thermodynamic Properties of Uranium Dioxide, J. Chem. Phys. 25, 1089 (1956).

17. R. W. Ohse, High Temperature Vapor Pressure Studies of $\mathrm{UO}_{2}$ by the Effusion Method, LUR-2166-E.

18. R. W. Dayton and S. J. Paprocki, Progress Relating to Civilian Applications during December 1964, BMI-1707, p. B8-9 (Jan 1, 1965).

19. J. A. Christiansen, Thermal Expansion and Change in Volume on Melting for Uranium Dioxide, J. Amer. Ceram. Soc. 46, 607 (1963).

20. R. A. Meyer and B. E. Wolfe, High Temperature Equation of State of Uranium Dioxide, Trans. Am. Nucl. Soc. 7(1), 111 (June 1964).

21. E. G. Rauh and R. J. Thorn, Vapor Pressure of Uranium, J. Chem. Phys. 22, 1414 (1954).

22. E. K. Storms, A Mass Spectrometric Study of the Vapor Pressure of Uranium Gas and $\mathrm{UC}_{2}$ Gas over Various Compositions in the U-C System, Symposium on Thermodynamies with Emphasis on Nuclear Materials and Atomic Transports in Solids, International Atomic Energy Agency, Vienna 22-27, July 1965 (or see LADC-6953).

23. W. M. Olson and R. N. R. Mulford, The Decomposition and Melting Point of Uranium Mononitride, J. Phys. Chem. 167, $952(1956)$.

24. E. D. Cater, P. W. Gilles, and R. J. Thorn, Uranium Monosulfide. I. Vaporization, Thermodynamics and Phase Behavior, J. Chem. Phys. 35, 608 (1961).

25. T. E. Phipps, G. W. Sears, R. L. Seifert, and O. C. Simpson, Vapor Pressure of Plutonium, Proceedings of International Conference on Peaceful Uses of Atomic Energy, 1955, 7, 382 (1956), United Nations, New York.
26. R. N. Mulford, The Vapor Pressure of Plutonium, LA-DC6926, (1965).

27. T. E. Phipps, G. W. Sears, and O. C. Simpson, The Volatility of Plutonium Dioxide, J. Chem. Phys. 18, 724 (1950).

28. R. N. R. Mulford and L. E. Lamar, "Volatility of Plutonium Oxide," in: Plutonium, 1960, p. 411, Clever-Hume, London (1961), ed. by E. Grison and W. B. H. Lord.

29. W. N. Pardue and Donald L. Keller, Volatility of $\mathrm{PuO}_{2}$ in Nonreducing Atmospheres, J. Am. Ceram. Soc. 47, 610 (1964).

30. M. Palmfreyman and P. E. Potter, "The Volatility of Plutonium Carbide," in: Carbides in Nuclear Energy, Vol. I. p. 336, Macmillian, London (1964).

31. Quarterly Status Report on Advanced Reactor Technology, for the Period Ending July 31, 1965, LA-3370-MS.

32. R. K. Edwards, Chemical Engineering Division, Argonne National Laboratory, Private communication, October 1965.

33. S. G. Brush, Equation of State. A Survey of Thermodynamic Properties of Matter at High Pressures and Temperatures, UCRL-7437 (June 12, 1964).

34. L. Knopoff, "Solids: Equations of State of Solids at Moderately High Pressures," in: High Pressure Physics and Chemistry, Chapter 5.1, Vol. II, ed. by R. S. Bradley, Academic Press (1963).

35. II. A. Bethe and J. H. Tait, An Estimate of the Grder of Magnitude of the Explosion When the Core of a Fast Reactor Collapses, RHM(56)/113 (1956).

36. D. Okrent, J. M. Cook, D. Satkus, R. B. Lazarus, and M. B. Wells, $A X-1$, A Computing Program for Coupled Neutronics-Hydrodynamics Calculations on the IBM-704, ANL-5977 (May 1959).

37. V. Z. Jankus, A Modified Equation of State for Hydrodynamic Calculations in AXI Numerical Program, Paper in Session VIIA, these Proceedings.

38. P. A. Persson and I. Persson, Determination of State Data for Uranium at High Pressures by Means of Shock Wave Experiments, UCRL-Trans-1173-L (from FOA-2 Rept. A2299-222, Sept. 1964 of the Research Institute of National Defense, Stockholm, Sweden).

39. M. H. Rice, R. G. McQueen, and J. M. Walsh, Compression of Solids by Strong Shock Waves, Solid State Phys., 6, 1 (1958).

40. G. E. Duvall and G. R. Fowles, "Shock Waves," in High Pressure Physics and Chemistry, Vol. II, ed. by R. S. Bradley, Academic Press (1963).

41. O. L. Anderson, Two Methods for Estimating Compression and Sound Velocity at Very High Pressures, Proc. Natl. Acad. Sci. US, 84, 667 (1965).

42. R. Latter, Solutions of the Thomas-Fermi-Dirac Statistical Model of Atoms, J. Chem. Phys. 41, 2275 (1964).

43. N. N. Kalitkin, The Thomas-Fermi Model of the Atom with Quantum and Exchange Corrections, Soviet Physics JETP 11, 1106 (1960).

44. R. E. McKeighen, W. L. Chen, and T. G. Hersum (D. Miller, Advisor), Determination of the Equation of State of Uranium, Plutonium, Carbon and Oxygen from the Statistical Model of the Atom, Unpublished report of the AMU-ANL Summer Engineering Practice School, Argonne National Laboratory, August 15, 1965.

45. S. B. Kormer, V, D. Urlin, and L. T. Popova, Interpolation Equation of State and Its Application to Experimental Data on Impact Compression of Metals, Soviet Physics, Solid State 3, 1547 (1962). 
46. L. V. Altschuler, A. A. Bakanova, and R. F. Trunin, Shock Adiabats and Zero Isotherms of Seven Metals at High Pressures, Soviet Physics, JETP 15, 65 (1962).

47. L. V. Altschuler, S. B. Kormer, A. A. Bakanova, and R. F. Trunin, Equation of State for Aluminum, Copper and Lead in the High Pressure Region, Soviet Physics, JETP 11, $573(1960)$.

48. A. H. Kazi and B. I. Cherry, Survey of Current Methods for Analysis of Fast Reactor Transients Including Meltdowns, UNC-5092.

\section{Jiscussion}

wor

pounds?

Mr. Miller: For ordinary substances, one can use the law of corresponding states or techniques obtained from statistical mechanics to get the deviations from ideality in order to know the partial pressures of various materials above a mixture. However, in the case of the reactor substancess, we don't have enough information at high temperatures to have great confidence in predictions. We use these techniques because we don't have any others. We need additional data.

Mr. Zebroski (GE): In very rapid processes, a great deal of evidence indicates that the equilibrium equations of state, particularly as concerns vapor pressure, do not apply for rate of vaporization. Brewer and his coworkers at Berkeley, particularly for semiconductors and especially for cadmium sulfide, have shown that evaporation rates are lower by a factor of 10 than we calculate from equilibrium considerations. Secondly, I think in accident analysis we are concerned with the product of two uncertainties. Here we have an uncertainty in the thermodynamic data and an uncertainty in the assumption of isentropic expansion. Although we can use the theoretical approach, I think some integral experiments are needed, in which measured amounts of energy are dumped into the substance and we find how much work is done.

Mr. Miller: The work of Prof. Brewer is certainly quite interesting, but was done at low pressures where one is concerned if Langmuir evaporation is taking 


\title{
Theoretical Studies on the Fast Reactor Maximum Accident
}

\author{
E. P. Hicks ANd D. C. Menzies \\ Dounreay Experimental Reactor Establishment \\ Thurso, Caithness, Scotland
}

(PRESENTED BY E. P. HICKS)

\section{Introduction}

The fast reactor development programme of the UK Atomic Energy Authority includes the design of a prototype power-producing reactor. The ultimate safety of a large fast reactor is concerned with the possibility of a superprompt critical power excursion which causes the core to disintegrate with explosive violence. It has, therefore, been a major aim of the programme to ensure that the design incorporates features which make such an incident incredible. At the same time, however, the usual practice of estimating the magnitude and consequences of various hypothetical explosive releases of energy has been followed, and calculations of this nature have been in progress for several years.

The present paper attempts to summarize the work done on several particular problems, chosen because the conclusions appear to be of fairly general applicability and importance. These problems were originally tackled at various times over a period of several years, and as a result there was considerable variation in the reactor parameters used in the original calculations. However, it is believed that the most significant conclusions would remain true for a substantial range of parametric values corresponding to a sodium-cooled $\mathrm{PuO}_{2} / \mathrm{UO}_{2}$-fuelled fast reactor operating at about $500-1,000 \mathrm{MWW}(\mathrm{H})$, so it was not thought necessary to repeat the calculations for a consistent set of parameters.

Three main problems are discussed. The first of these is the calculation of the total amount of energy generated in the assumed hypothetical excursion, and the lesser amount which is available to do mechanical work on the reactor structure; the second is a semiquantitative discussion of a mechanism involving sotium boiling, which could increase the amount of snergy available to do work; the third is a mainly qualitative comparison of the immediate mechanical sffects of the nuclear excursion and of an "equivalent" 
chemical explosion, together with some brief comments on longer-term mechanical effects.

The calculation of energy release starts from a known core configuration and a given rate of reactivity addition, usually derived from a model of gravitational collapse, as in the Bethe-Tait theory. A comprehensive treatment clearly requires a more realistic study of the mode of core collapse, and several attempts have been made to follow the course of events during core collapse in detail. Unfortunately, it appears to be extremely difficult to make calculations on core collapse which preserve at least a modicum of realism. In any case, the ralculations reported below indicate that the effective energy release, that is, the energy available to do work, of the primary nuclear excursion will normally be small, and that a SPICRT-type explosive interaction of the hot fuel with liquid sodium may be of greater eoncern. If this is so, the rate of core collapse could be a fairly unimportant parameter, provided of course that the amount and rate of collapse were large enough to give the relatively small nuclear explosion needed to disrupt and disperse the core.

\section{Calculations of Energy Release}

Calculations of the energy release during a superprompt critical excursion have been based on a mathematical model which retains the essential features of the Bethe-Tait model, ${ }^{(1,2)}$ that is, reactivity changes during the excursion are estimated by perturbation theory and mechanical wave propagation is ignored. ${ }^{(3)}$ The two main modifications which have been made to the simple Bethe-Tait theory are the inclusion of a prompt Doppler reactivity coefficient and the use of a more realistic equation of state of the fuel. We have also distinguished between the fucl density relevant to the equation of state and the total density used in the equation of motion in exactly the same manner as described by Nicholson. ${ }^{(4)}$ The most significant recent results have been obtained with a programme entitled PHOENIX, written for a Ferranti Mercury computer. PHOENIX assumes spherical symmetry, but in other respects is considered to be of adequate generality and flexibility for routine calculations of energy release. Its principal features are outlined as follows.

The thermodynamic properties of the fuel have been estimated from the principle of corresponding states applied to nonmetals by use of tables published by Hougen, Watson, and Ragatz. ${ }^{(5)}$ The fuel was assumed to be $\mathrm{UO}_{2}$, and the critical constants were estimated to be: $T_{c}=8,000^{\circ} \mathrm{K}, p_{c}=2,000 \mathrm{~atm}, V c=0.27 R T_{c} / p_{c}$; the ideal gas specific heat was taken to be $6.5 R$. All these values, which were derived independently, are in quite good agreement with corresponding estimates by IIeyer and Wolfe. ${ }^{(6)}$ The vapour pressure law, which determines the pressure at lower temperatures, was derived by extrapolating published data for $\mathrm{UO}_{2}$ in a manner which was in satisfactory agreement with corresponding-states tabulated data for a two-phase mixture of saturated liquid and vapour, whereas the singlephase tabulations gave the pressure and temperature as functions of the specific volume and energy density. A set of empirical analytic expressions were then derived for the pressure and temperature as functions of the energy density at constant volume. These expressions were in most cases simply straight lines which fitted the more detailed calculations to within a few per cent for all energy densities and sperific volumes likely to occur in practice. In particular, the pressure-energy density relationship was approximated by a straight line in the single-phase region. A modification of the calculation which effectively extended this linear relationship down to zero pressure enabled PHOENIX to be used for calculations of energy release with the threshold-type equation of state of the simple BetheTait theory. The zero for energy density has been taken arbitrarily at $0^{\circ} \mathrm{C}$.

The work done during the adiabatic expansion of the rore, after the (assumed) constani-volume energy release has terminated, was calculated as the decrease of internal cnergy from the compressed state to an expanded state at the same entropy and at temperature $3,450^{\circ} \mathrm{K}$, the estimated boiling temperature of $\mathrm{C}^{\top} \mathrm{O}_{2}$ at atmospherie pressure. In the case of the threshold equation of state, it was assumed that the work done was equal to the excess of the energy generated over the threshold energy. It is obvious from a pressure-volume diagram that the energy remaining as heat after the adiabatic expansion has been completed must increase with the energy generated, and hence the excess of energy gencrated over thre hold energy is an upper-limit estimate of the work done for the threshold-type equation of state. However, the calculations reported below show that the work done is often increased when the equation of state is modified by allowing for vapour pressure at lower energy densities, even though the energy generated is always reduced.

Figure 1 shows the pressure-energy relation at constant volume. All curves follow the vapour pressure law until they reach the saturation point, and then depart from it in a linear manner up to the maximum pressure shown in the figure; for the threshold-pressure-law calculations, the straight lines were extrapolated to zero pressure. The empirical curves agree well with the calculated points, the worst fit being near the transition points.

The temperature-energy relation at constant volume, which is required for the calculation of the Doppler reactivity feed-back, is shown in Fig. 2, together with 


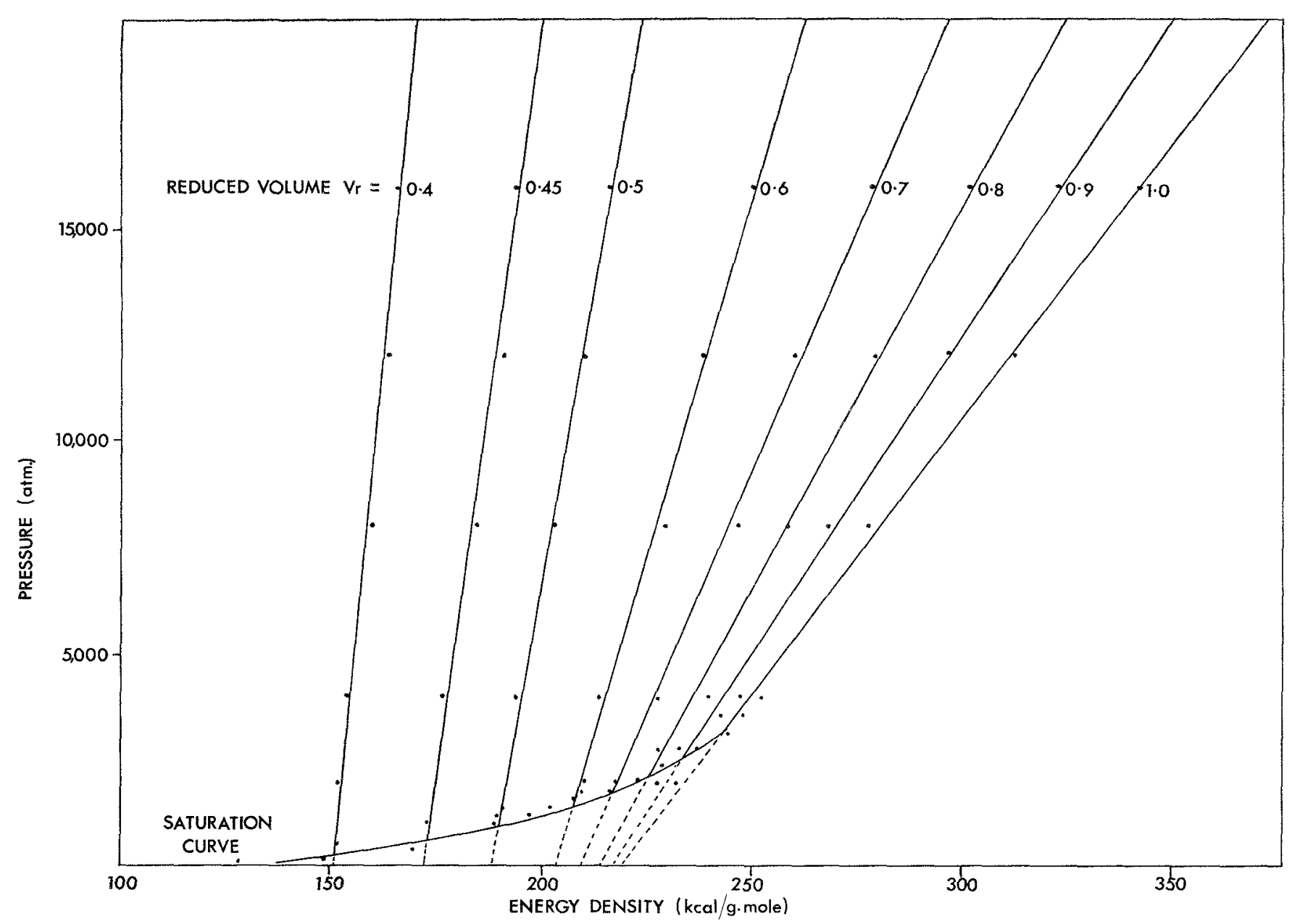

Frg. 1. Pressure and Energy Density at Constant Volume for $\mathrm{UO}_{2}$.

the empirical curves which were fitted to the calculated points. Two features are worthy of note. First, the empirical relations agree well with the calculated points, the worst departures again being at the transition points. Secondly, the additional curve displayed at low energies and temperatures up to $5,000^{\circ} \mathrm{K}$ was calculated by extrapolation of measured specific heat data in the region from 1,000 to $2,000^{\circ} \mathrm{K}$; the agreement at low temperatures with the corresponding-states theory is remarkably good.

Adiabatic relations were required for the calculation of energy release, and Fig. 3 shows the adiabats of the vapour pressure equation of state on a pressure-energy diagram.

Following Jankus, ${ }^{(7)}$ the reactivity change due to core motion satisfies the equation

$$
d^{2} k / d t^{2}=-\iiint(\operatorname{grad} p \cdot \operatorname{grad} w) d V
$$

where $p$ is the pressure and $w$ is the worth of unit mass of core material. The pressure can be expressed as a sum of two terms:

$$
p=P+p^{\prime},
$$

where $P$ is the pressure calculated from the energy density via the constant-volume equation of state, and $p^{\prime}$ is the pressure perturbation due to the waves of compression and rarefaction originating at the core boundary. The reactivity effect of the perturbation pressure can be expressed as an integral over the core boundary, and similar surface integrals arise when the expression (1) is integrated by parts. However, numerous calculations have shown that these surface integrals are relatively small and have little effect on the energy release in typical examples. The PHOENIX programme therefore neglects the surface integrals and calculates the reactivity change due to core motion from the expression

$$
d^{2} k / d t^{2}=\iiint P \nabla^{2} w d V
$$




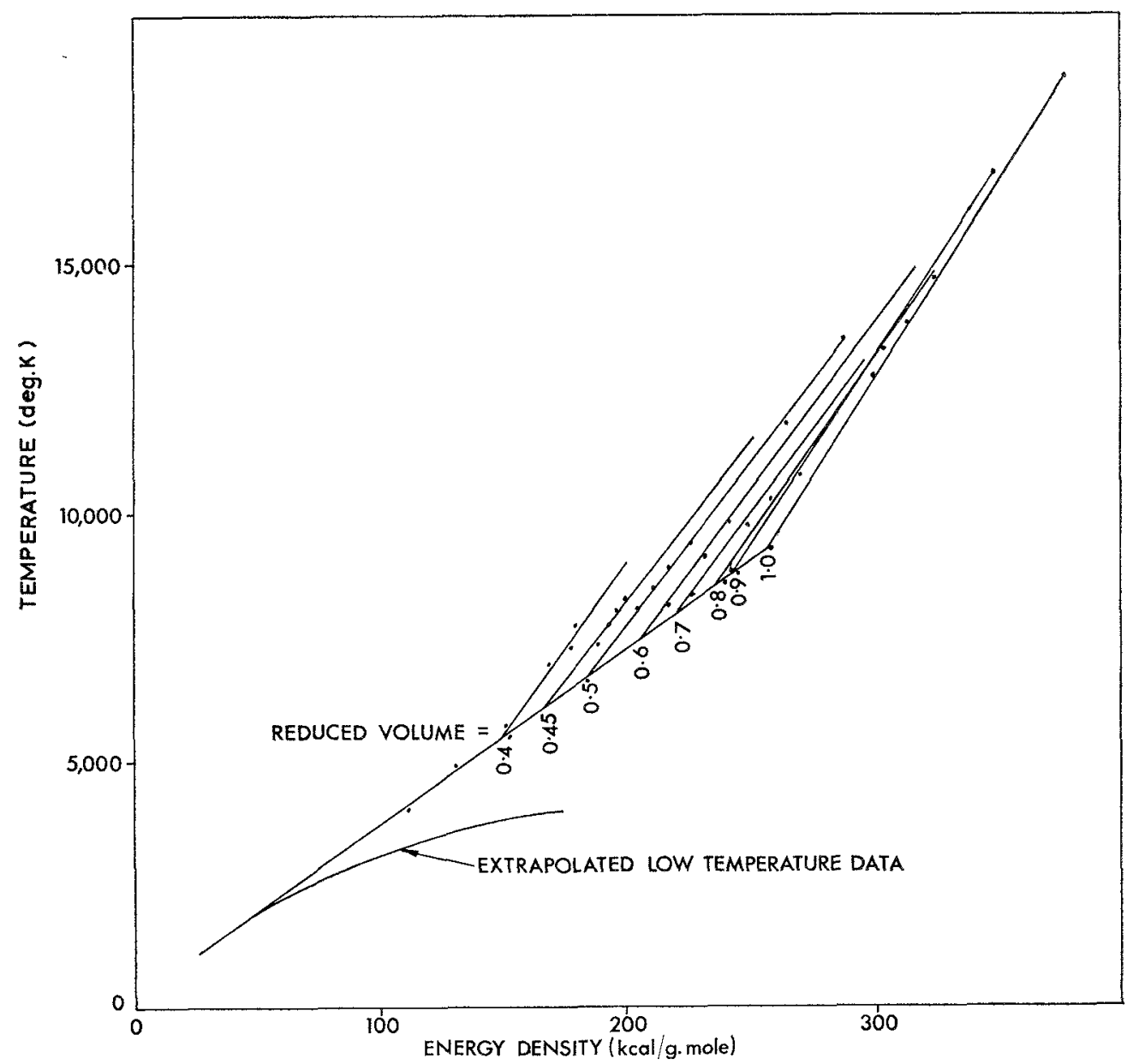

FIG. 2. Temperature and Energy Density at Constant Volume for $\mathrm{CO}_{2}$.

For a homogeneous spherical core of radius $b$,

$$
d^{2} k / d t^{2}=4 \pi \int_{0}^{b} P(d / d r)\left(r^{2} d w / d r\right) d r
$$

and the integral is evaluated in the PHOENIX programme by an 11-point Gaussian quadrature formula at each step in time. The material worth function $w(r)$ is estimated from a detailed neutronics calculation and approximated by a fourth-order polynomial, from which appropriate values of $(d / d r)\left(r^{2} d w / d r\right)$ are calculated and supplied to the programme as input data. A similar numerical procedure could be devised for other not too complicated geometries, such as a homogeneous finite cylinder.

The method used to incorporate a fairly realistic equation of state in the calculation of energy release has been described in some detail because of its novelty, but the same detail is not necessary elsewhere. The Doppler reactivity coefficient has been assumed to be of the form $\delta / T$, where $\delta$ is called the Doppler constant and $T$ is a suitably weighted average of the temperature throughout the core; a straightforward uniformly weighted average core temperature is in fact used in PHOENIX. The calculation is started at delayed critical and the reactor kinetic equation is taken to be ${ }^{(8)}$

$$
l d y_{1} / d t=(1-\beta) k(t)-1+\beta \exp \left(-y_{1}\right),
$$

where

$$
y_{1}=\ln \left(n / n_{0}\right)
$$

and $n$ is the reactor power density at the core centre, $n_{0}$ is the corresponding power density at delayed critical, $l$ is the prompt-neutron lifetime, $\beta$ is the delayedneutron fraction, and $k(t)$ is the reactivity, allowing for the imposed step or ramp rate and for feedback due to core expansion and the Doppler effect. The distribution of reactor power is expressed as a fourth-order polynomial, fitted to a preliminary neutronics calculation. Other equations are as in orthodox versions of the Bethe-Tait theory, ${ }^{(1,2)}$ and their numerical integration presents no serious problems. 


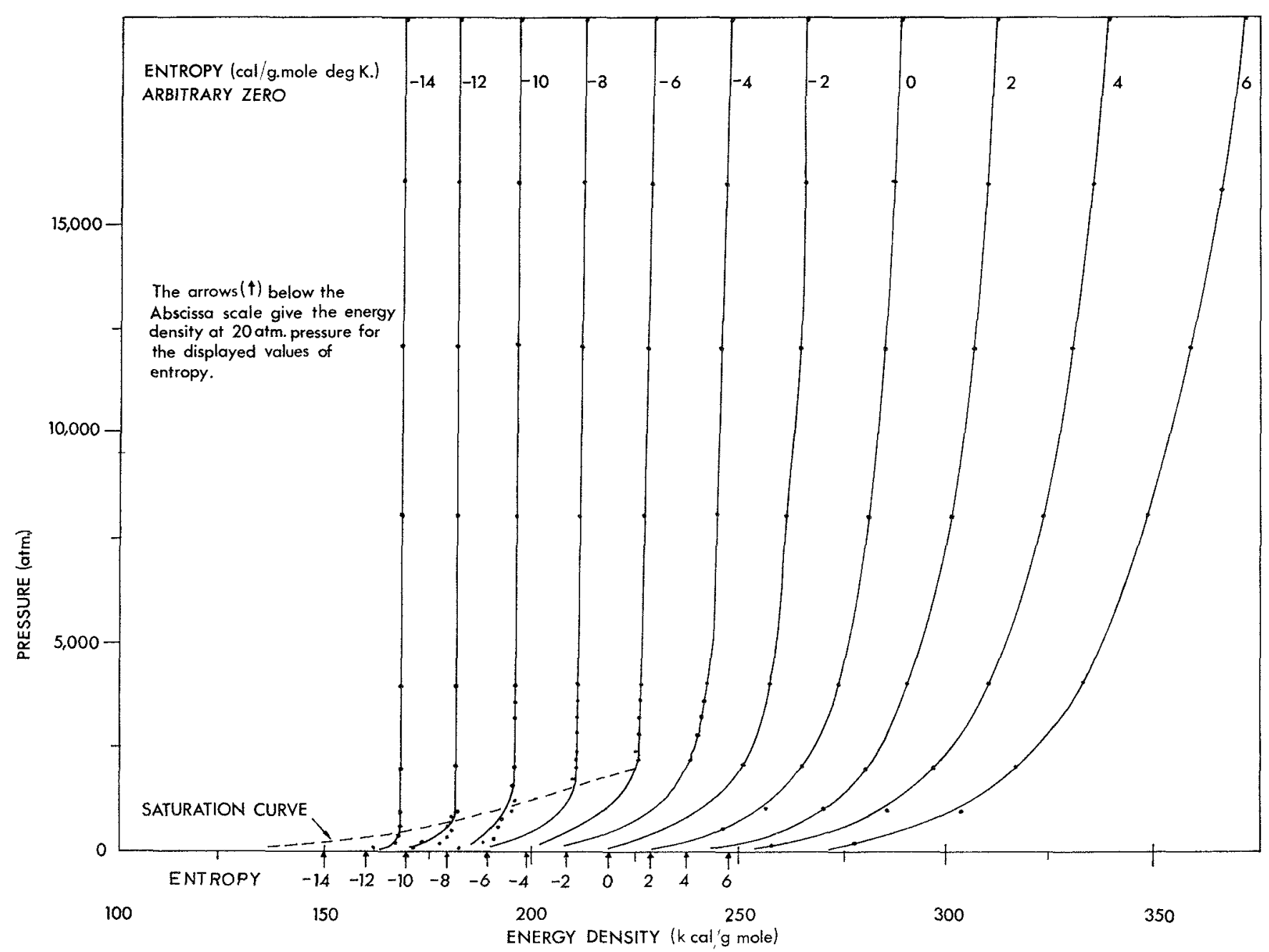

Fic: 3. Pressure and Energy Jensity at Constant Entropy for $\mathrm{UO}_{2}$.

To illustrate the effects of the Doppler reactivity coefficient and the equation of state on the energy release, and in particular on the work done during an adiabatic expansion of the core debris, a series of calculations of energy release have been performed for a spherically symmetric system simulating a fairly typical oxide-fuelled fast power reartor. The basic neutronics data were calculated assuming complete loss of core sodium, and, in particular, the Doppler constant $\delta$ was estimated to be $-0.24 \%$. However, in view of present uncertainties in Dopplor estimates, the energy release was calculated for a series of values of $\delta$ in the range from 0 to $-0.5 \%$. Similarly, in view of the uncertainties in our knowledge of core-collapse mechanisms, several rates of reactivity addition over the range from 0 to $200 \% / \mathrm{sec}$ were considered. The distributions of power and material worth were taken from detailed neutronies calculations and were fairly flat over the core. Other significant parameters used in the calculations of energy relcase were:

$$
\begin{aligned}
& \text { Prompt-neutron lifetime }=10^{-7} \text { sec } \\
& \begin{aligned}
& \text { Threshold energy density in } \\
& \text { the threshold equation of } \\
& \text { state }=3.35 \text { kjoules } \mathrm{g}^{-1} \text { of } \\
& \text { oxide fuel }
\end{aligned} \\
& \begin{aligned}
\text { Initial peak power density } & =100 \mathrm{~W} \mathrm{~g}^{-1} \\
\text { Equivalent spherical core } & \\
\text { radius } & =65 \mathrm{~cm} \\
\text { Actual core mass } & =4 \text { tonnes of oxide fuel. }
\end{aligned}
\end{aligned}
$$

The results of these calculations are summarized in Table 1 which gives the peak values of the energy density, temperature, and pressure at the core centre, and also the total work done by adiabatic expansion of the whole core, expressed both in energy units and in the mass of TNT of equivalent work capability (1 lb $T N T \sim 2$ Mjoules). It must be stressed however that the comparison with TNT is very questionable when the peak pressure is only a few atmospheres, as in many cases with vapour pressure included in the equation of state. Also, when the total work is small, significant 
Table 1. Calculations of Energy Release for a Fast Power Reactor

\begin{tabular}{|c|c|c|c|c|c|c|c|c|c|c|c|}
\hline \multirow{3}{*}{$\begin{array}{l}\text { Reactivity } \\
\text { Ramp Rate, } \\
\% \mathrm{sec}^{-1}\end{array}$} & \multirow{3}{*}{$\begin{array}{c}\text { Doppler } \\
\text { Constant, } \%\end{array}$} & \multicolumn{5}{|c|}{ Vapour Pressure Law } & \multicolumn{5}{|c|}{ Threshold Pressure Law } \\
\hline & & \multicolumn{3}{|c|}{ Central Core } & \multicolumn{2}{|c|}{ Total Effective Energy } & \multicolumn{3}{|c|}{ Central Core } & \multicolumn{2}{|c|}{ Total Effective Energy } \\
\hline & & $\begin{array}{c}\text { Energy } \\
\text { Density, } \\
\text { kjoule } \\
\mathrm{g}^{-1}\end{array}$ & $\begin{array}{c}\text { Temper- } \\
\text { at ure, } \\
{ }^{\circ} \mathrm{K}\end{array}$ & $\begin{array}{c}\text { Pressure, } \\
\text { atm }\end{array}$ & Mjoule & lb TNT & $\begin{array}{c}\text { Energy } \\
\text { Density, } \\
\text { kjoule } \\
\mathrm{g}^{-1}\end{array}$ & $\begin{array}{l}\text { Temper- } \\
\text { ature, } \\
{ }^{\circ} \mathrm{K}\end{array}$ & $\begin{array}{c}\text { Pressure, } \\
\text { atm }\end{array}$ & Mjoule & Ib TNT \\
\hline \multirow[t]{5}{*}{25} & 0 & 3.52 & 7,790 & 3,850 & 1,375 & 725 & 4.58 & 12,900 & 31,100 & 558 & 294 \\
\hline & -0.05 & 235 & 5,600 & 342 & 116 & & 3.64 & 8,380 & 7,410 & 24 & 23 \\
\hline & -0.1 & 1.81 & 4,370 & 21 & 10 & & 3.60 & 8,180 & 6,360 & 15 & 8 \\
\hline & -0.2 & 1.71 & 4,140 & 10 & 4 & & 3.57 & 8,050 & 5,710 & 9 & 5 \\
\hline & -0.3 & 1.58 & 3,830 & 3 & 1 & & 3.57 & 8,020 & 5,550 & 8 & 4 \\
\hline \multirow[t]{7}{*}{50} & 0 & 4.11 & 10,600 & 19,100 & 1,955 & 1,030 & 4.96 & 14,700 & 40,600 & 877 & 462 \\
\hline & -0.05 & 3.00 & 7,090 & 2,200 & 676 & 356 & 4.07 & 10,400 & 18,100 & 122 & 64 \\
\hline & -0.1 & 1.99 & 4,780 & 64 & 32 & & 3.81 & 9,170 & 11,500 & 60 & 30 \\
\hline & -0.15 & 2.16 & 5,170 & 156 & 64 & & 3.59 & 8,130 & 6,120 & 13 & 7 \\
\hline & -0.2 & 1.90 & 4,560 & 37 & 19 & & 3.67 & 8,520 & 8,170 & 30 & 16 \\
\hline & -0.3 & 1.82 & 4,390 & 22 & 11 & & 3.60 & 8,140 & 6,160 & 13 & 7 \\
\hline & -0.4 & 1.70 & 4,120 & 9 & 4 & & 3.62 & 8,280 & 6,910 & 20 & 10 \\
\hline \multirow[t]{6}{*}{100} & 0 & 4.85 & 14,200 & 37,800 & 3,150 & 1,660 & 5.48 & 17,200 & 53,800 & 1,630 & 860 \\
\hline & -0.1 & 2.82 & 6,680 & 1,470 & 570 & 300 & 3.79 & 9,080 & 11,100 & 56 & 30 \\
\hline & -0.2 & 2.39 & 5,450 & 265 & 95 & & 3.71 & 8,670 & 8,960 & 37 & 20 \\
\hline & -0.3 & 190 & 4,560 & 36 & 19 & & 3.67 & 8,520 & 8,150 & 30 & 16 \\
\hline & -0.4 & 2.02 & 4,830 & 73 & 36 & & 3.65 & 8,410 & 7,610 & 26 & 14 \\
\hline & -0.5 & 1.64 & 3,960 & 5 & 2 & & 3.64 & 8,330 & 7,180 & 22 & 12 \\
\hline \multirow[t]{6}{*}{200} & 0 & 5.75 & 18,600 & 61,100 & 4,680 & 2,470 & 6.23 & 20,800 & 72,610 & 2,850 & 1,500 \\
\hline & -0.1 & 3.82 & 9,250 & 11,960 & 1,660 & 870 & 4.56 & 12,800 & 30,600 & 542 & 286 \\
\hline & -0.2 & 2.26 & 5,400 & 243 & 89 & & 4.02 & 10,200 & 16,800 & 106 & 56 \\
\hline & -0.3 & 2.64 & 6,300 & 900 & 290 & 152 & 3.68 & 8,560 & 8,380 & 32 & 17 \\
\hline & -0.4 & 2.14 & 5,120 & 139 & 59 & & 3.84 & 9,330 & 12,400 & 67 & 35 \\
\hline & -0.5 & 1.79 & 4,300 & 17 & 7 & & 3.76 & 8,940 & 10,350 & 49 & 26 \\
\hline
\end{tabular}

pressures are only generated near the core centre, and most of the fuel makes no contribution to the work done.

As the Doppler constant increases, the work done, which we propose to call the effective energy release, varies in the damped oscillatory manner which has been reported and explained in earlier papers. ${ }^{(4,8)} \mathrm{It}$ is clearly not realistic to rely on such a phase-sensitive mechanism to limit the explosive energy release, so the detailed calculations have been used to construct approximate upper-bound envelopes of the effective energy as a function of Doppler constant and reactivity ramp rate; results for the vapour pressure equation of state are shown in Fig. 4. Earlier calculations with a threshold-type equation of state showed that the effect of the Doppler coefficient on the effective energy release could be expressed in terms of a critical Doppler constant $\delta_{0}$ at which the effective energy first becomes relatively small, let us say an order of magnitude less than the value for $\delta=0$; a simple approximate analytic formula for $\delta_{0}$ is quoted by R. D. Smith et al. ${ }^{(8)}$ The present calculations have indicated that a similar con- cept is valid for the vapour pressure equation of state and that the same equation for $\delta_{0}$ still roughly holds. It would appear an adequate overall rule-of-thumb approximation to assert that the effective energy release is negligible if $(-\delta) \geqslant 2\left(-\delta_{0}\right)$.

To demonstrate more clearly the differences between the excursions with the two types of equation of state, a series of graphs have been drawn showing the time variation of particular parameters during the excursions produced by a reactivity ramp rate of $25 \% / \mathrm{sec}$. Figure 5 shows the excess reactivity; the vapour pressure causes a steady reduction in excess reactivity after the pressure becomes significant, whereas the threshold-type equation of state causes a much sharper reactivity reduction when the threshold energy density is reached. The effect on reactor power is very similar.

The temperature at the core centre is plotted as a function of time in Fig. 6. The temperature corresponding to the threshold energy is about $7,500^{\circ} \mathrm{K}$, and all "threshold" excursions must reach temperatures exceeding this value. In contrast, temperatures around 


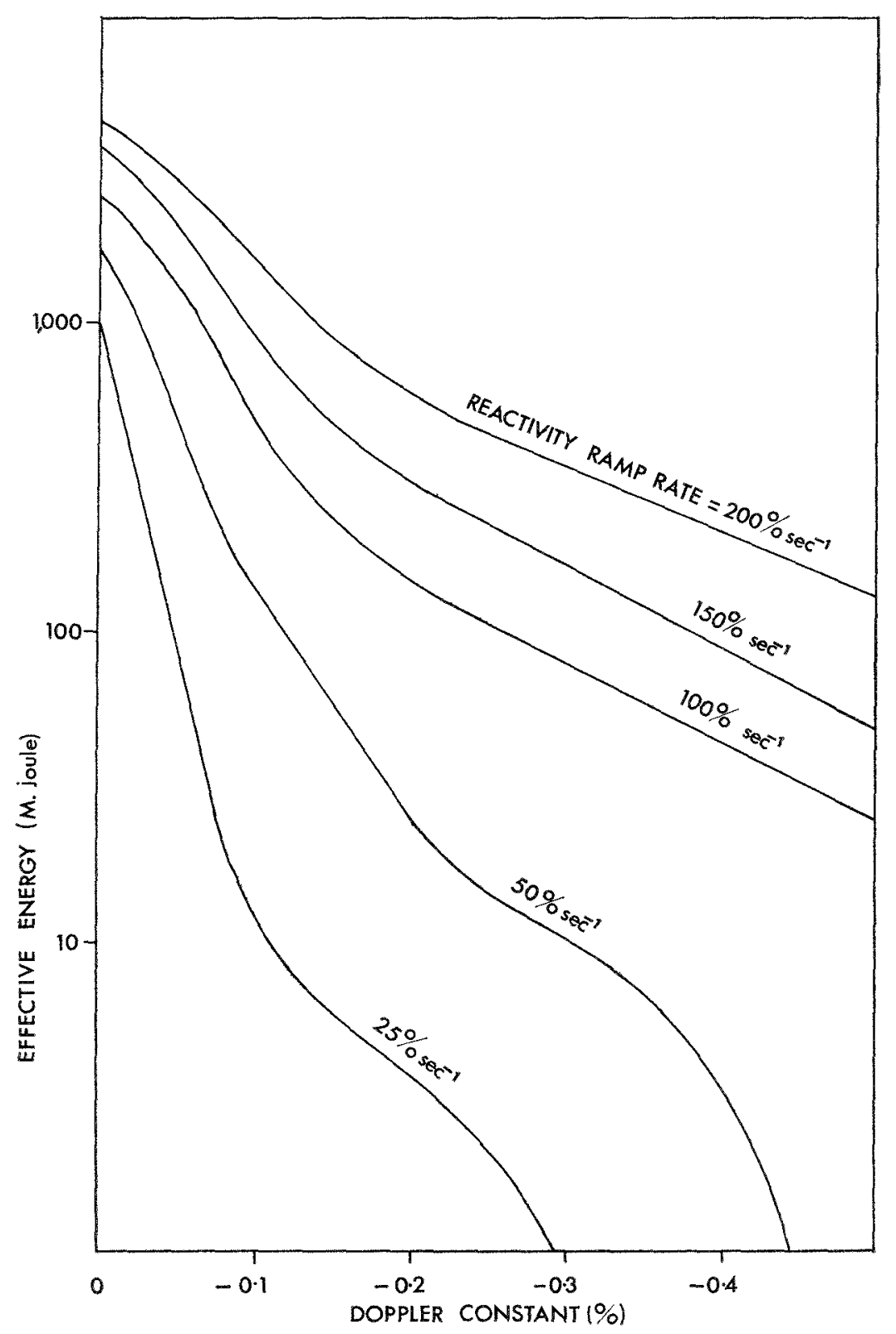

Fxg. 4. The Effective Energy Release.

$7,500^{\circ} \mathrm{K}$ only occur with a vapour pressure equation of state if the Doppler constant is small, and are generally much lower; in some cases the core barely reaches the boiling temperature of $\mathrm{UO}_{2}$. These relatively low temperatures are, of course, associated with correspondingly low core pressures.

The broad physical picture illuminated by these calculations would appear quite clear. The thresholdtype equation of state generally leads to core pressures which remain zero until appreciable excess reactivity has been built up and then increase rapidly; thus the core is dispersed and the excursion terminated by fairly large pressures acting for quite short times. In contrast, the vapour pressure becomes significant while the power is varying much less rapidly, and core dispersion is then due to much lower pressures acting for much longer times. The difference is particularly marked with a large Doppler constant, which increases the duration of the excursion and so allows greater time for a relatively low pressure to produce significant dispersion of the core.

\section{Autocatalytic Power Excursions in a Two-zone Core}

A super-prompt critical energy release in a fast reactor, of the type described by the Bethe-Tait theory, is 


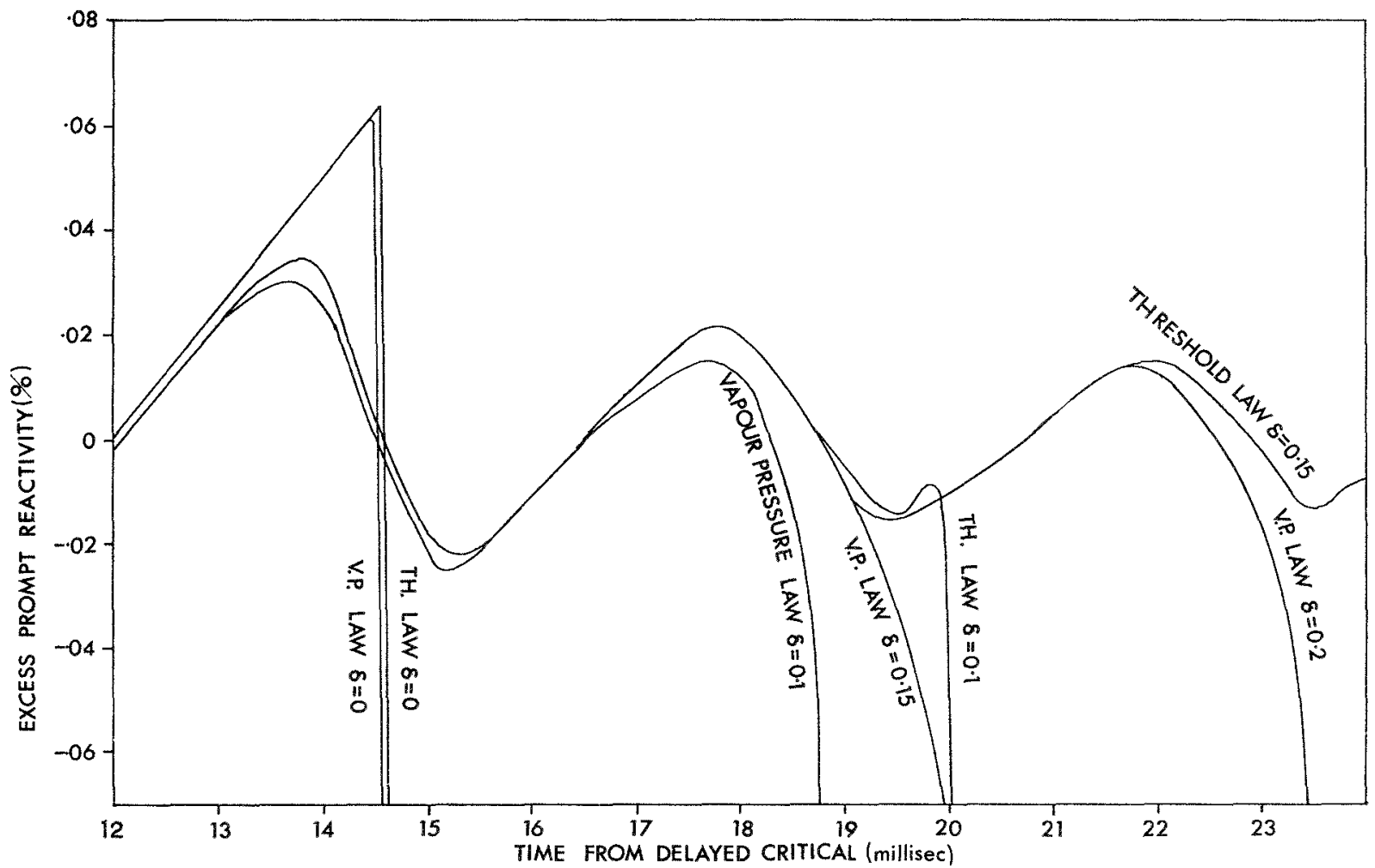

Frg. 5. The Excess Reactivity during Some Typical Power Excursions.

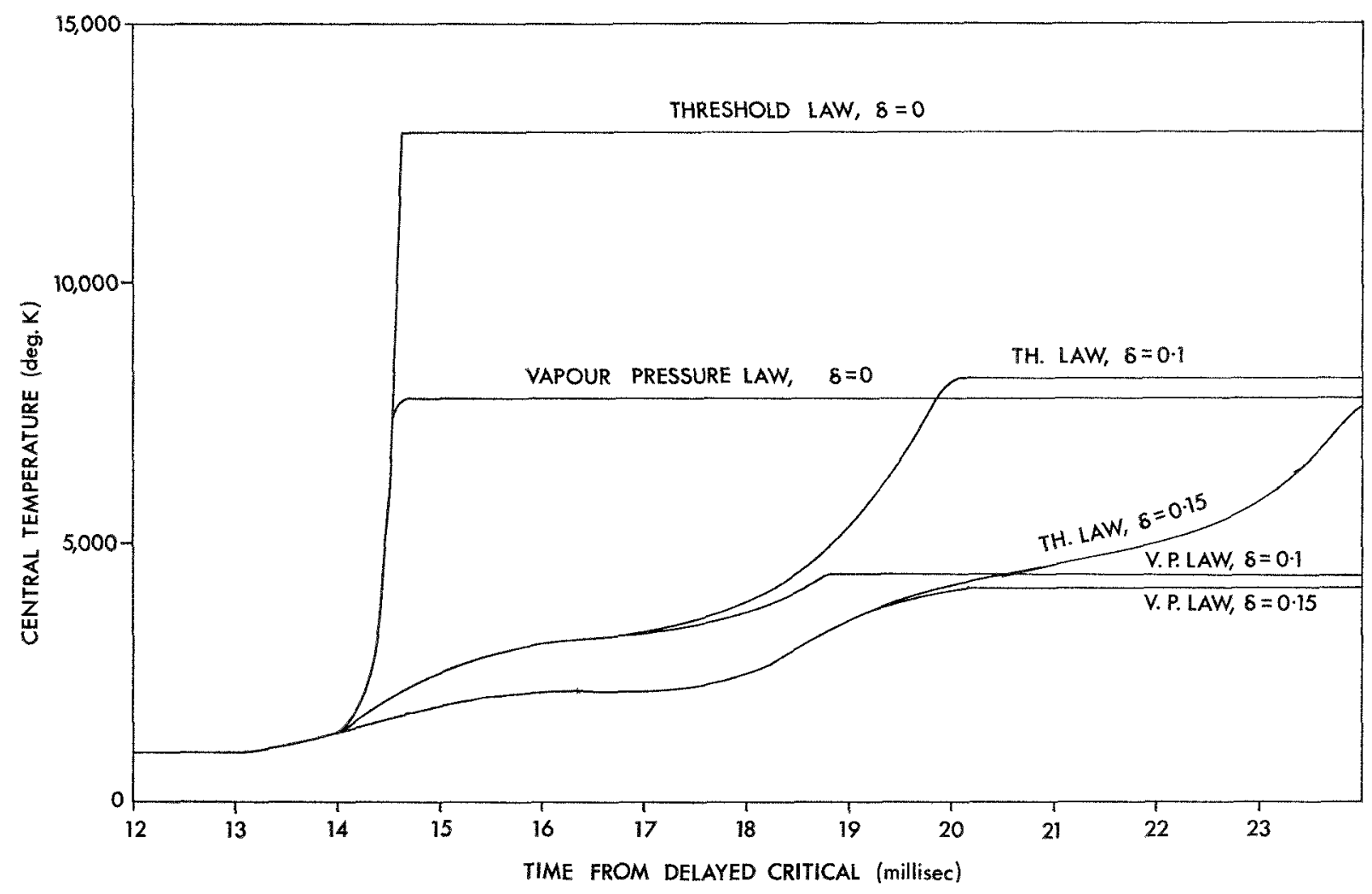

FIG. 6. The Core Central Temperature during Some Typical Power Excursions. 
terminated by the motion of the core material as a result of pressures created by the energy generation. In its simplest form, the theory assumes that this motion always tends to reduce reactivity, but the converse is physically possible in special circumstances. This effect, known as autocatalysis, could in principle greatly increase the energy released during the excursion.

Within a homogeneous region of the core, the pressure increases with energy generated and hence with the flux. Thus, except possibly near interzonal boundaries where additional considerations apply, the motion of the core material is down the flux gradient. In the onegroup approximation, the flux and neutron-importance distributions coincide so that motion down the flux gradient always tends to reduce reactivity. More generally, it is possible that importance can increase along directions of decreasing flux in limited parts of a core configuration, so giving a locally autocatalytic contribution to the total reactivity change.

A second autocatalytic effect can occur at the boundary between two core zones. Suppose that, at this boundary, both flux and importance decrease with increasing radial distance, but that the power density is discontinuous and is greater in the outer zone. Then the energy generation tends to cause a pressure discontinuity which relieves itself as a wave of compression spreading inwards through the inner zone and a wave of rarefaction spreading outwards through the outer zone. Both waves cause inwards motion of core material near the interzonal boundary, that is, in a direction opposed to the motion due to flux gradient.

In each type of autocatalysis, the autocatalytic effect is due to the motion of only a limited part of the core and is opposed by the bulk of the core motion. The net effect may thus be either to reduce or to increase reactivity. If the former, the transient will develop in a manner qualitatively similar to a normal Bethe-Tait excursion, and autocatalysis merely increases the energy release. If the latter, the excursion would continue with ever-increasing violence and would only be terminated by effects which are not at present taken into account in the calculation. In all cases computed to date, the overall effect of core motion has always been a reactivity reduction.

Calculations to investigate the autocatalytic effect due to the boundary between the two core zones of a fairly typical fast power reactor were based on a slightly modified version of the American digital computer programme ASTRA 417--3.0, in which the Doppler reactivity effect is not taken into account. The ASTRA programme calculates the energy release during a super-prompt critical excursion in a finite cylindrical assembly consisting of either one homogeneous zone or two zones of differing composition. For the present calculations, these zones were taken as the inner and outer core zones, that is, an inner solid cylinder and a surrounding annular cylinder of the same length. The flux and worth distributions in the core are supplied as input data to the programme, and can thus be derived from a calculation which allows correctly for the surrounding blanket material.

On the other hand, the neglect of the blanket from the ASTRA calculation implies that the dynamical boundary conditions at each core-blanket interface are not correctly simulated. The programme, in fact, allows the option of one of two simple boundary conditions at each core-blanket interface, and general considerations indicate that these alternatives usually bracket the correct condition. Moreover, it is found in practice that the choice of core-blanket boundary condition makes little difference to the energy release, thus justifying the approximation a posteriori. Similarly, the programme does not use the physically correct boundary conditions at the interface between the two core zones but allows the same option as at the other boundaries. In this case, however, the difference between the resulting energy releases may not be negligible, and is in fact a measure of the autocatalytic effect of the interzonal boundary.

Generalization of eq. (1) to a reactor composed of several homogeneous zones leads to the following expression for the reactivity change due to core motion:

$$
\begin{aligned}
d^{2} k / d t^{2}= & -\sum_{n} \iiint\left(\operatorname{grad} p \cdot \operatorname{grad} w_{n}\right) d V_{n} \\
= & -\sum_{n} \iiint\left(\operatorname{grad} P \cdot \operatorname{grad} w_{n}\right) d V_{n} \\
& \quad-\sum_{\imath, j} \iint p^{\prime}\left(\operatorname{grad}, w_{\imath}\right) d S_{\imath \jmath},
\end{aligned}
$$

where the volume integrals are summed over all zones $n$, and the surface integrals are evaluated and summed for all boundaries of every zone; grad, $w_{2}$ is the component of grad $w_{2}$ at a boundary in the direction normal to the boundary from zone $i$ to zone $j$. The ASTRA programme assumes that all surfaces are free, so that the total surface pressure is zero and $p^{\prime}$ is replaced by $-P$ in each surface integral. More accurately, the common interface pressure is given by

$$
p_{\imath \jmath}=\left(P_{\imath \rho_{\jmath}} c_{\jmath}+P_{\jmath} \rho_{\imath} c_{\imath}\right) /\left(\rho_{\imath} c_{\imath}+\rho_{\jmath} c_{\jmath}\right),
$$

where $\rho$ and $c$ are the densities and acoustic speeds, respectively, in the two regions. Thus, the contribution of the common surface $S_{\imath \jmath}$ of regions $i$ and $j$ to the rate of reactivity change is

$d^{2} k_{\imath \jmath} / d t^{2}=\iint\left[P_{\imath}\left(\operatorname{grad}_{\jmath} w_{\imath}\right)+P_{\jmath}\left(\operatorname{grad}_{\imath} w_{\jmath}\right)\right] d S_{\imath \jmath}$ 
in the ASTRA approximation, and

$$
\begin{aligned}
d^{2} k_{\imath \jmath} / d t^{2}=\iint & \left(P_{\imath}-P_{3}\right)\left[\rho_{\imath} c_{\imath}\left(\operatorname{grad}_{3} w_{\imath}\right)\right. \\
& \left.-\rho_{\jmath} c_{\jmath}\left(\operatorname{grad}_{\imath} w_{\jmath}\right)\right] d S_{\imath \jmath} /\left(\rho_{\imath} c_{\imath}+\rho_{\jmath} c_{\jmath}\right)
\end{aligned}
$$

exactly. These two expressions are equal if the gradient of the worth function is continuous across the interface, that is, if $\operatorname{grad}_{\imath} w_{\jmath}=-\operatorname{grad}_{1} w_{2}$, but in general they are different.

The only important difference between the two zones of a typical mixed oxide-fuelled fast power reactor is a greater plutonium enrichment in the outer zone, and thus the two zones have essentially the same density and acoustic velocity. The pressure in the outer zone, say $P_{\jmath}$, is greater than $P_{2}$. The worth distribution is such that $\operatorname{grad}_{2} w_{3}$ is positive and $\operatorname{grad}_{3} w_{2}$ is negative. Thus formulae (9) and (10) both give a positive contribution to $d^{2} k / d t^{2}$, that is, an autocatalytic effect. Moreover, if $\left(\operatorname{grad}_{2} w_{3}+\operatorname{grad}, w_{2}\right)$ is positive, it is easy to show that the ASTRA approximation gives the larger value, and so overestimates the autocatalytic effect; this was found to be the case in the calculation reported below.

As an illustration, we have calculated the energy release from a two-zone core, a central solid cylinder surrounded by an annulus of roughly equal volume, in the form of a cylinder of overall diameter of $56 \mathrm{in}$. and height, after partial core collapse, of $25 \mathrm{in}$. The composition of the core was taken to be: oxide fuel (density $11 \mathrm{~g} / \mathrm{cm}^{3}$ ) $35 \mathrm{v} / \mathrm{o}$, steel (density $8 \mathrm{~g} / \mathrm{cm}^{3}$ ) $25 \mathrm{v} / \mathrm{o}$, and void $40 \mathrm{v} / \mathrm{o}$. The plutonium fractions were about $18 \%$ and $23 \%$ in the inner and outer zones, respectively. Other parametric values used in the calculation were:

$$
\begin{array}{ll}
\text { Rate of reactivity addition } & =50 \% / \mathrm{sec} \\
\text { Prompt-neutron lifetime } & =5 \times 10^{-7} \mathrm{sec} \\
\text { Threshold encrgy density in } & \\
\text { the threshold equation of } & =2 \times 10^{10} \mathrm{erg} / \mathrm{g} \text { of } \\
\text { state } & \text { oxide fuel } \\
\text { Initial peak power density } & =750 \mathrm{~W} / \mathrm{g} \text { at prompt } \\
\text { critical } & \\
\text { Core mass } & =4 \text { tonnes oxide fuel. }
\end{array}
$$

The distributions of power generation and material worth throughout the core were determined by a multigroup calculation for the assumed cylindrical configuration of core and blankets.

The energy release during the power excursion was calculated by the ASTRA programme and converted to an equivalent mass of TNT as described above for the threshold equation of state. The results obtained were as follows:
Example
(a) Including all surface effects
(b) Excluding interface surface effecto

In both examples the outer surfaces of the core were treated as free surfaces; this neglect of the blankettamping effect reduces the explosive yield, but experience has shown that the effect is small in cores of similar size. Fixample (b) ignores the integrals over the interface between the two core zones in the expression for reactivity reduction, and may thus be considered as ignoring the autocatalytic effects of this boundary. Example (a) treats the interface as a free surface for each core zone and thus overestimates the autocatalytic effect, which therefore increases the explosive yield by no more than $20 \%$. It is considered that this result is indicative of the effect to be expected in any large fast reactor, namely, that this type of autocatalysis is unimportant.

\section{The Residual Energy}

It would seem quite clear from the calculations reported above and elsewhere ${ }^{(9)}$ that the immediate effective energy release will be small in a large fast power reactor, provided, of course, that the Doppler reactivity coefficient is of the order currently estimated. In contrast, the total energy release is always likely to be substantial, at least equal to the energy required to boil part of the oxide fuel, say $1 \mathrm{kjoule} / \mathrm{g}$. Thus the energy generated in a core containing 4 tonnes of fuel is at least about $4 \times 10^{3}$ Mjoule; if the whole of this energy could be converted into work in an explosive manner, the explosive effects would be comparable to those from the detonation of $2,000 \mathrm{lb}$ TNT.

One can visualize a mechanism which would allow part of the heat energy in the core debris to be converted into work. We suppose that the primary energy release is concentrated in the fuel and that the other core constituents, steel and residual sodium, remain relatively cool. The primary explosion may provide enough energy to disperse the fuel and increase the effective rate of heat transfer to the cooler materials, both in and surrounding the initial core. The cooler materials, in particular the sodium, then boil and the expanding vapour does work on the environment. If the fuel and sodium are sufficiently finely dispersed, the boiling process could produce pressures of explosive violence. The details of the process we have outlined are obviously very complicated, and we have only found it possible to make some simple calculations which illuminate a few particular points. The following discussion summarizes our work on three aspects of the overall problem which appear to be of general interest. First, how finely must the fuel and sodium be mixed to generate high 
pressures? Secondly, is such a degree of intermixing energetically possible? Thirdly, what fraction of the heat energy in the fuel is converted into work?

Consider a sphere of liquid sodium, radius $r_{0}$ and initial temperature $T_{0}$, surrounded by an infinite sea of liquid fuel at initial temperature $T_{1}>T_{0}$. It is straightforward to calculate the subsequent temperature distributions and heat flow rates due to thermal conduction, assuming that the system remains at rest, and it is found that the sodium temperature increases about $80-90 \%$ of the range $\left(T_{1}-T_{0}\right)$ in time

$$
t \sim \rho_{c} s_{c} r_{0}^{2} / 3 k_{f} \sim 10 r_{0}^{2} \mathrm{sec},
$$

where $\rho, s$, and $k$ are, respectively, the density, specific heat, and thermal conductivity of the sodium coolant (suffix $c$ ) and fuel (suffix $f$ ), and $r_{0}$ is in $\mathrm{cm}$; in other words, $10 r_{0}^{2}$ sec is a measure of the time scale of the process of heat transfer from the fuel to the sodium. The vapour pressure of sodium is quoted $\mathrm{as}^{(10)}$

$$
\log _{10} p(\mathrm{~atm})=4.521-\left(5,220 / T^{\circ} \mathrm{K}\right)
$$

up to $1,135^{\circ} \mathrm{C}$. Assuming that this relation continues to hold to much higher temperatures, we deduce vapour pressures up to about $1,000 \mathrm{~atm}$ at temperatures up to the boiling temperature of $\mathrm{UO}_{2}$. Thus pressures of this order are needed to prevent expansion of the sodium bubble; alternatively, pressures of this order will occur in the bubble if its expansion is small during time $10 r_{0}^{2}$ sec.

The rate of bubble expansion can be estimated from a simple theory due to Rayleigh. ${ }^{(11)}$ A sensible measure of the time scale of the expansion is the time taken to double the bubble radius, and is found to be about $10^{-3}$ $r_{0}$ sec for an average driving pressure of $100 \mathrm{~atm}$ within the bubble. Thus the dynamical time scale is less than the thermal time scale if $r_{0}$ is less than about 1 micron. In the case of sodium bubbles of submicron radius, an appreciable temperature, and hence pressure, increase could thus occur before there is any significant bubble expansion. The pressure created, of up to 1,000 atm, would then be relieved in an explosive manner. These pressures are rather lower than those associated with conventional explosives, such as TNT, and would probably cause mechanical damage to the reactor structure somewhat less than a TNT explosion of the same effective energy release. In contrast, bubbles of order, say, $1-\mathrm{mm}$ radius would merely boil vigorously at insignificant pressure.

The simple dynamical theory, which relates to the expansion of a single spherical bubble, can be extended rather elegantly to allow for interactions among a large number of bubbles. ${ }^{(12)}$ Consider a sphere of radius $R_{0}$ containing $\mathrm{N}$ sodium bubbles, each of radius $r_{0}$, in a sea of incompressible $\mathrm{UO}_{2}$ and surrounded by an effec- tively infinite volume of $\mathrm{CO}_{2}$. The expansion of each sodium bubble is governed by Rayleigh's equation

$$
p_{b}=p_{0}(t)+\rho\left(r_{0} \ddot{r}_{0}+1.5 \dot{r}_{0}^{2}\right),
$$

where $\rho$ is the density of $\mathrm{CO}_{2}, p_{b}$ is the (assumed constant) bubble pressure, and $p_{0}(t)$ is the pressure in the $\mathrm{UO}_{2}$ well away from a bubble; $p_{0}$ is determined by a similar equation governing the expansion of the sphere of radius $R_{0}$, namely,

$$
p_{0}(t)=P_{0}+\rho\left(R_{0} \ddot{R}_{0}+1.5 \dot{R}_{0}^{2}\right),
$$

where $P_{0}$ is the constant pressure at infinity. In addition the volume of $\mathrm{UO}_{2}$ in the sphere $R_{0}(t)$ is constant, so that

$$
R_{0}^{3}=N r_{0}^{3}+\text { constant. }
$$

It is straightforward to integrate these equations numerically and determine the time variation of bubble radius and of $\mathrm{UO}_{2}$ pressure. As is physically obvious, the bubble interaction increases the back pressure $p_{0}$ and so slows down the rate of bubble expansion. Typical calculations have shown that the effect is quite large and could, for example, reduce the rate of bubble expansion by about two orders of magnitude. Thus significant pressures can oceur with sodium bubbles of radius up to several tens of microns.

We visualize that the relatively small sodium bubbles needed to cause a secondary explosion are formed by fragmentation of more massive sodium by forces due to the primary explosion. This process increases the surface area and hence the surface energy of the sodium, but the increase is not large. For example, the surface energy of 1 tonne of sodium in the form of bubbles of 0.1-micron radius is only of the order $10 \mathrm{Mjoule,} \mathrm{equiva-}$ lent to an explosion of $5 \mathrm{lb}$ TNT. It would thus appear that more than adequate energy is available to fragment enough sodium, but this does not of course imply that large-scale fragmentation would actually occur. A realistic dynamical calculation does not appear possible at the present time.

An estimate, which is almost certainly pessimistic, of the thermal efficiency of the process of converting heat to work by sodium vapourization can be made on thermodynamic grounds. Suppose that a mass $m$ of sodium at absolute temperature $T_{c}$ mixes intimately with unit mass of fuel at absolute temperature $T_{f}$ and that thermodynamic equilibrium is established effectively instantaneously. Suppose that the mixture then begins to expand adiabatically, remaining in thermodynamic equilibrium throughout the expansion. At a general time during the expansion, let the mixture contain unit mass of fuel, mass $x$ of sodium vapour, and mass $(m-x)$ of liquid sodium, all at absolute temperature $T^{r}$ and pressure $p_{\imath}(T)$, the vapour pressure of 
sodium. The adiabatic relation ran be written down for a quite general equation of state, and calculations using a sodium equation of state derived from the principle of corresponding states are planned. However, the following calculations, based on a simple approximate twophase cquation of state for sodium, are thought to give at reasonably adequate representation of the correct results even though they predict temperatures and pressures which lie within the critical region.

Assuming that liquids are incompressible and of negligible sperific volume compared with the vapour phase, that sodium vapour behaves as a perfect gas, and that specific and latent heats are constant, the adiabatic relation for the fuel-sodium mixture is

$$
\left(m s_{l}+s_{j}\right)(d T / T)+L d(x / T)=0,
$$

where $s_{l}$ and $s_{f}$ are the specific heats of liquid sodium and fuel, respectively, and $L$ is the latent heat of evaporation of sodium; this integrates to

$$
L x / T=\left(m s_{l}+s_{f}\right) \ln \left(T_{m} / T\right),
$$

where $T_{m}$ is the initial equilibrium temperature, given by

$$
\mathrm{T}_{m}=\left(m s_{l} T_{c}+s_{f} T_{j}\right) /\left(m s_{l}+s_{f}\right) .
$$

Equation (15) determines the sodium vapour content of the mixture as a function of temperature. The work done during the adiabatic expansion is

$$
W=\left(m s_{l}+s_{f}\right)\left(T_{m}-T\right)-x(L-R T) .
$$

These results hold until $x=m$ at $T=T_{1}$, when all the sodium has been vapourized. During the further adiabatic expansion of the system, the appropriate adiabatic relation is

$$
m R \ln \left[p_{\imath}\left(T_{1}\right) / p\right]=\left(m s_{g p}+s_{j}\right) \ln \left(T_{1} / T\right),
$$

where $s_{g p}$ is the constant-pressure specific heat of sodium vapour, and the additional work done is

$$
W-W_{1}=\left(m s_{g v}+s_{f}\right)\left(T_{1}-T\right),
$$

where $s_{g}$ is the constant-volume specific heat.

Numerical results for a range of values of $m$ are given in Table 2 and I'ig. 7. Table 2 gives corresponding values of temperature, pressure, and mass $x$ of vapour, vapour volume $(x R T / p)$, and the work function $W$, all per unit mass of fuel. Figure 7 shows how the work done in an adiabatic expansion to several specified final pressures varies with $m$. Parametric values used in the calculations were:

$$
\begin{aligned}
T_{f} & =3,450^{\circ} \mathrm{K} ; & T_{c} & =1,150^{\circ} \mathrm{K} ; \\
s_{f} & =0.3 \text { joules } / \mathrm{g}^{-}{ }^{\circ} \mathrm{C} ; & s_{l} & =1.2 \text { joules } / \mathrm{g}-{ }^{\circ} \mathrm{C} ; \\
s_{g p} & =0.9 \text { joules } / \mathrm{g}^{\circ}{ }^{\circ} \mathrm{C} ; & R & =1 / 3 \text { joules } / \mathrm{g}-{ }^{\circ} \mathrm{C} ;
\end{aligned}
$$

$$
\begin{aligned}
& L=4,000 \text { joules } / g ; \quad P_{v}=A \exp (-B I T) \\
& A=4.85 \times 10^{5} \mathrm{lb} / \mathrm{in}^{2}, \\
& B=12,000^{\circ} \mathrm{K} \text { in the vapour prossure equation. }
\end{aligned}
$$

These values are not entirely consistent thermodynamially, but the discrepancies are not large and do not affect the broad conclusions to be drawn from the ralculations.

Figure 7 shows that, as $m$ increases, the work done first increases to a maximum and then decreases. The initial increase is due to the formation of an increasing volume of sodium vapour; the later decrease is due to the loss of energy in heating sodium which remains in liquid form. The maximum oceurs at a value of $m$ which increases with the final pressure from about 0.04 at $5,000 \mathrm{lb} /$ in. $^{2}$ to 0.10 at one atmosphere, that is, the maximum work is done when the fuel mixes with a slightly smaller volume of sodium. Secondly, it is apparent that a substantial part of the work is done at relatively low pressures, say, below $1,000 \mathrm{lb} / \mathrm{in} .{ }^{2}$. In contrast, the permanent gases produced by a chemical explosive perform relatively much less work, at low pressure, let us say typically only about $2.5 \%$ of the total at pressures below $1,000 \mathrm{lb} / \mathrm{in}^{2}{ }^{2}$. The total heat energy in the fuel is $s_{f} T_{f} \sim 10^{3}$ joule $/ \mathrm{g}$; thus the work done in an adiabatic expansion down to, say, 1,000 $\mathrm{lb} / \mathrm{in}^{2}$ is only about $10 \%$ of the total energy.

\section{The Equivalent Chemical Explosion}

The direct calculation of the effects of a hypothetical nuclear explosive release of energy on the reactor structure and its environment is practically impossible on account of the complicated geometry. It is, therefore, common practice to estimate these effects by means of experiments with scale models of the reactor and the use of conventional high explosives to simulate the nuclear explosion. We are not here concerned with the validity of the modelling technique for chemical explosives, so we assume that the experiments determine the effects of a chemical explosion on the full-scale reactor. The effects on the reactor of a given nuclear excursion can therefore be deduced if one can determine an "equivalent" size of conventional explosion.

Previously we briefly commented on one difference between a chemical explosion and a SPERT-type sodium-boiling incident. The following discumsion compares the chemical explosion with a nuclear excur-ion giving a fairly substantial primary effective encrgy release, say at least several hundred megajoules. Other calculations reported above have indicated the unlikelihood of such an excursion in a typical fast power reactor as currently conceived, but we considered the following comments were still of sufficient general interest to warrant inclusion in this survey. 


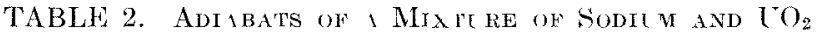

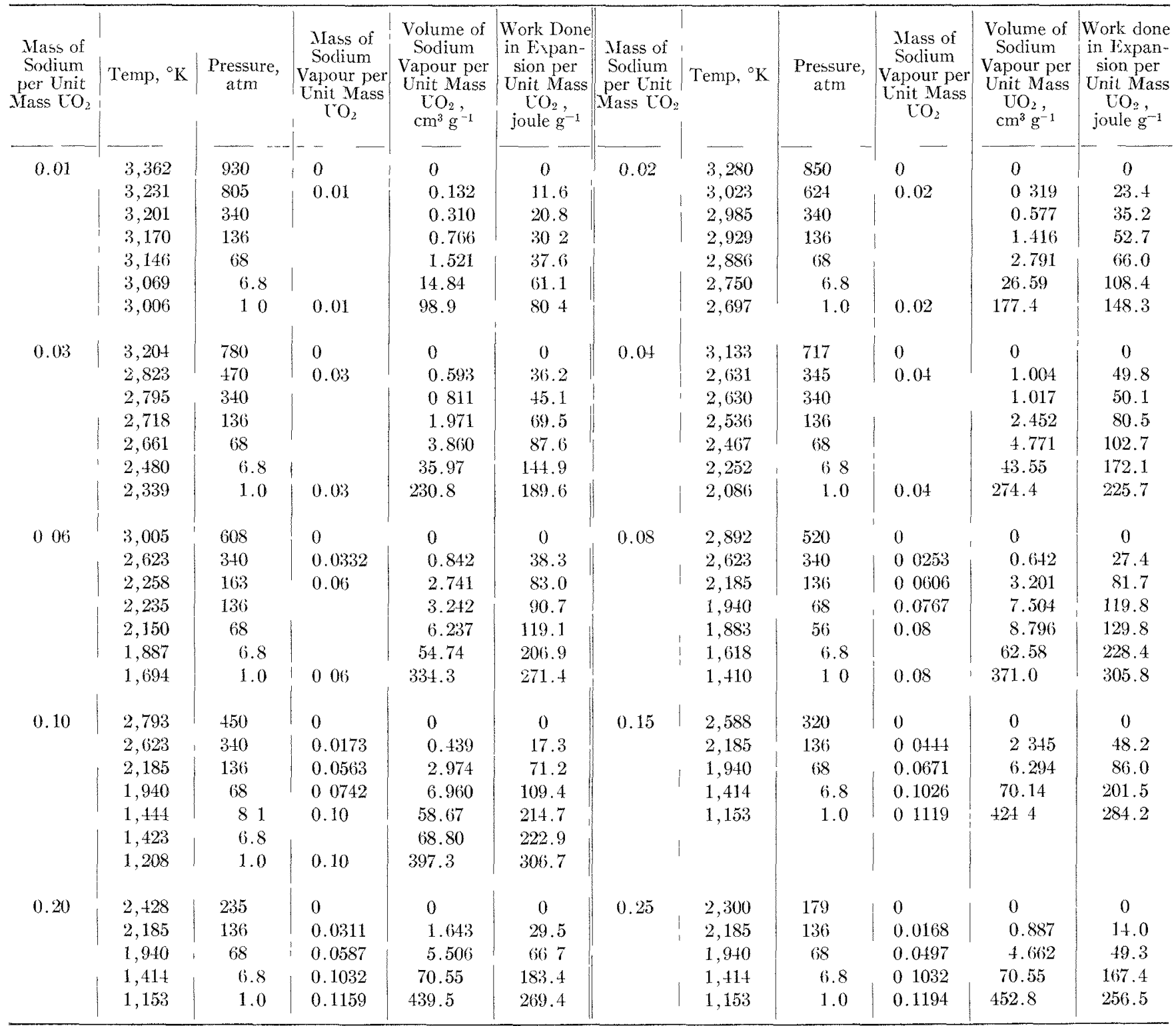

The concept of an equivalent charge of high explowive is at best only approximate. Even if it were possible to match exartly the effects at one point in the reactor, it is far from obvious that there would be similar matehing elsewhere. Moreover, matching in detail at even one point is not obviously possible since the response of a structure must in principle depend on the detailed time history of the applied load, which is unlikely to be completely matched. On the other hand, we are interested in practice mainly in broad effects, such as the energy absorbed by a particular structure, rather than in fine details. Fxperience with differing masses and types of rhemical explosive has shown that such broad effects correlate well with a few simple overall properties of the explosion, in particular the peak pressure and impulse of the blast wave, both of which depend largely on the energy released, the time scale of the release, and on the distance from the explosion. It has therefore appeared useful to compare and contrast the chemical and nuclear explosions in some detail, to try and assess the value of the concept of an equivalent charge of high explosive.

We first consider the effects of the explowion of a mass of chemical explosive, say several hundred lb TNT, placed at the position of the core in a geometrieal arrangement which is ot herwise the same as that of a large fast power reactor. The detonation of this explosive charge produces, in a time scale of order $10^{-4}$ sec, a mass of gascous products occupying essentially the ame volume as the solid explosive, at a temperature of 


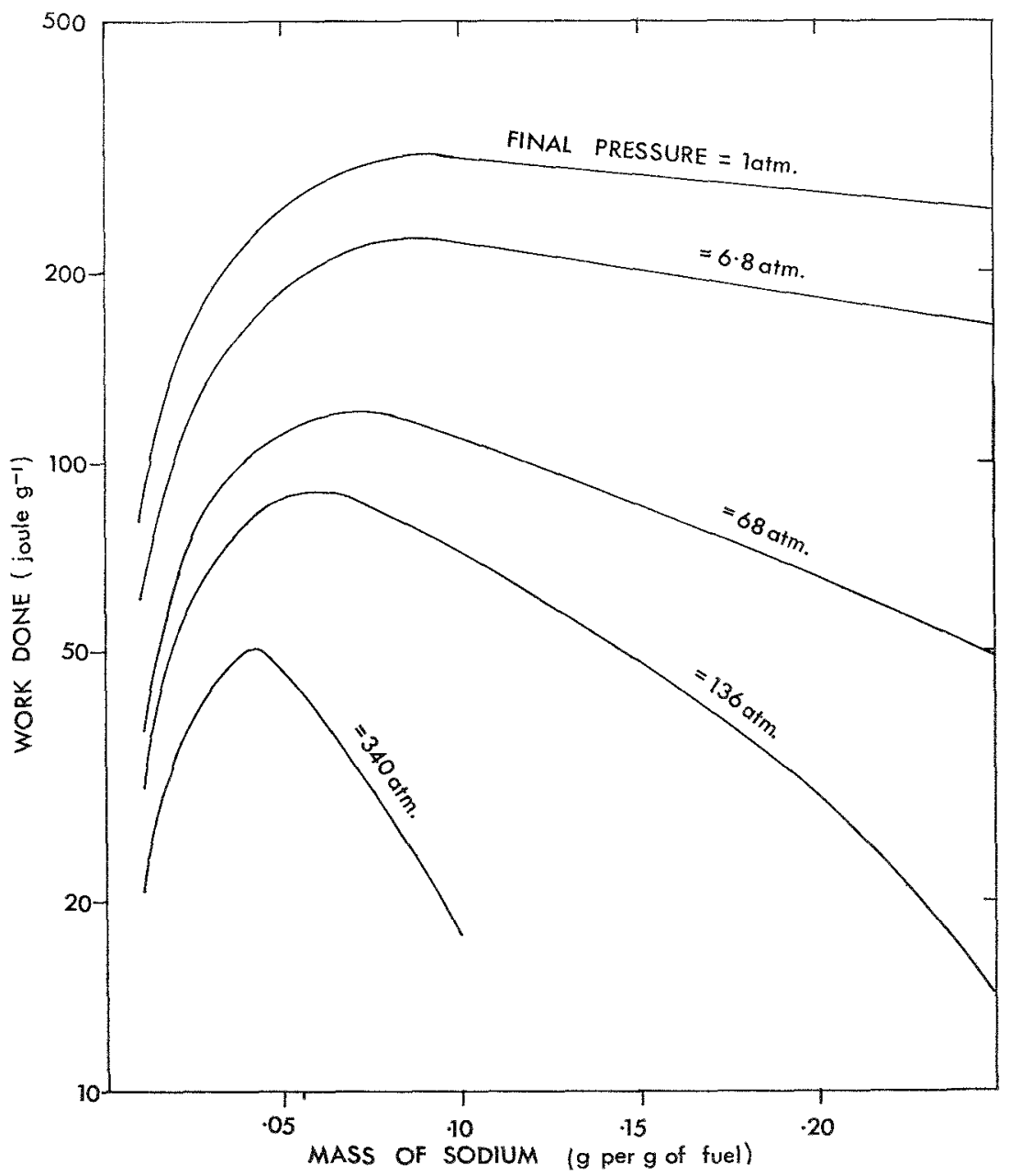

FIG. 7. The Work Done in a Spert-type Incident with Sodium and $\mathrm{UO}_{2}$.

the order of $3,000^{\circ} \mathrm{C}$, a peak pressure of the order of $10^{5} \mathrm{~atm}$, and in motion at velocities of the order of 5,000 $\mathrm{ft} / \mathrm{sec}$. It should be noted that the precise values, and particularly the particle velocity, depend on the detonation procedure and would, for example, be very different for central and surface points of detonation; this difference would be reflected in quite different primary shock waves close to the explosive surface. British model-scale tests use approximately central detonation of a right cylindrical explosive charge, which would seem a reasonable simulation of a rapid nuclear energy release spreading outwards from the core centre.

The mechanical interaction of the products of detonation with the surrounding medium creates a wave of compression headed by a shock which propagates out into the medium. Very close to the explosion, the properties of this wave (such as peak pressure and duration) are very dependent on the pressure and velocity of the detonation products. It is well known, for example, that explosives of the same energy content can differ in their ability to cause damage at very short ranges. At greater distances, however, this dependence on the detailed initial conditions dies out, and the strength and duration of the primary shock wave become ultimately dependent only on the total available energy. In our opinion, the pressure loading on, say, the roof of the reactor vault would not be very dependent on fine details of the energy release.

Whenever the shock wave meets an interface between different materials, part of the wave energy is transmitted, usually as a shock wave, and part reflected as either a shock or rarefaction wave. The wave energy is also steadily reduced by dissipation processes. The shock wave propagates at a velocity of $5,000-10,000$ $\mathrm{ft} / \mathrm{sec}$, and the linear dimension of the reactor vault is, say, $50 \mathrm{ft}$, so that the wave transit time is $5-10 \mathrm{~ms}$. Thus, after a time of, say, $0.1 \mathrm{sec}$, the original wave has been reflected at least tens of times from the multiple structural components within the vault. We think it probable that, by this stage, the shock-wave energy has very largely been dissipated as heat or as permanent structural damage. 
After the initial shock wave has been emitted, about $50 \%$ of the available energy remains in the bubble of high explosive gases. To fix orders of magnitude, consider a spherical bubble of gas, about $3 \mathrm{ft}$ in diameter, of density $1.5 \mathrm{~g} / \mathrm{cm}^{3}$, and weighing $1,000 \mathrm{lb}$. If the gas has an internal energy of 2,000 joules $/ g$, and assuming a perfect gas with $\gamma=1.25$, the bubble pressure would be about $7,500 \mathrm{~atm}$. The bubble temperature depends on the gas composition; for air it would be about $3,000^{\circ} \mathrm{K}$. Now suppose that the bubble expands adiabatically down to atmospheric pressure. The corresponding density would be $0.0012 \mathrm{~g} / \mathrm{cm}^{3}$, temperature, say, $500^{\circ} \mathrm{K}$, and internal energy 340 joules $/ \mathrm{g}$. The calculation has therefore shown that over $80 \%$ of the total energy (about $90 \%$ if the zero of energy is taken at $0^{\circ} \mathrm{C}$ instead of $0^{\circ} \mathrm{K}$ ) is available to do work on the surrounding media; corresponding results for TNT detonation products are similar. The expanded volume is about $13,400 \mathrm{ft}^{3}$, corresponding to a spherical diameter of about $30 \mathrm{ft}$. Thus, if the reactor vault remains intact, the bubble would not expand fully but would oscillate about an equilibrium size determined by the available free volume. For example, if the free volume is $2,000 \mathrm{ft}^{3}$, the equilibrium bubble pressure would be of order $150 \mathrm{lb} / \mathrm{in}^{2}$, if all heat losses are neglected.

The pressure in the medium surrounding the expanding bubble can be calculated for a deep underwater explosion of $1,000 \mathrm{lb}$ TNT and is quite large-for example, about 50 atm at a 15 -ft range when the bubble has expanded to about a 10-ft diameter. The problem is much more complex in a reactor structure on account of the complicated geometrical arrangement, the confinement, and the free surface of the coolant, but modelscale experiments have shown that pressures of hundreds of $\mathrm{lb} /$ in. $^{2}$ will persist for at least several tenths of a second. It seems likely that these pressures make a greater contribution than the shock waves to the total impulse on the vault roof, and so are the main cause of plug jump and perhaps also of failure of the roof itself. On the other hand, they would seem too small to cause damage to structural members which have survived the initial shock wave.

A fast reactor nuclear excursion, of the type described in the Bethe-Tait theory, is effectively a nuclear detonation spreading out from the core centre in a manner qualitatively similar to a centrally detonated chemical explosion. The nuclear excursion creates a volume of high-pressure, high-temperature fluid, quite analogous to the ball of gaseous detonation products formed by a chemical explosion. In typical Bethe-Tait calculations, assuming that the Doppler reactivity coefficient is small, the peak pressure is of the order of $10,000-50,000$ atm, and the particle velocity is estimated to be of the order of $10^{3} \mathrm{ft} / \mathrm{sec}^{2}$. The time scale of the bulk of the nuclear-energy generation is also quite comparable with that of the chemical explosion. We therefore consider that the shock-damage characteristics of the nuclear excursion are comparable with, although probably less severe than, those of an equivalent high explosive charge of the same energy, except perhaps very close to the point of detonation. It would seem obvious that the only energy which is physically relevant in this context is the energy available to do work, so that the realistic comparison is between effective energics rather than total energies.

The most useful basis of comparison between the bubble oscillation phases of the nuclear and chemical energy releases would appear to be the pressure-energy relationships during adiabatic expansion of the bubbles, and a typical comparison is illustrated in Fig. 8. This shows the work done as a function of bubble pressure during adiabatic expansions of bubbles simulating the detonation products of the two events. As discussed above, the adiabats of $\mathrm{CO}_{2}$ have been estimated from an equation of state deduced from the principle of corresponding states. The chemical products of combustion have been approximated by a perfect gas of initial density $1.5 \mathrm{~g} / \mathrm{cm}^{3}$, initial pressure about $7,500 \mathrm{~atm}$, and adiabatic constant $\gamma=1.25$. The work done during an adiabatic expansion down to atmospheric pressure is 1,000 Mjoule in each case.

In this particular example, the initial pressure is substantially lower in the nuclear detonation products, and Fig. 8 shows that much more work is done at lower pressures by the release of nuclear energy; for example, the conventional explosion does about twice as much work in expanding to a pressure of $1000 \mathrm{lb} / \mathrm{in} .{ }^{2}$. The corresponding effect would be less for larger onergy releases, but even greater than a factor of two for the smaller effective energy releases which seem more probable. To sum up, the present comparison appears to show that there is a sufficiently close similarity between the shock and bubble expansion phases of the two types of energy release to justify use of the concept of an equivalent chemical explosion defined on an equal work basis. In general, the nuclear excursion will generate somewhat smaller pressures and will therefore be a rather less effective explosion; however, the magnitude of the difference in damage will depend on the type of structure under consideration.

A few seconds after the initiation of either the chemical or the nuclear energy release, the heavily damped bubble oscillations will have effectively ceased, and we are then concerned with the residual heat energy of the debris, together with a quasi-steady residual pressure if the explosion is still contained. The most important differences between the two types of energy release seem likely to be associated with the dissipation of these 


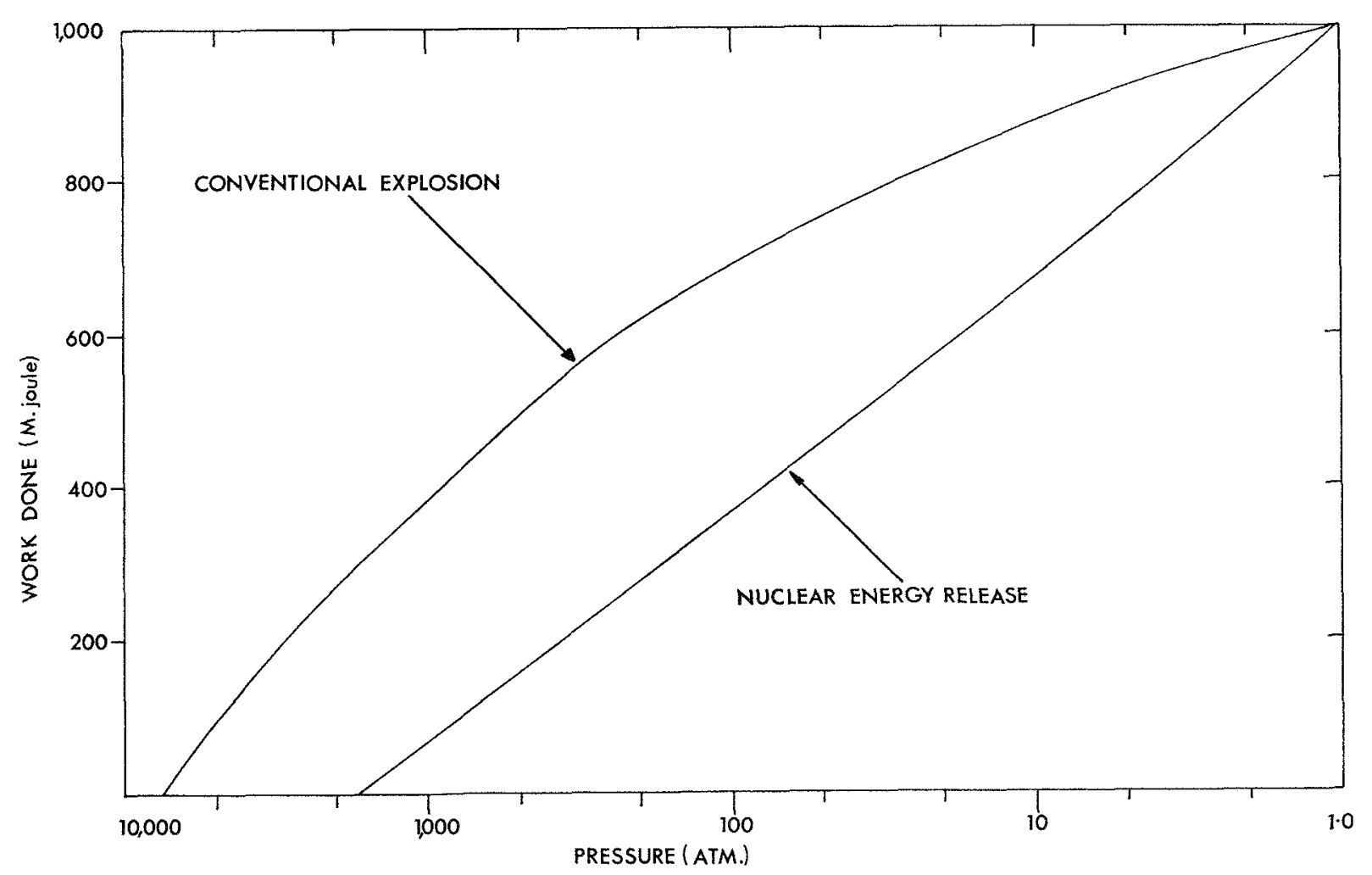

Fic. 8. Comparison between the Adiabatic Expansion of $\mathrm{CO}_{2}$ and Chemical Detonation Products.

residual pressures and temperatures. It is not the purpose of the present paper to discuss these longer time-scale phenomena in any detail. Rather, we propose merely to mention some of the factors which contribute to these differences.

In general, a very much larger proportion of the energy generated remains as residual heat in the nuclear debris, and the residual temperature of the nuclear debris is correspondingly higher. As discussed above, this allows the possibility of an additional explosive energy release as a result of sodium vapourization. In addition, continued sodium vapourization on a longer time scale may lead to further problems, such as an enhanced residual pressure in the reactor vault. The size of the increase will be determined by a complicated dynamic balance between the rates of sodium boiling at the hot core debris and of recondensation of vapour bubbles in the cooler bulk sodium. On an even longer time scale, a similar cycle of sodium boiling and recondensation will probably be necessary to remove the nuclear decay heat from the debris. These considerations indicate that the longer-term courses of events following the two types of energy release, and in particular the sizes of the residual pressures in the reactor vault, are affected by quite different phenomena, and that the concept of an equivalent chemieal explosion is no longer applicable.

\section{Concluding Remarks}

Estimates of the magnitude and consequences of hypothetical maximum accidents in a large fast power reactor were for many years dominated by calculations based on various modifications of the Bethe-Tait model. Such calculations are still an important part of the work but are no longer of dominating importance. This has resulted from an appreciation of the role played by the Doppler reactivity coefficient in limiting the Bethe-Tait energy release to such an extent that the pressures generated are insignificant in comparison with those due to a conventional chemical explosion. The emphasis has consequently shifted to a consideration of the fate of the energy generated as heat in solid and liquid fuel, and which may be partially converted into work, possibly in an explosive manner, by such processes as vapourization of liquid sodjum.

The possibility of such a secondary effective energy release cannot be ruled out on simple energy considerations because a quite small primary explosion, equivalent to perhaps only a few tens of $1 \mathrm{~b}$ TNT, would appear energetically able to bring the fuel and coolant into the necessary close contact. Fortunately, the explosive efficiency of the sodium-boiling process appears small. The ideal thermodynamic effieiency calculations show that no more than about $10 \%$ of the energy is converted into work at pressures of explosive magnitude, and heat 
transfer time lags will almost rertainly greatly reduce this value. On the other hand, the relatively large amounts of sodium vapour generated at lower pressures may be significant on a longer time scale, particularly in relation to the reartor vault pressure.

\section{REHETENCES}

1. MeCarthy, W. J., et al., Studies of Nuclear lccidents in Fast Power Reactors, Second Geneva Conference Paper $\mathrm{A} / \mathrm{CONF} .15 / \mathrm{P} / 2105$ (1958).

2. Fraser, A. R., and Taylor, J. B., I Version of the Bethe and Tait ifethod for 'alculating Fast Reactor 1ccidents. Appendix C to Morris, E., AWRE Report No. 0-61/62 (1962).

3. Nicholson, R. B., Methods for Determining the Energy Release in Hypothetical Fast Reactor Meltdown Accidents, Nucl. Sci. Eng., 18, 207 219 (1964).

4. Nicholson, R. B., Methods for Determining the Energy Release in Hypothetical Reactor Meltdown Accidents, API)A150 (1962).

5. Hougen, O. A., et al., Chemical Process Principles, Part II, Second Edition, John Wiley and Sons (1959).

6. Meyer, R. A., and Wolfe, B. E., High Temperature Equation of State of C'ranium Dioxide, Trans. ANs, (June 1964).

7. Jankus, V. Z., A Theoretical Study of Destructive Nuclear Bursts in Fast Power Reactors, ANL-6512 (1962).

8. Smith, R. D., et al., Fast Reactor Physics, including Results from U.K. Zero Power Reactors, Third Geneva Conference Paper A/CONF,28/A/166 (1964).

9. Wolfe, B., et al., Hypothetical Fast Cxide Reactor Veltdown Accident with Doppler Effect, Trans. ANS (Jume 1964).

10. Jackson, C. B., (ed), Liquid Vetals Handbook: SodiumNaK Supplement, TID-5277 (1955).

11. Lamb, H., Hydrodynamics, Six th Edition, Cambridge I"niversity Press, (1932), pp. 122-3.

12. Mather, I). J., unpublished.

\section{Discussion}

Mr. McCarthy (PRDC): You discussed one form of possible autoratalysis. Have you considered other cores, which are now getting to be very large in terms of surface area, and very, very thin? In the early days of the Bethe-Tait model, when cores were $2 \mathrm{ft}$ or so in diameter, one could relatively sensibly think of the whole core melting down at once. It seems to me much more likely that in these larger cores some smaller fraction of the core will melt down, some area of, say, a foot in diameter. Have you considered the possible influence on the unmelted part of the core of an explosion due to the melted part, say the compression of the fuel pins into a more compact geonetry in the unmelted section?

Mr. Hicks: In detail, no. There have been a number of speculations about the powibility of compressing parts of cores and producing secondary critical masses. Мy personal opinion is that it is so extremely unlikely as to be totally incredible.

Mr. Wolfe (GE): It appears necessary to assume that a large amount of sodium remains in the vessel and that although in principle one could generate the high pressures, one would expert that the sodium bubbles themselves would condense in much the same way that the vapor suppression type of containment condenses stean that goes through subsaturated liquid. Have you considered this?

Mr. Hicks: Partly. I think that if the sodium vapor bubbles (I'm thinking of the longer time scale now) are formed underneath a substantial depth of subcooled liquid sodium, they would unquestionably recondence as they rose to the surface. We've done a certain amount of calculations along those lines. But where the core debris is at the end of the primary explosion, I for one would not like to say. I don't see how one can be absolutely certain that the vapor is produeed with a substantial track in highly subcooled liquid before it comes out, let's say, in the cover gas.

Mr. Fenech (MITT): How did you establish the rate of reactivity insertion for the arcident, and on what basis have you determined the prompt reactivity at which the explosion would start?

Mr. Hicks: The usual approach of a Bethe-Tait type of uniform collapse under gravity is the basis of rate of reactivity addition, just to get an order of magnitude, and then one does calculations for a range of values around the estimated value. 


\title{
A Parameter Study of Large Fast Reactor Meltdown Accidents
}

\author{
R. A. MeYer, B. WOLFE, A\D X. F. HRLEDMA \\ General Electnc Company \\ A dvanced Products Operation \\ San Jose, Californa \\ (PRESENTEU BY B. WOLFE)
}

\section{ABsTRaCT}

The energy release from a fast reactor meltdown accident was examined to asses the importance of the various parameters involved. Modified Bethe-Tait calculations for a spherical 1000-1IWVe oxide-fueled reactor have been performed as a function of Doppler coefficient, neutron lifetime, pressure-energy relationship, core composition, power flattening, and initial starting conditions. Calculations have also been performed for a cylindrical version of the Bethe-Tait equations.

It is shown that with a large Doppler coefficient, one becomes relatively insensitive to the reactor parameters except for the heat capacity of the fuel, and that for reactivity inputs of the order of tens of dollars per second, a large but attainable Doppler effect can limit the explosive energy release to a magnitude for which containment has been engineered in presently operating fast reactors.

\section{Introduction}

The fast reactors which have been built in the United States to date have been designed to accommodate a maximum hypothetical accident which predicates gross melting and consequential compaction of the core. As a result of the reactivity introduced by the core compaction, the reactor is put on a prompt critical period which is finally terminated by explosive disassembly of the core. In the cases of Fermi and EBR-II, the explosive energy release was calculated, under pessimistic assumptions, to be of the order of $1000 \mathrm{MW}$-sec. Since the energy release for a given set of assumptions is roughly proportional to core mass, the possibility of a meltdown aceident poses a serious problem for the power reactors of the future which are expected to have more fuel in the core by over a factor of 10 than the present generation of experimental breeder reactors. In a paper presented at the last Argonne Conference ${ }^{(1)}$ and in subsequent papers, ${ }^{(2,3)}$ we have pointed out that the presence of a large Doppler effect can substantially reduce the energy release from a postulated meltdown accident. Similar conclusions have been reached by Nicholson ${ }^{(4)}$ and by smith et al.(5) Indeed, it should be pointed out that at the second Geneva Conference, Stratton et al. ${ }^{\left({ }^{(}\right)}$ showed that the energy release from a meltdown accident could be substantially reduced in the presence of a negative temperature coefficient. At the time, of course, it was not recognized that fast reactors could be designed with large negative Doppler coefficients and Stratton's work considered only prompt expansion coefficients. The basic mechanism is, however, the same.

In the present paper, the energy release from a meltdown accident is examined with reference to a 1000 MWe fast ceramic-fueled power reactor. The effect of the basic parameters on the energy release were examined. $N_{0}$ attempt was made to find a series of events which would lead to a given reactivity ramp; instead, we have assumed various reactivity insertion rates or reactivity steps, and have investigated the energy release as a function of the reactor parameters to assess the importance of these parameters and the sensitivity of the results to the assumptions.

The basic conclusion of the study is that if the Doppler coefficient is made large enough, the energy release from a large power reactor can be made of the same order of magnitude as that for which containment has already been engineered in the present generation of experimental reactors. It should be noted, however, that the price of containment may be high for a power reactor which is measured by economic performance. Although it is certainly possible to contain accidents releasing explosive energy of the order of $1000 \mathrm{MW}$-sec, the necessary holddown structures may make operations (especially refueling) cumbersome. Also, although the explosive disassembly of the core terminates the meltdown accident, one must still provide heat-removal equipment which will function reliably after the disassembly to remove tens of megawatts of decay heat. Finally, the need for a large Doppler coefficient may, itself. require a core design which penalizes economic performance.

This paper does not touch on these latter points, and the authors wish to state that this paper is not intended to imply that an accident ending in explosive disassembly of the core is a realistic consideration for 
a particular reactor design, or to imply a recommendation as to the safety philosophy which should be adopted.

\section{Description of the Calculations}

The calculations to be deseribed retain the essential assumptions of the Bethe-Tait formalism, namely, that the pressure is a function only of the energy density in the fuel, and that the reactivity effects of fuel movement can be calculated by perturbation techniques. However, instead of the linear pressure-energy density relationship above a threshold which was used in early Bethe-Tait work, a consistent equation of state has been derived by use of the principle of corresponding states. ${ }^{(7)}$ The derived equation of state relates temperatures, energy density, and pressure, and also permits a calculation of the arailable work (explosive energy) for a given energy density assuming an isentropic expansion. The method which was used to obtain these equation of state relations for $\mathrm{UO}_{2}$ is an extension of work originally performed by Brout. ${ }^{(8)}$ In the meltdown calculations, these relations were approximated by power series. The Doppler effect is included in the calculations, under the assumption that its temperature dependence is $1 / \mathrm{T}$, where $\mathrm{T}$ is the average fuel temperature in the core, i.e., no flux weighting was used. The reactor kinetics equations include the effects of delayed neutrons. Most of the calculations to be described involve a spherieal reactor core. However, we have also derived the BetheTait equations in cylindrical geometry and a number of calculations have been carried out for a cylindrically-shaped reactor core. The derivation of the equation of state and some of the methods used in the calculations have been deseribed previously. ${ }^{(1-3,7)}$ In this paper, attention will be directed primarily to the results of the calculations. A further, more detailed description of the calculational techniques is in preparation.

\section{Calculations for a 1000-MWe Spherical Reac- tor}

The reactor model chosen for these calculations is a spherical core with the same composition and same mass as that of the $1000-\mathrm{MWe}$ fast power reactor described in GEAP-4418. Its characteristics are as follows:

$\begin{array}{lc}\text { Material Composition: } & \\ \mathrm{CO}_{2}-\mathrm{PuO}_{2} & 22 \mathrm{v} / \mathrm{o} \\ \text { Steel } & 19 \mathrm{v} / \mathrm{o} \\ \text { Sodium } & 47 \mathrm{v} / \mathrm{o} \\ \text { Radius of equivalent sphere } & 113 \mathrm{~cm} \\ \text { Reflector savings } & 20 \mathrm{~cm} \\ \text { Neutron lifetime } & 5.7 \times 10^{7} \mathrm{sec} \\ \text { Peak-to-average power } & 171\end{array}$

In the calculations, it was assumed that the sodium was not in the core and that the void left by the sodium was available for fuel expansion. The equation of state for the $\mathrm{PuO}_{2}-\mathrm{UO}_{2}$ mixture was assumed to be the same as that of $\mathrm{UO}_{2}$ calculated on the basis of the law of corresponding states. ${ }^{(\tau)}$

In a given meltdown accident, the reactor is put on a fast period, and the power and energy density in the core rise until the point is reached at which there is a significant pressure buildup which tends to cause fuel movement and, ultimately, core disassembly and shutdown of the reactor. In the calculations it has been assumed that the clad is molten so that pressure tending toward fuel movement starts at the normal boiling point of the fuel. The course of the accident is completely determined if one gives the initial conditions at the time the boiling point of the fuel in the core is first reached. Prior to this time, it is assumed that the reactor behavior is described by the standard kinetics equations.

In order to gain an understanding of the behavior of the reactor in the disassembly process itself, a number of problems have been considered starting at the time that fuel boiled. In these problems (in order to start with conditions somewhat representative of a power excursion), the reactor was started from normal full power $(2200 \mathrm{MW}$ t) and a reactivity step or ramp was inserted, but no reactor feedback, i.e., Doppler effect, was included. The conditions existing at the time when the fuel first reached the boiling point were then used as the starting point for a number of parametric analyses described in the following sections.

\section{Influence of the Doppler Effect}

The curves on Figure 1 show the results of calculations in which various reactivity steps were inserted. The ordinate is the available work resulting from the excursion as a function of the Doppler coefficient. Since the Doppler coefficient $[T(d k / d T)]$ is assumed to vary as $1 / T$, the parameter $T(d k / d T)$ is a constant (referred to as $A_{\text {Dop }}$ throughout the paper). The results of Fig. 1 are consistent with previous analyses which have shown that a large Doppler coefficient can reduce the energy release by more than a factor of 10 . For calibration, it should be noted that the calculated Doppler coefficient for the $1000-\mathrm{MWe}$ reactor of GEAP-4418 is $A_{\text {Dop }}=-0.008$. Also, for calibration, it is worth noting that the reactivity steps (above prompt critical) of $0.001 \Delta k / k, 0.0005 \Delta k / k$, and $0.00025 \Delta k / k$ would be approximately equivalent to ramps of $45 \$ / \mathrm{sec}, 15 \$ / \mathrm{sec}$, and $6 \$ /$ sec in the absence of a Doppler coefficient.

The ability of the Doppler coefficient to reduce significantly the energy release during disassembly can be understood on the following basis. The pressure generated in the core ultimately shuts down the reac- 


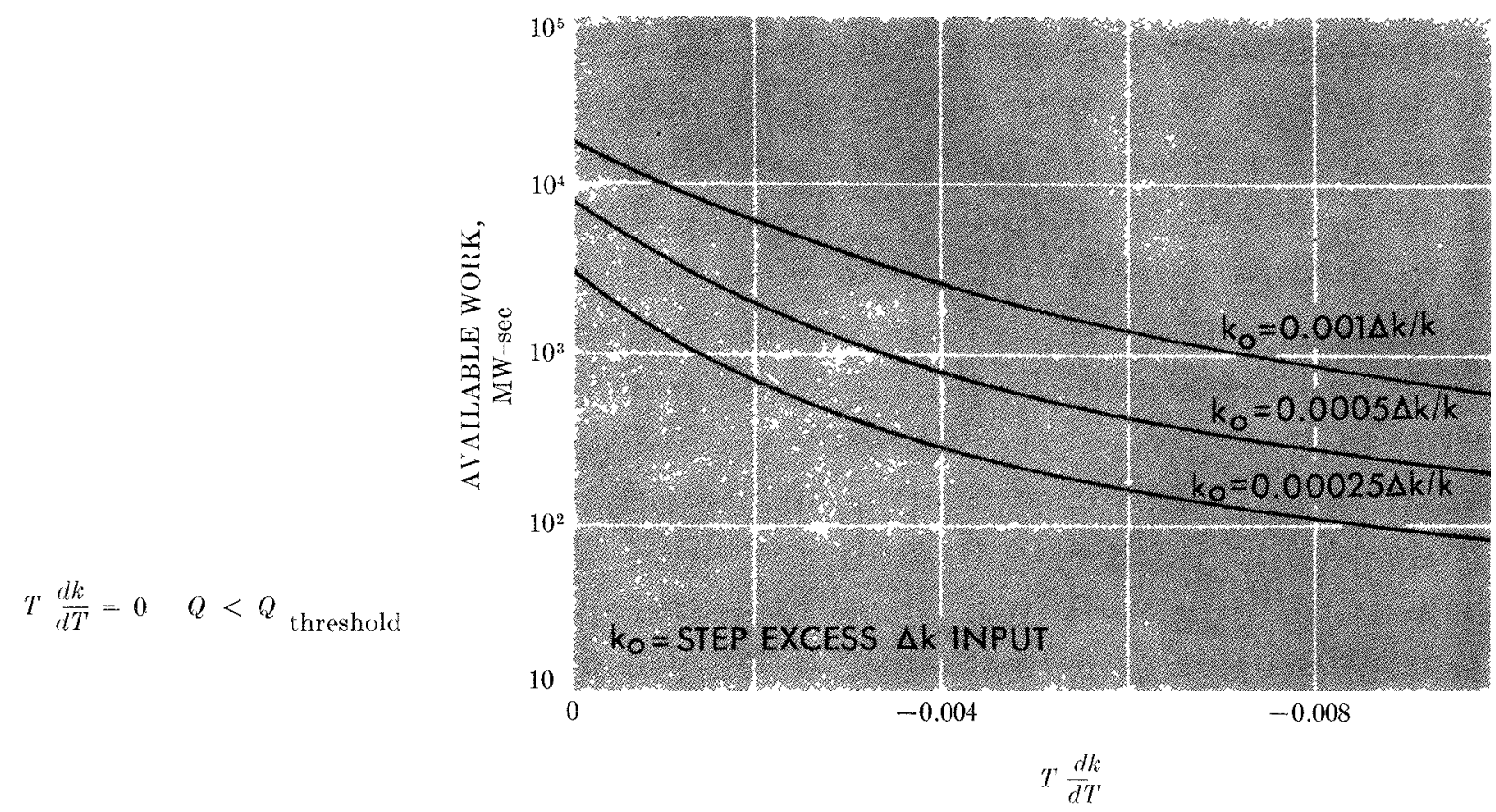

Fra. 1. Available work as a function of I)oppler coefficient $\left(A_{\text {nop }}\right)$ for various reactivity steps above prompt critical $\left(k_{0}\right)$. Excursions started from normal operating conditions (2200-MWt power) but without Doppler feedback until the peak temperature in the core reached the threshold for core disassembly. (Spherical meltdown model.)

tor. However, because of the inertia of the core, there is a significant time lapse between pressure buildup and core motion so that the power (and thus the energy density) continues to increase even after the pressure tends to move fuel out of the center of the core. On the other hand, when there is a strong Doppler effect, the transient is turned around and the power is reduced by the Doppler effect. This provides time for the core to be disassembled by relatively low pressure. This point is illustrated by Fig. 2, which is a plot of the reactivity feedback at the time that the power transient goes through its maximum. As can be seen in the case of zero or small Doppler coefficients, the major reactivity feedback at the time of the excursion turnaround is provided by the disassembly of the core. On the other hand, with a large Doppler coefficient, the major feedback is due to the Doppler coefficient, and the feedback due to the core disassembly occurs later in time.

\section{Effect of Neutron Lifetime}

For a given reactivity step, the period is decreased if the neutron lifetime is shortened. In this case, more energy will be generated during the period when the core is being disassembled. On the other hand, in the presence of a large Doppler effect, one would expect an insensitivity to the neutron lifetime since the reactivity feedback from Doppler depends only on the energy density in the core. That these speculations are, in fact, valid ean be seen from Fig. 3, where the available work is plotted for excursions initiated by a reactivity step at operating conditions as a function of the Doppler coefficient and the neutron lifetime. One notes that for $A_{\text {Dop }}$ of the order of -0.005 and above, the energy release becomes essentially independent of the neutron lifetime.

\section{Effect of Pressure-Energy Density Relationship}

Although considerable effort has been expended in deriving the equation of state and thermodynamically consistent equations have been derived, one must point out that both the experimental data underlying the work and the theoretical assumptions do not have a very firm basis. To check the sensitivity of the results to the pressure-energy density relationship, a number of calculations hare been performed in which the pressure-energy density relationship was arbitrarily varied. Again, one would expect that with the large Doppler coefficient, one might not be very sensitive to the pressure-energy density relationship. This point is made in Fig. 4 which shows the explosive energy release for a step excess reactivity input of $0.0005 \Delta k / k$ as a function of the Doppler coefficient for cases where the pressure-energy density relationship has been varied by factors of 10 . With a Doppler coefficient of $A_{\mathrm{Dop}}=-0.005$, the explosive energy release is only increased by $20 \%$ when the pressure is reduced by a factor of 100.

With zero Doppler coefficient, the energy release increases approximately inversely as the square root 


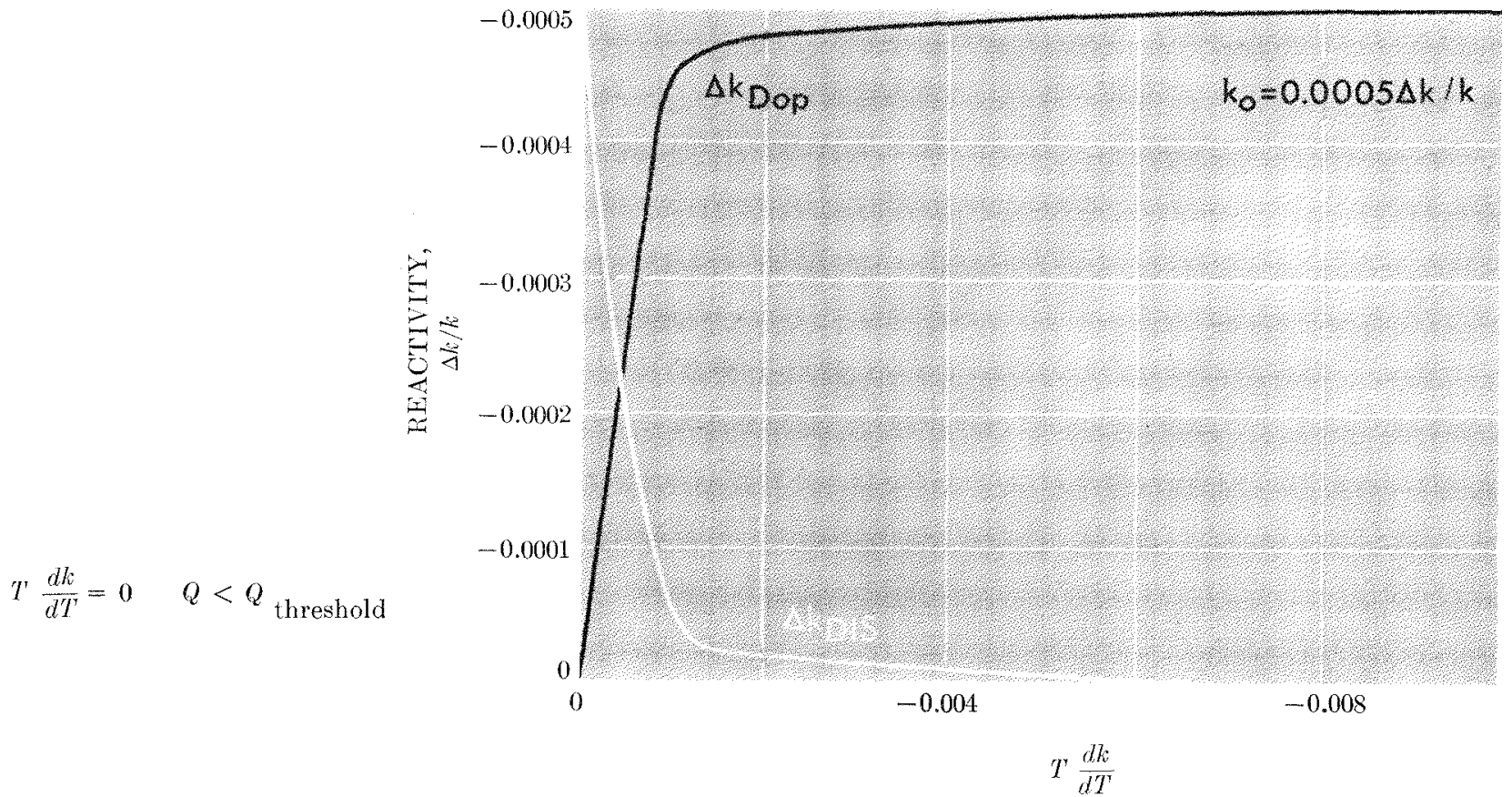

Fra. 2. Doppler and disassembly reactivity feedbacks at the turn-around point in an excursion for various values of $A_{\mathrm{D} o p}$ for the $0.0005-\Delta k / k$ step excess reactivity input shown in Fig. 1. (Spherical meltdown model.)

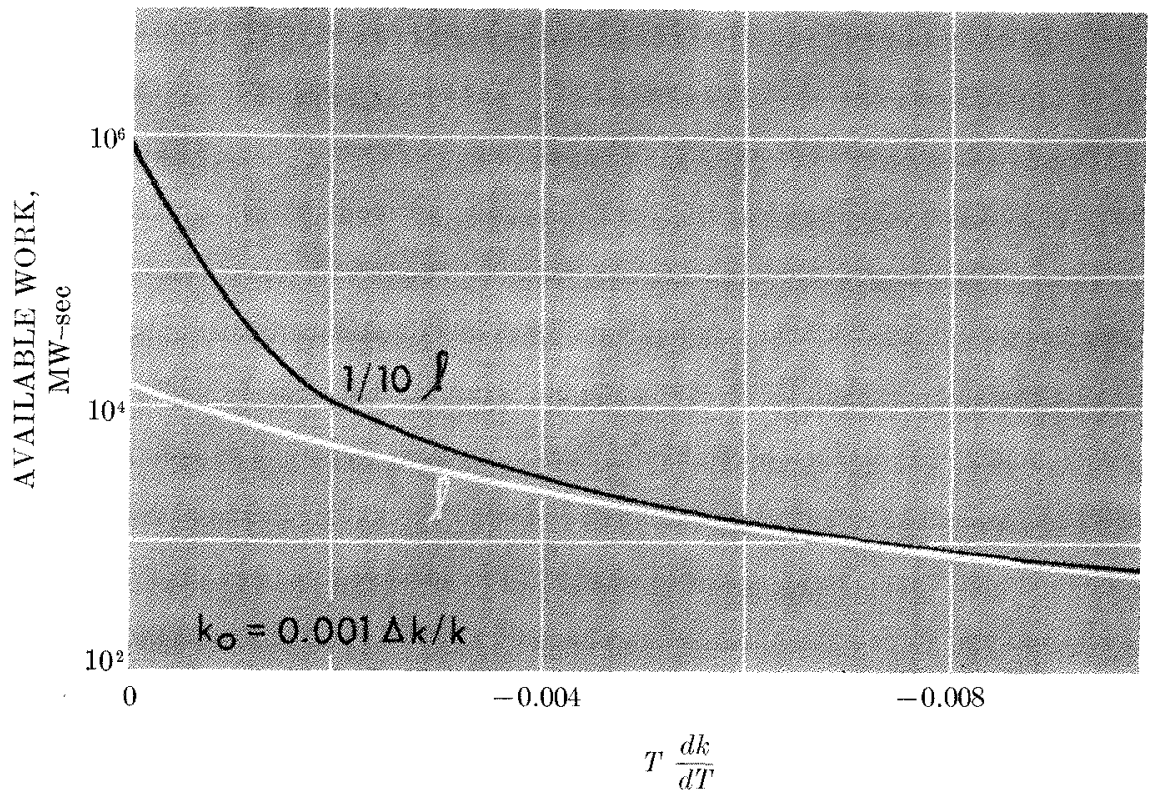

Fig. 3. Available work as a function of $A_{\text {Dop }}$ for excursions initiated by a $0.001-\Delta \mathrm{k} / k$ step excess reactivity input at operating conditions with normal neutron lifetime $\left(5.7 \times 10^{-7} \mathrm{sec}\right)$ and one-tenth lifetime. Doppler feedback was not included until the peak temperature in the core reached the threshold for core disassembly. (Spherical meltdown model.)

of the factor by which the pressure is reduced; reduction of pressure by a factor of 100 causes an increase somewhat less than a factor of 10 in explosive energy release. A brief discussion is in order, which we will find of use in a later discussion.

In the Bethe-Tait analysis, the disassembly reactivity can be written as

$$
\ddot{D}=\frac{1}{\rho} \int P\left(\nabla^{2} F\right) d V
$$

where $\ddot{D}$ is the second time derivative of the reactivity feedback $D$ due to disassembly, $P$ is the pressure at a point in the core, $\rho$ is the smeared density of the core material, and $F$ is a function which gives the impor- 


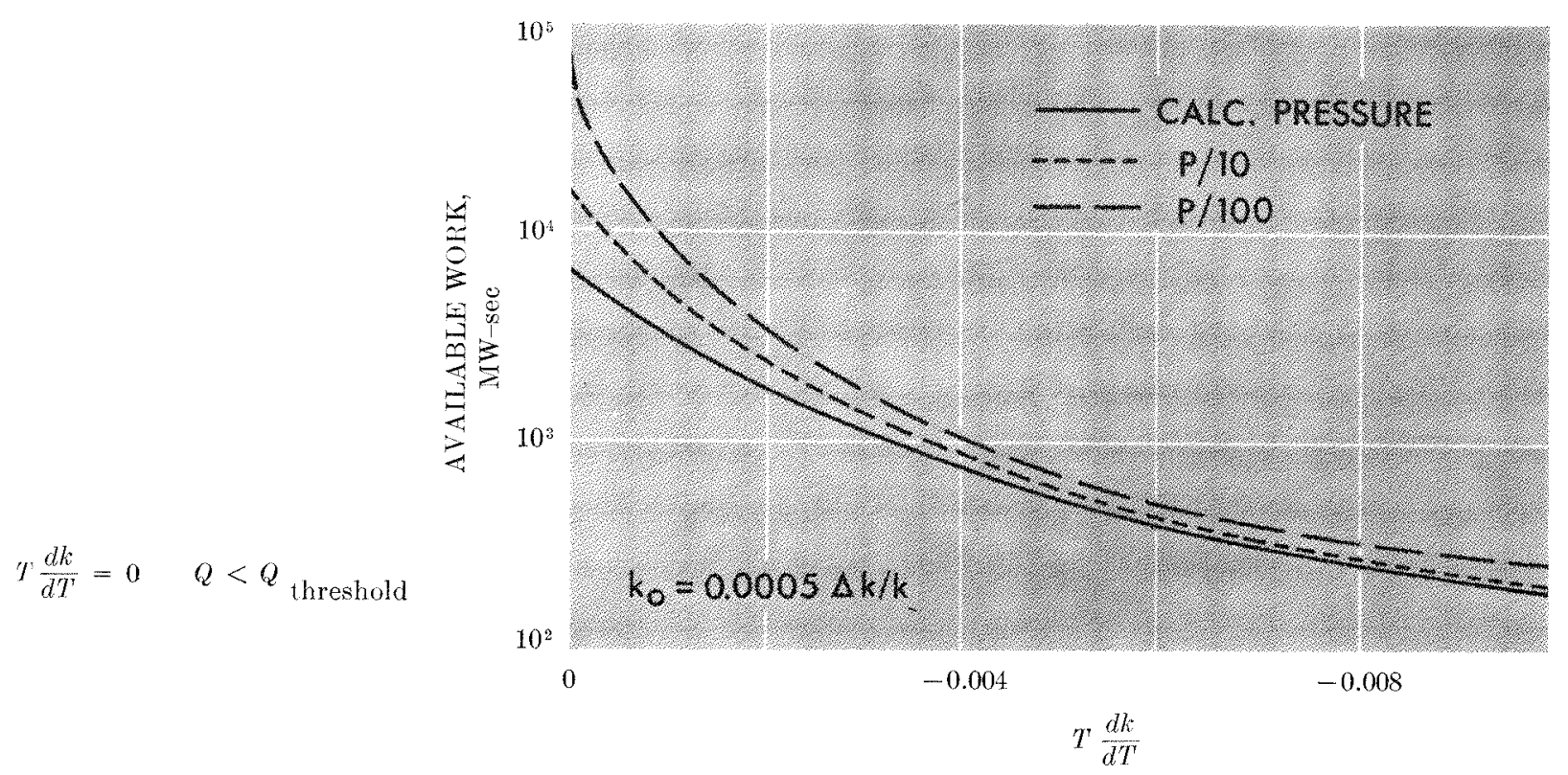

Frg. 4. Effect of the pressure-energy density relationship on the available work obtained from an excursion for various values of $A_{D \text { op }}$. Excursions initiated by a $0.0005-\Delta k / k$ step excess reactivity input at operating conditions with no Doppler feedback until the peak fuel temperatures reached the threshold for core disassembly. (Spherical meltdown model.)

tance of a unit of fuel as a function of core position. In addition to this term, there are normally included boundary terms. However, in the case of the large reactors considered here, the excursion is terminated well before the fuel at the boundary reaches the boiling point, so that these boundary terms do not enter into the calculations. (An alternative way of stating this is that where the boundary terms enter in, the accident is of such a magnitude that the possibility of containment is remote.) The previous results (see Fig. 4) were obtained by multiplying the integral on the right by a factor which reduces the pressure and therefore the disassembly reactivity feedback by factors of 10 .

If it is assumed for the moment that the pressure is constant in time, then a given reactivity feedback $D$ would be proportional to $t^{2}$, where $t$ is the time. Thus reduction of $P$ by a factor of 100 can be accommodated by an increase in $t$ by a factor of 10 . If the power were constant, this would result in an increase in the energy by a factor of 10 . Actually, during the transient the power is increasing with time, as is the pressure and the volume of the core where the pressure is greater than zero. Our results indicate that, despite this complicated behavior, the energy release does depend approximately inversely as the square root of the pressure; in fact, for small changes in pressure, the dependence is not quite this strong. Figure 5, plotted for pressure reductions of 2 and 5 , shows that a reduction of pressure by a factor of two increases the energy release by only about $30 \%$.

\section{The Effect of Power Flattening}

In the calculations discussed above, the power shape was assumed to be characteristic of a uniform spherical reactor, $226 \mathrm{~cm}$ in diameter, with an extrapolation length of $20 \mathrm{~cm}$. In order to increase the average specific power of the fuel in power reactors, it is sometimes proposed that either enrichment or compositions be varied so that the proper shape in the core is flattened. Such power flattening has an adverse effect on the energy release in a meltdown accident, i.e., it increases the energy release for a given accident. Figure 6 illustrates the physical reason for this. Shown is the power distribution during the excursion in the case where there is no power flattening. At the time when the core disassembly takes place, substantial pressures have built up in the central region of the core at the power peak; however, as indicated in the figure, the regions in the outer part of the core which make up most of the core mass have not reached the energy density characteristic of boiling. Thus, most of the core does not contain vaporized fuel which would generate explosive forces. On the other hand, when the core is flattened, a much larger percentage of the core will be in the vaporized state at the time that sufficient pressures have built up near the core center to cause disassembly of the core.

In principle, one would like very high pressures to be generated in a very small portion of the core in order to terminate the accident with a minimum amount of explosive energy release. Flattening the core does the 


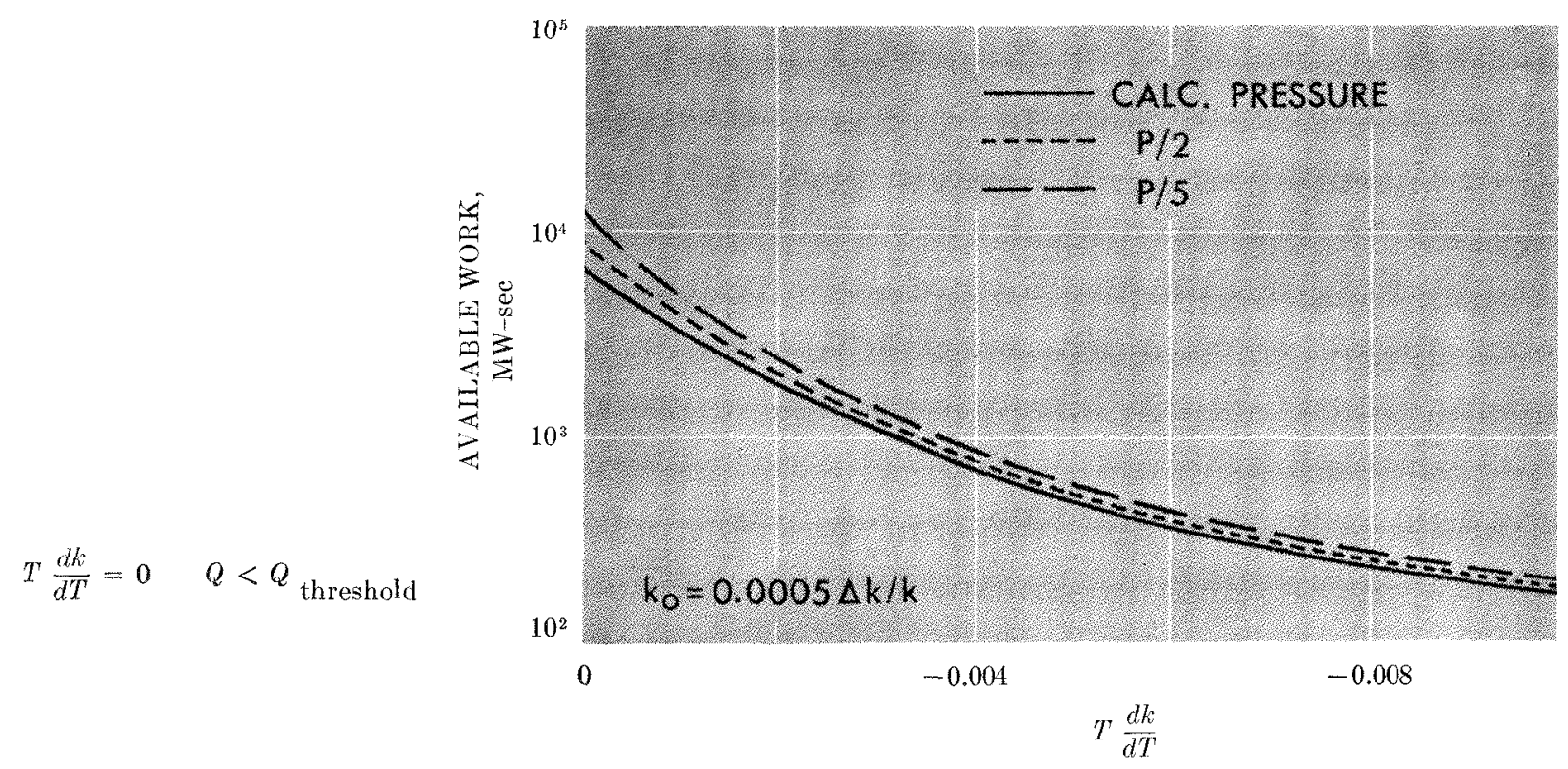

FIG. 5. Effect of the pressure-energy density relationship on the available work obtained from an excursion for various values of $A_{\text {Dop }}$. Excursions initiated by a $0.0005-\Delta k / k$ step excess reactivity input at operating conditions with no Doppler feedback until the peak fuel temperatures reached the threshold for core disassembly. (Spherical meltdown model.)

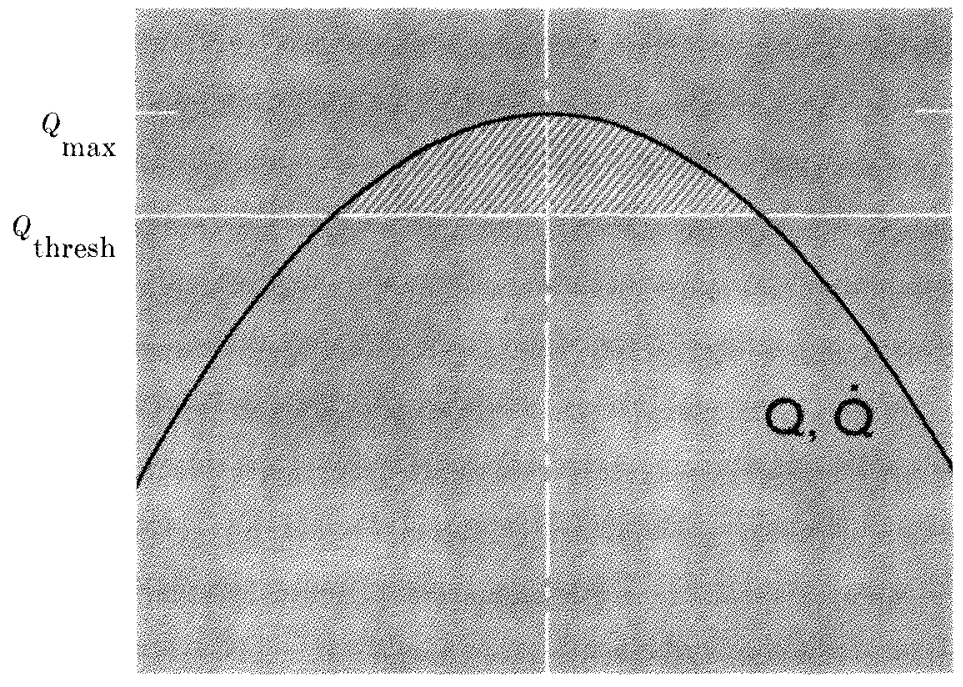

0

DISTANCE FROM CORE CENTER

FIc. 6. Typical energy-density distribution in a spherical core during an excursion terminated by core disassembly.

reverse of this. Figure 7 shows the results of calculations which were performed on cores with various assumed peak-to-average power distributions. It can be seen that decreasing the peak-to-average power from the unflattened value of 1.98 to a value of $1.33 \mathrm{in}$ creases the explosive energy release by a factor of 4 with no Doppler effect. Decreasing the peak-to-average further to a value of 1.03 increases the explosive yield by another factor of 9 . Further, it can be seen that the Doppler effect is not as effective in reducing the energy yield for the flattened core. In the unflattened case, the Doppler coefficient has the effect of reducing the power level while only a small part of the core is involved in the disassembly process. In the case of the flattened core, such a large portion of the core is already involved in the disassembly process that core disassembly occurs before the Doppler effect can exert much influence toward shutting down the reactor. In addition, the average temperature is higher in the flattened case during disassembly so that the Doppler feedback is lower for a given value of $A_{\text {Dop }}$.

It should be noted that the spherical case considered 
$10^{5}$

$$
P_{\max } / P_{\text {avg }}=1.03
$$

$T \frac{d k}{d T}=0 \quad Q<Q_{\text {threshold }}$

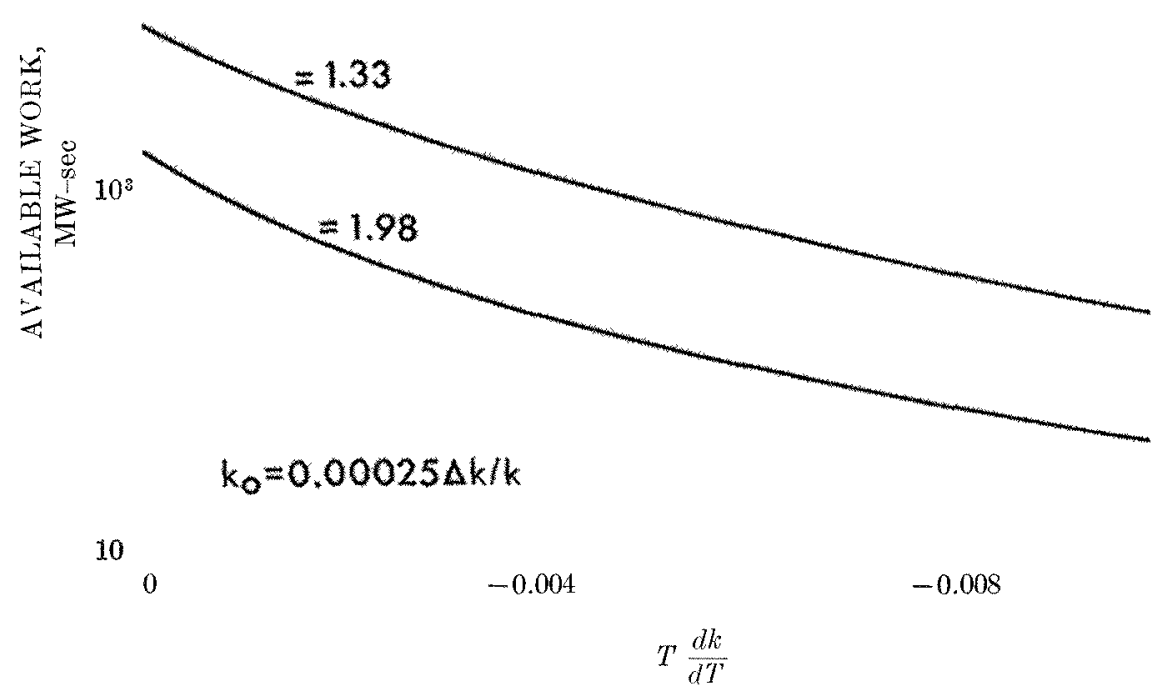

FIg. 7. Available work as a funetion of $A_{10 p}$ for varioun degrees of power flattening Excursions started from operating conditions but without Doppler feedback untl the peah temperature in the core reached the threshold for core disassembly.

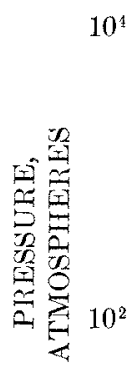
0

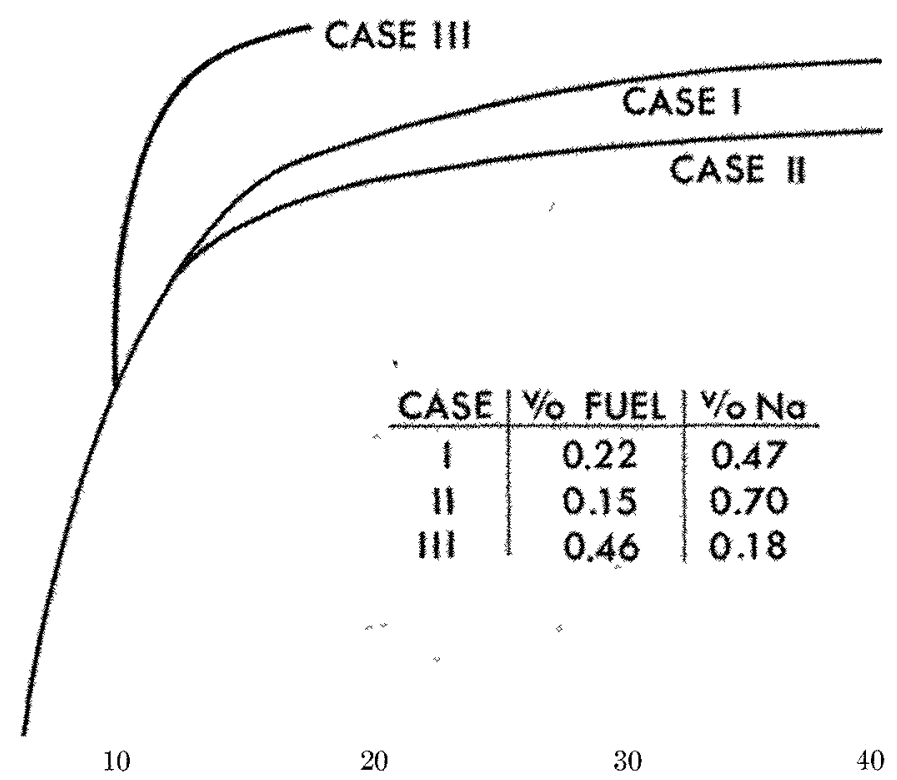

TEMPERATCRE, ${ }^{\circ} \mathrm{F} \times 10^{3}$

FI . 8. Pressure-temperature relation for three reactor core compositions. overpredicts the effect of flattening since it implies flattening in all three dimensions. In actual reactor design, it is normally the practice to flatten only in the radial direction and not to attempt power shaping in the axial direction. This point will be discussed later with reference to the calculations on the cylindrical core.

\section{Variation in Core Composition}

Equations of state were developed for several core compositions. The pressure-temperature relationship is shown in Fig. 8 for three cases. The first corresponds to the 1000-MWe reactor, as described above (GEAP. 4418 ), the second to a $1000-\mathrm{MWe}$ reactor of composition different than the one of GEAP-4418, and the 


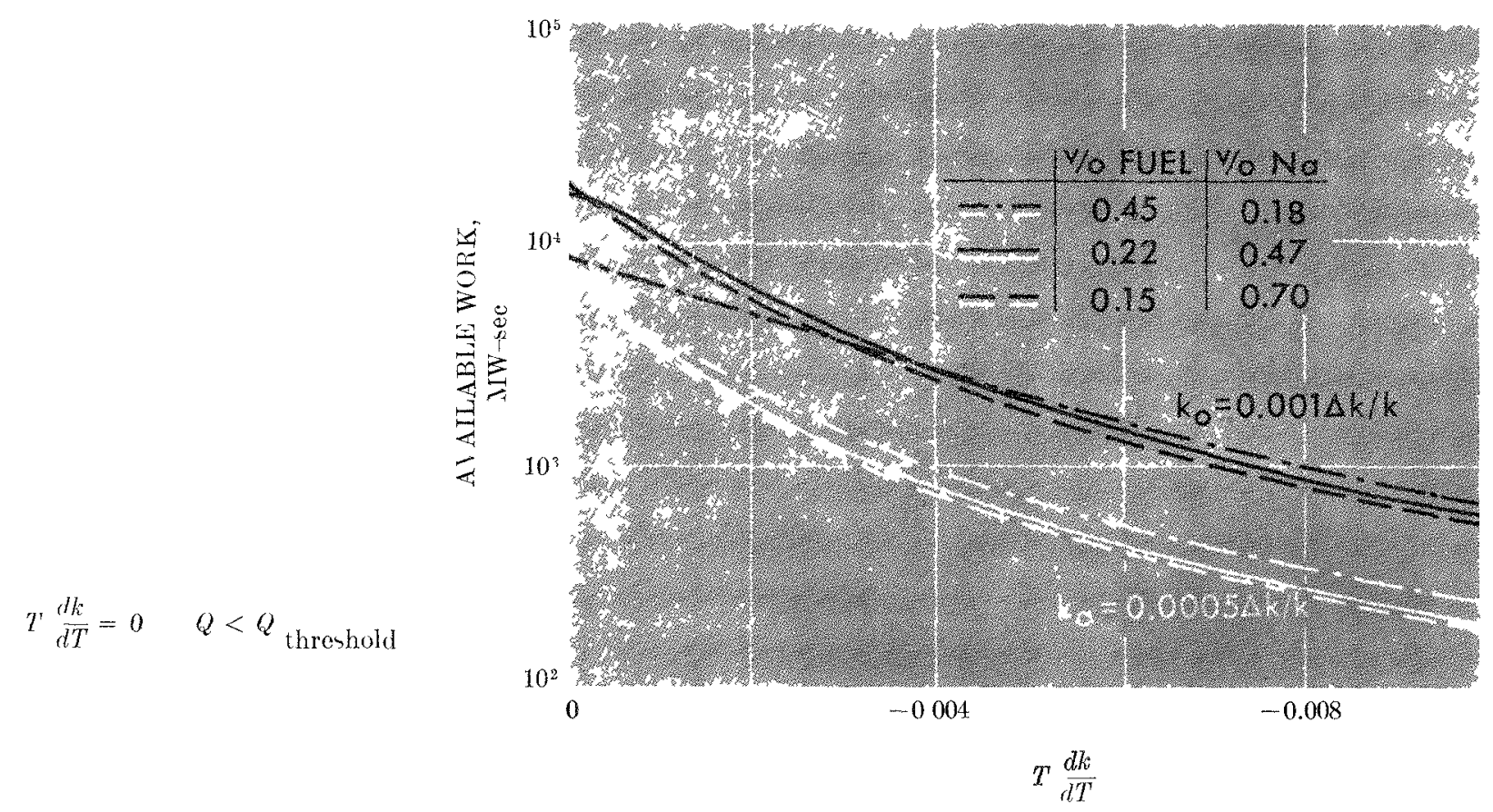

Fis 9 Avalable work a a function of $1_{\text {Inop }}$ for varous eativity btep al ove prompt critical for three reactors having duferent compositions but with equal fuel mass Excursions started from operating condition but without Dopplex feedback until the peak temperature in the core reached the threshold for core disansembly (Spherical melt dow n model)

third to the Southwest Experimental Fast Oxide Reaetor (SEFOR). The compositions for SEFOR and the alternate $1000-\mathrm{MWe}$ reactor are shown on Fig. 8. In all cases, the first part of the curve corresponds to the saturated vapor pressure of the fuel. The breakaway from the saturated vapor pressure curve corresponds to the situation when there is one phase which completely occupies the available space. It should be pointed out that the use of the SEFOR equation of state above the break point is questionable, since the sharp rise in pressure is due to hydrostatic forces of the liquid. Thus only a small increase in volume would relieve these pressures and cause the pressure to revert back to the saturation curve. In the SEFOR hazards analysis, therefore, we did not use the break in the curve, but assumed that the pressure continued along the saturation curve.

However, in order to gain some insight into the effect of the different equations of state, we have performed calculations with the three curves of Fig 8 . Figure 9 shows the result of these calculations. As one would expect from the previous discussion regarding the pressure-energy density relationship, the energy releare is not very sensitive to the equation of state. For low Doppler effect, one can see the effect of the sharp break in the SEFOR curve. On the other hand, the 1000-IWWe reactors have virtually the same energy release for this reactivity step because the peak temperatures did not reach the point at which the pressure-energy density curves for the two cases direrge. In performing these calculations, cores of equal mass were assumed. Thus the conclusion is that the energy release from a meltdown accident depends predominantly on the core mass and is relatively insensitive to the core composition for reactors using the same fuel material.

\section{Cylindrical Reactors}

The equations for the reactrity effect of disassembly of the core have been derived in cylindrical geometry by mean of the Bethe-Tait approximations, with the additional assumption that the fux is separable in the radial and axial directions. The derivation of the mathematical expressions will be described in detail in a paper now under preparation.

\section{Reactors of Various Herght-to-Diameter Ratios}

One would expect that a cylindrical core with a drameter-to-height $(\mathrm{D} / \mathrm{H})$ ratio of 1 would give result similar to that for a spherical reactor with the same core volume As a check on the cylindrical derivations, calculations were performed comparing a spherical reactor and a cylindrical reactor with $\mathrm{D} / \mathrm{H}$ $=1$. These calculations show good agreement betwcen the two models. One example of such a comparison is shown in Figure 11 to be discussed shortly.

One might also expect that where the Doppler effect is controlling, the energy release from a pancaked 


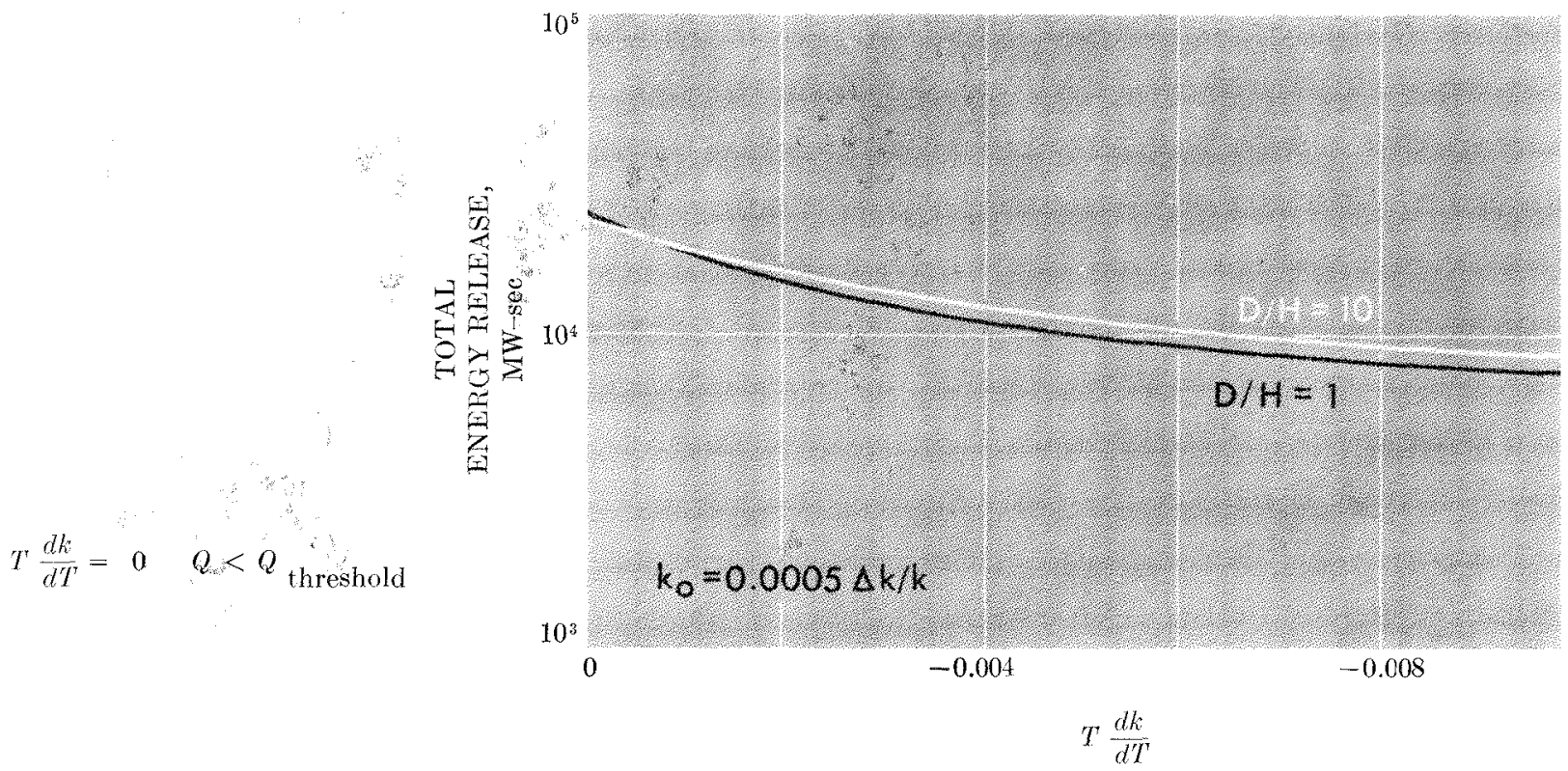

Fic. 10. Total energy release as a function of $A_{\text {Dop }}$ for various degrees of pancaking calculated with the cylindrical meltdown model. Excursions started from operating conditions but without Doppler feedback until the peak temperature in the core reached the threshold for core disassembly.

cylindrical core would be little different from that of a spherical core of the same mass. That this is so is shown in Figure 10, which compares the total energy of an excursion for cylindrical cores of the same mass with various $\mathrm{D} / \mathrm{H}$ ratios. One can see that for high Doppler coefficients, the total energy is very close. What is surprising is that the energy release is also very close in the case in which the Doppler coefficient is zero. In order to explain this rather unexpected result, we have looked at the disassembly equation and compared the value of $\nabla^{2} F$ at the core center for the cases in which $\mathrm{D} / \mathrm{H}=1$ and $\mathrm{D} / \mathrm{H}=10$. It turned out that there is only about a $30 \%$ difference in these two cases, with that for the $\mathrm{D} / \mathrm{H}=10$ being the larger.

In the previous discussion of the effect of reducing the pressure-energy density relation by large factors, we concluded that the energy release was relatively insensitive to a constant factor multiplying the disassembly equation. Thus the insensitivity to core shape can be explained from the mathematics involved. Physically, what appears to be the explanation is that the pressure and the reactivity worth function $\mathrm{F}$ are both spread out spatially by changes in core geometry such that the effect on reactivity by motion of the fuel caused by the pressure buildup is relatively insensitive to the core geometry.

\section{Power Flattering in the Cylindrical Geometry}

Figure 11 shows the total energy release for cases in which the core power distribution has been flattened in the radial direction. This is to be compared to the power-flattening calculations previously performed for the spherical core. As one would expect, the adverse effect of power flattening is not as great in the cylindrical case as in the spherical case, since in the cylindrical case the peak-to-average ratio in the axial direction was not changed. Further, as can be seen, the Doppler effect is still quite effective in reducing the energy release for a power-flattened cylindrical core as compared to the spherical core, since it acts to shut down the reactor before a very large part of the core is above the boiling point, and at a lower average temperature as compared to the flattened spherical case.

\section{Excursions Starting below the Threshold for Core Disassembly}

The previous calculations have considered the meltdown accident from the point in time at which boiling of fuel first starts. This was done so that one could gain a physical understanding of the factors which influence the disassembly process. In addition, the behavior of the reactor (although complicated prior to the time when boiling begins) is determined by the standard kinetics equations which themselves are well understood. However, a number of calculations have been performed in which the course of the accident has been followed from operating temperature. The results are different from those previously discussed only in the case in which a large Doppler effect acts.

Where a prompt negative coefficient is present, there are two major effects. First, in going from a low energy density to that at which boiling begins, the negative 


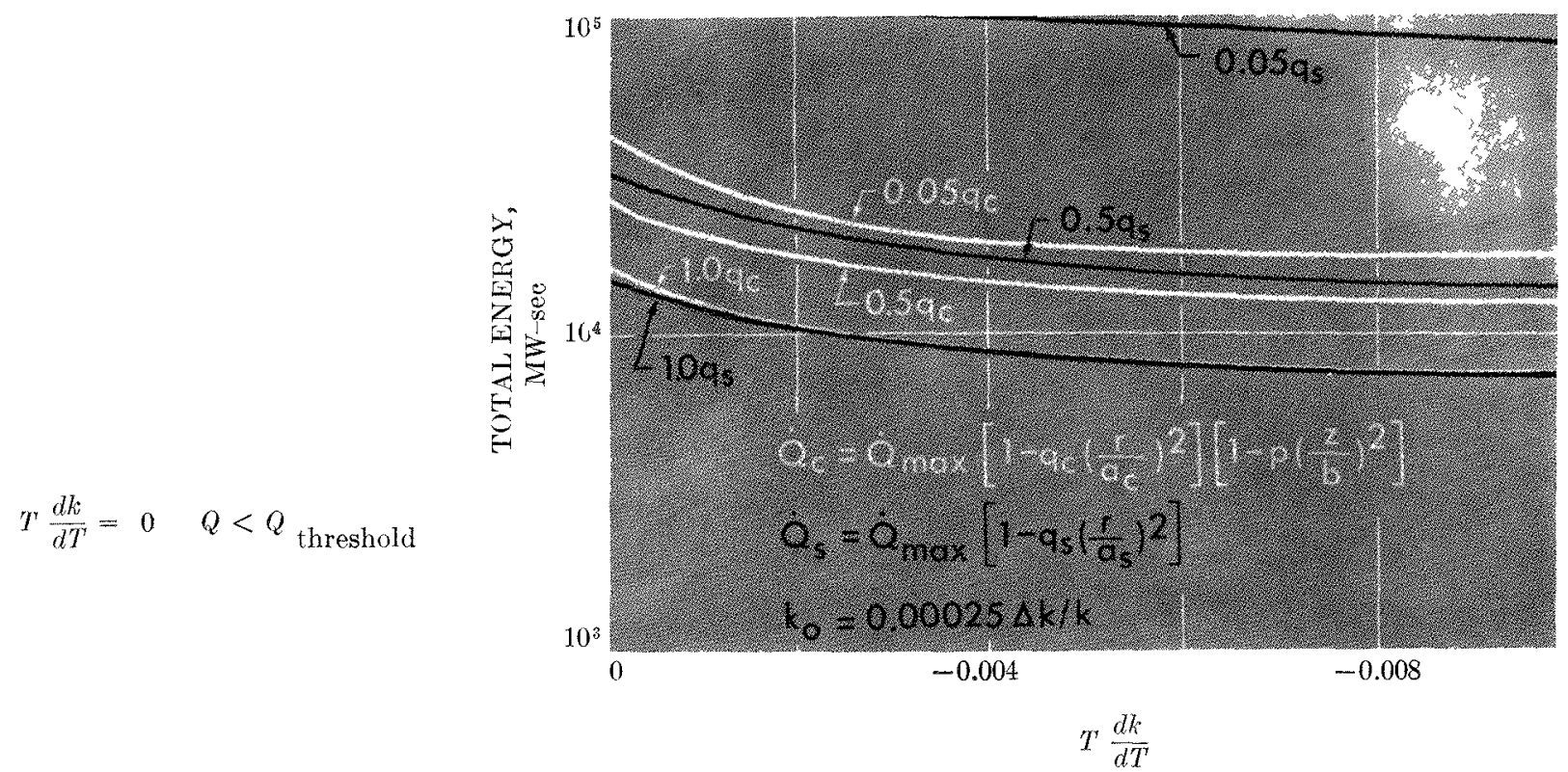

Frt. 11. Total energy release as a function of $A_{\text {Dop }}$ for various degrees of power flattening. Curves show the difference in power flattening in one dimension (cylindrieal meltdown model) and in three dimensions (spherical meltdown model). Excursions started from operatmg conditions but without Doppler feedback unt the peak temperature in the core reached the threshold for core disassembly.

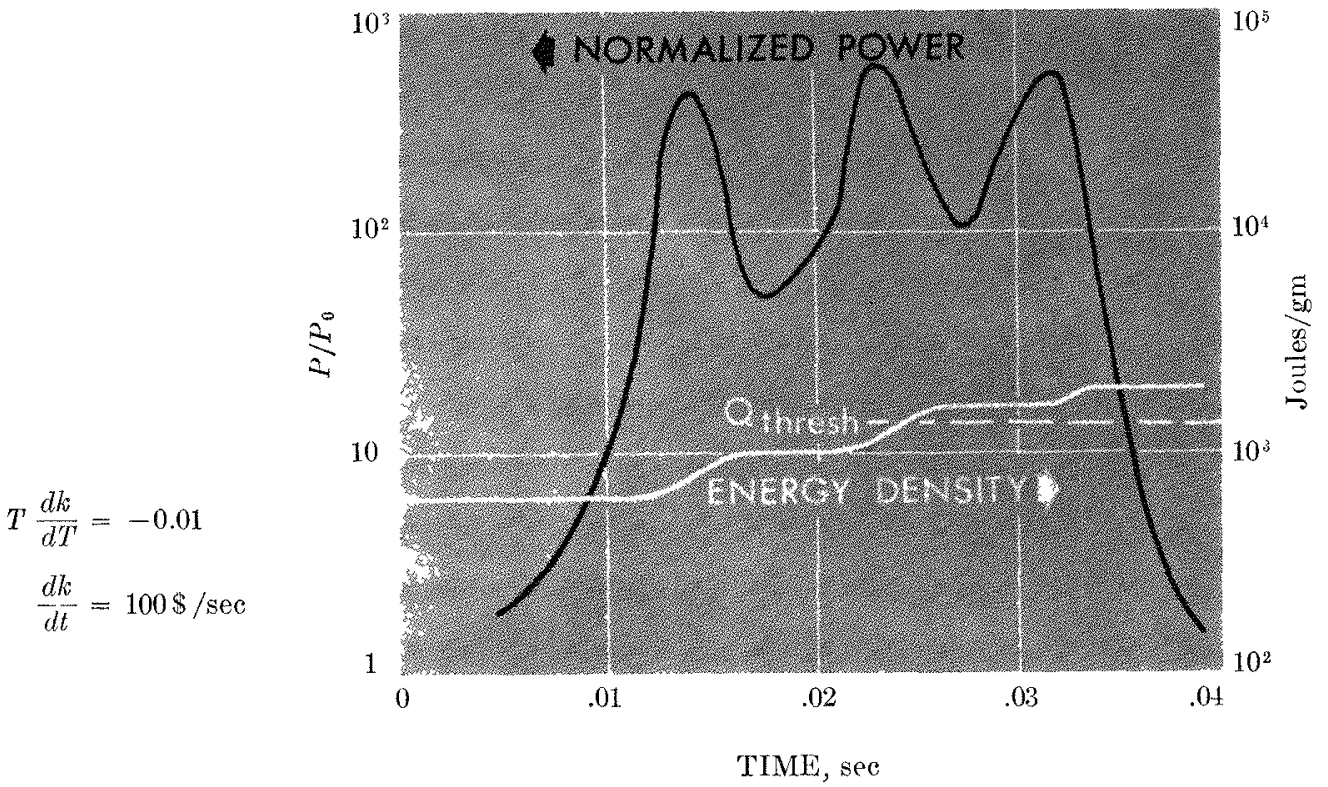

Fit. 12. Poner and energy densits as a function of tme for excursions initiated by a ramp reactivity incertion from normal operating conditions with Doppler feedback from the beginning of the excursion

coefficient will reduce the reactivity so that the disassembly process will start with a smaller net reactivity than is the case without a negative coefficient. However, the more interesting beharior is the well-known oscillation in power which can occur with the presence of a large negative coefficient. This is illustrated in Fig. 12, which shows the power oscillations for a particular case. If these oscillations occur before fuel boils, the energy release during disassembly will depend strongly upon the part of the power oscillation at which core disassembly first starts. For example, if disassembly should start near the peak of an oscillation, the reactivity at the time that boiling begins will be small and there will be timo for the core to start disasscmbling before the reactivity increases on the next cycle. Thus there exists a sensitivity to the fine structure in the power trace and, in turn, to the magnitude of the Doppler effect, the reactivity insertion rate, and 


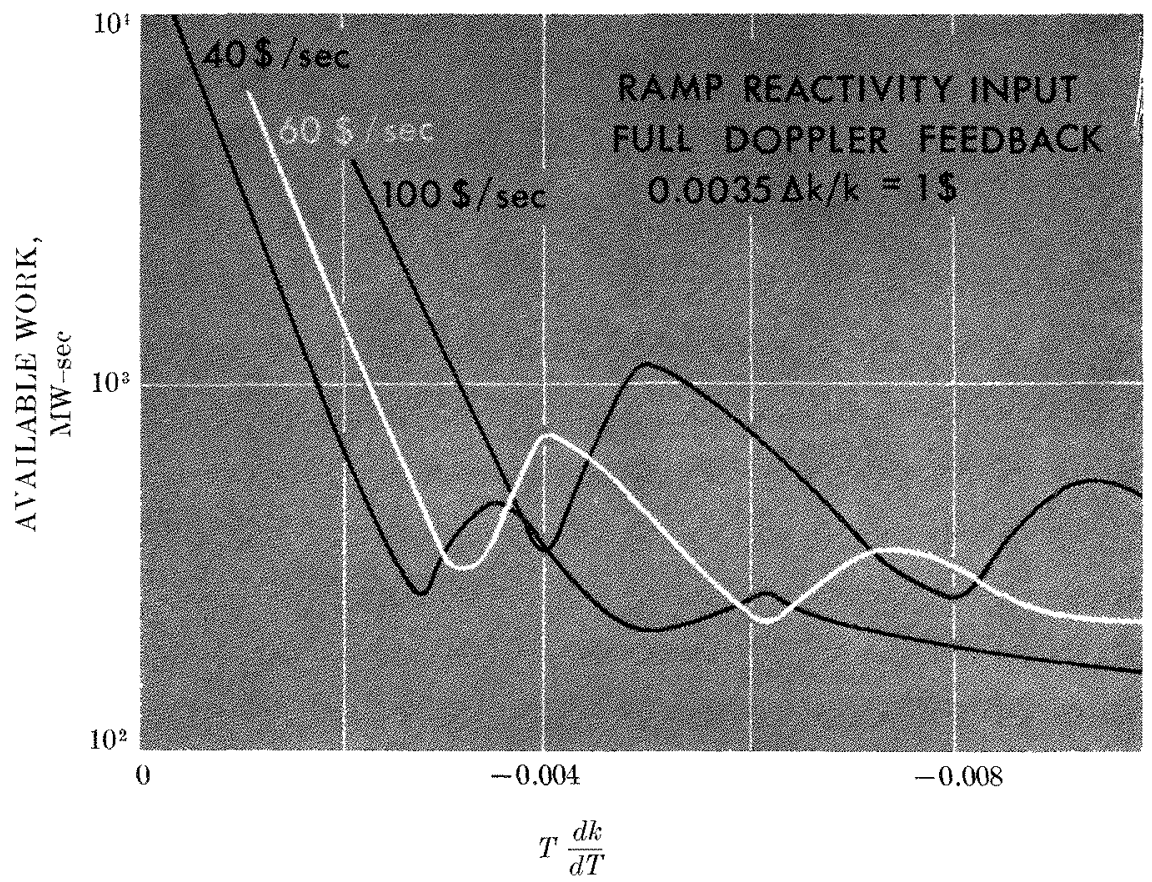

Foc. 13. Available work as a function of $A_{\text {Dop }}$ for excursions initiated by ramp reactivity insertions from normal operating conditions with Doppler feedback from the beginning of the excursion.

the lifetime of the core. Figure 13 is a plot of the explosive energy release for the spherical 1000-MWe reactor assumed to be operating at the steady-state power of $2200-\mathrm{MWt}$ at the time of a reactivity insertion. Reactivity insertion rates of $40 \$, 60 \$$, and $100 \$$ per second are considered. One notes from the figure that even small Doppler coefficients have a substantial effect prior to the point of core disassembly. However, it can also be seen that for high Doppler coefficients, where more than one oscillation in the power can take place, the energy release can be higher for a lower rate of reactivity insertion. Alternatively, one can see that the energy release for a given reactivity insertion can be higher in some cases for a higher Doppler coefficient.

Similar beharior is observed when one examines the effect of initial power level on energy release. Although reducing the initial power level generally results in an increase in energy release, one can see from Figure 14 that in the oscillatory situation with a large Doppler coefficient, a reversal of this behavior can occur.

Figure 15 shows results of calculations in which the average initial temperature of the fuel was changed. It can be seen that with no Doppler effect an increase of initial temperature at the start of the reactivity ramp will cause a decrease in energy release. This is because les reactivity is inserted before core disassembly occurs. On the other hand, with a large Doppler effect, a reduction in starting temperature reduces the energy release since the Doppler effect causes a larger reduction in the net reactivity as the tenperature is increased to the point of core disassembly. This more than compensates for the added reactivity inserted by the ramp. Again, when power oscillations occur, this behavior may be reversed.

Figure 16 shows results of calculations in which the neutron lifetime has been reduced by a factor of 10 for an accident initiated by a ramp reactivity input and starting from the $2200-1 M W t$ power level. In the case in which we considered the accident only from the time when core disassembly first occurred, a smaller neutron lifetime increased the energy release for small or zero Doppler effects, whereas with a high Doppler effect, there is an insensitivity to the neutron lifetime. In looking at the accident with Doppler feedback starting from a lower energy density in the core, one notes that there is a crossover. For zero Doppler effect, a small neutron lifetime increases the energy release. However, in the presence of a large Doppler effect, a short neutron lifetime reduces the energy release. A short neutron lifetime causes the reactor power to increase at a greater rate for a given reactivity insertion. Thus, at the time that core disassembly starts, the inserted reactivity is smaller; with a large Doppler coefficient, the resultant disassembly energy is reduced. On the other hand, for zero Doppler effect, the short period during disassembly results in an increased energy release. When one counts on a negative temperature coefficient to reduce the explosive energy release in a meltdown accident, as appears necessary for large power reactors, it is of no advantage to have 


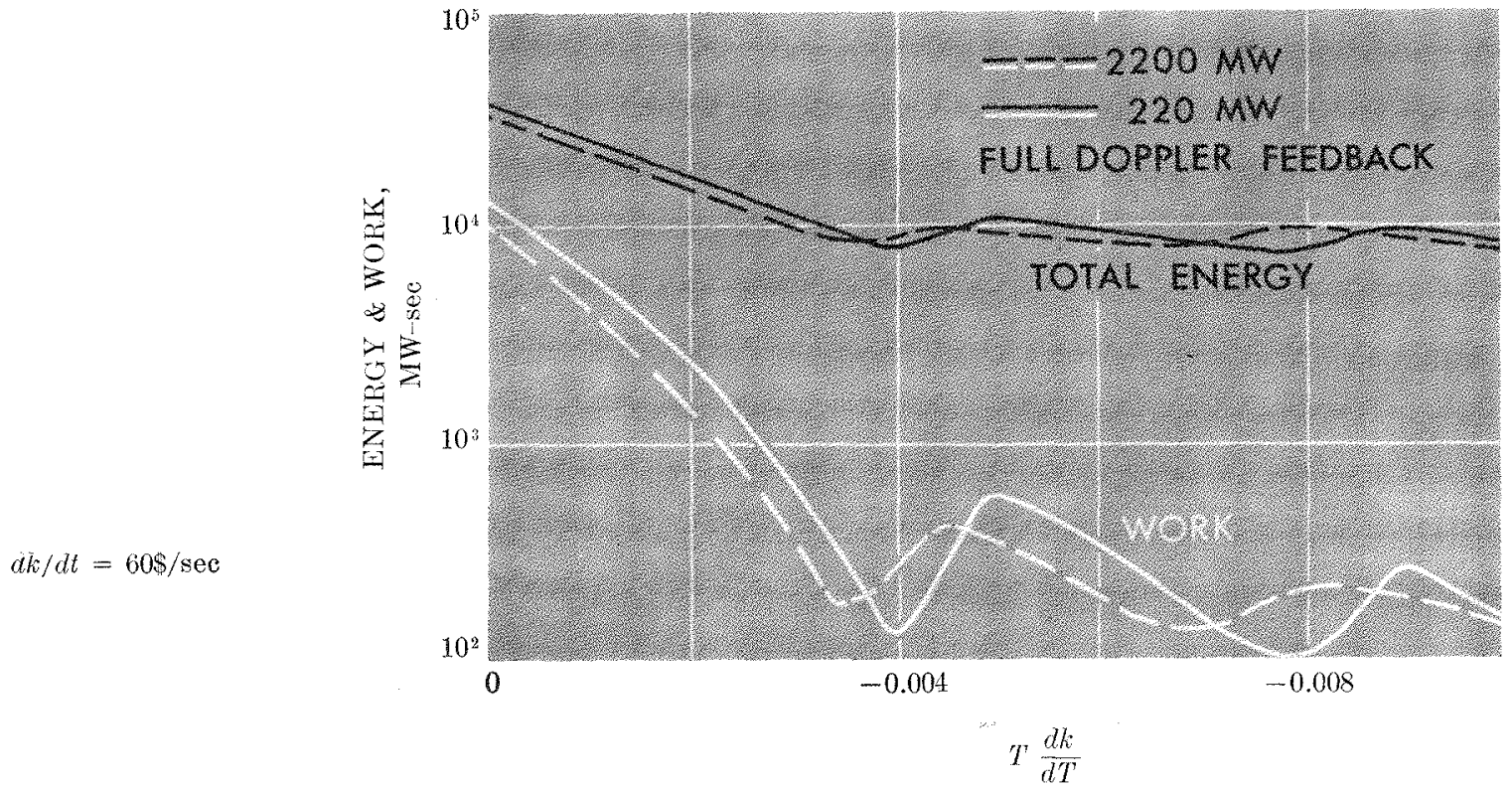

FIG. 14. Available work as a function of $A_{\text {pop }}$ for exeursions initiated by ramp reactivity insertions from normal operating power $(2200 \mathrm{MWt})$ and one-tenth power $(220 \mathrm{MWt})$ with Doppler feedback from the beginning of the excursion. These calculations used a peak-to-average power ratio of 1.91 .

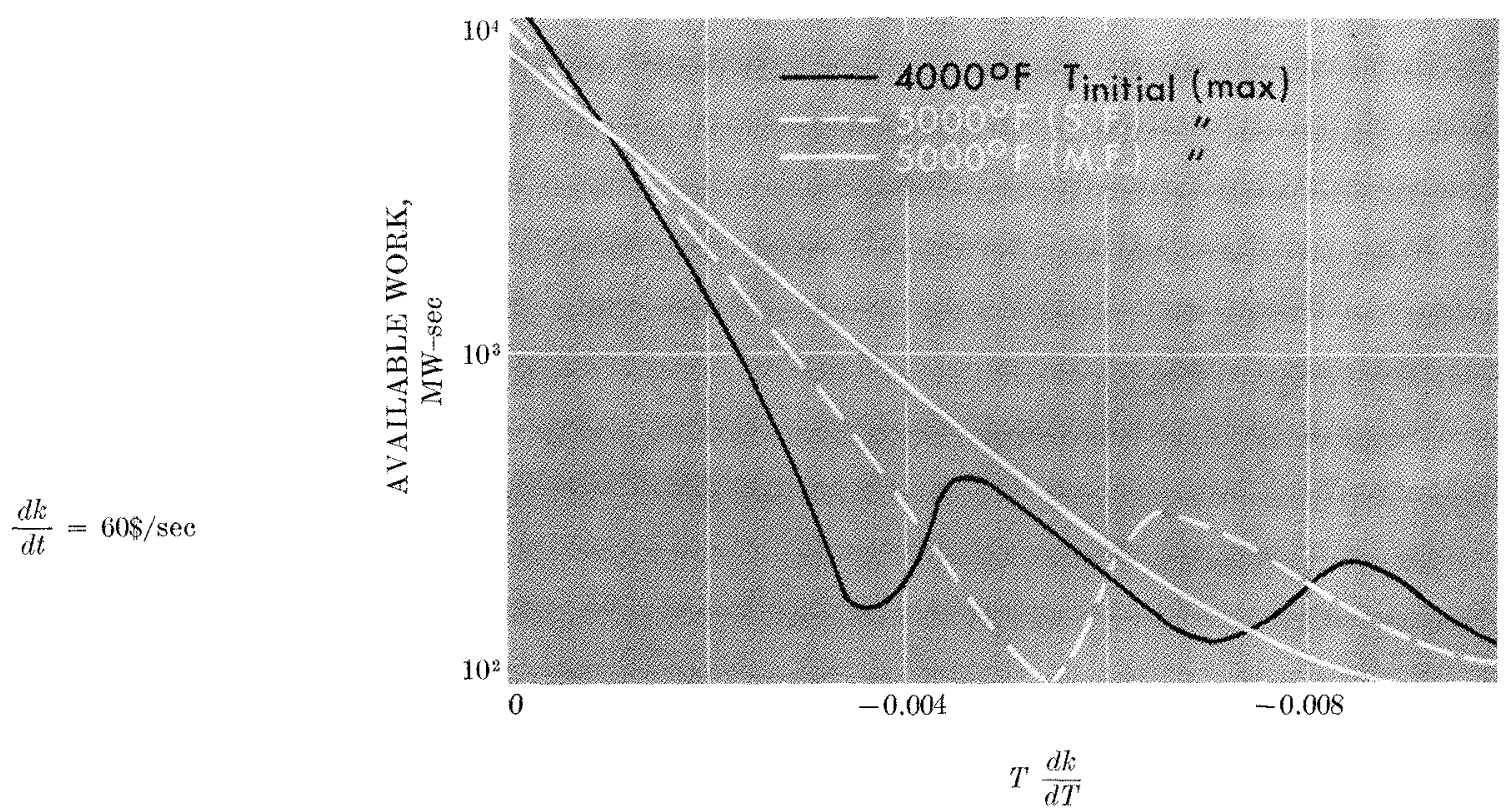

FIg. 15. Available work as a function of $A_{D o p}$ for excursions initiated by ramp reactivity insertions starting from various initial temperatures and normal operating power $(2200 \mathrm{MWt}$ ) with Doppler feedback from the beginning of the excursion. These calculations used a peak-to-average power ratio of 1.91 .

a long neutron lifetime. In fact, it is a disadvantage. Schemes involving coupled fast-thermal reactors, which claim benefit because of the longer resulting neutron lifetime, should therefore be looked at with suspicion. This point was made at the last Argonne Conference. (1)
The preceding comments are general in nature and do not consider the fine structure which results from the oscillatory nature of the power when one starts excursions from low energy density in the presence of a large Doppler coefficient. As can be seen from Figure 16 , in the region of oscillations, one can sometimes 


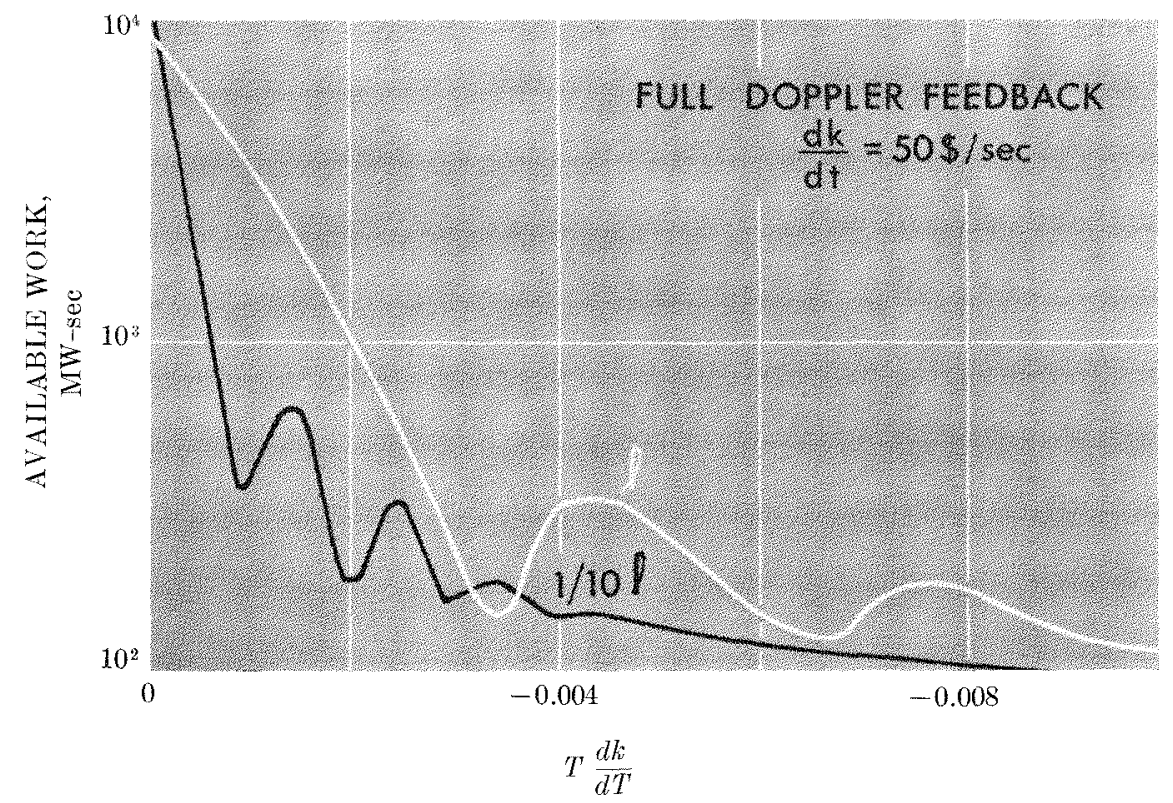

FIg. 16. Available work as a function of $A_{\text {Dop }}$ for excursions initiated by a ramp reactivity insertion with normal neutron lifetime and one-tenth hifetime. Excursions started from normal operating conditions with Doppler feedback from the beginning of the excursion.

get reversals such that a shorter neutron lifetime produces a greater energy release than a longer neutron lifetime.

\section{Conclusions and Observations}

The calculations described were intended to permit an understanding of the meltdown accident under various conditions. Aside from the points made in the discussion above, the authors would like to conclude with the following observations:

1. For meltdown rates of the order of tens of dollars per second in large power reactors, the presence of a large Doppler effect can reduce the energy release to values for which containment capability has already been engineered in the present generation of experimental reactors.

2. The fine structure produced by power oscillations in the presence of a large negative temperature coefficient must be taken into account in an accident analysis. The designer should be sure that he has designed for the envelope defining the maximum energy release if a meltdown and compaction accident is chosen as a design basis.

3. We have shown that in the presence of a large Doppler effect, there is an insensitivity to most of the parameters involved in the meltdown calculations. However, there is a sensitivity to the heat capacity of the fuel. Doubling the heat capacity has about the same effect as reducing the Doppler effect by a factor of 2 . Although it would be of value to have more defini- tive information on the pressure-energy density relationship, it is the recommendation of the authors that attention first be applied to obtaining heat capacity information. Fortunately, this may be easier to obtain than the full equation of state. (In the calculations described above, a value of $13 / 2 R$ was assumed for the heat capacity of the fuel above the melting point.)

It should also be noted that, in terms of minimizing energy release from a meltdown accident, it is advantageous to have a fuel with a low heat capacity.

4. The energy release calculated in the examples given above involve the assumption of an isentropic expansion of the vaporized fuel. This is a very pessimistic assumption, of course, and calculations by Jankus ${ }^{(9)}$ have indicated that in some cases, the energy release would be overestimated by as much as a factor of 10 . We have looked at the results of the one experiment performed in this field, the KIWI experiment. Based on the data which has been released and discussions with W. R. Stratton of Los Alamos, we estimate that the assumption of an isentropic expansion would have overestimated the energy release from KIWI by a factor of approximately 6 . If this factor, or a similar factor, could be verified for situations appropriate to power reactors, this would greatly ease the design of containment.

5. As noted in the Introduction, one should not infer from this investigation a conclusion that the meltdown accident is necessarily a credible accident. 


\section{REFERENCES}

1. B. Wolfe, N. Friedman, and 1). Riley, Influence of the Doppler Effect on the Heltlown 1ccudent, ANL-6792, Proceedings of the Conference on Breeding, Eeonomies and Safety in Large Fast Power Reactors, October 1963.

2. B. Wolfe, N. Friedman, and R. Meyer, Hypothetcal Fast Oxude Reactor Meltdoun Accudent with Doppler Effect, Trans. ANs (June 1964).

3. R. Meyer, B. Wolfe, and N. Friedman, Fast Reactor Meltdown Accidents with Doppler Effect, Trans. ANS (June 1965).

4. R. B. Nicholson, Methods for Determming the Energy Release in Hypothetical Reactor Meltdown Acridents, APDA-150 (Dec 1962).

5. R. D. Smith et al., Fast Reactor Physics, Including Results from U.K. Zero Power Reactors, Third United Nations Conference on the Peaceful Uses of Atomic Energy, A/Conf. 28/P/166, Lnited Kingdom, May 1964.

6. W. R. Stratton, T. H. Calvin, and R. B. Lazarus, Analysis of Prompt Excursions in Simple Systems and Idealized Fast Reactors, Proc. 2nd IN Conf. on Peaceful Uses of Atomic Energy, 12, 196 (1958).

7. R. Meyer and B. Wolfe, High Temperature Equation of State of Uranium Dioxide, Trans. ANS (June 1964).

8. R. H. Brout, Equation of State and Heat ('ontent of ('ranium, APIDA-118 (Feb 20, 1957).

9. V. Z. Jankus, 1 Theoretucal Study of Destructive Nuclear Bursts in Fast Power Reactors, ANL (5512 (Keb 1963).

\section{Discussion}

Mr. Schlechtendahl (Karlsruhe): You mentioned that a flat core and a compact core give about the same energy release for the same Doppler. I would presume that these calculations wore made with the same peak-to-average value. Isn't it the case that with the flat core you have a lesser peaking of the power profile in the core than with the compact core, so that from this as a whole the flat core would look less farorable than the compact core?

$M r$. Wolfe: Yes, I think in general that's right. Actually, we did these calculations with a constant reflector savings; thus, some of the effect you're talking about is already included. I don't know what the difference would have been if we had made the flux go to zero at the core boundary rather than an extrapolated boundary. But I think the interesting thing is that even from the 10 to 1 case, which is rather extreme, we didn't see very much difference; we do have a lower peak-to-average ratio due to the fact that we have the $20-\mathrm{cm}$ extrapolation distance in the axial direction, so the vertical peak to average is not as big as it would be if we made it go to zero. I want to point out that these calculations only give the total energy and not the work for the cylindrical case.

Mr. Campise (AI) : How did you handle the difference in the importance functions for the axial and radial cases in the cylindrical model? What volume of the core was involved in this accident?

Mr. Wolfe: In the cylindrical case we made the assumption that axial and radial fluxes would be separated, and we did a calculation taking first-order terms in the Bethe-Tait analysis. Incidentally I might point out that if you go through previous developments in the Bethe-Tait equations, you find they are not correct to first order. But the first order correction is not very large.

Mr. Häfele (Karlsruhe): I would like to make a comment in this respect for coupled reactors. I think that in evaluating the maximum credible accidents in the present state of the art one does realize that a flat power distribution gives considerably larger energy releases and a flat power distribution exists in coupled reactors. Therefore, what originally started out to be a safe design could perhaps be a somewhat more dangerous design. Secondly, a longer lifetime might be perhaps disadvantageous. Therefore the coupledreactor concept should be re-evaluated in the light of these two facts.

$M r$. Okrent (ANL): In view of the relatively weak dispersal mechanisms which may exist in the presence of a large negative Doppler effect, and since the core just oscillates about a high power for some accidents, I wonder whether one has not the potential source for a new difficulty, namely, a rather long-term, relatively large heat source which provides a serious problem of pressure and heat removal.

Mr. Wolfe: I should point out that you don't get the oscillator behavior in the disassembly. Once enough energy is in above the threshold, disassembly occurs. It's just a question of whether that happens on the first pulse or the second pulse or in between. Secondly, I think it's very improbable that you can have the core cooking away at very low power. It seems to me there are two possibilities. If the sodium is lost, the power is limited because of melting. If melting occurs, another criticality accident is obtained, but usually in this kind of analysis, the first one occurs at tens of dollars a second and the second one would be very minor relative to that.

Mr. Okrent: There was one virtue to the highpressure accident: there was only one and there was a finite amount of energy to contain. I wonder whether one is so confident that there isn't a longer-range heat source in this case.

Mr. Stachura (AI) : At the time when the burst was turning over, about what range of dollars had been put in? If it's substantial, the question may be whether this insertion can be realistically placed in terms of control rod, whether there are some mechanisms to do 
that, or whether it really requires material distribution. If it requires material redistribution, then the possibility is that flux shapes are not at all normal. With unusual flux shapes the pressure could be greatly altered and thus the mode of disassembly.

$M r$. Wolfe: On the amount that gets involved in some of these accidents, my recollection is that something like 30 to $50 \%$ of the core gets above the melting point in these accidents for reasonable power shapes in the core, that is, for a peak-to-average ratio of the order of 1.8 or so. Less than $10 \%$ of the core actually vaporizes.

$M r$. Stachura: I was thinking in terms of dollars of reactivity.

$M r$. Wolfe: The reactivity of $0.001 \Delta k$ is equivalent to $\$ 50$ a second or so. 


\title{
A Modified Equation of State for Hydrodynamic Calculations in AX1 Numerical Program
}

\author{
I. Z. JANKUS \\ Argonne Natzonal Laboratory \\ Argonne, Illinois
}

\section{Introduction}

An investigation of a hypothetical excursion in a fast reactor is concerned not only with the total energy yield but also with the fraction of it available for the damage of reactor containment structure. This fraction decreases quite rapidly with decreasing energy yield and usually is between one and ten percent for the accidents presently considered even remotely possible. Work available adiabatically and eventual kinetic cnergy yield in an uncontained reactor are often considered to be pessimistic and optimistic estimates of destructive effectiveness of an exr'ursion. Both estimates depend strongly upon the behavior of the equation of state at large volumes. Although in a contained excursion large expansions are improbable, the damage to the reactor containment is expected to require consideration of larger specific volumes than are noeded for the shutdown of power generation and calculation of total energy yicld. With this point of view the equation of state presently used in $\mathbf{X X 1}$ program has been examined. To calculate both large and small excursions two analytical formulations are needed. For large pressures, the equation of state is assumed to be in generalized Bethe-Tait form. For lower pressures, utilization of equation of state for safurated vapor seems necessary.

\section{Equation of State for Large Pressures}

The ralculation of enorgy yield with $\Lambda X 1$ code usually is made with a simple equation of state proposed first by W. R. Stratton. This equation assumes that pressure $p$ is a linear function of density $\rho$ and temperature $\theta$, given by

$$
p=\tau+\beta \theta+\alpha \rho,
$$

where $\tau$ is a negative quantity. Pressure is considered to be a positive quantity, so Fq. (1) is used only for sufficiently high values of $\theta$ and $\rho$. For lower values, when $\mathrm{Fq}$. (1) gives a negative number, pressure is supposed to vanish, that is

$$
p=0 \text { for } \tau+\beta \theta+\alpha \rho<\theta .
$$

In addition to pressure an expression for specific heat is needed. It is assumed to be a linear function of tempcrature

$$
C_{r}=A+B \theta
$$

both for positive and vanishing pressure. The latter assumption is logically inconsistent; the equation of state is overdefined.

From the second law of thermodynamics, we know that

$$
\left(\frac{\partial e}{\partial u^{\prime}}\right)_{\theta}=\theta\left(\frac{\partial p}{\partial \theta}\right)_{v}-p .
$$

Thus pressure given in terms of absolute temperature and specific volume determines also variation of internal energy $e$ with specific volume. Differentiating Wq. (3) with temperature, we obtain

$$
\frac{\partial^{2} e}{\partial \imath \partial \theta}=\left(\frac{\partial C_{v}}{\partial v}\right)_{\theta}=\theta \frac{\partial^{2} p}{\partial \theta^{2}} .
$$

Thus variation of specific heat with volume is already prescribed by the "thermal" equation of state Lq. (1) and Eq. (1a). Equation (4), of course, is satisfied by Eq. (2) in each of the regions of positive and vanishing pressures. However, it is not satisfied at the boundary between them. If we disregard this and calculate energy in two regions separately by use of $\mathrm{Eq}$. (1) with Eq. (2) for positive pressures and of Eq. (1a) with Eq. (2) for vanishing pressures, we would find a discrepancy in the internal energy at the intersection of those two regions. Now if we assume that Fq. (2) is correct for positive pressure, we must take

$$
C_{v}=A+B \theta+\frac{\alpha \beta^{2} \theta}{(\tau+\overline{\beta \theta})^{2}}
$$

when $\tau+\beta \theta+\alpha \rho<0$. This modification usually would have little effect on determination of total energy yield with $\mathrm{AX} 1$ program, if threshold energy density and other parameters are properly adjusted. However, numerical inconsistencies occur when the calculation is continued after the power burst and reactor material expands to the densities appreciably different from initial.

One certainly could modify Stratton's equations by 
assuming that (2) is valid for vanishing pressure. Then specific heat is decreased by a proper amount for positive pressures. Now the expression for sperifie heat at vanishing pressure would have a simple form. However, specific heat at positive pressures becomes negative when $\theta \rightarrow-\tau / \beta$, and the equation of state departs from physical reality.

Although the adoption of $\mathrm{Eq}$. (2a) when pressures vanish removes logical inconsistency from Stratton's equation, it still leaves us with an equation of state that is physically unsound when appreciable variation of density is allowed. The use of $\mathrm{Eq}$. (2a) for vanishing pressures gives a threshold energy density $Q^{*}$ that increases indefinitely with decreasing density. In addition, the rate of initial rise in pressure with excess energy is a constant $(\beta / A)$ independent of the density. Thus the corresponding Bethe-Tait parameter, $\gamma-1=$ $v(\beta / A)$, increases with increasing specific volume $v$ contrary to simple common sense. These two physical reasons seem to demand further modification of Stratton's equation.

Consider a general situation in which specifie heat depends on the temperature only (except at the threshold of pressure generation ):

$$
\left(\frac{\partial e}{\partial \theta}\right)_{v}=C_{v}=A^{\prime}(\theta) .
$$

From $\mathrm{Eq}$. (4) we see that pressure is a linear function of temperature:

$$
p=\beta(v) \theta-f^{\prime}(v),
$$

where the coefficients $\beta$ and $f^{\prime}$ may be any function of specific volume. Equation (7) is now quite general. It encompasses not only Stratton's equation but also the commonly used Mie-Grüneisen and van der Waals equations. Integrating (6) with respect to $\theta$ and recalling Eq. (3), we obtain in straight-forward fashion

$$
e=A(\theta)+f(v) \text {. }
$$

If we eliminate $\theta$ by means of Eq. (7), we obtain energy expressed in terms of pressure and density. In particular, if specific heat is constant, $A(\theta)=A \theta$, energy be('omes a linear function of the pressure:

$$
e=\left(f+{ }_{\beta}^{A} f^{\prime}\right)+\frac{A}{\beta} p .
$$

Thus the comnection between pressure and internal energy is in Bethe-Tait form

$$
e_{B T}=Q^{*}(v)+\frac{A}{\beta} p,
$$

where the threshold energy density $Q^{*}$ is given by

$$
Q^{*}=f+\frac{A}{\beta} f^{\prime}
$$

and the rate of pressure rise $(\beta / A)$ may depend on specific volume. Given $Q^{*}=Q^{*}(v)$ from Eq. (11) we ("an determine $f$, though not uniquely:

$$
\int \exp \int^{v} \frac{\beta}{A} d v=\int^{v} d v^{\prime} Q^{*} \frac{\beta}{A} \exp \int^{v^{\prime}} \frac{\beta}{A} d v^{\prime \prime} .
$$

The entropy is also a relatively simple expression:

$$
s=A \ln \theta+\int \beta(v) d v .
$$

Thus, an isentropie process is described by the equation

$$
A \theta \exp \int \frac{\beta}{A} d v=\text { const, }
$$

or, if $\theta$ is eliminated by use of Fq. (8),

$$
\begin{aligned}
& (e-f) \exp \int \frac{\beta}{A} d t^{\prime} \\
& \equiv\left(p+f^{\prime}\right)_{\beta^{-}}^{\left.\frac{1}{-} \text { ex] }\right)} \int \frac{\beta}{A} d v=\text { const. }
\end{aligned}
$$

The velocity of wave propagation, $c$, is given by

$$
c^{2} / v^{2}=(\beta / A)\left\{Q^{* \prime}+p\left[(A / \beta)^{\prime}+1\right]\right\} .
$$

The equations of state (6) and (7) for positive pressures determine again the sperific heat in the region of vanishing pressure. The computation of it, however, is even more complicated. Therefore, it seems advantageous to eliminate temperature from equation of state analytically, and let the numerical program do simpler calculations operating only with the relationship among pressure, internal energy, and density. After all, the temperature does not enter explicitly in isentropic wave propagation nor in shock conditions that are governed by the Hugoniot-Rankine equations. All that is necessary is the relationship among internal energy, pressure, and specific volume. Moreover, this relationship ean be checked by experiments involving shock propagation, but not the temperature. However, having accepted Eq. (10) as an only equation of state, we eamnot find the absolute temperature uniquely, and we acquiesce in the possibility that the specific heat may depend on volume and the relationship between pressure and temperature may be nonlinear.

\section{Equation of State for Saturated Vapor}

Some experimental measurements as well as theoretical calculations ${ }^{(1)}$ seem to indicate that for uranium pressure is linear function of internal energy (at least if the specific volume is not too large). For larger specifie volumes the linearity is valid only at larger pressure. Indeed, one should expect this behavior since at larger specifie volume part of the uranium can be found in liquid phase, another in the vapor phase. For 
a two-phase mixture the pressure depends on temperature only:

$$
\theta=\theta(p),
$$

and using Eq. (3) we obtain internal energy in terms of specific volume and pressure:

$$
e_{s v}=v\left(\frac{\theta}{\theta^{\prime}}-p\right)+F(p) .
$$

The expression for entropy in this system is quite simple:

$$
s=\left(v / \theta^{\prime}\right)+\int d p F^{\prime}(p) / \theta
$$

and an isentropic process is described by the equation

$$
\begin{aligned}
&\left(v / \theta^{\prime}\right)+\int d p F^{\prime}(p) / \theta \equiv(e-F) /\left(\theta-p \theta^{\prime}\right) \\
&+\int d p F^{\prime}(p) / \theta=\text { const. }
\end{aligned}
$$

The velocity of wave propagation, $c$, is obtained from the expression

$$
v^{2} / c^{2}=\left(\theta^{\prime} / \theta\right) F^{\prime}(p)-v^{\prime}\left(\theta^{\prime \prime} / \theta^{\prime}\right) .
$$

If one assumes (for moderately low temperatures) that the heat of vaporization is constant and the vapor behaves like an ideal gas, one can demonstrate that

$$
\theta=(L / R) \ln \left(p_{1} / p\right),
$$

where $L$ is the heat of vaporization, $R$ the gas constant, and $p_{1}$ an adjustable parameter. Fxperimentally this relationship is found valid over a relatively large range of pressures. For moderate specific volumes the energy is given essentially by the second term of Eq. (17). For "low" temperatures it can be assumed to be proportional to the absolute temperature, and the equation of state becomes

$$
e_{s v}=v p\left(\ln \frac{p_{1}}{p}-1\right)+\frac{A^{l} L}{R \ln \left(p_{1} / p\right)},
$$

where $A^{l}$ is the specific heat of the liquid at constant pressure. The velocity of wave propagation is calculated to be

$$
\begin{aligned}
1 / c^{2}=\left(A^{l} L / R\right) \ln ^{-3}\left(p_{1} / p\right)(p v)^{-2} \\
+\left[1-2 / \ln \left(p_{1} / p\right)\right](p v)^{-1} .
\end{aligned}
$$

For moderate specific volumes the first term is strongly dominant, and $c$ is practically proportional to the pressure. The work done in an isentropic expansion, $w$, can be calculated in straight-forward manner by use of Eq. (18):

$$
\begin{array}{r}
\frac{w}{A^{l}\left(\theta_{i}-\theta_{f}\right)}=\left[1-\left(1-\frac{R \theta_{f}}{L}\right) \frac{\theta_{f} \ln \left(\theta_{i} / \theta_{f}\right)}{\theta_{\imath}-\theta_{f}}\right] \\
+\frac{v_{\imath} p_{\imath}}{A^{l} \theta_{i}}\left[\frac{L}{R \theta_{i}}-1-\frac{\theta_{f}}{\theta_{\imath}}\right],
\end{array}
$$

where the subscript $i$ refers to the initial conditions and subscript $f$ to the final temperature (and pressure). For uranium the first term is dominant. It has been obtained previously by Russell and Morris ${ }^{(2)}$ by use of physical arguments. We notice, however, that a very largo expansion is required to achieve this efficiency. Thus the theoretical efficiency may be somewhat pessimistic.

Since for moderate specific volumes the first term is very small, pressure can be obtained as an explicit function of internal energy, i. e.,

$$
p \approx B \exp \left[-A /\left(e+e_{0}\right)\right] .
$$

Using this relationship Nicholson ${ }^{(3)}$ has calculated energy yield by generalized Bethe-Tait method, i.e., neglecting the propagation of pressure wave. Compared with threshold equation of state, these calculations have shown a significant decrease of energy yield for low reactivity input. $A$ disadvantage of this equation of state is that it is clearly valid only for a limited range of pressures. According to this equation, internal energy blows up when pressure approaches $p_{1}$. Actually, one can hardly trust this equation for pressure that makes $\left(\partial^{2} e / \partial p^{2}\right)$ negative, i.e., $p>p_{1} \exp (-2)$. Moreover, the number of parameters used in Nicholson's equation is small, and when one adjusts parameters to represent a 1 wo-phase region, one is unable (in general) to fit behavior of uranium in the BetheTait region, where the relationship between pressure and internal energy depends strongly upon specific volume. Numerical programs using this equation of state, ${ }^{(4)}$ however, can be easily amended by introducing linear dependence of pressure upon internal energy after the voids have been filled. Although the introduction of saturated vapor pressure yields smaller total energy, this is partially compensated by a larger efficiency of conversion of this energy into the isentropic work. For the threshold equation of state at small yield, available work is given by $w=p^{2} v^{2} / 2 c^{2}$, where the velocity of wave propagation is a fairly large constant. The efficiency of conversion in the saturated vapor system is at least $R \theta_{f} / L$ [see Eq. (23)] or significantly larger. In addition, we know that Bethe-Tait method usually is less valid for the excursions of lower initial period.

Speaking qualitatively, a large deviation is caused by the ability of the pressure wave to traverse the core during the initial period of the excursion. Although the velocity of wave propagation in saturated vapor is small compared with the wave velocity in the threshold 
equation of state, it.increases with increasing pressure. Thus, in some cases complete numerical calculation may result in a significant increase also of total energy yield. Such coupled neutronic-hydrodynamic calculations have been done in connection with reactor VERA. ${ }^{(2)}$ However, in these calculations the first term in the equation of state (21) has been neglected. This has resulted in an effective velocity of wave propagation, $c_{\text {eff }}$, given by

$$
1 / c_{\mathrm{eff}}^{2}=\left(A^{l} L / R\right) \ln ^{-2}\left(p_{1} / p\right)(p v)^{-2},
$$

several times smaller than calculated by means of $\mathrm{Eq}$. (22). Thus full deviation from the Bethe-Tait type of calculation has not been realized.

To approximate the behavior of uranium during power bursts of medium size and after, we need both the Bethe-Tait and saturated vapor equations of state. Each gives an internal energy as an explicit function of pressure and specific volume. Equation (17) should be used when it gives lower energy than Lq. (10). We have seen, however, that Eq. (21) deviates strongly from reasonable behavior when the pressure is large. Indeed, comparing Eq. (21) and Eq. (10) for large specifie volumes, we find a region, at fairly high pressures, when expression Eq. (21) yields lower values than Eq. (10). To eliminate this fictitious region in order to use Eq. (17) we must have not only $e_{B T}>e_{s v}$ but also $\partial e_{B T} / \partial p<\partial e_{s_{2}} / \partial p$ and $\partial^{2} e_{s i} / \partial p^{2}>0$. The last condition can be replaced by a stricter requirement $\ln \left(p_{1} / p\right)>2$.

To use our equations of state in the numerical program we need only a minor modification of the calculational scheme. Instead of using $e$ and $v$ as independent variables, as has been done in COOT, ${ }^{(2)}$ we use $p$ and $v$ as independent variables. Instead of romputing the (hange in energy, we compute the change in pressure:

$$
d p=\left(\frac{\partial p}{\partial e}\right)_{v} d Q-\frac{c^{2}}{v^{2}} d v
$$

where $(\partial e / \partial p)_{0}$ and

$$
\frac{c^{2}}{v^{2}}=\left[\left(\frac{\partial e}{\partial v}\right)_{p}+p\right] /\left(\frac{\partial e}{\partial p}\right)_{v}
$$

are now computed by use of either Fq. (10) or Ha. (17). Fach of these equations still contains two explicit functions. For the saturated vapor region we could use approximations:

$$
\frac{\theta}{\bar{\theta}^{\prime}}-p=p\left[\ln \frac{p_{1}}{p}-1+\frac{c_{1}}{\ln \left(p_{1} / p\right)}\right]
$$

and

$$
F=\frac{A^{l}}{R}-\frac{L}{\ln } \frac{L}{\left(p_{1} / p\right)}\left[1+\frac{c_{2}}{\ln \left(p_{1} / p\right)}\right]
$$

However, only the parameters $.^{l}, L / R$, and $p_{1}$ usually are known quite accurately from "low" temperature measurements. Similarly, two functions in the BetheTait equation of state could be approximated by the use of several parameters:

$$
\frac{A}{\beta}=\frac{v-b_{1}}{\gamma_{\infty}-1}\left[1+\frac{\gamma_{\infty}-\gamma_{s} b_{1}}{\gamma_{s}-1} \bar{v}\right] .
$$

The approximation of threnhold energy density over the whole range scems to require more parameters. The expression

$$
\begin{aligned}
& Q^{*}=L(1-b \rho)\left[\alpha_{0}+\alpha_{1}(1-b \rho)\right. \\
& \left.+\alpha_{2}(1-b \rho)^{2}+\alpha_{3}(1-b \rho)^{3}\right],
\end{aligned}
$$

with $\alpha_{0}+\alpha_{1}+\alpha_{2}+\alpha_{3}=1$, may be of sufficient complexity but still manageable from a practical point of view. Here also only the value of $\alpha_{0} L$ is known quite well from measurements at moderate temperatures. There are indications that $\alpha_{1}$ is quite small. Other parameters could be inferred from shock measurements with the help of some edueated arguments about corresponding states. The thermodynamic arguments, however, should be carried out to numerical evaluation of our four functions $\theta / \theta^{\prime}-p, F(p), A / \beta$, and $Q^{*}$, ignoring approximation by our forms $(26),(27),(28)$, and (29). We find that the critical point is quite sensitive to minor changes in these functions. Thus our approximations usually will shift their location. This, however, is not experted to influence significantly either the total energy yield or the work available. For calculation of power burst we believe that the leading terms in Eq. (28) and Eq. (29) with parameters adjusted for initial density are adcquate. For calculation of subsequent pressure wave other terms will have some influcnee.

\section{The Absolute Temperature}

The hydrodynamics calculations can be performed if we know the relationship among internal energy, pressure, and specific volume. In large reactors, however, shutdown is accomplished not only by bulk motion of core material. In a large reactor the Doppler effect is appreciable, and a small excursion may be shutdown mainly by changing effective cross sections which depend mainly on the temperature of the material. Since the temperature does not come into hydrodynamic calculation, we may postulate it separately as a function of internal energy and specific volume. Some arbitrariness in the choice of this function may be unavoidable if there is no thermodynamic equilibrium among various reartor materials.

However, if we assume existence of a two-phase region, the temperature there is determined presumably 
by Eq. (16). If we consider the Bethe-Tait region alone, formally, the temperature (or rather $1 / \theta$ ) there ran be defined as an integrating multiplier in the second law of thermodynamies. The determination is not unique, and temperature can be varied by an "arbitrary" factor on each adiabat without formal contradiction. From our $\mathrm{Eq}$. (13) and (14) we see then that the temperature obtained from relation

$$
\theta \exp \int \frac{\beta}{A} d v=\mathscr{F}\left\{(e-f) \exp \int \frac{\beta}{A} d v\right\},
$$

where $\mathscr{F}$ is an "arbitrary" function, will be consistent with Eq. (9). This arbitrary function can be found at the boundary line with another region where the temperature is known. In our case the temperature at the boundary of two-phase region is given, say, by liq. (20), and the arbit rary function in Lq. (30) can be evaluated.

When the Bethe-Tait region is joined with a saturated vapor region even the pressure on the boundary line $\left(p^{*}\right)$ cannot be found analytically as a function of specific volume, and analytical determination of $F$ in Eq. (30) is hard 10 contemplate. lor any given value of $v$ expansion of $F$ in power series is straightforward if $p^{*}(v)$ is known. If one limits this expansion to two terms, the resulting thermal equation gives a linear relationship between internal energy and temperature. In general, this relationship is valid only in the vicinity of the adiabat considered. The corresponding specific heat $A$ varies with the particular adiabat chosen. It is relatively easy to obtain this specific heat for low temperatures. If one assumes that Eq. (20) is valid for low temperatures and considers large volumes, one finds that the specific heat for a gas is given by

$$
A_{g}=\frac{Q^{*}(\infty)}{L} \frac{R}{\gamma_{\infty}-1}
$$

where

$$
\left(\gamma_{\infty}-1\right)=\lim _{\nu \rightarrow \infty}[v(\beta / A)] .
$$

If the gas is ideal the spercific heat should be

$$
R /\left(\gamma_{\infty}-1\right) .
$$

Thus $Q^{*}(\infty)$ should be equal to the heat of vaporization. This requirement certainly ean be compromised somewhat in order to obtain a better fit of the equation of state at more important specific volume; likewise, one finds that the specific heat of the liquid is

$$
A_{l}=A^{l} \gamma_{l}
$$

where

$$
\gamma_{l}-1=\lim _{v \rightarrow b}[(v-b)(\beta / A)]
$$

and $b$ is the value at which the threshold enorgy density vanishes:

$$
Q^{*}(b)=0 .
$$

The specific heat $A$ along each adiabat can be calculated quite in general. However, the resulting expressions are rather complex. Let us suppose we have expanded Eq. (30) and used the first two terms:

$$
\theta=\Im_{0} \exp \left(-\int^{v} \frac{\beta}{A} d v\right)+\frac{1}{A}(e-f) .
$$

The two unknown coefficients can be determined for any given $v$ by realizing that along the boundary between saturated vapor and the Bethe-Tait region both the temperature and its first variation coincide. The temperature in a saturated vapor region is a function of pressure alone and is given by Eq. (20) or, in general, by Eq. (16). Thus, along the boundary both pressure $p^{*}=p^{*}\left(v^{*}\right)$ and temperature $\theta^{*}=\theta^{*}\left(v^{*}\right)$ are functions of specific volume $v^{*}$. Substituting these values into $\mathrm{Eq}$. (31), we obtain

$$
\theta^{*}=\widetilde{\Im}_{0} \exp \left(-\int^{v *} \frac{\beta}{A} d v\right)+\frac{1}{A}\left(e^{*}-f^{*}\right) .
$$

From $E$. (31) and $E$. . (32) we ran eliminate one unknown constant, say $\mathfrak{F}_{0}$, obtaining as an equation of state in the vicinity of the adiabat, and going through the point $\left[v^{*}, p^{*}\left(v^{*}\right)\right]$,

$$
\begin{array}{r}
(e-f-A \theta)=\left(e^{*}-f^{*}-A \theta^{*}\right) \\
\cdot \exp \int_{v}^{v *} \frac{\beta}{A} d v,
\end{array}
$$

where the specific heat 1 is still to be determined. Given $Q^{*}=Q^{*}(v)$, then $f$ is not determined uniquely, as we have seen in Eq. (12). Equation (33), however, is not affected by this arbitrariness (as it should) if the same definition of $f$ is used on both side of the equation.

The specific heat $A$ now is determined by considering the variation of $\mathrm{Eq}$. (32) along the boundary line. If energy $e$ is eliminated from $\mathrm{Eq}$. (32) by means of kq. (9), one has

$$
\begin{aligned}
& \theta^{*}=\oiint_{0} \exp \left(-\int^{p *} \frac{\beta}{A} d v\right) \\
&+\frac{1}{A}\left(\frac{A}{\beta}\right)^{*}\left(p^{*}+f^{*}\right) .
\end{aligned}
$$

Differentiating this with respect to $v^{*}$, we have 


$$
\begin{aligned}
\frac{d \theta^{*}}{d p^{*}} \frac{d p^{*}}{d v^{*}} & =-\Im_{0} \frac{\beta}{A} \exp \left(-\int^{v^{*}} \frac{\beta}{A} d v\right) \\
+ & \frac{1}{A}\left\{\frac{d}{d v^{*}}\left[\left(\frac{A}{\beta}\right)^{*} f^{*}\right]+p \frac{d}{d v^{*}}\left(\frac{A}{\beta}\right)^{*}\right. \\
& \left.+\left(\frac{A}{\beta}\right)^{*} \frac{d p^{*}}{d v^{*}}\right\} .
\end{aligned}
$$

Eliminating $\widetilde{F}_{0}$ from Eq. (34) and (35), we have (omitting the asterisks for simplicity)

$$
\begin{aligned}
{\left[\frac{d \theta}{d p} \frac{d p}{d v}+\frac{\beta}{A} \theta\right]=\frac{1}{A} } & {\left[f^{\prime}+\left(\frac{A}{\beta} f^{\prime}\right)^{\prime}\right.} \\
& \left.+p+p\left(\frac{A}{\beta}\right)^{\prime}+\frac{A}{\beta} \frac{d p}{d v}\right] .
\end{aligned}
$$

Here we are considering various quantities only along the boundary line of two regions. The variation of pressure with volume along this boundary is obtained simply by differentiation of the relationship defining the boundary line $\left(e_{B T}=e_{s 1}\right)$ :

$$
\begin{aligned}
{\left[\frac{A}{\beta}+v \frac{\theta \theta^{\prime \prime}}{\theta^{12}}-F^{\prime}\right] \frac{d p}{d v}=- } & f^{\prime}-\left(\frac{A}{\beta} f^{\prime}\right)^{\prime} \\
& -\left(\frac{A}{\beta}\right) p-p+\frac{\theta}{\theta^{\prime}} .
\end{aligned}
$$

Now substituting $(d p / d v)$ from this expression into Eq. (36) and combining the terms, we obtain an expression for the specific heat $A$ :

$$
\begin{aligned}
A \theta^{*}\left[\frac{c_{B T}^{2}}{c_{\varepsilon v}^{2}}\left(\frac{\partial p}{\partial e_{s v}}\right)^{2}\right. & +\left(\frac{\partial p}{\partial e_{B T}}\right)^{2} \\
& \left.-2\left(\frac{\partial p}{\partial e_{s v}}\right)\left(\frac{\partial p}{\partial e_{B T}}\right)\right]=\frac{c_{B T}^{2}}{v^{2}}-\frac{c_{s v}^{2}}{v^{2}} .
\end{aligned}
$$

The quantities involved in this expression are routinely obtained during regular hydrodynamics calculations of $d p$. The Doppler effect can be expected to exert the greatest importance at the carliest stages of the excursion. Thus it seems sufficient to evaluate specific heat $A$ at the first time core material reaches the BetheTait region and keep it the same for the remainder of the power burst.

The temperature can now be calculated from $\mathrm{Eq}$. (33). It is strictly correct only along the adiabat going through the point $\left(v^{*}, p^{*}\right)$. Thic is rusits ... 


\title{
A Model Investigation of Explosion Containment in Single Tank Fast Reactors
}

\author{
N. J. M. REes \\ U. K. A tomic Energy Authorty \\ Foulness, Southend-on-Sea, Essex, England
}

\section{Introduction}

The possibility of an accidental super-prompt critical excursion causing the core of fast reactor to disintegrate with explosive violence and the subsequent effect on the reactor structure has to be considered. Such an explosion may breach the containment structure, and so the ultimate safety of the reactor system will depend on the adequate design of the system to withstand the anticipated explosive effects. Thus the magnitude of the nuclear explosion must be related to its explosive effects on the reactor under consideration.

The explosion of fast reactor cores has been examined in excursion calculations by Hicks, ${ }^{(1)}$ which gave the energy required to expand the fuel to fill all the voids in the reactor core and the energy released in excess of this during the excursion, together with the times taken. The direct calculation of the mechanical effects of this type of nuclear explosion would be prohibitively complicated, and its simulation by the detonation of a charge of chemical high explosive is discussed by Samuels. ${ }^{(2)}$ He concludes that if the excess encrgy of the nuclear excursion is equal to the energy of detonation of the chemical explosion, then in each type of explosion the mechanical effects will be very similar up to the end of the first expansion of the explosion cavity.

In this paper a series of model experiments is described which provide data for containment design in a single-tank, pool-type fast reactor. The models repsent a simple reactor geometry, in which the core and breeder are supported in the middle of a cylindrical pool. This arrangement has been examined to provide both an understanding of the physical processes occurring and quantitative information about the pressure loading produced on the walls and roof of the model reactor tank. Piezo-electric pressurc gauges have been used to measure the dynamic pressure loading (from a bare change of explosive) on the walls, roof, and core-access plug of an over-strong model tank partly filled with water. The influence on these parameters of enclosing the explosive charge in a model core and breeder structure, of varying the air gap between the roof of the model tank and the 
water surface, and of replacing the overstrong tank by a weak one, have all been examined.

\section{Scale Models and Their Limitations}

\subsection{Scale Models}

A mechanical system and a model of it are said to be dynamically similar when scaled forces are exerted at scaled times on similar portions of the system and the model. If the model has been properly designed and constructed, then dynamic measurements of physical parameters made with the model will have simple scale relationships to those that would be measured on the full-scale system. However, models by their very nature must contain a number of approximations, and due consideration of these should be given when interpreting model results for full-scale conditions. ${ }^{(3)}$

Dynamic similarity will be maintained between the model and the full-scale event if the linear dimensions of explosive charge and the mechanical system are divided by a factor $S$ and the model constructed of similar materials. Thus, if $M$ is the mass of the

TABLle 1. Pirameters on Full SCile and on A

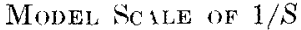

\begin{tabular}{|c|c|c|c|}
\hline Phy sical Quantity & Dimensions & $\underset{\text { Vull-scale }}{\text { Value }}$ & $\begin{array}{c}\text { Model-scale } \\
\text { Value }\end{array}$ \\
\hline $\begin{array}{l}\text { Charge Mass and Other } \\
\text { Masses }\end{array}$ & $M$ & $Z$ & $Z / S^{3}$ \\
\hline Shork-wave Pressures & $M L^{-1} T^{-2}$ & $p$ & $p$ \\
\hline $\begin{array}{l}\text { Length, e.g., Distance } \\
\text { from Charge }\end{array}$ & $L$ & $R$ & $R / S$ \\
\hline $\begin{array}{l}\text { Time, e.g., Duration of } \\
\text { shock Pressure }\end{array}$ & $T$ & $t$ & $t / S$ \\
\hline Area & $L^{2}$ & $A$ & $1 / S^{2}$ \\
\hline Impulse per Unit Area & $M L^{-1} T^{-1}$ & $I$ & $I / S$ \\
\hline Force & $11 L T^{-2}$ & $F$ & $F^{\top} / S^{2}$ \\
\hline Velocity & $L T^{-1}$ & $V$ & $V$ \\
\hline $\begin{array}{l}\text { Acceleration } \\
\text { (iravity) }\end{array}$ & $L T^{y-2}$ & $\alpha$ & $a S$ \\
\hline Stress & $M L^{-1} T^{-2}$ & $\sigma$ & $\sigma$ \\
\hline Strain & $\cdots$ & $\xi$ & $\xi$ \\
\hline Rate of strain & $T^{-1}$ & $\dot{\xi}$ & $\dot{\xi} S$ \\
\hline Wnergy & $M L^{2} T^{-2}$ & $E$ & $E / S^{3}$ \\
\hline
\end{tabular}

TABLE 2. COMPIHISON OF WIIER IND HOT SODI M COOLINT

\begin{tabular}{|c|c|c|c|}
\hline & $\begin{array}{l}\text { Water at } \\
20^{\circ} \mathrm{C}\end{array}$ & $\begin{array}{l}\text { Sodium at } \\
400^{\circ} \mathrm{C}\end{array}$ & $\begin{array}{l}\text { Sodium at } \\
600^{\circ} \mathrm{C}\end{array}$ \\
\hline$--\square-\cdots$ & $6-$ & $-\cdots$ & 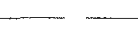 \\
\hline $\begin{array}{l}\text { Velocity of Sound } C_{0} \text {, } \\
\text { m see }\end{array}$ & 1484 & 2420 & 2310 \\
\hline Density $\rho, \mathrm{g} \mathrm{cm}^{-3}$ & 0.998 & 0.856 & 0.808 \\
\hline $\begin{array}{l}\text { Acoustic Impedance } \\
\rho \mathrm{C}_{\mathrm{n}}, \mathrm{g} \mathrm{cm}^{-2} \mathrm{sec}^{-1}\end{array}$ & $148 \times 10^{3}$ & $206 \times 10^{3}$ & $186 \times 10^{3}$ \\
\hline
\end{tabular}

TABle 3. Published Properties of Śrinless Steed Type FN58B and MHa Sted EN2

\begin{tabular}{|c|c|c|c|c|c|}
\hline \multicolumn{2}{|c|}{ Steel } & Temp, ${ }^{\circ} \mathrm{C}$ & $0.5 \%$ Proof & $\begin{array}{l}\text { Maximum } \\
\text { Stress, }\end{array}$ & Elongation \\
\hline $\begin{array}{l}\text { Stainless } \\
\text { EN58B }\end{array}$ & Steel & $\begin{array}{r}20 \\
100 \\
200 \\
300 \\
400 \\
500\end{array}$ & $\begin{array}{l}16.7 \\
13.7 \\
13.0 \\
12.2 \\
12.4 \\
11.2\end{array}$ & $\begin{array}{l}42.6 \\
34.0 \\
30.7 \\
300 \\
30.2 \\
27.9\end{array}$ & $\begin{array}{l}53.4 \\
48.8 \\
40.7 \\
38.4 \\
407 \\
38.3\end{array}$ \\
\hline Mild Steo & $\mathrm{EN} 2$ & 20 & 13.2 & 20.1 & 36 \\
\hline
\end{tabular}

TABLE 4. Seriar Nimbers of Experiments Performed in Cylindricll Tanks, $24 \mathrm{In}$. in Diameter AND 24 IN. DEEP

Explosive Charge Weight, 2 oz RDX/TNT 60/40

\begin{tabular}{|c|c|c|c|c|c|}
\hline \multirow[b]{2}{*}{$\begin{array}{c}\text { Air Gap } \\
\text { between } \\
\text { Water and } \\
\text { Roof of } \\
\text { Tank, in. }\end{array}$} & \multirow[b]{2}{*}{$\begin{array}{l}\text { Depth of } \\
\text { Charge } \\
\text { Centre } \\
\text { below } \\
\text { Surface of } \\
\text { Water, in. }\end{array}$} & \multicolumn{2}{|c|}{ Overstrong Tank } & \multicolumn{2}{|c|}{ Weak Tank } \\
\hline & & Bare Charge & $\begin{array}{l}\text { Charge } \\
\text { Enclosed } \\
\text { in Core } \\
\text { Support } \\
\text { Struc- } \\
\text { ture }\end{array}$ & $\begin{array}{l}\text { Bare } \\
\text { Charge }\end{array}$ & $\begin{array}{c}\text { Charge } \\
\text { Enclosed } \\
\text { in Core } \\
\text { Support } \\
\text { Struc- } \\
\text { ture }\end{array}$ \\
\hline None & 16.6 & 19 & & & \\
\hline $\begin{array}{l}4.0 \\
8.3\end{array}$ & $\begin{array}{r}12.6 \\
8.3\end{array}$ & $\begin{array}{c}3^{*}, 4^{*}, 5 \\
10^{*}, 11 \\
16 \\
17,18 \\
8,9\end{array}$ & 6,7 & $12^{*}, 13^{*}$ & $14^{*}, 15^{*}$ \\
\hline
\end{tabular}

* Tank severely damaged.

full-scale charge, $R$ is a full-scale length, and $t$ a fullscale time, then the corresponding model scale values are $H / S^{3}, R / S$, and $t / S$; shock-wave pressures on the full and model scales will be the same.

If $p$ is the peak shock overpressure and $E$ the energy released by detonation of the explosive, then

$$
p=f\left\{\frac{R}{E^{1 / 3}}, \frac{t}{E^{1 / 3}}\right\} .
$$

Similar shock pressures will be observed at scale distances from two charges of different energy release; the shock will arrive at a scaled time and will have a sealed time duration. Thus, for the same type of explosive and constant ambient conditions in the transmission medium, the shock pressure is completely defined by any two of the three parameters $E, R$, and $T$, where $T$ is a time characteristic of the explosion such as shock arrival time or duration. Some physical parameters that are commonly measured on full and model scales have been derived and are shown in Table 1. 
Certain parameter's cannot be correcty scaled in a model which has been derived from a mechanical system by simple linear scaling. The acceleration due to gravity is an important quantity which does not change between the model and full scales. Thus, in a linearly scaled system, although the masses will be correctly scaled, the model weight will not. In these experiments only the free flight of the model core plug is controlled by gravity. The impulsive explosion forces acting on the plug are large and of short dura-

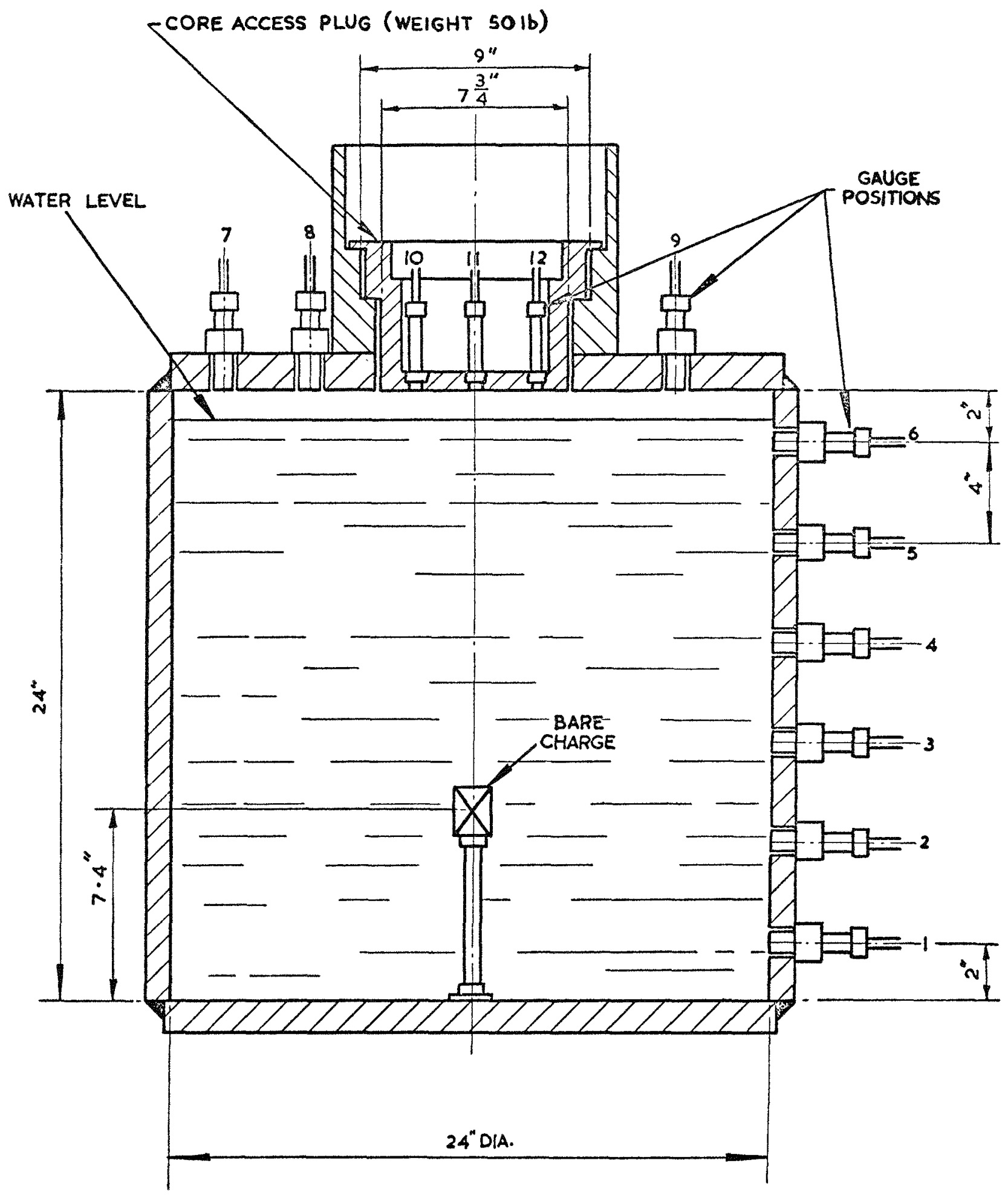

Frg. 1. Cross Section of Model Reactor Tank, Showing Positions of Pressure Gauges and the Bare Charge. 
tion; thus the plug quickly acquires a speed $V$. Since this quantity will be the same on the full and model scales, and the acceleration due to gravity is the same for both, the full- and model-scale trajectories will be the same.

\subsection{Simulation of Sodium Coolant}

In fast reactors the usual coolant is liquid sodium. Sometimes sodium-potassium eutectic mixture ( $\mathrm{NaK}$ ), which is liquid at room temperature, is substituted, but both are difficult liquids to handle experimentally. In work using explosive charges, safety problems become important and are difficult to solve when hot liquid sodium or $\mathrm{NaK}$ are used as the coolant in the model. Also in the case of hot sodium the safe introduction of a temperature-sensitive explosive charge into the model reactor would require a major modification of the model structure around the charge. The dynamic similarity of the model and the full-scale structure might be seriously impaired by this, and it is usual to simulate the hot sodium coolant by watcr at room temperature. This is not an exact simulation, however, since the important parameters: density $\rho$,

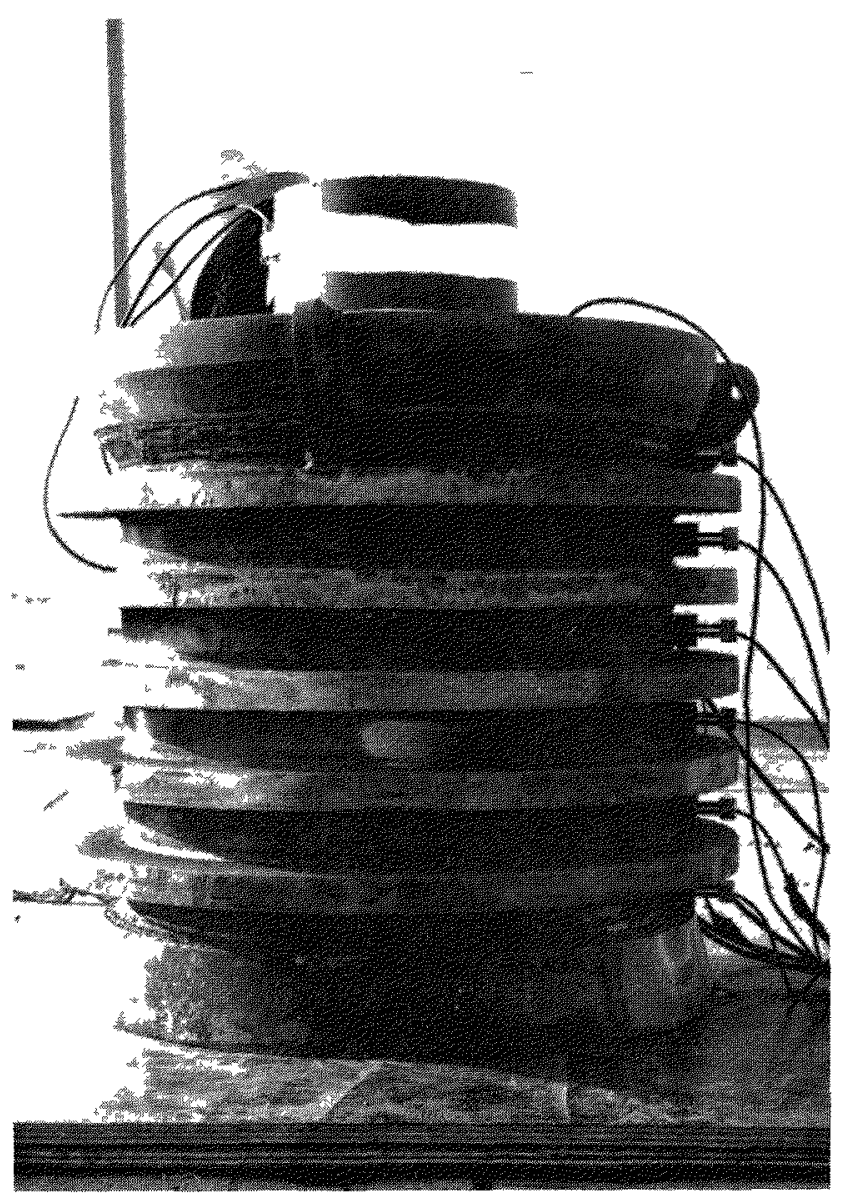

Fic 2 Strong Model Reactor Tank.

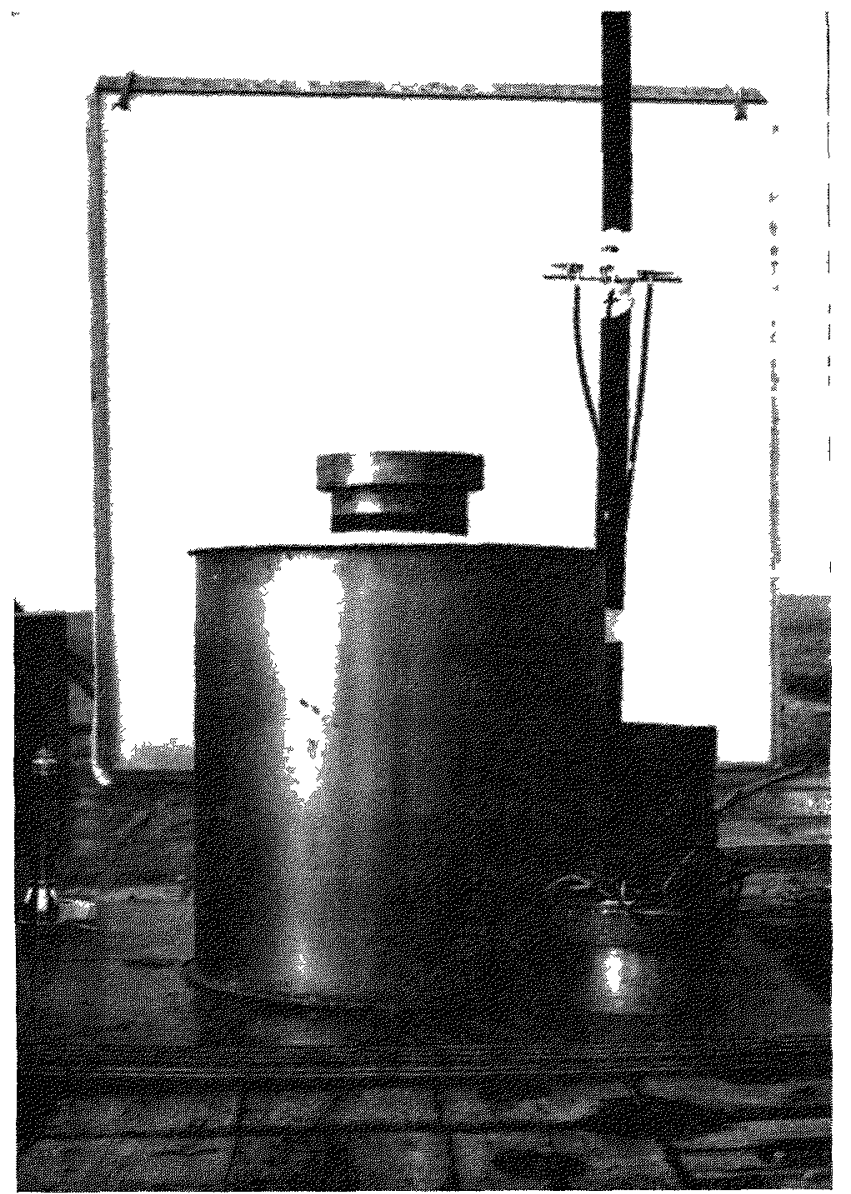

FIg 3 Model Reactor Tank of Minmum Strength.

speed of sound $c$, and acoustic impedance $\rho c$, do not exactly match for the two liquids (see Table 2). The effect of replacing hot sodium coolant by water on the pressure loading produced in small models of fast reactors has been examined separately. ${ }^{(4)}$

\subsection{Properties of Reactor Materials}

It is clear that the energy absorbed from the shock wave by the breeder and core support structure will have an important effect on the strength of the shock reaching the outer parts of the model, such as the primary sodium tank. Thu the parts of the model that will be deformed by the explosion should be made of a steel having a similar (stress-strain) curve as that of the steel of the full-scale structure at its operating temperature. Cnfortunately, suitable dynamic (stress-strain) curves for stainless and other steels are not eurrently available, and the best that can be done is to match the statically measured yield point, ultimate tensile strength, and the permanent elongation at the ultimate tensile stress. The substitution of cold water for the hot sodium coolant in the 


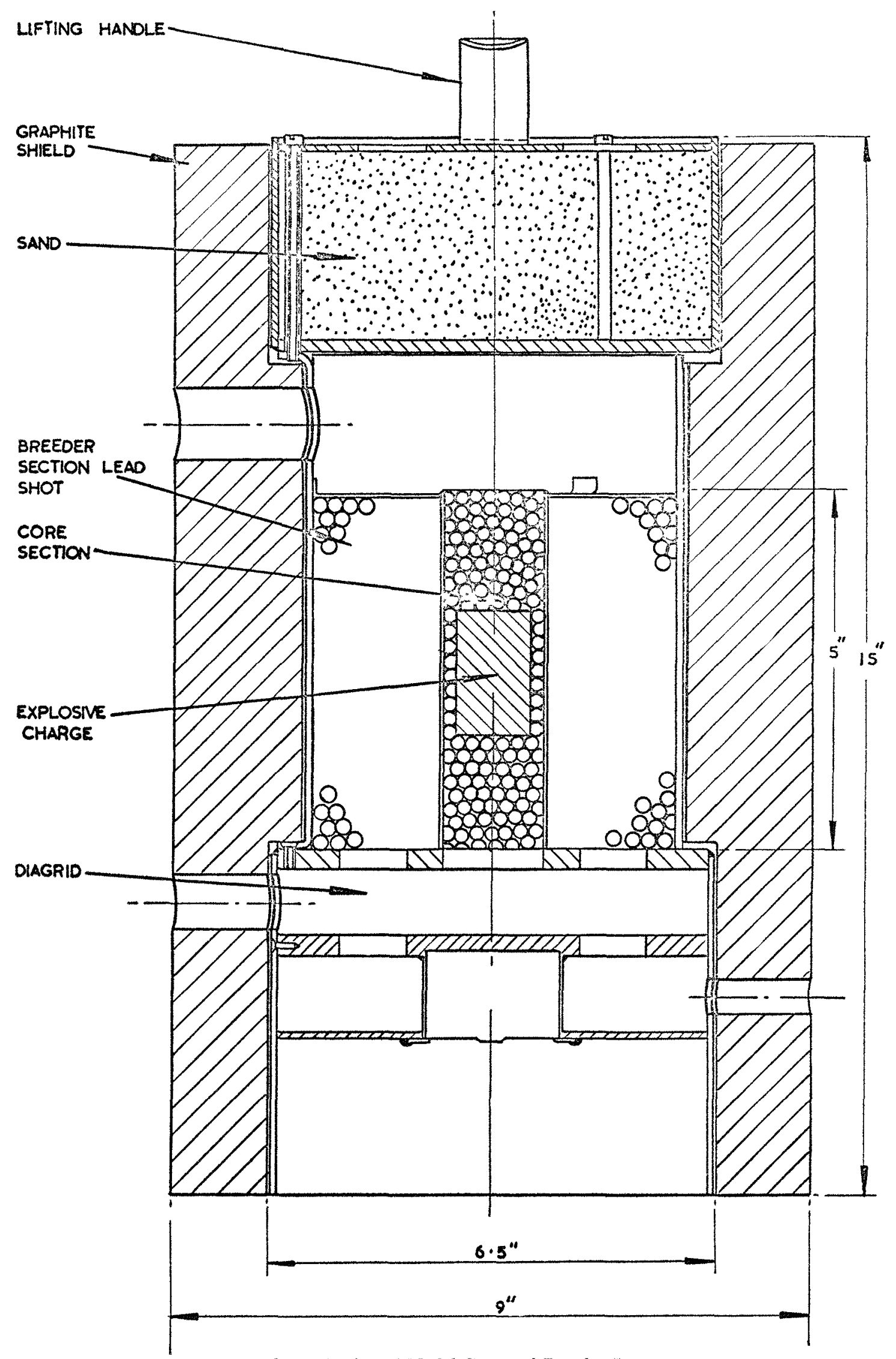

FIg. 4 Cross Section of Model Core and Breeder Structure. 
model means that the model structure will now be at $20^{\circ} \mathrm{C}$ instead of approximately $400^{\circ} \mathrm{C}$. If the model reactor were constructed of stainless steel, then it would be substantially stronger at room temperature and have a greater elongation before maximum stress occurs (see Table 3). The properties of mild steel $\mathrm{EN} 2$ at $20^{\circ} \mathrm{C}$ are very similar to a commonly used stainless steel (EN58B) in the temperature range from 300 to $500^{\circ} \mathrm{C}$ and the latter was used to make the model core support structures used in the experiments. The overall density of the breeder section was simulated by lead shot. A graphite cylinder was used to represent graphite shielding blocks and sand to represent the filling of the upper graphite reflector.

\section{Experimental Method}

In all, nineteen experiments have been performed in cylindrical model tanks. The experimental conditions for 17 are given in Table 4 . The closed, cylindrical model tanks were $24 \mathrm{in}$. in internal diameter and 24 in. deep; explosive charges of $2 \mathrm{oz} R D X / T N T$ $60 / 40$ were detonated inside them (see Fig. 1). The overstrong model reactor tank was used to measure the maximum shock reflexion pressure to be expected. The first model tanks with 1 -in-thick walls and $11 / 2$ in.-thick base and roof were not strong enough to withstand more than one explosion and were replaced by the reinforced type shown in Fig. 2. Here the base and roof were 3 in. thick, and the 1 -in.-thick walls were reinforced by 1.5 -in.-thick bands, 3 in. wide. This reinforcement enabled the tank to be used for three experiments before serious damage occurred. A number of weak vessels (see Fig. 3) made of thin (0.022 in. thick) sheet steel were also used to give the minimum containment of the explosion, to examine the effect of this on the core-plug pressure loadings.

A model of a hypothetical core support structure (see Figures 4 and 5) was made of suitable mild steel sheet. This was used with the explosive charge in the position of the core. The air gap between the surface of the water and the roof of the ressel was varied, but the bare charge was kept at the same height above the floor of the tank. All the possible combinations of the above parameters were not examined; the actual experiments performed are summarised in Table 4.

The experiments with the 24-in.-diameter cylindrical tank were supplemented with experiments at half this scale by use of a square section tank (see Fig. 19 below) with Perspex windows to enable the growth of the explosion products bubble to be examined by high-speed photography.

Most of the pressure measurements were made with use of piezo-electric transducers type MQ20 designed and made at Foulness. The gauge has a natural

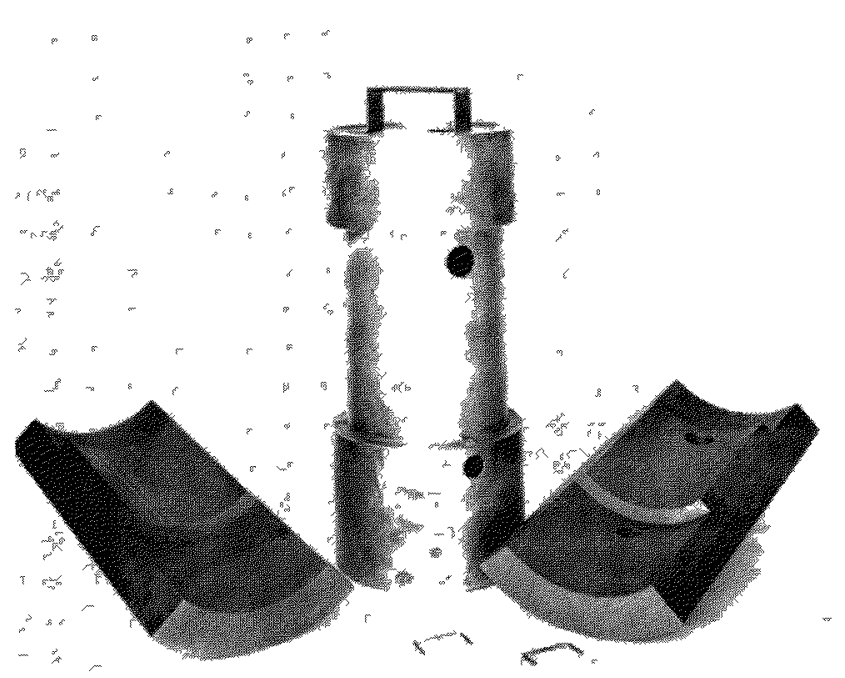

FIG. 5. Model Reactor Core with Part of Graphite shield.

resonance at about $300 \mathrm{kc} / \mathrm{sec}$ and is intended to have a good high-frequency response; it is $0.44 \mathrm{in}$. in diameter, $0.88 \mathrm{in}$. long, and contains a quartz crystal $0.25 \mathrm{in}$. in diameter by $0.125 \mathrm{in}$. thick. The transducer is well damped and is not prone to spurious oscillation. The output of this gauge, which is about 0.5 picocoulomb/psi, was fed to a recording system consisting of amplifiers and display oscilloscopes; the time sweep was provided by a rotating drum camera, and the system had an overall frequency response of about DC-20 kc/s. The impulse per unit area of the measured (pressure, time) curve is defincd as

$$
I=\int_{t_{1}}^{t_{2}} p(t) d t
$$

where $t_{1}$ and $t_{2}$ are the times between which the impulse is measured.

In all the experiments the movement of the plug was observed by means of a high-speed cine camera operating at about 700 frames/sec. In the half-scale experiments, the expansion of the explosion products bubble was photographed at 12,000 half-frames/sec by means of a Fastax camera.

\section{5 . Results}

\subsection{Initial Experiments}

In the first experiment a model tank without a roof was used so that the ejection of the water spray from the tank could be observed by cine cameras. No pressure records were taken. The initial spray thrown upwards by reflexion of the shock wave at the water surface had a speed of $175 \mathrm{ft} / \mathrm{sec}$; this was followed 70 msec later by a more solid body of water moving at $20 \mathrm{ft} / \mathrm{sec}$. No pressure records were obtained from 
the second experiment in a model tank with roof, plug, and a bare charge (see Fig. 1). Useful results were, however, obtained from 17 subsequent experiments in the 24-in.-diameter cylindrical tanks and the three half-scale experiments to observe the bubble growth of the explosion products.

During the early experiments ( 3 and 4 ) it was clear that very high stresses were being produced in the walls of the model reactor tank, since the tanks were extensively damaged after only one or two explosions. In order to see whether large spurious signals were propagated from the tank wall and gauge mount to the body of the gauge, an MQ20 pressure gauge was put in a blind mount for two experiments. The gauge was supported in the vessel wall in the same way as the other gauges, but the sensitive surface was pro-

Table 5. First Peak Presstres Measured in Oyerstrong Tank with an Air Gap of 1.1 In. Between Water Sulrace and Tank Roof

\begin{tabular}{|c|c|c|c|c|c|c|c|c|c|c|c|}
\hline \multirow{3}{*}{$\begin{array}{l}\text { Location of } \\
\text { Pressure Gauge } \\
\text { in Model }\end{array}$} & \multirow{3}{*}{$\begin{array}{l}\text { Position } \\
\text { Number of } \\
\text { Pressure } \\
\text { Gauge }\end{array}$} & \multicolumn{10}{|c|}{ First Peak Pressure (psi) (Peaks A or E of Figures 6,7 , and 8) } \\
\hline & & \multicolumn{7}{|c|}{ Bare Charge } & \multicolumn{3}{|c|}{$\begin{array}{l}\text { Charge Enclosed in Core } \\
\text { Support Structure }\end{array}$} \\
\hline & & $\operatorname{Rd} 3$ & Rd 4 & $\mathrm{Rd} 5$ & $\operatorname{Rd} 10$ & $\operatorname{Rd} 11$ & Rd 16 & $\begin{array}{l}\text { Mean, } \\
\text { psi }\end{array}$ & $\operatorname{Rd} 6$ & $\operatorname{Rd} 7$ & $\begin{array}{c}\text { Mean, } \\
\text { psi }\end{array}$ \\
\hline Tank Roof & $\begin{array}{l}7 \\
8 \\
9\end{array}$ & & 4,360 & $\begin{array}{l}>4,060 \\
>4,530\end{array}$ & $\begin{array}{r}6,060 \\
>6,440 \\
>4,980\end{array}$ & $\begin{array}{r}7,710 \\
15,900 \\
>7,490\end{array}$ & $\begin{array}{r}11,700 \\
>14,900 \\
13,100\end{array}$ & $\begin{array}{r}7,460 \\
15,900 \\
13,100\end{array}$ & $\begin{array}{l}3,390 \\
5,890\end{array}$ & $\begin{array}{l}6,690 \\
5,870\end{array}$ & $\begin{array}{l}5,040 \\
5,880\end{array}$ \\
\hline
\end{tabular}

Table 6. Impulse Menstred by Pressure Gavges in Overstrong Tank wite an Air Gap of 1.1 In. between Water SURFace and TiNk RoOF

\begin{tabular}{|c|c|c|c|c|c|c|c|c|c|c|c|}
\hline $\begin{array}{l}\text { Location of Pressure } \\
\text { Gauge in Model }\end{array}$ & \multirow{2}{*}{$\begin{array}{l}\text { Position } \\
\text { Number of } \\
\text { Pressure } \\
\text { Gauge }\end{array}$} & \multicolumn{7}{|c|}{ Bare Charge } & \multicolumn{3}{|c|}{$\begin{array}{l}\text { Charge Enclosed in Core } \\
\text { Support Structure }\end{array}$} \\
\hline & & $\mathrm{Rd} 3$ & $\operatorname{Rd} 4$ & $\operatorname{Rd} 5$ & $\operatorname{Rd} 10$ & $\mathrm{Rd} 11$ & $\operatorname{Rd} 16$ & $\begin{array}{l}\text { Mean, } \\
\text { psi-msec }\end{array}$ & $\mathrm{Rd} 6$ & $\operatorname{Rd} 7$ & $\begin{array}{l}\text { Mean, } \\
\text { psi-msec }\end{array}$ \\
\hline Tank Roof & $\begin{array}{l}7 \\
8 \\
9\end{array}$ & & 2,430 & $\begin{array}{r}>1,490 \\
1,010\end{array}$ & $\begin{array}{l}\text { Over } \\
1,000 \\
>850 \\
>700\end{array}$ & 1,450 & $\begin{array}{l}2,090 \\
\\
2,340 \\
2,290\end{array}$ & $\begin{array}{l}1,990 \\
2,340 \\
1,650\end{array}$ & $>1,800$ & $\begin{array}{r}2,740 \\
>1,610\end{array}$ & $\begin{array}{r}2,740 \\
>1,610\end{array}$ \\
\hline Core-access Plug & $\begin{array}{l}10 \\
11 \\
12\end{array}$ & & & & & $\begin{array}{l}>1,300^{*} \\
>1,100^{*}\end{array}$ & $\begin{array}{l}1,540 \\
1,900 \\
1,830\end{array}$ & $\begin{array}{l}1,540 \\
1,900 \\
1,830\end{array}$ & $\begin{array}{l}>1,300 \\
>1,500\end{array}$ & 2,220 & $\begin{array}{r}2,220 \\
>1,300 \\
>1,500\end{array}$ \\
\hline
\end{tabular}

* Estimated. 
tected from the pressure pulse. Only very small oscillations of negligible amplitude were recorded from this gauge; the pressure records obtained are therefore thought to be free from spurious interference of this kind.

\subsection{Results from Experiments in an Overstrong Tank} with an Air Gap of 1.1 In. between Water Surface and Tank Roof

The measurements of peak pressure and impulse obtained are given in Tables 5 and 6 . (Summaries of the mean peak pressures and impulses of core plug and tank roof, and the core-plug ejection velocities are given in Table 10 below.) The results of Round 3, 4, and 10 are given although the tanks were severely damaged, allowing venting of the internal pressures early in the experiment. The impulses measured by the pressure gauges in these rounds are low compared with those from Rounds 5, 11, and 16. The plug-ejection velocities measured in Rounds 3,4 , and 10 are also low.

The shock-reflexion and liquid-impact effects are clearly illustrated by the pressure records; a complete set of records was obtained from Round 16, for which a bare charge was used. Photographs of these are shown in Figures 6, 7, and 8; the direct shock wave from the explosion (A), the reflected shock from the base of the model (B), and the rarefaction from the free surface (C) can be seen. These shock waves were propagated at a velocity of about $6,100 \mathrm{ft} / \mathrm{sec}$. The first direct shock (A) striking the tank produced a shock which was transmitted through the steel to the gauges in the roof in gauge positions 7,8 , and 9 , producing the small oscillations due to body signal which start at (D). These oscillations were not recorded in positions 10,11, and 12 in the plug, due to the attenuation of the shock in the wall of the tank by the small clearance gap around the plug. The main pressure loading on the roof starts at (E), and this was probably due to a layer of water or spray thrown up by the reflection of (A) at the water surface. This pressure pulse occurred $0.62 \mathrm{msec}$ after the arrival of the initial shock (A) at the water surface, and the water or spray would have to travel $1.1 \mathrm{in}$. in this time, giving a velocity of $149 \mathrm{ft} / \mathrm{sec}$; this agrees approximately with the initial spray velocity of $174 \mathrm{ft} / \mathrm{sec}$ measured photographically in Round 1 . There is a second pulse (G) recorded by the gauges in the plug, and this may be due to the impact of a second layer of water thrown up by the reflection of $(B)$ at the water surface. The shock-pressure loading on the roof which starts at (E) appears to be reflected back
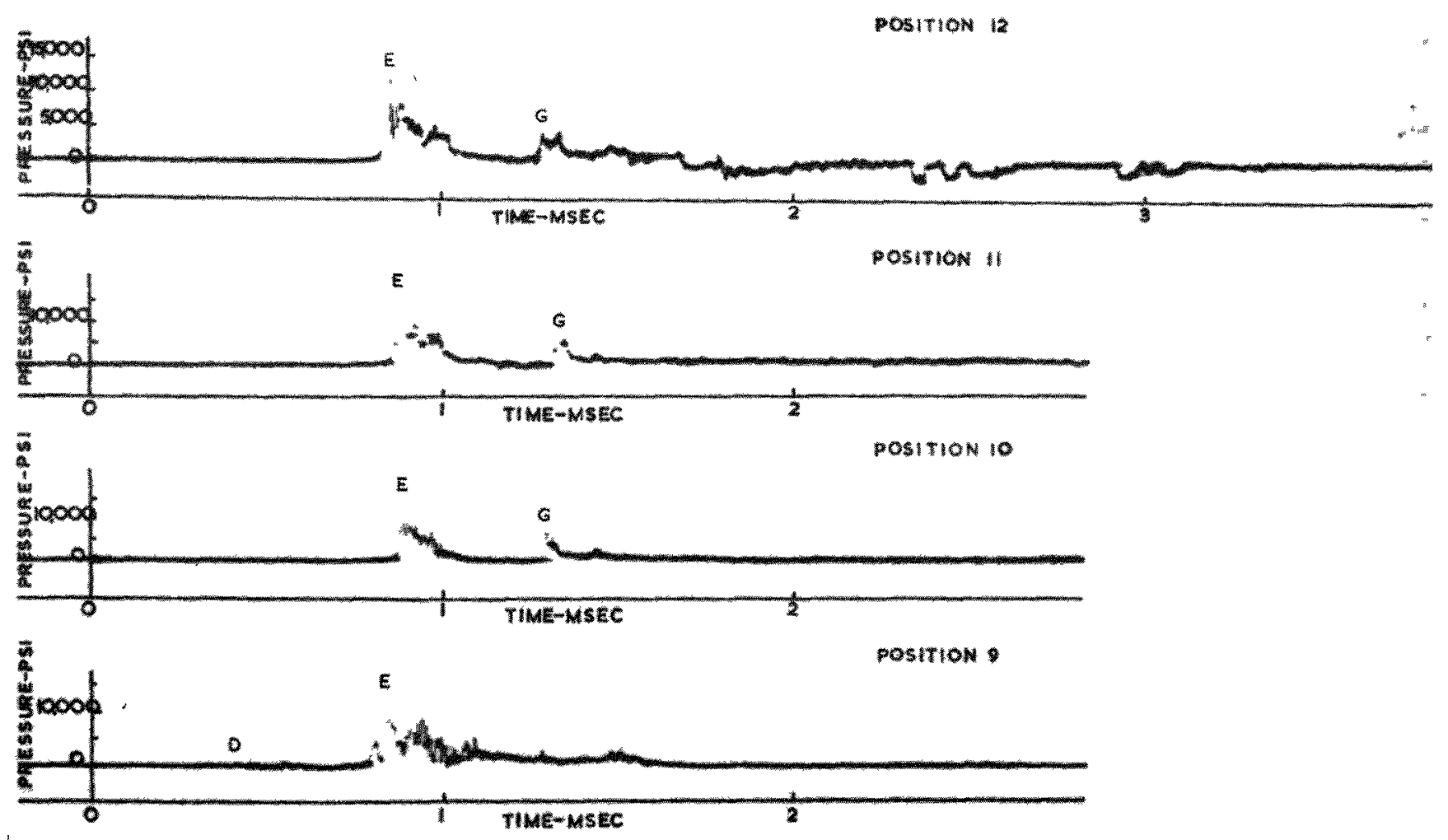

Fıg. 6. Records from Round 16 at Positions 9-12. 


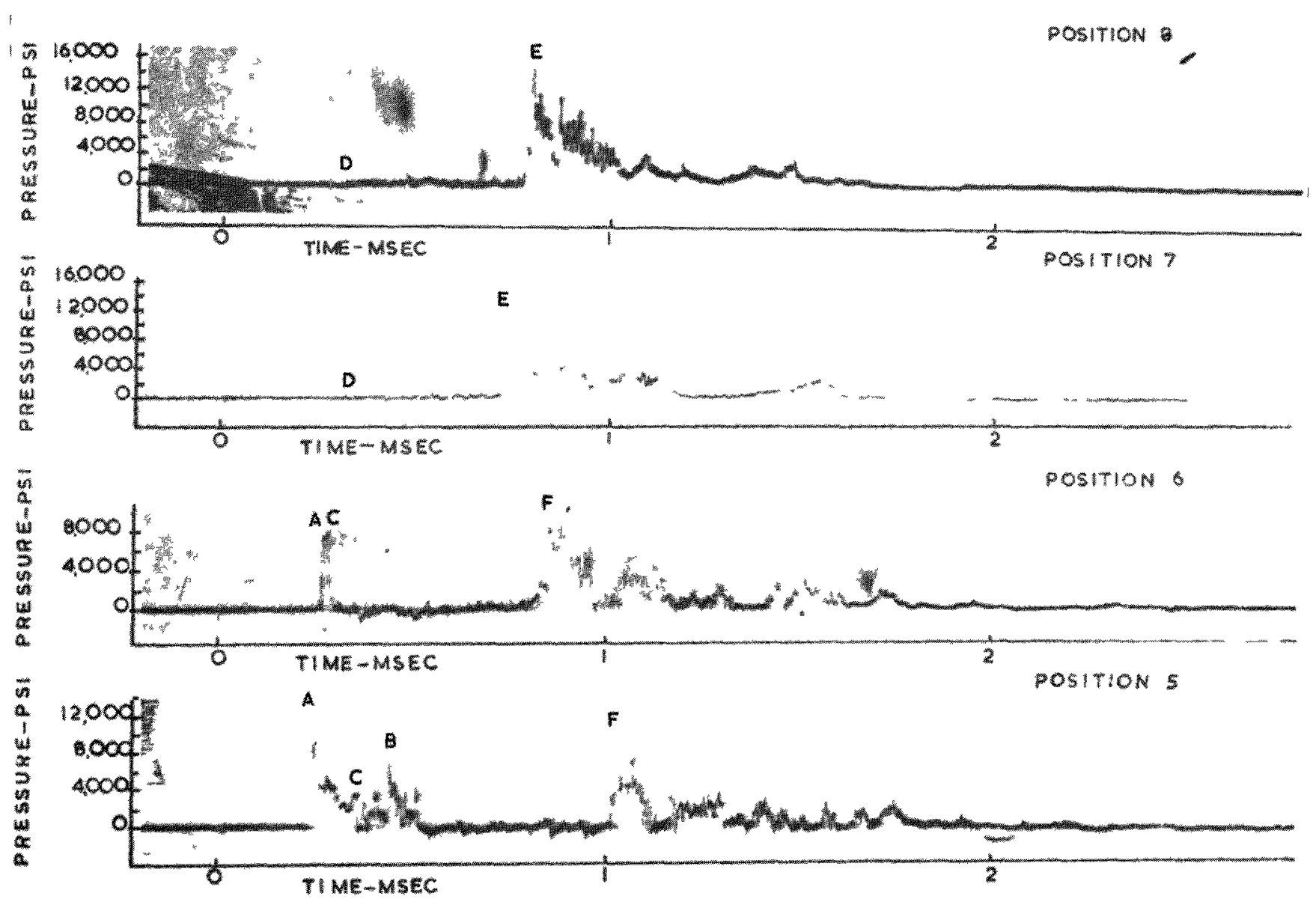

FIG 7 Records from Round 16 at Positions 5-8.

through the contents of the ressel, producing a second pulse (F) on the gauges in the side of the model. This pulse has an apparent velocity of about 1,600 $\mathrm{ft} / \mathrm{sec}$ between gauge positions 6 and 5 , falling to $1200 \mathrm{ft} / \mathrm{sec}$ between positions 5 and 4 ; at position 3 the amplitude of the pulse is very small.

The effect of enclosing the explosive charge in a model core support structure (see Figures 4 and 5 ) on the shock waves transmitted to the walls and roof of the tank is shown by Round 7 (see Frgures 9, 10, and 11).

The initial drect shocks measured at positions 1 to 6 lost their elassical shape after passing through the simulated breeder and reflector as compared to the results from Round 16 The peak pressures and the impulses were lower, except for the plug gauge positions. There was also a second pressure pulse some time after the first, probably caused by the bursting core support structure after it had been distorted and expanded by the explosion products

Some of the charge detonation energy has been used up in disrupting the core structure; the propor- tion of charge energy imparted to the plug has fallen from $1.8 \%$ for a bare charge to $1.4 \%$ for the enclosed charge (see Tables 10 and 11 below).

\subsection{Results from Experiments in an Overstrong Tank with Air Gaps from Zero to 8.3 In.}

The measurements of peak impact pressures and impulse are given in Tables 7 and 8 and summarised together with plug ejection velocities in Table 10 below. As would be expected, the highest peak pressures and impulses and the highest core-plug ejection velocity were measured in Round 19, in which no air gap was used.

The effect of increasing the air gap is shown in the pressure records from Round 17 (see Figures 12, 13, and 14). In the gauge positions below the water surface ( 1 to 4$)$ the records are similar to those from Round 16. At position 5 the rapid cutoff due to the rarefaction wave from the free water surface is seen. In the case of the $83-1 n$. air gap, there is a long delay in the arrival of the spray at the roof, though the pressure records are of similar shape. 


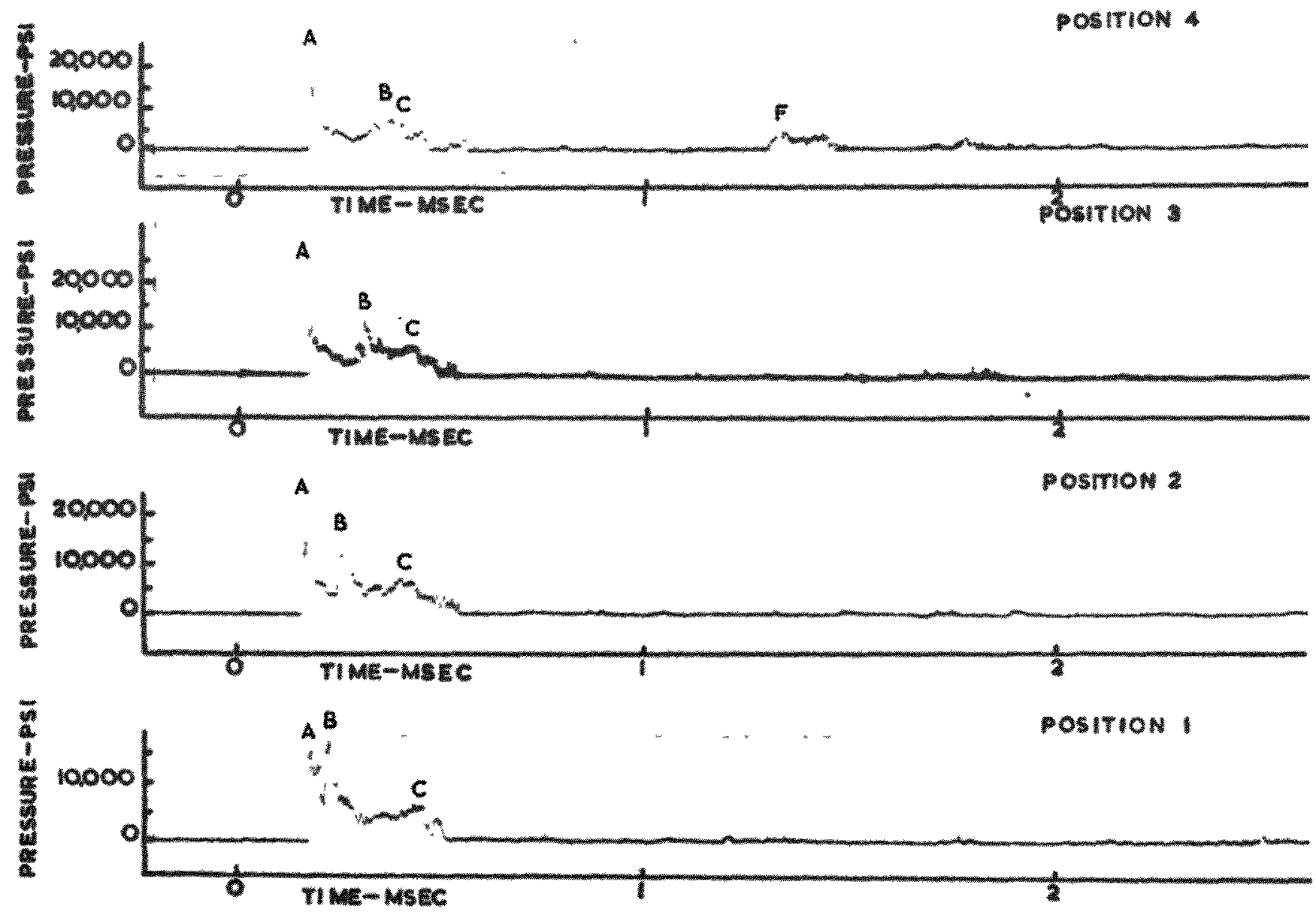

Fic. 8. Records from Round 16 at Positions 1-4.
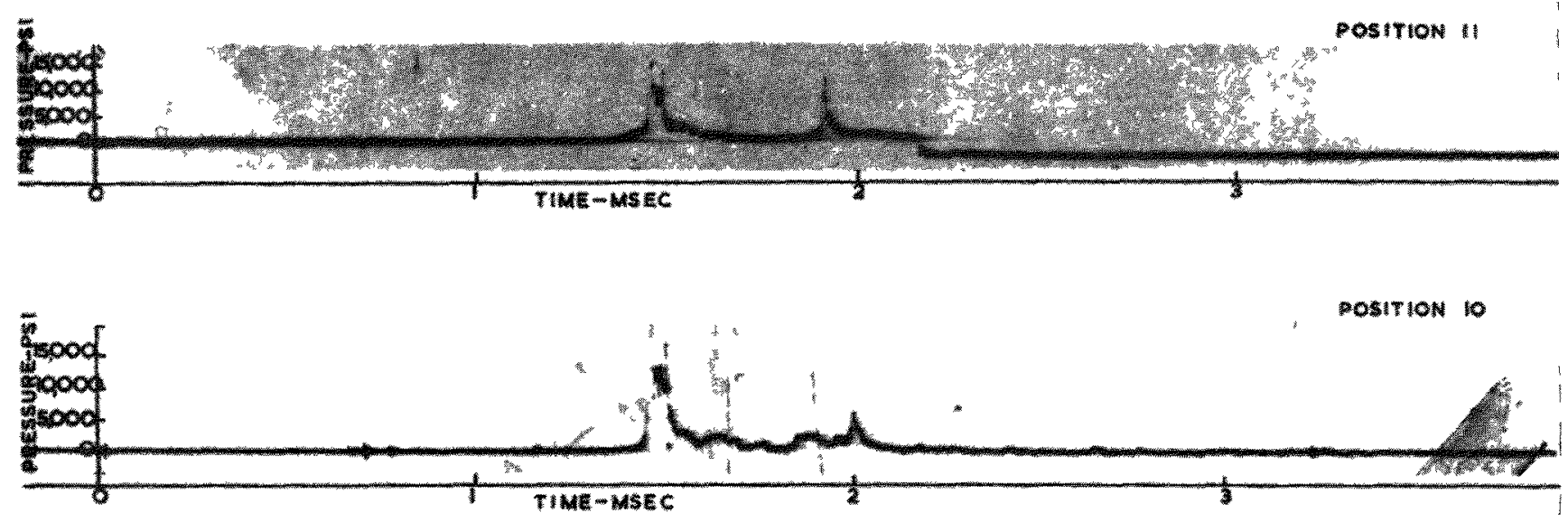

FIG. 9. Records fron Round 7 at Positions 10 and 11. 


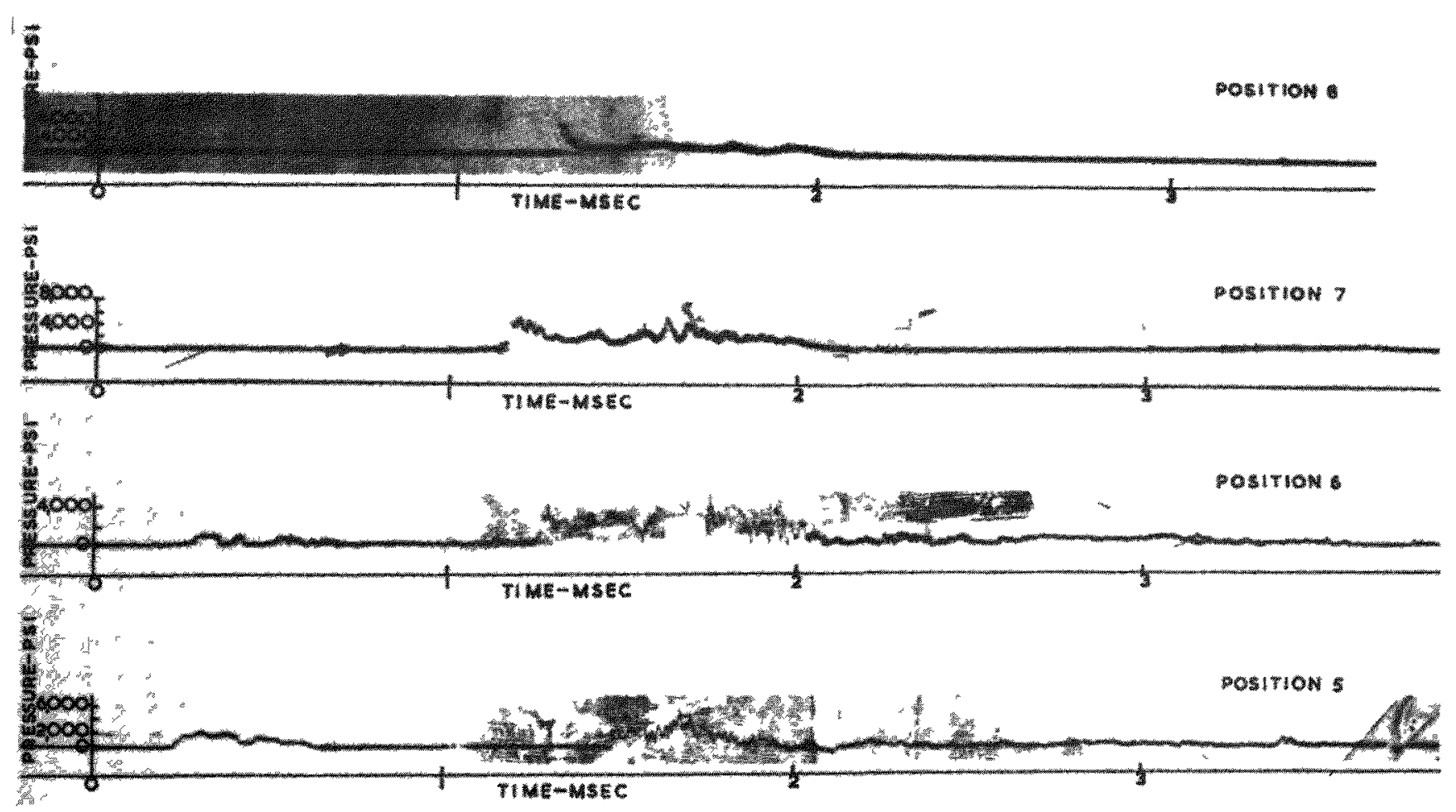

F1G. 10. Records from Round 7 at Positions 5-8.
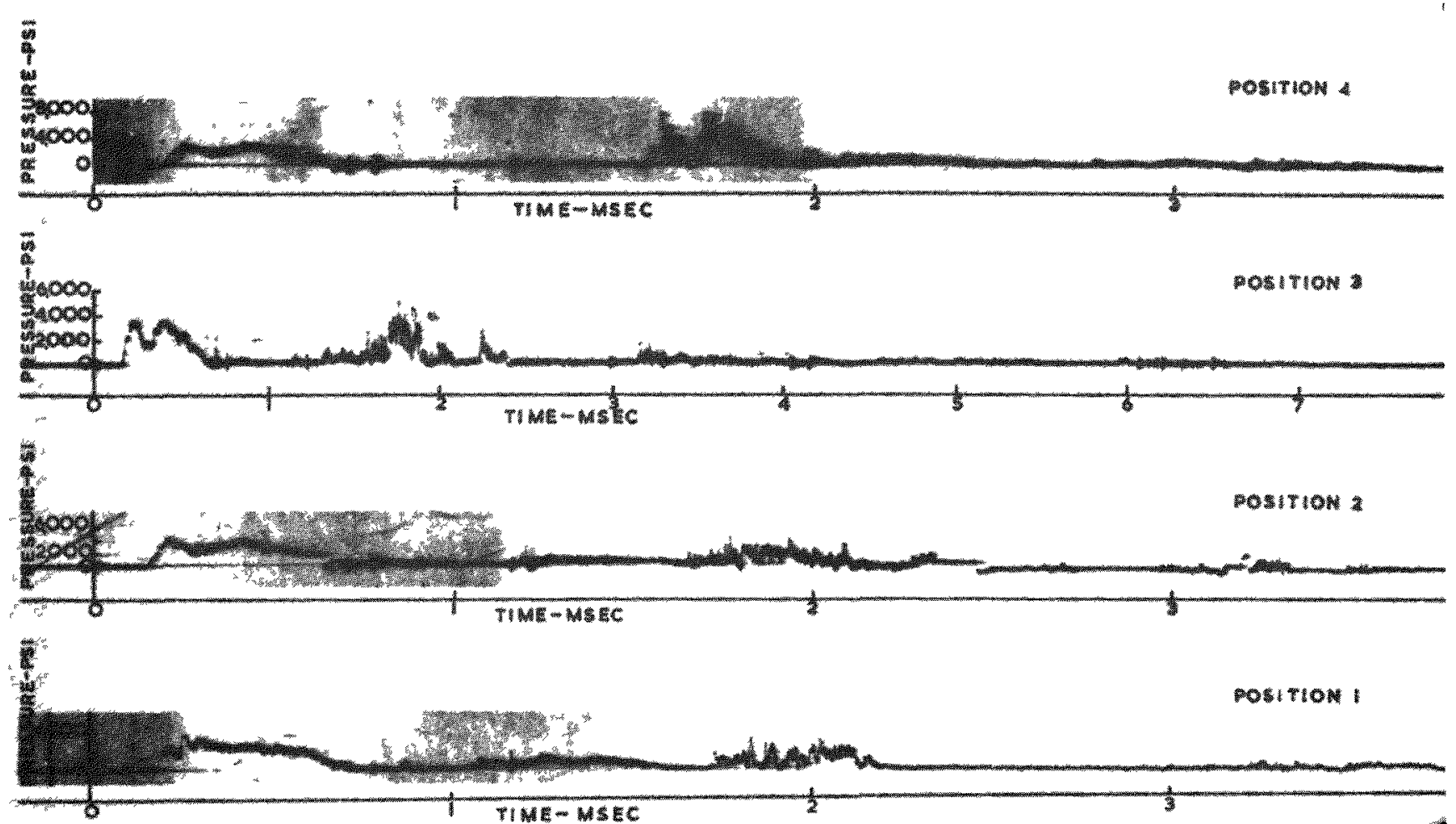

poratroul|

Frg. 11. Records from Round 7 at Positions 1-4. 
Table 7. First Peak Presstres Measlrei in Overstrong Tank Using a B ire Explosive Charge for Difierent Air Gaps BETWEEN WATER Sit RFICE AND TANK ROOF

\begin{tabular}{|c|c|c|c|c|c|c|c|c|c|c|c|c|c|c|c|}
\hline \multirow{3}{*}{$\begin{array}{c}\text { Location of Pressure } \\
\text { Gauge in Model }\end{array}$} & \multirow{3}{*}{$\begin{array}{l}\text { Position } \\
\text { Number of } \\
\text { Pressure } \\
\text { Gauge }\end{array}$} & \multicolumn{14}{|c|}{ Fïrst Peak Pressure (psi) (Peaks A or E of Figure 6, 7, and 8) } \\
\hline & & \multirow{2}{*}{$\frac{\text { No Gap }}{\operatorname{Rd} 19}$} & \multicolumn{7}{|c|}{ 1.1-in. Air Gap } & \multicolumn{3}{|c|}{ 4-in. Air Gal, } & \multicolumn{3}{|c|}{ 8.3-in. Air Gap } \\
\hline & & & $\mathrm{Rd} 3$ & $\mathrm{Rd} 4$ & $\operatorname{Rd} 5$ & $\operatorname{Rd} 10$ & Rd 11 & Rd 16 & $\begin{array}{l}\text { Mean, } \\
\text { psi }\end{array}$ & Rd 17 & Rd 18 & $\begin{array}{l}\text { Mean, } \\
\text { psi }\end{array}$ & $\operatorname{Rd} 8$ & $\mathrm{Rd} 9$ & $\begin{array}{l}\text { Mean, } \\
\text { psi }\end{array}$ \\
\hline Tank Side Wall & $\begin{array}{l}1 \\
2 \\
3 \\
4 \\
5 \\
6\end{array}$ & $\begin{array}{r}20,300 \\
9,740 \\
19,800 \\
23,700 \\
10,100 \\
13,100\end{array}$ & $\begin{array}{c}12,500 \\
11,800 \\
12,600 \\
\text { Blanked } \\
12,100 \\
9,850\end{array}$ & $\begin{array}{c}13,000 \\
15,200 \\
13,600 \\
16,700 \\
\text { Blanked } \\
9,080\end{array}$ & $\begin{array}{r}15,100 \\
9,630 \\
12,000 \\
9,530 \\
7,460\end{array}$ & $\begin{array}{r}12,400 \\
19,100 \\
17,400 \\
12,400 \\
>6,410 \\
6,780\end{array}$ & $\begin{array}{r}15,500 \\
22,400 \\
22,700 \\
21,800 \\
>9,950 \\
10,500\end{array}$ & $\begin{array}{r}15,300 \\
21,200 \\
23,100 \\
20,400 \\
12,900 \\
7,380\end{array}$ & $\begin{array}{r}13,900 \\
16,600 \\
16,900 \\
16,200 \\
12,500 \\
8,510\end{array}$ & $\begin{array}{r}13,100 \\
17,100 \\
25,900 \\
21,800 \\
11,600 \\
5,280\end{array}$ & $\begin{array}{r}14,600 \\
24,700 \\
24,100 \\
21,800 \\
10,400 \\
6,160\end{array}$ & $\begin{array}{r}13,850 \\
20,900 \\
25,000 \\
21,800 \\
11,000 \\
5,720\end{array}$ & $\begin{array}{r}19,800 \\
12,300 \\
13,700 \\
8,500 \\
\\
1,310\end{array}$ & $\begin{array}{r}20,700 \\
7,110 \\
13,200 \\
13,800 \\
4,520 \\
3,010\end{array}$ & $\begin{array}{r}20,200 \\
9,700 \\
13,400 \\
11,200 \\
4,520 \\
2,160\end{array}$ \\
\hline Tank Roof & $\begin{array}{l}7 \\
8 \\
9\end{array}$ & $\begin{array}{l}10,000 \\
11,000 \\
10,600\end{array}$ & & 4,360 & $\begin{array}{l}>4,000 \\
>4,530\end{array}$ & $\begin{array}{r}6,060 \\
>6,440 \\
>4,980\end{array}$ & $\begin{array}{r}7,710 \\
15,900 \\
>7,490\end{array}$ & $\begin{array}{r}11,700 \\
>14,900 \\
13,100\end{array}$ & $\begin{array}{r}7,460 \\
15,900 \\
13,100\end{array}$ & $\begin{array}{r}6,390 \\
8,000 \\
10,300\end{array}$ & $\begin{array}{r}8,750 \\
13,900 \\
8,970\end{array}$ & $\begin{array}{r}7,570 \\
10,900 \\
9,635\end{array}$ & $\begin{array}{l}7,260 \\
1,580\end{array}$ & $\begin{array}{l}4,590 \\
2,120 \\
2,080\end{array}$ & $\begin{array}{l}5,920 \\
2,120 \\
1,830\end{array}$ \\
\hline Core-access Plug & $\begin{array}{l}10 \\
11 \\
12\end{array}$ & $\begin{array}{l}20,200 \\
17,500 \\
15,800\end{array}$ & & & $>6,420$ & $\begin{array}{l}>5,110 \\
>5,410\end{array}$ & $\begin{array}{l}>11,100 \\
>12,800 \\
>12,800\end{array}$ & $\begin{array}{l}14,200 \\
14,900 \\
12,500\end{array}$ & $\begin{array}{l}14,200 \\
14,900 \\
12,600\end{array}$ & $\begin{array}{l}6,770 \\
4,000 \\
7,770\end{array}$ & $\begin{array}{r}7,410 \\
7,020 \\
>8,850\end{array}$ & $\begin{array}{r}7,090 \\
\mathbf{5}, 510 \\
>8,310\end{array}$ & $\begin{array}{l}3,210 \\
4,060 \\
4,440\end{array}$ & $\begin{array}{l}4,630 \\
3,600 \\
4,420\end{array}$ & $\begin{array}{l}3,920 \\
3,830 \\
4,430\end{array}$ \\
\hline
\end{tabular}

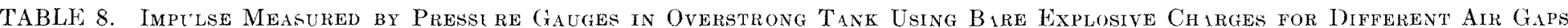
Between Water Surface IND TINK RoOF

\begin{tabular}{|c|c|c|c|c|c|c|c|c|c|c|c|c|c|c|c|}
\hline \multirow{3}{*}{$\begin{array}{l}\text { Location Pressure } \\
\text { Gauge in Model }\end{array}$} & \multirow{3}{*}{$\begin{array}{c}\text { Position } \\
\text { Number of } \\
\text { Pressure Gauge }\end{array}$} & \multicolumn{14}{|c|}{ Impulse from pressure-gauge records, psi-msec. } \\
\hline & & \multirow{2}{*}{$\begin{array}{l}\text { No Gap } \\
\text { Exp } 19\end{array}$} & \multicolumn{7}{|c|}{ 1.1-in. Air Gap } & \multicolumn{3}{|c|}{ 4-in Air Gap } & \multicolumn{3}{|c|}{ 8.3-in. Air Gap } \\
\hline & & & $\operatorname{Exp} 3$ & Epx 4 & $\operatorname{Exp} 5$ & Exp 10 & $\operatorname{Exp} 11$ & Fixp 16 & $\begin{array}{c}\text { Mean, } \\
\text { psi-msec }\end{array}$ & $\operatorname{Exp} 17$ & $\operatorname{Fip} 18$ & $\begin{array}{c}\text { Mean. } \\
\text { psi-msec }\end{array}$ & Exp 8 & $\operatorname{Exp} 9$ & $\begin{array}{l}\text { Mean, } \\
\text { psi-msec }\end{array}$ \\
\hline Tank Side Wall & $\begin{array}{l}1 \\
2 \\
3 \\
4 \\
5 \\
6\end{array}$ & $\begin{array}{l}1,810 \\
1,340 \\
1,330 \\
1,290 \\
2,150 \\
3,620\end{array}$ & $\begin{array}{r}1,420 \\
1,350 \\
1,180 \\
\\
917 \\
194\end{array}$ & $\begin{array}{r}1,490 \\
1,320 \\
1,540 \\
1,390 \\
\\
197\end{array}$ & $\begin{array}{l}2,580 \\
1,690 \\
1,630 \\
1,240\end{array}$ & $\begin{array}{r}908 \\
1,090 \\
710 \\
639 \\
319\end{array}$ & $\begin{array}{r}1,200 \\
1,820 \\
1,700 \\
980 \\
908 \\
348\end{array}$ & $\begin{array}{r}2,030 \\
2,180 \\
1,840 \\
1,540 \\
807 \\
182\end{array}$ & $\begin{array}{r}1,740 \\
1,540 \\
1,500 \\
1,170 \\
818 \\
248\end{array}$ & $\begin{array}{r}1,880 \\
1,740 \\
2,030 \\
1,090 \\
264 \\
773\end{array}$ & $\begin{array}{r}1,830 \\
2,080 \\
1,740 \\
1,200 \\
272 \\
597\end{array}$ & $\begin{array}{r}1,855 \\
1,910 \\
1,885 \\
1,145 \\
268 \\
685\end{array}$ & $\begin{array}{r}1,240 \\
961 \\
539 \\
159 \\
\\
877\end{array}$ & $\begin{array}{r}1,840 \\
1,140 \\
1,050 \\
385 \\
176 \\
1,900\end{array}$ & $\begin{array}{r}1,540 \\
1,050 \\
794 \\
272 \\
176 \\
1,390\end{array}$ \\
\hline Tank Roof & $\begin{array}{l}7 \\
8 \\
9\end{array}$ & $\begin{array}{l}2,000 \\
2,300 \\
1,800\end{array}$ & & 2,430 & $\begin{array}{r}>1,490 \\
1,010\end{array}$ & $\begin{array}{r}>1,000 \\
>850 \\
>700\end{array}$ & $\begin{array}{r}1,450 \\
>1,160\end{array}$ & $\begin{array}{l}2,090 \\
2,340 \\
2,290\end{array}$ & $\begin{array}{l}1,990 \\
2,340 \\
1,650\end{array}$ & $\begin{array}{l}1,740 \\
1,900 \\
1,700\end{array}$ & $\begin{array}{l}2,150 \\
2,200 \\
1,900\end{array}$ & $\begin{array}{l}1,950 \\
2,050 \\
1,800\end{array}$ & $\begin{array}{l}1,820^{*} \\
1,220^{*}\end{array}$ & $\begin{array}{l}1,320^{*} \\
2,140^{*} \\
1,810^{*}\end{array}$ & $\begin{array}{l}1,570^{*} \\
2,140^{*} \\
1,520^{*}\end{array}$ \\
\hline Core-access Plug & $\begin{array}{l}10 \\
11 \\
12\end{array}$ & $\begin{array}{r}>630 \\
>1,250 \\
>1,990\end{array}$ & & & & & $\begin{array}{l}>1,300^{*} \\
>1,100^{*}\end{array}$ & $\begin{array}{l}1,540 \\
1,900 \\
1,830\end{array}$ & $\begin{array}{l}1,540 \\
1,900 \\
1,830\end{array}$ & $\begin{array}{l}1,650 \\
2,250 \\
2,280\end{array}$ & $\begin{array}{r}>1,130 \\
1,570 \\
1,600\end{array}$ & $\begin{array}{l}1,650 \\
1,910 \\
1,940\end{array}$ & $\begin{array}{r}1,900^{*} \\
600^{*} \\
1,600^{*}\end{array}$ & $\begin{array}{l}1,000^{*} \\
1,750^{*} \\
1,680\end{array}$ & $\begin{array}{l}1,450^{*} \\
1,170^{*} \\
1,640\end{array}$ \\
\hline
\end{tabular}

* Estimated. 

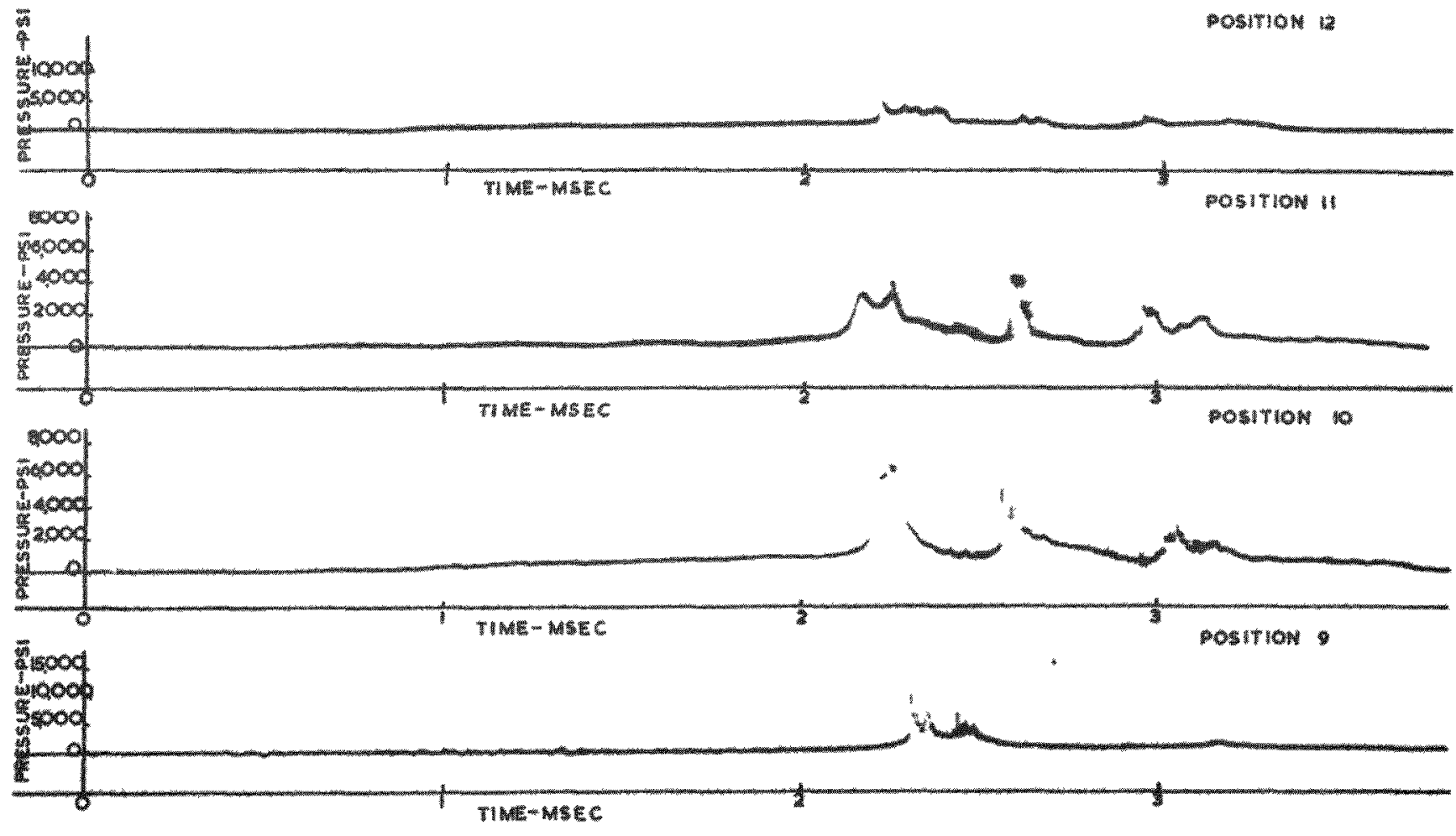

FIG 12 Records from Round 17 at Positions 9-12
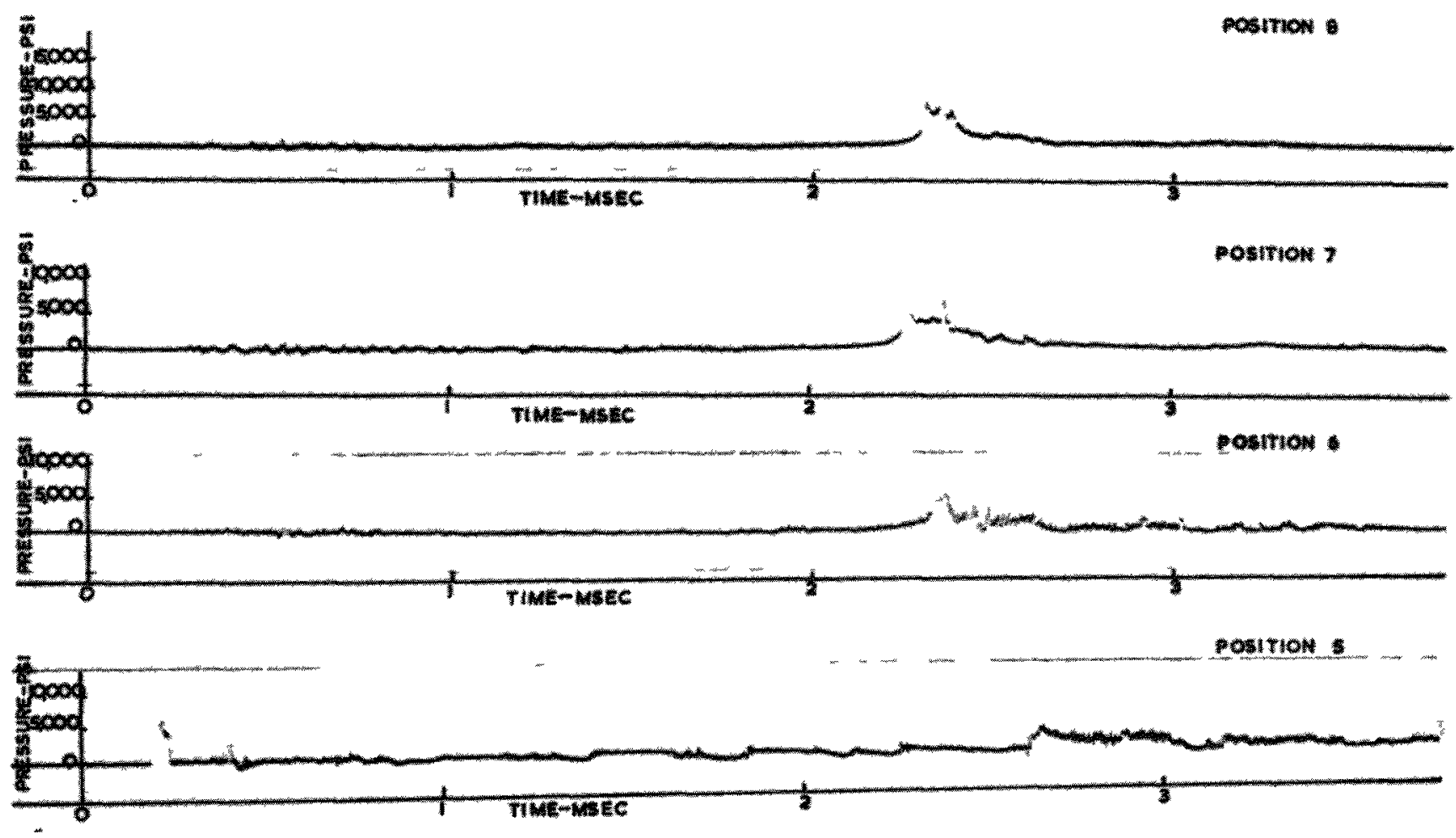

FIt 13 Records from Round 17 at Postions 5-8. 

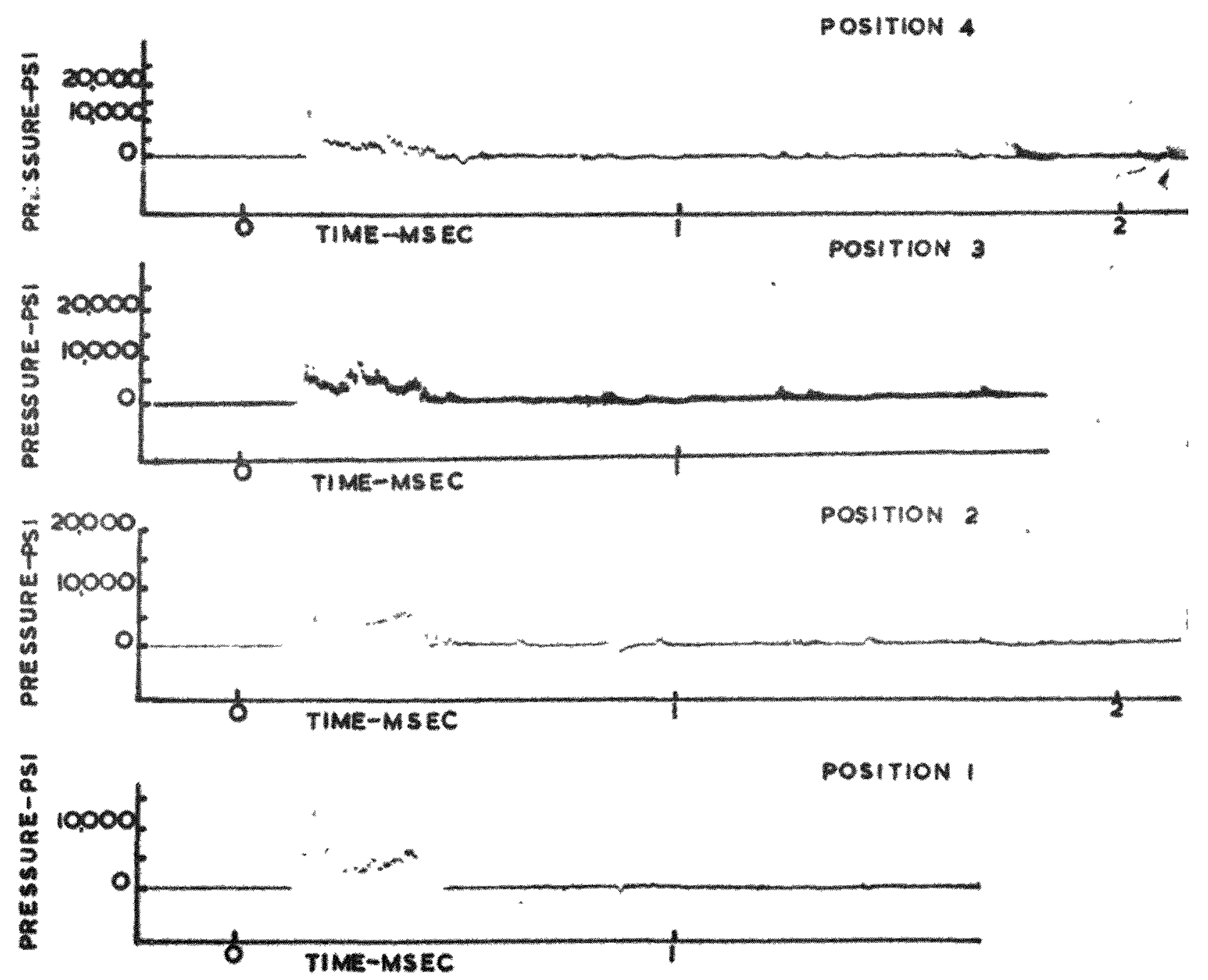

Fig. 14. Records from Round 17 at Positions 1-4.

The mean peak impact pressures on the core plug and tank roof are plotted in Fig. 15 versus air gap and the mean impulse per unit area versus air gap in Fig. 16. The peak pressures decrease rapidly with increasing air gap, though the impulse decreases only slowly. The measured plug ejection velocities also decrease with increasing air gap.

\subsection{Results of Experiments with a Weak Tank and 1.1-in. Air Gap}

The peak pressures and impulses measured on the core plug are given in Table 9. The plug ejection velocities given in Table 10 are much lower than the values obtained using the strong tank. The plug pressure records obtained are shown in Fig. 17 for the bare charge and Fig. 18 for the enclosed charge. The shocks incident on the side walls and base of the weak tank would be reflected as rarefactions, and since these are rapidly attenuated by cavitation the main pulse is shorter (see Fig. 17A for effect of explosion on weak-model reactor tank). The second pulse (G) seen in Round 16 does not appear in the bare-charge experiment. In the enclosed-charge case the pressure records are similar to those obtained in Round 7 , though again of smaller amplitude.

Some of the explosion energy released was absorbed in distorting and bursting the core support structure. Howerer, the velocity of plug ejection was increased, and it appears that of the remaining energy more was channelled upwards than before.

\subsection{Results of Half-scale Experiments to Examine Bubble Growth}

Cross sections of the observation tank are shown in Fig. 19 and a photograph in Fig. 20. Two preliminary experiments (Rounds 20 and 21) to establish 


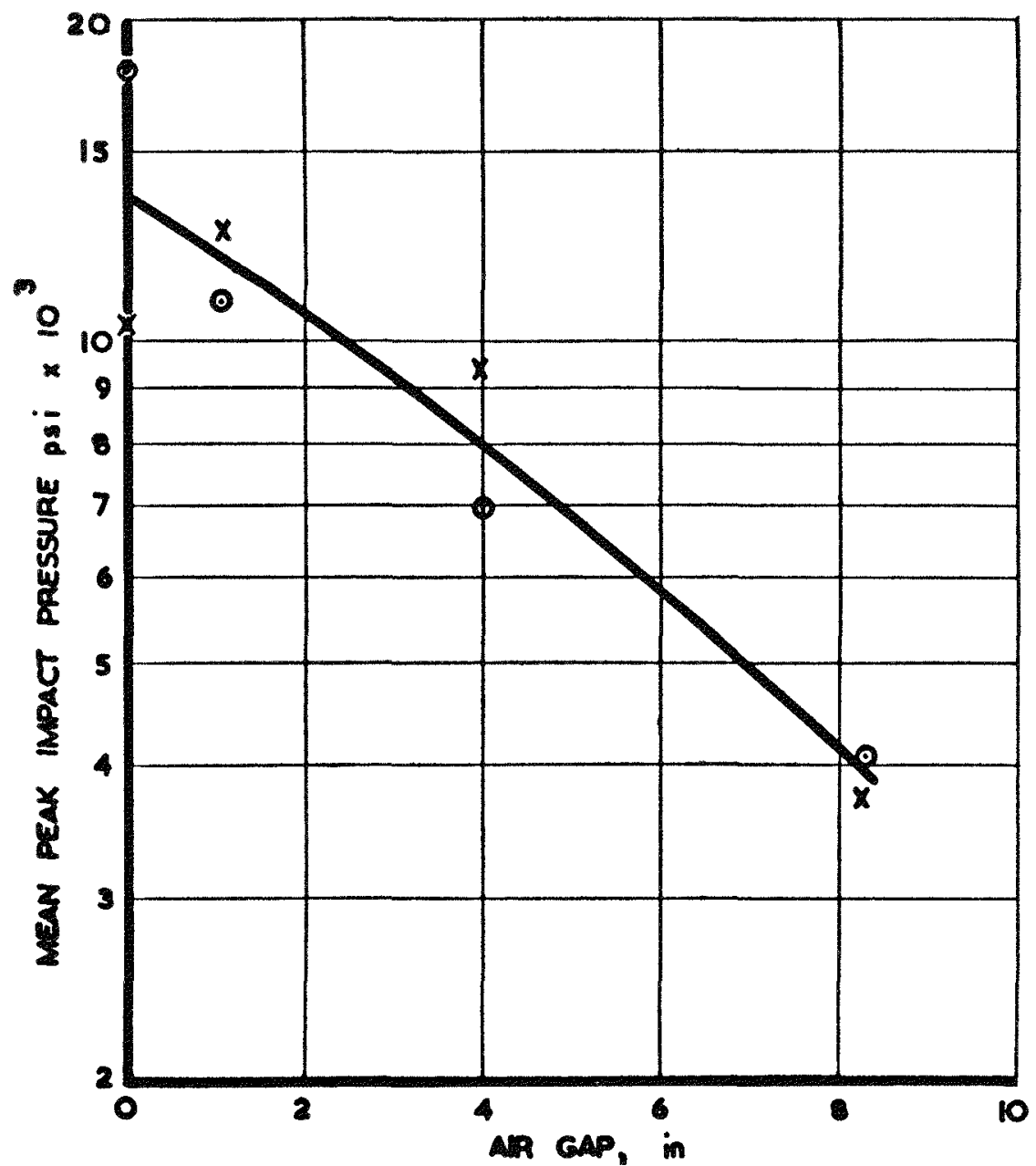

Fig. 15. Mean Peak Impact Pressure on Core Plug and Tank Roof versus Air Gap between Water Surface and Tank Roof.

suitable experimental methods were carried out. One experiment (Round 22) was done with a half-scale charge ( 6 g RDX/TNT plus a No. 8 detonator), after which the observation tank was severely damaged.

Prints from the Fastax cine film taken of Round 22 are given in Fig. 21, and the growth of the bubble plotted in Fig. 22 together with similar curves for two smaller charge weights. The impact pressure measured at the roof (positions $\mathrm{A}$ and $\mathrm{C}$ ) is shown in Fig. 23. Frame 3 of Fig. 21 shows the cavitation of the water produced just below the surface by the reflected rarefaction; by Frame 5 this was propagated to the base of the tank, and the cavitation bubbles became visible all the way down. In Frame 5 small jets of water can be seen striking the underside of the roof; this corresponds to the first large peak on the pressure record at $0.45 \mathrm{msec}$ after firing. The spray has reached the roof between Frames 6 and 7 , and this corresponds to the second peak in the pressure record at 0.6 msec. In Frame 7 cracks in the Perspex window can be seen; the cavitation has started to clear upwards from the base of the tank and has completely gone by Frame 10. This corresponds to the arrival of the third and highest peak in the pressure 
- PLUG

$X$ TANK ROOF

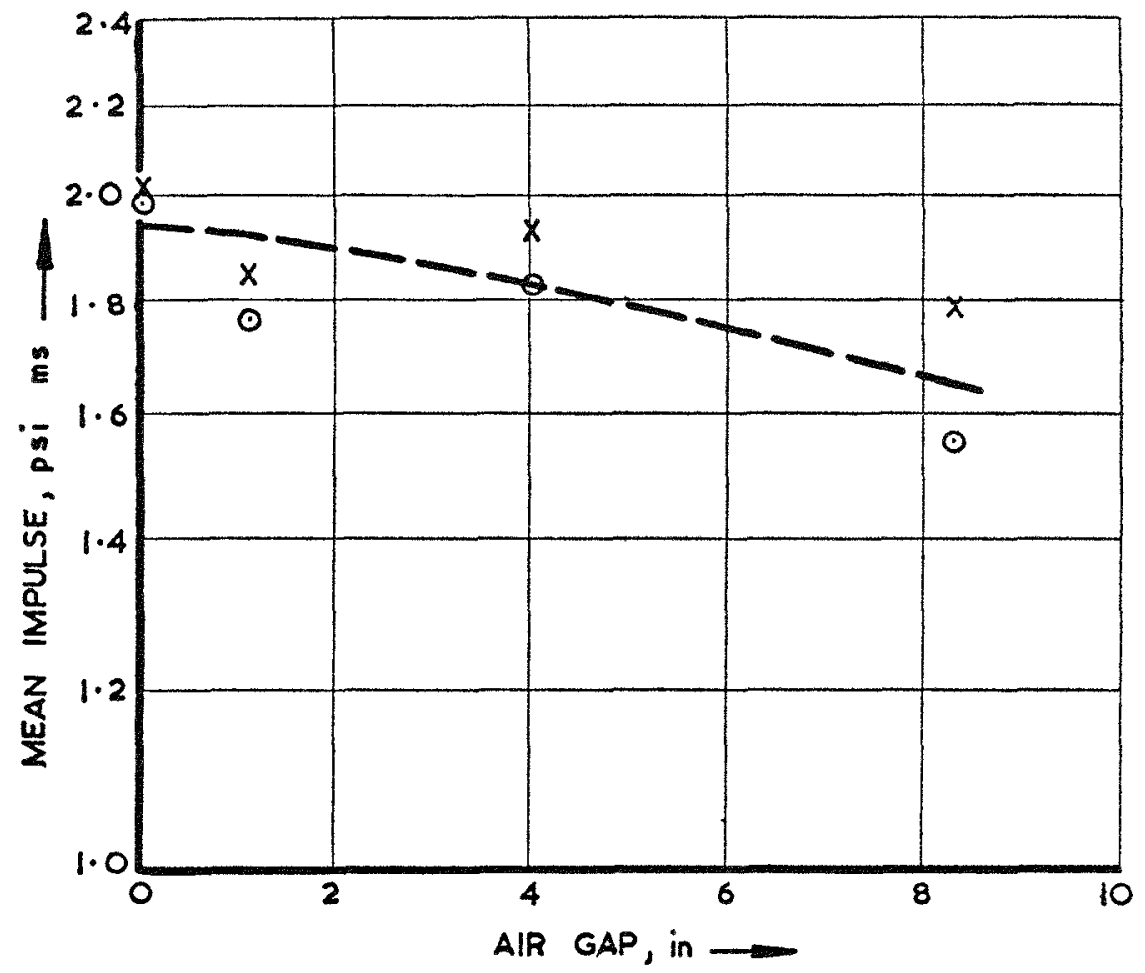

FItx. 16. Mean Impulse per Cnit Area on Core Plug and Tank Roof versus Air Gap between Water Surface and Tank Roof.

record at $0.8 \mathrm{msec}$. This suggests that the cavitation was cleared by a compression wave travelling up from the base of the tank.

The expansion of the explosion bubble (see Fig. 22) halts at about $1.1 \mathrm{msec}$ and does not restart until 1.7 msec. A small pressure rise occurs at 1.1 msec at gauge position A (see Fig. 23) and at 1.5 msec at position $\mathrm{C}$; the extensive cracking of the model windows is completed about this time, and large losses of water from the model begin. It seems probable that the pressure in the air gap becomes equal to the bubble pressure at about this time.

\section{Discussion of Results}

The experimental results of the pressure loading on the tank roof and core plug are summarised in Table 10. This pressure loading was caused by the impact of the water thrown upwards when the direct shock was reflected at the free surface. The impact-pressure loading on the roof and plug was large, and a pulse appears to be reflected and propagated back down into the contents of the vessel.

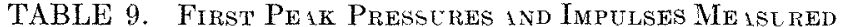
on Core Plig Using a WeiK REactor TANK WITH AN AIR Gap OF 1.1 IN. BETWEEN WATER SURFACE IND TANK ROOF

\begin{tabular}{|c|c|c|c|c|c|c|}
\hline \multirow{3}{*}{$\begin{array}{c}\text { Position } \\
\text { Number } \\
\text { of Pressure } \\
\text { Gauge }\end{array}$} & \multicolumn{6}{|c|}{$\begin{array}{l}\text { First Peak Pressure (psi) (Peaks A or E of Figs. 6, 7, } \\
\text { and } 8 \text { ) }\end{array}$} \\
\hline & \multicolumn{3}{|c|}{ Bare Charge } & \multicolumn{3}{|c|}{$\begin{array}{c}\text { Charge Enclosed in Core } \\
\text { Support Structure }\end{array}$} \\
\hline & $\mathrm{Rd} 12$ & Rd 13 & Mean, psi & $\operatorname{Rd} 14$ & $\operatorname{Rd} 15$ & Mean, psi \\
\hline 10 & 3,730 & 2,840 & 3,280 & 1,940 & 1,350 & 1,640 \\
\hline 11 & 2,530 & 2,550 & 2,540 & 2,170 & 816 & 1,490 \\
\hline 12 & 2,790 & 3,590 & 3,190 & 2,320 & 962 & 1,640 \\
\hline \multirow{3}{*}{$\begin{array}{c}\text { Position } \\
\text { Number } \\
\text { of Pressure } \\
\text { Gauge }\end{array}$} & \multicolumn{6}{|c|}{ Impulse from Pressure Records } \\
\hline & \multicolumn{3}{|c|}{ Bare Charge } & \multicolumn{3}{|c|}{$\begin{array}{l}\text { Charge Enclosed in Core } \\
\text { Support Structure }\end{array}$} \\
\hline & $\operatorname{Rd} 12$ & Rd 13 & $\begin{array}{c}\text { Mean, } \\
\text { psi-msec }\end{array}$ & $\operatorname{Rd} 14$ & $\operatorname{Rd} 15$ & $\begin{array}{l}\text { Mean } \\
\text { psi-msec }\end{array}$ \\
\hline 10 & 360 & 180 & 270 & 280 & 180 & 230 \\
\hline 11 & 300 & 240 & 270 & 330 & 190 & 260 \\
\hline 12 & 300 & 220 & 260 & 220 & 200 & 210 \\
\hline
\end{tabular}


TABLE 10. Summar of EXPerimentil Restlts

\begin{tabular}{|c|c|c|c|c|c|c|c|c|c|c|c|c|c|c|c|}
\hline \multirow{2}{*}{$\begin{array}{l}\text { Experi- } \\
\text { ment } \\
\text { Number }\end{array}$} & \multirow{2}{*}{$\begin{array}{c}\text { Type of } \\
\text { Tank }\end{array}$} & \multirow{2}{*}{$\begin{array}{l}\text { Type of } \\
\text { Explosive } \\
\text { Charge }\end{array}$} & \multirow{2}{*}{$\begin{array}{c}\text { Air Gap, } \\
\text { in. }\end{array}$} & \multicolumn{2}{|c|}{$\begin{array}{l}\text { Mean Peak } \\
\text { Pressure p, psi }\end{array}$} & \multicolumn{2}{|c|}{$\begin{array}{l}\text { Mean Impulse I, } \\
\text { psi-msec }\end{array}$} & \multirow{2}{*}{$\begin{array}{c}\text { Mean Time } \\
\text { of Arrival of } \\
\text { Shock on } \\
\text { Plug after } \\
\text { Detonation, } \\
\text { msec }\end{array}$} & \multirow{2}{*}{$\begin{array}{l}\text { Transit Time } \\
\text { of Spray in } \\
\text { Air Gap, msec }\end{array}$} & \multirow{2}{*}{$\begin{array}{l}\text { Spray } \\
\text { Velocity, } \\
u, \mathrm{ft} / \mathrm{sec}\end{array}$} & \multirow{2}{*}{$\left|\begin{array}{l}p / u, l b \mathrm{ft}^{-2} \\
\mathrm{sec}^{-1} \times 10^{5}\end{array}\right|$} & \multirow{2}{*}{$\begin{array}{c}\text { Velocity of } \\
\text { Plug Calcu- } \\
\text { lated from } \\
\text { Mean Im- } \\
\text { pulse, } V_{\mathrm{c}}= \\
(I A / M) \mathrm{ft} / \mathrm{se}\end{array}$} & \multirow{2}{*}{$\begin{array}{c}\text { Measured } \\
\text { Velocity of } \\
\text { Plug } V_{\mathrm{m}}, \\
\mathrm{ft} / \mathrm{sec}\end{array}$} & \multirow{2}{*}{$V_{m} / V_{c}$} & \multirow{2}{*}{$\begin{array}{c}\text { \%o of } \\
\text { Charge } \\
\text { Energy } \\
\text { Imparted } \\
\text { to Plug }\end{array}$} \\
\hline & & & & $\begin{array}{l}\text { Core } \\
\text { Plug }\end{array}$ & $\begin{array}{l}\text { Tank } \\
\text { Roof }\end{array}$ & $\begin{array}{l}\text { Core } \\
\text { Plug }\end{array}$ & $\begin{array}{l}\text { Tank } \\
\text { Rocf }\end{array}$ & & & & & & & & \\
\hline 19 & Strong & Bare & 0 & 17,800 & 10,500 & $1990^{*}$ & 2030 & 0.41 & - & - & 2.97 & $>60$ & 74 & 1.23 & 2.09 \\
\hline 3 & Strong & Bare & 1.1 & - & - & - & - & - & - & - & & - & 51 & - & 0.99 \\
\hline 4 & Strong & Bare & 1.1 & - & 4,400 & - & - & 0.70 & 0.48 & 191 & & - & 62 & - & 1.47 \\
\hline 5 & Strong & Bare & 1.1 & 6,400 & $>4,500$ & - & $>1490$ & 0.81 & 0.59 & 155 & 1.91 & -- & 71 & - & 1.92 \\
\hline 10 & Strong & Bare & 1.1 & $>5,400$ & $>6,300$ & - & - & 0.98 & 0.75 & 122 & $>2.05$ & - & 40 & - & 0.61 \\
\hline 11 & Strong & Bare & 1.1 & $>12,800$ & 11,800 & $>1300^{*}$ & $1450^{*}$ & 0.80 & 0.60 & 153 & $>3.90$ & $>39$ & about 66 & 1.69 & 1.66 \\
\hline 16 & Strong & Bare & 1.1 & 13,500 & $>13,200$ & 1760 & 2240 & 0.85 & 0.62 & 149 & 4.20 & 53 & 70 & 1.32 & 1.87 \\
\hline 17 & Strong & Bare & 4.0 & 6,200 & 8,200 & $2060^{*}$ & 1780 & 2.17 & 2.04 & 163 & 1.76 & 62 & 68 & 1.10 & 1.76 \\
\hline 18 & Strong & Bare & 4.0 & 7,800 & 11,900 & 1590 & 2080 & 2.13 & 1.97 & 169 & 2.14 & 48 & 65 & 1.35 & 1.61 \\
\hline 8 & Strong & Bare & 8.3 & 3,900 & 4,400 & $1370^{*}$ & $>1820^{*}$ & 2.79 & 2.69 & 257 & 0.704 & $>41$ & 60 & 1.46 & 1.38 \\
\hline 9 & Strong & Bare & 8.3 & 4,200 & 2,900 & $1720^{*}$ & $>1760^{*}$ & 3.32 & 3.22 & 215 & 0.904 & $>52$ & 63 & 1.21 & 1.52 \\
\hline 6 & Strong & Enclosed & 1.1 & 9,410 & 4,610 & $>1400$ & $>1800$ & 1.28 & 1.06 & 87 & 5.01 & $>42$ & 60 & 1.43 & 1.38 \\
\hline 7 & Strong & Enclosed & 1.1 & 14,100 & 6,280 & 2200 & 2740 & 1.45 & 1.22 & 75 & 8.73 & 67 & 62 & 0.93 & 1.47 \\
\hline 12 & Weak & Bare & 1.1 & 3,020 & - & 320 & - & 0.82 & 0.60 & 153 & 0.915 & 9.7 & 21 & 2.17 & 0.17 \\
\hline 13 & Weak & Bare & 1.1 & 2,990 & - & 213 & - & 0.82 & 0.60 & 153 & 0.906 & 6.5 & 24 & 3.70 & 0.22 \\
\hline 14 & Weak & Enclosed & 1.1 & 2,140 & -- & 277 & - & 2.29 & 2.07 & 44 & 2.26 & 8.4 & 41 & 4.9 & 0.64 \\
\hline 15 & Weak & Enclosed & 1.1 & 1,040 & - & 190 & -- & 3.08 & 2.86 & 32 & 1.51 & $\tilde{5} .8$ & 36 & 6.2 & 0.50 \\
\hline
\end{tabular}

* Estimated. $C=$ Speed of sound in water $-4760 \mathrm{ft} / \mathrm{sec}$.

Total Energy of charge taken as $203 \times 10^{3} \mathrm{ft}-\mathrm{lb}$. 

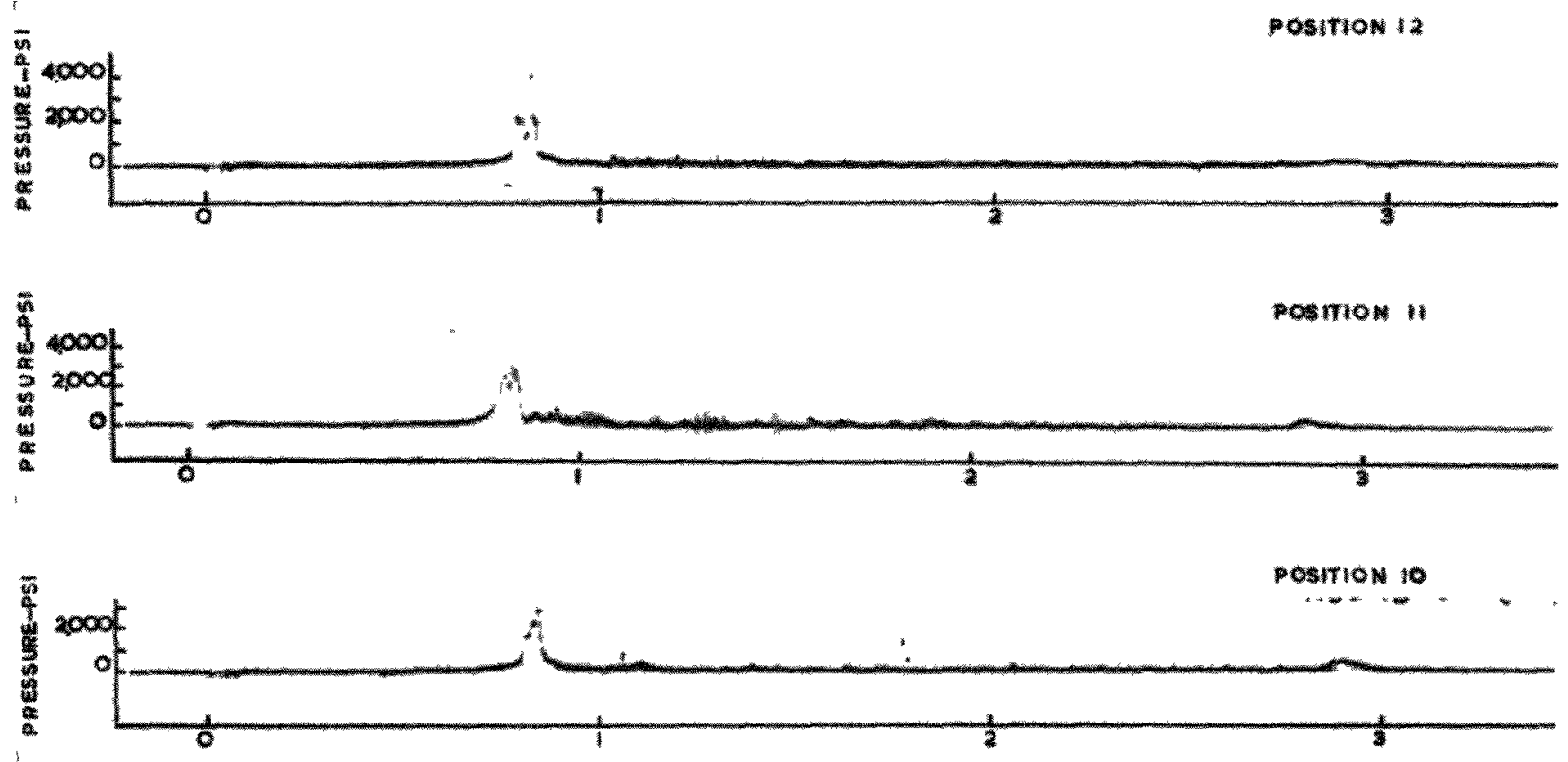

FIG. 17. Records from Round 13

The spray impact pressure $p$ is given by the equation

$$
p=\rho c u,
$$

where $\rho$ is the density of the spray, $c$ is the relocity sound in the spray, and $u$ is the spray velocity. The product $\rho c$ is known as the acoustic impedance of the medium. For water this is $2.97 \times 10^{5} \mathrm{lb} \mathrm{ft}^{-2} \mathrm{sec}^{-1}$. The spray velocities have been calculated from the observed transit times between the water surface and the plug, and values of the ratio $p / u$ are given in Table 10. For Rounds 11 and 16 this ratio $p / u$ exceeded the acoustic impedance of water, so it would appear that either $p$ is high or $u$ is low, or both.

Disregarding these two results, the mean value is $1.98 \times 10^{5} \mathrm{lb} \mathrm{ft}^{-2}$ for a 1.1-in. air gap and bare charge; for a 4.0 -in. air gap the value is $1.95 \times 10^{5}$, falling to $0.804 \times 10^{5} \mathrm{lb} \mathrm{ft}^{-2} \mathrm{sec}^{-1}$ for an 8.3 -in. air gap. In the last case the impact pressure is reduced though the spray velocity is higher; this implies that the density has been reduced, possibly by the venting of the explosion bubble.

The ratio $p / u$ calculated for the spray in Rounds 6 and 7 is very high; this may have been caused by the debris of the core support structure striking the plug. Low values of $p / u$ were obtained for the weak-vessel experiments of Rounds 12 and 13; this was again increased when the explosion was enclosed in the core support structure.

The velocity of plug ejection, $V_{c}$, has been calcu- lated from the equation

$$
V_{o}=I A / M
$$

where $A$ is the area of the plug, $I$ the mean impulse per unit area acting on the core plug, and $M$ is the plug weight. The area of the stepped plug (see Fig. 1) corresponding to its smallest diameter of $7.75 \mathrm{in}$. Was used to calculate the values of $V_{c}$ given in Table 10 . With the exception of Round 7 , these values are all smaller than the velocities measured from the cine film, and the ratios $V_{m} / l_{c}$ are given in Table 10. The mean value of the ratio for experiments with a bare charge in a strong vessel is 1.34 , and assuming the values of $I$ are correct this implies that the impulse was acting over a larger area than the value of 47 in. ${ }^{-2}$ used in the calculations. The increased area corresponds to a circle of 9 -in. diameter, which equals the intermediate plug diameter (see Fig. 1). The low value for Round 7 may have been caused by the plug jamming in the plug guide and increasing the effective value of $M$, the plug weight. The large values for Rounds 12,13,14, and 15 were probably caused by ejection of the plug, guide, and vessel roof as one unit, thereby increasing the ratio $\mathrm{A} / M$.

The conditions in the overstrong tank seem to have a limited effect on the percentage of the explosion energy imparted to the plug (see Tables 10 and 11). The variation was from $1.4 \%$ in the case of the enclosed charge to $2.1 \%$ in the case of a large charge with no air gap. Increase of the air gap decreased the 


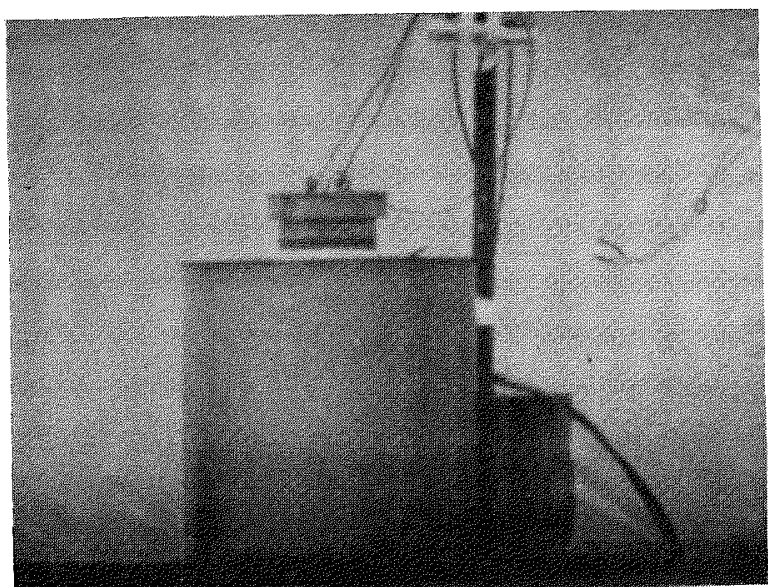

0

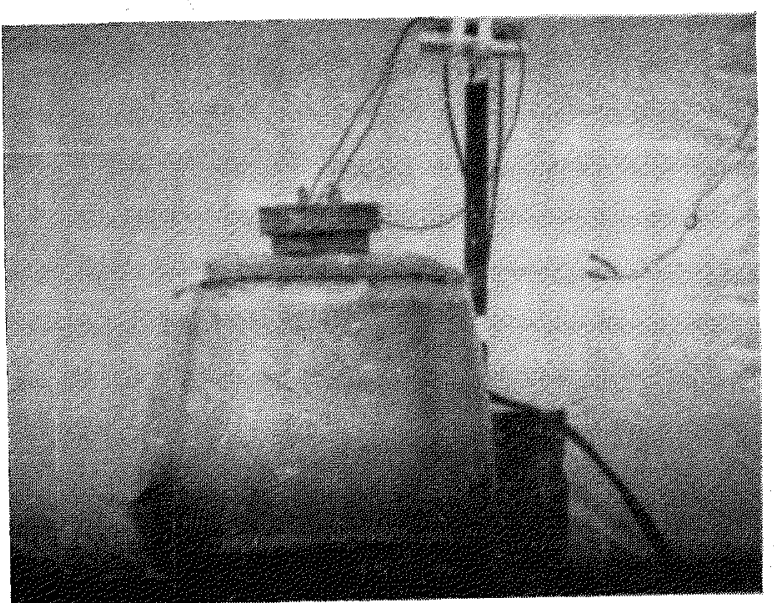

2

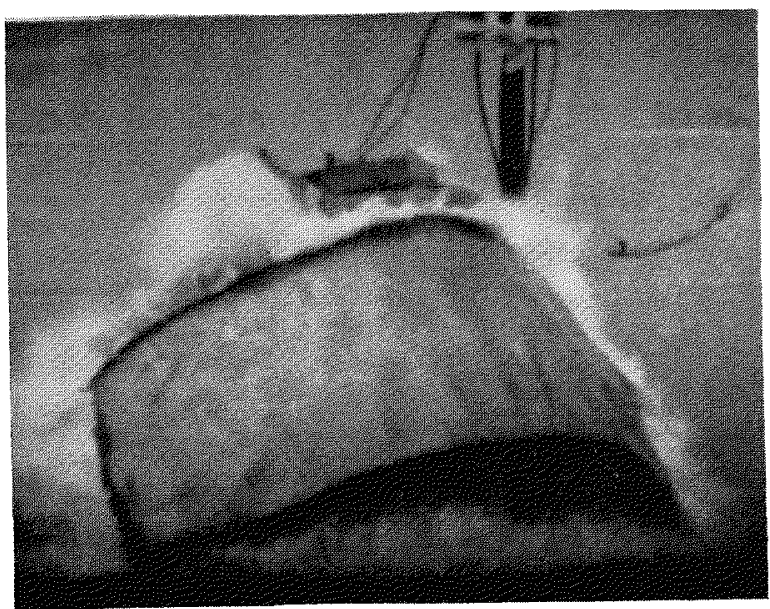

4

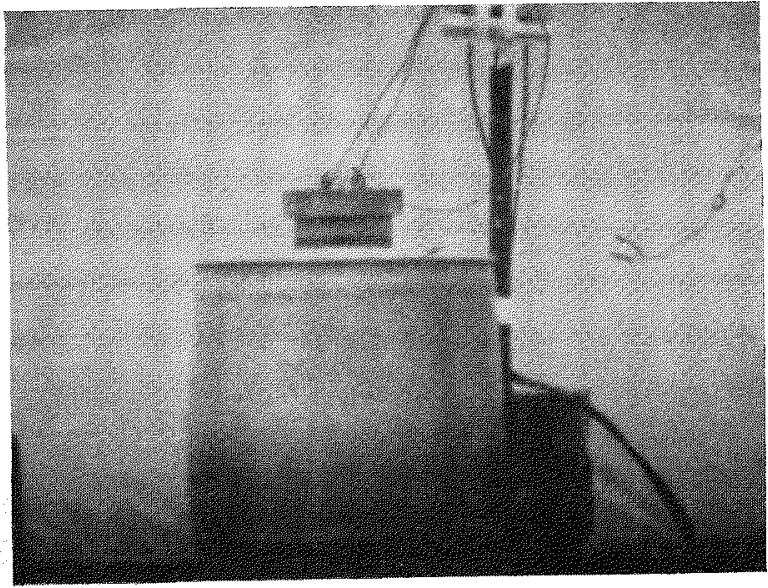

I

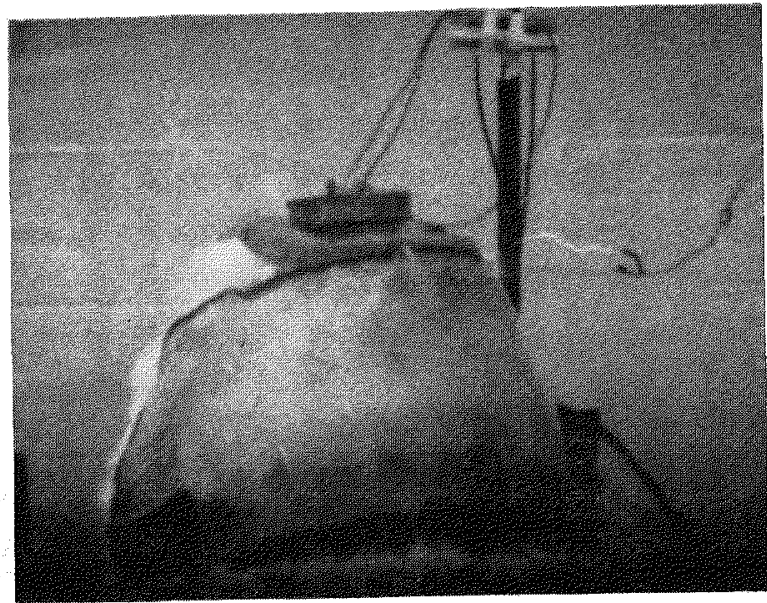

3

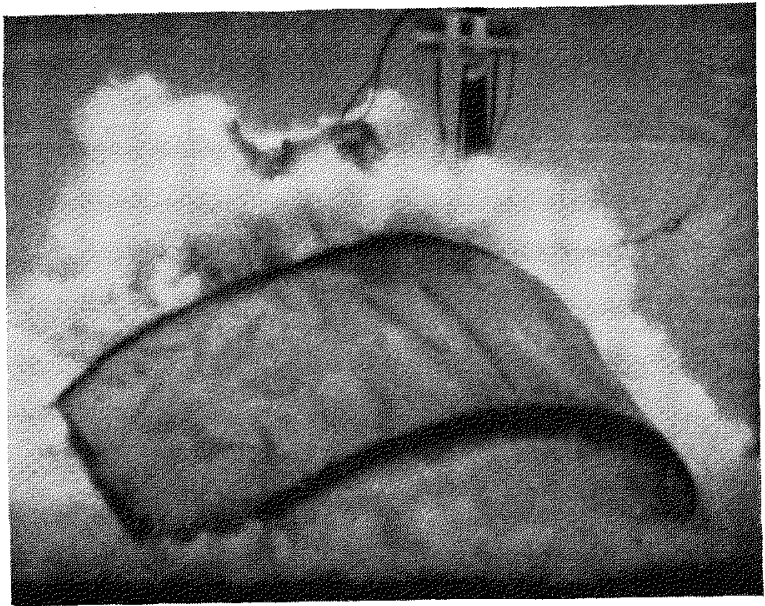

5

TIME BETWEEN FRAMES $151.32 \mathrm{~ms}$

Frg. 17A. Round 13. (Bare Charge) Effect of Explosion in Weak Model Reactor Tank. 

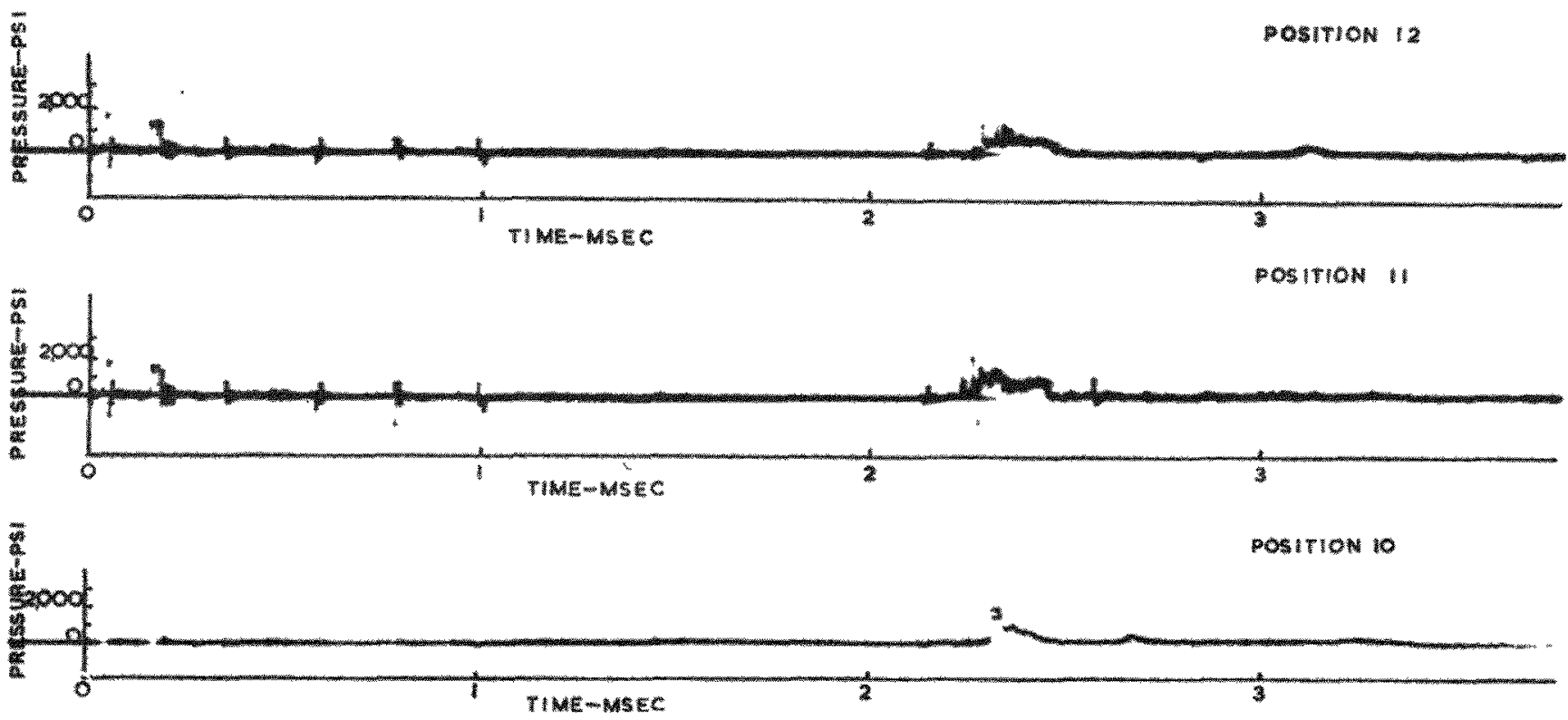

FIG. 18. Records from Round 14 .

imparted energy but only to $1.5 \%$ (see Fig. 24). Premature failure of the overstrong tank allowed early venting of the explosion pressures and decreased plug energy to $1.0 \%$. This was confirmed by experiments in weak tanks. However, the effect of a core support structure in a weak tank was to direct the energy released upwards to the tank roof and core plug, resulting in higher plug-ejection velocities. Similar effects on the plug total impulse were observed.

The methods and results reported here are included in a general discussion of fast reactor containment design by Samuels. ${ }^{(2)}$

\section{Acknowledgments}

The author wishes to thank T. P. O'Brien, D. O. Wells, and Mrs. H. A. Taylor for carrying out the experiments reported here.

\section{REFERENCES}

1. Hicks, E. P., and Menzies, D. C., see Paper in Session VII A of This Conference.

2. Samuels, I). E. J., see Paper in Session VII A of This Conference.

3. Cole, R. H., Underwater Explosions, Princeton University Press.

4. Drevon, (x. A. V., Falgayrettes, M., and Walford, F. J., see Paper in Session VII A of This Conference. 

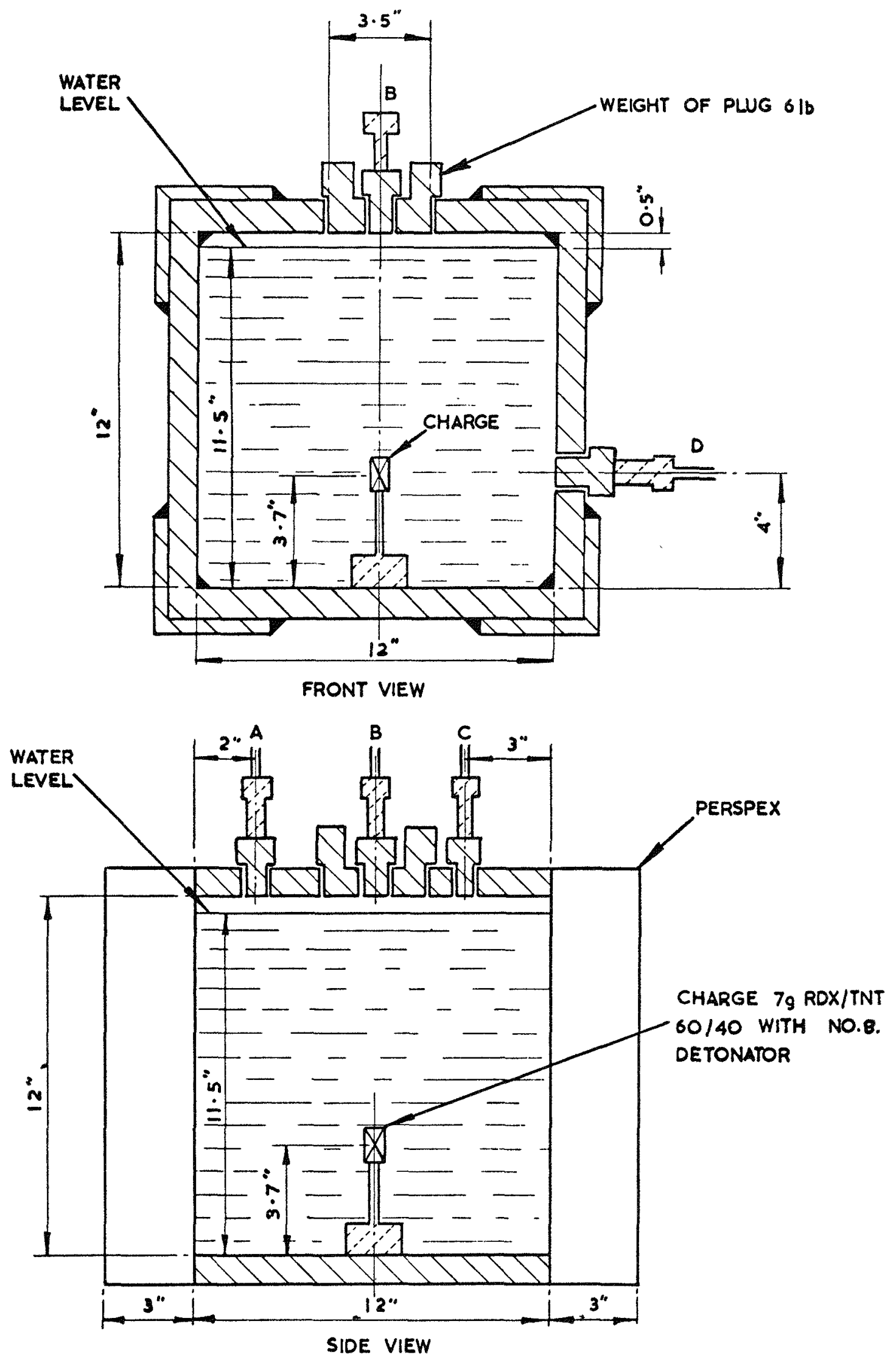

FIG. 19. Cross Sections of Half-scale Tank for Bubble Photography Showing Pressure-gauge Positions. 


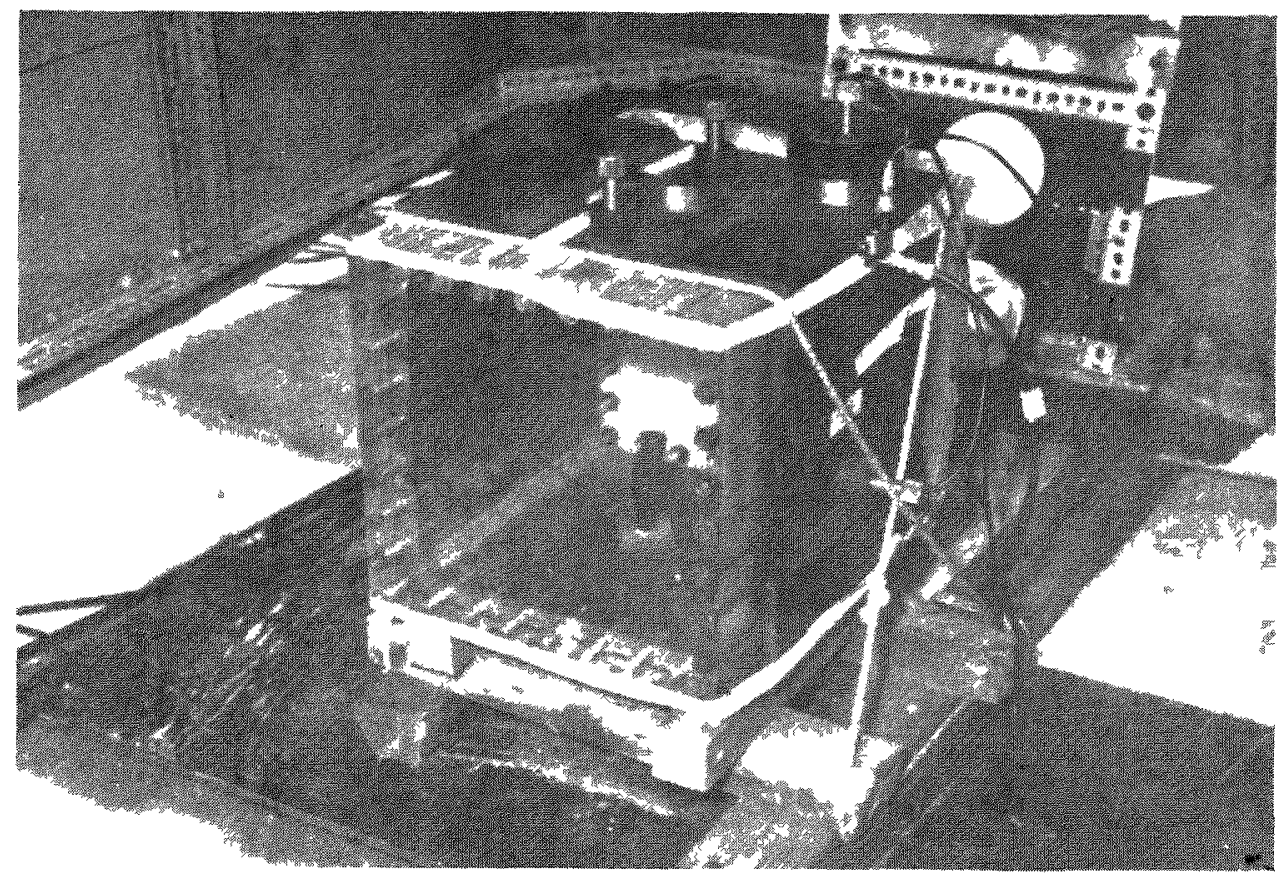

Fig 20 Before Firng Round 22 Half scale Model Used to Photograph Expansion of Bubble of Explosive Products. 


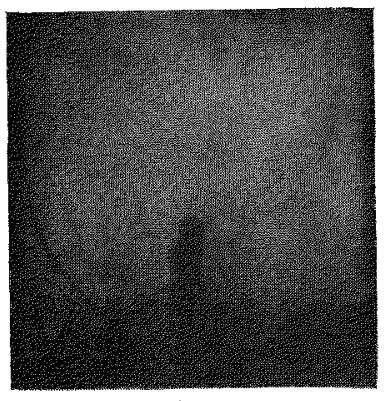

0

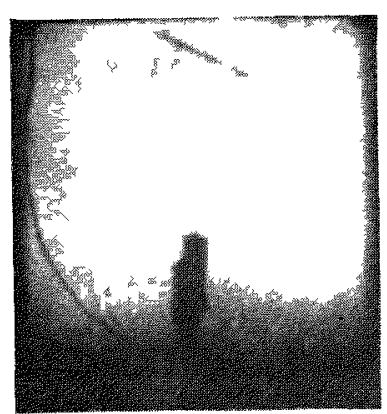

1

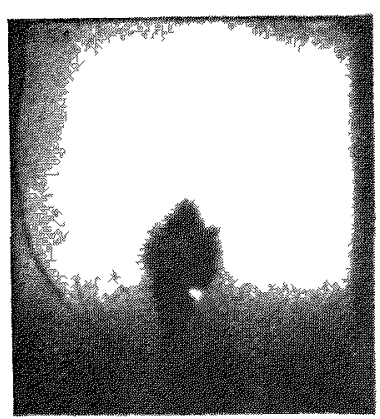

2

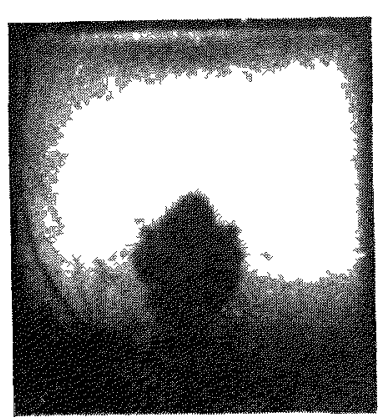

3

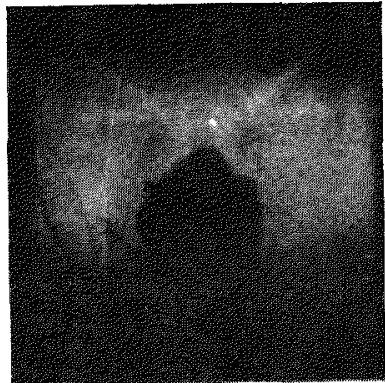

4

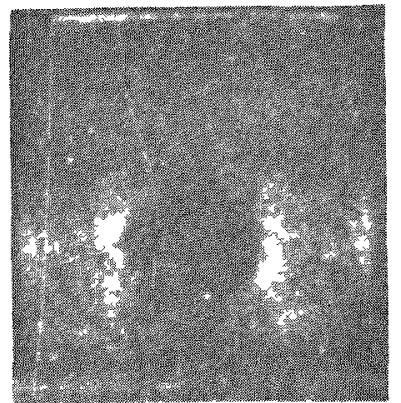

5

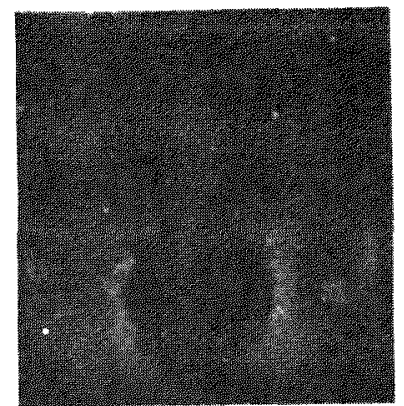

6

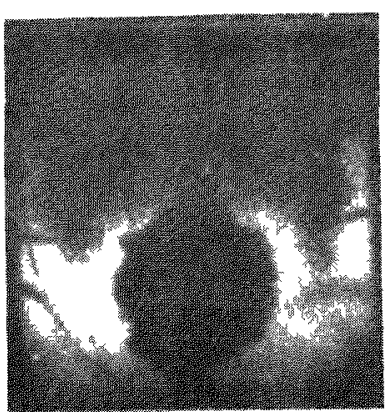

7
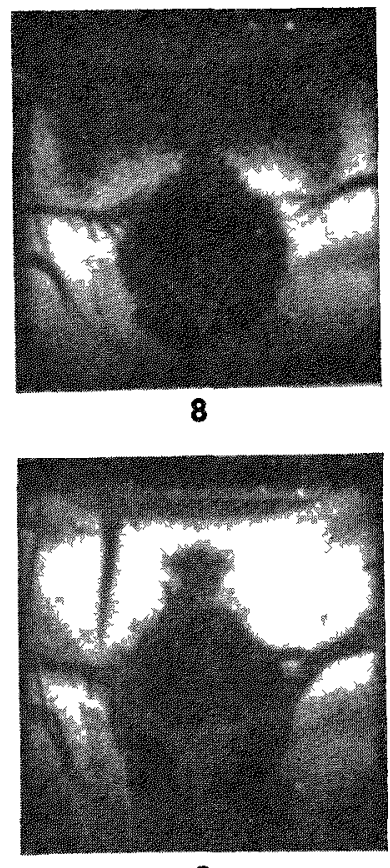

9

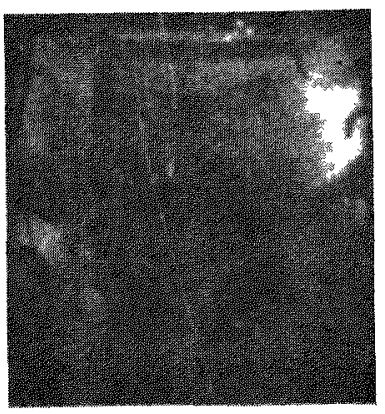

10

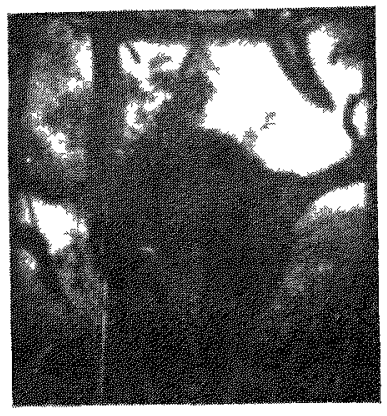

11

TIME BETWEEN FRAMES $150.033 \mathrm{~ms}$

HIG 21 Round 22 Expansion of Gas Bubble 


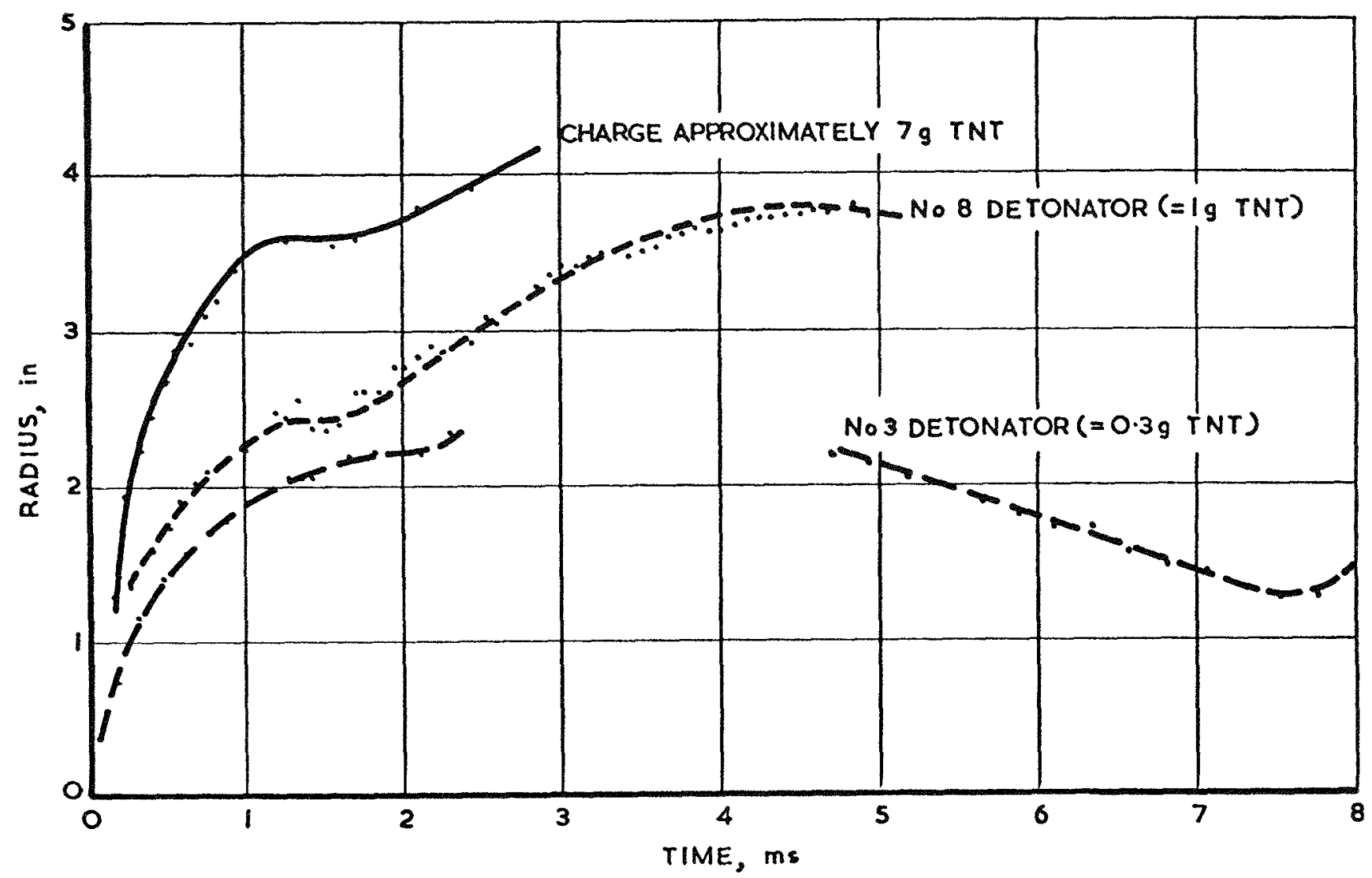

I IG 22 Rounds 20,21, and 22 Raduu time Vedsurements of Gas Bubbles in HaIf scale Reactor Models
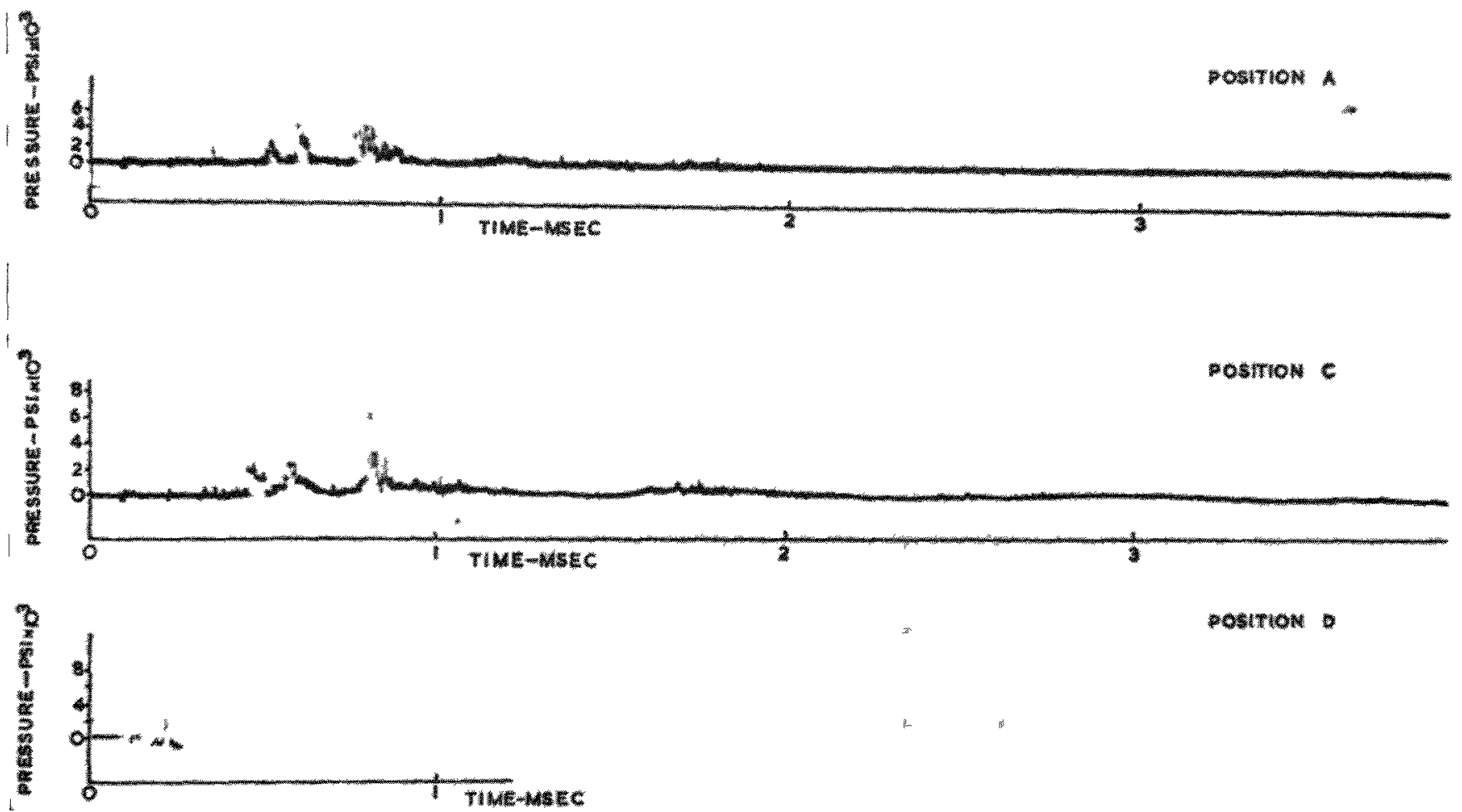

FIG 23 Records from Round 22 . 
TABle 11. Summatry of Mean Percentage of Charge Energy and Mean Total Imprise Given to Plug under Different Experimental Conditions

Total impulse of core plug $=M V_{m} l b-s e c$.

Total Energy of charge $203 \times 10^{3} \mathrm{ft}-\mathrm{lb}$.

\begin{tabular}{|c|c|c|c|c|}
\hline \multirow{3}{*}{$\begin{array}{c}\text { Air Gap } \\
\text { between Water } \\
\text { Surface and } \\
\text { Roof of } \\
\text { Tank, in. }\end{array}$} & \multicolumn{4}{|c|}{$\begin{array}{c}\text { Mean } \% \text { of Charge Energy Imparted to } \\
\text { Core-access Plug }\end{array}$} \\
\hline & \multicolumn{2}{|c|}{ Overstrong Tank } & \multicolumn{2}{|c|}{ Weak Tank } \\
\hline & Bare Charge & $\begin{array}{l}\text { Charge } \\
\text { Enclosed } \\
\text { in Core } \\
\text { Support } \\
\text { Structure }\end{array}$ & Bare Charge & $\begin{array}{l}\text { Charge } \\
\text { Enclosed } \\
\text { in Core } \\
\text { Support } \\
\text { Structure }\end{array}$ \\
\hline $\begin{array}{c}\text { None } \\
1.1 \\
4.0 \\
8.3\end{array}$ & $\begin{array}{l}2.09 \\
1.84^{*} \\
1.70 \\
1.45\end{array}$ & 1.43 & 0.20 & 0.57 \\
\hline \multirow{3}{*}{$\begin{array}{c}\text { Air Gap } \\
\text { between Water } \\
\text { Surface and } \\
\text { Roof of } \\
\text { Tank, in. }\end{array}$} & \multicolumn{4}{|c|}{$\begin{array}{c}\text { Total Impulse of Core-access Plug from Measured } \\
\text { Plug Velocity, lb-sec }\end{array}$} \\
\hline & \multicolumn{2}{|c|}{ Overstrong Tank } & \multicolumn{2}{|c|}{ Weak Tank } \\
\hline & Bare Charge & $\begin{array}{l}\text { Charge } \\
\text { Enclosed } \\
\text { in Core } \\
\text { Support } \\
\text { Structure }\end{array}$ & Bale Charge & $\begin{array}{l}\text { Charge } \\
\text { Enclosed } \\
\text { in Core } \\
\text { Support } \\
\text { Structure }\end{array}$ \\
\hline $\begin{array}{c}\text { None } \\
1.1 \\
4.0 \\
8.3\end{array}$ & $\begin{array}{l}115 \\
109^{*} \\
104 \\
96.0\end{array}$ & 95.2 & 35.1 & 60.1 \\
\hline
\end{tabular}

* Results of Experiments 3, 4, and 10 omitted, since overstrong tank severly damaged, allowing venting before plugejection process complete. 


$$
\begin{aligned}
& \text { MASS OF PLUG }=50 \mathrm{lb} \\
& \text { AREA OF PLUG }=47 \mathrm{in}^{2}
\end{aligned}
$$

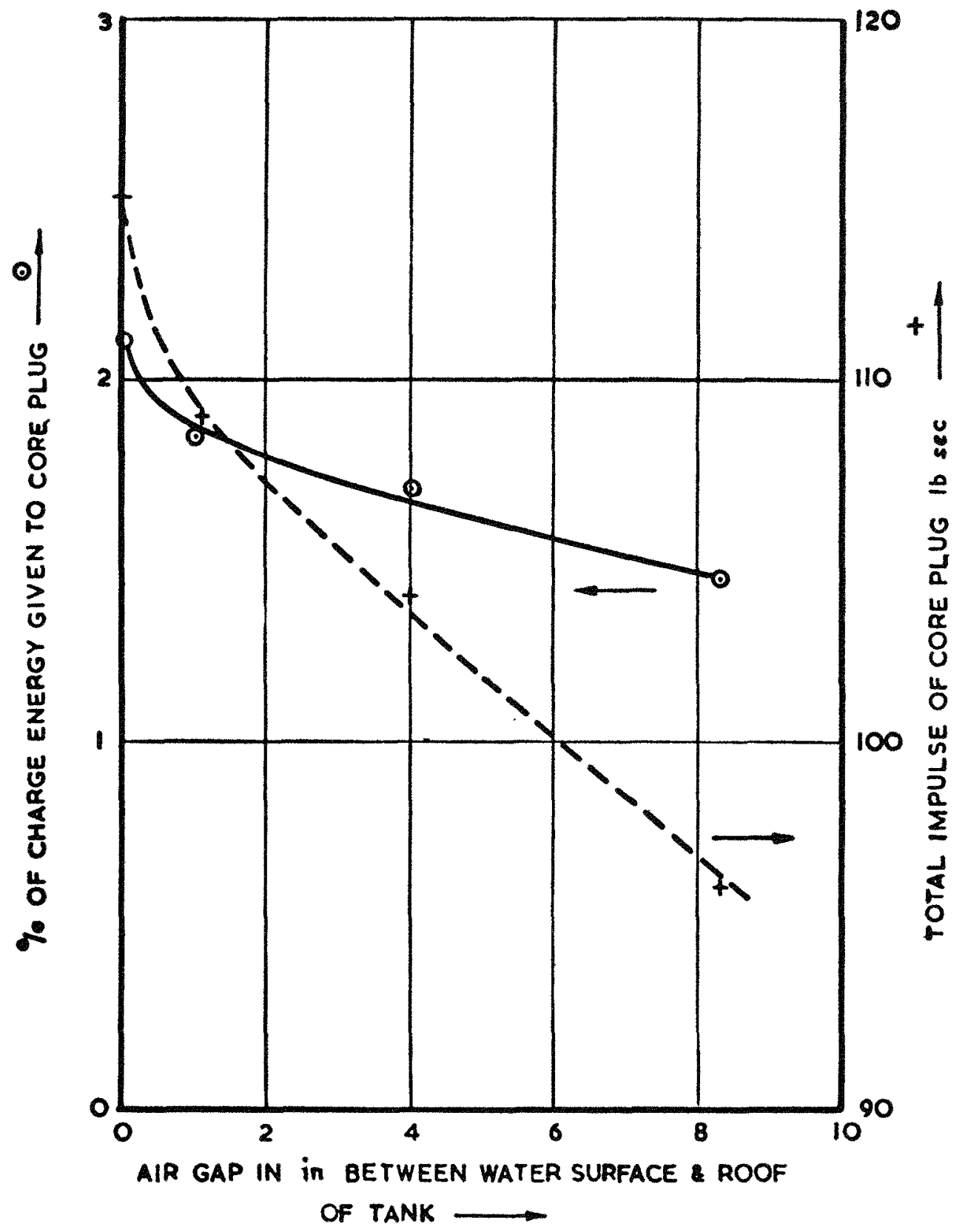

Frg. 24. Percentage of Charge Energy Imparted to Core Plug; Also Total Impulse Given to Core Plug versus Air Gap between Roof of Tank and Water Surface. 


\section{Discussion}

Mr. McCarthy (PRDC): Considerable work on this same problem has been done in the United States, but which has not been reported widely in the reactor community. I refer to work that was done by the U. S. Naval Ordnance Laboratory on the response of the Enrico Fermi reactor to $1000-1 b-T N T$ releases. A report, NOLTR-62-207, has been published. I was struck by the coincidence of results between Mr. Rees' paper and that in the NOLTR-62-207. In particular, the fraction of the energy released by the explosive, which appeared as kinetic energy of the plug, was identical in both of those studies which were done completely independently-in both cases being about $2 \%$, which is a remarkable agreement. The NOL work was done, as a matter of fact, with both hot sodium and with hot water, and some of their results indicated that the jump when sodium is used is somewhat higher than with water.

$M r$. Wolfe (GE) : Do I understand that the major energy imparted in plug jump is not due to the shock wave but due to the pressure of the gas which remains after the initial shock wave hits the plug?

$M r$. Rees: The actual pressures measured on the plug are due to waves that are rather longer than the classical shock ware from an explosion of this size. If you take the impulse measured in the pressurc loading on the plug and work out the plug velocity, then this does agree reasonably well with the velocities measured in the ciné records.

$M r$. Wolfe: If I understand it correctly, the pressures that are important are the so-called quasistatic pressures that remain after the initial shock, the pressure of the expanding gas. If that viewpoint is right, how relevant are the TNT cases to the actual case in which the gases may condense?

$M r$. Rees: The results that we give are probably correct up to the end of the first expansion phase of the bubble, which means that they are probably reasonably correct up to about perhaps 2 msec on this type of scale. After that there are probably very considerable deviations because of the different nature of expanding gases. This will be discussed rather more fully in Samuels' paper.

Mr. Denielou (CEA, France): I did not understand clearly whether or not the type of explosive charge was varied, especially with respect to the yield of gaseous products from the explosion.

$M r$. Rees: The explosive charge was the same throughout, 2 oz RDX TNT.

$M r$. Schikarski (Karlsruhe): Can you comment on the problem of shock waves travelling along pipe lines attached to the reactor tank? Did you study those effects?
Mr. Rees: A shock wave in a pipe usually breaks the pipe at the first bend. It depends very much on what strength of shock you get in the pipe in a particular arrangement, but in general this is the normal effect.

Mr. Fistedis (ANL): Is it actually the sodium momentum which follows the shock wave, timewise, which causes the plug to accelerate?

Mr. Rees: It's the pressure loading caused by the spray acting on the surface, which in this case can take some time. After all, there is the original shock, the expansion, and the explosion from its bubble; in some cases this is modified by the breakup of the core structure, so that there is an initial shock followed by a pressure loading which will vary due to the circumstances of origin.

Mr. Fistedis: What do you think, as far as the overall containment is concerned, if we somehow depart from the accepted, or shall we say, prevailing, pressure-volume computation? Can we start working directly with energy to be contained against strained energy available in the material which is to do the containing, provided now that the time intervals have been investigated sufficiently to satisfy that plasticity can occur? In other words, the strain rates are such that the material can flow. Don't you think that this will be a neater approach that will give us much more reliable factors of safety?

$M r$. Rees: It depends on where the strain energy will be absorbed. If the absorption is very near the charge, then I think you have a very difficult design problem. If the energy is absorbed further from the charge, that is, if you only aim at producing a leaktight containment structure at some distance from the charge, then I think you probably would be able to do this.

Mr. Fistedis: Containment generally comes in two steps: a primary and a secondary containment. The secondary containment is intended to prevent the escape of gases to the surrounding atmosphere. Now, why not make primary containment capable of absorbing the entire explosive energy through plastic deformation in material sufficiently far from the primary vessel or adjacent in such a way that the strain rates can exhibit this plasticity?

Mr. Rees: I think this is quite reasonable.

Mr. Fistedrs: Do you think this is possible?

Mr. Rees: Yes.

Mr. Klickman (APDA): Was the selection of explosive made because of convenience or availability, or because it was satisfactory for a simulant for uranium or $\mathrm{UO}_{2}$ ?

Mr. Rees: We chose it as an arbitrary representa- 
tion of energy release. This particular mixture was used because it was readily available and known to be reliable; TNT as such is a very tricky explosive to detonate completely and properly, whereas RDX TNT is completely detonable.

$M r$. Schlechtendahl (Karlsruhe): Is there any indication from your experiments that the impulse that is generated by the explosion does not propagate in a symmetrical way around the charge? It would be very difficult to provide blast shielding and to analyze blast shielding in the design if this were not the case.

Mr. Rees: Are you referring to the bare charge experiments?

Mr. Schlechtendahl: Yes. In one of the pictures taken with a high-speed camera it appeared that the vessel ruptured on one side. I wonder whether this effect could propagate further out.

$M r$. Rees: The reason why the weak tank ruptured on one side was because it was constructed of thin sheet metal welded on one side and the weld-affected zone had not been treated. Of course, this failed rather sooner than the unaffected zone, so you have a tearing occurring down the welded seam. It wasn't because the explosion was unsymmetrical.

Mr. McCarthy: I might add a point, too, that in a shell which doesn't fail, the damage is almost spherical. If it's a marginal failure, it fails in one place obviously, and that makes it look asymmetric. I might again refer to the NOL report for more data on that particular point. 


\section{Comparison of Pressure Loading Produced by Contained Explosions in Water and Sodium}

\author{
G. A. V. Drevon \\ C. E. A. de Saclay \\ Seine-toise, France
}

M. F. G. Falgayrettes

C. E. A. de Cadarache

Bouches-du-Rhône, France

AND

F. J. WALFORD

U. K. Atomic Energy Authority

Foulness, Southend-on-Sea, Essex, England

(PRESENTED BY F. J.WALFORD) 
sure transients and further measurements of dynamic strain. The experiments were performed at the C.E.N. de Caradache in three separate series. This was done so that the results from one series could be processed and their trend analysed, enabling any necessary modifications to be made to the programme and to the experimental rig prior to the commencement of the succeeding series.

\section{Experimental Details}

The intention in designing the apparatus was to maintain, as far as possible, a typical fast reactor geometry within the core vessel and also to permit an explosive charge to be placed in the centre of the core after the model had been assembled. The essential features of the apparatus are shown in Fig. 1. The simulated core vessel, of $250-\mathrm{mm}$ diameter and 424-mm height, was made of stainless steel (type $316)$, and the base of the vessel contained a re-entrant cylinder into which a charge could be inserted remotely. The radial and upper axial blankets were simulated by closely packed uranium and steel rods to give an average density of $12.7 \mathrm{~g} / \mathrm{cc}$. Surrounding the breeder were a number of steel rods and six concentric stainless steel cylinders of $2.5-\mathrm{mm}$ wall thickness which represented the neutron reflectors; surrounding these were four longer concentric cylinders $(1 \mathrm{~mm}$ thick) simulating the heat shields. There were $1-\mathrm{mm}$ spaces between each adjacent cylinder. The core vessel was mounted inside a strong containment vessel. Bolted to the thick flange at the top of the outer vessel was a heavy lid in which were mounted four type MQ.20 piezo-electric pressure transducers. These are single quartz crystal transducers developed by the UKAEA to measure high-pressure transients with a response up to $250 \mathrm{kc} / \mathrm{s}$. It was necessary to mount the transducers in cooled holders since they were not designed to operate above about $100^{\circ} \mathrm{C}$. Cooling was effected by pumping liquid paraffin through the holders. It was possible to keep the temperature of the body of the transducers below $90^{\circ} \mathrm{C}$ by this method.

The thick lid was held down to the flanges of the strong containment vessel by five stainless steel bolts which had pairs of Microdot weldable strain gauges (type SG120) mounted on them. One strain gauge was mounted on the side of the bolt facing the centre of the lid and the other was diametrically opposite, on the outside of the bolt. An additional strain gauge was mounted on the upper face of the thick lid. The nuts at each end of the hold-down bolts had a hemispherical seating which mated onto the hemispherically shaped spacing washers. The seatings were lubicated with molybdenum disulphide. The purpose of this method of mounting the nuts was to reduce the amount of bending in the hold-down bolts resulting from flexing of the lid. In order to obtain a uniform loading of the hold-down bolts, they were all pretensioned by the same amount by use of a torque spanner.

For each experiment a core vessel was filled either with water or with the appropriate volume of sodium so that when heated to the required temperature the liquid surface was $5 \mathrm{~cm}$ below the lid of the containment vessel. The vessels were filled with sodium at $150^{\circ} \mathrm{C}$, the volume of sodium required in each vessel being calculated from the results of a subsidiary experiment in which the differential expansion of core vessels and liquid sodium was measured. To prevent oxidation of the surface of the sodium during the experiment, the free volume above the surface was swept continuously with argon at atmospheric pressure. To allow for differential thermal expansion there was a $3-\mathrm{mm}$ gap between the top of the core vessel and the underside of the thick lid when the apparatus was at ambient temperature.

Three different charge weights were used in these experiments: 15-, 30-, and 60-g of Hexogene, corresponding to energy releakes of $2 \cdot 10^{4}, 4 \cdot 10^{4}$, and $8 \cdot 10^{4}$ cal.

The procedure in each experiment was to heat the liquid to the required temperature by means of the heating coil wrapped around the core vessel. Temperatures were recorded continuously by means of sheathed thermocouples immersed in the liquid. When steadystate conditions had been obtained, the explosive charge (which was enclosed within a weak asbestos container) was mounted on a plinth which stood on top of an hydraulic ram. The ram was operated remotely to raise the charge into the cavity in the base of the core vessel, and the plinth was then rotated, locking it into the base of the outer containment vessel. The ram was then lowered, leaving the charge and plinth in position in the model. This last operation triggered a relay which initiated the firing sequence: starting the recording apparatus, inserting calibration pulses, detonating the charge, and switching off the apparatus at the end of the recording period. An ionisation probe was placed on the detonator fuse to give a time-zero pulse on all the recording traces obtained with the UK equipment. A correlating pulse was inserted on the UK and the French recording traces so that zero time could be determined on all records.

The UK recording apparatus consisted of three four-channel oscillograph recorders equipped with high-speed rotating drum cameras, enabling a writing speed of up to $2 \mathrm{in} . / \mathrm{ms}$ for about $15 \mathrm{~ms}$ after time zero. The output from each of the pressure trans- 


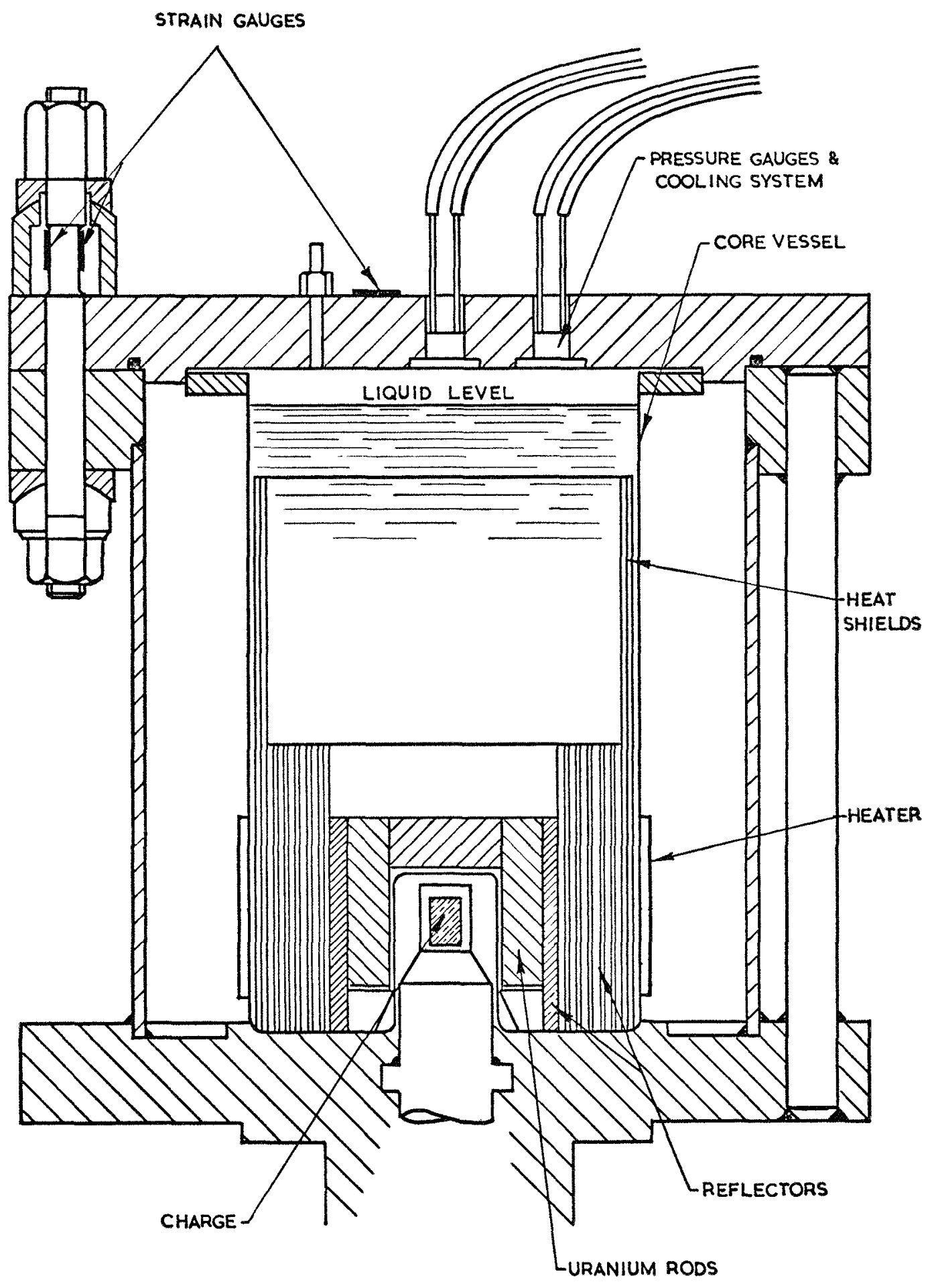

NOT TO SCALE

Frg. 1. Sketch Showing Main Components of the Model Reactor Assembly.

ducers was fed through two parallel amplifying channels, one set on a low gain to observe the highest impact pressures and the second on a higher gain to observe the lower pressure levels. The re- maining four channels were used to record strains from two of the hold-down bolts. The remaining seven strain gauges were connected to Wheatstone bridge networks and mirror-galvanometer recorders 
which were operated by the CEA team. This enabled a comparison to be made of the two strain-gauge systems. The nominal useful frequency range of the UK recording system was from DC to $15 \mathrm{kc} / \mathrm{s}$, but it is intended to carry out a frequency test of the equipment at a later date to check this data. The CEA equipment had a useful frequency range from $10 \mathrm{c} / \mathrm{s}$ to $8 \mathrm{kc} / \mathrm{s}$, and a writing speed of $0.4 \mathrm{in} . / \mathrm{ms}$.

The programme as planned was to measure the impact loading on the lid of the containment vessel for three different charge weights in water and in hot sodium, covering temperatures in the range from 150 to $550^{\circ} \mathrm{C}$. Strain measurements were made over the whole range, but the highest temperature at which impact pressures were recorded was $450^{\circ} \mathrm{C}$. Of the twenty-seven experiments, seventeen were performed with the $30 \mathrm{-g}$ charges and five each with the other charge weights. More experiments were performed with $30-\mathrm{g}$ charges because the effects of liquid temperature upon the impact loading were investigated with this weight of charge. Columns 1, 2, and 3 of Table 1 summarise the experimental conditions.

\section{Results}

In presenting the results it is convenient to consider the pressure and strain-gauge records separately. They will be considered jointly in the discussion towards the end of the paper together with the observations of vessel damage.

\subsection{Pressure Measurements}

Figure 2 shows a pressure-time curve obtained with the $15-\mathrm{g}$ charge in sodium at $150^{\circ} \mathrm{C}$ and one from $15 \mathrm{~g}$ in water, chosen at random to indicate the shape of individual signals from the pressure transducer. Salient points were measured on the oscillograph traces and the data were recorded on punched cards suitable for processing in an IBM 7030 'Stretch' computer. The curres in Fig. 2 are specimens of the computer output. Time zero on these curves corresponds to the signal from the ionisation probe on the detonator.

It will be seen in Table 1 that the experiments have been arranged in groups. Most of the analysis was performed on the average results of each group. The mean peak pressures quoted in Table 1 are the arithmetic means of the maximum pressures recorded by each of the four pressure transducers in each experiment. Figure 3 shows graphically the variation of mean peak pressure with charge weight. For the $15-\mathrm{g}$ charge the mean peak pressures in sodium were about 1.7 times higher than in water, whereas they were only 1.5 times higher with the $60-\mathrm{g}$ charges. It can be inferred from Fig. 3 that within the range of charge weights and for this particular
TABLE 1. Summary of Experimental Conditions and Measured Peak Pressures

\begin{tabular}{|c|c|c|c|c|c|}
\hline \multirow{2}{*}{$\begin{array}{l}\text { Expt } \\
\text { No. }\end{array}$} & \multirow[b]{2}{*}{ Group Title } & \multirow{2}{*}{$\begin{array}{l}\text { Actual } \\
\text { Temp, } \\
{ }^{\circ} \mathrm{C}\end{array}$} & \multirow{2}{*}{$\begin{array}{l}\text { Mean } \\
\text { Peak } \\
\text { Pressure } \\
\text { (psi) }\end{array}$} & \multicolumn{2}{|c|}{$\begin{array}{c}\text { Mean Peak } \\
\text { Pressure of Group }\end{array}$} \\
\hline & & & & $\begin{array}{l}\text { Pressure } \\
(\mathrm{psi})\end{array}$ & $\begin{array}{l}\text { Stand- } \\
\text { ard } \\
\text { Error } \\
\text { (psi) }\end{array}$ \\
\hline $\begin{array}{l}3.1 \\
3.7\end{array}$ & Water $15 \mathrm{~g}$ & $\begin{array}{l}20 \\
20\end{array}$ & $\begin{array}{l}3,200 \\
5,100\end{array}$ & 4,050 & \pm 810 \\
\hline $\begin{array}{l}1.2 \\
1.5 \\
1.8 \\
2.1 \\
2.2\end{array}$ & Water $30 \mathrm{~g}$ & $\begin{array}{l}20 \\
90 \\
20 \\
26 \\
90\end{array}$ & $\begin{array}{l}6,060 \\
7,700 \\
6,770 \\
6,460 \\
6,910\end{array}$ & 6,780 & \pm 300 \\
\hline $\begin{array}{l}3.2 \\
3.9\end{array}$ & Water $60 \mathrm{~g}$ & $\begin{array}{l}20 \\
20\end{array}$ & $\begin{array}{l}10,890^{b} \\
14,460^{b}\end{array}$ & 12,930 & \pm 990 \\
\hline $\begin{array}{l}3.10 \\
3.12\end{array}$ & Sodium $150^{\circ} \mathrm{C} 15 \mathrm{~g}$ & $\begin{array}{l}160 \\
170\end{array}$ & $\begin{array}{l}8,050 \\
7,210\end{array}$ & 7,630 & \pm 820 \\
\hline 3.6 & Sodium $400^{\circ} \mathrm{C} 15 \mathrm{~g}$ & 310 & 6,430 & 6,430 & \pm 1140 \\
\hline $\begin{array}{l}1.1 \\
1.3 \\
1.7 \\
2.4 \\
2.7 \\
3.11\end{array}$ & Sodium $150^{\circ} \mathrm{C} 30 \mathrm{~g}$ & $\begin{array}{l}160 \\
175 \\
160 \\
138 \\
150 \\
220\end{array}$ & $\begin{array}{l}10,380 \\
12,480 \\
11,020 \\
10,090^{b} \\
12,450^{b} \\
13,610^{b}\end{array}$ & 11,580 & \pm 530 \\
\hline $\begin{array}{l}1.4 \\
2.3\end{array}$ & Sodium $250^{\circ} \mathrm{C} 30 \mathrm{~g}$ & $\begin{array}{l}230 \\
260\end{array}$ & $\begin{array}{l}10,390 \\
11,150\end{array}$ & 10,770 & \pm 460 \\
\hline $\begin{array}{l}2.5 \\
2.6 \\
2.8\end{array}$ & Sodium $400^{\circ} \mathrm{C} 30 \mathrm{~g}$ & $\begin{array}{l}350 \\
450 \\
450\end{array}$ & $\begin{array}{l}13,240^{b} \\
12,620^{b} \\
11,400^{b}\end{array}$ & 12,420 & \pm 680 \\
\hline 2.9 & Sodium $550^{\circ} \mathrm{C} 30 \mathrm{~g}$ & 530 & $\begin{array}{r}\text { No } \mathrm{Me} \\
\text { tained }\end{array}$ & asuremen & ts Ob- \\
\hline $\begin{array}{l}3.3 \\
3.8\end{array}$ & Sodium $150^{\circ} \mathrm{C} 60 \mathrm{~g}$ & $\begin{array}{l}160 \\
160\end{array}$ & $\begin{array}{l}17,220^{b} \\
17,200^{b}\end{array}$ & 17,210 & \pm 1250 \\
\hline 3.5 & Sodium $400^{\circ} \mathrm{C} 60 \mathrm{~g}$ & 410 & $19,250^{\circ}$ & 19,250 & \pm 1240 \\
\hline
\end{tabular}

(a) The group mean peak pressures have been ealculated from the individual pressure records and not from the mean peak pressures of each experiment.

(b) Indicates that the core vessel ruptured.

geometry the peak pressure produced by a charge in sodium can be obtained by using twice the weight of explosive in water.

a) Calculation of Group-averaged PressureTime Curves. An average pressure-time curve was obtained for each experiment by calculating the arithmetic mean of the pressures measured by the four gauges. This was done at intervals of 0.01 ms, starting at the common time zero. There was 

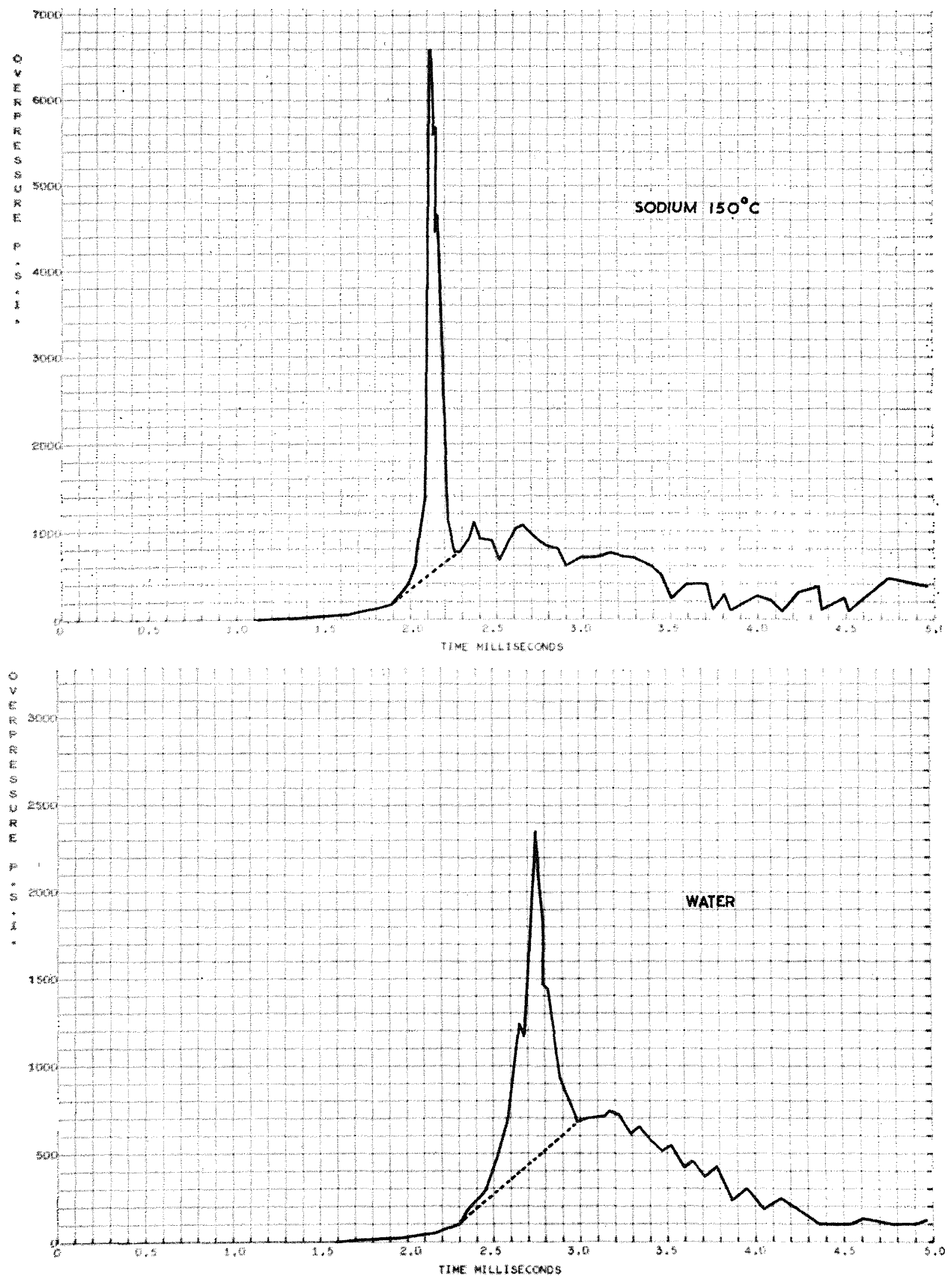

FI(x. 2. Typical Pressure-time Curves for a 15-g Charge. 


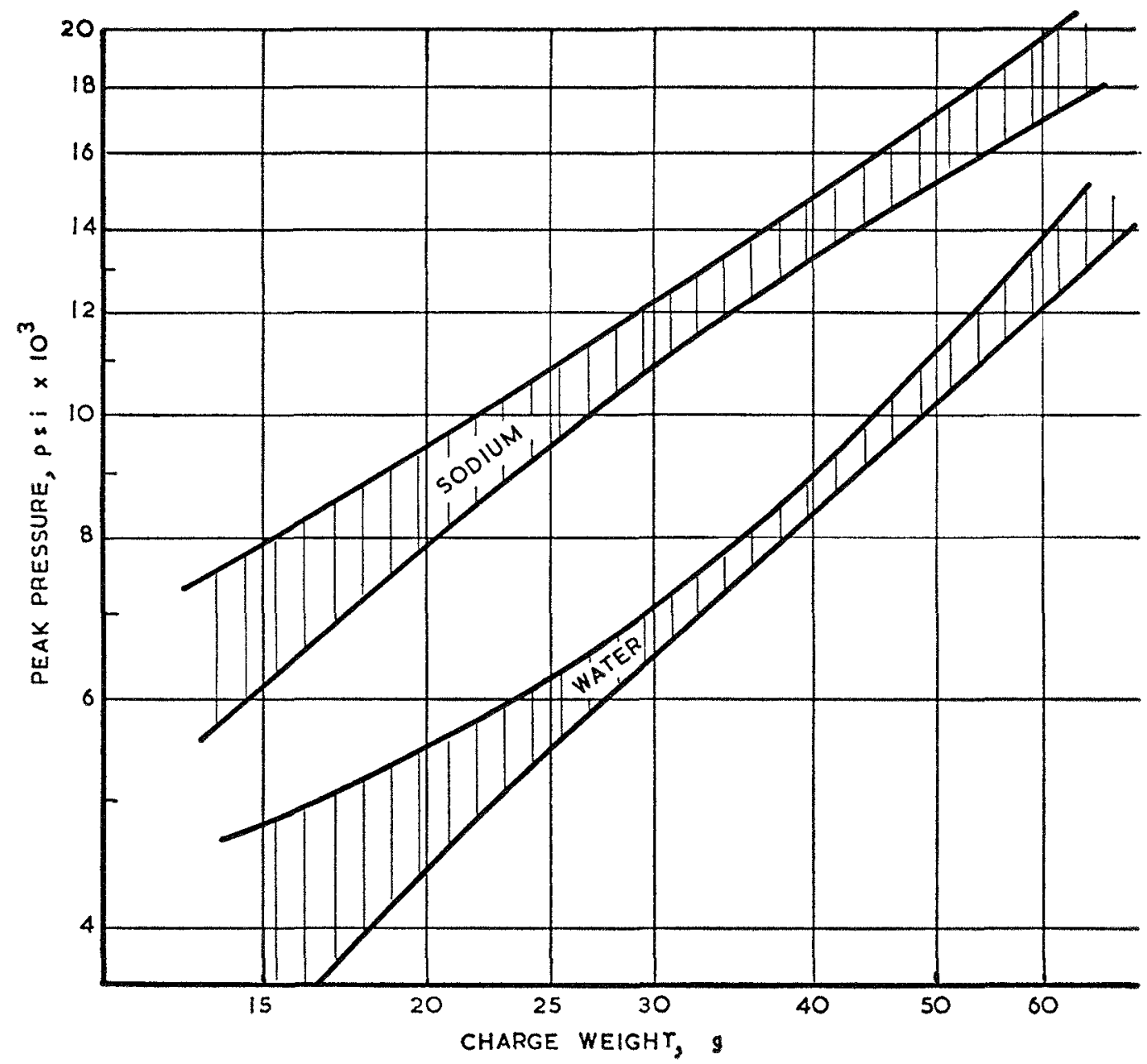

FIg. 3. Variation of Mean Peak Pressure with Charge Weight.

some small random scatter in the arrival times of the high peak at each gauge position in any single experiment, indicating that the liquid surface was not plane when it impacted upon the lid. There was also some variation in the arrival times of the peak pressures between experiments performed under the same conditions. This suggests that the liquid levels were not consistent from model to model. This fault was corrected after the first few experiments.

In order to produce a valid mean pressure-time curve for each group, it was decided to introduce a shift of the time seales of the average curves of each experiment so that the steep leading edge of the pressure peaks coincided in time. This was done in the computer programming by making the time at which the pressure first reached $600 \mathrm{psi}$ common to all curves in the group and equal to the average time of all the experiments in that group.

Figure 4 shows the average pressure-time curve for the water experiments and for the group of experiments using sodium at $150^{\circ} \mathrm{C}$, each with a $30-\mathrm{g}$ charge. The peak pressures shown in Fig. 4 are lower than the average of the peak pressures quoted in Table 1 because of the slight scatter in arrival times of the peaks within any one experiment. The averaging process has produced a lower peak pressure of longer duration containing the correct impulse. The averaged curves represent the forcing pressure integrated over the whole lid, but it must be remembered that any single point on the lid experienced peak pressures as high as those quoted in Table 1.

The averaging process has also produced pressure-time curves in which random fluctuations of pressure on individual traces have become smoothed out whilst systematic fluctuations have become more apparent. Figure 2 shows random fluctuations whilst the averaged curves in Fig. 4 show periodic oscillations. The average pressure-time curves of each 

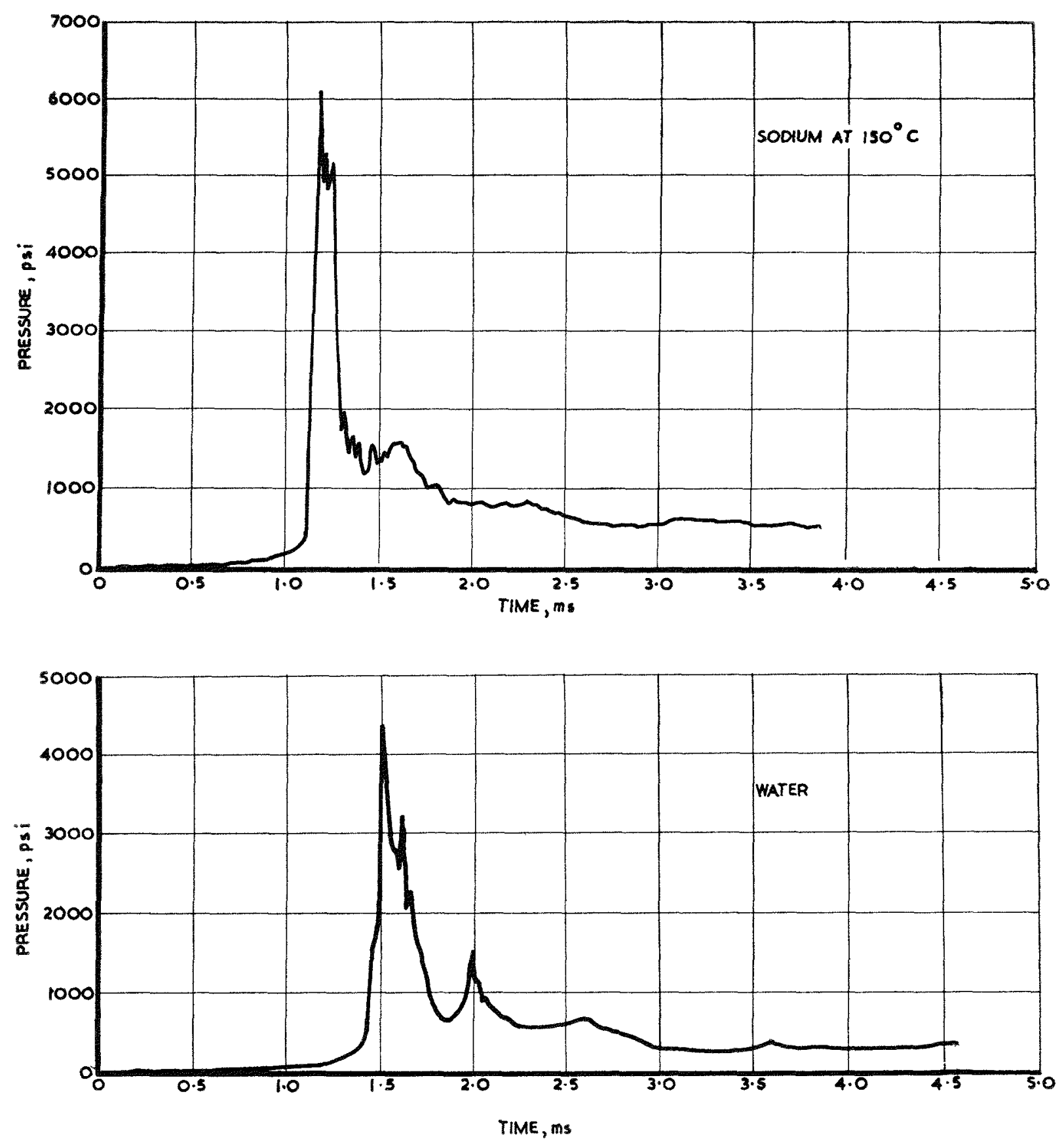

Fra. 4. Average Pressure-time Curves for 30-Charge.

group of experiments were similar in shape to those shown in Fig. 4.

In programming the analysis of the pressure measurements, the loading on the lid of the core vessel was considered to have two components: a high-pressure phase of $0.2-0.4-\mathrm{ms}$ duration, superimposed upon a more slowly rising pressure pulse which proceeded to oscillate with a period of $0.6-0.8 \mathrm{~ms}$. It was assumed that the peak pressure phase was caused by the impact of the surface layer of liquid which was accelerated upwards by the shock wave from the charge, whilst the oscillatory pressure phase was due to the oscillation of the bubble of detonation products and vapourised liquid.

In order to compare the oscillatory pressure loadings, the peak pressure regions were subtracted from the average pressure-time curves for each group. This was achieved by referring to the original pressure-time data from the gauge records and extracting the data points between the initial lead-in on the steep front of the peak and the pressure minimum after the high peak. The computer programme was 


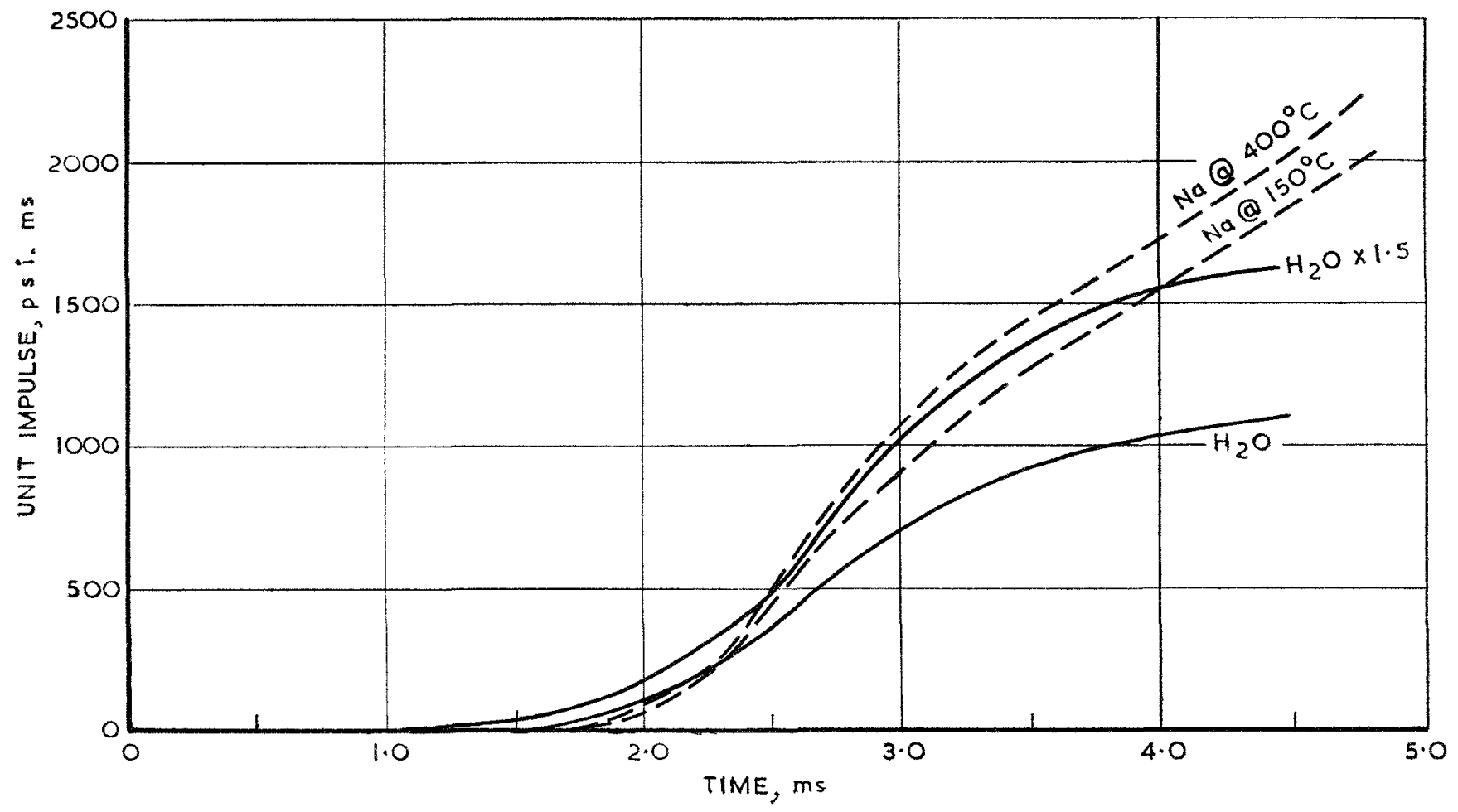

Fig. 5. Unit Impulse for $15-\mathrm{g}$ Charge without Impact Peaks

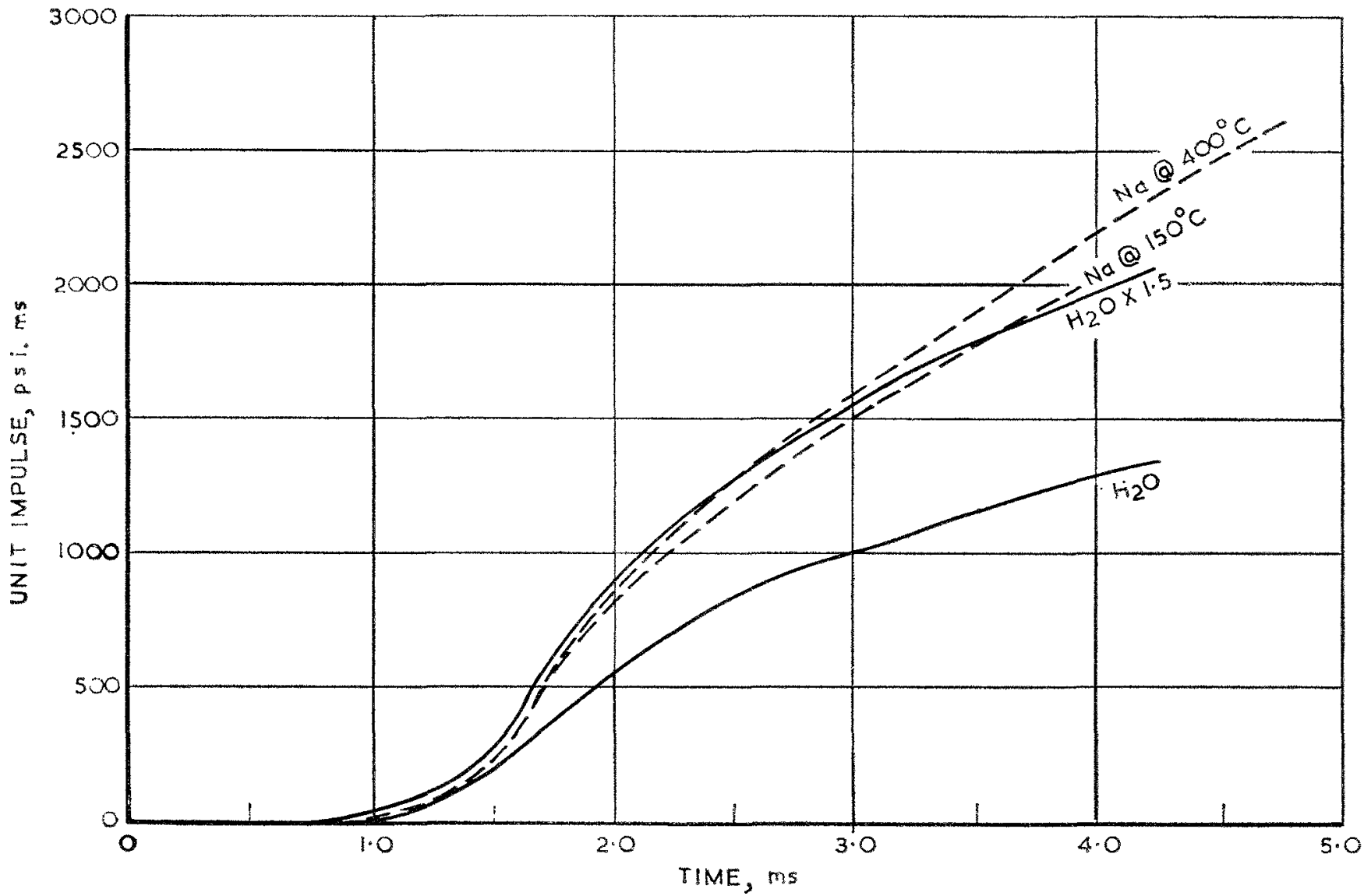

Fig. 6. Unit Impulse for 30-g Charge without Impact Peaks. 


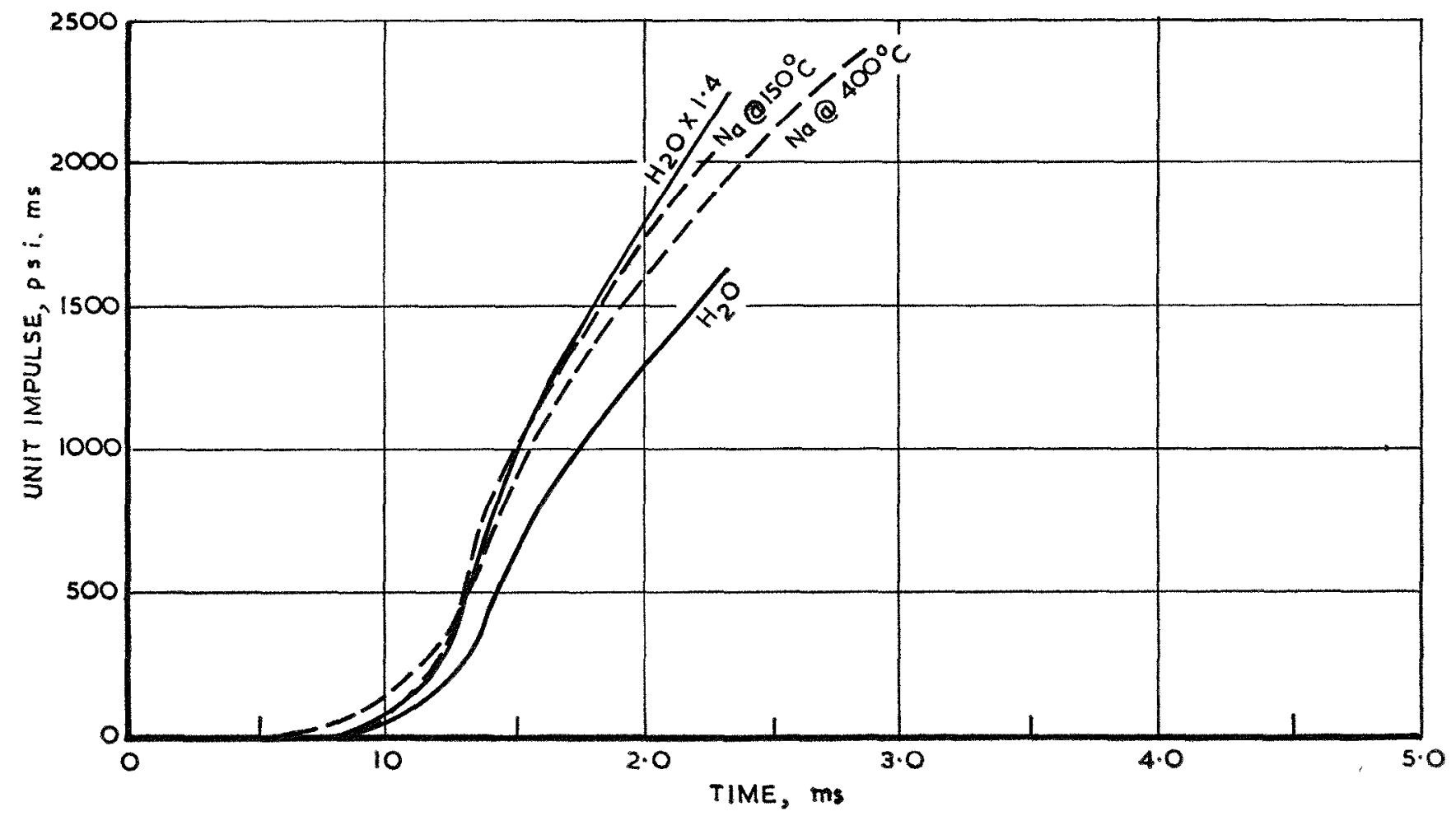

Fla. 7. Unit Impulse for 60-g Charge without Impact Peaks.

TABle 2. Inplose Contaned in Peik Phase (With Standard Error)

\begin{tabular}{|c|c|c|c|c|}
\hline \multicolumn{2}{|c|}{ Experimental Conditions } & \multirow{2}{*}{\multicolumn{2}{|c|}{$\begin{array}{l}\text { Impulse }\left(I_{p}\right), \text { psi-ms, and } \\
\text { Standard Error }\end{array}$}} & \multirow{2}{*}{$\begin{array}{l}230 \mathrm{~W}^{1 / 3} \\
(\mathrm{~W} \text { in } \mathrm{g})\end{array}$} \\
\hline Charge, & Liquid & & & \\
\hline 15 & $\begin{array}{l}\text { Water } \\
\text { Sodium } 150^{\circ} \mathrm{C} \\
\text { Sodium } 400^{\circ} \mathrm{C}\end{array}$ & $\begin{array}{l}465 \\
750 \\
662\end{array}$ & $\begin{array}{l} \pm 148 \\
\pm 198 \\
\pm 182\end{array}$ & 570 \\
\hline 30 & $\begin{array}{l}\text { Water } \\
\text { Sodium } 150^{\circ} \mathrm{C} \\
\text { Sodium } 250^{\circ} \mathrm{C} \\
\text { Sodium } 400^{\circ} \mathrm{C}\end{array}$ & $\begin{array}{l}670 \\
729 \\
737 \\
691\end{array}$ & $\begin{array}{r} \pm 50 \\
\pm 38 \\
\pm 126 \\
\pm 44\end{array}$ & 715 \\
\hline 60 & $\begin{array}{l}\text { Water } \\
\text { Sodium } 150^{\circ} \mathrm{C} \\
\text { Sodium } 400^{\circ} \mathrm{C}\end{array}$ & $\begin{array}{r}1,093 \\
848 \\
1,235\end{array}$ & $\begin{array}{r} \pm 136 \\
\pm 51 \\
\pm 79\end{array}$ & 900 \\
\hline
\end{tabular}

then re-run with a linear interpolation to cover the gap made in the pressure-time curve. The range of data to be extracted was chosen so that the linear interpolation was tangential to the lead-in of the complete pressure-time curve. The revised curves are indicated by the dotted lines in Fig. 2.

It was found convenient when making comparisons to consider the sodium and water impulse curves, impulse being defined as

$$
I(t)=\int_{0}^{t} P d t
$$

The impulse-time curves from the re-run of the computer programme (using the data without the peak pressure phase) are shown in Figures 5, 6, and 7 for the $15-, 30-$, and $60-\mathrm{g}$ charges, respectively. The sodium and the water curves are shown, together with the water curve multiplied by a factor of 1.5 in Figures 5 and 6 and by a factor of 1.4 in Fig. 7. The factors were chosen to give the best agreement with the sodium curves.

Table 2 shows the values of impulse subtracted from the pressure records in making the above comparisons (i.e., the impulse contained in the peak pressure phase above the linear interpolation). The standard errors are large, but the trend of these figures suggests that the impulse in this phase is the same for sodium and water for a given charge weight. This is shown particularly by the 30 -g-charge results for which most information was obtained. The impulse does not vary significantly from the relationship $I_{p}=230 \mathrm{~W}^{1 / 3}$, values of which are included in Table 2.

\subsection{Strain Measurements}

Figure 8 shows typical strain-gauge records from one bolt in experiment 3.11 with a $30-\mathrm{g}$ charge in sodium at $220^{\circ} \mathrm{C}$. Some bending of the hold-down bolt occurred, since the maximum strain measured by the gauge on the inside face was greater than that measured by the outer gauge. The shape of the strain-gauge signals was not at all like that of the 


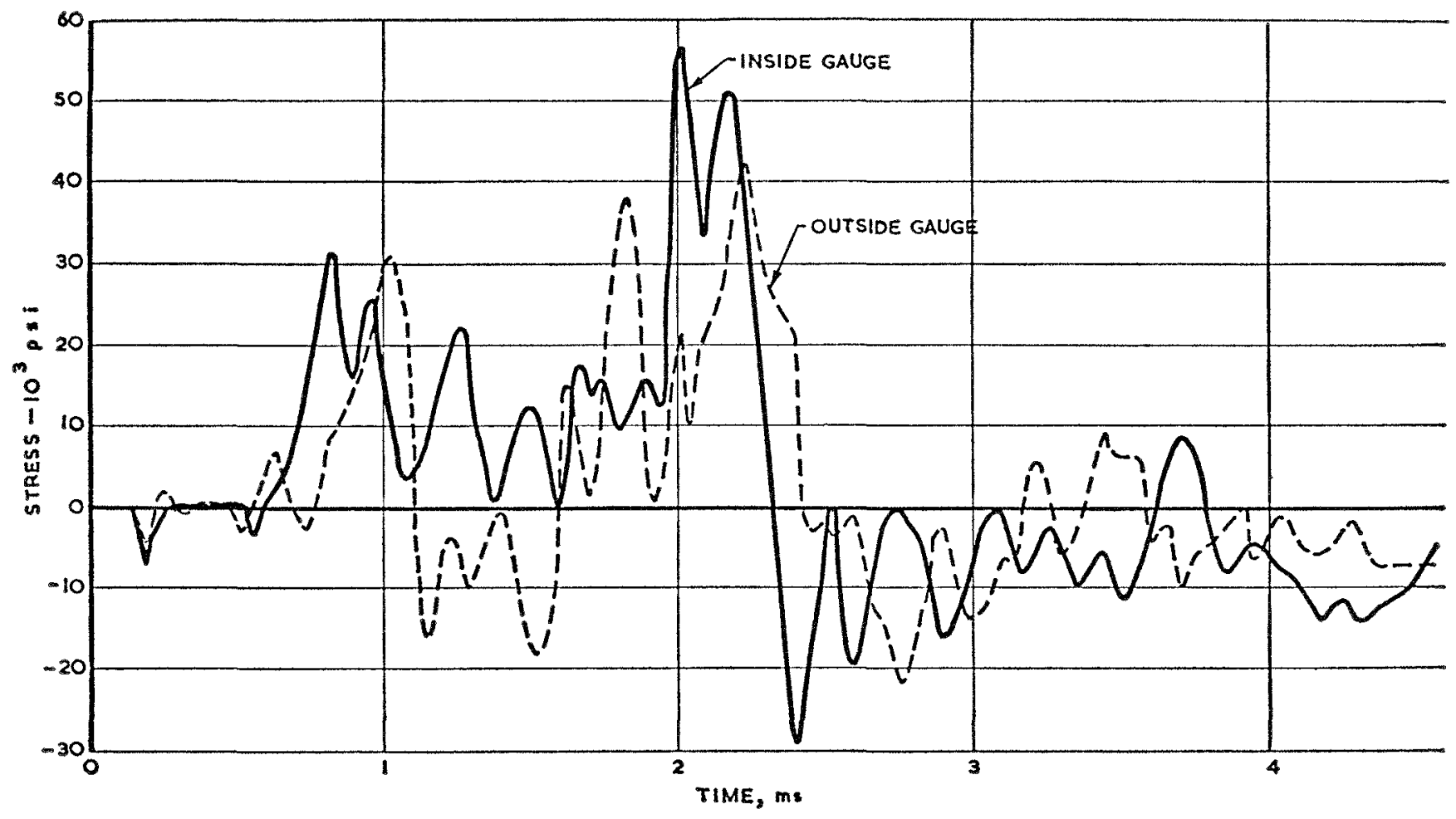

Fig. 8. Strain-gauge Records from One Hold-down Bolt, Experiment 3.11, 30-g Sodium at $220^{\circ} \mathrm{C}$.

pressure-time records. The dynamic response of the model can be regarded as analogous to the response of a mass-spring system. The strain gauges are mounted on a spring attached to a mass (i.e., the lid of the containment vessel) to which is applied the forcing function (as defined by the pressure-loading curves). The analogy is further complicated, however, by the fact that the whole experimental assembly could lift, so that the spring cannot be regarded as being attached to an infinite mass.

One thing common to all the strain-gauge records was that the first strain signals occurred before the first liquid impact was recorded upon the lid. In experiment 3.11 the maximum pressure was recorded $1.25 \mathrm{~ms}$ after time zero, whereas the first maximum strain in this expcriment (as shown in Fig. 8) arrived at $0.8 \mathrm{~ms}$. The probable explanation for this is that the base of the containment vessel received an initial downward impulse from the detonation, and this was transmitted by stress waves through the walls to the flange of the containment vessel which, in turn, received a downward impulse.

Table 3 summarises the peak values of the forees measured on the hold-down bolts, and Fig. 9 is a plot of these peak forces versus temperature of the sodium for experiments with $30-\mathrm{g}$ charges.

The strains measured by the U.K. team were generally greater than the strains measured by the C.E.A. team, but the general shapes of the signals were always very similar. The discrepancy has yet to be resolved, but it is thought that the difference in frequency response and writing speeds of the two recording systems may account for the differences in

Table 3. Mean Values of the Miximem Upward Force Measured in the Hold - DOWN Bolts for Each EXPERIMENTAL CONDITION

\begin{tabular}{l|c|c}
\hline \multirow{2}{*}{ Group Title } & \multicolumn{2}{|c|}{$\begin{array}{c}\text { Group Mean Value of Maximum } \\
\text { Force, } 10^{3} \mathrm{lb}\end{array}$} \\
\cline { 2 - 3 } & CEA & UKAEA \\
\hline Water $15 \mathrm{~g}$ & 81 & 92 \\
Water $30 \mathrm{~g}$ & 101 & 158 \\
Water $60 \mathrm{~g}$ & 97.8 & 148 \\
\hline Sodium $150^{\circ} \mathrm{C} 15 \mathrm{~g}$ & 110 & 149 \\
Sodium $400^{\circ} \mathrm{C} 15 \mathrm{~g}$ & 144 & 152 \\
\hline Sodium $150^{\circ} \mathrm{C} 30 \mathrm{~g}$ & 148 & 183 \\
Sodium $250^{\circ} \mathrm{C} 30 \mathrm{~g}$ & 112 & 173 \\
Sodium $400^{\circ} \mathrm{C} \mathrm{30 \textrm {g }}$ & 140 & 168 \\
Sodium $550^{\circ} \mathrm{C} 30 \mathrm{~g}$ & 177 & 195 \\
\hline Sodium $150^{\circ} \mathrm{C} 60 \mathrm{~g}$ & 169 & 263 \\
Sodium $400^{\circ} \mathrm{C} 60 \mathrm{~g}$ & 141 & 227 \\
\hline
\end{tabular}

Note. The maximum force measured in each experiment differed from the above mean values by no more than the $5 \%$ accuracy which the manufacturers quote for the strain gauges. 


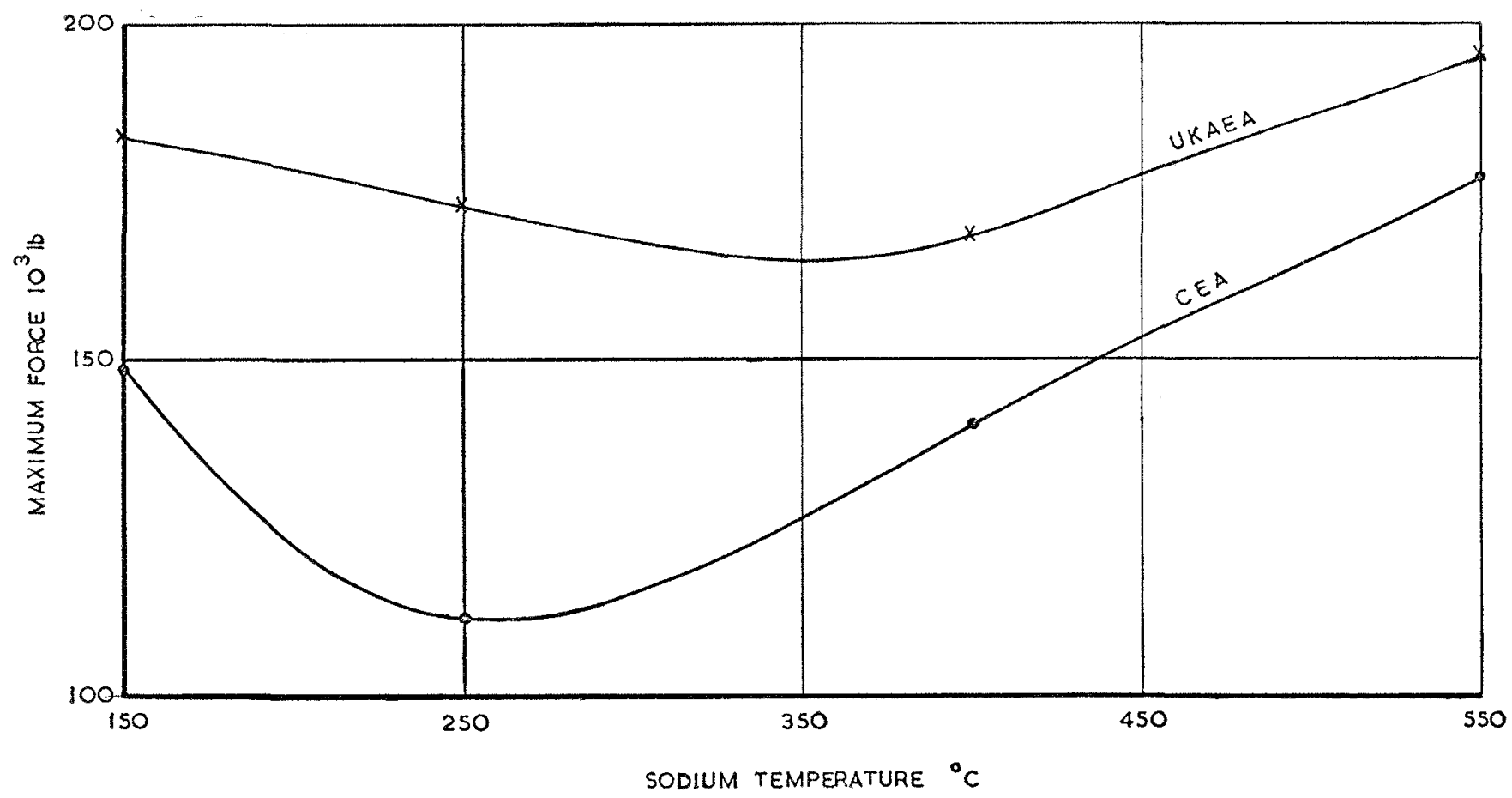

FIG. 9. Maximum Force Measured in Hold-down Bolts versus Sodium Temperature--30-g Charge.

measured strain levels. This hypothesis has to be checked.

\subsection{Damage to Vessels}

Detailed measurements were made of the distortions to all vessels after each experiment. It is not intended to include all this information here, however. Figure 10 is a photograph of the damaged vessel from Experiment $1.3\left(30-\mathrm{g}\right.$ charge in sodium at $\left.175^{\circ} \mathrm{C}\right)$ compared with an undamaged vessel. The damaged vessel has been reduced in height, and a series of corrugations has been formed in the walls, including one below the level of the heating coil. The cap was also torn off the top of the re-entrant cylinder, and the base of the core vessel dished upwards. This was the pattern of damage to all vessels.

For a given charge weight the vessels containing sodium were more severely distorted than those containing water and the distortions increased with increasing temperature. In all experiments with $60-\mathrm{g}$ charges and in some with $30-\mathrm{g}$ charges the vessels split vertically; the initiation of the split appears to have been at the point where the thermocouple insert was welded into the vessel wall.

\section{Discussion of Results}

From the analysis of the pressure-time records, as described above, an empirical method has been derived for constructing an approximate pressure-time curve for sodium from the averaged pressure-time curve obtained with the same weight of charge in water. The water pressures are multiplied by a fac-

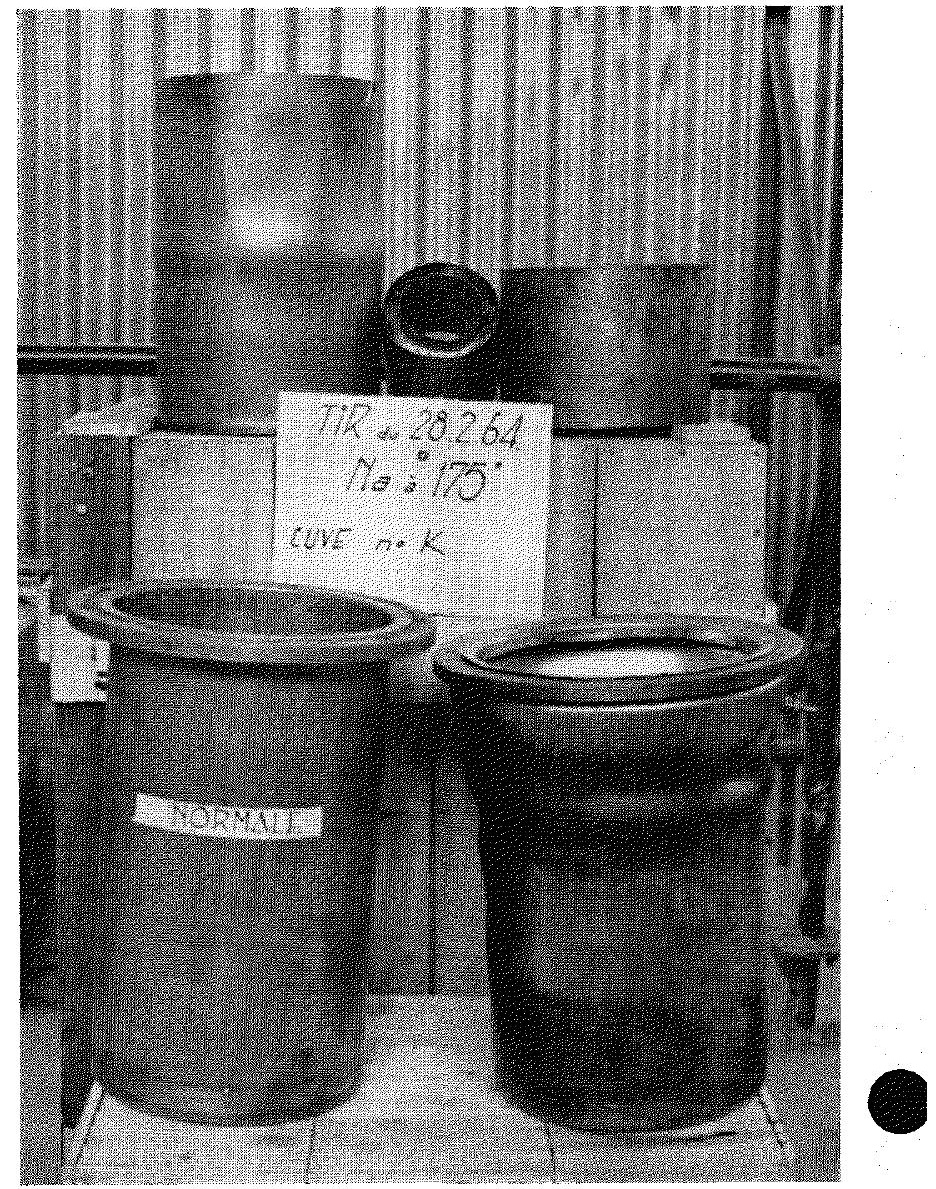

FIc. 10. Typical Core-vessel Damage from 30-g Charge in Sodium. 

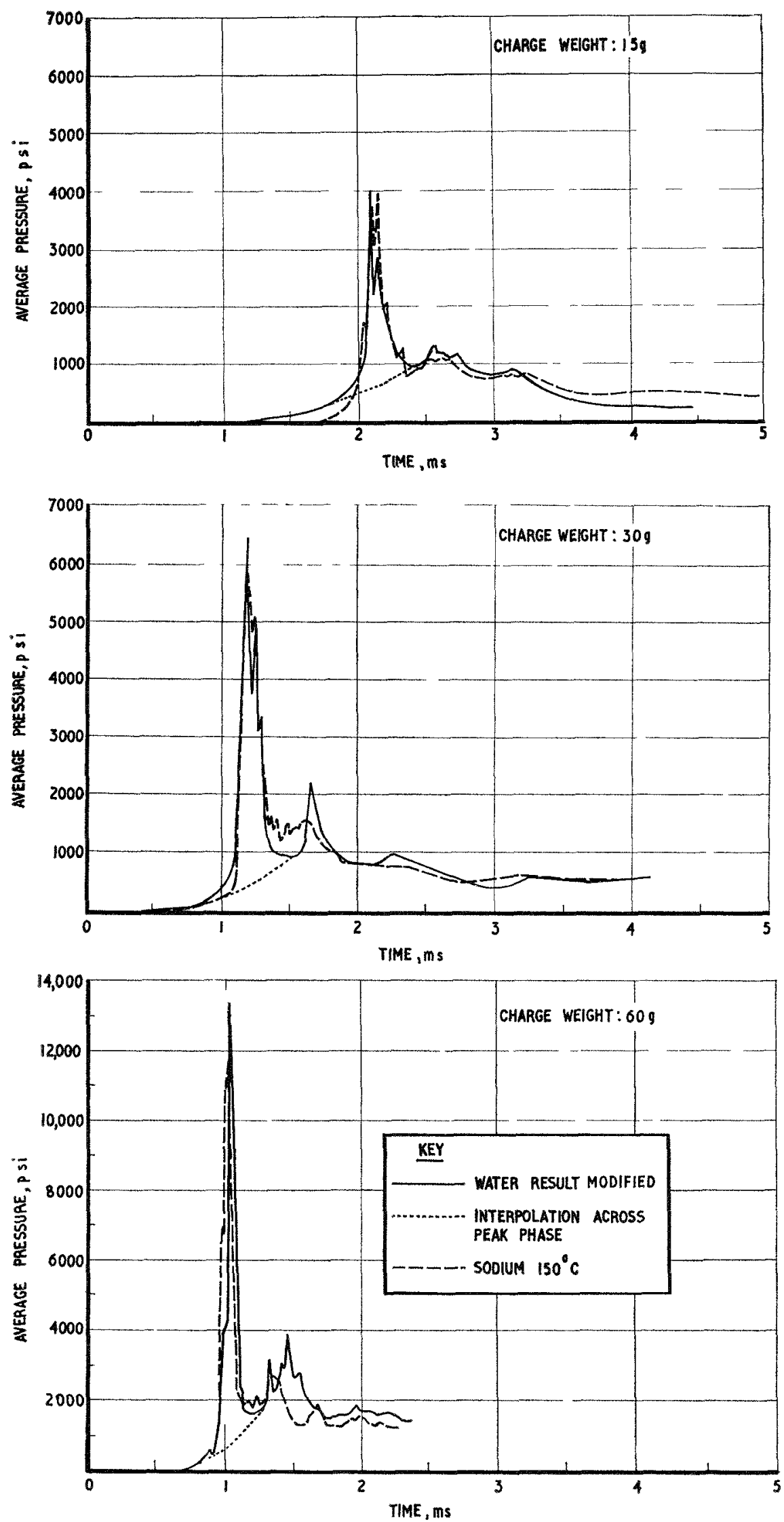

FIg. 11. Comparison of Pressures from Sodium at $150^{\circ} \mathrm{C}$ with the Curves Derived from the Water Results. 
TABle 4. Calculated ind Measured Impact Pressireses

\begin{tabular}{c|c|c|c}
\hline $\begin{array}{c}\text { Experimental } \\
\text { Conditions }\end{array}$ & $\begin{array}{c}\text { Transit Time } \\
\text { across 5 cm, ms }\end{array}$ & $\begin{array}{c}\text { Calculated } \\
\text { Impact } \\
\text { Pressure, psi }\end{array}$ & $\begin{array}{c}\text { Average } \\
\text { Measured } \\
\text { Impact } \\
\text { Pressure, psi } \\
(=\rho c v)\end{array}$ \\
\hline Sodium $15 \mathrm{~g}$ & 1.83 & 8,800 & 7,000 \\
$30 \mathrm{~g}$ & 1.00 & 16,200 & 11,600 \\
$60 \mathrm{~g}$ & 0.77 & 21,000 & 18,200 \\
\hline Water $15 \mathrm{~g}$ & 2.30 & 4,700 & 4,000 \\
$30 \mathrm{~g}$ & 1.22 & 8,800 & 6,800 \\
$60 \mathrm{~g}$ & 0.81 & 13,200 & 13,000 \\
\hline
\end{tabular}

For Sodium $\rho$ at $250^{\circ} \mathrm{C}=0.891 \mathrm{~g} / \mathrm{cm}^{3}$ (reference 3 ) $c$ at $250^{\circ} \mathrm{C}=2,507 \mathrm{~m} / \mathrm{s}$ (reference 4$)$

For Water $\quad \rho$ at $20^{\circ} \mathrm{C}=0.998 \mathrm{~g} / \mathrm{cm}^{3}$ (reference 5) $c$ at $20^{\circ} \mathrm{C}=1,484 \mathrm{~m} / \mathrm{s} \quad$ (reference 5)

tor of 1.5 and the duration of the impact peak is divided by the same factor (to keep the impulse constant in this phase). This has been done for the average results of each charge weight as shown in Fig. 11 where the calculated results are compared with the results in sodium at $150^{\circ} \mathrm{C}$. This method appears to be accurate (at least for this geometry) within the scatter of the results.

Ideally, we would like to have obtained from these experiments a method of estimating the weight of charge that would have to be used in water to simulate a given size of charge exploded in sodium, since in our scaled-model investigations we generally use water in place of the sodium coolant. Figure 3 shows that there is a simple factor of two in the weights of charge necessary to produce a given peak pressure in sodium and in water. There is no simple factor connecting any of the other explosive effects, however. For example:

1. The duration of the impact-loading phase is charge-dependent, so that doubling the weight of charge in water, although producing the same peak pressure as a charge in sodium, will produce a greater impulse. This can be seen from Table 2 .

2. It appears that the distortion produced by a given weight of charge in sodium is less than that produced by twice the charge weight in water.

The pressure loading on the lid of the containment vessel is relieved by venting of the core vessel. The failure of the vessels with some of the $30 \mathrm{~g}$ and $60 \mathrm{-g}$ charges was probably caused by the initial shock wave from the charge so that venting occurred in the early stages of the loading phase. Further venting could have occurred around the top of the core vessel when the top flange was forced out of the locating recess in the lid by the downward thrust on the flange.

In Fig. 5, the water result times 1.5 diverges from the sodium curves after the first bubble oscillation, indicating a higher pressure level in sodium. This may be due to reaction of sodium with the water vapour in the explosion products. If this is so, one would expect to observe that the impulse curves for sodium and water diverge at later times with the larger charges. Unfortunately, the period for which the pressures were recorded was terminated by the uranium rods above the charge impacting upon the transducers. The period of the oscillation does not vary significantly with charge weight and is probably a function of the model configuration. In an infinite medium the bubble period increases with charge weight, but the period is reduced by increased confinement. ${ }^{(2)}$ In this particular arrangement the two effects appear to be self-cancelling.

Examination of the results of the experiments with 30-g charges (see Fig. 6) shows that the total impulse is slightly higher at $400^{\circ} \mathrm{C}$ than at $150^{\circ} \mathrm{C}$ in sodium. Additionally, the strain-gauge results showed a minimum impulse at $250^{\circ} \mathrm{C}$ (see Fig. 9), but in both cases the differences are small compared to that between sodium and water. There was no observable variation of pressure loading with temperature in the water experiments. Nor was there any apparent variation of pressure loading in the few experiments for which the lcvel of the sodium was not correctly adjusted. The early arrival of the first impact pressures on the lid suggests that the space above the liquid in these experiments was only about $3 \mathrm{~cm}$ instead of the intended $5 \mathrm{~cm}$.

The delay times between detonation and the first impact have been measured, and the transit times of the detonation and shock waves through the explosive and liquid have been estimated. The difference between the two times is the transit time of the liquid surface from its rest position to the lid. If the surface layer of liquid (which is accelerated by the shock wave) acquires its terminal velocity almost instantaneously (i.e., in a few $\mu$ sec) and travels at uniform velocity, then an expected impact pressure can be calculated from the relation

$$
P=\rho c v
$$

where $\rho$ is the liquid density, $c$ the acoustic velocity of liquid, and $v$ the impact velocity of liquid.

Average values of the transit time and the calculated impact pressures are shown in Table 4 together with the average values of the measured pressure. The calculated pressures are higher than the measured pressures. The ratio 
peak pressure measured in sodium

peak pressure measured in water

is less with $60-\mathrm{g}$ charges than with $30-\mathrm{g}$ and $15-\mathrm{g}$ charges (see Fig. 3), and the ratio of impact velocities ( $c_{n}$ d hence calculated pressure ratios) shows the same trend. Hence we can assume that the pressure transducers functioned correctly. Individual pressuretime curves show a random variation of arrival time for the first impact signal in any single experiment, indicating that the liquid surface is not plane. There is also some scatter in the height of the impact pressurc peaks, so it is likely that the surface irregularities are comparable with the diameter of the sensitive face of the pressure transducers (namely, $0.26 \mathrm{in}$.). This could explain why the measured impact pressu'es are less than the calculated vales. Alternatively, an inadequate response of the amplifiers to high frequencies would reduce observed peak pressures.

\section{Conclusions}

It has been established that the forces transmitted to the lid of the core vessel are significantly higher with sodium than with water. By comparison, varying, the temperature of the sodium has a much smaller effect.

It was not possible to obtain a simple formula relating the effects in sodium to those in water because the forces on the top closure depend upon the deformation produced in the core vessel as well as upon the physical properties of the coolant. However, the physical processes involved in producing the forces in a fast reactor geometry are now better understood. This should enable us to derive the equations of motion which describe the response of the model to explosive loading, thus providing a direct comparison of the measurements of pressure and force. We should then be able to resolve the differences in the two methods of strain recording and also check the validity of the equations of motion.
In order to interpret model experiments using water in other reactor geometries, one needs to know the extent of the deformation in these systems, since any pressure relief resulting from deformation has a marked influence upon the loading of the top closure in the bubble-oscillation phase.

The initial impact loading is unlikely to be influenced by this deformation, so the early loading phase in sodium may be obtained directly from the results presented in this paper.

\section{REFERENCES}

1. Rees, N. J. M., see the preceding Paper.

2. Cole, R. H., I'nderwater Explosions, Princeton University Press.

3. CSAEC TID5277, Liquid Metals Handbook (Na. NaK1955).

4. Trelin, Yu. S., Vasil'ev, I. N., and Roshchupkin, V. V., Measurement of C'trasound Veloculy in Molten Alkali Metals, Atomnaya Energiya 9, No. 5 (Nov. 1960).

5. Kaye, G. W. C., and Laby, T. H., Tables of Physical and Chemical Constants, Longmans (1956).

\section{Discussion}

Mr. McCarthy (PRDC): What were the temperatures of the sodium and the water?

Mr. Walford: The sodium temperatures were within the range of 150 to $550^{\circ} \mathrm{C}$. The temperature of the sodium seemed to affect the pressure loading very little. Much more important is the liquid that is used. We did water experiments at 20 and $90^{\circ} \mathrm{C}$, and we could detect no significant difference at all.

Mr. McCarthy: The NOL work (see discussion in preceding Paper) showed quite a substantial difference which we attributed to the proximity of the highertemperature water to the boiling point and easier generation of steam by the heat from the explosion.

$M r$. Walford: This is what we expected. This was really half of the object of the experiment, and we were rather surprised to find that this didn't happen. 


\title{
Design Factors Influencing the Containment of Internal Explosion in Fast Reactors
}

\author{
D. E. J. Samuels \\ U. K. Atomic Energy Authority \\ Foulness, Southend-on-Sea, Essex, England
}

\section{STNOPSIS}

In recent years the Foulness Establishment of the U.K.A.E.A. has been engaged in a continuing investigation of the containment of core explosions in fast reactor systems. In this work small charges of chemical high explosive have been detonated in scaled models of the reactor structure. Beginning with the investigation of the containment potential of existing reactors, the work progressed to basic studies of different containment geometries (the conference paper by N.J. M. Rees is an example), and the model approach has now become an integral part of the design process for large fast reactor systems. Geometrical arrangements are studied in instrumented, overstrong models, while information on modes of deformation and energy absorption is obtained from models having correctly scaled structural strength.

The aim of this work has been to establish the sequence of events following a core explosion and to identify the loading and response mechanisms occurring in the structure. Processes studied include the plastic flow and rupture of metals, shock-wave interactions, venting and pressure rise in gas spaces, crushing of porous materials, and the impact of shock-driven liquids. This has led to the possibility of adjusting the balance between these processes by appropriate design so as to achieve the necessary structural integrity with the least economic penalty or interference with normal working.

\section{Introduction}

Although it is likely that large fast reactors of the future can be designed so that a core meltdown is impossible, in the present state of knowledge such an event cannot be entirely ruled out. We must, therefore, consider the ability of the reactor system to contain the effects of an explosion in the core which, pessimistically, may be equivalent to several hundred pounds of TNT. It is not sufficient to surround the system by a continuous outer containment strong enough to withstand the immediate forces of the explosion; the radioactive and corrosive debris must be contained for many years. A large reactor will con- tinue to produce several megawatts of fission product decay heat for a period of hours or days after an explosion, and it is necessary to provide some means of removing this heat so that the core debris does not melt its way through the containment. The designer, therefore, needs to know in some detail the loads imposed on different parts of the reactor structure by explosions of various magnitudes, and also the damage produced by these loads. A design which is balanced for normal operational loads is unlikely to be properly balanced for the containment of explosions. The early failure of components close to the core can markedly affect the loading of more outlying parts of the structure, and some geometrical arrangements can effectively channel the explosion energy and produce the hazard of local failure.

In this paper I consider the various mechanisms by which forces can be transmitted from the explosion to the structure and ways in which the energy of the explosion can be absorbed within the structure. A proper understanding of these mechanisms should enable designers to produce an economical balanced design from the point of view of explosion containment. This discussion is based on a number of model investigations of the kind described by Rees ${ }^{(1)}$ and by Drevon, Falgayrettes, and Walford. ${ }^{(2)}$

I shall restrict my discussion to liquid-cooled fast reactors. For the sake of definiteness $I$ shall consider oxide fuel contained in stainless steel pins supported in stainless steel fuel boxes and cooled by liquid sodium. I shall assume that the sodium is covered by an inert gas blanket and is held in a steel tank within a concrete vault which has a rotatable shield plug in the roof. These features are not essential, but will provide a framework within which to discuss explosion containment.

\section{Comparison with Chemical Explosion}

Before we can generalise the results obtained in model experiments, we must decide how closely chemical high explosive simulates a meltdown excursion. The gravity-controlled collapse model has already been discussed in detail by Hicks and Menzies. ${ }^{(3)}$ At 
the start of the power excursion, the core is partially molten, and consists of solid and molten uranium and/or plutonium oxide and stainless steel. The sodium will have been vapourised and expelled from the core region. The breeder is assumed to be intact. The energy release in the excursion is so rapid (it is complete in less than $1 \mathrm{~ms}$ ) that some fraction of the oxide fuel is rapourised and superheated to a high temperature and pressure, with virtually no heat transfer to the stainless steel or the remainder of the fuel. At this stage the core consists of high-pressure oxide vapour in which molten and solid fucl and stainless steel are dispersed. It is surrounded by the breeder structure and liquid sodium in their pre-excursion state. As the fuel vapour expands it drives a shock wave through the breeder assembly into the coolant, converting some of its internal energy into compression and kinetic energy of the surroundings. This is qualitatively similar to the situation when chemical high explosive detonates. The detonation products, which consist mainly of gaseous oxides of carbon and nitrogen and of water rapour, replace the fuel vapour. The initial pressure and temperature are similar in the two cases. The main difference is that in the case of the chemical explosive virtually all the energy relcased in the detonation is transferred to the surrounding medium as cornpression and kinetic energy, whereas in the nuclear excursion a large proportion (perhaps more than $90 \%)$ of the total energy remains in the core materials whin these recondense. Heat transfer rates from the liquid phase are so low that this energy (denoted by $Q^{*}$ by Hicks and Menzies) plays no part in the explosion process. When performing model experiments with chemical explosive, therefore, it is necessary to relite the detonation energy of the charge to the excess energy, $Q-Q^{*}$, of the nuclear excursion.

\section{Damage Mechanisms}

For successful containment of a meltdown explosion, all the energy has to be degraded into heat in the solid and liquid phases without breaching the outermost containment, essentially through processes of internal friction. Any medium is irreversibly heated by the passage of an intense shock wave through it. The amount of heat lost is a function of the shock strength; below a certain strength the loss is negligible, and the shock is said to have become acoustic. For high explosive in water this occurs at about ten charge radii, and within this distance about $25 \%$ of the detonation energy is deposited in the water. As the acoustic properties of liquid sodium are similar to those of water, a similar proportion of the excursion energy will be absorbed in a fast reactor having a sufficiently large volume of coolant. A similar amount of energy is carried to large distances by the acoustic wave. The remainder of the energy is associated with the oscillatory flow near the explosion cavity and appears in subsequent pressure pulses.

If a shock wave reaches a discontinuity in the medium through which it is travelling (either a change of phase or of material), a partial reflection occurs. If the acoustic impedance (the product of density $\rho$ and sound velocity, $\rho c$ ) increases at the discontinuity, the pressure at the interface is increased, the transmitted shock is increased in strength, and a reflected shock wave travels back into the region already traversed by the incident shock. Conversely, if the acoustic impedance decreases, as at a liquid-gas interface, the transmitted shock is weakened, and an expansion wave travels back into the shocked region. This expansion wave may set up tensile forces in the material which exceed its tensile strength, producing the phenomenon of scabbing in solids, or of cavitation in liquids. Momentum trapped in the separated layer causes it to fly off. A similar process may accelerate a membrane, such as a tank wall, but this would be restrained by tensile forces in the direction of the membrane surface, set up by eurvature. Such tensile forces are the major cause of structural failure.

When the separated layer just described, be it liquid sodium or a portion of the reactor vessel, reaches a rigid boundary - the concrete vault or roof plug-a high impact pressure will be produced. For impact on a completely rigid boundary the instantaneous pressure $P$ is cqual to the product of the acoustic impedance $\rho c$ and the velocity $u$ of the impacting material:

$$
P=\rho c u \text {. }
$$

If the impact is localised, this pressure will be rapidly relieved by sideways expansion. If the reactor vessel fails under the shock loading, the contents will vent into the concrete vault, and unless the free volume available in the vault is large a substantial pressure rise will result. On the other hand, if the vessel is strong enough to survive the shock loading, the kinetic energy of the coolant will be re-directed upwards, lifting the coolant and compressing the blanket gas. Further impact pressures may be produced by this process.

\section{The Sequence of Events}

A number of different processes have been described which can lead to damage: shock compression, scabbing and cavitation, membrane rupture, impact, and compression of vault and blanket gases. These are transient processes and they do not all occur simultaneously. Also, they are interdependent; for example, the vault gas is compressed only if the reactor vessel 


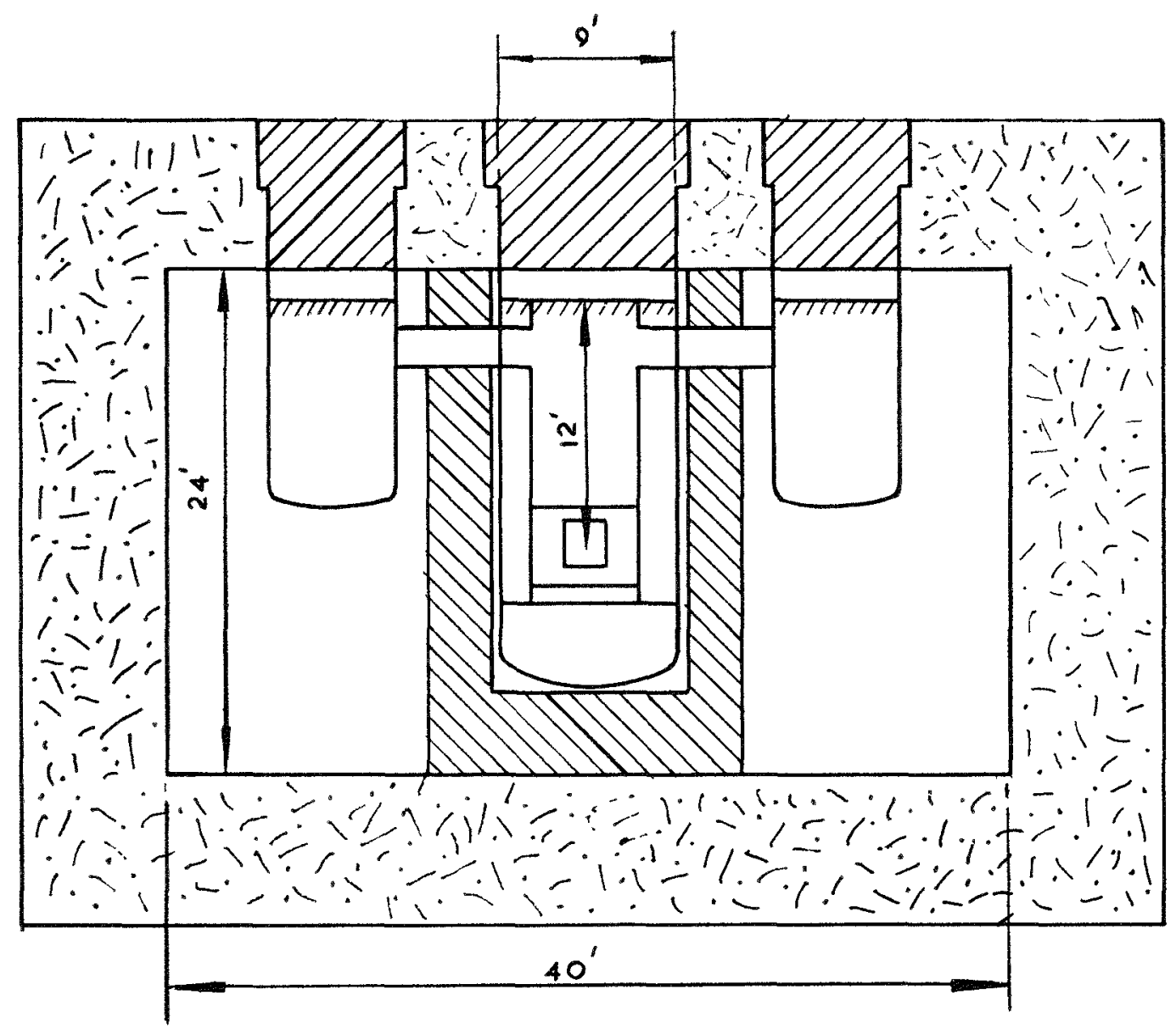

Fig. 1. Schematic Representation of Reactor System.

wall fails. It is the task of the reactor designer to adjust the geometry of his layout and the strength of components of the structure to produce a planned sequence of failures which will ensure that the energy of the explosion is dissipated with the least hazard to containment. Both the time of action and the intensity of the forces are important to proper containment design. It is, therefore, important to establish the sequence of events following an explosion in the particular system being considered.

A typical sequence is given below. It corresponds to a core explosion of excess cnergy equivalent to 250 $1 b$ TNT in the reactor system shown schematically in Fig. 1. The core is immersed to a depth of $12 \mathrm{ft}$ in sodium within a reactor vessel $9 \mathrm{ft}$ in diameter. This is surrounded by a graphite-block neutron shield and is connected to heat exchangers by ducts. The whole is contained in a concrete rault $40 \mathrm{ft}$ in diameter and $24 \mathrm{ft}$ high internally. The top of the reactor vessel is closed by a rotatable plug weighing 70 tons, the upward movement of which is restrained by an energyabsorbing device. There is a gas blanket $1.5 \mathrm{ft}$ deep above the sodium in the reactor vessel and heat ex- changers. The following sequence of events was derived from measurements in $1 / 20$-scale models.

0 End of excursion

$2 \mathrm{~ms}$ Shock at sodium surface

$9 \mathrm{~ms}$ Reactor vessel fractured

$17 \mathrm{~ms}$ Sodium impact on core plug $(3,000 \mathrm{psi})$

$24 \mathrm{~ms}$ Beginning of pressure rise on vault wall

$30 \mathrm{~ms}$ Pressure rise in heat exchangers, pressure decays on core plug

$100 \mathrm{~ms}$ Vault pressure maximum (3.5 psig)

$140 \mathrm{~ms}$ Heat exchanger pressure maximum (10 psig)

$180 \mathrm{~ms}$ Core plug brought to rest (1.5 ft displacement)

The model analogy cannot be carried much beyond this time as the residual heat in the core debris and heat effects from fission product decay predominate at later times. The figures can be scaled to larger or smaller reactor systems of similar geometry. The time scale will vary in proportion to the linear dimensions, the pressure values being unchanged. The events will correspond to an equivalent weight of TNT which is proportional to the cube of the linear scale ratio. 


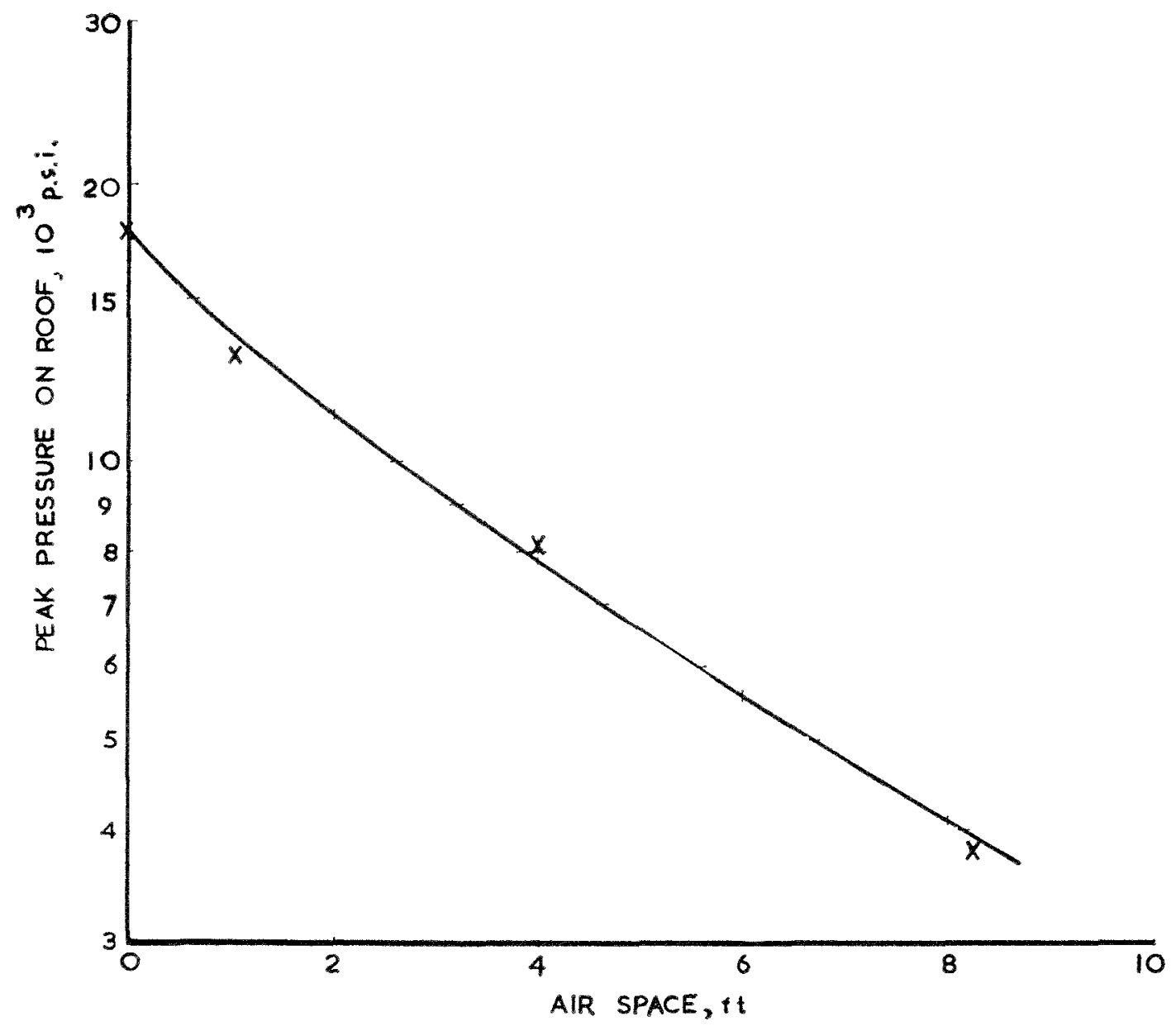

FIG. 2. Effect of Peak Impact Pressure on Tank Roof due to Variation of Air Space.

\section{Examples from Model Tests}

The sequence of events and relative magnitude of the loads will be influenced by the following design features:

(1) core confinement (mass and rigidity of the breeder structure);

(2) quantity of sodium in core vessel (distance to vessel wall);

(3) height of sodium above core;

(4) depth of gas blanket;

(5) strength of thermal shield;

(6) strength of core vessel;

(7) presence of graphite neutron shield within core vessel and crushability of shield;

(8) free volume in vault.

The effect of varying several of these factors has been illustrated by Rees. ${ }^{(1)}$ In one series of tests he varied the water level in his tank, keeping all other factors constant. Thus, both the height of water above the charge and the depth of air space were varied. The effect on the peak impact pressure on the roof of the tank is shown in Fig. 2, in which the length scale has been multiplied by 12 to correspond to an explosion of $250 \mathrm{lb}$ TNT in a tank $24 \mathrm{ft}$ in diameter and $24 \mathrm{ft}$ high. In addition to the steady decrease of impact pressure with increasing air space, the time between detonation and impact was increased. Rees also demonstrated the effects of surrounding the explosive charge by a model breeder structure, and of replacing rigid tank walls by a light-gauge tank. The breeder structure, although it was ruptured, considerably reduced the shock pressure on the side wall of the tank and channelled the energy upwards towards the core plug. The light-gauge tank was ruptured by the shock wave, relieving the pressure within the tank. This cut short the duration of loading on the roof and core plug, considerably reducing the plug jump.

The effect of having a crushable barrier within the reactor vessel has been demonstrated in models of the assembly shown in Fig. 3 , which is similar in principle to the EBR-II reactor. The graphite neutron shield was enclosed between two steel cylinders. In one model the graphite was in the form of well-compacted powder and chips, while in another model it con- 


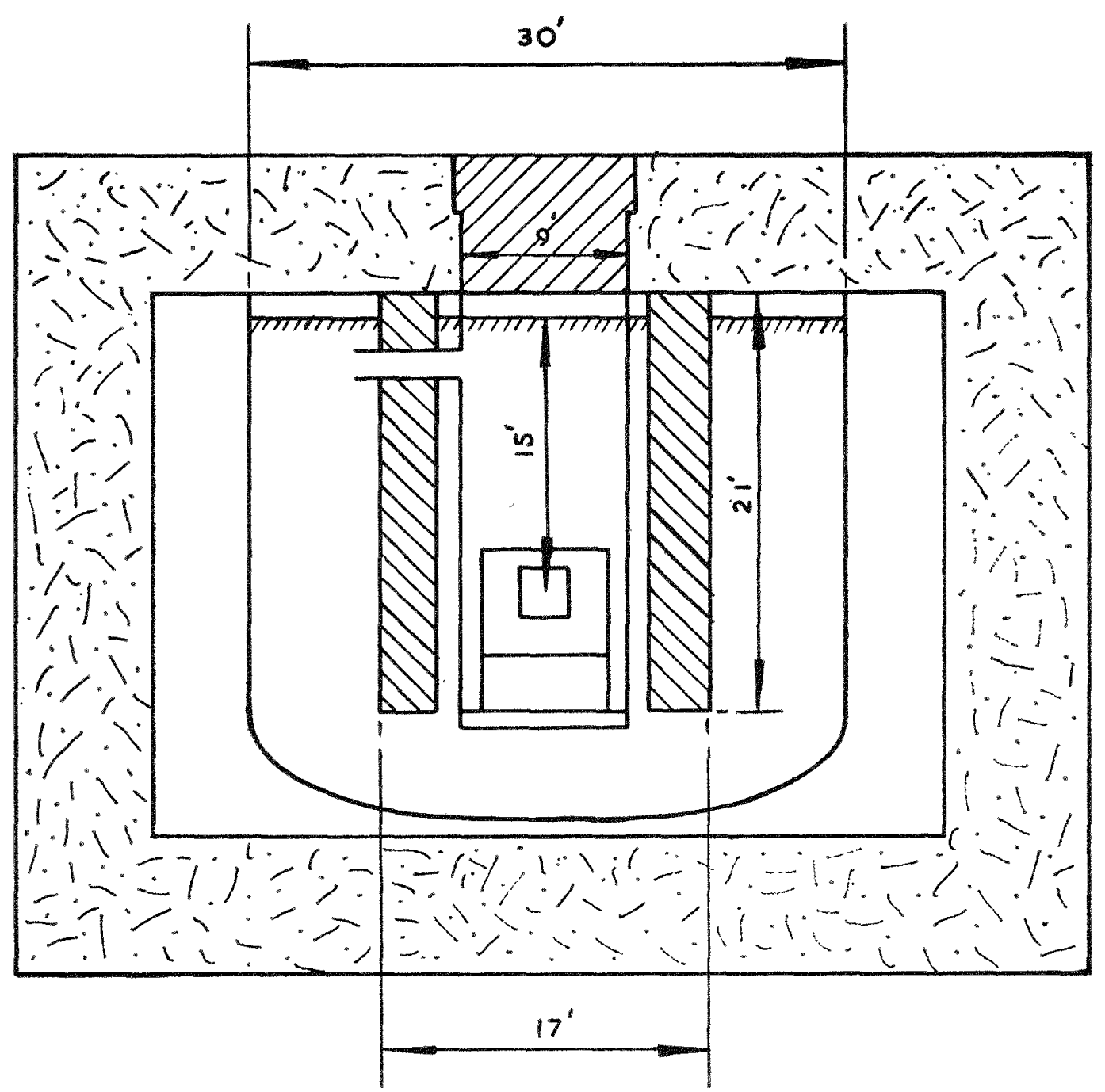

FIG. 3. Schematic Representation of Reactor Vessel with Crushable Barrier.

sisted of closely fitting solid blocks. The explosive change was equivalent to $250 \mathrm{lb}$ TNT full scale. The results of the two tests are compared below.

\begin{tabular}{l|c|c}
\hline & \multicolumn{2}{|c}{ Form of Graphite } \\
\hline & $\begin{array}{c}\text { Compacted } \\
\text { Powder }\end{array}$ & Solid Blocks \\
\hline Max eircumferential strain: & & \\
Neutron shield inner wall & $20 \%$ & $5 \%$ \\
Neutron shield outer wall & $3 \%$ & $5 \%$ \\
Peak impact pressure: & & \\
Core plug & $4,600 \mathrm{psi}$ & $8,200 \mathrm{psi}$ \\
Outer roof & $140 \mathrm{psi}$ & $370 \mathrm{psi}$ \\
Core plug stopping distance & $1.8 \mathrm{ft}$ & $2.4 \mathrm{ft}$ \\
\hline
\end{tabular}

The 70-ton core plug was restrained by a yielding device which exerted an average force of 1,300 tons.
The more rigid neutron shield deformed much less than the other and so absorbed less energy of the explosion. It acted as a gun barrel, directing the coolant flow upwards towards the core plug, and downwards into the outer part of the reactor vessel, compressing the gas blanket and producing more than twice the pressure rise on the outer roof.

The dependence of the pressure rise in the concrete vault on the free volume available for expansion has been demonstrated in the system shown in Fig. 1. In one model the graphite neutron shield was enclosed in a strong inner containment which reduced the free volume around the reactor vessel by a factor of 14 . A rise in pressure of 50 psig was measured in this containment, compared to $3.5 \mathrm{psig}$ formerly. It can, therefore, be said that over quite a wide range of volumes, the pressure rise in the vault is inversely proportional to the expansion volume. Other tests have shown that, in a given geometry, the pressure rise in 
the vault is also directly proportional to the explosion energy.

Finally, the interdependence of different effects of the explosion has been demonstrated, also in models based on the system shown in Fig. 1. In these tests the reactor vessel was only half filled with coolant. Two sizes of explosive charge were used, equivalent to 200 $1 \mathrm{~b}$ and $400 \mathrm{lb}$ TNT. With the smaller charge the reactor vessel was distorted but did not rupture; with the larger charge the vessel was split wide open and the neutron shield demolished. A smaller core plug was used for these tests, haring a full-scale weight of 20 tons. It was secured to the reactor vessel by bolts which failed in tension, and for both sizes of explosion the plug rose to a height of $80 \mathrm{ft}$. Although the peak pressure acting on the base of the plug was much greater for the larger explosion, the rapid failure of the reactor vessel wall cut short the load, and the total impulse applied to the plug was the same in both cases.

\section{Conclusion}

The main thesis of this paper is that, in order to design a reactor system capable of containing a meltdown explosion, one must have a clear understanding of the sequence of events and structural failure resulting from such an explosion. By having a clear qualitative picture of the pattern of events in mind, one can ensure there is no direct path by which the explosion energy can be conducted to the outer boundaries of the containment. By this means one can outline a structural system which is potentially capable of absorbing all the explosion energy, and which can be modified, refined, and optimised in a series of small-scale model tests.

\section{REFERENCES}

1. N. J. M. Rees, A Model Investigation of Explosion ('ontainment in Single-tank Fast Reactors, see paper in Session VII-A of This Conference.

2. G. A. V. Urevon, M. Falgayrettes, and F. J. Walford, Comparison of Pressure Loading Produced by Contained Explosions in Water and Sodium, see paper in Session VII-A of This Conference.

3. E. P. Hicks and D. C. Menzies, Theoretical Studies on the Fast Reactor Maximum Accident, see Paper in Session VIIA of This Conference.

\section{Discussion}

Mr. Merckx (Battelle-Northwest): We established a design nightmare the other day; in all cases we voided the core. If we're now establishing the design explosion, shouldn't we start from some sort of roided type of material, with the first wave impulses having to go through some sort of vapor phase instead of the liquid phase? What are your considerations on this as far as design?

Mr. Samuels: We did assume here that the sodium had been expelled from the core, but not from the whole vessel, by the start of the excursion. The driving material was vaporized fuel and not sodium. Mr. Hicks has looked in some detail at the comparison of the initial effects. He finds that after the end of the first bubble expansion, TNT or other high explosive does follow quite closely what we would expect from expanding fuel vapor.

$M r$. Wolfe (GE) : I'm still confused. In the American work to which Mr. McCarthy referred before, they calculated the plug jump for Fermi on the basis of the maximum pressure which the vessel could contain before it ruptured, and then assumed that that pressure accelerated the plug. In the British papers, both yours and the ones before, I gather that that's not the mechanism which you assume for acceleration of the plug. Am I right or wrong on this?

Mr. Samuels: It's partly that. I think that in the calculations before Wise's work it is assumed that the driving pressure was just the unsteady pressure you get by putting that much energy into the gas in the vault. When you have a small core vessel in a very large volume, this is not very far wrong. If the arrangement is such that there is direct impact on the plug by the sodium, more than half the total particle energy comes from that very impact and not from the unsteady pressure. The balance does come from the unsteady pressure. Both are important. The arrangement of the system determines which is more important.

Mr. Denielou (CEA, France): Has the same type of study been conducted anywhere in the case of a reactor having a fuel-handling cell overhead?

Mr. Bump (ANL): I don't know whether this is directly connected or not. In the FARET design at the moment we think it will not be necessary to hold down the cover on the reactor vessel. Assuming that the explosion occurs and that the energy is absorbed by the roof of the fuel-handling cell, the problem for FARET is that the cover has poison control rods attached to it; thus, we have to worry about what happens following the maximum accident if the poison rods are possibly pulled out. In our present approach we're trying to show that the core will have to be permanently disassembled, if enough energy is released in order to raise the cover.

Mr. Klickman (APDA): Could you elaborate on the plug-stopping distances that you gave? How did you arrive at them?

Mr. Samuels: This was in our small model. We used a very simple system, using plastic deformation of mild steel to fight the force. The 70-ton core plug was restrained by this yielding device, which exerted an average force of 1300 tons. Study of our results showed 
that this corresponded to the particular case where we had different neutron shields; the one was $1.8 \mathrm{ft}$ and the other $3.4 \mathrm{ft}$. Coming back to the question of Dr. Wolfe, these plugs are not normally solid structure. They are fairly uncrushable and deformable themselves, and it seems quite possible in some reactors to absorb the energy in the plug structure and to bolt the top of the plug down firmly to the containment. This will provide a method of complete containment inside the primary system.

Mr. Wolfe: On both your point and Mr. Denielou's, at SEFOR we took the latter course of action and bolted the head down; we then provided the bolts with enough energy absorption in the stretch to absorb the energy that the head would see from shock. Going back to the other point, in Wise's work he found that the shock energy imparted to the head in at least the small type of vessel, say the Fermi type of vessel, is only a small part of the energy from the expansion of the gases, even when sodium is right in contact with the plug. When an air gap exists between the sodium and the plug, he found that even less energy was imparted in the initial shock. So I'm still not sure whether or not there is a difference between his work and yours.

$M r$. Samuels: I'm not sure whether you're referring to the experimental measurements by Wise or to the earlier calculations for Fermi. There seems to be a difference in our results. I suspect it does depend on the geometry and how much venting into the vault there is in cutting short of the time which the impact lasts.

Mr. Nims (APDA): How much stretching can the ressel withstand before rupture?

$M r$. Samuels: This depends very much on the uniformity of loading, and in any practical reactor the structure just isn't all that uniform. We have our bits and pieces stuck in various places. We have measured permanent deformations up to $20 \%$ in the event of the rupture. On the other hand, in certain places we've only measured $1-3 \%$ stretch before the thing splits. It does depend on uniformity of construction and the uniformity of molding. 


\title{
Response of a Helium-cooled Fast Reactor to Changes in Coolant Flow and Reactivity*
}

\author{
R. S. Carlsuith and J. G. Delene \\ Oak Rudge National Laboratory \\ Oak Ridge, T'ennessee
}

(PRESENTED) BY J. (i. DELENE)

\begin{abstract}
The ORNL design of a helium-cooled fast reactor was studied with respect to its response to various types of accident conditions. These accident conditions included step and ramp reactivity insertions, and loss of coolant pressure and flow. The objective was to determine what kind of accidents this reactor could withstand before fuel melting began, without regard to questions of accident eredibility.

A Doppler coefficient $T(d k / d T)$ of -0.0053 and a total helium loss coefficient of $+0.0051 \delta k$ were calculated for this reactor. These coefficients proved adequate to prevent melting for a step reactivity addition of less than 29 cents, if no scram occurs, and a step change of $\$ 1.35$ if a power-level scram occurs. For reasonable rates of coolant loss (half-life in the vicinity of $20 \mathrm{sec}$ ), the reactor would not approach prompt criticality at any time during the transient. The ability of the reference reactor to remove delayed fission product heat in the event of two loss-of-coolant accidents was determined.
\end{abstract}

Interest in gas-cooled fast reactors as a possible alternative route to successful plutonium breeding is due in part to differences in physics characteristics between sodium-cooled and gas-cooled reactors. One would expect, for example, that the reactivity effect of the coolant would be much smaller with gas, so that the core geometry could be optimized without regard to void or coolant-loss coefficients of reactivity. One would also expect a harder neutron spectrum in the gas-cooled case, resulting on the one hand in a higher breeding ratio, but on the other hand in a lower Doppler coefficient. In this paper we point out first that the reactivity effect of a gas coolant may not in fact be negligible and should be considered in the reactor design. Secondly, we inquire what degree of protection may be provided by the smaller Doppler coefficient that does exist. Finally, we examine the

* Research sponsored by the U.S. Atomic Energy Commission under contract with the Union Carbide Corporation. power and temperature excursions to be expected in the event of loss of coolant flow and pressure.

We take as our reference design a 1000-MWe helium-cooled fast reactor design prepared by ORNL in a recent study. ${ }^{(1)}$ Some of the principal features of the reactor are given in Table 1. This design was obtained by assuming a maximum linear heat rating of $40,000 \mathrm{Btu} / \mathrm{hr}-\mathrm{ft}(12 \mathrm{~kW} / \mathrm{ft})$, a maximum cladding surface temperature of $1500^{\circ} \mathrm{F}$, and a maximum pumping power of about $10 \%$ of the net electrical output. The coolant pressure was limited to 1000 psi on the basis that higher pressures would be likely to introduce unknown problems in component technology. Dimensions of the fuel pin, the core, and the blanket were obtained by optimizing with regard to fuel-cycle costs.

We used one-dimensional multigroup-diffusion calculations for the static analysis of the core physics. We found it important to do both axial and radial calculations and to iterate the transverse buckling until consistent results were obtained. We checked one such result against a two-dimensional multigroup calculation and found excellent agreement in $k_{\text {eff }}$ and power distribution. The 16-group cross sections were obtained by averaging, for most nuclides, over the spectrum for a composition similar to that of the reference core. The isotopic content of the fuel was determined by doing equilibrium recycle burnup calculations and choosing the average core composition during a $100,000 \mathrm{MWd} / \mathrm{T}$ cycle as representative. The isotopic composition of the plutonium came out to be $61: 31: 5: 3\left({ }^{239} \mathrm{Pu}:{ }^{240} \mathrm{Pu}:{ }^{241} \mathrm{Pu}:{ }^{242} \mathrm{Pu}\right)$.

The isothermal Ioppler coefficient was estimated by the use of Doppler-broadened cross sections for several different temperatures in diffusion-theory calculations. The Doppler-broadened cross sections were obtained with the General Electric Rapture ${ }^{(2)}$ code and also with the General Atomic GAM-II ${ }^{(3)}$ code which uses somewhat different approximations. The two codes gave essentially the same result as shown in Figure 1. We obtained the effective neutron lifetime and delayed-neutron fractions by first-order pertur- 
table 1. ORNL Helit m-cooled Fast Reactor Design

Fuel Material

Blanket Material

Cladding Material

Total Thermal Power, MW

Gross Electrical Power, MW

Coolant Pressure, psi

Effective Core Iength, in.

Hffective Core Diameter, in.

Radial Blanket Thickness, in.

Axial Blanket Thickness, in.

Fuel-pin Diameter, in.

Cladding Thickness, in.

Pin-spacing Triangular Pitch, in.

Method of Pin Spacing

Average Core Fuel Enrichment, $/ c$

Average Core Power Density, W/ $\mathrm{cm} \mathrm{m}^{3}$

Average Core Specific Power, $\mathrm{kW} / \mathrm{kg}$ Fis

sile

Interual Breeding Ratio

Total Breeding Ratio

Doubling Time, $y x$

$$
\left(\mathrm{Pu},{ }^{238} \mathrm{U}\right) \mathrm{O}_{2}
$$

${ }^{238} \mathrm{UO}_{2}$

Stainless steel

2500

993

1000

72

133

18

15

0.250

0.015

0.432

Helical fins

10

145

761

0.92

1.39

11

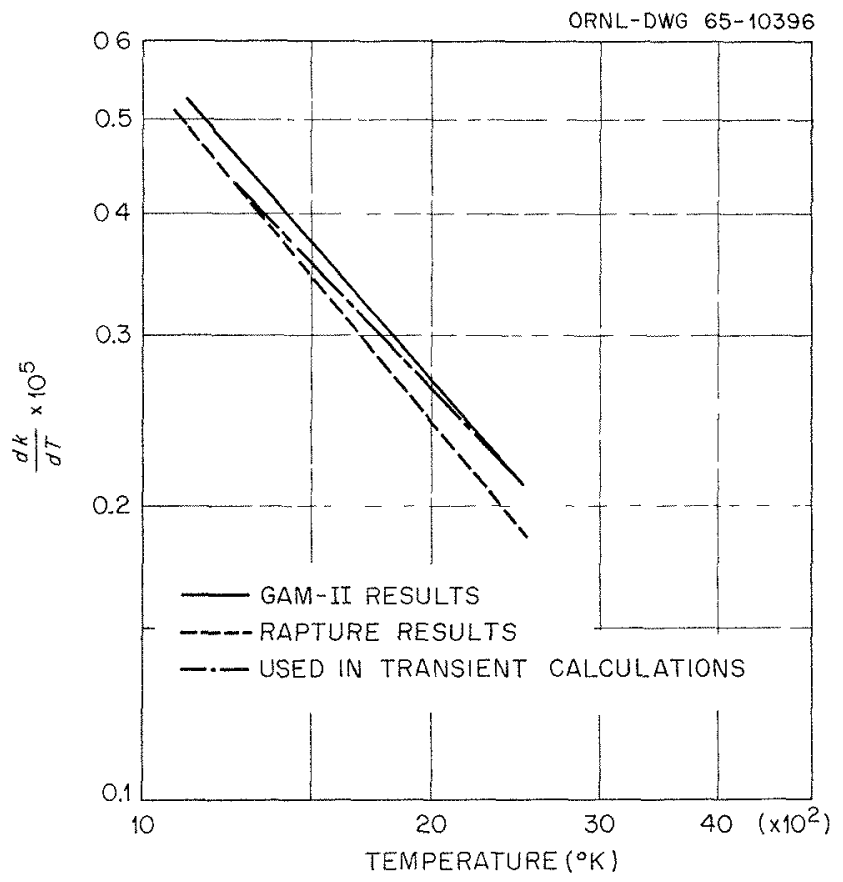

Fic. 1. Reference Reactor Doppler Coefficient.

bation-theory calculations. Direct calculations of reactivity with and without helium gave the value for the reactivity associated with helium loss. The values of these quantities for the reference reactor are shown in Table 2.

The helium-density reactivity coefficient deserves some further comment. It has two components. The transport component, which is always negative, depends principally on the core size and shape, and on the core coolant fraction and density. The down- scatter component is likely to be positive unless a considerable amount of moderating material is present in the core. In our reference reactor the transport component was $-0.06 \% \delta k$ while the downscatter component was $+0.57 \% \delta k$, giving a net reactivity gain of $0.51 \delta k$ for complete loss of helium. Since the transport component is so much smaller in magnitude than the downscatter component, it appears that changing the shape of the core would have little effect on the net coefficient. This is in contrast to the situation of liquid metal-cooled reactors for which the core shape in many of the designs has been selected so that a large transport component of the coolant coefficient would be obtained. The downscatter component, which is the important part of the net coefficient, can vary considerably from one helium-cooled core design to another. It can obviously be decreased by having a smaller core-coolant fraction. It also depends very sensitively on the shape of the neutron-energy spectrum and on the energy dependence of the absorption and fission cross sections of both the fuel and structural materials in the core. In calculations of somewhat different helium-cooled cores we have found helium-loss coefficients lower by as much as a factor of three than in the reference core. Thus the magnitude of the net coefficient is at least partially under the control of the reactor designer. The dependence of the helium coefficient on the exact high-energy spectrum and cross sections means that there is probably considerable uncertainty in our calculations of this quantity.

We have also considered $\mathrm{CO}_{2}$ as a fast reactor coolant. In our design we obtained the same fuel heat rating as for the helium-cooled reactor with a slightly lower fraction of coolant volume $(0.59 \mathrm{vs}$ 0.67 for helium) and slightly higher pressure (1300 psi vs 1000 psi for helium). The downscatter and

TABLE 2. Kinetics Parameters for Helium-cooled

REACTOR

\begin{tabular}{|c|c|c|}
\hline \multicolumn{2}{|c|}{$\begin{array}{l}\text { Doppler Coefficient at } 965^{\circ} \mathrm{C}, \delta k /{ }^{\circ} \mathrm{C} \\
\text { Reactivity Gain for Complete Coolant } \\
\text { Loss, } \delta k \\
\text { Effective Neutron Lifetime, sec } \\
\text { Delayed-neutron Yield Fractions: }\end{array}$} & $\begin{array}{c}-0.43 \times 10^{-5} \\
0.0051 \\
0.8 \times 10^{-6}\end{array}$ \\
\hline Group & $\lambda, \sec ^{-1}$ & Yield $\left(\times 10^{-3}\right)$ \\
\hline $\begin{array}{l}1 \\
2 \\
3 \\
4 \\
5 \\
6\end{array}$ & $\begin{array}{l}0.0129 \\
0.0311 \\
0.1335 \\
0.3316 \\
1.262 \\
3.208\end{array}$ & $\begin{array}{l}0.0833 \\
0.7093 \\
0.6425 \\
1.2517 \\
0.5843 \\
0.1909\end{array}$ \\
\hline Total & & 3.462 \\
\hline
\end{tabular}


absorption component of the coolant-loss coefficient was then $+0.81 \% \delta k$ while the transport component was $-0.52 \% \delta k$. With different core design a $\mathrm{CO}_{2}$ cooled fast reactor in this size range could have a negative coolant coefficient.

We have studied the response of the reference reactor to a varicty of reactivity changes and to coolant flow and pressure changes. The computer program that we used solves the point kinetics equations and the hot-channel heat transfer equations in alternating successive finite time steps in order to determine the axial and radial temperature distribution in the hot-channel fuel pin and the axial temperature distribution in the coolant gas. We take into account the reactivity feedback from the fuel Doppler coefficient and the reactivity effect of any changes in coolant density. For convenience it was assumed that $\delta k / d T$ varies as $T^{-1}$, whereas our calculations indicate an actual variation as $T^{-112}$. An importanceweighted effective Doppler coefficient to be used with the hot-channel temperature was obtained by modifying the actual Dopper coefficient to take into account the ratio of hot-channel temperature to coreaverage temperature and the initial ratio of hotchannel temperature rise to core-average temperature rise. The fission product heating was computed as a function of time by integrating the data of Shure. ${ }^{(4)}$ We did not include the negative reactivity feedback from fuel or structural expansion.

We defined as the "failure" point for the reactor the occurrence of either a $\mathrm{CO}_{2}$ temperature above $6500^{\circ} \mathrm{F}$ or a stainless steel cladding temperature above $2250^{\circ} \mathrm{F}$. Our basis for these choices was that the $\mathrm{UO}_{2}$ vapor pressure at $6500^{\circ} \mathrm{F}$ is high enough so that rupture of the cladding is likely, ${ }^{(5)}$ and that this condition or the failure of the cladding at about $2250^{\circ} \mathrm{F}$ would cause damage to the core.

The effect of the size of the Doppler coefficient in limiting the excursion resulting from a step reactivity addition without control action is shown in Fig. 2. In this figure the maximum allowable reactivity insertion is the one which produces temperatures just short of our failure point. The maximum step insertion for our reference reactor (without a scram) is $\$ 0.29$. If the coolant-loss coefficient were zero, the allowable step insertion would be increased to $\$ 0.46$. Hence, in this type of transient, the positive reactivity effect associated with the decrease in helium density in the core is about 17 cents.

Figures 3, 4, and 5 are plots of the heat-deposition rate and peak temperatures in the fuel and cladding as a function of time after a step reactivity insertion. Figure 3 shows the response of the reference reactor to a step insertion of slightly less than that

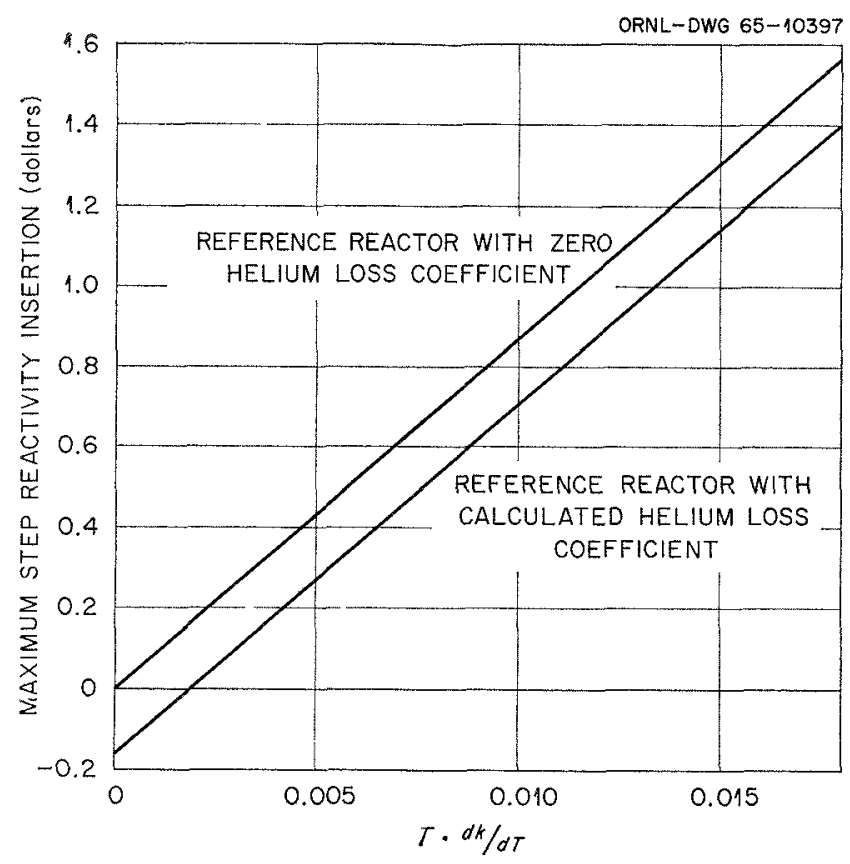

Fxc. 2. Maximum step Reactivity Insertion as a Function of $T d k / d T$.

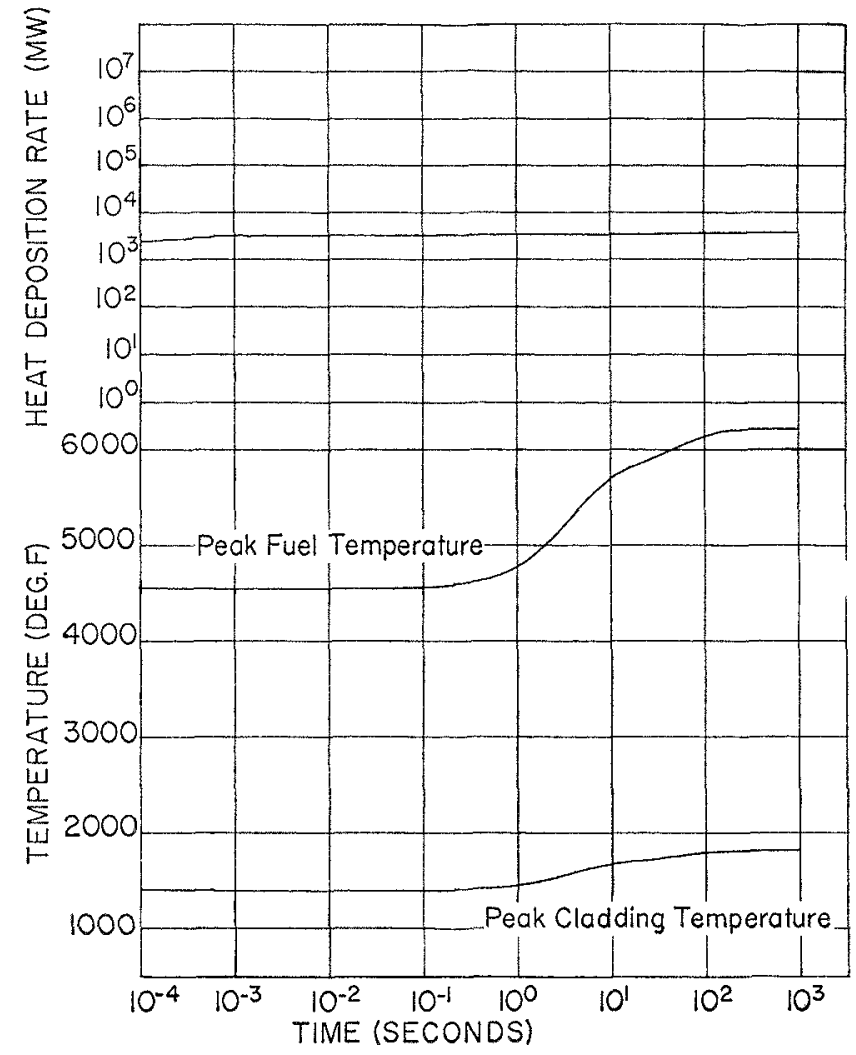

FIG. 3. Response of Reference Reactor to a $\$ 0.25$ Step Reactivity Addition without Control Rod Action.

required for fucl-pin failure. Here the peak power was approximately 1.9 times steady-state power.

We repeated this set of calculations but with a much 


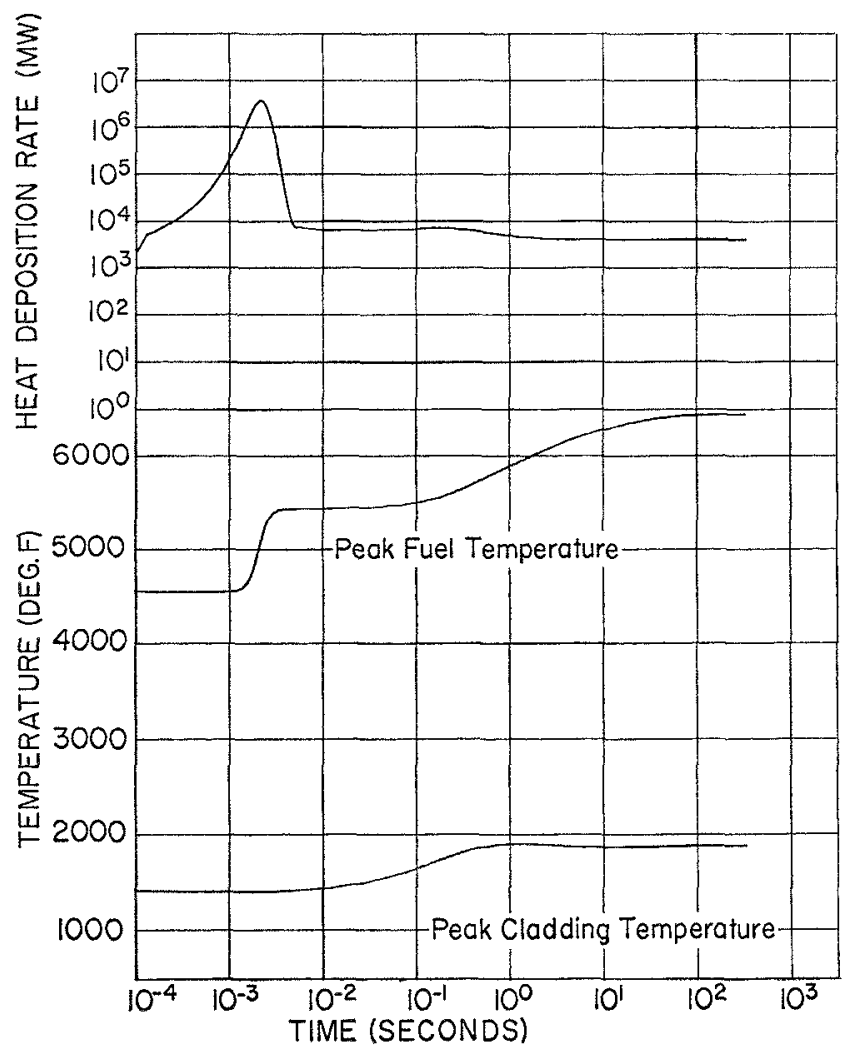

Fig. 4. Response of Reactor with $T d k / d T=0.0235$ to a $\$ 1.85$ Step Reactivity Addition without Control Rod Action.

higher Doppler coefficient in order to show the effect of the magnitude of the Doppler coefficient. Figure 4 shows the response with a Doppler coefficient of about four times the calculated Doppler coefficient, to a step reactivity insertion of $\$ 1.85$. The prompt period caused the power deposition rate to rise over three orders of magnitude. The fuel temperature rose very fast during the power peak. This temperature rise caused the excess reactivity to fall due to the strong Doppler coefficient. The prompt period was terminated when the excess reactivity fell below one dollar. The power level then returned to steady-state power within a factor of two or three. The rates of change of power and temperature were more gradual after the prompt power peak was terminated.

If the coolant coeffieient were equal to zero, the response shown in Fig. 4 is changed to that shown in Fig. 5. Figures 4 and 5 are virtually identical during the prompt excursion. They deviate only after sufficient time clapsed for the heat to be transferred from the fuel to the coolant gas. In this kind of transient the coolant-density coefficient is a delayed effect whereas the Doppler coefficient is a prompt effect. The difference in the final peak temperatures was approximately $180^{\circ} \mathrm{F}$. We conclude that the coolantdensity coefficient becomes less important as the Doppler coefficient is increased.
The maximum allowable reactivity insertion is of course significantly larger if control-rod action is taken. We assumed control-rod insertion starting 50 ms after the step reactivity insertion, producing a linear decrease in reactivity at the rate of $\$ 173$ per second for $250 \mathrm{~ms}$. The assumed $50-\mathrm{ms}$ delay is intended to include the times required for the scram signal to be given, for the rod actuation, and for an initial relatively slow rate of reactivity insertion. In this case the maximum allowable step reactivity insertion was $\$ 1.35$, with excessive cladding temperatures occurring rather than excessive fuel temperatures. Figure 6 shows the power and temperature responses as a function of time for a $\$ 1.35$ step reactivity insertion with control rod action. This plot illustrates the importance of the various time constants. The prompt burst was completed before control action was started. The only mechanism controlling this burst was the fuel Doppler coefficient, which was assumed to act instantaneously. Within a few milliseconds after control action began, the fuel temperature virtually stopped rising. Because of the relatively long time constants for heat transfer, the peak fuel temperature did not begin to fall until nearly a second after the control rods were inserted. As the heat flowed outward from the fuel, the cladding temperature

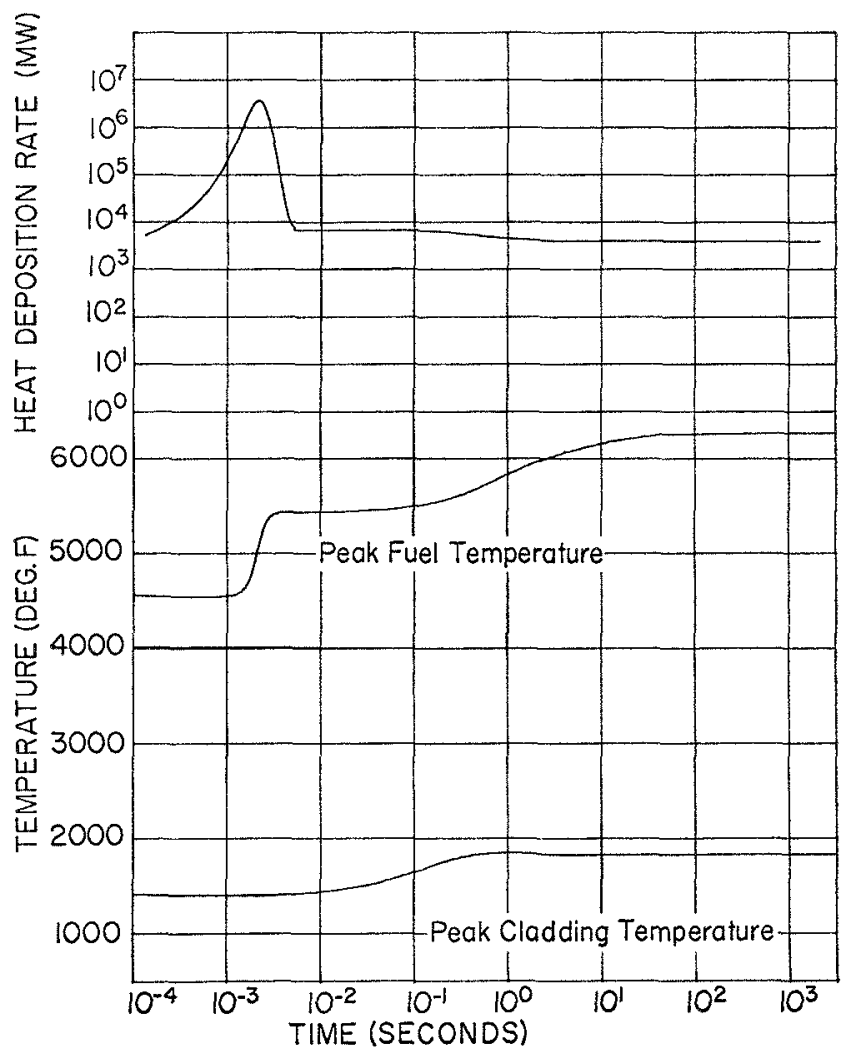

Fik. 5. Response of Reactor with $T d k / d T=0.0235$ and Coolant Density Coefficient $=0$ to a $\$ 1.85$ Step Reartivity Addition without Control Rod Action. 


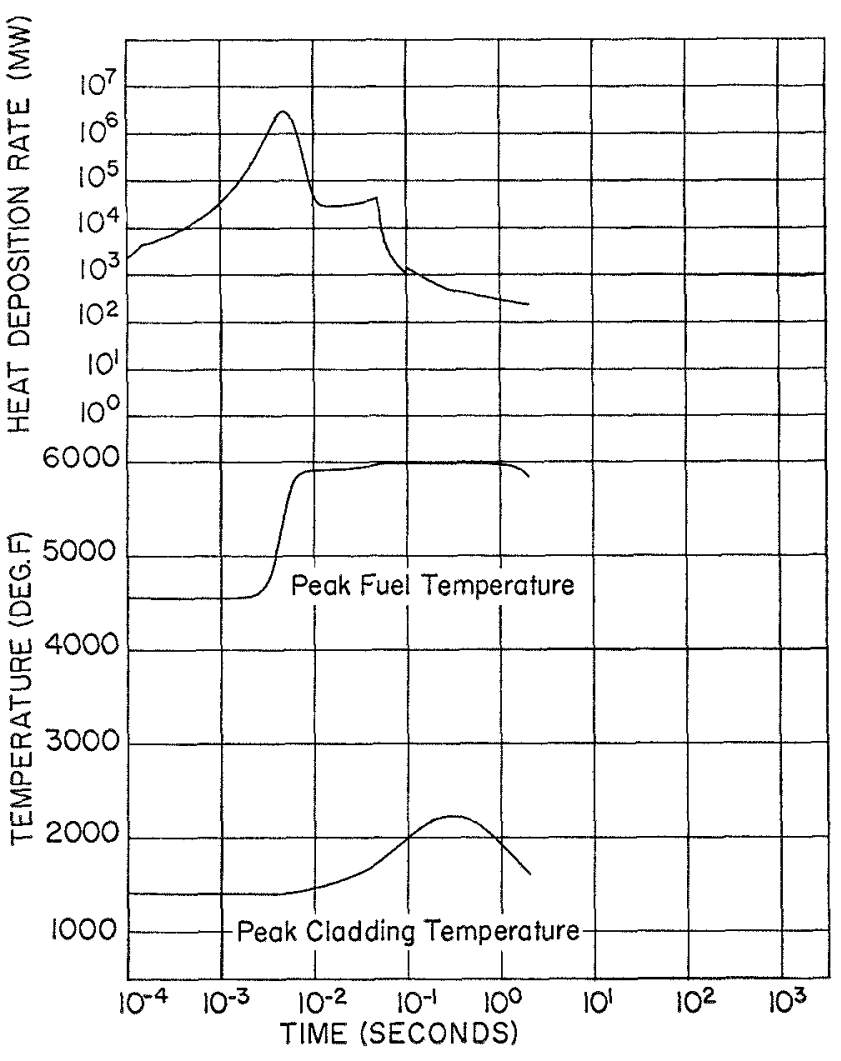

FIG. 6. Response of Reference Reactor to a $\$ 1.35$ Step Reactivity Addition with Control Rod Action at 0.05 Seconds.

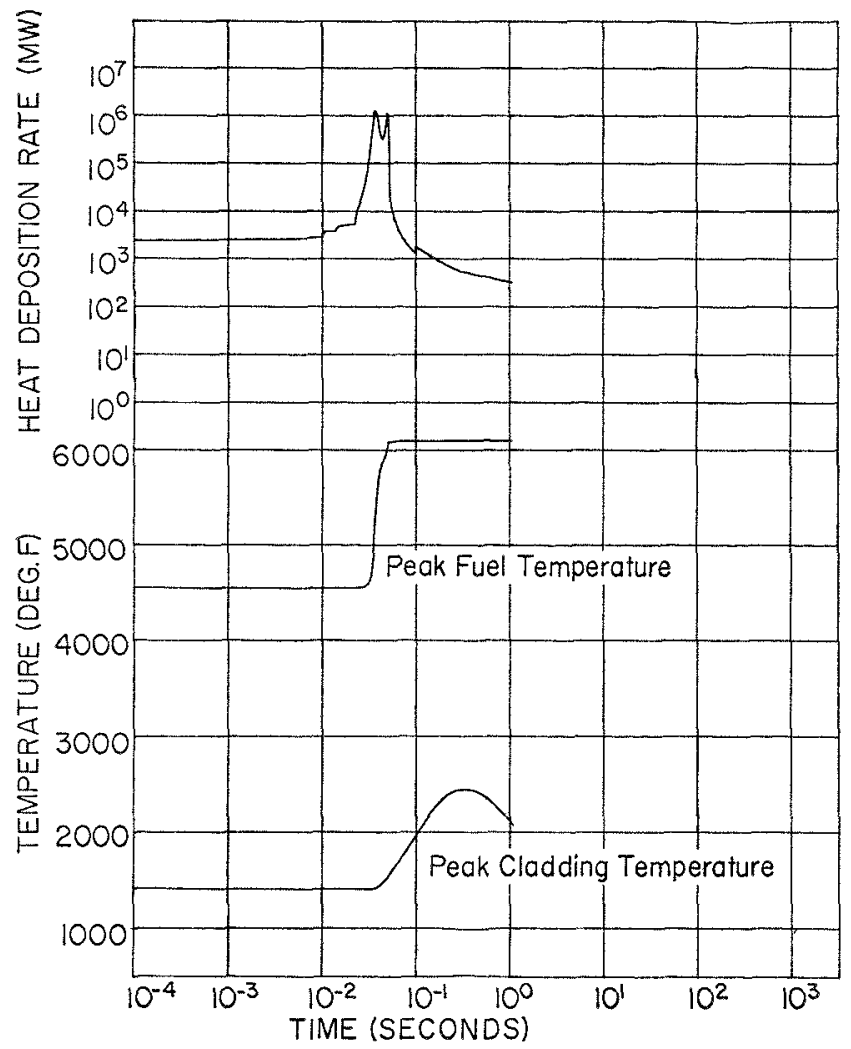

Fig. 7. Response of Reference Reactor to a $\$ 35 /$ sec Ramp Reactivity Addition with Control Rod Action at 0.05 Seconds.

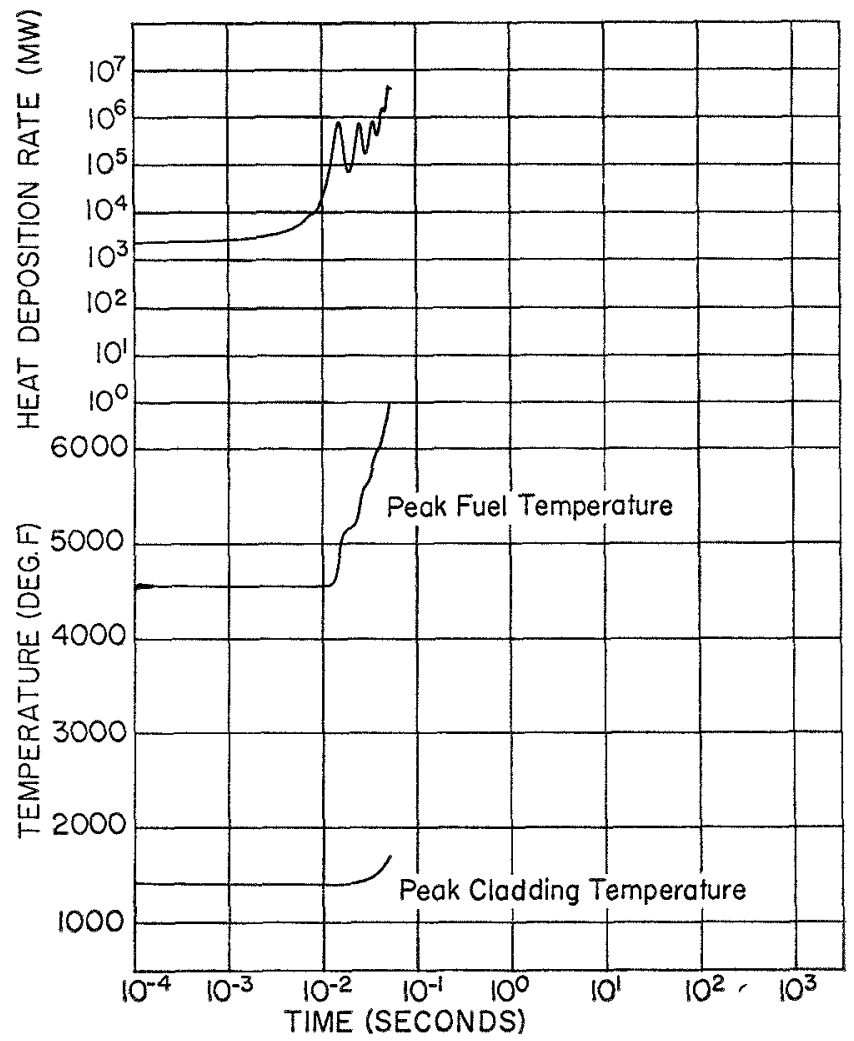

Frg. 8. Response of Reactor with $T d k / d T=0.0235$ to a $\$ 100 /$ sec Continuous Ramp Reactivity Addition without Control Rod Action.

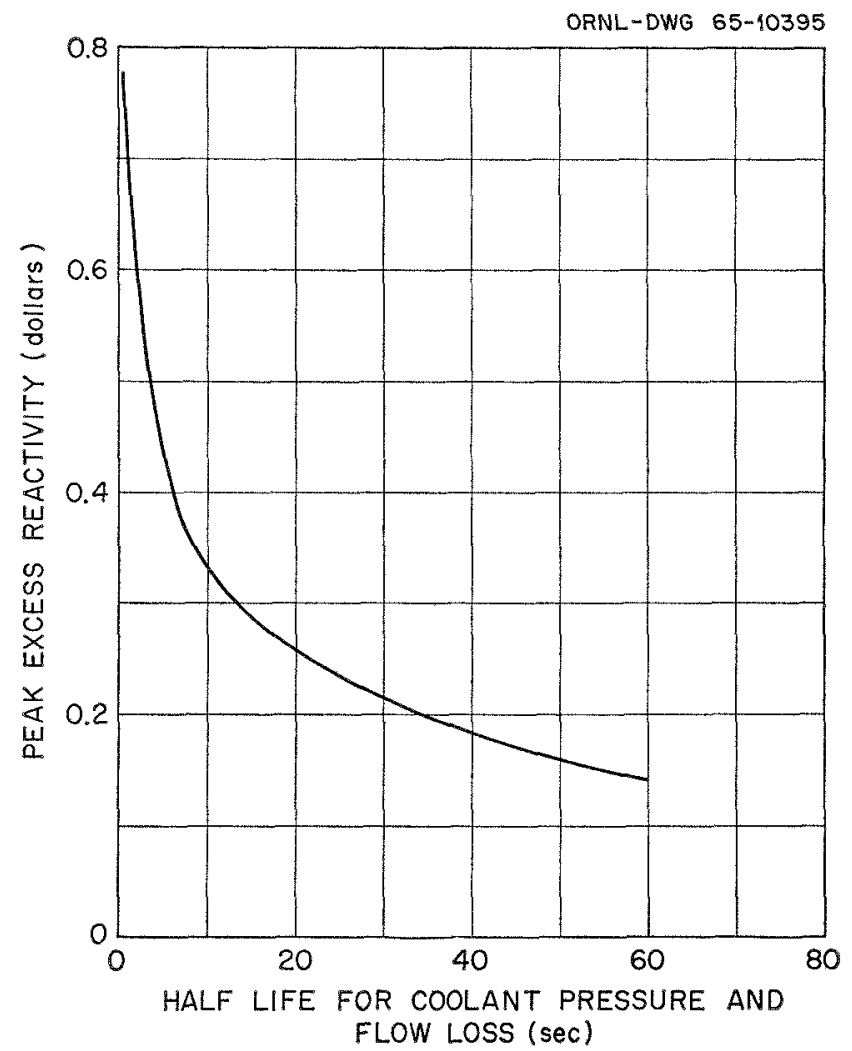

Fig. 9. Peak Excess Reactivity on Loss of Coolant as a Function of Half-Life for Coolant Pressure and Flow Loss. 


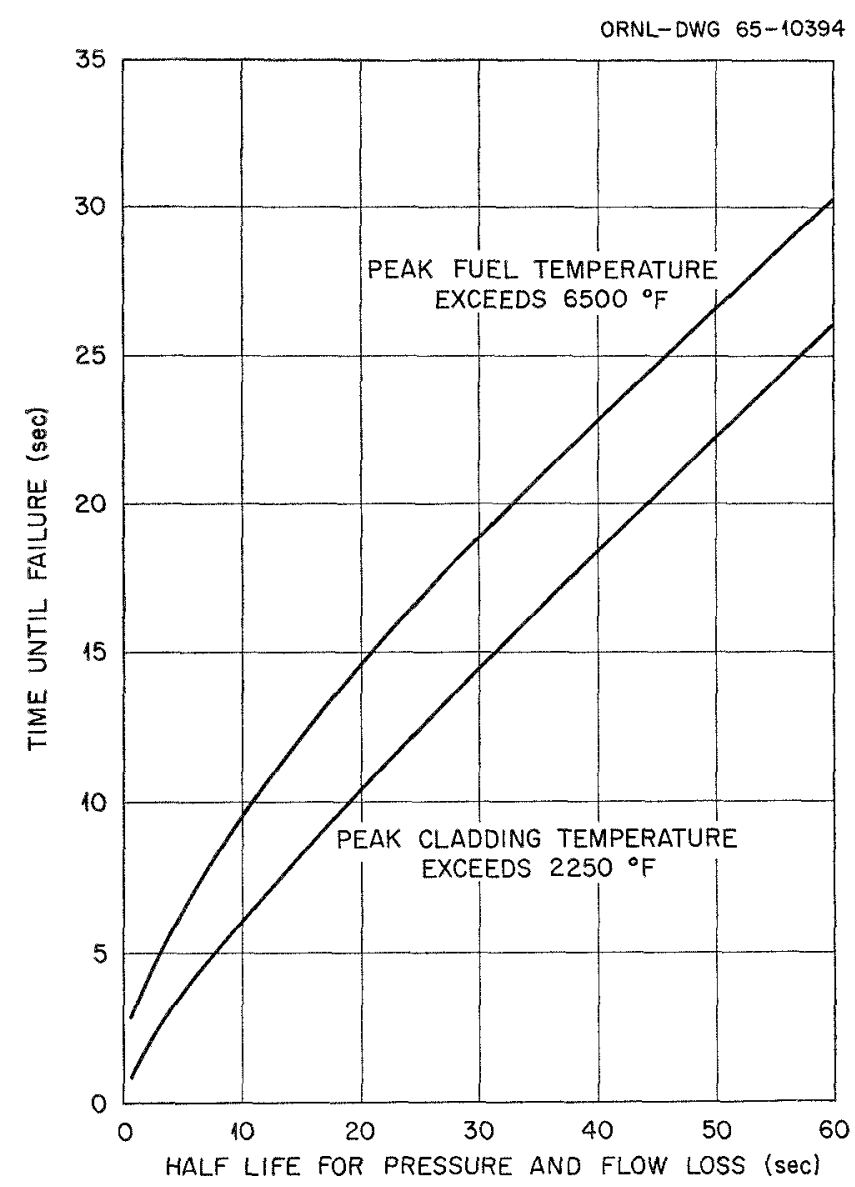

Fig. 10. Time Until Failure of Fuel Pin as a Function of Rate of Loss of Pressure and Flow.

started to rise, reaching its peak several hundred milliseconds after the control action began.

With control-rod action, the maximum allowable reactivity insertion by a ramp was somewhat larger than by a step. The reference reactor could tolerate a maximum ramp reactivity insertion of $\$ 33.00$ per second until control-rod action was initiated at $50 \mathrm{~ms}$. The total reactivity insertion by the ramp was thus $\$ 1.64$. Figure 7 shows the power and temperature responses as a function of time to a $\$ 35$ per second ramp reactivity insertion with control rod action. The phenomenon of multiple fast bursts is illustrated here. The first power burst occurs when the reactivity inserted by the ramp is enough to cause the reactor to become prompt eritical. The resulting swift temperature rise causes the Doppler coefficient to return the reactor to below prompt critical. The power level then drops and the temperature rises at a slower rate. The rate of reactivity removal by the Doppler coefficient becomes less than the addition rate from the ramp. This causes the reactor to become prompt critical again, initiating a second burst. After con- trol action is taken, the temperature behaves in the same manner as for step reactivity insertions. In Fig. 7 the limiting factor was a cladding temperature above $2250^{\circ} \mathrm{F}$.

Figure 8 also illustrates the phenomenon of multiple bursts. Here we are looking at the response of the reactor with a Doppler coefficient of about four times actual size to a $\$ 100$ per second ramp. The power was in the middle of its fifth burst when the fuel temperature exceeded $6500^{\circ} \mathrm{F}$.

The positive coolant-density coefficient implies that a sudden reduction in coolant inventory would lead to a reactivity increase as well as a reduction in capability for heat removal. Of course, the negative Doppler coefficient reduces the net reactivity increase which would otherwise occur. In Fig. 9 the maximum reactivity occurring during a coolant-loss transient is plotted as a function of the coolant loss rate (assuming no control rod action). The minimum credible half-life for coolant loss will need further study, but it appears likely that it would be in the neighborhood of 20 sec. If so, the peak reactivity during the transient would be $\$ 0.26$ instead of the $\$ 1.47$ associated with instantaneous coolant loss.

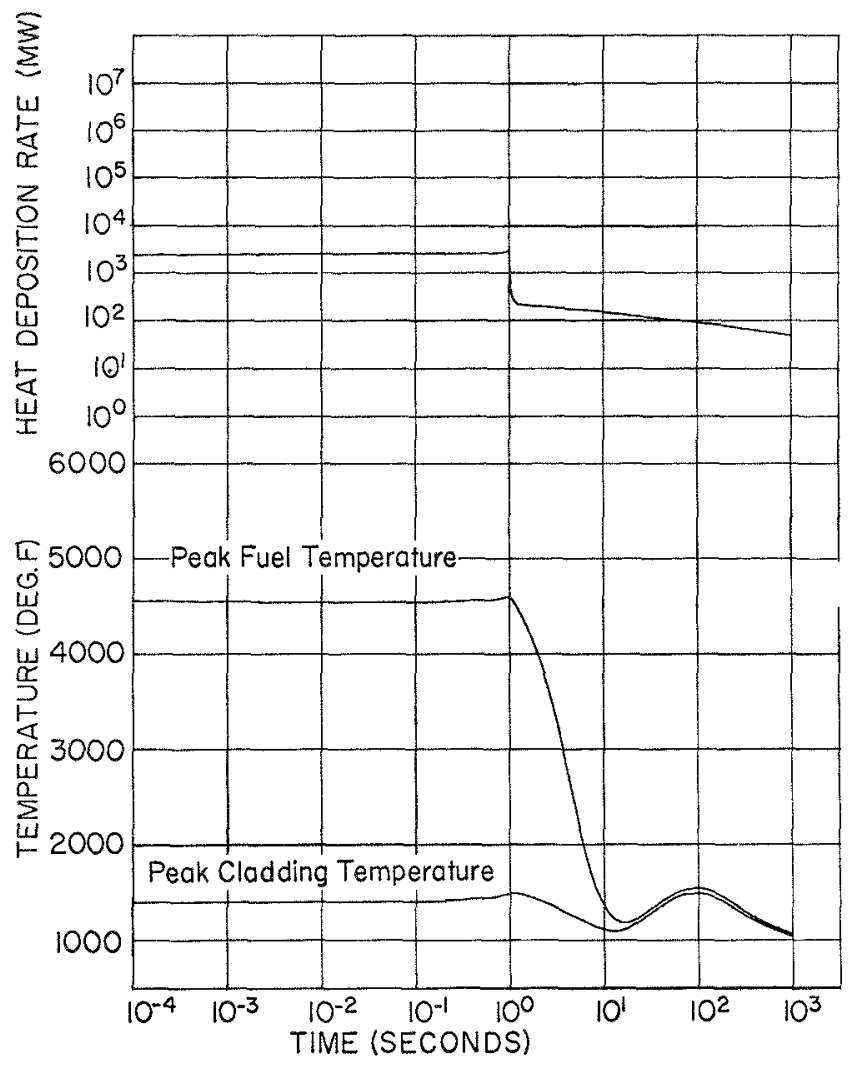

FIg. 11. Response of Reference Reactor with Control Rod Action at One Second to Partial Depressurization and Loss of Three Blowers. 


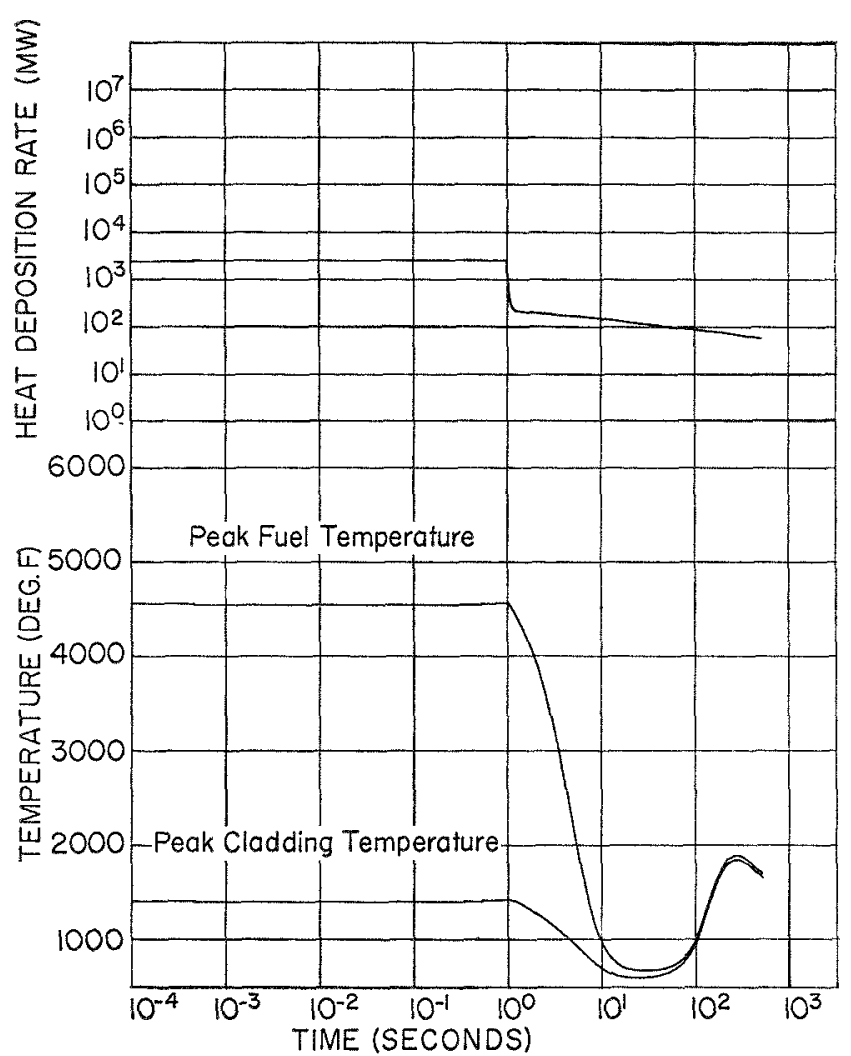

Frg. 12. Response of Reference Reactor nith Control Rod Action at One Second to Complete Depressurization and Loss of Three Blowers.

Figure 10 shows a more significant aspect of coolant loss, namely, the time available for corrective action after the coolant loss is started, again assuming that no control rod action is taken. If the coolant-loss half-life is no less than $20 \mathrm{sec}$, then the time available before the fuel cladding starts to melt is about $10 \mathrm{sec}$. The implication is that a relatively slow type of emergency cooling system would be effective.

We have studied two situations in which coolant pressure and flow loss were followed by control-rod action. In the first situation we assumed prossure equalization between the primary and secondary containment vessels, which reduces the coolant pressure to $10 \%$ of normal. It was assumed also that, in this accident, three of the four blowers would stop. We allowed the pressure equalization and blower coastdown to be completed in 20 sec, and the control-rod insertion to start $1.0 \mathrm{sec}$ after the start of the transient. The power and temperature responses to those conditions is shown in Fig. 11. Immediately following the scram the heat production decreased faster than the heat removal, allowing the temperatures to fall. As the coolant flow and pressure continued to decrease, the temperatures went up again until the afterheat production fell below the final heat-removal capability of the coolant. Maximum allowable temperatures were not exceeded at any time during the transient.

In Fig. 12 we have the more severe case of the coolant pressure being reduced to atmospheric with stoppage of three blowers on a 20 -sec half-life. In this instance, also, the maximum allowable temperatures were not exceeded.

Further analysis of such situations is required with particular emphasis on cases involving stoppage of all four blowers. The analysis here would involve the investigation of one or more types of emergency cooling system.

\section{RFFERENCES}

1. Gas-cooled Reactor Project Staff, Gas-cooled Fast Reactor Concepts, ORNL-3642 (Sept 1964).

2. J. II. Ferziger et al., Resonance Integral Calculations for Evaluation of Doppler Coeffcients-The Rapture, Code, GEAP-3923 (July 1962).

3. G. D. Joanou and J. S. Dudek, GAM-II: A B. Code for the Calculation of Fast-neutron Spectra and Associated:Multigroup Constants, GA-4265 (Sept 16, 1963).

4. K. Shure, Fission-product Decay Energy, WAPD-BT-214, pp. 1-17 (1)ee 1961).

5. M. J. MeNelly, Liquid Metal Fast Breeder Reactor Design Study, GEAP-4418 (Jan 1964).

\section{Discussion}

Mr. Morelle (Belgo-Nucleaire): I would like to comment on the rod-drop time and reactivity insertions. Many times during this meeting it has been stated or mentioned or used as an assumption that the insertion of reactivity or inception of reactivity insertion could occur in something like 50 msec. This implies that between the time that something is detected and the scram system operates after reactivity is inserted into the reactor, only $50 \mathrm{msec}$ is available. I think that this number is much, much too small. A realistic time would be about $200 \mathrm{msec}$ for most practical cases. Quite a few numbers presented in the meeting might be quite drastically changed if you pass from 50 to 200 msec.

Mr. Carlsmith: I don't know how drastic the change would be, and in some of the cases I don't think it would be quite as drastic as might have been implied. The prompt burst is already terminated by the time the control rods have been inserted and things are acting at a much slower rate at that time, at least in the cases I showed. 
0

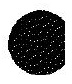




\title{
Session VII-B \\ October 14, 1965
}

\section{Other Systems and Concepts}

\author{
Chairman: K. P. CoHeN
}

General Electric

Secretary: J. T. Madell

Argonne National Laboratory 
0 


\title{
Mixed Fuel-cycle Fast Breeder Reactors Nuclear, Safety, and Materials Considerations
}

\author{
W. B. Loewenstein and B. Blumenthal \\ Argonne National Laboratory \\ Argonne, Illinois \\ (PRESENTEI) BY W. B. LOEWENSTEIN)
}

Much of the emphasis in recent 1000-MWe fast breeder reactor studies ${ }^{(1,2)}$ was related to reactor safety. Those aspects connected with the sodium coefficient of reactivity and loss of core sodium appeared to have a profound influence upon the various reactor configurations. To obtain a small core-sodium reactivity coefficient requires some significant compromises on nuclear performance. In particular, the core converson ratio tends to be lower, the fissile loading in the fuel higher, and the specific power may be lower than for the corresponding system with optimum nuclear performance having a considerably larger core-sodium reactivity coefficient. Furthermore, for metal-fueled systems, the choice of a modular array, with relatively higher plutonium content in the fuel alloy than for the optimum geometric configuration, directly affects the maximum possible operating temperature.

Quite apart from these considerations, it has been reported $^{(3)}$ that unmoderated U-233-Th-fueled systems generally have more negative sodium reactivity coefficients than corresponding $\mathrm{Pu}-\mathrm{U}-238$-fucled systems. Conceptually it is then possible to replace part of a $\mathrm{Pu}-\mathrm{U}-238$ fuel system with $\mathrm{U}-233-\mathrm{Th}$ fuel with some advantages as regards the sodium reactivity coefficient. If this replacement is carried out in a system with reasonably optimum nuclear performance and geometry, some advantages might be realized relative to the $\mathrm{Pu}-\mathrm{U}-238$ systems that have been specifically designed to counteract a passible deleterious positive sodium reactivity coefficient.

Generally, the sodium coefficient is a rapidly varying function of position, being positive near the center of the core and becoming negative near the core boundary. The crossover point from positive to negative is a sensitive function of core volume, composition, and geometry. Nevertheless, it seems reasonable to introduce the U-233-Th fuel in those regions of the core where the $\mathrm{Pu}-\mathrm{U}-238$ fuel gives the more positive contributions to the sodium reactivity coefficients.

It is assumed desirable to have the overall system operating on the $\mathrm{Pu}-\mathrm{U}-238$ cycle. Therefore, the breeding blankets are assumed to contain primarily natural or depleted uranium. The core will be constituted of two types of fuel subassemblies. One will be the standard type which contains $\mathrm{Pu}-\mathrm{U}-238$ fuel elements with axial segments of fertile material. The other subassembly will be similar to the standard subassembly, having essentially the same fuel element or pin height. However, the central region of unspecified height is constituted of $\mathrm{U}-233-\mathrm{Th}$ fuel while the axial core extremities consist of $\mathrm{Pu}-\mathrm{U}-238$ fuel.

The core that is assembled in this manner will have a central region of U-233-Th fuel which is surrounded on all sides by a larger region of Pu-U-238 fuel. The core is reflected by a blanket of natural or depleted uranium with the necessary engineering compromises. These usually imply a lower atom density of uranium in the axial blanket than is acceptable in the radial reflector.

This overall core-assembly principle can be applied to any of those major fuel types (e.g., oxide, carbide, and metal) as well as others that are not as obvious, e.g., mixtures of ceramic and metal as well as nitride and sulfides. The simplest application assumes that whatever type of fuel is used for the $\mathrm{Pu}-\mathrm{U}-238$ material is also used in the U-233-Th region. However, the rather good irradiation stability ${ }^{(4)}$ of U-233-Th metal does suggest that this material may be used in conjunction with a carbide or oxide fuel bearing $\mathrm{Pu}-\mathrm{U}-238$. On the other hand, a central region of U-233-Th oxide in a metallic $\mathrm{Pu}-\mathrm{U}-238$ system has safety advantages above those provided by the acceptable sodium coefficient. This particular approach would provide a significant negative Doppler coefficient of reactivity with a magnitude that is significantly larger than that associated with the more conventional design of a metallic-fueled system.

\section{Conceptual Considerations}

A number of multigroup diffusion-theory calculations were carried out in spherical geometry to simulate the optimum neutronic configuration for the reactor illustrated in Fig. 1. The analyses were made with 22 energy-group constants of the ANL26 group set. ${ }^{(5)}$ 


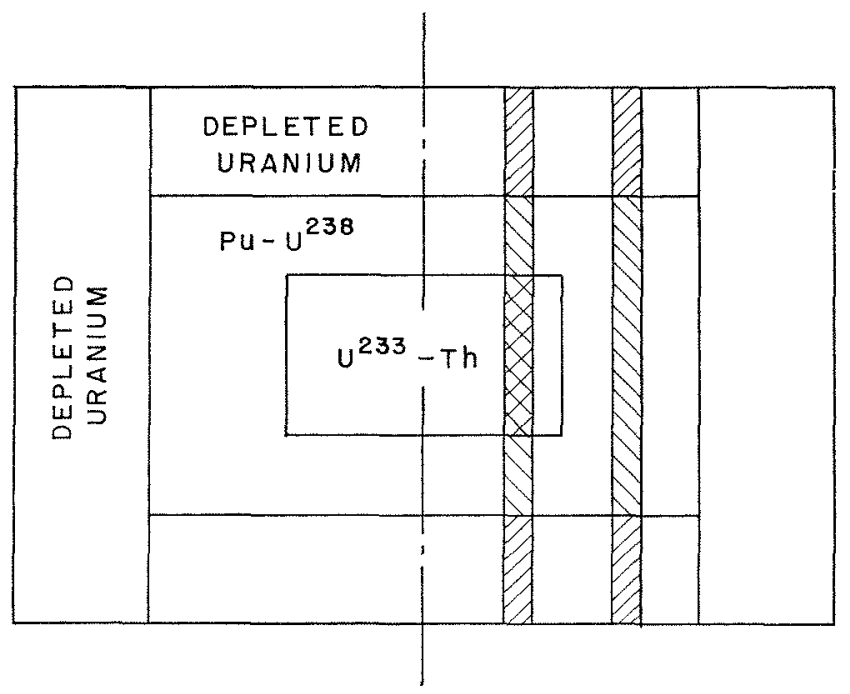

Frg. 1. Vertical Section through Cunceptual Reactor.

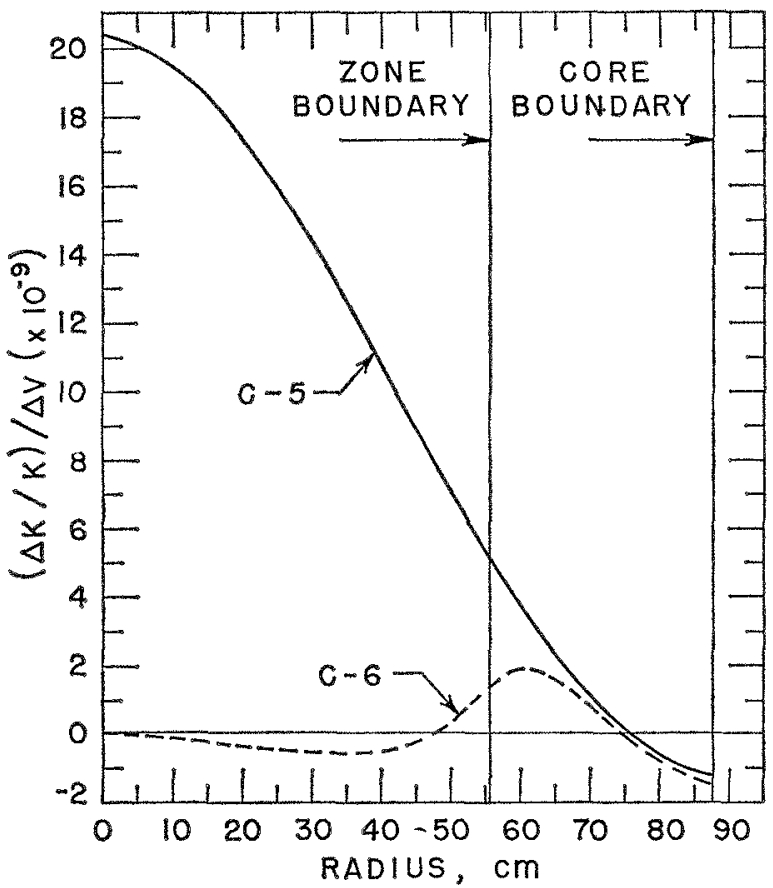

FiG. 2. Spatial Distribution of Sodium Worth (Carbide Reactors; C-5 Reference, C-6 with Central Zone).

The constants for the light-element materials in this set were obtained in the same manner as described in Ref. 3. A few comparative calculations with more detailed generation procedures for light-element cross sections exhibited about the same small uncertainties as indicated in Ref. 3. Calculations were also carried out to ascertain the significance, if any, on the conclusions when utilizing the recently measured plutonium fission cross sections. ${ }^{(6)}$ These calculations indicated small changes in numerical values but no significant changes in the principle of the reactor concept considered here.

Most of the analyses focused upon a core volume of 3000 liters. This was chosen as typical and in the range of interest. The choice is not intended to imply any particular capability for heat removal nor are the various configurations studied here assumed to have the same thermal performance. Some ancillary studies indicated, as expected, that increasing core volume required larger central $\mathrm{U}-233-\mathrm{Th}$ regions than for the reference 3000 -liter core volumes to achieve the same magnitude of the core-sodium reactivity coefficient.

Figure 2 shows the spatial distribution of sodium reactivity worth in the core of a 3000 -liter carbide core. Case $\mathrm{C}-5$ is the distribution for a 3000-liter $\mathrm{Pu}-\mathrm{U}-238$ system and Case C-6 for the same system with a central 800-liter core of U-233-Th fuel. Figure 3 is a similar comparison for typical oxide-fueled fast breeder reactors. Case $0-\mathrm{I}$ is the $\mathrm{Pu}-\mathrm{U}-238$ system. Cases 0-II and 0-III are similar systems with a central 800-liter core of U-Th. Case 0-II assumes that the central core is fueled by U-233 only. Case 0-III speculatively assumes a rather high concentration $(-20 \%)$ of $\mathrm{U}-234$ in the uranium of the U-Th fuel. This content was chosen not only to simulate U-234, but also to provide some pessimism in the analysis regarding uncertainties in the U-234 cross sections. Qualitatively, Fig. 2 and Fig. 3 indicate that the magnitude of the positive sodium reactivity can be

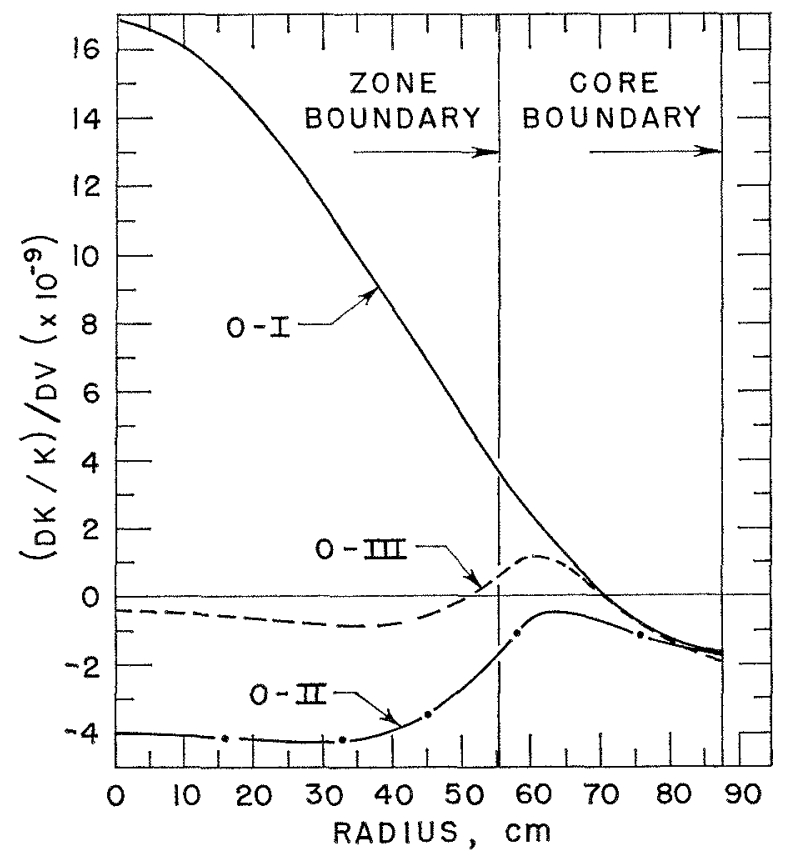

FIG. 3. Spatial Distribution of Sodium Worth (Oxide Reactors; O-I Reference, O-II with U-233, and O-III with U-233 and U-234 Central Zone). 
Mixed Fuel-cycle Fast Breeders

Table 1. Metala-Fueled Reactors (3000-hter Cores)

\begin{tabular}{|c|c|c|c|c|c|c|c|c|c|c|c|c|c|}
\hline \multirow{2}{*}{ Case } & \multirow{2}{*}{$\begin{array}{c}\text { Inner Core } \\
\text { Volume } \\
\text { (liters) }\end{array}$} & \multicolumn{3}{|c|}{ Core Conversion Ratio } & \multirow{2}{*}{$\begin{array}{l}\text { Breeding } \\
\text { Ratio }\end{array}$} & \multicolumn{2}{|c|}{$\begin{array}{l}\text { Core-sodium Void } \\
(\% \Delta k / k)^{(a)}\end{array}$} & \multirow{2}{*}{$P / A^{(d)}$} & \multicolumn{2}{|c|}{ Core Mass Ratios } & \multicolumn{3}{|c|}{$\begin{array}{l}\text { Core Fuel Material }{ }^{(e)} \\
(\mathrm{kg})\end{array}$} \\
\hline & & Inner & Outer & Total & & Inner & Outer & & $\begin{array}{c}\text { Inner } \\
M^{\mathrm{u}} / M^{\text {Th }}\end{array}$ & $\begin{array}{c}\text { Outer } \\
M^{\mathrm{Pu}} / M^{\mathrm{U}}\end{array}$ & Inner & Outer & Total \\
\hline 1 & 0 & - & - & 1.02 & 1.67 & - & +0.79 & 2.03 & - & 0.14 & - & 1410 & 1410 \\
\hline 2 & 600 & 0.82 & 0.92 & 0.89 & 1.50 & +0.14 & +0.11 & 1.62 & 0.110 & 0.157 & 220 & 1225 & 1445 \\
\hline 5 & $800^{(b)}$ & 0.95 & 0.82 & 0.87 & 1.51 & +0.27 & +0.08 & 1.31 & 0.125 & 0.176 & 328 & 1235 & 1563 \\
\hline 6 & 1200 & 0.77 & 0.87 & 0.81 & 1.39 & +0.001 & -0.15 & 1.71 & 0.117 & 0.167 & 464 & 970 & 1434 \\
\hline 7 & $800^{(e)}$ & 0.58 & 1.01 & 0.81 & 1.38 & -0.15 & -0.10 & 1.98 & 0.157 & 0.147 & 247 & 1061 & 1308 \\
\hline
\end{tabular}

(a) $40 \%$ removal of core sodium.

(b) With U231.

(e) U233-Th oxide.

(d) Peak-to-average power density in core.

(e) Inner Uranium (233 or $233+234)$; Outer $\sim$ Plutonium.

TABle 2. Carbide-féled Reactors (3000-liter Cores; 800-litel Central Cores)

\begin{tabular}{|c|c|c|c|c|c|c|c|c|c|c|c|c|c|}
\hline \multirow{2}{*}{ Case } & \multirow{2}{*}{ Inner Core Type ${ }^{(a)}$} & \multicolumn{3}{|c|}{$\begin{array}{c}\text { Core Conversion } \\
\text { Ratio }\end{array}$} & \multirow{2}{*}{$\begin{array}{l}\text { Breeding } \\
\text { Ratio }\end{array}$} & \multicolumn{2}{|c|}{$\begin{array}{c}\text { Core-sodium } \\
\text { Void }(\% \Delta k / k)^{(b)}\end{array}$} & \multirow{2}{*}{$\mathrm{P} / \mathrm{A}^{(\mathrm{e})}$} & \multicolumn{2}{|c|}{ Core Mass Ratios } & \multicolumn{3}{|c|}{$\begin{array}{l}\text { Core Fuel Material } \\
\qquad(\mathrm{kg})^{(d)}\end{array}$} \\
\hline & & Inner & Outer & Total & & Inner & Outer & & $\begin{array}{c}\text { Inner } \\
\mathcal{M}^{\mathrm{U}} / M^{\mathrm{Th}}\end{array}$ & $\begin{array}{c}\text { Outer } \\
M^{\mathrm{Pu}} / M^{\mathrm{U}}\end{array}$ & Inner & Outer & Total \\
\hline 1 & Reference $\mathrm{Pu}-\mathrm{UT}$ & - & - & 0.97 & 1.49 & 一 & +0.92 & 2.05 & $\ldots$ & 0.151 & - & 1375 & 1375 \\
\hline 2 & $\mathrm{~T} 233_{-} \mathrm{Th}$ & 0.70 & 0.94 & 0.83 & 1.33 & +0.002 & +0.013 & 1.79 & 0.131 & 0.156 & 275 & 1037 & 1312 \\
\hline
\end{tabular}

(a) Carbide unless otherwise indicated.

(b) $40 \%$ removal of core sodium.

(c) Peak-to-average power density in core.

(d) Inner $\sim$ Uranium (233 or $233+234)$; Outer $\sim$ Plutonium.

TABLF 3. Oxide-FUeled Reactors (3000-liter Cores; 300-later Central Cores)

\begin{tabular}{|c|c|c|c|c|c|c|c|c|c|c|c|c|c|}
\hline \multirow{2}{*}{ Case } & \multirow{2}{*}{ Inner Core Type } & \multicolumn{3}{|c|}{$\begin{array}{c}\text { Core Conversion } \\
\text { Ratio }\end{array}$} & \multirow{2}{*}{$\begin{array}{l}\text { Breeding } \\
\text { Ratio }\end{array}$} & \multicolumn{2}{|c|}{$\begin{array}{c}\text { Core-sodium } \\
\text { Void }^{(b)}(\% \Delta k / k)\end{array}$} & \multirow{2}{*}{$P / A^{(0)}$} & \multicolumn{2}{|c|}{ Core Mass Ratios } & \multicolumn{3}{|c|}{ Core Fuel Material $_{(\mathrm{kg})}^{(\mathrm{d})}$} \\
\hline & & Inner & Outer & Total & & Inner & Outer & & $\underset{M^{\mathrm{U}} / M^{\mathrm{Th}}}{\text { Inner }}$ & $\begin{array}{c}\text { Outer } \\
M^{\mathrm{Pu}} / M^{\mathrm{U}}\end{array}$ & Inner & Outer & Total \\
\hline 1 & Reference $\mathrm{Pu}-\mathrm{U}$ & - & - & 0.80 & 1.29 & 一 & +0.55 & 2.02 & - & 0.261 & 一 & 1555 & 1555 \\
\hline 2 & $\mathrm{U}^{233}-\mathrm{Th}$ & 0.56 & 0.85 & 0.70 & 1.14 & -0.27 & -0.26 & 2.14 & 0.163 & 0.246 & 256 & 1087 & 1343 \\
\hline 5 & $\mathrm{U}^{233}-\mathrm{Th}$ Metal & 0.76 & 0.77 & 0.76 & 1.17 & -0.28 & -0.13 & 2.19 & 0.119 & 0.273 & 312 & 1182 & 1494 \\
\hline
\end{tabular}

(a) Oxide unless otherwise indicated.

(b) $40 \%$ removal of core sodium.

(c) Peak to average power density in core.

(d) Inner $\sim$ Uranium $(233$ or $233+234)$; Outer $\sim$ Plutonium.

significantly lowered by introducing a central core of U-233-Th fuel. In fact, it is apparent that there may be a significant reactivity loss upon removal of core sodium under some conditions.

Tables 1, 2, and 3 show parameters for primarily metals, carbide-, and oxide-fueled reactors, respec- tively. Detailed comparisons of the data in the tables are difficult since fission distributions are not always continuous across the inner-outer core boundary. However, the cited peak-to-average core power densities give some indication of the extent of effective "power flattening" that has been factored into the analyses. 
Furthermore, the ceramic and metallic systems were assumed to contain fission products for an average of $5 \%$ and $2.75 \%$ burnup, respectively, consistent with the $1000-\mathrm{MWe}^{(1,2)}$ studies. The metallic systems all utilize the "high void" fucl elements ${ }^{(2)}$ to accommodate irradiation growth. The latter assumption may be somewhat pessimistic for the $\mathrm{U}-\mathrm{Th}$ metal since its irradiation growth is believed to be somewhat less than that for the corresponding $\mathrm{Pu}-\mathrm{U}$ metal fuel element. The plutonium isotopic content is generally consistent with that of the corresponding 1000-MWe studies.

In general, it can be seen from the preliminary results of Tables 1, 2, and 3 that the potentially deleterious core-sodium reactivity may be significantly altered in a favorable direction by utilizing the U-233Th cycle over a relatively small region near the center of the core. The size of the region will depend upon basic core volume, geometry, composition, and higher isotope content in both plutonium and fissile uranium. Current uncertainties in the $\mathrm{Pu}-240$ and $\mathrm{U}-234$ cross sections could have significant bearing upon the ultimate configurations. The effect of the 27-day $\mathrm{Pa}$ 233 half-life relative to the shorter $\mathrm{Np}-239$ half-life has not been taken into consideration; this will present some unique control problems. A related problem arises because the core conversion ratio of inner and outer core regions are usually different. However, as one attempts to "flatten" the power distributions radially, by decreasing and increasing fissile content in inner and outer cores, respectively, this difference tends to be minimized. Cases III and IV of Table 3 are typical of such power "flattening" effects.

Case 7 of Table 1 is the metallic core configuration that would have a significant negative Doppler coefficient of reactivity due to thorium that is larger than usually associated with a metallic core.

The breeding-ratio penalty associated with the more desirable configurations appears to not exceed 0.2 except for the Case 7 cited above. The total requirements for core fucl material (fissile plus higher isotopes) is relatively insensitive to the system complexity.

Since the conversion ratio of the U-233-Th inner core is always less than unity, makeup U-233 could be obtained by placing fertile thorium in the blankets, presumably away from the core boundary so that the low Th-232 fission cross section does not significantly alter the fertile fission fraction.

There may be distinct engineering advantages to not building a three-segment fuel element. There is no reason why the central core, including the axial extremites, could not be constituted of only U-233-Th fuel. The major disadvantages of this approach are reduced breeding ratio and increased requirements for U-233.

It is also conceivable that U-235 fuel can be used instead of $\mathrm{C}$-233 in a thorium base to obtain the same relatively small-magnitude sodium reactivity worths. This substitution would lower the initial central core conversion ratio by $\sim 0.2$ and aggravate the previously mentioned control problems. The initial requirements for fuel material for the central core would increase, but not quite double in this case. This approach may be a basis for gradually providing U-233 fuel in the central core.

The central core might also be constituted of U-235U-238 or U-233-U-238 fuel. This approach would provide initial conditions that may be acceptable, but would require a larger central core than that for the corresponding U-233-Th system. The major problem here is plutonium buildup, which would tend to alter the reactivity coefficients of the coolant.

The use of the three-segment fuel element may be viewed in a number of ways. Such an element may certainly be used on a long-range basis. It may also be viewed as an interim fuel element to gain operating experience with a large fast reactor until one may confidently operate a $\mathrm{Pu}-\mathrm{U}-238$ system, presumably after some current uncertainties regarding coolant reactivity are resolved. Finally, the use of a threesegment fuel element might be a basis for reducing significantly the required number of uncoupled core modules in a given application. This could be in the design of a 1000-MWe system as well as similar systems of higher power for desalination.

\section{Materials Considerations}

The combination of a uranium-base and thoriumbase fuel in a fuel element obviously presents a number of problems which do not exist for fuel elements containing only a single fuel material. For economic reasons it is probably most desirable to produce a fuel pin of multiple composition in a single cladding. It should, however, be mentioned that it might be entirely feasible to combine individual fuel pin sections in which each section is made from materials that are best suited for its particular function. A sodiumbonded section may therefore be neighboring a heliumbonded section of different fuel composition. The complexities introduced by such an approach may be unattractive economically, but may provide a great, deal of flexibility.

To produce an integrated fuel element of multiple composition its is desirable

1. that the cladding material for both the thoriumand uranium-base fuel be the same. Preferably this should be an austenitic type of stainless steel 
or a similar material of higher strength. Refractory alloy cladding materials are acceptable, but because of cost are less desirable;

2. that the outside dimensions of the cladding for all parts of the fuel pins be the same so that a constant coolant flow can be maintained in an individual channel;

3. that the attainable burnup level for the thorium core and the surrounding uranium-plutonium fuel can be matched. Since the power level in the inner zone of the reactor core is higher than in the outer zone, e.g., the axial extremities, the inner zone will reach its maximum burnup at a time when the burnup of the outer zone is still relatively low. Therefore, if both fuels are capable of attaining the same high-burnup level, the fuel element will be removed from the reactor for reprocessing long before the fuel capability of the ends has been fully utilized. The resulting waste or handling complications should be avoided. It is therefore desirable to select two fuels which simultaneously will reach their maximum burnup capability.

The fuel materials that are available for a composite fuel element are the metallic fuels, the oxides, and the carbides. For the metals and oxides there exist applicable technologies. For the carbides the technology is still in the process of development.

The following summary of the pertinent fuel properties is given solely for the purpose of providing a basis for discussion of suitable fuel, cladding, and bond combinations.

The uranium-plutonium-base metallic fuels have been evaluated by Kittel, Sebilleau and Rateick, ${ }^{(7)}$ and more recently by Kelman et al. ${ }^{(8)}$ and by Beck et $a l .{ }^{(9)}$ The most promising metallic fuels are the uranium-plutonium-zirconium and the uranium-plutonium-titanium alloys. These alloys are superior to the uranium-plutonium-molybdenum alloys and the uranium-plutonium-fizzium alloys because of their much higher solidus temperatures. The uranium-plutoniumzirconium alloys are compatibile with stainless steel and with sodium. An experimental alloy with $18.5 \mathrm{w} / \mathrm{o}$ $\mathrm{Pu}$ and $14.1 \mathrm{w} / \mathrm{o} \mathrm{Zr}$ has reached a burnup of $4.2 \%$ of the fissionable atoms in CP-5 at a central metal temperature of $820^{\circ} \mathrm{C}$ and an outside cladding temperature of $600^{\circ} \mathrm{C} .{ }^{(10)}$ A titanium alloy with $15 \mathrm{w} / 0$ plutonium and $10 \mathrm{w} / \mathrm{o}$ titanium has reached a burnup of $4.6 \%$ at a central metal temperature of $760^{\circ} \mathrm{C}$ and a cladding temperature of $560^{\circ} \mathrm{C} .{ }^{(10)}$ The titanium alloys, however, are not compatible with stainless steel and require a vanadium- $20 \%$ titanium alloy cladding.

The thorium-uranium alloys with up to $20 \%$ uranium are probably the best metallic fuels from a standpoint of irradiation resistance. (4) Thorium-uranium alloys with up to $31 \%$ uranium were irradiated to burnups as high as $10 \mathrm{a} / \mathrm{o}$ at the maximum irradiation temperatures of $750^{\circ} \mathrm{C}$; the average rate of swelling was less than $6 \%$ volume increase per a/o burnup. Unfortunately, these alloys are not compatible with stainless steel, but they are compatible with the vanadiumtitanium cladding alloys. (11)

Among the ceramic materials, $\mathrm{CO}_{2}$ has received the most extensive evaluation. The fabrication technology is well-established. Very high burnups are attainable. A good summary of our present knowledge is given by Frost. ${ }^{(12)}$

It is generally assumed that $\mathrm{UO}_{2}-\mathrm{PuO}_{2}$ solid solutions behave similarly to $\mathrm{CO}_{2}$. There are still a number of unsolved questions where predictions are difficult to make. They concern the volatility of the nongaseous fission products of high vapor pressure, like tellurium, iodine, cesium, rubidium, and the alkaline earth metals, and their redistribution, compound formation, and effect on swelling. There are also the changes in stoichiometry caused by the presence of the fission products, their chemical reactions with the $\mathrm{UO}_{2}-\mathrm{PuO}_{2}$ matrix, particularly at high central fuel temperatures when melting occurs. Our knowledge of the compatibility of the oxides with sodium as a bonding material, effects such as the penetration of the sodium into the cracks of irradiated fuel pellets, ${ }^{(13)}$ and the effect of a high internal vapor pressure of sodium on the cladding still require evaluation. In designing an oxide fuel element it is usually assumed that all the fission gases are released and that an equivalent void volume should be provided for. Also provision must be made for the prevention of gross movement of the fuel which might cause a reactivity increase. At the present stage of development it is probably advisable not to bond with sodium but to design with a helium bond, accepting its inherently poorer heat-transfer properties.

Rahin and Ullmann ${ }^{(14)}$ have irradiated $\mathrm{ThO}_{2}-\mathrm{CO}_{2}$ pellets to burnups of $95,000 \mathrm{MWD} /$ ton $\left(2.43 \times 10^{21}\right.$ fissions $/ \mathrm{cm}^{3}$ ). The centerline temperature was $1370^{\circ} \mathrm{C}$ $\left(2500^{\circ} \mathrm{F}\right)$ and the surface cladding temperature was $94^{\circ} \mathrm{C}\left(200^{\circ} \mathrm{F}\right)$. The results indicate so far that $\mathrm{ThO}_{2}-$ $\mathrm{UO}_{2}$ fuel should not be inferior to the pure $\mathrm{UO}_{2}$ fuel.

An extensive evaluation of the U-Pu carbides was recently made by Strasser, Wheelock, and Neimark. (15) An important variable is the carbon content of the fuel. Perfectly stoichiometric single-phase carbides are compatible with sodium. So are bypostoichiometric carbides containing the carbide phase and free metal which, however, cause problems of fuel-cladding compatibility. Sodium in contact with hyperstoichiometric material transfers carbon to the cladding and causes embrittlement. Hyperstoichiometric carbides require a 
TABle 4. Potentilly Usefic Fuel Combinations for Mixed Fuel-cycle Fast Breeder Re actor

\begin{tabular}{|c|c|c|c|}
\hline \multicolumn{2}{|c|}{ Iivel Zone } & \multirow{2}{*}{ Cladding } & \multirow{2}{*}{ Bond } \\
\hline Center & Outer & & \\
\hline \multicolumn{4}{|c|}{ A } \\
\hline Th-U & $\begin{array}{c}\text { L-Pu-Zr or } \\
\text { U-Pu-Ti }\end{array}$ & $\mathrm{V}-20 \mathrm{w} / 0 \mathrm{Ti}$ & $\mathrm{Na}$ \\
\hline $\mathrm{ThO}_{2}-\mathrm{UO}_{2}$ & $\mathrm{UO}_{2}-\mathrm{PuO}_{2}$ & Stainless Steel & He \\
\hline $\mathrm{Th} \mathrm{C}-\mathrm{UC}^{(\mathrm{t})}$ & $\mathrm{UC}-\mathrm{PuC}$ & Stainless Steel & $\mathrm{He}$ \\
\hline $\mathrm{ThC}^{-\mathrm{UC}^{(b)}}$ & $\mathrm{UC}-\mathrm{PuC}$ & Stainless Steel & $\mathrm{Na}$ \\
\hline \multicolumn{4}{|c|}{$B$} \\
\hline $\mathrm{ThO}_{2}-\mathrm{UO}_{2}$ & $\mathrm{U}-\mathrm{Pu}-\mathrm{Zr}$ & Stainless Steel & $\mathrm{He}$ \\
\hline $\mathrm{ThO}_{2}-\mathrm{UO}_{2}$ & $\mathrm{U}-\mathrm{Pu}-\mathrm{Ti}$ & $\mathrm{V}-20 \mathrm{w} / \mathrm{o} \mathrm{Ti}$ & $\mathrm{He}$ \\
\hline$T h C-U C^{(a)}$ & $\mathrm{U}-\mathrm{Pu}-\mathrm{Zr}$ & Stainless Steel & $\mathrm{He}$ \\
\hline ThC-UC(s) & $\mathrm{U}-\mathrm{Pu}-\mathrm{Ti}$ & $\mathrm{V}-20 \mathrm{w} / 0 \mathrm{Ti}$ & $\mathrm{He}$ \\
\hline $\mathrm{ThC}^{\mathrm{U}} \mathrm{UC}^{(\mathrm{b})}$ & $\mathrm{U}-\mathrm{Pu}-\mathrm{Zr}$ & Stainless Steel & $\mathrm{Na}$ \\
\hline
\end{tabular}

(a) Hyperstoichiometric.

(b) Single phase.

helium bond. The important open question, therefore, pertains to the change in stoichiometry and the effect on the stability of the desirable single-phase structure that may occur with fission product formation. The fission products may easily shift the phase relationships in the U-Pu-C system in an unfavorable sense. Recent work by Boucher and Barthelemy ${ }^{(16)}$ indicates that it might be possible to widen the stability range of the UC-PuC solid solution by TiC additions, which then would permit a variation in carbon and oxygen content (oxygen as an impurity). It would enable some control to be exercised over the effect of the fission products.

There is very little information arailable on the ThC-UC fuels. The ternary Th-U-C phase diagram was investigated by Benesovsky and Rudy. ${ }^{(17)}$ ThC and UC form a continuous series of solid solutions. The authors note, however, that the fcc phase rich in ThC is considerably less stable in air at room temperature than is the UC-rich solid solution. It can only be guessed that the properties of ThC-UC fuels are comparable to those of the UC-PuC fuels.

This very short resume is sufficient to permit an initial evaluation of the potentially useful fuel combinations.

As a first approach, it appears to be reasonable to adhere either to a metallic, oxide, or carbide fuel throughout each fuel pin. In such a case a Th-U metallic center section would be placed between a U$\mathrm{Pu}-\mathrm{Zr}$ or U-Pu-Ti alloy. Such a combination would permit sodium bonding and sodium cooling, but would eliminate stainless steel as a uniform cladding material for the entire fuel pin. A V-20 w/o Ti alloy cladding would be suitable. At this time it cannot be predicted that the Th-U alloys will permit a higher burnup than the U-Pu-Zr or U-Pu-Ti alloys, since neither material has been subjected to a high-enough burnup to permit evaluation of its full potential.

If the center zone contains $\mathrm{ThO}_{2}-\mathrm{UO}_{2}$ and the outer zone $\mathrm{UO}_{2}-\mathrm{PuO}_{2}$, stainless steel can be used as a cladding material for both zones. Both would require helium bonding. At this time it must be assumed that the burnup capabilities for both materials are the same and that, therefore, the material in the outer zone will not be fully utilized when the fuel pins are removed for reprocessing.

Similar conditions apply to the carbides if hyperstoichiometry should prevail throughout the irradiation. Stainless steel cladding and a helium bond are preferred. Again, at the time of maximum burnup of the center zone the material in the outer zone would not be fully utilized. Similar considerations apply to single-phase and stoichiometric carbides, for which a sodium bond might be feasible. These cases are summarized in Table $4 \mathrm{~A}$.

To satisfy the condition of a burnup capability that is higher in the center than in the outer zone, the combinations listed in Table $4 \mathrm{~B}$ are potentially useful. In these cases the outer zone has a U-Pu-Zror a U-PuTi metal fuel, the center zone either an oxide or a carbide fuel. Stainless steel can be used as a cladding material for the UT-Pu-Zr alloy, but a helium bond would have to be used throughout unless complications are permitted by use of a different bonding material for each zone. With a $\mathrm{U}-\mathrm{Pu}-\mathrm{Ti}$ alloy in the outer zone the $\mathrm{V}-20 \mathrm{~W} / \mathrm{O}$ Ti alloy would be the cladding. If, on the other hand, the stoichiometry of the ThC-UC fuel can be controlled well enough to permit sodium bonding, the last of the combinations listed in Table $4 \mathrm{~B}$ would become very attractive.

Other possible combinations would include a thorium-uranium metallic center zone together with an oxide or carbide outer zone. This is probably the least desirable combination as can readily be seen from the bonding and cladding and burnup requirements.

One other potential combination which cannot be ruled out entirely is an oxide center zone with a carbide outer zone, or vice versa, a carbide center zone with an oxide outer zone, since well-matching burnup capabilities might be obtainable in such a way. A much more detailed knowledge of the respective burnup capabilities is required than is presently available even from our most advanced studies, to evaluate such a combination.

For all of these reasons the combinations listed in Table 4 are those that, with the present technology, meet most closely the requirements of a fuel element 
for a power breeder reactor in which a thorium-uranium center zone is surrounded by a uranium-plutonium outer zone.

\section{REFERENCES}

1. An Evaluation of Four Design Studies of a 1000-MWe Ceramic-fueled Fast Breeder Reactor, COO-279 (Dec 1964).

2. L. L. Link et al., 1000-MWe Metal-fueled Fast Breeder Reactor, ANL-7001 (to be published).

3. D. Okrent, K. Cohen, and W. Loewenstein, Some Nuclear and Safety Considerations in the Design of Large Fast Power Reactors, Paper No. 267 presented at the 1964 Geneva Conference.

4. J. H. Kittel, J. A. Horak, W. F. Murphy, and S. H. Paine, Effects of Irradiation on Th and Th-Cranium Alloys, ANL-5674 (1963).

5. D. M. O'Shea, H. Il. Hummel, W. B. Loewenstein, D. Okrent, and C. N. Kelber, Twenty-six Group Cross-sectzons, ANL-6858 (to be published).

6. P. II. White, J. G. Hodgkinson, and G. J. Wall, Measurements of Fussion Cross Section for Neutrons of Energies in the Range 40-500 KeV, IAEA Symp. on Physics and Density of Fission, Salzburg, Austria (March 1965), Paper SM 60/14.

7. J. H. Kittel, F. Sebilleau, and R. G. Rateick, Metallic Fuel Elements for Fast Reactors, Proc. Am. Nucl. Soc., Meeting on Fast Reactor Technology, Detroit, April 26-28, 1965, p. $157-159$.

8. L. R. Kelman, II. Savage, C. M. Walter, B. Blumenthal, R. J. Dunworth, and H. V. Rhude, Status of Metallic Plutonium Fast Power Breeder Fuels, 3rd Internat. Conf. on Plutonium, London 22-26 November, 1965 (to be published).

9. W. N. Beck, W. F. Murphy, J. II. Kittel, and F. G. Foote, Irradhation Behavior of Plutonium Alloys for Fast Reactors, 3rd Internat. Conf. on Plutonium, London, 22-26 November 1965 (to be published).

10. W. N. Beck, private communication.

11. B. Blumenthal, Thorium-Uranium Plutonium Alloys as Potential Fast Power Breeder Fuels, Trans. Am. Nucl. Soc., 8(2) (1965) (to be published).

12. B. R. T. Frost, Behavior of Fuels at High Burnup Levels. Part I. Fast Reactor Fuels, Nucl. Eng. 9, 55-67 (Feb 4, 1964).

13. G. L. O'Neill, P. E. Novak, M. L. Johnson, and W. E. Bailey, Experimental Studies on Sodium Logging in Fast Reactor Fuels, GEAP-4283 (1963).

14. S. A. Rabin, J. W. Ullmann, E. L. Long, Jr., M. F. Osborne, and A. E. Goldman, Irradiation Behavior of High Burnup $\mathrm{ThO}_{2}-4.45 \%$ UO $\mathrm{O}_{2}$ Fuel Rods, ORNL-3837 (1965).

15. A. Strasser, C. Wheelock, and L. A. Neimark, UraniumPlutonum Carbide Fuels for Fast Breeder Reactors, Proc. Am. Nucl. Soc., Meeting on Fast Reactor Technology, Detroit, April 26-28, 1965, pp. 126-156.

16. R. Boucher and P. Barthelemy, Etudes d'Alliages à Base de Plutonium à Fontenay-aux-Roses, 3rd Internat, Conf. on Plutonium, London 22-26, November 1965 (to be published).

17. F. Benesowky and E. Rudy, Cntersuchungen im System Uran-Thorium-Kohlenstoff, Montashefte der Chemie 92(6), 1176-1183 (1961).

\section{Discussion.}

Mr. Sesonske (Purdue): Do you have any fear of of possible complications in fuel management and fuel cycle because of these different isotopic arrangements?

Mr. Loewenstein: I think it's quite obvious that there will have to be two separate streams of fabrication or reprocessing. Whether or not one can put these two types of fuel elements in the same clad or whether one has to provide three separate axial fuel elements is not clear at this time. I don't mean to be facetious, but I think this is an engineering detail at this point.

$M r$. Sesonske: I was thinking more of possible economic penalties.

Mr. Loewenstein: I am sure that there are obvious economic penalties, but I think there are also some economic advantages depending on whether you compare big apples with little apples. For example, here is a way to build a system with a trivial sodium coefficient of reactivity and in some cases with an enhanced Doppler coefficient of reactivity with a slightly higher core conversion ratio and, generally speaking, a significantly higher total breeding ratio than some of the systems that have been specifically designed to overcome the positive sodium coefficient.

Mr. Heck (WAPD) : I am curious if you considered the possibility of simply using a radially zoned loaded core with the thorium- $\mathrm{U}-233$ in the central region to avoid the possibility of having the multiple rods axially. In a cylindrical core you might be able to achieve somewhat the same effect by loading the whole central fuel, all the central fuel assemblies with thorium-U-233, and then put plutonium normal fuel assemblies around it instead of having to split assemblies.

Mr. Loewenstein: Yes, we have looked at this, but we believed that there was a premium paid to try to utilize plutonium-U-238 as much as possible, because this seems to have been the ground rule for the studies of large systems. The U-233-thorium effect was used only as a tool here. Certainly if one did what you suggest the core conversion ratio would decrease, the total breeding ratio would decrease, and the sodium coefficient, generally speaking, would be slightly more negative, but not much more.

Mr. Weiner (USAEC, Washington): Did I understand you to say that you got the same sodium voiding effect with U-235 at the beginning of life?

$M r$. Loewenstein: I did not do the calculations specifically for this system, but we have a number of calculations of U-233-thorium systems compared with U-235-thorium systems. Generally speaking, they exhibit about the same sodium reactivity coefficients.

$M r$. Weiner: Then wouldn't it be worthwhile to examine the possibility of using U-235 in the central zone? I would expect that you would get some worse sodium voiding effects towards the end of life, but I am wondering how much worse. 
Mr. Loewenstein: Again the question comes up, what are you comparing: clean U-235 or U-235 that has U236 in it? Generally speaking, I think you are probably right that U-235 would aggravate the situation slightly, but would not begin to approach the problem with plutonium.

Mr. Weiner: Then wouldn't this be a more economical way of getting the effect you are looking for rather than by the use of $\mathrm{C}-233$.

Mr. Loewenstein: It might very well be, except that in these studies we were primarily concerned with having something that would be self-regenerative. In other words, you put enough thorium in the blankets to make up the U-233 that you needed in the core, whereas it's kind of hard to breed U-235. Your comments are quite valid if one has a lot of U-235.

$M r$. Cohen $(\mathrm{GE})$ : You showed the core breeding ratios themselves to be somewhat less than one. Have you investigated the breeding ratio of the thorium per se?

Mr. Loewenstein: In the oxide system, offhand, the regeneration of the thorium region by itself is around 0.55 . In the carbide and in metal it would be somewhat higher. I would guess about 0.7 in the thorium system, whereas the carbide conversion ratio of just the plutonium-U-238 is around 0.9 . When you average over the whole core, you obtain something around 0.75 .

$M r$. Cohen: That number would suggest, of course, that you would have a reactivity swing to deal with.

Mr. Loewenstein: Yes. I think the fact that you have a three-day half-life of neptunium and a thirty-day half-life in protactinium will present a real problem to the control people. 


\title{
Nuclear Design of MPBE*
}

\author{
W. H. Hannum \\ Los Alamos Scientific Laboratory \\ Los Alamos, New Mexico
}

\section{Introduction}

A conceptual design of a large molten plutonium fast breeder reactor has previously been described. ${ }^{(1)} \mathrm{Al}-$ though the general performance of such a system can be oxpected to be quite favorable, there are a number of areas in which there are inadequte bases for defining performance limits. One such major area is the burnup limit for molten plutonium fuel. In the present paper, we will describe the design of a Molten Plutonium Burnup Experiment (MPBE) which will be used to determine the properties of a molten plutonium fuel during and after high burnup.

We first give a very brief review of the large reactor design concept. Then, some of the relevant parameters of the Fast Reactor Core Test Facility (FRCTF), ${ }^{(2)}$ in which this experiment will be done, are given. In the context set by the concept and by the facility, we then consider the criteria used in the design of the MPBE. Some of the expected performance features of this core are presented.

In a related paper, ${ }^{(3)}$ the materials program and the results of experimental work relative to the molten plutonium program have been considered. In another paper, ${ }^{(4)}$ one of the several unique safety aspects of a molten fuel has been discussed.

\section{Design Concept}

The reactor concept(1) is illustrated schematically in Fig. 1. The general configuration is an array of reactor modules, containing a sufficient number of modules to produce 500-1500 MW(e) (typically 10-30 modules).

It is assumed that the power level of the core is limited by the available heat transfer area. For a capsule core, this assumption-without reference to what the limiting value is-is adequate to determine a ratio of plutonium density in the fuel to container area which is optimum with respect to doubling time. ${ }^{(5)}$ As an example, with tantalum capsules of the order of a 1-cm diameter and a $0.05-\mathrm{cm}$ wall, the optimum plutonium density is in the range of $3-6 \mathrm{~g} \mathrm{Pu} / \mathrm{cc}$ fuel.

A specific and realistic case based on the current best

\footnotetext{
* Work performed under the auspices of the U. S. Atomic Energy Commission.
}

estimates for limiting factors yields the parameters given in Table 1.

\section{Facility}

The MPBE will be operated in the FRCTF, which will provide an environment in which a sequence of fast cores may be tested. As with any facility, however, there are certain limits which are relevant to the design of experiments. There are three factors which are potentially limiting, as shown in Table 2 .

This facility is presently under construction. The building is nearing completion, and the present schedule for installation of equipment is directed toward initial operation of MPBE in 1968.

\section{Design Criteria}

A prime consideration in the design of MIPBE, as for any experiment, is safety. Within this constraint, the intent is to investigate areas which strongly affect the economics of a large system and for which substantial uncertainty exists.

The unique and impressive safety features resulting from the use of a molten metal fuel (such as large expansion coefficient, negative sodium void effect, and potential for low fission product inventory) have been discussed elsewhere. ${ }^{(6)}$

Two fuel-cycle costs may be singled out, which are common to all reactors and which are frequently the dominant factors: fuel charges (burnup or buy-back, plus use charge), and fabrication charges. The fuel charges are related to the system doubling time. Fabrication charges are related to total burnup capability of the fuel elements (per unit fabrication cost).

Lifetime (burnup) capability for most fast reactor fuel elements is determined by structural changes of the fuel. With a molten fuel, this is not likely to be an early limit, for no structural damage to the fuel itself can occur. Consideration of reactivity lifetime can (conceptually) be avoided. The burnup limit is thus likely to be determined by fuel-containment life, by gross chemical fuel changes due to fission products, or by economics.

One other point relative to the lifetime of a fuel element should be mentioned. No matter what the con- 


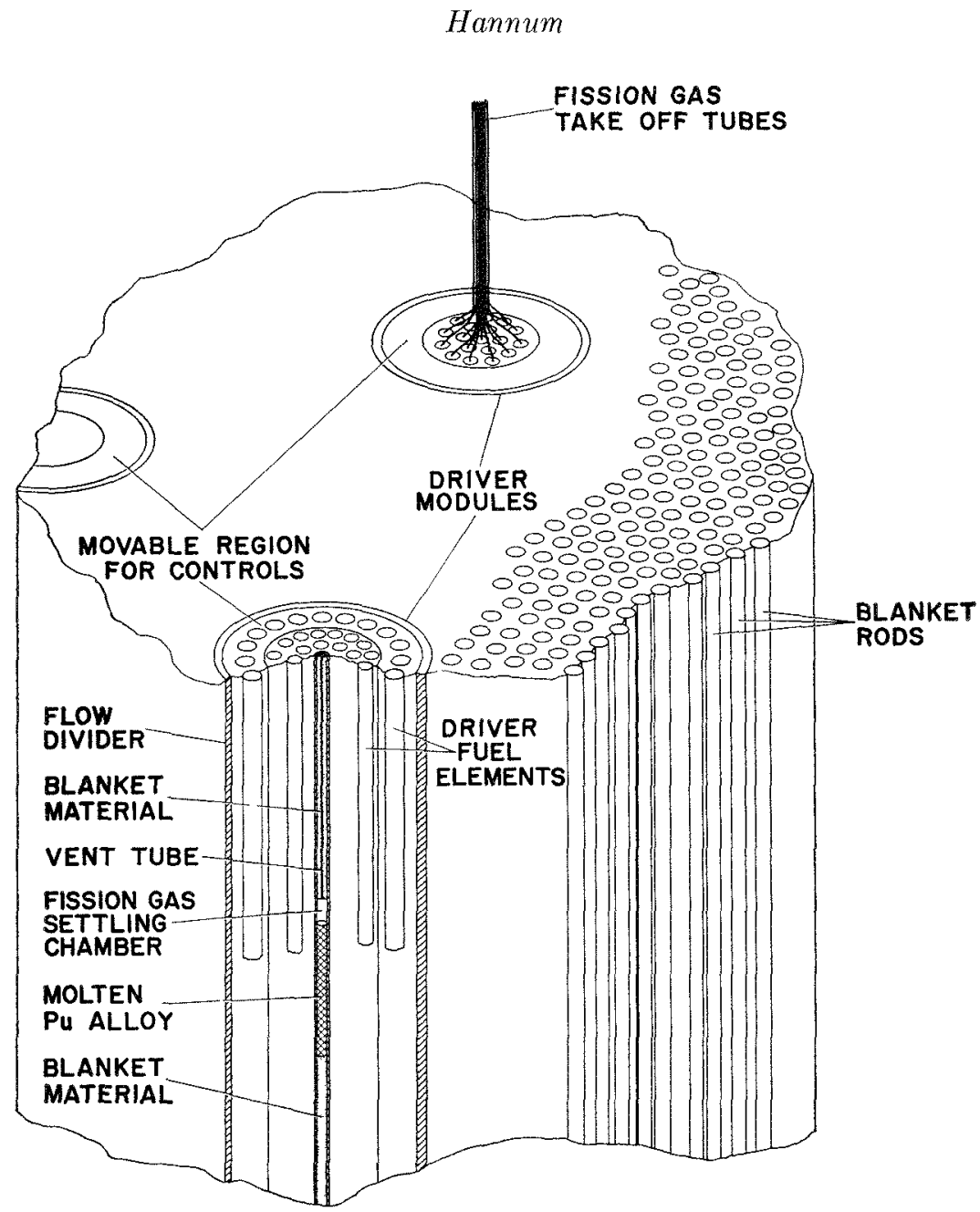

FIG. 1. Conceptual Layout of Large Modular Core.

TABLE 1. ILLestretive F ICTORS For a Heat-TransferLIMITED LARgE CORE

\begin{tabular}{l|c}
\hline Doubling Time (Core Inventory) & $7 \mathrm{yr}$ \\
Breeding Ratio (at Beginning of & 1.5 \\
Life) & \\
Specific Power (Core Inventory) & $0.9 \mathrm{MW} / \mathrm{kg} \mathrm{Pu}$ \\
Power Density & $4.0 \mathrm{MW} /$ liter fuel \\
Loading/Module & $120.0 \mathrm{~kg} \mathrm{Pu}$ \\
Power/Module & $110.0 \mathrm{MW}(\mathrm{th})$ \\
\hline
\end{tabular}

TABLE 2. SOME FRCTF Operating Limits

\begin{tabular}{l|c}
\hline Meat Dump and IfHX Capacity & $20 \mathrm{MW}(\mathrm{th})$ \\
Max Flow of Sodium & $132 \mathrm{~kg} / \mathrm{sec}$ \\
Max Mixed Mean Outlet Tempera- & $650^{\circ} \mathrm{C}$ \\
ture of Sodium & \\
\hline
\end{tabular}

cept, it is necessary to hold a fluid fuel in some type of container, which may or may not be a heat transfer medium. In principle, the use of a fluid fuel permits the changing of fuel without the replacement of the container. However, the container will also have a finite lifetime. The design choice as to whether to refill or to replace containers must consider the lifetime of the container as compared with that of the fuel. For a molten plutonium system of the type we are considering, it is not clear whether the lifetime capability of the fuel or of the container (if either) will be limiting. Thus, an ultimate decision as to whether to pursue a plumbed or a fixed fluid concept would be premature at this stage. It could be that the ability to handle fertile blanket materials in a fluid form will be a more significant advance.

The present design uses sealed capsules, but is also compatible with the use of vented capsules. The selection of this type of fuel containment for the present experiment has several distinct practical advantages: the technology developed for solid fuel in thermal and mechanical designs is available and useful; the performance limits are more readily defined; a failure of containment has limited and tolerable consequences; and the design is well suited to collection of data on properties of materials as a function of burnup. In addition, actual successful operational experience on such a core exists (LAMPRE I). ${ }^{(7)}$ 
The prineipal purpose of the Molten Plutonium Burnup Experiment is to extend the available data on the properties of the fuel and, to some degree, on the properties of tantalum containers, during and after high burnup. Upon completion of this experiment, it may be feasible to define realistic burnup limits.

\section{Selection of Core Design Parameters}

The performance potential of a molten plutonium system will clearly be affected by burnup in many ways. For example,

(a) The change in plutonium content by burnup and by accumulation of soluble fission products will change the physical character of the fuel (melting point, ete.).

(b) The presence of gross amounts of fission products in the fuel may affect the integrity of the containment.

(c) The volatile fission products will cause a pressure buildup in sealed capsules.

(d) The rate of gas generation may affect the equilibrium dilution (bubble content) of the fuel (the detailed character of this dilution a high burnup at high burnup is not fully known).

(e) The presence of insoluble fission products may result in mechanical changes to fuel performance (e.g., interference with natural circulation of the fuel).

(f) The burnup of plutonium and dilution of the fuel by fission products will determine the required reactivity compensation.

With regard to these considerations, we may inquire as to the parameters which are to be optimized in the design of the experiment. It may be noted that power is fission or burnup rate, specific power is fractional burnup rate, and power density is burnup rate per unit volume. Fractional burnup, or integrated specific power, is a reasonable measure of flux time $(n v t)$.

The change in neutronic properties is likely to be described in terms of fractional burnup or integrated specific power. Likewise, if neutron irradiation damage to the container is limiting, integrated specific power is the factor of interest. Gross fission product content, however, represents integrated power density. Thus, fuel changes are more likely to be characterized by this parameter. Further, container damage from fission product recoil is measured in terms of integrated fission or power density. To the extent that there are reasons to differentiate between power density and specific power, the emphasis in the design is on obtaining a high power density. This separation relies on the assumption that plutonium density in the fuel and the effects of burn up are not strongly related; an assumption which will be verified by selected special tests.

\section{Core Design Model and Calculational Results}

In the scoping studies, preliminary design studies, and nuclear design calculations described here, the two-dimensional $S_{n}$ code DDK ${ }^{(8)}$ was used. The use of two-dimensional calculations at this stage is feasible only if the calculational model is held to modest size, both in detail and in extent. The availability of the DPC code, ${ }^{(9)}$ however, has permitted the accounting for a considerable amount of detail in determining the material content of various regions in the design. The core cross section is shown in Fig. 2. Surrounding the region shown will be a reflector-blanket region. The initial operation (MPBE) will utilize a nickel reflector, with perhaps some special experiments in the reflector region.

In the calculations, the compositions of control segments are taken to include all of the material between the two structural members shown. Two control-segment compositions are considered: that with the control segment being nickel and that with tantalum. In both cases, an extra $10 \%$ of the total region volume is assumed to be sodium for internal cooling of the control segments.

The core sleeve and core-sleeve liner are solid 304 stainless steel.

All core regions are constructed of stainless steel module cans in sodium, each containing seven tantalum capsules. Two distinct types of core compositions are used: fueled regions and gas-space regions, representing the fueled and the void portions of the capsules. Figure 3 shows the cross section and dimensions of core modules.

In the core area are both fueled and reflector modules. The reflector compositions may further be separated into axial reflectors (physically part of the fueled-core modules), radial reflector modules, and fueled-control followers (upper sections of fueled-control clusters). All modules have the same configuration as shown in Fig. 3, with the following exceptions. Rather than capsules, each contains seven solid rods. For axial reflectors, these rods are of nickel, with the same diameter as the core capsules. For fueled-control followers, the rods are of solid tantalum, with the same diameter as the core capsules. The radial reffector rods are solid nickel of 0.500 -in. diameter.

The core design is compatible with fuel of either 6.2 or $8.0 \mathrm{~g} \mathrm{Pu} / \mathrm{cc}$. In addition, some margin in size is required for several purposes. Experimental objectives at the time of operations could dictate an increase or a decrease in the density of the plutonium in the fuel. Beyond this, since this is a new fuel, a substantial margin for analytical uncertainty is advisable. Further, compensation for depletion by increasing size may be desired. Thus, a wide range of loadings may be of 


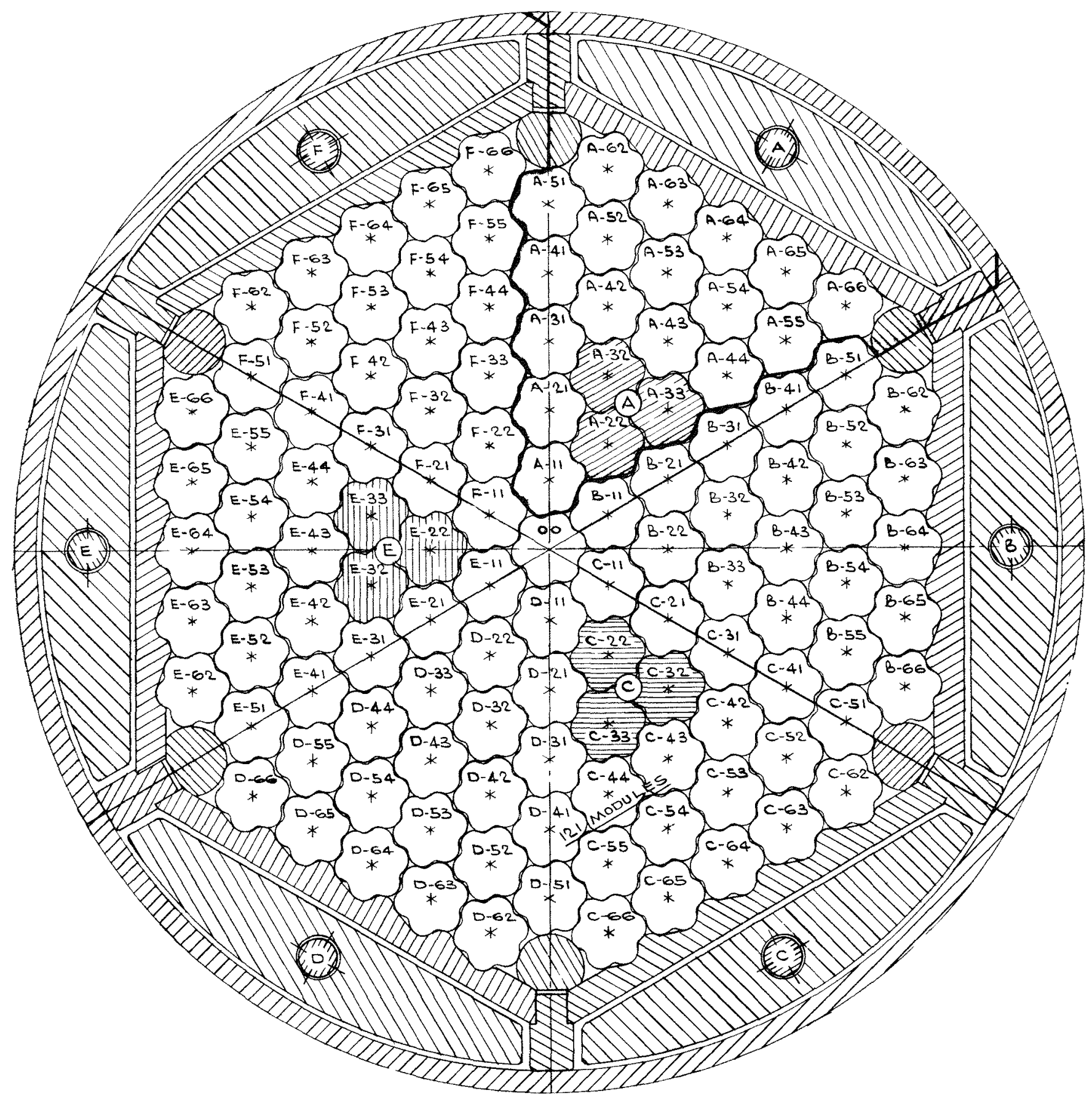

Fyg. 2. Cross section of MPBE Core.

interest. Table 3 shows the estimated size and loading for a few cores.

Cases 92 and 95 of Table 3 are considered to be the reference cases. Case 98 represents a hot, critical case with the $6.2 \mathrm{~g} \mathrm{Pu} / \mathrm{cc}$ fuel. Case 10 is included as a measure of the minimum fuel density which might be tolerable in this core geometry without severely compromising the available gas space.

\section{Control Worths}

Table 4 shows the calculated reactivity and reactivity swing for various control configurations.

For the $6.2 \mathrm{~g} \mathrm{Pu} / \mathrm{cc}, 530^{\circ} \mathrm{C}$ cases, the following observations may be made: The total control swing $(11.3 \% \Delta k)$ and the fueled control swing $(7.3 \% \Delta k)$ both appear to be ample. The worth of the inert control segments is slightly less than might be desired $(4.6 \%)$ 


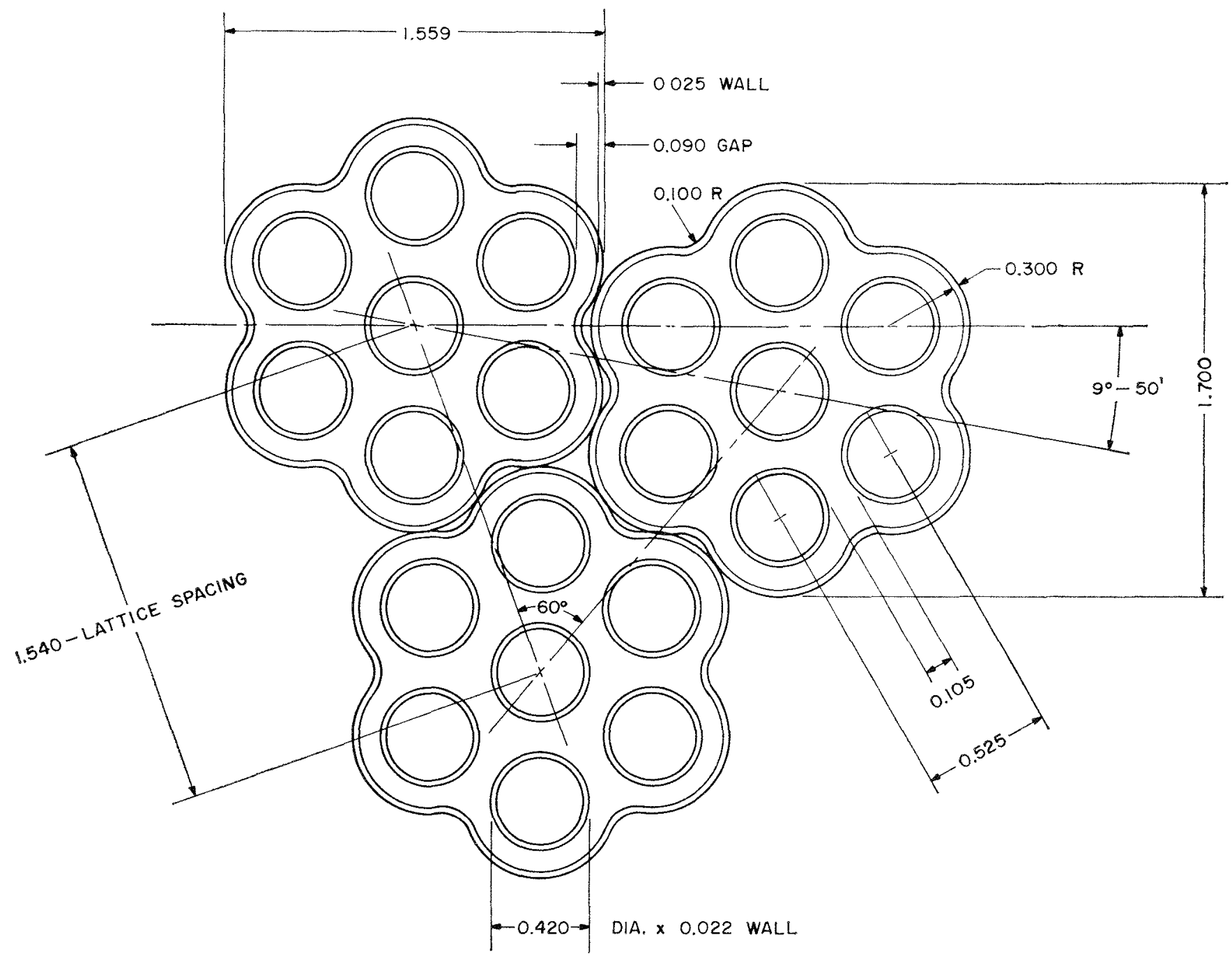

Fig. 3. Core Module of MPBE.

TABLE 3. Critical SIZE AND LoAding

\begin{tabular}{|c|c|c|c|c|c|c|c|}
\hline \multirow{2}{*}{ Case } & \multirow{2}{*}{$\begin{array}{c}\text { Fuel } \\
(\mathrm{g} \mathrm{Pu} / \mathrm{cc})\end{array}$} & \multirow{2}{*}{$\begin{array}{l}\text { Temp } \\
\left({ }^{\circ} \mathrm{C}\right)\end{array}$} & \multicolumn{2}{|c|}{$\begin{array}{l}\text { Fraction of } \\
\text { Control Up }\end{array}$} & \multicolumn{3}{|c|}{ Loading } \\
\hline & & & Fuel & Inert & $\begin{array}{l}\text { No. of } \\
\text { Mod- } \\
\text { ules }\end{array}$ & $\begin{array}{l}\text { Height } \\
\text { (in.) }\end{array}$ & $\mathrm{kg} \mathrm{Pu}$ \\
\hline 92 & 6.2 & 530 & $2 / 3$ & $1 / 2$ & 91 & 13.3 & 95.8 \\
\hline 98 & 6.2 & 530 & $3 / 3$ & $2 / 2$ & 91 & 11.7 & 84.7 \\
\hline 09 & 6.2 & 160 & $3 / 3$ & $2 / 2$ & 91 & 11.3 & 81.4 \\
\hline 95 & 8.0 & 530 & $2 / 3$ & $1 / 2$ & 91 & 8.4 & 793 \\
\hline 10 & 5.0 & 530 & $2 / 3$ & $1 / 2$ & 121 & 16.1 & 124.2 \\
\hline
\end{tabular}

but should be fully adequate. Some small interaction among various control motions may be inferred from the fact that the worths of fueled and of inert control do not add up to the combined worth. Part of this difference may be associated with the relatively loose convergence criteria used for these calculations.
The control worths at $160^{\circ} \mathrm{C}$ are seen to be roughly comparable to those at $530^{\circ} \mathrm{C}$.

For the $8 \mathrm{~g} \mathrm{Pu} / \mathrm{ec}$ fuel core, some loss in control worth may be noted. The fueled-control worth is not greatly reduced, in spite of the shortened stroke relative to that of the $6.2 \mathrm{~g} \mathrm{Pu} / \mathrm{cc}$ fuel case. The inert control is of somewhat more concern. This reduced worth is such as to suggest the possibility that different modes of operation may be required for the $8 \mathrm{~g} \mathrm{Pu} / \mathrm{ec}$ fuel case than for the $6.2 \mathrm{~g} \mathrm{Pu} / \mathrm{cc}$ fuel case, with somewhat greater reliance being placed on the fueled control.

\section{Reactivity Coefficients}

A few of the basic reactivity changes associated with normal changes in composition are given in Table 5. A considerable extention of this table, both from displacement and from perturbation calculations, is underway. It will be noted that none of these coefficients indicates serious difficulties. 
TABLE 4. Control-worth Calculations

\begin{tabular}{|c|c|c|c|c|c|c|}
\hline \multirow{2}{*}{ Case } & \multirow{2}{*}{$\begin{array}{c}\text { Fuel } \\
(\mathrm{g} \mathrm{Pu} / \mathrm{cc})\end{array}$} & \multirow{2}{*}{$\begin{array}{l}\text { Temp } \\
\left({ }^{\circ} \mathrm{C}\right)\end{array}$} & \multicolumn{2}{|c|}{$\begin{array}{l}\text { Fraction } \\
\text { Control Up }\end{array}$} & \multirow{2}{*}{$k$} & \multirow{2}{*}{$\Delta k(\%)$} \\
\hline & & & Fuel & Inert & & \\
\hline 92 & 6.2 & 530 & $2 / 3$ & $1 / 2$ & 0.995 & $\ldots$ \\
\hline $99 \mathrm{~A}$ & & & $0 / 3$ & $0 / 2$ & 0.924 & -7.1 \\
\hline 98 & & & $3 / 3$ & $2 / 2$ & 1.037 & +4.2 \\
\hline 99 & & & $0 / 3$ & $1 / 2$ & 0.946 & -4.9 \\
\hline 98 & & & $3 / 3$ & $1 / 2$ & 1.019 & +2.4 \\
\hline 94 & & & $2 / 3$ & $0 / 2$ & 0.971 & -2.5 \\
\hline 93 & & 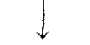 & $2 / 3$ & $2 / 2$ & 1.016 & +2.1 \\
\hline 02 & & 160 & $2 / 3$ & $1 / 2$ & 1008 & - \\
\hline 04 & & & $0 / 3$ & $0 / 2$ & 0.936 & -7.2 \\
\hline 09 & & & $3 / 3$ & $2 / 2$ & 1.043 & +3.5 \\
\hline 95 & 8.0 & 530 & $2 / 3$ & $1 / 2$ & 0.991 & - \\
\hline 11 & & & $0 / 3$ & $0 / 2$ & 0.933 & -5.8 \\
\hline 12 & & & $3 / 3$ & $2 / 2$ & 1.026 & +3.5 \\
\hline 97 & & & $2 / 3$ & $0 / 2$ & 0.973 & -1.8 \\
\hline 96 & $\downarrow$ & $\downarrow$ & $2 / 3$ & $2 / 2$ & 1.006 & +1.5 \\
\hline
\end{tabular}

TABLE 5. Reacturity Worths of Certhe Chinges

\begin{tabular}{|c|c|c|c|}
\hline Cases & $\begin{array}{l}\text { Fuel, } \\
\mathrm{g} \mathrm{Pu} / \mathrm{cc}\end{array}$ & Change & Inferred Coefficient \\
\hline $92 \sim 00$ & 6.2 & Isothermal $\Delta T$ & $-65 \times 10^{-6} \Delta k /{ }^{\circ} \mathrm{C}$ \\
\hline $92-13$ & & Fuel-expansion & $\begin{aligned} & -3.6 \times 10^{-3} \Delta k / \% \\
= & -34 \times 10^{-6} \Delta k /{ }^{\circ} \mathrm{C}\end{aligned}$ \\
\hline $92-05$ & & Sodium Removal & $\begin{array}{c}-4.7 \times 10^{-4} \Delta k / \% \\
\text { (uniform) }\end{array}$ \\
\hline $92-02$ & & $\begin{array}{l}\text { All except fuel ex- } \\
\text { panded }\end{array}$ & $-34 \times 10^{-6} \Delta k /{ }^{\circ} \mathrm{C}$ \\
\hline $\begin{array}{l}92,04 \\
09,98\end{array}$ & & $\begin{array}{l}\text { Change in fuel } \\
\text { height (con- } \\
\text { stant density) }\end{array}$ & $+0.33 \Delta k /(\Delta H / H)$ \\
\hline $02-92$ & & $\begin{array}{l}\text { Temperature de- } \\
\text { fect }\end{array}$ & $-1.3 \% \Delta k$ \\
\hline $92-13$ & & $\begin{array}{l}\text { Power defect } \\
\qquad\left(100^{\circ} \mathrm{C}\right)\end{array}$ & $-0.4 \% \Delta k$ \\
\hline $\begin{array}{c}95-01 \\
95\end{array}$ & 8.0 & $\begin{array}{c}\text { Isothermal } \Delta T \\
\text { Change in fuel } \\
\text { height (con- } \\
\text { stant density) }\end{array}$ & $\begin{array}{l}-66 \times 10^{-6} \Delta k /{ }^{\circ} \mathrm{C} \\
+0.30 \Delta k /(\Delta H / H)\end{array}$ \\
\hline
\end{tabular}

\section{Summary}

The general prospect for the use of molten plutonium as a fast breeder fuel is favorable, but several major extrapolations are involved in such assessments. A particular case, for example, has power density, $\sim 4$ $\mathrm{MW} /$ liter fuel; specific power, $\sim 1 \mathrm{MW} / \mathrm{kg} \mathrm{Pu}$; with burnup capability assumed to be $\widetilde{\lessgtr} 10 \%$. None of these have been suitably demonstrated. The MPBE will investigate this range of burnup, with high power densities (1.3-2 MW/liter fuel). The design presented
TABle 6. Summalz of Some Propertes of the Reference Cores

\begin{tabular}{|c|c|c|}
\hline & \multicolumn{2}{|c|}{$\mathrm{g} \mathrm{Pu} / \mathrm{cc}$ Fuel } \\
\hline & 6.2 & 8.0 \\
\hline Operational Loading (kg Pu) & 95.8 & 81.5 \\
\hline Specific Power $(\mathrm{kW} / \mathrm{kg})$ & 210.0 & 245.0 \\
\hline Power Density (MW/liter fuel) & 1.3 & 2.0 \\
\hline Minimum Critical Loading ( $\mathrm{kg})$ & 84.7 & - \\
\hline $\begin{array}{l}\text { Available Total Control Swing }(\%) \\
\Delta \pi)\end{array}$ & 11.3 & 9.3 \\
\hline $\begin{array}{l}\text { Available Fueled-control Suing } \\
(\% \Delta k)\end{array}$ & 4.6 & 3.3 \\
\hline Power (Peak to Average) & 1.46 & 1.43 \\
\hline $\begin{array}{l}\text { Medium Energy of Neutrons } \\
\text { Causing Fission }(\mathrm{MeV})\end{array}$ & 0.65 & 0.69 \\
\hline Medium Flux Energy (MeV) & 0.55 & 0.61 \\
\hline Average Flux $\left(n / \mathrm{cm}^{2}\right.$-sec $)$ & $1.7 \times 10^{15}$ & $2.0 \times 10^{15}$ \\
\hline
\end{tabular}

appears ot have adequate control, very favorable reactivity coefficients, and conservative thermal design.

A summary of some of the more important properties of this core is given in Table 6 .

\section{REFERENCES}

1. W. H. Hannum, B. M. Carmichael, and G. L. Ragan, "An Application of Molten Plutonium Fuel to Fast Breeders," in Proceedings of the Conference on Breeding, Economics, and Safety in Large Fast Power Reactors, ANL-6792 (Dec 1963).

2. A Preliminary Study of a Fast Reactor Core Test Facility, LA-2332 (1959).

3. L. D. Kirkbride, Molten Plutonium Alloys as Fast Reactor Fuels, see Paper in Session VI A of This Conference.

4. B. M. Carmichael, Effect of Fission Gas Bubbles on the Response of Molten Fuel to Reactor Transients, see Paper in Session VIII B of This Conference.

5. W. H. Hannum and L. D. Kirkbride, The Molten Plutonium Burnup Experiment, LA-3384-MS, in preparation.

6. D. B. Hall, "Los Alamos Molten Plutonium Program," in Fast Reactor Technology, ANS-100 (April 1965).

7. LAMPRE I C perations Report, in preparation.

8. B. G. Carlson, Difference Equations in Neutron Transport Theory, Trans. ANS, 6, 1 (June 1963).

9. B. M. Carmichael and W. H. Hannum, "Two-dimensional Data Preparation Code," in The International Conference on the Application of Computing Methods in Reactor Problems, ANL-7050 (Aug 1965).

\section{Discussion}

Mr. Leitz (GE) : In earlier reports on the molten plutonium system when the circulation of the molten fuel through the core was considered, it was observed that the generation of fission gases could be a limit to fuel lifetime or, perhaps better, core-residence time; the concern was less one of just simple dilution of the fuel than the fact that the gases could interfere with 
heat transfer from the fuel to the coolant. I was wondering if you developed a solution to this problem or if there is some reason, which isn't immediately obvious to me, why the sealed-pin molten plutonium concept would not be subject to this difficulty of fission gases, particularly those which might be held up on the interior of the cladding interfering with the heat transfer.

$M r$. Hannum: It is expected that the bulk of the fission gas bubbles which have not yet been released from the fuel will, in fact, imply only that the fuel temperature will be higher than it normally is. In this concept the limiting temperature is not the centerline of the fuel; we would be much happier to run it much hotter. The limiting criterion is that the corrosion is worse at high temperatures. We worry about the interface temperature between the plutonium and the container, and that should not be affected by bubbles.

Mr. Bartels (USAEC, Washington) : If you take the core of the Fast Core Test Facility as being reactivity limited, what would your fuel lifetime be?
M. Hannum: The major factor which changes the reactivity is simply the retention of fission gas bubbles within the fuel diluting the fuel, and thereby decreasing the reactivity. We expect to attain several percent depletion between partial refuelings even if a major fraction of the bubbles are retained. There is some preliminary indication from thermal reactor radiations that the dilution of fuel by bubbles saturates very quickly at about the amount it did in LAMPRE-I, namely, about $5 \mathrm{v} / \mathrm{O}$ of the fuel, in which case we would take an initial reactivity loss and after that the reactivity loss would be very slow.

$M r$. Chernick (BNL): Can you tell us what your experience, if any, has been with single elements on reactivity swings you might get with gas foaming, and the changes in density during operation?

Mr. Hannum: Nothing. No short-term reactivity changes have been detectable that can be associated with release of fission gases. If there is anything, it is a matter of a fraction of a cent. The bubbles come off continuously or in small quanta. 


\title{
Faret Core-I Reference Design
}

\author{
P. J. Persiani, T. R. Bump, and W. J. Kanx \\ Argonne National Laboratory \\ Argonne, Illinois
}

(PRESENTEI) BY P. J. PERSIANI)

The Fast Reactor Test Facility (FARET) being designed at Argonne is described in the literature. ${ }^{(1-3)}$ Briefly, however, the FARET facility has a heatremoval capacity of $50 \mathrm{MWt}$ and a mean outlet coolant temperature of up to $650^{\circ} \mathrm{C}$. It can accommodate a variety of core configurations, coolant flow rates, and core sizes ranging from less than 40 liters to about 500 liters. The smaller cores would be used for high-power-density experiments in the order of 1.5 MW/liter. The facility design includes provisions for the instrumentation of in-core components, and it can accommodate re-entrant loops for irradiation studies such as vented fuel elements, corrosion studies, or new fuel concepts.

\section{Introduction}

The purpose of this paper is to present the fuel-irradiation program and the reference core planned for the first experiment in the FARET facility. The objective of the experiment is to test fast reactor fuel elements over a range of operating conditions and design specifications associated with large fast power breeders. It is planned to study simultaneously the performance of several different designs of ceramicand metal-fuel assemblies in a small (70-liter) core. With the small core, the design and geometry of a prototype reactor can be simulated, but the fuel enrichment will be different. The plutonium content in the mixed fuel is made similar to that of the large reactor, with $U^{235}$ replacing $U^{238}$ to achieve criticality. By selection of core position and/or enrichment, the fuel assemblies can then be tested over the desired range of operating conditions.

The basis for utilizing this enrichment technique is that the substitution of $\mathrm{C}^{235}$ for $\mathrm{U}^{238}$ would not alter the metallurgical or physical properties of the fuels, at least to a first-order approximation. The $\mathrm{U}^{235}$-enriched uranium-plutonium system is chemically identical to a nonenriched uranium-plutonium system.

This technique is to be compared with the method in which a nonenriched uranium-plutonium system is irradiated in a higher neutron-flux environment to achicve the same fission rate or heavy atom burnup.
Phenomena such as diffusion or segregation of atoms which depend on temperature gradients are expected to differ, if at all, only to second order between the two fuel systems. ${ }^{(4)}$ Fission product yields for $U^{235}$ and $\mathrm{Pu}^{239}$ reported for thermal neutrons ${ }^{(5)}$ and estimates for fast neutrons ${ }^{(6)}$ indicate that the total yield of the more prevalent gascous fission products would be similar to within $10 \%$ for the enriched and nonenriched fuels. Differences in the fission-gas release rate and fission produce migration are not expected in solidsolution fuels.

The advantages of the enrichment technique as used in FARET are:

1. Many different fuel element designs may be tested simultaneously in a flexible and accessible small-core loading.

2. The hard neutron spectrum and the magnitude of the flux in FARET would subject the clad material to neutron dose rates comparable to those that would be obtained in the typical and somewhat softer spectrum of a large power reactor at the same fuel ratings.

3. The fuel inventory is determined only by the number of test assemblies required in the experiment.

4. The flexibility in core size allows generating experimental data at accelerated rates of heavy atom burnup.

5 . The frequency of adding or replacing fuel elements is determined only by the experimental requirements of these same test elements.

6. Finally, and most important, along with these low fuel loadings is the advantage of necding only a low-heat-removal system.

\section{Basic Core Plan and Test Conditions}

The FARET Core I reference design assumes that as the subassemblies are removed for inspection and destructive testing, they will be replaced with fresh subassemblies of the same design. This need not be the case. Improved fuel-element designs resulting from the continuing fast reactor fuel-development program ean be inserted into the core whenever available, replacing types showing a reduced performance 
TABLE 1. Fuel-element Data

\begin{tabular}{|c|c|c|c|}
\hline Fuel Material & $\mathrm{UO}_{\mathrm{z}}-15 \mathrm{w} / \mathrm{o} \mathrm{PuO}_{2}$ & $\mathrm{UC}-15 \mathrm{w} / \mathrm{o}$ & PuC Metal (b) \\
\hline Elements per Subassembly & 37 & 37 & 61 \\
\hline Fuel Neight, in. & 18 & 18 & 18 \\
\hline Clad Outside Dia, in. & 0.290 & 0.290 & 0.220 \\
\hline Clad Thickness, in. & 0.020 & $0.020^{(a)}$ & 0.016 \\
\hline Spacer Wire Dia, in. & 0.057 & 0.057 & 0.052 \\
\hline Axial Blanket Height (each end), in. & 6 & 6 & 6 \\
\hline Gas Reservoir, in. & 25 & 25 & 25 \\
\hline Element Height, in. & 55 & 55 & 55 \\
\hline Effertive Fuel Density, \% & 80 & 80 & 70 \\
\hline Bond Material & Flelium & Helium & Sodium \\
\hline Fuel U/Pu Atom Ratio & $6 / 1$ & $6 / 1$ & $5 / 1$ \\
\hline Fuel Jetails & Coprecipitated; O/M 1.97-2.00 & Single Phase; Solid Solution & - \\
\hline
\end{tabular}

(a) 0.015 in. in C4 subassemblies.

(b) U-15 w/o Pu-6.5 w/o Ti; U-15 w/o Pu-10 w/o $\mathrm{Zr}$.

capability. Further, should some originally fabricated designs prove to be of less interest in the early stages of the FARET experiment, they would be replaced by additional subassemblies of other designs having greater potential. Extra subassemblies of several types will be provided in the original fuel inventory. The physics of the core permits such flexibility.

The reference core will consist of carbide, metal, and oxide fuels and will test simultaneously ten different fuel element designs with good statistics. The fuel-element design data are presented in Table 1. The elements have a 6 -in. axial blanket at each end, with a 25-in. fission-gas reservoir to allow for 15 $a / 0$ burnup, if design and fuel performance permits. The ceramic fuels are helium gas-bonded. A conceptual instrumented fuel subassembly in illustrated in Fig. 1.

The fuel-element types and designs and the tentative test conditions are presented in Table 2. The elements are typed according to fuel material, fuel fabrication method, clad material, and thermal and power conditions. The reference core contains six mixed-oxide types, four mixed-carbide types, two metal alloy types, and three subassemblies for special experiments, if desired, making an overall total of 51 subassemblies. Two of the oxide designs will be run at two different linear heat-power ratings, and hence different maximum fuel and cladding temperatures. Each subassembly type is to be tested up to about 15 heavy a/o burnup, if design permits. The elements are to be destructively tested at predetermined intervals of burnup. The suggested periods of approximately $5,9,12$, and 14 or $15 \mathrm{a} / \mathrm{o}$ reflect the current confidence of the metallurgists that most of these elements will be able to achieve at least the first two burnup pcriods, say up to $9 \mathrm{a} / \mathrm{o}$. Also reflected in the frequency of inspection is the attempt to maximize full-power operating time. As will be subsequently discussed, other important constraints on the frequency of testing and irradiation intervals are the maintaining of criticality and the worth of the control system.

The core composition and configuration designed to approach the thermal conditions outlined are presented in Fig. 2 and Table 3 . The overall plan view of the core loading (sce Fig. 2) includes the placement of the control rods in the stainless steel reflector, and the core edge and reflector loops. The preliminary placement of some of the instrumented assemblies and the manner in which the cables may be laid out are also shown. The nomenclature, 056I, for a subassembly is to be read as 05 type oxide, removal at the end of the sixth irradiation period, instrumented.

Several conditions were considered in the positioning of the subassemblies. Symmetry was maintained to subject the elements of each type to similar power conditions throughout the experiment. The positioning was also influenced by the sequence of removal and replacement of the subassemblies. The instrumented assemblies are located so that the loading and unloading of other assemblies are not made cumbersome by having instrument leads located over subassemblies to be removed. For example, the instrument lead from the C4SI assembly is passed over subassemblies which are scheduled to be removed after the C4SI.

It is planned to remove the instrumented subassemblies in the latter stages of burnup for each type of element. This makes possible the continual recording of fuel and coolant temperature, and possibly of coolant flow rates, neutron flux, and fission gas release throughout most of the core life of each fuel element design.

In addition to measuring and recording engineering 

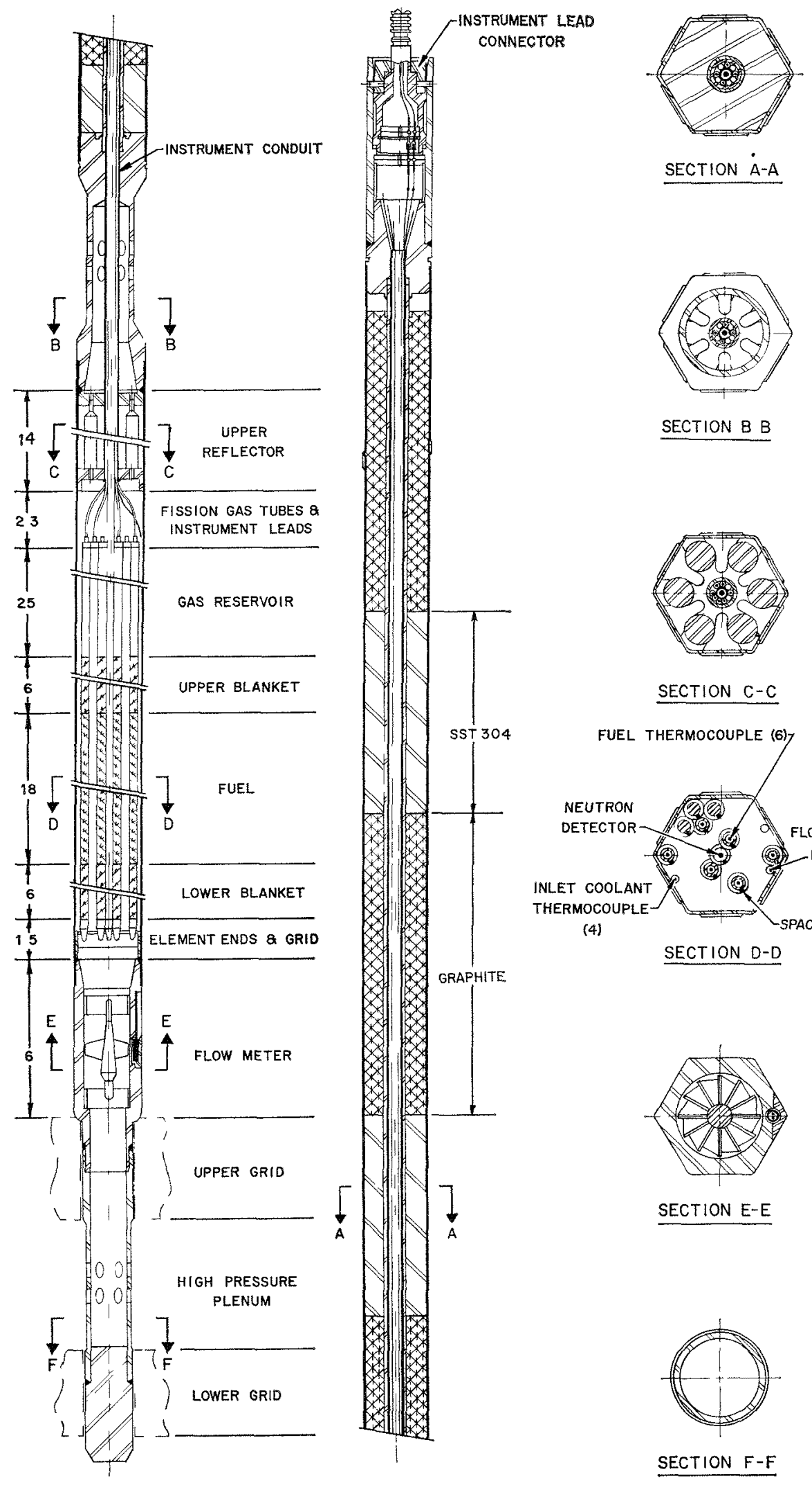

SECTION C-C

FUEL THERMOCOUPLE (6)
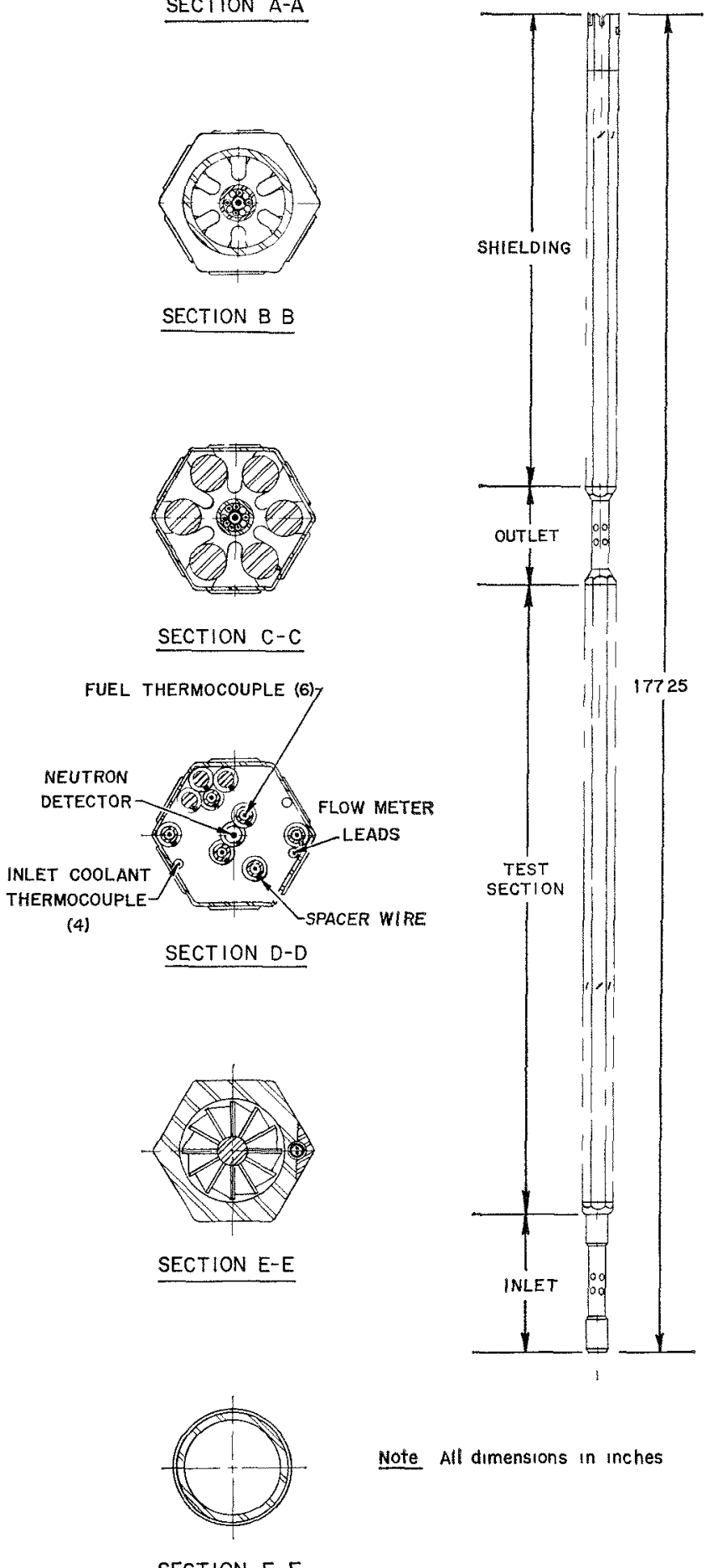

Note All dimensions in inches

FiG 1 Instrumented Core Subassembly 
TABLE 2. Subassembly Data

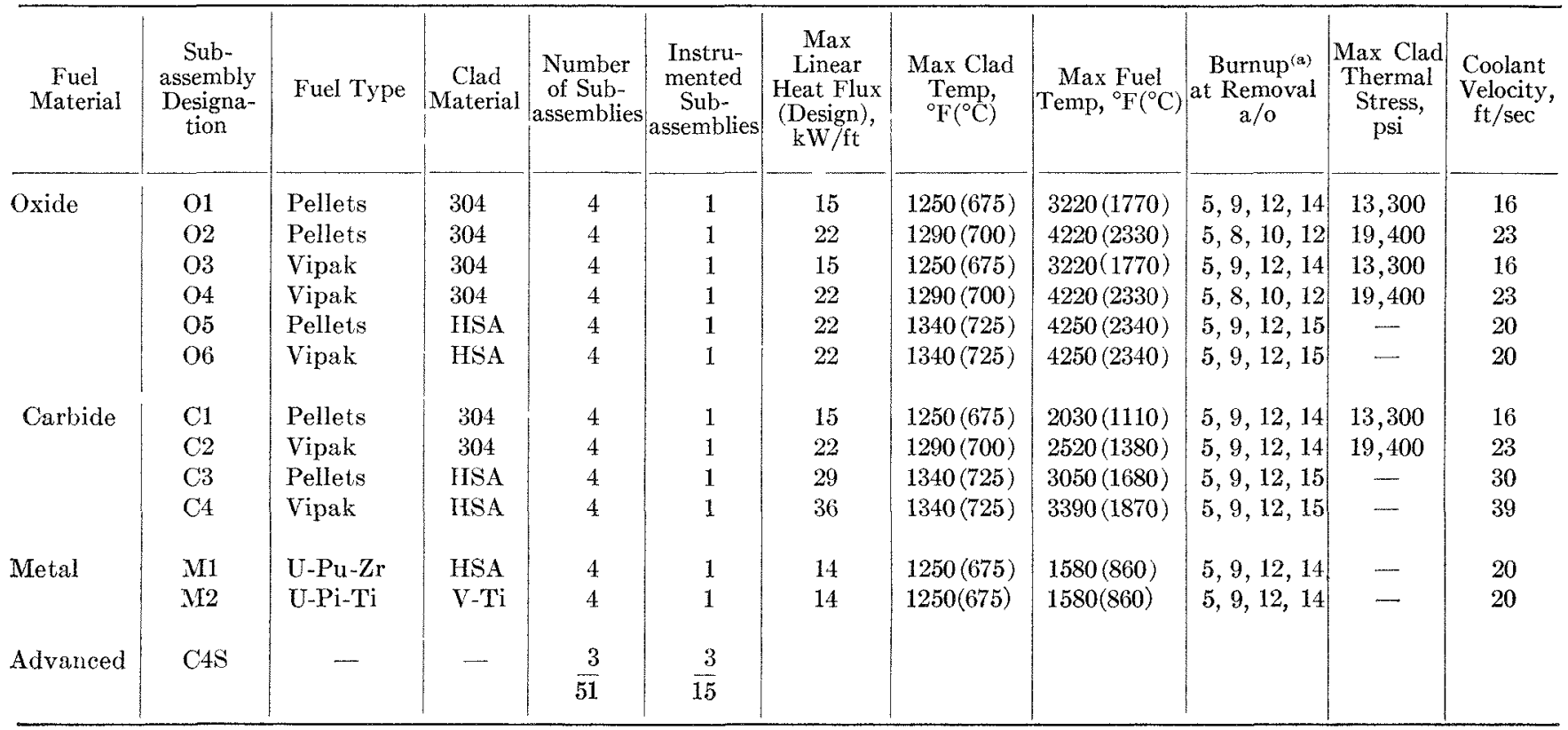

Notes: 1. Vipak means "vibratory compacted;" HSA means "high strength alloy," not necessarily the same for oxide as for carbide; etc.

2. Coolant inlet temp is $810^{\circ} \mathrm{F}$. Average outlet temp from each subassembly is $1050^{\circ} \mathrm{F}$. (Exception: outlet temp from 05 and 06 subassemblies is $1085^{\circ} \mathrm{F}$.) Axial peaking factor $=1.2$.

3. Max clad temp include both a 1.33 factor for flow and power maldistribution effects on coolant temp rise, and a $>1.10$ uncertainty factor. Max fuel temp include the 1.33 factor but no uncertainty factor.

(a) Approximate burnup intervals.

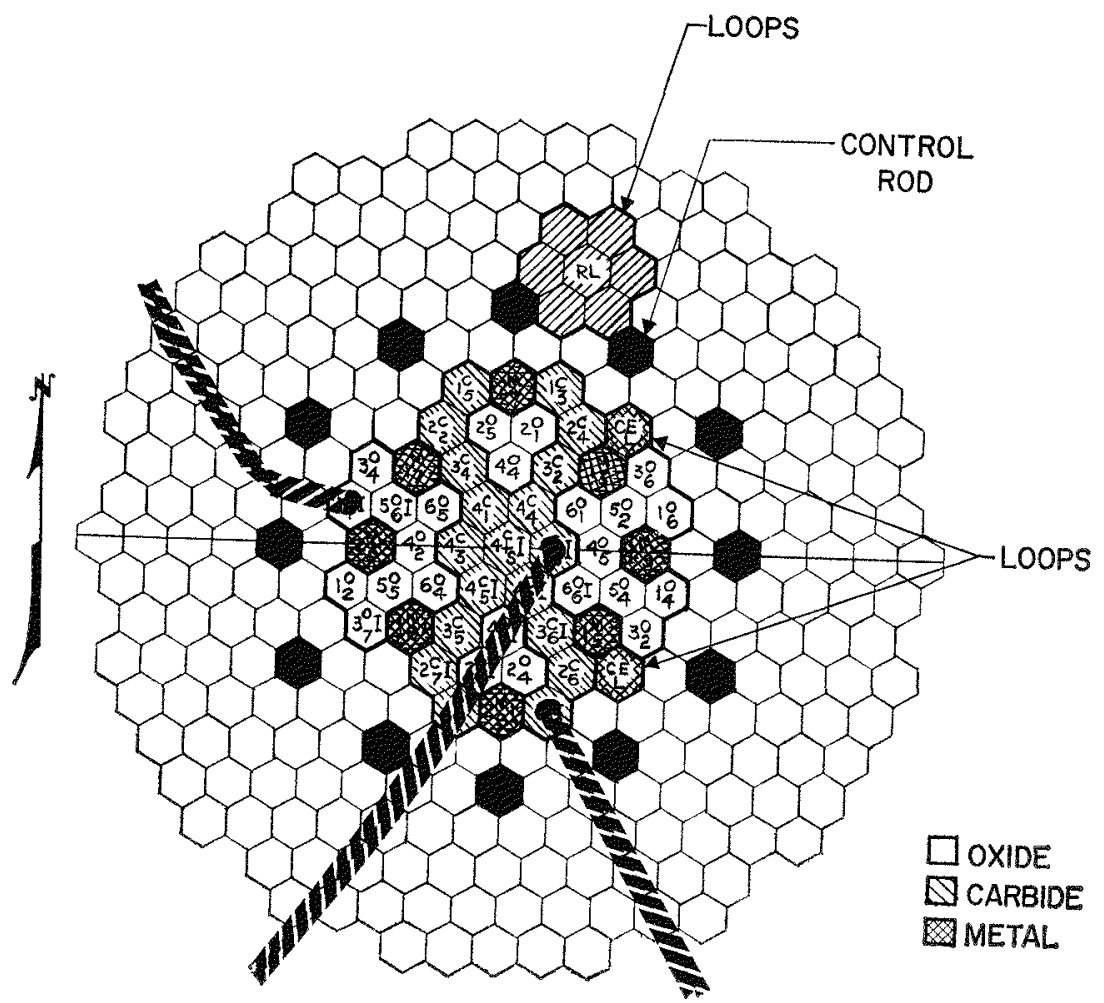

FIg, 2a FARET Core I. 
table 3. Perform ince Parameters of Faret Core I. Reactor Power: 50 MW( $\mathrm{t}$ )

Core Composition (\%): Ceramic Fuel 37/Sodium 40/Structure 22;

U/Pu Atom Ratio 6/1

Metallic Fuel 30/ Sodium 48/Structure 22;

$\mathrm{U} / \mathrm{Pu}$ Atom Ratio 5/1

Core Volume: $\quad 70$ Liters

Ileight: $45.7 \mathrm{~cm}$ (18 inches)

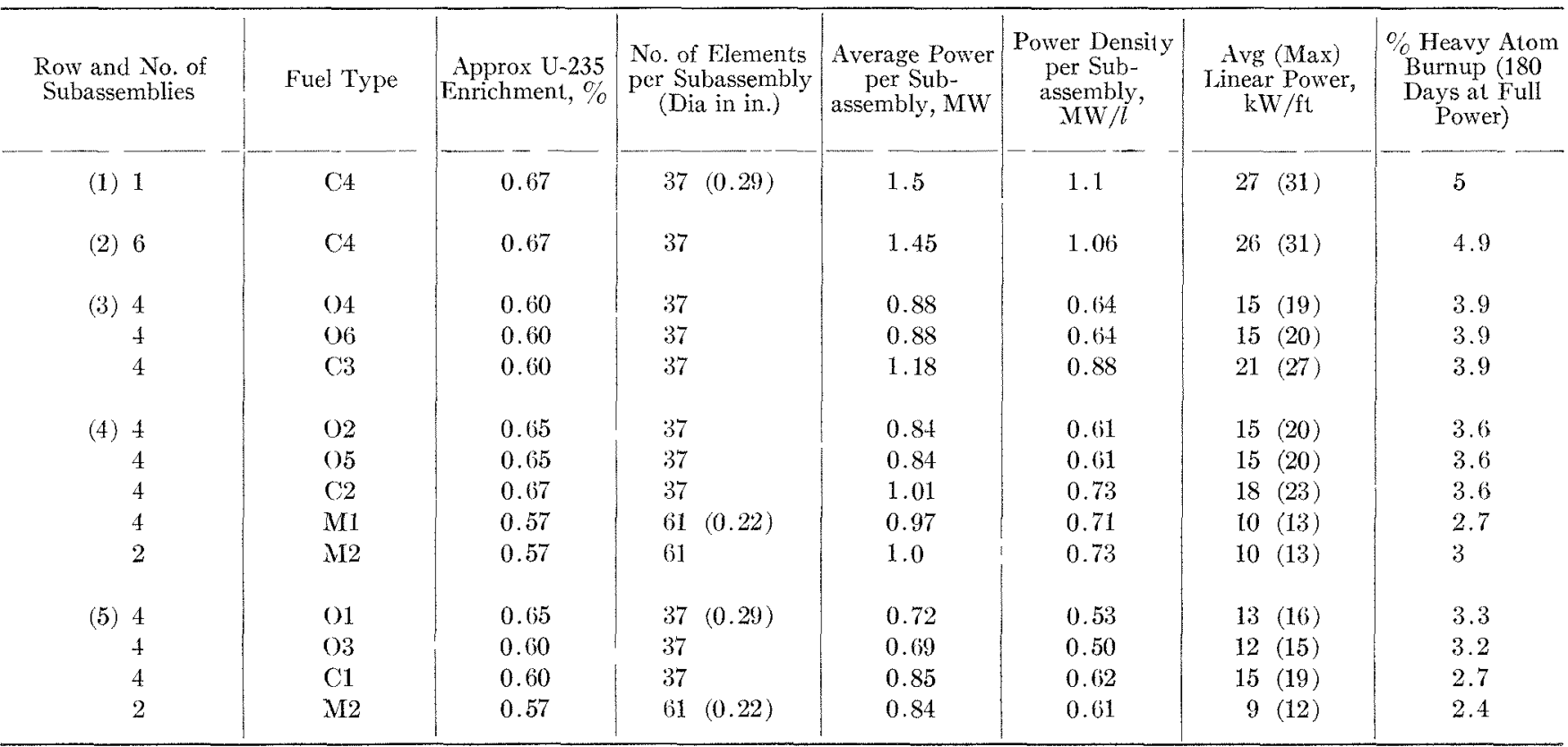

data, the instrumentation has now added a dimension of safety to the fuel-irradiation expcriment. Fuelelement failure can probably be anticipated by the continuous surveillance of these instrument readings.

In Table 3 are listed the enrichments and linear power rating for the loading shown in Fig. 2. A condition imposed on the obvious flexibility of enrichment was that the uranium enrichment be specified and held fixed for each method of fuel fabrication. Thus, the $\mathrm{C} 1$ and $\mathrm{C} 3$ pellets have the same enrichment, although the clad materials and thermal conditions are different.

The uranium enrichments also include the effect of control rods on the power-density distribution. The power density in the carbide $\mathrm{C} 1$ assembly may still be higher than that specified in the test conditions. If this is the case, the $\mathrm{C} 1$ elements may be moved. into the "corner" positions of Row 5. Computations indicate that about a $5 \%$ variation in the power densities can be expected between these two positions.

\section{Considerations in Planning the Experimental Pro- gram}

In planning the experimental program an important factor in establishing the sequence of fuel removal and replacement was the total worth of the control system. The optimum experiment with respect to time would be one in which the excess reactivity needed for the maximum burnup is accommodated by the initial fuel enrichment. The approach to the optimum experiment then is to design the control system with the maximum worth.

In Table 4 are listed the calculated nuclear and control paramcters for the reference core. The total neutron flux ranges from about 2 to $4 \times 10^{15} \mathrm{n} / \mathrm{cm}^{2}$-sec over the core. The percentage of total neutrons having energies above $1.35 \mathrm{MeV}$ at the center assembly and in the outer row are $22 \%$ and $13 \%$, respectively. As will be discussed later, the quality of the spectrum may be an important factor in the study of radiation damage to cladding material.

Also listed are the expansional reactivity coefficients. The signs are all negative, and the magnitudes are of the same order as those computed for similar sized but uniformly loaded cores. The total worth of the 12 boron carbide control rods are estimated to range between $7.5 \%$ and up to almost $10 \%$.

Included in Table 4 are the reactivity effects due to the expansion of coolant, structure, and fuel systems for the anticipated operational temperature change. If a range of excess reactivity of $3.5 \%$ to $6 \%$ for burnup, along with a $2 \%$ shutdown margin and the 


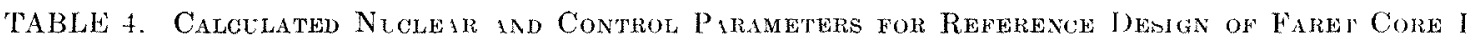

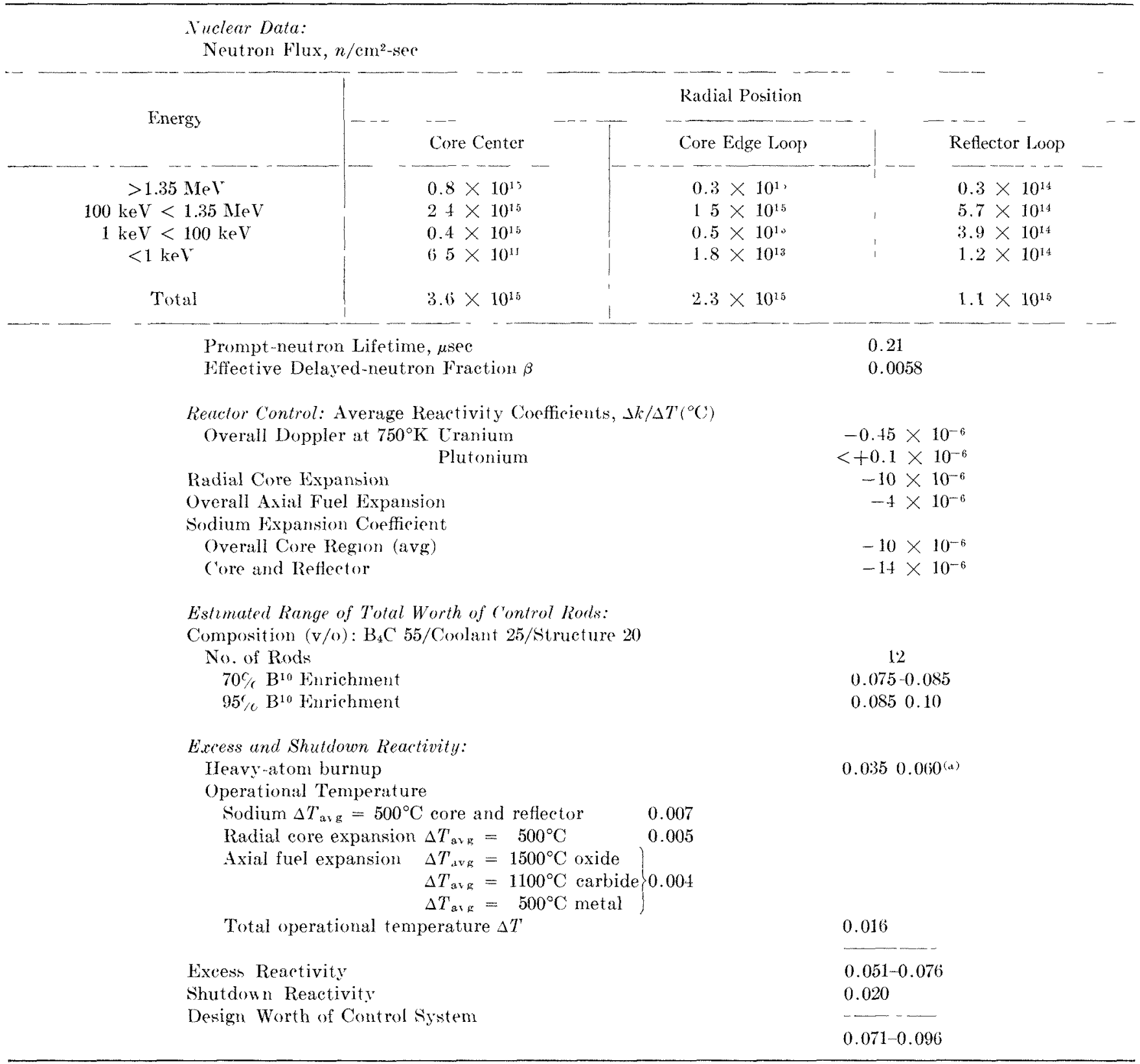

(a) Depending on the manner of compensating for burnup.

$1.6 \%$ margin for the operational temperature change, is taken, the required range of control of $7.1 \%$ to $9.6 \%$ is within the range of the computed worth of the control system.

\section{FARET Critical Experiments}

A series of FARET critical experiments in ZPR-3 was completed to firm up the physies analysis of multifueled, nonuniform core loadings. In Fig. 3 is presented the final core loading of the series which con- sisted of an inner region (first two rows) of oxidefueled assemblies followed by a row of carbide and a row of oxide assemblies, with the fifth row comprised of alternating alloy and oxide assemblies. The 93liter core had a height of $51 \mathrm{~cm}$. The $\mathrm{U}^{235}$ enrichment in the fuels ranged from $11 \%$ in the alloy to about $40 \%$ in the carbides and about $30 \%$ in the oxides.

Of primary interest was the establishment experimentally of the predicted control-rod reactivity worths of FARET core systems. Of equal importance was 


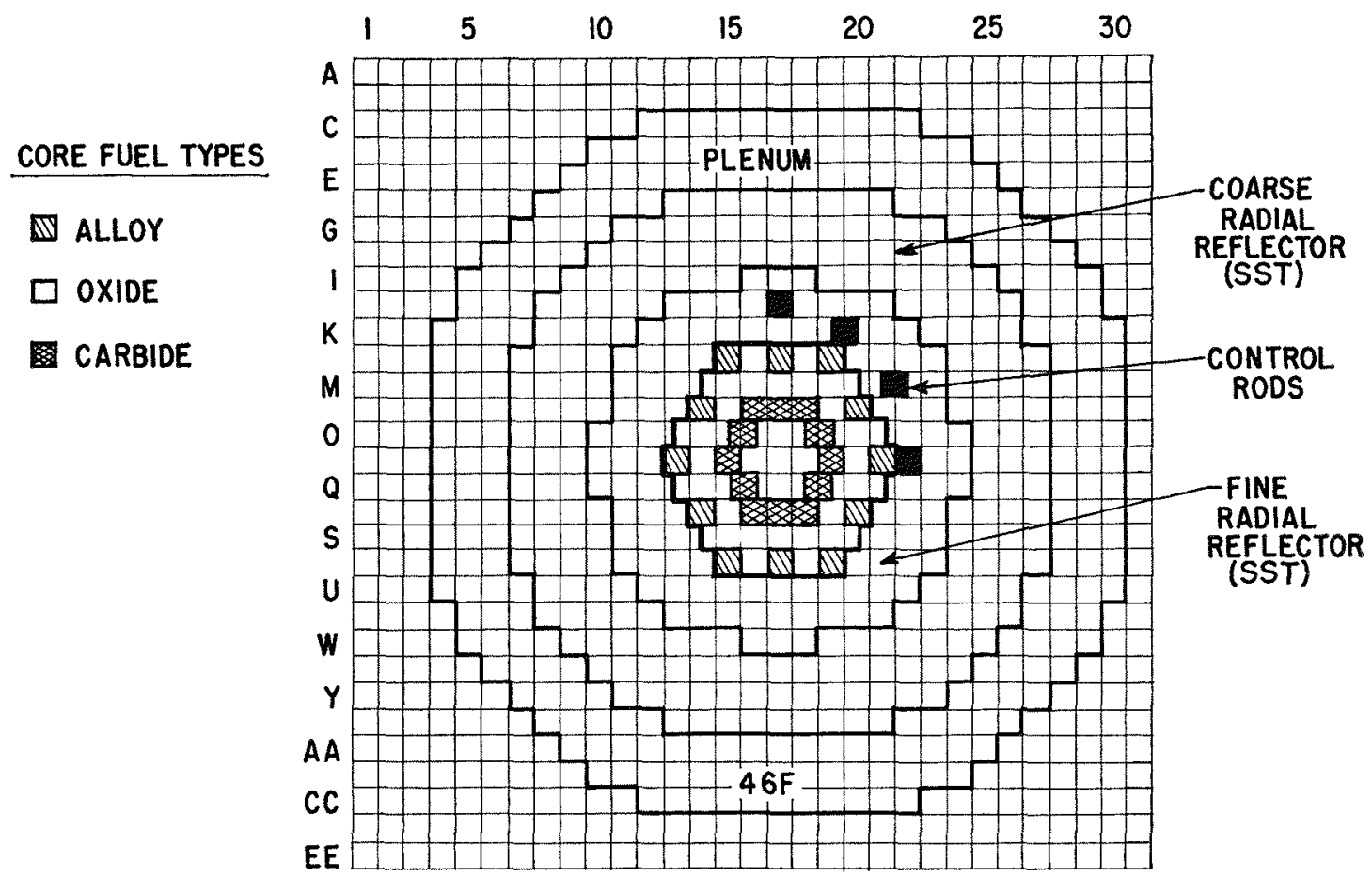

Fig. 3. ŻPR-3 FARET Critical Loading.

TABLE 5. WORIH OF CON IROL RODS IN TIIE FARET CRITICAL Experiments

Oxide-Carbide-Metal Loading $B^{10}$ Enrichment $\sim 70 \%$

\begin{tabular}{l|r|r|r|r}
\hline & \multicolumn{3}{|c}{ Reactivity } \\
\cline { 2 - 4 } & \multicolumn{2}{|c|}{ Experimental } & \multicolumn{2}{|c}{ Calculated } \\
& Inhours & $\Delta k$ & Inhours & $\Delta k$ \\
\hline P-22 & -425 & 0.0076 & -520 & 0.0093 \\
P-22, J-17 & -690 & 0.012 & -850 & 0.015 \\
All Rods (12) & -3705 & 0.067 & -4125 & 0.074 \\
\hline
\end{tabular}

the substantiation of the neutronic behavior of a mixed core when subassemblies of different type fucls are interchanged or replaced. Other completed measurements included the distributed worths of fuel and structural materials, reaction-rate traverses, and power periods.

The preliminary results of the experiments have been published. ${ }^{(7)}$ Some analytical results for the control-rod experiments in the nonuniform loading are summarized in Table 5. Banked-rods as well as singlerod experiments were performed to study the interaction effects. The preliminary values of the computations and the experiments compare to within $11 \%$ for the banked rods and to about $20 \%$ for the single and paired rods. The reasonable agreement establishes at least the range of control to be expected in the FARET cores.

In Table 6 , the experimental data on the substitution of fueled assemblies in cach of the rows are summarized and compared with the computed values. Except for the interchange between the alloy and oxide assemblies in Rows 4 and 5 , the computations agree to well within $10 \%$ of the experimental values.

TABLE 6. SLAASEMBLY REPLICEMENTS IN THE FARET CRITIC al Experiment

\begin{tabular}{l|c|c|c}
\hline \multicolumn{2}{c}{ Mixed Core (93 liters) } & \multicolumn{3}{c}{559 Inhours $=1 \% k$} \\
\hline $\begin{array}{c}\text { Replacement (1.5-liter } \\
\text { assembly) }\end{array}$ & Row & $\begin{array}{c}\text { Reactivity Change, } \\
\text { inhours }\end{array}$ \\
& & $\begin{array}{c}\text { Experi- } \\
\text { ment }\end{array}$ & $\begin{array}{c}\text { Calcula- } \\
\text { tions }\end{array}$ \\
\hline Carbide for Oxide & 1 & +396 & +375 \\
Alloy for Oxide & 1 & +214 & +195 \\
Carbide for Oxide & 2 & +382 & +370 \\
Alloy for Carbide & 3 & -150 & -154 \\
Carbide for Oxide & 4 & +264 & +237 \\
Alloy for Oxide & 4 & +140 & +114 \\
Oxide for Alloy & 5 & -84 & -66 \\
Alloy for Oxide & 5 & +82 & +63 \\
Oxide for Reflector & 6 & +150 & +148 \\
Carbide for Reflector & 6 & +272 & +248 \\
Reflector for I/2 Oxide & 5 & -214 & -234 \\
\hline
\end{tabular}




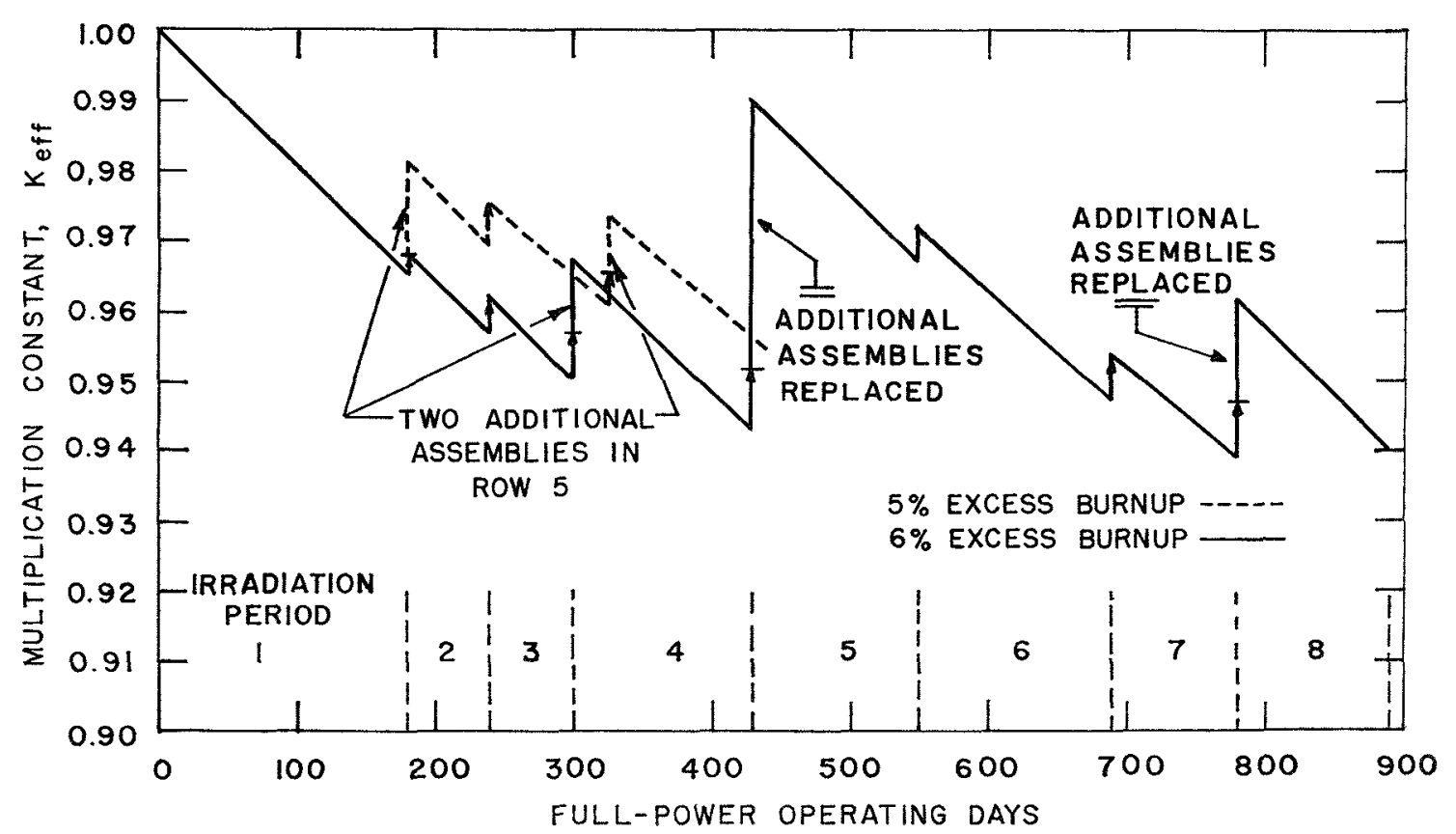

Fuc. 4. Iariation of Reactivity versus Fuel-irradiation Periods (1)ays) at Full Power for FARET ('ore-I Experiment.

The critical experiments are still in the process of being analyzed; however, the significanee that may bo attached to the preliminary results thus far is that the behavior of a mixed core loading can be understood as well as that of a uniform core loading.

The maximum allowable excess reactivity for burnup studies in the FARET core will be determined from the critical experiments on the inockup of the final design.

\section{The Experimental Program}

The uranium enrichments quoted in this paper include the $6 \%$ excess reactivity for burnup.

In Table 7 are presented the atom percent burnup, the irradiation periods, and the number of subassemblies removed and replaced. The carbide $\mathrm{C} 4$ fuel assembly is expected to reach a 5 a/o burnup in 180 full-power operating days. At this time, two of the higher-performance oxide assemblies would also be removed for investigation. These two types of ascemblies would then be allowed to go directly to about 9 $a / o$ burnup, if the investigation shows satisfactory performanee. An intermediate step, in which the other type of oxides have burned to about $5 \mathrm{a} / 0$, would require some 60 additional days.

After the third irradiation period $(305$ operating days), a reactivity loss of almost $5 \%$ is expected. If, at this time, two additional fuel assemblies are added in the fifth row, the reactivity loss could be reduced to $3.2 \%$. The excess reactivity would now allow an additional irradiation period of 125 days, at the end of which most of the fuel types would have incurred between 8 and $12 \mathrm{a} / \mathrm{o}$ burnup.

In Fig. 4 is plotted the reactivity as a function of operating days. The dashed curve is an alternative plan to illustrate the case where the control system allows less than $6 \%$ excess reactivity for burnup. A $5 \%$ excess reactivity would require a control system having a total worth of $8.6 \%$. The only difference between the two cases is the addition of two assemblies in the initial replacement step. The burnup interval becomes somewhat longer, so that the 8 to $12 \mathrm{a} / 0$ burnup would occur at about 460 days.

At the end of the 430-day operating period at full power, the performance of close to 1000 fuel elements would have been investigated at advanced thermal and burnup conditions. During this irradiation period, the fuel-pin cladding material would have been subjected to a total neutron exposure of $1.4 \times 10^{23} \mathrm{nvt}$. The exposure from neutrons above $1.35 \mathrm{MeV}$, that is, in the range of the $(n, p)$ and $(n, \alpha)$ threshold reactions, would have been about $3 \times 10^{22}$ nvt. These threshold reactions may prove to be a factor in limiting the life of the fuel cladding. In this ease, the harder neutron spectrum in FARET would subject the clad material to a dose rate comparable to that attained in the softer spectrum of a large reactor having a total flux of $10^{16} \mathrm{n} / \mathrm{cm}^{2}$-sec. In some power systems studied the percent neutrons above $1.35 \mathrm{MeV}$ ranges from 8 to 


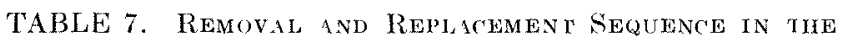
HXPeriventa Progrim for FARlet CORE I

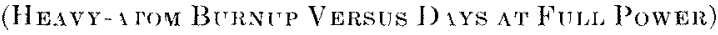

\begin{tabular}{|c|c|c|c|c|c|c|c|c|c|}
\hline \multirow{4}{*}{$\begin{array}{c}\text { Row and } \\
\text { Number of } \\
\text { Subassemblies }\end{array}$} & \multirow{4}{*}{ Fuel Type } & \multicolumn{8}{|c|}{ Irradiation Period (Days) } \\
\hline & & 1 & 2 & 3 & 4 & 5 & 6 & 7 & \multirow[t]{2}{*}{8} \\
\hline & & \multicolumn{7}{|c|}{ a/o Burnup } & \\
\hline & & 180 & 240 & 305 & 430 & 550 & 690 & 780 & 890 \\
\hline 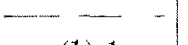 & $-\infty$ & 一 & - & - & - & - & - & - & - \\
\hline (1) 1 & $\mathrm{C} 4$ & & & & & & & & \\
\hline (2) 6 & $\mathrm{C} 4$ & 5 & & \multicolumn{2}{|c|}{8.512} & 15.2 & & & \\
\hline (3) 4 & 04 & & 5.2 & & 9.5 & 12 & 15 & & \\
\hline 1 & 06 & 3.9 & & & 9.5 & 12 & 15 & & \\
\hline 4 & $\mathrm{C} 3$ & & 5.2 & & 9.5 & 12 & 15 & & \\
\hline (4) 4 & $\mathrm{O} 2$ & 3.6 & & & 9 & 11 & 14.3 & & \\
\hline \pm & 05 & & 4.8 & & 9 & 11 & 14.3 & & \\
\hline \pm & $\mathrm{C} 2$ & & 4.4 & & 8 & & 12.5 & 14.3 & \\
\hline 4 & M1 & & & 4.6 & & 8.3 & & 12 & 15 \\
\hline 2 & M2 & & & 5 & & 9 & & & \\
\hline (5) 4 & 01 & & 4.4 & & 8 & & 12.5 & 14.3 & \\
\hline 4 & 03 & & 4.4 & & 8 & & 12.5 & 14.3 & \\
\hline 4 & $\mathrm{C} 1$ & & & 4.6 & & 8.3 & & 12 & 15 \\
\hline 2 & $\mathrm{M} 2$ & & & & & & & 10.5 & 14 \\
\hline \multicolumn{2}{|c|}{$\begin{array}{l}\text { No. of Subassemblies Re- } \\
\text { moved }\end{array}$} & 3 & 6 & 4 & 9 & 9 & 8 & 6 & 3 \\
\hline \multicolumn{2}{|c|}{$\begin{array}{l}\text { No. of Subassemblies Re- } \\
\text { placed }\end{array}$} & 3 & 6 & 4 & $15^{(\mathrm{a})}$ & 9 & 8 & $6^{(b)}$ & \\
\hline \multicolumn{2}{|c|}{$\begin{array}{l}\text { No. of Subabsemblies } \\
\text { Added in Row } 5\end{array}$} & & & 2 & & & & & \\
\hline
\end{tabular}

(a) Replace 6 Ct-Subassemblies or others as required to maintain desired reactivity for burnup berond $10 \mathrm{a} / \mathrm{o}$.

(b) Irradiated Subassemblies M1, M2, and $\mathrm{C} 1$ remaining may be moved into Row 3 .

$10 \%$ for ceramic- and metal-fueled reactors. This is to be compared with $22 \%$ in the FARET core.

In conclusion, the performance limits of a fuel-element design, including the effect of radiation on the clad, as determined in the FARET experiment, could then be assigned directly to a fuel-element design of a large power reactor with a high degree of confidence.

\section{REFERINCES}

1. P. J. Persiani, T. R. Bump, and W. J. Kann, $F A R E T$ ('ore $I$ Fuel Irradiation Program and Reference Design, ANI-7106 (Oct 1965).

2. W. R. Simmons, P. J. Persiani, and T. R. Bunp, Fast Reactor Test Factity (FARET) Initial Experimental Program, Am. Nucl. Soc. Fast Reactor Technology, National Topical Meeting, Detroit, Michigan, April 26-28, 1965, ANS 100.

3. A. Smaardyk, et al., Interim Report, FARET Experimental Program, ANL-6708 (April 1963).
4. N. I. Peterson, Isotope Erffect in Self-diffusion in Palladum, Phys. Rev. 136, A568 (1964).

5. F. K. IIs de, I. Perlman, and (:. T. Seakorg, The Nuclear Properacs of the Heary Elements, Prentice-Hall, Ine, New Jersey (1964), Vol. III, 4.

6. I. Burris, Jr., and I. ( X. Dillon, Estimation of Fission Produce Specira in Discharged Fuel from Fast Reartors, Nucl. Sci. Eng., 2, No. 5, 567-581 (Sept 1957). See also ANL-5742 (Dec. 1957).

7. Argonne National Jaboratory Reactor Development Program Progress Reports: ANL-7017, Feb., 19;5; ANL-7028, Mar. 1965; ANI-7045, April 1965; ANL-7046, May 1965; ANL-7071, June 1965; ANL-7082, July 1965; ANL-7090, August 1965.

\section{Discussion}

Mr. Zebroski (GE): Will the core and the system be designed to permit accommodating vented fuel? I would like to comment, if it doesn't detract from the very fine overall design picture, that the linear powers and central temperatures given for the oxide fuel seem inconsistent to me. I think, for example, the vibratory compacted fuel showed similar linear powers and central temperatures, and there should be something like a $15 \%$ penalty in conductivity on the vibratory compacted powder if it behaves as it does in "watercooled reactors. Also, the central temperatures seem generally low. Also, can you take vented fuel?

Mr. Persiani: Yes, we have tentatively set up some loop-type experiments. We also have considered allowing certain assemblies to vent directly from the element. We can do pin-element experiments and rent these directly out of the reactor system. If we decide to vent into the coolant, we might use a loop in the first phase of the experiment.

Ir. Kittel (ANL) : I'm not sure what Mr. Zebroski is getting at about the penalty for vibratory compacted fuel. In the FARET Core-I design, both the pellet fuel and the vibratory compacted fuel have the same smear density, so the amount of oxide material per inch of fuel rod length is the same in either case. The penalty that is paid for loss of thermal conductivity in the compacted powder lasts, we think, for only a rather short time until sintering begins to occur. 'This has been borne out at other sites working with $\mathrm{UO}_{2}$.

Mr. Bump (ANL): In the written paper it is pointed out that fucl temperatures don't include any uncertainty factors. The reason is that today I'm not quite sure what I want to use; but our final design will have some sort of uncertainty factor. Some changes from the Table presented will result. I could say that I am doing the same as General Electric. I am assuming that the oxide forms a central void, and I'm assuming the roid eventually will represent the total amount of void originally included. In other words, if the fuel goes out against the clad and is es- 
sentially $100 \%$ dense, all the original void occurs in the central hole. As a result you get a savings on central temperature from the geometrical effect.

Mr. Zebroski: I think you should. There are some data which indicate that even after the sintering process has occurred, there is still a systematic penalty you will pay for the powdered fuel, even at the same smear density.

Mr. Chernick (BNL): Could somebody from Argonne explain the philosophy on fuel failures? How many do you think you're going to have during the 900-day operating period? Do you expect to monitor flux? What are you going to do to replace the failed element? To what extent are you going to depend on calculations for this and to what extent on instrumentation?

Mr. Persiani: First I want to say that there is a fuel-irradiation program. Information will be fed back into the fuel system considered for FARET. These fuel pins in FARET will not be untested systems. They will probably have been irradiated in single elements in the EBR-II program. The goals listed were tentative conditions, incidentally. We expect to integrate into this program new ideas on fuels as well as modifications if we find, between now and two years from now when we have to firm up the core, that some of these elements are not performing properly. If not out they go. The frequency of failures to be expected is difficult to predict. We will monitor fuel behavior destructively. A lot of the elements will be instrumented to measure temperatures and perhaps other parameters. We would have fuel-element detection systems like those at EBR-II, or better.

Mr. Chernick: I assume that you're going to pursue these to the extent that you would expect some failure, not catastrophic failure, but some. When you get your first failure and have to remove your first bundle, what is your design philosophy at this noint? Do you stop and do the criticals over again? Do you check your calculations? Do you do a flux scan? To what extent do you depend on your calculations that you've done to date? Will you move fucl elements around?

Mr. Persiani: No, these will be replaced. First of all, we hope to minimize any failed fuel elements by removing them before they fail. We have a constant inspection scheme where we feed back this information into the experiment. If we are not satisfied with the performance of a particular type of fuel element, out it goes and in go the new fuel elements that have performed well up until that day. We will have a fuel inventory, other than the core loading shown. The flux distribution may vary a little, but this is a reactor with large neutron mean free paths. And little trouble is expected in predicting and maintaining flux distributions.

Mr. Leitz (GE): I'd like to shift attention to the positive side. I think a very good point has been made relative to the utility of small fast fueled test reactors. Their deficiency in flux can be compensated for in the case of the technique of enriching fuel and in the case of the clad. The test reactors tend to have a harder spectrum than the fast power reactors, at least those that will be fueled by coramic fuels. I'd like also to observe that both of those principles could be used now in available fast reactors, namely, EBRII, Fermi, and of course they are in use at Dounreay.

Mr. Persiani: Yes. except that FARET has the feature of instrumenting the fuel elements. (This is engineering data that has not been heretofore available.) We hope to be able to test more than 1000 fuel pins, and that should provide good statistics. 


\title{
Transient Response of Coupled Fast Reactor Cores
}

\author{
W. E. Gunson, F. M. Heck, and R. S. Daleas \\ Westinghouse Electric Corporation \\ Atomic Power Division \\ Pittsburgh, Pennsylvania \\ (PRESHNTED BY W. F. (GUNSON)
}

\section{Srmbol TABLE}

C Precursor concentration

$k_{\imath i}$ Internal reactivity

$k_{i j}$ Cross coupling reactivity

$l_{\imath}$ Internal lifetime

$l_{\imath}$ Cross coupling lifetime

$\&$ Laplace Transform dummy variable

$S$ Neutron source

$t$ Time

$T$ Temperature

$\beta$ Delayed-neutron fraction

$\gamma$ Delayed-neutron worth

$r$ Delayed-neutron time constant

\section{Introduction}

The reactor core presented by Westinghouse at the Argonne National Taboratory panel diseussion on fast reactor safety ${ }^{(1)}$ and in the Westinghouse-ALC 1000 MWe FBR Design Study ${ }^{(2)}$ is comprised of seven identical hexagonal modules (see Fig. 1). Wach module is composed of a central region of fuel with axial blankets and an annular radial blanket which in turn is surrounded by a barrier of graphite. A detailed description of the core is given in reference (2).

The arrangement of the core results in a small amount of neutronic coupling between the modulos. In the steady state, the leakage of neutrons into each module from the adjacent modules just balances the fraction by which the module would be subcritical if it were out of the array. The dynamic behavior of each module is also affected via the cross coupling with its neighboring modules. Because of this effect, a reactivity insertion into one module is divided among the other modules by some function of the neutronic coupling. The effect of the neutronic cross coupling on the dynamic behavior of a perturbed module was erident in the experiments conducted at Los Alamos ${ }^{(3,4)}$ with two loosely coupled KIWI-4B reactors. In these pulsed-power experiments, the driver core was closely followed at a lower power level by the passive core. No neutronic instabilities were encountered in the system.

The theory for neutronically coupled systems has received considerable attention during the past several years for clustered nuclear rockets. The intent of thi, paper is not to conclude which of the mathematical models, that is, the one employing neutron lifetimes, (i) time delays, ${ }^{(6)}$ or neutron waves, ${ }^{(7)}$ is correct, but to present analytical results and trends obtained from one model with regards to the ability of neutronic coupling apparently to enhance the safety characteristics of the modular reactor presented in references (1) and (2). The safety chararteristies of the modular core based on an isolated module using the Westinghouse EORE computer code are given in reference (1). A simplified study of the neutronic coupling effect on the safety (haracteristics of the modular core in given in reference (2).

Two computer codes are being developed at Wontinghouse in order to study the modular system. Both codes use point kineties with six groups of delayed neutrons. The first code uses the mathematical model of Avery ${ }^{(5)}$ and is capable of handling nineteen coupled cores. The serond code handles two cores and is a simplification of Avery's model. In this model, the crosswcoupling lifetimes are made equal to the internal lifetimes. Both codes consider Doppler feedback and are capable of handling sinusoidal and ramp reactivity insertions. In addition, the first code has provisions for scramming the reactor as well as for cousidering other feedback mechanims (due to clad, coolant, and fuel).

\section{The Kinetics Model}

The kinetics model of Avery ${ }^{(5)}$ was used in developing the computer rodes at Westinghouse. This basic model has several rirtues. It is conceptually simple. The equations are similar in form to the single-core kineties equations. The physical quantities required an be determined without recourse to transient methods.

As a brief introduction to the Avery model, consider' a two-core problem and neglect the delayed neutrons. Divide the total rate at which neutrons are generated in each reactor into two parts. Let one part represent the neutrons resulting from neutrons produced in the same reactor (one generation earlier) and the other represent the neutrons resulting from neutrons produced in the other reactor (one generation earlier). 
Let

$S_{1}(t)=$ total neutrons sec produced in reactor 1 ;

$S_{2}(t)=$ total neutrons, sec produced in reactor 2 .

Then

$S_{1}(t)=S_{11}(t)+S_{12}(t)$

$S_{2}(t)=S_{21}(t)+S_{22}(t)$

where $S_{12}(t)$ is that portion of the total neutrons/sec being produced in reactor 1 by neutrons born in reactor 2 in the previous generation. The other $S_{2 j}$ 's have a similar definition.

Now, if $S_{11}(t)$ neutrons second are produced at time $t$ from neutrons originally produced in reactor 1 , what is the magnitude of $S_{11}\left(t+t_{g}\right)$, where $t_{g}$ is one promptneutron generation time? Neglecting second-order terms, we may write $M_{1}^{11}-S_{11}(t)$, where $M_{1}^{11}$ is the singlegeneration multiplication factor for a neutron source having the distribution of $S_{11}$ producing new fission neutrons in reactor 1 . But this is not sufficient. There is also a nout ron source in reactor 1 at time $t$, identified as $S_{12}(t)$, which has a different spacial distribution than $S_{11}$, since it is the first-generation source resulting from the sources in reactor 2 . The quantity which we require is the source, with the same spacial distribution as $S_{11}$, which results from the previous generation of $S_{12}$, since we wish to treat them henceforth an $S_{11}$ neutrons.

The $S_{12}$ source does not produce a new source in one generation which has the spacial distribution of $S_{11}$. Hence, the simple splitting of the total source into $S_{11}$ and $S_{12}$ components is valid only in the steady state. In the transient case there are a series of sources in reactor 1 , representing successive generations of neutrons from previous $S_{12}$-type sources. Each of these has a slightly different multiplication factor and a slightly different generation time. Thus the assumption that the source of neutrons can be divided into two, and only two, components is not ralid.

This has been verified by attempting to treat two parts of a single large thermal core as a pair of coupled reactors with very high coupling. For the purpose of studying coupled modular cores where the degree of coupling is small, the inaceuracy arising from this source may be tolerated.

Of course, if this problem were unique to this one approach to coupled-core kinetics, then this approach would be abandoned. Cnfortunately, this is a fundamental problem associated with any attempt to portray coupled cores in which separability of time and space is assumed. Time and space are not separable in the coupled-core problem. There are problems with convergence of very loosely coupled regions in static calculations; hence, one may expect considerable difficulty in achieving successful kinetic calculations, even with adequate funds and computing equipment. Therefore, it does not seem practical at this time to attempt to perform spacial kinetic calculations.

The multiple coupled-core kinetic equations solved in the computer program are in the form proposed by Avery:

$$
\begin{aligned}
l_{\jmath k} \frac{\partial S_{\jmath k}}{\partial t} & =k_{\jmath k}\left(1-\beta^{*}\right) \sum_{m=1}^{N} S_{k m}-S_{\jmath k}+k_{\jmath k} \sum_{i=1}^{M} \tau_{i} C_{i} \gamma_{i} ; \\
\frac{\partial C_{k i}}{\partial t} & =\beta_{i} \sum_{m=1}^{N} S_{k m}=\tau_{\imath} C_{k i} ; \\
\beta^{*} & =\sum_{i=1}^{M} \beta_{\imath} \gamma_{i} ; \quad S_{\jmath}=\sum_{m=1}^{N} S_{\jmath m} .
\end{aligned}
$$

The symbols and equations are those used by Avery with the exception of the introduction of the delayedneutron worth values, $\gamma_{2}$.

The shift from the $S_{12}$ mode to the $S_{11}$ mode can be handled by several approaches. One is to ignore the fact that an $S_{12}$ source distribution does not become an $S_{11}$ distribution within one prompt generation time and simply treat all fissions resulting from $S_{12}$ as being in the $S_{11}$ distribution without any importance weighting. This overestimates the change in $S_{1}$.

A second approach is to importance weight $S_{12}$ and assume that all fissions resulting from the importanceweighted $S_{12}$ are in the $S_{11}$ distribution. The source arising from the importance-weighted $S_{12}$ distribution is the correct asymptotic $S_{11}$ distribution; therefore this is nearly correct. Since the approximate time to reach the asymptotic value is actually several prompt-neutron generations, this slightly underestimates the change in $S_{1}$. A third possibility is to permit the $S_{12^{-}}$type source to reproduce itself partially as an $S_{12}$-type source and partially as an $S_{11}$-type source.

These possibilities represent progressively more complex systems of equations, and longer running times for computer solutions. Our initial program to investigate coupled-core kinetics uses the first (simplest) type of representation. The results will eventually permit an estimate of the magnitude of the errors and a decision as to the advisability of introducing further complexity for multiple coupled-core analyses.

\section{The Computer Program}

In addition to the neutron kinetics equations, simplified heat transfer equations are included. The differential equations are handled in the program as simple forward difference equation.

The program will accept up to 19 distinet regions or modules. Reactivity insertions may be made independently in any or all regions, and may have the form of continuous ramp changes, terminated ramps, or sine waves. Temperature-dependent feedbacks in linear form are provided for coolant, clad, and fuel tempera- 
tures. Temperature feedback is provided for the Doppler effert based on the average fuel temperature, with the Doppler effect having a $1 / T$ variation.

Provision is made for the insertion of control rods (seram) in several modes. Control-rod seram maty be initiated by total power or by period from any region. Seram may then be initjated either in all regions or only in the region from which the scram signal originated. Once a scram is initiated, a time delay is provided before actual insertion occurs. The insertion is in the form of a terminated negative ramp.

Initial total power in thermal megawat ts is a seyrate input for each region. In order to insure corred initial conditions, the program assumes that the $k_{z q}$ values are absolute, and that the cross-coupling $k$ 's are correct relative to each other, and are adjusted if required for steady state. Provision is provided for up to seven delayed-neutron groups.

A time-step size is input. The program will print out results when ever the power has changed by $5 \%$ or after 10 time steps have gone by without a printout. The program treats the input time step as a trial value and continuously recomputes a time-step size to satisfy input accuracy reriteria for the neutron kinetic equations.

\section{Neutronic Cross-coupling Analysis}

In checking the solution given by the computer code, insight into the behavior of two coupled cores as described by the Avery model was obtained by linearizing and simplifying the equations for analytic solutions. The details of this analysis are given in Appendix A. The assumptions made to obtain solutions are as follows:

1. Reactivity is held constant after zero time.

2. Temperature-dependent reartivity feedbarks are set to zero.

3. A two-core system is used.

4. One group of delayed neutrons is employed.

5. All neutron lifetimes, as well as internal and crows 'coupling, are equal.

6. In the cross-roupling effeet, the delayed neutrons are neglected.

7. $k \beta \simeq \beta$.

The basic equations are 1 through 6 in Appendix $\Lambda$. Applying the above assumptions, one obtains equations 9 through 13, which provide solutions of the form

$$
S_{\imath}(t)=\sum_{m=1}^{4} A_{m \imath} e^{B_{n n} t} .
$$

In a real two-core system, it would be expected that the core with a rearetivity insertion would pull the second (passive) core along with it and simultaneously tend to be restrained somewhat by the passive rore. This results from a change in the ratio of neutron leakage to the total neutron production in each of the two cores. Thi neutron cross-coupling effect ran be interpreted as a reartivity sharing effert. This effect an be illustrated by rewriting equations (9) and (10):

$$
\begin{aligned}
& \frac{d S_{1}}{d t}=\left[\frac{k_{11}-1-\beta+\left(k_{12} \frac{S_{2}}{S_{1}}\right)}{l}\right] S_{1}+\frac{k_{11}}{l} \tau C_{1} ; \\
& \frac{d S_{2}}{d t}=\left[\frac{k_{22}-1-\beta+\left(k_{21} \frac{S_{1}}{S_{2}}\right)}{l}\right] S_{2}+\frac{k_{22}}{l} \tau C_{2},
\end{aligned}
$$

that is, the cross-coupling term can be interpreted as a reactivity effect which is directly proportional to the ratio of the powers in the two regions. For example, if $k_{11}$ is increased such that at some point in time $S_{1}=$ $1.1 S_{2}$, then the effective reactivity in core one is reduced by $k_{12}[1-(1,1.1)]$, and the reactivity in core two is increased by list $(1.1-1)$.

One result of the reactivity sharing effect is that there is no clear definition of the meaning of reactivity worth for an insertion, a control rod, or a fuel assembly in a modular system. The effective worth to a module of an insertion in one module out of 1 wo, for example, lies somewhere between the worth calculated as if that module were isolated and one-half of that value, depending upon the neutronic aross coupling and the relative power in the second (passive) module. This reactivity sharing phenomena will be illustrated in the following analyses. The neutronic cross-coupling effect on the dynamic behavior of one perturbed module out of two was evident in the pulsed-power experiments conducted at Los Alamos on the KIWI-4B reactor. The driver core was closely followed by the passive rore even with 0.8 cents of neutronic cross coupling.

\section{Analyses Performed}

The importance of the neutronic coupling to the reactivity sharing effect between coupled cores was indieated in the analytical solutions (see Appendix A) derived to obtain an insight into the behavior of the kinctic equations. In addition, if was also considered that the coupling neutron lifetime may be an important parameter in determining the power split in a coupledcore system. To investigate these two parameters, a reactor system was selected which ronsisted basically of two modules of the modular core depicted in Fig. 1. The cross-coupling reactirity and cross-coupling lifetime were raried by an order of magnitude for a large and a small finite ranu and sine wave reactivity insertions into one core. $A$ Doppler effect, $T(d K / d t)$, of 
-0.00383 was the only reactivity feedback included in the parametric study. Table 1 gives the selected combinations of coupling coefficients, neutron lifetimes, and reactivity insertions.
The $0.4 \%$ coupling reactivity, $k_{12}$ and $k_{21}$, represents beginning-of-life modules (see ref. 1), with depleted fuel in the radial blanket. The $4 \%$ coupling reactivity is approximately twice the coupling which would exist

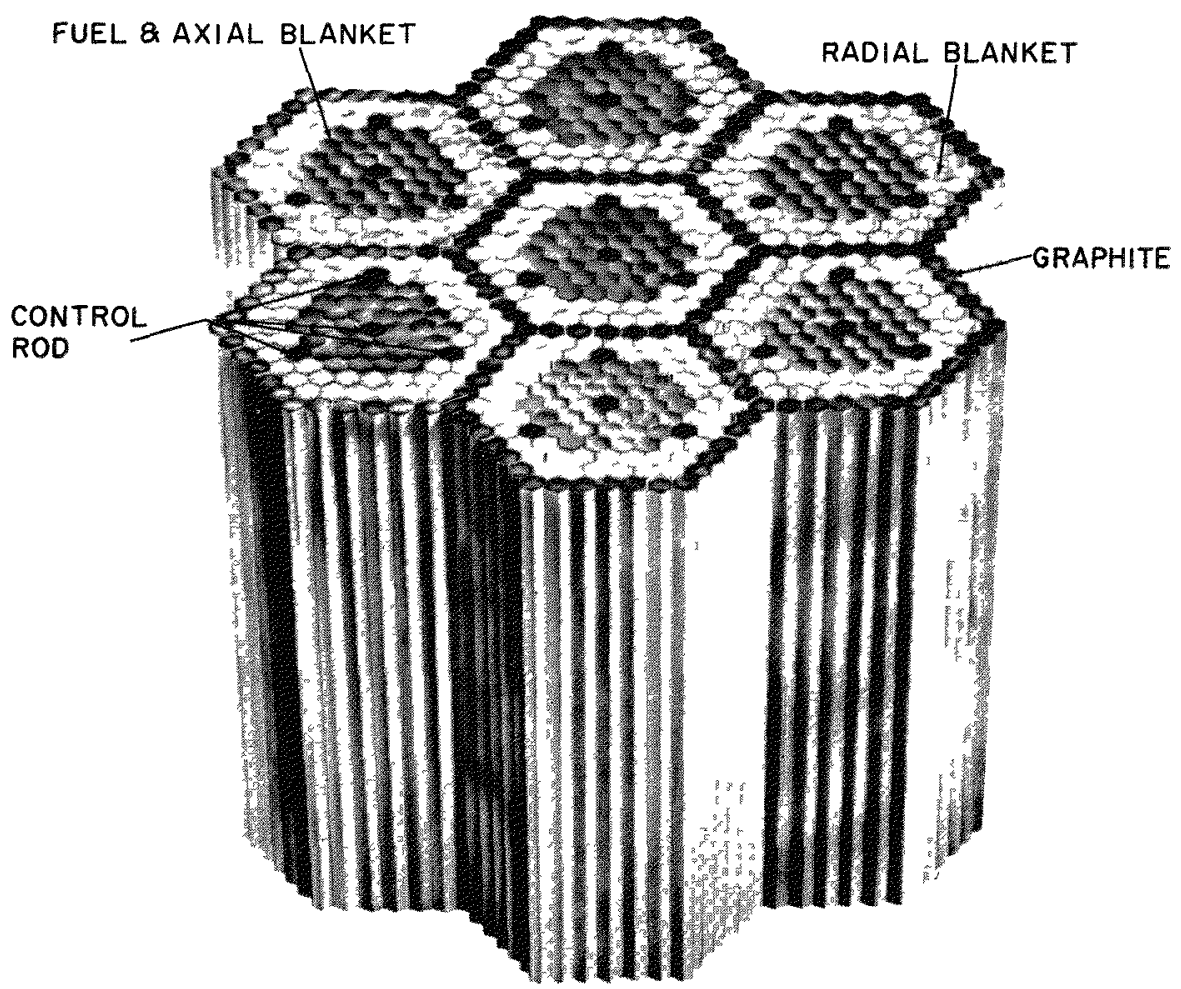

Fiq. 1. Modular Core Array.

TABle 1. Parametric Stldy: Combination of Coefficients Representing Two Loosely Coupled Cores Doppler $T(d K / d T)=-0.00383$

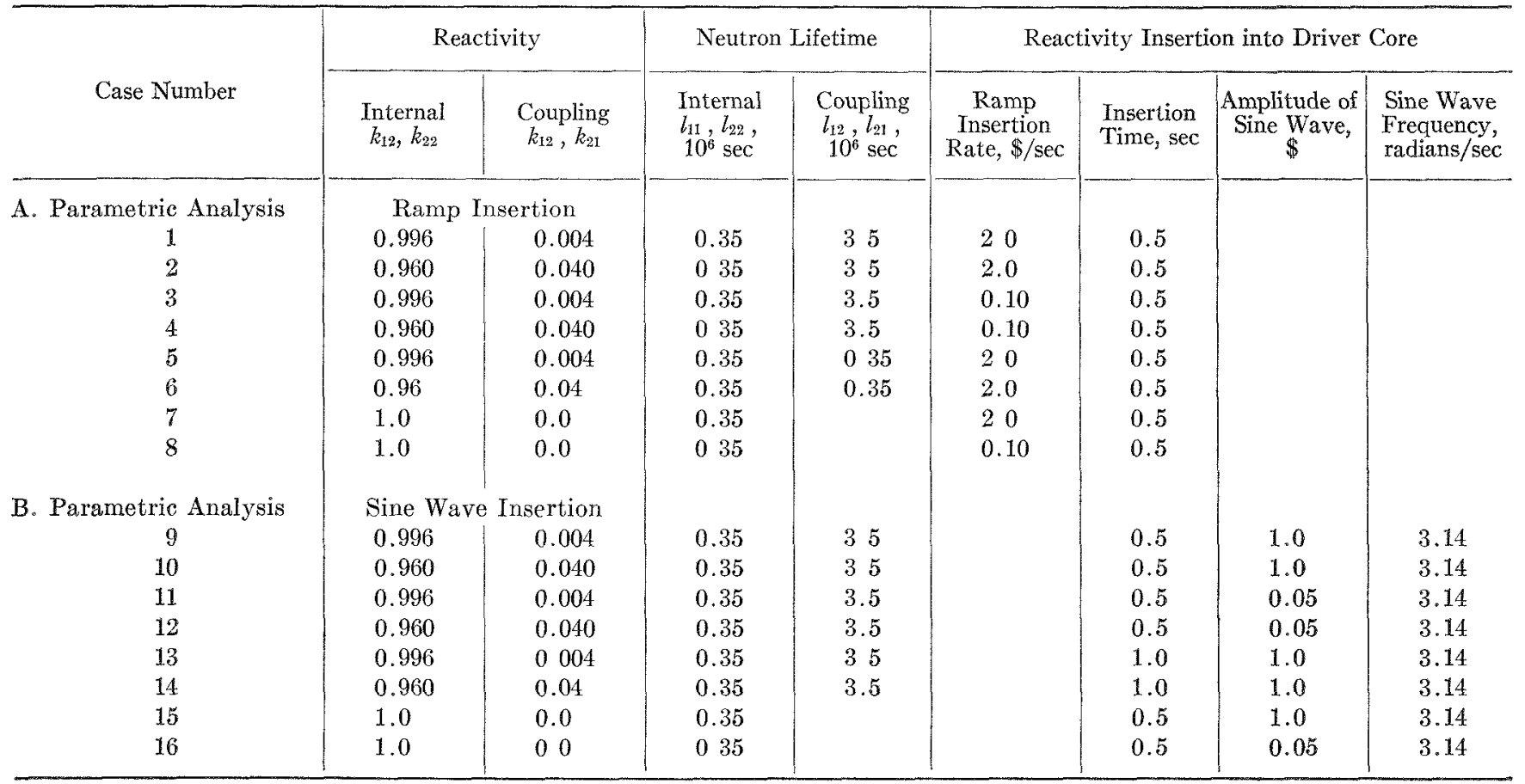


Table 2. Combination of Coefficients for Two loosely Coupled Carbide-fueled Cores with $3 \%$ Plutonium in the Radial Blanket

$$
\begin{aligned}
& k_{11}=k_{12}=0.9783 \\
& k_{12}=k_{21}=0.0217 \\
& l_{11}=l_{22}=0.3325 \times 10^{-6} \mathrm{sec} \\
& l_{12}=l_{21}=3.524 \times 10^{-6} \mathrm{sec}
\end{aligned}
$$

\begin{tabular}{|c|c|c|c|c|c|c|}
\hline & \multicolumn{2}{|c|}{ Reactivity Feedbacks } & \multicolumn{4}{|c|}{ Ramp Reactivity Insertion } \\
\hline & $\frac{\text { Doppler }}{T \frac{d K}{d T}}$ & $\frac{\text { Axial Expansion }}{\frac{d K}{d T} \times 10^{6}{ }^{\circ} \mathrm{F}}$ & \multicolumn{2}{|c|}{ Driver Core } & \multicolumn{2}{|c|}{ Passive Core } \\
\hline $\begin{array}{l}\text { 1. Inherent Stability } \\
\text { a. One core perturbed } \\
\text { b. Both cores perturbed } \\
\text { c. Both cores perturbed }\end{array}$ & $\begin{array}{l}-0.00338 \\
-0.00338 \\
-0.00338\end{array}$ & $\begin{array}{l}0.0 \\
0.0 \\
-3.896\end{array}$ & $\begin{array}{l}0.10 \\
0.10 \\
0.10\end{array}$ & $\begin{array}{l}1.0 \\
1.0 \\
1.0\end{array}$ & $\begin{array}{l}0.0 \\
0.10 \\
0.10\end{array}$ & $\begin{array}{l}0.0 \\
1.0 \\
1.0\end{array}$ \\
\hline $\begin{array}{l}\text { 2. Expulsion at } 100 \% \text { power } \\
\text { from one module a control } \\
\text { rod worth } 80 \text { cents }\end{array}$ & -0.00338 & 0.0 & 2.29 & 0.35 & 0.0 & 0.0 \\
\hline
\end{tabular}

in the selected system with $3 \%$ plutonium in the radial blankets.

The behavior of an isolated module was obtained with each reactivity insertion to provide a basis for assessing the reactivity sharing between the two coupled modules. In the isolated module, the internal reactivity of course is 1.0. In addition, analyses were performed for the two-module system using the coupling reactivity corresponding to the end-of-life radial blanket plutonium content, $3 \%$, given in ref. 1 . The cases studied are typical of those selected for accident analyses and are as follows:

1. inherent stability for a small reactivity insertion in one module;

2. inherent stability for a small reactivity insertion into both modules;

3. expulsion at $100 \%$ power from one module of a control rod worth 80 cents.

These cases are listed in Table 2 along with the coefficients calculated for the coupled core design of ref. 1.

\section{Discussion of Results}

\section{Parametric Study}

The analyses for the parametric study were performed with the use of two forms of reactivity insertion, finite ramps and sine waves; two amounts of reactivity were inserted, 5 cents in 0.5 sec and one dollar in 0.5 sec. For clarity in the following discussion, the module being perturbed, core 1 , is designated as the driver module and the unperturbed module, core 2 , as the passive module.

The power rises for both the finite ramp insertion and the sinusoidal insertion of 5 cents in 0.5 sec are shown in Figures 2 and 3, respectively. The power rise for an isolated module is also shown to provide a basis for assessing the neutronic coupling effect. The coupling lifetime is an order of magnitude greater than the internal lifetime (see Table 1, Cases 3, 4, and 8 for the ramp, and Cases 11,12 , and 16 for the sine wave).

The power rise in the driver module decreases with increasing neutronic coupling. With $0.4 \%$ coupling, the power rise for the driver core is $63 \%$ of that of the isolated module; with $4 \%$ coupling it is $52 \%$. The passive module power follows the driver module power but at a lower level. The spread between them is strongly dependent upon the magnitude of the coupling reactivity. For example, with $0.4 \%$ coupling the passive module power rise is $50 \%$ of the driver module power rise. Increasing the coupling reactivity to $4 \%$ causes the passive module power rise to be $90 \%$ of that of the driver core.

The results for the one-dollar reactivity insertion in 0.5 sec for both the finite ramp and the sine wave insertions are shown in Figures 4 and 5, respectively. The combinations of coefficients are given in Table 1 , Cases 1, 2, and 7 for the ramp, and Cases 9, 10, and 15 for the sine wave. The neutronic coupling greatly reduces the peak power in the driver module over that obtained in the isolated module for this amount of reactivity insertion. With $0.4 \%$ coupling reactivity, the peak power rise for the driver module with the finite ramp was $33 \%$ and for the sinusoidal insertion $38 \%$ of the power rise for the isolated module peak. This is a greater reduction than was obtained with the 5 -cent insertions shown in Figures 2 and 3. At 1.2 sec for both one-dollar insertions, the power rise for 


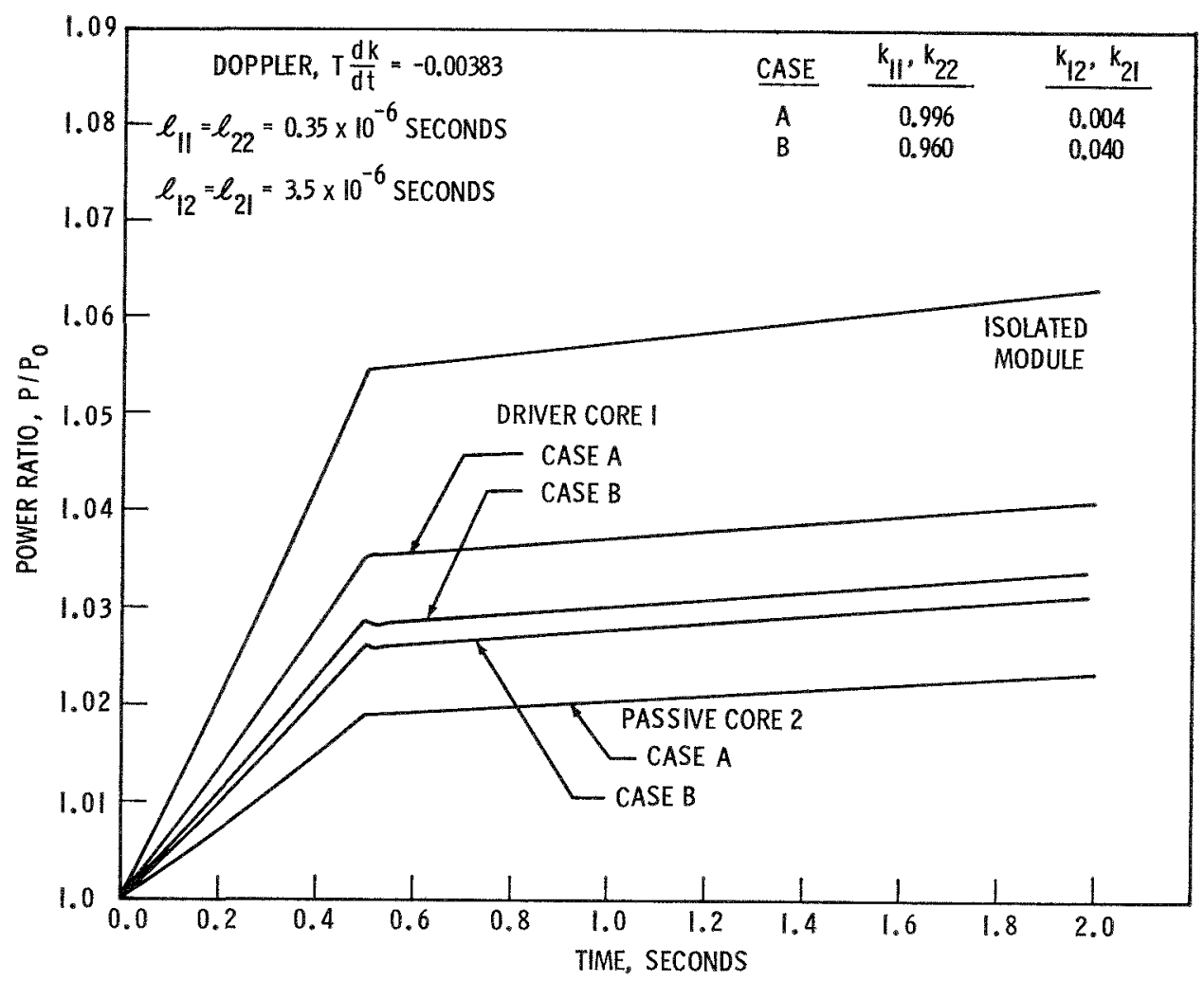

FIg. 2. Parameter Study: Response of Two Coupled Cores to a Ramp Reactivity Insertion of 5 Cents in 0.5 Sec.

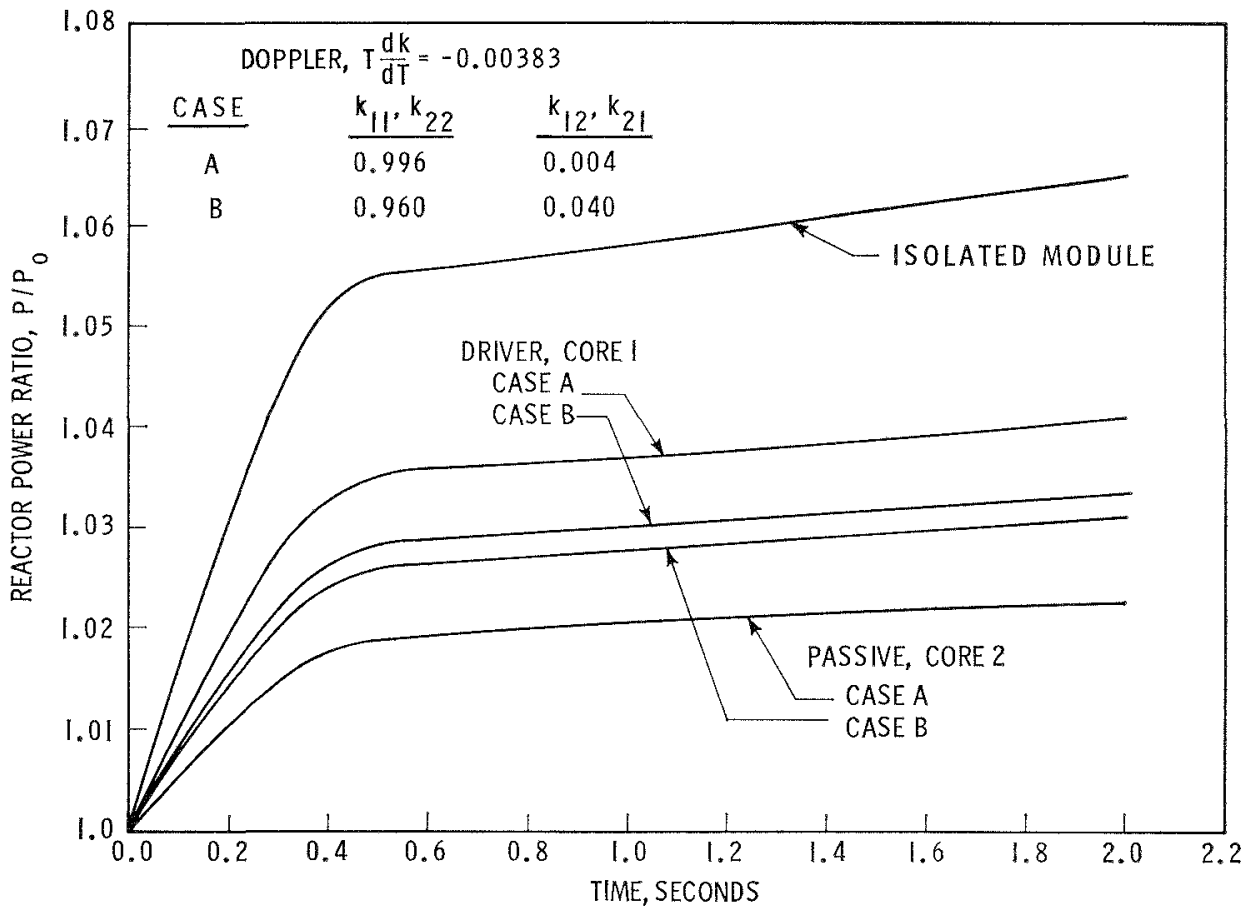

Fia. 3. Parameter Study: Response of Two Coupled Cores to a Sinusoidal Insertion of 5 Cents at 0.5 Cycles per Second.

the driver module for $0.4 \%$ coupling was $50 \%$ and for $4.0 \%$ coupling $33 \%$ of that of the isolated module.

The effect of neutronic coupling in limiting the power rise in a modular system relative to that in an isolated core appears to be more predominant with a large than with a small reactivity insertion. Undoubtedly, with larger reactivity insertions and hence larger temperature increases, the Doppler coefficient of the passive core 


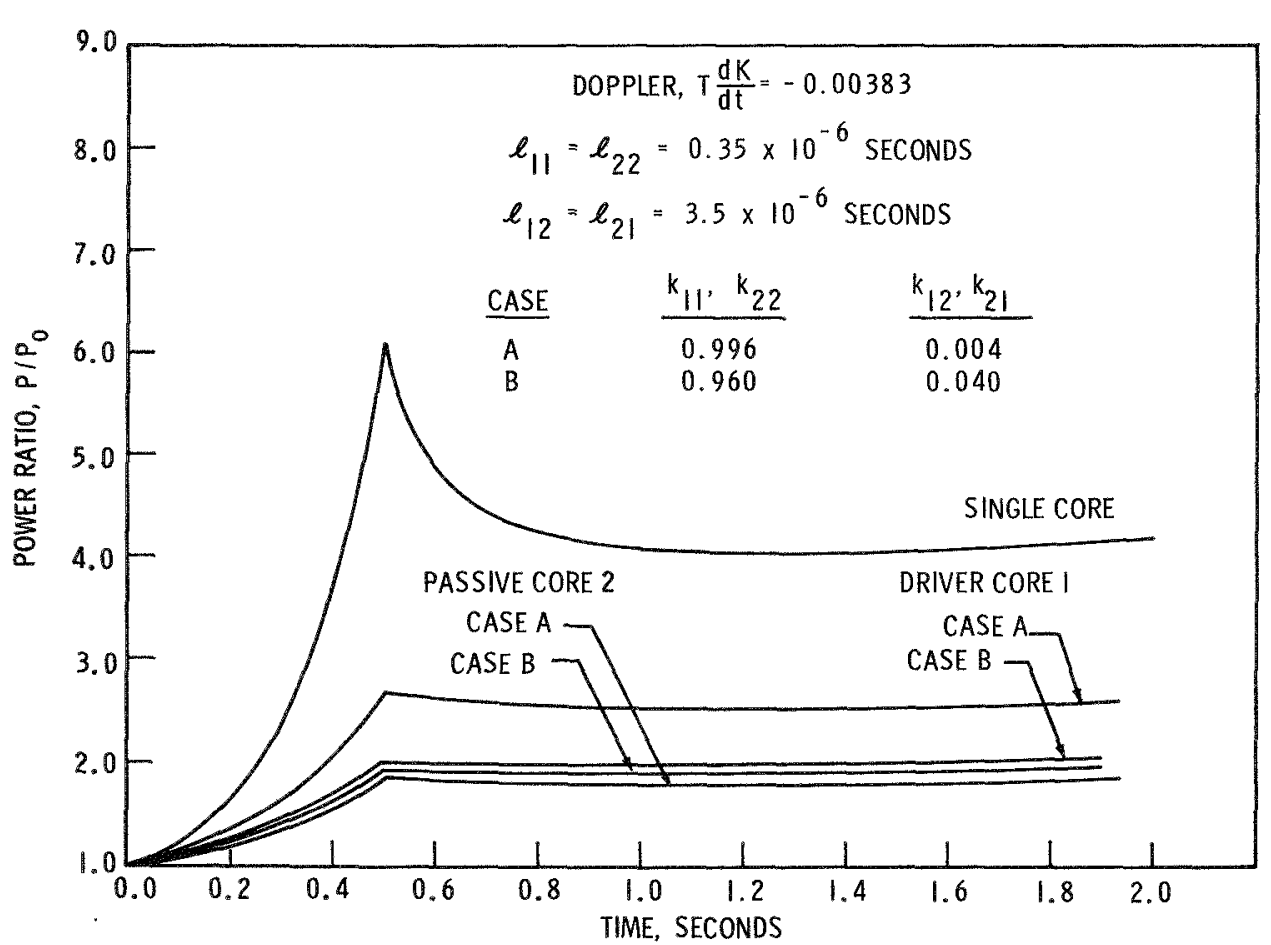

FtG. 4. Parameter Study: Response of Two Coupled Cores to a Ramp Reactivity Insertion of One Dollar in 0.5 Sec.

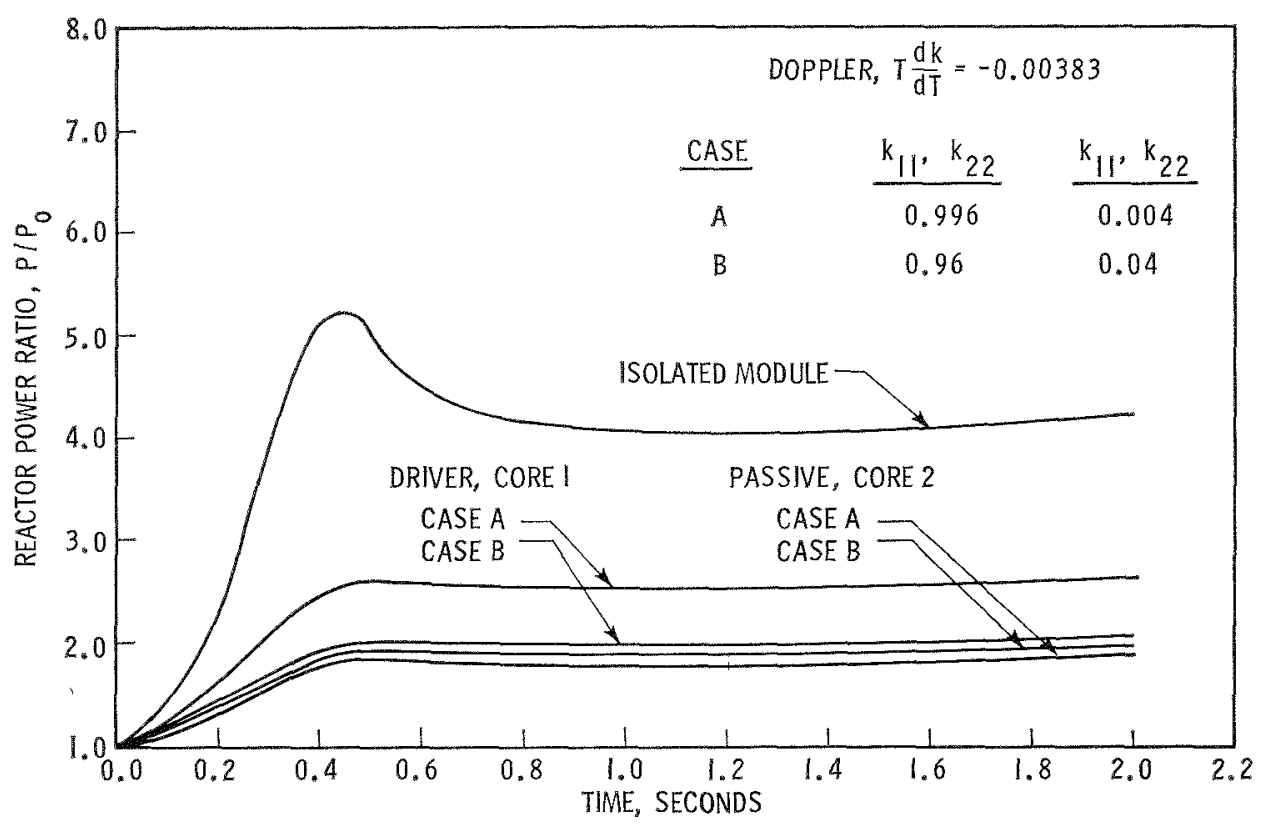

Frg. 5. Parameter Study: Response of Two Cores to a Sinusoidal Reactivity Insertion of One Dollar at 0.5 Cycles per Second for $0.5 \mathrm{Sec}$.

becomes correspondingly more important in assisting the Doppler coefficient of the driver core in reducing the total power rise of the system. Moreover, with the one-dollar insertions, when the coupling reactivity is increased from $0.4 \%$ to $4.0 \%$, the power rise of the driver module is reduced much more than the corresponding increase in power in the passive module.
However, with the 5 -cent insertions, the passive module power rose about the same amount as the driver module power decreased when the coupling reactivity was varied from $0.4 \%$ to $4.0 \%$.

The sinusoidal reactivity insertions discussed above were for only a quarter-cycle duration. The response of the two modules to a half-cycle of the one-dollar 
sinusoidal reactivity insertion is shown in Fig. 6 . These results are presented to show that the passive core follows the driver core in the same fashion regardless of whether the reactivity insertion into the driver core has a positive or negative slope.

The neutron coupling lifetime was found to have a negligible effect on the power split between the driver core and the passive core. The combinations of coefficients for this part of the parametric study are given in Table 1, Cases 5 and 6 . The results are given in Table 3.

In summary, the neutronic coupling reactivity in a modular reactor is effective in spreading among all the modules any reactivity insertion into one of the modules. The neutron coupling lifetime apparently has a negligible effect on the power split. The power rise of the driver module, relative to an isolated module power rise, is shown in Fig. 7 as a function of the coupling reactivity and the magnitude of the reactivity insertion. From Fig. 7 it can be seen that even a small amount of coupling reactivity, such as $0.1 \%$, will have a pronounced effect in reducing the power rise in a

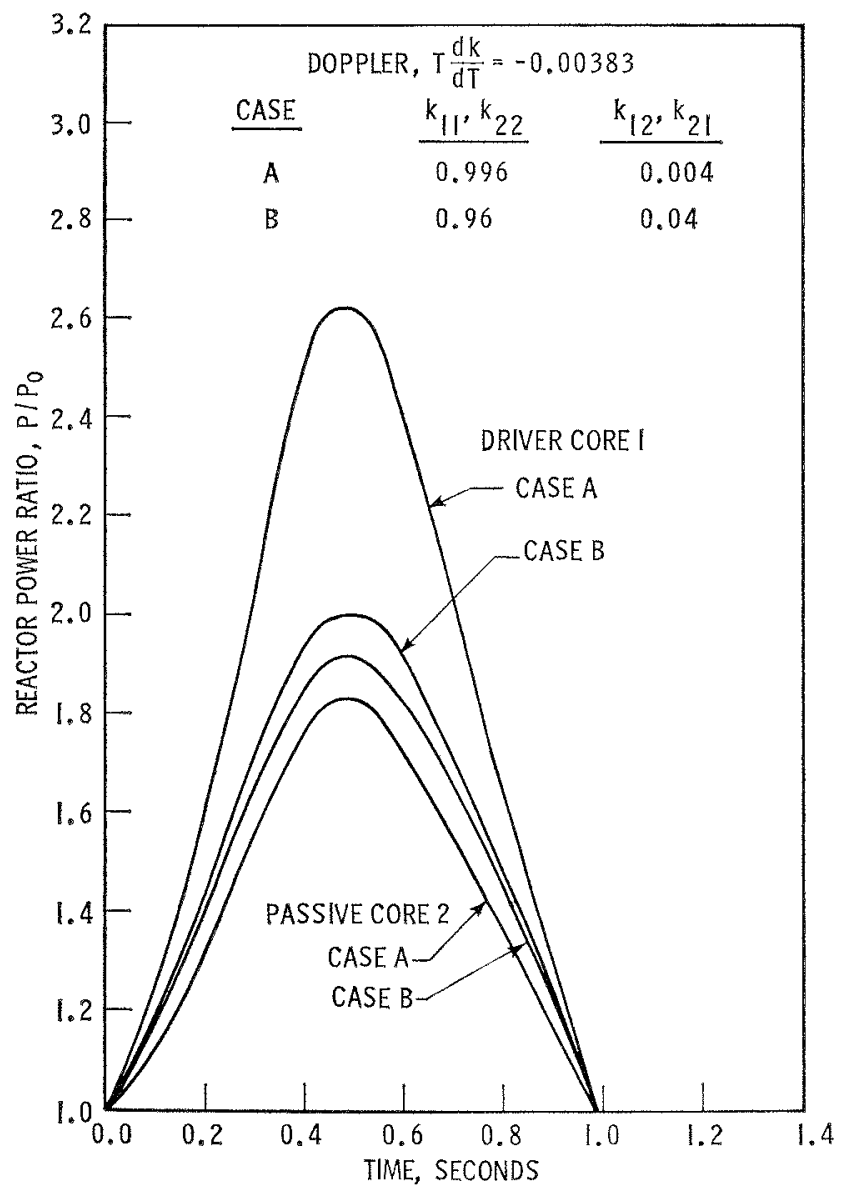

Frg. 6. Response of Two Coupled Cores to a Sinusoidal Reactivity Insertion of One Dollar at 0.5 Cycles per Second.
TABLE 3. EFFegt of Cross-coupling Lifetime

Rate of reactivity insertion $=2.0 \$ / \mathrm{sec}$.

Insertion time is $0.5 \mathrm{sec}$.

$l_{11}=l_{22}=0.35 \times 10^{-6} \mathrm{sec}$.

$P_{0}=100 \%$ power

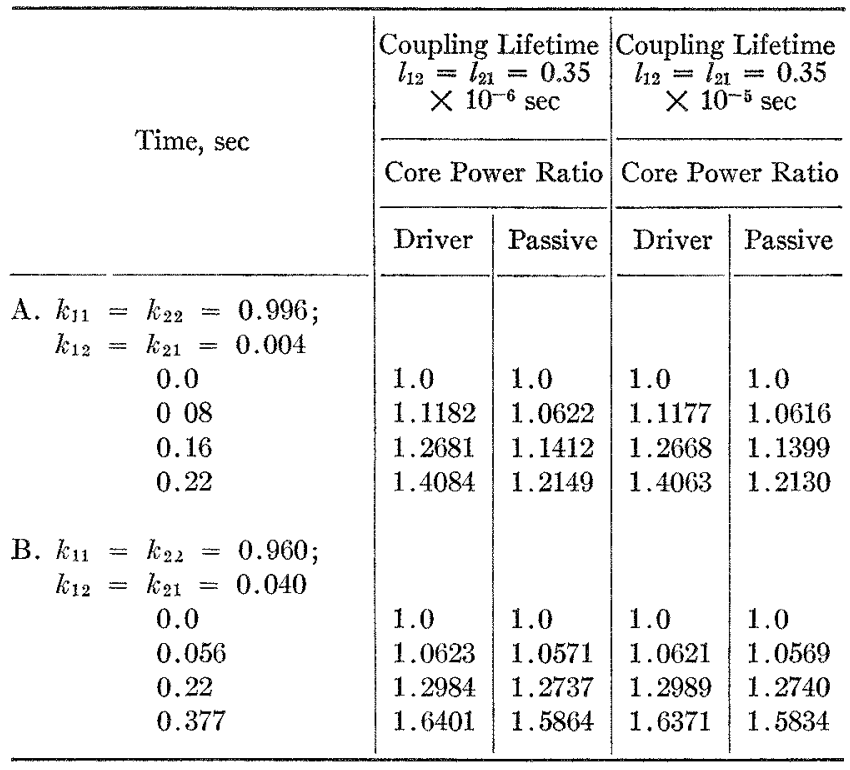

modular system. Moreover, the effectiveness of the coupling reactivity in limiting the excursion increases with increasing reactivity insertion due to the negative feedback of the Doppler coefficient of the passive core.

\section{Analyses of a Specific Modular Reactor}

The analyses listed in Table 2 were performed for the Westinghouse modular reactor described in ref. 1. The coefficients used in the analyses were calculated for this system with end-of-life conditions, that is, with $3 \%$ plutonium in the radial blanket. The coupling reactivity for this case is $2.17 \%$. The analyses are typical of those required for an accident study.

The inherent stability of two modules to a ramp insertion of 10 cents (10 cents/sec) into each modũle is given in Fig. 8. The analyses were performed with Doppler and with Doppler-plus-axial-expansion feedback. As expected, when both modules are simultaneously and equally perturbed, the system responds in the same manner as would an isolated module.

In the case that only one of the modules is perturbed by this reartivity insertion, the inherent stability with only Doppler feedback would be as shown in Fig. 9. The power rise in the driver core after $1.2 \mathrm{sec}$ is $53 \%$ of the power rise when both modules are perturbed equally (see Fig. 8). It can also be seen from these curves that the $2.17 \%$ coupling reactivity in a modular system is worth slightly more than an axial expansion coefficient of $-3.896 \times 10^{-6} /{ }^{\circ} \mathrm{F}$ would be in reducing the power rise in an isolated core. 


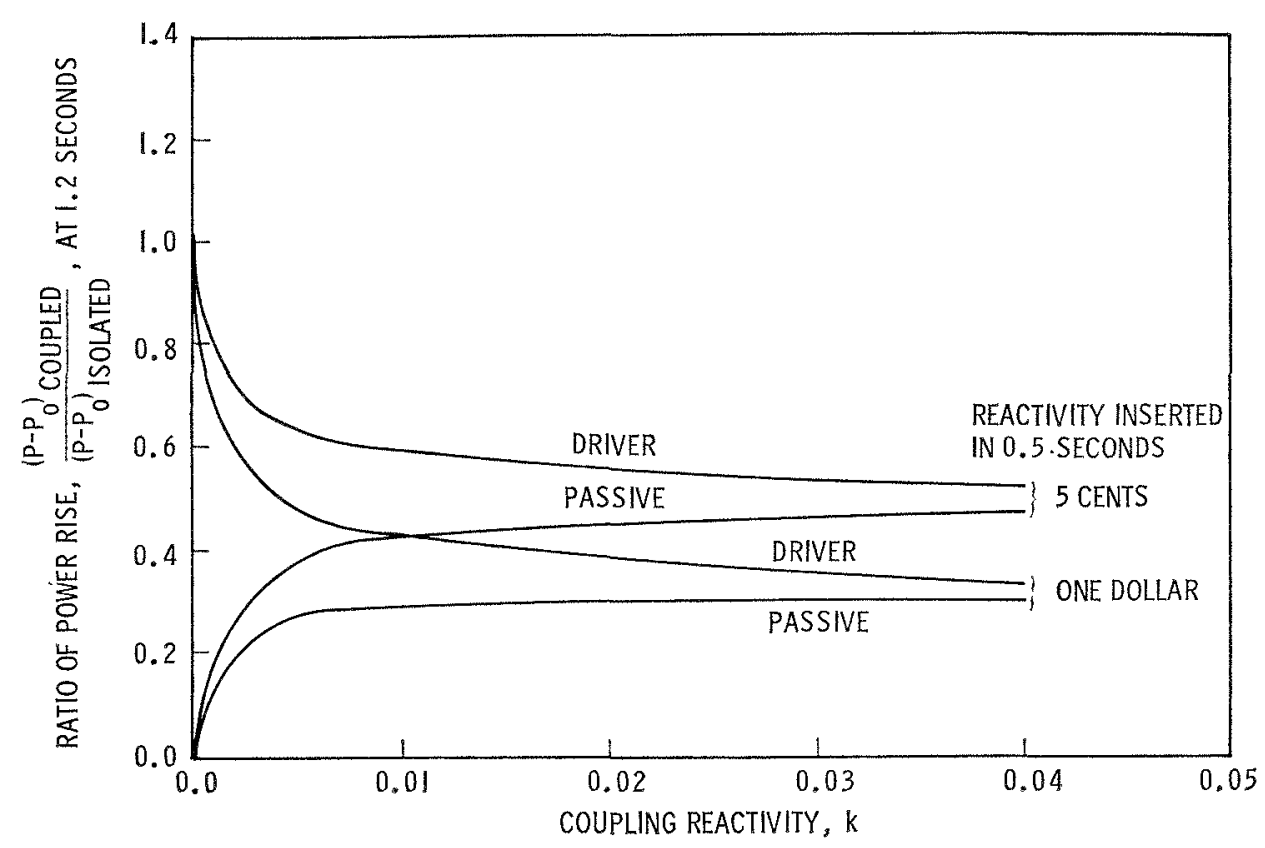

FIG. 7. Parameter Study: Ratio of Power Rise in Two Coupled Modules to an Isolated Module.

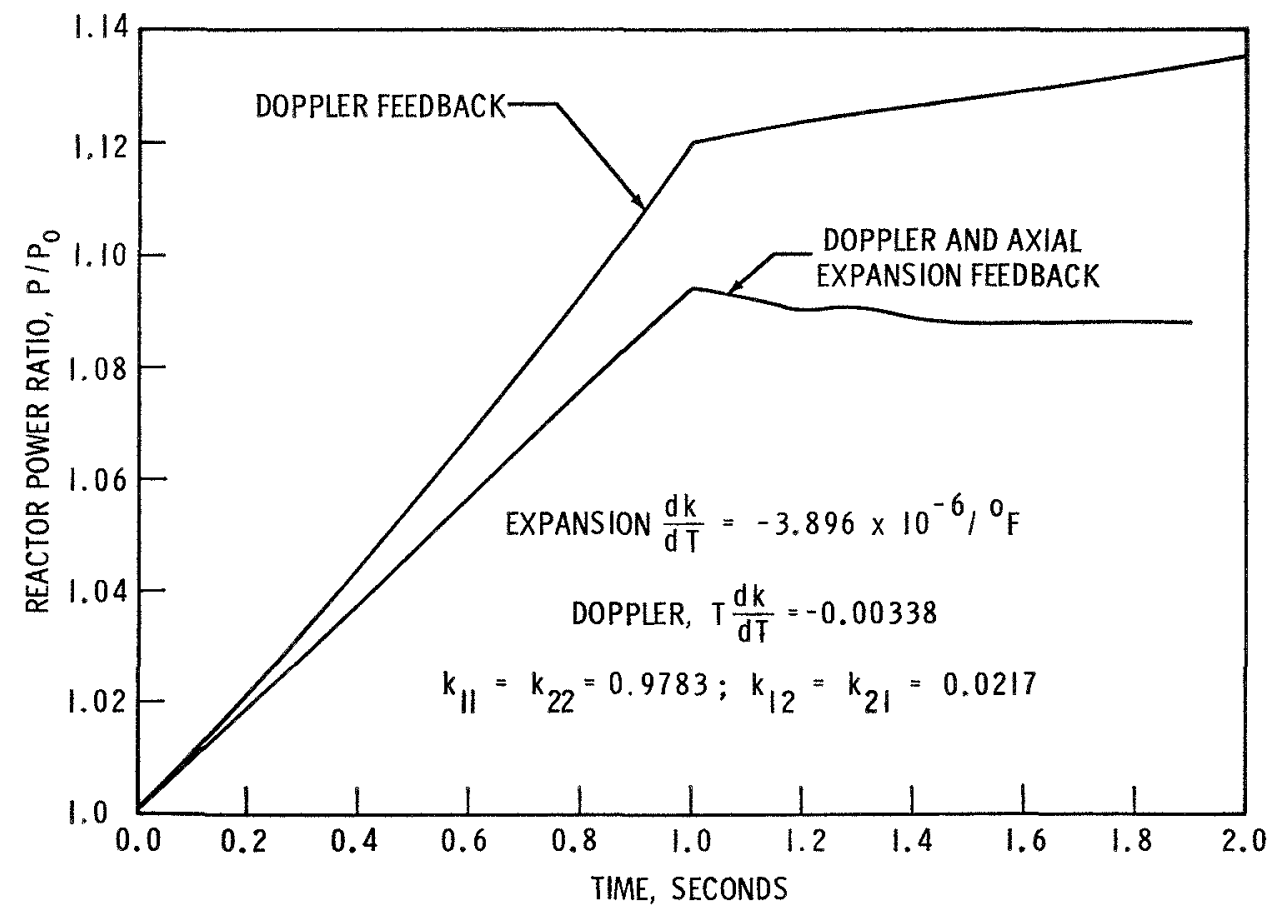

Fik. 8. Response of Two Coupled Cores with a Ramp Reactivity Insertion of 10 Cents in Each Core.

The response with Doppler feedback of a two-module system to a ramp reactivity insertion of 80 cents in $0.35 \mathrm{sec}$ is given in Fig. 10. This much reactivity (but with a parabolic distribution and thus less total reactivity inserted over the first $0.35 \mathrm{sec}$ ) was used for the shim control-rod expulsion accident analysis in ref. 1. The resultant power rise in the driver module is some- what lower than the power rise in the analysis of ref. 1 in which both Doppler and axial expansion feedback were considered.

Thus, due to the division of the reactivity insertion among all the modules, the severity of an accident initiated in one module will be much less than the same accident in an isolated core. 


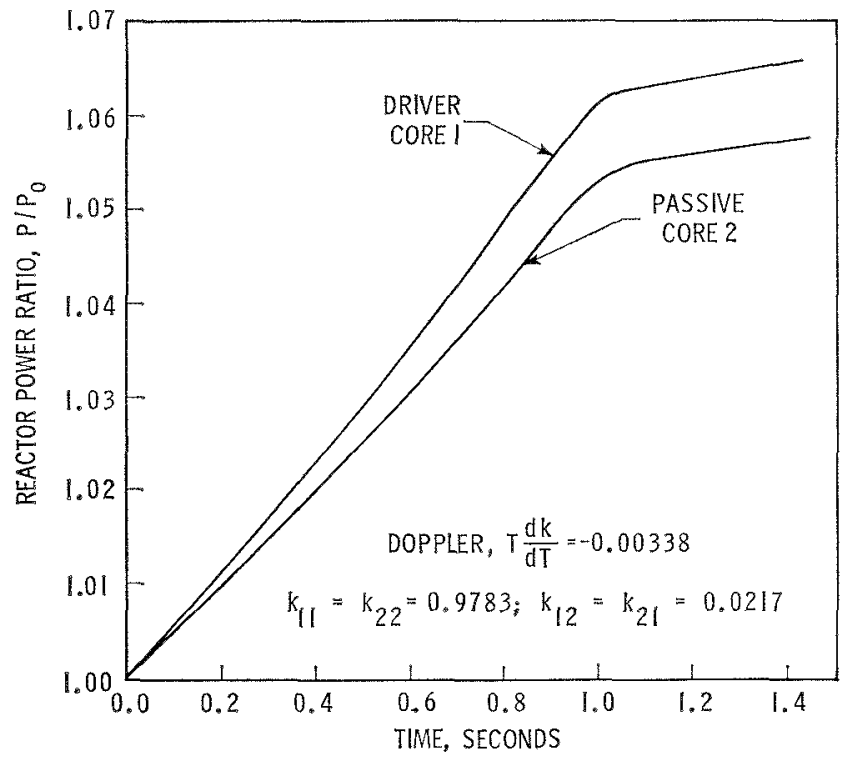

Frg. 9. Response of Two Coupled Cores for a Ramp Insertion Worth 10 Cents into One Core. cores inherently possesses a promptly acting safety mechanism through the neutronic coupling effect. It was shown that in a two-core system with only a few tenths per cent coupling coefficient a reactivity insertion into the driver core will result in a significant split of the resultant rise in power between the driver and passive cores. The power rise in the driver core is considerably less than the level which would be attained in an isolated core subjected to the same amount of reactivity insertion.

The Westinghouse reactor system of ref. 1 consists of seven modules. Reactivity insertions into one module out of seven will result in lower power rises in the driver core than those exhibited in the two-core analyses of this paper. Thus, it may very well be that the sevenmodule array is an overly conservative design. Future investigations may reveal that a smaller number of larger modules might still be a very conservative design but economically even more attractive. This would be a result of the higher specific powers, and internal

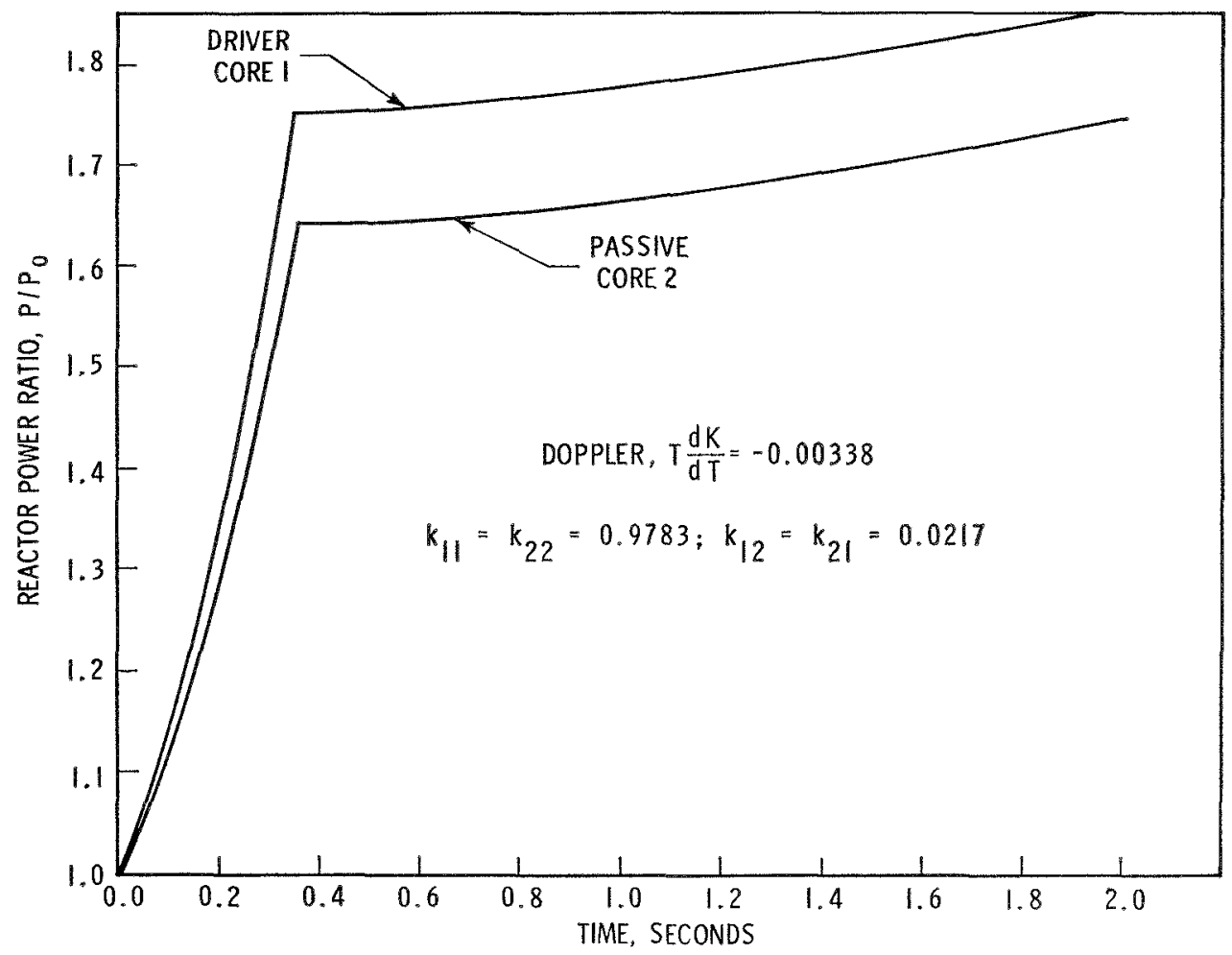

FIG. 10. Response of Two Coupled Cores to a Ramp Reactivity Insertion of 80 Cents in 0.35 Sec.

\section{Conclusions}

From the results presented herein, it can be seen that a reactor system consisting of a multi-modular array of

\section{Appendix}

Some insight into the behavior of coupled cores as described by the Avery model can be obtained by breeding ratios and lower inventory requirements doubling times and fuel-cycle costs which could conceivably be obtained in fewer modules.

linearizing and simplifying the equations in order to obtain analytic solutions. For this purpose we make the 
following assumptions:

1) constant reactivity values after time zero;

2) no temperature feedback to reactivity;

3) only two core regions;

4) only one group of delayed neutrons;

5) all neutron lifetimes are equal;

6) in cross coupling, delayed neutrons will be neglected;

7) $k \beta \simeq \beta$.

The basic equations are:

$$
\begin{aligned}
l_{11} \frac{d S_{11}}{d t} & =k_{11}(1-\beta) S_{1}-S_{11}+k_{11} \tau C_{1} ; \\
l_{12} \frac{d S_{12}}{d t} & =k_{12}(1-\beta) S_{2}-S_{12}+k_{12} \tau C_{2} ; \\
l_{21} \frac{d S_{21}}{d t} & =k_{21}(1-\beta) S_{1}-S_{21}+k_{21} \tau C_{1} ; \\
l_{22} \frac{d S_{22}}{d t} & =k_{22}(1-\beta) S_{2}-S_{22}+l_{22} \tau C_{2} ; \\
\frac{d C_{1}}{d t} & =\beta S_{1}-\tau C_{1} ; \\
\frac{d C_{2}}{d t} & =\beta S_{2}-\tau C_{2} .
\end{aligned}
$$

By assumption 5), $l_{11}=l_{12}=l_{21}=l_{22}=l$. Addition of equation (1) to (2), and of (3) to (4) yields

$$
\begin{aligned}
l \frac{d S_{1}}{d t}=\left(k_{11}-1-\beta\right) S_{1}+ & k_{11} \tau C_{1}+k_{12} \\
& {\left[\frac{(1-\beta)}{k_{12}} S_{2}+\tau C_{2}\right] } \\
l \frac{d S_{2}}{d t}=\left(k_{22}-1-\beta\right) S_{2}+ & k_{22} \tau C_{2}+k_{21} \\
\cdot & {\left[\frac{(1-\beta)}{k_{21}} S_{1}+\tau C_{1}\right] . }
\end{aligned}
$$

Then by application of assumption (6), which assumes that $(\tau C-(\beta / k) S)$ in the cross term can be neglected, we have

$$
\begin{aligned}
& \frac{d S_{1}}{d t}=\frac{\left(k_{11}-1-\beta\right)}{l} S_{1}+\frac{k_{11}}{l} \tau C_{1}+\frac{k_{12}}{l} S_{2} ; \\
& \frac{d S_{2}}{d t}=\frac{\left(k_{22}-1-\beta\right)}{l} S_{2}+\frac{k_{22}}{l} \tau C_{2}+\frac{k_{21}}{l} S_{1} ; \\
& A_{m 1}=\frac{\left[\frac{\beta S_{1}^{0}}{l\left(B_{m}+\tau\right)}+S_{1}^{0}\right]\left[B_{m}-\frac{k_{22}-1-\beta}{l}-\frac{\tau \beta}{l\left(B_{m}+\tau\right)}\right]+\frac{k_{12} \beta S_{2}^{0}}{l_{2}\left(B_{m}+\tau\right)}+\frac{k_{12} S_{2}^{0}}{l}}{\left[B_{m}-\frac{k_{22}-1-\beta}{l}-\frac{\tau \beta}{l\left(B_{m}+\tau\right)}\right]\left[1+\frac{\tau \beta}{l\left(B_{m}+\tau\right)^{2}}\right]} \\
& +\left[B_{m}-\frac{k_{11}-1-\beta}{l}-\frac{\tau \beta}{l\left(B_{m}+\tau\right)}\right]\left[1+\frac{\tau \beta}{l\left(B_{m}+\tau\right)^{2}}\right] ;
\end{aligned}
$$

$$
\begin{aligned}
& \frac{d C_{1}}{d t}=\beta S_{1}-\tau C_{1} \\
& \frac{d C_{2}}{d t}=\beta S_{2}-\tau C_{2} .
\end{aligned}
$$

Taking the Laplace transform of equations (9), (10), (5), and (6), combining and applying boundary conditions consisting of

$$
\begin{aligned}
S_{1}(0) & =S_{1}^{0} \\
S_{2}(0) & =S_{2}^{0} \\
\frac{d C_{1}(0)}{d t} & =\frac{d C_{2}(0)}{d t}=0
\end{aligned}
$$

we obtain the following tansform solutions for the sources:

$$
\begin{aligned}
S_{1}^{*}(s)= & \frac{\frac{S_{1 \beta}^{0} \beta}{l(s+\tau)}+\frac{k_{12} S_{2}^{*}(s)}{l}+S_{1}^{0}}{s-\frac{k_{11}-1-\beta}{l}-\frac{\tau \beta}{l(s+\tau)}} ; \\
S_{2}^{*}(s)= & \frac{\frac{S_{2 \beta}^{0}}{l(s+\tau)}+\frac{k_{21} S_{1}^{*}(s)}{l}+S_{2}^{0}}{s-\frac{k_{22}-1-\beta}{l}-\frac{\tau \beta}{l(s+\tau)}} .
\end{aligned}
$$

This provides solutions of the form

$$
S_{i}(t)=\sum_{m} A_{m i} e^{B_{m} t},
$$

where the $B_{m}$ are the roots of

$$
\begin{aligned}
& {\left[s-\left(\frac{k_{22}-1-\beta}{l}\right)-\frac{\tau \beta}{l(s+\tau)}\right]} \\
& {\left[\left[s-\left(\frac{k_{11}-1-\beta}{l}\right)-\frac{\tau \beta}{l(s+\tau)}\right]-\frac{k_{12}}{l} \frac{k_{21}}{l}=0 .\right.}
\end{aligned}
$$

This is a quartic equation in $s$, yielding, in general, four roots, all four of which appear in the solutions for both sources.

The coefficients $A_{m i}$ are the residues at the poles of 


$$
\begin{array}{r}
A_{m 2}=\frac{\left[\frac{\beta S_{2}^{0}}{l\left(B_{m}+\tau\right)}+S_{2}^{0}\right]\left[B_{m}-\frac{k_{11}-1-\beta}{l}-\frac{\tau \beta}{l\left(B_{m}+\tau\right)}\right]+\frac{k_{21} \beta S_{1}^{0}}{l^{2}\left(B_{m}+\tau\right)}+\frac{k_{21} S_{1}^{0}}{l}}{\left[B_{m}-\frac{k_{22}-1-\beta}{l}-\frac{\tau \beta}{l\left(B_{m}+\tau\right)}\right]\left[1+\frac{\tau \beta}{l\left(B_{m}+\tau\right)^{2}}\right]} \\
\quad+\left[B_{m}-\frac{k_{11}-1-\beta}{l}-\frac{\tau \beta}{l\left(B_{m}+\tau\right)}\right]\left[1+\frac{\tau \beta}{l\left(B_{m}+\tau\right)^{2}}\right] .
\end{array}
$$

The results are typically one mall positive exponent and three large negative exponents, with a slightly larger coefficient for the positive exponential for the core in which reactivity is inserted. Thus the passive region tend to follow the region with the insertion, but at a lower level.

It is of some interest to cherk these solutions for limiting cases. If the cores are uncoupled, then $k_{21}$ and $k_{12}$ go to zero. Then the equation for the roots, (16), reduces to

$$
\begin{aligned}
& {\left[s-\frac{k_{22}-1-\beta}{l}-\frac{\tau \beta}{l(s+\tau)}\right]} \\
& \cdot\left[s-\frac{k_{11}-1-\beta}{l}-\frac{\tau \beta}{l(s+\tau)}\right]=0 .
\end{aligned}
$$

Then there are two pairs of roots, one for each eore. These pairs are independent, since either bracket being zero will satisfy the equation. Hence the solutions limit correctly as the coupling vanishes.

At maximum coupling, we would expeet $k_{11}=k_{12}=$ $k_{22}=k_{21}$. Then the equation for the roots becomes

$$
\begin{aligned}
& {\left[s+\frac{1+\beta}{l}-\bar{l}\left(s+\frac{\tau \beta}{+\tau)}\right]^{2}\right.} \\
& \quad-2\left[s+\frac{1+\beta}{l}-\bar{l}(s+\tau)\right] \frac{k_{11}}{l}=0,
\end{aligned}
$$

and either

$$
s+\frac{1+\beta}{l}-\frac{\tau \beta}{l(s+\tau)}=0
$$

or

$$
s+\frac{1+\beta}{l}-\frac{\tau \beta}{l(s+\tau)}-2 \frac{k_{11}}{l}=0 .
$$

licquation (21) may be discarded since it would give roots independent of reactivity. Therefore, the solution effectively reduces to a single quadratic equation, (22), having two roots, just as one finds for a single core. Therefore, the equations at least approximately limit to the behavior of a single core at maximum coupling.

Physically, one experts that the rore with a reactivity insertion would tend to pull the other core along with it, and simultaneously tend to be somewhat restrained by the other core. This results from a change in the ratio of in-leakage to total neutron production in the
1 wo cores. This can be interpreted as a reactivity sharing effect. Equations (9) and (10) can be rewritten to illustrate this:

$$
\begin{aligned}
& \frac{d S_{1}}{d t}=\left[\frac{k_{11}-1-\beta+\left(k_{21} S_{2} / S_{1}\right)}{l}\right] S_{1}+\frac{k_{11}}{l} \tau C_{1} ; \\
& \frac{d S_{2}}{d t}=\left[\frac{k_{22}-1-\beta+\left(k_{21} S_{1} / S_{2}\right)}{l}\right] S_{2}+\frac{k_{22}}{l} \tau C_{2},
\end{aligned}
$$

that is, the cross-coupling term can be interpreted as a reactivity effect which is directly proportional to the ratio of the powers in the two regions. For example, if $k_{11}$ is increased such that at some point in time $S_{1}=$ $1.1 S_{2}$, then the effective reactivity in core one is redueed by $k_{12}[1-(1,1.1)]$, and the reactivity in core two is increased by $k_{21}(1.1-1)$.

One result of this reactivity sharing is that there is no clear definition of the meaning of reactivity worth for a modular core. The effective worth of a controlrod insertion in one module of seven, for example, lies somewhere between the worth calculated for the module isolated and one-seventh of that value, depending upon the coupling and the relative power in the other modules.

\section{REFERENCES}

1. Wright, J. H., et al., Conceptual Design and Preliminary Accident Analysis of a Sodium-cooled, Carbide-fueled, Large Modular Fast Reactor, Panel Discussion on Fast Reactor Safety at Argonne National Laboratory, October $11-14,1965$.

2. Steck, R. B., Liquid Metal Fast Breeder Reactor Design Study, WCAP-3251-1 (Jan 1964).

3. Chezem, C. G., and seale, R. L., Temperature-dependent Kinetics of Coupled Core Reactors, IAAMS-3148.

4. Chezem, C. G., Helmick, H. I., and Seale, R. L., The Los Alamos Coupled KIWI-B4 Experiments, American Institute of Aeronautics and Astronauties Propulsion Joint Specialists Conference, Colorado Springs, June 14-18, 1965.

5. Avery, R., Theory of ('oupled Reactors, Procedings of the Second United Nations International Conference on the Peaceful Uses of Atomic Lnergy, (Yeneva, 1958, P/1858.

6. Seale, R. L., Coupled Core Reactors, LAMS-2967.

7. Baldwin, G. C., Kinetics of a Reactor Composed of Two loosely Coupled Cores, Nucl. Sci. Fing., 6, 320-327 (1959).

\section{Discussion}

$M r$. Kohler (Texas A\&M): Have you tricd to derive your kineties equations from the Boltzmann equations 
directly? At Karlsruhe, in investigating the safety of roupled fast thermal reactors, it was found that when very strong reactivity changes occur in one of the cores, which makes the space-time nonseparability worse, your kind of model is not satisfactory any more to describe the kinetic behavior or a fast-thermal system. I would think this would apply to fast reactors, too.

Mr. Heck (WAPD): There is some discussion of this in the paper, in fact, particularly where you get very large excursions in one core relative to the other. It is clear that the simple assumption that the driven neutron source goes immediately in one generation into the first mode, which is what is assumed in those equations, is not going to correctly describe the situation. One of the possibilities we've been looking into is to split that particular equation, so that the driven source can go both into the first mode and back into the driven source as a first approximation to adjust for the correct spatial distribution.

$M r . G i b s o n(\mathrm{AI}):$ We, too, have looked at the modular type of core, and we seem to differ a little bit on the value of strong coupling coefficients. Some of our studies demonstrate that for a module which is $\$ 30$ subcritical when you pull it out of the complete array, you can get significant power shifts from local reactivity perturbations. How extensively have you investigated this? Secondly, Mr. Wright the other day mentioned that there was a possible Achilles heel of the modular concept in terms of the secondary criticality considerations when one module melts down. I would be interested in your comments.

Mr. Gunson: What happens when one module melts down has not really been studied. From our analysis one might draw the conclusion that the severity when a single module melts is reduced; but as the next modules go off, you might get a whole series of "bangs."

Mr. Lellouche (BNL): Do you have any expectation that such a model with a constant coupling would be valid for very fast excursions initiated in one module? In fact, to get any decent results, do you have to go to a space-dependent examination?

Mr. Gunson: At Westinghouse we have discussed this space-dependency aspert, and the change in the flux shape, but this is a "hairy" problem. We took the simple approach, just to get an engineering feel.

Mr. Spinrad (ANL): I would like to point out what's different in the calculations presented from essentially pseudo-static calculations for a single core which might, for example, be divided into two parts quite arbitrarily; the motion of a rod locally would have a bigger reactivity effect than it would if you tried to make a global reactivity. It seems to me that this is just a calculation of that effect and that to that extent it is, simply, a so-called modal expansion set of experiments. Can you comment on the difference between those two?

Mr. Heck: I'm not quite sure I know how to answer the question as it was posed. Yes, this is a sort of a modal approach to a system. The reason that one expects at least reasonable results from this is that in the kind of system we were looking at the roupling was extremely low, so that the spatial variations were reasonably small. We have looked at grossly unbalanced cores, but this work is still in progress.

Mr. Madell (ANL): Have you compared your method with Westinghouse's WIGLE code?

Mr. Gunson: No. 


\title{
Enhanced Safety Characteristics of a Sodium-cooled Coupled Reactor*
}

\author{
D. Eg(iex, A. Gibson, R. Sevt, anid M. Silberberc \\ .1 tomie's International \\ Canoga Park, California
}

(PRESENTEI) BY D). E( $($ IEN)

The importance of a strong negative Doppler reactivity effect and a weakly positive sodium void reactivity effect on the safety of fast reactor systems has been recognized by numerous investigators. As an outgrowth of a study of coupled reactors, a reference design was completed by Atomics International under AEC sponsorship of a fast reactor which was optimized to obtain a very strong negative Doppler effect and a negative sodium void reactivity effect. The design consists of modular fast regions surrounded by low-enriched flux-trap blankets. For a 1000-MWe plant, the core includes a hexagonal array of ten identical regions (see Fig. 1). The blanket regions are formed by two rings of low-enriched fuel elements ( $31 / 2 \%$ average plutonium enrichment on equilibrium cycle), separated by a ring of graphite moderator elements. These regions and the associated control rods can be seen in Fig. 1.

By the utilization of blanket regions with an equilibrium enrichment of $3 \frac{1}{2} \%$, the power extracted from these regions is large, specifically, $40 \%$ of the total reactor power. Similarly, the Doppler contribution from the blanket regions is large due to the large nuclear importance of the enriched blankets and the softened spectrum in the blanket region. Table 1 shows the negative isothermal Doppler constant by region and the "effective" isothermal Doppler constant when weighted by the appropriate temperature rise in each region.

Figure 2 shows the reactivity change due to sodium voiding as a function of the radial fraction of the core voided for a flux-trap blanket arrangement compared with a single-blanket arrangement. As noted from the figure, voiding of the single-blanket arrangement results in a slightly positive initial reactivity addition followed by a strongly negative effect as the blanket regions are roided. In the reference design arrangement, neutrons born in the fast region which penetrate to the outer blanket region of the module have a low probability for re-entering the fast region. This is due to their low energy state and the large

* Work performed under Contract AT(11-1)GEN-8 for the U. S. Atomie Energy Commission. mass of moderating material between the blanket and the fast core. This neutron-trapping effect and the leaky nature of the modular arrangement are the principal reasons for the negative sodium void effect observed.

Table 2 presents some of the principal nuclear characteristics of the reference design compared with a conventional fast breeder reactor in which no concerted effort is made to improve upon the Doppler or sodium reactivity coefficients. The Doppler effect shown in Table 2 represents the total reactivity change resulting from an increase from normal operating temperature to the point at which fuel damage occurs. The sodium roid reactivity effect represents the maximum possible reactivity addition obtained during voiding of the core. As noted from the table, some compromises in economic characteristics result from the enhancement of the safety characteristics. The breeding ratio is reduced and the fissile mass is increased from that of the conventional fast breeder reactor.

The kinetic behavior of the reference design reactor was studied in a series of hypothetical accident situations. Conditions in which the reactor protective system was both operative and disabled were studied. A criterion for fuel damage was established at $6500^{\circ} \mathrm{F}$ center fuel temperature. Reactivity insertion rates were programmed at up to $\$ 100 / \mathrm{sec}$. It was found that the strong Doppler effect of this design $(0.008)$ is sufficient to control a reactivity insertion of $\$ 1$ without rapid scram. With normal action of the protective system, a reactivity insertion of $\$ 1.65$ can be tolerated with no fuel damage. This is sufficient to control such hypothetical accidents as control-rod ejcction, dropped fuel element, and flow-channel blockage.

To determine the potential for damage in a hypothetical situation in which coolant flow is arbitrarily and instantaneously reduced to zero with no immediate compensating action by the protective system, the reference design was compared with a different fast reactor having a similar Doppler reactivity effect but a slightly positive roid reactivity effect. The results of this analysis are shown in Fig. 3. For the reactor 


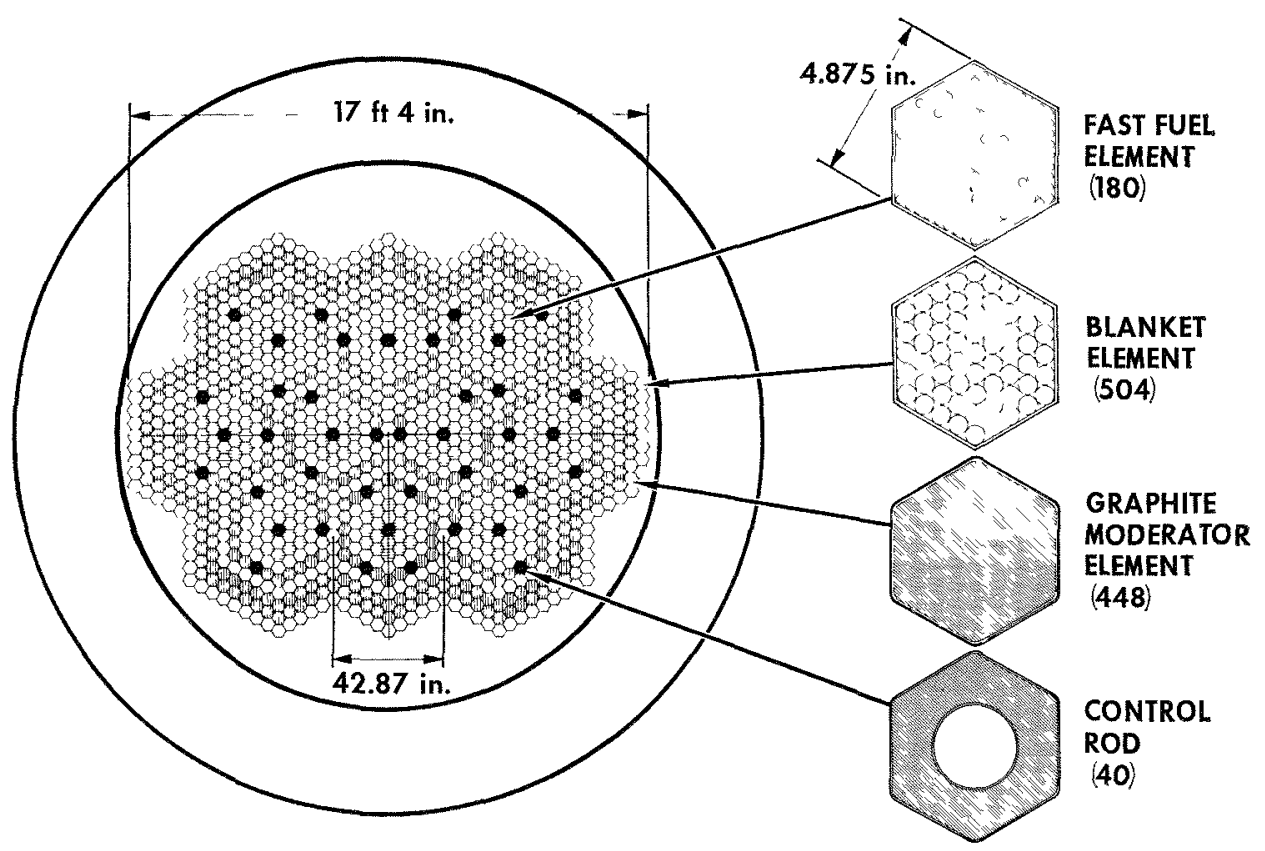

Fig 1 Modular Flux-trap Concept.

TABLE 1. ISOHERMAL DOPplar CONSTINT(A) $T\left(d k / d T^{\prime}\right)$ :

\begin{tabular}{l|l}
\hline Fast Region & -0.002298 \\
Inner Blanket Region & -0.009024 \\
Outer Blanket Region & -0006782 \\
$\quad$ Total & -0.0181 \\
"Effective" Total Weighted by Regional & -0.008 \\
Temperature Rise & \\
\hline
\end{tabular}

(a) $\mathrm{U}^{238}$ effect only.

with the positive void reactivity, it was assumed that the maximum void effect during loss of coolant from the core would be approximately $\$ 3.50$ positive and that complete voiding of the core would result in a slightly negative effect. As can be seen from Fig. 3, the net reactivity to the core becomes rapidly negative in the reference design arrangement due to both Doppler and void reactivity effects. In the case of the other fast reactor, although the Doppler effect reduces the total reactivity addition, the positive effect is more pronounced. The resulting temperature rise in the centerline of the hot fuel is also shown on Fig. 3.

Another study was performed to determine the compromise made in economics by emphasizing the sodium void reactivity effect. This study utilized an arrangement in which an additional row of fuel elements was added to the fast region. This increased the diameter of the module. In a 1000-MWe plant, an array of 7 such modules with an active height of
$5 \mathrm{ft}$ was compared with the 10-module array for the small module dexign. The core reartivity effects and economic for these designs are shown in Table 3 . It can be seen from the table that the Doppler effects are similar for the two designs, but the large module has a significantly larger positive sodium void effect. The fuel-cycle costs of the large-module design are roughly $1 / 4$ mill $/$ kWh less than those of the smallmodule design.

Potential long-term improvements in both safety and economics are obtained by using a modified version of the reference design with mixed-carbide fuel. Due to its lower operating temperature, the sodiumbonded, high-power-density, carbide-fueled core can tolerate reactivity insertions in excess of $\$ 2.00$ with protective system action and in excess of $\$ 1.50$ without rapid scram. Coolant boiling occurs more rapidly with the carbide fuel than with the oxide fuel because of the improved heat transfer characteristics. Therefore, it is necessary to suppress boiling by reducing coolant temperature drop or by some other means in order to take advantage of the improved carbide characteristics.

To determine the magnitude of additional costs associated with conservative safety criteria and conservative design practice for a first-generation plant, a cost estimate of two $1000-\mathrm{MWe}$ fast reactor plants was made. The results of this cost estimate are shown in Table 4. As shown in the table, the total costs of energy generation range from a low of 3.2 mills $/ \mathrm{kWhr}$ to a high of 4.4 mills $/ \mathrm{kWhr}$. This corresponds to a capitalized cost difference of $\$ 58,000,000$. The high 

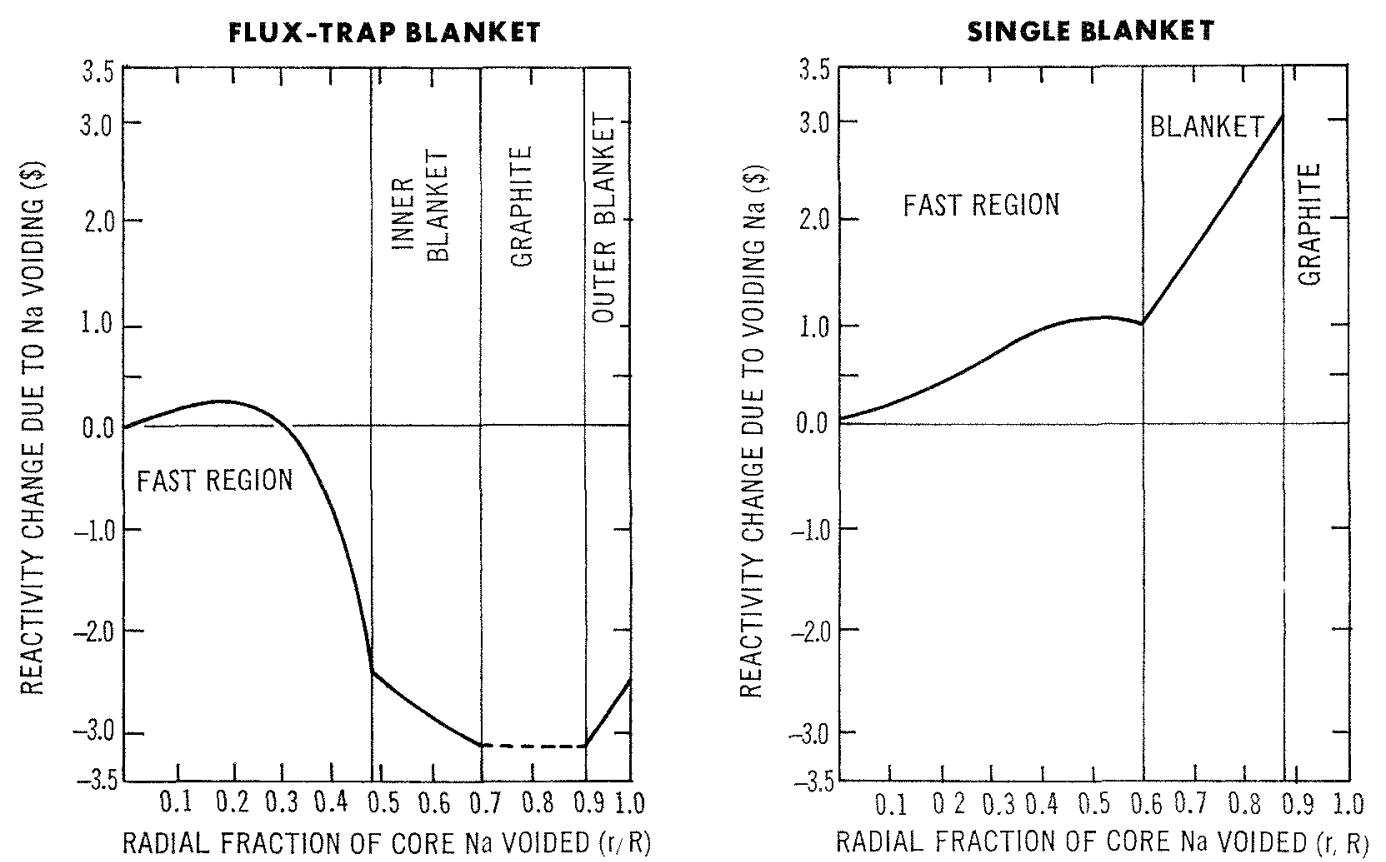

FIG. 2. Void Reactivity Effects.

value shown represents the probable costs for a firstgeneration plant with rather stringent safety requirements. For example, this plant would probably include a steel structure for containment of the entire primary system. Additionally, inert-gas-filled vaults would be utilized to house equipment components in the primary system. Both primary and secondary sodium systems would be used.

In addition to high figures for capital cost, the fuelcycle and operating and maintenance cost figures of a first-generation plant are higher. The fuel costs shown represent those associated with the smallmodule design previously discussed. The fuel-cycle costs also include a conservative estimate for fabrication costs. The inventory interest rates are computcd at $10 \%$ per annum, and plutonium is valued at $\$ 10 /$ $\mathrm{gm}$.

The low costs shown are believed to be representative of values which could be obtained after demonstration of certain safety concepts. This demonstration could be accomplished by a successful research and development program and by the construction of large prototype and/or operating fast reactor plants. The low costs include the kinds of reduction in capital costs which would occur from elimination of the secondary sodium system, from the possible use of a low-leakage tilt-up concrete building structure, from the elimination of inert-gas-filled equipment cells, and from a better definition of contingencies. The fuel-cycle costs shown in Table $4(0.45 \mathrm{mill} / \mathrm{kWhr})$ represent those obtained for the large-module design
TABLE 2. Physics Results

Doppler Compensates in Event of Sodium Loss

\begin{tabular}{l|c|c}
\hline & $\begin{array}{c}\text { Modular } \\
\text { Flux Trap }\end{array}$ & $\begin{array}{c}\text { Conventional FBR } \\
\text { (Typical) }\end{array}$ \\
\hline Doppler Effect (\$) & $\begin{array}{c}-1.50 \\
+0.53\end{array}$ & $\begin{array}{r}-0.50 \text { to }-0.75 \\
+8.0 \text { to }+12.0\end{array}$ \\
\hline Sodium Loss (\$) (max) & +0.50
\end{tabular}

Doppler Coefficient Insensitive to Sodium Loss Enriched Blankets Stabilize:

Power Distribution

Sodium Void

Doppler Coefficients

Single Module Criticality Requires $\$ 30$ Perturbation Safe Design Requires Modest Economic Compromises

\begin{tabular}{l|c|c}
\hline & $\begin{array}{c}\text { Modular } \\
\text { Flux Trap }\end{array}$ & $\begin{array}{c}\text { Conventional FBR } \\
\text { (Typical) }\end{array}$ \\
\hline Breeding Ratio & 1.30 & 1.4 to 1.5 \\
Fissile Mass (kg) & 3050 & 2000 to 2500 \\
8 to 12
\end{tabular}

and include optimistic assumptions about fabrication cost for mixed oxide fuel.

In summary, certain economic and safety trade-offs are possible in fast reactor design. These trade-offs are capable of increasing or decreasing the plant cost by many millions of dollars. The arrangement of the reference design provides a method for enhancing the Doppler reactivity effect and reducing the positivity of the sodium void effect while still maintaining an 

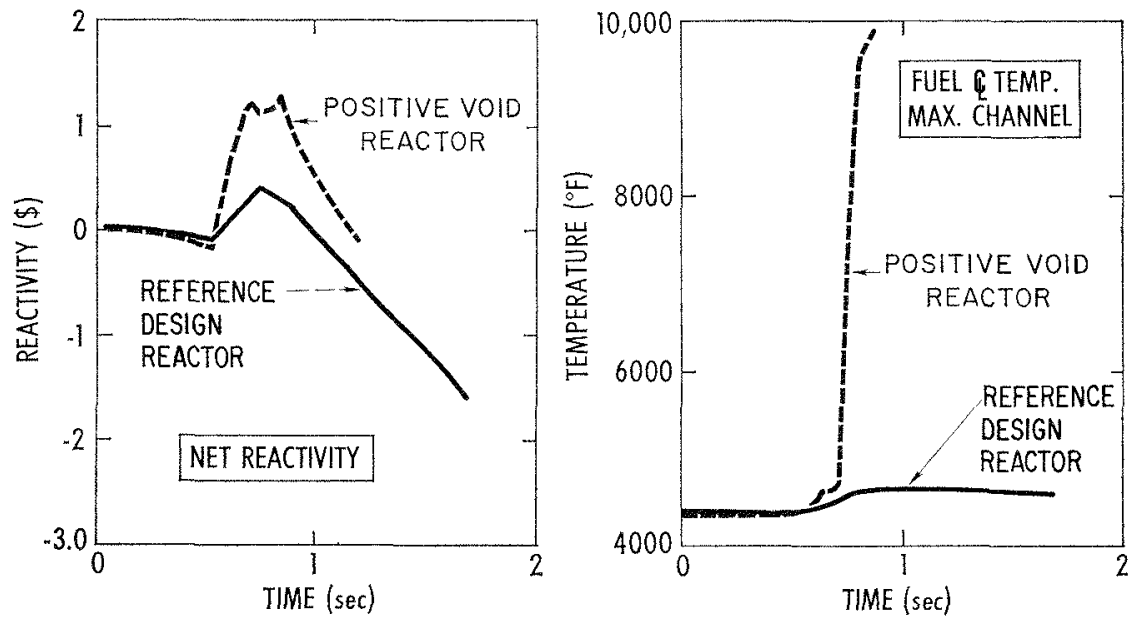

FIa. 3. Voiding Accident Behavior.

TABLE 3. Core Renctivity Coefrichents va Economics

\begin{tabular}{l|c|c}
\hline & $\begin{array}{c}\text { Small } \\
\text { Module }\end{array}$ & $\begin{array}{c}\text { Large } \\
\text { Module }\end{array}$ \\
\hline Active Height (ft) & 4.2 & 5.0 \\
Diameter across Flats (ft) & 3.5 & 4.0 \\
Doppler Reactivity Change to Fuel & -1.56 & -1.55 \\
$\quad$ Damage (\$) & & \\
Maximum Sodium Voiding Reactivity & 0.25 & 2.00 \\
$\quad$ Change (\$) & 0.76 & 0.55 \\
\hline
\end{tabular}

TABLE 4. EnERgY Costs of 1000-MWe Fast Reactor, Investor-owned Pubiac Utility

\begin{tabular}{|c|c|c|c|c|c|c|c|}
\hline \multirow{2}{*}{ Cost Item } & \multicolumn{2}{|c|}{$\begin{array}{c}\text { Capital } \\
\text { Cost }\left(\$ 10^{6}\right)\end{array}$} & \multirow{2}{*}{$\begin{array}{l}\text { Rate } \\
(\%)\end{array}$} & \multicolumn{2}{|c|}{$\begin{array}{c}\text { Annual } \\
\text { Cost }\left(\$ 10^{6}\right)\end{array}$} & \multicolumn{2}{|c|}{$\begin{array}{l}\text { Unit Cost } \\
\text { (mill/ } \\
\text { kWhr) }\end{array}$} \\
\hline & $\begin{array}{l}8 \\
90\end{array}$ & $\stackrel{3}{3}$ & & $\frac{50}{30}$ & 草 & 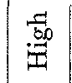 & 苞 \\
\hline $\begin{array}{l}\text { Fixed Charges } \\
\text { Capital }\end{array}$ & 150.6 & 120.6 & 14.5 & 21.8 & 17.5 & 3.12 & 2.50 \\
\hline $\begin{array}{l}\text { Nuclear Liability } \\
\text { Insurance } \\
\text { Operating Costs }\end{array}$ & - & - & $-\cdots$ & - & - & - & - \\
\hline Fuel-cycle Cost & - & $\ldots$ & - & 6.02 & 3.15 & 0.86 & 0.45 \\
\hline $\begin{array}{l}\text { Operating and } \\
\text { Maintenance }\end{array}$ & - & - & -- & 2.3 & 1.58 & 0.33 & 0.22 \\
\hline $\begin{array}{c}\text { Total Cost of Energy } \\
\text { Generation }\end{array}$ & & & & 30.45 & 22.56 & 4.36 & 3.22 \\
\hline
\end{tabular}

economic system with a fuel-cycle cost of $3 / 4$ mill/ $\mathrm{kWhr}$ (at $\$ 10 / \mathrm{gm}$ plutonium and $80 \%$ load factor). These particular safety-oriented parameters (Doppler and voids) can be strengthened or weakened in the reference design by suitable rearrangement of the geometric array of the reactor. In general, inereased safety results in increased cost.

\section{Discussion}

Mr. Peterson (Battelle-Northwest): A couple of years ago we investigated a similar concept and we found that indeed it did help our safety problems tremendously, but we traded this off for a poor power distribution across the core. We also found quite a power shift during the core lifetime. Would you care to comment on these characteristics?

Mr. Eggen: I'm sorry I forgot to give acknowledgment to you. We are aware of some of your printed reports. The indications of power distribution across the core are satisfactory as far as I can remember. We find that there is, of course, loading of depleted fuel in the blanket region and taking out of fuel which has derived about $7 \%$ plutonium by end of life, which amounts to something to the order of about 50,000 $\mathrm{MWd} / \mathrm{T}$ equivalent irradiation. In the blanket region there will be, of course, local power shifts. Investigation has shown that the temperature swings were relatively small; the problems associated with the structure, etc., could be accommodated, and hydraulic factors were introduced to accommodate this in the reactor design. Our complete report will soon be published as NAA-SR-11378.

$M r$. Chernick (BNL): To what extent do you pay for the fact that your coefficients don't apply to isothermal conditions, that the blanket coefficient Doppler effect is indeed delayed relative to the core, and that you again have problems of flux shape during possible accidents?

Mr. Eggen: We carried these through the various kinetic calculations with appropriate time constants using the Avery type of coupling analysis. We have also looked at it from the standpoint of using an analog system in which we put certain components. Because of the smallness of the system and the fact 
that most of the reactors operate with very fast neutrons, the time delays are not of concern.

Mr. Chernick: In really big accidents the short time constants of the fast reactor above prompt critical would be of concern.

Mr. Sevy (AI): In evaluating the Doppler feedback the specific power in the blanket which is lower than that in the core was taken into account. I believe what Mr. Chernick is asking about is the effect rising from the difference of prompt-neutron lifetimes in the thermalized blanket as compared to fast sections.

Mr. Eggen: In this case the Doppler is down by a factor of 3 or 4 .

Mr. Wirtz (Karlsruhe): Did you say how your power is distributed between the fast and thermal parts of your reactor?

Mr. Eggen: We do not have a thermal part, but the blanket area has about $40 \%$ of the total power of the system. About $25 \%$ of the power is in the inner blanket and about 13 or $14 \%$ in the outer blanket region; $60 \%$ is in the fast core sections.

$M r$. Wirtz: Did you say that in this case you have a breeding ratio of about 1.3 ?

Mr. Eggen: Overall, yes.

Mr. Wirtz: From my memory of the Karlsruhe calculation this seems to be fairly high.

$M r$. Eggen: Some people are inclined to say that the results of our calculations are a little higher than the average. Yet we had access to four fast reactor reports. We cross calculated such systems and derived approximately the same characteristics and the same breeding ratio. We thus assume we are on a comparable basis.

$M r$. Häfele (Karlsruhe): I think that in such a coupled concept as yours there is a very flat power distribution in the core and I think an accidental excursion gives a relatively large energy release. The energy release might be higher by a factor of 60 or so in the case of a very flat power distribution. Although you do get advantages with respect to Doppler coefficients and sodium void coefficients, in the case of a maximum hypothetical accident your energy releases might be higher by a factor of 50. How do you balance that against each other?

Mr. Eggen: One factor, of course, is that we have isolated the different modules. We believe that you can mechanically shut down the rest of the reactor system. For our excursion, a Bethe-Tait type of analysis indicated energy releases only something of the order of $70 \mathrm{lb}$ of TNT.

$M r$. Greebler (GE) : One aspect of your calculation that is a little disturbing is that the mode of the decoupling has appeared to make each module look like a very small reactor for sodium voiding purposes. In other words, neutrons along the edge have a low importance; yet for the Doppler effect it appears that the neutrons along the edge must have high importance. There is a bit of a contradiction here, but let's be specific. How did you treat the question of coupling, and how did the coupling between the modules change when sodium is removed? If you did this by studying a single module with adjustable boundary conditions, one can get very large errors from what one assumes about the boundary conditions.

Mr. Eggen: The power affects the Doppler coefficient and the temperature rise in the system, etc. Most of the Doppler effect comes from the inner part of the inner blanket, the outer blanket contributing primarily to the sodium void coefficient. The sodium void coefficient is significantly reduced by trapping neutrons from adjacent module units and also those that might have been reflected back into the system. Thus it is the outer blanket which effectively holds down the sodium void coefficient and it is the inner module that contributes most strongly towards the Doppler coefficient where it has more importance.

Mr. Sevy: In regard to Mr. Greebler's second question, we evaluated coupling in the normal operating case and determined that the self-criticality in the case of $k_{11}$ for any one of the modules was approximately 0.90 . This was done by having the eriticalsource modes in one module but no fissioning in the others.

Mr. Eggen: I think the answer is that we did not study that particular problem.

Mr. Avery (ANL): My comment relates to the previous paper mostly, but it was touched upon here. I would like to make a few qualitative statements about when we can expect the equations of the previous paper to be good and when they may fail. Let's talk about two modules, take the case where neutrons from one module will give rise to a distribution in the other module which is essentially of the same shape regardless of where the neutrons originated in the first one. Then I believe we can expect the equations as they are stated to be quite good, and I think that in two relatively isolated modules this will be the case. Quite clearly if we take a large system and arbitrarily cut it in two, this condition would not be met, and one would be involved in the situation in which one must treat the space-dependent equations. Thus by relatively intuitive thinking I think one can tell when the conditions are satisfied. This can be tested by straightforward static test if one does a single iteration starting with different source neutrons at different positions and looks at the shape at which they arrive. 


\title{
Some Safety Considerations Based on Design Experiences in the BNL Settled-bed Fast Reactor Concept*
}

\author{
Gerald S. Lellouche, Melvin M. Levine, and Jack Chernick \\ Brookhaven National Laboratory \\ Upton, New York
}

(PRESENTED BY M. M. LE\ INE)

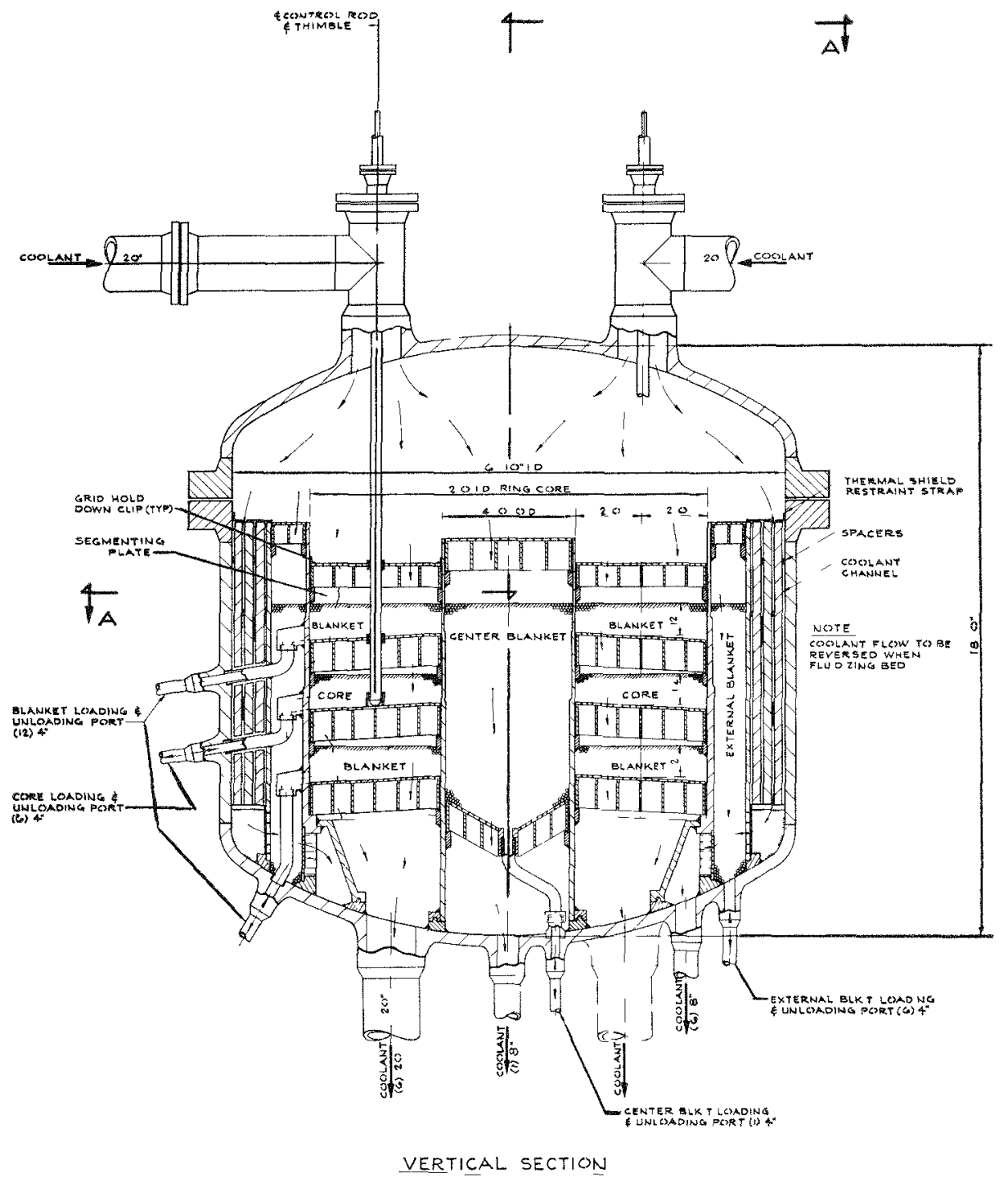

FIG. 1 Axıal Flow Settled Bed Reactor.

The mechanical design of a fast reactor can greatly affect its safety under a loss-of-coolant accident. In the short time available, we shall discuss one of the

* Work performed under the auspices of the U.S Atomic Energy Commission dominant safety features of the Settled Bed Fast Reactor (SBFR) concept, a concept we introduced at the conference two years ago. This safety feature can be of importance not only for reactors having fuel elements consisting of packed beds of fuel particles in random 


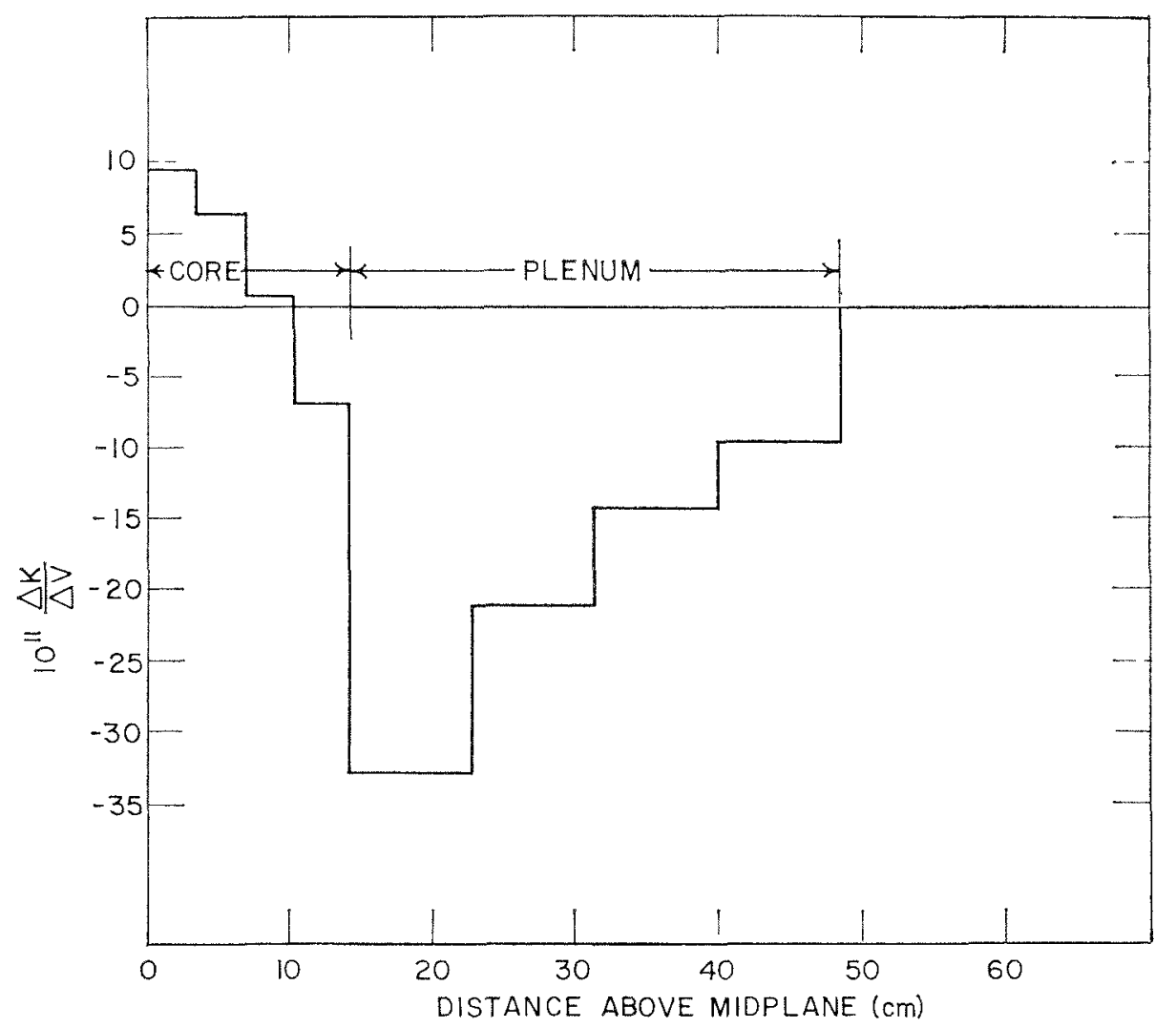

Frc. 2. Sodium Temperature Coefficient vs Position in Axial Flow Reactor.

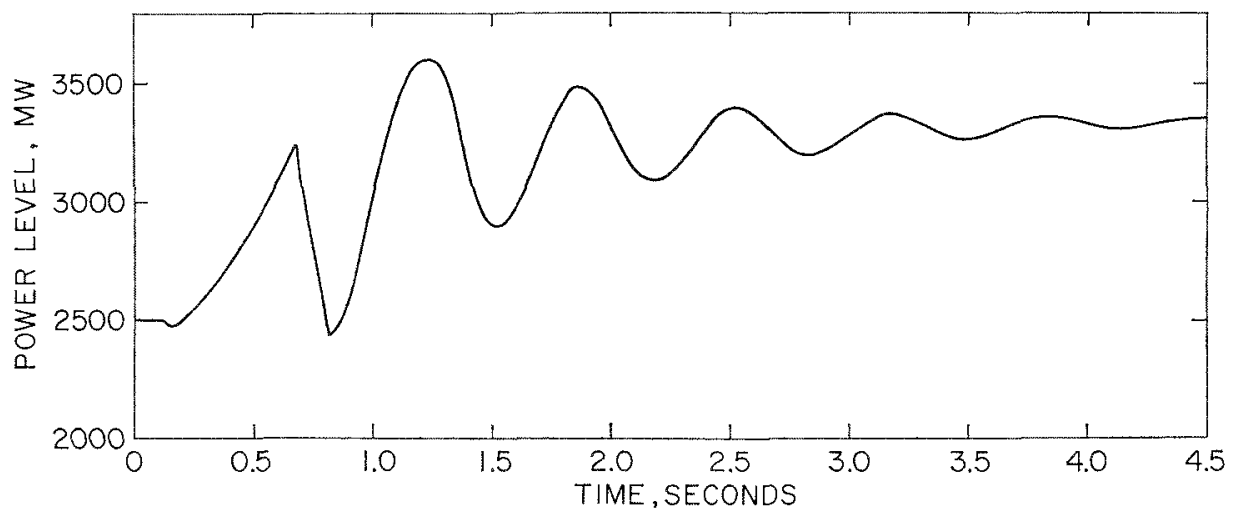

Frg. 3. Power Transient due to a Permanent 1)rop of $100^{\circ} \mathrm{C}$ in Reactor Lulet Sodium Temperature.

or ordered arrangement, but also for other reactor concepts.

In the SBFR there is a sodium region above the core and each of the axial blankets to provide head room for fluidization. In Fig. 1 is shown an elevation of one conceptual design. Immediately above and below the core are the sodium regions which are considered here. For both of these regions there is a strong negative reactivity effect when the sodium expands.

In Fig. 2 the local sodium expansion coefficient at power is shown as a function of position in this re- actor. As in most fast reactors, there is a local positive effect at the center of the core, and this becomes negative at the core edge. Here, however, there is a still stronger negative effect as we move into the sodium region. The overall temperature coefficient in this sodium region is many times larger than the core sodium and Doppler coefficients.

The temperature coefficient in the sodium region is a delayed one, due to the sodium transit time through the core, of the order of magnitude of $0.1 \mathrm{sec}$. In the present case this is a powerfully stabilizing influence. 


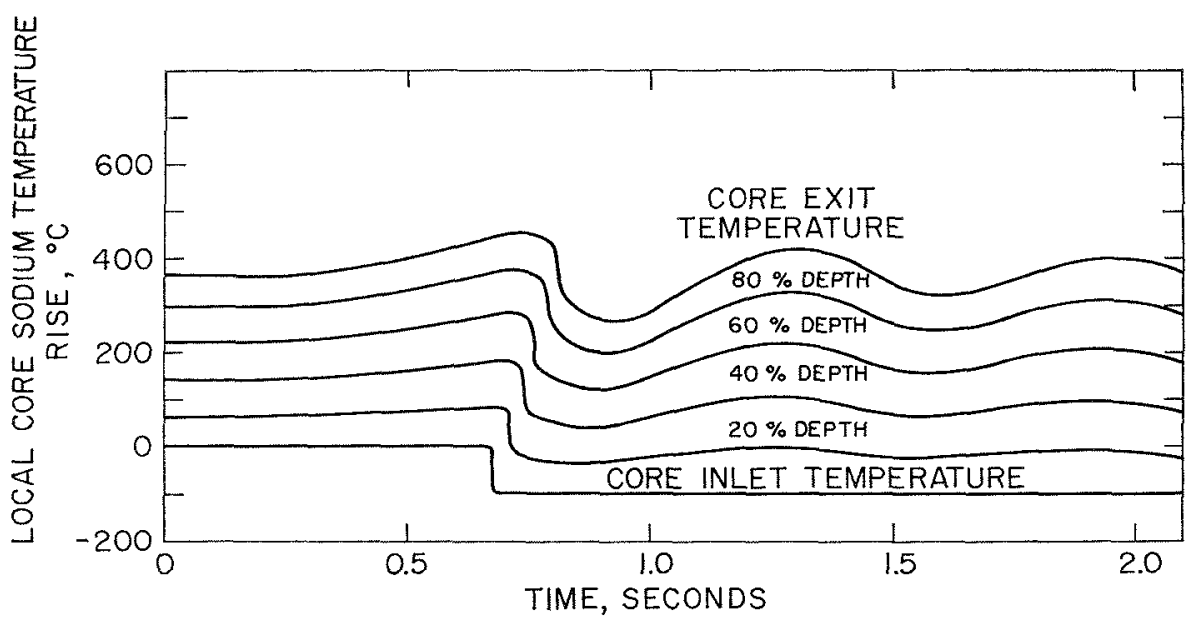

Fig. 4. Tariation of Local Core Sodium Temperatures after $100^{\circ} \mathrm{C}$ Drop in Reactor Inlet Sodium Temperature.

Figure 3 shows the time behavior of the reactor power when the inlet sodium temperature is reduced $100^{\circ} \mathrm{C}$ and maintained at that lower temperature; this corresponds to a reactivity increase of $\sim \$ 0.4$. Even for this drastic temperature change, the peak power exceeds the original power level by less than $50 \%$. The various turning points of this curve can be related to the passage of the cold front through various regions of the reactor, but our time limit here prevents discussing this.

Figure 4 shows the core sodium temperature at several positions as a function of time for the same perturbation. The peak rise of exit temperature exhibited is only $25 \%$ above the initial value. The time behavior exhibited was obtained from a multiregion space-andtime solution of the reactor equations, which were programmed at Brookhaven National Laboratory.

The sodium regions were introduced into the SBFR because of bed engineering requirements. However, a similar high-leakage region might well be of benefit in other reactor designs as well. The location, the size, and the composition of such a region can all be ad- justed to obtain desirable stability properties. This might be considered for use in many fast reactor designs. For fuel elements containing ordered arrays of fuel particles, such as in the Brookhaven National Laboratory Countercurrent Reactor concept presently under study, the values of the parameters of location, size, and composition are set by the fashion in which the individual fuel particles are stacked in the fuel element. Since the ordered bed fuel element can be designed so to be disassembled and reassembled periodically, there parameters can be altered when necessary.

\section{Discussion}

Mr. Kohler (Texas A\&M) : I noticed that on your curve for the active power when inserting cold sodium into the reactor, there was initially a very small dip in the reactor power. Could you explain that?

Mr. Levine: Yes. This very very small dip is a real effect. It is a very slightly positive reactivity coefficient from the blanket of the reactor. 


\title{
Comparison of $\mathrm{He}, \mathrm{CO}_{2}$, and Steam as Coolants of a 1000-MWe Fast Reactor*
}

\author{
M. Dalle-Donne $\dagger$ \\ Kernforschungszentrum \\ Karlsruhe, Germany
}

\section{Introduction}

The safety problems arising because of the sodium void coefficient in big fast reactor cores make the possibility of using a much less dense coolant, like a gas, very interesting. The recent discovery of the explosive character of sodium boiling(1,2) also makes these problems even more serious.

In the present paper the performances of three fluids: helium, carbon dioxide, and steam, as coolant of a big fast reactor core are compared.

\section{Equations Governing the Heat Transfer in the Coreł}

Assuming a chopped-cosine, axial power distribution and a core geometry given by a cluster of parallel smooth fuel rods, the hydraulic diameter of the cooling channel is given by

$$
d_{h}=K_{0}\left(\frac{P^{\prime}}{P}\right)^{0.8} \frac{H P^{\prime 0.2}}{Q^{0.2}}
$$

where

$$
\begin{gathered}
K_{0}=0.0193\left(T_{2}-T_{1}\right)^{0.2} \frac{1}{\nu}\left(\frac{\rho_{w}}{\rho}\right)^{0.8} \\
\cdot\left(\frac{c_{p m}^{0.2} c_{p w}^{0.8}}{c_{p M}}\right) \frac{k_{w}^{0.2}}{P r_{w}^{0.4}}\left(\frac{H^{\prime}}{H}\right) ; \\
\sqrt{\left(2 \varphi \frac{c_{p M}}{c_{p m}}-1\right)^{2} \sin ^{2} \frac{\pi}{2 H}-1}
\end{gathered}
$$

The factor $K_{0}$ includes all the parameters depending on the physical properties of the coolant, but it depends also on the ratio $H^{\prime} / H$, on the coolant temperatures at the inlet and outlet of the core, and on the maximum

* This paper has been prepared within the framework of the association EURATOM-Gesellschaft für Kernforschung mbH., in the field of fast breeder development.

$f$ Delegated from EURATOM to the "Fast Breeder Project," Kernforschungszentrum Karlsruhe.

$\ddagger$ The formulae shown in this paragraph are obtained in Appendix I. A table of nomenclature follows the appendices. surface temperature of the fuel element. However, once these parameters are chosen, $K_{0}$ is fixed and characteristic of the coolant chosen. It is interesting to notice at this point, that $K_{0}$ is pressure-independent for a perfect gas.

The total pressure drop in the core is the sum of various different contributions:

$\Delta p_{1}$, due to friction of the coolant against the wall of the coolant channel;

$\Delta p_{2}$, due to turbulent dissipation caused by the grids supporting the fuel rods;

$\Delta p_{3}$, due to the momentum loss necessary to accelerate the coolant in the cooling channel (acceleration due to increase of temperature and decrease of pressure), and acceleration losses and recoveries at the inlet and outlet of the channel.

If we assume a core geometry given by smooth fuel rods supported by transversal grids, the analytical expressions of these pressure drops are

$$
\begin{aligned}
\Delta p_{1}= & \frac{1.115}{\left(T_{2}-T_{1}\right)^{1.8}} \frac{\mu_{m}^{0.2}}{\rho_{m} c_{p m}^{1.8}} \frac{\left(P^{\prime} / P\right)^{1.8} H}{d_{h}^{3} P^{\prime 1.8}} Q^{1.8} \\
\Delta p_{2}= & \frac{16 \xi}{\left(T_{2}-T_{1}\right)^{2}} \frac{1}{\rho_{m} c_{p m}^{2}} \frac{\left(P^{\prime} / P\right)^{2} Q^{2}}{d_{h}^{2} P^{\prime 2}} ; \\
\Delta p_{3}= & \frac{16}{\left(T_{2}-T_{1}\right)^{2}}\left(\frac{T_{2}-T_{1}}{T_{m}}+\ln \frac{p_{1}}{p_{2}}\right. \\
& \left.\quad+\frac{1.1 T_{1}-0.55 T_{2}}{2 T_{m}}\right) \frac{1}{\rho_{m} c_{p m}^{2}} \frac{\left(P^{\prime} / P^{2} Q^{2}\right.}{d_{h}^{2} P^{\prime 2}} .
\end{aligned}
$$

To these pressure drops correspond three contributions to the total pumping power required to circulate the coolant in the core. The pumping power, as usual, is referred to the heat quantity to be abducted:

$$
\begin{aligned}
& \frac{N_{1}}{Q_{i h}}=K_{1}\left(P / P^{\prime}\right)^{0.6} \frac{Q^{2.4}}{H^{2} P^{\prime 2.4}} ; \\
& \frac{N_{2}}{Q_{l h}}=K_{2}\left(P^{\prime} / P\right)^{0.4} \frac{Q^{2.4}}{H^{2} P^{\prime 2.4}} ; \\
& \frac{N_{3}}{Q_{t h}}=K_{3}\left(P^{\prime} / P\right)^{0.4} \frac{Q^{2.4}}{H^{2} P^{\prime 2.4}},
\end{aligned}
$$


where

$$
\begin{aligned}
K_{1}= & \frac{1.553 \cdot 10^{5}}{\eta} \frac{\nu^{3}}{\left(T_{2}-T_{1}\right)^{3.4}} \frac{\left(\rho / \rho_{w}\right)^{2.4}}{\rho_{1} \rho_{m}} \\
& \cdot \frac{c_{p M}^{3}}{c_{p m}^{3.4} c_{p w}^{2.4}} \frac{\mu_{m}^{0.2} P r_{w}^{1.2}}{k_{w}^{0.6}}\left(\frac{H}{H^{\prime}}\right)^{3} ; \\
K_{2}= & \frac{4.29 \cdot 10^{4} \xi}{\eta} \frac{\nu^{2}}{\left(T_{2}-T_{1}\right)^{3.4}} \frac{\left(\rho / \rho_{w}\right)^{1.6}}{\rho_{1} \rho_{m}} \\
& \cdot \frac{c_{p M}^{2}}{c_{p m}^{3.4} c_{p w}^{1.6}} \frac{P r_{w}^{0.8}}{k_{w}^{0.4}}\left(\frac{H}{H^{\prime}}\right)^{2} ; \\
& \frac{4.29 \cdot 10^{4}}{\eta}\left(\frac{T_{2}-T_{1}}{T_{m}}+\ln \frac{p_{1}}{p_{2}}+\frac{1.1 T_{1}-0.55 T_{2}}{2 T_{m}}\right) \\
& \cdot \frac{\nu^{2}}{\left(T_{2}-T_{1}\right)^{3.4}} \frac{\left(\rho / \rho_{w}\right)^{1.6}}{\rho_{1} \rho_{m}} \frac{c_{p M}^{2}}{c_{p m}^{3.4} c_{p w}^{1.6}} \frac{P r_{w}^{0.8}}{k_{w}^{0.4}}\left(\frac{H}{H^{\prime}}\right)^{2} .
\end{aligned}
$$

Therefore the total pumping power required by the core is

$$
\begin{aligned}
& \frac{N_{t}}{Q_{t h}}=\left[K_{1}\left(P / P^{\prime}\right)^{0.6}+K_{2}\left(P^{\prime} / P\right)^{0.4}\right. \\
& \left.\quad+K_{3}\left(P^{\prime} / P\right)^{0.4}\right] \frac{Q^{2.4}}{H^{2} P^{\prime 2.4}}=K_{4} \frac{Q^{2.4}}{H^{2} P^{\prime 2.4}} .
\end{aligned}
$$

\section{General Comparison Based on Heat Transfer Properties}

For a certain fuel-element rod (constant $P^{\prime} / P, P^{\prime}$, $H$ ) and a certain amount of heat $Q$ to be carried away from any coolant channel of the core, $d_{h}$ is proportional to $K_{0}$.

We assume that the core is formed by parallel fuel rods without extended heat transfer surfaces (fins, etc.). The coolant volume fraction in the fuel boxes is given by

$$
\alpha=\frac{1}{1+\frac{d}{d h}} .
$$

Equation (19) of Ref. 3 shows that the optimum diameter of the fuel rod from the point of view of fuel-cycle considerations is proportional to $\chi^{0.25} / H^{0.25}$ for constant density, enrichment, and average burn-up of the fuel. The quantity $\chi H$ is the heat produced by each fuelelement rod, and it is equal to $Q$, the heat abducted in any coolant channel, in the case of square array of rods, or equal to $1 / 2 Q$ in the case of a triangular array. Thus we can write

$$
d=c_{1} \frac{Q^{0.25}}{H^{0.5}} .
$$

From (1), (9), and (10) we obtain

$$
\alpha=\frac{1}{1+\frac{d^{2.8}}{K_{0} c_{1}^{2} c_{2}^{0.2} Q^{0.3}}},
$$

where $P^{\prime}=c_{2} d$. Equation (11) gives the physical meaning of $K_{0}$ : for constant fuel-rod diameters, an increase in $K_{0}$ means an increase in coolant volume fraction; for constant coolant volume fractions, an increase in $K_{0}$ requires an increase in fuel-rod diameter.

In the previous reasoning $\chi H$, or $Q$, is held constant: this is significant because, as shown in Ref. $3, \chi H$ is one of the main parameters upon which the fuel cycle depends.

The physical meaning of $K_{4}$ is easier to understand: for a certain fuel-element rod and certain thermal output $Q$ for a coolant channel, $K_{4}$ is proportional to the total pumping power required to circulate the coolant in the core, referred to the total thermal output.

In order to compare the values of $K_{0}$ and $K_{4}$ for $\mathrm{He}$, $\mathrm{CO}_{2}$, and steam as coolant of a fast reactor core, we have to make a series of estimates in choosing the numerical values of some of the parameters which appear in the expressions.

\section{Coolant Pressure at the Core Inlet}

The physical properties of the coolant depend on the coolant pressure. The pressure variation along the cooling channels is generally quite small in comparison with the absolute value (of the order of a few percent for acceptable reactors). Accordingly we can take the pressure at only one section of the core as a characteristic of the pressure field. The most significant pressure is the maximum, because it influences the design of the pressure vessel, and it is generally at the inlet of the core. We have performed calculations for three values of $p_{1}$, namely, 70, 100, and $150 \mathrm{~atm}$. Such high pressures are necessary with gas cooling and are technically feasible with the use of prestressed concrete pressure vessels.

\section{Maximum Cladding Surface Temperature}

This temperature depends on the material used for cladding and on the can concept used. In terms of stainless steel, a maximum surface temperature of $650^{\circ} \mathrm{C}$ would be probably appropriate with free-standing clad; and $700^{\circ} \mathrm{C}$ with collapsed clad. (It is interesting to notice that these values are nominal maximum and that they would correspond to maximum hot spot temperatures of $750-800^{\circ} \mathrm{C}$ and $800-850^{\circ} \mathrm{C}$, respectively.) These figures, however, are only indicative; the cladding problem requires a lot of experimental investigation yet. Calculations were performed for both temperature values. 


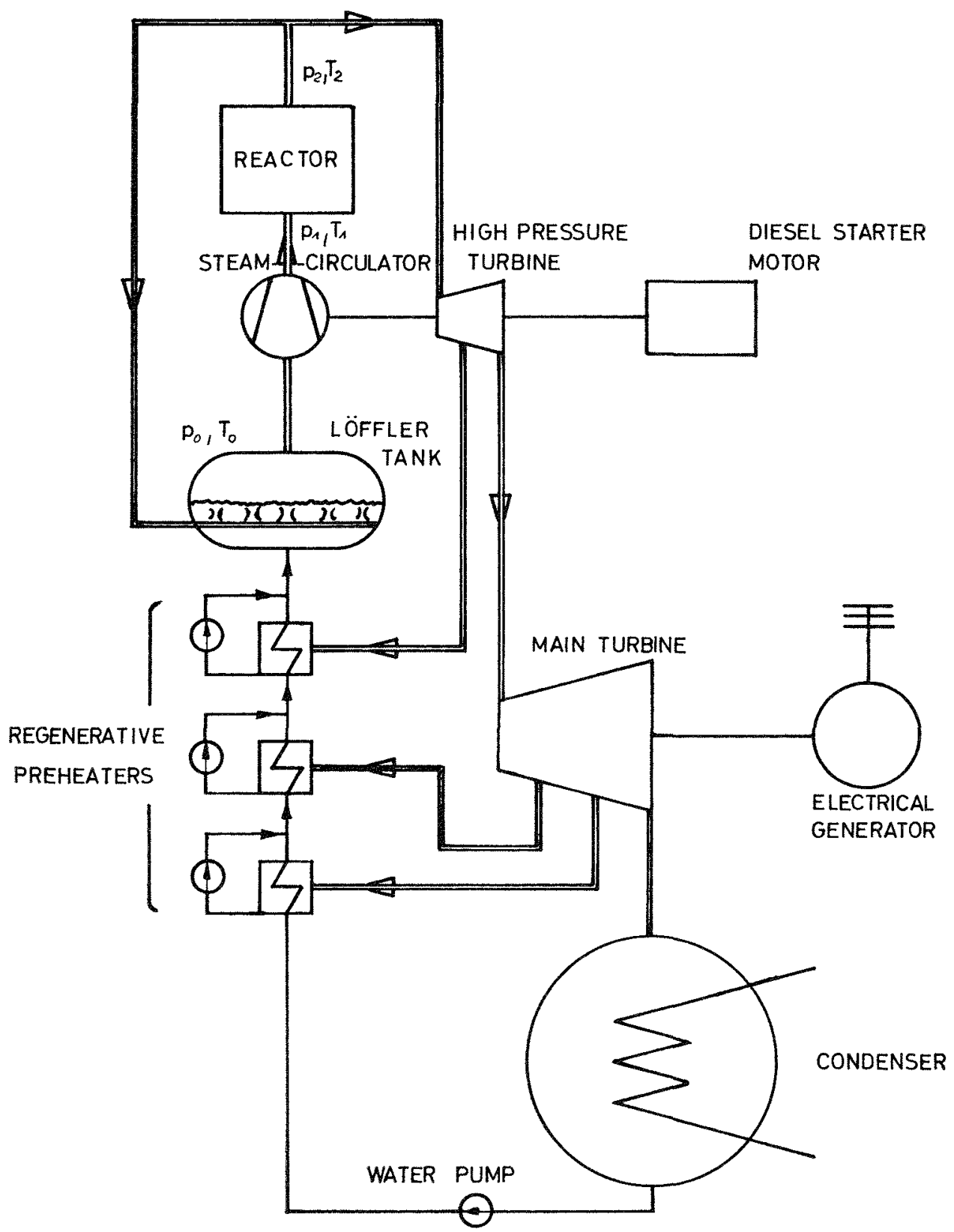

FIg. 1. Schematic Flow Diagram of Steam-cooled Power Plant.

\section{Coolant Temperature at the Core Outlet}

In the case of steam cooling no secondary circuit is used (see Fig. 1). Thus the coolant temperature at the core outlet is practically equal to the maximum superheated steam temperature required by the turbines. Modern big turbines require temperatures in the range from $538^{(4,5)}$ to $565^{\circ} \mathrm{C}$ or more. ${ }^{(6)}$ At the lower temperature ferritic steels can still be used; with the higher austenitic steels are necessary. In our calculations we used an average value of $550^{\circ} \mathrm{C}$. In the case of $\mathrm{He}$ or
$\mathrm{CO}_{2}$, heat exchangers are required. A difference between outlet gas temperature and maximum steam temperature in the heat exchangers often used is $30^{\circ} \mathrm{C} .{ }^{(7)} \mathrm{In}$ recent studies of gas-cooled fast reactors such a difference has been assumed equal to $60^{\circ} \mathrm{C}^{(4)}$ or to $83^{\circ} \mathrm{C} .{ }^{(5)}$ As a reasonable compromise we chose $50^{\circ} \mathrm{C}$. The resulting He and $\mathrm{CO}_{2}$ outlet temperature is $600^{\circ} \mathrm{C}$. In reality this temperature should be obtained by an optimisation process based on the cost of the kWhe, because the greater is $T_{2}$ (for a constant maximum steam tempera- 
ture) the smaller are the sizes of the heat exchangers and (for a constant value of $T_{w M}$ ) the bigger the pumping power.

\section{Coolant Temperature at the Core Inlet}

The gas inlet temperature (for $\mathrm{He}$ and $\mathrm{CO}_{2}$ ) should also be obtained by an optimisation process. Indeed, the higher it is, the higher is the efficiency of the steam cycle, but the coolant mass flow must be increased to carry away the same quantity of heat from the core and the pumping power increases considerably. In the studies indicated above, ${ }^{(4,5)} T_{1}$ has been chosen equal to $260^{\circ} \mathrm{C}$, which seems a reasonable value in terms of the considerations just mentioned. The same value was chosen in the present study. However, it is believed that the range of variation of $T_{1}$ is much larger than that of $T_{2}$, and it depends on the coolant pressure (more preeisely, higher values of $p_{1}$ allow higher $T_{1}$ 's). In a very recent study, ${ }^{(8)}$ released when the majority of our calculations were already performed, $T_{1}$ was assumed equal to $300^{\circ} \mathrm{C}$ for $p_{1}=70 \mathrm{~atm}$ and equal to $340^{\circ} \mathrm{C}$ for $p_{1}=140 \mathrm{~atm}$.

For steam the choice of $T_{1}$ is not free. Indeed, Fig. 1 clearly shows that $T_{1}$ depends on the pressure $p_{1}$ and the pumping power $N_{t} / Q_{t h}$, which fixes the degree of superheating of the steam at the core inlet. In Appendix II the relationship between $T_{1}, p_{1}$, and $N_{t} / Q_{t h}$ is studied. The values of $T_{1}$ shown in Table 1 were ob- tained from the values of $N_{t} / Q_{t h}$ in the numerical example shown in the next paragraph. The indicated values of $T_{1}$ are then less general than those for the other two gases. However, the variation of $T_{1}$ with $N_{t} / Q_{t h}$ in the range of practical values is quite small.

Other Parameters Appearing in the Expressions of $K_{0}$ and $K_{4}$

We assume:

$P^{\prime} / P=1$, because we refer to fuel element without heat transfer extended surfaces.

$H / H^{\prime}=0.821$, which refers to the numerical example of the next paragraph.

$\xi=0.21$, which corresponds to a fuel-element grid support studied at Karlsruhe. ${ }^{(9)}$

$\eta=0.726 .{ }^{(10)}$

The coolant pressure at the core outlet, $p_{2}$, appears also in the expression for $K_{4}$, but the influence on $K_{4}$ is very weak. Thus, as in the case of $T_{1}$ for steam, the values of $p_{2}$ have been obtained from the numerical example of the next paragraph. The physical properties of helium were taken from References 11, 12, and 13. The transport properties of carbon dioxide are from Ref. 14 and the thermodynamic properties from Ref. 15. The properties of steam were taken from References $16-20$.

Table 1 below shows the values of $K_{0}$ and $K_{4}$ calculated on the basis of the above assumptions. These

TABLE 1

\begin{tabular}{|c|c|c|c|c|c|c|}
\hline Coolant & $\begin{array}{c}\text { Maximum Surface } \\
\text { Fuel-element } \\
\text { Temperature, } \\
T_{M}\left({ }^{\circ} \mathrm{C}\right)\end{array}$ & $\begin{array}{c}\text { Core Inlet } \\
\text { Pressure } p_{1} \text { (atm) }\end{array}$ & $\begin{array}{c}\text { Core Inlet } \\
\text { Coolant } \\
\text { Temperature } \\
T_{1}\left({ }^{\circ} \mathrm{C}\right)\end{array}$ & $\begin{array}{c}\text { Core Outlet } \\
\text { Coolant } \\
\text { Temperature } \\
T_{2}\left({ }^{\circ} \mathrm{C}\right)\end{array}$ & $\begin{array}{l}\text { Collant Channel } \\
\text { Size Factor } K_{0} \cdot 10^{2} \\
\left(\mathrm{cal}^{0.2} \mathrm{~cm}^{-0.2} \mathrm{sec}^{-0.2}\right.\end{array}$ & $\begin{array}{l}\text { Pumping Power to } \\
\text { Thermal Output } \\
\text { Ratio Factor } K_{4} \cdot 10^{6} \\
\left(\text { cal }^{-2.4} \mathrm{~cm}^{4.4} \mathrm{sec}^{2.4}\right.\end{array}$ \\
\hline $\mathrm{He}$ & 650 & $\begin{array}{r}70 \\
100 \\
150\end{array}$ & $\begin{array}{l}260 \\
260 \\
260\end{array}$ & $\begin{array}{l}600 \\
600 \\
600\end{array}$ & $\begin{array}{l}1.468 \\
1.468 \\
1.468\end{array}$ & $\begin{array}{l}4.133 \\
1.816 \\
0.7706\end{array}$ \\
\hline $\mathrm{CO}_{2}$ & 650 & $\begin{array}{l}100 \\
150\end{array}$ & $\begin{array}{l}260 \\
260\end{array}$ & $\begin{array}{l}600 \\
600\end{array}$ & $\begin{array}{l}1.040 \\
1.039\end{array}$ & $\begin{array}{l}4.575 \\
1.331\end{array}$ \\
\hline \multirow{2}{*}{ Steam } & 650 & $\begin{array}{r}70 \\
100 \\
150\end{array}$ & $\begin{array}{l}310.4 \\
319.1 \\
344.5\end{array}$ & $\begin{array}{l}550 \\
550 \\
550\end{array}$ & $\begin{array}{l}1.666 \\
1.644 \\
1.584\end{array}$ & $\begin{array}{l}3.066 \\
0.9439 \\
0.3028\end{array}$ \\
\hline & 700 & $\begin{array}{r}70 \\
100 \\
150\end{array}$ & $\begin{array}{l}295.6 \\
315.2 \\
342.6\end{array}$ & $\begin{array}{l}550 \\
550 \\
550\end{array}$ & $\begin{array}{l}1.972 \\
1.981 \\
1.856\end{array}$ & $\begin{array}{l}1.228 \\
0.5080 \\
0.1791\end{array}$ \\
\hline
\end{tabular}


values are fairly general because they depend mainly on the gas chosen, and on the pressure and temperatures indicated in the table.

One can see that:

$K_{0}$ is practically not affected by the pressure.

Higher values of $T_{w}$ mean higher coolant volume fractions for constant fuel-rod diameters or higher fucl-rod diameters for constant coolant volume fractions.

Carbon dioxide requires smaller coolant volume fractions or smaller fuel-rod diameters than those for helium or steam.

In any case, steam requires the minimum amount of pumping power.

Helium is the second best.

\section{Numerical Example}

The above considerations, although useful and rather general, are not really conclusive. We know, for instance, that steam requires less pumping power than the other two gases, but this doesn't really mean much if we don't know something about the core dimensions, plant efficiency, fuel rating, etc.

We therefore made calculations for particular reactors using the above values of $K_{0}$ and $K_{4}$, and the following parameters: plant net electrical output: $1000 \mathrm{MWe}$

pin diameter in the core: $0.635 \mathrm{~cm}$

core height: $183 \mathrm{~cm}$ (chosen in a way to have reactors with $\mathrm{H} / \mathrm{D} \cong 0.7$ )

cladding material: stainless steel

cladding thickness: $0.38 \mathrm{~mm}$

average power per pin length: $211 \mathrm{~W} / \mathrm{cm}$

maximum power per pin length: $384 \mathrm{~W} / \mathrm{cm}$ (that is, a maximum oxide fuel temperature equal to $2200^{\circ} \mathrm{C}$ )

pin length due to axial blanket and gaseous fission products store chamber: the same as the core height

pin diameter in gaseous fission products store chamber: $80 \%$ of that in the core

pumping power required by the primary circuit other than reactor: $2 \%$ of the total thermal output for $\mathrm{He}$ and $\mathrm{CO}_{2} ; 1 \%$ for steam at 70 and 100 atms, $0.5 \%$ for steam at 150 atms

core blockage factor: $14 \%$ in volume, of which $13 \%$ made up of structural material and $1 \%$ of coolant The steam cycle thermodynamic efficiencies (which depend mainly on $T_{1}$ and $T_{2}$ ) have been calculated with the optimum number of regenerative preheatings. ${ }^{(21)}$ Auxiliary power other than that required by the primary coolant circulators and by the feedwater

TABLE 2

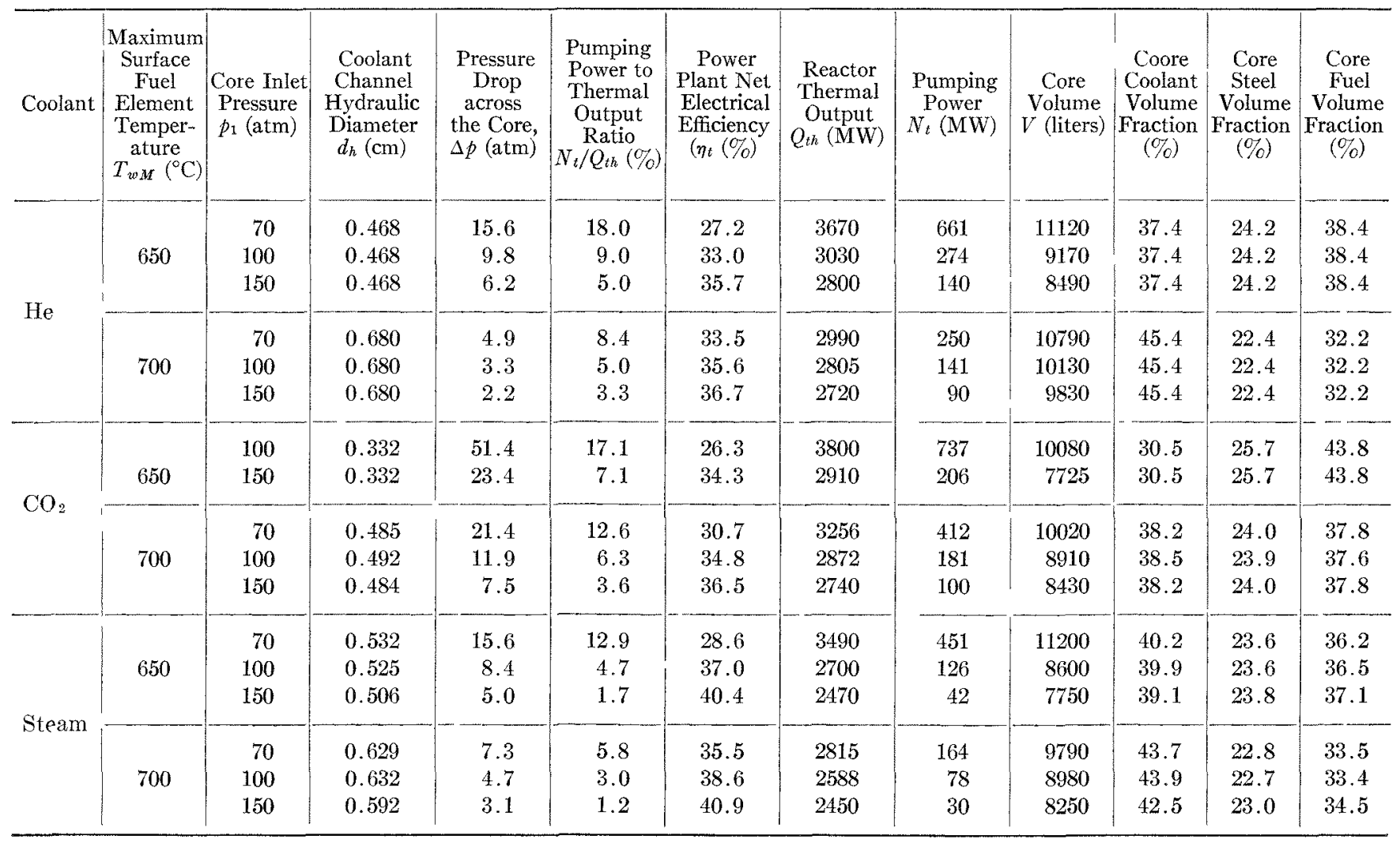




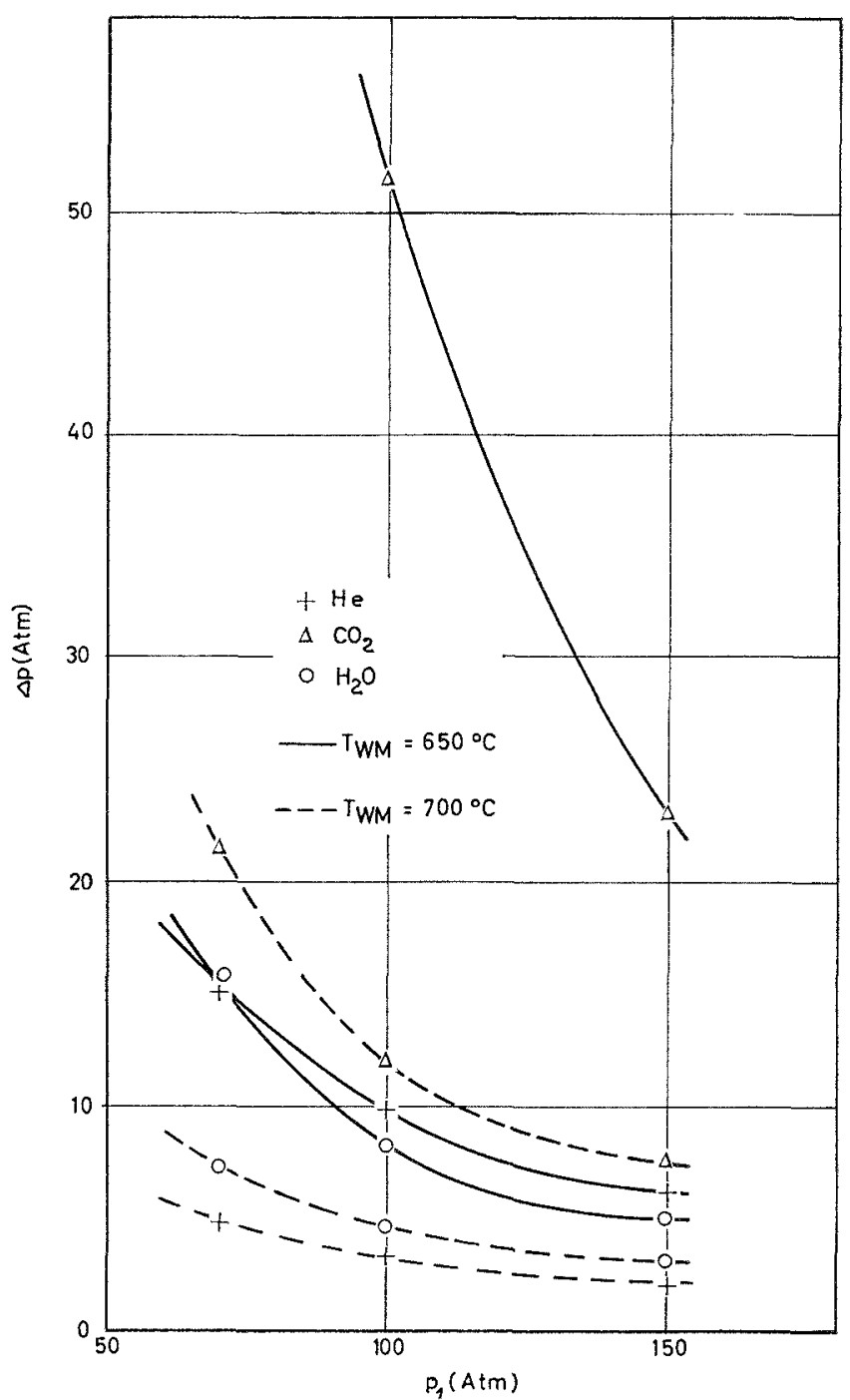

Fia. 2. Pressure Drop across the Core for a Constant Net Electrical Output of $1000 \mathrm{MWe}$.

pumps in the turbine circuit has been neglected. The primary-coolant circulators were assumed to be driven by high-pressure steam turbines.

These parameter's and assumptions can be considered typical of big gas-cooled reactors. Some of them are from previous studies on the subject; $;^{(4,5,8)}$ some come from estimates of the best or most likely values of these parameters.

The necessity for choosing values for such parameters reduces considerably the generality of the comparison performed in this paper. Indeed, the three coolants should be compared, not for the same parameters, but in the conditions in which their different properties can be used best. In practice this is very difficult, especially because of the great uncertainties in estimating the variations of plant capital cost when the above parameters, plus temperatures and pressure, vary. Nevertheless, it is believed that this type of comparison is still significant, because the physical properties of the three coolants compared are not extremely different. A comparison with sodium in these conditions would naturally be less significant.

The results of the calculations are reported in Table 2 and Figures 2 to 6 . As expected, carbon dioxide requires the minimum amount of coolant volume fraction, but produces the highest pressure drops in the core. As far as pumping power and plant efficiency are concerned, steam is always the best, at parity of $p_{1}$ and $T_{w M}$, followed by helium and then by carbon dioxide.

The comparison performed was for constant core height and therefore constant fuel-rod diameter [sce equation (10)]. The results of a comparison in which $H$ and $d$ vary, but always remain in the relationship (10), especially for carbon dioxide (where the values of $K_{0}$ are so much different from those of the other two coolants) are shown in Table 3 for $\mathrm{CO}_{2}$; the coolant-

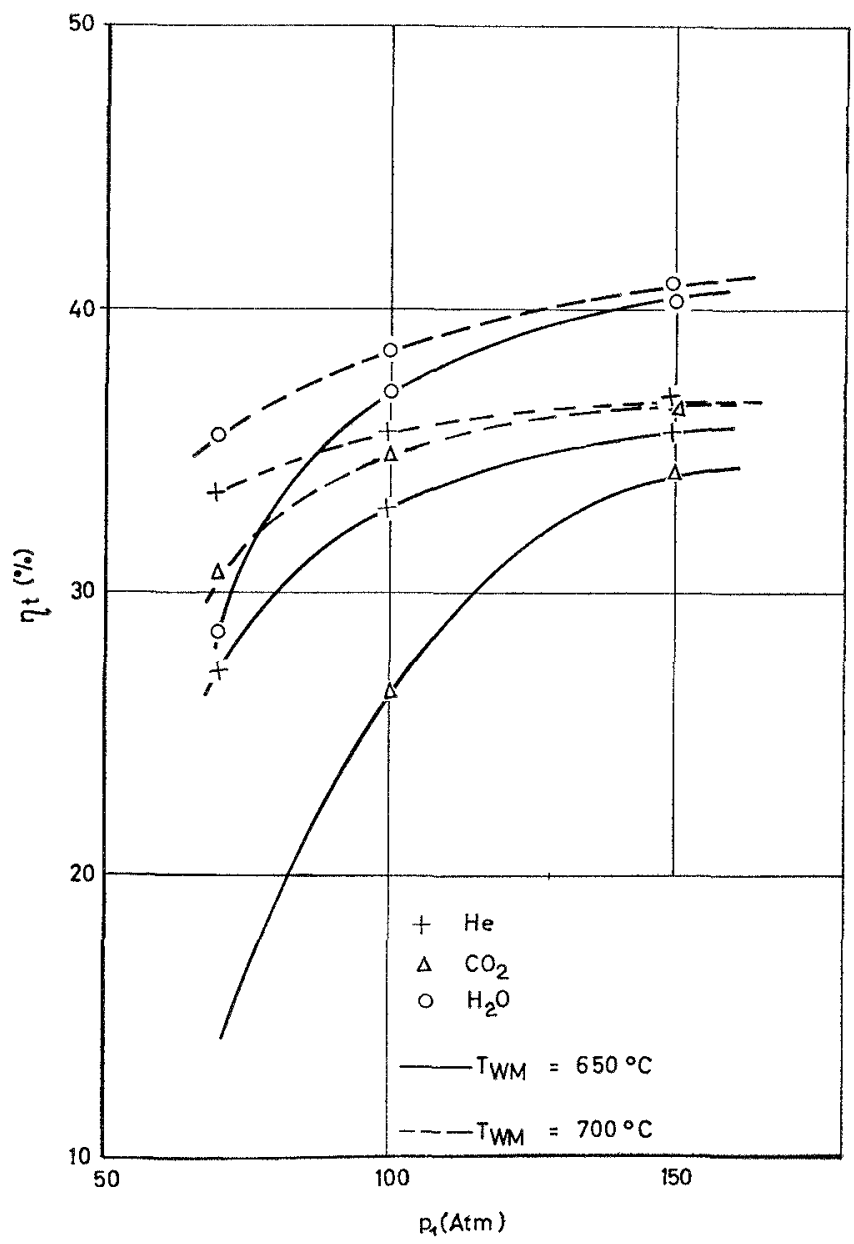

FIx. 3. Net Electrical Efficiency for a Constant Net Electrical Output of $1000 \mathrm{MWe}$. 
channel hydraulic diameter was held equal to that obtained for helium $(0.68 \mathrm{~cm})$. One can see that the performance of $\mathrm{CO}_{2}$ improves with respect to the case with $H=$ constant, although it is still not as good as that of steam, but this improvement is paid for by a considerable reduction in fuel-rod diameter. Thi- would probably produce an increase in the number of supporting grids (increase in $\xi$ ) which was not taken into account in the calculation and which would probably rompletely eancel the improvement in $N_{t} / Q_{t h}$ and $\eta_{\iota}$. Besides, H/D becomes about 1.1, and the core volume increases more than $20 \%$. Thus we thought that the improvement was not real and we did not pursue this way further.

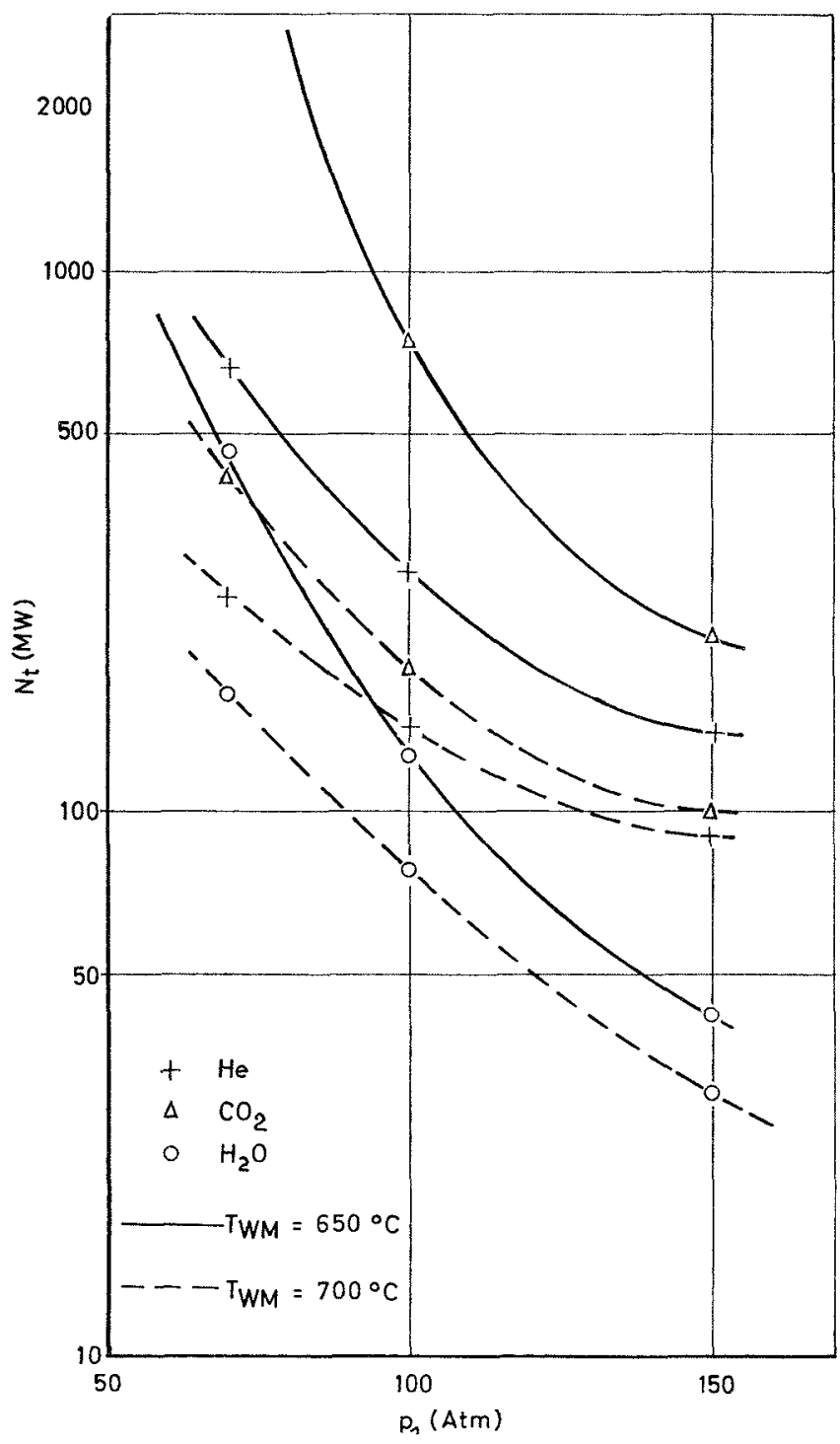

Fic. 4. Pumping Power Required by the Coolant Primary Circuit for a Constant Net Electrical Output of $1000 \mathrm{MWe}$.

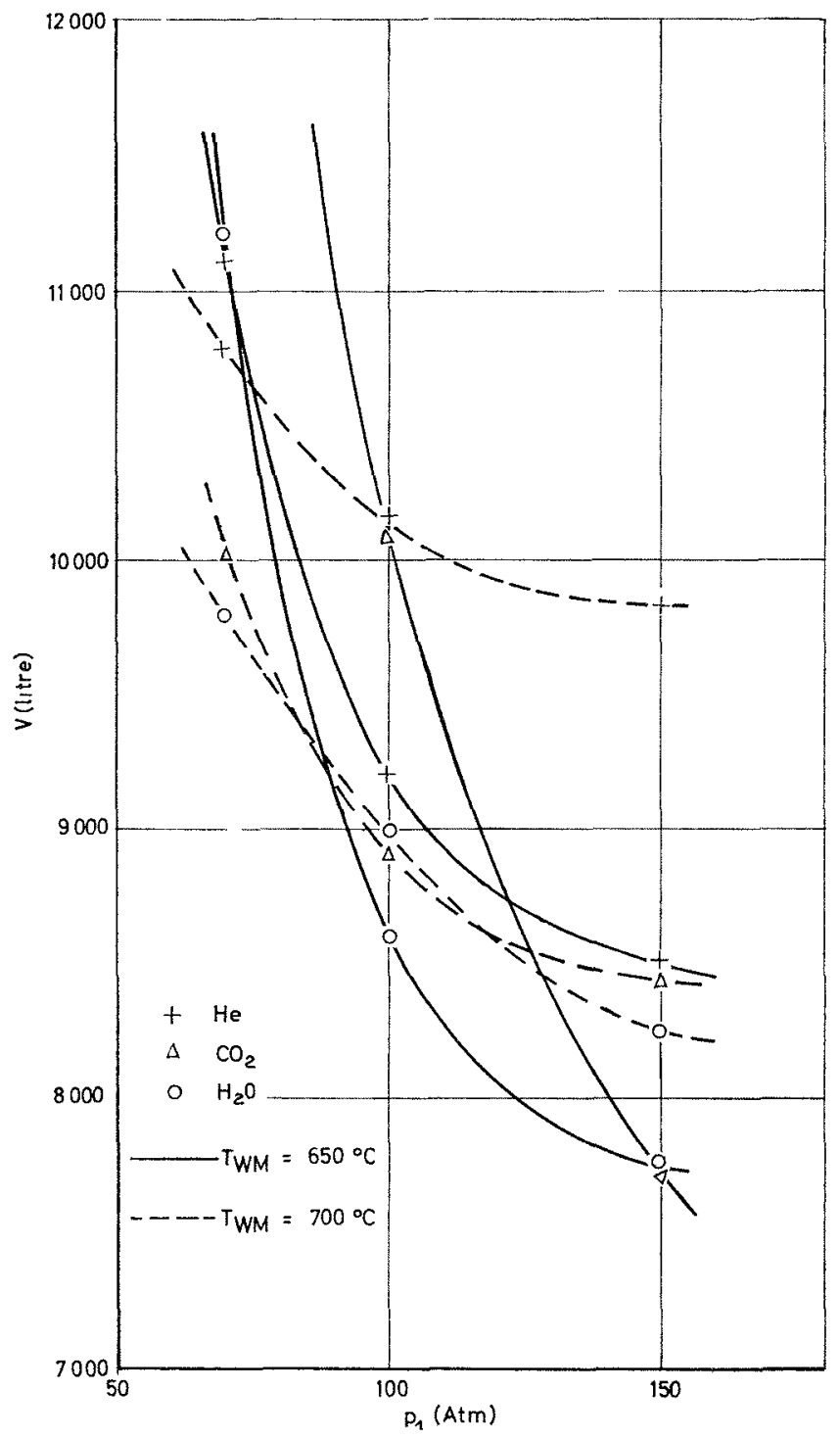

Fra. 5. Core Volume for a Constant Net Electrical Output of $1000 \mathrm{MWe}$.

\section{Neutronic Computations}

The thermal calculations allowed us to fix the dimensions, coolant, structural material, and fuel volume fractions in the core. With these parameters known, it is possible with neutronic calculations to obtain the fuel cnrichment, fuel rating, core internal and total breeding ratio, coolant void, and Doppler coefficients.

These calculations are described in more detail in Appendix III. The results are shown in 'Table 4 and in Figures 7 to 11 . The rating does not vary very much because of the assumptions that $Q, d$, and $H$ are constant. Thus for constant total plant efficiency the fuelrycle cost is approximately the same for all reactors. The Doppler coefficient constant $A_{\text {Dop }}$ is proportional 


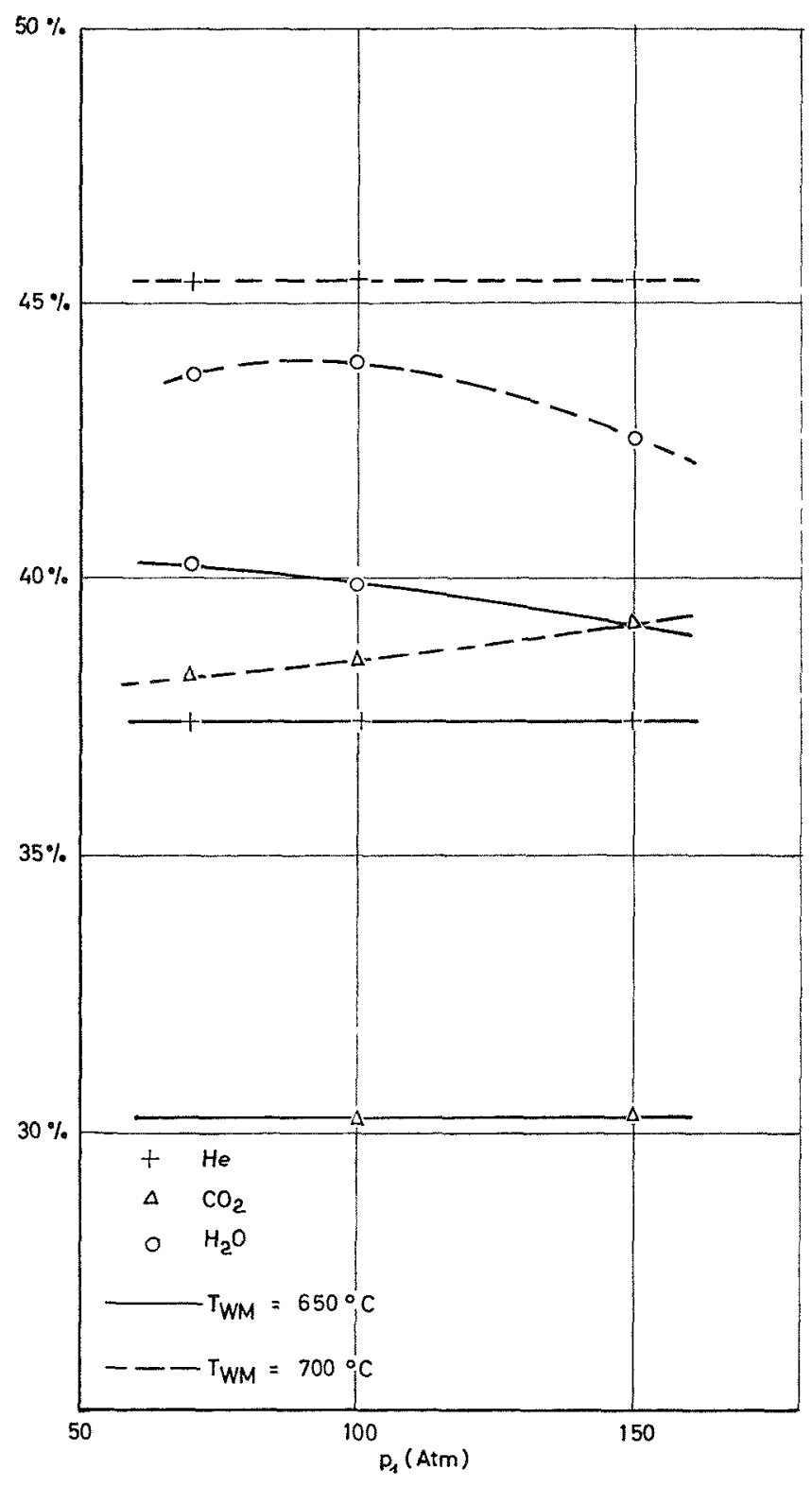

FIG, 6. Core Coolant Fraction for a Constant Net Electrical Output of $1000 \mathrm{MWe}$. to the Doppler coefficient because the fuel element temperature is approximately the same for all the cases considered.

The difference between the Doppler coefficient, calculated between the maximum fuel temperature of the average element and the fuel melting tomperature, and the roolant void roefficient is shown in Fig. 11. We consider this difference as an indicating roefficient of safety. In this one makes reference to a hypothetical accident due to instantaneous outflow of the coolant from the core and takes as a danger signal the melting of all the fuel with temperature above the peak temperature of the average fuel element. This coefficient of safety is only indicative, because such an arcident is in practice impossible; the action of the negative Doppler coefficient is always much faster than that of the positive void coefficient in any possible forseen accident, especially if one contemplates the use of a prestressed pressure vessel. A real safety evaluation could be derived only by a detailed analysis of any possible credible aceoident.

\section{Conclusions}

The results of this study have not a definitive character, since too many assumptions have been made. Some are somewhat arbitrary, for instance, the fixing of the height of the core, of the coolant temperature at the core inlet for helium and carbon dioxide, and of the pumping power required by the primary circuit other than reactor.

Furthermore, the assumed fuel-element geometry can be improved. For instance, partial roughening of the fuel-element rods or use of heat-transfer-extended surfaces for mechanical support would probably allow the use of rods with bigger diameters. It is clear also that partial roughening will improve the performance more with carbon dioxide than with helium or steam because of the tendency of that gas to require very small rod diameters with smooth rods. On the other hand, use of extended surfaces will improve the performance

TABLE 3

\begin{tabular}{|c|c|c|c|c|c|c|c|c|c|}
\hline \multicolumn{10}{|c|}{$\mathrm{CO}_{2}: T_{1}=260^{\circ} \mathrm{C} ; T_{2}=600^{\circ} \mathrm{C} ; T_{w M}=700^{\circ} \mathrm{C} ; d_{h}=0.680 \mathrm{~cm}$} \\
\hline $\begin{array}{l}\text { Core Inlet } \\
\text { Pressure } \\
p_{1}(\mathrm{~atm})\end{array}$ & $\begin{array}{c}\text { Core Height } \\
H(\mathrm{~cm})\end{array}$ & $\begin{array}{l}\text { Fuel Rod } \\
\text { Diameter } \\
d(\mathrm{~cm})\end{array}$ & $\begin{array}{l}\text { Pressure } \\
\text { Drop across } \\
\text { the core } \\
p(\mathrm{~atm})\end{array}$ & $\begin{array}{c}\text { Pumping } \\
\text { Power } 10 \\
\text { Thermal } \\
\text { Output } \\
\text { Ratio } \\
N_{B} / Q_{t h}(\%)\end{array}$ & $\begin{array}{c}\text { Power Plant } \\
\text { Aet } \\
\text { Electrical } \\
\text { Efficiency } \\
\eta_{i}(\%)\end{array}$ & $\begin{array}{l}\text { Reactor } \\
\text { Thermal } \\
\text { Output } \\
Q_{t h}(\mathrm{MW})\end{array}$ & $\begin{array}{l}\text { Pumping } \\
\text { Power } \\
N_{t} \text { (MW) }\end{array}$ & $\begin{array}{c}\text { Core Volume } \\
V \text { (liters) }\end{array}$ & $\begin{array}{c}\text { Core Coolant } \\
\text { Volume } \\
\text { Fraction } \\
(\%)\end{array}$ \\
\hline 70 & 266 & 0.526 & 15.9 & 9.6 & 32.7 & 3061 & 294 & 12250 & 49.5 \\
\hline 100 & 262 & 0.540 & 8.9 & 4.7 & 35.8 & 2790 & 132 & 11360 & 48.9 \\
\hline
\end{tabular}


TABLE 4

\begin{tabular}{|c|c|c|c|c|c|c|c|c|c|}
\hline Coolant & $\begin{array}{c}\text { Maximum } \\
\text { Surface Fuel } \\
\text { Element } \\
\text { Temperature } \\
T_{w M}\left({ }^{\circ} \mathrm{C}\right)\end{array}$ & $\begin{array}{c}\text { Core Inlet } \\
\text { Pressure } \\
p(\text { atm })\end{array}$ & $\begin{array}{l}\text { Enrichment } \\
\text { (Number of } \\
\text { Atoms of } \\
\text { Fissile } \\
\text { Plutonium } \\
\text { Over Total } \\
\text { Fuel) }\end{array}$ & $\begin{array}{c}\text { Rating } \\
\text { (MWth/kg } \\
\text { fiss) }\end{array}$ & $\begin{array}{c}\text { Core } \\
\text { Internal } \\
\text { Breeding } \\
\text { Ratio }\end{array}$ & $\begin{array}{l}\text { Total } \\
\text { Breeding } \\
\text { Ratio }\end{array}$ & $\begin{array}{c}\text { Void } \\
\text { Coefficient } \\
(\$)\end{array}$ & $\begin{array}{c}\text { Doppler } \\
\text { Coefficient } \\
\text { Constant } \\
A_{D \circ p}\end{array}$ & $\begin{array}{c}\text { Indicative } \\
\text { Safety } \\
\text { Coefficient } \\
(\$)\end{array}$ \\
\hline \multirow{2}{*}{$\mathrm{He}$} & 650 & $\begin{array}{r}70 \\
100 \\
150\end{array}$ & $\begin{array}{l}0.0958 \\
0.0976 \\
0.0986\end{array}$ & $\begin{array}{l}1.03 \\
1.01 \\
1.00\end{array}$ & $\begin{array}{l}1.108 \\
1.083 \\
1.071\end{array}$ & $\begin{array}{l}1.382 \\
1.382 \\
1.384\end{array}$ & $\begin{array}{l}0.413 \\
0.608 \\
0.922\end{array}$ & $\begin{array}{l}-0.00892 \\
-0.00870 \\
-0.00859\end{array}$ & $\begin{array}{l}-1.040 \\
-0.810 \\
-0.478\end{array}$ \\
\hline & 700 & $\begin{array}{r}70 \\
100 \\
150\end{array}$ & $\begin{array}{l}0.1037 \\
0.1050 \\
0.1050\end{array}$ & $\begin{array}{l}0.952 \\
0.939 \\
0.939\end{array}$ & $\begin{array}{l}1.015 \\
1.001 \\
1.000\end{array}$ & $\begin{array}{l}1.336 \\
1.332 \\
1.330\end{array}$ & $\begin{array}{l}0.555 \\
0.789 \\
1.183\end{array}$ & $\begin{array}{l}-0.00810 \\
-0.00795 \\
-0.00850\end{array}$ & $\begin{array}{l}-0.764 \\
-0.506 \\
-0.202\end{array}$ \\
\hline $\mathrm{CO}_{2}$ & 650 & $\begin{array}{l}100 \\
150\end{array}$ & $\begin{array}{l}0.0925 \\
0.0949\end{array}$ & $\begin{array}{l}1.07 \\
1.04\end{array}$ & $\begin{array}{l}1.152 \\
1.121\end{array}$ & $\begin{array}{l}1.407 \\
1.400\end{array}$ & $\begin{array}{l}0.703 \\
1.185\end{array}$ & $\begin{array}{l}-0.00944 \\
-0.00937\end{array}$ & $\begin{array}{l}-0.835 \\
-0.342\end{array}$ \\
\hline \multirow{2}{*}{ Steam } & 650 & $\begin{array}{r}70 \\
100 \\
150\end{array}$ & $\begin{array}{l}0.1014 \\
0.1050 \\
0.1068\end{array}$ & $\begin{array}{l}0.972 \\
0.939 \\
0.924\end{array}$ & $\begin{array}{l}1.004 \\
0.947 \\
0.899\end{array}$ & $\begin{array}{l}1.257 \\
1.209 \\
1.148\end{array}$ & $\begin{array}{l}5.40 \\
6.89 \\
8.71\end{array}$ & $\begin{array}{l}-0.0133 \\
-0.0141 \\
-0.0152\end{array}$ & $\begin{array}{l}+3.24 \\
+4.59 \\
+5.23\end{array}$ \\
\hline & 700 & $\begin{array}{r}70 \\
100 \\
150\end{array}$ & $\begin{array}{l}0.1068 \\
0.1092 \\
0.1099\end{array}$ & $\begin{array}{l}0.924 \\
0.902 \\
0.899\end{array}$ & $\begin{array}{l}0.944 \\
0.899 \\
0.859\end{array}$ & $\begin{array}{l}1.214 \\
1.162 \\
1.100\end{array}$ & $\begin{array}{l}5.92 \\
7.47 \\
9.06\end{array}$ & $\begin{array}{l}-0.0131 \\
-0.0141 \\
-0.0155\end{array}$ & $\begin{array}{l}+3.78 \\
+5.17 \\
+6.54\end{array}$ \\
\hline
\end{tabular}

more with steam and helium, because there is more space to accommodate them in the core than with carbon dioxide.

These are the limitations of the present study. However, we believe that the similarity of the physical properties of the coolants considered allows a fairly accurate comparison although the absolute values may be not the best for the three coolants.

The conclusions can be summarized as follows:

1. When the maximum coolant pressure and the maximum fuel-element clad surface temperature are held constant:

a) Steam is by far the best coolant from a thermal point of view, i.e., the most economical, followed by helium. Carbon dioxide is the worst coolant.

b) Carbon dioxide-cooled reactors are the best breeders. Steam-cooled reactors have the smallest breeding ratios.

c) If one takes the available Doppler minus the total coolant void reactivity as a criterion indicative for safety, then helium is the safest coolant, steam the most dangerous, with the difference between helium and carbon dioxide being very small.
2. The passage from maximum fuel cladding surface temperature of $700^{\circ} \mathrm{C}$ (indicative for collapsed fuel clad) to a temperature of $650^{\circ} \mathrm{C}$ (free standing clad) produces a considerable decrease in the total plant efficiency at low pressures, the effect being almost negligible at high pressures. The effect on breeding and safety coefficient is very small; the $650^{\circ} \mathrm{C}$ temperature is in any case better.

3. In Figures 12 and 13 the total breeding ratios and the safety coefficients (Doppler minus void) are plotted versus the total plant efficiency $\eta_{t}$, thus giving an indication of breeding versus economy, and safety versus economy. From these figures it appears that for a constant plant efficiency $\eta_{t}$ the carbon dioxide- and helium-cooled reactors are better breeders and safer than the steam-cooled reactors. However, since steam is a better coolant from a thermal point of view, the comparison at constant $\eta_{t}$ is much closer than at constant coolant pressure. Furthermore, it should be stressed that these diagrams are biased rather strongly against steam because for a constant $\eta$ and approximately the same fuel-cycle cost, implicit in the assumptions made in this compari- 


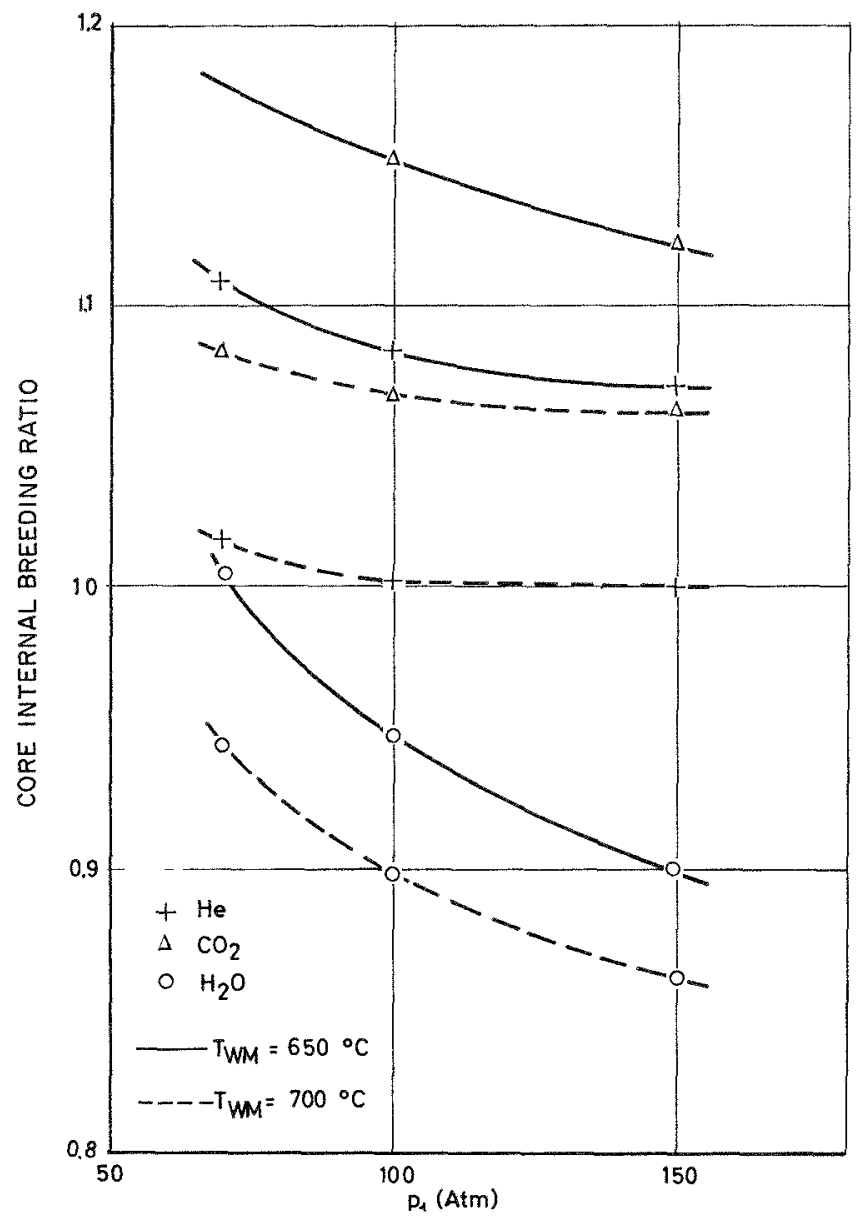

FIg. 7. Core Internal Breeding Ratio.

son, steam is more economical than carbon dioxide or helium. Indeed, the capital costs are considerably lower with steam because the required coolant pressure is smaller, and no heat exchangers are necessary. On the other hand, the turbine costs would probably be less with carbon dioxide or helium, especially if a low coolant pressure is chosen.

Until these capital costs are known with a sufficient precision, it is not possible to draw a definitive conclusion regarding the choice of a gas coolant for a large fast reactor.

\section{Appendix I}

\section{Equations Governing the Heat Transfer in the Core}

All the following considerations are valid for a central core subassembly where the maximum amount of heat will be produced. For the other subassemblies a certain amount of orificing is required.

\section{Temperature Distribution in Cooling Channels}

We assume that heat flux distribution in the core is given axially by a cosine law:

$$
q=q_{0} \cos \frac{\pi z}{H^{\prime}} .
$$

Then, the coolant enthalpy along the cooling channel is given by

$$
\begin{aligned}
G\left[I(z)-I_{1}\right]=\int_{-H / 2}^{z} q_{0} \cos \frac{\pi z}{H^{\prime}} d z \\
=\frac{q_{0} H^{\prime}}{\pi}\left(\sin \frac{\pi z}{H^{\prime}}+\sin \frac{\pi H}{2 H^{\prime}}\right) .
\end{aligned}
$$

The variation of pressure in a cooling channel is generally small in comparison with the absolute pressure value (of the order of a few percent), so it is possible to write with good approximation in place of equation (13),

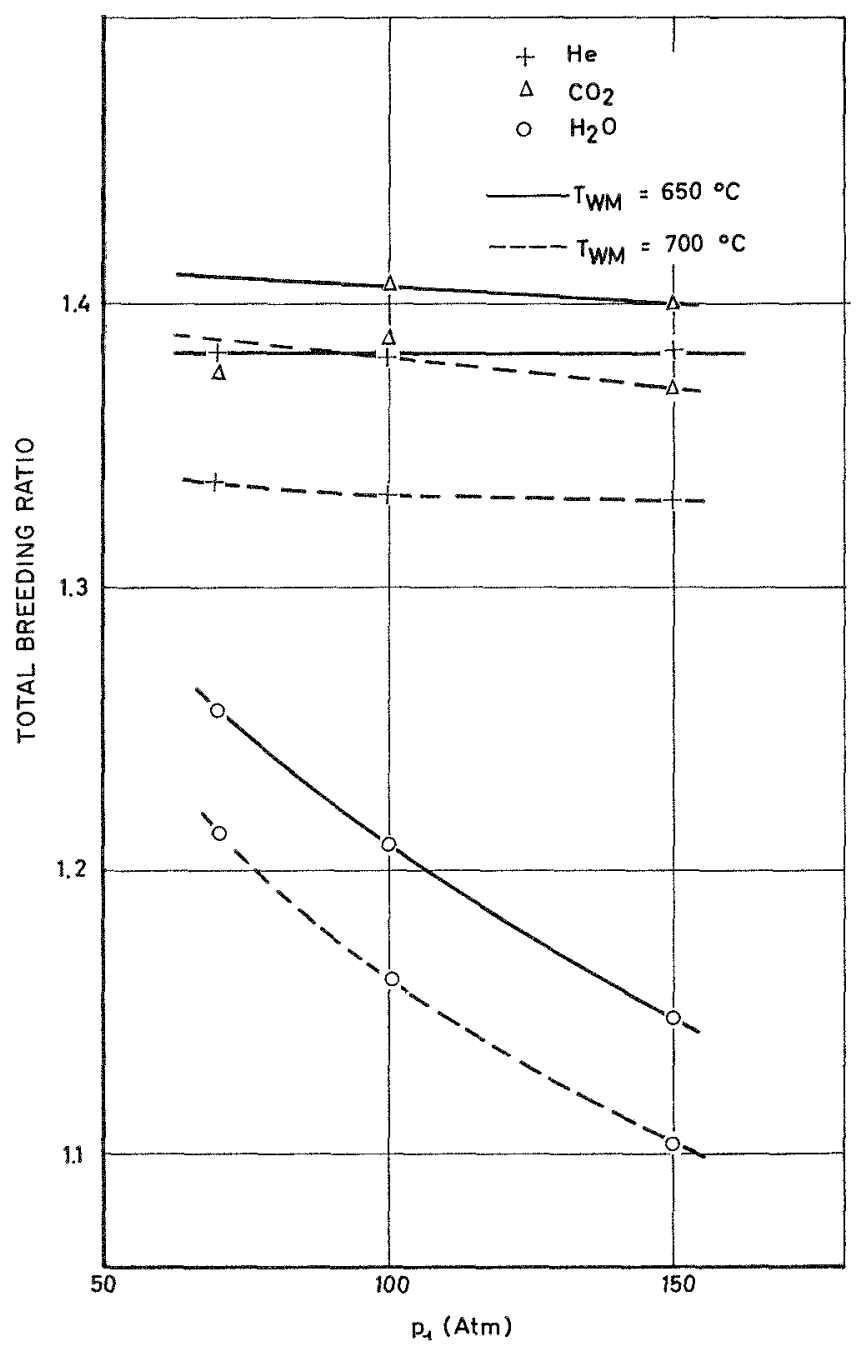

Fug. 8. Total Breeding Ratio. 


$$
G c_{p}\left[T(z)-T_{1}\right]=\frac{q_{0} H^{\prime}}{\pi}\left(\sin \frac{\pi z}{\overline{H^{\prime}}}+\sin \frac{\pi H}{2 H^{\prime}}\right),
$$

where $c_{p}$ is the sperifie heat averaged in the interval $T^{\prime}(z)-T_{1}$. This approximation is, of course, better for helium and $\mathrm{CO}_{2}$ than for steam.

For $T(z)=T_{2}$ eq. (14) beromes

$$
Q=G c_{p m}\left(T_{2}-T_{1}\right)=\frac{2 q_{0} H^{\prime}}{\pi} \sin \frac{\pi H}{2 H^{\prime}} .
$$

Therefore,

$$
q_{0}=\frac{\pi G c_{p m}\left(T_{2}-T_{1}\right)}{2 H^{\prime} \sin \frac{\pi H}{2 H^{\prime}}} .
$$

By definition one has

$$
P^{\prime} h\left[T_{w}(z)-T(z)\right]=q=q_{0} \cos \frac{\pi z}{H^{\prime}} .
$$

Therefore,

$$
\begin{array}{r}
T_{w}(z)=T_{1}+\frac{q_{0} H^{\prime}}{\pi G c_{p}}\left(\sin \frac{\pi z}{H^{\prime}}+\sin \frac{\pi H}{2 H^{\prime}}\right) \\
+\frac{q_{0}}{P^{\prime} h} \cos \frac{\pi z}{I^{\prime}},
\end{array}
$$

Now, $c_{p}$ and $h$ are not independent of $z$ because they depend on the temperature, which is not constant along the channel. However, except in rases of very high temperature gradients, this dependence is rather weak, so, for simplicity and to obtain general laws independent of temperature distributions, we assume in the following differentiation that $c_{p}$ and $h$ are independent of $z$. Differentiating (17) with respect to $z$ and setting the derivative equal to zero, we obtain the section $z_{M}$ where $T_{u}$ has a maximum:

$$
\left(\frac{\partial T_{w}}{\partial z}\right)_{z=z_{M}}=\frac{q_{0}}{G c_{p M}} \cos \frac{\pi z_{M}}{H^{\prime}}-\frac{q_{0} \pi}{P^{\prime} h H^{\prime}} \sin \frac{\pi z_{M}}{H^{\prime}}=0 .
$$

Therefore

$$
\tan \frac{\pi z_{M}}{H^{\prime}}=\frac{P^{\prime} h H^{\prime}}{G c_{p}} .
$$

We have from eq. (17),

$$
\begin{aligned}
T_{w M}=T_{1}+\frac{q_{0} H I^{\prime}}{\pi G c_{p M}}\left(\sin \frac{\pi z_{M}}{H^{\prime}}+\right. & \left.\sin \frac{\pi H}{2 H^{\prime}}\right) \\
& +\frac{q_{0}}{P^{\prime} h} \cos \frac{\pi z_{M}}{H^{\prime}} .
\end{aligned}
$$

Replacing equations (15) and (18) in (19), we obtain $\varphi=\frac{T_{w M}-T_{1}}{T_{2}-T_{1}}=\frac{1}{2} \frac{c_{p m}}{c_{p M}}\left(1+\frac{1}{\sin \frac{\pi z_{M}}{H^{\prime}} \sin \frac{\pi H}{2 H^{\prime}}}\right)$.

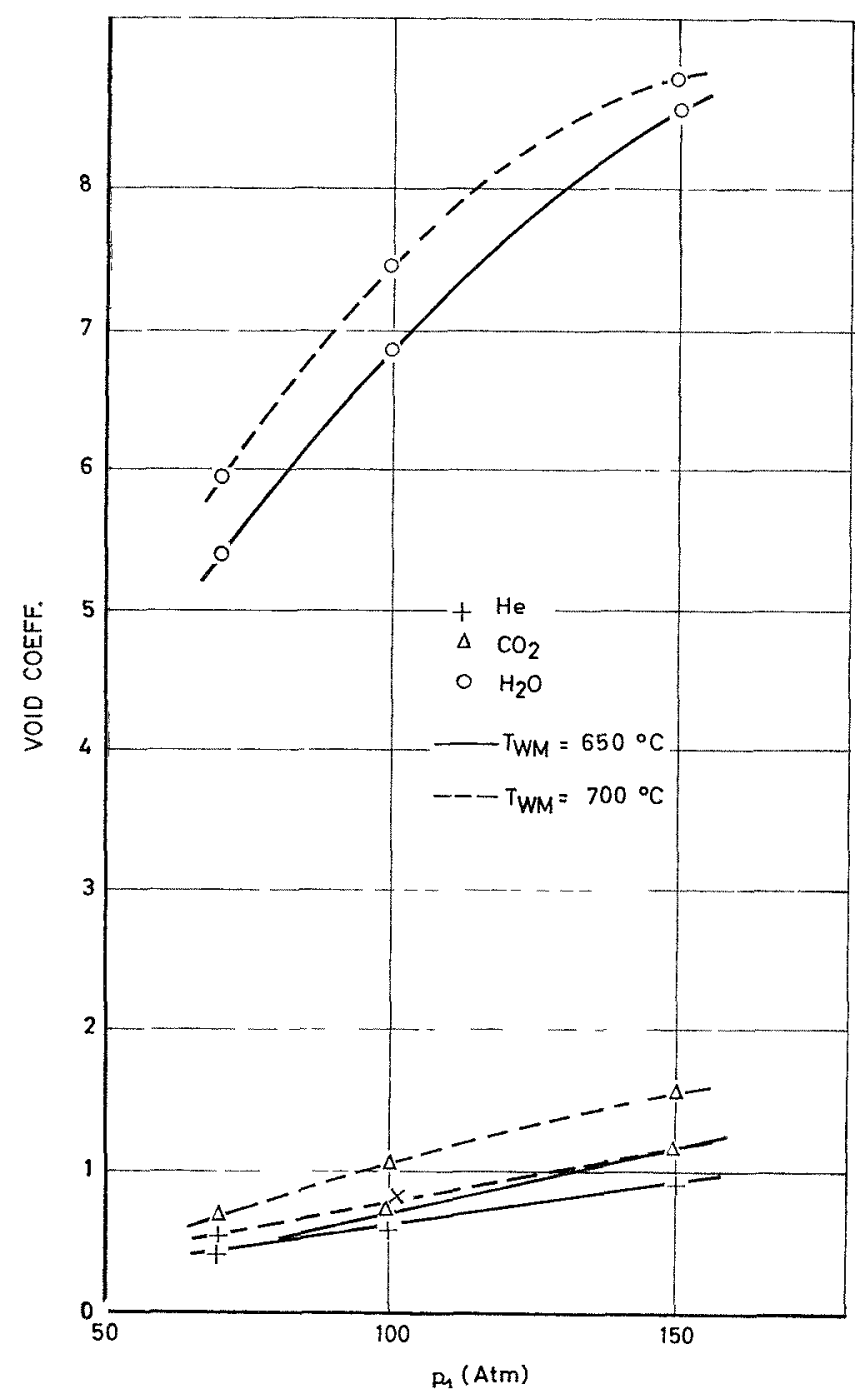

FIG. 9. Void Coefficient (\$).

Therefore

$$
\tan \frac{\pi z_{M}}{H^{\prime}}=\frac{1}{\sqrt{\left(2 \varphi \frac{c_{p M}}{c_{p m}}-1\right)^{2} \sin ^{2} \frac{\pi H}{2 H^{\prime}}-1}}
$$

and

$$
\frac{h P^{\prime} H^{\prime}}{\pi G c_{p M}}=\frac{1}{\sqrt{\left(2 \varphi \frac{c_{p M}}{c_{p m}}-1\right)^{2} \sin ^{2} \frac{\pi H}{2 H^{\prime}}-1}}=\nu .
$$

Replacing equations (15) and (18) in (14) for $z=z_{M}$, we obtain

$$
\begin{aligned}
T\left(z_{M}\right)=T_{1}+ & \frac{c_{p m}}{c_{p M}} \frac{T_{2}-T_{1}}{2} \\
& {\left[\frac{1}{\sin ^{2} \frac{\pi H}{2 H^{\prime}}\left(2 \varphi \frac{c_{p M}}{c_{p m}}-1\right)}+1\right] . }
\end{aligned}
$$




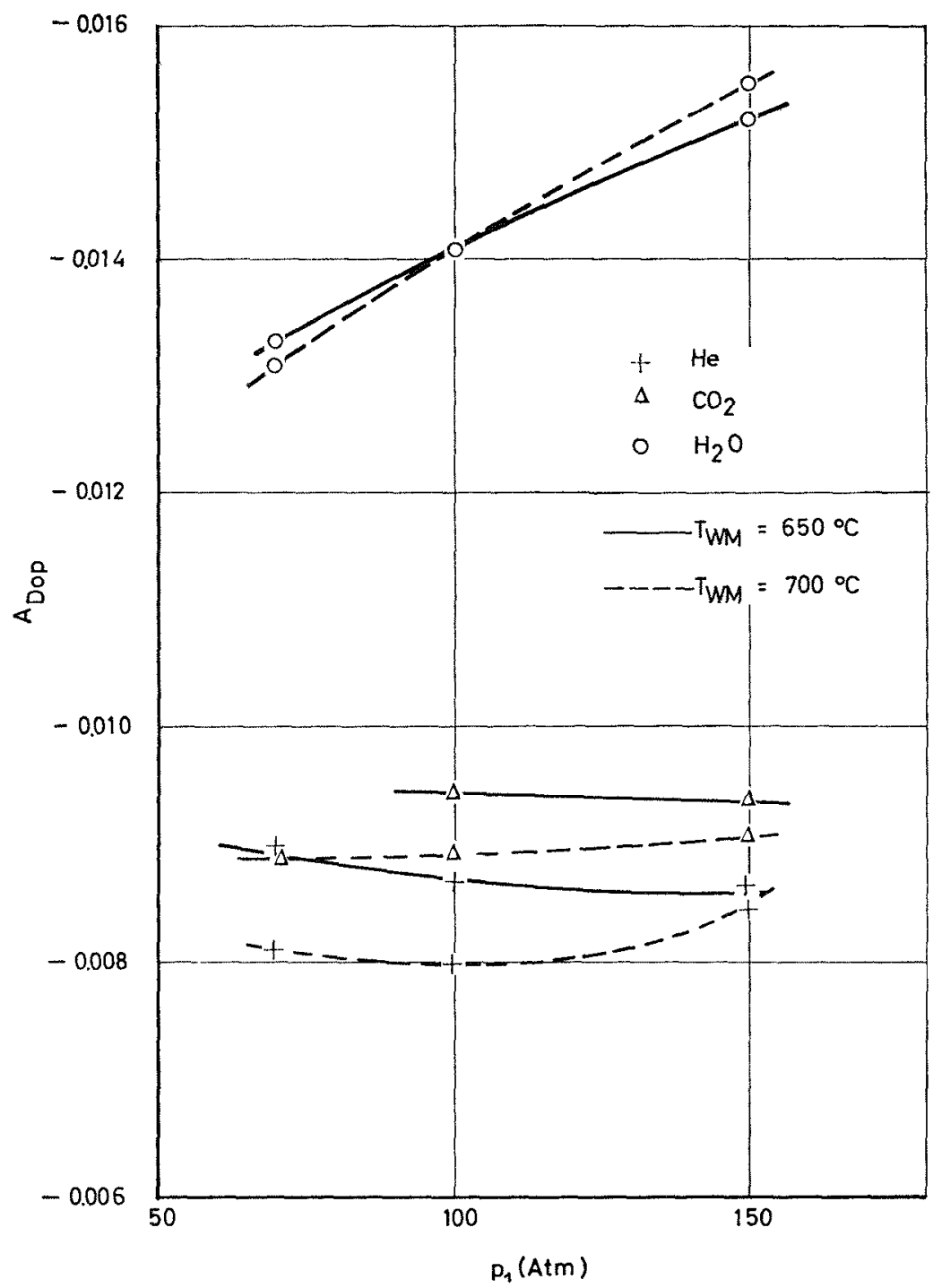

Fix. 10. Doppler Coefficient Constant Apop.

Heat Transfer Condition in the Section of Maximum Cladding Temperature

The heat transfer coefficient under forced convection of gases in tubes at high temperature has been extensively studied in the last 15 years. The conclusions of these studies and of experimental investigations is that the heat transfer coefficient is given ${ }^{(22)}$ by the equation

$$
N u_{w}=0.020 R e_{w}^{0.8} P r_{w}^{0.4} .
$$

Equation (24) is valid for tubes and for ducts which do not present very acute corners. So it is also valid in the case of a parallel-rod array when the pitch-to-rod diameter ratio $p / d$ is not too near to one. Fortunately, for big reactor cores the optimum $p / d$ is considerably higher than one and the cooling channel doesn't pre- sent acute corners. Consequently eq. (24) is still valid for this geometry provided that the inner diameter of the tube is replaced by the hydraulic diameter $d_{h}$.

By definition, eq. (24) (an be written as

$$
\frac{h d_{h}}{k_{w}}=0.020\left(\frac{\rho_{w} v_{h}}{\mu_{w}}\right)^{0.8} P r_{w}^{0.4}=0.020\left(\frac{\rho_{w}}{\rho} \frac{4 A \rho v}{P \mu_{w}}\right)^{0.8} P r_{w}^{0.4} .
$$

Therefore,

$$
d_{k}=\frac{0.020}{h} k_{w} P r_{k}^{0.4}\left(\frac{\rho_{w}}{\rho}\right)^{0.8}\left(\frac{4 G}{P \mu_{w}}\right)^{0.8} .
$$

But from eq. (22) we have

$$
h=\frac{\pi G c_{p M}}{P^{\prime} H^{\prime}}
$$

and 


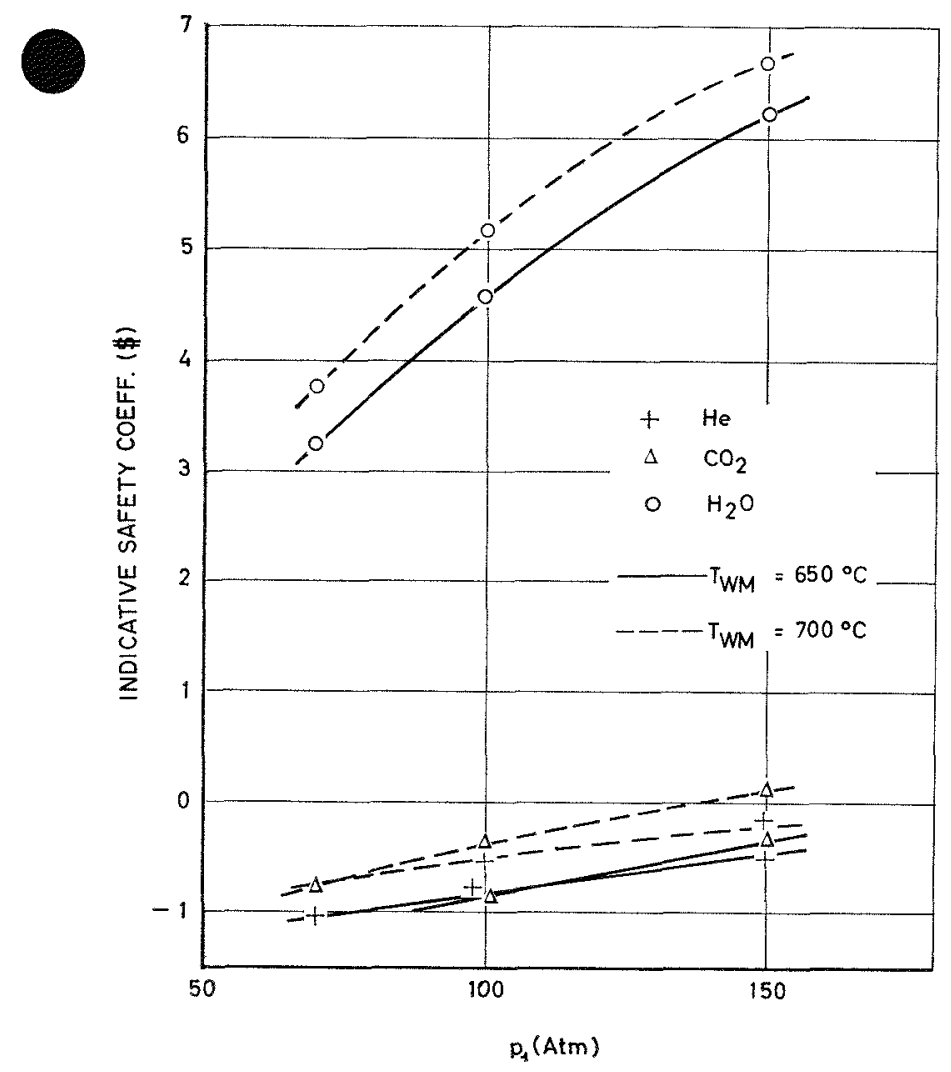

FIG. 11. Indicated Safety Coefficient.

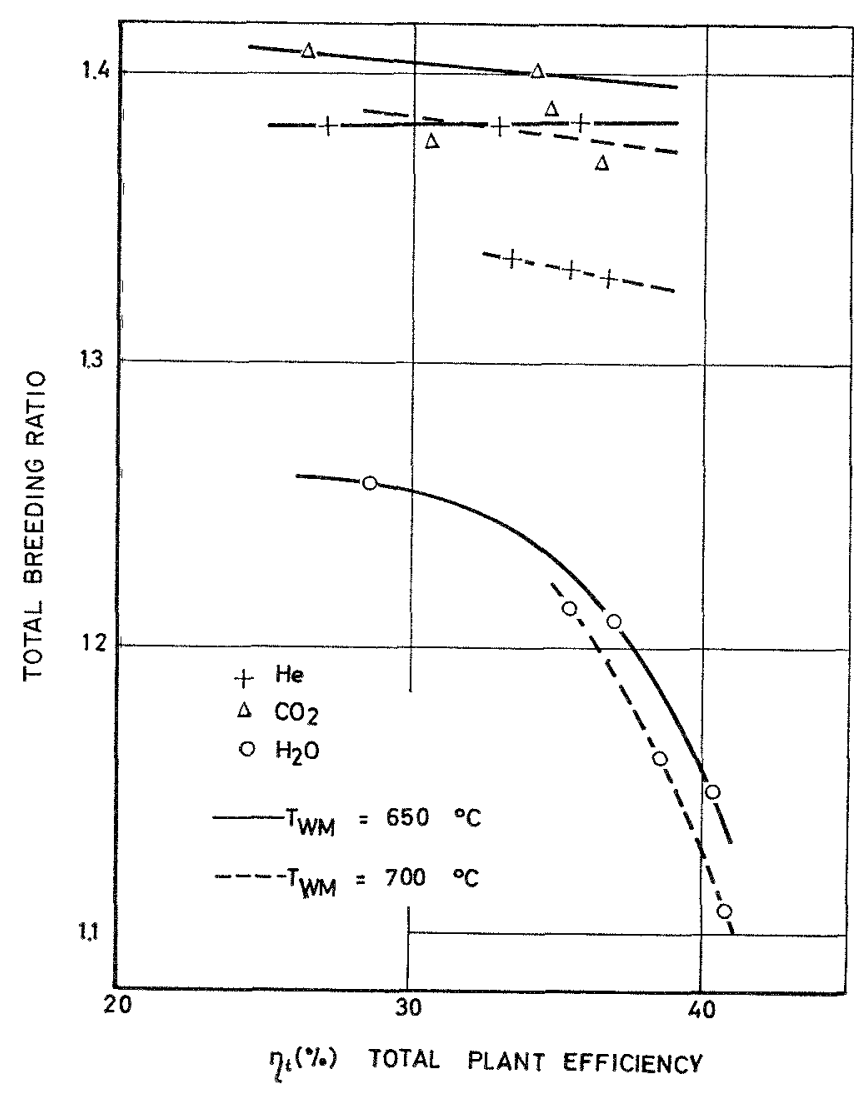

FIG. 12. Breeding versus Economy.

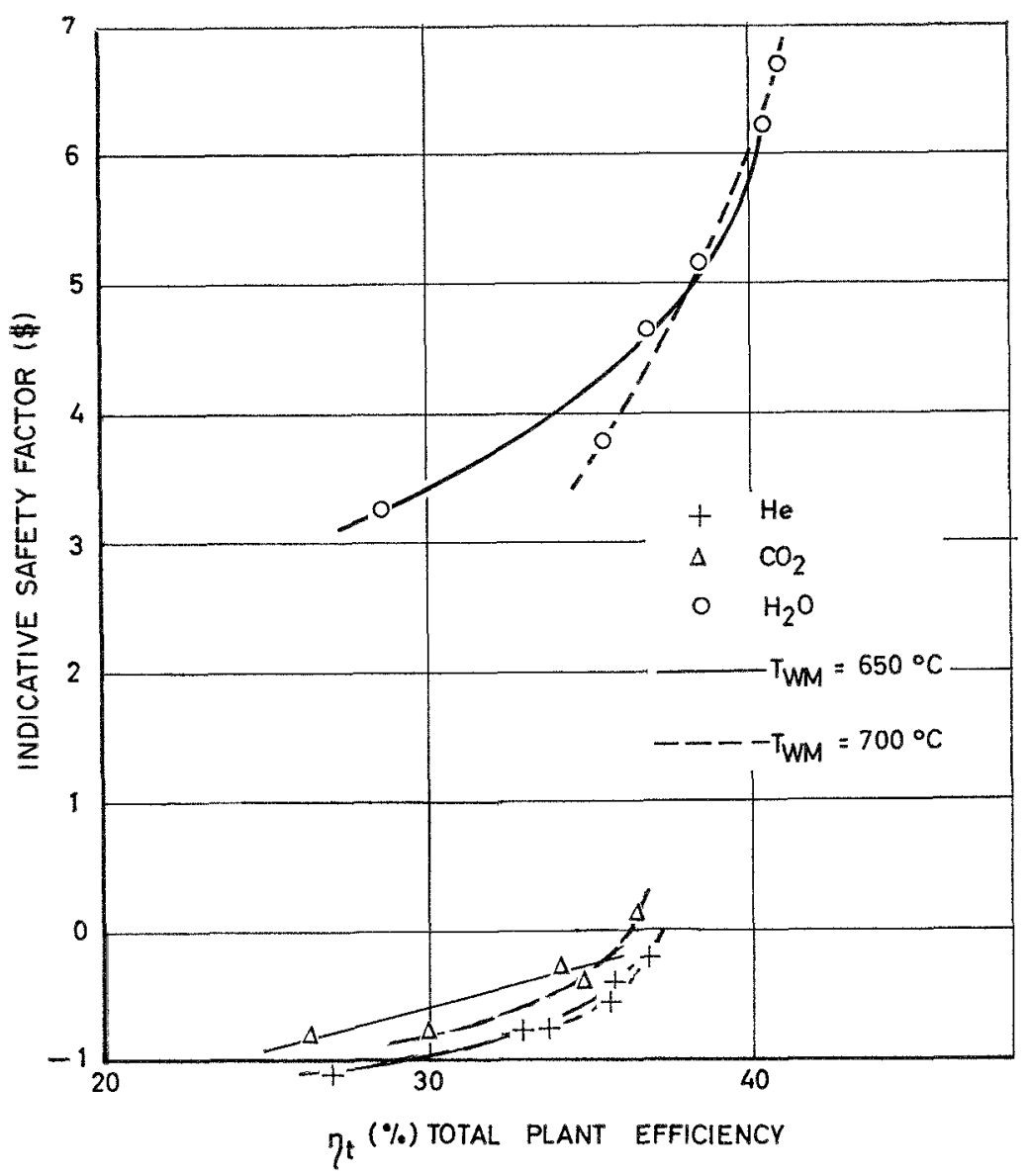

Fra. 13. Safety vs Economy. 


$$
G=\frac{Q}{c_{p m}\left(T_{2}-T_{1}\right)} .
$$

By replacing equations (26) and (27) in eq. (25) and remembering that

$$
\operatorname{Pr}_{w}=\frac{\mu_{w} c_{p w}}{k_{w}},
$$

one obtains eq. (1)

\section{Pressure Drop in the Cooling Channels}

The equations giving the pressure drop are as follows:

$$
\begin{aligned}
\Delta p_{1} & =f_{m}\left(2 H / d_{h}\right) \rho_{m} v_{m}^{2} \\
\Delta p_{2} & =\xi \rho_{m} v_{m}^{2} \\
\Delta p_{3} & =F\left(T_{1}, T_{2}, p_{1}, p_{2}\right) \rho_{m} v_{m}^{2},
\end{aligned}
$$

where

$$
\begin{array}{r}
f_{m}=\frac{0.046}{\left(\frac{\rho_{m} v_{m} d_{h}}{\mu_{m}}\right)^{0.2}} \quad \text { (Blasius's equation); } \\
F\left(T_{1}, T_{2}, p_{1}, p_{2}\right)=\frac{T_{2}-T_{1}}{T_{m}}+\ln \frac{p_{1}}{p_{2}} \\
+\frac{1.1 T_{1}-0.55 T_{2}}{2 T_{m}}
\end{array}
$$

(see Ref. 23, p. 40) and $\xi$ is a dimensionless constant depending on the shape, size, and number of supporting grids.

Replacing equations (27), (31), and (32) in equations (28), (29), and (30), and taking into account that $d_{h}=4 A / P$ and $G=A \rho_{m} v_{m}$, one obtains equations (2), (3), and (4). When the units of the physical parameters on the right side of these equations are those of the cgs system, the resulting pressure differences are in dyne $/ \mathrm{cm}^{2}$. To have them in atmospheres it is necessary to multiply by $0.9869 \cdot 10^{-6}$.

\section{Pumping Power}

The total pumping power required to circulate the coolant in the primary coolant circuit is established by the characteristics of the whole primary circuit. The part of the total pumping power relative to the core is given by

$$
\begin{aligned}
N_{\iota} & =\frac{1}{\eta} \frac{G_{i}}{\rho_{\mathrm{I}}}\left(\Delta p_{1}+\Delta p_{2}+\Delta p_{3}\right) \\
& =\frac{1}{\eta} \frac{Q_{t h}}{c_{p m}\left(T_{2}-T_{1}\right) \rho_{1}}\left(\Delta p_{1}+\Delta p_{2}+\Delta p_{3}\right) .
\end{aligned}
$$

We define $N_{1}, N_{2}$, and $N_{3}$ as follows:

$$
N_{1}=\frac{1}{\eta} \frac{Q_{i h}}{c_{p m}\left(T_{2}-T_{1}\right) \rho_{1}} \Delta p_{1}
$$

$$
\begin{aligned}
& N_{2}=\frac{1}{\eta} \frac{Q_{t h}}{c_{p m}\left(T_{2}-T_{1}\right) \rho_{1}} \Delta p_{2} ; \\
& N_{3}=\frac{1}{\eta} \frac{Q_{t h}}{c_{p m}\left(T_{2}-T_{1}\right) \rho_{1}} \Delta p_{3},
\end{aligned}
$$

so that $N_{i}=N_{1}+N_{2}+N_{3}$.

Using equations (2), (3), and (4), one obtains

$$
\begin{gathered}
\frac{N_{1}}{Q_{t h}}=\frac{1.115}{\eta} \frac{\left(P^{\prime} / P\right)^{1.8}}{\left(T_{2}-T_{1}\right)^{28}} \frac{\mu_{m}^{0.2}}{\rho_{1} \rho_{m} c_{p m}^{2.8}} \frac{H Q^{1.8}}{P^{\prime 18} d_{h}^{3}} ; \\
\frac{N_{2}}{Q_{t h}}=\frac{16 \xi}{\eta} \frac{\left(P^{\prime} / P\right)^{2}}{\left(T_{2}-T_{1}\right)^{3}} \frac{1}{\rho_{1} \rho_{m} c_{p m}^{3}} \frac{Q^{2}}{P^{\prime 2} d_{h}^{2}} ; \\
\frac{N_{3}}{Q_{t h}}=\frac{16}{\eta}\left(\frac{T_{2}-T_{1}}{T_{m}}+\ln \frac{p_{1}}{p_{2}}\right. \\
\left.\quad+\frac{1.1 T_{1}-0.55 T_{2}}{2 T_{m}}\right) \frac{\left(P^{\prime} / P\right)^{2}}{\left(T_{2}-T_{1}\right)^{3}} \\
\cdot \frac{1}{\rho_{1} \rho_{m} c_{c m}^{3}} \frac{Q^{2}}{P^{\prime 2} d_{h}^{2}} .
\end{gathered}
$$

Replacing equation (1) in (34), (35), and (36), one obtains equations (5), (6), and (7). When the units of the physical parameters on the right side of these equations are those of the cgs system and the heat is in calories, the resulting $N / Q$ 's are in erg/cal. To obtain the $N / Q$ 's in dimensionless form it is necessary to divide by $4.187 \cdot 10^{7}$.

\section{Appendix II}

Relationship between $T_{1}, p_{1}$, and $N_{t} / Q_{t h}$ for Steam

With reference to Fig. 1 one can write that the power of the steam circulator is given by

$$
N_{t}=\frac{\left(I_{1}-I_{0}\right) G_{t}}{\eta_{M}},
$$

where $\eta_{M}$ is the ratio between the power given to the steam in the circulator and the power at the shaft of the driving turbine. The ratio $\eta_{M}$ takes into account of the mechanical losses in the power transmission from turbine to circulator and of the mechanical losses in the circulator itself. We assume $\eta_{M}=0.91$. On the other hand, the core thermal output is given by

$$
Q_{t h}=G_{t}\left(I_{2}-I_{1}\right) \text {. }
$$

Combining equations (37) and (38), one obtains

$$
I_{1}=\frac{I_{0}+\eta_{M} \frac{N_{t}}{Q_{t h}} I_{2}}{1+\eta_{M} \frac{N_{t}}{Q_{t h}}} .
$$

Once $T_{1}, T_{2}$, and $p_{1}$ are known, $N_{t} / Q_{t h}, p_{2}$, and $p_{0}$ must be estimated. From $p_{0}$ and the condition $x=1$ it is possible to obtain $I_{0}$, and from $T_{2}$ and $p_{2}$ one has 
$I_{2}$. It is possible then to calculate $I_{1}$ from eq. (39), and from $p_{1}$ and $I_{1}$ one obtain $T_{1}$. One can then with this value of $T_{1}$ make a calculation of the pressure drop $\Delta p$ and pumping power necessary to rireulate the steam in the primary cireuit (nee Appendix I) and rherk if the assumed values of $N_{t} / Q_{t h}$ and $p_{2}$ are correct. If not, the calculation must be repeated until they are.

The difference $p_{2}-p_{0}$ takes into ale ount the pressure drop in the primary cireuit other than in the reactor, and it can be obtained by the relationship:

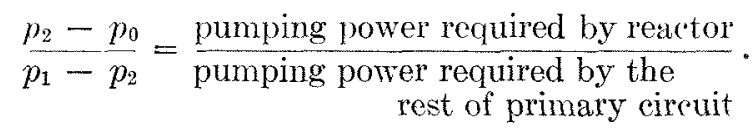

\section{Appendix III}

\section{Neutronic Computations}

The objertives of the neutronic computations were to obtain the enrichment, breeding ratio, and basic safety parameters for each of the 17 ases. Since this involves a considerable amount of computation, simplifying approximations were applied which primarily effect the coolant-loss reactivity effect, but which, it is believed, give sufficient aceurary for this type of comparative study. These simplifying approximations are the following:

\section{Calculations Were Performed in Spherical Geometry}

This approximation is imporiant to the computation of the coolant-lose reactivity effects, where core leakage makes an important contribution. The cylindrical cores considered (with $\mathrm{H} / \mathrm{D} \cong 0.7$ ) (an be well simulated in spherical geometry.

\section{No Changes Were Made in the Microscopic Group Constants Going from Coolant-in to Coolant-out Conditions.}

With sodium coolint the elastic transfer (ross' sertions of all materials in the range of the large sodium resonance $(\cong 3 \mathrm{keV}$ ) change considerably when the sodium is removed. For the gas coolants considered here such strong resonances do not exist, so it is expected that this contribution to the coolant loss effect will be small.

\section{The Total Coolant Loss Effects Will be in the Same Proportions as the Maximum Positive Coolant Loss Effects}

The greatest positive reartivity effect due to coolant loss most likely oc'urs when only the center portion of the core is void of coolant. However, for the purpose of comparing coolants, the effect of total coolant removal from the core is sufficiently representative of the maximum positive value.
The plutonium inotopir romposition used in the computations is that representative of fast reactor, namely, recycled plutonium which has reached equilibrium levels. ${ }^{(24)}$ The fractions of P'u-239, Pu-240, and Pu-241 in the plutonium are $0.823,0.159$, and 0.018 , respectivcly. I fission product pseudo-element representing long-lived fission products was included with an atom density corresponding to $50000 \mathrm{MWD} / 1$ burnup in the fuel. The cross-section set used is the 26-group set generated in the Soviet Union and adapted for use at Karlsruhe. ${ }^{(25)}$ Plutonium "enrichments" were set to give a $k_{\text {tff }}$ value of 1.01 , and the values given in Table 4 are based on total fuel plus fission product nuclear densities. In the computations of the Doppler coeffirient, both a uniform temperature distribution and change in the fuel (i.c., an isotopic coefficient) were assumed.

Conversion from the actual rylindrical dimensions of the reactor cores to equivalent spherieal dimensions was done by conserving core leakage in the fundamental-mode approximation, that is, the axial and radial bucklings, $B_{2,}^{2}$ and $B_{1 \text { ad }}^{2}$, were estimated from

$$
B_{\mathrm{dx}}^{2}=\left(\frac{\pi}{H+40}\right)^{2}
$$

and

$$
B_{\mathrm{rad}}^{2}=\left(\begin{array}{c}
2.404 \\
\frac{D}{2}+20
\end{array}\right)^{2}
$$

where $H$ and $D$ are the core height and diameter, respectively, and the 20 -cm extrapolation lengths are approximate for fast reactors. The spherical core radii $R_{\mathrm{sp}}$ are then given by

$$
\left(\frac{\pi}{R_{\mathrm{sp}}+20}\right)^{2}=B_{\mathrm{ax}}^{2}+B_{\mathrm{rad}}^{2} .
$$

A spherical blanket $40 \mathrm{~cm}$ thick was used. The composition of this blanket was taken to be a leakageweighted average of the axial and radial blanket compositions. Tetting $N_{\mathrm{sp}, m}, N_{\mathrm{ax}, m}$, and $N_{\mathrm{rad}, m}$ be the nuclear densities for material $m$ in the spherical, axial, and radial blankets, respertively, the value of $N_{\mathrm{sp}, m}$ was obtained from

$$
N_{\mathrm{sp}, m}=\frac{N_{\mathrm{dx}, m} B_{\mathrm{ax}}^{2}+N_{\mathrm{rad}, m} B_{\mathrm{rdd}}^{2}}{B_{\mathrm{ax}}^{2}+B_{\mathrm{rad}}^{2}} .
$$

The quantities $N_{a x, m}$ and $N_{\mathrm{rad}, m}$ wore determined by considering the axial blanket to have the same volume fractions as the core, and the radial blanket to have one-half the core coolant volume fraction and the same volume ratio of steel to fuel as in the core. Blanket fuel was taken to be pure $\mathrm{C}^{\top}-238$ oxide. 
The coolant-loss reactivity effects were computed by running one-dimensional diffusion-theory problems with and without the coolant present in the core. Doppler computations were accomplished with the $900^{\circ} \mathrm{C}$ and $2100^{\circ} \mathrm{C}$ values of the P'u-239 and U-238 cross sections available in the 26-group set. The small Doppler contribution due to Pu-240 was not included. It was then assumed that the Doppler coefficient follows the expression

$$
\frac{d k}{d T_{f}}=\frac{A_{\text {Dop }}}{T_{f}}
$$

where $A_{\text {Dop }}$ is a constant and $T_{f}$ js the average absolute temperature of the fuel. The values of $A_{\text {Dop }}$ have been reported for the 17 cases considered in this study and form a basis for comparison of the Doppler coefficients.

\section{Nomenclature}

Geometrical Parameters:

$$
\begin{array}{ll}
A & =\text { coolant-channel cross section }\left(\mathrm{cm}^{2}\right) \\
B_{\mathrm{ax}}^{2} & =\text { core axial buckling }\left(\cdot \mathrm{m}^{-2}\right) \\
B_{\mathrm{rad}}^{2} & =\text { core radial buckling }\left(\mathrm{cm}^{-2}\right) \\
d & =\text { fuel-element rod diameter }(\mathrm{cm}) \\
d_{h} & =\text { coolant-channel hydraulic diameter }(\mathrm{cm}) \\
D & =\text { core diameter }(\mathrm{cm}) \\
H & =\text { core height }(\mathrm{cm}) \\
H^{\prime} & =\text { core extrapolated height }(\mathrm{cm}) \\
P & =\text { coolant-channel wet ted perimeter }(\mathrm{cm}) \\
P^{\prime} & =\text { coolant-channel heat transfer perimeter } \\
& \text { (cm) } \\
R_{\mathrm{sp}} & \text { radius of equivalent spherical core }(\mathrm{cm}) \\
z & =\text { distance from the core inlet of the coolant } \\
& \text { cross section considered (cm) } \\
z_{M}= & \text { distance from the core inlet of the coolant } \\
& \text { cross section where the fuel surface tem- } \\
& \text { perature is maximum }(\mathrm{cm})
\end{array}
$$

Coolant Physical Properties:

$c_{p m}=$ specific heat at constant pressure at temperature $T_{m}$ and pressure $p_{m}$ (cal/ gm- ${ }^{\circ} \mathrm{C}$ )

$c_{p M}=$ specific heat at constant pressure at temperature $T\left(z_{M}\right)$ and pressure $p_{m}$ $\left(\mathrm{cal} / \mathrm{gm}-{ }^{\circ} \mathrm{C}\right)$

$c_{p w}=$ specific heat at constant pressure at tenperature $T_{w, M}$ and pressure $p_{m}$ (cal/ gm- ${ }^{\circ} \mathrm{C}$

$I(z)=$ enthalpy at cross section $z(\mathrm{cal} / \mathrm{gm})$

$I_{0} \quad=$ enthalpy upstream the coolant circulator (cal/gm)

$I_{1} \quad=$ enthalpy at core inlet $(\mathrm{cal} / \mathrm{gm})$

$I_{2} \quad=$ enthalpy at core outlet $(\mathrm{cal} / \mathrm{gm})$

$x \quad=$ steam quality $k_{w}=$ thermal conductivity at temperature $T_{w M}$ and pressure $p_{m}\left(\mathrm{cal} / \mathrm{cm}\right.$-sec- $\left.{ }^{\circ} \mathrm{C}\right)$

$\mu_{m} \quad=$ dynamic viseosity at temperature $T_{m}$ and pressure $p_{m}(\mathrm{gm} / \mathrm{cm}-\mathrm{sec})$

$=$ dynamic viscosity at temperature $T_{w M}$ and pressure $p_{m}(\mathrm{gm} / \mathrm{cm}-\mathrm{sec})$

$=$ density at temperature $T\left(z_{M}\right)$ and pressure $p_{m}\left(\mathrm{gm} / \mathrm{cm}^{3}\right)$

$\rho_{1} \quad=$ density at temperature $T_{1}$ and pressure $p_{1}\left(\mathrm{gm} / \mathrm{cm}^{3}\right)$

$=$ density at temperature $T_{m}$ and pressure $p_{m}\left(\mathrm{gm} / \mathrm{cm}^{3}\right)$

$\rho_{w} \quad=$ density at temperature $T_{w M}$ and pressure $p_{m}\left(\mathrm{gm} / \mathrm{cm}^{3}\right)$

\section{Other Physical Parameters:}

$G=$ coolant mass flow in the central coolant channels $(\mathrm{gm} / \mathrm{sec})$

$G_{t}=$ total coolant mass flow through core $(\mathrm{gm} / \mathrm{sec})$

$h=$ heat transfer coefficient between fuelelement surface and coolant (cal/cmser. ${ }^{\circ} \mathrm{C}$ )

$N_{1} \quad=$ pumping power required by friction losses in the core $(\mathrm{MW})$

$N_{2} \quad=$ pumping power required by losses due to supporting grids in the core (MW)

$N_{3} \quad=$ pumping power required by acceleration losses in the core (IIW)

$N_{t} \quad=$ total pumping power required by the core (MW)

$N_{\mathrm{sp}, m}=$ nuclear density of material $m$ in spherical blanket (atoms/b-cm)

$N_{\mathrm{ax}, m}=$ nuclear density of material $m$ in axial blanket (atoms $/ \mathrm{b}-\mathrm{cm}$ )

$N_{\text {rad }, m}=$ nuclear density of material $m$ in radial blanket (atoms/b-cm)

$p_{0} \quad=$ coolant pressure in the coolant circulator upstream (atm)

$\begin{array}{ll}p_{1} & =\text { coolant pressure at core inlet (atm) } \\ p_{2} & =\text { coolant pressure at core outlet (atm) }\end{array}$

$p_{2} \quad=1 / 2\left(p_{1}+p_{2}\right)(\mathrm{atm})$

$\Delta p_{1} \quad=$ pressure drop required by frietion losses in the core (atm)

$\Delta p_{2} \quad=$ pressure drop due to grids supporting fuel rods (atm)

$\Delta p_{3} \quad=$ pressure drop due to acceleration losses in the core (atm)

$\Delta_{p} \quad=\Delta p_{1}+\Delta p_{2}+\Delta p_{3}(\mathrm{~atm})$

$q \quad=$ heat to coolant per coolant channel and per unit length (eal/em-sec)

$q_{0}=$ maximum value of $q(\mathrm{cal} / \mathrm{cm}$-sec $)$

$Q=$ heat output per coolant channel $(\mathrm{cal} / \mathrm{sec})$

$Q_{t h} \quad=$ total core heat output (MW) 


$$
\begin{aligned}
T_{1}= & \text { absolute coolant temperature at core } \\
& \text { inlet }\left({ }^{\circ} \mathrm{K}\right) \\
T_{2}= & \text { absolute coolant tempcrature at core } \\
& \text { outlet }\left({ }^{\circ} \mathrm{K}\right) \\
T_{m}= & 1 / 2\left(T_{1}+T_{2}\right)\left({ }^{\circ} \mathrm{K}\right) \\
T(z)= & \text { absolute coolant temperature at coordi- } \\
& \text { nate } z\left({ }^{\circ} \mathrm{K}\right) \\
T_{w}(z)= & \text { absolute fuel-element surface tempera- } \\
& \text { ture at coordinate } z\left({ }^{\circ} \mathrm{K}\right) \\
T_{w M}= & \text { maximum absolute fuel-clement surface } \\
& \text { temperature }\left({ }^{\circ} \mathrm{K}\right) \\
T_{f}= & \text { average absolute temperature of fuel }\left({ }^{\circ} \mathrm{K}\right) \\
v & \text { coolant velocity }(\mathrm{cm} / \mathrm{sec}) \\
\chi & \text { heat produced per unit length of fuel rod } \\
& \text { (cal/cm-sec) } \\
\tau_{w}= & \text { shear stress at the wall (dynes } \left./ \mathrm{cm}^{2}\right)
\end{aligned}
$$

Dimensionless Groups:

$$
\begin{aligned}
& f_{m} \quad=\tau_{w / \rho v} / 2=\text { Fanning friction factor } \\
& N u_{w}=h d_{h} / k_{w}=\text { Nusselt number } \\
& P \gamma_{w}=\mu_{v o} c_{p w} / k_{w}=\text { Prandtl number } \\
& R e_{w} \quad=\rho_{w} v d_{h} / \mu_{v}=\text { Reynolds number } \\
& \alpha \quad=\text { coolant fraction in fuel boxes } \\
& \eta \quad=\text { efficiency of coolant circulator } \\
& \eta_{M}=\text { mechanical efficiency of coolant circula- } \\
& \text { tor } \\
& \eta_{t}=\text { total net efficiency of power plant } \\
& \varphi \quad=\left(T_{w M}-T_{1}\right) /\left(T_{2}-T_{1}\right) \\
& \nu=\frac{1}{\sqrt{\left(2 \varphi \frac{c_{p M}}{c_{p m}}-1\right)^{2} \sin ^{2} \frac{\pi H}{2 H^{\prime}}-1}} \\
& \xi \quad=\Delta p_{2} /\left(\rho_{m} v^{2}\right)=\text { pressure drop factor due to } \\
& \text { grids supporting fuel elements }
\end{aligned}
$$

Constants:

$$
\begin{aligned}
& \Delta_{\text {Dop }}=\text { constant for } 1 / T_{j} \text { variation of Doppler } \\
& \text { coefficient } \\
& c_{1}=d H^{0.5} / Q^{025} \\
& =P^{\prime} / d=\left\{\begin{array}{l}
\pi \text { (square fuel-element rod } \\
\text { array) } \\
\pi / 2 \text { (triangular fuel-element rod } \\
\text { array) }
\end{array}\right. \\
& K_{0} \quad=\text { defined in equation (1) } \\
& \begin{array}{ll}
K_{1} & = \\
K_{2} & =
\end{array} \text { defined in equations }(6),(7), \text { and }(8) \\
& K_{3}= \\
& K_{4}=K_{1}\left(P / P^{\prime}\right)^{06} \\
& +K_{2}\left(P^{\prime} / P\right)^{04}+K_{3}\left(P^{\prime} / P\right)^{0.4}
\end{aligned}
$$

\section{Acknowledgment}

The author wishes to thank Professor K. Wirtz, Director of the Institut für Neutronenphysik und Reaktortechnik, who encouraged him to perform the work.

\section{REFERENCLS}

1. H. W. Hoffmann and A. I. Krakoviak, "Convective boiling with liquid potassium," Proceedings of the 1964 Heal Transfer and Fluid Mechanics Institute, Stanford University Press, Stanford, Calif. (1964).

2. M. Dalle-Donne, 1 New and Simple Method of Estimating the Laquid Superheat due to Surface Conditions in Arucleate Boiling and Its Application to Sodium to be published in "Nukleonik."

3. W. Häfele, Principles and Probtems of the Development of a Fast Reactor Fuet Element, ANL-6742, p. 265.

4. P. Forteseue, R. Shanstrom, L. Mayer, W. Simon, and P. Fischer, Gas Cooling for Fast Reactors, A/Conf 28/P/694, May 1964.

5. The Gas-cooled Reactor Project Staff, Gas-cooled Fast Reactor Concepts, ORNL-3642 (Sept. 1964).

6. G. Wensch, United States Fast Reactor Fuel Element Development, Conference held at Kernforschungszentrum Karlsruhe, Nov. 8, 1963.

7. D. Smidt et al., Kernreaktor Internal Report (1962).

8. P. Fortescue, R. Shanstrom, and H. Fenech, Development of the Gas-cooled Fast Reactor Concept, ANS-100, p. 89 (April 1965).

9. B. de Temple, Interual Kernreaktor Report (1963).

10. H. Franze, Internal Kernreaktor Report (1964).

11. J. B. Mann and N. C. Blais, Thermal Conductivity of Helium and Hydrogen at High Temperatures, LA-2316 (1959).

12. N. V. Tsederberg, Thermal Conductivnty of Gases and Liquids, The M. I. T. Press, Cambridge, Mass. (1965).

13. P. D. Stroom, W. E. Ihele, and T. F. Irvine, Jr., Helium Prandtl Number leasurements and ralculated Viscosity and Thermal Conductivity, 1961 International Heat Transfer Conference, Cuiversity of Colorado.

14. K. Sullivan, Thermal Conductovty and Viscosity of $\mathrm{CO}_{2}$ TRG. Report 438(R) (1963).

15. J. Hilsenrath ef al., Tables of Thermodynamic and Transport Properties, Pergamon Press (1960).

16. M. P. Vukalovitch, Thermodynamic Properties of Water and Steam, VEB Verlag Technik Berlin (1958).

17. VDI Wasserdampftafel, 5 . Auflage.

18. VDI Wàrmeatlas, $\mathrm{Db} 3,1953$.

19. Vargaftik and Tarasimanov, Teploenergetika, 8 (1961).

20. Mayinger, Int. J. Heat and Mass Transfer, 5 (1962).

21. H. Franze, private communication, Kernreaktor Karlsuhe (1965).

22. M. Dalle Donne and F. H. Bowditeh, Experimental Local Heat Transfer and Fruction (oefficients for Subsonic Laminar, Transitional and Turbulent Flow of Air or Helium in a Tube at High T'emperatures, Dragon Project Report 181 (April 1963), A. E. E. Winfrith, Dorset, England.

23. W. Hall, Reactor Heat Transfer, Nuclear Engineering Monographs, Temple Press Itd, London (1958).

24. A. Jansen, Private communication, Kernreaktor Karlsruhe (1965).

25. L. B. Abagjan et al., Gruppenkonstanten schneller und intermediarer Veutronen für die Berechnung von Kernreaktoren, KFK-tr-144 (Translation from Russian), 1964

\section{Discussion}

Mr. Fell (Winfrith): I wonder if you would comment on the question that pressure drop is not fully a matter of thermal calculations of this kind. In particular, 
with a stean-cooled reactor you're much more interested in keeping the hydrogen content down; you are going to want to work with smaller passages.

Mr. Dalle-Donne: As far as steam in coneerned, nuclear considerations will be dircussed in detail in the next paper from Karlsruhe, by Dr. Ott. I think he will be able to answer your question.

Mr. Wirta (Karlsruhe): Could you tell us, if you have a choice from an economical point of view of the three coolants, which one would you select now?

MIr. Dalle-Donne: From consideration of the heat transfer performance in the core the steam is the best.

Mr. Fenech (MIT): I think it's a little meaningless to make a study of a choice of coolants on the basis of heat removal from the core because of other important factors that come into it, such as economics and safety; one cannot dissociate one from the other. Therefore, the selection of coolant should be done as a result of an overall study of reactor design and organization.

Mr. Dalle-Donne: Yes, this is only one point of the picture, but it is something which is in favor of steam.

Mr. Haffele (Karlsruhe): I think the paper of DalleDonne indieates that the differences between the cases of several surface temperatures smoothes out in case of high pressure, and particularly for steam. Therefore, it was important to have such studics to fix the situation. We have decided that in the case of steamcooled breeders you should go to high pressures.

Mr. Dalle-Donne: I forgot to say one thing. The pressure drops in the core are very important, not only because of pumping power, but because if you have high pressure drops there are important problems concerned with vibration of the fuel elements. The rods have to be supported firmly, and this leads again to an increase of pressure drops; you have a sort of divergency, I think.

Mr. Fenech: There is a minimum pressure drop in the core required for a good distribution of coolant across the core itself. The question of pressure drop, which has entered through the percentile that you use for pumping power, is not very important from the point of view of economies if your fuel-cycle cost is low; then you just don't are too much how much energy you're spending for pumping.

Mr. Dalle-Donne: I agree that the pressure drops themselves are not an economic question. The more vital question is one of safety. The fuel elements must not vibrate too much.

Mr. Fell: I wonder if I can come back to the point on roughened fuel elements. I think il's unlikely that one would design a fuel element for gas cooling without roughening. Therefore I am not quite sure whether I understand your conclusions to the differences between helium and steam, if you roughen the fuel element.

Mr. Dalle-Donne: The roughening is a rather curious thing in the sense that it does not allow us to decrease the pressure in pumping power. If you roughen, you increase the Fanning friction factor about three times, but the Stanton number only about two times. Thus, roughening completely the fuel element leads to a relation of pumping power to thermal power output which is worse than without roughening. But by partial roughening the ratio of Fanning friction factor to Stanton number remains about the same. As far as pumping power is concerned, there is no gain, but you can use bigger rods.

MIr. Cohen (GW): Would you care to comment on the possibility that perhaps different coolants might tolerate slightly different maximum temperatures?

MIr. Hafele: This is a very touchy and crucial point indeed. According to our present evaluation it makes a big difference whether you run the cladding material at 650,700 , or more than $700^{\circ} \mathrm{C}$. At higher temperatures the helium created by $(n, \alpha)$ processes can move inside the structure materials. These formidable helium bubbles lead to a sudden decrease of ductility, but this can be avoided according to our latest results and irradiation experiments in BR-II, the Belgian Reactor, if you stay within certain temperature limits. A reactor running at 700 or $750^{\circ} \mathrm{C}$ not only has to face the difficulty of these lower ereep strengths but also of the higher radiation damages. Therefore, I think it does have a strong feedback on the reactor design. 


\title{
Survey Parameter Study of Large Steam-cooled Fast Power Reactors*
}

\author{
F. KiefHaber and K. OTT \\ Kernforschungszentrum. \\ Karlsruhe, Germany \\ (PRLSENTED BY K. OTT)
}

\section{INTRODUCTION}

This paper gives an excerpt of our neutronic studies on steam-cooled fast power reactors dealing mainly with the reactivity behaviour at different coolant densities and its dependence on various parameters. It is the physies part of a feasibility study.

At the beginning of this study, and even nowadays, information from the literature concerning steam-cooled fast reactors was very sparse, a situation quite different compared to that for sodium, for which some extensive theoretical and experimental studies have been published. Furthermore, there are additional parameters of larger influence on steam rooling. For example neutron poisons and fission products may change the safety behaviour considerably. Also, the steam density is not as well-defined as the density of fluid sodium.

Bearing in mind all these facts, we started our work on large steam-cooled fast reactors with an extensive survey parameter study to get an idea of the influence of the important parameters on the reactivity behaviour and to determine, at least with certain approximations the meaningful regions in the parameter field within which feasible combinations of parameters may be found. Since the number of parameters involved is still large, even if one excludes those which were supposed to be of minor importance, we began with zerodimensional calculations. With the aid of a few onedimensional calculations we determined the physical differences between zero- and one-dimensional calculations so that we are able to take into account these differences in further calculations by means of a variation of the savings depending on the variation of the mean steam density. In the presentation of our zerodimensional results we have disregarded this effect for simplicity reasons, since the main conclusions drawn from these results will be unchanged or only slightly modified.

All results prenented here have been obtained with use of the group-constant set of Bondarenko et $a l^{(1)}$ with minor corrections. All calculations were done for

* This paper has been prepared within the framework of the association LURATOM-Gesellschaft für Kernforschung mbH. in the field of fast breeder development. the homogeneous case only. The inclusion of heterogeneity effects will mainly alter the results for the flooded case. For the normal and roided state the influence of this effect is small.

In this paper we discuss the influence of the following parameters on the reactivity behaviour of large fast steam-cooled reactors:

1. choice of the coolant $\left(\mathrm{H}_{2} \mathrm{O}\right.$ or $\left.\mathrm{D}_{2} \mathrm{O}\right)$;

2. isotopic composition of plutonium;

3. addition of small amounts of poisons;

4. accumulation of fission products.

We will analyse mostly the results for different reasonable values of

a) the geometric buckling $B^{2}$;

b) the coolant volume fraction $\alpha$;

c) the mean steam density under normal operating conditions $\left(\rho_{N}\right)$.

Since the feasibility of large steam-cooled fast reactors will mainly be influenced by their safety behaviour, a set of safety conditions (section II) was used to find the limitations in the parameter field. The procedure for this is described in section III. In section IV the results are discussed.

\section{I. DISCUSSION OF SAFETY CRITERIA}

The conditions outlined in Table 1 are based on the following arguments:

1. In a loss-of-coolant aceident the reactor should remain at least below the prompt critical level. In this reactivity range, shutdown rods are normally fast enough. Since in an accident the pressure may decrease rapidly, this condition seems to be the most important one.

2. Flooding of the reactor may be desirable in some situations (emergency cooling, afterheat removal, refueling). The reactor can be considered as safe if it is subcritical in this state. One could also discuss $\Delta k_{F^{r}}<$ $\beta$, but this condition would give only a small enlargement of the allowed parameter region. The possibility of safe shutdown, so as 10 compensate for a larger positive $\Delta k_{F}$, is not assumed in this paper.

3. At normal mean steam density, $\rho_{N}$, the density coefficient of reactivity, should be positive to get a 
TABLE 1. The SET OF A AFETy Conditions

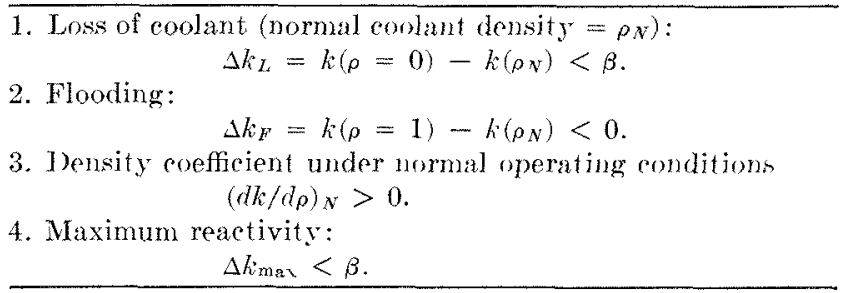

(We assumed in our analysis for the effective fraction of delaved neutrons $\beta=0.0035$, disregarding a system dependence for simplicity.)

self-stabilizing behaviour of the reactor with respect to small density or temperature fluctuations. In areidents a negative temperature coefficient of the coolant may also be helpful.

4. The reactivity will have a relative maximum between the normal density and the flooded case if conditions 2. and 3. are satisfied. It may be desirable to have this maximum reactivity lower than $\$ 1$. However, the increase of the coolant density from normal operation density to the flooded state can presumably oceur only artificially, so that sufficient control during this procedure may be guaranteed. Therefore, condition (4) is the weakest one and is ignored in most cases.

\section{TYPE OF ANALYSIS OF THE RESULTS}

It seemed instructive to represent the subregions in which the safety conditions are fulfilled basically in an $\left(\alpha, B^{2}\right)$-plane, where $\alpha$ is the coolant volume fraction and $B^{2}$ the buckling. All the other parameters are kept constant for such a diagram. Different combinations of the other parameters lead to different diagrams in the $\left(\alpha, B^{2}\right)$-plane. Criticality is achieved by a suitable fissile-to-fertile ratio.

Determining the curves in the diagrams we proceeded along the following line:

1. Calculation of $k(\rho)$, the multiplication factor for different coolant densities in the range of $0 \leq$ $\rho \leq 1 \mathrm{~g} / \mathrm{cm}^{3}$ for any set of the parameters $\alpha, B^{2}$, etc.

2. From these curves we read the quantities involved in the safety conditions $\left[\Delta k_{L}, \Delta k_{F}, \Delta k_{\text {max }},(d k /\right.$ $\left.d \rho)_{N}\right]$. From a plot of these quantities as functions of $B^{2}$, the special values of $B^{2}$ where the safety conditions are matched are determined by extrapolation or interpolation.

3. A plot of these values of $B^{2}$ for different $\alpha$-values then gives the limiting curve in the $\left(\alpha, B^{2}\right)$-plane.

We have used here only two different valucs of $\alpha$, so the limiting curves are straight lines in this approximation. However, this may be improved easily.

\section{DISCESSIOY OF THE RESULTS}

\section{A. Zero-dimensional Results \\ 1) Choice of ('oolant $\left(\mathrm{H}_{2} \mathrm{O}\right.$ or $\left.\mathrm{D}_{2} \mathrm{O}\right)$}

The two most important safety conditions are $\Delta k_{L}<$ $\beta$ and $\left(d k_{i}^{\prime} d \rho\right)_{N}>0$. $\Lambda$ s the example in Fig. 1 shows, these two most import ant conditions are heavily shifted in an unfavourable direction by replacing $\mathrm{H}_{2} \mathrm{O}$ with $\mathrm{D}_{2} \mathrm{O}$. Contrary to this, $\Delta k_{F}$ is influenced favourably. However, this can better be reached by accumulation of fission products or by additional neutron poisons.

From these results we decided to consider only $\mathrm{H}_{2} \mathrm{O}$ in the further investigation.

The physical reasons for this different behaviour of $\mathrm{H}_{2} \mathrm{O}$ and $\mathrm{D}_{2} \mathrm{O}$ may be understood from the following arguments:

At a first glance, one should expect a somewhat lower value for $\Delta k_{2}$ in the case of $\mathrm{D}_{2} \mathrm{O}$ than in the case of $\mathrm{H}_{2} \mathrm{O}$ because of the less pronounced spectral shift. The calculations showed that for $k_{\infty}$ (i.e., buckling $B^{2}=0$ ) one indeed gets the expected result. For a reasonable value of $B^{2}$, however, the change in the leakage probability, which gives a negative contribution to $\Delta k_{L}$, is much smaller for $\mathrm{D}_{2} \mathrm{O}$ than for $\mathrm{H}_{2} \mathrm{O}$ because of the smaller $\mathrm{D}_{2} \mathrm{O}$ transport cross section and the less softened spectrum in the case of $\mathrm{D}_{2} \mathrm{O}$ steam. This effect prevails over the $k_{\infty}$-effect, and in this way we get better results with respect to $\Delta k_{L}$ and $(d k / d \rho)_{N}$ for $\mathrm{H}_{2} \mathrm{O}$ than for $\mathrm{D}_{2} \mathrm{O}$ steam as the coolant. This was the main reason for preferring $\mathrm{H}_{2} \mathrm{O}$ to $\mathrm{D}_{2} \mathrm{O}$ as the coolant for our study, with the costs being a second argument.

In connection with Fig. 1 it should be noted that the addition of $\mathrm{D}_{2} \mathrm{O}$ decreases $\Delta k_{F}$ to a large extent. This is mainly due to the fact that in the flooded case for $\mathrm{D}_{2} \mathrm{O}$ the bulk of the neutron spectrum is in the range of low $\eta$ values, whereas for $\mathrm{H}_{2} \mathrm{O}$ a large number of neutrons has passed this range and is able to take advantage of the higher $\eta$ value in the thermal range. A second fact which acts in the same direction is the higher leakage rate for the flooded $\mathrm{D}_{2} \mathrm{O}$ reactor.

\section{2) Isotopic Composition of Plutonium}

The influence of the isotopic composition of plutonium on the reactivity and safety bchaviour of steamcooled reactors may be illustrated by comparison of the curves in Fig. 2. The calculations were performed for the same volume ratio of fissile to fertile atoms, all parameters other than the plutonium composition being equal. It can be seen that for pure $\mathrm{Pu}-239$ there is the well-known reactivity increase in the flooded case of about $20-30 \%$, whereas for the "dirty" plutonium in a certain range of isotopic compositions the reactivity 


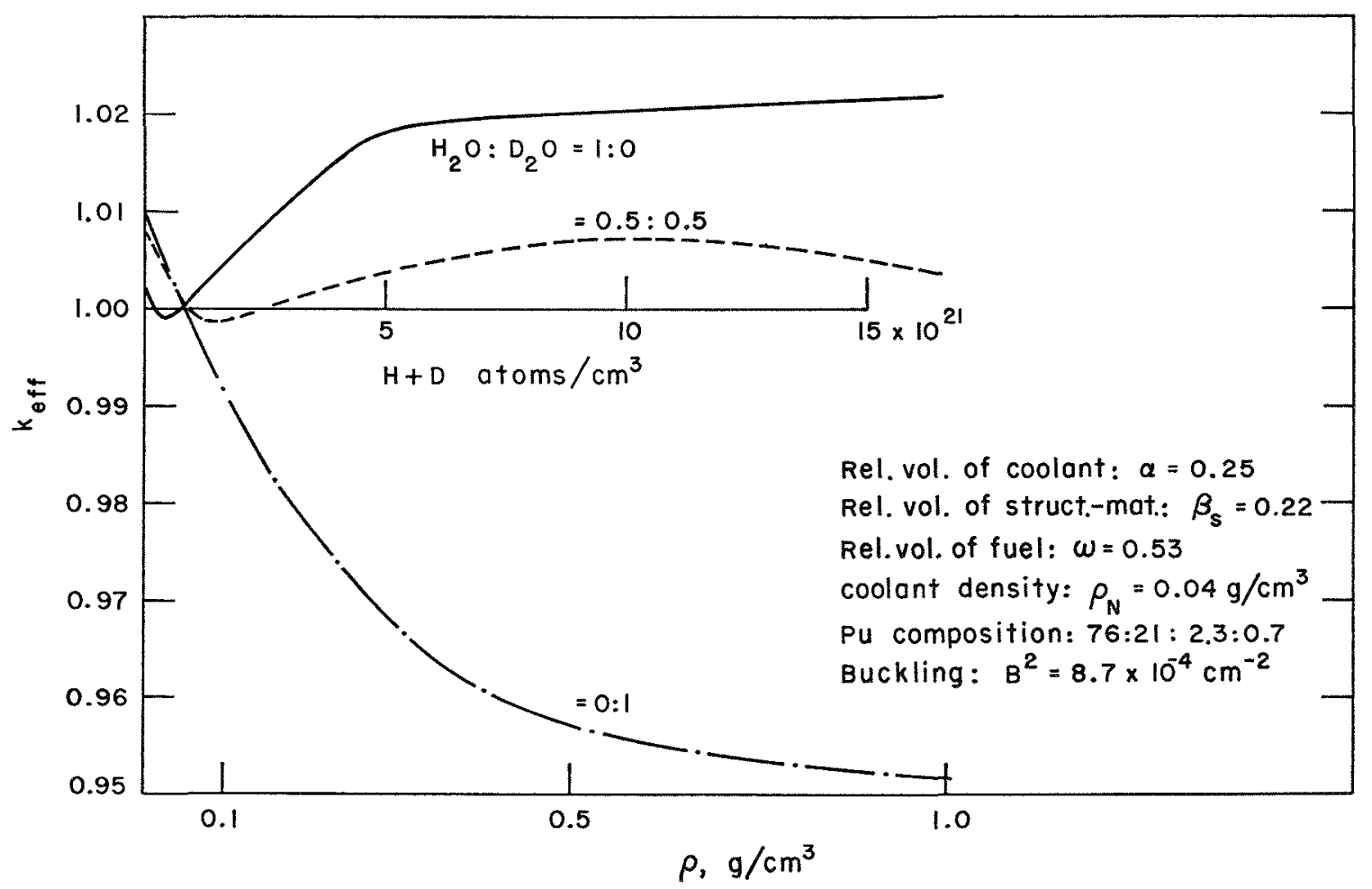

Fra. 1. heff as Function of Steam Density for Different $\left.\mathrm{H}_{2} \mathrm{O}-\mathrm{I}\right)_{2} \mathrm{O}$ Mlistures.

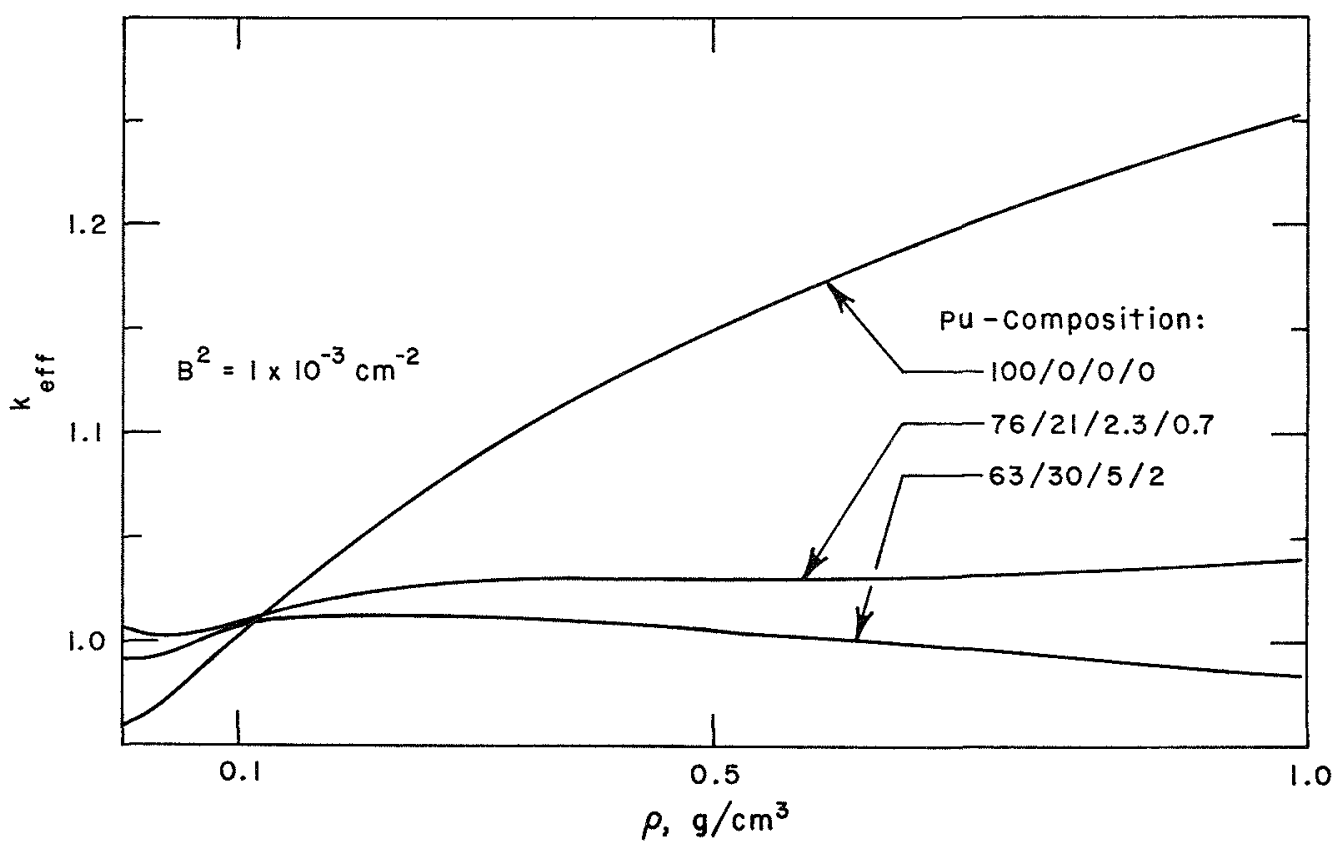

FI(x. 2. $k_{\text {eff }}$ as Function of Steam Density for Different Plutonium Compositions.

behaviour is essentially flat for all coolant densities. This effect is mainly due to the high absorption rate in the $1-\mathrm{eV}$ resonance of $\mathrm{Pu}-240$, as has been shown by detailed calculations. In the early study of Sofer, ${ }^{(2)}$ this effect was not found, perhaps because of a too coarse group structure.

Calculations were made for two isotopic compositions $\mathrm{Pu}^{239}: \mathrm{Pu}^{240}: \mathrm{Pu}^{241}: \mathrm{Pu}^{242}=63: 30: 5: 2$ 


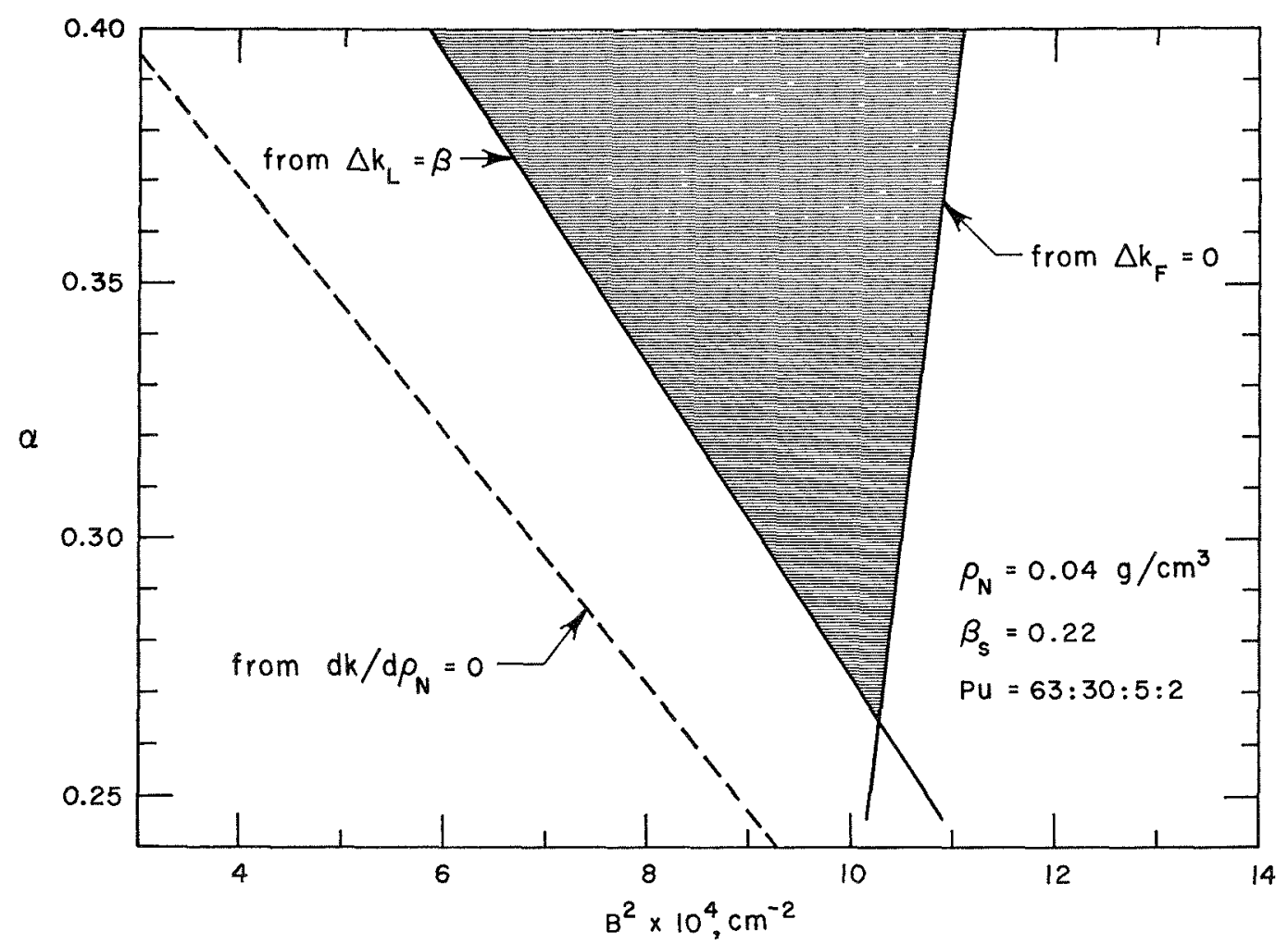

Fig. 3. In the Shadowed Area of This $\left(\alpha, 3^{2}\right)$ Plane the Safety Conditions for Flooding and Loss of Coolant Are Fulfilled, $\left(\alpha=\right.$ volume fraction of coolant; $\mathrm{B}^{2}=$ buckling; operational steam density $\left.\rho_{\mathrm{N}}=0.04 \mathrm{~g} / \mathrm{cc}.\right)$

and

$$
\mathrm{Pu}^{239}: \mathrm{Pu}^{240}: \mathrm{Pu}^{241}: \mathrm{Pu}^{242}=76: 21: 2.3: 0.7 \text {. }
$$

These compositions have been calculated for two representative refueling conditions: ${ }^{*}$ one in which plutonium bred in the blanket is fed to the core composition in an amount which is necessary to keep the reactor just critical, and one in which plutonium of the core and all the plutonium bred in the blanket is mixed during reprocessing, this mixture giving the new plutonium composition of the core.

As a result of our calculations and the evaluation schema described above, we obtained Figures 3, 4, and 5 , for mean steam densities of $0.04,0.0735,0.125$ $\mathrm{g} / \mathrm{cm}^{3}$, respectively, corresponding to pressures of 100 , 150, and 250 atm. The plutonium composition used was $63: 30: 5: 2$. The other one gave a positive flooding coefficient in all interesting rases and was therefore disregarded in this first step.

The fact that the safety behaviour (of $\Delta k_{L}, \Delta k_{F} \ldots$ ) is essentially combined with the long-time behaviour (of the plutonium composition) gives rise to further complications in the design of steam-cooled reactors.

Each of these figures shows the $\left(\alpha, B^{2}\right)$ region in which

* For these calculations the formulae of Ref. 3 were specialized for 1000-MWe fast reactors by the use of improved cross sections. the conditions concerning $\Delta k_{x}$ and $\Delta k_{F}$ can be satisfied. The density coefficient is positive in all these areas, thus giving no additional limitation. The wish to have also $\Delta k_{\max }<\beta$ ean only be realized for the highest steam pressure in a small subregion of the area shown in Fig. 5 .

The main results of these three figures are:

a) Csing very dirty plutonium, it is possible to satisfy the conditions $\Delta k_{L}<\beta$ and $\Delta l_{F}<0$ without using poisons.

b) The area where it is possible to fulfil conditions 1-3 of Table 1 grows considerably with increasing stcam pressure; at the highest pressure all conditions 1-4 may be fulfilled in a small subregion of the interesting $\left(\alpha, B^{2}\right)$ region.

The results of our calculations for the other plutonium composition showed that, apart from the fact that $\Delta k_{F}>0$, it is the more favoured one with respect to $\Delta k_{L}$ and $\left(d k_{i} d \rho\right)_{N}$, a result which may already be concluded from lig. 2.

\section{3) Addition of Small A mounts of Absorbers}

Plutonium of an isotopic composition in the neighbourhood of $76: 21: 2.3: 0.7$ is interesting since it belongs to a combined core-blanket reprocessing. As was mentioned in the last section, such a plutonium composition gives a reactivity increase in the flooded case due to the 


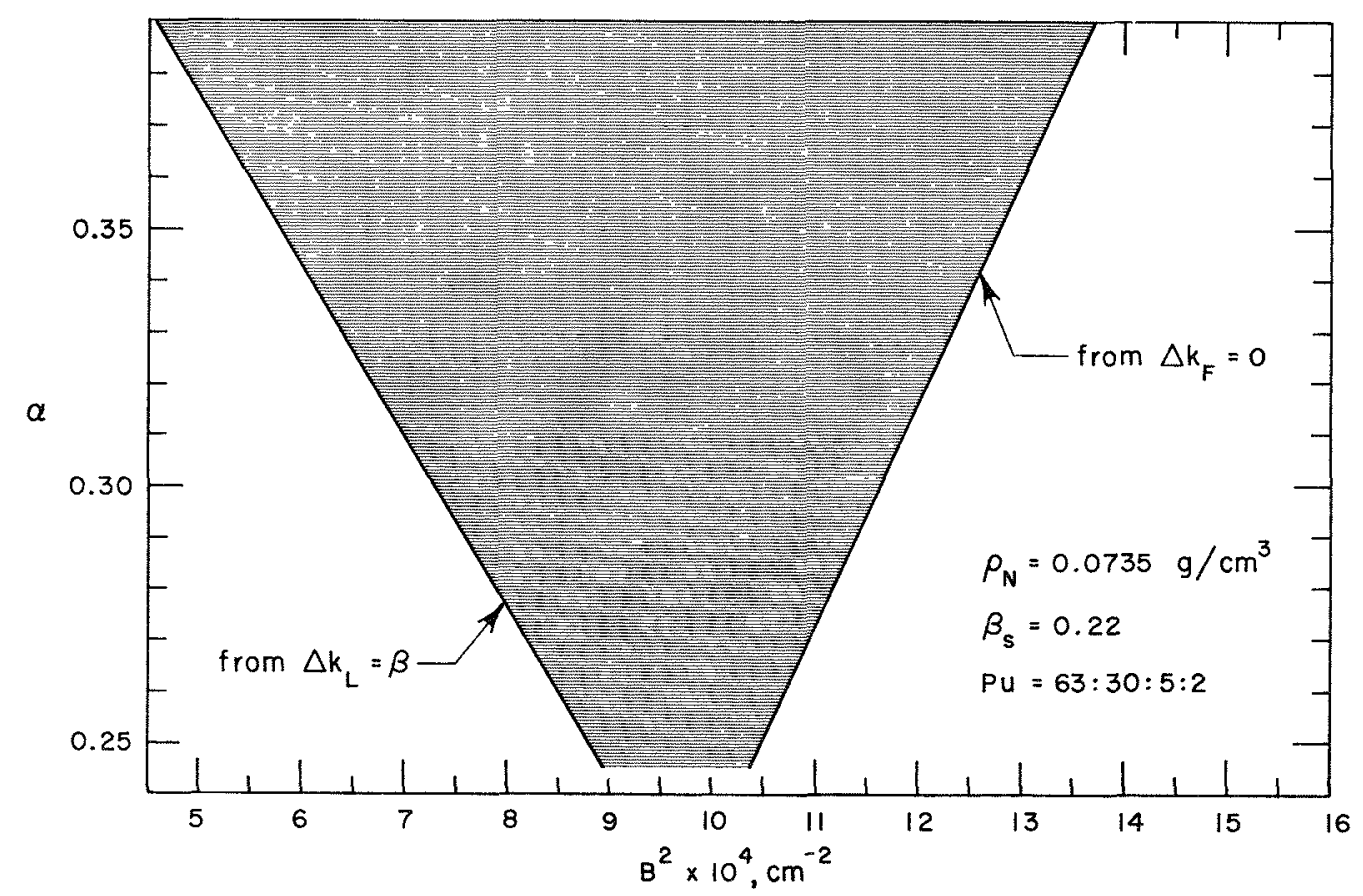

Fra. 1. In the Shadoned Area of This $\left(\alpha, B^{2}\right)$ Plane the Safety Conditions for Flonding and Loss of Coolant Are Fulfilled. $\left(\alpha=\right.$ volume fraction of coolant; $l^{2}=$ buckling; operational steam density $\rho_{\mathrm{N}}=0.0735 \mathrm{~g} / \mathrm{ce}$.)

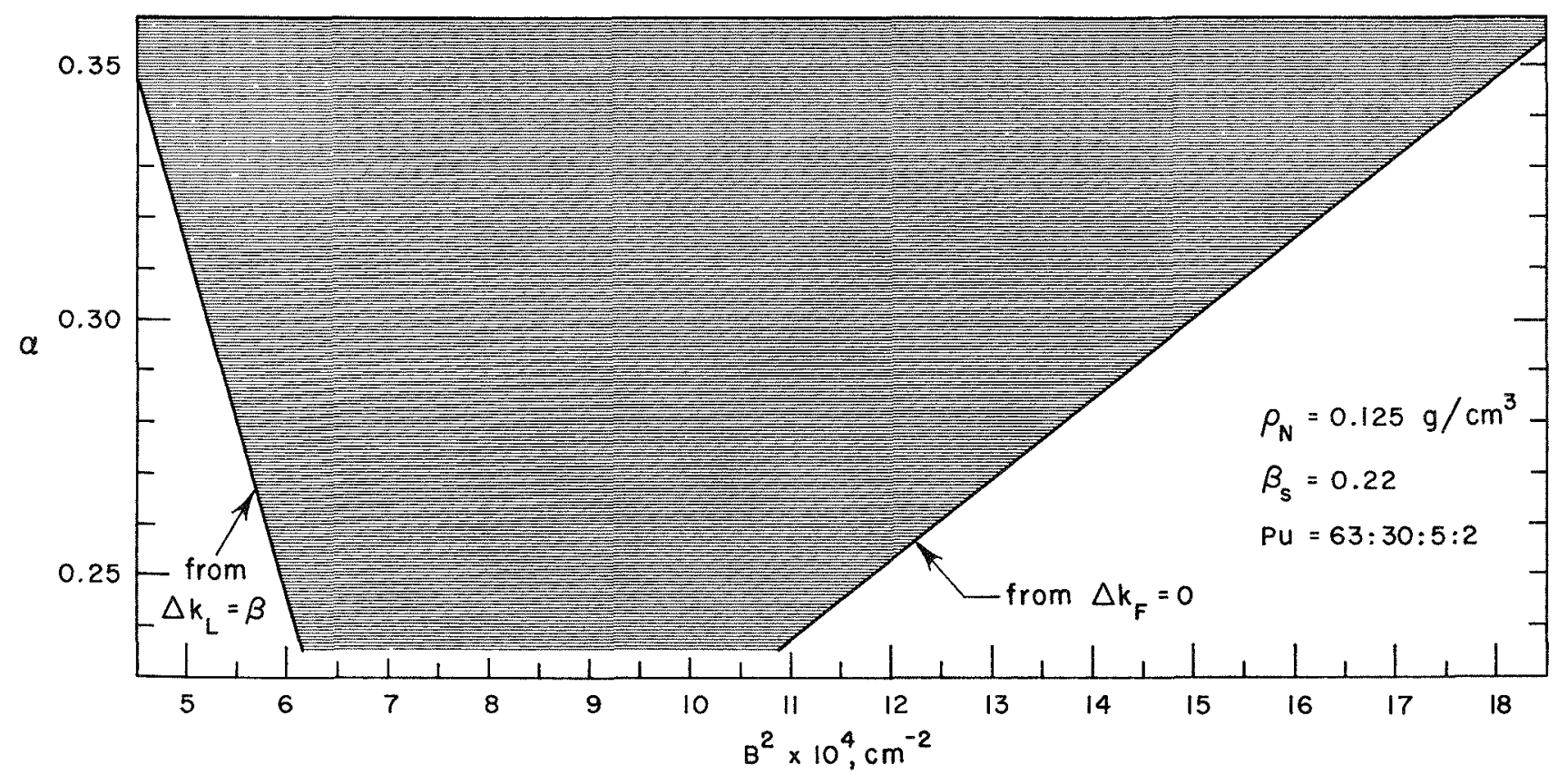

Fig. 5. In the shadowed Area of This $\left(\alpha, B^{2}\right)$ Plane the Safety Conditions for Flooding and Loss of Coolant Are Fulfilled. ( $\alpha=$ volume fraction of coolant; $B^{2}=$ buckling; operational steam density $\rho_{N}=0.125 \mathrm{~g} / \mathrm{cc}$.)

smaller amount of Pu-240 present. It is natural that one looks for other thermal or epithermal resonance absorbers which will produce a similar effect as Pu-240 when the coolant density is near or equal to the maximum value of $1 \mathrm{~g} / \mathrm{cm}^{3}$. Such poisons may be, for example, B, Cd, Eu, Gd, Hf, and Sm. We studied the changes in the reactivity behaviour caused by the addition of $\mathrm{Eu}, \mathrm{Gd}$, and $\mathrm{Hf}$, and will present hore only the results obtained for $\mathrm{Gd}_{2} \mathrm{O}_{3}$. Gadolinium has been (hosen for several reasons:

a) It has the best influence from the physics point of view, i.e., high influence on $\Delta k_{p}$, low influence on $\Delta k_{i_{L}}$-this fact also indicates a low reactivity penality in the normal states. 
b) The amount neressary to obtain the desired influence on $\Delta k_{F}$ is the smallest one compared with Hf and Eu; in the calculations discunsed below the volume fraction of $\mathrm{Gd}_{2} \mathrm{O}_{3}$ used was $.5 \cdot 10^{-4}$ of the whole core volume.

c) It is comparatively cheap poison, with respect to the fraction necenary perhap the cheapent one.

The result sobtained for the addition of $0.0 .5 \%\left(\mathrm{xd}_{2} \mathrm{O}_{3}\right.$ are given in the Figures 6,7 , and 8 . As in Figures 3,4 , and 5 , the results are hown for three mean steam densitics corresponding to three different pressures (100, 1.50 , and $2.50 \mathrm{~atm})$. It ran be seen that by the addition of poison a region in the $\left(\alpha, B^{2}\right)$ plane can be found where it is possible to fulfil the safety eriteria 1-3 or 1-4 of Table 1. Comparing Fig. 3 with Fig. 6, or 4 with 7 , or 5 with 8 , one recognizes that the "Jess dirty" plu1onium together with some suitable poison has a larger "allowed" region in the $\left(\alpha, B^{2}\right)$ plane than the "more

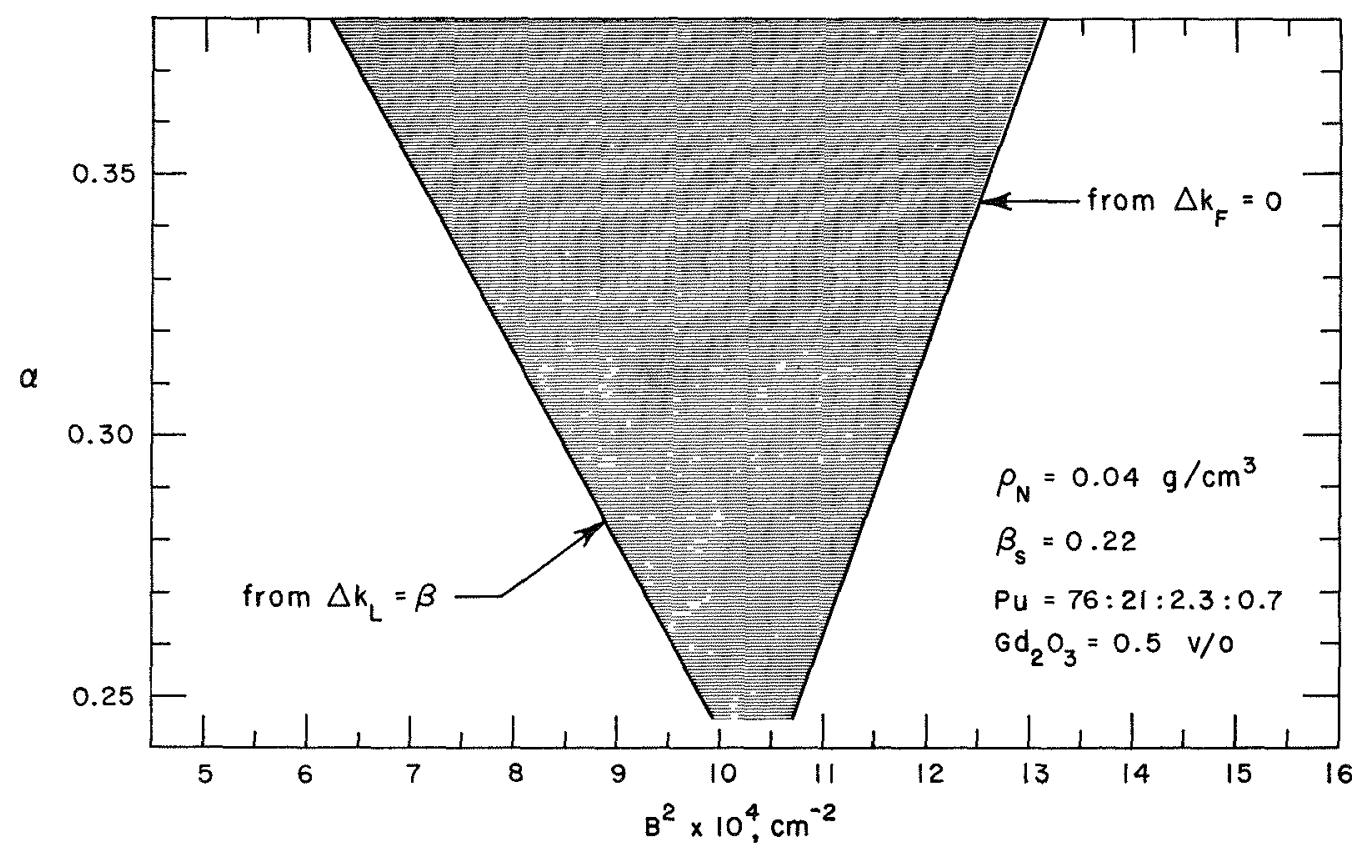

FIG. 6. In the shadoned Area of This $\left(\alpha, B^{2}\right)$ Plane the Safety Conditions for Flooding and Loss of Coolant Are Fulfilled. $\left(\alpha=\right.$ volume fraction of coolant; $B^{2}=$ buckling; operational steam density $\left.\rho_{N}=0.04 \mathrm{~g} / \mathrm{cc}.\right)$

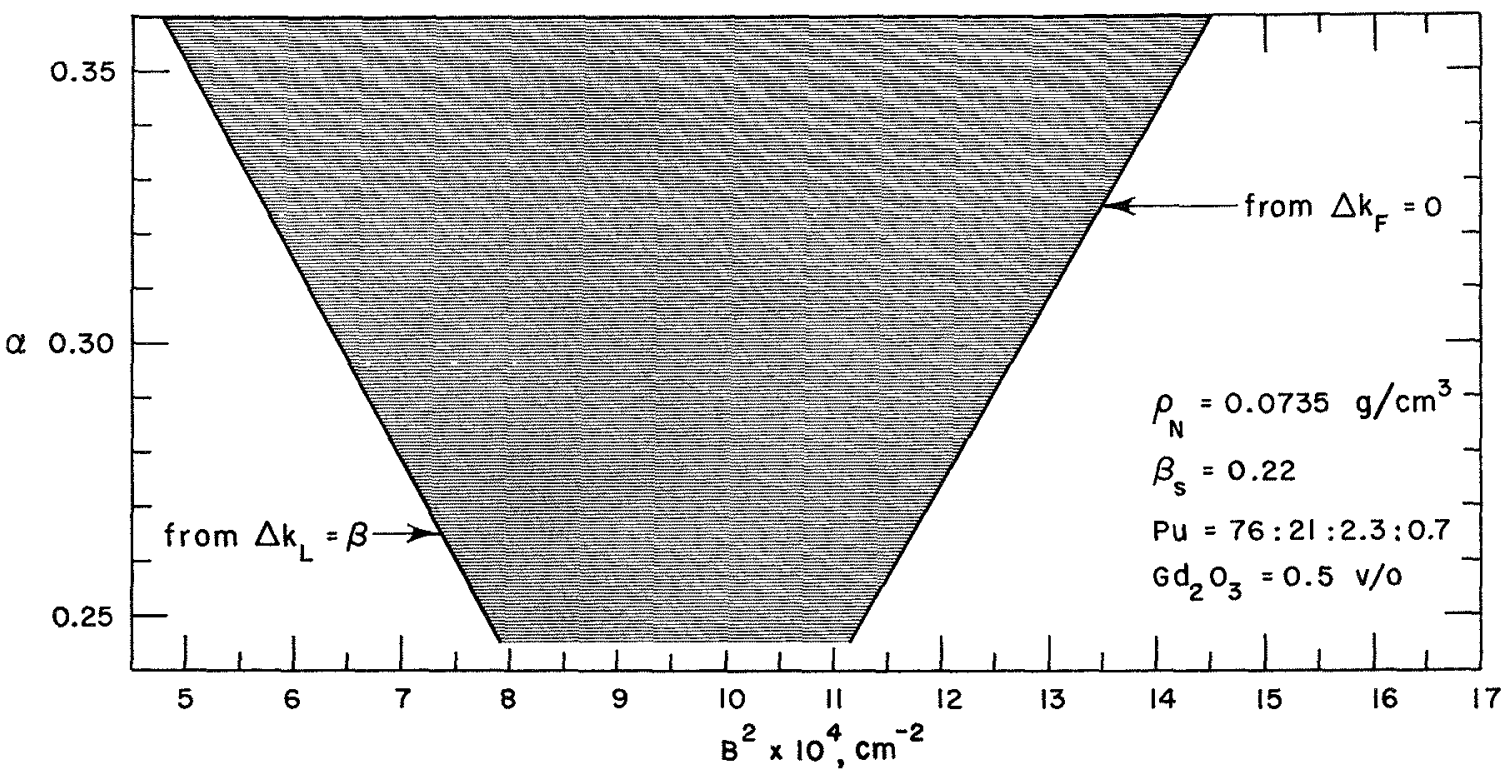

Fig. 7. In the shadowed Area of This $\left(\alpha, B^{2}\right)$ Plane the Safety Conditions for Flooding and Loss of Coolant Are Fulfilled. $\left(\alpha=\right.$ volume fraction of coolant; $B^{2}=$ buckling; operational steam density $\left.\rho_{N}=0.0735 \mathrm{~g} / \mathrm{cc}.\right)$ 


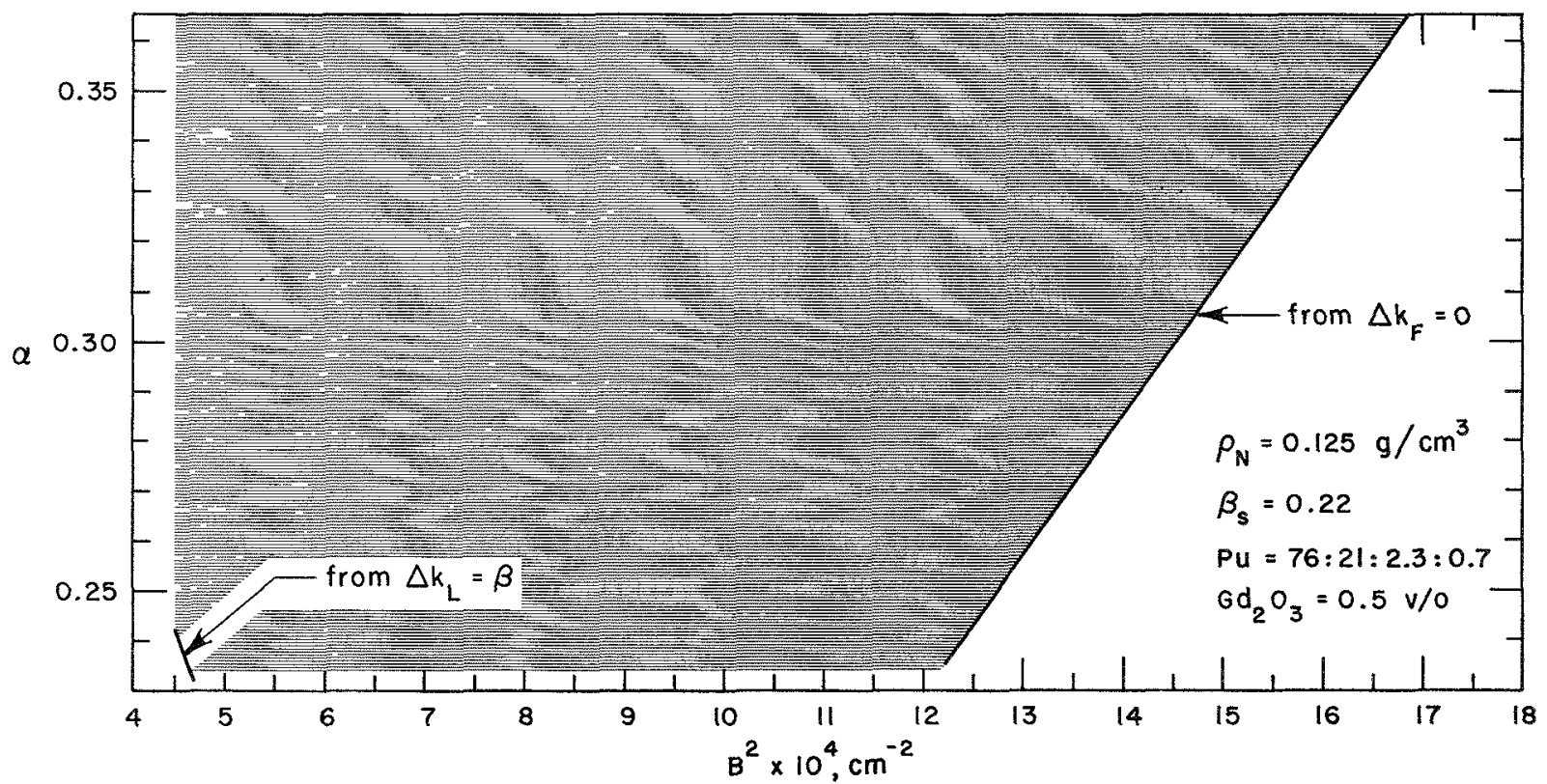

FIG. 8. In the Shadowed Area of This $\left(\alpha, B^{2}\right)$ Plane the Safety Conditions for Flooding and Loss of Coolant Are Fulfilled. $\left(\alpha=\right.$ volume fraction of coolant; $B^{2}=$ buckling; operational steam density $\left.\rho_{N}=0.125 \mathrm{~g} / \mathrm{cc}.\right)$

dirty" plutonium under the same safety conditions. This fact is due to the fast fission in Pu-240.

\section{4) Accumulation of Fission Products}

In the discussion above we have excluded the influence of fission products. These have partially similar nuclear properties as the poisons considered-some fission products are nuclei identical or very similar to the poison material. Because the burnup of ceramicfueled fast power reactors is very high, one should include the fission products in the consideration of the reactivity behaviour under normal operating conditions. In the following calculations $50,000 \mathrm{MWd} /$ to is used as an average burnup of the fuel in the core.

We give the following comments with respect to this section: The group constants used for the fission products of Pu-239 have been taken from the group-constant set of Bondarenko. ${ }^{(1)}$ As cited there, the following isotopes were omitted: Cd-113, Sm-149, Sm-151, Gd155 , and Gd-157. These fission products have very high thermal cross sections and are stable or long-lived. Their concentrations in an equilibrium state depend strongly on the spectrum of the reactor. Detailed calculations for every reactor considered will be necessary to get the right concentrations. Since we are at present only looking for a feasible reactor and even the pressure, which influences the spectrum markedly, is not yet fixed, it seems an unnecessary complication to determine these concentrations exactly.

Bearing in mind the partially large uncertainty connected with our knowledge of the cross sections for fission products in the fast-neutron range, we proceeded in the following manner. First, we tried to determine a favourable region in the $\left(\alpha, B^{2}\right)$ plane with the same method as for the addition of poison. As we expected, the buckling had to be increased considerably to satisfy the condition $\Delta k_{L}<\beta$. With increasing buckling the value of $\Delta k_{F}$ increases, too, keeping all the other parameters but the enrichment constant. At these high values of the buckling (about $2 \cdot 10^{-3} \mathrm{~cm}^{-2}$ ), it turned out that even with the addition of the fission products corresponding to $50000 \mathrm{MWd} /$ to but without the above-mentioned isotopes (with high thermal cross sections), there may occur a slight reactivity increase upon flooding. The omission of these isotopes gives a too pessimistic value for $\Delta k_{F}$ and a slightly too optimistic one for $\Delta k_{x}$. We simulate these thermal poisons by an addition of $1.6 \cdot 10^{-2} \mathrm{v} / \mathrm{o} \quad \mathrm{Gd}_{2} \mathrm{O}_{3}$ of natural isotopic composition. In other words, this means that about one per cent of the fission products are assumed to be natural gadolinium or about $0.3 \%$ to be either Gd-155 or Gd-157, a figure not too unrealistic if one considers that the other thermal poisons have been neglected.

The results for high pressure $(250 \mathrm{~atm})$ are given in Fig. 9 as an allowed region in the $\left(\alpha, B^{2}\right)$ plane. For low pressure there does not exist such a region, and for the intermediate pressure the region is very small.

For the exart determination of the reactivity behaviour of a steam-cooled fast reactor with moderate or high burnup, the cross sections for the fission prod- 


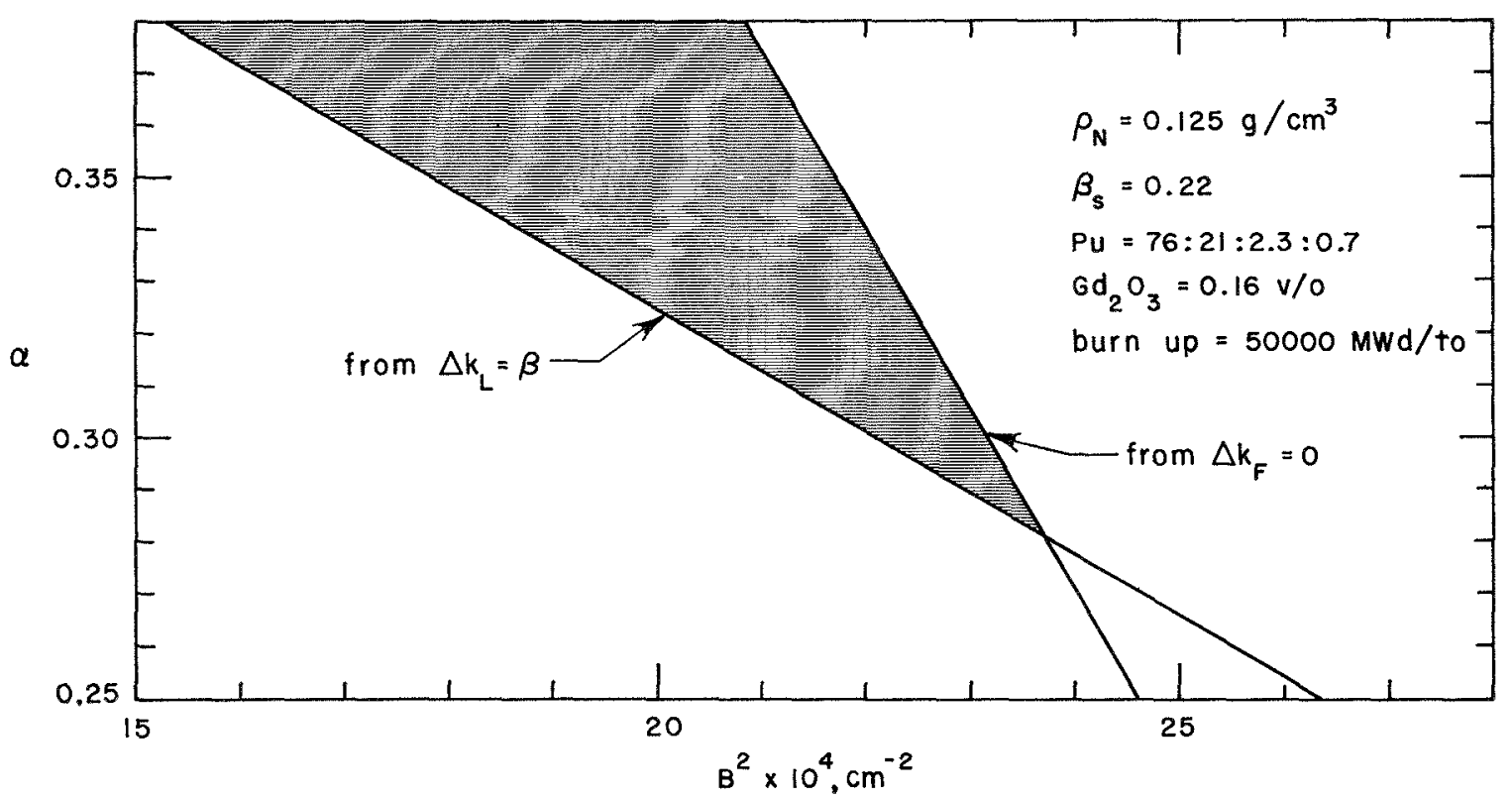

Fig. 9. In the shadowed Area of This $\left(\alpha, B^{2}\right)$ Plane the Safety Conditions for Flooding and Loss of Coolant Are Fulfilled. ( $\alpha=$ volume fraction of coolant; $B^{2}=$ buckling; operational steam density $\rho_{N}=0.125 \mathrm{~g} / \mathrm{cc}$.). Average burnup $=50,000 \mathrm{MWd} /$ to.)

ucts should be known with higher accuracy. Especially for the flooded case the yield of fission products which leads to thermal or epithermal absorbers should be known reasonably well, so that by means of the known cross sections and the spectrum of the reactor considered their concentrations may be calculated.

\section{B. One-dimensional Results}

\section{The Variation of Savings with Coolant Density}

We confine ourselves only to that part of our onedimensional calculations concerned with or to be interpreted as modifications of the zero-dimensional calculations discussed above. As is well-known, one may calculate at least approximately the criticality of a blanketed reactor by means of zero-dimensional calculations using the right savings corresponding to the blanket considered. Unfortunately, the savings varies appreciably with coolant density, so that instead of a constant buckling one should use in the zerodimensional calculations a buckling varying with the density of the coolant. For the determination of the buckling or savings variation, we performed one-dimensional calculations for a few standard blankets having the same core composition and varying only the coolant density.

The variation of the savings with steam density has been determined by adjusting at each coolant density the buckling in the zcro-dimensional calculations to give the same value of $k_{e \text { if }}$ as was obtained by the one-dimensional calculations. Knowing the buckling, it is quite easy to calculate the saving and its variation with coolant density for the special blanket considered. Two typical results are shown in Fig. 10 .

It is very instructive to recognize how sensitively the Pu-239 accumulated in the blanket influences the savings at high coolant densities. In the flooded case the blanket containing Pu-239 acts mainly as a thermal "core," thus producing a considerable reactivity increase. Since buildup of $\mathrm{Pu}-239$ and some $\mathrm{Pu}-240$ in the blanket will occur during one core life, the dependence of the savings upon coolant density will change during one core life too. This will cause an increase in $\Delta k_{F}$ and a decrease of $\Delta k_{L}$ during the core life. However, this will be compensated or over compensated by the simultaneous build-up of fission products in the core.

\section{FINAL DISCUSSIONS}

\section{A. Remarks Concerning the Accuracy}

It has been mentioned in section III that we have used only two values of the coolant volume fraction and two or three values of the buckling to determine the allowed regions in Figures 3-9, so that the boundaries are straight lines.

The value of $\Delta k_{F}$ is important for the determination of the allowed region in the $\left(\alpha, B^{2}\right)$ plane. This implies that one has to take into account quite accurately the heterogeneity effect, since it tends to increase the reactivity in the flooded case and in this way also $\Delta k_{F}$. In the normal and voided cases the influence of the heterogeneity effect will be smaller and, fortunately, tend to decrease $\Delta k_{L}$. 


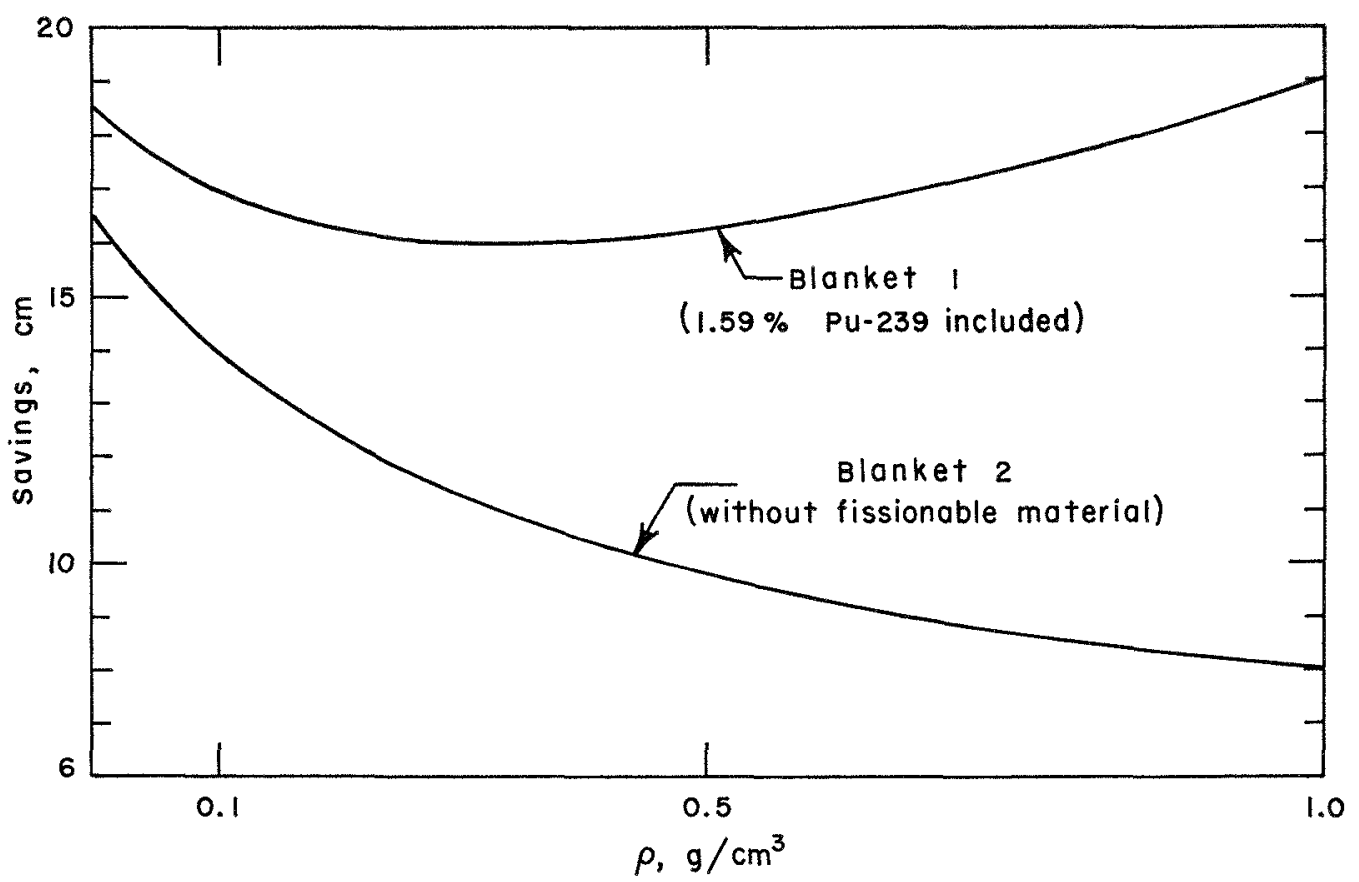

Fig. 10. Savings as Function of Steam Density for a Typical Large Steam-cooled Fast Reactor. Blanket 1 includes $1.59 \%$ bred plutonium-239; blanket 2 is without fissionable material.

Apart from these simplifications, which may be easily improved, there are other effects which result in inaccuracies or influence the reliability of the results. Two important ones will be discussed in the following.

1. From Fig. 10 one recognizes that the buckling is not really independent of coolant density as it was assumed to be in all our calculations. If a density-dependent buckling is considered by use of density-dependent savings, it is possible to improve the accuracy of the zero-dimensional results. However, as may be seen from Fig. 10, the savings strongly depend on the plutonium content of the blanket. The amount and the isotopic composition of plutonium bred in the blanket vary remarkably during core life, and this variation is not known a priori. To determine this plutonium variation in the blanket one has to treat the full burnup problem. Since it seems unreasonable to do so in a parameter study, there remains some uncertainty due to this effect which may not simply be eliminated using one- or twodimensional calculations.

2. With respect to the fission products one should bear in mind that the cross sections are fairly uncertain in the high-energy region. With respect to quantities as $\Delta k_{L}$ and $(d k / d \rho)_{N}$ this generates uncertainties coming mainly from this energy region. Furthermore, the yield and concentration of long-lived fission products has to be known with higher accuracy to get reliable results for $\Delta k_{F}$.

\section{B. Summary of Results and Conclusions}

The most important quantities with respect to the safety behaviour of a steam-cooled fast power reactor in our opinion are $\Delta k_{L}$ and $(d k / d \rho)_{N}$, although $\Delta k_{F}$ also has great importance.

Since $\mathrm{H}_{2} \mathrm{O}$ gives more favourable values for $\Delta k_{L}$ and $(d k / d \rho)_{N}$ then does $\mathrm{D}_{2} \mathrm{O}$, we chose $\mathrm{H}_{2} \mathrm{O}$-steam as the coolant medium for further investigations.

We found that the presence of $\mathrm{Pu}-240$ has a marked influence upon the quantities used for the safety criteria: $\Delta k_{L}$ and $(d k / d \rho)_{N}$ will be changed in the unfavoured, $\Delta k_{F}$ in the favoured direction with increasing $\mathrm{Pu}-240$ concentration.

The desired change in $\Delta k_{F}$ which is caused by the epithermal resonance absorption in Pu-240 may also be obtained by the addition of poisons or the accumulation of fission products, but with a less pronounced unfavourable influence on $\Delta k_{L}$ and $(d k / d \rho)_{N}$. The last fact is due to the absence of fast fission in these materials.

The results have shown that one may influence the two most important quantities mentioned above in the desired direction if one

1. increases the buckling;

2. increases the coolant volume fraction;

3. increases the pressure or the mean steam density under normal operation conditions; 
4. uses "less dirty" plutonium;

5 . reduces the average concentration of fission products.

As a general result one concludes that the allowed region increases with increasing operating pressure and is shifted towards higher values of buckling with increasing burnup. The last fact presumably leads to three alternatives:

1. At high power and high burnup, an annular or modular core has to be used.

2. With a normal core at high power, the burnup has to be reduced.

3 . With a normal core at high burnup, the power has to be reduced.

\section{REFHRENCEN}

1. L. P. Abagjan, N. O. Bazazjane, I. I. Bondarenko, and M. N. Nikolaev, Gruppenkonstanten schneller und intermediarer Neutronen fur die Berechnung von Kernreaktoren, KFK-tr144.

2. (x. Sofer $t$ t al., NDA-2148-4 (1961).

3. K. Ott and A. Jansen, "Thong-time behaviour of fast breeders," Proceedings of the Seminar on the Physics of Fast and Intermediate Reactors, IAKA, Vienna (1962), Vol. II, p. 213.

\section{Discussion}

Mr. Fell (Winfrith): From the earlier meetings I understand that you have replaced the Russian set by a new data set. Were the conclusions of this study stable to the exchange of data sets? Secondly, in any analysis of coolant condition requirements, I would have expected to see some requircment for maintaining a breeding gain. This could leave you, for example, with bigger areas if you increase the density of the coolant as you increase the pressure.

Mr. Ott: The Karlsruhe group-constant set was prepared by weighting a spectrum typical for a 1000 MW sodium-rooled reactor, and here we have a steamcooled reactor. Such a spectrum is also somewhat different from zero steam density to the full flooding state. Therefore, in this respect it is not easy to make a good group-constant set for such reactors by use of a typical weighting spectrum. We started these calculations before the Karlsruhe group-eonstant set was in operation; that is why, for all these calculations, the Russian group-constant set was used. Clearly the overall criticality will change if the Karlsruhe cross sections are used; however, the density coefficients would presumably not change too much. To your second question, we are fully aware of the fact that the breeding ratio and especially the breeding gain are very important quantities for fast reactors, and that all of our calculations are zero-dimensional. By these we can only get the internal breeding ratio, and we have in this ase calculated that the internal breeding ratio is larger than one. Therefore, we hope that a reasonable blanket will give at least more than 1.2 for the breeding ratio. However, the real value depends on the rhoice of shape. if annular or not. The two-dimensional calculations are under way.

$M r$. Greebler (GK): The void calculations you made were all made with a constant buckling, that is, the buckling with the steam in and the steam out were the same. When we did such ralculations and then went to one dimension, the effert of the steam on the so-called reflector effectiveness was very very large. One gets a substantial increase of the buckling when one considers the removal of steam from the core, and we found that while core thicknesses of the order of $11 / 2$ to $2 \mathrm{ft}$ were adequate, to get zero steam void 'onditions, one had to go to much thinner cores depending on the volume fraction of steam and pressure, etc., than what we had estimated from the zero-dimensional calculations. Do you have results?

Mr. Ott: Those are the results shown in Fig. 10. The savings are given as a function of the stearn density in the core between zero and one gram per cubic centimeter for two kinds of blankets. The savings as a function of steam density for a clean blanket is given in the lower curre. Going from normal operation density to the voided state one has a decrease in savings; this means a decrease in reactivity and an increase in voiding $\Delta l$. However, if one considers the rase in which a reasonable amount of plutonium is already bred in the blanket, one gets the upper curve of this diagram, so that savings as a function of the density is strongly dependent on the plutonium content of the adjacent blanket, that is, of the blanket which is rery near the core.

Mr. Heck (WAPD): I wanted to concur with Dr. Gecblex; we found the same thing in voiding calculations. It was necessary to adjust the buckling for steam, and we also found fairly significant changes when we switched from a sodium-suitable set to a water-suitable set of cross sections. Not only did we have to change the magnitude of the cross sections, we found it was also quite worthwhile to change the entire energy structure of the group set in order to describe better what was going on in this sort of a pseudo-thermalized system.

Mr. Ott: We also have a group structure which goes to thermal energies.

$M r$. Wall $(\mathrm{GF})$ : In our studies we find that the savings or the change in the bucklings when one goes from the voided state to the operating state depend on the energy: the high-energy bucklings do not 
change very significantly, but the low-energy bucklings do. What were your savings and your $B^{2}$ changes, thermal, fast, and average? Secondly, when you allow for this, does your system still look attractive?

$M r$. Ott: We have used only one effective average savings.

Mr. Thompson (MIT): I gather that at some time in its life, the reactor goes from the completely voided conditions for normal operation into the flooding region. If so, as a practical matter, how will you determine that you don't get a reactivity jump greater than the delayed neutron fraction in the reactor after you've actually built it?

Mr. Ott: In most cases presented here, $\Delta k_{\max }$ is larger than a dollar. Some are two dollars or more, and this may be still more if you have not a homogeneous density distribution as we calculated here. However, these cases can occur only if a controlled flooding is carried out, so that good control during this short procedure may be guaranteed. 


\title{
The Flood Safety of Steam- and Gas-cooled Fast Reactors*
}

\author{
J. W. Hallam, R. K. Haling, P. Kilitan, $\dagger$ and G. T. Peterson \\ General Electric Company \\ Vallecitos Atomic Laboratory \\ Pleasanton, California
}

(PRESENTED BY J.W. HALLAM)

\section{Introduction}

An important safety consideration in steam- and gas-cooled fast reactors is the change in reactivity for various degrees of steam-water density. Fast reactors employing these coolants have a potential flooding accident, and deliberate flooding of the reactor core is frequently proposed for refueling. Thus, the safety feasibility of these concepts depends upon the neutronics of flooding and the degree to which the associated reactivity effects can be minimized.

The reactivity effects resulting from an increase in hydrogen density are twofold: a decrease in neutron leakage from the core, and a softening of the neutron spectrum. The neutron-leakage effect is always positive and is most important in small systems with their attendant high leakage. The sign and magnitude of the spectrum effect depends upon the fuel type (uranium or plutonium), composition (Pu-240 fraction), and the floodable fraction of the core. Hence, the magnitude and even the sign of the net resultant reactivity change depends on a subtle balance between contributing terms, and its accurate prediction for a wide variety of cases is, therefore, a stringent test of calculational methods and basic cross-section data.

For most first-generation steam- and gas-cooled fast reactors of potential economic interest, ${ }^{(1-4)}$ the system sizes are such that upon uniform flooding, the reactivity would increase appreciably because the decrease in leakage is the predominant effect. In order to reduce or eliminate such a reactivity increase, the incorporation of resonance and/or thermal poisons into the fueled regions has been proposed..$^{(5-8)}$ The addition of poison materials greatly increases the overall capture-to-fission ratio in the softer flooded spectra. It is believed in principle that by the incorporation of suitable poisons in the correct amounts and distributions, the reactivity of any reasonable system can be held within tolerable limits for all degrees of uniform flooding.

\footnotetext{
* Work sponsored by U. S. Atomic Energy Commission under Contract AT(04-3)-189, P.A. 13.

$\dagger$ A.E.G., Frankfurt, Kernenergieanlage.
}

Figure 1 illustrates some of the effects involved. We have compared calculated results from various sources for three systems of varying sizes:

1. a relatively small, steam-cooled Mixed Spectrum Superheat Reactor (MSSR) $;^{(9)}$

2. a large MSSR; (10)

3. a gas-cooled fast reactor studied by General Atomics. ${ }^{(2)}$

The effect of various amounts of uniformly distributed poisons such boron, europium oxide, and gadolinium is shown in Fig. 1. The choice of control poison depends upon the fuel type and the system under consideration. The ideal poison should have zero reactivity worth under operating conditions and a high absorption under flooded conditions. A low concentration is also a very desirable feature.

In a prototype steam-cooled reactor, due to its small size, the decrease in leakage predominates, and flooding without poison present would cause a large reactivity increase. In such a system the floodable volume fraction would not be very large, so that upon flooding the spectrum softens into the resonance region, but the thermal flux remains relatively insignificant. Thus, poisons having large epithermal resonances, such as europium and hafnium, are more effective than thermal poisons. "Effective" here means high hold-down under the flooded condition with a relatively small penalty in the operating condition.

In a large steam-cooled fast reactor fueled with equilibrium recycle plutonium $(\sim 30 \% \mathrm{Pu}-240)$, the neutron-spectrum effect is much more important than for a smaller U-235-fueled system. The coolant volume fraction would probably be higher in such a system, which, in turn, causes substantial thermal flux in the flooded condition; for U-235 or pure $\mathrm{Pu}-239$ this gives rise to a reactivity increase. However, calculations show that the presence of $\mathrm{Pu}-240$, with its $1-\mathrm{eV}$ resonance, effectively reduces reactivity change to a small value. Finally, a gas-cooled fast reactor has an even larger coolant volume fraction, and the spectrum change from the operating state to fully flooded is very great. Calculations have suggested that gadolinium is probably the most effective poison for such systems. 


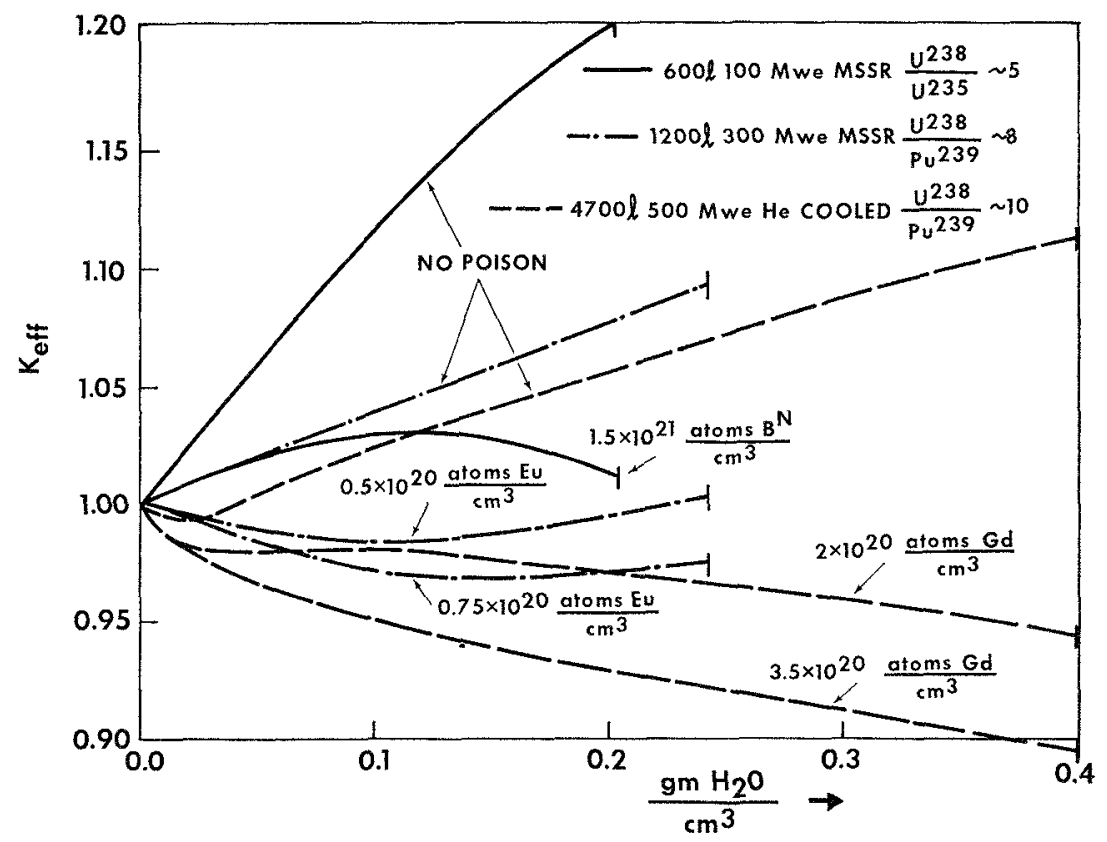

Fig. 1. Flooding Effect in Fast Reactors.

Calculations of the flooding effects are very difficult to perform with any great reliability because of all the different effects involved; handling the spectrum calculations is itself a large problem. The uncertainty in resonance poison cross sections and the generation of reliable spectrum-averaged group constants are the two main difficulties.

The above discussion shows that the reactivity change upon flooding of steam- or gas-cooled fast reactors is a complicated effect. The sign and magnitude of the reactivity changes influence the design and even the fcasibility of a concept. It is, therefore, obvious that before any serious consideration is given to this use of resonance poisons, sufficient detailed experimental information should be obtained to test thoroughly the feasibility of the technique and the calculational methods. The only experimental information available to date is that generated as part of the Mixed Spectrum Superheat Critical Experiment (MSSCE) program. ${ }^{(11)}$

\section{Experimental Information}

\subsection{Measurement System}

In the work on the Mixed Spectrum Critical Assembly (MSCA), the incorporation of resonance poisons into a U-235-fueled fast core has becn studied experimentally and theoretically as a means of ensuring flooding safety. Although the experiments and calculations were directed toward a specific reactor concept, the results are of general interest in the field of flood safety of steam- and gas-cooled fast reactors. In particular, these are the first experimental measurements demonstrating the relative effectiveness of resonance poisons such as hafnium, europium, and. boron.

The experiments have ranged from relatively small poisoned-flooded regions within a fast core to the flooding of relatively large fractions of the fast core containing resonance poisons.

Figures 2 and 3 show the general features of the MSCA which was built at Vallecitos Atomic Laboratory in support of the Mixed Spectrum Superheat Concept. It contains four distinct fuel regions:

a. a central fast core, nominally $23 \mathrm{w} / \mathrm{o}$ enriched uranium as $\mathrm{UO}_{2}$, Inconel clad;

b. a fast buffer, $1.3 \mathrm{w} / \mathrm{o}$ enriched uranium as $\mathrm{UO}_{2}$, stainless steel clad;

c. a thermal buffer, natural $\mathrm{UO}_{2}$ elad with aluminum and slightly boron poisoned;

d. a thermal core, $1.8 \mathrm{w} / \mathrm{o}$ enriched uranium as $\mathrm{CO}_{2}$, aluminum clad.

Figure 4 shows a typical fast-core fuel bundle when loaded with polyethylene and poison rods. The total floodable volume within a fast-core bundle is about $19 \%$. Polyethylene in the form of small-diameter rods and tubes could be introduced into the interstitial regions between fuel rods. In many cases, the bundles were flooded with water. By using combinations of polyethylene and water, the reactivity of the system could be measured with various volume fractions of water equivalent between 0 and $\sim 19 \%$.

Three types of poison rods were used in the MSCA fast-core flooding experiments. The poisons were boron 


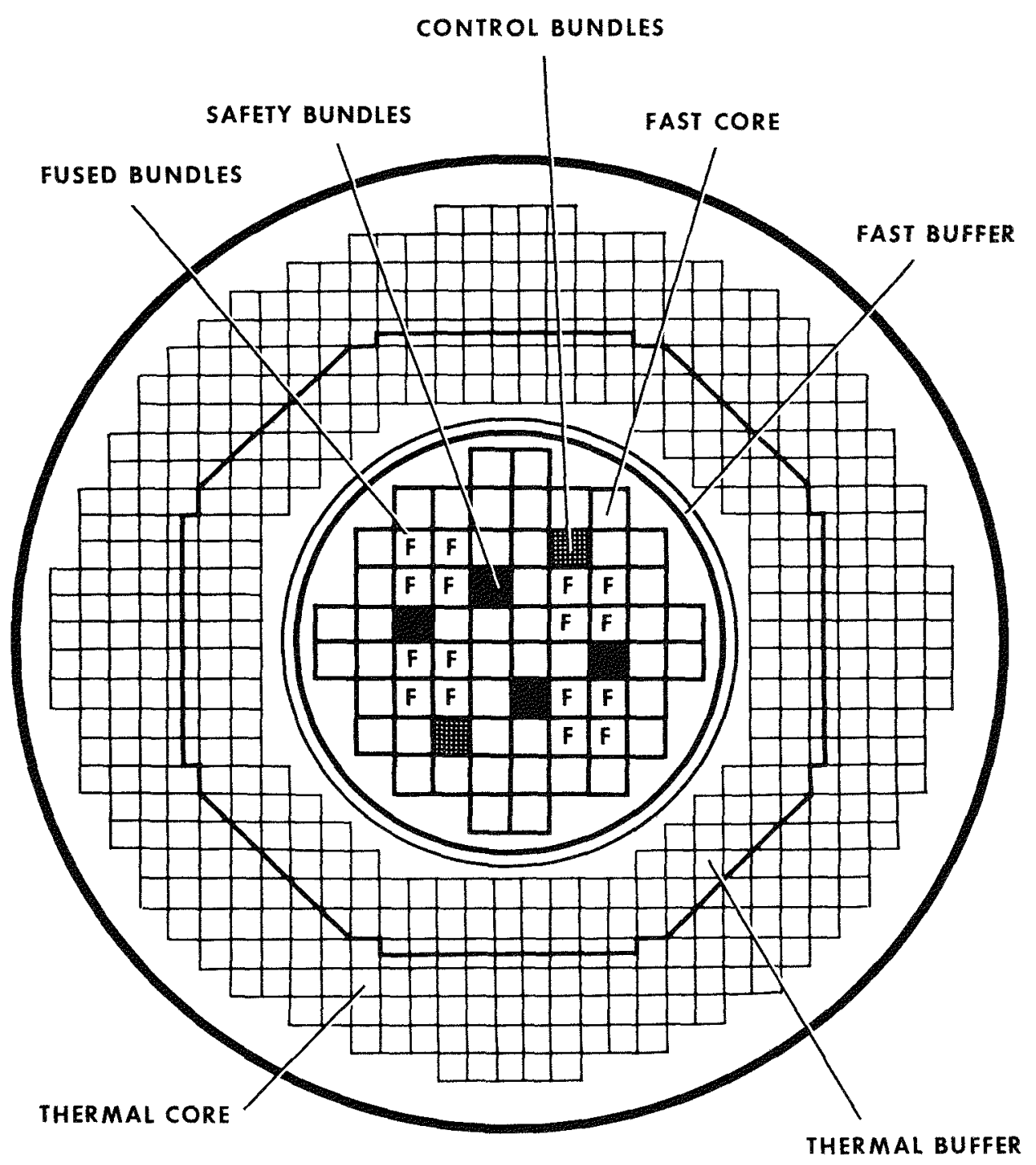

Fig. 2. Plan View of MSCA.

carbide clad in stainless steel, europium oxide similarly clad, and hafnium in metal wire form. Figure 5 shows the construction of a typical poison rod. The poison length was $30 \mathrm{in}$. corresponding to the length of the fueled region in the fast core. Each end was closed by 9-in.-long stainless steel filler plugs, corresponding to the axial Inconel reflector regions of the fast core.

\subsection{Results of Measurements and Calculations}

In the initial experiments, polyethylene was inserted in various radial and axial locations in the fast core, The polyethylene was in the form of sixteen rods $(64.97$ $\mathrm{gm}$ total), each 6 in. long and of 0.235 -in. diameter. The polyethylene was placed in the interstitial regions between fuel rods as shown in Fig. 4. Pieces of the regular Inconel filler rods of various lengths were used to support the polyethylene at different axial locations.
Reactivity measurements were made with the polyethylene sections at levels 1 ( $0-6$ in.), 3 (12-18 in.), 5 (24-30 in.), and 7 (36-42 in.) as shown in Fig. 6, and were repeated at different radial locations to determine the radial dependence.

Figure 7 shows the data obtained along with corresponding values obtained from four-group, twodimensional calculations. The reactivity values have been expressed in cents/kg polyethylene. Both experiment and calculation show that the worth of hydrogenous material is a very strong function of location within the fast core. It is also of interest to note that the worth of hydrogenous material actually goes negative in the axial reflector regions. This effect has been noticed before for other fast systems ${ }^{(12)}$ and is due to the thermalization of neutrons in the reflector region. Once a neutron is thermalized in the reflector, 


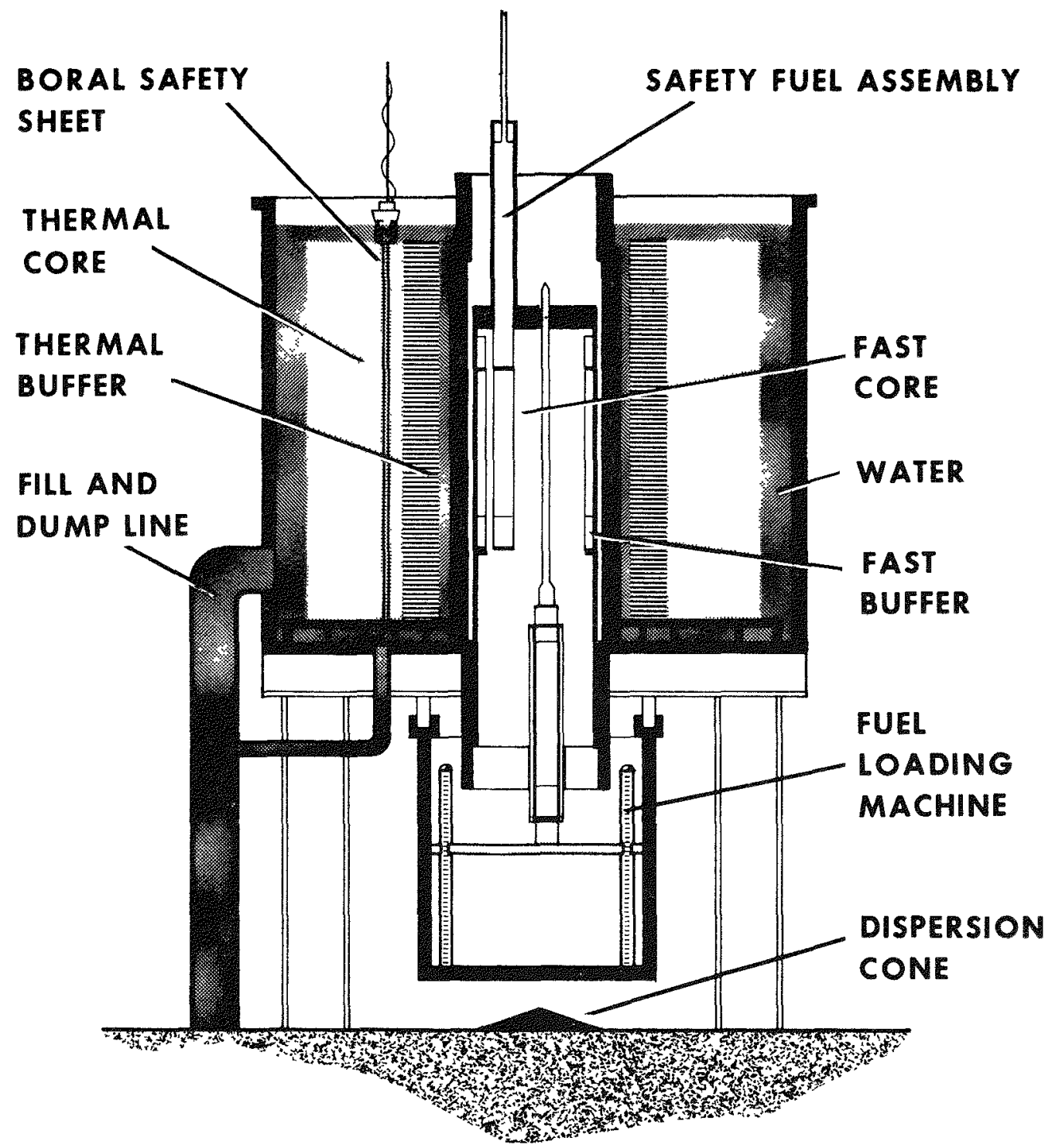

FIg. 3. Elevation of Mised Spectrum ('ritical Assembly.

its chances of parasitic capture are very great, especially in the MSCA, where the reflector is Inconel.

Figure 8 shows the results of four-central-bundle flooding measurements, with hafnium as a poison, for reactivity hold-down. It is clear that the flooding characteristic is favorable in that for a relatively small reactivity penalty, the amount of reactivity hold-down is very large. Figure 9 shows similar sets of data when boron carbide and europium oxide were used. The calculated data are shown as squares on the graph; the excellent agreement between calculation and experiment is most encouraging. Perturbation-theory calculations were found to be satisfactory for flooding small regions without poison, but a separate diffusion calculation was required for the flooded-poisoned state, especially for the larger perturbed regions.
The results of the measurements with four central bundles make a comparison of the effectiveness of the three poisons possible. It is clear that hafnium has the greatest flooding reactivity hold-down power and also the least penalty in the dry case; therefore, the "figure of merit" on this basis is highest for hafnium. Europium oxide appears somewhat less effective than hafnium, whereas boron carbide seems to have a relatively large penalty and relatively less hold-down power.

Table 1 shows various aspects of these poisons. Although hafnium looks best from the physics standpoint, it is relatively weak, that is to say, the required volume is quite large, so that if this poison were to be used in the form of hafnium oxide, 5 to $6 \%$ of the total core volume would be occupied by poison, which is clearly a large economic penalty in that proportionally 


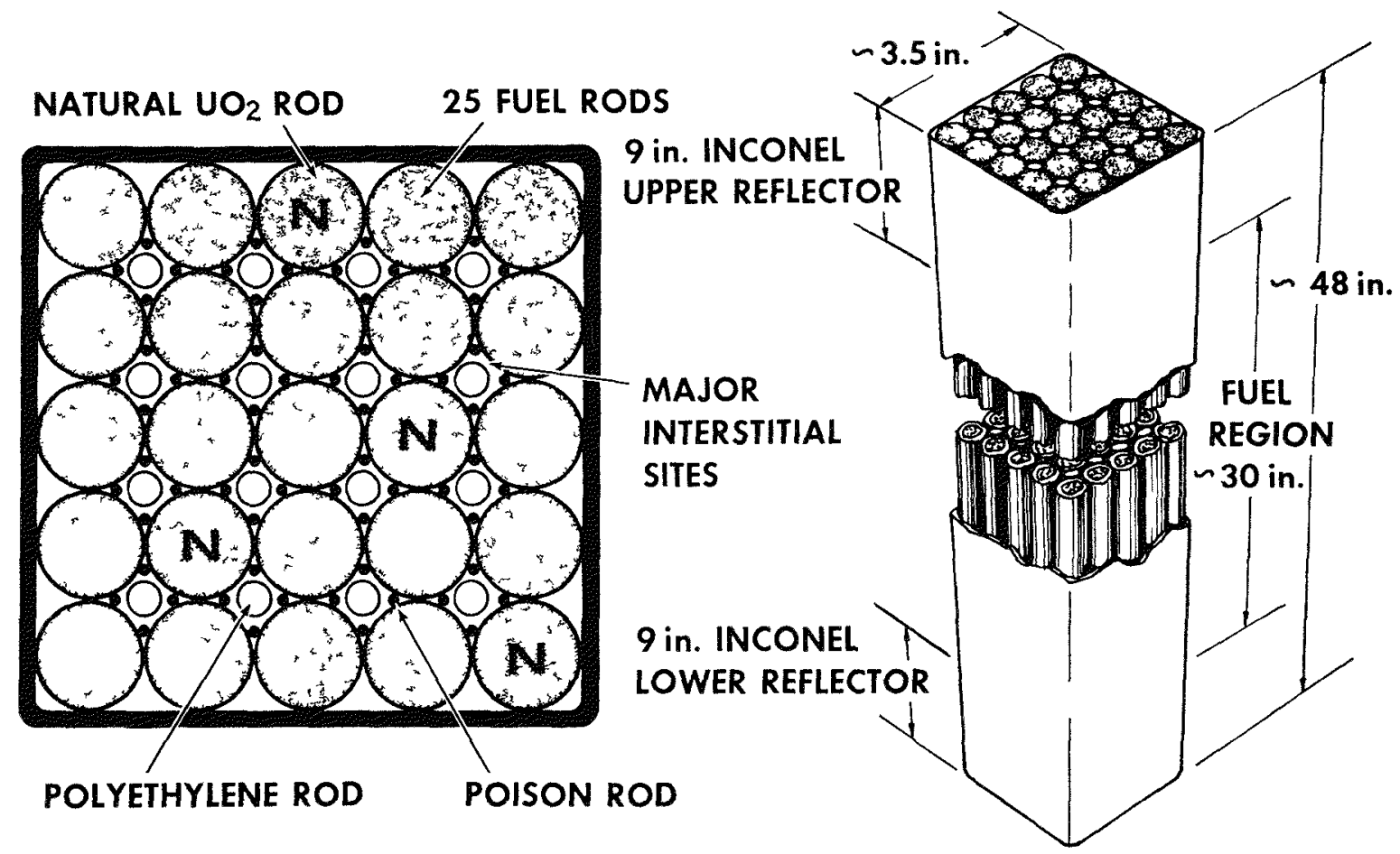

FIG 4 Typical Bundle Loadıng

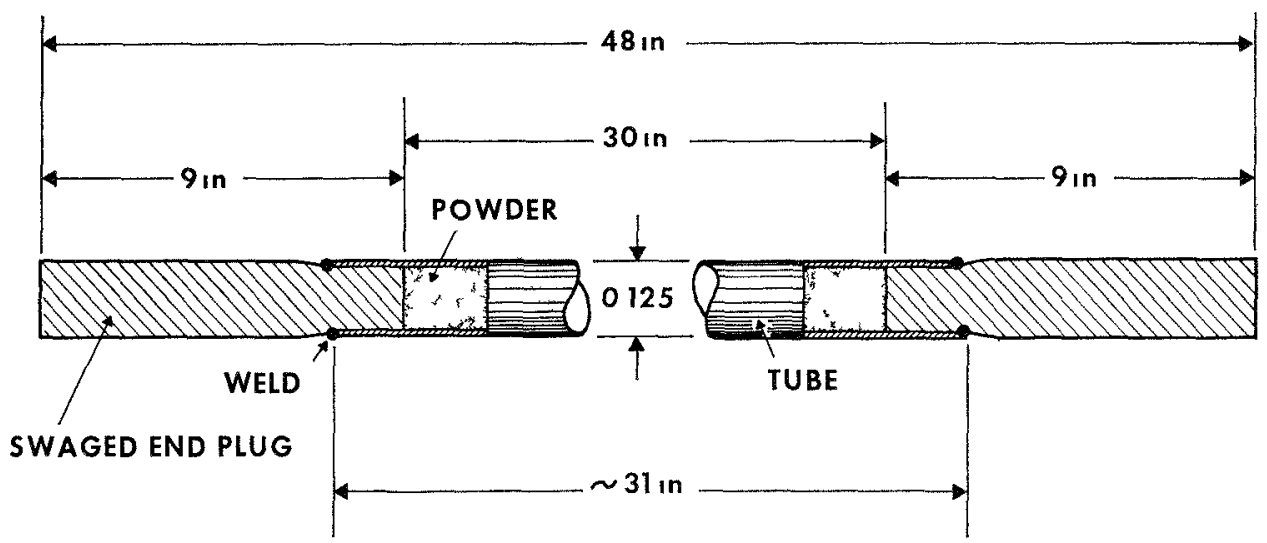

Hig 5 Details of MSCA Poison Rods

less fuel can be aecommodated The same table also presents some rough estimates of the cost effectiveness of the three poisons (cost multiplied by the amount required for a given reactivity hold-down) On this basis $\mathrm{B}_{4} \mathrm{C}$ is very favorable, however, there are doubts about the compatibility of $\mathrm{B}_{4} \mathrm{C}$ in fuels, and its migration during fuel manufacture and under operating conditions Perhaps an arrangement whereby the poison and fuel are kept separate, as suggested recently by Edlund, ${ }^{(3)}$ would be most appropriate for application of $\mathrm{B}_{4} \mathrm{C}$

The next series of experiments investigated the flooding effects in sixteen cential bundles as shown in Fig
10 These experiments determined how the flooding compensation effect of europium oxide varied as a function of the size and shape of central regions which were both poisoned and flooded The results are shown in Fig 11 Each standard bundle was loaded with about $0093 \mathrm{gm} \mathrm{Eu} \mathrm{O}_{3} / \mathrm{cm}^{3}$ and about $18 \mathrm{v} / 0$ water The reactivity change (per bundle), from the unperturbed condition, is plotted as a function of the size and shape of the flooded-poisoned region At the top of the figure the various flooded regions are illustrated relative to core center It is clear that as the size of the flooded region increases the effectiveness of the poison gets gi eater On this basis, it is expected that a considerably 


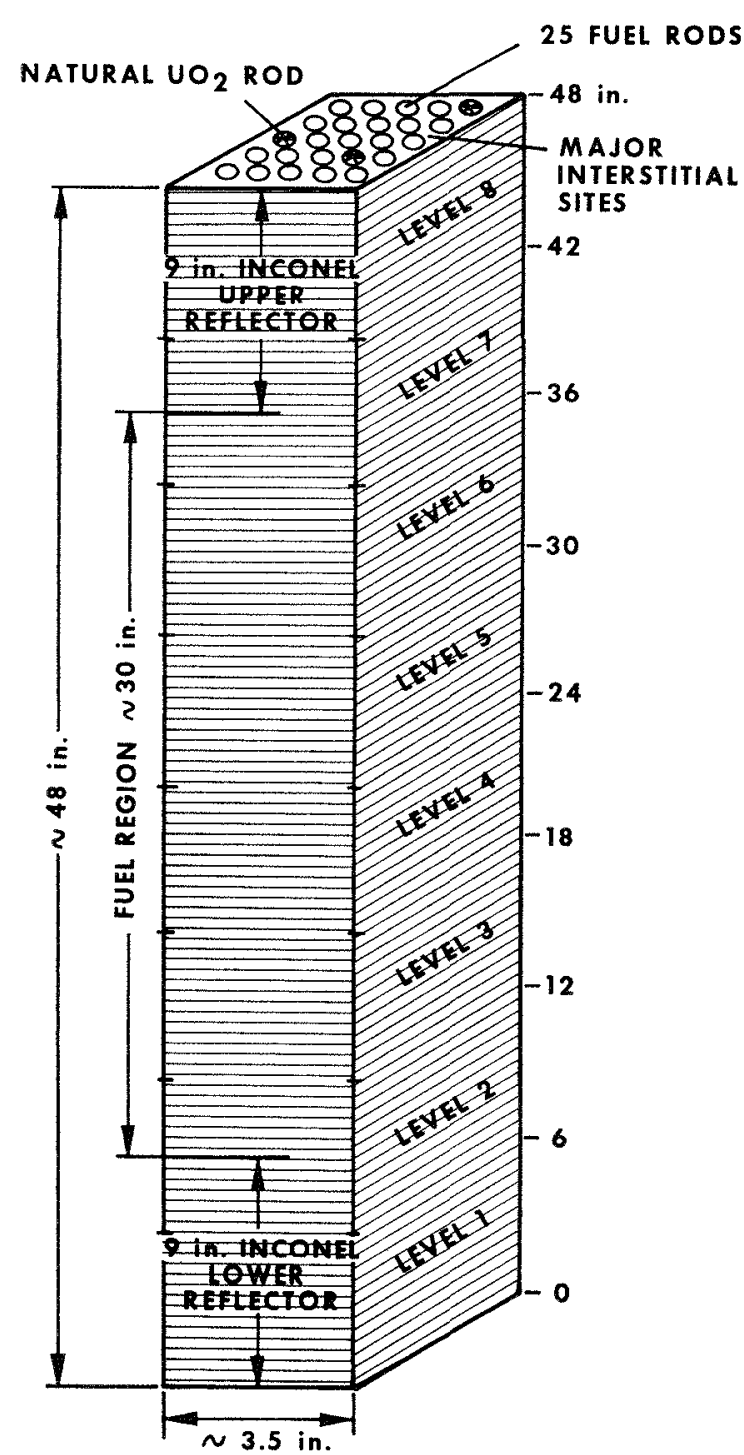

Fic. 6. Schematic Diagram of Experimental Bundle Used or Flooding-Poisoning Experiments.

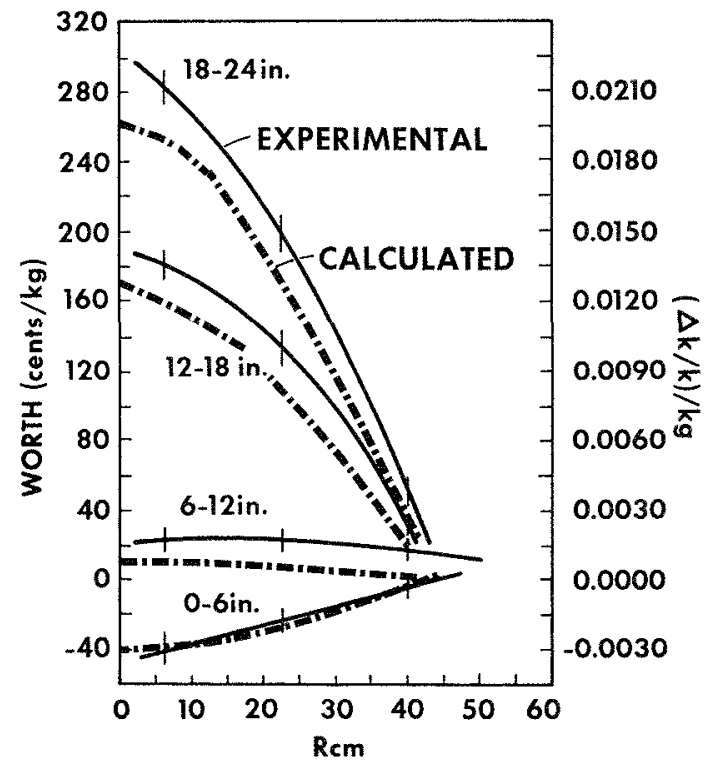

Fia. 7. Worth of Polyethylene vs Loeation.

smaller poison concentration would be needed for the case of whole-core flooding.

In order to verify that less poison density would be needed for reactivity hold-down at the edge of the fast core than at the center, an annular region of flooded and poisoned bundles was constructed as shown in Fig. 12. The net (per bundle) reactivity change due to flooding and poisoning a bundle at the edge of the fast core was only about +2 cents even though a much smaller poison concentration was used.

A summary of some experimental and calculated results is presented in Table 2. The calculations were performed with the use of 24-group diffusion theory in one-dimensional cylindrical geometry. The fissile and fertile cross-section data used were essentially those reported up to the 1964 Geneva Conference. Due to the

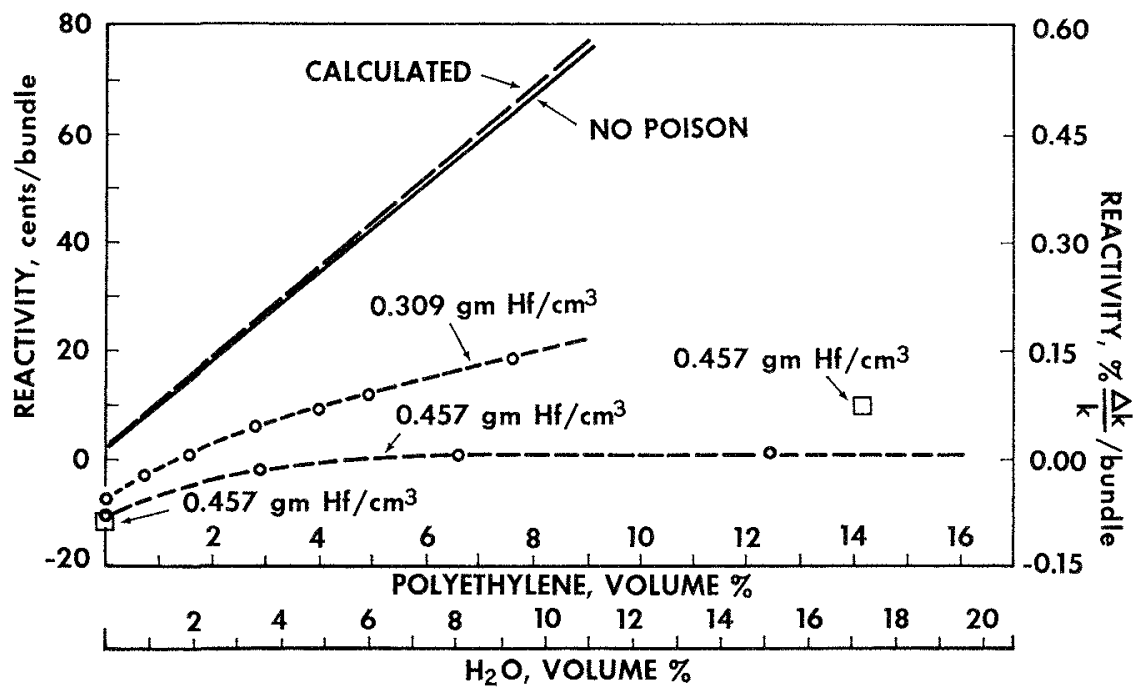

FIG. 8. Four-bundle Flooding with Hafnium Poison. 


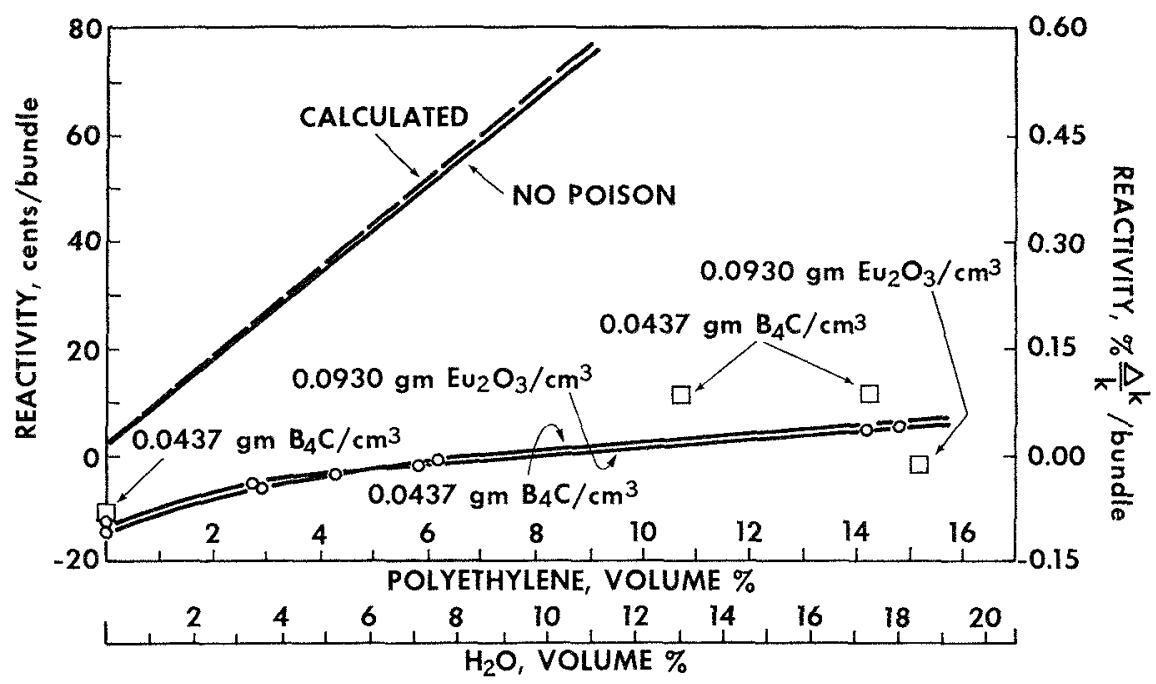

FI(x. 9. Four-bundle Flooding with $\mathrm{Eu}_{2} \mathrm{O}_{3}$ and $\mathrm{B}_{4} \mathrm{C}$ Poisons.

table 1. Resun ince Poison Ffactiveness (Based on Data from 4-central-bundle Flooding Experiments)

\begin{tabular}{l|c|c|c|c}
\hline Poison & $\begin{array}{c}\text { Amount(a) } \\
\left(\mathrm{gm} / \mathrm{cm}^{3}\right)\end{array}$ & $\begin{array}{c}\text { Relative } \\
\text { Penalty }\end{array}$ & $\begin{array}{c}\text { Volume } \\
\text { Fraction } \\
(\%)\end{array}$ & $\begin{array}{c}\text { Relative } \\
\text { Cost }^{(\mathrm{d})}\end{array}$ \\
\hline $\mathrm{Hf}$ & 0.46 & 1.0 & 3.5 & 120 \\
$\mathrm{Eu}_{2} \mathrm{O}_{3}$ & 0.098 & 1.3 & 1.3 & 190 \\
$\mathrm{~B}_{4} \mathrm{C}$ & 0.047 & 1.5 & 1.9 & 1.0 \\
$\mathrm{HfO}$ & 0.54 & 1.0 & 5.5 & 120 \\
\hline
\end{tabular}

(a) Denotes amount for same reactivity hold-down at 19 $\mathrm{v} / \mathrm{O} \mathrm{H}_{2} \mathrm{O}$.

(b) Relative reactivity penalties in dry condition.

(c) Derived assuming theoretical densities.

(d) Relative cost of material multiplied by the amount required for equivalent hold-down.

hardness of the spectrum, even when flooded, a conventional fast reactor group structure with only one thermal group was employed. The resonance fuel isotopic cross sections were shielded according to the single-level narrow-resonance approximation over the resolved regions. The resonance poison cross sections were mostly based on BNL-325 data with blacknesstheory self-shielding applied to the predominant resonances.

In all the experiments described above the entire height of the fuel bundles was flooded; however, flooding of an actual reactor would occur continuously from the bottom to the top of the core. It is therefore important to establish that during this process no large, unpredicted reactivity swings occur. For this reason, the change in reactivity as the water level in four central bundles was increased from zero to full core height was studied. The experimental technique is shown in Fig. 13. Each of the four bundles was poisoned with hafnium so that the flooding reactivity swing was con- trolled to a reasonable magnitude. The water height was increased in increments of $3 \mathrm{in}$. at a time from 0 to $48 \mathrm{in}$.

Figure 14 shows the results obtained along with a corresponding calculation for the case of flooding without poison present. Both the experimental and calculated results show that the axial reflector regions contribute a significant negative reactivity component. In the poisoned case, flooding the fueled region alone increased the reactivity about 85 cents, whereas flooding both axial reflectors and fueled region resulted in an increase of only about 59 cents. These results confirm the earlier data which showed the worth of polyethylene to be negative in the axial reflector regions. Again, both experiment and calculation show the same basic trends.

\section{Conclusions}

As a result of our experimental and calculational effort it can be concluded that it is feasible to achieve flood safety through incorporation of selected resonance poisons into small U-235-fueled steam-cooled fast reactors. The rather good agreement between calculated and experimental data confirms the validity of the multigroup-diffusion-theory approach. It should be noted, however, that although reactivity differences between unflooded and flooded conditions are fairly accurately predicted, the absolute values are not so well predicted, and there may be systematic errors in all cases which then disappear on taking differences. Furthermore, until data are obtained and evaluated for softer spectra and for plutonium fuel, it is not possible to guarantee the validity of the model for all regimes of flooding which can be encountered in practical systems. 


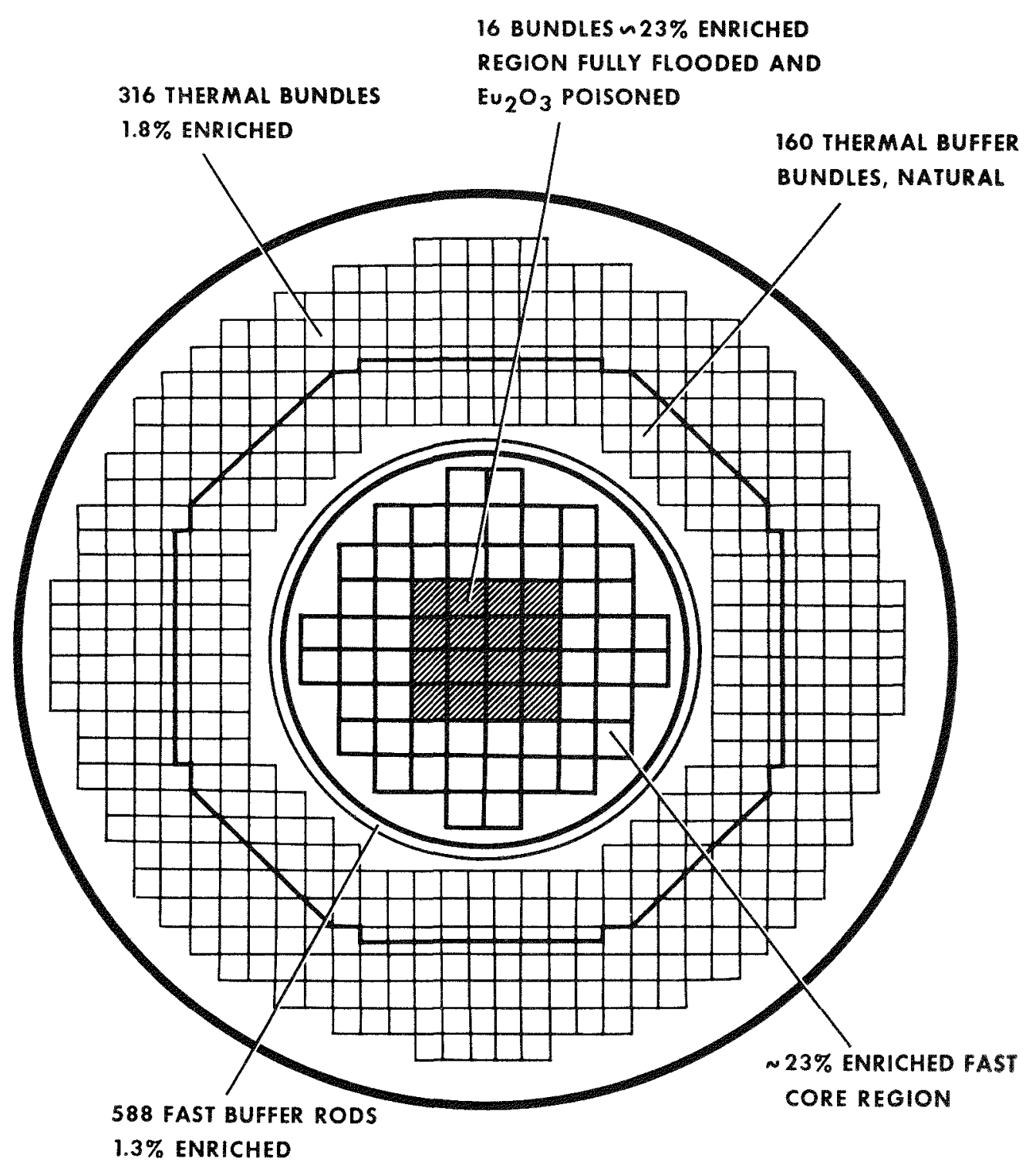

Fra. 10. 16-Central-bundle Flooding Measurement.

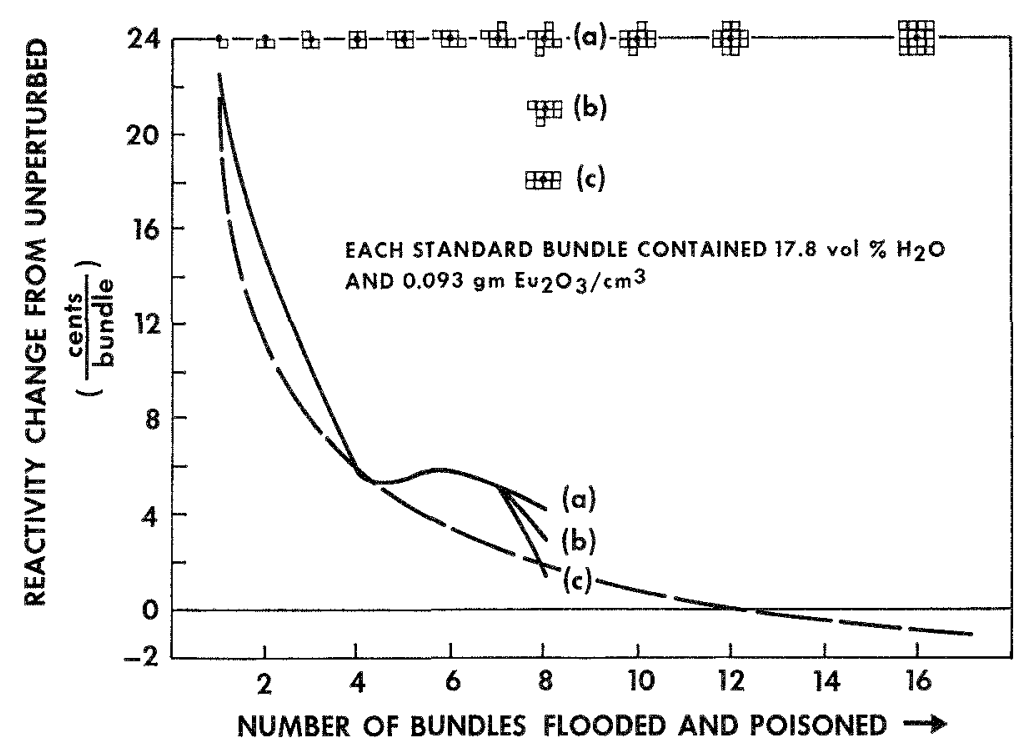

Frg. 11. Flooding Compensation as a Function of the Size and Shape of Central Flooded-poisoned Region. 
TABLE 2. MSSCE Frooding EFfects

Experimental and Calculated Values-33\% Power Split

\begin{tabular}{|c|c|c|c|c|c|}
\hline \multicolumn{3}{|c|}{ Case } & \multicolumn{3}{|c|}{ Reactivity Change (cents) } \\
\hline Location & $\begin{array}{l}\mathrm{v} / \mathrm{o} \\
\mathrm{H}_{2} \mathrm{O}\end{array}$ & $\begin{array}{l}\mathrm{gm} / \mathrm{cm}^{3} \\
\text { Poison }\end{array}$ & $\begin{array}{c}\text { Experi- } \\
\text { ment }\end{array}$ & $\begin{array}{l}\text { Calcul- } \\
\text { ation }\end{array}$ & $\begin{array}{l}\text { Differ- } \\
\text { ence } \\
\text { (Exp- } \\
\text { Calc) }\end{array}$ \\
\hline $\begin{array}{l}\text { One Central } \\
\text { Bundle }\end{array}$ & $\begin{array}{l}18.4 \\
18.4 \\
18.4 \\
18.2\end{array}$ & $\begin{array}{l}\text { None } \\
0.36 \mathrm{Hf} \\
0.093 \mathrm{Hu}_{2} \mathrm{O}_{3} \\
0.031 \mathrm{~B}_{4} \mathrm{C}\end{array}$ & $\begin{array}{r}+129 \\
+37 \\
+21 \\
+42\end{array}$ & $\begin{array}{r}+110 \\
+59 \\
+25 \\
+72\end{array}$ & $\begin{array}{l}+19 \\
-22 \\
-4 \\
-30\end{array}$ \\
\hline $\begin{array}{l}\text { Four Central } \\
\text { Bundles }\end{array}$ & $\begin{array}{l}17.0 \\
18.0 \\
17.3\end{array}$ & $\begin{array}{l}0.46 \mathrm{HIf} \\
0.093 \mathrm{Fu}_{2} \mathrm{O}_{3} \\
0.044 \mathrm{~B}_{4} \mathrm{C}\end{array}$ & $\begin{array}{l}+4.4 \\
+24 \\
+20\end{array}$ & $\begin{array}{l}+25 \\
-17 \\
+53\end{array}$ & $\begin{array}{l}-20 \\
+41 \\
-33\end{array}$ \\
\hline $\begin{array}{l}\text { Twelve Cen- } \\
\text { tral Bundles }\end{array}$ & 17.8 & $0.093 \mathrm{Eu}_{2} \mathrm{O}_{3}$ & +4.6 & -95 & +100 \\
\hline $\begin{array}{l}\text { Sixteen Bundle } \\
\text { Annulus }\end{array}$ & 18.7 & $0.070 \mathrm{Eu}_{2} \mathrm{O}_{3}$ & +32 & -0.7 & +33 \\
\hline
\end{tabular}

316 THER MAL BUNDLES

\section{$1.8 \%$ ENRICHED}

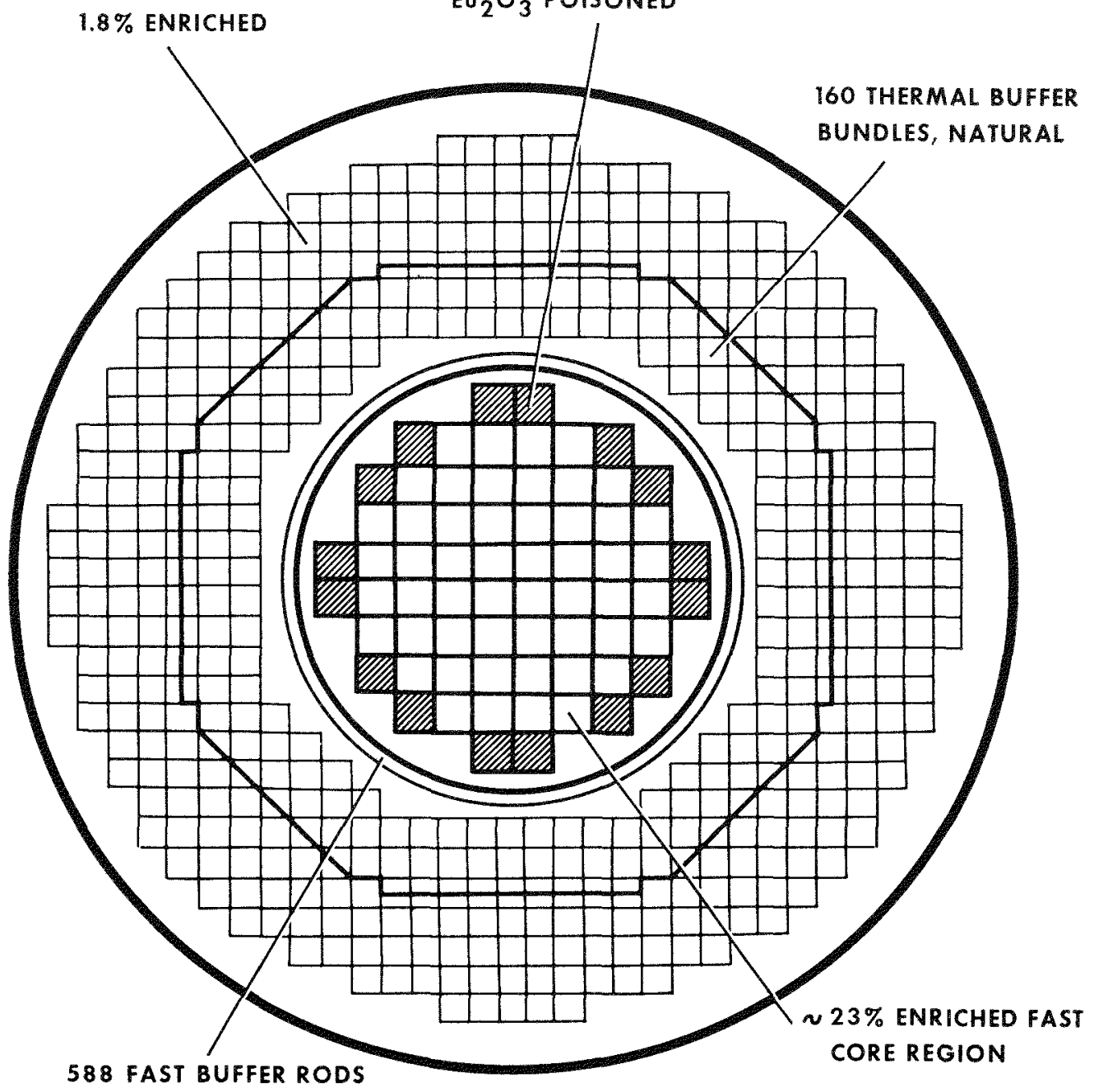

16 BUNDLES $~ 23 \%$ ENRICHED REGION FULLY FLOODED AND $\mathrm{Eu}_{2} \mathrm{O}_{3}$ POISONED 


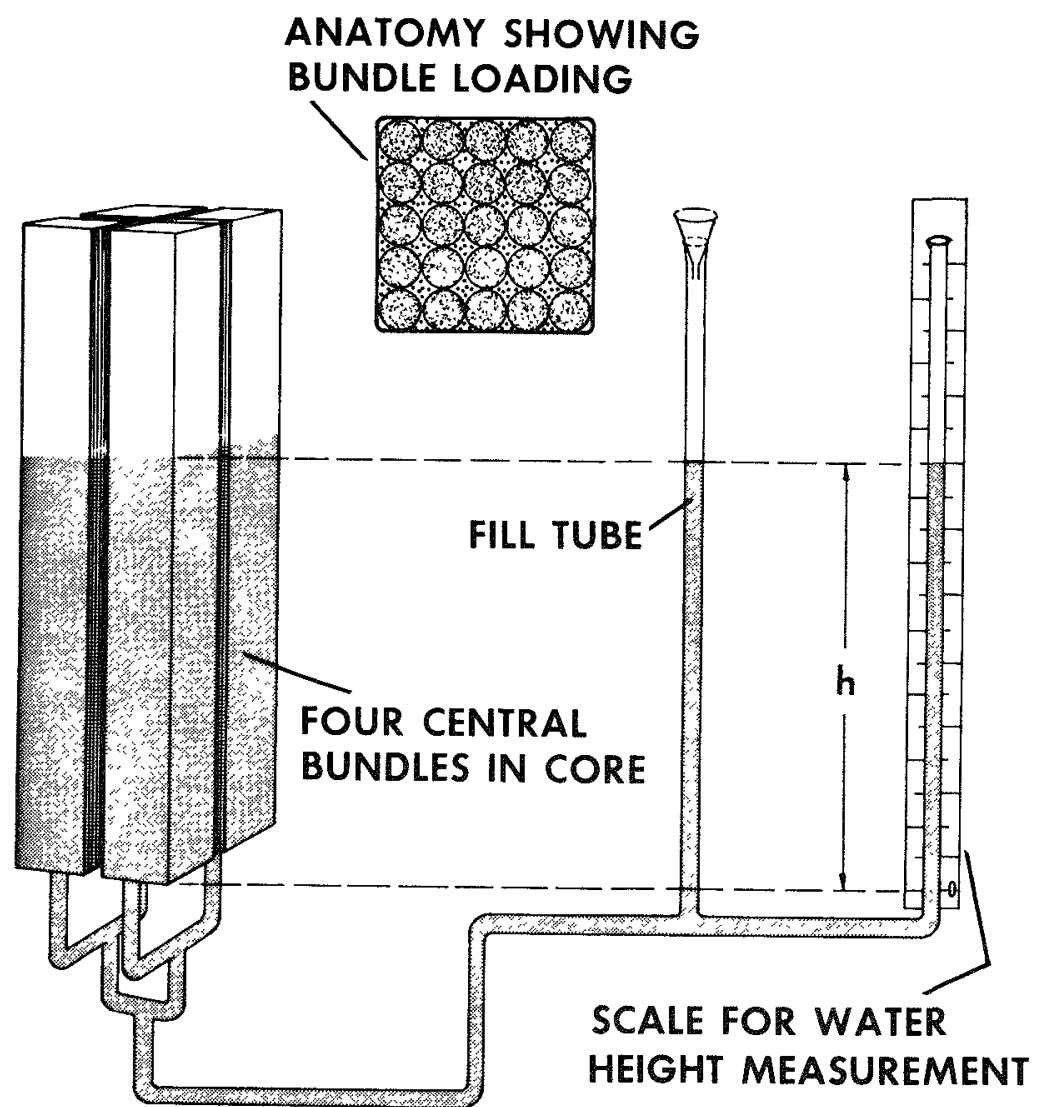

FIg. 13. Water-handling System.

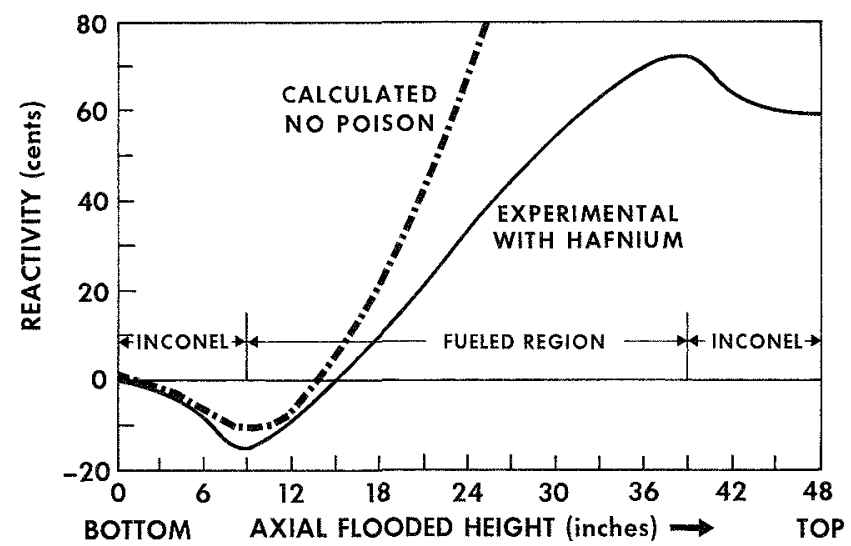

Fug. 14. Reactivity vs Flooded Height of Four Centrally Located MSCA Bundles.

\section{REFERENCES}

1. D. Smidt, Gptimization and Safety of Helium-cooled Fast Breeders, ANI-6792 (1963).

2. P. Fortescue, R. T. Shanstrom, and H. French, Development of the Gas Cooled Fast Reactor Concept, Nucleonies, (May 1965).

3. M. C. Edlund, J. H. MacMillan, and D. C. Schluderberg, Cooling Fast Breeders with Steam, Nucleonics (May 1965).

4. B. Wolfe, Economic Study of the Iixed Spectrum Superheater, GHAP-3590 (1960).
5. B. J. Toppel and R. Avery, Coupled Sleam Superheater Concept, Trans. ANs, 2, $149(1959)$.

6. B. Wolfe, (x. Brymsold, K. Hikido, D. Riley, and A. Silvester, The Conceptual Design of the Wixed Specirum Superheat Reactor, Trans, ANs, 5, 122 (1962).

7. A. B. Reynolds and B. Wolfe, Physics A nalysis of the Mixed Spectrum Superheater, Trans. ANS, 5, 123 (1962).

8. F. J. Thomas, Methods of Designing Compact Gas-cooled Reaclors to Accommodate Flooding, Trans. ANS, 7, 164 (1964).

9. W. L. Fiock, Nuclear Superheat Project - 19th Quarterly Report, (XHAP-4619 (1964).

10. A. B. Reynolds, Flood Safety of the Mixed Spectrum Superheater, (GEAP-3737 (1961).

11. I. B. Wall, et al., lixed Spectrum Superheat Critical $E_{x-}$ ment-Final Report, ( $\mathrm{X} \mathrm{HAP}-4680$ (Nov 1965).

12. G. E. Branyon, Core-1 Critical Studies for the Enrico Fermi Atomic Power Plant on ZIPR-III, AN1,-6629 (1962).

\section{Discussion}

Mr. Peterson (Battelle-Northwest): I was wondering, in view of your present experimental data, how you would consider the calculations of the previous papers that were presented and the precision of the indicated void and flood calculations.

Mr. Hallam: I think the experiments show that there are a lot of effects involved which may not be caught 
in the kind of calculations which were shown previously. I think the method of displaying the results in the previous paper was quite new to me, and I haven't quite digested exactly what it all means. I think the size effect was the one which bothered me most about the last paper. I was never quite sure what size of system was being considered.

Mr. Mäfele (Karlsruhe): The question of the accuracy of our calculation is indeed more or less open. We indicated that there are large areas of uncertainties: however, attention should be paid to the effect of the $1-\mathrm{eV}$ resonance of $\mathrm{Pu}-240$. If you deal with that correctly, then you are already in rather good shape.

Mr. Hallam: Yes, it is definitely of very great importance.

Mr. Wall (GE): I would like to point out to Mr. Peterson that the calculations for the axial and radial polyethylene worth were two-dimensional calculations. To Dr. Ott I point out that we have found in our calculations the two-dimensional effect in the loss of coolant is very important. You can be grossly misled with zero-dimensional calculations. 


\title{
Session VIII-A October 14, 1965
}

\section{Fission Products, Reactions and Interactions}

\author{
Chairman: J. A. LIEBerman
}

U. S. Atomic Energy Commission

Secretary: L. Baker, Jr.

Argonne National Laboratory 
-

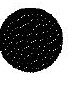




\title{
Experimental Studies of the High-temperature Interaction of Fuel and Cladding Materials with Liquid Sodium
}

\author{
David L. Swift and Louis Baker; Jr. \\ Argonne National Laboratory \\ Argonne, Illinois
}

(PRESENTED BY L. BAKER, JR.)

A series of experiments were performed to determine what interactions occur when abnormally hot fast reactor materials contact liquid sodium. The purpose of the investigation was to observe chemical or physical effects which would occur in a fast reactor meltdown accident. The program, thus far, has been a survey study to diseover unusual interactions such as exothermic chemical reactions, extensive surface attack, frothing disintegration, or alloy formation. In order to simulate the contact of coolant with reactor materials in a meltdown accident, small samples of reactor material were heated to temperatures as high as $2400^{\circ} \mathrm{C}$ and dropped into a pool of liquid sodium. The quenched sample of reactor material was removed from the sodium, cleaned with alcohol, and examined for evidence of interaction.

Figure 1 shows the glovebox in which the experiments were performed. The glovebox contained an argon atmosphere in which the concentrations of oxygen and water vapor were maintained at about 15 ppm. Thus far, the reactor materials studied have been metals and $\mathrm{UO}_{2}$ pellets. The metals were supported and heated in an induction-levitation coil shown in the figure. Samples were heated to as high as $2400^{\circ} \mathrm{C}$ and then dropped into a vessel containing sodium at $250^{\circ} \mathrm{C}$. The samples were generally short cylinders which on melting formed nominal $1 / 4-\mathrm{in}$. spheres. $\mathrm{UO}_{2}$ pellets could not be levitated; after heating by radiation from an inductively heated tantalum susceptor they were similarly dropped into the sodium container. In most of the experiments, the molten sodium was contained in a two-liter beaker placed on the floor of the glovebox rather than in a depression as shown in the figure. Sample temperatures were measured by a recording two-color optical pyrometer (Pyro-eye). Heat transfer calculations indicated that pellet temperatures decreased only about $100^{\circ} \mathrm{C}$ during the nominal 24 -in. drop into the sodium.

The first metals studied were not melted during levitation and heating. These were potential cladding metals as shown in Figure 2. The specimens shown were heated to the indicated temperature, $\mathrm{V}-20 \mathrm{w} / \mathrm{o}$ Ti to $1800^{\circ} \mathrm{C}, \mathrm{Nb}$ to $2250^{\circ} \mathrm{C}, \mathrm{Nb}-1 \mathrm{w} / \mathrm{o} \mathrm{Zr}$ to $2175^{\circ} \mathrm{C}$, and $\mathrm{V}$ to $1950^{\circ} \mathrm{C}$, while remaining in the solid state. As these solid specimens were dropped into the molten sodium, there was a brief period of bubbling, but the samples immediately disappeared from view. Fastax motion pictures showed only moderate surface turbulence as the solid metal samples entered the sodium. The quenched samples showed no evidence of chemical attack by the sodium. Samples were sectioned and mounted for metallographic examination. The photomicrographs gave no evidence of interaction. Similar samples of tantalum and molybdenum at $2200^{\circ} \mathrm{C}$ also gave no evidence of interaction.

We next studied some of the same metals, but at termperatures where they were molten. Figure 3 shows the residue from $\mathrm{V}$ at $2200^{\circ} \mathrm{C}, \mathrm{Ti}$ at $2050^{\circ} \mathrm{C}$, and $\mathrm{V}-20$ $\mathrm{w} / \mathrm{o} \mathrm{Ti}$ at $2100^{\circ} \mathrm{C}$. These materials fragmented upon contact with the liquid sodium. The contact brought about the expulsion of some of the sodium. The fragmented material, after being freed of sodium by immersion in alcohol, had a shiny metallic appearance, suggesting that no chemical reaction had occurred.

Figure 4 shows the results of drop tests with zirconium at a series of initial zirconium temperatures. Violent fragmentation occurred only at $2350^{\circ} \mathrm{C}$, the highest drop temperature studied in this series. At $2100^{\circ} \mathrm{C}$, the particle was hollow and some material had been ejected from the center. At $2000^{\circ} \mathrm{C}$, the residue was a nearly perfect sphere. At lower temperatures melting had been incomplete. In all cases the residue showed no evidence of chemical interaction.

The most violent interactions that we have observed occurred with stainless stcel. The residue from some drops with stainless steel and mild steel are shown in Figure 5. In one of the early experiments in which a $2100^{\circ} \mathrm{C}$ molten stainless steel sphere was dropped into a small sodium receiver (see Fig. 1), a ragged plate-like residue was formed. At $2300^{\circ} \mathrm{C}$, the stainless steel formed particles having an average diameter of about 10 mils. A number of experiments with 


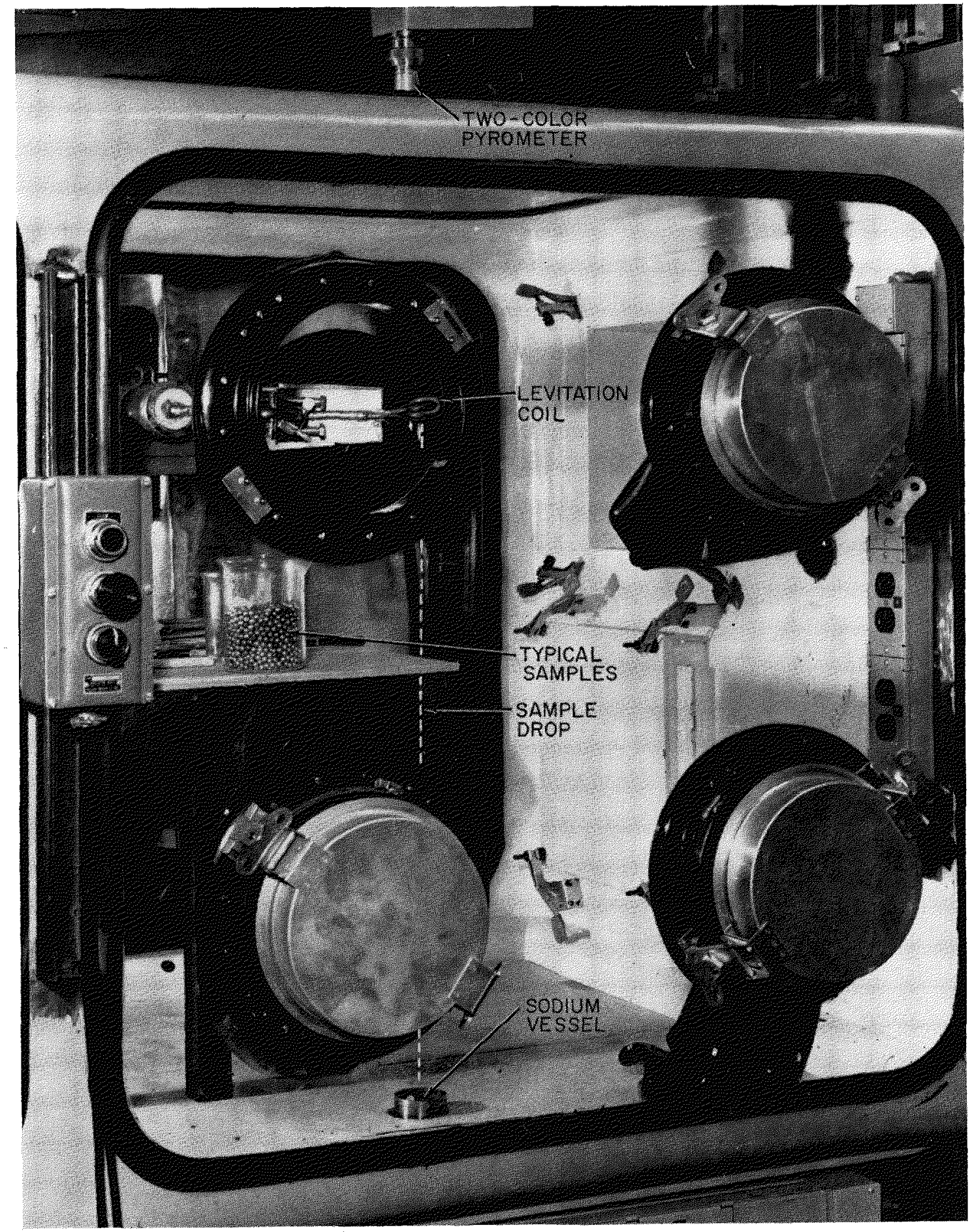

Frg. 1. Glovebox for Studies of Interaction of Molten Fuel and Cladding Materials with Liquid Sodium.

stainless steel have been performed at drop temperatures of about $2300^{\circ} \mathrm{C}$, and the results were quite reproducible. Many small particles of sodium, $2-3 \mathrm{~mm}$ in diameter, were expelled from the molten sodium container and adhered to the walls and ceiling of the glovebox, a maximum distance of $4 \mathrm{ft}$ from the container. Up to $15 \%$ of the stainless steel in a given experiment was also ejected during the violence created by the fragmentation. Experiments with stainless steel were performed not only with the standard 2-ft drop from the levitation coil into the sodium, but also with a 6 -in. and a 2 -in. drop. 
Equally volent and extensive fraginentation occuried regardless of the drop distance It is therefore concluded that the impact velocity is not a critical parameter for drop fragmentation

Experiments in which molten mild stcel and molten nickel were diopped $2 \mathrm{ft}$ into $250^{\circ} \mathrm{C}$ sodium showed similar behavior; however, the fragmentation appeared to be slightly less violent Chemical analyses of residue from drop experiments with zirconium, stainless steel, mild steel, and nickel indicated that no sodium was retamed by the metal This, combined with the shiny metalice appearance of the particles, indicated that there had been no chemical interaction

The only fuel materials studied to date have been uranum metal and $\mathrm{CO}_{2}$ pellets Uranum metal spheres fragmented over a wide range of temperatures, as shown in Fig 6 The fragmentation was somewhat less violent than with stamless steel Although stamless steel drops broke up into more or less spherical particles, the uranum samples formed a fragle filamentary net work
$\mathrm{CO}_{2}$ dropped as solid pellets, as shown in Fig 7, did not fragment, however, extensive cracking occurred in an experiment in which the $\mathrm{UO}_{2}$ pellet was heated to $2100^{\circ} \mathrm{C}$ Sodrum was absorbed into the cracks and was not removed by a brief alcohol rinse Sodium oxide appeared to exude from the cracks for several days after the experment

The principal finding of these experments has been the violent fragmentation which occurs with molten metals heated well above their melting points Thi spontaneous breakup was surprising because it appeared to contradict our previous experience upon dropping very hot molten metals into water. Molten pellets of zirconium, uranium, stainless steel, and aluminum smply dropped into water do not fragment The lesult of dropping zirconium, uranium, and stainless steel into $250^{\circ} \mathrm{C}$ sodium have already been described

The results with aluminum are shown in $F_{1 g} 8$ Aluminum at $800^{\circ} \mathrm{C}$ formed a sheet-like resıdue; at higher temperatures, very extensive fragmentation occuned It was beleved, at first, that the oxide film

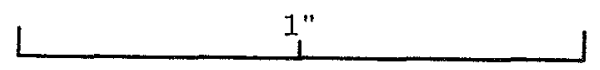

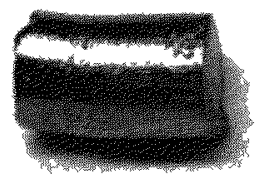

$\mathrm{V}-20 \% \mathrm{~T}$ $1800^{\circ} \mathrm{C}$

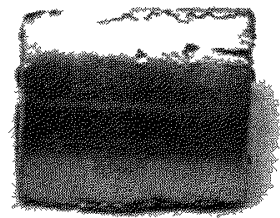

$\mathrm{Nb}$ $2250^{\circ} \mathrm{C}$

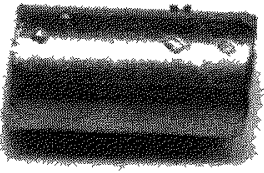

$\mathrm{Nb}-1 \% \mathrm{Zr}$ $2175^{\circ} \mathrm{C}$

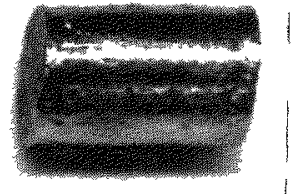

$\mathrm{V}$ $1950^{\circ} \mathrm{C}$

Fix 2 Residues of Several holid Metals Dropped into Molten Sodum

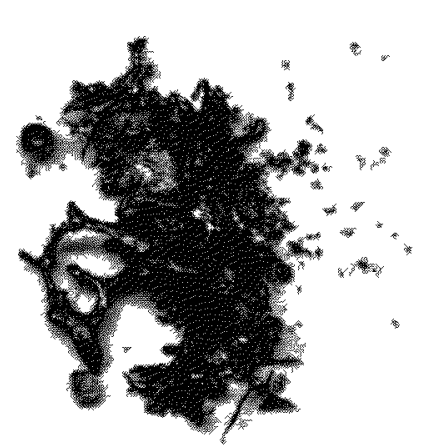

V, $2200^{\circ} \mathrm{C}$ Drop

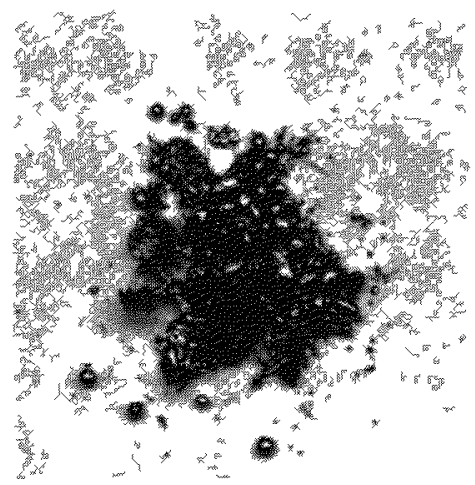

$\mathrm{T} 1,2050^{\circ} \mathrm{C}$ Drop

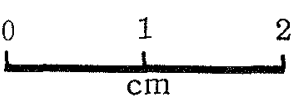

Fis 3 Residues of Several Molten Metals Dropped into Molten Sodrum 


\section{Zirconium (M. P. $\left.1860^{\circ} \mathrm{C}\right)$}

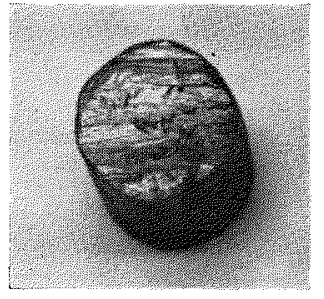

$1700^{\circ} \mathrm{C}$

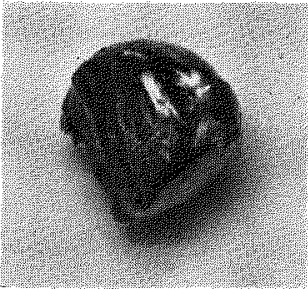

$1860^{\circ} \mathrm{C}$

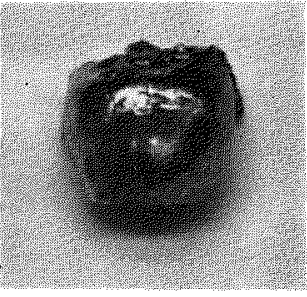

$\sim 1900^{\circ} \mathrm{C}$

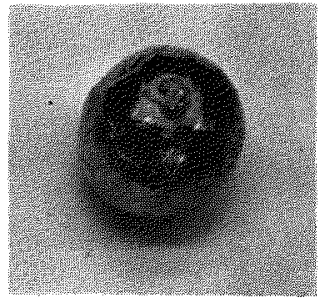

$\sim 2000^{\circ} \mathrm{C}$

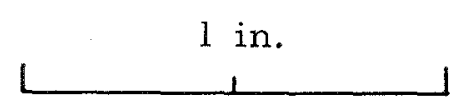

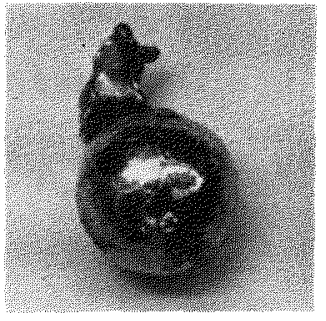

$\sim 2100^{\circ} \mathrm{C}$

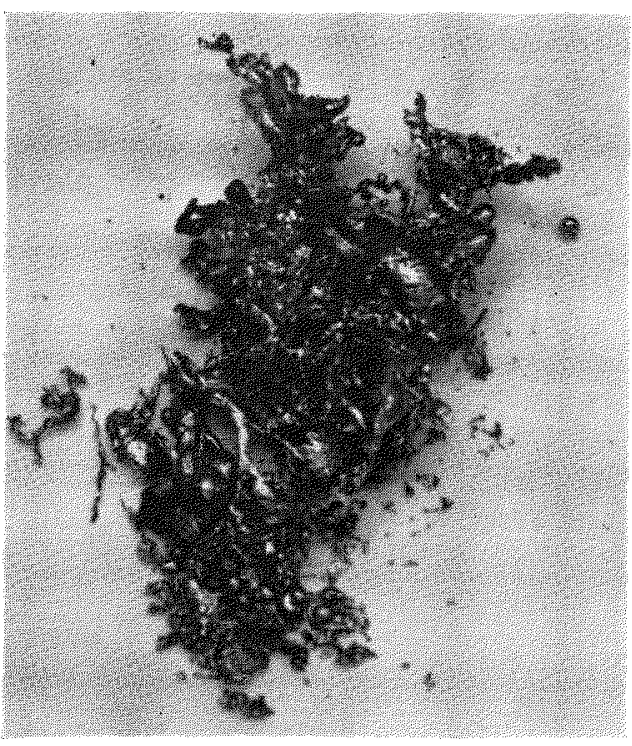

$2350^{\circ} \mathrm{C}$

FIG. 4. Zirenium Residues from Sodium-interaction Experiments at Various Drop Temperatures.

formed in drops into water may have helped to prevent fragmentation in that medium. To test this hypothesis we dropped spheres of silver both into water and into sodium; the results are indicated in Fig. 9. Although silver and gold do not form oxide films in water, they did not fragment; however, silver dropped into sodium from $1900^{\circ} \mathrm{C}$ did suffer fragmentation. Thus we concluded that the difference in behavior between water and liquid sodium is not caused by oxide films and is due to differences in physical properties of water and sodium.

In looking for an explanation for the fragmentation process, it occurred to us that the driving force for breakup might be the violent growth and collapse of sodium vapor bubbles which are characteristic of subcooled boiling in the transition and nucleate regimes. In general, it would be expected that the transition and nucleate boiling regimes would extend from nearly the boiling point to around the critical point. For sodium, this is a lange of temperature from 900 to about $2300^{\circ} \mathrm{C}$. All fragmentations occurred with molten metals cooling through some part of this temperature range. According to this hypothesis, fragmentation would also be expected to occur with molten metals in water but only in the temperature region of violent boiling. For water at one atm, the boiling point is $100^{\circ} \mathrm{C}$ and the critical point is $373^{\circ} \mathrm{C}$. Figure 10 shows the residue from some experiments in which small samples of some low-melting metals were dropped into water. All samples were initially molten at $825^{\circ} \mathrm{C}$. Bismuth, tin, and lead (with melting points below the critical temperature for water) did fragment, while zinc and aluminum (with melting points above the critical temperature for water) did not fragment.

According to our present view, the fragmentation process occurs as a molten material cools through the region of boiling where there is violent growth and collapse of vapor bubbles. Metals useful for con- 


\section{Stainless Steel}

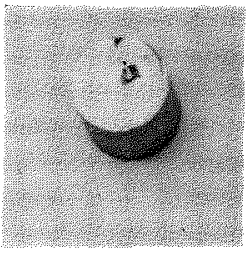

Sample

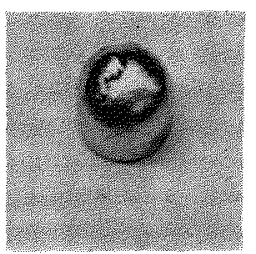

Incipient Melting

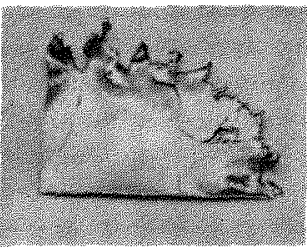

$2100^{\circ} \mathrm{C}$

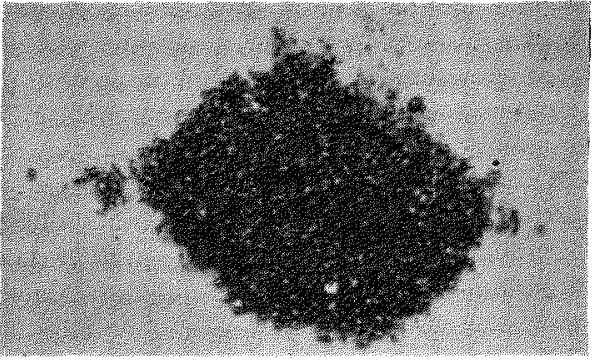

$2300^{\circ} \mathrm{C}$

\section{Mild Stee1--Cold Rolled}
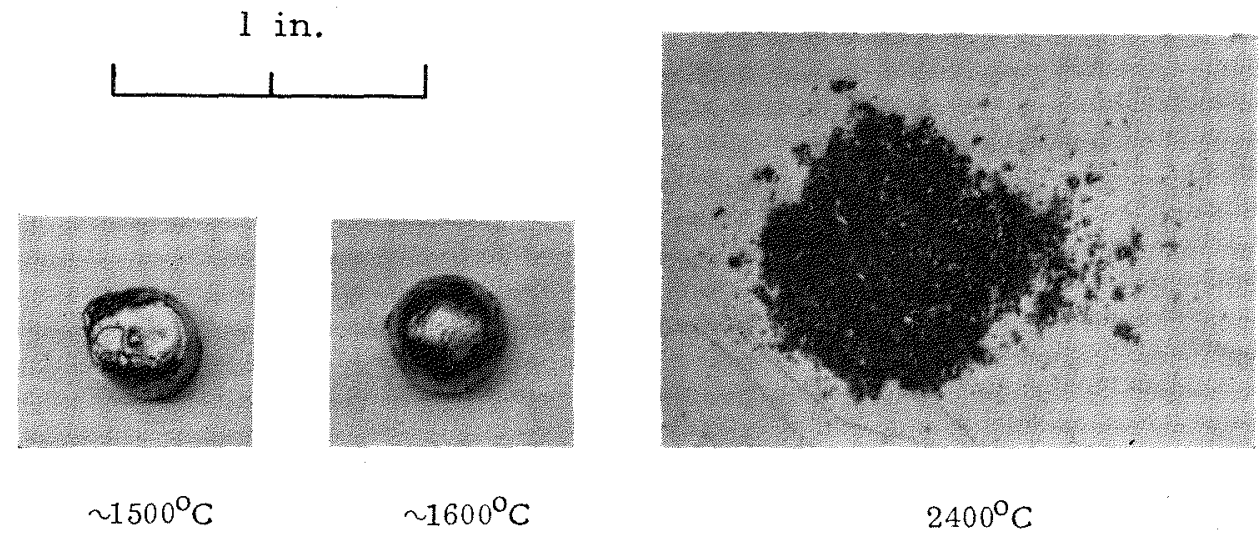

$2400^{\circ} \mathrm{C}$

Fra. 5. Stainless Steel and Mild Steel Residues from Sodium-interaction Experiments at Various Drop Temperatures.

struction, such as aluminum, stainless steel, zirconium, and some of the more refractory metals, will fragment on cooling in molten sodium. However, they will not fragment on quenching in water because cooling and solidification occur in a nonviolent film-boiling regime, and these metals are fully solidified before they reach the temperature of violent boiling in water.

A detailed sequence of events during the quenching and fragmentation of stainless steel in sodium is given in Fig. 11. Here are recorded the times corresponding to observations made with high-speed motion pictures of the molten pellet entering sodium. Beginning with the contact of molten stainless steel with the sodium, there is the appearance of a parabolic depression in the sodium which deepens over a period of about 5 msec. Then there is the appearance of a cloud which is presumably sodium vapor. It is at this time that it is presumed fragmentation occurs. The small particles of hot steel then transfer heat rapidly to a localized region of the sodium, resulting in a burst of vapor which causes ejection of some of the sodium and some of the hot steel pellets. At $5.5 \mathrm{msec}$, there is the first sign of particle ejection. Violence reaches a peak at about 7 msec and is completely subsided at about 12 msec. Fastax films of several other stainless steel-sodium interactions at similar drop temperatures indicate, however, that the timing of events is somewhat variable.

In conclusion, it should be pointed out that these preliminary studies of the interaction of abnormally hot reactor materials with liquid sodium have identified a fragmentation mechanism which results in extensive subdivision of molten cladding metals. This knowledge should be useful in the interpretation of the results of in-pile meltdown tests of fast reactor fuel elements. It appears that chemical attack and alloy formation do not occur to a significant degree during the brief time available in a rapid quench with the reactor materials studied. 


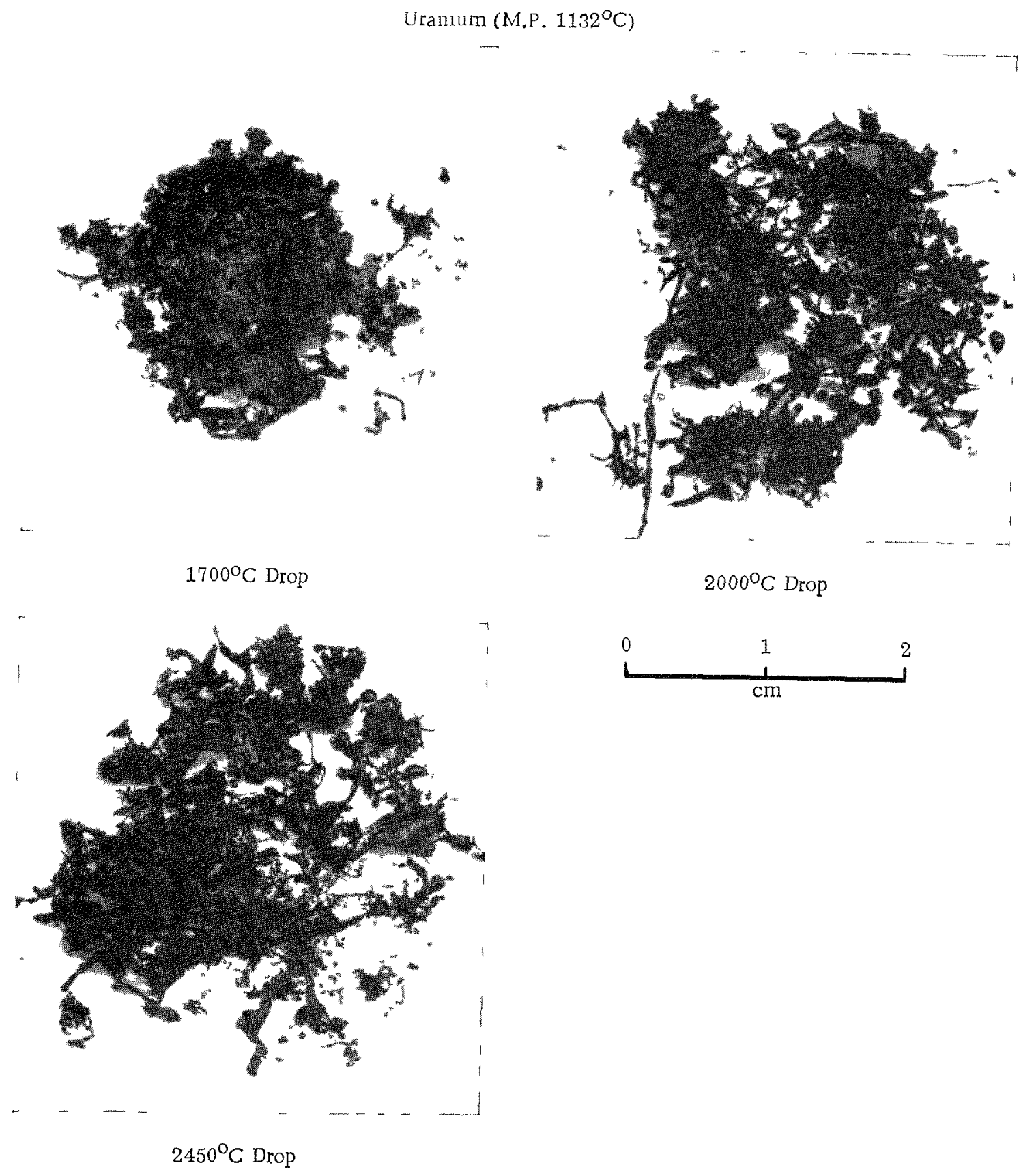

FIg 6 Uranum Resldues from Sodıum-nteraction Experments at Various Drop Temperature

\section{Discussion}

$M r$. Stachura (AI): Do you have any motion pictures of the water tests? Was the time sequence similar to the sodium?

$M r$. Baker: The water was not nearly as violent. We had a more or less quiet breakup. There was no ejection of hot material.

Mr. Stachura: Our considerations have led us to think that radiation at the high temperatures is quite an additional contribution of thermal energy transfer from fuel to coolant, one that is unique at the melting points of these refractory materials. Perhaps this may explain why the water tests were not quite as violent.

Mr. Baker: I think that is a very likely point.

Mr. Knight (GE): You had a summary statement with regard to the interaction of sodium with cladding material. Can you make any summary statement about the tests with $\mathrm{UO}_{2}$ ?

Mr. Baker: The number of our experiments has 


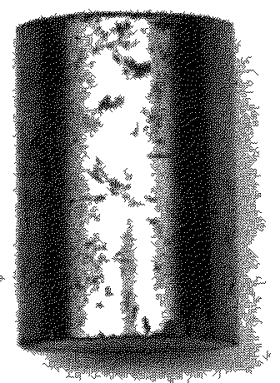

Sample

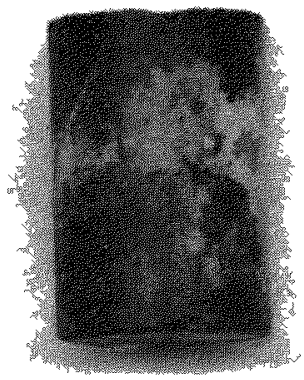

$900^{\circ} \mathrm{C}$ Drop

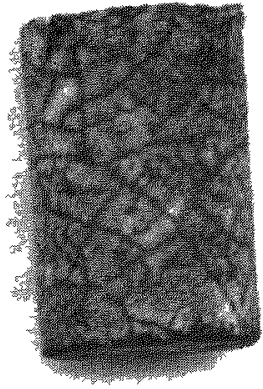

$2100^{\circ} \mathrm{C}$ Drop

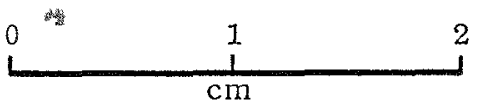

II 7 Tranum Dioxide Residues from Sodium interaction Experiments at Varous Drop Temperatures

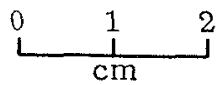

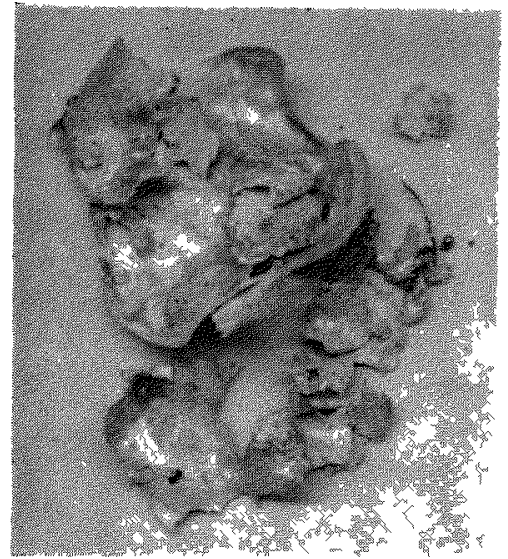

$800^{\circ} \mathrm{C}$ Drop

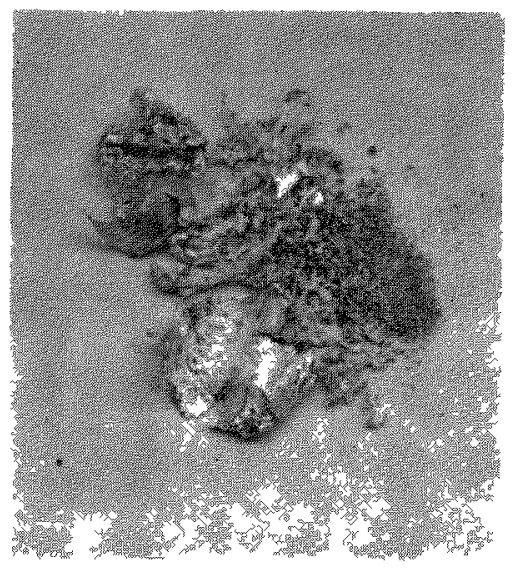

$1200^{\circ} \mathrm{C}$ Drop

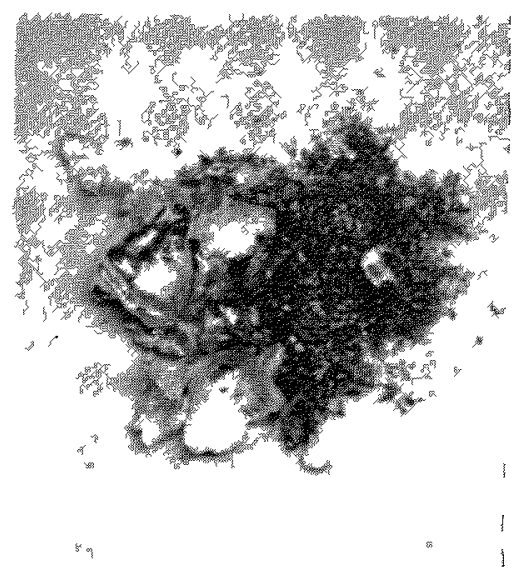

$1500^{\circ} \mathrm{C}$ Drop

Aluminum (M.P. $659^{\circ} \mathrm{C}$ ) in $250^{\circ} \mathrm{C}$ Sodium

FIG 8 Aluminum Residues fiom Sodium interaction Experiments at Valious I) op Temperature-

been quite limited However, it has been observed by other people that there is a temperature to which $\mathrm{UO}_{2}$ may be heated in soduum when wotting begins If there are cracks, sodium will be absorbed in a slow experiment. We had sufficient temperature to permit wetting and we did get sodium absorbed in the cracks

Mr Knght: Would you anticipate or have you considered that there might be some difference in the interaction of sodium with $\mathrm{UO}_{2}$ or mixed oxide as a function of the storchiometry, either on a transıent situation like this or a long-duration test?

Mr Baker We haven't studied mixed oxides, but
I wouldn't expect any marked difference unless perhaps the mixed oxide were more plastic and could withstand the quench without developing cracks.

$M r$ Knight: I was a little surprised that the $\mathrm{UO}_{2}$ pellet hadn't fragmented on this sudden quench. Would you care to comment on that?

Mr Baker: No. I have no comment

Mr Stachura. There is one comment we might make about $\mathrm{UO}_{2}$ When heat transfer begins to occur from a molten pellet to the coolant, so that surface cooling occurs, the conductivity of the material itself may not produce quite the same sustained surface temperatures on the sold oxide that it might on 


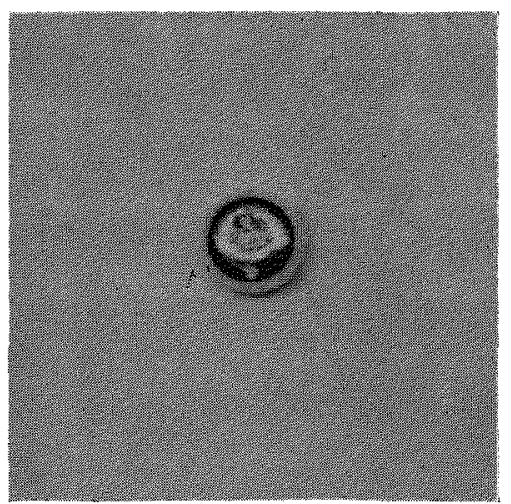

Gold (M.P. $1063^{\circ} \mathrm{C}$ )

$2600^{\circ} \mathrm{C}$ Drop into $25^{\circ} \mathrm{C}$ Water

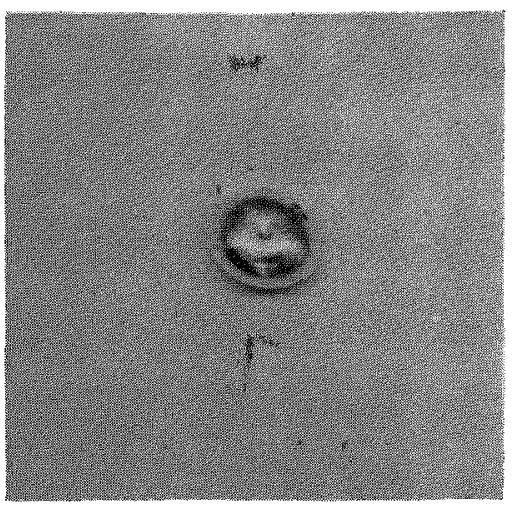

Silver (M.P. $961^{\circ} \mathrm{C}$ )

$1900^{\circ} \mathrm{C}$ Drop into $25^{\circ} \mathrm{C}$ Water

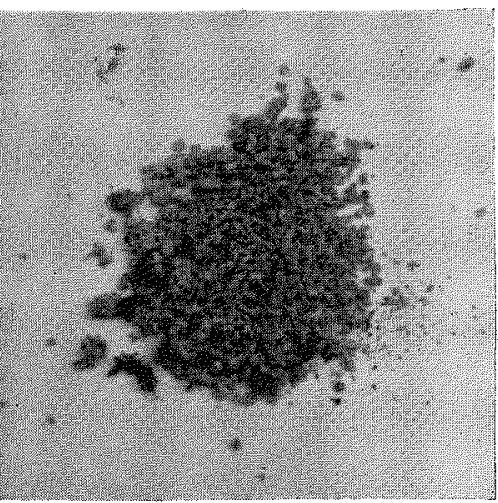

Silver $1900^{\circ} \mathrm{C}$ Drop

into $250^{\circ} \mathrm{C}$ Sodium

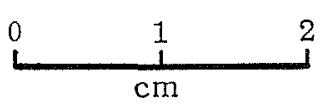

FIG. 9. Residues of Gold or Silver Dropped into Either Water or Molten Sodium.

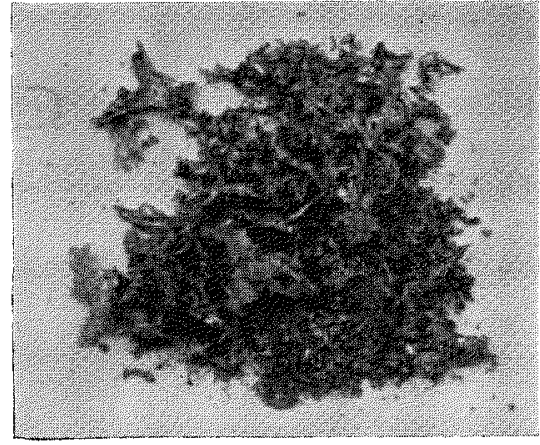

Bismuth (M.P. $271^{\circ} \mathrm{C}$ )

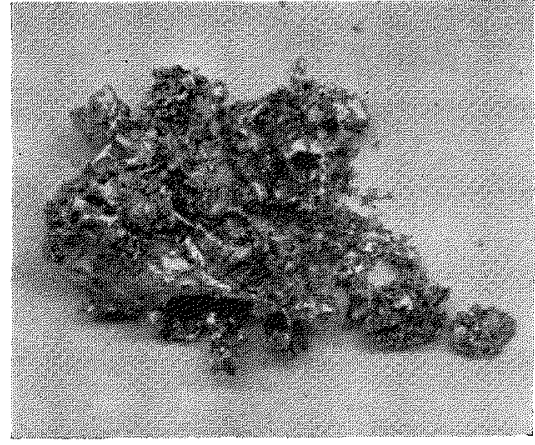

Tin (M.P. $\left.232^{\circ} \mathrm{C}\right)$

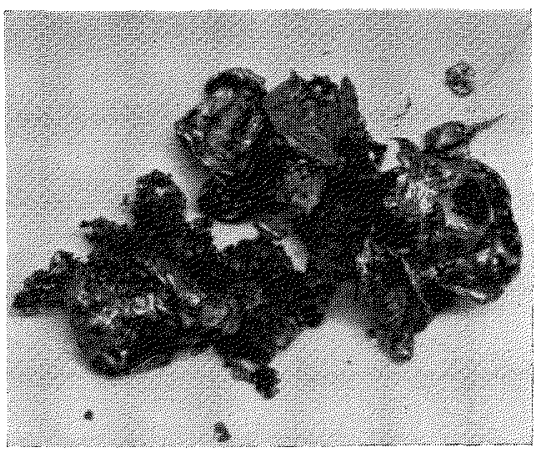

Lead (M.P. $327^{\circ} \mathrm{C}$ )

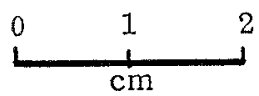

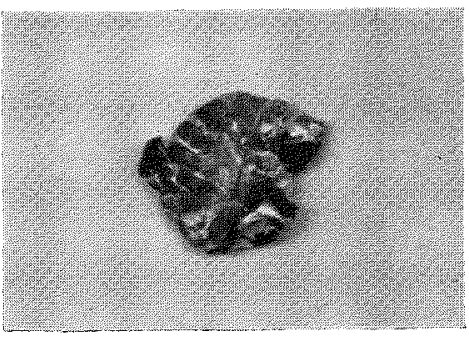

Zinc (M.P. $420^{\circ} \mathrm{C}$ )

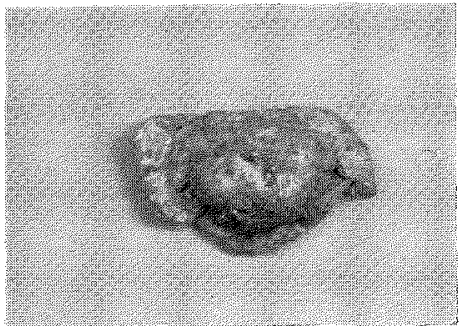

Aluminum (M.P. $659^{\circ} \mathrm{C}$ )

FIG. 10. Residues of Low-melting Metals Dropped into $0^{\circ} \mathrm{C}$ Water (Drop Temperature $825^{\circ} \mathrm{C}$ in Each Case).

materials of higher conductivity. It would be interesting to get the $\mathrm{UO}_{2}$ molten and see whether it disperses similarly as do the metals.

Mr. Blumenthal (ANL): Did you have any experiments in which you varied the volume of the pellet?

Mr. Baker: No, the levitation method is fairly specific with a given coil design and you can't vary the pellet size very much.

$M r$. O'Neill (GE): You seem to make a point about the sodium entering the cracks of the fuel of the $\mathrm{UO}_{2}$. We have had some experience with sodium interactions with fuel under reactor operating condi- 
High-temperature Interactions of Liquid Sodium

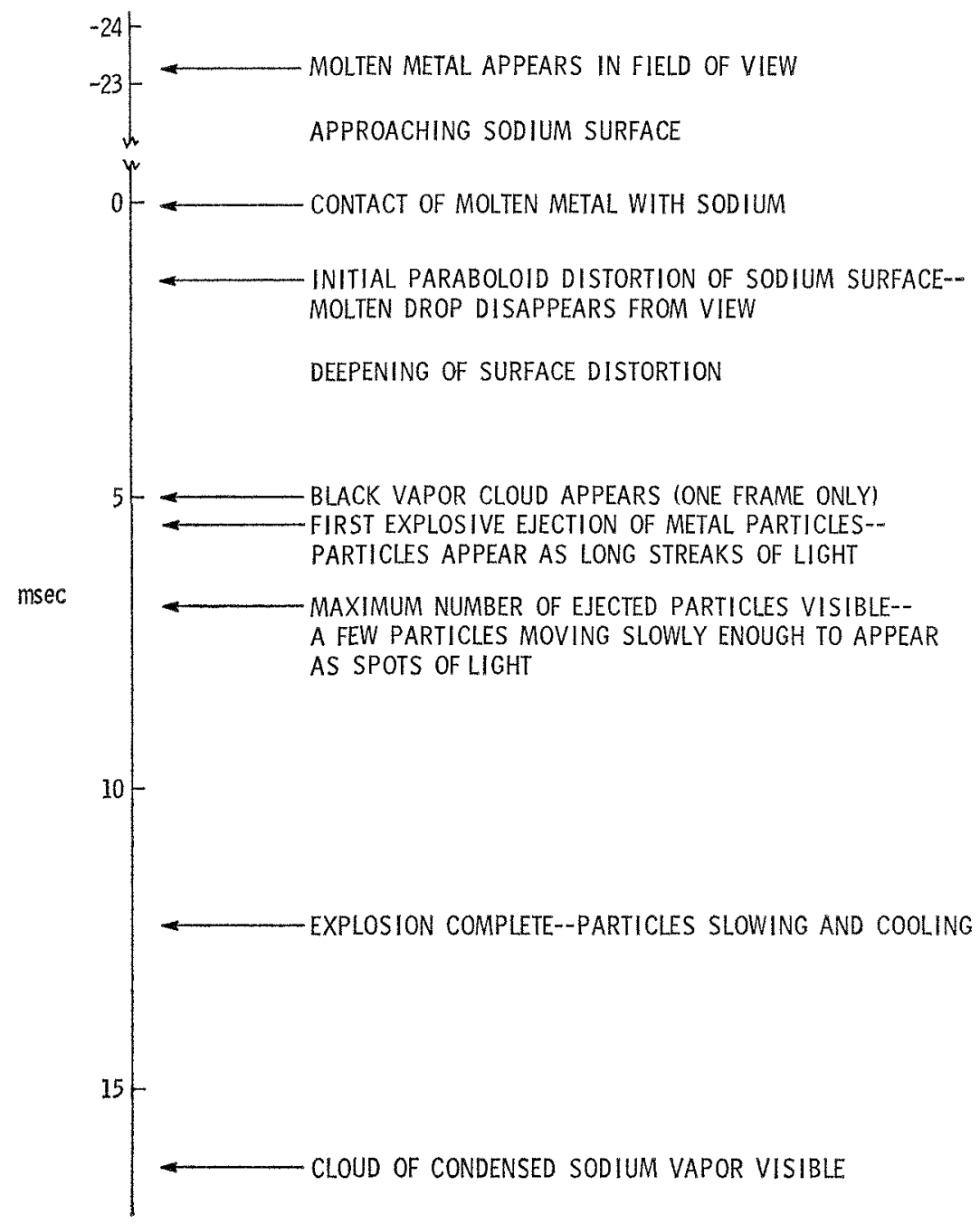

Fig. 11. Time sequence of Interaction of Type 304 Stainless Steel $\left(2400^{\circ} \mathrm{C}\right)$ and Sodium $\left(250^{\circ} \mathrm{C}\right)$ (from Fastax Film of Event).

tions, and there is, of course, a very high thermal gradient which forces the sodium in the opposite direction.

Mr. Levenson (ANL): Do you think there's any significance to the fact that the oxide pellets appeared to survive this test since all the metals survived when they weren't molten and the only things that fragmented were the things that went in molten? Would you expect the oxides to react any differently if they were molten?

Mr. Baker: If we could get well above the melting point, I would expect fragmentation; on the other hand, if we're over the critical temperature of sodium such that we could freeze the $\mathrm{UO}_{2}$ before getting into a violent boiling region (in other words, in a slow boiling region), then it might not fragment.

Mr. Kelman (ANL) : I didn't understand the comment that was just made here about the temperature gradient as related to the sodium getting into these eracks. It seems to me that the material cracks under the sodium. It cools off. By the time the sodium enters, there is no longer a temperature gradient to be concerned about. If fact, I would think it might create a kind of a vacuum situation which sucks the sodium back in.

Mr. Baker: I think that Mr. O'Neill referred to a reactor in which there is a gradient during normal operation. 


\title{
Chemical Considerations in Fast Reactor Safety*
}

\author{
A. W. Castleman, Jr. and I. N. Thivg \\ Brookhaven National Laboratory \\ Upton, New York
}

(PRESENTEI) BY A. W. CASTLEMAN, JR.)

\section{Introduction}

The hazard associated with any reactor depends, to a large extent, on the nature of the radioactive nuclides likely to be released in the event of an accident. A thorough knowledge of the chemical states of the fission products as a function of temperature, concentration, and environment can be used to predict behavior under a wide variety of conditions which would be virtually impossible to investigate in large-scale tests.

This paper is divided into two sections. The first summarizes the results of our studies on the chemical states of iodine released from uranium and $\mathrm{I}^{\top} \mathrm{C}$ into helium between 1200 and $2500^{\circ} \mathrm{C}$. The second section gives the chemical states of iodine formed upon relcase from these fucls into air and stcam. In addition, calculations, based on thermodynamic considerations, are included which predict the behavior of iodine in sodium.

\section{Chemical State of Iodine Released from Metallic Uranium and Uranium Monocarbide}

The studies presented in this section deal primarily with fission product-fuel reactions at elevated temperatures in a helium atmosphere. Samples of metallic uranium and hypostoichiometric $\mathrm{CC}(4.6 \mathrm{w} / \mathrm{o}$ carbon) irradiated over the range from $1.0 \times 10^{15}$ to $5.4 \times 10^{19}$ nvt were used. The apparatus and the experimental procedure have been described elsewhere. ${ }^{(1-3)}$ Briefly, the method consists of separating the fission products roleased from a heated fuel sample by condensing them or their compounds in a quartz tube having a linearly descending temperature profile along the wall. The deposition temperature of the fission products was determined by a gamma scan of the tube at the completion of a release experiment.

The experimental deposition profiles shown in Figure 1 indicate that at least three distinct iodine species are released from fuel samples heated to temperatures ranging from 1200 to $2500^{\circ} \mathrm{C}$. It is evident that the relative quantity of each released species varies with the fuel temperature. Most of the iodine released from molten uranium at $1240^{\circ} \mathrm{C}$ deposits at $275^{\circ} \mathrm{C}$. As the fuel

\footnotetext{
* Work performed under the auspices of the U. S. Atomic Energy Commission.
}

temperature is raised to $\approx 2000^{\circ} \mathrm{C}$, a second iodine species which collects below $100^{\circ} \mathrm{C}$ forms at the expense of the $27.5^{\circ} \mathrm{C}$ deposit. A third iodine species, which deposits at $\approx 200^{\circ} \mathrm{C}$, becomes progressively predominant at fuel temperatures above $2150^{\circ} \mathrm{C}$.

Although Fig. 1 shows only the results from the studies with metallic uranium, the same general trend was observed upon heating $\mathrm{ICC}$ fuels within the temperature range from 2300 to $\approx 2500^{\circ} \mathrm{C}$. In the case of UC $\left(\mathrm{m} . \mathrm{p} ., \approx 2350^{\circ} \mathrm{C}\right)$ no experiments wcre made at temperatures below $2300^{\circ} \mathrm{C}$. At temperatures far exceeding $2500^{\circ} \mathrm{C}$, the released iodine was observed to collec t largely below $100^{\circ} \mathrm{C}$.

A specific identification of each of the released species by conventional analytical methods is hindered since the quantities are generally below the picogram level. A combination of techniques involving neutron activation, observing the behavior of known species and the effect of varying chemical environment, together with a thermodynamic analysis of the system, have proved useful in their identification.

The nature of the deposition profiles suggested that the iodine was either in chemical combination with uranium or some other fission product. If the iodine had reacted with another fission product, combination with cesiun would be the most likely possibility, a conclusion based on free energy of formation values and relative fission product concentrations. Recent studies have shown that cesium and iodine are not released as CsI in these experiments but are transported independently. ${ }^{(4)}$ The presence of uranium in the 250 to $350^{\circ} \mathrm{C}$ iodine deposition zone was detected by means of neutron activation analysis, though a definite $\mathrm{U} / \mathrm{I}$. ratio was not established.

Although largely speculative, thermodynamic calculations based on estimated values given by Brewer ${ }^{(5)}$ indicate that a uranium subiodide such as LI or $\mathrm{UI}_{2}$ should be stable under the conditions of these experiments and, indeed, should be the major species at $1200^{\circ} \mathrm{C} . \mathrm{A}$ uranium subiodide having a $\mathrm{U} / \mathrm{I}$ ratio of 1.1 was successfully synthesized by passing elemental iodine tagged with I-131 over nonirradiated uranium. maintained at $1200^{\circ} \mathrm{C}$. This subiodide, carried by a 


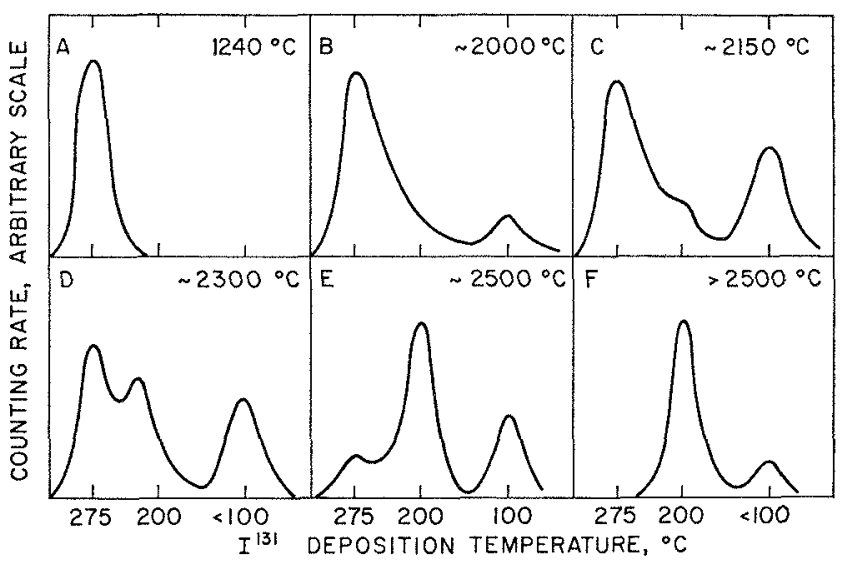

Fxc. 1. Deposition of Fission-product Iodine Released into Ile.

helium stream into the deposition tube, was found to deposit at $275^{\circ} \mathrm{C}$. This result suggested that the $275^{\circ} \mathrm{C}$ fission product iodine deposit was UI or some multiple thereof. The $200^{\circ} \mathrm{C}$ deposit has not yet been identified, but it is also probably a uranium subiodide. The deposit at $100^{\circ} \mathrm{C}$ and below was found to be essentially elemental iodine. This was concluded from the behavior of known samples of elemental iodine passed into the deposition tube and from experiments conducted in air, as discussed in the next session.

The temperature dependence of the species discussed above is in qualitative agreement with the behavior of many high-temperature systems as explained by Brewer's Rule. ${ }^{(6,7)} *$ This is seen by referring to Fig. 2 , which is a logarithmic plot of the relative partial pressures of the released iodine species versus $1 / T$. This was constructed by taking the relative partial pressures to be proportional to the peak areas in Fig. 1. The three straight lines in Fig. 2 show that all of the experimental results up to $\approx 2500^{\circ} \mathrm{C}$ can be explained solely on the basis of a different heat of vaporization for the individual species. Based on this plot, the species deposited at $275^{\circ} \mathrm{C}$ (which predominates at lower temperatures) should have the lowest heat of vaporization, whereas the species deposited at $200^{\circ} \mathrm{C}$ should have the highest heat of vaporization. Therefore, as the temperature is increased, the species which deposited at $100^{\circ} \mathrm{C}$ should become progressively more important until a point is reached where its partial pressure is equivalent to that of the $200^{\circ} \mathrm{C}$ species $\left(\approx 2400^{\circ} \mathrm{C}\right)$. At temperatures $>2400^{\circ} \mathrm{C}$ the $200^{\circ} \mathrm{C}$ deposit should then be the predominant species. This

* This rule, based on the fact that the entropies of evaporation of most gaseous species are approximately equal, states that in general, for the case of a saturated vapor, the relative concentration of a minor species with respect to the major species can often be expected to increase with temperature. model offers a satisfactory explanation for the general behavior of the iodine species released from metallic uranium and $\mathrm{CC}$ heated to $\approx 2500^{\circ} \mathrm{C}$. The reason why primarily atomic iodine is released from UC when heated to a temperature greatly exceeding its melting point is not certain. However, on the basis of the above dimension, this would be expected where the partial pressures of the iodine species are below their equilibrium saturation pressures.

A summary of these results is given in Table 1. In an incrt atmosphere both elemental iodine and iodine in combined forms are released; the relative quantity of each depends on the fuel temperature. At temperatures slightly above the melting point of metallic uranium, most of the iodine is converted to UI, which plates out at $275^{\circ} \mathrm{C}$. At temperatures in excess of $2000^{\circ} \mathrm{C}$, partial dissociation of UI to elemental iodine occurs. As the temperature is further increased to $\approx 2500^{\circ} \mathrm{C}$, a second released iodide, which plates out at $200^{\circ} \mathrm{C}$, becomes the major iodine species. Primarily atomic iodine is released in the case of UC heated far above its melting point.

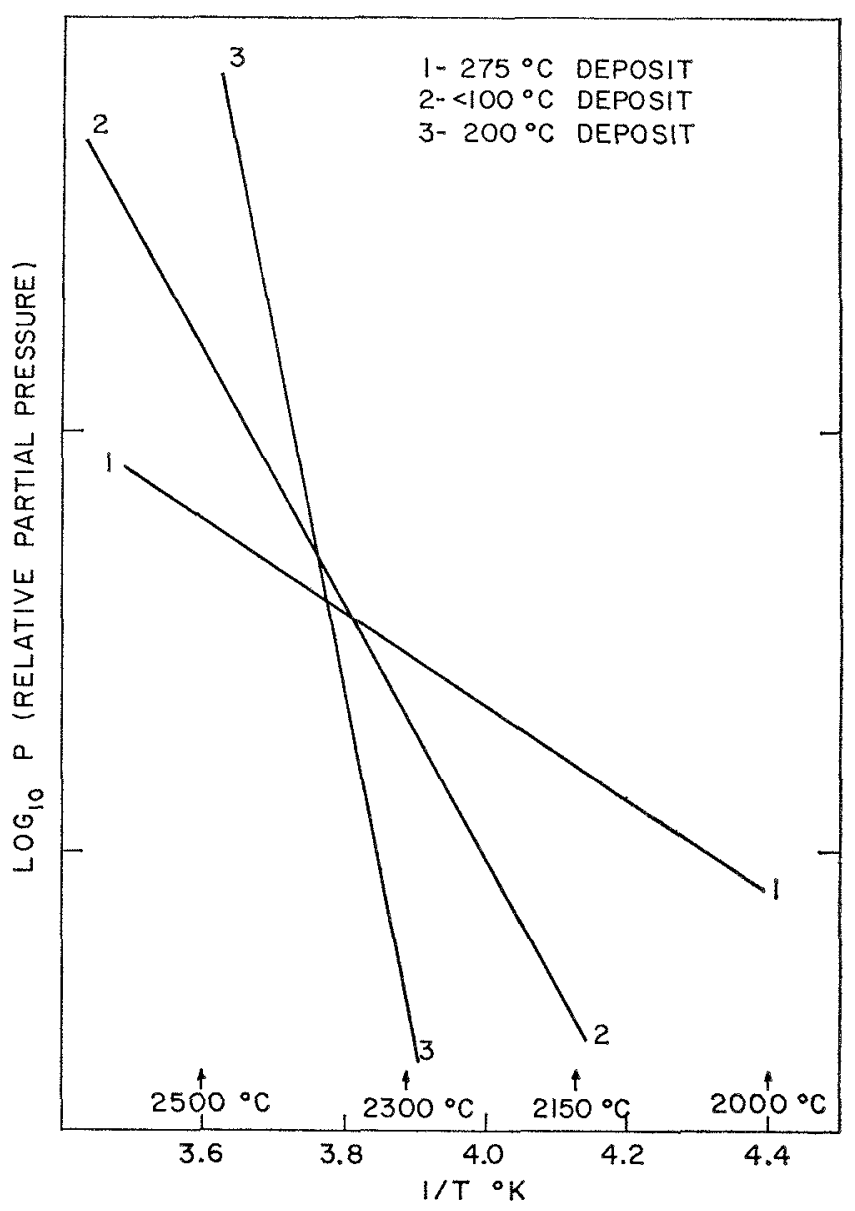

Fig. 2. Relative Partial Pressures of Released Iodine Species as a Function of Temperature. 
Table 1. Summary of Studies with Metallic Uranium AND UC IN INERT ATMOsphere

\begin{tabular}{c|c|c}
\hline $\begin{array}{c}\text { Fuel } \\
\text { Sample }\end{array}$ & Probable Iodine States & Conditions and Remarks \\
\hline $\mathrm{U}$ & $\mathrm{UI}$ & $\begin{array}{c}\sim 1200-2000^{\circ} \mathrm{C} \\
\text { Plate-out temperature: } 275^{\circ} \mathrm{C} \\
\text { Synthesized deposit composi- } \\
\text { tion } \mathrm{UI}_{0.9}\end{array}$ \\
\hline $\mathrm{UC}$ & $\begin{array}{c}\text { Species generally } \\
\text { similar to metal- } \\
\text { lic } \mathrm{U}\end{array}$ & $\begin{array}{c}\sim 2100-2500^{\circ} \mathrm{C} \\
\text { Primarily atomic iodine re- } \\
\text { leased at temperatures in } \\
\text { great excess of the melting } \\
\text { point of UC }\end{array}$ \\
\hline
\end{tabular}

\section{Environmental Behavior}

This section deals with the effect of the environment on the chemical states of fission products. The iodides released into an inert environment, as discussed above, were tenaciously held to surfaces at their respective deposition temperatures. Additional experiments were carried out to determine the effect of flowing air over these iodides. In these experiments, the iodide was nearly completely dissociated and the evolved elemental iodine was carried into the cooler regions of the deposition apparatus. At ambient temperature the iodides were also oxidized; however, free iodine was only very slowly evolved. These results are consistent with the known behavior of the uranium iodides, which readily oxidize in air by the following reactions:

$$
\begin{aligned}
\mathrm{UI}_{x}+\mathrm{O}_{2} & \rightarrow \mathrm{UO}_{2} \mathrm{I}_{x} ; \\
\mathrm{UO}_{2} \mathrm{I}_{x} & \rightarrow \mathrm{UO}_{2}+x / 2 \mathrm{I}_{2} .
\end{aligned}
$$

Since the uranium iodides are quite unstable in air, only elemental iodine was expected to be released upon heating the irradiated fuels directly in air. This was verified experimentally.

The effect of steam on the chemical states of iodine was also investigated. Experiments were carried out with metallic uranium fuels irradiated over the range from $3.0 \times 10^{14}$ to $5.4 \times 10^{19}$ nvt and heated within the temperature range from 1000 to $1300^{\circ} \mathrm{C}$. The uranium iodides are quite unstable in the presence of moisture and would readily hydrolyze, liberating elemental iodine. Therefore, these iodides would not be expected to be important in this case. In this system, the important species are those formed by reactions of the evolved iodine with the hydrogen produced by the metal-water reactions. ${ }^{\left({ }^{8}\right)}$ The relative distribution of the iodine $\left(\mathrm{I}^{0}\right)$, iodide $\left(\mathrm{I}^{-}\right)$, and iodate and periodate
$\left(\mathrm{I}^{+5}, \mathrm{I}^{+7}\right)$ states released from metallic uranium are shown in the first column of Table 2 . It is evident that the iodine released from metallic uranium is primarily in the reduced state (avg of $83.2 \%$ ) with only an average of $13.4 \%$ present as elemental iodine. In the case of the $\mathrm{UO}_{2}$ fuels where little hydrogen is present in the environment, primarily elemental iodine is released, as shown in the second column of this table.

The majority of the jodine released from uranium is rapidly converted to HI by the reactions shown in Fig. 3. The first reaction expresses the release of fission products (F.P.) from uranium during its oxidation in steam. The released iodine reacts with hydrogen to form HI by reaction (2). At high temperature, the equilibrium expressed in reaction (3) is far to the left, so that little molecular iodine is present within the cell. A small quantity of iodine dissolves in accordance with reaction (4); however, this is reversible and most is analyzed as elemental iodine. In solution, some HIO disproportionation takes place at elevated temperatures, giving rise to iodates and periodates via reaction (5). About 1 to $2 \%$ of the iodine released from uranium reacts to form methyl iodide.

The equilibrium between $\mathrm{H}_{2}$, $\mathrm{I}$, and $\mathrm{HI}$ was calculated for the iodine concentration range from $10^{-14}$ to $10^{-8}$ mole/liter, $\mathrm{H}_{2}$ partial pressures ranging from $10^{-5}$ to $1 \mathrm{~atm}$, and temperatures between 298 and $2000^{\circ} \mathrm{K}$ (see Fig. 4). This figure is useful for predicting con-

Table 2. Aver dge Chemical Srate of Iodine Released INTO STEAM

\begin{tabular}{c|c|c}
\hline \multirow{2}{*}{ State } & \multicolumn{2}{|c}{ Relative Amount, \% } \\
\cline { 2 - 3 } & $\mathrm{U}$ & $\mathrm{UO}_{2}$ \\
\hline $\mathrm{I}^{0}$ & 13.4 & 84.6 \\
$\mathrm{I}^{-}$ & 83.2 & 10.5 \\
$\mathrm{I}^{+5}, \mathrm{I}^{+7}$ & 1.8 & 0.5 \\
Others & 1.6 & 4.4 \\
\hline
\end{tabular}

$$
\begin{gathered}
\mathrm{U}(\mathrm{F.P.})+2 \mathrm{H}_{2} \mathrm{O} \longrightarrow \mathrm{UO}_{2}+2 \mathrm{H}_{2}+\mathrm{F} . \mathrm{P} . \\
\mathrm{H}_{2}+2 \mathrm{I} \rightleftharpoons 2 \mathrm{HI} \\
\mathrm{I}_{2}+\mathrm{H}_{2} \mathrm{O} \rightleftharpoons \mathrm{I}_{2} \\
=\mathrm{HOO}+\mathrm{I}^{-}+\mathrm{H}^{-} \\
3 \mathrm{IO}^{-} \stackrel{\Delta}{\rightleftharpoons} \mathrm{IO}_{3}^{-}+2 \mathrm{I}^{-}
\end{gathered}
$$

FIG. 3. Iodine Reactions in a Steam Environment. 


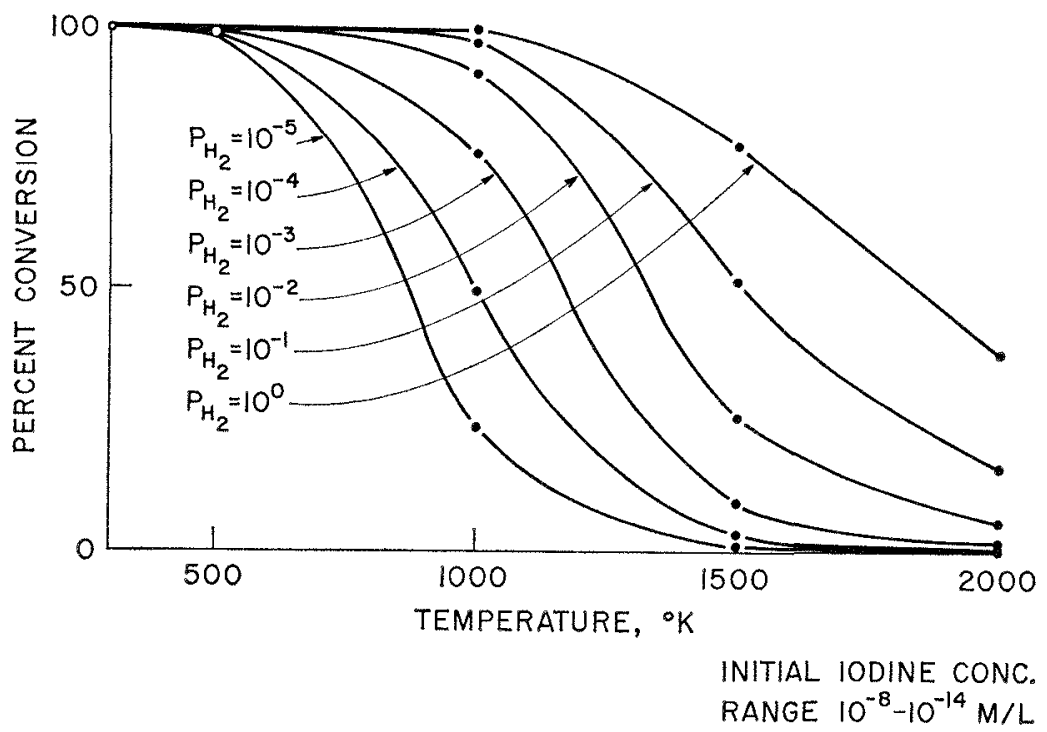

Fra. 4. Hydrogen Iodide Equilibrium.

version of iodine to hydrogen iodide. The experimental results of the aforementioned study are consistent with these calculations.

Although primarily elemental iodine is released from $\mathrm{U}^{\top} \mathrm{O}_{2}$ into steam, this has been observed to react with organic impurities, forming substantial quantities of methyl iodide. In these experiments, in which distilled water boiled from an alkaline permanganate bath was used, only small quantities of the iodine were found to be converted to organic compounds as listed in Table 2 under "Other" states. Similar experiments carried out under less stringent conditions produced up to $21 \%$ of these organic compounds. However, in other studies, we have shown that methyl iodide is relatively unstable in a combined oxidizing and radiation environment, and these compounds are expected to be substantially less significant under reaction accident conditions.

Most of the fast reactor concepts under study in this country involve the use of liquid metal coolants. At present sodium is the primary liquid metal under consideration. Some studies of fission product retention in sodium have been carried out elsewhere. However, there has been no satisfactory interpretation of the data, and the quantitative retention characteristics of sodium are still uncertain. We have recently calculated the probable chemical states and volatility of iodine in sodium to predict its behavior at high temperatures. These results are summarized below.

A comparison of the estimated standard free energies of formation of the uranium iodides and sodium iodide shows that $\mathrm{CI}_{4}, \mathrm{UI}_{3}$, and $\mathrm{UI}_{2}$ would undoubtedly react with sodium to form NaI. However, this cannot be stated with complete certainty in the case of some other subiodide such as UI. In considering reactions of iodine with other fission products, compound formation with cesium is found to be among the most probable based on relative concentrations and thermodynamic values. However, because of the low chemical potential of fission-product cesium in a large volume of sodium, compound formation with cesium would be minimal where concentrations are of the order of parts per million. Therefore, the iodine is almost certainly combined with sodium in dilute solutions.

Further calculations were done to predict the behavior of sodium iodide in sodium. In these calculations the standard free energy of formation values were taken. from the JANAF tables; ${ }^{(9)}$ the activity coefficient of sodium iodide in sodium was estimated by Wagner's $\operatorname{method}^{(10)}$ and the Na-NaI phase diagram published by Bredig and Sutherland. (11)

The free energy of mixing $\left(F^{m}\right)$ for $\mathrm{NaI}$ in sodium with mole fractions of $X_{2}$ and $X_{1}$, respectively, was represented by a power series. By use of the phase diagram, values of the four arbitrary constants were evaluated to give the following equation:

$$
\begin{aligned}
F^{m}=2062 & X_{1}^{4} X_{2}+61180 X_{1}^{3} X_{2}^{2}+1788 X_{1}^{2} X_{2}^{3} \\
& +8 \tilde{25} X_{1} X_{2}^{4}+R T\left(X_{1} \ln X_{1}+X_{2} \ln X_{2}\right) .
\end{aligned}
$$

The free energy of mixing of $\mathrm{NaI}$ is related to the total free energy of mixing, concentration, and activity coefficient in the following manner:

$$
F_{2}^{m}=F^{m}+\left(1-X_{2}\right)\left(\frac{\partial F^{m}}{\partial X_{2}}\right)=R T \ln \gamma_{2} X_{2} .
$$

The activity coefficient of NaI at infinite dilution was calculated from these two equations. This value, to- 
gether with the vapor pressure of pure NaI, was used to develop the following expression for the partial pressure of $\mathrm{NaI}$ in terms of its mole fraction, $X_{2}$, and temperature, $T$, in degrees Kelvin:

$P_{\text {NaI }}=\left[1.316 \times 10^{-3} \exp \left(-\frac{18822}{T}+19.28\right)\right] X_{2}$.

This equation can be used to calculate the partial pressure of sodium iodide in the gas phase, which in turn can be related to percentage iodine release knowing the volumes of the gas phase and liquid phase, respectively. Attempts to measure percent release as an intensive property of the system are obviously meaningless. A consideration of the standard free energy of

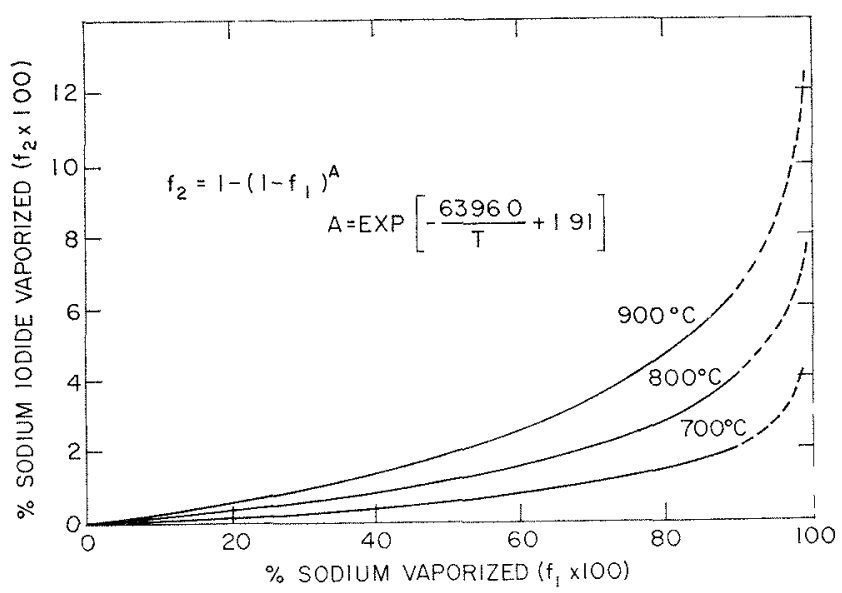

Fra. 5. Calculated Equilibrium Vaporization of Dilute Sodium Iodide Solutions in Sodium. formation for NaI shows that dissociation is negligible at least up to $1500^{\circ} \mathrm{K}$, and thus the iodine in the gas will be almost entirely $\mathrm{NaI}$.

Using this data, a Rayleigh equation expressing the percent vaporization of $\mathrm{NaI}$ as a function of the percent sodium vaporized was developed. The equation and a plot for 700,800 , and $900^{\circ} \mathrm{C}$ are shown in Fig. 5 . It is readily apparent that a significant fraction of the sodium can be vaporized without large losses of $\mathrm{NaI}$.

Finally, it is of interest to consider the consequences of oxidizing sodium containing iodine. Three possible reactions with the environment (oxygen and water vapor) are shown in Fig. 6. The first reaction has a more positive standard free energy of reaction than the latter two and can be neglected. The standard free energy for the second reaction is the smallest, and the reaction appears to be more favorable than the third. It turns out, however, unless a large fraction of the environment is water vapor, reaction 3 actually predominates.

The usual assumption of taking the activity of the two components $\mathrm{Na}_{2} \mathrm{O}_{2}$ and $\mathrm{NaI}$ as unity, suggests that no appreciable decomposition to free iodine takes place. However, it was found experimentally that during the initial stages of $\mathrm{NaI}$ oxidation, its activity was far less than unity, and decomposition of the iodide did indeed take place. An addition of excess $\mathrm{Na}_{2} \mathrm{O}_{2}$ substantially suppressed decomposition. Therefore, in the rase of a reactor environment containing large quantities of sodium also being oxidized to $\mathrm{Na}_{2} \mathrm{O}_{2}$, the decomposition of $\mathrm{X}$ aI should be negligible.

\begin{tabular}{|c|c|}
\hline Chemical Reaction & Equilibrium Constant \\
\hline 1) $\begin{aligned} & \mathrm{NaI}+ \frac{1}{4} \mathrm{O}_{2} \rightleftharpoons \frac{1}{2} \mathrm{Na}_{2} \mathrm{O}+\mathrm{I} \\
& \Delta \mathrm{F}_{1000^{\circ} \mathrm{K}}=28.82 \mathrm{kcal} / \mathrm{mole}\end{aligned}$ & $K_{1}=\frac{a_{\mathrm{Na}_{2} \mathrm{O}^{1 / 2}}^{a_{I}}}{a_{\mathrm{NaI} \mathrm{a}_{2}^{1 / 4}}^{1 / 4}}=4.9 \times 10^{-7}$ \\
\hline 2) $\begin{aligned} \mathrm{NaI}+\frac{1}{4} \mathrm{O}_{2}+\frac{1}{2} \mathrm{H}_{2} \mathrm{O} \rightleftharpoons \mathrm{NaOH}+\mathrm{I} \\
\Delta \mathrm{F}_{1000^{\circ} \mathrm{K}}^{\circ}=17.56 \mathrm{kcal} / \mathrm{mole}\end{aligned}$ & $\mathrm{K}_{2}=\frac{\mathrm{a}_{\mathrm{NaOH}} \mathrm{a}_{\mathrm{I}}}{\mathrm{a}_{\mathrm{NaI}} \mathrm{a}_{2}^{1 / 4} \mathrm{a}_{\mathrm{H}_{2} \mathrm{O}}^{1 / 2}}=1.4 \times 10^{-4}$ \\
\hline \multirow[t]{2}{*}{ 3) $\begin{aligned} \mathrm{NaI}+\frac{1}{2} \mathrm{O}_{2} \rightleftharpoons \frac{1}{2} & \mathrm{Na}_{2} \mathrm{O}_{2}+\mathrm{I} \\
\Delta \mathrm{F}_{1000^{\circ} \mathrm{K}}^{\circ} & =26.93 \mathrm{kcal} / \mathrm{mole}\end{aligned}$} & $K_{3}=\frac{a_{\mathrm{Na}_{2} \mathrm{O}_{2}}^{1 / 2} \mathrm{a}_{\mathrm{I}}}{\mathrm{aNaI}^{1 / 2} \mathrm{O}_{2}}=1.2 \times 10^{-6}$ \\
\hline & $P_{I_{a t m}} \cong 5.5 \times 10^{-7}\left[\frac{a \mathrm{NaI}}{a^{1 / 2} \mathrm{Na}_{2} \mathrm{O}_{2}}\right]$ \\
\hline
\end{tabular}

FIG. 6. Reactions and Thermodynamic Values for the Oxidation of Sodum Iodide.(a) (a) JANAF Thermochemical Tables, The Dow Chemical Company, Midland, Michigan. 


\section{Conclusions}

In an inert atmosphere, two iodides and atomic iodine are released from metallic uranium and $\mathrm{CC}$ fuels. The relative quantities of each species are primarily a function of fuel temperature. The two iodides are tenaciously held on surfaces at temperatures of $\approx 275$ and $\approx 200^{\circ} \mathrm{C}$ in an inert atmosphere, but readily evolve elemental iodine into an oxidizing environment.

Where iodine is released during a metal-water reartion, conversion to HI can be validly estimated from thermodynamic considerations. In a sodium environment, essentially all of the iodine will be converted to $\mathrm{NaI}$ when the fission product concentrations are of the order of parts per million. Expressions are given for calculating the volatility of NaI in sodium. Subsequent exposure to an oxidizing environment should not result in substantial $\mathrm{NaI}$ oxidation in the presence of excess $\mathrm{Na}_{2} \mathrm{O}_{2}$.

\section{Acknowledgments}

The authors wish to thank Mr. Harry R. Munkelwitz and Mr. George Hardman for assistance in performing many of the experiments. The authors are indebted to Dr. J. J. Egan for valuable discussions regarding thermodynamic considerations in these studies.

\section{REFERHNCES}

1. Castleman, A. W., Jr., TID-7641, pp. 155-68 (Oct 1962).

2. Castleman, A. W., Jr., Trans. ANS 6(1), 128 (June 1963).

3. Castleman, A. W., Jr., and Salzano, F. J., TID-7677, p. 16.

4. Castleman, A. W., Jr., Tang, I., and Munkelwitz, H. R., CONF-650407, 1, p. 325 (April 1965).

5. Brenor, Leo, Bromley, L. A., (xilles, P. W., and Lofgren, N. L., Chemistry of ('ranium, Book 1, pp. 21y-68 (1958).

6. Searcy, A. W., Proc. Intern. Symp. on High Temperature Technology, Asilomar, 1963, p. 105, Butterworths, London (1964).

7. Brenex, L., Pranciples of Hugh Temperalure Chemistry, Proc. Robert Wolch Foundation Conf. on Modern Inorganic Chemistry, Houston, November 1962.

8. Castleman, A. W., Jr., Tang, I., and Munkelwitz, II. R., CONF-650407, 2, p. 1118 (April 1965).

9. JANAF Thermochemical Tables, The Dow Chemical Company, Midland, Michigan.

10. Wagner, C., Thermodynamacs of Alloys, Addison-Wesley Publishing Co., Inc., Reading, Mass. (1952).

11. Bredig, M. A., and Sutherland, J. E., ORNI -2548 , p. 9 (1959).

\section{Discussion}

Mr. Stachura (AI): What were the values of the activity coefficient of $\mathrm{NaI}$ in the $500-1000^{\circ} \mathrm{K}$ range?

Mr. Castleman: They were of the order of something less than 10 , perhaps about 3 to 5 .

MIr. Stachura: I remember secing an Argonne report on the EBR-II refining process in which they discussed some of their differences and the possibility that they observed cesium iodide.

Mr. Castleman: Their work actually led us into this. We had originally also noticed that cesium and iodine come down together. We spread out our thermal gradient in a much larger apparatus and were able to show that they are transported independently. This doesn't mean that you can't get reaction on the surface when they do come together, but we don't believe they are released from the fuel together. Many of the Argonne studies were with synthetic mixtures of a uranium iodide and a cesium iodide; this may not lead to the same release as from a very dilute solution which one has in a fuel.

Mr. Stachura: I thought their contention was just the opposite--that since they had microbubbles and highly irradiated fuel the opportunity for the formation of the iodide might exist. The other point is in the case of your studies of the activities of jodine and sodium. Would not cesium be likely to influence the behavior you observed?

Mr. Castleman: Actually we looked into this on paper. It turns out that even though the free energy of reaction between cesium and iodine is more negative, the chemical potential of the cesium is so low at the concentrations involved that the cesium-iodine reactions would not predominate over those of sodium and iodine.

MIr. Stachura: In other words, you determined the activity coefficients for cesium and sodium?

MIr. Castleman: No, but we estimated that they would have to be of the order of at least over $10^{4}$. I know of no activity coefficient of anything in anything that is above 100 .

Mr. Stachura: In other words this occurs because the cesium concentrations are so dilute.

Mr. Castleman: The cesium concentrations are so dilute, and also there is a negative deviation with respect to an jdeal solution for cesium in sodium rather than a positive deviation. One would need a very large positive deviation to expect these reactions to be predominant when we're talking of the order of parts per million now and not diseussing higher concentrations. With an excess of cesium, we have nearly unit activity of cesium, and the cesium-iodine free energy of reaction is much more favorable than that between sodium and iodine.

Mr. Runnalls (AECL): Could you account for the shape of the cesium distribution you obtained?

Mr. Castleman: In the case of quartz, the cesium is highly adsorbed. We are seeing the movement of an adsorbtion wave, and by extending the length of the run, the cesium will move down and peak at about 
$450^{\circ} \mathrm{C}$. In the particular run plotted, the run was stopped before all of the cesium had desorbed, moved down, and given one peak position as the iodine did. However, we've run these experiments for fairly long periods of time and have found that the uranium iodide is very tenaciously held to the surface at $275^{\circ} \mathrm{C}$. The cesium will work its way down, but it is not moving with the iodine.
Mr. Runnalls: Well, the puzzle was that the cesium did not seem to be moving appreciably from the hot end. Do you not expect that it would evolve as cesium gas?

Mr. Castleman: Yes, but it is adsorbing on the surface. On that particular plot it was starting out high and it was peaking at around $450^{\circ} \mathrm{C}$. It was actually moving as an adsorption wave down the tube, 


\title{
Fission Product Retention in Sodium and Applications to Vented Fuel Element Design*
}

\author{
W. Kunkel and S. Berger \\ Atomics International \\ Canoga Park, California \\ (IRESENTED BY W. KUNKEL)
}

The use of a vented fuel element reduces cladding strain from released fission gas pressure, thereby increasing the potential of the fuel for extended burnup. Since there is a gradual release of fission gas as a result of venting, the possibility of a sudden, abrupt fission product release from the element upon failure during operation, handling, storage, or shipping is eliminated. In addition, for sodium-bonded fucl elements, the bond is not lost as a result of a cladding failure and pressure differential, thus eliminating the overheating problem associated with bond loss. Finally, the consequence of the fuel-slumping accident is reduced since the fuel density can be increased as a result of the decreased requirement for gas-holdup space.

Although there are some radiological problems in most vented fuel-element designs, the successful operation of the Dounreay Fast Reactor as reported recently has indicated that these problems can be satisfactorily resolved. ${ }^{(1)}$ This paper will indicate some of the safety problems associated with the ventedfuel-element concept and apply the results of recent experimental and analytical studies to those problems.

\section{A. Some Problems Associated with Vented Fuel Element Design}

Figure 1 illustrates schematically some of the various paths by which fission products may be transported from a vented fuel element. Also shown are some of the inherent and engineered control measures which might be utilized in a reactor employing vented fuel elements.

The fission products released from a vented fuel element to the sodium coolant may increase the operational radioactivity levels of the sodium or reduce the accessibility of the system for component replacement or maintenance operations after shutdown. The high specific activity of the sodium during operation would mask most of fission product radiation until the system is shut down. However, an increase in the fission product contamination

* Work performed under Contract AT(11-1) GEN-8 for the U. S. Atomic Energy Commission. level of the sodium in the reactor must be considered in the accident evaluation of a sodium spill or a sodium fire. The radiological effect of the fission product isotopes would probably greatly increase the environmental hazard as compared to normal sodium activation.

In a similar manner, the operational contamination of the reactor cover gas resulting from the release of fission products from a vented fuel element can be transported to the high bay as a result of small but finite leakage. This constitutes an environmental hazard in the region of the operating floor. In addition, the increase in the fission product gas in the holdup system necessitates careful design of holdup and filtration devices. Of special significance in this case is the possible effect of the decay of short-lived xenon and krypton isotopes into daughters of relatively lower volatility, cesium and strontium, with consequent increase in the radiological hazard. This effect was noted in analysis of the radioactivity levels in the Dounreay sphere. (1) Analysis of the airborne radioactivity showed that rubidium- 88 and cesium-138 were the principal eonstituents.

\section{B. Application of Available Data to these Problems}

Many of the results of recent AI studies ${ }^{(2)}$ of fission product retention in sodium are applicable to the behavior of fission products released into sodium from a vented fuel element. For example, the extent of isotopic retention in sodium is shown in Table 1. These data, which were acquired from several experiments, indicate the considerable extent to which iodine, and the relatively lower volatility isotopes such as barium, zirconium, etc., are retained in a sodium environment following release from a fuel rod. These data were obtained from a variety of experiments ranging from the in-pile melting and vaporization of uranium dises in sodium, to out-of-pile experiments in which iodine and sodium iodide were injected into sodium columns and the off-gas sampled for the presence of iodine.

In a typical fast reactor system, there are many 


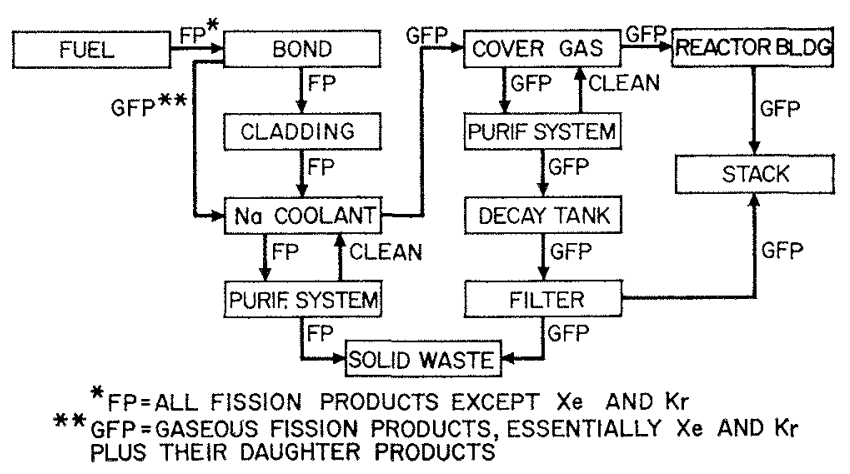

Fia. 1. Fission Product Control from Vented Fuel Elements. additional factors which may influence the transport behavior of fission products released from a vented fuel element. Some of these may be deliberately designed into the system, such as the coolant- and gascleanup systems shown sehematically in Fig. 1. Other factors are associated with sodium reactor components and piping. For example, past experience with fission products released into the Sodium Reactor Experiment (SRE) as a result of a partial fuel melting indicated that there is a considerable tendency for radioactivity to deposit at various points throughout the sodium system, thus depleting the amount available in the sodium in the event of fires or spills. Table 2 shows the radioactivity levels measured by Hart from sodium samplen in the SRE primary system. (5)

TABLE 1. COMP

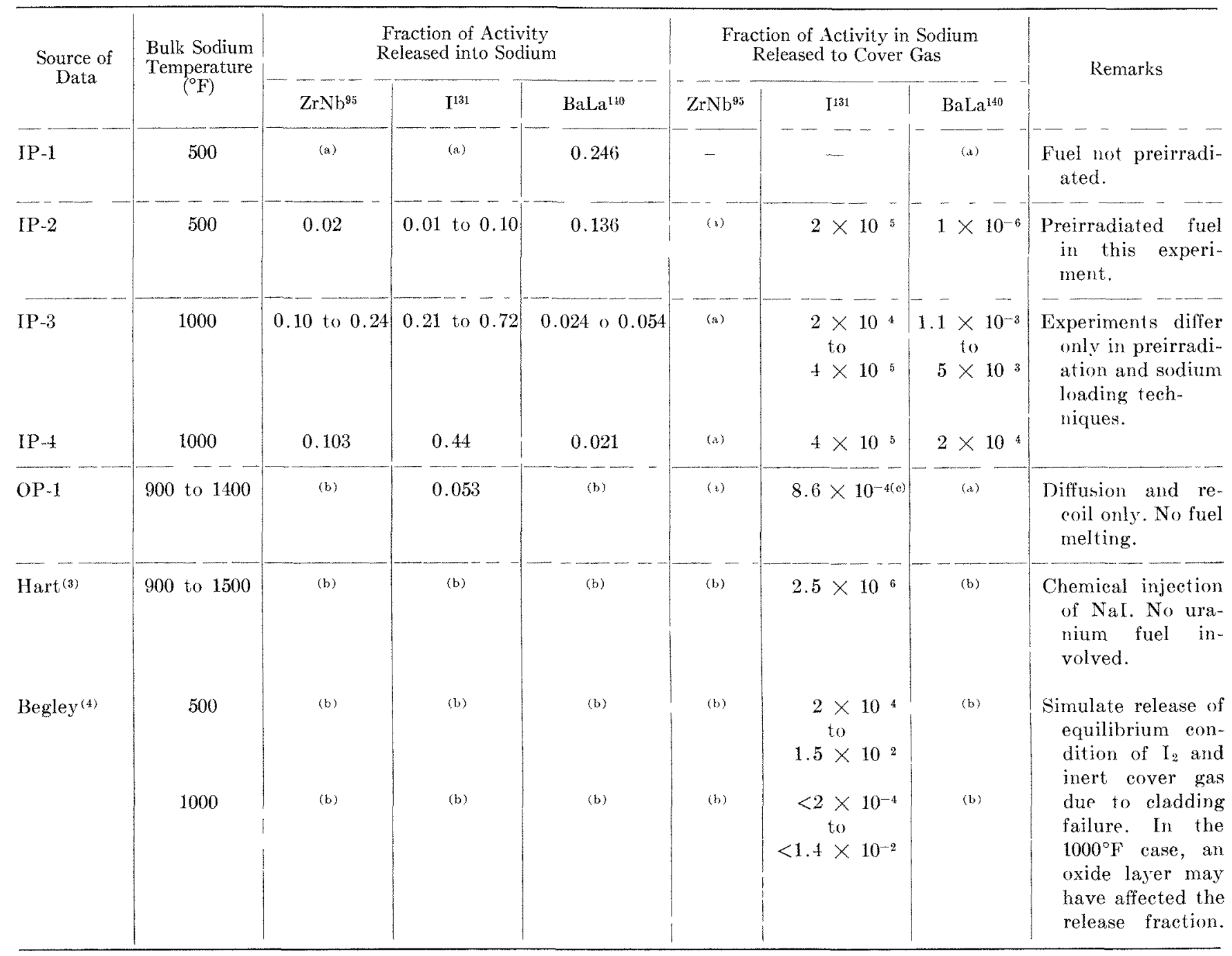

(a) Not observed.

(b) Not measured.

(c) This amount was recovered on the heated portion of the tubing. The amount actually mobile was $2 \times 10^{-\hat{6}}$ of the activity in the sodium. 
Based on the normal radioactive decay for each isotope, the predicted value after approximately three months is shown in Table 3 , along with actual sodium concentration measured at the later date. ${ }^{(5)}$

It was also observed shortly after the ineident that the fission product activity appeared to be associated very markedly with the several pounds of carbonaceous material which had originally caused the melting of the fuel element. Table 4 shows the difference in specific activity between sodium samples and flecks of the carbon material obtained from the system.

A larger sample of the carbonaceous material was obtained from the tops of the moderator cans and was compared to the sodium specific activity shown

TABLE 2. InItite Fission Prod Xet An alysis of SRE Primate hodicm

\begin{tabular}{|c|c|c|c|c|}
\hline Isotope & $\begin{array}{c}\text { Primary } \\
\text { Coolant } \\
\text { Activity } \\
(\mu \mathrm{Ci} / \mathrm{g} \\
\left.\mathrm{Na}^{\top}\right)^{(a)}\end{array}$ & $\begin{array}{c}\text { Total } \\
\text { Coolant } \\
\text { Inven- } \\
\text { tory } \\
\text { (Ci })^{(\mathrm{a})}\end{array}$ & $\begin{array}{l}\text { Total } \\
\text { Reactor } \\
(\mathrm{Ci})^{(a)}\end{array}$ & $\begin{array}{l}\text { Fraction of } \\
\text { Inventory } \\
\text { Released }^{(b)}\end{array}$ \\
\hline Cs-137 & 1.26 & $2.77 \times 10^{1}$ & $8.70 \times 10^{3}$ & $3.18 \times 10^{-3}$ \\
\hline Cs-134 & 0.02 & $4 \times 10^{-1}$ & $2 \times 10^{2}$ & $2 \times 10^{-3(c)}$ \\
\hline Sr-89 & 20.0 & $4.44 \times 10^{2}$ & $1.60 \times 10^{5}$ & $2.78 \times 10^{3}$ \\
\hline Sr -90 & 097 & $2.14 \times 10^{1}$ & $8.15 \times 10^{3}$ & $2.63 \times 10^{-3}$ \\
\hline $\mathrm{I}-131$ & 0.74 & $1.63 \times 10^{1}$ & $1.68 \times 10^{4}$ & $0.97 \times 10^{-3}$ \\
\hline $\mathrm{Ce}-141$ & 4.38 & $9.65 \times 10^{1}$ & $1.27 \times 10^{5}$ & $0.76 \times 10^{-3}$ \\
\hline $\mathrm{Ce}-144$ & 5.18 & $1.41 \times 10^{2}$ & $1.69 \times 10^{5}$ & $0.67 \times 10^{-3}$ \\
\hline $\mathrm{Ba}(\mathrm{La})-140$ & 1.65 & $3.63 \times 10^{1}$ & $5.61 \times 10^{4}$ & $0.65 \times 10^{-3}$ \\
\hline $\mathrm{ZrNb}-95$ & 139 & $3.06 \times 10^{2}$ & $5.53 \times 10^{5}$ & $0.55 \times 10^{-3}$ \\
\hline $\mathrm{Ru}-103$ & 0.95 & $2.09 \times 10^{1}$ & $7.52 \times 10^{4}$ & $0.28 \times 10^{-3}$ \\
\hline
\end{tabular}

(9) As of July 26, 1959.

(b) Multiply values in this column by 3 to adjust fraction released to average values for those fuel elements which suffered cladding failures ( 14 of 43 elements failed).

(c) From neutron capture in Cs-133; estimated.

TABle 3. Fission Prodect Contamin ition Decre ise in Primiry Sontey

(August \& to October 31, 1959)

\begin{tabular}{l|c|c|c|c}
\hline \multirow{1}{*}{ Isotope } & \multicolumn{2}{|c|}{$\begin{array}{c}\text { Fission Product Contamination } \\
(\mu \mathrm{Ci} / \mathrm{g} \text { Na) }\end{array}$} & \multicolumn{1}{|c|}{$\begin{array}{c}\text { Ratio: } \\
\text { Oct 31 (actual)/ } \\
\text { Oct 31 } \\
\text { (predicted) }\end{array}$} \\
\cline { 2 - 3 } Cs-137 & Aug 2 & $\begin{array}{c}\text { Oct 31 } \\
\text { (pre- } \\
\text { dicted) }\end{array}$ & $\begin{array}{c}\text { Oct 31 } \\
\text { (actual) }\end{array}$ & \\
Cs-134 & 1.26 & 1.26 & 0.45 & 0.36 \\
Sr-90 & 0.02 & 0.02 & 0.006 & 0.3 \\
Sr-89 & 0.97 & 0.97 & 0.060 & 0.062 \\
I-131 & 19.0 & 5.8 & 0.25 & 0.043 \\
Ce-141 & 0.42 & 0.00019 & 0.00012 & 0.63 \\
Ce-144 & 3.7 & 0.54 & 0.000088 & 0.00016 \\
ZrNb-95 & 5.1 & 3.9 & 0.00031 & 0.00008 \\
Ru-103 & 13.0 & 5.2 & 0.0067 & 0.0013 \\
& 0.85 & 0.19 & 0.0045 & 0.024 \\
\hline
\end{tabular}

(a) On the basis of radioactive decay of August 2 sample.
TABLE 4. RADrodetrvity An 4 Lysis of Typical Sampie

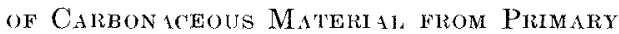
SODIUM S+MPLE

\begin{tabular}{l|c|c}
\hline Isotope & $\begin{array}{c}\text { Activity of Car- } \\
\text { bonaceous Material(i) } \\
(\mu \mathrm{Ci} / \mathrm{g} \text { of particles })^{(b)}\end{array}$ & $\begin{array}{c}\text { Activity of } \\
\text { Sodium } \\
(\mu \mathrm{Ci} / \mathrm{g} \mathrm{Na})^{(\mathrm{b})}\end{array}$ \\
\hline $\mathrm{Cs}-137$ & $1.1 \times 10^{3}$ & 0.45 \\
$\mathrm{Cs}-134$ & $2.9 \times 10^{1}$ & 0.006 \\
$\mathrm{Ce}-141$ & $2.3 \times 10^{2}$ & 0.0007 \\
$\mathrm{Ce}-144$ & $2.9 \times 10^{2}$ & 0.00039 \\
$\mathrm{ZrNb}-95$ & $2.2 \times 10^{2}$ & 0.018 \\
\hline
\end{tabular}

(a) Obtained from reactor on $10 / 31 / 59$.

(b) As of $7 / 26 / 59$.

TABLE 5. Radionctive Analysis of CARBONaCEOUS MATERI 14 TiKeN From TOP OF MIODER 1 TOR C ANS

\begin{tabular}{l|l|l}
\hline Isotope & $\begin{array}{c}\text { Activity of } \\
\text { Carbonaceous Material } \\
(\mu \mathrm{Ci} / \mathrm{g})\end{array}$ & $\begin{array}{c}\text { Activity of } \\
\text { Sodium } \\
(\mu \mathrm{Ci} / \mathrm{g})\end{array}$ \\
\hline $\mathrm{ZrNb}-95$ & $6.8 \times 10^{3}$ & 0.018 \\
$\mathrm{Cs}-137$ & $5.0 \times 10^{3}$ & 0.45 \\
$\mathrm{Cs}-134$ & $1.3 \times 10^{2}$ & 0006 \\
$\mathrm{Ce}-144$ & $3.6 \times 10^{3}$ & 0.00039 \\
\hline
\end{tabular}

(a) As of $7 / 29 / 59$.

TABla 6. Impurity Leveis in Cold Trap as Complred TO SODIYM COOLAN'T IN THE SRL

\begin{tabular}{l|c|c|c}
\hline \multicolumn{1}{c|}{ Impurity } & $\begin{array}{c}\text { Level in } \\
\text { Cold Trap }\end{array}$ & $\begin{array}{c}\text { Level in } \\
\text { Sodium Coolant }\end{array}$ & $\begin{array}{c}\text { Collection } \\
\text { Ratio } \\
A_{\text {trap }}\end{array}$ \\
\hline $\begin{array}{l}A_{\text {coolant }} \\
\text { Carbon }\end{array}$ & $144-1550 \mathrm{ppm}$ & $18-60 \mathrm{ppm}$ & 77 \\
$\mathrm{Cs-137^{(a) }}$ & $4.0 \times 10^{2} \mu \mathrm{Ci} / \mathrm{g}$ & $1.5 \times 10^{-2} \mu \mathrm{Ci} / \mathrm{g}$ & $2.7 \times 10^{4}$ \\
$\mathrm{Sb}-125^{(a)}$ & $4.3 \mu \mathrm{Ci} / \mathrm{g}$ & $0.63 \times 10^{-2} \mu \mathrm{Ci} / \mathrm{g}$ & $6.8 \times 10^{2}$ \\
$\mathrm{Fe}^{(b)}$ & $200->500 \mathrm{ppm}$ & $50 \mathrm{ppm}$ & $>10$ \\
$\mathrm{Si}^{(b)}$ & $200->500 \mathrm{ppm}$ & $50 \mathrm{ppm}$ & $>10$ \\
$\mathrm{Mn}^{(b)}$ & $50-500 \mathrm{ppm}$ & $<5 \mathrm{ppm}$ & $>100$ \\
$\mathrm{~Pb}^{(b)}$ & $5->500 \mathrm{ppm}$ & $10 \mathrm{ppm}$ & $>50$ \\
$\mathrm{Cr}^{(b)}$ & $5->500 \mathrm{ppm}$ & $5 \mathrm{ppm}$ & $>100$ \\
$\mathrm{Ni}^{(b)}$ & $10-300 \mathrm{ppm}$ & $5 \mathrm{ppm}$ & 60 \\
\hline
\end{tabular}

(d) Measured by gamma spectroscopy.

(b) Measured by emission spectroscopy.

in Table 5. As far as the activity of the cover gas is concerned, only xenon and krypton isotopes were observed in the reactor cover gas following the fuelelement release.

Work by Hansen ${ }^{(6)}$ has shown that the system cold trap, which is designed to keep the oxide level to a lower limit of $10 \mathrm{ppm}$, is also a very effective device for coolant purification. These results are shown in Table 6.

The high collection ratio of the Cs-137 is particularly interesting. Hansen speculates that cold trap- 
ping of the cesium by saturation is not likely since the cesium concentration by weight is $10^{-4} \mathrm{ppm}$ and it is soluble in sodium to $50 \mathrm{w} / \mathrm{O}$ at $250^{\circ} \mathrm{F}$. He further suggests that the high oxidation potential of cesium may result in the reduction of sodium oxide as the retention mechanism. The much higher collection ratio for cesium-137 indicates cesium-trapping mechanisms other than a trapping of carbon particles carrying cesium.

Earlier results also have been corroborated with more recent data which were obtained as the primary system piping of the SRE was dismantled for reactor modification. Figure 2 show's a photograph of a black deposit which was noted on the SRE spool piece (the piping section of the primary system adjacent to the reactor). For comparison the figure includes an autoradiograph of the same sector showing the marked correlation of the black carbonaceous
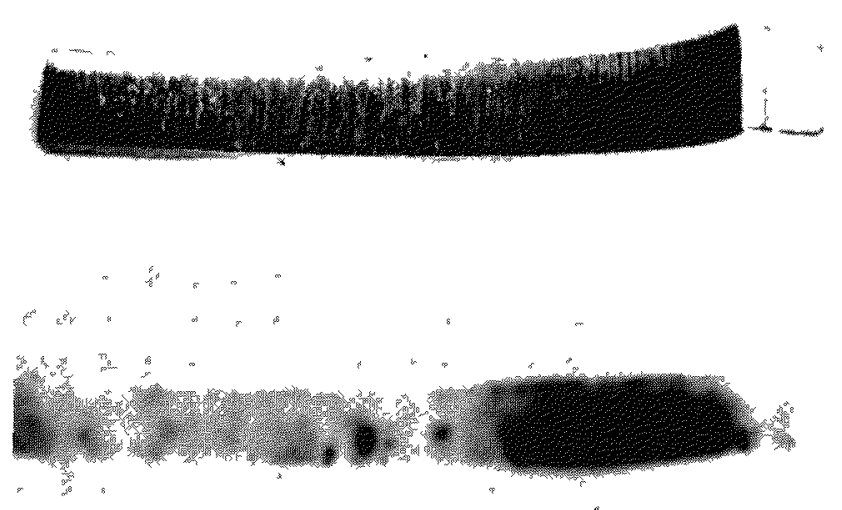

FIG. 2. Photo and Autoradiograph of SRE Spool L-103 after 26-hr HCl Ftch.

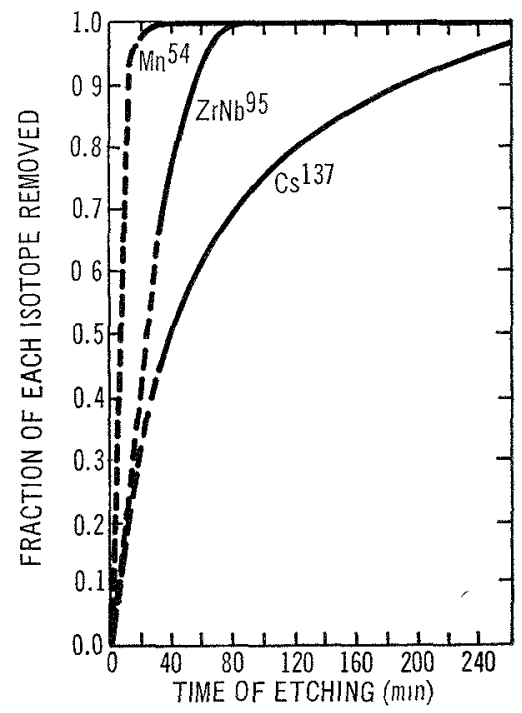

Fig. 3. Relative Efficiency for Isotope Removal by Nitric Acid Rinsing.
TABLE 7. Relative Isocopic Activity Inventory of $A$ migh-buRnep UC Capsule

\begin{tabular}{l|c|c|r}
\hline \multirow{2}{*}{ Isotope } & \multicolumn{2}{|c|}{ Cover Gas } & Fuel Rod \\
\cline { 1 - 3 } & $\begin{array}{c}\text { Measured } \\
\mu \mathrm{Ci}\end{array}$ & Relative $^{(a)}$ & \\
\hline $\mathrm{Kr}-85$ & $1.0 \times 10^{-5}$ & 10 & 10 \\
$\mathrm{Xe}-131 \mathrm{~m}$ & $1.2 \times 10^{-5}$ & 1.2 & 17 \\
$\mathrm{I}-131$ & $36 \times 10^{-7}$ & 0.036 & 15.3 \\
$\mathrm{Xe}-133$ & $11 \times 10^{-4}$ & 11.0 & 354.0 \\
\hline
\end{tabular}

(a) Normalized to $\mathrm{Kr}-85$.

stain with deposition of radioactivity. Sectors of the same pipe spool piece were repetitively etched with acid to remove the deposited radioactivity. These data are typified by the graph shown in Fig. 3. It is of interest to note that this activity was not merely lying on the pipe surface, but, as indicated by the tenacity of the deposition removal, was rather firmly affixed to the pipe surface.

An important factor in assessing safety considerations when vented fuel elements are used is the extent of decay during transport time of the rare gases released into the cover gas. Very little experimental data are arailable regarding the transport delay times of xenon and krypton isotopes from formation in the fuel to the release into the sodium bond, and subsequent appearance in the cover gas. Some information applicable to this problem was recently obtained from post-irradiation examination of covergas and sodium-bond activity in a high-burnup UC test fuel rod irradiated to $20,000 \mathrm{Mwd} / \mathrm{MTC}$. The fuel rod was sodium bonded to 10 -mil stainless-steel cladding, and the sodium bond extended approximately $1 / 2$ in. above the active fuel region. The gas space above the sodium was filled initially with helium. The fuel was hypostoichiometric UC enriched to approximately $11 \%$ in $\mathrm{U}-235$. The fuel rod was $18 \mathrm{in}$. long and $0.5 \mathrm{in}$. in diameter, and contained $14.6 \mathrm{~g}$ of sodium in the bond and $749.6 \mathrm{~g}$ of $\mathrm{CC}$.

The radioactive inventory of a cover-gas sample obtained from this fuel rod is shown in Table 7. The actual amounts measured have no significance since some of the gas activity was apparently lost due to a leak in a thermocouple weld; thus, it was necessary to use a normalization procedure to evaluate the results.

The data from the cover-gas sample were extrapolated back to reactor out-time and were normalized to the longest-lived isotope present (Kr-85). These data were compared with the estimated inventory of fission products anticipated from fuel under these burnup conditions. The calculated activity ratios in the fucl-rod inventory would indicate 
that there should be approximately 354 times as much $\mathrm{Xe}-133$ in the cover gas as there would be Kr-85, whereas the measurements showed only 11 times. There was, therefore, an indicated depletion of $\mathrm{Xe}-133$ by a factor of 32 relative to the krypton. If is is assumed that one may associate this depletion with half-life, it represents a decay while in transport equivalent to $2^{\sim}=32$, or five half-lives. Similarly, comparing the Xe-131m release, a 0.49 half-life is indicated. The half-life of $\mathrm{Xe}-133$ is approximately 5.3 days; that of $\mathrm{Xe}-131 \mathrm{~m}$ is 12 days. Threfore, an apparent delay of xenon release compared to krypton release could account for these ancmalies if it is assumed that this delay is within the range of $5 \times 5.3=27$ days to $0.49 \times 12=6$ days.

The data presented in Table 7 also provided some insight into the transport behavior of I-131 under steady-state conditions of release. Apparently, there should have been 15.3 times as much I-131 in the gas space as krypton-85 if they were released in the same fraction. The data indicate that there was only 0.036 times as much I-131 as Kr-85. Therefore, the iodine must have been depleted by passage through the sodium bond, plating, or some other phenomena. These data, while admittedly sparse, indicate an apparent figure for iodine release from the sodium of $0.2 \%$. These results compare with the other experimental release figures under controlled laboratory conditions of $0.02 \%$.

\section{Parameter Survey for Hypothetical Sodium Re- actor System}

An analytical study was recently conducted to determine the activity in the high-bay area and in the reactor cover gas during normal operation of a

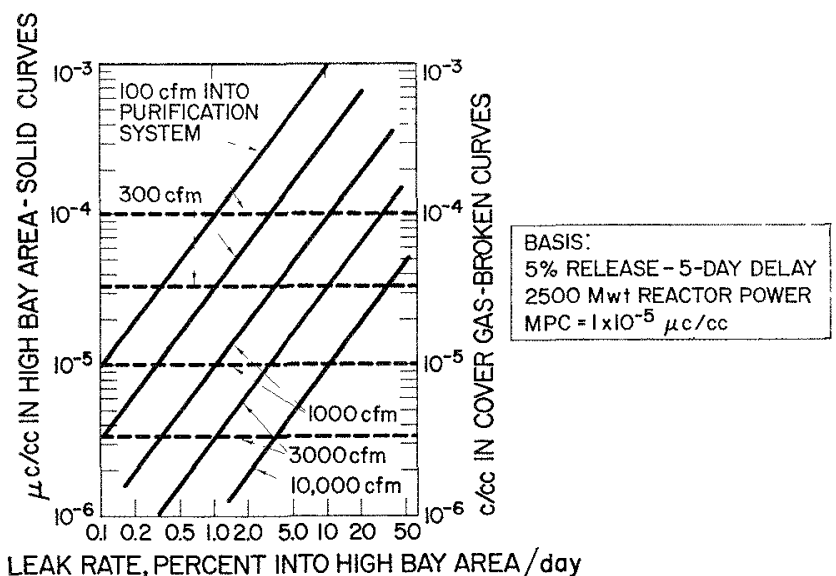

Fig. 4. Concentration of $\mathrm{Xe}^{133}$ in High-bay Area and in Cover Gas vs Leak Rate. (Parameters Represent Flow Rate into Purification System.)

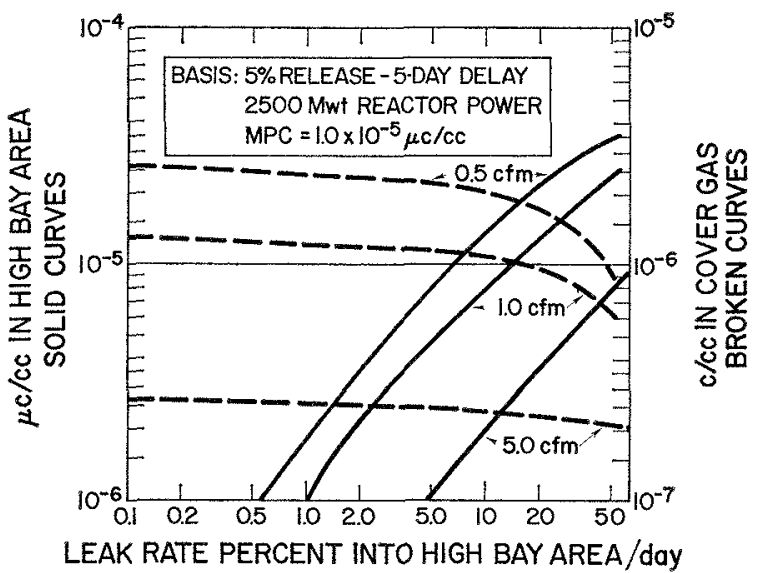

Fig. 5. Concentration of $\mathrm{Kr}^{85}$ in High-bay Area and Cover Gas vs Leak Rate. (Parameters Represent Flow Rates into Purification System.)

power reactor containing vented fuel elements. As pointed out above, it is probable that during the operation of such a reactor, only the noble gases ( $\mathrm{Xe}$ and $\mathrm{Kr}$ ) and their daughter products will reach the reactor cover gas and the high-bay area. In this study, a conservative delay time of 5 days was assumed. With these considerations in mind, only two isotopes become significant contributors to the activity: $\mathrm{Kr}-85$ and Xe-133. A further, rather arbitrary, condition was imposed, namely, that $5 \%$ of all fission gases formed leave the fuel and migrate through the sodium pool into the cover gas region.

Figures 4 and 5 show the estimated activities in the high-bay and cover-gas areas based on various leak rates from the cover gas into the high-bay area, and for various purification rates of the cover gas. The following conditions were kept constant during the parameter study:

1) a high-bay-area ventilation of 2 changes/hr;

2) a cover gas volume of $2500 \mathrm{ft}^{3}$;

3) a high-bay volume of $750,000 \mathrm{ft}^{3}$;

4) thermal reactor power of $2500 \mathrm{MW}$.*

It becomes obvious that a system to purify the cover gas will be necessary. The size of the purification system is, of course, dependent on the leak rate into the high-bay area. For a $0.1 \%$ /day flow rate into the high-bay area from the cover gas, a purification rate of $100 \mathrm{cfm}$ will be required to maintain $\mathrm{MPC}$ $\left(1 \times 10^{-5} \mu \mathrm{Ci} / \mathrm{cc}\right)$ in the high-bay area. Of the two isotopes considered, $\mathrm{Xe}-133$ and $\mathrm{Kr}-85$, the former is by far the most significant.

The ultimate disposal of these radioactive gases (now contained within the purification system) must also be considered. A reasonable period of time, say

* Although the study was originally done for a thermal reacfor, the results are applicable to the fast reactor system. 
one to two months, can be allowed for the decay of Xe-133, since its half-life is only 5.2 days. Krypton-85, with a half-life of $10 \mathrm{yr}$, will not significantly decay within a reasonable period of time. Figure 6 indicates the dose rate at the surface of a shielded cylinder containing a one-year supply of $\mathrm{Kr}-85$. From these data, it is apparent that just under 6 in. of steel will be required to maintain a surface dose rate of $200 \mathrm{mR} / \mathrm{hr}$. The dose rate at $3 \mathrm{~m}$ from the surface will be less than $10 \mathrm{mR} / \mathrm{hr}$.

\section{Summary and Recommendations}

An attempt has been made in this paper to correlate the results of limited information, obtained from several sources, which are applicable to some safety problems anticipated in designs of vented fuel elements. It is obvious that considerable information of a specific nature is required to substantiate the assumptions made. For example, the noble gases and their daughters probably constitute the primary radioactivity concern as far as routine operation and maintenance are concerned. There also appears to be considerable evidence to suggest that a suitably designed sodium-cleanup system employing a cold trap or some form of carbon, such as charcoal, may be an effective means of removing fission products from the primary coolant for vented-fuel-element concepts. The observed deposition and plating of long-lived isotopes on piping surfaces may otherwise constitute a maintenance and replacement problem.

It appears that the radiation problems associated with the vented-fuel-element concept can be solved by the use of inherent and enginecring safeguard devices. A comprehensive and detailed experimental and analytical evaluation will be necessary to determine the adequacy of these safeguard mechanisms for assuring safe operation.

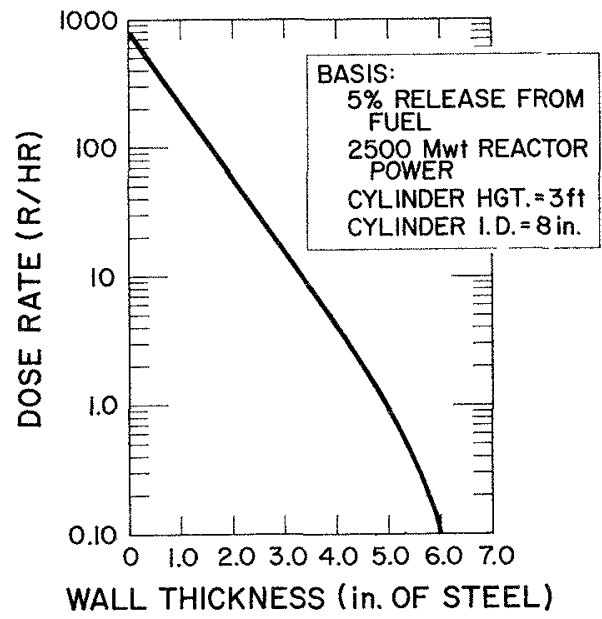

Fro. 6. Dose Rate at Surface of Cylinder Containing a Oneyear Accumulation of $\mathrm{Kr}^{85}$.

\section{REFHRENCES}

1. J. L. Phillips, Sustained Full Power Operation of the Dounreay Fast Reactor, Nucl. Eng. (Aug 1965).

2. W. P. Kunkel et al., High Temperature Experiments on Fission Product Retention in Sodium, NAA-SR-9287.

3. R. S. Hart and C. T. Nelson, Fission Product Retention Characteristics of Sodium at High Temperatures, NAA-SRMemo 8712.

4. R. J. Begley, personal communication, Atomics International.

5. R. A. Hart, Distribution of Fission Product Contamination in the SRE, NAA-SR-6890 (March 1962).

6. A. 1. Hansen, The Effects of Long-term Gperation on $S R E$ Sodium System Components, NAA-SR-11396 (Aug 1965).

\section{Discussion}

Mr. Leitz (GE): I think the results reported are very interesting and a very useful contribution to to the entire ficld of fast reactor technology, and to the vented-fuel concept in particular. It's comforting to know that there is a second line of defense provided by the sodium coolant relative to the escape of fission products to the cover-gas system and perhaps beyond. However, I think this in no way reduces the importance and desirability of having the first line of defense function properly, that is, to have the fission products insofar as possible retained within the fuel itself. There seems to have been a misconception developed in the course of this conference that those who are considering vented-to-coolant fuel are somehow in favor of contaminating the sodiumcoolant system. This is far from being the case. Quite to the contrary, the objective of venting a ceramic fuel to coolant is specifically to release selectively the long-lived fission gases so that the pressure stress on the fuel pin can be reduced; hopefully this will lead to a less frequent failure of fuel pins, which could in turn lead to the contamination of the coolant through a leaching phenomena. We think it's altogether credible that the venting to coolant may, in fact, reduce the burden of fission product contamination in the coolant rather than increasing it. It was this mechanism of leaching of fission products out of fuel that had been irradiated that led finally to the shutting down of the Russian BR-5 reactor, if I understand things correctly, the leaching of cesium-137 to the coolant.

$M r$. Kunkel: I would like to add that we'd be interested in some of the data from Dounreay on the actual activity levels that they have observed in their sodium coolant during their vented fuel operation.

Mr. Porges (ANL): Do I understand that you might exploit this carbonaceous deposit further and possibly use carbonaceous material of some sort as a means of removing fission products from the core in a purifying loop?

Mr. Kunkel: I would not like to say categorically 
that the carbon traps the cesium. It hasn't been sufficiently demonstrated. I merely propose it as a rather interesting observation that perhaps coolantcleanup devices of this sort might be a credible contribution to the remainder of the reactor system.
Mr. Porges: What specific thing are you doing now in this purifying loop?

Mr. Kunkel: We're using cold trap devices primarily. I don't know that there is any intent to use a carbon trap at the present time. 


\title{
Fuel-failure Detection in Sodium-cooled Reactors
}

\author{
K. G. Porges \\ Argonne National Laboratory \\ Argonne, Illinois
}

A discussion of the present status and future possibilities of cladding-failure detection in connection with fast, sodium-cooled reactors may usefully start with a circumspective review of the rationale of fuelfailure monitoring of reactors of different types. For metal-fueled gas- and water-cooled reactors, the basic purposes of providing impervious cladding are the prevention of chemical attack of the coolant on the fuel and prevention of contamination of the coolant by fission products. The design of this cladding inevitably represents some compromise between extreme reliability on one hand, and efficiency as well as cost on the other; thus, occasional cladding failures must be expected to occur with any practical core, and provision should therefore be made to detect these at an early stage of development. Moreover, the high cost of shut-down time of a power reactor justifies the installation of very elaborate systems of failure location. These circumstances led to the development of ever larger cladding-failure monitoring complexes, ${ }^{(1-3)}$ with hundreds of sampling tubes installed at the exit of the core, working into programmed multiple-port valves which direct coolant sample streams into batteries of detectors sensitive to the presence of fission products. In some of these installations, ${ }^{(3)}$ the detector outputs, as well as the reactor instrumentation, are processed by a special digital computer which calculates the probable size of the cladding failure and decides whether to continue operating while keeping a watchful eye on the leaking fuel element, or to scram in order to replace it. In all modern cladding-failure monitors, the occurrence of a leak in a fuel element is inferred from the presence of fission products in the coolant, since even bulky coolant-sampling manifolds are in practice more compatible with various dosign requirements than the alternative, direct attachment of sensors to each element or subassembly, provided in some early reactors.

The advent of sodium cooling puts an entirely differcnt emphasis on the rationale of cladding-failure monitoring. Sodium does not attack the fuel, nor is the increase of the coolant activity which would result from fission product contamination (over and above the $\mathrm{Na}^{24}$ activity, possibly up to $10 \mathrm{mCi} / \mathrm{cm}^{3}$, already present) cause for concern. Thus, fast reac- tors may well have vented fuel elements, as discussed earlier in this conference, while, in the same vein, satisfactory operation of a fast core with a large number of failed elements has been reported. ${ }^{(4)}$

In contrast, it may be pointed out that a number of meltdowns, whose exact cause could not in all cases be ascertained, have occurred in sodium-cooled reactor experiments. It is perhaps not unreasonable to suppose that a severe failure of a fuel element, resulting in emission of debris which might lodge in adjacent flow channels, thus blocking the coolant and leading to a rapid temperature rise, could provide the starting mechanism for a partial meltdown. This would indicate a need for monitoring cladding failure at least for the immediate future of fast reactor technology; moreover, the failure monitor must be very fast-responding and, if possible, provide at least qualitative information regarding the type of failureassuming that the dectector signal can be correlated with the degree of danger to the structure for a given core and fuel. More generally, location of a potentially dangerous failed element, which must be removed, at least within a group of subassemblies will be needed if this type of failure cannot be reduced to insignificance through advances in the art of fuel design.

Turning now to consider the technical means available for implementing these design goals, it is evident that much depends here on the specific design of the reactor, and particularly the freedom of designing the coolant-sampling system afforded by it. As concerns the choice of detection equipment, the most rapid detection system appears to be delayed-neutron monitoring; other fast-responding detection schemes are eliminated by the strong gamma activity of the coolant. Since the delayed-neutron signal varies strongly with both power level and coolant throughput, a confirming detector, based on a different scheme, is desirable. For this purpose, precipitation detectors, in connection with gas-processing equipment which will be discussed below, are most suitable. Pilot systems of these two types are, in fact, installed in all fast reactors currently in operation or under construction. By way of illustration, the system now in operation in EBR-II will be briefly described here, together 
with some possible future additions; this system provides gross monitoring only, as well as a limited amount of qualitative information on the type of failure. As concerns the next generation of fast reactors, more ambitious cladding-detection schemes, which would have at least partial location capability, will be considered, in broad outline, further on.

The EBR-II cladding-failure monitor consists of a fast delayed-neutron detector, FERDL (Fuel Element Rupture Detection Loop), as well as a wire precipitator presently monitoring the gas blanket. The FERDL is installed on a loop which samples the coolant at the heat exchanger, with a maximum loop flow of $100 \mathrm{gpm}$ out of a total of about $8000 \mathrm{gpm}$. At a site well shielded from core neutrons, a graphite stack surrounds a section of this loop, in order to moderate the fast neutrons emitted by the delayed-neutron emitters down to the region where cross sections useful for detection are large. The size of the graphite stack, the location and efficiency of embedded detectors, determine the number of counts per input neutron per detector. Cost of graphite and shielding must be considered versus cost of additional detection channels as well as, of course, available space for either, for a given efficiency. The unit shown in Fig. 1, a 20-cu ft stack with eight detectors, thus represents only one of many possible compromises between the above-mentioned factors. The loop pipe is surrounded by a vacuum jacket inside the stack, shown here before electric heaters were attached and heat insulation packed onto the whole length of exposed pipe. A lead radiation shield of 8-in. minimum thickness was then built around the whole system. As may be seen in the figure, the stack as well as the electromagnetic pump is designed to move several inches with the thermal expansion of the pipe. The neutron detectors and four preamplifiers are accessible through a small port in the shielding, covered in operation with a removable lid, through which cables as well as forced-air ducts emerge.

The signals from the neutron detectors are processed through electronic circuitry of $20 \mathrm{Mc}$ bandwidth, designed to minimize pileup of the numerous small background pulses, and providing from two to four output channels. Some of this output information is temporarily stored in a buffer magnetic-tape memory and displayed by a fast oscillograph in the control room on command of a count-rate discriminator and alarm. The system discriminates very adequately against gamma background; detection efficiency per fast neutron emitted within the 2-liter loop section effectively surveyed approaches $1 \%$. The more important criterion of the minimum extent of clearly distinguishable cladding failures depends, however, on two largely unknown additional factors: sampling efficiency and neutron background.
Considering, first, the sampling efficiency, there are indications that dilution in the coolant, as well as transit time to the detector of a "slug" of fission products, depends very considerably on its point of origin in the core; these uncertainties result in even larger variations in signal strength and duration through the short half-life of most delayed-neutron precursors. Even a precise knowledge of these factors would, moreover, apply only to a reactor with clean sodium, whereas various causes, inter alia cladding leaks, contribute in practice to a steady buildup of tramp uranium through which the signal-to-background ratio of the detector deteriorates. As a concomitant of this loss of discrimination, one must expect an increase of false alarms, or excursions in the count rate which are not clearly identifiable. In this situation, a confirming detector of integrating type, and thus an inherently better signal-to-background ratio as well as slower response time, effectively complements the delayed-neutron unit. Such a detector is now installed in EBR-II, monitoring the gas blanket for volatile fission products; the integrating feature consists of a trap viewed by a gamma detector in which the fission products, previously precipitated onto a traveling wire, are retained.(5)

At present, the signal from this detector is delayed by a time of the order of $20 \mathrm{~min}$, largely due to the unusually large amount of coolant and extensive gasblanket characteristic of EBR-II.

Considering now possible future improvements in this instrumentation, it is evident that a chargecollection fission product detector could yield a much faster return-of the order of a few minutes-if it were located on the sampling loop. The inefficiency of sampling would be more than compensated for by the high concentration of fission products in the coolant, provided that a highly efficient method of gas stripping can be developed. Gas-stripping devices of very good efficiency have been designed recently for this very purpose in connection with water-cooled reactors, and could probably be adapted to function with sodium. ${ }^{(6)}$ For continuous operation over long periods, it will further be necessary to strip the sparging-gas stream of remaining small droplets of sodium, perhaps through a system of mesh and baffle traps which must be designed so as to shed excess sodium, through trapping in lowvapor-pressure liquids, such as $\mathrm{NaK}$ or mercury, or else by sonic precipitation. Alternatively, it may be preferable to design a fast-acting valve which would take a sample of the very portion of the coolant which resulted in an excursion of the delayed-neutron monitor count rate. This sample may then be sparged very thoroughly and the sparging stream analyzed by a number of methods.

A second possible future addition to the cladding- 


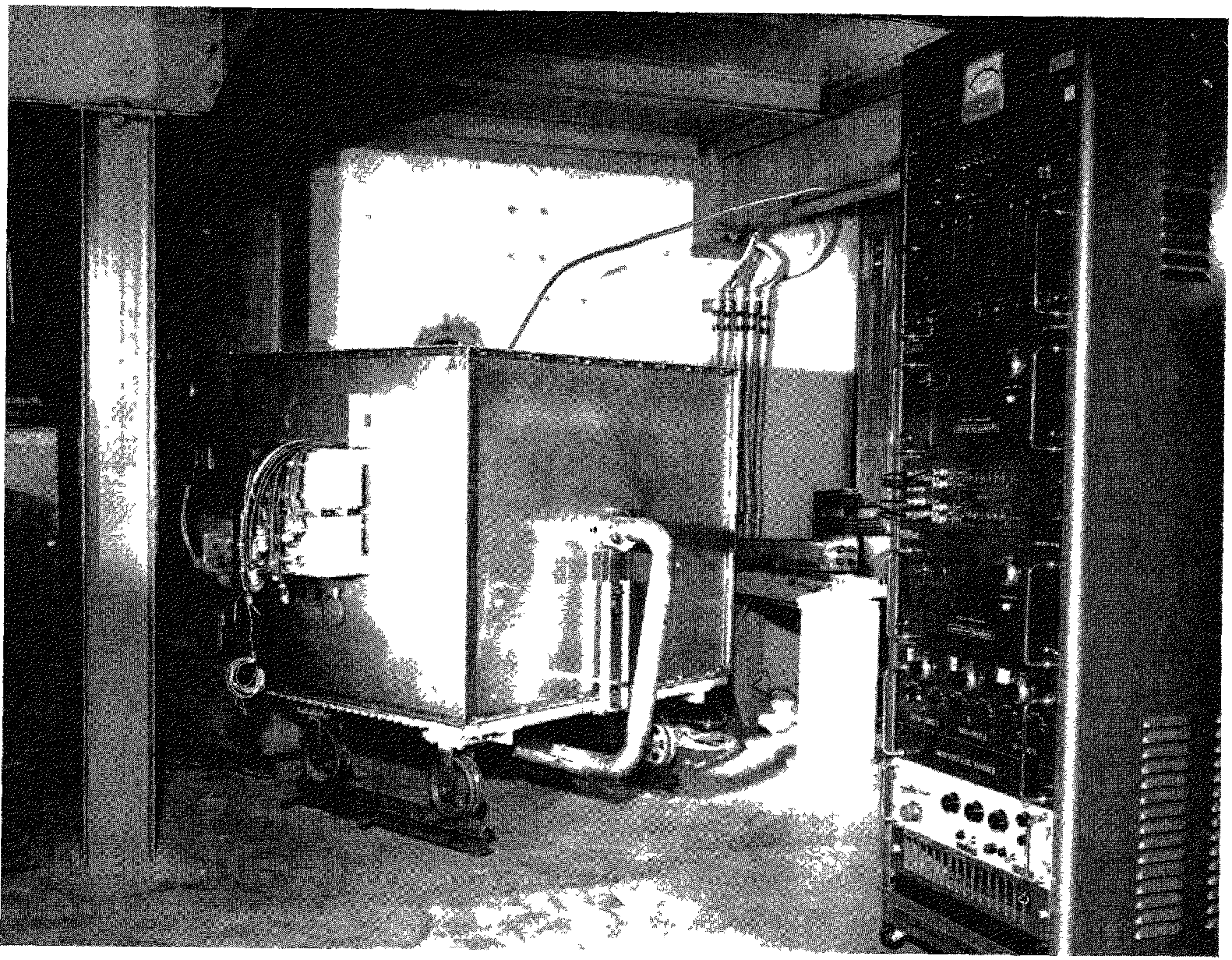

FI(3. 1, FERJ)L Jetector Station, EBR-II.

failure detection facilities of EBR-II would be a monitor installed on the charging machine for the purpose of identifying subassemblies containing failed elements during reactor-unloading operations. In its simplest form, such a monitor would pump gas in contact with the subassembly inside the coffin through a cold trap at $-110^{\circ} \mathrm{C}$ so as to retain fission product xenon. The trap might then be viewed by a gamma detector such as a NaI scintillation spectrometer, adjusted to some strong line in the $\mathrm{Xe}$ fission product component, say the $250-\mathrm{keV}$ line of 9 -hr $\mathrm{Xe}$ 135 (a more sophisticated detector installation would employ a $\mathrm{Li}$ drifted PIN junction detector inside the trap, with correspondingly better resolution, hence discrimination against background). A further refinement would be a duplicate unit downstream from the first trap, reading background only, for purposes of subtraction.

Considering now what can be done to improve claddingmailure detection in the future, certain possible improvements in detection efficiency may be briefly discussed, before turning to the more important question of improvements in sampling and other means of partial location of cladding failures. The slgnal strength of a delayed-neutron monitor can evidently be boosted by the simple means of sampling closer to the core, through a pipe of relatively small diameter, so as to shorten the transit time; by the same token, the section of the sampling loop passing through the detector may be cnlarged, up to a limit determined by considerations of moderating efficiency. Discrimination against random background excursions may be enhanced through installation of a second monitor downstream and signal processing based on delayed coincidence. Finally, the statistics may be considerably improved through the insertion of fission plates or otherwise loading the graphite stacks, up to a multiplication of the order $10-20 .{ }^{(7)}$ 
Although all these stratagems would require considerable development work, even more elaborate tests would be required to develop a sampling system capable of locating the core region in which the cladding failure has occurred. The design of the reactor may allow a certain degree of this location by relatively simple means, from the time of arrival of fission products at a number of detectors sampling the whole core at different points. ${ }^{(8)}$ Improvement of the precision of this method through increasing the number of detectors is, however, limited by the large size of detector stations and requirement of proximity to the core.

A more practical system might be based on sampling manifold and valving station, which would go into action only after a cladding failure signal is established by means as described above, while the reactor runs at half power. The cost of designing such a sampling manifold, which may have to be removable for loading, is possibly beyond a justifiable limit. However, a single, teleseopically retractable, partially articulated sampling tube might be easier to work out. Such a sampling tube could eventually be programmed to sweep across the core to seek a maximum signal and thus to locate the cladding failure.

In principle, the complete location of a faulty subassembly is therefore not necessarily impossible technically. As mentioned earlier, however, it is conceivable that reactors will be allowed to operate with a number of small cladding leaks; on the other hand, a partially debris-blocked channel may, after the initial burst signal, deliver an insufficient amount of coolant for clear identification by local sampling as suggested above. Thus, the practical problem is not necessarily solved by a single sampling system.

This circumstance lends some interest to another scheme of burst-slug location, which has the advantage of detecting largely fresh cladding leaks. In this scheme, fuel elements of EBR-II type would be coded with different gases or solids. Suitable gases (which must be chemically inert and, of course, absent elsewhere in the reactor) might be nitrogen, neon, krypton, and xenon; solids, which may be alloyed into the fuel or onto its surface, might include gold, arsenic, or antimony. ${ }^{(9)}$ Detection would be effected by taking a sample of the coolant on command of the delayedneutron detector, and separating the code element from the sodium. In the case of solids, this would require a certain amount of chemistry, starting with aqueous solution, addition of carrier, etc.; the resultant sample would be gamma counted. For gases, sparging and various methods of detection might be tried; it may be pointed out that in comparison with the coding with solids, coding with gases is a less reliable means of failure indication since relatively little gas may be emitted in a cladding failure near the bottom of a fuel element. Although the number of available codes is necessarily limited, it is clear that the combination of coding and delayed-neutron channel-sampling techniques provides a far more powerful means of establishing and locating a dangerous type of slug burst than either method alone.

At the present time, all these suggestions are necessarily highly conjectural. Whether the cost of implementing some of the measures considered here, or others of a similar nature, is justified by their possible usefulness depends to a large extent on the experience which will become available as a result of operating facilities such as EBR-II, Dounreay, Rhapsodie, BR-5, and others.

Meanwhile, however, some preliminary tests and calculations on a more modest scale might be undertaken, and other concepts developed, which these remarks are hoped to stimulate.

\section{REFERENCES}

1.a. F. Harlen, Nucl. Eng., 158 (April 1958); b. D. Iggulden, Nucl. Power, 6, 71 (May 1961).

2. M. W. Jervis, Nuclear Reactor Instrumentation, Temple Press, London (1961).

3.a. J. Megy, A. Chapelot, J. P. Satre, and J. Tranchant, B.T.S.T. No. 61, 68 (May 1962); b. P. Desneiges and A. Roguin, ibid., 58; c. J. Megy and A. Roguin, CEA-2051 (1961).

4.a. D. Smidt and W. Sommer, F. J. Leitz, F. W. Knight, and D. B. Scherer, see Papers in Sessions II and VI A of this Conference; $b$. Nuclear News, 8, No, 6, 24 (June 1965).

5.a. R. R. Smith and C. B. Doe, ANL 6788 (Jan 1964); b. R. R. Smith and C. B. Doe, ANL-7067 (to be published).

6.a. D. Aliaga-Kelly, Nucl. Power, 77 (July 1959); b. H. Saeufferer, Kerntechnik 4, 453 (Oct 1962).

7. K. G. Porges, internal memorandum, Argonne National Laboratory.

8. A. Paziaud, EPR.R. 050/MFF (Nov 1963).

9. R. Larsen, private communication, Argonne National Laboratory.

\section{Discussion}

Mr. Hannum (LASL): It may be of some interest to say what we did in LAMPRE, the Los Alamos Molten Plutonium Reactor, and what we are planning to do in our next one. First, there is somewhat a different philosophy in that we do not seek to find any small leaks at all, but we are concerned about large leaks for the very reason they may propagate. They certainly will dump molten plutonium into the sodium stream. There were two major failures in LAMPRE-I. These were first detected by gross increases in the gamma activity of the cover gas, and this was correlated with a loss in reactivity due to the loss in the fuel from the reactor. These were located simply by checking to see which fuel element 
didn't have enough reactivity. In the core test facility we added another complication in that we set as a criterion that the detection system must work with vented fuel. In this case, in contrast to Mr. Leitz, we release all our fission products when we vent. So we cannot use fission products at all. Again, we are going to rely on the gross loss of fuel. We use a module can (seven pins to a can) with thermocouples in the outlet of each. If we assume the fuel leaves the high-flux region, that will cause a loss in power in that module, and this will locate our failurc.

Mr. Porges: That would be essentially a system in which each module or subassembly has permanent sensors attached, which brings one back to the old days. For instance, at the Brookhaven pile all the fuel elements were pressurized, and one detected element failure simply on a large board which had hundreds of Bourdon gauges on it.

Mr. Hannum: Quite true, except we use thermocouples.

Mr. Porges: Would this be practical in a large reactor?

Mr. O'Neill (GE) : I'm confused by the remark relating to fragmented release associated with vented fuel. Do you mean with any fuel?

Mr. Porges: Yes, I mean really with any fuel. We want a sure indication that the type of failure is indeed something to worry about and not just a pinhole. We've thought of putting sieves or something into the sample tube, but so far this is really quite an engineering problem.

Mr. O'Neill: You failed to mention one thing that I consider a disadvantage of fuel-failure location systems, and that is the simple fact that you have to place it in the way of refueling. This system is another system in between the core and the refueler, and that is a disadvantage.

Mr. Porges: I had this in mind; that's why I would say that there is really no hope of having a permanent manifold. I would have a remote-control engineer make an articulated tube which can normally be folded out of the way so it does not get in the way of refueling; then you have a gross fuel-element failure monitor which tells you that you have to stop when you get to half power. Of course, this is something which doesn't exist. It's a kind of a Buck Rogers thing.

Mr. O'Neill: I hope he works for G.E.

$M r$. Leitz (GE): I would just like to comment relative to the specifications which you suggested that a vented fuel should attempt to meet to permit the detection or at least no interference with the present planned detection. The delayed time for the inert gases (and I would assume the halides would be held up at least as long) was observed to be about 5 days, so this would seem to be no problem. When you suggested the use of charged-wire methods of observing the escape of fission products, perhaps you were thinking of the detection of 9-hr xenon; may I just observe that 5 days are equivalent to a $10^{4}$ decay period. The noise from that source should be fairly low. You'll have to look for fresh fission product gases as opposed to those that are old relative to 5 days. The discrimination between these two should be no great chore I would think. Is that right?

Mr. Porges: Yes, in sort of an amplification of this, you want to multiply the number of fuel elements in the reactor. This gives you an idea of the attenuation you require for the activity that is being released by the vented pin in order to be able to detect the same activity from a fresh break. If you have a thousand fuel elements, then you want to go 10 half-lives roughly so as to provide a one-to-one signal-to-background ratio. With a 60-sec half-life, this is very manageable.

Mr. Jackson (Risley): What confidence do you have in your fast delayed-neutron monitor? What kind of response time do you have, and what kind of contamination is in the circuit anyway?

Mr. Porges: For a fast neutron emitted in about 2 liters of the pipe that is being viewed by the detectors, one can get of the order of 0.1 to $1 \%$ detection. It depends really how large you want to go: the number of detectors, graphite, etc. One can improve this by having a thin pipe coming up from where the sampling takes place and then have the pipe widen out in the detection volume. One can further improve this by having many, many detectors. Finally, there is the possibility of loading graphite with fission plates and getting neutron multiplication. With all this means one can certainly always get a very good neutron signal. There are, however, two unknown factors in all these detection schemes. One is the mixing. You release a certain slug of contamination into the coolant. How well does this mix and what is the shape of the signal? The second problem is, of course, that the flow pattern of many reactors is clearly unknown. There is also some indication from model tests from Cadarache that if you have a fuel-element failure in certain parts of the reactor it may take considerable time to reach a given sampling tube. It doesn't, perhaps, do much good to improve the neutron-detection signal because as time goes on the neutron background will build up, so that you have to keep on increasing the discriminator level; where this can end nobody knows. It's very difficult at this stage to say what kind of fuel-element failures we can detect with 
any certainty. I'm afraid that doesn't show a very high degree of confidence.

$M r$. Jackson: You suggested one might be able to locate a defective fuel pin by enclosing a pin in some way. We've certainly looked at this in the U.K. and are using it in a limited way. I'm surprised that you think you can use solids. Are you suggesting that the whole of the inside of the fuel pin be coated with a solid? I don't see how else you make it comprehensive. Am I missing a point?

Mr. Porges: This is still a very open question. It would seem that gold at least can be quite nicely bonded on the surface of the fuel pin and then over that would go the cladding. This was suggested by Bob Larsen as an answer to the uncertainty of coating with gas and also because the chemistry is extremely easy and well understood. I'm afraid that's about as far as this idea has gone. The rest of the ideas haven't even proceeded that far.

Mr. Kirn (ANL): Perhaps I could answer your question about the sensitivity of delayed neutrons. There will be a paper at the Washington ANS meeting with respect to a bare-pin experiment in which a bare EBR-II pin was put into the reactor and both the delayed-neutron monitor and the fission gas monitor response was investigated. The background from the sodium was very low. I think the background from what we would call tramp uranium in this system was something like half a count per megawatt, and the signal from a bare pin, which is equivalent to about $50 \mathrm{~cm}$ of exposed surface, was something of the order. of 2 counts per megawatt with the delayed neutron monitor. The fission gas monitor had about a 4 to 1 signal-to-noise ratio, and delay time was something like 20 to 30 minutes. 


\title{
Preliminary Evaluation of a Technique to Study Expulsion of Sodium into Air
}

\author{
I. Chatrak and F. A. Smith \\ Argonne National Laboratory \\ Argonne, Illinois
}

(PRESENTED BY F. A. SMITH)

\section{Introduction}

A technique has been developed to study the expulsion of molten sodium into air. This technique features an impact mechanism presently using a 0.38cal Tefion pellet fired from a revolver and is limited to sodium ejections of about $2 \mathrm{gm}$. The work was initiated as a laboratory effort to determine if small quantity ejections of sodium into air could produce data capable of being extrapolated to full-size systems. If the small-scale experiments are appropriate to define the physical nature of the sodium-air reaction, then experiments on a larger scale with their associated costs in time and effort may be avoided.

The need to conduct these specific sodium-air reaction tests is related to the FARET containment design (see Fig. 1). Specifically, the containment design should accommodate a maximum pressure associated with the sodium-air reaction following explosive ejection of sodium into the air-filled containment.

The FARET Preliminary Safety Analysis Report(1) indicates that argon will be maintained initially within the containment structure. It is believed that at certain times during the life of the plant it will be desirable to provide air above the FARET reactor vessel, both in the cell and the cell annex (see Fig. 1). The most likely time at which one would desire to use air above the reactor vessel is during the regular, normal maintenance of control-rod drives and instrumentation when the reactor is shut down. Since hold-down of the vessel cover is presently not contemplated, one must concede that for containmentdesign purposes all of the sodium in the reactor might react with all of the available oxygen in the cell and/ or cell annex above the vessel. The vault and reactor cavity will normally contain argon, so those volumes need not be considered to contribute to a sodiumoxygen reaction.

\section{Criteria for the Experiments}

In order to obtain engineering data related to possible FARET cell pressure, the following criteria for a small experiment were developed:

(1) All of the sodium available in a model core would be ejected into a model air cell.
(2) The sodium available would be limited to gram-sized quantities.

(3) The sodium temperature should be variable as desired up to $1200^{\circ} \mathrm{F}$.

(4) The sodium in its capsule should be blanketed with an argon atmosphere separate from the air-cell atmosphere to prevent oxidation of the sodium during preheating.

(5) The velocity of sodium ejection should be about $100 \mathrm{ft} / \mathrm{sec}$, corresponding to estimates of a maximum possible rate of ejection of the entire core contents.

(6) Air-cell pressure should be monitored with fast-response instrumentation.

(7) The sequence of sodium-air reactions should be photographed at high speed in an effort to gain some insight into the physical phenomena which accompany the expulsion of sodium into air.

\section{Equipment Developed to Conduct Experiments}

The earlier developed criteria were met with the apparatus shown in Fig. 2.

(1) The volume of the sodium capsule and the volume of available air were chosen to provide an approximate theoretical stoichiometric reaction $\left(\mathrm{Na} / \mathrm{O}_{2}\right.$ mole ratio of 4.0$)$.

(2) 1-2-gm quantities of sodium were contained in a thimble or capsule which is an upright cylinder with an open top and closed bottom.

(3) An external heater consisting of a Hevi Duty Electric Nichrome heater wire in a ceramic circular form was used to heat the sodium sample to temperatures up to $1200^{\circ} \mathrm{F}$.

(4) The sodium-sample capsule rested on a circular-sheet, spring-steel impact diaphragm gasketed to a retainer ring. This provided for an argon seal at the bottom of the Sodium Capsule Barrel. A thin aluminum foil formed its own gasket at the top of the Sodium Capsule Barrel. Small ( $1 / 8$-in.-OD) gas inlet and outlet lines were welded to the Sodium Capsule Barrel to provide for a positive purge of argon during heatup of the sodium sample. The Sodium Capsule Barrel was maintained near at- 


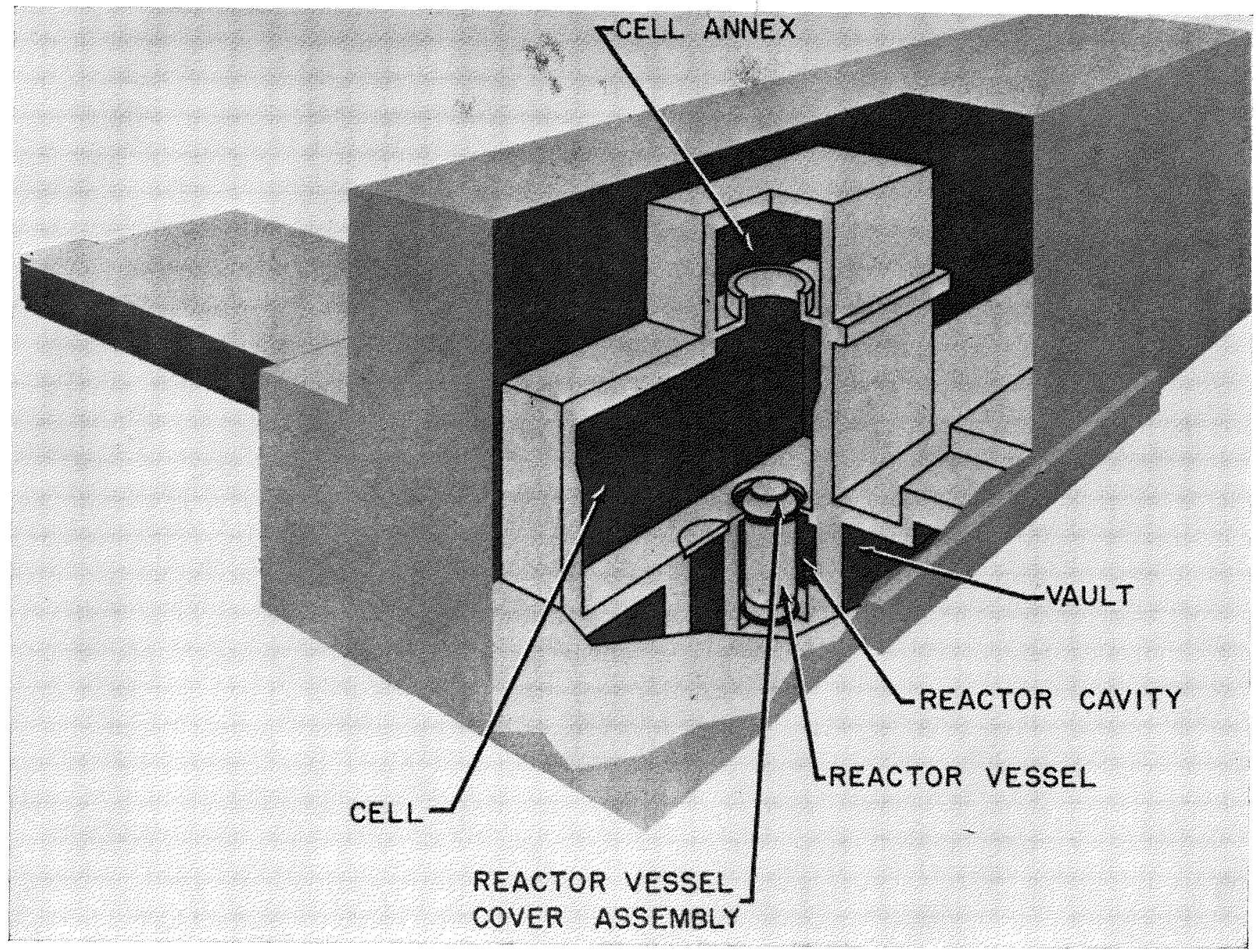

FIG. 1. FARET Containment.

mospheric pressure during heatup, and just before "firing" the gas supply was turned off.

(5) A separate set of preliminary experiments indicated that a 0.38 -cal shell with a charge of 3.2 grains of Hercules "Bullseye" powder was capable of firing a Teflon pellet against the spring-steel diaphragm so that the resultant velocity of the sodium sample was about $100 \mathrm{ft} / \mathrm{sec}$. The velocity of the sodium capsule was measured by a Hewlett Packard Electronic Counter with readout on a 502 Tektronic Dual Beam Scope. Two light screens by Industrial Instruments provided the source of light to photocells which was broken by the flight of the Sodium Capsule.

During the preliminary measurements of velocity, the sodium-containing capsule was permitted to enter the air cell. In subsequent tests, a mechanical stop was used to prevent the sodium capsule from entering the air cell. The capsule remained in the argon firing chamber but on impact against the stop, the molten sodium was expelled into the air chamber. The Sodium Capsule acted as a "cookie cutter" to break the aluminum foil separating the argon atmosphere from the air cell.

(6) Air-cell pressure was measured by a 150.0 psig Consolidated Strain Gauge pressure transducer with a frequency response of $40 \mathrm{kc}$. The pressure signal was recorded on a frequency-modulated Precision Instrument magnetic-tape recorder with a DC response to $10 \mathrm{kc}$.

(7) High-speed photography was employed with a Fastax WF-3 camera with Ektachrome Commercial color film shot at 6000 frames per second.

\section{Experimental Approach}

Initially a large $(20 \mathrm{in}$.) sheet metal cube for the Air Chamber was constructed. The box contained two gasketed Lucite windows opposing each other in the 


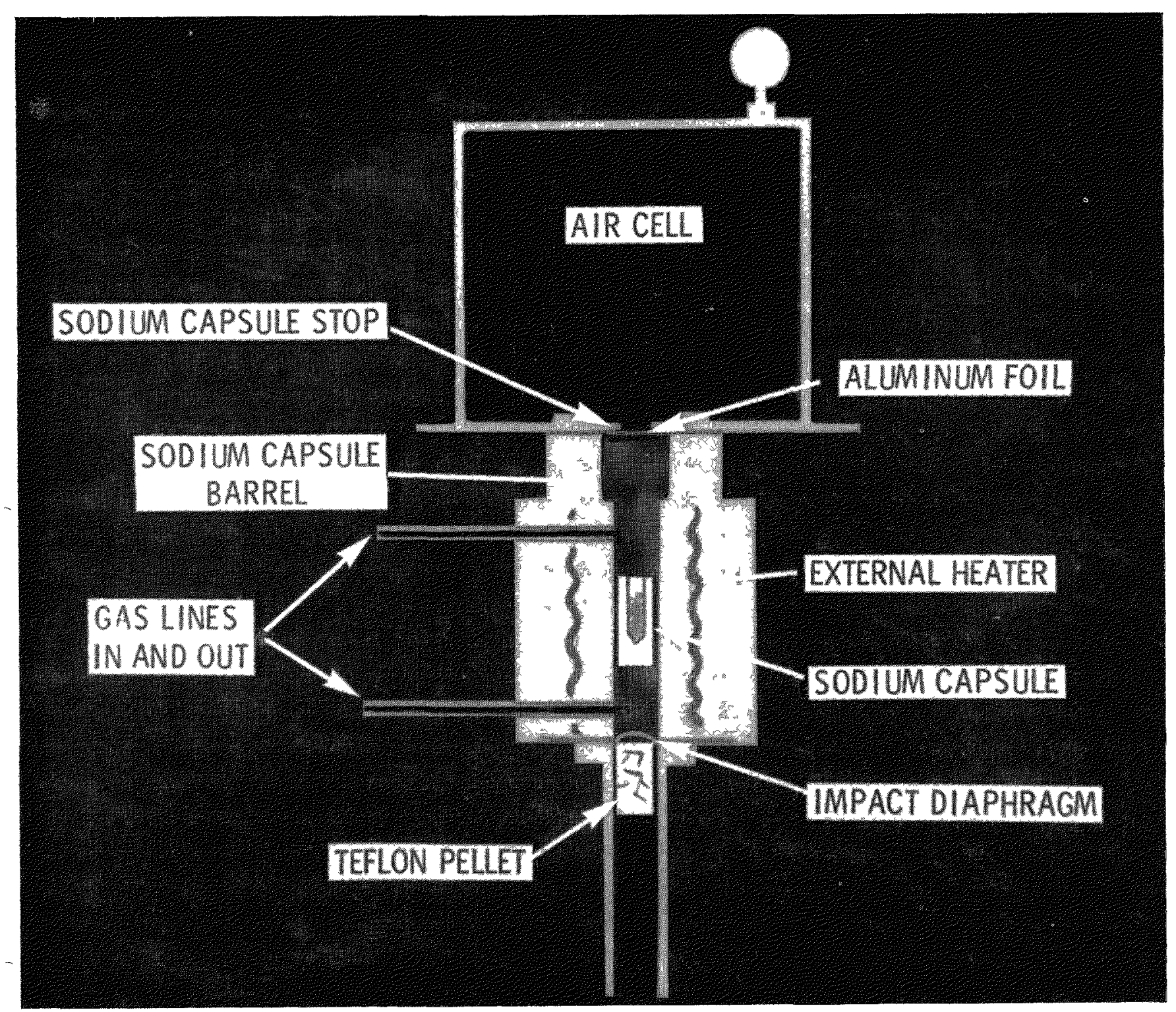

Hrg 2 Experimental Apparatus.

vertical plane of the box. The front window allowed for the high-speed photography and the back window permitted back lighting of high intensity.

To form a comparative basis to observe the expulsion of liquid sodrum into air, water was expelled into air so that water droplet size and the "cone" or "shape" of the ejected water particles might be compared with those of ejected sodium Also, a sodiumargon expulsion was conducted to determine if sodium droplets, dispersed into argon, behave similarly to water droplets dispersed into air.

Next, sodium was expelled into the same 20 -in. air chamber.

These preliminary experiments indicated that molten sodium could successfully be expelled into an air cell and that high-speed photography was very useful in observing the nature of the sodium-air reactions.

Early attempts at pressure measurement indicated a rapidly occurring peak pressure sufficiently high to cause leakage at the gaskets of the cube. Therefore steps were taken to provide for a more positive seal of the air chamber in following studes A typical pressure-time trace is shown in Fig. 3.

Finally, three Iucte cylinders were fabricated for use as air cells These cylunders were all of the same volume but of different $\mathrm{H} / \mathrm{D}$ ) ratios. The volume was chosen to provide an approximate stoichiometric ratio of oxygen to about $2.0 \mathrm{gm}$ of sodium.

Still photographs taken from the film are shown in Figures 4 through 6 . 


\section{Results and Preliminary Conclusions}

Preliminary conclusions and observations are as follows:

(1) It was observed in the water-air test that the particle size of ejected water was smaller than the particle size of ejected sodium into argon. Also it was observed that the "cone" of the water-air

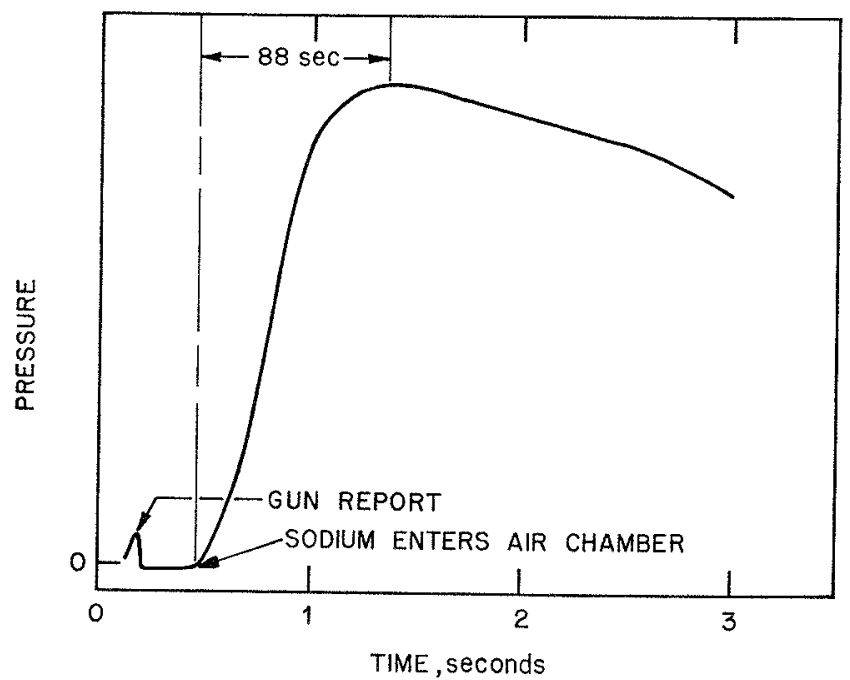

Fix. 3. Typical Pressure-Tme Response.

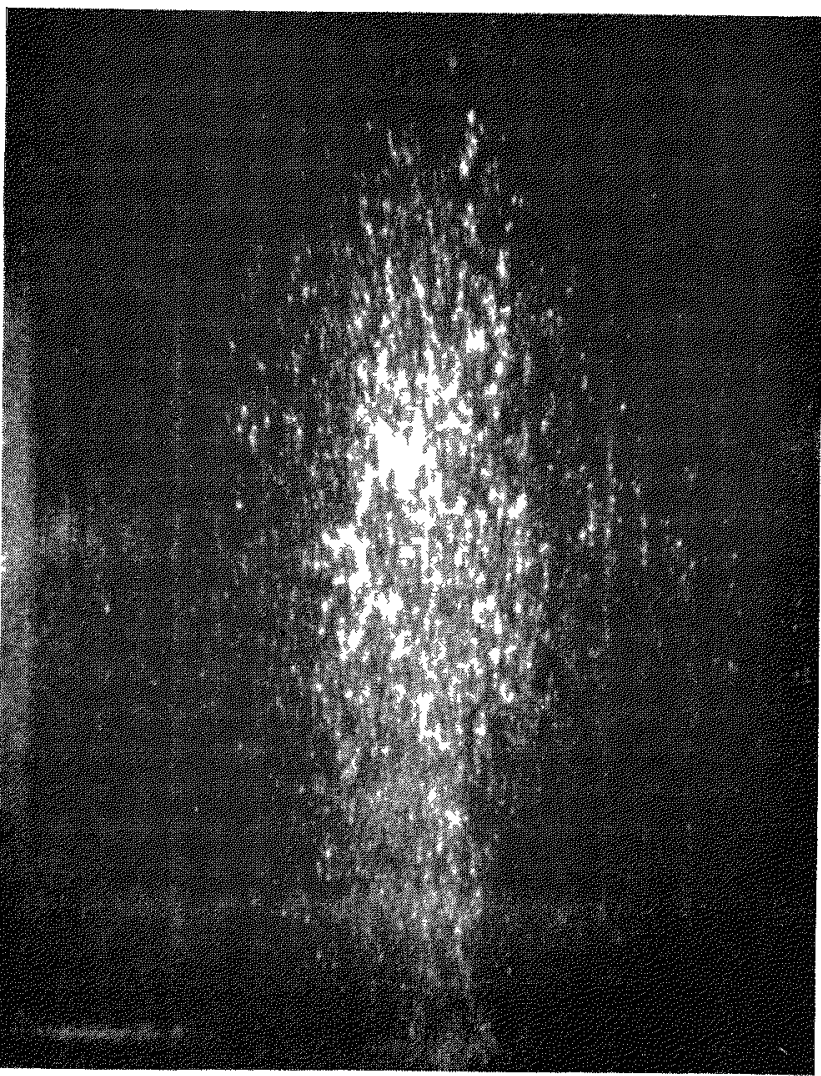

FIG. 4. Water into Air.

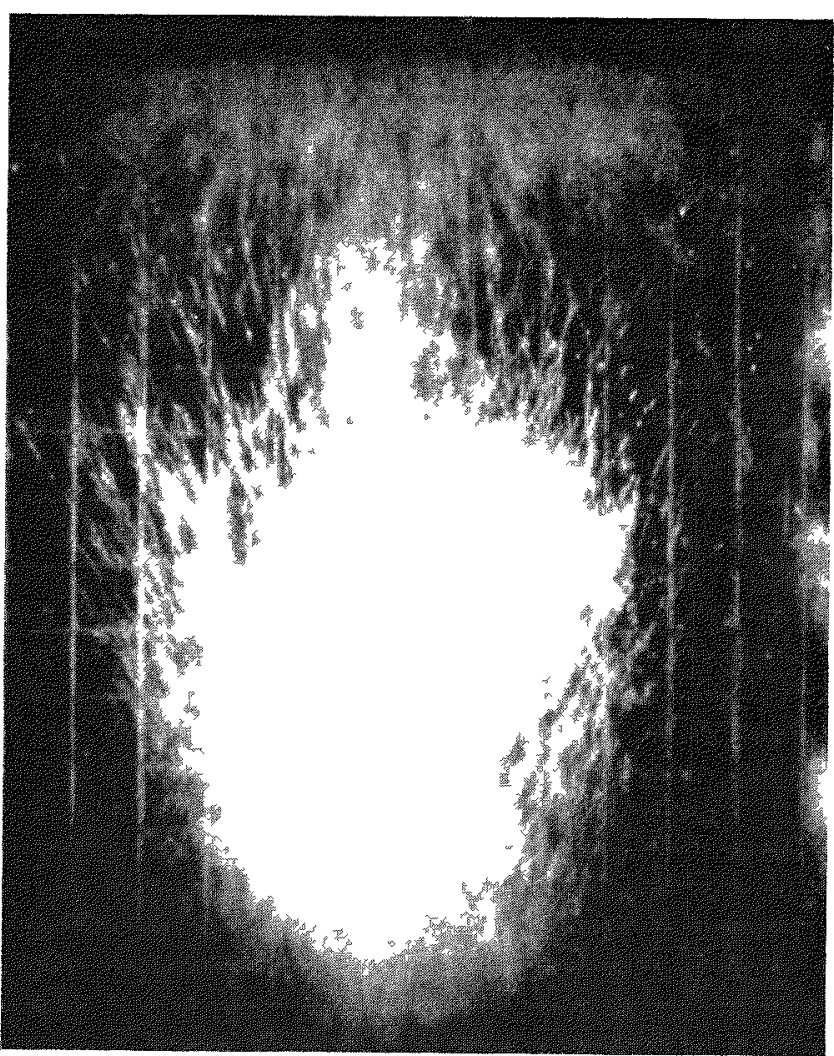

Fig 5 Sodium into Air (2) x $20-\mathrm{m}$ Cube).

expulsion was wider than the "cone" of the sodium-argon reaction. These observations may be due to the fact that the surface tension of sodium is greater than that of water.

(2) It was observed, in the first sodium-air reaction in the $20-1 n$. cube, that all of the sodium did not react instantaneously with the available air supply. There appeared to be an initial sodium reaction with the arr immediately adjacent to the junction of the argon firing chamber with the air chamber. There was a conıiderable fraction of sodium which did not react until this sodium contacted air near the top of the air chamber. There was also evidence that for a finite period of time a small quantity of sodlum did not react with air until the sodiun droplets returned by gravity to the bottom of the air cell.

(3) The preliminary pressure measurements indicated that for constant-volume air cells, the measured pressure was greater for cells of smaller diameter.

\section{Future Work}

We are now starting to perform a statistically designed experiment to show that the geometry of the air container has a significant effect on the peak pres- 


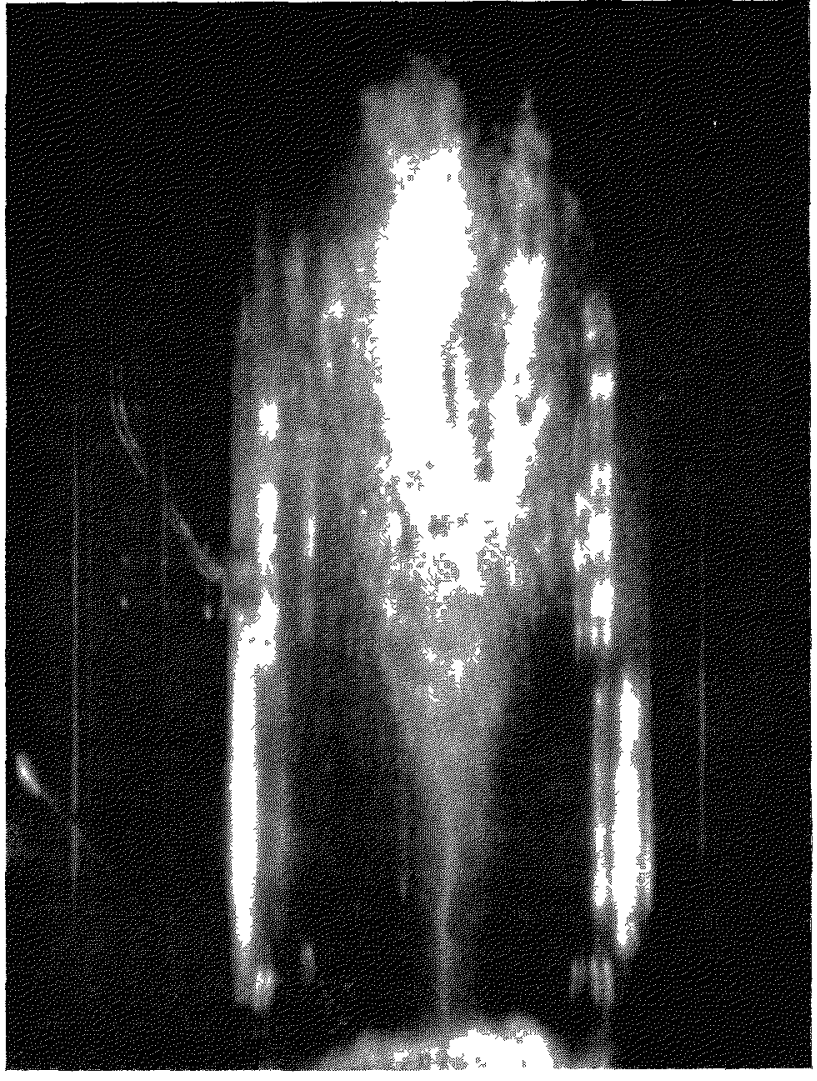

Fig 6 Sodium to Air (Nmall Cylinder)

sure attained. A two-factor analysis with three levels of each factor with replication is being performed. The two factors to be considered initially are sodium weight and $\mathrm{H} / \mathrm{D}$ ratio, with the air volumes being held constant. The sequence of conducting these tests will be randomized so that statistical inference may be drawn from the results.

\section{Acknowledgment}

The authors wish to acknowledge the services of retired ANI. Sgt. Roy Andrews who assisted with the use of the hand gun, Mr. John McBride of the Reactor Engineering Instrument group, Mr. Lester Bohne, Reactor Engineering, and Mr. Daniel Giroux of Information Services who provided the high-speed photography.

\section{REFERENCF}

(1) W. R. Simmons et al, Preluminary Safety Analysts of the Fast Reactor T'est Facalnty $\left(F_{\alpha} 1 R E T\right), \mathrm{ANL}_{1}-6813$, to be published

\section{Discussion}

Mr. Suift (ANL): Don't you think that the larger size of the sodium breakup is due to its higher surface tension? I think sodium has a surface tension of about 200 dynes $/ \mathrm{cm}$, whereas water has about onethird of this value.

Mr. Smath: That may be very true; it's a good point.

Mr. Bernard (AEC): Do you have any intentions of varying the velocity of the injected sodium and the ratio of oxygen to sodium?

$M r$. Smith: Yes. The velocity is very easy to vary just by changing the charge of our 38 shell. We have tried to standardize on accurate loading of shells to get some preliminary data. We're also interested in what happens if there is a depletion of oxygen, particularly with respect to the effect on the ultimate pressure buildup.

$M r$. Liimatainen (ANL): Can your data be compared to the work in an opaque system of Ross Humphreys and if so, are the pressures that you observe in the same ball park?

$M r$. Smith: Our work is so preliminary that I would not like to comment on the absolute accuracy of our pressure measurements as compared with Ross's work. The asset of our system is that we can study many variables rather quickly because of the small size.

Mr. Dietrich (Combustion Engineering): How do the measured pressures compare with what might be expected from approximately complete reaction?

Mr. Charak (ANL): All one can do, as far as I know, is to compute so-called theoretical maximum pressures. To my knowledge there is little if any data on the reaction kinetics between sodium and oxygen. The theoretical maximum pressure computed for a $4: 1$ mole ratio of sodium to oxygen yields pressures of the order of 100-150 Ib, depending upon atmospheric pressure. However, peak pressures occur at different mole ratios than one might expect from the basic stoichiometry. The pressures that we're getting are of the order of 20 to $80 \mathrm{lb}$. 


\section{Session VIII-B \\ October 14, 1965}

\section{Mostly Sodium Boiling}

Chairman: P. A. Lotres

Argonne National Laboratory

Secretary: R. M. Singer

Argonne National Laboratory 


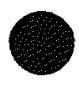

- 


\title{
Effect of Fission Gas Bubbles on the Response of Molten Fuel Reactors to Fast Transients*
}

\author{
B. M. CaRmirhael \\ Los _llamos Scientific Laboratory \\ Los Alamos, New Mexico
}

Molten plutonium as a fast reactor fucl has been under development at Los Alamos for a number of years. The low-melting-point $\left(411^{\circ} \mathrm{C}\right)$ alloy, $\mathrm{Pu}-\mathrm{Ke}$, was tested in LAMPRE I (Los Alamos Molten Plutonium Reactor Experiment, No. I) to about $0.5 \%$ burnup. ${ }^{(1)}$ More recently, the ternary alloy $\mathrm{Pu}-\mathrm{Co}-\mathrm{Ce}$ is being tested to higher burnups in an in-pile loop. ${ }^{(2)}$ The latter alloy has the advantage that the plutonium fraction may be varied over wide limits without significant change of the melting point $\left(\sim 450^{\circ} \mathrm{C}\right)$. A core utilizing $\mathrm{Pu}-\mathrm{Co}-\mathrm{Ce}$ is in an advanced design stage in preparation for a test in the Fast Reactor Core Test Facility (FRCTF). ${ }^{(3,4)}$

Interest in molten fuels has, in part, been stimulated by the safety advantages offered by these fuels in fast reactor applications. These advantages derive from the large thermal expansion coefficients available in liquid metals. Linear expansion coefficients for Pu-Fe and $\mathrm{Pu}-\mathrm{Co}-\mathrm{Ce}$ are compared in Table 1.

The low expansion coefficient of tantalum, included in Table 1, plays an important role in amplifying the expansion effects occurring in tantalum capsules containing molten fuel. The type of fuel element under consideration is illustrated in Fig. 1. As the fuel is heated, the liquid fuel expands into the space provided above the fuel for the collection of fission gases. Since the tantalum expansion coefficient is less by about a factor of four than that of the fucl, the radial expansion of the capsule is small, and nearly the full volumetric expansion of the fuel is effective in increasing the core height, thus reducing reactivity. $t$ Hence, the fuel temperature coefficient of reactivity is expected to be about three times larger for the liquid fuel than it would be if the fuel were in the solid state. The actual

\footnotetext{
* Work done under the auspices of the U. S. Atomic Energy Commission.

$\dagger$ The effective expansion coefficient is thus

$$
\alpha_{\mathrm{eff}}=\frac{1}{H} \frac{\partial H}{\partial T}=3 \alpha_{\mathrm{Pu}}-2 \alpha_{\mathrm{Ta}}
$$

where $H$ is an increment of fuel height, and $\alpha_{P_{u}}$ and $\alpha_{\mathrm{T}_{c t}}$ are the linear expansion coefficients of fuel and tantalum, respectively.
}

isothermal temperature coefficients measured in LAMPRE are given in Table 2. (The constituent coefficients for the fuel and sodium were determined by assuming the total coefficient is resolvable into three components: fuel, sodium, and "structure.")

The effectiveness of the expansion coefficients in controlling transients in clean unirradiated fuel is illustrated in Fig. 2. The parameters plotted are the peak temperature, peak pressure, and energy release in the first burst caused by each given reactivity insertion rate in a LAMPRE-type core. In the analysis, it was assumed that the fuel-capsule walls and bottoms are fixed and rigid, and heat transfer from the fuel was neglected. For sufficiently rapid heating of the fuel, its expansion is inertially retarded, so that a pressure transient is induced. The pressure rise is at a maximum at the lower fixed end of the capsules and decreases to zero at the upper free surface of the fuel. The mechanisms are well adapted to the analytic methods for treating transients in fuel elements developed by Daane and Jens. ${ }^{(5)}$

The aim in the analysis of the clean-fuel transients was to investigate what range of reactivity insertion rates might lead to damage of the fuel eapsule. In the results shown in Fig. 2, it is seen that the temperature rises $\left(\leq 10^{\circ} \mathrm{C}\right)$ for reactivity insertion rates up to $\$ 100 /$ see should have no adverse effects. However, the threshold for capsule damage due to the pressure rise might be expected to lie in the vicinity of the 7500 psi associated with the $\$ 100 /$ sec ramp.

It would be expected that the transients shown in Fig. 2 would be significantly altered in irradiated fuel containing fission product gas bubbles. Long-term reactivity effects due to these bubbles were observed in LAMPRE, and the presence of the bubbles in the fuel was verified directly in postmortem capsule examination.

The history of reactivity losses occurring during the initial runs of a LAMIPRE core is plotted in Fig. 3. The record shows some perturbations due to power level changes and reactor startups, but the overall trend is caused by the accumulation of fission gas bubbles. At the end of the irradiation, the total reactivity loss was about $\$ 10$, corresponding to $4.7 \mathrm{v} / \mathrm{o}$ 
bubbles in the fuel. No short-term power instabilities due to the bubbles were observed.

Photographs taken of sectioned fuel samples after the irradiation are displayed in Fig. 4. The upper photograph shows bubbles collected along the wall of a capsule, and the lower photograph shows bubbles in the meniscus at the upper surface of the fuel. No bubbles are observed in the interior of the fuel. The maximum bubble size is about one millimeter in diameter, but the bubbles average about $0.1 \mathrm{~mm}$ in diameter.

TABLE 1. LINEAR Expansion COEFTCHENTS

\begin{tabular}{l|c|c|c}
\hline Material & Temperature & State & $\begin{array}{c}\text { Linear } \\
\text { Expansion } \\
\text { Coefficient } \\
\left(\times 10^{6}\right)\end{array}$ \\
\hline $\mathrm{Pu}-\mathrm{Fe}$ & $500^{\circ} \mathrm{C}$ & liquid & 28.8 \\
$\mathrm{Pu}-\mathrm{Co}-\mathrm{Ce}$ & & liquid & 28.7 \\
$\mathrm{Ta}$ & & solid & 6.5 \\
\hline
\end{tabular}

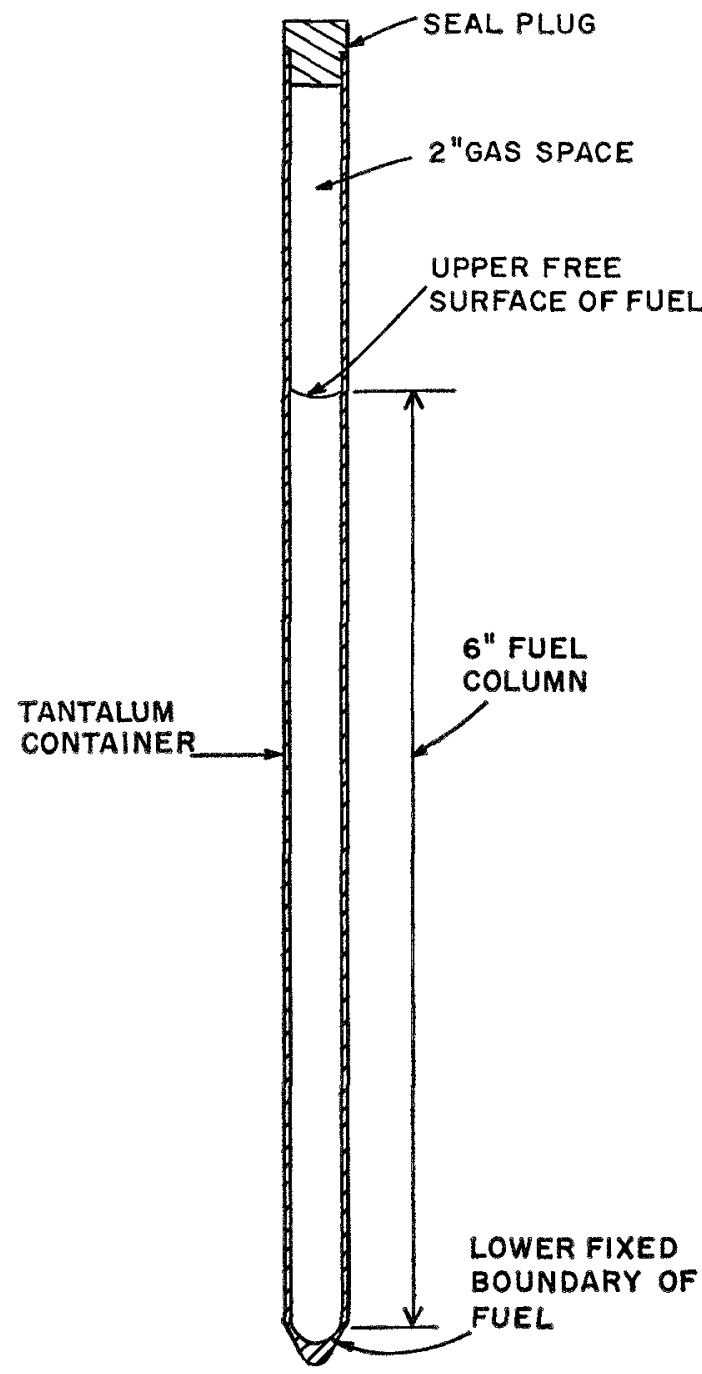

Fra. 1. Molten Plutonium Fuel Element.
TABLE 2. LAMPRE Temperature COEFforents

\begin{tabular}{c|c|c|c|c|c}
\hline $\begin{array}{c}\text { Tempera- } \\
\text { ture, }{ }^{\circ} \mathrm{C}\end{array}$ & Fuel State & $\begin{array}{c}\text { Sodium } \\
\text { Coolant }\end{array}$ & $\begin{array}{c}\text { Total Co- } \\
\text { efficient } \\
\left(-\mathrm{c} /{ }^{\circ} \mathrm{C}\right)\end{array}$ & $\begin{array}{c}\text { Fuel Co- } \\
\text { efficient } \\
\left(-\mathrm{c} /{ }^{\circ} \mathrm{C}\right)\end{array}$ & $\begin{array}{c}\text { Sodium } \\
\text { Coeff- } \\
\text { cient } \\
\left(-\mathrm{c} /{ }^{\circ} \mathrm{C}\right)\end{array}$ \\
\hline 80 & solid & out & 1.1 & 0.8 & \\
160 & solid & in & 1.3 & 0.5 & 0.5 \\
480 & liquid & in & 2.9 & 2.0 & 0.5 \\
\hline
\end{tabular}

To evaluate the effects of the bubbles on fast transients, a first approximation to the equation of state of the medium consisting of a mixture of liquid fuel and bubbles is considered. For a small volume, $V_{0}$, of the mixture, the ehange in volume is the sum of changes in the liquid volume $V_{0 l}$ and gas volume $V_{0 g}$ :

$\Delta V=-\frac{V_{0 g}}{\gamma_{c} P_{0}} p(x, t)+V_{0 l} \alpha T(x, t)-V_{0 l} K_{l} p(x, t)$.

The adiabatic case is considered only, i.e., it is assumed no heat is lost from the liquid, either to the bubbles or to the external environment of the capsule. This will tend to overestimate the effects of the bubbles, since heating of the gas in the bubbles is an effect opposing the compression of the bubbles. In Eq. (1), $p(x, t)$ and $T(x, t)$ are the pressure and temperature rises at a point $x$ measured from the bottom of the capsule; $\alpha$ and $K_{l}$ are the volume expansion coefficient and compressibility of the liquid; $\gamma_{c}$ is the ratio of specific heats in the gas.

During the course of an irradiation of the fuel, the steady-state pressure $P_{0}$ increases continuously in the sealed capsules as the inventory of fission gases increases. A pressure of $500 \mathrm{psi}$ (corresponding to the maximum pressure observed in LAMPRE capsules) was assumed in the calculations for purposes of illustration.

In terms of the fuel displacement $E(x, t)$, Eq. (1) becomes

$$
p(x, t)=\frac{1}{K}\left[\alpha T(x, t)-\frac{\partial E(x, t)}{\partial x}\right],
$$

where $K$, the total compressibility, is

$$
K=\frac{\beta_{0 g}}{\gamma_{c} I_{0}}+K_{l}
$$

The liquid compressibility is about 1 part in $10^{7}$ per psi of applied pressure. For a volume fraction of gas in the fuel, $\beta_{0 g}$, of 0.047 , taken from LAMIPRE experience, compressibility of about one part in $10^{4}$ is found from the first term in Eq. (3). Thus, the bubbles, as expected, enormously increase the compressibility of the fuel.

The hydrodynamic wave equation for the fuel mixture is 


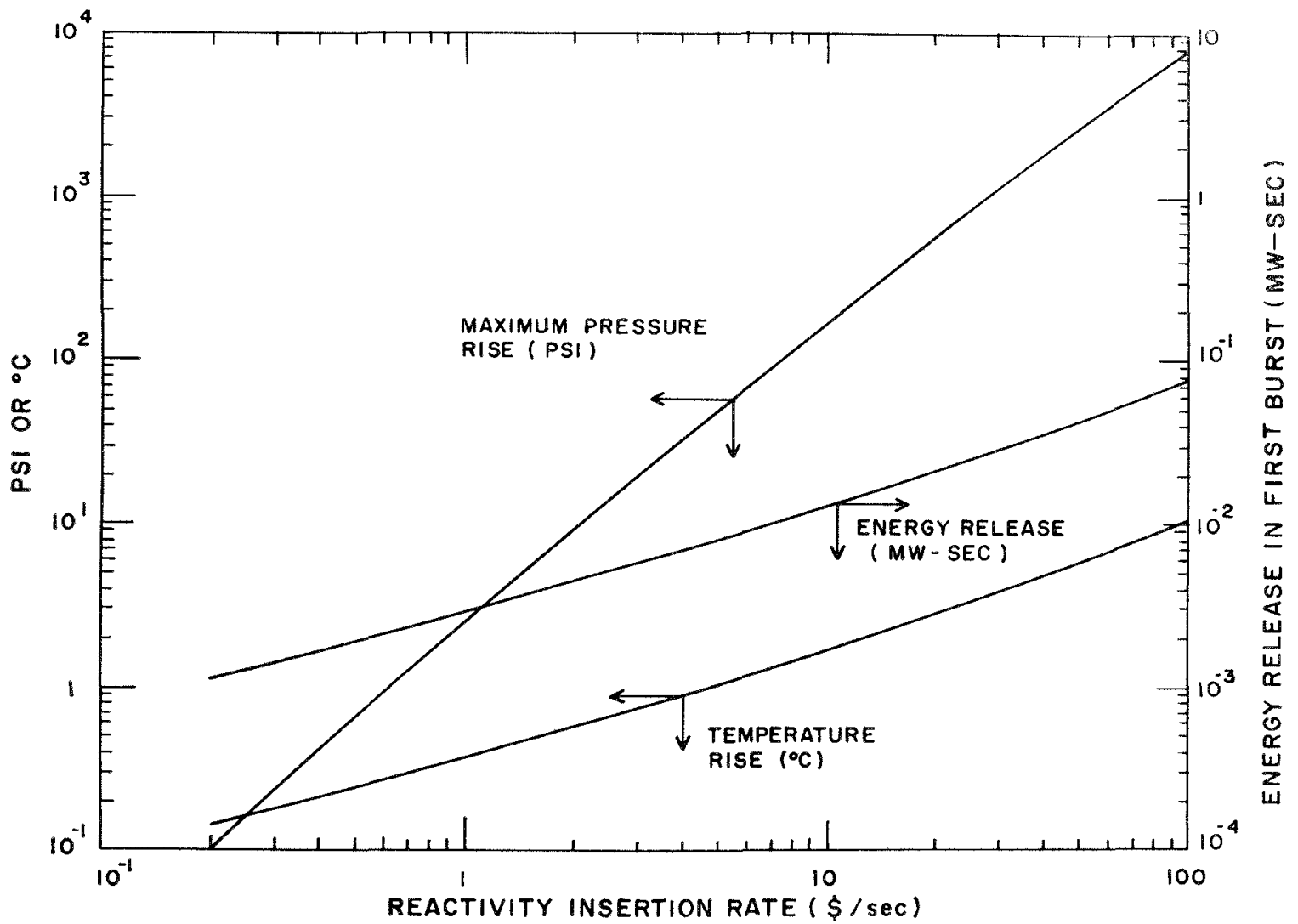

Frg. 2. Transients in Unirradiated Capsules.

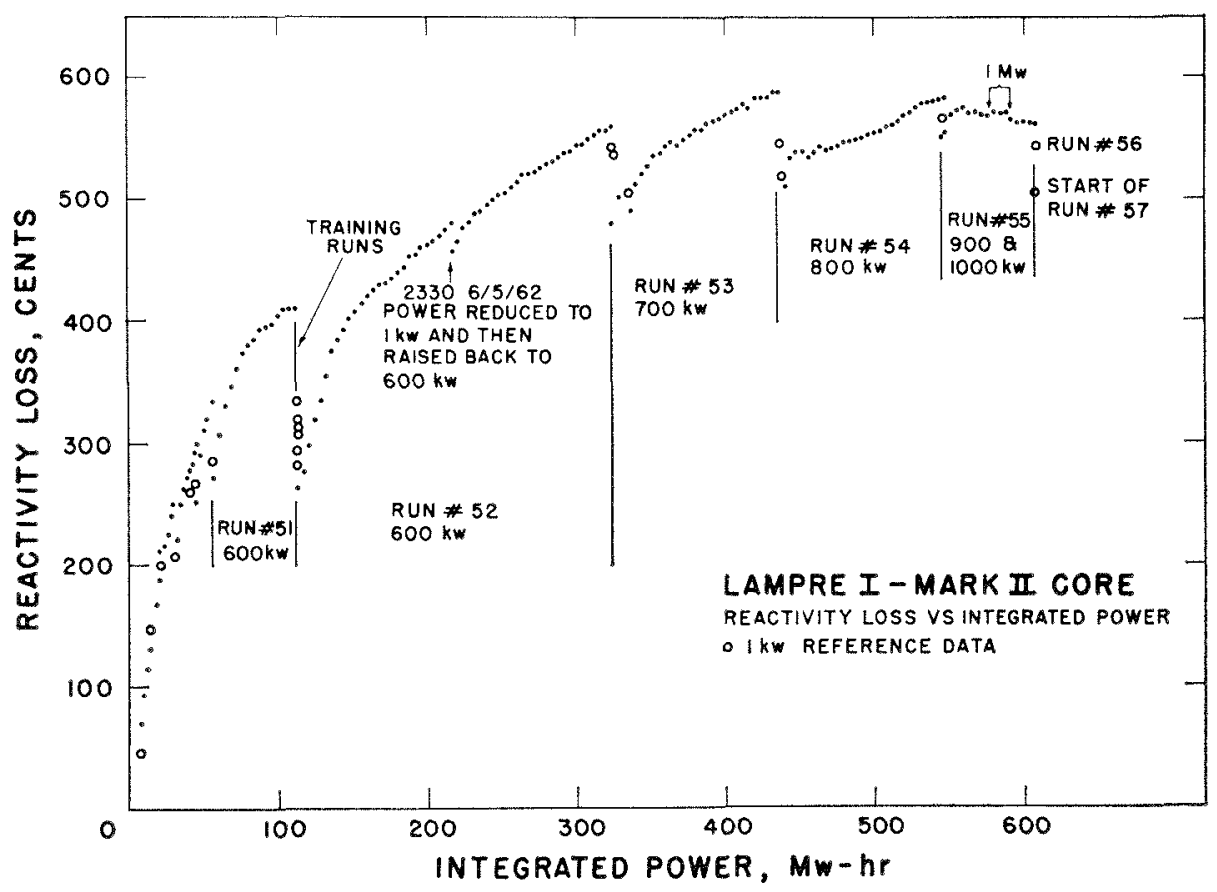

FIG. 3. LAMPRE I: Reactivity Loss vs Integrated Power. 


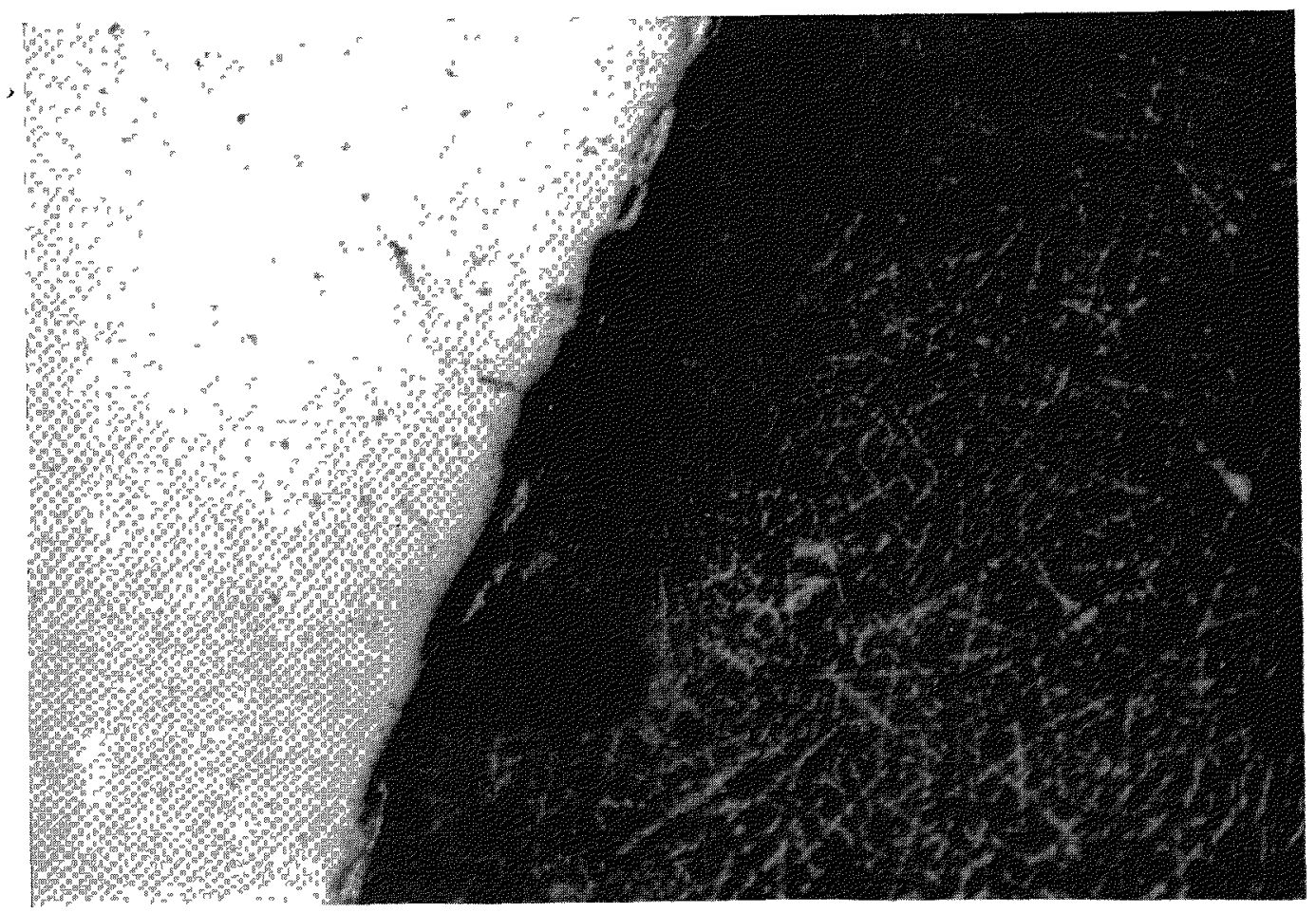

\section{Below Meniscus}

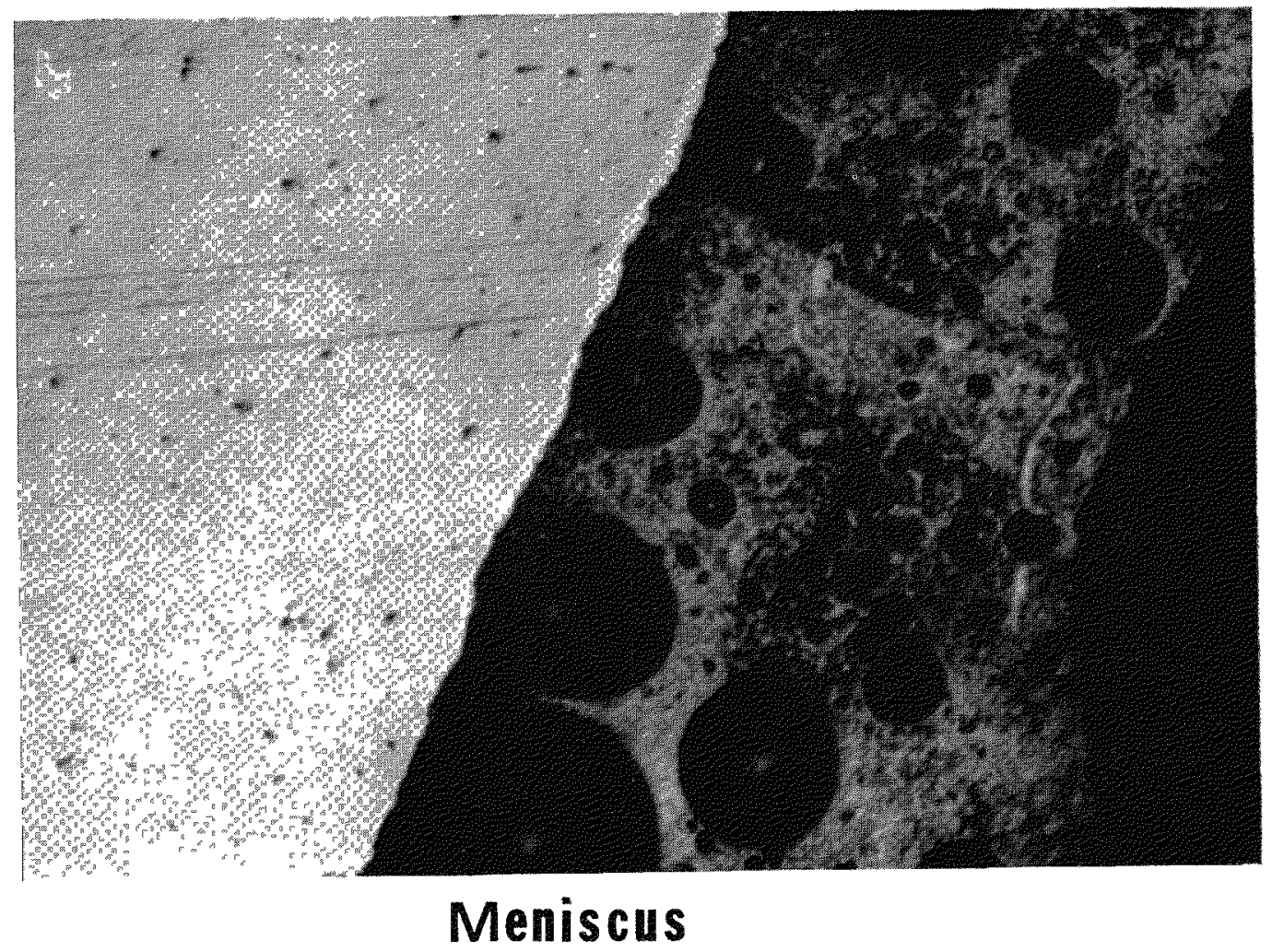

FIG. 4. Photomicrographs of Fission Gas Bubbles. 


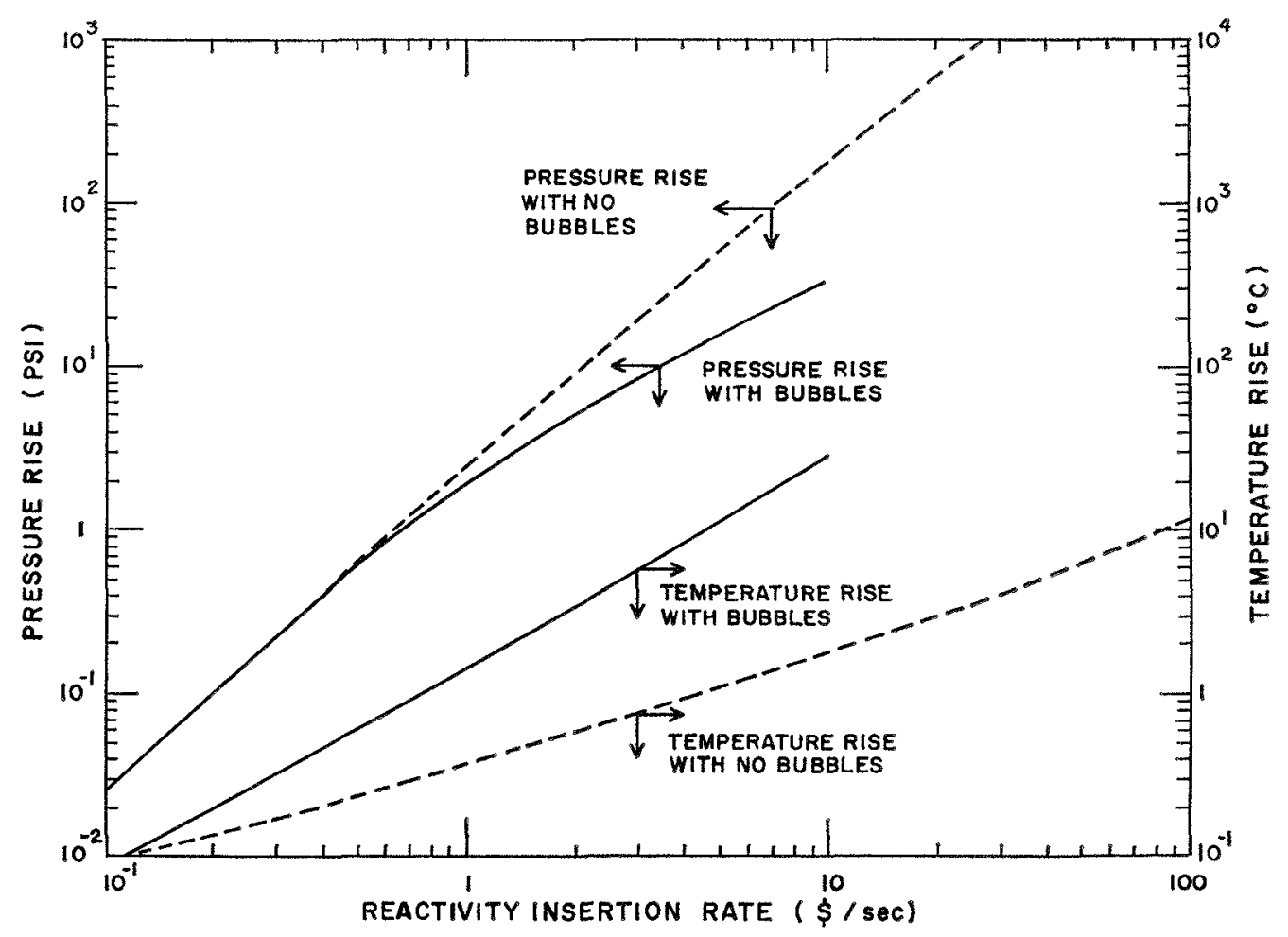

FIG. 5. Transients in Irradiated Capsules.

$$
\ddot{E}(x, t)=c^{2}\left[\frac{\partial^{2} E(x, t)}{\partial x^{2}}-\alpha \frac{\partial T(x, t)}{\partial x}\right],
$$

where the acoustie speed is

$$
c=\sqrt{\frac{1}{\rho_{0} K}}=\sqrt{\frac{\gamma_{c} P_{0}}{\rho_{0} \beta_{0_{g}}}} .
$$

The acoustic speed from Eq. (5) is $7100 \mathrm{~cm} / \mathrm{sec}$, slower by about a factor of 5 than the speed in air. This slow speed is characteristic of a medium possessing both a high liquid metal density, $\rho_{0}=16 \mathrm{~g} / \mathrm{cc}$, and a high compressibility.

Simultaneous solutions of Eqs. (2) and (4) and the neutron kinetic equations are depieted in the transients of Fig. 5. Because of the retardation of the fuel expansion due to the bubble compression, transients are prolonged, and the energy releases and temperature rises are larger. However, in the range of reactivity insertion rates up to $\$ 100 /$ sec, no damage to capsules would be expected because of the temperature rise.

On the other hand, the pressure rise is strongly inhibited by the bubbles. The bubbles, in effect, provide a protective cushion or internal shock absorber along the walls of the capsules. Thus, it is expected that irradiated capsules will withstand larger reactivity insertion rates than unirradiated capsules. It will be noted in Fig. 5 that transients for the bubble case are extended only up to a reactivity insertion rate of $\$ 10 /$ sec. For larger rates where the pressure rise $p(x, t)$ becomes significant compared to the initial pressure $P_{0}$, $\mathrm{Eq}$. (3) is no longer a valid description of the compressibility. Both the compressibility and the acoustic speed vary during the course of such larger transients, and Eqs. (2) and (4) accordingly become nonlinear and intractable to the analytic methods used.

It may also be noted that the flexing of the capsule walls during transients in unirradiated fuel will produce effects similar, but smaller in magnitude, to the bubble effects. Wall flexing will lead to an increase in the effective compressibility or a decrease in the effective acoustic speed. Thus, the pressure rises for clean fuel given in Fig. 2 are overestimated, since they are based upon the assumption of rigid walls. Similar to the bubble case, the decrease in pressure rises is accompanied by an increase in temperature rises and energy releases.

\section{RFFERENCES}

1. LAMPRE I Operations Report, in preparation.

2. L. D. Kirkbride, Molten Plutonium Alloys as Fast Reactor Fuels, see paper in Session VI,A of this Conference.

3. W. H. Hannum, Nuclear Design of MPBE, see paper in Session VII,B of this Conference.

4. Fast Reactor Core Test Facility Safety Analysis Report, LA-2735 (Aug. 30, 1962).

5. R. A. Daane and W. H. Jens, Fast Reactor Accident Scaling Laws, NDA-14-84 (May 18, 1955). 


\section{Discussion}

Mr. Sesonske (Purdue): I missed a point whether or not you concluded that the collapse of the bubble did not inhibit linear expansion of the fuel because of the compressibility effects.

Mr. Carmichael: The compressibility of the fuel mixture with bubbles changes in character because it's much easier to compress the bubbles. On the other hand, the energy releases and temperature rises are much greater with bubbles present since the transient is prolonged and expansion is retarded.

Mr. Morelle (Belgo-Nucleaire): What type of model did you use for calculating the speed of sound in the mixture of liquid fuel containing gas bubbles?

Mr. Carmichael: In the model it was assumed that the bubbles were spread homogeneously. 


\title{
The Development and Growth of In-core Voids Due to Boiling during Fast Reactor Transients*
}

\author{
R. C. Foyes, H. Lurie, and A. A. Jarrett \\ Atomics International \\ ('anoga Park, California
}

(PRESENTED BY R. C. NOYES)

An improved understanding of some of the characteristics of transient boiling in fast reactors has been achieved as the result of recent studies. Particularly significant among these characteristics are the following:

1) Voiding in a reactor channel starts near the upper boundary of the core and grows axially along the fuel channel.

2) Vapor production is so rapid that transient selfpressurization of the fuel channel oceurs.

3) The resulting two-phase flow is strongly unstable hydrodynamically.

These findings have been developed from experiments and from studies with two digital codes developed at Atomies International for this purpose. The actual experiments and codes are described in detail elsewhere; $;^{(1,2)}$ selected results of some of the digital studies and experiments are presented here to support and explain our main conclusions about the nature of transient boiling in fast reactors. Other significant results of the studies are developed in the discussion.

\section{A. Single-channel Boiling}

Steady-state boiling under forced-convection conditions has been studied and characterized by many investigators. Figure 1 shows the general picture for both metals and nonmetals that has been developed to explain many of the observed phenomena. The vapor first appears as small bubbles attached to the heated surface in a region where the bulk of the fluid is subcooled. As heat is added downstream and the bulk coolant temperature reaches saturation, large vapor agglomerations appear; then, an annular-flow region develops; finally, most of the liquid is evaporated and the heated walls dry out. This type of twophase flow is characterized by constant mass flow rate everywhere in the channel and, in downstream regions, by relatively large differentials between the phase velocities (slip). Slip has an important influence on two-phase void fraction and pressure drop.

* Work performed under Contract AT(11-1)GEN-8 for the U. S. Atomic Energy Commission.
Recent measurements of these quantities made in a vertical, annular test section at AI are shown in Figures 2,3 , and 4 . Note that steady-state slip ratios for sodium can be quite high.

Much steady-state heat transfer data can be applied directly to transients with confidence; however, the application of steady-state hydrodynamic data must be approached with considerable caution. It is quite certain that the condition of constant mass flow rate with position does not hold for transients and that the interpretation of slip ratios and void fractions for transients must be considerably different than for steady-state analysis.

The general picture of void configuration during transients developed from our studies is shown in Fig. $5 \mathrm{~B}$. The roid fraction in the vapor region is near $100 \%$, and this region is confined by two liquid slugs which are subcooled except in a narrow region near the void. The void grows primarily by pushing the liquid phase out of the channel rather than by evaporating the liquid. Because axial acceleration is constant for the incompressible liquid, pressure in the liquid regions increases linearly from the channel ends to the boundary of the void. The temperature distribution in the coolant depends on the history of the transient; however, there is always some sort of peak in the void region. Because vapor pressure is related exponentially to temperature, the vapor pressure curves corresponding to the bulk coolant temperature drop more rapidly than the total pressure curves as one moves away from the void region. The relation between the vapor pressure and total pressure curves is such that the liquid becomes more subcooled the farther it is from the void.

Other pictures of the void pattern during these transients might be postulated, and it is worth discussing these since the model used for analysis depends to a certain extent on basic assumptions about the vapor structure. A series of quasi-steady-state conditions could be considered as a possible description of transient boiling (see Fig. 5A). This approach is grossly in error, even for slow transients, because of 

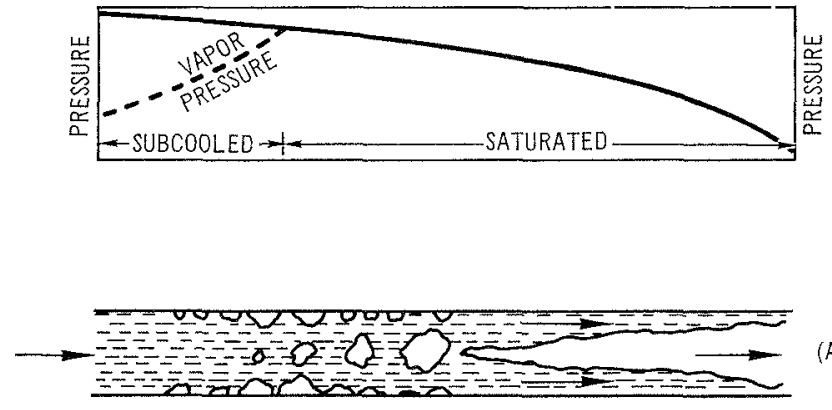

FIG. 1. Stable Steady-state Boiling.

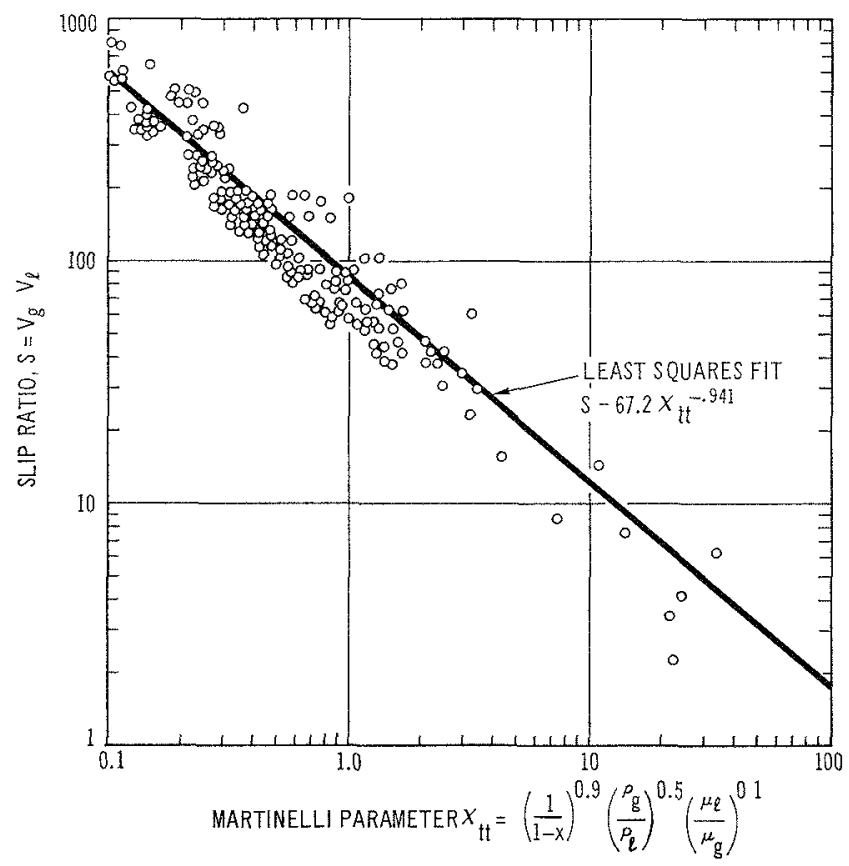

Fra. 2. Measurements of Slip Ratio for Sodium.

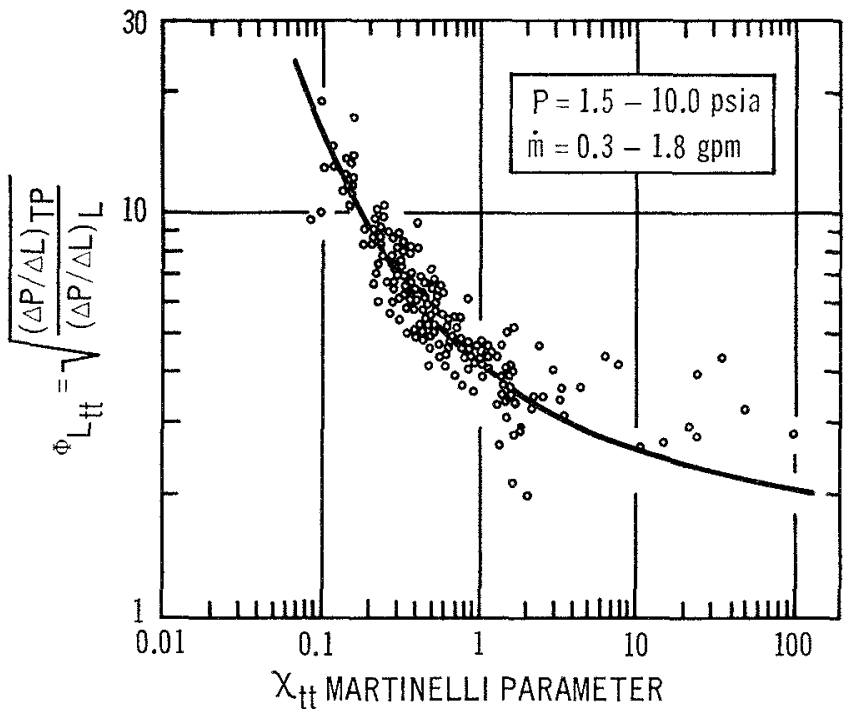

Fra. 3. Two-phase Pressure Drop for Vertical Upflow of Sodium.

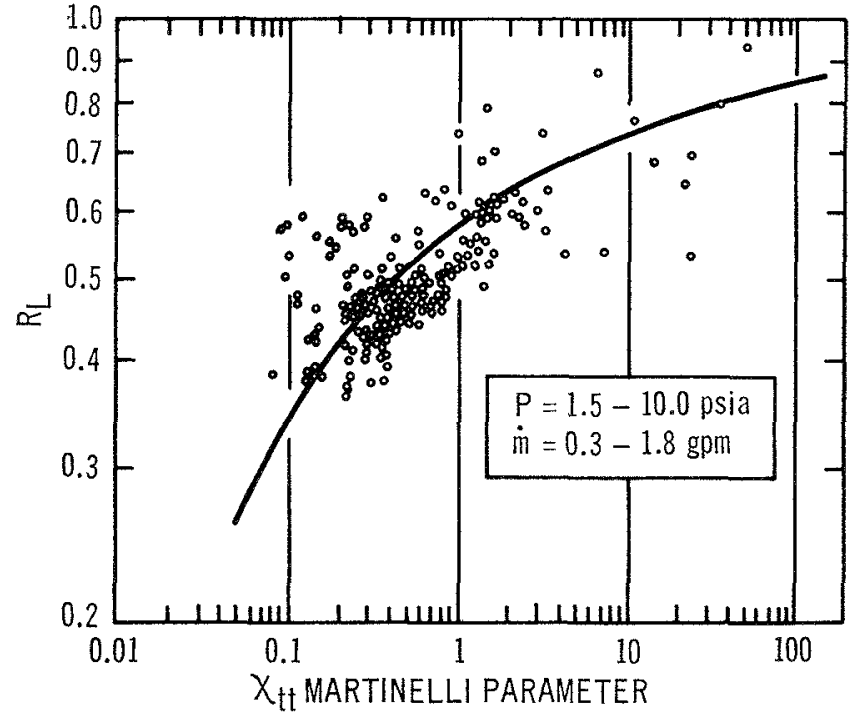

Fra. 4. Liquid Fraction in Vertical Upflow of Sodium.
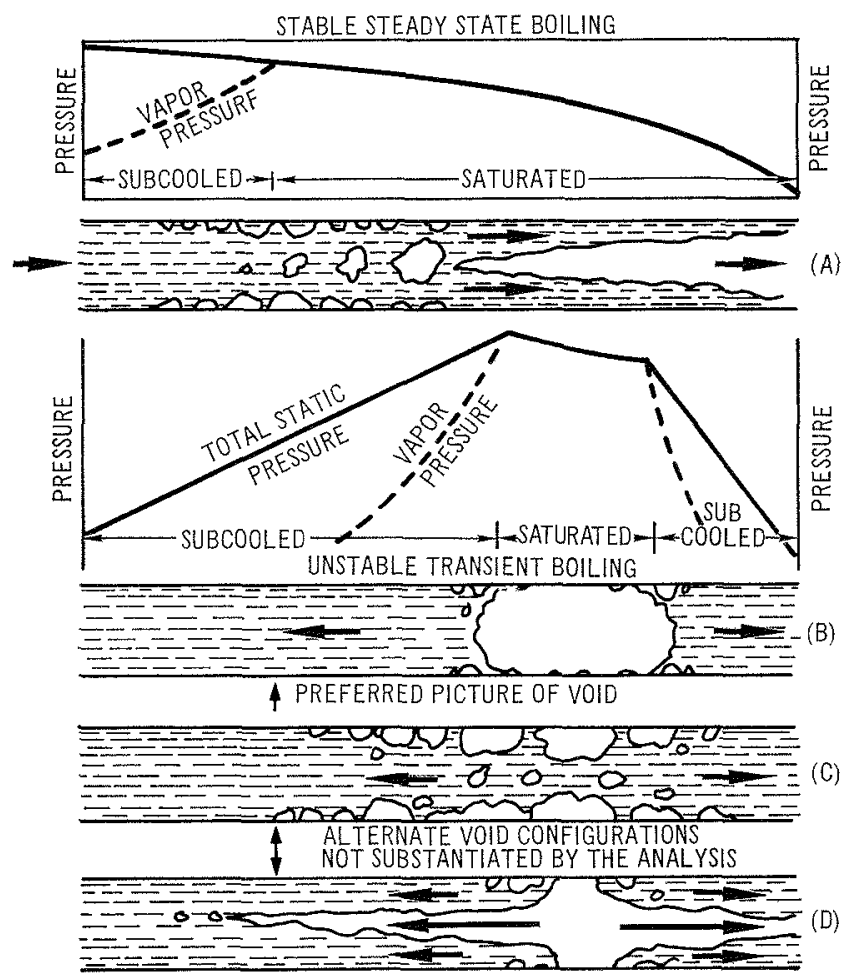

Fı (x. 5. Idealized Picture of Single-channel Boiling.

the strongly unstable nature of the hydrodynamic situation if any boiling occurs in these channels. This applies for all power levels except perhaps for decay heat levels; if boiling should occur due only to decay heat, it could be relatively stable in some fast reactor configurations. As another alternate, it might be supposed that, because of superheating near the walls, bubbles would grow outward from the walls over a significant length of the channel (see Fig. 5C); or, 
because of liquid-vapor interfacial instability, vapor from the high-void region might tend to slip out through the liquid, illustrated in Fig. 5D, as it does in steady state. These alternate pictures are not supported by the results of calculations primarily because of the relatively high calculated inertial pressures generated by the expanding void and the consequent tendency for quenching of rapor bubbles in regions away from the primary roid. Also, the high heat transfer coefficient of the liquid metal minimizes wall-to-bulk temperature differences and reduces the axial region in which subcooled boiling can occur to a narrow range. The self-pressurizing effect keeps most of the liquid below its saturation temperature and probably prevents rapor formation or intrusion in most of the liquid region. Therefore, two-phase flow in the ordinary manner (parallel flow of the phases) probably is not present to any significant dcgree during the important initial phase of the transient. Since such two-phase flow is not present, it is not essential to calculate void fraction or slip ratio accurately in order to determine transient hydrodynamic behavior.

The preceding is not meant to imply that the calculation of void fraction is not an important result of the analysis, since it obviously is. However, the important transient fluid motions are more a function of transient buildup of vapor pressure than of the potential void generation. The void develops as a consequence of the motion of the liquid phase away from the vapor region and not vice versa. The important analytical problem is the determination of pressures.

\section{B. Heat-transfer-limited Void Growth}

Our first approach to the problem of calculating these transients was to assume that vapor formation was primarily determined by the heat input to the coolant, but that it might be modified by pressure buildup that occurred as a result of acceleration. The code TRANSFUGUE-I was developed from this point of view as a method of solution of the classical hydrodynamic differential equations for one-dimensional, variable-density flow. The solution is programmed in a general form which in principle does not rely on any assumptions about the limiting factors in void growth.

However, problems with numerical instabilities have thus far prevented its application without some artificial restrictions on certain parameters which tend to contribute to these instabilities. These restrictions apply to the factors that modify vapor production as a result of inertial pressure buildup and therefore tend to cause overestimates of roid growth, that is, the calculated inertial pressures are effectively decoupled from the solution of the rest of the problem and, therefore, do not affect the calculated void volume. This is accomplished by holding vapor density and saturation temperature constant everywhere in the channel. The stable solutions that have been obtained with this approximation are valid for the limiting case in which inertial self-pressurization is not sufficient to alter the rate of growth of voids significantly. Because of this fact, studies with this code have provided much of the quantitative data which have formed the basis of the picture of void growth outlined above.

Figure 6 shows schematically the hydraulic and heat transfer characteristics of a sodium-cooled, fast reactor fuel channel that has been studied with the TRANSFCGCE-I code. The transient started from full-flow, full-power conditions, and loss of flow was simulated by suddenly reducing the inlet pressure to a value corresponding to the natural convection head of the system. Rapid flow decay occurred, leading to boiling in about $0.8 \mathrm{sec}$ as power was held constant. Figure 7 shows sodium temperature profiles calculated during the transient using 85 axial nodes.

Note that boiling does not start at the center of the core but near the outlet interface between core and blanket. A number of power and loss-of-flow transients have been studied recently, and this type of voiding pattern has been found always to occur in transients that start from full-power conditions. There is little doubt about the ralidity of this result because it does not depend in any way on the boiling model used. Prediction of the initiation point for the void depends almost exclusively on the transient heat transfer and single-phase flow solution to the problem. Some uncertainty of the exact timing does result from uncertainty about the degree of superheat required to nucleate the first bubble, and some statistical uncertainty must be associated with the precise location of a favorable nucleation site. However, the sharp peaking of the rapor pressure curve guarantees that the void will start at a location near the core outlet.

Description of the subsequent growth pattern and growth rate of a void is more difficult and uncertain because of the complexity of the situation and the limited experimental data available. Certain characteristics are clear, however. One is the presence of strong hydrodynamic instabilities. These were predicted for boiling in the Enrico Fermi Reactor as far back as 1957.(3)

All studies of boiling in sodium-cooled fast reactors are in agreement with the conclusion that the twophase hydrodynamics are strongly unstable. This natural instability has two important consequences: it 

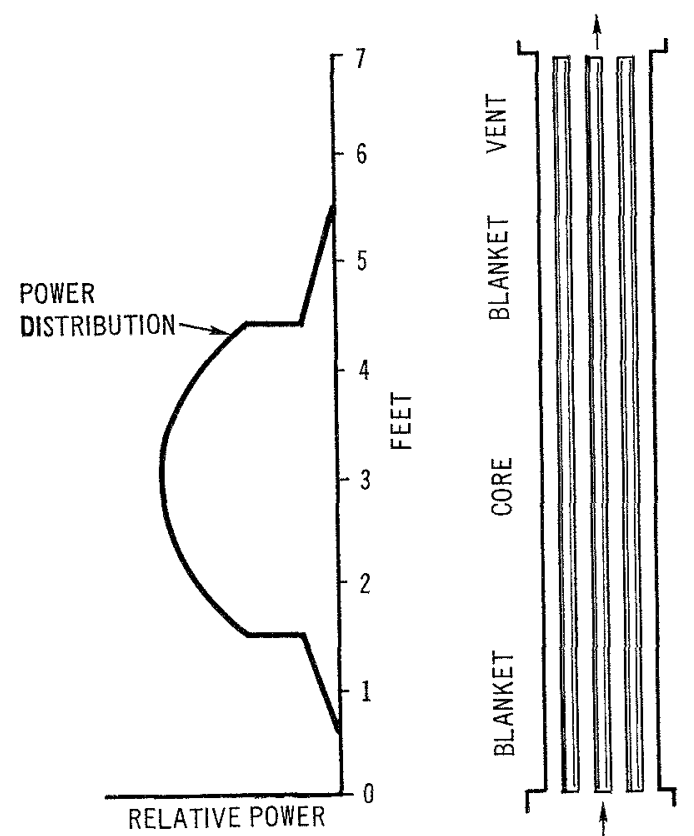

CHANNEL FLOW AREA $=0.099 \mathrm{ft}^{2}$

HEAT TRANSFER AREA $=17.7 \mathrm{ft}^{2} / \mathrm{ft}$

OUTLET PRESSURE $=19$ psia

HYDRAULIC DIAM $=0.011 \mathrm{ft}$

FUEL PIN DIAM $=0.25 \mathrm{in}$.

NO. PINS $=271$

FIG. 6. Schematic of Fuel Channel.

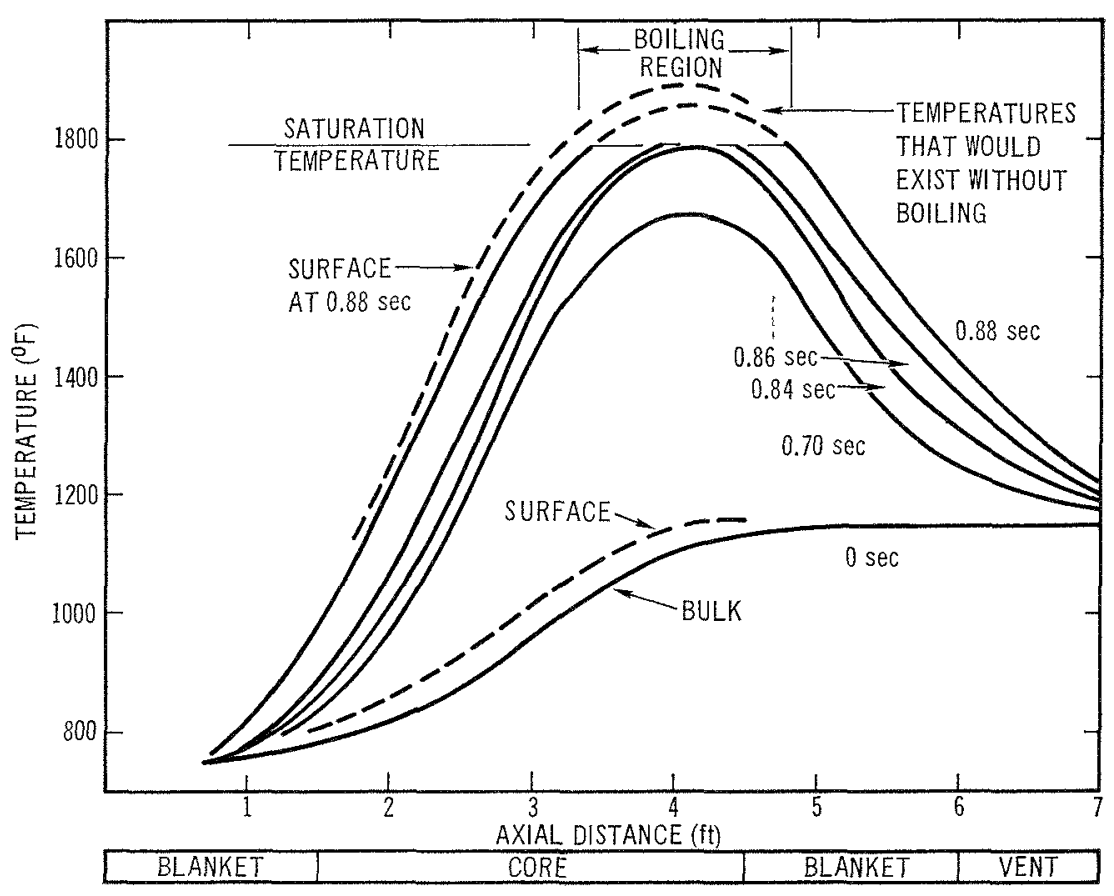

FIG. 7. Sodium Temperatures during Loss-of-flow Transient.

moans that void growth is (1) rapid and (2) difficult to analyze numerically. Problems with numerical instabilities in the TRANSFUGUE-I calculation method are closely related to this characteristic. Rapid void growth is a consequence of the unstable flow-choking effect of boiling, which is autocatalytic. Under these circumstances, power sufficient just to initiate boiling results in complete voiding of the channel without further increase in power. There is a tendency for sodium to re-enter a channel after the initial voiding transient. The unstable nature of the hydrodynamics means that the re-entrant flow will be unstable and some sort of chugging behavior will occur. Such behavior may have important influences on the ultimate outcome of a given transient and will be the subject of significant research in the future. 


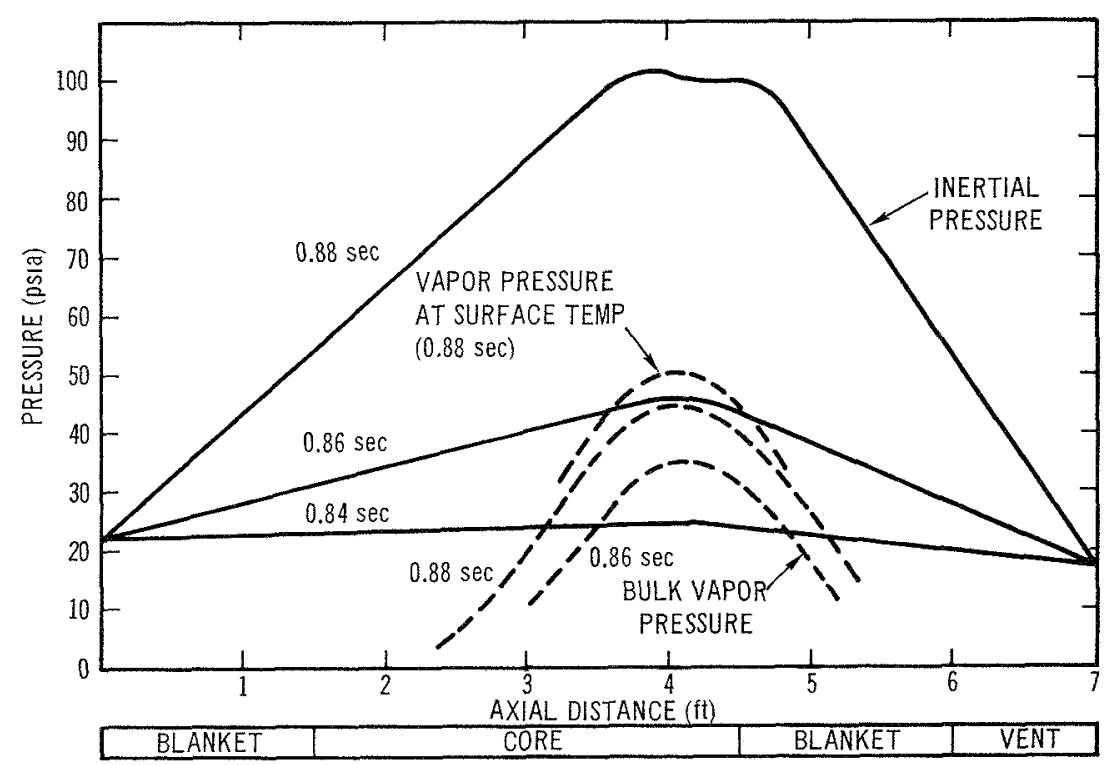

Fig. 8. Results of Calculations of Sodium Pressure for Loss-of-flow Transient.

\section{Pressure-limited Void Growth}

The inertial pressures calculated by TRANSFUGUE-I for the loss-of-flow transient are plotted in Fig. 8 together with curves of sodium vapor pressure corresponding to the temperatures shown in Fig. 7 . Study of plots of this type for a number of loss-offlow and power transients has led to the general conclusion that self-pressurization occurs in most transients initiated from full power. As indicated previously, saturation temperature and vapor density were held constant in this calculation for reasons of numerical stability, thus yielding a heat-transferlimited rate of growth of voids. The calculated inertial pressure is the pressure required to move the liquid out of the way to make room for the growing void under the assumed circumstances. The calculation clearly shows that more pressure is required for this type of void growth than is available from the only source of pressure, namely, the vapor pressure of the sodium. This means, simply, that the void will not grow at a heat-transfer-limited rate, but will grow at a slower rate controlled by the rate of increase of vapor pressure at the hottest spot. It also means that the maximum pressure will be no less than the maximum vapor pressure of the hottest liquid sodium in the channel. This is the self-pressurization effect to which we have referred.

Figure 8 also shows the typical relationship between the shapes of the vapor pressure and inertial pressure curves. The total pressure gradients in the liquid regions are constant because acceleration is uniform. The inertial pressure curve at 0.86 see and the vapor pressure curve at 0.88 sec together are a fair represen- tation of these quantities for a solution at $0.88 \mathrm{sec}$ corrected for the effect of self-pressurization. In other words, the true inertial pressure curve for $0.88 \mathrm{sec}$ probably should be about in the position of the 0.86 sec curve. Comparison of these curves then shows the rapid development of subcooling in regions away from the void. Both power and loss-of-flow transients show this type of behavior, and it is believed to be fairly general for fast reactors at substantial power levels.

In certain power transients initiated by relatively large reactivity insertions $(>\$ 1.00 / \mathrm{sec})$, the power level will rise so rapidly that the critical (burnout) heat flux will be exceeded before bulk boiling starts. Because of the relatively high heat transfer resistance introduced by film boiling under such circumstances, it is possible that heat transfer limitations then would be the controlling factor in determining void growth and that the self-pressurization effect would be secondary. Our studies of this aspect of the problem are not complete; however, they do indicate that film boiling can, under the proper circumstances, have an important influence on void growth and pressure buildup. A number of experimental measurements of critical heat flux for sodium in forced convection have recently been completed at our laboratory. Figure 9 shows critical heat flux trends in both the subcooled and saturated boiling regions. The details of this work are being published. (1)

Void growth characteristics determined from interpretation of results of TRANSFUGUE-I calculations of power and loss-of-flow transients in typical fast reactors have been used as the theoretical basis of 


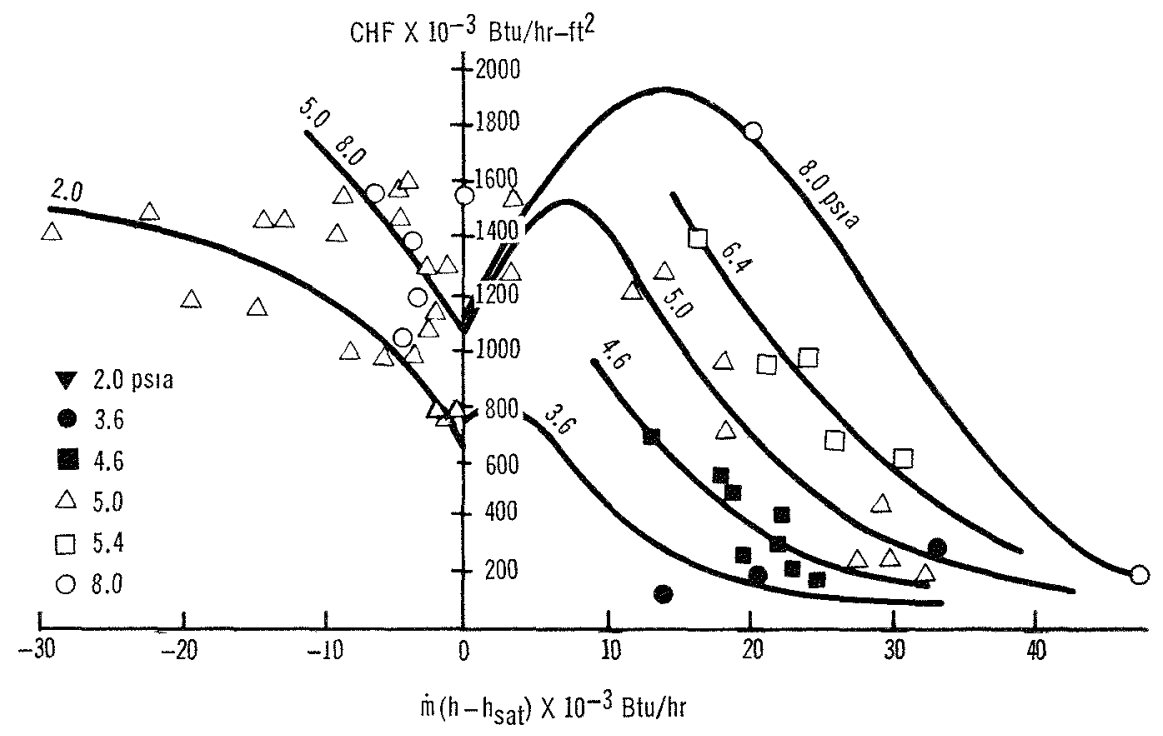

FIc. 9. Critical Heat Flux for Sodium under Foreed Convection.
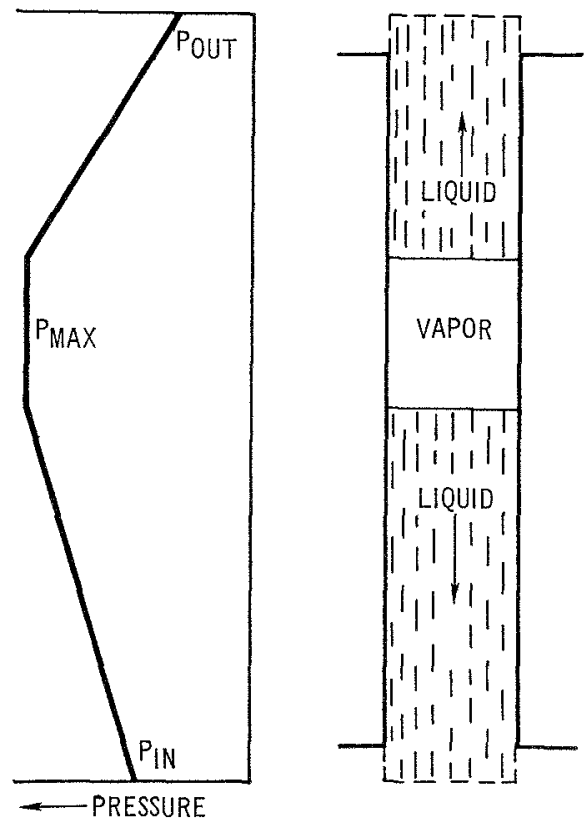

FIG. 10. Geometry Assumed in BURP Model.

another calculational method which avoids some of the numerical problem areas of TRANSFUGUE-I. The fundamental assumptions that are used to construct this model are:

1) The void spreads axially along the channel from a single starting point, as shown in Fig. 10.

2) Pressure in the void region is equal to the highest vapor pressure of the bounding liquid.

3) The self-pressurization effect supresses boiling away from the growing void region.

4) Void fraction within the vapor region is $100 \%$.

The second assumption regarding pressure in the void region is the key factor in the analysis and is also subject to the greatest uncertainty. In the digital code based on this model (BURP), temperatures in the liquid regions are calculated numerically by the usual finite-difference equations and searched for a maximum which usually occurs at the lower liquidvapor interfacial node. This temperature is converted to pressure by use of the vapor pressure relationship for sodium, and this pressure is then assumed to exist in the vapor region. This ignores possible pressure sources in the vapor region and also ignores the condensing effect on the vapor pocket that would tend to occur when the upper boundary moves into relatively cool blanket and upper plenum regions. Determination of better assumptions to use in determining pressure in the void must await the completion of appropriate experiments.

In any event, the BURP method yields the best available estimates of void growth rate and pattern and pressure buildup for the class of fast reactor transients to which it applies. As examples of results of calculations based on this method, a loss-of-flow and a power transient have been selected. The loss-offlow transient (see Fig. 11) is for the same system and conditions described previously for the study with TRANSFUGUE-I. The ramp power transient (see Fig. 12) is for a similar reactor which has a $4.3-\mathrm{ft}-$ long core instead of the 3.0 -ft core studied in the lossof-flow situation.

Results confirm previous calculations indicating that the void starts near the outlet core-blanket interface and tends to work into the upper blanket before the core. This characteristic is most pronounced for the power transient in which the pump keeps running. 


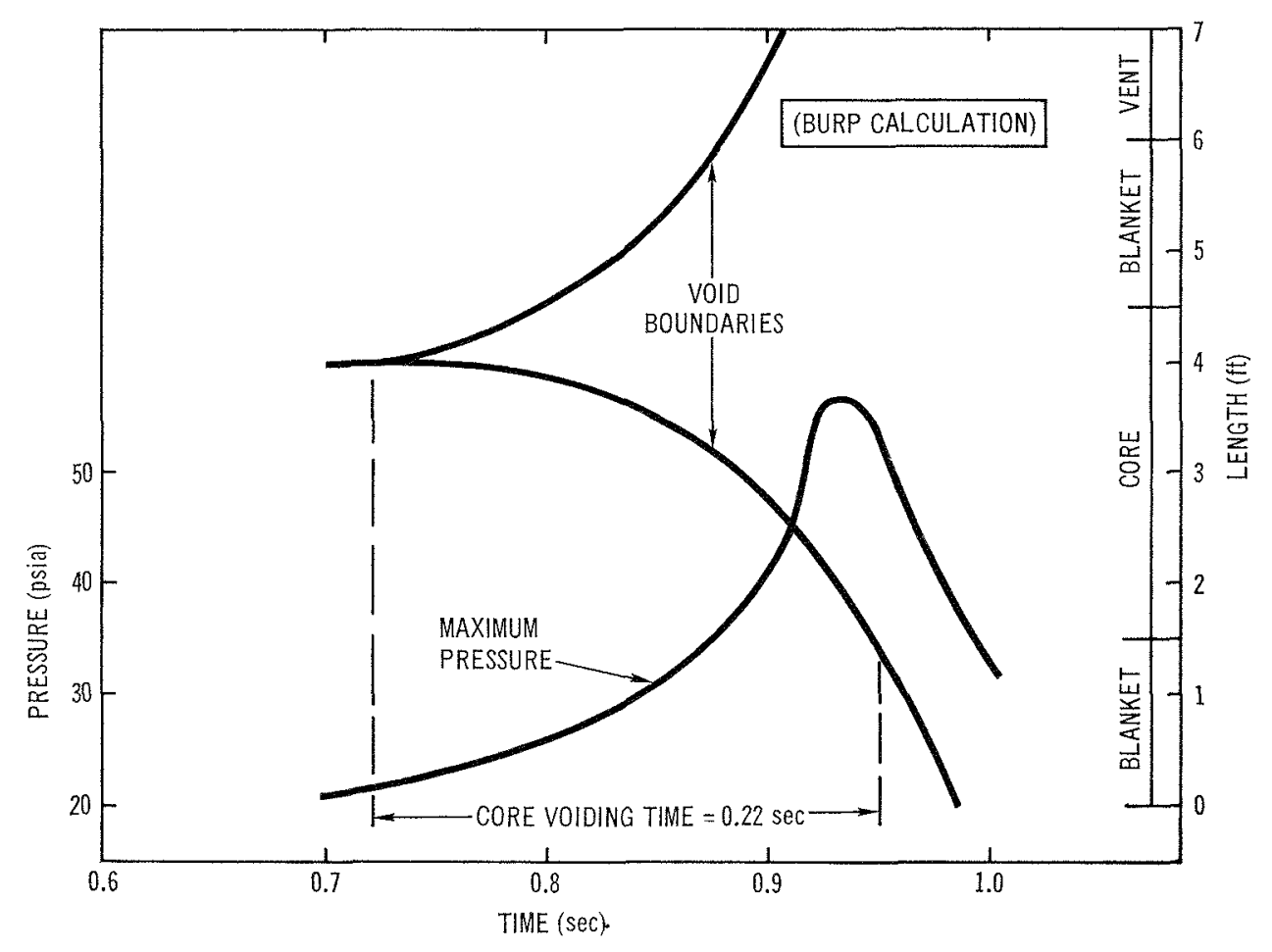

Frg. 11. Maximum Pressure and Location of Void Boundaries after Boiling in Flow Transient.

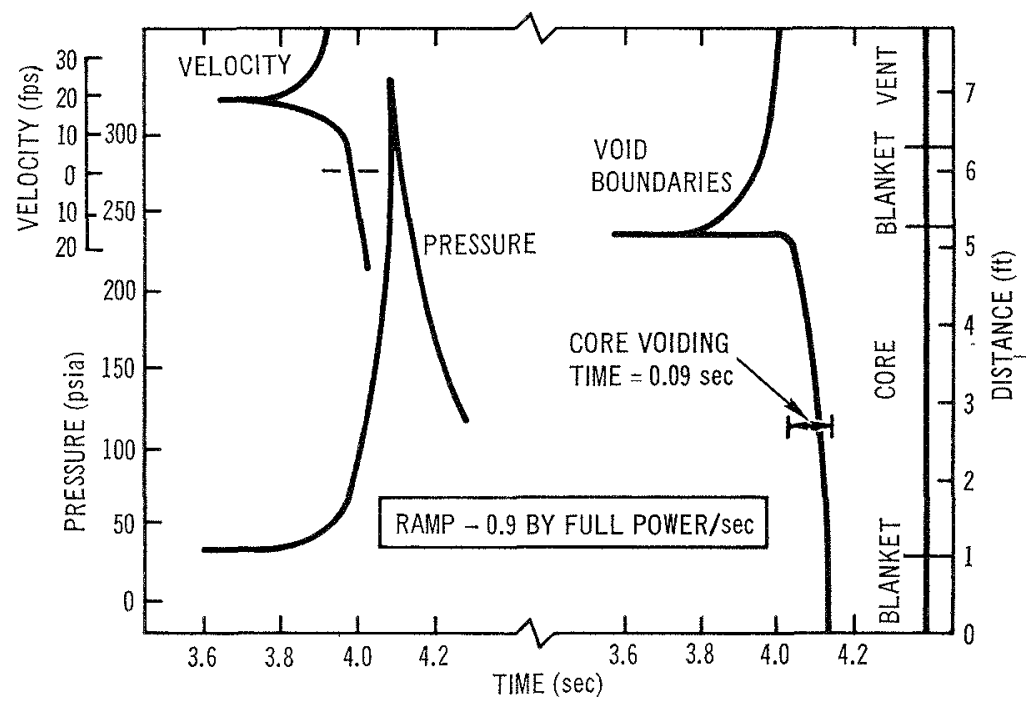

FIG. 12. Velocity, Maximum Pressure, and Void Boundaries after Boiling in Ramp Power Transient.

An approximately 0.15 -see delay occurs between the starting of the void into the upper blanket and starting of the void into the core. The reason for this delay is explained best by study of the velocity plot shown in Fig. 12. Coolant moving through the core must turn around, i.e., go from a positive to a negative velocity, before the void can grow downward into the core. If a relatively high upward velocity exists when boiling starts, a certain time will be required to stop the lower liquid column and get it moving downward, and the core will remain flooded during this time. No such conditions apply to the upper blanket region, so the void will develop more rapidly there.

With the exception of the consideration of surfaceto-bulk radial temperature variations, this discussion and analysis has been limited to the one-dimensional problem. It is believed that void-growth patterns and pressures calculated by this method can be applied with reasonable confidence to single fuel elements typical of most proposals for fast reactor design. 
However, further investigation of the effects of pinto-pin power variations and local flow variations certainly is required. In order to obtain void effects for the core as a whole, the multichannel problem must be solved together with nuclear feedback effects. Although complicated in application, the multichannel problem is relatively simple in principle once a satisfactory method for single-channel analysis is in hand. It is expected that continued development along the lines outlined here will yield the desired result.

\section{REFERENCES}

1. H. Lurie, Steady-state Sodium Bonling and Hydrodynames, NAA-SR-11586 to be published.

2. R. C. Noyes, J. G. Morgan, and H. H. Cappel, TR.1MSFCGUE-I, A Digital Code for Transient Two phase Flow and Heat Transfer, NAA-SI-11008 (July 1965).

3. H. R. Kroeger and J. W. Stephenson, "The Effect of Sodium Vapor Formation on the Operation of the Enrico Fermi Reactor," ASTRA, Ine., Milford, Conn., July 1957.

\section{Discussion}

Mr. Sanathanan (ANL): You mentioned that the slip ratios could be very different during transients. Could you explain how you derived the transient slip ratios?

Mr. Noyes: With the picture I postulated of transient boiling in a fast reactor with substantial power, the slip ratio almost has no meaning at all. The vapor bubble just grows as a unit, and there is really no parallel flow of liquid in the vapor that is significant in the problem until the initial bubble reaches the boundary of the core. Then with reentry and so forth you get a situation in which slip would be involved. Another factor is that when you start the transient, the slip ratios will tend to be lower because the starting condition is essentially a slip ratio of one. On generating the bubbles have the same velocity initially as the liquid, and the acceleration has to occur over some period of time. Somehow during the transient, the slip, if there is a slip, would tend to be lower. We haven't calculated the slip ratios or measured them during any transient. This is qualitative speculation.

Mr. Sanathanan: Would it not be better to go with the hydrodynamic equations themselves rather than introducing the slip ratio?

Mr. Noyes: Homogeneous model?

Mr. Sanathanan: No. I don't expect a rigorous solution but you have to have something to help specify what happens at the time boiling starts. This was your difficulty, was it not?

Mr. Noyes: No, not really. It's a homogeneous model, and I think that the arguments that I just mentioned tend to justify using a homogeneous model.
You essentially assume the slip ratio is one, and it makes the problem a lot more tenable mathematically.

Mr. Sanathanan: Yes, but mathematical difficulty should not prevent a physical explanation.

Mr. Noyes: Well, I didn't mean to imply that. I hope I didn't.

Mr. MacFarlane (ANL): I'm a little confused on your comments about the unstable nature of twophase liquid metal boiling, in particular, your comments about numerical instability. Does this indicate anything physical about the unstable nature of boiling in the transient?

Mr. Noyes: Two things are involved: numerical instabilities and physical instabilities. I tried to distinguish between them. So far as the physical instabilities are concerned, under fast reactor conditions with substantial power, at anything above decay heat level the flow is as strongly unstable. This is related to the numerical instability. The numerical problem depends on the numerical approach used. We are using Euler integration. In sequential integrating procedures, very often the physical instabilities tend to be amplified by the numerical technique.

Mr. MacFarlane: People postulate that the instabilities in loops are concerned with things like liquid supcrheat, nucleation sites, and the like. Now, any model you could develop to do a transient two-phase calculation is by definition going to be somewhat limited and cannot include these effects. Therefore I think that any conclusions about unstable flow from these numerical results is somewhat subject to question.

Mr. Noyes: I disagree. First of all, you don't have to make transient calculations to predict instabilities. The calculations that were made in 1957 and for Fermi were steady-state calculations. They showed a very strong negative slope and check curves which have always been shown to be associated with hydrodynamic instabilities. I am not talking about nucleation instabilities or superheat instabilities; those are another type of instability.

Mr. MacFarlane: But that's a steady state analysis, isn't it?

Mr. Noyes: That's right.

Mr. MacFarlane: I think this is a very weak approach.

Mr. Noyes: It always works.

Mr. Häfele (Karlsruhe): I would like to point out that numerical instabilities can be avoided principally by a certain technique of integration of the Eulerian equations. For example, you are given a curve which contains some initial conditions, say of the velocity, density, or whatever. You have to integrate along the characteristics. On the inside of the area the flow pattern is determined by the initial conditions. What one 
does usually in the numerical procedure is to work along a network. Then it can easily happen that the flow pattern is determined numerically although it cannot be determined physically from the starting conditions. Therefore, if one applies such a technique of integration, these numerical instabilities can be avoided. One has to have in hand the velocity of sound, because the slope of the characteristic is dictated by the velocity of sound, and therefore one has to make an evaluation of what the velocity of sound is in this two-phase flow. I agree that there are a number of physical reasons for instabilities and we feel partly that they are connected with the question of shock waves. If one investigates the velocity of sound in the presence of bubbles, it isn't too difficult to handle the question of shock waves at the same time.

$M r$. Noyes: I generally agree and we have started on this sort of approach to the problem so far as detailed calculations are concerned. However, I think that for making calculations for safety studies, where you want to get an idea primarily of the voiding rate or the rate of ejection of sodium, the simple model that I presented probably will be good enough.

$M r$. Fischer (Karlsruhe): Did you take into account the kinetic energy in the basic differential equations?

Mr. Noyes: Yes.

$M r$. Fischer: Did you consider the difference between the entropy and the internal energy in these equations?

Mr. Noyes: Yes. I did a separate analysis of that problem which is really not contained in the writeup on TRANSFUGUE and tentatively came to the conclusion that it was not important in these problems to differentiate between enthalpy in that way.

$M r$. Fischer: The difference is always the work of displacement, and I believe that as expansion progresses this difference is essential.

Mr. Cobb (Nuclear Power Group, England): In your description of the first model you talked about inertial pressure drop; I didn't hear you mention frictional pressure drop at all. Do you allow for that in the model?

$M r$. Noyes: Yes, the friction is there. It isn't very important in these terms because the dominant term during the transient is the inertial pressure.

$M r$. Cobb: I would have thought that you were talking about voiding in less than $0.1 \mathrm{sec}$; in that case the velocities would be quite high.

$M r$. Noyes: Yes, and inertial pressures are quite high, too.
Mr. Cobb: I thought the friction might even be equal to the inertial pressure drop.

Mr. Weills (ANL): I would like to make one little comment after seeing the pressure pulse going up to 300 psi. This is enough in many designs to cause a ballooning of the hexagonal tube, which would make the modular type of reactor shut itself down due to radial expansion. This should be part of a complete analysis of the reactor kinetics coupled with the hydrodynamies.

Mr. Noyes: Yes, it could. The existence of that pressure is over a very short period of time, though.

Mr. Carmichael (LASL): I'd like to comment on Dr. Häfele's remark about the speed of sound. Isn't the problem essentially one that some fraction of the energy may be transmitted directly through the liquid without affecting the bubbles? It's sort of like a heary spring that is impacted and you get a high frequency ping that's transmitted directly through the metal whereas probably the bulk of the energy goes into compressing the spring. In the calculations we did recognize this; and certainly neglecting this sort of energy overestimates the effect of the bubbles. In our application, also, there will be bubbles clinging to the wall and not homogeneous through the liquid.

Mr. Fauske (ANL): I would like to point and something of importance about this sonic velocity business. If you do perform a calculation of the sonic velocity in sodium at atmospheric pressure, assuming equilibrium, you find out that the sonic velocity is on the order of 5 fect a second, which is very low. Now, another way of calculating this is to assume complete metastable conditions where the sonic velocity does not see a phase change. In this case you get a sonic velocity of the order of 80 feet a second. In any case, or in both cases, I think this is of very much importance in the expulsion of sodium from the tube. As a matter of fact, in the instant you form vapor you probably have a choking type phenomena. This can cause another hydrodynamic instability. There is an experimental observation of the velocity of sound in a two-phase mixture which indicated these low velocities.

Mr. Lottes (ANL) : Do you have a reference?

Mr. Fauske: There is a Russian paper. I don't remember the author. Kasmin or something like that. It concerns work on steam-water where they actually show that the velocity of sound propagates with a metastable behavior. They also have the newest measurements on the velocity of sound in a two-component system. Air-water mixtures with primarily bubble flow show an extremely low velocity of sound. 


\title{
Coolant-ejection Studies with Analogy Experiments
}

\author{
G. Friz \\ ELRATOM-C.C.R. \\ Ispra, Italy
}

\section{Introduction}

An important part of the Karlsruhe Fast Breeder Program* deals with safety questions. The coolant behaviour during a reactor accident is of particular interest, especially the ejection of the coolant from the channel. At Ispra, the experimental program is concerned with liquid metals and with analogy experiments with other liquids. Preliminary experimental results are presented in this paper.

Among the potential causes of boiling excursion with consequent coolant ejection are the following:

1) an increase of heat production;

2) a decrease of coolant velocity;

3) a decrease of pressure.

The first series of experiments dealt with a special combination of 2) and 3 ): the ejection of primary stagnant coolant from a single vertical channel as a consequence of a sudden depressurization. Water was taken as test liquid. The process was observed by taking high-speed movies.

\section{Experimental}

The test section is a simple glass apparatus (see Fig. 1) consisting of a central test tube of $7.2-\mathrm{mm}$ diameter, surrounded by a jacket for preheating with glycerine. The top of the test tube expands to a $20-\mathrm{mm}$ diameter tube which is surrounded by a water-cooled jacket. The whole assembly is closed at the top by a thin plastic diaphragm (the cooling is necessary to protect this diaphragm from the water vapor). An electromagnetic rupture device perforates the diaphragm at a fixed delay time after the camera starts. The liquid column can be pressurized up to $4 \mathrm{~atm}$; the diaphragm withstands this pressure until it is perforated, and then ruptures immediately and completely.

The movies were taken with a Fairchild camera using photography by transmitted light. Illumination was provided by three 500- IV lamps with a sereen behind the heating jacket. On the film, the vertical channel has a horizontal orientation. This was done to obtain a larger image of the object. With the $35-\mathrm{mm}$ objective, the camera-object distance was $1.2 \mathrm{~m}$.

* This work was done within the framework of the Karlsruhe Fast Breeder Project (Association EURATOM/ (resellschaft fur Kernforschung).
Two series of experiments werc made, one with double-distilled, thoroughly degassed water and the other with normal non-degassed water. The starting temperatures $T_{1}$ in both cases were: $105 / 107.5 / 110 /$ $112.5 / 115 / 117.5 / 120 / 125^{\circ} \mathrm{C}$. The temperature was measured with a thermometer at the top of the heating jacket. The temperature fall along the jacket was less than $0.3^{\circ} \mathrm{C}$.

Before starting a test the water column was pressurized up to $3.5 \mathrm{~atm}$, then heated up from $100^{\circ} \mathrm{C}$ to the desired temperature. Immediately after arriving at this temperature, the camera was started. After a fixed delay time of $1 \mathrm{sec}$, necessary for allowing the camera to arrive at the desired speed of ca. 3,000 frames/sec, the diaphragm was perforated by the rupture device.

The rupture results in a depressurization wave which arrives at the surface of the water column about $10^{-3}$ sec later. Taking into account that the pressure ratio from starting to atmospheric pressure is supercritical, about 3 reflections at the outlet (diaphragm position) and closed end (top of the water column) of the "pipe" are necessary to obtain atmospheric pressure at the surface of the water column. That is to say we can assume atmospheric pressure from 3 to $5 \cdot 10^{-2}$ sec after the diaphragm rupture. This is still a short time with respect to the duration of the ejection process itself, which is about 0.1 to 0.2 sec.

Thus we can state the following boundary conditions:

$$
\begin{aligned}
t<0 ; & p=3.5 \mathrm{~atm} ; \\
t \geqq 0 & \left\{\begin{array}{l}
p=1.0 \mathrm{~atm} ; \\
T_{1}=105 / 107.5 / 110 / 112.5 / \\
115 / 117.5 / 120 / 125^{\circ} \mathrm{C}
\end{array}\right\} 0<x<1,
\end{aligned}
$$

that is to say, the whole column is equally superheated with respect to the atmospheric pressure.

\section{Evaluation of the Film}

The liquid phase appears on the image as a black shadow, whereas the vapor phase is white. Thus, the liquid-phase velocity and the movement of vapor bubbles can be distinguished. When the flow pattern has transformed into mist flow (see point 2), one can still observe the movement of water droplets but no 
longer the vapor-phase velocity. The slip ratio cannot be measured in this region.

Observing the film, one can give a qualitative answer on the question of how the ejection process occurs:

1) Immediately after arrival of the depressurization wave a great number of bubbles is formed over the whole length of the water column. The bubble density diminishes with the distance from tube exit. The medium bubble density grows with the superheating temperature. The bubbles are more numerous in the case of non-degassed water than with degassed water.

2) The acceleration of the water-bubble mixture starts at the exit of the tube in form of an "acceleration front" which propagates along tube against flow direction. The depth of this front is several centimeters. When the acceleration front passes a certain position, the flow pattern changes from a liquid-phase flow (with enclosed small vapor bubbles) of low velocity to a mist flow of high velocity. The movement of the acceleration front is changed by statistically distributed disturbances, but the medium speed of the front is practically constant.

3) This phenomenon becomes more regular with higher superheating temperatures, i.e., with a greater number of previously formed bubbles. The gas content of the test liquid has the same effect.

4) In the case of double-distilled degassed water up to superheating of $20^{\circ} \mathrm{C}$, a certain amount of superheated liquid remains within the tube (see Fig. 2) after the initial ejection. Depending on occasional disturbances, it is expelled some seconds later, similar to the retarded boiling phenomenon.

5) The downstream flow (behind the acceleration front) appears to be critical flow. This is consistent with the observation at the tube exit that the velocity increases in a region of expanding flow area.

The medium velocity of the acceleration front was measured by observing the time span $\tau$ over the tube length. In the case of degassed water, the distance between exit and surface of this rest column was taken and divided by the respective time span.

The results are presented in Fig. 3. There is an obvious difference between the behaviour of degassed and non-degassed water. At superheating temperatures less than $15^{\circ} \mathrm{C}$, the velocity is markedly higher with nondegassed water than with degassed water. At higher superheatings, the influence of gas content diminishes and the experimental points join to one curve.

\section{Theoretical Considerations}

To simplify the calculations, we take the transfer from liquid-phase flow to mist flow to occur in a front of negligible depth (as in shock or detonation fronts).

With this assumption we can obtain a simple station-

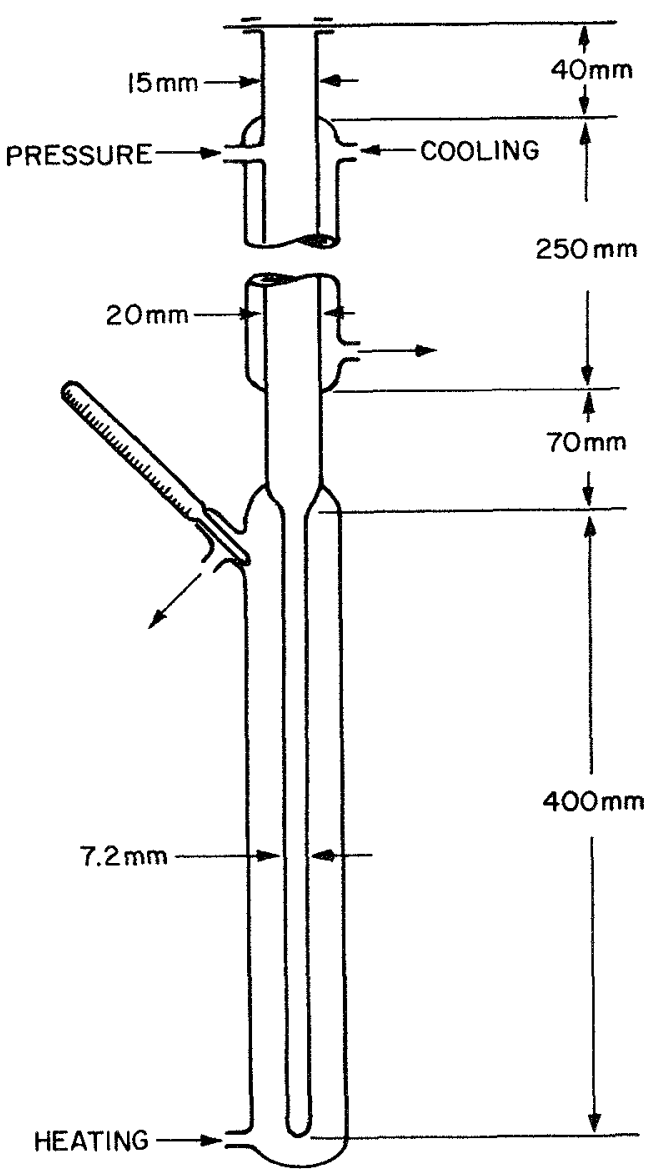

Fig. 1. Test Section.

ary problem, taking a coordinate system travelling with the front and applying the energy, momentum, and mass balances. Figure 4 presents the basic equations. We have a system of 3 equations in 4 unknown quantities:

$$
u_{1}, u_{2}, T_{2} \text {, and } x \text {. }
$$

The values of pressure and vapor density are given by temperature relations assuming thermodynamic equilibrium. For the density $\rho_{1}$, the liquid density $\rho_{l}$ was taken, i.e., we neglect the influence of the little bubbles on density. By reason of the fact that we have only 3 equations for 4 unknowns, one additional assumption on the downstream conditions is required.

Firstly, one can make the assumption - which is always made in subsonic flow-that the pressure at the tube exit is equal to atmospheric pressure. (The pressure drop along the test tube and the "pipe" between test tube exit and diaphragm shall be neglected.) The results of the calculations with this assumption are presented in Fig. 3 (lower curve).

However, the film evaluation suggests using the critical condition in the downstream region (behind the 


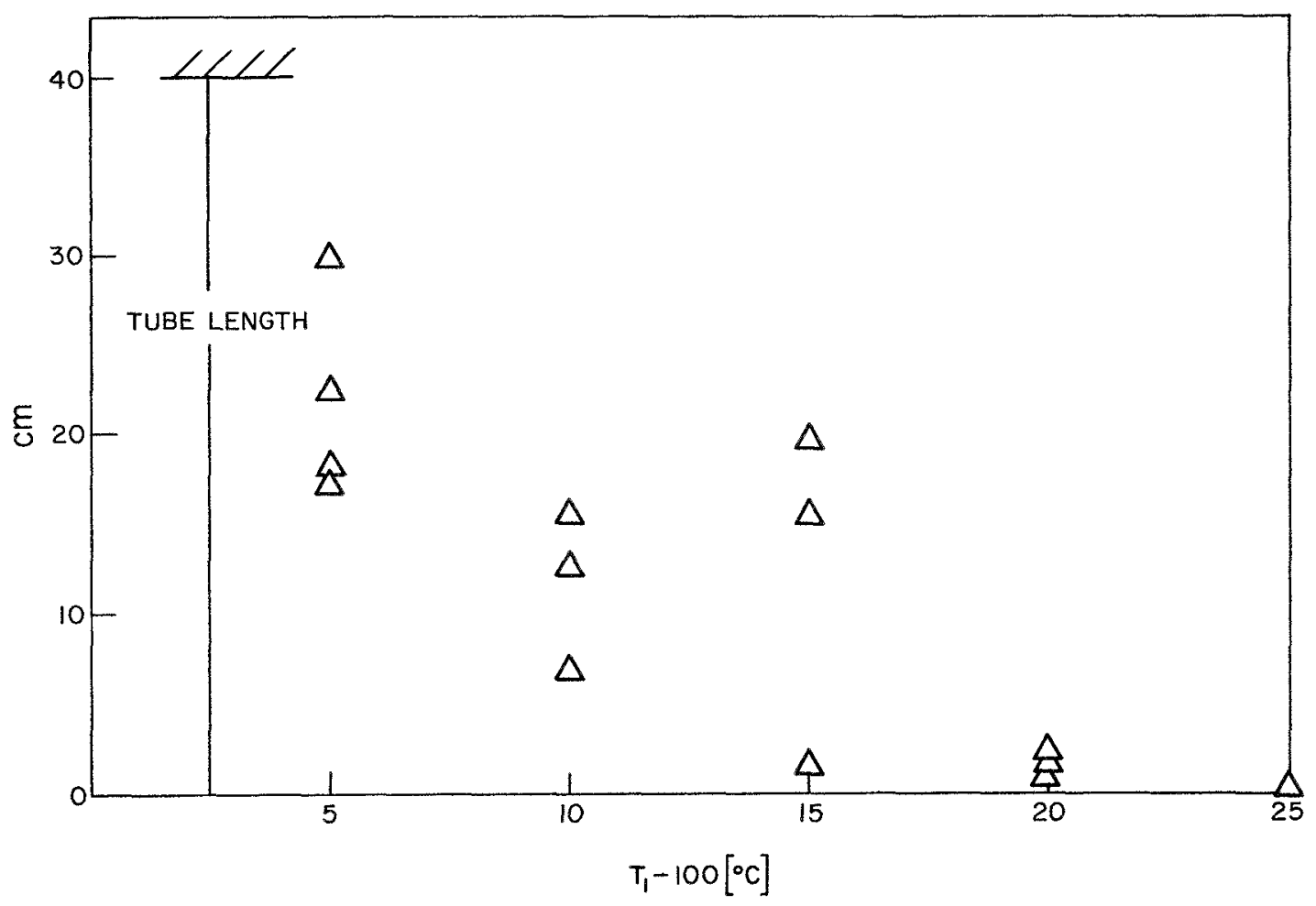

FIG. 2. Remaining Column after Ejection.

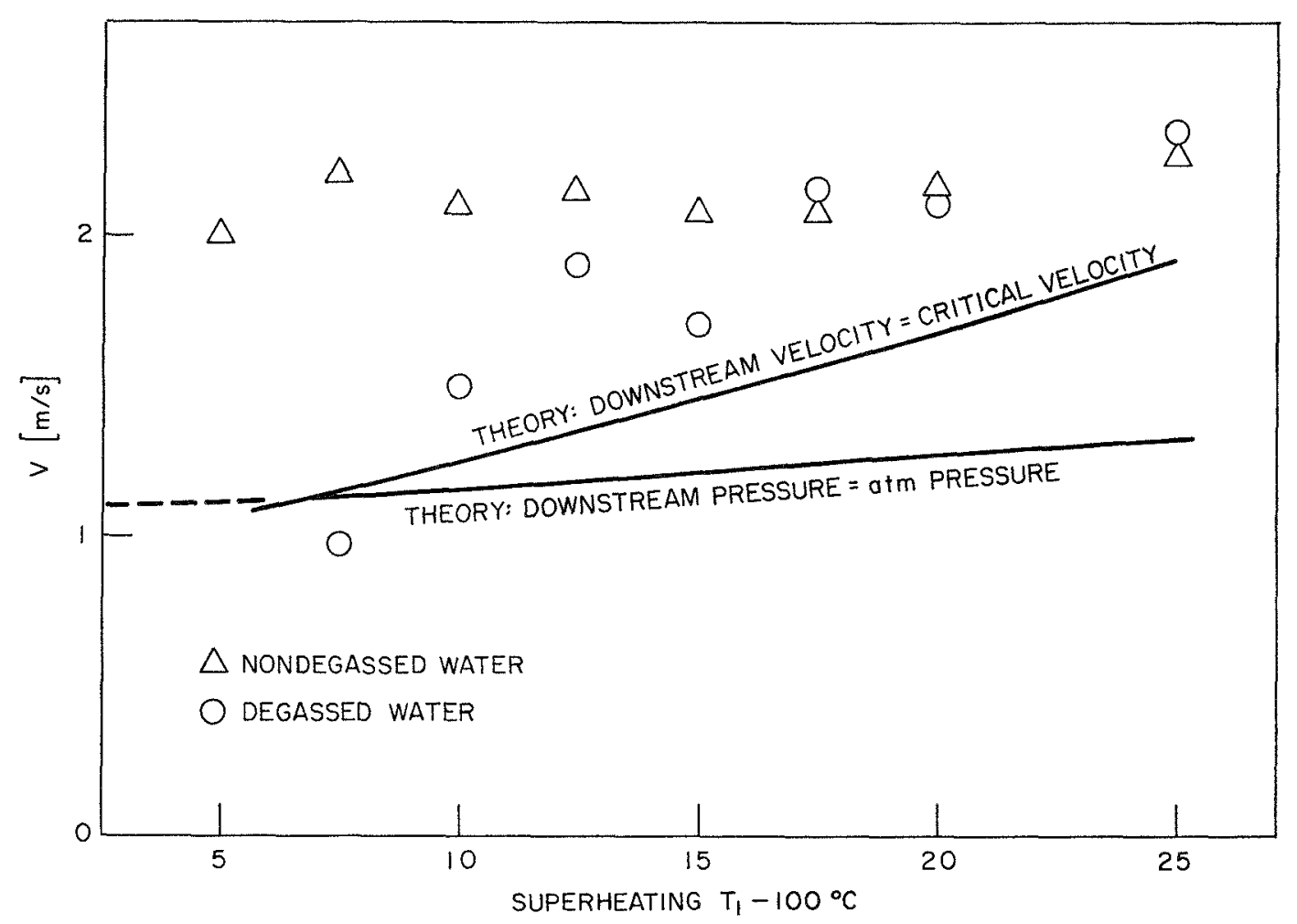

FIG. 3. Medium Velocity $V$ of the Acceleration Front. 
front). Critical velocity is defined by

$$
C_{c}=\sqrt{\frac{d p}{d \rho_{\mathrm{adiab}}}} .
$$

The results of the calculations for a vapor-waterdroplet mixture are presented in Fig. 5. The criticalflow relation is based on the assumption of unit slip ratio (vapor and liquid phases have the same velocity). The critical velocity of a mixture depends on two parameters: temperature and steam quality $x$ or an-

(2)

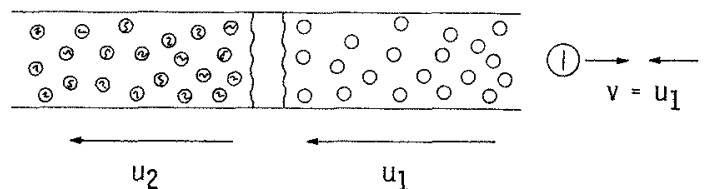

ENERGY:

$$
\frac{1}{2}\left(u_{1}^{2}-u_{2}^{2}\right)+\frac{1}{\rho_{1}}\left(P_{1}-P_{2}\right)+C\left(T_{1}-T_{2}\right)=X \cdot L
$$

MOMENTUM: $P_{1}+\rho_{1} u_{1}^{2}=P_{2}+\rho_{2} u_{2}^{2}$

CONTINUITY: $\rho_{1} u_{1}=\rho_{2} U_{2}$

$P=p(T) \quad \rho_{1} \approx \rho_{l} \quad \rho_{2}=\rho_{1} /\left(1+X \cdot \rho_{1} / \rho_{V}\right)$

$\rho_{V}=\rho_{V}(\mathrm{~T}) \quad C_{C}=\sqrt{(\mathrm{dp} / \mathrm{d} \rho)_{\text {adiab. }}}$

$\rho_{V}=$ VAPOUR DENSITY $X=$ STEAM QUALITY

FIG. 4. Acceleration-front Velocity $V$; Basic Equations. other quantity related to $x$. In Fig. 5 the specific volume of the mixture was taken for abscissa.

A graphical method was used to solve the system of equations. The critical velocity and absolute downsteam velocity were plotted against the downstream temperature $T_{2}$. The intersection point gives the desired values of $T_{2}$. The result is presented in Fig. 3 (upper curve).

Comparing now the experimental points with the curves, we must prefer the critical velocity assumption, especially with higher superheatings, where the deviation is about $20 \%$. Taking into account the simplicity of the model, the agreement is satisfactory.

Figure 6 shows the results of another very simple calculation with a model which can be called the "column ejection model." In this model we assumed that the pressure $p_{1}$ related to the starting temperature $T_{1}$ expels the whole liquid column with only one expanding bubble at the bottom of the tube. It is to be seen that, at least from the theoretical standpoint, the cjection in form of big slugs should occur quicker than in form of a propagating acceleration front.

On the other hand, we must take into consideration that the assumption of thermodynamic equilibrium may not be valid. The nucleation and evaporation rates must play a significant role, especially with lower superheating and with a small number of bubbles. The diserepaney between the results obtained with degassed and non-degassed water confirms this.

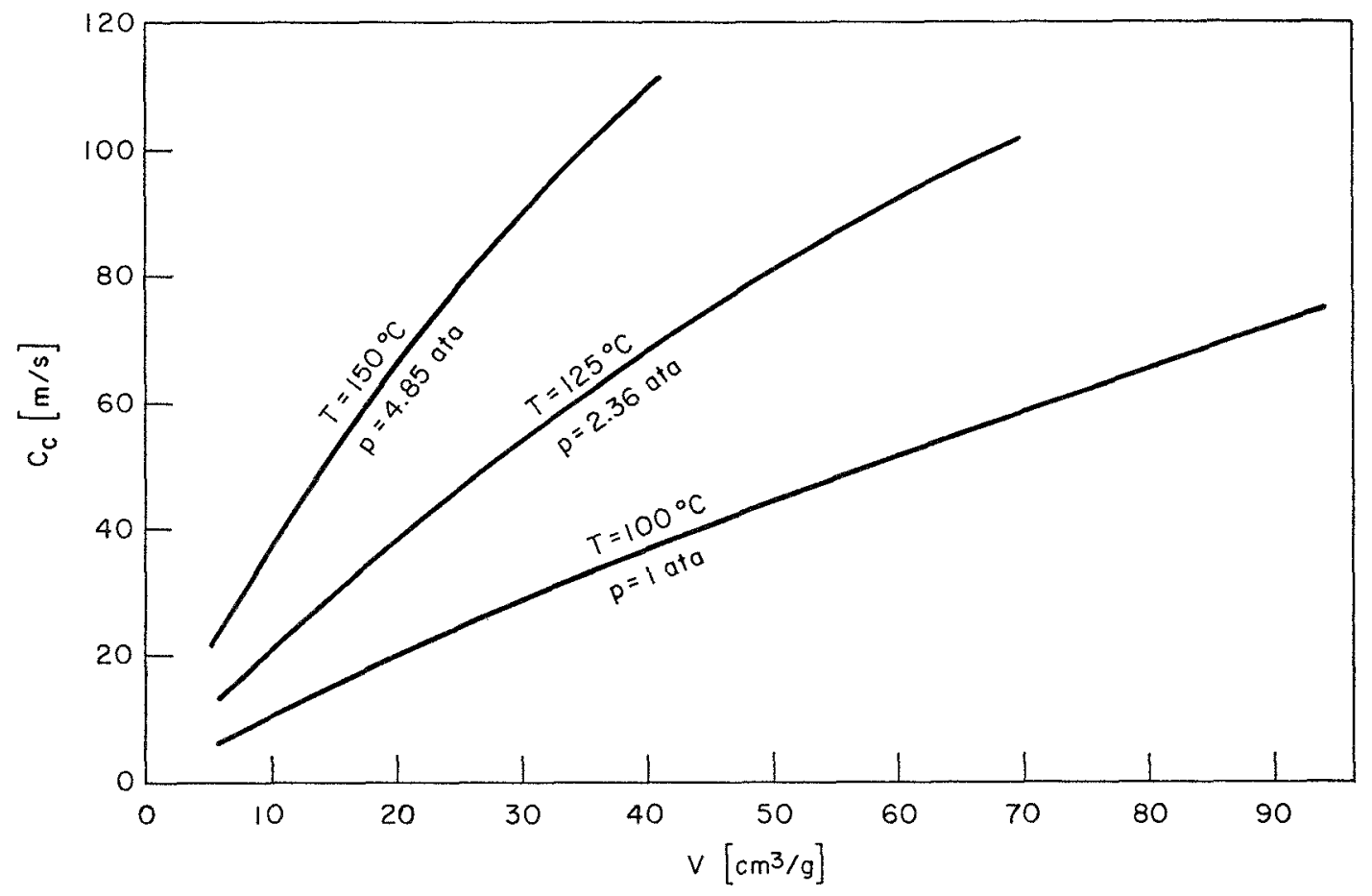

Frg. 5. Critical Velocity of a Water-Vapour Mixture. 


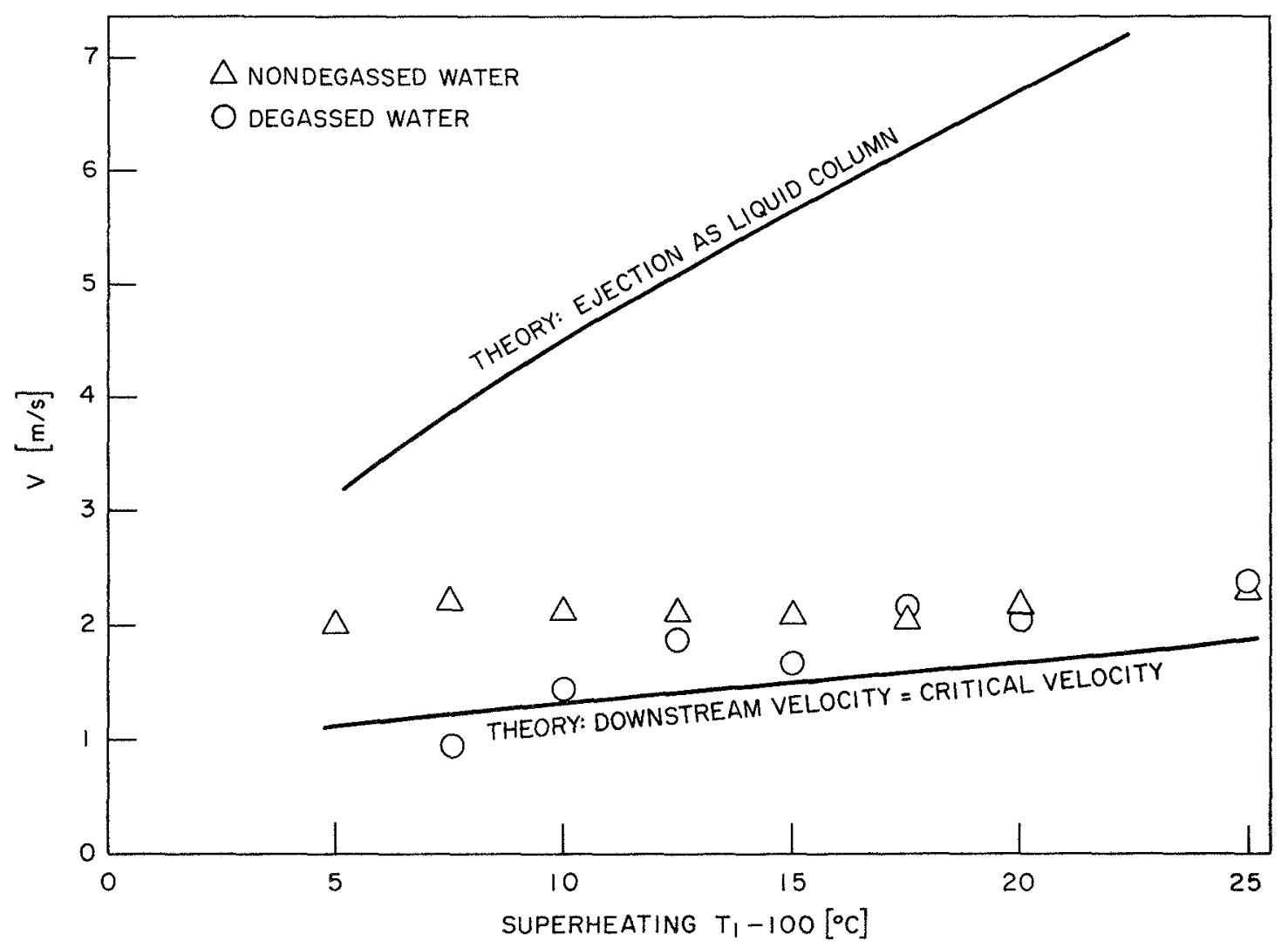

Fic. 6. Medium Velocity $V$ of the Acceleration Front.

\section{View on Future Program}

In the future, the experiments will be extended in the following directions:

1) variation of geometrical dimensions and test liquid;

2) extending the superheating range;

3) partial superheating of the column.

These experiments are directed toward extending the range of variables so that the formulation of mechanisms and analytical models might be adequately established, and subsequently applied to liquid metals.

\section{Acknowledgment}

The author wants to express his thanks to Prof. Häfele and Dr. Grass for sponsoring this work and helpful discussion.

\section{Discussion}

Mr. Noyes (AI): Have you done any experiments, or do you intend to do any experiments, in which there is an axial temperature gradient along the channel?

Mr. Friz: Not up to now. We plan to use a longitudinal temperature gradient in our next experiment, that is, a part superheated and another part not. In this case we start with stagnant liquid. We cannot maintain a radial temperature gradient; otherwise we should have a loop and this is a much more complicated device. For the next year we shall probably continue to work with stagnant liquids.

$M r$. Cobb (Nuclear Power Group, England): Two very simple points I seem to have missed. First I take it the image on the film was reversed. Were the voids the dark areas?

$M r$. Friz: The dark area was the water and the clear area was the vapor.

$M r$. Cobb: And the other thing was, you started with a column of superheated liquid. How did the boiling start? What initiated it?

Mr. Friz: It started by the rupture of the diaphragm. The column was first pressurized to $3.5 \mathrm{~atm}$. The diaphragm was then ruptured, producing depressurization down to atmospheric pressure and instantaneous superheating.

$M r . C o b b$ : That superheat referred to the pressure after you passed the diaphragm.

Mr. Friz: Yes, with respect to the external pressure. 


\title{
Shock Front Conditions in Two-phase Flow Including the Case of Desuperheat*
}

\author{
M. Fischer ANd W. HÄFELE \\ Kernforschungszentrum \\ Karlsruhe, Germany \\ (PRESENTED BY M. FISCHER)
}

\section{Introduction}

The investigation of the safety of large fast sodiumcooled reactors leads to the problem of coolant behaviour in the cooling channels during a power excursion. (1) This is primarily a hydrodynamic problem. But it is linked to the neutron behaviour of the core via the coolant void effect. The time characteristic for the expulsion of coolant together with the reactivity value of voiding the coolant channels result in a ramp rate of a positive reactivity insertion. The reactivity value of voiding the coolant channels was calculated in a recent study. The first value calculated was $\$ 3 ;^{(2)}$ more recent results indicate a value of nearly $\$ 7 .{ }^{(2,3)}$ But the duration of the expulsion of the coolant is probably even more uncertain. Its calculation is a problem of twophase sodium hydrodynamics. Attacking this problem, one realizes soon the crucial importance of shorkfront phenomena. The appearance of shocks tends to bring the integral process of expulsion to a time scale which is dictated by the velocity of sound, because after having passed a shock front, the velocity of the medium is subsonic. Therefore, both the investigation of the shock-front conditions and the velocity of sound are necessary as first steps for a more complete theoretical analysis of the whole process of the expulsion of the coolant.

A recent analysis of Smidt ${ }^{(4)}$ indicated that superheating and desuperheating may play an important role in the safety analysis. In particular, the plugging and superheating accident of a subassembly located near the center might lead to a severe accident even if the safety system is operative. (1) Therefore, it was important to include in this analysis the phenomena of superheat and desuperheat.

It should be mentioned that parallel to the described theoretical effort of the Karlsruhe group, experimental studies have been started and will be increasingly pursued. In particular, the work of the Ispra group should

* This paper has been prepared within the framework of the association EURATOM-Gesellschaft fur Kernforschung mbFI. in the field of fast breeder development. be mentioned. Friz ${ }^{(5)}$ reported the first such experimental results.

\section{The Euler Equation of Two-phase Flow}

The conservation of mass, momentum, and energy of a homogeneous two-phase flow is expressed in differential, one-dimensional form as follows:

Continuity:

$$
\frac{\partial \rho}{\partial t}+\frac{\partial(\rho u)}{\partial z}=0
$$

Momentum:

$$
\frac{\partial(\rho u)}{\partial t}+\frac{\partial(\rho u \cdot u)}{\partial z}=-\frac{\partial p}{\partial z}
$$

Energy:

$$
\frac{\partial}{\partial t}\left[\rho\left(e+\frac{u^{2}}{2}\right)\right]+\frac{\partial}{\partial z}\left[\rho u\left(h+\frac{u^{2}}{2}\right)\right]=\frac{q}{d}
$$

where $\rho$ is the density, $u$ the velocity along the $z$ axis, $p$ the pressure, $e$ the internal energy, $h$ the enthalpy, $q$ the heat flux per $\mathrm{cm}^{2}$ rod surface, $d$ a characteristic width of the cooling channel, $t$ the time, and $z$ the coordinate along the cooling channel.

Sone remarks on these equations should be made:

1. The only independent variable introduced apart from the time $t$ is the axial coordinate $z$ along the cooling channel. The radial gradients of the densities, velocities, and pressures are not taken into account here.

2 . In the transient expulsion process which is of interest here the gravity forces are neglected in the equation of momentum.

3. In the energy equation the contributions from the internal energy, kinetic energy, the heat input at the wall, and the work of displacement are taken into account. The enthalpy appears only as an abbreviation of the term $u+(p / \rho)$, as usual.

Lurie and Noyes ${ }^{(6)}$ neglect the difference between internal energy and enthalpy. Both Noyes and Becker et al. ${ }^{(7)}$ also neglect the term involving kinetic energy. 
But the contribution of the kinetic energy to eq. (2.3) is essential for the expulsion mechanism which is considered here.

In concurrence with Lurie and Noyes and Becker et al. the contributions of forces of dissipation and gravity to the energy balance (2.3) are neglected.

The energy equation (2.3) may now be brought into a simpler form by the introduction of the entropy. This offers advantages for the solution of the set of eq. (2.1)-(2.3). One obtains

$$
\frac{\partial(\rho s)}{\partial t}+\frac{\partial(\rho u s)}{\partial z}=\frac{q}{d T},
$$

where $s$ is the entropy and $T$ the temperature.

Consistent with this set of differential equations are the integral conditions of the next section. They can be obtained for the stationary case, $\partial / \partial t=0$, by integrating once along the $z$ axis.

\section{Shock Fronts in Two-phase Flow}

The motion of the shork fronts considered here shall be quasi-stationary. By transformation of the reference system to the shock front, stationary conditions are the result which are described by the following set of shock equations:

Continuity:

$$
u_{1} \rho_{1}=u_{2} \rho_{2}
$$

Momentum:

$$
u_{1}^{2} \rho_{1}+p_{1}=u_{2}^{2} \rho_{2}+p_{2} ;
$$

Energy:

$$
\frac{u_{1}^{2}}{2}+h_{1}=\frac{u_{2}^{2}}{2}+h_{2} .
$$

Equations (3.1)-(3.3) are valid close to the shock front from the left side (1) and the right side (2). Within the shock front, that is, over some mean free paths only, friction also has to be taken into account. But we idealize the shock front as of zero thickness. Therefore the effect of friction is taken into account only indirectly; if eqs. (3.1)-(3.3) have a solution, the state 2 comes out to be different from 1 .

\subsection{Analytical Treatment of the Shock Conditions in Two-phase Flow}

In this paper homogeneous two-phase mixtures shall be considered. Starting from eqs. (3.1)-(3.3), it is possible to write the initial conditions as

$$
u_{1} \frac{\rho_{1}^{\prime} \rho_{1}^{\prime \prime}}{\rho_{1}^{\prime \prime}+x_{1}\left(\rho_{1}^{\prime}-\rho_{1}^{\prime \prime}\right)}=u_{2} \frac{\rho_{2}^{\prime} \rho_{2}^{\prime \prime}}{\rho_{2}^{\prime \prime}+x_{2}\left(\rho_{2}^{\prime}-\rho_{2}^{\prime \prime}\right)}
$$

$$
\begin{aligned}
& u_{1}^{2} \frac{\rho_{1}^{\prime} \rho_{1}^{\prime \prime}}{\rho_{1}^{\prime \prime}+x_{1}\left(\rho_{1}^{\prime}\right.}-\frac{\left.\rho_{1}^{\prime \prime}\right)}{\prime \prime}+p_{1} \\
& =u_{2}^{2} \frac{\rho_{2}^{\prime \prime} \rho_{2}^{\prime \prime}}{\rho_{2}^{\prime \prime}+x_{2}\left(\rho_{2}^{\prime}-\rho_{2}^{\prime \prime}\right)}+p_{2} ; \\
& \frac{u_{1}^{2}}{2}+h_{1}^{\prime}+x_{1}\left(h_{1}^{\prime \prime}-h_{1}^{\prime}\right) \\
& =\frac{u_{2}^{2}}{2}+h_{2}^{\prime}+x_{2}\left(h_{2}^{\prime \prime}-h_{2}^{\prime}\right) .
\end{aligned}
$$

Here $\rho_{1}^{\prime}$ and $\rho_{1}^{\prime \prime}$ are the densities of the saturated liquid and vapor before the front, respectively, $\rho_{2}^{\prime}$ and $\rho_{2}^{\prime \prime}$ those behind the front; $x_{1}$ and $x_{2}$ are the qualities of the mixture before and behind the front, respectively; corresponding definitions are valid for the enthalpies $h_{1}^{\prime}, h_{1}^{\prime \prime}$ and $h_{2}^{\prime}, h_{2}^{\prime \prime}$, respectively.

We further assume thermal equilibrium of the two phases of the homogeneous mixture, but that implies that the Clausius-Clapeyron equation relates the pressure to the temperature or vice versa:

$$
\left(\frac{d T}{d p}\right)_{c}=\frac{T}{h^{\prime \prime}-h^{\prime}}\left(\frac{1}{\rho^{\prime \prime}}-\frac{1}{\rho}\right)
$$

where $\rho^{\prime}, \rho^{\prime \prime}, h^{\prime}$, and $h^{\prime \prime}$ are now functions of the pressure $p$ only. A homogeneous two-phase flow, therefore, is completely defined by $u, p$, and $x$. Hence, the three eqs. (3.4)-(3.6) are available to determine the three unknown quantities $u_{2}, p_{2}$, and $x_{2}$.

We look for conditions which give a nontrivial solution for $u_{2}, p_{2}$, and $x_{2}$, apart from the trivial solution $u_{2}=u_{1}, p_{2}=p_{1}$, and $x_{2}=x_{1}$. From eqs. (3.4) and (3.5) it follows that

$$
x_{2}=\frac{1}{\rho_{1} \rho_{2}^{\prime}-\rho_{2}^{\prime} \rho_{2}^{\prime \prime}}-\frac{p_{2}-p_{1}}{\rho_{1}^{\prime \prime} u_{1}^{2}} \frac{\rho_{2}^{\prime} \rho_{2}^{\prime \prime}}{\rho_{2}^{\prime}-\rho_{2}^{\prime \prime}}-\frac{\rho_{2}^{\prime \prime}}{\rho_{2}^{\prime}-\rho_{2}^{\prime \prime}},
$$

and with eq. (3.6)

$$
\begin{aligned}
& \frac{\left(p_{2}-p_{1}\right)^{2}}{2 \rho_{1}^{2} u_{1}^{2}}-\frac{p_{2}-p_{1}}{\rho_{1}} \\
& +\left[\left(\frac{1}{\rho_{1}}-\frac{p_{2}-}{\rho_{1}^{2} u_{1}^{2}}\right) p_{1}^{\prime} \rho_{2}^{\prime}-1\right] \\
& -\frac{\rho_{2}^{\prime \prime}}{\rho_{2}-\rho_{2}^{\prime \prime}}\left(h_{2}^{\prime \prime}-h_{2}^{\prime}\right)+h_{2}^{\prime}-h_{1}=0 \text {. }
\end{aligned}
$$

Equation (3.8) is a transcendental equation for the variable $p_{2}$ which depends on $u_{1}, p_{1}$, and $x_{1}$. In addition to the trivial solution $p_{2}=p_{1}$, whose existence is necessary in all cases, eq. (3.8) has a second root characterizing a shock, i.e., $p_{2}=p_{2}\left(u_{1}, p_{1}, x_{1}\right) \neq p_{1}$. Thus, eq. (3.8) represents the exact shock condition for homogeneous two-phase mixtures, which is hardly very illuminating in this form. It is useful in a direct 
form only for a numerical evaluation of the shock strength $p_{2} / p_{1}$.

But it is not too difficult to arrive rather quickly at physically meaningful results in the cases

a) of differentially small shock strengths considered leading to the velocity of sound;

b) of shock strengths which are small enough to allow for a finite but linear approximation of $p_{2} / p_{1}$.

\subsection{The Velocity of Sound}

In order to arrive at the velocity of sound, the following simplifications in eq. (3.8) must be made:

$$
\begin{aligned}
h_{2}^{\prime \prime}-h_{2}^{\prime}=h_{1}^{\prime \prime}-h_{1}^{\prime}= & \Delta h_{1} ; \\
& c_{p l 2}=c_{p l 1} ; \quad \rho_{2}^{\prime}=\rho_{1}^{\prime},
\end{aligned}
$$

where $c_{p l}$ is the specific heat of the liquid. Moreover, we have $\rho^{\prime} \gg \rho^{\prime \prime}$.

These simplifications are the more valid the smaller is the shock strength.

For getting the velocity of sound only differentially small changes have to be considered. Therefore, one obtains from eq. (3.8),

$$
\begin{aligned}
& \frac{\left(p_{2}-p_{1}\right)^{2}}{2 \rho_{1}^{2} u_{1}^{2}}-\frac{p_{2}-p_{1}}{\rho_{1}}+c_{p l 1}\left(T_{2}-T_{1}\right) \\
& \quad+\left(\frac{x_{1}}{\rho_{1}^{\prime \prime}}-\frac{p_{2}-p_{1}}{\rho_{1}^{2} u_{1}^{2}}\right) \rho_{2}^{\prime \prime} \Delta h_{1}-x_{1} \Delta h_{1}=0 .
\end{aligned}
$$

The temperature difference $T_{2}-T_{1}$ is expressed as a pressure difference by means of the Clausius-Clapeyron equation:

$$
T_{2}-T_{1}=\left(\frac{d T}{d p}\right)_{1}\left(p_{2}-p_{1}\right) .
$$

One should realize that for getting the velocity of sound it is sufficient to have a Taylor expansion of first order only. For the case of weak but finite shocks, the Taylor development must be at least of second order.

For the vapor density $\rho_{2}^{\prime \prime}$ in eq. (3.9),

$$
\rho_{2}^{\prime \prime}=\rho_{1}^{\prime \prime}+\delta \rho^{\prime \prime} \text {, }
$$

with

$$
\delta \rho^{\prime \prime}=\left(\frac{\delta \rho^{\prime \prime}}{\delta p}\right)_{T} \delta p+\left(\frac{\delta \rho^{\prime \prime}}{\delta p}\right)_{p} \delta T .
$$

With a modified gas equation $\rho^{\prime \prime}=p / z^{\prime} R T$; therefore, the following relation results:

$$
\delta \rho^{\prime \prime}=\rho_{1}^{\prime \prime} \frac{p_{2}-p_{1}}{p_{1}}\left(1-\frac{p_{1}}{\bar{T}_{1}} \frac{\delta T}{\delta p}\right) .
$$

Here $\delta T / \delta p$ is taken from the Clausius-Clapeyron relation. This gives the relation

$$
\kappa_{2}^{\prime \prime}=\frac{p_{1}}{z^{\prime} R T_{1}^{\prime}}\left[1+\frac{p_{2}-p_{1}}{p_{1}}\left\{1-\left(\frac{d T}{d p}\right)_{1} \frac{p_{1}}{T_{1}}\right\}\right] .
$$

By means of eqs. (3.10) and (3.11) it follows from eq. (3.9) that

$$
\begin{gathered}
\frac{\left(p_{2}-p_{1}\right)^{2}}{2_{\mu_{1}}^{2} u_{1}^{2}}-p_{2} \frac{p_{1}}{\rho_{1}}+c_{p l 1}\left(\frac{d T}{d p}\right)_{1}\left(p_{2}-p_{1}\right) \\
+\left\{( \frac { x _ { 1 } } { \rho _ { 1 } ^ { \prime \prime } } - \frac { p _ { 2 } - p _ { 1 } } { \rho _ { 1 } ^ { 2 } u _ { 1 } ^ { 2 } } ) \frac { p _ { 1 } } { z ^ { \prime } R T _ { 1 } } \left[1+\frac{p_{2}-p_{1}}{p_{1}}\right.\right. \\
\left.\left.\cdot\left(1-\left(\frac{d T}{d p}\right)_{1} \frac{p_{1}}{T_{1}}\right)\right]-x_{1}\right\} \Delta h_{1}=0
\end{gathered}
$$

After some transformations this gives

$$
\begin{aligned}
&\left(p_{2}-p_{1}\right)^{2} {\left[\frac{z^{\prime} R T_{1}-2\left\{1-\left(\frac{d T}{d p}\right)_{1} \frac{p_{1}}{T_{1}}\right\} \Delta h_{1}}{2 \rho_{1}^{2} u_{1}^{2} z^{\prime} R T_{1}}\right] } \\
&-\left(p_{2}-p_{1}\right)\left[\frac{1}{\rho_{1}}-c_{p l 1}\left(\frac{d T}{d p}\right)_{1}+\frac{p_{1} \Delta h_{1}}{{ }_{1}^{2} u_{1}^{2} z^{\prime} R T_{1}}\right. \\
&\left.-\frac{x_{1} \Delta h_{1}}{p_{1}}\left\{1-\left(\frac{d T}{d p}\right)_{1} \frac{p_{1}}{T_{1}}\right\}\right]=0 .
\end{aligned}
$$

Now it is possible to separate the trivial solution $p_{2}=p_{1}$. If the difference between $p_{2}$ and $p_{1}$ becomes infinitely small for the remaining nontrivial root $\left(p_{2}-p_{1}\right)$, this results in the velocity of sound $a$ in homogeneous two-phase mixtures according to eq. (3.13) of

$$
\begin{aligned}
& u_{1}^{2}=a_{1}^{2} \\
& =\frac{\frac{\rho_{1}^{\prime \prime}}{\rho_{1}^{2}} \Delta h_{1}\left(\frac{d p}{d T}\right)_{1}}{c_{p l 1}+x_{1}\left[\frac{\Delta h_{1}}{p_{1}}\left(\frac{d p}{d T}\right)_{1}-\frac{\Delta h_{1}}{T_{1}}-\frac{1}{x_{1} \rho_{1}}\left(\frac{d p}{d T}\right)_{1}\right]} .
\end{aligned}
$$

The propagation of weak disturbances is nearly isentropic also in two-phase mixtures, so that the derivation of the velocity of sound may just as well be made from the general expression

$$
a^{2}=\left(\frac{\partial p}{\partial \rho}\right)_{s}=-\left(\frac{\partial p}{\partial v}\right)_{s} v^{2}
$$

where $v$ is the specific volume. In order to evaluate this expression a reduction of eq. (3.15) to known functions in the two-phase region is necessary. From the total differentials of the entropy $s$ and the specific volume $v$ :

$$
\begin{aligned}
& d s(p, x)=\left(\frac{\partial s}{\partial p}\right)_{x} d p+\left(\frac{\partial s}{\partial x}\right)_{p} d x \\
& d v(p, x)=\left(\frac{\partial v}{\partial p}\right)_{x} d p+\left(\frac{\partial v}{\partial x}\right)_{p} d x
\end{aligned}
$$


after brief calculation the differential quotient in question is the following:

$$
\left(\frac{\partial p}{\partial v}\right)_{s}=\frac{\left(\frac{\partial s}{\partial x}\right)_{p}}{\left(\frac{\partial v}{\partial p}\right)_{x}\left(\frac{\partial s}{\partial x}\right)_{p}-\left(\frac{\partial v}{\partial x}\right)_{p}\left(\frac{\partial s}{\partial p}\right)_{x}} .
$$

If the functions for two-phase mixtures

$$
\begin{aligned}
& s(p, x)=s_{l}+x\left(s_{v}-s_{l}\right) ; \\
& v(p, x)=v_{l}+x\left(v_{v}-v_{l}\right),
\end{aligned}
$$

where the subseripts $l$ and $v$ denote liquid and vapor, are known, the differential quotients in eq. (3.16) are also known, as was also shown by Levy. ${ }^{(8)}$ Thus the velocity of sound in homogeneous two-phase mixtures can also be written as

$$
\begin{aligned}
a^{2}=-\left(\frac{\partial p}{\partial v}\right)_{s} v^{2} & -\frac{v^{2}}{(1-x) \frac{d v_{l}}{d p}+x \frac{d v_{v}}{d p}} \\
& -\frac{v_{v}-v_{l}}{s_{v}-s_{l}}\left[(1-x) \frac{d s_{l}}{d p}+x \frac{d s_{v}}{d p}\right]
\end{aligned}
$$

By using the entropy differential

$$
d s=\frac{d h}{T}-v \frac{d p}{T}
$$

and the conditions which resulted in eq. (3.14), it is possible to transform eq. (3.17) into eq. (3.14).

In eq. (3.17) it becomes apparent that in the plane of thermal states it is not possible to differentiate across the curves bounding the two-phase region but only along the boundaries $x=0$ and $x=1$, respectively, as these lines form discontinuities in the plane of thermal states. Thus, the velocity of sound in homogeneous two-phase mixtures and also in saturated and superheated liquids (i.e., $x \rightarrow 0$, but different from zero) is obtained.

The derivation of the velocity of sound was presented in considerable detail since it is of general importance for the understanding of shock conditions.

In the next section it will be shown that for velocities $u_{l}>a_{l}$ the existence of shocks is possible.

One should be aware of the fact that the velocity of sound obtained here implies differentially small condensations and vaporisations, $x_{2}-x_{1} \neq 0$. This requires an adequately quick heat transfer and, therefore, an adequately high degree of homogeneity. If this condition is not fulfilled it may happen that the process of condensation and vaporization cannot follow fast enough because of delays by heat transfer. The velocity of sound in that case finds a limit in the case in which there are no phase changes $\left(x_{2}=x_{1}\right)$. For that case one can evaluate a different velocity of sound. This will be done in section ,3.5.2.

\subsection{Conditions for Weak but Finite Shocks}

The exact shock equation (3.8) is transcendental, and this prevents the insight into the conditions and mechanisms of shock fronts in flowing two-phase mixtures. Therefore, it is first transformed:

$$
\begin{aligned}
& \frac{\left(p_{2}-p_{1}\right)^{2}}{2 \rho_{1}^{2} u_{1}^{2}}-p_{2}-\frac{p_{1}}{\rho_{1}}+\left(\frac{x_{1}}{\rho_{1}^{\prime \prime}}-\frac{p_{2}-p_{1}}{\rho_{1}^{2} u_{1}^{2}}\right) \\
& \cdot \frac{\Delta h_{1}}{z^{\prime} R} \frac{p_{2}}{T_{2}}+c_{p l 1} T_{2}-c_{p l 1} T_{1}-x_{1} \Delta h_{1}=0,
\end{aligned}
$$

where the modified gas equation was used for the steam density:

$$
\rho_{2}^{\prime \prime}=\frac{p_{2}}{z^{\prime} R T_{2}} .
$$

Further, $\rho_{2}^{\prime} \gg \rho_{2}^{\prime \prime}$ as well as $\rho_{2}^{\prime} \approx \rho_{1}^{\prime}$ were taken into account.

In eq. (3.18) we now expand the temperature $T_{2}$ and the function $1 / T_{2}$ into a Taylor series as a function of pressure around state 1 :

$$
\begin{aligned}
& T_{2}=T_{1}+\left(\frac{d T}{d p}\right)_{1}\left(p_{2}-p_{1}\right) \\
& \quad+\frac{1}{2}\left(\frac{d^{2} T}{d p^{2}}\right)_{1}\left(p_{2}-p_{1}\right)^{2}+\cdots \\
& \frac{1}{T_{2}}=\frac{1}{T_{1}}-\frac{1}{T_{1}^{2}}\left(\frac{d T}{d p}\right)_{1}\left(p_{2}-p_{1}\right) \\
& -\left[\frac{1}{2 T_{1}^{2}}\left(\frac{d^{2} T}{d p^{2}}\right)_{1}-\frac{1}{T_{1}^{3}}\left(\frac{d T}{d p}\right)_{1}^{2}\right]\left(p_{2}-p_{1}\right)^{2}+\cdots
\end{aligned}
$$

The series are truncated after the second order, one order more than necessary in the case of flow near sound conditions. From eqs. (3.18), (3.19), and (3.20) an algebraic equation of the fourth order in $p_{2} / p_{1}$ is formed:

$$
\begin{aligned}
& \left(p_{2}-p_{1}\right)^{3} \frac{p_{2}}{p_{1}^{2}}\left[\frac{\Delta h_{1} z^{\prime} R \rho_{1}^{\prime \prime}}{2 \rho_{1}^{2} u_{1}^{2}}\left(\frac{d^{2} T}{d p^{2}}\right)_{1}\right. \\
& \left.-\frac{\Delta h_{1} z^{\prime} R \rho_{1}^{\prime \prime}}{\rho_{1}^{2} u_{1}^{2} T_{1}}\left(\frac{d T}{d p}\right)_{1}^{2}\right]+\left(p_{2}-p_{1}\right)^{2} \\
& \cdot \frac{p_{2}}{p_{1}}\left[-\frac{x_{1} \Delta h_{1}}{2 T_{1}}\left(\frac{d^{2} T}{d p^{2}}\right)_{1}+\frac{x_{1} \Delta h_{1}}{T_{1}^{2}}\left(\frac{d T}{d p}\right)_{1}^{2}\right. \\
& \left.+\frac{\Delta h_{1} \rho_{1}^{\prime \prime}}{\rho_{1}^{2} u_{l}^{2} T_{1}}\left(\frac{d T}{d p}\right)_{1}\right]+\left(p_{2}-p_{1}\right)^{2} \\
& \cdot\left[\frac{1}{2 \rho_{1}^{2} u_{1}^{2}}+\frac{c_{p l}}{2}\left(\frac{d^{2} T}{d p^{2}}\right)_{1}\right]
\end{aligned}
$$




$$
\begin{aligned}
& +\left(p_{2}-p_{1}\right) \frac{p_{2}}{p_{1}}\left[-\frac{x_{1} \Delta h_{1}}{T_{1}}\left(\frac{d T}{d p}\right)_{1}-\frac{\rho_{1}^{\prime \prime} \Delta h_{1}}{\rho_{1}^{2} u_{1}^{2}}\right] \\
& +\left(p_{2}-p_{1}\right)\left[-\frac{1}{\rho_{1}}+c_{p l}\left(\frac{d T}{d p}\right)_{1}+\frac{x_{1} \Delta h_{1}}{p_{1}}\right]=0,
\end{aligned}
$$

or, in an abbreviated form (where $A, B, C, D$, and $E^{\prime}$ replace the square brackets in eq. (3.21),

$$
\begin{aligned}
\left(p_{2}-p_{1}\right)^{3} \frac{p_{2}}{p_{1}^{2}} A+\left(p_{2}-p_{1}\right)^{2} & \frac{p_{2}}{p_{1}} B \\
+\left(p_{2}-p_{1}\right)^{2} & \left(+\left(p_{2}-p_{1}\right)\right. \\
& \quad \frac{p_{2}}{p_{1}} D+\left(p_{2}-p_{1}\right) E^{\gamma}=0 .
\end{aligned}
$$

After separation of the trivial solution $p_{2}=p_{1}$ and multiplication by $1 / p_{1}$ there is a cubic equation for the nontrivial second solution of the basic shock equation:

$$
\begin{aligned}
& \left(\frac{p_{2}}{p_{1}}\right)^{3} A+\left(\frac{p_{2}}{p_{1}}\right)^{2}(-2 A+B) \\
& +\frac{p_{2}}{p_{1}}\left(A-B+C-\frac{D}{p_{1}}\right)-C^{y}+\frac{E}{p_{1}}=0 .
\end{aligned}
$$

In full detail,

$$
\begin{aligned}
\left(\frac{p_{2}}{p_{1}}\right)^{3} & {\left[\frac{\Delta h_{1} z^{\prime} R \rho_{1}^{\prime \prime 2}}{2 \rho_{1}^{2} u_{1}^{2}}\left(\frac{d^{2} T}{d p^{2}}\right)_{1}-\frac{\Delta h_{1} z^{\prime} R \rho_{1}^{\prime \prime 2}}{\rho_{1}^{2} u_{1}^{2} T_{1}}\left(\frac{d T}{d p}\right)_{1}^{2}\right] } \\
+ & \left(\frac{p_{2}}{p_{1}}\right)^{2}\left[-\frac{\Delta h_{1} z^{\prime} R \rho_{1}^{\prime \prime}}{\rho_{1}^{2} u_{1}^{2}}\left(\frac{d^{2} T}{d p^{2}}\right)_{1}+\frac{2 \Delta h_{1} z^{\prime} R \rho_{1}^{\prime 2}}{\rho_{1}^{2} u_{1}^{2} T_{1}}\right. \\
& \cdot\left(\frac{d T}{d p}\right)_{1}^{2}-\frac{x_{1} \Delta h_{1}}{2 T_{1}}\left(\frac{d^{2} T}{d p^{2}}\right)_{1}+\frac{x_{1} \Delta h_{1}}{T_{1}^{2}}\left(\frac{d T}{d p}\right)_{1}^{2} \\
& \left.+\frac{\Delta h_{1} \rho_{1}^{\prime \prime}}{\rho_{1}^{2} u_{1}^{2} T_{1}}\left(\frac{d T}{d p}\right)_{1}\right]+\frac{p_{2}}{p_{1}}\left[\frac{\Delta h_{1} z^{\prime} R \rho_{1}^{\prime \prime 2}}{2 \rho_{1}^{2} u_{1}^{2}}\left(\frac{d^{2} T}{d p^{2}}\right)_{1}\right. \\
& -\frac{\Delta h_{1} z^{\prime} R \rho_{1}^{\prime \prime 2}}{\rho_{1}^{2} u_{1}^{2} T_{1}}\left(\frac{d T}{d p}\right)_{1}^{2}+\frac{x_{1} \Delta h_{1}}{2 T_{1}}\left(\frac{d^{2} T}{d p^{2}}\right)_{1}-\frac{x_{1} \Delta h_{1}}{T_{1}^{2}} \\
& \cdot\left(\frac{d T}{d p}\right)_{1}^{2}-\frac{\Delta h_{1} \rho_{1}^{\prime \prime}}{\rho_{1}^{2} u_{1}^{2} T_{1}}\left(\frac{d T}{d p}\right)_{1}+\frac{1}{2 \rho_{1}^{2} u_{1}^{2}}+\frac{c_{p l}}{2}\left(\frac{d^{2} T}{d p^{2}}\right)_{1} \\
& \left.-\frac{x_{1} \Delta h_{1}}{p_{1} T_{1}}\left(\frac{d T}{d p}\right)_{1}-\frac{\Delta h_{1} \rho_{1}^{\prime \prime}}{p_{1} \rho_{1}^{2} u_{1}^{2}}\right]+\left[-\frac{1}{2 \rho_{1}^{2} u_{1}^{2}}\right. \\
& \left.-\frac{c_{p l}}{2}\left(\frac{d^{2} T}{d p^{2}}\right)_{1}-\frac{1}{\rho_{1} p_{1}}+\frac{c_{p l}}{p_{1}}\left(\frac{d T}{d p}\right)_{1}+\frac{x_{1} \Delta h_{1}}{p_{1}^{2}}\right]=0 .
\end{aligned}
$$

The following points have to be observed in cq. (3.22)

1 . If $p_{2} / p_{1} \rightarrow 1$, this again results in a nontrivial solution of the velocity of sound in the form of eq. (3.14).

2. Truncating the Taylor expansion gives additional roots, which are without any physical meaning, however. Only that root which is contained in the original transcendental eq. (3.8) is of interest.

In order to obtain this root, $p_{2} / p_{1}$ is set up as a linear function in eq. (3.22):

$$
\frac{p_{2}}{p_{1}}=1+\alpha ; \quad\left(\frac{p_{2}}{p_{1}}\right)^{2}=1+2 \alpha ; \quad\left(\frac{p_{2}}{p_{1}}\right)^{3}=1+3 \alpha .
$$

Using the abbreviations of eq. (3.21a) we obtain

$$
\alpha=\frac{-\frac{D}{p_{1}}-\frac{E}{p_{1}}}{B+C+\frac{D}{p_{1}}} .
$$

In spite of its approximate nature this solution gives the desired insight into the physies of the original shock conditions.

Now it is evident that the flow velocity $u_{1}$ (apart, from the thermal quantities $p_{1}$ and $x_{1}$ ) plays an important role also in two-phase flow when conditions for shock fronts are concerned. The characteristic quantity of every consideration of shock fronts in the sense of general gas dynamics is the Mach number $M=u / a$.

After multiplication of numerator and denominator in eq. (3.23) by $p_{\mathrm{t}}(d p / d T)_{1}$ and by using the relations

$$
\left(\frac{d T}{d p}\right)_{1}=\frac{T_{1}^{\prime}}{\Delta h_{1} \rho_{1}^{\prime \prime}}
$$

and

$$
\frac{p_{1}}{\rho_{1}^{\prime \prime}}=z^{\prime} R T_{1}^{\prime},
$$

we obtain the desired shock condition:

$$
\begin{gathered}
\alpha=\frac{\frac{\rho_{1}^{\prime \prime} \Delta h_{1}}{\rho_{1}^{2} a_{1}^{2}}\left(\frac{d p}{d T}\right)_{1}\left(1-\frac{1}{M_{1}^{2}}\right)}{\frac{1}{M_{1}^{2}} \frac{\rho_{1}^{\prime \prime}}{\rho_{1}^{2} a_{1}^{2}}\left(h_{1}-\frac{3}{2} \frac{p_{1}}{\rho_{1}^{\prime \prime}}\right)\left(\frac{d p}{d T}\right)_{1}+\frac{c_{p l 1}}{2 \Delta h_{1}} \cdot} \\
\cdot\left(\Delta h_{1}-2 \frac{p_{1}}{\rho_{1}^{\prime \prime}}\right)-\frac{x_{1}}{2 \rho_{1}^{\prime \prime}}\left(\frac{d p}{d T}\right)_{1}+\frac{x_{1} \Delta h_{1}}{T_{1}}
\end{gathered}
$$

Equation (3.24) shows that in addition to the trivial solution $p_{2}=p_{1}$ there is indeed a second solution if $M_{1} \neq 1$. In the following section it is shown that the entropy criterion excludes dilution shocks also in twophase flow. However, this means that only in cases of $M_{1}>1$ discontinuities, i.c., shocks, may be experted.

Now it is important that in the homogeneous twophase flow of low quality this limiting value may be reached and exceeded very easily. In section 3.6 this is discussed in great detail both phenomenologically and numerically.

The conditions for shock fronts in flowing homogeneous two-phase mixtures and for $x_{1} \rightarrow 0$ (but different from zero, saturated fluids) and $x_{1} \rightarrow 1$ (but different, from one, vapors) are, therefore, as follows: 


$$
\begin{gathered}
\frac{p_{2}}{p_{1}}=1+\alpha=1 \\
(3.25)+\frac{\frac{\rho_{2}^{\prime \prime} \Delta h_{1}}{\rho_{1}^{2} a_{1}^{2}}\left(\frac{d p}{d T}\right)_{1}\left(1-\frac{1}{M_{1}^{2}}\right)}{\frac{1}{M_{1}^{2}} \frac{\rho_{1}^{\prime \prime}}{\rho_{1}^{2} a_{1}^{2}}\left(\Delta h_{1}-\frac{3 p_{1}}{2 \rho_{1}^{\prime \prime}}\right)\left(\frac{d p}{d T}\right)+\frac{c_{p l 1}}{2 \Delta h_{1}} \cdot} \\
\qquad\left(\Delta h_{1}-2 \frac{p_{1}}{\rho_{1}^{\prime \prime}}\right)-\frac{x_{1}}{2 \rho_{1}^{\prime \prime}}\left(\frac{d p}{d T}\right)_{1}+\frac{x_{1} \Delta h_{1}}{T_{1}} \\
\text { (3.26) } \frac{x_{2}}{x_{1}}=1+\alpha\left[1-\frac{p_{1}}{T_{1}}\left(\frac{d T}{d p}\right)_{1}-\frac{\rho_{1}^{\prime \prime} p_{1}}{\rho_{1}^{2} a_{1}^{2}} \frac{1}{M_{1}^{2}} \frac{1}{x_{1}}\right] \\
\text { (3.27) } \frac{u_{2}}{u_{1}}=1-\frac{p_{1}}{\rho_{1} u_{1}^{2}}\left(\frac{p_{2}}{p_{1}}-1\right)=1-\frac{p_{1}}{\rho_{1}^{2} a_{1}^{2}} \frac{1}{M_{1}^{2}} \alpha .
\end{gathered}
$$

Equations (3.25)-(3.27) are the complete shock conditions in a linear approximation.

The conditions for density and enthalpy, $\rho_{2} / \rho_{1}$ and $h_{2} / h_{1}$, are functions of

$$
\frac{p_{2}}{p_{1}}, \frac{u_{2}}{u_{1}}, \text { and } \frac{x_{2}}{x_{1}} \text {. }
$$

For the density ratio before and after the front one obtains from eqs. (3.1) and (3.27),

$$
\frac{\rho_{2}}{\rho_{\mathrm{I}}}=\frac{1}{1-\frac{p_{1}}{\rho_{1} a_{1}^{2}} \frac{1}{M_{1}^{2}} \alpha},
$$

and for the enthalpy condition

$$
\frac{h_{2}}{h_{1}}=1+\frac{p_{1}}{h_{1} \rho_{1}} \alpha .
$$

Consistent with the linear approximation for $p_{2} / p_{1}$ higher powers of $p_{2} / p_{1}$ appearing in eqs. (3.28) and (3.29) are not taken into account.

\subsection{The Entropy Criterion for Shacks}

The criterion for the existence of shocks is the increase of entropy in the shock.

For all homogeneous media and also for homogeneous two-phase mixtures, the following relation holds: ${ }^{(9)}$

$$
T_{1}\left(s_{2}-s_{1}\right)=\frac{1}{12}\left(\frac{\partial^{2}}{\partial p^{2}} \frac{1}{\rho}\right)_{s, 1}\left(p_{2}-p_{1}\right)^{3}+\cdots
$$

This change of entropy was derived for states close to state 1 . With a slight pressure increase, the entropy grows with the third power of $p_{2}-p_{1}$ if the second derivative of $1 / \rho$ with respect to $p$ is positive at constant entropy.

A more elaborate consideration shows that the second derivative is positive, not only for gases or liquids, but also for two-phase mixtures. Therefore, also in the case considered here, only shock fronts are possible which are associated with pressure increases.
However, this implies that the shock conditions eqs. (3.25)-(3.27) have a physical meaning only for flow rates $M_{1}>1$; that means there are no dilution shocks in the case of two-phase flow either.

\subsection{Changes of Phase in Shocks}

Equation (3.26) shows directly that, depending upon the quantities of state $u_{1}, p_{1}$, and $x_{1}$, the quality $x_{2}$ after the shock is larger, equal, or smaller than $x_{1}$. According to eq. (3.29), however, the enthalpy after the shork is always higher than before the shock:

$$
h_{2}=h_{1}+\frac{p_{1}}{\rho_{1}} \alpha_{\text {. }}
$$

Associated with the increase with enthalpy is an increase of pressure and temperature. The ClausiusClapeyron equation now shows that pressure increase favors condensation and temperature increase favors vaporization. Therefore, pressure and temperature increase, both dictated by the increase of enthalpy, are competing influences on the question of phase change in the shock front. If the quality is low ( $x_{1}$ small), the specific heat of the mixture is high; the temperature increase for a given increase of enthalpy is then low and leads therefore to condensation.

If on the other hand the quality is high $\left(x_{1}\right.$ large), the specific heat of the mixture is low; the temperature increase for the given increase of enthalpy prevails and leads to vaporization. For certain conditions pressure increase and temperature increase counterbalance each other and no change of phase takes place.

Therefore, all three cases: condensation, no change of phase, and vaporization, can be associated with shocks.

This turns out to be a main feature of the dynamics of two-phase flow.

3.5.1 The Limit between Shocks with Condensation and Shocks with Vaporization. Again it is useful to apply the linear approximation. From eq. (3.26) for $x_{1}=x_{2}$ it follows that

$$
\alpha\left[1-\frac{p_{1}}{T_{1}}\left(\frac{d T}{d p}\right)_{1}-\frac{\rho_{1}^{\prime \prime} p_{1}}{\rho_{1}^{2} a_{1}^{2}} \frac{1}{M_{1}^{2}} \frac{1}{x_{1}}\right]=0 .
$$

For $\alpha \neq 0\left(M_{1}>1\right)$ this equation requires

$$
1-\frac{p_{1}}{T_{1}}\left(\frac{d T}{d p}\right)_{1}-\frac{\rho_{1}^{\prime \prime} p_{1}}{\rho_{1}^{2} a_{1}^{2}} \frac{1}{M_{1}^{2}} \frac{1}{x_{1}}=0 .
$$

Using (3.14), one obtains:

$$
\begin{aligned}
x_{1} & =\frac{\left[\frac{1}{\rho_{1}^{\prime}}-c_{p 1}\left(\frac{d T}{d p}\right)_{1}\right] \frac{p_{1}}{M_{1}^{2} \Delta h_{1}}}{\left[\frac{1}{M_{1}^{2}}-1\right]\left[1-\frac{p_{1}}{T_{1}}\left(\frac{d T}{d p}\right)_{1}\right]-\frac{p_{1}}{M_{1}^{2} \Delta h_{1}} \frac{1}{\rho_{1}^{\prime \prime}}} \\
& =x_{1}\left(p_{1}, M_{1}\right) .
\end{aligned}
$$


Shock fronts in two-phase mixtures of this special composition result in a higher level of pressure and enthalpy, but not in a change of quality. Fquation (3.34) is subject to the following limitations:

a. The relation $\rho^{\prime} \gg \rho^{\prime \prime}$ must hold; the vicinity of the critical point has to be avoided.

b. $M_{1}>1$ must be in the range of the linear approximation.

However, this equation holds exactly for a flow near sound conditions, i.e., $M_{1} \rightarrow 1$. There it is possible to define a limiting curve depending only on $p_{1}$.

For $M_{1} \rightarrow 1$ it follows from eq. (3.34) that

$$
x_{1}=c_{p l 1} \rho_{1}^{\prime \prime}\left(\frac{d T}{d p}\right)_{1}-\frac{\rho_{1}^{\prime \prime}}{\rho_{1}}=x_{1}\left(p_{1}\right) .
$$

Equation (3.35) therefore is the limit between shocks with condensation and those with vaporization in the flow near sound conditions. It can be interpreted as a two-phase flow without heat transfer between the two involved phases.

3.5.2 The Velocity of Sound in Case of Phase Constancy. Now it will be assumed that there is no heat transfer between the two involved phases of a twophase flow. This implies that no longer a ClausiusClapeyron coupling of temperature and pressure exists.

We now derive the velority of sound for that case. Assuming $x_{2}=x_{1}$ in the three basic equations (3.4)(3.6), we may determine the quantities $u_{2}, p_{2}$, and $T_{2}$ without referring to the above-mentioned direct coupling between $p$ and $T$. that

After separation of the trivial root $p_{2}=p_{1}$, it follows

$$
\frac{p_{2}}{p_{1}}=\frac{\frac{1}{2 \rho_{1}^{2} u_{1}^{2}}+\frac{1}{\rho_{1} p_{1}}-\frac{c_{p l}}{z^{\prime} R \rho_{1}^{\prime \prime} p_{1}}}{\frac{1}{2 \rho_{1}^{2} u_{1}^{2}}-\frac{c_{p l}}{x_{1} z^{\prime} R \rho_{1}^{2} u_{1}^{2}}} .
$$

In this case the quantities $\Delta h, c_{p l}$, and $\rho^{\prime}$ are again treated as constants. Again allowing $p_{2} / p_{1} \rightarrow 1$ now gives the velocity of sound in the two-phase mixtures; we have used a compressibility mechanism only and not considered condensation and vaporization:

$$
u_{1}^{2}=a_{1}^{2}=\frac{1}{\frac{x_{1} \rho_{1}}{p_{1}}\left(\frac{\rho_{1}}{\rho_{1}^{\prime \prime}}-\frac{z^{\prime} R}{c_{p l 1}}\right)} .
$$

According to eq. (3.37), which has been evaluated numerically in Fig. 1, the velocity of sound of the twophase mixtures at phase constancy converges rapidly but steadily to the corresponding velocities of sound of the one-phase conditions for $x_{1} \rightarrow 0$ (including zero) as well as $x_{1} \rightarrow 1$ (including one).

Formally, arcording to eq. (3.37), the velocity of sound tends towards infinity for $x_{1} \rightarrow 0$. In that case,

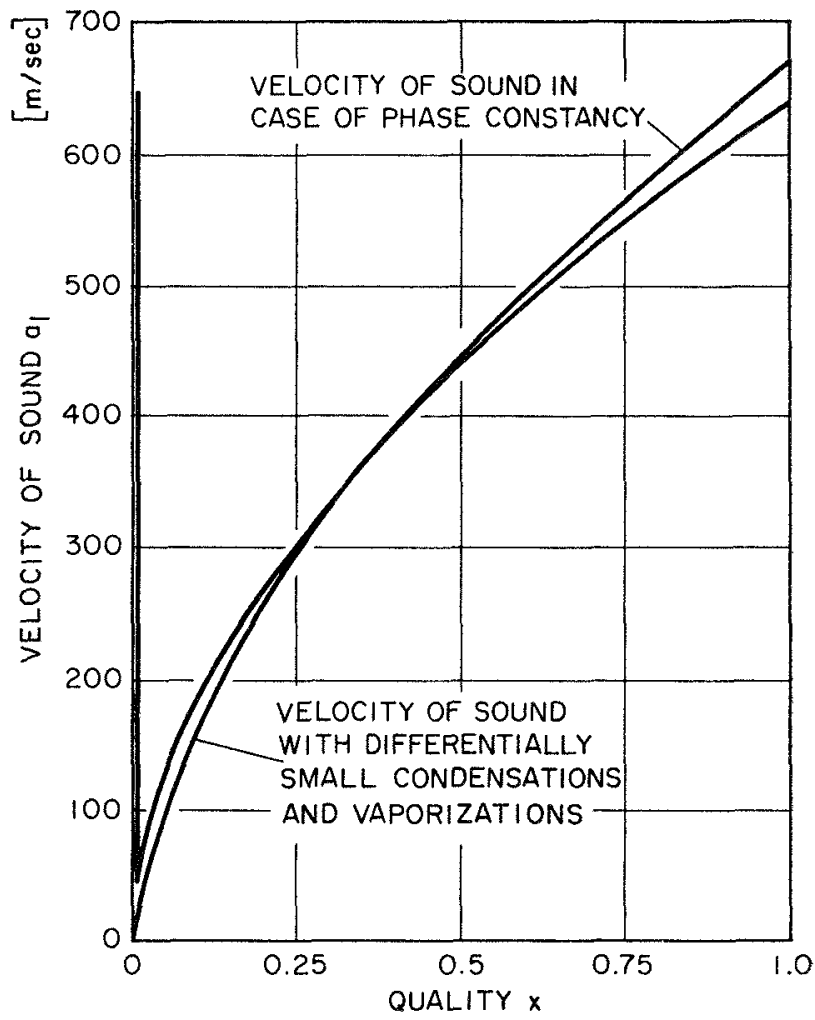

Fif, 1 . Velocity of Sound in a Sodium Two-phase Mixture at $1000^{\circ} \mathrm{C}$ as Function of Quality.

of course, the equation of state of the liquid has to be introduced into the basic equations (3.4)-(3.6).

\subsection{Shock Probability in the Two-phase Flow}

Acrording to section 3.3 , shock fronts are possible in two-phase flow by exceeding the critical velocity, i.e., for $u_{1}>a_{1}$ or $M_{1}>1$. The critical velocity is represented by the velocity of sound, which is not only dependent on the thermal state, but also on the degree of homogeneity of the mixtures.

There is a lower and an upper limit to the velocity of sound in two-phase mixtures as function of the homogeneity. The lower limit implies differentially small condensations and vaporizations in the sound wave and is given by eq. (3.14). These phase changes require an adequately quick heat transfer and therefore an adequately high degree of homogeneity. The upper limit is reached when there are no phase changes in the sound wave and the process of sound propagation is only determined by the compressibility of the mixture. In this case the velocity of sound is given by eq. (3.37).

However, in two-phase mixtures of the special composition according to eq. (3.35), no changes of phase are possible in a sound wave. Therefore in mixtures of such a kind only one velocity of sound is possible, 


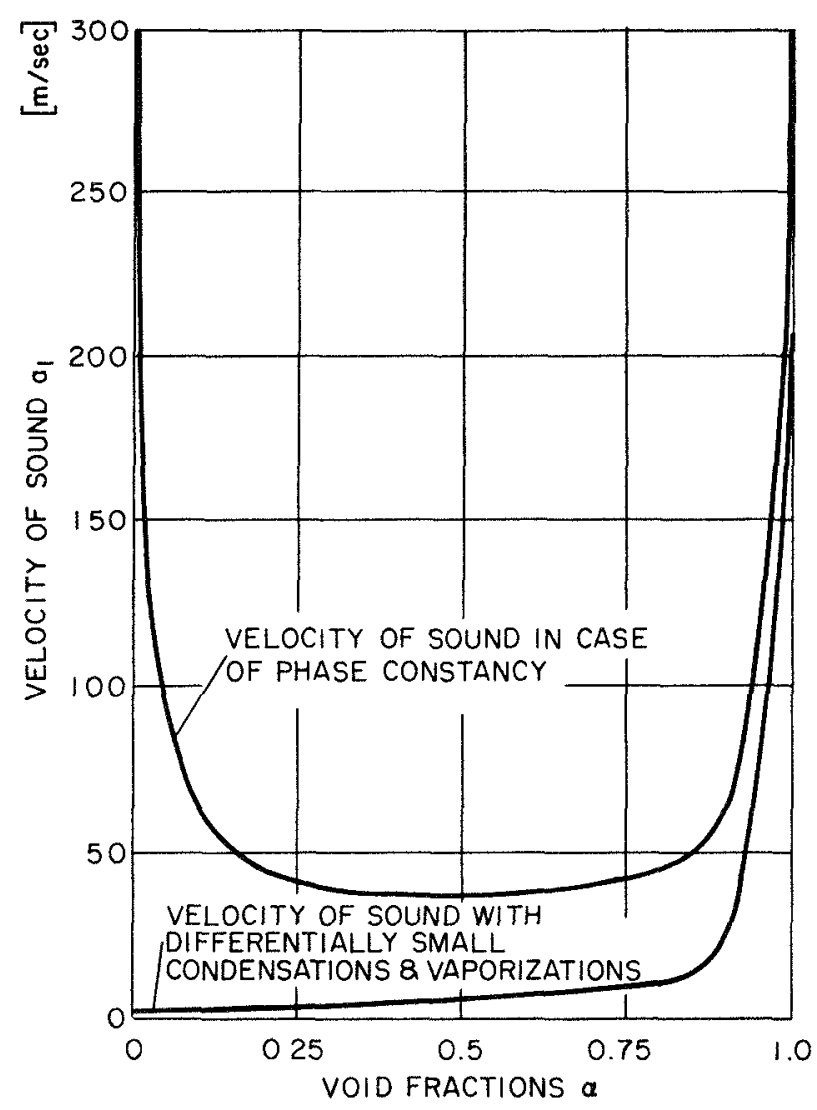

Frg. 2. Velocity of Sound in a Sodium Two-phase Mixture at $1000^{\circ} \mathrm{C}$ as Function of Void Fraction.

i.e., the above-defined lower and upper limits must agree exactly.

Semenov and Kosterin ${ }^{(10)}$ paid no attention in their work to this fact.

In Figures 1 and 2, respectively, these limits of the critical velocity of two-phase mixtures, given by eq. (3.14) and eq. (3.37), are plotted for sodium versus quality and void fraction, respectively.

Most important for the reactivity ramp rate due to voiding the coolant channels are low qualities, because for sodium at a quality of $1 \%$ the vapor volume fraction already reaches nearly $91 \%$. However, in homogeneous mixtures of this low quality, the lower limit of the critical velocity may be reached very easily (see Fig. 1), whereas the upper limit tends very rapidly towards the high value of the liquid.

Associated with the large difference between the two possible critical velocities at low qualities is the effect of dispersion, i.e., the dependence of the propagation of sound waves in two-phase mixtures on the wave frequency.

Let $\tau$ be a characteristic time for heat transfer:

$$
\tau=\frac{\rho c r^{2}}{\lambda}
$$

where $\rho c$ is the heat capacity per $\mathrm{cm}^{3}, r$ is a characteristic length describing the degree of homogeneity, and $\lambda$ is the thermal conductivity. Then the quantity $\tau f$ (where $f$ is the frequency of the sound wave) governs the dispersion. If

$$
\tau f \gg 1,
$$

then the mixture appears to be homogeneous, corresponding to the low velocity of sound [see eq. (3.14)]. If

$$
\tau f \ll 1,
$$

then the mixture does not appear to be homogeneous and the high velocity of sound applies [see eq. (3.37)].

The dispersion phenomenon increases the complexity of the process of coolant ejection.

\section{Shock Fronts in the Case of Superheated Fluids}

The consideration of superheat in connection with shock fronts only makes sense if $x_{1} \rightarrow 0$, because $x_{1} \neq 0$ would immediately lead to desuperheating. But this automatically implies that this consideration is important because in this region $\left(x_{1} \rightarrow 0\right)$ the velocity of sound is low and the shock probability is high (see section 3.6). Therefore, this case shall be considered now, always putting $x_{1}=0$.

\subsection{Desuperheating with a Simultaneous Condensation Shock}

The superheat energy is now introduced in the energy equation (3.6):

$$
\frac{u_{1}^{2}}{2}+c_{p l 1} T_{l b}+c_{p l 1} \Delta T=\frac{u_{2}^{2}}{2}+h_{2}^{\prime}+x_{2}\left(h_{2}^{\prime \prime}-h_{2}^{\prime}\right),
$$

where $T_{l b}$ is the boiling tempcrature and $\Delta T$ is the fluid superheat.

With eq. (4.1) and the functions and abbreviations of sections 3.1 and 3.3 , the following polynominal for the pressure ratio $p_{2} / p_{1}$ is obtained:

$$
\begin{aligned}
& \left(p_{2}-p_{1}\right)^{3} \frac{p_{2}}{p_{1}^{4}} A+\left(p_{2}-p_{1}\right)^{2} \frac{p_{2}}{p_{1}^{3}} B \\
& +\left(p_{2}-p_{1}\right)^{2} \frac{1}{p_{1}^{2}} C+\left(p_{2}-p_{1}\right) \frac{p_{2}}{p_{1}^{2}} \frac{D}{p_{1}} \\
& \quad+\left(p_{2}-p_{1}\right) \frac{1}{p_{1}} \frac{E}{p_{1}}=\frac{c_{p l} \Delta T}{p_{1}^{2}}
\end{aligned}
$$

For eq. (4.2) again the temperature $T_{2}$ was expanded as a function of pressure (see section 3.3).

A trivial solution $p_{2}=p_{1}$ no longer exists in the case of superheating according to eq. (4.2). There are always two solutions which now are nontrivial:

The first root is the so-called quasitrivial solution $\left(p_{2} / p_{1}\right)_{q t}$. It describes desuperheating without a 
condensation shock. The second root characterises desuperheating with a simultaneous condensation shock.

In order to obtain the second root of eq. (4.2), the quasitrivial root $\left(p_{2} / p_{1}\right)_{q t}$ also has to be separated. This is done by replacing in eq. (4.2) the sum of coefficients

$$
C-\frac{E}{p_{1}}-\frac{c_{p l} \Delta T}{p_{1}^{2}}
$$

by the quasitrivial solution:

$$
\begin{aligned}
C-\frac{E}{p_{1}}-\frac{c_{p l} \Delta T}{p_{1}^{2}} & =-\left(\frac{p_{2}}{p_{1}}\right)_{q t}^{4} A-\left(\frac{p_{2}}{p_{1}}\right)_{q t}^{3}(-3 A+B) \\
& -\left(\frac{p_{2}}{p_{1}}\right)_{q t}^{2}\left(3 A-2 B+C+\frac{D}{p_{1}}\right) \\
& -\left(\frac{p_{2}}{p_{1}}\right)_{q t}\left(-A+B-2 C-\frac{D}{p_{1}}+\frac{E}{p_{1}}\right) .
\end{aligned}
$$

In this way it is possible to form differences of the type

$$
\left(\frac{p_{2}}{p_{1}}\right)^{n}-\left(\frac{p_{2}}{p_{1}}\right)_{q t}^{n}
$$

which permits separation. After some calculations the result is

$$
\begin{aligned}
{\left[\left(\frac{p_{2}}{p_{1}}\right)\right.} & \left.-\left(\frac{p_{2}}{p_{1}}\right)_{q t}\right]\left\{\left(\frac{p_{2}}{p_{1}}\right)^{3} A+\left(\frac{p_{2}}{p_{1}}\right)^{2}\right. \\
& \cdot\left[\left(\frac{p_{2}}{p_{1}}\right)_{q t} A-3 A+B\right] \\
& +\frac{p_{2}}{p_{1}}\left[\left(\frac{p_{2}}{p_{1}}\right)_{q t}^{2} A+\left(\frac{p_{2}}{p_{1}}\right)_{q t}\right. \\
& \left.\cdot(-3 A+B)+3 A-2 B+C+\frac{D}{p_{1}}\right] \\
& +\left[\left(\frac{p_{2}}{p_{1}}\right)_{q t}^{3} A+\left(\frac{p_{2}}{p_{1}}\right)_{a t}\right. \\
& \cdot\left(3 A-2 B+C+\frac{D}{p_{1}}\right) \\
& \left.\left.+\left(-A+B-2 C-\frac{D}{p_{1}}+\frac{E}{p_{1}}\right)\right]\right\}=0 .
\end{aligned}
$$

This separates the first root, desuperheating without shock:

$$
\frac{p_{2}}{p_{1}}=\left(\frac{p_{2}}{p_{1}}\right)_{q t} .
$$

What remains is to find the second root of eq. (4.3).
If there is no superheat, i.e., if $\left(p_{2} / p_{1}\right)_{q t}=1$, eq. (4.3) converges into eq. (3.23).

In eq. (4.3) $p_{2} / p_{1}$ is again set up as a linear function:

$$
\frac{p_{2}}{p_{1}}=1+\alpha^{*} ; \quad\left(\frac{p_{2}}{p_{1}}\right)^{2}=1+2 \alpha^{*} ; \quad\left(\frac{p_{2}}{p_{1}}\right)^{3}=1+3 \alpha^{*} .
$$

For desuperheating with a simultaneous condensation shock, it then follows for the intensity of the shock in a linear approximation and with the abbreviations used in section 3.3 that [see eq. (4.4) at bottom of page].

According to the entropy criterion, shock fronts are again always connected with an increase in pressure. Hence the possibility of condensation shocks with a simultaneous desuperheating in superheated fluids is directly described by eq. (4.4). It will be always a condensation shock.

The condensation in the shock front is weakened by vaporization due to desuperheating. Therefore, shock fronts with simultaneous desuperheating according to eq. (4.4) are restricted to regions with superheated fluids in which the effects of condensation and vaporization balance out partially or totally.

The condensation effect is determined by the flow velocity, the associated vaporization effect by the degree of supcrheat.

We now consider a second kind of critical velocity leading to $\alpha^{*}=0$; that means condensation (due to the shock) and vaporization (due to desuperheating) compensate totally. This critical velocity $a^{*}$ is a function of the degree of superheat and the Mach number (involving the velocity of sound with phase changes).

Desuperheating with a simultaneous condensation shock can happen only for flow velocities larger than $a^{*}$, because only then condensation prevails.

In Fig. 3 this second critical velocity $a^{*}$ is plotted for sodium versus the degree of superheat.

\subsection{Desuperheating without a Simultaneous Condensation Shock}

The first root of eq. (4.2) is the so-called quasitrivial solution $\left(p_{2} / p_{1}\right)_{q t}$. It is generated in the case of superheating evolving from the original trivial solution $p_{2}=p_{1}$. It describes the desuperheating without a condensation shock.

The quasitrivial solution is now given as a linear expansion with respect to the superheating term of eq. (4.2):

$$
\alpha^{*}=\frac{A\left[\left(\frac{p_{2}}{p_{1}}\right)_{q t}^{3}-2\left(\frac{p_{2}}{p_{1}}\right)_{q t}^{2}+\left(\frac{p_{2}}{p_{1}}\right)_{q t}\right]+B\left[\left(\frac{p_{2}}{p_{1}}\right)_{q t}^{2}-\left(\frac{p_{2}}{p_{1}}\right)_{q t}\right]+C\left[\left(\frac{p_{2}}{p_{1}}\right)_{q t}-1\right]+\left(\frac{p_{2}}{p_{1}}\right)_{q t} \frac{D}{p_{1}}+\frac{E}{p_{1}}}{-\left\{A\left[\left(\frac{p_{2}}{p_{1}}\right)_{q t}^{2}-\left(\frac{p_{2}}{p_{1}}\right)_{q t}\right]+\left(\frac{p_{2}}{p_{1}}\right)_{q t} B+C+\frac{D}{p_{1}}\right\}} .
$$




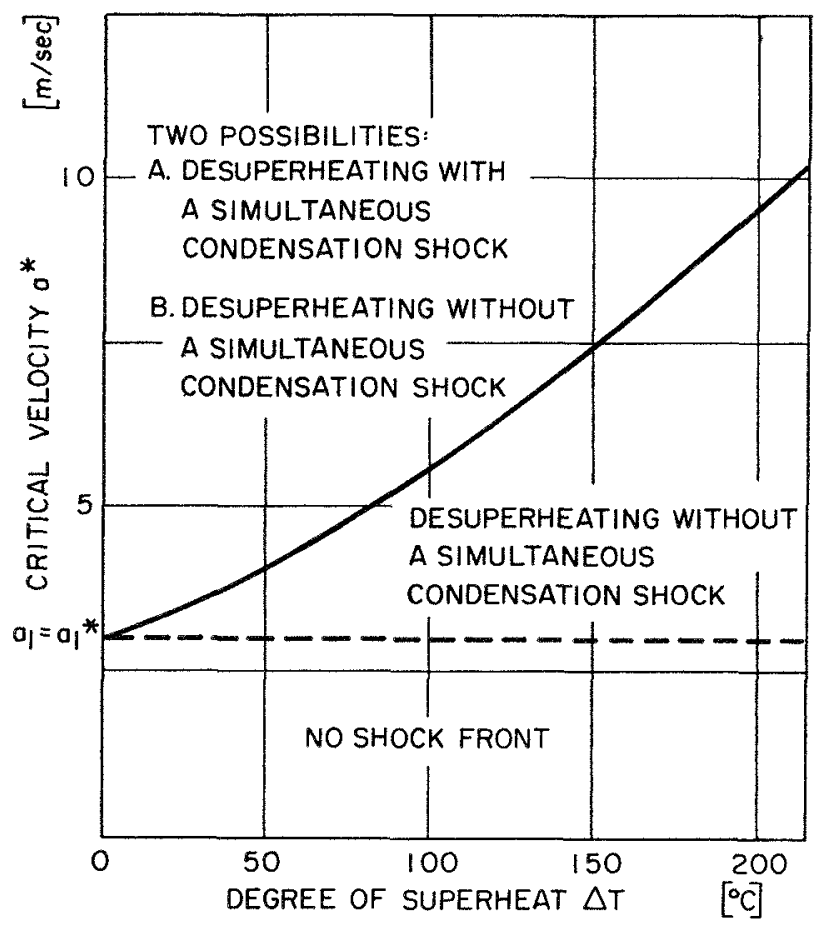

FIG. 3. Critical Velocity in Superheated Sodium at a Pressure of $2.71 \mathrm{~atm}$ (i.e., a Boiling Temperature of $1000^{\circ} \mathrm{C}$ ).

$$
\left(\frac{p_{2}}{p_{1}}\right)_{q t}=1+\frac{\frac{c_{p l 1} \Delta T}{p_{1}}}{\frac{\Delta h_{1} \rho_{1}^{\prime \prime}}{\rho_{1}^{2} \alpha_{1}^{2}}\left(1-\frac{1}{M_{1}^{2}}\right)} .
$$

Also, this shock condition is physically meaningful for $M_{1}>1$ only. $\left(p_{2} / p_{1}\right)_{q t}-1$ is inversely proportional to $1-\left(1 / M_{1}^{2}\right)$, a term which appeared up to now only as a numerator [see eq. (3.24)]. It refers to the velocity of sound with phase changes [see eq. (3.14)]. This critical velocity is the proper measure for the flow velocity also in this case. If $M_{1} \rightarrow 1$ the quantity $\left(p_{2} / p_{1}\right)_{q t}-1$ tends towards infinity. The reason is that the pressure increase associated with the process of desuperheating cannot dissipate, because such dissipation would have to use the velocity of sound and the flow of the fluid just counteracts such a dissipation.

At flows for which $M_{1}>1$, the energy of the front is indeed dissipated over larger flow areas in the direction of flow, which results in a removal of the otherwise infinitely high pressure at the original place of the shock.

In Fig. 4 the two possible shock fronts are plotted for superheated sodium:

1. desuperheating without a condensation shock and according to section 4.1 ;

2. desuperheating with a simultaneous condensation shock.

Figure 3 also evaluates the limits for desuperheating with and without simultaneous shocks.
It is useful to apply the following arguments:

Again the mechanism of state 1 involved here is that $x_{1} \rightarrow 0$ but is different from zero. This can be seen from the nature of $a$ [see eq. (3.14)], which appears in the definition of $M$ in eq. (4.5). It is implied also by the fact that, according to the shock conditions, the increase of pressure is accompanied by an increase of density as it is in the front phenomena without superheat. This is true in spite of the occurrence of boiling.

But one does expect a dilution if boiling occurs. This cannot take place in form of a shock phenomenon; it spreads out over a finite interval and, therefore, becomes a boiling wave and not a boiling front. The experiments of Friz ${ }^{(5)}$ also seem to indicate this.

\section{Conclusions}

The state of liquid is described by $x_{1}=0$; if boiling starts it therefore evolves from $x_{1}=0$. According to the results of this paper one has to face a somewhat complex situation. On the one hand, there exists the dispersion phenomenon. But if the quality $x$ increases somewhat,

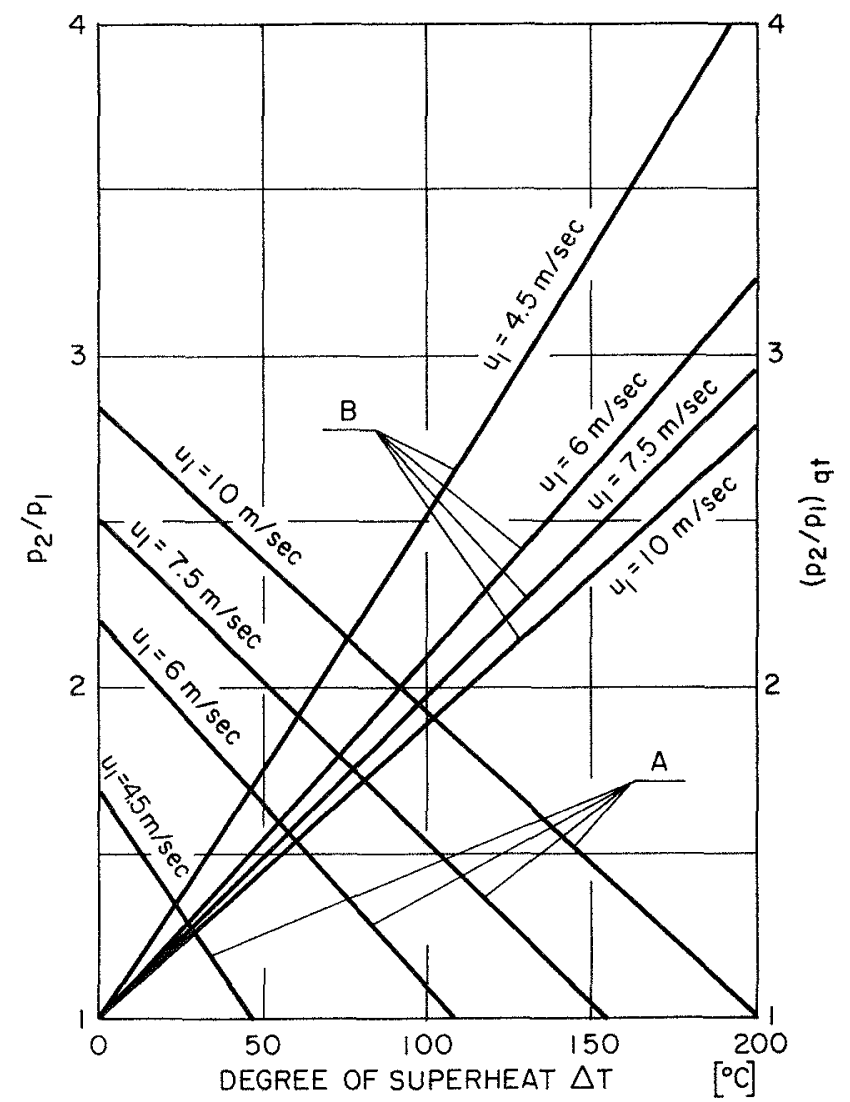

Fig. 4. The Two Possible Shock Fronts in the Case of Superheated Sodium at a Pressure of $2.71 \mathrm{~atm}$ (i.e., a Boiling Temperature of $1000^{\circ} \mathrm{C}$ ): A. Desuperheating with a Simultaneous Condensation Shock: $p_{2} / p_{1} ; \mathrm{B}$. Desuperheating without a Simultaneous Condensation Shock, i.e., the Quasitrivial Solution $\left(p_{2} / p_{1}\right)_{q t}$. 
the two critical velocities which are possible come close to each other again. Then in the general case there is practically only one value for the velocity of sound, i.e., the critical velocity. The possibility for shock waves, on the other hand, increases as the velocity of sound decreases. Therefore, in general the low velocities of sound obtained in this study indicate that there is a rather large probability for shocks.

The occurrence of shocks in turn reduces the flow velocity to subcritical values again. Therefore, the velocity of sound is a rather general indication for the voiding of a coolant channel.

A typical situation could be the following:

Saturated sodium at $1000^{\circ} \mathrm{C}(2.71 \mathrm{~atm})$

\begin{tabular}{l|c|c|c}
\hline quality, $x_{1}$ & 0.0001 & 0.001 & 0.01 \\
\hline \begin{tabular}{l} 
void fraction, $\beta_{1}$ \\
\hline $\begin{array}{l}\text { lower limit of the critical velocity, } \\
a_{1} \text { (m/sec) }\end{array}$
\end{tabular} & 0.087 & 0.49 & 0.91 \\
\hline $\begin{array}{l}\text { upper limit of the critical velocity, } \\
a_{2}(\mathrm{~m} / \mathrm{sec})\end{array}$ & 69.0 & 39.0 & 66.0 \\
\hline
\end{tabular}

We conclude that in the sense of the arguments given above and with regard to the already large void fraction at still very small qualities, an overall effective expulsion velocity could be of the order of at least 4 $\mathrm{m} / \mathrm{sec}$ and at most $60 \mathrm{~m} / \mathrm{sec}$.

Together with the more recent value of a maximum sodium void effect of the Karlsruhe 1000-MWe study of $\$ 7$, this leads to ramp rates of at least $\$ 56 /$ sec to several hundred $\$ /$ sec, with a minimum expulsion time of 125 msec.

The ramp rate due to voiding the coolant channels is, therefore, indeed an open problem. It must be realized that with the present study only the lower and upper limits of the ramp rate were investigated. A detailed. study of the ejection taking into account all kinds of phenomena described here will follow as mentioned earlier.

\section{REFERENCES}

1. W. Häfele, D. Smidt, and K. Wirtz, The Karlsruhe Reference Design of a 1000-MWe Sodium-cooled Fast Breeder Reactor, see paper in Session III of this Conference.

2. D. Smidt et al., Safety and Cost Analysis of a 1000-MWe Sodium-cooled Fast Breeder Reactor, see paper in Session I of this Conference.

3. K. H. Küsters and W. Metzenroth, The Influence of Some Important Group Constants on Integral Fast Reactor Quantities, see paper on Session V,B of this Conference.

4. D. Smidt, Der Siedevorgang und seine Auswirkungen in grossen natriumgekühlten schnellen Brutreaktoren, Kernforschungszentrum Karlsruhe, Internal IRE-Report No. $18 / 65$.
5. G. Friz, Coolant-ejection Studies with Analogy Experiments, See paper in Session VIII,B of this Conference.

6. H. Lurie and R. C. Noyes, Boiling Studies for Sodium Reactor Safety, Part II, NAA-SR-9477.

7. K. M. Becker et al., Hydrodynamic Instability and Dynamic Burnout in Vatural Circulation Two-phase Flow. An Experimental and Theoretical Study, III. International Conference on the Peaceful Uses of Atomic Energy, Geneva (1964), A/Conf.28/P/607.

8. S. Levy, Prediction of Two-phase Critical Flow Rate, GEAP4395 (1963).

9. K. Oswatitsch, Gasdynamik, J. Springer, Berlin (1952).

10. N. J. Semenov and S. J. Kosterin, Results of an Investigation of the Speed of Sound in Moving Gas-Liquid Mixtures, Teploenergetika, 11, No. 6 (1964) and WAPDTrans-15.

\section{Discussion}

Mr. MacFarlane (ANL): What type of flow do you have? Is it a homogeneous model? Secondly, if this is an expediency for calculation, what effect would a slip type of model have on the results you have calculated?

Nr. Fischer: I think in the expulsion process the slip ratio is not essential. Therefore, we are limited only by the upper and lower limits of the two derivatives; this implies that the homogeneity of the mixture is only essential for the critical velocity.

Mr. Fauske (ANL): As far as the upper and lower limits of the critical sonic velocity are concerned, measurements have been made recently of a critical flow in air-water systems compared to steam-water systems which give an excellent comparison between metastability and equilibrium. From these results the metastable sonic velocity apparently is the one controlling. This work was also presented at the recent Oxford meeting on two-phase flow.

Mr. Fischer: Yes. This work was done by the Rusrians.

Mr. Häfele (Karlsruho): In the Russian work it is indicated that the velocity of sound follows the scheme in which no change of phases appear. However, one should be careful because it depends on the degree of homogeneity. The characteristic time is defined as $\rho c r^{2} / \lambda$ as in the paper just given. For a given heterogeneity one can dream of sufficiently low frequencies that heat transfer is possible, and one can think of frequencies which are high enough to avoid heat transfer. Therefore, it should always be made clear what frequencies are being considered. This dispersion phenomenon really adds to the complexity.

$M r$. Cohen (GE): What pressures were involved in your calculations?

Mr. Häfele: In our investigations the amount of pressure built up was not yet explicit. It will be investigated in a second step.

$M r$. Fell (Winfrith): If I regarded this as a problem 
of transmission of sound through a mixture of materials with two sound velocities, I'd recognize there were two problems: the question of the differing velocity of sound in the two media, and the question of impedance matching between the two media. I'd expect to find, in fact, that you just can't transmit a sound through this material, just as, for example, a bottle of glass beads can' $t$ be seen through. Why is it that you can ignore the actual physical heterogeneity and the matching between the phases when you do a model of this kind? You certainly can't if you tried to transmit light through the mixture of material of different dielectric constants.

Mr. Fischer: I would say if you take into account the inhomogeneity, the upper limit is defined by the inhomogeneity.

Mr. Fell: Surely in that case you would expect to see an attenuation effect. If you have a mixture of materials, the two associated velocities have different impedances. You get strong attenuation in a medium. of this kind. I just wonder how valid caleulations of this kind are, which say that there is a different velocity, and you have a statement of the velocity as a function of frequency, but you have no statement of attenuation. I thought that the actual physical heterogeneity itself did produce a strong attenuation of sounds, of velocities of this kind.

Mr. Fischer: The degree of homogeneity of the mixture is described by the factor $\tau$, where $\tau$ is the characteristic time for heat transfer and $f$ is the frequency of the sound wave. Therefore, we take both the inhomogeneity and the frequency into account.

Mr. Zivi (TRW): Some years ago, at what was then Armour Research Foundation, Karplus developed a theory for the propagation of sound through two-phase mixtures and shock fronts. Can you tell me how your theory differs from his? Are you familiar with his work?

Mr. Fischer: No.

Mr. Lottes: The report which was published as an Armour Research Bulletin, I believe, wasn't widely publicized. If I might, I would like to add one comment about Karplus' work that amplifies some of the things that have been said here. His initial experiments were with gas-water systems and the gas bubbles were of very small diameters, of the order of a fraction of a millimeter. He calculated a bulk modulus of the mixture in two ways: Case A was for an isothermal process, and Case B for an adiabatic one. The results of the two calculations differed by $20 \%$. He couldn't force his data to fit the adiabatic relation; his case was isothermal. This raised the question of what do you do in a steamwater mixture-should you allow heat transfer, condensation, vaporization to take place? He proposed certain experiments along this line but lost support for his project. We never saw any final results.
Mr. Zivi: He did make some measurements and in fact has data on the propagation of very strong shocks through two-phase mixtures. Shocks that are so strong that the pressure completely collapses the voids. I know he did get extremely low acoustic velocities, much lower for the steam-water than for the air-water mixtures.

Mr. Wolfe (GE): I'd like to ask a question of the previous speaker. Is it possible to get pressures in a channel which are higher than the equivalent saturation pressure when you have superheated liquid? If so, what is the physical cause of these higher pressures?

Mr. Zivi: I think the answer is it's possible. The physical mechanism is the water-hammer type of impact. You have a relatively long time to put impulse into a slug of liquid. It can hit a rigid boundary or other liquid, and the impulse is converted into pressure in a short time.

Mr. Fauske: The question was raised earlier in regard to what effect slip might have on the sonic velocity. If you look, not at bubble flow, but at droplet flow, just the complete opposite, in the gas phase, the continuous phase, it turns out you have to take into account slip in order to explain the sonic velocities. Furthermore, in the limiting case the liquid droplet is not seen at all by the sound wave. In other words, you have a constant void fraction there as the sound is propagated through the two-phase system. In calculation you find that the sonic velocity is independent of quality in a high-quality range. Data have been taken in this region that satisfy the calculations.

Mr. Fischer: No, I disagree. The sound velocity in the case of constant phase is dependent upon the compressibility, and the compressibility is a function of the void fraction or quality.

Mr. Fauske: I'm talking about droplet flow primarily at low pressure, where the void fraction is practically $100 \%$.

Mr. Fischer: But there is a large dependence upon the quality.

Mr. Fauske: Not in this region.

$M r$. Fischer: In that region, of course. With a quality of $1 \%$ the void fraction is $90 \%$. If so, and you saw the first limiting curves as function of quality, there is a large dependence upon the quality.

$M r$. Fauske: I'm talking about a different quality region now. I'm talking about 50,60 , or $70 \%$ quality, which you don't have on the plot at all. If you assume thermodynamic equilibrium or no slip at all, you cannot explain the sonic velocity obtained because it does appear as a function of quality and this will show up experimentally.

Mr. Hafele: I think one should be aware of the fact that after the reflection of a shork wave or after inter- 
action of shock waves together, pressure can increase tremendously. This can provide a mechanism to increase pressure besides what can happen in a regular flow. Just because the velocity of sound is in many cases so very small, the probability of shock waves has increased, and by the same token there is an increase of the probability of a pressure increase. Thus the picture becomes even more complex. Mr. Fischer and I were very astonished to see that after a shock front passes, both vaporization and condensation can takc place. The more you look at the problem the more complex it becomes.

$M r$. Bump (ANL): In the study of coolant ejection by Friz, was there any vertical temperature gradient in the water? In other words, the hotter material would tend to go to the top and therefore superheat would be greater at the top.

Mr. Friz (EURATOM, Ispra): The temperature difference was less than $0.3^{\circ} \mathrm{C}$ because we could control the temperature in the interior and in the thermostat itself. The only reason that acceleration starts at the exit is the big fall in pressure related to the superheating temperature. On the other hand, it is impossible or nearly impossible that the acceleration start at the bottom, because the whole column must then be accelerated, which takes a much greater dynamic pressure gradient than when it starts at the exit.

Mr. Fischer (ANL): What I'm concerned about is the kinetics which initiate a fast reactor accident. The point I'm concerned about is as we go towards pancakes or o1her types of large fast reactors, we get very different power gradients axially. Now suppose you have, for example, a pancake reactor. What does a very flat axial gradient do to the kineties of sodium ejection?

Mr. Hafele: We have been working many months to get the answer and don't have it yet. 


\section{Non-ANL Attendees}

Abiko, S., Atomic Fuel Corp. of Japan

Adler, F. T., University of Illinois

Allgeier, H., Atomic Power Development Associates

Alter, H., Atomics International

Amorosi, A., Atomic Power Development Associates

An, S., Tokyo University

Anderson, C., Los Alamos Scientific Laboratory

Astley, E. R., Battelle-Northwest Laboratories

Baily, W. E., General Electric Co.

Baroch, C., Babcock \& Wilcox Company

Bartels, W. C., U. S. Atomic Energy Commission

Bauer, A., Battelle-Columbus

Bauser, Capt. E. J., Joint Congressional Committee on Atomic Energy

Beeley, R. J., Atomics International

Benedict, M., Massachusetts Institute of Technology

Blessing, W., U. S. Atomic Energy Commission

Blomstrand, J. H., CKAEA, Winfrith

Bradbury, B. T., CKAEA, Harwell

Branyan, C. E., Atomic Power Development Associates

Broido, J, General Atomic

Broomfield, A. M., UKAEA, Winfrith

Bryan, R. H., U. S. Atomic Energy Commission

Burton, E. J., CKAEA, Risley

Bustraan, M., Reactor Centrum Nederland

Campise, A. V., Atomics International

Carlsmith, R. S., Oak Ridge National Laboratory

Carmichael, B. M., Los Alamos Scientific Laboratory

Carpenter, S. G., Atomies International

Castleman, A. W., Jr., Brookhaven National Laboratory

Chernick, J., Brookhaven National Laboratory

Cherry, B., United Nuclear Corp.

Christianson, H., Institute for Atomic Energy, Kjeller

Cicognani, G., CNEN, Bologna

Cobb, E. C., Nuclear Power Group, England

Codd, J., UKAEA, Winfrith

Cohen, K., General Electric Co.

Comprelli, F. A., General Electric Co.

Critoph, E., Atomic Energy of Canada Ltd.

Cybulskis, P., U.S. Atomic Energy Commission

Dales, R., Westinghouse Electric Corp.

Dalle-Donne, M., Kernforschungszentrum, Karlsruhe

Davis, J. F., American Electric Power Service Corp.

Delene, J. G., Oak Ridge National Laboratory

Denielou, G., CEN, Cadarache

Dietrich, J. R., Combustion Engineering

Dingee, D. A., Battelle-Columbus
Doan, R. L., U. S. Atomic Energy Commission

Doyle, T. A., Euratom, Brussels

Dunenfeld, M., Allison Division, General Motors Corp.

Edlund, M. C., Babcock \& Wilcox Co.

Eggen, D. T., Atomies International

Engelder, T. C., Babcock \& Wilcox Co.

Engelmann, P., Kernforschungszentrum, Karlsruhe

Falcinelli, G., CNEN, Bologna

Farmer, W., Allis-Chalmers Manufacturing Co.

Fell, J., UKAEA, Winfrith

Fenech, H., Massachusetts Institute of Technology

Field, H., U. S. Atomic Lnergy Commission

Fischer, M., Kernforschungszentrum, Karlsruhe

Flora, J., U. S. Atomic Energy Commission

Fortescue, P., General Atomic

Fricke, E. F., Bell Aerosystems

Friedland, A. J., Atomic Power Development Associates

Friz, G., Euratom, Ispra

Fry, W. E., U.S. Atomic Energy Commission

Gandini, A., CNEN, Rome

Gardiner, D. M., U. S. Atomic Energy Commission

Gariboldi, R., L. S. Atomic Energy Commission

Gast, K., General Electric Co.

Gavigan, F. X., U. S. Atomic Energy Commission

Gee, W., U. S. Atomic Energy Commission

Gibson, A. S., Atomics International

Godts, J. E., Westinghouse Electric Corporation

Greebler, P., General Electric Co.

Green, F. P., Union Carbide Corp.

Gueron, J., Euratom, Brussels

Gunson, W. E., Westinghouse Electric Corp.

Häfele, W., Kernforschungszentrum, Karlsruhe

Hall, D. B., Los Alamos Scientific Laboratory

Hallam, J. W., General Electric Co.

Hannum, W. H., Los Alamos Scientific Laboratory

Harde, R., Interatom $\mathrm{GmbH}$

Heck, F. M., Westinghouse Electric Corp.

Hellstrand, E., AB Atomenergi

Hennig, R. J., Battelle-Northwest Laboratories

Herbst, R., Westinghouse Electric Corp.

Hicks, E. P., UKAEA, Dounreay

Hill, E. E., U. S. Atomic Energy Commission

Hirota, J., Japan Atomic Energy Research Institute

Hofmann, P. L., Battelle-Northwest Laboratories

Hogan, W. S., Battelle-Columbus

Honeck, H., U. S. Atomic Energy Commission

Hovorka, G., Commonwealth Associates, Inc. 
Jackson, G. O., UKAEA, Risley

Jens, W. H., Atomic Power Development Associates Jirlow, K., AB Atomenergi

Jones, R. H., U. S. Atomic Energy Commission

Katman, T. S., Babeock \& Wilcox Co.

Karanagh, G. M., C. S. Atomic Energy Commission

Keller, D. L., Battelle-Columbus

Keller. W., Siemens-Schuckertwerke AG

Kilian, P., Allgemeine Elektricitats-Gesellschaft

Kirchenmayer, A., AEG, Kernenergieanlagen

Kirkbride, L. D., Los Alamos Scientific Laboratory

Klickınan, A. E., Atomic Power Development Associates

Knight, F. W., General Electric Co.

Kohler, W. H., Texas A\&M University

Küchle, M., Kernforschungszentrum, Karlsruhe

Kummerer, K., Kernforschungszentrum, Karlsruhe

Kunkel, W. P., Atomies International

Kuroi, H., Japan Atomic Energy Research Institute

Küsters, H., Kernforschungszentrum, Karlsruhe

Kutchey, J., U. S. Atomic Energy Commission

Leitz, F. J., Jr., General Electric Co.

Lellouche, G. S., Brookhaven National Laboratory

Levine, M. M., Brookhaven National Laboratory

Levine, S., U. S. Atomic Energy Commission

Lichtenberger, H., Combustion Engineering

Lieberman, J. A., C. S. Atomic Energy Commission

Little, W. W., Jr., Battelle-Northwest Laboratories

Maerkl, Dr., Siemens-Schuckertwerke AG

Malmberg, T., Kernforschungszentrum, Karlsruhe

Markley, R. A., Westinghouse Electric Corp.

Martin, J., Electricite de France

McAnally, J., Purdue Lniversity

MeCarthy, W. J., Power Reactor Development Co.

Mercier, C. E., CEN, Cadarache

Merckx, K. R., Battelle-Northwest Laboratories

Meunier, A., CEN, Saclay

Miki, R., Central Research Institute, Electric Power Ind.

Miller, C. E., Jr., Oak Ridge National Laboratory

Milliot, B., Euratom, Brussels

Morelle, J. M., Belgo-Nucleaire

Morrison, D. L., Battelle-Columbus

Moss, D. A., U. S. Atomic Energy Commission

Motoda, Y., IAEA, Vienna

Mravea, A. E., U. S. Atomic Energy Commission

Murphy, P. M., General Electric Co.

Mustelier, J-P., CEN, Fontenay-aux-Roses

Nakagawa, P., Tokyo Electric Power Co., Ine.

Niestlie, J. W., Allison Division, General Motors Corp.

Nims, J. B., Atomic Power Development Associates

Novak, P., General Electric Co.

Noyes, R. C., Atomics International

Nozawa, M., Japan Atomic Energy Research Institute
Olhoeft, J. E., Westinghouse Electric Corp.

O'Neill, G. L., General Electric Co.

Osterkamp, W. J., Philips Research Laboratories, Eindhoven

Ott, K., Kernforschungszentrurn, Karlsruhe

Page, E. M., Atomic Power Development Associates

Pardue, W., Battelle-Columbus

Paul, D. J., Nuclear Materials \& Equipment Corp.

Perry, A. M., Oak Ridge National Laboratory

Petersen, G. T., General Electric Co.

Peterson, R. E., Battelle-Northwest Laboratories

Pflasterer, G. R., General Electric Co.

Piskarev, E., IAEA, New York

Pitterle, T., Atomic Power Development Associates

Poulsen, N. B., Texas A\&M University

Pressesky, A., U. S. Atomic Energy Commission

Pursel, C., U. S. Atomic Energy Commission

Quere, Y., CEN, Fontenay-aux-Roses

Rabin, S. A., General Electric Co.

Ragan, G. L., Los Alamos Seientific Laboratory

Rees, N. J. M., UKAEA, Foulness

Restelli, E., Westinghouse Electric Corp.

Reynolds, A. B., General Electric Co.

Rough, F., Battelle-Columbus

Runnalls, O. J. C., Atomic Energy of Canada Ltd.

Samuels, D. E. J., UKAEA, Foulness

Schikarski, W., Kernforschungszentrum, Karlsruhe

Schlectendahl, E. G., Kernforschungszentrum, Karlsruhe

Schluderberg, D. C., Babcock \& Wilcox Co.

Segal, B. M., Atomic Power Development Associates

Sesonske, A., Purdue University

Sevy, R. H., Atomics International

Shanstrom, R., General Atomic

Shaw, M., U. S. Atomic Energy Commission

Sherer, D. B., General Electric Co.

Shoudy, A. A., Jr., Atomic Power Development Associates

Shutt, P., Babcock \& Wilcox Co.

Sicgel, S., Atomics International

Silverman, L., Harvard University

Skavdahl, R. E., Battelle-Northwest Laboratories

Smidt, D., Kernforschungszentrum, Karlsruhe

Sofer, G., United Nuclear Corp.

Stachura, S. J., Atomics International

Stahl, D., United Nuclear Corp.

Stitt, R. K., Phillips Petroleum Co.

Stoker, D., Atomies International

Strauss, S., Nucleonies Magazine

Strawson, D. G., Allis-Chalmers Manufacturing Co.

Swartout, J. A., U. S. Atomic Energy Commission

Szawlewicz, S. A., U. S. Atomic Energy Commission

Teague, H. J., UKAEA, Risley

Testa, G., CNEN, Rome 
Thomas, D., Commonwealth Associates

Thomas, I. D., Nuclear Materials \& Equipment Corp.

Thompson, T. J., Massachusetts Institute of Technology

Tower, S. N., Westinghouse Electric Corp.

Turner, S. E., Southern Nuclear Engineering, Inc.

Ullmann, J., Oak Ridge National Laboratory

Vestergaard, R., AB Atomenergi

Vorderbrueggen, I., U. S. Atomic Energy Commission

Wagschal, J. J., Hebrew University

Walford, F. J., UKAEA, Foulness

Wall, I. B., General Electric Co.

Watanabe, H., Japan Atomic Energy Research Institute

Webb, R. A., Babcock \& Wilcox Co.

Weinberg, A. M., Oak Ridge National Laboratory

Weiner, M., U. S. Atomic Energy Commission
Wensch, G. W., U. S. Atomic Energy Commission

Wheeler, R. C., UKAFA, Winfrith

Whittenbrach, M. G., Battelle-Northwest Laboratories

Wilhelm, J., Kernforschungszentrum, Karlsruhe

Wirtz, K., Kernforschungszentrum, Karlsruhe

Wise, J., U.S. Atomic Energy Commission

Wisner, S., Combustion Engineering

Wolfe, B., General Electric Co.

Woodhouse, J., U.S. Atomic Energy Commission

Wright, J. H., Westinghouse Electric Corp.

Yiftah, S., Israel Atomic Energy Conmission

Zartman, I., U.S. Atomic Energy Commission

Zebroski, E. I., General Electric Co.

Ziegler, A., Siemens-Schuckertwerke AG

Zinn, W. H., Combustion Engineering

Zivi, S., TRW, Inc. 